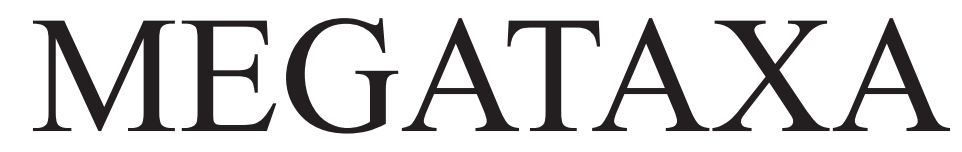

\title{
5
}

\section{New concepts and methods for phylogenetic taxonomy and nomenclature in zoology, exemplified by a new ranked cladonomy of recent amphibians (Lissamphibia)}

\footnotetext{
ALAIN DUBOIS ${ }^{1}$, ANNEMARIE OHLER $^{2} \&$ R. ALEXANDER PYRON ${ }^{3}$

${ }^{1}$ Institut de Systématique, Evolution, Biodiversité (ISYEB), Muséum national d'Histoire naturelle,

CNRS, Sorbonne Université, EPHE, Université des Antilles, Paris, France.

”"alain.dubois@mnhn.fr; ○ https://orcid.org/0000-0002-6463-3435

${ }^{2}$ Institut de Systématique, Evolution, Biodiversité (ISYEB), Muséum national d'Histoire naturelle,

CNRS, Sorbonne Université, EPHE, Université des Antilles, Paris, France.

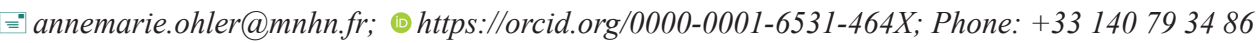

${ }^{3}$ Department of Biological Sciences, The George Washington University, Washington DC, USA.

"”rpyron@colubroid.org; @ https://orcid.org/0000-0003-2524-1794; Phone: +1 (202) 9946616
}

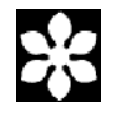

Magnolia Press

Auckland, New Zealand 
ALAIN DUBOIS, ANNEMARIE OHLER \& R. ALEXANDER PYRON

New concepts and methods for phylogenetic taxonomy and nomenclature in zoology, exemplified by a new ranked cladonomy of recent amphibians (Lissamphibia)

(Megataxa 5)

738 pp.; $30 \mathrm{~cm}$.

26 February 2021

ISBN 978-1-77688-192-5 (Paperback)

ISBN 978-1-77688-193-2 (Online edition)

FIRST PUBLISHED IN 2021 BY

Magnolia Press

P.O. Box 41-383

Auckland 1041

New Zealand

e-mail: magnolia@mapress.com

https://www.mapress.com/j/mt

(C) 2021 Magnolia Press

ISSN 2703-3082 (Print edition)

ISSN 2703-3090 (Online edition) 


\section{Table of Contents}

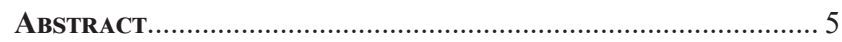

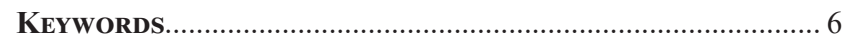

TERMINOLOGY, CONVENTIONS AND ABBREVIATIONS ...................... 6

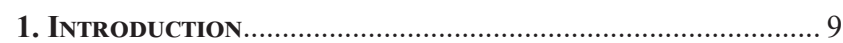

2. MATERIALS AND METHODS: PHYLOGENY, TAXONOMY AND

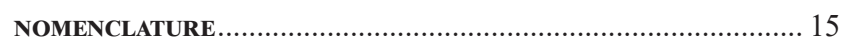

2.1. Phylogeny ........................................................ 15

2.1.1. Phylogenetic reconstruction......................... 15

2.1.2. The lability of phylogenetic hypotheses and the use of the term clade .................................. 17

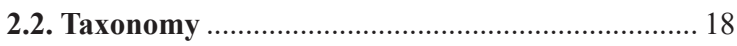

2.2.1. Taxonomic paradigms ................................... 18

2.2.2. Phylogenetic taxonomies............................. 20

2.2.3. Taxonomic categories and nomenclatural ranks ............................................. 21

2.2.4. Taxonomic concepts and Criteria ................ 22

2.2.4.1. General situation .................................. 22

2.2.4.2. Species taxonominal level ....................... 23

2.2.4.3. Genus taxonominal level........................ 23

2.2.4.4. Suprageneric taxonominal levels ........... 25

2.2.5. Taxonomic scope of the present work .......... 25

2.2.5.1. Species taxonominal level ....................... 25

2.2.5.2. Genus taxonominal level........................ 26

2.2.5.3. Suprageneric taxonominal levels ........... 27

2.3. Nomenclature ............................................................. 30

2.3.1. Introduction ................................................... 30

2.3.2. Nomenclatural Rules, recommendations

and conventions ....................................................... 31

2.3.3. Nominal-series, nominal-sets and

nomenclatural ranks ............................................... 32

2.3.4. Nomenclatural availability ............................ 35

2.3.4.1. Publication availability .......................... 38

2.3.4.2. Nominal-series assignment of

suprageneric nomina ............................................ 38

2.3.4.3. Nomen and onomatergy availability ...... 45

2.3.4.3.1. General situation ............................... 45

2.3.4.3.2. Metagraphs...................................... 51

2.3.4.3.3. Categories of homonymy................ 53

2.3.4.3.4. Ectonyms....................................... 58

2.3.4.3.4.1. Oligocaconyms:

non-binominal specific nomenclature ...... 59

2.3.4.3.4.2. Hypercaconyms:

plurinominal nomina above the

species-series

2.3.4.3.4.3. Anemonyms: nomina

unassignable to a nominal-series. 59

2.3.4.3.4.4. Notharchonyms: alternative

nomenclatural systems

2.3.4.3.4.5. Anhypsonyms: unranked or pseudoranked nomenclatural systems,

mero-systems or pseudo-systems

2.3.4.3.5. Acceptable tolerance for

borderline gymnonymy ..................................... 63

2.3.4.3.5.1. Polythetic diagnoses............... 64
2.3.4.3.5.2. Non-differential diagnoses .... 65

2.3.4.3.5.3. Diagnosis unconnected with

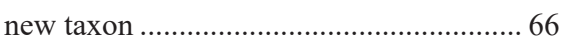

2.3.4.3.5.4. Notes for the future ............... 67

2.3.4.4. Conclusion .............................................. 67

2.3.5. Categories of usage of nomina ....................... 68

2.3.6. Taxonomic allocation of nomina .................. 68

2.3.6.1. Family-series nomina............................... 68

2.3.6.2. Class-series nomina ................................ 69

2.3.7. Validity and correctness of nomina ............... 70

2.3.7.1. Introduction .............................................. 70

2.3.7.2. Priority ........................................................ 71

2.3.7.3. Airesy (first reviser) ............................... 71

2.3.7.4. Proedry (rank precedence)...................... 71

2.3.7.5. Eugraphy ................................................ 72

2.3.7.6. Reversal of precedence ........................... 74

2.3.7.6.1. Article 23.9................................... 74

2.3.7.6.2. Other Articles resulting in

reversal of precedence................................... 75

2.3.7.7. Sozoidy ................................................... 78

2.3.7.8. Archoidy (Commission's Plenary

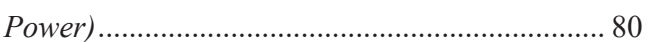

2.3.7.9. Conclusion ................................................ 80

2.3.8. Comprehensive lists of supraspecific

nomina of LISSAMPHIBIA .......................................... 80

2.3.9. 'Vernacular', 'common' and scientific

names.....

2.4. A new methodology for taxonomic and nomenclatural transcription of a tree into a

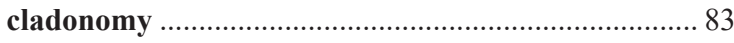

2.4.1. Introduction ................................................ 83

2.4.2. Allocation, assignment, attribution and

allotment of nomina .............................................. 86

2.4.3. The two basic mandatory ranks between

genus and class: family and order ........................ 87

2.4.4. Nominal-series saturation .............................. 88

2.4.5. Recognition of suprageneric taxa and

their rank attribution: the 'Ten Criteria

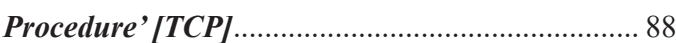

2.4.5.1. General Criteria ....................................... 89

2.4.5.1.1. Criterion [CNC]: the

'Consistent Naming Criterion' ......................... 89

2.4.5.1.1.1. Statement of Criterion ........... 89

2.4.5.1.1.2. Rationale and use of this

Criterion

89

2.4.5.1.2. Criterion $[\mathrm{NPC}]$ : the

'Nomenclatural Precedence Criterion'............ 90

2.4.5.1.2.1. Statement of Criterion ............ 90

2.4.5.1.2.2. Rationale and use of

Criterion

90

2.4.5.1.3. Criterion $[\mathrm{CHC}]$ : the 'Consistent

Hierarchy Criterion' .........................................91

2.4.5.1.3.1. Statement of Criterion ............ 91

2.4.5.1.3.2. Rationale and use of this

Criterion .................................................... 9

2.4.5.1.3.3. Examples of use of this 
Criterion 91 2.4.5.1.4. Criterion [FPC]: the 'Family-

Series Precedence Criterion' 92

2.4.5.1.4.1. Statement of Criterion ... 92

2.4.5.1.4.2. Rationale and use of this

Criterion 92

2.4.5.1.4.3. Examples of use of this

Criterion

2.4.5.2. Criteria applying only or particularly to families ... 93

2.4.5.2.1. Criterion [UQC]: the 'Upper

Quartile Criterion' ............................................. 93

2.4.5.2.1.1. Statement of Criterion ........... 93

2.4.5.2.1.2. Rationale and use of this

Criterion ..................................................... 93

2.4.5.2.1.3. Consequences of the use of this Criterion .95

2.4.5.2.2. Criterion [STC]: the 'Sister-

Taxa Criterion' ……........................................... 95

2.4.5.2.2.1. Statement of Criterion ............ 95

2.4.5.2.2.2. Rationale and use of this

Criterion ................................................... 95

2.4.5.2.2.3. Examples of use of this

Criterion 96

2.4.5.2.2.4. Consequences of the use of

this Criterion. 96

2.4.5.2.3. Criterion [CPC]: the 'Conflict

of Precedence Criterion'.................................... 97

2.4.5.2.3.1. Statement of Criterion ............ 97

2.4.5.2.3.2. Rationale and use of this Criterion .. .97

2.4.5.2.3.3. Consequences of the use of this Criterion. 97

2.4.5.2.4. Criterion [NRC]: the "Non-

Redundancy Criterion' .................................... 97

2.4.5.2.4.1. Statement of Criterion ............ 97

2.4.5.2.4.2. Rationale and use of this

Criterion

98

2.4.5.2.4.3. Examples of use of this

Criterion

98

2.4.5.2.4.4. Consequences of the use of

this Criterion. ... 99

2.4.5.5.5. Criterion [MRC]: the 'Mandatory

Rank Criterion'................................................. 99

2.4.5.2.5.1. Statement of Criterion ........... 99

2.4.5.2.5.2. Rationale and use of this Criterion ... 99

2.4.5.2.5.3. Examples of use of this Criterion 100

2.4.5.2.5.4. Consequences of the use of this Criterion 100

2.4.5.2.6. Criterion [NTC]: the

'Nomenclatural Thrift Criterion'. 100

2.4.5.2.6.1. Statement of Criterion 100

2.4.5.2.6.2. Rationale and use of this Criterion 100
2.4.5.2.6.3. Consequences of the use

of this Criterion 103

2.4.6. Implementation of the [TCP] and rank

attribution of suprageneric taxa. 103

2.4.6.1. A general summary of the Ten

Criteria Procedure [TCP]................................... 103

2.4.6.2. Theoretical examples ............................ 105

2.4.6.2.1. Example T1 ...................................... 105

2.4.6.2.2. Example T2 …............................... 106

2.4.6.3. Real examples ......................................... 108

2.4.6.3.1. Example R1: the taxonominal

hierarchy in the three orders of extant

LISSAMPHIBIA ……........................................ 108

2.4.6.3.2. Example R2: genera Telmatobius

and Rhinoderma .......................................... 109

2.4.6.3.3. Example R3: genus Epidalea ....... 109

2.4.6.3.4. Example R4: genus

Cycloramphus ………............................... 110

2.4.6.3.5. Example R5: genus Polypedates........

110

2.4.6.3.6. Example R6: genus

Odontobatrachus........................................... 110

2.4.6.3.7. Example R7: genus Litoria .............111

2.4.6.3.8. Example R8: genera Alytes,

Bombina and Discoglossus .............................111

2.4.6.3.9. Example R9: genera Ambystoma

and Dicamptodon ..........................................111

2.4.6.3.10. A few other examples and

comments ................................................. 112

2.5. Some comments on the new nomina

introduced in the present work 113

2.6. Definition of character states used for

diagnosis 120

3. RESULTS: PHYLOGENY, TAXONOMY AND NOMENCLATURE..... 120

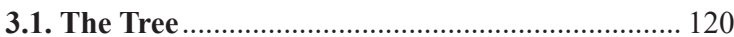

3.2. The nomina .......................................................... 122

3.3. The cladonomy .................................................... 122

4. DISCUSSION AND CONCLUSION …………………................... 294

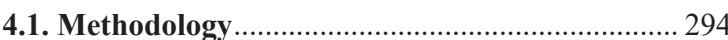

4.1.1. Phylogeny ...................................................... 294

4.1.2. Taxonomy .................................................... 295

4.1.3. Nomenclature ................................................ 295

4.1.4. The Ten Criteria Procedure ........................ 296

4.1.4.1. General Criteria ................................... 296

4.1.4.1.1. [CNC]. Consistent Naming

Criterion $\{\mathrm{TN}\}$.......................................... 296

4.1.4.1.2. [NPC]. Nomenclatural

Precedence Criterion $\{\mathrm{N}\}$.......................... 296

4.1.4.1.3. [CHC]. Consistent Hierarchy

Criterion $\{\mathrm{N}\}$.......................................... 296

4.1.4.1.4. [FPC]. Family-Series

Precedence Criterion $\{\mathrm{N}\}$............................ 296

4.1.4.2. Criteria applying only or particularly

to families .......................................................... 297

4.1.4.2.1. [UQC], [Q]. Upper Quartile

Criterion $\{\mathrm{TN}\}$.............................................. 297 
4.1.4.2.3. [CPC], [P]. Conflict of

Precedence Criterion $\{\mathrm{N}\}$ 297

\subsubsection{4. [NRC], [N]. Non-Redundancy}

Criterion $\{\mathrm{N}\}$ 297

4.1.4.2.5. [MRC], [M]. Mandatory Rank

Criterion $\{\mathrm{N}\}$.

4.1.4.2.6. [NTC], [T]. Nomenclatural

Thrift Criterion. $\{\mathrm{N}\}$..................................... 298

4.1.5. Comments on the concept of 'stability'...... 298

4.2. Findings and proposals

... 299

4.3. What remains to be done

301

4.3.1. Regarding the analysis

301

4.3.1.1. Taxonomic sampling......................... 301

4.3.1.2. Nomenclatural sampling .................. 302

4.3.1.3. Tomoidy ......................................... 304

4.3.1.4. Cladistic methodology..................... 305

4.3.2. Regarding the taxonomic completeness .... 305

4.3.2.1. Introduction ....................................... 306

4.3.2.2. Exhaustive taxonomy ....................... 307

4.3.2.3. Preventive taxonomy ........................ 308

4.3.2.4. Time is for field work and

collections 309

5. ACKNOWLEDGEMENTS.

310

6. REFERENCES .

310

7. APPENDICES

.. 366

APPEndix A1.GLO. Glossary . 366

APPENDix A2.TREE-1. Detailed phylogenetic tree of

LisSAMPHIBIA, showing all species and higher

supraspecific taxa recognised here as valid. 407

APPENDix A3.TREE-2. Simplified phylogenetic tree of

LiSSAMPHIBIA, showing all genera and higher

supraspecific taxa recognised here as valid.

APPENDIX A4.RNK. Abbreviations for ranks of taxa cited in

Appendices A6.NFS, A7.NCS and A8.ECT.....

APPENDix A5.NGS. Genus-series nomina and taxa of LISSAMPHIBIA

APPENDIX A6.NFS. Family-series nomina and taxa of LISSAMPHIBIA.

APPENDix A7.NCS. Class-series nomina and taxa of LISSAMPHIBIA.

APPENDix A8.ECT. Ectonyms of LisSAMPHibia.

APPENDix A9.CLAD-1. Complete cladonomy and nomenclature of LISSAMPHIBIA proposed here..... 674

APPENDIX A10.CLAD-2. Simplified cladonomy and nomenclature of LISSAMPHIBIA proposed here, showing all taxa from classis to subfamily and all genera.

APPENDIX A11.CLAD-3. Families and subfamilies of

LisSAMPHIBIA here considered valid.

APPENDIX A12.CLAD-4. Class-series cladonomy and nomenclature of LISSAMPHIBIA proposed here.

APPENDIX A13.QUA. Usage of nomina of families of extant LiSSAMPHiBia from 1796 to 2014.

APPENDIX A14.AIR. New airesies.

APPENDIX A15.MIS. Missing molecular data.

APPENDIX A16.BUF. The Buffon Declaration.
APPENDIX A.17.ADD. Notes added in proofs 738

\section{Abstract}

Although currently most taxonomists claim to adhere to the concept of 'phylogenetic taxonomy', in fact most of the zoological classifications currently published are only in part 'phylogenetic' but include also phenetic or gradist approaches, in their arbitrary choices of the nodes formally recognised as taxa and in their attribution of ranks to these taxa. We here propose a new approach to 'phylogenetic taxonomy and nomenclature', exemplified by a phylogenetic classification or cladonomy of the extant amphibians (subclass LiSSAMPHIBIA of the class AMPHIBIA) derived from a supermatrix-based phylogenetic analysis using 4060 amphibian species, i.e. about half of the 8235 species recognised on 31 October 2020. These taxa were represented by a mean of 3029 bp (range: 197-13849 bp) of DNA sequence data from a mean of 4 genes (range: $1-15)$. The cladistic tree thus generated was transferred into a classification according to a new taxonomic and nomenclatural methodology presented here, which allows a bijective or isomorphic relationship between the phylogenetic hypothesis and the classification through a rigorous use of suprageneric ranks, in which their hierarchy mirrors the structure of the tree. Our methodology differs from all previous ones in several particulars: [1] whereas the current International Code of Zoological Nomenclature uses only three 'groups of names' (species, genus and family), we recognise four nominal-series (species, genus, family and class); [2] we strictly follow the Code for the establishment of the valid nomen (scientific name) of taxa in the three lower nominal-series (however, in a few situations, we suggest improvements to the current Rules of the Code); [3] we provide precise and unambiguous Criteria for the assignment of suprageneric nomina to either the family- or the class-series, excluding nomina proposed expressly under unranked or pseudoranked nomenclatural systems; [4] in the class-series, for which the Code provides only incomplete Rules concerning availability, we provide precise, complete and unambiguous Criteria for the nomenclatural availability, taxonomic allocation and nomenclatural validity and correctness of nomina; [5] we stress the fact that nomenclatural ranks do not have biological definitions or meanings and that they should never be used in an 'absolute' way (e.g., to express degrees of genetic or phenetic divergence between taxa or hypothesised ages of cladogeneses) but in a 'relative' way: two taxa which are considered phylogenetically as sister-taxa should always be attributed to the same nomenclatural rank, but taxa bearing the same rank in different 'clades' are by no means 'equivalent', as the number of ranks depends largely on the number of terminal taxa (species) and on the degree of phylogenetic resolution of the tree; [6] because of this lack of 'equivalence', some arbitrary criteria are necessary 
to fix a starting point for assigning a given suprageneric rank to some taxa, from which the ranks of all other taxa will automatically derive through a simple implementation of the hierarchy of ranks: for this purpose we chose the rank family and we propose a 'Ten Criteria Procedure' allowing to fix the position of this rank in any zoological classification. As a result of the implementation of this set of Criteria, we obtained a new ranked classification of extant lissamphibians using 25 suprageneric ranks below the rank class (11 class-series and 14 family-series ranks), and including 34 class-series and 573 family-series taxa, and where the 575 genera we recognise are referred to 69 families and 87 subfamilies. We provide new nomina and diagnoses for 10 class-series taxa, 171 family-series taxa, 14 genus-series taxa and 1 species. As many new species of amphibians are permanently described, this classification and its nomenclature will certainly have to change many times in the future but, using the clear, explicit, complete, automatic and unambiguous methodology presented here, these changes will be easy to implement, and will not depend on subjective and arbitrary choices as it has too often been the case in the last decades. We suggest that applying this methodology in other zoological groups would improve considerably the homogeneity, clarity and usefulness of zoological taxonomy and nomenclature.

\section{KEYWORDS}

Amphibia, Lissamphibia, classification, phylogeny, cladonomy, ergotaxonomy, taxonomic concepts, taxonomic category, Code, Duplostensional Nomenclatural System, nomenclatural rank, mandatory rank, nomenclatural availability, taxonomic allocation of nomina, usage of nomina, nomenclatural validity, nomenclatural correctness, comprehensive list of nomina, class-series, family-series, genus-series, synonymy load, nomenclatural parsimony, taxonomic completeness, preventive taxonomy

\section{TERMINOLOGY, CONVENTIONS AND ABBREVIATIONS}

In the present work, we use a very detailed and precise technical terminology for nomenclatural, taxonomic, evolutionary, biological and other concepts mentioned in the text, Figures, Tables and Appendices. We are conscious that this unusual terminology will be found tedious or hard to follow by some our readers. Although most of it has been largely adopted by the Linz Zoocode Committtee (Dubois et al. 2019) and a few of these new terms have already entered the common language of taxonomy and nomenclature, we do not expect most of this terminology to be adopted soon by the taxonomic community at large and incorporated into the Code. We use this terminology for the reasons already highlighted by Dubois $(2000 \mathrm{~b}$, 2011a, 2013) and Dubois et al. (2016, 2019), mostly because the terminology of the current Code is often unclear, ambiguous and misleading. Our terminology has two very important advantages: $\{\mathrm{A} 1\}$ it provides non-ambiguous definitions of the concepts used in our work; and $\{\mathrm{A} 2\}$ it allows an important parsimony in the expression of ideas in our text, using a single term to express a concept, even if complex, instead of a long periphrase. As is well known by all those who have worked on writing a glossary or dictionary, the exercise of writing definitions for technical terms used in a particular, specialised, domain, is very difficult and demanding, but it allows considerable clarification of one's ideas and is beneficial to both its authors and readers. In the text below, we will encounter many cases of semantic disambiguation concerning 'common' terms of taxonomy and nomenclature, such as nomen/paronym, author/scriptor, taxon/ taxomen, rank/category or type/onomatophore. For those who have difficulties reading our text, we suggest to have a printed version of our Glossary at hand. After some time, they might become accustomed to some of our new terms and even appreciate them.

Appendix A1. GLO below provides definitions and etymology for many terms and formulae used in this work. These terms are printed in bold italics at least on the occasion of their first or most important uses in the text, whereas simple bold is used to call attention to important terms or expressions.

The term nomen (plural nomina) is used here for 'scientific name', and the expression nominalseries for 'groups of names' as used in the Code.

Simple italics are used for species- and genus-series nomina, for titles of publications and websites, for anatomical structures (e.g. musculus semitendinosus) and for Latin-derived terms or expressions (such as idem or hoc loco).

Today, more and more scientific information is made publicly available on websites, blogs, etc., but not as genuine scientific publications (often submitted to peer review and formally published as permanent printed or online documents). No guarantee exists that such electronic-only databases, applications and other 'gray' documents will still exist and be available to the scientists of the future, even in the short term. For this reason, whenever the same information could be found in genuine publications, we refrained here from giving such references for scientific information relevant to our work. However, in the cases no such permanent publications exist, we provided 
the electronic address of the online document, designated by an abbreviation (e.g. $<A W b 2020>$ for Amphibian Web). Such references are given separately from those of duly published works at the beginning of our list of references, before anonymous works (defined according to Dubois $2015 b)$.

The following abbreviations and conventions are used below, particularly in the chapter 3.3 presenting our cladonomy.

\section{Nominal-series}

CS. - Class-series (no term in the Code).

FS. • Family-series (family group in the Code).

GS. - Genus-series (genus group in the Code).

NS. - Nominal-series (group of names in the Code).

SS. Species-series (species group in the Code).

\section{Mode of writing of nomina}

Species-series nomina.

Genus-series nomina.

FAMILY-SERIES NOMINA.

Class-SERIES NOMINA.

\section{Numbering of nomina}

C.n.n. • Class-series nomen.

F.n.n. • Family-series nomen.

G.n.n. $\bullet$ Genus-series nomen.

S.n.n. $\bullet$ Species-series nomen.

\section{Numbers of taxa (see A.CLAD-1)}

$\boldsymbol{n} \mathbf{C} \dot{\dagger}$. $\bullet$ Number of all-fossil class-series taxon or taxa, not listed here.

$\boldsymbol{n} \mathbf{F} \uparrow$. $\bullet$ Number of all-fossil family-series taxon or taxa, not listed here.

$\boldsymbol{n} \mathbf{G} \dagger$. • Number of all-fossil genus or genera, not listed here.

$\boldsymbol{n}$ GIS. • Number of extant genera incertae sedis.

\section{Etymology of nomina}

G. • Etymology derived from classical Greek.

L. • Etymology derived from classical Latin.

N. • Etymology derived from an available nomen.

P. • Etymology derived from the name of a person.

R. • Etymology derived from a modern language.

Homonymy, synonymy and synotaxy

Homonym, homonymous, homonymy. • Concerning any nomen that has to be considered a homonym of another one of the same nominal-series according to the Code (in the genus- or family-series) or to DONS Criteria (in the class-series).

Synonym, synonymic list, synonymous, synonymy, synonymy load. $\bullet$ Concerning any nomen of the same nominal-series that applies to the same taxon according to the Code (in the genus- or family-series) or to DONS Criteria (in the class-series).

Synotaxic, synotaxic list, synotaxon. - Concerning any nomen of the same or different nominal-series, or unassigned to any nominal-series (ectonym), that applies to the same taxon.

Categories of airesy

EPITA. • Explicit Internal Airesy.

ETA. • External Airesy.

IPITA. • Implicit Internal Airesy.

\section{Nomenclatural systems}

AONS. • Ambiostensional Nomenclatural System.

DONS. • Duplostensional Nomenclatural System.

LSNS. • Linnaean-Stricklandian Nomenclatural System.

MONS. • Metrostensional Nomenclatural System.

OONS. • Orostensional Nomenclatural System.

Ten Criteria Procedure for attribution of a nomen to the rank family

CHC. $\bullet$ Consistent Hierarchy Criterion.

CNC. $\bullet$ Consistent Naming Criterion.

CPC. $\bullet$ Conflict of Precedence Criterion.

FPC. • Family-Series Precedence Criterion.

LR. • Lowest ranked nomen/taxon.

MRC. • Mandatory Rank Criterion.

NPC. $\bullet$ Nomenclatural Precedence Criterion.

NRC. $\bullet$ Non-Redundancy Criterion.

NTC. $\bullet$ Nomenclatural Thrift Criterion.

STC. $\bullet$ Sister-Taxa Criterion.

TCP. • Ten Criteria Procedure.

UQ. • Upper (third) Quartile.

UQC. • Upper Quartile Criterion.

UQN. • Upper Quarter of Nomina.

Auctorship and date of nomina

|LEPOSPONDYLI|, etc. • Class-series nomen used following current tradition, but without auctorship and date, for not having been validated according to DONS Criteria (see Dubois 2006a).

|Noble, 1931|, etc. • Secondary auctorship validated through Article 35.4.1 (see Dubois 2015a).

||Bonaparte, $1850 \|$, etc. - Primary auctorship validated through Article 40.2 (see Dubois 2015a).

Astrodactylus [Hogg, 1838] Hogg, 1839, etc. • The nomen Astrodactylus does not appear in the work of Hogg (1838), but is implied by the presence in this work of the family-series ASTRODACTYLIDAE; the nomen Astrodactylus appeared for the first time in the work of Hogg (1839).

Various abbreviations and conventions

BZN. • Bulletin of Zoological Nomenclature.

DOP. • Part of the identifier of a nomen established as new 
in the present work ('Dubois, Ohler \& Pyron').

Glossary. • 'Glossary' section of the present work (Appendix A1.GLO).

Keratodont formulae of tadpoles. $\bullet$ Given here according to the conventions of Dubois (1995a).

LLS. - Latonia-like situation, in which a single welldiagnosable (by clear morphological, behavioural, ecological or other characters, but not merely by its position in a tree) species $\mathrm{S}$ is cladistically sister to a group of several or many species G1 being itself welldiagnosable from $\mathrm{S}$, which leads to recognise a distinct genus $\mathrm{G} 2$ for the latter (see details under M\&M).

M\&M. - 'Material and methods' section of the present work.

Phalangeal formulae of digits (fingers and toes). $\bullet$ They are given under the form 2-2-3-3 for hands and 2-2-3-4-3 for feet, starting from the axial digit (closest to body axis).

SVL. - Snout-vent length of a specimen.

The Code. $\bullet$ The edition currently in force of the International Code of Zoological Nomenclature (Anonymous 1999, 2012, 2014).

The Commission. - The International Commission on Zoological Nomenclature (see Anonymous 1999).

The LZC. $\bullet$ The Linz Zoocode Committee (see Dubois et al. 2019).

TL. $\bullet$ Total length of a specimen.

TREE. - The molecular cladistic tree shown in A2.TREE1.

\section{Other conventions}

\{Boulenger 1882\}, etc. $\bullet$ Sources of diagnoses of new taxa. $\{\mathrm{A} 1\},\{\mathrm{a} 1\}$, etc. $\bullet$ Items in a series of related items.

" ". • Anoplonym.

' ' - Family-series nomen being redundant to a superordinate class-series nomen in a given ergotaxonomy, that should therefore not be used under the nomenclatural Criteria used in the present work.

«» - Nomen expressly proposed as unranked (anhypsonym).

$<>$ - Nomen expressly proposed as following the International Code for Phylogenetic Nomenclature (Cantino \& Queiroz 2020) (notharchonym).

“". • Exact quotation from publication.

' '. • Highlighted, questionable or problematic term.

\section{Figures in text}

F1.MOR. - Figure 1. MOR. Mandatory and optional nomenclatural ranks in zoological nomenclature. (Page 29).

F2.MPT. - Figure 2. MPT. Monothetic and polythetic classes. (Page 65).

F3.NDD. - Figure 3. NDD. A non-differential diagnosis for a new taxon. (Page 66).

F4.TCP-1. - Figure 4. TCP-1. The Ten Criteria Procedure.
Example T1. (Page 106).

F5.TCP-2. • Figure 5. TCP-2. The Ten Criteria Procedure. Example T2. (Page 107).

F6.TREE-3. - Figure 6. TREE-3. Oversimplified phylogenetic tree of LisSAMPHibia on which the present taxonomy is based, showing the families and subfamilies recognised here as valid and their relationships (Page 121).

Tables in text

T1.HIE. - Table 1. HIE. Hierarchical taxonominal levels used in this work. (Page 33).

T2.SEQ. - Table 2. SEQ. Sequences of steps of allocation of ranks. (Page 34).

T3.AVP. - Table 3. AVP. Criteria of unavailability of publications. (Pages 36-37).

T4.AVN. - Table 4. AVN. Criteria of unavailability of classseries and family-series nomina, and of nomenclatural acts concerning them. (Pages 39-41).

T5.RHI. - Table 5. RHI. Categories of rhizonymy in the family-series and class-series with their standard endings used here. (Pages 43-45).

T6.ASN. - Table 6. ASN. Criteria of assignment of nomina to the class-series or to the family-series. (Pages 46$50)$.

T7.NS-1. • Table 7. NS1. Nomina and spellings. Definitions of categories. (Pages 52-53).

T8.NS-2. - Table 8. NS2. Nomina and spellings. Criteria of distinction between categories. (Pages 54-56).

T9.ENZ. - Table 9. ENZ. Endings based on the stems form, morph and zoo used for class-series nomina in Zhang (2011a, 2013a). (Page 73).

T10.ENL. - Table 10. ENL. Endings used in the protographs of panrhizonyms of class-series nomina of LisSAMPHibia. (Page 74).

T11.LEG. - Table 11. LEG. Legethographs of class-series nomina of AмpнiвIA. (Pages 76-78).

T12.ZYG. • Table 12. ZYG. Zygoidy. (Page 79).

T13.NOD. - Table 13. NOD. Resolution of suprageneric polytomies in extant LiSSAMPHIBIA. (Page 102).

T14.NUM. • Table 14. NUM. Number of suprageneric taxa and nomina below class in LissamphiBIA. (Page 113).

T15.NEW. • Table 15. NEW. New nomina and paronyms of LisSAMPHIBIA introduced in the present work. (Pages 114-119).

T16.SYN. - Table 16. SYN. Synonymy load in extant LisSAMPHibIa (i.e., excluding all-fossil supraspecific taxa) according to the taxonomy adopted here. (Page 123).

T17.PAR. - Table 17. PAR. Family-series paronymy in extant LissampHibia (i.e., excluding all-fossil supraspecific taxa) according to the taxonomy adopted here. (Page 124). 


\section{Appendices}

A1.GLO. • Appendix 1. GLO. Glossary. (Pages 366-406).

A2.TREE-1. - Appendix 2. TREE-1. Detailed phylogenetic

tree of Lissamphibia, showing all species and higher supraspecific taxa recognised here as valid. (Pages 407-447).

A3.TREE-2. - Appendix 3. TREE-2. Simplified phylogenetic tree of LISSAMPHIBIA, showing all genera and higher supraspecific taxa recognised here as valid. (Pages 448-452).

A4.RNK. - Appendix 4. RNK. Abbreviations for ranks of taxa cited in Appendices A6.NFS, A7.NCS and A8.ECT. (Page 453).

A5.NGS. - Appendix 5. NGS. Genus-series nomina and taxa of LisSAMPHibia (Pages 454-557).

A6.NFS. - Appendix 6. NFS. Family-series nomina and taxa of Lissamphibia. (Pages 558-613).

A7.NCS. - Appendix 7. NCS. Class-series nomina and taxa of LissaMPhibia (Pages 614-662).

A8.ECT. Appendix 8. ECT. Ectonyms of Lissamphibia (Pages 663-673).

A9.CLAD-1. • Appendix 9. CLAD-1. Complete taxonomy and nomenclature of LISSAMPHIBIA proposed here. (Pages 674-708).

A10.CLAD-2. - Appendix 10. CLAD-2. Simplified cladonomy and nomenclature of LISSAMPHIBIA proposed here, showing all taxa from classis to subfamily and all genera. (Pages 709-718).

A11.CLAD-3. - Appendix 11. CLAD-3. Families and subfamilies of LisSAMPHibia here considered valid. (Pages 719-723).

A12.CLAD-4. - Appendix 12. CLAD-4. Class-series taxonomy and nomenclature of LISSAMPHIBIA proposed here. (Pages 724-725).

A13.QUA. - Appendix 13. QUA. Usage of nomina of families of extant LisSAMPHibia from 1796 to 2014. (Pages 726-729).

A14.AIR. • Appendix 14. AIR. New airesies. (Pages 730732).

A15.MIS. - Appendix 15. MIS. Missing molecular data. (Pages 733-735).

A16.BUF. - Appendix 16. BUF. The Buffon Declaration. (Pages 736-737).

A17.ADD. - Appendix 17. ADD. Notes added in proofs. (Page 738).

\section{Introduction}

Frequent are the laments over the instability of our systematic nomenclature; bitter the complaints against those who change names. But surely such complaints are unjust when urged against those who range themselves under laws. We are forcibly reminded by such complaints of the ancient apologue of the wolf and the lamb. The stream of nomenclature has indeed been much muddied, but it is due to the acts of those who refuse to be bound by laws or reason. The only way to purify the stream is to clear out all the disturbing elements. In doing so, mud that has settled for a time may be disturbed, but this is at worst anticipating what would have inevitably happened sooner or later. We are suffering from the ignorance or misdeeds of the past. In opposing the necessary rectifications and the enforcement of the laws, extremes may meet; conservatives and anarchists agree. But the majority may be depended upon in time to subscribe to the laws, and the perturbed condition will then cease to be.

Theodore Gill 1896: 600

The purpose of this work is double: $\{\mathrm{B} 1\}$ to propose new concepts and a new methodology for phylogenetic taxonomy and nomenclature in zoology, particularly at higher levels; and $\{\mathrm{B} 2\}$ to exemplify these proposals in detail by a new ranked suprageneric cladonomy of recent amphibians.

One might argue that, despite the structural interrelationships among these two topics, a better choice might have been to devote one separate work to each of them. However, in this respect our work has one major classic precedent (Simpson 1945), not to mention the many works of lesser ambition where new taxonomic and nomenclatural concepts and methods were offered in the context of revisionary works dealing with precise zoological groups. General theoretical proposals in these domains (e.g., Dubois 2005b, 2006a), are more difficult to grasp by newcomers than the same proposals illustrated by detailed examples (e.g., Kluge 2010), and on the reverse publishing new taxonomic proposals but without a detailed explanation of the taxonomic and nomenclatural concepts and methods they rely upon (e.g., Frost et al. 2006 for their suprageneric classification and nomenclature) does not allow their clear understanding and discussion.

We provide below an updated phylogenetic classification (cladonomy) and nomenclature of recent amphibians (LISSAMPHIBIA), i.e., the group of the class AMPHIBIA (including all-fossil taxa) that is represented in the extant fauna of our planet. This requires an updated evaluation of the taxonomy (formal recognition of taxa) of the group, based on the most recent phylogenetic hypotheses, and an updated nomenclature (identification of the valid nomina for these taxa), based on unambiguous and universal Rules or Criteria. We here use the term recent to designate all lissamphibians, the term 'allfossil' for lissamphibian taxa that do not include 
a single extant species, and the term 'extant' for all lissamphibian taxa that are represented today by at least one species in the living fauna. Species recently extinct (during the anthropocene), such as Rheobatrachus silus, are here referred to the category 'extant'.

Today, the classification of Eucaryotes cannot but be 'phylogenetic'. This means that only groups that are hypothesised, on the basis of morphological, molecular and/or other evidence, to be 'monophyletic' (sensu Hennig 1950) or holophyletic (Ashlock 1971), should be recognised as valid taxa, and that the sequence of nodes in the phylogenetic tree should be reflected in the taxonomic hierarchy, more basal nodes corresponding to higher, more inclusive taxa (Hennig 1950, 1966; Wiley 1981). As phylogenetic hypotheses are permanently modified (in most cases improved) by the addition of new taxa and new characters and the implementation of new methods of analysis of the data, no classification is or will ever be 'final', and taxonomists must become accustomed to the fact that we work only on 'provisional' or 'working' classifications, more shortly ergotaxonomies (Dubois 2005c). But, to be fully 'phylogenetic', it is not enough for a given ergotaxonomy to include only holophyletic taxa: it must also reflect in all details the structure of the tree, each node of the latter being formally recognised as a taxon and named according to a device that allows to identify its place in the tree. This is currently not the case in any of the classifications currently used in the literature, particularly in the group of amphibians, for two distinct reasons: $\{\mathrm{C} 1\}$ only some nodes are currently considered 'worthy' of being formally recognised taxonomically and named; and $\{\mathrm{C} 2\}$ the ranks attributed to these taxa are arbitrary and inconsistent, thus precluding their use for communicating the structure of the phylogenetic tree.

The building of the ergotaxonomy of a group of organisms has to go through three stages, steps or 'floors': $\{\mathrm{D} 1\}$ a phylogenetic analysis leading to a phylogenetic hypothesis for the group (a cladistic 'tree'); $\{\mathrm{D} 2\}$ a transcription of this tree into a classification of taxa; and $\{D 3\}$ the naming of these taxa, following fixed sets of international Rules of nomenclature or explicit Criteria for nomina at ranks which are not regulated by the Code. We detail below the methodology we used for each of these three steps, and which leads to the three results of this study presented and discussed below: $\{\mathrm{E} 1\}$ a cladistic tree of amphibians, designated below as TREE (Appendices A2-3.TREE-1 to A.TREE-3; Figure F6.TREE-3); $\{$ E2 $\}$ a cladistic suprageneric ergotaxonomy of amphibians, CLAD (Appendices A9.CLAD-1 to A12.CLAD4); and $\{E 3\}$ a nomenclature of suprageneric taxa of amphibians (Appendices A9.CLAD-1 to A12. CLAD-4).

Amphibians are a very diverse and charismatic vertebrate group (Vitt \& Caldwell 2009). The recent amphibians (LISSAMPHibia) comprise three groups, currently considered as orders: frogs (ANURA), salamanders (URODELA) and caecilians (GYMNOPHIONA). More than 8,200 species (8235 on 31 October 2020 according to $<A W b 2020>$ ) are currently recognised in this group. Today, they are found in almost every habitat on every land mass except Antarctica and various islands and archipelagoes (Duellman 1999). Amazingly, nearly half of the known species have been described only in the last 25 years (Dubois 2004c; $<A W b 2020>$ ), for two main reasons: $\{\mathrm{F} 1\}$ many groups exhibit staggeringly diverse radiations in poorly explored areas of the globe (such as the Andes, the Amazon and Congo basins, the Oriental region and New Guinea); and $\{\mathrm{F} 2\}$ the methods and concepts used to distinguish species have shown a major change in the recent decades (due in particular to the development of nucleic acid sequencing and of the cladistic methodology, but also, in some groups at least, by improvements in the methods of morphoanatomical study). This diversity is also currently in crisis, as many of these hyperdiverse regions have experienced major population crashes in recent years, due to factors such as habitat loss, destruction or degradation, climate change and infectious diseases, and faunistic and genetic pollution (Stuart et al. 2004). Thus, an updated classification of these organisms is critically necessary, to understand their diversity and distribution, evolutionary history and conservation needs.

Contrary to a widespread belief, it is not true that the classification of amphibians has long shown only historical inertia and informal consensus of researchers. In fact, all along the history of biology, and even long before taxonomy became 'phylogenetic' and 'molecular', the classification of amphibians has witnessed permanent and considerable changes, mostly through the identification of homoplasy and polyphyly, and more recently paraphyly, but 'incidentally' and 'intuitively', before these concepts were clearly identified and named, indeed before the concept of evolution was adopted as the paramount concept of biology, or even accepted as being scientific. The current classification of the amphibians is the result of this long progress of knowledge about these animals. This process started by the use 
of morphological characters of adults, initially external and later internal (mostly sketetal), then by the consideration of larval characters, then by the use of data from behaviour, karyology, protein electrophoresis and nucleic acid hybridisation, and finally (first mitochondrial and later nuclear) nucleic acid sequencing. The Hennigian 'revolution' was followed by the introduction of the cladistic methodology based on morphoanatomical characters in the 1970s, and molecularbased phylogenies started being produced in the early 2000 s.

A complete and detailed review of the history of the taxonomy of amphibians would be beyond the scope of the present work, as it would require not only comparisons of classifications but also, and more significantly, of the characters on which these classifications were based, and on the way these characters were used, that showed several dramatic changes over two centuries and a half.

In amphibians like in all other groups, taxonomy began by using 'overall resemblance' (expressed through the use of vernacular terms to designate the taxa: 'frog', 'toad', 'treefrog', etc.), and only later started to analyse this 'similarity' in terms of characters. In many cases this showed that 'overall resemblance' was not, as could be understood through the claims in some recent works, 'completely stupid'. Despite the much repeated statements of Hennig and some of his disciples, as soon as classifications started being based on explicit characters, no classification has ever been 'completely phenetic', as all authors have always classified the males and the females as members of the same species, and rejected as 'unnatural' taxa in which 'resemblance' was clearly due to 'convergence'. The Hennigian 'revolution' was a methodological revolution the most important novelties of which were the introduction of the concepts of plesiomorphy and apomorphy and of an explicit methodology of cladistic analysis, but, when one considers the classifications, the transition from so-called phenetic to so-called phylogenetic classifications was much smoother and progressive than it has often been claimed. What is clear is that before the time of molecular studies, researchers interested in the evolution and taxonomy of amphibians had to examine specimens and 'read' their phenotype in terms of characters, which is far from being always the case today.

'Overall resemblance' as understood in many old works usually consisted in a set of characters that often appear correlated within the organisms (see examples below), not necessarily for being inherited from a common ancestor, but often for constituting a set of features that allow a good adaptation to a certain mode of life, therefore reflecting convergence. But subsequent studies of other characters, independent from this set of correlated ones, often allowed to show that homoplasy was at stake. In frogs for example, from the very early days of systematics, various 'general morphotypes' were identified which show adaptation to aquatic, terrestrial, burrowing, arboreal, hypogeous, etc., modes of life. These groups correspond in fact to the concept of 'guild' as used initially in birds' ecology and more recently, with much success, in larval anurans (Altig \& Johnson 1989), but quite strangely not in adult amphibians, although it could be of great use in the understanding of their evolution and adaptations.

A few examples will be enough to show that, even before the introduction of 'phylogenetic taxonomy' and molecular sequencing, major reevaluations of the taxonomy had taken place, based on morphological characters alone.

In what is often considered the first scientific classification of animals, Linnaeus (1758a) showed a very poor understanding of 'lower' vertebrates. He recognised only two of the three groups of recent amphibians that we still recognise today, but simply as genera: Rana for the anurans, which he placed in a group also including Lacerta, Testudo and Draco; and Caecilia for the caecilians, which he placed within the snakes. As for the urodeles, he did not even recognise the group, as his genus Lacerta lumped as 'lizards' several other groups of 'reptiles' and the urodeles. The frogs were recognised as a group of its own already by Scopoli (1777). The salamanders were removed from the lizards by Brongniart ( $1800 a-b)$, who was also the first one to point to their close relationship with frogs, and to remove caecilians from the snakes, but without referring them to the amphibians. This was formally done by Duméril's student Oppel $(1811 a-b)$, who was the first author to recognise the three groups of recent amphibians we recognise today.

Among the urodeles, some retain branchiae in the adult stage and were long considered as 'branchiate' salamanders. The first discovered ones were described as the genera Siren Österdam, 1766, Proteus Laurenti, 1768, Gyrinus Shaw, 1798 (later renamed 'Axolotl Oken, 1821' and Axolotus Jarocki, 1822) and Amphiuma Garden in Smith, 1821. For a while two schemes were in force in parallel in the literature for the classification of these genera: $\{\mathrm{G} 1\}$ following Sonnini \& Latreille $(1801 d)$, placing them in a special taxon, sister to that accommodating the frogs and salamanders; and $\{\mathrm{G} 2\}$ following Duméril (1805), placing them 
in the urodeles. Latreille (1825) was the first author to realise that axolotls were larval salamanders and to separate them from the other three genera.

The concept of 'treefrog' (recognised in many languages under widely different terms such as 'rainette' in French or 'Laubfrosch' in German) is particulartly enlightening to show how, on the basis of morpho-anatomy alone, the taxonomy of amphibians progressively freed itself from the 'overall resemblance' paradigm. The term 'treefrog', indicating in most cases (but not always) an arboreal mode of life, corresponds to a rather well defined morphotype or 'syndrome' that would allow recognition of a 'guild', including; $\{\mathrm{H} 1\}$ enlarged adhesive digital tips (identified from the early days of frogs's systematics); $\{\mathrm{H} 2\}$ presence of intercalary additional elements before the last phalanx of digits (first mentioned apparently by Gadow 1901: 27 and Noble 1922: $22,59,71) ;\{\mathrm{H} 3\}$ granular 'treefrog belly skin' (Ohler 1999: 40; first mentioned apparently by Cope 1889a: 321); $\{\mathrm{H} 4\}$ absence of latero-dorsal folds; $\{\mathrm{H} 5\}$ short hind limbs; $\{\mathrm{H} 6\}$ incomplete webbing; and $\{\mathrm{H} 7\}$ often uniform green colour. As now documented by molecular methods, we know that this 'syndrome' appeared independently in a number of evolutionary groups (Manzano et al. 2007), but by itself this finding is not in the least new, as it had already been made through careful purely morphological observations and without recourse to the Hennigian concept of synapomorphy. What the modern methods allow is to go deeper, more reliably and in more details into the resolution of such cases of homoplasy, but this does not constitute a 'conceptual revolution'.

The concept of 'treefrog' is missing in Linnaeus, but both Garsault (1764) and Laurenti (1768) erected a genus (respectively Ranetta and Hyla) for the frogs having enlarged adhesive pads at the extremities of digits, which were first all referred to the latter genus for decades. This genus was then progressively dismantled into several genera or/and subgenera but which were long left in the same higher group as all other frogs: three in Fitzinger (1826), nine in Wagler (1830), twelve in Bonaparte (1831a), twenty-two in Tschudi (1838), sixteen in Duméril \& Bibron (1841), thirty-seven in Fitzinger (1843), etc. Günther (1858) was the first author to dispatch the treefrogs genera in nine families placed in three 'sections'.

Cope $(1864 b, 1875)$ went a step further, in distinguishing two main groups of frogs based on the 'arciferous' or 'firmisternous' structure of the pectoral girdle, which led him to sort the 'treefrogs' in two distinct families (HYLIDAE and RANIDAE) referred respectively to these two groups long called 'hyloids' and 'ranoids'. Noble (1931: 524) restricted the latter family to the frogs devoid of intercalary cartilage and erected a distinct family for those having this character. Subsequent works have shown that the ranoids with enlarged adhesive pads but missing this intercalary element were in fact not 'treefrogs' and were missing other characters of the 'treefrog syndrome', pointing to other modes of life: for example, the members of several ranid genera (e.g. Amolops Cope, 1865, Meristogenys Yang, 1991 and Odorrana Fei, Ye \& Huang, 1990) having smooth bellies, long limbs, and often dorsolateral folds and polychromous coloration, are rheophilous and not arboreal. But even among these frogs, careful examination of the morphology of these enlarged digit tips allowed homoplasy to be uncovered (Ohler \& Dubois 1989).

Another major step in the identification of homoplasy in 'treefrogs' was Laurent's (1951) splitting of 'ranoid' treefrogs into two families (RHACOPHORIDAE and HYPEROLIIDAE) which are only remotely related. Other more recent findings were supported by molecular data, so that today more than ten different higher taxa correspond to the initial phenetic concept of 'treefrog' (Manzano et al. 2007) but, as shown by this quick survey, part of this result was already obtained by 'traditional' morphological studies and did not rely on molecular phylogeny. In fact, whereas purely morphological methods have proved to be quite efficient to detect polyphyly, they have been much less so to detect paraphyly, and for this purpose molecular phylogenetic methods have been much more useful.

For more than a century, a number of authors proposed an overall classification of all extant amphibians (Laurenti 1768; Brongniart 1800a- $b$; Daudin 1800, 1803a- $b$; Sonnini \& Latreille 1801 $a-$ $d$; Duméril 1805; Oppel 1811a-b; Blainville 1816a-b, 1835; Merrem 1820; Gray 1825, 1831a; Latreille 1825; Fitzinger 1826, 1843; Bory de Saint-Vincent 1828; Ritgen 1828; Wagler 1830; Bonaparte 1831 $a-b, 1840 a-b, 1850$; Tschudi 1838; Hogg 1838, 1839a-b, 1841; Haeckel 1866b; Lataste $1878 a, 1879 a, 1888$; Sarasin \& Sarasin 1887, 1890; Zittel 1888; Gadow 1901; Stejneger 1907; Noble 1931; Laurent 1948a-b; Kuhn 1961, 1962, 1965; etc.) or of one of their three groups: frogs (Duméril \& Bibron 1841; Günther 1858; Cope 1864b, 1865, 1866, 1867; Mivart 1869; Boulenger 1882b, 1888; Nicholls 1916; Bolkay 1919; Fejérváry 1921b; Miranda-Ribeiro 1926; Laurent 1967; Reig 1958; Griffiths 1959, 1963; Tatarinov 1964a; etc.), salamanders (Gray 1850; Duméril et al. 1854; Boulenger 1882c; Brame 1957, 1958; Thorn 1969; etc.) and caecilians (Duméril 
\& Bibron 1841; Boulenger 1882c; etc.). However, as the number of species of other taxa increased dramatically, it began more difficult to embrace the taxonomy of the whole group. Many authors then concentrated their work on subsamples of the group, defined either taxonomically (Parker 1934; Fuhn 1960; Wake 1966; Taylor 1968; Duellman 1970, 1977; etc.) or geographically (Kellogg 1932; Rivero 1961; Cei 1962, 1980; Liu \& Hu 1961; Zweifel 1972; Dubois 1981b, 1987a, 1992; etc), and fewer and fewer authors endeavoured to provide a comprehensive classification of the whole group. Interest in such a more complete approach was triggered by the renewed approach of phylogeny initiated by Hennig (1950, 1966), and morphology-based phylogenetic hypotheses concerning some groups started being produced (Liem 1970; Lynch 1971, 1973b; Duellman 1975; Clarke 1981; Roček 1981; Channing 1989; etc.), followed by new general phylogenies and classifications of the extant amphibians (Inger 1967; Sokol 1977; Dowling \& Duellman 1978; Goin et al. 1978; Laurent 1980, 1986; Dubois 1983b, 1984b, 1985, 2005d; Duellman \& Trueb 1985; Lynch 1971, 1973b; Trueb 1971; Starrett 1973; Heyer 1975; Heyer \& Liem 1976; Milner 1988; Fei et al. 1990; Trueb \& Cloutier 1991; Blommers-Schlösser 1993; Cannatella \& Hillis, 1993; Ford \& Cannatella, 1993; Larson \& Dimmick, 1993; Duellman \& Trueb, 1994; etc.).

With the advent and increasing popularity of molecular systematics starting in the 1990s, phylogenetic estimates of many amphibian groups started to appear, and also began to illustrate numerous problems of the prevailing classification, including newly recognised lineages through the resolution of paraphyletic and polyphyletic groupings, often due to homoplasy and morphological convergence (Graybeal 1993; Marmayou et al. 2000; Biju \& Bossuyt 2003; Darst \& Cannatella 2004; Faivovich et al. 2005; Roelants \& Bossuyt 2005; San Mauro et al. 2005; Wiens et al. 2005a-b; Grant et al. 2006).

Since the 2000s, there have been several attempts to utilise this information to stabilise the taxonomy and nomenclature of amphibians. Dubois $(2005 d)$ proposed a synthesis of available phylogenetic and taxonomic information, though he noted that many groups were poorly diagnosed and delimited. Frost et al. (2006) produced the landmark work "The Amphibian Tree of Life", containing for the first time a comprehensive phylogeny for amphibians based on DNA sequence data, and a taxonomy that transcribed this estimate of evolutionary relationships into a unified scheme for extant taxa. Subsequent works have refined the phylogeny of several groups (Sá et al. 2012; Grant et al. 2006; etc.), but overall the phylogenetic framework was corroborated, and the taxonomy represented a robust framework for future revision. It should be noted however that, in the recent decades, except in a few groups like the HYLOIDEA, much more information has been obtained on the molecular relationships of recent groups than on their morphology and anatomy, a domain which remains largely unexplored and which in the future might disclose some important problems regarding phylogenetic hypotheses.

As for the nomenclature of the amphibians, it long remained based on obsolete interpretations based on a very incomplete review of the existing literature and on a largely shared ignorance of the Rules of the Code, especially for the nomina of taxa above the rank genus. To give just one example, in most works before 1981, the family including the genus Rana was named 'RANIDAE Bonaparte, 1831 ', because this author was (wrongly) believed to have been the first to use the spelling RANIDAE for this nomen, which ignored the fact that, under the Rules, its author was Batsch (1796) who had first named it as RANINI (Dubois \& Bour 2011) and that it had been mentioned under various aponyms (avatars) of the latter before 1831 (Dubois 1984b: 41). Dubois (1981b, 1983b, 1984b, 1987a, 1992, 2005d) clarified the historical and nomenclatural status of many nomina of amphibians, and in particular (Dubois 2004b) those of higher taxa above the rank superfamily, for the taxonomic allocation and the nomenclatural validity of nomina of which the Code does not provide Rules. Unfortunately, following Frost et al. (2006), these analyses were largely ignored or challenged in subsequent works, and the higher nomenclature of amphibians used in recent websites (e.g. $\angle A S W$ 2020a, AWb 2020, GBIF 2020, ITIS 2020, SN2020, Taxonomicon 2020, ToL 2020, uBio 2020>), which is based on several factual errors and inconsistent nomenclatural Criteria, requires correction (see Dubois \& Ohler 2019).

In 2011, Pyron \& Wiens presented the first large-scale (i.e., with the aim of representing species-level diversity) phylogenetic estimate for amphibians containing 2,871 species (Pyron \& Wiens 2011), 5.5 times more than the 522 species sampled by Frost et al. (2006). These species represented essentially all major lineages of amphibians, including $432(86 \%)$ of the 504 genera recognised at that time. The results were actually fairly similar to those of Frost et al. (2006), resolving a few lingering issues such as the holophyly of South American marsupial frogs (family HEMIPHRACTIDAE), and naming 
additional lineages within the former family LEPTODACTYLIDAE (see Fouquet et al. 2013). This study has since been widely used as a baseline in studies of amphibian systematics, including both taxonomy and evolutionary analyses, given the usefulness of the topology and branch lengths for phylogenetic comparative analyses (Bell \& Zamudio 2012; Fritz \& Rahbek 2012).

Despite the enormous advances and apparent robustness and stability of the phylogenetic estimates and taxonomy and nomenclature of Frost et al. (2006) and Pyron \& Wiens (2011), they cannot be considered the final word in amphibian systematics, for a number of reasons. The first is that no taxonomy will ever be final, as accumulation of data and knowledge will continue endlessly. Numerous new lineages of amphibians have been recognised since 2011 that alter our interpretation of evolutionary history, phylogenetic relationships and nomenclatural allocations (Kamei et al. 2012; Barej et al. 2014). The second is that the inevitable errors that will plague any large-scale scientific study, in this case mostly misidentified or erroneous sequences or insufficient taxonomic or character sampling within some lineages, has changed the phylogeny in some places (Blotto et al. 2013), necessitating further taxonomic clarification.

The third reason is perhaps the most important, which is that both Frost et al. (2006) and Pyron \& Wiens (2011), as well as most taxonomic studies in any group of animals, were inconsistent or erroneous in their application of some Rules of the Code, and above all did not present a unified set of explicit, unambiguous and objective Criteria for the allocation of nomina to higher taxa and/ or ranks based on the phylogenetic analyses. Recognition of most taxa, such as superfamilies, families and subfamilies, was based primarily on recent tradition, but not under any particular standard of usage (e.g., use by at least 10 authors in 25 publications in the preceding 50 years). Thus, recognition of a family in one lineage or a genus in another does not indicate any kind of consistency or equivalence in the application of those ranks.
This is not to say that these ranks by themselves are biologically meaningful, which they generally are not, but that their application in amphibians (as in most groups) is based neither on a robustly defined historical tradition or on recent conventions, nor on a meaningful division of the taxonomic hierarchy to reflect the structure of the tree. Thus, the current amphibian taxonomy in many ways represents the worst of all possible worlds.

A thorough revision can easily alleviate these problems, and form the basis of a stable nomenclature that is objectively Rules-and Criteriabased, and which can be interpreted meaningfully by researchers working in any amphibian subgroup as a platform for new species descriptions and further revision as new data become available. We attempt to provide such a revision here, based on several data and Criteria.

\{I1\} First, we utilise a recent phylogenetic analysis (Jetz \& Pyron 2018) containing 4060 species (about $50 \%$ of the currently known, extant amphibian species), sampled for up to 15 genes (5 mitochondrial and 10 nuclear, $15091 \mathrm{bp}$ total).

\{I2\} Then, we implement a set of ten objective Criteria that allow for the unambiguous fixation of the rank family in a suprageneric classification, and Criteria for the availability, allocation and validity of nomina of taxa above the rank superfamily (i.e., ranks which are not covered by the Code).

\{I3\} We provide an unprecedented complete review of all the supraspecific nomina ever proposed for lissamphibians in the 262 years of taxonomy from 1758 to 2020 and establish their status.

\{I4\} Finally, we apply our Criteria and these data on the nomina to our tree, to generate an objective, Rules- and Criteria-based taxonomic and nomenclatural revision of all lisamphibians, which reflects in a bijective manner our tree for all suprageneric ranks. This scheme is certainly by no means the last word on amphibian systematics, but we hope that it will serve to further stabilise amphibian taxonomy, and provide a solid basis for future researchers. 


\section{MATERIALS AND METHODS: PHYLOGENY, TAXONOMY AND NOMENCLATURE}

\subsection{Phylogeny}

\subsubsection{Phylogenetic reconstruction}

The phylogenetic tree we have based our classification on was published by Jetz \& Pyron (2018), in their analysis of the historical evolutionary factors driving diversification in amphibians, and the interplay therein with present-day extinction risk. These authors presented a sparsely-sampled supermatrix, which was very similar to recent efforts in numerous groups, including plants (McMahon \& Sanderson 2006; Hinchliff \& Roalson 2013), birds (Jetz et al. 2012), amphibians (Pyron \& Wiens 2011) and squamates (Pyron et al. 2011, 2013; Tonini et al. 2016). In short, they attempted to compile all available DNA sequence data for extant amphibian species from a set of broadly sampled loci, for a concatenated, partitioned analysis using Maximum Likelihood (ML). They then assessed node support using the increasingly well-established Shimodaira-Hasegawa Like (SHL) approximate LikelihoodRatio Test (aLRT), which shows high precision and accuracy with respect to traditional non-parametric bootstrap methods, while being computationally efficient and quick to calculate (see Anisimova et al. 2011; Pyron et al. 2011).

This approach has been well validated both empirically (Pyron et al. 2011) and theoretically (Queiroz \& Gatesy 2007), at least with respect to consistency in topology and branch-length estimates across studies, and with regard to the expectation of relationships derived from other sources, such as morphological data (Frost et al. 2006). Amphibian relationships have been remarkably consistent across $\{\mathrm{J} 1\}$ studies sampling many loci and fewer taxa to infer higher-level relationships (Feng et al. 2017), $\{\mathrm{J} 2\}$ studies examining species-level relationships of particular groups in detail (Duellman et al. 2016) and $\{\mathrm{J} 3\}$ supermatrix approaches to large-scale inference of amphibian phylogeny (Pyron \& Wiens 2011).

Thus, concerns about the potential impacts of missing data, which have generally been shown to be negligible in most cases (Wiens 2003), should be alleviated based on this congruence. In particular, the amount of 'missing data' is a function of the number of loci chosen for analysis, and the amount of data present is in reality the most important parameter (Wiens \& Morrill 2011). If a study of a particular family samples 50 species for 5 genes with $0 \%$ missing data, and those data are then added into a supermatrix with a scaffold of 15 genes, each of those species then has $67 \%$ 'missing data' by default. Yet, the same phylogenetic signal is still present. As has been seen empirically (Pyron \& Wiens 2011, Sanderson et al. 2011), the supermatrix approach used here has typically recovered the same topology and branch lengths as the original smaller-scale studies. This is not to say that the results are necessarily correct, but if poor or misleading phylogenetic signal characterises the underlying data, this is not a problem of the supermatrix approach per se, and would have to be addressed separately.

Thus, the artificial increase in 'missing data' introduced by inclusion in the supermatrix does not seem to negatively impact the existing phylogenetic signal in the existing data (but see Sanderson et al. 2015). What is more important is instead the amount of phylogenetically informative DNA sequence data present for each species. This is necessarily heterogeneous given the nature of the supermatrix approach due to different levels of sequencing effort and overlap among different groups. Thus, we have increased our sampling of both species and genes over previous efforts, to gather as much available data as possible for as many terminals as possible.

Another empirical confirmation of the statements above came from this study itself. The final TREE on which this whole study is based was produced in August 2014, and a first skeleton of our taxonomy $C L A D$ was produced in the immediately following months. Then, we worked for several years on the completion of the nomenclatural survey of all the existing supraspecific nomina of amphibians and above all of their nomenclatural and taxonomic status according to the taxonomic and nomenclatural Rules and Criteria adopted here (and detailed below), up to the final stage presented here, which was reached at the end of 2019. During all this time, the incorporation of all the taxonomic changes required by phylogenetic novelties published by colleagues (discussed below in the 'Taxonomic changes' section) did not require any significant change in the structure of CLAD. Several new taxa (mostly genera) that we had recognised in the preliminary part of our work were recognised and named by colleagues in the meanwhile, and of course we adopted their nomina when they were taxonomically justified and nomenclaturally available. In a few cases (discussed below), the addition of missing species allowed the 
resolution of some phylogenetic ambiguities concerning these species and to improve our taxonomy, but in no case did these new data result in challenging the main taxa we had recognised at any rank above subtribe. Thus the TREE on which this study is based, with the SHL-aLRT support value of $90 \%$ that we adopted (see below) showed a remarkable, and unexpected, even for us, robustness. This robustness will no doubt be challenged in the future with the addition of large numbers of species or sequencing of additional genes, but so far it has not been so for a period of five years despite the addition of about 1000 species since the beginning of the present work.

The previous iteration of this matrix used for taxonomic revision (Pyron \& Wiens 2011) contained data from 2871 species sampled for up to 12,712 bp from up to 12 genes, three mitochondrial and nine nuclear. Substantial increases in the number of described species and associated sequencing efforts since then have drastically broadened the data available in GenBank for large-scale phylogenetic inference. Their methods for incorporating these data into an updated supermatrix closely followed previous studies (Jetz et al. 2012; Tonini et al. 2016), which we reiterate here, from Jetz \& Pyron (2018).

As we were primarily interested in a taxonomic revision of extant AMPHIBIA, it was necessary first to have a reference taxonomy representing current usage. We refer to this as our 'naïve' taxonomy. Until very recently (see Frost et al. 2006), amphibian taxonomy was largely a matter of social consensus, with few rigorous analytical studies defining taxa as holophyletic groups based on shared, derived characters and strict application of nomenclatural Rules. In the recent decades, much progress has been made, and most higher-level amphibian taxa currently recognised (Blackburn \& Wake 2011) represent wellsupported holophyletic groups.

Amphibian taxonomy is curated in two separate online resources: Amphibian Species of the World $(<A S W 2020 a>)$ and AmphibiaWeb $(<A W b>)$, both of which maintain up-to-date species lists of extant taxa and are broadly similar in reflecting recent updates to higher-level classifications. Although they are relatively equivalent taxonomically, the AmphibiaWeb interface was easier to extract data from, and Jetz \& Pyron (2018) thus used the update of 19 February 2014 of this website as reference. This contained 7238 recognised extant amphibian species. These were classified into families and subfamilies generally following the most recent large-scale revision (Pyron \& Wiens 2011), with a few recent updates from recently recognised higher taxa.

In general, this taxonomy reflected recent updates that have shifted higher-ranked taxa (e.g., families) towards the tips. Examples include recognition of groups previously considered subfamilies of RANIDAE (e.g., RHACOPHORINAE) or CAECILIIDAE (e.g., DERMOPHIINAE) as families (RHACOPHORIDAE, DERMOPHIIDAE). In addition, they curated several updates to the AmphibiaWeb taxonomy, such as new evidence for the placement of Crossodactylodes and Rupirana. Thus, our naïve reference taxonomy represents a general recent consensus, rather than an 'AmphibiaWeb' or 'Amphibian Species of the World' taxonomy specifically. When we do make specific reference to a taxonomic position taken by those references, it is made explicit.

A final important note is that, at this point, we also adopt the updated taxonomies at the genus level from these sites, including many of those introduced recently (Frost et al. 2006) for traditionally recognised groups such as Bufo, Hyla and Rana. Although not adopted by some recent authors (Pauly et al. 2009; see Frost et al. 2009), these are clearly holophyletic based on those results and others. This does not affect our higher-level taxonomic revision directly, but it does indicate that progress has been made in the generic taxonomy of extant amphibians (but see below 'Genus taxonominal level'). Although we recommend only a few taxonomic changes at genus level in this work, we noted all instances of nonholophyly at the genus level, as a guide for future revisions. As it does not provide any clue on whether a holophyletic taxon is a genus, a subgenus, a tribe or a family, holophyly is not by itself a sufficient Criterion for genus recognition, but this question is not tackled in detail in the present work, where we tend to follow the current 'consensus' regarding amphibian genera, except in a few cases which we make explicit.

To generate the updated supermatrix, Jetz \& Pyron (2018) first took the 2871-species, 12-gene matrix and updated the sequence of species to the naïve reference taxonomy (e.g., breaking up Bufo, Hyla and Rana). They also removed a few instances of misidentified specimens and mislabeled sequences identified by D. R. Frost in $A S W<2020 b>$ and other recent authors (e.g., Blotto et al. 2013). They then identified two additional mitochondrial genes (NADH subunits 1 and 2; ND1 and ND2) and one nuclear locus (brain-derived neurotrophic factor; BDNF) for which a large number of species $(>500)$ were available on GenBank, and could thus add significant data to the matrix.

They thus had a total of 15 genes: long- and short-subunit rRNAs (12S/16S), brain-derived 
neurotrophic factor (BDNF), C-X-C chemokine receptor type 4 (CXCR4), cytochrome $b$ (CYTB), histone $3 \mathrm{a}$ (H3A), NADH subunits 1 and 2 (ND1 and ND2), sodium-calcium exchanger (NCX1), proopiomelanocortin (POMC), recombination-activating gene 1 (RAG1), rhodopsin (RHOD), seventh-inabsentia (SIA), solute-carrier family 8 (SLC8A3) and tyrosinase (TYR). For each gene, they searched GenBank exhaustively (e.g., 'Amphibia AND BDNF'), adding in all available data for species in the naïve reference taxonomy. The protein-coding genes were aligned using the 'Translation Align' option in Geneious (Biomatters Ltd.), with the MAFFT algorithm under the default parameters (Katoh \& Standley 2013). This ensured that all sequences were coding and in open reading frame.

The ribosomal RNAs (12S/16S) were aligned en masse using the default parameters in MAFFT. Other approaches such as SATé (Liu et al. 2011) have generally shown good performance for datasets such as these, by co-estimating phylogeny and alignment to arrive at an optimised static alignment. However, preliminary use of these tools showed relatively poorer performance (e.g., lower pairwise identity) than the en masse strategy. In general, the SATé-type approach yielded large block of taxa that were well aligned to each other, but not to other such blocks (e.g., seemingly erroneous frame shifts between apparently homologous sites among different blocks).

The final matrix contained sequence for 4060 amphibian species and the outgroup Homo sapiens. These taxa were represented by a mean of $3030 \mathrm{bp}$ (range: 197-13,849) of DNA sequence data from a mean of 4 genes. The total matrix was $15091 \mathrm{bp}$ long. The individual genes were sampled as follows: 16S, 3717 species; 12S, 3062; CYTB, 1770; RAG1, 1594; ND1, 1045; TYR, 1041; RHOD, 1001; ND2, 826; POMC, 758; SIA, 512; H3A, 483; CXCR4, 471; BDNF, 433; NCX1, 429; SLC8A3: 299. Thus, some mitochondrial genes (e.g., $12 \mathrm{~S}$ and $16 \mathrm{~S}$ ) were sampled for the majority of species, providing a scaffold for species-level relationships, whereas many nuclear genes were sampled for major lineages, providing a scaffold for higher-level relationships.

In terms of sampling, this includes 4060 , i.e. $49.3 \%$ of the 8235 total currently (as of 31 October $2020,<A W b 2020>$ ) recognised extant species: 3449 of 7263 frogs (47.5\%), 549 of 759 salamanders $(72.3 \%)$ and 62 of 213 caecilians $(29.1 \%)$. We sampled 524 of 575 extant genera of amphibians recognised in CLAD (91.1 \%): 425 of 468 genera for frogs $(90.0 \%), 77$ of 77 for salamanders (100\%) and 22 of 30 for caecilians $(73.3 \%)$. Thus, the sampling represents a relatively complete overview of the extant diversity in AMPHIBIA, including essentially all major lineages.

Phylogenetic inference using this dataset took a two-step approach, as in recent studies (Jetz et al. 2012; Tonini et al. 2016). To infer trees using ML, Jetz \& Pyron (2018) used the program ExaML, an update of RA $\times$ ML (Kozlov et al. 2015) which is specially designed for analysis of large-scale datasets such as this one on high-performance computing clusters. ExaML executes a single search on a starting tree, and typical ML inference requires a large number of searches to adequately explore treespace and assure convergence on a global ML estimate. Thus, they used RA $\times$ MLv8.0.14 to generate 100 randomised maximum-parsimony starting trees. They then executed $100 \mathrm{ML}$ searches on these trees using ExaML, and selected the one with the best likelihood score.

Estimating SHL support values requires a single ML tree, which is then NNI-optimised to calculate the aLRT at each node. Thus, Jetz \& Pyron (2018) took the best ML tree from the 100 ExaML searches, and passed it back to RA $\times$ MLv8.0.14 using the '-f J' algorithm, which does an additional ML search to optimise topology and branch lengths via NNI. The SHL values are then calculated at each branch. Thus, the final TREE used here (Appendix A2.TREE-1) from Jetz \& Pyron (2018) is an NNI-optimised version of the highest-scoring tree from $100 \mathrm{ML}$ searches, with SHL-aLRT support values at each node. These are roughly equivalent to the probability that the branch has been resolved optimally compared to the next four suboptimal NNI rearrangements (i.e., that the branch can be resolved unambiguously based on the signal present in the data, and is not optimally represented as a polytomy). For more security and stability of our taxonomic decisions, we chose $90 \%$ as a cutoff for 'strong' support, roughly equivalent to $\mathrm{BS}=70$ or $\mathrm{Pp}=95$ (see Pyron et al. 2011). This is even higher than the cutoff of $85 \%$ recommended as a result from simulations and empirical results.

\subsubsection{The lability of phylogenetic hypotheses and the use of the term clade}

In the present work, we refrained from using the term clade, as it is highly confusing (see Glossary below). It has been used in the literature in at least four distinct meanings, in zoological taxonomy and nomenclature to designate a nomenclatural CS rank and more recently as a CS and FS preudo-rank, 
and in evolutionary biology as a homophyletic or holophyletic group of organisms. In many recent publications it is used simultaneously in both the second and fourth of the meanings above.

For many recent biologists, this term carries a misleading message of 'reality', as if our cladistic trees were an exact representation of the evolutionary relationships. This 'reality' of clades is also hightighted by some authors even going as far as crediting clades with the status of 'individuals'. This is a clear abuse of language. 'Clades', just like 'species' or 'genera', are not 'observed' (as are facts or real individuals) but hypothesised to match biological concepts. These hypotheses are doubtless 'scientific', as they rely on explicit concepts and refutable methodologies, but they nevertheless remain hypotheses. Like all other hypotheses, they may be subjected to test, refutation and abandonment, to replace them by better ones, which in their turn may suffer the same fate. This is not a quibble, a simple matter of language, this is a basic conceptual, scientific question.

Examples are numerous and frequent, of groups of species once considered as forming a clade, where the addition of either taxa, individuals or molecular data, or changes in the methods for building trees and considering them robust, result in challenging this hypothesis. Furthermore, many so-called 'clades' are hypothesised to include many more species than those actually studied, and addition of species to the analyses not rarely results in showing that the 'clade' so far assumed to exist was in fact heterogeneous and artificial (see e.g. Delorme et al. 2004). In such frequent cases, what had been considered for a while as a 'reality' turns out to be just an abstraction, a concept that did not reflect the real relationships. There is nothing shocking or contemptuous in stating this, this is just the way science progresses. This misunderstanding is strikingly exemplified by the frequent statement appearing in papers, including in their titles (e.g., Van Dyken et al. 2006; Maddison et al. 2008; Fang et al. 2016), that a new clade has been 'discovered'. Objects, organisms or facts can be discovered, but concepts and hypotheses are formulated, not discovered.

But there is another reason, the importance of which is only beginning to be really appreciated and acknowledged by the community of biologists, for being reluctant to use the concept of 'clade' in evolutionary biology. This is the fact that evolution has not only involved splitting (cladogeneses), that can be expressed in the form of a 'tree', but also reticulation (mixogeneses), that should be expressed as a complex multidimensional 'network'. We must recognise that "the history of life cannot properly be represented as a tree" (Doolitlle 1999a), because "events such as meiotic and sexual recombination, horizontal gene transfer and hybrid speciation cannot be modeled by bifurcating trees" (Linder et al. 2004: [2]), so that "life's history is sometimes like a tree and sometimes like a net" (Doolittle 1999b: M8). Therefore the concept of 'clade' corresponds to a gross simplification of evolutionary patterns and should certainly not be taken for a 'fact'. It is merely a tool aiming at facilitating our analysis of evolution, until better tools are conceived, tested and adopted.

For all these reasons, we think that the permanent use in the phylogenetic and taxonomic literature of the term 'clade', understood as designating real objects or individuals, is misleading and should be abandoned. In the present work, in most cases, we used instead the term branch, which clearly refers to a human construction as it designates simply a portion of a cladistic tree, built here on the basis of nucleic acid sequencing, adopted as a hypothesis for the construction of our ergotaxonomy pending a better hypothesis. We do not claim that 'branches' do exist in the 'real world', we just consider them as tools for the establishment of a provisional and refutable ergotaxonomy. In a few cases, we used the term lineage to designate the evolutionary biology concept of holophyletic group of organisms, as this term has apparently never been used to designate a taxonominal rank and is thus less confusing than 'clade'.

Anyway, whatever progresses are made in the study of the cladistic relationships between the species known to us (both extant and extinct), we should realise that we will never know the 'true' relationships between them, as many more species have existed on earth and will never be known to us, and incomplete species sampling has a major impact on cladistic inference (Lecointre et al. 1993), so the trees we are building will remain forever, at best, approximations and will never reflect accurately the detailed course of evolution.

\subsection{Taxonomy}

\subsubsection{Taxonomic paradigms}

The term taxonomy, as first introduced by Candolle (1813), simply meant classification of organisms 
into particular units later called taxa (Meyer 1926), which initially had no phylogenetic meaning. The science of taxonomy relies on concepts (mostly taxa, categories and ranks) and Criteria (allowing to recognise that the data fit with the concepts). The first basic question, which is entirely scientific, is to know when a group of organisms 'deserves' to be formally recognised as a taxon: the reply to this question depends on the taxonomic paradigm adopted. The second question is how to arrange all the taxa into a unique hierarchical nomenclatural system which allows to store and retrieve the information relative to taxa. This second question is both scientific (deciding which Criteria should be used to build this hierarchy) and ergonomic: as taxonomy has to deal with millions of objects (the taxa), it cannot do without an ergonomic system of indexation of the information, just like in an encyclopaedia or a database. While it would not be so in a domain dealing with a few hundreds or thousands of known objects (like the planets), the need of a hierarchical system is very strong in zoological taxonomy.

So far, after its initial empirism, which did not rely on any theoretical framework, two main schools of taxonomy have played a leading role during the history of taxonomy: the phenetic and the cladistic ones.

To put the things schematically, the phenetic approach aims at measuring the resemblance/similarity or the differences or 'distance' between organisms or groups of organisms. Although not contradictory in its tenets with the concept of evolution, it does not use this concept for the building of classifications. On the basis of character analysis and of comparison on the data on characters concerning several individuals, it produces hierarchical classifications using taxonomic ranks. Of course, the main problem with this approach is that, in many cases, it fails to recognise homoplasy and parallel evolution, and produces polyphyletic or paraphyletic taxa, which do not reflect evolution.

In contrast, the concept of evolution is central to the cladistic approach of taxonomy. Relying also on character analysis, but additionally on the cladistic analysis methodology and therefore on the concept of synapomorphy, this approach aims at avoiding the formal recognition of polyphyletic and paraphyletic taxa. The main problem with this approach is that it only allows to recognise taxa that are considered monophyletic/holophyletic, but does not produce hierarchical classifications, as was understood and stressed by the supporters of the Phylocode system (Cantino \& Queiroz 2020), which only recognises 'clades' nested among each other, but no ranks.

A third approach to taxonomy has strangely seldom been identified as such although it has been used in thousands of taxonomic publications, mostly dealing with species-level taxonomy (microtaxonomy of Mayr \& Ashlock 1991). It relies only partly on character analysis, however not to measure 'similarity' or 'kinship' but to understand the genuine genetic or other interactions between organisms in nature or in artificial conditions. It makes use of a particular taxonomic concept which has been termed relacter (Dubois 2004d). A relacter is a relationship or interaction that may exist between organisms in the real word, and is not construed by man through intellectual phenetic or cladistic comparisons of data concerning the characters of organisms. It does not characterise the individuals taken separately but their dynamic biological interaction, and therefore can be used only for syntopic and synchronic organisms.

A well-known example of relacter concerns the study of sympatry between two sets or organisms or of contact zones between two such entities (parapatry). In such cases, two sets of organisms are first conceptualised on the basis of several independent characters observed on numerous individuals. Then, if in sympatry these two sets remain always distinct (e.g. set A with character states A1 to A5, and set B with character states B1 to B5, without composite individuals), every biologist, under whatever taxonomic paradigm and independently from the 'species concept' used, will deduce that there is no gene flow between the two entities and recognise them as distinct species. The same will apply when there are no hybrids in a contact zone between two well characterised entities. The situation is more complex when hybrids exist in this zone, as then the dynamics of the gene flow between both entities must be studied, and, even more problematic, such Criteria are not usable in allopatry or allochrony. In other words, this Criterion is asymmetrical: it works to distinguish species but not, at least in isolation, to lump them. This approach is neither phenetic nor cladistic and may be designated as relational. It is meaningful only at low taxonomic levels, i.e. mostly for species, but its use has also been advocated at genus level (Dubois 1988b; see below). This approach of taxonomy can be compatible with both the phenetic and cladistic approaches but is perpendicular to them. It does not contribute to the measurement of either resemblance or kinship, but it reflects an inescapable feature of evolution, i.e. the fact that the latter is possible only through the permanent emergence of genetic incompatibility between groups of organisms that were previously interfertile. It should therefore not be ignored in theoretical analyses of taxonomy, but it is indeed often so. 


\subsubsection{Phylogenetic taxonomies}

It is only after the spreading of the works of Hennig $(1950,1966,1974)$ that it became clear that two major 'schools' of taxonomy, 'phylogenetic' and 'phenetic', had to be distinguished. Today, few authors would advocate the latter approach, and most taxonomists claim to adhere to a 'phylogenetic' school of taxonomy. However, the widely known term 'phylogenetic taxonomy' is confusing because it has been employed in the recent years with several meanings. As pointed out by Mayr \& Ashlock (1991), the confusion stems from that surrounding the terms phylogenesis and phylogeny. As first introduced by Haeckel (1866a), these terms were meant at accounting for the evolutionary history of organisms and for the emergence and complexification of what is now called biodiversity. This phenomenon was viewed as consisting in three different but complementary processes: $\{\mathrm{K} 1\}$ branching or cladogenesis; $\{\mathrm{K} 2\}$ diversification or anagenesis; and $\{\mathrm{K} 3\}$ stabilisation and persistence or stasigenesis. However, starting with Hennig (1950), the meaning of the term phylogenesis has derived, becoming largely synonymous with one only of these two processes, cladogenesis.

The best known meaning of 'phylogenetic taxonomy', which was termed cladification by Mayr (1997) and cladonomy independently by both Brummitt (1997) and Dubois (1997), aims at producing a classification that best reflects strictly the structure of the cladistic tree, the latter being strictly understood as a cladogram of taxa, i.e., a tree showing a succession of nodes corresponding to cladogenetic events resulting in independent branches, often called 'clades' or 'lineages'. But two other approaches at least could claim to be 'phylogenetic', if the term is taken in its original sense in Haeckel $(1866 a)$. In the 'eclectic' or 'synthetic' approach advocated e.g. by Mayr (1974), which could be called phylonomy, the classification is based on a phylogram, i.e. a cladogram which incorporates 'distances', meant at measuring the 'divergences' or 'resemblances' between taxa. A third approach, initially considered but later rejected by Hennig himself but later supported by several authors (e.g. Kiriakoff 1954, 1965; Crowson 1970; Sibley \& Ahlquist 1982, 1990; Avise \& Johns 1999; Avise \& Mitchell 2007), consists in incorporating in cladograms estimates of the absolute geological age of taxa to determine their rank in the taxonomic hierarchy. This approach, using what could be called chronogram, could be designated as chrononomy.

Because cladonomy, phylonomy and chrononomy all three start from and rely on a cladogram to build up their classifications, they qualify all three as 'phylogenetic' but they are not equivalent, neither in their methods nor in their results. To avoid confusion, we use below the terms 'cladonomy' and 'cladonomic' to designate the approach we adopted here for suprageneric taxonomy.

Taxa recognised under a cladonomic approach should be strictly monophyletic (sensu Hennig 1950, not Haeckel 1866b; concept renamed holophyletic by Ashlock 1971), i.e., they should include a unique hypothetical ancestral species and all its descendants.

The recognition of monophyletic/holophyletic groups as valid taxa is independent from their naming and from their rank. Taxa can be diagnosed or defined without being named, e.g., through the use of explicit expanded diagnoses (as was the case before and even after Linnaeus 1758a) or of numericlatures or other systems (see Dubois 2005c). But verbal communication between humans is made easier by the use of names, and this requires nomenclatural Rules. Furthermore, recognition of all holophyletic groups as taxa is only the first step of the transcription of a cladistic tree into a classification. The second step is the organisation of the taxa into a hierarchy that transcribes unambiguously the topology of the branching pattern of all well-supported nodes in the tree. Various non-nomenclatural methods have been proposed for this purpose, such as the indentation of lines respective to the margin in a table presenting a classification (Wiley 1981), but these methods do not allow this information to be carried by the names themselves. They require the inclusion of such tables in any publication dealing with an ergotaxonomy, which is very heavy and often unpracticable, or the citation of works providing them, i.e., relying on external information for the understanding of the relationships between taxa. As shown below, this information can be carried by the names themselves, if they are unambiguously assigned to ranks, in the frame of Linnaean-derived nomenclatural Rules.

Transcription of a cladistic tree under the form of an ergotaxonomy requires conventions. The most often used convention, and so far the most efficient one for this purpose, is a hierarchical arrangement of taxa (classificatory units) corresponding to the successive nodes of the tree. Ideally, these nodes should be dichotomous, but in cases of partially unresolved trees they may be polytomous. In both cases the taxa immediately resulting from the division can be called sister-taxa.

There are four kinds of relationships between taxa (topotaxy) in a phylogenetic hierarchical 
classification: $\{\mathrm{L} 1\}$ parordinate taxa are sister-taxa resulting from a dichotomy or polytomy in the tree adopted as basis for the ergotaxonomy; $\{\mathrm{L} 2\}-\{\mathrm{L} 3\}$ superordinate taxa are hierarchically above their subordinate taxa (or, to put the same idea differently, they include the latter); any two parordinate taxa always have a single immediately superordinate taxon (their getangiotaxon; Dubois \& Berkani 2013) and may have (but do not always have) two or more immediately subordinate taxa (their getendotaxa; Dubois \& Berkani 2013); and \{L4\} alienordinate taxa are taxa that are not in a direct relation of ordination (i.e., of parordination, superordination or subordination).

As will be shown below, if used with caution, the system of nomenclatural ranks is appropriate to reflect directly in the nomina of taxa the hierarchical classification which reflects the topology of the tree and therefore these relationships between taxa. For this system to be efficient, universal and unambiguous, it must rely on precise nomenclatural Rules and Criteria.

\subsubsection{Taxonomic categories and nomenclatural ranks}

The nomenclatural system of the Code relies on the use of nomenclatural ranks, such as genus, family or class. Many different ranks have been used by zootaxonomists during the two and a half centuries of history of the discipline (Dubois 2006a; Appendice A4.RNK). In the recent decades, some authors, who often ignored their respective works (e.g., Smith 1988; Sundberg \& Pleijel 1994; Minelli 2000; Pleijel \& Rouse 2003; Kluge 2005; Bertrand et al. 2006; Laurin 2010; Avise \& Liu 2011) as well as works expressing different opinions, claimed that ranks should be abandoned as they are subjective and arbitrary, are not equivalent throughout zoology and do not warrant comparisons between taxa of same rank in different groups. The latter is quite true if ranks are considered to have an absolute meaning, as if they were permanently attached to taxa and expressed their 'nature' or 'essence', in biological or historical-chronological terms (Dubois 2006c, 2007a), but this essentialist interpretation is based on a misunderstanding and on a confusion between the concepts of nomenclatural rank and taxonomic category which takes its roots in a gradist/phenetic, non evolutionary, conception of taxonomy (for details see Dubois 2005b, 2006a, 2007a, 2008f, 2011a; Dubois \& Raffaëlli 2012).

The same nomen, referring to the same taxon, often moves from one rank to another within its nominalseries to follow the changes in our phylogenetic hypotheses and taxonomic hierarchies. Taxonomic hierarchies as reflected in nomenclatural ranks are 'organisational models of relationships' (Knox 1998) that are extremely useful for keeping track of inter-level relationships among entities in a hierarchical system, as understood at a given moment of the history of the phylogenetic and taxonomic work on a zoological group. Whether a given higher taxon is treated as a superfamily, an order or a class is a matter of tradition and of general consensus among specialists of the group concerned at a given time, but ranks do not, cannot and should not carry any information on the 'amount of divergence' between taxa (measured by whatever Criterion), on their 'biological diversity' (Van Valen 1973; Giribet et al. 2016), on their 'patterns of evolution' (Dubois 1988b) or on the 'time elapsed since separation' between taxa throughout the tree of life (Schaefer 1976; Dubois 1988b, 2008f: 56-57; Avise \& Johns 1999).

There would be no point in discussing whether a taxon 'is' a class or an order, as there is no concept of class or order, no theoretical background for defining ranks: ranks used by zootaxonomists are not meant at providing any information on the taxa themselves, but only on their hierarchical relationships and, through them, on the structure of the cladistic tree used as a reference for the building of a classification. They only point to a place in a hierarchy, and this place is highly labile according to the frequent additions to the available information and changes in taxonomic arrangements. The question is not to suppress ranks, but to realise that they only have a relative meaning, informing us on the hierarchical structure of the (provisional) taxonomy, i.e., on hypothesised cladistic relationships, but carry no further information.

The concept of taxonomic category, on the other hand, points to the fact that, at the lowest levels of the taxonomic hierarchy, it is possible to use different concepts for the recognition and delimitation of taxa, such as the different (and much discussed) 'species concepts' but also 'genus concepts' used by different authors. In this case, 'definitions' are indeed used for categories, which therefore do not only carry cladistic information.

An important distinction must therefore be made between two situations. In most of the nomenclatural hierarchy, i.e. above the rank genus, taxa can indeed be attributed to nomenclatural ranks, which carry only information on the structure of the tree, but this is different at the lowest end of this hierarchy, i.e. 
for the ranks species and genus and related ones. In these cases, these terms refer to both a nomenclatural rank and a taxonomic category, a double qualification which can be termed a taxonominal level. The term 'species' can designate both a rank in a hierarchy, having no proper 'meaning' or definition, and a taxonomic concept relying on a definition. The same is true, although this is often ignored, for the term genus. For this reason, Dubois (2007a) distinguished the terms species and genus (for the nomenclatural ranks) and the terms specion and genion (for the taxonomic concepts). However, as these latter terms have not gained common acceptance so far, below we follow the tradition and we use the traditional terms in both cases, the context allowing in most cases to distinguish them.

\subsubsection{Taxonomic concepts and Criteria}

The concepts and Criteria used to recognise and distinguish taxa are not the same at different taxonominal levels. Let us briefly review them successively, starting with the general situation, then in the four main taxonominal levels used in the present work: the species-, genus- and family-series recognised by the Code, and the class-series as defined by Dubois (2000b). We do not use here the formula 'integrative taxonomy' (Dayrat 2005; Vences et al. 2013), as it is ambiguous, having been employed in the literature with different meanings, and anyway it is poorly informative, as "taxonomy has been integrative for most of its history" (Valdecasas et al. 2008: 211). We prefer to identify the different taxonomic concepts (species concepts, genus concepts, etc.) and criteria used by the authors to recognise, define and delimitate the taxa of different kinds.

\subsubsection{General situation}

As we have seen, relational taxonomic Criteria are of no use for higher ranked taxa: they can be and have been used only at species and genus taxonominal levels.

Only two approaches are common to all taxonominal levels: the phenetic one, which recognises phenons as taxa, and the cladistic one, which recognises 'lineages', 'clades' or better cladons (Mayr 1995) as taxa. After a period of extensive use of the first approach at the time of flourishing of 'numerical taxonomy', by the end of the $20^{\text {th }}$ century the cladistic approach had become largely dominant. However, in recent years, and following the introduction of the 'Barcode of Life' methodology, the phenetic approach has shown an impressive new youth. This approach uses 'genetic distances' and a priori fixed 'thresholds' to decide 'objectively' that two groups of organisms should be recognised as distinct taxa and at which ranks these taxa should be attributed. Such taxa have received the designation of boleons (Dubois 2017c: 17). They can be and are used at all taxonominal levels.

In zoological groups where a calibration by external methods such as palaeochronology has been carried out, 'genetic distances' based on barcoding can be considered to provide more or less accurate estimates of the ages of the cladogeneses that were at the origin of two branches, and this has been used by some to ascribe a rank to such sister-taxa. Beside the uncertainty that still exists regarding the accuracy of these datings, which will certainly be improved in the future, this method which allows to recognise what could be called chronotaxa is not tenable at the scale of the whole animal kingdom, because rates of evolution are widely different in different branches of the tree of life, as was definitively demonstrated by Avise \& Johns (1999) and Avise \& Mitchell (2007). It would not be acceptable to associate different ages to the same rank in different zoological groups, and a homogeneisation of the use of ranks following this Criterion would require considerable changes in the 'taxonomic tradition'. In this respect, Dubois (2007a: 33) wrote: "adopting the age of taxa as a basis for allocating taxa to ranks would result in major changes in the ranks traditionally given to many taxa (...), and it is unlikely that most taxonomists would be willing to take this step today. They might change their mind in a few decades, as many more data on the ages of taxa will then be available, but, even then, it is unlikely that this could be obtained by an addition of individual actions. It would probably require holding one or several large international meetings specially for this purpose." However, beside tradition and accuracy of the information, Dubois (2008f: 57) raised another problem of this approach, which is that it applies only to organisms living synchronically, e.g., today, as otherwise all fossil taxa would have to be given higher ranks simply because they lived long ago! As molecular and palaeontological data increase, it will be possible to estimate the absolute age of all major taxa at any epoch of the earth's history, 
and therefore to use this method for rank assignation of taxa, but these ranks would be valid only for comparisons of synchronic taxa, or different Criteria should be used to attribute ranks to taxa in the living fauna and in the faunae at different epochs of this history, which would not be workable.

\subsubsection{Species taxonominal level}

A considerable literature has been devoted to discussing the 'species concept'. Many distinct 'species concepts' have been proposed and several are still in force in zootaxonomy. It is possible to compile lists of 92 'definitions' of 'species' (Lherminier \& Solignac 2000) or to distinguish 22 'species concepts' (Mayden 1997), and certainly more, but many of these definitions and concepts are largely equivalent, and the useful number can be reduced to a few main categories (Mayr \& Ashlock 1991). This literature is in part confusing, as it often does not distinguish between 'species' as a nomenclatural rank and 'species' (specion) as a taxonomic category. Three taxonomic species concepts have by far been most frequently used in the zoological literature (Dubois 2008c, 2009c, 2011b): the phenetic (morphospecies, or better phenospecies), mixiological (mayron) and phylogenetic/cladogenetic (simpson) concepts. Although the implementation of these different concepts often results practically in the recognition of the same taxonomic units (i.e., including the same organisms), this is not always the case. Furthermore, they do not cover all the situations found in nature, as they ignore the cases of 'strange species' or kyons, i.e. entities having unusual modes of formation of gametes, of initiation of development and of genetic transmission across generations, thus illustrating the reticulate dimension of evolution mentioned above, such as kleptons (like the frog 'species' Pelophylax esculentus or the salamander 'species' Ambystoma platineum) or klonons (like the lizard 'species' Cnemidophorus uniparens) (for details, see Dubois $2008 c, 2009 c, 2011 b)$.

The recent trend to use a 'threshold value' of 'molecular distance' as measured by the barcode methodology to 'delimitate species' (boleospecies) is nothing but a recent avatar of the phenetic species concept already used, and criticised, before the onset of nucleic acid sequencing, under the form of 'genetic distance' based on the results of electrophoretic comparisons of proteins from different populations (Dubois 1977). It sometimes gives results which are widely distinct from that obtained through the use of the nondimensional 'mixiological species concept' (mayron) based on the relational approach of taxonomy making use of relacters and not only of characters (Dubois 2007a). The latter however can be used strictly only in sympatry and parapatry and cannot be so in allopatry or allochrony, which requires to have recourse to other concepts in such situations.

\subsubsection{Genus taxonominal level}

As highlighted by Dubois (1988b), in contrast with the 'species concept', the number of publications dealing with the 'genus concept' has been ridiculously low. This is highly surprising, in view of the fact that the generic substantive is part of the scientific binomen which designates every species according to the Principle of Binomina. Most zootaxonomists would probably argue that there is no problem in this respect as there is no 'genus concept', as if genera were given empirically and did not require any theoretical elaboration.

For a long time, genera were only recognised on the basis of phenetic Criteria, mostly shared morphological characters. Such a morphogenus, or more widely phenogenus (when non-morphological characters were included in the diagnosis) concept had a great heuristic value for taxonomists, particularly for helping in recognising new species: genera so defined were often quite homogeneous morphologically, which limited the number of species with which any potential new species had to be compared to confirm or infirm that an undescribed species was involved. But of course this approach had the basic two complementary traps of all phenetic analyses: the exclusion from the genus of closely related species having divergent characters or the inclusion in the genus of remotely related species resembling those of the genus by homoplasy.

Inger (1958) proposed a concept of genus, that can be termed ecogenus, which considered genera as both morphological units and ecological units, sharing closely related ecological niches and adaptive zones. This was an improved phenetic concept of genus, having a good heuristic value, but strangely few subsequent authors adopted it (even its own author abandoned it without explanation in his subsequent works). 
The idea that genera should be holophyletic evolutionary units (cladogenus concept) followed the onset of cladistic thinking, but few authors cared to propose Criteria to fix the limits of such cladistic units, that would distinguish them from taxa at other lower or higher ranks, and complying with an intensional, objective and nonarbitrary concept (as defined by Simpson 1961). Therefore, in a way, the cladogenus concept is a partially empty, or at least incomplete, one, as it allows to reject polyphyletic and paraphyletic genera but does not provide information allowing to identify the node in the tree where a given genus 'stops'. In practice, most authors just rely in this respect on 'tradition', keeping 'well-known' genera, but this methodology soon reaches its limits when many new species are added to a former well-known genus, allowing to distinguish several lineages within it. A common practice is then to erect a new genus if a new species is discovered which appears to be the sister-species to all the species already known, but this is often arbitrary and unsubstantiated, giving particularly undue importance to recent discoveries. An alternative to these poorly argued practices is possible only if, additionally to being 'holophyletic groups', genera are understood as taxonomic units complying with other Criteria implied by a more elaborated 'genus concept'.

In this perspective, Dubois (1981a,c, 1983a, 1988a,c, 2004d) supported and developed a 'mixiological genus concept' earlier formulated by Van Gelder (1977) and that could be designated more briefly as mixogenus. This concept requires to maintain in the same genus all species documented to have produced, whether in natural or in artificial conditions, true viable adult diploid hybrids, as well as their closely related species. This concept takes evolutionary information into account to define genera, recognising that this category, unlike all higher ranks, "is the classificatory level above the species where reticulate evolution just begins to stop" (Böhme \& Köhler 2005: 294). In order to fit with the requirement of holophyly, this relational Criterion may lead to lump two or more 'traditional' genera that had been defined previously on the basis of cladistic Criteria. This use of a relacter to define genera is parallel in a way to the use of the mixiological concept at species level. Just like the latter but in the reverse way, this Criterion is not symmetrical: it can be used to group species in the same genus, but not to separate them in distinct genera, which would require to place in different genera closely related species that have developed mechanisms of interspecific isolation in sympatry and parapatry. Crossability (or its absence) between two species is not a 'character' of any of these species, but a characteristic of their relationship. It is therefore neither plesiomorph nor apomorph (if it were so, we would have to consider that each species bears billions of such characters, according to its potential crossability with all other living species of the planet) and is therefore useless in cladistic analysis.

The use of the mixogenus concept in zootaxonomy would have many important advantages (see Dubois 1988b: 72-75). In particular, and contrary to what has been written (e.g., Vences et al. 2013: 222), the use of this Criterion would lead to a much stronger stability in generic classifications of animals than all other Criteria, even taken together: it is very economical in use, as it is enough to have reliable information on the crossability between two cladistically remotely related species to refer permanently to the same genus both these species and all those previously referred to the most cladistically basal genus including one of them, as well as to its sister-group including the second one (see figures 4-6 in Dubois \& Bour 2010a and figure 7 in Vences et al. 2013, clearly derived from the latter although it was not cited). In such cases, a single positive cross would allow to fix permanently the generic classification of the whole group. 'Permanently' means that this would stop the 'back and forth' movement between two generic classifications that has often been observed in such cases. Use of this concept would no doubt result, in some cases, in much larger genera, but this would be a true relief in the zoological groups which are currently much oversplit, because more studied, compared to the rest of zootaxonomy, like the birds (Dubois 1988b: 70-71, 76-78). In such cases, the 'traditional' genera could continue to be used, as least for some time, as subgenera. The only real problem with this genus concept is that it cannot be implemented in some zoological groups where the interspecific crossability cannot be tested, either because of some of the biological characteristics of their members (e.g., for being unisexual), or of the impossibility to cross them in captivity (e.g., for living in inaccessible environments or for being allochronous), but the fact that a concept or Criterion cannot be used universally should not bar us from using it when this is possible: otherwise the whole taxonomy of animals, covering extant and fossil taxa, should be based only on data obtained from the fossilisable parts of animals (Dubois 1988b: 73).

It is quite clear that today zootaxonomists are not ready to adopt the mixogenus concept, probably for fear of having to abandon the sacrosanct 'taxonomic stability', but also because studies of artificial crosses between amphibian species, which were very frequent in amphibians after the middle of the $20^{\text {th }}$ century where they had proved very informative (see e.g. Blair 1972), have stopped being so because 
of the current 'fashion' for molecular phylogeny. But no other genus concept would allow for a real homogeneisation of generic taxa straddling most zoological groups. The recent use of the boleogenus concept, using barcode data and arbitrary thresholds, cannot play this role: it does not rely on a welldefined genus concept and belongs fully, as mentioned above, in the realm of phenetic taxonomy. The chronogenus concept is not better, as it cannot have any universality throughout the animal kingdomor it could have some only at the expense of much greater challenges to the sacrosanct 'taxonomic stability' than the mixogenus concept (see e.g. Avise \& Johns 1999).

In the absence of genus concept that would be fully satisfying from a theoretical point of view, an empirical approach is inescapable. The concept of diagnogenus seems then an acceptable compromise. It rests on two Criteria: \{M1\} genera should be groups of species considered on the basis of robust cladistic information to be strictly holophyletic; $\{\mathrm{M} 2\}$ they should be diagnosable (and preferably, if possible, apognosable) through characters accessible to the external examination of specimens or to the study of the animals in their natural habitat, i.e. mostly morphological, behavioural and ecological, but excluding internal anatomical characters, cytogenetic or molecular data. Genera so defined through their diagnosability (Guayasamin et al. 2009; Vences et al. 2013; Araujo-Vieja et al. 2020) would have a great heuristic value, as they would include 'similar' species that could be readily ascertained by phenetic survey, even in the field without dissection or other technical treatments. This would be much appreciated by all biologists working in the field with natural populations of animals (taxonomists, ecologists, conservation biologists) and it could help as an important guide for the collection of specimens, especially in our time when this has become more and more difficult for administrative and legal reasons. Genera so defined would be of extremely varied sizes (in terms of numbers of species included), which is not a problem in itself and even provides useful information on the degree of stasigenesis (evolutionary stasis) of the group concerned (Dubois 1988b).

\subsubsection{Suprageneric taxonominal levels}

In the family- and class-series, as we have seen, nomenclatural ranks do not qualify as taxonomic categories, that could be defined biologically, historically or otherwise. They only reflect the structure of the taxonomy adopted, which at these levels, according to the taxonomic paradigm adopted, can be based only on a phenetic quantitative measurement of similarity or distance, or on a cladistic tree. Relational Criteria cannot be used at these levels. Some recent authors used the concepts of boleon or chronotaxon to attribute ranks to presumably holophyletic higher taxa, but this approach cannot be used universally, because as we have seen this can be meaningful only within limited groups of the tree of life and could not be used throughout the latter.

\subsubsection{Taxonomic scope of the present work}

The present work does not provide a complete revision of the taxonomy and nomenclature of recent amphibians (LISSAMPHIBIA), but only a revision of their suprageneric taxonomy and nomenclature. A few changes are proposed here at generic and infrageneric levels, but they are very limited, for the reasons given above and below. A good understanding of these questions requires to grasp fully the distinction between the concepts of nomenclatural rank and of taxonomic category, which are often confounded in the literature.

In the present revisionary work, which deals mostly with the phylogenetic relationships among extant amphibians, we paid attention primarily to the definition of suprageneric taxa and their hierarchical relationships but we did not challenge the currently 'accepted' or 'dominant' species (or specion) taxa, nor, except in a few cases, the 'accepted' or 'dominant' genus (or genion) taxa.

\subsubsection{Species taxonominal level}

In the present work, we did not challenge the currently dominant species classification of extant amphibians, as given for example in the websites $A S W<2020 a>$ and $A W b<2020>$. We note however that this current classification is highly heterogeneous as, depending on the genus, family or other 
higher taxon, it relies on different species concepts, or more exactly on different 'Criteria' which do not even always rely on explicit concepts, particularly in the case of boleospecies. For the time being, the current specific classification of extant amphibians is far from having 'homogeneised' the 'species concepts' used in different subgroups and in different parts of the world. Revising the whole specific taxonomy of all extant amphibians in order to use a single 'species concept' or at least a homogeneous approach to species level taxonomy (e.g., recognising or not taxa attributed to the ranks and categories subspecies, aggregate of species and aggregate of subspecies accepted by the Code; see e.g. Dubois \& Raffaëlli 2009, 2012) would be a huge work by itself, which is far beyond the scope of the present endeavour, and we did not introduce changes in this respect in our work.

\subsubsection{Genus taxonominal level}

We also refrained from introducing major changes in the currently 'accepted' generic classification of extant amphibians, although we are conscious of the fact that it is highly heterogeneous in terms of 'genus concepts' used, generally surreptitiously, as if genera were 'given' by the (often molecular) data, without any taxonomic analysis or justification, by different authors in different taxonomic groups and regions of the world. At this level, the heterogeneity of the taxonomic treatment in different groups is probably even greater than at species level. This treatment straddles from an unquestionably much too lumped approach to an unquestionably much too split one. In the first category, some genera are 'auberges espagnoles' ('potlucks') which are heterogeneous by all criteria (morphology, development, life history, behaviour, bioacoustics, etc.), whereas others, in the second category, are monospecific but differ only very slightly, or even not at all, from their sister-genera or even more distantly related genera. There are clearly some trends in this respect, which are related to the authors involved in the study, to their country of work, to the region of the world and/or to the higher taxon concerned. But the cause of these discrepancies is the same in most cases, being the absence not only of a 'genus concept' but also of well-defined and explicit Criteria for the recognition of a supraspecific taxon as a genus.

In most publications dealing with generic classifications, authors insist on the requirement of monophyly/holophyly, which is not exclusive to genera but concerns all supraspecific taxa, but, as we have seen, by itself this Criterion does not provide the slightest clue for recognising a taxon as a genus and not a subgenus, a family or an order. The only reason that appears in many such papers is the sacrosanct 'taxonomic stability', but even this fuzzy Criterion becomes less convincing as more and more new species are described, which leads to modify the diagnoses and contents of the 'traditional' genera. Using the Criterion of holophyly alone does not allow in the least to decide whether Leiopelma and Leioaspetos, or Nanorana and Paa, or Gastrophrynoides, Siamophryne and Vietnamophryne, or Eurycea and Urspelerpes, or Epicrium and Ichthyophis, should be recognised as different genera or as synonyms or subgenera, as in all these cases the genus or genera would be holophyletic. Even concerning the European fauna, the taxonomy of which has been studied for more than 250 years, some 'mysteries' (or more exactly inconsistencies) remain: why is the genus Latonia recognised as distinct from Discoglossus, although they are virtually indistinguishable by their external morphology and ecology, whereas the genus Ammoryctis is not recognised as valid for the single species Ammoryctis cisternasii, which is readily distinguishable by several external morphological characters and its ecology from all the other species of Alytes, or Pelodytopsis not distinguished from Pelodytes on the same grounds? There are dozens of similar cases in the classification of extant amphibians. In order to progress towards a better (if not perfect) generic taxonomy of this group, other Criteria should be added to holophyly. Suggestions in this respect have been given above in our brief survey of the main 'genus concepts' or at least Criteria, that can be considered for this purpose.

A supplementary difficulty exists in the taxonomy of the amphibians, which does not exist in many other zoological groups: it is the long-standing rejection by many taxonomists (e.g. Duellman 1977) of the category of subgenus, despite the efforts of some authors (e.g. Dubois 1987b, 1988b; Smith \& Chiszar 2006) to 'rehabilitate' it. The fact that the well-known website $A S W<2020 a>$, which many, despite its numerous weaknesses, consider as 'authoritative', does not provide a formal classification of subgenera, and also of subspecies, of amphibians, but treats them as 'synonyms' (although mentioning these taxa sometimes in the discussions of the genera and species concerned), has certainly played a great role in this respect. This has led many authors to consider that they were 'obliged', when they found two or more well characterised holophyletic groups within a genus or within a species, to choose 
between two 'black and white' solutions: either recognising a single genus or a single species, or two genera or two species. Thus doing they failed to use all the possibilities offered by the Code to have a fine grained taxonomy for amphibians at low taxonomic levels, which has many theoretical and practical advantages and allows to express fine-scaled evolutionary processes and patterns (Bernardi 1956, 1957, 1980; Dubois 1988b, 2008c, 2009c, 2011b).

As concerns the rank 'supergenus', which has been used as a valid rank in amphibians by some authors (e.g., Vieites et al. 2007), and even expressly stated by Vences et al. (2013: 208) to be Codecompliant (!), although it would certainly be useful (Dubois 2006b), it is currently not recognised in the Code and should not be used in zootaxonomy until the Code is improved in this respect (Dubois 2008f).

In the present work, except in one case to solve a persisting irritating nomenclatural problem, as there exists currently no consensus either on the use of the subgenus category or on which taxa should be recognised at this level, we refrained from mentioning the subgenera in CLAD, and we listed them as 'synonyms' in Appendices A5.NGS and A9.CLAD-1, although we consider that a good number of subgenera should be recognised in amphibian taxonomy, but treating this question would have been outside the framework of this work.

\subsubsection{Suprageneric taxonominal levels}

We adopted a drastically different approach for our suprageneric taxonomy of extant amphibians. In this case, for extant taxa we relied on a strict cladonomic methodology. Our unique arbitrary decisions were the choice (largely supported by empirical observations in various zoological groups) of a SHLaLRT support value of $90 \%$ or more as the basis for the recognition of a distinct taxon, the recognition of seven mandatory ranks in the taxonominal hierarchy and our uncompromising rejection of taxonomic redundancy, except at the rank family, for reasons explained below.

As addition of taxa and genes and changes in methods of analysis may result in changes in this respect, we relied on each node having a SHL-aLRT support value of $90 \%$ or more as the basis for the recognition of a distinct taxon. Although this threshold is arbitrary, we followed it consistently, even in cases where it results in significant changes in 'traditional' classifications. Not doing so in some cases because of subjective 'suspicions of error', not based on evidence of erroneous species allocation, of nucleic acid contamination or of errors in sequences, would be even more arbitrary and scientifically untenable.

The original method of Hennig (1950) required to recognise each well-supported node of the tree as a distinct taxon. It was abandoned by subsequent workers not on theoretical but on 'practical' grounds, simply because it resulted in a very high and 'unmanageable' number of taxa and, above all, of ranks over the whole animal kingdom. Most taxonomists then agreed that 'some' nodes only should be recognised as taxa. But then, which ones? The 'most important' ones? But on which grounds will this 'importance' be evaluated? It may appear tempting in this respect to afford more weight to the taxa traditionally recognised at the main ranks, such as class, order, family and tribe. But, as we have seen, these ranks are arbitrary, lacking biological or other 'definitions' and are just a reflection of the structure of the tree. The only Criterion that remains here is 'tradition', admittedly a poor scientific Criterion - all the more that it is itself very imprecise. In extant anurans, it seems inescapable to recognise a family RANIDAE, as this was the first family ever recognised in frogs, but then, should we also recognise a family RHACOPHORIDAE, a family MANTELLIDAE, a family DICROGLOSSIDAE, etc.? Where will 'tradition' start and end? 'Tradition' by itself is an imprecise guide.

To solve this problem, we devised a completely new method, the 'Ten Criteria Procedure' [TCP], which is presented in detail below. This procedure allows one to determine objectively which familyseries nomina will have to be fixed at the rank family in the classification of extant amphibians, and would allow it even in a much larger taxonomic group. Then, starting from this fixed rank, the position of all other well-supported taxa in the taxonominal hierarchy is given automatically step by step, both above and below the rank family. This allocation is objective and indisputable as soon as a single Criterion is used for the recognition of a node as a distinct taxon, the chosen support value in our tree, without any exception in one direction or another (e.g., either continued recognition of a 'wellknown' taxon when this is contradictory with the data, or refusal to erect a new taxon for a previously undetected and possibly poorly characterised lineage). No other Criterion (such as phenetic or genetic 
distance, or estimated age of common ancestor) is taken into account for the recognition of taxa. The resulting classification is an exact reflection of the tree on which it is based: in other words, both sets of data are in a relation of bijection or isomorphism, in which each well-supported node of the tree is paired with exactly one taxon in the classification, and vice versa.

In our classification of all extant amphibians presented below, strict usage of this methodology, without paying any attention to other Criteria, led to the recognition of 23 ranks between genus and order. This number, which allows to reflect completely and unambiguously our TREE, may be considered by many as much too high. It is then fully possible to reduce the number of ranks used for a given purpose (e.g., for a local or regional fauna or for a phylogenetic work bearing on a small proportion of extant amphibians): one may for example decide to keep only, above genus, the ranks order, suborder, superfamily, family and subfamily, or even less, e.g. order and family, but then the taxa corresponding to these ranks will not be chosen arbitrarily but imposed by the Ten Criteria Procedure, the chosen support value for nodes and the rejection of taxonomic redundancy. As we will see, the result is largely at variance with both the traditional and recent classifications of extant amphibians, although based on similar and largely compatible phylogenetic data. This is because the assignation of ranks to taxa in these classifications did not follow any consistent Criterion.

A few points deserve additional comments. In order for our classification CLAD to be fully bijective with our TREE, the former must consistently reject taxonomic redundancy, i.e. the situation in which a given taxon has a single getendotaxon (immediately subordinate taxon). Therefore, every time that our data led us to recognise a taxon having only two getendotaxa, e.g. two genera in a family, we refused the recognition of intermediate taxa between them, even if these had been consistently used in all recent taxonomies. An example will make this clear. The salamander superfamily SALAMANDROIDEA has long been considered to include two main lineages, one (traditionally known as the family SALAMANDRIDAE) including the genus Salamandra and many other genera, and one including only the two genera Ambystoma and Dicamptodon. In all recent classifications (e.g. Zhang \& Wake 2009), these two latter genera were placed in two 'monotypic' families, AmBYSTOMATIDAE and DICAMPTODONTIDAE, because "Dicamptodon has a long fossil record dating to the Paleocene (...), it differs from Ambystoma in easily visible features of morphology (...), and [it has] perennial, stream-adapted larvae rather than generally short-lived (except for neotenic populations) pond larvae as in most Ambystoma" (Zhang \& Wake 2009: 503). In other words, these two families were based on the 'absolute age of taxa' and the 'phenetic distance' between them, two Criteria that we explicitly reject as irrelevant in the frame of our taxonomic (cladonomic) paradigm. We did not accept this familial arrangement, which obscures the fact that Ambystoma + Dicamptodon represent the sister-group to the SALAMANDRIDAE. In order to reflect this fact, both these genera should be grouped in a single family AMBYSTOMATIDAE. Would then it be possible to 'save' the taxon DICAMPTODONTIDAE by ascribing it the rank subfamily within the AMBYSTOMATIDAE? No, because the taxa AMBYSTOMATINAE and DiCAMPTODONTINAE would then be strictly redundant with the genera Ambystoma and Dicamptodon. Their recognition would not bring any relevant additional information on the cladistic relationships between salamandroid taxa. The result is that there is apparently an important change in the overall classification of salamandroids, with complete suppression of a family (without even downgrading it to the rank subfamily), but looking closer at the data shows that it has in fact strictly no impact on our cladistic interpretation of the data, which remains the same as in previous works.

There is however a single situation in which we accept redundancy in our classification: it is when a suprafamilial taxon includes a single getendotaxon. In this case this taxon is always afforded the rank family. Let us consider the taxon that we recognise below as the superphalanx Archaeosalientia. It includes two superfamilies, the PELOBATOIDEA and the SCAPHIOPODOIDEA. The former includes two getendotaxa, which we recognise as the epifamilies PELOBATOIDAE and PELODYTOIDAE. The Pelobatoidae include again two getendotaxa, the families PELOBATIDAE and MEGOPHRYIDAE, but the second one includes only two extant genera, Pelodytes and Pelodytopsis. In the latter case, recognising a family PELODYTIDAE is strictly redundant with the epifamily PELODYTOIDAE and it does not bring any additional cladonomic information. The same is true for the superfamily SCAPHIOPODOIDEA, which only incudes two extant genera, Scaphiopus and Spea. Nevertheless, we recognise the families PELODYTIDAE and SCAPHIOPODIDAE. Their function here is not to bring cladonomic information but to contribute to information storage and retrieval.

Dubois (2007a: 48-50) discussed this question in detail and illustrated it in his figure 1 (reproduced here as Figure F1.MOR). He showed that to have a fully informative taxonomy and nomenclature, only two kinds of taxa required taxonomic and nomenclatural recognition: those including several supraspecific 


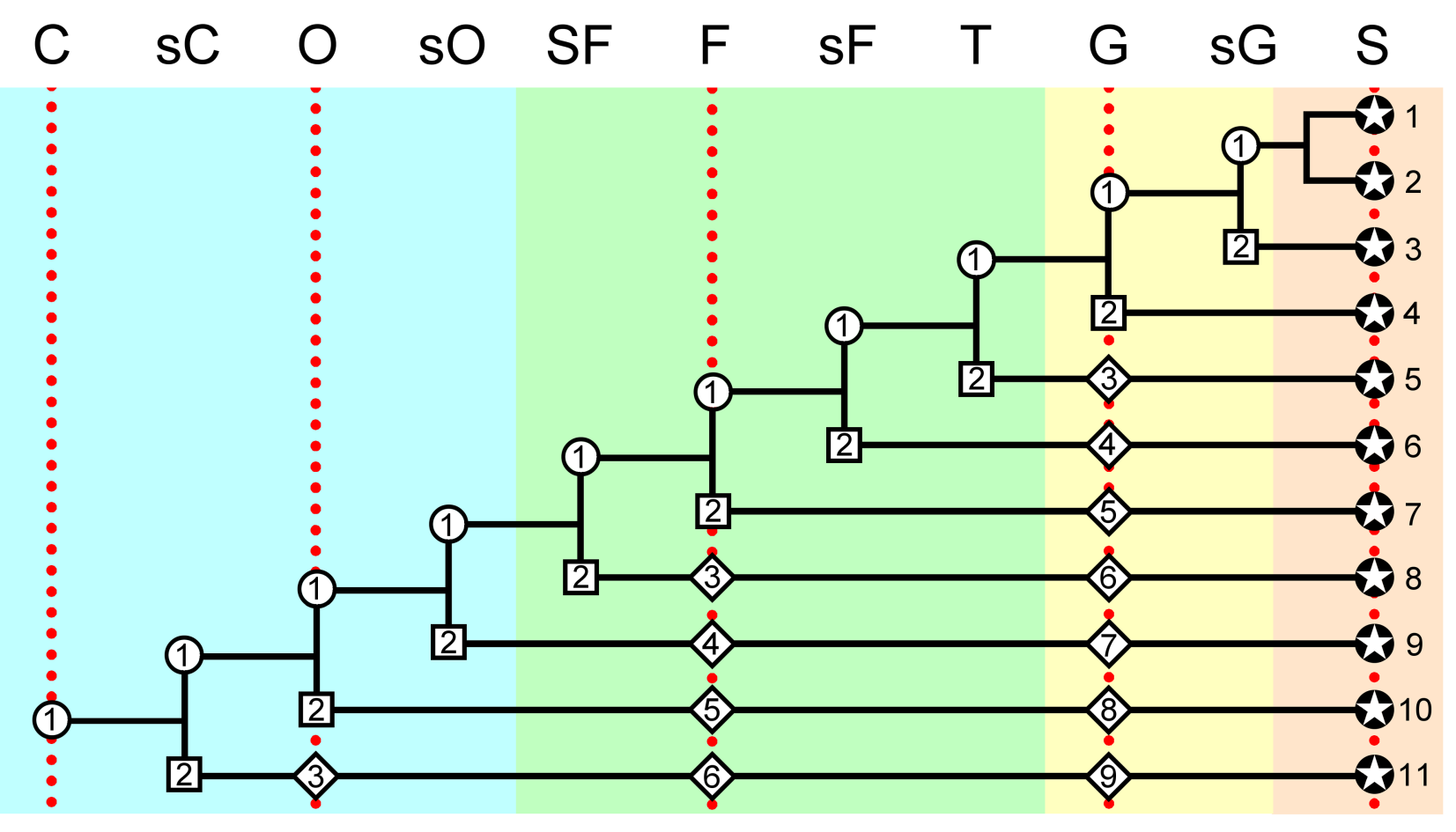

FIGURE 1.MOR. Mandatory and optional nomenclatural ranks in zoological nomenclature.

Nomenclatural ranks as designated in the upper line are as follows: C, classis; $\mathrm{sC}$, subclassis; $\mathrm{O}$, ordo; sO, subordo; $\mathrm{SF}$, superfamilia; F, familia; sF, subfamilia; T, tribus; G, genus; sG, subgenus; $\mathrm{S}$, species.

Background colours indicate the nominal-series in which these ranks belong: blue, class-series; green, family-series; yellow, genus-series; salmon, species-series.

Red stippled lines correspond to five major ranks that must be named in all cases, according to the guidelines supported by Dubois (2007a) and adopted here.

The reasons for naming these taxa are as follows: [1] star in circle, terminal taxa (species); [2] circles, taxa that must be named because they include several subtaxa; [3] squares, taxa that must be named, although they include only one species, because, according to the phylogeny presented, they are sister-groups of taxa indicated by circles; [4] diamonds, taxa that are not supported by cladistic data, but that must be named for purpose of allowing the nomenclatural hierarchy to play its role of system of storage and retrieval of information.

Figure reproduced from Dubois (2007a).

subtaxa and those that are their sister-groups, even if they do not include supraspecific subtaxa. But he added that a third situation requires taxonomic and nomenclatural recognition, deriving from the need to refer all animal organisms to taxa attributed to the seven primary key ranks (regnum, phylum, classis, ordo, familia, genus, species), which should thus be considered virtually 'mandatory' in all classifications. This proposal follows a long tradition in zootaxonomy. It is supported by the important idea that biological classifications have two major and distinct functions (Mayr 1982, 1997), a practical one (i.e., providing a universal system of storage and retrieval of information) and an explanatory one (i.e., providing an evolutionary interpretation and explanation of the diversity of organisms). Ignoring the first of these functions to concentrate only on the second may seem appealing as a 'purer' approach and may please some professional taxonomists and theoreticians, but is not doing a service to taxonomy and its innumerable users in all domains of human activity (Cracraft 1974; Ashlock 1984; Benton 2000; Dubois 2005b; Kuntner \& Agnarsson 2006). For this reason, in our classification all genera (except those which are incertae sedis) are referred to a taxon of rank family, even when the latter is redundant with its getangiotaxon.

The classification of extant amphibians we present here is based on our TREE which relies on data of nucleic acid sequencing. Although our sampling of the extant amphibian species is considerable (4060 species out of 8235 recognised on 31 October 2020), it is not complete. In order to include the missing species, we had to rely either on morphological data or on recent publications based on sequencing that 
were published after the building of our matrix. When they are included, some of our conclusions may have to change.

Although, for purpose of completeness, we mentioned the fossil taxa, we did so entirely on the basis of the recent literature, as had already been done by Dubois (2005d), but we did not propose changes in their current taxonomic allocation and we did not discuss them in our text below.

For sake of completeness, and following Dubois (2005d), we included in our classification, and in our Appendices A5.NGS, A6.NFS, A7.NCS and A9.CLAD-1 to A12.CLAD-4, all the all-fossil taxa of LISSAMPHIBIA currently recognised as valid in the literature, with their currently accepted synonyms. For this we relied on the most recent publications dealing with these taxa (not listed in our References). We considered as valid all the nomina that have not been recently synonymised, although some of them are likely to be synonyms. We were also conservative for their taxonomic allocation, so that we referred them to the lowest ranked taxon in which, according to the current knowledge, this taxon appears to belong. We consider that in many cases the validity and taxonomic allocation of their nomina is just tentative, and above all we take no stand on whether these taxa would have to be recognised as valid within the frame of our classification if they could be submitted to a molecular analysis. Thus their implementation in our scheme did not interfere with our taxonomic treatment of the extant taxa. For example, as we have seen above, we recognise a single family AMBYSTOMATIDAE for the two extant genera Ambystoma and Dicamptodon to avoid taxonomic redundancy. In fact, in this case five all-fossil genera are currently also referred to this family, but this had no impact on our taxonomic decision, as we implement the Criterion of non-redundancy only among members of the extant (living and recently extinct) fauna.

The inclusion of nomina of taxa based initially on fossil specimens may also be useful for the taxonomy and nomenclature of extant taxa as, in a few cases (Andrias, Latonia, and possibly in other so far undetected cases), the valid nomen of a taxon still represented in the extant fauna may be one such nomen of 'fossil' taxon.

As we have seen above, except in a few cases, we did not challenge the currently accepted generic classification of extant amphibians. This of course has an impact on our suprageneric classification and nomenclature. Let us just consider the family BUFONIDAE, which in our classification is the family having the highest number of subordinate ranks (ten, from subfamily to catoclanus). This is the result imposed on us if we accept all the genera currently considered as valid in the literature, on the basis of unclear and heterogeneous decisions regarding the genus concept implemented. If another, clearer genus concept, was adopted, the suprageneric classification could change drastically. For example, if the mixogenus concept was applied to this family, the number of genera would be considerably reduced (see Dubois \& Bour 2010a: 12-25), and by way of consequence the number of family-series taxa in this family too. The implementation of the diagnogenus concept throughout this family would also most probably reduce the number of genera but much less than with the mixogenus concept. A similar impact of taxonomic decisions regarding generic classification exists across the whole suprageneric classification of extant amphibians, but will have to persist as long as the decision to afford the rank genus to a branch remains largely arbitrary, as it is currently in most of amphibian groups.

\subsection{Nomenclature}

\footnotetext{
No one wants to alter the language of common sense, any more than we wish to give up talking of the sun rising and setting. But astronomers find a different language better, and I contend that a different language is better in philosophy. (...) I conclude that common sense, whether correct or incorrect in the use of words, does not know in the least what words are. I wish I could believe that this conclusion would render it speechless.
}

Bertrand Russell 1953: 306-307

\subsubsection{Introduction}

Efficient and universal communication about scientific classifications requires to use a scientific nomenclature. Scientific names or nomina (Dubois 2000b) are "the key of the big new biology" (Patterson et al. 2010). Because they rely on theoretical formalisation of empirical data (characters) and their correspondence among taxa through homology statements, which are the basis for building 
models of relationships, they have conceptual, explanatory and predictive powers (Mayr 1982, 1997), and they also allow information storage and retrieval, so that "No other way of naming in science is so powerful" (Valdecasas et al. 2014). But in order to play fully this role, their allocation to taxa must follow strict and universal Rules, not vague 'consensus' based solely upon 'usage' or 'authority'. Such Rules are provided by the 'Linnaean-Stricklandian nomenclatural system' (Dubois 2006c), or more briefly 'LSNS', implemented in the International Code of Zoological Nomenclature (Anonymous 1999), which we strictly follow in this work except for a few points, explicitly mentioned below, for which we follow the Linz Zoocode Proposals (LZP) recently published by he Linz Zoocode Committee (LZC), in particular their 17 explicit Principles (see Dubois \& Aescht 2017b; Dubois et al. 2019). The latter include two general ones (Zoological Nomenclature Independence and Nomenclatural Foundation), four dealing with the nomenclatural assignment and availability of nomina (NominalSeries, Binomina, Coordination and Neonymy), one dealing with the taxonomic allocation of nomina (Onomatophores), nine dealing with the validity of nomina and the correctness of paronyms (Zygoidy, Homonymy, Synonymy, Priority, Airesy, Proedry, Nomography, Sozoidy and Archoidy) and one dealing with the registration of nomina and onomatergies (Registration). Six of these Principles are present as such in the Code, although sometimes under different designations; eight do not appear there as Principles but are implemented as Rules in some articles; and three are not mentioned as Principles or Rules but are implied by some of the Rules of the Code (for details see our Glossary).

An important (and often ignored or misunderstood) characteristic of the zoological Code, not shared with other codes like the botanical Code (Turland et al. 2018), the Phylocode (Cantino \& Queiroz 2020) or the project of Biocode (Greuter et al. 2011), is that the nomina of taxa are assigned to three 'groups of names', better called nominal-series (Dubois 2000b), each of which consists of several nomenclatural ranks, and within which slightly different Rules apply: the species-series (with four ranks only, species, subspecies, 'aggregate of species' and 'aggregate of subspecies'; nomina written here in italics), the genus-series (with two ranks only, genus and subgenus; nomina here in italics) and the family-series (from subtribe to superfamily, with as many additional ranks below superfamily as needed; nomina here in CAPITAL ITALICS). Additionally, following Dubois (2000b) and the LZP, a class-series, not recognised by the Code, can be used for nomina of taxa above the rank superfamily (order, class, phylum, and as many additional ranks as needed; nomina here in BOLD CAPITALS). In zoological nomenclature, nomina are not attached to ranks but to nominal-series, and the latter play a role much more important than the ranks themselves in the functioning of the nomenclatural system.

In each zoological ergotaxonomy, allocation of nomina to taxa follows strict and automatic Rules, but their allocation to nominal-series and ranks is largely arbitrary, being linked to tradition and 'consensus' rather than to a well-defined methodology. There is nothing in the Code or in the taxonomic literature to indicate why any given taxon should be attributed to the rank family, superfamily, suborder or order, and in fact the whole history of taxonomy is a succession of changes in this respect, with a clear tendency towards the progressive upgrading of the ranks of taxa, even when the latter do not change in their definitions and contents. Most of the genera recognised by Linnaeus (1758a) correspond now to taxa of rank family, order or even higher.

This peculiar characteristic of the LSNS, with four (in fact three + one) nominal-series that lack biological or other definitions is a result of the history of zoological nomenclature. It is justified only by the need to organise the taxonomic information in a convenient and ergonomic way, allowing easy and efficient storage and retrieval of taxonomic and bibliographic information (Mayr \& Ashlock 1981), but it has no 'theoretical' justification. However, suppressing this system today to replace it by a 'better' (still to be defined) system of organisation of ranks and nomina (with different requirements and forms for the nomina in the different nominal-series) would be a very cumbersome endeavour and would cause endless problems. Its implementation would require considerable working time, care and funding, and would probably entail many mistakes that would have to be corrected later on. It should certainly not be considered as appropriate now, in this time of 'taxonomic urgency' (for details see Dubois 2011a, $2015 a)$.

\subsubsection{Nomenclatural Rules, recommendations and conventions}

Once taxa have been defined and arranged into a hierarchy, they must be named. Note that the process goes this way (from taxa to nomina), not the reverse way (from nomina to taxa). For this to be done consistently, and in a way that preserves an unambiguous relationship between the ergotaxonomy and the nomenclature, nomenclatural Rules must be strictly followed. Here we follow the Rules of the Code for all nomina of taxa at the rank superfamily and below, and the Duplostensional Nomenclatural 
System or DONS (Dubois 2015c, 2016, 2020a) for all nomina of ranks above the rank superfamily, which are not regulated by the Code.

The Nomenclatural Process which leads ultimately to the establishment of the valid nomen of a taxon under these Rules is a three-step process consisting in: $\{\mathrm{N} 1\}$ the availability of nomina and nomenclatural acts; $\{\mathrm{N} 2\}$ the taxonomic allocation of nomina; and $\{\mathrm{N} 3\}$ the validity and correctness of nomina (Dubois 2005b). As we will see in more detail below, when first published, a nomen may be nomenclaturally available (hoplonym) or unavailable (anoplonym) (Dubois 2000b). It is usually then or more rarely later taxonomically allocated through a name-bearing type of onomatophore. It may then be valid (kyronym), if it has precedence over all other available nomina allocated to the taxon at stake (synonyms) or having the same spelling (homonyms), or invalid (akyronym), if not.

As the present work deals mostly with suprageneric classification and nomenclature, we will give below some details on the nomenclatural Rules, recommendations and conventions that we follow here, first in the family-series (regulated by the Code) and then in the class-series (regulated by the DONS Criteria).

\subsubsection{Nominal-series, nominal-sets and nomenclatural ranks}

The Code is both imprecise and restrictive concerning the number and designation of ranks usable in zoological nomenclature. In the species- and genus-series, the number of ranks complying with the Code is strictly limited, respectively to four and two. In the family-series, it is limited only at the top, the highest rank allowed being superfamily. Above this rank, i.e., in the class-series, no precision is given in the Code, so one is led to consider that no limitation or rule exists.

It would be misleading to believe that each 'group or level' of nomina is limited to the rank that is designated by the same basic or 'key' term, possibly combined with another 'qualifying' term, such as 'family', 'subfamily' and 'superfamily'. Under such an interpretation, ranks based on different 'key' terms, such as family and tribe, or phylum, class and order, should be referred to different nominalseries. This is obviously wrong in the case of family and tribe, and using such a rule in the case of nomina at ranks above superfamily, in recognising e.g. a 'phylum-series' and an 'order-series' distinct from the 'class-series', as suggested by Hemming (1953), Levine (1958), Blackwelder (1967), Rodendorf (1977a-b), Brothers (1983a-b) or Starobogatov $(1984,1991)$, would only unnecessarily but considerably complicate the nomenclature of higher-ranked taxa (for details see Dubois, 1984b, 2005b, $2006 a$ ). Such proposals ignore the fact that the ranks of taxa are completely arbitrary and merely based, in each zoological group, on tradition and consensus, as they provide by themselves no information on the biological characteristics of taxa or on their evolutionary history. In order to remove this ambiguity, the LZC proposed to use the new expression nominal-set to designate the gathering of all the ranks the designations of which are based on the same 'key' term-e.g., family, tribe, phylum, class, order (Dubois \& Aescht 2017c). All members of the same nominal-set belong of course in the same nominalseries, but a given nominal-series may include several nominal-sets (e.g., family and tribe in the familyseries, or regnum, phylum, class and order in the class-series).

In the family-series, the Code states that as many ranks as needed ("that may be desired" according to Article 35.1) may be used from the rank superfamily downwards to the rank genus. No explanation or rationale is given for this upward limitation, which forbids the use of ranks like hyperfamily above superfamily, but as long as the Code has not been modified in this respect (Dubois 2006a, 2011a), this limitation must be followed.

As we will see, to be available, a family-series nomen must be a rhizonym, i.e., a nomen formed by adding an ending indicating plural to the stem of an available genus-series nomen, its 'type genus' or better nucleogenus (Dubois 2005b). Five FS ranks are fully regulated by the Code regarding their endings: one rank regarded here as mandatory (family, ending in -IDAE) and four ranks regarded here as optional (superfamily, ending in -OIDEA; subfamily, ending in -INAE; tribe, ending in -INI; and subtribe, ending in -INA). Although their use is explicitly allowed in the Code, no guideline is offered in this text regarding the endings to be used for the other optional ranks in the FS, which are unlimited in number, except that these endings should indicate plural in Latin. This is made mandatory by the fact that all FS nomina are "nouns in the nominative plural" (Article 11.7.1.1), or should be treated as such.

This question was addressed by Bour \& Dubois $(1985,1986)$, Dubois $(2006 a)$ and Dubois \& Aescht (2019j), who made proposals which we largely follow here, with slight modifications. As we will see 
below, in order to express fully the cladistic relationships among extant amphibians disclosed by our analysis, we need 25 ranks above genus and below classis: 11 ranks in the class-series and 14 ranks in the family-series. In other zootaxonomic groups, more ranks would be necessary and for this we refer to Dubois (2006a), but here we will limit our discussion to these 25 ranks.

As we will see also, in our proposed methodology the mandatory rank family plays a central role, its position in the hierarchy being fixed by objective Criteria. Of course, in the FS we need additional ranks both above and below the rank family. The situation above the rank family offers little freedom as, according to the Code, the hierarchy must stop at the rank superfamily. We inserted two ranks between superfamily and family, namely epifamily (as used in Bour \& Dubois 1985, 1986, Lescure et al. 1986 and Dubois 2005d, but not in Dubois 2006a, which was not Code-compliant in this respect) and apofamily, but we refrained to add more as the class-series provides many additional ranks that allow expanding the hierarchy to the top. Below family, Dubois (2006a: 208) proposed a hierarchy of 38 ranks, but as we need only 10 of these ranks for the extant amphibians we used only a small subsample of this virtual hierarchy. In particular, we did not use intermediate ranks between family and the three subordinate ranks below family fully regulated by the Code (subfamily, tribe and subtribe), so that they follow each other in descending order.

TABLE 1.HIE. Hierarchical relationships between the ranks in the species-, genus-, family- and class-series used in this work.

This Table provides the first mentions of these ranks in the herpetological literature, and the prefixes (modifiers) used to designate these ranks in the present work, as well as the standard endings [between square brackets] used here in the family-series for the nomina referred to these ranks. The family-series ranks for which the Code mentions standard endings are shown on light gray background. The places in the hierarchy of the ranks clanus and phalanx follow Dubois (2006b). INR: information not relevant here.

\begin{tabular}{|c|c|c|c|c|c|c|c|c|}
\hline Modifier & Species & Genus & Clanus & Tribus & Familia & Phalanx & Ordo & Classis \\
\hline Super & INR & INR & INR & INR & $\begin{array}{l}\text { Günther 1858; } \\
\text { Gill } 1884 \\
\text { [-OIDEA }]\end{array}$ & Hoc loco & INR & INR \\
\hline Epi & INR & INR & INR & INR & $\begin{array}{l}\text { Bour \& Dubois } \\
1985 \\
{[-O I D A E]}\end{array}$ & Hoc loco & INR & INR \\
\hline Apo & INR & INR & INR & INR & $\begin{array}{l}\text { Hoc loco } \\
{[-E I D A E]}\end{array}$ & INR & INR & INR \\
\hline- & $\begin{array}{l}\text { Linnaeus } \\
1758 a\end{array}$ & $\begin{array}{l}\text { Linnaeus } \\
1758 a\end{array}$ & $\begin{array}{l}\text { Dubois } \\
2008 f \\
{[-I T E S]}\end{array}$ & $\begin{array}{l}\text { Latreille } 1825 \\
{[-I N I]}\end{array}$ & $\begin{array}{l}\text { Batsch } 1788 \\
{[-I D A E]}\end{array}$ & Hoc loco & Linnaeus $1758 a$ & Linnaeus $1758 a$ \\
\hline Sub & $\begin{array}{l}\text { Linnaeus } \\
1758 a ; \\
\text { Bedriaga } 1881\end{array}$ & $\begin{array}{l}\text { Linnaeus } \\
1758 a ; \\
\text { Gray } 1825\end{array}$ & $\begin{array}{l}\text { Hoc loco } \\
{[- \text { ITIES }]}\end{array}$ & $\begin{array}{l}\text { Lescure }^{+2} 1986 \\
{[-I N A]}\end{array}$ & $\begin{array}{l}\text { Rafinesque } 1815 \\
{[-I N A E]}\end{array}$ & Hoc loco & $\begin{array}{l}\text { Linnaeus } 1758 a \\
\text { Rafinesque } 1815\end{array}$ & $\begin{array}{l}\text { Linnaeus } 1758 a \\
\text { Rafinesque, } 1814\end{array}$ \\
\hline Infra & INR & INR & $\begin{array}{l}\text { Hoc loco } \\
{[- \text { ITOES }]}\end{array}$ & $\begin{array}{l}\text { Lescure }^{+2} 1986 \\
{[-I N I A]}\end{array}$ & INR & Hoc loco & Hoc loco & INR \\
\hline Нуро & INR & INR & $\begin{array}{l}\text { Hoc loco } \\
{[- \text { ITUES }]}\end{array}$ & $\begin{array}{l}\text { Hoc loco } \\
{[-I N O A]}\end{array}$ & INR & Hoc loco & Hoc loco & INR \\
\hline Cato & INR & INR & $\begin{array}{l}\text { Hoc loco } \\
\text { [-ITYES }]\end{array}$ & INR & INR & INR & INR & INR \\
\hline
\end{tabular}




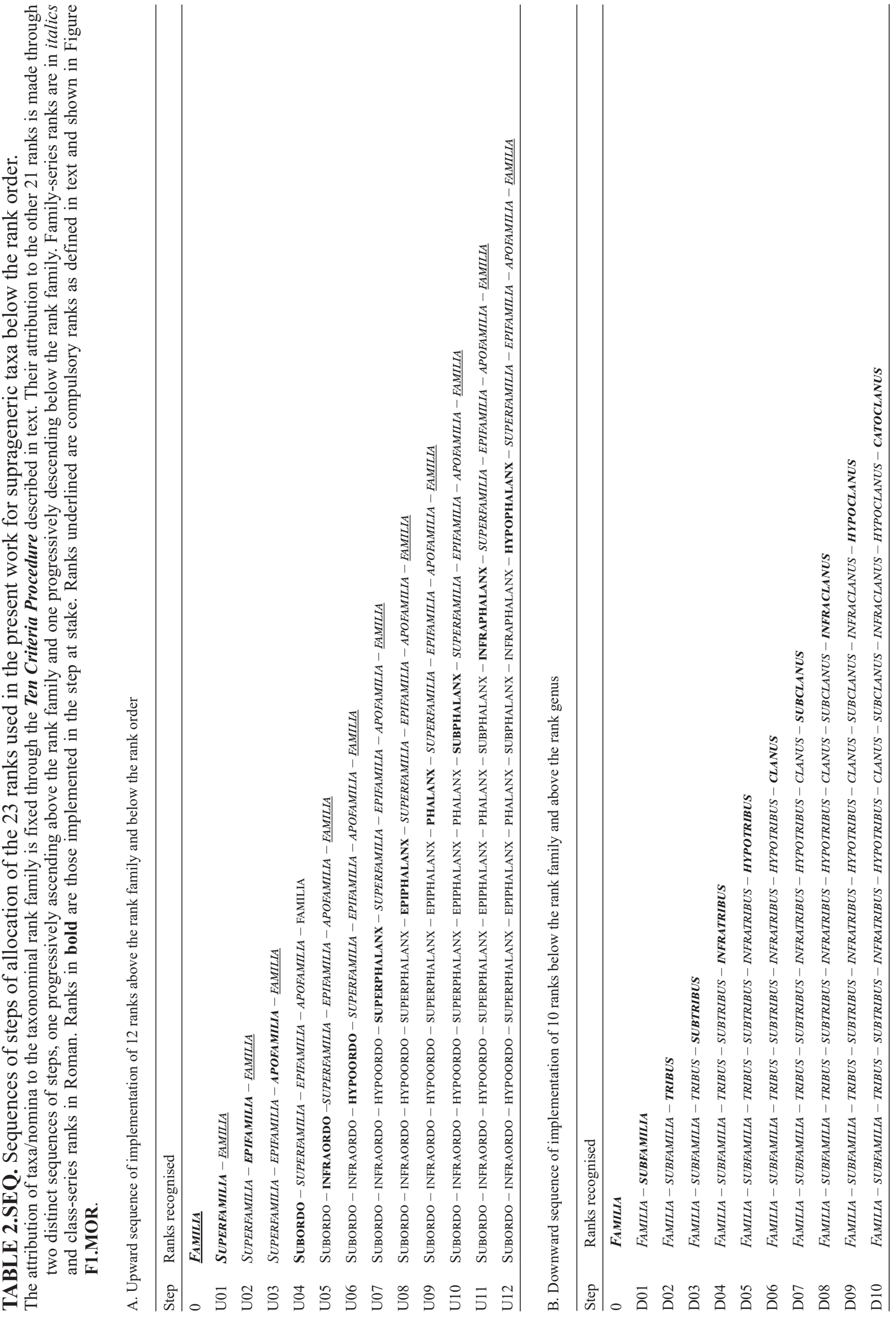


As concerns the CS ranks, Dubois (2006a) provided a hierarchy of 99 ranks, but we only need to use 11 of them (from subclass to hypophalanx) for the extant amphibians. They are referred to three distinct nominal-sets, those of class (one rank), order (four ranks) and phalanx (six ranks).

Starting from the key rank family, the fixation of which is detailed below, in order to deal with all the suprageneric taxa recognised here in extant amphibians we need 15 superordinate ranks below class and above family (12 in the CS and 3 in the FS) and 10 subordinate ranks below family (all in the FS). To avoid arbitrary and chaotic allocation of ranks to taxa, the use of these 25 ranks needs to follow in all cases the same, fixed, sequence, giving priority to some 'common' ranks over 'rarer' ones. The simplest situation is when there is a need of a single rank above family and below order, and of only three ranks between family and genus. In such cases, priority will be given to the four ranks fully regulated by the Code (superfamily above family, and subfamily, tribe and subtribe below family). But as soon as more ranks have to be added, we need an a priori fixed sequence for the addition of ranks into the hierarchy. In most cases, this sequence will simply follow a descending order (e.g., tribe, subtribe, infratribe, hypotribe, or order, suborder, infraorder, hypoorder). But there are a few exceptions. For example, we did not use any rank between subfamily and tribe (such as infrafamily or supertribe), in order to allow the four 'official' (i.e., fully regulated by the Code) ranks (family, subfamily, tribe and subtribe) to follow directly each other - but such additional ranks might be necessary in another zoological group whose taxonomy would require more ranks than the amphibians. For the same reason, the ranks epifamily and apofamily will be used only after the rank superfamily, as the latter is part of the ranks recommended by the Code.

Table T1.HIE presents all the ranks used in this work, with their respective nominal-series and nominalsets, and reference to the first works where they were mentioned in herpetology. For the 14 FS ranks we used, it shows the standard endings adopted here, which differ slightly in a few cases from those suggested in the previous works cited above.

Table T2.SEQ provides the two sequences of allocation of ranks to taxa that we implemented in this work, both above and below the rank family. This excludes the ranks class, subclass and order, for which here we simply followed the tradition, as given e.g. in the database of the Zoological Record $(<Z R$ 1864-2020>).

On the whole, a total of 1389 (766 generic and 623 suprageneric) lissamphibian taxa, are recognised in this work (Table T.14.NUM), attributed to 14 family-series and 11 class-series ranks below class, some of these ranks being used very often and some very rarely. The following list provides the number of taxa using each of these ranks in CLAD presented here. Ranks underlined in this list are part of the seven key ranks considered here mandatory in zoological nomenclature for reasons stated above.

Numbers of taxa attributed to class-series ranks below class $(34+1 \dagger)$ : C03 SubCLASSIS (1); $\underline{\text { C04 }}$ Ordo ( $3+1 \dagger$ ); C05 Subordo (7); C06 Infraordo (2); C07 Hypoordo (2); C08 SuperphalanX (2); C09 Epiphalanx (2); C10 Phalanx (3); C11 Subphalanx (5); C12 Infraphalanx (4); C13 HYPOPHALANX (3).

Numbers of taxa attributed to family-series ranks $(573+15 \dagger)$ : F14 SUPERFAMILIA (18); F15 EPIFAMILIA (12); F16 APOFAMilia (9); F17 FaMiLIa $(69+13 \dagger) ;$ F18 SubFAMILIa $(87+2 \dagger) ;$ F19 TRIBUS (89); F20 Subtribus (92); F21 InFRatribus (65); F22 Hypotribus (44); F23 Clanus (32); F24 SubCLANUs (17); F25 INFRACLANUS (23); F26 HYPOCLANUS (14); F27 CATOCLANUS (2).

Numbers of taxa of rank genus $(566+200 \dagger)$.

\subsubsection{Nomenclatural availability}

As we have seen, the nomenclatural process which leads to the establishment of the valid nomen of a taxon in a given ergotaxonomy is a three-step process (availability, allocation, validity). Let us consider first the step nomenclatural availability.

To be usable in zoological taxonomy, a nomen must have been introduced in the taxonomic literature following strict Criteria of availability, resulting from the process of nomenclatural promulgation (Dubois 2020b). These Criteria include in fact four distinct levels, three concerning availability proper (Dubois 2005b) and one concerning nominal-series assignment. They must be implemented in the following order: $\{\mathrm{O} 1\}$ publication availability or p-availability; $\{\mathrm{O} 2\}$ nominal-series assignment of nomen; $\{\mathrm{O} 3\}$ nomen availability or $\boldsymbol{n}$-availability; and, whenever necessary, $\{\mathrm{O} 4\}$ nomenclatural act availability or a-availability. The Criteria of p-availability apply indiscriminately to all publications 


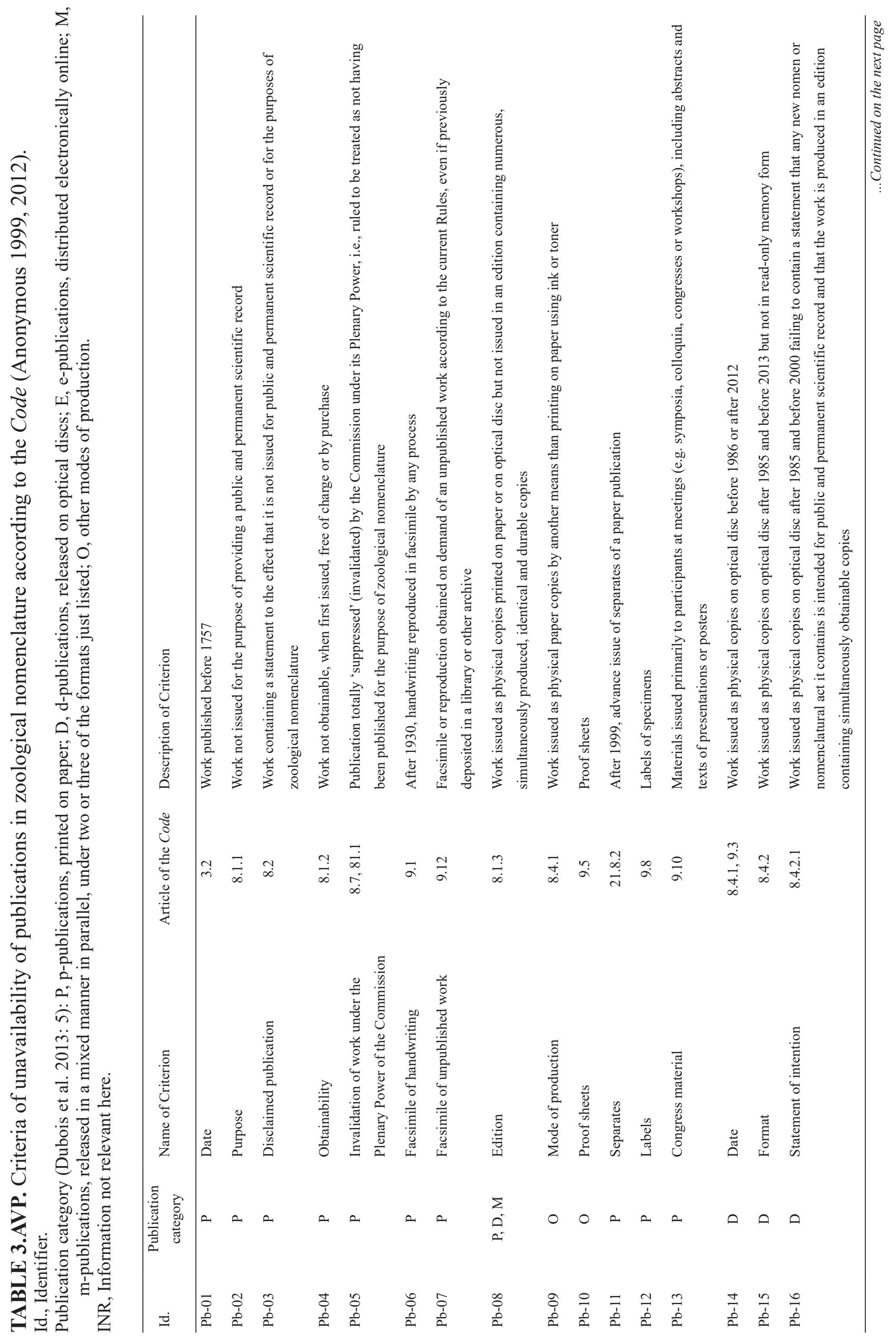




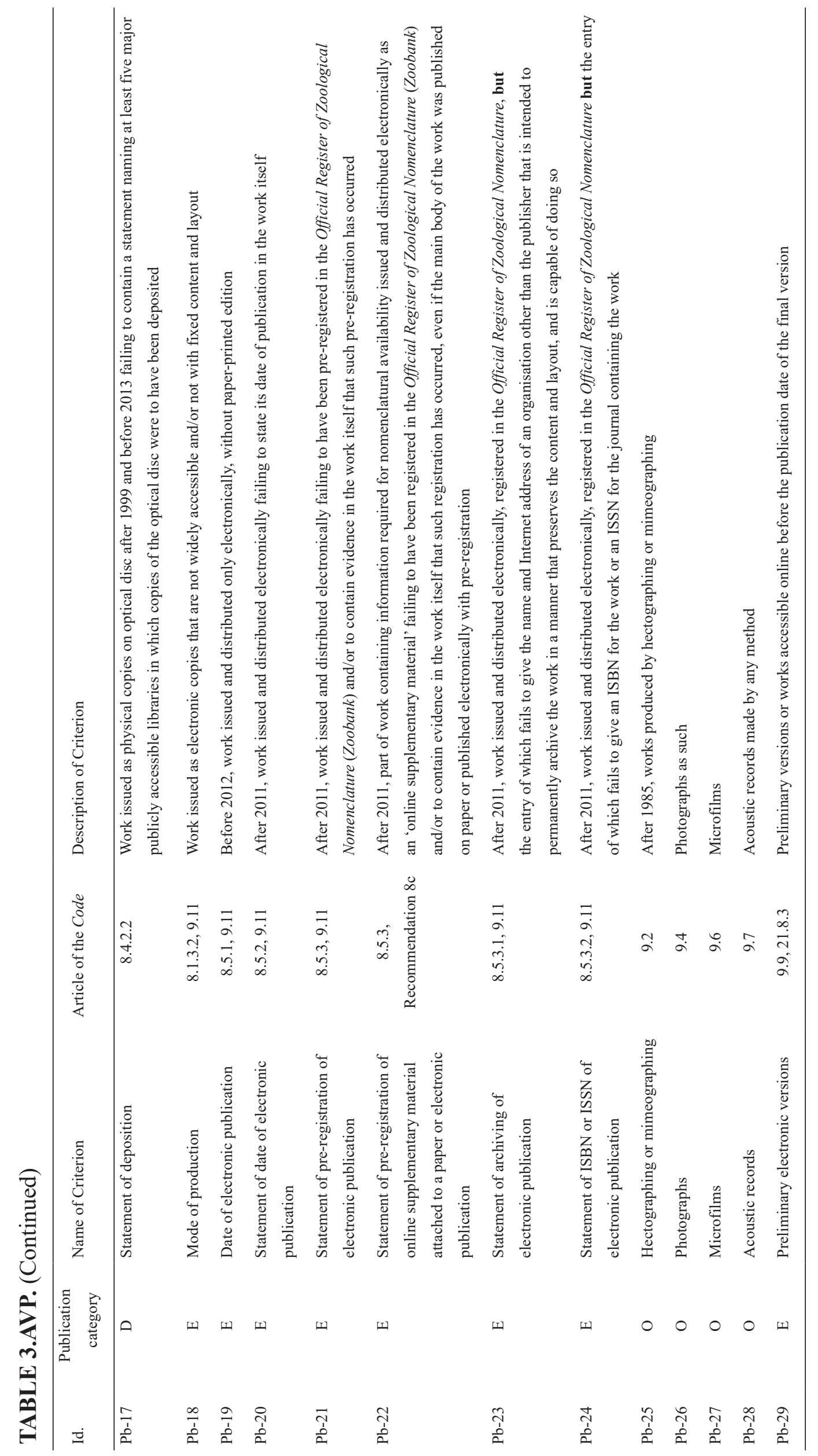


that contain new nomina, whatever their nominal-series, whereas the Criteria of n-availability and aavailability differ slightly in each nominal-series.

In what follows, available nomina are designated as hoplonyms and unavailable ones as anoplonyms (Dubois 2000b). The latter are presented below" between straight quotation marks ".

\subsubsection{Publication availability}

Before examining the Criteria of availability of nomina themselves, all the Criteria of nomenclatural availability of the works where these nomina were published should also be checked. Table T3.AVP presents the 29 situations described in the Code or in DONS as leading to unavailability of publications for nomenclatural purposes (see Dubois 2015c: 83-84). Following the 2012 amendment of the Code (Anonymous 2012), three kinds of works can now be potentially available in zoological nomenclature: paper-printed publications (p-publications) since 1758; optical discs (CD-Roms, DVDs; d-publications) from 1986 to 2011; and online electronic publications (e-publications) since 2012. No other kind of document can be available in zoological nomenclature.

Dubois et al. (2013: 61-64) pointed to a list of 40 works which are nomencaturally unavailable for having been published by BMC periodicals from 2001 to 2013 either only online before 2012, or after 2011 but without statement of Zoobank pre-registration. These works proposed 97 new SS, GS, FS and CS nomina which turned out to be anoplonyms, as well as 4 nomenclatural acts which are unavailable, in many zoological groups. Since then, many more such situations occurred. Among the cases listed, two publications and four nomina concerned the extant AMPHIBIA, but more appeared since then, and more will inevitably occur in the future, as long as the Commission has not faced the problems raised by Dubois et al. (2013) and modified the Code in order to suppress or limit them (see Dubois et al. 2019).

The following works are nomenclaturally unavailable according to the relevant Criteria in T3.AVP, so that all the new nomina of AMPHIBIA they contain are unavailable and should never be used:

(Pb-02) Work not issued for the purpose of providing a public and permanent scientific record. - Lowe, 1950: " Aneides flavipunctatus quercetorum", "Aneides flavipunctatus sequoiensis" .

(Pb-05) Invalidation of work under the Plenary Power of the Commission. • [1] La Cepède 1788:" Buffo". [2] Oken 1816:" Calamita", "Phryne".

(Pb-13) Congress material. • Liu 1964:" Paramegophrys".

(Pb-19) Date of electronic publication. - [1] Perez-Ramos \& Saldana de la Riva 2000: "Pseudoeurycea amuzgd" (see Dubois et al. 2005: 50). [2] Stöck et al. 2008:" Bufo siculus" (see Dubois et al. 2013). [3] Biju et al. 2009: "Ghatophryne", "Xanthophryne", "Xanthophryne tigerinus" (see Dubois et al. 2013).

(Pb-21) Statement of pre-registration of electronic publication. • [1] Tissier et al. 2015: "Phosphotriton" †," Phosphotriton sigel" $\dagger$. [2] Chen et al. 2016:" Prospea "†," Prospea holoserisca" $\dagger$. [3] Sá et al. 2018:" Relictus". [4] Souza Carvalho et al. 2019a:" Cratopipa". [5] Agnolin et al. 2020a: "Kururubatrachus". [6] Skutschas et al. 2020a:" Balveherpeton".

(Pb-22) Statement of pre-registration of online supplementary material attached to a paper or electronic publication. • Li et al. 2008:" Liuixalus".

\subsubsection{Nominal-series assignment of suprageneric nomina}

The nominal-series assignment of zoological nomina is usually straightforward when it concerns species- and genus-series nomina, except in very old publications, where for example some SS nomina were uninomina, but then the work is unavailable by virtue of Article 11.4 (see Table T4.AVN). But the distinction between family-series and class-series nomina is more difficult, at least in some cases. This question was discussed at length by Dubois \& Bour (2010b), Dubois (2015c) and Dubois \& Ohler (2019), and will only be summarised here. 
TABLE 4.AVN. Criteria of unavailability of class-series (CS) and family-series (FS) nomina, and of nomenclatural acts concerning them, according to the Code (Anonymous 1999) for FS nomina and nomenclatural acts, and to the DONS Criteria (Dubois 2015e) for CS nomina (indicated as DONS in column 5).

For details and terminology, see Glossary and Dubois (2000b, 2010a, 2013). [Ex], Example. Id., Identifier of Rule or Criterion.

General domain: CS, nomen of the class-series; FS, nomen of the family-series; ON, onomatergy (nomenclatural act).

\begin{tabular}{llllll}
\hline Id. & $\begin{array}{l}\text { General } \\
\text { domain }\end{array}$ & Precise domain & Name of criterion & $\begin{array}{l}\text { Article of } \\
\text { the Code }\end{array}$ & Description of criterion \\
\hline Av-01 & $\begin{array}{l}\text { CS, FS, } \\
\text { ON }\end{array}$ & Date & $\begin{array}{l}\text { Anecdidonym 1: } \\
\text { absence of } \\
\text { publication }\end{array}$ & 11.1 & Nomen or nomenclatural act anterior to 1758. \\
Av-02 & $\begin{array}{l}\text { CS, FS, } \\
\text { ON }\end{array}$ & Publication & $\begin{array}{l}\text { Anecdidonym 1: } \\
\text { absence of } \\
\text { publication }\end{array}$ & 11.1 & $\begin{array}{l}\text { Nomen or nomenclatural act not published, after 1757, in the meaning of } \\
\text { Articles 3.2, 8-9 and 21.8 (see Table T3.AVP). }\end{array}$ \\
Av-03 & CS, FS & Publication & $\begin{array}{l}\text { Anecdidonym 2: } \\
\text { anonymous } \\
\text { publication }\end{array}$ & 14 & Nomen published after 1950 with anonymous authorship. \\
Av-04 & FS & $\begin{array}{l}\text { Nomenclatural } \\
\text { system }\end{array}$ & $\begin{array}{l}\text { Ectonym 1: } \\
\text { non-binominal } \\
\text { specific nomenclature }\end{array}$ & 11.4 & $\begin{array}{l}\text { Species-, genus- or family-series nomen (oligocaconym) published } \\
\text { but unavailable within the framework of zoological nomenclature as } \\
\text { regulated by the Code, for having been published in a work that is not } \\
\text { consistently binominal for nomina of rank species, not even in its index. } \\
\text { Comment. } \bullet \text { Article 11.4 expressly states that CS nomina are not } \\
\text { concerned by this Rule. }\end{array}$
\end{tabular}

\begin{tabular}{|c|c|c|c|c|c|}
\hline Av- 05 & $\mathrm{CS}, \mathrm{FS}$ & $\begin{array}{l}\text { Nomenclatural } \\
\text { system }\end{array}$ & $\begin{array}{l}\text { Ectonym 2: } \\
\text { plurinominal } \\
\text { suprageneric nomen }\end{array}$ & 4.1 & $\begin{array}{l}\text { Nomen (hypercaconym) published but unavailable within the framework } \\
\text { of zoological nomenclature as regulated by the Code, for being a } \\
\text { plurinominal suprageneric nomen. } \\
\text { Comment. • In contrast with Rule Av- } 04 \text { for FS nomina, this does not } \\
\text { result in making all other FS and CS nomina proposed in the same work } \\
\text { unavailable. }\end{array}$ \\
\hline Av-06 & $\mathrm{CS}, \mathrm{FS}$ & $\begin{array}{l}\text { Nomenclatural } \\
\text { system }\end{array}$ & $\begin{array}{l}\text { Ectonym 3: nomen } \\
\text { unassignable to } \\
\text { nominal-series }\end{array}$ & $\begin{array}{l}1.2 .2 \\
\text { DONS }\end{array}$ & $\begin{array}{l}\text { Nomen (anemonym) published but unavailable within the framework of } \\
\text { zoological nomenclature as regulated by the Code or by DONS, for being } \\
\text { unassignable to a nominal-series in the original publication. }\end{array}$ \\
\hline Av- 07 & $\mathrm{CS}, \mathrm{FS}$ & $\begin{array}{l}\text { Nomenclatural } \\
\text { system }\end{array}$ & $\begin{array}{l}\text { Ectonym } 4: \\
\text { alternative } \\
\text { nomenclatural system }\end{array}$ & $\begin{array}{l}\text { Code, } \\
\text { DONS }\end{array}$ & $\begin{array}{l}\text { Nomen (notharchonym) published but unavailable within the framework } \\
\text { of zoological nomenclature as regulated by the Code or by DONS, for } \\
\text { being proposed within the framework of an alternative nomenclatural } \\
\text { system distinct from that of the Code and incompatible with it (e.g., the } \\
\text { Phylocode or the Biocode). }\end{array}$ \\
\hline Av- 08 & CS, FS & $\begin{array}{l}\text { Nomenclatural } \\
\text { system }\end{array}$ & $\begin{array}{l}\text { Ectonym 5: unranked } \\
\text { or pseudoranked } \\
\text { nomenclatural system } \\
\text { or pseudo-system }\end{array}$ & DONS & $\begin{array}{l}\text { Nomen (anhypsonym) published but unavailable within the framework } \\
\text { of zoological nomenclature as regulated by the Code, for being proposed } \\
\text { within a fully or partially unranked nomenclatural system (e.g., using } \\
\text { unranked 'taxa', 'phyla' or 'clades' above the FS). }\end{array}$ \\
\hline Av-09 & $\mathrm{CS}$ & Purpose & Taxonomic system & DONS & $\begin{array}{l}\text { Nomen not respecting the requirement to be proposed expressly within } \\
\text { the frame of a taxonomic system, i.e. a hierarchical classification } \\
\text { recognising several other taxa, whether named in the publication at stake, } \\
\text { or implied by reference to other works. }\end{array}$ \\
\hline Av-10 & $\mathrm{CS}, \mathrm{FS}$ & Purpose & $\begin{array}{l}\text { Temporary or } \\
\text { informal reference }\end{array}$ & $\begin{array}{l}\text { 1.3.5, } \\
8.1 .1 \\
11.7 .1 .2\end{array}$ & $\begin{array}{l}\text { Nomen proposed as temporary reference or as a plural noun referring } \\
\text { to the members of a taxon (e.g., 'testudines' for the members of the } \\
\text { genus Testudo in Linnaeus } 1758 a \text { ), not for formal, public and permanent } \\
\text { taxonomic use to designate a taxon. }\end{array}$ \\
\hline Av-11 & $\begin{array}{l}\text { CS, FS, } \\
\text { ON }\end{array}$ & Purpose & Conditional proposal & $\begin{array}{l}11.5 .1 \\
15.1\end{array}$ & $\begin{array}{l}\text { Nomen (eulabonym) or nomenclatural act proposed conditionally after } \\
1960 .\end{array}$ \\
\hline Av-12 & $\mathrm{CS}, \mathrm{FS}$ & Purpose & Synonym & 11.6 & $\begin{array}{l}\text { Nomen introduced as junior synonym of a nomen considered valid. } \\
\text { Exception: this Rule does not apply if the nomen was treated as available } \\
\text { in the scientific literature between } 1757 \text { and 1961. [Ex] LEPTODACTYLIDAE } \\
\text { Werner, } 1896 \text {. }\end{array}$ \\
\hline
\end{tabular}

......continued on the next page 
TABLE 4. (Continued)

\begin{tabular}{|c|c|c|c|c|c|}
\hline Id. & $\begin{array}{l}\text { General } \\
\text { domain }\end{array}$ & Precise domain & Name of criterion & $\begin{array}{l}\text { Article of } \\
\text { the Code }\end{array}$ & Description of criterion \\
\hline Av-13 & CS, FS & Purpose & Invalidity & $11.5,11.6$ & $\begin{array}{l}\text { Nomen introduced as invalid. } \\
\text { Exceptions: } \\
\text { [1] nomina proposed conditionally before } 1961 \text { (see Av-11); } \\
\text { [2] nomina introduced as junior synonyms and having been validated } \\
\text { before } 1961 \text { (see Av-12). }\end{array}$ \\
\hline Av-14 & CS, FS & Purpose & Explicit intentionality & 16.1 & $\begin{array}{l}\text { Nomen published after } 1999 \text { without explicit statement that it is a new } \\
\text { nomen. }\end{array}$ \\
\hline Av-15 & $\mathrm{CS}, \mathrm{FS}$ & Intension & Taxonomic allocation & 1.1 & $\begin{array}{l}\text { Nomen proposed for a taxon explicitly excluded from the animal } \\
\text { kingdom. }\end{array}$ \\
\hline Av-16 & $\mathrm{CS}, \mathrm{FS}$ & Intension & Hypothetical concept & 1.3 .1 & $\begin{array}{l}\text { Nomen proposed for a hypothetical taxonomic concept, not based on } \\
\text { actual specimen(s). }\end{array}$ \\
\hline Av-17 & CS, FS & Intension & $\begin{array}{l}\text { Individual specimens } \\
\text { as such }\end{array}$ & $\begin{array}{l}1.3 .2 \\
1.3 .3\end{array}$ & $\begin{array}{l}\text { Nomen proposed for teratological specimen(s) as such or for hybrid(s) as } \\
\text { such (i.e., not for formal taxa). }\end{array}$ \\
\hline Av-18 & $\mathrm{CS}, \mathrm{FS}$ & Intension & Gymnonym & 12,13 & $\begin{array}{l}\text { Nomen (gymnonym or nomen nudum) introduced } \\
\text { [1] before 1931, without a description or definition of the taxon it denotes } \\
\text { or an indication; } \\
\text { [2] after 1930, without [a] a description or definition that states in } \\
\text { words characters that are purported to differentiate the taxon, or [b] a } \\
\text { bibliographic reference to such a statement, or [c] a statement that the } \\
\text { new nomen is a neonym (nomen novum) for an available nomen. } \\
\text { Exception: a FS nomen published after } 1930 \text { and before } 1961 \text { which does } \\
\text { not satisfy the provisions of [2] above and was not rejected after } 1960 \\
\text { and before } 2000 \text { by an author expressly mentioning these provisions, } \\
\text { and which was used as valid before 2000, is available from its original } \\
\text { publication. }\end{array}$ \\
\hline $\mathrm{Av}-19$ & $\mathrm{CS}, \mathrm{FS}$ & Intension & Works of animals & 1.3.6 & Nomen proposed after 1930 for the work of extant animal(s). \\
\hline Av-20 & FS & Rank & $\begin{array}{l}\text { Nomenclatural } \\
\text { hierarchy: availability } \\
\text { of FS nomen }\end{array}$ & 35.1 & $\begin{array}{l}\text { Family-series nomen expressly proposed as superordinate to the rank } \\
\text { superfamily. }\end{array}$ \\
\hline Av-21 & CS & Rank & $\begin{array}{l}\text { Nomenclatural } \\
\text { hierarchy: availability } \\
\text { of CS nomen }\end{array}$ & DONS & $\begin{array}{l}\text { Class-series nomen expressly proposed as parordinate or subordinate to at } \\
\text { least one nomen of the family-series (i.e., of rank superfamily or below). }\end{array}$ \\
\hline Av-22 & CS, FS & Language & Zoological formula & 1.3 .7 & $\begin{array}{l}\text { 'Zoological formula' (see Anonymous 1922), i.e., nomen proposed as } \\
\text { modification of an available nomen by addition of a standard prefix or } \\
\text { suffix (e.g., prefix Pan- to indicate 'total-clades'; see Louchart et al. } \\
\text { 2014). }\end{array}$ \\
\hline Av- 23 & CS, FS & Language & Latin alphabet & 11.2 & $\begin{array}{l}\text { Nomen not spelled in the } 26 \text { letters of the expanded Latin alphabet (taken } \\
\text { to include the letters } j, k, w \text { and } y \text { ). }\end{array}$ \\
\hline Av-24 & CS, FS & Language & $\begin{array}{l}\text { Grammatical case and } \\
\text { number }\end{array}$ & $\begin{array}{l}11.7 .1 \\
\text { DONS }\end{array}$ & $\begin{array}{l}\text { Nomen not respecting the requirement to be a noun in the nominative } \\
\text { plural when introduced. }\end{array}$ \\
\hline$A v-25$ & FS & Language & $\begin{array}{l}\text { 'Non-latinised' FS } \\
\text { nomen }\end{array}$ & $\begin{array}{l}\text { 11.7.1.1, } \\
11.7 .2\end{array}$ & $\begin{array}{l}\text { Barbaronym: } \\
\text { [1] Before 1900, FS nomen originally published in 'non-latinised' form } \\
\text { and failing to have been validated through [a] subsequent latinisation, } \\
\text { [b] 'general acceptance' by 'authors interested in the group concerned' } \\
\text { as [b1] valid and [b2] dating from that first publication in 'non-latinised' } \\
\text { form. } \\
\text { [2] After 1899, any FS nomen originally published in 'non-latinised' } \\
\text { form. } \\
\text { Comment: Article } 11.7 .2 \text { of the Code is in fact not operational as the } \\
\text { Criteria listed above are quite imprecise and of difficult interpretation and } \\
\text { implementation (see Dubois } 2015 e: 8-9 \text { ). }\end{array}$ \\
\hline Av-26 & FS & Language & Suffixes & 11.7.1.4 & $\begin{array}{l}\text { FS nomen based on a GS nomen applied only to fossils and ending in the } \\
\text { suffix -ites, -ytes or -ithes }\end{array}$ \\
\hline
\end{tabular}


TABLE 4. (Continued)

\begin{tabular}{|c|c|c|c|c|c|}
\hline Id. & $\begin{array}{l}\text { General } \\
\text { domain }\end{array}$ & Precise domain & Name of criterion & $\begin{array}{l}\text { Article of } \\
\text { the Code }\end{array}$ & Description of criterion \\
\hline $\mathrm{Av}-27$ & $\mathrm{CS}, \mathrm{FS}$ & Language & $\begin{array}{l}\text { Metagraph 1: } \\
\text { leipoprotograph }\end{array}$ & $\begin{array}{l}19,24.2 \\
32.2 .1\end{array}$ & $\begin{array}{l}\text { Incorrect original spelling after its rejection through airesy among } \\
\text { multiple original spellings (symprotographs). }\end{array}$ \\
\hline $\mathrm{Av}-28$ & CS, FS & Language & $\begin{array}{l}\text { Metagraph 2: } \\
\text { nomographic } \\
\text { correction }\end{array}$ & $\begin{array}{l}19,33.2 .2 \\
34,50.4\end{array}$ & $\begin{array}{l}\text { Spelling rejected as a result of a mandatory spellings or ending } \\
\text { correction. }\end{array}$ \\
\hline$A v-29$ & CS, FS & Language & $\begin{array}{l}\text { Metagraph 3: } \\
\text { ameletograph }\end{array}$ & $19,33.3$ & $\begin{array}{l}\text { Incorrect subsequent spelling. } \\
\text { Comment: a careful analysis is needed to avoid confusion between this } \\
\text { concept and those of autoneonym and alloneonym (see Tables T7.NS-1 } \\
\text { and T8.NS-2). }\end{array}$ \\
\hline $\mathrm{Av}-30$ & FS & Rhizonymy & $\begin{array}{l}\text { Family-series } \\
\text { arhizonym or } \\
\text { quasirhizonym }\end{array}$ & 11.7 .1 & FS nomen not based on a then available genus-series nomen. \\
\hline$A v-31$ & FS & Rhizonymy & $\begin{array}{l}\text { Family-series } \\
\text { cenorhizonym or } \\
\text { xenorhizonym }\end{array}$ & $\begin{array}{l}\text { 11.7.1, } \\
13.2\end{array}$ & $\begin{array}{l}\text { FS nomen based on an available generic nomen but the latter not being } \\
\text { used as valid in the FS taxon adopted in the work where the FS nomen } \\
\text { was introduced. }\end{array}$ \\
\hline $\mathrm{Av}-32$ & FS & Rhizonymy & $\begin{array}{l}\text { Family-series } \\
\text { auxorhizonym }\end{array}$ & 11.7 .1 & $\begin{array}{l}\text { FS nomen based on an available generic nomen used as valid in the } \\
\text { taxonomy adopted in the publication where the FS nomen is introduced, } \\
\text { but not being formed directly from the stem of this nomen but on this } \\
\text { nomen to which a suffix (e.g., -formes or -morpha) has been added. }\end{array}$ \\
\hline Av-33 & CS, FS & Ostension & Original aphory & $\begin{array}{l}\text { 11.7.1.5, } \\
13.2,16.2 \\
39, \text { DONS }\end{array}$ & $\begin{array}{l}\text { Nomen originally published without proper fixation of nucleogenus (type } \\
\text { genus): } \\
\text { [1] After } 1930 \text { and before 2000: [1a] FS nomen based on an unavailable } \\
\text { GS nomen (Av-30); [1b] FS nomen based on a GS nomen not used } \\
\text { as valid in the FS taxon adopted in the work where the FS nomen is } \\
\text { introduced (Av-31). } \\
\text { [2] After 1999, FS nomen published without explicit statement of } \\
\text { nucleogenus. } \\
\text { [3] At all times, FS nomen based on a GS nomen which has } \\
\text { been invalidated by the International Commission on Zoological } \\
\text { Nomenclature. } \\
\text { [4] After 2015, CS nomen published without explicit designation of } \\
\text { conucleogenera or of a uninucleogenus. }\end{array}$ \\
\hline $\mathrm{Av}-34$ & CS, FS & Neonymy & $\begin{array}{l}\text { Neonym of } \\
\text { anoplonym }\end{array}$ & 12,13 & $\begin{array}{l}\text { Nomen introduced as a neonym (nomen novum) for an anoplonym } \\
\text { (unavailable nomen). }\end{array}$ \\
\hline$A v-35$ & FS & Registration & $\begin{array}{l}\text { Absence of } \\
\text { registration }\end{array}$ & $\begin{array}{l}10.7 \\
79.4 .3\end{array}$ & $\begin{array}{l}\text { Nomen not listed in a part of the List of Available Names in Zoology } \\
\text { adopted by the International Commission on Zoological Nomenclature, } \\
\text { despite any previous availability. }\end{array}$ \\
\hline$A v-36$ & FS & Invalidation & $\begin{array}{l}\text { Invalidation under the } \\
\text { Plenary Powers }\end{array}$ & $\begin{array}{l}10.1,78, \\
81\end{array}$ & $\begin{array}{l}\text { Availability of FS nomen removed by the International Commission on } \\
\text { Zoological Nomenclature under its Plenary Power (exoplonym). }\end{array}$ \\
\hline
\end{tabular}

Two independent suprageneric nominal-series exist in zoological nomenclature: the family-series (nomina of families, tribes and related ranks), whose nomina are fully regulated by the Code, and the class-series (nomina of classes, orders and taxa attributed to other higher ranks), whose nomina are only partially regulated by the Code (mostly concerning their nomenclatural availability). However, there is no biological or other Criterion to decide whether a given suprageneric nomen should be assigned to either nominal-series, and the Code fails to provide any unambiguous Criterion to distinguish between FS and CS nomina. It just states in its Article 11.7.1.1 that, to be an available FS nomen, a nomen "must be a noun in the nominative plural formed from the stem of an available generic name", i.e., must be a rhizonym (Dubois 2006c), but unfortunately it does not exclude the possibility that a CS nomen can also be a rhizonym, which is a strong source of potential confusion.

Furthermore, Linnaeus (1758a), in the book that was later fixed as the starting point of zoological nomenclature, only used five named ranks below reign (class, order, genus, species, variety) and 12 unnamed ranks (Dubois 2007c), but not the ranks family and tribe, which were introduced only later, 
and whose position in the hierarchy wandered for a while before becoming fixed between order and genus only around 1825 (Dubois 2006a).

It results from this complex situation that, in the early texts of zootaxonomy, the fact that an author used the denomination 'family' or 'tribe' for a suprageneric taxon is not an acceptable evidence that this nomen should be assigned to the family-series as understood today. Unambiguous Criteria are needed. Such Criteria were first proposed by Dubois (2006a) and Dubois \& Bour (2010b), and refined by Dubois $(2015 c)$ and Dubois \& Ohler (2019).

Some terminological clarifications are needed. The first useful distinction is between rhizonyms (Dubois 2006c), arhizonyms (Dubois 2006c), pseudorhizonyms (Dubois 2015c) and quasirhizonyms (Dubois \& Frétey 2020a), concepts presented in detail here in Table T5.RHI:

$\{\mathrm{P} 1\}$ An arhizonym is a suprageneric nomen which is not based on the stem of an existing nomen, whether of the genus-series or of another nominal-series.

$\{\mathrm{P} 2\}$ A rhizonym is a suprageneric nomen $\mathbf{H N}$ proposed for a suprageneric taxon $\mathbf{H T}$ and complying with three conditions: $\{\mathrm{P} 2 \mathrm{a}\}$ it is based on the stem of a then available GS nomen GN included in HT; $\{\mathrm{P} 2 \mathrm{~b}\}$ this stem is followed by a simple plural ending, that can be construed as being derived from the

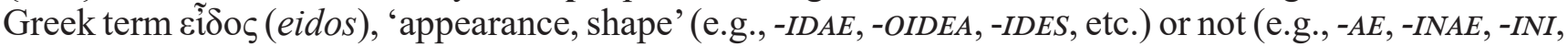
$-I N A,-I T I,-I T E S$, etc.); $\{\mathrm{P} 2 \mathrm{c}\}$ the nomen GN is allocated as valid to the taxon HT in the ergotaxonomy adopted in the publication where $\mathbf{H N}$ is introduced.

\{P3\} A pseudorhizonym is a suprageneric nomen based on the stem of a genus-series nomen failing to comply with one at least of the three conditions $\{\mathrm{P} 2 \mathrm{a}-\mathrm{c}\}$. Three categories of pseudorhizonyms (auxorhizonyms, cenorhizonyms and xenorhizonyms) were distinguished by Dubois (2015c), Dubois \& Aescht (2019j) and Dubois \& Frétey (2020a), who provided detailed discussions of these concepts.

$\{\mathrm{P} 4\}$ A quasirhizonym is a suprageneric nomen based on the stem of either a nomen of the species-, family- or class-series or of a non-scientific name of animal, this stem being combined with an ending derived from another or several other terms.

Altogether, rhizonyms, pseudorhizonyms and quasirhizonyms, which are based on the stems of other nomina or names, which opposes them to arhizonyms, qualify as panrhizonyms,

The Code only deals with some of the situations that are encountered in zoological nomenclature:

$\{$ Q1 $\}$ To be acceptable as an available FS nomen under the Code, a suprageneric nomen HN must be a rhizonym as defined above under $\{\mathrm{P} 2\}$.

$\{$ Q2 $\}$ Any nomen unambiguously assigned to the FS in the original publication that does not comply with the conditions of $\{\mathrm{Q} 1\}$ is an unavailable FS nomen.

Arhizonyms, pseudorhizonyms and quasirhizonyms therefore fail to comply with the Code's Criteria of nomenclatural availability of FS nomina. But this does not mean that they are automatically available CS nomina: they can be so only if proposed clearly for taxa at ranks above superfamily.

After a detailed analysis of the literature dealing with amphibians, Dubois (2015c: 87-89) concluded that 10 situations can be encountered regarding the nominal-series assignment of suprageneric nomina (see Table T6.ASN): 5 which result in assignment to the CS, 3 which result in assignment to the FS and 2 which result in unassignment to a nominal-series and unavailability.

Six Criteria can be used to ascertain the nominal-series assignment of suprageneric nomina: $\{\mathrm{R} 1\}$ original rank attribution of nomen, which applies only for the original Linnaean ranks reign, class and order and their subsidiary ranks (whose names start with sub-, super-, etc.); $\{\mathrm{R} 2\}$ rhizonymy, which is mandatory for family-series nomina but can occur also in the class-series; $\{\mathrm{R} 3\}$ coordination and polysemy, which apply only to family-series nomina; $\{\mathrm{R} 4\}$ topotaxy, i.e. the place of taxa in the taxonomic hierarchy; $\{\mathrm{R} 5\}$ historical Criterion, taking into account the first date of appearance of the family-series nomina in the zoological group considered; and $\{\mathrm{R} 6\}$ taxonomic consistency, which requires to give pre-eminence to the family-series in case of heretogeneity of the nominal-series assignment of parordinate nomina in a publication. In many cases, none of these Criteria is sufficient alone to reach a clear-cut decision, but their combination allows it. These Criteria were examined in details, with examples, in Dubois (2015c: 29-36) and in Dubois \& Ohler (2019: 19-23) and it would be redundant to repeat all this information here, so we refer to these publications.

It is important to note that these Criteria apply only and strictly in the original publication in which the nomen is introduced, not in any subsequent work, whether by its original author or by another author.

In the present work, we applied carefully these Criteria to establish the nominal-series assignment of all suprageneric nomina ever proposed for taxa of extant amphibians, which allowed us to assign clearly all of them to a nominal-series and to state whether they are nomenclaturally available or not. 
TABLE 5.RHI. Categories of rhizonymy in the family-series and class-series with their standard endings used here.

In the family-series, standard endings are imposed by the Code for 5 ranks, and in the present work we use standard endings for 9 additional ranks (see Table T1.HIE). In the class-series, under DONS, the Criteria for standard endings shown below are those of the Code for FS rhizonyms, and those proposed by Dubois (2015c) and emended by Dubois \& Frétey (2020a) in the frame of DONS for CS arhizonyms and panrhizonyms (rhizonyms, cenorhizonyms, auxorhizonyms, xenorhizonyms and quasirhizonyms). In the fourth column, whenever appropriate the radiconomen of the nomen HN is given between square brackets and followed by: (I) if the radiconomen is a radicogenus nomen included in the CS taxon and therefore plays the role of onomatophore for the latter; $(\mathrm{N})$ if the radicomen is a radicogenus nomen not included in the CS taxon and therefore does not play this role; (V) if the radiconomen is not a genus-series nomen but a species-series or a class-series nomen, or a non-scientific name of animal, and therefore does not play this role; (Z) if the $\mathrm{HN}$ nomen is not based on a radiconomen.

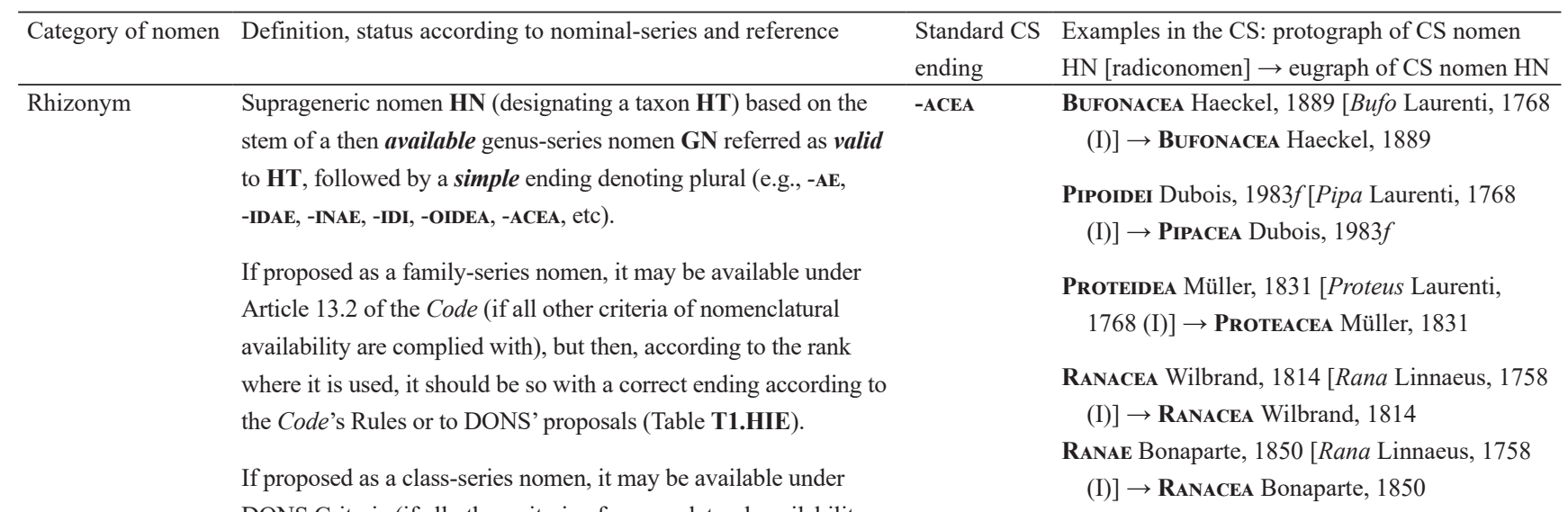

DONS Criteria (if all other criteria of nomenclatural availability are complied with), but then, it should be so with the standard ending -ACEA, which is not in a relation of hierarchy and may be used at whatever rank.

Dubois 2006c: 8, 2015c: 80

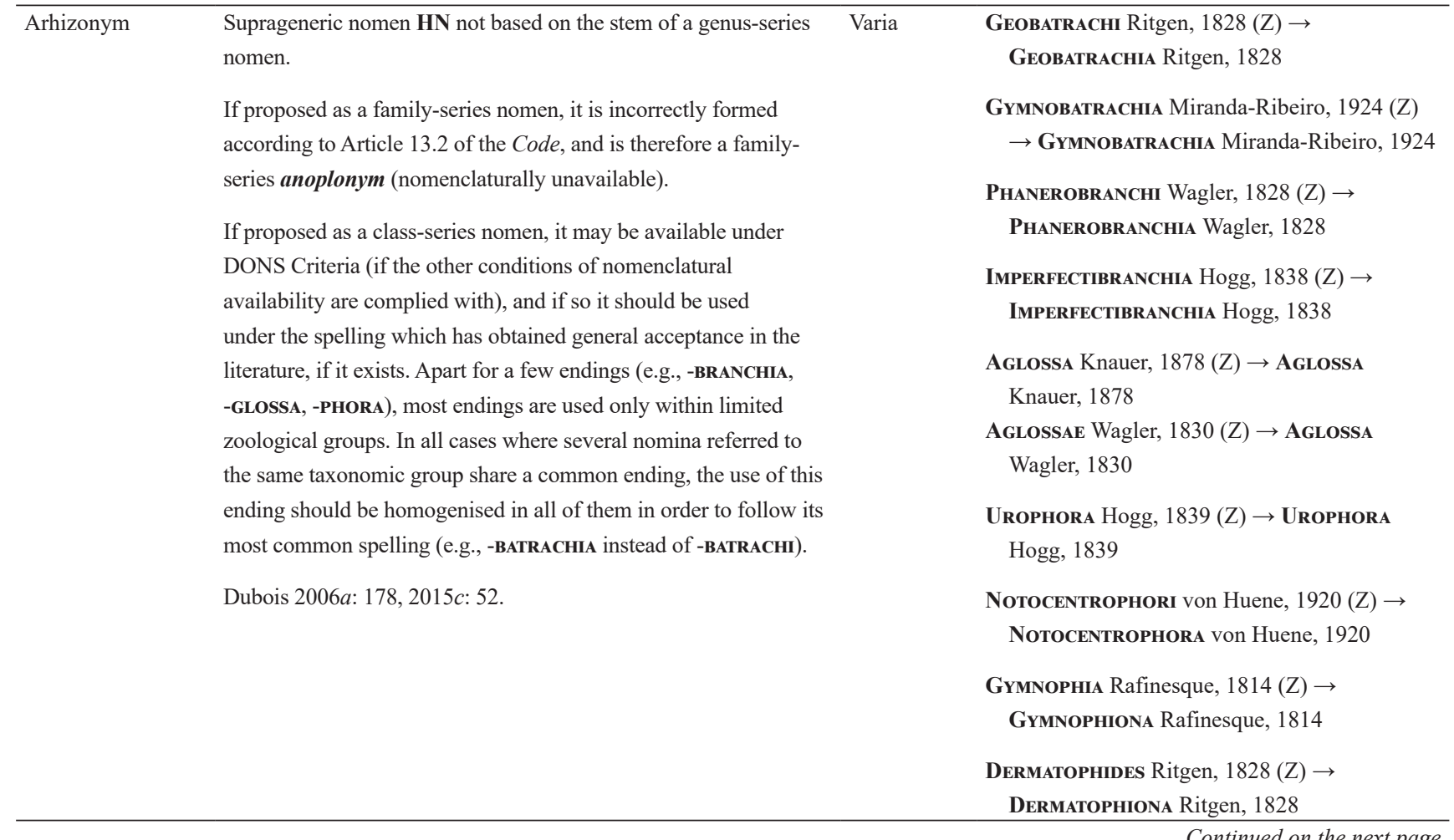


TABLE 5.RHI. (Continued)

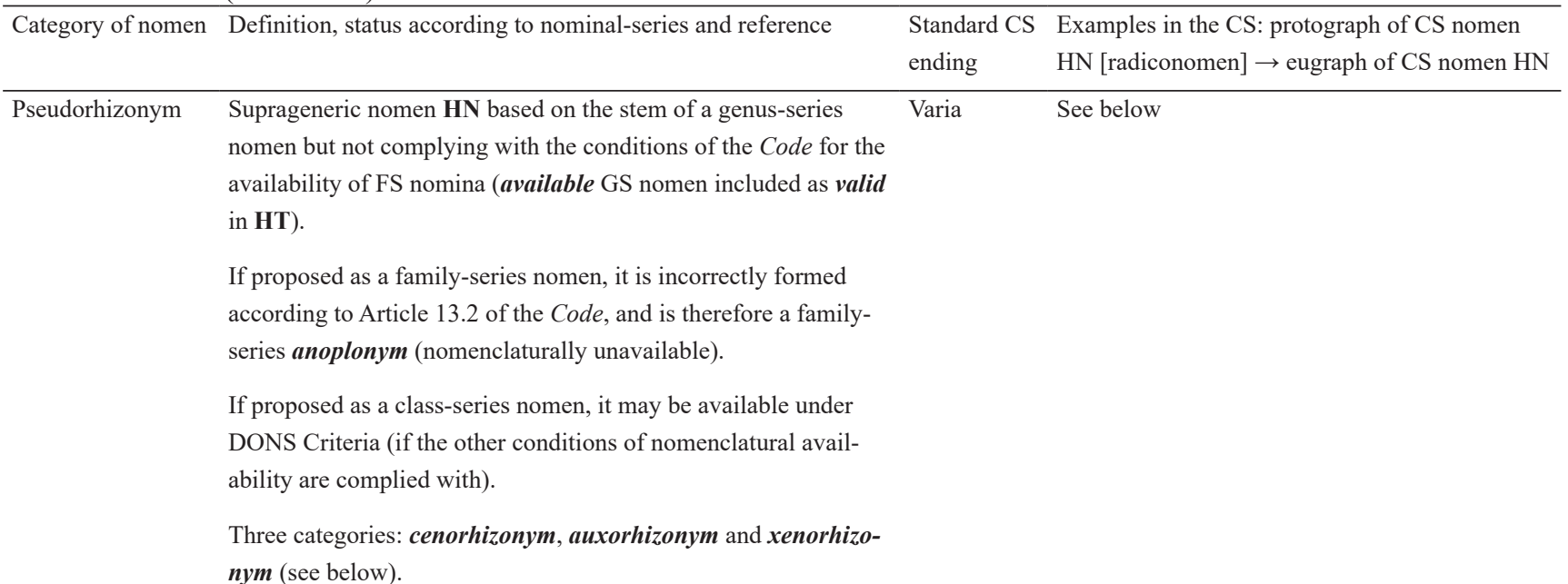

Dubois 2015c: 22, 79.

\begin{tabular}{|c|c|c|c|}
\hline Cenorhizonym & $\begin{array}{l}\text { A subcategory of pseudorhizonym: suprageneric nomen HN } \\
\text { (designating a taxon HT) [1] based on the stem of an available } \\
\text { or unavailable generic nomen GN, followed by a simple ending } \\
\text { denoting plural (e.g., -AE, -IDAE, -INAE, -IDI, -OIDEA, -ACEA, etc), } \\
\text { but [2] this nomen not being referred as valid to the taxon HT } \\
\text { in the ergotaxonomy adopted in the publication where HN was } \\
\text { introduced. } \\
\text { If proposed as a family-series nomen, it is incorrectly formed } \\
\text { according to the Code, and is therefore a FS anoplonym. } \\
\text { If proposed as a class-series nomen and available, it should be } \\
\text { used with the standard ending -ACEI, which is not in a relation of } \\
\text { hierarchy and may be used at whatever rank. } \\
\text { Dubois \& Bour 2011: } 157 \text {; Dubois } 2015 c \text { : 53; Dubois \& Frétey } \\
2020 a \text {. }\end{array}$ & -ACEI & $\begin{array}{l}\text { ANGUINEA Wiegmann \& Ruthe, } 1832 \text { [Anguis } \\
\text { Linnaeus, } 1758 \text { (N)] } \rightarrow \text { ANGUINACEI } \\
\text { Wiegmann \& Ruthe, } 1832 \\
\text { CALAMITAE Link, } 1807 \text { [Calamita Schneider, } \\
1799 \text { (N)] } \rightarrow \text { CALAMITACEI Link, } 1807 \\
\text { LACERTINI Gray, } 1850 \text { [Lacerta Linnaeus, } 1758 \\
(\text { N)] } \rightarrow \text { LACERTACEI Gray, } 1850\end{array}$ \\
\hline Auxorhizonym & $\begin{array}{l}\text { A subcategory of pseudorhizonym: suprageneric nomen HN } \\
\text { (designating a taxon HT) [1] based on the stem of a then avail- } \\
\text { able generic nomen GN referred as valid to the taxon included in } \\
\text { HT in the ergotaxonomy adopted in the publication where HN } \\
\text { was introduced, but [2] combined with an ending derived from } \\
\text { another or several other terms (e.g., -formes, -morpha, -phora, } \\
\text { etc.). } \\
\text { If proposed as a family-series nomen, it is incorrectly formed } \\
\text { according to the Code, and is therefore a FS anoplonym. } \\
\text { If proposed as a class-series nomen and available, common } \\
\text { particular cases are those of such nomina the original endings of } \\
\text { which were derived from the roots forma (Latin) or } \mu \text { oppŕ, mor- } \\
\text { phe (Greek) meaning 'form, shape': under DONS as emended by } \\
\text { Dubois \& Frétey (2020a), it should be used under the respective } \\
\text { standard endings -IFORMIA or -OMORPHA, which are not in a } \\
\text { relation of hierarchy but may be both used at whatever rank. } \\
\text { Dubois } 2015 c \text { : } 22 \text {; Dubois \& Frétey } 2020 a \text {. }\end{array}$ & -OMORPHA & $\begin{array}{l}\text { BUFONIFORMES Cope, } 1864 b \text { [Bufo Laurenti, } \\
1768 \text { (I)] } \rightarrow \text { BUFONIFORMIA Cope, } 1864 b \\
\text { HYLAEFORMIA Cope, } 1863 b \text { [Hyla Laurenti, } \\
1768 \text { (I)] } \rightarrow \text { HYLIFORMIA Cope, } 1863 b \\
\text { PIPAEFORMES Brocchi, } 1881 \text { [Pipa Laurenti, } \\
1768 \text { (I)] } \rightarrow \text { PIPIFORMIA Brocchi, } 1881 \\
\text { RANIFORMIA Hogg, } 1839 a \text { [Rana Linnaeus, } \\
1758(\mathrm{I})] \rightarrow \text { RANIFORMIA Hogg, } 1839 a \\
\text { ASTEROPHRYOMORPHA Fejérváry, } 1923 \\
\text { [Asterophrys Tschudi, } 1838 \text { (I)] } \rightarrow \\
\text { ASTEROPHRYOMORPHA Fejérváry, } 1923 \\
\text { PIPAEMORPHA Fejérváry, } 1921 b[\text { Pipa Laurenti, } \\
\text { 1768 (I)] } \rightarrow \text { PIPOMORPHA Fejérváry, } 1921 b \\
\text { RANOMORPHA Fejérváry, } 1921 b[\text { Rana } \\
\text { Linnaeus, } 1758 \text { (I)] } \rightarrow \text { RANOMORPHA } \\
\text { Fejérváry, } 1921 b\end{array}$ \\
\hline
\end{tabular}


TABLE 5.RHI. (Continued)

\begin{tabular}{|c|c|c|c|}
\hline Category of nomen & Definition, status according to nominal-series and reference & $\begin{array}{l}\text { Standard CS } \\
\text { ending }\end{array}$ & $\begin{array}{l}\text { Examples in the CS: protograph of CS nomen } \\
\mathrm{HN} \text { [radiconomen] } \rightarrow \text { eugraph of CS nomen } \mathrm{HN}\end{array}$ \\
\hline Xenorhizonym & $\begin{array}{l}\text { A subcategory of pseudorhizonym: suprageneric nomen HN } \\
\text { (designating a taxon HT) [1] based on the stem of an available } \\
\text { or unavailable genus-series nomen GN, but [2] this nomen not } \\
\text { being referred as valid to the taxon HT in the ergotaxonomy } \\
\text { adopted in the publication where HN was introduced and [3] its } \\
\text { stem being combined with an ending derived from another or } \\
\text { several other terms (e.g., -formes, -morpha, -phora, etc.). } \\
\text { If proposed as a family-series nomen, it is incorrectly formed } \\
\text { according to the Code, and is therefore a FS anoplonym. } \\
\text { If proposed as a class-series nomen and available, common }\end{array}$ & -IFORMI & $\begin{array}{l}\text { ANGUIFORMES Gouriet, } 1868 \text { [Anguis Linnaeus, } \\
1758 a(\mathrm{~N})] \rightarrow \text { ANGUIFORMI Hogg, } 1839 \\
\text { ANGUIFORMIA Hogg, } 1839 a \text { [Anguis Linnaeus, } \\
\quad 1758 a(\mathrm{~N})] \rightarrow \text { ANGUIFORMI Hogg, } 1839 \\
\text { LACERTIFORMIA Jarocki, } 1822 \text { [Lacerta } \\
\text { Linnaeus, } 1758 a(\mathrm{~N})] \rightarrow \text { LACERTIFORMI } \\
\text { Jarocki, } 1822 \\
\text { LACERTIFORMIA Hogg, } 1839 a[\text { Lacerta } \\
\quad \text { Linnaeus, } 1758 a(\mathrm{~N})] \rightarrow \text { LACERTIFORMI } \\
\text { Hogg, } 1839\end{array}$ \\
\hline
\end{tabular}
particular cases are those of such nomina the original endings of

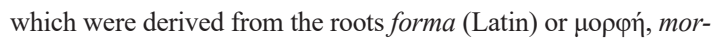
phe (Greek) meaning 'form, shape': under DONS as emended by Dubois \& Frétey (2020a), it should be used under the respective standard endings -IFORMI or -OMORPHI, which are not in a relation of hierarchy but may be both used at whatever rank.

Dubois 2015c: 22, 82, 90; Dubois \& Frétey 2020a.

\begin{tabular}{|c|c|}
\hline Quasirhizonym & $\begin{array}{l}\text { Suprageneric nomen HN based on the stem of either a nomen of } \\
\text { the species-, family- or class-series or of a non-scientific name of } \\
\text { animal, this stem being combined with an ending derived from } \\
\text { another or several other terms (e.g., -formes, -morpha, -phora, } \\
\text { etc.). } \\
\text { If proposed as a family-series nomen, it is incorrectly formed } \\
\text { according to Article } 13.2 \text { of the Code, and is therefore a family- } \\
\text { series anoplonym (nomenclaturally unavailable). } \\
\text { If proposed as a class-series nomen and available, common } \\
\text { particular cases are those of such nomina the original endings of } \\
\text { which were derived from the roots forma (Latin) or } \mu \text { oppŕ, mor- } \\
\text { phe (Greek) meaning 'form, shape': under DONS as emended by } \\
\text { Dubois \& Frétey ( } 2020 a \text { ), it should be used under the respective } \\
\text { standard endings -IFORMES or -OMORPHES, which are not in a } \\
\text { relation of hierarchy but mav be both used at whatever rank }\end{array}$ \\
\hline
\end{tabular}

-IFORMES

(1)
Pisciformia Hogg, $1839 a($ V $) \rightarrow$ Pisciformes
Hogg, 1839
SERPENTIFORMIA Leuckart, $1840(\mathrm{~V}) \rightarrow$
SERPENTIFORMES Leuckart, 1840

-OMORPHES ICHTHYOMORPHA Owen, $1866(\mathrm{~V}) \rightarrow$

ICHTHYOMORPHES Owen, 1866

OPHIOMORPHa Van der Hoeven, $1855(\mathrm{~V}) \rightarrow$ Ophiomorphes Van der Hoeven, 1855

Theriomorpha Owen, $1866(\mathrm{~V}) \rightarrow$

THERIOMORPHES Owen, 1866

ThERIOMORPHA Hoffmann, $1878(\mathrm{~V}) \rightarrow$

THERIOMORPHES Hoffmann, 1878

Dubois \& Frétey $2020 a$.

\subsubsection{Nomen and onomatergy availability}

\subsection{General situation}

Table T4.AVN presents the 36 situations leading to unavailability of higher ranked nomina or of nomenclatural acts concerning them (see Dubois 2015c: 85-86). Three of these situations apply to the onomatergies (nomenclatural acts) and 34 to FS nomina under the Code, while 27 apply to CS nomina according to the DONS Rules (Dubois 2015c). These situations are much more varied than many taxonomists believe. Many authors think that the formula nomen nudum applies to all anoplonyms (unavailable nomina), but this is incorrect. The Glossary of the Code clearly defines nomen nudum as referring to a nomen that, if published before 1931, fails to conform to Article 12, or, if published after 1930, fails to conform to Article 13. This applies to only three of the 36 situations described in Table T4.AVN (Av-16, Av-31, Av-32). Rather than using the formula nomen nudum in an improper manner, it is therefore preferable to use the general terms anoplonym for the 36 situations, gymnonym for nomen nudum as defined in the Code, and atelonym for all other cases of anoplonyms, which include several subcategories not discussed here (see Dubois 2011 $a$ and Glossary below for details). 

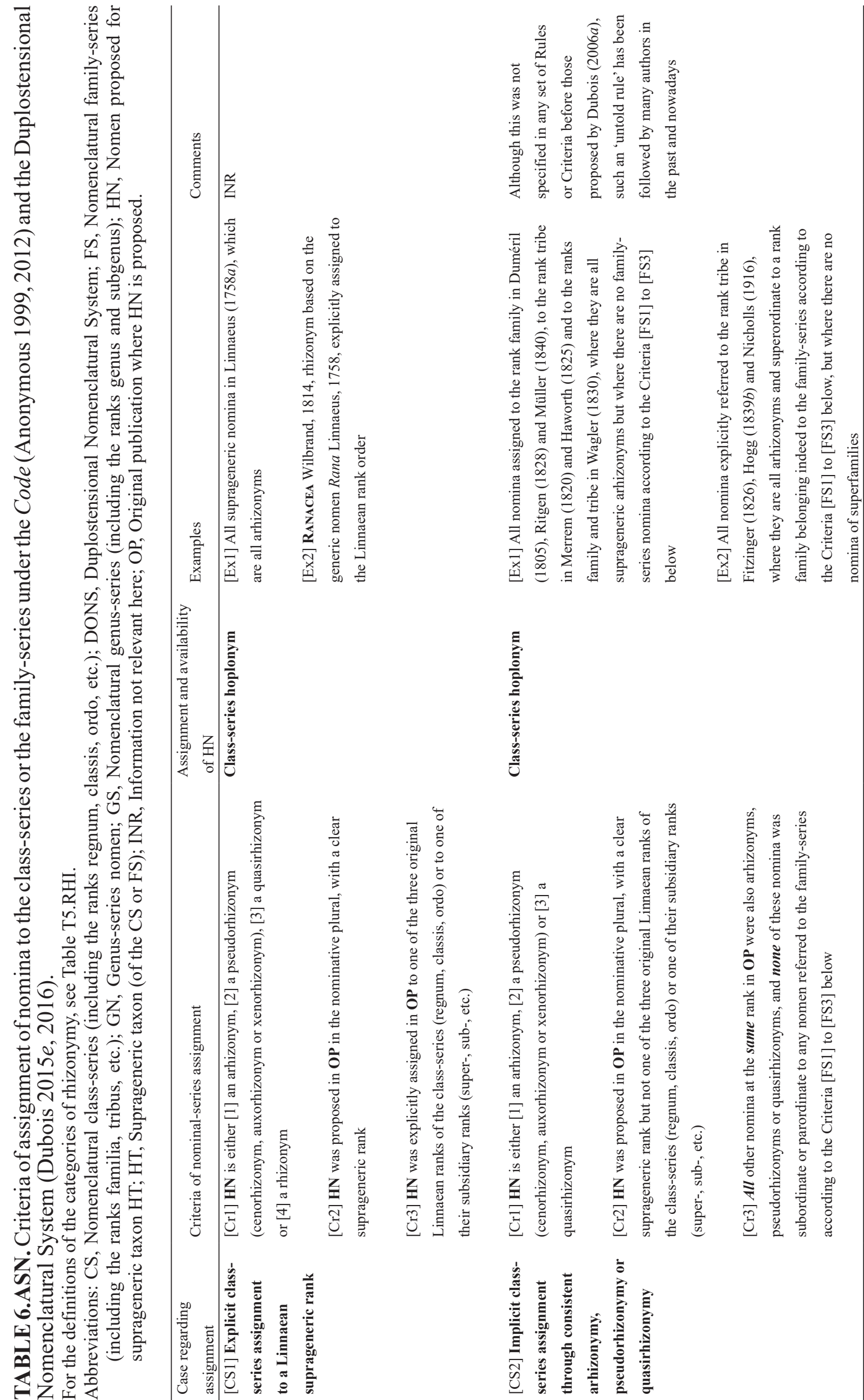

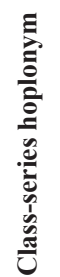
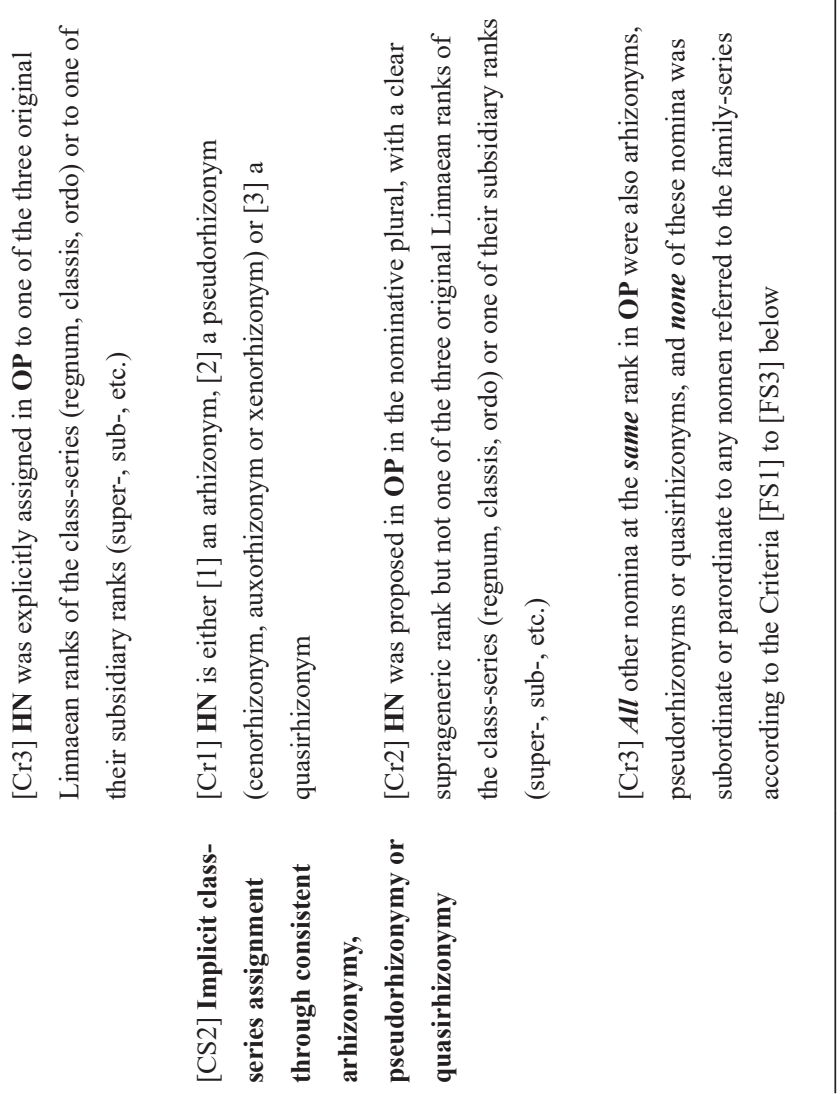


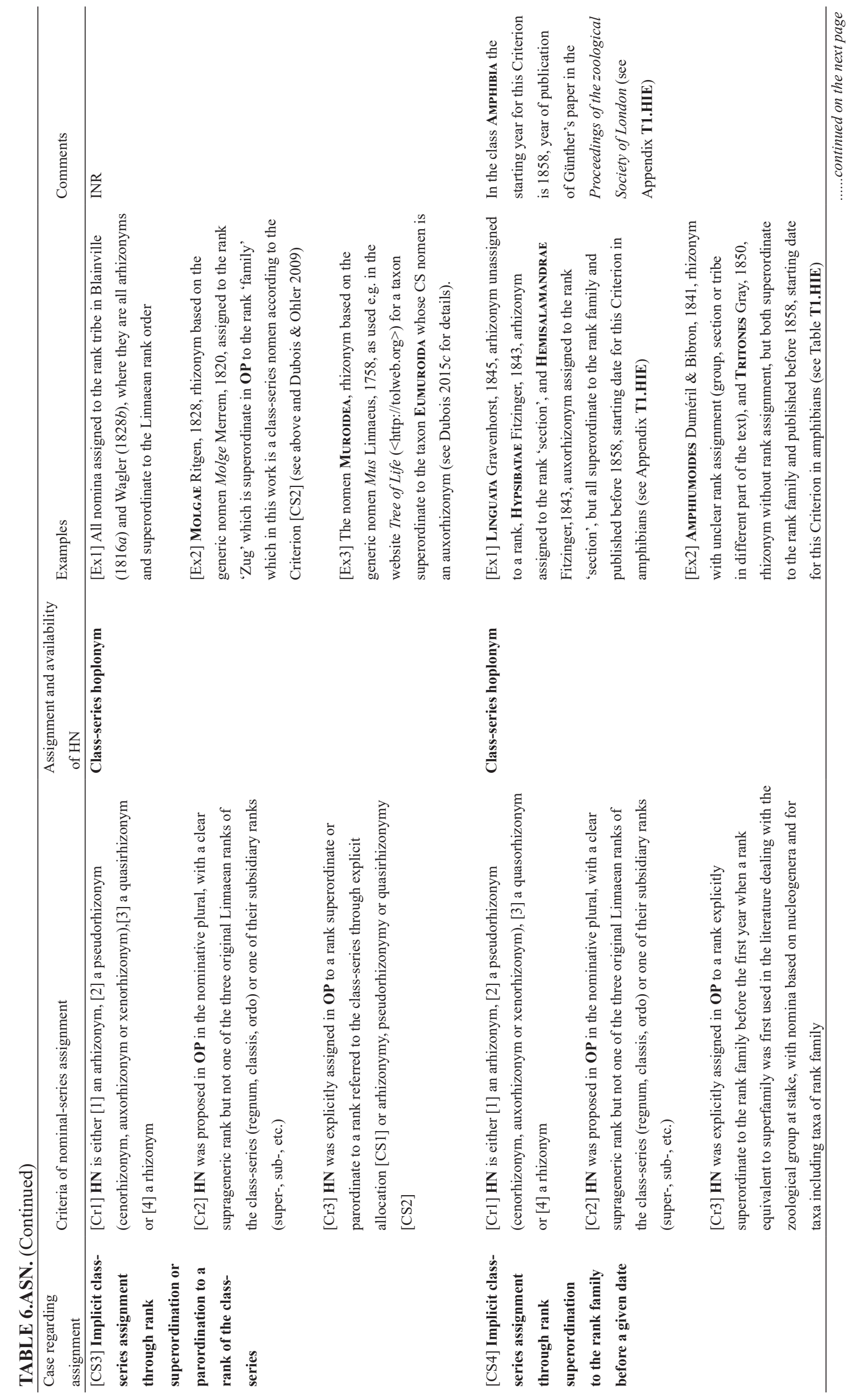




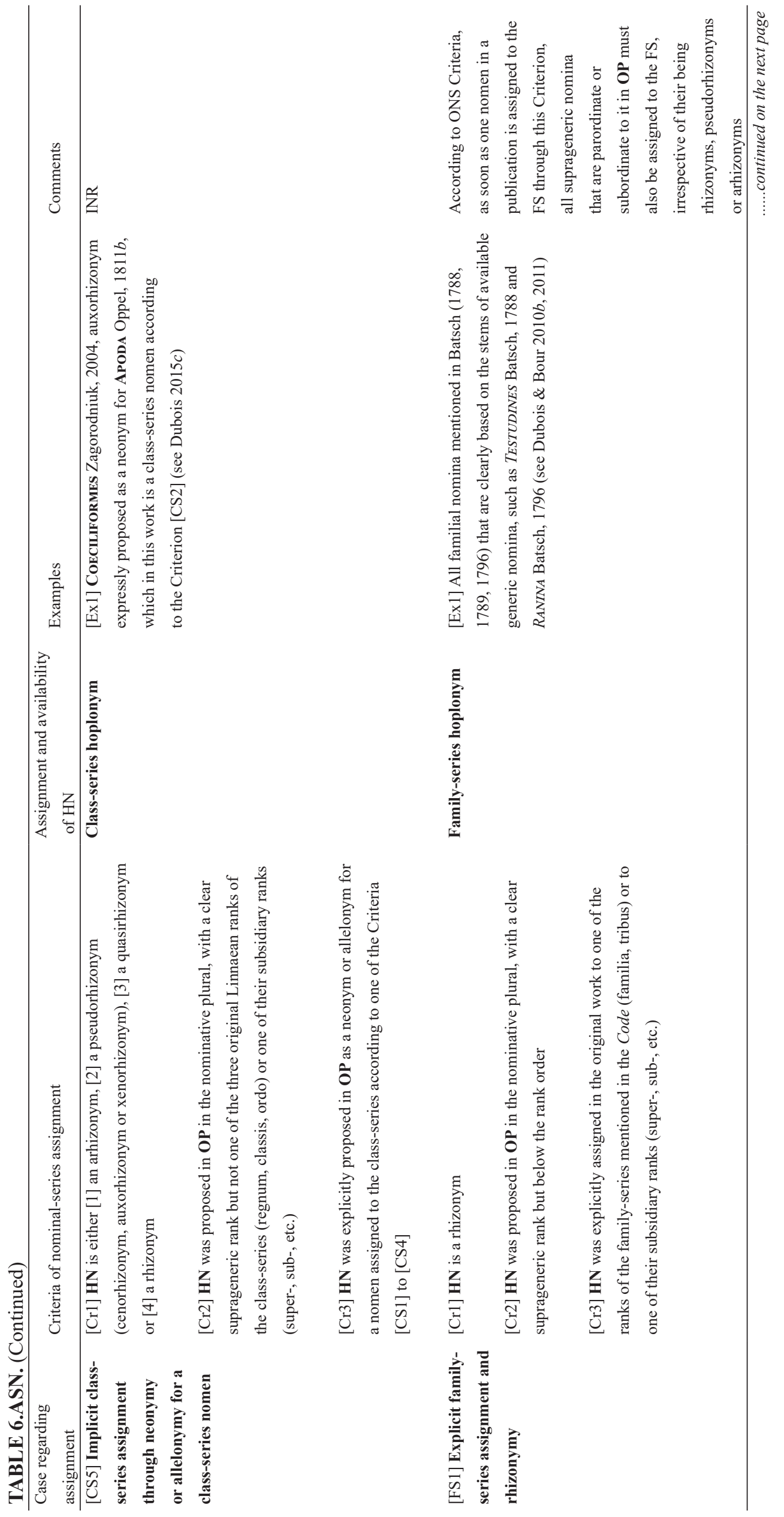




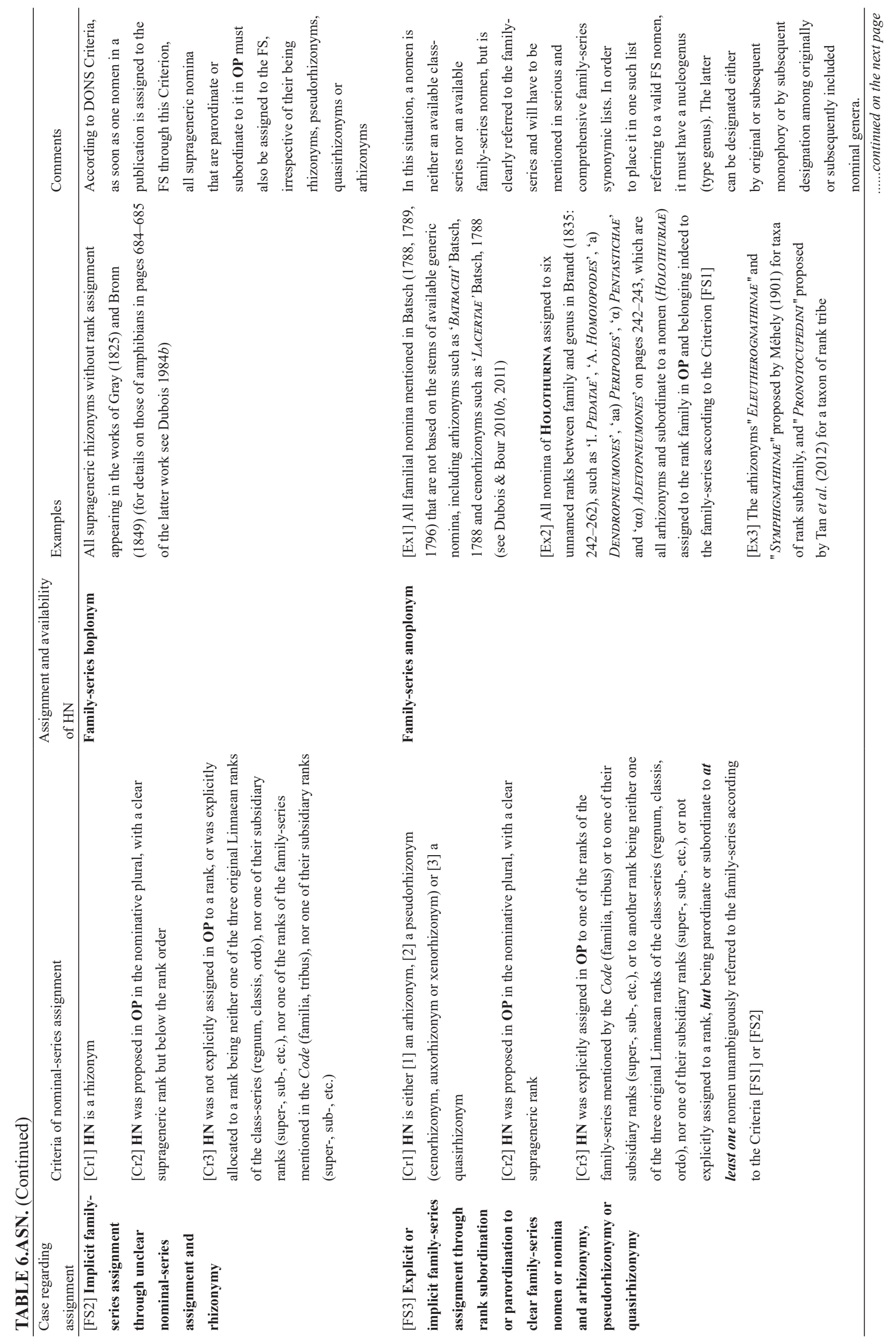




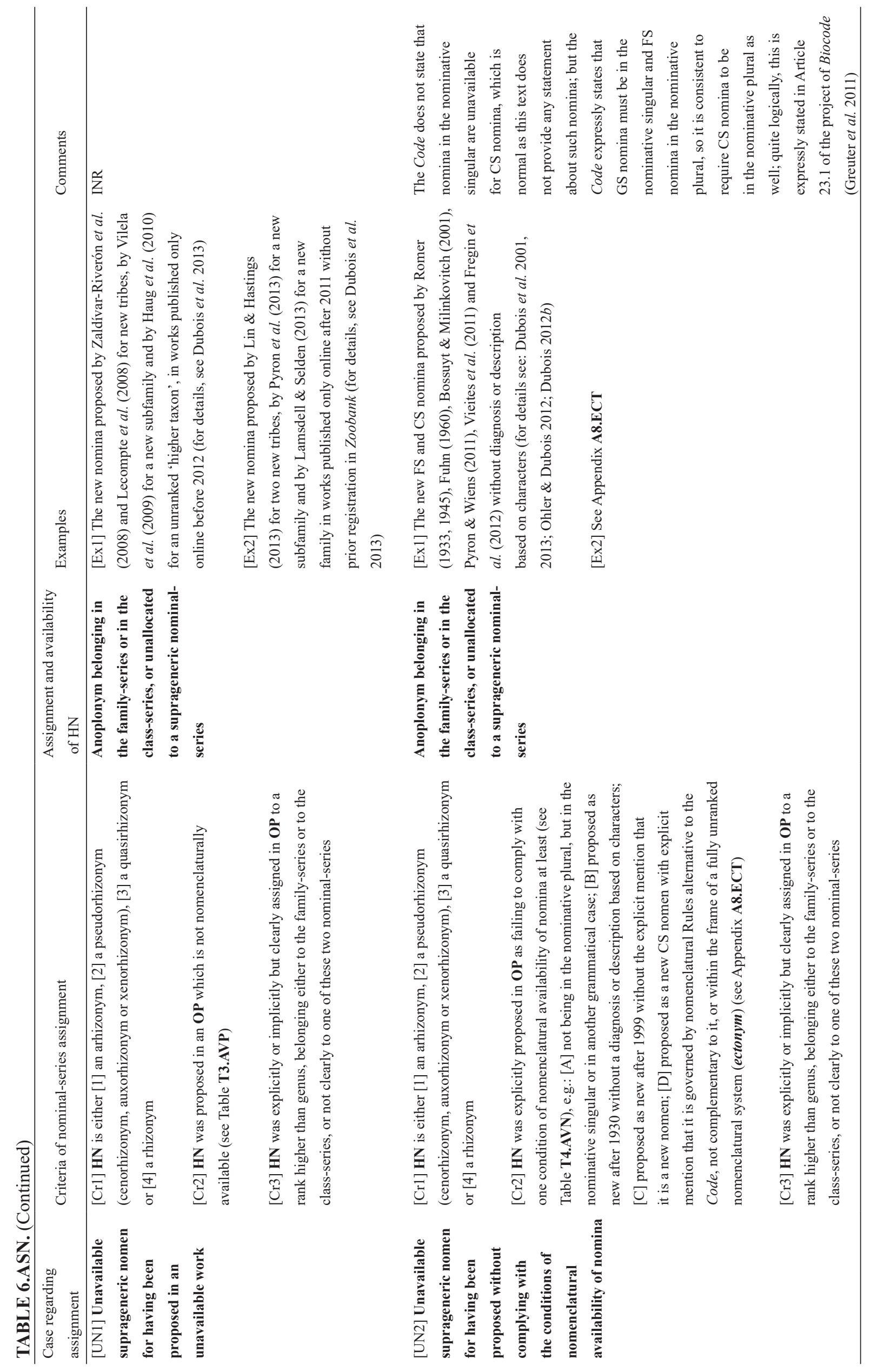


Careful examination of Table T4.AVN shows that a number of amphibian FS and CS nomina published in the past, and for some of them recently, are nomenclaturally unavailable and cannot be used in zoological nomenclature. Here are some examples of nomina which are nomenclaturally unavailable according to the relevant Criteria in Table T4.AVN, and therefore should never be used:

(Av-11) Conditional proposal. • "LEPTOBRACHIINI Dubois, 1980 ", validated later as LEPTOBRACHIINAE Dubois, 1983c.

(Av-18) Gymnonym. • [1] " AllophrynidaE Savage, 1973", validated later as ALLOPHRYNIDAE Goin, Goin \& Zug, 1978 (see Dubois 1986a). [2] " ANEIDINI Vieites, Nieto Román, Wake \& Wake, 2001", validated later as ANEIDINI Wake, 2012 (see Dubois 2012b). [3] "LIUIXALINI Li, Nieto Román, Wake \& Wake, 2001", validated in the present work as RoMERINA nov. [4] "MICRIXALINAE Bossuyt \& Milinkovitch, 2001", validated later as MICRIXALINAE Dubois, Ohler \& Biju, 2001 (see Dubois et al. 2001). [5] " Paratelmatobinnae Pyron \& Wiens, 2011", validated later as ParatelmatobiInae Ohler \& Dubois, 2012. [6]"Relictus Sá et al. 2018 ", validated in the present work as Relictocleis nov. [7] "Unicus Sá et al. 2019a", same as preceding. [8]" Unicus Sá et al. 2019b", same as preceding.

(Av-29) Metagraph 3: ameletograph. • "DICROGLOSSIDAE Anderson, 1871 ", ameletograph of DisCOGLOSSIDAE Günther, 1858 (see Ohler \& Dubois 2014 and Ohler et al. 2014).

(Av-30) Family-series arhizonym or quasirhizonym. • [1] "BATRACHI Batsch, 1788 ". [2] "ICHTyoida Latreille, 1825 ". [3] " TRItonides Tschudi, 1838" . [4] " Eleutherognathinae Méhely, 1901". [5]" HemignathodontinaE Miranda-Ribeiro, 1926".

(Av-31) Family-series cenorhizonym or xenorhizonym. • [1] " TRITONIA Rafinesque, 1815 ", validated later as TRItones Tschudi, 1838. [2] "PRoteInA Gray, 1825 ", validated later as PRoteinA Bonaparte, 1831 (see Dubois \& Ohler 2015).

(Av-32) Family-series auxorhizonym. • [1] "BUFONIFORMES Duméril \& Bibron, 1841 ". [2] " Pipinomorpha Báez \& Pugener, 2003 ". [3]" Allocentroleniae Guayasamin et al. 2009".

Four particular domains regarding the availability of nomina require particular attention because of the existence of several situations which may be confounded if not enough attention is paid to their complexity: $\{\mathrm{S} 1\}$ that of metagraphs, i.e. the distinction between autoneonyms (available) and some categories of apographs (which, being spellings and not nomina, can qualify neither as available nor as unavailable); $\{\mathrm{S} 2\}$ that of the categories of homonymy; $\{\mathrm{S} 3\}$ that of ectonyms, i.e. nomina which are proposed under a nomenclatural system different from that of the Code and incompatible with it; $\{\mathrm{S} 4\}$ that of the acceptable tolerance for borderline gymnonymy.

\subsection{Metagraphs}

A really tricky problem of zoological nomenclature is that of the distinction between different kinds of alternative spellings for nomina and of the distinction between different spellings of nomina and different nomina. This question was discussed at full length in several papers (Dubois 1987b, 2010a, 2012a, 2015c, 2017b; Dubois \& Ohler 2019; Dubois \& Aescht 2019o) which should be consulted for details. Here the conclusions of these discussions are shown in Tables T4.AVN, T7.NS-1 and T8.NS-2. Table T7.NS-1 presents the categories and subcategories of nomina and spellings we distinguish in this work, whereas T8.NS-2 presents the Criteria of distinction between some of these categories.

To make these matters short, once a new nomen has been made available in zoological nomenclature, it is liable either to be replaced by another available nomen (neonym) according to the Principle of Neonymy, or to have its original spelling (protograph) modified. This modification may be either intentional (meletograph) or not (ameletograph), being then due to inadvertence from the part of the 'author' of the publication (more precisely, the nomenclatural auctor of the nomen or the subsequent scriptor of the spelling) or of its editor, publisher or printer. As long as this situation has not been clarified by a careful analysis, this modified spelling may be designated as that of a metagraph. The latter may later turn out to be either a voluntary change in spelling (meletograph), i.e. an 'unjustified emendation' or autoneonym, therefore a distinct nomen with its own auctor and date, or simply an ameletograph (involuntary change in spelling), which does qualify as a distinct nomen and does not have its own auctor but only a scriptor. There are then several possibilities: this spelling may be a symprotograph or a leipoprotograph, a nomographic correction or an ameletograph. Among all the existing or potential spellings of a given nomen, in the end only one (the eugraph) can qualify as the correct one for this nomen to designate a given taxon in a given ergotaxonomy. It is important to realise that the relevant 
TABLE 7.NS-1. Nomina and spellings. Definitions of categories.

Categories of nomina are designated by terms ending in -onym, whereas categories of spellings are designated by terms ending in -graph.

Column $4 \mathrm{~N} / \mathrm{S}$ : N, nomen, which may be available or unavailable; S, spelling, devoid of independent availability.

\begin{tabular}{|c|c|c|c|}
\hline $\begin{array}{l}\text { Category of } \\
\text { nomen or spelling }\end{array}$ & $\begin{array}{l}\text { Subcategory of } \\
\text { nomen or spelling }\end{array}$ & Definition & $\mathrm{N} / \mathrm{S}$ \\
\hline Nomen & - & Scientific name as defined and regulated by the zoological Code. & $\mathrm{N}$ \\
\hline Nomen & Poieonym & Brand new nomen, not proposed to replace an existing one. & $\mathrm{N}$ \\
\hline Nomen & Homonym & $\begin{array}{l}\text { One of two (or several) nomina deemed to be homonyms under the Rules of the Code } \\
\text { (for SS, GS or FS nomina) or under the DONS Criteria (for CS nomina). }\end{array}$ & $\mathrm{N}$ \\
\hline Nomen & Homograph & One of two (or several) nomina having exactly the same spelling. & $\mathrm{S}$ \\
\hline Homonym & Hadromonym & Permanent homonym. & $\mathrm{N}$ \\
\hline Homonym & Asthenomonym & Conditional homonym. & $\mathrm{N}$ \\
\hline Nomen & Synonym & $\begin{array}{l}\text { One of two (or several) nomina deemed to denote the same taxon in a given ergotaxonomic } \\
\text { frame under the Rules of the Code (for SS, GS or FS nomina) or under the DONS Criteria } \\
\text { (for CS nomina). }\end{array}$ & $\mathrm{N}$ \\
\hline Synonym & Isonym & Objective synonym. & $\mathrm{N}$ \\
\hline Synonym & Doxisonym & Subjective synonym. & $\mathrm{N}$ \\
\hline Synonym & Allelonym & $\begin{array}{l}\text { One of two (or several) synonymous nomina used both (or all) as valid for the same taxon } \\
\text { (having the same content) in the same publication. }\end{array}$ & $\mathrm{N}$ \\
\hline Nomen & Isomonym & $\begin{array}{l}\text { Any of two or more distinct nomina being both homonyms and isonyms under the Rules of } \\
\text { the Code (for SS, GS or FS nomina) or under the DONS Criteria (for CS nomina). }\end{array}$ & $\mathrm{N}$ \\
\hline Nomen & Archaeonym & Original nomen that has been replaced by a neonym. & $\mathrm{N}$ \\
\hline Nomen & Neonym & $\begin{array}{l}\text { Nomen proposed expressly to replace an available nomen (its archaeonym), and having the } \\
\text { same onomatophore (Articles 12.2.3,13.1.3,33.2.3) and also the same onomatostasis in } \\
\text { some cases of CS sozonymorphs (Dubois 2015c) as the latter. }\end{array}$ & $\mathrm{N}$ \\
\hline Neonym & Autoneonym & $\begin{array}{l}\text { Neonym having the same etymology as its archaeonym, i.e., directly derived from it through } \\
\text { unjustified emendation (Article } 33.2 .3 \text { ). }\end{array}$ & $\mathrm{N}$ \\
\hline Neonym & Alloneonym & $\begin{array}{l}\text { Neonym having a partially or totally different etymology from that of its archaeonym, i.e., } \\
\text { not directly derived from it through unjustified emendation (Articles } 12.2 .3,13.1 .3 \text { ). }\end{array}$ & $\mathrm{N}$ \\
\hline Neonym & Mesoneonym & Neonym whose etymology is not clearly different or the same as that of its archaeonym. & $\mathrm{N}$ \\
\hline Neonym & Haploneonym & $\begin{array}{l}\text { Subsequent spelling of a nomen which, being clearly a meletograph, must be considered a } \\
\text { neonym although it does not meet the restrictive Criteria of Article 33.2.1 (see NH1-NH5 in } \\
\text { column } 3 \text { of Table T8.NS-2). }\end{array}$ & $\mathrm{N}$ \\
\hline Neonym & Archoneonym & $\begin{array}{l}\text { Ameletograph which has been afforded the status of available neonym by the Commission } \\
\text { under the Plenary Power. }\end{array}$ & $\mathrm{N}$ \\
\hline Spelling & - & $\begin{array}{l}\text { The arrangement of letters that compose a word. In nomenclature, the same nomen can take } \\
\text { different spellings, its parographs. }\end{array}$ & S \\
\hline Spelling & Parograph & $\begin{array}{l}\text { Any spelling, either original (protograph) or subsequent (apograph), ever used in the } \\
\text { literature for a nomen. }\end{array}$ & $\mathrm{S}$ \\
\hline Spelling & Protograph & Original parograph of a nomen in the publication where it was originally introduced. & S \\
\hline Protograph & Holoprotograph & A category of protograph: unique original spelling of a nomen. & S \\
\hline Protograph & Symprotograph & A category of protograph: one of two or more alternative original spellings of a nomen. & S \\
\hline Protograph & Lectoprotograph & The symprotograph validated by an airesy under Article 24.2 . & $\mathrm{S}$ \\
\hline Protograph & Leipoprotograph & Any symprotograph rejected by an airesy under Article 24.2 . & $\mathrm{S}$ \\
\hline Spelling & Apograph & Any subsequent parograph of an existing nomen. & $\mathrm{S}$ \\
\hline
\end{tabular}


TABLE 7. (Continued)

\begin{tabular}{|c|c|c|c|}
\hline $\begin{array}{l}\text { Category of } \\
\text { nomen or spelling }\end{array}$ & $\begin{array}{l}\text { Subcategory of } \\
\text { nomen or spelling }\end{array}$ & Definition & $\mathrm{N} / \mathrm{S}$ \\
\hline Spelling & Eugraph & Correct spelling of a nomen for a given taxon in a given ergotaxonomy. & S \\
\hline Spelling & Nothograph & Incorrect spelling of a nomen for a given taxon in a given ergotaxonomy. & $\mathrm{S}$ \\
\hline Spelling / Nomen & Metagraph & $\begin{array}{l}\text { Any spelling of a nomen different from the correct original spelling and which may be } \\
\text { either an apograph (a symprotograph or a leipoprotograph, a nomographic correction, } \\
\text { a meletograph or an ameletograph) or an autoneonym. }\end{array}$ & $\mathrm{N} / \mathrm{S}$ \\
\hline Metagraph & Meletograph & $\begin{array}{l}\text { Spelling of a nomen used voluntarily in a publication by an author, scriptor, editor, printer or } \\
\text { publisher. }\end{array}$ & $\mathrm{N} / \mathrm{S}$ \\
\hline Metagraph & Ameletograph & Spelling of a nomen used inadvertently in a publication by an author, editor or publisher. & $\mathrm{S}$ \\
\hline Metagraph & $\begin{array}{l}\text { Nomographic } \\
\text { correction }\end{array}$ & $\begin{array}{l}\text { Any correction in the spelling, stem or ending of a nothograph required by the } \\
\text { nomenclatural Rules, which may be either a mandatory ending correction } \\
\text { (Article 32.5; Dubois 2013) or a mandatory spelling correction (Article 34; Dubois 2013). }\end{array}$ & $\mathrm{S}$ \\
\hline Metagraph & Archapograph & $\begin{array}{l}\text { Autoneonym which has been given the status of apograph by the Code } \\
\text { (Articles } 33.2 .3 .1,35.4 .1 \text { ) or by the Commission under the Plenary Powers. }\end{array}$ & $\mathrm{S}$ \\
\hline
\end{tabular}

criterion to distinguish a neonym from an ameletograph is not the fact that the new spelling is 'justified' (as implied by the Code's terminology) but whether it is intentional (voluntary) or not.

We followed the Criteria summarised in these tables throughout the present work to establish the status of all amphibian nomina regarding their availability and spelling. In particular, following Dubois (2017b), we adopted a wider acceptation of the concept of autoneonym than that implemented in the Code, which we consider questionable and non-operational. Rather than on the concepts of 'justified' or 'unjustified' emendations, our interpretation relies on the distinction between 'intentional' and 'inadvertent' spelling changes which we consider more relevant. As a consequence, following the Criteria described in T7.NS-1 and under HN-1 to HN-5 in T8.NS-2, we afford here nomenclatural availability to a few nomina (sigoneonyms) which are denied this status in some recent publications and databases, as well as in some recent decisions of the Commission. However, as none of these nomina has precedence over its synonyms or homonyms, this difference of interpretation does not result in any changes in the nomina recognised as valid in our ergotaxonomy, and is therefore not liable to raise new nomenclatural problems.

\subsection{Categories of homonymy}

In zoological nomenclature, although designated by a term used since the $19^{\text {th }}$ century, homonymy is not 'simple homonymy' as understood in common language or even in linguistics. It is a precise technical qualification of nomina that is not equivalent to strict homography (identical spelling). First of all, homonymy only applies to nomina of the same nominal-series: two homographic nomina in different nominal-series are hemihomonyms (Starobogatov 1984, 1991) and are not concerned by the Rules of homonymy (see Shipunov 2011). Second, under the Code, homonymy is defined differently according to the nominal-series considered: $\{\mathrm{T} 1\}$ in the genus-series, homonymy exists only in case of absolute homography (a single one-letter difference being enough to prevent homonymy); $\{\mathrm{T} 2\}$ in the species-series, nomenclatural homonymy occurs more widely than between homographs, as it only requires 'almost absolute' homography, i.e., paromography or rhizomography (a few 'variant spellings' being 'deemed to be identical'); $\{\mathrm{T} 3\}$ in the family-series, homonymy only requires rhizomography (homography of the stems of the nomina, which qualify then as rhizomonyms).

The traditional Code's subcategories of homonyms were redefined by Dubois (2000b) as hadromonyms (which cover two categories of the Code, 'simple homonyms' in the GS and FS and primary homonyms in the SS) and asthenomonyms (SS secondary homonyms in the Code). Later, Dubois (2012a) proposed to recognise two additional subcategories of homonyms in particular situations. 


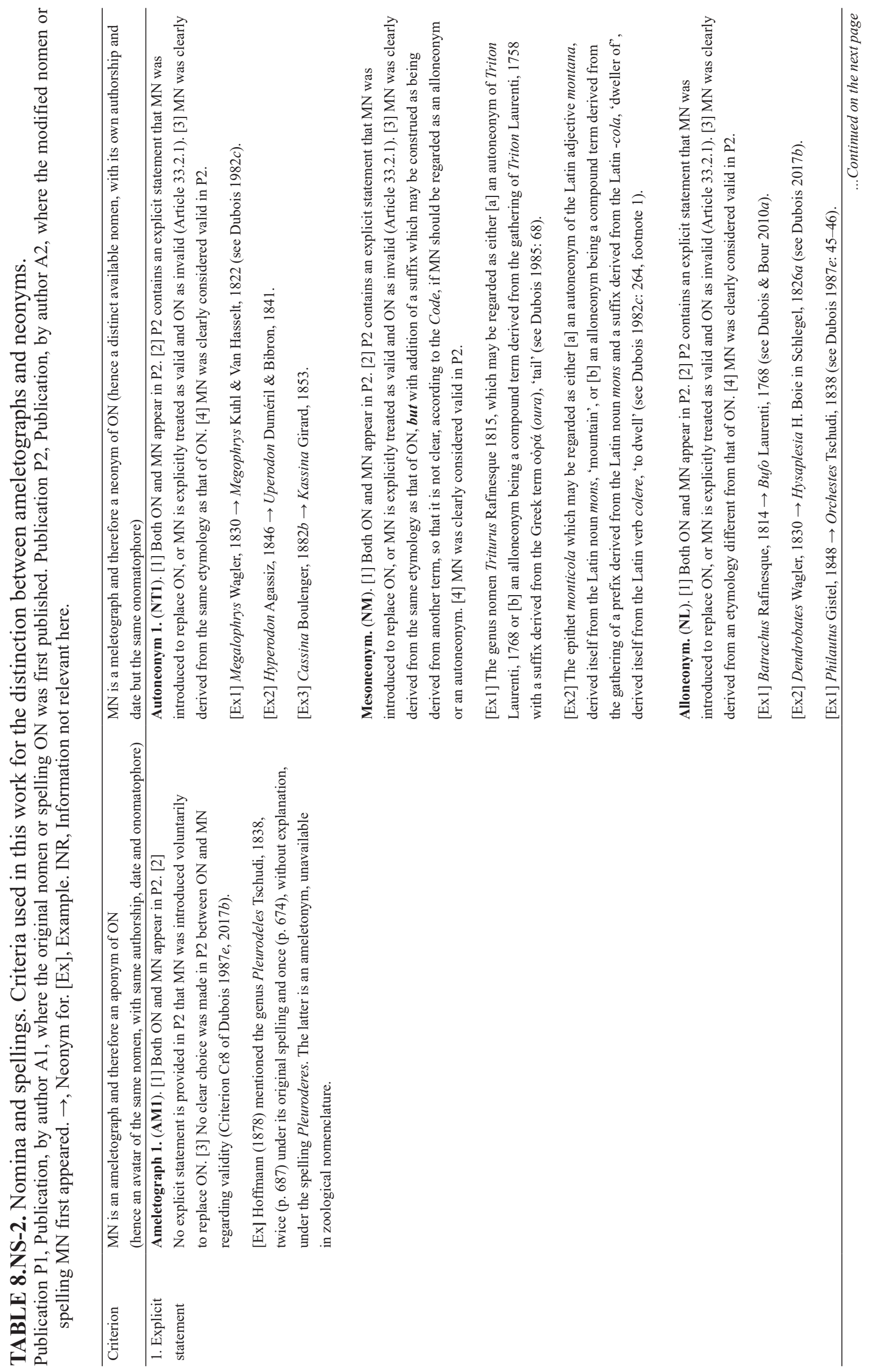




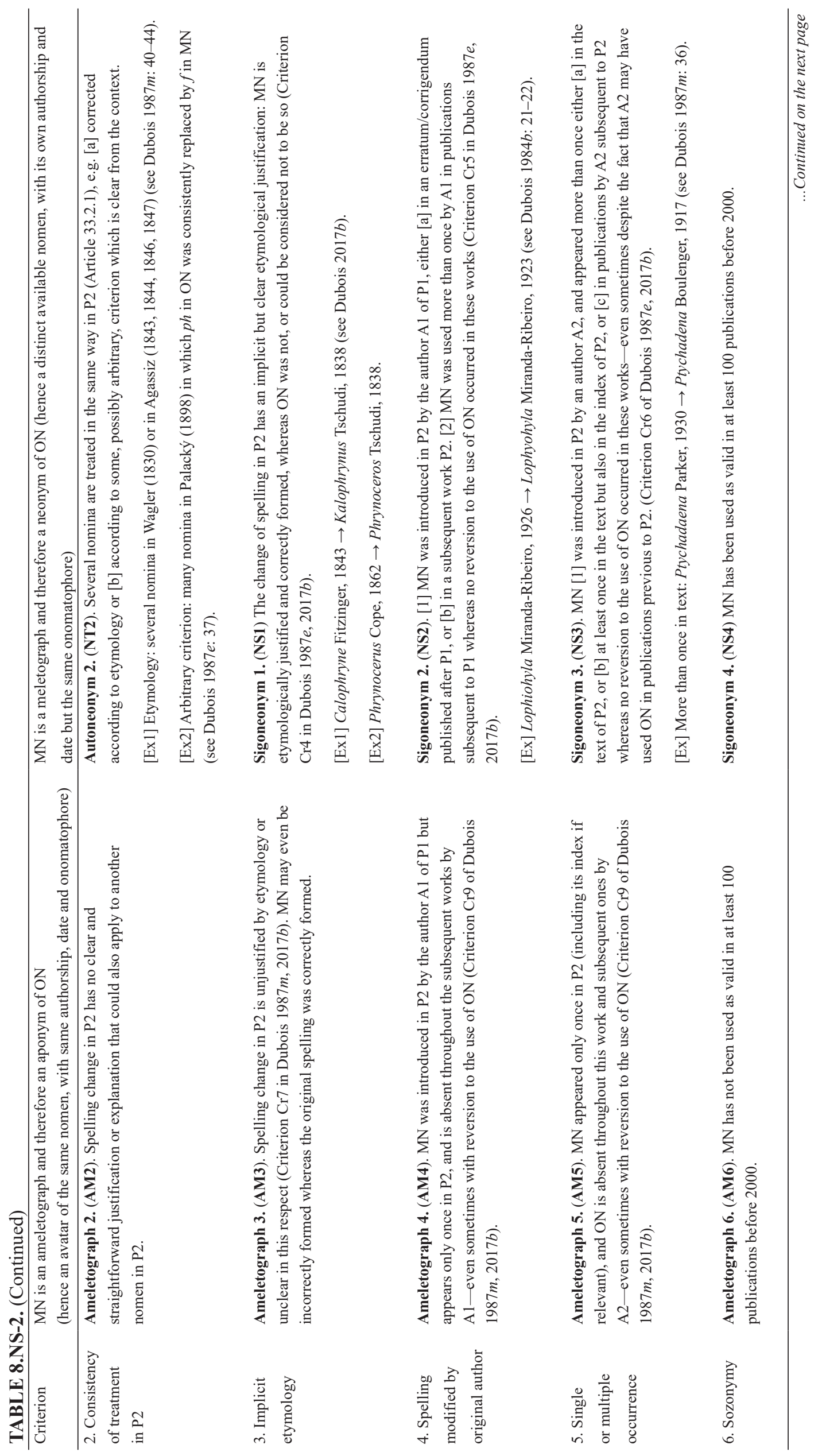




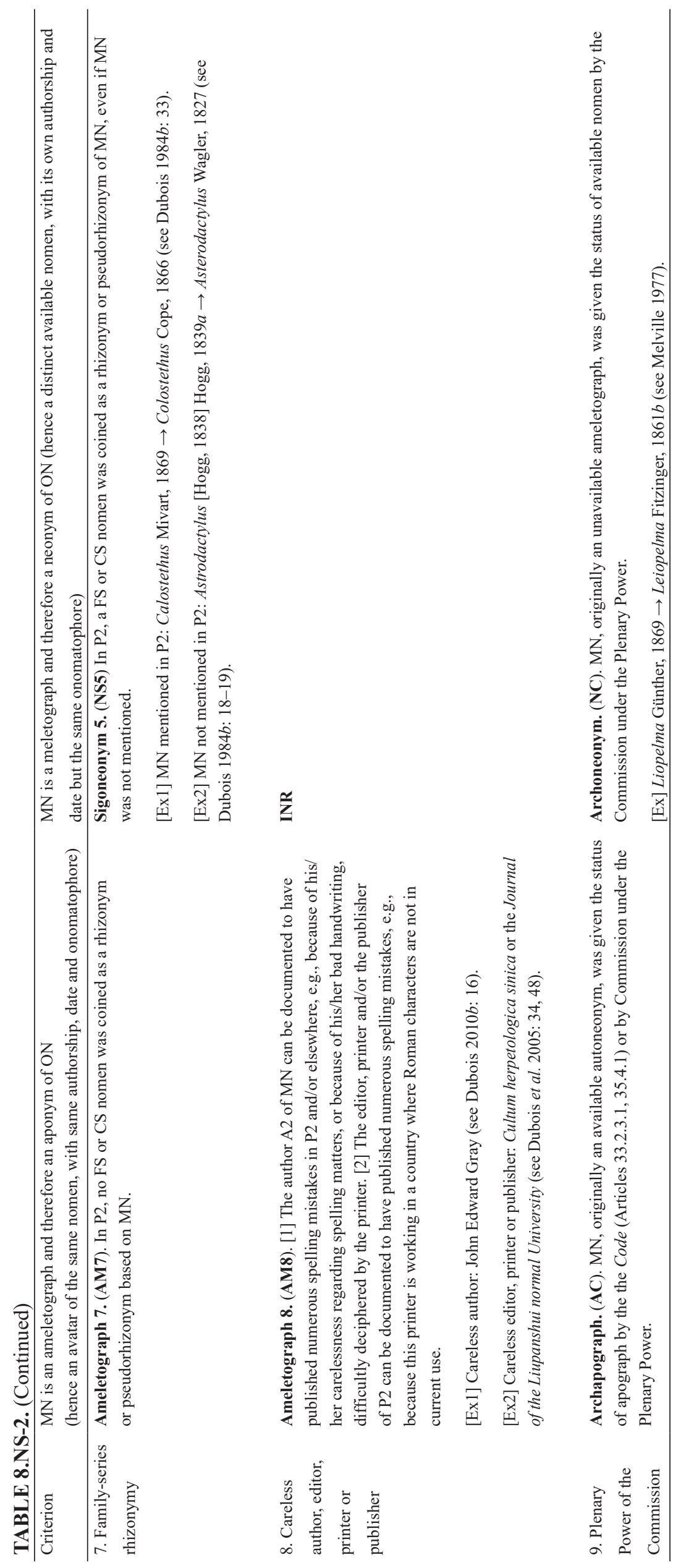


A metomonym is a junior homonym that results from a 'redefinition' of a nomen, through unwarranted modification or replacement of the onomatophore of a previously introduced nomenwhich in fact results in the promulgation of a new nomen. Thus, citing 'AMPHIBIA Linnaeus, 1758' but for a taxon having a much more restricted extension than the original one amounts to the promulgation or recognition of a new homonymous nomen AMPHIBIA. Metomonyms are particularly frequent in the class-series but also sometimes occur in other nominal-series. For this situation to apply however, the change in onomatophore should be explicit, or at least indisputable, for example through explicit mention of the original onomatophore or of part of it as being then referred to a distinct taxon: the mere misuse of a nomen, for example for a taxon not explicitly including the onomatophore but without mention of the taxonomic allocation of the latter, does not qualify as the promulgation of a metomonym (see Dubois \& David 2020). Otherwise, any misidentification of a specimen or of a taxon would qualify as the promulgation of a new nomen, and the taxonomic literature would have to recognise millions of such 'junior homonyms'. Dubois (2012a: 67) gave several examples of metomonyms and of misuses of previously introduced nomina that do not qualify as metomonyms.

An isomonym is a new nomen which has the same onomatophore as a previously (and independently) introduced nomen and which is a homonym of the latter according to the nomenclatural Rules. This situation is very common in the family-series. Quite often, in the old literature, but also sometimes in more recent works, different auctores introduced independently, i.e., without knowledge of their respective works, homonymous family-series nomina. This is an automatic consequence of the fact that, to be available, a family-series nomen must be a rhizonym, and that if two auctores decide independently, perhaps for different reasons, to erect a new FS taxon and to base its nomen on the same nucleogenus (type genus), the resulting nomina will be homonymous. In most publications which mention CS and FS nomina, the auctorship and date of the nomina are not given, and in the old literature it was quite frequent to erect new taxa and to introduce new nomina without stating that the taxa and nomina were new, but this did not impede the availability of the new nomina - it became so only in the current edition of the Code, the Article 16 of which requires explicit mention of the intention to introduce a new nomen, and also explicit mention of its nucleogenus. But then, when mention of the intention is missing in the original text, it is often difficult or impossible, especially in the older works, to ascertain whether this is a subsequent use of an existing nomen, possibly modified in its ending, or a new isomonymous nomen. However, in most cases it is of no practical nomenclatural consequence and it would even be a futile endeavour to try to ascertain this, because: $\{\mathrm{U} 1\}$ if the junior use of the nomen is based on the senior nomen or is slightly different from it but based on the same stem (e.g., RANINA and RANIDAE), it is just a mere citation (chresonym) or a subsequent avatar (aponym) of the latter (e.g., following a change of rank) and it has the same auctor, date and onomatophore; $\{\mathrm{U} 2\}$ if the junior nomen is independent of the senior nomen, it is simply its junior isomonym, i.e. both its junior isonym (objective synonym) and hadromonym, and anyway it will not have any chance to be valid. It is therefore justified, in synonymic lists of family-series nomina, to consider all subsequent mentions of a family-series nomen, whether under its protograph or under one of its apographs, as subsequent uses of the original nomen as its chresonym or aponym and not as its isomonym. This practice greatly simplifies the reading of such lists (see e.g. Dubois 1984b) and it avoids heavy and useless researches to ascertain whether the user of the junior nomen had cited it or had 'established' it again, believing he/she was the first to use it. However before doing so, two elements should be ascertained:

\{V1\} If an isomonym is introduced with the explicit statement that it is a new nomen or applies to a new taxon, it is available as a new junior homonym and synonym with its own auctor and date, not a mere citation of an existing nomen.

\{V2\} If the content of the taxon is not compatible with the onomatophore (and onomatostasis if relevant, see below) of the original taxon, it is also available as a new junior metomonym with its own auctor and date. This applies even if the nomen was credited to a previous auctor.

Note however that this situation is quite different from the situation in the other three nominalseries (species-, genus- and class-series), where the fact that two nomina are homographs does not automatically mean that they are the same nomen, with the same auctor, date and onomatophore: in such cases a careful study of all the information available is necessary to establish the status of the junior nomen.

Dubois $(2012 a$ : 59-60, 67) gave several examples of isomonyms and of cases of subsequent usage of identical or slightly modified nomina that do not qualify as isomonyms. The FS nomen RANIDAE provides a good example of the frequent situation where no evidence exists that the auctor had created 
an isomonym and had not just used an existing nomen without mentioning its auctor. The family nomen RANIDAE was the first one introduced in the scientific literature for a family of anuran amphibians, which has nothing surprising as it was based on the nomen Rana, the only generic nomen of anurans in Linnaeus (1758a). However, it was re-introduced on repeated occasions, presumably as 'new', at least by auctores who did not quote each other, and who used different spellings for it: RANINA Batsch, 1796; RANAE Goldfuss, 1820; RANADAE Gray, 1825; RANOIDEA Fitzinger, 1826; RANIDAE Boie, 1828; etc. From a purely formal point of view, all these nomina should probably be considered isomonyms, but this would only make the synonymy of this family nomen, which is already very heavy, even more cumbersome and difficult to read: it is therefore much simpler and clearer to consider them all as aponyms of RANINA Batsch, 1796, which is the valid nomen (as RANIDAE) of the family.

Opposite examples can be given. Dubois \& Raffaëlli (2012: 113) explicitly established a new salamander tribe RANODONTINI, based on the nucleogenus Ranodon Kessler, 1866. They were not aware of the existence of the nomen RANODONTINAE, previously established by Thorn (1966: 108) on the basis of the same nucleogenus. Their nomen is therefore an invalid junior isomonym of Thorn's nomen. The same applies to their nomina ANEIDINI, BATRACHOSEPINI and HYDROMANTINA, which were in press when Wake (2012) hurried to publish identical nomina for three tribes based on the same nucleogenera (see Dubois 2012b).

In the present work, we have strictly limited the recognition of isomonyms to the cases where the new use of the isomonymous nomen was accompanied by the explicit statement that the latter was new-which in most cases was due to the ignorance of the existence in the literature of the senior isomonym or to almost synchronous publication of both works.

\section{$\underline{2.3 .4 .3 .4 . ~ E c t o n y m s ~}$}

Among the 36 situations that lead to nomenclatural unavailability of zoological nomina and nomenclatural acts listed in Table T4.AVN, 31 concern cases of unavailability due to errors made within the nomenclatural system of the Code or of the Code-compatible Duplostensional Nomenclatural System for class-series nomenclature. But five of them concern cases of nomina which were proposed within the framework of alternative and incompatible nomenclatural systems, or at least which do not respect some of the basic requirements of the Code such as binominal nomenclature for species, the assignment of nomina to nominal-series and ranks, or the taxonomic allocation of nomina through ostension with onomatophores but not through verbal intensional definitions (see e.g. Dubois 2011a).

At the beginning of the $20^{\text {th }}$ century, when zoologists from various countries agreed to adopt international Rules for zoological nomenclature (Blanchard 1905), one of their first decisions was to draw clear lines of delimitation between works respecting these Rules and works ignoring them. For this purpose, they had to take quite drastic decisions. For example, one of the first Rules adopted concerned the requirement that specific nomina should be binomina, not uninomina or plurinomina. They could have restricted themselves to state that species nomina that did not respect this Rule were unavailable, and therefore cast aside the domain of zoological nomenclature. But they went further, and stated that works that were not consistently binominal for nomina of rank species were expelled altogether for this reason from zoological nomenclature, so that even the genus-series and family-series nomina or the nomenclatural acts in such works were also unavailable. Note that they did not go as far concerning plurinominal genus-, family- or class-series nomina, or family-series nomina not being rhizonyms: in these cases, the Code only states that the ill-formed nomina are unavailable, but this has no impact on the availability of the other new nomina in the same works - a possibility which would indeed have made sense then, but which could not be implemented today as it would have catastrophic consequences on nomenclatural stability. Other 'barriers' exist between Code-compliant nomenclature and other possible nomenclatural systems, e.g. concerning the date, the language and alphabet used or the kind of documents providing nomenclatural availability (excluding e.g. manuscripts, unpublished works or non-pre-registered electronic publications). These barriers are very important, as if they did not exist it would be impossible to have strict Rules leading to international, unambiguous and automatic recognition of the valid nomina of taxa.

In the recent years, a renewed interest in zoological nomenclature has led to various proposals of changes in the nomenclatural Rules. Some of these proposals, like that of DONS, were compatible with the basic Principles and Rules of the Code, but others, like that of the Phylocode or that to abandon 
the use of ranks for the nomina of higher taxa, were not. The latter proposals are in fact 'immiscible' with the Code and should be considered as amounting to the implementation, either fully conscious and elaborated or not, of alternative nomenclatural systems. As such proposals and practices tend to become more and more common, we think it is high time for zootaxonomists who wish to follow strictly the Rules of the Code, or Criteria compatible with the latter for questions not addressed by the Code (such as class-series nomenclature), to erect new explicit barriers to 'protect' the Code from such alternative systems, just like when works that were not binominal for species were expelled from Code-compliant zoological nomenclature. For example, we think that the Code should clearly state that works using totally or partially unranked, or pseudo-ranked, nomenclature for higher taxa, should be considered as nomenclaturally unavailable, at least for the new unranked nomina that they contain, and that such nomina should not be used in taxonomic works respecting the Code. We implemented this proposal in the present work.

We designate here such nomina, which we consider as unavailable under Code-compliant zoological nomenclature, as ectonyms. For the time being, we recognise five categories of ectonyms (see T4.AVN), but this does not preclude the possible recognition of further categories later on.

\subsection{Oligocaconyms: non-binominal specific nomenclature}

Article 11.4 of the Code denies nomenclatural availability to all species-, genus- and familyseries nomina (here called oligocaconyms) established in works that are not consistently binominal for nomina of rank species. Such works were still quite frequent after the publication of Linnaeus' $(1758 a)$ tenth edition of the Systema Naturae. In some of these works (e.g., concerning amphibians, Rösel von Rosenhof 1758 or Gronovius 1763), the nomina of species were plurinomina (in fact rather diagnoses than nomina, just like in many pre-1758 Linnaean zoological books), whereas in others they were uninomina and in still others (e.g., Linnaeus 1758b) they were variable (uninomina, binomina and plurinomina).

Note that Article 11.4 expressly states that class-series nomina are not concerned by this Rule, so that for example the class-series nomina that appeared in Linnaeus (1758b) but were absent in Linnaeus (1758a), such as ACANTHOPTERYGII, must be considered nomenclaturally available (see Dubois 2010a, 2012a).

\subsection{Hypercaconyms: plurinominal nomina above the species-series}

Article 4.1 of the Code denies nomenclatural availability to genus-, family- and class-series nomina which are not uninomina (i.e. which are binomina or plurinomina). Concerning amphibians, this was the case of some class-series nomina published until late in the $19^{\text {th }}$ century (e.g., by Daubenton 1782 , La Cepède 1788, Cuvier 1797, Shaw 1802, Latreille 1804, Pallas 1814, Wilbrand 1829, Wagler 1830, Bronn 1853, Stannius 1856, Günther 1858, Wright \& Huxley 1866, Strauch 1870). We did not include these nomina in our survey of amphibians' class-series nomina.

Note that in this case, in contrast with the preceding, the unavailability concerns only these hypercaconyms themselves but does not apply to the other new nomina proposed in the same work.

\subsection{Anemonyms: nomina unassignable to a nominal-series}

Article 1.2.2 of the Code states that this text regulates the nomina of taxa of the species-, genus- and family-series, and that some Articles also provide partial regulation (mainly concerning their availability) for class-series nomina. Then, in the rest of the Code, details are given on various Rules which, although obeying the same general Principles, are different, and sometimes quite so, according to the nominalseries. It is therefore fully clear that, although this is not stated in full words in the Code, in order to be recognised as available in zoological nomenclature, a nomen must be either explicitly assigned or implicitly assignable to a nominal-series in the work where it is first proposed, as otherwise it could not be regulated by the Code. We call such nomina anemonyms.

As we have seen (Table T6.ASN), following the works of Dubois (2015c and references therein), 
in most cases this assignment is possible through the use of objective Criteria, but there are a few exceptions, and in such cases the nomen must be considered as unavailable. One such example is the nomen "Porcellana", proposed by Costa (1776) for a taxon called family on page 177 but genus on page 297 (Dubois 2015c: 32).

Anhypsonyms, discussed below, represent a particular category of anemonyms.

\subsection{Notharchonyms: alternative nomenclatural systems}

In the recent years, several nomenclatural systems alternative to the current Code have been proposed by different authors. Most of these systems claim to be 'phylogenetic nomenclatural systems' and show two main differences with the Code:

\{W1\} These systems do not rely, for the allocation of nomina to taxa, to ostension through onomatophores (see below subchapter 'Taxonomic allocation of nomina'), but to intensional definitions of nomina. Nomina established under such systems may be qualified as diorismonyms.

\{W2\} They do not use nominal-series and nomenclatural ranks but treat all supraspecific taxa indiscriminately as unranked 'taxa' or 'clades'. This question is discussed further below under the subchapter on anhypsonyms.

The best advertised and famous of these intensional nomenclatural systems is the Phylocode (Cantino \& Queiroz 2020), which has both characteristics $\{\mathrm{W} 1\}$ and $\{\mathrm{W} 2\}$.

The project of Biocode (Greuter et al. 2011) relies on ostensional allocation of nomina to taxa but follows $\{\mathrm{W} 2\}$ and is therefore also incompatible with the Code (see Dubois 2011c).

The Phylocode has elicited a number of severe criticisms (see e.g. Dubois 2005b: 387-398, and references therein). Nevertheless, it has had a limited success among some taxonomists, particularly palaeontologists, for a reason that is easy to understand: the fact that the Code fails to provide Rules for the nomenclature of taxa above the rank superfamily, whereas 'phylogenetic nomenclatural systems', including the Phylocode, do not have such limitations, as they cover the whole nomenclatural hierarchy. It is comprehensible in such conditions that some authors (e.g., Kuntner \& Agnarsson 2006) proposed a compromise solution, 'maintaining' the nomina of lower taxa in the Code and 'offering' the nomina of higher taxa to the Phylocode. This solution is not only flawed and shaky, it is not viable in the long run. The modes of functioning of the two nomenclatural systems are fundamentally different and incompatible, as they rely on widely different systems of allocation of nomina to taxa, and their association in a unique nomenclatural system could not function harmoniously for long. Nomenclatural ranks as used in the Code carry most useful information on the structure of a taxonomic hierarchy (and thus also, through the latter and following some conventions, about a phylogenetic tree), but are fully arbitrary, having by themselves no biological meaning concerning the 'kind of taxon' at stake, so that not rarely a taxon has to shift from a rank to another, in order to allow a better expression of phylogenetic relationships within a group. But this can often be done without any change in its intensional and extensional definition. In a system based on a chimera between the Code and the Phylocode, what would occur if a nomen had to shift, e.g., from the rank superfamily to suborder, or vice versa? It would also have to shift from an ostensional definition based on an onomatophore to an intensional 'phylogenetic' definition, or vice versa. As the nomenclatural status of nomina depends on their original taxonomic allocation in the original work where they are introduced, this would be fully unmanageable. The proper solution to the problem of higher taxa nomenclature is not in an unholy marriage but in a widening of the domain of competence of the Code in order to include all taxa at all ranks.

For a long time, the Phylocode did not have a deep impact on amphibian nomenclature, as only two ectonyms explicitly based on its Rules (notharchonyms) had been published before 2020 (see Appendix A8.ECT): < GYMNOPHIONIFORMES $>$ Marjanović \& Laurin, 2008 and $<$ GYMNOPHIONOMORPHA $>$ Marjanović \& Laurin, 2008. But four additional ones were proposed in the book Phylonyms (Queiroz et al. 2020), and it can be expected that many more will be published in the coming years.

Besides, many nomina have been coined, before the implementation of the Phylocode, within the frame of unranked nomenclatural systems or pro-systems, as we will now see. 
2.3.4.3.4.5. Anhypsonyms: unranked or pseudoranked nomenclatural systems, mero-systems or pseudosystems

Dubois (2015c: 7-9) discussed the concept of 'nomenclatural system' and proposed to distinguish several kinds of such systems. He defined a comprehensive nomenclatural system or nomenclatural holo-system as a set of Principles, Rules and Criteria that allows to find the valid and correct nomen of any taxon of a given group of organisms under any taxonomic arrangement, in all situations and in an unambiguous, automatic, repeatable and universal manner. This means that such a system does not leave room for interpretations, discussions and debates. It must therefore cover all particular cases and situations that may be encountered in the taxonomic literature and give precise instructions in such cases, including in some particular situations the need to resolve an ambiguity through an airesy (revisionary nomenclatural act). It cannot accept imprecisions regarding the terms used and the Rules. A good Criterion to recognise such a system is that its Rules are automatic enough to allow their potential computerisation aiming at solving any nomenclatural question, provided all necessary information has been entered in a database, without leaving room for personal decision, except in a very limited set of situations which require recourse to an airesy. A nomenclatural system which does not comply with these requirements cannot be stated to be comprehensive, and may be more appropriately described as an incomplete nomenclatural system or nomenclatural pro-system. Under holo-systems, two different authors working seriously on different sides of the planet, confronted to the same nomenclatural situation or problem, should come to the same conclusion without having to contact each other, to rely on 'consensus' or to appeal to a committee, board or court, whereas this is not the case under prosystems.

Pro-systems themselves are of two kinds, nomenclatural mero-systems that provide Rules or Criteria for some taxonomic or nomenclatural situations only, e.g. not covering the whole nomenclatural hierarchy, and nomenclatural pseudo-systems that are not internally consistent and leave room for personal interpretations and subjective decisions even in the situations supposedly covered by the system.

As we have seen above in 2.2.2, the widespread misunderstanding which consists in equating nomenclatural ranks with taxonomic categories is at the basis of the recent practice of using no supraspecific rank at all (unranked nomenclatural systems) or of a mixture of ranked taxa (e.g., genera, subfamilies, families and superfamilies) and unranked taxa, all the latter being simply designated as 'taxa' or 'clades'. This latter mixture was designated by Dubois (2007a: 34) as pseudoranked nomenclatural pro-systems. Dubois (2008f: 69-80) discussed such systems in detail and illustrated them (tables 5-10) with two examples, taken from the works of Frost et al. (2006) and Vieites et al. (2007).

Unranked nomina established under such systems or pro-systems (anhypsonyms), cannot be assigned to nominal-series and as such cannot be available under the Code or under a nomenclatural system for class-series nomina that would be compatible with the Code. We propose the following convention to write such ectonyms: «PARATOIDEA» Queiroz \& Gauthier, 1992; «STEGOKROtAPHIA» Cannatella \& Hillis, 1993; «NATATANURA» Frost et al., 2006.

The first such ectonyms were proposed by Queiroz \& Gauthier (1992) under a nomenclatural system 'announcing' the Phylocode. These authors, followed by Cannatella \& Hillis (1993) and Ford \& Cannatella (1993), made no distinction between taxonomic categories and nomenclatural ranks. Their criticism of the latter in fact applied to taxonomic categories but did not in the least address the question of the appropriateness of using a hierarchy of ranks and nomina to express the structure of the hypothetic phylogenetic tree on which their taxonomy and nomenclature were based. They distinguished 'stem-names' and 'node-names', which they both treated as 'singular nouns', because "taxa are historical entities" (Ford \& Cannatella 1993: 95). This by itself is sufficient to remove the nomenclatural availability of these nomina under the Code or DONS.

In amphibians, many such anhypsonyms (48) were later proposed by a single research team (45 in the work of Frost et al. 2006 and 3 in that of Grant et al. 2006). Some of these nomina were fully new, whereas others were borrowed from the literature but redefined, thus in fact establishing new hemihomonymous nomina. These authors found some merits in the recommendations of Queiroz \& Gauthier (1992) but did not follow them consistenly. They used a mixed nomenclatural pro-system "based on common sense" in which they respected the Code for taxa of the ranks species to superfamily (which they called 'regulated taxa'), but used the indiscrimate general term 'taxon' for all higher taxa, stating that they applied "an unranked taxonomy for unregulated taxa (above family group), the hypotheses 
for these taxa being derived from their included content and diagnostic synapomorphies." (Frost et al. 2006: 143). This sentence by itself shows again the common confusion between taxonomy and nomenclature, as the Code regulates nomina, not taxa. They did not justify this difference of treatment between both kinds of taxa. Furthermore, this statement is misleading because the unranked taxa they recognised were not all "above family group" as most of them were parordinate to family-series taxa or to taxa being themselves subordinate to family-series taxa (see table 9 in Dubois 2008f: 77). Besides, the Criteria they used to 'validate' some ancient nomina under their system were inconsistent (Dubois \& Ohler 2019). Their nomenclatural pro-system is therefore both a mero- and a pseudo-system.

Dubois (2015c) attempted to 'save' some of these ectonyms by applying to them the Criteria of assignment of nomina to nominal-series listed above under $\{\mathrm{R} 1\}$ to $\{\mathrm{R} 6\}$. As we have seen, nomina introduced within the frame of a fully unranked nomenclatural system like the Phylocode cannot be referred to ranks and nominal-series, or could be so only arbitrarily, and must therefore anyway be treated as unavailable in Code-regulated zoological nomenclature (Criterion Av-07 in Table T4.AVN). But some nomina introduced under pseudoranked nomenclatural pro-systems could possibly be referred to ranks (and consequently to nominal-series) through the Criterion of topotaxy $\{\mathrm{R} 4\}$ : in such cases, all nomina parordinate or subordinate to nomina which are clearly assigned to the FS according to the Criteria [FS1] to [FS3] of Table T6.ASN, would have to be assigned to the FS, whereas all those that were introduced for taxa superordinate to the latter should be assigned to the CS. Then, all the unranked 'taxa' of caecilians and frogs introduced by Frost et al. (2006), being parordinate or subordinate to families, would belong in the FS. Most of these nomina, being arhizonyms or pseudorhizonyms, would therefore be unavailable in zoological nomenclature, except for two of them («HYLOIDES» and «RANOIDES») which, being rhizonyms, could be available - although invalid for being junior homonyms, respectively of HYLINA Rafinesque, 1815 (1825) and RANINA Batsch, 1796. Finally, in salamanders, two nomina of 'higher' taxa, «CRYPTOBRANCHOIDEI» (just superordinate to the families CRYPTOBRANCHIDAE and HyNOBIIDAE) and "DiadeCtOSALAMANDROIDEI», parordinate to the latter, would belong in the CS. However, these two CS nomina would then be invalid junior synonyms of much older CS nomina (respectively ImPerfectibranchia Hogg, 1838 and Pseudobranchia Sonnini \& Latreille, 1801; see Dubois \& Raffaëlli 2012). In the end, there would be no way to 'save' the 45 new higher nomina of 'taxa' introduced by Frost et al. (2006), some of which are quite long and unpalatable (Dubois \& Raffaëlli 2009, Dubois 2010e) and in fact this is fully justified, as the authors of these nomina had clearly proposed them outside the regular system of the Code, as those of unranked taxa.

Because of the unwarranted mistrust in ranks, based on a misunderstanding, that has been spread by a few recent authors, a number of papers using pseudoranked nomenclature were published in the last 25 years. Appendix A8.ECT lists the 96 such nomina that were established for amphibian taxa from 1992 to 2020 and which are unavailable both in the FS according to the Code and in the CS according to DONS. Few of the authors of these works justified their use of such unranked nomina for these higher taxa, and when they did so their explanations were sometimes quite strange indeed. Thus, Guayasamin et al. (2009: 20) established an 'unranked taxon' "ALLOCENTROLENIAE», whose nomen is unavailable under the Code, in the superfamily HYLOIDEA for the two families CENTROLENIDAE and ALLOPHRYNIDAE, instead of using an intermediate family-series nomen for this taxon, because this would have created "nomenclatural instability by shifting the ranks of taxa"-a phenomenon which occurs frequently in zootaxonomy as a result of the progress of research and is not a problem as there exists nothing like a 'stability of ranks'. For their part, Streicher et al. (2018: 142) wrote: "We take this opportunity to propose new names for some of the more well-supported clades of [sic] families (note that taxa above the family level do not require formal diagnoses). Even if these clades prove to be incorrect in the future, these names at least allow us to reference these groups." The idea that suprafamilial taxa "do not require formal diagnoses" clearly takes its root in the Phylocode ideology, but is not justified under a Codecompatible conception of nomenclature (see Articles 1.2.2, 10.1 and 13.1.1).

As a matter of fact, among the 96 anhypsonyms and notharchonyms listed in Appendix A8.ECT, 18 were published accompanied only by 'phylogenetic definitions' ('node-' or 'stem-based' nomina) but no diagnosis, definition or description in words allowing to make them nomenclaturally available under the Code, so that even if some authors wished to use, against all evidence, the ectonyms of this table under a Code-compliant taxonomy, this could not apply to these 18 diorismonyms. 
One of the main, if not the main, reasons for the unavailability of nomina is gymnonymy, i.e., in most cases, the fact that the new nomen was originally published without "a description or definition that states in words characters that are purported to differentiate the taxon" (Article 13.1.1), or even, before 1931, an indication, e.g., an illustration of the taxon being named (Article 12.2.7). On the other hand, a vernacular name, a locality, a geological horizon, the mention of a host, a label or a specimen do not in themselves constitute a description, definition or indication and do not provide nomenclatural availability. The same applies, although this is not mentioned in the Code, to the position of a taxon in a hypothetic phylogenetic tree or to its geographical distribution. Dubois (2017d) discussed this matter in detail and showed that the important point here is the presence of this description, definition or indication in the original publication, not its accuracy or completeness. He also argued that the term character in this definition designated in fact character states (e.g., eye colour blue), not the characters by themselves (e.g., eye colour).

In the recent decades, much confusion has been introduced in the taxonomic literature by the supporters of so-called 'phylogenetic taxonomies' like the Phylocode, which in fact are systems mingling phylogeny, taxonomy and nomenclature. The purposes of these three domains are distinct. That of phylogeny is to establish the historical kinship between organisms and to formulate hypotheses about the existence of lineages, that of taxonomy is to classify these organisms into evolutionary meaningful units, the taxa, and that of nomenclature is to give universal and unambiguous nomina to these taxa. Although today it is clear to all zoologists that the taxa we recognise should ultimately correspond to groups considered to be independent lineages, this is not an absolute necessity. In some cases, particularly at the species level, it may be fully justified to erect a new taxon and to name it on the basis of its fixed differences in taxonomic characters with all other known taxa, even before its phylogenetic position is clarified. Species delimitation is a concept different from species relationships. The recent idea that taxa should not be named until their phylogenetic position is 'known' (in fact hypothesised) is misleading (see in this respect Páll-Gergely 2017 and Dubois 2020c).

Dubois (2017d) listed different kinds of taxognoses (definition of taxon, whether based on characters or on hypothesised cladistic relationships between taxa) that can be used in taxonomy and nomenclature. The most often used kinds of taxognoses in Code-compliant nomenclature are idiognoses, called 'descriptions' in the Code (taxognosis based on character states that are considered to provide a brief description or characterisation of a taxon, including both diagnostic character states and character states shared with other taxa, but without mentioning its comparison with other taxa), diagnoses s.str., called 'definitions' in the Code (intensional taxognosis providing character states considered to allow a nonambiguous distinction of a taxon from other taxa with which it is compared, irrespective of any cladistic hypothesis) and apognoses (intensional taxognosis providing a definition of a taxon based on character states that are considered to be shared by all members of the taxon and absent in all non-members, and that are considered, on the basis of a cladistic analysis and hypothesis, to be autapomorphic for the taxon). These three kinds of taxognoses, one of which only refers to a cladistic hypothesis, comply with the requirement to provide "characters that are purported to differentiate the taxon" for its naming.

On the other hand, coinognoses (extensional taxognoses based directly on hypothesised cladistic relationships derived from a cladistic analysis), which include the 'phylogenetic definitions' of the Phylocode, and which do not refer to character states, do not provide nomenclatural availability under the Code. Although this has been pointed out 20 years ago (Dubois 1999), and acknowledged by a number of taxonomists (Bauer et al. 2010), this is still not understood by many authors, who continue to 'describe' and name new taxa without stating any diagnostic character of the latter but only referring to the topology of a molecular tree.

Until recently, there has been a permanent increase in the quality of idiognoses and diagnoses during the history of taxonomy. Anyone who has worked with the old taxonomic publications of the $18^{\text {th }}$ and $19^{\text {th }}$ centuries knows that many old descriptions were extremely laconic and, in fact, clearly insufficient to characterise the taxon. However, this did not impede them to make the new nomina nomenclaturally available under the Code's Rules, as shown by an example.

The nomen of the European frog species Rana dalmatina was made available through the following sentence in Bonaparte (1838a) in his account of Rana temporaria Linnaeus, 1758: "La Rana dalmatina, nuova especie del Fitzinger a noi incognita, seppur non è une gigantesca varietà della presente, la somiglia moltissimo, secundo lui medesimo, che altra differenza non vi ritrova fuor della statura 
maggiore, e i piedi posteriori proporzionatamente anco più lunghi." [The Rana dalmatina, a new species of Fitzinger unknown to us, even if it is not a gigantic variety of the present species, resembles it very much, according to himself, which besides its greater size has the rear legs proportionately quite longer.] According to the Code, this description undoubtedly makes the nomen Rana dalmatina Fitzinger in Bonaparte, 1838 available. However, it was ignored by Günther (1859) and Boulenger $(1882 b)$ who used for this species its junior synonym Rana agilis Thomas, 1855. Boulenger (1898: 332) then wrote: "The strict application of the law of priority would require the adoption of this name in preference to that proposed by Thomas sixteen years later, as the former was accompanied by a definition ('Gigantea, pedibus posticis longissimus'), however inadequate, and specimens so labelled by Fitzinger are preserved in the Vienna Museum. However, this is one of those cases in which, it appears to me, conservatism is desirable, as the name agilis was the first to appear in connexion with a proper description, and has been so generally in use within the last half-century. Similar considerations have guided me in the naming of the two species of the genus Bombinator, and I hope, in the interest of the stability of nomenclature, they will commend themselves to future workers." This is an excellent example of the weakness of the argument of 'nomenclatural stability', because more than one century later, the nomen Rana dalmatina, resurrected by Stejneger (1907: 108), has been used consistently for this species - and the generic nomen Bombinator Merrem, 1820 is now universally considered an invalid synonym of Bombina Oken, 1816, and the two species mentioned by Boulenger (1898) are now known under other epithets.

This example also highlights the fact that nomenclatural availability should not rely on the accuracy and completeness of the original diagnosis. If we looked at the original 'definition' of Rana dalmatina with the eyes of today, we would say that it cannot allow to distinguish this species, as we now know several species of Rana that have longer legs than Rana temporaria - and furthermore the latter tends to be larger than Rana dalmatina in many populations. But at the time of this description, these characters could be considered sufficient to characterise the new species. Considering today that this diagnosis is insufficient to "differentiate the taxon" and does not provide nomenclatural availability would not only challenge again the nomenclatural stability in this group, it would also open the door to many other similar 'revisionary' actions. For the sake of nomenclatural consistency and stability, the availability of nomina published long ago, or even more recently, with clearly insufficient diagnoses, should not be challenged. The important point is not the quality of the diagnosis but the fact that a description or definition was provided, with the intention to allow recognition of the taxon.

Although this is quite clear concerning the 'historical' works of early taxonomy, this does not mean that today we should not require from taxonomists a more 'serious' work regarding taxognoses. For the sake of quality of taxonomic research, the threshold of tolerance for 'unprofessional' work should be lowered. In particular, three peculiar situations deserve special consideration: $\{\mathrm{X} 1\}$ polythetic diagnoses; $\{\mathrm{X} 2\}$ absence of characters distinguishing the new taxon from the taxa with which it is compared; and $\{\mathrm{X} 3\}$ absence of direct connexion between the taxognosis and the taxon being described.

\subsection{Polythetic diagnoses}

Diagnoses are abstractions based on generalisations derived from several observations. A diagnosis concerns a taxon, i.e. a concept, not a fact. It is not necessarily associated with a cladistic hypothesis. It is often based on character states shared by all members of the taxon and absent in the non-members, but this is not always the case, as polythetic diagnoses (Sneath 1962; Van Regenmortel 2016: 6; see Figure F2.MPT) are acceptable under some taxonomic paradigms: whereas a monothetic diagnosis includes a unique combination of character states and relies only on properties that are both necessary and sufficient for membership in the taxon, a polythetic diagnosis involves a variable, but unique to the taxon, combination of alternative properties, none of which is necessarily present in every member of the class. In fact, the Aristotelian requirement for 'necessary and sufficient' properties shared by all members of a taxon refers to an 'essence' of the latter, which makes no sense within the framework of an evolutionary understanding of biodiversity.

A polythetic diagnosis is fully appropriate to make a new nomen available, as it allows to characterise the taxon. But it does not allow to identify its members, as none of them shares all its character states with all the other ones. For example, the diagnosis of both taxa AMPHIBIA and SQUAMata may include the mention of four chiridian members being present or absent, but combined with other diagnostic 


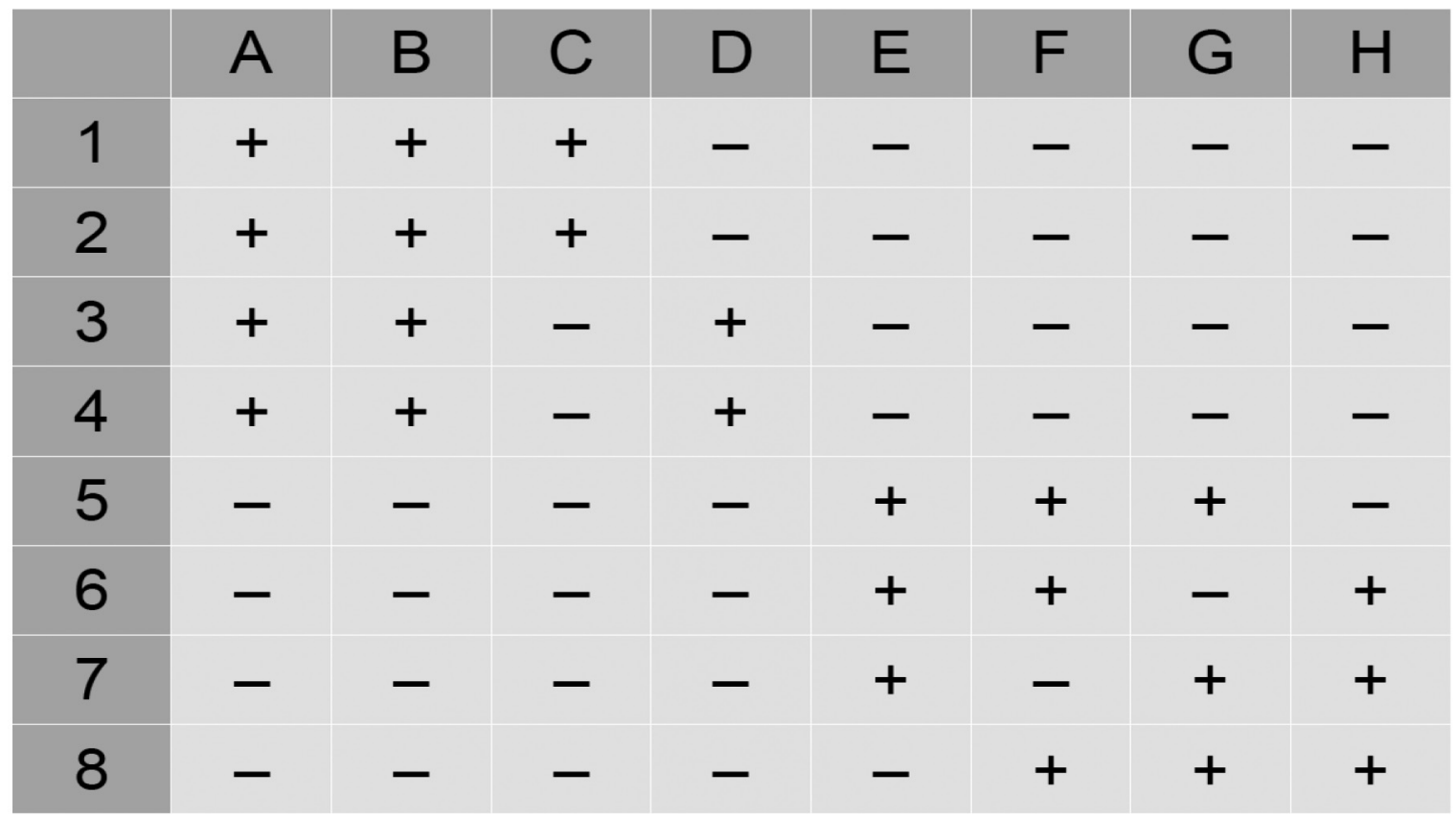

FIGURE 2.MPT. Monothetic and polythetic classes.

Hypothetic example with 8 individuals (1-8) and 8 properties (A-H). The possession of a property (character state) is indicated by a plus sign. Individuals $1-2,3-4$ and 1-2-3-4 form three monothetic classes with respectively 3,3 and 2 properties present in all the members. Individuals 5-8 constitute a polythetic class, each member possessing 3 out of 4 properties with no common property being present in all the members (Van Rijsbergen 1979; Van Regenmortel 2016).

characters in these two taxa. The identification of the members of a taxon may rely on detailed descriptions, or on non-purely dichotomic identification keys, or better on tables showing the variability within the taxon of character states for some characters. Although this variability is a source of complexity for the building of matrices of characters and for phenetic or cladistic analyses, it is a biological reality that should not be ignored by taxonomists.

From a nomenclatural point of view, it is therefore misleading to require that the diagnosis provided to make a new nomen available includes only character states shared by all members of the taxon, let alone synapomorphies of the taxon, as a diagnosis does not need to include cladistic information to provide nomen availability. But of course, when phylogeny and taxonomy are at stake, more information is necessary.

\subsection{Non-differential diagnoses}

As we have seen, the Code allows to make a new nomen available through the publication of a simple idiognosis, i.e. a description of the taxon or even of a specimen, without comparison with other taxa. What the formula "purported to differentiate the taxon" means in such cases is that the author of the nomen thinks that these characters states or their combination are diagnostic of the taxon, i.e. allow its distinction from all other taxa.

However, as soon as an author provides, instead of an idiognosis, a real diagnosis s.str. of a taxon, in order to make the new nomen available it is necessary to mention not only characters (e.g., colour or tympanum) but also character states (colour blue or red, tympanum present or absent, or round or oval), and that the combination of these character states be unique among the taxa with which the new taxon is compared. Whenever, considering all the character states cited for a new taxon and for other taxa with which it is expressly compared in the original publication, the new taxon has a strictly identical list of characters, there is no "character purported to differentiate the taxon" and the new nomen is unavailable. Although rare, this situation exists in the literature, as exemplified by the case of the amphibian generic nomen Paradactylodon Risch, 1987 discussed by Dubois \& Raffälli (2012: 114) and misunderstood in 


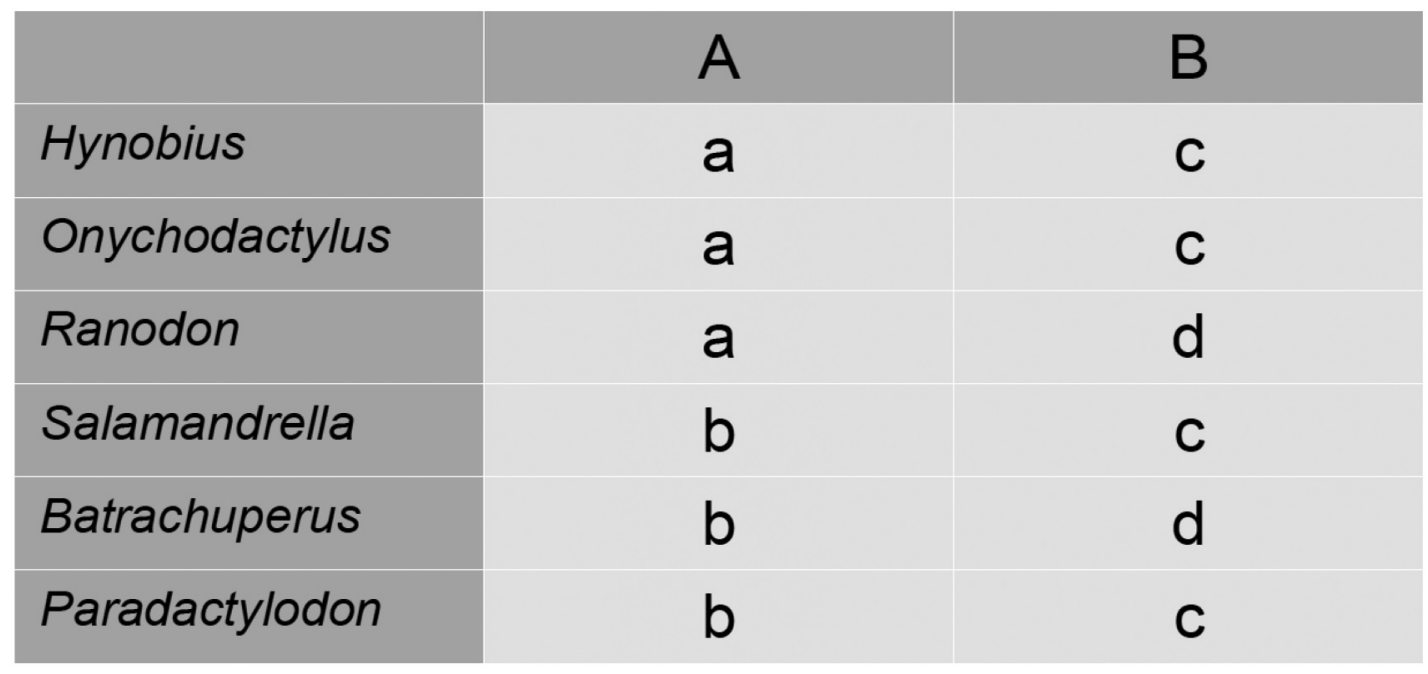

FIGURE 3.NDD. A non-differential diagnosis for a new taxon.

Diagnosis, based on two characters (A-B) and two character states for each (respectively a-b and c-d) proposed by Risch (1984) for the new salamander genus Paradactylodon, compared with the genera Hynobius, Onychodactylus, Ranodon, Salamandrella and Batrachuperus.

$A S W<2020 a>$ and by Stöck et al. (2019). As shown here in Figure F3.NDD, in this case the information provided does not qualify as a polythetic diagnosis allowing to provide nomenclatural availability to the new nomen, as the character states mentioned for the new taxon are identical to those mentioned for the genus Salamandrella.

To evaluate the availability of a new nomen, the diagnosis or 'definition' provided for a new taxon must therefore be compared in detail with those given in the same work for the taxa considered by the author to be closely related.

There is a case when nomenclatural Rules are not enough to prevent the erection of unwarranted taxa: it is when the new taxon is referred to a wrong superordinate taxon, e.g. when a purported new species is placed in a 'wrong' genus. In this case, the diagnosis provided for the new taxon may well be accurate, but, as it is based on misleading comparisons with taxa which are not closely related to it, this diagnosis is irrelevant, or amounts to a diagnosis of the new taxon relatively to the higher taxon to which it was wrongly referred. This was a very common situations in the early days of taxonomy, but it stills occurs from time to time, as shown by the cases of the occidozygine Ingerana charlesdarwini, described by Das (1998) as a member of the ranid genus Rana (see Dubois et al. 2005, Dinesh et al. 2009), of the discroglossine Paa mokokchungensis, described by Das \& Chanda (2000) as a member of the megophryid genus Scutiger (see Dubois 2002), or of the arthroleptid Arthroleptis nonakoensis, described by Plath et al. (2006) as a member of the phrynobatrachid genus Phrynobatrachus (see Frétey 2008). Of course, in such cases, it may appear 'easy' to find diagnostic characters for the 'new species', but they are often irrelevant and useless to characterise the taxon-so that it is not surprising that in some such cases the transfer of the taxon to its proper genus results in its immediate synonymisation, as in the case of the lizard Geophis alasukai Gasc \& Rodrigues, 1979, which, once transferred to the genus Atractus Wagler, 1828, proved to be a mere synonym of A. flammigerus (Boie, 1827) (see Chippaux 1986).

In such cases the Code is useless to avoid the potential publication of a junior synonym, as formally the new nomen has been validated by a 'diagnosis'. This is why the role of competent referees may be important in taxonomic publications, as they may avoid such failures.

\subsection{Diagnosis unconnected with new taxon}

The last point raised here is a bit subtle to understand. Let us come back to the wording of article 13.1.1, which formulates the main condition for the availability of a new nomen as follows: this nomen must "be accompanied by a description or definition that states in words characters that are purported 
to differentiate the taxon [stressed by us]". This means that the diagnosis must concern the new taxon being described, not its members, whether individuals or subordinate taxa. Thus, when for example a new genus is erected, the diagnosis provided to make its nomen available should be clearly attached to this taxon of rank genus, not to one of its included specimens or species, even its nucleospecies (type species), and even if the latter is the only species referred to this genus in the original description. The two taxa at stake, the genus and the species, have different taxonominal functions: the first one points to the need to recognise a taxon of rank genus, which may be sister to one or several other taxa of the same rank, whereas the second plays the same role at species level.

To the best of our knowledge, this subtlety in the reading of Article 13.1.1 was raised for the first time, aptly in our opinion, by Arribas (2016) in his discussion of the availability of the lizard generic nomen Caucasilacerta Harris et al., 1998. It applies also to the 'Relictus case', and possibly to other cases that have not yet been identified. Sá et al. (2018) erected the frog subgenus Relictus for the single species Chiasmocleis gnoma, without providing a formal diagnosis of it. Dubois et al. (2018: 55-56) listed a few characters extracted from the original description which could be construed as constituting a polythetic diagnosis in order to try to 'save' this genus-series nomen. But in the original publication these characters were attached to the species Chiasmocleis gnoma, not to the taxon Relictus, so that the latter nomen remains unavailable, and with it also its neonym Unicus proposed by Sá et al. (2019a), who did not seize this opportunity to provide a real diagnosis for this taxon, as suggested by Dubois et al. (2018).

\subsection{Notes for the future}

In the recent decades, several papers provided recommendations for a modern 'integrative taxonomy' and the use of 'best practices' in this domain (Dayrat 2005; Padial et al. 2010; Kaiser et al. 2013; Vences et al. 2013). However, nomenclature has remained the 'poor relative' of this taxonomic 'revolution'. We think that this should change, and that taxonomists should pay more attention to 'nomenclatural accuracy' (Dubois 2017e) in their works, particularly in their descriptions of new taxa (Dubois et al. 2018).

In particular, we think it is intolerable that, at the beginning of the $21^{\text {th }}$ century, be still published descriptions of new taxa missing formal diagnoses or idiognoses, or other basic elements allowing to ascertain the nomenclatural status of the new nomina, including the actual publication date of the final version of a work in case of electronic publication, compelling subsequent workers to carry out heavy inquiries to obtain this information. We suggest the Code should be improved in adding, after a starting date still to be defined, several requirements acting as 'barriers' (as defined above) and being indispensable for the nomenclatural availability of any new nomen: $\{Y 1\}$ a formal idiognosis, diagnosis or apognosis, identified as such in a special paragraph; $\{\mathrm{Y} 2\}$ a precise onymotope ('type locality') for any new species-series taxon; $\{Y 3\}$ collection numbers for onymophoronts of species-series taxa; $\{Y 4\}$ the etymology of the new nomen, including its mode of derivation (according to Article 31.1.1 or 31.1.2) in case of species-series taxon dedicated to a person; $\{Y 5\}$ basic grammatical information on the nomen, such as the grammatical gender and the stem for a genus-series nomen, or the grammatical status as adjective, participle or noun in apposition for a species-series epithet. We applied these recommendations in the taxonomic part of the present work for all the new nomina introduced here.

\subsubsection{Conclusion}

Throughout the present work, we followed the Rules and Criteria presented above to establish the status regarding availability of all the nomina of the genus-, family- and class-series nomina of recent amphibians ever published. Most of these Rules are those of the Code, but when the latter does not provide Rules (concerning class-series nomina) or in a few cases when the Rules of the Code appear to us as grossly inappropriate (e.g., for the distinction between autoneonyms and apographs), we followed consistently the Criteria presented above. 


\subsubsection{Categories of usage of nomina}

As discussed by Dubois (2010a), the Code makes numerous references to the concept of 'usage' or 'prevailing usage' but does not provide a general operational definition of these terms, which are defined differently in different parts of the text (e.g. in Article 23.9 and in the Glossary). In the present work, in the three nominal-series covered by the Code, we had to follow these imprecise Rules and we did this as much as possible. But for class-series nomina, for which the Code does not provide Rules or Recommendations, we adopted the precise categories of usage defined by Dubois (2006a, 2010a), limiting ourselves to the three main ones, as implemented in DONS (Dubois \& Raffaëlli 2012; Dubois 2015c), as follows:

$\{\mathrm{Z} 1\}$ A sozonym is a CS nomen that has had since a given date a real massive usage in the scientific literature at large, i.e., not limited to the specialised taxonomic literature, to designate a given taxon, whereas no other nomen has been used significantly for the same taxon or closely related taxa after that date. The quantitative requirements adopted here, following Dubois (2016), are: $\{Z 1 \mathrm{a}\}$ for the landmark starting date, 31 December 1899; $\{\mathrm{Z} 1 \mathrm{~b}\}$ for the definition of real massive usage, the presence of the nomen in the titles of at least 100 scientific publications.

\{Z2\} A sozodiaphonym is a CS nomen that has also had such a large usage in the scientific literature at large, but alternatively to another competing nomen or several other nomina which also had a large usage for the same taxon.

$\{Z 3\}$ A distagmonym is a CS nomen that has not had such a large usage in the scientific literature.

The term sozonymorphs designates both sozonyms and sozodiaphonyms, as opposed to distagmonyms.

As we will see below, these terms and definitions will be useful both for the taxonomic allocation of CS nomina and for their taxonomic validity.

\subsubsection{Taxonomic allocation of nomina}

The LSNS is a theory-free ostensional nomenclatural system in which the allocation of nomina to taxa is made through onomatophores (Simpson 1940), i.e., through the objective link established between specimens and nomina, not subjectively through verbal definitions of taxa. We here use the verb to anchor to designate the nomenclatural act of designation of an onomatophore for a taxon and the noun anchorage to designate the result of this act. Species-series nomina are connected to taxa through nomen-bearing 'type specimens' (onymophoronts), genus-series nomina are so through nomen-bearing 'type-species' (nucleospecies) and family-series nomina are so through nomen-bearing 'type-genera' (nucleogenera) (for details and terminology see Dubois 2005b, 2011a). Concerning class-series nomina, the Code does not give any clue for their taxonomic allocation. Let us therefore consider separately the situation in the FS and in the CS.

\subsubsection{Family-series nomina}

Whereas in the SS and GS the designation of onomatophores may be done by several procedures and is sometimes quite complex, in the FS this designation is straightforward because available FS nomina are based on the stems of GS nomina (the 'stem' being sometimes the entire nomen itself; see Article 29), which are therefore automatically their onomatophores.

Until 2000, the identification of the nucleogenera of FS nomina did not have to be expressly stated in the original publication, as it could usually be easily deduced from the similarity between the FS nomen and the stem of one of its included genera. This kind of indication (according to the Code) was called implicit etymological designation (Dubois 1984b). However, in a few cases, a doubt was possible when the nomina of two genera included in a new FS taxon had the same stem, so that in the current version of the Code, to be valid the designation must be explicit, and if this designation is missing the nomen is not available, as stated in Criterion (Av-33) in Table T4.AVN.

Family-series nomina which are not based on the stems of available nomina of included genera considered valid are nomenclaturally unavailable and therefore cannot be valid. However, it may be useful to allocate each of these nomina to a given synonymy, as anyone finding one of these nomina in 
the literature or in databases may wish to know to which taxon it applies. Given the fact that, under the Code, nomina are allocated to taxa through their onomatophore, this allocation requires the designation of a nucleogenus for any such FS anoplonym. This is similar to the designation of nucleospecies for GS anoplonyms, which was implemented for example by Dubois \& Raffaëlli (2009) in salamanders. There is nothing in the Code that forbids to do so. In the case of FS anoplonyms, there are three situations. In the first one $\{\mathrm{a} 1\}$, the nomen of the FS anoplonym is clearly a rhizonym based on the stem of an available genus nomen of the taxonomic group concerned: we consider this as a nucleogenus designation by implicit etymological designation, just like for hoplonyms. In the second situation $\{\mathrm{a} 2\}$, the FS anoplonym is an arhizonym, but one or several generic nomina were allocated to the taxon in the original work (situation of original symphory): in such cases one of them has to be chosen and designated as nucleogenus, except when a single genus nomen was mentioned, which is therefore nucleogenus by original monophory. In the third situation $\{a 3\}$, no available genus-series nomen was explicitly associated with the new FS anoplonym in the original publication (situation of original aphory): in such cases a nucleogenus should be designated in order to fit with the taxon that appears to have corresponded to the taxon intended by the new unavailable nomen. The nucleogenera hereby established or designated for FS nomina are shown in Appendix A6.NFS below.

\subsubsection{Class-series nomina}

As explained by Dubois \& Ohler (2019), in the absence of Rules in the Code for the allocation of CS nomina to taxa, any taxonomist who wishes to use such nomina has to adopt a system for this purpose, and none of such systems can be claimed to be more 'Code-compliant' than any other. However, for such a system to be 'Code-compatible', it should follow some of the basic characteristics of the Code: regarding CS nomina, their taxonomic allocation should be done through an ostensional system using onomatophores, not through a system of verbal definitions, be them 'phylogenetic' or not.

In this work, we used the Duplostensional Nomenclatural System (DONS) as described in Dubois (2015c, 2016), which is derived through simplification from the Ambiostensional Nomenclatural System (AONS) initially proposed by Dubois (2006a). We refer to the works of Dubois (2006c, 2007a, 2011a, 2015c, 2016, 2020a; Dubois \& Raffaëlli 2012) for detailed explanations of the rationale of this nomenclatural system, which are not repeated here. An important difference between this system and the system of the Code in the three lowest nominal-series is that this system is monosemic, i.e., it does not use a Principle of Coordination: therefore, in a given ergotaxonomy a given CS nomen can apply only to a single taxon, not to a set of coordinated nomina.

Allocation of CS nomina to taxa under the DONS Rules is simple and straightforward. It depends however on the category of usage to which the nomen is referred. According to this category of usage, a different nomenclatural subsystem of DONS will be used for the taxonomic allocation of the CS nomen.

\{b1\} Metrostensional Nomenclatural Subsystem (MONS). If the CS nomen is a distagmonym, its taxonomic allocation relies solely on its onomatophore, i.e. on the list of its conucleogenera (or on its single uninucleogenus), i.e., all the available nominal genus-series nomina originally and unambiguously referred as valid to the taxon for which the CS nomen was proposed. This list is an indissoluble set of available nomina which act altogether as the onomatophore of the CS nomen at stake. Then, within any ergotaxonomy adopted as valid, this nomen is a nesonym, which applies to the metronym, i.e. the least inclusive CS taxon which contains all these nucleogenera (the metrotaxon of the nomen in this ergotaxonomy). This provides an unambiguous allocation of the nomen to a single CS taxon in the ergotaxonomy adopted.

\{b2\} Orostensional NomenclaturalSubsystem(OONS). If theCSnomen is asozonymorph(sozonym or sozodiaphonym), its taxonomic allocation is made through a combination of its onomatophore and its onomatostasis, which provides the external limits of the taxon. The onomatostasis of a CS nomen consists in the list of its alienogenera, i.e., the indissoluble set of available generic nomina originally explicitly listed as valid but as non-members of the taxon. Then, within any ergotaxonomy adopted as valid, this nomen is a choronym, which applies to the oronym, i.e. the most inclusive CS taxon which contains all its nucleogenera and excludes all its coalienogenera (the orotaxon of the nomen in the ergotaxonomy adopted as valid). This also provides an unambiguous allocation of the nomen to a single CS taxon in the ergotaxonomy adopted. 
Three particular cases must be considered separately. More details on these situations were given in Dubois (2015c: 37-42).

$\{b 3\}$ In some rather rare cases, a new CS nomen, either sozonymorph or distagmonym, is proposed for a taxon that is defined or diagnosed, which makes the nomen available, but to which no genus is referred. In such cases, just like for SS and GS nomina that happen to be in the same situation of original aphory (absence of onomatophore), the first subsequent author who listed nominal genera as included in the CS taxon fixed the nucleogenera (and if available the alienogenera) of the nomen by subsequent designation (for more details see Dubois 2006c). In the present work, we did several such designations The nucleogenera and alienogenera hereby established or designated for FS nomina are shown in Appendix A7.NCS below.

$\{b 4\}$ In the rare cases where a CS nomen has only original or subsequent conucleogenera (or a single uninucleogenus) but no original alienogenus, it also only has a metronym and cannot have an oronym, but in this case this is because of incompleteness of information, not of overlap between taxa. Such a CS nomen is an ellitonym and it must be treated as a metronym: it can be taxonomically allocated only through its metrotaxon. This can be formulated differently in stating that in this case its metronym is also its oronym in the ergotaxonomy adopted.

$\{b 5\}$ In the (rather frequent) cases where some (or even a single one) of the original alienogenera are/is now included in the metrotaxon of a CS sozonymorph, the latter, although available, cannot be valid in this ergotaxonomy. It is then a gephyronym, a particular case of anaptonym, i.e., a nomen that cannot be allocated to a taxon and therefore cannot be valid.

In practice, for sozonymorphs, it is not necessary to look for the taxonomic allocation of all the alienogenera of a choronym to determine the extension of the taxon it designates. It is enough to find the (phylogenetically) 'closest' alienogenera that will allow to ascertain the external limits of the taxon and therefore identify the taxon to which the CS nomen under consideration applies in the ergotaxonomy chosen. These immediate 'neighbours' are the getextragenera of the CS nomen and they are sufficient to allocate unambiguously the CS nomen to a taxon. A single getextragenus is enough for this purpose. This has important ergonomic advantages as long as the analysis is done 'by hand' and is not computerised, because it is not necessary to ascertain the taxonomic allocation of all the alienogenera, including those that are clearly 'remote' from the orotaxon but may belong in taxonomic groups with which the taxonomist doing the nomenclatural analysis is not well acquainted. Of course, it would not be the case if all zoological nomina were taxonomically allocated, but this is far from being the case, as it is easy to check by looking at any large and 'comprehensive' database. Even among the nomina of Linnaeus (1758a), a few are still taxonomically unallocated or only at very high taxonomic levels.

The OONS system for sozonymorphs has two main advantages: $\{\mathrm{c} 1\}$ it allows to validate the sozonym or sozodiaphonym for a taxon identical or very close to that recognised under this nomen in the literature; $\{\mathrm{c} 2\}$ but it also allows to expand the content of the taxon to include more basal taxa recently discovered (often as fossils) without having to coin a new nomen for the taxon including the traditional taxon but also the more basal taxon ('stem-' or 'pan-'taxon). Finally, this system is liable to be entirely computerised, so that in the future all the rather complex and tedious verifications and actions described above can be automatised, which will avoid the mistakes that no rarely occur when the analysis is carried out 'by hand'.

\subsubsection{Validity and correctness of nomina}

\subsubsection{Introduction}

The final aim of the nomenclatural work is to establish unambiguously the valid nomen which must be used by all biologists worldwide for a given taxon under a given classification and its correct spelling. In order to be potentially valid, a nomen must have gone through the first two stages of the nomenclatural process: it must be available (promulgated) and anchored (it must have an onomatophore).

The 'ideal' situation in zoological nomenclature is that where a single available nomen was proposed, during the whole history of zoological taxonomy, for a taxon now recognised as valid in an ergotaxonomy. Unfortunately, this situation is not general, and various problems of nomenclatural conflict are observed in most genuine situations. These conflicts can occur between nomina, between spellings of nomina or between onomatergies (nomenclatural acts). Dubois (2013) surveyed these 
situations of conflict which he termed zygoidy. We here refer to this work for details and explanations. Below, we summarise the Principles (including some 'untold' ones which were first described and named by Dubois in 2013 and later adopted among the LZP), Rules, Criteria and 'codified exceptions' which, according to the Code, allow the resolution of the conflicts of zygoidy in the SS, GS and GS, as well as those used here under the DONS Criteria in the CS.

\subsubsection{Priority}

The Principle of Priority is explicitly mentioned in Article 23 of the Code. It is by far the most often used system of resolution of zygoidy whenever the two competing items were published at different dates. According to this Principle, in any situation of allochronous zygoidy and in the three nominalseries recognised and covered by the Code, the first published zygonym (competing homonym or synonym), zygograph (competing parograph) or zygonomatergy (competing nomenclatural act) has precedence, except if the Principle of Nomography or the Principle of Archoidy applies. The same Principle with its limitations is recognised in the CS according to DONS, except that here the Principle of Sozoidy may also prevail over the Principle of Priority.

Two particular cases regarding Priority must be pointed out in the family-series: those covered by Articles 35.4.1 and 40.2 of the Code. In a few special cases, the valid nomen of a FS taxon is not the oldest one but a more recent one. In order to indicate such exceptions, such nomina bear 'double auctorships', one being that of the earliest published nomen and the other one the nomen validated through the relevant Article. This question was treated in detail by Dubois (2015a) and we refer to this work for details. In the present work, we used the following presentations for such nomina: $\{\mathrm{d} 1\}$ LEPTODACTYLIDAE ||Tschudi, 1838|-Werner, 1896 for nomina validated through Article 35.4.1; and \{d2\} MEGOPHRYIDAE Bonaparte, 1850-|Noble, 1931| for nomina validated through Article 40.2.

\subsubsection{Airesy (first reviser)}

The Principle of Airesy is explicitly mentioned in Article 24.2.1 of the Code as the 'Principle of the First Reviser', a needless cumbersome formulation as the Code does not recognise any 'second' or subsequent revisers. It states that in any situation of synchronous zygoidy and in the three nominalseries covered by the Code, precedence among zygoids (zygonyms, zygographs or zygonomatergies) is fixed by the action of the arbiter ('first reviser') publishing an explicit airesy ('first reviser action') of seniorisation removing this ambiguity. DONS also recognises this Principle in the CS. Such airesies are definitive and irreversible by subsequent actions of individual zoologists. They may however be superseded by the Principles of Proedry and the Principle of Archoidy, and in the CS according to DONS by the Principle of Sozoidy. Code:

Three distinct categories of Airesies can be distinguished in the three nominal-series covered by the

\{e1\} External Airesy (ETA): explicit Airesy made by the arbiter who mentioned both competing synchronous nomina, spellings or onomatergies and expressly chose one as valid (Article 24.2.3). This kind of Airesy is also recognised in the CS according to DONS.

\{e2\} Explicit Internal Airesy (EPITA): same as ETA, but made by the original auctor(s) and concerning only competing synchronous spellings published in the same original work (Article 24.2.4). This kind of Airesy is also recognised in the CS according to DONS.

\{e3\} Implicit Internal Airesy (IPITA): implicit Airesy made by the original auctor(s) of competing synchronous spellings published in the same original work, through mentioning only one of them in a subsequent publication (Article 24.2.4). This kind of Airesy does not concern nomina or onomatergies, and is not implemented in the CS according to DONS (in this respect see Dubois 2010a: 14-18).

\subsubsection{Proedry (rank precedence)}

In the three nominal-series covered by the Code, whenever zygonyms are introduced simultaneously, but proposed at different ranks within their nominal-series, the nomen proposed at higher rank has 
precedence (Articles 24.1, 55.5, 56.3, 57.7). The same applies to the cases of simultaneous onomatophore fixations for a nominal taxon (situation of zygophory): the fixation for the taxon at higher rank takes precedence (Article 61.2.1).

The Code just mentions these Rules in the Articles cited above, but does not recognise a special Principle for these onomatergies. Dubois (2013) proposed to recognise it as the Principle of Proedry, which was adopted among the LZP (Dubois \& Aescht 2019n; Dubois et al. 2019) where it also applies to CS nomina.

\subsubsection{Eugraphy}

In the three nominal-series covered by the Code, the correct spelling (eugraph) of a given nomen in a given ergotaxonomy may be different from its original spelling (protograph or lectoprotograph) when this nomen is a nomograph and requires either mandatory spelling correction (justified emendation or eunomograph) of an inadvertent spelling error (Articles 32.2.2, 32.5.1, 33.2.2), or mandatory ending correction (mandatory change or legonomograph) in the case of a species-series nomen (to comply with agreement in grammatical gender with the generic nomen) (Articles 31.2, 34.2) or of a family-series nomen (to comply with the mandatory spellings indicating the rank of some family-series nomina) (Articles 29.2, 34.1). The protograph of a nomen must also be modified when this follows a decision of the Commission under the Plenary Power (archograph). The Code just mentions these Rules in the Articles cited above, but does not recognise a special Principle for these onomatergies. Dubois (2013) proposed to recognise it as the Principle of Nomography, which was adopted by the LZC (Dubois \& Aescht 2019o, Dubois et al. 2019).

These Rules apply in the three nominal-series covered by the Code, the SS, GS and FS, but they cannot apply directly in the class-series, so that Criteria for fixing the correct spelling (legethograph) of these nomina had to be devised (Dubois 2015c; Dubois \& Frétey 2020a). For the spelling of the stems of the nomina, the Code's Rules concerning the mandatory spelling correction are appropriate, but the Rules concerning their endings cannot be used, especially in the cases of panrhizonyms, i.e., class-series nomina based of the stems of other nomina.

Alonso-Zarazaga (2005) proposed that all nomina above the rank superfamily be referred to a single 'upper uninominal group', including also the 'family-subgroup', and be based on the stems of genus-series nomina, combined with endings derived from the words zoo (for the 'phylum-subgroup'), morph (for the 'class-subgroup') and form (for the 'order-subgroup'). Dubois (2006c) provided a detailed criticism and rebuttal of this proposal which does not need to be repeated here. Adopting it would entail gigantic and catastrophic changes in the higher nomenclature of animals, for no benefits in counterpart. Currently, panrhizonyms are used in the class-series nomenclature of only a small proportion of the zoological groups. This can be ascertained for example by simply looking at the two volumes of review of the higher taxonomy of animals published by Zhang $(2011 a, 2013 a)$ : of the 49 groups surveyed in these volumes, only 18 used panrhizonyms to name a few of their higher taxa (see Table T9.ENZ). As for the endings used for these nomina, three were based on form, seven on morph and one on zoo, but there was no correlation between the use of a given ending and the rank of the taxon. For example, the ending -OMORPHA was used in 11 works, but for the following ranks or pseudoranks: 'phylum' (in a pseudoranked nomenclatural system), phylum, subphylum, class, subclass, superorder, order, suborder and infraorder. For the classseries ranks and below, the most used of these endings in these works based on form and morph are, respectively, -IFORMES and -IFORMIA, and -OMORPHA and -OMORPHI. Various other endings, not based on these terms and simpler (one to five letters), have been used for class-series nomina in these works: some (e.g. -OIDEA, -INA or -INI) are identical to endings imposed by the Code in the family-series, or acceptable in this nominal-series (e.g. -OIDEI, -OIDES or -IDEI), and are therefore liable to cause confusion, whereas others (e.g. -ACEA or -ACEI) are less prone to ambiguity.

Dubois $(2015 c)$ proposed a different system, in which the endings of the panrhizonyms are not supposed to reflect their rank but their category of panrhizonymy, according to a set of Criteria detailed in Table T5.RHI. Considering the facts above, he chose the following standard endings for the four categories of panrhizonyms that he distinguished: -ACEA for rhizonyms, -ACEI for cenorhizonyms, IFORMIA and -OMORPHA for auXorhizonyms, and -IFORMES and -OMORPHI for xenorhizonyms. These endings replace the original endings with minimal perturbation. Dubois \& Frétey $(2020 a)$ distinguished the category of quasirhizonyms and proposed for it the standard endings -IFORMES and -OMORPHES. 


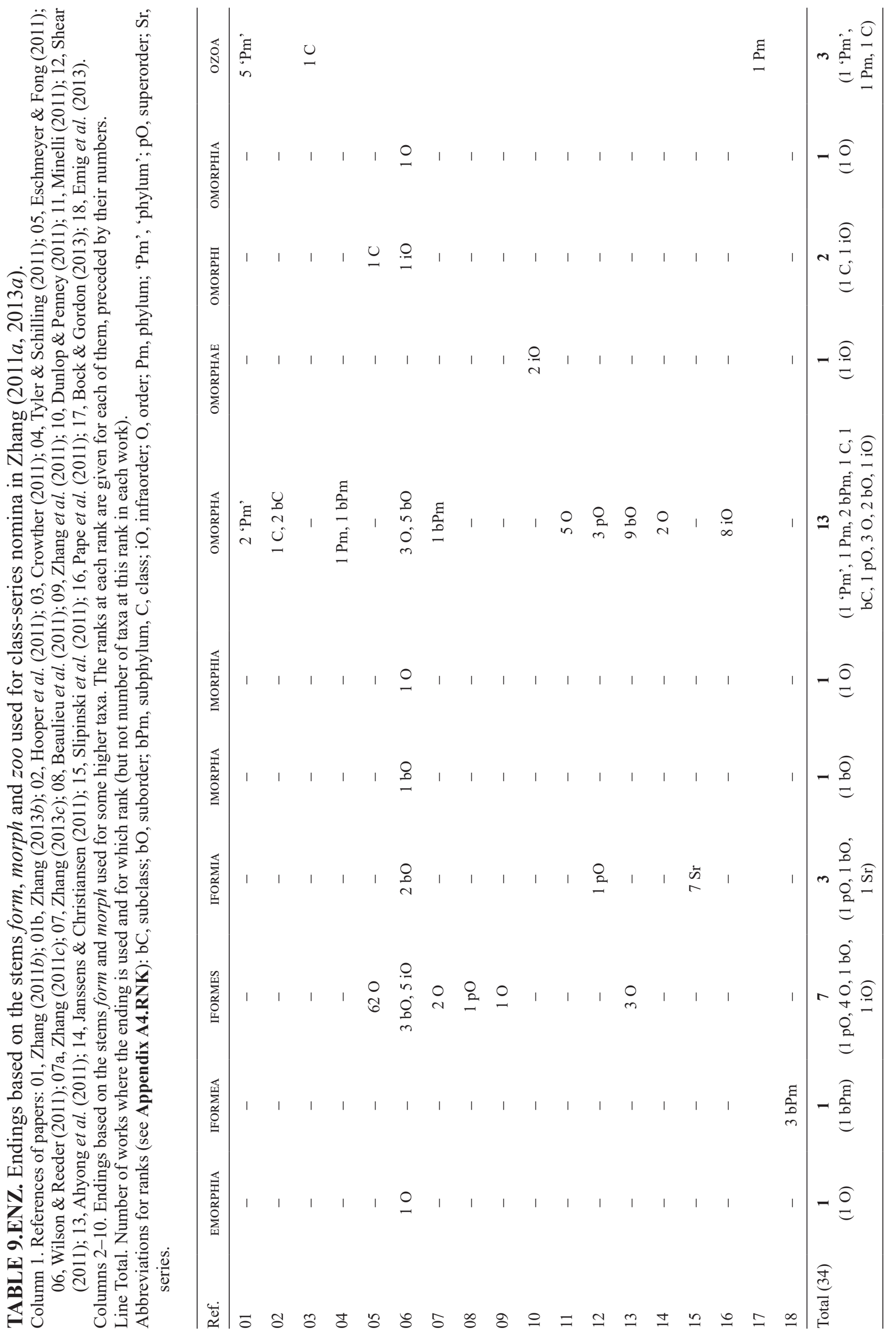


TABLE 10.ENL. Endings used in the protographs of panrhizonyms of class-series nomina of LISSAMPHIBIA in the literature according to Appendix A7.NCS.

The lines provide the original endings of the protographs of these 105 nomina, and the columns their standard endings following the system proposed by Dubois (2015c) and Dubois \& Frétey $(2020 a)$.

\begin{tabular}{|c|c|c|c|c|c|}
\hline Original ending & $\begin{array}{c}\text { Rhizonyms } \\
\text {-ACEA }\end{array}$ & $\begin{array}{c}\text { Cenorhizonyms } \\
\text {-ACEI }\end{array}$ & $\begin{array}{c}\text { Auxorhizonyms } \\
\text {-IFORMIA } \\
\text {-OMORPHA }\end{array}$ & $\begin{array}{c}\text { Xenorhizonyms } \\
\text {-IFORMI } \\
\text {-OMORPHI }\end{array}$ & $\begin{array}{c}\text { Quasirhizonyms } \\
\text {-IFORMES } \\
\text {-OMORPHES }\end{array}$ \\
\hline$-\mathbf{A}(1)$ & 1 & 0 & 0 & 0 & 0 \\
\hline -ACEA (4) & 4 & 0 & 0 & 0 & 0 \\
\hline$-\mathbf{A E}(5)$ & 4 & 1 & 0 & 0 & 0 \\
\hline -AEFORMES (1) & 0 & 0 & 1 & 0 & 0 \\
\hline -AEFORMIA (1) & 0 & 0 & 1 & 0 & 0 \\
\hline -AEMORPHA (1) & 0 & 0 & 1 & 0 & 0 \\
\hline$-\operatorname{DES}(1)$ & 1 & 0 & 0 & 0 & 0 \\
\hline$-\mathbf{E A}(1)$ & 0 & 1 & 0 & 0 & 0 \\
\hline -ES (4) & 4 & 0 & 0 & 0 & 0 \\
\hline$-\mathbf{I A}(2)$ & 2 & 0 & 0 & 0 & 0 \\
\hline- IDA (5) & 5 & 0 & 0 & 0 & 0 \\
\hline- IDEI $(2)$ & 2 & 0 & 0 & 0 & 0 \\
\hline -IFORMES (10) & 0 & 0 & 9 & 1 & 0 \\
\hline -IFORMIA (11) & 0 & 0 & 6 & 3 & 2 \\
\hline -INA (2) & 2 & 0 & 0 & 0 & 0 \\
\hline$-\mathbf{I N I}(1)$ & 0 & 1 & 0 & 0 & 0 \\
\hline -OIDEA (32) & 32 & 0 & 0 & 0 & 0 \\
\hline -OIDEI (5) & 5 & 0 & 0 & 0 & 0 \\
\hline -OIDES (1) & 1 & 0 & 0 & 0 & 0 \\
\hline -OMORPHA (15) & 0 & 0 & 7 & 0 & 8 \\
\hline Total (105) & 63 & 3 & 25 & 4 & 10 \\
\hline
\end{tabular}

For xenorhizonyms based on -form, they proposed the ending -IFORMI. Tables T10.ENL and T11.LEG present information on the original endings of all the panrhizonyms so far published for LiSSAMPHIBIA (listed in Appendix A7.NCS). Among these 105 nomina of LiSSAMPHIBIA listed in this Table, 23 had original endings based on the term form, 16 had endings based on morph and 66 had 'simple' endings like -OIDEA, -OIDEI, -IDA or -AE. Only two of these 105 nomina (HEMIPHRACTIFORMIA and RANOMORPHA) are used as valid in the taxonomy of LISSAMPHIBIA adopted here (Appendices A9.CLAD-1 to A12. CLAD-4).

For sake of completeness, the term khoristarhizonym (distinct from $\boldsymbol{k h o r i s t o r h i z o n y m}$ as defined by Dubois \& Frétey 2020a) is here proposed for arhizonyms ending in form or morph. To avoid confusion with panrhizonyms, the endings -IFORMIES and -OMORPHIES are here proposed for such CS nomina.

\subsubsection{Reversal of precedence}

In the Code, several exceptions to the Principles of Priority and 'first reviser' are allowed in the species-, genus- and family-series, in 9 situations involving so-called 'prevailing usage'. However, as discussed by Dubois (2010a: 13-14, 2017b: 24) and Löbl (2015), this formula is used inconsistently in the Code.

\subsection{Article 23.9}

Article 23.9 on 'reversal of precedence' allows in certain conditions to protect 'prevailing usage' through validation of a nomen protectum (protected nomen) against a nomen oblitum (forgotten nomen) that would have precedence over the former according to the Code. The conditions for such an 
onomatergy are that the former nomen must have been used by at least 10 authors in 25 works published during at least 10 years in the immediately preceding 50 years, whereas the latter has never been used as valid after 1899. In this Article, the expression 'prevailing usage' is given a precise definition which, although it raises several problems (see Ohler \& Dubois 2018 and Dubois \& Ohler 2018), is operational. This Article results in validating some junior zygonyms against Priority. It applies only to the three nominal-series covered by the Code. In the CS, it would correspond partially to the Principle of Sozoidy discussed below.

\subsection{Other Articles resulting in reversal of precedence}

However, in the 'Glossary' of the Code (which fails to mention the formula 'reversal of precedence'), 'prevailing usage' of a nomen is defined differently, as the usage "which is adopted by at least a substantial majority of the most recent authors concerned with the relevant taxon, irrespective of how long ago their work was published". This vague definition is based on undefined terms. When is a majority 'substantial'? How is 'most recent' defined? Who are the authors 'concerned with the relevant taxon'? This definition is therefore not operational and the mention of the formula 'prevailing usage' in some Articles of the Code is confusing. The 8 following Articles, which apply only in the three nominalseries covered by the Code, are concerned by such exceptions:

\{f1\} Article 23.12 reads: "A name that was rejected between 6 November 1961 and 1 January 1973, by an author who explicitly applied Article $23 \mathrm{~b}$ in force between those dates under the then current editions of the Code, on the grounds that it was a nomen oblitum (...) is not to be given precedence over a junior synonym in prevailing usage, unless the Commission rules that the older but rejected name is to take precedence". This Article results in validating some junior synonyms against Priority.

\{f2\} Article 29.5 reads: "If a spelling of a family-group name was not formed in accordance with Article 29.3 but is in prevailing usage, that spelling must be maintained, whether or not it is the original spelling". This Article results in validating some family-series apographs against Priority and Nomography.

\{f3\} Article 33.2.3.1 reads: "when an unjustified emendation is in prevailing usage and is attributed to the original author and date it is deemed to be a justified emendation". This Article results in transforming some autoneonyms into apographs and in validating the latter against Priority.

\{f4\} Article 33.3.1 reads: "when an incorrect subsequent spelling is in prevailing usage and is attributed to the publication of the original spelling, the subsequent spelling and attribution are to be preserved and the spelling is deemed to be a correct original spelling". This Article results in validating some apographs against Priority.

$\{$ f5\} Article 35.4.1 reads: "A family-group name based upon an unjustified emendation (...) or an incorrect spelling of the name of the type genus must be corrected, unless it is preserved under Article 29.5 or unless the spelling of the genus-group name used to form the family-group name is preserved under Articles 33.2.3.1 or 33.3.1". This Article results in transforming some family-series autoneonyms into apographs and in validating the latter against Priority, and in validating some familyseries apographs against Priority and Nomography.

\{f6\} Article 35.5 reads: "If after 1999 a name in use for a family-group taxon (...) is found to be older than a name in prevailing usage for a taxon at higher rank in the same family-group taxon [which could be stated more briefly: "than a superordinate name in prevailing usage"], the older name is not to displace the younger name". This Article results in validating some family-series junior synonyms against Priority.

\{f7\} Article 40.2 reads: "If (...) a family-group name was replaced before 1961 because of the synonymy of the type genus, the substitute name is to be maintained if is in prevailing usage". This Article results in validating some family-series junior synonyms against Priority.

\{f8\} Article 59.3 reads: "A junior secondary homonym replaced before 1961 is permanently invalid unless the substitute name is not in use and the relevant taxa are no longer considered congeneric (...)". This Article results in validating some species-series junior nomina against Priority.

All these Articles of the Code present in fact 'codified exceptions' that do not correspond to a Principle. They rely on a vague concept of 'usage' which is highly questionable in a text which is supposed to play a regulatory role in millions of scientific publications (Dubois $2010 b-c$ ). This is why Dubois $(2005 b)$ proposed to replace this ambiguous and undefined concept by well-defined categories of usage on the basis of which he proposed to implement a new nomenclatural Principle, the Principle of Sozoidy (Dubois 2013). 
TABLE 11.LEG. Legethographs (Latin standard endings or full spellings) adopted here for the classseries nomina of LISSAMPHIBIA.

Column 1: Categories of CS nomina regarding rhizonymy (see Table T5.RHI). Column 2: Standard Latin ending, full spelling or both adopted here for nomina of this category (see text for explanations). Column 3: Original ending, full spelling or both used for this nomen in the literature (see Appendix A6.NFS), which should be replaced by the ending or spelling in column $2 ;-\mathbf{X X X}$, standard spelling of ending adopted here; $\mathbf{Y Y Y}$, standard spelling of full nomen adopted here; $\div \mathbf{z z z}$, standard spelling of either full nomen or ending adopted here. The numbers given after the nomina are those of the latter bearing this ending in the category, with the following distinctions: [ ], number of nomina that had originally this ending; ( ), number of nomina the original ending of which had to be corrected; ||, number of nomina established as new in the present work; \{\} , total number of nomina of this category. A, Arhizonyms: Latin standard ending consistent with usage in other zoological class-series nomina based on the same final stem. C, Cenorhizonyms: Latin standard ending in -ACEI, avoiding confusion with FS nomina with standard FS endings in -IDAE, -INAE, -INA, -INI and -OIDEA. P, Pararhizonyms: Latin standard ending in -ACEAE, avoiding confusion with FS nomina with standard FS endings in $-I D A E,-I N A E,-I N A,-I N I$ and $-O I D E A$. Q, Quasirhizonyms: Latin standard ending in -IFORMES or -OMORPHES. R, Rhizonyms: Latin standard ending in - ACEA, avoiding confusion with FS nomina with standard FS endings in -IDAE, INAE, $-I N A,-I N I$ and -OIDEA. U, Auxorhizonyms: Latin standard ending in -IFORMIA or-OMORPHA. X, Xenorhizonyms: Latin standard ending in -IFORMI or - OMORPHI.

\begin{tabular}{|c|c|c|}
\hline $\begin{array}{l}\text { Category of nomen } \\
\text { regarding rhizonymy }\end{array}$ & Spelling of word or standard ending adopted here & $\begin{array}{l}\text { Original spellings or endings } \\
\text { that had to be corrected }\end{array}$ \\
\hline $\mathbf{A}\{2\}$ & -ALIA [2] & - \\
\hline \multirow[t]{3}{*}{$\mathbf{A}\{10\}$} & $\div$ AMPHIBIA [7] + (3) & AMPHIBIENS (1) \\
\hline & & AMPHIBIES (1) \\
\hline & & AMPHYBIENS (1) \\
\hline $\mathbf{A}\{2\}$ & -BATAE [2] & - \\
\hline \multirow[t]{5}{*}{$\mathbf{A}\{32\}$} & $\div$ BATRACHIA $[15]+(17)$ & -BATRACHI (8) \\
\hline & & BATRACHIER (1) \\
\hline & & BATRACHOIDEA (2) \\
\hline & & BATRACHOIDEI (1) \\
\hline & & BATRACIENS (5) \\
\hline \multirow[t]{5}{*}{$\mathbf{A}\{28\}$} & -BRANCHIA [14] + (14) & -BRANCHES (5) \\
\hline & & -BRANCHI (2) \\
\hline & & -BRANCHIALES (1) \\
\hline & & -BRANCHIATA (5) \\
\hline & & -BRANCHIENS (1) \\
\hline $\mathbf{A}\{2\}$ & BRANCHIATA [2] & - \\
\hline \multirow[t]{3}{*}{$\mathbf{A}\{18\}$} & $\div$ CAUDATA $[11]+(7)$ & -CAUdATAE (1) \\
\hline & & $\div$ CAUDATI $(4)$ \\
\hline & & -CAUDES (2) \\
\hline $\mathbf{A}\{1\}$ & -CERA [1] & - \\
\hline $\mathbf{A}\{1\}$ & - CERCI $[1]$ & - \\
\hline $\mathbf{A}\{1\}$ & CERCOPI [1] & - \\
\hline $\mathbf{A}\{1\}$ & - CHELATA [1] & - \\
\hline \multirow[t]{2}{*}{ A $\{24\}$} & - COELA $[20]+(4)$ & $-\operatorname{COELI}(2)$ \\
\hline & & -COELIDAE (2) \\
\hline $\mathbf{A}\{3\}$ & $\div$ COSTATA $[1]+(2)$ & $\div$ COSTATI $(2)$ \\
\hline \multirow[t]{3}{*}{$\mathbf{A}\{7\}$} & -DACTYLA [2] + (5) & -DACTYLES (2) \\
\hline & & -DACTYLI (2) \\
\hline & & -DACTYLia (1) \\
\hline $\mathbf{A}\{5\}$ & -DELA [4] + (1) & -DELES (1) \\
\hline $\mathbf{A}\{4\}$ & $\div$ DENTATA [4] & - \\
\hline $\mathbf{A}\{7\}$ & -DERA [2] + (5) & -DERES (5) \\
\hline $\mathbf{A}\{3\}$ & -DERMA [3] & - \\
\hline $\mathbf{A}\{1\}$ & -DYTAE [1] & - \\
\hline $\mathbf{A}\{3\}$ & -ECHMIA [3] & - \\
\hline $\mathbf{A}\{10\}$ & -ENTIA [10] & - \\
\hline
\end{tabular}


TABLE 11. (Continued)

\begin{tabular}{|c|c|c|}
\hline $\begin{array}{l}\text { Category of nomen } \\
\text { regarding rhizonymy }\end{array}$ & Spelling of word or standard ending adopted here & $\begin{array}{l}\text { Original spellings or endings } \\
\text { that had to be corrected }\end{array}$ \\
\hline \multirow[t]{2}{*}{$\mathbf{A}\{3\}$} & -FERA [1] + (2) & -FERES $(1)$ \\
\hline & & -FERI (1) \\
\hline $\mathbf{A}\{5\}$ & -GLENA [2] + (3) & -GLENIDES (3) \\
\hline \multirow[t]{3}{*}{$\mathbf{A}\{12\}$} & - GLOSSA $[6]+(6)$ & -GLOSSAE (2) \\
\hline & & -GLOSSES (2) \\
\hline & & -GLOSSI (2) \\
\hline \multirow[t]{2}{*}{$\mathbf{A}\{5\}$} & -GYRINIA (5) & -GYRINIDAE (3) \\
\hline & & -GYRINIDES (2) \\
\hline \multirow[t]{5}{*}{$\mathbf{A}\{7\}$} & 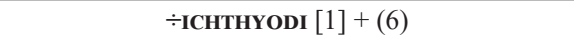 & -ICHTHYENS (1) \\
\hline & & ICHTHYOIDEA (2) \\
\hline & & -ІСНтнYI (1) \\
\hline & & ICHTYOIDA (1) \\
\hline & & ICTYOIDES (1) \\
\hline $\mathbf{A}\{2\}$ & LINGUATA [2] & - \\
\hline $\mathbf{A}\{3\}$ & MEANTES [2] + (1) & MEANTIA (1) \\
\hline $\mathbf{A}\{4\}$ & -MELA (4) & -MELES (4) \\
\hline $\mathbf{A}\{8\}$ & -MOLGAE $[5]+(3)$ & -MOLGAEI (3) \\
\hline $\mathbf{A}\{5\}$ & $\div$ MUTABILIA $[5]$ & - \\
\hline $\mathbf{A}\{2\}$ & -NECTAE [2] & - \\
\hline $\mathbf{A}\{4\}$ & - NUDA [3] + (1) & - NUDS (1) \\
\hline $\mathbf{A}\{2\}$ & -ONYXIA [2] & - \\
\hline \multirow[t]{5}{*}{$\mathbf{A}\{8\}$} & -OPHIONA $(7)+|1|$ & -OPHIA (1) \\
\hline & & -OPHIDES (3) \\
\hline & & -OPHIDIA (1) \\
\hline & & -OPHILIA (1) \\
\hline & & -OPHYDIENS (1) \\
\hline $\mathbf{A}\{2\}$ & -PARES [2] & - \\
\hline $\mathbf{A}\{1\}$ & PAROTOIDIA [1] & - \\
\hline $\mathbf{A}\{1\}$ & PEDATA [1] & - \\
\hline $\mathbf{A}\{1\}$ & -PHARA [1] & - \\
\hline $\mathbf{A}\{2\}$ & -PHILI [2] & - \\
\hline $\mathbf{A}\{2\}$ & -PHORA [1] + (1) & -PHORI (1) \\
\hline $\mathbf{A}\{2\}$ & $\div$ PHRYNIA [2] & - \\
\hline $\mathbf{A}\{2\}$ & -PLEURAE [2] & - \\
\hline A $\{4\}$ & -PNEUMa [1] + (3) & -PNEUMENA (3) \\
\hline $\mathbf{A}\{1\}$ & -PNEUSTA (1) & -PNEUSTA (1) \\
\hline $\mathbf{A}\{5\}$ & -PNOA [5] & - \\
\hline $\mathbf{A}\{5\}$ & -PODA [5] & - \\
\hline \multirow[t]{2}{*}{$\mathbf{A}\{2\}$} & $\div$ PULMONATA $(2)$ & -PULMONADOS (1) \\
\hline & & PULMONES (1) \\
\hline $\mathbf{A}\{1\}$ & $-\operatorname{ROSA}[1]$ & - \\
\hline $\mathbf{A}\{2\}$ & -SACRALIA [2] & - \\
\hline $\mathbf{A}\{2\}$ & -SALAMANDRAE [2] & - \\
\hline $\mathbf{A}\{4\}$ & -SAURIA [3] + (1) & -SAURIENS (1) \\
\hline $\mathbf{A}\{1\}$ & -SCOLECODES [1] & - \\
\hline $\mathbf{A}\{2\}$ & -SIPHONA [2] & - \\
\hline $\mathbf{A}\{1\}$ & -SOMA (1) & -SOMES (1) \\
\hline $\mathbf{A}\{7\}$ & -STERNIA [7] & - \\
\hline $\mathbf{A}\{1\}$ & -STOMATA [1] & - \\
\hline $\mathbf{A}\{3\}$ & -TARSATA (3) & -TARSIDEN (3) \\
\hline $\mathbf{A}\{1\}$ & -TREMATA [1] & - \\
\hline $\mathbf{A}\{1\}$ & -TRETA [1] & - \\
\hline
\end{tabular}


TABLE 11. (Continued)

\begin{tabular}{|c|c|c|}
\hline $\begin{array}{l}\text { Category of nomen } \\
\text { regarding rhizonymy }\end{array}$ & Spelling of word or standard ending adopted here & $\begin{array}{l}\text { Original spellings or endings } \\
\text { that had to be corrected }\end{array}$ \\
\hline $\mathbf{A}\{23\}$ & - URA [9] $+(5)+|9|$ & $\begin{array}{c}\text {-OURA }(2) \\
- \text { OURES }(1) \\
- \text { URES }(2)\end{array}$ \\
\hline $\mathbf{A}\{1\}$ & VERTEBRATA [1] & - \\
\hline \multicolumn{3}{|l|}{ Total A $\{315\}$} \\
\hline $\mathbf{R}\{63\}$ & -ACEA [4] + (59) & $\begin{array}{c}- \text {-A (1) } \\
- \text {-AE (4) } \\
\text {-DES (1) } \\
- \text { ES (4) } \\
\text {-IA (2) } \\
\text {-IDA (5) } \\
\text {-IDEI (2) } \\
\text {-INA (2) } \\
\text { - OIDEA (32) } \\
\text {-OIDEI (5) } \\
\text { - OIDES (1) }\end{array}$ \\
\hline \multicolumn{3}{|l|}{ Total R $\{63\}$} \\
\hline $\mathbf{C}\{2\}$ & - ACEI (2) & $\begin{array}{l}-\mathbf{A E}(1) \\
-\mathbf{I N I}(1)\end{array}$ \\
\hline $\mathbf{P}\{1\}$ & -ACEAE (1) & $-\mathbf{A E}(1)$ \\
\hline \multicolumn{3}{|l|}{ Total $\mathbf{C}+\mathbf{P}\{3\}$} \\
\hline $\mathbf{U}\{17\}$ & -IFORMIA [6] + (11) & $\begin{array}{l}\text {-AEFORMES (1) } \\
\text {-AEFORMIA (1) } \\
\text {-IFORMES (9) }\end{array}$ \\
\hline $\mathbf{U}\{8\}$ & -OMORPHA [7] + (1) & -AEMORPHA (1) \\
\hline \multicolumn{3}{|l|}{ Total U $\{25\}$} \\
\hline $\mathbf{X}\{4\}$ & -IFORMI [1] + (3) & -IFORMIA (3) \\
\hline $\mathbf{X}\{0\}$ & -OMORPHI $\{0\}$ & - \\
\hline \multicolumn{3}{|l|}{ Total $X\{4\}$} \\
\hline $\mathbf{Q}\{2\}$ & -IFORMES (2) & -IFORMIA (2) \\
\hline $\mathbf{Q}\{8\}$ & -OMORPHES (8) & -OMORPHA (8) \\
\hline \multicolumn{3}{|l|}{ Total Q $\{10\}$} \\
\hline TOTAL $\{420\}$ & & \\
\hline
\end{tabular}

\subsubsection{Sozoidy}

So far, the Principle of Sozoidy is not part of the Code and cannot be applied to the nomenclature of the three nominal-series covered by the Code. However, as the Code does not provide any clue for establishing the validity of class-series nomina, for these nomina any zoologist is entitled to use for this purpose the Criteria or conventions that he/she considers the best, provided he/she justifies this choice (Dubois \& Ohler 2019; Dubois et al. 2019). In this respect, in the present work we follow the DONS Criteria (Dubois 2015c, 2016). These Criteria might also apply later in the other three nominal-series if the LZP (Dubois et al. 2019) were adopted, but we refrained from doing this here. They consist in three points:

\{g1\} Among two or more zygonyms, whenever one qualifies as a sozonym, i.e., has been used since 1900 either universally (symphonym) or significantly whereas none of its zygonyms has been used so for the same taxon or closely related taxa (paneurydiaphonym), it must be given precedence for validity (if not invalid for another reason) over its senior or seniorised zygonym(s). The same applies $\{\mathrm{g} 1 \mathrm{a}\}$ to two or more zygographs if one of them qualifies as a sozograph, i.e., complies with the same Criteria, or $\{\mathrm{g} 1 \mathrm{~b}\}$ to two or more onomatergies if one of them qualifies as a sozairetophory, i.e. results in the validation of an airetophory. 


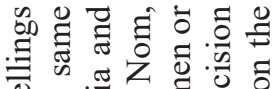
क्षे

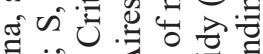

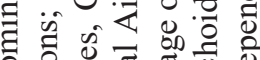

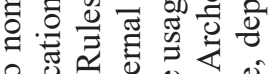

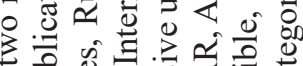

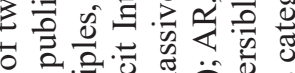

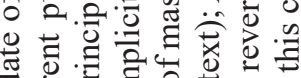

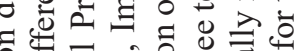
형

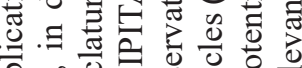

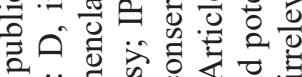

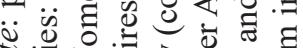

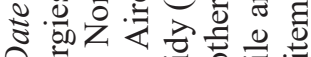

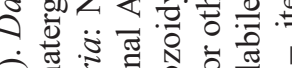

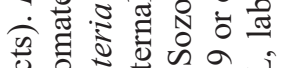
ठั

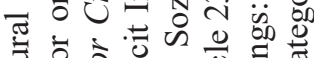
焉

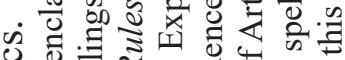

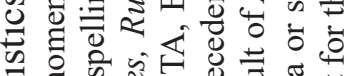
क्ष

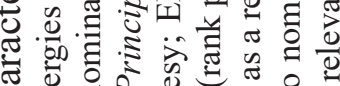

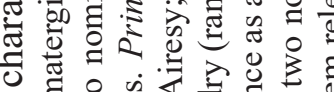

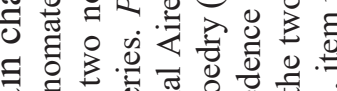

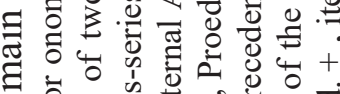

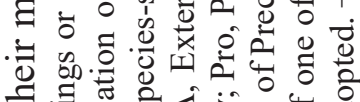

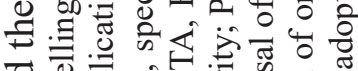
过

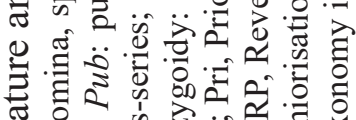

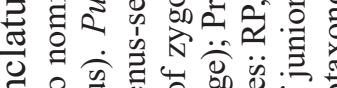

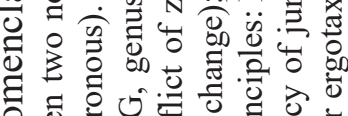

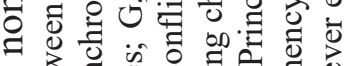

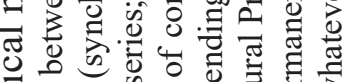

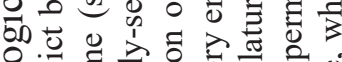

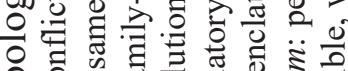

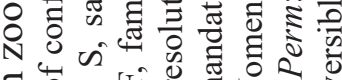

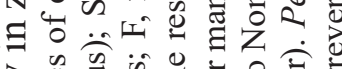

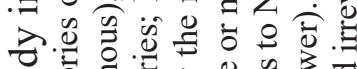
응 㐘

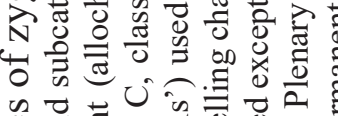

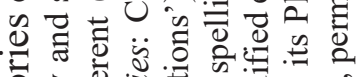

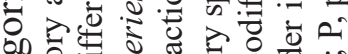

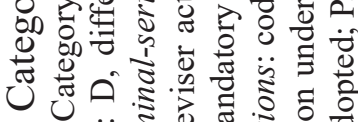

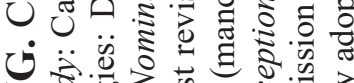

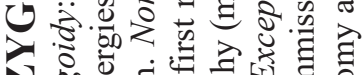

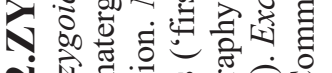
工

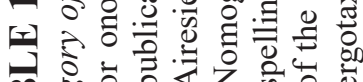

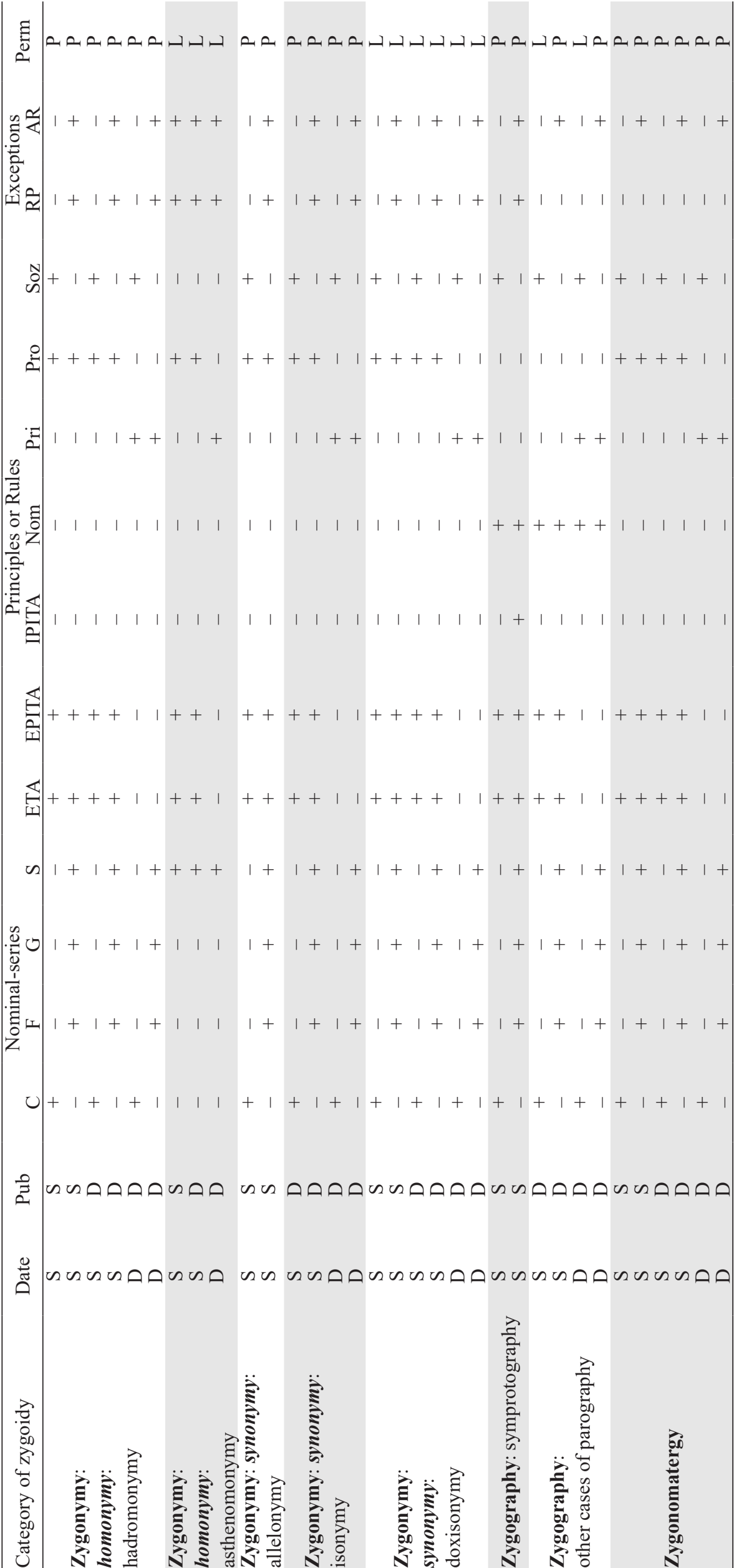


$\{\mathrm{g} 2\}$ Nomina and spellings that are neither sozonyms nor sozographs can be either $\{\mathrm{g} 2 \mathrm{a}\}$ sozodiaphonyms or sozodiaphographs (nomina or spellings that have also been used significantly in the non-systematic literature but alternatively to other nomina which have also had such a large usage) or \{ $\mathrm{g} 2 \mathrm{~b}$ \} distagmonyms or distagmographs (nomina or spellings that have not been used significantly in the non-systematic literature). If no sozonyms or sozographs are available, the normal Principles of precedence of the Code (Priority, Airesy, etc.) apply first among sozodiaphonyms or sozodiaphographs if available, then among distagmonyms or distagmographs.

\{g3\} For the purpose of this Principle, the term significantly is to be understood as qualifying a nomen or a spelling that has been used in the titles of at least 100 scientific works published after 31 December 1899.

\subsubsection{Archoidy (Commission's Plenary Power)}

Although the Principles, Rules and codified exceptions presented above allow to solve the main problems, confusions or conflicts that may arise during the application of the nomenclatural Rules in zootaxonomy, in a few particular situations the normal Rules of the Code do not allow to do this. In such cases, in the interest of nomenclatural universality, unambiguity and stability, the Code allows the International Commission on Zoological Nomenclature to use its Plenary Power to promulgate decisions circumventing some of the Principles and Rules of the Code (except those concerning its own powers and duties). The use of the Plenary Power is treated in the Code just as a 'codified exception', but it should rather be viewed as the implementation of a Principle, the Principle of Archoidy, as suggested by the LZC (Dubois \& Aescht 2019; Dubois et al. 2019).

So far, it does not seem that the Commission has ever used its Plenary Power to invalidate or validate a class-series nomen or an onomatergy concerning a CS nomen-which is consistent with the fact that the Code currently does not include any Rule concerning the validity of such nomina. Until it decides to change explicitly its practices in this respect, the Principle of Archoidy should be considered not to apply to CS nomina. This means that when the Commission imposes the use of some CS nomina in the applications published in the $B Z N$ or in the Opinions and Declarations it issues, this should not be construed as a decision taken under the Plenary Power and having force of law but just as a non-binding editorial decision. This is an important point, as the Commission regularly imposes such uses, as for example those of the invalid (in our opinion) nomina CAUdata (for Urodela) or Testudines (for CheloniI) in such texts or at least in their titles (see respectively Dubois \& Bour $2010 b$ and Dubois \& Ohler 2019).

\subsubsection{Conclusion}

Table T12.ZYG provides a survey of all the categories of conflicts of zygoidy in zoological nomenclature presented above, with their main characteristics and information on the Principles, Rules, Criteria or codified exceptions allowing to solve them.

Dubois (2015c: 91-108), Dubois (2020a) and Dubois \& Frétey (2020b-d, 2021a-d) presented detailed analyses exemplifying the use of the DONS Criteria for the resolution of nine quite complex problems of zygoidy in the class-series concerning the recent amphibians. These analyses are not repeated here but their conclusions were implemented in the present work, along with many others not detailed here.

\subsubsection{Comprehensive lists of supraspecific nomina of LISSAMPHIBIA}

In order to establish the valid nomina of all the suprageneric lissamphibian taxa that our phylogenetic analysis and our taxonomic methodology lead us to recognise, we had to rely on comprehensive lists of all the available nomina of the species-, genus-, family- and class-series, of all the aponyms ever used for them in the literature since 1758, on reliable information on their taxonomic allocation and nomenclatural validity and correctness (according to the Rules of the Code for SS, GS and FS nomina, and to DONS for CS nomina), as well as on lists of nomina that cannot be used as valid for being either anoplonyms, ectonyms or anaptonyms. As such comprehensive lists did not exist, we had to build them, 
and in order to do so we surveyed virtually all the relevant publications since 1758 where new nomina or aponyms could have been published.

Our methodology was as follows. We started from the recent works of Dubois $(1981 b, 1984 b$, 1987a,e, $m, 1992,2004 b, 2005 d$ ), Frost (1985), Frost et al. (2006) and $A S W<2020 a>$ to build preliminary 'skeleton lists' of lissamphibian nomina of the GS, FS and CS, as well as of ectonyms. We then built a list of all the references cited as sources of these nomina and we ordered them chronologically. We then surveyed all these works in the chronological order for new nomina and aponyms. We progressed very slowly, year by year, using as many sources as possible, including the references mentioned in these works, as well as the website Biodiversity Heritage Library $<B H L 2020>$. This lead us to 'rediscover' many references of publications not being 'classically' mentioned in the literature on amphibian systematics. This allowed us not only to rediscover plenty of nomina and spellings that had sunk into partial or complete oblivion, but also to establish the chronological appearance of all the aponyms for each nomen, which turned out be quite different in many cases from that which appeared in previous works (Dubois 1984b; Fouquette \& Dubois 2014; $\angle A S W$ 2020a $>$ ). This very difficult and painstaking work kept us busy for about five years, but now we think these lists are very close to absolute completeness and will not require further verification, except perhaps in a few borderline cases. We would be very grateful to any colleagues who could indicate to us references, nomina and aponyms that we would have missed, and we plan to keep these lists updated in the future and to devote an online database to this information.

These lists are presented in Appendices A5.NGS, A6.NFS, A7.NCS and A8.ECT, where the nomina are presented in alphabetical order. They contain a great deal of nomenclatural information, presented in a standardised abbreviated manner, explained in detail in their legends. For a full benefit of these lists, some time should first be devoted to a careful study of these conventions. Despite these conventions and abbreviations, these lists cover 219 pages in the present work, but an expanded and explicit presentation of the same data would have required several times more pages.

We used these lists to establish the valid nomina, with their correct spellings, of all the taxa recognised as valid in the present work. In the course of this work, we found that no nomina were available for 200 $(14.4 \%)$ of the 1389 extant supraspecific taxa below the rank classis here recognised, and we provided new nomina for them, including 14 new genus-series nomina and one new species-series nomen. In fact, during our work we had planned to establish 18 new generic nomina, but five of these new genera (Firouzophrynus, Leucostethus, Nesorohyla, Rentapia and Zhangixalus) were named by other authors during the six years and half of our work. We did not mention the new nomina that we had coined for these taxa, although most of them were shorter and would have been preferable in our opinion (see Dubois \& Raffaëlli 2009, Dubois 2010e), especially when they may have to be used as nucleogenera of family-series nomina.

\subsection{9. 'Vernacular', 'common' and scientific names}

The following lines were borrowed in part from Dubois \& Ohler (2019: 12-13).

Science is an international endeavour, the aim of which is universality. Every time in history that science has been put to the narrow service of a country, a culture, a language, an ideology, this has entailed declines in knowledge and in the usefulness of science for mankind (Raposo et al. 2017). Biological taxonomy makes sense only as an international approach: if the same organisms were given different nomina in different countries or in different villages, no communication would be possible among biologists worldwide and this would have dramatic consequences on our understanding of biodiversity and our ability to use it, act upon it, manage or protect it, or protect us from it. This aim requires the use of a single language in all countries to designate the taxa recognised by taxonomists. At the beginning of taxonomy, some taxonomists of different countries and cultures tended to use scientific names in their own languages. Latin was chosen as the unique language of nomenclature because it is a 'dead' language that uses the same letters as many 'living' languages and because it was the language of the scholars throughout medieval and Renaissance Europe, where modern zoology began.

Today, keeping Latin as the 'neutral' language of taxonomy is important as it avoids the imposition to this discipline of the linguistic idiosyncrasies of a few countries, which are not shared in other parts of the world. It is also important to keep a single corpus of scientific nomina for biological taxa. Because of the many problems posed by the availability, allocation, synonymy and homonymy of 
nomina, nomenclature is a complex domain and its proper management is time- and effort-consuming. Developing in parallel other nomenclatural corpora, sometimes with their own 'rules', certainly cannot be seen as contributing to the continuity, unity and universality of science, and this should not be supported by taxonomists.

As a matter of fact, beside the 'official' Latin nomenclatures regulated by the International Codes, a tendency has developed in the recent decades to develop 'parallel' biological nomenclatures in different modern languages. Committees have been established, lists published, and more and more biologists now tend to use these non-Latin names to designate the taxa they study, for example in the texts and even in the titles of their publications. Thus, many recent taxonomic checklists and databases provide, besides the Latin nomina (scientific names) of taxa, their 'modern' names, either in a single language (often English) or several. These lists are usually presented as lists of 'common', 'trivial' or even 'vernacular' names of the taxa, which they are not in fact. Such names are of two different kinds.

A few of them do indeed qualify as 'vernacular' names, i.e. names used used in "the language or dialect spoken by the ordinary people in a particular country or region" (Pearsall 2001: 2054). They have often been employed by the persons speaking or writing the language at stake for centuries and before the onset of scientific language. This applies to common terms like 'frog' in English, 'Frosch' in German, 'grenouille' in French or 'rana' in Italian, Portuguese or Spanish. Such names will appear for example in usual dictionaries of the language concerned. But then, most of these genuine vernacular names do not correspond to taxonomic concepts. For example, vernacular designations like 'common frog', 'green frog' or 'brown frog' are used by local people in Europe, North America, South Africa or Australia, where they do not designate the same, or even related, biological taxa. Citing such names as 'synonyms' of scientific names is therefore misleading and confusing.

But the vast majority, if not the totality in many cases, of the names in such lists of 'common' or 'vernacular' names are not 'common' at all, being completely unknown of the 'ordinary people' of the countries concerned. Most of these names are in fact recent alternative scientific names for the Latin nomina now recognised by the Codes, provided by some dedicated 'committees' or simply coined by the authors of the lists. This is in fact similar to the so-called 'vernacular' names used in many early taxonomic publications in various European languages. Despite a frequent misunderstanding of this term, these names were not 'vernacular' in the least, they were genuine scientific names, but in languages others than Latin, derived by literate scientists from scholarly etymologies (usually from Greek or Latin roots). The recent tendency, supported in zoology by the absence of a clear definition of this term in the Glossary of the Code, which applies it in fact indiscriminately to any 'non-Latin' name, ignores these facts, which poses problems regarding the nomenclatural availability of some nomina (see e.g. Dubois 2015c: 26-27 and references therein).

Therefore, the recent flourishing of lists of so-called 'common names' of taxa in modern languages, used in parallel with the scientific names, is certainly not to be welcomed as a progress for taxonomy. Particularly problematic is the recent tendency to designate taxa in the titles of scientific publications by English names or names in other recent languages, not accompanied by the valid Latin nomina of these taxa, and we think this practice should be abandoned by taxonomists and editors.

Real vernacular names for animal 'species' (which in fact in many cases are used by the local people to designate several closely related or similar species, or different sexes or life stages of the same species), or based on the local names of the regions or localities where these animals are known to occur, may find and have found their way to scientific zoological nomenclature, having been borrowed by taxonomists to name genus-series or species-series taxa of animals (e.g., in amphibians, respectively Paa Dubois, 1975 or Beduka nov., and Aubria masako Ohler \& Kazadi, 1990 or Beduka amboli nov.). But then these names leave the world of vernacular names designating 'kinds of animals' to enter that of scientific names designating taxa, i.e. scientific concepts. Then, they must comply with all the Rules of availability, allocation, validity and correction of the Code. Recently, a trend has appeared in some borderline 'scientific' literature, to mix both worlds, and to suggest abandoning some available and valid zoological nomina for so-called 'ethical' reasons (e.g., Shiffman 2019) and even replacing them by vernacular names given to 'kinds of animals' (covering often several scientific taxa) in local languages (Gilman \& Wright 2020). Such proposals do not need serious discussion, as they just express the complete ignorance of their authors, and of the journals which accepted to publish them, of the nature of scientific taxonomy and nomenclature, of the concepts of taxon, nomen, availability, priority, validity and stability. In this respect, a 'non-scientific' journal like The New York Times recently showed more understanding of these questions (Roach 2020) than 'scientific' journals like Scientific American 
or Nature. If some authors in the future decided to use such vernacular names as valid in zoological nomenclature to replace available nomina currently considered valid, these non-scientific names would simply have to be rejected as unavailable for being ectonyms.

Unambiguous naming for all animal taxa is the main issue of zoological nomenclature, as regulated by the Code, so multiple names for taxa cannot be promoted within the system. Nevertheless it is important to acknowledge that in our world exist various systems of naming natural kinds depending on the aims, on the places and on the usage. But this has to be considered elsewhere.

\subsection{A new methodology for taxonomic and nomenclatural transcription of a tree into a cladonomy}

\subsubsection{Introduction}

Some have argued that a 'phylogenetic taxonomy' requires a 'phylogenetic nomenclature' (Queiroz \& Gauthier 1990, 1994), such as the Phylocode (Cantino \& Queiroz 2020), as the LSNS Rules would not allow to express nomenclaturally the phylogenetic relationships between taxa, in particular because of their use of arbitrary nomenclatural ranks. As we have seen, we disagree with this point of view, which is based on a confusion between taxonomy and nomenclature, and particularly between taxonomic categories and nomenclatural ranks. Because it uses "phylogenetic definitions of taxon names" for the allocation of nomina to taxa, the Phylocode is an intensional system which is theory-bound regarding taxonomy. In contrast, the Code, which uses onomatophores for this allocation, is an ostensional and theory-free system. This has allowed it to adapt to the changes in taxonomic paradigms that have been frequent in the history of biology, and it should remain able to do so as no one knows today what will be the taxonomic paradigms of the future. But we agree that the LSNS Rules are not enough by themselves to ensure that nomenclature will reflect the cladistic hypotheses on which the classification is based. Several conditions should be respected for the LSNS being able to do so. The first one is taxonomic: $\{\mathrm{h} 1\}$ that only groups hypothesised to be monophyletic/holophyletic should be recognised as taxa. Two other ones are taxonominal: $\{\mathrm{h} 2\}$ that sister-taxa (according to the tree adopted as valid) be always afforded the same nomenclatural rank (i.e., be parordinate); and $\{\mathrm{h} 3\}$ that nominal-series strictly follow each other when going upwards or downwards in the hierarchy, without overlap between them, and that ranks do the same within nominal-series.

The first condition concerns the recognition, contents and diagnosis of taxa, i.e., formal groups of organisms considered to be holophyletic (i.e., including one ancestor species and all its descendants). The definition and composition of taxa is fully independent both from their nomina and from the ranks given to the latter.

The second condition concerns the ranks afforded to these taxa. These ranks are relative, not absolute as believed by some. This means that they are not part of the definition of taxa or of nomina. As we have seen, nomenclatural ranks just provide information on the structure of the tree, i.e., on the hypothesised cladistic relationships between the taxa, not on their characters, 'degree of divergence' or age, as would taxonomic categories, which have long been confused with them. In order to account for changes in the topology of the tree, the same taxon may freely shift from one rank to another within a nominalseries without any change in its definition and content. This has indeed occurred very frequently in 'real taxonomies' throughout the history of biology.

Before going further however, it is important to remember that taxonomy and nomenclature are not meant at being useful only to specialised phylogeneticists and taxonomists, but also to all users of scientific nomina, including other biologists and non-biologists. This means that, as far as possible, 'verywell-known' taxa and nomina should remain in use, at least for taxa that do not contradict the requirement for holophyly of taxa, which requires in some cases to use them for taxa different (less inclusive) than those for which they had been originally proposed, as it is the case for the nomen AMPHIBIA. There should exist both some robustness and some lability or adaptability of nomina relative to the content of taxa, as long as nomina remain anchored through a stable objective reference, their onomatophores, which do not refer to verbal definitions of the taxa for which they were proposed or are now used, but simply to inclusion of one or a few specimens in the latter.

As we have seen, in order to act as an efficient information storage and retrieval system, the nomenclatural system should rely first on a few 'mandatory' or 'compulsory' ranks, namely kingdom, 
phylum, class, order, family, genus and species (Wiley 1979, 1981; Dubois 2006a, 2007a, Kuntner \& Agnarsson 2006): all organisms on earth should be referable to taxa attributed to these seven ranks. Nomenclatural ranks do not carry biological, historical or other information, and they are not, and cannot be made, 'equivalent' by any Criterion across the whole animal kingdom. In practice, taxonomic assignment of these seven ranks therefore relies only upon 'tradition' and 'consensus' among specialists of the main zoological groups: an order of mammals is by no Criterion equivalent to an order of insects, molluscs, nematodes or ciliates.

Beside these seven mandatory ranks, all other nomenclatural ranks are optional. Their use should not be based on trying to carry some information on the taxa themselves, their characters, their 'degree of divergence' or their age, but only to reflect the structure of the tree adopted as the basis for the classification of any given group, i.e. the topology of the succession of their well-supported nodes. Cladistic trees may appear as 'well-resolved', at least in part, when they include 'well-supported' dichotomies, or 'poorly resolved', at least in their portions that include polytomies.

If taxonomy and nomenclature are to act as a device carrying information on the evolutionary history of a group, not in terms of adaptations, convergences or innovations in characters, but in terms of successions of dichotomies or cladogeneses (resulting in separate 'clades' between which no gene flow occurs any more), these dichotomies should be reflected in the classification and nomenclature. This can be done in a non-ambiguous manner by giving the same nomenclatural rank to the two taxa resulting from each dichotomy (or, provisionally, by the several taxa resulting from an unresolved polytomy). Not doing so would reflect a 'gradist' or 'gradonomic' conception of taxonomy, in which some taxa resulting from dichotomies would be more 'important' than others by some criterion, and therefore would merit to be given a 'higher rank', than their sister-taxa.

This is indeed what is done in all 'pseudoranked' ergotaxonomies and nomenclatures, which are in fact the common standard in the current taxonomic literature (see Dubois 2007a, 2008f) although few authors would provide theoretical justification for their use. For example, Vieites et al. (2007) recognised a salamander subfamily HEMIDACTYLINAE with four immediately subordinate taxa (corresponding to an unresolved polytomy): genus Batrachoseps, 'supergenus' Bolitoglossa (with twelve genera), genus Hemidactylium and tribe SPELERPINI (with four genera). The genera recognised by this ergotaxonomy are therefore not all sister-taxa and this nomenclature does not carry any message concerning their cladistic relationships. To transform this pseudoranked ergotaxonomy into a genuine ranked ergotaxonomy, these four taxa should be afforded the same rank, e.g. tribe, two tribes including a single genus and the other two being composed of several genera (Dubois 2008f). Similarly, Frost et al.'s (2006) classification of the AмPHIBIA recognised pairs of sister-taxa such as 'taxon'/familia or familia/superfamilia, so that the nomenclatural hierarchy in this work is devoid of cladistic meaning.

In contrast, if all well-supported nodes are recognised as taxa, if all sister-taxa are always given the same nomenclatural rank, and if successive nodes are given different ranks, the latter carry cladistic information. This was argued for by Hennig $(1950,1966,1974)$ and many of his successors. The important point in ranking is not the absolute rank given to any taxon, which has no meaning by itself and is fully labile, but the relative ranks of the different taxa, and in particular the fact that sister-taxa in a phylogenetic taxonomy have the same rank, as pointed out long ago, for example by Raikow (1985: 195): "In any Linnaean classification, the taxa are arranged in a nested hierarchy of progressively more inclusive ranks or categories. In cladistic classification, the pattern of cladistic relationships, usually taken to hypothesise genealogy, is the basis for ranking. The clades are recognised as taxa and their rank is determined by their position. More inclusive groups are ranked at higher category levels than less inclusive groups. (...) This is totally unambiguous; the classification exactly expresses the genealogy."

This system allows a fully bijective or isomorphic relationship between the tree and the ergotaxonomy: the latter derives directly from the tree, and reciprocally it allows to reconstruct the tree automatically in all its details. But, for this to be possible, this requires to use as many different ranks as successive dichotomies (or polytomies) in the tree. A particular problem is caused here by the fact that the current Code limits arbitrarily the number of ranks allowed in the genus-series (with only two ranks, genus and subgenus, but for example no rank supergenus) and in the species-series (with only four ranks, 'aggregate of species', species, 'aggregate of subspecies' and subspecies). Hopefully these arbitrary limitations will later finally be cancelled (see Dubois 2006a, 2011a), but in the meanwhile the detailed nomenclatural expression of cladistic trees through a hierarchy of ranks will have to rely mostly on nomina of the family- and class-series. In the former, the number of ranks is indefinite below the rank superfamily, but, strangely enough, additional ranks above superfamily are forbidden by the Code. In 
the latter, the number of ranks is fully unlimited, which allows as many ranks as needed to express in detail the cladistic relationships even in trees displaying very high numbers of nodes at successive levels.

Few cladistic trees are 'balanced', with equal or subequal numbers of levels in both branches originating from a single basal dichotomy. The usual situation is to have two widely unbalanced branches, one being much richer in terminal taxa (species or subspecies) and in intermediate nodes than the other one. In such cases, the number of ranks in each branch will be widely different and these ranks will not be equivalent between branches, thus carrying no phylogenetic signal at this level, but they will carry such a signal within branches, whenever the same rank is given to two sister-branches. This raises no theoretical problem as soon as it is acknowledged that ranks are meaningless by themselves and only useful to express hypothesised cladistic relationships between taxa.

This system thus makes use of two different 'kinds' of ranks, shown in Figure F1.MOR. Mandatory ranks (kingdom, phylum, class, order, family, genus, species) have a double function: $\{11\}$ that of allowing information storage and retrieval in bibliographic (such as the Zoological Record $<$ ZR 1864$2020>$ ) and taxonomic (such as the Integrated Taxonomic Information Service $<$ ITIS 2020>, or the Universal Biological Indexer and Organiser $<u$ Bio 2020 $>$ ) databases; $\{$ i2 $\}$ that of providing information on the structure of the tree, i.e., on the cladistic relationships between taxa. This second function is the only one performed by optional ranks. The latter are potentially unlimited in number. However, in real taxonomies, which never cover the whole animal kingdom in all details, but are either very general (limited to higher rank taxa) or quite specialised (limited to rather lowly ranked taxa, as in the present work), they will rarely exceed a few dozens or less. Optional ranks are therefore needed only to express sister-taxa relationships, so they must be used only for taxa that include several subtaxa. Therefore, in unbalanced taxonomies, less ranks will be used in taxa-poor branches than in taxa-rich ones. Optional ranks do not allow useless taxonomic redundancy (inclusion in one taxon of a single subtaxon of next lower rank in the same nominal-series having the same content/extension and characters/intension). In contrast, in the case of mandatory ranks, taxonomic redundancy is imposed by their function $\{\mathrm{i} 1\}$. For example, although the frog family RHINOPHRYNIDAE contains a single extant genus Rhinophrynus with a single species Rhinophrynus dorsalis, so that these three taxa are redundant in terms of taxonomy and phylogeny, they are not so for the purpose of information retrieval, and all three should be recognised as distinct taxa. But there is no need, and it would be a mistake, to recognise a subfamily RHINOPHRYNINAE, a tribe RHINOPHRYNINI, a subgenus Rhinophrynus (Rhinophrynus) or a subspecies Rhinophrynus dorsalis dorsalis, as these taxa would have no sister-taxa.

So far, in zootaxonomy, most authors, even without clear formulation of the concept of 'mandatory rank', have in practice acted in agreement with the recommendation above to attribute well-known taxa to these seven ranks. But no explicit and detailed methodology has ever, to the best of our knowledge, been proposed to serve as a guideline for the choice of the taxa to which these seven ranks should be attributed. We here propose such a methodology. As reminded above, taxonomy should be at service of numerous users of various kinds and should not comply only with the aims and preferences of specialised taxonomists and phylogeneticists. Therefore, this methodology is meant at standardising the use of ranks in zoological nomenclature, not at 'revolutionising' it. In particular, it should allow to maintain as much as possible the long traditions regarding the nomina used for the best known animal taxa. Because of the widely different traditions used in different branches of zootaxonomy, we insist that, for the time being, this methodology should be applied separately in the different major animal groups as traditionally recognised - except when these have been shown to be polyphyletic or paraphyletic, such as in the case of the 'REPTILIA' or 'PISCES'. A good source for establishing these traditions is provided by the numerous volumes of the Zoological Record ( $<$ ZR 1864-2020 $>$ ), a yearly updated database that exists since 1864 and which is now available online.

As we have seen, at this stage we excluded terminal taxa (species and subspecies) as well as genera and subgenera from our recommendations, because today several distinct species/subspecies and genus/ subgenus concepts are used by different taxonomists or groups of taxonomists, in part following different taxonomic traditions in different animal taxonomic groups, e.g., with or without frequent use of the ranks subgenus and subspecies, or in groups including organisms with peculiar reproductive modes like parthenogenesis, gynogenesis and 'hybridogenesis' (see Dubois 2011b). At this stage we recommend to follow such traditions in the groups at stake, or to propose changes but based on explicit concepts and methodology.

Regarding extant amphibians, our recommendations concern in the first place $\{\mathrm{j} 1\}$ the two mandatory 
suprageneric ranks family and order, and $\{j 2\}$ their nomina. As will be shown below, fixing the position in TREE of the two ranks family and order, which usually is fully arbitrary and does not correspond to any biological or historical Criterion, will be a starting point that will allow to determine the assignment of all other ranks, in any given partial taxonomic hierarchy (restricted to a defined higher taxon), to the holophyletic taxa recognised.

\subsubsection{Allocation, assignment, attribution and allotment of nomina}

In zoological taxonomy, nomina are allocated to taxa, assigned to nominal-series and attributed to nomenclatural ranks, and taxa are referred to taxonominal ranks. These four kinds of attachment or connexion are distinct and independent. Some are permanent, others are labile. So far, in the literature no clear distinction has been made between them, and no specific term has been fixed for any of them: in most cases, these processes are not distinguished, and are designated by general terms like 'allocation' or 'attribution'. In order to avoid confusions and misunderstandings, we propose here a formal terminology for these four distinct concepts.

$\{\mathrm{k} 1\}$ Following Dubois (2005b), we propose to use the verb to allocate and the substantive allocation for the process of connexion between a nomen and a taxon. This taxonomic allocation is effected through two tools specific to zoological nomenclature, the onomatophore and the onomatostasis. The onomatophore is usually designated in the original publication where the nomen is established, but in some cases in a subsequent airesy effected by a taxonomist of by the Commission under its Plenary Power. Once designated, it is fixed and permanent, not liable to change. In contrast, the onomatostasis is usually (in the SS, GS, FS and for doxisonyms in the CS) labile, depending on the structure of the taxonomy (see Dubois 2020a), but it is fixed and permanent in the case of sozonymorphs in the CS under DONS Criteria.

$\{\mathrm{k} 2\}$ Following Dubois $(2015 \mathrm{c})$, we propose to use the verb to assign and the substantive assignment for the process of attachment of a nomen to one of the four nominal-series (SS, GS, FS and CS). This nominal-series assignment must be effected in the original publication where the nomen is established. Failing to do renders the new nomen nomenclaturally unavailable (Tables T4.AVN and T6.ASN). Once done, this assignment is permanent and non-modifiable.

$\{\mathrm{k} 3\}$ Zoological nomina are thus permanently attached to nominal-series but, contrary to the situation in botanical nomenclature, not in the least to ranks. Owing to the Principle of Coordination, once established at any rank in a nominal-series, a nomen is deemed to have been established simultaneously, with the same auctor, date and onomatophore, at any other rank in the same nominal-series. In a given ergotaxonomy however, a given nomen will be used as valid only at certain ranks, depending on the taxonomic arrangement. For the process leading to decisions in this domain, we hereby propose to use the verb to attribute and the substantive attribution. The rank attribution of a nomen is highly labile, being liable to change whenever the phylogenetic hypotheses and ergotaxonomic arrangements change. Furthermore, in any given ergotaxonomy, in the species-, genus- and family-series, the same nomen may be used as valid at several distinct ranks that are immediately super/subordinate to each other.

$\{\mathrm{k} 4\}$ In most cases, when describing a taxon, an author refers it to a nominal-series and a rank within this nominal-series. Subsequent works may lead to modify the rank of this nomen, which poses no nomenclatural problem as long as one remains within the same nominal-series, as the nomen will keep its auctor, date and onomatophore. However, in certain cases, and particularly when suprageneric taxa are concerned, the choice between a FS rank (e.g. superfamily) and a CS rank (e.g. infraorder), which as we have seen does not rely on taxonomic concepts but largely on tradition and consensus, may be challenged in subsequent works. But then transfer of a taxon from the FS to the CS or vice versa will require a change of nomen for the taxon, even if its intension and extension are not modified, because nomina in different nominal-series obey different nomenclatural rules and nomina can never be transferred from one nominal-series to another one. This change of nomen may be a source of ambiguities and confusions. In order to limit the occurrence and the negative consequences of such situations, it is useful to dispose of operational Criteria allowing to choose the nominal-series to which a new nomen will be assigned. We propose such Criteria below. For this process, which is distinct from the three processes described above, we hereby propose to use the verb to allot and the substantive allotment. More details on nominal-series allotment are given below. 


\subsubsection{The two basic mandatory ranks between genus and class: family and order}

In amphibians, the introduction of explicit phylogenetic analysis as a basis for taxonomic (partly cladonomic and partly gradonomic) classification has resulted in a tendency for higher-ranked taxa (e.g., families) to be moved toward the tips (i.e., making families less inclusive). This movement was justified by the fact that the number of traditional higher taxa in extant amphibians was clearly too low to be able to express conveniently the complexity of the relationships within the group, which had been underestimated in the past. However, we think this movement should not continue forever, and that drastic changes in the higher taxonomy of the group should now be restricted to genuine major discoveries or changes in the structure of the tree, but not occur as a simple result of mere increase in the number of known species and genera which do not change the basic pattern of relationships between taxa.

In amphibians, regarding the mandatory rank order, the tradition is very entrenched in the literature and it should in our opinion not be challenged. In innumerable works, the extant amphibians are referred to three higher taxa that are usually given the rank order: the frogs, the salamanders and the caecilians. They correspond exactly to the three main holophyletic groups disclosed by our cladistic analysis (see below), where they form an unresolved trichotomy. The valid nomina of these three orders, following the DONS methodology (Dubois 2015c, 2020a; Dubois \& Ohler 2019; Dubois \& Frétey, 2020b-c, 2021b), are respectively ANURA Duméril, 1805, Urodela Duméril, 1805 and GyMnophiona Rafinesque, $1814^{1}$.

Stating that the ranks order and family are mandatory means that every animal species must be referred to a taxon at each of these two ranks. But these two ranks are the only ones that are mandatory below class and above genus. All the other ranks are facultative, and their implementation in any branch of TREE will depend on the structure of this branch, i.e. on the number of well-supported nodes between the rank family and the rank order.

The situation is much more complex concerning the rank 'family'. Here, 'tradition' only is not enough, as new families are constantly added in the extant amphibians as in most other zoological groups: there were 8 families in Duméril \& Bibron's $(1841,1854)$ classification of this group, 19 in Boulenger's (1882a-b), 20 in Noble's (1931), 42 in Duellman \& Trueb's (1985) and 54 in Frost et al.'s (2006). This process might continue indefinitely, with a permanent increase in the number of families which does not always correspond to an increase of knowledge but sometimes only to a particular focus given by some recent authors to 'their' groups, at the expense of less studied groups. Some Criteria are needed to limit this constant increase in the number of families and unjustified upgrade of lower-ranked taxa to the rank family. This led us to devise a completely new methodology for fixing the level of the rank 'family' in a zoological classification. We present below in detail the rationale and the Criteria of this methodology, the 'Ten Criteria Procedure' [TCP] which we used as the basis for our attribution of all amphibian suprageneric taxa, not only to this rank but also, by way of consequence, to all other suprageneric and infraordinal ranks. As we will show, having established the nomen to which the rank family is attributed, the entire suprageneric classification of the group at stake (our CLAD) is automatically generated by our Criteria.

Most current taxonomists highly praise 'nomenclatural stability', which is even stated in the Code as one of its main purposes. However, this concept is a complex one (Dubois 2005b) which is usually poorly defined, including in the Code (Dubois 2010c). In the absence of scientific Criteria to define nomenclatural ranks, we agree that nomenclatural stability in the main ranks is a laudable goal, but we insist that $\{11\}$ it should be based on precise Criteria and $\{12\}$ it cannot concern all taxa and ranks but only some of them, as otherwise taxonomy and nomenclature would have to be frozen forever and could not evolve with new concepts and new data as they have always done and should continue to do (Dubois 1998). Therefore, whereas we agree that the taxonomy of amphibians should always include 'well-known' nomina like those of the order ANURA, of the family RANIDAE and of the genus Rana, it is important to realise that this stability of nomina does not always imply a stability of the corresponding taxa: while the taxon ANURA has the same meaning today as it had in the work of Duméril (1805), both taxa RANIDAE and Rana now have intensions and extensions very different from that which they had in the original works of respectively Batsch (1796) and Linnaeus (1758a). Here we do not use the concept of 'nomenclatural stability' in the imprecise sense it usually has in the literature but in a technical, welldefined and operational meaning, described in detail below.

1. For the correct authorship of the paper where this nomen and others appeared for the first time, see Dubois \& Frétey (2021b). 


\subsubsection{Nominal-series saturation}

As we have seen, the Code restricts the number of ranks that can be used in the three nominal-series it governs: they are two in the GS (genus and subgenus), four in the SS (species, subspecies, 'aggregate of species' and 'aggregate of subspecies') and they are limited in the upward direction in the FS by the rank superfamily. In the first two three nominal-series, using additional ranks in not Code-compliant and should be corrected whenever found in a publication.

At genus level, some recent authors used nomenclatures which are not Code-compliant, such as implementing a rank 'supergenus' above genus (e.g. Vieites et al. 2007 in the HEMIDACTYLIINAE) or several ranks between genus and species (e.g. Hillis \& Wilcox 2005). In order to transfer such nomenclatures to Code-compliant practices, in the first case the rank supergenus should be replaced by a low family-series rank (such as subtribe or below): in the present case for example we used eight ranks between subfamily and genus in the HEMIDACTYLIINAE. Concerning the second situation, the Code allows to use a single additional rank between subgenus and species, that of 'aggregate of species' (or better supraspecies, see Dubois \& Raffaëlli 2009), but no other rank is to be used in order to remain in a Code-compliant nomenclature.

In the family-series, all ranks above superfamily that may or have been used are not Code-compliant and should be abandoned, but they may be replaced by low ranks of the class-series, as this nominalseries, not being regulated by the Code, has no limitation in the number and names of ranks

We call nominal-series saturation the situation in which all the ranks allowed by the Code in a given nominal-series have been used in a formal ergotaxonomy and nomenclature. This saturation is soon reached, after two ranks, in the GS, and after four ranks in the SS. If more ranks are used in these two NS, they are not acceptable under the Code and their nomina are not submitted to the Rules of homonymy, synonymy and priority of the Code.

In the FS, the situation is special as the Code provides a list of five 'main' ranks but states that "any other rank bekow superfamily and above genus that may be desired" is acceptable (Article 35.1). There is therefore no limitation in the addition of ranks in the downward direction below family. In the present work, we make use of 10 ranks below family and this is Code-compliant. In the upward direction above family, the Code just fixes an upper limit (superfamily) but does not state that no ranks are allowed between family and superfamily. Saturation exists there only in the upward hierarchy above family when all the ranks between family and superfamily accepted as valid in a given work have been used. In the present work, the maximum number we used, in some groups only, is three (apofamily, epifamily and superfamily). This is also Code-compliant, but the use of ranks above superfamily (such as hyperfamily) would not be so. In the group of extant amphibians where the structure of our TREE imposes more than three ranks above family and below order, starting with the fourth rank above family the transition to the CS must be effected.

\subsubsection{Recognition of suprageneric taxa and their rank attribution: the 'Ten Criteria Procedure' [TCP]}

Our procedure relies on ten Criteria, which may be implemented in any suprageneric zoological cladonomy for the attribution of a suprageneric nomen to the rank family. This will allow to reflect bijectively a cladistic tree and allow back and forth equivalence between them. The ten Criteria of the $[\mathrm{TCP}]$ rely either only on nomenclatural Rules $\{\mathrm{N}\}$ or on both taxonomic and nomenclatural Criteria $\{\mathrm{TN}\}$. The following three-letter abbreviations are used in the text below to designate these ten Criteria, and one-letter abbreviations between square brackets are used in Appendix A9.CLAD-1 for five of them:

$[\mathrm{CHC}]$ Consistent Hierarchy Criterion. $\{\mathrm{N}\}$.

$[\mathrm{CNC}]$ Consistent Naming Criterion. $\{\mathrm{TN}\}$.

$[\mathrm{CPC}]$ or $[\mathrm{P}]$ Conflict of Precedence Criterion. $\{\mathrm{N}\}$.

$[\mathrm{FPC}]$ Family-Series Precedence Criterion. $\{\mathrm{N}\}$.

$[\mathrm{MRC}]$ or $[\mathrm{M}]$ Mandatory Rank Criterion. $\{\mathrm{N}\}$.

[NPC] Nomenclatural Precedence Criterion. $\{\mathrm{N}\}$.

$[N R C]$ or $[N]$ Non-Redundancy Criterion. $\{N\}$.

$[N T C]$ or $[\mathrm{T}]$ Nomenclatural Thrift Criterion. $\{\mathrm{N}\}$. 
[STC] Sister-Taxa Criterion. $\{\mathrm{TN}\}$.

[UQC] or [Q] Upper Quartile Criterion. \{TN\}.

The Criterion [CNC] provides necessary conditions for the recognition of suprageneric taxa, the Criterion [NTC] allows to settle potential conflicts between the allotment of a taxon to the FS or the CS, and the other eight provide general Criteria for the attribution of ranks to taxa.

Below we describe in detail these Criteria, and we provide some information on our cladonomy of extant amphibians which we built using these Criteria and which includes 69 families (55 of frogs, 9 of salamanders and 5 of caecilians). A full understanding of what follows requires to refer to our tree and our cladonomy, shown in Appendices A2.TREE-1 and A9.CLAD-1, which are presented and discussed in more details in our section Results below.

Note that the use of the methodology described below can be used consistently only on the basis of a robust cladistic tree, such as TREE in the present work, which is based on a thorough analysis of numerous molecular data. Today it cannot be used for all-fossil taxa, even when these have been submitted to careful morphological and anatomical analyses, as many of them rely largely on incomplete specimens and therefore result in many 'missing data' in matrices (see e.g.: Ruta et al. 2003; Ruta \& Coates 2007; Sigurdsen \& Green 2011; Marjanović \& Laurin 2015, 2019). In the present work, we adopted uncritically the all-fossil families of lissamphibians recognised by paleontologists, which simply correspond to tradition and consensus. The status and rank of these data were not challenged here and they were not submitted to the methodology described in detail below for extant taxa.

\subsubsection{General Criteria}

\subsection{Criterion [CNC]: the 'Consistent Naming Criterion'}

\subsection{Statement of Criterion}

"In any given cladonomy, all sister-branches resulting from nodes having a support value equal to or higher than a given a priori threshold must be recognised as distinct taxa, whereas no branch resulting from nodes having a support below this threshold should be so. However, for two sister-branches to be taxonomically recognised, one of them at least must include more than one supraspecific subtaxon (i.e., of rank genus or above)."

\subsection{Rationale and use of this Criterion}

As explained above, our aim here is to propose for the first time a complete 'phylogenetic taxonomy' or more exactly cladonomy of the extant amphibians above the rank genus, here designated as CLAD, being an exact and reversible transcription of the cladogenetic tree, here designated as TREE, based on nucleic acid sequencing data, that we here adopt as (provisionally) valid. For this to be possible and reliable, all suprageneric branches resulting from nodes having a SHL-aLRT support value of $90 \%$ or more must be recognised as distinct taxa, whereas no branch resulting from nodes having a support below this threshold, even if 'close' to it (e.g., $89 \%$ ), should be so. If some such nodes were purely subjectively retained, despite being unsupported, for corresponding to 'well-known' or 'important' taxa, or on the contrary nodes meeting this Criterion rejected for being 'less known' or 'less meaningful' than others, or simply for the purpose of reducing the number of ranks in our hierarchy in order to follow 'tradition', the resulting taxonomy would not entertain a bijective relationship with our TREE any more-i.e., it would not allow alone to reconstruct TREE.

Of course, in subsequent works, it will not be necessary to mention always all these ranks and taxa and it may be sufficient, according to the purpose of the publication, to mention a few 'traditionally important' ranks (such as order, superfamily, family and subfamily), but the taxa attributed to these ranks will not be given haphazardly but will correspond to a well-defined taxonominal paradigm. And of course, when dealing with the complete classification, all these ranks and taxa should be mentioned.

This means that even in very unbalanced situations, e.g., when a single species comes out as the sister-taxon of a large taxon including hundreds of species, both branches of the dichotomy should nevertheless be named and be attributed to the same nomenclatural rank. By itself, the resulting 
'unbalanced taxonomy' will be very informative regarding the pattern and processes of evolution of the group at stake, as it will point to very different rates of diversification and speciation, or of extinction, in these two branches, thus allowing to ask meaningful evolutionary questions, whereas this would be 'masked' in a taxonomy that would care mostly for equilibrated numbers in related taxa in the futile and hopeless search for quantitatively 'equivalent' taxa at the same rank.

The only case where well-supported nodes in TREE should not be used as evidence for distinct taxa is when the results are 'strange' enough, on the basis of robust previously published information, to suggest the existence of a problem regarding the reliability of either the identification of the voucher or the molecular sequence. Such problems should be solved if possible, and if impossible these specimens and sequences should be removed from TREE and from CLAD until more is known, but this decision should be made clear to all readers. As a matter of fact, so far we did not identify any such case in the data we used, or we were able to correct them.

Therefore, in the present work, this Criterion was followed strictly for all suprageneric nomina/taxa, whatever its consequences regarding 'usage' and 'consensus'.

Stating that a taxon must be recognised and named on the basis of TREE means that this taxon will have to be taken into account when it comes to establish the ranks and nomina of taxa but does not tell us which rank and nomen it should bear. This information will be derived from the other nine Criteria below.

\subsection{Criterion [NPC]: the 'Nomenclatural Precedence Criterion'}

\subsection{Statement of Criterion}

"In zoological nomenclature, precedence between family-series nomina is established through the same Rules as for species-series and genus-series nomina, i.e., publication priority, airesy, proedry, sozoidy or archoidy. In the class-series, according to the DONS Criteria, it is established through sozonymy, or through priority, airesy or proedry among sozodiaphonyms, or through priority, airesy or proedry among distagmonyms."

\subsection{Rationale and use of Criterion}

In the family-series, the mention of this Criterion here can be considered superfluous as it is just part of the standard Rules of the Code. A few decades ago, a number of taxonomists simply ignored that the Principle of Priority, sometimes tempered by other Principles or Rules, did indeed apply to family-series nomina, but in this respect the situation has improved in the recent decades.

However, in the class-series, as the Code does not provide Criteria of validity, a chaotic situation currently prevails in the literature. Various Criteria, or more often inconsistent 'pseudo-Criteria' (see Dubois \& Ohler 2019), have been used by different authors, who generally did not care for explaining the rationale for their choices. Few do so, and propose consistent Principles and Rules for this purpose, under the form of explicit proposals of 'nomenclatural systems' (e.g. Dubois 2006a, 2015c; Kluge 2010). As the Code does not take a stand on these matters, none of these explicit nomenclatural systems, or inexplicit 'pseudo-systems' (see Dubois 2015c) can be stated to be more 'Code-compliant' than others. The only requirement that can be expected to be met from authors in publications dealing with these matters is to state in full words which Principles, Rules, Criteria or at least guidelines they follow in this respect (Dubois \& Ohler 2019). Here we clearly state that, for the nomenclature of all taxa of the classseries (i.e., above the rank superfamily), we followed the Criteria of the Duplostentional Nomenclatural System (DONS) as explained by Dubois (2015c, 2016, 2020a).

In the present work, these Rules (for FS nomina) and Criteria (for CS nomina) were followed strictly, whatever their consequences regarding 'usage' and 'consensus'. 


\subsection{Criterion $[\mathrm{CHC}]$ : the 'Consistent Hierarchy Criterion'}

\subsection{Statement of Criterion}

"In any given cladonomy, in one branch at least resulting from a node, subordinate and superordinate taxa should be attributed to immediately successive nomenclatural ranks in the taxonominal hierarchy, but some of these ranks may be lacking in its sister-branch(es)."

\subsection{Rationale and use of this Criterion}

As we will see, the Criteria [UQC] and [STC] below allow to fix the position of many taxa in the taxonominal hierarchy as belonging to the rank family. This will then allow to fix the ranks of most other suprageneric taxa, simply by following the hierarchical succession of ranks. This would seem to be a straightforward operation but it is not so, because some ranks are optional and are not always used in ergotaxonomies. In this respect, the situation is different below and above the rank family.

$\{\mathrm{m} 1\}$ Below the rank family and above the rank genus, as the Code currently forbids to use any rank above genus in the GS, all ranks belong in the FS and the attribution of ranks to taxa is simply automatic, by just descending progressively the hierarchy of ranks. But when relatively few ranks are needed, it is even possible to ignore the potential intermediate ranks between subfamily and tribe in order to keep an isolated 'block' for the ranks for which the Code imposes fixed endings (family, subfamily, tribe and subtribe). It was the case in the present work, where we needed only ten ranks to account for all our hierarchy below family. If in another zoological group more ranks are needed, these potential intermediate ranks (e.g., infrafamily or supertribe) may be used and the downward progression may be indefinite. Dubois (2006a) proposed a system with 38 distinct ranks between family and genus, and the Code does not forbid to have even more. Therefore, the downward hierarchical succession of rank depends on the context, i.e. of the ranks taken in consideration in a given work.

$\{\mathrm{m} 2\}$ The situation is different above the rank family, because here two distinct nominal-series must be distinguished, the FS and the CS. As we have seen, in the FS, the Code imposes a limitation in the number of ranks above family, as no rank is allowed above superfamily. In the present work, we indulged ourselves in implementing when necessary two optional intermediate ranks between family and superfamily, namely first epifamily below superfamily if just one such rank is needed and second apofamily below epifamily if a second such rank is needed (see Table T2.SEQ above). But the implementation of these two ranks is optional and required only when there is a need for more than one FS rank above superfamily, and it would seem inappropriate to increase indefinitely this number, all the more that there is no upward limitation to the number of ranks that can be implemented above superfamily in the CS. In the CS, there is no limitation of any kind and a descending hierarchy may be smoothly followed without disruption. Dubois (2006a) proposed a system with 16 distinct CS ranks between order and superfamily, and if necessary more could be used, but in the present work we needed only eleven ranks to account for the hierarchy of CS taxa required by TREE below the rank class.

\subsection{Examples of use of this Criterion}

The number of ranks may be different in distinct sub-branches of the same branch. This affects particularly the two ranks epifamily and apofamily, which need to be used in a few cases only. Because of the Criterion [STC] (see below), in a given branch all sister-taxa must bear the same rank, but when a given taxon has no sister-taxon, some intermediate ranks between the mandatory rank family and higher ranks won't be used in its hierarchy. Thus, the FS nomen RANIDAE and its parordinate RHACOPHORIDAE constitute together an apofamily RANEIDAE, which has four parordinate nomina/ taxa (CERATOBATRACHEIDAE, DISCOGLOSSEIDAE, NYCTIBATRACHEIDAE and RANIXALEIDAE). Altogether, these five apofamilies constitute an epifamily $R A N O I D A E$ which has five parordinate epifamilies (Conrauoidae, ERICABatrachoidae, Micrixaloidae, Petropedetoidae and PyXicephaloidae). Altogether these six epifamilies constitute a superfamily RANOIDEA which has two parordinate superfamilies (ODONTOBATRACHOIDEA and PHRYNOBATRACHOIDEA). At this stage, we have reached the nominal-series saturation for this set of taxa and the transition to the class-series must be effected, at the 
lowest rank needed to account for the next dichotomies above, and at this stage the three superfamilies will have to constitute a hypophalanx ECAUDATA. However, the number of FS ranks between the ranks family and superfamily is variable in this branch according to the subbranch: whereas it is three for the RANIDAE, it is only two for the other four apofamilies, and one for the five other epifamilies. In this case, the NS saturation is reached although some only of the three FS ranks above family are used.

\subsection{Criterion [FPC]: the 'Family-Series Precedence Criterion'}

\subsection{Statement of Criterion}

"In any given suprafamilial cladonomy, whenever the other Criteria allow it, the nominalseries allotment of the suprafamilial taxa should be made giving precedence to the FS over the CS, and allotment to the CS should start only when all the available FS ranks above family have been used (nominal-series saturation), at least in one branch of the ergotaxonomy."

\subsection{Rationale and use of this Criterion}

This Criterion concerns the transition between the family-series and the class-series.

The Criteria that will be examined below allow to fix automatically, without recourse to subjective decisions, to 'consensus' or 'tradition', the place of the rank family in the hierarchical taxonomy of any zoological group. They also allow to fix the positions of most other ranks, but in some particular cases there may exist a problem of allotment (as defined above under 2.4.2) of taxa to nominal-series. As we have seen under Criterion [CHC], the situation here is different below and above the rank family.

$\{\mathrm{n} 1\}$ Below the rank family and above the rank genus, a single nominal-series, the FS, is represented, so there is never any problem of taxonominal series allotment of taxa, and the Criterion [CHC] is sufficient to fix the ranks of taxa.

\{n2\} The situation is different above the rank family, because here two distinct nominal-series must be distinguished, the FS and the CS, and we will need Criteria to know where the transition between them occurs. This situation is complexified by the fact that, according to the Code, no FS rank is allowed above superfamily. Even if, as suggested here, two additional optional ranks epifamily and apofamily are implemented between family and superfamily, which is Code-compliant, the number of FS ranks above family is much lower than that in the CS. In such conditions, two situations may occur above family:

\{n2a\} In many cases, the implementation of the six Criteria [STC] to [NTC] below allows to exclude any ambiguity, because the combination of upper quartile, sister-taxa, consistent-hierarchy and mandatory rank allows to refer clearly all taxa between family and superfamily to a precise rank, including, in some branches at least, epifamily and apofamily, and then, continuing the progression upwards, there is no other possibility than to start using the class-series just above superfamily. In the $\mathrm{CS}$, there is no special requirement except that ranks must follow smoothly each other, without ignoring some intermediate ranks.

$\{\mathrm{n} 2 \mathrm{~b}\}$ But it is not the case when the order contains much less taxonomic diversity. In such cases, the numbers of suprafamilial ranks needed are much lower, and the transition between the FS and the CS is not given automatically. In such cases, where should we put the transition? Or, in other words, which Criterion should be used for the nominal-series allotment of these suprafamilial taxa? As amply discussed above, no scientific Criterion would allow to decide in this respect, as ranks have no biological or other meaning. However, two 'practical' arguments allow to make the case in favour of one possibility: $\left\{\mathrm{n}_{2} \mathrm{~b}_{1}\right\}$ the FS being regulated by the Code, the nomina in the three FS suprafamilial ranks will be imposed by the regular Rules, and will not be liable to be challenged by authors who would refuse to follow the DONS Criteria for CS nomenclature; $\left\{\mathrm{n} 2 \mathrm{~b}_{2}\right\}$ more importantly, the FS being submitted to the Principle of Coordination, using this nominal-series for three ranks would involve more nomenclatural parsimony. 


\subsection{Examples of use of this Criterion}

\{02a\} This situation was observed in the present work in the order ANURA. The suprafamilial ergotaxonomy of all the 55 taxa of rank family that were imposed by the Criteria described here shows a clear and smooth transition between the rank superfamily and the lowest CS rank (which is not the same in different branches). In this order, all ranks from subtribe and superfamily, including epifamily and apofamily, are used in a few branches, so that the transition to the CS is automatic when the progression upwards continues. This is due to the fact that, the more there are species, genera and suprageneric taxa in a group, the more suprafamilial and infraordinal ranks are needed (up to 12 in the present case), and the more there are constraints on these ranks through the Criteria [STC] and [CHC]. In this order, there is therefore no problem of nominal-series allotment of suprafamilial taxa.

$\{\mathrm{o} 2 \mathrm{~b}\}$ The situation is different in the amphibian orders URODELA and GYMNOPHIONA.

In the salamanders, as in some branches there are up to 4 suprafamilial ranks, the three ranks superfamily, epifamily and apofamily will not be sufficient for all the suprafamilial taxa and the recourse to CS ranks will anyway have to be implemented. The four needed ranks could then be distributed in four different ways between the two nominal-series FS and CS: respectively 3 and 1, or 2 and 1, or 1 and 2, or 0 and 4. In the present case, in the URODELA the three available FS suprafamilial ranks superfamily, epifamily and apofamily are used above the taxa AMPHIUMIDAE, PLETHODONTIDAE and RHYACOTRITONIDAE, which imposes the use of the $3+1$ solution, with a single CS rank above superfamily and below URODELA, namely suborder. According to the Criterion [STC], this rank will have to be attributed to the three taxa subordinate to this order as they are part of an uresolved trichotomy.

Finally, in the caecilians, the number of suprafamilial taxa above the five families are only 1 or 2 , so that, according to the Criterion [FPC], they could all be allotted to the FS, at the ranks superfamily and epifamily. But in this case, for sake of homogeneity with the other two orders which have suborders, we decided to recognise two suborders in the GyMnophiona, and then only the rank superfamily above family in the FS. This is the only case in the whole CLAD where we did not follow 'blindly' our a priori Criteria, and we concede that this decision can be rejected by others. They should then replace our two suborders by two superfamilies (whose nomina CAECILIOIDEA and RHINATREMATOIDEA will be imposed by simple priority), and then dowgrade by one step the ranks of all other suprageneric taxa of the order.

\subsubsection{Criteria applying only or particularly to families}

\subsection{Criterion [UQC]: the 'Upper Quartile Criterion'}

\subsection{Statement of Criterion}

"In any given cladonomy, any UQ-nomen (family-series nomen designating a taxon considered valid and having had a number of usages above the upper quartile of usages since 1758) must be maintained as valid at the nomenclatural rank family, irrespective whether it is also used at other superordinate or subordinate ranks".

\subsection{Rationale and use of this Criterion}

This new device is the key Criterion of the [TCP].

As we have seen, because nomina are useful for the communication not only among taxonomists but also between them and other biologists and even the whole society, it is important that 'well-known' nomina of higher taxa, especially at mandatory ranks (class, order, family), remain in use, even in a renewed taxonomy resulting from new cladistic data. But this requirement does not exist for nomina that have been seldom used. By 'stability of use', we understand long-term stability covering the whole history of the taxonomy of the group since Linnaeus $(1758 a)$, or a really massive usage in a significant and recent part of it (e.g., since 1950).

In order to measure this, in 2014 we surveyed 101 publications (followed by $\{Q\}$ in our list of references), from 1758 to 2014, presenting complete familial classifications of all extant amphibians or 
of at least of one of the three extant orders of the class (ANURA, Gymnophiona, Urodela). In each of these publications, we noted the nomina of all the families recognised as valid. We then distributed these publications into five periods: 1796-1849 (starting with Batsch 1796, the first publication where an available family nomen was proposed for amphibians), 1850-1899, 1900-1949, 1950-1999 and 2000-2014 (although this last period is shorter, it deserves to be considered separately, as it corresponds to the expansion of the use of molecular cladistic works in amphibians). We then treated separately the nomina in the three extant orders. For each order, we computed the frequency of use of each familial nomen in each period among the works presenting a complete or subcomplete (e.g., missing the fossil taxa) familial classification of the order, and then we averaged these frequencies over the five periods. The results, presented in detail in Appendix A13.QUA, show that the average frequency of use of a nomen over the five periods varied from 1.0 to $100 \%$. We then divided in each order the complete list of familial nomina according to their usage in four equal parts (containing each one quarter of all these nomina), and we decided that all the nomina with a number of citations in these works being above the Upper (third) Quartile (UQ-nomina) should be considered 'well-known' for having been used consistently during the two and half centuries of zoological nomenclature, or at least massively in the recent periods, and that for this reason any ergotaxonomy of the group at stake should recognise one family bearing each of these nomina. This threshold (upper quartile deliminating the most used quarter of nomina, the Upper Quarter of nomina or $\boldsymbol{U Q N}$ ) is doubtless arbitrary, but its implementation as a Criterion is fully automatic and objective. It can be implemented independently by all zoologists in any country of the planet and, if the sample of publications is large enough (we suggest a minimum of 100), it should result in the same list of nomina in all cases.

We think this arbitrary Criterion should be applied 'blindly', without any qualms, as if exceptions are haphazardly tolerated the Criterion vanishes altogether. So, a nomen just above the threshold should always be kept in the list, whereas a nomen just below it should not (which of course does not forbid its use in the rank family if this is required by the other Criteria proposed here).

However, we suggest two exceptions in the implementation of this Criterion:

$\{\mathrm{p} 1\}$ Even if it belongs in the upper quarter, a familial nomen should not be placed in the list of 'mandatory valid family nomina' if it does not appear in any of the publications analysed for the last period (2000-2014). This is because the sudden disappearance of this well-known nomen in recent publications calls attention to a drastic and significant change, due to either nomenclatural or taxonomic reasons. Nomenclatural reasons may include the rejection of a nomen for being an invalid synonym (this is the case here of CYSTIGNATHIDAE) or for having been considered so in error (this is the case here of ENGYSTOMATIDAE). Taxonomic reasons include a drastic change in the taxonomic status of a group resulting from molecular surveys: this would apply for example to the nomen PSEUDIDAE, which was long used as a valid familial nomen until it was found to apply to an aquatic specialised group of the family HYLIDAE (Darst \& Cannatella 2004) and then abandoned by all authors at the rank family (in this case, although widely used in the past, this nomen is not part of the upper quarter, but even if it was it should be rejected from the list for not having been used for a family after 1999).

\{p2\} A reverse exception, or more exactly tolerance, should be accepted for a nomen which, although not being part of the Upper Quarter of usages over the period 1758-2014, has been used in 90\% or more of the publications in the period 2000-2014, thus pointing to an almost universal acceptance of the use of this nomen/taxon at the rank family in the most recent period. The acceptance of this tolerance, with the data of Appendix A13.QUA, resulted in the incorporation in the set of UQ-nomina of three additional nOmina: MEGOPHRYIDAE (90.7\%), CRYPTOBRANCHIDAE (100\%) and RHYACOTRITONIDAE (100\%).

The implementation of this Criterion as the first step for the building of a new suprageneric cladonomy is a guarantee of strong nomenclatural stability in zootaxonomy, which will be applauded by most users of classifications who are not specialists of the zoological groups at stake. It will facilitate the communication between taxonomists and non-taxonomists, whereas the recent permanent changes in suprageneric zoological taxonomies tends to discourage non-specialists and to develop a bad image of taxonomy and nomenclature in the biological community at large. Furthermore, as we will see below, it does not impede in the least the implementation of drastic changes in classifications whenever genuine discoveries or changes in phylogenetic hypotheses occur (e.g., the recent recognition of the ODONTOBATRACHIDAE). 


\subsection{Consequences of the use of this Criterion}

Implementation of this Criterion in the three orders of extant amphibians provided the following three lists of 36 FS nomina which, having in each order a number of usages above the upper quartile, or above $90 \%$ for the period 2000-2014 (marked [Q+] below), must apply at least to a family:

Order ANURA (24): BOMBINATORIDAE; BRACHYCEPHALIDAE; BUFONIDAE; CENTROLENIDAE; DENDROBATIDAE; DISCOGLOSSIDAE; HELEOPHRYNIDAE; HEMIPHRACTIDAE; HEMISOTIDAE; HYLIDAE; HYPEROLIIDAE; LEIOPELMATIDAE; LEPTODACTYLIDAE; MEGOPHRYIDAE [Q+]; Microhylidae; Myobatrachidae; Pelobatidae; Pelodytidae; Pipidae; Ranidae; Rhacophoridae; Rhinodermatidae; RHINOPHRYNIDAE; SOOGLOSSIDAE

Order GYMNOPHIONA (3): CAECILIIDAE; ICHTHYOPHIIDAE; RHINATREMATIDAE.

Order URodela (9): AmbYSTOMATIDAE; AMPHIUMIDAE; CRYPTOBRANCHIDAE [Q+]; HYNOBIIDAE; PLETHODONTIDAE; PROTEIDAE; RHYACOTRITONIDAE [Q+]; SALAMANDRIDAE; SIRENIDAE.

\subsubsection{Criterion [STC]: the 'Sister-Taxa Criterion'}

\subsection{Statement of Criterion}

"In any given cladonomy, parordinate taxa (i.e., taxa that are considered sister-taxa according to the cladistic hypothesis adopted) should always be attributed to the same nomenclatural rank."

\subsection{Rationale and use of this Criterion}

This Criterion applies to all pairs of taxa resulting from a dichotomy but also to all taxa involved in a polytomy as long as their relationships are partially unresolved. Although very simple in its formulation, and deriving directly from the basic principles of 'phylogenetic taxonomy', this Criterion is very rarely used in recent taxonomic works. In fact, apart from the works of Lescure et al. (1986), Dubois (2005b, $2006 a$ ) and Dubois \& Raffaëlli $(2009,2012)$, we are not aware of any comprehensive taxonomic work dealing with the amphibians where it would have been consistently implemented. This has important consequences on the taxonomic hierarchies used by most authors and in most revisionary works, taxonomic and faunistic checklists and databases. Striking examples of ignorance of this Criterion can be found in Bossuyt \& Milinkovitch (2001), Frost et al. (2006), Grant et al. (2006), Zhang et al. (2008), Van Bocxlaer et al. (2009), Blackburn \& Wake (2011) or Vieites et al. (2011), as well as in many other recent works. All these taxonomies fail to follow consistently this Criterion and qualify therefore at least in part as 'gradist' and 'pseudo-ranked' because they afford higher ranks to some sister-taxa than to others for mere reasons of 'anagenetic divergence' or 'geological age' —or sometimes only of 'tradition', which is even less justifiable scientifically.

Although it applies at all ranks, the consequences of the Criterion [STC] are particularly important regarding the use of the rank family in zoological nomenclature. Whereas the Criterion [UQC] requires that in our taxonomy all the family-series UQ-nomina be used as valid at the rank family, it does not state for which taxa. In some groups, when the hierarchy required by the structure of the phylogeny counts few ranks, there is no choice and the rank family will apply to the only taxon of the FS that has to be recognised. But in other cases, when the hierarchy is expanded, this nomen will apply to several taxa at different ranks which all include its nucleogenus (e.g., RANOIDEA, RANIDAE, RANINAE, RANINI, RANINA, which are all paronyms of the same FS nomen based on the GS nomen Rana). In such cases, the Criterion [STC] requires that the paronym of rank family be attached to a taxon parordinate to a familial UQ-nomen. This is because the rank family is crucial in the taxonomy of a group, and its use is more informative if it is given to a taxon having a sister-taxon than to a taxon having none. As we have seen, the fully expanded taxonomy presented here will be seldom mentioned, and many authors will only mention the ranks which they view as 'the most important', like order, family and genus, and sometimes superfamily and subfamily. So recognising taxonomically a dichotomy at the rank family is more informative than having a redundancy here.

The first consequence of consistent use of the Criterion [STC] is therefore that all the taxa which turn out to be, under the phylogeny adopted, sister-taxa of the families adopted under the Criterion 
[UQC], must also be recognised as families. The second consequence is that this also applies to their getendotaxa (immediate subordinate taxa) and to their getangiotaxa (immediate superordinate taxa), and step by step this applies to many taxa in the hierarchy. Thus, combined with the Criterion [UQC], the application of the Criterion [STC] allows to fix the ranks of an important proportion of nomina in a given ergotaxonomy.

In the present work, this Criterion was followed strictly for all suprageneric nomina/taxa, whatever its consequences regarding 'usage' and 'consensus'.

\subsection{Examples of use of this Criterion}

The following examples concern cases of taxa which, according to our data, require erection of a taxon of higher rank to account for the fact that they are parordinate to taxa including numerous species and supraspecific taxa.

As tackled above, the current treatment in the literature of the concept of genus is highly heterogeneous. This is particularly striking in the cases where a 'cladistically isolated' species is found to be parordinate to a well-supported branch containing several, or sometimes many, species. There is no general treatment of this situation in the current generic ergotaxonomy of amphibians. In some cases, the 'external' species is referred to its own monospecific genus and all the other ones to their own genus, whereas in other cases they are incorporated into the same genus as the other ones. In general, this difference of treatment reflects mostly 'tradition' but is not justified by any non-cladistic Criterion, such as phenetic divergence or hypothesised geological age. In several cases in the present work, in agreement with the diagnogenus concept mentioned above, we supported the recognition of a distinct genus for the 'external' species when the latter can be easily diagnosed from the larger genus by clear external morphological characters and/or occupancy of a distinct ecological niche, or even by fully disjunct geographical distribution: this is the case for Leioaspetos vs. Leiopelma, Ammoryctis vs. Alytes, Pelodytopsis vs. Pelodytes or Boreorana vs. Lithobates. The respective situations of the two genera in each pair are similar to those of other pairs currently accepted by the international community such as Latonia vs. Discoglossus, Blythophryne vs. Bufoides, Chaltenobatrachus vs. Atelognathus or Urspelerpes vs. Eurycea.

The species Ceuthomantis smaragdina appears in TREE as the only sequenced representative of a small group of six species in two genera that constitute one of the two branches resulting from a node having a support of $100 \%$. In TREE, the sister-branch of this group includes 482 species in 29 genera and 23 suprageneric taxa of AMPHiBia. Nevertheless, despite their huge disparity, both branches should be recognised as taxa of the same rank, i.e., family in this case (respectively CEUTHOMANTIDAE and BRACHYCEPHALIDAE) to comply with the [STC].

Although it has been a long time since Laurent (1943b) showed that the 'traditional' family RHACOPHORIDAE was an arboreal specialised group of RANIDAE, long confused with the HYPEROLIIDAE, and that the latter family occupies a similar situation relative to the ARTHROLEPTIDAE, both families RHACOPHORIDAE and HYPEROLIIDAE have remained in use in taxonomic works since then and they are now part of the upper quarter, so they should be stabilised at familial rank, and the same should apply to the families RANIDAE and ARTHROLEPTIDAE. In contrast, this does not apply to the nomen MANTELLIDAE, which has been used for a taxon of rank family only recently and not universally, and does not appear in the upper quarter.

The two salamander genera Siren and Pseudobranchus are the only living representatives of one of the three branches of a trichotomy. Although the other two branches include many more species, genera and suprageneric taxa, the three branches must be attributed to the same rank, which in this case is suborder to comply with the Criterion [STC].

\subsection{Consequences of the use of this Criterion}

Implementation of this Criterion in the three orders of extant amphibians provided the following two lists of 17 FS nomina that, being parordinate with FS nomina above the upper quartile for each order, must apply at least to a family (preceded below by the nomina of their sister-families between square brackets, followed by $\rightarrow$ ): 
Order ANura (16): [BRACHYCEPHALIDAE $\rightarrow$ ] CEUTHOMANTIDAE; [BUFONIDAE $\rightarrow$ ] ODONTOPHRYNIDAE; [CENTROLENIDAE $\rightarrow$ ] Allophrynidae; [DENDROBATIDAE $\rightarrow$ ] ARomobatidaE; [DISCOGLOSSIDAE $\rightarrow$ ] ALYTIDAE; [HEMISOTIDAE $\rightarrow$ ] BREVICIPITIDAE;

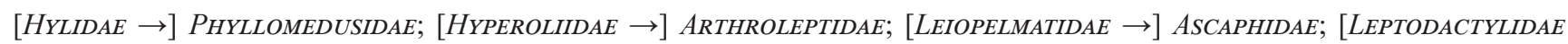

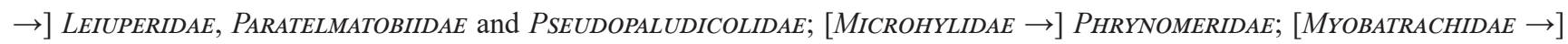
CALYPTOCEPHALELLIDAE; [RHINODERMATIDAE $\rightarrow$ ] TELMATOBIIDAE; [SOOGLOSSIDAE $\rightarrow$ ] NASIKABATRACHIDAE.

Order GYMNOPHIONA (1): [ICHTHYOPHIIDAE $\rightarrow$ ] URAEOTYPHLIDAE.

The Criterion [STC] therefore allows to fix the ranks of 17 families additional to the 36 which had been settled by the Criterion [UQC]. There remain then only 16 unsettled situations, which will be settled by the following Criteria.

\subsection{Criterion $[\mathrm{CPC}]$ : the 'Conflict of Precedence Criterion'}

\subsection{Statement of Criterion}

"In any given cladonomy, whenever a taxon that could be cladistically subordinate to a UQnomen has nomenclatural precedence over it according to the Criterion [NPC], it should be raised to the rank family as parordinate to the UQ-nomen at stake."

\subsection{Rationale and use of this Criterion}

The Criteria that we devised to attribute the ranks to taxa in CLAD are not meant at replacing or ignoring the basic Rules of the Code, in particular those of precedence among nomina for validity. They must be compatible with them and respect them. Therefore, the fact that the Criterion [UQC] requires to recognise a nomen as valid at the rank family cannot lead to affording it precedence over another nomen which according to the Rules has nomenclatural precedence over it, but to accept both nomina as valid at the rank family. This then requires to upgrade both of them as sister-taxa from a lower rank which would be compatible with TREE up to the rank family, and then to adapt the ranks of taxa superordinate and subordinate to them.

\subsection{Consequences of the use of this Criterion}

Five taxa were raised at the rank family in order to be parordinate to UQ-families as their nomina had precedence over them (the latter are mentioned after them in the following list):

Order Anura (4): Alytidae (Discoglossidae); ARthroleptidae (HyperoliddaE); BreVICIPITIDae (Hemisotidae); TELMATOBIIDAE (RHINODERMATIDAE).

Order URODELA (1): CRYPTOBRANCHIDAE (HYNOBIIIDAE).

\subsection{Criterion [NRC]: the 'Non-Redundancy Criterion'}

\subsection{Statement of Criterion}

"In any given cladonomy, within a given nominal-series, redundant taxa, i.e., having the same intension and extension as their immediate superordinate or subordinate taxon, should be avoided if possible. If allowed by the data, subordinate taxa should be divided in two sister-taxa of the same rank (see Criterion [STC]). This Criterion does not apply automatically to taxa belonging to different nominal-series, if one of the ranks involved in the redundancy is one of the seven mandatory ranks (see text and Criterion [MRC]). It applies to taxa of the rank family relatively to their just superordinate taxon, except in the situation where this rank corresponds hierarchically to an unresolved polytomy (see Criterion [NTC])." 


\subsubsection{2 . Rationale and use of this Criterion}

Under the taxonomic paradigm adopted here, the purpose of the use of ranks is not to carry any message regarding the characters of the taxa, their 'degree of anagenetic divergence', their age or any other biological or historical information. Ranks as we use them are useful only for two reasons: $\{\mathrm{q} 1\}$ to reflect the topology of TREE and in particular to identify sister-taxa; $\{\mathrm{q} 2\}$ additionally, but mostly in the case of the four mandatory ranks used in the present work (species, genus, family, order), to facilitate storage and retrieval of taxonominal information. So, except in the latter case, there is no need to recognise taxa at all ranks in all classifications. The only useful taxa and ranks in a given cladonomy are those which correspond to well-supported dichotomies (or by default polytomies) in TREE. As discussed already above in the case of the family RHINOPHRYNIDAE which contains a single genus Rhinophrynus and a single species Rhinophrynus dorsalis, as long as no additional species or subspecies of this group are recognised, there is no need to recognise redundant taxa like subfamily, tribe, subgenus or subspecies. Applied to taxa, the term redundant is understood here strictly as meaning coordinated taxa at different ranks sharing the same intension and extension.

This suggests that redundant taxa (and therefore ranks) should be banned from phylogenetic taxonomy. But there is a limitation to this Criterion: it should apply only within nominal-series, not between them, as if it were not the case it would be impossible to have monospecific genera, monogeneric families or monofamilial orders, situations which are quite frequent and justified in zootaxonomy, and accounted for in the next Criterion [MRC].

This Criterion has important consequences in several cases. It avoids the useless recognition of redundant taxa and therefore results in nomenclatural and taxonomic parsimony (see Dubois $2006 a-c$, $2007 a, 2008 f$ ). For example, if a rather isolated group (having no close relatives) is composed of two sister-genera, according to the Criterion $[\mathrm{NRC}]$ it is not justified to erect for them two distinct families, even if these genera have been cladistically separated 'long ago' or if they show a 'strong anagenetic divergence'. Such Criteria would be relevant only if nomenclatural ranks were taxonomic categories and had the function to carry information on phenetic divergence or on the chronology of evolutionary events but, if they are regarded as carrying only information on the structure of the tree, the two families carry no additional information and are fully redundant with the genera.

Our cladonomy CLAD reflects our phylogenetic analysis TREE, which is based exclusively on nucleic acid sequencing. For the purpose of completeness and information retrieval, we included the all-fossil taxa of extant amphibians in CLAD, but, as stated above, their position there is not supported by such molecular taxa, and we therefore consider it as only tentative. In consequence, we did not take all-fossil taxa into account for the establishment of the ranks of taxa.

Therefore, the Criterion [NRC] forbids redundancy within one nominal-series, but allows it in some cases between different nominal-series. In fact, the strength of tradition in taxonomy is very high, and it may be predicted that, in some cases and possibly for some time only, some authors will prefer to continue to use a well-known superordinate nomen in one nominal-series rather than (or in addition to) a redundant superordinate nomen in the next higher nominal-series. Such perissonyms can be 'tolerated' for purposes of perpetuation of tradition but they are useless for pure reasons of communication about the structure of the tree and are therefore not justified under the [TCP]. In such cases, to point to this redundancy, we suggest that the nomen of the lowest redundant taxon be written between simple straight quotation marks ('...'), and the corresponding taxon should be removed from the analysis if the cladonomy is used to reconstruct the tree. Although we here point to its possibility, we did not implement this awkward concession to 'tradition' in the present work.

\subsection{Examples of use of this Criterion}

As we have seen, the family CEUTHOMANTIDAE contains only two genera and six species, whereas its sister-family BRACHYCEPHALIDAE contains more than 500 taxa. As we will see, in CLAD these two families together make up the hypophalanx GAIANURA, parordinate to two other hypophalanges which are required by the rest of TREE. It would be useless to recognise a superfamily BRACHYCEPHALOIDEA for these two families, as it would be redundant with the hypophalanx, but if some authors prefer superfamilial nomina, for example because, unlike class-series nomina, these nomina are fully regulated by the Code, they should mention this nomen as 'BRACHYCEPHALOIDEA'. At any rate, as long as it 
contains a single genus with a single undivided species, the family CEUTHOMANTIDAE should not have subfamilies or tribes, and its single genus should not have subgenera. This is a common and basic situation in zoological nomenclature, which does not require special comments here.

Nevertheless, consistent implementation of the Criterion [NRC] results in challenging some long established traditions. A good example of this is the case of the salamander genera Ambystoma and Dicamptodon, already mentioned above (2.2). In various recent works, these two genera are referred to two distinct families, AMBYSTOMATIDAE and DICAMPTODONTIDAE, but this arrangement does not bring any cladistic information additional to the distinction of two genera. The recognition of a single FS taxon, the family AMBYSTOMATIDAE, for these two genera as well as five all-fossil genera for which we have no molecular cladistic information, is enough to provide the information available. This family should be divided in two subfamilies only if one of the three additional pieces of information became available: $\{\mathrm{r} 1\}$ erection of a third new genus of extant ambystomatid (based on an explicit genus concept), resulting either $\{\mathrm{rla}\}$ from a splitting of the genus Ambystoma or of the genus Dicamptodon in two or more distinct genera or $\{\mathrm{rlb}\}$ from genuine discovery of a new extant species sister to all other members of one of these two genera; or $\{$ r2 $\}$ obtention of reliable data allowing to refer the all-fossil genera or at least one of them to the same branch as either Ambystoma or Dicamptodon. As long as this is not the case, the two extant and five fossil genera should be referred to a single getangiotaxon, the family AMBYSTOMATIDAE, sister to the SALAMANDRIDAE.

In most cases, redundancy between taxa referred to different nominal-series can be avoided by suppressing one of the two redundant taxa. This will doubtless be seen by some authors as a problem, even if they adopt our taxonomy, in the case of sister-families that together make up a class-series taxon, and they may thus perpetuate the 'tradition' in this respect. For example, although unnecessary from the viewpoint of the transcription of TREE into a taxonomic hierarchy, it is quite possible that some taxonomists may wish to continue to use the superfamilial nomen PIPOIDEA for the taxon including the two extant families PIPIDAE and the RHINOPHRYNIDAE, as subordinate to the nomen of hypoordo DORSIPARES, although both nomina DORSIPARES and PIPOIDEA are redundant. In such cases, in order to point to this imprecision, we suggest that the superfamilial nomen be written 'PIPOIDEA'.

\subsection{Consequences of the use of this Criterion}

This Criterion allowed to validate 17 family nomina in our work:

Eleven families were validated for being parordinate of UQ-families (the latter are mentioned after them in the following list):

Order ANuRa (9): AlLOPHRYNIDAE (CENTROLENIDAE); ARomobatidaE (DENDROBATIDAE); AsCAPHIDAE (LeIOPELMATIDAE); CALYPTOCEPHALELLIDAE (MYobatraChIDAE); CEUTHOMANTIDAE (BRACHYCEPHALIDAE); NASIKABATRACHIDAE (SOOGLOSSIDAE); Odontophrynidae (Bufonidae); Phrynomeridae (Microhylidae); Phyllomedusidae (HyLidae).

Order GYMNOPHIONA (2): SCOLECOMORPHIDAE (CAECILIIDAE); URAEOTYPHLIDAE (ICHTHYOPHIIDAE).

Three pairs of families were both validated by this Criterion [NRC]:

Order ANura (6): AstrobatrachidaE and NyCTIBATRACHIDAE; CACOSTERNIDAE and PyXICEPHALIDAE; DicRogLOSSIDAE and OCCIDOZYGIDAE.

\subsection{Criterion [MRC]: the 'Mandatory Rank Criterion'}

\subsection{Statement of Criterion}

"In any given cladonomy, all zoological species recognised as valid should be referred formally (at least provisionally) to one taxon of the following mandatory taxonominal ranks: genus, family, order, class, phylum and kingdom."

\subsection{Rationale and use of this Criterion}

The rationale for this Criterion was explained above and is illustrated in Figure F1.MOR. According to this Criterion, all terminal taxa (species or subspecies) recognised in any ergotaxonomy must be 
referred at least to four taxa attributed to the four mandatory ranks of the zootaxonomic hierarchy concerned by the present work: species, genus, family and order. This can be put differently in stating that, in any given taxonomic hierarchy to which a species is referred, there should always exist at least one taxon at each of these four ranks, even if it has no known sister-taxon and even if this implies nomenclatural redundancy with taxa in other nominal-series. So, it is unacceptable to have a genus or a group of genera directly included in a taxon of rank order. A family must always be recognised between the order and the genus/genera, even if this family is redundant with the order and/or the genus, i.e., if it has the same intensional definition and the same taxonomic content (extension) as the latter. This is required for purposes of information storage and retrieval in databases, not of cladistic information.

\subsection{Examples of use of this Criterion}

Among the frogs including the genus Pelobates, the Criterion [UQC] requires to recognise two families, the PELOBATIDAE and PELODYTIDAE. These two families are not sister-taxa, the cladistic relationships established by TREE among these frogs being as follows: (Scaphiopus)(Pelodytes (Pelobates+Megophrys)). Climbing up TREE from the genera, the taxon including the genus Pelobates must be recognised first as the family PELOBATIDAE, and its sister-taxon including the genus Megophrys as the family MEGOPHRYIDAE. Together, the PELOBATIDAE and the MEGOPHRYIDAE make up a superordinate taxon, the epifamily PELOBATOIDAE, parordinate to the PELODYTOIDAE which include the single family PELODYTIDAE. Altogether, the PELOBATOIDAE and the PELODYTOIDAE constitute the superfamily PELOBATOIDEA, which is sister to the SCAPHIOPODOIDEA. This superfamily includes only two extant genera, but these cannot be directly placed in the superfamily: a taxon SCAPHIOPODIDAE at the mandatory rank family must be recognised between the genera and the superfamily, despite being fully redundant with the latter.

Note that in this case the Criterion [STC] requires to recognise an epifamily PELODYTOIDAE for the single family PELODYTIDAE, but that there is no such requirement for the family SCAPHIOPODIDAE, which should be referred directly to the superfamily SCAPHIOPODOIDEA, without intermediate rank epifamily. This case exemplifies the fact that the concept of 'consistent-hierarchy' does not imply necessarily that all successive ranks be represented in all the branches of a tree.

\subsection{Consequences of the use of this Criterion}

Implementation of this Criterion in the three orders of extant amphibians provided the following list of 17 FS nomina that need to apply at least to a family:

Order Anura (17): Cacosternidae; Ceratobatrachidae; Ceratophryidae; ConRauidaE; CycloramphidaE; DicRoglossidae; ERICABATRACHIDAE; MicRIXALIDAE; NYCTIBATRACHIDAE; OCCIDOZYGIDAE; ODONTOBATRACHIDAE;

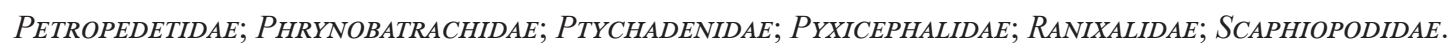

\subsection{Criterion [NTC]: the 'Nomenclatural Thrift Criterion'}

\subsection{Statement of Criterion}

"In any given cladonomy, whenever according to the data the rank family should be granted to several taxa forming together an unresolved polytomy (more than two sister-taxa), a single family should be provisionally recognised and the polytomy should be downgraded to the rank subfamily."

\subsection{Rationale and use of this Criterion}

The purpose of our work is to homogenise and clarify the hierarchical relationships between taxa and nomina to make them compatible with our current cladistic hypotheses. But we are conscious that the latter are labile and will change in the future, when more species have been collected, distinguished 
and sequenced and molecular data obtained for more complete genomes. Because out threshold Criteria are quite demanding, we recognise nodes only when the support for them is robust and quite unlikely to change easily. But in many cases our data do not allow complete resolution of the relationships among closely related taxa.

In TREE, suprageneric nodes supported by our a priori threshold are of two kinds: 'suprageneric' and 'infrageneric' (or 'intrageneric') ones. Because of the absence of an explicit 'genus concept' followed consensually by current amphibian taxonomists, this distinction is largely arbitrary. As we decided to comply with the current generic classifications of extant amphibians (with a few exceptions) and with the non-recognition of subgenera by most recent authors (which derives in part from their non-recognition in the database $A S W<2020 a>$ ), we did not recognise taxonomically (and therefore nomenclaturally) the well-supported nodes of the second kind. This is a provisional situation which will hopefully be improved when more solid concepts and Criteria are adopted by the community for the taxonomic category genus.

After exclusion of the nodes whose support is beyond our threshold, and of the nodes which are considered as infrageneric, there remain 393 nodes in our TREE (Table T13.NOD). Among them, 278 (70.7 $\%$ ) are dichotomies and $115(29.3 \%$ ) are polytomies (from trichotomies, with three branches, to enneatomies, with nine branches). All these nodes are recognised as suprageneric taxa, and each of them includes at least two genera. Besides, 214 suprageneric taxa are recognised for 'achotomic' branches, i.e. branches that do not include any suprageneric dichotomy or polytomy but that are sister-branches to dichotomic or polytomic branches. On the whole, therefore, 214 suprageneric taxa $(35.3 \%)$ include a single genus, $278(45.8 \%)$ include at least two subordinate suprageneric taxa and $115(18.9 \%)$ from three to nine such taxa.

Except for dichotomies, the 115 polytomies mentioned above have vocation to be resolved in the future, when more information is available. In order for CLAD to remain bijective, each resolution will increase the number of ranks and therefore of taxa that will have to be recognised. A trichotomy with three genera $(\mathrm{A})(\mathrm{B})(\mathrm{C})$ requires the recognition of only one suprageneric taxon $\mathrm{ABC}$, but its resolution as $((A)(B))(C)$ requires the recognition of three suprageneric taxa, $A B, C$ and $A B C$. Therefore, we are confident that, except in cases where it will be shown that our TREE included genuine errors (of taxonomic identification of specimens or of sequencing, alignment or analysis), the number of taxa that will have to be recognised in order to keep a bijective suprageneric taxonomy will increase as compared to our scheme, and that most of the nomina of suprageneric taxa here recognised as valid will remain so, but at ranks which will be higher than those used here. This led us to introduce in this work 171 new FS suprageneric extant taxa below the rank class $(29.8 \%$ of the total of 573 such taxa considered valid in $C L A D$ ), as we expect them to remain valid, at least for taxonomists interested in having a completely bijective cladonomy.

However, we followed a slightly differential approach in the case of families. This is because the rank family is mandatory over the whole of animal taxonomy and will be used in many works, even having no phylogenetic or taxonomic dimension, and in many databases. Therefore, we refrained from coining new family nomina, or upgrading to the rank family nomina already available, in all the cases where according to the nine Criteria above we should have had a polytomy at the rank family. All polytomies at the rank family should be resolved in the shorter or longer term into several hierarchised taxa: two families, each of which may include two subfamilies, each of which may include two tribes, etc. Recognising all the branches of these polytomies as families would draw the attention to these taxa and give them an undue importance, for example in the light of the Criterion [UQC], and we think that in such cases it is better to wait. This approach called nomenclatural thrift (Dubois 2019) was implemented here only in the cases of polytomies at the rank family but not at higher or lower ranks.

Therefore, in all cases of polytomies which, according to the structure of TREE, should have been recognised as families, we recognised provisionally a single family and we downgraded all the branches/ taxa of the polytomy at the rank subfamily. When research progresses, one of these subfamilial nomina will be raised at family rank, except when fully new species are discovered which require the erection of a new family as sister-taxon of that recognised in the present work.

Because of the Criterion [CPC], this procedure cannot be applied if the polytomy at rank family includes a single taxon designated by a UQ-nomen that does not have nomenclatural precedence over one of the other members of the polytomy, or if the polytomy involves two branches corresponding to UQ-nomina or more. In such cases, all the members of the polytomy must be granted the rank family. However, none of these situations occurred in CLAD. 
TABLE 13.NOD. Resolution of suprageneric polytomies among extant lissamphibian taxa in CLAD (allfossil taxa excluded).

Id, Identifier of rank or series of ranks. Abbreviations for higher taxa: A, ANura; G, Gymnophiona; U, Urodela; L,

Lissamphibia (A $+\mathrm{G}+\mathrm{U}+$ incertae sedis $\mathrm{L}$ ). Categories of tomoidy: 1, Taxonomic achotomy (a single subordinate genus); 2, Dichotomy (two subordinate taxa of next lower suprageneric rank); 3, Polytomy (three to nine subordinate taxa of next lower suprageneric rank). Structure of information in each cell: Abbreviation of higher taxon; Number of nomina of this rank in line [\% of sum in line].

\begin{tabular}{|c|c|c|c|c|c|}
\hline Id & Rank & 1 & 2 & 3 & Total \\
\hline \multirow[t]{4}{*}{$\mathbf{A}$} & \multirow{4}{*}{$\begin{array}{l}\text { C.03. SubCLASSIS - } \\
\text { C.13. HypophaLANX }\end{array}$} & A: $0[0]$ & A: $20[74.0]$ & A: 7 [25.9] & A: 27 \\
\hline & & G: 1 [33.3] & G: 2 [66.7] & G: $0[0]$ & G: 3 \\
\hline & & $\mathrm{U}: 0[0]$ & $\mathrm{U}: 2$ [66.7] & $\mathrm{U:} 1$ [33.3] & $\mathrm{U}: 3$ \\
\hline & & $\mathrm{L}: 1[2.9]$ & L: 24 [70.6] & L: $9[26.5]$ & L: 34 \\
\hline \multirow[t]{4}{*}{ B } & \multirow{4}{*}{$\begin{array}{l}\text { F.14. SUPERFAMILIA- } \\
\text { F.16. APOFAMILIA }\end{array}$} & A: 4 [12.9] & A: $22[71.0]$ & A: $5[16.1]$ & A: 31 \\
\hline & & G: $0[0]$ & G: 2 [100] & G: $0[0]$ & G: 2 \\
\hline & & $\mathrm{U}: 1[16.7]$ & U: 5 [83.3] & $\mathrm{U}: 0[0]$ & $\mathrm{U}: 6$ \\
\hline & & L: 5 [12.8] & L: $29[74.4]$ & L: 5 [12.8] & L: 39 \\
\hline \multirow[t]{4}{*}{ C } & \multirow[t]{4}{*}{ F.17. FAMILIA } & A: $13[23.6]$ & A: $27[49.1]$ & A: 15 [27.3] & A: 55 \\
\hline & & G: $2[40.0]$ & G: $3[60.0]$ & G: $0[0]$ & G: 5 \\
\hline & & $\mathrm{U}: 2$ [22.2] & $\mathrm{U}: 6[66.7]$ & $\mathrm{U}: 1[11.1]$ & $\mathrm{U}: 9$ \\
\hline & & L: 17 [24.6] & $\mathrm{L}: 36[52.2]$ & L: 16 [23.2] & L: 69 \\
\hline \multirow[t]{4}{*}{ D } & \multirow[t]{4}{*}{ F.18. SUBFAMILIA } & A: $28[35.9]$ & A: 36 [46.2] & A: 14 [17.9] & A: 78 \\
\hline & & G: $0[0]$ & G: 2 [100] & G: $0[0]$ & G: 2 \\
\hline & & U: 2 [28.6] & $\mathrm{U}: 4$ [57.1] & U: 1 [14.3] & U: 7 \\
\hline & & L: 30 [34.5] & L: 42 [48.3] & L: 15 [17.2] & L: 87 \\
\hline \multirow[t]{4}{*}{$\mathbf{E}$} & \multirow[t]{4}{*}{ F.19. TRIBUS } & A: $26[35.1]$ & A: 31 [41.9] & A: $17[23.0]$ & A: 74 \\
\hline & & G: $1[25.0]$ & G: 3 [75.0] & G: $0[0]$ & G: 4 \\
\hline & & $\mathrm{U}: 1[9.1]$ & U: 8 [72.7] & $\mathrm{U}: 2$ [18.2] & $\mathrm{U}: 11$ \\
\hline & & L: 28 [31.5] & L: $42[47.2]$ & L: 19 [21.3] & L: 89 \\
\hline \multirow[t]{4}{*}{$\mathbf{F}$} & \multirow[t]{4}{*}{ F.20. Subtribus } & A: 32 [45.7] & A: $27[38.6]$ & A: $11[15.7]$ & A: 70 \\
\hline & & G: $0[0]$ & G: $3[75.0]$ & G: $1[25.0]$ & G: 4 \\
\hline & & U: 8 [44.4] & $\mathrm{U}: 9[50.0]$ & $\mathrm{U}: 1[5.6]$ & $\mathrm{U}: 18$ \\
\hline & & L: 40 [43.5] & L: 39 [42.4] & L: 13 [14.1] & L: 92 \\
\hline \multirow[t]{4}{*}{ G } & \multirow[t]{4}{*}{ F.21. INFRATRIBUS } & A: 27 [52.9] & A: 17 [33.3] & A: $7[13.7]$ & A: 51 \\
\hline & & G: $0[0]$ & G: $3[75.0]$ & G: 1 [25.0] & G: 4 \\
\hline & & U: 3 [30.0] & U: 5 [50.0] & $\mathrm{U}: 2[20.0]$ & $\mathrm{U}: 10$ \\
\hline & & L: 30 [46.2] & L: 25 [38.5] & L: $10[15.4]$ & $\mathrm{L}: 65$ \\
\hline \multirow[t]{4}{*}{ H } & \multirow[t]{4}{*}{ F.22. HYPOTRIBUS } & A: 13 [43.3] & $\mathrm{A}: 10[33.3]$ & A: $7[23.3]$ & A: 30 \\
\hline & & G: $2[50.0]$ & G: $2[50.0]$ & G: $0[0]$ & G: 4 \\
\hline & & $\mathrm{U}: 4[40.0]$ & $\mathrm{U}: 1[10.0]$ & $\mathrm{U}: 5[50.0]$ & $\mathrm{U}: 10$ \\
\hline & & L: 19 [43.2] & L: 13 [29.5] & L: 12 [27.3] & L: 44 \\
\hline \multirow[t]{4}{*}{ I } & \multirow[t]{4}{*}{ F.23. CLANUS } & A: $10[47.6]$ & A: $6[28.6]$ & A: $5[23.8]$ & A: 21 \\
\hline & & G: $0[0]$ & G: $0[0]$ & G: $0[0]$ & G: 0 \\
\hline & & $\mathrm{U}: 5$ [45.5] & $\mathrm{U}: 5$ [45.5] & $\mathrm{U}: 1[9.1]$ & $\mathrm{U}: 11$ \\
\hline & & L: 15 [46.9] & L: 11 [34.4] & L: $6[18.8]$ & L: 32 \\
\hline \multirow[t]{4}{*}{$\mathbf{J}$} & \multirow{4}{*}{$\begin{array}{l}\text { F.24. SUBCLANUS - } \\
\text { F.27. CATOCLANUS }\end{array}$} & A: $26[52.0]$ & A: $14[28.0]$ & A: $10[20.0]$ & A: 50 \\
\hline & & G: $0[0]$ & G: $0[0]$ & G: $0[0]$ & G: 0 \\
\hline & & $\mathrm{U}: 3[50.0]$ & $\mathrm{U}: 3[50.0]$ & $\mathrm{U}: 0[0]$ & $\mathrm{U}: 6$ \\
\hline & & L: $29[41.1]$ & L: 17 [23.5] & L: 10 [35.3] & L: 56 \\
\hline \multirow[t]{4}{*}{ A-J } & TOTAL TAXA & A: $179[36.8]$ & A: $210[43.1]$ & A: 98 [20.1] & A: 487 \\
\hline & \multirow{3}{*}{$\begin{array}{l}\text { C.03. SubCLASSIS - } \\
\text { F.27. CATOCLANUS }\end{array}$} & G: 6 [21.4] & G: 20 [71.4] & G: $2[7.1]$ & G: 28 \\
\hline & & U: 29 [31.9] & U: 48 [52.7] & U: 14 [15.4] & $\mathrm{U:} 91$ \\
\hline & & L: 214 [35.3] & L: $278[45.8]$ & L: 115 [18.9] & L: 607 \\
\hline $\mathbf{A - J}$ & TOTAL NODES & - & A: $210[68.2]$ & A: $98[31.8]$ & A: 308 \\
\hline & C.03. SubClassis - & & G: 20 [90.9] & G: $2[9.1]$ & G: 22 \\
\hline & F.27. CATOCLANUS & & U: 48 [77.4] & U: 14 [22.6] & $\mathrm{U}: 62$ \\
\hline & & & L: 278 [70.7] & L: 115 [29.3] & L: 393 \\
\hline
\end{tabular}




\subsection{Consequences of the use of this Criterion}

This Criterion applies to four family nomina in our work, two of which are UQ-nomina.

Order ANura (4): CERATOBATRACHIDAE; CYCLORAMPHIDAE; HeMIPHRACTIDAE [Q]; LEPTODACTYLIDAE [Q].

\subsubsection{Implementation of the [TCP] and rank attribution of suprageneric taxa}

The ten Criteria detailed above allow to fix the ranks in any given cladonomic hierarchy in an objective and repeatable manner. Some of these Criteria, like the [UQC] or the [MRC], are doubtless arbitrary, but if adopted by the community of taxonomists and used consistently, they would allow two independent taxonomists, working in different places on the globe and having no contact with each other, to come out with the same taxonomic hierarchy, the same taxa and the same nomina if they start from the same tree, and this cladonomy could be transcribed automatically exactly into the same tree by anyone despite having never seen this tree previously.

In order for this Ten Criteria Procedure to be fully clear to all readers, after a summary of the latter, we detail below a few hypothetical and real (based on TREE) examples of their implementation in a few different situations, and then on the use of the nomenclatural Rules reminded above.

\subsubsection{A general summary of the Ten Criteria Procedure [TCP]}

In order to be successful, the Ten Criteria Procedure of assignment of ranks to suprageneric taxa in a given zoological group should follow a series of steps. Until a software is devised and made available allowing an automation of this procedure, it has to be implemented 'by hand', which is quite heavy and requires care and attention.

The first important point is that, in our proposed system, the ranks of suprageneric taxa cannot be fixed separately. This fixation must be done altogether for all the suprageneric taxa recognised within a zoological group in a given ergotaxonomy. Any change to this taxonomy required by new data, e.g. through resolving polytomies or correcting errors (e.g. due to misidentification of voucher specimens), must therefore imply, before its implementation, a re-examination of the whole taxonominal hierarchy. The procedure then relies on three basic 'feet' which are made possible by the concept of 'mandatory rank': the procedure starts from the nomina/taxa attributed to three 'fixed landmarks', the mandatory ranks $\{\mathrm{s} 1\}$ genus and $\{\mathrm{s} 2\}$ order, and $\{\mathrm{s} 3\}$ the set of nomina fixed as valid by the Criterion [UQC] for the mandatory rank family.

Regarding orders and genera, as explained above, in our work the attribution of extant amphibian taxa to these two ranks is given by two a priori unchallenged (in the present work) facts, i.e. tradition (in the case of the three orders) or current 'consensus', even if based on unclear and non-universal concepts and Criteria (in the case of genera). The Ten Criteria Procedure allows to attribute automatically to nomenclatural ranks all the taxa intermediate in the hierarchy between these two references, starting with the rank family and processing from it both upwards and downwards. The taxonominal hierarchy between these two references is composed in fact of two independent and successive hierarchies, in the FS and in the CS. The transition between them does not occur always between the same ranks, depending on the number of ranks implemented in each of them in each section of TREE. Let us call $\boldsymbol{C S}$-branch (class-series branch) any section of TREE below the rank order and above the rank superfamily, upperFS-branch (upper-family-series branch) any section of TREE below the lowest CS rank and above the rank family, and lower-FS-branch (lower-family-series branch) any section of TREE below the rank family rank and the rank genus. These three kinds of partial hierarchies can be designated collectively as $\boldsymbol{N S}$-branches (nominal-series branches).

In order to simplify the presentation below, although the concepts of node (phylogenetic dichotomy or polytomy), taxon (classificatory unit) and nomen (label designating such a classificatory unit) are distinct, for more simplicity they will often be designated collectively here by the expressions 'node/ taxon' or 'taxon/nomen' which mean 'the node, the taxon designating it and the nomen applying to it'.

To apply this procedure, a number of data and Criteria must be available and respected.

$\{\mathrm{t} 1\}$ Some of these steps are general and must be completed before starting the analysis itself:

$\{t 1 a\}$ Build up a database of all available genus-series (GS) nomina in the group studied, with their 
nucleospecies ('type species') and a database of all available family-series (FS) nomina in the group, with their nucleogenera.

$\{\mathrm{t} 1 \mathrm{~b}\}$ Build up a database of all the FS nomina of the group ever used as valid at the rank family in at least one of 100 published comprehensive classifications or more since 1758, count their respective numbers of usages, sort them into four quarters and list those belonging in the upper quarter (UQ).

\{t1c\} Build up a TREE showing all the species involved in the cladistic analysis and all the wellsupported nodes according to a chosen a priori threshold value. Each of these nodes will be recognised in CLAD as a suprageneric taxon, including subordinate nodes/taxa and terminal taxa (species).

$\{\mathrm{t} 1 \mathrm{~d}\}$ Transcribe exactly the TREE into a cladonomic hierarchy CLAD of well-supported nodes, from the rank genus upwards, which correspond to more and more inclusive suprageneric taxa recognised in $C L A D$ as valid, but at this stage have neither nomina nor ranks. For each of these nodes/taxa, at the end of this procedure, all the parordinate taxa will have to be attributed to the same rank, but at this stage this rank is not known.

Starting from this point, the following steps of the procedure should be followed. This includes 8 points $\{\mathrm{t} 2\}$ that have to be followed in all situations, and three points $\{\mathrm{t} 3\}$ that have to be applied only in particular situations.

\{t2a\} Upper Quartile Criterion [UQC]. Point among all the genera considered valid in this group all the genera (or their synonyms) that are nucleospecies of UQ family-series nomina. The Criterion [UQC] requires that, at the end of this procedure, all these family-series nomina will be used as valid at least for one taxon of rank family (and possibly for other subordinate and/or superordinate taxa), but it does not tell us at this stage for which taxa.

$\{\mathrm{t} 2 \mathrm{~b}\}$ Upper Quartile Criterion [UQC]. Identify the pairs of UQ-taxa/nomina that appear parordinate at some level in the taxonomic hierarchy of CLAD. At the end of this procedure, some of them will remain parordinate, whereas others will not, if they must be referred to different superordinate taxa.

\{t2c\} Consistent Hierarchy Criterion [CHC], Sister-Taxa Criterion [STC] and Family-Series Precedence Criterion [FPC]. Identify the lowest ranked pair(s) (LRP) of parordinate UQ-taxa/nomina at rank family, i.e. the one or those which in CLAD has/have the highest number of superordinate taxa/ nomina below order. This/these lowest ranked pair(s) of UQ-nomina will provide the upward hierarchy of ranks superordinate to it/them used in CLAD: the suprafamilial FS ranks (in the upper-FS-branch) should be fixed in order to saturate the FS (i.e., using the ranks apofamily, epifamily and superfamily) if enough ranks are available, and then the hierarchy of CS ranks (in the CS-branch) should be implemented if more ranks are needed.

\{t2d\} Sister-Taxa Criterion [STC], Non-Redundancy Criterion [NRC] and Upper Quartile Criterion [UQC]. In each branch subordinate to a parordinate taxon/nomen resulting from $\{\mathrm{t} 2 \mathrm{c}\}$, the rank family should be attributed to the highest ranked taxon and its parordinate taxon/a, except if the $[\mathrm{NTC}]$ requires to attribute it to a lower ranked taxon.

\{t3a\} Nomenclatural Thrift Criterion [NTC]. Check that, in the nomenclature adopted following the preceding Criteria, there does not exist any polytomy at the rank family. If such a polytomy exists, recognise it taxonomically as a single family, even if this is contradictory with the [NRC] (i.e., if this family is redundant with its superordinate super-, epi- or apofamily), and downgrade all the other 'families', and by way of consequence all their subordinate taxa, by one rank.

\{t3b\} Family-Series Precedence Criterion [FPC]. If there remain some unnamed taxa in CLAD, name them and, if their allotment to the FS or CS is unclear, give precedence to the FS if this is compatible with the other ranks in the hierarchy imposed by the previous steps of this procedure.

\{t3c\} Nomenclatural Precedence Criterion [NPC] and Conflict of Precedence Criterion [CPC]. Check that, in the nomenclature adopted following the preceding Criteria, the Code's or DONS Rules of nomenclatural precedence among nomina are respected, and if not correct the nomenclature accordingly.

\{t2e\} Mandatory Rank Criterion [MRC]. Check that all species/terminal taxa are indeed referred to a taxon of the rank family, and if it is missing implement it, even if it is redundant with a superordinate taxon.

\{t2f Non-Redundancy Criterion [NRC]. Check that, in the taxonomic hierarchy now obtained, there are no redundant taxa (i.e., taxa of different ranks having no parordinate taxa and having the same intension and extension) within the same nominal-series (FS or CS). If such redundant taxa/nomina exist, delete them, except those that are attributed through the implementation of the [NTC] or of the [MRC]. 
$\{\mathrm{t} 2 \mathrm{~g}\}$ Consistent Hierarchy Criterion [CHC]. Proceed similarly for the downward hierarchies below each taxon/nomen fixed at the rank family in $\{\mathrm{t} 2 \mathrm{~b}\},\{\mathrm{t} 2 \mathrm{~d}\},\{\mathrm{t} 2 \mathrm{e}\}$ and $\{\mathrm{t} 3\}$ and below each of their parordinate taxon/nomen.

$\{\mathrm{t} 2 \mathrm{~h}\}$ Consistent Naming Criterion [CNC]. Check that all nodes/taxa in TREE have been allocated a nomen and attributed a rank, and that the hierarchy of ranks is consistent, with all parordinate taxa sharing the same rank. If this is not the case, go back step by step in the procedure until the source of the error has been found and corrected.

Theoretical and real examples will allow to illustrate this procedure and point to some of its possible traps.

\subsubsection{Theoretical examples}

Let us start with two examples based on two hypothetical partial trees shown in Figures F4.TCP-1 and F5.TCP-2. Both concern 22 genera and show only well-supported nodes according to our threshold. The hypothesised phylogenetic relationships are the same in the upper part of both examples (genera G1 to G10), so that the taxa and the taxonomic hierarchies in this part are also the same in both cases, but they are different in the lower part (genera G11 to G22). These examples illustrate the importance of the Ten Criteria Procedure, and particularly in this case of the Upper Quartile, Sister-taxa and Nomenclatural Thrift Criteria, in fixing the ranks of taxa.

\subsection{Example T1}

This case is shown in the partial tree of Figure F4.TCP-1. The following steps allow to attribute ranks and allocate nomina to all the nodes/taxa of this partial tree between the 22 genera G1 to G22 and the order MO1.

\{t2a\} In TCP-1, four FS nomina QF1, QF2, QF3 and QF4, based respectively on the nucleogenera G1, G5, G11 and G16, belong in the Upper Quarter of usages. These four UQ- nomina must therefore be allocated to four taxa attributed to the rank family, and possibly to others if required by the Principle of Coordination.

\{t2b Among the sister-taxa relationships shown in Figure F4.TCP-1, two involve pairs of parordinate UQ-taxa/nomina: QF1 and QF2, and QF3 and QF4. The family QF1 is therefore defined as including G1-G3 and its sister-family QF2 as including G4-G6. The family QF3 is defined as including G11-G15 and its sister-family QF4 as including the single genus G16.

$\{\mathrm{t} 2 \mathrm{c}\}$ There are five infraordinal ranks above the pair QF1 and QF2 whereas there are only four above QF3 and QF4. Therefore, according to the [FPC], the taxonominal hierarchy above the first of these two pairs provides the family-series saturation: the taxa/nomina at the five ranks between these two families and the order MO1 are fixed first at the three suprafamilial ranks in the FS (HA1, HE1 and HP1) and then in the CS (HI1 and HU1). This applies also to their parordinate taxa/nomina (SA2, SE2 and SP2 in the FS; SI2 and SU2 in the CS).

$\{\mathrm{t} 2 \mathrm{~d}\}$ The families QF3 and QF4 are subordinate to four infraordinate taxa. In this hierarchy, the ranks HU1, HI1 and SP2 are imposed by the hierarchy above the pair of families QF3-QF4. Therefore, the taxon below the superfamily SP2 and above the families QF3 and QF4 must be attributed to the rank epifamily (see Table T2.SEQ) as DE3, and this also applies to its sister-taxon SE4, including the genera G17 and G18.

$\{\mathrm{t} 3 \mathrm{a}\}$ This step has been respected.

$\{\mathrm{t} 3 \mathrm{~b}\}$ to $\{\mathrm{t} 3 \mathrm{c}\}$ These steps are irrelevant here.

\{t2e\} So far, the genera G7-G10 and G17-G22 have not been referred to any taxon at family rank. According to the [MRC], six additional families should be recognised for these 10 genera. Two of them, DF5 and DF6, are sister-families, but the other four, MF7, MF8, MF9 and MF10 have no sister-families. They must nevertheless be recognised, although they are redundant with their immediate superordinate taxa which belong for the first two of them in the same nominal-series (the FS), and for the other two in a different nominal-series (the CS).

$\{\mathrm{t} 2 \mathrm{f}\}$ There are only two redundant taxa within the FS in this cladonomy, MF7 and MF8, and both are imposed by the $[\mathrm{MRC}]$. 


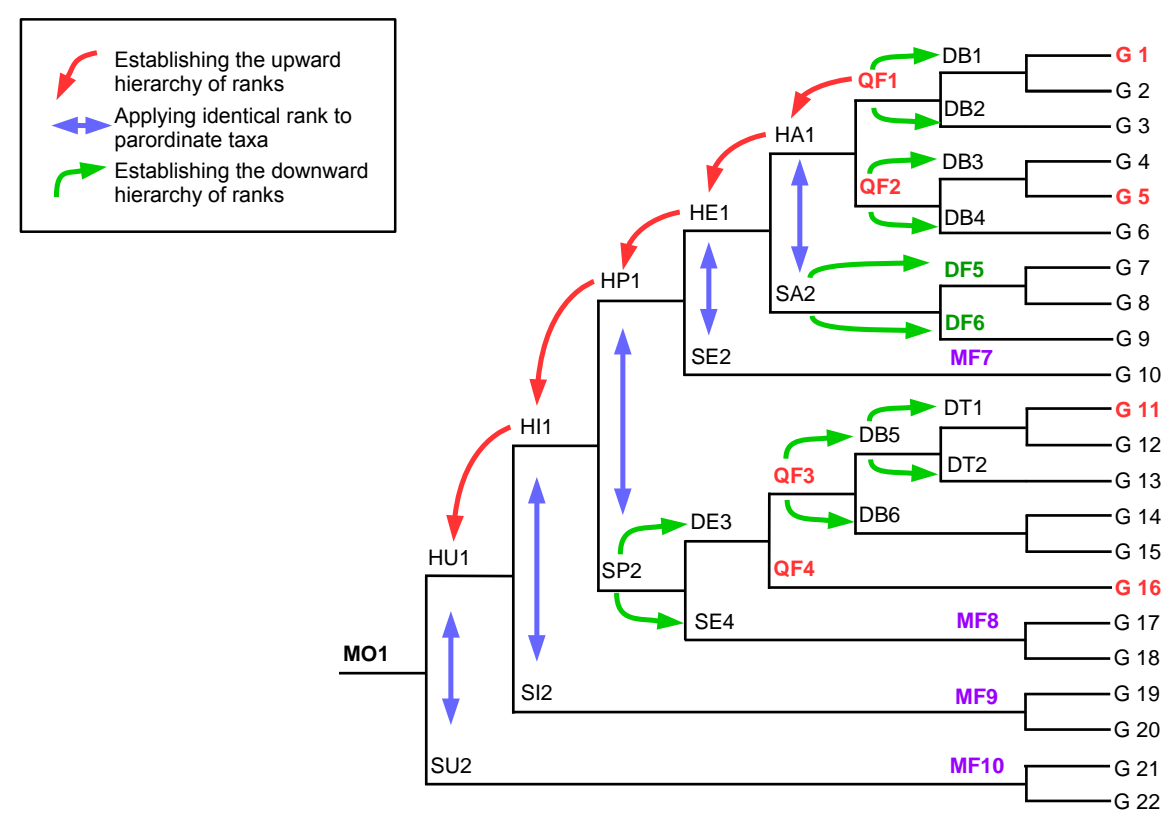

FIGURE 4. TCP-1. The Ten Criteria Procedure. Example T1.

Meaning of letters in identifiers for nomina/taxa:

Two-letter identifiers: First of two letters (using the Ten Criteria Procedure for the rank attribution of the nomen/taxon): D, downward hierarchy; $\mathrm{H}$, upward hierarchy; $\mathrm{M}$, mandatory rank; Q, upper quartile; S, sister-taxon.

Second of two letters (rank attributed to nomen/taxon through the Criteria [CHC], [STC] and [MRC]): A, apofamilia; B, subfamilia, E, epifamilia; F, familia; I, infraordo; O, ordo; P, superfamilia; R, subtribus; T, tribus; U, subordo. Colours for families: red, nomen attributed to this rank through the Upper Quartile Criterion; green, nomen attributed to this rank through downward hierarchy; violet: nomen attributed to this rank through the Mandatory Rank Criterion.

Single letter identifiers (rank of nomen/taxon): G, genus.

Generic identifiers in red refer to genera which are nucleospecies of family-series nomina belonging in the Upper Quarter of usages.

$\{\mathrm{t} 2 \mathrm{~g}\}$ The downward hierarchy requires to recognise the subordinate taxa DB1 to DB6, and DT1 and DT2.

$\{\mathrm{t} 2 \mathrm{~h}\}$ This step has been respected.

In conclusion, this case is quite simple and straightforward, as the ranks of all the suprageneric nodes/ taxa derive automatically, through parordination, superordination or subordination, from four pieces of information, the fact that the nomina QF1, QF2, QF3 and QF4 are part of the Upper Quarter of usages. Additionally, in this case two family taxa/nomina are redundant with their immediate superordinate FS taxa. As a consequence, the cladonomy derived from this information requires the recognition of 10 families in this partial tree. Note that this conclusion derives only from the implementation of the $a$ priori Criteria defined above, and did not indulge any subjective decision.

\subsection{Example T2}

This case is shown in the partial tree F.TCP-2. The following steps allow to attribute ranks and allocate nomina to all the nodes/taxa of this partial tree between the 22 genera G1 to G22 and the order MO1. Contrary to the preceding, this example applies the Nomenclatural Thrift criterion [NTC].

\{t2a\} In TCP-2, two FS nomina QF1 and QF2, based respectively on the nucleogenera G1 and G5, belong in the Upper Quarter of usages. These two UQ-taxa/nomina must therefore be attributed to two taxa attributed to the rank family, and possibly to others if required by the Principle of Coordination. 


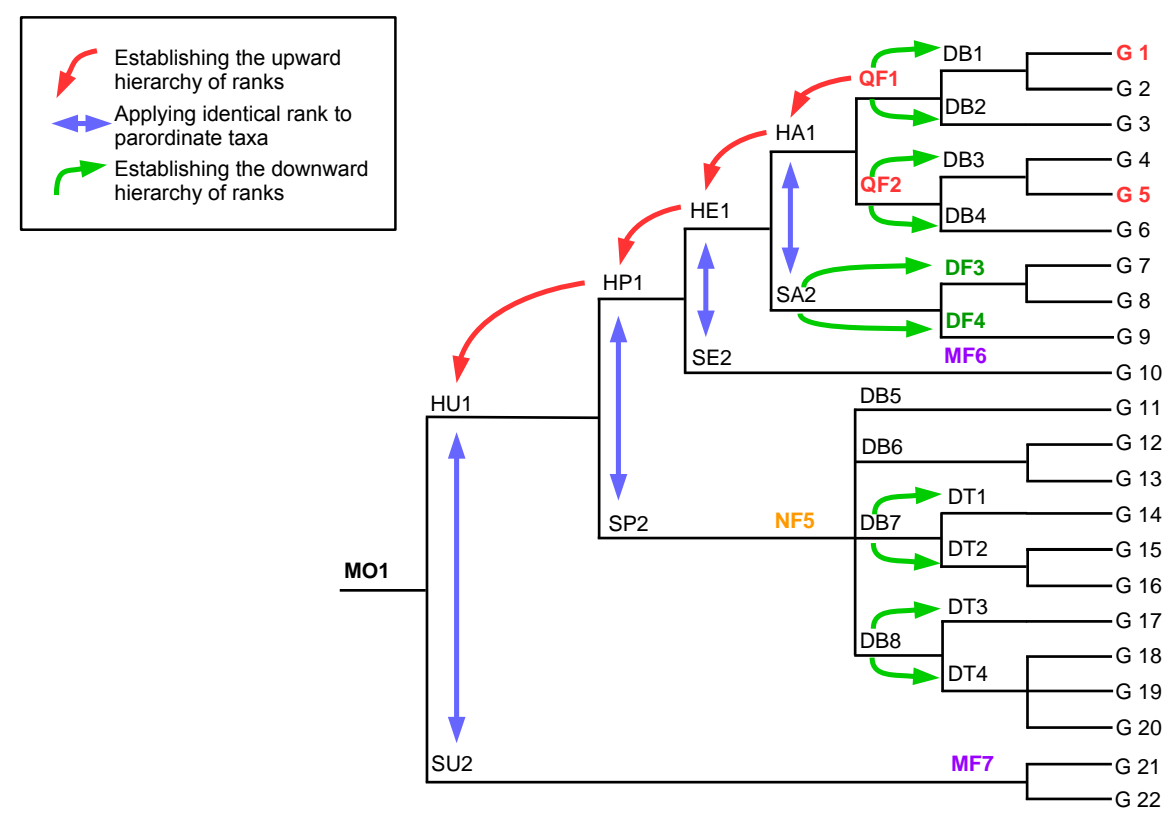

FIGURE 5. TCP-2. The Ten Criteria Procedure. Example T2.

Meaning of letters in identifiers for nomina/taxa:

Two letter identifiers: First of two letters (using the Ten Criteria Procedure for the rank attribution of the nomen/taxon): D, downward hierarchy; H, upward hierarchy; $\mathrm{M}$, mandatory rank; N, nomenclatural thrift; $\mathrm{Q}$, upper quartile; S, sistertaxon.

Second of two letters (rank attributed to nomen/taxon through the Criteria [CHC], [STC] and [MRC]): A, apofamilia; B, subfamilia, E, epifamilia; F, familia; I, infraordo; O, ordo; P, superfamilia; R, subtribus; T, tribus; U, subordo.

Colours for families: red, nomen attributed to this rank through the Upper Quartile Criterion; green, nomen attributed to this rank through downward hierarchy; orange: nomen attributed to this rank through the Nomenclatural Thrift Criterion; violet: nomen attributed to this rank through the Mandatory Rank Criterion.

Single letter identifiers (rank of nomen/taxon): G, genus.

Generic identifiers in red refer to genera which are nucleospecies of family-series nomina belonging in the Upper Quarter of usages.

\{t2b Among the sister-taxa relationships shown in Figure F5.TCP-2, a single one involves UQnomina as parordinate, QF1-QF2. The family QF1 is defined as including G1-G3 and its sister-family QF2 as including G4-G6.

$\{\mathrm{t} 2 \mathrm{c}\}$ There are four infraordinal ranks above the pair QF1-QF2. Therefore, according to the [FPC], the taxonominal hierarchy above this pair provides the family-series saturation: the taxa/nomina at the four ranks between these two families and the order MO1 are fixed first at the three suprafamilial ranks in the FS (HA1, HE1 and HP1) and then in the CS (HU1). This applies also to their parordinate nomina/ taxa (SA2, SE2 and SP2 in the FS; SU2 in the CS).

$\{\mathrm{t} 2 \mathrm{~d}\}$ At this stage, there remain four main branches for which the position of the rank family has not been fixed: SA2, SE2, SP2 and SU2. No UQ-nomen is available for any of them. The first of these four branches, SP2, includes a dichotomy, one branch of which also includes a dichotomy: the first dichotomy corresponds to the highest ranked taxa subordinate to SA2 and should be taxonomically recognised as a pair of families DF3-DF4. Two other branches include only one genus (G10) or two genera (G21 and G22), but no node/taxon that could be attributed to the family-series: they will be discussed further below. The fourth branch is more complex, as it consists in a tetratomy and includes 10 genera G11-G20. According to the 'normal' situation in $\{\mathrm{t} 2 \mathrm{~d}\}$, the four branches of this tetratomy, which are the highest ranked taxa in the superfamily SP2, should be attributed to the rank family, but this is hindered by the [NTC].

$\{\mathrm{t} 3 \mathrm{a}\}$ As the tetratomy under SP2 is not resolved, the Nomenclatural Thrift Criterion requires to 
downgrade its four branches at the subfamily level as DB5 to DB8 and to recognise above them a single family NF5, despite the fact that it is redundant within the family-series with the superfamily SP2.

$\{\mathrm{t} 3 \mathrm{~b}\}$ This step is irrelevant here.

$\{t 3 c\}$ This step cannot be but irrelevant here as we did not take into account the publication dates of the FS nomina in this hypothetical example.

$\{\mathrm{t} 2 \mathrm{e}\}$ This step has been respected.

\{t2f\} So far, the genera G10, and G21 and G22 have not been allocated to any taxon at family rank. According to the [MRC], two families MF6 and MF7 should therefore be recognised, although they are redundant with their immediate superordinate taxa which belong for the first in the same nominal-series (the family-series), and for the other one in a different nominal-series (the class-series).

$\{\mathrm{t} 2 \mathrm{~g}\}$ The downward hierarchy requires to recognise the subordinate taxa DB1 to DB4, and DT1 to DT4.

$\{\mathrm{t} 2 \mathrm{~h}\}$ This step has been respected.

Although this case is a bit more complex than the preceding, it is also straightforward, as the ranks of all the suprageneric nodes/taxa derive automatically, through parordination, superordination or subordination, from two pieces of information, the fact that the nomina QF1 and QF2 are part of the upper quartile. Additionally, in this case a family is erected for a polytomy which should 'normally' have been taxonominally accounted for by four parordinate families, the latter being downgraded at the rank subfamily. This family, as well as another one including a single genus, is redundant within the family-series with its immediate superordinate taxon. In conclusion, the cladonomy derived from this information and these Criteria requires the recognition of 7 families in this partial tree. Here also this conclusion derives only from the implementation of the a priori Criteria defined above, and did not imply any arbitrary decision.

\subsubsection{Real examples}

\subsection{Example R1: the taxonominal hierarchy in the three orders of extant LISSAMPHIBIA}

As we have seen above, the number of ranks is often quite dissimilar in different parts of a tree. According to the Ten Criteria Procedure, the nominal-series and the names of these ranks are determined by the maximum number of suprafamilial and infraordinal ranks between the rank order and the rank family. Therefore, under the [TCP] Criteria the taxonominal hierarchy will have to be fixed independently in each zoological order.

In the subclass Lissamphibia, excluding the all-fossil Allocaudata whose position in TREE and rank are unclear, we recognise three orders including extant species: the ANURA, UrodeLA and GyMnOPHiona. Let us consider them successfully.

\{u1\} Example R1a: order ANURA. In this order, a careful survey of TREE and CLAD allows to find that the lowest ranked pair (LRP) of parordinate UQ-taxa/nomina fixed at rank family by the [UQC] is the pair RANIDAE-RHACOPHORIDAE. This pair has 11 superordinate taxa below the rank order, which is the highest number in this order. According to the Family-Series Precedence Criterion [FPC] step and the Criterion $\{\mathrm{t} 2 \mathrm{c}\}$ above, this pair will allow to fix the upward hierarchy of ranks superordinate to it. This hierarchy starts in the upper-FS-branch with the three ranks apofamily (RANEIDAE), epifamily (RANOIDAE) and superfamily (RANOIDEA), which saturates the family-series, and then follows with eight ranks in the CS-branch, from infraphalanx (ECAUDATA) to suborder (HYDROBATRACHIA). Starting from this hierarchy and using first the Sister-Taxa Criterion and then all the other Criteria of the [TCP], the ranks of all other taxa of ANURA derive unambiguously. For example, if we consider the lower-FSrank between the rank familia (RANIDAE) and the rank genus (Rana), the structure of TREE requires to have eight ranks, from subfamilia RANINAE to infraclanus RANITOES. Therefore the [FPC] is a very parsimonious and powerful Criterion to fix automatically the taxonominal hierarchy in an order.

The same result could have been obtained differently, starting from the genera rather than the pair of families. The generic nomen Rana Linnaeus, 1758 is the nucleogenus of the family UQ-nomen RANIDAE Batsch, 1796. Therefore, CLAD has to include a family RANIDAE. Climbing up CLAD above Rana leads then first to a series of taxa including this genus and therefore potentially bearing the nomen RANIDAE under another paronym at a higher rank, then to the UQ-nomen RHACOPHORIDAE, based on Rhacophorus Kuhl \& van Hasselt, 1822. Both RANIDAE and RHACOPHORIDAE, being UQ-nomina, must be recognised 
as parordinate families, so the common taxon that will include them both will have to be at a higher rank. Above the rank family, to account for all well-supported nodes in TREE we need 11 ranks below order: three in the FS above family, the FS ranks being then saturated, and eight in the CS, from infraphalanx to suborder. Below the rank family, we need eight suprageneric ranks. Therefore, altogether, to fix unambiguously the place of Rana in CLAD, we need to use 22 of the 23 suprageneric ranks that are employed here in the subclassis LISSAMPHIBIA including all extant amphibians.

\{u2\} Example R1b: order Urodela. The same methodology can be used in this order, where it is much quicker, as the number of genera involved is much lower. In this case, the lowest ranked pair (LRP) of parordinate UQ-taxa/nomina fixed at rank family by the [UQC] is the pair AMPHIUMIDAEPLETHODONTIDAE. This pair has only 4 superordinate taxa below the rank order, which is the highest number in this order. In order to saturate the family-series, from this pair the hierarchy starts in the upperFS-branch with the three ranks apofamily (AMPHIUMEIDAE), epifamily (AMPHIUMOIDAE) and superfamily (AMPHIUMOIDEA), and then we only need one rank in the CS-branch, suborder (PseUdosAuria). These four ranks will therefore be the only suprafamilial ranks used in the URODELA.

\{u3\} Example R1c: order Gymnophiona. Finally, if we turn to this order, the number of genera and suprageneric taxa is still much lower. In this order, only three FS-nomina belong in the Upper Quarter: the CAECILIIDAE, ICHTHYOPHIDAE and RHINATREMATIDAE. Among them, the lowest ranked pair is CAECILIIDAE-ICHTHYOPHIDAE. If we relied on this pair as above, as the starting point for fixing the taxonominal hierarchy in this order, we would need to use only one rank, superfamily, above family in this order, with a superfamily RHINATREMATOIDEA including a single family RHINATREMATIDAE and a superfamily CAECILIOIDEA with two families CAECILIIDAE and ICHTHYOPHIIDAE. This would not bother us much, but we are aware that most taxonomists have an immoderate fondness for 'taxonomic stability', a non-scientific concept, and would probably be very 'shocked' by a move from 10 families of caecilians as advocated by San Mauro et al. (2014) to three families! For this reason, we decided to derogate, at least provisionally, from our general Criteria in this case, and to recognise for the time being five families within this order. For this to be possible, it is necessary to add one 'superfluous' rank to the taxonominal hierarchy in this order, and, by symmetry with the other two orders, we recognised two suborders in the latter. Then, we have one suborder with a single family and a second suborder with two superfamilies including two families each, which allows to respect the [UQC].

\subsection{Example R2: genera Telmatobius and Rhinoderma}

The nomen RHINODERMATIDAE Bonaparte, 1850, based on the genus Rhinoderma Duméril \& Bibron, 1841, being part of the UQN, a family must bear this nomen. Climbing up TREE above this genus leads first to the nomen TELMATOBIIDAE Fitzinger, 1843, based on Telmatobius Wiegmann, 1834, which would have nomenclatural priority if Rhinoderma and Telmatobius were placed in the same family. To comply with the Criterion [CPC], we must recognise a family TELMATOBIIDAE, parordinate to RHINODERMATIDAE. To respect sister-taxa relationships, they both constitute the apofamily TELMATOBIEIDAE, which is part of the epifamily TELMATOBIOIDAE, and the latter of the superfamily CERATOPHRYOIDEA. The latter has four parordinate superfamilies, and together they constitute a taxon which has to be in the class-series as FS nomenclatural saturation has been reached in this branch: this turns out to be the hypophalanx Hylobatrachia.

\subsection{Example R3: genus Epidalea}

The genus Epidalea Cope, 1864 belongs in the UQ-family BUFONIDAE which is part of the superfamily BUFONOIDEA, one of the five branches that make up the hypophalanx HYLOBATRACHIA and for which the rank superfamily is required because of FS rank saturation in one of them, the CERATOPHRYNOIDEA. Therefore, the upward subordinal hierarchy above BUFONIDAE includes ten ranks, nine CS ranks (from hypophalanx to suborder) and one FS rank (superfamily).

Below BUFONIDAE, the hierarchical placement of the genus Epidalea (as well as of 11 other genera) requires nine ranks (from subfamily to hypoclanus).

Therefore, the unambiguous hierarchical placement of the genus Epidalea requires 21 of the 23 of the suprageneric ranks that we use here below the rank suclassis. 


\subsection{Example R4: genus Cycloramphus}

This genus is part of a taxon for which the first available nomen is CYCLORAMPHIDAE Bonaparte, 1850, which belongs in the UQN, and which has four sister-taxa. Altogether, these five taxa make up a group which is parordinate to the apofamily TELMATOBIEIDAE mentioned above and must therefore be known as the apofamily CYCLORAMPHEIDAE. Following the Criterion [NTC] requires to recognise in this apofamily a single family CYCLORAMPHIDAE, with five subfamilies, despite the fact that this makes the apofamily and the family nomina redundant. The present solution is provisional and will last only until the cladonomic relationships within this group are better resolved, allowing to have only two families in this apofamily.

As shown by Dubois (1984b), the first nomen available for the family including the genus Cycloramphus Tschudi, 1838 is CYCLORHAMPHINA Bonaparte, 1850, based on Cyclorhamphus Agassiz, 1847, an unjustified emendation of Cycloramphus Tschudi, 1838, whereas the spelling based on Cycloramphus appeared only later, in Bonaparte (1852). However, the spelling CYCLORAMPHIDAE must be preserved, and credited (misleadingly) to Bonaparte (1850) where it did not appear, by virtue of Article 35.4.1, whose pertinence is questionable (Dubois 2010a).

\subsection{Example R5: genus Polypedates}

The first superordinate FS taxon/nomen of Polypedates Tschudi, 1838 among the UQN is RHACOPHORIDAE, so the implementation of the ranks here is parallel to that of examples R1a and R1b above, however with a small but significant and noteworthy difference, which is not due to the Criterion [FPC] but to the nomenclatural Rules of the Code.

The first family-series nomenclaturally available for this genus is POLYPEDATIDAE Günther, 1858, but this nomen was invalidated before 1961 by usage of Article 40.2 in order to validate the 'wellknown' (in fact, then mostly by a few specialists) nomen RHACOPHORIDAE Hoffman, 1932, so that according to the Code the latter nomen should be known as RHACOPHORIDAE Hoffman, 1932 (1858).

In the meantime however, the genus Polypedates Tschudi, 1838 was revalidated as applying to a genus distinct from Rhacophorus Kuhl \& Van Hasselt, 1822. Today, the nomen RHACOPHORIDAE must therefore be used at all ranks for taxa that include both Rhacophorus and Polypedates. In CLAD, following the Principle of Coordination it applies to 7 taxa, from familia RHACOPHORIDAE to subclanus RHACOPHORITIES.

However, the situation is different regarding the two taxa that include Polypedates but exclude Rhacophorus, at ranks subclanus and infraclanus. Article 40.2.1 reads: "A name maintained by virtue of this Article retains its own author but takes the priority of the replaced name, of which it is deemed to be the senior synonym." Taking strictly these words would lead to strange consequences: in this case the family and all its endotaxa including the genus Rhacophorus would bear paronyms based on the genus Rhacophorus, dated 1858, but no taxon including the genus Polypedates could bear a FS nomen based on Polypedates, even if it excludes Rhacophorus. In fact, at low taxonomic levels, the nomina RHACOPHORIDAE and POLYPEDATIDAE cannot be synonyms when they apply to taxa mutually exclusive regarding their nucleogenera. Solving this nomenclatural problem would require to establish a new FS nomen. This would be possible on the basis of the genus nomen Taruga Meegaskumbura et al., 2010, although a nomen based on Polypedates already exists. This would clearly not be a good solution in terms of nomenclatural parsimony.

We think this Article, which is already problematic for other reasons (Dubois 2010a) should be reworded or even better suppressed from the Code, as Article 23.9 on Reversal of precedence is sufficient to solve problems of this kind when they arise. In the meantime, we consider that these two nomina cannot be synonyms in this situation and we recognise two subclans POLYPEDATITIES and RHACOPHORITIES, and an infraclan POLYPEDATITOES.

\subsection{Example R6: genus Odontobatrachus}

This case is much simpler than the preceding ones. According to TREE, this genus is parordinate to two other taxa, the genus Phrynobatrachus and the large taxon for which it was shown above that the proper 
rank and nomen were superfamily RANOIDEA. Therefore, two more superfamilies PHRYNOBATRACHOIDEA and ODONTOBATRACHOIDEA must be recognised. Each of them contains a single family, and the latter are indeed redundant, in this case, with both the superfamily and the genus, but these families should be recognised to comply with the Criterion [MRC]. Discrepancies in the number of subordinate taxa in the three superfamilies mentioned here reflect quite accurately the different structures of TREE in its different parts, so they are phylogenetically informative and not random or arbitrary and they should be recognised taxonomically, as provided by the Criterion [STC].

\subsection{Example R7: genus Litoria}

The first FS nomen available above the generic nomen Litoria Tschudi, 1838 is PELOBII Fitzinger, 1843, which is invalid for being based on the generic nomen Pelobius Fitzinger, 1843, an invalid junior homonym (Dubois 1984b; Dubois \& Frétey 2016). The next available FS nomen for this genus is PELODRYADIDAE Günther, 1859, a junior synonym of PHYLLOMEDUSIDAE Günther, 1858. None of the latter nomina is part of the UQN. The sister-taxon of this family is HYLIDAE Rafinesque, 1815, which belongs in the UQN, therefore both taxa should be recognised as families by virtue of the Criterion [STC]. Treating the PHYLLOMEDUSIDAE as a subfamily of the HYLIDAE would make the latter redundant relative to the superfamily HYLOIDEA, which has four parordinate superfamilies, and this should be avoided according to the Criterion [NRC].

The nomen PELODRYADIDAE, here retained as valid but at the rank subfamily, is based on the generic nomen Pelodryas Günther, 1858, which is currently considered as a doxisonym of Ranoidea Tschudi, 1838 (Dubois \& Frétey 2016). According to Article 40.1, the fact that Pelodryas is currently considered as an invalid junior synonym of another valid generic nomen has no impact on the validity of the FS nomen, as the latter was not replaced before 1961, contrary to the situation in the example R5 above.

\subsection{Example R8: genera Alytes, Bombina and Discoglossus}

In the examples mentioned above, starting from the genus and moving upwards in the taxonominal hierarchy always led to a point where we encountered a FS nomen being part of the UQN. But this is not always the case, as the following example will show.

The getangiotaxon A1a of the genus Alytes Wagler, 1829 is parordinate to a taxon A1b accommodating the genera Discoglossus Otth, 1837 and Latonia Meyer, 1845. Both taxa A1a and A1b constitute the taxon A2a which is parordinate to A2b, that contains the genera Barbourula Taylor \& Noble, 1924 and Bombina Oken, 1816. Three FS nomina can be used for these taxa (see Dubois 1987e): ALYTAE Fitzinger, 1843; Bombinatorina Gray, 1825; and DisCOGLOSSIDAE Günther, 1858. The last two belonging in the UQN, they must be recognised at the rank family. One could a priori consider the possibility to use the nomen DISCOGLOSSIDAE either for A1b or for A2a, but in the latter case it could not be kept at the rank family because the nomen ALYTIDAE has nomenclatural priority upon it. Therefore, Alb must be DISCOGLOSSIDAE to comply with the Criteria [STC], [NPC] and [CPC]. ALYTIDAE then applies to A1a, ALYTOIDEA to A2a and BOMBINATOROIDEA to its parordinate superfamily, with a single family BOMBINATORIDAE imposed by the Criterion [MRC] despite being redundant. This example shows that: $\{\mathrm{v} 1\}$ to follow strictly the Criterion [STC], one has also to pay attention to nomenclatural priority among nomina, which sometimes precludes the use of some possible taxonominal solutions (this is similar to example R2 above); $\{\mathrm{v} 2\}$ at any rate, in all cases even the position in the hierarchy of the families whose nomina are not among the UQN is fixed automatically by the application of the Criterion [CPC]. In this case, the position of the DISCOGLOSSIDAE is fixed by the Criterion [UQC], that of the ALYTIDAE by the Criteria [STC], [NPC] and [CPC], and that of the BOMBINATORIDAE by the Criteria [UQC] and [MRC].

\subsection{Example R9: genera Ambystoma and Dicamptodon}

The family nomina AMBYSTOMATIDAE and SALAMANDRIDAE both belong in the UQN and, according to the structure of TREE and CLAD, must therefore be used at family rank to designate sister-taxa. The 
family SALAMANDRIDAE includes more than 20 genera and has a rather complex taxonomic structure, but the family AмBYSTOMATIDAE includes only two extant genera Ambystoma and Dicamptodon and five all-fossil genera, the status of which is still uncertain. As explained above, in the present work the taxonominal hierarchy is fixed only on the basis of the extant taxa, and does not take the all-fossil ones into account. The Consistent Naming Criterion [CNC] contains the following precision: "for two branches to be taxonomically recognised, one of them at least must include more than one supraspecific subtaxon (i.e., of rank genus or above)". This is not the case here and, as long as no third extant genus is cladistically supported and taxonomically recognised in this family, the latter should include no subfamily but only these two sister-genera.

\subsection{A few other examples and comments}

As mentioned above, the family rank plays a crucial role in our system because it is mandatory. A cladonomy will be more informative if as many families as possible have parordinate families. This imposes sometimes to recognise at the same rank family some sister-taxa which are highly unbalanced in terms of numbers of included taxa, such as CRYPTOBRANCHIDAE and HYNOBIIDAE, BREVICIPITIDAE and HEMISOTIDAE Or BRACHYCEPHALIDAE and CEUTHOMANTIDAE.

This latter example shows that the Criterion [UQC] does not fix the taxonomic hierarchy in a rigid manner. As a matter of fact, one might fear that the implementation of this Criterion could forbid the recognition of new families when brand new species are discovered that represent not only new genera but also new higher taxa. In such cases, as shown by a few recent examples, there exists indeed a 'temptation' for the authors who describe the new species or genus to 'overrate' their finding and to erect immediately a new family for it. However, not all recently discovered organisms require the erection of such high-ranked taxa, whatever exciting their discovery may have been for the biologists who found them. The analysis presented in the present work shows that it is sometimes the case and sometimes not. When genuine phylogenetic discoveries lead to the taxonomic recognition of brand new branches in TREE, these can be recognised at the rank family even if their nomina are very recent and then of course not members of the UQN. Let us consider in this respect the fate of the eight last nomina of families of extant amphibians that have been established in the literature, from 2003 to 2014. They can be sorted in four categories:

\{w1 The most extreme example in this respect, R6 above, is the genus Odontobatrachus, described in 2014 but the isolated cladistic position of which requires its recognition in CLAD not only as a new family ODONTOBATRACHIDAE but also as a superfamily ODONTOBATRACHOIDEA, the latter being parordinate to two long known taxa, now the superfamilies PHRYNOBATRACHOIDEA and RANOIDEA. In this case not only the new family appears warranted under our Criteria but it is even an 'understatement' of the uniqueness of this lineage.

\{w2\} Twomonogeneric familieserectedin2003(NASIKABATRACHIDAE) andin2009(CEUTHOMANTIDAE, discussed above in 2.4.5.2.2.3-4 and 2.4.5.2.4.3-4) are maintained at the rank family in CLAD, although of course they do not belong in the UQN.

\{w3 Two families erected in 2006 (CRYPTOBATRACHIDAE) and 2008 (CRAUGASTORIDAE) are here downgraded to the rank subfamily, and two other ones established in 2008 (STRABOMANTIDAE) and 2012 (CHIKILIDAE) to the rank tribe.

$\{\mathrm{w} 4\}$ The last one, THOROPIDAE, erected in 2006, is a strict synonym of a nomen established in 1850, CYCLORAMPHIDAE, and does not even deserve to be downgraded to a lower rank.

The heterogeneity of these situations, for a few taxa established over a short period of 12 years, highlights the fact that, in the absence of an explicit methodology for fixing the position of the rank family in the taxonominal hierarchy, decisions are bound to be largely arbitrary and a great heterogeneity of treatment for similar situations cannot but exist from one group to another. The mere fact that a new genus is 'cladistically isolated', i.e. referred alone (with no sister-genus) to its getangiotaxon, does not require by itself to give the latter a high rank in the taxonominal hierarchy. This all depends on the other taxa required by the cladistic tree. In CLAD, in order to reflect bijectively TREE, the 'cladistically isolated' genera described after 2000 require indeed the recognition of new FS taxa, whose nomina are based on theirs, but these are referred here to a vast array of ranks, including superfamily (Odontobatrachus), family (Astrobatrachus, Nasikabatrachus), subfamily (Astrobatrachus), tribe (Chikila), subtribe (Karsenia) and infratribe (Hypodactylus). Therefore, unlike under a phenetic paradigm under a cladistic paradigm the discovery of a 'brand new kind of organisms' does not entail necessarily the erection of highly ranked new taxa. 
TABLE 14.NUM. Number of generic and suprageneric taxa of LISSAMPHIBIA below class recognised as valid in the present work.

\begin{tabular}{|c|c|c|c|c|c|c|}
\hline Rank & $\begin{array}{l}\text { Total number } \\
\text { of extant } \\
\text { taxa }\end{array}$ & $\begin{array}{c}\text { Number of } \\
\text { new extant taxa }\end{array}$ & $\begin{array}{c}\% \text { of } \\
\text { new extant } \\
\text { taxa }\end{array}$ & $\begin{array}{l}\text { Total number of } \\
\text { fossil taxa }\end{array}$ & $\begin{array}{l}\text { Total number of } \\
\text { extant }+ \\
\text { fossil taxa }\end{array}$ & $\begin{array}{l}\text { Unnamed } \\
\text { incertae sedis }\end{array}$ \\
\hline Subclassis & 1 & 0 & 0 & - & 1 & - \\
\hline Ordo & 3 & 0 & 0 & 1 & 4 & - \\
\hline Subordo & 7 & 1 & 14.3 & - & 7 & 3 \\
\hline Infraordo & 2 & 0 & 0 & - & 2 & - \\
\hline Hypoordo & 2 & 0 & 0 & - & 2 & - \\
\hline Superphalanx & 2 & 0 & 0 & - & 2 & 1 \\
\hline Epiphalanx & 2 & 1 & 50.0 & - & 2 & - \\
\hline Phalanx & 3 & 2 & 66.7 & - & 3 & - \\
\hline Subphalanx & 5 & 2 & 40.0 & - & 5 & 1 \\
\hline Infraphalanx & 4 & 3 & 75.0 & - & 4 & - \\
\hline Hypophalanx & 3 & 1 & 33.3 & - & 3 & - \\
\hline TOTAL Class-series & 34 & 10 & 29.4 & 1 & 35 & 5 \\
\hline Superfamilia & 18 & 0 & 0 & - & 18 & 3 \\
\hline Epifamilia & 12 & 1 & 8.3 & - & 12 & - \\
\hline Apofamilia & 9 & 0 & 0 & - & 9 & - \\
\hline Familia & 69 & 1 & 1.4 & 13 & 82 & 12 \\
\hline Subfamilia & 87 & 11 & 12.6 & 2 & 89 & 6 \\
\hline Tribus & 89 & 21 & 23.6 & - & 89 & 6 \\
\hline Subtribus & 92 & 29 & 31.5 & - & 92 & 3 \\
\hline Infratribus & 65 & 26 & 40.0 & - & 65 & 2 \\
\hline Hypotribus & 44 & 24 & 54.5 & - & 44 & 2 \\
\hline Clanus & 32 & 22 & 68.8 & - & 32 & - \\
\hline Subclanus & 17 & 10 & 58.8 & - & 17 & - \\
\hline Infraclanus & 23 & 14 & 60.9 & - & 23 & 1 \\
\hline Hypoclanus & 14 & 11 & 78.6 & - & 14 & 1 \\
\hline Catoclanus & 2 & 1 & 50.0 & - & 2 & - \\
\hline TOTAL Family-series & 573 & 171 & 29.8 & 15 & 588 & 36 \\
\hline Genus & 575 & 13 & 2.2 & 191 & 766 & - \\
\hline TOTAL CS, FS \& GS & 1182 & 194 & 16.4 & 207 & 1389 & 41 \\
\hline
\end{tabular}

\subsection{Some comments on the new nomina introduced in the present work}

In the present work, we recognise 573 extant taxa of the family-series, including 171 new ones (29.8\%), from rank familia to catoclanus (Table T14.NUM). To name these taxa, because of the nomenclatural parsimony provided by the Principle of Coordination, we needed to coin only 154 new FS nomina, as well as 17 hyponymous paronyms of some of the latter (having the same nucleogenera, authors and dates, and being therefore not distinct nomina). To make these new nomina available, anchored and valid, we followed strictly the rules of the Code, in particular: $\{\mathrm{x} 1\}$ we mentioned the fact that their nomina were new nomina provided for new taxa; $\{\mathrm{x} 2\}$ we provided character-based diagnoses for these taxa; $\{\mathrm{x} 3\}$ we explicitly designated the nucleogenera of these nomina. There were two situations regarding the designation of the nucleogenera for these 154 new FS nomina (Table T15. NEW): in 110 cases $(71.4 \%)$, we had no choice, because the new FS taxon included a single valid genus according to CLAD; but in 44 cases $(28.6 \%)$, a choice had been made between two or more included genera. For such choices, we followed two basic Criteria. The first one, $\{\mathrm{y} 1\}$, relies on the important idea that nomina coined by taxonomists should not be so to 'please themselves' or to show the breadth and depth of their 'classical culture', but to act as convenient devices for unambiguous universal communication 


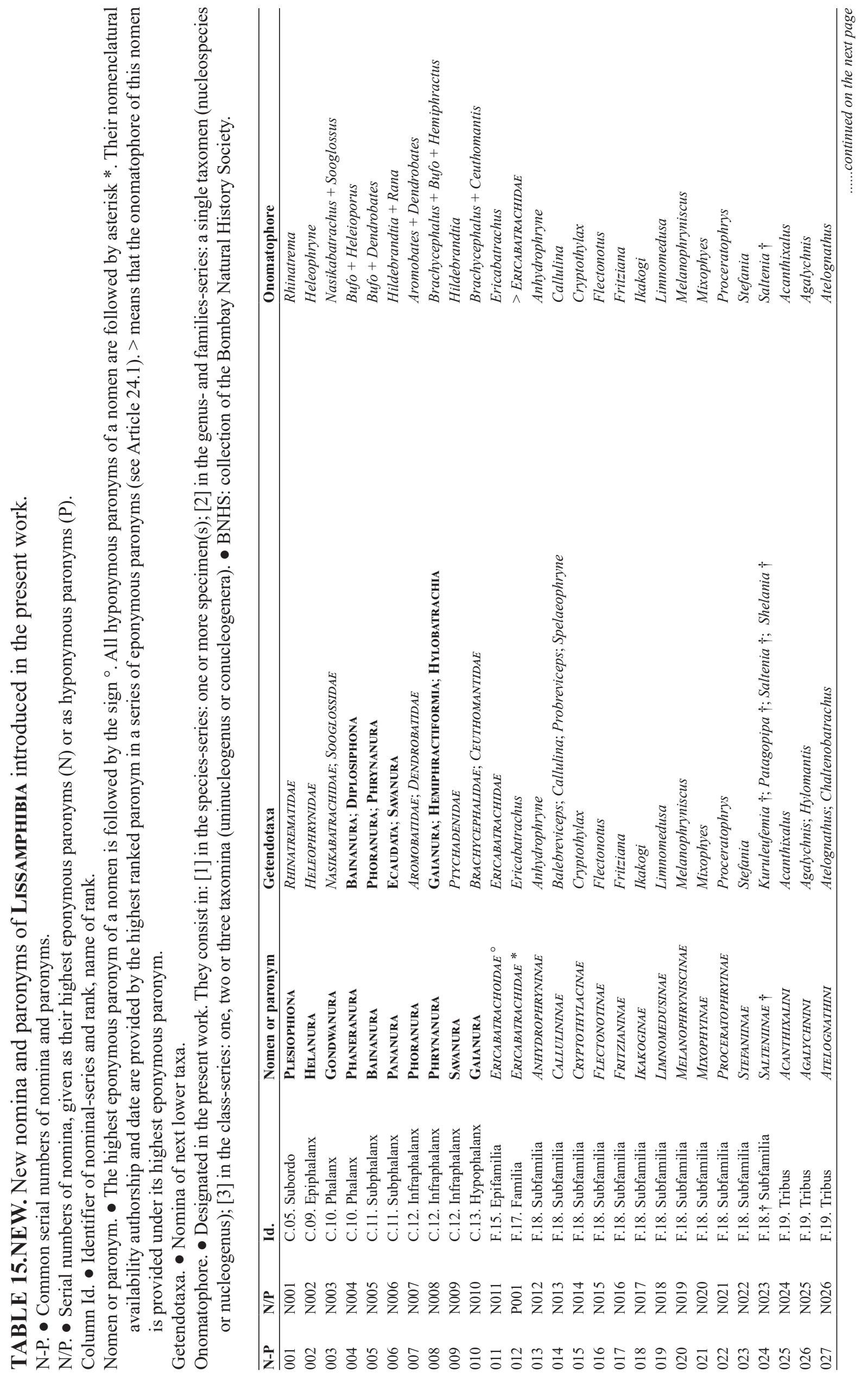




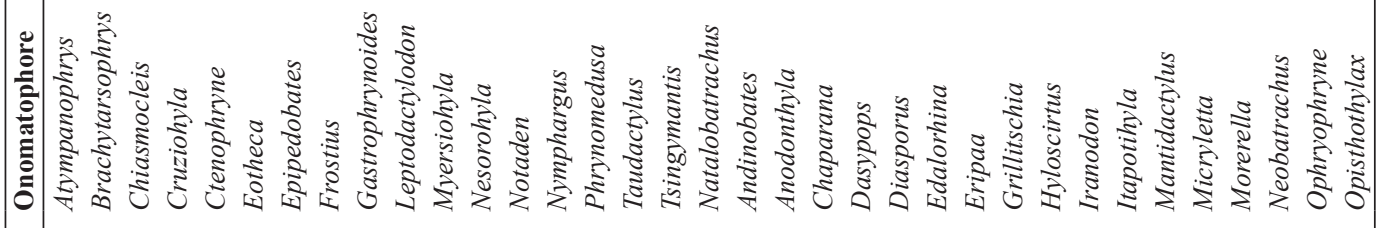
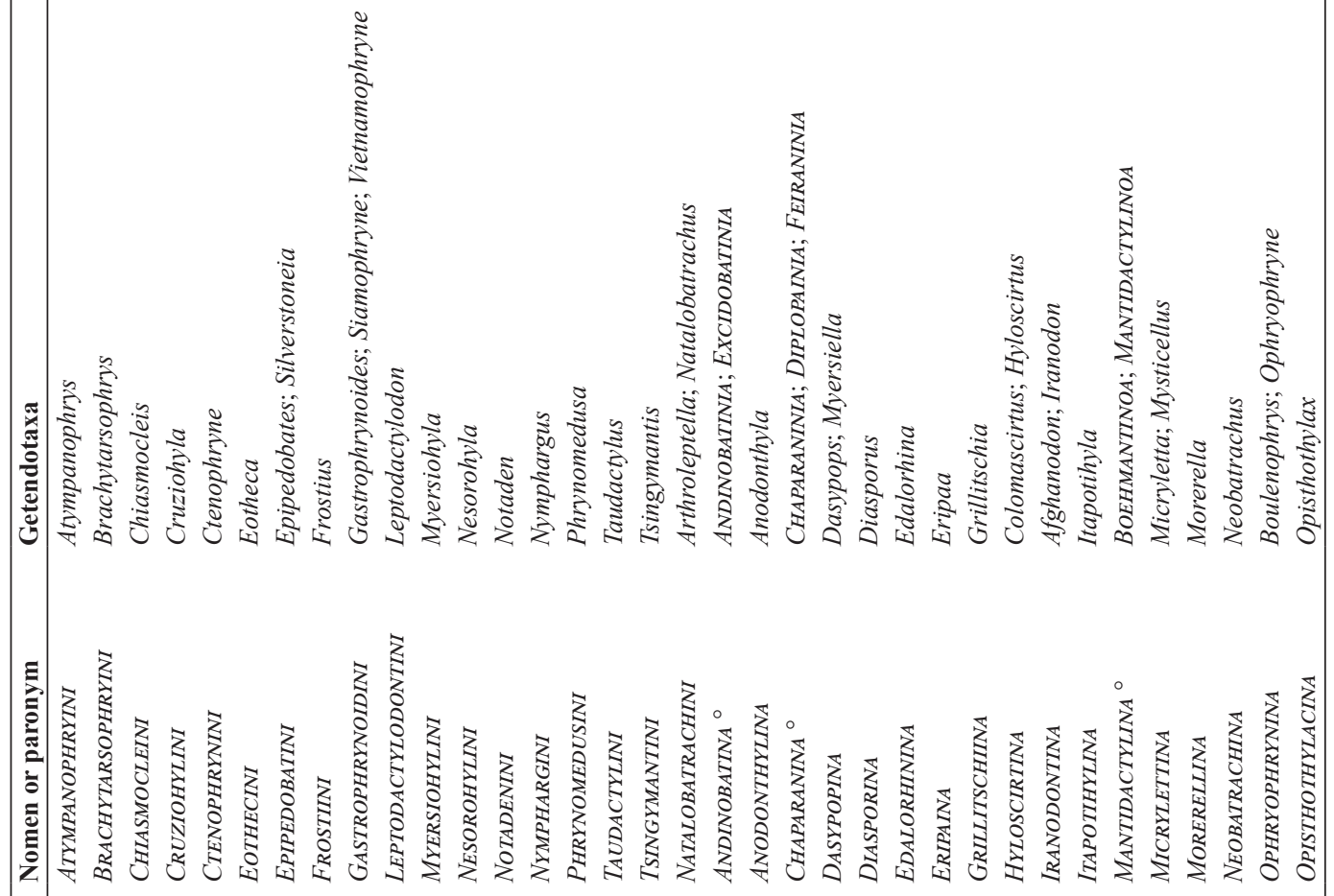

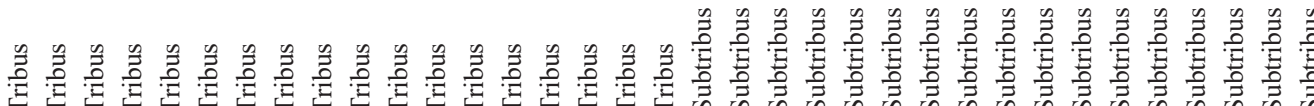

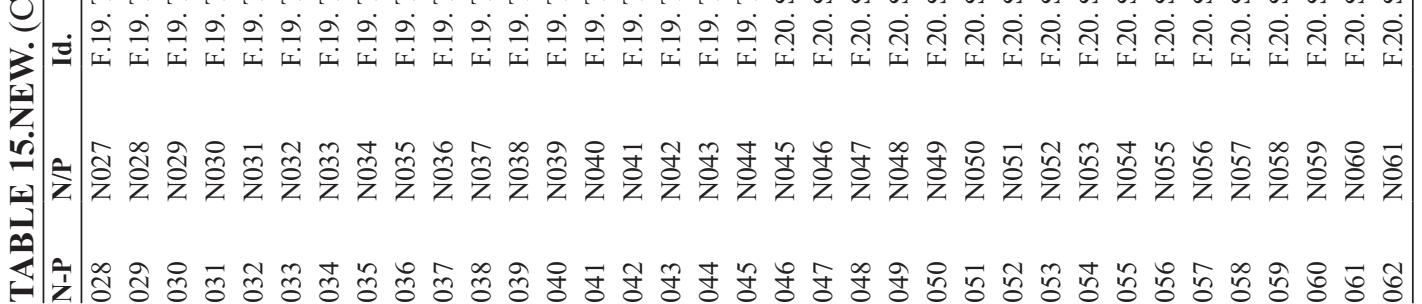




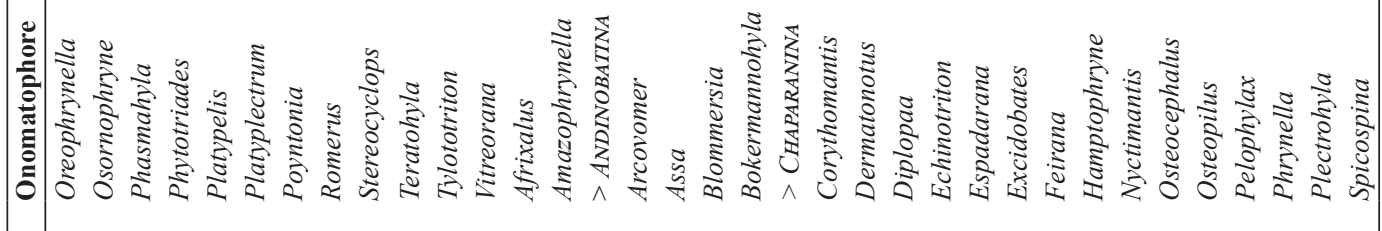

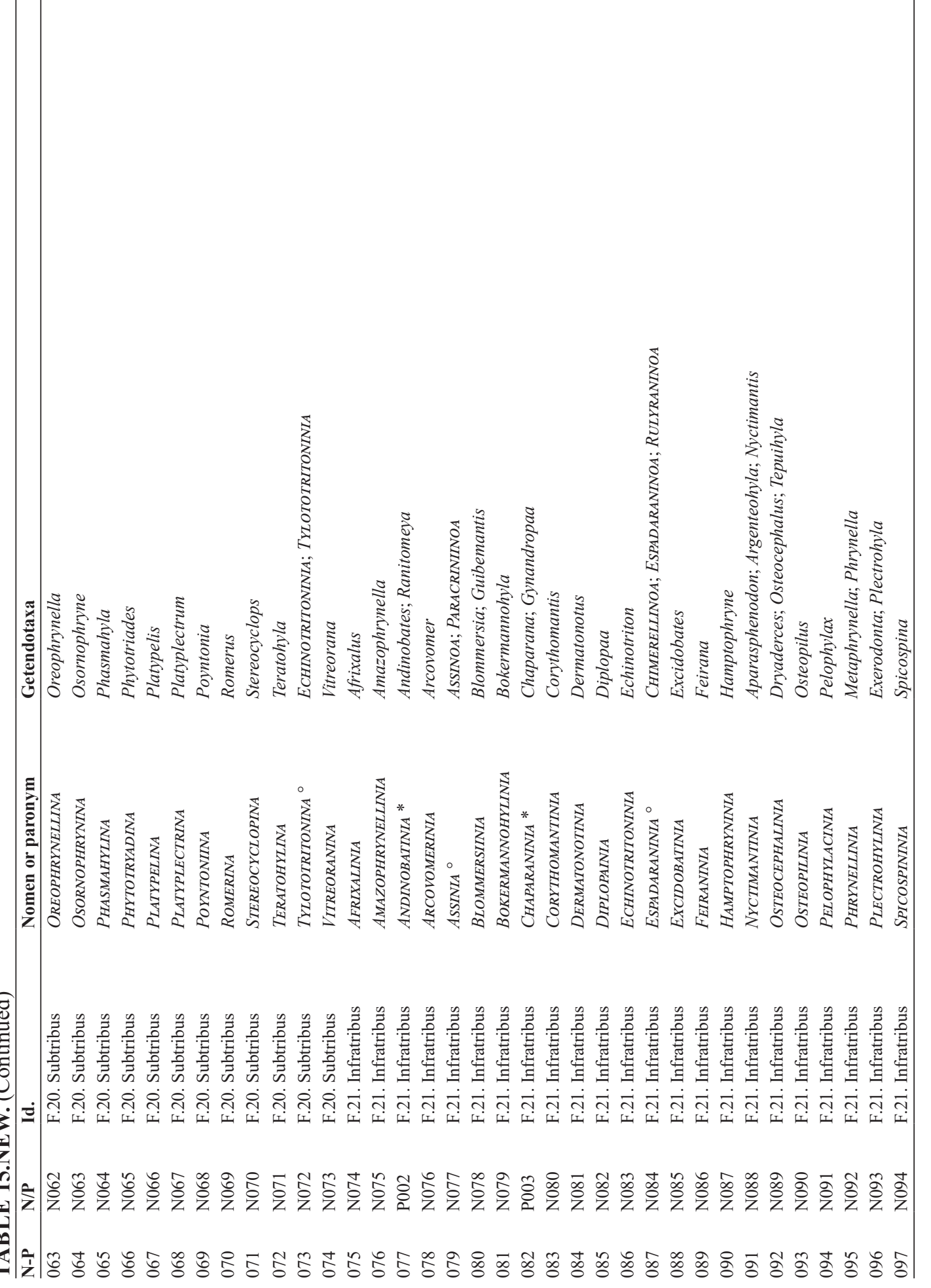




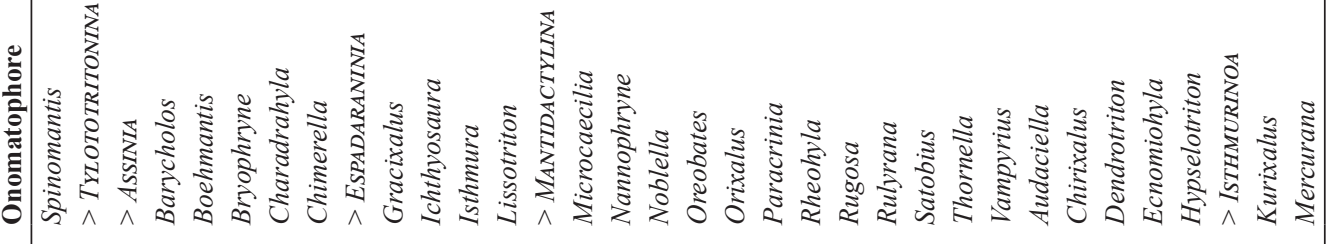

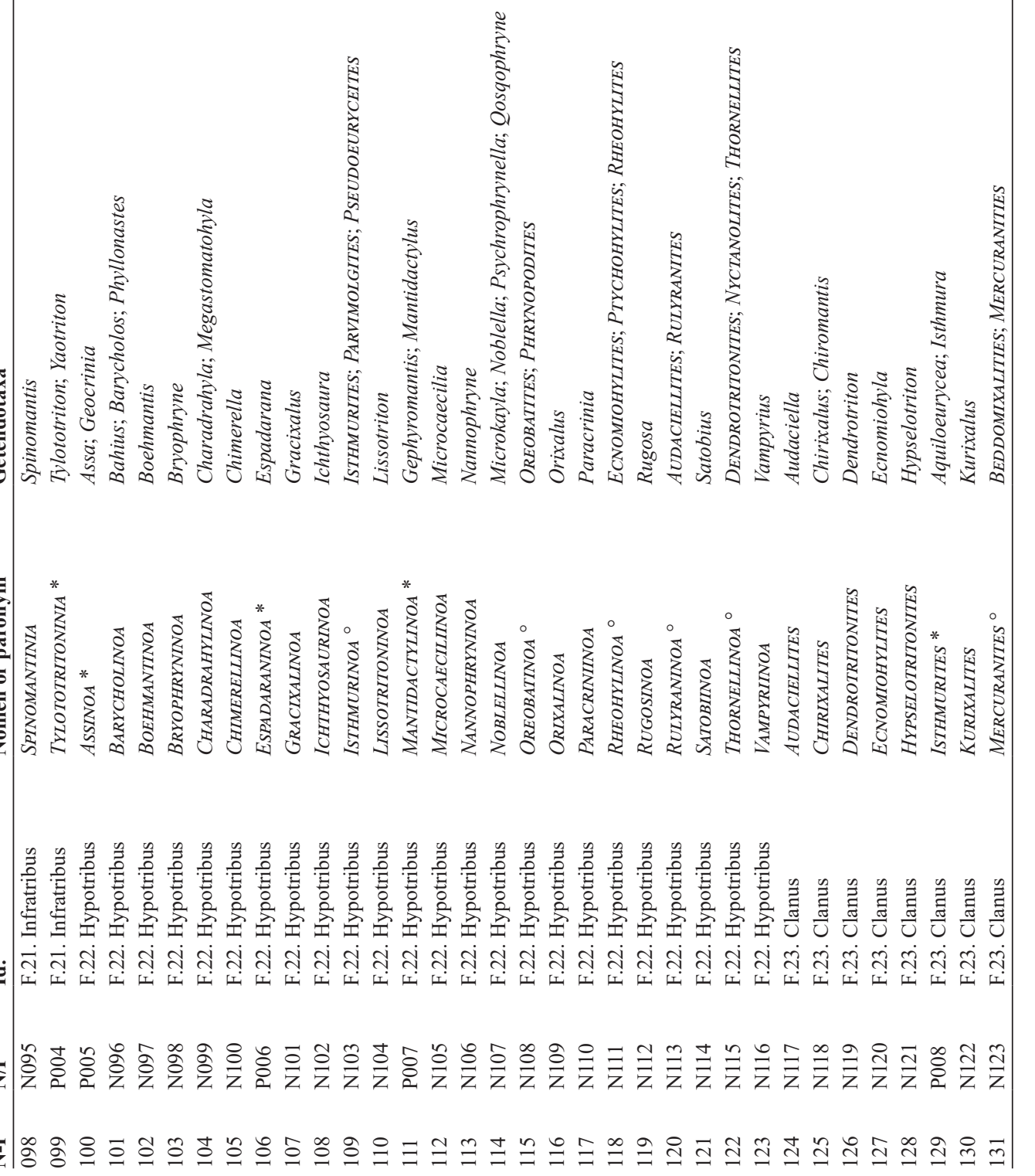




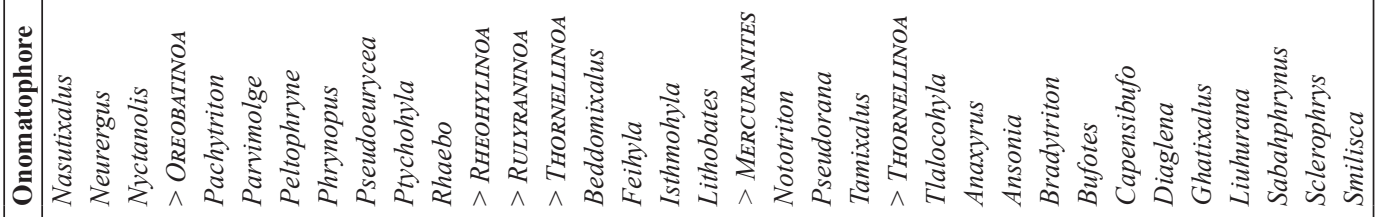

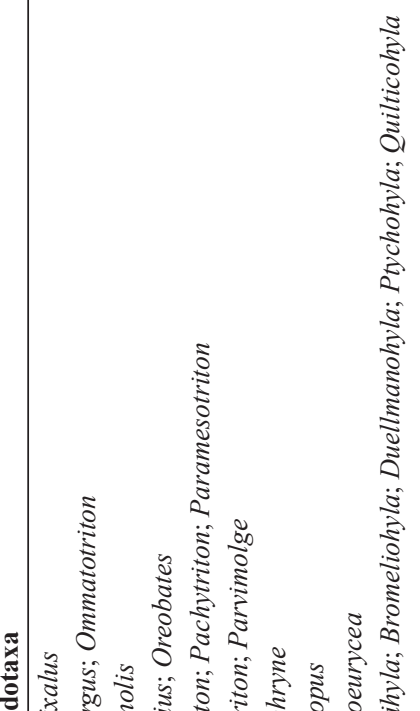

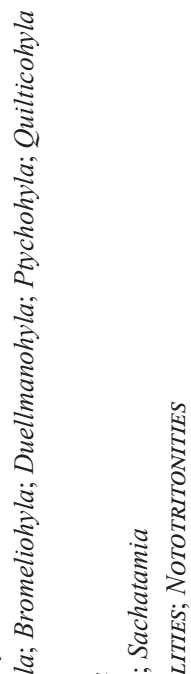

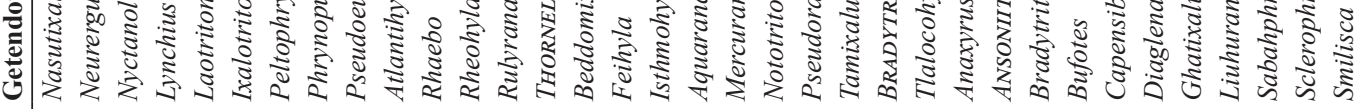

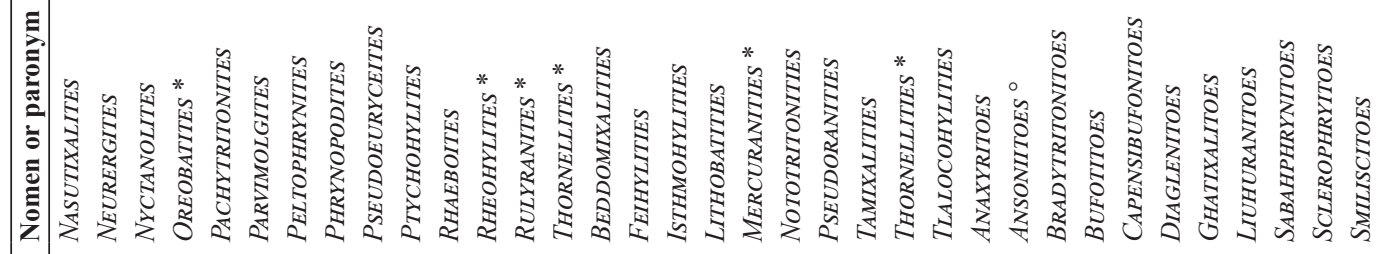




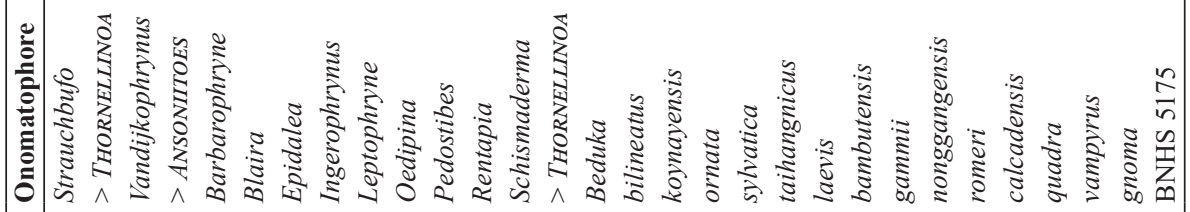

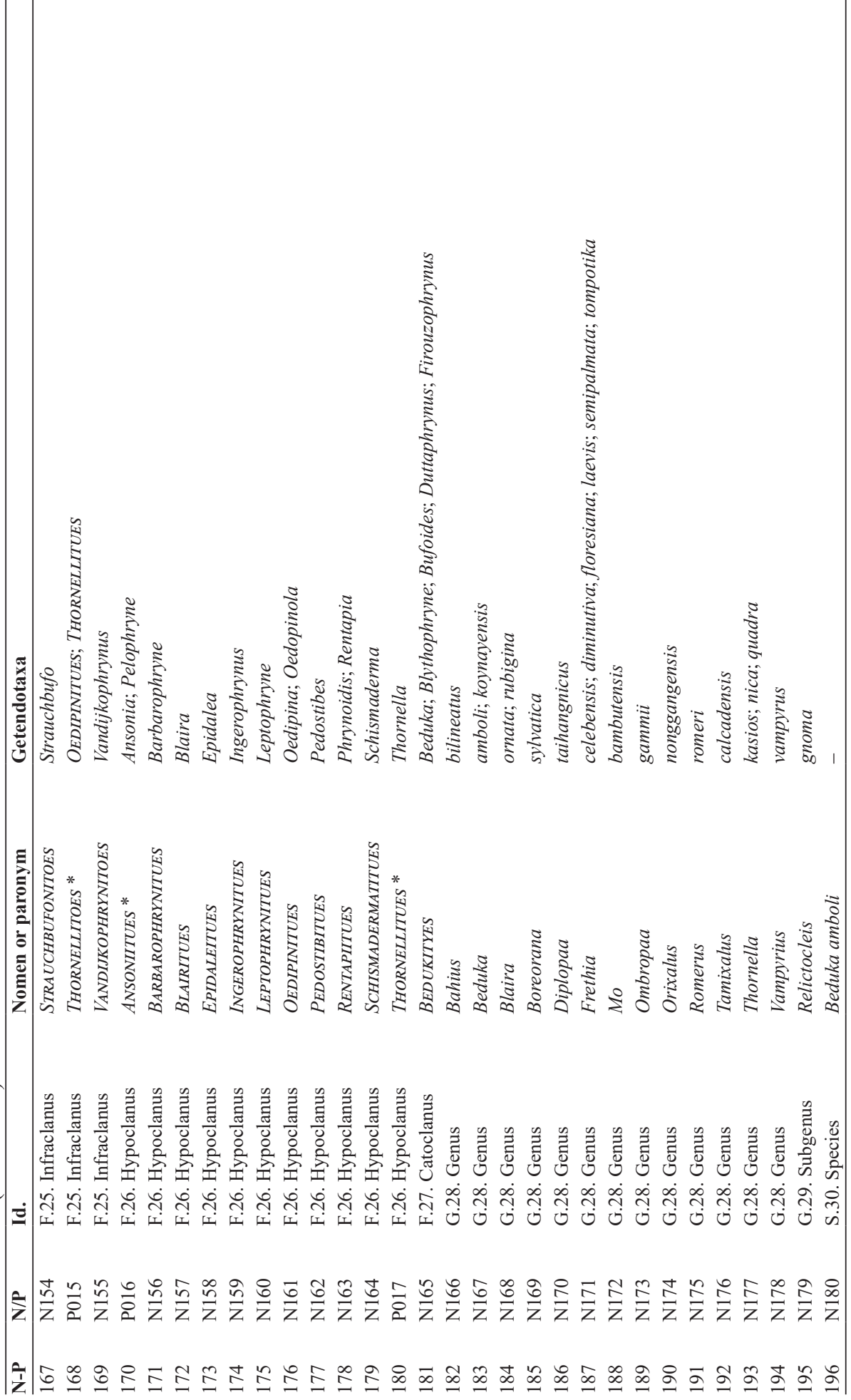


about taxa, so that short, euphonious nomina should be preferred to oversized, unpronounceable and unmemorable ones like HYDATINOSALAMANDROIDEI, CALYPTOCEPHALELLIDAE, Sigalegalephrynus or huehuetenanguensis (Dubois \& Raffaëlli 2009, Dubois 2010e). Thus we preferred a FS nomen based on Assa to one based on Geocrinia, on Mercurana to one based on Pseudophilautus, or on Oedipina to one based on Oedopinola. However, in most cases, Criterion $\{\mathrm{y} 1\}$ did not impose a clear choice, so we used Criterion $\left\{\mathrm{y}_{2}\right\}$ : we tended to base the new FS nomen on the oldest GS nomen, as more recent generic nomina run more risk to be synonymised in the future. Although this would not invalidate the FS nomen, this situation should be avoided if possible.

We followed the Criterion $\{\mathrm{y} 1\}$ to name new genus- and class-series taxa. Our 13 new genus-series nomina have 2 to 12 letters and 1 to 5 syllables, and our 10 new class-series nomina 8 to 12 letters and 4 to 6 syllables.

\subsection{Definition of character states used for diagnosis}

Most characters and character states used for diagnosis of new taxa were taken from publications, in particular taxonomic revisions, as indicated in the references, and a few only from personal observations. We provide below details on some morphological characters used in our diagnoses of a large array of taxa and that have no general acceptation in the literature.

The elements of the pectoral girdle are named following Duellman \& Trueb (1994) and RobovskáHavelková (2010). The latter work gives a recent review of these elements in an ontogenetic and phylogenetic perspective. The prezonal element that is unique to anurans is named omosternum. Some works distinguish the part that is ossified as omosternum and the cartilaginous distal element as episternum but as both have an identical ontogenetic origin and vary according to developmental stage and taxonomic group, this distinction is not significant. The situation is similar for the postzonal element, the sternum, but this element has two different ontogenetic origins with either a paired or a single rudiment. In adults, a bony mesosternum and a cartilaginous metasternum (unique structure) or xiphisternum (showing two branches) are distinguished but, similarly to the omosternum, these two parts are distinguished by ossification or absence of it.

Three conditions of the adductor mandibulae muscle and the pathway of the mandibular ramus of the trigeminal nerve were defined by Starrett (1968) and discussed by Lynch (1986a) for eleutherodactyline frogs. The trigeminal nerve passing lateral to the adductor mandibulae muscle which is extending from the zygomatic ramus of the squamosal to the posterior part of the maxilla, defines the ' $S$ ' condition, whereas the nerve passing medial to the muscle that extends from the squamosal to the angular is the ' $E$ ' condition. There may be also a ' $S+E$ ' condition where the muscles share origin and insertion but the nerve pass between them (Lynch 1986a).

The paired macroglands behind the eyes and tympana in amphibians are here called 'parotoids' following Tyler et al. (2001).

\section{RESULTS: PHYLOGENY, TAXONOMY AND NOMENCLATURE}

\subsection{The Tree}

Appendices A2.TREE-1 and A3.TREE-2 and Figure F6.TREE-3 show the TREE on which our analyses are based with different levels of precisions. Our Appendix A2.TREE-1 displays all the 4060 species on which our analysis is based and all the nodes resulting from this analysis, but the values of these nodes are given only when they are equal or superior to our a priori threshold SHL-aLRT support value of $90 \%$. It shows also the nomina of all the suprageneric taxa recognised as valid here. Appendix A3.TREE-2 provides a simplified version of TREE, showing only the genera and all the suprageneric taxa accepted as valid in this work. Figure F6.TREE-3 provides an oversimplified version of TREE, showing only the families and subfamilies accepted as valid in this work. 


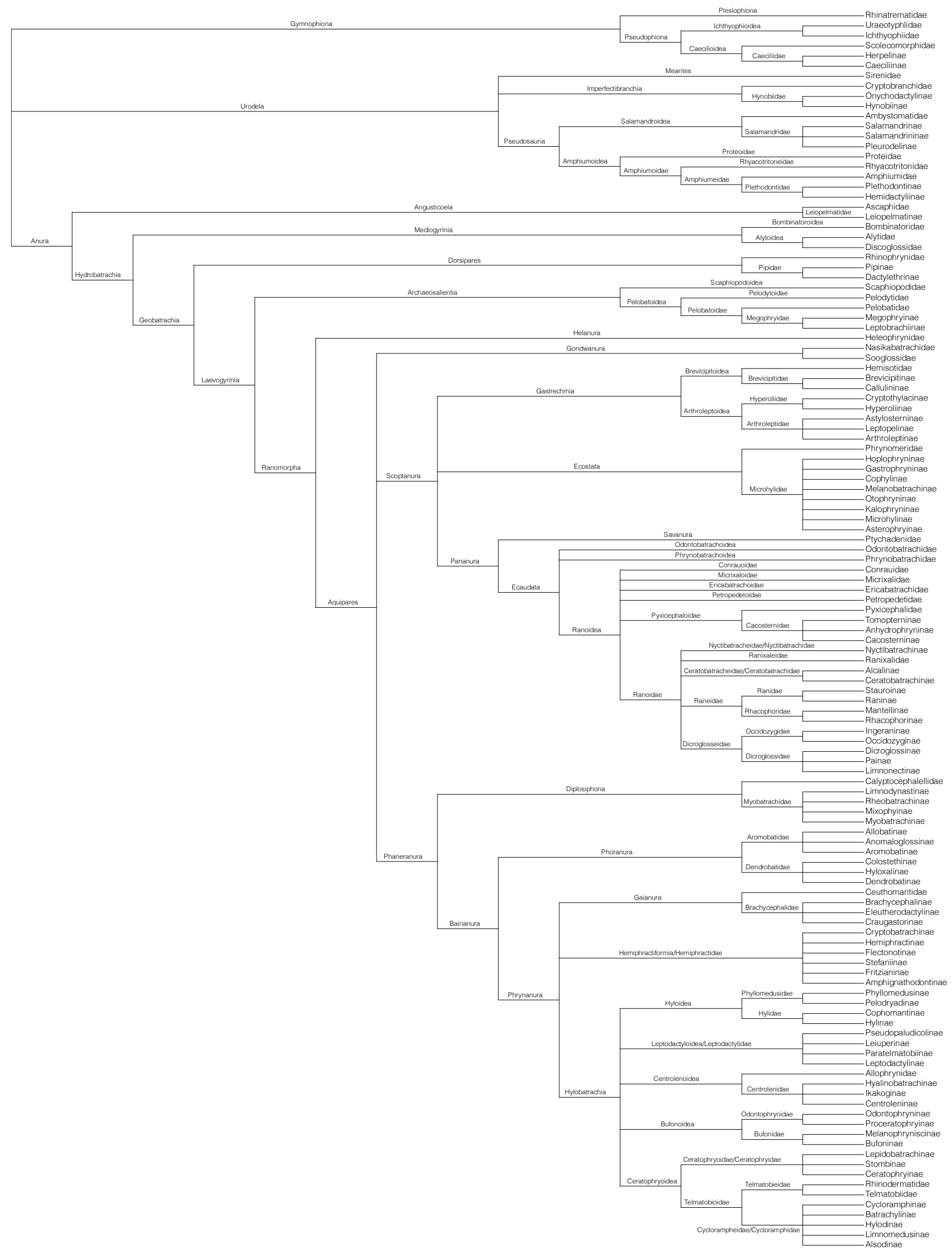

FIGURE 6.TREE-3. Oversimplified phylogenetic tree of LISSAMPHIBIA on which the present taxonomy is based, showing the families and subfamilies recognised here as valid and their relationships. 


\subsection{The nomina}

Appendices A5.NGS, A6.NFS and A7.NCS provide the lists of all the lissamphibian nomina of the genus-, family- and class-series ever proposed in the literature, as well as of all their aponyms. As explained above, we think these lists, which cannot be absolutely complete of course, are very close to being so. As such, they will be useful to all forthcoming amphibian taxonomists as a 'mine' for existing nomina that can be potentially used to name some taxa that will have to be recognised in the future. With the existence of these lists, there will be 'no excuse' for proposing new junior synonyms or homonyms of these nomina.

In each of these three tables, we provide a great deal of information concerning these nomina: references, status regarding availability, allocation, validity and correctness according to CLAD, homonymy, airesies, proedry, archoidy and miscellanea. A few airesies (new nucleospecies designations, fixation of precedence between synchronous doxisonyms or symprotographs) are effected in the present work: they are listed in Appendix A14.AIR.

All the references listed in Appendices A6.NFS and A7.NCS are listed in our list of References, but this is not the case for the 1921 nomina of A5.NGS, as this would add about 1300 references to our list of 1458 references, and for this purpose we refer to the existing online databases, although all of them contain mistakes. We plan to publish these data later elsewhere.

Altogether, as shown in A5.NGS, A6.NFS and A7.NCS, we identified 2828 available supraspecific nomina of LiSSAMPHIBIA: 1827 in the genus-series (1642 available and 185 unavailable ones), 592 in the family-series (488 available and 104 unavailable ones) and 409 in the class-series (402 available and 7 unavailable ones).

In Appendix A8.ECT, we provide a list of the 96 lissamphibian ectonyms published since 1992, which, for reasons explained above, we consider unavailable both under the Code and DONS, and which should not be used in taxonomic publications following these Rules.

\subsection{The cladonomy}

TREE is based exclusively on molecular data. Although in CLAD we incorporated the nomina of all the all-fossil generic and suprageneric taxa of LiSSAMPHIBIA, we did this only for the record and for completeness of lissamphibian nomina, on the basis of the recent literature, but, except in one case (subfamily SALTENIINAE), we incorporated no new data on these taxa and we do not take a stand on the validity of the cladistic and taxonomic allocation of these taxa in CLAD. These all-fossil taxa and nomina are not further addressed in the discussions below.

For every suprageneric taxon listed as valid in CLAD for which we use an already available nomen, we provide below its protonym (original spelling and rank) with its auctorship and rank, as well as the reference to the first use of its eunym (valid nomen with its correct spelling and rank), and its getangiotaxon (immediately superordinate taxon), adelphotaxon/a (parordinate taxon/a) and getendotaxon/a (immediately subordinate taxon). For every new nomen, we provide its getangiotaxon, adelphotaxon/a and getendotaxon/a, its onomatophore (nucleospecies, nucleogenus or conucleogenera), its etymology, stem and, for genus-series nomina, grammatical gender, and a diagnosis of the taxon. If necessary, taxonomic, nomenclatural or other comments are provided.

We only gave diagnoses for the taxa for which we provide new nomina, in order to make the latter available. Most diagnoses were built on the basis of a careful analysis of the literature concerning the included taxa. They are therefore very heterogeneous in terms of quality and completeness. In fact, this huge work allowed us to measure how incomplete and superficial is the state of knowledge concerning the morphology and anatomy of adults and larvae, the cytogenetics, bioacoustics, ethology and life history of most species in most amphibian groups. More worrying is the fact that, as today most phylogenetic information used for the classification of amphibians derives from nucleic acid sequencing, very few cladistic analyses based on non-molecular characters have been carried out recently. Such analyses, sometimes very enlightening, had been produced in the eighties and nineties, but with the rise and success of molecular phylogeny most of them were not updated and are now obsolete. As a consequence, in a vast majority of cases we were only able to provide diagnoses, as defined by Dubois $(2017 d$ ), but not apognoses, or, to put it differently, we do not know which characters in our diagnoses are indeed synapomorphies of the taxon and which ones are only 'differential' characters providing no cladogenetic information. 
In the present work, we introduce 10 new class-series nomina, 171 new family-series nomina, 14 new genus-series nomina and one new species-series nomen (Table T15.NEW), and we revalidate (resurrect) many other nomina that had been considered invalid either for a long time or just in the recent years. All these nomina are necessary to comply with our initial aims and requirements, which were to produce an ergotaxonomy being strictly bijective with the tree on which it is based. We have no doubt that the present work is only a progress report that will soon be obsolete when more species and more genes are sequenced, and possibly also with an increase in the number and quality of non-molecular characters of extant amphibians. This will be followed by a triple process of $\{z 1\}$ synonymisation of some of the nomina considered valid here, $\{z 2\}$ modification of the contents and diagnoses of the taxa designated by some of these nomina, and $\{z 3\}$ erection of new taxa and introduction of new nomina for the latter. This has nothing surprising, strange or negative: this is the normal process of taxonomic research, which supports the idea that fighting for 'taxonomic and nomenclatural stability' is a counterproductive approach, that should not be backed (Dubois 1998, 2010c). Taxonomy can only progress this way, by successive trials and errors. The double process of synonymisation and revalidation (so-called 'resurrection') of nomina is a permanent one in taxonomy, and testifies to the progress of our knowledge on the biodiversity of our planet. Trying articially to stop it would lead to 'freezing' research in this domain. Such a freezing might be appreciated by administrators, technocrats, lawyers and even some conservation biologists, whose dream is to have 'final' lists of taxa with 'fixed' nomina, but this would be at the expense of both our knowledge and of our actions for the preservation of biodiversity - even if the latter is already largely a lost cause.

Table T14.NUM provides the numbers of generic and suprageneric taxa and nomina below class of extant LiSSAMPHiBia recognised as valid here. Among them, the number of new taxa introduced in the present work for extant lissamphibian taxa is high: 13/575 (2.3\%) at the rank genus (Appendix A5.NGS), 171/573 (29.8 \%) in the family-series (Appendix A6.NFS) and 10/34 (29.4 \%) in the classseries below the rank class (Appendix A7.NCS), i.e. 194/1182 (16.4\%) in total. But this number is of a much lower magnitude than the total number of available extant lissamphibian nomina already published but now considered invalid synonyms (Table T16.SYN): 869/1824 (47.6\%) in the genusseries, $204 / 487(41.8 \%)$ in the family-series and $367 / 409(89.7 \%)$ in the class-series, i.e. 1344/2825 $(47.6 \%)$ in total. So, even if half of them were synonymised this would have a feeble impact on the Synonymy Load Index (SLI = number of invalid nomina / number of available nomina) in amphibians, while the progress in the resolution of the taxonomy of amphibians provided by the other ones would be substantial. Furthermore, as discussed below (see our section 4.3.1.3 Tomoidy), it can be quite safely expected that the progressive resolution of the polytomies still present in TREE will be followed by an increase in the number of taxa/nomina rather than a decrease.

The Principle of Coordination allows nomenclatural parsimony in the family-series. In this nominal-series, a given nomen can be used at several ranks (these are different parohypses of the same nomen). This can be measured by a Nomenclatural Parsimony Index (NPI = number of nomina / number of parohypses). If we consider only the nomina of extant lissamphibian taxa (Table T17.PAR), we need only 356 nomina for 573 parohypses, many of them being used as valid at two to 12 ranks, which amounts to a NPI of $62.1 \%$, an impressive figure indeed, which speaks in favour of the nomenclatural Principle of Coordination. The same would apply in the genus-series if a comprehensive ergotaxonomy of all extant LISSAMPHIBIA using consistently the rank subgenus was implemented, and in the speciesseries if the four ranks recognised by the Code (supraspecies, species, exerge and subspecies) were used, as proposed by Dubois \& Raffaëlli $(2009,2012)$ in the Urodela. But in the class-series, where the Principle of Coordination cannot apply (for reasons explained above), the number of nomina needed is the same (34) as the number of taxa (Table T14.NUM).

TABLE 16.SYN. Synonymy load in extant LISSAMPHIBIA. (i.e., excluding all-fossil supraspecific taxa) according to the taxonomy adopted here.

Synonymy load index: SLI = number of akyronyms / number of hoplonyms.

In this Table, exoplonyms are included in anoplonyms.

\begin{tabular}{lllllll}
\hline Nominal-series & Nomina & Hoplonyms & Anoplonyms & Kyronyms & Akyronyms & SLI \\
\hline Class-series & 409 & 402 & 7 & 35 & 367 & $91.3 \%$ \\
Family-series & 592 & 488 & 104 & 380 & 108 & $22.1 \%$ \\
Genus-series & 1826 & 1641 & 185 & 770 & 871 & $53.1 \%$ \\
Total & 2827 & 2531 & 296 & 1185 & 1346 & $53.2 \%$ \\
\hline
\end{tabular}


TABLE 17.PAR. Family-series paronymy in the extant LiSSAMPHIBIA (i.e., excluding all-fossil supraspecific taxa) according to the taxonomy adopted here.

The table gives the numbers (from 1 to 12) of parohypses of all family-series nomina of extant LissaMPHIBIA nomina having from 1 to 12 parohypses in CLAD. Nomina are listed according to their acrohypses (highest ranked taxon bearing the nomen).

\begin{tabular}{lccccccccccc}
\hline Acrohypse & 1 & 2 & 3 & 4 & 5 & 6 & 8 & 10 & 12 & $\begin{array}{c}\text { Subtotal } \\
2-12\end{array}$ & $\begin{array}{c}\text { Total } \\
1-12\end{array}$ \\
\hline 14. Superfamilia & - & 6 & 3 & 5 & 1 & - & 1 & 1 & 1 & 18 & 18 \\
15. Epifamilia & - & 7 & 1 & - & - & - & - & - & - & 8 & 8 \\
16. Apofamilia & - & 3 & 2 & - & 1 & - & - & - & - & 6 & 6 \\
17. Familia & 20 & 7 & 1 & 5 & 1 & 2 & 1 & - & - & 17 & 37 \\
18. Subfamilia & 38 & 10 & 6 & 3 & - & - & - & - & - & 19 & 57 \\
19. Tribus & 38 & 8 & 3 & 2 & 1 & - & - & - & - & 14 & 52 \\
20. Subtribus & 43 & 10 & 2 & - & - & - & - & - & - & 12 & 55 \\
21. Infratribus & 32 & 5 & - & - & - & - & - & - & - & 5 & 37 \\
22. Hypotribus & 21 & 6 & - & - & 1 & - & - & - & - & 7 & 28 \\
23. Clanus & 18 & 1 & 1 & - & - & - & - & - & - & 2 & 20 \\
24. Subclanus & 8 & 3 & - & - & - & - & - & - & - & 3 & 11 \\
25. Infraclanus & 13 & 2 & 1 & - & - & - & - & - & - & 3 & 16 \\
26. Hypoclanus & 10 & - & - & - & - & - & - & - & - & - & 10 \\
27. Catoclanus & 1 & - & - & - & - & - & - & - & - & - & 1 \\
\hline Total nomina & 242 & 68 & 20 & 15 & 5 & 2 & 2 & 1 & 1 & 114 & 356 \\
Total parohypses (taxa) & 242 & 136 & 60 & 60 & 25 & 12 & 16 & 10 & 12 & 331 & 573 \\
\hline
\end{tabular}

\section{C.01.01. Subphylum Vertebrata Cuvier, 1800}

Protonym: VerTÉBRÉs Cuvier, 1800: first unnumbered table [UC].

Eunym: Cuvier, 1816: 58.

Getangiotaxon: Not treated here.

Adelphotaxa: Not treated here.

Getendotaxa: AмpHIBIA Blainville, 1816; all other vertebrate taxa not treated here.

\section{C.02.01. Classis Aмpнibia Blainville, 1816}

Protonym: AMPHYBIEns Blainville, 1816: '107' [115] [C].

Eunym: Macleay, 1821: 275.

Getangiotaxon: Not treated here.

Adelphotaxa: Not treated here.

Getendotaxa: LisSAMPHIBIA Gadow, 1898; all-fossil non-lissamphibian amphibian taxa not treated here.

Comments: Following the rationale of Dubois (2015c), the Duplostentional Nomenclatural System and the tradition in the Zoological Record for one and a half century, we apply this nomen, which is a sozodiaphonym (Dubois \& Frétey 2021d), with this auctorship and date, to the holophyletic group, usually attributed to the rank class (e.g. Cannatella \& Hillis 2004, Marjanović \& Laurin 2015), covering all the anamniote tetrapods subsequent to the 'lissamphibian-amniote phylogenetic split' (Ruta et al. 2003a), including the Palaeozoic groups |LEPOSPONDYLI| and |TEMnOSPONDYLI|. The present work deals only with the subclass LissampHIBIA of this class, as defined below. The cladistic relationships between this subclass and the other, all-fossil, groups of anamniote vertebrates are still controversial (see e.g. Schoch 2009, Marjanović \& Laurin 2015) and therefore their relative hierarchical relationships are not stabilised. 
Protonym and eunym: LissaMPHiBia Gadow, 1898: xii, 13 [bC].

Getangiotaxon: AMPHiBIa Blainville, 1816.

Adelphotaxa: All-fossil non-lissamphibian amphibian taxa not treated here.

Getendotaxa: ANUra Duméril, 1805; Gymnophiona Rafinesque, 1814; Urodela Duméril, $1805 ; 1 \mathrm{C} \uparrow$.

Comments: Following the rationale of Dubois (2020a) and the recent tradition in the literature (see Dubois 2015c), we apply this nomen, which is a sozodiaphonym (Dubois \& Frétey 2021c), with this auctorship and date, to the subclass accomodating all the recent amphibians, distributed in the three orders Anura, Gymnophiona and Urodela, as well as the fossil order Allocaudata, whose cladistic relationships with these three taxa are unresolved (see e.g. Marjanović \& Laurin 2015).

Based on the molecular data of TREE, the three extant orders ANURA, GYMNOPHIONA and URODELA are well supported holophyletic taxa, and it is the case also for these three groups altogether, but our data do not allow to resolve the relationships between these three groups, which for the time being constitute therefore an unresolved trichotomy. In general, morphological and molecular analyses tend to agree that salamanders and frogs are sister-lineages, and several recent authors (e.g. Frost et al. 2006; Roelants et al. 2007; San Mauro et al. 2010) credited the ANURA and URODELA with a sister-group relationship excluding the GyMnOPHIONA. If this hypothesis was supported by future data and analyses, the taxon accommodating the former two taxa should bear the nomen BATRACHIA Brongniart, 1800, which is currently invalid for being a hypnokyronym (see Dubois 2015c, 2016; Dubois \& Frétey 2020a,d). However, the phylogenetic signal in these datasets has long been noted to be inconsistent or incongruent among partitions (see Siu-Ting et al. 2019 and Hime et al. 2020, and historical references therein). The most recent genome-scale analysis (Hime et al. 2020) recovered 'strong' support for BATRACHIA in combined analyses of 220 nuclear loci, but noted that only 67 individual gene trees actually support this node. While we (and probably most other batrachologists) believe that the dichotomy BATRACHIAGymnophiona is likely the 'true' topology, at this stage we continue to reflect this uncertainty in our cladonomy.

Addition of BATRACHIA to the system proposed here would merely require the insertion of an additional rank superorder for this taxon and for its sister-taxon GYMNOPHIONA. The latter nomen would therefore apply both to the rank superorder and to the rank order, a rare situation where coordination can be used in the class-series (see Dubois 2015c). On the other hand, if, as suggested by Feller \& Hedges (1998), the Gymnophiona and Urodela were found to be sister-taxa excluding the ANURA, they should be grouped under the nomen DEROTRETA Van der Hoeven, 1833, whereas no nomen would be available for a taxon including the ANURA and GYMNOPHIONA but excluding the Urodela (Dubois 2015c: 103-104, 108). The synonymies of all these class-series (CS) nomina are provided in Appendix A7.NCS.

\section{C.04.01. Ordo Anura Duméril, 1805}

Protonym: ANoures Duméril, 1805: 91 ['F'].

Eunym: Ficinus \& Carus, 1826: plate.

Getangiotaxon: LISSAMPHIBIA Gadow, 1898.

Adelphotaxa: Gymnophiona Rafinesque, 1814; Urodela Duméril, 1805; $1 \mathrm{C} \uparrow$.

Getendotaxa: Angusticoela Reig, 1958; Hydrobatrachia Ritgen, 1828; 3 F†; 39 G†.

Comments: The holophyly of all extant anurans is supported by all phylogenetic studies based on morphology and on molecular data. Numerous CS nomina are available for this taxon (Appendix A7.NCS) but the valid one under DONS Criteria is the sozodiaphonym ANURA Duméril, 1805 (Dubois 2004b, 2005b, 2015c, 2020a; Dubois \& Ohler 2019; Dubois \& Frétey 2020b).

As explained in our M\&M section, our assignment of the rank family to a taxon relies on a series of Criteria. The first Criterion is the long-term usage of this rank for a valid taxon as documented by the analysis of about 100 ergotaxonomies (classifications adopted as valid) from the late $18^{\text {th }}$ century to 2014. Twenty-five family-series nomina fall into the first quarter of usage employed in 23 to $99 \%$ of the ergotaxonomies. Based on TREE and our Criteria, 55 taxa were assigned to the rank family in 
the ANURA, which is slightly more than in the recent classifications published. Detailed synonymies of the valid nomina applied to these families are provided in Appendix A6.NFS. Syntaxic nomina that apply to the same taxa are listed in Appendices A7.NCS and A8.ECT. These nomina are unavailable respectively because they were not based on available genus-series (GS) nomina then considered valid (anoplonyms) or because they were purposely proposed outside the frame of the Code (ectonyms).

To transpose TREE into the ergotaxonomy CLAD, we retained up to 12 ranks to describe the relationships below the rank order and above the rank family, nine in the CS and three in the FS.

According to TREE, the ANURA are divided into two highly supported branches, here recognised as suborders: $\{\alpha 1\}$ the ANGUSTICOELA, synotaxic with the superfamily LEIOPELMATOIDEA of Dubois (2005d) and Zhang et al. (2013), a redundant and therefore useless rank in CLAD, including the families ASCAPHIDAE and LEIOPELMATIDAE; and $\{\alpha 2\}$ its sister-taxon the HYDrobatraCHIA, including all other anurans.

\section{C.05.01. Subordo Angusticoela Reig, 1958}

Protonym and eunym: ANGuSTiCoela Reig, 1958: 111 [bO].

Getangiotaxon: ANURA Duméril, 1805.

Adelphotaxon: Hydrobatrachia Ritgen, 1828.

Getendotaxa: ASCAPHIDAE Fejérváry, 1923; LEIOPELMATIDAE Mivart, 1869-|Turbott, 1942|.

Comments: The cladistic relationship of this branch to the other anurans and within this branch is stable and recognised in all recent works (Roelants \& Bossuyt 2005; Frost et al. 2006; Bossuyt \& Roelants 2009; Pyron \& Wiens 2011; Irisarri et al. 2012; Zhang et al. 2013; Feng et al. 2017).

The nomen AMPHICOEla Noble, 1931 is a distagmonym and a junior homonym of the nomina Amphicoela Meyer, 1860 and Amphicoela Owen, 1860, and thus cannot be valid for this group (see Appendix A7.NCS).

As the FS taxon and nomen LEIOPELMATIDAE is retained by the 'Upper Quartile Criterion' [UQC] at the rank family, its sister-taxon ASCAPHIDAE must be attributed to the same rank according to the 'SisterTaxa Criterion' [STC].

\section{F.17.01. Familia ASCAPHIDAE Fejérváry, 1923}

Protonym and eunym: ASCAPHIDAE Fejérváry, 1923: 178 [F].

Getangiotaxon: AnguSTiCoela Reig, 1958.

Adelphotaxon: LeIOPELMATIDAE Mivart, 1869-|Turbott, 1942|.

Getendotaxon: Ascaphus Stejneger, 1899.

F.17.02. Familia LEIOPELMATIDAE Mivart, 1869-|Turbott, 1942|

Protonyms: LIOPELMATINA Mivart, 1869: 291 [bF]; |LEIOPELMIDAE Turbott, 1942: 247| [F].

Eunym: Stephenson 1951: 18.

Getangiotaxon: Angusticoela Reig, 1958.

Adelphotaxon: AsCAPHIDAE Fejérváry, 1923.

Getendotaxa: LeIOPELMATINAE Mivart, 1869-|Turbott, 1942|; 1 F†.

F.18.01. Subfamilia LeIOPELMatinaE Mivart, 1869-|Turbott, 1942|

Eunym: Kuhn 1965: 86.

Getangiotaxon: LEIOPELMATIDAE Mivart, 1869-|Turbott, 1942|.

Adelphotaxon: $1 \mathrm{~F} \dagger$.

Getendotaxa: Leioaspetos Wells \& Wellington, 1985; Leiopelma Fitzinger, 1861. 
Getangiotaxon: LEIOPELMATINAE Mivart, 1869-|Turbott, 1942|.

Adelphotaxon: Leiopelma Fitzinger, 1861.

Getendotaxon: Leioaspetos hamiltoni (McCulloch, 1919).

Comments: The species originally described as Liopelma hamiltoni McCulloch, 1919 is a striking example of 'Latonia-like situation' (LLS) relatively to all other species currently referred to the genus Leiopelma Fitzinger, 1861 (see M\&M section). The generic nomen Leioaspetos Wells \& Wellington, 1985 is available for this species, and we recognise this genus as distinct from Leiopelma Fitzinger, 1861.

\section{C.05.02. Subordo Hydrobatrachia Ritgen, 1828}

Protonym: Hydrobatrachi Ritgen, 1828: 278 ['F'].

Eunym: Hoc loco.

Getangiotaxon: ANURA Duméril, 1805.

Adelphotaxon: Angusticoela Reig, 1958.

Getendotaxa: Geobatrachia Ritgen, 1828; Mediogyrinia Lataste, 1878; 2 G†.

Comments: The suborder Hydrobatrachia includes all the AnURa except the Angusticoela. The synonymic list of this distagmonym includes ARCHEOBATRACHIA Reig, 1958 as originally defined, and its synotaxic list includes the ectonym «LALAGOBATRACHIA», a name coined explicitly as 'unregulated' (Frost et al. 2006: 143), i.e. unavailable under the Code and DONS.

This taxon includes two well supported branches recognised here as the infraorders GEOBATRACHIA and Mediogyrinia.

\section{C.06.01. Infraordo Geobatrachia Ritgen, 1828}

Protonym: Geobatrachi Ritgen, 1828: 278 ['F'].

Eunym: Hoc loco.

Getangiotaxon: Angusticoela Reig, 1958.

Adelphotaxon: Mediogyrinia Lataste, 1878.

Getendotaxa: Dorsipares Blainville, 1816; LAEVogYrinia Lataste, 1878; 1 G†.

Comments: The valid nomen under DONS of the well supported branch that includes the DoRSIPARES (families PIPIDAE and RHINOPHRYNIDAE) and the LAEvogYRINIA (all other frogs) is the distagmonym GEOBATRACHIA Ritgen, 1828. This branch was not retrieved in Frost et al. (2006), where their «Xenoanura», our Dorsipares, was sister-taxon to all frogs but ANGuSTiCoela. In Roelants \& Bossuyt (2005), Bossuyt \& Roelants (2009) and Pyron \& Wiens (2011), this branch («XenOANURA» or PIPOIDEA) found its position as the sister-branch to what is here called the LAEvOGYRINIA.

\section{C.07.01. Hypoordo DorsiPaRes Blainville, 1816}

Protonym: DorsiPares Blainville, 1816: '111' [119] [bO].

Eunym: Hoc loco.

Getangiotaxon: Geobatrachia Ritgen, 1828.

Adelphotaxon: LAEVOGYRINIA Lataste, 1878.

Getendotaxa: PIPIDAE Gray, 1825-|Fitzinger, 1826|; RHINOPHRYNIDAE Günther, 1858; 1 F†; 7 G†.

Comments: This taxon is recognised in all phylogenies and taxonomies based on morphological or molecular data. Besides the all-fossil PALAEOBATRACHIDAE, it groups two extant families, the PIPIDAE and RHINOPHRYNIDAE.

The distagmonym DorsiPARES, being the first available nomen for this taxon, is its valid nomen 
under DONS. Its long synonymic list (see Appendix A7.NCS) includes XENOANURA Starrett, 1973, and its synotaxic to the FS nomen PIPOIDEA used by Roelants \& Bossuyt (2005) but which is redundant in CLAD.

Frost et al. (2006) used for this taxon the ectonym «XENOANURA», erroneously credited to Savage (1973: 352) where it had been borrowed from Starrett (1973: 251), and which is an ectonym in their work, like all their other subordinal names above the rank superfamily as they were not attributed to ranks.

Both PIPIDAE and RHINOPHRYNIDAE are retained at the family rank on account of the [UQC]. Whereas the RHINOPHRYNIDAE include a single genus Rhinophrynus, the PIPIDAE include five extant genera. Pipa, the only member of the PIPINAE, is sister-taxon to the other genera, for which the subfamilial nomen DACTYLETHRINAE is available. Within this branch, the tribe DACTYLETHRINI (genera Silurana and Xenopus) constitutes a well-supported sister-branch to the tribe HYMENOCHIRINI (genera Hymenochirus and Pseudhymenochirus).

\title{
F.17.03. Familia PIPIDAE Gray, 1825-|Fitzinger, 1826|
}

\author{
Protonyms: PIPRINA Gray, 1825: 214 [UC]; |PIPOIDEA Fitzinger, 1826: 37| [F]. \\ Eunym: Swainson 1839: 88. \\ Getangiotaxon: DorSIPARES Blainville, 1816. \\ Adelphotaxa: RHINOPHRYNIDAE Günther, 1858; 1 F†. \\ Getendotaxa: DACTYLETHRINAE Hogg, 1838; PIPINAE Gray, 1825-|Fitzinger, 1826|; 1 bF $\dagger$; 6 G†.
}

Comments: Aranciaga-Rolando et al. (2019) introduced two ectonyms for all-fossil taxa of this group: «PANPIPIDAE» and «SHELANIINAE». Although they 'look like' nomina of respectively familial and subfamilial rank, they are unavailable because these authors did not mention these ranks but designated these names as 'stem-based nomina', because they proposed these new nomina under the designation of 'nomen novum', which applies to neonyms, not to poieonyms, and because they did not explicitly designate nucleogenera ('type genera') for these nominal taxa (the former not being even based on an available genus-series nomen).

The unavailable nomen «SHELANIINAE» applies to a well-diagnosed taxon which we recognise here as a third, all-fossil, subfamily of the family PIPIDAE besides the PIPINAE and the DACTYLETHRINAE. Kuhn (1965: 88) mentioned a family "SALTENIIDAE", based on the oldest genus nomen of this group (Saltenia Reig, 1959) but without any diagnostic element that could make it nomenclaturally available. He credited 'Kuhn 1963' with authorship of this nomen, but to the best of our knowledge Kuhn did not publish any scientific paper in 1963. In his 1962 work, he placed the genus Saltenia in a "Fam. nov." which he did not name but for which he provided a diagnosis and a figure (borrowed from Reig 1959). It is impossible to use Article 13.1.2 of the Code to provide nomenclatural availability to the nomen "SALTENIIDAE" proposed by Kuhn (1965), because in this work he did not provide the reference of his 1962 work. Furthermore, in his comprehensive list of herpetological higher taxa nomina (Kuhn 1967b), he did not mention this nomen. We are therefore led to provide nomenclatural availability to this nomen below.

Beside the two ectonyms mentioned above, Aranciaga-Rolando et al. (2019) introduced two new available nomina, the genus nomen Patagopipa and the nomen of its nucleospecies ('type species') for the epithet of which they used two alternative spellings: corsolinii (pages 727, 728, 730, 731) and corsolini (pages 728, 729, 732). Among these two symprotographs ('multiple original spellings'), we hereby designate corsolinii as the lectoprotograph ('correct original spelling') of this nomen.

\section{F.18.†02. Subfamilia SALTENIINAE nov.}

Getangiotaxon: PIPIDAE Gray, 1825-|Fitzinger, 1826|.

Adelphotaxa: DaCtylethrinae Hogg, 1838; PIPINAE Gray, 1825-|Fitzinger, 1826|.

Getendotaxa: Kuruleufemia Gómez, 2016 †; Saltenia Reig, 1959 †; Shelania Casamiquela, 1960 †; Patagopipa Aranciaga Rolando, Agnolin \& Corsolini, $2019 \uparrow$.

Nucleogenus, by present designation: Saltenia Reig, 1959. • Etymology of nomen: R: Salta, province of Argentina. • Stem of nomen: Salteni-. 
Diagnosis: Anterior ramus of pterygoid reaching the antorbital plane; eight presacral vertebrae; presacral vertebrae I-II not fused but imbricated medially; marked forward orientation of the transverse process of presacra vertebrae IV; cross-section of distal iliac shaft flattened, dorsoventrally compressed; second pair of ribs anterolaterally oriented. \{Aranciaga-Rolando et al. 2019: 727\}.

F.18.02. Subfamilia DACTYLETHRINAE Hogg, 1838

Protonym: DACTYLETHRIDAE Hogg, 1838: 152 [F].

Eunym: Metcalf 1923: 391.

Getangiotaxon: PIPIDAE Gray, 1825-|Fitzinger, 1826|.

Adelphotaxon: PIPINAE Gray, 1825-|Fitzinger, 1826|.

Getendotaxa: DACTYLETHRINI Hogg, 1838; HYMENOCHIRINI Bolkay, 1919.

\section{F.19.01. Tribus DACTYLETHRINI Hogg, 1838}

Eunym: Hoc loco.

Getangiotaxon: DACTYLETHRINAE Hogg, 1838.

Adelphotaxon: HyMENOCHIRINI Bolkay, 1919.

Getendotaxa: Silurana Gray, 1864; Xenopus Wagler in Boie, 1827.

F.19.02. Tribus Hymenochirini Bolkay, 1919

Protonym: HYMENOCHIRIDAE Bolkay, 1919: 343 [F].

Eunym: Bewick, Chain, Heled \& Evans 2012: 914.

Getangiotaxon: DACTYLETHRINAE Hogg, 1838.

Adelphotaxon: DACTYLETHRINI Hogg, 1838.

Getendotaxa: Hymenochirus Boulenger, 1896; Pseudhymenochirus Chabanaud, 1920.

F.18.03. Subfamilia PIPINAE Gray, 1825-|Fitzinger, 1826|

Eunym: Metcalf 1923: 3.

Getangiotaxon: PIPIDAE Gray, 1825-|Fitzinger, 1826|.

Adelphotaxon: DACTYLETHRINAE Hogg, 1838.

Getendotaxon: Pipa Laurenti, 1768.

F.17.04. Familia RHINOPHRYNIDAE Günther, 1858

Protonym and eunym: RHINOPHRYNIDAE Günther, 1858: 348 [F].

Getangiotaxon: DorsiPARES Blainville, 1816.

Adelphotaxa: PIPIDAE Gray, 1825-|Fitzinger, 1826|; 1 F†.

Getendotaxon: Rhinophrynus Duméril \& Bibron, 1841.

\section{C.07.02. Hypoordo LAevogyrinia Lataste, 1878}

Protonym: LAevogyrinidae Lataste, 1878: 491 [UC].

Eunym: Hoc loco.

Getangiotaxon: GEOBATRACHIA Ritgen, 1828.

Adelphotaxon: Dorsipares Blainville, 1816.

Getendotaxa: Archaeosalientia Roček, 1981; Ranomorpha Fejérváry, 1921; 1 GIS (Colodactylus Tschudi, 1845). 
Comments: This highly supported taxon accomodates the ARCHAEOSALIENTIA and the RANOMORPHA. The sister-group relationship of these two branches was documented by Roelants \& Bossuyt (2005), Frost et al. (2006), Bossuyt \& Roelants (2009) and Pyron \& Wiens (2011). Frost et al. (2006) used for this taxon the ectonym «ACOSMANURA» derived from the nomen ACOSMANURA Starrett, 1973 (credited in error to Savage 1973), which is a junior synonym of the distagmonym LAEVOGYRINIA Lataste, 1878.

\section{C.08.01. Superphalanx ARChaeosalientia Roček, 1981}

Protonym: Archaeosalientia Roček, 1981: 1 [O].

Eunym: Hoc loco.

Getangiotaxon: LAEVOGYRINIA Lataste, 1878.

Adelphotaxon: RaNomorpha Fejérváry, 1921.

Getendotaxa: PELOBATOIDEA Bonaparte, 1850; SCAPHIOPODOIDEA Cope, 1865; 4 G†

Comments: The ArCHAEOSALIENTIA, a highly supported taxon, contains two superfamilies and is sistergroup to the RANOMORPHA. The nomen Anomocoela Nicholls, 1916, used by Frost et al. (2006) as the ectonym "ANOMOCOELA», cannot be applied to this taxon as in the original publication Nicholls (1916) included in this taxon the genus Palaeobatrachus which is now a member of the DoRSIPARES, thus making his Anomocoela a junior synonym of Geobatrachia. Anomocoela Noble, 1922 is indeed a synonym of ARCHAEOSALIENTIA Roček, 1981, but it is invalid under DONS for being a distagmonym and a junior homonym of ANOMOCOELA Nicholls, 1916.

This taxon includes four extant families, with the following relationships: ((MEGOPHRYIDAE, Pelobatidae) (PELODYTIDAE)) (SCAPHIOPODIDAE). The PELOBATIDAE and the PELODYTIDAE are attributed family-rank following the [UQC]. In consequence, the MEGOPHRYIDAE, sister-taxon of the PELOBATIDAE, is also attributed this rank by the [STC], and two epifamilies PELOBATOIDAE and PELODYTOIDAE must be recognised in the superfamily PELOBATOIDEA. The latter is sister to the SCAPHIOPODOIDEA which contains a single 'redundant' family SCAPHIOPODIDAE, whose rank is imposed by the Mandatory-Rank Criterion [MRC].

\section{F.14.01. Superfamilia PELOBATOIDEA Bonaparte, 1850}

Protonym: PelobatidaE Bonaparte, 1850: plate [F].

Eunym: Bolkay 1919: 348.

Getangiotaxon: ArChaeosalientia Roček, 1981.

Adelphotaxon: SCAPHIOPODOIDEA Cope, 1865.

Getendotaxa: Pelobatoidae Bonaparte, 1850; PeLODYToIDAE Bonaparte, 1850.

Comments: For the nomen of this superfamily, the precedence of PELOBATIDAE Bonaparte, 1850 over PeLODYTINA Bonaparte, 1850 was fixed by the Principle of Proedry, as the first one was established for a taxon of rank family and the second one for a taxon of rank subfamily (Dubois 1983b: 271).

\section{F.15.01. Epifamilia PELOBATOIDAE Bonaparte, 1850}

Eunym: Hoc loco.

Getangiotaxon: PELOBATOIDEA Bonaparte, 1850.

Adelphotaxon: PELODYTOIDAE Bonaparte, 1850.

Getendotaxa: MEGophryidaE Bonaparte, 1850-|Noble, 1931|; PelobatidaE Bonaparte, 1850; 1 G†.

Comments: For the nomen of this epifamily, the precedence of PELOBATIDAE Bonaparte, 1850 over MEGALOPHREIDINA Bonaparte, 1850 was fixed by the Principle of Proedry, as the first one was established for a taxon of rank family and the second one for a taxon of rank subfamily (Dubois 1983b: 271). 
Protonyms: MEGALOPHRYIDINA Bonaparte, 1850: plate [bF]; |MEGOPHRYINAE Noble, 1931: 492| [bF].

Eunym: Špinar 1983: 55.

Getangiotaxon: PELOBATOIDAE Bonaparte, 1850.

Adelphotaxon: PELOBATIDAE Bonaparte, 1850.

Getendotaxa: LePToBRACHIINAE Dubois, 1983; MeGOPHRYINAE Bonaparte, 1850-|Noble, 1931|.

Comments: This family is the species-richest group within the ARCHAEOSALIENTIA. We recognise in our classification 11 genera, one of which has to be named as new to resolve paraphyly when dismantling the genus Megophrys. The relationships within the family MEGOPHRYIDAE are transcribed by the following scheme. The two well-supported branches within the family are recognised as the subfamilies LEPTOBRACHIINAE and MEGOPHRYINAE. Within the LEPTOBRACHIINAE, two supported branches form the tribes LEPTOBRACHIINI and LEPTOLALAGINI. The latter only includes a single genus Leptobrachella (Chen et al. 2018), an assemblage that probably will be dismantled when more data on the included species are available. Within the LEPTOBRACHIINI, the two taxa are the LEPTOBRACHIINA with a single genus Leptobrachium and the OREOLALAGINA including the genera Oreolalax and Scutiger.

F.18.04. Subfamilia LEPTOBRACHIINAE Dubois, 1983

Protonym and eunym: LEPTOBRACHIINAE Dubois, 1983c: 147 [bF].

Getangiotaxon: MEGOPHRYIDAE Bonaparte, 1850-|Noble, 1931|.

Adelphotaxon: MEGopHrYINAE Bonaparte, 1850-|Noble, 1931|.

Getendotaxa: Leptobrachinin Dubois, 1983; Leptolalagini Delorme, Dubois, Grosjean \& Ohler, 2006.

F.19.03. Tribus LEPTOBRACHIINI Dubois, 1983

Eunym: Hoc loco.

Getangiotaxon: LEPTOBRACHIINAE Dubois, 1983.

Adelphotaxon: LeptolalaGiNi Delorme, Dubois, Grosjean \& Ohler, 2006.

Getendotaxa: Leptobrachina Dubois, 1983; Oreolalagina Tian \& Hu, 1985.

F.20.01. Subtribus LEPTOBRACHIINA Dubois, 1983

Eunym: Hoc loco.

Getangiotaxon: LEPTOBRACHIINI Dubois, 1983.

Adelphotaxon: OREOLALAGINA Tian \& Hu, 1985.

Getendotaxon: Leptobrachium Tschudi, 1838.

F.20.02. Subtribus OREOLALAGINA Tian \& Hu, 1985

Protonym: OREOLALAXINAE Tian \& Hu, 1985: 221 [bF].

Eunym: Hoc loco.

Getangiotaxon: LEPTOBRACHIINI Dubois, 1983.

Adelphotaxon: LePTOBRACHIINA Dubois, 1983.

Getendotaxa: Oreolalax Myers \& Leviton, 1962; Scutiger Theobald, 1868.

F.19.04. Tribus LePtolalagini Delorme, Dubois, Grosjean \& Ohler, 2006

Protonym: LePtolalaGinae Delorme, Dubois, Grosjean \& Ohler, 2006: 7 [bF].

Eunym: Hoc loco. 
Getangiotaxon: LEPTOBRACHIINAE Dubois, 1983.

Adelphotaxon: LePTOBRACHIINI Dubois, 1983.

Getendotaxon: Leptobrachella Smith, 1925.

\section{F.18.05. Subfamilia MEGOPHRYINAE Bonaparte, 1850-|Noble, 1931|}

Eunym: Noble 1931: 492.

Getangiotaxon: MEGOPHRYIDAE Bonaparte, 1850-|Noble, 1931|.

Adelphotaxon: MEGOPHRYINAE Bonaparte, 1850-|Noble, 1931|.

Getendotaxa: ATYMPANOPHRYNINI nov.; BRACHYTARSOPHRYNINI nov.; MEGOPHRYINI Bonaparte, 1850-|Noble, 1931|; XENophryini Delorme, Dubois, Grosjean \& Ohler, 2006.

Comments: This branch received high support in all recent cladistic analyses (Frost et al. 2006; Pyron \& Wiens 2011; Li et al. 2011; Chen et al. 2017; Mahony et al. 2017). Within this group some relationships are poorly resolved, so we recognise four taxa as tribes. The tribes ATYMPANOPHRYINI, BRACHYTARSOPHRYINI and MEGOPHRYINI each correspond to a single genus, whereas the XENOPHRYINI includes four genera of unresolved relationships. This genus level classification follows that adopted by Chen et al. (2017) and Deuti et al. (2017).

\section{F.19.05. Tribus ATYMPANOPHRYINI nov.}

Getangiotaxon: MEGOPHRYINAE Bonaparte, 1850-|Noble, 1931|.

Adelphotaxa: Brachytarsophrynini nov.; MEgophryini Bonaparte, 1850-|Noble, 1931|; XENOPHRYINI Delorme, Dubois, Grosjean \& Ohler, 2006.

Getendotaxon: Atympanophrys Tian \& Hu, 1983.

Nucleogenus, by present designation: Atympanophrys Tian \& Hu, 1983. • Etymology of nomen: G:

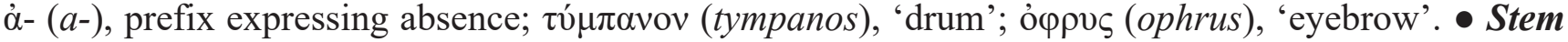
of nomen: Atympanophry-.

Diagnosis: Medium to large sized megophryids (males SVL 34-90 mm; females SVL 47-110 mm); vomerine teeth absent; tympanum concealed or very small; finger and toe tips rounded; web rudimentary or small; finger I longer or shorter than finger II; finger II shorter than finger IV; inner metatarsal tubercle relatively long (more than half length of toe I); hindlimb long, reaching eye; dorsal skin relatively smooth, with few tubercles; dorsal coloration usually including a triangular spot between eyes and $\mathrm{X}$-shaped dark marking; ventral coloration with reddish pattern. Breeding males with blackish nuptial spines on fingers I and II; internal subgular vocal sacs present or absent. Eggs about $3 \mathrm{~mm}$ large, creamy yellow. Larvae with funnel-like mouth, body thin and long, tail tip bluntly pointed, dorsal and ventral body dark coloured. $\{$ Fei \& Ye 2016\}.

\section{F.19.06. Tribus BRACHYTARSOPHRYINI nov.}

Getangiotaxon: MEGOPHRYINAE Bonaparte, 1850-|Noble, 1931|.

Adelphotaxa: Atympanophrynini nov.; Megophryini Bonaparte, 1850-|Noble, 1931|; Xenophryini Delorme, Dubois, Grosjean \& Ohler, 2006.

Getendotaxon: Brachytarsophrys Tian \& Hu, 1983.

Nucleogenus, by present designation: Brachytarsophrys Tian \& Hu, 1983. • Etymology of nomen: G:

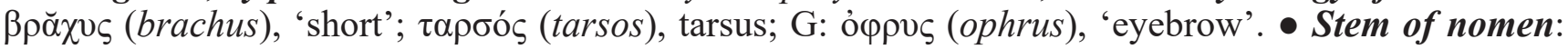
Brachytarsophry-.

Diagnosis: Very large sized megophryids, males (SVL 78-122 mm) smaller than females (SVL 91$137 \mathrm{~mm}$ ); skin smooth; subarticular tubercles and ridges on fingers and toes absent; inner metacarpal 
tubercle elliptical; 1-4 large conic tubercles on outer margin of upper eyelid; tongue large, round, slightly incurved; pupil vertical; iris entirely dark brown in life; toes small or moderately webbed; snout rounded, not projecting beyond lower lip; loreal region obviously flared; hind limbs short, heels not meeting; axillary glands small, on sides of chest; femoral glands not visible. Breeding males with nuptial spines on fingers; vocal sac and lineae masculinae present. Eggs entirely cream yellow or cream white. Tadpoles with relatively small body, total length reaching $40 \mathrm{~mm}$; tail muscles well developed; upper caudal fins starting posterior to first muscle node of tail muscle; anterior part of fins low; lateral lymph sacs not dilated; mouth of tadpole funnel shaped; lip margin extremely wide, covered with papillae; labial teeth and horny beaks absent; anal opening located in middle of tail base and anal tube free in lower caudal fins; spiracle located on left side of body; dorsally between body and tail no Y-shaped mark; ventral side purple blue covered with light spots. Skull broad, its width obviously larger than its length, and highly ossified; maxilla overlapping with quadratojugal; maxillary teeth well developed; vomerine ridges present; nasal process of premaxilla inclining slightly backward; nasal bones large, in contact with each other and with frontoparietal; central part of frontoparietal very narrow; ethmoid cartilage only reaching premaxilla; otic ramus of squamosal having a posterior process; prootic separated from exoccipital; dentary and angular bone narrow; pterygoid of moderate size; tympanum hidden, tympanic anulus and columella present; pores of Eustachian tube large; equal in length to coracoid; sacral diapophyse wide and large; urostyle articulation monocondyle. Chromosomes: 2 n 26-30; nf 44-52. \{Fei \& Ye 2016\}.

\section{F.19.07. Tribus MEGOPHRYINI Bonaparte, 1850-|Noble, 1931|}

Eunym: Dubois 1980: 471.

Getangiotaxon: MEGOPHRYINAE Bonaparte, 1850-|Noble, 1931|.

Adelphotaxa: ATYMPANOPHRYNINI nov.; BRACHYTARSOPHRYNINI nov.; XENOPHRYINI Delorme, Dubois, Grosjean \& Ohler, 2006.

Getendotaxon: Megophrys Kuhl \& Hasselt, 1822.

F.19.08. Tribus XENOPHRYINI Delorme, Dubois, Grosjean \& Ohler, 2006

Protonym and eunym: XENOPHRYINI Delorme, Dubois, Grosjean \& Ohler, 2006: 7 [T].

Getangiotaxon: MEGOPHRYINAE Bonaparte, 1850-|Noble, 1931|.

Adelphotaxa: ATYMPANOPHRYNINI nov.; BRACHYTARSOPHRYNINI nov.; MEGOPHRYINI Bonaparte, 1850-|Noble, 1931|.

Getendotaxa: GRILLITSCHIINA nov.; OPHRYOPHRYNIINA nov.; XENOPHRYINA Delorme, Dubois, Grosjean \& Ohler, 2006.

\section{F.20.03. Subtribus GRILLITSCHIINA nov.}

Getangiotaxon: XENOPHRYINI Delorme, Dubois, Grosjean \& Ohler, 2006.

Adelphotaxa: OPHRYophrynina nov.; XENOPHRYINA Delorme, Dubois, Grosjean \& Ohler, 2006.

Getendotaxon: Grillitschia nov.

Nucleogenus, by present designation: Grillitschia nov. • Etymology of nomen: Patronym Grillitsch (see below). • Stem of nomen: Grillitschi-.

Diagnosis: See below under Grillitschia nov.

\section{G.28.022. Genus Grillitschia nov.}

Getangiotaxon: GRILLITSCHIINA nov.

Adelphotaxon: None.

Getendotaxa: Grillitschia aceras (Boulenger, 1903); Grillitschia longipes (Boulenger, 1885).

Etymology of nomen: This genus is dedicated to Britta Grillitsch (1952-) and Heinz Grillitsch (1951-) 
(Wien, Austria) in appreciation of their work on amphibians, particularly on larvae. $\bullet$ Stem of nomen: Grillitschi-. • Grammatical gender of nomen: feminine.

Nucleospecies, by present designation. • Megophrys longipes Boulenger, 1885.

Diagnosis: Medium sized species (males SVL 40-60 mm; females SVL 50-86 mm); feebly notched tongue, vomerine teeth present, a moderately enlarged head, a narrow, sharply bent supratympanic fold without a posterior glandular swelling, a pair of dorsolateral folds and a V-or X-shaped fold in shoulder region, upper eyelid with a single horn-like tubercle and a coloration pattern including vertical bars on upper lip. \{Boulenger, 1885, 1903; Taylor 1962; Manthey \& Grossmann 1997\}.

\section{F.20.04. Subtribus OPHRYOPHRYNINA nov.}

Getangiotaxon: XeNOPHRYINI Delorme, Dubois, Grosjean \& Ohler, 2006.

Adelphotaxa: GriLlitschina nov.; Xenophryina Delorme, Dubois, Grosjean \& Ohler, 2006.

Getendotaxa: Boulenophrys Fei, Ye \& Jiang in Fei \& Ye, 2016; Ophryophryne Boulenger, 1903.

Nucleogenus, by present designation: Ophryophryne Boulenger, 1903. • Etymology of nomen: G:

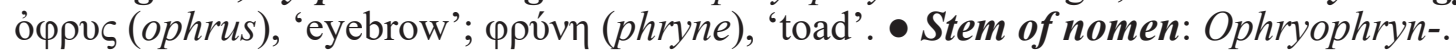

Diagnosis. - Small to medium sized megophryids (males SVL 26-43 mm, females SVL 34-50); skin usually smooth; snout shape shield-shped; canthus rostralis sharp; upper-lip without white stripe; a tubercle or skin folds on outer margin of upper lid; iris entirely dark, brown in life; web on toes absent or weak; subarticular tubercles absent; longitudinal ridges under toes absent; tibia slightly longer than femur; groin without crescent mark; small white axillary glands on side of chest. Skull weakly or strongly ossified; maxilla overlapping with quadratojugal; vomerine teeth and vomerine ridges absent; nasal bones separated from each other, but in contact with sphenethmoid and separated from frontoparietal; otic ramus of squamosal with a posterior process; tympanum and tympanic anulus present; columella present, pores of Eustachian tube large; cartilaginous mesosternum, equal or longer than coracoid; xiphisternum slender; sacral diapophyses wide and large; sacral-coccygeal articulation monocondyle. Eggs entirely creamy white or creamy yellow; larva with a small body, well developed tail muscles, caudal fins not reaching base of tail, lateral lymph sacs not dilated, funnel shaped mouth, horny jaws absent; 4 pairs of spoon-like prelingual papillae present on mouth floor. In males, nuptial spines on fingers I or I and II; no spines on chest of lip margin; a single internal subgular vocal sac; no lineae masculinae. $\{$ Fei \& Ye 2016\}.

\section{F.20.05. Subtribus XENOPHRYINA Delorme, Dubois, Grosjean \& Ohler, 2006}

Eunym: Hoc loco.

Getangiotaxon: XeNOPHRYINI Delorme, Dubois, Grosjean \& Ohler, 2006.

Adelphotaxa: GRILLITSCHIINA nov.; OPHRYOPHRYNINA nov.

Getendotaxon: Xenophrys Günther, 1864.

\section{F.17.06. Familia PELOBATIDAE Bonaparte, 1850}

Eunym: Bonaparte 1850: plate.

Getangiotaxon: PELOBATOIDAE Bonaparte, 1850.

Adelphotaxon: MEGOPHRYIDAE Bonaparte, 1850-|Noble, 1931|.

Getendotaxa: Pelobates Wagler, 1830; 1 G†. 
Protonym: PeLODYTINA Bonaparte, 1850: 7 [bF].

Eunym: Hoc loco.

Getangiotaxon: PELOBATOIDEA Bonaparte, 1850.

Adelphotaxon: PelobatoIdaE Bonaparte, 1850.

Getendotaxon: PELODYTIDAE Bonaparte, 1850.

\section{F.17.07. Familia PELODYTIDAE Bonaparte, 1850}

Eunym: Cope 1866: 68.

Getangiotaxon: PELODYTOIDAE Bonaparte, 1850.

Adelphotaxon: None.

Getendotaxa: Pelodytes Bonaparte, 1838; Pelodytopsis Nikolskii, 1896; 2 G†.

\section{G.28.028. Genus Pelodytopsis Nikolskii, 1896}

Getangiotaxon: PELODYTIDAE Bonaparte, 1850.

Adelphotaxa: Pelodytes Bonaparte, 1838; 2 G†.

Getendotaxon: Pelodytopsis caucasicus (Boulenger, 1896).

Comments: The species originally described as Pelodytes caucasicus Boulenger, 1896 is another striking example of 'Latonia-like situation' (LLS) relatively to all other species currently referred to the genus Pelodytes Bonaparte, 1838 (see M\&M section). The generic nomen Pelodytopsis Nikolskii, 1896 is available for this species, and we recognise this genus as distinct from Pelodytes Bonaparte, 1838.

\section{F.14.02. Superfamilia SCAPHIOPODOIDEA Cope, 1865}

Protonym: SCAPHIOPODIDAE Cope, 1865: 104 [F].

Eunym: Hoc loco.

Getangiotaxon: ArChaEOSAlientia Roček, 1981.

Adelphotaxon: PELOBATOIDEA Bonaparte, 1850.

Getendotaxon: SCAPHIOPODIDAE Cope, 1865.

\section{F.17.08. Familia SCAPHIOPODIDAE Cope, 1865}

Eunym: Cope 1865: 104.

Getangiotaxon: SCAPHIOPODOIDEA Cope, 1865.

Adelphotaxon: None.

Getendotaxa: Scaphiopus Holbrook, 1836; Spea Cope, 1866.

\section{C.08.02. Superphalanx RANomorPHA Fejérváry, 1921}

Protonym and eunym: RANOMORPHA Fejérváry, 1921: 16 [Gs].

Getangiotaxon: LAEVOGYRINIA Lataste, 1878.

Adelphotaxon: Archaeosalientia Roček, 1981.

Getendotaxa: AquiPares Blainville, 1816; Helanura nov.

Comments: This highly supported branch is recovered in all molecular analyses of anurans (Roelants \& Bossuyt 2005; Frost et al. 2006; Roelants et al. 2007; Bossuyt \& Roelants 2009; Pyron \& Wiens 2011) and has been designated in these works as NeOBATRACHIA Reig, 1958, a distagmonym which is both a 
junior homonym of NeOBATRACHIA Sarasin \& Sarasin, 1890 and a junior synonym of RANOMORPHA Fejérváry, 1921, and is therefore invalid under DONS. It includes two branches, the taxon-rich AQUIPARES and its sister-taxon, the HeLANURA, which corresponds to the single family HELEOPHRYNIDAE.

\section{C.09.01. Epiphalanx AquiPares Blainville, 1816}

Protonym: AQUIPARES Blainville, 1816: '111' [119] [bO].

Eunym: Hoc loco.

Getangiotaxon: RanomorPHa Fejérváry, 1921.

Adelphotaxon: Helanura nov.

Getendotaxa: Gondwanura nov.; Phaneranura nov.; Scoptanura Starrett, 1973.

Comments: The AQUIPARES are a highly supported branch of RANOMORPHA that contains three taxa, the relationships between which are not resolved in our TREE. The name used by Frost et al. (2006) for this taxon, «PHTHANOBATRACHIA», was explicitly presented as an 'unregulated' name outside the Code (Frost et al. 2006: 143) and is thus an unavailable ectonym. Even if it was available, it would anyway be an invalid junior synonym of the distagmonym AQUIPARES.

\section{C.10.01. Phalanx Gondwanura nov.}

Getangiotaxon: AQUIPARES Blainville, 1816.

Adelphotaxa: Phaneranura nov.; Scoptanura Starrett, 1973.

Getendotaxa: NasikabatrachidaE Biju \& Bossuyt, 2003; SooglossidaE Noble, 1931.

Comments: A highly supported branch in TREE accommodates the families NASIKABATRACHIDAE and SOOGLOSSIDAE. This relationship was recovered in all recent molecular cladistic analyses. In Frost et al. (2006), this taxon was named SOOGLOSSIDAE, of which NASIKABATRACHIDAE was considered a synonym, whereas in Bossuyt \& Roelants (2009) it was recognised as the superfamily SOOGLOSSOIDEA. Here we credit SOOGLOSSIDAE with the rank family according to the [UQC], and its sister-taxon NASIKABATRACHIDAE is afforded the same rank to follow the Non-Redundancy Criterion [NRC], i.e. to avoid redundancy between the nomina of phalanx and family. As no class-group nomen is available for this taxon, we name it GoNDWANURA, which points to its biogeographical origin.

Conucleogenera, by present designation: Nasikabatrachus Biju \& Bossuyt, 2003; Sooglossus Boulenger, 1906.

Etymology of nomen: Sanskrit: गोण्डवन (gondavana, from wana, 'forest' and Goondu, name of a Dravidian hill people), 'Gondwana'; N: ANURA Duméril, 1805, derived from G: $\alpha v$ - (an-), 'without'; oüpá (oura), 'tail'. This nomen refers to the Gondwanian distribution of this relict group of frogs (Biju \& Bossuyt 2003).

Diagnosis: Very small to large (SVL 9-90 mm) sized frogs; smooth or tubercular skin; absence of columella; presence of a neopalatine bone; coracoids slender, lateral ends as wide or wider than medial ends; presence of a small supplementary bony element on tarsus; sharply pointed terminal phalanges; inguinal amplexus. \{Biju \& Bossuyt 2003; Van der Meijden et al. 2007\}.

\section{F.17.09. Familia NASIKABATRACHIDAE Biju \& Bossuyt, 2003}

Protonym: NASIKABATRACHIDAE Biju \& Bossuyt, 2003: 711 [F].

Getangiotaxon: GoNDWANURA nov.

Adelphotaxon: SooglossidaE Noble, 1931.

Getendotaxon: Nasikabatrachus Biju \& Bossuyt, 2003. 


\section{F.17.10. Familia SooglosSIDAE Noble, 1931}

Protonym: Sooglossinae Noble, 1931: 492 [bF].

Eunym: Griffiths 1963: 273.

Getangiotaxon: GoNDWANURA nov.

Adelphotaxon: NASIKABATRACHIDAE Biju \& Bossuyt, 2003.

Getendotaxa: Sechellophryne Nussbaum \& Wu, 2007; Sooglossus Boulenger, 1906.

\section{C.10.02. Phalanx Phaneranura nov.}

Getangiotaxon: AQUIPARES Blainville, 1816.

Adelphotaxa: Gondwanura nov.; Scoptanura Starrett, 1973.

Getendotaxa: Bainanura nov.; Diplosiphona Günther, 1859.

Comments: This highly supported branch is a member of an unresolved trichotomy with GoNDWANURA and Scoptanura. It includes the Bainanura and the Diplosiphona. This taxon was named «Notogaeanura» in Frost et al. (2006) but as this name was explicitly coined outside the Code, it is an unavailable ectonym. As no nomen is available for this taxon, we name it here.

Conucleogenera, by present designation: Bufo Garsault, 1764; Heleioporus Gray, 1841.

Etymology of nomen: G: pavepós (phaneros), 'visible, conspicuous'; N: ANURA Duméril, 1805, derived from G: $\alpha v-($ an-), 'without'; oüpó (oura), 'tail'. This nomen refers to the behaviour of many of these frogs, which often do not hide and are therefore visible in their natural habitat, even in the day time.

Diagnosis: Very small to large (SVL 10-110 mm) sized frogs; terrestrial breeding with direct development of terrestrial eggs (ovoviviparity in Eleutherodactylus jasperi); an embryonic egg teeth present; arciferal or rarely pseudofirmisternal pectoral girdle; calcanea and astragali partially fused; usually with T-shaped terminal phalanges; intercalary elements of phalanges always lacking. \{Hedges et al. 2008; Heinicke et al. 2009$\}$.

\section{C.11.01. Subphalanx BaInANURA nov.}

Getangiotaxon: Phaneranura nov.

Adelphotaxon: Diplosiphona Günther, 1859.

Getendotaxa: Phoranura nov.; Phrynanura nov.

Comments: This branch is within the Phaneranura the sister-group of the Diplosiphona and has high statistical support. It has been recovered in all cladistic analyses based on molecular data (Bossuyt \& Roelants 2009; Pyron \& Wiens 2011; Irisarri et al. 2012; Zhang et al. 2013; Feng et al. 2017; Hutter et al. 2017; Streicher et al. 2018). It includes two highly supported branches, the Phoranura (Dendrobatoidea) and the Phrynanura. In Frost et al. (2006), this taxon is termed the «NobleObatrachia» (an ectonym); in more recent works (Zhang et al. 2013; Feng et al. 2017; Streicher et al. 2017), it is named HYLOIDEA, a nomen here applied to a much less inclusive taxon under DONS Criteria. As there is no class-series nomen available for this taxon, we name it here.

Conucleogenera, by present designation: Bufo Garsault, 1764; Dendrobates Wagler, 1830.

Etymology of nomen: G: ßaívw (baino), 'I walk'; N: ANURa Duméril, 1805, derived from G: àv- (an-), 'without'; oüpó (oura), 'tail'. This nomen refers to the behaviour of many of these frogs, which often walk rather than they jump.

Diagnosis: Very small to very large sized frogs (SVL 12-230 mm); morphology frog-, toad- or treefroglike; sternum present, ossified or cartilaginous; pectoral girdle arciferal or firmisternal; terminal 
phalanges variable; intercalary elements absent or present; fibulare and tibiale fused at proximal and distal end, or very rarely completely fused; Bidder's organs absent or present; amplexus axillary or absent, rarely inguinal; free living tadpoles, but also various modes of independence from water (nests, dorsal transport of tadpoles, body cavities, endotrophy, viviparity); tadpole with keratinised mouthparts, branchial chambers fused, spiracle positioned on left side of body. \{Mendelson et al. 2000; Hedges et al. 2008; Heinicke et al. 2009; Vitt \& Caldwell 2014\}.

\section{C.12.01. Infraphalanx Phoranura nov.}

Getangiotaxon: BaINANURA nov.

Adelphotaxon: Phrynanura nov.

Getendotaxa: АRомоватіDAE Grant, Frost, Caldwell, Gagliardo, Haddad, Kok, Means, Noonan, Schargel \& Wheeler, 2006; DENDROBATIDAE ||Bonaparte, 1850\|-Cope, 1865.

Comments: The position of this branch in the phylogeny of anurans has been highly unstable and debated. It was recognised as a family DENDROBATIDAE or as a superfamily DENDROBATOIDEA grouping the AROMOBATIDAE and DENDROBATIDAE. This taxon was proposed to be within the RANOIDEA which included also the MICROHYLIDAE, the ARTHROLEPTIDAE, the RANIDAE, the HYPEROLIIDAE, the RHACOPHORIDAE and the genus Hemisus (Ford \& Cannatella 1993), but in recent phylogenies it was part of the BainanuRa (Darst \& Cannatella 2004; Frost et al. 2006; Grant et al. 2006). Its position within this taxon is not fixed and it was recovered as sister-taxon to hyline frogs (Darst \& Cannatella 2004), to Thoropa, together being sister-taxon to the BUFONIDAE (Frost et al. 2006), as sister-taxon to the HYLODIDAE within the «ATHESPHATANURA》 or as sister-taxon to the BUFONIDAE (Bossuyt \& Roelants 2009; Pyron \& Wiens 2011; Irisarri et al. 2012; Frazão et al. 2015). Zhang et al. (2013) recovered the DENDROBATIDAE within the HYLOIDEA as sister-taxon to all other hyloid frogs, whereas in Streicher et al. (2017) it was considered sister-taxon to the LEPTODACTYLIDAE and in Feng et al. (2017) as outgroup to a branch including some leptodactyloid and bufonid families. Hutter et al. (2017) recovered the DENDROBATIDAE in the same position as our infraphalanx PHORANURA, as sister-taxon to a large taxon, here named PHRYNANura, grouping the families BRACHYCEPHALIDAE, CEUTHOMANTIDAE, HEMIPHRACTIDAE, BUFONIDAE, ODONTOPHRYNIDAE, ALLOPHRYNIDAE,

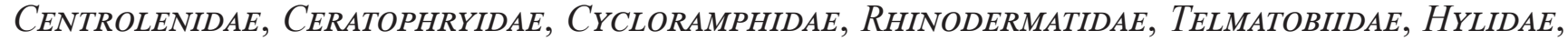
PHYLLOMEDUSIDAE and LEPTODACTYLIDAE. As no class-series nomen has been given to this taxon so far, we name it here.

The Phoranura consist in the Aromobatidae and the Dendrobatidae. Santos et al. (2009) recognised a single family DENDROBATIDAE including the ALLOBATINAE and DENDROBATINAE and they synonymised all the genera of DENDROBATINAE under Dendrobates. Brown et al. (2011) and Grant et al. (2017) argued for maintaining a classification that reflects more precisely the variation within the taxon here named Phoranura. Here the DENDRobatidae are attributed family rank based on the [UQC] and consequently the AROMOBATIDAE are also afforded this rank based on the [STC] and on the [NRC], i.e. to avoid redundancy between the family and the infraphalanx.

Conucleogenera, by present designation: Aromobates Myers, Paolillo \& Daly, 1991; Dendrobates Wagler, 1830.

Etymology of nomen: G: $\varphi \varepsilon ́ p \omega$ (phero), 'I bear'; N: AnuRa Duméril, 1805, derived from G: ảv- (an-), 'without'; oüpó (oura), 'tail'. This nomen refers to the fact that in the species of this group the adult male or female carries the tadpoles on its back from a small water collection to another one (Grant et al. 2006).

Diagnosis: Small to medium sized frogs (SVL 13-50 mm); supernumerary tubercles on hand present; tarsal ridge present; a weak metatarsal ridge; insertion of distal tendon of musculus semitendinosus dorsal to $m$. gracilis; presence of a binding tendon straping $m$. semitendinosus to outer edge of $m$. gracilis; dorsal flap of $m$. depressor mandibulae present; tympanum conceiled superficially by $m$. depressor mandibulae; $m$. intermandibularis supplementary elements oriented anteromedially; amplexus absent; presence of dorsal transport of tadpoles; epicoracoids completely fused, non-overlapping; omosternum medially ossified; maxillary teeth nonpedicellate; retroarticular process of mandible present; chromosome 
number 24. \{Silverstone 1975; Myers \& Daly 1976; Myers 1982; Savage 2002; Grant et al. 2006; PaezVacas et al. 2010; Brown et al. 2011; Grant \& Myers 2013 .

F.17.11. Familia ARомоватіDAE Grant, Frost, Caldwell, Gagliardo, Haddad, Kok, Means, Noonan, Schargel \& Wheeler, 2006

Protonym and eunym: ARomoвatidae Grant, Frost, Caldwell, Gagliardo, Haddad, Kok, Means, Noonan, Schargel \& Wheeler, 2006: 4 [F].

Getangiotaxon: Phoranura nov.

Adelphotaxon: DENDROBATIDAE ||Bonaparte, 1850\|-Cope, 1865.

Getendotaxa: Allobatinae Grant, Frost, Caldwell, Gagliardo, Haddad, Kok, Means, Noonan, Schargel \& Wheeler, 2006; Anomaloglossinae Grant, Frost, Caldwell, Gagliardo, Haddad, Kok, Means, Noonan, Schargel \& Wheeler, 2006; Aromobatinae Grant, Frost, Caldwell, Gagliardo, Haddad, Kok, Means, Noonan, Schargel \& Wheeler, 2006.

Comments: Three highly supported branches are recognised within the AROMOBATIDAE but the relationships between them are not resolved. They are transposed in the current ergotaxonomy as the subfamily ALLOBATINAE, with the single genus Allobates, the subfamily ANOMALOGLOSSINAE, with the genera Anomaloglossus and Rheobates, and the subfamily AROMOBATINAE, with the genera Aromobates and Mannophryne (Grant et al. 2006, 2017).

F.18.06. Subfamilia AlLobatinae Grant, Frost, Caldwell, Gagliardo, Haddad, Kok, Means, Noonan, Schargel \& Wheeler, 2006

Protonym and eunym: AllobatinaE Grant, Frost, Caldwell, Gagliardo, Haddad, Kok, Means, Noonan, Schargel \& Wheeler, 2006: $4[\mathrm{bF}]$.

Getangiotaxon: Allobatidae Grant, Frost, Caldwell, Gagliardo, Haddad, Kok, Means, Noonan, Schargel \& Wheeler, 2006.

Adelphotaxa: Anomaloglossinae Grant, Frost, Caldwell, Gagliardo, Haddad, Kok, Means, Noonan, Schargel \& Wheeler, 2006; Авомоватіnae Grant, Frost, Caldwell, Gagliardo, Haddad, Kok, Means, Noonan, Schargel \& Wheeler, 2006.

Getendotaxon: Allobates Zimmermann \& Zimmermann, 1988.

F.18.07. Subfamilia ANOMALOGLOSSINAE Grant, Frost, Caldwell, Gagliardo, Haddad, Kok, Means, Noonan, Schargel \& Wheeler, 2006

Protonym and eunym: Anomaloglossinae Grant, Frost, Caldwell, Gagliardo, Haddad, Kok, Means, Noonan, Schargel \& Wheeler, 2006: 4 [bF].

Getangiotaxon: AllobatidaE Grant, Frost, Caldwell, Gagliardo, Haddad, Kok, Means, Noonan, Schargel \& Wheeler, 2006.

Adelphotaxa: Allobatinae Grant, Frost, Caldwell, Gagliardo, Haddad, Kok, Means, Noonan, Schargel \& Wheeler, 2006; Aromobatinae Grant, Frost, Caldwell, Gagliardo, Haddad, Kok, Means, Noonan, Schargel \& Wheeler, 2006.

Getendotaxa: Anomaloglossus Grant, Frost, Caldwell, Gagliardo, Haddad, Kok, Means, Noonan, Schargel \& Wheeler, 2006; Rheobates Grant, Frost, Caldwell, Gagliardo, Haddad, Kok, Means, Noonan, Schargel \& Wheeler, 2006.

F.18.08. Subfamilia ARомоватіNAE Grant, Frost, Caldwell, Gagliardo, Haddad, Kok, Means, Noonan, Schargel \& Wheeler, 2006

Eunym: Grant, Frost, Caldwell, Gagliardo, Haddad, Kok, Means, Noonan, Schargel \& Wheeler, 2006: 4.

Getangiotaxon: AllobatidaE Grant, Frost, Caldwell, Gagliardo, Haddad, Kok, Means, Noonan, Schargel \& Wheeler, 2006.

Adelphotaxa: AllobatinaE Grant, Frost, Caldwell, Gagliardo, Haddad, Kok, Means, Noonan, Schargel \& Wheeler, 2006; Anomaloglossinae Grant, Frost, Caldwell, Gagliardo, Haddad, Kok, Means, Noonan, Schargel \& Wheeler, 2006.

Getendotaxa: Aromobates Myers, Paolillo \& Daly, 1991; Mannophryne La Marca, 1992. 


\section{F.17.12. Familia DENDROBATIDAE ||Bonaparte, 1850\|-Cope, 1865}

Protonyms and eunym: ||EUBAPHIDAE Bonaparte, 1850: plate|| [F]; DENDROBATIDAE Cope, 1865: 100 [F]. Getangiotaxon: PhORANURA nov.

Adelphotaxon: АвомоватіDAE Grant, Frost, Caldwell, Gagliardo, Haddad, Kok, Means, Noonan, Schargel \& Wheeler, 2006.

Getendotaxa: Colostethinae Cope, 1867; DendrobatinaE ||Bonaparte, 1850||-Cope, 1865; HyloXaLINAE Grant, Frost, Caldwell, Gagliardo, Haddad, Kok, Means, Noonan, Schargel \& Wheeler, 2006.

Comments: The family DENDROBATIDAE reveals three branches of unresolved relationships, recognised here as the subfamilies COLOSTETHINAE, DENDROBATINAE and HYLOXALINAE.

Within the Colostethinae, the tribes Colostethini (genera Ameerega, Colostethus and Leucostethus of unresolved mutual relationships) and EPIPEDOBATINI (genera Epipedobates and Silverstoneia; relationships agreeing with Grant et al. 2017) are here recognised.

The DENDRobatinaE are here divided into two tribes DENDROBATINI and PhYLLOBATINI. The first covers two branches regognised as the subtribes ANDINOBATINA and DENDROBATINA. The ANDINOBATINA includes the infratribes ANDINOBATINIA, with the genera Andinobates and Ranitomeyia, and EXCIDOBATINIA, with the single genus Excidobates. The relationships within the subtribe DENDROBATINA are not resolved and four genera, Adelphobates, Dendrobates, Minyobates and Oophaga, are recognised within this taxon. The positions of the genus-series taxa within this subtribe are not fixed in our classification as the relationships between the branches do not have sufficient support in our tree. This is also reflected by the variable position of Minyobates in the recent phylogenies published. Thus in the tree of Grant et al. $(2006,2017)$ it is sister-taxon to the branch encompassing Ranitomeya, Adelphobates, Oophaga and Dendrobates, whereas Santos et al. (2009) included it into their Dendrobates galactonotus group which groups species of Adelphobates and Minyobates, and Brown et al. (2011) recognised a genus Minyobates as sister to Adelphobates, Oophaga and Dendrobates. The second tribe of Dendrobatinae, the PhYLLOBATINI, includes a single genus Phyllobates.

The subfamily HYLOXALINAE is represented in TREE by a single genus Hyloxalus, but it also includes the genera Ectopoglossus and Paruwrobates (Grant et al. 2017), not represented in TREE. This arrangement corresponds in the relationships and in the proposed classification to those presented by Grant et al. $(2006,2017)$ and Brown et al. (2011). This differs from the classification proposed by Santos et al. (2009) in that these authors synonymised all the genera of DENDROBATINAE under Dendrobates. Brown et al. (2011) and Grant et al. (2017) argued for maintaining a classification that reflects more precisely the variation within Dendrobates s.1. and we follow them. The family-series taxa of this classification are formally named below.

\section{F.18.09. Subfamilia Colostethinae Cope, 1867}

Protonym: Colostethidae Cope, 1867: 191 [F].

Eunym: Bauer 1987: 5.

Getangiotaxon: DENDROBATIDAE ||Bonaparte, 1850||-Cope, 1865.

Adelphotaxa: DendrobatinaE ||Bonaparte, 1850\|-Cope, 1865; HyloXalinaE Grant, Frost, Caldwell, Gagliardo, Haddad, Kok, Means, Noonan, Schargel \& Wheeler, 2006.

Getendotaxa: Colostethini Cope, 1867; EPIPEDobatini nov.

\section{F.19.09. Tribus Colostethini Cope, 1867}

Eunym: Hoc loco.

Getangiotaxon: ColostethinaE Cope, 1867.

Adelphotaxon: EPIPEDOBATINI nov.

Getendotaxa: Ameerega Bauer, 1986; Colostethus Cope, 1866; Leucostethus Grant, Rada, Anganoy-Criollo, Batista, Dias, Jeckel, Machado \& Rueda-Almonacid, 2017. 


\section{F.19.10. Tribus EPIPEDOBATINI nov.}

Getangiotaxon: ColostethinaE Cope, 1867.

Adelphotaxon: Colostethini Cope, 1867.

Getendotaxa: Epipedobates Myers, 1987; Silverstoneia Grant, Frost, Caldwell, Gagliardo, Haddad, Kok, Means, Noonan, Schargel \& Wheeler, 2006.

Nucleogenus, by present designation: Epipedobates Myers, 1987. • Etymology of nomen: G: غ̀ $\pi i ́ \pi \varepsilon \delta \circ \varsigma$

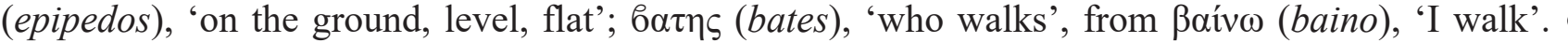
Stem of nomen: Epipedobat-.

Diagnosis: Small, cryptic colored frogs; skin smooth, granular or tubercular; pale oblique lateral stripe present; ventrolateral stripes present or absent; narrow to moderately expanded finger discs; median lingual process absent; larval vent tube dextral; testes entirely pigmented; no dark throat collar. \{Grant et al. 2006\}.

\section{F.18.10. Subfamilia DENDROBATINAE ||Bonaparte, 1850||-Cope, 1865}

Eunym: Gadow 1901: xi, 272.

Getangiotaxon: DENDROBATIDAE $\|$ Bonaparte, 1850\|-Cope, 1865.

Adelphotaxa: Colostethinae Cope, 1867; HyloxalinaE Grant, Frost, Caldwell, Gagliardo, Haddad, Kok, Means, Noonan, Schargel \& Wheeler, 2006.

Getendotaxa: Dendrobatini ||Bonaparte, 1850||-Cope, 1865; Phyllobatini Fitzinger, 1843.

\section{F.19.11. Tribus DENDROBATINI ||Bonaparte, 1850||-Cope, 1865}

Eunym: Grant, Rada, Anganoy-Criollo, Batista, Dias, Jeckel, Machado \& Rueda-Almonacid 2017: 27.

Getangiotaxon: DENDROBATINAE ||Bonaparte, 1850\|-Cope, 1865.

Adelphotaxon: PHYLLOBATINI Fitzinger, 1843.

Getendotaxa: ANDINOBATINA nov.; DENDROBATINA ||Bonaparte, 1850||-Cope, 1865.

\section{F.20.06. Subtribus ANDINOBATINA nov.}

Getangiotaxon: DENDROBATINI ||Bonaparte, 1850||-Cope, 1865.

Adelphotaxon: DENDROBATINA ||Bonaparte, 1850||-Cope, 1865.

Getendotaxa: ANDINOBATINIA nov.; EXCIDOBATINIA nov.

Nucleogenus, by present designation: Andinobates Twomey, Brown, Amézquita \& Mejía-Vargas in Brown, Twomey, Amézquita, Souza, Caldwell, Lötters, May, Melo-Sampaio, Mejía-Vargas, Pérez-Peña, Pepper, Poelman, Sanchez-Rodriguez \& Summers, 2011. E Etymology of nomen: Spanish: andino, 'Andean' (of or from the Andes); G: Baívw (baino), 'I walk'. • Stem of nomen: Andinobat-.

Diagnosis: Small, darkly or brilliantly colored frogs; head narrower than body; vocal slits in males; lateral and dorsal stripes usually absent or incomplete; finger discs expanded or narrow; median lingual process absent; larval vent tube dextral or medial; larval oral disc emarginate; lipophilic alkaloids secreted in the skin in most species; testes pigmented in most species; dark throat collar absent. \{Grant et al. 2006; Twomey \& Brown, 2008; Brown et al. 2011\}.

\section{F.21.01. Infratribus ANDINOBATINIA nov.}

Getangiotaxon: ANDINOBATINA nov.

Adelphotaxon: EXCIDOBATINIA nov. 
Getendotaxa: Andinobates Twomey, Brown, Amézquita \& Mejía-Vargas in Brown, Twomey, Amézquita, Souza, Caldwell, Lötters, May, Melo-Sampaio, Mejía-Vargas, Pérez-Peña, Pepper, Poelman, Sanchez-Rodriguez \& Summers, 2011; Ranitomeya Bauer, 1985.

\section{F.21.02. Infratribus EXCIDOBATINIA nov.}

Getangiotaxon: ANDINOBATINA nov. Adelphotaxon: ANDINOBATINIA nov.

Getendotaxon: Excidobates Twomey \& Brown, 2008.

Nucleogenus, by present designation: Excidobates Twomey \& Brown, 2008. • Etymology of nomen:

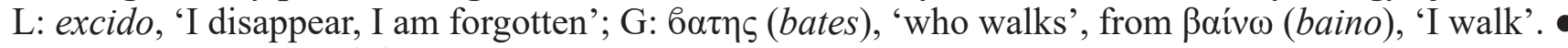
Stem of nomen: Excidobat-.

Diagnosis: Small, darkly colored frogs; dark dorsal spots present; dorsal stripes absent or incomplete; skin smooth or granular; pale spots under the chin and on the ventral surface of thighs; head narrower than body; vocal slits in males; tongue ovoid; finger discs moderately expanded; labial tooth rows in tadpoles following formula $2: 2+2 / 1+1: 3$; well developed keratinised jaw sheaths; medial indentation in posterior jaw sheath present or absent; vent dextral; spiracle sinistral. \{Twomey \& Brown, 2008\}.

\section{F.20.07. Subtribus DENDROBATINA ||Bonaparte, 1850||-Cope, 1865}

Eunym: Hoc loco.

Getangiotaxon: DENDROBATINI ||Bonaparte, 1850||-Cope, 1865.

Adelphotaxon: ANDINOBATINA nov.

Getendotaxa: Adelphobates Grant, Frost, Caldwell, Gagliardo, Haddad, Kok, Means, Noonan, Schargel \& Wheeler, 2006; Dendrobates Wagler, 1830; Minyobates Myers, 1987; Oophaga Bauer, 1994.

\section{F.19.12. Tribus PhyLlobatini Fitzinger, 1843}

Protonym: PhYLlOBATAE Fitzinger, 1843: 32 [F].

Eunym: Hoc loco.

Getangiotaxon: DENDROBATINAE ||Bonaparte, 1850|--Cope, 1865.

Adelphotaxon: DENDROBATINI ||Bonaparte, 1850||-Cope, 1865.

Getendotaxon: Phyllobates Duméril \& Bibron, 1841.

F.18.11. Subfamilia HyloXaLINAE Grant, Frost, Caldwell, Gagliardo, Haddad, Kok, Means, Noonan, Schargel \& Wheeler, 2006

Protonym and eunym: HYLOXALINAE Grant, Frost, Caldwell, Gagliardo, Haddad, Kok, Means, Noonan, Schargel \& Wheeler, 2006: $4[\mathrm{~F}]$.

Getangiotaxon: DENDROBATIDAE \|Bonaparte, 1850\|-Cope, 1865.

Adelphotaxa: Colostethinae Cope, 1867; Dendrobatinae ||Bonaparte, 1850||-Cope, 1865.

Getendotaxa: Ectopoglossus Grant, Rada, Anganoy-Criollo, Batista, Dias, Jeckel, Machado, and Rueda-Almonacid, 2017; Hyloxalus Jiménez de la Espada, 1870; Paruwrobates Bauer, 1994.

\section{C.12.02. Infraphalanx Phrynanura nov.}

Getangiotaxon: BAINANURA nov.

Adelphotaxon: Phoranura nov.

Getendotaxa: Gaianura nov.; Hemiphractiformia Brocchi, 1881; Hylobatrachia Ritgen, 1828. 
Comments: This taxon, retrieved in the tree of Hutter et al. (2017), accommodates all 'hyloid' frogs except the Phoranura (Dendrobatoidea). It includes the Gaianura, Hemiphractiformia and HylobatraChia. The relationships between these three well supported branches are unresolved, even the sister-taxon relationship between GaIANURA and HEMIPHRACTIFORMIA not having significant support (SHL 65).

Conucleogenera, by present designation: Brachycephalus Fitzinger, 1826; Bufo Garsault, 1764; Hemiphractus Wagler, 1828.

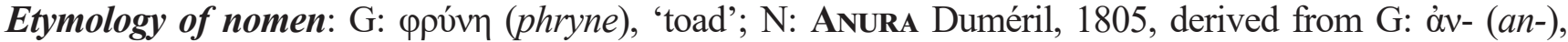
'without'; oüpó (oura), 'tail'. This nomen refers to the fact that this taxon includes the BUFONIDAE, a family many members of which are often designated by the common language 'toad', and many nominal genera of which are based on the Greek root $\varphi \rho v ́ v \eta$.

Diagnosis: Very small to very large sized frogs (SVL 12-230 mm); morphology frog-, toad- or treefroglike; sternum present, ossified or cartilaginous; pectoral girdle arciferal, rarely pseudofirmisternal; terminal phalanges variable; intercalary elements absent or present; transverse process of sacral vertebra cylindrical or moderately expanded, bicondylar articulation; palatines and frontoparietals paired; fibulare and tibiale fused at proximal and distal end, or rarely completely fused; Bidder's organs absent or present; amplexus axillary, rarely inguinal; breeding free living tadpoles, but also various modes of independence from water (nests, body cavities, endotrophy, viviparity); tadpole with keratinised mouthparts, a unique branchial chamber, spiracle positioned on left side of body. \{Mendelson et al. 2000; Vitt et al. 2014; Castroviejo-Fisher 2015\}.

\section{C.13.01. Hypophalanx Gaianura nov.}

Getangiotaxon: Phrynanura nov.

Adelphotaxa: Hemiphractiformia Brocchi, 1881; Hylobatrachia Ritgen, 1828.

Getendotaxa: BRACHYCEPHaLIDAE Günther, 1858; CeuthomantidaE Heinicke, Duellman, Trueb, Means, MacCulloch \& Hedges, 2009.

Comments: The sister-taxon relationship of this highly supported branch with the HEMIPHRACTIFORMIA has poor support, so we treat these two taxa as hypophalanges, along with the HYLOBATRACHIA. The GaIANURA include the families BRACHYCEPHALIDAE (with the subfamilies BRACHYCEPHALINAE, CRAUGASTORINAE and ELEUTHERODACTYLINAE) and CEUTHOMANTIDAE. The BRACHYCEPHALIDAE are attributed family rank by the [UQC] and consequently the CEUTHOMANTIDAE as well, according to the [STC].

The taxon Gaianura was recognised by Darst \& Cannatella (2004), Heinicke et al. (2007), Bossuyt \& Roelants (2009), Pyron \& Wiens (2011), Zhang et al. (2013), Feng et al. (2017), Hutter et al. (2017) and Streicher et al. (2018) without formally naming it. Frost et al. (2006) used the family nomen BRACHYCEPHALIDAE for this taxon. Hedges et al. (2008) created for this taxon the unranked ectonym «TERRARANA». Heinicke et al. (2009) created a junior homonymous ectonym having the same spelling «TERRARANA» but a distinct etymology, hence being a distinct name, which was used by Taboada et al. (2013) and under the form "TERRARANAE», first suggested by Dubois (2009a), who however had considered it in error as a class-series nomen, by Duellman et al. (2016) and Heinicke et al. (2018). Padial et al. (2014) used the superfamilial nomen BRACHYCEPHALOIDEA for this taxon. Under DONS, there is no class-series nomen available for this taxon and we hereby name it GAIANURA.

Conucleogenera, by present designation: Brachycephalus Fitzinger, 1826; Ceuthomantis Heinicke, Duellman, Trueb, Means, MacCulloch \& Hedges, 2009.

Etymology of nomen: G: yaĩ (gaia), 'earth' (as opposed to water); N: ANURA Duméril, 1805, derived from G: $\dot{\alpha} v-(a n-)$, 'without'; oüpá (oura), 'tail'. This nomen refers to the fact that the species of this group of frogs (except Eleutherodactylus jasperi) lay their eggs under some shelter on the ground, where they undergo direct development (Hedges et al. 2008). 
Diagnosis: Very small to large (SVL 10-110 mm) sized species; terrestrial breeding with direct development of terrestrial eggs (ovoviviparity in Eleutherodactylus jasperi); an embryonic egg teeth present; arciferal or rarely pseudofirmisternal pectoral girdle; calcanea and astragali partially fused; usually with T-shaped terminal phalanges; intercalary elements of phalanges always lacking; Bidder's organs absent. \{Hedges et al. 2008; Heinicke et al. 2009\}.

\section{F.17.13. Familia BRACHYCEPHALIDAE Günther, 1858}

Protonym: BRACHYCEPHALINA Günther, 1858: 344 [Sc].

Eunym: Günther 1858: 346.

Getangiotaxon: GaIANURA nov.

Adelphotaxon: CEUthomantidae Heinicke, Duellman, Trueb, Means, MacCulloch \& Hedges, 2009.

Getendotaxa: BRACHYCEPHALINAE Günther, 1858; CRAUGASTORINAE Hedges, Duellman \& Heinicke, 2008; Eleutherodactylinae Lutz, 1954; 2 GIS (Atopophrynus Lynch \& Ruíz-Carranza, 1982; Geobatrachus Ruthven, 1915).

Comments: We recognise the three well supported taxa within the BRACHYCEPHALIDAE as the subfamilies BRACHYCEPHALINAE (with two valid genera Brachycephalus and Ischnocnema), CRAUGASTORINAE and ELEUTHERODACTYLINAE that both show a more complex structure. For the genera Atopophrynus and Geobatrachus, no molecular data are available and so far these brachycephalid taxa have not been allocated in the subfamilial classification.

The CRAUGASTORINAE show two tribes, the CRAUGASTORINI, including the genera Craugastor and Haddadus, and the tribe STRABOMANTINI. The latter tribe is divided into two subtribes, the Strabomantina and Pristimantina. The Strabomantina show two supported branches, the HOLOADENINIA and the STRABOMANTINIA which correspond to the genus Strabomantis. The relationships within the HOLOADENINIA are not resolved, so the branches with high support are recognised here as four hypotribes: BARYCHOLINOA nov., for the genera Bahius nov., Barycholos and Phyllonates; BRYOPHRYNINOA nov., for the genus Bryophryne; HOLOADENINOA for the genera Euparkerella and Holoaden; and NobleLLINOA nov., for the genera Microkayla, Noblella, Psychrophrynella and Qosqophryne; the genera Niceforonia and Tachiramantis, for which no molecular data are available, are referred to this infratribe on morphological grounds but have not been so far allocated to any of these four taxa. The supported branches of the second subtribe PRISTIMANTINA are recognised as the infratribe HYPODACTYLINIA, corresponding to the genus Hypodactylus, and the infratribe PRISTIMANTINIA. In the latter taxon two well supported taxa are recognised as the hypotribes OREOBATINOA nov. and PristimantinOA, holding the genera Pristimantis and Yunganastes. The hypotribe OREOBATINOA contains two supported taxa recognised as OREOBATITES nov., for the genera Lynchius and Oreobates, and PHRYNOPODITES nov., for the genus Phrynopus.

The ELEUTHERODACTYLINAE include two highly supported branches recognised as the tribes ELEUTHERODACTYLINI, with two subtribes DIASPORINA nov. (genus Diasporus) and ELEUTHERODACTYLINA (genera Eleutherodactylus and Euhyas), and PHYZELAPHRYNINI with the genera Adelophryne and Phyzelaphryne.

In the recent literature, the main relationships within the BRACHYCEPHALIDAE have been rather stable, but different taxonomic interpretations have been provided. The structure of the trees has varied, in particular concerning the position of the genera Ceuthomantis and Dischidodactylus (but see below for the history of the classification of this group) which are here considered to constitute the sister-taxon to the BRACHYCEPHALIDAE. Darst \& Cannatella (2004) were the first to show that Brachycephalus belonged in the same branch as Eleutherodactylus and related genera but did not propose a formal naming for this branch, for which they used the incorrect designation 'ELEUTHERODACTYLINI: LEPTODACTYLIDAE' in their figure 2. This taxon was named LEPTODACTYLIDAE BRACHYCEPHALINAE by Dubois (2005d) and BRACHYCEPHALIDAE by Frost et al. (2006). Hedges et al. (2008) and Heinicke et al. (2018) recognised four families that correspond to the two subfamilies BRACHYCEPHALINAE and ELEUTHERODACTYLINAE and to the two tribes (CRAUGASTORINI and STRABOMANTINI) of the CRAUGASTORINAE in our classification. Padial et al. (2014) recognised three families, including the genus Ceuthomantis within the CRAUGASTORIDAE PristimantinaE. The sister-taxon relationships of Phyzelaphryne and Adelophryne, Diasporus and Eleutherodactylus, Craugastor and Haddadus, Ischnocnema and Brachycephalus, and Yunganastes and 
Pristimantis, have been confirmed in all recent works, but the other relationships between genera are not consensual.

\section{F.18.12. Subfamilia BRACHYCEPHALINAE Günther, 1858}

Eunym: Noble 1931: 507.

Getangiotaxon: BRACHYCEPHALIDAE Günther, 1858.

Adelphotaxa: CRAUGASTORINAE Hedges, Duellman \& Heinicke, 2008; ELEUTHERODACTYLINAE Lutz, 1954.

Getendotaxa: Brachycephalus Fitzinger, 1826; Ischnocnema Reinhardt \& Lütken, 1862.

F.18.13. Subfamilia CRAUGASTORINAE Hedges, Duellman \& Heinicke, 2008

Protonym: CRAUGASTORIDAE Hedges, Duellman \& Heinicke, 2008: 3 [F].

Eunym: Pyron \& Wiens 2011: 547.

Getangiotaxon: BRACHYCEPHALIDAE Günther, 1858.

Adelphotaxa: BRACHYCEPHALINAE Günther, 1858; ELEUTHERODACTYLINAE Lutz, 1954.

Getendotaxa: CRAUGASTORINI Hedges, Duellman \& Heinicke, 2008; STRABOMANTINI Hedges, Duellman \& Heinicke, 2008.

\section{F.19.13. Tribus CRAUGASTORINI Hedges, Duellman \& Heinicke, 2008}

Eunym: Hoc loco.

Getangiotaxon: CRAUGaStorinaE Hedges, Duellman \& Heinicke, 2008.

Adelphotaxon: STRABOMANTINI Hedges, Duellman \& Heinicke, 2008.

Getendotaxa: Craugastor Cope, 1862; Haddadus Hedges, Duellman \& Heinicke, 2008.

F.19.14. Tribus Strabomantini Hedges, Duellman \& Heinicke, 2008

Protonym: StRabomantidaE Hedges, Duellman \& Heinicke, 2008: 5 [F].

Eunym: Hoc loco.

Getangiotaxon: CRAUGaStorinaE Hedges, Duellman \& Heinicke, 2008.

Adelphotaxon: CRAUGASTORINI Hedges, Duellman \& Heinicke, 2008.

Getendotaxa: Strabomantina Hedges, Duellman \& Heinicke, 2008; Pristimantina Ohler \& Dubois, 2012.

\section{F.20.08. Subtribus StRABOMANTINA Hedges, Duellman \& Heinicke, 2008}

Eunym: Hoc loco.

Getangiotaxon: STRABOMANTINI Hedges, Duellman \& Heinicke, 2008.

Adelphotaxon: PRISTIMANTINA Ohler \& Dubois, 2012.

Getendotaxa: Holoadeninia Hedges, Duellman \& Heinicke, 2008; StRabomantinia Hedges, Duellman \& Heinicke, 2008.

\section{F.21.03. Infratribus HoLOADENINIA Hedges, Duellman \& Heinicke, 2008}

Protonym: HolOADENINAE Hedges, Duellman \& Heinicke, 2008: 5 [bF].

Eunym: Hoc loco.

Getangiotaxon: STRABOMANTINA Hedges, Duellman \& Heinicke, 2008.

Adelphotaxon: STRABOMANTINIA Hedges, Duellman \& Heinicke, 2008.

Getendotaxa: BARYCHOLINOA nov.; BRYOPHRYNINOA nov.; HOLOADENINOA Hedges, Duellman \& Heinicke, 2008; NOBLELLINOA nov.; 2 GIS (Niceforonia Goin \& Cochran, 1963; Tachiramantis Heinicke, Barrio-Amoros \& Hedges, 2015). 


\section{F.22.01. Hypotribus BARYCHOLINOA nov.}

Getangiotaxon: HoLOADENINIA Hedges, Duellman \& Heinicke, 2008.

Adelphotaxa: Bryophryninoa nov.; Holoadeninoa Hedges, Duellman \& Heinicke, 2008; NobleLlinOA nov.; 2 GIS (Niceforonia Goin \& Cochran, 1963; Tachiramantis Heinicke, Barrio-Amoros \& Hedges, 2015).

Getendotaxa: Bahius nov.; Barycholos Heyer, 1969; Phyllonastes Heyer, 1977.

Nucleogenus, by present designation: Barycholos Heyer, 1969. • Etymology of nomen: G: $\beta \alpha \rho u ́ \chi 0 \lambda ం \varsigma$ (barycholos), 'savage'. Named in honor of Jay M. Savage (Heyer 1969). • Stem of nomen: Barychol-.

Diagnosis: Small sized direct-developing frogs; head narrower than body; pupil horizontal; tympanum distinct or absent; dentigerous process absent or present; condition of adductor mandibulae muscle ' $\mathrm{S}$ '; terminal phalanges knob-shaped; finger I shorter or longer than finger II; toe III about equal in length of toe V; tubercle finger IV in some species reduced to a single tubercle; supernumerary tubercles on palm present; toe tips pointed or enlarged, then forming discs with grooves; inner tarsal tubercle present; inner metatarsal tubercle large, rounded not cornified; outer metatarsal tubercle present; feet not webbed; dorsum smooth or finely areolate; venter granulate; nuptial pads usually absent on male thumb. \{Bokermann 1975; Lynch 1986; Hedges et al. 2008; Lehr \& Catenazzi 2009; Dias et al. 2017\}.

\section{G.28.063. Genus Bahius nov.}

Getangiotaxon: BARYCHOLINOA nov.

Adelphotaxa: Barycholos Heyer, 1969; Phyllonastes Heyer, 1977.

Getendotaxon: Bahius bilineatus (Bokermann, 1975).

Etymology of nomen: Portuguese: bahia, obsolete spelling of baia, 'bay'. This nomen refers to the name 'Bahia' of the state of Brazil where these frogs occur. $\bullet$ Stem of nomen: Bahi-. $\bullet$ Grammatical gender of nomen: masculine.

Nucleospecies, by present desigation: Eleutherodactylus bilineatus Bokermann, 1975.

Diagnosis: Small sized (SVL 20-30 mm) species with a white dorsolateral stripe on either side of the dark-colored dorsum, throat and chest dark with white speckles, reduced adhesive toe pads, and welldeveloped acuminate subarticular tubercles. \{Bokermann 1975\}.

\section{F.22.02. Hypotribus BRYOPHRYNINOA nov.}

Getangiotaxon: HolOADENINIA Hedges, Duellman \& Heinicke, 2008.

Adelphotaxa: BarYCHolinoa nov.; HoloadeninoA Hedges, Duellman \& Heinicke, 2008; NobleLLINOA nov.; 2 GIS (Niceforonia Goin \& Cochran, 1963; Tachiramantis Heinicke, Barrio-Amoros \& Hedges, 2015).

Getendotaxon: Bryophryne Hedges, Duellman \& Heinicke, 2008.

Nucleogenus, by present designation: Bryophryne Hedges, Duellman \& Heinicke, 2008. • Etymology

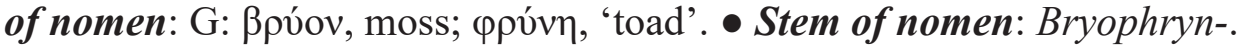

Diagnosis: Small, direct-developing frogs with head narrower than body, lack of tympanic membrane, tympanic anulus, columella, cavum tympanicum, cranial crests, and dentigerous process of vomers; ' $\mathrm{S}$ ' condition of adductor mandibulae muscle; knob-shaped terminal phalanges; finger I shorter than finger II; toes III and V about equal in length; subarticular tubercles not projecting; dorsum finely areolate; venter coarsely areolate. \{Hedges et al. 2008\}. 
Eunym: Hoc loco.

Getangiotaxon: HoLOADENINIA Hedges, Duellman \& Heinicke, 2008.

Adelphotaxa: BARYCHOLINOA nov.; BRYOPHRYNINOA nov.; NOBLELLINOA nov.; 2 GIS (Niceforonia Goin \& Cochran, 1963; Tachiramantis Heinicke, Barrio-Amoros \& Hedges, 2015).

Getendotaxa: Euparkerella Griffiths, 1959; Holoaden Miranda-Ribeiro, 1920.

\section{F.22.04. Hypotribus NOBLELLINOA nov.}

Getangiotaxon: HoloAdENINIA Hedges, Duellman \& Heinicke, 2008.

Adelphotaxa: BARYCHOLINOA nov.; BRYophryninoa nov.; HoloadeninoA Hedges, Duellman \& Heinicke, 2008 ; 2 GIS (Niceforonia Goin \& Cochran, 1963; Tachiramantis Heinicke, Barrio-Amoros \& Hedges, 2015).

Getendotaxa: Microkayla Riva, Chaparro, Castroviejo-Fisher \& Padial, 2017; Noblella Barbour, 1930; Psychrophrynella Hedges, Duellman \& Heinicke, 2008; Qosqophryne Catenazzi, Mamani, Lehr \& May, 2020.

Nucleogenus, by present designation: Noblella Barbour, 1930. • Etymology of nomen: derived from the patronym of G. K. Noble (1894-1940). • Stem of nomen: Noblell-.

Diagnosis: Small sized frogs (SVL 14-34 mm); head not wider than body; tympanum visible of hidden; cranial crests absent; vomerine ridges usually absent; ' $S$ ' condition of adductor mandibulae muscle; terminal discs narrow or slightly expanded; toe V longer than III. \{Hedges et al. 2008\}.

F.21.04. Infratribus StRABOMANTINIA Hedges, Duellman \& Heinicke, 2008

Eunym: Hoc loco.

Getangiotaxon: StRABOMANTINA Hedges, Duellman \& Heinicke, 2008.

Adelphotaxon: HoLOADENINIA Hedges, Duellman \& Heinicke, 2008.

Getendotaxon: Strabomantis Peters, 1863.

F.20.09. Subtribus Pristimantina Ohler \& Dubois, 2012

Protonym: PRistimantinaE Ohler \& Dubois, 2012: 165 [bF].

Eunym: Hoc loco.

Getangiotaxon: STRABOMANTINI Hedges, Duellman \& Heinicke, 2008.

Adelphotaxon: STRABOMANTINA Hedges, Duellman \& Heinicke, 2008.

Getendotaxa: HypodaCtYlinia Heinicke, Lemmon, Lemmon, McGrath \& Hedges, 2018; Pristimantinia Ohler \& Dubois, 2012.

F.21.05. Infratribus HYPODACTYLINIA Heinicke, Lemmon, Lemmon, McGrath \& Hedges, 2018

Protonym: HyPoDACTYLINAE Heinicke, Lemmon, Lemmon, McGrath \& Hedges, 2018: 152 [bF].

Eunym: Hoc loco.

Getangiotaxon: PRISTIMANTINA Ohler \& Dubois, 2012.

Adelphotaxon: Pristimantinia Ohler \& Dubois, 2012.

Getendotaxon: Hypodactylus Hedges, Duellman \& Heinicke, 2008. 
Eunym: Hoc loco.

Getangiotaxon: PRISTIMANTINA Ohler \& Dubois, 2012.

Adelphotaxon: HYPODACTYLINIA Heinicke, Lemmon, Lemmon, McGrath \& Hedges, 2018.

Getendotaxa: Oreobatinoa nov.; Pristimantinoa Ohler \& Dubois, 2012.

\section{F.22.05. Hypotribus OREOBATINOA nov.}

Getangiotaxon: Pristimantinia Ohler \& Dubois, 2012.

Adelphotaxon: PRISTIMANTINOA Ohler \& Dubois, 2012.

Getendotaxa: OREOBATITES nov.; PHRYNOPODITES nov.

Nucleogenus, by present designation: Oreobates Jiménez de la Espada, 1872. • Etymology of nomen: G: ópos (oros), ‘mountain'; $\beta \alpha \tau \varepsilon \dot{\omega} \omega$ (bateo), 'to walk'. • Stem of nomen: Oreobat-.

Diagnosis: Small, direct-developing frogs with head narrower than or as wide as body; tympanic membrane and anulus present or absent; cranial crests absent; dentigerous process of vomers prominent or absent; ' $\mathrm{S}$ ' condition of adductor mandibulae muscle; terminal digits narrow, rounded or bulbous; and knob- or T-shaped terminal phalanges. \{Hedges et al. 2008\}.

\section{F.23.01. Clanus OREOBATITES nov.}

Getangiotaxon: OREOBATINOA nov.

Adelphotaxon: PHRYNOPODITES nov.

Getendotaxa: Lynchius Hedges, Duellman \& Heinicke, 2008; Oreobates Jiménez de la Espada, 1872.

\section{F.23.02. Clanus PHRYNOPODITES nov.}

Getangiotaxon: OREOBATINOA nov.

Adelphotaxon: OREOBATITES nov.

Getendotaxon: Phrynopus Peters, 1873.

Nucleogenus, by present designation: Phrynopus Peters, 1873. • Etymology of nomen: G: $\varphi \rho v ́ v \eta$ (phryne), 'toad'; toús (pous), 'foot'. • Stem of nomen: Phrynopod-.

Diagnosis: Small, direct-developing frogs with head narrower than body; differentiated tympanic membrane; tympanic anulus usually absent; cranial crests absent; dentigerous process of vomers usually absent; ' $S$ ' condition of adductor mandibulae muscle; terminal digits narrow, rounded or bulbous; and knob-shaped terminal phalanges. \{Hedges et al. 2008\}.

\section{F.22.06. Hypotribus PRISTIMANTINOA Ohler \& Dubois, 2012}

Eunym: Hoc loco.

Getangiotaxon: PRISTIMANTINIA Ohler \& Dubois, 2012.

Adelphotaxon: OREOBATINOA nov.

Getendotaxa: Pristimantis Jiménez de la Espada, 1870; Yunganastes Padial, Castroviejo-Fisher, Köhler, Domic \& Riva, 2007. 
Protonym and eunym: ELEUTHERODACTYLINAE Lutz, 1954: 157 [bF].

Getangiotaxon: BRACHYCEPHALIDAE Günther, 1858.

Adelphotaxa: BRACHYCEPHALINAE Günther, 1858; CRAUGASTORINAE Hedges, Duellman \& Heinicke, 2008.

Getendotaxa: Eleutherodactylini Lutz, 1954; Phyzelaphrynini Hedges, Duellman \& Heinicke, 2008

\section{F.19.15. Tribus ELEUTHERODACTYLINI Lutz, 1954}

Eunym: Lynch 1969: 3.

Getangiotaxon: ELEUTHERODACTYLINAE Lutz, 1954.

Adelphotaxon: PhyZelaPHRYNINI Hedges, Duellman \& Heinicke, 2008.

Getendotaxa: DIASPORINA nov.; ELEUTHERODACTYLINA Lutz, 1954.

\section{F.20.10. Subtribus DIASPORINA nov.}

Getangiotaxon: ELEUTHERODACTYLINI Lutz, 1954.

Adelphotaxon: ELEUTHERODACTYLINA Lutz, 1954.

Getendotaxon: Diasporus Hedges, Duellman \& Heinicke, 2008.

Nucleogenus, by present designation: Diasporus Hedges, Duellman \& Heinicke, 2008. • Etymology

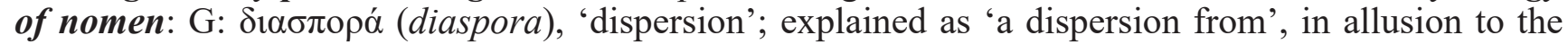
close relationship of this mainland group to the Caribbean branch, inferring an ancient dispersal event (Hedges et al. 2008). • Stem of nomen: Diaspor-.

Diagnosis: Small sized frogs (SVL 10.9-26 mm); head distinct from body; head width 32-41 \% of SVL; tympanic membrane usually differentiated; cranial crests absent; vomerine ridges usually prominent; ' $S$ ' condition condition of adductor mandibulae musculature; toe pads expanded with or without lanceolate or papillate tips; circumferential grooves present; terminal phalanges T-shaped; finger I shorter than finger II; toe V much longer than toe III; subarticular tubercles not prominent; dorsum smooth to rugose; venter roughly areolate. $\{$ Hedges et al. 2008\}.

\section{F.20.11. Subtribus ELEUTHERODACTYLINA Lutz, 1954}

Eunym: Hoc loco.

Getangiotaxon: ELEUTHERODACTYLINI Lutz, 1954.

Adelphotaxon: DIASPORINA nov.

Getendotaxa: Eleutherodactylus Duméril \& Bibron, 1841; Euhyas Fitzinger, 1843.

F.19.16. Tribus PhyzeLAPHRYNini Hedges, Duellman \& Heinicke, 2008

Protonym: Phyzelaphrininae Hedges, Duellman \& Heinicke, 2008: 5 [bF].

Eunym: Hoc loco.

Getangiotaxon: ELEUTHERODACTYLINAE Lutz, 1954.

Adelphotaxon: ELEUTHERODACTYLINI Lutz, 1954.

Getendotaxa: Adelophryne Hoegmood \& Lescure, 1984; Phyzelaphryne Heyer, 1977.

F.17.14. Familia Ceuthomantidae Heinicke, Duellman, Trueb, Means, MacCulloch \& Hedges, 2009

Protonym and eunym: CEUTHOMANTIDAE Heinicke, Duellman, Trueb, Means, MacCulloch \& Hedges, 2009: 1 [F]. 


\title{
Getangiotaxon: GAIANURA nov.
}

Adelphotaxon: BRACHYCEPHALIDAE Günther, 1858.

Getendotaxa: Ceuthomantis Heinicke, Duellman, Trueb, Means, MacCulloch \& Hedges, 2009; Dischidodactylus Lynch, 1979.

Comments: This family was erected by Heinicke et al. (2009) because of its sister-group relationship to all other GaIANURA, the BRACHYCEPHALIDAE. This position was confirmed by Pyron \& Wiens (2011) and accepted by Blackburn \& Wake (2011). The position of the genus Ceuthomantis is quite different in Padial et al. (2014), where it is sister-branch to the branch formed by Pristimantis and Yunganastes within their PRISTIMANTINAE. TREE supports this lineage as sister-branch to our BRACHYCEPHALIDAE, therefore it is recognised as a family following the [STC].

\section{C.13.02. Hypophalanx HeMiPHRaCTIFORMia Brocchi, 1881}

\author{
Protonym: HeMiPHRACTIFORMES Brocchi, 1881: 9 [UC]. \\ Eunym: Hoc loco. \\ Getangiotaxon: Phrynanura nov. \\ Adelphotaxa: Gaianura nov.; Hylobatrachia Ritgen, 1828. \\ Getendotaxon: HEMIPHRACTIDAE Peters, 1862.
}

Comments: The HeMiphractiformia englobe a single family HeMiphraCtidaE. This taxon is recognised in all recent phylogenies based on molecular data but its position is debated. In Frost et al. (2006), it is a holophyletic group but it is sister-taxon to the «MERIDIANURA» (ectonym) that group all other BAINANURA. In Zhang et al. (2013), Feng et al. (2017) and Streicher et al. (2018), it is within the BAINANURA but in various positions. In Hutter et al. (2017), it is outgroup of a group formed by the Gaianura and Hylobatrachia, but this relationship has only a weak support. Its position in TREE and CLAD is well supported and similar to that in Pyron \& Wiens (2011).

\section{F.17.15. Familia HEMIPHRACTIDAE Peters, 1862}

\author{
Protonym and eunym: HEMIPHRACTIDAE Peters, 1862: 146 [F]. \\ Getangiotaxon: HeMIPHRACTIFORMIA Brocchi, 1881. \\ Adelphotaxon: None. \\ Getendotaxa: Amphignathodontinae Boulenger, 1882; CRYPtobatrachinaE Frost, Grant, Faivovich, Bazin, Haas, \\ Haddad, Sá, Channing, Wilkinson, Donnellan, Raxworthy, Campbell, Blotto, Moler, Drewes, Nussbaum, Lynch, Green \\ \& Wheeler, 2006; Flectonotinae nov.; Fritzianinae nov.; Hemiphractinae Peters, 1862; Stefanimae nov.
}

Comments: Darst \& Cannatella (2004) found the HEMIPHRACTIDAE (their HEMIPHRACTINAE) being polyphyletic and not in close relationship with the HYLIDAE. In Faivovich et al. (2005), Hemiphractus, as the only representative of the HEMIPHRACTIDAE, appears as sister-taxon to BRACHYCEPHALIDAE species. Wiens et al. $(2005 b, 2006,2007)$ found molecular evidence that hemiphractid taxa are related to part of the polyphyletic LEPTODACTYLIDAE. Frost et al. (2006) confirmed the distant relationship of these taxa to the HYLIDAE and revealed three distant branches recognised as the families AMPHIGNATHODONTIDAE (Flectonotus and Gastrotheca), CRYPTOBATRACHIDAE (Cryptobatrachus and Stefania) and HEMIPHRACTIDAE (Hemiphractus). Guayasamin et al. (2008) and Pyron \& Wiens (2011) recovered a holophyletic branch and consequently recognised a single family HEMIPHRACTIDAE. This family was accepted by Blackburn \& Duellman (2013) and Duellman (2015), and supported by a larger sampling including representatives of all genera by Castroviejo-Fisher et al. (2015). The position of the family in TREE is different from the relationships obtained by Castroviejo-Fisher et al. (2015) although the branch can also be described as being within the hypophalanx PHaneranura (their «NoBLEOBATRACHIA»). In our classification it is one of three hypophalanges, but the relationships among these three cannot be resolved.

Although the branches recognised as genera in the proposed classification have significant support (above 90 in TREE), the relationships between them cannot be considered as stable. In Blackburn 
\& Duellman (2013), Flectonotus is outgroup to all other HEMIPHRACTIDAE. Within the remaining genera, Hemiphractus is outgroup to an aggregate that holds Fritziana, Gastrotheca and Stephania without statistically support to the relationships between these groups. The classification within the HEMIPHRACTIDAE proposed by Castroviejo-Fisher et al. (2015) is based on a larger sampling and more genes and recognises the five genera and Cryptobatrachus with a different relationship. Based on a dataset with the complete sampling, Flectonotus and Cryptobatrachus are sister-taxa to a taxon that groups the other four genera. Within these groups, Stefania is sister-taxon to a group that includes Fritziana, as sister-taxon to Hemiphractus and Gastrotheca. In TREE, we did not find high statistical support for the relationships among these six branches, but between Duellman's (2015) subgenera Eotheca, Cryptotheca, Gastrotheca and Australotheca, which we recognise at the genus level (the latter, being preoccupied, under its neonym Alainia). Among these branches, Cryptotheca is sistertaxon to Amphignathodon, and Alainia to Gastrotheca. To account provisionally for these partly unresolved relationships, we recognise six subfamilies: the AMPHIGNATHODONTINAE including three tribes, AMPHIGNATHODONTINI (with Amphignathodon and Cryptotheca), EothECINI (with Eotheca) and GASTROTHECINI (with Alainia and Gastrotheca), the CRYPTOBATRACHINAE (with Cryptobatrachus), the FLECTONOTINAE (with Flectonotus), the FRITZIANINAE (with Fritziana), the HEMIPHRACTINAE (with Hemiphractus) and the STEFANIINAE (with Stefania). The reason why we recognise these taxa as subfamilies and not as families is explained in the M\&M section above as the Nomenclatural Thrift Criterion [NTC].

F.18.15. Subfamilia AmPHigNATHODontinAE Boulenger, 1882

Protonym: AmphignatodontidaE Boulenger, 1882: xvi, 449 [F].

Eunym: Gadow 1901: xi, 188.

Getangiotaxon: HeMIPHRACTIDAE Peters, 1862.

Adelphotaxa: CRYPtobatrachinaE Frost, Grant, Faivovich, Bazin, Haas, Haddad, Sá, Channing, Wilkinson, Donnellan, Raxworthy, Campbell, Blotto, Moler, Drewes, Nussbaum, Lynch, Green \& Wheeler, 2006; FLECTONOTINAE nov.; Fritzianinae nov.; Hemiphractinae Peters, 1862; StefaniInaE nov.

Getendotaxa: AmpHignathodontini Boulenger, 1882; EotheCINI nov.; GaStRotheCINI Noble, 1927.

\section{F.19.17. Tribus AMPHIGNATHODONTINI Boulenger, 1882}

Eunym: Hoc loco.

Getangiotaxon: AMPHIGNATHODONTINAE Boulenger, 1882.

Adelphotaxa: EotheCINI nov.; GASTROTHECINI Noble, 1927.

Getendotaxa: Amphignathodon Boulenger, 1882; Cryptotheca Duellman, 2015.

\section{F.19.18. Tribus EOTHECINI nov.}

Getangiotaxon: AMPHIGNATHODONTINAE Boulenger, 1882.

Adelphotaxa: AMPHIGNATHODONTINI Boulenger, 1882; GASTROTHECINI nov.

Getendotaxon: Eotheca Duellman, 2015.

Nucleogenus, by present designation: Eotheca Duellman, 2015. • Etymology of nomen: G: ć̣̂̀os(eoos), 'early'; Өท́кๆ (theke), 'box, chest'; referring to the basal position of this taxon relative to Gastrotheca (Duellman 2015). • Stem of nomen: Eothec-.

Diagnosis: Small to large sized (males SVL 28-90 mm; females SVL 33-110 mm) hemiphractid frogs; head large, with co-ossified skin of the dermal roof bones of the skull; lack of dermal ornamentation; dorsum tan or greenish tan, with or without brown ornamentation; osteological synapomophies include a complete temporal arcade over the ortic region; alary process of premaxillae nearly vertical; lateral profile of snout high and truncate; presence of a massive postorbital process on maxilla with a horizontal articulation with wide zygomatic ramus of squamosal; otic plate of squamosal barely developed and 
narrowly overlapping cartilaginous lateral margin of crista parotica; neopalatines separated; vomerine ridges between the anterior part of choanae; eggs undergoing direct development into froglets. \{Duellman $2015\}$.

\section{F.19.19. Tribus GASTROTHECINI Noble, 1927}

Protonym: GASTROTHECINAE Noble, 1927: 93 [bF].

Eunym: Hoc loco.

Getangiotaxon: AMPHIGNATHODONTINAE Boulenger, 1882.

Adelphotaxa: AMPHIGNATHODONTINI Boulenger, 1882; EOTHECINI nov.

Getendotaxa: Alainia Duellman \& Cannatella, 2018; Gastrotheca Fitzinger, 1843.

F.18.16. Subfamilia CRYPTOBATRACHINAE Frost, Grant, Faivovich, Bazin, Haas, Haddad, Sá, Channing, Wilkinson, Donnellan, Raxworthy, Campbell, Blotto, Moler, Drewes, Nussbaum, Lynch, Green \& Wheeler, 2006

Protonym: CRYPTobatrachidae Frost, Grant, Faivovich, Bazin, Haas, Haddad, Sá, Channing, Wilkinson, Donnellan, Raxworthy, Campbell, Blotto, Moler, Drewes, Nussbaum, Lynch, Green \& Wheeler, 2006: 6 [F].

Eunym: Castroviejo-Fisher, Padial, Riva, Pombal, Silva, Rojas-Runjaic, Medina-Méndez \& Frost 2015: 20.

Getangiotaxon: HEMIPHRACTIDAE Peters, 1862.

Adelphotaxa: AMPHIGNATHODONTINAE Boulenger, 1882; FLECTONOTINAE nov.; FRITZIANINAE nov.; HEMIPHRACTINAE Peters, 1862; STEFANIINAE nov.

Getendotaxon: Cryptobatrachus Ruthven, 1916.

\section{F.18.17. Subfamilia FLECTONOTINAE nov.}

Getangiotaxon: HEMIPHRACTIDAE Peters, 1862.

Adelphotaxa: Amphignathodontinae Boulenger, 1882; CRYptobatrachinae Frost, Grant, Faivovich, Bazin, Haas, Haddad, Sá, Channing, Wilkinson, Donnellan, Raxworthy, Campbell, Blotto, Moler, Drewes, Nussbaum, Lynch, Green \& Wheeler, 2006; FritZIANINAE nov.; HEMIPHRACTINAE Peters, 1862; STEFANIINAE nov.

Getendotaxon: Flectonotus Miranda-Ribeiro, 1926.

Nucleogenus, by present designation: Flectonotus Miranda-Ribeiro, 1926. • Etymology of nomen: L: flecto, 'bend'; G: võ̃os (notos), 'the back'. • Stem of nomen: Flectonot-.

Diagnosis: Small sized (males SVL 16-26 mm; females SVL 19-32 mm) hemiphractid frogs; dermal bones of skull not co-ossified with overlying skin; frontoparietals medially articulated throughout their lengths; nasal small, not articulated; neopalatines edentate and not serrated; procoelous presacral vertebrae lacking elongate neural spines; adhesive pad on subarticular tubercle of antepenultimate articulation absent; vocal slits and vocal sac absent; fleshy proboscis on tip of snout and fleshy tubercles on upper eyelids absent; first finger shorter than second; nuptial pads present; eggs developing into nonfeeding tadpoles in a pouch with a longitudinal opening on back of female. \{Duellman 2015\}.

\section{F.18.18. Subfamilia FRITZIANINAE nov.}

Getangiotaxon: HEMIPHRACTIDAE Peters, 1862.

Adelphotaxa: Amphignathodontinae Boulenger, 1882; CRYptobatrachinaE Frost, Grant, Faivovich, Bazin, Haas, Haddad, Sá, Channing, Wilkinson, Donnellan, Raxworthy, Campbell, Blotto, Moler, Drewes, Nussbaum, Lynch, Green \& Wheeler, 2006; Flectonotinae nov.; Hemiphractinae Peters, 1862; Stefaninae nov.

Getendotaxon: Fritziana Mello-Leitão, 1937. 
Nucleogenus, by present designation: Fritziana Mello-Leitão, 1937. • Etymology of nomen: P: Fritz Müller (1821-1897), Brasilian zoologist and naturalist; L: -iana, feminine suffix. • Stem of nomen: Fritzian-.

Diagnosis: Small sized (males SVL 18-34 mm; females SVL 25-39 mm) hemiphractid frogs; dermal bones of skull not co-ossified with overlying skin; frontoparietals medially articulated throughout their lengths; nasal large, nearly in contact anterior to sphenethmoid; neopalatines edentate and not serrated; procoelous presacral vertebrae lacking elongate neural spines; adhesive pad on subarticular tubercle of antepenultimate articulation absent; vocal slits and vocal sac present; a fleshy proboscis on tip of snout and fleshy tubercles on upper eylids absent; first finger shorter than second; nuptial pads present; eggs developing into non-feeding tadpoles in a basin between lateral folds of skin on back of female. $\{$ Duellman 2015\}.

\section{F.18.19. Subfamilia Hemiphractinae Peters, 1862}

Eunym: Gadow 1901: xi, 210.

Getangiotaxon: HeMIPHRACTIDAE Peters, 1862.

Adelphotaxa: AmpHignathodontinaE Boulenger, 1882; CRYPTOBATRACHINAE Frost, Grant, Faivovich, Bazin, Haas, Haddad, Sá, Channing, Wilkinson, Donnellan, Raxworthy, Campbell, Blotto, Moler, Drewes, Nussbaum, Lynch, Green \& Wheeler, 2006; FLECTONOTINAE nov.; FRITZIANINAE nov.; STEFANIINAE nov.

Getendotaxon: Hemiphractus Wagler, 1828.

\section{F.18.20. Subfamilia STEFANIINAE nov.}

Getangiotaxon: HEMIPHRACTIDAE Peters, 1862.

Adelphotaxa: Amphignathodontinae Boulenger, 1882; CRYptobatrachinae Frost, Grant, Faivovich, Bazin, Haas, Haddad, Sá, Channing, Wilkinson, Donnellan, Raxworthy, Campbell, Blotto, Moler, Drewes, Nussbaum, Lynch, Green \& Wheeler, 2006; Flectonotinae nov.; Fritzianinae nov.; Hemiphractinae Peters, 1862.

Getendotaxon: Stefania Rivero, 1968.

Nucleogenus, by present designation: Stefania Rivero, 1968. • Etymology of nomen: P: Luis Stefani Raffucci (1901-1971), Chancellor of the University of Costa Rica. • Stem of nomen: Stefani-.

Diagnosis: Small to large sized (males SVL 34-67 mm; females SVL 37-96 mm) hemiphractid frogs; dermal bones of skull not co-ossified with overlying skin; frontoparietals with lateral elevated edges, medially articulated throughout their lengths; nasal large, in contact anterior to sphenethmoid; neopalatines without ventral spur; procoelous presacral vertebrae lacking elongate neural spines; adhesive pad on subarticular tubercle of antepenultimate articulation absent; vocal slits and vocal sac absent; fleshy proboscis on tip of snout and fleshy tubercles on upper eyelids absent; first finger shorter than second; nuptial pads present; males much smaller than females; eggs developing into froglets on back of female. \{Duellman 1970, 2015\}.

\section{C.13.03. Hypophalanx Hylobatrachia Ritgen, 1828}

Protonym: Hylobatrachi Ritgen, 1828: 278 ['F'].

Eunym: Hoc loco.

Getangiotaxon: Phrynanura nov.

Adelphotaxa: Hemiphractiformia Brocchi, 1881; Gaianura nov.

Getendotaxa: Bufonoidea Gray, 1825; Centrolenoidea Taylor, 1951; CERATophryoidea Tschudi, 1838; Hyloidea Rafinesque, 1815-|Gray, 1825|; LEPTODACTYLOIDEA ||Tschudi, 1838||-Werner, 1896; 1 GIS (Ancudia Philippi, 1902).

Comments: This branch has high support in TREE. It was recognised with this content first by Pyron \& Wiens (2011) and more recently by Hutter et al. (2017). It includes five branches having each high 
support but their pentatomy is not resolved, as the relationships between them are not supported by values of SHL of 90 or above. They are therefore attributed here to the rank superfamily: BUFONOIDEA, CENTROLENOIDEA, CERATOPHRYOIDEA, HYLOIDEA and LEPTODACTYLOIDEA.

\title{
F.14.03. Superfamilia BUFONOIDEA Gray, 1825
}

\author{
Protonym: BUFONINA Gray, 1825: 214 [UC]. \\ Eunym: Gill 1884: 621. \\ Getangiotaxon: HyLOBATRACHIA Ritgen, 1828 \\ Adelphotaxa: CENTRolenoIdea Taylor, 1951; CERATophryoIdeA Tschudi, 1838; HyloIdeA Rafinesque, 1815-|Gray, 1825|; \\ LEPTODACTYLOIDEA ||Tschudi, 1838||-Werner, 1896; 1 GIS (Ancudia Philippi, 1902). \\ Getendotaxa: BuFonIDAE Gray, 1825; ODONTOPHRYNIDAE Lynch, 1971.
}

Comments: This branch, recognised in CLAD as the superfamily BUFONOIDEA, accommodates two highly supported taxa, the families BUFONIDAE and ODONTOPHRYNIDAE. The family rank is attributed to the taxon named BUFONIDAE through the [UQC] and to the ODONTOPHRYNIDAE through the [STC]. Whereas the ODONTOPHRYNIDAE show a relatively simple structure including two highly supported taxa, recognised as the subfamilies ODONTOPHRYNINAE for the genera Macrogenioglottus and Odontophrynus, and PROCERATOPHRYINAE for the genus Proceratophrys, the BUFONIDAE, including more than 50 genuslevel taxa, have a very complex hierarchical structure, and require nine FS ranks, from subfamily to hypoclanus, which is the highest number of infrafamilial FS ranks used in CLAD in a family.

\section{F.17.16. Familia BUFONIDAE Gray, 1825}

Eunym: Bell 1839: 105.

Getangiotaxon: BUFONOIDEA Gray, 1825.

Adelphotaxon: ODONTOPHRYNIDAE Lynch, 1971.

Getendotaxa: BUFONINAE Gray, 1825; MELANOPHRYNISCINAE nov.

Comments: The BUFONIDAE (true toads) are an interesting group concerning character evolution as they consist in numerous taxa worldwide having a conservative morphology and life history, intermingled with other taxa that show a wide array of adaptations to various habitats, life histories and breeding modes combined with derived morphology. Traditionally, the toad-like forms were kept in a large genus Bufo, whereas various other genera were erected to account for this diversity of adaptations, but this made the traditional genus Bufo largely paraphyletic. There were two possible taxonomic solutions to this situation: either, as suggested e.g. by Dubois \& Bour (2010a), to increase the coverage of the genus $B u f o$ in order to include several of these 'specialised lineages' either as synonyms or, for some of them at least, as (holophyletic) subgenera; or to dismantle the traditional genus Bufo. Given the absence of collective reflection on the 'genus concept' in zoology discussed above in the M\&M section, starting with Frost et al. (2006) the second solution was implemented without real discussion, and in complete contradiction with the attitude adopted in the same work in other amphibian groups, for example the 'genus' Nanorana, which showed a similar morphological and ecological heterogeneity but for which lumping was preferred to dismantlement without discussion.

As a matter of fact, the holophyly of the extensive genus Bufo, as understood e.g. by Blair (1972), excluding these 'satellite specialised genera', was challenged by a series of authors (e.g. Graybeal 1997, Pauly et al. 2004), until Frost et al. (2006) took the decision to propose a classification replacing the paraphyletic genus Bufo and its 'satellites' by a series of redefined holophyletic genera. Their family BUFONIDAE included 17 genera but did not recognise groups among these taxa. In fact, their tree showed several groupings that we recovered again in TREE but with a wider sampling of genera. In TREE, Melanophryniscus is sister-branch to all other BUFONIDAE (as found in all subsequent molecular phylogenies) and Atelopus is with Osornophryne (but Oreophrynella is missing). The following relationships found by Frost et al. (2006) are confirmed: Bufo margaritifer is close to Rhamphophryne (Chaparro et al. 2007; our data), thus the latter is a junior subjective synonym of Rhinella; a branch with Bufo asper, now Pedostibes asper, and Pedostibes hosei, now Rentapia hosei, appears as the 
hypoclanus RENTAPIITUES in our classification; and a branch groups the African bufonids, Sclerophrys (as Amietophryne), Mertensophryne, Vandijkophryne and Capensibufo, as the subclanus STEPHOPAEDITIES in our classification. But other associations proposed by Frost et al. (2006) within the BUFONIDAE were not confirmed by further studies (Van Bocxlaer et al. 2009; Pyron \& Wiens 2011; Liedtke et al. 2016; our data).

The recently published phylogenies recovered a similar pattern concerning the relationships of the basal genera but the relationships within the 'Old World toads' remain largely unresolved (Matsui et al. 2007; Van Bocxlaer et al. 2009; Pyron \& Wiens 2001; Portik \& Papenfuss 2015). In a phylogenetic study on mainly African bufonids, Liedtke et al. (2016) could resolve some of these relationships. They proposed a two-fold origin of African bufonids but one of their branches only has a support of $60 \%$. Most of the groups of Eurasian bufonids have low support, so that few relationships within these toads appear robust, leading to a poorly resolved classification when submitted to our Criteria.

Melanophryniscus, the sister-branch to all other BUFONIDAE (Van Bocxlaer et al 2009; Pyron \& Wiens 2011; Portik \& Papenfuss 2015; Liedtke et al. 2016) requires erection of a subfamily MELANOPHRYNISCINAE opposed to the BUFONINAE. Then Frostius is the highly supported sister-branch to all other members of the subfamily BUFONINAE, deserving erection of the tribe FROSTIINI, represented by a single species, opposed to the tribe BUFONINI.

\section{F.18.21. Subfamilia BUFONINAE Gray, 1825}

Eunym: Fejérváry 1917: 26.

Getangiotaxon: BUFONIDAE Gray, 1825.

Adelphotaxon: MELANOPHRYNISCINAE nov.

Getendotaxa: BUFONINI Gray, 1825; FROSTIINI nov.

\section{F.19.20. Tribus BUFONINI Gray, 1825}

Eunym: Hoc loco.

Getangiotaxon: BUFONINAE Gray, 1825.

Adelphotaxon: FROSTIINI nov.

Getendotaxa: ATELOPODINA Fitzinger, 1843; BUFONINA Gray, 1825; OREOPHRYNELLINA nov., OSORNOPHRYNINA nov.; 2 GIS (Metaphryniscus Señaris, Ayarzagüena \& Gorzula, 1994; Truebella Graybeal \& Cannatella, 1995).

Comments: The tribe BUFONINI holds four highly supported branches (SHL 100) recognised here as the subtribes ATELOPODINA, BUFONINA, OREOPHRYNELLINA and OSORNOPHRYNINA, but the relationships between them does not have sufficient statistical support. As previous studies did not include members of Frostius, Oreophrynella and Amazophrynella, the relationships among the branches shared by all analyses cannot be compared without important assumptions. Therefore we will only present results of our study. The OREOPHRYNELLINA, for the genus Oreophrynella, is the sister-branch to the OSORNOPHRYNINA, for the genus Osornophryne, but with a SHL of only 86; the ATELOPODINA, including the genus Atelopus, is sister-branch to this taxon with a SHL of only 70. Members of the genera Metaphryniscus and Truebella have not been sequenced, so they cannot be allocated to a group within the tribe BUFONINI.

\section{F.20.12. Subtribus ATELOPODINA Fitzinger, 1843}

Protonym: ATELOPODA Fitzinger, 1843: 32 [F].

Eunym: Hoc loco.

Getangiotaxon: BUFONINI Gray, 1825.

Adelphotaxa: Bufonina Gray, 1825; OREOPhrynELLINA nov., Osornophrynina nov.; 2 GIS (Metaphryniscus Señaris, Ayarzagüena \& Gorzula, 1994; Truebella Graybeal \& Cannatella, 1995).

Getendotaxon: Atelopus Duméril \& Bibron, 1841 
Getangiotaxon: ATELOPODINA Fitzinger, 1843.

Adelphotaxon: None.

Getendotaxa: About a hundred species.

Comments: We hereby designate Hylaemorphus dumerilii Schmidt, 1857 as type-species of the nominal genus Hylaemorphus Schmidt, 1857. The origin was indicated as 'Neu-Granada' [ViceRoyalty of New Granada], a political unit which included the northern part of South America and the southern part of Central America. As announced in Schmidt (1857), the genus Hylaemorphus and the two included species were redescribed in detail as new by Schmidt in 1858. He then indicated a more precise origin for the symphoronts of Hylaemorphus dumerilii, namely 'Provinz Verugua' [Veraguas, now in Panama] (Schmidt 1858). This is near the onymotope of the neophoront of Phrynidium varium Lichtenstein, Weinland \& Martens, 1856, written as 'Veragoa': both refer to the province Veragua in western Panama. Savage (1972) designated ZMB 3379 as neotype of both Hylaemorphus dumerilii and Hylaemorphus bibronii, although in Schmidt (1858) these species do not have the same origin, in particular in altitudinal distribution: Hylaemorphus dumerilii was collected at 8000 feet [2530 m] altitude and Hylaemorphus bibronii from an unprecise place between 2000 and 3000 feet [630-950 m], 'unweit Panama' [near Panama]. In 1928, Dunn could study the original type specimens deposited in the Krakau collection (Savage 1972), but Henryk Szarski could not find these specimens in the early seventies (Savage 1972: 89). Thus the neotype designation of Savage (1972) is valid and the onymotope for both species is now 'Veragua', Panama. Savage (1972) argued that the specimen of Hylaemorphus bibronii figured by Schmidt (1858) resembles populations of Atelopus varius from the Pacific slopes of Volcan Chiriqui. A frog from this population would have been a much better choice for a neotype.

When describing Phrynidium varium, Lichtenstein et al. (1856) established the new genus Phrynidium with four included nominal species-series taxa: Phrynidium varium, Phrynidium varium var. (a) maculatum, Phrynidium varium var. (b) adspersum and Phrynidium crucigerum. In his historical survey of the classification of Atelopus, McDiarmid (1971) did not designate a type-species for Phrynidium, but Lötters et al. (1998) did so by mentioning Phrynidium varium as 'type-species'. This designation is valid although these authors were not aware that the genus was described on the basis of several nominal taxa (see Article 69.1.1 of the Code).

If in the future this genus was to be dismantled as two genera (or subgenera), the nomen Phrynidium would be available for the Andean-Choco-Central American branch of Lötters et al. (2011).

\section{F.20.13. Subtribus BUFONINA Gray, 1825}

Eunym: Hoc loco.

Getangiotaxon: BUFONINI Gray, 1825.

Adelphotaxa: Atelopodina Fitzinger, 1843; Oreophrynellina nov., Osornophrynina nov.; 2 GIS (Metaphryniscus Señaris, Ayarzagüena \& Gorzula, 1994; Truebella Graybeal \& Cannatella, 1995).

Getendotaxa: AmazophrynelliNia nov; BUFONINIA Gray, 1825; DENDRopHrYNiSCINIA Jiménez de la Espada, 1870.

Comments: The branch named BUFONINA includes three highly supported branches, attributed to the infratribes AMAZOPHRYNELLINIA for the genus level taxon Amazophrynella, DENDROPHRYNISCINIA for the genus Dendrophryniscus, and BUFONINIA for 43 genera. Within these three, AMAZOPHRYNELLINIA and DENDROPHRYNISCINIA appear in TREE as sister-branches, but only with a SHL of 77, below the significance level retained.

\section{F.21.07. Infratribus AMAZOPHRYNELLINIA nov.}

Getangiotaxon: BUFONINA Gray, 1825.

Adelphotaxa: Bufoninia Gray, 1825; DendRophryniscinia Jiménez de la Espada, 1870.

Getendotaxon: Amazophrynella Fouquet, Recoder, Teixeira, Cassimiron Amaro, Camacho, Demasceno, Carnaval, Moritz \& Rodrigues, 2012. 
Nucleogenus, by present designation: Amazophrynella Fouquet, Recoder, Teixeira, Cassimiron Amaro, Camacho, Demasceno, Carnaval, Moritz \& Rodrigues, 2012. Etymology of nomen: R: Amazonia, for

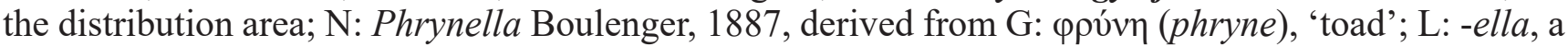
feminine suffix indicating a diminutive form. $\bullet$ Stem of nomen: Amazophrynell-.

Diagnosis: Small toads without parotoid glands, no external tympanum, no cranial crests, uniformly granular skin, basally webbed feet, long hind-limbs, no vocal slits, snout pointed in profile, longitudinally elliptical subarticular tubercles, blotches or spots on venter, proportionally large limbs and eyes, and short snout. \{Fouquet et al. 2012\}.

\section{F.21.08. Infratribus BUFONINIA Gray, 1825}

Eunym: Hoc loco.

Getangiotaxon: BUFONINA Gray, 1825.

Adelphotaxa: AmaZophrynellinia nov.; DENDROPHRYNISCINIA Jiménez de la Espada, 1870.

Getendotaxa: BUFONINOA Gray, 1825; NANNOPHRYNINOA nov.

Comments: Within the infratribe BUFONINIA, Nannophryne, allocated to the hypotribe NANNOPHRYNINOA, is sister-branch to the remaining genera, which form the hypotribe BUFONINOA.

\section{F.22.07. Hypotribus BUFONINOA Gray, 1825}

Eunym: Hoc loco.

Getangiotaxon: BUFONINIA Gray, 1825.

Adelphotaxon: NANNOPHRYNINOA nov.

Getendotaxa: BUfONITES Gray, 1825; PELTOPHRYNITES nov.; RHAEBOITES nov.

Comments: This hypotribe BUFONINOA holds three highly supported branches with poorly supported mutual relationships, attributed to the clans BUFONITES, PELTOPHRYNITES for Peltophryne, and RHAEBOITES for Rhaebo.

\section{F.23.03. Clanus BuFONITES Gray, 1825}

Eunym: Hoc loco.

Getangiotaxon: BUFONINOA Gray, 1825.

Adelphotaxa: PeltophryNites nov.; RHAEBoItes nov.

Getendotaxa: Bufonities Gray, 1825; PhrYNisCITIES Günther, 1858; STEPHopaEdities Dubois, 1987.

Comments: The clan BUFONITES includes three branches attributed to the hierarchical rank subclan, BUFONITIES, PHRYNISCITIES and STEPHOPAEDITIES.

\section{F.24.01. Subclanus BuFONITIES Gray, 1825}

Eunym: Hoc loco.

Getangiotaxon: BUFONITES Gray, 1825.

Adelphotaxa: Phryniscities Günther, 1858; STEPHopaedities Dubois, 1987.

Getendotaxa: Adenomitoes Cope, 1861; Ansonitties nov.; Bufonitoes Gray, 1825; Bufotitoes nov.; NECTOPHRYNitoes Laurent, 1942; SABAHPHRYNITOES nov.; STRAUCHBUFONITOES nov.; ToRNIERIOBATITOES Miranda-Ribeiro, 1926; 1 G†; 2 GIS (Altiphrynoides Dubois, 1987; Parapelophryne Fei, Ye \& Jiang, 2003).

Comments: Matsui et al. (2007), on the basis of an analysis of a small sample of Asian taxa, confirmed the holophyly of a branch that corresponds to our subclanus BUFONITIES, within which the relationships were poorly supported. 
This taxon was confirmed and called 'Old World toads' by Van Bocxlaer et al. (2009), Liedtke et al. (2016) and our work, but did not have significant support in Pyron \& Wiens (2011) and Portik \& Pappenfuss (2015). The relationships within this taxon are poorly supported (see below). The genera Altiphrynoides, Blythophryne, Bufoides, Palaeophrynos, Parapelophryne and Pseudobufo can be allocated to the subclan BUFONITIES or some of its subordinate CS taxa on morphological evidence, but no molecular data are available to propose more precise relationships within this subclan. The other genera can be allocated molecularly to eight branches with high support, recognised here as infraclans.

Adenomus, Duttaphrynus and four other genera form a highly supported taxon (Pyron \& Wiens 2011), here called the hypoclan ADENOMITUES. This taxon is sister-group to Bufotes in Pyron \& Wiens (2011) but here it is sister-group to Pedostibes, the hypoclan PEDOSTIBITUES, both forming the infraclan ADENOMITOES in CLAD. The latter does not include Ansonia and Pelophryne as proposed by Van Bocxclaer et al. (2009). In our classification, the infraclan ANSONIITOES includes a series of genera that have low support concerning their relationships, except for Ansonia and Pelophryne, for which we define the hypoclan ANSONIITUES, and Phrynoidis and Rentapia, for which we erect the hypoclan RENTAPIITUES. As to the remaining genera of this infraclan, their relationships remaining unclear, they have to be recognised at the same hierarchical level, as the hypoclans BARBAROPHRYNITUES for Barbarophryne, INGEROPHRYNITUES for Ingerophrynus and BLAIRITUES for Blaira.

The infraclan NECTOPHRYNITOES accommodates three supported taxa, the hypoclans EPIDALEITUES for Epidalea, LEPTOPHRYNITUES for Leptophryne and NECTOPHRYNITUES for the genera Didynamipus, Laurentophryne, Mo, Nectophryne, Nimbaphrynoides, Werneria and Wolterstorffina. There is no significant support for a holophyletic Werneria (SHL 84) and the members of these two taxa can be diagnosed morphologically, so we formally attribute below to the lineage of Werneria bambutensis the new genus nomen $M o$.

As already revealed by Van Bocxlaer et al. (2009), there is a close phylogenetic relationship between Churamiti, Nectophrynoides and Schismaderma, presented in the following classification scheme. The infraclan TORNIERIOBATITOES represents three genera, Churamiti being sister-taxon of Nectophrynoides, recognised as the hypoclan TORNIERIOBATITUES, and Schismaderma being the sister-taxon to this group is subsequently recognised as the hypoclan SCHISMADERMATITUES.

Each of the remaining genera forms an independent lineage as the phylogenetic relationships in TREE do not show sufficient support. These lineages are recognised as the hypoclans BUFONITOES for Bufo, Bufotitoes for Bufotes, SABAHPHRYNitoes for Sabahphrynus and StRAUCHBUfonitoes for Strauchbufo.

Three nomina ("Ghatophryne", "Xanthophryne" and "Xanthophryne tigerinus") currently in use in the literature for taxa referred to the subclan BUFONITIES are nomenclaturally unavailable for having been published before 2012 by Biju et al. (2009) in the online-only journal BMC Research Notes. The deposition of facsimiles of this work in six libraries, announced in this publication, does not make it available, because these facsimiles do not comply with the requirement to have been "obtainable, when first issued, free of charge or by purchase" (Article 8.1.2) and because "facsimiles or reproductions obtained on demand of an unpublished work [Art. 8], even if previously deposited in a library or other archive" do not constitute published works (Article 9.12). Dubois et al. (2013) published a clear warning in this respect, but it was ignored by all subsequent authors who cited these nomina, none of which provided available nomina for these taxa. We therefore propose here new nomina for these three taxa.

F.25.01. Infraclanus ADENOMITOES Cope, 1861

Protonym: Adenominae Cope, 1861: 371 [F].

Eunym: Hoc loco.

Getangiotaxon: BUFONITIES Gray, 1825.

Adelphotaxa: Ansonittoes nov.; Bufonitoes Gray, 1825; BUfotitoes nov.; NeCtopHRYNitoes Laurent, 1942; SABAHPhryntoes nov.; StraUChbUfonitoes nov.; ToRnieriobatitoes Miranda-Ribeiro, 1926; 1 G†; 2 GIS (Altiphrynoides Dubois, 1987; Parapelophryne Fei, Ye \& Jiang, 2003).

Getendotaxa: Adenomitues Cope, 1861; Pedostibitues nov. 
Eunym: Hoc loco.

Getangiotaxon: AdENOMITOES Cope, 1861.

Adelphotaxon: PEDOSTIBITUES nov.

Getendotaxa: ADENOMITYES Cope, 1861; BEDUKITYES nov.

Comments: The nomen "Xanthophryne", introduced in a online-only journal before 2012, is not available according to the Code, as pointed out by Dubois et al. (2013) but ignored by all subsequent authors who cited this nomen. Besides, recognition of a taxon "Xanthophryne" makes the genus Duttaphrynus paraphyletic. We are therefore led to introduce two genus-series nomina in this group, which are here used at rank genus but could also be so at rank subgenus: Beduka for "Xanthophryne" and Firouzophrynus for the 'Bufo stomaticus group' of Inger (1972) and Dubois \& Ohler (1999).

\section{F.27.01. Catoclanus ADENOMITYES Cope, 1861}

Eunym: Hoc loco.

Getangiotaxon: ADENOMITUES Cope, 1861.

Adelphotaxon: BEDUKITYES nov.

Getendotaxa: Beduka nov.; Blythophryne Chandramouli, Vasudevan, Harikrishnan, Dutta, Janani, Sharma, Das \& Aggarwal, 2016; Bufoides Pillai \& Yazdani, 1973; Duttaphrynus Frost, Grant, Faivovich, Bazin, Haas, Haddad, Sá, Channing, Wilkinson, Donnellan, Raxworthy, Campbell, Blotto, Moler, Drewes, Nussbaum, Lynch, Green \& Wheeler, 2006; Firouzophrynus Safaei-Mahroo \& Ghaffari, 2020.

Nucleogenus, by present designation: Beduka nov. • Etymology of nomen: Marathi language of Maharashtra: beduka, 'toad'. • Stem of nomen: Beduk-.

Diagnosis: Small to very large toads (males SVL 22-103 mm, females SVL 22-133 mm); dorsal skin with keratinised tips on tubercles; canthal, preorbital, supraorbital and postorbital ridge present or absent; tympanum present, but may be hidden; parotoid glands present; fingers free, rarely with basal webbing; that of toes very variable, from free toes to completely webbed toes; finger and toe tips rounded, rarely dilated into discs, grooves always absent; tarsal folds absent; eggs small to large sized with dark pigmented animal pole; a single median, external vocal sac in adult males; tadpoles with keratodonts, but no ventral sucker as in sister-taxon Adenomus. \{Inger 1972; Dubois 1974; Sarkar 1984; Chanda 1994; Manamendra-Arachchi \& Pethiyagoda 1998; Dubois \& Ohler 1999; Wogan et al. 2003; Biju et al. 2009; Chandramouli et al. 2011, 2016; Deuti et al. 2012; Meegaskumbura et al. 2015a; Gaitonde et al. 2016; Chandramouli \& Amarasinghe 2016; personal observations by AD and AO\}.

\section{G.28.105. Genus Beduka nov.}

Getangiotaxon: BEDUKITYES nov.

Adelphotaxa: Blythophryne Chandramouli, Vasudevan, Harikrishnan, Dutta, Janani, Sharma, Das \& Aggarwal, 2016; Bufoides Pillai \& Yazdani, 1973; Duttaphrynus Frost, Grant, Faivovich, Bazin, Haas, Haddad, Sá, Channing, Wilkinson, Donnellan, Raxworthy, Campbell, Blotto, Moler, Drewes, Nussbaum, Lynch, Green \& Wheeler, 2006; Firouzophrynus Safaei-Mahroo \& Ghaffari, 2020.

Getendotaxa: Beduka amboli nov.; Beduka koynayensis (Soman, 1963).

Nucleospecies, by present desigation: Bufo koynayensis Soman, 1963. • Etymology of nomen: Marathi language of Maharashtra: beduka, 'toad'. This nomen points to the geographic distribution of this genus, in the Western Ghats of southern India. • Stem of nomen: Beduk-. • Grammatical gender of nomen: feminine.

Diagnosis: Small sized toads (males SVL 26.5-32.9 mm, females SVL 33.3-35.3 mm); dorsal coloration light brown and chrome-yellow; flanks and sides of abdomen with chrome-yellow patches 
or bands; dorsal skin with keratinised tubercles; canthal ridge discontinuous and weak; preorbital ridge discontinuous and weak; tympanum indistinct; parotoid glands present, but rather weak; fingers and toes without webbing; finger and toe tips rounded; tarsal folds absent; eggs in clutches, black and white color; a single, median, external vocal sac; tadpoles with keratodonts present; habitat on lateritic rock. \{Biju et al. 2009; Meegaskumbura et al. 2015; Gaitonde et al. 2016\}.

Comments: As explained above, the nomen "Xanthophryne", published online only before 2012 by Biju et al. (2009), is nomenclaturally unavailable. We provide here a new nomen for this taxon, which is much shorter than the original one as it does not end with the six-letter ending -phryne currently overused in bufonid nomenclature in our opinion (see in this respect Dubois \& Raffaëlli 2009 and Dubois 2010).

\section{S.29.01. Species Beduka amboli nov.}

Getangiotaxon: Beduka nov.

Adelphotaxon: Beduka koynayensis (Soman, 1963).

Getendotaxon: None.

Holophoront (holotype), by present desigation: BNHS 5175, adult male, SVL $30.5 \mathrm{~mm}$. $\bullet$ Etymology of nomen: Amboli, name of onymotope (type locality) of this species in Maharashtra, India. $\bullet$ Grammatical status of epithet: noun in apposition.

Diagnosis: See the diagnosis of "Xanthophryne tigerinus" in Biju et al. (2009: 4).

Comments: As explained above, the nomen "Xanthophryne tigerinus", published online only before 2012 by Biju et al. (2009), is nomenclaturally unavailable. We provide here a new nomen for this taxon. We did not take over the epithet tigerina (misspelt tigerinus in the original description), because it is already used in the nomen of a common frog species of southern India, Hoplobatrachus tigerinus (Daudin, 1802), and as such is liable to cause confusion in faunistic lists, ecological works or even in careless taxonomic publications.

\section{F.26.02. Hypoclanus PEDOSTIBITUES nov.}

Getangiotaxon: ADENOMITOES Cope, 1861.

Adelphotaxon: ADENOMITUES nov.

Getendotaxon: Pedostibes Günther, 1876.

Nucleogenus, by present designation: Pedostibes Günther, 1876. • Etymology of nomen: G: $\pi \varepsilon \delta o \sigma \tau i ß j u s$ (pedostibes), 'walking on the earth'. • Stem of nomen: Pedostib-.

Diagnosis: Small, arboreal toads with horizontal pupils, elliptical tongue, partially webbed fingers and toes, terminal digits expanded into truncated discs, and united outer metatarsals. \{Boulenger 1890b; Graybeal \& Cannatella 1995\}.

\section{F.25.02. Infraclanus ANSONIITOES nov.}

Getangiotaxon: BUFONITIES Gray, 1825.

Adelphotaxa: Adenomitoes Cope, 1861; Bufonitoes Gray, 1825; Bufotitoes nov.; NeCtophrYNITOES Laurent, 1942; Sabahphrynitoes nov.; StRauchbufonitoes nov.; ToRnieriobatitoes Miranda-Ribeiro, 1926; 1 G†; 2 GIS (Altiphrynoides Dubois, 1987; Parapelophryne Fei, Ye \& Jiang, 2003).

Getendotaxa: ANSONIITUES nov.; BARBAROPHRYNITUES nov.; BLAIRITUES nov.; INGEROPHRYNITUES nov.; RENTAPIITUES nov.; 2 GIS (Pseudobufo Tschudi, 1838; Sigalegalephrynus Smart, Sarker, Arifin, Harvey, Sidik, Hamidy, Kurniawan \& Smith, 2017). 
Nucleogenus, by present designation: Ansonia Stoliczka, 1870. Etymology of nomen: P: Dedicated to Archibald Edward Harbond Anson (1826-1925), Lieutenant Governor of Penang from 1867 to 1882.

- Stem of nomen: Ansoni-.

Diagnosis: Very small to large sized toads (males SVL 13-80 mm; females SVL 13-105 mm); vocal sac present; webbing on hand present or absent; webbing foot often large; iris golden to red-brown colours, rarely green; tympanum distinct or absent; skin of belly coarsely granular; toe tips rounded, in some groups expanded; tarsal ridge present or absent; skin head cranial crests usually absent; parotoid glands present, often roundish, or absent; skin on dorsum with scattered warts, sometimes bearing horny structures; colour of dorsum brownish, usually with spotted pattern; eggs pigmented or non-pigmented, small to large sized $(1.2-2.8 \mathrm{~mm})$, numerous or few in number; tadpoles stream-living, usually of general bufonid type but also with adaptations to this habitat; one genus showing phytotelm breeding. \{Barbour 1938; Grismer 2006; Pramuk 2006; Matsui et al. 2007; Biju et al. 2009; Beukema et al. 2013 \}.

\section{F.26.03. Hypoclanus ANSONIITUES nov.}

Getangiotaxon: ANSONIITOES nov.

Adelphotaxa: BARBAROPHRYNITUES nov.; BLAIRITUES nov.; INGEROPHRYNITUES nov.; RENTAPITUES nov.; 2 GIS (PSEUdobufo Tschudi, 1838; Sigalegalephrynus Smart, Sarker, Arifin, Harvey, Sidik, Hamidy, Kurniawan \& Smith, 2017).

Getendotaxa: Ansonia Stoliczka, 1870; Pelophryne Barbour, 1938.

\section{F.26.04. Hypoclanus BARBAROPHRYNITUES nov.}

Getangiotaxon: ANSONITOES nov.

Adelphotaxa: ANSONIITUES nov.; BLAIRITUES nov.; INGEROPHRYNITUES nov.; RENTAPIITUES nov.; 2 GIS (Pseudobufo Tschudi, 1838; Sigalegalephrynus Smart, Sarker, Arifin, Harvey, Sidik, Hamidy, Kurniawan \& Smith, 2017).

Getendotaxon: Barbarophryne Beukema, Pous, Donaire-Barroso, Bogaerts, Garcia-Porta, Escoriza, Arribas, El Mouden \& Carranza, 2013.

Nucleogenus, by present designation: Barbarophryne Beukema, de Pous, Donaire-Barroso, Bogaerts, Garcia-Porta, Escoriza, Arribas, El Mouden \& Carranza, 2013. - Etymology of nomen: L: barbaro,

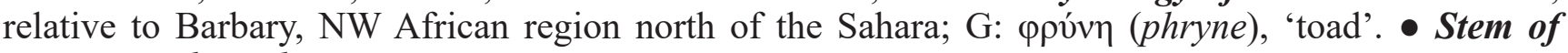
nomen: Barbarophryn-.

Diagnosis: Small toads, lacking warts on the dorsal surface of the head, nearly circular parotoid glands, nearly round tympanum, lacking gland on the tibia, and paired distal subarticular tubercles on the fourth toe. $\{$ Beukema et al. 2013$\}$.

\section{F.26.05. Hypoclanus BLAIRITUES nov.}

Getangiotaxon: ANSONIITOES nov.

Adelphotaxa: ANSONIITUES nov.; BARBAROPHRYNITUES nov.; INGEROPHRYNITUES nov.; RENTAPIITUES nov.; 2 GIS (PSEUdObUfo Tschudi, 1838; Sigalegalephrynus Smart, Sarker, Arifin, Harvey, Sidik, Hamidy, Kurniawan \& Smith, 2017).

Getendotaxon: Blaira nov.

Nucleogenus, by present designation: Blaira nov. • Etymology of nomen: P: Dedicated to William Franklin Blair, (1912-1984), zoologist, for his contribution to the knowledge about the evolution of toads. • Stem of nomen: Blair-. Grammatical gender of nomen: feminine.

Diagnosis: See below under Blaira nov. 


\section{G.28.115. Genus Blaira nov.}

Getangiotaxon: BLAIRITUES nov.

Adelphotaxon: None.

Getendotaxa: Blaira ornata (Günther, 1876); Blaira rubigina (Pillai \& Pattabiraman, 1981).

Nucleospecies, by present designation: Ansonia ornata Günther, 1876. • Etymology of nomen: P: Dedicated to William Franklin Blair, (1912-1984), zoologist, for his contribution to the knowledge about the evolution of toads. • Stem of nomen: Blair-. • Grammatical gender of nomen: feminine.

Diagnosis: Small toads, reddish-brown dorsal coloration, dark brownish-black ventral coloration with prominent yellowish-orange spots, no cranial ridges, no parotoid glands evident, no webbing on fingers, moderate webbing on toes, sparse granular projections on dorsal skin, non-pigmented eggs, and tadpoles with suctorial disc. \{Biju et al. 2009\}.

Comments: As explained above, the nomen "Ghatophryne", published online only before 2012 by Biju et al. (2009), is nomenclaturally unavailable. We provide here a new nomen for this taxon, which is much shorter than the original one as it does not end with the six-letter ending -phryne currently overused in bufonid nomenclature in our opinion (see in this respect Dubois \& Raffaëlli 2009 and Dubois 2010).

\section{F.26.06. Hypoclanus INGEROPHRYNITUES nov.}

Getangiotaxon: ANSONIITOES nov.

Adelphotaxa: Ansonittues nov.; BARbarophrynttues nov.; BlaIRITUES nov.; RentaPiItUeS nov.; 2 GIS (PSeudobufo Tschudi, 1838; Sigalegalephrynus Smart, Sarker, Arifin, Harvey, Sidik, Hamidy, Kurniawan \& Smith, 2017).

Getendotaxon: Ingerophrynus Frost, Grant, Faivovich, Bain, Haas, Haddad, de Sá, Channing, Wilkinson, Donnellan, Raxworthy, Campbell, Blotto, Moler, Drewes, Nussbaum, Lynch, Green \& Wheeler, 2006.

Nucleogenus, by present designation: Ingerophrynus Frost, Grant, Faivovich, Bain, Haas, Haddad, Sá, Channing, Wilkinson, Donnellan, Raxworthy, Campbell, Blotto, Moler, Drewes, Nussbaum, Lynch, Green \& Wheeler, 2006. • Etymology of nomen: P: Robert Frederick Inger (1920-2019); G: $\varphi \rho v ́ v \eta$ (phryne), 'toad'. • Stem of nomen: Ingerophryn-. • Grammatical gender of nomen: masculine.

Diagnosis: Small to medium sized toads; granular dorsal skin; most species with brownish dorsal coloration; tympanum present or absent; cranial crests absent or well-developed; parotoid glands lacking or distinct; and reduced to moderately-developed toe webbing. \{Matsui et al. 2007\}.

\section{F.26.07. Hypoclanus RENTAPIITUES nov.}

Getangiotaxon: ANSONIITOES nov.

Adelphotaxa: ANSONItTUES nov.; BARBAROPHRYNITUES nov.; BLAIRITUES nov.; INGEROPHRYNITUES nov.; 2 GIS (PSeudobufo Tschudi, 1838; Sigalegalephrynus Smart, Sarker, Arifin, Harvey, Sidik, Hamidy, Kurniawan \& Smith, 2017).

Getendotaxa: Phrynoidis Fitzinger in Treitschke, 1842; Rentapia Chan, Grismer, Zachariah, Brown \& Abraham, 2016.

Nucleogenus, by present designation: Rentapia Chan, Grismer, Zachariah, Brown \& Abraham, 2016. - Etymology of nomen: P: After the legendary Iban warrior Libau Rentap, Borneo, Malaysia. • Stem of nomen: Rentapi-. • Grammatical gender of nomen: masculine.

Diagnosis: Medium sized toads; most species with rugose or granular or smooth skin and brownish or greenish dorsal coloration; horizontal pupil; conspicuous channeled groove on posterior margin of neural arch; transverse process of vertebra VII oriented perpendicularly; posteromedial margin of the sacrum relatively smooth; and dorsal crest of ilial shaft present and well-developed in medial view. $\{$ Pramuk 2006\}. 
Eunym: Hoc loco.

Getangiotaxon: BUFONITIES Gray, 1825.

Adelphotaxa: ADENOMITOES Cope, 1861; ANSONITTOES nov.; BUfotitoes nov.; NECTOPHRYNITOES Laurent, 1942; SABAHPHRYNITOES nov.; STRAUCHBUfonitoes nov.; ToRNIERIOBATITOES Miranda-Ribeiro, 1926; 1 G†; 2 GIS (Altiphrynoides Dubois, 1987; Parapelophryne Fei, Ye \& Jiang, 2003).

Getendotaxon: Bufo Garsault, 1764.

\section{F.25.04. Infraclanus BUFOTITOES nov.}

Getangiotaxon: BUFONITIES Gray, 1825.

Adelphotaxa: Adenomitoes Cope, 1861; Ansonittoes nov.; Bufonitoes Gray, 1825; NeCtophrYNitoes Laurent, 1942; SABahphryntoes nov.; Strauchbufonitoes nov.; Tornieriobatitoes Miranda-Ribeiro, $1926 ; 1$ G†; 2 GIS (Altiphrynoides Dubois, 1987; Parapelophryne Fei, Ye \& Jiang, 2003).

Getendotaxon: Bufotes Rafinesque, 1815.

Nucleogenus, by present designation: Bufotes Rafinesque, 1815. • Etymology of nomen: N: Bufo Laurenti, 1768, derived from L: bufo, 'toad'; G: - $\tau \varepsilon \varsigma$ (-tes), suffix meaning 'one who does'. • Stem of nomen: Bufot-.

Diagnosis: Medium to large sized toads (SVL 38-97 mm); ratio of seventh to third transverse process of vertebrae $0.575-0.725$; vertebral crest median; main slip from humerodorsalis muscle to $4^{\text {th }}$ finger present; supinator manus humeralis present; cranial crest none; tibia gland absent; tarsal ridge present; vocal sac present, with gular pigmentation; subarticular tubercles on forth toe usually single; mating call series of notes with well defined internote intervals (type IIIa of Martin 1972); release calls pulsed structure with distinct interpulse intervals; chromosomses viridis-like chromosome set. \{Inger 1972; Eiselt \& Schmidtler 1973; Stöck et al. 2001a-b; Fei \& Ye 2016).

\section{F.25.05. Infraclanus NECTOPHRYNITOES Laurent, 1942}

Protonym: NECTOPHRYNIDAE Laurent, 1942: 6 [F].

Eunym: Hoc loco.

Getangiotaxon: BUFONITIES Gray, 1825.

Adelphotaxa: AdENOMITOES Cope, 1861; ANSONIITOES nov.; BUFONITOES Gray, 1825; BUFOTITOES nov.; SABAHPHRYNITOES nov.; Strauchbufonitoes nov.; ToRnieriobatitoes Miranda-Ribeiro, 1926; 1 G†; 2 GIS (Altiphrynoides Dubois, 1987; Parapelophryne Fei, Ye \& Jiang, 2003).

Getendotaxa: EPIDALEITUES nov.; LEPTOPHRYNITUES nov.; NECTOPHRYNITOES Laurent, 1942.

\section{F.26.08. Hypoclanus EPIDALEITUES nov.}

Getangiotaxon: NECTOPHRYNITOES Laurent, 1942.

Adelphotaxa: LEPTOPHRYNITUES nov.; NECTOPHRYNITOES Laurent, 1942.

Getendotaxon: Epidalea Cope, 1864.

Nucleogenus, by present designation: Epidalea Cope, 1864. • Etymology of nomen: G: żđí (epi),

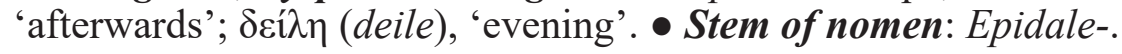

Diagnosis: Medium sized toads (SVL 41-66 mm), cranial crests absent, tarsal ridge present, vocal sac present, surrounding muscle and connective tissue with melanophores, tibia gland present, supinator manus humeralis present, humerodorsalis with main slips to third and fourth fingers and an accessory slip to fourth metacarpal, adductor longus present, vertebral column with a single median crest, seventh transverse process $0.576-0.725$ of third, occipital canal exposed, dorsal surface of skull smooth or 
weakly pitted, squamosal without a dorsal otic plate, transverse parasphenoid ridge absent, and palatine usually smooth. \{Inger 1972\}.

F.26.09. Hypoclanus LEPTOPHRYNITUES nov.

Getangiotaxon: NECTOPHRYNITOES Laurent, 1942.

Adelphotaxa: EPIDALEITUES nov.; NECTOPHRYNITUES Laurent, 1942.

Getendotaxon: Leptophryne Fitzinger, 1843.

Nucleogenus, by present designation: Leptophryne Fitzinger, 1843. • Etymology of nomen: G: $\lambda \varepsilon \pi \tau o ́ s$ (leptos), 'thin, delicate'; $\varphi$ pv́vๆ (phryne), 'toad'. • Stem of nomen: Leptophryn-.

Diagnosis: Small, slender toads with long limbs; no bony crests on head; short snout projecting slightly over the mouth; distinct tympanum smaller than eye; small round discs on tips of fingers and toes; no webbing on fingers; toes webbed over half their lengths; Bidder's organ; epicoracoid cartilage partially fused; elongated subarticular tubercle at the base of each toe. \{Graybeal \& Cannatella 1995; Malkmus et al. 2002\}.

F.26.10. Hypoclanus NECTOPHRYNITUES Laurent, 1942

Eunym: Hoc loco.

Getangiotaxon: NECTOPHRYNITOES Laurent, 1942.

Adelphotaxa: EPIDALEITUES nov.; LEPTOPHRYNITUES nov.

Getendotaxa: Didynamipus Andersson, 1903; Laurentophryne Tihen, 1960; Mo nov.; Nectophryne Buchholz \& Peters in Peters, 1875; Nimbaphrynoides Dubois, 1987; Werneria Poche, 1903; Wolterstorffina Mertens, 1939.

\section{G.28.125. Genus Mo nov.}

Getangiotaxon: NECTOPHRYNITUES Laurent, 1942

Adelphotaxa: Didynamipus Andersson, 1903; Laurentophryne Tihen, 1960; Nectophryne Buchholz \& Peters in Peters, 1875; Nimbaphrynoides Dubois, 1987; Werneria Poche, 1903; Wolterstorffina Mertens, 1939.

Getendotaxon: Mo bambutensis (Amiet, 1972).

Nucleospecies, by present designation: Bufo bambutensis Amiet, 1972. • Etymology of nomen: P: 'Mo', the nickname of Mark-Oliver Rödel (1965-), German herpetologist, to whom this genus is dedicated in appreciation of his contribution to the progress of our knowledge on African amphibians.

- Stem of nomen: Mo-. • Grammatical gender of nomen: masculine.

Diagnosis: Small sized toads (males SVL 28-33 mm; females SVL 30-38 mm); body stout; snout rounded; skin with micro-reticulations; dorsolateral lines absent; belly without spotted pattern; hindlegs short and thick, without black bars; webbing large, toes with broad fringes; terminal phalange enlarged; males bearing minute spines on head; first finger with smooth subdigital pad; 380-480 unpigmented eggs, $2 \mathrm{~mm}$ in diameter; probably rather aquatic habits. \{Amiet 1976; Rödel et al. 2004; Hirschfeld et al. 2012\}.

\section{F.25.06. Infraclanus SABAHPHRYNITOES nov.}

Getangiotaxon: BUFONITIES Gray, 1825.

Adelphotaxa: Adenomitoes Cope, 1861; AnsonitToes nov.; BUFONITOES Gray, 1825; Bufotitoes nov.; NECTOPHRYNITOES Laurent, 1942; Strauchbufonitoes nov.; ToRnieriobatitoes Miranda-Ribeiro, 1926; 1 G†; 2 GIS (Altiphrynoides Dubois, 1987; Parapelophryne Fei, Ye \& Jiang, 2003).

Getendotaxon: Sabahphrynus Matsui, Yambun \& Sudin, 2007. 
Nucleogenus, by present designation: Sabahphrynus Matsui, Yambun \& Sudin, 2007. • Etymology of nomen: R: Sabah, state of Malaysia; G: $\varphi$ pv́vๆ (phryne), 'toad'.• Stem of nomen: Sabahphryn-.

Diagnosis: Small sized toads (males SVL 40-42 mm; females SVL 45-50 mm); lacking tympanic anulus, columella and Eustachian tube; cranial crests on head absent; parotoid glands absent; fingers expanded into distinct pads; distal phalanges T-shaped; webbing on feet moderate; male without vocal sac opening; ova numerous (1000), small and unpigmented; coccyx articulated to sacrum; eight presacral vertebrae; quadratojugal complete; pectoral girdle arciferal. \{Matsui et al. 2007\}.

\section{F.25.07. Infraclanus STRAUCHBUFONITOES nov.}

Getangiotaxon: BUFONITIES Gray, 1825.

Adelphotaxa: AdENOMItoEs Cope, 1861; ANSONIITOES nov.; BUfONITOES Gray, 1825; BUfotitoes nov.; NECTOPHRYNITOES Laurent, 1942; SABahphrynitoes nov.; Tornieriobatitoes Miranda-Ribeiro, 1926; 1 G†; 2 GIS (Altiphrynoides Dubois, 1987; Parapelophryne Fei, Ye \& Jiang, 2003).

Getendotaxon: Strauchbufo Fei, Ye \& Jiang, 2012.

Nucleogenus, by present designation: Strauchbufo Fei, Ye \& Jiang, 2012. • Etymology of nomen: P: Alexander Strauch (1832-1893); L: bufo, 'toad'. • Stem of nomen: Strauchbufon-.

Diagnosis: Small toads with prominent parotoid glands, horizontal pupil, tympanic membrane not visible, male guttural resonator present, longitudinal skin fold on internal edge of tarsus, singular subarticular tubercles on toes, tip of $4^{\text {th }}$ finger does not reach $1^{\text {st }}$ articulation of $3^{\text {rd }}$ finger, dorsal coloration olive or greenish-gray wit large dark spots and narrow middorsal line, and belly light gray with few dark spots. \{Inger 1972; Fei \& Ye 2016\}.

F.25.08. Infraclanus ToRnieriobatitoes Miranda-Ribeiro, 1926

Protonym: ToRNIERIOBATIDAE Miranda-Ribeiro, 1926: 19 [F].

Eunym: Hoc loco.

Getangiotaxon: BUFONITIES Gray, 1825.

Adelphotaxa: Adenomitoes Cope, 1861; ANSONIITOES nov.; Bufonitoes Gray, 1825; Bufotitoes nov.; NECTOPHRYNITOES Laurent, 1942; SABAhPhryNitoes nov.; StRAUChbUfonitoes nov.; 1 G†; 2 GIS (Altiphrynoides Dubois, 1987; Parapelophryne Fei, Ye \& Jiang, 2003).

Getendotaxa: SChISMadermatitues nov.; ToRnieriobatitues Miranda-Ribeiro, 1926.

\section{F.26.11. Hypoclanus SCHISMADERMATITUES nov.}

Getangiotaxon: ToRNIERIOBATITOES Miranda-Ribeiro, 1926.

Adelphotaxon: Tornieriobatitues Miranda-Ribeiro, 1926.

Getendotaxon: Schismaderma Smith, 1849.

Nucleogenus, by present designation: Schismaderma Smith, 1849. • Etymology of nomen: G: $\sigma \chi i \sigma \mu \alpha$ (schisma), 'division'; $\delta \varepsilon \dot{\rho} \mu \alpha$ (derma), 'skin'; referring to the ridge separating dorsal surface from flanks (Du Preez \& Carruthers 2009). • Stem of nomen: Schismadermat-.

Diagnosis: Medium sized toads with flaps on head, tarsal fold, single subarticular tubercles under the fingers, large externally visible tympanum, no parotoid glands, glandular ridge running dorsolaterally from tympanum to leg insertions outlined in black, reddish-brown dorsal coloration, usually a pair of round markings on sacral region, vocal sac and nuptial pads in breeding males, and U-shaped fold on the back of the larvae. \{Graybeal \& Cannatella 1995; Frost et al. 2006; Mercurio 2011). 
Eunym: Hoc loco.

Getangiotaxon: ToRNIERIOBATITOES Miranda-Ribeiro, 1926.

Adelphotaxon: SCHISMADERMATITUES nov.

Getendotaxa: Churamiti Channing \& Stanley, 2002; Nectophrynoides Noble, 1926.

F.24.02. Subclanus PHRYNISCITIEs Günther, 1858

Protonym: PHRYNISCIDAE Günther, 1858: 346 [F].

Eunym: Hoc loco.

Getangiotaxon: BUFONITES Gray, 1825.

Adelphotaxa: Bufonities Gray, 1825; STEPHOPAEDITIES Dubois, 1987.

Getendotaxa: ANAXYRITOES nov.; PhrYNISCITOES Günther, 1858.

Comments: This branch and its internal relationships were revealed by Pramuk (2006) and Pramuk et al. (2008) but their relationship in these works is different fom those found by Van Bocxlaer et al. (2009). Within the subclan PHRYNISCITNIES, Anaxyrus is sister-taxon to Incilius; together they constitute the infraclan ANAXYRITOES. Its sister-taxon, the infraclan PHRYNISCIOTES, includes only the genus Rhinella.

\section{F.25.09. Infraclanus ANAXYRITOES nov.}

Getangiotaxon: PHRYNISCITIES Günther, 1858.

Adelphotaxon: PHRYNISCITOES Günther, 1858.

Getendotaxa: Anaxyrus Tschudi, 1845; Incilius Cope, 1863.

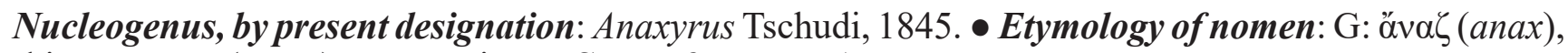
'king'; ov̄бos (oyros), 'mountain'. • Stem of nomen: Anaxyr-.

Diagnosis: Medium sized toads with warty or granular dorsal skin; primarily grayish, brownish, or yellowish dorsal coloration; no known morphological synapomorphies; numerous molecular synapomorphies. \{Frost et al. 2006\}.

\section{F.25.10. Infraclanus PHRYNISCITOES Günther, 1858}

Eunym: Hoc loco.

Getangiotaxon: PHRYNISCITIES Günther, 1858.

Adelphotaxon: ANAXYRITOES nov.

Getendotaxon: Rhinella Fitzinger, 1826.

\section{F.24.03. Subclanus STEPHOPAEDITIES Dubois, 1987}

Protonym: STEPHOPAEDINI Dubois, 1987: 27 [T].

Eunym: Hoc loco.

Getangiotaxon: BUFONITES Gray, 1825.

Adelphotaxa: BUfONITIES Gray, 1825; PhRYNISCITIES Günther, 1858.

Getendotaxa: CAPENSIBUfONITOES nov.; SCLEROPHRYITOES nov.; STEPHOPAEDITOES Dubois, 1987; VANDIJKOPHRYNITOES nov.

Comments: This taxon was revealed by Liedtke et al. (2016). This subclan STEPHOPAEDITIES accommodates five genus level taxa. Mertensophryne is sister-taxon to Poyntonophrynus, forming the infraclan STEPHOPAEDITOES. The relationships between this taxon and the other taxa do not have high 
support. These lineages are therefore recognised provisionally as taxa at the same level, the hypoclans CAPENSIBUfonitoes for Capensibufo, SCLEROPHRYITOES for Sclerophrys and VANDIJKOPHRYNITOES for Vandijkophrynus.

\section{F.25.11. Infraclanus CAPENSIBUFONITOES nov.}

Getangiotaxon: STEPHOPAEDITIES Dubois, 1987.

Adelphotaxa: SCLEROPHRYITOES nov.; STEPHOPAEDITOES Dubois, 1987; VANDIJKOPHRYNITOES nov.

Getendotaxon: Capensibufo Grandison, 1980.

Nucleogenus, by present designation: Capensibufo Grandison, 1980. • Etymology of nomen: R: Cap, region of South Africa; L: -ensis, suffix meaning 'originating from'; N: Bufo Laurenti, 1768, derived from L: bufo, 'toad'. • Stem of nomen: Capensibufon-.

Diagnosis: Small to medium sized toads; toes without webbing; large, pigmented eyes; small clutch sizes; omosternum present; paired subarticular tubercles in most species; reduced palatine; pterygoid not contacting parasphenoid; and large frontoparietal fontanelle. \{Graybeal \& Cannatella 1995\}.

\section{F.25.12. Infraclanus SCLEROPHRYITOES nov.}

Getangiotaxon: STEPHOPAEDITIES Dubois, 1987.

Adelphotaxa: CAPENSIBUfonitoes nov.; STEPHOPAEDITOES Dubois, 1987; VANDIJKophrynitoes nov.

Getendotaxon: Sclerophrys Tschudi, 1838.

Nucleogenus, by present designation: Sclerophrys Tschudi, 1838. • Etymology of nomen: G: $\sigma \kappa \lambda \varepsilon \rho o ́ \varsigma$ (skleros), 'hard'; ỏ $\rho v \varsigma$ (ophrus), 'eyebrow'. - Stem of nomen: Sclerophry-.

Diagnosis: Medium sized toads; karyotype $2 \mathrm{n}=20$ or 22; no known morphological synapomorphies; molecular transformations in several genes can be used to diagnose taxon. \{Frost et al. 2006\}.

\section{F.25.13. Infraclanus STEPHOPAEDITOES Dubois, 1987}

Eunym: Hoc loco.

Getangiotaxon: STEPHOPAEDITIES Dubois, 1987.

Adelphotaxa: CAPENSIBUFONITOES nov.; SCLEROPHRYITOES nov.; VANDIJKOPHRYNITOES nov.

Getendotaxa: Mertensophryne Tihen, 1960; Poyntonophrynus Frost, Grant, Faivovich, Bazin, Haas, Haddad, Sá, Channing, Wilkinson, Donnellan, Raxworthy, Campbell, Blotto, Moler, Drewes, Nussbaum, Lynch, Green \& Wheeler, 2006.

\section{F.25.14. Infraclanus VANDIJKOPHRYNITOES nov.}

Getangiotaxon: STEPHOPAEDITIES Dubois, 1987.

Adelphotaxa: CAPENSIBUfonitoes nov.; SCLEROPHRYITOES nov.; STEPHOPAEDITOES Dubois, 1987.

Getendotaxon: Vandijkophrynus Frost, Grant, Faivovich, Bain, Haas, Haddad, Sá, Channing, Wilkinson, Donnellan, Raxworthy, Campbell, Blotto, Moler, Drewes, Nussbaum, Lynch, Green \& Wheeler, 2006.

Nucleogenus, by present designation: Vandijkophrynus Frost, Grant, Faivovich, Bain, Haas, Haddad, Sá, Channing, Wilkinson, Donnellan, Raxworthy, Campbell, Blotto, Moler, Drewes, Nussbaum, Lynch, Green \& Wheeler, 2006. - Etymology of nomen: P: David Eduard (Eddie) Van Dijk (1925-), South

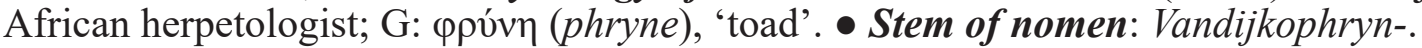

Diagnosis: Small to medium sized toads with robust body and limbs; morphologically confused and difficult to distinguish; poorly to well-developed parotoid glands; numerous small, flattened warts scattered over smooth dorsal skin; distinct, pale vertebral stripe in some species; dark, reticulate dorsal color-pattern; horizontal pupil; small, distinct tympanum; axillary amplexus. \{Tandy \& Keith 1972\}. 


\section{F.23.04. Clanus PELTOPHRYNITES nov.}

Getangiotaxon: BUFONINOA Gray, 1825.

Adelphotaxa: BUFONITES Gray, 1825; RHAEBOITES nov.

Getendotaxon: Peltophryne Fitzinger, 1843.

Nucleogenus, by present designation: Peltophryne Fitzinger, 1843. • Etymology of nomen: G: $\pi \varepsilon ́ \lambda \tau \eta$ (pelte), 'small shield'; $\varphi$ púvๆ (phryne), 'toad'. • Stem of nomen: Peltophryn-.

Diagnosis: Small to medium sized, robustly built toads; primarily brown to reddish-brown or yellowishbrown dorsal coloration; maxillae extend anteriorly, meeting in front of the premaxillae; zygomatic ramus of squamosal extending ventrally, abutting maxilla; zygomatic ramus connected by a flange to ventral ramus of squamosal. \{Graybeal \& Cannatella 1995\}.

\section{F.23.05. Clanus RHAEBOITES nov.}

Getangiotaxon: BUFONINOA Gray, 1825.

Adelphotaxa: BUFONITES Gray, 1825; PELTOPHRYNITES nov.

Getendotaxon: Rhaebo Fitzinger, 1843.

Nucleogenus, by present designation: Rhaebo Fitzinger, 1843. • Etymology of nomen: G: paıßós (raibos), with bent legs. - Comments: To avoid homonymy with the family-series nomen RHAEBINAE based on Rhaebus Fischer de Waldheim, 1824 (COLEOPTERA), we use the entire nomen Rhaebo, of unclear etymology, as the stem for this nomen, following Recommendation 29A of Article 29.6. • Stem of nomen: Rhaebo-.

Diagnosis: Medium to large sized toads with smooth, glandular skin; dark brown, yellowish-brown, or reddish-brown dorsal coloration; lacking cephalic crests; yellowish-orange skin secretions; omosternum present; and hypertrophied testes. \{Frost et al. 2006; Pramuk 2006\}.

\section{F.22.08. Hypotribus NANNOPHRYNINOA nov.}

Getangiotaxon: BUFONINIA Gray, 1825.

Adelphotaxon: BUFONINOA Gray, 1825

Getendotaxon: Nannophryne Günther, 1870.

Nucleogenus, by present designation: Nannophryne Günther, 1870. • Etymology of nomen: G: vóvvos

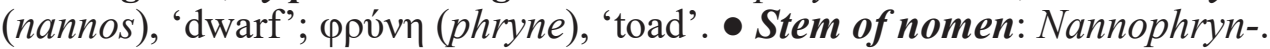

Diagnosis: Small to medium sized toads with blunt snouts, tympanum not visible externally, ovoid parotoid, skin smooth and glandular, moderately to lightly ossified skulls lacking dermal sculpturing and exostosing, otic ramus usually not enlarged, and cranial crests lacking. \{Pramuk 2006\}.

\section{F.21.09. Infratribus DENDROPHRYNISCINIA Jiménez de la Espada, 1870}

Protonym: DENDRophrynisCINA Jiménez de la Espada, 1870: 65 [Sc].

Eunym: Hoc loco.

Getangiotaxon: BUFONINA Gray, 1825.

Adelphotaxa: AMAZOPHRYNELLINIA nov.; BUFONINIA Gray, 1825.

Getendotaxon: Dendrophryniscus Jiménez de la Espada, 1870. 


\title{
F.20.14. Subtribus OREOPHRYNELLINA nov.
}

Getangiotaxon: BUFONINI Gray, 1825.

Adelphotaxa: ATELopodina Fitzinger, 1843; BUFONINA Gray, 1825; OsornophrYNINA nov.; 2 GIS (Metaphryniscus Señaris, Ayarzagüena \& Gorzula, 1994; Truebella Graybeal \& Cannatella, 1995).

Getendotaxon: Oreophrynella Boulenger, 1895.

Nucleogenus, by present designation: Oreophrynella Boulenger, 1895. • Etymology of nomen: G: ópos (oros), 'mountain'; N: Phrynella Boulenger, 1887, derived from G: $\varphi$ púvๆ (phryne), 'toad'; L: -ella, a feminine suffix indicating a diminutive form. $\bullet$ Stem of nomen: Oreophrynell-.

Diagnosis: Small, stout toads with robust limbs; rugose or granular dorsal skin; generally dark brown to black dorsal coloration; stubbed fingers and toes; first toe elongate and opposable to the remaining three; first two vertebrae fused; six presacral vertebrae; significantly reduced frontoparietals. \{Graybeal \& Cannatella 1995\}.

\section{F.20.15. Subtribus OSORNOPHRYNINA nov.}

\author{
Getangiotaxon: BUFONINI Gray, 1825. \\ Adelphotaxa: Atelopodina Fitzinger, 1843; Bufonina Gray, 1825; Oreophrynellina nov.; 2 GIS (Metaphryniscus \\ Señaris, Ayarzagüena \& Gorzula, 1994; Truebella Graybeal \& Cannatella, 1995). \\ Getendotaxon: Osornophryne Ruiz-Carranza \& Hernández-Camacho, 1976.
}

Nucleogenus, by present designation: Osornophryne Ruiz-Carranza \& Hernández-Camacho, 1976. - Etymology of nomen: P: Ernest and Hernando Osorno Mesa, Columbian herpetologists; G: $\varphi \rho v ́ v \eta$ (phryne), 'toad'. • Stem of nomen: Osornophryn-.

Diagnosis: Small, robust toads with stout limbs; generally brownish ventral coloration with incomplete lighter-colored glandular ridges dorsolaterally in some species; skin roughly granular; inguinal amplexus; six presacral vertebrae; absence of alary and posterolateral processes of hyoid; epicoracoid cartilages fused, parotoids absent; palmate hands and feet; coccyx expanded laterally; hand formula 2-2-3-2; small clutches of large, unpigmented eggs. \{Cannatella 1986; Graybeal \& Cannatella 1995\}.

\section{F.19.21. Tribus FROSTIINI nov.}

Getangiotaxon: BUFONINAE Gray, 1825.

Adelphotaxon: BUFONINI Gray, 1825.

Getendotaxon: Frostius Cannatella, 1986.

Nucleogenus, by present designation: Frostius Cannatella, 1986. • Etymology of nomen: P: Darrel Frost (1951-). • Stem of nomen: Frosti-.

Diagnosis: Small, stout toads; dark brown to blackish dorsal coloration; epicoracoid cartilages fused, typanum visible externally, tadpoles not gastromyzophorous. \{Cannatella 1986; Graybeal \& Cannatella $1995\}$.

Comments: When describing the genus Frostius, Cannatella (1986) showed that it shared morphological characters with Atelopus, Melanophryniscus, Dendrophryniscus, Oreophrynella and Osornophryne, and his phylogenetic analysis proposed it to be sister-taxon either to Atelopus or to the taxon grouping Atelopus and Osornophryne. In a phylogeny based on molecular data (Peloso et al. 2012), Frostius is sister-taxon to Oreophrynella, this group being sister-taxon to Amazophrynella. In TREE, this taxon is sister-taxon to all other BUFONINAE and we propose it as a new tribe FROSTIINI in our classification. 


\section{F.18.22. Subfamilia MELANOPHRYNISCINAE nov.}

Getangiotaxon: BUFONIDAE Gray, 1825.

Adelphotaxon: BUFONINAE Gray, 1825.

Getendotaxon: Melanophryniscus Gallardo, 1961.

Nucleogenus, by present designation: Melanophryniscus Gallardo, 1961. • Etymology of nomen:

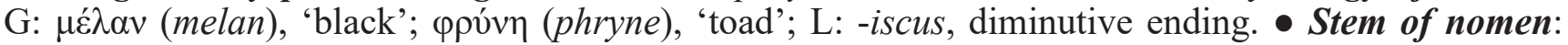
Melanophrynisc-.

Diagnosis: Small, stout toads; granular projections dorsal skin; frequently dark dorsal coloration with brilliant yellow and red ventral blotches; tadpoles with one pair of subhyoid muscles until Gosner's (1960) larval stage 44, elongated processus anterior dorsalis of the suprarostral alae, and absence of a chondrified commissura quadratoorbitalis (diagnostic for Melanophryniscus against all other bufonids); diploid karyotype $2 \mathrm{n}=22$ (six large and five small pairs); absence of the zygomatic ramus of the squamosal, exostosed frontoparietals diverging anteriorly, ossified orbitosphenoid cartilage, frontoparietals fused posteriorly, and parasphenoid fused to the chondrocranium. \{Graybeal \& Cannatella 1995; Larson P. et al. 2003; Baldo et al. 2012, 2014).

Comments: The sister-group relationship of Melanophryniscus to all other BUFONIDAE was found by several recent molecular studies (Van Bocxlaer et al. 2007; Pyron \& Wiens 2011; Portik \& Papenfuss 2015; Liedtke et al. 2016). In our classification, being the sister-taxon to the BUFONINAE, it is recognised as the new subfamily MELANOPHRYNISCINAE.

\section{F.17.17. Familia ODONTOPHRYNIDAE Lynch, 1971}

Protonym: ODONTOPHRYNINI Lynch, 1971: 130 [T].

Eunym: Pyron \& Wiens 2011: 543.

Getangiotaxon: BUFONOIDEA Gray, 1825.

Adelphotaxon: BUFONIDAE Gray, 1825.

Getendotaxa: ODONTOPHRYNINAE Lynch, 1971; PROCERATOPHRYINAE nov.

Comments: This family-level taxon was first defined based on morphological similarity as the tribe ODONTOPHRYNINI by Lynch (1971) including the same taxa as the present family, Odontophrynus, with Macrogenioglottus as synonym, and Proceratophrys. Heinicke et al. (2009) found Odontophrynus as sister-group to the BUFONIDAE. The strong support for a holophyletic grouping of the genera Macrogenioglottus and Odontophrynus, with Proceratophrys as sister-taxon, was found by Pyron \& Wiens (2011). But it was only Streicher et al. (2018) who found support to the sister-taxon relationship of ODONTOPHRYNIDAE and BUFONIDAE, as we confirm in TREE. Here we recognise this taxon at the family rank based on the Sister-Taxa Criterion [STC]. The taxon grouping Macrogenioglottus and Odontophrynus is recognised as the subfamily ODONTOPHRYNINAE, and consequently the sister-taxon including the unique genus Proceratophrys as the subfamily PROCERATOPHRYINAE nov.

F.18.23. Subfamilia ODONTOPHRYNINAE Lynch, 1971

Eunym: Hoc loco.

Getangiotaxon: ODONTOPHRYNIDAE Lynch, 1971.

Adelphotaxon: PROCERATOPHRYINAE nov.

Getendotaxa: Macrogenioglottus Carvalho, 1946; Odontophrynus Reinhardt \& Lütken, 1862.

\section{F.18.24. Subfamilia PROCERATOPHRYINAE nov.}

Getangiotaxon: ODONTOPHRYNIDAE Lynch, 1971.

Adelphotaxon: ODONTOPHRYNINAE Lynch, 1971

170 - Megataxa 005 (1) (C) 2021 Magnolia Press

DUBOIS ET AL. 
Nucleogenus, by present designation: Proceratophrys Miranda-Ribeiro, 1920. • Etymology of

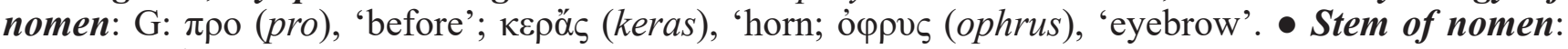
Proceratophry-.

Diagnosis: Adults SVL 30-95 mm; skin granular; body lacking glands; toes free of webbing, usually with lateral fringes, outer metatarsal tubercle present, inner metatarsal tubercle small or enlarged and spade-like, digital tips narrow, no finger webbing, numerous conical supernumerary thenar and plantar tubercles, first finger longer than second; males lacking nuptial asperities on thumb; cervical cotylar arrangement type II (Lynch 1971), cotyles closely approximated; sacral diapophyses rounded; alary processes of premaxillae long, strongly directed posterodorsally, except in the Proceratophrys bigibbosa group, relatively narrow at base; palatal shelf of maxilla broad, pterygoid process prominent; maxillae slightly expanded posteriorly; nasals relatively narrow, keeled, separated medially or in contact medially; nasals in contact with frontoparietals; frontoparietals bear lateral crests which meet posteriorly; frontoparietal crests heavily exostosed posteriorly in $P$. cristiceps and probably in $P$. bigibbosa; zygomatic ramus of squamosal broad and elongate, in sutural contact with maxilla, weakly exostosed; otic ramus of squamosal large, exostosed, expanded medially into relatively large otic plate; squamosal-maxillary angle $40-50^{\circ}$; occipital condyles large, not stalked, closely juxtaposed. \{Lynch 1971; Martins \& Giaretta 2011\}.

\section{F.14.04. Superfamilia CENTROLENOIDEA Taylor, 1951}

Protonym: CENTROLENIDAE Taylor, 1951: 36 [F].

Eunym: Hoc loco.

Getangiotaxon: HyLOBATRACHIA Ritgen, 1828

Adelphotaxa: Bufonoidea Gray, 1825; CERATophryoidea Tschudi, 1838; HyLOIDEA Rafinesque, 1815-|Gray, 1825|; LEPTODACTYLOIDEA ||Tschudi, 1838\|-Werner, 1896; 1 GIS (Ancudia Philippi, 1902).

Getendotaxa: AllophrynidaE Goin, Goin \& Zug, 1978; CENTRolenidAE Taylor, 1951.

Comments: The superfamily CENTROLENOIDEA, named «ALLOCENTROLENIAE», an unranked ectonym, by Guayasamin et al. (2009), is one branch within an unresolved ensemble that is recognised here as Hylobatrachia. It contains the AlLOPHRYNIDAE and the CENTROLENIDAE. The branch named CENTROLENIDAE is attributed to the rank family because it has been highly used [UQC]; consequently its sister-taxon is recognised at the same rank. Although this relationship was suggested by Noble (1931), it was highly debated and not recognised until evidence from molecular data confirmed it (Austin et al. 2002; Frost et al. 2006; Guayasamin \& Trueb 2007; Guayasamin et al. 2009; Pyron \& Wiens 2011).

\section{F.17.18. Familia ALLOPHRYNIDAE Goin, Goin \& Zug, 1978}

Protonym and eunym: ALLOPHRYNIDAE Goin, Goin \& Zug, 1978: 240 [F].

Getangiotaxon: CENTROLENOIDEA Taylor, 1951.

Adelphotaxon: CENTROLENIDAE Taylor, 1951.

Getendotaxon: Allophryne Gaige, 1926.

\section{F.17.19. Familia CENTROLENIDAE Taylor, 1951}

Eunym: Taylor 1951: 36.

Getangiotaxon: CENTROLENOIDEA Taylor, 1951.

Adelphotaxon: ALLOPHRYNIDAE Goin, Goin \& Zug, 1978.

Getendotaxa: CentroleninaE Taylor, 1951; HyalinobatraChinaE Guayasamin, Castroviejo-Fisher, Trueb, Ayarzagüena, Rada \& Vilà, 2009; IKAKOGINAE nov. 
Comments: The classification within the family CENTROLENIDAE is still not settled, as indicated by the changes between the classifications recently published and that proposed here. Several relationships within this family do not have sufficient support to be recognised in the proposed classification. Species sampling in some works on molecular relationships including members of this family is insufficient to give well supported results on relationships within the taxa (Darst \& Cannatella 2004; Faivovich et al. 2005; Frost et al. 2006; Streicher et al. 2018). Guayasamin et al. (2008, 2009), Hutter et al. (2013) and Castroviejo-Fisher et al. (2014) provided a phylogeny of the family and a classification based on these relationships (considering a boostrap of $70 \%$ as sufficient for support). They recognised two subfamilies CENTROLENINAE and HyaliNOBATRACHINAE, and included the genus Ikakogi as incertae sedis. An unnamed taxon, including the genera Centrolene and Nymphargus, was recognised as sistertaxon to the tribe COCHRANELLINI which included the genera ((((Cochranella (Espadrana, Chimerella)) (Rulyrana, Sachatamia) Teratohyla) Vitreorana).

The relationships within the subfamily CENTROLENINAE found in TREE are different from those in Guayasamin et al. (2009), Pyron \& Wiens (2011), Hutter et al. (2013) and Castroviejo-Fisher et al. (2014). In our classification, the family includes three highly supported taxa, recognised as the subfamilies CENTROLENINAE, HYALINOBATRACHINAE and IKAKOGINAE. The relationships between these three taxa do not have sufficient support to be formally recognised. The subfamily IKAKOGINAE includes a single genus Ikakogi, and HYALINOBATRACHINAE englobes the genera Celsiella and Hyalinobatrachus, as found in the previous works. The subfamily CENTROLENINAE has a more complex structure, including three taxa of poorly supported relationships, recognised as tribes, the CENTROLENINI for Centrolene, the NYMPHARGINI for Nymphargus and the COCHRANELLINI. The latter tribe holds three well supported subtribes of unsupported mutual relationships, the COCHRANELLINA, TERATOHYLINA and VITRORANINA. The two latter stand each for a single genus, but the COCHRANELLINA split into two well supported taxa, the infratribe COCHRANELLINIA for Cochranella, and the infratribe ESPADARANINIA. The latter infratribe includes three taxa of unsupported mutual relationships, recognised as the hypotribes CHIMERELLINOA for Chimerella, ESPADARANINOA for Espadarana, and RULYRANINOA. The genera in the latter hypotribe form two well supported taxa, the new genus Audaciella being the sister-group to a taxon holding the genera Rulyrana and Sachatamia, formally recognised as the clans AUDACIELLIONES and RULYRANIONES.

\section{F.18.25. Subfamilia CENTROLENINAE Taylor, 1951}

Eunym: Barrio 1968: 165; Lutz 1968: 22.

Getangiotaxon: CENTROLENIDAE Taylor, 1951.

Adelphotaxa: HyalinobatrachinaE Guayasamin, Castroviejo-Fisher, Trueb, Ayarzagüena, Rada \& Vilà, 2009; IKAKOGINAE nov.

Getendotaxa: CENTRolenini Taylor, 1951; CochranelLINI Guayasamin, Castroviejo-Fisher, Trueb, Ayarzagüena, Rada \& Vilà, 2009; NYMPHARGINI nov.

\section{F.19.22. Tribus CENTROLENINI Taylor, 1951}

Eunym: Hoc loco.

Getangiotaxon: CENTROLENINAE Taylor, 1951.

Adelphotaxa: CochranelLINI Guayasamin, Castroviejo-Fisher, Trueb, Ayarzagüena, Rada \& Vilà, 2009; NYMPHARGINI nov.

Getendotaxon: Centrolene Jiménez de la Espada, 1872.

F.19.23. Tribus CoCHRANELLINI Guayasamin, Castroviejo-Fisher, Trueb, Ayarzagüena, Rada \& Vilà, 2009

\footnotetext{
Protonym and eunym: CoCHRANELLINI Guayasamin, Castroviejo-Fisher, Trueb, Ayarzagüena, Rada \& Vilà, $2009: 3$ [T]. Getangiotaxon: CENTROLENINAE Taylor, 1951.

Adelphotaxa: CENTROLENINI Taylor, 1951; NYMPHARGINI nov.

Getendotaxa: Cochranellina Guayasamin, Castroviejo-Fisher, Trueb, Ayarzagüena, Rada \& Vilà, 2009; TERATOHYLINA nov.; VITREORANINA nov.
} 
F.20.16. Subtribus CochranelLINA Guayasamin, Castroviejo-Fisher, Trueb, Ayarzagüena, Rada \& Vilà, 2009

Eunym: Hoc loco.

Getangiotaxon: CochranelLINI Guayasamin, Castroviejo-Fisher, Trueb, Ayarzagüena, Rada \& Vilà, 2009.

Adelphotaxa: TERATOHYLINA nov.; VITREORANINA nov.

Getendotaxa: CoChranelLINIA Guayasamin, Castroviejo-Fisher, Trueb, Ayarzagüena, Rada \& Vilà, 2009; ESPADARANINIA nov.

F.21.10. Infratribus CoCHRANELLINIA Guayasamin, Castroviejo-Fisher, Trueb, Ayarzagüena, Rada \& Vilà, 2009

Eunym: Hoc loco.

Getangiotaxon: Cochranellina Guayasamin, Castroviejo-Fisher, Trueb, Ayarzagüena, Rada \& Vilà, 2009.

Adelphotaxon: ESPADARANINIA nov.

Getendotaxon: Cochranella Taylor, 1951.

\section{F.21.11. Infratribus ESPADARANINIA nov.}

Getangiotaxon: Cochranellina Guayasamin, Castroviejo-Fisher, Trueb, Ayarzagüena, Rada \& Vilà, 2009. Adelphotaxon: Cochranellinia Guayasamin, Castroviejo-Fisher, Trueb, Ayarzagüena, Rada \& Vilà, 2009. Getendotaxa: CHIMERELLINOA nov.; ESPADARANINOA nov.; RULYRANINOA nov.

Nucleogenus, by present designation: Espadarana Guayasamin, Castroviejo-Fisher, Trueb, Ayarzagüena, Rada \& Vilà, 2009. • Etymology of nomen: P: Marcos Jiménez de la Espada (18311898), a Spanish zoologist; L: rana, 'frog'. • Stem of nomen: Espadaran-.

Diagnosis: Small glassfrogs with humeral spines present or absent; green bones; dentigerous process of vomer present or absent, teeth present or absent; males calling from upper surfaces of leaves. \{Guayasamin et al. 2009\}.

\section{F.22.09. Hypotribus CHIMERELLINOA nov.}

Getangiotaxon: ESPADARANINIA nov.

Adelphotaxa: ESPADARANINOA nov.; RULYRANINOA nov.

Getendotaxon: Chimerella Guayasamin, Castroviejo-Fisher, Trueb, Ayarzagüena, Rada \& Vilà, 2009.

Nucleogenus, by present designation: Chimerella Guayasamin, Castroviejo-Fisher, Trueb,

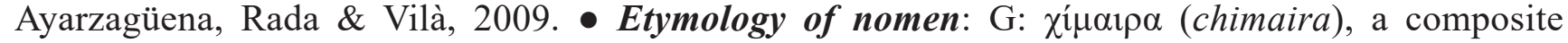
creature; L: suffix -ella, a diminutive form. $\bullet$ Stem of nomen: Chimerell-.

Diagnosis: Small glassfrogs with small humeral spines in adult males; lobed liver covered by a white hepatic peritoneum; ventral parietal peritoneum completely transparent; webbing reduced or absent between inner fingers, moderate between outer fingers; pale green bones; dentigerous process of vomer present, lacking teeth; males calling from upper surfaces of leaves. \{Guayasamin et al. 2009\}.

\section{F.22.10. Hypotribus ESPADARANINOA nov.}

Getangiotaxon: ESPADARANINIA nov.

Adelphotaxa: CHIMERELLINOA nov.; RULYRANINOA nov..

Getendotaxon: Espadarana Guayasamin, Castroviejo-Fisher, Trueb, Ayarzagüena, Rada \& Vilà, 2009. 


\title{
F.22.11. Hypotribus RULYRANINOA nov.
}

Getangiotaxon: ESPADARANINIA nov.

Adelphotaxa: ESPADARANINOA nov.; RULYRANINOA nov.

Getendotaxa: AUDACIELLITES nov.; RULYRANITES nov.

Nucleogenus, by present designation: Rulyrana Guayasamin, Castroviejo-Fisher, Trueb, Ayarzagüena, Rada \& Vilà, 2009. • Etymology of nomen: P: 'RuLy', concatenation of the first two letters of the names of Pedro Ruiz-Carranza (1932-1998) and John D. Lynch (1941-); L: rana, 'frog'. • Stem of nomen: Rulyran-.

Diagnosis: Small glassfrogs with or without humeral spines; ventral parietal peritoneum white anteriorly and transparent posteriorly; green bones; vomerine teeth usually present; males calling and females depositing clutches on the upper surfaces of leaves or rocks. \{Lynch \& Duellman 1973; Duellman \& Schulte 1993; Cisneros-Heredia \& McDiarmid 2007; see also Guayasamin et al. 2009; Twomey et al. $2014\}$.

\section{F.23.06. Clanus AUDACIELLITES nov.}

\author{
Getangiotaxon: RULYRANINOA nov. \\ Adelphotaxon: RULYRANITES nov. \\ Getendotaxon: Audaciella nov.
}

Nucleogenus, by present designation: Audaciella nov. • Etymology of nomen: L: audax, 'daring'; -ella, a feminine suffix indicating a diminutive form. $\bullet$ Stem of nomen: Audaciell-.

Diagnosis: Small glassfrogs with vomerine teeth; green bones; parietal peritoneum white; visceral peritoneum clear; most species with green dorsal coloration, with golden or bluish-white flecks; snout rounded or truncate in profile; dorsal skin shagreened with or without spicules; arms and legs lacking dorsal folds; humeral spines; distinct tympanum; enlarged prepollex; no prepollical spine; pair of enlarged tubercles below vent; first finger longer than second. \{Lynch \& Duellman 1973; CisnerosHeredia 2007; Duellman \& Schulte 1993; see also Guayasamin et al. 2009; Twomey et al. 2014\}.

\section{G.28.159. Genus Audaciella nov.}

Getangiotaxon: AUDACIELLITES nov.

Adelphotaxon: None.

Getendotaxa: Audaciella audax (Lynch \& Duellman, 1973); Audaciella durrellorum (Cisneros-Heredia, 2007); Audaciella fernandoi (Duelman \& Schulte, 1993).

Nucleospecies, by present designation: Centrolenella audax Lynch \& Duellman, 1973. • Etymology of nomen: L: audax, 'daring'; -ella, a feminine suffix indicating a diminutive form. $\bullet$ Stem of nomen: Audaciell-. • Grammatical gender of nomen: feminine.

Diagnosis: Small glassfrogs with vomerine teeth; green bones; parietal peritoneum white; visceral peritoneum clear; most species with green dorsal coloration, with golden or bluish-white flecks; snout rounded or truncate in profile; dorsal skin shagreened with or without spicules; arms and legs lacking dorsal folds; humeral spines; distinct tympanum; enlarged prepollex; no prepollical spine; pair of enlarged tubercles below vent; first finger longer than second. \{Lynch \& Duellman 1973; Duellman \& Schulte 1993; Cisneros-Heredia 2007; see also Guayasamin et al. 2009; Twomey et al. 2014\}. 


\section{F.23.07. Clanus RULYRANITES nov.}

Getangiotaxon: RULYRANINOA nov.

Adelphotaxon: AUDACIELLITES nov.

Getendotaxa: Rulyrana Guayasamin, Castroviejo-Fisher, Trueb, Ayarzagüena, Rada, and Vilà, 2009; Sachatamia Guayasamin, Castroviejo-Fisher, Trueb, Ayarzagüena, Rada, and Vilà, 2009.

\section{F.20.17. Subtribus TERATOHYLINA nov.}

Getangiotaxon: CochranelLINI Guayasamin, Castroviejo-Fisher, Trueb, Ayarzagüena, Rada \& Vilà, 2009.

Adelphotaxa: Cochranellina Guayasamin, Castroviejo-Fisher, Trueb, Ayarzagüena, Rada \& Vilà, 2009; VITREORANINA nov.

Getendotaxon: Teratohyla Taylor, 1951.

Nucleogenus, by present designation: Teratohyla Taylor, 1951. • Etymology of nomen: G: $\tau \varepsilon \rho \alpha o ́ \varsigma$ (teratos), monster, anormal production; N: Hyla, of debated etymology. • Stem of nomen: Teratohyl-.

Diagnosis: Small glassfrogs without humeral spines; liver covered by a white or transparent hepatic peritoneum; digestive tract translucent or white; ventral parietal peritoneum white anteriorly and transparent posteriorly, or completely transparent; moderate to extensive webbing between fingers III and IV; bones pale to dark green in life; dentigerous process of vomer present, with or without teeth; males calling and females depositing eggs on upper surfaces and tips of leaves; prepollical spine protruding or not. \{Guayasamin et al. 2009\}.

\section{F.20.18. Subtribus VITREORANINA nov.}

Getangiotaxon: CochraneLLINI Guayasamin, Castroviejo-Fisher, Trueb, Ayarzagüena, Rada \& Vilà, 2009.

Adelphotaxa: Cochranellina Guayasamin, Castroviejo-Fisher, Trueb, Ayarzagüena, Rada \& Vilà, 2009; TERATOHYLINA nov.

Getendotaxon: Vitreorana Guayasamin, Castroviejo-Fisher, Trueb, Ayarzagüena, Rada \& Vilà, 2009.

Nucleogenus, by present designation: Vitreorana Guayasamin, Castroviejo-Fisher, Trueb, Ayarzagüena, Rada \& Vilà, 2009. • Etymology of nomen: L: vitreum, 'glass'; N: Rana Linnaeus, 1758 , derived from rana, 'frog'. • Stem of nomen: Vitreoran-.

Diagnosis: Small glassfrogs with a white hepatic peritoneum covering or partially covering the liver, and most species with white gastrointestinal peritoneum. \{Guayasamin et al. 2009\}.

\section{F.19.24. Tribus NYMPHARGINI nov.}

Getangiotaxon: CENTROLENINAE Taylor, 1951.

Adelphotaxa: CochranelLINI Guayasamin, Castroviejo-Fisher, Trueb, Ayarzagüena, Rada \& Vilà, 2009; NYMPHARGINI nov.

Getendotaxon: Nymphargus Cisnero-Heredia \& McDiarmid, 2007.

Nucleogenus, by present designation: Nymphargus Cisnero-Heredia \& McDiarmid, 2007. • Etymology

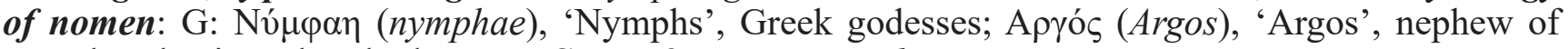
nymph Io having a hundred eyes. • Stem of nomen: Nympharg-.

Diagnosis: Small glassfrogs with reduced webbing between fingers III and IV; humeral spines absent except in males of Nymphargus grandisonae; tri- or tetra-lobed liver with transparent hepatic peritoneum; ventral parietal peritoneum white anteriorly and transparent posteriorly; bones green, or white in $N$. rosadus and N. anomalus; conspicuous spinules on dorsum of most breeding males; type I nuptial pads 
in breeding males; male advertisement call and female clutch deposition on upper sides of streamside leaves. \{Guayasamin et al. 2009\}.

\section{F.18.26. Subfamilia HyalinobatRaCHINAE Guayasamin, Castroviejo-Fisher,} Trueb, Ayarzagüena, Rada \& Vilà, 2009

Protonym and eunym: HYALINOBATRACHINAE Guayasamin, Castroviejo-Fisher, Trueb, Ayarzagüena, Rada \& Vilà, 2009: 36 $[\mathrm{F}]$.

Getangiotaxon: CENTROLENIDAE Taylor, 1951.

Adelphotaxa: CENTROLENINAE Taylor, 1951; IKAKOGINAE nov.

Getendotaxa: Celsiella Guayasamin, Castroviejo-Fisher, Trueb, Ayarzagüena, Rada \& Vilà, 2009; Hyalinobatrachium RuízCarranza \& Lynch, 1991.

Comments: The original spelling of this subfamial nomen is incorrect, missing an I, but it should not be corrected because of the new Article 29.4, which states that now such incorrect spellings should not be corrected, a highly confusing Rule (see Dubois \& Aescht 2019o: 125-126).

\section{F.18.27. Subfamilia IKAKOGINAE nov.}

Getangiotaxon: CENTROLENIDAE Taylor, 1951.

Adelphotaxa: CENTROLENINAE Taylor, 1951; HyalinobatraChinaE Guayasamin, Castroviejo-Fisher, Trueb, Ayarzagüena, Rada \& Vilà, 2009.

Getendotaxon: Ikakogi Guayasamin, Castroviejo-Fisher, Trueb, Ayarzagüena, Rada \& Vilà, 2009.

Nucleogenus, by present designation: Ikakogi Guayasamin, Castroviejo-Fisher, Trueb, Ayarzagüena, Rada \& Vilà, 2009. • Etymology of nomen: P: Ika (or Ijka) and Kogi people, descendants of the Tayrona, who inhabit the Sierra Nevada de Santa Marta, Colombia. • Stem of nomen: Ikakog-.

Diagnosis: Small glassfrogs with conspicuous humeral spines and enlarged crista medialis extending the entire length of the humerus, white bones, ventral parietal peritoneum white anteriorly and transparent posteriorly, and transparent hepatic and visceral peritonea. \{Guayasamin et al. 2009 \}.

\section{F.14.05. Superfamilia CERATOPHRYOIDEA Tschudi, 1838}

Protonym: CERATOPHRYDES Tschudi, 1838: 26 [F].

Eunym: Hoc loco.

Getangiotaxon: HYLOBATRACHIA Ritgen, 1828.

Adelphotaxa: BufonoIdeA Gray, 1825; CENTROLENoIdeA Taylor, 1951; HyloIDEA Rafinesque, 1815-|Gray, 1825|; LEPTODACTYLOIDEA ||Tschudi, 1838\|-Werner, 1896; 1 GIS (Ancudia Philippi, 1902).

Getendotaxa: CERAtophryoidae Tschudi, 1838; Telmatobioidae Fitzinger, 1843.

\section{F.15.03. Epifamilia CERATOPHRYOIDAE Tschudi, 1838}

Eunym: Hoc loco.

Getangiotaxon: CERATOPHRYOIDEA Tschudi, 1838.

Adelphotaxon: TELMATOBIOIDAE Fitzinger, 1843.

Getendotaxon: CERATOPHRYIDAE Tschudi, 1838. 
Eunym: Parker 1935: 12.

Getangiotaxon: CERATOPHRYOIDAE Tschudi, 1838.

Adelphotaxon: None.

Getendotaxa: CERATOPhryinae Tschudi, 1838; LePIDObatraChinaE Bauer, 1987; Stombinae Gallardo 1965.

Comments: Within the family CERATOPHRYIDAE, on the basis of TREE we recognise the four genera Ceratophrys, Chacophrys, Lepidobatrachus and Stombus (which includes the species Stombus cornutus and Stombus calcaratus). In Faivovich et al. (2014), Ceratophrys stolzmanni was sister-group to the taxon recognised as Stombus. The low support of this relationship does not allow further taxonomic decision. This result may point to absence of informative characters or sampling gaps.

\section{F.18.28. Subfamilia CERATOPHRYINAE Tschudi, 1838}

Eunym: Parker 1935: 511.

Getangiotaxon: CERATOPHRYIDAE Tschudi, 1838.

Adelphotaxa: LePIDobatraChinAE Bauer, 1987; STOMBINAE Gallardo 1965.

Getendotaxa: Ceratophrys Neuwied, 1824; 1 G†.

\section{F.18.29. Subfamilia LEPIDOBATRACHINAE Bauer, 1987}

Protonym: LEPIDOBATRACHIDAE Bauer, 1987: 5 [F].

Eunym: Hoc loco.

Getangiotaxon: CERATOPHRYIDAE Tschudi, 1838.

Adelphotaxa: CeratophryinaE Tschudi, 1838; Stombinae Gallardo 1965.

Getendotaxa: Chacophrys Reig \& Limeses, 1963; Lepidobatrachus Budgett, 1899; 1 G†.

\section{F.18.30. Subfamilia STOMBINAE Gallardo 1965}

Protonym and eunym: STOMBINAE Gallardo 1965: 5 [bF].

Getangiotaxon: CERATOPHRYIDAE Tschudi, 1838.

Adelphotaxa: CERATOPHRYINAE Tschudi, 1838; LePIDOBATRACHINAE Bauer, 1987.

Getendotaxon: Stombus Gravenhorst, 1825.

\section{F.15.04. Epifamilia TELMATOBIOIDAE Fitzinger, 1843}

Protonym: TELMATовII Fitzinger, 1843: 32 [F].

Eunym: Hoc loco.

Getangiotaxon: CERATOPHRYOIDEA Tschudi, 1838.

Adelphotaxon: CERATOPHRYOIDAE Tschudi, 1838.

Getendotaxa: CYCLORAMPHEIDAE Bonaparte, 1850-|Bonaparte, 1852|; TELMATOBIEIDAE Fitzinger, 1843.

Comments: Within the epifamily TELMATOBIOIDAE, two well supported branches are recognised here, the apofamilies CYCLORAMPHEIDAE and TELMATOBIEIDAE.

\section{F.16.01. Apofamilia CYCLORAMPHEIDAE Bonaparte, 1850-|Bonaparte, 1852|}

Protonyms: CYCLORHAMPHINA Bonaparte, 1850: plate [bF]; |CYCLORAMPHINA Bonaparte, 1852: 477| [bF].

Eunym: Hoc loco.

Getangiotaxon: TELMATOBIOIDAE Fitzinger, 1843. 
Adelphotaxon: TeLMATOBIEIDAE Fitzinger, 1843.

Getendotaxon: CYCLORAMPHIDAE Bonaparte, 1850-|Bonaparte, 1852|.

Comments: The major relationships within this taxon are not highly supported in TREE, but high support exists for the taxa Alsodes and Eupsophus, Atelognathus and Chaltenobatrachus with its sister-taxon including Batrachyla and Hylorina, Cycloramphus and Thoropa, Crossodactylus and Hylodes, whereas Limnomedusa forms a lineage that cannot be grouped with any of the other groups with sufficient support. Pending the resolution of this polytomy, these branches are provisionally recognised here as the subfamilies ALSODINAE, BATRACHYLINAE, CYCLORAMPHINAE, HYLODINAE and LIMNOMEDUSINAE of a single family CYCLORAMPHIDAE, by virtue of the Nomenclatural Thrift Criterion [NTC]. Within the BATRACHYLINAE, two sister-taxa are recognised as the tribes ATELOGNATHINI and BATRACHYLINI.

\section{F.17.21. Familia CYCLORAMPHIDAE Bonaparte, 1850-|Bonaparte, 1852|}

Eunym: Frost, Grant, Faivovich, Bain, Haas, Haddad, Sá, Channing, Wilkinson, Donnellan, Raxworthy, Campbell, Blotto, Moler, Drewes, Nussbaum, Lynch, Green \& Wheeler 2006: 6.

Getangiotaxon: CYCLORAMPHEIDAE Bonaparte, 1850-|Bonaparte, 1852|.

Adelphotaxon: None.

Getendotaxa: AlsodinaE Mivart, 1869; BATRACHYLINAE Gallardo, 1965; CYCLORAMPHINAE Bonaparte, 1850-|Bonaparte, 1852|; HYLODINAE Günther, 1858; LIMNOMEDUSINAE nov.

\section{F.18.31. Subfamilia ALSODINAE Mivart, 1869}

Protonym: ALSODINA Mivart, 1869: 290 [bF].

Eunym: Pyron \& Wiens 2011: 546.

Getangiotaxon: CYCLORAMPHIDAE Bonaparte, 1850-|Bonaparte, 1852|.

Adelphotaxa: BATRACHYLINAE Gallardo, 1965; CYCLORAMPHINAE Bonaparte, 1850-|Bonaparte, 1852|; HYLODINAE Günther, 1858; LIMNOMEDUSINAE nov.

Getendotaxa: Alsodes Bell, 1843; Eupsophus Fitzinger, 1843.

Comments: Pyron \& Wiens (2011) and Streicher et al. (2018) found a relationship between Alsodes and Eupsophus but Pyron and Wiens (2011) also included Limnomedusa in the family ALSODIDAE. This classification cannot be retained as a taxon with Limnomedusa, Alsodes and Eupsophus does not have sufficient support in TREE.

\section{F.18.32. Subfamilia BATRACHYLINAE Gallardo, 1965}

Protonym and eunym: BATRACHYLINAE Gallardo, 1965: 83 [bF].

Getangiotaxon: CYCLORAMPHIDAE Bonaparte, 1850-|Bonaparte, 1852|.

Adelphotaxa: Alsodinae Mivart, 1869; CYClORAMPhINAE Bonaparte, 1850-|Bonaparte, 1852|; HyLODINAE Günther, 1858; LIMNOMEDUSINAE nov.

Getendotaxa: ATELOGNATHINI nov.; BATRACHYLINI Gallardo, 1965.

Comments: Streicher et al. (2018) found a sister-group relationship between Atelognathus and Chaltenobatrachus, as well as between Batrachyla and Hylorina. Here we recognise these two groups as the tribes ATELOGNATHINI and BATRACHYLINI.

\section{F.19.25. Tribus ATELOGNATHINI nov.}

Getangiotaxon: BATRACHYLINAE Gallardo, 1965.

Adelphotaxon: BATRACHYLINI Gallardo, 1965.

Getendotaxa: Atelognathus Lynch, 1978; Chaltenobatrachus Basso, Úbeda, Bunge \& Martinazzo, 2011. 


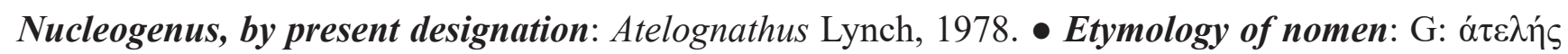

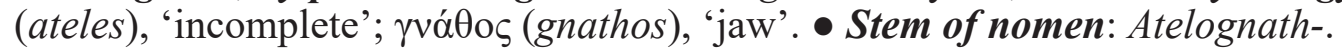

Diagnosis: Patagonian frogs formerly assigned to the LEPTODACTYLIDAE TELMATOBIINAE, with large frontoparietal fontanelles, short palatine bones not contacting the maxilla or calcified sphenethmoid, large nasal bones in median contact, no quadratojugals, columellar plectra, tympanic anuli and cavi tympani. $\{$ Lynch 1978$\}$.

\section{F.19.26. Tribus BATRACHYLINI Gallardo, 1965}

Eunym: Lynch 1971: 123.

Getangiotaxon: BATRACHYLINAE Gallardo, 1965.

Adelphotaxon: ATELOGNATHINI nov.

Getendotaxa: Batrachyla Bell, 1843; Hylorina Bell, 1843.

\section{F.18.33. Subfamilia CYCLORAMPHINAE Bonaparte, 1850-|Bonaparte, 1852|}

Eunym: Ardila-Robayo 1979: 455.

Getangiotaxon: CYCLORAMPHIDAE Bonaparte, 1850-|Bonaparte, 1852|.

Adelphotaxa: AlsodinaE Mivart, 1869; BATRACHYLINAE Gallardo, 1965; HylodinaE Günther, 1858; LIMNOMEDUSINAE nov.

Getendotaxa: Cycloramphus Tschudi, 1838; Thoropa Cope, 1865.

Comments: The sister-group relationship between Cycloramphus and Thoropa was confirmed by Pyron \& Wiens (2011) and by Streicher et al. (2018). As, in TREE, the position of Cystignathus parvulus Girard, 1853, onomatophore of the generic nomen Zachaenus, would render Cycloramphus polyphyletic, we treat here Zachaenus Cope, 1866 as a subjective junior synonym of Cycloramphus Tschudi, 1838

\section{F.18.34. Subfamilia HYLODINAE Günther, 1858}

Protonym: HYLODIDAE Günther, 1858: 346 [F].

Eunym: Savage 1973: 354.

Getangiotaxon: CYCLORAMPHIDAE Bonaparte, 1850-|Bonaparte, 1852|.

Adelphotaxa: AlsodinaE Mivart, 1869; BATRACHYLINAE Gallardo, 1965; CYCLORAMPHINAE Bonaparte, 1850-|Bonaparte, 1852|; LIMNOMEDUSINAE nov.

Getendotaxa: Crossodactylus Duméril \& Bibron, 1841; Hylodes Fitzinger, 1826.

Comments: The holophyly of the group composed of the genera Crossodactylus and Hylodes was found by Frost et al. (2006), who recognised the genus Megaelosia Miranda-Ribeiro, 1923, here considered as a synonym of Hylodes Fitzinger, 1826.

\section{F.18.35. Subfamilia LIMNOMEDUSINAE nov.}

Getangiotaxon: CYCLORAMPHIDAE Bonaparte, 1850-|Bonaparte, 1852|.

Adelphotaxa: ALsodinaE Mivart, 1869; BATRACHYLINAE Gallardo, 1965; CyCLORAMPHINAE Bonaparte, 1850-|Bonaparte, 1852|; HYLODINAE Günther, 1858

Getendotaxon: Limnomedusa Fitzinger, 1843.

Nucleogenus, by present designation: Limnomedusa Fitzinger, 1843. • Etymology of nomen: G: $\lambda$ í $\mu \mathrm{\eta}$

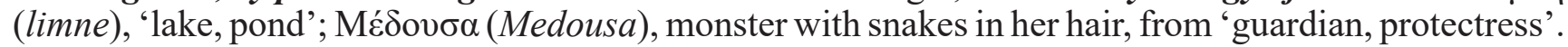

- Stem of nomen: Limnomedus-. 
Diagnosis: Medium sized frogs formerly assigned to the ALSODIDAE, diagnosable based primarily on larval morphology, with small oral disc, intra-angular margins, rostral gap, intra-marginal lateral papillae present only in supra-angular region, lacking intra-marginal mental papillae, marginal papillae present in multiple rows mentally, rostrodonts wider than deep, normal keratodont formula, level of nostril aperture not raised, lateral sinistral spiracle, proctodeal tube, medial vent opening, normal tail fins, lacking oral disc sucker, lacking abdominal sucker, eggs are laid and hatch out of water, larval development occurs out of water, and larvae are active feeders. \{Lavilla 1988\}.

\title{
F.16.02. Apofamilia TELMATOBIEIDAE Fitzinger, 1843
}

Eunym: Hoc loco.

Getangiotaxon: TeLMATOBIOIDAE Fitzinger, 1843.

Adelphotaxon: CYCLORAMPHEIDAE Bonaparte, 1850-|Bonaparte, 1852|.

Getendotaxa: Rhinodermatidae Bonaparte, 1850; TELMATOBIIDAE Fitzinger, 1843.

Comments: The holophyly of a branch including Insuetophrynus and Rhinoderma has been recognised using molecular evidence by Pyron \& Wiens (2011) and Streicher et al. (2018). According to the Criterion [UQC], this taxon must be referred to the rank family, and this also applies to its sister-taxon TELMATOBIIDAE.

\section{F.17.22. Familia RHINODERMATIDAE Bonaparte, 1850}

Protonym: RHINODERMINA Bonaparte, 1850: plate [bF].

Eunym: Günther 1858: 346.

Getangiotaxon: TELMATOBIEIDAE Fitzinger, 1843.

Adelphotaxon: TeLMATOBIIDAE Fitzinger, 1843.

Getendotaxa: Insuetophrynus Barrio, 1970; Rhinoderma Duméril \& Bibon, 1841.

\section{F.17.23. Familia TELMATOBIIDAE Fitzinger, 1843}

\author{
Eunym: Miranda-Ribeiro 1920: 320. \\ Getangiotaxon: TELMATOBIEIDAE Fitzinger, 1843. \\ Adelphotaxon: RHINODERMATIDAE Bonaparte, 1850. \\ Getendotaxa: Telmatobius Wiegmann, 1834; 1 G†.
}

Comments: This branch is recognised at the rank family TELMATOBIIDAE in our classification by virtue of the Criterion [STC]. Frost et al. (2006) referred this taxon to the rank subfamily, whereas Bossuyt \& Roelants (2009), Pyron \& Wiens (2011), Zhang et al. (2013), Feng et al. (2017), Hutter et al. (2017) and Streicher et al. (2018) used the family rank for it.

\section{F.14.06. Superfamilia HyLOIDEA Rafinesque, 1815-|Gray, 1825|}

Protonyms: HyLARINIA Rafinesque, 1815: 78 [F]; |HYLINA Gray, 1825: 213| [UF].

Eunym: Dubois 1983: 272.

Getangiotaxon: HyLOBATRACHIA Ritgen, 1828.

Adelphotaxa:BUfonOIDEA Gray, 1825; CENTROLENOIDEA Taylor, 1951; CERATOPHRYOIDEA Tschudi, 1838; LEPTODACTYLOIDEA ||Tschudi, 1838||-Werner, 1896; 1 GIS (Ancudia Philippi, 1902).

Getendotaxa: HylidAE Rafinesque, 1815-|Gray, 1825|; PHYLLOMEDUSIDAE Günther, 1858.

Comments: In our classification, the superfamily HYLOIDEA groups two taxa of rank family, the HYLIDAE and the PHYLLOMEDUSIDAE. The extension of this taxon corresponds to the HYLIDAE of Faivovich et al. (2005), Frost et al. (2006), Pyron \& Wiens (2011), Fouquet et al. (2013), Zhang et al. (2013), Feng et 
al. (2017), Hutter et al. (2017) and Streicher et al. (2018), and to the «ARBORANAE» of Duellman et al. (2016).

\title{
F.17.24. Familia HYLIDAE Rafinesque, 1815-|Gray, 1825|
}

Eunym: Bonaparte 1850: plate.

Getangiotaxon: HYLOIDEA Rafinesque, 1815-|Gray, 1825|.

Adelphotaxon: PHYLLOMEDUSIDAE Günther, 1858.

Getendotaxa: Cophomantinae Hoffmann, 1878; Hylinae Rafinesque, 1815; 3 G†.

Comments: The extension of this taxon corresponds to that of the HYLINAE of Faivovich et al. (2005), Frost et al. (2006), Pyron \& Wiens (2011), Zhang et al. (2013), Feng et al. (2017), Hutter et al. (2017) and Streicher et al. (2018).

\section{F.18.36. Subfamilia COPHOMANTINAE Hoffmann, 1878}

\author{
Protonym: Cophomantina Hoffmann, 1878: 614 [F]. \\ Eunym: Hoc loco. \\ Getangiotaxon: HYLIDAE Rafinesque, 1815-|Gray, 1825|. \\ Adelphotaxon: HYLINAE Rafinesque, 1815-|Gray, 1825|. \\ Getendotaxa: COPHOMANTINI Hoffmann, 1878; MYERSIOHYLINI nov.; NESOROHYLINI nov.
}

Comments: This taxon corresponds to the subfamily COPHOMANTINAE of Duellman et al. (2016) and to the tribe CopHomantini of Faivovich et al. (2005) and Pinheiro et al. (2019). As the relationships within this branch are not resolved, we recognise three tribes, the COPHOMANTINI with two subtribes COPHOMANTINA and HYLOSCIRTINA, the MYERSIOHYLINI for the genus Myersiohyla and the NESOROHYLINI for the genus Nesorohyla.

\section{F.19.27. Tribus Cophomantini Hoffmann, 1878}

Eunym: Faivovich, Haddad, Garcia, Frost, Campbell \& Wheeler 2005: 3.

Getangiotaxon: COPHOMANTINAE Hoffmann, 1878.

Adelphotaxa: MYERSIOHYLINI nov.; NESOROHYLINI nov.

Getendotaxon: Cophomantina Hoffmann, 1878; HyLOSCIRTINA nov.

Comments: Within this tribe, we recognise two highly supported branches as the subtribes HYLOSCIRTINA, for the genera Colomascirtus and Hyloscirtus, and COPHOMANTINA, including two sister-groups described as infratribes, BOKERMANNOHYLINIA for Bokermannohyla and COPHOMANTINIA for Aplastodiscus and Boana. The relationship between these groups have been revealed by Faivovich et al. (2005), Wiens et al. (2010), Brunetti et al. (2015) and Duellman et al. (2016).

\section{F.20.19. Subtribus COPHOMANTINA Hoffmann, 1878}

Eunym: Hoc loco.

Getangiotaxon: COPHOMANTINI Hoffmann, 1878.

Adelphotaxon: HYLOSCIRTINA nov.

Getendotaxa: BoKERMANNOHYLINIA nov.; COPHOMANTINIA Hoffmann, 1878.

F.21.12. Infratribus BOKERMANNOHYLINIA nov.

Getangiotaxon: COPHOMANTINA Hoffmann, 1878. 
Adelphotaxon: CoPHOMANTINIA Hoffmann, 1878.

Getendotaxon: Bokermannohyla Faivovich, Haddad, Garcia, Frost, Campbell \& Wheeler, 2005.

Nucleogenus, by present designation: Bokermannohyla Faivovich, Haddad, Garcia, Frost, Campbell \& Wheeler, 2005. • Etymology of nomen: P: Werner Carlos Augusto Bokermann (1929-1995); N: Hyla, of debated etymology. • Stem of nomen: Bokermannohyl-.

Diagnosis: Small to large sized treefrogs (males SVL 30-104 mm; females SVL 42-88 mm); vocal sac subgular, or rarely laterally extended; tympanum small, large in a few species; dorsal pattern generally with presence of transverse dark brown cross bars; thighs and flanks with dark bars; color of groin and concealed surfaces of arms and legs uniform light or yellow or reddish; prepollex always well developed, with curved spine exposed or not. \{Cochran 1955; Bokermann 1965; Caramaschi \& Feio 1990; Faivovich et al. 2005; Lugli \& Haddad 2006; Carvalho et al. 2012\}.

\section{F.21.13. Infratribus COPHOMANTINIA Hoffmann, 1878}

Eunym: Hoc loco.

Getangiotaxon: COPHOMANTINA Hoffmann, 1878.

Adelphotaxon: BOKERMANNOHYLINIA nov.

Getendotaxa: Aplastodiscus Lutz, 1950; Boana Gray, 1825.

\section{F.20.20. Subtribus HyLOSCIRTINA nov.}

Getangiotaxon: COPHOMANTINI Hoffmann, 1878.

Adelphotaxon: CoPHomantina Hoffmann, 1878

Getendotaxa: Colomascirtus Duellman, Marion \& Hedges, 2016; Hyloscirtus Peters, 1882.

Nucleogenus, by present designation: Hyloscirtus Peters, 1882. Etymology of nomen: N: Hyla, of

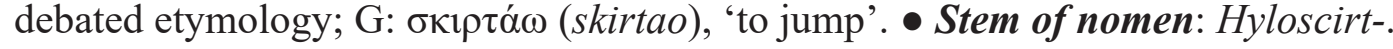

Diagnosis: Medium sized, primarily South American treefrogs (a group generally lacking clear morphological synapomorphies); apognosable by 56 transformations in DNA sequences from regions in the nucleus and mitochondrion, and by wide dermal fringes on the fingers and toes. \{Faivovich et al. $2005\}$.

Comments: Pinheiro et al. (2019) did not recognise Colomascirtus, but they obtained a tree of similar topology and recognised three species groups, the bogotensis group corresponding to Hyloscirtus, and the armatus and larinopygion groups, corresponding to Colomascirtus. The two groups that we recognise here as genera are holophyletic and have high support.

\section{F.19.28. Tribus MYERSIOHYLINI nov.}

Getangiotaxon: COPHOMANTINAE Hoffmann, 1878.

Adelphotaxa: Cophomantini Hoffmann, 1878; NeSOROHYLINI nov.

Getendotaxon: Myersiohyla Faivovich, Haddad, Garcia, Frost, Campbell \& Wheeler, 2005.

Nucleogenus, by present designation: Myersiohyla Faivovich, Haddad, Garcia, Frost, Campbell \& Wheeler, 2005. • Etymology of nomen: P: Charles W. Myers (1936-); N: Hyla, of debated etymology. - Stem of nomen: Myersiohyl-.

Diagnosis: South American treefrogs (a group generally lacking clear morphological synapomorphies) apognosable at present only by 48 transformations in DNA sequences for mitochondrial and ribosomal genes, with no known morphological synapomorphies. \{Faivovich et al. 2005\}. 
Comments: The taxon named MYERSIOHYLINI is sister-group to all other CopHomantinAE except Nesorohyla kanaima (Faivovich et al. 2005; Wiens et al. 2010; Duellman et al. 2016), but this relationship does not have a strong support in TREE, so we are bound to recognise three tribes in the subfamily

\section{F.19.29. Tribus NESOROHYLINI nov.}

Getangiotaxon: COPHOMANTINAE Hoffmann, 1878.

Adelphotaxa: MYERSIOHYLINI nov.; NESOROHYLINI nov.

Getendotaxon: Nesorohyla Pinheiro, Kok, Noonan, Means \& Haddad, 2018.

Nucleogenus, by present designation: Nesorohyla Pinheiro, Kok, Noonan, Means \& Haddad, 2018. - Etymology of nomen: G: ṽ̃бos (nesos), ‘island'; G: öpos (oros), ‘mountain'; N: Hyla, of debated etymology. • Stem of nomen: Nesorohyl-.

Diagnosis: As for the genus Nesorohyla (the former 'Hyla geographica group'), diagnosable by moderately slender body with distinct head, smooth dorsal skin, skin on head not co-ossified with underlying dermal elements, distinct tympanum, prepollex not modified as a projecting spine, unwebbed fingers and reduced fringes, moderate $(\sim 1 / 3)$ webbing of toes and reduced fringes, lacking limb fringes, two small and blunt calcar tubercles, lacking axillary membrane, long diagonal vomerine odontophores, dorsal coloration brown, iris dark; nuptial pads light colored, on inner margin of finger I and prepollex; tadpole with oral disc showing short anterior and posterior gaps on marginal papillae, three emarginations on posterior labium, keratodont formula $2+2 / 1+1: 3$ and pigmented eggs. \{Duellman \& Hoogmoed 1992; Faivovich et al. 2005; Pinheiro et al. 2019\}.

Comments: The onomatophore of Nesorohyla is sister-group to all other COPHOMANTINAE in all recent trees (Wiens et al. 2010; Duellman et al. 2016) but this relationship does not have strong support in TREE.

\section{F.18.37. Subfamilia HYLINAE Rafinesque, 1815-|Gray, 1825|}

Eunym: Gadow 1901: 189.

Getangiotaxon: HYLIDAE Rafinesque, 1815-|Gray, 1825|.

Adelphotaxon: CoPHomantinaE Hoffmann, 1878.

Getendotaxa: DENDROPSOPHINI Fitzinger, 1843; HYLINI Rafinesque, 1815-|Gray, 1825|; LOPHYOHYLINI Miranda-Ribeiro, 1926-|Fouquette \& Dubois, 2014|; SCINAXINI Duellman, Marion \& Hedges, 2016.

Comments: The relationships within the branch here recognised as subfamily HYLINAE are poorly resolved. In consequence, we transcribe the relationships revealed by TREE by attributing the rank tribe to the four highly supported taxa in this group: DENDROPSOPHINI, HYLINI, LOPHYOHYLINI and SCINAXINI.

\section{F.19.30. Tribus DENDROPSOPHINI Fitzinger, 1843}

Protonym: DENDROPSOPHI Fitzinger, 1843: 31 [F].

Eunym: Faivovich, Haddad, Garcia, Frost, Campbell \& Wheeler 2005: 3.

Getangiotaxon: HYLINAE Rafinesque, 1815-|Gray, 1825|.

Adelphotaxa: HyLINI Rafinesque, 1815-|Gray, 1825|; LорнуонYLINI Miranda-Ribeiro, 1926-|Fouquette \& Dubois, 2014|; SCINAXINI Duellman, Marion \& Hedges, 2016.

Getendotaxa: DENDROPSOPHINA Fitzinger, 1843; PSEUDINA Fitzinger, 1843.

Comments: This group reveals two highly supported branches, recognised here as the subtribes DENDROPSOPHINA, containing the genera Dendropsophus and Xenohyla, and PSEUDINA, for the genera Pseudis and Scarthyla. 
Eunym: Hoc loco.

Getangiotaxon: DENDROPSOPHINI Fitzinger, 1843.

Adelphotaxon: PSEUDINA Fitzinger, 1843.

Getendotaxa: Dendropsophus Fitzinger, 1843; Xenohyla Izecksohn, 1998.

\section{F.20.22. Subtribus PSEUDINA Fitzinger, 1843}

Protonym: PSEUDAE Fitzinger, 1843: 33 [F].

Eunym: Hoc loco.

Getangiotaxon: DENDROPSOPHINI Fitzinger, 1843.

Adelphotaxon: DENDROPSOPHINA Fitzinger, 1843.

Getendotaxa: Pseudis Wagler, 1830; Scarthyla Duellman \& Sá, 1988.

\section{F.19.31. Tribus HYLINI Rafinesque, 1815-|Gray, 1825|}

Eunym: Faivovich, Haddad, Garcia, Frost, Campbell \& Wheeler 2005: 3.

Getangiotaxon: HYLINAE Rafinesque, 1815-|Gray, 1825|.

Adelphotaxa: DendRopsophinI Fitzinger, 1843; LopHYohYLINI Miranda-Ribeiro, 1926-|Fouquette \& Dubois, 2014|; SCINAXINI Duellman, Marion \& Hedges, 2016.

Getendotaxa: ACRISINA Mivart, 1869; HYLINA Rafinesque, 1815-|Gray, 1825|.

Comments: The tribe HYLINI is composed of two sister-groups, recognised as the subtribes ACRISINA and HYLINA. The subtribe ACRISINA is composed of the sister-genera Hyliola and Pseudacris, recognised as the infratribe HYLIOLINIA, and their sister-taxon Acris, recognised as the infratribe ACRISINIA. The proposed classification is consistent with Faivovich et al. (2005), Wiens et al. (2006, 2010), Lemmon et al. (2007a-b), Pyron \& Wiens (2011), Barrow et al. (2014), Duellman et al. (2016) and Dubois et al. (2017). Hyliola corresponds to the 'West Coast clade' of Barrow et al. (2014). Faivovich et al. (2018) discussed the available evidence for recognition of this genus, and in favour of 'stability' and in absence of 'taxonomic utility' did not recognise this taxon, but did not formally synonymise its nomen with Pseudacris.

\section{F.20.23. Subtribus ACRISINA Mivart, 1869}

Protonym: ACRIDINA Mivart, 1869: 292 [bF].

Eunym: Hoc loco.

Getangiotaxon: HYLINI Rafinesque, 1815-|Gray, 1825|.

Adelphotaxon: HYLINA Rafinesque, 1815-|Gray, 1825|.

Getendotaxa: ACRISINIA Mivart, 1869; HyLIOLINIA Dubois, Duellman \& Ohler, 2017.

Comments: Dubois et al. (2017) provided a detailed discussion of the status of the nomen ACRIDINA, introduced by Mivart (1869: 299) for a subfamily, and emended by Kuhn (1965: 96) into ACRIDINAE. In order to resolve the nomenclatural problem posed by the homonymy between this nomen and the nomen ACRIDIDAE Macleay, 1821 (ORTHOPTERA), they emended the amphibian nomen into ACRISINAE, using the whole generic nomen Acris as stem for this family-series nomen. In order to validate formally this emendation, they announced their intention to submit the case to the Commission, but did not do it yet, in view of the slowness or failure of the latter to deal with cases submitted to it, as had already been the case for many other nomenclatural problems concerning amphibians, in the past but even recently (see Dubois 2005b: 417-418; Dubois et al. 2019: 52). This action should now be undertaken by anyone having more trust in the Commission's efficiency. 
Eunym: Hoc loco.

Getangiotaxon: ACRISINA Mivart, 1869.

Adelphotaxon: HyLIOLINIA Dubois, Duellman \& Ohler, 2017.

Getendotaxon: Acris Duméril \& Bibron, 1841.

\title{
F.21.15. Infratribus HYLIOLINIA Dubois, Duellman \& Ohler, 2017
}

Protonym: HyLIOLINAE Dubois, Duellman \& Ohler, 2017: 55 [bF].

Eunym: Hoc loco.

Getangiotaxon: ACRISINA Mivart, 1869.

Adelphotaxon: ACRISINIA Mivart, 1869.

Getendotaxa: Hyliola Mocquard, 1899; Pseudacris Fitzinger, 1843.

F.20.24. Subtribus HyLINA Rafinesque, 1815-|Gray, 1825|

\author{
Eunym: Hoc loco. \\ Getangiotaxon: HYLINI Rafinesque, 1815-|Gray, 1825|. \\ Adelphotaxon: ACRISINA Mivart, 1869. \\ Getendotaxa: Hylinia Rafinesque, 1815-|Gray, 1825|; PLECTROHYLINIA nov.
}

Comments: This subtribe accommodates two highly supported branches recognised in our classification as the infratribes HYLINIA and PLECTROHYLINIA. The latter includes the genera Exerodonta and Plectrohyla, whereas the former includes three hypotribes, CHARADRAHYLINOA, HYLINOA and RHEOHYLINOA, of unresolved relationships. The first of these hypotribes includes the genera Charadrahyla and Megastomatohyla. The other hypotribes are discussed below.

\section{F.21.16. Infratribus HYLINIA Rafinesque, 1815-|Gray, 1825|}

\author{
Eunym: Hoc loco. \\ Getangiotaxon: HYLINA Rafinesque, 1815-|Gray, 1825|. \\ Adelphotaxon: PLECTROHYLINIA nov. \\ Getendotaxa: CHARADRAHYLINOA nov.; HYLINOA Rafinesque, 1815-|Gray, 1825|; RHEOHYLINOA nov.
}

Comments: The infratribe HYLINIA includes three well supported branches that are recognised as the hypotribes CHARADRAHYLINOA, HYLINOA and RHEOHYLINOA.

Pyron \& Wiens (2011), Smith et al. (2007), Wiens et al. (2010), Duellman et al. (2016), and Hutter et al. (2017) found highly supported relationships between Charadrahyla and Megastomatohyla, our CHARADRAHYLINOA, whereas in Faivovich et al. (2005) Chararahyla was outgroup to the taxon including Hyla, and Megastomatohyla outgroup to the rest of our HYLINIA but Charadrahyla.

The hypotribe HYLINOA splits into two well supported branches, the clans HYLITES, for Dryophytes and Hyla, and TRIPRIONITES, with the subclans ISTHMOHYLITIES, for Isthmohyla, TLALOCOHYLITIES, for Tlalocohyla, and TRIPRIONITIES. This latter taxon holds three subgroups of unsupported relationships, recognised as infraclans in our classification: Diaglenitoes for Diaglena, Smiliscitoes for Smilisca, and TRIPRIONITOES for Anotheca and Triprion.

In Faivovich et al. (2005), Smith et al. (2007), Duellman et al. (2010), Wiens et al. (2010) and Duellman et al. (2016), as well as in TREE, Isthmohyla is sister-taxon to a group that includes (Anotheca and Triprion) and Smilisca. As Triprion is rendered paraphyletic by the position of Anotheca (Smith et al. 2007; Pyron \& Wiens 2011), we recognised, as did Wiens et al. (2010) and Duellman et al. (2016), the genus Diaglena for Triprion spatulatus; others synonymised Anotheca and Diaglena with Triprion, a genus that then encloses our TRIPRIONITIES, and did not take into account long recognised morphological differentiations within this group. The highly supported sister-group relationship of 
Smilisca with TRIPRIONITOES in the tree of Pyron \& Wiens (2011) is not recovered in TREE. Inversely, Pyron \& Wiens (2011) only had a bootstrap support of 77 for the relationship within two taxa of their Hyla, our HyLITES, but this taxon has high support in Smith et al. (2007) and Duellman et al. (2016). Here we follow the latter authors, who recognised the highly supported subgroups as genera.

The RHEOHYLINOA, third taxon within the HYLINIA includes three clans: ECNOMIOHYLITES for Ecnomiohyla, PTYCHOHYLITES for Atlantihyla, Bromeliohyla, Duellmanohyla, Ptychohyla and Quilticohyla, and RHEOHYLITES for Rheohyla. The relationships within them are not sufficiently supported for recognising further taxa. The recognition of the genera Rheohyla for Hyla miotympanum and Bromeliohyla for Hyla bromeliacia rendered Ecnomiohyla holophyletic (Duellman et al. 2016). We also follow Wiens et al. (2010) in transferring Hyla salvadorensis from Ptychohyla to Duellmanohyla.

This group allows to stress a point that applies indeed to most of the phylogeny and taxonomy of amphibians, i.e. the fact that molecular evidence is growing quickly while morphological, anatomical or etho-ecological characters are not studied and analysed in the same path. As a result, the argumentation in support of new taxa, although they fulfil the first command of holophyly, is poor concerning biological and evolutionary significance. This explains the weak concepts used in many taxonomic decisions, particularly at low taxonominal ranks. Some authors (e.g. Faivovich et al. 2005) have complained about this in hylids, but this applies to most of the taxonomy of amphibians, in which most taxa are just defined by diagnoses or idiognoses but not by apognoses (for the distinctions between these concepts, see Dubois $2017 d$ and the M\&M section above).

\section{F.22.12. Hypotribus CHARADRAHYLINOA nov.}

Getangiotaxon: HYLINIA Rafinesque, 1815-|Gray, 1825|.

Adelphotaxa: HyLINOA Rafinesque, 1815-|Gray, 1825|; RHEOHYLINOA nov.

Getendotaxa: Charadrahyla Faivovich, Haddad, Garcia, Frost, Campbell \& Wheeler, 2005; Megastomatohyla Faivovich, Haddad, Garcia, Frost, Campbell \& Wheeler, 2005.

Nucleogenus, by present designation:Charadrahyla Faivovich, Haddad, Garcia, Frost, Campbell \&

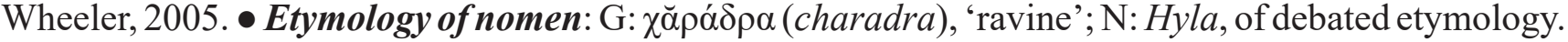

- Stem of nomen: Charadrahyl-.

Diagnosis: Medium to large sized frogs; dorsum color green or brown with darker blotches or spots; limbs banded; palpebral membrane clear; fingers one-third to two thirds webbed; toes about three-forth webbed; dermal appendages absent; fringes absent; an axillary membrane usually present but sometimes absent; vocal sac absent or a barely distensible single, median subgular, vocal sac; nuptial pads usually present; anterior arm of squamosal not extending to the maxillary; prevomerine teeth present; tadpoles mouth ventral; 2-7 upper and 3-11 lower keratodont rows. \{Duellman 1970; Canseco-Marquez et al. 2017; Jiménez-Arcos et al. 2019\}.

As a study of morphological synapomorphies is lacking, the taxon is apognosable by a number of molecular synapomorphies in the DNA sequence of several nuclear, mitochondrial, and ribosomal genes. \{Faivovich et al. 2005\}.

F.22.13. Hypotribus HyLINOA Rafinesque, 1815-|Gray, 1825|

Eunym: Hoc loco.

Getangiotaxon: HYLINIA Rafinesque, 1815-|Gray, 1825|.

Adelphotaxa: CHARADRAHYLINOA nov.; RHEOHYLINOA nov.

Getendotaxa: HyLITES Rafinesque, 1815; TRIPRIONITES Miranda-Ribeiro, 1926.

F.23.08. Clanus HyLITES Rafinesque, 1815-|Gray, 1825|

Eunym: Hoc loco.

Getangiotaxon: HYLINOA Rafinesque, 1815-|Gray, 1825|. 
Adelphotaxon: TRIPRIONITES Miranda-Ribeiro, 1926.

Getendotaxa: Dryophytes Fitzinger, 1843; Hyla Laurenti, 1768.

\title{
F.23.09. Clanus TRIPRIONITES Miranda-Ribeiro, 1926
}

\author{
Protonym: TRIPRIONINAE Miranda-Ribeiro, 1926: 64 [F]. \\ Eunym: Hoc loco. \\ Getangiotaxon: HYLINOA Rafinesque, 1815-|Gray, 1825|. \\ Adelphotaxon: HYLITES Rafinesque, 1815-|Gray, 1825|. \\ Getendotaxa: ISTHMoHyLITIES nov.; TLALOCOHYLITIES nov.; TRIPRIONITES Miranda-Ribeiro, 1926.
}

\section{F.24.04. Subclanus ISTHMOHYLITIES nov.}

Getangiotaxon: TRIPRIONITES Miranda-Ribeiro, 1926.

Adelphotaxa: TLALOCOHYLITIES nov.; TRIPRIONITES Miranda-Ribeiro, 1926.

Getendotaxon: Isthmohyla Faivovich, Haddad, Garcia, Frost, Campbell \& Wheeler, 2005.

Nucleogenus, by present designation: Isthmohyla Faivovich, Haddad, Garcia, Frost, Campbell \&

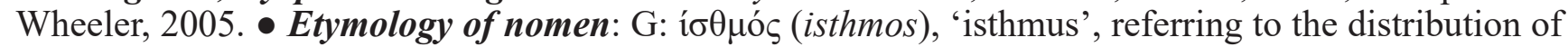
the genus; N: Hyla, of debated etymology. • Stem of nomen: Isthmohyl-.

Diagnosis: Medium sized frogs, dorsum brownish or green, usually mottled or marked by blotches; transverse bands on limbs usually lacking; palpebral membrane clear; fingers up to one-third webbed; toes half to three-forth webbed; axillary membrane usually absent; dermal folds on hindlimbs absent; a single, median subgular vocal sac present; horny nuptial pads on prepollex usually present. \{Duellman $2001\}$.

Morphological characters have not been studied by phylogenetic methods, so synapomorphic characters are not defined but the taxon is apognosable by 42 molecular synapomorphies in the DNA sequences of several nuclear, mitochondrial and ribosomal genes. \{Faivovich et al. 2005\}.

\section{F.24.05. Subclanus TLALOCOHYLITIES nov.}

Getangiotaxon: TRIPRIONITES Miranda-Ribeiro, 1926.

Adelphotaxa ISTHMOHYLITIES nov.; TRIPRIONITES Miranda-Ribeiro, 1926.

Getendotaxon: Tlalocohyla Faivovich, Haddad, Garcia, Frost, Campbell \& Wheeler, 2005.

Nucleogenus, by present designation: Tlalocohyla Faivovich, Haddad, Garcia, Frost, Campbell \& Wheeler, 2005. • Etymology of nomen: R: Tlaloc, the Olmec god of the rain; N: Hyla, of debated etymology. • Stem of nomen: Tlalocohyl-.

Diagnosis: Small to medium sized frogs; dorsum yellowish or light gray; hidden surfaces of legs and webbing or thigh yellow or red; palpebral membrane clear; fingers one-fourth to tree-fifth webbed; toes two-third to three-fourth webbed; dermal appendages and fringes of limbs absent; an axillary membrane present; tympanum visible; vocal sac single, median, subgular; nuptial pads absent or present; skulls weakly to moderately ossified; nasals separated medially; quadratojugals bony and in contact with maxillary; anterior arm of squamosal no more than half of the distance to maxillar; prevomerine teeth present, but may be absent; tadpoles with an anteroventral mouth; two upper three lower keratodont rows; tail with rather deep, terminally pointed fins. \{Duellman 2001 .

Taxon apognosable by 92 molecular synapomorphies in the DNA sequences of several nuclear, mitochondrial and ribosomal genes. \{Faivovich et al. 2005\}. 
Eunym: Hoc loco.

Getangiotaxon: TRIPRIONITES Miranda-Ribeiro, 1926.

Adelphotaxa: ISTHMOHYLITIES nov.; TLALOCOHYLITIES nov.

Getendotaxa: DIAGLENITOES nov.; SMILISCITOES nov.; TRIPRIONITOES Miranda-Ribeiro, 1926.

\section{F.25.15. Infraclanus DIAGLENITOES nov.}

Getangiotaxon: TRIPRIONITIES Miranda-Ribeiro, 1926.

Adelphotaxa: SMILISCITOES nov.; TRIPRIONITOES Miranda-Ribeiro, 1926.

Getendotaxon: Diaglena Cope, 1887.

Nucleogenus, by present designation: Diaglena Cope, 1887. • Etymology of nomen: G: Sió (dia), 'accross'; $\gamma \lambda \eta \dot{v \eta}$ (glene), 'pupilla'; referring to the horizontal shape of the pupilla as stated in the original description (Cope 1887). • Stem of nomen: Diaglen-.

Diagnosis: Large sized species (males SVL 69-87 mm, females SVL 90-101 mm); dorsum color greenish to yellowish with green to yellow flecks of variable extend; tympanum partly hidden; axillary membrane absent; horny nuptial pad on prepollex in breeding males; webbing between fingers I and II absent, between fingers III and IV rudimentary; tarsal fold present; toes about two-thirds webbed; anal flap absent; large prenasals, greatly expanded maxillaries, odontoids on palatines, spines on top of head absent and no dermal sphenethmoid. \{Duellman 2001\}.

\section{F.25.16. Infraclanus SMILISCITOES nov.}

Getangiotaxon: TRIPRIONITIES Miranda-Ribeiro, 1926.

Adelphotaxa: Diaglenitoes nov.; TRIPRIONITOES Miranda-Ribeiro, 1926.

Getendotaxon: Smilisca Cope, 1865.

Nucleogenus, by present designation: Smilisca Cope, 1865. • Etymology of nomen: G: $\sigma \mu \bar{\imath} \lambda \eta($ smile),

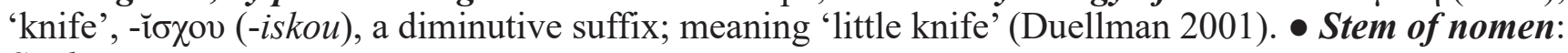
Smilisc-.

Diagnosis: Medium to large sized frogs; a blotched or barred dorsal pattern in green or brown; flanks are mottled, spotted or veined; ventrally white; pupil horizontally elongated; palpebral membrane clear; toes at least three-fourths webbed; paired, subgular greatly distensible vocal sacs; breeding males with horny brown nuptial pads; skull broad, well ossified; lacks dermal co-ossification; nasals moderately slender, separated medially; frontoparietal fontanelle usually present; vomerine teeth present; tadpoles with two upper and three lower keratodont rows; mouth bordered by papillae. \{Faivovich et al. 2005\}.

No morphological synapomorphies (Duellman 2001), but taxon apognosable by 92 molecular synapomorphies in the DNA sequences of several nuclear, mitochondrial and ribosomal genes. $\{$ Faivovich et al. 2005$\}$.

\section{F.25.17. Infraclanus TRIPRIONITOES Miranda-Ribeiro, 1926}

Eunym: Hoc loco.

Getangiotaxon: TRIPRIONITIES Miranda-Ribeiro, 1926.

Adelphotaxa: SMILISCITOES nov.; TRIPRIONItoEs Miranda-Ribeiro, 1926.

Getendotaxa: Anotheca Smith, 1939; Triprion Cope, 1866. 
Getangiotaxon: HYLINIA Rafinesque, 1815-|Gray, 1825|.

Adelphotaxa: HYLINOA Rafinesque, 1815-|Gray, 1825|; CHARADRAHYLINOA nov.

Getendotaxa: ECNOMIOHYLITES nov.; PTYCHOHYLITES nov.; RHEOHYLITES nov.

Nucleogenus, by present designation: Rheohyla Duellman, Marion \& Hedges, 2016. • Etymology of nomen: G: j́ćos (rheos), 'stream', referring to the breeding site of Rheohyla species; N: Hyla, of debated etymology. • Stem of nomen: Rheohyl-.

Diagnosis: Small to medium sized species; dorsum greenish to brownish usually with various markings; palpebral membrane unmarked but some species with pigmentation; fingers one-forth to two-thirds webbed; toes one-third to four-fifth webbed; fringes absent or tubercles in rows, or indented dermal fringes (Ecnomiohyla); an axillary membrane usually absent; a single, median, subgular vocal sac usually present; nuptial pads present; skull moderately ossified; frontoparietal fontanelle present; quadratojugals present, reduced or absent; anterior arm of squamosal extend to one-half of the distance to the maxillary; vomerine teeth present; tadpoles with ventral mouth (funnel-shaped in Duellmanohyla); 1-7 upper, 3-7 lower keratodont rows; moderately long tails, with low web. \{Duellman 2001; Campbell \& Duellman 2000; McCranie \& Castaneda 2006; Duellman et al. 2016; Canseco-Marquez et al. 2017\}.

\section{F.23.10. Clanus ECNOMIOHYLITES nov.}

Getangiotaxon: RHEOHYLINOA nov.

Adelphotaxa: PTYCHOHYLITES nov.; RHEOHYLITES nov.

Getendotaxon: Ecnomiohyla Faivovich, Haddad, Garcia, Frost, Campbell \& Wheeler, 2005.

Nucleogenus, by present designation: Ecnomiohyla Faivovich, Haddad, Garcia, Frost, Campbell \&

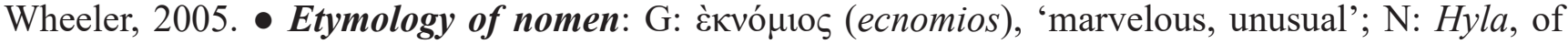
debated etymology. $\bullet$ Stem of nomen: Ecnomiohyl-.

Diagnosis: Medium to large sized species; dorsum green or brownish mottled or not with brown or dark green; palpebral membrane clear or pigmented; hands and feet very large; toe pads large; finger web at least two-thirds webbed; toes web more than three-fourths webbed; indented dermal fringes on outer edge of forearm and fourth finger, and on the outer edge of the foot and fifth toe; a single, median, subgular vocal sac (absent in one species); first finger of adult males with a variously modified propollex; skull moderately well ossified; frontoparietal fontanelle present; in some species co-ossification of skin with the fronto-parietals and squamosals; quadratojugals in bony contact with the maxillary; anterior arm of squamosal extend no more than one-half of the distance to the maxillary; vomerine teeth present \{Duellman 2001; Batista et al. 2014\}.

The included genus can be apognosed by molecular synapomorphies ( 37 transformations in nuclear and mitochondrial protein and ribosomal genes). \{Faivovich et al. 2005\}.

\section{F.23.11. Clanus PTYCHOHYLITES nov.}

Getangiotaxon: RHEOHYLINOA nov.

Adelphotaxa: ECNOMIOHYLITES nov.; RHEOHYLITES nov.

Getendotaxa: Atlantihyla Faivovich, Pereyra, Luna, Hertz, Blotto, Vásquez-Almazán, McCranie, Sánchez, Baêta, AraujoVieira, Köhler, Kubicki, Campbell, Frost, Wheeler \& Haddad, 2018; Bromeliohyla Faivovich, Haddad, Garcia, Frost, Campbell \& Wheeler, 2005; Duellmanohyla Campbell \& Smith,1992; Ptychohyla Taylor, 1944; Quilticohyla Faivovich, Pereyra, Luna, Hertz, Blotto, Vásquez-Almazán, McCranie, Sánchez, Baêta, Araujo-Vieira, Köhler, Kubicki, Campbell, Frost, Wheeler \& Haddad, 2018. 


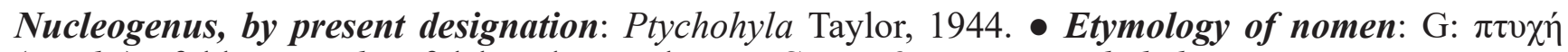
(ptyche), 'fold'; N: Hyla, of debated etymology. • Stem of nomen: Ptychohyl-.

Diagnosis: Small to medium sized species; dorsum green or shades of brown, usually with some markings; palpebral membrane unmarked or with pigmentation; fingers one-fourth to one half webbed; toes onethirds to four-fifths webbed; fringes with a row of tubercles on forearm or without such fringe; axillary membrane absent; vocal sac present, sometimes absent; nuptial pads present; ventrolateral macroglands (but absent in Bromeliohyla and Duellmanohyla); skull moderately ossified; frontoparietal fontanella present; nasals slender, separated medially; quadratojugals usually present; anterior arm of squamosal extending one-third or one-half of distance to the maxillary; vomerine teeth present; tadpoles with a ventral mouth (funnel-shaped in Duellmanohyla); 2-6 upper, 5-7 lower keratodont rows; long tails with low fins. \{Duellman 2001; Campbell \& Duellman 2000; McCranie \& Castaneda 2006; CansecoMarquez et al. 2017\}.

This taxon is apognosable by a number of molecular synapomorphies in the DNA sequences of several nuclear, mitochondrial and ribosomal genes; diagnostic morphological characteristics include a well-developed lingual flange of the pars palatina of the premaxillary (Ptychohyla); tadpoles with dorsoventrally flattened bodies and elongated tails hatching from eggs laid in bromeliad cavities (Bromeliohyla); and red irises, a labial stripe expanded below orbit, lack of nuptial excrescences, ventrally oriented funnel-shaped oral disc in the tadpoles, labial tooth rows reduced in length, and lateral processes on upper jaw sheath absent in Duellmanohyla. \{Faivovich et al. 2005\}.

\section{F.23.12. Clanus RHEOHYLITES nov.}

Getangiotaxon: RHEOHYLINOA nov.

Adelphotaxa: ECNOMIOHYLITES nov.; PTYCHOHYLITES nov.

Getendotaxon: Rheohyla Duellman, Marion \& Hedges, 2016.

\section{F.21.17. Infratribus PLECTROHYLINIA nov.}

Getangiotaxon: HYLINA Rafinesque, 1815-|Gray, 1825|.

Adelphotaxon: HYLINIA Rafinesque, 1815-|Gray, 1825|.

Getendotaxa: Exerodonta Brocchi, 1879; Plectrohyla Brocchi, 1877.

Nucleogenus, by present designation: Plectrohyla Brocchi, 1877. • Etymology of nomen: G: $\pi \lambda \eta े \kappa \tau \rho \circ v$ (plektron), 'spur', referring to the shape of the prepollex; N: Hyla, of debated etymology. $\bullet$ Stem of nomen: Plectrohyl-.

Diagnosis: Small to large frogs (adults SVL 20-90 mm); fingers long with small or absent webbing and rounded pads; toes largely to extensively webbed; tadpoles with moderately depressed body and long, muscular tail with moderately developed fins; oral disc with several rows of papillae; $2-3$ upper and 3-7 lower keratodont rows. \{Duellman \& Campbell 1992; Mendelson \& Campbell 1994; Campbell \& Duellman 2000; Duellman 2001\}.

Apognosable by a number of molecular synapomorphies in the DNA sequences of several nuclear, mitochondrial and ribosomal genes; no morphological synapomorphies are known at present. \{Faivovich et al. 2005$\}$.

\section{F.19.32. Tribus LopHYohyLINI Miranda-Ribeiro, 1926-|Fouquette \& Dubois, 2014|}

Protonyms and eunym: LoPHIOHYLINAE Miranda-Ribeiro, 1926: 64 [F]; |LOPHYOHYLINI Fouquette \& Dubois, 2014: 7| [T]. Getangiotaxon: HYLINAE Rafinesque, 1815-|Gray, 1825|.

Adelphotaxa: DENDROPSOPHINI Fitzinger, 1843; HYLINI Rafinesque, 1815-|Gray, 1825|; SCINAXINI Duellman, Marion \& Hedges, 2016.

Getendotaxa: ITAPOHYLINA nov.; LOPHYOHYLINA Miranda-Ribeiro, 1926-|Fouquette \& Dubois, 2014|; PHYTOTRYADINA nov.; TRACHYCEPHALINA Lutz, 1969. 
Comments: The relationships within the tribe LOPHYOHYLINI are poorly resolved and consequently four subtribes are recognised in our classification: the ITAPOTIHYLINA for Itapotihyla, the LOPHYOHYLINA, detailed below, the PHYTOTRYADINA for Phytotriades and the TRACHYCEPHALINA, detailed below. Due to support values below our limit, within the subtribe LOPHYOHYLINA we recognise three infratribes: the LOPHYOHYLINIA for Phyllodytes (the valid nomen for Lophyohyla), the OSTEOCEPHALINIA for Dryaderces, Osteocephalus and Tepuihyla, and the OsteopiLINIA for Osteopilus. The TRACHYCEPHALINA include three infratribes: the CORYTHOMANTINIA for Corythomantis, the NYCTIMANTINIA for Aparasphenodon, Argenteohyla and Nyctimantis, and the TRACHYCEPHALINIA for Trachycephalus.

Faivovich et al. (2010) found Phyllodytes to be sister-taxon to all other LoPHYOHYLINI (their LOPHIOHYLINI) which is in TREE sister-taxon to a taxon grouping Osteopilus, Tepuihyla and Osteocephalus. Although there are numerous poorly supported taxa in the LoPHYOHYLINI, some relationships seem rather stable. As in TREE, Tepuihyla was recovered sister-taxon to Osteocephalus (Faivovich et al. 2010; Wiens et al. 2010; Pyron \& Wiens 2011; Duellman et al. 2016). Most recent works found Aparasphenodon, Argenteohyla and Nyctimantis forming a holophyletic taxon (Faivovich et al. 2010; Wiens et al. 2010; Pyron \& Wiens 2011; Duellman et al. 2016) as does this group with the genera Trachycephalus and Corythomantis, but the relationships within this taxon shown in these trees have not been confirmed in TREE.

\section{F.20.25. Subtribus ITAPOTIHYLINA nov.}

Getangiotaxon: LорнуонтLINI Miranda-Ribeiro, 1926-|Fouquette \& Dubois, 2014|.

Adelphotaxa: Lophyohylina Miranda-Ribeiro, 1926-|Fouquette \& Dubois, 2014|; PHYTOTRYADINA nov.; TRACHYCEPHALINA Lutz, 1969.

Getendotaxon: Itapotihyla Faivovich, Haddad, Garcia, Frost, Campbell \& Wheeler, 2005.

Nucleogenus, by present designation: Itapotihyla Faivovich, Haddad, Garcia, Frost, Campbell \& Wheeler, 2005. - Etymology of nomen: R: Itapoti (Tupi-Guarani term), itá, 'rock' and poti, 'flower or to flourish', which means lichen or moss, referring to the skin of the frog; N: Hyla, of debated etymology. • Stem of nomen: Itapotihyl-.

Diagnosis: Large sized frogs; dorsum of males bearing small tubercles; skin of flanks tubercular, on forearms web extending to base of penultimate phalange of finger III; presence of indented dermal folds on outer edges of hands and feet; a row of tubercles on posterior edge of jaw; a white subanal fold; dorsum greenish or brownish with darker shades; belly and ventral surfaces of thighs yellowish orange; lips unmarked; tadpole with robust and elongated body, eyes positioned laterally, tail muscle high and obtusely pointed, dorsal fin higher than ventral fin, oral disc anteroventral, with 2 upper and 5 lower keratodont rows. \{Duellman 1974; Pimenta \& Canedo 2007\}.

Apognosable by 122 molecular synapomorphies in the DNA sequences of several nuclear, mitochondrial, and ribosomal genes; a potential morphological synapomorphy is a prominent subcloacal flap. \{Faivovich et al. 2005\}.

\section{F.20.26. Subtribus LOPHYOHYLINA Miranda-Ribeiro, 1926-|Fouquette \& Dubois, 2014|}

Eunym: Hoc loco.

Getangiotaxon: LOPHYOHYLINI Miranda-Ribeiro, 1926-|Fouquette \& Dubois, 2014|.

Adelphotaxa: Itapotihylina nov.; PHYTOTRYAINA nov.; TRACHYCEPHALINA Lutz, 1969.

Getendotaxa: LophYoHYLINIA Miranda-Ribeiro, 1926-|Fouquette \& Dubois, 2014|; OsteOCEPHALINIA nov.; OSTEOPILINIA nov.

F.21.18. Infratribus LOРнYонYLINIA Miranda-Ribeiro, 1926-|Fouquette \& Dubois, 2014|

Eunym: Hoc loco.

Getangiotaxon: LOPHYOHYLINA Miranda-Ribeiro, 1926-|Fouquette \& Dubois, 2014|. 
Adelphotaxa: OSTEOCEPHALINIA nov.; OSTEOPILINIA nov.

Getendotaxon: Phyllodytes Wagler, 1830.

\section{F.21.19. Infratribus OSTEOCEPHALINIA nov.}

Getangiotaxon: LорнуонуLINA Miranda-Ribeiro, 1926-|Fouquette \& Dubois, 2014|.

Adelphotaxa: LopнуонуLINIA Miranda-Ribeiro, 1926-|Fouquette \& Dubois, 2014|; OSTEOPILINIA nov.

Getendotaxa: Dryaderces Faivovich, Padial, Castroviejo-Fisher, Lyra, Berneck, Iglesias, Kok, MacCulloch, Rodrigues, Verdade, Torres-Gastello, Chaparro, Valdujo, Reichle, Moravec, Gvoždík, Gagliardi-Urrutia, Ernst, Riva, Means, Lima, Señaris, Wheeler \& Haddad, 2013; Osteocephalus Steindachner, 1862; Tepuihyla Ayarzagüena, Señaris \& Gorzula, 1993.

Nucleogenus, by present designation: Osteocephalus Steindachner, 1862. • Etymology of nomen: G:

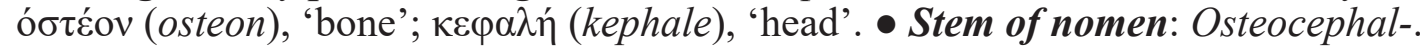

Diagnosis: Small to large sized; dorsal skin with tubercles, which in males usually bear spinules, but females often smooth; dorsum color brownish or green; palpebral membrane clear; pupil horizontal; skull usually broader than long; discs large; fingers basic to half webbed; toes half to almost entirely webbed; tympanum large; vocal sac single or paired, sometimes absent; nuptial pads usually present; skulls well ossified, exostosed and/or co-ossified in some species; dentigerous processus of vomer angular, but also straight; in larvae two upper and three to six lower keratodont rows. \{Duellman \& Trueb 1971; Ayarzagüena et al. 1993; Jungfer \& Hödl 2002; Jungfer et al. 2013; Hoogmoed 2013 .

Apognosable by a number of molecular synapomorphies in the DNA sequences of several nuclear, mitochondrial and ribosomal genes. \{Faivovich et al. 2005\}.

\section{F.21.20. Infratribus OSTEOPILINIA nov.}

Getangiotaxon: LOPHYOHYLINA Miranda-Ribeiro, 1926-|Fouquette \& Dubois, 2014|.

Adelphotaxa: LOPHYOHYLINIA Miranda-Ribeiro, 1926-|Fouquette \& Dubois, 2014|; OsteocePHALINIA nov.

Getendotaxon: Osteopilus Fitzinger, 1843.

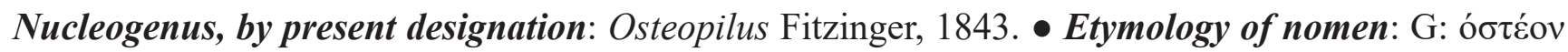
(osteon), 'bone'; זì $\lambda$ os (pilos), 'felt', referring to the finely granular bones of the skull. $\bullet$ Stem of nomen: Osteopil-.

Diagnosis: Medium to large sized frogs; skulls about as long as broad; dermal oofing bones well ossified, exostosed and co-ossified; prenasal and internasal bones absent; dermal sphenethmoid present large, curved vomerine ridge bearing teeth; vocal sac single and subgular; tympanum large; finger and toe pads large and round; in breeding males nuptial pads present; no fringes on hind or forelimbs. \{Trueb \& Tyler 1974$\}$.

Apognosable by 43 molecular synapomorphies in the DNA sequences of several nuclear, mitochondrial and ribosomal genes. \{Faivovich et al. 2005\}.

\section{F.20.27. Subtribus PHYTOTRYADINA nov.}

Getangiotaxon: LOРнYОНYLINI Miranda-Ribeiro, 1926-|Fouquette \& Dubois, 2014|.

Adelphotaxa: ITAPOTIHYLINA nov.; LOPHYOHYLINA Miranda-Ribeiro, 1926-|Fouquette \& Dubois, 2014|; TRACHYCEPHALINA Lutz, 1969.

Getendotaxon: Phytotriades Jowers, Downie \& Cohen, 2009.

Nucleogenus, by present designation: Phytotriades Jowers, Downie \& Cohen, 2009. • Etymology

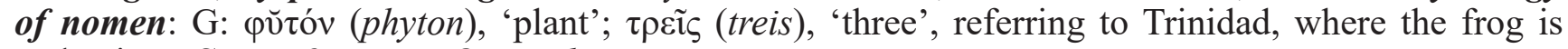
endemic. $\bullet$ Stem of nomen: Osteopil-. 
Diagnosis: Small sized frogs, head slightly broader than long, snout truncate, tympanum hidden; finger and toe tips dilated into well-developed pads; fingers free, toes slightly webbed; skin smooth, brown and gold, with golden longitudinal stripes; on lower jaw a series of fine bony tooth-like serrations, decreasing in size from the symphysis; single subgular vocal sac. \{Boulenger 1917; Kenny 1969; Jowers et al. 2008 \}.

\section{F.20.28. Subtribus TRACHYCEPHALINA Lutz, 1969}

Protonym: TRACHYCEPHALINAE Lutz, 1969: 275 [bF].

Eunym: Hoc loco.

Getangiotaxon: LOPHYOHYLINI Miranda-Ribeiro, 1926-|Fouquette \& Dubois, 2014|.

Adelphotaxa: Itapotihylina nov.; Lophyohylina Miranda-Ribeiro, 1926-|Fouquette \& Dubois, 2014|; PhYTOTRYADINA nov.

Getendotaxa: CoRYthomantinia nov.; NYCTIMANTINIA nov.; TRACHYCEPHALINIA Lutz, 1969.

\section{F.21.21. Infratribus CORYTHOMANTINIA nov.}

Getangiotaxon: TRACHYCEPHALINA Lutz, 1969.

Adelphotaxa: NYCTIMANTINIA nov.; TRACHYCEPHALINIA Lutz, 1969.

Getendotaxon: Corythomantis Boulenger, 1896.

Nucleogenus, by present designation: Corythomantis Boulenger, 1896. • Etymology of nomen: G:

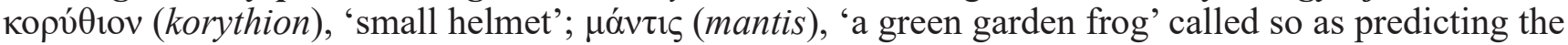
weather. $\bullet$ Stem of nomen: Corythomant-.

Diagnosis: Large sized frogs with depressed head, skull longer than broad, with projecting labial borders, surface of dermal roofing bones consisting of reticulate network of ridges; nasals concealed with alary process of premaxillaries; vomerine teeth present; tympanum distinct; fingers free, toes two-thirds webbed; tips dilated into pads; single, median, subgular vocal sac. \{Boulenger 1896; Trueb 1970a; Pombal et al. 2012\}.

Apognosable by 132 molecular transformations in the DNA sequences of several nuclear, mitochondrial and ribosomal genes. \{Faivovich et al. 2005\}.

\section{F.21.22. Infratribus NYCTIMANTINIA nov.}

Getangiotaxon: TRACHYCEPHALINA Lutz, 1969.

Adelphotaxa: CORYTHOMANTINIA nov.; TRACHYCEPHALINIA Lutz, 1969.

Getendotaxa: Aparasphenodon Miranda-Ribeiro, 1920; Argenteohyla Trueb, 1970; Nyctimantis Boulenger, 1882.

Nucleogenus, by present designation: Nyctimantis Boulenger, 1882. • Etymology of nomen: G: vó $\xi$

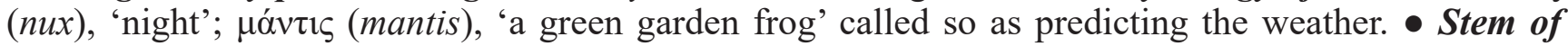
nomen: Nyctimant-.

Diagnosis: Medium sized frogs; dorsum skin smooth; skull longer than broad or slightly broader than long; discs moderate; fingers with reduced webbing; toes up to two-thirds webbed; vocal sac single or paired (Argenteohyla); dermal ornation on skulls present; canthal ridges distinct; palatine bones present; vomerine teeth present. \{Trueb 1970a-b; Duellman \& Trueb 1976\}.

Apognosable by a number of molecular transformations in the DNA sequences of several nuclear, mitochondrial and ribosomal genes; diagnostic morphological characters include a prenasal bone (Aparasphenodon); articulation of the zygomatic ramus of the squamosal with the pars fascialis of the maxillary, and reduced finger and toe discs (Argenteohyla); and an irregular orbital flange in the frontoparietal, and sphenethmoid concealed dorsally by frontoparietals and nasals in Nyctimantis. $\{$ Faivovich et al. 2005\}. 
Eunym: Hoc loco.

Getangiotaxon: TRACHYCEPHALINA Lutz, 1969.

Adelphotaxa: CoRYTHOMANTINIA nov.; NYCTIMANTINIA nov.

Getendotaxon: Trachycephalus Tschudi, 1838.

\section{F.19.33. Tribus SCINAXINI Duellman, Marion \& Hedges, 2016}

Protonym: SCINAXINAE Duellman, Marion \& Hedges, 2016: 3, 25 [bF].

Eunym: Hoc loco.

Getangiotaxon: HYLINAE Rafinesque, 1815-|Gray, 1825|.

Adelphotaxa: DENDROPSOPHINI Fitzinger, 1843; HYLINI Rafinesque, 1815-|Gray, 1825|; LoPHYOHYLINI Miranda-Ribeiro, 1926-|Fouquette \& Dubois, 2014|.

Getendotaxa: SCINAXINA Duellman, Marion \& Hedges, 2016; SpHaEnorhynCHINA Faivovich et al., 2018.

Comments: This taxon was documented by Wiens et al. (2010), Pyron \& Wiens (2011) and Faivovich et al. (2018), and recognised as a subfamily by Duellman et al. (2016). These authors recognised the genera Ololygon Fitzinger, 1843 and Julianus Duellman, Marion \& Hedges, 2016, as distinct from Scinax, but Lourenço et al. (2016) and Faivovich et al. (2018) considered these two nomina as synonyms of the latter. We follow them.

The recent erection of the well-supported genus Gabohyla, for the species Sphaenorhynchus pauloalvini not represented in TREE, leads us to recognise two subtribes SCINAXINA and SPHAENORHYNCHINA in this tribe.

Wagler (1830: 201) provided the etymology of his generic nomen Scinax: " $\Sigma \kappa \imath \alpha \xi$ agilis ad

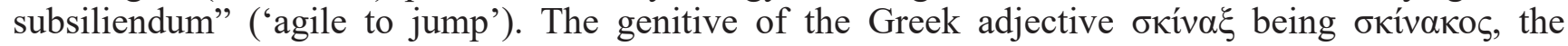
subfamilial nomen coined by Duellman et al. (2016) should have been spelt SCINACINAE, and the incorrect original spelling should have been corrected before 2000, but it is no more the case under the 1999 Code because of the new Article 29.4, which states that now such incorrect spellings should not be corrected, a highly confusing Rule (see Dubois \& Aescht 2019o: 125-126).

\section{F.20.29. Subtribus SCINAXINA Duellman, Marion \& Hedges, 2016}

Protonym: SCINAXINAE Duellman, Marion \& Hedges, 2016: 3, 25 [bF].

Eunym: Hoc loco.

Getangiotaxon: SCINAXINI Duellman, Marion \& Hedges, 2016.

Adelphotaxon: SpHaENorhynChina Faivovich, Pereyra, Luna, Hertz, Blotto, Vásquez-Almazán, McCranie, Sánchez, Baêta, Araujo-Vieira, Köhler, Kubicki, Campbell, Frost, Wheeler \& Haddad, 2018.

Getendotaxon: Scinax Wagler, 1830.

\section{F.20.30. Subtribus SPHAENORHYNCHINA Faivovich et al., 2018}

Protonym: Sphaenorhynchini Faivovich, Pereyra, Luna, Hertz, Blotto, Vásquez-Almazán, McCranie, Sánchez, Baêta, Araujo-Vieira, Köhler, Kubicki, Campbell, Frost, Wheeler \& Haddad, 2018: 25 [T].

Eunym: Hoc loco.

Getangiotaxon: SCINAXINI Duellman, Marion \& Hedges, 2016.

Adelphotaxon: SCINAXINA Duellman, Marion \& Hedges, 2016.

Getendotaxa: Gabohyla Araujo-Vieira, Luna, Caramaschi \& Haddad, 2020; Sphaenorhynchus Tschudi, 1838.

\section{F.17.25. Familia PHYLLOMEdusidaE Günther, 1858}

Protonym and eunym: PHYLLOMEDUSIDAE Günther, 1858: 346 [F]. 
Getangiotaxon: HYLOIDEA Rafinesque, 1815-|Gray, 1825|.

Adelphotaxon: HYLIDAE Rafinesque, 1815-|Gray, 1825|.

Getendotaxa: PelodRYAdinaE Günther, 1859; PHYLLOMEDUSINAE Günther, 1858.

Comments: Most recent authors (e.g. Bossuyt \& Roelants 2009) considered the PELODRYADIDAE and the PHYLLOMEDUSIDAE as two distinct families. However, on the basis of TREE, we consider that they constitute together the sister-taxon to the family HYLIDAE whose rank is fixed by the [UQC]. By virtue of the [STC], they should therefore be lumped as two subfamilies of a single family for which the valid nomen, according to the Principle of Priority, is PHYLLOMEDUSIDAE.

\section{F.18.38. Subfamilia PELODRYADINAE Günther, 1859}

Protonym: PELODRYADIDAE Günther, 1859: ix, 119 [F].

Eunym: Dowling \& Duellman 1978: 37.1.

Getangiotaxon: PHYLLOMEDUSIDAE Günther, 1858.

Adelphotaxon: PHYLLOMEDUSINAE Günther, 1858.

Getendotaxa: Litoria Tschudi, 1838; Nyctimystes Stejneger, 1916; Ranoidea Tschudi, 1838.

Comments: As stated by Faivovich et al. (2010), we encounter "almost complete ignorance about phylogenetic relationships within the PELODRYADINAE", and therefore, as we have no evidence for further resolution of the relationships within this taxon, here we recognise the three highly supported groups as the genera Litoria, Nyctimystes and Ranoidea. Numerous currently synonymous genus- and family-series nomina (see Dubois \& Frétey 2016 and Appendices A5.NGS and A6.NFS) are available for further subdivisions of this species-rich assemblage, but they must await further taxonomic and phylogenetic studies to be re-evaluated.

\section{F.18.39. Subfamilia PHYLLOMEDUSINAE Günther, 1858}

Eunym: Miranda-Ribeiro 1926: 64.

Getangiotaxon: HYLOIDEA Rafinesque, 1815-|Gray, 1825|.

Adelphotaxon: PELODRYADINAE Günther, 1859.

Getendotaxa: AgalyChini nov.; CRUZiohylini nov.; Phrynomedusini nov.; Phyllomedusini Günther, 1858.

Comments: The relationships within this subfamily do not have sufficient support for a resolved classification, so four groups must be recognised as tribes: AGALYCHNINI for the genera Agalychnis and Hylomantis, CRUZIOHYLINI for the genus Cruziohyla, PHRYNomedusini for the genus Phrynomedusa, and PHYLLOMEDUSINI. This latter tribe shows resolved relationships, with a subtribe PHASMAHYLINA for the genus Phasmahyla, being sister-taxon to a subtribe PHYLLOMEDUSINA. Within this latter subtribe, the intratribe PHYLLOMEDUSINIA, corresponding to genus Phyllomedusa, is sister-group to the PItHECOPODINIA, which groups the genera Callimedusa and Pithecopus. These relationships are largely confirmed by all recent studies. Faivovich et al. (2010) and Duellman et al. (2016) found sister-taxa relationship between Agalychnis and Hylomantis, our AGALYCHNINI, and similar relationships between the taxa that we recognise as PHYLLOMEDUSINI.

\section{F.19.34. Tribus AGALYCHNINI nov.}

Getangiotaxon: PHYLLOMEDUSINAE Günther, 1858.

Adelphotaxa: CruZiohylini nov.; Phrynomedusini nov.; Phyllomedusini Günther, 1858.

Getendotaxa: Agalychnis Cope, 1864; Hylomantis Peters, 1873.

Nucleogenus, by present designation: Agalychnis Cope, 1864. • Etymology of nomen: G: áya (aga),

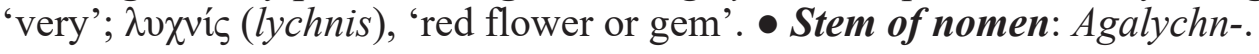


Diagnosis: Small to large sized frogs with a green dorsum; white, yellow or orange ventrally; pupil vertical, iris red or yellow; palpebral membrane reticulated; fingers and toes at least half webbed; toe pads large; first toe shorter than second and not opposable to the others; a single, median subgular vocal sac; skin of dorsum smooth or granulate; if present, poorly developed parotoid glands; no cranial coossification; breeding males with horny brown nuptial pads on finger I; skull shallow, parietal slopes downward anteriorly; large frontoparietal fontanelle, moderately developed squamosals with short anterior arms not extending beyond one-half distance to maxillary; nasals large, narrowly separated medially; sphenethmoid only moderately ossified; teeth on premaxillaries, maxillaries and vomers; pelagic tadpoles; with terminal mouth, anteriorly directed; mouth with two or three rows of papillae, but median part of upper lip free of papillae; 2 upper and 3 lower rows of keratodonts; caudal musculature slender, ventral fin deeper than dorsal fin; apognosable by a number of molecular transformations in the DNA sequences of several nuclear, mitochondrial, and ribosomal genes; diagnostic morphological characters include extensive webbing on the hands and feet and a yellow, red, or dark red iris in Agalychnis. \{Duellman 1970; Faivovich et al. 2005\}.

\section{F.19.35. Tribus CRUZIOHYLINI nov.}

Getangiotaxon: PhYLLOMEDUSINAE Günther, 1858.

Adelphotaxa: AgalyChnini nov.; Phrynomedusini nov.; Phyllomedusini Günther, 1858.

Getendotaxon: Cruziohyla Faivovich, Haddad, Garcia, Frost, Campbell \& Wheeler, 2005.

Nucleogenus, by present designation: Cruziohyla Faivovich, Haddad, Garcia, Frost, Campbell \& Wheeler, 2005. • Etymology of nomen: P: Carlos Alberto Gonçalves da Cruz (1944-), herpetologist, Brasil; N: Hyla, of debated etymology. • Stem of nomen: Cruziohyl-.

Diagnosis: Medium to large sized frogs; moderate to large distinct tympanum; fingers and toes moderately to extensively webbed; snout in profile sloping or truncate; green dorsum with speckles or spots; barring of various extension on lateral surfaces of flanks; dermal flaps on heel, tarsus or forelimbs and lower jaw; morphological synapomorphies include the extensive hand and foot webbing and the development of tadpoles in water-filled depressions on fallen trees. \{Faivovich et al. 2005$\}$.

Apognosable by 171 molecular transformations in the DNA sequences of several nuclear, mitochondrial and ribosomal genes. \{Faivovich et al. 2005; Gray 2018\}.

\section{F.19.36. Tribus PHRYNOMEDUSINI nov.}

Getangiotaxon: PHYLLOMEDUSINAE Günther, 1858.

Adelphotaxa: AgALYCHNINI nov.; CRUZIOHYLINI nov.; PHYLLOMEDUSINI Günther, 1858.

Getendotaxon: Phrynomedusa Miranda-Ribeiro, 1923.

Nucleogenus, by present designation: Phrynomedusa Miranda-Ribeiro, 1923. • Etymology of nomen: G: $\varphi \rho v ́ v \eta$ (phryne), 'toad'; $\mu \varepsilon ́ \delta o v \sigma \alpha$ (medousa), name of a Gorgon, from $\mu \varepsilon ́ \delta \omega$ (medo), 'rule over'. • Stem of nomen: Phrynomedus-.

Diagnosis: Small treefrogs; iris bicolored with a diffuse horizontal dark stripe; palpebral reticulation absent; dorsum smooth; parotoid glands absent; dorsolateral glands absent; vocal sacs present; nuptial pads keratinised and covering metacarpus and proximal phalanx; webbing absent between finger I and II, reduced between others; flanks, medial and lateral regions of thighs without flash color ornamentation; cloacal opening at upper level of thighs; calcar triangular on tarsus; webbing between toes reduced; U-shaped aponeurosis of musculus intermandibularis and musculus interhyoideus; posterolateral elements of musculus intermandibularis inserting on aponeurosis; posterolateral elements of musculus intermandibularis triangular; third ramus of depressor mandibulae absent; tadpoles with complete row of marginal papillae in oral disc. \{Faivovich et al. 2005\}.

Apognosable by 171 molecular transformations in the DNA sequences of several nuclear, mitochondrial, and ribosomal genes; diagnostic morphological characters include extensive webbing 
on the hands and feet, and development of larvae in the cavities of fallen trees. \{Faivovich et al. 2005; Baêta et al. 2016\}.

\section{F.19.37. Tribus PHYLLOMEDUSINI Günther, 1858}

Eunym: Hoc loco.

Getangiotaxon: PHYLLOMEDUSINAE Günther, 1858.

Adelphotaxa: AGALYCHNINI nov.; CRUZIOHYLINI nov.; PHRYNOMEDUSINI nov.

Getendotaxa: Phasmahylina nov.; PhyLlomedusina Günther, 1858.

\section{F.20.31. Subtribus Phasmahylina nov.}

Getangiotaxon: PhYLLOMEDUSINI Günther, 1858.

Adelphotaxon: PhyLLOMEdusina Günther, 1858.

Getendotaxon: Phasmahyla Cruz, 1991

Nucleogenus, by present designation: Phasmahyla Cruz, 1991. • Etymology of nomen: G: pó $\sigma \mu \alpha$ (phasma), 'monster, phantom'; N: Hyla, of debated etymology. • Stem of nomen: Phasmahyl-.

Diagnosis: Small sized phyllomedusids (SVL 29-46 mm); dorsal skin showing moderate rugosity; arms and legs with bluish rounded spots; ventral parts whitish; slits of vocal sacs absent; two superior branches of squamosal present, about half the length of inferior branch, articulated with posterior branch of pterygoid at level of occipital condyles; quadratojugal present; processus cultriform of parasphenoid truncate and serrated; prevomer poorly developed without teeth; alar processus of premaxillar poorly developed and dorsally directed; parotoid glands absent, but a pair of dorsolateral glands; digital pads rounded, moderate; webbing on hand absent or rudimentary, on feet rudimentary; nuptial pad of males composed of numerous horny granules distributed to antepenultimate phalange of first finger; carpal tubercle developed and oval, subarticular tubercles developed, conical and slightly projected; internal metatarsal tubercle small and oval; presence of a small rounded appendix on tibiotarsal articulation; tibia slim, longer than femur; spawning in rolled or gathered leaves above water surface; tadpoles with mouth in anterodorsal position with a dermal funnel-shaped fold, its surface covered with papillae of different size; one series of keratodonts superior to mouth, two series inferior; tadpoles living in mountain creeks and streams in forested mountains. \{Cruz 1991\}.

F.20.32. Subtribus PHYLLOMEDUSINA Günther, 1858

Eunym: Hoc loco.

Getangiotaxon: PHYLLOMEDUSINI Günther, 1858.

Adelphotaxon: PhASMAHYLINA nov.

Getendotaxa: Phyllomedusinia Günther, 1858; PItheCopodinia Lutz, 1969.

F.21.24. Infratribus PHYLLOMEDUSINIA Günther, 1858

Eunym: Hoc loco.

Getangiotaxon: PhyLlomedusina Günther, 1858.

Adelphotaxon: PITHECOPODINIA Lutz, 1969.

Getendotaxon: Phyllomedusa Wagler, 1830.

F.21.25. Infratribus PITHECOPODINIA Lutz, 1969

Protonym: PITHECOPINAE Lutz, 1969: 274 [bF]. 
Eunym: Hoc loco.

Getangiotaxon: PHYLLOMEDUSINA Günther, 1858.

Adelphotaxon: PHYLLOMEDUSINIA Günther, 1858.

Getendotaxa: Callimedusa Duellman, Marion \& Hedges, 2016; Pithecopus Cope, 1866.

F.14.07. Superfamilia LEPTODACTYLOIDEA ||Tschudi, 1838||-Werner, 1896

Protonyms: ||CYSTIGNATHI Tschudi, 1838: 25|| [F]; LEPTODACTYLIDAE Werner, 1896: 357 [F].

Eunym: Reig 1972: 29.

Getangiotaxon: HyLOBATRACHIA Ritgen, 1828.

Adelphotaxa: Bufonoidea Gray, 1825; Centrolenoidea Taylor, 1951; Ceratophryoidea Tschudi, 1838; Hyloidea Rafinesque, 1815-|Gray, 1825|; 1 GIS (Ancudia Philippi, 1902).

Getendotaxon: LEPTODACTYLIDAE ||Tschudi, 1838\|-Werner, 1896.

Comments: The extent of the family LEPTODACTYLIDAE has much changed following the results of recent phylognetic studies. Pyron \& Wiens (2011) proposed three subfamilies in this family, the LEIUPERINAE, LEPTODACTYLINAE and PARATELMATOBIINAE. Here, following our rationale, this branch is recognised as the superfamily LEPTODACTYLOIDEA and includes four subfamilies. The taxon LEPTODACTYLIDAE is referred to the rank family because of the [UQC], whereas the four taxa it contains are recognised as the subfamilies LEIUPERINAE, LEPTODACTYLINAE, PARATELMATOBIINAE and PSEUDOPALUDICOLINAE on account of the [NTC]. Although we confirm the holophyly of the LEPTODACTYLOIDEA, on the contrary of Grant et al. (2017), the relationships between the four main taxa it contains here recognised as subfamilies are not sufficiently supported to allow for a resolved classification.

\section{F.17.26. Familia LEPTODACTYLIDAE ||Tschudi, 1838\|-Werner, 1896}

Eunym: Werner 1896: 357.

Getangiotaxon: LEPTODACTYLOIDEA ||Tschudi, 1838||-Werner, 1896.

Adelphotaxon: None.

Getendotaxa: LEIUPERINAE Bonaparte, 1850: LEPTODACTYLINAE ||Tschudi, 1838||-Werner, 1896; PARATELMATOBIINAE Ohler \& Dubois, 2012; PSEUDOPALUDICOLINAE_Gallardo, 1965.

\section{F.18.40. Subfamilia LEIUPERINAE Bonaparte, 1850}

Protonym: LEIUPERINA Bonaparte, 1850: plate [bF].

Eunym: Pyron \& Wiens 2011: 574.

Getangiotaxon: LEPTODACTYLIDAE \|Tschudi, 1838\|-Werner, 1896.

Adelphotaxa:LEPTODACTYLINAE||Tschudi, 1838||-Werner, 1896; PARATELMATOBIINAEOhler\&Dubois, 2012; PSEUDOPALUDICOLINAE Gallardo, 1965.

Getendotaxa: LEIUPERINI Bonaparte, 1850; PALUDICOLINI Mivart, 1869.

Comments: Grant et al. (2006) showed Pleurodema to be sister-taxon to a taxon grouping Edalorhina and Physalaemus. In TREE and in Lourenço et al. (2015), Pleurodema is sister-group to all other members of the LEIUPERINAE, and these two groups are recognised here as the tribes LEIUPERINI and PALUDICOLINI. In our taxonomy, the latter tribe includes two taxa, the subtribe EDALORHININA for Edalorhina, sistergroup to the PALUDICOLINA, including the genera Engystomops, Eupemphix and Physalaemus, of poorly supported relationships. In Lourenço et al. (2015), Edalorhina appears as sister-genus to Engystomops, and together they are sister-group to Physalaemus which includes two taxa, their 'Physalaemus signifer clade', our Eupemphix, and their 'Physalaemus cuvieri clade', our Physalaemus. In TREE, we do not have enough support for a genus Physalaemus including both groups to be recognised as a single taxon, so we validate Eupemphix. 
Eunym: Hoc loco.

Getangiotaxon: LEIUPERINAE Bonaparte, 1850.

Adelphotaxon: PALUDICOLINI Mivart, 1869.

Getendotaxon: Pleurodema Tschudi, 1838.

F.19.39. Tribus PALUdICOLINI Mivart, 1869

Protonym: PALUDICOLINA Mivart, 1869: 290 [bF].

Eunym: Hoc loco.

Getangiotaxon: LEIUPERINAE Bonaparte, 1850.

Adelphotaxon: LEIUPERINI Bonaparte, 1850.

Getendotaxa: EDALORHININA nov.; PALUDICOLINA Mivart, 1869.

\section{F.20.33. Tribus EDALORHININA nov.}

Getangiotaxon: PALUDICOLINI Mivart, 1869.

Adelphotaxon: PALUDICOLINA Mivart, 1869.

Getendotaxon: Edalorhina Jiménez de la Espada, 1870.

Nucleogenus, by present designation: Edalorhina Jiménez de la Espada, 1870. • Etymology of nomen:

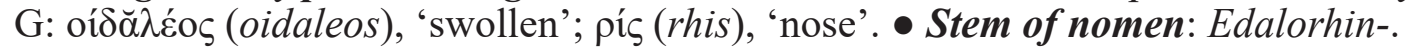

Diagnosis: South American foam-nesting frogs diagnosable by macroglands present, flash coloration on the thighs, eggs laid in a foam nest during amplexus, spiky dermal projections over the eye, and diploid karyotype $2 \mathrm{n}=22$. \{Lourenco et al. 2000; Faivovich et al. 2012\}.

\section{F.20.34. Tribus PALUDICOLINA Mivart, 1869}

Eunym: Hoc loco.

Getangiotaxon: PALUDICOLINI Mivart, 1869.

Adelphotaxon: EDALORHININA nov.

Getendotaxa: Engystomops Jiménez de la Espada, 1872; Eupemphix Steindachner, 1863; Physalaemus Fitzinger, 1826.

\section{F.18.41. Subfamilia LEPTODACTYLINAE ||Tschudi, 1838||-Werner, 1896}

Eunym: Metcalf 1926: 272.

Getangiotaxon: LEPTODACTYLIDAE ||Tschudi, 1838\|-Werner, 1896.

Adelphotaxa: LeIUPERINAE Bonaparte, 1850; PARATELMATOBIINAE Ohler \& Dubois, 2012; PSEUDOPALUDICOLINAE_Gallardo, 1965.

Getendotaxa: ADENOMERINI Hoffmann, 1878; LEPTODACTYLINI ||Tschudi, 1838||-Werner, 1896.

\section{F.19.40. Tribus ADENOMERINI Hoffmann, 1878}

Protonym: ADENOMERIDAE Hoffmann, 1878: 613 [bF].

Eunym: Hoc loco.

Getangiotaxon: LEPTODACTYLINAE ||Tschudi, 1838\|-Werner, 1896.

Adelphotaxon: LEPTODACTYLINI ||Tschudi, 1838||-Werner, 1896.

Getendotaxa: Adenomera Steindachner, 1867; Lithodytes Fitzinger, 1843. 
Eunym: Hoc loco.

Getangiotaxon: LEPTODACTYLINAE ||Tschudi, 1838||-Werner, 1896.

Adelphotaxon: ADENOMERINI Hoffmann, 1878.

Getendotaxon: Leptodactylus Fitzinger, 1826.

F.18.42. Subfamilia PaRatelmatobinate Ohler \& Dubois, 2012

Protonym and eunym: PARATELMATOBIINAE Ohler \& Dubois, 2012: 613 [bF].

Getangiotaxon: LEPTODACTYLIDAE ||Tschudi, 1838||-Werner, 1896.

Adelphotaxa: LEIUPERINAE Bonaparte, 1850; LEPTODACTYLINAE ||Tschudi, 1838||-Werner, 1896; PSEUDOPALUDICOLINAE Gallardo, 1965.

Getendotaxa: Crossodactylodes Cochran, 1938; Rupirana Heyer, 1999.

\section{F.18.43. Subfamilia PSEUdOPALUDICOLINAE Gallardo, 1965}

Protonym and eunym: PSEUDOPALUDICOLINAE Gallardo, 1965: 84 [bF].

Getangiotaxon: LEPTODACTYLIDAE ||Tschudi, 1838\|-Werner, 1896.

Adelphotaxa: LEIUPERINAE Bonaparte, 1850; LEPTODACTYLINAE ||Tschudi, 1838||-Werner, 1896; PARATELMATOBIINAE Ohler \& Dubois, 2012.

Getendotaxon: Pseudopaludicola Miranda-Ribeiro, 1926.

Comments: Pseudopaludicola was included by Grant et al. (2006) in the family LEIUPERIDAE, whereas in Lourenço et al. (2015) it was outgroup to a branch including Leptodactylus and the genera here included in the LEIUPERINAE. Pyron \& Wiens (2011) found Pseudopaludicola being sister-taxon to all other species of their LEIUPERINAE, although with rather feeble support. In TREE, we did not find sufficient support to confirm the holophyly of a group including the LEIUPERINAE and the PSEUDOPALUDICOLINAE. Therefore, following the [STC], the group corresponding to the genus Pseudopaludicola is recognised at the subfamily rank pending further results concerning the relationships within the LEPTODACTYLIDAE.

\section{C.11.02. Subphalanx DiPLOSIPHONa Günther, 1859}

Protonym: DiplosiPhona Günther, 1859: vii, 3 [Sr].

Eunym: Hoc loco.

Getangiotaxon: Phaneranura nov.

Adelphotaxon: BaINanura nov.

Getendotaxa: CALYPTOCEPHALELLIDAE_Reig, 1960; MYOBATRACHIDAE Schlegel, 1850.

Comments: The subphalanx Diplosiphona is sister-taxon of the BaInanura and includes two family rank taxa, the CALYPTOCEPHALELLIDAE, for the South American genera Calyptocephalella and Telmatobufo, and the MYOBATRACHIDAE, an Australian and New Guinean group. This relationship was first obtained by San Mauro et al. (2005) and then confirmed by Frost et al. (2006) and Pyron \& Wiens (2011). This group named MYOBATRACHIDAE is attributed to the family rank because of the [UQC] and consequently its sister-group CALYPTOCEPHALELLIDAE is attributed the same rank following the [STC].

\section{F.17.27. Familia CALYPTOCEPHALELLIDAE Reig, 1960}

Protonym: CALYPTOCEPHALELLINAE Reig, 1960: 113 [bF].

Eunym: Bossuyt \& Roelants 2009: 359.

Getangiotaxon: DiPLOSIPHONA Günther, 1859.

Adelphotaxon: MYOBATRACHIDAE Schlegel, 1850.

Getendotaxa: Calyptocephalella Strand, 1928; Telmatobufo Schmidt, 1952. 
Protonym and eunym: MYOBATRACHIDAE Schlegel, 1850: 10 [F].

Getangiotaxon: DiPLOSIPHONA Günther, 1859.

Adelphotaxon: CALYPTOCEPHALELLIDAE Reig, 1960.

Getendotaxa: LimnODYNASTINAE Lynch, 1971; MiXOPHYINAE nov.; MYOBATRACHINAE Schlegel, 1850; RHEOBATRACHINAE Heyer \& Liem, 1976; 1 G†.

Comments: This family includes four taxa of insufficiently supported relationships, the subfamilies LIMNODYNASTINAE and MYOBATRACHINAE, discussed in detail below, and the subfamilies MIXOPHYINAE, for the genus Mixophyes, and RHEOBATRACHINAE, for the genus Rheobatrachus. In recent classifications, this family was attributed the rank superfamily and thus the subfamilies below were recognised at the family rank, but this does not follow the rationale applied here throughout. Frost et al. (2006) recovered Mixophyes as sister-group to Rheobatrachus and included this genus in their MYOBATRACHIDAE, whereas Bossuyt \& Roelants (2009) recognised three family rank taxa LIMNODYNASTIDAE, MYOBATRACHIDAE and RHEOBATRACHIDAE. In Pyron \& Wiens (2011), Rheobatrachus was sister-group to all other MYOBATRACHINAE, and Mixophyes sister-group to all MYOBATRACHINAE except Rheobatrachus, but these relationships had rather low support, resulting in fact in relationships similar to TREE.

F.18.44. Subfamilia LIMNODYNASTINAE Lynch, 1971

Protonym: LIMNODYNASTINI Lynch, 1971: 83 [T].

Eunym: Heyer \& Liem 1976: 5.

Getangiotaxon: MYOBATRACHIDAE Schlegel, 1850.

Adelphotaxa: Mixophyinae nov.; MyobatraChinaE Schlegel, 1850; RheObatraCHinaE Heyer \& Liem, $1976 ; 1$ G†.

Getendotaxa: LIMNODYNASTINI Lynch, 1971; NotADENINI nov.

Comments: Within this subfamily two tribes are recognised, the NOTADENINI for Notaden, sister-group to the LIMNODYNASTINI. The latter tribe includes four subtribes of unresolved relationships: HELEIOPORINA for Heleioporus, Limnodynastina for Adelotus, Limnodynastes and Philoria, NEOBATRACHINA for Neobatrachus, and PLATYPLECTRINA for Platyplectrum. As Lechriodus melanopyga, the onomatophore of Lechriodus Boulenger, 1882, and L. fletcheri render Platyplectrum paraphyletic, Lechriodus is here considered a subjective junior synonym of Platyplectrum Günther, 1863.

\section{F.19.42. Tribus LIMNODYNASTINI Lynch, 1971}

Eunym: Lynch 1971: 83.

Getangiotaxon: LIMNODYNASTINAE Lynch, 1971.

Adelphotaxon: NOTADENINI nov.

Getendotaxa: Heleioporina Bauer, 1987; LimNodynastina Lynch, 1971; NeObatraCHINA nov.; PLATYPLECTRINA nov.

F.20.35. Subtribus HELEIOPORINA Bauer, 1987

Protonym: HELEIOPORIDAE Bauer, 1987: 52 [F].

Eunym: Hoc loco.

Getangiotaxon: LIMNODYNASTINI Lynch, 1971.

Adelphotaxa: LimnodynaStina Lynch, 1971; NEOBATRACHINA nov.; PlatyPLECTRINA nov.

Getendotaxon: Heleioporus Gray, 1841.

F.20.36. Subtribus LIMNODYNASTINA Lynch, 1971

Eunym: Hoc loco. 
Getangiotaxon: LIMNODYNASTINI Lynch, 1971.

Adelphotaxa: HelEIOPORINA Bauer, 1987; NeOBATRACHINA nov.; PLATYPLECTRINA nov.

Getendotaxa: Adelotus Ogilby, 1907; Limnodynastes Fitzinger, 1843; Philoria Spencer, 1901.

\section{F.20.37. Subtribus NEOBATRACHINA nov.}

Getangiotaxon: LIMNODYNASTINI Lynch, 1971.

Adelphotaxa: Heleioporina Bauer, 1987; LIMNODYNASTINA Lynch, 1971; PLATYPLECTRINA nov.

Getendotaxon: Neobatrachus Peters, 1863.

Nucleogenus, by present designation: Neobatrachus Peters, 1863. • Etymology of nomen: G: véos

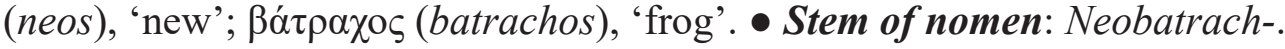

Diagnosis: Small sized, heavy bodied frogs, diagnosable by fusion of cervical and second vertebrae; minute omosternum; toothed maxillary arch, teeth blunt and pedicellate; long alary processes of premaxillae directed posterodorsally, relatively wide at base; palatal shelf of premaxilla narrow, palatal process long; facial lobe of maxilla deep, not exostosed; palatal shelf of maxilla narrow, no pterygoid process; nasals small and separated medially; nasals in contact with maxillae, not pterygoids; nasals not in contact with frontoparietals; frontoparietal fontanelle medium sized; frontoparietals not ornamented; epiotic eminences prominent; cristae paroticae long and narrow; carotid artery lying in a deep groove, exposed dorsally; zygomatic ramus of squamosal minute; otic ramus of squamosal very small, developed medially into a small otic plate; squamosal-maxillary angle $50-55^{\circ}$; prevomers of moderate size, entire, toothed, narrowly separated medially; palatines thin and widely separated medially; sphenethmoid entire, extending anteriorly beneath nasals; anterior ramus of parasphenoid narrow, weakly keeled; parasphenoid alae oriented at right angles to anterior ramus, narrowly overlapped laterally by median rami of pterygoids; pterygoids relatively large, anterior rami in long contact with maxillae, not reaching palatines; occipital condyles relatively small, not stalked, narrowly separated medially; mandible lacking odontoids; $m$. depressor mandibulae in two slips; pupil vertical; males with median subgular vocal sac; nuptial pad (callosities) on thumb and second finger; lacking glands on body; tongue large, round, free behind; toes one-fourth to fully webbed, outer metatarsal tubercle absent, inner metatarsal tubercle spade-like, tips of digits narrow, first finger longer than second; larvae with dextral vent, $3 / 3$ tooth rows, labial papillae interrupted anteriorly; amplexus inguinal; eggs deposited in long strings in slow moving streams and temporary ponds; adult $\mathrm{SVL}<50 \mathrm{~mm}$; and tympanum indistinct externally, concealed beneath skin. \{Lynch 1971\}.

\section{F.20.38. Subtribus PLATYPLECTRINA nov.}

Getangiotaxon: LIMNODYNASTINI Lynch, 1971.

Adelphotaxa: HelEIOPORINA Bauer, 1987; LIMNODYNASTINA Lynch, 1971; NEOBATRACHINA nov.

Getendotaxon: Platyplectrum Günther, 1863.

Nucleogenus, by present designation: Platyplectrum Günther, 1863. • Etymology of nomen: G: $\pi \lambda \alpha \tau u ́ \varsigma$

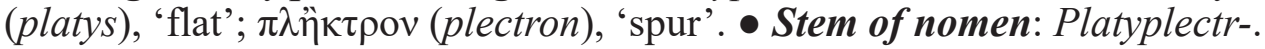

Diagnosis: Medium sized, heavy-bodied frogs, apognosable by numerous molecular synapomorphies (Frost et al. 2006) and cervical and second vertebrae free; omosternum present, moderate sized; toothed maxillary arch, teeth blunt and pedicellate; alary processes of premaxillae directed posterodorsally, wide at base; palatal shelf of premaxilla moderate in width and deeply incised; facial lobe of maxilla deep, not exostosed; palatal shelf of maxilla relatively narrow, small pterygoid process; nasals moderate sized, apparently in median contact; nasals in contact with maxillae, not with pterygoids; nasals not in contact with frontoparietals; frontoparietal fontanelle lacking; frontoparietals not ornamented; epiotic eminences poorly defined; cristae paroticae long and relatively broad; carotid artery enclosed in long, roofed, bony canal; zygomatic ramus of squamosal slightly shorter than otic ramus; otic ramus of squamosal of moderate length, expanded medially into small otic plate; squamosal-maxillary angle 
about $50^{\circ}$; prevomers entire, toothed, large, dentigerous rami in tenuous median contact; palatines large, narrowly separated medially; sphenethmoid entire, extending anteriorly beneath posterior edge of nasals; anterior ramus of parasphenoid narrow, not keeled; parasphenoid alae oriented at right angles to anterior ramus, overlapped laterally by median rami of pterygoids; pterygoids relatively large, anterior rami in long contact with maxillae, nearly reaching palatines; occipital condyles moderately large, not stalked, narrowly separated medially; mandible lacking odontoids; $m$. depressor mandibulae in two slips; horizontal pupils; males with median subgular vocal sac; nuptial asperities of many small spines on thumb; body lacking glands; tongue round, posterior edge free; toes lack webbing, outer metatarsal tubercle absent, digital tips narrow, first finger as long as second; larvae with dextral vent, 2/3 tooth rows, labial papillae broadly interrupted anteriorly; amplexus inguinal; eggs laids in foam nest in temporary ponds and puddles; and tympanum visible externally. \{Lynch 1971\}.

\section{F.19.43. Tribus NotadENINI nov.}

Getangiotaxon: LIMNODYNASTINAE Lynch, 1971.

Adelphotaxon: LIMNODYNASTINI Lynch, 1971.

Getendotaxon: Notaden Günther, 1873.

Nucleogenus, by present designation: Notaden Günther, 1873. • Etymology of nomen: G: võ̃os (notos), 'back'; ádév (aden), 'gland'. • Stem of nomen: Notaden-.

Diagnosis: Small sized, heavy bodied frogs, diagnosable by inguinal amplexus; lack of foam nesting; cervical and second vertebrae fused; omosternum absent; maxillary arch edentate; alary processes of premaxillae elongate, directed dorsally, narrow at base; palatal shelf of premaxilla narrow, palatal process relatively short; facial lobe of maxilla shallow; palatal shelf of maxilla absent; incomplete maxillary arch, maxilla not contacting quadratojugal or premaxilla, quadratojugal present; nasals small and separated medially; nasals not in contact with maxillae or pterygoids; nasals barely in contact with frontoparietals; large frontoparietal fontanelle; frontoparietals not ornamented; epiotic eminences prominent; cristae paroticae short, stocky; carotid artery lies in a shallow groove exposed dorsally; zygomatic and otic rami of squamosal lacking; squamosal-maxillary angle $\sim 80^{\circ}$; prevomers moderately large, entire, toothed, separated medially; palatines reduced in size, not contacting maxillae and widely separated medially; sphenethmoid entire, small, not extending anteriorly to nasals; anterior ramus of parasphenoid broad, short, not keeled; parasphenoid alae oriented at right angles to anterior ramus, not overlapped laterally by median rami of pterygoids; pterygoids small, anterior rami in long contact with maxillae, usually contacting palatines; occipital condyles large, not stalked, narrowly separated medially; mandible lacking odontoids; $m$. depressor mandibulae in two slips; pupil horizontal; males with median subgular vocal sac; nuptial pad on thumb; at least two ill-defined glands on dorsum, less discrete but more extensive than in Heleioporus; tongue large, round, not free behind; toes one-half to two-thirds webbed, outer metatarsal tubercle absent, inner metatarsal tubercle spade-like, tips of digits narrow, first finger as long as second; larvae with median vent, $3 / 3$ tooth rows, labial papillae interrupted anteriorly; and tympanum concealed. \{Lynch, 1971; Frost et al. 2006\}.

\section{F.18.45. Subfamilia MIXOPHYINAE nov.}

Getangiotaxon: MYOBATRACHIDAE Schlegel, 1850.

Adelphotaxa: Limnodynastinae Lynch, 1971; MyobatrachinaE Schlegel, 1850; Rheobatrachinae Heyer \& Liem, 1976; $1 \mathbf{G \dagger}$.

Getendotaxon: Mixophyes Günther, 1864.

Nucleogenus, by present designation: Mixophyes Günther, 1864. • Etymology of nomen: G: $\mu$ í $\alpha$ (miga), 'mixed'; pví (phye), 'stature, shape'. • Stem of nomen: Mixophy-.

Diagnosis: Medium sized, heavy bodied frogs, diagnosable by cervical and second vertebrae free; omosternum present and relatively large; maxillary arch toothed, teeth blunt and pedicellate; alary 
processes of premaxillae directed posterodorsally, broad at base; palatal shelf of premaxilla narrow, palatal process elongate; facial lobe of maxilla deep with a slight squamosal process, not exostosed; palatal shelf of maxilla very narrow, no pterygoid process; nasals large, in median contact anteriorly, separated posteriorly, exposing sphenethmoid; nasals in contact with maxillae, not in contact with pterygoids; nasals in tenuous contact with frontoparietals; frontoparietal fontanelle absent; frontoparietals not ornamented; epiotic eminences prominent; cristae paroticae long and narrow; carotid artery enclosed in a complete bony canal; zygomatic ramus of squamosal elongate, tendon contacting squamosal process of maxilla; otic ramus of squamosal long, developed medially into otic plate; squamosal-maxillary angle $\sim 5-50^{\circ}$; prevomers small, entire, toothed, separated medially; palatines thin, separated medially, bearing odontoid ridges; sphenethmoid entire, extending anteriorly to anterior edge of nasals; anterior ramus of parasphenoid narrow, not keeled; parasphenoid alae deflected posteriorly, overlapped laterally by median rami of pterygoids; pterygoids large, anterior rami in long contact with maxillae, nearly reaching palatines and nasals; occipital condyles moderate sized, not stalked, separated medially; mandible lacking odontoids; $m$. depressor mandibulae in two slips; vertical pupils; males with median subgular vocal sac, nuptial asperities on thumb; lacking glands on dorsum; tongue large, rounded, only posterior edge free; toes two-thirds webbed, outer metatarsal tubercle absent, inner metatarsal tubercle not spade-like, tips of digits narrow; larvae with dextral vent, 6/3 tooth rows, labial papillae not interrupted anteriorly; inguinal amplexus; eggs laid in terrestrial situations and hatch upon flooding; males and females $\sim 50-100 \mathrm{~mm}$ SVL as adults; and tympanum visible externally. \{Lynch, 1971; Frost et al. 2006\}.

\section{F.18.46. Subfamilia MYobatRACHINAE Schlegel, 1850}

Eunym: Parker 1940: 2.

Getangiotaxon: MYOBATRACHIDAE Schlegel, 1850.

Adelphotaxa: Limnodynastinae Lynch, 1971; MiXophyinaE nov.; RheobatraChinaE Heyer \& Liem, $1976 ; 1$ G†.

Getendotaxa: MYoBATRACHINI Schlegel, 1850; TAUDACTYLINI nov.

Comments: The branch recognised as the tribe TAUDACTYLINI, corresponding to the genus Taudactylus, is sister-group to all other MYOBATRACHINAE, forming the tribe MYOBATRACHINI. Within this tribe we recognise the subtribes CRINIINA and MYOBATRACHINA. Within the subtribe CRINIINA, the genus Crinia, within the infratribe CRINIINIA, is sister-group of the ASSINIA. The later infratribe hold two sister-groups here treated as hypotribes, ASSINOA for Assa and Geocrinia, and PARACRINIINOA for Paracrinia. The subtribe MYOBATRACHINA includes three infratribes of unresolved relationships, the MYOBATRACHINIA, the SPICOSPININIA, for the genus Spicospina, and the UPEROLEIINIA, for the genus Uperoleia. In the MYOBATRACHINIA, the genus Pseudophryne, in the hypotribe PSEUDOPHRYNINOA, is sister-group to the other genera (Arenophryne, Metacrinia and Myobatrachus) placed in the MYOBATRACHINOA. The ASSINOA (Assa and Geocrinia) and Paracrinia form a holophyletic group in Pyron \& Wiens (2011), corresponding to our ASSINIA. The other relationships of Pyron \& Wiens (2011) are not recognised here formally, as in TREE they do not have sufficient support according to our Criteria.

F.19.44. Tribus MYobatRaCHINI Schlegel, 1850

Eunym: Hoc loco.

Getangiotaxon: MYOBATRACHINAE Schlegel, 1850.

Adelphotaxon: TAUDACTYLINI nov.

Getendotaxa: CRINIINA Cope, 1866; MyobatraChina Schlegel, 1850.

\section{F.20.39. Subtribus CRINIINA Cope, 1866}

Protonym: CRINIAE Cope, 1866: 89 [Gr].

Eunym: Hoc loco.

Getangiotaxon: MYOBATRACHINI Schlegel, 1850. 
Adelphotaxon: MYOBATRACHINA Schlegel, 1850.

Getendotaxa: ASSINIA nov.; CRINIINIA Cope, 1866.

F.21.26. Infratribus ASSINIA nov.

Getangiotaxon: CRINIINA Cope, 1866.

Adelphotaxon: CRINIINIA Cope, 1866.

Getendotaxa: ASSINOA nov.; PARACRINIINOA nov.

Nucleogenus, by present designation: Assa Tyler, 1972. • Etymology of nomen: L: assa, 'dry-nurse', referring to the breeding behaviour of the species, carrying the young but not feeding them. $\bullet$ Stem of nomen: Ass-.

Diagnosis: Small, heavy bodied frogs, diagnosable by outer metatarsal tubercle small and not compressed and prevomerine bones small but complete (Paracrinia); or outer metatarsal tubercle absent (Assa, Geocrinia); prevomerine teeth usually missing (Assa); prevomerine bones small but complete, prevomerine teeth present, skin of venter smooth, toe fringes absent; eggs laid out of water; larvae entering water after early development in Geocrinia. \{Lynch, 1971; Frost et al. 2006\}.

F.22.15. Hypotribus ASSINOA nov.

Getangiotaxon: ASSINIA nov.

Adelphotaxon: PARACRINIINOA nov.

Getendotaxa: Assa Tyler, 1972; Geocrinia Blake, 1973.

F.22.16. Hypotribus PARACRINIINOA nov.

Getangiotaxon: ASSINIA nov.

Adelphotaxon: ASSINOA nov.

Getendotaxon: Paracrinia Heyer \& Liem, 1976.

Nucleogenus, by present designation: Paracrinia Heyer \& Liem, 1976. • Etymology of nomen: G:

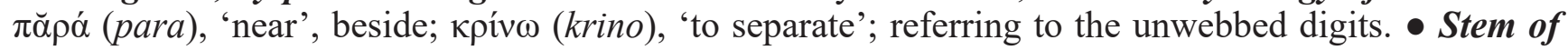
nomen: Paracrin-.

Diagnosis: Small, heavy-bodied frogs, diagnosable by outer metatarsal tubercle small and not compressed and prevomerine bones small but complete. \{Lynch 1971; Frost et al. 2006\}.

\title{
F.21.27. Infratribus CRINIINIA Cope, 1866
}

Eunym: Hoc loco.

Getangiotaxon: CRINIINA Cope, 1866.

Adelphotaxon: ASSINIA nov.

Getendotaxon: Crinia Tschudi, 1838.

\section{F.20.40. Subtribus MYobatrachina Schlegel, 1850}

\author{
Eunym: Hoc loco. \\ Getangiotaxon: MYOBATRACHINI Schlegel, 1850. \\ Adelphotaxon: CRINIINA Cope, 1866. \\ Getendotaxa: MYobatraChINIA Schlegel, 1850; SPICOSPININIA nov.; UPEROLEIINIA Günther 1858.
}


Eunym: Hoc loco.

Getangiotaxon: MYOBATRACHINA Schlegel, 1850.

Adelphotaxa: SPICOSPININIA nov.; UPEROLEIINIA Günther 1858.

Getendotaxa: MyobatraChINOA Schlegel, 1850; PSEUdophryninoa Bauer, 1987.

\section{F.22.17. Hypotribus MYobatRACHINOA Schlegel, 1850}

Eunym: Hoc loco.

Getangiotaxon: MYOBATRACHINIA Schlegel, 1850.

Adelphotaxon: PSEUdOPHRYNINOA Bauer, 1987.

Getendotaxa: Arenophryne Tyler, 1976; Metacrinia Parker, 1940; Myobatrachus Schlegel, 1850.

\section{F.22.18. Hypotribus PSEUDOPHRYNINOA Bauer, 1987}

Protonym: PSEUDOPHRYNOIDEA Bauer, 1987: 51 [pF].

Eunym: Hoc loco.

Getangiotaxon: MYOBATRACHINIA Schlegel, 1850.

Adelphotaxon: MYOBATRACHINOA Schlegel, 1850.

Getendotaxon: Pseudophryne Fitzinger, 1843.

\section{F.21.29. Infratribus SPICOSPININIA nov.}

Getangiotaxon: MYOBATRACHINA Schlegel, 1850.

Adelphotaxa: MyobatraChinIa Schlegel, 1850; UPERoleIINIA Günther 1858.

Getendotaxon: Spicospina Roberts, Horwitz, Wardell-Johnson, Maxson \& Mahony, 1997.

Nucleogenus, by present designation: Spicospina Roberts, Horwitz, Wardell-Johnson, Maxson \& Mahony, 1997. • Etymology of nomen: L: spicus, 'spike'; spina, 'vertebra'; referring to the spines on the posterior margins and the transverse process of the vertebrae. $\bullet$ Stem of nomen: Spicospin-.

Diagnosis: Small, heavy bodied frogs, diagnosable by pectoral girdle arciferal; alary process of hyoid plate broad; cricoid cartilage divided ventrally; eight amphicoelous, non-imbricate, presacral vertebrae; $M$. intermandibularis not underlying the M. submentalis; prevomer absent; sphenethmoid complete, ossified; cervical cotyles widely separated; moderately broad sacral diapophyses; tympanum and columella present; all presacral vertebrae with a shallow dorsal keel-more marked on first three; small irregular spines on posterior, dorsal margin of first four vertebrae; third pre-sacral vertebra with flat, broad, triangular, arrow-head shaped spine directed upwards and backward on proximal, dorsal, posterior margin of both transverse processes; xiphisternum large, ossified centrally in an arrow-head shape; massive parotoid glands; ventral skin smooth; knobbed terminal phalanges; dentate maxillary arch; maxillary teeth pedicellate; anterior corn of hyoid with inward directed hook on anterior margin; nasals narrow, small and widely separated; toes and fingers free, no fringe or web; phalangeal formula of hand 2-2-3-3; phalangeal formula of foot 2-2-3-4-3; amplexus inguinal; eggs deposited singly in water. \{Roberts et al. 1997$\}$.

\section{F.21.30. Infratribus UPEROLEIINIA Günther 1858}

Protonym: UPEROLIIDAE Günther 1858: 346 [F].

Eunym: Hoc loco.

Getangiotaxon: MYOBATRACHINA Schlegel, 1850.

Adelphotaxa: MyobatraCHINIA Schlegel, 1850; SPICOSPININIA nov.

Getendotaxon: Uperoleia Gray, 1841. 


\title{
F.19.45. Tribus TAUDACTYLINI nov.
}

Getangiotaxon: MYOBATRACHINAE Schlegel, 1850.

Adelphotaxon: MYoBATRACHINI Schlegel, 1850.

Getendotaxon: Taudactylus Straughan \& Lee, 1966.

Nucleogenus, by present designation: Taudactylus Straughan \& Lee, 1966. • Etymology of nomen: G:

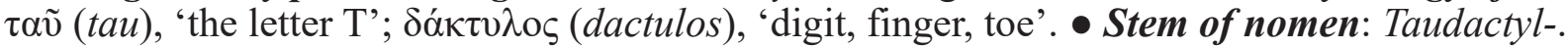

Diagnosis: Small, heavy-bodied frogs; omosternum absent; maxillary arch toothed, teeth blunt and pedicellate; alary processes of premaxillae directed very slightly posterodorsally, narrow at base; palatal shelf of premaxilla relatively broad laterally, narrow medially, bearing greatly elongated palatal process; facial lobe of maxilla shallow; palatal shelf of maxilla of moderate width, narrowing posteriorly, pterygoid process minute; quadratojugal shallow, long and thin; nasals very small, widely separated medially; nasals not in contact with maxillae or pterygoids; nasals not in contact with frontoparietals; frontoparietal fontanelle present, small and narrow; frontoparietals not ornamented; epiotic eminences obsolete; cristae paroticae short, stocky; carotid artery passing dorsal to skull bones; zygomatic ramus of squamosal short, thin, about one-third length of otic ramus, therefore proportionately longer than zygomatic rami of other myobatrachines; otic ramus of squamosal long, not expanded medially into otic plate; squamosal-maxillary angle $\sim 55^{\circ}$; columella present; prevomers minute, fragmented, dentigerous rami absent, restricted to medial edges of choanae; palatines narrow, widely separated medially; sphenethmoid divided; anterior ramus of parasphenoid long, narrow, reaching level of palatines, not keeled; parasphenoid alae short, deflected posteriorly, not overlapped by median rami of pterygoids; pterygoids comparatively large, anterior rami in short contact with maxillae, not reaching palatines; occipital condyles small, stalked, widely separated medially; terminal phalanges T-shaped; $m$. depressor mandibulae in two slips; pupil horizontal; males with median, subgular vocal sac; diffuse nuptial pad on thumb; body lacking glands; tongue long, narrow, posterior edge free; toes not webbed, bearing distinct lateral fringes, outer metatarsal tubercle absent; and tympanum concealed. \{Lynch 1971\}.

\section{F.18.47. Subfamilia RHEOBatRaChinae Heyer \& Liem, 1976}

\author{
Protonym and eunym: RHEOBATRACHINAE Heyer \& Liem, 1976: 11 [bF]. \\ Getangiotaxon: MYOBATRACHIDAE Schlegel, 1850. \\ Adelphotaxa: LimnodynaStinaE Lynch, 1971; MiXOPHYINAE nov.; MyobatraCHINAE Schlegel, 1850; 1 G†. \\ Getendotaxon: Rheobatrachus Liem, 1973.
}

\section{C.10.03. Phalanx Scoptanura Starrett, 1973}

\author{
Protonym: ScoptanURa Starrett, 1973: 251 [UC]. \\ Eunym: Hoc loco. \\ Getangiotaxon: AQUIPARES Blainville, 1816. \\ Adelphotaxa: Gondwanura nov.; Phaneranura nov. \\ Getendotaxa: Ecostata Lataste, 1879; Gastrechmia Cope, 1867; Pananura nov.; 1 G†.
}

Comments: The phalanx SCOPTANURA is one of the three branches in the AQUIPARES, sister-group to the Gondwanura and Phaneranura. For this taxon, that is recognised in all phylogenetic analyses, the superfamilial nomen RANOIDEA was used by Ford \& Cannatella (1993), Darst \& Cannatella (2004), Pyron \& Wiens (2011), Zhang et al. (2013) and Feng et al. (2017), whereas Frost et al. (2006), Bossuyt \& Roelants (2009) and Irisarri et al. (2012) used the ectonym «RANOIDES». It includes three taxa, here treated as the subphalanges Ecostata, Gastrechmia and Pananura, as the relationship between EcostaTA and GASTREChMia has only a SHL-aLRT value of 70, thus below the set threshold. 


\title{
C.11.03. Subphalanx Ecostata Lataste, 1879
}

\author{
Protonym: Ecostati Lataste, 1879: 339 ['bT']. \\ Eunym: Hoc loco. \\ Getangiotaxon: SCOPTANURA Starrett, 1973. \\ Adelphotaxa: Gastrechmia Cope, 1867; Pananura nov.; 1 G†. \\ Getendotaxa: MiCRoHYLIDAE ||Fitzinger, 1843||-Noble, 1931; PhrYNOMERIDAE Noble, 1931.
}

Comments: The highly supported branch ECOSTATA groups the MICROHYLIDAE and the PHRYNOMERIDAE. It would be named MICROHYLOIDEA if the use of the superfamily level was warranted, which is not the case here as it would be redundant with the subphalanx. It was recovered by Bossuyt \& Roelants (2009), Pyron \& Wiens (2011) and Feng et al. (2017), and named MICROHYLOIDEA or MICROHYLIDAE, depending if PHRYNOMERIDAE was recognised at the family level, or as subfamily within the MICROHYLIDAE. Here we refer both taxa to the rank family level as MICROHYLIDAE is imposed at this rank by the [UQC], and PHRYNOMERIDAE has to be at the same rank according to the [STC].

\section{F.17.29. Familia MiCRoHYLIDAE ||Fitzinger, 1843\|-Noble, 1931}

Protonyms: ||GASTROPHRYNAE Fitzinger, 1843: 33\| [F]; MiCROHYLINAE Noble, 1931: 451 [bF].

Eunym: Parker 1934: i.

Getangiotaxon: Ecostata Lataste, 1879.

Adelphotaxon: PHRYNOMERIDAE Noble, 1931.

Getendotaxa: AdelaStinaE Peloso, Frost, Richards, Rodrigues, Donnellan, Matsui, Raxworthy, Biju, Lemmon, Lemmon \& Wheeler, 2016; Asterophryinae Günther, 1858; Cophylinae Cope, 1889; Gastrophryninae Fitzinger, 1843; Hoplophryninae Noble, 1931; Kalophryninae Mivart, 1869; Melanobatrachinae Noble, 1931; MicrohylinaE ||Fitzinger, 1843\|-Noble, 1931; OtophrYNINAE Wassersug \& Pyburn, 1987.

Comments: In TREE, the relationships within this family are poorly resolved, resulting in the recognition of nine subfamily rank taxa: the ADELASTINAE for Adelastes; the HOPLOPHRYNINAE for Hoplophryne and Parhoplophryne; the KALOPHRYNINAE for Kalophrynus; the MELANOBATRACHINAE for Melanobatrachus; the OTOPHRYNINAE for Otophryne and Synapturanus; and the ASTEROPHRYINAE, the COPHYLINAE, the GASTROPHRYNINAE and the MICROHYLINAE, discussed in more details below.

Similar subfamilial classifications were proposed by most authors (Frost et al. 2006, Van der Meijden et al. 2007, Pyron \& Wiens 2011, Kurabayashi et al. 2011, Sá et al. 2012, Frazão et al. 2015, Feng et al. 2017). However, Bossuyt \& Roelants (2009) recognised these groups at the family level, which was not followed later. Peloso et al. (2016) found Chaperina as sister-group to all other MICROHYLIDAE and thus recognised a new subfamily for this taxon. In Tu et al. (2018), Chaperina was downgraded to the status of a genus within the subfamily MICROHYLINAE. Here we recognise for this genus a subtribe within the MICROHYLINAE. In TREE, we found Phrynomantis as sister-group to all other MICROHYLIDAE, so that we recognised this taxon as the family PHRYNOMERIDAE. This position was found by Kurabayashi et al. (2011) and Tu et al. (2018) but in other phylogenies Phrynomantis was within the MICROHYLIDAE in various positions. Van der Meijden et al. (2007) and Matsui et al. (2011) recovered it as sister-group to the GASTROPHRYNINAE, whereas it was within a taxon grouping KALOPHRYNINAE and OTOPHRYNINAE in the tree of Sá et al. (2012), and Peloso et al. (2016) found it sister-group to the MELANOBATRACHINAE.

\section{F.18.48. Subfamilia AdELASTINAE Peloso, Frost, Richards, Rodrigues, Donnellan,} Matsui, Raxworthy, Biju, Lemmon, Lemmon \& Wheeler, 2016

Protonym and eunym: Adelastinae Peloso, Frost, Richards, Rodrigues, Donnellan, Matsui, Raxworthy, Biju, Lemmon, Lemmon \& Wheeler, 2016: $131[\mathrm{bF}]$.

Getangiotaxon: MICROHYLIDAE |Fitzinger, 1843\|-Noble, 1931.

Adelphotaxa:AsterophryinaE Günther, 1858; CopHYLINAE Cope, 1889; GASTRoPHRYNINAE Fitzinger, 1843; HoPlopHRYNINAE Noble, 1931; Kalophryninae Mivart, 1869; MelanobatraChinae Noble, 1931; MicrohylinaE ||Fitzinger, 1843\|Noble, 1931; Otophryninae Wassersug \& Pyburn, 1987.

Getendotaxon: Adelastes Zweifel, 1986. 


\section{F.18.49. Subfamilia ASTEROPHRYINAE Günther, 1858}

Protonym: ASTEROPHRYDIDAE Günther, 1858: 346 [F].

Eunym: Fejérváry 1923: 181.

Getangiotaxon: MICROHYLIDAE ||Fitzinger, 1843\|-Noble, 1931.

Adelphotaxa: AdELASTINAE Peloso, Frost, Richards, Rodrigues, Donnellan, Matsui, Raxworthy, Biju, Lemmon, Lemmon \& Wheeler, 2016; Cophylinae Cope, 1889; Gastrophryninae Fitzinger, 1843; HoplophryninaE Noble, 1931; Kalophryninae Mivart, 1869; Melanobatrachinae Noble, 1931; MicrohylinaE ||Fitzinger, 1843\|-Noble, 1931; OTOPHRYNINAE Wassersug \& Pyburn, 1987.

Getendotaxa: ASTEROPHRYINI Günther, 1858; GASTROPHRYNOIDINI nov.

Comments: Within the subfamily ASTEROPHRYINAE, two tribes are recognised here, the ASTEROPHRYINI for Asterophrys, and the GASTROPHYNOIDINI for Gastrophrynoides, Siamophryne and Vietnamophryne. Our TREE does not allow to build a clear generic classification within the tribe ASTEROPHRYINI. Although several groups in this assemblage have high support, within most of them the species are currently allocated in the literature to several genera, whereas no nomina are available for other groups, so that the current generic classification does not reflect the well supported phylogenetic hypotheses. As a consequence, we provisionally synonymise all genus group nomina available in this tribe under a single genus Asterophrys. Recent works on this group by Rivera et al. (2017) and Tu et al. (2018) did not resolve paraphyly and polyphyly of generic taxa but continue to recognise about 15 poorly diagnosed such taxa on weak phylogenetic grounds. This group needs a fundamental taxonomic revision before robust inter-group phylogenetic relationships can be proposed.

\section{F.19.46. Tribus ASTEROPHRYINI Günther, 1858}

Eunym: Burton 1986: 444.

Getangiotaxon: ASTEROPHRYINAE Günther, 1858.

Adelphotaxon: GASTROPHRYNOIDINI nov.

Getendotaxon: Asterophrys Tschudi, 1838.

\section{F.19.47. Tribus GASTROPHYNOIDINI nov.}

Getangiotaxon: ASTEROPHRYINAE Günther, 1858.

Adelphotaxon: ASTEROPHRYINI Günther, 1858

Getendotaxa: Gastrophrynoides Noble, 1926; Siamophryne Suwannapoom, Sumontha, Tunprasert, Ruangsuwan, Pawangkhanant, Korost \& Poyarkov, 2018; Vietnamophryne Poyarkov, Suwannapoom, Pawangkhanant, Aksornneam, Duong, Korost \& Che, 2018.

Nucleogenus, bypresent designation: Gastrophrynoides Noble, 1926. • Etymology of nomen: G: $\gamma \alpha \sigma \tau \eta \jmath$

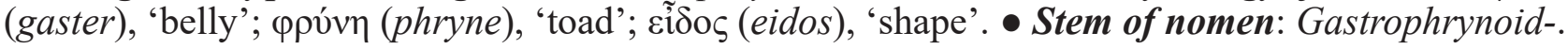

Diagnosis: Small sized microhylid frogs $(15-40 \mathrm{~mm})$; vomeropalatine small, no vomerine teeth; clavicles absent or present as slender tiny bones; omosternum absent; sternum cartilaginous or only partly calcified cartilage; vertebrae procoelous with eight presacral vertebrae lacking neural crests; terminal phalanges T-shaped or bobbin-shaped; pupil rounded; tympanum distinct; tongue entire, spatulate or oval; two or one transverse palatal fold; digits enlarged to small discs, or rounded; webbing reduced or absent; a distinct inner metatarsal tubercle, outer metatarsal tubercle absent; skin granular to smooth. \{Noble 1926; Parker 1934; Inger 1966; Poyarkov et al. 2018; Suwannapoom et al. 2018\}.

Comments: As the recently described genera Siamophryne and Vietnamophryne are not represented in TREE, we do not propose formal classification within the GASTROPHYNOIDINI. 
Protonym: COPHYLIDAE Cope, 1889: 248 [F].

Eunym: Parker 1934: v.

Getangiotaxon: MICROHYLIDAE \|Fitzinger, 1843\|-Noble, 1931.

Adelphotaxa: AdelastinaE Peloso, Frost, Richards, Rodrigues, Donnellan, Matsui, Raxworthy, Biju, Lemmon, Lemmon \& Wheeler, 2016; AsterophryinaE Günther, 1858; GASTRophrYNINAE Fitzinger, 1843; HoplophrYNINAE Noble, 1931; Kalophryninae Mivart, 1869; Melanobatrachinae Noble, 1931; Microhylinae ||Fitzinger, 1843||-Noble, 1931; Otophryninae Wassersug \& Pyburn, 1987.

Getendotaxa: Cophylini Cope, 1889; SCAPHIOPHRYNINI Laurent, 1946.

Comments: Here we include Paradoxophyla and Scaphiophryne, often recognised as the subfamily SCAPHIOPHRYNINAE, as a tribe SCAPHIOPHRYNINI in the subfamily COPHYLINAE. In TREE, this tribe is sistergroup to the COPHYLINI. Within the latter tribe, four subtribes, the mutual relationships of which are not sufficiently supported, are recognised: the ANODONTHYLINA for Anodonthyla, the CoPHYLINA for Cophyla and Mantipus, the PLATYPELINA for Platypelis, and the RHOMBOPHRYNINA for Rhombophryne. In the recent phylogenies, Madecassophryne is sister-taxon to all other COPHYLINI. In TREE, many groups have poor support so we could not identify sister-group relationships. In Scherz et al. (2019), Cophyla is sister-taxon to Platypelis, Plethodontohyla to the new genus Mini, and these four are sister-group to Anodonthyla. Scherz et al. (2019) also recognised a holophyletic Rhombophryne, sister-group to Stumpffia. In TREE, the Rhombophryne serratopalpebrosa group (Scherz et al. 2017) is sister-group to Stumpffia with low support (87). In order to recognise a highly supported holophyletic taxon, Stumpffia is here considered synonym of Rhombophryne. Another important difference with our taxonomy is the position of Anilany which is sistergroup to Rhombophryne and Stumpffia in Scherz et al. (2019) but here treated as a synonym of Cophyla as we did with Plethodontohyla and Mini, in order to have holophyletic and highly supported taxa.

\section{F.19.48. Tribus Cophylini Cope, 1889}

Eunym: Hoc loco.

Getangiotaxon: COPHYLINAE Cope, 1889.

Adelphotaxon: SCAPHIOPHRYNINI Laurent, 1946.

Getendotaxa: Anodonthylina nov.; Cophylina Cope, 1889; Platypelina nov.; Rhombophrynina Noble, $1931 ; 1$ GIS (Madecassophryne Guibé, 1974).

\section{F.20.41. Subtribus ANODONTHYLINA nov.}

Getangiotaxon: CoPHYLINI Cope, 1889.

Adelphotaxa: Cophylina Cope, 1889; Platypelina nov.; Rhombophrynina Noble, 1931; 1 GIS (Madecassophryne Guibé, 1974).

Getendotaxon: Anodonthyla Müller, 1892.

Nucleogenus, by present designation: Anodonthyla Müller, 1892. • Etymology of nomen: G:

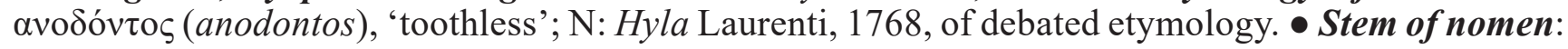
Anodonthyl-.

Diagnosis: Small sized microhylids (SVL 22-38 mm); maxillary teeth present; prevomer small, absence of postchoanal vomer; clavicle and procoracoid present, well developed, reaching scapula and mid-line of girdle; omosternum and sternum well developed, cartilaginous; terminal phalanges Tshaped; pupil horizontal; tympanum distinct or indistinct about half eye length; tongue slightly notched; tips of digits dilated; first finger much shorter than second; a large, cultriform prepollex present; toes web absent; an inner but no outer metatarsal tubercle; skin finely granular at least on belly. \{Müller 1892; Parker 1934; Scherz et al. 2019 \}. 
Eunym: Hoc loco.

Getangiotaxon: COPHYLINI Cope, 1889.

Adelphotaxa: Anodonthylina nov.; Platypelina nov.; Rhombophrynina Noble, 1931; 1 GIS (Madecassophryne Guibé, 1974).

Getendotaxa: Cophyla Boettger, 1880; Mantipus Peters, 1883.

\section{F.20.43. Subtribus PLATYPELINA nov.}

Getangiotaxon: COPHYLINI Cope, 1889.

Adelphotaxa: Anodonthylina nov.; Cophylina Cope, 1889; Rhombophrynina Noble, 1931; 1 GIS (Madecassophryne Guibé, 1974).

Getendotaxon: Platypelis Boulenger, 1882.

Nucleogenus, by present designation: Platypelis Boulenger, 1882. • Etymology of nomen: G: $\pi \lambda \alpha \tau \varsigma \varsigma$

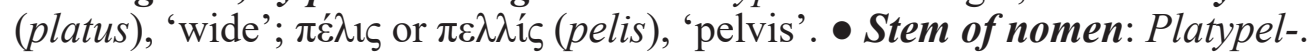

Diagnosis: Small sized microhylids (SVL 26-40 mm); maxillary teeth present; prevomer divided, postchoanal portion long in contact medially, overlying palatine and bearing teeth; clavicle present, but reduced, not reaching mid-line of girdle or scapula; procoracoid broad, curved, insertion on middle of anterior border of coracoid; sternum large, cartilaginous; omosternum small and cartilaginous or absent; vertebrae procoelous; terminal phalanges expanded; pupil horizontal; tympanum hidden or distinct, about half eye length; tongue oval, large, entire; palate without dermal folds; tips of digits broadly dilated; first finger much shorter than second; toes feebly webbed; a feeble inner metatarsal tubercle; outer metatarsal tubercle absent; skin smooth or with warts. \{Parker 1934\}.

\section{F.20.44. Subtribus RHOMворнRYNINA Noble, 1931}

Protonym: RHombophryninae Noble, 1931: 529 [bF].

Eunym: Hoc loco.

Getangiotaxon: COPHYLINI Cope, 1889.

Adelphotaxa: Anodonthylina nov.; Cophylina Cope, 1889; Platypelina nov.; 1 GIS (Madecassophryne Guibé, 1974).

Getendotaxon: Rhombophryne Boettger, 1880.

\section{F.19.49. Tribus SCAPHIOPHRYNINI Laurent, 1946}

Protonym: SCAPHIOPHRYNINAE Laurent, 1946: 337 [bF].

Eunym: Hoc loco.

Getangiotaxon: CopHYLINAE Cope, 1889.

Adelphotaxon: Cophylini Cope, 1889.

Getendotaxa: Paradoxophyla Blommers-Schlösser, 1991; Scaphiophryne Boulenger, 1882.

\section{F.18.51. Subfamilia GASTROPHRYNINAE Fitzinger, 1843}

Protonym: GASTROPHRYNAE Fitzinger, 1843: 33 [F].

Eunym: Metcalf 1923: 294.

Getangiotaxon: MICROHYLIDAE ||Fitzinger, 1843\|-Noble, 1931.

Adelphotaxa: AdelastinaE Peloso, Frost, Richards, Rodrigues, Donnellan, Matsui, Raxworthy, Biju, Lemmon, Lemmon \& Wheeler, 2016; Asterophryinae Günther, 1858; Cophylinae Cope, 1889; HoplophrYNinaE Noble, 1931; Kalophryninae Mivart, 1869; Melanobatrachinae Noble, 1931; MicrohylinaE ||Fitzinger, 1843||-Noble, 1931; Otophryninae Wassersug \& Pyburn, 1987. 
Getendotaxa: CHIASMOCLEINI nov.; CTENOPHRYNINI nov.; GASTROPHRYNINI Fitzinger, 1843.

Comments: The subfamily GASTROPHRYNINAE includes three tribes, the CHIASMOCLEINI for Chiasmocleis, the CTENOPHRYNINI for Ctenophryne, and the GASTROPHRYNINI. The latter tribe has a complex structure, including three subtribes, the DASYPOPINA for Dasypops and Myersiella, the STEREOCYCLOPINA for Stereocyclops, and the GASTROPHRYNINA, including five infratribes of unsupported relationships, the ARCOVOMERINIA for Arcovomer, the DERMATONOTINIA for Dermatonotus, the ENGYSTOMATINIA for Engystoma, the GASTROPHRYNINIA for Gastrophryne and Hypopachus, and the HAMPTOPHRYNINIA for Hamptophryne. The sister-branch relationships of these groups were found by Greenbaum et al. (2011) and Pyron \& Wiens (2011) but in both works some of the genera of this subfamily were missing in the analysis. In Tu et al. (2018), most of the intra-generic relationships have high support. The sister-group relationship between Dasypops and Myersiella is confirmed, as well as the sister-group relationship between Gastrophryne and Hypopachus. This latter taxon is sister-taxon to Engystoma (a genus often called by error Elachistocleis). These authors found also a highly supported relationship between Dermatonotus and Stereocyclops, which is not supported in our TREE.

\section{F.19.50. Tribus CHIASMOCLEINI nov.}

Getangiotaxon: GASTROPHRYNINAE Fitzinger, 1843.

Adelphotaxa: CTENOPHRYNINI nov.; GASTROPHRYNINI Fitzinger, 1843.

Getendotaxon: Chiasmocleis Méhelÿ, 1904.

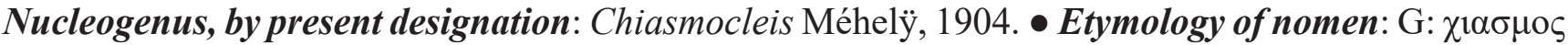
(chiasmos), 'in shape of X'; $\kappa \lambda \varepsilon 1 \delta$ íos (cleidos), 'clavicle'; referring to the position of the clavicles in relation to the coracoid (see Méhelÿ 1904, plate 103 figure 4). • Stem of nomen: Chiasmocle-.

Diagnosis: Small sized microhylids (males SVL 13-32 mm, females SVL 11-42 mm); vomerine teeth absent; maxillary and jugal widely separated; prevomer divided, post-choanal portion absent; neopalatine not distinguishable; clavicles present, short, reaching mid-line of the girdle, but, meeting coracoid in its lateral half and not reaching scapula; epicoracoids long; procoracoid present, short, reaching mid-line of girdle, but, meeting coracoid in its lateral half, and not reaching glenoid region; sternum cartilaginous; omosternum absent; vertebrae diplasiocoelous; terminal phalanges simple; occipital fold absent; pupil round; tongue oval, entire and free behind; two smooth dermal ridges on palate; tympanum hidden; finger tips without or with very small terminal discs; web between fingers with more or less distinct fleshy webbing or fringes; first finger much shorter than second, second shorter than fourth; web between toes very variable, often absent or small, but varying up to large web; toe tips rounded or swollen; outer metatarsals fused; outer metatarsal tubercle absent; inner metatarsal tubercle small; hind limb length short to moderately long; skin smooth, scattered spines sometimes on some body parts; sexual dimorphism developed; eggs usually small and pigmented, but also large and unpigmented; larva aquatic, free living, of typical microhylid morphology, rarely endotrophic. \{Parker 1934; Zweifel 1986; Caramaschi \& Cruz 1997; Cruz et al. 1999, 2007; Canedo et al. 2004; Funk \& Cannatella 2009; Morales \& McDiarmid 2009; Peloso et al. 2014; Tonini et al. 2014; Sá et al. 2018 .

\section{G.28.290. Genus Chiasmocleis Méhelÿ, 1904}

Getangiotaxon: CHIASMOCLEINI nov.

Adelphotaxon: None.

Getendotaxa: Chiasmocleis Méhelÿ, 1904; Relictocleis nov.; Syncope Walker, 1973.

Comments: Peloso et al. (2014) synonymised the gastrophrynine generic nomen Syncope Walker, 1973 with Chiasmocleis Méhelÿ, 1904. Sá et al. (2018) provided a molecular phylogenetic analysis of this group and recognised three well-supported branches in it. For two of them they used the nomina Chiasmocleis and Syncope at subgeneric rank and they erected a new subgeneric nomen "Relictus" for the latter. However, this nomen was shown by Dubois et al. (2018) to be both unavailable (for having 
been published online without Zoobank designation and for missing a diagnosis) and invalid (for being a junior homonym). This was followed by three other publications by the original authors or part of them trying without success to make the nomen "Relictus " and later the nomen "Unicus " available for this subgenus (see Dubois \& Frétey 2020a: 26, footnote 1): in Sá et al. (2019a), the nomen "Relictus" was still unavailable (for still missing a diagnosis); in Sá et al. (2019b), the nomen "Unicus "was also unavailable (both for still missing a diagnosis, but also for being presented as a neonym for an unavailable nomen, therefore unavailable itself according to Article 13.1.3); and in Sá et al. (2019c), with a different auctorship, the nomen "Unicus " was still unavailable (for missing the explicit intention of the authors to establish a new nominal taxon, as required by Article 16.1 and crediting this nomen to a previous work, but also for being presented as a neonym for an unavailable nomen, therefore unavailable itself). The following sentence in the latter paper shows that the authors, referees and editor of this paper have still not understood what availability and auctorship are in zoological nomenclature: "With this publication, we therefore render the nomen Unicus de Sá, Tonini, van Huss, Long, Cuddy, Forlani, Peloso, Zaher and Haddad, 2019 available for Chiasmocleis (Unicus) gnoma." Had this sentence been missing in this paper, it could be debated whether the latter had provided nomenclatural availability to a nomen "Unicus Sá, Tonini, Huss, Zaher \& Haddad, 2019", but unfortunately it is not the case. Errare humanum est, perseverare diabolicum: it is now time to provide an available nomen for this taxon, for those who wish to use the subgeneric rank in amphibian taxonomy. For this purpose, we propose the nomen Relictocleis.

\section{G.29.001. Subgenus Relictocleis nov.}

Getangiotaxon: Chiasmocleis Méhelÿ, 1904.

Adelphotaxa: Chiasmocleis Méhelÿ, 1904; Syncope Walker, 1973.

Getendotaxon: Chiasmocleis (Relictocleis) gnoma Canedo, Dixo \& Pombal, 2004.

Etymology of nomen: L: relictus, 'left, remaining', from relinquo, 'I leave, I abandon'; N: ending of Chiasmocleis Méhelÿ, 1904. • Stem of nomen: Relictocle-. • Grammatical gender of nomen: feminine.

Nucleospecies, by present designation: Chiasmocleis gnoma Canedo, Dixo \& Pombal, 2004.

Diagnosis: Small sized species (males SVL 12.8-15.5 mm, females SVL 13.1-17.9 mm); nasals fused with each other along the most of their mid line, an autapomorphic trait that differentiates Relictocleis from Chiasmocleis and Syncope in which the nasals are separated along their medial length; neopalatine bones present, elongated and thin, slightly beneath the anterior margin of the planum anterorbitale and fused with the vomers and the underlying and well-ossified sphenethmoid, whereas in Chiasmocleis and Syncope they are reduced or absent; zygomatic ramus of the squamosal absent, whereas it is present in most species of Chiasmocleis and Syncope; pars facialis of the maxilla well-developed and bearing a rounded opening anteriorly; phalangeal formula of the manus of Relictocleis 1-2-3-3, whereas it is 2-2-3-3 in Chiasmocleis and 1-2-3-2 in Syncope except in C. hudsoni which has the formula 1-2-3-3; presence in Relictocleis of several autapomorphic substitutions in mitochondrial and nuclear markers. \{Canedo et al. 2004; Sá et al. 2019c).

Comments: Article 11.8 of the Code states that, to be available, a generic nomen "must be, or be treated as, a noun in the nominative singular". This is a fully 'ineffective' and 'void' statement, that could well be removed from the Code, because we know of no case where a generic zoological nomen would have been considered unavailable for being originally an adjective, a rather common situation indeed (e.g., in amphibians, Rugosa Fei, Ye \& Huang, 1990) or for 'looking like' a Latin plural for having an ending that did not exist in any Latin declension (see Dubois 2018) in the nominative singular (e.g., in amphibians, Churamiti Channing \& Stanley, 2002 or Ikakogi Guayasamin, Castroviejo-Fisher, Trueb, Ayarzagüena, Rada \& Vilà, 2009). In all such cases, even if they did not mention it in the original publication, it may be agreed that, by using it as a generic nomen, the original author had ipso facto "treated it as a noun in the nominative singular". Despite this tolerance of the Code, we are not in favour of establishing generic nomina which are clearly based on Latin adjectives or on terms that look like plural Latin terms, and we 
prefer to use from the start genuine nouns in the nominative singular. The new nomen we provide for this taxon is clearly a noun in the nominative singular, not an adjective, as were the first two unavailable nomina originally given to this genus.

\title{
F.19.51. Tribus CTENOPHRYNINI nov.
}

Getangiotaxon: GASTROPHRYNINAE Fitzinger, 1843.

Adelphotaxa: ChIASMOCLEINI nov.; GASTROPHRYNINI Fitzinger, 1843.

Getendotaxon: Ctenophryne Mocquard, 1904.

Nucleogenus, by present designation: Ctenophryne Mocquard, 1904. • Etymology of nomen: G: $\tau \tau \varepsilon 1 \sigma$ (cleis), 'comb'; $\varphi$ vv́v (phryne), 'toad'; referring to the shape of the posterior transversal fold on the pharingial roof (Moquard 1904: 308). • Stem of nomen: Ctenophryn-.

Diagnosis: As for the single genus, small to medium sized microhylids (SVL $43 \mathrm{~mm}$ ); clavicles, procoracoids and omosternum absent; sternum cartilaginous; terminal phalanges pointed or dilated; pupil vertical; tympanum hidden; tongue oval, large, notched, entirely adherent with a median furrow; two dermal ridges across palate anterior to pharynx, a shorter and a longer denticulate; digits slightly dilated; toes largely webbed; a flat inner metatarsal tubercle; outer metatarsal tubercle absent; skin smooth. \{Parker 1934\}.

\section{F.19.52. Tribus GASTROPHRYNINI Fitzinger, 1843}

Eunym: Dubois 2005: 15.

Getangiotaxon: GASTROPHRYNINAE Fitzinger, 1843.

Adelphotaxa: CHIASMOCLEINI nov.; CTENOPHRYNINI nov.

Getendotaxa: DASYPOPINA nov.; GASTROPHRYNINA Fitzinger, 1843; STEREOCYCLOPINA nov.

\section{F.20.45. Subtribus DASYPOPINA nov.}

Getangiotaxon: GASTROPHRYNINI Fitzinger, 1843.

Adelphotaxa: GASTROPHRYNINA Fitzinger, 1843; STEREOCYCLOPINA nov.

Getendotaxa: Dasypops Miranda-Ribeiro, 1924; Myersiella Carvalho, 1954.

Nucleogenus, by present designation: Dasypops Miranda-Ribeiro, 1924. • Etymology of nomen:

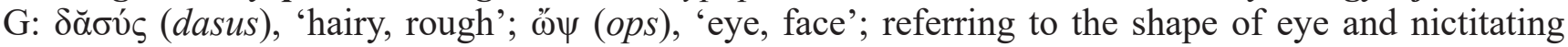
membrane. • Stem of nomen: Dasypop-.

Diagnosis: Small to medium sized microhylid frogs (males SVL 20-46 mm, females SVL 25-33 mm); vomerine and maxillary teeth absent; clavicles absent or reduced; procoracoid absent; coracoid ossified, short and broad, arched; sternum cartilaginous, large semicircular; omosternum absent; pupil circular; tongue large, free posteriorly; dermal folds on palate present; tympanum indistinguishable or absent; finger tips cylindrical or enlarged; web between fingers absent; first finger shorter than second, fourth shorter or equal to second; toe tips enlarged; web between toes absent or reduced; outer metatarsals fused; outer metatarsal tubercle absent; inner metatarsal tubercle small and distinct, or indistinct; hind limbs short; skin smooth or rough. \{Miranda-Ribero 1924; Carvalho 1954; Bokermann 1952; Nelson \& Lescure 1975\}.

\section{F.20.46. Subtribus GASTROPHRYNINA Fitzinger, 1843}

\author{
Eunym: Hoc loco. \\ Getangiotaxon: GASTROPHRYNINI Fitzinger, 1843.
}


Adelphotaxa: DASYPOPINA nov.; STEREOCYCLOPINA nov.

Getendotaxa: ARCOVOMERINIA nov.; DERMATONOTINIA nov.; ENGYSTOMATINIA Bonaparte, 1850; GASTROPHRYNINIA Fitzinger, 1843; HAMPTOPHRYNINIA nov.

\section{F.21.31. Infratribus ARCOVOMERINIA nov.}

Getangiotaxon: GASTROPHRYNINA Fitzinger, 1843.

Adelphotaxa: DERmatonotinia nov.; ENGYSTOMatinia Bonaparte, 1850; GaStrophrYNINIA Fitzinger, 1843; HAMPTOPHRYNINIA nov.

Getendotaxon: Arcovomer Carvalho, 1954.

Nucleogenus, by present designation: Arcovomer Carvalho, 1954. • Etymology of nomen: L: arcus, arched; vomer, 'ploughshare'; referring to the particular shape of the prevomer (Carvalho 1954). $\bullet$ Stem of nomen: Arcovomer-.

Diagnosis: Very small sized microhylid (male SVL $16 \mathrm{~mm}$ ); prevomer divided, postchoanal parts fused on mid-line forming a single arc-like element, center lying in front of anterior tip of parasphenoid, lateral wings curving forward under ethmoids and supporting cartilage of ethmoids; ethmoids separate; palatine absent; quadratojugal not in contact with maxillary; vertebrae diplasiocoelous; clavicle curved, not extending to glenoid cartilage, resting at mid-point of coracoid on a block-like vestige of procoracoid and separating clavicle from coracoid; terminal phalanges T-shaped; fingers and toes not webbed, tips truncate; inner metatarsal tubercle present, outer metatarsal tubercle absent; pupil rounded; tongue narrow, long, entire. \{Carvalho 1954\}.

\section{F.21.32. Infratribus DERMATONOTINIA nov.}

Getangiotaxon: GASTROPHRYNINA Fitzinger, 1843.

Adelphotaxa:ARCovomerinia nov.; ENGYSTOMATINIA Bonaparte, 1850; GASTROPHRYNINIA Fitzinger, 1843; HAMPTOPHRYNINIA nov.

Getendotaxon: Dermatonotus Méhelÿ, 1904.

Nucleogenus, by present designation: Dermatonotus Méhelÿ, 1904. • Etymology of nomen: G:

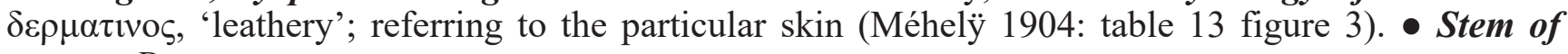
nomen: Dermatonot-.

Diagnosis: Large sized microhylids (males SVL 52-62 mm, females SVL 62-73 $\mathrm{mm}$ ); vomerine teeth absent, but a ridge between choanae; premaxillaries separate from maxillary bones; clavicles straight, almost reaching midline of girdle; procoracoid ossified, in middle united with a rhomboidal cartilaginous plate; sternum as an anchor-shaped cartilaginous plate; omosternum absent; diapophyses of sacral vertebra strongly dilated; lower surface of terminal phalanges with a shovel-shaped dilatation; pupil vertical; tongue large, elliptic, entire, free in its posterior half; tympanum hidden; finger tips not dilated; web between fingers absent; first finger much shorter than second, fourth subequal to second; web between toes absent; toe tips blunt; outer metatarsals united; outer metatarsal tubercle absent; inner metatarsal tubercle oval, very prominent; hind limbs short; skin smooth, strongly thickened on dorsum, leather-like, porous. \{Méhelÿ 1904; Giaretta et al. 2013\}.

\section{F.21.33. Infratribus ENGYSTOMATINIA Bonaparte, 1850}

Protonym: ENGYSTOMIDAE Bonaparte, 1850: plate [F].

Eunym: Hoc loco.

Getangiotaxon: GASTROPHRYNINA Fitzinger, 1843.

Adelphotaxa: ARCovomerinIa nov.; DERMATONOTINIA nov.; GASTROPHRYNINIA Fitzinger, 1843; HAMPTOPHRYNINIA nov. Getendotaxon: Engystoma Fitzinger, 1826. 
Getangiotaxon: ENGYSTOMATINIA Bonaparte, 1850.

Adelphotaxon: None.

Getendotaxa: Engystoma bicolor (Guérin-Méneville, 1838); Engystoma bumbameuboi (Caramaschi, 2010); Engystoma carvalhoi (Caramaschi, 2010); Engystoma cesarii Miranda-Ribeiro, 1920; Engystoma corumbaense (Piva, Caramaschi \& Albuquerque, 2017); Engystoma erythrogaster (Kwet \& Di-Bernardo, 1998); Engystoma haroi (Pereyra, Akmentins, Laufer \& Vaira, 2013); Engystoma helianneae (Caramaschi, 2010); Engystoma magnum (Toledo, 2010); Engystoma matogrosso (Caramaschi, 2010); Engystoma muiraquitan (Nunes-de-Almeida \& Toledo, 2012); Engystoma ovale (Schneider, 1799); Engystoma panamense (Dunn, Trapido \& Evans, 1948); Engystoma pearsei (Ruthven, 1914); Engystoma piauiense (Caramaschi \& Jim, 1983); Engystoma skotogaster (Lavilla, Vaira \& Ferrari, 2003); Engystoma surinamense (Daudin, 1802); Engystoma surumu (Caramaschi, 2010).

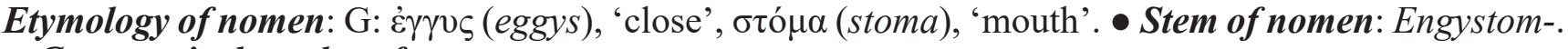
- Grammatical gender of nomen: neuter.

Comments: The generic nomen Engystoma was established by Fitzinger (1826), who did not designate a type species for it. Duméril \& Bibron (1841: 740) designated the nominal species Rana ovalis Schneider, 1799 as type of this genus, and this nomen was consistently used for a genus including this species by various authors until Stejneger (1910) stated in error that the type species of this genus was Rana gibbosa Linnaeus, 1758, then (and still now) referred to the genus Breviceps Merrem, 1820. Parker (1927) established the genus nomen Elachistocleis for Rana ovalis, and this generic nomen was used for this and related species by various authors since then. In 1982, Dubois discovered that Stejneger's (1910) statement was wrong, and, in order to maintain nomenclatural stability, submitted a detailed application to the Commission asking it to use its plenary power to designate Rana gibbosa as type species of Engystoma, thus making it an invalid objective junior synonym of Breviceps. The secretariat of the Commission acknowledged reception of this application and announced it in the Bulletin of Zoological Nomenclature (Anonymous 1982: 230), although with a misprint in the nomen of the genus (Elachistocles), but never published the application and the latter was never submitted to the Commission for vote. After several mails to this secretariat asking for this publication, Dubois (1987f) finally published it elsewhere, but this case was never settled by the Commission. This refusal to address this problem cannot but be interpreted as meaning that for the Commission there existed, in fact, no nomenclatural problem, and that the regular Rules of the Code must apply in this case. The first idea that comes to mind then is to use Article 23.9 of the Code on reversal of precedence, but this is not possible, as the condition of Article 23.9.1.1 is not met, the generic nomen having been used as valid after 1899, and even after 1910, in several publications, either under its protograph and eugraph Engystoma (e.g.: Strecker 1909; Brimley 1915; Nieden 1926; Stabler \& Chen 1936; Metcalf 1940) or under its autoneonym Engistoma Peracca, 1904. The replacement of Elachistocleis by Engystoma, which we implement here, just restores a common practice from 1841 to 1910, and has another nomenclatural advantage, in terms of nomenclatural parsimony: it allows to use for the infratribe here recognised for this genus the nomen ENGYSTOMIDAE Bonaparte, 1850 for which no synonym would be available to replace it if its type species was modified (see Appendix A6.NFS), which would require the introduction of a new FS nomen. This nomen also has been used as valid after 1899 (e.g.: Méhelÿ 1901; Nieden 1926) and could also not be rejected by Article 23.9.

\section{F.21.34. Infratribus GASTROPHRYNINIA Fitzinger, 1843}

Eunym: Hoc loco.

Getangiotaxon: GASTROPHRYNINA Fitzinger, 1843.

Adelphotaxa: ARCovomerinia nov.; DERMATONOTINIA nov.; ENGYSTOMATINIA Bonaparte, 1850; HAMPTOPHRYNINIA nov.

Getendotaxa: Gastrophryne Fitzinger, 1843; Hypopachus Keferstein, 1867. 


\section{F.21.35. Infratribus HAMPTOPHRYNINIA nov.}

Getangiotaxon: GASTROPHRYNINA Fitzinger, 1843.

Adelphotaxa: ArCovomerinia nov.; DeRMATONOTINIA nov.; ENGYSTOMATINIA Bonaparte, 1850; GASTROPHRYNINIA Fitzinger, 1843.

Getendotaxon: Hamptophryne Carvalho, 1954.

Nucleogenus, by present designation: Hamptophryne Carvalho, 1954. • Etymology of nomen: N: Hampton, in honor of Hampton Wildman Parker (1897-1968), London, specialist of microhylids; G:

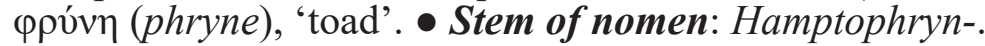

Diagnosis: Medium sized microhylids (males SVL 34-50 mm, females SVL 39-44 mm); vomerine teeth absent; maxillary arcade incomplete, maxilla and quadratojugal not in contact; prevomer divided, posterior part reduced to a small osseous plate lying more or less free in mucosa of palate; quadratojugal in contact with maxillary; clavicles not reaching glenoid cartilage, resting at distal end on coracoid, at proximal end on tip of reduced vertebrae diplasiocoelous; terminal phalanges slightly expanded; pupil round; tympanum hidden; finger tips blunt; web between fingers absent or basal, fingers with or without narrow fringes; web between toes absent or small; toe tips blunt; first finger much shorter than second, fourth subequal to second; outer metatarsal tubercle absent; inner metatarsal tubercle distinct or prominent; hind limbs short; skin smooth or shagreened. \{Parker 1927, 1934; Carvalho 1954; Wild 1995; Funk \& Cannatella 2009\}.

\section{F.20.47. Subtribus STEREOCYCLOPINA nov.}

Getangiotaxon: GASTROPHRYNINI Fitzinger, 1843.

Adelphotaxa: DASYPOPINA nov.; GASTROPHRYNINA Fitzinger, 1843.

Getendotaxon: Stereocyclops Cope, 1870.

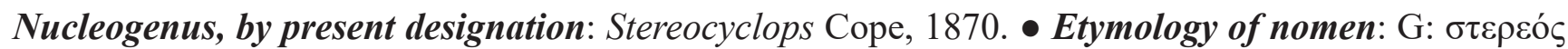

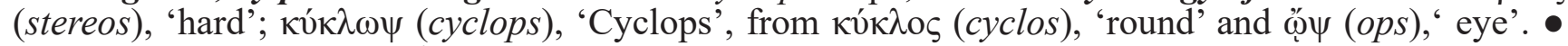
Stem of nomen: Stereocyclop-.

Diagnosis: Small to medium sized GASTROPHRYNINI (males SVL 24-49 mm; females SVL 25-57 mm); vomerine and maxillary teeth absent; quadratojugal and maxilla in firm bony contact; palatine palatal folds present; clavicles long or short, when short not reaching glenoid cartilage; procoracoid cartilage well developed, extending from mid-line of girdle to glenoid cartilage, touching mesial part of coracoid and supporting clavicle in its entire length; two fenestrae on each side of pectoral girdle between procoracoid and coracoid; xiphisternum cartilaginous and broad; vertebrae diplasiocoelous; terminal phalanges simple or slightly expanded; occipital fold present; pupil oval horizontal; tongue large not notched; dermal fords on palate present; tympanum indistinct; finger tips rounded, not enlarged; web on hand absent, narrow fringes sometimes present; first finger shorter than second; fourth longer or subequal to second; web on feet absent or a small webbing present; toe tips rounded, not enlarged; outer metatarsal tubercle absent or indistinct; inner metatarsal tubercle well developed; hindlimbs short to rather long; skin smooth, but dermal spines present in some body parts; larva aquatic, free living, of typical microhylid morphology. \{Cope 1869a; Wettstein 1934; Carvalho 1948, 1954; Targino \& Pombal 2011; Caramaschi et al. 2012\}.

\section{F.18.52. Subfamilia HoPLOPHRYNINAE Noble, 1931}

Protonym and eunym: HoPLOPHRYNINAE Noble, 1931: 539 [bF].

Getangiotaxon: MICROHYLIDAE \|Fitzinger, 1843\|-Noble, 1931.

Adelphotaxa: AdeLASTINAE Peloso, Frost, Richards, Rodrigues, Donnellan, Matsui, Raxworthy, Biju, Lemmon, Lemmon \& Wheeler, 2016; Asterophryinae Günther, 1858; Cophylinae Cope, 1889; Gastrophryninae Fitzinger, 1843; Kalophryninae Mivart, 1869; MELANobatrachinae Noble, 1931; Microhylinae ||Fitzinger, 1843\|-Noble, 1931; OtophryninaE Wassersug \& Pyburn, 1987.

Getendotaxa: Hoplophryne Barbour, 1928; Parhoplophryne Barbour, 1928. 


\section{F.18.53. Subfamilia KALOPHRYNINAE Mivart, 1869}

Protonym: KALOPHRYNINA Mivart, 1869: 289 [bF].

Eunym: Noble 1931: 536.

Getangiotaxon: MICROHYLIDAE ||Fitzinger, 1843\|-Noble, 1931.

Adelphotaxa: AdelastinaE Peloso, Frost, Richards, Rodrigues, Donnellan, Matsui, Raxworthy, Biju, Lemmon, Lemmon \& Wheeler, 2016; Asterophryinae Günther, 1858; CophylinaE Cope, 1889; Gastrophryninae Fitzinger, 1843; Hoplophryninae Noble, 1931; Melanobatrachinae Noble, 1931; Microhylinae ||Fitzinger, 1843||-Noble, 1931; OTOPHRYNINAE Wassersug \& Pyburn, 1987.

Getendotaxon: Kalophrynus Tschudi, 1838.

\section{F.18.54. Subfamilia MELANOBATRACHINAE Noble, 1931}

Protonym and eunym: MELANOBATRACHINAE Noble, 1931: 538 [bF].

Getangiotaxon: MICROHYLIDAE \|Fitzinger, 1843\|-Noble, 1931.

Adelphotaxa: Adelastinae Peloso, Frost, Richards, Rodrigues, Donnellan, Matsui, Raxworthy, Biju, Lemmon, Lemmon \& Wheeler, 2016; Asterophryinae Günther, 1858; CophylinaE Cope, 1889; GastrophrYNINAE Fitzinger, 1843; Hoplophryninae Noble, 1931; Kalophryninae Mivart, 1869; MicrohylinaE ||Fitzinger, 1843\|-Noble, 1931; OtophryninaE Wassersug \& Pyburn, 1987.

Getendotaxon: Melanobatrachus Beddome, 1878.

F.18.55. Subfamilia MICROHYLINAE \|Fitzinger, 1843\|-Noble, 1931

Eunym: Noble 1931: 451.

Getangiotaxon: MICROHYLIDAE \|Fitzinger, 1843\|-Noble, 1931.

Adelphotaxa: Adelastinae Peloso, Frost, Richards, Rodrigues, Donnellan, Matsui, Raxworthy, Biju, Lemmon, Lemmon \& Wheeler, 2016; Asterophryinae Günther, 1858; Cophylinae Cope, 1889; Gastrophryninae Fitzinger, 1843; Hoplophryninae Noble, 1931; Kalophryninae Mivart, 1869; Melanobatrachinae Noble, 1931; OtophrYninae Wassersug \& Pyburn, 1987.

Getendotaxa: DYSCOPHINI Boulenger, 1882; MICROHYLINI ||Fitzinger, 1843||-Noble, 1931.

Comments: In the subfamily MICROHYLINAE, according to TREE, the tribe DYSCOPHINI (credited with the rank subfamily by Tu et al. 2018) for Dyscophus, is sister-group to all other MICROHYLINAE, recognised as the tribe MICROHYLINI. Within this tribe four subtribes of unsupported relationships are recognised, the CHAPERININA for Chaperina, the MICROHYLINA for Glyphoglossus and Microhyla, the MiCRYLETTINA for Micryletta and Mysticellus, and the HyLAEDACTYLINA, that include three infratribes, the CACOPINIA for Uperodon, the HYLAEDACTYLINIA for Kaloula, and the PHRYNELLINIA for Metaphrynella and Phrynella.

Tu et al. (2018) found in our tribe MICROHYLINI two highly supported branches, grouping in a taxon the subtribes CHAPERININA and MICROHYLINA, and in a second taxon the subtribes HYLAEDACTYLINA and MICRYLETTINA.

Matsui et al. (2011) found the species attributed to the genus Microhyla forming two paraphyletic groups, including one composed of Calluella and Glyphoglossus. These three groups are highly supported but their relationships are poorly resolved. Tu et al. (2018) and Garg \& Biju (2019) found the same relationships between the last two genera and considered Calluella as a junior subjective synonym of Glyphoglossus. In TREE, Glyphoglossus is sister-taxon to a holophyletic Microhyla, and we maintain both genera.

\section{F.19.53. Tribus DYSCOPHINI Boulenger, 1882}

Protonym: DYSCOPHIDAE Boulenger, 1882: 179 [F].

Eunym: Hoc loco.

Getangiotaxon: MICROHYLINAE ||Fitzinger, 1843\|-Noble, 1931. 
Adelphotaxon: MICROHYLINI ||Fitzinger, 1843\|-Noble, 1931.

Getendotaxon: Dyscophus Grandidier, 1872.

F.19.54. Tribus MICROHYLINI ||Fitzinger, 1843\|-Noble, 1931

Eunym: Dubois 2005d: 15.

Getangiotaxon: MiCROHYLINAE ||Fitzinger, 1843\|-Noble, 1931.

Adelphotaxon: DYSCOPHINI Boulenger, 1882.

Getendotaxa: Chaperinina Peloso, Frost, Richards, Rodrigues, Donnellan, Matsui, Raxworthy, Biju, Lemmon, Lemmon \& Wheeler 2016; HYLAEDACTYLINA Fitzinger, 1843; MICROHYLINA ||Fitzinger, 1843||$-$ Noble, 1931; MiCRYLETTINA nov.

F.20.48. Subtribus CHAPERININA Peloso, Frost, Richards, Rodrigues, Donnellan, Matsui, Raxworthy, Biju, Lemmon, Lemmon \& Wheeler 2016

Protonym: Chaperininae Peloso, Frost, Richards, Rodrigues, Donnellan, Matsui, Raxworthy, Biju, Lemmon, Lemmon \& Wheeler 2016: 135 [bF].

Eunym: Hoc loco.

Getangiotaxon: MICROHYLINI ||Fitzinger, 1843\|-Noble, 1931.

Adelphotaxa: HyLAEDACTYLINA Fitzinger, 1843; MiCROHYLINA ||Fitzinger, 1843||-Noble, 1931; MiCRYLETTINA nov.

Getendotaxon: Chaperina Mocquard, 1892.

\section{F.20.49. Subtribus HYLAEDACTYLINA Fitzinger, 1843}

Protonym: HyLAEDACTYLI Fitzinger, 1843: 33 [F].

Eunym: Hoc loco.

Getangiotaxon: MICROHYLINI ||Fitzinger, 1843\|-Noble, 1931.

Adelphotaxa: Chaperinina Peloso, Frost, Richards, Rodrigues, Donnellan, Matsui, Raxworthy, Biju, Lemmon, Lemmon \& Wheeler 2016; MiCRoHYLINA ||Fitzinger, 1843\|-Noble, 1931; MiCRYLETtina nov.

Getendotaxa: CACOPINIA Noble, 1931; HylaEdACTYLINIA Fitzinger, 1843; PHRYNELLINIA nov.

\section{F.21.36. Infratribus CACOPINIA Noble, 1931}

Protonym: CACOPINAE Noble, 1931: 532 [bF].

Eunym: Hoc loco.

Getangiotaxon: HYLAEDACTYLINA Fitzinger, 1843.

Adelphotaxa: HyLAEDACTYLINIA Fitzinger, 1843; PHRYNELLINIA nov.

Getendotaxon: Uperodon Duméril \& Bibron, 1841.

\section{F.21.37. Infratribus HYLAEDACTYLINIA Fitzinger, 1843}

Eunym: Hoc loco.

Getangiotaxon: HYLAEDACTYLINA Fitzinger, 1843.

Adelphotaxa: CACopinia Noble, 1931; PhrYNELLINIA nov.

Getendotaxon: Kaloula Gray, 1831.

\section{F.21.38. Infratribus PHRYNELLINIA nov.}

Getangiotaxon: HYLAEDACTYLINA Fitzinger, 1843.

Adelphotaxa: CACOPINIA Noble, 1931; HYLAEDACTYLINIA Fitzinger, 1843.

Getendotaxa: Metaphrynella Parker, 1934; Phrynella Boulenger, 1887. 
Nucleogenus, by present designation: Phrynella Boulenger, 1887. • Etymology of nomen: G: $\varphi \rho v ́ v \eta$ (phryne), 'toad'; L: -ella, a feminine suffix indicating a diminutive form. • Stem of nomen: Phrynell-.

Diagnosis: Small to medium sized microhylids (SVL males 19-45 mm; females 23-45 mm); vomerine and maxillary teeth absent; prevomer divided; palatine, clavicles and procoracoid absent; sternum cartilaginous; omosternum absent or small; vertebrae procoelous; terminal phalanges Y-shaped; pupil horizontal; tongue oval, scarcely free; two transverse ridges on palate; tympanum hidden; finger tips strongly dilated; subarticular tubercles of hands enlarged to form accessory adhesive organs; rudiment of web on hand; feet largely webbed; toe tips with distinct discs; first finger shorter than second, second little shorter than fourth; outer metatarsal tubercle absent; inner metatarsal tubercle present; hind limb length short; skin smooth or pustular; dorsal coloration dark with light lines or dark pattern; mid-dorsal stripe absent; ventral coloration light with or without spots. \{Parker 1934; Inger 1966; Manthey \& Grossmann 1997\}.

\title{
F.20.50. Subtribus MiCROHYLINA ||Fitzinger, 1843\|-Noble, 1931
}

\author{
Eunym: Hoc loco. \\ Getangiotaxon: MICROHYLINI ||Fitzinger, 1843\|-Noble, 1931. \\ Adelphotaxa: Chaperinina Peloso, Frost, Richards, Rodrigues, Donnellan, Matsui, Raxworthy, Biju, Lemmon, Lemmon \& \\ Wheeler 2016; HYLAEDACTYLINA Fitzinger, 1843; MICRYLETTINA nov. \\ Getendotaxa: Glyphoglossus Günther, 1869; Microhyla Tschudi, 1838.
}

\section{F.20.51. Subtribus MiCRYLETTINA nov.}

Getangiotaxon: MICROHYLINI ||Fitzinger, 1843\|-Noble, 1931.

Adelphotaxa: Chaperinina Peloso, Frost, Richards, Rodrigues, Donnellan, Matsui, Raxworthy, Biju, Lemmon, Lemmon \& Wheeler 2016; HyLAEDACTYLINA Fitzinger, 1843; MICROHYLINA ||Fitzinger, 1843||-Noble, 1931.

Getendotaxa: Micryletta Dubois, 1987; Mysticellus Garg \& Biju, 2019.

Nucleogenus, by present designation: Micryletta Dubois, 1987. • Etymology of nomen: N: Micrhyla

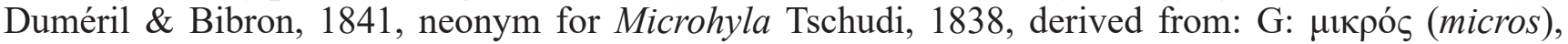
'small'; N: Hyla, of debated etymology; -etta, a feminine suffix indicating jumping behaviour of these frogs. $\bullet$ Stem of nomen: Micrylett-.

Diagnosis: Small sized microhylid (SVL males 19-28 mm; females SVL 22-29 mm); vomerine teeth absent; maxillary teeth absent; prevomer divided; palatine absent; ethmoid strongly developed; clavicles absent; procoracoid absent; sternum cartilaginous; omosternum absent; vertebrae diplasiocoelous; terminal phalanges simple; occipital fold absent; pupil oval or rounded; finger tips blunt; supernumerary tubercles present, distinct; first finger shorter than second; web between fingers absent; web between toes absent or with small rudiment; toe tips blunt; outer metatarsals fused; inner metatarsal tubercle prominent; hind limbs short; skin smooth or shagreened; ventral coloration clear, more or less spotted. \{Boulenger 1909; Parker 1934; Tarkhnishvili 1994; Manthey \& Grossmann 1997; Garg \& Biju 2019 \}.

\section{F.18.56. Subfamilia Otophryninae Wassersug \& Pyburn, 1987}

Protonym and eunym: OTOPHRYNINAE Wassersug \& Pyburn, 1987: 532 [bF].

Getangiotaxon: MICROHYLIDAE ||Fitzinger, 1843\|-Noble, 1931.

Adelphotaxa: AdelastinaE Peloso, Frost, Richards, Rodrigues, Donnellan, Matsui, Raxworthy, Biju, Lemmon, Lemmon \& Wheeler, 2016; AsterophryinaE Günther, 1858; Cophylinae Cope, 1889; GastrophryninaE Fitzinger, 1843; Hoplophryninae Noble, 1931; KalophryninaE Mivart, 1869; MelanobatraChinaE Noble, 1931; MicrohylinaE ||Fitzinger, 1843\|-Noble, 1931.

Getendotaxa: Otophryne Boulenger, 1900; Synapturanus Carvalho, 1954. 
Protonym: PhrYNomerinaE Noble, 1931: 538 [bF].

Eunym: Parker 1934: 9.

Getangiotaxon: Ecostata Lataste, 1879.

Adelphotaxon: MICROHYLIDAE ||Fitzinger, 1843||-Noble, 1931.

Getendotaxon: Phrynomantis Peters, 1867.

Comments: The position of Phrynomantis is highly variable in the phylogenetic trees published but in the recent works including exhaustive sampling (Bossuyt \& Roelants 2009; Pyron \& Wiens 2011; Tu et al. 2019) as well as in TREE it shows to be the sister-taxon to all microhylid taxa. Thus, here we recognise it on the basis of the [STC] at the family rank as PHRYNOMERIDAE.

\section{C.11.04. Subphalanx Gastrechmia Cope, 1867}

Protonym: Gastrechmia Cope, 1867: 190 [bO].

Eunym: Hoc loco.

Getangiotaxon: ScoptanURA Starrett, 1973.

Adelphotaxa: Ecostata Lataste, 1879; Pananura nov.; 1 G†.

Getendotaxa: ARthroleptoIdeA Mivart, 1869; BREVICIPITOIDEA Bonaparte, 1850.

Comments: This highly supported branch groups the families ARTHROLEPTIDAE, BREVICIPITIDAE, HEMISOTIDAE and HYPEROLIIDAE. The branch was first recovered for Arthroleptis, Leptopelis, Heterixalus, Hyperolius, Kassina and Breviceps by Van der Meijden et al. (2004) and recognised by them as ARTHROLEPTOIDAE. It was documented by Frost et al. (2006), Bossuyt \& Roelants (2009), Frazão et al. (2015), Feng et al. (2017) and Portik \& Blackburn (2016), and given the ectonym «AFrobatrachia» by Frost et al. (2006). Zhang et al. (2013) recognised this taxon as BREVICIPITOIDAE. All recent works, as well as TREE, find a sister-group relationship between a taxon grouping the ARTHROLEPTIDAE and HYPEROLIIDAE and a second taxon grouping the BREVICIPITIDAE and HEMISOTIDAE. Here this first taxon is named ARTHROLEPTOIDEA and the second BREVICIPITOIDEA. HYPEROLIIDAE and HEMISOTIDAE are attributed to the family rank by virtue of the [UQC], and all the other ranks derive from this.

\section{F.14.08. Superfamilia ARTHROLEPTOIDEA Mivart, 1869}

Protonym: ARTHROLEPTINA Mivart, 1869: 294 [bF].

Eunym: Hoc loco.

Getangiotaxon: GaSTREChMia Cope, 1867.

Adelphotaxon: BREVICIPITOIDEA Bonaparte, 1850.

Getendotaxa: ARthroleptidAE Mivart, 1869; HyPERoLIIDAE Laurent, 1943.

\section{F.17.31. Familia ARTHROLEPTIDAE Mivart, 1869}

Eunym: Laurent 1972: 200.

Getangiotaxon: ARTHROLEPTOIDEA Mivart, 1869.

Adelphotaxon: HyPEROLIIDAE Laurent, 1943.

Getendotaxa: Arthroleptinae Mivart, 1869; Astylosterninae Noble, 1927; LEPTOPELINAE Laurent, 1972.

Comments: The family ARTHROLEPTIDAE is recognised at the family rank according to the sister-taxon Criterion [STC]. It includes three taxa of unresolved relationships that are recognised as the subfamilies ARTHROLEPTINAE for the single genus Arthroleptis, LEPTOPELINAE for Leptopelis, and ASTYLOSTERNINAE. This latter subfamily includes two taxa, the tribe LEPTODACTYLODONTINI for Leptodactylodon, and the tribe ASTYLOSTERNINI, for the sister-taxa Nyctibates and Scotobleps, and their sister-taxon Astylosternus. 
To keep a holophyletic taxon with high support for Astylosternus, we synonymised Trichobatrachus with the latter. As there is no support for the holophyly of Cardioglossa, it is synonymised with Astylosternus. The analysis of Portik \& Blackburn (2016) results in similar relationships within the ARTHROLEPTIDAE but they obtained holophyletic groups for Astylosternus, with Trichobatrachus as sister taxon, and Cardioglossa, with Arthroleptis as sister-taxon.

\title{
F.18.57. Subfamilia ARTHROLEPTINAE Mivart, 1869
}

Eunym: Noble 1931: 515.

Getangiotaxon: ARTHROLEPTIDAE Mivart, 1869.

Adelphotaxa: ASTYLOSTERNINAE Noble, 1927; LEPTOPELINAE Laurent, 1972.

Getendotaxon: Arthroleptis Smith, 1849.

\section{F.18.58. Subfamilia ASTYLOSTERNINAE Noble, 1927}

Protonym and eunym: AstYLOSTERNINAE Noble, 1927: 110 [bF].

Getangiotaxon: ARTHROLEPTIDAE Mivart, 1869.

Adelphotaxa: ARthroleptinae Mivart, 1869; LePtopelinaE Laurent, 1972.

Getendotaxa: ASTYLOSTERNINI Noble, 1927; LEPTODACTYLODONTINI nov.

\section{F.19.55. Tribus ASTYLOSTERNINI Noble, 1927}

Eunym: Frost, Grant, Faivovich, Bain, Haas, Haddad, Sá, Channing, Wilkinson, Donnellan, Raxworthy, Campbell, Blotto, Moler, Drewes, Nussbaum, Lynch, Green \& Wheeler 2006: 234.

Getangiotaxon: ARTHROLEPTINAE Mivart, 1869.

Adelphotaxon: LEPTODACTYLODONTINI nov.

Getendotaxa: Astylosternus Werner, 1898; Nyctibates Boulenger, 1904; Scotobleps Boulenger, 1900.

\section{F.19.56. Tribus LEPTODACTYLODONTINI nov.}

Getangiotaxon: ARTHROLEPTINAE Mivart, 1869.

Adelphotaxon: AsTYLOSTERNINI Noble, 1927.

Getendotaxon: Leptodactylodon Andersson, 1903.

Nucleogenus, by present designation: Leptodactylodon Andersson, 1903. • Etymology of nomen: G:

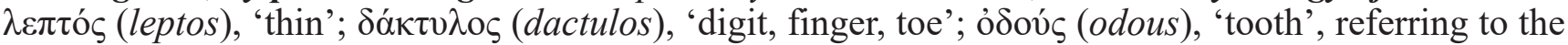
shape of the terminal phalanges. • Stem of nomen: Leptodactylodont-.

Diagnosis: Small to medium sized frogs (males SVL 20-44 mm; females SVL 22-42 mm); stocky habitus, legs relatively short, small head, snout rounded; small eyes with oval horizontal pupilla; webbing absent on foot; terminal phalanges not exsertile, slightly pointed; males with two groups of nuptial spines on inner side of hand and on first finger; omosternum not forked, enlarged; tadpoles with large buccal labia, keratodonts absent, mouth sheath with long tooth sharp serrations. \{Amiet 1981\}.

\section{F.18.59. Subfamilia LEPTOPELINAE Laurent, 1972}

\author{
Protonym: LEPTOPELINI Laurent, 1972: 201 [T]. \\ Eunym: Dubois 1981: 227. \\ Getangiotaxon: ARTHROLEPTIDAE Mivart, 1869. \\ Adelphotaxa: Arthroleptinae Mivart, 1869; AstylosterninaE Noble, 1927. \\ Getendotaxon: Leptopelis Günther, 1859.
}


Protonym: HyPEROLIINAE Laurent, 1943: 16 [bF].

Eunym: Laurent 1951: 116.

Getangiotaxon: ARTHROLEPTOIDEA Mivart, 1869.

Adelphotaxon: ARTHROLEPTIDAE Mivart, 1869.

Getendotaxa: CRYPtothylaCinaE nov.; Hyperolinae Laurent, 1943; 3 GIS (Arlequinus Perret, 1988; Callixalus Laurent, 1950; Chrysobatrachus Laurent, 1951).

Comments: This taxon is attributed to the family rank following the [UQC]. Within this family, two groups are recognised as subfamilies: the CRYPTOTHYLACINAE for Cryptothylax, the sister-group to the rest of the HYPEROLIIDAE, recognised as the HYPEROLIINAE. Within the HYPEROLIINAE there is support for three taxa with unresolved mutual relationships, recognised as the tribes ACANTHIXALINI for Acanthixalus, KASSININI for Hylambates, Kassina, Kassinula, Paracassina and Semnodactylus, and the HYPEROLIINI. This is quite different from the results of Portik \& Blackburn (2016) as their tree of HYPEROLIIDAE consists in two taxa, one for our KASSININI, sister-group to all other HYPEROLIIDAE, which include our CRYPTOTHYLACINAE, ACANTHIXALINI and HYPEROLIINI. Other groups supported in TREE find also support in Portik \& Blackburn (2016), as the sister-taxon relationship of Heterixalus and Tachycnemis, that form a holophyletic relationship with Afrixalus, recognised in our classification as TACHYCNEMINIA. In TREE, the inclusion of Kassina maculata within a group of species that were attributed to the genus Phlyctimantis by Portik \& Blackburn (2016) is confirmed. However, this taxon should be named Hylambates Duméril, 1853, following the Principle of Priority, as Phlyctimantis Laurent \& Combaz, 1950 is its subjective junior synonym. Contrary to what appears in $A S W<2020 a>$, the fact that Opinion 849 (Anonymous 1968) have afforded priority to Kassina Girard, 1853 over Hylambates Duméril, 1853 when both nomina are considered synonyms has not resulted in the 'suppression' (invalidation) of the latter and has no bearing on its validity when it is not considered synonym of Kassina! Therefore the species included in this genus should be known as Hylambates boulengeri (Perret, 1986), Hylambates keithae (Schiøtz, 1975), Hylambates leonardi Boulenger, 1906, Hylambates maculatus Duméril, 1953 and Hylambates verrucosus Boulenger, 1912.

\section{F.18.60. Subfamilia CRYPTOTHYLACINAE nov.}

Getangiotaxon: HYPEROLIIDAE Laurent, 1943.

Adelphotaxa: Hyperolinat Laurent, 1943; 3 GIS (Arlequinus Perret, 1988; Callixalus Laurent, 1950; Chrysobatrachus Laurent, 1951).

Getendotaxon: Cryptothylax Laurent \& Combaz, 1950.

Nucleogenus, by present designation: Cryptothylax Laurent \& Combaz, 1950. • Etymology of nomen:

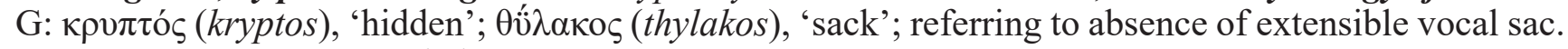
- Stem of nomen: Cryptothylac-.

Diagnosis: Large sized hyperoliids (males SVL 39-54 mm; females SVL 48-58 mm); body slender, skin shagreen with small warts on dorsum; gular glands very large, obscuring gular region; vocal sac absent; finger and toes with enlarged pads with ventro-marginal groove anteriorly; fingers slightly webbed; toes largely webbed; tadpole with 1/3 rows of keratodonts. \{Liem 1970; Drewes 1984\}.

\section{F.18.61. Subfamilia HYPEROLIINAE Laurent, 1943}

Eunym: Laurent 1943: 16.

Getangiotaxon: HYPEROLIIDAE Laurent, 1943.

Adelphotaxa: CRYPTothylaCinae nov.; 3 GIS (Arlequinus Perret, 1988; Callixalus Laurent, 1950; Chrysobatrachus Laurent, 1951).

Getendotaxa: ACANTHIXALINI nov.; HYPEROLIINI Laurent, 1943; KASSININI Laurent, 1972. 


\section{F.19.57. Tribus ACANTHIXALINI nov.}

Getangiotaxon: HYPEROLIINAE Laurent, 1943.

Adelphotaxa: HyPEROLIINI Laurent, 1943; KASSININI Laurent, 1972.

Getendotaxon: Acanthixalus Laurent, 1944.

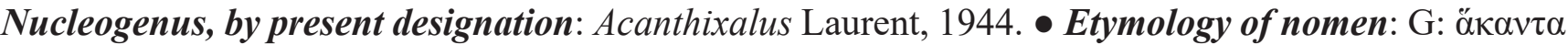

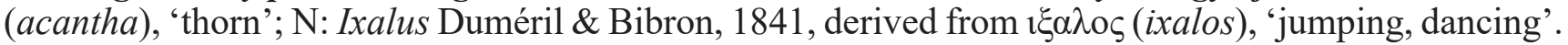
- Stem of nomen: Acanthixal-.

Diagnosis: Small sized frogs (SVL 32-36 mm); pupil horizontal; paired oval gular glands; tarsal spines present; tympanum absent; vertebrae procoelous; posterolateral process of hyoid present. \{Drewes $1984\}$.

\section{F.19.58. Tribus HYPEROLIINI Laurent, 1943}

Eunym: Laurent 1972: 201.

Getangiotaxon: HYPEROLIINAE Laurent, 1943.

Adelphotaxa: ACANTHIXALINI nov.; KASSININI Laurent, 1972.

Getendotaxa: HyPERolitna Laurent, 1943; Morerellina nov.; OPISTHOthYLACINA nov.; TACHYCNEMINA Channing, 1989.

Comments: In TREE, the relationships within the HYPEROLIINI are poorly resolved, so we recognise four subtribes, the HYPEROLIINA for Hyperolius, the MORERELLINA for Morerella, the OPISTHOTHYLACINA for Opisthothylax, and the TACHYCNEMINA. In the latter subtribe, Heterixalus is sister-genus to Tachycnemis, constituting together the infratribe TACHYCNEMINIA, and Afrixalus, the infratribe AFRIXALINIA, is sistertaxon to this group.

\section{F.20.52. Subtribus HYPEROLIINA Laurent, 1943}

Eunym: Hoc loco.

Getangiotaxon: HYPEROLIINI Laurent, 1943.

Adelphotaxa: Morerellina nov.; OpisthothylaCina nov.; TACHYCNEMINA Channing, 1989.

Getendotaxon: Hyperolius Rapp, 1842.

\section{F.20.53. Subtribus MORERELLINA nov.}

Getangiotaxon: HYPEROLIINI Laurent, 1943.

Adelphotaxa: Hyperolina Laurent, 1943; OpISTHothylaCina nov.; TACHYCNEMINA Channing, 1989.

Getendotaxon: Morerella Rödel, Kosuch, Grafe, Boistel \& Veith in Rödel, Kosuch, Grafe, Boistel, Assemian, Kouamé, Tohé, Gourène, Perret, Henle, Tafforeau, Pollet \& Veith, 2009.

Nucleogenus, by present designation: Morerella Rödel, Kosuch, Grafe, Boistel \& Veith in Rödel, Kosuch, Grafe, Boistel, Assemian, Kouamé, Tohé, Gourène, Perret, Henle, Tafforeau, Pollet \& Veith, 2009. • Etymology of nomen: P: Jean-Jacques Morère (1947-), French batrachologist. • Stem of nomen: Morerell-.

Diagnosis: Medium sized tree-frogs (males mean SVL $29 \mathrm{~mm}$; females mean SVL $32 \mathrm{~mm}$ ); slender body; large protruding eyes; pupil horizontal; tympanum small but distinct; males with medium sized, medioposterior gular gland without dilatable skin of vocal sac; males with small spines on back and limbs; sphenethmoid not visible dorsally; ventroanterior portion of sphenethmoid unfused, consisting of two elements; non-imbricate neural arches not completely roofing spinal canal; transverse processes of eighth vertebra not angled markedly forward; a greatly forked omosternum; space between arms more than twice width of one arm; posterolateral process of hyoid absent; sternum completely ossified; pads on finger and toe tips round; intercalary elements of phalanges completely mineralised; short 
advertisement call, tonal grouped and not pulsed; arboreal eggs and aquatic larval stages. \{Rödel et al. $2009\}$.

\section{F.20.54. Subtribus OPISTHOTHYLACINA nov.}

Getangiotaxon: HYPEROLIINI Laurent, 1943.

Adelphotaxa: HyPEROLIINA Laurent, 1943; MORERELLINA nov.; TACHYCNEMINA Channing, 1989.

Getendotaxon: Opisthothylax Perret, 1966.

Nucleogenus, by present designation: Opisthothylax Perret, 1966. • Etymology of nomen: G: ó

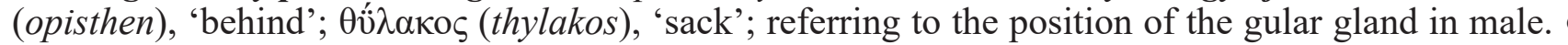
Stem of nomen: Opisthothylac-.

Diagnosis: Small sized frogs (SVL 30-33 mm); pupil vertical, tympanum absent; skin of dorsum and limbs very warty; males with a medioposterior gular gland on non-distensible skin of vocal sac; tympanum absent; chromosome complement $2 \mathrm{n}=24$ with the presence of a distinctive pair of subtelocentric chromosomes; eggs large, not pigmented, 8-10 per clutch in foam nest deposited in folded leaves; tadpole without keratodonts. \{Amiet 1974; Drewes 1984\}.

\section{F.20.55. Subtribus TACHYCNEMINA Channing, 1989}

Protonym: TACHYCNEMINAE Channing, 1989: 116 [bF].

Eunym: Hoc loco.

Getangiotaxon: HYPEROLIINI Laurent, 1943.

Adelphotaxa: HyPEROLIINA Laurent, 1943; MORERELLINA nov.; OPISTHOTHYLACINA nov.

Getendotaxa: AFRIXALINIA nov.; TACHYCNEMINIA Channing, 1989.

\section{F.21.39. Infratribus AFRIXALINIA nov.}

Getangiotaxon: TACHYCNEMINA Channing, 1989.

Adelphotaxon: TACHYCNEMINIA Channing, 1989.

Getendotaxon: Afrixalus Laurent, 1944.

Nucleogenus, by present designation: Afrixalus Laurent, 1944. • Etymology of nomen: R: Africa; N:

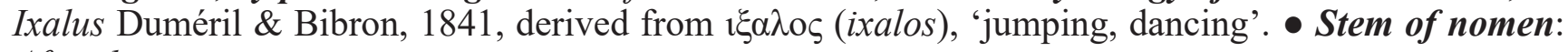
Afrixal-.

Diagnosis: Small sized frogs (SVL 20-35 mm); pupil vertical; skin of males usually spinulous; tympanum usually distinct; toes usually half-webbed; posterolateral process of hyoid absent; keratodont formula usually 0/10. \{Liem 1970; Drewes 1984\}.

\section{F.21.40. Infratribus TACHYCNEMINIA Channing, 1989.}

Eunym: Hoc loco.

Getangiotaxon: TACHYCNEMINA Channing, 1989.

Adelphotaxon: AFRIXALINIA nov.

Getendotaxa: Heterixalus Laurent, 1944; Tachycnemis Fitzinger, 1843. 
Getangiotaxon: HYPEROLIINAE Laurent, 1943.

Adelphotaxa: ACANTHIXALINI nov.; HYPEROLIINI Laurent, 1943.

Getendotaxa: Hylambates Duméril, 1853; Kassina Girard, 1853; Kassinula Laurent, 1940; Paracassina Peracca, 1907; Semnodactylus Hoffman, 1939.

F.14.09. Superfamilia BREVICIPITOIDEA Bonaparte, 1850

Protonym: BREVICIPITINA Bonaparte, 1850: plate [bF].

Eunym: Hoc loco.

Getangiotaxon: GaSTREChMia Cope, 1867.

Adelphotaxon: ARTHROLEPTOIDEA Mivart, 1869.

Getendotaxa: BREVICIPITIDAE Bonaparte, 1850; HemisotidaE Cope, 1867.

\section{F.17.33. Familia BREVICIPITIDAE Bonaparte, 1850}

Eunym: Cope 1867: 191.

Getangiotaxon: BREVICIPITOIDEA Bonaparte, 1850.

Adelphotaxon: HeMISOTIDAE Cope, 1867.

Getendotaxa: BREVICIPITINAE Bonaparte, 1850; CALLULININAE nov.

Comments: Within the family BREVICIPITIDAE, two groups have high support, recognised here as the subfamily BREVICIPITINAE for Breviceps, and CALLULININAE for four branches of unsupported relationships, the genus-group taxa Balebreviceps, Callulina, Probreviceps and Spelaeophryne. These two groups were confirmed in Pyron \& Wiens (2011) and Portik \& Blackburn (2016). Further studies are needed for clarifying the relationships within the CALLULININAE.

\section{F.18.62. Subfamilia BREVICIPITINAE Bonaparte, 1850}

Eunym: Van Kampen 1923: x.

Getangiotaxon: BREVICIPITIDAE Bonaparte, 1850.

Adelphotaxon: CALLULININAE nov.

Getendotaxon: Breviceps Merrem, 1820.

\section{F.18.63. Subfamilia CALLULININAE nov}

Getangiotaxon: BREVICIPITIDAE Bonaparte, 1850.

Adelphotaxon: BREVICIPITINAE Bonaparte, 1850.

Getendotaxa: Balebreviceps Largen \& Drewes, 1989; Callulina Nieden, 1911; Probreviceps Parker, 1931; Spelaeophryne Ahl, 1924.

Nucleogenus, by present designation: Callulina Nieden, 1911. Etymology of nomen: N: Callula Günther, 1864, autoneonym for Kaloula Gray, 1831, derived from G: ká $\lambda \lambda$ os (kallos), 'beautiful'; L: -ina, diminutive suffix for feminine nouns. • Stem of nomen: Callulin-.

Diagnosis: Small to medium sized frogs (males SVL 28-52 mm; females SVL 42-60 mm); horizontal pupilla; large subcircular not incurved tongue; tips of fingers and toes pointed or enlarged; distal metatarsals not separated by web; omosternum very small to moderately sized, cartilaginous; sternum absent; apophyses of sacral vertebra enlarged; coccyx and sacrum fused; distal phlanges blunt or tshaped. \{Nieden 1911; Ahl 1924; Parker 1931; Largen \& Drewes 1989; Channing \& Howell 2006; Loader et al. 2010\}. 


\title{
F.17.34. Familia HemisotidaE Cope, 1867
}

\author{
Protonym: HeMISIDAE Cope, 1867: 198 [F]. \\ Eunym: Frost \& Savage 1987: 24. \\ Getangiotaxon: BREVICIPITOIDEA Bonaparte, 1850. \\ Adelphotaxon: BREVICIPITIDAE Bonaparte, 1850. \\ Getendotaxon: Hemisus Günther, 1859.
}

\section{C.11.05. Subphalanx Pananura nov.}

Getangiotaxon: SCOPTANURA Starrett, 1973.

Adelphotaxa: Ecostata Lataste, 1879; Gastrechmia Cope, 1867; 1 G†.

Getendotaxa: Ecaudata Scopoli, 1777; SAVANURA nov.

Comments: This highly supported taxon has been recognised in all recent classifications (Frost et al. 2006; Roelants et al. 2007; Bossuyt \& Roelants 2009; Pyron \& Wiens 2011; Irisarri et al. 2012; Zhang et al. 2013; Feng et al. 2017) and named «NATATANURA» by Frost et al. (2006) and RANOIDAE by Zhang et al. (2013). It includes two major taxa, the ECAUdATA and the SAVANURA. As the name «NatatanURA» is an ectonym expressly proposed outside the Code, it is not available and we here name the new infraphalanx Pananura.

Conucleogenera, by present designation: Hildebrandtia Nieden, 1907; Rana Linnaeus, 1758.

Etymology of nomen: G: $\pi \tilde{\alpha} \varsigma$ (pas), 'all, every'; N: ANURA Duméril, 1805, derived from G: àv- (an-), 'without'; oüpó (oura), 'tail'. This nomen refers to the very wide distribution of this group of frogs, which covers most land masses except central and southern Australia and New Zealand (Frost et al. 2006).

Diagnosis: Small to very large sized frogs (SVL 14-320 mm); tongue present; pectoral girdle firmisternal; omosternum generally ossified; metasternum ossified or not; scapula not covered by clavicle; astragal and calcaneum separate; parahyoid not ossified; eight presacral vertebrae, usually biconcave, often procoelous; ribs absent; transverse process of presacral vertebrae generally long; transverse process of sacral vertebra cylindical or feebly dilated; sacrum not fused to urostyle, bicondylar articulation; urostyle without transversal process; articulations of atlas largely separated; Bidder's organs absent; amplexus usually axillary, rarely inguinal; parasphenoid without posterio-lateral processes; free living tadpoles or different adaptations to independence from water, also direct development in several groups; tadpoles with horny beak and keratodonts; spiracle unique, on left side of body. \{Laurent 1986; Frost et al. 2006; Vitt \& Caldwell 2014\}.

Scott (2005) presented the following morphological apomorphies for this group: relative length of transverse processes of presacral vertebra VIII roughly equal in length to transverse processes of presacral vertebra IV; neural spines of presacral vertebrae II-IV present; dorsal ridge of urostyle well developed, extending more than half length of urostyle; anterodorsal process at anterior edge of dorsal ridge of urostyle strongly developed, large and distinct; sacral diapophyses undilated; omosternum style present, large and well ossified; frontoparietal fenestra reduced to merely a suture, frontoparietals large and touching centrally; femoral granules obvious, well-defined, extending $1 / 2$ to $3 / 4$ length of thigh from vent. Haas (2003) proposed as synapomorphies for this taxon: anterior insertion of musculus subarcualis rectus II-IV on ceratobranchial III; commissura proximalis II and III absent.

\section{C.12.03. Infraphalanx Ecaudata Scopoli, 1777}

Protonym: ECAUDATA Scopoli, 1777: 464 [O].

Eunym: Hoc loco.

Getangiotaxon: Pananura nov.

Adelphotaxon: Savanura nov.

Getendotaxa: OdontobatraChoidea Barej, Schmitz, Günther, Loader, Mahlow \& Rödel, 2014; PhRYNOBATRACHOIDEA Laurent, 1941; RANOIDEA Batsch, 1796. 
Comments: The ECAUDATA groups all the genera that were previously in the RANIDAE or RANOIDEA, to the exclusion of the PTYCHADENIDAE. Frost et al. (2006) used the ectonym "VictoranURA» for this taxon but two Code-compliant nomina, ECAUDATA Scopoli, 1777 being the oldest, were already available for this taxon. It has been recognised in most recent molecular phylogenies (Frost et al. 2006; Bossuyt \& Roelants 2009; Pyron \& Wiens 2011; Yuan et al. 2018). This group includes three taxa, attributed here to the rank superfamily, the ODONTOBATRACHOIDEA, PHRYNOBATRACHOIDEA and RANOIDEA, the relationships between which are not resolved in TREE. The PHRYNOBATRACHOIDEA and RANOIDEA appear as sister-groups but with a SHL support of 75 only, these two being sister-group to the ODONTOBATRACHOIDEA. The ECAUDATA include now 16 families of poorly resolved mutual relationships. Both the PHRYNOBATRACHOIDEA and ODONTOBATRACHOIDEA include a single family rank taxon, respectively the PHRYNOBATRACHIDAE and the ODONTOBATRACHIDAE. Within the superfamily RANOIDEA, the branches RANIDAE and RHACOPHORIDAE are sister-groups and have long been given family rank in a large number of classifications, and thus are credited with this rank here on account of the Upper Quartile Criterion. The ranks of all the other suprageneric taxa derive directly from this and from the topology of TREE. These two families constitute together the apofamily RANEIDAE, which is part of an unresolved tetratomy with the apofamilies DICROGLOSSEIDAE, NYCTIBATRACHEIDAE and RANIXALEIDAE. Altogether, these four taxa make up the epifamily RANOIDAE, which is part of an unresolved hexatomy with the epifamilies ConRaUidae, ERICABATRACHOIDAE, MiCRIXALOIDAE, PETROPEDETOIDAE and PYXICEPHALOIDAE which altogether constitute the RANOIDEA.

Among the latter, the DICROGLOSSEIDAE incorporate the families DICROGLOSSIDAE and OCCIDOZYGIDAE, whereas the CERATOBATRACHEIDAE include the families ALCALIDAE and CERATOBATRACHIDAE, assigned to the rank family due to the Non-Redundancy Criterion [NRC]. Frost et al. (2006) retained 10 families in their classification within our PANANURA. The relationships between these families are quite different from ours in their work, as the CERATOBATRACHIDAE are outgroup to all others, the PHRYNOBATRACHIDAE are sister-taxon to the PYXICEPHALOIDEA, which include the PETROPEDETIDAE and PYXICEPHALIDAE (with CACOSTERNINAE as subfamily), and the NYCTIBATRACHIDAE are sister-groups to the RANIDAE, and together sister-group to their RHACOPHOROIDEA. Bossuyt \& Roelants (2009) recovered a sister-group relationship between MANTELLIDAE and RHACOPHORIDAE, and between DICROGLOSSIDAE and RANIDAE, these two taxa forming a taxon with NYCTIBATRACHIDAE as sister-group. In their tree, MICRIXALIDAE and RANIXALIDAE form a taxon with CERATOBATRACHIDAE as outgroup. These two assemblages form a taxon with PETROPEDETIDAE and PYXICEPHALIDAE, having PHRYNOBATRACHIDAE as outgroup. Finally, the PTYCHADENIDAE, as in TREE, are sister-group to the ECAUDATA. Pyron \& Wiens (2001) recognised ten families, with very similar relationships as found in TREE. The differences come mainly from divergences in the methodology of transcription of these relationships into a classification. The PTYCHADENIDAE are within the ECAUDATA in the trees presented by Zhang et al. (2013), Frazão et al. (2015), Feng et al. (2017) and Yuan et al. (2018). In these trees, the MANTELLIDAE and RHACOPHORIDAE are sister-group to RANIDAE, but the positions of other groups are highly variable. The family classification derived from TREE is discussed below under the respective family nomina concerned.

\section{F.14.10. Superfamilia ODONTOBATRACHOIDEA Barej, Schmitz, Günther, Loader, Mahlow \& Rödel, 2014}

Protonym: Odontobatrachidae Barej, Schmitz, Günther, Loader, Mahlow \& Rödel, 2014: 1 [F].

Eunym: Hoc loco.

Getangiotaxon: ECAUDATA Scopoli, 1777.

Adelphotaxa: Phrynobatrachoidea Laurent, 1941; RANOIDEa Batsch, 1796.

Getendotaxon: OdontobatrachidaE Barej, Schmitz, Günther, Loader, Mahlow \& Rödel, 2014.

F.17.35. Familia ODontobatRaChidae Barej, Schmitz, Günther, Loader, Mahlow \& Rödel, 2014

Eunym: Barej, Schmitz, Günther, Loader, Mahlow \& Rödel, 2014: 1.

Getangiotaxon: ODontobatraChoIdEA Barej, Schmitz, Günther, Loader, Mahlow \& Rödel, 2014. 
Adelphotaxon: None.

Getendotaxon: Odontobatrachus Barej, Rödel, Loader \& Schmitz in Barej, Rödel, Loader, Menegon, Gonwouo, Penner, Gvoždík, Günther, Bell, Nagel \& Schmitz, 2014.

Comments: One of the most interesting discoveries of the recent years, this family has been defined by Barej et al. (2014) in resolving the paraphyly of the PETROPEDETIDAE. In their tree, this branch is sistertaxon to the Dicroglossidae within the Pananura. The difference with our taxonomy clearly comes from the sampling of taxa by these authors, mainly limited to African species. The single genus holds now five species (Barej et al. 2015).

\section{F.14.11. Superfamilia PhrYNobatraCHOIDEA Laurent, 1941}

Protonym: PhrYNobatrachinae Laurent, 1941: 192 [bF].

Eunym: Hoc loco.

Getangiotaxon: ECAUDATA Scopoli, 1777.

Adelphotaxa: Odontobatrachoidea Barej, Schmitz, Günther, Loader, Mahlow \& Rödel, 2014; RANOIDEA Batsch, 1796.

Getendotaxon: PhrinobatraChIDAE Laurent, 1941.

\section{F.17.36. Familia PhrYNOBATRACHIDAE Laurent, 1941}

Eunym: Laurent, 1941: 192.

Getangiotaxon: PHRYNOBATRACHOIDEA Laurent, 1941.

Adelphotaxon: None.

Getendotaxa: Phrynobatrachus Günther, 1862; Phrynodon Parker, 1935.

Comments: Zimkus et al. (2010, 2012) identified three taxa within the PHRYNOBATRACHIDAE. Further studies are requested for the taxonomic recognition of these groups as genera (Zimkus et al. 2010). At this stage, these data support at least the resurrection of the genus Phrynodon as distinct from Phrynobatrachus, but for a more comprehensive taxon than the monotypic genus traditionally recognised under this nomen.

\section{F.14.12. Superfamilia RANOIDEA Batsch, 1796}

Protonym: RANINA Batsch, 1796: 179 [F].

Eunym: Bolkay 1929: 58.

Getangiotaxon: ECAUDATA Scopoli, 1777.

Adelphotaxa: OdontobatraChoIdeA Barej, Schmitz, Günther, Loader, Mahlow \& Rödel, 2014; PhRYNOBATRACHOIDEA Laurent, 1941.

Getendotaxa: Conrauoidae Dubois, 1992; ERICABatraChoidaE nov.; MiCRIXaloidae Dubois, Ohler \& Biju, 2001; Petropedetoidae Noble, 1931; PyXICEPHaloidae Bonaparte, 1850; RanoidaE Batsch, 1796.

Comments: Within this superfamily, six highly supported branches are recognised as epifamilies: the Conrauoidae, ERICabatrachoidae, Micrixaloidae, Petropedetoidae, PyXicephaloidae and RANOIDAE. The relationships between these groups are not resolved.

\section{F.15.05. Epifamilia CONRAUOIDAE Dubois, 1992}

Protonym: ConRAUOINI Dubois, 1992: 314 [T].

Eunym: Hoc loco.

Getangiotaxon: RANOIDEA Batsch, 1796.

Adelphotaxa: ERICABATRACHOIDAE nov.; MiCRIXALOIDAE Dubois, Ohler \& Biju, 2001; PETROPEDETOIDAE Noble, 1931; PYXICEPHALOIDAE Bonaparte, 1850; RANOIDAE Batsch, 1796. 
Eunym: Pyron \& Wiens 2011: 547.

Getangiotaxon: CONRAUOIDAE Dubois, 1992.

Adelphotaxon: None.

Getendotaxon: Conraua Nieden, 1908.

Comments: The epifamily CONRAUOIDAE includes a single genus, Conraua, whose relationships with the five other epifamilies of RANOIDEA are not clarified. This taxon is recognised at the family rank as CONRAUIDAE by application of the Consistent Naming Criterion [CNC] to the single genus Conraua. The position of this genus within the RANOIDEA is highly variable in recent phylogenies: it has been included in the PETROPEDETIDAE by Frost et al. (2006), found as sister-group to Petropedetes (Zimkus et al. 2010), sister-group, given the rank family, to all other RANOIDEA (Pyron \& Wiens 2011), sister-group to the PYXICEPHALIDAE and PETROPEDETIDAE (Barej et al. 2014) or sister-group to the PETROPEDETIDAE (Feng et al. 2017; Yuan et al. 2018). Here we recognise it provisionally as an independent lineage as the support for its relationships with other ranoid groups is below our Criteria.

\section{F.15.06. Epifamilia ERICABATRACHOIDAE nov.}

Getangiotaxon: RANOIDEA Batsch, 1796.

Adelphotaxa: Conrauoidae Dubois, 1992; MicriXaloidae Dubois, Ohler \& Biju, 2001; Petropedetoidae Noble, 1931; PYXICEPHALOIDAE Bonaparte, 1850; RANOIDAE Batsch, 1796.

Getendotaxon: ERICABATRACHIDAE nov.

Nucleogenus, by present designation: Ericabatrachus Largen, 1991. • Etymology of nomen: N: Erica,

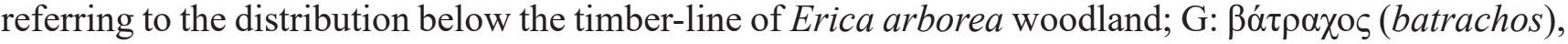
'frog'. • Stem of nomen: Ericabatrach-.

Diagnosis: Small sized frogs (males SVL 19-22 mm, females SVL 23-27 mm); vomerine teeth absent; maxillary teeth present; terminal phalanges simple; tongue deeply notched bearing a pointed median papilla; pupil oval, horizontal; tympanum poorly distinct; finger tips moderately dilated, bifid discs; first finger distinctly reduced; web between fingers absent; web between toes rudimentary; outer metatarsals fused; outer metatarsal tubercle absent; inner metatarsal tubercle small, oval; hind limbs moderately long; skin rugose, densely covered with tiny warts and scattered small tubercles; dorsal coloration grayish with obscure darker pattern; mid-dorsal stripe absent; ventral coloration dark graybrown and whitish mottling; females with large unpigmented eggs; males with oval femoral glands and with subgular vocal sac. \{Largen 1991\}.

\section{F.17.38. Familia ERICABATRACHIDAE nov.}

Getangiotaxon: ERICABATRACHOIDAE nov.

Adelphotaxon: None.

Getendotaxon: Ericabatrachus Largen, 1991.

Comments: The epifamily ERICABATRACHOIDAE includes a single genus, Ericabatrachus, whose relationships with the five other epifamilies of RANOIDEA are not clarified. This taxon is recognised at the family rank as ERICABATRACHIDAE by application of the Consistent Naming Criterion [CNC] to the single genus Ericabatrachus. The position of this genus within the RANOIDEA is not resolved in TREE. Siu-Ting et al. (2014) proposed it as sister-group to Petropedetes, a relationship also found in TREE, but with poor support. 
Protonym: MiCRIXALINAE Dubois, Ohler \& Biju, 2001: 56 [bF].

Eunym: Hoc loco.

Getangiotaxon: RANOIDEA Batsch, 1796.

Adelphotaxa: Conrauoidae Dubois, 1992; ERICABatraChoidae nov.; Petropedetoidae Noble, 1931; PyXICEPHALOIDAE Bonaparte, 1850; RANOIDAE Batsch, 1796.

Getendotaxon: MICRIXALIDAE Dubois, Ohler \& Biju, 2001.

\section{F.17.39. Familia MICRIXALIDAE Dubois, Ohler \& Biju, 2001}

Eunym: Frost, Grant, Faivovich, Bain, Haas, Haddad, Sá, Channing, Wilkinson, Donnellan, Raxworthy, Campbell, Blotto, Moler, Drewes, Nussbaum, Lynch, Green \& Wheeler 2006: 7.

Getangiotaxon: MICRIXALOIDAE Dubois, Ohler \& Biju, 2001.

Adelphotaxon: None.

Getendotaxon: Micrixalus Boulenger, 1888.

Comments: Within the epifamily MICRIXALOIDAE, the rank family is given to the lowest FS taxon including the genus Micrixalus by application of the [CNC]. This taxon was recognised as a family by Frost et al. (2006) within the «TELMATOBATRACHIA», as sister-group of the «AMETROBATRACHIA». In Bossuyt \& Roelants (2009), it was sister-taxon to the RANIXALIDAE. In the tree of Pyron \& Wiens (2011) it was sister-taxon to all the ECAUDATA, including Phrynobatrachus. It was sister-group to our epifamilia RANOIDAE in Barej et al. (2014).

\section{F.15.08. Epifamilia PetropedetoIdAE Noble, 1931}

Protonym: Petropedetinae Noble, 1931: 520 [bF].

Eunym: Hoc loco.

Getangiotaxon: RANOIDEA Batsch, 1796.

Adelphotaxa: ConRauoIdaE Dubois, 1992; ERICABatraChOIDAE nov.; MiCRIXALOIDAE Dubois, Ohler \& Biju, 2001; PYXICEPHALOIDAE Bonaparte, 1850; RANOIDAE Batsch, 1796.

Getendotaxon: PETRopedETIDAE Noble, 1931.

F.17.40. Familia Petropedetidae Noble, 1931

Eunym: Bauer 1985: 3.

Getangiotaxon: PETROPEDETOIDAE Noble, 1931.

Adelphotaxa: Conrauoidae Dubois, 1992; ERICABatraChoIdaE nov.; MiCRIXaloidae Dubois, Ohler \& Biju, 2001; PYXICEPHALOIDAE Bonaparte, 1850; RANOIDAE Batsch, 1796.

Getendotaxa: Arthroleptides Nieden, 1911; Petropedetes Reichenow, 1874.

Comments: This is another holophyletic lineage which has to be recognised both as an epifamily and a family according to the [CNC]. It includes two genera, Arthroleptides and Petropedetes (Barej et al. 2014).

\section{F.15.09. Epifamilia PYXICEPHALOIDAE Bonaparte, 1850}

Protonym: PYXICEPHALINA Bonaparte, 1850: plate [bF].

Eunym: Hoc loco.

Getangiotaxon: RANOIDEA Batsch, 1796.

Adelphotaxa: ConRaUoIdaE Dubois, 1992; ERICABATRACHOIDAE nov.; MiCRIXALOIDAE Dubois, Ohler \& Biju, 2001; PETROPEDETOIDAE Noble, 1931; RANOIDAE Batsch, 1796. 
Getendotaxa: CACOSTERnIDAE Noble, 1931; PYXICEPHALIDAE Bonaparte, 1850.

Comments: This epifamily includes two taxa, the family PYXICEPHALIDAE, for the genera Aubria and Pyxicephalus, and the family CACOSTERNIDAE. These two taxa are recognised at the rank family on account of the [MRC] and the [NRC]. Frost et al. (2006) and Pyron \& Wiens (2011) gave them the rank subfamily.

\section{F.17.41. Familia CACOSTERNIDAE Noble, 1931}

Protonym: CACOSTERNINAE Noble, 1931: 527 [bF].

Eunym: Hoc loco.

Getangiotaxon: PYXICEPHALOIDAE Bonaparte, 1850.

Adelphotaxon: PYXICEPHALIDAE Bonaparte, 1850.

Getendotaxa: AnHydrophryninae nov.; Cacosterninae Noble, 1931; Tomopterninae Dubois, 1987.

Comments: Most authors recognise this taxon as a subfamily of the PYXICEPHALIDAE. The family as here understood includes three well supported branches, with poor support concerning their mutual relationships, recognised here as the sufamilies ANHYDROPHRYNINAE for Anhydrophryne, TOMOPTERNINAE for Nothophryne and Tomopterna (Bittencourt-Silva et al. 2016) and the CACOSTERNINAE. The relationships within the latter subfamily are discussed below.

\section{F.18.64. Subfamilia ANHYDROPHRYNINAE nov.}

Getangiotaxon: CACOSTERNIDAE Noble, 1931.

Adelphotaxa: Cacosterninae Noble, 1931; Tomopterninae Dubois, 1987.

Getendotaxon: Anhydrophryne Hewitt, 1919.

Nucleogenus, by present designation: Anhydrophryne Hewitt, 1919. • Etymology of nomen: G:

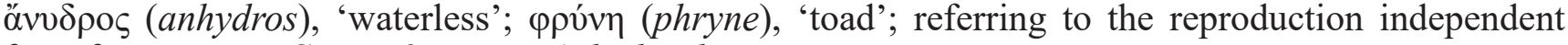
from free water. $\bullet$ Stem of nomen: Anhydrophryn-.

Diagnosis: Small sized frogs (males mean SVL $17 \mathrm{~mm}$, females mean SVL $20 \mathrm{~mm}$ ); horizontal pupillae; distinct rather large tympanum; broad dark band on canthal and tympanic region; subarticular tubercles poorly developed on hands and feet; webbing absent on hands and feet; metatarsal tubercles poorly developed or absent; dorsal skin rather smooth; terrestrial nest, development within egg envelopes, no free living tadpole. \{Bishop 1985; Du Preez \& Carruthers 2009\}.

\section{F.18.65. Subfamilia CACOSTERNINAE Noble, 1931}

Eunym: Noble 1931: 527.

Getangiotaxon: CACOSTERNIDAE Noble, 1931.

Adelphotaxa: ANHYDROPHRYNINAE nov.; TOMOPTERNINAE Dubois, 1987.

Getendotaxa: CACOStERNINI Noble, 1931; NATALOBATRACHINI nov.; STRONGYLOPINI Scott, 2005.

Comments: The relationships between three highly supported branches within the subfamily are poorly resolved. These three taxa are recognised here as the tribe CACOSTERNINI, including the sistertaxa Cacosternum and Microbatrachella constituting the subtribe CACOSTERNINA, and their sistertaxon, the subtribe PoYNTONIINA for Poyntonia, the tribe NATALOBATRACHINI for Arthroleptella and Natalobatrachus, and the tribe STRONGYLOPINI for Amietia and Strongylopus. 
Eunym: Hoc loco.

Getangiotaxon: CACOSTERNINAE Noble, 1931.

Adelphotaxa: NATALOBATRACHINI nov.; STRONGYLOPINI Scott, 2005.

Getendotaxa: CACOSTERnINA Noble, 1931; PoyntoniIna nov.

F.20.56. Subtribus CACOSTERNINA Noble, 1931

Eunym: Hoc loco.

Getangiotaxon: CACOSTERNINI Noble, 1931.

Adelphotaxon: POYNTONIINA nov.

Getendotaxa: Cacosternum Boulenger, 1887; Microbatrachella Hewitt, 1926.

\section{F.20.57. Subtribus POYNTONIINA nov.}

Getangiotaxon: CACOSTERNINI Noble, 1931.

Adelphotaxon: CACOSTERNINA Noble, 1931.

Getendotaxon: Poyntonia Channing \& Boycott, 1989.

Nucleogenus, by present designation: Poyntonia Channing \& Boycott, 1989. • Etymology of nomen: P: John Charles Poynton (1931-), South African herpetologist. • Stem of nomen: Poyntoni-.

Diagnosis: Small sized frogs $(23-30 \mathrm{~mm})$; maxillary and premaxillary teeth present; vomerine teeth absent; pupil horizontal; tympanum not visible; white or orange stripes under tympanic ridge; glandular region behind eyes; fingers and toes blunt, without discs; tarsal tubercle and outer metatarsal tubercle absent; rudimentary web extending with fringes on fingers; moderate webbing between toes; dorsal skin with warts bearing granules; dorsal colour gray-brown, often with middorsal line; tadpoles brownish, long and streamlined; keratodont formulae $1 / 2$ or $2: 2+2 / 2$, free living in shallow seepage areas. \{Channing \& Boycott 1989; Du Preez \& Carruthers 2009\}.

\section{F.19.61. Tribus NATALOBATRACHINI nov.}

Getangiotaxon: CACOSTERNINAE Noble, 1931.

Adelphotaxa: CACOSTERNINI Noble, 1931; STRONGYLOPINI Scott, 2005.

Getendotaxa: Arthroleptella Hewitt, 1926; Natalobatrachus Hewitt \& Methuen, 1912.

Nucleogenus, by present designation: Natalobatrachus Hewitt, 1912. • Etymology of nomen: L: natalis, 'relating to birth', referring to the date of discovery, Christmas day, of the region now known as the South African province Natal; G: ßá $\rho \alpha \chi 0 \varsigma$ (batrachos), 'frog'. • Stem of nomen: Natalobatrach-.

Diagnosis: Very small to small sized cacosternids (males SVL 12-30 mm, females SVL 14-37 mm); metasternum with a well developed bony rod; pupillae horizontal; tympanum indistinct or distinct; tympanic ridge present; finger tips swollen to expanded; short limbs; tips of toes slighly expanded; toes and fingers without webbing; tubercles on hand indistinct; moderately developed subarticular tubercles; inner metatarsal tubercle distinct; outer metatarsal tubercle very weak or absent; web on toes absent or extending half; dorsum dark colored; ventral body light or dark colored but throat and chest in males dark; vocal sacs present or absent; egg slightly pigmented or white; direct development or free swimming larvae. \{Hewitt \& Methuen, 1912; Hewitt 1926, 1927; Turner \& Channing 2008; Du Preez \& Carruthers 2009\}. 
Protonym: STRONGYLOPINAE Scott, 2005: 507 [bF].

Eunym: Hoc loco.

Getangiotaxon: CACOSTERNINAE Noble, 1931.

Adelphotaxa: CACOSTERNINI Noble, 1931; NATALOBATRACHINI nov.

Getendotaxa: Amietia Dubois, 1987; Strongylopus Tschudi, 1838.

\section{F.18.66. Subfamilia ToMopterniNaE Dubois, 1987}

Protonym: ToMOPTERNINI Dubois, 1987: 56 [T].

Eunym: Dubois 1992: 336.

Getangiotaxon: CACOSTERNIDAE Noble, 1931.

Adelphotaxa: AnHYdrophryninae nov.; CACosterninae Noble, 1931.

Getendotaxa: Nothophryne Poynton, 1963; Tomopterna Duméril \& Bibron, 1841.

\section{F.17.42. Familia PYXICEPHALIDAE Bonaparte, 1850}

Eunym: Roelants, Gower, Wilkinson, Simon, Biju, Guillaume, Moriau \& Bossuyt 2007: 889.

Getangiotaxon: PYXICEPHALOIDAE Bonaparte, 1850.

Adelphotaxon: CACOSTERNIDAE Noble, 1931.

Getendotaxa: Aubria Boulenger, 1917; Pyxicephalus Tschudi, 1838.

F.15.10. Epifamilia RANOIDAE Batsch, 1796

Eunym: Dubois 1992: 309.

Getangiotaxon: RANOIDEA Batsch, 1796.

Adelphotaxa: Conrauoidae Dubois, 1992; ERICABatrachoidae nov.; MiCRIXaloidae Dubois, Ohler \& Biju, 2001; Petropedetoidae Noble, 1931; PyXICEPHaLOIDAE Bonaparte, 1850.

Getendotaxa: CeratobatraCheidae Boulenger, 1884; Dicroglosseidae Dubois, 1987; NyCtibatraCheIDAE BlommersSchlösser, 1993; RANEIDAE Batsch, 1796; RANIXALEIDAE Dubois, 1987.

Comments: This epifamily was recognised in Frost et al. (2006) as the «SAUKROBATRACHIA», including the DiCROGLOSSIDAE and the "AgLAIOANURA» which included the RHACOPHOROIDEA and the RANOIDEA, which in their turn included the NYCTIBATRACHIDAE and the RANIDAE, but not the CERATOBATRACHIDAE. In Bossuyt \& Roelants (2009), two taxa were proposed within this group, one with CERATOBATRACHIDAE being sister-group to a branch formed of the MICRIXALIDAE and RANIXALIDAE, and a second with NYCTIBATRACHIDAE as sister-branch of (MANTELLIDAE and RHACOPHORIDAE) and (DICROGLOSSIDAE and RANIDAE). The branch including the CERATOBATRACHIDAE was confirmed by Pyron \&Wiens (2011), who found high support for the NYCTIBATRACHIDAE, CERATOBATRACHIDAE, RANiXalidae, Dicroglossidae, MANTELLIDAE, RHACOPHORIDAE and RANIDAE. Whereas sister-group relationships between (RHACOPHORIDAE and MANTELLIDAE) and RANIDAE, and DICROGLOSSINAE and OCCYDOZYGINAE were confirmed in most recent phylogenies (Zhang et al. 2013, Feng et al. 2017; Yuan et al. 2018), the position of the NYCTIBATRACHIDAE and RANIXALIDAE is not stable. Brown et al. (2015) proposed a classification of the CERATOBATRACHEIDAE that was expanded here to include the genus Liurana following Yan et al. (2016).

In TREE, the relationships between the five branches that constitute the epifamily RANOIDAE are not resolved and these five branches are here recognised at the same rank, as apofamilies Ceratobatracheidae, Dicroglosseidae, NyCtibatraCheidae, RANEIDAE and RaNiXaleidae. The apofamily CERATOBATRACHEIDAE includes three branches, here recognised as subfamilies of a single family CERATOBATRACHIDAE based on the [NTC], the subfamily ALCALINAE with the only genus Alcalus, the subfamily CERATOBATRACHINAE with the genera Cornufer and Platymantis, and the subfamily LIURANINAE for the single genus Liurana. The apofamily DICROGLOSSEIDAE has two highly supported 
branches recognised as the families DICROGLOSSIDAE and OCCIDOZYGIDAE following the [NRC]. The detailed classification is provided below. The apofamily NYCTIBATRACHEIDAE includes a single family, the NyCTIBATRACHIDAE, with two genera, Lankanectes and Nyctibatrachus. The fourth group, the apofamily RANEIDAE, includes two highly supported groups recognised as the families RANIDAE and RHACOPHORIDAE, whose detailed classification is presented below. Finally, the apofamily RANIXALEIDAE accommodates a single family rank taxon, the RANIXALIDAE, with the genera Indirana and Walkerana.

\section{F.16.03. Apofamilia CERATOBATRACHEIDAE Boulenger, 1884}

Protonym: CERATOBATRACHIDAE Boulenger, 1884: 212 [F].

Eunym: Hoc loco.

Getangiotaxon: RANOIDAE Batsch, 1796.

Adelphotaxa: Dicroglosseidae Dubois, 1987; NyCtibatraCheIdaE Blommers-Schlösser, 1993; RaneIDAE Batsch, 1796; RANIXALEIDAE Dubois, 1987.

Getendotaxon: CERATOBATRACHIDAE Boulenger, 1884.

\section{F.17.43. Familia CERATOBATRACHIDAE Boulenger, 1884}

Eunym: Boulenger, 1884: 212.

Getangiotaxon: CERATOBATRACHEIDAE Boulenger, 1884.

Adelphotaxon: None.

Getendotaxa: AlCALINAE Brown, Siler, Richards, Diesmos \& Cannatella, 2015; CeratobatraChinaE Boulenger, 1884; LiURANINAE Fei, Ye \& Jiang, 2010.

F.18.67. Subfamilia ALCALINAE Brown, Siler, Richards, Diesmos \& Cannatella, 2015

Protonym and eunym: ALCALINAE Brown, Siler, Richards, Diesmos \& Cannatella, 2015: 142 [bF].

Getangiotaxon: CERATOBATRACHIDAE Boulenger, 1884.

Adelphotaxa: CeratobatrachinaE Boulenger, 1884; LiURaninae Fei, Ye \& Jiang, 2010.

Getendotaxon: Alcalus Brown, Siler, Richards, Diesmos \& Cannatella, 2015.

\section{F.18.68. Subfamilia CERATOBATRACHINAE Boulenger, 1884}

Eunym: Gadow 1901: xi, 237.

Getangiotaxon: CERATOBATRACHIDAE Boulenger, 1884.

Adelphotaxa: AlCALINAE Brown, Siler, Richards, Diesmos \& Cannatella, 2015; LiURANINAE Fei, Ye \& Jiang, 2010.

Getendotaxa: Cornufer Tschudi, 1838; Platymantis Günther, 1859.

\section{F.18.69. Subfamilia LiURANINAE Fei, Ye \& Jiang, 2010}

Protonym and eunym: LIURANINAE Fei, Ye \& Jiang, 2010: 12 [bF].

Getangiotaxon: CERATOBATRACHIDAE Boulenger, 1884.

Adelphotaxa: AlCALINAE Brown, Siler, Richards, Diesmos \& Cannatella, 2015; CeratobatraChinaE Boulenger, 1884.

Getendotaxon: Liurana Dubois, 1987.

\section{F.16.04. Apofamilia DiCRoglosseIDAE Dubois, 1987}

Protonym: DiCRoglossini Dubois, 1987b: 57 [T].

Eunym: Hoc loco.

Getangiotaxon: RANOIDAE Batsch, 1796. 
Adelphotaxa: CERATobatraCHeIdaE Boulenger, 1884; NyCtibatraCHEIDAE Blommers-Schlösser, 1993; RANEIDAE Batsch, 1796; RANIXALEIDAE Dubois, 1987.

Getendotaxa: DicRoglossidaE Dubois, 1987; OCCIDOZYGIDAE Fei, Ye \& Huang, 1990.

\section{F.17.44. Familia DiCROGLOSSIDAE Dubois, 1987}

Eunym: Frost, Grant, Faivovich, Bain, Haas, Haddad, Sá, Channing, Wilkinson, Donnellan, Raxworthy, Campbell, Blotto, Moler, Drewes, Nussbaum, Lynch, Green \& Wheeler 2006: 7.

Getangiotaxon: DiCROGLOSSEIDAE Dubois, 1987.

Adelphotaxon: OCCIDOZYGIDAE Fei, Ye \& Huang, 1990.

Getendotaxa: Dicroglossinae Dubois, 1987; Limnonectinae Dubois, 1992; Painae Dubois, 1992; 1 GIS (Chrysopaa Ohler \& Dubois, 2006).

Comments: The family DiCRoGLOSSIDAE here corresponds to the DICROGLOSSINAE of Roelants et al. (2004), Frost et al. (2006), Pyron \& Wiens (2011) and Yuan et al. (2018). Its recognition at the family rank is a consequence of application of consistent Rules throughout TREE. The relationships between the three highly supported taxa obtained within this family are not resolved, so they are recognised equally as the subfamilies DiCRoGLOSSINAE, LIMNONECTINAE for the single genus Limnonectes, and PAINAE. These three groups have been revealed by Roelants et al. (2004) but in their tree the branch here named LIMNONECTINAE was sister-group to a branch combining the DICROGLOSSINAE and PAINAE.

The nomen DICROGLOSSIDAE first appeared in the literature in Anderson (1871: 38), who used it without stating that it was a new nomen and without diagnosis, comment or included species. Dubois (1987b: 57-58) guessed that it was based on the generic nomen Dicroglossus Günther, 1860, a junior synonym of Euphlyctis Fitzinger, 1843 (see Dubois 1975), and used it, under the apograph DiCRoGLOSSINI, for a tribe including this genus and a few others. Since then, this nomen and its parographs have had a large use in the literature for several taxa from the rank tribe to the rank superfamily. However, Ohler \& Dubois (2014) provided evidence that Anderson's (1871) nomen was not a new nomen but just a misspelling for DISCOGLOSSIDAE Günther, 1858, based on the generic nomen Discoglossus Otth, 1837. In order not to upset nomenclatural stability, they proposed to maintain the nomen DICROGLOSSIDAE and its parographs as valid, but credited to Dubois (1987b), who had first used it explicitly as a family-series nomen based on the generic nomen Dicroglossus. Ohler et al. (2014) submitted to the Commission an application asking it to use its Plenary Power to implement this nomenclatural act. This application was published in the $B Z N$, and the Case 3666 first announced on the Commission website as under study by the Commission, but later withdrawn from this website without explanation, although no vote on this case, and even no comment on it, was published in the $B Z N$. In the absence of decision of the Commission on this case, we simply consider the nomen "DiCROGLOSSIDAE Anderson, 1870" as unavailable and 'non-existent', and we credit the nomen DICROGLOSSINI to Dubois (1987b).

\section{F.18.70. Subfamilia DiCRogLOSSINAE Dubois, 1987}

Eunym: Dubois 1992: 313.

Getangiotaxon: DiCRoglossidae Dubois, 1987.

Adelphotaxa: Limnonectinae Dubois, 1992; Painae Dubois, 1992.

Getendotaxa: Dicroglossini Dubois, 1987; FeJervaryini Fei, Ye \& Jiang, 2010.

Comments: The two highly supported branches within this subfamily are recognised here as the tribes DICROGLOSSINI and FEJERVARYINI. Within the DICROGLOSSINI, two subtribes are erected: NANNOPHRYINA, including the single genus Nannophrys, is sister-group to DICROGLOSSINA, recognised for the genera Euphlyctis, Hoplobatrachus and Phrynoderma. The validation of Phrynoderma Fitzinger, 1843 for Phrynoderma hexadactylum and Phrynoderma aloysii is necessary as Euphlyctis including these two species has very poor support in TREE (SHL 11\%). The relationships among the three genera of FEJERVARYINI are not resolved. 
Eunym: Dubois 1987b: 57.

Getangiotaxon: DiCRoglossinaE Dubois, 1987.

Adelphotaxon: FEJERVARYINI Fei, Ye \& Jiang, 2010.

Getendotaxa: Dicroglossina Dubois, 1987; Nannophryina Fei, Ye \& Jiang, 2010.

F.20.58. Subtribus DiCRoglossina Dubois, 1987

Eunym: Hoc loco.

Getangiotaxon: DICROGLOSSINI Dubois, 1987.

Adelphotaxon: NANNOPHRYINA Fei, Ye \& Jiang, 2010.

Getendotaxa: Euphlyctis Fitzinger, 1843; Hoplobatrachus Peters, 1863; Phrynoderma Fitzinger, 1843.

Comments: As noted by Kosuch et al. (2001), the original description of Rana chinensis Osbeck, 1765 clearly applies to the species long designated in the literature as Rana rugulosa Wiegmann, 1834 or Hoplobatrachus rugulosus, so this species should be known as Hoplobatrachus chinensis. Contrary to the remarks in $A S W<2020 a>$, the designation of the specimen CIB 980505 from near Guangzhou City, Guangdong, China by Fei et al. (2009: 1320) as neotype for this nominal species was not unwarranted but was necessary to identify objectively the taxon to which the nomen Rana chinensis applies. As this nomen was used as valid after 1899 by several authors, including non-taxonomists, it cannot and should not be rejected under Article 23.9 and it should be used as valid.

\section{G.28.372. Genus Phrynoderma Fitzinger, 1843}

Getangiotaxon: DiCRoglossina Dubois, 1987.

Adelphotaxa: Euphlyctis Fitzinger, 1843; Hoplobatrachus Peters, 1863.

Getendotaxa: Phrynoderma aloysii (Joshy, Alam, Kurabayashi, Sumida \& Kuramoto, 2009); Phrynoderma hexadactylum (Lesson, 1834); Phrynoderma karaavali (Priti, Naik, Seshadri, Singal, Vidisha, Ravikanth \& Gururaja, 2016).

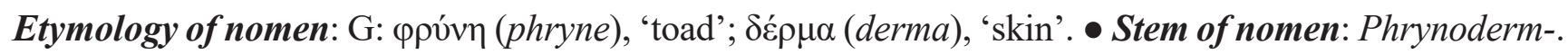
- Grammatical gender of nomen: neuter.

Comments: The nomen Phrynoderma Sturm, 1843 (COLEOPTERA) was published as a synonym of Zopherus Gray, 1832 and was not treated as available before 1961, so that it was not made available through Article 11.6.1. Therefore, it does not threaten the nomen Phrynoderma Fitzinger, 1843 as a potential senior homonym, and the latter can be used as valid.

\section{F.20.59. Subtribus NANNOPHRYINA Fei, Ye \& Jiang, 2010}

Protonym: NANNOPHRYINI Fei, Ye \& Jiang, 2010: 17 [T].

Eunym: Hoc loco.

Getangiotaxon: DiCROGLOSSINI Dubois, 1987.

Adelphotaxon: Dicroglossina Dubois, 1987.

Getendotaxon: Nannophrys Günther, 1869.

\section{F.19.64. Tribus FEJERVARYINI Fei, Ye \& Jiang, 2010}

Protonym and eunym: FEJERVARYINI Fei, Ye \& Jiang, 2010: 17 [T].

Getangiotaxon: DiCRogLOSSINAE Dubois, 1987.

Adelphotaxon: DiCRoglossini Dubois, 1987.

Getendotaxa: Fejervarya Bolkay, 1915; Minervarya Dubois, Ohler \& Biju, 2001; Sphaerotheca Günther, 1859. 
Protonym: LIMNONECTINI Dubois, 1992: 315 [T].

Eunym: Fei, Ye \& Jiang, 2010: 27.

Getangiotaxon: DiCRoGLOSSIDAE Dubois, 1987.

Adelphotaxa: Limnonectinae Dubois, 1992; PAINAE Dubois, 1992.

Getendotaxon: Limnonectes Fitzinger, 1843.

\section{F.18.72. Subfamilia PAINAE Dubois, 1992}

Protonym: PAINI Dubois, 1992: 317 [T].

Eunym: Fei, Ye \& Jiang, 2010: 17.

Getangiotaxon: DiCRoglossidae Dubois, 1987.

Adelphotaxa: Dicroglossinae Dubois, 1987; Limnonectinae Dubois, 1992.

Getendotaxa: PaINI Dubois, 1992; QuasipaInI Fei, Ye \& Jiang, 2010; 1 GIS (Allopaa Ohler \& Dubois, 2006).

Comments: TREE confirmed the holophyly of the subfamily PAINAE which includes two highly resolved branches (Roelants et al. 2004; Jiang et al. 2005; Ohler \& Dubois 2006; Che et al. 2010; Pyron \& Wiens 2011), recognised here as the tribes PAINI and QUASIPAINI. The holophyly of Quasipaa has been confirmed in several analyses (Jiang et al. 2005; Ohler \& Dubois 2006; Che et al. 2010; Pyron \& Wiens 2011), in which it appears as sister-group to a jumble called Nanorana in these works, but on the basis of a very incomplete taxonomic sample studied molecularly. Our conclusions combine phylogenetic relationships based on molecules with the morphological data obtained on far more taxa within this group (Ohler \& Dubois 2006). Here within the PAINI two subtribes correspond to the highly supported groups, recognised formally as the PAINA for the genera Nanorana and Paa, and the CHAPARANINA, with poorly resolved internal relationships, which leads us to recognise three infratribes, the CHAPARANINIA for Chaparana and Gynandropaa, the DIPLOPAINIA for Diplopaa, and the FEIRANINIA for Feirana. The tribe QUASIPAINI contains three taxa with poorly supported mutual relationships, the subtribes ANNANDIINA for Annandia, ERIPAINA for Eripaa and QUASIPAINA for Quasipaa and Yerana.

\section{F.19.65. Tribus PAINI Dubois, 1992}

Eunym: Dubois 1992: 317.

Getangiotaxon: PAINAE Dubois, 1992.

Adelphotaxa: QuasipaInI Fei, Ye \& Jiang, 2010; 1 GIS (Allopaa Ohler \& Dubois, 2006).

Getendotaxa: Chaparanina nov.; PAINA Dubois, 1992.

\section{F.20.60. Subtribus CHAPARANINA nov.}

Getangiotaxon: PAINI Dubois, 1992.

Adelphotaxon: PAINA Dubois, 1992.

Getendotaxa: ChapaRANINIA nov.; DiplopaINIA nov.; FeIRANINIA nov.; 1 GIS (Ombropaa nov).

Nucleogenus, by present designation: Chaparana Bourret, 1939. • Etymology of nomen: R: Chapa, French writing for Sapa, town in northern Vietnam, in the distribution area of the species; N: Rana Linnaeus, 1758, from L: rana, 'frog'. • Stem of nomen: Chaparan-. • Grammatical gender of nomen: feminine.

Diagnosis: Large sized dicroglossids (males 65-107 mm, females 70-97 mm); tympanum indistinct or distinct; length of first finger shorter or longer than second; webbing between fingers absent; finger tips blunt; proximal subarticular tubercles of fingers relatively small; leg length longer or shorter than half snout vent length; toe tips blunt; webbing between toes very variable, complete to very incurved between extremities of adjacent toes; flap of skin along toe $\mathrm{V}$ from tip of toe to first subarticular tubercle 
or beyond; tarsal fold absent or present; skin on dorsum smooth or with warts; laterodorsal folds narrow, continuous, discontinuous or absent; skin on belly smooth; large black spines potentially present on fingers I to II, throat and chest but always absent on arm and belly; vent of male without or with spines or with dermal flap; vocal sacs absent or present; forearm in adult breeding male enlarged or not; dorsal colour brown or greenish with darker pattern; chevron potentially present; mid-dorsal line absent; ventral colour light, uniform or with spots; eggs dark animal pole; tadpoles keratodont formula 7-9/3. \{Boulenger 1920b; Fei 1999; Dubois \& Ohler 2005; Ohler \& Dubois 2006 \}.

\section{G.28.380. Genus Ombropaa nov.}

Getangiotaxon: CHAPARANINA nov.

Adelphotaxon: None.

Getendotaxon: Ombropaa gammii (Anderson, 1871).

Nucleospecies, by present designation: Rana gammii Anderson, 1871. • Etymology of nomen: G:

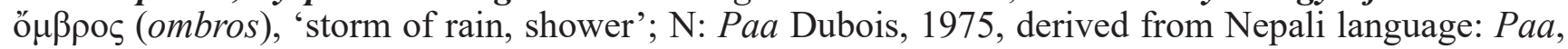
'frog'; referring to the tendency of these frogs to be active by heavy rainy nights (Dubois 1976: 206207, 1992: 318). • Stem of nomen: Ombropa-. • Grammatical gender of nomen: feminine.

Diagnosis: Medium sized dicroglossids (males 55-63 mm; females 61-88 mm); snout length distinctly longer than eye length; internarial distance larger than distance between eyelids; tympanum distinct; length of first finger shorter than second; tips of toes blunt; proximal subarticular tubercles small; shank longer than half body length; webbing between toes complete, without incurvation between toes; flap of skin along toe $\mathrm{V}$ extending to the proximal subarticular tubercle; tarsal fold absent; skin on dorsum smooth; laterodorsal folds narrow, as a continuous line; skin belly smooth; dorsal colour olive graybrown; middorsal chevron present in some individuals; mid-dorsal line absent; ventral colour yellowish; in adult male nuptial spines absent on fingers I to III, prepollex, forearm, chest and belly, and forearm not enlarged; adult breeding males with a large zone bearing spines, around and inside vent, and vocal sacs present; eggs with dark animal pole; tadpoles with a bunch of papillae at the corner of mouth. \{Anderson 1871; Dubois 1976, 1992; Ohler \& Dubois 2006; personal observations by AD and AO\}.

Comments: Dubois (1976) described the only known species of this genus as Rana (Paa) sikimensis Jerdon, 1870, but he stated that he had not found the type specimen(s) of this species in the collection of the Zoological Survey of India (ZSI) in Calcutta. He considered the nomina Rana gammii Anderson, 1871 and Rana assamensis Sclater, 1892 as synonyms of this nomen. Chanda et al. (2000) reported having identified the specimen ZSI 9580 from Darjeeling as one of the syntypes (symphoronts) of Rana sikimensis Jerdon, 1870. In 2000, Annemarie Ohler and Stéphane Grosjean visited the ZSI and had the opportunity to examine this specimen, which we hereby designate as lectotype (lectophoront) of this nominal species. It is an adult male (SVL $84.0 \mathrm{~mm}$ ) with nuptial spines on the chest and on the first three fingers, which does not belong in the species described by Dubois (1976) under this nomen but in the 'form' described by this author (pages 61-62) as 'Rana (Paa) liebigii with vocal sacs' but which we now regard as a species of Paa distinct from Paa liebigii, present in eastern Nepal and in Sikkim. The nomen Paa sikimensis (Jerdon, 1870) is therefore available for this species, which differs from Paa liebigii, the males of which do not have vocal sacs, by several constant characters (Dubois \& Ohler, unpublished).

Dubois (1976: 191-192) also reported having looked for the syntypes of Rana gammii Anderson, 1871 in the ZSI. He gave arguments for considering that the specimen ZSI 9173, designated by Annandale (1917: 138-139) as lectotype of this nominal species, was not one of these syntypes, so that this lectotype designation is invalid. On the other hand, he suggested that the four specimens ZSI 9664-9667, kept then under the nomen Rana vicina Stoliczka, 1872 and without mention of origin, could be the syntypes of Rana gammii. One of them, ZSI 9667 (adult male, SVL $62 \mathrm{~mm}$ ), has a large spiny zone around vent, corresponding to the figure 4 of Annandale (1917: 137). This male secondary character, also illustrated by Dubois (1976: 201, figure 76) on the basis of a 'Rana sikimensis' specimen from Nepal, exists only, in the Himalayas, in the latter species - but also in the genus Chaparana from northern Indochina and southern China (Dubois \& Ohler 2005) and in the genus Diplopaa (Fei 1999; Yang et al. 2011). Chanda et al. (2000: 109) stated that the four specimens ZSI 9664-9667, from "Darjeeling, Alt. 4000 ft. to 6000 
ft.", were indeed the syntypes of Rana gammii. We hereby designate the specimen ZSI 9667, figured by Annandale (1917), as lectotype of Rana gammii Anderson, 1871.

Dubois (1992: 318) erected a subgenus Ombrana of the genus Chaparana Bourret, 1939 for the species Rana sikimensis Jerdon, 1870. The biological species for which this genus-series nomen was intended is that for which the valid nomen was established above to be Rana gammii Anderson, 1871, but the nominal species actually designated is in fact a member of the taxon now known as Paa Dubois, 1975, of which Ombrana is therefore an invalid junior synonym. No other nomen being available for the genus including Rana gammii Anderson, 1871, we are led to propose the new nomen Ombropaa for this taxon.

\section{F.21.41. Infratribus CHAPARANINIA nov.}

Getangiotaxon: CHAPARANINA nov.

Adelphotaxa: Diplopainia nov.; FeIRaninia nov.; 2 GIS (Chrysopaa Ohler \& Dubois, 2006; Ombropaa nov.).

Getendotaxa: Chaparana Bourret, 1939; Gynandropaa Dubois, 1992.

\section{F.21.42. Infratribus DIPLOPAINIA nov.}

Getangiotaxon: CHAPARANINA nov.

Adelphotaxa: Chaparaninia nov.; FEIRANINIa nov.; 2 GIS (Chrysopaa Ohler \& Dubois, 2006; Ombropaa nov).

Getendotaxon: Diplopaa nov.

Nucleogenus, by present designation: Diplopaa nov. • Etymology of nomen: G: $\delta 1 \pi \lambda \operatorname{os}$ (diploos), 'double'; N: Paa Dubois, 1975, derived from Nepali language: Paa, 'frog'; referring to the presence of spines both on fingers and vent of adult breeding male. $\bullet$ Stem of nomen: Diplopa-.

Diagnosis: Large sized dicroglossids (males SVL 51-90 mm, females SVL 50-103 mm); snout length longer than eye length; internarial distance larger than distance between eyelids; tympanum small, poorly distinct; first finger longer than second; webbing between fingers absent; finger tips rounded; proximal subarticular tubercles of fingers enlarged; leg length longer snout-vent length; toe tips rounded; webbing between toes full; flap of skin along toe V extending near base of metatarsus; tarsal fold absent; skin on dorsum with small rounded warts or spinules, more dense in posterior part; laterodorsal folds as row of warts in a line; skin belly smooth; adult breeding male with nuptial pads absent or present on first finger and prepollex, forearm not enlarged; vent of breeding male with spines around and inside; vocal sacs present or absent; dorsal colour brownish with indistinct markings; chevron absent; mid-dorsal line absent; ventral colour grayish white with or without spots; eggs with dark animal pole; tadpoles with gray body, with darker or lighter spots, lower labial papillae in two rows, lower corners of mouth with additional papillae; tadpoles keratodont formula 5-7/3. \{Fei 1999; Yang et al. 2011\}.

\section{G.28.383. Genus Diplopaa nov.}

Getangiotaxon: DIPLOPAINIA nov.

Adelphotaxon: None.

Getendotaxa: Diplopaa kangxianensis (Yang, Wang, Hu \& Jiang, 2011); Diplopaa taihangnica (Chen \& Jiang, 2002).

Nucleospecies, by present designation: Paa (Feirana) taihangnica Chen \& Jiang, 2002. • Etymology

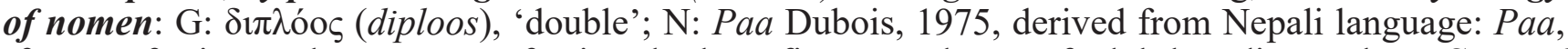
'frog'; referring to the presence of spines both on fingers and vent of adult breeding male. $\bullet$ Stem of nomen: Diplopa-. • Grammatical gender of nomen: feminine.

Diagnosis: Large sized dicroglossids (males SVL 51-90 mm, females SVL 50-103 mm); snout length longer than eye length; internarial distance larger than distance between eyelids; tympanum small, poorly distinct; first finger longer than second; webbing between fingers absent; finger tips rounded; 
proximal subarticular tubercles of fingers enlarged; leg length longer snout-vent length; toe tips rounded; webbing between toes full; flap of skin along toe V extending near base of metatarsus; tarsal fold absent; skin on dorsum with small rounded warts or spinules, more dense in posterior part; laterodorsal folds as rows of warts in a line; skin belly smooth; adult breeding male with nuptial pads absent or present on first finger and prepollex, forearm not enlarged; vent of male with spines around and inside; vocal sacs present or absent; dorsal colour brownish with indistinct markings; chevron absent; mid-dorsal line absent; ventral colour grayish white with or without spots; eggs with dark animal pole; tadpoles with gray body, with darker or lighter spots, lower labial papillae in two rows, lower corners of mouth with additional papillae; tadpoles keratodont formula 5-7/3. \{Fei 1999; Yang et al. 2011\}.

\title{
F.21.43. Infratribus FEIRANINIA nov.
}

Getangiotaxon: CHAPARANINA nov.

Adelphotaxa: Chaparaninia nov.; Diplopainia nov.; 2 GIS (Chrysopaa Ohler \& Dubois, 2006; Ombropaa nov.).

Getendotaxon: Feirana Dubois, 1992.

Nucleogenus, by present designation: Feirana Dubois, 1992. • Etymology of nomen: P: Fei Liang (1936-), Chinese herpetologist; N: Rana Linnaeus, 1758, from L: rana, 'frog'. - Stem of nomen: Feiran-.

Diagnosis: Large sized dicroglossids (males SVL 79.0-89.0 mm, females SVL 85.0-97.0 mm); tympanum small, poorly distinct; length of finger shorter than second; webbing between fingers absent; finger tips rounded; proximal subarticular tubercles of fingers relatively small; leg length longer than half snout-vent length; toe tips rounded; webbing between toes complete; flap of skin along toe $\mathrm{V}$ from tip of toe to between subarticular tubercle of toe $\mathrm{V}$ and base of metatarsus; tarsal fold absent; skin on dorsum relatively smooth, with rounded tubercles, particular in lateral part; laterodorsal folds discontinuous; skin belly smooth; adult breeding male with nuptial pads spines absent on fingers, arm, forearm, throat, chest, belly, and forearm not enlarged; vent of breeding male with square dermal flap; vocal sacs absent; dorsal colour dull green with lighter flecks; chevron sometimes present; mid-dorsal line absent; eggs with dark animal pole; tadpoles body brownish-green, tail with dark spots; two rows of lower labial papillae; tadpoles keratodont formula 7-9/3. \{Fei 1999; Ohler \& Dubois 2006\}.

\section{F.20.61. Subtribus PaINA Dubois, 1992}

\author{
Eunym: Hoc loco. \\ Getangiotaxon: PAINI Dubois, 1992. \\ Adelphotaxon: CHAPARANINA nov. \\ Getendotaxa: Nanorana Günther, 1896; Paa Dubois, 1975.
}

F.19.66. Tribus QUASIPAINI Fei, Ye \& Jiang, 2010

Protonym and eunym: QUASIPAINI Fei, Ye \& Jiang, 2010: 17 [T].

Getangiotaxon: PAINAE Dubois, 1992.

Adelphotaxa: PAINI Dubois, 1992; 1 GIS (Allopaa Ohler \& Dubois, 2006).

Getendotaxa: AnNANDIINA Fei, Ye \& Jiang, 2010; ERIPAINA nov.; QuasipaIna Fei, Ye \& Jiang, 2010.

\section{F.20.62. Subtribus ANNANDIINA Fei, Ye \& Jiang, 2010}

\author{
Protonym: ANNANDIINI Fei, Ye \& Jiang, 2010: 17 [T]. \\ Eunym: Hoc loco. \\ Getangiotaxon: QUASIPAINI Fei, Ye \& Jiang, 2010. \\ Adelphotaxa: ERIPAINA nov.; QuasipaIna Fei, Ye \& Jiang, 2010.
}




\section{F.20.63. Subtribus ERIPAINA nov.}

Getangiotaxon: QUASIPAINI Fei, Ye \& Jiang, 2010.

Adelphotaxa: AnNandina Fei, Ye \& Jiang, 2010; QuasipaIna Fei, Ye \& Jiang, 2010.

Getendotaxon: Eripaa Dubois, 1992.

Nucleogenus, by present designation: Eripaa Dubois, 1992. • Etymology of nomen: L: ericius, hedgehog; N: Paa Dubois, 1975, derived from Nepali language: Paa, 'frog'; referring to the spines on forearm and chest of these frogs. $\bullet$ Stem of nomen: Eripa-.

Diagnosis: Large sized dicroglossids (up to $106 \mathrm{~mm}$ ); snout shorter than eye; tympanum indistinct in external observation; first finger longer than second; webbing between fingers absent; finger tips enlarged; proximal subarticular tubercles of fingers large; leg length longer than half of snout-vent length; toe tips distinctly enlarged; webbing between toes complete, not incurved between extremities of adjacent toes; flap of skin along toe V from tip of toe to base of metatarsus of toe V; tarsal fold present, well developed; skin on dorsum longitudinally elongate, regularly arranged warts on mid-dorsal skin; laterodorsal folds absent; adult breeding male with large, distinct, black spines on finger I, sometimes on fingers II and III, forearm, breast and belly, but absent on throat; spines of forearm, chest and belly in a unique patch arranged in clusters; vent of breeding male without morphological differentiation; vocal sacs absent; forearm in breeding male enlarged; dorsal colour brownish; chevron always absent; mid-dorsal line always absent; ventral colour dirty white; eggs with coloured animal pole; tadpoles large, body stout, oval; tail fin with black spots but without a transverse bar between tail and body; beak undivided, outer surface of lower beak smooth, upper beak dimpled in middle; tadpoles keratodont formula 2:5+5/1+1:2; three rows of papillae on lower labium. \{Ohler \& Dubois 2006; Inthara et al. $2009\}$.

\section{F.20.64. Subtribus QUASIPAINA Fei, Ye \& Jiang, 2010}

Eunym: Hoc loco.

Getangiotaxon: QUASIPAINI Fei, Ye \& Jiang, 2010.

Adelphotaxa: ANNANDIINA Fei, Ye \& Jiang, 2010; ERIPAINA nov.

Getendotaxa: Quasipaa Dubois, 1992; Yerana Jiang, Chen \& Wang, 2006.

\section{F.17.45. Familia OCCIDOZYGIDAE Fei, Ye \& Huang, 1990}

Protonym: OCCIDOZYGINAE Fei, Ye \& Huang, 1990: 4, 123 [bF].

Eunym: Borah, Bordoloi, Purkayastha, Das, Dubois \& Ohler 2013: 39.

Getangiotaxon: DiCRoGlosseIDAE Dubois, 1987.

Adelphotaxon: DiCRoGlossidaE Dubois, 1987.

Getendotaxa: Ingeraninae Fei, Ye \& Jiang, 2010; OCCIDozyginaE Fei, Ye \& Huang, 1990.

Comments: This family includes two subgroups here recognised as the subfamilies INGERANINAE for Ingerana, and OCCIDOZYGINAE for a group of genera of unresolved relationships. For reasons previously given (Dubois 1987a; Dubois \& Ohler 2001), we do not synonymise Phrynoglossus with Occidozyga, and we now recognise within the subfamily two other genera, Oreobatrachus Boulenger, 1896, for Oreobatrachus baluensis, and Frethia nov., for the species F. celebensis, F. diminutiva, F. floresiana, F. laevis, F. semipalmata and F. tompotika, in order to have only holophyletic genera. 
Getangiotaxon: OCCIDOZYGIDAE Fei, Ye \& Huang, 1990.

Adelphotaxon: OCCIDOZYGINAE Fei, Ye \& Huang, 1990.

Getendotaxon: Ingerana Dubois, 1987.

\section{F.18.74. Subfamilia OCCIDOZYGINAE Fei, Ye \& Huang, 1990}

Eunym: Fei, Ye \& Huang 1990: 4, 123.

Getangiotaxon: OCCIDOZYGIDAE Fei, Ye \& Huang, 1990.

Adelphotaxon: INGERANINAE Fei, Ye \& Jiang, 2010.

Getendotaxa: Frethia nov.; Occidozyga Kuhl \& Van Hasselt, 1822; Oreobatrachus Boulenger, 1896; Phrynoglossus Peters, 1867.

\section{G.28.392. Genus Frethia nov.}

Getangiotaxon: OCCIDOZYGINAE Fei, Ye \& Huang, 1990

Adelphotaxa: Occidozyga Kuhl \& Van Hasselt, 1822; Oreobatrachus Boulenger, 1896; Phrynoglossus Peters, 1867.

Getendotaxa: Frethia celebensis (Smith, 1927); Frethia diminutiva (Taylor, 1922); Frethia floresiana (Mertens, 1927); Frethia laevis (Günther, 1859); Frethia semipalmata (Smith, 1927); Frethia tompotika (Iskandar, Arifin \& Rachmanasah, 2011).

Nucleospecies, by present designation: Oxyglossus laevis Günther, 1859. • Etymology of nomen: P: nomen composed of the first three letters of the patronym and the first three letters of the forename of Thierry Frétey (1963-), French herpetologist, to whom we are indebted for his valuable help throughout the years and particularly during the preparation of this work. $\bullet$ Stem of nomen: Frethi-. $\bullet$ Grammatical gender of nomen: feminine.

Diagnosis: Small to medium sized frogs (males SVL 25.5-41.6 mm; females SVL 31.6-61.8 mm); internarial distance larger than distance between upper eyelids, which is shorter than width of upper eyelids; distance of nostrils to snout shorter then distance from nostril to eye; tympanum hidden; tongue ovoid, not pointed and not notched; vomerine ridge and vomerine teeth absent; first finger as long as second or shorter; no web between fingers; tips of fingers pointed or slightly swollen; shanks short; toe tips with distinctly swollen tips but no grooves; webbing complete or incurved but with fringes up to discs; inner metatarsal tubercle oval, rather prominent; outer metatarsal tubercle absent; tarsal tubercle absent; dorsal skin smooth, with a few scattered, small, smooth tubercles or white spinules on posterior portion of back and dorsal surface of legs; ventral body smooth; males with nuptial pads extending from terminal joint of finger to the wrist on dorsal and median surfaces of finger I; small white spinules on throat, chest and belly variously developed; medium subgular internal vocal sacs, with elongated openings; females known to show male sexual characters; amplexus axillar; brownish with darker more or less distinct patterns on back, including a middorsal line or band in some specimens; ventral body whitish, immaculate or with various darker pattern; chest from cream colour with a few dark spots to almost solid black; limbs with dark crossbars or spots; occur usually in water bodies, like pools, streams and creeks, rice paddies, small mud puddles. \{Günther 1859; Mertens 1927; Smith 1927; Inger 1954, 1966; personal observations by $\mathrm{AO}$ and $\mathrm{AD}\}$.

Comments: Nicholls (1916: 82) had coined the nomen "Oxyrhachis" for the species Oxyglossus laevis Günther, 1859. Unfortunately, this nomen is both an anoplonym and a junior homonym of Oxyrhachis Germar, 1833 (HEMIPTERA). Therefore it cannot be used for an anuran genus, and a new nomen had to be coined for the latter. 
Protonym: NYCTIBATRACHINAE Blommers-Schlösser, 1993: 199 [bF].

Eunym: Hoc loco.

Getangiotaxon: RANOIDAE Batsch, 1796.

Adelphotaxa: CeratobatraCheidae Boulenger, 1884; DiCRoglosseidae Dubois, 1987; RaneIDAE Batsch, 1796; RANIXALEIDAE Dubois, 1987.

Getendotaxa: AstrobatraCHIDAE Vijayakumar, Pyron, Dinesh, Torsekar, Srikanthan, Swamy, Stanley, Blackburn \& Shanker, 2019; NYCTIBATRACHIDAE Blommers-Schlösser, 1993.

F.17.46. Familia AstrobatrachidaE Vijayakumar, Pyron, Dinesh, Torsekar, Srikanthan, Swamy, Stanley, Blackburn \& Shanker, 2019

Protonym: Astrobatrachinae Vijayakumar, Pyron, Dinesh, Torsekar, Srikanthan, Swamy, Stanley, Blackburn \& Shanker, 2019: $1[\mathrm{bF}]$.

Eunym: Hoc loco.

Getangiotaxon: NYCTIBATRACHEIDAE Blommers-Schlösser, 1993.

Adelphotaxon: NyCTIBATRACHIDAE Blommers-Schlösser, 1993.

Getendotaxon: Astrobatrachus Vijayakumar, Pyron, Dinesh, Torsekar, Srikanthan, Swamy, Stanley, Blackburn \& Shanker, 2019.

\section{F.17.47. Familia NyCTIBATRACHIDAE Blommers-Schlösser, 1993}

Eunym: Blommers-Schlösser 1993: 199.

Getangiotaxon: NYCTIBATRACHEIDAE Blommers-Schlösser, 1993.

Adelphotaxon: AstrobatrachidaE Vijayakumar, Pyron, Dinesh, Torsekar, Srikanthan, Swamy, Stanley, Blackburn \& Shanker, 2019.

Getendotaxa: Lankanectes Dubois \& Ohler, 2001; Nyctibatrachus Boulenger, 1882.

\section{F.16.06. Apofamilia RANEIDAE Batsch, 1796}

Eunym: Hoc loco.

Getangiotaxon: RANOIDAE Batsch, 1796.

Adelphotaxa: CERATobatraCheIDAE Boulenger, 1884; DicroglosseidaE Dubois, 1987; NyCTIBATRACHEIDAE BlommersSchlösser, 1993; RANIXALEIDAE Dubois, 1987.

Getendotaxa: RANIDAE Batsch, 1796; RHACOPHORIDAE \|Günther, 1858||-Hoffman, 1932.

\section{F.17.48. Familia RANIDAE Batsch, 1796}

Eunym: Boie 1828: 363.

Getangiotaxon: RANEIDAE Batsch, 1796.

Adelphotaxon: RHACOPHORIDAE $\|$ Günther, 1858\|-Hoffman, 1932.

Getendotaxa: Raninae Batsch, 1796; StaURoInAE Dubois, 2005.

Comments: The rank family and the nomen RANIDAE are attributed to this branch based on the [UQC]. This taxon includes two branches, the subfamily STAUROINAE and its sister-branch, composed of all other RANIDAE, formally named as the subfamily RANINAE. This branch includes two highly supported branches, recognised in this classification as the tribes MERISTOGENYINI, for the genera Clinotarsus and Meristogenys, and RANINI, for the other genera of the subfamily. Beside identification errors for some ranid species, Frost et al. (2006)'s tree shows a different relationship within this family but confirms its sister-group relationship with the RHACOPHORIDAE, including the MANTELLINAE. In their work, the NYCTIBATRACHINAE are sister-group to the other RANIDAE, and Staurois has a similar positon as sister- 
group of the remaining RANIDAE. Within their RANINAE the relationships are obscured by small sampling, specific identification or generic allocation problems. The relationships of the major groups in the RANIDAE are similar in Wiens et al. (2009), Pyron \& Wiens (2011) and Yuan et al. (2018). Huang \& $\mathrm{Tu}$ (2016) found different relationships within the RANIDAE, but their sampling was biased and support values for their branches were not indicated.

\section{F.18.75. Subfamilia RANINAE Batsch, 1796}

Eunym: Boulenger 1888: 205.

Getangiotaxon: RANIDAE Batsch, 1796.

Adelphotaxon: STAUROINAE Dubois, 2005.

Getendotaxa: MeristogenYini Fei, Ye \& Jiang, 2010; RANINI Batsch, 1796; 1 GIS (Pterorana Kiyasetuo \& Khare, 1986).

\section{F.19.67. Tribus MERIStogenYini Fei, Ye \& Jiang, 2010}

Protonym: MeristogenYINAE Fei, Ye \& Jiang, 2010: 18 [bF].

Eunym: Fei, Ye \& Jiang 2010: 18.

Getangiotaxon: RANINAE Batsch, 1796.

Adelphotaxa: RANINI Batsch, 1796; 1 GIS (Pterorana Kiyasetuo \& Khare, 1986).

Getendotaxa: Clinotarsus Mivart, 1869; Meristogenys Yang, 1991; Sumaterana Arifin, Smart, Hertwig, Smith, Iskandar \& Haas, 2018.

Comments: As in TREE the species attributed to Huia Yang, 1991 do not form a highly supported branch, they are included in Meristogenys, of which the nomen Huia is considered a subjective junior synonym.

\section{F.19.68. Tribus RANINI Batsch, 1796}

Eunym: Dubois 1992: 320.

Getangiotaxon: RANINAE Batsch, 1796.

Adelphotaxa: Meristogenyini Fei, Ye \& Jiang, 2010; 1 GIS (Pterorana Kiyasetuo \& Khare, 1986).

Getendotaxa: Amolopina Fei, Ye \& Huang, 1990; Ranina Batsch, 1796; 1 G†.

Comments: The genus Amolops, only member of the subtribe AMOLOPINA, is the sister-taxon to all other members of the tribe RANINI, formally recognised here as the subtribe RANINA. Within this subtribe, the infratribe PELOPHYLACINIA, for the single genus Pelophylax, is the sister-group to the RANINIA with five lineages of unresolved relationships, assigned to the hypotribes GLANDIRANINOA for Glandirana, LIMNODYTINOA for Abavorana and Hylarana, RUGOSINOA for Rugosa, SANGUIRANINOA for Sanguirana and RANINOA for the other genera. Oliver et al. (2015) proposed a classification of Hylarana sensu lato with more or less supported genera. As the relationships within the species assigned to this group are not resolved with high support in our classification, all genus-series nomina available within this group are here considered synonyms of Hylarana (see Appendices A5.NGS and A9.CLAD-1). Within the hypotribe RANINOA, three clans of unresolved relationships are taxonomically recognised here, namely the NIDIRANITES for Babina and Nidirana, the ODORRANITES for Odorrana, and the RANITES. This latter branch groups three subclans, the LITHOBATITIES for Aquarana, Boreorana and Lithobates, the PSEUDORANITIES for Pseudorana and the RANITIES for an infraclan LIUHURANITOES, for the single genus Liururana, and an infraclan RANITOES, for the genera Amerana and Rana.

The holophyly of the AMOLOPINA recovered here seems confirmed by recent results (Cai et al. 2007, Wiens et al. 2009, Pyron \& Wiens 2011), but the study of Huang \& Tu (2016) found a paraphyletic Amolops. The holophyly of the PELOPHYLACINIA is stable but its position is not stable: in TREE, Wiens et al. (2009) and Pyron \& Wiens (2011), Pelophylax is member of a group that is sister-group to Amolops, whereas in Yuan et al. (2018) it is sister-taxon to the group that includes Amolops and the other ranids, in Cai et al. (2007) it is sister-group to a taxon including Meristogenys and Huia, and in Huang \& Tu (2016) it is sister-taxon to some Amolops species. 
In the remaining ranids, here formally named RANINOA, few highly supported relationships are confirmed in TREE. Thus, the relationship of Babina and Nidirana was confirmed by Lyu et al. (2017) who, analysing Babina and Nidirana sensu stricto, could resurrect the genus Nidirana, previously well defined morphologically and ethologically by several synapomorphies (Dubois 1992), but strangely synonymised with Babina by Frost et al. (2006). Finally, the close relationship of the genus Rana with a part of North American ranids, the Amerana (the 'boylii group') is supported here, as it was in Wiens et al. (2009), and as is the sister-group relationship of this group to Liuhurana.

We recognise a new genus Boreorana for Rana sylvatica which is in a 'Latonia-like situation' (LLS) relatively to Lithobates, with which its relationship does not have high support. In Frost et al. (2006), Rana sylvatica was recovered as sister-group to a taxon that includes Lithobates, Typheropsis, Sierrana and Pantherana species, whereas in Wiens et al. (2009) and Pyron \& Wiens (2011) it was sister-taxon to Aquarana.

\section{F.20.65. Subtribus AmolopINA Fei, Ye \& Huang, 1990}

Protonym: AmolopinaE Fei, Ye \& Huang, 1990: 4, 123 [bF].

Eunym: Hoc loco.

Getangiotaxon: RANINI Batsch, 1796.

Adelphotaxa: RANINA Batsch, 1796; 1 G†.

Getendotaxon: Amolops Cope, 1865.

\section{F.20.66. Subtribus RANINA Batsch, 1796}

Eunym: Hoc loco.

Getangiotaxon: RANINI Batsch, 1796.

Adelphotaxa: Amolopina Fei, Ye \& Huang, 1990; 1 G†.

Getendotaxa: PelophylaCINIA nov.; RaNiNIA Batsch, 1796.

\section{F.21.44. Infratribus PELOPHYLACINIA nov.}

Getangiotaxon: RANINA Batsch, 1796.

Adelphotaxon: RANINIA Batsch, 1796.

Getendotaxon: Pelophylax Fitzinger, 1843.

Nucleogenus, by present designation: Pelophylax Fitzinger, 1843. • Etymology of nomen: G: $\pi \varepsilon \lambda o ́ \varsigma$ (pelos), 'mud'; $\varphi v ́ \lambda \alpha \xi$ (phylax), 'guardian'. • Stem of nomen: Pelophylac-.

Diagnosis: Medium to large sized ranids (males SVL 38-106 mm; females SVL 38-103 mm) with long limbs; first finger longer than second; tips of digits pointed; external metatarsal tubercle present or absent; web on toes usually large; metatarsals widely separated by web; large, prominent latero-dorsal folds present; male with or without external vocal sac; nuptial pads on first finger; tympanum smaller than eye in both sexes; dorsal pattern without black chevron but with large spots; a mediodorsal line present or absent; tadpoles without ventral sucker; tadpoles keratodont formula 1-3/2-3. \{Dubois 1992; Fei 1999; Gül et al. 2011\}.

\section{F.21.45. Infratribus RANINIA Batsch, 1796}

Eunym: Hoc loco.

Getangiotaxon: RANINA Batsch, 1796.

Adelphotaxon: PELOPHYLACINIA nov.

Getendotaxa: GLANDIRANINOA Fei, Ye \& Jiang, 2010; LIMNODYTINOA Fitzinger, 1843; RANINOA Batsch, 1796; RUGOSINOA nov.; SANGUIRANINOA Fei, Ye \& Jiang, 2010. 


\section{F.22.19. Hypotribus GLANDIRANINOA Fei, Ye \& Jiang, 2010}

Protonym: GLANDIRANINI Fei, Ye \& Jiang, 2010: 18 [T].

Eunym: Hoc loco.

Getangiotaxon: RANINIA Batsch, 1796.

Adelphotaxa: LimnodytinoA Fitzinger, 1843; RANinOA Batsch, 1796; Rugosinoa nov.; SANGUiRAninoA Fei, Ye \& Jiang, 2010.

Getendotaxon: Glandirana Fei, Ye \& Huang, 1990.

\section{F.22.20. Hypotribus LIMNODYTINOA Fitzinger, 1843}

Protonym: LIMNODYTAE Fitzinger, 1843: 31 [F].

Eunym: Hoc loco.

Getangiotaxon: RANINIA Batsch, 1796.

Adelphotaxa: Glandiraninoa Fei, Ye \& Jiang, 2010; Raninoa Batsch, 1796; Rugosinoa nov.; SanguiRaninoa Fei, Ye \& Jiang, 2010.

Getendotaxa: Abavorana Oliver, Prendini, Kraus \& Raxworthy, 2015; Hylarana Tschudi, 1838.

\section{F.22.21. Hypotribus RANINOA Batsch, 1796}

Eunym: Hoc loco.

Getangiotaxon: RANINIA Batsch, 1796.

Adelphotaxa: GLANDIRANinoa Fei, Ye \& Jiang, 2010; LimnODYTINOA Fitzinger, 1843; RUGOSINOA nov.; SANGUIRANINOA Fei, Ye \& Jiang, 2010.

Getendotaxa: NidiRanites Fei, Ye \& Jiang, 2010; OdorRanites Fei, Ye \& Jiang, 2010; Ranites Batsch, 1796.

\section{F.23.13. Clanus NidiRANITES Fei, Ye \& Jiang, 2010}

Protonym: NIDIRANINI Fei, Ye \& Jiang, 2010: 18 [T].

Eunym: Hoc loco.

Getangiotaxon: RANINOA Batsch, 1796.

Adelphotaxa: OdorRanites Fei, Ye \& Jiang, 2010; RANITES Batsch, 1796.

Getendotaxa: Babina Thompson, 1912; Nidirana Dubois, 1992.

\section{F.23.14. Clanus ODORRANITES Fei, Ye \& Jiang, 2010}

Protonym: ODORRANINI Fei, Ye \& Jiang, 2010: 18 [T].

Eunym: Hoc loco.

Getangiotaxon: RANINOA Batsch, 1796.

Adelphotaxa: NIDIRANITES Fei, Ye \& Jiang, 2010; RANITES Batsch, 1796.

Getendotaxon: Odorrana Fei, Ye \& Huang, 1990.

\section{F.23.15. Clanus RANITES Batsch, 1796}

Eunym: Hoc loco.

Getangiotaxon: RANINOA Batsch, 1796.

Adelphotaxa: NidiRANITES Fei, Ye \& Jiang, 2010; OdoRRANITES Fei, Ye \& Jiang, 2010.

Getendotaxa: Lithobatities nov.; PSEUdORANITIES nov.; RANITIES Batsch, 1796. 


\section{F.24.07. Subclanus LITHOBATITIES nov.}

Getangiotaxon: RANITES Batsch, 1796.

Adelphotaxa: PSEUdoRANITIES nov.; RANITIES Batsch, 1796.

Getendotaxa: Aquarana Dubois, 1992; Boreorana nov.; Lithobates Fitzinger, 1843.

Nucleogenus, by present designation: Lithobates Fitzinger, 1843. • Etymology of nomen: G: $\lambda i \theta$ os

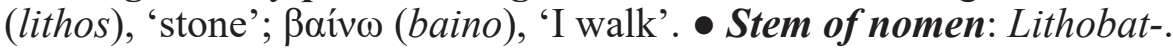

Diagnosis: Ranid frogs of medium to very large, rarely small size (males SVL 34-158 mm; females SVL 32-200 mm); snout as long or little longer than eye; internarial distance larger or as large as interorbital distance; tympanum in adult males highly variable, attaining in many species sizes larger than eye length but always larger than half eye diameter; first finger usually longer or of same size as second, rarely shorter; webbing between fingers absent; tips of fingers pointed or obtuse rarely with small dicks; legs short, medium or long; toe tips variable, pointed obtuse, expanded and some species showing small discs; webbing usually full to large, rarely less; metatarsals separated from distal half to base; inner metatarsal tubercle usually short and feebly prominent, rarely prominent and hard or of moderate length; outer metatarsal tubercle usually absent, rarely a small tubercle present; dorsal skin smooth or bearing granules, round or elongate warts or a combination of these structures; laterodorsal folds absent or present as a narrow to broad, flat or prominent glandular band, sometimes not continuous or only extending on anterior dorsum; skin on abdomen smooth; nuptial pads present or absent (not expressly stated in numerous species descriptions); external or internal vocal sacs usually present; dorsum brown, olive or green, uniform, or anterior part of brighter colour, or with mottling, with marbling or with small or large spots, set irregularly or in a line, sometimes outlined by a light halo; chevron on back absent; abdomen usually white, cream or yellow but marbling or spots quite often present on throat and chest, more rarely on abdomen, few species dark coloured ventral surface. \{Boulenger 1883, 1920a; Günther 1900; Taylor 1939, 1942; Goin \& Netting 1940; Zweifel 1957; Smith 1959; Sanders 1973; Pace 1974; Frost \& Bagnara 1976; Hillis et al. 1984; Platz \& Frost 1984; Moler 1985; Hillis \& Frost 1985; Hillis \& Sá 1988; Dubois 1992; Webb 2001\}.

\section{G.28.412. Genus Boreorana nov.}

Getangiotaxon: LITHOBATITIES nov.

Adelphotaxa: Aquarana Dubois, 1992; Lithobates Fitzinger, 1843..

Getendotaxon: Boreorana sylvatica (Le Conte, 1825).

Nucleospecies, by present designation: Rana sylvatica Le Conte, 1825. • Etymology of nomen: G: Bopéas (boreas), Greek god of North wind; N: Rana Linnaeus, 1758, from L: rana, 'frog'. • Stem of nomen: Boreoran-. • Grammatical gender of nomen: feminine.

Diagnosis: Medium sized ranid frogs (males SVL 32-63 mm; females SVL 42-83 mm); umbraculum of iris absent; tips of digits and toes not enlarged, not bearing pads; humeral gland in males absent; suprabrachial glands in male absent; fissura metotica dorsalis absent; no fusion of sacral and presacral vertebrae; mid-dorsal chevron potentially present; mediodorsal line potentially present; chromosome complement $2 \mathrm{n}=26$; eggs pigmented, moderate in size $(2 \mathrm{~mm})$, clutch size from 300 to 1500 eggs; tadpoles with 3 keratodont rows on upper lip and 4 on lower lip; keratodont on margin of oral disc absent; ventral sucker absent. \{Case 1979; Dubois 1992; Dodd 2013 \}.

\section{F.24.08. Subclanus PSEUDORANITIES nov.}

Getangiotaxon: RANITES Batsch, 1796.

Adelphotaxa: Lithobatities nov.; RANITIES Batsch, 1796.

Getendotaxon: Pseudorana Fei, Ye \& Huang, 1990. 
Nucleogenus, by present designation: Pseudorana Fei, Ye \& Huang, 1990. • Etymology of nomen:

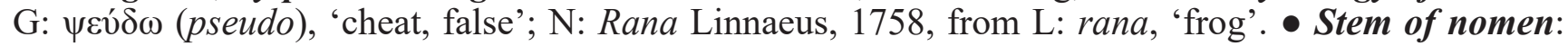
Pseudoran-.

Diagnosis: Medium sized ranids (males SVL 32-50 mm; females SVL 43-53 mm); first finger longer than second; long legs; thin dorsolateral folds; smooth dorsal and ventral skin; tips of fingers dilated without grooves, tips of toes dilated with ventrolateral grooves; inner metatarsal tubercle distinct, outer one small; web between toes large; metatarsals only shortly separated; presence of a dark middorsal chevron; nuptial pads on first finger, vocal sacs absent or present; tadpoles with ventral sucker but without dermal glands; tadpoles keratodont formula 5-7/5-8. \{Dubois 1992; Fei 1999\}.

F.24.09. Subclanus RANITIES Batsch, 1796

Eunym: Hoc loco.

Getangiotaxon: RANITES Batsch, 1796.

Adelphotaxa: LITHOBATITIES nov.; PSEUDORANITIES nov.

Getendotaxa: LIUHURANITOES nov.; RANITOES Batsch, 1796.

F.25.18. Infraclanus LIUHURANITOES nov.

Getangiotaxon: RANITIES Batsch, 1796.

Adelphotaxon: RANITOES Batsch, 1796.

Getendotaxon: Liuhurana Fei, Ye, Jiang, Dubois \& Ohler in Fei, Ye \& Jiang 2010.

Nucleogenus, by present designation: Liuhurana Fei, Ye, Jiang, Dubois \& Ohler in Fei, Ye \& Jiang 2010. - Etymology of nomen: P: concatenation of the names of Liu Chengchao (1900-1976) and Hu Shuqin (1914-1992), Chinese herpetologists; N: Rana Linnaeus, 1758, from L: rana, 'frog' • Stem of nomen: Liuhuran-.

Diagnosis: Small sized ranids (males SVL 30-32 mm; female SVL $39 \mathrm{~mm}$ ); first finger longer than second; short legs; well developed dorsolateral folds present; smooth dorsal and ventral skin; tips of fingers and toes pointed, inner metatarsal tubercle distinct, outer one absent; web on toes moderate; metatarsals only distally separated; large glands on tibia, tarsus and metatarsus, smaller glands on forearm, a suprabrachial gland; a dark middorsal chevron absent; nuptial pads on first finger, paired subgular vocal sacs present; tadpoles without ventral sucker; tadpoles keratodont formula 2/4. \{Liu 1950; Fei et al. 2010\}.

F.25.19. Infraclanus RANITOES Batsch, 1796

Eunym: Hoc loco.

Getangiotaxon: RANITIES Batsch, 1796.

Adelphotaxon: LIUHURANITOES nov.

Getendotaxa: Amerana Dubois, 1992; Rana Linnaeus, 1758.

\section{F.22.22. Hypotribus RUGOSINOA nov.}

Getangiotaxon: RANINIA Batsch, 1796.

Adelphotaxa: GLANDIRANINOA Fei, Ye \& Jiang, 2010; LIMNODYTINOA Fitzinger, 1843; RANINOA Batsch, 1796; SANGUIRANINOA Fei, Ye \& Jiang, 2010.

Getendotaxon: Rugosa Fei, Ye \& Huang, 1990. 
Nucleogenus, by present designation: Rugosa Fei, Ye \& Huang, 1990. • Etymology of nomen: L: rugosus, 'wrinkled'. • Stem of nomen: Rugos-.

Diagnosis: Medium sized ranids (males SVL 37-51 mm; females SVL 44-59 mm) with short limbs; first finger longer than second; tips of digits rounded; external metatarsal tubercle present; toes entirely webbed; metatarsals widely separated by web; latero-dorsal folds absent, but upper parts of body strongly granular and showing numerous short longitudinal folds; male with or without internal vocal sac; nuptial pads on base of first finger; tympanum smaller than eye in both sexes; dorsal pattern without black chevron or large spots. \{Dubois 1992; Fei 1999; Maeda \& Matsui 1999\}.

\title{
F.22.23. Hypotribus SANGUIRANINOA Fei, Ye \& Jiang, 2010
}

Protonym: SANGUIRANINI Fei, Ye \& Jiang, 2010: 18 [T].

Eunym: Hoc loco.

Getangiotaxon: RANINIA Batsch, 1796.

Adelphotaxa: Glandiraninoa Fei, Ye \& Jiang, 2010; Limnodytinoa Fitzinger, 1843; Raninoa Batsch, 1796; RUGosinoA nov.

Getendotaxon: Sanguirana Dubois, 1992.

F.18.76. Subfamilia STAUROINAE Dubois, 2005

Protonym: StaURoINI Dubois, 2005: 5 [T].

Eunym: Hoc loco.

Getangiotaxon: RANIDAE Batsch, 1796.

Adelphotaxon: RANINAE Batsch, 1796.

Getendotaxon: Staurois Cope, 1865.

\section{F.17.49. Familia RHACOPHORIDAE \|Günther, 1858\|-Hoffman, 1932}

\author{
Protonyms and eunym: ||POLYPEDATIDAE Günther, 1858: 346\|| [F]; RHACOPHORIDAE Hoffman, 1932: 562 [F]. \\ Getangiotaxon: RANEIDAE Batsch, 1796. \\ Adelphotaxon: RANIDAE Batsch, 1796. \\ Getendotaxa: MANTELLINAE Laurent, 1946; RHACOPHORINAE ||Günther, 1858||-Hoffman, 1932.
}

Comments: The sister-group relationship of the branches here named MANTELLINAE and RHACOPHORINAE has been confirmed in all recent works (Bossuyt \& Milinkovitch 2000; Emerson et al. 2000; Roelants et al. 2004; Frost et al. 2006; Bossuyt \& Roelants 2009; Pyron \& Wiens 2011; Yuan et al. 2018). As the RHACOPHORIDAE and their sister-taxon RANIDAE are both attributed to the rank family by the [UQC], the immediately included taxa, which are not credited with the rank family by the [UQC], are attributed to the just subordinate rank, which is subfamily.

\section{F.18.77. Subfamilia MANTELLINAE Laurent, 1946}

Protonym and eunym: MANTELLINAE Laurent, 1946: 336 [bF].

Getangiotaxon: RHACOPHORIDAE \|Günther, 1858\|-Hoffman, 1932.

Adelphotaxon: RHACOPHORINAE \|Günther, 1858\|-Hoffman, 1932.

Getendotaxa: BoopHINI Vences \& Glaw, 2001; LALIOSTOMINI Vences \& Glaw, 2001; MANTELLINI Laurent, 1946; TSINGYMANTINI nov.

Comments: In TREE, the relationships within the four included branches of this subfamily do not have enough support, so we recognise them at the same rank, as the tribes BOOPHINI for Boophis, LALIOSTOMINI for Aglyptodactylus and Laliostoma, TSINGYMANTINI for Tsingymantis, and MANTELLINI. The latter tribe 
includes two branches, taxonomically recognised as the subtribes MANTELLINA and MANTIDACTYLINA. The MANTELLINA include the infratribe BLOMMERIINIA for Blommersia and Guibemantis, and the infratribe MANTELLINIA for Mantella and Wakea. Within the subtribe MANTIDACTYLINA, the infratribe SPINOMANTINIA, for Spinomantis, is sister-taxon to the infratribe MANTIDACTYLINIA, with BOEHMANTINOA for Boehmantis, and MANTIDACTYLINOA for Gephyromantis and Mantidactylus.

The taxon here recognised as the subfamily MANTELLINAE was since the beginning of molecular studies identified as a holophyletic group (Bossuyt \& Milinkovitch 2000; Richards et al. 2000; Vences et al. 2000, 2003; Frost et al. 2006; Bossuyt \& Roelants 2009; Pyron \& Wiens 2011; Wollenberg et al. 2011; Yuan et al. 2018), but the relationships within this group have changed. Glaw \& Vences (2006) recognised a family rank taxon MANTELLIDAE with three subfamilies, whereas in the trees of Frost et al. (2006) and Wollenberg et al. (2011) the BOOPHINAE appeared as the sister-taxon of the MANTELLINAE composed of the LALIOSTOMINI and MANTELLINI, whereas in Richards et al. (2000) and Kurabayashi et al. (2008) the BoOPHINAE were sister-taxon to the LALIOSTOMINAE. The position of the TSINGYMANTINI was also disputed. Considered basal to the MANTELLINAE (our MANTELLINI) based on morphological characters (Raselimanana et al. 2007), it was sister-taxon to this group in Wollenberg et al. (2011), whereas in Kurabayashi et al. (2008) it appeared as sister-taxon to their BooPHINAE and LALIOSTOMINAE.

F.19.69. Tribus BoophinI Vences \& Glaw, 2001

Protonym: Boophinae Vences \& Glaw, 2001: 85 [bF].

Eunym: Dubois 2005: 16.

Getangiotaxon: MANTELLINAE Laurent, 1946.

Adelphotaxa: LALIOSTOMINI Vences \& Glaw, 2001; MANTELLINI Laurent, 1946; TSINGYMANTINI nov.

Getendotaxon: Boophis Tschudi, 1838.

\title{
F.19.70. Tribus LALIOSTOMINI Vences \& Glaw, 2001
}

\author{
Protonym: LALIOSTOMINAE Vences \& Glaw, 2001: 85 [bF]. \\ Eunym: Dubois 2005: 16. \\ Getangiotaxon: MANTELLINAE Laurent, 1946. \\ Adelphotaxa: Boophini Vences \& Glaw, 2001; MANTELLINI Laurent, 1946; TSINGYMANTINI nov. \\ Getendotaxa: Aglyptodactylus Boulenger, 1919; Laliostoma Glaw, Vences \& Böhme, 1998.
}

Comments: The generic nomen Laliostoma was derived from the Greek roots $\lambda \alpha \lambda$ ió (lalia), 'chat' and $\sigma \tau$ ó $\mu \alpha$ (stoma), 'mouth' (Glaw et al. 1998). The genitive of $\sigma \tau o ́ \mu \alpha$ being $\sigma \tau$ ó $\mu \alpha \tau o \zeta$, the subfamilal nomen introduced by Vences \& Glaw (2001) based on this generic nomen should have been spelt LALIOSTOMATINAE, just like in the case of AmBYSTOMATIDAE or ENGYSTOMATIDAE. Before 2000, the incorrect original spelling should have been corrected, but it is no more the case under the 1999 Code because of the new Article 29.4, which states that such incorrect spellings should be maintained, a highly confusing Rule, especially in this case, as several family-series nomina, based on the same final stem like -stoma, must now have different endings according to whether they were made available before 2000 or after 1999 (see Dubois 2005e: 74-75; Dubois \& Aescht 2019o: 125-126).

\section{F.19.71. Tribus MANTELLINI Laurent, 1946}

Eunym: Dubois 2005: 16.

Getangiotaxon: MANTELLINAE Laurent, 1946.

Adelphotaxa: Boophini Vences \& Glaw, 2001; LaLIostomini Vences \& Glaw, 2001; TSingYmantini nov.

Getendotaxa: MANTELLINA Laurent, 1946; MANTIDACTYLINA nov. 
Eunym: Hoc loco.

Getangiotaxon: MANTELLINI Laurent, 1946.

Adelphotaxon: MANTIDACTYLINA nov.

Getendotaxa: BLOMMERSIINIA nov.; MANTELLINIA Laurent, 1946.

\section{F.21.46. Infratribus BLOMMERSIINIA nov.}

Getangiotaxon: MANTELLINA Laurent, 1946.

Adelphotaxon: MANTELLINIA Laurent, 1946.

Getendotaxa: Blommersia Dubois, 1992; Guibemantis Dubois, 1992.

Nucleogenus, by present designation: Blommersia Dubois, 1992. • Etymology of nomen: P: Rose Marie Antoinette Blommers-Schlösser (1944-), Dutch herpetologist. • Stem of nomen: Blommersi-.

Diagnosis: Small to medium sized frogs (SVL 15-60 mm); webbing between toes rudimentary to extended; metatarsalia connected or separated; inner and outer metatarsal tubercle present; finger tips slightly to distinctly enlarged; femoral glands type 1 (Glaw et al. 2000) in male, absent in female; tibial glands absent; vocal sac single, subgular; maxillary teeth present; vomerine teeth present or absent; tongue weakly or distinctly bifid; vertebral column diplasiocoelous or procoelous; tympanum same size in male and female; habits terrestrial, arboreal or phytotelmic; nocturnal or diurnal activity; eggs pigmented, brownish or greenish, laid above the water or hidden in cavities on the ground; tadpoles free swimming and feeding; keratodont formula 1:(2+2)-(6+6)/3. \{Blommers-Schlösser \& Blanc 1991; Dubois 1992; Glaw \& Vences 1994, 2006, 2007; Lehtinen et al. 2012\}.

\section{F.21.47. Infratribus MANTELLINIA Laurent, 1946}

Eunym: Hoc loco.

Getangiotaxon: MANTELLINA Laurent, 1946.

Adelphotaxon: BLOMMERSIINIA nov.

Getendotaxa: Mantella Boulenger, 1882; Wakea Glaw \& Vences, 2006.

\section{F.20.68. Subtribus MANTIDACTYLINA nov.}

Getangiotaxon: MANTELLINI Laurent, 1946.

Adelphotaxon: MANTELLINA Laurent, 1946.

Getendotaxa: MANTIDACTYLINIA nov.; SPINOMANTINIA nov.

Nucleogenus, by present designation: Mantidactylus Boulenger, 1895. • Etymology of nomen: G:

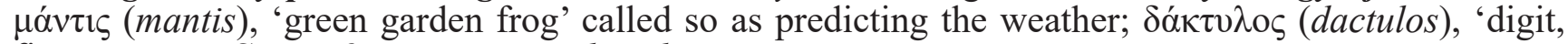
finger, toe'. • Stem of nomen: Mantidactyl-.

Diagnosis: Small to large sized frogs (SVL 17-120 mm); webbing between toes often moderately extended, but also full, rudimentary or absent; metatarsalia connected or separated; inner metatarsal tubercle present or absent; outer metatarsal tubercle generally present; finger tips moderately enlarged, but also slightly or strongly enlarged; femoral glands type 2, 3 or 4 in male, small or absent in female, or not recognisable externally; tibial glands present or absent; vocal sac single, subgular paired or bilobate; maxillary teeth present or absent; vomerine teeth present or absent; tongue bifid; vertebral column diplasiocoelous or procoelous; tympanum moderate or very small, in male mostly larger then in female, or of same size; habits terrestrial or arboreal, along torrents, small streams or stagnant water bodies, or independent from water bodies; diurnal and nocturnal activity; eggs terrestrial or arboreal; parental care in some species; tadpoles free swimming and exotrophic, or endotrophic with direct development or non-feeding larvae, nests known for some species. \{Guibé 1978; Glaw \& Vences 2006\}. 


\section{F.21.48. Infratribus MANTIDACTYLINIA nov.}

Getangiotaxon: MANTIDACTYLINA nov.

Adelphotaxon: SPINOMANTINIA nov.

Getendotaxa: BOEHMANTINOA nov.; MANTIDACTYLINOA nov.

\section{F.22.24. Hypotribus BOEHMANTINOA nov.}

Getangiotaxon: MANTIDACTYLINIA nov.

Adelphotaxon: MANTIDACTYLINOA nov.

Getendotaxon: Boehmantis Glaw \& Vences, 2006.

Nucleogenus, by present designation: Boehmantis Glaw \& Vences, 2006. • Etymology of nomen: P:

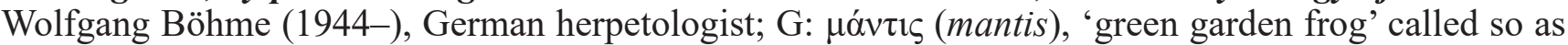
predicting the weather. $\bullet$ Stem of nomen: Boehmant-.

Diagnosis: Large sized frogs (SVL 60-80 mm); webbing between toes extended; metatarsalia separated; inner metatarsal tubercle present; outer metatarsal tubercle absent; finger tips strongly enlarged; femoral glands not recognisable externally in males and females; tibial glands absent; vocal sac single, subgular; maxillary teeth present; vomerine teeth present; tongue bifid; tympanum very small; habits terrestrial in torrents; nocturnal activity; eggs pigmented; parental care not observed; tadpoles exotrophic; tadpoles keratodont formula 4:(2+2)-(4+4)/3. \{Glaw \& Vences 2006; Andreone \& Nussbaum 2006\}.

\section{F.22.25. Hypotribus MANTIDACTYLINOA nov.}

Getangiotaxon: MANTIDACTYLINIA nov.

Adelphotaxon: BoEHMANTINOA nov.

Getendotaxa: Gephyromantis Methuen, 1920; Mantidactylus Boulenger, 1895.

\section{F.21.49. Infratribus SPINOMANTINIA nov.}

Getangiotaxon: MANTIDACTYLINA nov.

Adelphotaxon: MANTIDACTYLINIA nov.

Getendotaxon: Spinomantis Dubois, 1992.

Nucleogenus, by present designation: Spinomantis Dubois, 1992. • Etymology of nomen: L: spina, 'spine'; G: $\mu$ óv $\tau \iota \varsigma$ (mantis), 'green garden frog' called so as predicting the weather. $\bullet$ Stem of nomen: Spinomant-.

Diagnosis: Small to medium sized frogs (SVL 22-60 $\mathrm{mm}$ ); webbing between toes rudimentary to moderate; metatarsalia connected or separated; inner metatarsal tubercle present; outer metatarsal tubercle generally present; finger tips distinctly enlarged; femoral glands type 2 in male, absent in female; tibial glands absent; vocal sac single, subgular, or paired or slightly bilobed; maxillary teeth present; vomerine teeth generally present; tongue bifid; vertebral column diplasiocoelous; tympanum same size in male and female; in arboreal or terrestrial habitat along or in small streams; generally nocturnal or partly diurnal activity; eggs yellowish; tadpoles free swimming and feeding; keratodont formula $0-1:(2+2)-(3+3) /(1+1): 1-2$. \{Glaw \& Vences 2006; Vejarano et al. 2006\}.

\section{F.19.72. Tribus TSINGYMANTINI nov.}

Getangiotaxon: MANTELLINAE Laurent, 1946.

Adelphotaxa: Boophini Vences \& Glaw, 2001; LALIostomini Vences \& Glaw, 2001; MANTELLINI Laurent, 1946. 
Getendotaxon: Tsingymantis Glaw, Hoegg \& Vences, 2006.

Nucleogenus, by present designation: Tsingymantis Glaw, Hoegg \& Vences, 2006. • Etymology of nomen: R: tsingy, Malagasy word for eroded karstic limestone formations where these frogs live; G:

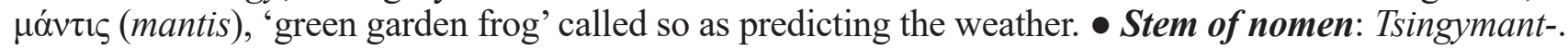

Diagnosis: Medium sized mantellids (SVL 53-67 mm); webbing between toes small; metatarsalia largely connected; inner metatarsal tubercle very distinct; outer metatarsal tubercle absent; finger tips strongly enlarged; femoral glands not recognisable in both sexes; tibial glands absent; vocal sac not observed externally; maxillary teeth present; vomerine teeth present; tongue bifid; tympanum large, slightly larger in males; habits terrestrial; nocturnal activity; eggs pigmented; tadpoles free swimming and feeding; keratodont formula 1:4+4/1+1:2. \{Glaw et al. 2006; Raselimanana et al. 2007; Randrianiaina et al. $2011\}$.

\title{
F.18.78. Subfamilia RHACOPHORINAE $\|$ Günther, 1858\|-Hoffman, 1932
}

\author{
Eunym: Laurent 1943: 16. \\ Getangiotaxon: RHACOPHORIDAE ||Günther, 1858\|-Hoffman, 1932. \\ Adelphotaxon: MANTELLINAE Laurent, 1946. \\ Getendotaxa: BuergeriINI Channing, 1989; RHACOPHoRINI ||Günther, 1858||-Hoffman, 1932; 1 G†; 1 GIS \\ (Dendrobatorana Ahl, 1927).
}

Comments: The subfamily RHACOPHORINAE consists in two tribes, the BUERGERIINI for Buergeria, which is sister-branch to the RHACOPHORINI, including all other RHACOPHORINAE. This relationship was first revealed by Bossuyt et al. (2006) and confirmed by Wilkinson et al. (2002), Grosjean et al. (2008), Li et al. (2008), Yu et al. (2009), Wiens et al. (2009), Pyron \& Wiens (2011), Hertwig et al. (2013), Meegaskumbura et al. (2015a) and Chan et al. (2018).

In the latter tribe, the subtribe ROMERINA, for Romerus, is sister-branch to the subtribe RHACOPHORINA, including all other members of the tribe. The position of Romerus within the RHACOPHORINAE was revealed by Li et al. (2008) who however proposed for this genus a nomen, "Liuixalus ", which is not available as no diagnostic characters were given in their work. We provide here an available nomen for this genus, the phylogenetic position of which was confirmed by other authors.

In the subtribe RHACOPHORINA, two branches are revealed, recognised as the infratribes NYCTIXALINIA for Nyctixalus and Theloderma, and RHACOPHORINIA for the remaining genera. The sister-group relationship of Nyctixalus and Theloderma was found by Wilkinson et al. (2002), Grosjean et al. (2008), Li et al. (2008), Yu et al. (2009), Wiens et al. (2009), Li et al. (2009), Pyron \& Wiens (2011), Hertwig et al. (2013), Meegaskumbura et al. (2015a) and Chan et al. (2018).

The relationship among the RHACOPHORINIA are not resolved and five hypotribes are recognised here: the GRACIXALINOA for Gracixalus, the ORIXALINOA for Orixalus, the VAMPYRIINOA for Vampyrus, the Philautinoa and the RhaCophorinoa. Within the hypotribe Philautinoa, the unresolved relationships lead to the recognition of four clans, the KURIXALITES for Kurixalus, the Nasutixalites for Nasutixalus, the Philautites for Philautus, and the Mercuranites, with two subclans, BEDDOMIXALITIES for Beddomixalus, and MERCURANITIES for Mercurana, Pseudophilautus and Raorchestes. Within the hypotribe RHACOPHORINOA, the clan CHIRIXALITES, for Chirixalus and Chiromantis, is sister-taxon to the RHACOPHORITES. This latter clan contains four taxa of unresolved relationships, the subclans FEIHYLITIES for Feihyla, RHACOPHORITIES for Leptomantis, Rhacophorus and Zhangixalus, the TAMIXALITIES for Tamixalus, and the POLYPEDATITIES, for the infraclans GHATIXALITOES for Ghatixalus, and PoLYPEDATITOES for Polypedates and Taruga.

The supported sister-taxa relationships of the species attributed to Chirixalus and Chiromantis, Polypedates and Taruga, and Leptomantis, Rhacophorus and Zhangixalus have been confirmed in most recent molecular phylogenies (Wilkinson et al. 2002; Grosjean et al. 2008; Li et al. 2008; Yu et al. 2009; Wiens et al. 2009; Li et al. 2009; Pyron \& Wiens 2011; Hertwig et al. 2013; Meegaskumbura et al. 2015b; Chan et al. 2018), although the taxonomic conclusions were often not formally done, as statistical support was low for many groupings or taxon sampling was not sufficient. The tree published by Chan et al. (2018) has resolved relationships for all the genera retained here, and might be the basis for of a better resolved classification of the subfamily RHACOPHORINAE. 
Protonym: BUERGERIINAE Channing, 1989: 116 [bF].

Eunym: Dubois 2005: 335.

Getangiotaxon: RHACOPHORINAE $\|$ Günther, 1858\|-Hoffman, 1932.

Adelphotaxa: RHACOPHORINI ||Günther, 1858\|-Hoffman, 1932; 1 G†; 1 GIS (Dendrobatorana Ahl, 1927).

Getendotaxon: Buergeria Tschudi, 1838.

F.19.74. Tribus RHACOPHORINI ||Günther, 1858||-Hoffman, 1932

Eunym: Dubois 1992: 336.

Getangiotaxon: RHACOPHORINAE \|Günther, 1858\|-Hoffman, 1932.

Adelphotaxa: BuERGERIINI Channing, 1989; 1 G†; 1 GIS (Dendrobatorana Ahl, 1927).

Getendotaxa: RHACOPHORINA ||Günther, 1858||-Hoffman, 1932; ROMERINA nov.

\section{F.20.69. Subtribus RHACOPHORINA ||Günther, 1858||-Hoffman, 1932}

Eunym: Hoc loco.

Getangiotaxon: RHACOPHORINI ||Günther, 1858||-Hoffman, 1932.

Adelphotaxon: ROMERINA nov.

Getendotaxa: NYCTIXALINIA Grosjean, Delorme, Dubois \& Ohler, 2008; RHACOPHORINIA ||Günther, 1858||-Hoffman, 1932.

F.21.50. Infratribus NyCTIXALINIA Grosjean, Delorme, Dubois \& Ohler, 2008

Protonym: NYCTIXALINI Grosjean, Delorme, Dubois \& Ohler, 2008: 174 [T].

Eunym: Hoc loco.

Getangiotaxon: RHACOPHORINA ||Günther, 1858||-Hoffman, 1932.

Adelphotaxon: RHACOPHORINIA ||Günther, 1858\|-Hoffman, 1932.

Getendotaxa: Nyctixalus Boulenger, 1882; Theloderma Tschudi, 1838.

F.21.51. Infratribus RHACOPHORINIA ||Günther, 1858||-Hoffman, 1932

Eunym: Hoc loco.

Getangiotaxon: RHACOPHORINA ||Günther, 1858||-Hoffman, 1932.

Adelphotaxon: NYCTIXALINIA Grosjean, Delorme, Dubois \& Ohler, 2008.

Getendotaxa: GRaCIXALINOA nov.; ORIXALINOA nov.; Philautinoa Dubois, 1981; RhaCophorinoA ||Günther, 1858||Hoffman, 1932; VAMPYRIINOA nov.

\section{F.22.26. Hypotribus GRACIXALINOA nov.}

Getangiotaxon: RHACOPHORINIA \|Günther, 1858\|-Hoffman, 1932.

Adelphotaxa: OriXalinoa nov.; PHILAUTINOA Dubois, 1981; RHACOPHORINOA ||Günther, 1858||-Hoffman, 1932; VAMPYRIINOA nov.

Getendotaxon: Gracixalus Delorme, Dubois, Grosjean \& Ohler, 2005.

Nucleogenus, by present designation: Gracixalus Delorme, Dubois, Grosjean \& Ohler, 2005. Etymology of nomen: L: gracilis, 'thin, slender'; N: Ixalus Duméril \& Bibron, 1841, derived from G: $1 \xi \alpha \lambda o \varsigma$ (ixalos), 'jumping, dancing'. • Stem of nomen: Gracixal-.

Diagnosis: Small sized rhacophorids (SVL $<25 \mathrm{~mm}$ ); no vomerine teeth; tongue notched; tympanum distinct; distance between nostrils smaller than distance between eyes; finger tips largely expanded with circumventral grooves, toe tips slightly smaller; web between fingers absent, web between toes small 
to moderate, no web between metatarsals; dorsal skin usually smooth with granules, in particular on eyelids; dorsum bearing patterns like an interorbital band or triangle and a X-shaped pattern between shoulders; dark canthal and tympanic band sometimes continued on flanks by a series of dark spots; eggs laid on vegetation overhanging water bodies; life cycle including free swimming and feeding larvae; keratodont formula 1:4+4/3. \{Delorme et al. 2005; Rowley et al. 2011\}.

\section{F.22.27. Hypotribus ORIXALINOA nov.}

Getangiotaxon: RHACOPHORINIA \|Günther, 1858\|-Hoffman, 1932.

Adelphotaxa: GraciXalinoa nov.; Philautinoa Dubois, 1981; Rhacophorinoa ||Günther, 1858||-Hoffman, 1932; VAMPYRIINOA nOv.

Getendotaxon: Orixalus nov.

Nucleogenus, by present designation: Orixalus nov. • Etymology of nomen: G: ópoৎ(oros), ‘mountain'; N: Ixalus Duméril \& Bibron, 1841, derived from G: $1 \xi \alpha \lambda o \varsigma$ (ixalos), 'jumping, dancing'; referring to the distribution across the mountains in northern Indochinese region. $\bullet$ Stem of nomen: Orixal-.

Diagnosis: Small to medium sized frogs (males SVL 23-42 mm; females SVL 29-43 mm); webbing between toes moderate or small, 2-3.5 phalanges free on toe IV; metatarsalia not separate or separate; inner metatarsal tubercle distinct, usually small; outer absent; finger and toe tips expanded into large discs; at least a few tubercles present on upper eyelids; dorsal skin smooth, with tubercles or granules; nuptial pads present on finger I; inner subgular vocal sacs present; maxillary teeth present; vomerine teeth absent; tongue usually deeply notched; tympanum distinct; distance between nostrils smaller than distance between upper eyelids; dorsal color brown, or green with blotches, with darker pattern including an interorbital triangle continued by paired bands on side of back; ventral color whitish or gray with darker markings or spots; living on vegetation, or in karst environment; active at night; eggs bicolored, but egg laying not observed; parental care not observed; tadpoles unknown. \{Boulenger 1893; Hu et al. 1978; Fei 1999; Matsui \& Orlov 2004; Mo et al. 2013; Nguyen et al. 2013$\}.$

\section{G.28.438. Genus Orixalus nov.}

Getangiotaxon: ORIXALINOA nov.

Adelphotaxon: None.

Getendotaxa: Orixalus ananjevae (Matsui \& Orlov, 2004); Orixalus carinensis (Boulenger, 1893); Orixalus jinxiuensis (Hu in $\mathrm{Hu}$, Fei \& Ye, 1978); Orixalus nonggangensis (Mo, Zhang, Luo, Zhou \& Chen, 2013); Orixalus waza (Nguyen, Le, Pham, Nguyen, Bonkowski \& Ziegler, 2013).

Nucleospecies, by present designation: Chirixalus nonggangensis Mo, Zhang, Luo, Zhou \& Chen, 2013. • Etymology of nomen: G: őpos (oros), 'mountain'; N: Ixalus Duméril \& Bibron, 1841, derived

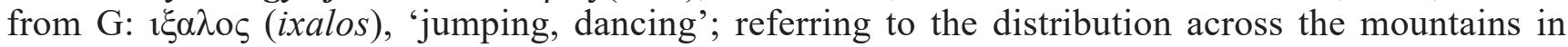
northern Indochinese region. • Stem of nomen: Orixal-. 'frog'. • Grammatical gender of nomen: masculine.

Diagnosis: Small to medium sized frogs (males SVL 23-42 mm; females SVL 29-43 mm); webbing between toes moderate or small, 2-3.5 phalanges free on toe IV; metatarsalia not separate or separate; inner metatarsal tubercle distinct, usually small; outer absent; finger and toe tips expanded into large discs; at least a few tubercles present on upper eyelids; dorsal skin smooth, with tubercles or granules; nuptial pads present on finger I; inner subgular vocal sacs present; maxillary teeth present; vomerine teeth absent; tongue usually deeply notched; tympanum distinct; distance between nostrils smaller than distance between upper eyelids; dorsal color brown, or green with blotches, with darker pattern including an interorbital triangle continued by paired bands on side of back; ventral color whitish or gray with darker markings or spots; living on vegetation, or in karst environment; active at night; eggs bicolored, but egg laying not observed; parental care not observed; tadpoles unknown. \{Boulenger, 1893; Hu et al. 1978; Fei 1999; Matsui \& Orlov 2004; Mo et al. 2013; Nguyen et al. 2013\}. 
Protonym: PhILAUTINAE Dubois, 1981: 227 [bF].

Eunym: Hoc loco.

Getangiotaxon: RHACOPHORINIA ||Günther, 1858||-Hoffman, 1932.

Adelphotaxa: GRACIXALINOA nov.; ORIXALINOA nov.; RHACOPHORINOA \|Günther, 1858\|-Hoffman, 1932; VAMPYRIINOA nov.

Getendotaxa: KuriXalites nov.; Mercuranites nov.; NasutiXalites nov.; PhilaUtites Dubois, 1981.

\section{F.23.16. Clanus KURIXALITES nov.}

Getangiotaxon: PhILAUTINOA Dubois, 1981.

Adelphotaxa: MercuRanites nov.; NASUtiXalites nov.; PhilaUtites Dubois, 1981.

Getendotaxon: Kurixalus Ye, Fei \& Dubois in Fei, 1999.

Nucleogenus, by present designation: Kurixalus Ye, Fei \& Dubois in Fei, 1999. • Etymology of nomen: P: Mitsuru Kuramoto (1934-), Japanese herpetologist; N: Ixalus Duméril \& Bibron, 1841, derived from G: $1 \xi \alpha \lambda o \varsigma$ (ixalos), 'jumping, dancing'. • Stem of nomen: Kurixal-.

Diagnosis: Small to medium sized rhacophorids (males SVL 23-37 mm; females SVL 29-50 mm); snout length usually subequal to eye but also shorter or much longer than eye; a more or less prominent conical projection on snout usually present; pupil horizontal; internaral distance smaller to larger than distance between upper eyelid; vomerine teeth in two small patches near choanae, widely separated from each other usually present; tongue emarginated; tympanum distinct, rarely hidden, about half eye size; a rudimentary web between fingers; well developed or small discs with circumferential grooves present on all toes and fingers; webbing between toes moderately developed; metatarsalia separated; a rather elongate, not very prominent inner metatarsal tubercle present; outer metatarsal tubercle absent; skin on dorsum smooth or bearing various densities of tubercles giving in many species a rough aspect; a fringe composed of tubercles or more developed dermal appendages on the edge of forearm and tarsus present in most species; some also showing dermal appendages below vent and on heels, in one species these structures all absent; skin on belly granular; white or yellowish nuptial pads present, forming large swellings in one species; presence of inner subgular vocal sac; dorsum of gray or brown, rarely green shades with darker patterns; ventrally whitish with or without darker spots; habits forests or swamps on low vegetation; nocturnal; large whitish eggs laid either on the ground or in phytotelm; aquatic tadpoles. \{Günther 1858; Boulenger 1893; Boettger 1895; Annandale 1912; Bourret 1942; Inger 1947, 1966; Taylor 1962; Kuramoto \& Wang 1987; Fei 1999; Inger et al. 1999; Matsui \& Orlov 2004; Nguyen et al. $2014 a-b$; personal observations by AO).

\section{F.23.17. Clanus MERCURANITES nov.}

Getangiotaxon: PHILAUTINOA Dubois, 1981.

Adelphotaxa: KuriXalites nov.; NASUtiXalites nov.; Philautites Dubois, 1981.

Getendotaxa: BEDDOMIXALITIES nov.; MERCURANITIES nov.

Nucleogenus, by present designation: Mercurana Abraham, Pyron, Ansil, Zachariah \& Zachariah, 2013. • Etymology of nomen: P: Freddie Mercury (1946-1991), lead singer of the British rock band Queen; N: Rana Linnaeus, 1758, derived from L: rana, 'frog'. - Stem of nomen: Mercuran-.

Diagnosis: Body length usually very small or small, rarely medium sized frogs (males SVL 11-42 mm; females SVL 15-65 mm); snout shorter, longer or subequal to eye length; pupil horizontal; internarial distance shorter than distance between upper eyelids; vomerine teeth absent or weekly developed; tongue emarginated, with or without papillae; tympanum distinct or indistinct, rarely hidden; upper eyelid usually smooth but sometimes bearing tubercles or granules; webbing between fingers absent or rarely rudimentary; fingers bearing distinctly enlarged discs; webbing between toes rudimentary to 
large; metatarsalia fused or slightly separated; inner metatarsal tubercle short, moderately developed or indistinct; outer metatarsal tubercle absent; dorsal skin in many species smooth or shagreened but often granular at least at some parts of body or in some species presence of horny spinules; rarely with horny ridges or with prominent symmetrical glandular swellings; skin on belly granular; nuptial pad present or absent; a large or rarely small subgular median vocal sac present (in all species for which indicated); dorsal coloration brownish or green usually with various patterns, including interorbital band, dorsolateral bands or various smaller patches or spots, rarely uniform; few species bright or uniformly coloured; ventral coloration usually light coloured including various shades of gray or yellow, either uniformly of with spots or variegations, few species with dark or bright coloured belly; arboreal forest dwelling species occurring in primary forests but also in disturbed habitats and plantations or grassland; observed on bushes and trees up to $20 \mathrm{~m}$ but often on forest floor in leaf litter or under stones; nocturnal rarely diurnal; eggs of large size, few to more rarly moderate in number, laid on ground or on leaves or phytotelms; usually direct development but two lineages with free living aquatic tadpoles. \{Boulenger 1882b, 1893, 1906; Smith 1924; Das \& Chanda 1998; Bossuyt \& Dubois 2001; Bossuyt et al. 2001; Bossuyt 2002; Biju 2003; Kuramoto \& Joshy 2003; Biju \& Bossuyt 2005a-b, 2006, 2009; Gururaja et al. 2007; Biju et al. 2010; Zachariah et al. 2011; Seshadri et al. 2012; Orlov et al. 2012; Abraham et al. 2013; Padhye et al. 2013; Wickramasinghe et al. 2013a-b; Vijayakumar et al. 2014\}.

\title{
F.24.10. Subclanus BEDDOMIXALITIES nov.
}

\author{
Getangiotaxon: MERCURANITES nov. \\ Adelphotaxon: MERCURANITIES nov. \\ Getendotaxon: Beddomixalus Abraham, Pyron, Ansil, Zachariah \& Zachariah, 2013.
}

Nucleogenus, by present designation: Beddomixalus Abraham, Pyron, Ansil, Zachariah \& Zachariah, 2013. • Etymology of nomen: P: Richard Henry Beddome (1830-1911), British working on herpetofauna

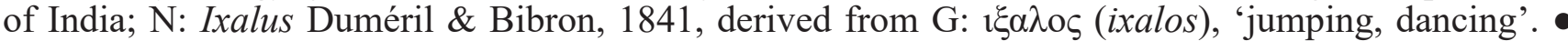
Stem of nomen: Beddomixal-.

Diagnosis: Slender, medium sized frogs (males SVL 40-42 mm; female SVL $61 \mathrm{~mm}$ ); snout longer than eye length; pupil horizontal; internarial distance shorter than distance between upper eyelids; vomerine teeth absent; tongue emarginated, without papillae; tympanum distinct; upper eyelid smooth; webbing between fingers absent; fingers bearing distinctly enlarged discs; webbing between toes moderate; metatarsalia slightly separated; inner metatarsal tubercle short, moderately developed; outer metatarsal tubercle absent; dorsal skin granular; skin on belly granular; nuptial pad absent; a small subgular vocal sac present; dorsal coloration brownish with a pair of distinct yellowish parallel longitudinal strips from eye to vent; ventral coloration uniformly white; an arboreal, forest dwelling species occurring arround seasonal swamps or marshes near mid to high-elevation forests; clutches of 175 large sized, white eggs laid on soil or grass and subsequently washed by rainwater to pools; tadpoles free living with oval and depressed body, blackish dorsally and pinkish ventrally; keratodont formula 1:4+4/3. \{Zachariah et al. 2011; Abraham et al. 2013\}.

\section{F.24.11. Subclanus MERCURANITIES nov.}

Getangiotaxon: MERCURANITES nov.

Adelphotaxon: BEDDOMIXALITIES nov.

Getendotaxa: Mercurana Abraham, Pyron, Ansil, Zachariah \& Zachariah, 2013; Pseudophilautus Laurent, 1943; Raorchestes Biju, Shouche, Dubois, Dutta \& Bossuyt, 2010.

\section{F.23.18. Clanus NASUTIXALITES nov.}

Getangiotaxon: PhILAUTINOA Dubois, 1981.

Adelphotaxa: KuriXalites nov.; MerCuranites nov.; Philautites Dubois, 1981. 
Getendotaxon: Nasutixalus Jiang, Yan, Wang \& Che in Jiang, Yan, Wang, Zou, Li \& Che, 2016.

Nucleogenus, by present designation: Nasutixalus Jiang, Yan, Wang \& Che in Jiang, Yan, Wang, Zou, Li \& Che, 2016. • Etymology of nomen: L: nasutus, 'large-nosed'; N: Ixalus Duméril \& Bibron,

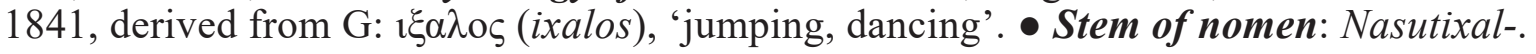

Diagnosis: Small sized rhacophorids (males SVL 37-45 mm; female SVL $47 \mathrm{~mm}$ ); snout rounded; canthus rostralis obtuse and raised prominently, forming a ridge from nostril to anterior corner of eyes; web rudimentary on hand; moderate webbing on foot; phalange ' $Y$ ' shaped, visible from dorsal side of fingers and toes; skin of dorsal surfaces relatively smooth with small tubercles; phytotelm-breeding; eggs non pigmented, creamy-white; oophagous tadpole lacking keratinised tooth rows. \{Jiang et al. 2016; Biju et al. 2016\}.

\section{F.23.19. Clanus Philautites Dubois, 1981}

Eunym: Hoc loco.

Getangiotaxon: PhILAUTINOA Dubois, 1981.

Adelphotaxa: KURIXALITES nov.; MERCURANITES nov.; NASUTIXALITES nov.

Getendotaxon: Philautus Gistel, 1848.

\section{F.22.29. Hypotribus RHACOPHORINOA \|Günther, 1858|-Hoffman, 1932}

Eunym: Hoc loco.

Getangiotaxon: RHACOPHORINIA ||Günther, 1858\|-Hoffman, 1932.

Adelphotaxa: GraciXalinOa nov.; OrIXALINOA nov.; PhILAUTINOA Dubois, 1981; VAMPYRIINOA nov.

Getendotaxa: CHIRIXALITES nov.; RHACOPHORITES ||Günther, 1858||-Hoffman, 1932.

\section{F.23.20. Clanus CHIRIXALITES nov.}

Getangiotaxon: RHACOPHORINOA ||Günther, 1858||-Hoffman, 1932.

Adelphotaxon: RHACOPHORITES ||Günther, 1858||-Hoffman, 1932.

Getendotaxa: Chirixalus Boulenger, 1893; Chiromantis Peters, 1854.

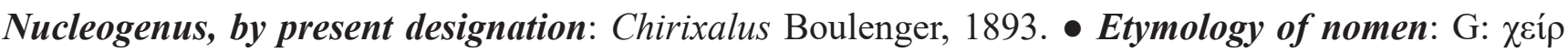
(cheir), 'hand'; N: Ixalus Duméril \& Bibron, 1841, derived from G: $1 \xi \alpha \lambda \circ \varsigma$ (ixalos), 'jumping, dancing'. - Stem of nomen: Chirixal-.

Diagnosis: Small to large size frogs (males SVL 22-75 mm, females SVL 29-92 mm); snout length shorter or longer than eye diameter; pupil horizontal; vomerine teeth absent or present; tongue notched; tympanum distinct; two inner fingers opposed to two outer ones; webbing between fingers rudimentary or small; intercalary elements present; finger tips dilated into large discs; webbing between toes half to full (1-2 phalanges free on toe IV); metatarsalia separate; inner metatarsal tubercles small, outer absent; skin on dorsum smooth or warty; skin belly granular; nuptial pads on fingers I and II; an internal subgular vocal sac; dorsal color uniform, often light colored, with darker pattern; ventral color whitish, with traces of pigmented spots; habits arboreal, on shrubs or trees, in forests or savannah; unpigmented rather large eggs in foam nests; described tadpoles uniformly gray with round body and rather short tail, keratodont formula $1:<2-4>\mid<0-3>: 2-3$; omosternum forked or unforked; sternum with bony style. \{Boulenger 1893; Annandale 1915; Cochran 1927; Taylor 1962; Schiøtz 1999\}.

F.23.21. Clanus RHACOPHORITES ||Günther, 1858||-Hoffman, 1932

Eunym: Hoc loco. 
Getangiotaxon: RHACOPHORINOA ||Günther, 1858||-Hoffman, 1932.

Adelphotaxon: CHIRIXALITES nov.

Getendotaxa: FeIHYLITIES nov.; PolYPedatities Günther, 1858; RHACOPHoRIties \|Günther, 1858||-Hoffman, 1932; TAMIXALITIES nov.

\section{F.24.12. Subclanus FEIHYLITIES nov.}

Getangiotaxon: RHACOPHORITES ||Günther, 1858\|-Hoffman, 1932.

Adelphotaxa: PolYPedatities Günther, 1858; RHACOPHORITIES ||Günther, 1858\|-Hoffman, 1932; TAMIXALITIES nov.

Getendotaxon: Feihyla Frost, Grant, Faivovich, Bain, Haas, Haddad, Sá, Channing, Wilkinson, Donnellan, Raxworthy, Campbell, Blotto, Moler, Drewes, Nussbaum, Lynch, Green \& Wheeler, 2006.

Nucleogenus, by present designation: Feihyla Frost, Grant, Faivovich, Bain, Haas, Haddad, Sá, Channing, Wilkinson, Donnellan, Raxworthy, Campbell, Blotto, Moler, Drewes, Nussbaum, Lynch, Green \& Wheeler, 2006. • Etymology of nomen: P: Fei Liang (1936-), Chinese herpetologist; N: Hyla Laurenti, 1768, of debated etymology. • Stem of nomen: Feihyl-.

Diagnosis: Small sized frogs (males SVL 18-29 mm; females SVL 23-32 mm); snout length equal or longer to eye; distance between nostrils shorter than distance between upper eyelids; vomerine teeth absent; tongue notched; tympanum may be hidden or distinct; upper eyelid spines absent; fingers I and II opposed to fingers III and IV; rudimentary webbing between fingers III and IV; finger tips widely enlarged; webbing between toes moderate to large (1.5 to 3 phalanges free on toe IV); metatarsalia separate at distal part; innermetatarsal tubercles usually small, outer absent; dorsal skin smooth, males usually showing fine spinucules; skin on belly granular; nuptial pads absent or whitish pod on finger I; an internal subgular vocal sac with bilateral slit-like openings; dorsally usually uniform brown or yellow, sometimes leave green, often with dorsolateral bands or spots on dorsum; ventral color uniformly unpigmented; found perched on low vegetation near water bodies; active at night; eggs unpigmented or pigmented, in clutches, sometimes in foam nests; tadpoles small sized (stage 36 about $35 \mathrm{~mm}$ ); pigmentation on body and tail; keratodont formula 4-5/3. \{Boulenger 1887; Smith 1924; Cochran 1927; Pope 1931; Bourret 1942; Taylor 1962; Dring 1983; Inger et al. 1999; Fei et al. 2010; Matsui et al. 2014\}.

\section{F.24.13. Subclanus POLYPEDATITIES Günther, 1858}

Protonym: PolYPEDATIDAE Günther, 1858: 346 [F].

Eunym: Hoc loco.

Getangiotaxon: RHACOPHORITES ||Günther, 1858\|-Hoffman, 1932.

Adelphotaxa: FEIHYLITIES nov.; RHACOPHORITIES ||Günther, 1858||-Hoffman, 1932; TAMIXALITIES nov.

Getendotaxa: GHATIXALITOES nov.; POLYPEDATITOES Günther, 1858.

\section{F.25.20. Infraclanus GHATIXALITOES nov.}

Getangiotaxon: POLYPEDATITIES Günther, 1858.

Adelphotaxon: PolyPEDATITOES Günther, 1858.

Getendotaxon: Ghatixalus Biju, Roelants \& Bossuyt, 2008.

Nucleogenus, by present designation: Ghatixalus Biju, Roelants \& Bossuyt, 2008. • Etymology of nomen: R: Ghat, Sanskrit, 'step', referring to the mountain range of the Western Ghats; N: Ixalus Duméril \& Bibron, 1841, derived from G: $1 \xi \alpha \lambda o \varsigma$ (ixalos), 'jumping, dancing'. • Stem of nomen: Ghatixal-.

Diagnosis: Medium sized frogs (males SVL 39-51mm; females SVL 58-67 mm); webbing between fingers moderate, between toes extensive; metatarsalia not separate; inner metatarsal tubercle short, rather distinct, outer absent; finger tips enlarged; nuptial pads present on finger I; vocal sacs indicated by 
a pair of openings; maxillary and vomerine teeth present; tongue emarginate; tympanum distinct; dorsal pattern with dominant blotches; ventral color uniform; life cycle associated with mountain streams and at higher altitudes; nocturnal activity; eggs laid in foam nests, entirely white; free swimming tadpoles. \{Biju et al. 2008\}.

\section{F.25.21. Infraclanus PoLYPEDATITOES Günther, 1858}

Eunym: Hoc loco.

Getangiotaxon: POLYPEDATITIES Günther, 1858.

Adelphotaxon: GHATIXALITOES nov.

Getendotaxa: Polypedates Tschudi, 1838; Taruga Meegaskumbura, Meegaskumbura, Bowatte, Manamendra-Arachchi, Pethiyagoda, Hanken \& Schneider, 2010.

\section{F.24.14. Subclanus RHACOPHORITIES ||Günther, 1858||-Hoffman, 1932}

Eunym: Hoc loco.

Getangiotaxon: RHACOPHORITES ||Günther, 1858\|-Hoffman, 1932.

Adelphotaxa: FeIHYLITIES nov.; PolyPeDATITIES Günther, 1858; TAMIXALITIES nov.

Getendotaxa: Leptomantis Peters, 1867; Rhacophorus Kuhl \& Van Hasselt, 1822; Zhangixalus Li, Jiang, Ren \& Jiang, 2019.

\section{F.24.15. Subclanus TAMIXALITIES nov.}

Getangiotaxon: RHACOPHORITES ||Günther, 1858\|-Hoffman, 1932.

Adelphotaxa: FeIhylities nov.; PolyPedatities Günther, 1858; RHACOPHoRITIES ||Günther, 1858||-Hoffman, 1932.

Getendotaxon: Tamixalus nov.

Nucleogenus, by present designation: Tamixalus nov. • Etymology of nomen: Tamil language: Tamil, referring to the name of the distribution area, Tamil Nadu; N: Ixalus Duméril \& Bibron, 1841, derived from G: $1 \xi \alpha \lambda o \varsigma$ (ixalos), 'jumping, dancing'. • Stem of nomen: Tamixal-.

Diagnosis: Medium sized frogs (males SVL 33-47 mm); dorsal skin with prominent granular projections; intercalary elements present; webbing between fingers and toes complete; metatarsalia separate; a distinct oval inner metatarsal tubercle; outer metatarsal tubercle absent; pads on fingers and toes well developed; nuptial pads present; maxillary and vomerine teeth present; tongue notched; tympanum distinct, rounded; observed high on leaves and stems of shrubs and trees; active at night; eggs of creamy white color laid in foam nest; parental care not known; tadpoles not described. \{Biju et al. 2013$\}$.

\section{G.28.455. Genus Tamixalus nov.}

Getangiotaxon: TAMIXALITIES nov.

Adelphotaxon: None.

Getendotaxon: Tamixalus calcadensis (Ahl, 1927).

Nucleospecies, by present designation: Rhacophorus calcadensis Ahl, 1927. • Etymology of nomen: Tamil language: Tamil, referring to the name of the distribution area, Tamil Nadu; N: Ixalus Duméril \& Bibron, 1841, derived from G: $1 \xi \alpha \lambda o \varsigma$ (ixalos), 'jumping, dancing'. • Stem of nomen: Tamixal-. Grammatical gender of nomen: masculine.

Diagnosis: Medium sized frogs (males SVL 33-47 mm); dorsal skin with prominent granular projections; intercalary elements present; webbing between fingers and toes complete; metatarsalia separate; a distinct oval inner metatarsal tubercle; outer metatarsal tubercle absent; pads on fingers and toes well 
developed; nuptial pads present; maxillary and vomerine teeth present; tongue notched; tympanum distinct, rounded; observed high on leaves and stems of shrubs and trees; active at night; eggs of creamy white color laid in foam nest; parental care not known; tadpoles not described. \{Biju et al. 2013 .

\section{F.22.30. Hypotribus VAMPYRIINOA nov.}

Getangiotaxon: RHACOPHORINIA \|Günther, 1858\|-Hoffman, 1932.

Adelphotaxa: GraciXalinoa nov.; OrIXalinoa nov.; Philautinoa Dubois, 1981; Rhacophorinoa ||Günther, 1858|Hoffman, 1932.

Getendotaxon: Vampyrius nov.

Nucleogenus, by present designation: Vampyrius nov. • Etymology of nomen: English: vampire, derived from the German Vampir, derived in turn from the Serbian vampire (Вампир), a nocturnal being feeding on life substance; referring to the two large keratinised labial teeth on lower lip of tadpole.

Stem of nomen: Vampyri-.

Diagnosis: Medium sized frogs (males SVL 42-45 mm; females SVL 39-53 mm); intercalary elements present; webbing between fingers moderate; webbing between toes moderate; metatarsalia separate; inner metatarsal tubercle low, oval; outer metatarsal tubercle absent; finger tips with well developed discs; nuptial pads absent; an external paired subgular vocal sac present; maxillary and vomerine teeth present; tongue deeply notched; tympanum barely visible; observed on trees near phytotelms where eggs are laid in foam nests; females observed to lay trophic eggs in phytotelms; tadpoles showing greatly reduced oral disc, only an upper jaw sheath and a pair of keratinised hooks on the edge of the lower labium; tadpoles feeding on eggs. \{Rowley et al. 2010, 2012; Vassilieva et al. 2013 \}.

\section{G.28.456. Genus Vampyrius nov.}

Getangiotaxon: VAMPYRIINOA nov.

Adelphotaxon: None.

Getendotaxon: Vampyrius vampyrus (Rowley, Le, Thi, Stuart \& Hoang, 2010).

Nucleospecies, by present designation: Rhacophorus vampyrus Rowley, Le, Thi, Stuart \& Hoang, 2010. • Etymology of nomen: English: vampire, derived from the German Vampir, derived in turn from the Serbian vampir (Вампир), a nocturnal being feeding on life substance; referring to the two large keratinised labial teeth on lower lip of tadpole. • Stem of nomen: Vampyri-. • Grammatical gender of nomen: masculine.

Diagnosis: Medium sized frogs (males SVL 42-45 mm; females SVL 39-53 mm); intercalary elements present; webbing between fingers moderate; webbing between toes moderate; metatarsalia separate; inner metatarsal tubercle low, oval; outer metatarsal tubercle absent; finger tips with well developed discs; nuptial pads absent; an external paired subgular vocal sac present; maxillary and vomerine teeth present; tongue deeply notched; tympanum barely visible; observed on trees near phytotelms where eggs are laid in foam nests; females observed to lay trophic eggs in phytotelms; tadpoles showing greatly reduced oral disc, only an upper jaw sheath and a pair of keratinised hooks on the edge of the lower labium; they are feeding on eggs. \{Rowley et al. 2010, 2012; Vassilieva et al. 2013\}.

\section{F.20.70. Subtribus ROMERINA nov.}

Getangiotaxon: RHACOPHORINI ||Günther, 1858||-Hoffman, 1932.

Adelphotaxon: RHACOPHORINA \|Günther, 1858||-Hoffman, 1932

Getendotaxon: Romerus nov. 
Nucleogenus, by present designation: Romerus nov. • Etymology of nomen: P: John D. Romer (19201982), British herpetologist who worked in Hongkong. • Stem of nomen: Romer-.

Diagnosis: Very small sized rhacophorids (males SVL 16-20 mm; females SVL 18-22 mm); head longer than wide; vomerine teeth absent; tympanum present; pads on fingers and toes relatively small; webbing on hand reduced; webbing on feet small; tibia relatively long (more than $50 \%$ of SVL); serrated ridges on forearm and tarsus absent; dorsal pattern with a more or less distinct darker X and interobital band; free living tadpoles. \{Milto et al. 2013; Qin et al. 2015\}.

\section{G.28.457. Genus Romerus nov.}

Getangiotaxon: ROMERINA nov.

Adelphotaxon: None.

Getendotaxa: Romerus calcarius (Milto, Poyarkov, Orlov \& Nguyen, 2013); Romerus hainanus (Liu \& Hu, 2004); Romerus jinxiuensis (Hu in $\mathrm{Hu}$, Fei \& Ye, 1978); Romerus ocellatus (Liu \& Hu, 1973); Romerus romeri (Smith, 1953); Romerus shiwandashan (Li, Mo, Xie \& Jiang in Qin, Mo, Jiang, Cai, Xie, Jiang, Murphy, Li \& Wang, 2015).

Nucleospecies, by present designation: Philautus romeri Smith, 1953. • Etymology of nomen: P: John D. Romer (1920-1982), British herpetologist who worked in Hongkong. • Stem of nomen: Romer-. Grammatical gender of nomen: masculine.

Diagnosis: Very small sized rhacophorids (males SVL 16-20 mm; females SVL 18-22 mm); head longer than wide; vomerine teeth absent; tympanum present; pads on fingers and toes relatively small; webbing on hand reduced; webbing on feet small; tibia relatively long (more than $50 \%$ of SVL); serrated ridges on forearm and tarsus absent; dorsal pattern with a more or less distinct darker $\mathrm{X}$ and interobital band; free living tadpoles. (Milto et al. 2013; Qin et al. 2015).

Comments: • Li et al. (2008) proposed the nomen" Liuxalus" for this genus without giving a diagnosis in the original description. The authors referred to a list of positions and nucleic acid name abbreviations that should be compared with the aligned matrix, but this information was not given in the publication or in a work published earlier. In consequence, the nomen is not available according to Article 13.1.1 of the Code. Here we propose formally a new nomen for this taxon.

\section{F.16.07. Apofamilia RANIXALEIDAE Dubois, 1987}

Protonym: RANIXALINI Dubois, 1987: 66 [T].

Eunym: Hoc loco.

Getangiotaxon: RANOIDAE Batsch, 1796.

Adelphotaxa: Ceratobatracheidae Boulenger, 1884; Dicroglosseidae Dubois, 1987; NyCtibatraCheidae BlommersSchlösser, 1993; RANEIDAE Batsch, 1796.

Getendotaxon: RANIXALIDAE Dubois, 1987.

\section{F.17.50. Familia RANIXALIDAE Dubois, 1987}

Eunym: Van Bocxlaer, Roelants, Biju, Nagaraju \& Bossuyt 2006: 2.

Getangiotaxon: RANIXALEIDAE Dubois, 1987.

Adelphotaxon: None.

Getendotaxa: Indirana Laurent, 1986; Walkerana Dahanukar, Modak, Krutha, Nameer, Padhye \& Molur, 2016.

\section{C.12.04. Infraphalanx SavanuRa nov.}

Getangiotaxon: Pananura nov. 
Uninucleogenus, by present designation: Hildebrandtia Nieden, 1907

Etymology of nomen: Spanish: çabana, from the Taïno (Haïti) zavana or zabana, 'savannah'; G: $\dot{\alpha} v$ (an-), 'without'; oüpó (oura), 'tail'. This nomen refers to the savannicolous life habits of many species of Ptychadena, its most speciose genus (Rödel 2000; Channing 2001; Du Preez \& Carruthers 2009).

Diagnosis: Small to medium sized frogs (SVL 25-85 mm); snout much longer than eye; dorsal skin usually with paired folds; external vocal sacs present; otic plate absent or rudimentary; neopalatines absent; 'point' overlap of medial ramus of pterygoid and anterior lateral border of parasphenoid ala in an anterior-posterior plane; clavicles reduced, well separated in midline; style of sternum ossified, short, compact; eighth presacral and sacral vertebrae fused; dorsal protuberance on ilium not or only slightly differentiated from dorsal prominence which is smooth surfaced and confluent with a well developed ilial crest; eggs floating in single layer on lentic water bodies; tadpole with reduced number of keratodont rows (2/2, 1/2 or 0/2). \{Clarke 1981, 1982; Rödel 2000; Frost et al. 2006; Du Preez \& Carruthers 2009\}.

Comments: The Savanura are sister-group to the Ecaudata within the Pananura. They include a single family, the PTYCHADENIDAE. This position of the PTYCHADENIDAE was first recovered by Frost $e t$ al. (2006), then confirmed by Bossuyt \& Roelants (2009) and Pyron \& Wiens (2011), but this taxon is within the ECAUDATA in Zhang et al. (2013) and Frazão et al. (2015).

\section{F.17.51. Familia PTYCHADENIDAE Dubois, 1987}

Protonym: PTYCHADENINI Dubois, 1987: 55 [T].

Eunym: Frost, Grant, Faivovich, Bain, Haas, Haddad, Sá, Channing, Wilkinson, Donnellan, Raxworthy, Campbell, Blotto, Moler, Drewes, Nussbaum, Lynch, Green \& Wheeler 2006: 7.

Getangiotaxon: SAVANURA nov.

Adelphotaxon: Ecaudata Scopoli, 1777.

Getendotaxa: Hildebrandtia Nieden, 1907; Lanzarana Clarke, 1982; Ptychadena Boulenger, 1917.

\section{C.09.02. Epiphalanx Helanura nov.}

Getangiotaxon: RANOMORPHA Fejérváry, 1921.

Adelphotaxon: AQUIPARES Blainville, 1816.

Getendotaxon: HELEOPHRYNIDAE Noble, 1931.

Uninucleogenus, by present designation: Heleophryne Sclater, 1898.

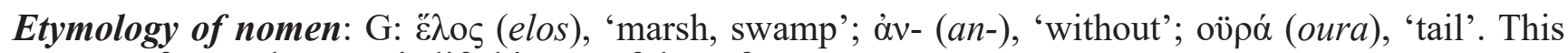
nomen refers to the aquatic life history of these frogs.

Diagnosis: Small to medium sized frogs (SVL 25-65 mm); morphology adapted to stream living; pupil vertical; expanded digital tips present; dorsal colour pattern showing large dark spots on paler, brown or green, background; inguinal amplexus; larvae adapted to living in rocky streams, showing numerous morphological apomorphies, in particular loss of upper jaw sheaths (lower present only in one species). \{Haas 2003; Du Preez \& Carruthers 2009\}.

Comments: The Helanura are sister-group to the AQUIPARES, the other group of the RANOMORPHA (the «NEOBATRACHIA» of some authors). This relationship was recovered by Frost et al. (2006), Bossuyt \& Roelants (2009), Pyron \& Wiens (2011), Irisarri et al. (2012), Zhang et al. (2013) and Feng et al. (2017). Although it holds a single family, the HELEOPHRYNIDAE, as sister-taxon to the AQUIPARES this taxon deserves to be named as a phalanx, to comply with the hierarchy of class-series ranks adopted here. 


\section{F.17.52. Familia HELEOPHRYNIDAE Noble, 1931}

Protonym: HeLEOPHRYNINAE Noble, 1931: 498 [bF].

Eunym: Hoffman 1935: 2.

Getangiotaxon: HelaNura nov.

Adelphotaxon: None.

Getendotaxa: Hadromophryne Van Dijk, 2008; Heleophryne Sclater, 1898.

\section{C.06.02. Infraordo Mediogyrinia Lataste, 1878}

Protonym: MediogYrinidae Lataste, 1878: 491 [UC].

Eunym: Hoc loco.

Getangiotaxon: Angusticoela Reig, 1958.

Adelphotaxon: Geobatrachia Ritgen, 1828.

Getendotaxa: AlYToIdeA Fitzinger, 1843; BombinatoroIdeA Gray, 1825; 1 F†; 5 G†.

Comments: The branch Mediogyrinia, grouping the families ALYTIDAE, DisCogLOSSIDAE and BOMBINATORIDAE, is recognised in all molecular phylogenies recently published. The relationships within this group are stable in the molecular phylogenies (Roelants \& Bossuyt 2005; Frost et al. 2006; Bossuyt \& Roelants 2009; Pyron \& Wiens 2011; Irisarri et al. 2012; Zhang et al. 2013; Feng et al. 2017) but the taxonomic interpretations varied. This taxon was named «CosTATA» by Frost et al. (2006) and Bossuyt \& Roelants (2009), and DISCOGLOSSOIDEA by Roelants \& Bossuyt (2005) and Pyron \& Wiens (2011). As a class-series nomen, Costata Lataste, 1879 would be invalid for this taxon for being a junior synonym of Mediogrrinia Lataste, 1878. As for DisCoglossoidea Günther, 1858, it is a family-group nomen that cannot be parordinate to a class-series nomen (and if used at this rank it should anyhow be replaced by the older nomen ALYTOIDEA Fitzinger, 1843).

In our classification, after applying the [UQC], we retained three family-rank taxa, the ALYTIDAE, DisCOGLOSSIDAE and BOMBINATORIDAE. The ALYTIDAE are sister-group to the DISCOGLOSSIDAE. As both ALYTIDAE and BOMBINATORIDAE are among the familial nomina retained by the [UQC], DISCOGLOSSIDAE as sister-taxon to ALYTIDAE must be attributed family rank by the [STC]. The taxon grouping ALYTIDAE and DISCOGLOSSIDAE therefore has to be referred to the rank superfamily, as ALYTOIDEA, and consequently its sister-group also, as BOMBINATOROIDEA.

\section{F.14.13. Superfamilia ALYTOIDEA Fitzinger, 1843}

Protonym: ALYTAE Fitzinger, 1843: 32 [F].

Eunym: Hoc loco.

Getangiotaxon: Mediogyrinia Lataste, 1878.

Adelphotaxon: BombinatoroIDEA Gray, 1825.

Getendotaxa: ALYTIDAE Fitzinger, 1843; DISCOGLOSSIDAE Günther, 1858.

\section{F.17.53. Familia ALYTIDAE Fitzinger, 1843}

Eunym: Günther, 1858: 346.

Getangiotaxon: ALYTOIDEA Fitzinger, 1843.

Adelphotaxon: DISCOGLOSSIDAE Günther, 1858.

Getendotaxa: Alytes Wagler, 1829; 1 G†.

F.17.54. Familia DisCoGLOSSIDAE Günther, 1858

Protonym and eunym: DISCOGLOSSIDAE Günther, 1858: 346 [F].

Getangiotaxon: ALYTOIDEA Fitzinger, 1843. 
Adelphotaxon: ALYTIDAE Fitzinger, 1843.

Getendotaxa: Discoglossus Otth, 1837; Latonia Meyer, 1843; 6 G†.

F.14.14. Superfamilia BombINATOROIDEA Gray, 1825

Protonym: BOMBINATORINA Gray, 1825: 214 [UF].

Eunym: Dubois 2005: 7.

Getangiotaxon: Mediogyrinia Lataste, 1878.

Adelphotaxon: ALYTOIDEA Fitzinger, 1843.

Getendotaxon: BOMBINATORIDAE Gray, 1825.

F.17.63. Familia Bombinatoridae Gray, 1825

Eunym: Gray 1831: 38.

Getangiotaxon: BombINATOROIDEA Gray, 1825.

Adelphotaxon: None.

Getendotaxa: Barbourula Taylor \& Noble, 1924; Bombina Oken, 1816; 1 G†.

\section{C.04.02. Ordo Gymnophiona Rafinesque, 1814}

Protonym: Gymnophia Rafinesque, 1814: 104 [O].

Eunym: Müller 1832: 198.

Getangiotaxon: LiSSAMPHIBIA Gadow, 1898.

Adelphotaxa: Anura Duméril, 1805; Urodela Duméril, 1805; 1 C†.

Getendotaxa: Plesiophiona nov.; Pseudophiona Blainville, 1816; 1 F $\dagger ; 1$ G $\dagger$.

Comments: The holophyly of all extant caecilians is supported by all phylogenetic studies based on morphology and on molecular data. Numerous CS nomina are available for this taxon (Appendix A7.NCS) but the valid one under DONS Criteria is the sozodiaphonym GyMnopHIONA Rafinesque, 1814 (Dubois 2004b, 2005b, 2015c, 2020; Dubois \& Ohler 2019; Dubois \& Frétey 2020d).

The phylogenetic relationships within TREE have the same structure as the previous phylogenies published (San Mauro et al. 2014). They show a well resolved, statistically supported and highly hierarchical structure. Its translation into a classification according to our above defined Criteria (see M\&M section) leads to major changes in the nomina of several taxa and also in the definitions and contents of some of them. As a consequence, the classification CLAD presented here is quite different from the previous classifications, for two main reasons: $\{\beta 1\}$ all hypothesised sister-groups have the same hierarchical rank; and $\{\beta 2\}$ through the [UQC], we applied statistical measure of usage of family level nomina to fix application of suprageneric ranks in the proposed classification.

Wilkinson et al. (2011) proposed a classification of the GYMNOPHIONA with nine families. San Mauro et al. (2014) and $A S W<2020 a>$ recognised 10 families, adding the recently described CHIKILIDAE. The present classification CLAD proposes five families with a complex infrafamilial classification. The family-ranked taxa CAECILIIDAE, ICHTHYOPHIDAE and RHINATREMATIDAE are kept for being supported by usage [UQC]. Then, through use of the Sister-Taxa Criterion [STC], the family SCOLECOMORPHIDAE is recognised as sister-taxon to CAECILIIDAE, and the family UREOTYPHLIDAE as sister-taxon to ICHTHYOPHIDAE. The contents of RHINATREMATIDAE and of SCOLECOMORPHIDAE remain unchanged. The former HERPELIDAE and CHIKILIDAE form a holophyletic group, here recognised as the subfamily HERPELINAE of CAECILIIDAE and containing two tribes, the HERPELINI and CHIKILINI. The hyponymous subfamily CAECILIINAE of the CAECILIIDAE accommodates two tribes, the CAECILIINI and SIPHONOPINI. The CAECILIINI incude two subtribes, the CAECILIINA (the former CAECILIIDAE) and TyPHLONECTINA (the former TYPHLONECTIDAE). The tribe SIPHONOPINI consists in two subtribes, the GRANDISONIINA (corresponding to the former INDOTYPHLIDAE) and SIPHONOPINA. The SIPHONOPINA include the DESMOPHINIA (the former DESMOPHIDAE) and SIPHONOPINIA (the former SIPHONOPIDAE).

These changes relative to recent usage may seem to be quite important modifications. Nevertheless 
we have to keep in mind that the modern classification of GYMNOPHIONA was founded in the work of Taylor (1968), not even five decades ago. This founding classification proposed four families. Successively the authors added family-rank taxa or synonymised other taxa, but the four families of Taylor (1968) were kept and are still valid in our much modified arrangement. Lescure et al. (1986) increased the number of families to ten but a new analysis of morphological data led to a proposal of a classification with six families (Nussbaum \& Wilkinson 1989). The classification proposed by Frost et al. (2006), based on molecular data, established three new families in order to resolve the paraphyly created by the placement of URAEOTYPHLIDAE, and recognised the former families SCOLECOMORPHIDAE and TYPHLONECTIDAE, deeply imbedded within the CAECILIIDAE, as their subfamilies SCOLECOMORPHINAE and TYPHLONECTINAE. However, in keeping the families TYPHLONECTIDAE and SCOLECOMORPHIDAE despite the paraphyly of the CAECILIIDAE, this amounted to using the phenetic argument of 'degree of distinctiveness' (Nussbaum \& Wilkinson 1989; Wilkinson et al. 2011). It is this distinctiveness that seems to have guided the use of the rank family for the highly embedded branches in the GYMNOPHIONA classification, instead of the hierarchical structure of relationships within the order.

In $C L A D$, the GYMNOPHIONA are divided into two suborders that both have high support in our phylogeny. The suborder Plesiophiona nov., which consists in a single family RHINatrematidae, is sister-group to suborder PSEUdOPHIONA including all other GyMnOPHIONA. The Appendix A9.CLAD-1 gives all details of classification, including fossil taxa, and the Appendices A6.NFS and A5.NGS provide information upon respectively family- and class-series nomina, in particular available synonyms and the status of available and unavailable nomina. In what follows, all the generic and specific nomina listed as valid designate taxa represented by at least one specimen in TREE, except those followed by ${ }^{\circ}$. For genus-series nomina, complete synonymies and homonymies are given in Appendix A5.NGS, but in the discussion below only the valid nomina are mentioned.

\section{C.05.03. Subordo Plesiophiona nov.}

Getangiotaxon: Gymnophiona Rafinesque, 1814.

Adelphotaxa: Pseudophiona Blainville, 1816; 1 F $\dagger ; 1$ G $\dagger$.

Getendotaxon: RHINATREMATIDAE Nussbaum, 1977.

Uninucleogenus, by present designation: Rhinatrema Duméril \& Bibron, 1841.

Etymology of nomen: G: $\pi \lambda \eta \sigma i o ́ \varsigma$ (plesios), 'near, close'; ő $\varphi ı \varsigma$ (ophis), 'snake'. This nomen is based on the same stem as the ordinal nomen GyMnOPHIONA and the subordinal nomen PSEUdophiona, and suggests the phylogenetic proximity of the species of this group with those of the latter.

Diagnosis: Presence of a posterior notch in the squamosal accommodating a distinct process of the os basale; lack of a distinct basipterygoid process; reduction of the posterior hyobranchial apperatus including reduction of absence of ceratobranchials 2 and 3, position of larynx posterior to glossal skeleton; absence of the musculus subarcualis rectus II and II; sinoatrial aperture partial divided; left pulmonary artery supplying oesophagus. \{Wilkinson \& Nussbaum 2006\}.

F.17.56. Familia RHINATREMATIDAE Nussbaum, 1977

Protonym and eunym: RhinAtrematidae Nussbaum, 1977: 1 [F].

Getangiotaxon: Plesiophiona nov.

Adelphotaxon: None.

Getendotaxon: Rhinatrema Duméril \& Bibron, 1841.

C.05.04. Subordo Pseudophiona Blainville, 1816

Protonym: PseUdophydiens Blainville, 1816: '111' [119] [O].

Eunym: Hoc loco. 
Getangiotaxon: GyMnophiona Rafinesque, 1814.

Adelphotaxa: Plesiophiona nov.; 1 F $\dagger ; 1$ G†.

Getendotaxa: CAECILIOIDEA Rafinesque, 1814-|Gray, 1825|; ICHтнYорніоIDEA Taylor, 1968.

Comments: This suborder includes two sister-groups, which both have high support, and that are recognised here as superfamilies: the CAECILIOIDEA and the ICHTHYOPHIOIDEA. These superfamilies include respectively the families CAECILIIDAE and SCOLECOPORPHIDAE, and ICHTHYOPHIDAE and URAEOTYPHLIDAE.

\section{F.14.15. Superfamilia CAECILIOIDEA Rafinesque, 1814-|Gray, 1825|}

Protonyms: CECILIINA Rafinesque, 1814: 104 [F]; |CAECILIADAE Gray, 1825: 217| [F].

Eunym: Lescure, Renous \& Gasc 1986: 167.

Getangiotaxon: Pseudophiona Blainville, 1816.

Adelphotaxon: ICHTHYOPHIOIDEA Taylor, 1968.

Getendotaxa: CAECILIIDAE Rafinesque, 1814-|Gray, 1825|; SCOLECOMORPHIDAE Taylor, 1969.

Comments: Wilkinson \& Nussbaum (2006) proposed the ectonym «TERESOMATA» "as a rankless name for [a] suprafamilial clade", sister-group to the UREOTYPHLOPIDAE and ICHTHYOPHIIDAE of their classification. In our classification this taxon is the superfamily CAECILIOIDEA.

\section{F.17.57. Familia CAECILIIDAE Rafinesque, 1814-|Gray, 1825|}

Eunym: Bonaparte 1850: plate.

Getangiotaxon: CAECILIOIDEA Rafinesque, 1814-|Gray, 1825|.

Adelphotaxon: SCOLECOMORPHIDAE Taylor, 1969.

Getendotaxa: CAECILIINAE Rafinesque, 1814-|Gray, 1825|; HERPELINAE Laurent, 1984.

F.18.79. Subfamilia CAECILIINAE Rafinesque, 1814-|Gray, 1825|

Eunym: Taylor 1969: 303.

Getangiotaxon: CAECILIIDAE Rafinesque, 1814-|Gray, 1825|.

Adelphotaxon: HERPELINAE Laurent, 1984.

Getendotaxa: CAECILIINI Rafinesque, 1814-|Gray, 1825|; SIPHONOPINI Bonaparte, 1850.

F.19.75. Tribus CAECILIINI Rafinesque, 1814-|Gray, 1825|

Eunym: Hoc loco.

Getangiotaxon: CAECILIINAE Rafinesque, 1814-|Gray, 1825|.

Adelphotaxon: SIPHONOPINI Bonaparte, 1850.

Getendotaxa: CAECILIINA Rafinesque, 1814-|Gray, 1825|; TYPHLONECTINA Taylor, 1968.

F.20.71. Subtribus CAECILIINA Rafinesque, 1814-|Gray, 1825|

Eunym: Hoc loco.

Getangiotaxon: CAECILIINI Rafinesque, 1814-|Gray, 1825|.

Adelphotaxon: TyPHLONECTINA Taylor, 1968.

Getendotaxa: Caecilia Linnaeus, 1758; Oscaecilia Taylor, 1968. 


\section{F.20.72. Subtribus TYPHLONECTINA Taylor, 1968}

Protonym: TYPHLONECTIDAE Taylor, 1968: xi, 231 [F].

Eunym: Hoc loco.

Getangiotaxon: CAECILIINI Rafinesque, 1814-|Gray, 1825|.

Adelphotaxon: CAECILIINA Rafinesque, 1814.

Getendotaxa: Atretochoana Nussbaum \& Wilkinson, 1995; Chthonerpeton Peters, 1880; Nectocaecilia Taylor, 1968; Potamotyphlus Taylor, 1968; Typhlonectes Peters, 1880.

Comments: In TREE, only two genera of this subtribe, Chthonerpeton and Typhlonectes, are represented. In the tree of San Mauro et al. (2014), a third genus, Potamotyphlus, was added to the molecular phylogeny, and Chthonerpeton appears as sister-group to a group formed by Potamotyphlus and Typhlonectes. Allocation of Atretochoana and Nectocaecilia to the TYPHLONECTINA is based on Wilkinson \& Nussbaum (1997, 1999).

\section{F.19.76. Tribus SIPHONOPINI Bonaparte, 1850}

Protonym: SiPHONOPINA Bonaparte, 1850: plate [bF].

Eunym: Lescure, Renous \& Gasc 1986: 166.

Getangiotaxon: CAECILIINAE Rafinesque, 1814-|Gray, 1825|.

Adelphotaxon: CAECILIINI Rafinesque, 1814-|Gray, 1825|.

Getendotaxa: GRANDISONIINA Lescure, Renous \& Gasc, 1986; SIPHONOPINA Bonaparte, 1850.

\section{F.20.73. Subtribus GRANDISONIINA Lescure, Renous \& Gasc, 1986}

Protonym: GRANDISONILAE Lescure, Renous \& Gasc, 1986: 163 [iF].

Eunym: Hoc loco.

Getangiotaxon: SIPHONOPINI Bonaparte, 1850.

Adelphotaxon: TYPHLONECTINA Taylor, 1968.

Getendotaxa: GRANDISONIINIA Lescure, Renous \& Gasc, 1986; IndotyPHLINIA Lescure, Renous \& Gasc, 1986 ; 1 GIS (Sylvacaecilia Wake, 1987).

Comments: This taxon corresponds to that named INDOTYPHLIDAE by San Mauro et al. (2014) but which should have been named GRANDISONIIDAE following the Code. The latter nomen had been created by Lescure et al. (1986) for a subfamily GRANDISONINAE with the same date as the tribe nomen INDOTYPHLINI. According to Article 24.1 of the Code, the nomen published at higher rank, GRANDISONIINAE, has permanent precedence over the nomen of lower rank published in the same work, INDOTYPHLINI (Principle of Proedry).

According to Wilkinson et al. (2011), the genus Sylvacaecilia should belong in their INDOTYPHLIDAE, our GRANDISONIINA, without more precision on its place in the hierarchy.

\section{F.21.52. Infratribus GRANDISONIINIA Lescure, Renous \& Gasc, 1986}

Eunym: Hoc loco.

Getangiotaxon: GRANDISONIINA Lescure, Renous \& Gasc, 1986.

Adelphotaxon: INDOTYPHLINIA Lescure, Renous \& Gasc, 1986.

Getendotaxa: Hypogeophis Peters, 1880; Idiocranium Parker, 1936; Praslinia Boulenger, 1909;.

Comments: In San Mauro et al. (2014), Idiocranium is sister-group to the other genera included in GRANDISONIINA (their INDOTYPHLIDAE), and Grandisonia (two species) is highly supported and has Hypogeophis as sister-group, represented by two specimens of a single species, thus insinuating a support for the genus. With a larger sample of species represented in TREE, the holophyly of these two genera is not supported, and we treat Grandisonia as a synonym of Hypogeophis. 
Protonym: INDOTYPHLINI Lescure, Renous \& Gasc, 1986: 164 [T].

Eunym: Hoc loco.

Getangiotaxon: GRANDISONIINA Lescure, Renous \& Gasc, 1986.

Adelphotaxon: GRANDISONIINIA Lescure, Renous \& Gasc, 1986.

Getendotaxa: Gegeneophis Peters, 1880; Indotyphlus Taylor, 1960.

\section{F.20.74. Subtribus SIPHONOPINA Bonaparte, 1850}

Eunym: Hoc loco.

Getangiotaxon: SIPHONOPINI Bonaparte, 1850.

Adelphotaxon: GRANDISONIINA Lescure, Renous \& Gasc, 1986.

Getendotaxa: Dermophinia Taylor, 1969; SipHonopinia Bonaparte, 1850.

\section{F.21.54. Infratribus DERMOPHIINIA Taylor, 1969}

Protonym: DERMophinaE Taylor, 1969: 303 [bF].

Eunym: Hoc loco.

Getangiotaxon: SIPHONOPINA Bonaparte, 1850.

Adelphotaxon: SIPHONOPINIA Bonaparte, 1850.

Getendotaxa: DermophinoA Taylor, 1969; GeotrypetinoA Lescure, Renous \& Gasc, 1986.

\section{F.22.31. Hypotribus DERMOPHIINOA Taylor, 1969}

Eunym: Hoc loco.

Getangiotaxon: DERMOPHIINIA Taylor, 1969.

Adelphotaxon: GeOtRYPETINOA Lescure, Renous \& Gasc, 1986.

Getendotaxa: Gymnopis Peters, 1874; Schistometopum Parker, 1941.

Comments: In their tree, San Mauro et al. (2014) did not find support for the holophyly of Gymnopis nor of Dermophis (represented by a single species). TREE supports the holophyly of a taxon grouping Gymnopis and Dermophis species. Accordingly, we consider that, pending additional data, these species should be grouped in a single genus for which the nomen Gymnopis has priority.

\section{F.22.32. Hypotribus GEOTRYPETINOA Lescure, Renous \& Gasc, 1986}

Protonym: GEOTRYPETIDAE Lescure, Renous \& Gasc, 1986: 145 [F].

Eunym: Hoc loco.

Getangiotaxon: DERMOPHIINIA Taylor, 1969.

Adelphotaxon: DERMOPHIINOA Taylor, 1969.

Getendotaxon: Geotrypetes Peters, 1880.

\section{F.21.55. Infratribus SIPHONOPINIA Bonaparte, 1850}

Eunym: Hoc loco.

Getangiotaxon: SIPHONOPINA Bonaparte, 1850.

Adelphotaxon: DERMOPHIINIA Taylor, 1969.

Getendotaxa: MiCROCAECILIINOA nov.; SIPHONOPINOA Bonaparte, 1850; 2 GIS (Brasilotyphlus Taylor, 1968; Mimosiphonops Taylor, 1968). 
Comments: According to Wilkinson et al. (2011), Brasilotyphlus and Mimosiphonops are members of their SIPHONOPIDAE, the present SIPHONOPINIA, without more precision on their place in the hierarchy.

\section{F.22.33. Hypotribus MICROCAECILIINOA nov.}

Getangiotaxon: SIPHONOPINIA Bonaparte, 1850.

Adelphotaxa: SIPHONOPINOA Bonaparte, 1850; 2 GIS (Brasilotyphlus Taylor, 1968; Mimosiphonops Taylor, 1968).

Getendotaxon: Microcaecilia Taylor, 1968.

Nucleogenus, by present designation: Microcaecilia Taylor, 1968. • Etymology of nomen: G: $\mu 1 \kappa \rho o ́ s$ (micros), 'small'; N: Caecilia Linnaeus, 1758, derived from L: caecilia, 'slow worm, blind snake'. • Stem of nomen: Microcaecili-.

Diagnosis: Eye under bone; temporal fossae absent; mesethmoid not exposed dorsally; no splenial teeth; secondary grooves usually present, absent in one species; scales present; tentacular opening closer to eye than to external naris; no unsegmented terminal shield; no narial plugs; no diastema between vomerine and palatine teeth; terminal keel present or absent. \{Wilkinson \& Nussbaum 2006; Wilkinson et al. 2013, 2014\}.

\section{F.22.34. Hypotribus SIPHONOPINOA Bonaparte, 1850}

Eunym: Hoc loco.

Getangiotaxon: SIPHONOPINIA Bonaparte, 1850.

Adelphotaxa: MICROCAECILIINOA nov.; 2 GIS (Brasilotyphlus Taylor, 1968; Mimosiphonops Taylor, 1968).

Getendotaxa: Luetkenotyphlus Taylor, 1968; Siphonops Wagler, 1828.

\section{F.18.80. Subfamilia HERPELINAE Laurent, 1984}

Protonym and eunym: HERPELINAE Laurent, 1984: 199 [bF].

Getangiotaxon: CAECILIIDAE Rafinesque, 1814-|Gray, 1825|.

Adelphotaxon: CAECILIINAE Rafinesque, 1814-|Gray, 1825|.

Getendotaxa: ChIKILINI Kamei, San Mauro, Gower, Van Bocxlaer, Sheratt, Thomas, Babu, Bossuyt, Wilkinson \& Biju, 2012; HERPELINI Laurent, 1984.

Comments: This taxon includes the tribes HERPELINI corresponding to the HERPELIDAE and CHIKILINI corresponding to the CHIKILIDAE of recent auhors (San Mauro et al. 2014). This sister-group relationship is strongly supported in TREE.

\section{F.19.77. Tribus CHIKILINI Kamei, San Mauro, Gower, Van Bocxlaer,} Sheratt, Thomas, Babu, Bossuyt, Wilkinson \& Biju, 2012

Protonym: ChikilidaE Kamei, San Mauro, Gower, Van Bocxlaer, Sheratt, Thomas, Babu, Bossuyt, Wilkinson \& Biju, 2012: $1[\mathrm{~F}]$.

Eunym: Hoc loco.

Getangiotaxon: HERPELINAE Laurent, 1984.

Adelphotaxon: HERPELINI Laurent, 1984.

Getendotaxon: Chikila Kamei, San Mauro, Gower, Van Bocxlaer, Sheratt, Thomas, Babu, Bossuyt, Wilkinson \& Biju, 2012. 


\section{F.19.78. Tribus HERPELINI Laurent, 1984}

Eunym: Lescure, Renous \& Gasc 1986: 163.

Getangiotaxon: HERPELINAE Laurent, 1984.

Adelphotaxon: Chikilini Kamei, San Mauro, Gower, Van Bocxlaer, Sheratt, Thomas, Babu, Bossuyt, Wilkinson \& Biju, 2012.

Getendotaxa: Boulengerula Tornier, 1896; Herpele Peters, 1880.

\section{F.17.58. Familia SCOLECOMORPHIDAE Taylor, 1969}

Protonym and eunym: SCOLECOMORPHIDAE Taylor, 1969: 297 [F].

Getangiotaxon: CAECILIOIDEA Rafinesque, 1814-|Gray, 1825|.

Adelphotaxon: CAECILIIDAE Rafinesque, 1814-|Gray, 1825|.

Getendotaxa: Crotaphatrema Nussbaum, 1985; Scolecomorphus Boulenger, 1883.

\section{F.14.16. Superfamilia ICHтнYOРнIOIDEA Taylor, 1968}

Protonym: ICHTHYOPHIIDAE Taylor, 1968: x, 46 [F].

Eunym: Hoc loco.

Getangiotaxon: Pseudophiona Blainville, 1816.

Adelphotaxon: CAECILIOIDEA Rafinesque, 1814-|Gray, 1825|.

Getendotaxa: ICHTHYOPHIIDAE Taylor, 1968; URAEOTYPHLIDAE_Nussbaum, 1979.

\subsection{Familia ICHTHYOPHIIDAE Taylor, 1968}

Eunym: Taylor 1968: x, 46.

Getangiotaxon: ICHTHYOРНIOIDEA Taylor, 1968.

Adelphotaxon: URAEOTYPHLIDAE Nussbaum, 1979.

Getendotaxa: Caudacaecilia Taylor, 1968; Ichthyophis Fitzinger, 1826.

F.17.60. Familia URAEOTYPHLIDAE Nussbaum, 1979

Protonym: URAEOTYPHLINAE Nussbaum, 1979: 14 [bF].

Eunym: Lescure, Renous \& Gasc 1986: 145.

Getangiotaxon: ICHTHYOРHIOIDEA Taylor, 1968.

Adelphotaxon: ICHTHYOPHIIDAE Taylor, 1968.

Getendotaxon: Uraeotyphlus Peters, 1880.

Comments: This branch is sister-group to the ICHTHYOPHIIDAE, a nomen validated at family rank through the [UQC]. Altogether, both groups form a taxon with high support.

The sister-group relationship of the species Ichthyophis bombayensis with the genus Uraeotyphlus has been recovered in all molecular phylogenies since Gower et al. (2002). It renders the genus Ichthyophis paraphyletic. We transfer this species to the genus Uraetyphlus as Uraetyphlus bombayensis (Taylor, 1960), which resolves the taxonomic incongruity, pending confirmation in further taxonomic works.

\section{C.04.03. Ordo Urodela Duméril, 1805}

Protonym: UrodèLes Duméril, 1805: 91 ['F'].

Eunym: Knauer 1878: 93.

Getangiotaxon: LisSAMPHIBIA Gadow, 1898. 
Adelphotaxa: Anura Duméril, 1805; Gymnophiona Rafinesque, 1814; 1 C†.

Getendotaxa: Imperfectibranchia Hogg, 1838; Meantes Linné, 1767; Pseudosauria Blainville, 1816; 5 F†; 21 G†.

Comments: The holophyly of all extant urodeles is supported by all phylogenetic studies based on morphology and on molecular data. Numerous CS nomina are available for this taxon (Appendix A7.NCS) but the valid one under DONS Criteria is the sozodiaphonym Urodela Duméril, 1805 (Dubois 2004b, 2005d, 2015c, 2020a; Dubois \& Raffaëlli 2012; Dubois \& Ohler 2019; Dubois \& Frétey 2020c). Frost et al. (2006: 356) tried to impose the use of the nomen CAUDATA for this order on the ground that this was the nomen used by "most working systematists" but they provided a single reference in support of this allegation (Duellman \& Trueb 1985, misquoted as '1986'), which was clearly wrong (see Dubois \& Raffaëlli 2012: 109). Quite strangely a number of authors uncritically followed this misleading statement, which indeed modified the 'usage' after 2006, but in case of nomenclatural conflict of zygonymy between two nomina which both have been used widely in the literature for two centuries, penny-pinching calculations cannot play the role of a 'justice of the peace' as suggested by some 'Google taxonomists' (see Dubois 2007b) and we need explicit Criteria to settle the conflict. In the present case all possible Criteria require to keep URODELA, the 'sister-nomen' to ANURA (while Caudata was the 'sister-nomen' to ECAUdaTa), as the valid nomen of this taxon (Dubois 2015c, Dubois \& Ohler 2019, Dubois \& Frétey 2020c).

Applying the [UQC], we retained 9 family-rank taxa of URODELA, distributed in three suborders, the Imperfectibranchia, the Meantes and the Pseudosauria. The suborder Imperfectibranchia includes two families, the CRYPTOBRANCHIDAE and the HyNOBIIDAE, the suborder Meantes includes a single family SIRENIDAE, and the suborder Pseudosauria includes 6 family-rank taxa, the

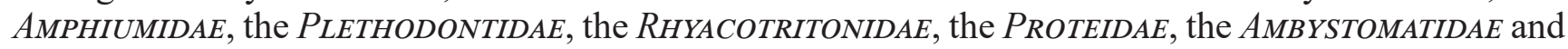
the SALAMANDRIDAE. These taxa are confirmed by morphological and molecular data (Larson A. et al. 2003) and are accepted by most authors today.

The relationship between the CRYPTOBRANCHIDAE and the HYNOBIIDAE found support in most recent studies (Gao \& Shubin 2001; Larson A. et al. 2003; Wiens et al. 2005a; Frost et al. 2006; Roelants et al. 2007; Pyron \& Wiens 2011; Dubois \& Raffaëlli 2012; Shen et al. 2013; Pyron 2014), and the taxon here recognised as the suborder IMPERFECTIBRANCHIA is called 'suborder CRYPTOBRANCHOIDEA' by some authors (Larson A. et al. 2003; Vieites et al. 2009). The position of MEANTES, and its only family SIRENIDAE has been highly variable in the recent literature. In their review, Larson A. et al. (2003) and Zhang \& Wake (2009) considered this family as basal to all other URODELA, whereas in Weisrock et al. (2005) it was sister-group to the SALAMANDROIDEA, and in Frost et al. (2006) and Gao \& Shubin (2012) it appeared as sister-taxon to the PROTEIDAE. However, already Wiens et al. (2005a) and later Roelants et al. (2009), Vieites et al. (2009), Shen et al. (2013) and Pyron (2014) had retrieved this taxon as sister to a taxon that groups all Pseudosauria taxa. As in TREE the support for this grouping is below the threshold retained, we recognise three groups as suborders.

\title{
C.05.05. Subordo IMPERFECTIBRANCHIA Hogg, 1838
}

\author{
Protonym: IMPERFECTIBRANCHIA Hogg, 1838: 152 [O]. \\ Eunym: Hoc loco. \\ Getangiotaxon: Urodela Duméril, 1805. \\ Adelphotaxa: Meantes Linné, 1767; Pseudosauria Blainville, 1816; 5 F†; 21 G†. \\ Getendotaxa: CRYPTOBRANCHIDAE Fitzinger, 1826; HYNOBIIDAE \|Hallowell, 1856\|-Cope, 1859; 2 G†.
}

Comments: Following Noble (1931), this group was recognised as a suborder by various recent authors, but named 'CRYPTOBRANCHOIDEA'. This paronym was initially an aponym, first-used by Dunn (1922), of the family-series nomen CRYPTOBRANCHIDAE Fitzinger, 1826, but it became then a new class-series nomen Cryptobranchoidea Noble, 1931. For this taxon the oldest available class-series nomen is IMPERFECTIBRANCHia Hogg, 1838, which should be used (Dubois \& Raffaëlli 2012). Two highly supported branches, found in all recent phylogenies (see Larson A. et al. 2003 for a review; Weisrock et al. 2005; Wiens et al. 2005a; Frost et al. 2006; Roelants et al. 2007; Vieites et al. 2009; Zhang \& Wake 2009; Shen et al. 2013) as well as in TREE, are here recognised as the families HYNOBIIDAE and 
CRYPTOBRANCHIDAE. In our classification the family rank is attributed to the HYNOBIIDAE by the [UQC], and to the CRYPTOBRANCHIDAE by the [STC].

\title{
F.17.61. Familia CRYPTOBRANCHIDAE Fitzinger, 1826
}

\author{
Protonym: CRYPTOBRANCHOIDEA Fitzinger, 1826: 41 [F]. \\ Eunym: Cope 1889: 18. \\ Getangiotaxon: IMPERFECTIBRANCHIA Hogg, 1838. \\ Adelphotaxa: HYNOBIIDAE ||Hallowell, 1856\|-Cope, 1859; 2 G†. \\ Getendotaxa: Andrias Tschudi, 1837; Cryptobranchus Leuckart, 1821; 7 G†.
}

Comments: This family includes two extant genera, Andrias and Cryptobranchus. It found support in most recent studies that included relevant samples (Larson A. et al. 2003; Wiens et al. 2005a; Frost et al. 2006; Zhang \& Wake 2009; Pyron \& Wiens 2011; Chen et al. 2011).

\section{F.17.62. Familia HYNOBIIDAE ||Hallowell, 1856||-Cope, 1859}

\author{
Protonyms: ||ELLIPSOGLOSSIDAE Hallowell, 1856: 11\| [bF]; HynobIINAE Cope, 1859: 125 [bF]. \\ Eunym: Cope 1866: 107. \\ Getangiotaxon: IMPERFECTIBRANCHIA Hogg, 1838. \\ Adelphotaxa: CRYPTOBRANCHIDAE Fitzinger, 1826; ONYCHODACTYLINAE Dubois \& Raffaelli, 2012; 2 G†. \\ Getendotaxon: HyNOBIINAE \|Hallowell, 1856\|-Cope, 1859.
}

Comments: The family HYNOBIIDAE includes two branches, recognised here as the subfamily ONYCHODACTYLINAE for the single genus Onychodactylus, and the subfamily HYNOBIINAE. The position of Onychodactylus in relation to the other HYNOBIIDAE was already presented by Larson A. et al. (2003) and found in all recent studies (Zhang et al. 2006; Peng et al. 2010; Pyron \& Wiens 2011; Chen G. et al. 2011; Weisrock et al. 2013; Chen M. Y. et al. 2015).

\section{F.18.81. Subfamilia HyNobIINAE \|Hallowell, 1856||-Cope, 1859}

\author{
Eunym: Cope 1859: 125. \\ Getangiotaxon: HYNOBIIDAE ||Hallowell, 1856||-Cope, 1859. \\ Adelphotaxa: ONYCHODACTYLINAE Dubois \& Raffaelli, 2012; 2 G†. \\ Getendotaxa: HYNOBIINI ||Hallowell, 1856||-Cope, 1859; RANODONTINI Thorn, 1966.
}

Comments: The subfamily HYNOBIINAE includes two branches recognised here as the tribes HYNOBIINI and RANODONTINI. Within the HYNOBIINI, three branches of unresolved relationships form the subtribes PACHYHYNOBIINA for Pachyhynobius, SALAMANDRELLINA for Salamandrella, and HyNOBIINA. This latter subtribe includes two infratribes, the PRoтонYNoBIINIA for the genera Batrachuperus, Liua and Pseudohynobius, and the HyNOBIINIA with two hypotribes, the SATOBIINOA for Satobius, and the HyNOBIINOA, including Hynobius, Pachypalaminus and Poyarius.

In fact, the relationships within this subfamily are still not settled. This may be partly due to the sampling which is incomplete in many studies due to the large geographic range of this taxon.

The sister-group relationship between Hynobius and Poyarius seems to be confirmed (Zhang et al. 2006; Xiong et al. 2007; Peng et al. 2010; Chen G. et al. 2011; Weisrock et al. 2013). The position of Pachypalaminus is close to these two genera, but either Poyarius is sister-branch of Pachypalaminus and both sister to Hynobius (Pyron \& Wiens 2011) or Hynobius and Pachypalaminus are sister-branches (Nishikawa et al. 2010). This group, named here the hypotribe HYNOBIINOA, is sister to the hypotribe SATOBIINOA, for the single genus Satobius. These two hypotribes form a holophyletic group here recognised as the infratribe HYNOBIINIA. Most taxonomists keep all the species of this group in a single genus Hynobius.

The three genera Batrachuperus, Liua and Pseudohynobius, forming a holophyletic group of high 
support, recognised in the present classification as the infratribe PRотонYNOBIINIA, are retained by all recent authors (Zeng et al. 2006; Xiong et al. 2007; Peng et al. 2010; Pyron \& Wiens 2011; Chen G. et al. 2011; Weisrock et al. 2013; Chen M. Y. et al. 2015).

There seems to exist an agreement of relationships within our tribe RANODONTINI (Zhang et al. 2006; Xiong et al. 2007; Pyron \& Wiens 2011; Weisrock et al. 2013), although the taxonomic and nomenclatural treatment is not much disputed. Some authors consider Paradactylodon as available (Stöck et al. 2019), whereas it is a nomen nudum (Dubois \& Raffaelli 2012) because no explicit diagnostic characters are mentioned in the original description (e.g., the latter states that there exists a differential character concerning the vomero-palatine ridge shape to separate the genus from Salamandrella, but this character is not given!; see above Figure F3.NDD).

The position of Pachyhynobius and Salamandrella is not fixed. Here these two genera are referred to two subtribes, the PACHYHYNOBIINA and the SALAMANDRELLINA, sister-taxa of unresolved relationships with the HYNOBIINA. In other phylogenies, Pachyhynobius is either sister-group to all other HYNOBIINAE (Xiong et al. 2007; Peng et al. 2010; Chan G. et al. 2011) or sister to ((PROTOHYNOBIINIA + Salamandrella) + HyNobiINOA) (Zhang et al. 2006; Chen M. Y. et al. 2015). In Pyron \& Wiens (2011), Pachyhynobius and Salamandrella are sister-group to all other HyNOBIINAE, whereas in Weisrock et al. (2013) Pachyhynobius is sister-group to the RANODONTINI. Similarly, the position of Salamandrella changes in the different phylogenies published. It has been considered as sister-taxon to the ProtoHYNOBIINIA (Zhang et al. 2006; Peng et al. 2010; Chen G. et al. 2011; Chen M. Y. et al. 2015), to the RANODONTINI (Xiong et al. 2007), to Hynobius (Nishikawa et al. 2010), to Pachyhynobius (Pyron \& Wiens 2011), or still sistergroup to all other HYNOBIINAE (Weisrock et al. 2013).

F.19.79. Tribus HYNOBIINI |Hallowell, 1856||-Cope, 1859

Eunym: Dubois \& Raffaëlli 2012: 113.

Getangiotaxon: HYNOBIINAE ||Hallowell, 1856\|-Cope, 1859.

Adelphotaxon: RANODONTINI Thorn, 1966.

Getendotaxa: HynobiIna \|Hallowell, 1856||-Cope, 1859; PACHYHYNOBIINA Dubois \& Raffaelli, 2012; SALAMANDRELLINA Dubois \& Raffaelli, 2012.

F.20.75. Subtribus HyNoвIINA ||Hallowell, 1856||-Cope, 1859

Eunym: Dubois \& Raffaëlli 2012: 113.

Getangiotaxon: HYNOBIINI ||Hallowell, 1856||-Cope, 1859.

Adelphotaxa: PACHYHYNobiIna Dubois \& Raffaelli, 2012; SALAMANDRELLINA Dubois \& Raffaelli, 2012.

Getendotaxa: Hynobinia ||Hallowell, 1856||-Cope, 1859; РRотонYNobinia Fei \& Ye, 2000.

F.21.56. Infratribus HYNOBIINIA ||Hallowell, 1856||-Cope, 1859

Eunym: Hoc loco.

Getangiotaxon: HYNOBIINA \|Hallowell, 1856\|-Cope, 1859.

Adelphotaxon: PRotohynobinia Fei \& Ye, 2000.

Getendotaxa: HynobiINoA ||Hallowell, 1856||-Cope, 1859; SATOBIINOA nov.

F.22.35. Hypotribus НyNOBIINOA ||Hallowell, 1856||-Cope, 1859

Eunym: Hoc loco.

Getangiotaxon: HYNOBIINIA ||Hallowell, 1856||-Cope, 1859.

Adelphotaxon: SATOBIINOA nov.

Getendotaxa: Hynobius Tschudi, 1838; Pachypalaminus Thompson, 1912; Poyarius Dubois \& Raffaelli, 2012. 


\section{F.22.36. Hypotribus SATOBIINOA nov.}

Getangiotaxon: HYNOBIINIA ||Hallowell, 1856||-Cope, 1859.

Adelphotaxon: HynobirnoA ||Hallowell, 1856||-Cope, 1859.

Getendotaxon: Satobius Adler \& Zhao, 1990.

Nucleogenus, by present designation: Satobius Adler \& Zhao, 1990. • Etymology of nomen: P: Ikio Sato (1902-1945), Japanese zoologist; G: Bíos (bios), 'life'. • Stem of nomen: Satobi-.

Diagnosis: Salamanders with very long limbs and toes (tips of digits of limbs adpressed along body in joining direction overlap up to 4 intercostal distances in adults); tail longer than head and body length; small head and long neck; no premaxillary fontanelle or basibranchial radii; two short series of vomerine teeth arranged in transverse arcs between internal nares; vomer sutured to anterior end of parasphenoid; lungs present; chromosome complement $2 \mathrm{n}=40$; duration of larval stage one year of more, sometimes neoteny; adults terrestrial and aquatic outside breeding season. \{Adler \& Zhao 1990\}.

\section{F.21.57. Infratribus PROTOHYNOBIINIA Fei \& Ye, 2000}

Protonym: PRotohynobinat Fei \& Ye, 2000: 64 [F].

Eunym: Hoc loco.

Getangiotaxon: HyNOBIINA ||Hallowell, 1856||-Cope, 1859.

Adelphotaxon: HYNOBIINIA ||Hallowell, 1856||-Cope, 1859.

Getendotaxa: Batrachuperus Boulenger, 1878; Liua Zhao, 1983; Pseudohynobius Fei \& Yang, 1983.

\section{F.20.76. Subtribus PACHYнYNobIINA Dubois \& Raffaëlli, 2012}

Protonym: PACHYHYNobIINI Dubois \& Raffaëlli, 2012: 113 [T].

Eunym: Hoc loco.

Getangiotaxon: HyNOBIINI ||Hallowell, 1856||-Cope, 1859.

Adelphotaxa: Hynobina ||Hallowell, 1856||-Cope, 1859; SALAMANDRELLINA Dubois \& Raffaelli, 2012.

Getendotaxon: Pachyhynobius Fei, Qu \& Wu, 1983.

F.20.77. Subtribus SALAMANDRELLINA Dubois \& Raffaëlli, 2012

Protonym and eunym: SALAMANDRELLINA Dubois \& Raffaëlli, 2012: 113 [bT].

Getangiotaxon: HYNOBIINI ||Hallowell, 1856||-Cope, 1859.

Adelphotaxa: HynobiIna ||Hallowell, 1856||-Cope, 1859; PACHYHYNoBIINA Dubois \& Raffaelli, 2012.

Getendotaxon: Salamandrella Dybowsky, 1870.

\section{F.19.80. Tribus RANODONTINI Thorn, 1966}

Protonym: RANODONTIDAE Thorn, 1966: 108 [F].

Eunym: Hoc loco.

Getangiotaxon: HYNOBIINAE ||Hallowell, 1856||-Cope, 1859.

Adelphotaxon: HyNOBIINI ||Hallowell, 1856||-Cope, 1859.

Getendotaxa: IRANODONTINA nov.; RANODONTINA Thorn, 1966.

\section{F.20.78. Subtribus IRANODONTINA nov.}

Getangiotaxon: RANODONTINI Thorn, 1966.

Adelphotaxon: RANODONTINA Thorn, 1966. 
Getendotaxa: Afghanodon Dubois \& Raffaelli, 2012; Iranodon Dubois \& Raffaelli, 2012.

Nucleogenus, by present designation: Iranodon Dubois \& Raffaëlli, 2012. • Etymology of nomen: R: Iran, name of country of origin; G: ódov́s (odous), 'tooth'. • Stem of nomen: Iranodont-.

Diagnosis: Small sized salamanders (up to $22 \mathrm{~cm}$ total length); rectangular or rounded head; vomerine ridges forming V; lungs present; 11-14 costal folds; hindlimbs with 4 toes; presence of keratinisation on digits; chromosome complement $2 \mathrm{n}=62$. \{Dubois \& Raffaëlli 2012\}.

F.20.79. Subtribus RANODONTINA Thorn, 1966

Eunym: Hoc loco.

Getangiotaxon: RANODONTINI Thorn, 1966.

Adelphotaxon: IRANODONTINA nov.

Getendotaxon: Ranodon Kessler, 1866.

F.18.82. Subfamilia ONYCHODACTYLINAE Dubois \& Raffaëlli, 2012

Protonym and eunym: ONYCHODACTYLINAE Dubois \& Raffaëlli, 2012: 108 [F].

Getangiotaxon: HYNOBIIDAE ||Hallowell, 1856||-Cope, 1859.

Adelphotaxa: HyNOBIINAE ||Hallowell, 1856\|-Cope, 1859; 2 G†.

Getendotaxon: Onychodactylus Tschudi, 1838.

\section{C.05.06. Subordo MeanTes Linné, 1767}

Protonym: MeANTES Linné, 1767: unnumbered additional page [O].

Eunym: Stejneger \& Barbour 1917: 24.

Getangiotaxon: Urodela Duméril, 1805.

Adelphotaxa: Imperfectibranchia Hogg, 1838; Pseudosauria Blainville, 1816; 5 F†; 21 G†.

Getendotaxa: SIRENIDAE Gray, 1825; 1 F†.

\section{F.17.63. Familia SIRENIDAE Gray, 1825}

Protonym and eunym: SIRENIDAE Gray, 1825: 108 [F].

Getangiotaxon: MeANTes Linnaeus, 1767.

Adelphotaxon: $1 \mathrm{~F} \dagger$.

Getendotaxa: Pseudobranchus Gray, 1825; Siren Österdam, 1766.

Comments: The family SIRENIDAE is the single extant family-rank taxon in the MeANTES. It includes two highly supported branches, the genera Pseudobranchus and Siren.

\section{C.05.07. Subordo Pseudosauria Blainville, 1816}

Protonym: Pseudo-Sauriens Blainville, 1816: '111' [119] [O].

Eunym: Hoc loco.

Getangiotaxon: Urodela Duméril, 1805.

Adelphotaxa: Imperfectibranchia Hogg, 1838; Meantes Linné, 1767; 5 F†; 21 G†.

Getendotaxa: AmpHIUMoIDEA Gray, 1825; SALAMANDROIDEA Goldfuss, 1820; 2 G†.

Comments: The Pseudosauria are divided in two highly supported branches, recognised here as the superfamilies AMPHIUMOIDEA and SALAMANDROIDEA. The AMPHIUMOIDEA split into two highly supported branches, the epifamilies PROTEOIDAE for the single family PROTEIDAE including the genera Necturus 
and Proteus, and the AMPHIUMOIDAE. The latter taxon includes two branches, allocated to the apofamilies RHYACOTRITONEIDAE, for the single family RHYACOTRITONIDAE with the single genus Rhyacotriton, and AMPHIUMEIDAE. The latter taxon includes two highly supported taxa, recognised as the families AMPHIUMIDAE, for the single genus Amphiuma, and PLETHODONTIDAE, whose classification is described below. The superfamily SALAMANDROIDEA splits into two highly supported branches, recognised as the family AMBYSTOMATIDAE, for the genera Ambystoma and Dicamptodon, and the SALAMANDRIDAE, whose classification is described below. Besides RHYACOTRITONEIDAE, assigned to the rank family by the Consistent Naming Criterium [CNC], all these families are recognised at the family rank by the Upper Quartile Criterium [UQC].

The relationship of ((AMPHIUMIDAE + PLETHODONTIDAE $)+$ RHYACOTRITONIDAE $)$, here named epifamily AMPHIUMOIDAE, was recovered with molecular data by Wiens et al. (2005a) and most of the subsequent studies (Frost et al. 2006; Roelants et al. 2007; Vieites et al. 2009; Zhang \& Wake 2009; Pyron \& Wiens 2011; Zheng et al. 2011; Shen et al. 2013). The position of the PROTEIDAE has been much disputed, but seems confirmed in recent studies as sister-group of the AMPHIUMOIDAE (Roelants et al. 2007; Vieites et al. 2009; Zhang \& Wake 2009; Pyron \& Wiens 2011; Zheng et al. 2011; Shen et al. 2013).

The sister-group relationship of the AMBYSTOMATIDAE (here including the genus Dicamptodon) and SALAMANDRIDAE was recognised already through morphological evidence (Larson 1991; Larson \& Dimmick 1993; Gao \& Shubin 2001) and later confirmed by molecular data (Wiens et al. 2005a; Frost et al. 2006; Roelants et al. 2007; Weisrock et al. 2005; Vieites et al. 2009; Zhang \& Wake 2009; Pyron \& Wiens 2011; Shen et al. 2013).

F.14.17. Superfamilia AMPHIUMOIDEA Gray, 1825

Protonym: AMPHIUMIDAE Gray, 1825: 216 [F].

Eunym: Dunn 1922: 426.

Getangiotaxon: Pseudosauria Blainville, 1816.

Adelphotaxa: SALAMANDROIDEA Goldfuss, 1820; 2 G†.

Getendotaxa: AmPHiUmoIdAE Gray, 1825; PROTEOIDAE Bonaparte, 1831.

\section{F.15.11. Epifamilia AMPHIUMOIDAE Gray, 1825}

Eunym: Dubois \& Raffaëlli 2012: 138.

Getangiotaxon: AMPHIUMOIDEA Gray, 1825.

Adelphotaxon: PROTEOIDAE Bonaparte, 1831.

Getendotaxa: AMPHIUMEIDAE Gray, 1825; RHYACOTRITONEIDAE Tihen, 1958.

F.16.08. Apofamilia AMPHIUMEIDAE Gray, 1825

Eunym: Hoc loco.

Getangiotaxon: AMPHIUMOIDAE Gray, 1825.

Adelphotaxon: RHYACOTRITONEIDAE Tihen, 1958.

Getendotaxa: AmpHidmidae Gray, 1825; PLETHodontidaE Gray, 1850.

\section{F.17.64. Familia AMPHIUMIDAE Gray, 1825}

Eunym: Gray 1825: 216.

Getangiotaxon: AMPHIUMEIDAE Gray, 1825.

Adelphotaxon: Plethodontidae Gray, 1850.

Getendotaxon: Amphiuma Garden in Smith, 1821. 


\title{
F.17.65. Familia PLETHODONTIDAE Gray, 1850
}

\author{
Protonym and eunym: PLETHODONTIDAE Gray, 1850: 5, 31 [F]. \\ Getangiotaxon: AMPHIUMEIDAE Gray, 1825. \\ Adelphotaxon: AMPHIUMIDAE Gray, 1825. \\ Getendotaxa: HemidaCtylinae Hallowell, 1856; PlethodontinaE Gray, 1850; 1 G†.
}

Comments: Within the family PLETHODONTIDAE two branches find high support, recognised as the subfamilies HEMIDACTYLIINAE and PleTHODONTINAE. This dichotomy was first proposed by Vieites et al. (2007) and confirmed by subsequent authors (Vieites et al. 2011; Kozak et al. 2009; Chen G. Y. et al. 2011; Pyron \& Wiens 2011; Shen et al. 2016), but the taxonomic treatment did not always reflect this relationship.

\section{F.18.83. Subfamilia HemidaCtYLIINAE Hallowell, 1856}

\author{
Protonym: HeMIDACTYLIDAE Hallowell, 1856: 11 [bF]. \\ Eunym: Chippindale, Bonett, Baldwin \& Wiens 2004: 2819. \\ Getangiotaxon: PlethodontidAE Gray, 1850. \\ Adelphotaxa: Plethodontinae Gray, 1850; 1 G†. \\ Getendotaxa: Bolitoglossini Hallowell, 1856; HemidaCtYlini Hallowell, 1856; SPELERPINI Cope, 1859.
}

Comments: The subfamily HEMIDACTYLIINAE contains three branches of unresolved relationships that are here attributed to the rank tribe, as the HEMIDACTYLIINI for Hemidactylium, the BoLITOGLOSSINI, and the SPELERPINI. The position of Hemidactylium has long been instable, but often it was close to Batrachoseps and Bolitoglossa (Mueller et al. 2004; Macey et al. 2005; Vieites et al. 2007, 2011; Kozak et al. 2009; Chen G. et al. 2011; Pyron \& Wiens 2011; Shen et al. 2016). In the recent work of Shen et al. (2016), based on a high number of nuclear markers, the relationship between the BOLITOGLOSSINI and the HEMIDACTYLIINI has high support which if confirmed would lead to the synonymisation of BOLITOGLOSSINI at the rank tribe.

\section{F.19.81. Tribus BoLitoglossini Hallowell, 1856}

\author{
Protonym: Bolitoglossidae Hallowell, 1856: 11 [bF]. \\ Eunym: Wake 1966: 1. \\ Getangiotaxon: HEMIDACTYLIINAE Hallowell, 1856. \\ Adelphotaxa: HeMIDACTYLIINI Hallowell, 1856; SPELERPINI Cope, 1859. \\ Getendotaxa: BAtrachosepina Wake, 2012; Bolitoglossina Hallowell, 1856.
}

Comments: In TREE, the relationships within the BOLITOGLOSSINI are resolved and form a series of hierarchical family-series taxa. Thus, the subtribe BATRACHOSEPINA, with the single genus Batrachoseps, is sister branch to Bolitoglossina. This relationship has been revealed by Vieites et al. (2011), Pyron \& Wiens (2011), Shen et al. (2016) and Rovito \& Parra-Olea (2016). This subtribe Bolitoglossina includes the infratribes BOLITOGLOSSINIA and THORIINIA. Here we propose a resolved taxonomy using our rationale.

The content of genus-level taxa of the subtribe BoLITOGLOSSINA corresponds to those of the works of Rovito et al. (2015) and Rovito \& Parra-Olea (2016), except that here we recognise the subgenera of Oedipina as genera and that the relationships between the genera are in part different. Rovito et al. (2015) and Rovito \& Parra-Olea (2016) had a holophyletic group including (Parvimolge + $(($ Isthmura + Aquiloeurycea $)+$ Bolitoglossa $)+($ Ixalotriton + Pseudoeurycea $))$, whereas in the infratribe BOLITOGLOSSINIA of TREE Bolitoglossa is the sister-branch to all other genera. In our taxonomy the grouping (Isthmura + Aquiloeurycea) is the only supported one within this infratribe and recognised as the clan ISTHMURITES. The sister-taxon THORIINIA of our BOLITOGLOSSINIA is not holophyletic in the studies of Rovito et al. (2015) and Rovito \& Parra-Olea (2016) where Chiropterotriton and Thorius are sistergroups of BoLITOGLOSSINIA. These authors found a holophyletic group (Dendrotriton + (Cryptotriton 
$+($ Nyctanolis $+($ Nototriton $+($ Bradytriton $+("$ "Oeditriton" $+($ Oedopinola + Oedipina $))))))$. The relationship Nyctanolis $+($ Nototriton $+($ Bradytriton $+($ Thornella $+($ Oedopinola + Oedipina $)))))$ is also supported in TREE and named as the clan THORNELLITES. The works on significant samples of this very speciose group are still in its beginnings and more data are needed to confirm phylogenetic relationships and taxonomic decisions.

\section{F.20.80. Subtribus BATRACHOSEPINA Wake, 2012}

Protonym: BATRACHOSEPINI Wake, 2012: 76 [T].

Eunym: Hoc loco.

Getangiotaxon: BolitoglossinI Hallowell, 1856.

Adelphotaxon: BoLITOGLOSSINA Hallowell, 1856.

Getendotaxon: Batrachoseps Bonaparte, 1839.

\section{F.20.81. Subtribus Bolitoglossina Hallowell, 1856}

Eunym: Hoc loco.

Getangiotaxon: BoLItoglossini Hallowell, 1856.

Adelphotaxon: BATRACHOSEPINA Wake, 2012.

Getendotaxa: Bolitoglossinia Hallowell, 1856; Thorinna Cope, 1869.

\section{F.21.58. Infratribus BoLITOGLOSSINIA Hallowell, 1856}

Eunym: Hoc loco.

Getangiotaxon: Bolitoglossina Hallowell, 1856.

Adelphotaxon: ThoriInIa Cope, 1869.

Getendotaxa: BolitoglossinoA Hallowell, 1856; ISTHMURINOA nov.

\section{F.22.37. Hypotribus BoLITOGLOSSINOA Hallowell, 1856}

Eunym: Hoc loco.

Getangiotaxon: BoLITOGLOSSINIA Hallowell, 1856.

Adelphotaxon: ISTHMURINOA nov.

Getendotaxon: Bolitoglossa Duméril, Bibron \& Duméril, 1854.

\section{F.22.38. Hypotribus ISTHMURINOA nov.}

Getangiotaxon: BoLITOGLOSSINIA Hallowell, 1856.

Adelphotaxon: Bolitoglossinoa Hallowell, 1856.

Getendotaxa: IsthmuRITES nov.; PARVIMolgITES nov.; PSEUdoeuryCEITES nov.

Nucleogenus, by present designation: Isthmura Dubois \& Raffaëlli, 2012. • Etymology of nomen: G: ì $\sigma \mu$ ó $($ (isthmos), 'isthmus', which evokes the constricted basis of the tail of these salamanders; oüpó, oura, 'tail'. • Stem of nomen: Isthmur-.

Diagnosis: Diminutive to very large sized plethodontids; body stout to slender; limbs and toes short to long; tails moderate to very long; webbing on hands and feet rudimentary to moderate; columella absent or present. \{Wake \& Elias 1983; Parra-Olea et al. 2005; Rovito et al. 2015\}. 


\section{F.23.22. Clanus ISTHMURITES-nov.}

Getangiotaxon: ISTHMURINOA nov.

Adelphotaxa: PARVIMolgItes nov.; PSEUDOEURYCEITES nov.

Getendotaxa: Aquiloeurycea Rovito, Parra-Olea, Recuero \& Wake, 2015; Isthmura Dubois \& Raffaëlli, 2012.

\section{F.23.23. Clanus PARVIMOLGITES nov.}

Getangiotaxon: ISTHMURINOA nov.

Adelphotaxa: ISTHMURITES nov.; PSEUDOEURYCEITES nov.

Getendotaxa: Ixalotriton Wake \& Johnson, 1989; Parvimolge Taylor, 1944.

Nucleogenus, by present designation: Parvimolge Taylor, 1944. • Etymology of nomen: L: parvus, 'small'; N: Molge Merrem, 1820, derived from L: molge, modern Latin from German Molch, 'amphibian'.

- Stem of nomen: Parvimolg-.

Diagnosis: Diminutive to relatively large salamanders; body rather strong; limbs, toes and tail relatively short to long; webbing on hand and foot moderate; teeth on maxilla, premaxilla and mandible present; premaxilla single or fused; sublingual fold present. \{Taylor 1944; Wake \& Johnson 1989\}.

\section{F.23.24. Clanus PSEUDOEURYCEITES nov.}

Getangiotaxon: ISTHMURINOA nov.

Adelphotaxa: IsCTHMURITES nov.; PARVIMOLGITES nov.

Getendotaxon: Pseudoeurycea Taylor, 1944.

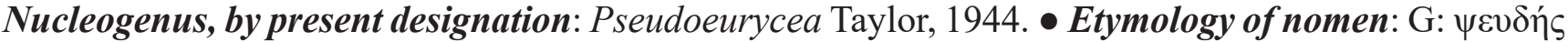

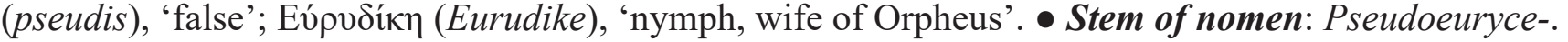

Diagnosis: Salamanders with middle digits of and foot free or with rudimentary webbing; vertebral articulation intermediate, lacking any trace of a rounded, terminal condyle; teeth on maxilla, premaxilla and mandible, pleurodont; premaxilla single, with frontal processes on a slight elevation; frontopremaxillary fontanelle well defined; columella absent from operculum; parasphenoid lacking a lateral notch; no septomaxilla; no lateral spine on posterior parts of centra, except on atlas; presence of a sublingual fold. \{Taylor 1944\}.

F.21.59. Infratribus THORIINIA Cope, 1869

Protonym: THORIIDAE Cope, 1869: 110 [F].

Eunym: Hoc loco.

Getangiotaxon: Bolitoglossina Hallowell, 1856.

Adelphotaxon: Bolitoglossinia Hallowell, 1856.

Getendotaxa: THORIINOA Cope, 1869; THORNELLINOA nov.

F.22.39. Hypotribus THORIINOA Cope, 1869

Eunym: Hoc loco.

Getangiotaxon: THORIINIA Cope, 1869.

Adelphotaxon: THORNELLINOA nov.

Getendotaxa: Chiropterotriton Taylor, 1944; Cryptotriton García-París \& Wake, 2000; Thorius Cope, 1869. 


\section{F.22.40. Hypotribus THORNELLINOA nov.}

Getangiotaxon: THORIINIA Cope, 1869.

Adelphotaxon: THORIINOA Cope, 1869.

Getendotaxa: DENDROTRITONITES nov.; NYCTANOLITES nov.; THORNELLITES nov.

Nucleogenus, by present designation: Thornella nov. • Etymology of nomen: P: Robert Thorn (19252011), Luxembourg specialist of salamanders; L: -ella, a feminine suffix indicating a diminutive form.

- Stem of nomen: Thornell-.

Diagnosis: Small to large sized plethodontid salamanders; body slender to stout, short or long; tail rounded, but also compressed or rectangular; legs long or short; hands and feet small but also broad; digits rounded, blunt, rarely broad tipped; premaxillary fused, rarely not fused; sublingual fold present; ulnare and intermedium fused or not fused; tarsals four and five fused or not fused; vertebrae short; prefrontals present or absent; tibial spurs present or absent; biology terrestrial, but also arboreal or fossorial. \{Wake \& Elias 1983; García-París \& Wake 2000; McCranie et al. 2008\}.

\section{F.23.25. Clanus DENDROTRITONITES nov.}

Getangiotaxon: THORNELLINOA nov.

Adelphotaxa: NYCTANOLITES nov.; THORNELLITES nov.

Getendotaxon: Dendrotriton Wake \& Elias, 1983.

Nucleogenus, by present designation: Dendrotriton Wake \& Elias, 1983. • Etymology of nomen: G:

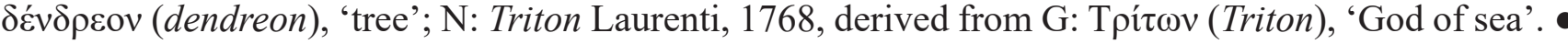
Stem of nomen: Dendrotriton-.

Diagnosis: Small sized plethodontid salamanders; body slender, short; tail long, rounded; legs long; hands and feet broad; digits long, broad-tipped; premaxillary simple; sublingual fold present; ulnare and intermedium not fused; tarsals four and five not fused; vertebrae short; prefrontals absent; tibial spurs absent; arboreal. \{Wake \& Elias 1983 \}.

\section{F.23.26. Clanus NyCTANOLITES nov.}

Getangiotaxon: THORNELLINOA nov.

Adelphotaxa: DENDROTRITONITES nov.; THORNELLITES nov.

Getendotaxon: Nyctanolis Elias \& Wake, 1983.

Nucleogenus, by present designation: Nyctanolis Elias \& Wake, 1983. • Etymology of nomen: G:

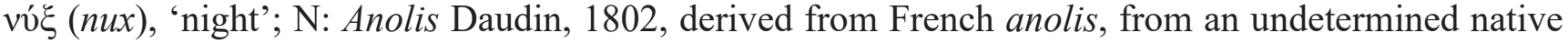
Caribbean language anoalli, anoli. • Stem of nomen: Nyctanoli-.

Diagnosis: Large sized plethodontid salamanders; body short, rather thin; tail long, rounded; legs, hands and feet long; digits blunt, slightly enlarged; premaxillary double; sublingual fold present; ulnare and intermedium not fused; tarsals four and five not fused; vertebrae short; prefrontals present; tibial spurs present; terrestrial and arboreal. \{Wake \& Elias 1983\}.

\section{F.23.27. Clanus THORNELLITES nov.}

Getangiotaxon: THORNELLINOA nov.

Adelphotaxa: DENDROTRITONITES nov.; NYCTANOLITES nov.

Getendotaxa: THORNELLITIES nov.; NOTOTRITONITIES nov. 


\section{F.24.16. Subclanus THORNELLITIES nov.}

Getangiotaxon: THORNELLITES nov.

Adelphotaxon: NototritoNities nov.

Getendotaxa: BRADYTRITONITOES nov.; THORNELLITOES nov.

\section{F.25.22. Infraclanus BRADYTRITONITOES nov.}

Getangiotaxon: THORNELLITIES nov.

Adelphotaxon: THORNELLITOES nov.

Getendotaxon: Bradytriton Wake \& Elias, 1983.

Nucleogenus, by present designation: Bradytriton Wake \& Elias, 1983. • Etymology of nomen: G:

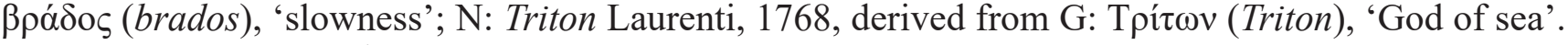

- Stem of nomen: Bradytriton-.

Diagnosis: Medium sized plethodontid salamanders; body stout; tail strongly compressed; legs short; hands and feet small; digits blunt; premaxillaries fused; sublingual fold present; ulnare and intermedium fused; tarsals four and five fused; vertebrae short; prefrontals present; tibial spurs present; terrestrial. \{Wake \& Elias 1983\}.

\section{F.25.23. Infraclanus THORNELLITOES nov.}

Getangiotaxon: THORNELLITIES nov.

Adelphotaxon: BRADYTRITONITOES nov.

Getendotaxa: OEDIPINITUES nov.; THORNELLITUES nov.

\section{F.26.13. Hypoclanus OEDIPINITUES nov.}

Getangiotaxon: THORNELLITOES nov.

Adelphotaxon: THORNELLITUES nov.

Getendotaxa: Oedipina Keferstein, 1868; Oedopinola Hilton, 1946.

Nucleogenus, by present designation: Oedipina Keferstein, 1868. • Etymology of nomen: G: oídítov $\varsigma$ (oidipous), 'swollen foot'; -ina, feminine suffix. • Stem of nomen: Oedipin-.

Diagnosis: Medium to large sized plethodontid salamanders; body long; tail long, rounded; legs relatively long; hands and feet small; premaxillary single; sublingual fold present; ulnare and intermedium fused; tarsals four and five fused; vertebrae short; prefrontals absent; tibial spurs absent; semi-fossorial or fossorial species. \{Wake \& Elias 1983; García-París \& Wake 2000\}.

\section{F.26.14. Hypoclanus THORNELLITUES nov.}

Getangiotaxon: THORNELLITOES nov. Adelphotaxon: OEDIPINITUES nov.

Getendotaxon: Thornella nov.

G.28.533. Genus Thornella nov.

Getangiotaxon: THORNELLITUES nov.

Adelphotaxon: None. 
Getendotaxa: Thornella kasios (McCranie, Vieites \& Wake, 2008); Thornella nica (Sunyer, Wake, Townsend, Travers, Rovito, Papenfuss, Obando \& Köhler, 2010); Thornella quadra (McCranie, Vieites \& Wake, 2008).

Nucleospecies, by present designation: Oedipina (Oeditriton) quadra McCranie, Vieites \& Wake, 2008. - Etymology of nomen: P: Robert Thorn (1925-2011), Luxembourg specialist of salamanders; L: -ella, a feminine suffix indicating a diminutive form. $\bullet$ Stem of nomen: Thornell-. • Grammatical gender of nomen: feminine.

Diagnosis: Small to medium sized (SVL 33-56 mm) plethodontid salamanders; body long and slender; tail very long, about twice body length, nearly rectangular or round in cross section; eyes directed frontolaterally; mental glands of males inconspicuous; suborbital groove not intercepting lip line; hands and feet tiny, narrow, elongate; digital tips rouned, blunt, with weak subdigital pads; coloration uniformly dark or with tiny light dots. \{McCranie et al. 2008; Sunyer et al. 2010\}.

Comments: • McCranie et al. (2008) proposed the nomen "Oeditriton " for this taxon (established as a subgenus) without designating a type species for it. The nomen is therefore nomenclaturally unavailable according to Article 13.3 of the Code. Here we propose formally a new nomen for this taxon.

\title{
F.24.17. Subclanus Nototritonities nov.
}

\author{
Getangiotaxon: THORNELLITES nov. \\ Adelphotaxon: THORNELLITIES nov. \\ Getendotaxon: Nototriton Wake \& Elias, 1983.
}

Nucleogenus, by present designation: Nototriton Wake \& Elias, 1983. • Etymology of nomen: G:

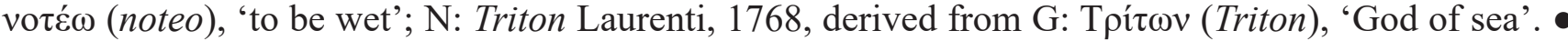
Stem of nomen: Nototriton-.

Diagnosis: Small sized plethodontid salamanders; body slender, trunc short; tail long, rounded; legs moderately long to short; hands and feet small; digits short, not enlarged; premaxillary fused; sublingual fold present; ulnare and intermedium fused; tarsals four and five fused; vertebrae short; prefrontals present; tibial spurs present; biology arboreal, terrestrial or semifossorial. \{Wake \& Elias 1983\}.

\section{F.19.82. Tribus HEMIDACTYLIINI Hallowell, 1856}

\author{
Eunym: Wake 1966: 1. \\ Getangiotaxon: HeMIDACTYLIINAE Hallowell, 1856. \\ Adelphotaxa: Bolitoglossini Hallowell, 1856; SPELERPINI Cope, 1859. \\ Getendotaxon: Hemidactylium Tschudi, 1838.
}

\section{F.19.83. Tribus SPELERPINI Cope, 1859}

Protonym: SPELERPINAE Cope, 1859: 123 [bF].

Eunym: Dubois 2005: 20.

Getangiotaxon: HeMIDACTYLIINAE Hallowell, 1856.

Adelphotaxa: Bolitoglossini Hallowell, 1856; HemidaCtyLini Hallowell, 1856.

Getendotaxa: PSEUdotritonina Dubois \& Raffaelli, 2012; SPELERPINA Cope, 1859.

Comments: The group here named tribe SPELERPINI was recognised in all molecular studies of Plethodontidae (Mueller et al. 2004; Chippindale et al. 2004; Macey 2005; Vieites et al. 2007, 2011; Camp et al. 2009; Kozak et al. 2009; Chen G. et al. 2011; Pyron \& Wiens 2011; Shen et al. 2016). In TREE, it shows two highly supported branches which are here allocated to the subtribe PSEUDOTRITONINA, including the genera Gyrinophilus, Pseudotriton and Stereochilus, with unresolved 
mutual relationships, and the subtribe SPELERPINA for Eurycea and Urspelerpes. The lineage here named the subtribe PSEUDOTRITONINA has been revealed by previous studies, which also recognised Eurycea as its sister-taxon (Mueller et al. 2004; Chippindale et al. 2004; Macey 2005; Vieites et al. 2007, 2011; Camp et al. 2009; Kozak et al. 2009; Chen G. et al. 2011; Pyron \& Wiens 2011). There is no consensus on the relative position of the other genus-series taxa.

\section{F.20.82. Subtribus PSEUDOTRITONINA Dubois \& Raffaëlli, 2012}

Protonym and eunym: PSEUdotRITONINA Dubois \& Raffaëlli, 2012: 115 [bT].

Getangiotaxon: SPELERPINI Cope, 1859.

Adelphotaxon: SPELERPINA Cope, 1859.

Getendotaxa: Gyrinophilus Cope, 1869; Pseudotriton Tschudi, 1838; Stereochilus Cope, 1869.

\section{F.20.83. Subtribus SPELERPINA Cope, 1859}

Eunym: Hoc loco.

Getangiotaxon: SPELERPINI Cope, 1859.

Adelphotaxon: PSEUdotRItonina Dubois \& Raffaelli, 2012.

Getendotaxa: Eurycea Rafinesque, 1822; Urspelerpes Camp, Peterman, Milanovich, Lamb, Maerz \& Wake, 2009.

\section{F.18.84. Subfamilia PlethodontinaE Gray, 1850}

Eunym: Boulenger 1882: vii, 51.

Getangiotaxon: PLETHODONTIDAE Gray, 1850.

Adelphotaxa: HeMIDACTYLIINAE Hallowell, 1856; 1 G†.

Getendotaxa: Hydromantini Wake, 2012; Plethodontini Gray, 1850.

Comments: The subfamily PlETHODONTINAE includes two tribes, the HydRomantini, with the subtribe HYDROMANTINA for Hydromantes and Speleomantes, the subtribe KARSENIINA for Karsenia, and the tribe PleTHODONTINI. Within this latter tribe, the subtribe DESMOGNATHINA holds the infratribe ANEIDINIA for Aneides, and the infratribe DESMOGNATHINIA for Desmognathus and Phaeognathus, whereas the subtribe ENSATININA includes the single genus Ensatina.

The relationships within this subfamily have not attained an agreement and various hypotheses on the relationships have been published. This may be a consequence of taxon sampling, as few works have representatives of all genera in their analysis. Wake (2012) recognised five tribes within the subfamily: the ANEIDINI for Aneides, the DESMOGNATHINI for Desmognathus and Phaeognathus, the ENSATINI for Ensatina, the HYDROMANTINI for Hydromantes (including the subgenera Atylodes, Hydromantes and Speleomantes) and Karsenia, and the PLETHODONTINI for Plethodon (with the subgenera Hightonia and Plethodon). The sister-group relationship of Desmognathus and Phaeognathus was revealed in most studies (Mueller et al. 2004; Chippindale et al. 2004; Macey 2005; Vieites et al. 2007, 2011; Camp et al. 2009; Kozak et al. 2009; Pyron \& Wiens 2011; Chen G. et al. 2011). The taxon HydRomanTINI was resolved by Vieites et al. (2007) as in TREE, but there is no support for Karsenia being sistertaxon to Hydromantes and Speleomantes in Vieites et al. (2011), Pyron \& Wiens (2011) and Shen et al. (2016), the other works that included this genus. The relationship Ensatina + (Desmognathus + Phaeognathus) has poor support, therefore their relation with Plethodon is not resolved and within the PLETHODONTINI three subtribes are here recognised. A similar arrangement had been obtained in some works (Chippindale et al. 2004; Vieites et al. 2007) but in other works Plethodon shows very different sister-group relationships. It is sister-group to all other PLETHODONTINAE in a number of phylogenies (Mueller et al. 2004; Macey 2005; Camp et al. 2009; Kozak et al. 2009; Pyron \& Wiens 2011; Chen G. Y. et al. 2011; Shen et al. 2016) but sister-group to Phaeognathus and Desmognathus in Vieites et al. (2011). 
Protonym and eunym: HYDROMANTINI Wake, 2012: 80 [T].

Getangiotaxon: PLETHODONTINAE Gray, 1850.

Adelphotaxon: Plethodontini Gray, 1850.

Getendotaxa: Hydromantina Wake, 2012; KARSENIINA Dubois \& Raffaelli, 2012.

\section{F.20.84. Subtribus HYDROMANTINA Wake, 2012}

Eunym: Hoc loco.

Getangiotaxon: HYDROMANTINI Wake, 2012.

Adelphotaxon: KARSENIINA Dubois \& Raffaelli, 2012.

Getendotaxa: Hydromantes Gistel, 1848; Speleomantes Dubois, 1984.

\section{F.20.85. Subtribus KaRSENIINA Dubois \& Raffaëlli, 2012}

Protonym: KARSENIINI Dubois \& Raffaëlli, 2012: 117 [T].

Eunym: Dubois \& Raffaëlli 2012: 118.

Getangiotaxon: HYDROMANTINI Wake, 2012.

Adelphotaxon: HydRomantiNA Wake, 2012.

Getendotaxon: Karsenia Min, Yang, Bonett, Vieites, Brandon \& Wake, 2005.

\section{F.19.85. Tribus Plethodontini Gray, 1850}

Eunym: Wake 1966: 1.

Getangiotaxon: PLETHODONTINAE Gray, 1850.

Adelphotaxon: HYDROMANTINI Wake, 2012.

Getendotaxa: Desmognathina Gray, 1850; Ensatinina Gray, 1850; Plethodontina Gray, 1850.

\section{F.20.86. Subtribus Desmognathina Gray, 1850}

Protonym: Desmognathina Gray, 1850: 40 [UF].

Eunym: Hoc loco.

Getangiotaxon: PLETHODONTINI Gray, 1850.

Adelphotaxa: ENSATININA Gray, 1850; PLETHODONTINA Gray, 1850.

Getendotaxa: ANEIDINIA Wake, 2012; DESMOGNATHINIA Gray, 1850.

F.21.60. Infratribus ANEIDINIA Wake, 2012

Protonym: ANEIDINI Wake, 2012: 79 [T].

Eunym: Hoc loco.

Getangiotaxon: DESMOGNATHINA Gray, 1850.

Adelphotaxon: Desmognathinia Gray, 1850.

Getendotaxon: Aneides Baird, 1851.

F.21.61. Infratribus DESMogNathinia Gray, 1850

Eunym: Hoc loco.

Getangiotaxon: Desmognathina Gray, 1850. 
Adelphotaxon: ANEIDINIA Wake, 2012.

Getendotaxa: Desmognathus Baird, 1850; Phaeognathus Highton, 1961.

F.20.87. Subtribus ENSATININA Gray, 1850

Protonym: ENSATININA Gray, 1850: 48 [UF].

Eunym: Hoc loco.

Getangiotaxon: PLETHODONTINI Gray, 1850.

Adelphotaxa: Desmognathina Gray, 1850; Plethodontina Gray, 1850.

Getendotaxon: Ensatina Gray, 1850.

\section{F.20.88. Subtribus Plethodontina Gray, 1850}

Eunym: Hoc loco.

Getangiotaxon: PLETHODONTINI Gray, 1850.

Adelphotaxa: Desmognathina Gray, 1850; ENSATININA Gray, 1850.

Getendotaxon: Plethodon Tschudi, 1838.

F.16.09. Apofamilia RHYACOTRITONEIDAE Tihen, 1958

Protonym: RHYACotritoninae Tihen, 1958: 1 [bF].

Eunym: Hoc loco.

Getangiotaxon: AMPHIUMOIDAE Gray, 1825.

Adelphotaxon: AMPHIUMEIDAE Gray, 1825.

Getendotaxon: RHYACOTRITONIDAE Tihen, 1958.

F.17.66. Familia RHYACOTRITONIDAE Tihen, 1958

Eunym: Good \& Wake 1992: v, xi, 1, 13.

Getangiotaxon: RHYACOTRITONEIDAE Tihen, 1958.

Adelphotaxon: None.

Getendotaxon: Rhyacotriton Dunn, 1920.

F.15.12. Epifamilia PROTEOIDAE Bonaparte, 1831

Protonym: PROTEINA Bonaparte, 1831: 781 [UF].

Eunym: Dubois \& Raffaëlli 2012: 98.

Getangiotaxon: AMPHIUMOIDEA Gray, 1825.

Adelphotaxon: AMPHIUMOIDAE Gray, 1825.

Getendotaxon: PROTEIDAE Bonaparte, 1831.

\section{F.17.67. Familia PRoteIdAe Bonaparte, 1831}

Eunym: Hogg 1838: 152.

Getangiotaxon: PROTEOIDAE Bonaparte, 1831.

Adelphotaxon: None.

Getendotaxa: Necturus Rafinesque, 1819; Proteus Laurenti, 1768; 3 G†. 


\title{
F.14.18. Superfamilia SALAMANDROIDEA Goldfuss, 1820
}

\author{
Protonym: SALAMANDRAE Goldfuss, 1820: 11 [F]. \\ Eunym: Garman 1884: 37. \\ Getangiotaxon: Pseudosauria Blainville, 1816. \\ Adelphotaxa: AMPHIUMOIDEA Gray, 1825; 2 G†. \\ Getendotaxa: AmbYSTOMATIDAE Gray, 1850; SALAMANDRIDAE Goldfuss, 1820.
}

Comments: This superfamily includes two branches, both recognised at the family rank on account of the [UQC], the AmBYSTOMATIDAE for the genera Ambystoma and Dicamptodon, and the family SALAMANDRIDAE. Several recent authors (Wiens et al. 2005a; Weisrock et al. 2005; Vieites et al. 2009; Zhang et al.2009; Pyron \& Wiens 2011; Shen et al.2013) recognised a separate family DICAMPTODONTIDAE for the latter genus, either on the basis of a criterion of morphological divergence or to account for the hypothesised geological age of the cladogenesis that separated these two genera, but such phenetic or chronological criteria do not have to be taken into account in a cladonomy like that presented here, the aim of which is just to reflect as accurately as possible the structure of the cladogram supposed to describe the patterns of relationships between the taxa studied, irrespective of other considerations.

\section{F.17.68. Familia AmbYSTOMATIDAE Gray, 1850}

Protonym: АмвуSTомina Gray, 1850: 32 [UF].

Eunym: Hay 1892: 415.

Getangiotaxon: SALAMANDROIDEA Goldfuss, 1820.

Adelphotaxon: SALAMANDRIDAE Goldfuss, 1820.

Getendotaxa: Ambystoma Tschudi, 1838; Dicamptodon Strauch, 1870; 5 G†.

\section{F.17.69. Familia SALAMANDRIDAE Goldfuss, 1820}

Eunym: Gray 1825: 215.

Getangiotaxon: SALAMANDROIDEA Goldfuss, 1820.

Adelphotaxon: AMBYSTOMATIDAE Gray, 1850.

Getendotaxa: Pleurodelinae Tschudi, 1838; SALAMANDrinaE Goldfuss, 1820; SalamandrininaE Fitzinger, 1843.

Comments: Within the family SALAMANDRIDAE, three branches of unresolved mutual relationships are recognised as the subfamilies PLEURODELINAE, SALAMANDRINAE and SALAMANDRININAE. The latter includes a single genus, Salamandrina. These main groups find general agreement, although the relationships between these groups are not consistent.

Recently, Veith et al. (2018) published a historical analysis of the classification of SALAMANDRIDAE. They underlined several points of disagreement with Pyron (2014), in particular the usage of both mitochondrial and nuclear data in a single analysis and non representative sampling for some data. Nevertheless this is a general flaw in systematic studies as methods and taxa discoveries lead to forever changing hypotheses on phylogenetic relationships and the classifications based on these hypotheses.

\section{F.18.85. Subfamilia Pleurodelinae Tschudi, 1838}

Protonym: PLEURODELES Tschudi, 1838: 56 [F].

Eunym: Brame 1957: 2.

Getangiotaxon: SALAMANDRIDAE Goldfuss, 1820.

Adelphotaxa: SALAMANDRINAE Goldfuss, 1820; SALAMANDRININAE Fitzinger, 1843.

Getendotaxa: Molgini Bonaparte, 1850; PLEURodeLINI Tschudi, 1838; 9 G†.

Comments: Within the subfamily PLEURODELINAE, the taxon here recognised as the tribe MoLGINI is sister-taxon to the PLEURODELINI. 
The MolGINI include two subtribes, the TARICHINA, including the genera Notophthalmus and Taricha, and the MOLGINA. In the MOLGINA, two branches are recognised as the infratribes EUPROCTINIA for Euproctus, and MoLGINIA. The relationships of the latter are unresolved and four hypotribes are recognised: the CYNOPINOA, including the clans CYNOPITES for Cynops, HyPSELOTRITONITES for Hypselotriton and PACHYTRITONITES for Laotriton, Pachytriton and Paramesotriton; the ICHTHYOSAURINOA for Ichthyosaura; the LISSOTRITONINOA for Lissotriton; and the MOLGINOA for the clans MoLGITES for Calotriton and Triturus, and NEURERGITES for Neurergus and Ommatotriton.

Within the tribe PLEURODELINI, the subtribe PLEURODELINA, for Pleurodeles, is sister-taxon to the TYLOTOTRITONINA, containing the infratribes ECHINOTRITONINIA for Echinotriton, and TYLOTOTRITONINIA for Tylototriton and Yaotriton.

The relationships within the PLEURODELINAE are much more discussed. Zhang et al. (2008) attributed nomina to some of their groups that Veith et al. (2018) used for the analysis of the relationships within this subfamily. Nevertheless these groups are not homologous by sister-group relationships and thus should not be recognised at similar taxonomic groups in a formal classification. All molecular studies obtain a dichotomy within the subfamily which corresponds to the PLEURODELINI, their 'primitive newts', and the MoLGINI. Within this latter group, the relationships are much disputed although several holophyletic subgroups are informally recognised. Thus the 'modern Asian newts', our CYNOPINOA, as well as the 'New World newts', our TARICHINA, are highly supported holophyletic groups (Titus \& Larson 1995; Weisrock et al. 2006; Steinfartz et al. 2007; Zhang et al. 2008; Chen G. et al. 2011; Pyron \& Wiens 2011). Within the CYNOPINOA, in TREE, no sufficient support for the holophyly of Cynops sensu lato has been found (see also Weisrock et al. 2006; Zhang et al. 2008), thus requiring to recognise two genera Cynops and Hypselotriton of poorly supported relationships (Dubois \& Raffaëlli 2009). In TREE, the holophyly of 'European modern newts' does not have sufficient support, but such a group has been revealed by Zhang et al. (2008), Chen G. et al. (2011) and Veith et al. (2018). In other studies this group is still paraphyletic (Titus \& Larson 1995; Weisrock et al. 2006; Steinfartz et al. 2007; Pyron \& Wiens 2011).

F.19.86. Tribus MolgINI Bonaparte, 1850

Protonym: MolginA Bonaparte, 1850: plate [bF].

Eunym: Dubois \& Raffaëlli 2012: 30.

Getangiotaxon: PLEURODELINAE Tschudi, 1838.

Adelphotaxa: PleurodelinI Tschudi, 1838; 9 G†.

Getendotaxa: Molgina Bonaparte, 1850; TARICHINA Dubois \& Raffaelli, 2009.

F.20.89. Subtribus MoLGINA Bonaparte, 1850

Eunym: Dubois \& Raffaëlli 2012: 30.

Getangiotaxon: MoLGINI Bonaparte, 1850.

Adelphotaxon: TARICHINA Dubois \& Raffaelli, 2009.

Getendotaxa: EuproctinIA Dubois \& Raffaelli, 2009; MolginIA Bonaparte, 1850.

F.21.62. Infratribus EUPROCTINIA Dubois \& Raffaëlli, 2009

Protonym: EuPRoctita Dubois \& Raffaëlli, 2009: 50 [iT].

Eunym: Hoc loco.

Getangiotaxon: MolgINA Bonaparte, 1850.

Adelphotaxon: MolginIA Bonaparte, 1850.

Getendotaxon: Euproctus Gené, 1839. 
Eunym: Hoc loco.

Getangiotaxon: MoLGINA Bonaparte, 1850.

Adelphotaxon: EUPROCTINIA Dubois \& Raffaelli, 2009.

Getendotaxa: CyNOPINOA Dubois \& Raffaelli, 2009; ICHTHYOSAURINOA nov.; LiSSOTRITONINOA nov.; MoLGINOA Bonaparte, 1850.

\section{F.22.41. Hypotribus CYNOPINOA Dubois \& Raffaëlli, 2009}

Protonym: CYNOPITA Dubois \& Raffaëlli, 2009: 44 [iT].

Eunym: Hoc loco.

Getangiotaxon: MOLGINIA Bonaparte, 1850.

Adelphotaxa: ICHTHYosaURINOA nov.; LISSOTRITONINOA nov.; MolGINOA Bonaparte, 1850.

Getendotaxa: CYNopites Dubois \& Raffaelli, 2009; HyPSELOTRITONITES nov.; PACHYTRITONITES nov.

\section{F.23.28. Clanus CYNOPITES Dubois \& Raffaelli, 2009}

Eunym: Hoc loco.

Getangiotaxon: CYNOPINOA Dubois \& Raffaelli, 2009.

Adelphotaxa: HyPSELOTRITONITES nov.; PACHYTRITONITES nov.

Getendotaxon: Cynops Tschudi, 1838.

\section{F.23.29. Clanus HyPSELOTRITONITES nov.}

Getangiotaxon: CYNOPINOA Dubois \& Raffaelli, 2009.

Adelphotaxa: CyNOPITES Dubois \& Raffaelli, 2009; PACHYTRITONITES nov.

Getendotaxon: Hypselotriton Wolterstorff, 1934.

Nucleogenus, by present designation: Hypselotriton Wolterstorff, 1934. • Etymology of nomen: G:

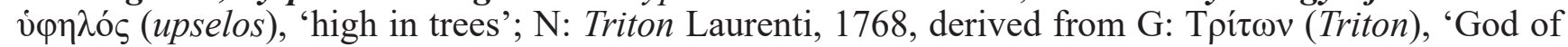
sea'. Stem of nomen: Hypselotriton-.

Diagnosis: Rather small sized newts with strongly developed sexual size dimorphism (males TL 70$120 \mathrm{~mm}$; females TL 90-160 mm); body high and laterally compressed; tail poorly differentiated from body; parotoid glands poorly developed; no vertebral crest or ridge; skin smooth or slightly rugose; premaxillary unique with short posterior processus; no internasal cavity; fronto-squamosal arc ossified rather strongly developed; paroccipital processes present; tongue small, slightly free on sides. \{Thorn 1969; Raffaëlli 2013\}.

\section{F.23.30. Clanus PACHYTRITONITES nov.}

Getangiotaxon: CYNOPINOA Dubois \& Raffaelli, 2009.

Adelphotaxa: CYNOPITES Dubois \& Raffaelli, 2009; HyPSELOTRITONITES nov.

Getendotaxa: Laotriton Dubois \& Raffaelli, 2009; Pachytriton Boulenger, 1878; Paramesotriton Chang, 1936.

Nucleogenus, by present designation: Pachytriton Boulenger, 1878. • Etymology of nomen: G: $\pi \alpha \chi v ́ \varsigma$

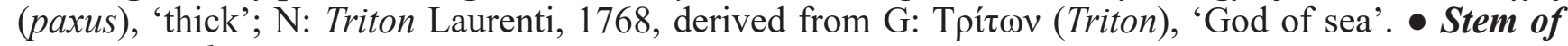
nomen: Pachytriton-.

Diagnosis: Small to large sized newts (TL 130-250 mm); habitus stout; skull wide; usually 12 vertebrae, but varying from 11 to 13; parotoids prominent; skin granular or smooth; vertebral ridge prominent or absent; lateral ridges present or absent; coloration dull, rarely bright. \{Dubois \& Raffaëlli 2009 . 


\section{F.22.42. Hypotribus ICHTHYOSAURINOA nov.}

Getangiotaxon: MoLGINIA Bonaparte, 1850.

Adelphotaxa: CynopinoA Dubois \& Raffaelli, 2009; LisSotritoninoA nov.; MolginoA Bonaparte, 1850.

Getendotaxon: Ichthyosaura Sonnini \& Latreille, 1801.

Nucleogenus, by present designation: Ichthyosaura Sonnini \& Latreille, 1801. • Etymology of nomen:

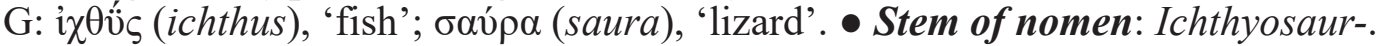

Diagnosis: Relatively small sized newts (males TL 80-100 mm; females TL 100-120 mm); frontosquamosal arc ossified, poorly developed; paroccipital processes poorly prominent; internasal cavity elongate and large; posterior process of premaxillary narrow and short; posterior process of axillary short; dermal crest on dorsum entire, not serrated; skin smooth or rugose on dorsum, completely smooth on ventral side; gular fold distinct; prominent sexual color dimorphism with males showing black and white pattern on dermal crest and flanks; ventral coloration orange or red in both sexes. \{Boulenger 1910; Thorn 1969\}.

\section{F.22.43. Hypotribus LISSOTRITONINOA nov.}

Getangiotaxon: MoLGINIA Bonaparte, 1850.

Adelphotaxa: CYNOPINOA Dubois \& Raffaelli, 2009; ICHTHYosaURINOA nov.; MolginoA Bonaparte, 1850.

Getendotaxon: Lissotriton Bell, 1839.

Nucleogenus, by present designation: Lissotriton Bell, 1839. • Etymology of nomen: G: $\lambda 1 \sigma \sigma o ́ s$

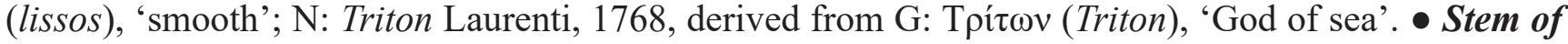
nomen: Lissotriton-.

Diagnosis: Small sized newts (males TL 45-110 mm; females TL 55-100 mm); fronto-squamosal arc strongly ossified or partly developed; paroccipital processes well developed; internasal fontanella large and oval; posterior process of premaxillary long and divided posteriorly in two branches surrounding internasal cavity. \{Bolkay 1928; Thorn 1969\}.

\section{F.22.44. Hypotribus MoLGINOA Bonaparte, 1850}

Eunym: Hoc loco.

Getangiotaxon: MoLGINIA Bonaparte, 1850.

Adelphotaxa: CYNOPINOA Dubois \& Raffaelli, 2009; ICHTHYOSAURINOA nov.; LISSOTRITONINOA nov. Getendotaxa: MoLGITES Bonaparte, 1850; NEURERGITES nov.

\section{F.23.31. Clanus Molgites Bonaparte, 1850}

Eunym: Hoc loco.

Getangiotaxon: MoLGINOA Bonaparte, 1850.

Adelphotaxon: NEURERGITES nov.

Getendotaxa: Calotriton Gray, 1858; Triturus Rafinesque, 1815.

\section{F.23.32. Clanus NEURERGITES nov.}

Getangiotaxon: MoLGINOA Bonaparte, 1850.

Adelphotaxon: MoLGITES Bonaparte, 1850.

Getendotaxa: Neurergus Cope, 1862; Ommatotriton Gray, 1850. 
Nucleogenus, by present designation: Neurergus Cope, 1862. • Etymology of nomen: G: vē̄pov (neuron), 'tendon'; '̌p $\gamma \omega$ (ergo), 'to shut in', referring to the fronto-parietal which is replaced by a ligament (Cope 1862). • Stem of nomen: Neurerg-.

Diagnosis: Medium to large sized newts (TL 140-190 mm); habitus stout, body flattened; sexual dimorphism moderate; premaxillary unique; fronto-squamosal arc ossified, incomplete; posterior processus of maxillary long, separated or linked to pterygoid; two series of vomero-palatine teeth anteriorly converging and diverging immediately to the posterior from this point; tongue rounded, small free on sides and on posterior part; parotoid glands scarcely distinct; tail long and compressed; reproduction in lotic or lentic habitat. \{Thorn 1969; Dubois \& Raffaëlli 2009\}.

F.20.90. Subtribus TARICHINA Dubois \& Raffaëlli, 2009

Protonym and eunym: TARICHINA Dubois \& Raffaëlli, 2009: 57 [bT].

Getangiotaxon: MoLGINI Bonaparte, 1850.

Adelphotaxon: MoLGINA Bonaparte, 1850.

Getendotaxa: Notophthalmus Rafinesque, 1820; Taricha Gray, 1850.

F.19.87. Tribus PleURodelini Tschudi, 1838

Eunym: Dubois \& Raffaëlli 2009: 30

Getangiotaxon: PLEURODELINAE Tschudi, 1838.

Adelphotaxa: MolgINI Bonaparte, 1850; 9 G†.

Getendotaxa: Pleurodelina Tschudi, 1838; TyLOtotritonina nov.

F.20.91. Subtribus PLEURODELINA Tschudi, 1838

Eunym: Hoc loco.

Getangiotaxon: PLEURODELINI Tschudi, 1838.

Adelphotaxon: TYLOTOTRITONINA nov.

Getendotaxon: Pleurodeles Michahelles, 1830.

F.20.92. Subtribus TYLOTOTRITONINA nov.

Getangiotaxon: PLEURODELINI Tschudi, 1838.

Adelphotaxon: PLEURODELINA Tschudi, 1838.

Getendotaxa: ECHINOTRITONINIA nov.; TYLOTOTRITONINIA nov.

Nucleogenus, by present designation: Tylototriton Anderson, 1871. • Etymology of nomen: G: $v \dot{\lambda} \lambda \mathrm{s}$

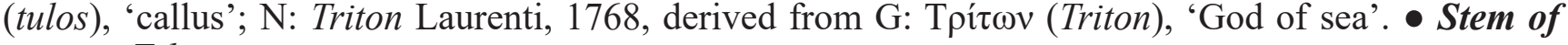
nomen: Tylototriton-.

Diagnosis: Small to medium sized salamanders (TL 120-230 mm); habitus stout; cephalic ridges present; vertebral ridge present; dorsal coloration dark, or with bright coloration; ventral coloration dark or light; aquatic during breeding, or completely terrestrial; eggs rather small to large; deposited in water or on land. \{Nussbaum \& Brodie 1982; Dubois \& Raffaëlli 2009; Raffaëlli 2013 \}.

F.21.64. Infratribus ECHINOTRITONINIA nov.

Getangiotaxon: TYLOTOTRITONINA nov.

Adelphotaxon: TYLOTOTRITONINIA nov. 
Getendotaxon: Echinotriton Nussbaum \& Brodie, 1982.

Nucleogenus, by present designation: Echinotriton Nussbaum \& Brodie, 1982. • Etymology of nomen:

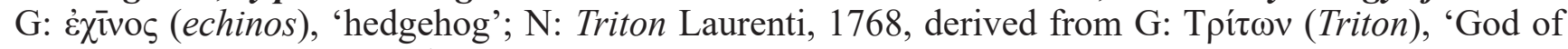
sea'. Stem of nomen: Echinotriton-.

Diagnosis: Medium sized salamanders (TL 130-160 mm); body stout; cephalic ridges poorly developed; vertebral ridge moderate; dorsal and ventral coloration dark; terrestrial, relatively large eggs (3.0$3.2 \mathrm{~mm}$ ) deposited on land; an anteriorly curved spine posterolaterally on each quadrate covered by enlarged granular glands; strong anterior ribs; ribs 3-9 elongated, sharp-tipped, and distally free of trunk musculature; ribs bearing $0-3$, usually one, dorsally projecting epipleural processes; one lateral row of large primary warts present; 0-3 medial rows of smaller secondary warts present. \{Nussbaum \& Brodie 1982; Dubois \& Raffaëlli 2009; Raffaëlli 2013\}.

\section{F.21.65. Infratribus TYLOTOTRITONINIA nov.}

Getangiotaxon: TYLOTOTRITONINA nov.

Adelphotaxon: ECHINOTRITONINIA nov.

Getendotaxa: Tylototriton Anderson, 1871; Yaotriton Dubois \& Raffaelli, 2009.

F.18.86. Subfamilia SALAMANDRINAE Goldfuss, 1820

Eunym: Cope 1859: 125.

Getangiotaxon: SALAMANDRIDAE Goldfuss, 1820.

Adelphotaxa: PleUrodelinaE Tschudi, 1838; SALAMANDRININAE Fitzinger, 1843.

Getendotaxa: Chioglossini Dubois \& Raffaelli, 2009; SALAMANDRINI Goldfuss, 1820; 1 G†.

Comments: Within the second branch of SALAMANDRIDAE, the subfamily SALAMANDRINAE, two branches of high support are recognised as the tribes CHIOGLOSSINI, for Chioglossa and Mertensiella, and SALAMANDRINI, for Lyciasalamandra and Salamandra. The relationship between the genera within this subfamily, called 'true salamanders' by Steinfartz et al. (2007), are consistent in most works (Titus \& Larson 1995; Weisrock et al. 2006; Steinfartz et al. 2007; Zhang et al. 2008; Chen G. et al. 2011; Pyron \& Wiens 2011; Veith et al. 2018).

F.19.88. Tribus CHIoglossini Dubois \& Raffaëlli, 2009

Protonym and eunym: CHIOGLOSSINI Dubois \& Raffaëlli, 2009: 60 [T].

Getangiotaxon: SALAMANDRINAE Goldfuss, 1820.

Adelphotaxa: SALAMANDRINI Goldfuss, 1820; 1 G†.

Getendotaxa: Chioglossa Bocage, 1864; Mertensiella Wolterstorff, 1925.

F.19.89. Tribus SALAMANDRINI Goldfuss, 1820

Eunym: Dubois \& Raffaëlli 2009: 60.

Getangiotaxon: SALAMANDRINAE Goldfuss, 1820.

Adelphotaxa: Chioglossini Dubois \& Raffaëlli, 2009; 1 G†.

Getendotaxa: Lyciasalamandra Veith \& Steinfartz, 2004; Salamandra Garsault, 1764. 


\title{
F.18.87. Subfamilia SALAMANDRININAE Fitzinger, 1843
}

\author{
Protonym: SALAMANDRINAE Fitzinger, 1843: 33 [F]. \\ Eunym: Dubois \& Raffaëlli 2009: 29. \\ Getangiotaxon: SALAMANDRIDAE Goldfuss, 1820. \\ Adelphotaxa: PleUrodelinaE Tschudi, 1838; SALAMANDRINAE Goldfuss, 1820. \\ Getendotaxon: Salamandrina Fitzinger, 1826.
}

Comments: Three recent works (Zhang et al. 2008; Pyron \& Wiens 2011; Veith et al. 2011) found the SALAMANDRININAE to be the sister-group to all other salamandrids but in TREE it is sister-group of PLEURODELINAE with a support value below our threshold value (SHL $84 \%$ ).

\section{Discussion AND CONCLUSION}

\subsection{Methodology}

This work had four basic aims: $\{\gamma 1\}$ to provide a new, explicit, consistent, rigorous and repeatable, methodology for the taxonomic and nomenclatural expression of a cladogenetic hypothesis in zoology; $\{\gamma 2\}$ to provide a new, updated, hypothesis of cladistic relationships among all the suprageneric taxa of extant amphibians, based on the sequencing of as many nuclear and mitochondrial genes from as many species as possible; $\{\gamma 3\}$ to provide an ergotaxonomy reflecting as closely and unambiguously as possible this phylogeny; $\{\gamma 4\}$ to provide a nomenclature following precise and consistent Rules and Criteria for this taxonomy. We have indeed reached these four aims. Following the explicit and rigorous methodology explained throughout the text above, we provided a cladogenetic hypothesis, a taxonomy and a nomenclature for all extant amphibians.

\subsubsection{Phylogeny}

We built a cladogram (TREE) based on a methodology which allows to produce a single tree on the basis of variable numbers of sequences, retrieved from Genbank, from 10 nuclear and 5 mitochondrial genes in specimens representing 4060 species currently considered valid in the literature. As with most studies using the 'supermatrix' approach to systematics (Queiroz \& Gatesy 2007) which combines multiple matrices of single genes (each from a single specimen) into a single, sparsely-sampled supermatrix, our terminals are 'composite', often consisting of gene sequences from more than one specimen. This means that the trees produced by such analyses are neither 'trees of specimens' nor even simple 'trees of sequences' but in fact 'trees of concepts' as they rely on taxonomic interpretations of different specimens as belonging to the same 'species', which requires the implementation of a 'species concept'. Even if, as discussed above under 2.2.4.2 and 2.2.5.1, different 'species concept' have no doubt governed the recognition of 'species' in different subgroups of amphibians, depending particularly on the period of the last revisionary work, it is likely that most of these 'species' are indeed well-defined holophyletic units. If all the specimens used in the supermatrix have been correctly 'identified', i.e. allocated to these units, then this approach will generally yield highly congruent results. Although this would be 'philosophically' more satisfying, little additional empirical accuracy would be gained by using single specimens for all genes. While we have attempted to minimise incorrectly labeled sequences from Genbank (see 4.3.1.2 below), there is no doubt that not all our 'species' are holophyletic, as a few of them are likely to be hybrid populations or clusters of cryptic species, and that some specimens have been misidentified. This represents an additional source of analytical errors in TREE. However, given the robust corroboration that TREE has brought to the structure of Pyron \& Wiens (2011)'s phylogeny, as well as the robustness the original 2014 version of TREE when confronted to more recent works noted above under 2.1.1, we suggest this is minimal, at least to the extent that it affects our CLAD. Indeed, simulation results (Campbell et al. 2009) suggest that the use of composite taxa uniformly increases phylogenetic accuracy over the alternative, which is to only use available sequences from a single specimen and accept a drastic increase in missing data. As 'phylogenomic' studies become the norm (e.g., Hime et al. 2020), it is now more common to generate entire matrices of hundreds or 
thousands of gene regions from single specimens, which should mitigate or eliminate this problem in future large-scale studies of this type.

The number of genes sequenced per species spanned from 1 to 15 . Our tree, built in 2014, includes 4060 species, i.e. $55 \%$ of the 7317 species recognised by taxonomists at the end of 2014 and $49.3 \%$ of the 8235 species recognised on 31 October $2020<A W b 2020>$. Among all the nodes produced by this analysis, we respected strictly a threshold of $90 \%$ SHL-aLRT support value as a minimum value for considering a node as robust, i.e. as indicating holophyly of all the branches resulting from it. Among the 393 robust suprageneric nodes of TREE, 278 (i.e. $70.7 \%$ ) result in dichotomies, i.e. indicate fully resolved sister-branches relationships, whereas 115 (i.e. $29.3 \%$ ) result in polytomies (trichotomies, tetratomies, etc.), i.e. indicate unresolved relationships between genera (see Table T13.NOD).

\subsubsection{Taxonomy}

We used these results to build a phylogenetic suprageneric taxonomy or cladonomy (CLAD) which reflects exactly (bijectively) the structure of TREE: we afforded the status of taxon to all suprageneric nodes meeting the requirement of our $90 \%$ threshold, and we denied it to all those which did not. This means that we did not take any subjective decision as to whether some nodes are 'more important' or 'more significant' than others but that our taxonomic conclusions were imposed by the data and only them. In the present work, we adopted as valid the species and almost all the genera recognised as such in the recent literature, although it is quite clear that, even in the recent years, different authors and different works implemented different species and genus concepts. In a few cases we erected or synonymised genera in order to comply with the requirement of holophyly. But the heart of our work was the suprageneric taxonomy. Based on the crucial distinction between taxonomic category and nomenclatural rank highlighted by Dubois (2005b and subsequent works up to Dubois et al. 2019), we consider that the hierarchical levels to which all taxa above the rank genus are referred (such as family, order or class) do not qualify as taxonomic categories (defined by biological, chronological or other criteria) but merely as nomenclatural ranks, the hierarchy of which only expresses the succession of nodes taxonomically recognised, i.e. the structure of the tree, irrespective of any phenetic criterion of amount of divergence or of geological age of cladogenesis. In other words, the names of all the ranks above the rank genus are purely arbitrary and fixed only by tradition and consensus but do not 'mean' anything regarding the characteristics of the taxa by themselves, but only refer to their cladistic relationships.

\subsubsection{Nomenclature}

We used a set of explicit Rules to attribute ranks and allocate nomina to taxa, following for this work the Nomenclatural Process involving three main steps (nomenclatural assignment and availability, taxonomic allocation, and nomenclatural validity and correctness of nomina) highlighted by Dubois (2005b: 380, 2011a, 2013) and Dubois et al. (2019). Regarding the nomenclatural assignment of ranks to taxa, which as we have seen above does not rely on biological, evolutionary or other criteria, so far, no explicit operational methodology of any kind has ever been proposed to fix these ranks in zootaxonomy, and we here propose one, the Ten Criteria Procedure (see 4.1.4). It is based on a series of ten explicit Criteria allowing to determine automatically (i.e., without subjective opinion or decision) at which level of the hierarchy, in any given branch of a tree, should the rank family be applied. The most important of these Criteria is the Upper Quartile Criterion [UQC], which relies on quantitative data on the usage of family nomina in the literature, not only in the recent one but during the whole history of scientific zoological taxonomy and nomenclature since 1758 . Once the rank family has been so fixed, all the other ranks for all other taxa derive automatically from it, following a procedure that we describe in detail. The allocation of nomina to taxa then follows. For all nomina of the nominal-series for which the International Code of Zoological Nomenclature (Anonymous 1999, 2012) provides a complete set or Rules, namely those of the species- (SS), genus- (GS) and family-series (FS), i.e. from the rank species to the rank superfamily, we followed strictly the Code, as well as the decisions of the International Commission on Zoological Nomenclature whenever appropriate. For all nomina of the class-series (CS, including all ranks above superfamily), for which the Code only provides a few Rules 
concerning nomenclatural availability, we used the Duplostensional Nomenclatural System (DONS) described in detail by Dubois $(2006 a, 2015 c, 2016,2020 a)$ and Dubois \& Frétey $(2020 a)$. In order to be able to express unambiguously and bijectively the structure of TREE, we had to use 31 ranks, two in the species-series, two in the genus-series, 14 in the family-series and 11 in the class-series below the rank class.

\subsubsection{The Ten Criteria Procedure}

The Ten Criteria Procedure is one of the main contributions of the present work to the theory and practice of zoological taxonomy and nomenclature. It consists in a set of ten Criteria aiming at reflecting bijectively a cladistic tree and allowing back and forth equivalence between them in any suprageneric zoological cladonomy. Among these ten Criteria, four have a general value and six apply specifically to the nomina of families. In both cases, the Criteria may rely only on nomenclatural Rules $\{\mathrm{N}\}$ or on both taxonomic and nomenclatural Criteria $\{\mathrm{TN}\}$. Three-letter abbreviations are used below to designate these ten criteria, and one-letter abbreviations between square brackets are used in Appendix A9.CLAD-1 for five of them. Let us remind here the definitions of these ten Criteria and their main consequences.

\subsubsection{General Criteria}

\subsubsection{1. $[\mathrm{CNC}]$. Consistent Naming Criterion $\{\mathrm{TN}\}$}

"In any given cladonomy, all sister-branches resulting from nodes having a support value equal to or higher than a given a priori threshold must be recognised as distinct taxa, whereas no branch resulting from nodes having a support below this threshold should be so. However, for two sister-branches to be taxonomically recognised, one of them at least must include more than one supraspecific subtaxon (i.e., of rank genus or above)".

\subsubsection{2. [NPC]. Nomenclatural Precedence Criterion $\{N\}$}

"In zoological nomenclature, precedence between family-series nomina is established through the same Rules as for species-series and genus-series nomina, i.e., according to the situation, publication priority, airesy, proedry, sozoidy or archoidy. In the class-series, according to the DONS criteria, it is established through sozonymy, or through priority, airesy or proedry among sozodiaphonyms, or through priority, airesy or proedry among distagmonyms."

\subsubsection{3. [CHC]. Consistent Hierarchy Criterion $\{\mathrm{N}\}$}

"In any given cladonomy, in one branch at least resulting from a node, subordinate and superordinate taxa should be attributed to immediately successive nomenclatural ranks in the taxonominal hierarchy, but some of these ranks may be lacking in its sister-branch(es)."

\subsubsection{4. [FPC]. Family-Series Precedence Criterion $\{\mathrm{N}\}$}

"In any given suprafamilial cladonomy, whenever the other Criteria allow it, the nominal-series allotment of the suprafamilial taxa should be made giving precedence to the FS over the CS, and allotment to the CS should start only when all the available FS ranks have been used (family-series saturation), at least in one branch of the ergotaxonomy." 


\subsubsection{Criteria applying only or particularly to families}

\subsubsection{1. [UQC], [Q]. Upper Quartile Criterion $\{\mathrm{TN}\}$}

"In any given cladonomy, any UQ-nomen (family-series nomen designating a taxon considered valid and having had a number of usages above the upper quartile of usages since 1758) must be maintained as valid at the nomenclatural rank family, irrespective whether it is also used at other superordinate or subordinate ranks."

This Criterion allowed to validate 34 family nomina in our work:

Order ANura (24): BombINATORIDAE; BRACHYCEPHALIDAE; BUFONIDAE; CENTROLENIDAE; DENDROBaTIDAE; Discoglossidae; HeLEophrynidae; Hemiphractidae; Hemisotidae; Hylidae; Hyperoliddae;

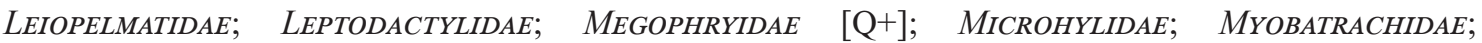
Pelobatidae; Pelodytidae; PipIDae; Ranidae; Rhacophoridae; Rhinodermatidae; Rhinophrinidae; SOOGLOSSIDAE.

ORDER GYMNOPHIONA (3): CAECILIIDAE; ICHTHYOPHIIDAE; RHINATREMATIDAE.

Order Urodela (9):AMbYSTOMATIDAE; AMPHIUMIDAE; CRYPTOBRANCHIDAE [Q+] ; HyNOBIIDAE; PleTHODONTIDAE; PROTEIDAE; RHYACOTRITONIDAE [Q+]; SALAMANDRIDAE; SIRENIDAE.

\subsubsection{2. [STC]. Sister-Taxa Criterion $\{\mathrm{TN}\}$}

"In any given cladonomy, parordinate taxa (i.e. taxa that are considered sister-taxa according to the cladistic hypothesis adopted) should always be attributed to the same nomenclatural rank".

Implementation of this Criterion in the three orders of extant amphibians provided the following two lists of 17 FS nomina that, being parordinate with FS nomina above the upper quartile for each order, must apply at least to a family (preceded below by the nomina of their sister-families between square brackets, followed by $\rightarrow$ ):

Order ANuRa (16): [BRACHYCEPHALIDAE $\rightarrow$ C CEUTHOMANTIDAE; [BUfONIDAE $\rightarrow$ ] ODONTOPHRYNIDAE;

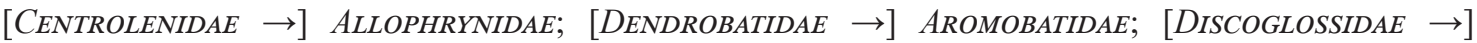

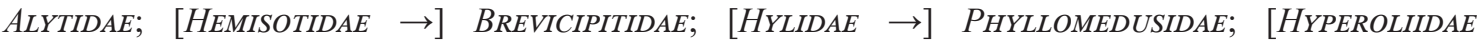
$\rightarrow$ ] ARTHROLEPTIDAE; [LEIOPELMATIDAE $\rightarrow$ ] ASCAPHIDAE; [LEPTODACTYLIDAE $\rightarrow$ ] LEIUPERIDAE,

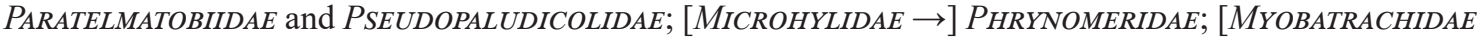
$\rightarrow$ Calyptocephalellidae; [Rhinodermatidae $\rightarrow]$ TelmatobiIdaE; [SOOglossidae $\rightarrow]$ NASIKABATRACHIDAE.

Order GYMNOPHIONA (1): [ICHTHYOPHIIDAE $\rightarrow$ ] URAEOTYPHLIDAE.

\subsubsection{3. [CPC], [P]. Conflict of Precedence Criterion $\{\mathrm{N}\}$}

"In any given cladonomy, whenever a taxon that could be cladistically subordinate to a UQ-nomen has nomenclatural precedence over it according to the Criterion [NPC], it should be raised to the rank family as parordinate to the UQ-nomen at stake."

Five taxa were raised at the rank family in order to be parordinate to UQ-families as their nomina had precedence over them (the latter are mentioned after them in the following list):

Order ANura (4): ALYTIDAE (DISCOGLOSSIDAE);ARTHROLEPTIDAE (HYPEROLIIDAE);BREVICIPITIDAE (HEMISOTIDAE); TELMATOBIIDAE (RHINATREMATIDAE).

Order URODELA (1): CRYPTOBRANCHIDAE (HYNOBIIDAE).

\subsubsection{4. [NRC], [N]. Non-Redundancy Criterion $\{\mathrm{N}\}$}

"In any given cladonomy, within a given nominal-series, redundant taxa, i.e., having the same intension and extension as their immediate superordinate or subordinate taxon, should be avoided if possible. If allowed by the data, they should be divided in two sister-taxa of the same rank (see Criterion [STC]). This Criterion does not apply automatically to taxa belonging to different nominalseries, if one of the ranks involved in the redundancy is one of the seven mandatory ranks (see text and 
Criterion [MRC]). It applies to taxa of the rank family relatively to their just superordinate taxon, except in the situation where this rank corresponds hierarchically to an unresolved polytomy (see Criterion [NTC])."

This Criterion allowed to validate 17 family nomina in our work.

Eleven families were validated for being parordinate of UQ-families (which are mentioned after them in the following list):

Order Anura (9): Allophrynidae (CENTRolenidae); Aromobatidae (DendrobatidaE); AscaphidaE (LEIOPELMATIDAE); CALYPTOCEPHALELLIDAE (MYOBATRACHIDAE); CEUTHOMANTIDAE (BRACHYCEPHALIDAE); NASIKABATRACHIDAE (SOOGLOSSIDAE); ODONTOPHRYNIDAE (BUfONIDAE); PHRYNOMERIDAE (MiCROHYLIDAE); PHYLLOMEDUSIDAE (HYLIDAE).

Order GYMNOPHIONA (2): SCOLECOMORPHIDAE (CAECILIIDAE); URAEOTYPHLIDAE (ICHTHYOPHIDAAE).

Three pairs of families were both validated by the [NRC]:

Order ANura (6): Astrobatrachidae and NyCtibatrachidae; CaCosternidae and PyXicephalidaE; DICROGLOSSIDAE and OCCIDOZYGIDAE.

\subsubsection{5. [MRC], [M]. Mandatory Rank Criterion $\{\mathrm{N}\}$}

"In any given cladonomy, all zoological species recognised as valid should be referred formally (at least provisionally) to one taxon of the following mandatory taxonominal ranks: genus, family, order, class, phylum and kingdom."

This Criterion allowed to validate 17 family nomina in our work.

Order Anura (17): CaCosternidae; CeratobatraChidaE; CeratophryidaE; ConRauidaE; CyCloramphidaE; DicRoglossidaE; ERICABATRACHIDAE; MICRIXALIDAE; NYCTIBATRACHIDAE; OCCIDOZYGIDAE; Odontobatrachidae; Petropedetidae; Phrynobatrachidae; PTychadenidae; Pyxicephalidae; RANIXALIDAE; SCAPHIOPODIDAE.

\subsubsection{6. [NTC], [T]. Nomenclatural Thrift Criterion. $\{\mathrm{N}\}$}

"In any given cladonomy, whenever according to the data the rank family should be granted to several taxa forming together an unresolved polytomy (more than two sister-taxa), a single family should be provisionally recognised and the polytomy should be downgraded to the rank subfamily."

This Criterion applies to four family nomina in our work, two of which are UQ-nomina.

Order ANura (4): CERATOBATRACHIDAE; CYCLORAMPHIDAE; HeMIPHRACTIDAE [Q]; LEPTODACTYLIDAE [Q].

\subsubsection{Comments on the concept of 'stability'}

It is easy to predict that the new ideas, concepts and terms, as well as the new classification and nomenclature of amphibians, presented herein, will meet resistance in the communities of taxonomists and batrachologists, and that a major criticism of these proposals will be that they threaten 'stability' in its various forms (of concepts and terms, of the Code, of classification and nomenclature of taxa).

The concept of 'stability', often expressed by the unclear terms of 'usage' or 'dominant usage', is a complex one, that can be considered from different points of view (Dubois 2005a: 383-386, 2010c). Let us first set aside the (important) fact that many recent statements about 'stability' and 'usage' are clearly misleading, being based only on considerations concerning very short recent periods or limited to a small number of authors, a situation well exemplified by the conflict UrodELA-CAUDATA (see Dubois \& Raffaëlli 2012: 109). Much more importantly, stability by itself is not a scientific aim, but a problem of communication and information. Science is not a dogma or a revealed truth that should be maintained unchanged for decades against all evidence. It is a permanent process of change, of production, refutation and replacement of hypotheses that result in an improvement of our ideas and knowledge. This is particularly true in taxonomy, a domain in which it is quite clear that "stability is ignorance" (Gaffney 1979: 103). Bremer et al. (1990) aptly stated: "Taxonomists should pursue their scientific venture and stop worrying about instability in classification. Taxonomy is not a service function for labelling organisms, but a science of its own, dealing with variation, relationships and phylogeny. 
Other biologists need to keep themselves informed, and should realize that removal of artificial groups and improvements in classification are desirable". The solution to many so-called problems of stability and usage lies in an improvement of communication systems allowing non-taxonomists to keep updated with taxonomic changes, and also with disagreements between taxonomists, which are normal and healthy in a lively scientific domain — rather than relying on so-called 'authoritative' lists and databases which only reflect the point of views of individuals or groups (see Raposo et al. 2017).

More largely, regarding our conceptual and terminological proposals, we think that they should not be rejected blindly or ignored simply on the ground that they are new or 'too complex', but submitted to examination and scientifically argumented agreement or rejection.

As for the attitude of some colleagues, who claim to be interested in 'biology' and 'evolution' but not in trivial matters like nomenclature, and consider that the latter should only be dealt with through tradition, consensus and 'common sense', and do not deserve formal Rules (e.g., regarding the nomenclature of higher taxa), they clearly show their ignorance in this domain and they should rather keep silent about these matters, instead of contributing to the growth of nomenclatural chaos that has been considerable in the recent decades (Dubois 2017e). It is quite clear that, in such matters, these three 'methods' do not work. Simple intellectual honesty requires to recognise that, currently, because of the absence of universal Rules for higher nomenclature, no one can know which taxon is designated in any scientific or non-scientific paper by 'controversial' nomina like INSECTA, AMPHibia, RePTILIA, AVES or MammaLiA, originally used in Linnaeus (1758a), to mention just a few among many. Who can pretend that this is not harmful for communication about biodiversity, both within the scientific community and between the latter and society as a whole? We just hope that, in the longer run, the necessary improvements in taxonomic and nomenclatural methodology will progressively be implemented, in the frame of permanently changing taxonomic paradigms and of growing information on the relationships between organisms.

\subsection{Findings and proposals}

The consistent application of the methodology outlined above led us to recognise 575 valid generic and 607 valid suprageneric taxa of extant lissamphibians below class with their valid nomina in our cladonomy. The distribution of these taxa among the generic and suprageneric ranks used here is provided in Table T14.NUM, which also gives the numbers of new nomina we had to establish to express this taxonomy. We allocated all the taxa and nomina of extant amphibians (subclass LiSSAMPHIBIA of the class Aмpнibia) recognised as valid here to three orders (ANURA, GYMnOPHiona and Urodela), the mutual phylogenetic relationships of which are not resolved. We also provide indications on the taxonomic placement of all the nomina proposed for all-fossil taxa of LISSAMPHIBIA in our classification, but only based on the current literature, without critical reassessment or validation, as our cladonomy of the extant taxa is almost exclusively based on molecular data, except for the taxa for which no molecular analysis is currently available.

In $C L A D$, we use 9 class-series ranks below order and 14 family-series ranks, i.e. 23 ranks between order and genus, for a group of about 8200 known species. Of course, the complete taxonomy of extant amphibians described in CLAD is much too complex and detailed to be mentioned in most publications dealing with the amphibians. In most such works, it will be useful to cite only the nomina of the main ranks of this taxonomy, i.e. those which are usually mentioned in standard scientific publications: the class (AMphibia), the three orders (AnUra, Gymnophiona, Urodela), the 69 families and the 575 genera, and additionally in a more limited number of works the subclass (LISSAMPHIBIA), the 18 superfamilies and the 87 subfamilies. Why, then, did we deem useful to provide this expanded hierarchy and these numerous nomina, including many new ones? As explained above, this is in order to comply with the requirement of having a bijective taxonomy, reflecting accurately the structure of the tree on which it is based. Whereas the ranks, particularly those of family and subfamily, used in traditional works, are fully arbitrary and subjective, the 'same' ranks used in CLAD result from a precise and repeatable rationale and methodology, detailed throughout our work, which could be used independently by any other taxonomists in the world and which would produce the exactly same results as ours if based on the same data.

The number of ranks used here is much higher than in most ergotaxonomies currently published for taxonomic groups of similar, or even much larger, size. Even classifications based on giant trees 
with thousands of species make rarely use of more than 20-30 ranks, which means that only such low numbers of nodes separating any terminal taxa from the root are taxonomically recognised. This is not because the tree contains only such numbers of well-supported nodes, but because most authors limit voluntarily the number of ranks to such low numbers for 'practical' reasons of parsimony and 'manageability' of ergotaxonomies. But this is at the expense of the clarity and thoroughness of the phylogenetic information provided by the ergotaxonomy, as the choices of the ranks to be accepted are arbitrary and such classifications reflect only partially the phylogenetic trees on which they are based. If our methodology was adopted in other taxonomic groups, the number of ranks would increase considerably in the most species-rich ones, but naming these ranks would not raise particular problems: the system of nomination of ranks proposed by Dubois (2006a: 206-225) uses 19 key ranks and 10 subsidiary ranks, thus allowing to distinguish 209 ranks, and this number could be increased easily if necessary by adding key ranks.

As our purpose here regarding the taxonomy of extant amphibians was limited to three precise aims (using explicit concepts and Criteria to produce a phylogenetic hypothesis, a taxonomy and a nomenclature of this group), we abstained from drawing conclusions or expressing opinions regarding evolutionary, adaptive, biological or ecological, bio- or phylogeographical questions concerning the evolution of amphibians, but our taxonomic and nomenclatural results, based on a consistent methodology, will allow such discussions much better than all the previous taxonomies of amphibians which followed no consistent and explicit rationale for the allocation of ranks and nomina to taxa and were largely of phenetic inspiration, despite their claim to follow a 'phylogenetic' approach.

Our repeatable methodology provides objective and repeatable Criteria allowing to fix the rank family in any given zootaxonomic group. This is particularly useful, because taxa attributed to this rank are very often used in the scientific literature for large scale comparisons and analyses, particularly in evolutionary biology, bio- and phylogeography, ecology and conservation biology. Let us give here just two examples.

$\{\delta 1\}$ Here we propose a treatment of the Australo-Papuan PELODRYADINAE and the Central and South American PHYLLOMEDUSINAE as two sister-subfamilies of a single family PHYLLOMEDUSIDAE, sister to the family HYLIDAE and then both families as the two sister-families of a superfamily HYLOIDEA, itself sister to four other subfamilies, etc. This is much more informative in evolutionary and phylogeographic terms than having the HYLIDAE, PELODRYADIDAE and PHYLLOMEDUSIDAE as three families 'sister' to 11 other families (Bossuyt \& Roelants 2009), or to 49 families and 3 superfamilies placed at the same level $\angle A S W 2020 a>$, or taxonomically overlumped by recognition of a single family HYLIDAE for these three groups (Faivovich et al. 2005; Frost et al. 2006; Blackburn \& Wake 2011; Pyron \& Wiens 2011; Borkin \& Litvinchuk 2014; Hime et al. 2020). In all these taxonomic schemes, the 'ranks' carry no clear phenetic or cladistic information, or more exactly no information at all, thus hindering any relevant phylogeographic or evolutionary considerations.

$\{\delta 2\}$ An opposite example, oversplit at the taxonomic level family, concerns three groups of salamanders, which have been considered by most recent authors as three families: the holarctic SALAMANDRIDAE, the nearctic AmBYSTOMATIDAE and the nearctic DiCAMPTODONTIDAE. They were considered as three families parordinate to seven others by Blackburn \& Wake (2011), Pyron \& Wiens (2011), Borkin \& Litvinchuk (2014), Hime et al. (2020) and $\langle A S W 2020 a\rangle$, as three families parordinate to four other families by Zhang \& Wake (2009), and as three families grouped in a superfamily in Vieites et al. (2009). Such arrangements lead to an overweighting of the 'distances' between branches, for example in biogeographic analyses. Frost et al. (2006) recognised two families, SALAMANDRIDAE and AMBYSTOMATIDAE, the latter with two subfamilies AMBYSTOMATINAE and DICAMPTODONTINAE. For reasons explained above we here recognise the same two families as these authors, but without subfamilies in the AMBYSTOMATIDAE which only include two extant sister-genera.

The main differences between our approach and the traditional (even recent) ones, which contrary to the latter produce in an objective manner repeatable results, derive from the following aspects of our methodology: $\{\varepsilon 1\}$ the strict respect of an a priori threshold $(90 \%)$ as the only basis for the decision to recognise, or not, a node of TREE as a formal taxon bearing a formal nomen; $\{\varepsilon 2\}$ the strict respect of the requirement that parordinate taxa (sister-branches in TREE) be always assigned to the same nominal-series and attributed to the same rank in the latter, all other superordinate and subordinate ranks in the same branch being automatically consistent with it; $\{\varepsilon 3\}$ the strict respect of an explicit set of Criteria for the fixation of the rank family in any given branch of TREE, these Criteria relying mainly, but not only, on usage, but the latter being precisely defined and quantified over the whole history of 
taxonomic literature, not based on a vague 'impression' and relying only on recent literature; $\{\varepsilon 4\}$ the strict respect of the Rules of the Code concerning availability, allocation and validity for SS, GS and FS nomina, and of the Criteria of DONS for CS nomina.

Despite the numerous clarifications brought by our work on the phylogeny, taxonomy and nomenclature of extant amphibians, a high number of questions remain unanswered and will require additional works, as stressed below.

\subsection{What remains to be done}

\subsubsection{Regarding the analysis}

\subsubsection{Taxonomic sampling}

Our cladonomy is based on molecular data obtained from 4060 specimens which are considered to belong in 4060 distinct biological species of extant amphibians. This represents $49.3 \%$ of the 8235 species recognised more or less consensually by the community of taxonomists worldwide on 31 October $2020<A W b 2020>$. Although this proportion is high, it does not allow to pretend that our analysis is a complete one concerning the extant amphibian species of the world, for two reasons: $\{\zeta 1\}$ the rate of descriptions of new species published each year in the last decades has been very high (Tapley et al. 2018): 140 species were described each year from 2000 (5206 species) to 2020 (8146), and from 2014 to 2020 this mean number raised to 151; therefore we are far from having collected, studied, distinguished, taxonomically recognised and named all the amphibian species still present on earth (this point is discussed in more detail below); $\{\zeta 2\}$ our analysis bears mainly on the suprageneric relationships among extant amphibians, and, for reasons explained above, except in a few cases we did not challenge the 'accepted' generic taxonomy of these animals, although it is clearly heterogeneous as it relies on different genus concepts in different higher taxonomic groups of amphibians and in different works. However, if we accept this situation as a provisional starting point, our suprageneric analysis would be fully reliable, or at least acceptable, only if our sample of sequenced species included at least one species unambiguously referred to every genera recognised in this 'consensual' generic taxonomy. This is far from being the case. In this work we recognise 579 valid genera of extant amphibians, but $52(9.0 \%)$ of them are not represented in our molecular tree. For the taxonomic placement of these 'missing' genera in CLAD, except in a few cases where molecular data on some of these genera were published after 2014, we could rely only on the available morphological information on these taxa, which is often very scanty and little reliable. Therefore, to increase the quality of our taxonomy, new collections will be required. The last column of Appendix A15.MIS lists the 52 genera which are not represented at all in TREE and for which specimens and sequences will have to be incorporated in our matrix (if published after 2014) or obtained from freshly collected specimens, or possibly in some cases from preserved museum collections.

A good sampling at species level is also indispensable for a good resolution of generic taxonomy. It is not appropriate to take taxonomic decisions on the basis of very small molecular samples of the species of genera which according to morphological and other non-molecular data are considered to include five, ten or many more species. As long as only some of these species are included in the analysis, the possibility exists that just one or a few of them are wrongly allocated to genera, which does not result in invalidating the latter (see in this respect Delorme et al. 2004). Particularly irrelevant is the decision to synonymise two genera on the basis of only one species of both genera (!) or even one species of one genus $v s$. two of the other one, as easily shown by a few examples in amphibians.

The genus Nidirana Dubois, 1992, well supported by several morphological and behavioural synapomorphies, was synonymised by Frost et al. (2006) with Babina Thompson, 1912 based on molecular data on two species of Nidirana only and none of Babina, but was revalidated using a molecular sample that included all but one species of the two genera (Lyu et al. 2017). The generic and subgeneric classification based on an extensive morphological work on the Hylarana sensu lato group (Dubois 1987b, 1992) was washed away by Frost et al. (2006) according to the molecular data on 11 species of the group (about $10 \%$ of the known species), but many of these taxa were revalidated and new genera added by a molecular study including more than $70 \%$ of the known species (Oliver et al. 2015). The members of the HEMIPHRACTIDAE based on strong morphological evidence (Duellman 1970) 
were distributed in three families (Frost et al. 2006) but then several studies (Guayasamin et al. 2008; Blackburn \& Duellman 2013; Castroviejo-Fischer et al. 2015) recovered this branch as holophyletic.

Some such unwarranted decisions based on insufficient sampling may have far-reaching consequences concerning our understanding of evolution and biogeography. The Chiromantis-Chirixalus case is particularly striking in this respect. Frost et al. (2006) showed that inclusion by Liem (1970: 95) of the species Ixalus vittatus Boulenger, 1887 in the genus Chirixalus Boulenger, 1893 rendered it paraphyletic, and they removed it from this genus to place it in their new genus Feihyla, which was justified on the basis of their data and genus concept, and supported by morphological and biological data (reproductive mode). But then they went one step further and placed the Asian genus Chirixalus in the synonymy of the African genus Chiromantis Peters, 1854 on the basis of their molecular data on only two species of the former (including its nucleospecies Chirixalus doriae Boulenger, 1893) vs. a single one of the latter (its nucleospecies Chiromantis xerampelina Peters, 1854), although both genera were then considered to include other species. By so doing they created out of nothing the only amphibian genus whose distribution straddled tropical Africa and tropical South-East Asia. Chen et al. (2020), using two African and two Asian species, which their analysis showed to be well-supported sister-branches, re-established the genus Chirixalus as distinct from Chiromantis. This decision is reinforced in TREE, based on three African and two Asian species. Admittedly, these two genera still appear as sister-taxa and constitute together our clan CHIRIXALITES, which shares with other suprageneric amphibian taxa an African-Asian distribution, but this is quite different from placing them in the same genus, given the key role given to the rank genus in many biogeographic and evolutionary analyses.

\subsubsection{Nomenclatural sampling}

The situation is even worse if the nomenclatural aspect of our sampling is concerned. In order to have a fully reliable nomenclature, the taxonomic allocation of all the available extant amphibian generic nomina, not only of those considered valid, should be ascertained, as the subsequent finding that a synonym was wrongly allocated may challenge the validity of another generic nomen. Therefore, the nomenclatural status of all the taxonomic genera recognised in CLAD on the basis of the structure of TREE depends on a thorough allocation of all these nomina, and the only strict way to reach this aim is to rely on sequences obtained from specimens that can be allocated without doubt to the nucleospecies (type species) of all these generic nomina. The best situation in this respect is when these sequences were obtained from the holophoronts (holotypes), lectophoronts (lectotypes) or neophoronts (neotypes) of these species, or rarely from symphoronts (syntypes) of the latter (which should then be designated as lectophoronts), but this is a rare situation, which applies only to species freshly collected and described as new in the recent years, or in exceptional cases to sequences which could be obtained from onymophoronts kept in collections (e.g. Rancilhac et al. 2020). The situation is less good, but still acceptable, when the specimen used for TREE had been collected in the original onymotope of the species, or when it was identified at species level by a competent taxonomist, well acquainted with the taxonomic group concerned. Although this is probably the case for a majority of species, it is not always true. Doubts are allowed when a publication reports on sequences stated to have been obtained from species that have been very rarely collected and reported above in the literature and for which no comments are present in the publication showing that the authors were conscious of this fact. In some cases, it may be wondered whether the identification was accurate, or based on a superficial work relying for example on photos in a field guide, on short descriptions or merely on identification keys or on labels on specimens in collections. Examples of such cases were provided by Dubois (2004a) concerning amphibians from Nepal and many others could be given.

Therefore, in case of doubt, especially when the position of a species in a tree appears 'strange' in the light of previous morphological or other data, care should always be taken for a competent taxonomist to re-examine the voucher from which the sequences were obtained. But, for this to be possible, this specimen should have been fixed, kept in a permanent collection and its collection location and number should have been published. Although this is more and more the case in recent publications, it has not always been so and this is still not true in some cases. In Jones \& Weisrock (2018), numerous species of Desmognathus were misidentified in the field, with no voucher information. This was noted by Pyron et al. (2020). Even on Genbank, not all sequences are connected with a number of voucher. When a return to the vouchers is indeed made, it is not exceptional to disclose wrong identifications, which 
may have important consequences on the nomenclatural interpretation of a molecular tree, even if the results of the molecular, cladistic and taxonomic analyses are correct: after all, a molecular cladogram is just a tree of sequences, not of taxa. The best example is in Pyron \& Wiens (2011), who included numerous misidentified Genbank vouchers in their analysis, discussed by Frost $<$ in $A S W 2020 a>$ and Blotto et al. (2013). Frost <in ASW 2020a> wrote in 2011: "Unfortunately, the study includes Genbank sequences that were previously noted to be misidentified. For examples that became evident due to the surprising placement of terminals in their tree, Poyntonophrynus vertebralis sequences included by Pyron \& Wiens were reidentified as Amietophrynus maculatus by Cunningham and Cherry (2004); sequences associated with Yunganastes pluvicanorus in the Frost et al. (2006) study and reused by Pyron and Wiens were reidentifed as Pristimantis pharangobates by Padial (2007) [presumably Padial et al. 2007 (AD's comment)]; the 12S and 16S sequences of Amolops daorum were reidentified as Odorrana hmongorum by Stuart et al. (2010)." Most of these errors were fixed in Jetz \& Pyron (2018). Other cases of wrong taxonomic allocation of sequences used in phylogenetic analyses were pointed out by Bridge et al. (2003) and Vilgalys (2003). For all these reasons, in Appendices A9.CLAD-1 and A5.NGS, we provided information on the quality and reliability of the specific identification of the specimens on which TREE is based by referring them to the five following categories regarding their genus-series nomina:

* The nominal genus is represented in TREE by specimens referred to its nucleospecies or to an isonym of the latter: Rana*.

${ }^{1}$ The nominal genus is represented in TREE by specimens referred to a doxisonym of its nucleospecies: Pipa ${ }^{1}$.

${ }^{2}$ The nominal genus is represented in TREE by specimens referred to the nucleospecies of a generic nomen being its doxisonym: Andrias ${ }^{2}$.

${ }^{3}$ The nominal genus is represented in TREE but only by specimens referred to the species that include neither its nucleospecies, nor a doxisonym of the latter, nor the nucleospecies of a doxisonym of the generic nomen at stake: Latonia ${ }^{3}$.

${ }^{\circ}$ The nominal genus is not represented at all in TREE: Dischidodactylus ${ }^{\circ}$. (This amounts to the situation of insufficient taxonomic sampling mentioned above).

The reliability of the nomen allocated to a genus in $C L A D$ decreases from the first to the last of these five categories. In order to obtain a fully reliable version of CLAD, it would be necessary that all available generic nomina nomina belong to the first category. This is of course impossible, but in the coming years the community of amphibian taxonomists should do its best to upgrade the quality of the vouchers on which all the sequences are based. Our Appendix A5.NGS establishes the existence in the literature of 1639 available generic nomina of lissamphibians, among which only 770 (47.0\%) are used as valid in CLAD including 575 (74.7\% o the valid ones) for extant genera. Appendix A15.MIS provides a complete list of the latter, among which the numbers and proportions of those referred to the five categories above are respectively, in the order of their presentation above, of 437 $(76.0 \%), 45(7.8 \%), 17(3.0 \%), 24(4.2 \%)$ and $52(9.0 \%)$. It is quite clear that we are still far from an 'ideal' situation where we would have $100 \%$ of the first category. This suggests that, although in the recent decades some efforts have been made to clarify and stabilise (sometimes through designation and sequencing of lectophoronts or neophoronts) the nomenclatural status of the generic nomina considered valid, this trend should be consolidated and amplified if we want to strive for a reliable and robust generic nomenclature of amphibians.

The situation is worse if we include in this count the 869 available nomina of extant genera (not listed in Appendix A15.MIS but appearing in Appendices A5.NGS and A9.CLAD-1) currently considered invalid synonyms, which include $731(84.1 \%)$ whose onomatophoronts are present and $138(15.9 \%)$ whose onomatophoronts are missing. Then the proportions for the total of 1444 nomina are respectively of $1168(80.9 \%)$ whose onomatophoronts are present and $276(19.1 \%)$ whose onomatophoronts are missing. As long as these nomina are considered invalid junior (or juniorised) synonyms, this is not a big problem, but it might become so whenever new data or new taxonomic interpretations of the current data lead to the dismantlement of some genera: then it is necessary to know reliably to which taxa do apply such synonyms, in order to avoid the useless establishment of invalid junior synonyms, thus contributing to nomenclatural instability and to avoidable increase of the synonymy load (Table T15.SYN). For these reasons, every time the opportunity appears, through study of ancient onymophoronts or through collection of fresh specimens (especially from onymotopes of available nomina), to reduce the number of valid and invalid nomina in the last four categories above, it should be seized. 
As first clearly stated by Hennig $(1950,1966)$, the process of evolution can be structurally described (i.e., irrespective of the processes at work), e.g. through a cladogram or a phylogenetic tree, as a succession of cladogenetic events, i.e. of dichotomies or divisions of one lineage or branch in two. These successive dichotomies are traditionally expressed, in taxonomies, by a hierarchical pattern, the most basal dichotomies being given the highest taxonominal ranks and the most terminal ones the lowest ranks (usually species or subspecies). However, most real trees produced by phylogeneticists, obtained either by classical analysis of morphological traits or by sequencing of nucleic acids, are not composed only of dichotomies but of three main patterns, here referred to as three categories of tomoidy: $\{\eta 1\}$ the pattern of dichotomy, which corresponds to the classical case of splitting of a branch in two, is usually interpreted as expressing a resolved cladogenetic relationship; $\{\eta 2\}$ the pattern of polytomy, which expresses an unresolved relationship (a 'comb', instead of a 'scale' of dichotomies), more than two branches resulting from the 'synchronous' splitting of a single branch; $\{\eta 3\}$ the third pattern, for which we propose the new term of achotomy, which describes an undivided branch, at least at a given level of a tree (or of the hierarchy expressing it), if not at a more terminal (in a tree) or lower one (in a hierarchy).

The situation of dichotomy is usually considered of clear interpretation, as reflecting a 'real' evolutionary event of cladogenesis. Even if the methodology of construction of the tree of reference is excellent (which is not always the case), this statement is certainly often misleading, given the gigantic incompleteness of the taxonomic record, not only regarding fossil species but also living ones (see below), but there is no way to avoid this difficulty except by increasing our effort of exploration of the planet and of collection of specimens (see also below). But the problems are even worse in the other two categories of tomoidy.

The Hennigian statement that evolution consists only in dichotomies is clearly a methodological 'trick' allowing to simplify, or even simply make possible, cladistic analyses following Hennig's (1950, 1966) proposals, but there is no theoretical reason to dismiss the possibility of real polytomies, e.g. whenever a geological catastrophic event results in the synchronous splitting of a single population into several. Even without needing to have recourse to such a gratuitous hypothesis, whatever the methods used (morphological, cytogenetic, molecular, etc.), it may be difficult to find apomorphies allowing to reconstruct the chronology of several dichotomous events having occurred in a short (in geological terms) period of time. This is true even using molecular markers, or cytogenetic, behavioural, ecophysiological or other biological characters having usually a quicker evolutionary rate than most morphological ones. In such cases, especially when few terminal taxa (species) are at stake, we may have no way to 'resolve' the polytomy, and the latter must be accepted as a final result. But in other cases, the polytomy may be resolved through an increase of the taxonomic sampling or of the number of genes sequenced. In amphibians, the following examples of such resolutions of polytomies through an increase of the taxonomic or molecular coverage illustrate this possibility. On the whole, it is reasonable to consider that a high number of unresolved polytomies in a tree reflects more the defects of our analyses than the existence of genuine polytomies in the evolution of a group. In the frame of a bijective taxonomy reflecting all the nodes of a tree, the progressive resolution of most of these polytomies will result automatically in an increase of the dichotomies and therefore of the number of suprageneric taxa in the group under study. The simplest example of this situation is that of the three orders of the subclass LisSAMPHIBIA discussed above: as long as the trichotomy is not resolved, we need only three taxa/nomina of rank order (ANURA, GYMNOPHIONA and URODELA) to account for the cladonomy, but as soon as a dichotomy between two of these orders is supported we will need for nomina and an additional rank, superorder (see above and Dubois 2015c: 108). As we will see below, even without an increase in the numbers of species and genera recognised by taxonomists, this is the general trend that what can be expected from an improvement of our cladistic analyses - and this effect will be increased by the expectable increase in the numbers of species and genera taxonomically recognised.

However, an opposite effect may be expected from the resolution of some polytomies: those which, beside one or several dichotomies and polytomies, involve more than one achotomy. Among the 214 achotomies taxonomically recognised in CLAD (179 in ANURA, 29 in URODELA and 6 in GYMNOPHIONA), only 100 , i.e. $46.7 \%$ (respectively 76, 18 and 6, i.e. $35.5,8.4$ and $2.8 \%$ ) are involved isolately in polytomies, whereas 114 (respectively 103, 11 and 0 ) belong in polytomies involving more than one achotomy (Table T13.NOD). The latter branches may appear so only because of insufficient taxonomic 
or genetic sampling, but may be united as a few dichotomies with better resolution. Depending on the cladistic relationships, the result might then lead to a reduction of the number of suprageneric taxa. Therefore, although it is impossible to model and predict in detail the future evolution of the number of suprageneric taxa/nomina in LisSAMPHIBIA, on the whole this number should increase rather than decrease, and, for the taxonomists who will adopt a bijective cladonomy, most of the new nomina proposed here will stand.

Table T13.NOD analyses the situation regarding tomoidy in the three orders of extant amphibians and in ten ranks or series of ranks which provide a partition in 10 groups of the 25 suprageneric ranks of extant lissamphibians below class used in CLAD. This table shows that the mean 'quality' of the resolution of the nodes of the tree (percentage of dichotomies among the 393 robust nodes of TREE) is of $70.7 \%$, but is much better in the caecilians $(90.9 \%)$ than in the salamanders $(77.4 \%)$ and then than in frogs $(68.2 \%)$. If taxa, including those based on achotomies, and not only nodes, are considered, the proportions of dichotomies drop to $45.8 \%$ for all extant lisamphibians, and respectively of $71.4 \%$ for caecilians, $52.7 \%$ for salamanders and $43.1 \%$ of 'well supported' taxa based on 'well resolved' dichotomies. These data suggest that we are still far from having a 'fully resolved' phylogeny of extant lissamphibians, even if limited to the incomplete subsample of the species that have so far been collected and taxonomically recognised.

\subsubsection{Cladistic methodology}

Studies such as those of Siu-Ting et al. (2019) and Hime et al. (2020) show the great promise that genome-scale phylogenetics has for resolving deep and intransigent branches in the Tree of Life. Concomitantly, they illustrate the dangers that can befall studies based on only a few loci, or limited taxon sampling. Gene duplication as well as incomplete lineage sorting are well-known processes via which the 'true' species tree (if such a thing exists) may not match individual gene trees (Edwards 2009). But broad sampling of the genome, careful assessment of orthology, and accounting for processes such as gene-tree error can resolve these disagreements in favor of a robust and strongly supported topology.

We must also keep in mind the mounting evidence for the general importance of the third major process driving genealogical discordance, that of reticulation between lineages. This may happen both at deep timescales (Burbrink \& Gehara 2018), and particularly among recently diverged lineages (Edwards et al. 2016). These may seriously affect both our understanding of species boundaries (and the integrity of their identity as distinct units), as well as relationships among species. For many parts of the Tree of Life, representing phylogenetic relationships as a bifurcating tree may not be accurate, but instead as a reticulating network showing gene-flow across lineages through time (see Pyron et al. 2020).

\subsubsection{Regarding the taxonomic completeness}

\footnotetext{
La culture ce n'est pas avoir le cerveau farci de dates, de noms ou de chiffres, c'est la qualité du jugement, l'exigence logique, l'appétit de la preuve, la notion de la complexité des choses et de l'arduité des problèmes. C'est l'habitude du doute, le discernement dans la méfiance, la modestie d'opinion, la patience d'ignorer, la certitude qu'on n'a jamais tout le vrai en partage; c'est avoir l'esprit ferme sans l'avoir rigide, c'est être armé contre le flou et aussi contre la fausse précision, c'est refuser tous les fanatismes et jusqu'à ceux qui s'autorisent de la raison; c'est suspecter les dogmatismes officiels mais sans profit pour les charlatans, c'est révérer le génie mais sans en faire une idole, c'est toujours préférer ce qui est à ce qu'on préférerait qui fût.
}

[Culture does not consist in having your brain stuffed with dates, names or numbers but in the quality of judgement, logical stringency, craving for evidence, the notion that things are complex and problems arduous. It means being used to doubting, discerning in mistrust, humble in one's opinions, patient in ignorance, and certain that not all the truth has ever been bestowed upon us. It means being firm in mind but not rigid, and being armed against vagueness as well as false precision. It means refusing all kinds of fanaticism, including those grounded upon reason, and suspecting all forms of official dogmatism, yet without profit for the charlatans. It means revering genius but without making an idol of it, and always prefering what is to what one would prefer it were.]

Jean Rostand 1963: 47 


\subsubsection{Introduction}

So far, we have concentrated our attention, results and recommendations, on the construction of a suprageneric cladonomy of all the known species of amphibians and on suggestions for improving these results. But this aim is of limited scope, for a simple reason: we are still far from having collected all the species of amphibians of our planet, perhaps not even half of them. The lines that follow derive largely from three papers that have not drawn attention from the community of batrachologists (Dubois 2003, $2008 e, 2009 b)$.

We have now fully entered the century of extinctions (Dubois 2003). In the coming decades, the order of magnitude of species extinctions on this planet, which qualifies as the sixth mass extinction (Wilson 1988), will be much higher than during the whole history of mankind. Although efforts are currently made to 'conserve' species, they have and will have little effect on the main cause of extinctions for many groups of organisms, namely the destruction, or major modification, of habitats and ecosystems, especially in tropical regions. As noted by Hoffmann et al. (2010: 1509), "conservation responses will need to be substantially scaled up to combat the extinction crisis", and in this sentence, "would need' would have been more appropriate than 'will need'. The scenarios that can be built, through duly considering the data and actions of 'conservation biology', make it quite doubtless that "biodiversity will continue to decline over the $21^{\text {st }}$ century" (Pereira et al., 2010: 1496). This statement is certainly more realistic than loud announcements like that of the '2010 Biodiversity Target' (Anonymous 2011). In April 2002, the Parties of the Convention on Biological Diversity (CBD) "committed themselves to achieve by 2010 a significant reduction of the current rate of biodiversity loss at the global, regional and national level as a contribution to poverty alleviation and to the benefit of all life on Earth". Although this target was not reached, which was quite predictable indeed, no perceptible change in the way of working of such corporates is evident. In fact, there is little doubt that millions of species will become extinct before the end of this century, whereas our taxonomic knowledge of most of them is terribly insufficient. This will have irreversible consequences on the incompleteness of our knowledge of biodiversity and evolution on earth: in contrast with regressions of populations, which, even if very drastic, would, at least theoretically, be liable to be reversed if the aggressions of our civilisation on the biosphere were reduced or disappeared, extinctions of species are definitive and 'without consolation'. The combination of this taxonomic impediment (Anonymous 1994) and its consequence the taxonomic gap (Dubois 2010c) with the biodiversity crisis (Wilson 1985) qualifies as a new paradigm for biology (Dubois 2007a: 27), the taxonomic urgency (Dubois 2010c), but the current taxonomic crisis (Dubois 2003, 2010c; Wheeler 2004; Wheeler et al. 2004) will make it difficult, if not fully impossible, for the international scientific community to answer adequately this challenge.

It may seem strange that, until now, the international community of biologists did not elaborate any action program for this century that would take the realistic prospective of a continuation of biodiversity decline into account, just as if ignoring these scenarios of extinction would be enough to avoid them. Against all evidence, most biologists interested in biodiversity 'do as if' the actions undertaken or proposed for limiting biodiversity losses were likely to succeed in conserving most species. Such a 'denial of reality' resembles a 'magic' attitude based on the idea that 'mass extinctions cannot occur because this would be too sad and dreadful'. This has little to do with a scientific attitude, for which, as Rostand's citation above reminds, it is always better to prefer "what is to what one would prefer it were".

Today, it would still be possible, by investing considerable manpower and budgets, to reduce drastically the taxonomic impediment, but this will be impossible in a few decades, when half or more of the species currently living on earth are extinct. This urgency has not yet been identified as such by most scientists, including biologists, and has not been included among the priorities recognised by academic institutions, governmental and international research programs. Until this is done, it will be too late, at least for a large proportion of the 'non-vertebrate' or 'lower vertebrate' species of our planet. This loss will have considerable consequences which are impossible to predict today, in many domains including ecology, forestry, agriculture, fisheries, economy, ethics and aesthetics, including human health. It will also result in a huge and definitive loss of information on the diversity and history of organisms on our planet, which comparative and evolutionary biologists will miss forever (see Dubois 2009d).

Amphibians are particularly exemplary in this respect. Although the number of known species of this group has more than doubled in a few decades, we have probably not yet discovered half of 
the living, or recently extinct, species. More than $30 \%$ of the total number of described amphibian species of the planet are threatened with extinction and $30 \%$ are data deficient (Stuart et al. 2004, 2008). Threats on the unknown species are impossible to estimate. However, the size of the group remains 'manageable', and these rather large animals can be efficiently discovered and collected by competent field taxonomists. This peculiar situation could allow for the possibility to develop in the coming decades two different projects regarding this group, which may be called exhaustive taxonomy and preventive taxonomy.

\subsubsection{Exhaustive taxonomy}

At any given time of the history of our planet, each taxonomic group has been represented by a certain number of species. It would theoretically be possible to make a complete list of these species, which could be called an exhaustive taxonomy of the group at stake at a given date. However, no exhaustive taxonomy will ever be possible for extinct species, as most of them disappeared without leaving fossils. This aim is also unrealistic for many extant groups, especially counting dozens or hundreds of thousands of species of small size and living in habitats of difficult access to man. However, such a project would appear more realistic for groups of relatively few large sized animals living usually in habitats readily accessible to man. Amphibians are such a group. Today, it would still be possible for the community of batrachologists to take a strong decision: that of considerably increasing the effort of taxonomic exploration of all the countries, ecosystems and habitats of the planet, in order to approach an 'almost complete inventory' of the amphibian species still present on earth. International meetings which would recognise this priority could decide to promote the objective of achieving an (almost) exhaustive taxonomy of amphibians in the first half of our century. This would require a strong 'political' decision, a shift in priorities and a modification in well-entrenched habits. Whatever interesting and informative they are, phylogenetic studies contribute only marginally to reducing the taxonomic impediment. Their major contribution to this work is through the recognition of relevant classificatory units at low levels of the nomenclatural hierarchy above species (genera, subgenera, species groups), which facilitate the relevant comparisons of newly discovered organisms with their close relatives, a work which is not possible when these units are not well defined. But phylogenetic data by themselves contribute only marginally to the discovery of new species, as this is not their main focus or target. To take only three examples, extraordinary taxonomic discoveries like those of Rheobatrachus and its unique reproductive mode, Nasikabatrachus and its unusual morphology and biogeographical affinities, or Karsenia and its unexpected distribution, did not result from phylogenetic analysis but from 'mere' exploratory work in poorly studied habitats: how many other discoveries of this kind are 'still' expecting us, and for how long still?

As stressed by May (2004), regarding the inventory of living species, collecting new specimens in the field everywhere on the planet will remain the rate-limiting step. New species are not in the computers and sequencers of the big cities of the 'North', but in the forests, savannahs, mountains, rivers, lakes and marshes of the whole planet and especially of the 'South'. No 'technical solution' will bring these species from the field to the laboratories, even as nucleic acid sequences for barcoding analysis. In particular, the "triumvirate adjoining a unitary taxonomic cyberstructure + automated DNA barcoding + molecular phylogeny" has been qualified as "a threefold myopia" (Carvalho et al. 2008). The search for 'magical solutions' will not be sufficient to solve the problem of the taxonomic urgency (Wheeler, 2004; Carvalho et al., 2005, 2007, 2008; Crisci, 2006a-b; Dubois, 2010c). They fail to address the core problems of the taxonomic impediment, which are $\{\theta 1\}$ the considerable manpower shortage of taxonomy and $\{\theta 2\}$ the many barriers put to the collection of specimens in natural habitats in many parts of the world. In order to face the taxonomic urgency, we would need a strong increase in the active field work by competent taxonomists worldwide. This would require an important increase in the number of positions of professional taxonomists (i.e., salaries) and in the funds allocated to field work, institutional collections of specimens, taxonomic revisions and publications. As well summarised by Carvalho et al. (2005), more than 'miracle solutions' (mostly based on technology instead of manpower), taxonomy requires "theoretical training, more professionals, a lasting commitment to collections, and recognition as a robust science by peers and policy-makers, without which taxonomy itself may fall victim to extinction".

Field collection of specimens, for large animals like amphibians and in terrestrial habitats accessible to man, does not require expensive techniques and equipment, but mostly manpower, brains and arms, 
i.e., salaries, plenty of working time, and the free access to natural habitats, with the possibility to collect and remove specimens from this habitat and store them in permanent collections. Although this may appear 'simple', such a 'program' has become complicated and difficult because of the shortage of salaries for such kinds of work in most countries, of legal restriction on collection and fixation of specimens in many parts of the world, and probably also, although this may appear paradoxical, of the absence of need for complex and costly techniques and equipments: all scientists know that it is much easier today to obtain large amounts of money for very expensive technical investments (which are always welcomed by the companies that produce them) than for salaries.

A recent, but important, problem that would have to be addressed before embarking on such a 'program' would be to deal with the legal aspects of collection of specimens, which is currently hampered by the many barriers put to this kind of research in many countries. Legislative problems barring the collection of specimens by taxonomists derive from a misunderstanding of the real impact of such collections on natural populations (Dubois 2003), and has been denounced by many taxonomists (Prathapan et al. 2018), without real effect so far. It is noteworthy and highly significant that the 'Buffon declaration' (Anonymous 2008; see Appendix A16.BUF below), adopted in 2007 in the Paris Museum by representatives of 93 natural history institutions from 36 countries and four continents, has never been advertised or even published by any of its promoters and authors.

Development of a strategy for deploying enough manpower for field work to approach an (almost) exhaustive taxonomy of amphibians worldwide would still be possible, but it would be a strong political act from the international scientific community, especially of batrachologists and herpetologists. A strategy could be devised to provide international support to all countries in the world for training amphibian taxonomists, for funding field work and taxonomic research, and for collection facilities.

Within such an international framework, with a strong public international support to this project, each country could endorse the aim of providing an (almost) exhaustive taxonomy of amphibians in its territory, in some cases with the support and contribution of specialists from other countries (at least for training new amphibian specialists).

Such an international strategy would require changes in the minds and habits of many taxonomists. For the time being, taxonomic research is largely an individual or institutional endeavour, and competition between colleagues, teams and institutions, if not countries, is an important characteristic of this work. Admittedly, in the last two decades more and more multi-authored studies have developed, involving often researchers and teams from the North and the South, especially in order to obtain large samples of specimens from various origins and taxa for cladistic analyses. But so far this has been mostly the result of agreements between individuals or institutions, not as an outcome of an international strategy or of cooperative programs carried out according to rational plans and transcending the traditional competitive approach of taxonomy, which is well illustrated by the predominance of a 'mihilist' approach to nomenclature (Dubois 2008a, 2015a). Therefore the impact of such studies on our knowledge of the amphibian diversity is very uneven from one country or one region of the world to another. This short-minded approach is certainly allowed, and even encouraged, by the current nomenclatural rules according to which the Latin scientific nomen of a species is attached to the name of its 'author', who is the first person (or group of persons) to have published a description of the species. A shift to a different attitude, promoting mutual training and collaboration between specialists worldwide, with a common aim for all, rather than competition for 'priority', would be a major change (Dubois 2008a, 2015a). But, at the time when species are becoming extinct by thousands in front of us in the almost complete indifference of our societies, would not this aim be more exciting for any taxonomist than to be the "first one' to describe and name a new species?

\subsubsection{Preventive taxonomy}

Even if such an international collaborative strategy may sound today a bit like a dream, it would appear possible immediately to develop a new 'culture' in taxonomy, at least regarding special urgencies. In many countries, destruction of natural habitats is progressing at a very fast pace, and leaves little time for long-term or medium-term programs for the collection and study of biodiversity. In such cases, it would be useful to define priorities for urgent taxonomic surveys, especially when the habitat destructions can be foreseen because of well-known collective decisions. From a taxonomic point of view, the urgency of exploration and specimen collection is particularly high for habitats and ecosystems 
which are known to be soon threatened by programmed deforestation, change in agricultural practices, construction of roads or buildings, flooding by dam lakes or various other expected habitat destructions. The community of taxonomists could consider developing special tools to deal with such situations. For this we could take advantage of the experiences developed in the recent years in other research fields.

The community of archaeologists has implemented preventive archaeology (see e.g. BozókiErnycy 2007) and rescue archaeology (see e.g. Demoule 2002), which provide methods, funding and manpower to allow rapid archaeological surveys of sites when their deposits are threatened with partial or total destruction. The community of conservation biologists has developed similar tools, for example the Rapid Assessment Programs (see e.g. Muchoney et al. 1991) which allow realising quick ecological surveys of little-known areas critical for biodiversity conservation. In a few cases, conservation biologists have proved able to carry out important programs to 'save' some of the species of some ecosystems before their destruction, e.g. by displacing them. Among many others, one such example is the program of 'ecological survey' and 'protection of the terrestrial fauna' developed in the nineties in French Guiana concerning $300 \mathrm{~km}^{2}$ of primary forest due to be flooded by the construction of the dam of Petit Saut on the river Sinnamary: this program produced interesting results concerning the consequences of the fragmentation of a humid tropical forest, mostly on the populations of endotherm vertebrates (Lecomte 1997; Forget 2002), but it did not include any taxonomic part, although the species of many groups of 'small organisms' of this area were far from being known. Actually, in some cases, participants of the program were even discouraged from collecting specimens for so-called 'conservation' and legal reasons, although it was clear that in the coming months many of these organisms with limited capacities of displacements were sure to be drowned, or, if displaced somewhere else, would enter in competition with resident populations or species.

Isn't it time for taxonomists to promote a preventive taxonomy? As soon as a threat on a habitat is identified, special field work could be organised, not to 'save' the species, which in most cases is fully unrealistic in most animal groups including amphibians, but to collect specimens, tissues and information, to index and store them in safe conditions. This would require the implementation of ad hoc techniques for rapid collection of specimens, tissues and data in all taxonomic groups. This would also require solving the legal problems associated with collection and fixation of specimens in such special circumstances. Implementation of preventive taxonomy would probably need, just like in archaeology, the special training of teams of field taxonomists ready for such interventions. Amphibians, being a limited group of rather large organisms, could be an excellent group to test these techniques and start such 'last minute' collections. Even if time and manpower are currently lacking for studying properly these specimens, at least the latter would not completely vanish forever. A testimony of their existence could be kept for the future generations. This material might possibly be used later on for some unexpected discoveries and for a better knowledge of the organisms that were present on the blue planet before mankind appeared and devastated it.

\subsubsection{Time is for field work and collections}

Taxonomists in the century of extinctions do not only need new data on the known taxa and on 'discovering' new branches of the tree of life. Such 'exciting' new findings do not make obsolete the need of obtention of new specimens and data which do not deserve erection of new taxa. Organic evolution is not teleological. It results from a variable combination of 'chance and necessity' (Monod 1970), i.e. of genetic variation and natural selection, and as such is not deterministic and predictable but statistical. The frequent use in the recent literature (e.g. Wheeler 2001) of the formula 'predictive classification' means that phylogenetic classifications may allow to predict some characters of known species that have not yet been studied, but not the characters, or a fortiori the mere existence, of species not yet collected by scientists. In this respect, phylogenetic taxonomy would be more accurately described as 'postdictive'. However accurate they can be, given the data then available, no 'model' or phylogenetic analysis would have allowed to anticipate the existence of Astrobatrachus, Nasikabatrachus, Rheobatrachus, Karsenia or Urspelerpes, or to foresee that adults of Barbourula kalimantanensis lack lungs, that some Amietia have 'invented' a corneal elygium, that Nymbaphrynoides toads are viviparous, that the tadpoles of Mertensophryne have dorsal crowns and that those of Amolops and other anurans are gastromyzophorous. The only way to know the biodiversity of our planet is to study it for itself, not only the phylogenetic relationships between its members. We have now reached a point where, for 
amphibians like for many other groups, "time is for field work and collections" (Dubois 2010-2014). If the international community of batrachologists continues to ignore this imperative and this urgency, it will not be exaggerated to state that it has contributed in its sphere, which is mostly that of knowledge, to the irreversible losses caused by our civilisation to the biodiversity of our planet.

\section{ACKNOWLEDGEMENTS}

Funding for RAP was provided by US NSF grants DBI-0905765 and DEB-1441719, and by a fellowship from the MNHN program Labex BCDiv ('Diversités biologiques et culturelles'). Thierry Frétey (Saint-Maugan) made important contributions to various stages of the manuscript; parts of his work had support from funds of the UMR 7205 ISYEB. Andrea Kourgli (Vienna) and Victoire Koyamba (Paris) continuousely supported literature search during the long period of this project. Many other colleagues have allowed us to access the complete literature or provided useful information or help on different matters: Kraig Adler (Ithaca), Franco Andreone (Torino), Aaron Bauer (Villanova), Roger Bour $\uparrow$ (Montgeron), Josslin Cayer (Lanrelas), Aubierge Desalme (Paris), Jean Dubois (Lanrelas), Marissa Fabrezzi (Salta), Martin Grillitsch (Wien), Oleksandr Kovalchuk (Kyiv), Gloria Lekaj (Wien), Stefan Lötters (Trier), Marco A. Mendez (Santiago de Chile), Andrew R. Milner (London), Violaine Nicolas (Paris), Fabio Petti (Trento), Elizabeth Prendini (New York), Thomas Prikryl (Praha), Ingrid Ramirer (Wien), Jean Raffaëlli (Penclen), Elie Mario Saliba (Paris), Rainer Schoch (Stuttgart), Oleksandr Yahin (Kyiv) and Hussam Zaher (São Paulo). We are grateful to Aaron Bauer and two anonymous referees for their useful comments on a first version of the manuscript of this work. Finally, we would like to commend the works of hundreds of taxonomists, zoologists and other biologists which over 250 years have permanently increased and improved our knowledge of the amphibians of the world, allowing taxonomists now to have a rather good overview of the evolution and classification of these fascinating animals.

\section{REFERENCES}

The list below gives the references of all the publications cited in this work, except those cited in Appendix A5.NGS and not elsewhere, for reasons of space parsimony (see 3.2 above).

One at least of the three authors of the present work personally examined each of the works cited below, except those we could not obtain, which are marked here as [Not seen].

\section{Information provided at the end of reference}

[1758.la], [1796.ba], etc. $\bullet$ Identifier of the publication used in the identifiers of nomina and their paronyms in Appendices A5.NGS, A6.NFS, A7.NCS and A8.ECT.

[1968] - Identifier of the publication used in Appendix A13.QUA

$\{\mathrm{Q}\} \bullet$ Publication used in the computation of the numbers of usages of nomina in Appendix A13.QUA.

$\lceil\mathrm{P} 00],\{\mathrm{P} 01\}$, etc. $\bullet$ Note providing actual publication date or relative chronology of publication of works published in the same year, according to either published evidence of more precise publication dates, or citation of one work in the other (first page of citation only mentioned):

Work published first $>$ work published second.

Work published second $<$ work published first.

See list of these notes at the end of the list of references. 


\section{Websites}

ASW (2020a) Frost, D. Amphibian Species of the World. Version 6.1. American Museum of Natural History, New York, U.S.A. https://amphibiansoftheworld.amnh.org/index.php

ASW (2020b) Frost, D. Amphibian Species of the World. The big changes in amphibian taxonomy (2006-2013): versions 5.6 and 6.0

https://amphibiansoftheworld.amnh.org/The-big-changes-inamphibian-taxonomy-2006-2013-versions-5.6-and-6.0

AWb (2020) Amphibia Web. University of California, Berkeley, U.S.A.

https://amphibiaweb.org

GBIF (2020) Global Biodiversity Information Facility. GBIF Secretariat, Copenhagen, Denmark. https://www.gbif.org

ITIS (2020) Integrated Taxonomic Information System. U.S. Geological Survey, Reston, Virginia, U.S.A. https://www.itis.gov

SN (2020) Brands, S.J. (Ed.), Systema Naturae 2000. Universal Taxonomic Services, Amsterdam (Zwaag). http://sn2000.taxonomy.nl

Taxonomicon (2020) Brands, S.J. (Ed.). The Taxonomicon. Universal Taxonomic Services, Zwaag, The Netherlands. http://taxonomicon.taxonomy.nl

ToL (2020) The Tree of Life Web Project. http://tolweb.org

uBio (2020) Universal Biological Indexer and Organiser. http://www.ubio.org/

ZR (1864-2020) Zoological Record. https://www.biodiversitylibrary.org/bibliography/119689\#/ summary

\section{Anonymous publications}

Anonymous (1922) Opinion 72. Herrera's zoological formulae. In: Opinions rendered by the International Commission on Zoological Nomenclature; in: Smithsonian miscellaneous Collections, 73 (1), 19-22.

Anonymous (1967) Lista actualizada de los trabajos, comentarios bibliograficos e informaciones publicados en la Revista de la Asociación geológica argentina, tomo I (1946) al tomo XXII (1967). Revista de la Asociación geológica argentina, 22 (4), 313-324. [1967].

Anonymous (1968) Opinion 849. Kassina Girard, 1853 (Amphibia): grant under the plenary powers of precedence over Hylambates Duméril, 1853. Bulletin of zoological Nomenclature, 25 (1), 20-21.

Anonymous (1976) Geographic distribution. Herpetological Review, 7 (3), 128. [1976.aa].

Anonymous (1982) Notices. Bulletin of zoological Nomenclature, 39 (4), 229-230.

Anonymous [International Commission on Zoological Nomenclature] (1993) Opinion 1749. Epicrium Wagler, 1828 and Ichthyophiidae Taylor, 1968 (Amphibia, Gymnophiona): conserved, and Epicriidae Berlese, 1885 (Arachnida, Acari): conserved by the emendation of Epicriidae Fitzinger, 1843 (Amphibia, Gymnophiona) to Epicriumidae. Bulletin of zoological Nomenclature, 50 (3), 261-263. [1993.aa].

Anonymous [Systematics Agenda 2000] (1994). Systematics Agenda 2000: Charting the biosphere: a global initiative to discover, describe and classify the world's species. Technical report. American Museum of Natural History, American Society of Plant Taxonomy, Society of Systematic Biologists, Willi Hennig Society, New York, 34 pp.

Anonymous [International Commission on Zoological Nomenclature] (1996) Opinion 1830. Caeciliidae Kolbe, 1880 (Insecta, Psocoptera): spelling emended to Caeciliusidae, so emoving the homonymy with Caeciliiidae Rafinesque, 1814 (Amphibia, Gymnophiona). Bulletin of zoological Nomenclature, 53, 68-69. [1996.aa].

Anonymous [International Commission on Zoological Nomenclature] (1997) Opinion 1873. Hemidactyliini Hallowell, 1856 (Amphibia, Caudata): conserved. Bulletin of zoological Nomenclature, 54 (2), 140-141. [1997.aa].

Anonymous [International Commission on Zoological Nomenclature] (1999) Opinion 1921. Petropedetinae Noble, 1931, Cacosterninae Noble, 1931 and Phrynobatrachinae Laurent, 1941 (Amphibia, Anura): given precedence over Hemimantidae Hoffmann, 1878, and Phrynobatrachinae, not given precedence over Petropedetinae Bulletin of zoological Nomenclature, 56 (1), 96-100. [1999.aa].

Anonymous [International Commission on Zoological Nomenclature] (1999) International code of zoological nomenclature. 'Fourth edition'. International Trust for zoological Nomenclature, London, $\mathrm{i}-\mathrm{xxix}+306 \mathrm{pp}$.

Anonymous [The Buffon Symposium] (2008) The Buffon Declaration. Natural History Institutions and the Environmental Crisis. Concluding Message from the Buffon Symposium - October 18th and 19th, 2007 Muséum National d'Histoire Naturelle, Paris.

http://www.mnhn.fr/museum/front/medias/pdf/13696_The Buffon_Declaration_CBDmay08_Eng.pdf

Anonymous [International Commission on Zoological Nomenclature] (2009) Opinion 2223 (Case 3345). Dendrobatidae Cope, 1865 (1850) (Amphibia, Anura): familygroup name conserved. Bulletin of zoological Nomenclature, 66 (1), 103-105. [2009.aa].

https://doi.org/10.21805/bzn.v66i1.a17

Anonymous [Convention on Biological Diversity] (2011) Convention on Biological Diversity.

http://www.cbd.int/

Anonymous [International Commission on Zoological Nomenclature] (2012) Amendment of Articles 8, 9, 10, 21 and 78 of the International Code of Zoological Nomenclature to expand and refine methods of publication. Bulletin of zoological Nomenclature, 69 (3), 161-169.

https://doi.org/10.21805/bzn.v69i3.a8.161 


\section{Onymous publications}

Abel, O. (1919) Die Stämme der Wirbelthiere. Walter de Gruyter \& Co., Berlin \& Leipzig, i-xviii + 914 pp. [1919.aa].

Abraham, R.K., Pyron, R.A., Ansil, B.R., Zachariah, A. \& Zachariah, A. (2013) Two novel genera and one new species of treefrog (Anura: Rhacophoridae) highlight cryptic diversity in the Western Ghats of India. Zootaxa, 3640 (2), 177-199. https://doi.org/10.11646/zootaxa.3640.2.3

Acloque, A. (1900) Faune de France. Poissons, Reptiles, Batraciens, Tuniciers. Baillière, Paris, i-ix + pp. 339-548. [1900.aa].

Adler, K. \& Zhao, E. (1990) Studies on hynobiid salamanders, with description of a new genus. Herpetological Research, 4, 37-45.

Agassiz, L. (1843) Nomina systematica generum Vermium (Annulatorum, Turbellarium et Entozoorum), tam viventium quam fossilium... Jent \& Gassmann, Soloduri, i-vi + 14 pp. + 5 pp. + [i-ii]. [P06] [1843.aa].

Agassiz, L. (1844) Nomina systematica generum Reptilium, tam viventium quam fossilium, secundum ordinem alphabeticum disposita, adjectis auctoribus, libris in quibus reperiuntur, anno editionis, etymologia et familiis, ad quas pertinent. Jent \& Gassmann, Soloduri, i-vii +48 pp. https://doi.org/10.5962/bhl.title.12046

Agassiz, L. (1845) Reptilia (Addenda et corrigenda.). Jent \& Gassmann, Soloduri, $10 \mathrm{pp}$.

Agassiz, L. (1847) Nomenclatoris zoologici. Index universalis continens nomina systematica classium, ordinum, familiarum et generum animalium omnium, tam viventium quam fossilium, secundum ordinem alphabeticum unicum disposita, adjectis homonymiis plantarum, nec non variis adnotationibus et emendationibus. '1846'. Jent \& Gassmann, Soloduri, i-viii $+393 \mathrm{pp}$.

Agassiz, L. (1848) Nomenclatoris zoologici. Index universalis, continens nomina systematica classium, ordinum, familiarum et generum animalium omnium, tam viventium quam fossilium, secundum ordinem alphabeticum unicum disposita, adjectis homonymiis plantarum. Jent \& Gassmann, Soloduri, $\mathrm{i}-\mathrm{x}+$ $1135 \mathrm{pp}$. https://doi.org/10.5962/bhl.title.1819

Ahl, E. (1924) Neue afrikanische Frösche. Zoologischer Anzeiger, 61, 99-103.

Ahl, E. (1930) Neuere Erkenntnisse über die systematische Einteilung der Amphibien. Sitzungsberichte der Gesellschaft naturforschender Freunde, 1930, 78-85. [1930.aa].

Ahl, E. (1931) Amphibia, Anura III, Polypedatidae. Das Thierreich, 55, i-xvi +477 pp. $\{\mathrm{Q}\}$ https://doi.org/10.1515/9783111625430

Ahyong, S.T., Lowry, J.K., Alonso, M., Bamber, R.N.,Boxshall, G.A., Castro, P., Gerken, S., Karaman, G.S., Goy, J.W., Jones, D.S., Meland, K., Rogers, D.C. \& Svavarsson, J. (2011) Subphylum Crustacea Brünnich, 1772. In: Zhang, Z.-Q. (Ed.), Animal biodiversity: an outline of higher-level classification and survey of taxonomic richness. Zootaxa, 3148 (1), 165191.

https://doi.org/10.11646/zootaxa.3148.1.33
Allmon, W. (2017) Species, lineages, splitting, and divergence: why we still need 'anagenesis' and 'cladogenesis'. Biological Journal of the Linnean Society, 120 (2), 474-479. https://doi.org/10.1111/bij.12885

Alonso-Zarazaga, M.A. (2005) Nomenclature of higher taxa: a new approach. Bulletin of zoological Nomenclature, 62 (4), 189-199.

Altig, R. \& Johnston, G.F. (1989) Guilds of anuran larvae: relationships among developmental modes, morphologies, and habitats. Herpetological Monographs, 3, 81-109. https://doi.org/10.2307/1466987

Amiet, J.-L.(1974) La ponte et la larve d'Opisthothylax immaculatus Boulenger (Amphibien Anoure). Annales de la Faculté des Sciences du Cameroun, 17, 121-130.

Amiet, J.-L. (1976) Observations anatomiques et biologiques sur le genre Werneria Poche, 1903. Revue de Zoologie et Botanique africaines, 90, 33-45.

Amiet, J.-L. (1981) Revision du genre Leptodactylodon Andersson (Amphibia, Anura, Astylosterninae). Annales de la Faculté des Sciences du Cameroun, 27, 69-224.

Ananjeva, N.B., Borkin, L.J., Darevsky, I.S. \& Orlov, N.L. (1988) Dictionary of animal names in five languages. Russky Yazyk Publishers, Moscow, 556 pp. $\{\mathrm{Q}\}$.

Anderson, J. (1871) A list of the reptilian accessions to the Indian Museum, Calcutta, from 1865 to 1870 , with a description of some new species. Journal of the Asiatic Society of Bengal, 40, 12-39. [1871.aa].

Andreone, F. \& Nussbaum, R.A. (2006) A revision of Mantidactylus microtis and M. microtympanum, and a comparison with other large Madagascan stream frogs (Anura: Mantellidae: Mantellinae). Zootaxa, 1105 (1), 49-68. https://doi.org/10.11646/zootaxa.1105.1.5

Anisimova, M., Gil, M., Dufayard, J.F., Dessimoz, C. \& Gascuel, O. (2011) Survey of branch support methods demonstrates accuracy, power, and robustness of fast likelihood-based approximation schemes. Systematic Biology, 60 (5), 685699.

https://doi.org/10.1093/sysbio/syr041

Annandale, N. (1912) Zoological results of the Abor Expedition, 1911-1912. I. Amphibia. Records of the Indian Museum, 8, 7-36, 4 pls. https://doi.org/10.5962/bhl.part.1186

Annandale, N. (1915) Herpetological notes and descriptions. Records of the Indian Museum, 11, 341-347, 1 pl.

Annandale, N. (1917) Zoological results of a tour in the Far East. Batrachia. Memoirs of the Asiatic Society of Bengal, 6, 119155.

Aranciaga-Rolando, A.M., Agnolin, F.L. \& Corsolini, J. (2019) A new pipoid frog (Anura, Pipimorpha) from the Paleogene of Patagonia. Paleobiogeographical implications. Comptes rendus Palevol, 18, 725-734. [2019.aa]. https://doi.org/10.1016/j.crpv.2019.04.003

Araujo-Vieira, K., Luna, M.C., Caramaschi, U. \& Haddad, C.F.B. (2020) A new genus of lime treefrogs (Anura: Hylidae: Sphaenorhynchini). Zoologischer Anzeiger, 286, 81-89. https://doi.org/10.1016/j.jcz.2020.04.002 
Ardila-Robayo, M.C. (1979) Status sistemático del género Geobatrachus Ruthven 1915 (Amphibia: Anura). Caldasia, 12, 383-495, 1 pl. [1979.aa].

Arribas, O.J. (2016) Why Caucasilacerta Harris, Arnold \& Thomas, 1998 is a nomen nudum? Russian Journal of Herpetology, 23 (4) 305-306.

Ashlock, P.D. (1971) Monophyly and associated terms. Systematic Biology, 20, 63-69. https://doi.org/10.1093/sysbio/20.1.63

Ashlock, P.D. (1984) Monophyly: its meaning and importance. In: Duncan, T. \& Stuessy, T.F. (Eds.), Cladistics: perspectives on the reconstruction of evolutionary history. Columbia University Press, New York, pp. 39-46. https://doi.org/10.7312/dunc90660-006

Aubekerova-Tleuberdina, P.A. (1977) Сопоставление Есекартканской фауны с гиппарионовыми и позднегиппарионовыми фаунами Азии. [The comparison of the Esekartkan fauna with Hipparion and late Hipparion faunas of Asia]. In: Мезокайнозойская фауна и флора северо-западного Казахстана [Fauna and flora of Mesozoic and Cainozoic [sic] periods in north-western Kazakhstan], Материалы по истории фауны и флоры Казахстана [Materials on the history of fauna and flora.of Kazakhstan], 7, 75-81. [In Russian]. [1977.ab].

Auffenberg, W. (1958) A new family of Miocene salamanders from the Texas coastal plain. Quarterly Journal of the Florida Academy of Sciences, 21 (2), 169-176. [1958.aa].

Auffenberg, W. \& Goin, C.J. (1959) The status of the salamander genera Scapherpeton and Hemitrypus of Cope. American Museum Novitates, 1979, 1-12. [1959.aa].

Austin, J., Lougheed, S., Tanner, K., Chek, A.A., Bogart, J. \& Boag, P. (2002) A molecular perspective on the evolutionary affinities of an enigmatic neotropical frog, Allophryne ruthveni. Zoological Journal of the Linnean Society, 134, $335-346$

https://doi.org/10.1046/j.1096-3642.2002.00011.x

Averianov, A. \& Sues, H.-D. (2012) Correlation of late Cretaceous continental vertebrate assemblages in middle and central Asia. Journal of Statigraphy, 36 (2), 462-485. [2012.aa].

Avise, J.C. (2008) Clonality. The genetics, ecology, and evolution of sexual abstinence in vertebrate animals. Oxford University Press, New York, i-xi +237 pp.

Avise, J.C. \& Johns, G.C. (1999) Proposal for a standardized temporal scheme of biological classification for extant species. Proceedings of the national Academy of Sciences, 96, 7358-7363.

https://doi.org/10.1073/pnas.96.13.7358

Avise, J.C. \& Liu, J.-X. (2011) On the temporal inconsistencies of Linnean taxonomic ranks. Biological Journal of the Linnean Society, 102, 707-714.

https://doi.org/10.1111/j.1095-8312.2011.01624.x

Avise, J.C. \& Mitchell, D. (2007) Time to standardize taxonomies. Systematic Biology, 56, 130-133. https://doi.org/10.1080/10635150601145365

Ayarzagüena, J., Señaris, J.C. \& Gorzula, S. (1993) Un nuevo género para les especies de grupo Osteocephalus rodriguezi (Anura:
Hylidae). Memoria de la Sociedad de Ciencias naturales La Salle, 52, 213-221.

Ax, P. (1984) Das phylogenetische System: Systematisierung der lebenden Natur aufgrund ihrer Phylogenese. Fischer, Stuttgart \& New York, 349 pp. [Not seen].

Baêta, D., Giasson, L., Pombal, J. \& Haddad, C. (2016) Review of the rare genus Phrynomedusa Miranda-Ribeiro, 1923 (Anura: Phyllomedusidae) with description of a new species. Herpetological Monographs, 30, 49-78.

https://doi.org/10.1655/HERPMONOGRAPHS-D-1500009.1

Báez, A.M. \& Pugener, L.A. (2003) Ontogeny of a new Palaeogene pipid frog from southern South America and xenopodinomorph evolution. Zoological Journal of the Linnean Society, 139, 439-476. [2003.ba]. https://doi.org/10.1046/j.1096-3642.2003.00085.x

Baird, S.F. (1850) Revision of the North American Tailed-Batrachia, with descriptions of new genera and species. Journal of the Academy of natural Sciences of Philadelphia, '1849', (2) 1, 281-294. [P07] [1850.ba].

Baird, S.F. (1851) Vertebrata. Class II. Reptilia. In: Heck, J.G. (Ed.), Iconographic encyclopedia of science, literature, and art. Vol. 2. Botany, zoology, anthropology, and surgery, Rudolph Garrigue, New York, pp. 244-289. [1851.ba].

Baldo, D., Cotichelli, L., Pereyra, M.O., Borteiro, C., Netto, F., Kolenc, F., Brusquetti, F. \& Bidau, C. (2012) A cytotaxonomic survey of the genus Melanophryniscus Gallardo, 1961 (Anura: Bufonidae). Journal of Herpetology, 46, 25-32. https://doi.org/10.1670/10-293

Baldo, D., Vera Candioti, F., Haad, B., Kolenc, F., Borteiro, C., Pereyra, M., Zank, C., Colombo, P., Bornschein, M., Netto, F., Brusquetti, F., Conte, C., Costa, P., Almeida-Santos, P. \& Pie, M. (2014) Comparative morphology of pond, stream and phytotelm-dwelling tadpoles of the South American redbelly toads (Anura: Bufonidae: Melanophryniscus). Biological Journal of the Linnean Society, 112, 417-441. https://doi.org/10.1111/bij.12296

Barbadillo, L.J., García-París, M. \& Sanchíz, B. (1997) Orígenes y relaciones evolutivas de la herpetofauna ibérica. In: Pleguezuelos, J.M. (Ed.), Distribución y biogeografia de los anfibios y reptiles en España y Portugal, Universidad de Granada \& Asociación Herpetológica Española, Granada, Spain, pp. 47-100. [1997.ba].

Barbour, T. (1938) Notes on 'Nectophryne'. Proceedings of the biological Society of Washington, 51, 191-196.

Barej, M.F., Schmitz, A., Günther, R., Loader, S.P., Mahlow, K. \& Rödel, M.-O. (2014) The first endemic West African vertebrate family - a new anuran family highlighting the uniqueness of the Upper Guinean biodiversity hotspot. Frontiers in Zoology, 11 (8), [i] + 1-10 + online additional file (1-27). [2014.ba]. https://doi.org/10.1186/1742-9994-11-8

Barej, M., Schmitz, A., Penner, J., Doumbia, J., SandbergerLoua, L., Petersen, M., Brede, C., Emmrich, M., Germain Kouamé, N., Hillers, A., Gonwouo, N., Nopper, J., Adeba, P., Bangoura, M., Gage, C., Anderson, G. \& Rödel, M.-O. (2015) Life in the spray zone- - overlooked diversity in West African 
torrent-frogs (Anura, Odontobatrachidae, Odontobatrachus). Zoosystematics and Evolution, 91, 115-149.

https://doi.org/10.3897/zse.91.5127

Barigozzi, C. (Ed.) (1982) Mechanisms of speciation. Liss, New York, i-xiv $+546 \mathrm{pp}$.

Barrio, A. (1968) Incorporación a la batracofauna argentina de la familia Centrolenidae (Anura). Physis, 28, 165-169. [1968. ba].

Barrow, L.N., Ralicki, H.F., Emme, S.A. \& Lemmon, E.M. (2014) Species tree estimation of North American chorus frogs (Hylidae: Pseudacris) with parallel tagged amplicon sequencing. Molecular Phylogenetics and Evolution, 75, 78-90.

https://doi.org/10.1016/j.ympev.2014.02.007

Batista, A., Hertz, A., Mebert, K., Köhler, G., Lotzkat, S., Ponce, M. \& Vesely, M. (2014) Two new fringe-limbed frogs of the genus Ecnomiohyla (Anura: Hylidae) from Panama. Zootaxa, 3826 (3), 449-474.

https://doi.org/10.11646/zootaxa.3826.3.2

Batsch, A.J.G.C. (1788) Versuch einer Anleitung, zur Kennniss und Geschichte der Thiere und Mineralien. Erster Theil. Akademischen Buchhandlung, Jena, i-viii + 528 pp., pls. 1-5. [1788.ba].

Batsch, A.J.G.C. (1789) Versuch einer Anleitung, zur Kenntniß und Geschichte der Thiere und Mineralien, für akademische Vorlesungen entworfen, und mit den nöthigsten Abbildungen versehen. Zweyter Theil. Besondre Geschichte der Insekten, Gewürme und Mineralien. Akademische Buchhandlung, Jena, [i] + 520-690 pp. + [i-ii].

https://doi.org/10.5962/bhl.title.79854

Batsch, A.J.G.C. (1796) Umriß der gesammten Naturgeschichte: ein Auszug aus den frühern Handbüchern des Verfassers für seine Vorlesungen. Christian Ernst Gabler, Jena \& Leipzig, $\mathrm{i}-\mathrm{xvi}+1-287 \mathrm{pp}+3-160 \mathrm{pp}+[1-32 \mathrm{pp}]+[\mathrm{i}-\mathrm{-vi}]+1-80 \mathrm{pp}$. $\{\mathrm{Q}\}$ [1796.ba].

Bauer, A.M., Parham, J.F., Brown, R.M., Stuart, B.L., Grismer, L., Papenfuss, T.J., Böhme, W., Savage, J.M., Carranza, S., Grismer, J.L., Wagner, P., Schmitz, A., Ananjeva, N.B. \& Inger, R.F. (2010) Availability of new Bayesian-delimited gecko names and the importance of character-based species descriptions. Proceedings of the royal Society, (B), Biological Sciences, 278 (1705), 490-492.

https://doi.org/10.1098/rspb.2010.1330

Bauer, L. (1985) Considerations about Rana with a proposal of new genera. / Overpeinzingen betreffende Rana met een voorstel van nieuwe genusnamen. Ripa, 1 November 1985, E 1-9 + D N1-N7. [1985.ba].

Bauer, L. (1986) The lightly armed mud soldier. Essay on some African anurans with a resurrection and a new genus. Ripa, April 1986, [i-viii]. [1986.ba].

Bauer, L. (1987a) Dikkoptypen. Herwaardering van bekende gegevens en een nieuwe typering van dikkopjes. Ripa, February 1987, 1-9. [1987.ba].

Bauer, L. (1987b) Maar het is geen Dendrobates! D. silverstonei hoort thuis in een ondergeslacht van Phyllobates. Aanvulling/ correctie bij twee eerdere artikelen. Ripa, April 1987, 1-8. [1987.bb].

Bauer, L. (1987c) Thoughts on phylogeny and classification of anurans. In: Van Gelder, J.J., Strijbosch H. \& Bergers, P.J.M. (Eds.), Proceedings of the 4th Ordinary General Meeting of the Societas Europaea Herpetologica, Nijmegen, 17-21 August 1987, pp. 49-54. [1987.bc].

Bauer, L. (1988) Pijlgifkikkers en verwanten: de familie Dendrobatidae. Het Paludarium November 1988, D 1-6, D-E 5-6. [1988.ba].

Bayer, F. (1885) O kostře žab z čeledi pelobatid. Příspěvek srovnávací osteilogii obojživelníkův. Abhandlungen der Königlichen Böhmischen Gesellschaft der Wissenschaften '1884', (6), 12 (13), 1-24, pl. 1-2. [In Czech]. [P00 ] [1885. ba].

Beaulieu, F., Dowling, A.P.G., Klompen, H., De Moraes, G.J. \& Walter, D.E. (2011) Superorder Parasitiformes Reuter, 1909. In: Zhang, Z.-Q. (Ed.), Animal biodiversity: an outline of higher-level classification and survey of taxonomic richness. Zootaxa, 3148 (1), 123-128.

https://doi.org/10.11646/zootaxa.3148.1.23

Bedriaga, J. v. (1881) Die Amphibien und Reptilien Griechenlands. Bulletin de la Société impériale des Naturalistes de Moscou, 56 (2), 242-310.

Bell, R.C. \& Zamudio, K.R. (2012) Sexual dichromatism in frogs: natural selection, sexual selection and unexpected diversity. Proceedings of the royal Society, (B), 279, 4687-4693. https://doi.org/10.1098/rspb.2012.1609

Bell, C.L. (1839) A history of British Reptiles. J. van Oorst, London, i-xxiv +142 pp. [1839.ba]. https://doi.org/10.5962/bhl.title.10835

Bell, T. (1836) Amphibia. In: Todd, R.B. (Ed.), The cyclopedia of anatomy and physiology. 1. A-DEA, Longman, Brown, Green, Longmans \& Roberts, London, pp. 90-107. [1836.ba].

Benton, M.J. (1993) The fossil record. 2. Chapman \& Hall, London, $\mathrm{i}-\mathrm{xvii}+529-845 \mathrm{pp} .\{\mathrm{Q}\}$.

Benton, M.J. (2000) Stems, nodes, crown clades, and rank-free lists: is Linnaeus dead? Biological Reviews, 75 (4), 633-648. https://doi.org/10.1111/j.1469-185X.2000.tb00055.x

Bernardi, G. (1956) Contribution à l'étude des catégories taxonomiques. I. Avant-propos, nomenclature et définitions. Bulletin de la Société entomologique de France, 61 (9-10), 194-200.

Bernardi, G. (1957) Contribution à l'étude des catégories taxonomiques. II. Les Règles internationales de la nomenclature zoologique et la notation des catégories taxonomiques. Bulletin de la Société entomologique de France, 62 (9-10), 224-250.

Bernardi, G. (1980) Les catégories taxonomiques de la systématique évolutive. In: Bocquet, C., Génermont, J. \& Lamotte, M. (Eds.), Les problèmes de l'espèce dans le règne animal 3, Mémoires de la Société zoologique de France, 40, pp. 373425.

Berthold, A.A. (1827) Latreille's natürliche Familien des Thierreichs aus dem aus dem Französischen mit Anmerkungen und Zusätzen. Landes-Industrie Comptoir, Weimar, $\mathrm{i}-\mathrm{x}+606$ pp. [1827.ba]. 
Bertrand, Y., Pleijel, F. \& Rouse, G.W. (2006) Taxonomic surrogacy in biodiversity assessments, and the meaning of Linnaean ranks. Systematics \& Biodiversity, 4 (2), 149-159. https://doi.org/10.1017/S1477200005001908

Betta, E. de (1857) Erpetologia delle provincie venete e del Tirolo meridionale. Vicentini \& Franchini, Verona, i-xvi $+1-365$. [1857.ba].

Betta, E. de (1864) Monografia degli anfibi urodeli italiani e più diffusamente delle specie viventi nelle provincie venete. Memorie dell'Istituto Veneto di Scienze Lettere ed Arti, (3) 11, 495-569, pl. 25. [1864.ba].

Beukema, W., De Pous, P., Donaire-Barroso, D., Bogaerts, S., Garcia-Porta, J., Escoriza, D., Arribas, O.J., El Mouden, E.H. $\&$ Carranza, S. (2013) Review of the systematics, distribution, biogeography and natural history of Moroccan amphibians. Zootaxa, 3661 (1), 1-60. https://doi.org/10.11646/zootaxa.3661.1.1

Bewick, A.J., Chain, F.J.J., Heled, J. \& Evans, B.J. (2012) The pipid root. Systematic Biology, 61 (6), 913-926. [2012.ba]. https://doi.org/10.1093/sysbio/sys039

Biju, S.D. (2003) Reproductive mode in the shrub frog Philautus glandulosus (Jerdon, 1853) (Anura: Rhacophoridae). Current Science, 84, 283-284.

Biju, S.D. \& Bossuyt, F. (2003) New frog family from India reveals an ancient biogeographical link with the Seychelles. Nature, 425, 711-714. [2003.bb]. https://doi.org/10.1038/nature02019

Biju, S.D. \& Bossuyt, F. (2005a) Two new Philautus (Anura: Ranidae: Rhacophorinae) from Ponmudi Hill in the Western Ghats of India. Copeia, 2005, 29-37. https://doi.org/10.1643/CH-04-194R1

Biju, S.D. \& Bossuyt, F. (2005b) New species of Philautus (Anura: Ranidae, Rhacophorinae) from Ponmudi Hill in the Western Ghats of India. Journal of Herpetology, 39, 349-353. https://doi.org/10.1670/133-04A.1

Biju, S.D. \& Bossuyt, F. (2006) Two new species of Philautus (Anura, Ranidae, Rhacophorinae) from the Western Ghats, India. Amphibia-Reptilia, 27, 1-9. https://doi.org/10.1163/156853806776051985

Biju, S.D. \& Bossuyt, F. (2009) Systematics and phylogeny of Philautus Gistel, 1848 (Anura, Rhacophoridae) in the Western Ghats of India, with description of 12 new species. Zoological Journal of the Linnean Society, 155, 374-444. https://doi.org/10.1111/j.1096-3642.2008.00466.x

Biju, S.D., Kamei, R.G., Mahony, S., Thomas, A. \& Garg, S. (2013) Taxonomic review of the tree frog genus Rhacophorus from the Western Ghats, India (Anura: Rhacophoridae), with description of ontogenetic colour changes and reproductive behaviour. Zootaxa, 3636 (2), 257-289.

https://doi.org/10.11646/zootaxa.3636.2.3

Biju, S.D., Roelants, K. \& Bossuyt, F. (2008) Phylogenetic position of the montane treefrog Polypedates variabilis Jerdon, 1853 (Anura: Rhacophoridae), and description of a related species. Organisms, Diversity and Evolution, 8, 267-276. https://doi.org/10.1016/j.ode.2007.11.004

Biju, S.D., Senevirathne, G., Garg, S., Mahony, S., Kamei, R.G.,
Thomas, A., Shouche, Y., Raxworthy, C.J., Meegaskumbura, M. \& Bocxlaer, I. Van (2016) Frankixalus, a new rhacophorid genus of tree hole breeding frogs with oophagous tadpoles. PloS One, 11 (e0145727), 1-17.

https://doi.org/10.1371/journal.pone.0145727

Biju, S.D., Shouche, Y., Dubois, A., Dutta, S.K. \& Bossuyt, F. (2010) A ground-dwelling rhacophorid frog from the highest mountain peak of the Western Ghats of India. Current Science, 98, 1119-1125.

Biju, S.D., Van Bocxlaer, I., Giri, V.B., Loader, S.P. \& Bossuyt, F. (2009) Two new endemic genera and a new species of toad (Anura: Bufonidae) from the Western Ghats of India. BMC Research Notes, 2, 1-6. https://doi.org/10.1186/1756-0500-2-241

Bishop, P.J. (1985) A new species of Arthroleptella in South Africa. South African Journal of Science, 81, 209.

Bittencourt-Silva, G., Conradie, W., Siu-Ting, K., Tolley, K., Channing, A., Cunningham, M., Farooq, H., Menegon, M. \& Loader, S. (2016) The phylogenetic position and diversity of the enigmatic mongrel frog Nothophryne Poynton, 1963 (Amphibia, Anura). Molecular Phylogenetics and Evolution, 99, 89-102. https://doi.org/10.1016/j.ympev.2016.03.021

Blackburn, D.C. \& Duellman, W.E. (2013) Brazilian marsupial frogs are diphyletic (Anura: Hemiphractidae: Gastrotheca). Molecular Phylogenetics and Evolution, 68, 709-714. https://doi.org/10.1016/j.ympev.2013.04.021

Blackburn, D.C. \& Wake, D.B. (2011) Class Amphibia, Gray, 1825. In: Zhang, Z.-Q. (Ed.), Animal biodiversity: an outline of higher-level classification and survey of taxonomic richness, Zootaxa, 3148 (1), pp. 39-55. \{Q\} [2011.ba]. https://doi.org/10.11646/zootaxa.3148.1.8

Blackwelder, R.E. (1967) Taxonomy: a text and reference book. John Wiley \& Sons, New York, $\mathrm{i}-\mathrm{xv}+698$ pp.

Blainville, [H.D.] de (1839) Notice historique sur la place assignée aux Cécilies dans la série zoologique. Compte rendu des Séances de l'Académie des Sciences, 9, 663-675. [1839.bb].

Blainville, H.D. de (1835) Description de quelques espèces de Reptiles de la Californie, précédée de l'analyse d'un système général d'erpétologie et d'amphibiologie. Nouvelles Annales du Muséum d'Histoire naturelle, 4, 233-296, 4 pl. [1835.ba].

Blainville, H. de (1816a) Prodrome d'une nouvelle distribution systématique du règne animal. Bulletin des Sciences de la Société philomatique de Paris, Juillet 1816, '105-112' [113120] + 121-124. [1816.ba].

Blainville, H. de (1816b) Prodrome d'une nouvelle distribution systématique du règne animal. Journal de Physique, de Chimie, d'Histoire naturelle et des Arts, Octobre 1816, 83, 244-267. [1816.bb].

Blainville, H. de (1818) Vorläufige Anzeige einer neuen systematischen Eintheilung des Thierreichs. Isis von Oken, 1818, 1365-1384. [1818.ba].

Blair, W.F. (Ed.) (1972) Evolution in the genus Bufo. University of Texas Press, Austin \& London, i-viii + 1-459, pls. 1-6.

Blanchard, R. (1885) Remarques sur la classification des Batraciens Anoures. Bulletin de la Société zoologique de France, 10, 
584-589. [1885.bb].

Blanchard, R.(Ed.)(1905)Règles internationales delanomenclature zoologique adoptées par les Congrès Internationaux de Zoologie. Rudeval, Paris, 64 pp.

Blommers-Schlösser, R.M.A. (1993) Systematic relationships of the Mantellinae Laurent 1946 (Anura Ranoidea). Ethology, Ecology and Evolution, 5, 199-218. [1993.ba]. https://doi.org/10.1080/08927014.1993.9523105

Blommers-Schlösser, R.M.A. \& Blanc, C.P. (1991) Amphibiens (première partie). Faune de Madagascar, 75, 1-379.

Blotto, B.L., Nunez, J.J., Basso, N.G., Ubeda, C.A., Wheeler, W.C. \& Faivovich, J. (2013) Phylogenetic relationships of a Patagonian frog radiation, the Alsodes + Eupsophus clade (Anura: Alsodidae), with comments on the supposed paraphyly of Eupsophus. Cladistics, 29 (2), 113-131. https://doi.org/10.1111/j.1096-0031.2012.00417.x

Bock, P.E. \& Gordon, D.P. (2013) Phylum Bryozoa Ehrenberg, 1831. In: Zhang, Z.-Q. (Ed.), Animal biodiversity: an outline of higher-level classificationand survey of taxonomic richness (Addenda 2013). Zootaxa, 3703 (1), 67-74.

https://doi.org/10.11646/zootaxa.3703.1.14

Boettger, C.R. (1952) Die Stämme des Tierreichs in ihrer systematischen Gliederung. Abhandlungen der Braunschweigischen Wissenschaftlichen Gesellschaft, 4, 238-300.

https://doi.org/10.1007/978-3-663-02236-7_1

Boettger, O. (1895) Neue Frösche und Schlangen von den LiukiuInseln. Zoologischer Anzeiger, 18: 266-270.

Bogart, J.P. \& Tandy, M. (1981) Chromosome lineages in African ranoid frogs. Monitore zoologico italiano, New Series, 15 (Supplement), 15, 55-91. [1981.ba]. https://doi.org/10.1080/03749444.1981.10736629

Böhme, M., Winklhofer, M. \& Ilg, A. (2011) Miocene precipitation in Europe: temporal trends and spatial gradients. Palaeogeography, Palaeoclimatology, Palaeoecology, 304, 212-218 + online supplementary [1-8]. [2011.bb]. https://doi.org/10.1016/j.palaeo.2010.09.028

Böhme, W. \& Köhler, J. (2005) Do endings of adjective flecticle species names affect stability? A final note on the gender of Podarcis Wagler, 1830 (Reptilia, Lacertidae). Bonner zoologische Beiträge, 53 (3-4), 293-295.

Boie, H. (1828) Bemerkungen über die Abtheilungen im natürlichen Systeme und deren Characteristik. Isis von Oken, 21 (4), 351364. [1828.ba].

Bokermann, W.C.A. (1952) Microhylidae da coleçao do Departamento de zoologia (Amphibia-Anura). Papeis avulsos do Departamento de Zoologia Secretaria de Agricultura São Paulo, 10, 271-292.

Bokermann, W.C.A. (1965) Hyla langei, a new frog from Paraná, Southern Brasil. Journal of the Ohio herpetological Society, $5,49-51$

https://doi.org/10.2307/1562630

Bokermann,W.C.A.(1975)Trêsespécies novas de Eleutherodactylus do sudeste da Bahia, Brasil (Anura, Leptodactylidae). Revista brasileira de Biologia, 34, 11-18.

Bolkay, S.J. (1919) Osnove uporedne osteologije anurskih batrahija sa dodatkom o porijeklu Anura i sa skicom naravnoga sistema istih. Glasnik Zemaljskog Muzeja u Bosni i Hercegovini, 31, 277-357. \{Q\} [1919.ba].

Bolkay, S.J. (1928) Die Schädel der Salamandrinen, mit besonderer Rücksicht auf ihre systematische Bedeutung. Zeitschrift für Anatomie und Entwicklungsgeschichte, Abtheilung 1, 86, 259-319. https://doi.org/10.1007/BF02117989

Bolkay, S.J. (1929) Die Amphibien und Reptilien von Sarajevo und Umgebung. Glasnik Zemaljskog Muzeja u Bosni $i$ Hercegovini, 41, 57-77, pl. 10. [1929.ba].

Bonaparte, C.L. (1831a) Saggio di una distribuzione metodica degli animali vertebrati. Antonio Boulzaler, Roma, 78 pp. [1831.ba]. https://doi.org/10.5962/bhl.title.48624

Bonaparte, C.L. (1831b) Saggio di una distribuzione metodica degli animali vertebrati a sangue freddo. Giornale Arcadico di Scienze, Lettere ed Arti, 52, 129-209. [1831.bb]. https://doi.org/10.5962/bhl.title.48624

Bonaparte, C.L. (1838a) Iconografia della fauna italica per le quattro classi degli animali vertebrati. Vol. 2. Fasciculo 22. Salviucci, Roma: (1) Hyla viridis, Raganella arborea: [193198], pl. [79]; (2) Rana esculenta, Ranocchia verde: [199202], pl. [79]; (3) Pelodytes punctatus, Pelodite puntato: [207-208], pl. [80]. [P05] [1838.ba].

Bonaparte, C.L. (1838b) Amphibiorum tabula analytica. Nuovi Annali delle Scienze naturali, 1 (1), 391-397. \{Q\} [1838. $\mathrm{bb}$.

Bonaparte, C.L. (1838c) Classification des animaux vertébrés. Compte rendu des Séances de l'Académie des Sciences, 7, 656-658. [1838.bc].

Bonaparte, C.L. (1838d) Synopsis vertebratorum systematis. Nuovi Annali delle Scienze naturali, 1 (2), 105-133. [1838.bd].

Bonaparte, C.L. (1839a) Iconografia della fauna italica per le quattro classi degli animali vertebrati. Vol. 2. Fasciculo 24. Salviucci, Roma: (1) Discoglossus sardus, Discogloso sardo: [pp. 219-221], pl. [81]; (2) Bufo vulgaris, Rospo comune: [pp. 223-228], pl. [82]. \{P05 ] [1839.bc].

Bonaparte, C.L. (1839b) Iconografia della fauna italica per le quattro classi degli animali vertebrati. Vol. 2. Fasciculo 26. Salviucci, Roma: Euproctus platycephalus, Euprotto del Rusconi: [pp. 259-266], pl. [85]. [1839.bd].

Bonaparte, C.L. (1839c) Synopsis vertebratorum systematis (proseguimento e fine). Annali medico-chirurgici, 1 (5), 268279. [1839.be].

Bonaparte, C.L. (1839d) Systema amphybiorum. Mémoires de la Société des Sciences naturelles de Neuchâtel, 2, 15-16. [1839.bf].

Bonaparte, C.L. (1840a) A new systematic arrangement of vertebrated animals. Transactions of the Linnean Society of London, 18 (3), 247-304. [1840.ba]. https://doi.org/10.1111/j.1095-8339.1838.tb00177.x

Bonaparte, C.L. (1840b) Amphibia Europaea ad systema nostrum vertebratorum ordinata. Memorie della reale Accademia delle Scienze di Torino, (2) 2, 385-456. [1840.bb]. https://doi.org/10.5962/bhl.title.4058 
Bonaparte, C.L. (1845) Specchio generale dei sistemi erpetologico ed anfibiologico. Atti della sesta Riunione degli Scienzati italiani, tenuta in Milano nel Settembre del MDCCCXLIV, Luigi di Giacomo Pirola, Milano, pp. 376-378. [1845.ba].

Bonaparte, C.L. (1850) Conspectus systematum herpetologiae et amphibiologiae. Editio altera reformata. E. J. Brill, Lugduni Batavorum, 1 pl. \{P07\} \{Q\} [1850.bb].

Bonaparte, C.L. (1852) Conspectus systematum herpetologiae et amphibiologiae. Editio altera reformata. (Continuazione). Nuovi Annali delle Scienze naturali, Bologna, (3) 5, 477-480. [1852.ba].

Borah, M.M., Bordoloi, S., Purkayastha, J., Das, M., Dubois, A. \& Ohler, A. (2013) Limnonectes (Taylorana) medogensis (Fei, Ye \& Huang, 1997) from Arunachal Pradesh (India), and the identity of some diminutive ranoid frogs (Anura, Dicroglossidae, Occidozygidae). Herpetozoa, 26 (1-2), 3948. [2013.ba].

Borkin, L.J. \& Litvinchuk, S.N. (2013) Амфибии Палеарктики: Таксономический состав. [Amphibians of the Palearctic: taxonomic composition]. Trudy zoologicheskogo Instituta, Rossiyskoy Akademii Nauk, [Proceedings of the zoological Institute of the Russian Academy of Sciences], 317, 494-541. [In Russian]. [2013.bb].

Borkin, L.J. \& Litvinchuk, S. (2014) Зоогеография северного полушария и амфибии: Палеарктика и неарктика или голарктика? [Zoogeography of the northern hemisphere and amphibians: the Palearctic and the Nearctic vs. the Holarctic?]. Trudy zoologicheskogo Instituta, Rossiyskoy Akademii Nauk, [Proceedings of the zoological Institute of the Russian Academy of Sciences], 318, 433-485. [In Russian].

Bory de Saint-Vincent, [J.B.G.M.] (1828) Résumé d'herpétologie, ou d'histoire naturelle des Reptiles. Bureau de l'Encyclopédie Portative, Paris, i-viii +292 pp. +20 pp., pl. 1-52 + front. [1828.bb].

Bossuyt, F. (2002) A new species of Philautus (Anura: Ranidae) from Western Ghats of India. Journal of Herpetology, 36, 656-661.

https://doi.org/10.1670/0022-1511(2002)036[0656: ANSOPA]2.0.CO;2

Bossuyt, F. \& Dubois, A. (2001) Areview of the frog genus Philautus Gistel, 1848 (Amphibia, Anura, Ranidae, Rhacophorinae). Zeylanica, 6, 1-112.

Bossuyt, F. \& Milinkovitch, M.C. (2000) Convergent adaptive radiations in Madagascan and Asian ranid frogs reveal covariation between larval and adult traits. Proceedings of the national Academy of Sciences, 97, 6585-6590. https://doi.org/10.1073/pnas.97.12.6585

Bossuyt, F. \& Milinkovitch, M.C. (2001) Amphibians as indicators of early Tertiary 'out-of-India' dispersal of Vertebrates. Science, 292, 93-95. [2001.ba]. https://doi.org/10.1126/science. 1058875

Bossuyt, F. \& Roelants, K. (2009) Frogs and toads (Anura). In: Hedges, S.B. \& Kumar, S. (Ed.), The Timetree of Life, Oxford University Press, New York, pp. 357-364. [2009.ba].

Bossuyt, F., Brown, R.M., Hillis, D.M., Cannatella, D.C. \& Milinkovitch, M.C. (2006) Phylogeny and biogeography of a cosmopolitan frog radiation: late Cretaceous diversification resulted in continent-scale endemism in the family Ranidae. Systematic Biology, 55, 579-594.

https://doi.org/10.1080/10635150600812551

Bossuyt, F., Roelants, K., Spithoven, L. \& Daro, M.-H. (2001) Philautus bombayensis (Bombay Oriental shrub-frog). Reproduction. Herpetological Review, 32, 34-35.

Boulenger, E.G. (1914) Reptiles and Batrachians. Dent \& Sons, Ltd., London, i-xiv +278 pp., 80 pls. $\{Q\}$.

Boulenger, G.A. (1882a) Reptilia and Batrachia. The zoological Record, 18, 1-16. [1882.ba].

Boulenger, G.A. (1882b) Catalogue of the Batrachia Salientia s. Ecaudata in the collection of the British Museum. Second edition. Trustees of the British Museum, Natural History, London, i-xvi +503 pp., pls. 1-30. \{Q\} [1882.bb].

Boulenger, G.A. (1882c) Catalogue of the Batrachia Gradientia s. Caudata and Batrachia Apoda in the collection of the British Museum. Second edition. Trustees of the British Museum, Natural History, London, i-ix + 127 pp., pls. 1-9. \{Q\} [1882. bc].

Boulenger, G.A. (1883) Descriptions of new species of lizards and frogs collected by Herr A. Forrer in Mexico. Annals and Magazine of natural History, (5), 11, 342-344. https://doi.org/10.1080/00222938309459162

Boulenger, G.A. (1884) Diagnoses of new Reptiles and Batrachians from the Solomon Islands, collected and presented to the British Museum by H. B. Guppy, Esq. Proceedings of the zoological Society of London, 1884, 210-213. [1884.ba].

Boulenger, G.A. (1885) Description of a new frog of the genus Megalophrys. Proceedings of the zoological Society of London, 1885, 850, 1 pl.

Boulenger, G.A. (1887) An account of the Batrachians obtained in Burma by M. L. Fea of the Genoa Civic Museum. Annali del Museo civico di Storia naturale di Genova, 25, 418-424, 3 pl.

Boulenger, G.A. (1888) Note on the classification of the Ranidae. Proceedings of the zoological Society of London, 1888, 204206. [1888.ba]. https://doi.org/10.1111/j.1469-7998.1888.tb06694.x

Boulenger, G.A. (1890a) Second report on additions to the batrachian collection in the Natural-History Museum. Proceedings of the zoological Society of London, 1890, 323-328, pls. 25-26. [1890.ba].

Boulenger, G. A. (1890b) The fauna of British India, including Ceylon and Burma. Reptilia and Batrachia. Taylor \& Francis, London, $\mathrm{i}-\mathrm{xv}$ iii $+541 \mathrm{pp}$.

Boulenger, G.A. (1893) Reptilia and Batrachia. The zoological Record, 29, 1-41. [1893.ba].

Boulenger, G.A. (1893) Viaggio di Leonardo Fea in Birmania e regioni vicine. LII. Concluding report on the Reptiles and Batrachians obtained in Burma by Signor L. Fea, dealing with the collection made in Pegu and the Karin Hills in 1887-88. Annali del Museo civico di Storia naturale di Genova, 33, 304-347, 6 pls.

Boulenger, G.A. (1896) Descriptions of new Batrachians in the British Museum. Annals and Magazine of natural History, 
(6), 17, 401-405.

https://doi.org/10.1080/00222939608680389

Boulenger, G.A. (1898) The tailless batrachians of Europe. Part II. Ray Society, London, 211-376 pp., 4 pls. + pls. 11-24.

Boulenger, G.A. (1903) Report on the Batrachians and Reptiles. In: Annandale, N. \& Robinson, H.C. (Ed.), Fasciculi malayenses, Anthropological and zoological results of an expedition to Perak and the Siamese Malay States 1901-1902 undertaken by Nelson Annandale and Herbert C. Robinson under the auspices of the University of Edinburgh and the University of Liverpool, Zoology, Part 1, Longmans, Green \& Co., London, pp. 127-176.

Boulenger, G.A. (1906) Description of two new Indian frogs. Journal and Proceedings of the Asiatic Society of Bengal, 2, 385-386.

Boulenger, G.A. (1909) Descriptions of four new frogs and a new snake discovered by Mr. H. Sauter in Formosa. Annals and Magazine of natural History, 4, 492-495.

https://doi.org/10.1080/00222930908692704

Boulenger, G.A. (1910) Les Batraciens et principalement ceux d'Europe. Octave Doin et Fils, Paris, $[\mathrm{i}-\mathrm{iv}]+305$ pp. $\{\mathrm{Q}\}$.

Boulenger, G.A. (1917) On a second species of the batrachian genus Amphodus. Annals and Magazine of Natural History, (8), 20, 184-185.

https://doi.org/10.1080/00222931709486988

Boulenger, G.A. (1920a) A monograph of the American frogs of the genus Rana. Proceedings of the American Academy of Arts and Sciences, 55, 413-480.

https://doi.org/10.2307/20025810

Boulenger, G.A. (1920b) A monograph of the South Asian, Papuan, Melanesian and Australian frogs of the genus Rana. Records of the Indian Museum, 20, 1-226.

https://doi.org/10.5962/bhl.title.12471

Bour, R. \& Dubois, A. (1985) Nomenclature ordinale et familiale des Tortues (Reptilia). Studia geologica salmanticensia. Vol. especial 1. Studia palaeocheloniologica. 1. Ediciones Universidad, Salamanca, pp. 77-86.

Bour, R. \& Dubois, A. (1986) Nomenclature ordinale et familiale des Tortues (Reptilia). Note complémentaire. Bulletin mensuel de la Société linnéenne de Lyon, 55, 87-90. https://doi.org/10.3406/linly.1986.10749

Bourbaki, N. (1970) Eléments de mathématique. Théorie des ensembles. Diffusion C.C.L.S., Paris, 1-51 pp + 1-52 pp. + 1-99 pp. + 1-101 pp. + 1-45 pp. + [I].

Bourret, R. (1942) Les Batraciens de l'Indochine. Institut Océanographique de l'Indochine, Hanoi, i-x + 547 pp., 4 pl.

Bowley, D.R. \& Smith, H.M. (1968) The dates of publication of Louis Agassiz's Nomenclator Zoologicus. Journal of the Society for the Bibliography of natural History, 5 (1), 35-36. [1968]. https://doi.org/10.3366/jsbnh.1968.5.1.35

Bozóki-Ernycy, K. (Ed.) (2007) European preventive archaeology. National Office of Cultural Heritage, Hungary \& Council of Europe, Directorate of Culture and Cultural and Natural Heritage, 222 pp.

Brame, A. (1967) A list of the world's recent and fossil salamanders.
Herpeton, 2 (1), 1-26. [1967.ba].

https://doi.org/10.2307/1441584

Brame, A.H. (1957) A list of the world's recent Caudata. University of Southern California, [i] + a-b +21 pp. [1957.ba].

Brame, A.H. (1958) A list of the world's fossil Caudata. University of Southern California, [i] + 5 pp. + a-b. [1958.ba].

Brame, A.H., Hochnadel, R., Smith, H.M. \& Smith, R.B. (1978) Bionumeric codes for Amphibians and Reptiles of the world. I. Salamanders. Transactions of the Kansas Academy of Sciences, 81 (1), 43-56. [1978.ba].

https://doi.org/10.2307/3627356

Brandt, J.F. (1835) Prodromus descriptionis animalium ab H. Mertensio observatorum. Fascic. I. Polypos, Acalephas Discophoras et Siphonophoras, nec non Echinodermata continens. Recueil des Actes des Séances publiques de l'Académie impériale des Sciences de St. Petersbourg, 1834, 201-275. https://doi.org/10.5962/bhl.title.10196

Breen, J.F. (1974) Encyclopedia of Reptiles and Amphibians. T.F.H. Publications, Neptune City, 576 pp. $\{Q\}$.

Bremer, K., Bremer, B., Karis, P.O. \& Källersjö, M. (1990) Time for change in taxonomy. Nature, 343, 202. https://doi.org/10.1038/343202a0

Bridge, P.D., Roberts, P.J., Spooner, B.M. \& Panchal, G. (2003) On the unreliability of published DNA sequences. New Phytologist, 160 (1), 43-48. https://doi.org/10.1046/j.1469-8137.2003.00861.x

Brimley, C.S. (1915) List of Reptiles and Amphibians of North Carolina. Journal of the Elisha Mitchell scientific Society, 30 (4), 195-206.

Brocchi, P. (1881) Mission scientifique au Mexique et dans l'Amérique Centrale. Recherches zoologiques. Troisième partie, deuxième section. Etude des Batraciens de l'Amérique Centrale. Imprimerie Nationale, Paris, 56 pp., pl. 1-5, 9, 10. ¡P10] [1881.ba].

Brongniart, A. (1800a) Essai d'une classification naturelle des Reptiles. Ire partie. Etablissement des ordres. Bulletin des Sciences, par la Société philomatique, 2 (35), 81-82. [P01 J [1800.ba].

Brongniart, A. (1800b) Essai d'une classification naturelle des Reptiles. II ${ }^{\mathrm{e}}$ partie. Formation et disposition des genres. Bulletin des Sciences, par la Société philomatique, 2 (36), 89-91.

Bronn, H.G. (1849) Handbuch einer Geschichte der Natur. Dritter Band. Zweite Abtheilung. Schweizerbart, Stuttgart, i-iii + 1106 pp. [1849.ba].

Bronn, H.G. (1853) Lethaea geognostica oder Abbildung und Beschreibung der für die Gebirgs-Formationen bezeichnendsten Versteinerungen. Dritter Band. Third edition. Schweizerbart, Stuttgart, i-viii + 1130 pp. https://doi.org/10.3931/e-rara-23714

Brookes, J. (1828) A prodromus of a Synopsis Animalium, comprising a Catalogue Raisonné of the zootomical collection of Joshua Brookes, Esq. F.R.S. etc. Gold \& Walton, London, 76 pp. [1828.bc].

Brothers, D.J. (1983a) Nomenclature at the ordinal and higher 
levels. Systematic Zoology, 32 (1), 34-42.

https://doi.org/10.1093/sysbio/32.1.34

Brothers, D.J. (1983b) Comments on Rasnitsyn's proposal to regulate the names of taxa above the family group. Bulletin of zoological Nomenclature, 40 (2), 72-73.

Brower, A.V.Z. (2016) What is a cladogram and what is not? Cladistics, 32, 573-576. https://doi.org/10.1111/cla.12144

Brown, J.L., Twomey, E., Amezquita, A., Souza, M.B. de, Caldwell, J.P., Loetters, S., May, R. von, Melo-Sampaio, P.R., Mejia-Vargas, D., Perez-Pena, P., Pepper, M., Poelman, E.H., Sanchez-Rodriguez, M. \& Summers, K. (2011) A taxonomic revision of the Neotropical poison frog genus Ranitomeya (Amphibia: Dendrobatidae). Zootaxa, 3083 (1), 1-120. https://doi.org/10.11646/zootaxa.3083.1.1

Brown, R.M, Siler, C.D., Richards, S.J., Diesmos, A.C. \& Cannatella, D.C. (2015) Multilocus phylogeny and a new classification for Southeast Asian and Melanesian forest frogs (family Ceratobatrachidae). Zoological Journal of the Linnean Society, 174, 130-168 + supplementary online information (Figure S1). [2015.ba]. https://doi.org/10.1111/zoj.12232

Bruch, C. (1862) Beiträge zur Naturgeschichte und Classification der nackten Amphibien. Würzburger Naturwissenschaftliche Zeitschrift, 3, 181-224. \{Q\} [1862.ba].

Brummitt, R.K. (1997) Taxonomy versus cladonomy, a fundamental controversy in biological systematics. Taxon, 46, 723-734. https://doi.org/10.2307/1224478

Brunetti, A., Hermida, G., Luna, M., Barsotti, A., Jared, C., Antoniazzi, M., Rivera-Correa, M., Von Muller Berneck, B. \& Faivovich, J. (2015) Diversity and evolution of sexually dimorphic mental and lateral glands in Cophomantini treefrogs (Anura: Hylidae: Hylinae). Biological Journal of the Linnean Society, 114, 12-34.

https://doi.org/10.1111/bij.12406

Burbrink, F.T \& Gehara, M. (2018) The biogeography of deep time phylogenetic reticulation. Systematic Biology, 67 (5), 743-755.

https://doi.org/10.1093/sysbio/syy019

Burton, M. \& Burton, R. (1970) Systematic index. In: The international wildife encyclopedia. 20. B.P.C. Publishing limited, Paris, pp. 2796-2800. \{Q\}.

Burton, T.C. (1986) A reassessment of the Papuan subfamily Asterophryinae (Anura: Microhylidae). Record of the South Australian Museum, 19 (19), 405-450. [1986.bb].

Cai, H.X., Che, J., Pang, J.F., Zhao, E.M. \& Zhang, Y.P. (2007) Paraphyly of Chinese Amolops (Anura, Ranidae) and phylogenetic position of the rare Chinese frog, Amolops tormotus. Zootaxa, 1531 (1), 49-55. https://doi.org/10.11646/zootaxa.1531.1.4

Cain, A.J. \& Harrison, G.A. (1960) Phyletic weighting. Proceedings of the zoological Society of London, 135, 1-31. https://doi.org/10.1111/j.1469-7998.1960.tb05828.x

Camp, C., Peterman, W., Milanovich, J., Lamb, T., Maerz, J. \& Wake, D. (2009) A new genus and species of lungless salamander (family Plethodontidae) from the Appalachian highlands of the south-eastern United States. Journal of Zoology, 279, 86-94.

https://doi.org/10.2307/2804896

Camp, W.H. \& Gilly, C.L. (1943) The structure and origin of species. Brittonia, 4 (3), 323-385.

Campbell, J. \& Duellman, W. (2000) New species of streambreeding hylid frogs from the northern versant of the highlands of Oaxaca, Mexico. Scientific Papers, Natural History Museum, University of Kansas, 16, 1-28. https://doi.org/10.5962/bhl.title.16165

Campbell, V. \& Lapointe, F.-J. (2009) The use and validity of composite taxa in phylogenetic analysis. Systematic Biology, 58, 560-572. https://doi.org/10.1093/sysbio/syp056

Candolle, A.P. de (1813) Théorie élémentaire de la botanique, ou exposition des principes de la classification naturelle et de l'art de décrire et d'étudier les végétaux. Déterville, Paris, i-viii +527 pp.

https://doi.org/10.5962/bhl.title.39705

Canedo, C., Dixo, M. \& Pombal, J.P. (2004) A new species of Chiasmocleis Mehely, 1904 (Anura, Microhylidae) from the Atlantic Rainforest of Bahia, Brazil. Herpetologica, 60, 495-501. https://doi.org/10.1655/03-66

Cannatella, D.C. (1986) Anew genus of bufonid (Anura) from South America, and phylogenetic relationships of the Neotropical genera. Herpetologica, 42, 197-205.

Cannatella, D.C. \& Hillis, D.M. (1993) Amphibian relationships: phylogenetic analysis of morphology and molecules. Herpetological Monographs, 7, 1-7. [1993.ca]. https://doi.org/10.2307/1466947

Cannatella, D.C. \& Trueb, L. (1988) Evolution of the pipoid frogs: intergeneric relationships of the aquatic frog family Pipidae (Anura). Zoological Journal of the Linnean Society, 94, 1-38. [1988.ca]. https://doi.org/10.1111/j.1096-3642.1988.tb00880.x

Cannatella, D.C. \& Hillis, D.M. (2004) Amphibians: leading a life of slime. In: Cracraft, J. \& Donoghue, M.J. (Eds.), Assembling the Tree of Life, Columbia University Press, New York, pp. 430-450.

Canseco-Márquez, L., Ramírez-González, C.G. \& González-Bernal, E. (2017) Discovery of another new species of Charadrahyla (Anura, Hylidae) from the cloud forest of northern Oaxaca, México. Zootaxa, 4329 (1), 64-72.

https://doi.org/10.11646/zootaxa.4329.1.2

Cantino P.D. \& Queiroz, K. de (2007) International code of phylogenetic nomenclature. Version $4 \mathrm{~b}$.

http://www.phylocode.org

Cantino, P.D. \& Queiroz, K. de (2010) International code of phylogenetic nomenclature. Version 4c. 102 pp. http:// www.ohio.edu/PhyloCode4c

Cantino, P.D. \& Queiroz, K. de (2020) International code of phylogenetic nomenclature (PhyloCode). Boca Raton, London $\&$ New York (CRC Press), $\mathrm{i}-\mathrm{xl}+149 \mathrm{pp}$. https://doi.org/10.1201/9780429446320

Caramaschi, U. \& Da Cruz, C.A.G. (1997) Redescription of 
Chiasmocleis albopunctata (Boettger) and description of a new species of Chiasmocleis (Anura: Microhylidae). Herpetologica, 53, 259-268.

Caramaschi, U. \& Feio, R. (1990) A new species of Hyla (Anura, Hylidae) from southern Minas Gerais, Brazil. Copeia, 1990, 542-546.

https://doi.org/10.2307/1446357

Caramaschi, U., Lula Salles, R.D.O. \& Goncalves Cruz, C.A. (2012) A new species of Stereocyclops Cope (Anura, Microhylidae) from southeastern Brazil. Zootaxa, 3583 (1), 83-88. https://doi.org/10.11646/zootaxa.3583.1.7

Carus, C.G. (1834) Lehrbuch der vergleichenden Zootomie. Erster Theil. Ernst Fleischer, Leipzig, i-xxxii + 414 pp. [1834.ca].

Carvalho, A.L. (1948) Sobre a validez de Stereocyclops incrassatus Cope, 1871 e Hypopachus mülleri (Boettger), 1885. Boletim do Museu nacional, 84, 1-13.

Carvalho, A.L. de (1954) A preliminary synopsis of the genera of American microhylid frogs. Occasional Papers of the Museum of Zoology, University of Michigan, 555, 1-19.

Carvalho, M.R. de, Bockmann, F.A., Amorim, D.S. \& Brandão, C.R.F. (2008) Systematics must embrace comparative biology and evolution, not speed and automation. Evolutionary Biology, 35, 150-157.

https://doi.org/10.1007/s11692-008-9018-7

Carvalho, M.R. de, Bockmann, F.A., Amorim, D.S., Brandão, C.R.F., Vivo, M. de, Figueiredo, J.L. de, Britski, H. A., Pinna, M.C.C. de, Menezes, N.A., Marques, F.P.L., Papavero, N., Cancello, E.M., Crisci, J.V., McEachran, J.D., Schelly, R.C., Lundberg, J.G., Gill, A.C., Britz, R., Wheeler, Q.D., Stiassny, M.L.J., Parenti, L.R., Page, L.M., Wheeler, W.C., Faivovich, J., Vari, R.P., Grande, L., Humphries, C.J., DeSalle, R., Ebach, M.C. \& Nelson, G. (2007) Taxonomic impediment or impediment to taxonomy? A commentary on systematics and the cybertaxonomic-automation paradigm. Evolutionary Biology, 34, 140-143.

https://doi.org/10.1007/s11692-007-9011-6

Carvalho, M.R. de, Bockmann, F.A., Amorim, D.S., Vivo, M. de, Toledo-Piza, M. de, Menezes, N.A., Figueiredo, J.L. de, Castro, R.M.C., Gill, A.C., McEachran, J.D., Compagno, L.J.V., Schelly, R.C., Britz, R., Lundberg, J.G., Vari, R.P. \& Nelson, G. (2005) Revisiting the taxonomic impediment. Science, 307, 353.

https://doi.org/10.1126/science.307.5708.353b

Carvalho, M.R. de., Ebach, M.C., Williams, D.M., Nihei, S.S., Rodrigues, M.T., Grant, T., Silveira, L.F., Zaher, H., Gill, A.C., Schelly, R.C., Sparks, J.S., Bockmann, F.A., Seret, B., Ho, H.-C.C., Grande, L., Rieppel, O., Dubois, A., Ohler, A., Faivovich, J., Assis, L.C.S., Wheeler, Q.D., Goldstein, P.Z., de Almeida, E.A.B., Valdecasas, A.G. \& Nelson, G. (2014) Does counting species count as taxonomy? On misrepresenting systematics, yet again. Cladistics, 30, 322-329. https://doi.org/10.1111/cla.12045

Carvalho, T.R. de, Giaretta, A.A. \& Magrini, L. (2012) A new species of the Bokermannohyla circumdata group (Anura: Hylidae) from southeastern Brazil, with bioacoustic data on seven species of the genus. Zootaxa, 3321 (1), 37-55. https://doi.org/10.11646/zootaxa.3321.1.3

Casamiquela, R.M. (1959) Un escuerzo fósil de Río Negro. Los anuros fósiles de la Argentina. Misiones culturales, Revista de la Dirección de Cultura de Río Negro, 2 (diciembre), 5-12. [1959.ca].

Casamiquela R.M. (1960) Datos preliminares sobre un pipoideo fósil de Patagonia. Actas y Trabajos del primer Congreso sudamericano de Zoología (La Plata, 12-24 October 1959), Universidad nacional de La Plata 4, pp. 17-22. [1960.ca].

Casamiquela, R.M. (1961) Un pipoideo fosil de Patagonia. Revista del Museo de la Plata, New Series, 4 (Paleontología 22), 71-123, pls. 1-3. [1961.ca].

Case, S.M. (1979) Observations on some cranial foramina in the Ranidae. Copeia, 1979, 346-348. https://doi.org/10.2307/1443424

Castroviejo-Fisher, S., Guayasamin, J., Gonzalez-Voyer, A. \& Vilà, C. (2014) Neotropical diversification seen through glassfrogs. Journal of Biogeography, 41, 66-80.

https://doi.org/10.1111/jbi.12208

Castroviejo-Fisher, S., Padial, J.M., Riva, I. de la, Pombal, J.P., Silva, H.R. da, Rojas-Runjaic, F.J.M., Medina-Méndez, E. \& Frost, D.R. (2015) Phylogenetic systematics of egg-brooding frogs (Anura: Hemiphractidae) and the evolution of direct development. Zootaxa, 4004 (1), 1-75. [2015.ca]. https://doi.org/10.11646/zootaxa.4004.1.1

Cei, J.M. (1958) Las láminas originales del suplemento a los batraquios chilenos de Philippi: primera impresión y comentario. Investigaciones zoologicas chilenas, 4, 265-288. [1958.ca].

Cei, J.M. (1962) Batracios de Chile. Ediciones de la Universidad de Chile, Santiago de Chile, 128 pp. + i-cviii. [1962.ca].

Cei, J.M. (1970) La posición filética de Telmatobiinae, su discusión reciente y significado crítico de algunos immunotests. Acta zoologica lilloana, 27, 181-192. [1970.ca].

Cei, J.M. (1980) Amphibians of Argentina. Monitore zoologico italiano, New Series, Monographia, 2, i-xii + 1-609.

Chaimanee, Y., Jager, J.J. \& Suteethorn, V. (1993) Pleistocene microvertebrates from fissure-fillings in Thailand. Journal of Southeast Asian Earth Sciences, 8 (1-4, Special volume), 45-48. [1993.cb]. https://doi.org/10.1016/0743-9547(93)90006-B

Chan, K.O., Grismer, L.L. \& Brown, R.M. (2018) Comprehensive multi-locus phylogeny of Old World tree frogs (Anura: Rhacophoridae) reveals taxonomic uncertainties and potential cases of over- and underestimation of species diversity. Molecular Phylogenetics and Evolution, 127, 1010-1019. https://doi.org/10.1016/j.ympev.2018.07.005

Chanda, S.K. (1994) Anuran (Amphibia) fauna of northeast India. Memoirs of the zoological Survey of India, 18, i-vi + 1-132.

Chanda, S.K., Das, I. \& Dubois, A. (2000) Catalogue of amphibian types in the collection of the Zoological Survey of India. Hamadryad, 25, 100-128.

Chandramouli, S.R. \& Amarasinghe, A.A.T. (2016) Taxonomic reassessment of the arboreal toad genus Pedostibes Günther 1876 (Anura: Bufonidae) and some allied oriental bufonid genera. Herpetologica, 72, 137-147. 
https://doi.org/10.1655/HERPETOLOGICA-D-15-00053

Chandramouli, S.R., Ganesh, S.R. \& Baskaran, N. (2011) On recent sightings of a little-known south Indian toad, Duttaphrynus hololius (Günther, 1876) with notes on its morphological characterization and ecology. Herpetological Notes, 4, 271274.

Chandramouli, S.R., Vasudevan, K., Harikrishnan, S., Dutta, S.K., Jegath Janani, S., Sharma, R., Das, I. \& Aggarwal, R. (2016) A new genus and species of arboreal toad with phytotelmonous larvae, from the Andaman Islands, India (Lissamphibia, Anura, Bufonidae). Zookeys, 555, 57-90.

https://doi.org/10.3897/zookeys.555.6522

Chang, M.L.Y. (1936) Contribution à l'étude morphologique, biologique et systématique des Amphibiens Urodèles de la Chine. Picart, Paris, 156 pp. [1936.ca].

Channing, A. (1989) A re-evaluation of the phylogeny of Old World treefrogs. South African Journal of Zoology, 24 (2), 116-131. [1989.ca]. https://doi.org/10.1080/02541858.1989.11448147

Channing, A. (2001) Amphibians of Central and Southern Africa. Cornell University Press, New York, $\mathrm{i}-\mathrm{x}+470$ pp., pls. 124.

https://doi.org/10.7591/9781501733697

Channing, A. \& Boycott, R.C. (1989) A new frog genus and species from the mountains of the Southwestern Cape, South-Africa (Anura, Ranidae). Copeia, 1989, 467-471.

https://doi.org/10.2307/1445445

Channing, A. \& Howell, K.M. (2006) Amphibians of East Africa. Comstock Books in Herpetology, Ithaca, $\mathrm{i}-\mathrm{xi}+418 \mathrm{pp}$.

Chaparro, J.C., Pramuk, J. \& Gluesenkamp, A. (2007) A new species of arboreal Rhinella (Anura: Bufonidae) from cloud forest of southeastern Peru. Herpetologica, 63, 203-212. https://doi.org/10.1655/0018-0831(2007)63[203: ANSOAR]2.0.CO;2

Chapuis, F. (1874) Histoire naturelle des Insectes. Genera des Coleoptères. Tome dixième. Famille des Phytophages. F. Lacordaire Th. \& Chapuis (Ed). Librairie Encyclopédique de Roret, Paris, [i-vi] + i-iv +455 pp.

Che, J., Zhou, W., Hu, J., Yan, F., Papenfuss, T.J., Wake, D.B. \& Zhang, Y. (2010) Spiny frogs (Paini) illuminate the history of the Himalayan region and Southeast Asia. Proceedings of the national Academy of Sciences, 107, 13765-13770.

https://doi.org/10.1073/pnas.1008415107

Chen, J., Bever, G.S., Yi, H.-Y. \& Norell, M.A. (2016) A burrowing frog from the late Paleocene of Mongolia uncovers a deep history of spadefoot toads (Pelobatoidea) in East Asia. Scientific Reports, 6 (19209), 1-7. https://doi.org/10.1038/srep19209

Chen, G., Wang, B., Liu, J., Xie, F. \& Jiang, J. (2011) Complete mitochondrial genome of Nanorana pleskei (Amphibia: Anura: Dicroglossidae) and evolutionary characteristics. Current Zoology, 57, 785-805.

https://doi.org/10.1093/czoolo/57.6.785

Chen, J.-M., Poyarkov, N.A., Suwannapoom, C., Lathrop, A., Wu, Y.-H., Zhou, W.-W., Yuan, Z.-Y., Jin, J.-Q., Chen, H.-M., Liu, H.-Q., Nguyen, T.Q., Nguyen, S.N., Duong, T. Van, Eto, K.,
Nishikawa, K., Matsui, M., Orlov, N.L., Stuart, B.L., Brown, R.M., Rowley, J.J.L., Murphy, R.W., Wang, Y.-Y. \& Che, J. (2018) Large-scale phylogenetic analyses provide insights into unrecognized diversity and historical biogeography of Asian leaf-litter frogs, genus Leptolalax (Anura: Megophryidae). Molecular Phylogenetics and Evolution, 124, 162-171. https://doi.org/10.1016/j.ympev.2018.02.020

Chen, J.-M., Prendini, E., Wu, Y.-H., Zhang, B.-L., Suwannapoom, C., Chen, H.-M., Jin, J.-Q., Lemmon, E. M., Lemmon, A.R., Stuart, B.L., Raxworthy, C.J., Murphy, R.W., Yuan, Z.-Y. \& Che, J. (2020) An integrative phylogenomic approach illuminates the evolutionary history of Old World tree frogs (Anura: Rhacophoridae). Molecular Phylogenetics and Evolution, 145, 1-9.

https://doi.org/10.1016/j.ympev.2019.106724

Chen, J.-M., Zhou, W.-W., Poyarkov, N.A., Stuart, B.L., Brown, R.M., Lathrop, A., Wang, Y.-Y., Yuan, Z.-Y., Jiang, K., Hou, M., Chen, H.-M., Suwannapoom, C., Nguyen, S.N., Duong, T. Van, Papenfuss, T.J., Murphy, R.W., Zhang, Y.-P. \& Che, J. (2017) A novel multilocus phylogenetic estimation reveals unrecognized diversity in Asian horned toads, genus Megophrys sensu lato (Anura: Megophryidae). Molecular Phylogenetics and Evolution, 106, 28-43.

https://doi.org/10.1016/j.ympev.2019.106724

Chen, M.-Y., Mao, R.-L., Liang, D., Kuro-o, M., Zeng, X.-M. \& Zhang, P. (2015) A reinvestigation of phylogeny and divergence times of Hynobiidae (Amphibia, Caudata) based on 29 nuclear genes. Molecular Phylogenetics and Evolution, $83,1-6$.

https://doi.org/10.1016/j.ympev.2014.10.010

Chippaux, J.P. (1986) Les Serpents de la Guyane Française. The snakes of French Guiana. Editions de l'ORSTOM, Paris, 165 pp.

Chippindale, P.T., Bonett, R.M., Baldwin, A.S. \& Wiens, J.J. (2004) Phylogenetic evidence for a major reversal of lifehistory evolution in plethodontid salamanders. Evolution, 58, 2809-2822. [2004.ca]. https://doi.org/10.1111/j.0014-3820.2004.tb01632.x

Cisneros-Heredia, D.F. \& McDiarmid, R.W. (2007) Revision of the characters of Centrolenidae (Amphibia: Anura: Athesphatanura), with comments on its taxonomy and the description of new taxa of glassfrogs. Zootaxa, 1572 (1), $1-82$.

https://doi.org/10.11646/zootaxa.1572.1.1

Clarke, B.T. (1981) Comparative osteology and evolutionary relationships in the African Raninae (Anura Ranidae). Monitore zoologico italiano, New Series, 15 (Supplement), 285-331. https://doi.org/10.1080/03749444.1981.10736638

Clarke, B.T. (1982) A new genus of ranine frog (Anura: Ranidae) from Somalia. Bulletin of the British Museum (Natural History), Zoology, 43, 179-183.

Claus, C. (1868) Grundzüge der Zoologie zum Gebrauche an Universitäten und höhern Lehranstalten. N. G. Elwert's Universitäts-Buchhandlung, Marburg \& Leipzig, i-viii + 839 pp. + [i]. [1868.cb]. 
Cochran, D.M. (1927) New reptiles and batrachians collected by Dr. Hugh M. Smith in Siam. Proceedings of the biological Society of Washington, 40, 179-191.

Cochran, D.M. (1955) Frogs of Southeastern Brazil. Bulletin of the United States national Museum, 206, 1-423. https://doi.org/10.5479/si.03629236.206.1

Cochran, D.M. (1962) Living Amphibians of the world. Doubleday \& Company Inc., New York, 199 pp. $\{Q\}$.

Cook, O.F. (1906) Factors of species-formation. Science, New Series, 23 (587), 506-507. https://doi.org/10.1126/science.23.587.506

Cook, O.F. (1908) Evolution without isolation. The American Naturalist, 42 (503), 727-731. https://doi.org/10.1086/279001

Cooper, J.G. (1859) No. 4. Report on the Reptiles collected on the survey. In: Cooper, J.G. \& Suckley, G. (Ed.), The natural history of Washington territory, with much relating to Minnesota, Nebraska, Kansas, Oregon, and California, between the thirty-sixth and forty-ninth parallels of latitude, being those parts of the final reports on the survey of the Northern Pacific railroad route, containing the climate and physical geography, with full catalogues and descriptions of the plants and animals collected from 1853 to 1857. Vol. 12 . Book 2. Part 3. Zoological report, Baillière Brothers, New York, Baillière \& Fils, Paris \& C. Bailly-Baillière, Madrid, pp. 292-306, pls. 12-30. [1859.ca].

Cope, E.D. (1859) On the primary divisions of the Salamandridae, with descriptions of two new species. Proceedings of the Academy of natural Sciences of Philadelphia, 11, 122-128. [1859.cb].

Cope, E.D. (1861) Descriptions of Reptiles from tropical America and Asia. Proceedings of the Academy of natural Sciences of Philadelphia, '1860' 12, 368-374. [P00 J [1861.ca].

Cope, E.D. (1863a) Notes upon some Reptiles of the Old World. Proceedings of the Academy of natural Sciences of Philadelphia, '1862', 14, 337-344. [P00] [1863.ca].

Cope, E.D. (1863b) Catalogues of the Reptiles obtained during the explorations of the Parana, Paraguay, Vermejo and Uruguay rivers, by Capt. Thos. J. Page, U. S. N.; and of those procured by Lieut. N. Michler, U. S. Top. Eng., Commander of the expedition conducting the Survey of the Atrato river. Proceedings of the Academy of natural Sciences of Philadelphia, '1862', 14, 346-359. |P00] [1863.cb].

Cope, E.D. (1864a) On Trachycephalus, Scaphiopus and other American Batrachia. Proceedings of the Academy of natural Sciences of Philadelphia, '1863', 15, 43-54. 〔P00] [1864. ca].

Cope, E.D. (1864b) On the limits and relations of the Raniformes. Proceedings of the Academy of natural Sciences of Philadelphia, 16, 181-183. [1864.cb].

Cope, E.D. (1865) Sketch of the primary groups of Batrachia Salientia. Natural History Review, New Series, 5 (17), 97120. $\{\mathrm{Q}\}$ [1865.ca].

Cope, E.D. (1866) On the structure and distribution of the genera of the arciferous Anura. Journal of the Academy of natural Sciences of Philadelphia, (2), 6, 67-112. \{Q\} [1866.ca].। https://doi.org/10.5962/bhl.title.13790

Cope, E.D. (1867) On the families of Raniformes Anura. Journal of the Academy of natural Sciences of Philadelphia, (2), 6, 189-206. \{Q\} [1867.ca].

Cope, E.D. (1868) Synopsis of the extinct Batrachia of North America. Proceedings of the Academy of natural Sciences of Philadelphia, 20, 208-221. [1868.ca]. https://doi.org/10.5962/bhl.title.60482

Cope, E.D. (1869a) Sixth contribution to the herpetology of tropical America. Proceedings of the Academy of natural Sciences of Philadelphia, '1868', 20, 305-312. [P09] [1869.ca].

Cope, E.D. (1869b) A review of the species of the Plethodontidae and Desmognathidae. Proceedings of the Academy of natural Sciences of Philadelphia, 21, 93-118. [1869.cb].

Cope, E.D. (1875) Checklist of North American Batrachia and Reptilia; with a systematic list of the higher groups, and an essay on geographical distribution. Based on the specimens contained in the U.S. national Museum. Bulletin of the United States national Museum, 1, [i-ii] + i-ii + 3-104. \{Q\} [1875. ca].

https://doi.org/10.5962/bhl.title.11851

Cope, E.D. (1888) The ossicula auditus of the Batrachia. The American Naturalist, 22, 464-467, pl. 6. [1888.ca]. https://doi.org/10.1086/274632

Cope, E.D. (1889a) The Batrachia of North America. Bulletin of the United States national Museum, 34, 1-525, pls. 1-86. \{Q\} [1889.ca].

Cope, E.D. (1889b) Synopsis of the families of Vertebrata. The American Naturalist, 23, 849-877. \{Q\} [1889.cb]. https://doi.org/10.1086/275018

Cowan, C.F. (1969) Cuvier's Règne Animal, first edition. Journal of the Society for the Bibliography of natural History, 5 (3), 219. [1969]. https://doi.org/10.3366/jsbnh.1969.5.3.219

Cracraft, J. (1974) Phylogenetic models and classification. Systematic Zoology, 23 (1), 71-90. https://doi.org/10.1093/sysbio/23.1.71

Crespo, E.G. (2001) Paleo-herpetofauna de Portugal. Publicações avulsas do Museu Bocage, (2) 7, 1-183. [2001.ca].

Crisci, J.V. (2006a) One-dimensional systematist: perils in a time of steady progress. Systematic Botany, 31 (1), 217-221. https://doi.org/10.1600/036364406775971859

Crisci, J.V. (2006b) Making taxonomy visible. Systematic Botany, $31(2), 439-440$. https://doi.org/10.1600/036364406777585829

Crosnier, A. \& Clark, P.F. (1998) Publication dates of the 'Recherches zoologiques pour servir à l'histoire de la faune de l'Amérique centrale et du Mexique'. Archives of natural History, 25 (1), 87-101. [1998]. https://doi.org/10.3366/anh.1998.25.1.87

Crowson, R.A. (1970) Classification and biology. Heinemann, London, $359 \mathrm{pp}$.

Crowther, A.L. (2011) Class Anthozoa Ehrenberg, 1834. In: Zhang, Z.-Q. (Ed.), Animal biodiversity: an outline of higher-level classification and survey of taxonomic richness. Zootaxa, 3148 (1), 19-23. 
https://doi.org/10.11646/zootaxa.3148.1.5

Cruz, C.A.G. (1990) Sobre as relacoes intergenericas de Phyllomedusinae da Floresta Atlantica (Amphibia, Anura, Hylidae). Revista brasileira de Biologia, 50, 709-726.

Cruz, C.A.G., Caramaschi, U. \& Freire, E.M.X. (1999) Occurrence of the genus Chiasmocleis (Anura: Microhylidae) in the State of Alagoas, north-eastern Brazil, with a description of a new species. Journal of Zoology, 249, 123-126. https://doi.org/10.1111/j.1469-7998.1999.tb01065.x

Cruz, C.A.G., Caramaschi, U. \& Napoli, M.F. (2007) A new species of Chiasmocleis (Anura, Microhylidae) from the Atlantic rain forest of northeastern Bahia, Brazil. South American Journal of Herpetology, 2, 47-52.

https://doi.org/10.2994/1808-9798(2007)2[47: ANSOCA]2.0.CO;2

Cuénot, L. \& Tétry, A. (1951) L'évolution biologique. Les faits, les incertitudes. Masson, Paris, $\mathrm{i}-\mathrm{ix}+588 \mathrm{pp}$.

Cunningham, M.J. \& Cherry, M.I. (2004) Molecular systematics of African 20-chromosome toads (Anura: Bufonidae). Molecular Phylogenetics and Evolution, 32, 671-685.

https://doi.org/10.1016/j.ympev.2004.03.003

Cuvier, G. (1797) Tableau élémentaire de l'histoire naturelle des animaux. Baudouin, Paris, i-xvi +710 pp., pls. 1-14. https://doi.org/10.5962/bhl.title.45918

Cuvier, G. (1800) [An VIII] Leçons d'anatomie comparée. Tome 1. Les organes du mouvement. Baudouin, Paris, $\mathrm{i}-\mathrm{xxxi}+522$ pp., tabs. 1-9. [1800.ca].

https://doi.org/10.5962/bhl.title.118777

Cuvier, G. (1802) Lectures on comparative anatomy. Translated from the French of G. Cuvier (...) by William Ross, under the inspection of James Macartney (...). Vol. 1. On the organs of motion. Longman \& Rees, London, $\mathrm{i}-\mathrm{xl}+542$ pp., tabs. 1-9. [1802.ca].

Cuvier, G. (1810) Vorlesungen über vergleichende Anatomie. Uebersetzt und mit Anmerkungen und Zusätzen vermehrt von I.F. Meckel. Dritter Theil, welcher die Organe der Verdauung enthält. Kummer, Leipzig, i-xxxii +743 pp., tab. 1-9, pls. 14-21. [1810.ca].

Cuvier, G. (1816) Le règne animal distribué d'après son organisation, pour servir de base à l'histoire naturelle des animaux et d'introduction à l'anatomie comparée. Tome 1, contenant l'introduction, les Mammifères et les Oiseaux. '1817'. Deterville, Paris, i-xxxvii + 540 pp. 〔P04] [1816. ca].

Da Costa, E.M. (1776) Elements of conchology: or, An introduction to the knowledge of shells. Benjamin White, London, i-viii + iii-vi +318 pp. + [i], pls. 1-7.

Darst, C.R. \& Cannatella, D.C. (2004) Novel relationships among hyloid frogs inferred from $12 \mathrm{~S}$ and $16 \mathrm{~S}$ mitochondrial DNA sequences. Molecular Phylogenetics and Evolution, 31, 462475 .

https://doi.org/10.1016/j.ympev.2003.09.003

Das, I. (1998) A remarkable new species of ranid (Anura: Ranidae), with phytotelmonous larvae, from Mount Harriet, Andaman Island. Hamadryad, 23, 41-49.

Das, I. \& Chanda, S.K. (1998) A new species of Philautus (Anura:
Rhacophoridae) from the Eastern Ghats, south-eastern India. Journal of South Asian natural History, 3, 103-112.

Das, I. \& Chanda, S.K. (2000) A new species of Scutiger (Anura: Megophryidae) from Nagaland, north-eastern India. Herpetological Journal, 10, 69-72.

Daubenton, M. (1782) Les animaux quadrupèdes ovipares, et les serpens. In: Encyclopédie méthodique-Histoire naturelle des animaux. Tome premier. Panckoucke, Paris, pp. 545-712.

Daudin, F.M. (1800) [an IX] Histoire naturelle des Quadrupèdes ovipares (...) avec des gravures faites et enluminées sur les dessins d'après nature, par J. Barraband. Fuchs \& Delalain, fils, Paris, and Treuttel \& Wurtz, Paris \& Strasbourg: two parts published, without page numbers, including 1, 9 and 12 text pages, with 6 and 6 non-numbered plates. https://doi.org/10.5962/bhl.title.5020

Daudin, F.M. (1803a) Histoire naturelle, générale et particulière, des Reptiles. Vol. 7. F. Dufart, Paris, 436 pp., pls. 81-92.

Daudin, F.M. (1803b) Histoire naturelle, générale et particulière, des Reptiles. Vol. 8. F. Dufart, Paris, 439 pp., pls. 93-100.

Davis, F.D. (1935) A new generic and family position for Bufo borbonica. Zoology Series of the Field Museum of natural History, 20, 87-92. [1935.da]. https://doi.org/10.5962/bhl.part.8625

Dayrat, B. (2005) Toward integrative taxonomy. Biological Journal of the Linnean Society, 85, 407-415. https://doi.org/10.1111/j.1095-8312.2005.00503.x

De Stefano, G. (1903) Sui Batraci Urodeli del Quercy. Bollettino della Societa geologica italiana, 22, 40-50, pl. 3. [1903.sa].

Delorme, M., Dubois, A., Grosjean, S. \& Ohler, A. (2005) Une nouvelle classification générique et subgénérique de la tribu des Philautini (Amphibia, Anura, Ranidae, Rhacophoridae). Bulletin mensuel de la Société Linnéenne de Lyon, 74, 165171.

https://doi.org/10.3406/linly.2005.13595

Delorme, M., Dubois, A., Grosjean, S. \& Ohler, A. (2006) Une nouvelle ergotaxinomie des Megophryidae (Amphibia, Anura). Alytes, 24 (1-4), 6-21. [2006.da].

Delorme, M., Dubois, A., Kosuch, J. \& Vences, M. (2004) Molecular phylogenetic relationships of Lankanectes corrugatus from Sri Lanka: endemism of South Asian frogs and the concept of monophyly in phylogenetic studies. Alytes, 22 (1-2), 53-64.

Demoule, J.-P. (2002) Rescue archaeology: the French way. Public Archaeology, 2 (3), 170-177.

https://doi.org/10.1179/pua.2002.2.3.170

Denton, R.K. \& O’Neill, R.C. (1998) Parrisia neocesariensis, a new batrachosauroidid salamander and other amphibians from the Campanian of Eastern North America. Journal of Vertebrate Paleontology, 18 (3), 484-494. [1998.da]. https://doi.org/10.1080/02724634.1998.10011076

Desmarest, E. (1856) Reptiles et poissons. In: Chenu, Dr. (Ed.), Encyclopédie d'histoire naturelle ou traité complet de cette science, Maresq \& Co. \& Gustave Havard, Paris, pp. [i-x] + 1-360, pl. 48. \{Q\} [1856.da].

Desmarest, E. (1857) Reptiles et poissons. In: Chenu, Dr. (Ed.), Tables alphabétiques de l'encyclopédie d'histoire naturelle, Maresq \& Co., Paris, pp. [i-iv] + 1-62. [1857.da]. 
Deuti, K., Nicolas, V., Grosjean, S. \& Ohler, A. (2017) Nomenclatural puzzle in early Xenophrys nomina (Anura, Megophryidae) solved with description of two new species from India (Darjeeling hills and Sikkim). Alytes, 34, 21-49.

Deuti, K., Ray, S. \& Dey, S.K. (2012) Status survey of the Khasi Hills rock toad (Bufoides maghalayana) at Cherrapunjee, Meghalaya. Records of the zoological Survey of India, 111, $21-25$.

Dias, I., Mira-Mendes, C., Souza-Costa, C., Juncá, F. \& Solé, M. (2017) The advertisement call and comments on the distribution of Eleutherodactylus bilineatus Bokermann, 1975, an endemic frog of Bahia State, Brazil (Amphibia, Anura). Zookeys, 677, 151-159. https://doi.org/10.3897/zookeys.677.12309

Dinesh, K.P., Radhakrishnan, C., Gururaja, K.V. \& Bhatta, G.K. (2009) An annotated checklist of Amphibia of India with some insight into the pattern of species discoveries, distribution and endemism. Occasional Papers, Zoological Survey of India, $302,1-153$

Dobzhansky, T. (1970) Genetics of the evolutionary process. Columbia University Press, New York \& London, 505 pp.

Dodd Jr., K.C. (2013) Frogs of the United States and Canada. John Hopkins University Press, Baltimore, i-xxvii +982 pp.

Doolittle, W.F. (1999a) Phylogenetic classication and the universal tree. Science, 284, 2124-2129.

https://doi.org/10.1126/science.284.5423.2124

Doolittle, W.F. (1999b) Lateral genomics. Trends in biological Sciences, 24 (12), M5-M8. https://doi.org/10.1016/S0968-0004(99)01471-1

Dowling, H.G. \& Duellman, W.E. (1978) Systematic herpetology: a synopsis of families and higher categories. Publications in Herpetology, 7, i-vii + 1.1-118.3 + i-viii. \{Q\} [1978.da].

Drewes, R.C. (1984) A phylogenetic analysis of the Hyperoliidae (Anura): treefrogs of Africa, Madagascar, and the Seychelles Islands. Occasional Papers of the California Academy of Sciences, $139, \mathrm{i}-\mathrm{x}+1-70$

Dring, J. (1983) Some new frogs from Sarawak. Amphibia-Reptilia, $4,103-115$. https://doi.org/10.1163/156853883X00021

Du Preez, L.H. \& Carruthers, V.C. (2009) A complete guide to the frogs of Southern Africa. Struik Nature, Cape Town, 488 pp + CD. [2009.pa].

Dubois, A. (1974) Liste commentée d'Amphibiens récoltés au Népal. Bulletin du Muséum national d'Histoire naturelle de Paris, (3) 213, 341-411.

Dubois, A. (1975) Un nouveau sous-genre (Paa) et trois nouvelles espèces du genre Rana. Remarques sur la phylogénie des Ranidés (Amphibiens, Anoures). Bulletin du Muséum national d'Histoire naturelle, (3) 324 (Zoologie 231), 1093-1115.

Dubois, A. (1976) Les grenouilles du sous-genre Paa du Népal. Famille Ranidae, genre Rana. Cahiers népalais, Documents, $6, i-v i+1-275$.

Dubois, A. (1977) Les problèmes de l'espèce chez les Amphibiens Anoures. In: Bocquet, C., Génermont, J. \& Lamotte, M. (Eds.), Les problèmes de l'espèce dans le règne animal, 2, Mémoires de la Société zoologique de France, 39, 161-284.
Dubois, A. (1980) Notes sur la systématique et la répartition des Amphibiens Anoures de Chine et des régions avoisinantes IV. Classification générique et subgénérique des Pelobatidae Megophryinae. Bulletin mensuel de la Société linnéenne de Lyon, 49, 469-482. [1980.da]. https://doi.org/10.3406/linly.1980.10444

Dubois, A. (1981a) Hybridation interspécifique et notion du genre en zoologie. Comptes rendus des Séances de l'Académie des Sciences, (3) 292 (A), 201-203.

Dubois, A. (1981b) Liste des genres et sous-genres nominaux de Ranoidea (Amphibiens, Anoures) du monde, avec identification de leurs espèces-types: conséquences nomenclaturales. Monitore zoologico italiano, New Series, 15 (Supplement), 225-284. [1981.da]. https://doi.org/10.1080/03749444.1981.10736637

Dubois, A. (1981c) Quelques réflexions sur la notion de genre en zoologie. Bulletin de la Société zoologique de. France, 106, 503-513.

Dubois, A. (1982a) Les notions de genre, sous-genre et groupe d'espèces en zoologie à la lumière de la systématique évolutive. Monitore zoologico italiano, New Series, 16, 9-65. [1982.da].

Dubois, A. (1982b) Phrynobatrachinae Laurent, 1940 (Amphibia, Anura): proposed conservation. Z.N.(S) 2362. Bulletin of zoological Nomenclature, 39 (2), 134-140. [1982.db]. https://doi.org/10.5962/bhl.part.23554

Dubois, A. (1982c) Le statut nomenclatural des noms génériques d'Amphibiens créés par Kuhl \& Van Hasselt (1822): Megophrys, Occidozyga et Rhacophorus. Bulletin du Muséum national d'Histoire naturelle, (4) 4 (A), 261-280.

Dubois, A. (1982d) Dendrobates Wagler, 1830 and Dendrobatidae Cope, 1965 (Amphibia, Anura): proposed conservation. Z.N.(S) 1930. Bulletin of zoological Nomenclature, 39 (4), 267-278. [1982.dc]. https://doi.org/10.5962/bhl.part.23594

Dubois,A.(1983a)Hybridation interspécifique, similarité génétique, parenté phylogénétique et classification supraspécifique en zoologie. L'Année biologique, (4) 22, 37-68.

Dubois, A. (1983b) Classification et nomenclature supragénérique des Amphibiens Anoures. Bulletin mensuel de la Société linnéenne de Lyon, 52, 270-276. [1983.da]. https://doi.org/10.3406/linly.1983.10607

Dubois, A. (1983c) Note préliminaire sur le genre Leptolalax Dubois, 1980 (Amphibiens, Anoures), avec diagnose d'une espèce nouvelle du Vietnam. Alytes, 2 (4), 147-153. [1983. $\mathrm{db}]$

Dubois, A. (1984a) Miscellanea nomenclatorica batrachologica (V). Alytes 3 (3), 111-116. [1984.da].

Dubois, A. (1984b) La nomenclature supragénérique des Amphibiens Anoures. Mémoires du Muséum national d'Histoire naturelle, A (Zoologie), 131, 1-64. \{Q\}.

Dubois, A. (1985) Miscellanea nomenclatorica batrachologica (VII). Alytes, 4 (2), 61-78. \{Q\} [1985.da].

Dubois, A. (1986a) Miscellanea nomenclatorica batrachologica (VIII). Alytes, 4, 94-96.

Dubois, A. (1986b) A propos de l'emploi controversé du terme 
'monophylétique': nouvelles propositions. Bulletin mensuel de la Société linnéenne de Lyon, 55, 248-254.

https://doi.org/10.3406/linly.1986.10766

Dubois, A. (1987a) Living amphibians of the world: a first step towards a comprehensive checklist. Alytes, 5 (3), 99-149.

Dubois, A. (1987b) Miscellanea taxinomica batrachologica (I). Alytes '1986', 5 (1-2), 7-95. [P16] [1987.da].

Dubois, A. (1987c) Miscellanea nomenclatorica batrachologica (XIV). Alytes '1986', 5 (4), 173-174. [P16] [1987.db].

Dubois, A. (1987d) Miscellanea taxinomica batrachologica (II). Alytes, 6 (1-2), 1-9.

Dubois, A. (1987e) Discoglossidae Günther, 1858 (Amphibia, Anura): proposed conservation. Alytes, 6 (1-2), 56-68.

Dubois, A. (1987f) Elachistocleis Parker, 1927 (Amphibia, Anura): proposed conservation. Alytes, 6 (1-2), 75-84.

Dubois, A. (1988a) Some comments on the genus concept in zoology. Monitore zoologico italiano, New Series, 22, 2744.

Dubois, A. (1988b) Dates de publication du journal Alytes (19821987). Alytes '1987', 6 (3-4), 116. [1988].

Dubois, A. (1988c) The genus in zoology: a contribution to the theory of evolutionary systematics. Mémoires du Muséum national d'Histoire naturelle, (A) 140, 1-123.

Dubois, A. (1988d) Miscellanea nomenclatorica batrachologica (XVII). Alytes, 7 (1), 1-5. [1988.da].

Dubois, A. (1991) Nomenclature of parthenogenetic, gynogenetic and 'hybridogenetic' vertebrate taxons: new proposals. Alytes, 8, 61-74.

Dubois, A. (1992) Notes sur la classification des Ranidae (Amphibiens, Anoures). Bulletin mensuel de la Société linnéenne de Lyon, 61 (10), 305-352. [1992.da]. https://doi.org/10.3406/linly.1992.11011

Dubois, A. (1995a) Keratodont formulae in anuran tadpoles: proposals for a standardization. Journal of zoological Systematics \& evolutionary Research, 33 (1), i-xv. https://doi.org/10.1111/j.1439-0469.1995.tb00207.x

Dubois, A. (1995b) The valid scientific name of the Italian treefrog, with comments on the status of some early scientific names of Amphibia Anura, and some articles of the Code concerning secondary homonyms. Dumerilia, 2, 55-71.

Dubois, A. (1997) An evolutionary biologist's view on the science of biology. Alytes, 15 (3), 133-136.

Dubois, A. (1998) List of European species of amphibians and reptiles: will we soon be reaching 'stability'? AmphibiaReptilia, 19 (1), 1-28.

https://doi.org/10.1163/156853898X00304

Dubois, A. (1999) Miscellanea nomenclatorica batrachologica. 19. Notes on the nomenclature of Ranidae and related groups. Alytes, 17 (1-2), 81-100.

Dubois, A. (2000a) Nomenclature zoologique: quelques problèmes et besoins actuels. Bulletin de la Société française de Systématique, Juin 2000, 24, 13-23.

Dubois, A. (2000b) Synonymies and related lists in zoology: general proposals, with examples in herpetology. Dumerilia, 4 (2), 33-98.

Dubois, A. (2002) The taxonomic status of Scutiger mokokchungensis Das \& Chanda, 2000 (Amphibia, Anura). Amphibia-Reptilia, 22 (1), 499-506.

https://doi.org/10.1163/15685380152770444

Dubois, A. (2003) The relationships between taxonomy and conservation biology in the century of extinctions. Comptes rendus Biologies, 326 (Supplement 1), S9-S21. https://doi.org/10.1016/S1631-0691(03)00022-2

Dubois, A. (2004a) Book review. Amphibians of Nepal: a few words of caution. Alytes, 21 (3-4), 174-180.

Dubois, A. (2004b) The higher nomenclature of recent amphibians. Alytes, 22 (1-2), 1-14.

Dubois, A. (2004c) Developmental pathway, speciation and supraspecific taxonomy in amphibians. 1. Why are there so many frog species in Sri Lanka? Alytes, 22 (1-2), 19-37.

Dubois, A. (2004d) Developmental pathway, speciation and supraspecific taxonomy in amphibians. 2. Developmental pathway, hybridizability and generic taxonomy. Alytes, 22 (1-2), 38-52.

Dubois, A. (2005a) Propositions pour l'incorporation des nomina de taxons de rang supérieur dans le Code international de nomenclature zoologique. In: Dubois, A., Poncy, O., Malécot, V. \& Léger, N. (Eds.), Comment nommer les taxons de rang supérieur en zoologie et en botanique?, Biosystema, 23, pp. 73-96.

Dubois, A. (2005b) Proposed Rules for the incorporation of nomina of higher-ranked zoological taxa in the International Code of Zoological Nomenclature. 1. Some general questions, concepts and terms of biological nomenclature. Zoosystema, 27 (2), 365-426.

https://doi.org/10.11646/zootaxa.1337.1.1

Dubois, A. (2005c) Proposals for the incorporation of nomina of higher-ranked taxa into the Code. Bulletin of zoological Nomenclature, 62 (4), 200-209.

Dubois, A. (2005d) Amphibia Mundi. 1.1. An ergotaxonomy of recent amphibians. Alytes, $23(1-2), 1-24$. \{Q\} [2005.da].

Dubois, A. (2005e) Amphibia Mundi. 1.3. Recent amphibians: suprageneric taxonomic additions (1967-2002). Alytes, 23 (1-2), 70-80.

Dubois, A. (2006a) Proposed Rules for the incorporation of nomina of higher-ranked zoological taxa in the International Code of Zoological Nomenclature. 2. The proposed Rules and their rationale. Zoosystema, 28 (1), 165-258.

https://doi.org/10.11646/zootaxa.1337.1.1

Dubois, A. (2006b) New proposals for naming lower-ranked taxa within the frame of the International Code of Zoological Nomenclature. Comptes rendus Biologies, 329 (10), 823840.

https://doi.org/10.1016/j.crvi.2006.07.003

Dubois, A. (2006c) Incorporation of nomina of higher-ranked taxa into the International Code of Zoological Nomenclature: some basic questions. Zootaxa, 1337 (1), 1-37. https://doi.org/10.11646/zootaxa.1337.1.1

Dubois, A. (2006d) Naming taxa from cladograms: a cautionary tale. Molecular Phylogenetics and Evolution, 42, 317-330. https://doi.org/10.1016/j.ympev.2006.06.007

Dubois, A. (2007a) Phylogeny, taxonomy and nomenclature: the 
problem of taxonomic categories and of nomenclatural ranks. Zootaxa, 1519 (1), 27-68.

https://doi.org/10.11646/zootaxa.1519.1.3

Dubois, A. (2007b) Naming taxa from cladograms: some confusions, misleading statements, and necessary clarifications. Cladistics, 23, 390-402. https://doi.org/10.1111/j.1096-0031.2007.00151.x

Dubois, A. (2007c) Nomina zoologica linnaeana. In: Zhang, Z.Q. \& Shear, W.A. (Eds.), Linnaeus tercentenary: progress in invertebrate taxonomy, Zootaxa, 1668 (1), pp. 81-106. https://doi.org/10.11646/zootaxa.1668.1.9

Dubois, A. (2008a) A partial but radical solution to the problem of nomenclatural taxonomic inflation and synonymy load. Biological Journal of the Linnean Society, 93, 857-863. https://doi.org/10.1111/j.1095-8312.2007.00900.x

Dubois, A. (2008b) Authors of zoological publications and nomina are signatures, not persons. Zootaxa, 1771 (1), 63-68. https://doi.org/10.11646/zootaxa.1771.1.6

Dubois, A. (2008c) Drôles d'espèces. Hybridation, perturbations de la méiose et spéciation dans le règne animal: quelques points délicats de terminologie, d'éidonomie et de nomenclature. In: Prat, D., Raynal-Roques, A. \& Roguenant, A. (Eds.), Peuton classer le vivant?, Linné et la systématique aujourd'hui, Belin, Paris, pp. 169-202.

Dubois, A. (2008d) Le Code international de nomenclature zoologique: présentation, philosophie, règles majeures, problèmes actuels. In: Prat, D., Raynal-Roques, A. \& Roguenant, A. (Eds.), Peut-on classer le vivant?, Linné et la systématique aujourd'hui, Belin, Paris, pp. 355-402.

Dubois, A. (2008e) I had a dream... Alytes, 25 (3-4), 89-92.

Dubois, A. (2008f) Phylogenetic hypotheses, taxa and nomina in zoology. In: Minelli, A. Bonato, L. \& Fusco, G. (Eds.), Updating the Linnaean heritage: names as tools for thinking about animals and plants, Zootaxa, 1950 (1), pp. 51-86. [2008.da]. https://doi.org/10.11646/zootaxa.1950.1.7

Dubois, A. (2009a) Miscellanea nomenclatorica batrachologica. 20. Class-series nomina are nouns in the nominative plural: Terrarana Hedges, Duellman \& Heinicke, 2008 must be emended. Alytes, 26 (1-4), 167-175. [2009.da].

Dubois, A. (2009b) The special message from threatened amphibians. Alytes, 27 (1), 26-36.

Dubois, A. (2009c) Qu'est-ce qu'une espèce animale? In: Aller à l'espèce: illusion ou nécessité, Mémoires de la Société entomologique de France, 8, 9-48.

Dubois, A. (2009d) Endangered species and endangered knowledge. Zootaxa, 2201 (1), 26-29. https://doi.org/10.11646/zootaxa.2201.1.5

Dubois, A. (2010-2014) Time is for field work and collections. Unpublished lecture given at the WNPATA workshop and seminar Amphibians of the Western Ghats, University of Delhi, India, 2-3 November 2010, and again at the CEFIPRA workshop. Strengthening capacity for inventory of fauna in biodiversity hotspots in India and neighbouring countries, Hyderabad, India, 14-17 October 2014.

Dubois, A. (2010a) Retroactive changes should be introduced in the
Code only with great care: problems related to the spellings of nomina. Zootaxa, 2426 (1), 1-42.

https://doi.org/10.11646/zootaxa.2426.1.1

Dubois, A. (2010b) Nomenclatural rules in zoology as a potential threat against natural history museums. Organisms, Diversity and Evolution, 10, 81-90. https://doi.org/10.1007/s13127-010-0015-1

Dubois, A. (2010c) Zoological nomenclature in the century of extinctions: priority vs. 'usage'. Organisms, Diversity and Evolution, 10, 259-274. https://doi.org/10.1007/s13127-010-0021-3

Dubois, A. (2010d) Bionomina, a forum for the discussion of nomenclatural and terminological issues in biology. Bionomina, 1, 1-10. https://doi.org/10.1007/s13127-010-0021-3

Dubois, A. (2010e) Describing new species. Taprobanica, 2 (1), $6-24$. https://doi.org/10.4038/tapro.v2i1.2703

Dubois, A. (2010f) [29 December] Editorial. Taxonomy in the century of extinctions: taxonomic gap, taxonomic impediment, taxonomic urgency. Taprobanica, 2 (1), 1-5. https://doi.org/10.4038/tapro.v2i1.2702

Dubois, A. (2011a) The International Code of Zoological Nomenclature must be drastically improved before it is too late. Bionomina, 2, 1-104. https://doi.org/10.11646/bionomina.2.1.1

Dubois, A. (2011b) Species and 'strange species' in zoology: do we need a 'unified concept of species'? Comptes rendus Palevol, 10, 77-94. https://doi.org/10.1016/j.crpv.2011.01.002

Dubois, A. (2011c) A zoologist's viewpoint on the Draft BioCode. Bionomina, 3, 45-62. https://doi.org/10.11646/bionomina.3.1.4

Dubois, A. (2012a) The distinction between introduction of a new nomen and subsequent use of a previously introduced nomen in zoological nomenclature. Bionomina, 5, 57-80. https://doi.org/10.11646/bionomina.5.1.2

Dubois, A. (2012b) New synonymies in the order Urodela Duméril, 1805 (Amphibia, Batrachia), with comments on the use of the formula 'new taxon' to designate new nomina. Zootaxa, 3563 (1), 65-68. https://doi.org/10.11646/zootaxa3563.1.5

Dubois, A. (2013) Zygoidy, a new nomenclatural concept. Bionomina, 6, 1-25. https://doi.org/10.11646/bionomina.6.1.1

Dubois, A. (2015a) Zoological nomina in the century of extinctions: new proposals. Bionomina, 8, 11-53. https://doi.org/10.11646/bionomina.8.1.2

Dubois, A. (2015b) What is an anonymous publication? Is the International Code of Zoological Nomenclature anonymous? Bionomina, 9, 27-34.

https://doi.org/10.11646/bionomina.9.1.2

Dubois, A. (2015c) The Duplostensional Nomenclatural System for higher zoological nomenclature. Dumerilia, 5, 1-108.

Dubois, A. (2016) The Duplostensional Nomenclatural System for higher zoological nomenclature: additional comments. 
Dumerilia, 6, 5-16.

Dubois, A. (2017a) A few problems in the generic nomenclature of insects and amphibians, with recommendations for the publication of new generic nomina in zootaxonomy and comments on taxonomic and nomenclatural databases and websites. Zootaxa, 4237 (1), 1-16.

https://doi.org/10.11646/zootaxa.4237.1.1

Dubois, A. (2017b) The nomenclatural status of Hysaplesia, Hylaplesia, Dendrobates and related nomina (Amphibia, Anura), with general comments on zoological nomenclature and its governance, as well as on taxonomic databases and websites. Bionomina, 11, 1-48.

https://doi.org/10.11646/bionomina.11.1.1

Dubois, A. (2017c) The need for reference specimens in zoological taxonomy and nomenclature. Bionomina, 12, 4-38. https://doi.org/10.11646/bionomina.12.1.2

Dubois, A. (2017d) Diagnoses in zoological taxonomy and nomenclature. Bionomina, 12, 64-88. https://doi.org/10.11646/bionomina.12.1.8

Dubois, A. (2017e) A plea for nomenclatural accuracy in taxonomic and faunistic checklists. Dumerilia, 7, 1-17.

Dubois, A. (2018) The correct spelling of the nomen Nyctimystes cheemani Tyler, 1964 (Amphibia, Anura), with some comments on Latin grammar and the Rules of the Code. Alytes, 35 (1-4), 75-84.

Dubois, A. (2019) The status of the generic nomina Palaeobufo Bolkay, 1919 and Neobufo Bolkay, 1919 (Amphibia, Anura). Alytes, 37 (1-2), 73-80.

Dubois, A. (2020a) Allocation of nomina to taxa in zoological nomenclature. Bionomina, 18, 1-43. https://doi.org/10.11646/bionomina.18.1.1

Dubois, A. (2020b) The status regarding publication date and availability of taxonomic works published online without proper Zoobank registration. Bionomina, 18, 44-55. https://doi.org/10.11646/bionomina.18.1.2

Dubois, A. \& Aescht, E. (Ed.) (2016) LZC Session 3. Proposal TER-01. The term nomen. Dumerilia, 6, 47-53.

Dubois, A. \& Aescht, E. (Ed.) (2017a) LZC Session 8. Proposal GEN-03. The Principles of the Zoocode. [1] The Principle of Nomenclatural Independence. Dumerilia, 7, 21-23.

Dubois, A. \& Aescht, E. (Ed.) (2017b) LZC Session 9. Proposal GEN-04. The Principles of the Zoocode. [2] The Principle of Nomenclatural Foundation. Dumerilia, 7, 24-25.

Dubois, A. \& Aescht, E. (Ed.) (2017c) LZC Session 10. Proposal TER-02. Nominal-series. Dumerilia, 7, 26-28.

Dubois, A. \& Aescht, E. (Ed.) (2017d) LZC Session 11. Proposal GEN-05. The Principles of the Zoocode. [3] The Principle of Nominal-Series. Dumerilia, 7, 29-31.

Dubois, A. \& Aescht, E. (Ed.) (2017e) LZC Session 12. Proposal AVA-03. Availability of new species-series nomina: the need of at least one name-bearer specimen preserved in a public permanent curated collection and available for study. Dumerilia, 7, 32-34.

Dubois, A. \& Aescht, E. (Ed.) (2017f) LZC Session 13. Proposal AVA-04. Problems with the 2012 Amendment of the Code. Dumerilia 7, 35-47.
Dubois, A. \& Aescht, E. (Ed.) (2019a) LZC Session 16. What is the meaning of 'fixed content and layout' in Article 8.1.3.2 of the 2012 Amendment of the Code? Consequences regarding this Amendment. Dumerilia, 8, 6-34.

Dubois, A. \& Aescht, E. (Ed.) (2019b) LZC Session 17. The Principles of the Zoocode. 4. The Principle of Binomina. Dumerilia, 8, 35-36.

Dubois, A. \& Aescht, E. (Ed.) (2019c) LZC Session 18. The term type. Dumerilia, 8, 37-41.

Dubois, A. \& Aescht, E. (Ed.) (2019d) LZC Session 19. The Principles of the Zoocode. 5. The Principle of Coordination. Dumerilia, 8, 42-47.

Dubois, A. \& Aescht, E. (Ed.) (2019e) LZC Session 20. The Principles of the Zoocode. 6. The Principle of Neonymy. Dumerilia, 8, 48-52.

Dubois, A. \& Aescht, E. (Ed.) (2019f) LZC Session 21. The Principles of the Zoocode. 7. The Principle of Onomatophores. Dumerilia, 8, 53-56.

Dubois, A. \& Aescht, E. (Ed.) (2019g) LZC Session 23. The Principles of the Zoocode. 8. The Principle of Zygoidy. Dumerilia, 8, 62-65.

Dubois, A. \& Aescht, E. (Ed.) (2019h) LZC Session 24. Subtelties of homonymy in zoological nomenclature. Dumerilia, 8, 66-84.

Dubois, A. \& Aescht, E. (Ed.) (2019i) LZC Session 25. The Principles of the Zoocode. 9. The Principle of Homynymy. Dumerilia, 8, 85-87.

Dubois, A. \& Aescht, E. (Ed.) (2019j) LZC Session 27. Suffixes in family-series nomenclature. Dumerilia, 8, 98-105.

Dubois, A. \& Aescht, E. (Ed.) (2019k) LZC Session 28. The Principles of the Zoocode. 10. The Principle of Synonymy. Dumerilia, 8, 106-109.

Dubois, A. \& Aescht, E. (Ed.) (2019l) LZC Session 29. The Principles of the Zoocode. 11. The Principle of Priority. Dumerilia, 8, 110-113.

Dubois, A. \& Aescht, E. (Ed.) (2019m) LZC Session 30. The Principles of the Zoocode. 12. The Principle of Airesy. Dumerilia, 8, 114-116.

Dubois, A. \& Aescht, E. (Ed.) (2019n) LZC Session 31. The Principles of the Zoocode. 13. The Principle of Proedry. Dumerilia, 8, 117-118.

Dubois, A. \& Aescht, E. (Ed.) (2019o) LZC Session 32. The Principles of the Zoocode. 14. The Principle of Nomography. Dumerilia, 8, 119-132. [2019.da].

Dubois, A. \& Aescht, E. (Ed.) (2019p) LZC Session 33. The Principles of the Zoocode. 15. The Principle of Sozoidy. Dumerilia, 8, 133-142.

Dubois, A. \& Aescht, E. (Ed.) (2019q) LZC Session 34. The Principles of the Zoocode. 16. The Principle of Archoidy. Dumerilia, 8, 143-146.

Dubois, A. \& Aescht, E. (Ed.) (2019r) LZC Session 35. The Principles of the Zoocode. 17. The Principle of Registration. Dumerilia, 8, 147-154.

Dubois, A. \& Aescht, E. (Ed.) (2019s) LZC Session 37. Diagrams of the Nomenclatural Process. Dumerilia, 8, 159-168.

Dubois, A., Aescht, E. \& Dickinson, E.C. (2016) Burning questions 
of zoological nomenclature. The Linz International Workshop on Zoological Nomenclature (9-10 July 2014). Dumerilia , 6, 24-34.

Dubois, A., Bauer, A.M., Ceríaco, L.M.P., Dusoulier, F., Frétey, T., Löbl, I., Lorvelec, O., Ohler, A., Stopiglia, R. \& Aescht, E. (2019) The Linz Zoocode project: a set of new proposals regarding the terminology, the Principles and Rules of zoological nomenclature. First report of activities (20142019). Bionomina, 17, 1-111.

https://doi.org/10.11646/bionomina.17.1.1

Dubois, A. \& Berkani, M. (2013) The misleading use of the terms parent, child, ancestor and descendant in databases dealing with biological evolution and taxonomy. Bionomina, 6, 5256.

https://doi.org/10.11646/bionomina.6.1.4

Dubois, A. \& Bour, R. (2010a) The nomenclatural status of the nomina of amphibians and reptiles created by Garsault (1764), with a parsimonious solution to an old nomenclatural problem regarding the genus Bufo (Amphibia, Anura), comments on the taxonomy of this genus, and comments on some nomina created by Laurenti (1768). Zootaxa, 2447 (1), 1-52. https://doi.org/10.11646/zootaxa.2447.1.1

Dubois, A. \& Bour, R. (2010b) The distinction between familyseries and class-series nomina in zoological nomenclature, with emphasis on the nomina created by Batsch $(1788,1789)$ and on the higher nomenclature of turtles. Bonn zoological Bulletin, 57 (2), 149-171.

Dubois, A. \& Bour, R. (2011) The authorship and date of the familial nomen Ranidae (Amphibia, Anura). Alytes, 27 (4), 154-160.

Dubois, A., Crochet, P.-A., Dickinson, E.C., Nemesio, A., Aescht, E., Bauer, A.M., Blagoderov, V., Bour, R., De Carvalho, M.R., Desutter-Grandcolas, L., Fretey, T., Jager, P., Koyamba, V., Lavilla, E.O., Loebl, I., Louchart, A., Malecot, V., Schatz, H. \& Ohler, A. (2013) Nomenclatural and taxonomic problems related to the electronic publication of new nomina and nomenclatural acts in zoology, with brief comments on optical discs and on the situation in botany. Zootaxa, 3735 (1), 1-94. https://doi.org/10.11646/zootaxa.3735.1.1

Dubois, A., Crombie, R.I. \& Glaw, F. (2005) Amphibia Mundi. 1.2. Recent amphibians: generic and infrageneric taxonomic additions (1981-2002). Alytes, 23 (1-2), 25-69.

Dubois, A., Duellman, W.E. \& Ohler, A. (2017) Taxonomic and nomenclatural notes on the subfamily Acridinae of the family Hylidae (Amphibia, Anura). Bionomina, 11, 49-61. [2017. da].

https://doi.org/10.11646/bionomina.11.1.2

Dubois, A. \& Frétey, T. (2016) A new nomen for a subfamily of frogs (Amphibia, Anura). Dumerilia, 6, 17-23. [2016.da].

Dubois, A. \& Frétey, T. (2020a) Herpetological higher taxa nomina. 1. Introduction and methodology. Bionomina, 19, 1-56. https://doi.org/10.11646/bionomina.19.1.1

Dubois, A. \& Frétey, T. (2020b) Herpetological higher taxa nomina. 2. Anura Duméril, 1805. Bionomina, 20, 1-16. https://doi.org/10.11 646/bionomina.20.1.1

Dubois, A. \& Frétey, T. (2020c) Herpetological higher taxa nomina. 3. Urodela Duméril, 1805. Bionomina, 20, 17-35. https://doi.org/10.11646/bionomina.20.1.2

Dubois, A. \& Frétey, T. (2020d) Herpetological higher taxa nomina.

4. Batrachia Brongniart, 1800. Bionomina, 20, 36-46.

https://www.mapress.com/j/bn

Dubois, A. \& Frétey, T. (2021a) Herpetological higher taxa nomina. 5. Anura, Urodela, Batrachia: addenda and corrigenda. Bionomina: in press.

Dubois, A. \& Frétey, T. (2021b) Herpetological higher taxa nomina. 6. Gymnophiona Rafinesque, 1814. Bionomina: in press.

Dubois, A. \& Frétey, T. (2021c) Herpetological higher taxa nomina. 7. Lissamphibia Gadow, 1898. Bionomina: in press.

Dubois, A. \& Frétey, T. (2021d) Herpetological higher taxa nomina. 8. Amphibia Blainville, 1816. Bionomina: in press

Dubois, A., Frétey, T. \& Ohler, A. (2018) The Relictus case: it is high time that taxonomists follow the Code's requirements for nomenclatural availability and validity of new zoological nomina. Bionomina, 13, 51-64.

https://doi.org/10.11646/bionomina.13.1.4

Dubois, A. \& Günther, R. (1982) Klepton and synklepton: two new evolutionary systematics categories in zoology. Zoologische Jahrbücher, Abteilung für Systematik, Ökologie \& Biologie der Tiere, 109, 290-305.

Dubois, A. \& Jiang, J.-P. (2005) The higher nomenclature of recent Amphibians. Herpetologica sinica, 10, 354-358. [In Chinese]. [2005.db].

Dubois, A. \& Malécot, V. (2005) Glossaire: termes de taxinomie et de nomenclature utilisés dans ce volume. In: Dubois, A., Poncy, O., Malécot, V. \& Léger, N. (Eds.), Comment nommer les taxons de rang supérieur en zoologie et en botanique?, Biosystema, 23, pp. 97-103.

Dubois, A. \& Ohler, A. (1999) Asian and Oriental toads of the Bufo melanostictus, Bufo scaber and Bufo stejnegeri groups (Amphibia, Anura): a list of available and valid names and redescription of some name-bearing types. Journal of South Asian natural History, 4, 133-180.

Dubois, A. \& Ohler, A. (2001) A new genus for an aquatic ranid (Amphibia, Anura) from Sri Lanka. Alytes, 19 (2-4), 81-106. [2001.da].

Dubois, A. \& Ohler, A. (2005) Taxonomic notes on the Asian frogs of the tribe Paini (Ranidae, Dicroglossinae: 1. Morphology and synonymy of Chaparana aenea (Smith, 1922), with a proposal of a new statistical method for testing homogeneity of small sample. Journal of natural History, 39, 1759-1778. https://doi.org/10.1080/00222930400023735

Dubois, A. \& Ohler, A. (2009) The status of the amphibian nomina created by Merrem (1820) and Ritgen (1828). Zootaxa, 2247 (1), 1-36. https://doi.org/10.11646/zootaxa.2247.1.1

Dubois, A. \& Ohler, A. (2015) An often overlooked Rule of the Code, and its bearing on the authorship and date of the nomen Proteidae (Amphibia, Urodela). Bionomina, 9, 41-49. [2015. da]. https://doi.org/10.11646/bionomina.9.1.4

Dubois, A. \& Ohler, A. (2018) The Hyla quoyi-Hyla prasina case (Amphibia, Anura), with comments on bibliographic and taxonomic databases and on Article 23.9 of the Code. 
Zoosystema, 40 (23), 501-506.

https://doi.org/10.5252/zoosystema2018v40a23

Dubois, A. \& Ohler, A. (2019) The nomina Anura, Urodela, Ecaudata and Caudata, credited to 'Fischer von Waldheim, 1813', do not exist, with comments on the nomenclature of higher zoological taxa and on the authorships and dates of other amphibian nomina. Bionomina, 14, 1-68. https://doi.org/10.11646/bionomina.14.1.1

Dubois, A., Ohler, A. \& Biju, S.D. (2001) A new genus and species of Ranidae (Amphibia, Anura) from south-western India. Alytes, 19 (2-4), 53-79. [2001.db].

Dubois, A. \& Raffaëlli, J. (2009) A new ergotaxonomy of the family Salamandridae Goldfuss, 1820 (Amphibia, Urodela). Alytes, 26 (1-4), 1-85. [2009.db].

Dubois, A. \& Raffaëlli, J. (2012) A new ergotaxonomy of the order Urodela Duméril, 1805 (Amphibia, Batrachia). Alytes, 28 (3-4), 77-161. [2012.da].

Ducrotay de Blainville, H.M. (1821) Note analytique sur les travaux anatomiques, physiologiques et zoologiques de $M$. H. M. Ducrotay de Blainville. Huzard-Courcier, Paris, 18 pp.. [1821.da].

Ducrotay de Blainville, H.M. (1822) De l'organisation des animaux, ou Principes d'anatomie comparée. Tome premier, contenant la morphologie et l'aistésologie. Levrault, Paris, [i-iii] + i-lix +574 pp., 10 tab. [1822.da].

https://doi.org/10.5962/bhl.title.110421

Duellman, W.E. (1970) The hylid frogs of Middle America. Monographs of the Museum of natural History, 1, 1-753, 72 $\mathrm{pl}$.

Duellman, W.E. (1974) A reassessment of the taxonomic status of some neotropical hylid frogs. Occasional Papers of the Museum of natural History, The University of Kansas, 27, $1-27$.

Duellman, W.E. (1975) On the classification of frogs. Occasional Papers of the Museum of natural History, The University of Kansas, 42, 1-14. $\{\mathrm{Q}\}$.

Duellman, W. E. (1977) Liste der rezenten Amphibien und Reptilien. Hylidae, Centrolenidae, Pseudidae. Das Tierreich, 95, 1-225. $\{\mathrm{Q}\}$. https://doi.org/10.2307/1443200

Duellman, W.E. (1979) The numbers of amphibians and reptiles. Herpetological Review, 10, 83-84. \{Q\}.

Duellman, W.E. (1988) Evolutionary relationships of the Amphibia. In: Fritzsch, B. (Ed.), The evolution of the Amphibian auditory system, John Wiley \& Sons, New York, pp. 13-34. \{Q\}.

Duellman, W.E. (1999) Patterns of distribution of amphibians: a global perspective. John Hopkins University Press, Baltimore, Maryland, $633 \mathrm{pp}$.

Duellman, W.E. (2001) Hylid frogs of Middle America. Society for the Study of Amphibians and Reptiles, Ithaca, New York, $\mathrm{i}-\mathrm{xvi}+1159 \mathrm{pp}$.

Duellman, W.E. (2003) An overview of anuran phylogeny, classification and reproductive modes. In: Reproductive biology and phylogeny of Anura. In: Jamieson, B.G.M. (Ed.), Reproductive biology and phylogeny 2, Science Publishers, Enfield \& Plymmouth, pp. 1-18. \{Q\}.
Duellman, W.E. (2015) Marsupial frogs: Gastrotheca \& allied genera. John Hopkins University Press, Baltimore, i-xviii + 408 pp.

Duellman, W.E. \& Adler, K. (2007) Chapter 1. The evolution of amphibian systematics: an historical perspective. In: Heatwole, H. \& Tyler, M. (Eds.), Amphibian biology, Volume 7, Systematics, Surrey Beatty, Chipping North, pp. 24072435. \{Q\} [2007.da].

Duellman, W.E. \& Campbell, J. (1992) Hylid frogs of the genus Plectrohyla: systematics and phylogenetic relationships. Miscellaneous Publications of the Museum of Zoology, University of Michigan, 181, 1-32.

Duellman, W.E. \& Hoogmoed, M.S. (1992) Some hylid frogs from the Guiana highlands, northeastern South America: new species, distributional records, and a generic reallocation. Occasional Papers of the Museum of natural History, The University of Kansas, 147, 1-21.

Duellman, W.E., Marion, A.B. \& Hedges, S.B. (2016) Phylogenetics, classification, and biogeography of the treefrogs (Amphibia: Anura: Arboranae). Zootaxa, 4104 (1), 1-109.

https://doi.org/10.11646/zootaxa.4104.1.1

Duellman, W.E. \& Schulte, R. (1993) New species of centrolenid frogs from northern Peru. Occasional Papers of the Museum of natural History, The University of Kansas, 155, 1-33.

Duellman, W.E. \& Trueb, L. (1971) A synopsis of neotropical hylid frogs, genus Osteocephalus. Occasional Papers of the Museum of natural History, The University of Kansas, 1, $1-47$.

Duellman, W.E. \& Trueb, L. (1976) The systematic status and relationships of the hylid frog Nyctimantis rugiceps Boulenger. Occasional Papers of the Museum of natural History, The University of Kansas, 58, 1-14.

Duellman, W. E. \& Trueb, L. (1985) Biology of Amphibians. McGraw-Hill, New York, i-xix +670 pp. $\{Q\}$.

Duellman, W.E. \& Trueb, L. (1994) Biology of Amphibians. Johns Hopkins University Press, Baltimore, $\mathrm{i}-\mathrm{xxi}+670 \mathrm{pp}$.

Duméril A.M.C. (1805) Zoologie analytique, ou méthode naturelle de classification des animaux, rendue plus facile à l'aide de tableaux synoptiques. '1806'. Allais, Paris i-xxxiii +544 pp. [P02] [1805.da]. https://doi.org/10.5962/bhl.title.44835

Duméril, [A.M.C.] (1839) Mémoire sur la classification et la structure des Ophiosomes ou Céciloïdes, famille de reptiles qui participent des Ophidiens et des Batraciens, relativement à la forme et à l'organisation. Compte rendu des Séances de l'Académie des Sciences, 9, 581-587. [1839.da].

Duméril, A. (1863) Catalogue méthodique de la collection des Batraciens du Muséum d'Histoire Naturelle de Paris. Mémoires de la Société impériale des Sciences naturelles de Cherbourg, 9, 295-321, pl. 1-2. [1863.da].

Duméril, A.-M.-C. \& Bibron G. (1839) Erpétologie générale ou Histoire naturelle complète des Reptiles. Tome 5. Roret, Paris, i-viii +855 pp. [1839.db].

Duméril, A.-M.-C. \& Bibron, G. (1841) Erpétologie générale ou Histoire naturelle complète des Reptiles. Tome 8. Roret, Paris, i-vii + 792 pp. \{Q\} [1841.da]. 
Duméril, A.-M.-C., Bibron, G. \& Duméril, A. (1854) Erpétologie générale ou histoire naturelle complète des Reptiles. Tome 9. Roret, Paris, i-xx + 440 pp. [1854.da]. https://doi.org/10.5962/bhl.title.118797

Dunlop, J.A. \& Penney, D. (2011) Order Araneae Clerck, 1757. In: Zhang, Z.-Q. (Ed.), Animal biodiversity: an outline of higher-level classification and survey of taxonomic richness. Zootaxa, 3148 (1), 149-153.

https://doi.org/10.11646/zootaxa.3148.1.27

Dunn, E.R. (1917) The salamanders of the genera Desmognathus and Leurognathus. Proceedings of the United States national Museum, 53, 393-433. [1917.da]. https://doi.org/10.5479/si.00963801.53-2211.393

Dunn, E.R. (1922) The sound-transmitting apparatus of salamanders and the phylogeny of the Caudata. The American Naturalist, 56 (646), 418-427. [1922.da]. https://doi.org/10.1086/279882

Dupuis, C. (1984) Willi Hennig's impact on taxonomic thought. Annual Review of Ecology and Systematics, 15, 1-25. https://doi.org/10.1146/annurev.es.15.110184.000245

Duvernoy, [G.L.] (1849) Cours d'histoire naturelle des corps organisés professé au Collège de France. Leçons sur l'embranchement des Vertébrés. Revue et Magasin de Zoologie pure et appliquée (2) 1, 179-189. [1849.da].

Edwards, J. (1976) Spinal nerves and their bearing on salamander phylogeny. Journal of Morphology, 148 (3), 305-327. [1976. ea].

https://doi.org/10.1002/jmor.1051480304

Edwards, S.V. (2009) Is a new and general theory of molecular systematics emerging? Evolution, 63, 1-19. https://doi.org/10.1111/j.1558-5646.2008.00549.x

Edwards, S.V., Potter, S., Schmitt, C.J., Bragg, J.G. \& Moritz, C. (2016) Reticulation, divergence, and the phylogeographyphylogenetics continuum. Proceedings of the national Academy of Sciences, 113 (29), 8025-8032. https://doi.org/10.1073/pnas.1601066113

Ehrenberg, C.G. (1831) Symbolae physicae. Animalia evertebrata exclusis insectis. Mittlero, Berolini, [128 pp. +10 pls.]. [1831. ea].

Eichwald, E. (1831) Zoologia specialis quam expositis animalibus tum vivis, tum fossilibus potissimum rossiae in universum, et poliniae in specie, in usum lectionum publicarum in Universitate Caesarea Vilnensi habendarum. Pars posterior. Voss, Lipsiae, i-iii + 404 pp., pl. 1. [1831.eb].

Eiselt, J. (1988) Krötenfrösche (Pelobates gen., Amphibia Salientia) in Türkisch-Thrakien und Griechenland. Annalen des Naturhistorischen Museums in Wien, (Serie B für Botanik und Zoologie), 90, 51-59. [1988.ea].

Eiselt, J. \& Schmidtler, J.F. (1973) Froschlurche aus dem Iran unter Berücksichtigung außeriranischer Populationsgruppen. Annalen des Naturhistorischen Museums in Wien, 77, 181243.

Emerson, S.B., Inger, R.F. \& Iskandar, D. (2000) Molecular systematics and biogeography of the fanged frogs of Southeast Asia. Molecular Phylogenetics and Evolution, 16, 131-142. https://doi.org/10.1006/mpev.2000.0778
Emig, C C., Bitner, M. A. \& Álvarez, F. (2013) Phylum Brachiopoda. In: Zhang, Z.-Q. (Ed.), Animal biodiversity: an outline of higher-level classificationand survey of taxonomic richness (Addenda 2013). Zootaxa, 3703 (1), 75-78.

https://doi.org/10.11646/zootaxa.3703.1.15

Erichson, W.F. (1837) Die Käfer der Mark Brandenburg. F. J. Morin, Berlin, i-viii +740 pp. [1837.ea]. https://doi.org/10.5962/t.172994

Eschmeyer, W.N. \& Fong, J.D. (2011) Pisces. In: Zhang, Z.-Q. (Ed.), Animal biodiversity: an outline of higher-level classification and survey of taxonomic richness. Zootaxa, 3148 (1), 26-38. https://doi.org/10.11646/zootaxa.3148.1.7

Estes, R. (1965) Fossil salamanders and salamander origins. American Zoologist, 5, 319-334. [1965.ea]. https://doi.org/10.1093/icb/5.2.319

Estes, R. (1969) Prosirenidae, a new family of fossil salamanders. Nature, 224, 87-88. [1969.ea]. https://doi.org/10.1038/224087a0

Estes, R. (1981) Gymnophiones, Caudata. In: Wellnhofer, P. (Ed.), Handbuch der Paläoherpetologie. Teil 2. Gustav Fischer, Stuttgart and New York, pp. i-Xv + 1-115. \{Q\} [1981.ea].

Faivovich, J., Ferraro, D., Basso, N., Haddad, C., Rodrigues, M., Wheeler, W.C. \& Lavilla, E. (2012) A phylogenetic analysis of Pleurodema (Anura: Leptodactylidae: Leiuperinae) based on mitochondrial and nuclear gene sequences, with comments on the evolution of anuran foam nests. Cladistics, 28, 460482.

https://doi.org/10.1111/j.1096-0031.2012.00406.x

Faivovich, J., Haddad, C.F.B., Baêta, D., Jungfer, K.-H., Alvres, G.F.R., Bandao, R.A., Sheil, C., Barrientos, L.S., BarrioAmoros, C.L., Cruz, C.A.G. \& Wheeler, W.C. (2010) The phylogenetic relationships of the charismatic poster frogs, Phyllomedusinae (Anura, Hylidae). Cladistics, 26, 227-261. https://doi.org/10.1111/j.1096-0031.2009.00287.x

Faivovich, J.C., Haddad, F.B., Garcia, P.C.A., Frost, D.R., Campbell, J.A. \& Wheeler, W.C. (2005) Systematic review of the frog family Hylidae, with special reference to Hylinae: a phylogenetic analysis and taxonomic revision. Bulletin of the American Museum of natural History, 294, 1-240. [2005.fa]. https://doi.org/10.1206/0003-0090(2005)294[0001: SROTFF]2.0.CO;2

Faivovich, J., Nicoli, L., Blotto, B.L., Pereyra, M.O., Baldo, D., Barrionuevo, J.S., Fabrezi, M., Wild, E.R. \& Haddad, C.F.B. (2014) Big, bad, and beautiful: phylogenetic relationships of the horned frogs (Anura: Ceratophryidae). South American Journal of Herpetology, 9, 207-227. https://doi.org/10.2994/SAJH-D-14-00032.1

Faivovich, J., Pereyra, M.O., Luna, M.C., Hertz, A., Blotto, B.L., Vásquez-Almazán, C.R., McCranie, J.R., Sánchez, D. A., Baêta, D., Araujo-Vieira, K., Köhler, G., Kubicki, B., Campbell, J.A., Frost, D.R., Wheeler, W.C. \& Haddad, C.F.B. (2018) On the monophyly and relationships of several genera of Hylini (Anura: Hylidae: Hylinae), with comments on recent taxonomic changes in hylids. South American Journal of Herpetology, 13 (1), 1-32. [2018.fa]. https://doi.org/10.2994/SAJH-D-17-00115.1 
Fatio, V. (1872) Faune des Vertébrés de la Suisse. Volume 3. Histoire naturelle des Reptiles et des Batraciens. Georg, Genève \& Bâle, i-iii + 603 pp., pl. 1-5. \{Q\} [1872.fa].

Fei, L. (1999) Atlas of Amphibians of China. Henan Press of Science and Technology, Zhengzhou, i-ii + $432 \mathrm{pp}$.

Fei, L., Hu S., Ye, C. \& Huang, Y. (2009) Fauna Sinica. Amphibia, Vol. 3. Anura Ranidae. Science Press, Beijing, $[\mathrm{i}-\mathrm{iv}]+\mathrm{i}-\mathrm{xii}+$ 959-1847 pp., 16 pl.

Fei, L., Jiang, J., Ye, C. \& Cheng, S. (1995) Electrophoresis analyses of crystalline [sic] lens protein of twentyone [sic] species (subspecies) of four genera of Peelobatidae [sic]. Acta herpetologica sinica, Guiyang, (3) 4-5, 230-237. [In Chinese]. [1995.fa].

Fei, L. \& Ye, C. (1990) Intergeneric relationships and diversification of the high-altitude pelobatid toads in Asia and their relation with the formation of Qinghai-Xizang Plateau (Amphibia: Pelabatidae [sic]). Acta zoologica sinica, 36, 420-428. [In Chinese]. [1990.fa].

Fei, L. \& Ye, C. (2016) Amphibians of China. Volume 1. Science Press, Beijing, i-xvi 1040 pp. [2016.fa].

Fei, L. \& Ye, C.-Y. (2000) A new hynobiid subfamily with a new genus and new species of Hynobiidae from west China (Amphibia: Caudata). Cultum herpetologica sinica, 8, 64-70. [In Chinese]. [2000.fa].

Fei, L., Ye, C. \& Huang, Y. (1990) Key to Chinese Amphibia. Editions of Sciences and Techniques, Chongqing, [i-iv] $+2+$ 364 pp. [In Chinese]. [1990.fb].

Fei, L., Ye, C.-Y. \& Jiang, J.-P. (2010) Phylogenetic systematics of Ranidae. Herpetologica sinica, 12, 1-43. [2010.fa].

Fei, L., Ye, C., Jiang, J., Xie, F. \& Huang, Y. (2005) An illustrated key to Chinese amphibians. Sichuan Publishing Group, Sichuan Publishing House of Science \& Technology, i-v + 340 pp., pls. 1-12. [2005.fb].

Fejérváry, A.M. v. (1918) Über die rudimentären Rippen der anuren Batrachier. Verhandlungen der zoologisch-botanischen Gesellschaft in Wien, 68, 114-128. [1918.fa].

Fejérváry, G.J. de (1917) Anoures fossiles des couches préglaciaires de Püspökfürdő en Hongrie, en considération spéciale du développement phylétique du sacrum chez les Anoures. Földtani Közlöny 47, 141-172, pl. 1-3. [1917.fa].

Fejérváry, G.J. de (1921a) Remarques sur la position systématique des genres Bufavus et Ranavus. Annales Musei nationalis hungarici, 18, 28-30. [1921.fa].

Fejérváry, G.J. von (1921b) Kritische Bemerkungen zur Osteologie, Phylogenie und Systematik der Anuren. Archiv für Naturgeschichte, 87, 1-30. \{Q\} [1921.fb].

Fejérváry, G.J. de (1923) Ascaphidae, a new family of the tailless batrachians. Annales Musei nationalis hungarici, 20, 178181. [1923.fa].

Feller, A.E. \& Hedges, S.B. (1998) Molecular evidence for the early history of living amphibians. Molecular Phylogenetics and Evolution, 9, 509-516. [1998.fa]. https://doi.org/10.1006/mpev.1998.0500

Feng, Y.-J., Blackburn, D.C., Liang, D., Hillis, D.M., Wake, D.B., Cannatella, D.C. \& Zhang, P. (2017) Phylogenomics reveals rapid, simultaneous diversification of three major clades of
Gondwanan frogs at the Cretaceous-Paleogene boundary. Proceedings of the national Academy of Sciences, 114, E5864-E5870.

https://doi.org/10.1073/pnas.1704632114

Ficinus, [H.D.A.] \& Carus [C.G.] (1826) Uebersicht des gesammten Thierreichs. Arnold, Dresden, 1 pl. [1826.fa].

Fischer, G. (1808) Tableaux synoptiques de zoognosie. Imprimerie de 1'Université Impériale, Moscou, [1-60] + 186 pp., 6 pl. [1808.fa].

Fischer, G. (1813) Zoognosia tabulis synopticis illustrata. Editio tertia, classium, ordinum, generum illustratione perpetua aucta. Volumen primum. Typis Nicolai Sergeidis Vsevolozsky, Mosquae, i-xiv +465 pp. [1813.fa].

Fischer de Waldheim, G. (1824) Entomographie de la Russie. Entomographia Imperii Rossici. Volume 2. Augustus Semen, Academia Caesari Medico-Chriurgica, Moscow, i-xx + 1264, 39 tables. https://doi.org/10.3931/e-rara-74024

Fitzinger, L. (1835) Entwurf einer systematischen Anordnung der Schildkröten nach den Grundsätzen der natürlichen Methode. Annalen des Wiener Museums der Naturgeschichte, 1, 103128. [1835.fa].

Fitzinger, L.I. (1826) Neue Classification der Reptilien. Heubner, Wien, i-viii + 66 pp., 1 tab. \{Q\} [1826.fb].

Fitzinger, L.I. (1843) Systema Reptilium. Fasc. 1. Amblyglossae. Braumüller \& Seidel, Vindobonae, 106 pp. + i-ix. \{Q\} [1843. fa].

Fitzinger, L.J. (1827) Neue Classification der Reptilien. Isis von Oken, 20, 261-267. [1827.fa].

Fitzinger, L.J. (1828) Erwiederung an Herrn Schlegel, Conservator am kön. naturhistorischen Museum zu Leyden, und Herrn Dr. Wagler, Professor an der kön. Universität zu München, in Betreff ihrer Angriffe gegen meine neue Classification der Reptilien. Isis von Oken, 21 (1), 3-24. [1828.fa].

Fitzinger, L.J. (1832) Ueber die Ausarbeitung einer Fauna des Erzherzogthumes Oesterreich, nebst einer systematischen Aufzählung der in diesem Lande vorkommenden Säugethiere, Reptilien und Fische, als Prodrom einer Fauna derselben. Beiträge zur Landeskunde Oesterreich's unter der Enns, 1, 280-340. [1832.fa].

Fitzinger, L.J. (1861a) Die Ausbeute der österreichischen Naturforscher an Säugethieren und Reptilien während der Weltumsegelung Sr. Majestät Fregatte Novara. Sitzungsberichte der kaiserlichen Akademie der Wissenschaften Wien, 42 (25), 383-416. [1861.fa].

Fitzinger, L.J. (1861b) Eine Neue Batrachier-Gattung aus NeuSeeland. Verhandlungen der zoologisch-botanischen Vereins in Wiens, 11, 217-220. [1861.fb].

Fitzinger, L.-J. (1826b) Nouvelle classification des Reptiles, établie d'après les affinités naturelles. Bulletin des Sciences naturelles et de Géologie, 9, 347-349. [1826.fc].

Ford, L.S. \& Cannatella, D.C. (1993) The major clades of frogs. Herpetological Monographs, 7, 94-117. [1993.fa]. https://doi.org/10.2307/1466954

Forget, P.-M. (Ed.) (2002) Fragmentation de la forêt tropicale humide: le barrage de Petit Saut, rivière Sinnamary, Guyane 
française. Revue d'Ecologie, La Terre \& la Vie, Supplement 2, 1-199.

Fouquet, A., Blotto, B.L., Maronna, M.M., Verdade, V.K., Juncá, F.A., De Sá, R. \& Rodrigues, M.T. (2013) Unexpected phylogenetic positions of the genera Rupirana and Crossodactylodes reveal insights into the biogeography and reproductive evolution of leptodactylid frogs. Molecular Phylogenetics and Evolution, 67, 445-457. [2013.fa].

https://doi.org/10.1016/j.ympev.2013.02.009

Fouquet, A., Loebmann, D., Castroviejo-Fisher, S., Padial, J.M., Orrico, V.G.D., Lyra, M.L., Roberto, I.J., Kok, P.J.R., Haddad, C.F.B. \& Rodrigues, M.T. (2012) From Amazonia to the Atlantic forest: molecular phylogeny of Phyzelaphryninae frogs reveals unexpected diversity and a striking biogeographic pattern emphasizing conservation challenges. Molecular Phylogenetics and Evolution, 65, 547-561.

https://doi.org/10.1016/j.ympev.2012.07.012

Fouquette, M.J. Jr. \& Dubois, A. (2014) A checklist of North American Amphibians and Reptiles. Vol. 1. Amphibians. Xlibris, Lexington, Kentucky, 613 pp. [2014.fa].

Fox, R.C. \& Naylor, B.G. (1982) A reconsideration of the relationships of the fossil amphibian Albanerpeton. Canadian Journal of Earth Sciences, 19, 118-128. [1982.fa]. https://doi.org/10.1139/e82-009

Frazão, A., Silva, H.R. da \& Moraes Russo, C.A. de (2015) The Gondwana breakup and the history of the Atlantic and Indian oceans unveils two new clades for early neobatrachian diversification. PloS One, 10 (11), [e0143926], 1-18 + online supporting information (7 documents). Online document not preregistered in Zoobank. [2015.fa].

https://doi.org/10.1371/journal.pone.0143926

Fregin, S., Haase, M., Olsson, U. \& Alström, P. (2012) New insights into family relationships within the avian superfamily Sylvioidea (Passeriformes) based on seven molecular markers. BMC evolutionary Biology, 12 (157), 1-12 + 1 additional online file. [Online document not preregistered in Zoobank]. https://doi.org/10.1186/1471-2148-12-157

Frétey, T. (2008) Revue des genres africains Arthroleptis Smith, 1849 et Phrynobatrachus Günther, 1862 (Amphibia, Anura). Alytes, 25 (3-4), 99-172.

Freytag, G.E. (1974) Exkursionsfauna für die Gebiete der DDR und der BRD. Band 3: Wirbeltiere. Sixth edition. Volk und Wissen, Berlin, 370 pp. $\{\mathrm{Q}\}$.

Fritz, S.A. \& Rahbek, C. (2012) Global patterns of amphibian phylogenetic diversity. Journal of Biogeography, 39, 1373 1382.

https://doi.org/10.1111/j.1365-2699.2012.02757.x

Frost, D.R. (1985) Amphibian species of the world, Allen Press \& Association of Systematics Collections, Lawrence, $[\mathrm{i}-\mathrm{iv}]+$ $\mathrm{i}-\mathrm{v}+732 \mathrm{pp} .\{\mathrm{Q}\}$.

Frost, D.R., Grant, T., Faivovich, J., Bain, R.H., Haas, A., Haddad, C.F.B., De Sa, R.O., Channing, A., Wilkinson, M., Donnellan, S.C., Raxworthy, C.J., Campbell, J.A., Blotto, B.L., Moler, P., Drewes, R.C., Nussbaum, R.A., Lynch, J.D., Green, D.M. \& Wheeler, W.C. (2006) The amphibian tree of life. Bulletin of the American Museum of natural History, 297, 1-370. \{Q\} [2006.fa].

https://doi.org/10.1206/0003-0090(2006)297[0001: TATOL]2.0.CO;2

Frost, D.R., McDiarmid, R.W. \& Mendelson, J.R., III (2009) Response to the Point of View of Gregory B. Pauly, David M. Hillis, and David C. Cannatella, by the anuran subcommittee of the SSAR/HL/ASIH scientific and standard English names list. Herpetologica, 65 (2), 136-153. https://doi.org/10.1655/09-009R1.1

Frost, D.R. \& Savage, J.M. (1987) Gender of Hemisus and correct formation of the family-group name. The Journal of the herpetological Association of Africa, 33, 24. [1987.fa]. https://doi.org/10.1080/04416651.1987.9650173

Frost, J.S. \& Bagnara, J.T. (1976) A new species of leopard frog (Rana pipiens complex) from northwestern Mexico. Copeia, 1976, 332-338. https://doi.org/10.2307/1443955

Fuhn, I.E. (1960) Amphibia. Fauna Republicii Populare Romîne, Academiei Republicii Populare Romîne Bucuresti 14, 1-288. $\{\mathrm{Q}\}$ [1960.fa].

Funk, W.C. \& Cannatella, D.C. (2009) A new, large species of Chiasmocleis Mehely 1904 (Anura: Microhylidae) from the Iquitos region, Amazonian Peru. Zootaxa, 2247 (1), 37-50. https://doi.org/10.11646/zootaxa.2247.1.2

Gadow, H. (1898) A classification of Vertebrata, recent and extinct. Adam \& Charles Black, London, i-xvii +82 pp. [1898.ga]. https://doi.org/10.5962/bhl.title.24205

Gadow, H. (1901) Amphibia and Reptiles. Macmillan \& Co., London, i-xiii + 668 pp., 1 pl. \{Q\} [1901.ga] https://doi.org/10.5962/bhl.title.160212

Gaffney, E.S. (1979) An introduction to the logic of phylogeny reconstruction. In: Cracraft, J. \& Eldredge, N. (Eds.), Phylogenetic analysis and paleontology. Columbia University Press, New York, pp. 79-111. https://doi.org/10.7312/crac92306-005

Gaitonde, N., Giri, V. \& Kunte, K. (2016) 'On the rocks': reproductive biology of the endemic toad Xanthophryne (Anura: Bufonidae) from the Western Ghats, India. Journal of natural History, 50, 2557-2572. https://doi.org/10.1080/00222933.2016.1200686

Gallardo, J.M. (1961) La ubicación sistemática y distribución geográfica de Brachycephalidae argentinos. I ${ }^{a}$ Reunión de Trabajos y Comunicaciones de Ciencias naturales y Geografia del Litoral argentino, Universidad nacional del Litoral, Santa Fe, Argentina, pp. 205-212. [1961.ga].

Gallardo, J.M. (1965) A propósito de los Leptodactylidae (Amphibia Anura). Papeis avulsos del Departamento de Zoologia de São Paulo, 17, 77-87. [1965.ga].

Gao, K.-Q. \& Shubin, N.H. (2001) Late Jurassic salamanders from northern China. Nature, 410, 574-577. https://doi.org/10.1038/35069051

Gao, K.-Q. \& Shubin, N.H. (2012) Late Jurassic salamandroid from western Liaoning, China. Proceedings of the national Academy of Sciences, 109, 5767-5772. https://doi.org/10.1073/pnas.1009828109 
García-París, M. \& Wake, D.B. (2000) Molecular phylogenetic analysis of relationships of the tropical salamander genera Oedipina and Nototriton, with descriptions of a new genus and three new species. Copeia, 2000, 42-70.

https://doi.org/10.1643/0045-8511(2000)2000[0042: MPAORO]2.0.CO;2

Gardiner, B.G. (1982) Tetrapod classification. Zoological Journal of the Linnean Society, 74, 207-232. [1982.ga]. https://doi.org/10.1111/j.1096-3642.1982.tb01148.x

Garg, S. \& Biju, S.D. (2019) New microhylid frog genus from Peninsular India with Southeast Asian affinity suggests multiple Cenozoic biotic exchanges between India and Eurasia. Scientific Reports, 9, 1906. https://doi.org/10.1038/s41598-018-38133-x

Garman, S. (1884) The North American Reptiles and Batrachians. A list of the species occurring north of the Isthmus of Tehuantepec, with references. Bulletin of the Essex Institute, 16, 1-46. [1884.ga].

Garsault, F.A.P. de (1764) Les Figures des plantes et animaux d'usage en médecine, décrits dans la Matière Médicale de Mr. Geoffroy Médecin. Mrs. Defehrt, Prevost, Duflos, Martinet \& Co., Paris, [i-v] + 20 pp., pls. 644-729. [1764.ga]. https://doi.org/10.5962/bhl.title.49481

Gaudant, J. (1997) Cinq nouveaux gisements de Pelobatidae (Amphibiens, Anoures) dans l'Oligocène d'Europe centrale. Neues Jahrbuch für Geologie und Paläontologie, Monatshefte, 1997 (7), 434-446. [1997.ga]. https://doi.org/10.1127/njgpm/1997/1997/434

Génermont, J. \& Lamotte, M. (1980) Le concept biologique de l'espèce dans la zoologie contemporaine. Mémoires de la Société zoologique de France, 40, 427-452.

Gervais, P. (1847) Phanéroglosses. Phaneroglossa. In: d'Orbigny, C., Dictionnaire universel d'histoire naturelle. Tome 9. Renard, Martinet \& Co., Paris, pp. 721. [1847.ga].

Gervais, P. (1848) Reptiles. Reptilia. In: d'Orbigny, C. (Ed.), Dictionnaire universel d'histoire naturelle. Tome 11. Renard, Martinet \& Co., Paris, pp. 1-65. [1848.ga]

Giaretta, A.A., De Andrade, F.S., Haga, I.A. \& Bernardes, C.D.S (2013) On the advertisement call of Dermatonotus muelleri (Boettger, 1885) (Anura, Microhylidae). Zootaxa, 3700 (4), 593-596. https://doi.org/10.11646/zootaxa.3700.4.8

Giebel, C.G. (1846) Paläozoologie. Entwurf einer systematischen Darstellung der Fauna der Vorwelt. Nulandt, Merseburg, i-viii + 360 pp. [1846.ga]. https://doi.org/10.5962/bhl.title.39515

Giebel, C.G. (1852) Allgemeine Palaeontologie. Entwurf einer systematischen Darstellung der Fauna und Flora der Vorwelt. Ambrosius Abel, Leipzig, i-viii + 414 pp. [1852.ga].

Gill, T. (1884) Zoology. In: Annual report of the board of regents of the Smithsonian Institution, showing the operations, expenditures, and condition of the institution for the year 1882, Government Printing Office, Washington, 565-632. [1884.gb].

Gill, T. (1896) Some questions of nomenclature. Science, New Series, 4, 581-601. https://doi.org/10.1126/science.4.95.581

Gill, T. (1903) Biographical memoir of John Edwards Holbrook 1794-1871. Biographical Memoirs, National Academy of Sciences, 1, 47-77 [1903.ga].

Girard, C. (1858) Herpetology. In: United States exploring expedition during the years 1838, 1839, 1840, 1841, 1842, under the command of Charles Wilkes, U.S.N. Vol. 20. Sherman \& Son, Philadelphia, i-xv +492 pp. [1858.ga].

Giribet, G., Hormiga, G. \& Edgecombe, G.D. (2016) The meaning of categorical ranks in evolutionary biology. Organisms, Diversity \& Evolution, 16, 427-430. https://doi.org/10.1007/s13127-016-0263-9

Gistel, J. (1848) Naturgeschichte des Thierreichs für höhere Schulen. Hoffmann, Stuttgart, i-xi +216 pp $+\mathrm{i}-\mathrm{iv}, \mathrm{pl}$. 1-32. [1848.gb].

Gistel, J. (1850) Handbuch der Naturgeschichte aller drei Reiche, für Lehrer and Lernende, für Schule und Haus. Hoffmann'sche Verlangs-Buchhandlung, Stuttgart, 1037 pp., pls. 1-48. \{Q\}. https://doi.org/10.5962/bhl.title.37040

Glaw, F. \& Vences, M. (1994) A fieldguide to the amphibians and reptiles of Madagascar. Second edition including mammals and freshwater fish. Vences \& Glaw, Köln, 480 pp.

Glaw, F. \& Vences, M. (2006) Phylogeny and genus-level classification of mantellid frogs (Amphibia, Anura). Organisms, Diversity and Evolution, 6, 236-253. [2006.ga]. https://doi.org/10.1016/j.ode.2005.12.001

Glaw, F. \& Vences, M. (2007) A field guide to the amphibians and reptiles of Madagascar. Third Edition. Vences \& Glaw Verlag, Cologne, 495 pp.

Glaw, F., Hoegg, S. \& Vences, M. (2006) Discovery of a new basal relict lineage of Madagascan frogs and its implications for mantellid evolution. Zootaxa, 1334 (1), 27-43. https://doi.org/10.11646/zootaxa.1334.1.2

Glaw, F., Köhler, J., Lötters, S. \& Vences, M. (1998) Vorläufige Liste und Bibliographie neubeschriebener Amphibienarten und -unterarten von 1993 bis 1997. Elaphe, 6, i-xxiv. \{Q\}.

Glaw, F., Vences, M. \& Gossmann, V. (2000) A new species of Mantidactylus (subgenus Guibemantis) from Madagascar, with a comparative survey of internal femoral gland structure in the genus (Amphibia: Ranidae: Mantellinae). Journal of natural History, 34, 1135-1154. https://doi.org/10.1080/00222930050020140

Goin, C.J. \& Goin, O.B. (1962) Introduction to herpetology. Freeman \& Co., San Francisco \& London, i-ix + 341 pp. $\{\mathrm{Q}\}$.

Goin, C.J., Goin, O.B. \& Zug, G.R. (1978) Introduction to herpetology. Third edition. Freeman \& Co., San Francisco, i-xiii + 378 pp. \{Q\} [1978.ga].

Goin, C.J. \& Netting, M.G. (1940) A new gopher frog from the Gulf Coast, with comments upon the Rana areolata group. Annals of the Carnegie Museum, 28, 137-168.

Goldfuss, A. (1832) Ausführliche Erläuterung des naturhistorischen Atlasses. Theil 4. Arnz \& Comp., Düsseldorf, i-xii + 560 pp. [1832.ga].

Goldfuss, A. (1834) Grundriss der Zoologie. Zweite vermehrte und verbesserte Auflage. Leonhard Schrag, Nürnberg, i-x + 673 
pp. [1834.ga].

Goldfuss, G.A. (1820) Handbuch der Zoologie. Dritter Theil, zweite Abtheilung. Johann Leonhard Schrag, Nürnberg, i-xxiv + 512 pp., pl. 3-4. \{Q\} [1820.ga].

Good, D.A. \& Wake, D.B. (1992) Geographic variation and speciation in the torrent salamanders of the genus Rhyacotriton (Caudata: Rhyacotritonidae). University of California Publications in Zoology, 126, i-xii + 1-91. [1992.ga].

Goodrich, E.S. (1930) Studies on the structure and development of vertebrates. Macmillan \& Co., London, $\mathrm{i}-\mathrm{xxx}+837 \mathrm{pp}$. [1930.ga]. https://doi.org/10.5962/bhl.title.82144

Gorham, S.W. (1966) Liste der rezenten Amphibien und Reptilien. Ascaphidae, Leiopelmatidae, Pipidae, Discoglossidae, Pelobatidae, Leptodactylidae, Rhinophrynidae. Das Tierreich, 85, 1-222. $\{\mathrm{Q}\}$.

Gorham, S.W. (1974) Checklist of world amphibians up to January 1, 1970. The New Brunswick Museum, Saint-John, 173 pp. $\{\mathrm{Q}\}$.

Gouriet, E. (1868) Essai sur la classification parallélique des Batraciens ou Amphibiens. Revue de Zoologie pure et appliquée, (2) 20, 199-210. \{Q\} [1868.ga].

Gower, D.J., Kupfer, A., Oommen, O.V., Himstedt, W., Nussbaum, R.A., Loader, S.P., Presswell, B., M, H., Krishna, S.B., Boistel, R. \& Wilkinson, M. (2002) A molecular phylogeny of ichthyophiid caecilians (Amphibia: Gymnophiona: Ichthyophiidae): out of India or out of South East Asia? Proceedings of the royal Society of London, (B) 269, 15631569.

https://doi.org/10.1098/rspb.2002.2050

Grant, T., Frost, D.R., Caldwell, J.P., Gagliardo, R., Haddad, C.F.B., Kok, P.J.R., Means, D.B., Noonan, B.P., Schargel, W.E. \& Wheeler, W.C. (2006) Phylogenetic systematics of dartpoison frogs and their relatives (Amphibia: Athesphatanura: Dendrobatidae). Bulletin of the American Museum of natural History, 299, 1-262. [2006.gb].

https://doi.org/10.1206/0003-0090(2006)299[1: PSODFA]2.0.CO;2

Grant, T. \& Myers, C.W. (2013) Review of the frog genus Silverstoneia, with, descriptions of five new species from the Colom Choco (Dendrobatidae: Colostethinae). American Museum Novitates, 3784, 1-58. https://doi.org/10.1206/3784.2

Grant, T., Rada, M., Anganoy-Criollo, M.A., Batista, A., Dias, P.H. dos S., Jeckel, A.M., Machado, D.J. \& Rueda-Almonacid, J.V. (2017) Phylogenetic systematics of dart-poison frogs and their relatives revisited (Anura: Dendrobatoidea). South American Journal of Herpetology, 12 (Special Issue), 1-90. [2017.ga]. https://doi.org/10.2994/SAJH-D-17-00017.1

Gravenhorst, J.L.C. (1817) Grundzüge der Systematischen Naturgeschichte für seine Zuhöhrer. Holdufer, Breslau, 76 pp., 11 tabs. [1817.ga]. https://doi.org/10.5962/bhl.title.96886

Gravenhorst, J.L.C. (1843) Vergleichende Zoologie. Graß, Barth \& Co., Breslau, i-xx + 687 pp., pls. 1-12. [1843.ga]. https://doi.org/10.5962/bhl.title.58729

Gravenhorst, J.L.C.(1845)Das Thierreichnach den Verwandtshaften und Uebergängen in den Klassen und Ordnungen desselben. Graß, Barth \& Co., Breslau, i-x + 1-254, 12 pls. [1845.ga]. https://doi.org/10.5962/bhl.title.115396

Gray, A. (2018) Review of the genus Cruziohyla (Anura: Phyllomedusidae), with description of a new species. Zootaxa, 4450 (4), 401-426. https://doi.org/10.11646/zootaxa.4450.4.1

Gray, J.E. (1825) A synopsis of the genera of Reptiles and Amphibia, with a description of some new species. Annals of Philosophy, (2), 10, 193-217. \{Q\} [1825.ga].

Gray, J.E. (1829) Synopsis generum reptilium et amphibiorum. Isis von Oken, 22 (2), 187-206. [1829.ga].

Gray, J.E. (1831a) A synopsis of the species of the class Reptilia. In: Griffith, E. \& Pidgeon, E. (Ed.), The class Reptilia arranged by the Baron Cuvier, with specific descriptions; in: Griffith, E. \& Pidgeon, E. (Eds.), The animal kingdom arranged in conformity with its organization, by the Baron Cuvier, member of the Institute of France, \&c. \&c. \&c., with additional descriptions of all the species hitherto named, and of many not before noticed, vol. 9, Whittaker, Treacher \& Co., London, pp. 1-110, pls. 1-55. [1831.ga].

Gray, J.E. (1831b) Description of two new genera of frogs discovered by John Reeves, Esq. in China. In: Gray, J.E. (Ed.), The zoological Miscellany. Part 1. Wurtz \& Co., Treuttel, pp. 38. [1831.gb].

Gray, J.E. (1842) The northern zoological gallery. In: Synopsis of the contents of the British Museum. $44^{\text {th }}$ Edition. British Museum, London, pp. 97-157. [1842.ga].

Gray, J.E. (1850) Catalogue of the specimens of Amphibia in the collection of the British Museum. Part II. Batrachia Gradientia, etc. Spottiswoodes \& Shaw, London, 72 pp., pls. 3-4. \{P07\}\{Q\} [1850.ga].

Gray, J.E. (1858) Proposal to separate the family of Salamandridae Gray, into two families, according to the form of the skull. Proceedings of the zoological Society of London, 1858, 136144. [1858.gb]. https://doi.org/10.1111/j.1469-7998.1858.tb06357.x

Graybeal, A. (1997) Phylogenetic relationships of bufonid frogs and tests of alternate macroevolutionary hypotheses characterizing their radiation. Zoological Journal of the Linnean Society, 119, 297-338.

https://doi.org/10.1111/j.1096-3642.1997.tb00139.x

Graybeal, A. \& Cannatella, D.C. (1995) A new taxon of Bufonidae from Peru, with descriptions of two new species and a review of the phylogenetic status of supraspecific bufonid taxa. Herpetologica, 51, 105-131.

Greenbaum, E., Smith, E.N. \& Sá, R.O. de (2011) Molecular systematics of the Middle American genus Hypopachus (Anura: Microhylidae). Molecular Phylogenetics and Evolution, 61, 265-277.

https://doi.org/10.1016/j.ympev.2011.07.002

Gregory, S.M.S. (2010) The two 'editions' of Duméril's Zoologie analytique, and the potential confusion caused by Froriep's translation Analytische Zoologie. Zoological Bibliography, 1 
(1), 6-8. [2010].

Greuter, W., Garrity, G., Hawksworth, D.L., Jahn, R., Kirk, P.M., Knapp, S., McNeill, S., Michel, E., Patterson, D.J., Pyle, R. \& Tindall, B.J. (2011) Draft BioCode (2011). Principles and Rules regarding the naming of organisms. New draft, revised in November 2010. Bionomina, 3, 26-44.

https://doi.org/10.11646/bionomina.3.1.3

Griffiths, I. (1959) The phylogeny of Sminthillus limbatus and the status of the Brachycephalidae. Proceedings of the zoological Society of London, 132, 457-487.

https://doi.org/10.1111/j.1469-7998.1959.tb05531.x

Griffiths, I. (1963) The phylogeny of the Salientia. Biological Reviews, 38, 241-292, pl. 1. \{Q\} [1963.ga]. https://doi.org/10.1111/j.1469-185X.1963.tb00784.x

Grismer, L.L. (2006) A new species of Ansonia Stoliczka, 1870 (Anura: Bufonidae) from a lowland rainforest in southern peninsular Malaysia. Herpetologica, 62, 466-475.

https://doi.org/10.1655/0018-0831(2006)62[466: ANSOAS]2.0.CO;2

Gronovius, L.T. (1763) Zoophylacii Gronoviani. Fasciculus primus. Lugduni Batavorum, Sumptibus Auctoris, [i-iii] + i-iv + 236 pp. $+[\mathrm{i}]$, pls. $1-17$

Grosjean, S., Delorme, M., Dubois, A. \& Ohler, A. (2008) Evolution of reproduction in the Rhacophoridae (Amphibia, Anura). Journal of zoological Systematics and evolutionary Research, 46, 169-176. [2008.ga].

https://doi.org/10.1111/j.1439-0469.2007.00451.x

Guayasamin, J.M., Castroviejo-Fisher, S., Ayarzagüena, J., Trueb, L. \& Vilà, C. (2008) Phylogenetic relationships of glassfrogs (Centrolenidae) based on mitochondrial and nuclear genes. Molecular Phylogenetics and Evolution, 48, 574-595. https://doi.org/10.1016/j.ympev.2008.04.012

Guayasamin, J. M., Castroviejo-Fisher, S., Trueb, L., Ayarzagüena, J., Rada, M. \& Vilà, C. (2009) Phylogenetic systematics of glassfrogs (Amphibia: Centrolenidae) and their sister taxon Allophryne ruthveni. Zootaxa, 2100, 1-97. [2009.ga]. https://doi.org/10.11646/zootaxa.2100.1.1

Guayasamin, J.M. \& Trueb, L. (2007) A new species of glassfrog (Anura: Centrolenidae) from the lowlands of northwestern Ecuador, with comments on centrolenid osteology. Zootaxa, 1447 (1), 27-45. https://doi.org/10.11646/zootaxa.1447.1.2

Gubin, Y.M. (1991) Палеоденовые саламандры юга Мюнголии. [Paleocene salamanders from southern Mongolia]. Paleontologischeskii Zhurnal, 1991 (1), 96-106, pls. 8-9. [In Russian]. [1991.ga].

Guibé, J. (1978) Les Batraciens de Madagascar. Bonner zoologische Monographien, 11, 1-144, pls. 1-82. [1978.gb].

Gül, S., Özdemir, N., Üzüm, N., Olgun, K. \& Kutrup, B. (2011) Body size and age structure of Pelophylax ridibundus populations from two different altitudes in Turkey. AmphibiaReptilia, 32, 287-292.

https://doi.org/10.1163/017353711X559094

Günther (1870) Reptilia. [1869]. Zoological Record, 6, 105-122. [1870.ga].

Günther, A. (1858) On the systematic arrangement of the Tailless
Batrachians and the structure of Rhinophrynus dorsalis. Proceedings of the zoological Society of London, 1858, 339352. [1858.gc]. https://doi.org/10.1111/j.1469-7998.1858.tb06387.x

Günther, A. (1859) Catalogue of the Batrachia Salientia in the collection of the British Museum. Taylor \& Francis, London '1858', i-xvi + 160 pp., pls. 1-12. [P08 \{ \{Q\} [1859.ga].

Günther, A. (1868) Reptilia. [1867]. Zoological Record, 4, 126149. [1868.gb].

Günther, A.C.L.G. (1900) Reptilia and Batrachia. In: Godman, F.D. \& Salvin, O. (Eds.), Biologia Centrali-Americana. R.H. Porter, London, pp. i-xx + 1-326, 76 pls.

Gururaja, K.V., Dinesh, K.P., Palot, M.J., Radhakrishnan, C. \& Ramachandra, T.V (2007) A new species of Philautus Gistel (Amphibia: Anura: Rhacophoridae) from southern Western Ghats, India. Zootaxa, 1621, 1-16.

https://doi.org/10.11646/zootaxa.1621.1.1

Haas, A. (2003) Phylogeny of frogs as inferred from primarily larval characters (Amphibia: Anura). Cladistics, 19, 23-89. [2003.ha]. https://doi.org/10.1016/S0748-3007(03)00006-9

Haeckel, E. (1866a) Generelle Morphologie der Organismen. Erster Band. Allgemeine Anatomie der Organismen. Georg Kramer, Berlin, i-xxxii + 574 pp., pl. 1.

Haeckel, E. (1866b) Generelle Morphologie der Organismen. Zweiter Band. Allgemeine Entwicklungsgeschichte der Organismen. Georg Kramer, Berlin, i-clx + 462 pp., pls. 1-8. [1866.ha].

Haeckel, E. (1874) Histoire de la création des êtres organisés d'après les lois naturelles. Reinfald, Paris, i-xxxi + 680 pp., $15 \mathrm{pls}$. https://doi.org/10.5962/bhl.title.159276

Haeckel, E. (1879) Natürliche Schöpfungs-Geschichte. Siebente umgearbeitete und vermehrte Auflage. Reimer, Berlin, i-xxx + 718 pp., pls. 1-15. [1879.ha].

Haeckel, E. (1889) Natürliche Schöpfungs-Geschichte. Achte umgearbeitete und vermehrte Auflage. Reimer, Berlin, i-xxxi + 832 pp., 1 pl. [1889.ha].

Haeckel, E. (1902) Natürliche Schöpfungs-Geschichte. Zehnte verbesserte Auflage. Zweiter Theil. Allgemeine StammesGeschichte (Phylogenie und Anthropogenie). Reimer, Berlin, [i-v] + 371-832 pp., pls. 4-30. [1902.ha]. https://doi.org/10.1515/9783111440392

Hallowell, E. (1856) Description of several species of Urodela, with remarks on the geographical distribution of the Caducibranchiate division of these animals and their classification. Proceedings of the Academy of natural Sciences of Philadelphia, 8, 6-11. [1856.ha].

Hallowell, E. (1858) Notice of a collection of Reptiles from the Gaboon country, West Africa, recently presented to the Academy of Natural Sciences of Philadelphia, by Dr. Henry A. Ford. Proceedings of the Academy of natural Sciences of Philadelphia, '1857', 9, 48-72. \{P00] [1858.ha].

Haug, J.T., Haug, C., Maas, A., Kutschera, V. \& Waloszek, D. (2010) Evolution of mantis shrimps (Stomatopoda, Malacostraca) in the light of new Mesozoic fossils. BMC evolutionary Biology, 
10 (290), $1-17+3$ additional online files.

https://doi.org/10.1186/1471-2148-10-290

Haworth, A.H. (1825) A binary arrangement of the class Amphibia. Philosophical Magazine and Journal, 65, 372-373. [1825. ha].

https://doi.org/10.1080/14786442508652879

Hay, O.P. (1892) The Batrachians and Reptiles. State of Indiana. In: Gorby, S.S. (Ed.), Indiana Department of Geology \& natural Resources, Seventeeth Annual Report, 1891. Burford, Indianapolis, pp. 409-611. [1892.ha]. https://doi.org/10.5962/bhl.title.9103

Hay, O.P. (1929) Second bibliography and catalogue of the fossil Vertebrata of North America. Vol. 1. Carnegie Institution of Washington, Washington, i-viii + 916 pp. [1929.ha].

Heatwole, H. \& Carroll, R.L. (2000) Appendix 2. Classification of the amphibian genera cited in this volume. In: Heatwole, H. \& Carroll, R.L. (Ed.), Amphibian biology. Vol. 4. Palaeontology, The evolutionary history of amphibians. Surrey Beatty, Chipping Norton, pp. 1462-1470. [2000.ha]

Hedges, S.B., Duellman, W.E., Heinicke, M.P. (2008) New World direct-developing frogs (Anura: Terrarana): molecular phylogeny, classification, biogeography, and conservation. Zootaxa, 1737 (1), 1-182. [2008.ha]. https://doi.org/10.11646/zootaxa.1737.1.1

Hedges, S.B., Nussbaum, R.A. \& Maxson, L.R. (1993) Caecilian phylogeny and biogeography inferred from mitochondrial DNA sequences of the 12s rRNA and 16S rRNA genes (Amphibia: Gymnophiona). Herpetological Monographs, 7, 64-76. [1993.ha]. https://doi.org/10.2307/1466952

Heinicke, M.P., Duellman, W.E. \& Hedges, S.B. (2007) Major Caribbean and Central American frog faunas originated by ancient oceanic dispersal. Proceedings of the national Academy of Sciences of the United States of America, 104, 10092-10097.

https://doi.org/10.1073/pnas.0611051104

Heinicke, M.P., Duellman, W.E., Trueb, L., Means, D.B., MacCulloch, R.D. \& Hedges, S.B. (2009) A new frog family (Anura: Terrarana) from South America and an expanded direct-developing clade revealed by molecular phylogeny. Zootaxa, 2211 (1), 1-35. [2009.ha]. https://doi.org/10.11646/zootaxa.2211.1.1

Heinicke, M.P., Lemmon, A.R., Lemmon, E.M., McGrath, K. \& Hedges, S.B. (2018) Phylogenomic support for evolutionary relationships of New World direct-developing frogs (Anura: Terraranae). Molecular Phylogenetics and Evolution, 118, 145-155. [2017.ha].

https://doi.org/10.1016/j.ympev.2017.09.021

Hellmich, W. (1957) Herpetologische Ergebnisse einer Forschungsreise in Angola. Veröffentlichungen der zoologischen Staatssammlung München, 5, 1-92, pls. 1-8. [1957.ha].

Hellmich, W. (1962) Reptiles and Amphibians of Europe. English edition by A. Leutscher. Blanford Press, London, i-viii + $1-160 .\{Q\}$.

Hellmich, W. (1963) Klasse Amphibia, Lurche. In: Bertalanffy, L. von \& Gessner, F. (Eds.), Handbuch der Biologie, 6 (24-25), pp. 629-670. [1963.sa].

Hemming, F. (Ed.) (1953) Copenhagen decisions on zoological nomenclature. Additions to, and modifications of, the Règles internationales de la nomenclature zoologique; approved and adopted by the Fourteenth International Congress of Zoology, Copenhagen, August, 1953. International Trust for Zoological Nomenclature, London, i-xxxi + 135 pp.

Hemprich, W. (1829) Grundriß der Naturgeschichte für höhere Lehranstalten. Zweite Auflage. August Rücker, Berlin, i-xxii, 535 pp. [1829.ha].

Hennig, W. (1950) Grundzüge einer Theorie der phylogenetischen Systematik. Deutscher Zentralverlag, Berlin, i-vii +370 pp.

Hennig, W. (1966) Phylogenetic systematics. University of Illinois Press, Urbana, Chicago \& London, i-vii +263 pp.

Hennig, W. (1974) Kritische Bemerkungen zur Frage "Cladistic analysis or cladistic classification?". Zeitschrift für zoologische Systematik und Evolutionsforschung, 12, 279-294. https://doi.org/10.1111/j.1439-0469.1974.tb00170.x

Herre, W. (1950) Schwanzlurche aus dem Paleocän von Walbeck. Zoologischer Anzeiger, Supplement 145, 286-301. [1950. ha].

Hertwig, S.T., Schweizer, M., Das, I. \& Haas, A. (2013) Diversification in a biodiversity hotspot-The evolution of Southeast Asian rhacophorid tree frogs on Borneo (Amphibia: Anura: Rhacophoridae). Molecular Phylogenetics and Evolution, 68, 567-581. [2013.ha]. https://doi.org/10.1016/j.ympev.2013.04.001

Hewitt, J. (1926) Some new or little-known Reptiles and Batrachians from South Africa. Annals of the South African Museum, 20, 473-490.

Hewitt, J. (1927) Further descriptions of reptiles and batrachians from South Africa. Record of the Albany Museum, Grahamstown, 3, 371-415.

Hewitt, J. \& Methuen, P.A. (1912) Descriptions of some new Batrachia and Lacertilia from South Africa. Transactions of the royal Society of South Africa, 3, 107-111. https://doi.org/10.1080/00359191309519682

Heyer, W.R. (1975) A preliminary analysis of the intergeneric relationships of the frog family Leptodactylidae. Smithsonian Contributions to Zoology, 199, i-iii + 1-55. [1975.ha]. https://doi.org/10.5479/si.00810282.199

Heyer, W.R. \& Liem, D.S. (1976) Analysis of the intergeneric relationships of the Australian frog family Myobatrachidae. Smithsonian Contributions to Zoology, 233, i-iii + 1-29. [1976.ha]. https://doi.org/10.5479/si.00810282.233

Highton, W.A. (1940) Preliminary remarks on the skeletons of salamanders of the family Hynobidae. Journal of Entomology \& Zoology, 38 (3), 40-45. [1940.ha].

Hillis, D.M. \& Frost, J.S. (1985) Three new species of leopard frogs (Rana pipiens complex) from the Mexican Plateau. Occasional Papers of the Museum of natural History University of Kansas, 117, 1-14.

Hillis, D.M., Frost, J.S. \& Webb, R.G. (1984) A new species of frog of the Rana tarahumarae group from Southwestern Mexico. 
Copeia, 1984, 398-403.

https://doi.org/10.2307/1445197

Hillis, D.M. \& Sa, de R. (1988) Phylogeny and taxonomy of the Rana palmipes group (Salientia: Ranidae). Herpetological Monographs, 2, 1-26.

https://doi.org/10.2307/1467024

Hillis, D.M. \& Wilcox, T.P. (2005) Phylogeny of the New World true frogs (Rana). Molecular Phylogenetics and Evolution, 34, 299-314.

https://doi.org/10.1016/j.ympev.2004.10.007

Hime, P.M., Lemmon, A.R., Lemmon, E.C.M., Prendini, E., Brown, J.M., Thomson, R.C., Kratovil, J.D., Noonan, B.P., Pyron, R.A., Peloso, P.L. V, Kortyna, M.L., Keogh, J.S., Donnellan, S.C., Mueller, R.L., Raxworthy, C.J., Kunte, K., Ron, S.R., Das, S., Gaitonde, N., Green, D.M., Labisko, J., Che, J. \& Weisrock, D.W. (2021) Phylogenomics reveals ancient gene tree discordance in the Amphibian Tree of Life. Systematic Biology, 70, 49-66.

https://oi.org/10.1093/sysbio/syaa034

Hinchliff, C. \& Roalson, E. (2013) Using supermatrices for phylogenetic inquiry: an example using the sedges. Systematic Biology, 62 (2), 205-219.

https://doi.org/10.1093/sysbio/sys088

Hirschfeld, M., Barej, M.F., Loader, S.P. \& Rödel, M. (2012) Description of two Werneria tadpoles from Cameroon (Amphibia: Anura: Bufonidae). Zootaxa, 3172 (1), 65-68. https://doi.org/10.11646/zootaxa.3172.1.5

Hoff, M. \& Daszkiewicz, P. (2001) Index faunistique de Guyane française. 1. Les Vertébrés. Patrimoines naturels, Muséum National d'Histoire Naturelle, Paris, 35, 1-66. [2001.ha].

Hoffman, A.C. (1932) Researches relating to the validity of the South African Polypedatidae (Rhacophoridae) as an autonomous family of the Anura. South African Journal of Science, 29, 562-583. [1932.ha].

Hoffman, A.C. (1935) Die sistematiese posiesie van Heleophryne. Soölogiese Navorsing van die nasionale Museum Bloemfonstein, 1, 1-2. [1935.ha].

Hoffmann, C.K. (1878) Klassen und Ordnungen der Amphibien wissenschaftlich dargestelldt in Wort und Bild. In: Bronn, H.G. (Ed.), Die Klassen und Ordnungen des Thier-Reichs wissenschaftlich dargestelldt in Wort und Bild. 6 (2). Winter, Leipzig \& Heidelberg, pp. 1-726, pls. 1-52. \{Q\} [1878.ha].

Hoffmann, M., Hilton-Taylor, C., Angulo, A., Böhm, M., Brooks, T.M., Butchart, S.H.M., Carpenter, K.E., Chanson, J., Collen, B., Cox, N.A., Darwall, W.R.T., Dulvy, N.K., Harrison, L.R., Katariya, V., Pollock, C.M., Quader, S., Richman, N.I., Rodrigues, A.S.L., Tognelli, M.F., Vié, J.-C., Aguiar, J.M., Allen, D.J., Allen, G.R., Amori, G., Ananjeva, N.B., Andreone, F., Andrew, P., Ortiz, A.L.A., Baillie, J.E.M., Baldi, R., Bell, B.D., Biju, S.D., Bird, J.P., Black-Decima, P., Blanc, J.J., Bolaños, F., Bolivar-G., W., Burfield, I.J., Burton, J.A., Capper, D.R., Castro, F., Catullo, G., Cavanagh, R.D., Channing, A., Chao, N.L., Chenery, A.M., Chiozza, F., Clausnitzer, V., Collar, N.J., Collett, L.C., Collette, B.B., Fernandez, C.F.C., Craig, M.T., Crosby, M.J., Cumberlidge, N., Cuttelod, A., Derocher, A.E., Diesmos, A.C., Donaldson,
J.S., Duckworth, J.W., Dutson, G., Dutta, S.K., Emslie, R.H., Farjon, A., Fowler, S., Freyhof, J., Garshelis, D.L., Gerlach, J., Gower, D.J., Grant, T.D., Hammerson, G.A., Harris, R.B., Heaney, L.R., Hedges, S.B., Hero, J.-M., Hughes, B., Hussain, S.A., Icochea M., J., Inger, R.F., Ishii, N., Iskandar, D.T., Jenkins, R.K.B., Kaneko, Y., Kottelat, M., Kovacs, K.M., Kuzmin, S.L., La Marca, E., Lamoreux, J.F., Lau, M.W.N., Lavilla, E.O., Leus, K., Lewison, R.L., Lichtenstein, G., Livingstone, S.R., Lukoschek, V., Mallon, D.P., McGowan, P.J.K., McIvor, A., Moehlman, P.D., Molur, S., Alonso, A.M., Musick, J.A., Nowell, K., Nussbaum, R.A., Olech, W., Orlov, N.L., Papenfuss, T.J., Parra-Olea, G., Perrin, W.F., Polidoro, B.A., Pourkazemi, M., Racey, P.A., Ragle, J.S., Ram, M., Rathbun, G., Reynolds, R.P., Rhodin, A.G.J., Richards, S.J., Rodríguez, L.O., Ron, S.R., Rondinini, C., Rylands, A.B., Sadovy de Mitcheson, Y., Sanciangco, J.C., Sanders, K.L., Santos-Barrera, G., Schipper, J., Self-Sullivan, C., Shi, Y., Shoemaker, A., Short, F.T., Sillero-Zubiri, C., Silvano, D.L., Smith, K.G., Smith, A.T., Snoeks, J., Stattersfield, A.J., Symes, A.J., Taber, A.B., Talukdar, B.K., Temple, H.J., Timmins, R., Tobias, J.A., Tsytsulina, K., Tweddle, D., Ubeda, C., Valenti, S. V, Paul van Dijk, P., Veiga, L.M., Veloso, A., Wege, D.C., Wilkinson, M., Williamson, E.A., Xie, F., Young, B.E., Akçakaya, H.R., Bennun, L., Blackburn, T.M., Boitani, L., Dublin, H.T., da Fonseca, G.A.B., Gascon, C., Lacher, T.E., Mace, G.M., Mainka, S.A., McNeely, J.A., Mittermeier, R.A., Reid, G.M., Rodriguez, J.P., Rosenberg, A.A., Samways, M.J., Smart, J., Stein, B.A. \& Stuart, S.N. (2010) The impact of conservation on the status of the World's Vertebrates. Science, 330, 1503-1509.

https://doi.org/10.1126/science. 1194442

Hogg, J. (1838) [On the classifications of the Amphibia]. Annals of natural History (1) 1 (2), 152. \{Q\} [1838.ha]. https://doi.org/10.1080/00222933809512254

Hogg, J. (1839a) On the classifications of the Amphibia. The Magazine of natural History, New Series, 3, 265-274. \{Q\} [1839.ha].

Hogg, J. (1839b) On the classifications of the Amphibia. (Continued). The Magazine of natural History, New Series, 3, 367-378. \{Q\} [1839.hb].

Hogg, J. (1841) On the existence of branchiae in the young caeciliae; and on a modification and extension of the branchial classification of the Amphibia. Annals and Magazine of natural History, (1) 7, 353-363. \{Q\} [1841.ha]. https://doi.org/10.1080/03745484109442709

Holbrook, J.E. (1842) North American herpetology; or, A description of the Reptiles inhabiting the United States. Vol. 4. J. Dobson, Philadelphia, i-vi +7-139 pp., pls. 1-35. [1842.ha]. https://doi.org/10.5962/bhl.title.12492

Holman, J.A. (1975) Herpetofauna of the Wakeeney local fauna (Lower Pliocene: Clarendonian) of Trego County, Texas. In: Smith, G.R. \& Friedland, N.E. (Ed.), Studies on cenozoic paleontology ans stratigraphy in honor of Claude $W$. Hibbard, Claude W. Hibbard memorial volume 3, Papers on Paleontology. Vol. 12. University of Michigan, Ann Arbor, 
pp. 49-66. [1975.hb].

Hoogmoed, M. (2013) Rediscovery of the rare tree frog Hyla inframaculata Boulenger, 1882 (Anura: Hylidae), in Amazonian Brazil with notes on variation and distribution, and its generic allocation. Amphibia-Reptilia, 34, 421-432. https://doi.org/10.1163/15685381-00002907

Hooper, J.N. A., Van Soest, R.W.M. \& Pisera, A. (2011) Phylum Porifera Grant, 1826. In: Zhang, Z.-Q. (Ed.), Animal biodiversity: an outline of higher-level classification and survey of taxonomic richness. Zootaxa, 3148 (1), 13-18. https://doi.org/10.11646/zootaxa.3148.1.4

Hu, S.-C., Fei, L. \& Ye, C.-Y. (1978) Three new amphibian species in China. Materials for herpetological Research, 4, 20.

Huang, Z. \& Tu, F. (2016) Characterization and evolution of the mitochondrial DNA control region in Ranidae and their phylogenetic relationship. Genetics and molecular Research, 15, 1-9. https://doi.org/10.4238/gmr.15038491

Hübner, J. (1816) Verzeichniß bekannter Schmettlinge. Augsburg (bey dem Verfasserr zu finden), 431 pp. + 72 pp. [1816.ha]. https://doi.org/10.5962/bhl.title.48607

Huene, F. von (1920) Systematische und genetische Betrachtungen über die Stegocephalen. Zeitschrift für induktive Abstammungsund Vererbungslehre, 23, 209-212. [1920.ha]. https://doi.org/10.1007/BF02099348

Huene, F. von (1952) Die Saurierwelt und ihre geschichtlichen Zusammenhänge. Gustav Fischer, Jena, i-iv + 64 pp. [1952. ha].

Huene, F.R. von (1931) Amphibia. (Paläontologie). In: Dittler, R., Joos, G., Korschelt, E., Linck, G., Oltmanns, F. \& Schaum, K. (Eds.), Handwörterbuch der Naturwissenschaften, Zweite Auflage. Erster Band. Abbau-Blut. Gustav Fischer, Jena, pp. 299-312. [1931.ha].

Huene, F.R. von (1948) Short review of the lower tetrapods. In: Du Toit, A.L. (Ed.), Robert Broom Commemorative Volume. Special Publication of the Royal Society of South Africa. Royal Society of South Africa, Cape Town, pp. 65-186. [1948.ha].

Huene, F.R. von (1956) Paläontologie und Phylogenie der Niederen Tetrapoden. Gustav Fischer, Jena, i-xii + 716 pp. [1956.ha].

Hunter, J. (1834) Descriptive and illustrated catalogue of the physiological series of comparative anatomy contained in the Museum of the Royal College of Surgeons in London. Volume II, including the absorbent, circulating, respiratory, and urinary systems. Richard Taylor, London, i-xii + 164 pp., pls. 1-30. [1834.ha].

Hutchins, M., Duellman, W.E. \& Schlager, N. (2003) Amphibians species list. Grzimek's Animal Life Encyclopedia. Vol. 6. Amphibians. Michael Hutchins, Farmington Hills, pp. 456489. $\{\mathrm{Q}\}$.

Hutter, C., Lambert, S. \& Wiens, J. (2017) Rapid diversification and time explain amphibian richness at different scales in the tropical Andes, earth's most biodiverse hotspot. The American Naturalist, 190, 828-843.

https://doi.org/10.1086/694319

Huxley, J.H. (1957) The three types of evolutionary process. Nature, 180, 454-455. https://doi.org/10.1038/180454a0

Huxley, T.H. (1863) Description of Anthracosaurus russelli, a new labyrinthodont from the Lanarkshire coal-field. The quarterly Journal of the geological Society of London, 19 (1), 56-68. [1863.ha]. https://doi.org/10.1144/GSL.JGS.1863.019.01-02.11

Huxley, T.H. (1871) A manual of the anatomy of vertebrated animals. J. \& A. Churchill, London, i-vii + 510 pp. [1871. ha].

https://doi.org/10.5962/bhl.title.49320

Inger, R.F. (1947) Preliminary survey of the Amphibians of the Riukiu Islands. Fieldiana: Zoology, 82, 297-352.

https://doi.org/10.5962/bhl.title.2991

Inger, R.F. (1954) Philippine Zoological Expedition 1940-1947. Systematics and zoogeography of Philippine Amphibia. Fieldiana: Zoology, 33, 181-531. https://doi.org/10.5962/bhl.title.5571

Inger, R.F. (1958) Comments on the definition of genera. Evolution, 12, 370-384. https://doi.org/10.2307/2405859

Inger, R.F. (1966) The systematics and zoogeography of the Amphibia of Borneo. Fieldiana: Zoology, 52, 1-402. https://doi.org/10.5962/bhl.title.3147

Inger, R.F. (1967) The development of a phylogeny of frogs. Evolution 21, 369-384. https://doi.org/10.1111/j.1558-5646.1967.tb00165.x

Inger, R.F. (1972) Bufo in Eurasia. In: Blair, W.F (Ed.), Evolution of the genus Bufo. University of Texas Press, Austin, Texas, pp. 102-118.

Inger, R.F., Orlov, N.L. \& Darevsky, I.S. (1999) Frogs of Vietnam: a report on new collections. Fieldiana: Zoology, New Series, $92,1-46$.

Inthara, C., Chuaynkern, Y., Duengkae, P. \& Grosjean, S. (2009) The tadpole of Quasipaa fasciculispina (Inger, 1970) from southeastern Thailand, with the description of its buccal anatomy. Alytes, 26, 86-96.

Irisarri, I., San Mauro, D., Abascal, F., Ohler, A. \& Vences, M. (2012) The origin of modern frogs (Neobatrachia) was accompanied by acceleration in mitochondrial and nuclear substitution rates. BMC Genomics, 13 [626], 1-19.

https://doi.org/10.1186/1471-2164-13-626

Ivachnenko, M.F. (1978) Хвостатые Амфибии из триаса и юры средней Азии. [Urodele amphibians from the Triassic and Jurassic of Soviet Central Asia]. Paleontologicheskii Zhurnal, 12, 84-89, pls. 9-10. [In Russian]. [1978.ia].

Jan, G. (1857) Cenni sul Museo civico di Milano ed indice sistematico die Rettili ad Anfibi espositi nel medesimo. Luigi di Giacomo Pirola, Milano, 61 pp., 1 pls. [1857.ja].

Jánossy, D. (1979) A magyarországi pleisztocén tagolása gerinces faunák alapján. Akadémiai Kiadó, Budapest, 207 pp. [1979. ja].

Janssens, F. \& Christiansen, K.A. (2011) Class Collembola Lubbock, 1870. In: Zhang, Z.-Q. (Ed.), Animal biodiversity: an outline of higher-level classification and survey of taxonomic richness. Zootaxa, 3148 (1), 192-194. https://doi.org/10.11646/zootaxa.3148.1.34

Jarocki, F.P. (1822) Zoologiia czyli zwierzetopismo ogolne podlug 
naynowszego systematu. Vol. 3. Gady i plaz. Latkiewicz, Warsaw, [i-vi] + 184 pp + [10 pp], 3 pls. [1822.ja].

Jenkins, F.A. \& Walsh, D.M. (1993) An early Jurassic caecilian with limbs. Nature, 365, 246-250. [1993.ja].

https://doi.org/10.1038/365246a0

Jetz, W. \& Pyron, R.A. (2018) The interplay of past diversification and evolutionary isolation with present imperilment across the amphibian tree of life. Nature Ecology \& Evolution, 2, 850-858. https://doi.org/10.1038/s41559-018-0515-5

Jetz, W., Thomas, G.H., Joy, J.B., Hartmann, K. \& Mooers, A.O. (2012) The global diversity of birds in space and time. Nature, 491 (7424), 444-448.

https://doi.org/10.1038/nature11631

Jiang, J., Dubois, A., Ohler, A., Tillier, A., Chen, X., Xie, F. \& Stöck, M. (2005) Phylogenetic relationships of the tribe Paini (Amphibia, Anura, Ranidae) based on partial sequences of mitochondrial 12S and 16S rRNA genes. Zoological Science, 22, 353-362.

https://doi.org/10.2108/zsj.22.353

Jiang, K., Yan, F., Wang, K., Dahu, Z., Li, C. \& Che, J. (2016) A new genus and species of treefrog from Medog, southeastern Tibet, China (Anura, Rhacophoridae). Zoological Research, 37, 15-20.

Jiménez [X.] de la Espada, [M.] (1870) Faunae neotropicalis species quaedam nondum cognitae. Jornal de Sciencias mathematicas, physicas e naturaes, Academia real das Sciencias de Lisboa, 3 (9), 57-65.

Jiménez-Arcos, V.H., Calzada-Arciniega, R.A., Alfaro-Juantorena, L.A., Váquez-Reyes, L.D., Blair, C. \& Parra-Olea, G. (2019) A new species of Charadrahyla (Anura: Hylidae) from the cloud forest of western Oaxaca, Mexico. Zootaxa, 4554 (2), 371-385.

https://doi.org/10.11646/zootaxa.4554.2.3

Jockusch, E.I., Martínez-Solano, I., Hansen, R.W. \& Wake, D.B. (2012) Morphological and molecular diversification of slender salamanders (Caudata: Plethodontidae: Batrachoseps) in the southern Sierra Nevada of California with descriptions of two new species. Zootaxa, 3190 (1), 1-30. [2012.ja].

https://doi.org/10.11646/zootaxa.3190.1.1

Jones, K. \& Weisrock, D. (2018) Genomic data reject the hypothesis of sympatric ecological speciation in a clade of Desmognathus salamanders. Evolution, 72 (11), 2378-2393.

https://doi.org/10.1111/evo.13606

Jones, T.R.J. (1841) A general outline of the animal kingdom and manual of comparative anatomy. John vanVoorst, London, $\mathrm{i}-\mathrm{xv}+732$ pp. [1841.ja].

https://doi.org/10.5962/bhl.title.152781

Jourdan, A.-J.-L. (1834a) Dictionnaire raisonné, étymologique, synonymique et polyglotte, des termes usités dans les sciences naturelles... Tome premier. A-K. J.-B. Baillière, Paris, i-xi + 675 pp. [1834.ja].

https://doi.org/10.5962/bhl.title.95409

Jourdan, A.-J.-L. (1834b) Dictionnaire raisonné, étymologique, synonymique et polyglotte, des termes usités dans les sciences naturelles... Tome second. L-Z. J.-B. Baillière, Paris, [i-iii] +
628 pp. [1834.jb].

https://doi.org/10.5962/bhl.title.95409

Jowers, M., Downie, J.R. \& Cohen, B.L. (2008) The Golden Tree frog of Trinidad, Phyllodytes auratus (Anura: Hylidae): systematic and conservation status. Studies on neotropical Fauna and Environment, 43, 181-188. https://doi.org/10.1080/01650520801965490

Jungfer, K.-H. \& Hödl, W. (2002) A new species of Osteocephalus from Ecuador and a redescription of $O$. leprieurii (Duméril \& Bibron, 1841) (Anura: Hylidae). Amphibia-Reptilia, 23, 21-46.

https://doi.org/10.1163/156853802320877609

Jungfer, K.-H., Faivovich, J., Padial, J., Castroviejo-Fisher, S., Lyra, M., Von Muller Berneck, B., Iglesias, P., Kok, P., MacCulloch, R., Rodrigues, M., Verdade, V., Torres-Gastello, C., Chaparro, J.C., Valdujo, P., Reichle, S., Moravec, J., Gvoždík, V., Gagliardi-Urrutia, G., Ernst, R. \& Haddad, C. (2013) Systematics of spiny-backed treefrogs (Hylidae: Osteocephalus): an Amazonian puzzle. Zoologica scripta, 42, 351-380. https://doi.org/10.1111/zsc.12015

Kaiser, H., Crother, B.I., Kelly, C.M.R., Luiselli, L., O'Shea, M., Ota, H., Passos, P., Schleip, W.D. \& Wüster, W. (2013) Best practices: in the 21 st century, taxonomic decisions in herpetology are acceptable only when supported by a body of evidence and published via peer-review. Herpetological Review, 44 (1), 8-23.

Kamei, R.G., San Mauro, D., Gower, D.J., Van Bocxlaer, I., Sheratt, E., Thomas, A., Babu, S., Bossuyt, F., Wilkinson, M. \& Biju D.D. (2012) Discovery of a new family of amphibians from northeast India with ancient links to Africa. Proceedings of the royal Society, (B), 279, 2396-2401. [2012.ka].

https://doi.org/10.1098/rspb.2012.0150

Karsch, F. (1893) Die Insecten der Berglandschaft Adeli im Hinterlande von Togo (Westafrika) nach dem von den Herren Hauptmann Eugen Kling (1888 und 1889) und Dr. Richard Büttner (1890 und 1891) gesammelten Materiale. I. Abtheilung. Apterygota, Odonata, Orthoptera Saltatoria, Lepidoptera Rhopalocera. Königliches Museum für Naturkunde, Berlin, [i] + 266 pp., pls. 1-6, map. [1893.ka]. https://doi.org/10.5962/bhl.title.8524

Katoh, K. \& Standley, D.M. (2013) MAFFT multiple sequence alignment software version 7: improvements in performance and usability. Molecular Biology and Evolution, 30 (4), 772780.

https://doi.org/10.1093/molbev/mst010

Keferstein, W. (1867) Ueber einige neue oder seltene Batrachia aus Australien und dem tropischen Amerika. Nachrichten von der königlichen Gesellschaft der Wissenschaften und der Georg August Universität zu Göttingen, 18, 341-361. [1867.ka].

Keller, R.A., Boyd, R.N. \& Wheeler, Q.D. (2003) The illogical basis of phylogenetic nomenclature. The botanical Review, $69,93-110$.

https://doi.org/10.1663/0006-8101(2003)069[0093: TIBOPN]2.0.CO;2

Kellogg, R. (1932) Mexican tailless amphibians in the United States 
National Museum. Bulletin of the United States national Museum, 160, i-iv+ 224 pp., pl. 1.

https://doi.org/10.5479/si.03629236.160.i

Kenny, J.S. (1969) The Amphibia of Trinidad. Studies on the Fauna of Curaçao and other Caribbean Islands, 29, 1-78.

Kirby, W. (1835) On the power, wisdom and goodness of God as manifested in the creation of animals and in their history, habits and instincts. Vol. 2. William Pickering, London, i-viii + 542 pp., 20 pls. [1835.ka].

https://doi.org/10.1037/11915-000

Kiriakoff, S.G. (1953) The nomenclature of the specific complex. Bulletin of zoological Nomenclature, 10 (14), 450-451.

Kiriakoff, S.G. (1965) La vicariance géographique et la taxonomie. Comptes rendus de la Société de Biogéographie, 41, 103 115.

Kluge, A.G. (2005) Taxonomy in theory and practice, with arguments for a new phylogenetic system of taxonomy. In: Donnelly, M.H., Crother, B.I., Guyer, C., Wake, M.H. \& White, M.E. (Eds.), Ecology and evolution in the tropics: a herpetological perspective. University of Chicago Press, Chicago, Illinois, pp. 7-47.

Kluge, N.J. (2010) Circumscriptional names of higher taxa in Hexapoda. Bionomina, 1, 15-55. https://doi.org/10.11646/bionomina.1.1.3

Knauer, F.K. (1878) Naturgeschichte der Lurche. (Amphibiologie). Pichler's Witwe \& Sohn, Wien, i-xx + 340 pp., 5 pls. [1878. ka].

Knox, E., (1998) The use of hierarchies as organizational models in systematics. Biological Journal of the Linnean Society, 63, $1-49$.

https://doi.org/10.1111/j.1095-8312.1998.tb01637.x

Kolbe, H. (1880) Das Flügelgeäder der Psociden und seine systematische Bedeutung. Entomologische Zeitung, Stettin 41, 179-186, 1 pls. [1880.ka].

Kosuch, J., Vences, M., Dubois, A., Ohler, A. \& Böhme, W. (2001) Out of Asia: mitochondrial DNA evidence for an Oriental origin of tiger frogs, genus Hoplobatrachus. Molecular Phylogenetics and Evolution, 21, 398-407. https://doi.org/10.1006/mpev.2001.1034

Kozak, K.H., Mendyk, R.W. \& Wiens, J.J. (2009) Can parallel diversification occur in sympatry? Repeated patterns of body-size evolution in coexisting clades of North American salamanders. Evolution, 63, 1769-1784.

https://doi.org/10.1111/j.1558-5646.2009.00680.x

Kozlov, A.M., Aberer, A.J. \& Stamatakis, A. (2015) ExaML version 3: a tool for phylogenomic analyses on supercomputers. Bioinformatics, 31 (15), 2577-2579. https://doi.org/10.1093/bioinformatics/btv184

Krefft, G. (1865) The frogs of Australia. Monthly Notices of Papers and Proceedings of the royal Society of Tasmania, 1865, 1620. [1865.ka].

https://doi.org/10.5962/bhl.part.1567

Krumbiegel, G., Rüffle, L. \& Haubold, H. (1983) Das eozäne Geiseltal. Ein mitteleuropäisches Braunkohlenvorkommen und seine Pflanzen- und Tierwelt. Die Neue Brehm-Bücherei, Wittenberg Lutherstadt, 237 pp. [1983.ka].
Kuhn, O. (1939) Die fossilen Amphibien und die Abstammung ihrer lebenden Vertreter. Gebrüder Borntraeger, Berlin, i-iv +98 pp. [1939.ka].

Kuhn, O. (1941) Die eozänen Anura aus dem Geiseltale nebst einer Übersicht über die fossilen Gattungen. Nova Acta leopoldina, 10 (71), 345-376, pls. 1-8. [1941.ka].

Kuhn, O. (1960) Fossilium catalogus. Pars 97, Amphibia supplementum I ad partes 61 et 84 . W. Junk, 's Gravenhage [Den Haag], 164 pp. \{Q\}.

Kuhn, O. (1961) Die Familien der rezenten und fossilen Amphibien und Reptilien. Meisenbach \& Oeben, Bamberg, 79 pp. [1961. $\mathrm{ka}]$. https://doi.org/10.2307/1440026

Kuhn, O. (1962) Die vorzeitlichen Frösche und Salamander, ihre Gattungen und Familien. Jahreshefte des Vereins für vaterländische Naturkunde in Württemberg, 117, 327-372. [1962.ka].

Kuhn, O. (1965) Die Amphibien. Oeben, Krailling bei München, 102 pp. \{Q\} [1965.ka].

Kuhn, O. (1967a) Namensänderungen bei einigen höheren Taxa der Amphibia und Reptilia. Neues Jahrbuch für Geologie und Paläontologie, Monatshefte, 1967, 185-187. [1967.ka].

Kuhn, O. (1967b) Amphibien und Reptilien. Katalog der Subfamilien und höheren Taxa mit Nachweis des ersten Auftretens. Gustav Fischer, Stuttgart, i-vii + 124 pp. \{Q\} [1967.kb]. https://doi.org/10.2307/1442049

Kuntner, M. \&Agnarsson, I. (2006)Are the Linnean and phylogenetic nomenclatural systems combinable? Recommendations for biological nomenclature. Systematic Biology, 55, 774-784. https://doi.org/10.1080/10635150600981596

Kurabayashi, A., Matsui, M., Belabut, D.M., Yong, H.-S., Ahmad, N., Sudin, A., Kuramoto, M., Hamidy, A. \& Sumida, M. (2011) From Antarctica or Asia? New colonization scenario for Australian-New Guinean narrow mouth toads suggested from the findings on a mysterious genus Gastrophrynoides. BMC evolutionary Biology, 11 [175], 1-12.

https://doi.org/10.1186/1471-2148-11-175

Kurabayashi, A., Sumida, M., Yonekawa, H., Glaw, F., Vences, M. \& Hasegawa, M. (2008) Phylogeny, recombination, and mechanisms of stepwise mitochondrial genome reorganization in mantellid Frogs from Madagascar. Molecular Biology and Evolution, 25, 874-891.

https://doi.org/10.1093/molbev/msn031

Kuramoto, M. \& Joshy, S.H. (2003) Two new species of the genus Philautus (Anura: Rhacophoridae) from the Western Ghats, southwestern India. Current Herpetology, 22, 51-60. https://doi.org/10.5358/hsj.22.51

Kuramoto, M. \& Wang, C.-S. (1987) A new rhacophorid treefrog from Taiwan, with comparisons to Chirixalus eiffingeri (Anura, Rhacophoridae). Copeia, 1987, 931-942. https://doi.org/10.2307/1445556

La Cepède, [B. G. E. L.] de (1788) Histoire naturelle des quadrupèdes ovipares et des serpens. Tome Premier. Hôtel de Thou, Paris, $1-17+651$ pp., pls. $1-41,1$ tab. [In $8^{\circ}$ ]. https://doi.org/10.5962/bhl.title.5036

Lameere, A. (1942) Précis de Zoologie. Tome VII. Les Vertébrés 
tétrapodes. Amphibiens et Reptiles suivi d'un Abrégé de la classification des Oiseaux et des Mammifères. Editions Desoer, Liège, [i-ii] + 357 pp. $\{\mathrm{Q}\}$.

Lamsdell, J.C. \& Selden, P.A. (2013) Babes in the wood-a unique window into sea scorpion ontogeny. BMC evolutionary Biology 13 (98), 1-46 + 1 additional online file. [Online document not preregistered in Zoobank]. https://doi.org/10.1186/1471-2148-13-98

Lankester, E.R. (1877) Notes on the embryology and classification of the animal kingdom: comprising a revision of speculations relative to the origin and significance of the germ-layers. Quarterly Journal of microscopical Science, 68, 399-454.

Lankester, E.R. (1911) Zoological nomenclature. In: Chisholm, H. (Ed.), The encyclopedia britannica, A dictionary of arts, sciences, literature and general information. Vol. 28. Eleventh Edition. Vetch to Zymotic diseases. The Encyclopaedia Britannica Company, New York, pp. 1021-1039.

Largen, M.J. (1991) A new genus and species of petropedetine frog (Amphibia Anura Ranidae) from high altitude in the mountains of Ethiopia. Tropical Zoology, 4, 139-152. https://doi.org/10.1080/03946975.1991.10539483

Largen, M.J. \& Drewes, R.C. (1989) A new genus and species of brevicipitine frog (Amphibia Anura Microhylidae) from high altitude in the mountains of Ethiopia. Tropical Zoology, 2, 13-30. https://doi.org/10.1080/03946975.1989.10539423

Larson, A. (1991) A molecular perspective on the evolutionary relationships of the salamander families. Evolutionary Biology, 25, 211-277.

Larson, A. \& Dimmick, W.W. (1993) Phylogenetic relationships of the salamander families: an analysis of congruence among morphological and molecular characters. Herpetological Monographs, 7, 77-93. https://doi.org/10.2307/1466953

Larson, A., Weisrock, D.W. \& Kozak, K.H. (2003) Phylogenetic systematics of salamanders (Amphibia: Urodela), a review. In: Sever, D.M. (Ed.), Reproductive biology and phylogeny of Urodela. Science Publishers, Enfield \& Plymouth, pp. 31-108. $\{\mathrm{Q}\}$.

Larson, P., Sá, R. de \& Arrieta, D. (2003) Chondrocranial, hyobranchial and internal oral morphology in larvae of the basal bufonid genus Melanophryniscus (Amphibia: Anura). Acta zoologica, 84, 145-154.

https://doi.org/10.1046/j.1463-6395.2003.00140.x

Lataste, F. (1878a) Division en familles naturelles des Batraciens Anoures d'Europe. Revue internationale des Sciences, 2 (42), 488-499. [1878.la].

Lataste, F. (1878b) Les Batraciens et particulièrement ceux d'Europe et de France. Feuille des jeunes Naturalistes, 9 (97) 2-5. [1878.1b].

Lataste, F. (1879a) Sur un nouveau genre de Batracien anoure d'Europe. Comptes rendus hebdomadaires des Séances de l'Académie des Sciences, 88, 983-985. [1879.1a].

Lataste, F. (1879b) Etude sur le Discoglossus pictus Otth. Actes de la Société linnéenne de Bordeaux, (4) 3, 275-342, pls. 3-5. [1879.1b].
Lataste, F. (1888) Sur la classification des Batraciens Anoures, à propos du système de M. le Dr. R. Blanchard. Zoologischer Anzeiger, 11, 237-240. [1888.la].

Latreille, [P. A.] (1824) Esquisse d'une distribution générale du règne animal. Agasse, Paris, 22 pp. [1824.la].

Latreille, [P. A.] (1825) Familles naturelles du règne animal. Baillière, Paris, [i-v] +570 pp. [1825.la].

Latreille, P. A. (1800) Histoire naturelle des salamandres de France, précédée d'un tableau méthodique des autres reptiles. Villier, Paris, i-xlvii + 63 pp., pls. 1-6. [P01 J [1800.la]. https://doi.org/10.5962/bhl.title.5045

Latreille, P.A. (1804) Tableaux méthodiques d'histoire naturelle. In: Nouveau dictionnaire d'histoire naturelle. Tome 24. Deterville, Paris, [i] +238 pp.

Latreille, P.A. (1806) Genera crustaceorum et insectorum secundum ordinem naturalem in familias disposita, iconibus exemplisque plurimis explicata. Tomus primus. Amand Koenig, Parisiis \& Argentorati, [i-v] + i-xviii +303 pp., pls. 1-16. [1806.la]. https://doi.org/10.5962/bhl.title.5093

Laurent, R. (1942) Note sur les Procoellens [sic] Firmisternes (Batrachia Anura). Bulletin du Musée royal d'Histoire naturelle de Belgique, 18 (43), 1-20. [1942.1a].

Laurent, R. (1943a) Sur la position systématique et l'ostéologie du genre Mantidactylus Boulenger. Musée royal d'Histoire naturelle de Belgique, 19 (5), 1-8. [1943.la].

Laurent, R. (1943b) Contribution à l'ostéologie et à la systématique des Rhacophoridés non africains. Musée royal d'Histoire naturelle de Belgique, 19 (28), 1-16, pls. 1-4. [1943.lb].

Laurent, R. (1946) Mises au point dans la taxonomie des Ranides. Revue de Zoologie et de Botanique africaines, 39 (4), 336338. [1946.la].

Laurent, R. (1948a) Clef des familles d'Anoures. Laurent, 5 pp. [1948.la].

Laurent, R. (1948b) Clef des familles d'Urodèles. Laurent, 3 pp. [1948.lb].

Laurent, R. (1967) Taxonomia de los anuros. Acta zoologica lilloana, 22, 207-2010. \{Q\} [1967.1a].

Laurent, R. (1975) La distribution des Amphibiens et les translations continentales. Mémoires du Muséum national d'Histoire naturelle, A (Zoologie), 88, 176-191. [1975.la].

Laurent, R.F. (1941a) Contribution à l'ostéologie et à la systématique des Ranides africains. Première note. Revue de Zoologie et de Botanique africaines, '1940', 34 (1), 74-97, pls. 3-5. [1941. la].

Laurent, R.F. (1941b) Contribution à l'ostéologie et à la systématique des Ranides africains. Deuxième note. Revue de Zoologie et de Botanique africaines, 34 (2), 192-235, pls. 6-7. [1941. lb].

Laurent, R.F. (1951) Sur la nécessité de supprimer la famille des Rhacophoridae mais de créer celle des Hyperoliidae. Revue de Zoologie et de Botanique africaines, 45, 116-122. [1951. la].

Laurent, R.F. (1972) The morphology, systematics, and evolution of the Old World treefrogs (Rhacophoridae and Hyperoliidae) by Sioe Sing Liem. Copeia, 1972 (1), 198-201. [1972.la]. https://doi.org/10.2307/1442811 
Laurent, R.F. (1979) Herpetofaunal relationships between Africa and South America. In: Duellman, W.E. (Ed.), The South American herpetofauna: its origin, evolution, and dispersal. Monograph No. 7. University of Kansas, Lawrence, Kansas, pp. 55-71. $\{\mathrm{Q}\}$.

Laurent, R.F. (1980) Esquisse d'une phylogenèse des Anoures. Bulletin de la Société zoologique de France, '1979', 104 (4), 397-422. [P14 J [1980.1a].

Laurent, R. F. (1984) Heterogeneidad de la familia Caeciliidae (Amphibia Apoda). Acta zoologica lilloana, 37, 199-200. [1984.la].

Laurent, R.F.(1986) Sous-classe des Lissamphibiens (Lissamphibia). Systématique. In: Grassé, P.-P. \& Delsol, M. (Eds.), Traité de Zoologie. Vol. 14. Amphibiens. Fascicule I-B. Masson, Paris, pp. 594-796. \{Q\} [1986.1a].

Laurent, R.F. (1991) Classification and phylogeny of ranoid frogs. Circalytes, 5 (1), 1-7. [1991.la].

Laurent, R.F. \& Teran, E.M. (1981) Lista de los Anfíbios y Reptiles de la provincia de Tucumán. Miscelánea, Fundación e Instituto Miguel Lillo, 71, 1-15. [1981.1a].

Laurenti, J.N. (1768) Specimen medicum, exhibens synopsin Reptilium emendatam cum experimentis circa venena et antidota Reptilium austriacorum. Joan. Thom. Nob. de Trattnern, Viennae, i-ii + 215 pp., pls. 1-5. [1768.la]. https://doi.org/10.5962/bhl.title.5108

Laurin, M. (2010) The subjective nature of Linnaean categories and its impact in evolutionary biology and biodiversity studies. Contributions to Zoology, 79 (4), 131-146. https://doi.org/10.1163/18759866-07904001

Lavilla, E.O.(1988) Lower Telmatobiinae(Anura: Leptodactylidae): generic diagnoses based on larval characters. Occasional Papers of the Museum of natural History, University of Kansas, 124, 1-19.

Lecointre, G., Philippe, H., Lê, H. L. V. \& Le Guyader, H. (1993) Species sampling has a major impact on phylogenetic inference. Molecular Phylogenetics \& Evolution, 2, 205224

Lecompte, E., Aplin, K., Denys, C., Catzeflis, F., Chades, M. \& Chevret, P. (2008) Phylogeny and biogeography of African Murinae based on mitochondrial and nuclear gene sequences, with a new tribal classification of the subfamily. $B M C$ evolutionary Biology, 8 (199), 1-21 + 4 additional online files.

https://doi.org/10.1186/1471-2148-8-199

Lecomte, J. (Ed.) (1997) [Untitled volume]. Hydroécologie appliquée, 9, 1-262.

https://doi.org/10.1051/hydro:1997001e

Lehr, E. \& Catenazzi, A. (2009) Three new species of Bryophryne (Anura: Strabomantidae) from the region of Cusco, Peru. South American Journal of Herpetology, 4, 125-138. https://doi.org/10.2994/057.004.0204

Lehtinen, R.M., Glaw, F., Andreone, F., Pabijan, M. \& Vences, M. (2012) A new species of putatively pond breeding frog of the genus Guibemantis from Southeastern Madagascar. Copeia, 2012, 648-662.

https://doi.org/10.1643/CH-11-128
Lemmon, E.M., Lemmon, A.R. \& Cannatella, D.C. (2007a) Geological and climatic forces driving speciation in the continentally distributed trilling Chorus frogs (Pseudacris). Evolution, 61, 2086-2103.

https://doi.org/10.1111/j.1558-5646.2007.00181.x

Lemmon, E., Lemmon, A., Collins, J., Lee-Yaw, J. \& Cannatella, D. (2007b) Phylogeny-based delimitation of species boundaries and contact zones in the trilling chorus frogs (Pseudacris). Molecular Phylogenetics and Evolution, 44, 1068-1082. https://doi.org/10.1016/j.ympev.2007.04.010

Lescure, J. \& Renous, S. (1988) Biogéographie des Amphibiens Gymnophiones et histoire du Gondwana. Compte rendu des Séances de la Société de Biogéographie, 64 (1), 19-40. [1988.la].

Lescure, J., Renous, S. \& Gasc, J.-P. (1986) Proposition d'une nouvelle classification des Amphibiens Gymnophiones. Mémoires de la Société zoologique de France, 43, 145-177, 1 tab. [1986.lb]

Leuckart, F.S. (1840) Einige zoologische Bemerkungen. 2. Ueber das Gen. Cryptobranchus. Neue Notizen aus dem Gebiete der Natur- und Heilkunde, 13 (2), 19-20. [1840.1a].

Leuckart, F.S. (1841) Zoologische Bruchstücke. II. F. L. Rieder \& Comp., Stuttgart, i-viii + 1-132, pls. 1-6. [1841.la].

Leuckart, S. (1821) Einiges über die fischartigen Amphibien. Isis von Oken, Litterarischer Anzeiger, 1821, 257-265, pl. 5. [1821.1a].

Leunis, J. (1844) Synopsis der drei Naturreiche. Erster Theil. Zoologie. Hahn'sche Hofbuchhandlung, Hannover, i-xxxii + 477 pp. [1844.la].

Leunis, J. (1851) Schul-Naturgeschichte. Erster Theil. Zoologie. Hahn'sche Hofbuchhandlung, Hannover, $\mathrm{i}-\mathrm{x}+316$ pp. [1851. la].

Leunis, J. (1860) Synopsis der Naturgeschichte der Thierreichs. Erster Theil. Zoologie. Hahn'sche Hosbuchhandlung, Hannover, i-lxvi + 1014 pp. [1860.la].

Leunis, J. (1883) Synopsis der Thierkunde. Erster Band. In: Leunis, J., Synopsis der drei Naturreiche, Erster Theil, Zoologie, Hahn'sche Hofbuchhandlung, Hannover, i-xvi + 1083 pp. [1883.la].

Levine, N.D. (1958). Uniform endings for the names of higher taxa. Systematic Zoology, 7 (3), 134-135. https://doi.org/10.2307/2411979

Lherminier, P. \& Solignac, M. (2000) L'espèce: définitions d'auteurs. Comptes rendus de l'Académie des Sciences de Paris, Sciences de la Vie, 323, 153-165. https://doi.org/10.1016/S0764-4469(00)00119-0

Li, C., Guo, X. \& Wang, Y.-Z. (2011) Tadpole types of Chinese megophryid frogs (Anura: Megophryidae) and implications for larval evolution. Current Zoology, 57, 93-100. https://doi.org/10.1093/czoolo/57.1.93

Li, J., Che, J., Bain, R.H., Zhao, E. \& Zhang, Y. (2008) Molecular phylogeny of Rhacophoridae (Anura): a framework of taxonomic reassignment of species within the genera Aquixalus, Chiromantis, Rhacophorus, and Philautus. Molecular Phylogenetics and Evolution, 48, 302-312. https://doi.org/10.1016/j.ympev.2008.03.023 
Li, J., Che, J., Murphy, R.W., Zhao, H., Zhao, E., Rao, D. \& Zhang, Y. (2009) New insights to the molecular phylogenetics and generic assessment in the Rhacophoridae (Amphibia: Anura) based on five nuclear and three mitochondrial genes, with comments on the evolution of reproduction. Molecular Phylogenetics and Evolution, 53, 509-522.

https://doi.org/10.1016/j.ympev.2009.06.023

Lichtenstein, H., Weinland, D. \& Martens, E. (1856) Nomenclator Reptilium et Amphibiorum Musei Zoologici Berolinensis. Namenverzeichniss der in der zoologischen Sammlung der Königlichen Universität zu Berlin aufgestellten Arten von Reptilien und Amphibien nach ihren Ordnungen, Familien und Gattungen. Königliche Academie der Wissenschaften, Berlin, i-iv + 48 pp. \{Q\} [1856.la]. https://doi.org/10.5962/bhl.title.45245

Liedtke, H.C., Müller, H., Rödel, M.-O., Menegon, M., Gonwouo, L.N., Barej, M.F., Gvoždík, V., Schmitz, A., Channing, A., Nagel, P. \& Loader, S.P. (2016) No ecological opportunity signal on a continental scale? Diversification and life-history evolution of African true toads (Anura: Bufonidae). Evolution, 70, 1717-1733.

https://doi.org/10.1111/evo.12985

Liem, S.S. (1970) The morphology, systematics, and evolution of the Old World treefrogs (Rhacophoridae and Hyperoliidae). Fieldiana: Zoology, 57, i-vii + 1-145. https://doi.org/10.5962/bhl.title.2939

Lin, H.-C. \& Hastings, P.A. (2013) Phylogeny and biogeography of a shallow water fish clade (Teleostei Blenniiformes). BMC evolutionary Biology, 13 (210), 1-18 + 4 additional online files. [Online document not preregistered in Zoobank]. https://doi.org/10.1186/1471-2148-13-210

Linder, C.R., Moret, B.M.E., Nakhleh, L. \& Warnow, T. (2004) Network (reticulate) evolution: biology, models, and algorithms. Infoscience, EPFL scientific publications: [4041].

https://www.researchgate.net/publication/37441563_ Network_Reticulate_Evolution_Biology_Models_and_ Algorithms

Link, H.F. (1807) Beschreibung der Naturalien-Sammlung der Universität zu Rostock. Abth. 2. Erben, Rostock, pp. 51-100. [1807.la]. https://doi.org/10.5962/bhl.title.69815

Linnaeus, C. (1758a) Systema Naturae per regna tria naturae, secundum classes, ordines, genera, species, cum characteribus, differentiis, synonymis, locis. Editio decima, reformata. Tomus 1. Laurentii Salvii, Holmiae, [i-iv] +824 pp. [1758.1a].

https://doi.org/10.5962/bhl.title.542

Linnaeus, C. (1758b) Opera varia in quibus continentur Fundamenta Botanica, Sponsalia Plantarum, et Systema Naturae, in quo proponuntur naturae regna tria secundum classes, ordines, genera et species. Typographia Juntiniana, Lucae, [i-iv] + 376 pp., 1 pl.

https://doi.org/10.5962/bhl.title.154190

Linné [Linnaeus], C. (1767) s.n. In: Systema Naturae. Editio duodecima, reformata. Tomus 1. Pars 2. Laurentii Salvii,
Holmiae, pp. 533-1327 + [i-xxxiv]. [1767.la].

Litvinchuk, S.N. \& Borkin, L.J. (2009) Evolution, systematics and distribution of crested newts (Triturus cristatus complex) in Russia and adjacent countries. Evropeisky Dom Press, Saint Petersburg, 589 pp. [2009.1a].

Liu, C. (1950) Amphibians of Western China. Fieldiana: Zoology Memoirs, 2, 1-400, 10 pls. https://doi.org/10.5962/bhl.part.4737

Liu, C.-C. (1964) Taxonomic discussion on Chinese Megohrynae [sic]. (Abstract). Unpublished page, distributed at the 30th Annual Congress of the Chinese Society of Zoology, Beijing, 18 July 1964. [In Chinese].

Liu, C.-C. \& Hu, S.-C. (1961) The tailless amphibians of China. Science Press, Beijing, [i-ii] + i-xvi +364 pp., pls. 1-6+ $1-28$.

Liu, K., Warnow, T.J., Holder, M.T., Nelesen, S.M., Yu, J., Stamatakis, A.P. \& Linder, C.R. (2011) SATe-II: very fast and accurate simultaneous estimation of multiple sequence alignments and phylogenetic trees. Systematic Biology, 61 (1), 90-106.

https://doi.org/10.1093/sysbio/syr095

Loader, S.P., Gower, D.J., Ngalasan, W. \& Menegon, M. (2010) Three new species of Callulina (Amphibia: Anura: Brevicipitidae) highlight local endemism and conservation plight of Africa's Eastern Arc forests. Zoological Journal of the Linnean Society, 160, 496-514.

https://doi.org/10.1111/j.1096-3642.2010.00652.x

Löbl, I. (2015) Stability under the International Code of Zoological Nomenclature: a bag of problems affecting nomenclature and taxonomy. Bionomina, 9, 35-40.

https://doi.org/10.11646/bionomina.9.1.3

Lotsy, J. P. (1918) La quintessence de la théorie du croisement. Archives néerlandaises des Sciences exactes et naturelles, (3B) 3, 351-353.

Lötters, S., Böhme, W. \& Günther, R. (1998) Notes on the type material of the neotropical Harlequin frogs Atelopus varius (Lichtenstein \& Martens, 1856) and Atelopus cruciger (Lichtenstein \& Martens, 1856) deposited in the Museum für Naturkunde of Berlin (Anura, Bufonidae). Zoosystematics and Evolution, 74, 173-184.

https://doi.org/10.1002/mmnz.19980740203

Lötters, S., Van der Meijden, A., Coloma, L., Boistel, R., Cloetens, P., Ernst, R., Lehr, E. \& Veith, M. (2011) Assessing the molecular phylogeny of a near extinct group of Vertebrates: the Neotropical harlequin frogs (Bufonidae; Atelopus). Systematics and Biodiversity, 9, 45-57. https://doi.org/10.1080/14772000.2011.557403

Lourenço, A., Zina, J., Catroli, G., Kasahara, S., Faivovich, J. \& Haddad, C.F.B. (2016) A new species of the Scinax catharinae group (Anura: Hylidae) from southeastern Brazil. Zootaxa, 4154 (4), 415-435.

https://doi.org/10.11646/zootaxa.4154.4.3

Lourenço, L., Cardoso, A. \& Recco-Pimentel, S. (2000) Cytogenetics of Edalorhina perezi (Anura, Leptodactylidae). Cytolocia, 65, 359-363.

https://doi.org/10.1508/cytologia.65.359 
Lourenço, L.B., Targueta, C.P., Baldo, D., Nascimento, J., Garcia, P.C.A., Andrade, G. V, Haddad, C.F.B. \& Recco-Pimentel, S.M. (2015) Phylogeny of frogs from the genus Physalaemus (Anura, Leptodactylidae) inferred from mitochondrial and nuclear gene sequences. Molecular Phylogenetics and Evolution, 92, 204-216.

https://doi.org/10.1016/j.ympev.2015.06.011

Lowe, C.H. (1950) Speciation and ecology in salamanders of the genus Aneides. PhD Dissertation, UCLA, Los Angeles, California, [i-vii] +81 pp., 3 pls.

Lugli, L. \& Haddad, C.F.B. (2006) New species of Bokermannohyla (Anura, Hylidae) from Central Bahia, Brazil. Journal of Herpetology, 40, 7-15.

https://doi.org/10.1670/67-05A.1

Lutz, A. (1929) Taxonomia y biologia del genero Cyclorhamphus. Memórias do Instituto Oswaldo Cruz, 11, 5-25. [1929.1a]. https://doi.org/10.1590/S0074-02761929000100001

Lutz, A. (1930) Observações sobre Batrachios brasileiros. Taxonomia e biologia das Elsoiinas. Memórias do Instituto Oswaldo Cruz, 24, 195-249, pls. 64-67. [1930.1a]. https://doi.org/10.1590/S0074-02761930000900001

Lutz, B. (1954a) Anfíbios Anuros do Distrito Federal. Memórias do Instituto Oswaldo Cruz, 52 (1), 155-197, pls. 1-19. [1954. la]. https://doi.org/10.1590/S0074-02761954000100009

Lutz, B. (1954b) The frogs of the Federal District of Brazil. Memórias do Instituto Oswaldo Cruz, 52 (1), 219-238. [1954. $\mathrm{lb}]$.

https://doi.org/10.1590/S0074-02761954000100009

Lutz, B. (1968) Taxonomy of the neotropical Hylidae. The PearceSellards Series, 11, 1-25. [1968.1a].

Lutz, B. (1969) Adaptações, especializações e linhagens nos Anuros neotropicais. Acta zoologica lilloana, 24, 267-291. [1969.la].

Lydekker, R. (1889) Part III. Palaeozoology. Vertebrata. In: Nicholson H.A. \& Lydekker, R. (Eds.), A manual of palaeontology for the use of students, with a general introduction on the principles of palaeontology. Vol. 2. William Blackwood \& Sons, Edinburgh \& London, pp. i-xi + 887-1624. \{Q\} [1889.la].

Lydekker, R. ed. (1896) The royal natural history. Volume 5. Frederick Warne \& Co, London \& New York, i-xvi +584 pp. $\{\mathrm{Q}\}$.

Lydekker, R., Cunningham, J.T., Boulenger, G.A. \& Thomson, A.J. (1912) Reptiles, Amphibia, Fishes and lower Chordata. Mehuen \& Co. Ltd., London, i-xvii + 510 pp., pls. 1-36. $\{\mathrm{Q}\}$.

Lynch, J.D. (1969) Program for the final public examination for the degree of Doctor of Philosophy. University of Kansas, Lawrence, Kansas, 4 pp. [1969.lb].

Lynch, J.D. (1971) Evolutionary relationships, osteology, and zoogeography of leptodacyloid frogs. University of Kansas Museum of natural History, Miscellaneous Publication, 53, 1-238. [1971.la].

Lynch, J.D. (1973a) Books. Evolution in the genus Bufo, edited by W. Frank Blair (1972). Bioscience, 23, 497. [1973.la]. https://doi.org/10.2307/1296616

Lynch, J.D. (1973b) The transition from archaic to advanced frogs. In: Vial, J.L. (Ed.), Evolutionary biology of the anurans. University of Missouri Press, Columbia, pp. 133-182. [1973. lb].

Lynch, J.D. (1978) A reassessment of the telmatobiine leptodactylid frogs of Patagónia. Occasional Papers of the Museum of natural History, The University of Kansas, 72, 1-57. [1978. la].

Lynch, J.D. (1986a) The definition of the Middle American clade of Eleutherodactylus based on jaw musculature (Amphibia: Leptodactylidae). Herpetologica, 42 (2), 248-258.

Lynch, J.D. (1986b) New species of minute leptodactylid frogs from the Andes of Ecuador and Peru. Journal of Herpetology, 20, 423-431. https://doi.org/10.2307/1564505

Lynch, J.D. \& Duellman, W.E. (1973) A review of the centrolenid frogs of Ecuador, with descriptions of new species. Occasional Papers of the Museum of natural History, The University of Kansas, 16, 1-66. https://doi.org/10.5962/bhl.part.29037

Lyu, Z.-T., Zeng, Z., Wang, J., Lin, C.-Y., Liu, Z. \& Wang, Y.-Y. (2017) Resurrection of genus Nidirana (Anura: Ranidae) and synonymizing $N$. caldwelli with $N$. adenopleura, with description of a new species from China. Amphibia-Reptilia, $38,483-502$.

https://doi.org/10.1163/15685381-00003130

Macey, J.R. (2005) Plethodontid salamander mitochondrial genomics: a parsimony evaluation of character conflict and implications for historical biogeography. Cladistics, 21 (2), 194-202.

https://doi.org/10.1111/j.1096-0031.2005.00054.x

Macleay W.S. (1821) Horae entomologicae: or Essays on the annulose animals. Volume 1. Part 2. Bagster, London, [i] + 161-524 pp. [1821.ma].

Maddison, W., Bodner, M. \& Needham, K. (2008) Salticid spider phylogeny revisited, with the discovery of a large Australasian clade (Araneae: Salticidae). Zootaxa, 1893 (1), 49-64. https://doi.org/10.11646/zootaxa.1893.1.3

Maeda, N. \& Matsui, M. (1999) Frogs and toads of Japan. Revised. Bun-ichi Sogo Shuppan, Tokyo, 223 pp.

Mahony, S., Foley, N.M., Biju, S.D. \& Teeling, E.C. (2017) Evolutionary history of the Asian Horned Frogs (Megophryinae): integrative approaches to timetree dating in the absence of a fossil record. Molecular Biology and Evolution 34, 744-771. https://doi.org/10.1093/molbev/msw267

Malkmus, R., Manthey, U., Vogel, G., Hoffmann, P. \& Kosuch, J. (2002) Amphibians \& Reptiles of Mount Kinabalu (North Borneo). Koeltz Scientific Books, Königstein, 1-424.

Manamendra-Arachchi, K. \& Pethiyagoda, R. (1998) A synopsis of the Sri Lankan Bufonidae (Amphibia: Anura), with description of two new species. Journal of South Asian natural History, 3, 213-246.

Manthey, U. \& Grossmann, W. (1997) Die Amphibien und Reptilien Südostasiens. [The amphibians and reptiles of south east 
Asia.]. Natur und Tier-Verlag, Münster, 512 pp.

Manzano, A.S., Fabrezi, M. \& Vences, M. (2007) Intercalary elements, treefrogs, and the early differentiation of a complex system in the Neobatrachia. The anatomical Record, 290, 1551-1567.

https://doi.org/10.1002/ar.20608

Marjanović, D. \& Laurin, M. (2008) A reevaluation of the evidence supporting an unorthodox hypothesis on the origin of extant amphibians. Contributions to Zoology, 77 (3), 149-199. [2008.ma]. https://doi.org/10.1163/18759866-07703002

Marjanović, D. \& Laurin, M. (2014) An updated palaeontological timetree of lissamphibians, with comments on the anatomy of crown-group salamanders (Urodela). Historical Biology, 26 (4), 535-550. [2014.ma].

https://doi.org/10.1080/08912963.2013.797972

Marjanović, D. \& Laurin, M. (2015) Reevaluation of the largest published morphological data 6149 matrix for phylogenetic analysis of Paleozoic limbed vertebrates. PeerJ PrePrints, 6150, e1995.

https://doi.org/10.7287/peerj.preprints.1596v1

Marjanović, D. \& Laurin, M. (2019) Phylogeny of Paleozoic limbed vertebrates reassessed through revision and expansion of the largest published relevant data matrix. PeerJ, 6 (e5565), 1191.

https://doi.org/10.7717/peerj.5565

Marmayou, J., Dubois, A., Ohler, A., Pasquet, E. \& Tillier, A. (2000) Phylogenetic relationships in the Ranidae. Independent origin of direct development in the genera Philautus and Taylorana. Comptes rendus de l'Académie des Sciences, Sciences de la Vie, 323, 287-297.

https://doi.org/10.1016/S0764-4469(00)00133-5

Martín, C., Alonso-Zarazaga, M.A. \& Sanchiz, B. (2012) Nomenclatural notes on living and fossil amphibians. Graellsia, 68 (1), 159-180. [2012.ma]. https://doi.org/10.3989/graellsia.2012.v68.056

Martins, L. \& Giaretta, A. (2011) A new species of Proceratophrys Miranda-Ribeiro (Amphibia: Anura: Cycloramphidae) from central Brazil. Zootaxa, 2880 (1), 41-50.

https://doi.org/10.11646/zootaxa.2880.1.4

Massalongo, A. (1854) Catalogo ragionato dei Rettili fino ad ora conosciuti nella provincia veronese. Memorie dell'Accademia d'Agricoltura, Arti e Commercio di Verona, 29, 383-434. [1854.ma].

Matsui, M., Hamidy, A., Belabut, D.M., Ahmad, N., Panha, S., Sudin, A., Khonsue, W., Oh, H., Yong, H., Jiang, J. \& Nishikawa, K. (2011) Systematic relationships of Oriental tiny frogs of the family Microhylidae (Amphibia, Anura) as revealed by mtDNA genealogy. Molecular Phylogenetics and Evolution, 61, 167-176.

https://doi.org/10.1016/j.ympev.2011.05.015

Matsui, M. \& Orlov, N. (2004) A new species of Chirixalus from Vietnam (Anura: Rhacophoridae). Zoological Science, 21, 671-676.

https://doi.org/10.2108/zsj.21.671

Matsui, M., Shimada, T. \& Sudin, A. (2014) First record of the tree- frog genus Chiromantis from Borneo with the description of a new species (Amphibia: Rhacophoridae). Zoological Science, $31,45-51$

https://doi.org/10.2108/zsj.31.45

Matsui, M., Yambun, P. \& Sudin, A. (2007) Taxonomic relationships of Ansonia anotis Inger, Tan, and Yambun, 2001 and Pedostibes maculatus (Mocquard, 1890), with a description of a new genus (Amphibia, Bufonidae). Zoological Science, 24, 1159-1166.

https://doi.org/10.2108/zsj.24.1159

Mattison, C. (1987) Frogs \& toads of the world. Blanford Press, Poole, New York \& Sydney, 191 pp. $\{$ Q $\}$.

May, R.M. (2004) Tomorrow's taxonomy: collecting new species in the field will remain the rate-limiting step. Philosophical Transactions of the royal Society of London, (B), Biological Sciences, 359, 733-734.

https://doi.org/10.1098/rstb.2003.1455

Mayden, R.L. (1997) A hierarchy of species concepts: the denouement in the saga of the species problem. In: Claridge, M.F., Dawah, H.A. \& Wilson, M.R (Eds.), Species: the units of biodiversity. Chapman \& Hall, London, pp. 381-424.

Mayer, F.J.C. (1849) System des Thier-Reichs oder Eintheilung der Thiere nach einem Princip. Verhandlungen des naturhistorischen Vereins der Preussischen Rheinlande and Westphalens, 6, 177-210. [1849.ma].

Mayr, E. (1965) Numerical phenetics and taxonomic theory. Systematic Zoology, 14, 73-97. https://doi.org/10.2307/2411730

Mayr, E. (1969) Principles of systematic zoology. McGraw-Hill, New York, i-xiii + 1-428.

Mayr, E. (1974) Cladistic analysis or cladistic classification? Zeitschrift für zoologische Systematik und Evolutionsforschung, 12, 94-128. https://doi.org/10.1111/j.1439-0469.1974.tb00160.x

Mayr, E. (1982) The growth of biological thought. Belknap Press, Cambridge, Mass. \& London, [i-xiii] + 974 pp.

Mayr, E. (1995) Systems of ordering data. Biology and Philosophy, $10,419-434$. https://doi.org/10.1007/BF00857592

Mayr, E. (1997) This is biology. The science of the living world. Belknap Press, Cambridge, Mass. \& London, i-xvii +327 pp.

Mayr, E. \& Ashlock, P.D. (1991) Principles of systematic zoology. Second edition. McGraw-Hill, New York, i-xx +475 pp.

Mayr, E., Linsley, E.G. \& Usinger, R.L. (1953) Methods and principles of systematic zoology. McGraw-Hill, New York, $\mathrm{i}-\mathrm{ix}+328 \mathrm{pp}$. https://doi.org/10.2307/1440379

McCranie, J.R. \& Castañeda, F.E. (2006) A new species of hylid frog from Northwestern Honduras. Herpetologica, 62, 318323.

https://doi.org/10.1655/0018-0831(2006)62[318: ANSOHF]2.0.CO;2

McCranie, J., Vieites, D. \& Wake, D. (2008) Description of a new divergent lineage and three new species of Honduran salamanders of the genus Oedipina (Caudata, Plethodontidae). 
Zootaxa, 1930 (1), 1-17.

https://doi.org/10.11646/zootaxa.1930.1.1

McDiarmid, R.W. (1971) Comparative morphology and evolution of frogs of the neotropical genera Atelopus, Dendrophryniscus, Melanophryniscus, and Oreophrynella. Bulletin of the Los Angeles County Museum of natural History, 12, 1-66.

McDiarmid, R.W. \& Altig, R. (1999) Tadpoles: the biology of anuran larvae. University of Chicago Press, Chicago \& London, i-xiv + 444 pp. $\{\mathrm{Q}\}$.

McMahon, M.M. \& Sanderson, M.J. (2006) Phylogenetic supermatrix analysis of GenBank sequences from 2228 papilionoid legumes. Systematic Biology, 55 (5), 818-836. https://doi.org/10.1080/10635150600999150

Meegaskumbura, M., Senevirathne, G., Biju, S.D., Garg, S., Boyagoda, S., Pethiyagoda, R., Hanken, J. \& Schneider, C. $(2015 b)$ Patterns of reproductive-mode evolution in Old World tree frogs (Anura, Rhacophoridae). Zoologica scripta, 44, 509-522.

https://doi.org/10.1111/zsc.12121

Meegaskumbura, M., Senevirathne, G., Wijayathilaka, N., Jayawardena, B., Bandara, C., Manamendra-Arachchi, K. \& Pethiyagoda, R. (2015a) The Sri Lankan torrent toads (Bufonidae: Adenominae: Adenomus): species boundaries assessed using multiple criteria. Zootaxa, 3911 (2), 245-261. https://doi.org/10.11646/zootaxa.3911.2.6

Méhelÿ, L. (1904) Investigations on Paraguayan batrachians. Annales Musei nationalis hungarici, 2, 207-232, 1 pl.

Méhelÿ, L. v. (1901) Beiträge zur Kenntnis der Engystomatiden von Neu Guinea. Természetrajzi Füzetek, Budapest, 24, 169271, pls. 4-12. [1901.ma].

Melville, R.V. (1977) Opinion 1071. Emendation under the plenary powers of Liopelmatina to Leiopelmatidae (Amphibia Salientia). Bulletin of zoological Nomenclature, 33 (3-4), 167-169. [1977.ma].

Melville, R.V. (1978) Opinion 1104. Relative precedence of Cornufer Tschudi, 1838, and Platymantis Günther, 1858 (Amphibia Salientia). Bulletin of zoological Nomenclature, 34, 224-233. [1978.ma].

Mendelson, J.R., Da Silva, H.R. \& Maglia, A.M. (2000) Phylogenetic relationships among marsupial frog genera (Anura: Hylidae: Hemiphractinae) based on evidence from morphology and natural history. Zoological Journal of the Linnean Society, 128, 125-148.

https://doi.org/10.1111/j.1096-3642.2000.tb00159.x

Mendelson, J.R. III \& Campbell, J.A. (1994) Two new species of the Hyla sumichrasti group (Amphibia: Anura: Hylidae) from Mexico. Proceedings of the biological Society of Washington, 107, 398-409.

Menke, C.T. (1828) Synopsis methodica molluscorum generum omnium et specierum earum, quae in Museo Menkeano adservantur; cum synonymia critica et novarum specierum diagnosibus. Henrici Gelpke, Pyrmonti, i-xii + 91 pp. [1828. ma].

https://doi.org/10.5962/bhl.title.13182

Mercurio, V. (2011) Amphibians of Malawi. An analysis of their richness and community diversity in a changing landscape.
Contributions to natural History, 49, 1-393.

Merrem, B. (1820) Versuch eines Systems der Amphibien. Tentamen systematis Amphibiorum. Iohann Christian Krieger, Marburg, [i-Xv +191 pp.] $\times 2,1$ pl. [1820.ma].

https://doi.org/10.5962/bhl.title.5037

Merrem, B. (1822) Tentamen systematis Amphibiorum. Isis von Oken, 10 (6), 688-704. [1822.ma].

Mertens, R. (1927) Neue Amphibien und Reptilien aus dem IndoAustralischen Archipel gesammelt während der SundaExpedition Rensch. Senckenbergiana biologica, 9, 234-242.

Metcalf, M.M. (1923) The opalinid ciliate infusorians. Bulletin of the United States national Museum, 120, i-vii + 484 pp. $\{\mathrm{Q}\}$ [1923.ma]. https://doi.org/10.5479/si.03629236.120.1

Metcalf, M.M. (1940) Further studies on the opalinid ciliate infusorians and their hosts. Proceedings of the United States national Museum, 87, 465-634. https://doi.org/10.5479/si.00963801.87-3077.465

Methuen, P.A. \& Hewitt, J. (1913) On a collection of Batrachia from Madagascar made during the year 1911. Annals of the Transvaal Museum, 4, 49-64, pls. 9-10. [1913.ma].

Meyer, A. (1926) Logik der Morphologie im Rahmen einer Logik der gesamten Biologie. Julius Springer, Berlin, i-vii $+1-$ 290. https://doi.org/10.1007/978-3-642-50733-5

Meyer, H. von (1832) Palaeologica zur Geschichte der Erde und ihrer Geschöpfe. Siegmund Schmerber, Frankfurt am Main, 560 pp. [1832.ma].

Meyer, H. von (1860a) Salamandrinen aus der Braunkohle am Rhein und in Böhmen. Palaeontographica, Beiträge zur Naturgeschichte der Vorwelt, 7 (2), 47-73, pls. 8-9. [1860. ma].

Meyer, H. von (1860b) [Untitled]. Neues Jahrbuch für Mineralogie, Geognosie, Geologie und Petrefaktenkunde 1860, 556-560. [1860.mb].

Meyer, H. von (1863) Heliarchon furcillatus, ein Batrachier aus der Braunkohle von Rott im Siebengebirge. Palaeontographica, Beiträge zur Naturgeschichte der Vorwelt, 10 (6), 292-298, pl. 1. [1863.ma].

Milner, A.R. (1988) The relationships and origin of living amphibians. In: Benton, M.J. (Ed.), The phylogeny and classification of the tetrapods. Vol. 1. Amphibians, reptiles, birds, The Systematics Association Special Volume $35 \mathrm{~A}$. Clarendon Press, Oxford, pp. 59-102. [1988.ma].

Milner, A.R. (2000) Chapter 18. Mesozoic and Tertiary Caudata and Albanerpetontidae. In: Heatwole, H. \& Carroll, R.L. (Eds.), Amphibian biology, 4, Paleontology, the evolutionary history of amphibians. Surrey Beatty, Chipping Norton, pp. 1412-1444. [2000.ma].

Milto, K.D., Poyarkov, N.A., Orlov, N.L. \& Nguyen, T.T. (2013) Two new rhacophorid frogs from Cat Ba Island, Gulf of Tonkin, Vietnam. Russian Journal of Herpetology, 20, 287300 .

Minelli, A. (2000) The ranks and the names of species and higher taxa, or a dangerous inertia of the language of natural history. In: Ghiselin, M.T. \& Leviton, A.E. (Eds.), Cultures 
and institutions of natural history: essays in the history and philosophy of sciences. California Academy of Sciences, San Francisco, pp. 339-351.

Minelli, A. (2011) Class Chilopoda, Class Symphyla and Class Pauropoda. In: Zhang, Z.-Q. (Ed.), Animal biodiversity: an outline of higher-level classification and survey of taxonomic richness. Zootaxa, 3148 (1), 157-158.

https://doi.org/10.11646/zootaxa.3148.1.31

Miranda-Ribeiro, A. de (1920) As Hylas coelonotas do Museu Paulista. Revista do Museu Paulista, 12, 321-328. [1920. ma]. https://doi.org/10.5962/bhl.part.22255

Miranda-Ribeiro, A. de (1923) Os hylodideos do Museu Paulista. Revista do Museu Paulista, 13, 825-846. [1923.mb].

Miranda-Ribeiro, A. de (1924) Notas batrachologicas. I. Boletim do Museu nacional do Rio de Janeiro, 2, 137-143. [1924.ma].

Miranda-Ribeiro, A. de (1926) Notas para servirem ao estudo dos Gymnobatrachios (Anura) Brasileiros. Arquivos do Museu nacional do Rio de Janeiro, 27, 1-227, pls. 1-22. \{Q\} [1926. ma].

Miranda Ribeiro, A. de (1937) Sobre una collecção de vertebrados dio nordeste brasileiro. Primeira parte: peixes e batrachios. $O$ Campo, Rio de Janeiro, Janeiro, 1937, 54-56. [1937.ma].

Mivart, S.G. (1869) On the classification of the anurous batrachians. Proceedings of the zoological Society, 1869, 280-295. \{Q\} [1869.ma]. https://doi.org/10.1111/j.1469-7998.1869.tb07329.x

Mo, Y., Zhang, W.E.I., Luo, Y.U., Zhou, S. \& Chen, W. (2013) A new species of the genus Gracixalus (Amphibia: Anura: Rhacophoridae). Zootaxa, 3616 (1), 61-72. https://doi.org/10.11646/zootaxa.3616.1.5

Moler, P.E. (1985) A new species of frog (Ranidae, Rana) from northwestern Florida. Copeia 1985, 379-383. https://doi.org/10.2307/1444847

Monod, J. (1970) Le hasard et la nécessité. Essai sur la philosophie naturelle de la biologie moderne. Seuil, Paris, $213 \mathrm{pp}$.

Morales, V.R. \& McDiarmid, R.W. (2009) A new species of Chiasmocleis (Anura: Microhylidae) from southern Amazonian Peru with comments on some other microhylids. Biotempo, 9, 71-76. https://doi.org/10.31381/biotempo.v9i0.69

Moreno, A.R. \& Alcaide, M.F. (1978) Histomorfologia del oviducto de Ceratophrys ornata (Bell) (Amphybia [sic], Anura). Acta zoologica lilloana, 33 (1), 93-106. [1978.mb].

Morescalchi, A. (1995) Les chromosomes. In: Delsol, M. (Ed.), Traité de zoologie, Anatomie, systématique, biologie, Amphibiens. 14 (1A). Masson, Paris, pp. 855-870. [1995. ma].

Muchoney, D.M., Grossman, D.H. \& Solomon, R. (1991) Rapid ecological assessment for conservation planning. Technical Papers of the ACSM-ASPRS Annual Convention, 4, 141145.

Mueller, R.L., Macey, J.R., Jaekel, M., Wake, D.B. \& Boore, J.L. (2004) Morphological homoplasy, life history evolution, and historical biogeography of plethodontid salamanders inferred from complete mitochondrial genomes. Proceedings of the national Academy of Sciences of the United States of America, 101, 13820-13825.

https://doi.org/10.1073/pnas.0405785101

Müller, [J.] (1840) Über den Bau des Pentacrinus Caput Medusae. Bericht über die zur Bekanntmachung geeigneten Verhandlungen der Königlich Preussischen Akademie der Wissenschaften zu Berlin, 1840, 88-106. [1840.ma].

Müller, F. (1892) Siebter Nachtrag zum Katalog der herpetologischen Sammlung des Basler Museums. Verhandlungen der Naturforschenden Gesellschaft in Basel, 10, 195-215.

Müller, J. (1831) Kiemenlöcher an einer jungen Coecilia hypocyanea, im Museum der Naturgeschichte zu Leyden beobachtet vom Prof. Joh Müller. Isis von Oken, 24, 709-711. [1831.ma].

Müller, J. (1832) Beiträge zur Anatomie und Naturgeschichte der Amphibien. Zeitschrift für Physiologie, 4, 190-275, pls. 1822. [1832.mb].

Myers, C.W. (1982) Spotted poison frogs: descriptions of three new Dendrobates from western Amazonia, and resurrection of a lost species from 'Chiriqui'. American Museum Novitates, 2721, 1-23.

Myers, C.W. \& Daly, J.W. (1976) Preliminary evaluation of skin toxins and vocalizations in taxonomic and evolutionary studies of poison-dart frogs (Dendrobatidae). Bulletin of the American Museum of natural History, 157, 175-262.

Naylor, B. (1978) The systematics of fossil and recent salamanders (Amphibia: Caudata), with special reference to the vertebral colum and trunk musculature. Ph. D. thesis, University of Alberta, i-xxxiv + 857 pp. [1978.na].

Nelson, C.E. \& Lescure, J. (1975) The taxonomy and distribution of Myersiella and Synapturanus (Anura: Microhylidae). Herpetologica, 31, 389-397.

Nessov, L.A. (1981) Хвостатые и бесхвостые земноводные Мела Кызылкумов. [Cretaceous salamanders and frogs of Kizylkum Desert]. In: Anan'eva, N.B. \& Borkin, L.J. (Eds.), Fauna i Ekologiâ Amfibij i Reptilij palearktičeskoj Azii, Trudy Zoologičeskogo Instituta. Vol. 101. Akademiâ Nauk SSSR, Moscow, pp. 57-88. [In Russian]. [1981.na].

Nessov, L.A. (1993) New salamanders from the Upper Jurassic Morrison fm. of Dinosaur National Monument. In: Santucci, V.L. (Ed.), National Park Service, Paleontological Research. Vol. 1. Technical Report, NPS/NRPEFO/NRTR-93/11, pp. 30. [1993.na].

Nguyen, T.Q., Le, M.D., Pham, C.T., Nguyen, T.T., Bonkowski, M. \& Ziegler, T. (2013) A new species of Gracixalus (Amphibia: Anura: Rhacophoridae) from northern Vietnam. Organisms, Diversity and Evolution, 13, 203-214. https://doi.org/10.1007/s13127-012-0116-0

Nguyen, T.T., Matsui, M. \& Duc, H.M. (2014a) A new tree frog of the genus Kurixalus (Anura: Rhacophoridae) from Vietnam. Current Herpetology, 33, 101-111.

https://doi.org/10.5358/hsj.33.101

Nguyen, T.T., Matsui, M. \& Eto, K. (2014b) A new cryptic tree frog species allied to Kurixalus banaensis (Anura: Rhacophoridae) from Vietnam. Russian Journal of Herpetology, 21, 295302. 
Nicholls, G.A. (1916) The structure of the vertebral colum in the Anura Phaneroglossa and its importance as a basis of classification. Proceedings of the Linnean Society of London, 128, 80-92. [1916.na]. https://doi.org/10.1111/j.1095-8312.1916.tb00038.x

Nieden, F. (1911) Verzeichnis der bei Amani in Deutschostafrika vorkommenden Reptilien und Amphibien. Sitzungsberichte der Gesellschaft Naturforschender Freunde Berlin, 1910, 441-452.

Nieden, F. (1923) Anura I. Subordo Aglossa und Phaneroglossa. Sectio 1. Arcifera. Das Tierreich, 46, i-xxxii +1-584. $\{\mathrm{Q}\}$. https://doi.org/10.1515/9783111434582-003

Nieden, F. (1926) Amphibia Anura II. Engystomatidae. Das Tierreich, 49, i-xvi + 1-110. https://doi.org/10.1515/9783111385464

Nishikawa, K., Jiang, J.-P., Matsui, M., Mo, Y.-M., Chen, X.-H., Kim, J.-B., Tominaga, A. \& Yoshikawa, N. (2010) Invalidity of Hynobius yunanicus and molecular phylogeny of Hynobius salamander from continental China (Urodela, Hynobiidae). Zootaxa, 2426 (1), 65-67.

https://doi.org/10.11646/zootaxa.2426.1.5

Noble, G.K. (1922) The phylogeny of the Salientia. I. The osteology and the thigh musculature; their bearing on classification and phylogeny. Bulletin of the American Museum of natural History, 46, 1-87, pls. 1-23. [1922.na].

Noble, G.K. (1924) A new spadefoot toad from the Oligocene of Mongolia with a summary of the evolution of the Pelobatidae. American Museum Novitates, 132, 1-15. [1924.na].

Noble, G.K. (1926) An analysis of the remarkable cases of distribution among the Amphibia, with descriptions of new genera. American Museum Novitates, 212, 1-24.

Noble, G.K. (1927) The value of life history data in the study of the evolution of the Amphibia. Annals of the New York Academy of Sciences, 30, 31-128, pls. 9. [1927.na].

https://doi.org/10.1111/j.1749-6632.1927.tb55359.x

Noble, G.K. (1931) The biology of the Amphibia. Dover, New York, i-Xviii + 577 pp. \{Q\} [1931.na].

https://doi.org/10.5962/bhl.title.82448

Nussbaum, R.A. (1977) Rhinatrematidae: a new family of caecilians (Amphibia: Gymnophiona). Occasional Papers of the Museum of Zoology of the University of Michigan, 682, 1-30. [1977.na].

Nussbaum, R.A. (1979) The taxonomic status of the caecilian genus Uraeotyphlus Peters. Occasional Papers of the Museum of Zoology of the University of Michigan, 687, 1-20. [1979.na].

Nussbaum, R.A. \& Brodie, E.D. (1982) Partitioning of the salamandrid genus Tylototriton Anderson (Amphibia: Caudata) with a description of a new genus. Herpetologica, 38, 320-332.

Nussbaum, R.A. \& Wilkinson, M. (1989) On the classification and phylogeny of caecilians (Amphibia: Gymnophiona), a critical review. Herpetological Monographs, 3, 1-42.

https://doi.org/10.2307/1466984

Ohler, A. (1999) The identity of Dendrobatorana Ahl, 1927 (Amphibia, Ranoidea). Zoosystematics and Evolution, 75, $37-45$. https://doi.org/10.1002/mmnz.19990750105

Ohler, A., Amarasinghe, A.A.T., Andreone, F., Bauer, A., Borkin, L., Channing, A., Chuaynkern, Y., Das, I., Deuti, K., Fretey, T., Matsui, M., Truong, N., Pyron, R.A., Roedel, M.O., Segniagbeto, G.H., Vasudevan, K. \& Dubois, A. (2014) Case 3666. Dicroglossidae Dubois, 1987 (Amphibia, Anura): proposed conservation. Bulletin of zoological Nomenclature, 71, 244-249.

https://doi.org/10.21805/bzn.v71i4.a15

Ohler, A. \& Dubois, A. (1989) Démonstration de l'origine indépendante des ventouses digitales dans deux lignées phylogénétiques de Ranidae (Amphibiens, Anoures). Comptes rendus de l'Académie des Sciences, (Série 3, Sciences de la Vie), 309, 419-422.

Ohler, A. \& Dubois, A. (2006) Phylogenetic relationships and generic taxonomy of the tribe Paini (Amphibia, Anura, Ranidae, Dicroglossinae), with diagnoses of two new genera. Zoosystema, 28, 769-784.

Ohler, A. \& Dubois, A. (2012) Validation of two familial nomina nuda of Amphibia Anura. Alytes, 28 (3-4), 162-167. [2012. oa].

Ohler, A. \& Dubois, A. (2014) Is Dicroglossidae Anderson, 1871 (Amphibia, Anura) an available nomen? Zootaxa, 3838 (5), 590-594.

https://doi.org/10.11646/zootaxa.3838.5.8

Ohler, A. \& Dubois, A. (2018) Article 23.9 of the Code cannot be used to reject the nomen Hyla quoyi Bory de Saint-Vincent, 1828 as a nomen oblitum. Zoosystema, 40 (6), 109-121. https://doi.org/10.5252/zoosystema2018v40a6

Oken, [L. von] (1816) Lehrbuch der Naturgeschichte. Dritter Theil, Zoologie. Zweite Abtheilung, Fleischthiere. Jena, Schmid, $\mathrm{i}-\mathrm{xvi}+1272 \mathrm{pp}$.

Oliver, L.A., Prendini, E., Kraus, F. \& Raxworthy, C.J. (2015) Systematics and biogeography of the Hylarana frog (Anura: Ranidae) radiation across tropical Australasia, Southeast Asia, and Africa. Molecular Phylogenetics and Evolution, 90, 176-192. https://doi.org/10.1016/j.ympev.2015.05.001

Oppel, M. (1811a) Ordre II. Reptiles à écailles. Section 2. Ophidiens. Annales du Muséum d'Histoire naturelle '1810', 16, 254-295 +376-393. 〔P03 J [1811.oa].

Oppel, M. (1811b) Second mémoire sur la classification des Reptiles. Annales du Muséum d'Histoire naturelle, 16, 394 418. [P03 」 [1811.ob].

Oppel, M. (1811c) Die Ordnungen, Familien und Gattungen der Reptilien als Prodrom einer Naturgeschichte derselben. Joseph Lindauer, München, i-xii + 87 pp. \{P03 J [1811.oc]. https://doi.org/10.5962/bhl.title.4911

Orlov, N.L., Poyarkov, N.A., Vassilieva, A.B., Ananjeva, N.B., Nguyen, T.T., Sang, N.N. \& Geissler, P. (2012) Taxonomic notes on rhacophorid frogs (Rhacophorinae: Rhacophoridae: Anura) of southern part of Annamite Mountains (Truong Son, Vietnam), with description of three new species. Russian Journal of Herpetology, 19, 23-64.

Ota, H. (1995) A review of introduced Reptiles and Amphibians of the Ryukyu Archipelago, Japan. Island Studies in Okinawa, 
13, 63-78. [1995.oa].

Owen, O. (1841) Odontography; or, a treatise on the comparative anatomy of the teeth; their physiological relations, mode of development, and microscopic structure, in the vertebrate animals. Volume 1. Text. Hippolyte Baillière, London, i-xxi $+\mathrm{i}-1$ xxiv +655 pp. [1841.oa]. https://doi.org/10.5962/bhl.title.114889

Owen, R. (1835) On the structure of the heart of the Perennibranchiate Batrachia. Transactions of the zoological Society of London, 1, 213-220, pl. 31. [1835.oa].

https://doi.org/10.1111/j.1096-3642.1835.tb00620.x

Owen, R. (1860) Paleontology, or A systematic summary of extinct animals and their geographical relations. Adam \& Charles Black, Edinburgh, i-xv + 420 pp. [1860.oa]. https://doi.org/10.5962/bhl.title. 153670

Owen, R. (1866) On the anatomy of vertebrates. Vol. 1. Fishes and Reptiles. Longmans, Green \& Co., i-xlii + 650 pp. [1866. oa].

https://doi.org/10.5962/bhl.title.33654

Pace, A.E. (1974) Systematic and biological studies of the Leopard frogs (Rana pipiens complex) of the United States. Miscellaneous Publications of the Museum of Zoology, University of Michigan, 148, [i-viii] + 1-140.

Padhye, A.D., Sayyed, A., Jadhav, A. \& Dahanukar, N. (2013) Raorchestes ghatei, a new species of shrub frog (Anura: Rhacophoridae) from the Western Ghats of Maharashtra. Journal of threatened Taxa, 5, 4913-4931. https://doi.org/10.11609/JoTT.03702.4913-31

Padial, J.M., Castroviejo-Fisher, S., Köhler, J., Domic, E. \& Riva, I. de la (2007) Systematics of the Eleutherodactylus fraudator species group (Anura: Brachycephalidae). Herpetological Monographs, 21, 213-240.

https://doi.org/10.1655/06-007.1

Padial, J., Grant, T. \& Frost, D. (2014) Molecular systematics of terraranas (Anura: Brachycephaloidea) with an assessment of the effects of alignment and optimality criteria. Zootaxa, 3825 (1), 1-132. [2014.pa]. https://doi.org/10.11646/zootaxa.3825.1.1

Padial, J.M., Miralles, A., de la Riva, I. \& Vences, M. (2010) The integrative future of taxonomy. Frontiers in Zoology, 7 (16), $1-14$. https://doi.org/10.1186/1742-9994-7-16

Paez-Vacas, M.I., Coloma, L.A. \& Santos, J.C. (2010) Systematics of the Hyloxalus bocagei complex (Anura: Dendrobatidae), description of two new cryptic species, and recognition of $H$. maculosus. Zootaxa, 2711 (1), 1-75. https://doi.org/10.11646/zootaxa.2711.1.1

Palacký, J. (1898) Die Verbreitung der Batrachier auf der Erde. Verhandlungen der kaiserlich-königlichen zoologischbotanischen Gesellschaft in Wien, 48, 374-382.

Pallas, P.S. (1814) Zoographia rosso-asiatica. Vol. 3. Animalia monocardia seu frigidi sanguinis imperii rosso-asiatici. Separate. Officina Caesaris Academiae Scientiarum Petropolis, Petropoli, 428 pp. [1814.pa].

Pape, T., Blagoderov, V. \& Mostovski, M. B. (2011) Order Diptera Linnaeus, 1758. In: Zhang, Z.-Q. (Ed.), Animal biodiversity: an outline of higher-level classification and survey of taxonomic richness. Zootaxa, 3148 (1), 222-229.

https://doi.org/10.11646/zootaxa.3148.1.42

Parker, H.W. (1927) The Brevicipitid frogs allied to the genus Gastrophryne. Occasional Papers of the Museum of Zoology University of Michigan Ann Arbor, 187, 1-6.

Parker, H.W. (1931) Some brevicipitid frogs from Tanganyika Territory. Annals and Magazine of natural History, (10), 8, 261-264. https://doi.org/10.1080/00222933108673395

Parker, H.W. (1933) A list of the frogs and toads of Trinidad. Tropical Agriculture, 10 (1), 8-12. [1933.pa].

Parker, H.W. (1934) A monograph of the frogs of the family Microhylidae. British Museum, London, i-viii + 208 pp. [1934.pa].

Parker, H.W. (1935) The frogs, lizards, and snakes of British Guiana. Proceedings of the zoological Society of London, 1935, 505-530. [1935.pa].

https://doi.org/10.1111/j.1096-3642.1935.tb01678.x

Parker, H.W. (1940) The Australasian frogs of the family Leptodactylidae. Novitates zoologicae, 42 (1), 1-106, pl. 1. [1940.pa].

Parra-Olea, G., García-París, M. \& Wake, D.B. (2004) Molecular diversification of salamanders of the tropical American genus Bolitoglossa (Caudata: Plethodontidae) and its evolutionary and biogeographical implications. Biological Journal of the Linnean Society, 81, 325-346. https://doi.org/10.1111/j.1095-8312.2003.00303.x

Patterson, D.J., Cooper, J., Kirk, P.M., Pyle, R.L. \& Rensen, D.P. (2010) Names are key to the big new biology. Trends in Ecology \& Evolution, 25 (12), 686-691. https://doi.org/10.1016/j.tree.2010.09.004

Pauly, G.B., Hillis, D.M. \& Cannatella, D.C. (2004) The history of a nearctic colonization: molecular phylogenetics and biogeography of the nearctic toads (Bufo). Evolution, 58, 2517-2535.

https://doi.org/10.1111/j.0014-3820.2004.tb00881.x

Pauly, G.B., Hillis, D.M. \& Cannatella, D.C. (2009) Taxonomic freedom and the role of official lists of species names. Herpetologica, 65 (2), 115-128. https://doi.org/10.1655/08-031R1.1

Pearsall, J. (Ed.) (2001) The new Oxford dictionary of English. Oxford University Press, Oxford, i-xxi + 2152 pp.

Pearse, A.S. (Ed.) (1936) Zoological names. A list of phyla, classes, and orders. Duke University, Durham, 24 pp. [1936.pa].

Pearse, A.S. (Ed.) (1948) Zoological names. A list of phyla, classes, and orders. Third Edition. [Duke University], Durham, 24 pp. [1948.pa].

Pearse, A.S. (Ed.) (1949) Zoological names. A list of phyla, classes, and orders. Fourth Edition. [Duke University], Durham, 24 pp. [1949.pa].

Peloso, P.L.V, Faivovich, J., Grant, T., Gasparini, J.L. \& Haddad, C.F.B. (2012) An extraordinary new species of Melanophryniscus (Anura, Bufonidae) from Southeastern Brazil. American Museum Novitates, 3762, 1-32. https://doi.org/10.1206/3762.2 
Peloso, P.L.V., Frost, D.R., Richards, S.J., Rodrigues, M.T., Donnellan, S.C., Matsui, M., Raxworthy, C.J., Biju, S.D., Lemmon, E.M., Lemmon, A.R. \& Wheeler, W.C. (2016) The impact of anchored phylogenomics and taxon sampling on phylogenetic inference in narrow-mouthed frogs (Anura, Microhylidae). Cladistics, 32 (2), 113-140. [2016.pa]. https://doi.org/10.1111/cla.12118

Peloso, P., Sturaro, M., Forlani, M., Gaucher, P., Motta, A. \& Wheeler, W.C. (2014) Phylogeny, taxonomic revision, and character evolution of the genera Chiasmocleis and Syncope (Anura, Microhylidae) in Amazonia, with descriptions of three new species. Bulletin of the American Museum of natural History, 386, 1-112. https://doi.org/10.1206/834.1

Peng, R., Zhang, P., Xiong, J., Gu, H.-J., Zeng, X. \& Zou, F.-D. (2010) Rediscovery of Protohynobius puxiongensis (Caudata: Hynobiidae) and its phylogenetic position based on complete mitochondrial genomes. Molecular Phylogenetics and Evolution, 56, 252-258.

https://doi.org/10.1016/j.ympev.2009.12.011

Peracca, M.G. (1886) Sulla bontà specifica del Triton Blasii de l'Isle e descrizione di una nuova forma ibrida di Triton francese. Bollettino dei Musei di Zoologia ed Anatomia comparata dell' reale Università di Torino, 1 (12), 1-13, 1 pl. https://doi.org/10.5962/bhl.part.4563

Pereira, H.M., Leadley, P.W., Proença, V., Alkemade, R., Scharlemann, J.P.W., Fernandez-Manjarrés, J.F., Araújo, M.B., Balvanera, P., Biggs, R., Cheung, W.W.L., Chini, L., Cooper, D., Gilman, E.L., Guénette, S., Hurtt, G.C., Huntington, H.P., Mace, G.M., Oberdorff, T., Revenga, C., Rodrigues, P., Scholes, R.J., Sumaila, U.R. \& Walpole, M. (2010) Scenarios for global biodiversity in the $21^{\text {st }}$ century. Science, 330, 1496-1501.

https://doi.org/10.1126/science.1196624

Perez-Ramos, E. \& Saldana de la Riva, L. (2000) Una nueva especie de salamandra Pseudoeurycea (Amphibia: Caudata: Plethodontidae) de la región Amuzga, al sureste de Guerrero, Mexico. Revista digital universitaria, 1 (1). Online document.

http://www.revista.unam.mx/vol.1/art1/index.html [accessed 28 October 2013]

Peters, W. (1862) Über der Batrachier-Gattung Hemiphractus. Monatsberichte der königlich preussischen Akademie der Wissenschaften zu Berlin, 1862, 144-152, pls. 1-2. [1862. pa].

Peters, W.C.H. (1882) Naturwissenschaftliche Reise nach Mossambique auf Befehl Seiner Majestät des Königs Friedrich Wilhelm IV. In den Jahren 1842 bis 1848 ausgeführt. Zoologie 3. Amphibien. G. Reimer, Berlin, $\mathrm{i}-\mathrm{xv}+1-191,33$ pls. [1882. pa].

Philippi, R.A. (1902) Suplemento a los Batraquios chilenos descritos en la Historia Física i Política de Chile de don Claudio Gay. Santiago de Chile (Enrique Blanchard-Chessi), i-xi +62 pp. [1902.pa]. https://doi.org/10.5962/bhl.title.104403

Pimenta, B. \& Canedo, C. (2007) Description of the tadpole of
Itapotihyla langsdorffii (Anura: Hylidae). Zootaxa, 1387, 39-46.

https://doi.org/10.11646/zootaxa.1387.1.3

Pinheiro, P.D.P., Kok, J.R., Noonan, B.P., Means, D.B. \& Haddad, C.F.B. (2019) A new genus of Cophomantini, with comments on the taxonomic status of Boana liliae (Anura, Hylidae). Zoological Journal of the Linnean Society, 185 (1), 226-245. [2019.pa]. https://doi.org/10.1093/zoolinnean/zly030

Piveteau, J. (1937) Paléontologie de Madagascar. XXIII. Un Amphibien du Trias inférieur: essai sur l'origine et l'évolution des Amphibiens Anoures. Annales de Paléontologie, 26, 135178, pls. 19-20. [1937.pa].

Plath, M., Hermann, H.-W. \& Böhme, W. (2006) New frog species of the genus Phrynobatrachus (Anura: Phrynobatrachidae) from Mt. Nlonako, Cameroon. Journal of Herpetology, 40, 486-495.

https://doi.org/10.1670/0022-1511(2006)40[486: NFSOTG]2.0.CO;2

Platz, J.E. \& Frost, J.S. (1984) Rana yavapaiensis, a new species of Leopard frog (Rana pipiens complex). Copeia, 1984, 940948. https://doi.org/10.2307/1445338

Pleijel, F. \& Rouse, G.W. (2003) Ceci n'est pas une pipe: names, clades and phylogenetic nomenclature Journal of zoological Systematic and evolutionary Research, 41, 162-174. https://doi.org/10.1046/j.1439-0469.2003.00236.x

Pombal, J., Menezes, V., Fontes, A., Nunes, I., Rocha, C. \& Van Sluys, M. (2012) A second species of the casque-headed frog genus Corythomantis (Anura: Hylidae) from northeastern Brazil, the distribution of C. greeningi, and comments on the genus. Boletim do Museu Nacional, (Nova Série Zoologia), $530,1-14$.

Pope, C.H. (1931) Notes on Amphibians from Fukien, Hainan, and other parts of China. Bulletin of the American Museum of natural History, 61, 397-610, 10 pls.

Porter, K.R. (1972) Herpetology. W. B. Saunders Company, Philadelphia, London \& Toronto, i-xii + 1-524. \{Q\}.

Portik, D. \& Blackburn, D. (2016) The evolution of reproductive diversity in Afrobatrachia: a phylogenetic comparative analysis of an extensive radiation of African frogs. Evolution, 70, 2017-2023.

https://doi.org/10.1111/evo.12997

Portik, D. \& Papenfuss, T. (2015) Historical biogeography resolves the origins of endemic Arabian toad lineages (Anura: Bufonidae): evidence for ancient vicariance and dispersal events with the Horn of Africa and South Asia. BMC evolutionary Biology, 15 (152), 1-19.

https://doi.org/10.1186/s12862-015-0417-y

Pough, F.H., Andrews, R.M., Cadle, J.E., Crump, M.L., Savitzky, A.H. \& Wells, K.D. (1998). Herpetology. Prentice Hall, Upper Saddle River, 1-577. \{Q\}.

Poyarkov, N., Suwannapoom, C., Pawangkhanant, P., Aksornneam, A., Duong, T., Korost, D. \& Che, J. (2018) A new genus and three new species of miniaturized microhylid frogs from Indochina (Amphibia: Anura: Microhylidae: Asterophryinae). 
Zoological Research, 39, 130-157.

https://doi.org/10.24272/j.issn.2095-8137.2018.019

Pramuk, J.B. (2006) Phylogeny of South American Bufo (Anura:

Bufonidae) inferred from combined evidence. Zoological Journal of the Linnean Society, 146, 407-452.

https://doi.org/10.1111/j.1096-3642.2006.00212.x

Pramuk, J.B., Robertson, T., Sites, J.W. \& Noonan, B.P. (2008)

Around the world in 10 million years: biogeography of the nearly cosmopolitan true toads (Anura: Bufonidae). Global Ecology and Biogeography, 17, 72-83.

https://doi.org/10.1111/j.1466-8238.2007.00348.x

Prathapan, K.D., Pethiyagoda, R., Bawa, K.S., Raven, P.H., Rajan, P.D., Acosta, L.E., Adams, B., Adl, S., Ahyong, S.T., Anderson, R., Arango, C.P., Arnedo, M.A., Armbruster, J.W., Avila, L.J., Azevedo, C.O., Baldo, D., Barclay, M.V.L., BaronSzabo, R., Bauer, A.M., Bentlage, B., Bezdek, A., Bird, G., Blagoderov, V., Bocak, L., Bonaldo, A., Bond, J.E., Borkent, C.J., Branham, M.A., Carranza, S., Carreno, R., de Carvalho, M.R., Castroviejo-Fisher, S., Chiba, H., Ciampor, F., Clarke, D.J., Collins, A.G., Constantino, R., Crespo, F.A., Daly, M., Dominiak, P., Dronen, N., Dubois, A., Duda, T.F., Eleaume, M., Erlacher, S., Estrela, P.C., Evenhuis, N., Fehlauer-Ale, K.H., Fery, H., Fritz, U., Gaimari, S.D., Garrison, R., Gaubert, P., Geiger, D.L., Gill, A.C., Gimmel, M.L., Goldschmidt, T., Goswami, R., Gonzalez, A.P., Gonzalez, V.H., Gordon, D., Gower, D.J., Greenslade, P., Gusarov, V.I., Hajdu, E., Harms, D., Heinicke, M.P., Hilton, E.J., Hodgson, C.J., Hormiga, G., Hughes, L.E., Hutchings, P., Jäger, P., Jennings, J.T., Kadej, M., Kaila, L., Kaminski, M.J., Karaman, G.S., Karanovic, T., Kathirithamby, J., Kerr, P.H., Kirkendall, L.R., Kitahara, M.V., Klautau, M., Kondratieff, B.C., Kroh, A., Labarque, F.M., Leavengood, J.M., Letardi, A., Liang, A.-P., Lima, F.C.T., Liu, Z., Löbl, I., Lohrmann, V., Malchus, N., Malipatil, M.B., Marques, A.C., Matzke-Karasz, R., Mayer, G., Mayoral, J.G., McInnes, S.J., Minelli, A., Moir, M.L., Monks, S., Morrone, J.J., Muster, C., Nagy, Z.T., Narayanan, K.S., Nearns, E.H., Nekola, J., Nihei, S.S., Nutzel, A., Ohler, A., Orrico, V.G.D., Padial, J.M., Page, L.M., Passos, P., Paulson, D., Perkins, P.D., Pfingstl, T., Prieto, C., Pinheiro, L.R., Pinto-da-Rocha, R., Prendini, L., Price, B., Prins, J.D., Ramirez, M., Rasmussen, C., Rasmussen, P., Redei, D., Ribera, I., Ricarte, A., Rivera, J., Rix, M.G., Rossaro, B., Roy, A.D., Ruiz, G.R.S., Salles, F.F., Sanborn, A.F., Sartori, M., Scholler, M., Schmelz, R.M., Schrödl, M., Segniagbeto, G.H., Serrano, J., Shimano, S., Shin, M.K., Sidorchuk, E., Siler, C.D., Sket, B., Smith, A.D., Smith, A.B.T., Smith, R., Smith-Pardo, A.H., Sparks, J., Sterrer, W.E., Stroinski, A., Svavarsson, J., Toledo, M., Twomey, E., Vasudevan, K., Vences, M., de Voogd, N., Wang, Q., Watson, G.W., Weiner, W.M., Weksler, M., Wesener, T., Whitmore, D., Wiklund, H., Williams, P.H., Winterton, S.L., Wood, T.S., Yen, S.-H., Zaher, H., Zhang, Z.-Q. \& Zhou, H.-Z. (2018) When the cure kills-CBD limits biodiversity research. Science, 360 (6396), 1405-1406 + supplementary materials (11 pages).

https://doi.org/10.1126/science.aat9844

Pyron, R.A. (2014) Biogeographic analysis reveals ancient continental vicariance and recent oceanic dispersal in amphibians. Systematic Biology, 63, 779-797.

https://doi.org/10.1093/sysbio/syu042

Pyron, R.A., Burbrink, F.T., Colli, G.R., De Oca, A.N.M., Vitt, L.J., Kuczynski, C.A. \& Wiens, J.J. (2011) The phylogeny of advanced snakes (Colubroidea), with discovery of a new subfamily and comparison of support methods for likelihood trees. Molecular Phylogenetics and Evolution, 58 (2), 329342.

https://doi.org/10.1016/j.ympev.2010.11.006

Pyron, R.A., Burbrink, F.T. \& Wiens, J.J. (2013) A phylogeny and revised classification of Squamata, including 4161 species of lizards and snakes. BMC evolutionary Biology, 13 (93), 1-53 + 2 additional online files. [Online document not preregistered in Zoobank]. https://doi.org 10.1186/1471-2148-13-93

Pyron, R.A., O'Connell, K.A., Lemmon, E.M., Lemmon, A.R. \& Beamer, D.A. (2020) Phylogenomic data reveal reticulation and incongruence among mitochondrial candidate species in dusky salamanders (Desmognathus). Molecular Phylogenetics and Evolution, 146, 1-13.

https://doi.org/10.1016/j.ympev.2020.106751

Pyron, R.A. \& Wiens, J.J. (2011) A large-scale phylogeny of Amphibia including over 2800 species, and a revised classification of extant frogs, salamanders, and caecilians. Molecular Phylogenetics and Evolution, 61, 543-583. \{Q\} [2011.pa]. https://doi.org/10.1016/j.ympev.2011.06.012

Qin, S., Mo, Y., Jiang, K., Cai, B., Xie, F., Jiang, J., Murphy, R.W., Li, J.-T. \& Wang, Y. (2015) Two new species of Liuixalus (Rhacophoridae, Anura): evidence from morphological and molecular analyses. PloS One, 10 [e0136134], 1-17. https://doi.org/10.1371/journal.pone.0136134

Queiroz, K. de (1998) The general lineage concept of species, species criteria, and the process of speciation: a conceptual unification and terminological recommendations. In: Howard, D.J. \& Berlocher, S.H. (Eds.), Endless forms: species and speciation. Oxford University Press, New York, pp. 57-75.

Queiroz, K. de, Cantino, P.D. \& Gauthier, J. A. (2020) Phylonyms. A companion to the PhyloCode.Boca Raton, London \& New York (CRC Press), i-xxvii + 1324 pp. [2020.qa]. https://doi.org/10.1201/9780429446276

Queiroz, A. de \& Gatesy, J. (2007) The supermatrix approach to systematics. Trends in Ecology \& Evolution, 22, 34-41. https://doi.org/10.1016/j.tree.2006.10.002

Queiroz, K. de \& Gauthier, J. (1990) Phylogeny as a central principle in taxonomy: phylogenetic definitions of taxon names. Systematic Zoology, 39 (4), 307-322. https://doi.org/10.2307/2992353

Queiroz, K. de \& Gauthier, J. (1992) Phylogenetic taxonomy. Annual Review of Ecology \& Systematics, 23, 449-480. [1992.qa].

https://doi.org/10.1146/annurev.es.23.110192.002313

Queiroz, K. de \& Gauthier, J. (1994) Toward a phylogenetic system of biological nomenclature. Trends in Ecology \& Evolution, 9, 27-31. 
Raffaelli, J. (2007) Les Urodèles du monde. Penclen Edition, Plumelec, [1-6]+1-377. \{Q\}.

Raffaëlli, J. (2013) Les Urodèles du monde. Second edition. Penclen Edition, Plumelec, 472 pp. $\{\mathrm{Q}\}$.

Rafinesque, C.S. (1814) Fine del prodromo d'erpetologia siciliana. Specchio delle Scienze o Giornale enciclopedico di Sicilia, 2 (10), 102-104. [1814.ra].

Rafinesque, C.S. (1815) Analyse de la nature ou Tableau de l'univers et des corps organisés. Jean Barravecchia, Palerme, 124 pp., 1 pl. \{Q\} [1815.ra]. https://doi.org/10.5962/bhl.title.106607

Rage, J.C. \& Roček, Z. (1989) Redescription of Triadobatrachus massinoti (Piveteau, 1936), an anuran amphibian from the early Triassic. Paleontographica, (Abteilung A), Paläozoologie, Stratigraphie, 206 (1-3), 1-16. [1989.ra].

Rage, J.C., Marshall, L.G. \& Gayet, M. (1993) Enigmatic Caudata (Amphibia) from the Upper Cretaceous of Gondwana. Geobios, 26 (5), 515-519. [1993.ra].

https://doi.org/10.1016/S0016-6995(06)80234-2

Raikow, R.J. (1985) Problems in avian classification. In: Johnston, R.F. (Ed.), Current Ornithology. Vol. 2. Plenum, New York, pp. 187-212. https://doi.org/10.1007/978-1-4613-2385-3_6

Rancilhac, L., Bruy, T., Scherz, M.D., Pereira, E.A., Preick, M., Straube, N., Lyra, M.L., Ohler, A., Streicher, J.W. Andreone, F., Crottini, A., Randrianantoandro, J. C., Rakotoarison, A., Glaw, F., Hofreiter, M. \& Vences, M. (2020) Target-enriched DNA sequencing from historical type material enables a partial revision of the Madagascar giant stream frogs (genus Mantidactylus). Journal of natural History, 54, [i] + 1-32. Published online with Zoobank preregistration. https://doi.org/10.1080/00222933.2020.1748243

Randrianiaina, R.-D., Koehler, J., Glos, J., Vences, M. \& Glaw, F. (2011) Where to grow in the Tsingy? Limestone rock pools as breeding habitats of the relict frog Tsingymantis antitra from Madagascar and description of its tadpole. Salamandra, 47, $77-89$.

Raposo, M.A., Stopiglia, R., Brito, G.R.R., Bockmann, F.A., Kirwan, G.M., Gayon, J. \& Dubois, A. (2017) What really hampers taxonomy and conservation? A riposte to Garnett and Christidis (2017). Zootaxa, 4317 (1), 179-184. https://doi.org/10.11646/zootaxa.4317.1.10

Raselimanana, A.P., Glaw, F. \& Vences, M. (2007) Lack of secondary sexual characters in a male of Tsingymantis antitra confirms its position as most basal mantelline frog lineage. Zootaxa, 1557, 67-68

https://doi.org/10.11646/zootaxa.1557.1.5

Regal, P.J. (1966) Feeding specializations and the classification of terrestrial salamanders. Evolution, 20 (3), 392-407. [1966. ra].

https://doi.org/10.1111/j.1558-5646.1966.tb03374.x

Reig, O.A. (1958) Proposiciones para una nueva macrosistematica de los anuros (Nota preliminar). Physis, 21, 109-118. \{Q\} [1958.ra].

Reig, O.A. (1959) Primeros datos descriptivos sobre los anuros del Eocretaceo de Ia provincia de Salta (Rep. Argentina).
Ameghiniana, 1, 3-7.

Reig, O.A. (1960) Les relaciones genéricas del Anuro chileno Calyptocephalella gayi (Dum. \& Bibr.). Actas y Trabajos del primer Congreso sudamericano de Zoología (La Plata, 12-24 octubre 1959), Universidad nacional de La Plata, La Plata, 4 , 113-131. [1960.ra].

Reig, O.A. (1961) Noticia sobre un nuevo Anuro fósil del Jurásico de Santa Cruz (Patagonia). Ameghiniana, 2 (5), 73-78. [1961. ra].

Reig, O.A. (1972) Macrogenioglottus and the South American bufonid toads. In: Blair, W.F. (Ed.), Evolution in the genus Bufo. University of Texas Press, Austin and London, pp. 1436. [1972.ra].

Reig, O.A. \& Limeses, C.E. (1963) Un nuevo género de Anuros Ceratofrínidos del distrito chaqueño. Physis, 24, 113-128. [1963.ra].

Rensch, B. (1947) Neuere Probleme der Abstammungslehre. Enke, Stuttgart, i-vi +407 pp.

Richards, C., Nussbaum, R. \& Raxworthy, C. (2000) Phylogenetic relationships within the Madagascan boophids and mantellids as elucidated by mitochondrial ribosomal genes. African Journal of Herpetology, 49, 23-32. https://doi.org/10.1080/21564574.2000.9650013

Richards, C.M., Carlson, B.M., Connelly, T.G., Rogers, S. \& Ashcraft, E. (1977) A scanning electron microscopic study of differentiation of the digital pad in regenerating digits of the Kenyan reed frog, Hyperolius viridiflavus ferniquei. Journal of Morphology, 153, 387-396. [1977.ra].

https://doi.org/10.1002/jmor.1051530305

Riley, M.D. \& China, W.E. (1963) Opinion 649. Ambystoma Tschudi, 1838 (Amphibia); validation under the plenary powers. Bulletin of zoological Nomenclature, 20 (2), 102 104. [1963.rb].

https://doi.org/10.5962/bhl.part.6587

Risch, J.-P. (1984) Brève diagnose de Paradactylodon, genre nouveau d'Urodèle de l'Iran (Amphibia, Caudata, Hynobiidae). Alytes, 3 (1), 44-46.

Ritgen, F.A. (1828) Versuch einer natürlichen Eintheilung der Amphibien. Nova Acta physico-medica Academiae Caesareae Leopoldino-Carolinae Naturae Curiosorum, 14, 245-284. [1828.ra].

Rivera, J., Kraus, F., Allison, A. \& Butler, M. (2017) Molecular phylogenetics and dating of the problematic New Guinea microhylid frogs (Amphibia: Anura) reveals elevated speciation rates and need for taxonomic reclassification. Molecular Phylogenetics and Evolution, 112, 1-11. https://doi.org/10.1016/j.ympev.2017.04.008

Rivero, J.A. (1961) Salientia of Venezuela. Bulletin of the Museum of comparative Zoology, 126, 1-207.

Roberts, D., Horwitz, P., Wardell-Johnson, G., Maxson, L. \& Mahony, M. (1997) Taxonomy, relationships and conservation of a new genus and species of myobatrachid frog from the high rainfall region of southwestern Australia. Copeia, 1997, 373-381.

https://doi.org/10.2307/1447757

Robovská-Havelková, P. (2010) How can ontogeny help us to 
understand the morphology of anuran pectoral girdle? Zoomorphology, 129, 121-132.

https://doi.org/10.1007/s00435-010-0105-9

Roček, Z. (1981) Cranial anatomy of frogs of the family Pelobatidae Stannius, 1856, with outlines of their phylogeny and systematics. Acta Universitatis Carolinae, (Biologica), 1980 (1-2), 1-164. \{P15 J [1981.ra].

Roček, Z. \& Nessov, L.A. (1991) Cretaceous Anurans from central Asia. In: Korsós, Z. \& Kiss, I. (Eds.), Abstract of the $6^{\text {th }}$ General Meeting of the Societas Europaea Herpetologica, Budapest, Hungary, 1991, pp. 78. [1991.ra].

Rödel, M.-O. (2000) Herpetofauna of West Africa. Vol. 1. Amphibians of the West African savanna. Edition Chimaira, Frankfurt am Main, 332 pp.

Rödel, M.-O., Kosuch, J., Grafe, T.U., Boistel, R., Assemian, N.E., Kouamé, N.G., Tohé, B., Gourène, G., Perret, J.-L., Henle, K., Tafforeau, P., Pollet, N. \& Veith, M. (2009) A new tree-frog genus and species from Ivory Coast, West Africa. Zootaxa, 2044 (1), 23-45.

https://doi.org/10.11646/zootaxa.2044.1.2

Rödel, M., Schmitz, A., Pauwels, O.S.G. \& Böhme, W. (2004) Revision of the genus Werneria Poche, 1903, including the descriptions of two new species from Cameroon and Gabon (Amphibia: Anura: Bufonidae). Zootaxa, 720 (1), 1-28. https://doi.org/10.11646/zootaxa.720.1.1

Rodendorf, B.B. (1977a) O ratsionalizatsii nazvanyi taksonov visokogo ranga $\mathrm{v}$ zoologii. Paleontologicheskij Zhurnal, 1977 (2), 14-22.

Rodendorf, B.B. (1977b) The rationalization of names of higher taxa in zoology. Paleontological Journal, 11, 149-155.

Roelants, K. \& Bossuyt, F. (2005) Archaeobatrachian paraphyly and pangaean diversification of crown-group frogs. Systematic Biology, 54, 111-126.

https://doi.org/10.1080/10635150590905894

Roelants, K., Gower, D.J., Wilkinson, M., Loader, S.P., Biju, S.D., Guillaume, K., Moriau, L. \& Bossuyt, F. (2007) Global patterns of diversification in the history of modern amphibians. Proceedings of the national Academy of Sciences of the United States of America, 104, 887-892. \{Q\} [2007. ra]. https://doi.org/10.1073/pnas.0608378104

Roelants, K., Jiang, J. \& Bossuyt, F. (2004) Endemic ranid (Amphibia: Anura) genera in southern mountain ranges of the Indian subcontinent represent ancient frog lineages: evidence from molecular data. Molecular Phylogenetics and Evolution, 31, 730-740.

https://doi.org/10.1016/j.ympev.2003.09.011

Romer, A.S. (1933) Vertebrate paleontology. The University of Chicago Press, Chicago, i-vii +491 pp. [1933.ra].

https://doi.org/10.2307/1437149

Romer, A.S. (1945) Vertebrate paleontology. Second edition. The University of Chicago Press, Chicago, i-ix +687 pp. $\{Q\}$ [1945.ra].

Romer, A.S. (1966) Vertebrate paleontology. Third edition. The University of Chicago Press, Chicago \& London, i-ix +468 pp. $\{Q\}$ [1966.rb].
Rösel von Rosenhof, A.I. (1758) Historia naturalis ranarum nostratium. Nürnberg, Johann Joseph Fleischmann, [i-viii] + i-viii + 116 pp., pls. I-XXIV + fronton.

Rostand, J. (1963) Le droit d'être naturaliste. Stock, Paris, 213 pp. Rovito, S.M. \& Parra-Olea, G. (2016) Neotropical plethodontid biogeography: insights from molecular phylogenetics. Copeia, 104, 222-232. https://doi.org/10.1643/CH-14-190

Rovito, S.M., Parra-Olea, G., Recuero, E. \& Wake, D.B. (2015) Diversification and biogeographical history of neotropical plethodontid salamanders. Zoological Journal of the Linnean Society, 175, 167-188.

https://doi.org/10.1111/zoj.12271

Rowe, T., Cifelli, R.L., Lehman, T.M. \& Weil, A. (1992) The Campanian Terlingua local fauna, with a summary of other vertebrates from the Aguja Formation, Trans-Pecos Texas. Journal of Vertebrate Paleontology, 12, 472-493. [1992.ra]. https://doi.org/10.1080/02724634.1992.10011475

Rowley, J.J.L., Tran, D.T.A, Le, D.T.T., Hoang, H.D. \& Altig, R. (2012) The strangest tadpole: the oophagous, treehole dwelling tadpole of Rhacophorus vampyrus (Anura: Rhacophoridae) from Vietnam. Journal of natural History, 46, 2969-2978. https://doi.org/10.1080/00222933.2012.732622

Rowley, J.J.L., Dau, V.Q., Nguyen, T.A.O.T., Cao, T.T. \& Nguyen, S.V.A.N. (2011) A new species of Gracixalus (Anura: Rhacophoridae) with a hyperextended vocal repertoire from Vietnam. Zootaxa, 3125, 22-38.

Rowley, J.J.L., Le, T.T.D., Tran Thi Anh Dao, Stuart, B.L. \& Hoang, D.H. (2010) A new tree frog of the genus Rhacophorus (Anura: Rhacophoridae) from southern Vietnam. Zootaxa, 2727 (1), 45-55. https://doi.org/10.11646/zootaxa.2727.1.4

Ruggiero, M.A., Gordon, D.P., Orrell, T.M., Bailly, N., Bourgoin T., Brusca, R.C., Cavalier-Smith, T., Guiry, M.D. \& Kirk, P.M. (2015) A higher level classification of all living organisms. PloS One, 10 (4), e0119248, 1-60. [2015.ra]. https://doi.org/10.1371/journal.pone.0119248

Rüppell, E. (1845) Verzeichniss der in dem Museum der Senckenbergischen naturforschenden Gesellschaft aufgestellten Sammlungen. Dritte Abtheilung. Amphibien. Museum Senckenbergianum, 3, 293-316. [1845.ra].

Russell, B. (1953) The cult of 'common usage'. British Journal for the Philosophy of Science, 3 (12), 303-307.

https://doi.org/10.1093/bjps/III.12.303

Ruta, M. \& Coates, M. I. (2007) Dates, nodes and character conflict: addressing the lissamphibian origin problem. Journal of systematic Palaeontology, 5, 69-122. https://doi.org/10.1017/S1477201906002008

Ruta, M., Coates, M. \& Quicke, D. (2003) Early tetrapod relationships revisted. Biological Reviews of the Cambridge philosophical Society, 78, 251-345. https://doi.org/10.1017/S1464793102006103

Sá, R.O. de, Streicher, J., Sekonyela, R., Forlani, M., Loader, S., Greenbaum, E., Richards, S. \& Haddad, C. (2012) Molecular phylogeny of microhylid frogs (Anura: Microhylidae) with 
emphasis on relationships among New World genera. $B M C$ evolutionary Biology, 12 [241], 1-21.

https://doi.org/10.1186/1471-2148-12-241

Sá, R.O. de, Tonini, J.F.R., Huss, H. van, Long, A., Cuddy, T., Forlani, M.C., Peloso, P.L.V., Zaher, H. \& Haddad, C.F.B. (2018) Multiple connections between Amazonia and Atlantic Forest shaped the phylogenetic and morphological diversity of Chiasmocleis Mehely, 1904 (Anura: Microhylidae: Gastrophryninae). Molecular Phylogenetics and Evolution, 130, 198-210. [Published online on 19 October 2018, without pre-registration in Zoobank].

https://doi.org/10.1016/j.ympev.2018.10.021

Sá, R.O. de, Tonini, J.F.R., Huss, H. van, Long, A., Cuddy, T., Forlani, M.C., Peloso, P.L.V., Zaher, H. \& Haddad, C.F.B. (2019a) Multiple connections between Amazonia and Atlantic Forest shaped phylogenetic and morphological diversity in the genus Chiasmocleis Méhely, 1904 (Anura: Microhylidae: Gastrophryne). Molecular Phylogenetics and Evolution, 130, 198-210. [Published on paper in January 2019].

https://doi.org/10.1016/j.ympev.2018.10.021

Sá, R.O. de, Tonini, J.F.R., Huss, H. van, Long, A., Cuddy, T., Forlani, M.C., Peloso, P.L.V., Zaher, H. \& Haddad, C.F.B. (2019b) Corrigendum to: 'Multiple connections between Amazonia and Atlantic Forest shaped the phylogenetic and morphological diversity of Chiasmocleis Méhely, 1904 (Anura: Microhylidae: Gastrophryninae)'. Molecular Phylogenetics and Evolution, 132, 321. [Published on paper in March 2019].

https://doi.org/10.1016/j.ympev.2019.01.016

Sá, R.O. de, Tonini, J.F.R., Huss, H. van, Zaher, H. \& Haddad, C.F.B. (2019c) The unique traits of the subgenus Unicus within Chiasmocleis Méhely, 1094 [sic] (Anura, Microhylidae). Zootaxa, 4646 (3), 585-590. [Published online, after Zoobank preregistration, on 25 July 2019].

https://doi.org/10.11646/zootaxa.4646.3.8

Salvadori, T. (1888) Le date della pubblicazione della 'Iconografia della fauna Italica' del Bonaparte ed Indice delle specie illustrate in detta opera. Bollettino dei Musei di Zoologia ed Anatomia comparata della reale Università di Torino, 3 (48), 1-25. [1888].

San Mauro, D. (2010) A multilocus timescale for the origin of extant amphibians. Molecular Phylogenetics and Evolution, $56,554-561$.

https://doi.org/10.1016/j.ympev.2010.04.019

San Mauro, D., Gower, D., Müller, H., Loader, S., Zardoya, R., Nussbaum, R. \& Wilkinson, M. (2014) Life-history evolution and mitogenomic phylogeny of caecilian amphibians. Molecular Phylogenetics and Evolution, 73, 177-189. https://doi.org/10.1016/j.ympev.2014.01.009

San Mauro, D., Vences, M., Alcobendas, M., Zardoya, R. \& Meyer, A. (2005) Notes and comments. Initial diversification of living amphibians predated the breakup of Pangaea. The American Naturalist, 165, 590-599.

https://doi.org/10.1086/429523

Sanchíz, B. (1984) Análisis filogenético de la tribu Alytini (Anura, Discoglosidae) mediante el estudio de su morfoestructura ósea.
In: Hemmer, H. \& Alcover, J.A. (Eds.), Història biològica del ferreret. Moll, Mallorca, pp. 61-108. [1984.sa].

Sanchiz, B. (1998) Salientia. Handbuch der Paläoherpetologie, Dr. Friedrich Pfeil, München, 4, i-xii + 275 pp. \{Q\} [1998].

Sanders, O. (1973) A new leopard frog (Rana berlandieri brownorum) from Southern Mexico. Journal of Herpetology, 7, 87-92. https://doi.org/10.2307/1563205

Sanderson, M.J., McMahon, M.M. Stamatakis, A., Zwickl, D.J. \& Steel, M. (2015) Impacts of terraces on phylogenetic inference. Systematic Biology, 64 (5), 709-726. https://doi.org/10.1093/sysbio/syv024

Sanderson, M.J., McMahon, M.M. \& Steel, M. (2011) Terraces in phylogenetic tree space. Science, 333 (6041), 448-450. https://doi.org/10.1126/science.1206357

Santamaría, R. \& Therón, R. (2009) Treevolution: visual analysis of phylogenetic trees. Bioinformatics, 25 (15), 1970-1971. https://doi.org/10.1093/bioinformatics/btp333

Santos, J.C., Coloma, L.A., Summers, K., Caldwell, J.P., Ree, R. \& Cannatella, D.C. (2009) Amazonian amphibian diversity is primarily derived from Late Miocene Andean lineages. PloS Biology, 7 (e1000056), 448-461.

https://doi.org/10.1371/journal.pbio. 1000056

Sarania, B., Devi, A., Kumar, A., Wang, K. \& Rakshit, K. (2015) A record of Scutiger nyingchiensis Fei, 1977 (Amphibia: Anuran [sic]: Megophryidae) in the Eastern Himalaya, North East India. Current Science, 109 (3), 413-414. [2015.sa].

Sarasin, P. \& Sarasin, F. (1887) Zur Entwicklungsgeschichte und Anatomie der ceylonesischen Blindwühle Ichthyophis glutinosus, L. Erster Theil. In: Ergebnisse naturwissenschaftlicher Forschungen auf Ceylon, Kreidel, Wiesbaden, Zweiter Band, 40 pp., pls. 1-5. [1887.sa]. https://doi.org/10.5962/bhl.title.112354

Sarasin, P. \& Sarasin, F. (1890) Zur Entwicklungsgeschichte und Anatomie der ceylonesischen Blindwühle Ichthyophis glutinosus, L. Vierter Theil. In: Ergebnisse naturwissenschaftlicher Forschungen auf Ceylon, Kreidel, Wiesbaden, Zweiter Band, pp. 151-263, pls. 15-24. [1890. $\mathrm{sa}$.

Sarkar, A.K. (1984) Taxonomic and ecological studies on the amphibians of Calcutta and its environs. Records of the zoological Survey of India, 81, 215-236.

Sauvage, E. (1885) Les Reptiles et les Batraciens. In: Brehm, A.E. (Ed), Merveilles de la Nature. Librairie J.-B. Baillière et fils, Paris, pp. $[\mathrm{i}-\mathrm{v}]+1-726 .\{\mathrm{Q}\}$.

Savage, J.M. (1972) The Harlequin frogs, genus Atelopus, of Costa Rica and Western Panama. Herpetologica, 28, 77-94.

Savage, J.M. (1973) The geographic distribution of frogs: patterns and predictions. In: Vial, J.L. (Ed.), Evolutionary biology of the anurans. University of Missouri Press, Columbia, Missouri, pp. 351-445. \{Q\} [1973.sa].

Savage, J.M. (2002) The amphibians and reptiles of Costa Rica: a herpetofauna between two continents, between two seas. University of Chicago Press, Chicago, i-xx $+934 \mathrm{pp}$.

Savage, J.M. \& Carvalho, A.L. de (1953) The family position of Neotropical frogs currently referred to the genus Pseudis. 
Zoologica, 38, 193-200. [1953.sa].

Säve-Söderbergh, G. (1935) On the dermal bones of the head in labyrinthodont stegocephalians and primitive Reptilia. Meddelelser om Grønland, 98 (3), 1-211, pls. 1-15. [1935. sa].

Savi, P. (1823) Sopra la Salamandra perspicillata. Nuovo Giornale dei Letterati, Pisa, 4 (7), 104-112, 1 pl.

Schaefer, W.W. (1976) The reality of the higher taxonomic categories. Zeitschrift für zoologische Systematik und Evolutionsforschung, 14, 1-10.

https://doi.org/10.1111/j.1439-0469.1976.tb00513.x

Schaeffer, I.C. (1760) De studii ichthyologici faciliori ac tutiori methodo. Typis Weissianis et impensis Montagii, Ratisbonae, 24 pp., 1 pl. [1760.sa].

Scherz, M.D., Hawlitschek, O., Andreone, F., Rakotoarison, A., Vences, M. \& Glaw, F. (2017) A review of the taxonomy and osteology of the Rhombophryne serratopalpebrosa species group (Anura: Microhylidae) from Madagascar, with comments on thevalue of volume rendering of micro-CT data to taxonomists. Zootaxa, 4273 (3), 301-340.

https://doi.org/10.11646/zootaxa.4273.3.1

Scherz, M.D., Hutter, C.R., Rakotoarison, A., Riemann, J.C., Rödel, M.-O., Ndriantsoa, S.H., Glos, J., Hyde Roberts, S., Crottini, A., Vences, M. \& Glaw, F. (2019) Morphological and ecological convergence at the lower size limit for vertebrates highlighted by five new miniaturised microhylid frog species from three different Madagascan genera. PloS One, 14 (e0213314), 1-45.

https://doi.org/10.1371/journal.pone.0213314

Schinz, H.R. (1833) Naturgeschichte und Abbildungen der Reptilien. Brodtmann, Schaffhausen, [i-iii] + i-iv +240 pp., pls. 1-102. [1833.sa].

Schiøtz, A. (1999) Treefrogs of Africa. Chimaira, Frankfurt am Main, 1-350.

Schlegel, H. (1850) Description of a new genus of Batrachians from Swan River. Proceedings of the zoological Society of London, 1850, 9-10. \{P07 J [1850.sa].

Schmidt, O. (1857) Diagnosen neuer Frösche des zoologischen Cabinets zu Krakau. Sitzungsberichte der kaiserlichen Akademie der Wissenschaften, MathematischNaturwissenschaftiche Classe, 24, 10-15.

Schmidt, O. (1858) Deliciae herpetologicae Musei zoologici Cracoviensis. Beschreibung der im k. k. Museum du Krakau befindlichen, von J.v. Warszewicz in Neu-Granada und Bolivia gesammelten ungeschwänzten Batrachier. Denkschriften der kaiserlichen Akademie der Wissenschaften, MathematischNaturwissenschaftliche Classe, 14, 237-258.

Schoch, R.R. (2009) Evolution of life cycles in early amphibians. Annual Review of Earth and planetary Sciences, 37, 135162. https://doi.org/10.1146/annurev.earth.031208.100113

Schreiber, E. (1875) Herpetologia europaea. Friedrich Vieweg \& Sohn, Braunschweig, i-xvii + 1-639. [1875.sa].

Schulze, E. (1891) Amphibia europaea. Jahresbericht und Abhandlungen des Naturwissenschaftlivchen Vereins in Magdeburg, 1889-1890, 163-178. [1891.sa].
Scopoli, I.A. (1777) Introductio ad historiam naturalem, sistens genera lapidum, plantarum, edt animalium hactenus detecta, caracteribus essentialibus donata, in tribus divisa, subinde ad leges naturae. Gerle, Pragae, $[\mathrm{i}-\mathrm{x}]+506$ pp. + i-xxxvi. [1777.sa]. https://doi.org/10.5962/bhl.title.10827

Scott, E. (2005) A phylogeny of ranid frogs (Anura: Ranoidea: Ranidae), based on a simultaneous analysis of morphological and molecular data. Cladistics, 21, 507-574. [2005.sa]. https://doi.org/10.1111/j.1096-0031.2005.00079.x

Seshadri, K.S., Gururaja, K.V. \& Aravind, N.A. (2012) A new species of Raorchestes (Amphibia: Anura: Rhacophoridae) from mid-elevation evergreen forests of the southern Western Ghats, India. Zootaxa, 3410 (1), 19-34.

https://doi.org/10.11646/zootaxa.3410.1.2

Shaw, G. (1802) General zoology, or systematic natural history. Vol. III, part I. Amphibia. London, Kearsley, i-viii + 312 pp., pls. $1-86$.

Shear, W. (2011) Class Diplopoda de Blainville in Gervais, 1844. In: Zhang, Z.-Q. (Ed.), Animal biodiversity: an outline of higher-level classification and survey of taxonomic richness. Zootaxa, 3148 (1), 159-164.

https://doi.org/10.11646/zootaxa.3148.1.32

Shen, X.-X., Liang, D., Chen, M.-Y., Mao, R.-L., Wake, D.B. \& Zhang, P. (2016) Enlarged multilocus data set provides surprisingly younger time of origin for the Plethodontidae, the largest family of Salamanders. Systematic Biology, '2015', $65,66-81$.

https://doi.org/10.1093/sysbio/syv061

Shen, X.-X., Liang, D., Feng, Y., Chen, M. \& Zhang, P. (2013) A versatile and highly efficient toolkit including 102 nuclear markers for Vertebrate phylogenomics, tested by resolving the higher level relationships of the Caudata. Molecular Biology and Evolution, 30, 2235-2248.

https://doi.org/10.1093/molbev/mst122

Sherborn, C.D. (1914) Attempt at a fixation of the dates of issue of the parts of the publications of the Musée d'Histoire Naturelle of Paris, 1802-1850. Annals and Magazine of natural History, (8), 13, 365-368. [1914]. https://doi.org/10.1080/00222931408693496

Sherborn, C.D. (1934) Dates of publication of catalogues of natural history (post 1850) issued by the British Museum. Annals and Magazine of natural History, (10), 13, 308-312. [1934].

https://doi.org/10.1080/00222933408654812

Shipunov, A. (2011) The problem of hemihomonyms and the on-line hemihomonyms database (HHDB). Bionomina, 4, 65-72. https://doi.org/10.11646/bionomina.4.1.3

Shubin, N.H. \& Jenkins, F.A. (1995) An early Jurassic jumping frog. Nature, 377, 49-52. [1995.sa]. https://doi.org/10.1038/377049a0

Sibley, C.G. \& Ahlquist, J.E. (1982) The relationships of the yellowbreasted chat (Icteria virens) and the alleged slowdown in the rate of macromolecular evolution in birds. Postilla, 187, $1-19$.

Sibley, C.G. \& Ahlquist, J.E. (1990) Phylogeny and classification of birds. A study in molecular evolution. Yale University 
Press, New Haven \& London, 999 pp.

https://doi.org/10.2307/j.ctt1xp3v3r

Sigurdsen, T. \& Green, D. M. (2011) The origin of modern amphibians: a re-evaluation. Zoological Journal of the Linnean Society, 162, 457-469. https://doi.org/10.1111/j.1096-3642.2010.00683.x

Silverstone, P.A. (1975) A revision of the poison-arrow frogs of the genus Dendrobates Wagler. Science Bulletin of natural History Museum of Los Angeles City, 21, 1-55.

Simpson, G.G. (1940) Types in modern taxonomy. American Journal of Science, 238, 413-431. https://doi.org/10.2475/ajs.238.6.413

Simpson, G.G. (1961) Principles of animal taxonomy. Columbia University Press, New York, i-xii +247 pp. https://doi.org/10.7312/simp92414

Siu-Ting, K., Gower, D.J., Pisani, D., Kassahun, R., Gebresenbet, F., Menegon, M., Mengistu, A.A., Saber, S.A., Sá, R. de, Wilkinson, M. \& Loader, S.P. (2014) Evolutionary relationships of the Critically Endangered frog Ericabatrachus baleensis Largen, 1991 with notes on incorporating previously unsampled taxa into large-scale phylogenetic analyses. $B M C$ evolutionary Biology, 14 [44], 1-24.

https://doi.org/10.1186/1471-2148-14-44

Siu-Ting, K., Torres-Sánchez, M., San Mauro, D., Wilcockson, D., Wilkinson, M., Pisani, D., O’Connell, M.J, \& Creevey, C.J. (2019) Inadvertent paralog inclusion drives artifactual topologies and timetree estimates in phylogenomics. Molecular Biology and Evolution, 36 (6), 1344-1356. https://doi.org/10.1093/molbev/msz067

Slipinski, S. A., R. Leschen, A. B. \& Lawrence, J. F. (2011) Order Coleoptera Linnaeus, 1758. In: Zhang, Z.-Q. (Ed.), Animal biodiversity: an outline of higher-level classification and survey of taxonomic richness. Zootaxa, 3148 (1), 203-208. https://doi.org/10.11646/zootaxa.3148.1.39

Skutschas, P.P. (2009) Re-evaluation of Mynbulakia Nesov, 1981 (Lissamphibia: Caudata) and description of a new salamander genus from the late Cretaceous of Uzbekistan. Journal of Vertebrate Paleontology, 29 (3), 659-664. [2009.sa]. https://doi.org/10.1671/039.029.0326

Smith, A. (1831) Contributions to the natural history of South Africa, \&c. South African quarterly Journal, 2 (5), 9-24. [1831.sa].

Smith, A.B. (1988) Patterns of diversification and extinction in early Palaeozoic echnioderms. Palaeontology, 31, 799-828.

Smith, H.M. (1959) Herpetozoa from Guatemala, I. Herpetologica, 15, 210-216.

Smith, H.M. \& Chiszar, D. (2006) Dilemma of name-recognition: why and when to use new combination of scientific names. Herpetological Conservation and Biology, 1 (1), 6-8.

Smith, H.M. \& Pérez-Higareda, G. (1986) Nomenclatural nameforms. Systematic Zoology, 35, 421-422. https://doi.org/10.2307/2413392

Smith, H.M. \& Polhemus, J. T. (1984) Caeciliidae in Amphibia and Insecta (Psocoptera): alternative proposals to remove the homonymy. Z.N.(S.) 2333. Bulletin of zoological Nomenclature, 41 (2), 108-109. [1984.sb].
Smith, H.M. \& Taylor, E. H. (1948) An annotated checklist and key to the Amphibia of Mexico. Bulletin of the United States national Museum, 194, i-iv + 1-118. [1948.sa]. https://doi.org/10.5479/si.03629236.194

Smith, H.M. \& Tihen, J. A. (1961) Tigrina (Salamandra) Green, 1825: proposed validation under the plenary powers (Amphibia, Caudata). Z.N.(S.)1460. Bulletin of zoological Nomenclature, 18, 214-216. [1961.sa].

Smith, M.A. (1924) New tree-frogs from Indo-China and the Malay Peninsula. Proceedings of the zoological Society of London, 1924, 225-235, 3 pls. https://doi.org/10.1111/j.1096-3642.1924.tb01499.x

Smith, M.A. (1927) Contributions to the herpetology of the Indo Australian region. Proceedings of the zoological Society of London, 1927, 199-225. https://doi.org/10.1111/j.1096-3642.1927.tb02255.x

Smith, M.A. (1939) XV. Amphibia and Reptilia. The zoological Record, 75, 1-45. [1939.sb].

Smith, S.A., Nieto, A., Oca, M. De, Reeder, T.W. \& Wiens, J.J. (2007) A phylogenetic perspective on elevational species richness patterns in Middle American treefrogs: why so few species in lowland tropical rainforests? Evolution, 61, 11881207. https://doi.org/10.1111/j.1558-5646.2007.00085.x

Smith, W.H. (1877) The Tailed Amphibians, including the Caecilians. A thesis presented to the Faculty of Michigan University. Herald Publishing House, Detroit, 158 pp., 6 tabs. [1877.sa]. https://doi.org/10.5962/bhl.title.53698

Sneath, P.H.A. (1962) The construction of taxonomic groups. In: Ainsworth, G.C. \& Sneath, P.H.A. (Eds.), Microbial classification, Twelfth Symposium of the Society for General Microbiology held at the Royal Institution, London, April 1962. Vol. 12. Cambridge University Press, Cambridge, pp. 289-332.

Sokol, O.M. (1977) A subordinal classification of frogs (Amphibia: Anura). Journal of Zoology, London, 182, 505-508. [1977. sa]. https://doi.org/10.1111/j.1469-7998.1977.tb04166.x

Sonnini, C.S. \& Latreille, P.A. (1801a) Histoire naturelle des Reptiles. Tome 1. Première partie. Quadrupèdes et Bipèdes Ovipares. Deterville, Paris, [i-iii] + i-xxii + 332 pp., 14 pls.

Sonnini, C.S. \& Latreille, P.A. (1801b) Histoire naturelle des Reptiles. Tome 2. Première partie. Quadrupèdes et Bipèdes Ovipares. Deterville, Paris, [i-iii] + 332 pp., 19 pl.

Sonnini, C.S. \& Latreille, P.A. (1801c) Histoire naturelle des Reptiles. Tome 3. Seconde partie. Serpens. Deterville, Paris, [i-iii] +335 pp., 5 pl., 1 tab.

Sonnini, C.S. \& Latreille, P.A. (1801d) Histoire naturelle des Reptiles. Tome 4. Seconde partie. Serpens. Deterville, Paris, [i-iii] +410 pp., 14 pls. [1801.sa].

Spadola, F. \& Insacco, G. (2010) Incomplete albinism in Discoglossus pictus (Otth, 1837). Acta herpetologica, 5 (2), 245-253. [2010.sa].

Špinar, Ž.V. (1972) Tertiary frogs from central Europe. Prague (Academia), 1-286, pls. 1-184. [1972.sa]. 
Špinar, Ž.V. (1976) Endolymphatic sacs and dorsal endocranial pattern: their significance for systematics and phylogeny of frogs. Věstnik Ústřredního Ústavu Geologického, 51, 285290. [1976.sa].

Špinar, Ž.V. (1979) Latonia kolebabi Špinar, 1976 (Amphibia) and remarks on the 'genus Miopelobates'. In: Paleonteologická Konference 1977, Univ. Karlova, Prague, Czechia, '1978', 289-303. [P13 J [1979.sa].

Špinar, Z.V. (1983) Paleogeography and its significance for the phylogeny of some European fossil frogs. Věstnik Ústředního ústavu geologického, 58 (1), 53-56. [1983.sa].

Špinar, Ž.V., Boubelik, M. \& Romanovsky, A. (1971) A contribution to the phylogeny of the family Pelobatidae (Anura). Acta Universitatis Carolinae, Geologica, 1971 (3), 279-285. [1971.sa].

Spix, J. de (1824) Animalia nova sive species novae Testudinum et Ranarum quas in itinere per Brasiliam annis MDCCCXVIIMDCCCXX jussu et auspiciis Maximiliani Josephi I Bavariae Regis suscepto collegit et descripsit Dr. J. B. de Spix. Hübschmann, Monachii, i-iii + 53 pp., pls. 1-22. [1824.sa]. https://doi.org/10.5962/bhl.title.5117

Stabler, R.M. \& Chen, T.-T. (1936) Observations on an Endamoeba parasitizing opalinid ciliates. Biological Bulletin, 70 (1), 5671. https://doi.org/10.2307/1537312

Stannius, H. (1856) Handbuch der Anatomie der Wirbelthiere. 2. Zootomie der Amphibien. In: Von Siebold C.T. \& Stannius, H., Handbuch der Zootomie, Vol. 1, i-vi + 270 pp. \{Q\} [1856. sa].

Starobogatov Y.I. (1984) O problemakh nomenklatury vysshikh taksonomicheskikh kategoriy. In: Tatarinov, L.P. \& Shimanskiy, V.N. (Eds.), Spravochnik po sistematike iskopayemykh organbizmov (taksony otryadnoy $i$ vyshchikh grupp). Izdatel'svo Nauka, Moscow, pp. 174-187. [In Russian; not seen; quoted after Starobogatov 1991].

Starobogatov, Y.I. (1991) Problems in the nomenclature of higher taxonomic categories. Bulletin of zoological Nomenclature, 48 (1), 6-18. [English translation of Starobogatov 1984]. https://doi.org/10.5962/bhl.part.663

Starrett, P.H. (1968) The phylogenetic significance of the jaw in anuran amphibians. Ph.D. Dissertation, University of Michigan, i-viii + 179 pp.

Starrett, P.H. (1973) Evolutionary patterns in larval morphology. In: Vial, J.L. (Ed.), Evolutionary biology of the anurans. University of Missouri Press, Columbia, Missouri, pp. 251271. [1973.sb].

Steindachner, F. (1867) Amphibien. In: Reise der Österreichischen Fregatte Novara um die Erde in den Jahren 1857, 1858, 1859 unter den Befehlen des Commodore B. von WüllerstorfUrbair, Zoologischer Theil, Wirbelthiere. 1 (4). KaiserlichKönigliche Hof- und Staatsdruckerei, Wien, pp. 1-70, pls. 1-5. [1867.sa].

Steinfartz, S., Vicario, S., Arntzen, J.W. \& Caccone, A. (2007) A Bayesian approach on molecules and behavior: reconsidering phylogenetic and evolutionary patterns of the Salamandridae with emphasis on Triturus newts. Journal of experimental
Zoology, 308B, 139-162.

https://doi.org/10.1002/jez.b.21119

Stejneger, L. (1907) Herpetology of Japan and adjacent territory. Bulletin of the United States national Museum, 58, i-xx + 577, pls. 1-35. [1907.sa]. https://doi.org/10.5479/si.03629236.58.i

Stejneger, L. (1910) The amphibian generic name Engystoma untenable. Proceedings of the biological Society of Washington, 23, 165-168.

Stejneger, L. \& Barbour, T. (1917) A check list of North American Amphibians and Reptiles. Harvard University Press, Cambridge, i-iv + 5-125 pp. [1917.sa]. https://doi.org/10.5962/bhl.title.54316

Stephenson, N.G. (1951) Observations on the development of the amphicoelous frogs, Leiopelma and Ascaphus. Journal of the Linnean Society of London, Zoology, 42, 18-28, pls. 1-3. [1951.sa]. https://doi.org/10.1111/j.1096-3642.1951.tb01851.x

Stipanicic, P.N. \& Reig, O.A. (1956) Breve noticia sobre el hallazgo de Anuros en el denominado 'Complejo porfírico de la Patagonia extraandina' con consideraciones acerca de la composición geológica del mismo. Revista de la Asociación geológica argentina, '1955', 10 (4), 215-233. [P11] [1956. sa].

Stöck, M., Fakharzadeh, F., Kuhl, H., Rozenblut-Kościsty, B., Leinweber, S., Patel, R., Ebrahimi, M., Voitel, S., Schmidtler, J., Kami, H.G., Ogielska, M. \& Förster, D. (2019) Shedding light on a secretive tertiary urodelean relict: hynobiid salamanders (Paradactylodon persicus s.1.) from Iran, illuminated by phylogeographic, developmental and transcriptomic data. Genes, 10 [306], 1-16. https://doi.org/10.3390/genes10040306

Stöck, M., Frynta, D., Grosse, W.-R., Steinlein, C. \& Schmid, M. (2001a) A review of the distribution of diploid, triploid and tetraploid Green toads (Bufo viridis complex) in Asia including new data from Pakistan. Asiatic herpetological Research, 9, 77-100. https://doi.org/10.5962/bhl.part.15562

Stöck, M., Günther, R. \& Böhme, W. (2001b) Progress towards a taxonomic revision of the Asian Bufo viridis group: Current status of nominal taxa and unsolved problems (Amphibia: Anura: Bufonidae). Zoologische Abhandlungen des Staatlichen Museums für Tierkunde Dresden, 51, 253-319.

Stöck, M., Sicilia, A., Belfiore, N.M., Buckley, D., Lo Brutto, S, Lo Valvo, M. \& Arculeo, M. (2008) Post-Messinian evolutionary relationships across the Sicilian channel: mitochondrial and nuclear markers link a new green toad from Sicily to African relatives. BMC evolutionary Biology, 19 (56), 1-19. https://doi.org/10.1186/1471-2148-8-56

Strauch, A. (1870) Revision der Salamandriden-Gattungen nebst beschreibung einiger neuen und weniger bekannten Arten dieser Familie. Mémoires de l'Académie impériale des Sciences de Saint Pétersbourg, (7) 16 (4), 1-110. [1870.sa].

Strecker, J.K. (1909) Notes on the narrow-mouthed toads (Engystoma) and the description of a new species from southeastern Texas. Proceedings of the biological Society of 
Washington, 22, 115-120.

Streicher, J.W., Miller, E.C., Guerrero, P.C., Correa, C., Ortiz, J.C., Crawford, A.J., Pie, M.R. \& Wiens, J.J. (2018) Evaluating methods for phylogenomic analyses, and a new phylogeny for a major frog clade (Hyloidea) based on 2214 loci. Molecular Phylogenetics and Evolution, 119, 128-143. [2017.sa]. https://doi.org/10.1016/j.ympev.2017.10.013

Stuart, B.L., Bain, R.H., Phimmachak, S. \& Spence, K. (2010) Phylogenetic systematics of the Amolops monticola group (Amphibia: Ranidae), with description of a new species from northwestern Laos. Herpetologica, 66, 52-66. https://doi.org/10.1655/08-073.1

Stuart, S.N., Chanson, J.S., Cox, N.A., Young, B.E., Rodrigues, A.S.L., Fischman, D.L. \& Waller, R.W. (2004) Status and trends of amphibian declines and extinctions worldwide. Science, 306, 1783-1786.

https://doi.org/10.1126/science.1103538

Stuart, S.N., Hoffmann, M., Chanson, J., Cox, N., Berridge, R., Ramani, P. \& Young, B. (Ed.) (2008) Threatened Amphibians of the world. Lynx Editions, Barcelona, $\mathrm{i}-\mathrm{xv}+758 \mathrm{pp}$. $\{\mathrm{Q}\}$.

Sullivan, R.M. \& Lucas, S.G. (2015) Cretaceous Vertebrates of New Mexico. In: Lucas, S.G. \& Sullivan, R.M. (Eds.), Fossil Vertebrates in New Mexico, New Mexico Museum of natural History and Science Bulletin, 68, 105-130. [2015.sb].

Sundberg, P. \& Pleijel, F. (1994) Phylogenetic classification and the definition of taxon names. Zoologica scripta, 23, 19-25. https://doi.org/10.1111/j.1463-6409.1994.tb00369.x

Sunyer, J., Wake, D.B., Townsend, J.H., Travers, S.L., Rovito, S.M., Papenfuss, T.J., Obando, L.A. \& Köhler, G. (2010) A new species of worm salamander (Caudata: Plethodontidae: Oedipina) in the subgenus Oeditriton from the highlands of northern Nicaragua. Zootaxa, 2613 (1), 29-39. https://doi.org/10.11646/zootaxa.2613.1.3

Suwannapoom, C., Sumontha, M., Tunprasert, J., Ruangsuwan, T., Pawangkhanant, P., Korost, D. V. \& Poyarkov, N.A. (2018) A striking new genus and species of cave-dwelling frog (Amphibia: Anura: Microhylidae: Asterophryinae) from Thailand. PeerJ, 6 (e4422), 1-42. https://doi.org/10.7717/peerj.4422

Swainson, W. (1839) The natural history of Fishes, Amphibians, \& Reptiles, or Monocardian Animals. Vol. 2. Longman \& Co., London, i-vi + 452 pp. [1839.sa]. https://doi.org/10.5962/bhl.title.62140

Taboada, C., Grant, T., Lynch, J. \& Faivovich, J. (2013) New morphological synapomorphies for the New World direct-developing frogs (Amphibia: Anura: Terrarana). Herpetologica, 69, 342-357. https://doi.org/10.1655/HERPETOLOGICA-D-13-00019

Tan, J., Wang, Y., Ren, D. \& Yang, X. (2012) New fossil species of ommatids (Coleoptera: Archostemata) from the Middle Mesozoic of China illuminating the phylogeny of Ommatidae. BMC evolutionary Biology, 12 (113), 1-19 + 3 additional files. [doi:10.1186/1471-2148-12-113]. [Online document not preregistered in Zoobank].

http://www.parasitesandvectors.com/content/pdf/1756-33054-229.pdf
Tancoigne, E. \& Dubois, A. (2013) Taxonomy: no decline, but inertia. Cladistics, 29 (5), 567-570.

https://doi.org/10.1111/cla.12019

Tandy, M. \& Keith, R. (1972) Bufo of Africa. In: Blair, W.F. (Ed.), Evolution in the genus Bufo, University of Texas Press, Austin, Texas, pp. 119-170.

Tapley, B., Michaels, C.J., Gumbs, R., Böhm, M., Luedtke, J., Pearce-Kelly, P. \& Rowley, J.J.L. (2018) The disparity between species description and conservation assessment: a case study in taxa with high rates of species discovery. Biological Conservation, 220, 209-214. https://doi.org/10.1016/j.biocon.2018.01.022

Tardieu, C. (2011) La bonne orthographe du mot taxinomie. Paleo, $22,331-334$

https://doi.org/10.4000/paleo.2201

Targino, M. \& Pombal Jr., J.P. (2011) Redescription and variation of Hyophryne histrio Carvalho, 1954, an enigmatic microhylid frog from the Atlantic Rainforest of Brazil. Amphibia-Reptilia, 32, 465-475.

https://doi.org/10.1163/156853811X598488

Tarkhnishvili, D. (1994) Amphibian communities of the southern Viet Nam: preliminary data. Journal of Bengal natural History Society, 13, 3-62.

Tatarinov, L.P. (1964a) Надотряд Salientia. Прыгающие, или бесхвостые. [Superorder Salientia. Jumping or tailless amphibians]. In: Rojdestvenskii, A.K. \& Tatarinov, L.P., Земноводные, Пресмыкающиеся и Птицы [Amphibians, Reptiles and Birds]. In: Orlov, J.A. (Ed.), Основы Палеонтологии [Fundamentals of Paleontology]. Vol. 12. (Издательство 'Наука'), Moskva pp. 125-133. [In Russian] \{Q\} [1964.ta].

Tatarinov, L.P.(1964b)Подкласc Lepospondyli. Лепоспондильные [Subclass Lepospondyli. Lepospondyls]. In: Rojdestvenskii, A.K. \& Tatarinov, L.P. Земноводные, Пресмыкающиеся и Птицы [Amphibians, Reptiles and Birds]. In: Orlov, J.A. (Ed.), Основы Палеонтологии [Fundamentals of Paleontology]. Vol. 12. (Издательство 'Наука'), Moskva, pp. 144-190. [In Russian]. [1964.tb].

Taylor, E.H. (1939) New species of Mexican tailless Amphibia. Kansas University Science Bulletin, 25, 385-405. https://doi.org/10.5962/bhl.part.1706

Taylor, E.H. (1942) New Caudata and Salientia from Mexico. University of Kansas Science Bulletin, 28, 295-323.

Taylor, E.H. (1944) The genera of plethodont salamanders in Mexico, pt. I. The University of Kansas Science Bulletin, 30, 189-232.

https://doi.org/10.5962/bhl.part.1706

Taylor, E.H. (1951) Two new genera and a new family of tropical American frogs. Proceedings of the biological Society of Washington, 64, 33-40. [1951.ta].

Taylor, E.H. (1960) On the caecilian species Ichthyophis monochrous and Ichthyophis glutinosus with descriptions of related species. The University of Kansas Science Bulletin, 40, 37-120.

https://doi.org/10.5962/bhl.part.18735

Taylor, E.H. (1962) The amphibian fauna of Thailand. The 
University of Kansas Science Bulletin, 43, 265-600. https://doi.org/10.5962/bhl.part.13347

Taylor, E.H. (1968) The Caecilians of the World. A taxonomic review. University of Kansas Press, Lawrence, i-xiv +848 pp. $\{Q\}$ [1968.ta].

Taylor, E.H. (1969) A new family of African Gymnophiona. The University of Kansas Science Bulletin, 48, 297-305. [1969. ta]. https://doi.org/10.5962/bhl.part.11226

Thorn, R. (1966) Salamandres et tritons. Aperçu sur l'ordre des Urodèles, avec quelques considérations sur la phylogénie de ces animaux. Bulletin de la Société des Naturalistes luxembourgeois, '1964', 69, 101-111. [P12] [1966.ta].

Thorn, R. (1969) Les salamandres d'Europe, d'Asie et d'Afrique $d u$ Nord. Description et mours de toutes les espèces et sousespèces d'Urodèles de la région paléarctique d'après l'état de 1967. Encyclopédie pratique du Naturaliste, Paul Lechevalier, Paris '1968' 35, i-iv + 376 pp.

Tian, W. \& Hu, Q. (1985) Taxonomical studies on the primitive anurans of the Hengduan mountains, with descriptions of a new subfamily and subdivision of Bombina. Acta herpetologica sinica, (2) 4 (3), 219-224. [In Chinese]. [1985.ta].

Tihen, J.A. (1958) Comments on the osteology and phylogeny of ambystomatid salamanders. Bulletin of the Florida State Museum, Biological Sciences, 3 (1), 1-50. [1958.ta].

Tilak, R. \& Mehta, H.S. (1977) Report on a collection of Amphibians from district Kangra, Himachal Pradesh. Newsletter of the zoological Survey of India, 3 (4), 196-198. [1977.ta].

Tissier, J., Rage, J.-C., Boistel, R., Fernandez, V., Pollet, N., Garcua, G. \& Laurin, M. (2015) Synchrotron analysis of a 'mummified' salamander (Vertebrata: Caudata) from the Eocene of Quercy, France. Zoological Journal of the Linnean Society Linnean Society, 177, 147-164.

https://doi.org/10.1111/zoj.12341

Titus, T.A. \& Larson, A. (1995) A molecular phylogenetic perspective on the evolutionary radiation of the salamander family Salamandridae. Systematic Biology, 44, 125-151. https://doi.org/10.2307/2413703

Tonini, J.F.R., Beard, K.H., Ferreira, R.B., Jetz, W. \& Pyron, R.A. (2016) Fully-sampled phylogenies of squamates reveal evolutionary patterns in threat status. Biological Conservation, 204, 23-31.

https://doi.org/10.1016/j.biocon.2016.03.039

Tonini, J.F.R., Forlani, M.C. \& de Sa, R.O. (2014) A new species of Chiasmocleis (Microhylidae, Gastrophryninae) from the Atlantic Forest of Espirito Santo State, Brazil. Zookeys, 428, 109-132. https://doi.org/10.3897/zookeys.428.7352

Troschel, F.H. (1848) Amphibien. In: R. Schomburgk, Reisen in Britisch-Guiana in den Jahren 1840-44, Theil 3, Versuch einer Zusammenstellung der Fauna und Flora von BritischGuiana, Leipzig (J. J. Weber), pp. 645-661. [1848.ta].

Trueb, L. (1970a) Evolutionary relationships of casque-headed tree frog with co-ossified skulls (family Hylidae). University of Kansas Publications, Museum of natural History, 18, 547716. https://doi.org/10.5962/bhl.part.19992

Trueb, L. (1970b) The generic status of Hyla siemersi Mertens. Herpetologica, 26, 254-267.

https://doi.org/10.2307/1442303

Trueb, L. (1971) Phylogenetic relationships of certain neotropical toads with the description of a new genus (Anura: Bufonidae). Contributions in Science, Natural History Museum of Los Angeles County, 216, 1-40.

Trueb, L. \& Cloutier, C. (1991) A phylogenetic investigation of the inter- and intrarelationships of the Lissamphibia (Amphibia: Temnospondyli). In: Schultze, H.-P. \& Trueb, L. (Eds.), Origins of the higher groups of tetrapods: controversy and consensus, Cornell University Press, Ithaca \& New York, pp. 223-313. [1991.ta].

https://doi.org/10.7591/9781501718335-010

Trueb, L. \& Tyler, M.J. (1974) Systematics and evolution the Greater Antillean hylid frogs. Occasional Papers of the Museum of natural History, University of Kansas, 24, 1-60.

https://doi.org/10.5962/bhl.part.11750

Tschudi, J.J. (1838) Classification der Batrachier, mit Berücksichtigung der fossilen Thiere dieser Abtheilung der Reptilien. Petitpierre, Neuchâtel, 98 pp., pls. 1-6+[i-ii]. \{Q\} [1838.ta]. https://doi.org/10.5962/bhl.title.4883

Tschudi, J.J. de (1845a) Reptilium conspectus quae in Republica Peruana reperiuntur et pleraque observata vel collecta sunt in itinerere a Dr. J. J. de Tschudi. Archiv für Naturgeschichte, 11 (1), 150-170. [1845.ta]. https://doi.org/10.5962/bhl.part.7963

Tschudi, J.J. von (1845b) Untersuchungen über die Fauna Peruana. Herpetologie. Druck und Verlag Scheitlin und Zollikofer, Saint Gallen, 80 pp., pls. 1-12. [1845.tb].

Tu, N., Yang, M., Liang, D. \& Zhang, P. (2018) A large-scale phylogeny of Microhylidae inferred from a combined dataset of 121 genes and 427 taxa. Molecular Phylogenetics and Evolution, 126, 85-91.

https://doi.org/10.1016/j.ympev.2018.03.036

Turbott, E.G. (1942) The distribution of the genus Leiopelma in New Zealand with a description of a new species. Transactions and Proceedings of the royal Society of New Zealand, 71, 247253, pl. 42. [1942.ta].

Turland, N.J., Wiersema, J.H., Barrie, F.R., Greuter, W., Hawksworth, D.L., Herendeen, P.S., Knapp, S., Kusber, W.-H., Li, D.-Z., Marhold, K., May, T.W., McNeill, J., Monro, A.M., Prado, J., Price, M.J. \& Smith, G.F. (Ed.) (2018) International Code of nomenclature for algae, fungi, and plants (Shenzhen Code) adopted by the Nineteenth International Botanical Congress, Shenzhen, China, July 2017. Regnum Vegetabile. (Koeltz Botanical Book), Glashuitten, 159 pp. https://doi.org/10.12705/Code.2018

Turner, A.A. \& Channing, A. (2008) A new species of Arthroleptella Hewitt, 1926 (Anura: Pyxicephalidae) from the Klein Swartberg Mountain, Caledon, South Africa. African Journal of Herpetology, 57, 1-12.

https://doi.org/10.1080/21564574.2008.9635564

Twomey, E. \& Brown, J.L. (2008) Spotted Poison Frogs: rediscovery 
of a lost species and a new genus (Anura: Dendrobatidae) from northwestern Peru. Herpetologica, 64, 121-137. https://doi.org/10.1655/07-009.1

Twomey, E., Delia, J.R.J. \& Castroviejo-Fisher, S. (2014) A review of northern Peruvian glassfrogs (Centrolenidae), with the description of four new remarkable species. Zootaxa, 3851 (1), 1-87. https://doi.org/10.11646/zootaxa.3851.1.1

Tyler, S. \& Schilling, S. (2011) Phylum Xenacoelomorpha Philippe, et al., 2011. In: Zhang, Z.-Q. (Ed.), Animal biodiversity: an outline of higher-level classification and survey of taxonomic richness. Zootaxa, 3148 (1), 24-25. https://doi.org/10.11646/zootaxa.3148.1.6

Valdecasas, A. G., Williams, D. \& Wheeler, Q. D. (2008) ‘Integrative taxonomy' then and now: a response to Dayrat (2005). Biological Journal of the Linnean Society, 93, 211-216.

Valdecasas, A.G., Peláez, M.L. \& Wheeler, Q.D. (2014) What's in a (biological) name? The wrath of Lord Rutherford. Cladistics, 30, 215-223.

https://doi.org/10.1111/cla.12035

Tyler, M.J., Burton, T. \& Bauer, A.M. (2001) Parotoid or parotid: on the nomenclature of an Amphibian skin gland. Herpetological Review, 32, 79-81.

Van Bocxlaer, I., Biju, S.D., Loader, S.P. \& Bossuyt, F. (2009) Toad radiation reveals into-India dispersal as a source of endemism in the Western Ghats-Sri Lanka biodiversity hotspot. BMC evolutionary Biology, 9 (131), 1-10.

https://doi.org/10.1186/1471-2148-9-131

Van Bocxlaer, I., Roelants, K., Biju, S. D., Nagaraju, J. \& Bossuyt, F. (2006) Late Cretaceous vicariance in Gondwanan amphibians. PloS One, 1, 1-6 + supporting information (Material \& Methods S1, Figures S1-S4, Tables S1-S10). [2006.va]. https://doi.org/10.1371/journal.pone.0000074

Van der Hoeven, J. (1828) Tabula regni animalis, additis classium ordinumque characteribus. J. C. Cyfveer, Lugduni Batavorum, 1 pl. [1828.va].

Van der Hoeven, J. (1833) Handboek der dierkunde, of grondbeginsels der natuurlijke geschiedenis van het dierenrijk. Tweede en laatse deels. Tweede stuk. Gewervelde tieren. Sulpke, Amsterdam, $\mathrm{i}-\mathrm{x}+\mathrm{v}-\mathrm{x}+$ pp. 115-698. [1833. va].

Van der Hoeven, J. (1855) Handboek der dierkunde. Tweede, vrbeterde en vermeerderde Uitgave. Tweede deel. J. C. A. Sulpke, Amsterdam, i-xxviii +1068 pp., pls. 1-24. [1855. va].

Van der Hoeven, J. (1864) Philosophia zoologica. E. J. Brill, Lugduni Batavorum, i-iv + 402 pp. [1864.va].

Van der Meijden, A., Boistel, R., Gerlach, J., Ohler, A., Vences, M. \& Meyer, A. (2007) Molecular phylogenetic evidence for paraphyly of the genus Sooglossus, with the description of a new genus of Seychellean frogs. Biological Journal of the Linnean Society, 91, 347-359.

https://doi.org/10.1111/j.1095-8312.2007.00800.x

Van der Meijden, A., Vences, M. \& Meyer, A. (2004) Novel phylogenetic relationships of the enigmatic brevicipitine and scaphiophrynine toads as revealed by sequences from the nuclear Rag-1 gene. Proceedings of the royal Society of London, (B), Biological Sciences, 271, S378-S381.

https://doi.org/10.1098/rsbl.2004.0196

Van Dyken, M. \& Brian, D. (2006) Discovery of a new clade of trypanosomatids in Culex tarsalis and Culex pipiens mosquitoes. American Journal of tropical Medicine and Hygiene, 75 S, 266.

Van Gelder, R.G. (1977) Mammalian hybrids and generic limits. American Museum Novitates, 2635, 1-25.

Van Kampen, P.N. (1923) The Amphibia of the Indo-Australian archipelago. Brill, Leiden, i-xii + 304 pp. [1923.va].

Van Regenmortel, M.H.V. (2016) Classes, taxa and categories in hierarchical virus classification: a review of current debates on definitions and names of virus species. Bionomina, 10, $1-21$. https://doi.org/10.11646/bionomina.10.1.1

Van Rijsbergen, K. (1979) Information retrieval. Second edition. Butterworths, London, 208 pp.

Van Valen, L. (1973) Are categories in different phyla comparable? Taxon, 22, 333-373.

https://doi.org/10.2307/1219322

Vanzolini, P.E. (1977) An annotated bibliography of the land and fresh-water reptiles of South America (1758-1975). Museu de Zoologia, Universidad de São Paulo, Brazil, i-iv + 186 pp. [1977]. https://doi.org/10.5962/bhl.title.100695

Vasilyan, D., Böhme, M., Chkhikvadze, V. M., Semenov, Y.A. \& Joyce, W.G. (2013) A new giant salamander (Urodela, Pancryptobrancha) from the Miocene of eastern Europe (Grytsiv, Ukraine). Journal of Vertebrate Paleontology, 33 (2), 301-318. [2013.va]. https://doi.org/10.1080/02724634.2013.722151

Vassilieva, A.B., Galoyan, E.A. \& Poyarkov, N.A.J. (2013) Rhacophorus vampyrus (Anura: Rhacophoridae) reproductive biology: a new type of oophagous tadpole in Asian treefrogs. Journal of Herpetology, 47, 607-614. https://doi.org/10.1670/12-180

Vaux, F., Trewick, S.A. \& Morgan-Richards, M. (2016) Lineages, splits and divergence challenge whether the terms anagenesis and cladogenesis are necessary. Biological Journal of the Linnean Society, 117 (2), 165-176. https://doi.org/10.1111/bij.12665

Vaux, F., Trewick, S.A. \& Morgan-Richards, M. (2017) Speciation through the looking-glass. Biological Journal of the Linnean Society, 120 (2), 480-488.

https://doi.org/10.1111/bij.12872

Veith, M., Bogaerts, S., Pasmans, F. \& Kieren, S. (2018) The changing views on the evolutionary relationships of extant Salamandridae (Amphibia: Urodela). PloS One, 13, 1-16. https://doi.org/10.1371/journal.pone.0198237

Vejarano, S., Thomas, M. \& Vences, M. (2006) Comparative larval morphology in Madagascan frogs of the genus Guibemantis (Amphibia: Mantellidae). Zootaxa, 1329 (1), 39-57. https://doi.org/10.11646/zootaxa.1329.1.3

Vellard, J. (1951) Estudios sobre Batracios andinos. I. El grupo Telmatobius y formas afines. Memorias del Museo de Historia 
natural 'Javier Prado', 1, 1-89, 8 pls. [1951.va].

https://doi.org/10.2307/1437653

Vences, M. \& Glaw, F. (2001) When molecules claim for taxonomic changes: new proposals on the classification of Old World treefrogs. Spixiana, 24 (1), 85-92. [2001.va]

Vences, M., Glaw, F., Kosuch, J., Das, I. \& Veith, M. (2000) Polyphyly of Tomopterna (Amphibia: Ranidae) based on sequences of the mitochondrial 16S and 12S rRNA genes, and ecological biogeography of malagasy relict amphibian groups. In: Diversité et Endémisme à Madagascar, Mémoires de la Société de Biogéographie, Paris, 2000, pp. 229-242.

Vences, M., Guayasamin, J., Miralles, A. \& De La Riva, I. (2013) To name or not to name: criteria to promote economy of change in Linnaean classification schemes. Zootaxa, 3636, 201-244. https://doi.org/10.11646/zootaxa.3636.2.1

Vences, M., Vieites, D.R., Glaw, F., Brinkmann, H., Kosuch, J., Veith, M. \& Meyer, A. (2003) Multiple overseas dispersal in amphibians. Proceedings of the royal Society, (B), 270, $2435-2442$

https://doi.org/10.1098/rspb.2003.2516

Vieites, D.R., Min, M.-S. \& Wake, D.B. (2007) Rapid diversification and dispersal during periods of global warming by plethodontid salamanders. Proceedings of the national Academy of Sciences, 104, 19903-19907.

https://doi.org/10.1073/pnas.0705056104

Vieites, D.R., Nieto, S., Wake, M.H. \& Wake, D.B. (2011) A multigenic perspective on phylogenetic relationships in the largest family of salamanders, the Plethodontidae. Molecular Phylogenetics and Evolution, 59, 623-635. [2011.va]. https://doi.org/10.1016/j.ympev.2011.03.012

Vieites, D.R., Zhang, P. \& Wake, D.B. (2009) Salamanders (Caudata). In: Hedges, S.B. \& Kumar, S. (Eds.), The timetree of life. Oxford University Press, New York, pp. 365-368.

Vijayakumar, S.P., Dinesh, K.P., Prabhu, M.V. \& Shanker, K. (2014) Lineage delimitation and description of nine new species of bush frogs (Anura: Raorchestes, Rhacophoridae) from the Western Ghats Escarpment. Zootaxa, 3893 (4), 451-488. https://doi.org/10.11646/zootaxa.3893.4.1

Vijayakumar, S.P., Pyron, R.A., Dinesh, K.P., Torsekar, V.R., Srikanthan, A.N., Swamy, P., Stanley, E.L., Blackburn, D.C. \& Shanker, K. (2019) A new ancient lineage of frog (Anura: Nyctibatrachidae: Astrobatrachinae subfam. nov.) endemic to the Western Ghats of Peninsular India. PeerJ, 7 (e6457), 1-28. [2019.va]. https://doi.org/10.7717/peerj.6457

Vilela, R.V., Machado, T., Ventura, K., Fagundes, V., Silva, M.J. de \& Yonenaga-Yassuda, Y. (2009) The taxonomic status of the endangered thin-spined porcupine, Chaetomys subspinosus (Olfers, 1818), based on molecular and karyologic data. BMC evolutionary Biology, 9 (29), 1-17+ 1 online additional file. https://doi.org/10.1186/1471-2148-9-29

Vilgalys, R. (2003) Taxonomic misidentification in public DNA databases. New Phytologist, 160 (1), 4-5. https://doi.org/10.1046/j.1469-8137.2003.00894.x

Vitt, L.J. \& Caldwell, J.P. (2009) Herpetology. An introductory biology of Amphibians and Reptiles. Elsevier \& Academic
Press, Amsterdam, Boston, Paris, etc., i-xiv +697 pp. $\{\mathrm{Q}\}$.

Vitt, L.J. \& Caldwell, J.P. (2014) Herpetology. An introductory biology of Amphibians and Reptiles. Fourth edition. Elsevier Inc., London, Waltham, San Diego, i-xiv + 757 pp. \{Q\}.

Vorobyeva, E. I. \& Hinchliffe, J.R. (1996) Developmental pattern and morphology of Salamandrella keyserlingii limbs (Amphibia, Hynobiidae) including some evolutionary aspects. Russian Journal of Herpetology, 3, 68-81. [1996.va].

Wagler, [J.] (1828b) Systema Amphibiorum. Isis von Oken, 21, 859-861. [1828.wb].

Wagler, J. (1828a) Über die an Coecilia annulata von ihm beobachteten Thränenhöhlen und über die Eckzähne eines Frosches (Hemiphractus Spixii). Isis von Oken, 21, 735-737, pl. 10. [1828.wa].

Wagler, J. (1830) Natürliches System der Amphibien, mit vorangehender Classification der Säugethiere und Vögel. Cotta, München, Stuttgart \& Tübingen, i-vi + 1-354. [1830. wa]. https://doi.org/10.5962/bhl.title.58730

Waite, E.R. (1927) The fauna of Kangaroo Island, South Australia. No. 3. The Reptiles and Amphibians. Transactions of the royal Society of South Australia, 51, 326-329. [1927.wa].

Wake, D.B. (1966) Comparative osteology and evolution of the lungless salamanders, family Plethodontidae. Memoirs of the Southern California Academy of Sciences, 4, [i-vii] + 1-111. [1966.wa]. https://doi.org/10.5962/bhl.title.146947

Wake, D.B. (2012) Taxonomy of salamanders of the family Plethodontidae (Amphibia: Caudata). Zootaxa, 3484 (1), 75-82. [2012.wa].

https://doi.org/10.11646/zootaxa.3484.1.5

Wake, D.B. \& Elias, P. (1983) New genera and a new species of Central American salamanders, with a review of the tropical genera (Amphibia, Caudata, Plethodontidae). Contributions in Science, Natural History Museum of Los Angeles County, $345,1-19$.

Wake, D.B. \& Johnson, J.D. (1989) A new genus and species of plethodontid salamander from Chiapas, Mexico. Contributions in Science, Natural History Museum of Los Angeles County, $411,1-10$.

Wassersug, R.J. \& Pyburn, W.F. (1987) The biology of the Peret' toad, Otophryne robusta (Microhylidae), with special consideration of its fossorial larva and systematic relationships. Zoological Journal of the Linnean Society, 91 (2), 137-169. [1987.wa]. https://doi.org/10.1111/j.1096-3642.1987.tb01726.x

Webb, R.G. (2001) Frogs of the Rana tarahumarae group in western Mexico. In: Mesoamerican herpetology: systematics, zoogeography and conservation. University of Texas at El Paso Centennial Museum Special Publication, No 1, pp. 20-43.

Weisrock, D., Harmon, L. \& Larson, A. (2005) Resolving deep phylogenetic relationships in salamanders: analyses of mitochondrial and nuclear genomic data. Systematic Biology, 54, 758-777. https://doi.org/10.1080/10635150500234641 
Weisrock, D., Macey, J., Matsui, M. \& Mulcahy, D. (2013) Molecular phylogenetic reconstruction of the endemic Asian salamander family Hynobiidae (Amphibia, Caudata). Zootaxa, 3626 (1), 77-93.

https://doi.org/10.11646/zootaxa.3626.1.3

Weisrock, D.W., Papenfuss, T.J., Macey, J.R., Litvinchuk, S.N., Polymeni, R., Ugurtas, I.H., Zhao, E., Jowkar, H. \& Larson, A. (2006) A molecular assessment of phylogenetic relationships and lineage accumulation rates within the family Salamandridae (Amphibia, Caudata). Molecular Phylogenetics and Evolution, 41, 368-383.

https://doi.org/10.1016/j.ympev.2006.05.008

Werner, F. (1896) Beiträge zur Kenntniss der Reptilien und Batrachier von Centralamerika und Chile, sowie einiger seltenerer Schlangenarten. Verhandlungen der kaiserlichköniglichen zoologisch-botanischen Gesellschaft in Wien, 46, 344-365, pl. 6. [1896.wa]. https://doi.org/10.5962/bhl.part.19752

Werner, F. (1912) Lurche und Kriechtiere. Erster Band. Brehms Tierleben, Allgemeine Kunde des Tierreichs, Bibliographisches Institut, Leipzig \& Wien, 4, i-xvi 572 pp. \{Q\}.

Wettstein, O. (1934) Hypopachus parkeri nov. spec., ein neuer Termitenfrosch aus Brasilien. Zoologischer Anzeiger, 105, 270-272.

Wheeler, Q.D. (2001) Systematics, Overview. In: Levin, S.A. (Ed), Encyclopedia of biodiversity, Volume 5, Elsevier, New York, pp. 569-588.

Wheeler, Q.D. (2004) Taxonomic triage and the poverty of phylogeny. Philosophical Transactions of the royal Society, (B) 359, 571-583.

https://doi.org/10.1098/rstb.2003.1452

Wheeler Q.D., Raven P.H. \& Wilson E.O. (2004) Taxonomy: impediment or expedient? Science, 303, 285. https://doi.org/10.1126/science.303.5656.285

Whitney, W.D. (1890) The century dictionary. The Centurry Co., New York 4 (16), 4485-4880. [1890.wa].

Wickramasinghe, M., Vidanapathirana, D., Rajeev, G., Ariyarathne, S., Chanaka, A.W., Priyantha, L.L., Wickramasinghe, N. \& Bandara, I.N. (2013b) Eight new species of Pseudophilautus (Amphibia: Anura: Rhacophoridae) from Sripada World Heritage Site (Peak Wilderness), a local amphibian hotspot in Sri Lanka. Journal of threatened Taxa, 5, 3789-3920. https://doi.org/10.11609/JoTT.o3099.3789-920

Wickramasinghe, L.J.M., Vidanapathirana, D.R., Airyarathne, S., Rajeev, G., Chanaka, A., Pastorini, J., Chathuranga, G. \& Wickramasinghe, N. (2013a) Lost and found: One of the world's most elusive amphibians, Pseudophilatus stellatus (Kelaart 1853) rediscovered. Zootaxa, 3620 (1), 112-128. https://doi.org/10.11646/zootaxa.3620.1.5

Wiechmann, M.F. (2003) Albanerpetontidae (Lissamphibia) aus dem Mesozoikum der Iberischen Halbinsel und dem Neogen von Süddeutschland. Ph. D. thesis, Freien Universität Berlin, 179 pp., pls. 1-27+ [1], 3 tab. + [1-2]. [2003.wa].

Wied, M., zu (1865) Verzeichnis der Reptilien, welche auf einer Reise im nördlichen America beobachtet wurden. Verhandlungen der kaiserlichen Leopoldino-Carolinischen deutschen Akademie der Naturforscher, 32 (1) [8], i-viii + 144 pp., pls. 1-7. [1865.wa].

Wiedersheim, R.E.E. (1877) Das Kopfskelet der Urodelen. Morphologisches Jahrbuch, 3, 352-448, pls. 19-23. [1877. wa].

Wiegmann, A.F.A. \& Ruthe, J.F. (1832) Handbuch der Zoologie. C. G., Lüderik, i-vi +622 pp. [1832.wa]. https://doi.org/10.5962/bhl.title. 115720

Wiegmann, A.F.A. \& Ruthe, J.F. (1843) Handbuch der Zoologie. Zweite Auflage. C. G. Lüderik, Berlin, i-iv + 670 pp. [1843. wa].

Wiens, J.J. (2003) Missing data, incomplete taxa, and phylogenetic accuracy. Systematic Biology, 52 (4), 528-538. https://doi.org/10.1080/10635150390218330

Wiens, J.J. \& Morrill, M.C. (2011) Missing data in phylogenetic analysis: reconciling results from simulations and empirical data. Systematic Biology, 60 (5), 719-731. https://doi.org/10.1093/sysbio/syr025

Wiens, J.J., Bonett, R. \& Chippindale, P. (2005a) Ontogeny discombobulates phylogeny: paedomorphosis and higherlevel salamander relationships. Systematic Biology, 54, 91110. https://doi.org/10.1080/10635150590906037

Wiens, J.J., Fetzner, J.W.J., Parkinson, C.L. \& Reeder, T.W. (2005b) Hylid frog phylogeny and sampling strategies for speciose clades. Systematic Biology, 54, 778-807. https://doi.org/10.1080/10635150500234625

Wiens, J.J., Graham, C.H., Moen, D.S., Smith, S.A. \& Reeder, T.W. (2006) Evolutionary and ecological causes of the latitudinal diversity gradient in hylid frogs: treefrog trees unearth the roots of high tropical diversity. The American Naturalist, 168, 579-596. https://doi.org/10.1086/507882

Wiens, J.J., Kuczynski, C.A., Duellman, W.E. \& Reeder, T.W. (2007) Loss and re-evolution of complex life cycles in marsupial frogs: does ancestral trait reconstruction mislead? Evolution, 61, 1886-1899. https://doi.org/10.1111/j.1558-5646.2007.00159.x

Wiens, J.J., Kuczynski, C.A., Hua, X. \& Moen, D.S. (2010) An expanded phylogeny of treefrogs (Hylidae) based on nuclear and mitochondrial sequence data. Molecular Phylogenetics and Evolution, 55, 871-882. https://doi.org/10.1016/j.ympev.2010.03.013

Wiens, J.J., Sukumaran, J., Pyron, R.A. \& Brown, R.M. (2009) Evolutionary and biogeographic origins of high tropical diversity in old world frogs (Ranidae). Evolution, 63, 12171231. https://doi.org/10.1111/j.1558-5646.2009.00610.x

Wilbrand, J.B. (1814) Ueber die Classification der Thiere. Taeché, Giesen, i-viii + 147 pp., 1 tab. [1814.wa].

Wilbrand, J.B. (1829) Handbuch der Naturgeschichte des Thierreichs. Georg Friedrich Heyer, Giessen, i-viii + 612 pp., 1 tab. [1829.wa].

Wild, E.R. (1995) New genus and species of Amazonian microhylid frog with a phylogenetic analysis of New World genera. Copeia, 1995, 837-849. 
https://doi.org/10.2307/1447032

Wiley, E.O. (1979) An annotated Linnaean hierarchy, with comments on natural taxa and competing systems. Systematic System, 28, 308-337.

https://doi.org/10.1093/sysbio/28.3.308

Wiley, E.O. (1981) Phylogenetics. The theory and practice of phylogenetic systematics. Wiley, New York, i-xv + 439 pp.

Wilkinson, J.A., Drewes, R.C. \& Tatum, O.L. (2002) A molecular phylogenetic analysis of the family Rhacophoridae with an emphasis on the Asian and African genera. Molecular Phylogenetics and Evolution, 24, 265-273.

https://doi.org/10.1016/S1055-7903(02)00212-9

Wilkinson, M. \& Nussbaum, R.A. (1997) Comparative morphology and evolution of the lungless caecilian Atretochoana eiselti (Taylor) (Amphibia: Gymnophiona: Typhlonectidae). Biological Journal of the Linnean Society, 62, 39-109. https://doi.org/10.1111/j.1095-8312.1997.tb01616.x

Wilkinson, M. \& Nussbaum, R.A. (1999) Evolutionary relationships of the lungless caecilian Atretochoana eiselti (Amphibia: Gymnophiona: Typhlonectidae). Zoological Journal of the Linnean Society, 126, 119-123.

https://doi.org/10.1111/j.1096-3642.1999.tb00153.x

Wilkinson, M. \& Nussbaum, R.A. (2006) Caecilian phylogeny and classification. In: Reproductive biology and phylogeny of Gymnophiona. Vol. 5. Science Publisher, Enfield, Jersey \& Plymouth, pp. 39-78. [2006.wa].

Wilkinson, M., Kok, P.J.R., Ahmed, F. \& Gower, D.J. (2014) Caecilita Wake \& Donnelly, 2010 (Amphibia: Gymnophiona) is not lungless: implications for taxonomy and for understanding the evolution of lunglessness. Zootaxa, 3779 (3), 383-388. https://doi.org/10.11646/zootaxa.3779.3.6

Wilkinson, M., Nussbaum, R. \& Hoogmoed, M. (2009) A new species of Microcaecilia (Amphibia: Gymnophona [sic]: Caeciliidae) from Suriname. Herpetologica, 65 (4), 413-418. [2009.wa]. https://doi.org/10.1655/08-030.1

Wilkinson, M., San Mauro, D., Sherratt, E. \& Gower, D.J. (2011) A nine-family classification of caecilians (Amphibia: Gymnophiona). Zootaxa, 2874 (1), 41-64. [2011.wa]. https://doi.org/10.11646/zootaxa.2874.1.3

Wilkinson, M., Sherratt, E., Starace, F. \& Gower, D.J. (2013) A new species of skin-feeding caecilian and the first report of reproductive mode in Microcaecilia (Amphibia: Gymnophiona: Siphonopidae). PloS One, 8 (e57756), 1-11. https://doi.org/10.1371/journal.pone.0057756

Williams, D., Schmitt, M. \& Wheeler, Q. (2016) The future of phylogenetic systematics: the legacy of Willi Hennig. Systematics Association Special Volume Series. Cambridge University Press, Cambridge, i-xvi +488 pp. https://doi.org/10.1017/CBO9781316338797

Wilson, E.O. (1985) The global biodiversity crisis: a challenge to science. Issues in Science \& Technology, 2, 20-29.

Wilson, E.O. (Ed.) (1988) Biodiversity. National Academy Press, Washington National Academy Press, i-xiii +521 pp.

Wilson, D.E. \& Reeder, D.M. (2011) Class Mammalia Linnaeus,
1758. In: Zhang, Z.-Q. (Ed.), Animal biodiversity: an outline of higher-level classification and survey of taxonomic richness. Zootaxa, 3148 (1), 56-60.

https://doi.org/10.11646/zootaxa.3148.1.9

Wilson, L.E. (2006) Comparative taphonomy and paleoecological reconstruction of two microvertebrate accumulations from the late Cretaceous (Maastrichtian) Hell Creek Formation, eastern Montana. MS Thesis, Montana State University, Bozeman, i-ix + 106 pp. [2006.wb].

Wogan, G.O.U., Win, H., Thin, T., Lwin, K.S., Shein, A.K., Kyi, S.W. \& Tun, H. (2003) A new species of Bufo (Anura: Bufonidae) from Myanmar (Burma), and redescription of the little-known species Bufo stuarti Smith 1929. Proceedings of the California Academy of Sciences, 54, 141-153.

Wollenberg, K.C. \& Measey, G.J. (2009) Why colour in subterranean vertebrates? Exploring the evolution of colour patterns in caecilian amphibians. Journal of evolutionary Biology, 22, 1046-1056 + supplementary information S1-S3. [2009.wb]. https://doi.org/10.1111/j.1420-9101.2009.01717.x

Wollenberg, K.C., Vieites, D.R., Glaw, F. \& Vences, M. (2011) Speciation in little: the role of range and body size in the diversification of Malagasy mantellid frogs. $B M C$ evolutionary Biology, 11 [217], 1-15. https://doi.org/10.1186/1471-2148-11-217

Wood, J.G. (1863) The illustrated natural history. Reptiles, Fishes, Molluscs, \&c. \&c. \&c. George Routledge \& sons, London, [i-ii] +810 pp., 1 pl. [1863.wa]. https://doi.org/10.5962/bhl.title.58683

Wright, E.P. \& Huxley, T.H. (1866) On a collection of fossils from the Jarrow Colliery, Kilkenny, Ireland. The geological Magazine, 3, 165-171.

Xiong, J.-L., Chen, Q., Zeng, X.-M., Zhao, E.-M. \& Qing, L.-Y. (2007) Karyotypic, morphological, and molecular evidence for Hynobius yunanicus as a synonym of Pachyhynobius shangchengensis (Urodela: Hynobiidae). Journal of Herpetology, 41, 664-671. https://doi.org/10.1670/07-054.1

Yan, F., Jiang, K., Wang, K., Jin, J., Suwannapoom, C., Li, C., Vindum, J., Brown, R. \& Che, J. (2016) The Australasian frog family Ceratobatrachidae in China, Myanmar and Thailand: discovery of a new Himalayan forest frog clade. Zoological Research, 37, 7-14.

Yang, D. (1989) Phylogenetic systematics of the Amolops group of ranid frogs of southeastern Asia and the greater Sunda islands. Proceedings of the 12th congress of China Zoological Association and its 55th annual academic meeting, 256-257. [1989.ya].

Yang, D. (Ed.) (1991) The Amphibia fauna of Yunnan. China Forestry Publishing House, Kunming, [i-vii] + i-iv + 1-259. [In Chinese]. [1991.ya].

Yang, X., Wang, B., Hu, J. \& Jiang, J. (2011) A new species of the genus Feirana (Amphibia: Anura: Dicroglossidae) from the Western Qinling Mountains of China. Asian herpetological Research, 2, 72-86. https://doi.org/10.3724/SP.J.1245.2011.00072

Ye, C., Fei, L. \& Hu, S. (1993) Rare and economic amphibians 
of China. Chengdu (Sichuan Publishing House of Science \& Technology), [i-iv] + 1-2 + 1-2+1-8+1-413. [1993.ya].

Yu, G., Rao, D., Zhang, M. \& Yang, J. (2009) Re-examination of the phylogeny of Rhacophoridae (Anura) based on mitochondrial and nuclear DNA. Molecular Phylogenetics and Evolution, 50, 571-579. https://doi.org/10.1016/j.ympev.2008.11.023

Yuan, Z.-Y., Zhang, B.-L., Raxworthy, C.J., Weisrock, D.W., Hime, P.M., Jin, J.-Q., Lemmon, E.M., Lemmon, A.R., Holland, S.D., Kortyna, M.L., Zhou, W.-W., Peng, M.-S., Che, J. \& Prendini, E. (2018) Natatanuran frogs used the Indian Plate to step-stone disperse and radiate across the Indian Ocean. National Science Review, 6, 10-14. https://doi.org/10.1093/nsr/nwy092

Zachariah, A., Dinesh, K. \& Kunhikrishnan, E. (2011) Nine new species of frogs of the genus Raorchestes (Amphibia: Anura: Rhacophoridae) from southern Western Ghats, India. Biosystematica, 5, 25-48.

Zagorodniuk, I.V. (2004) Поняttя Apoda в зоології та його омонімія. [Name Apoda in zoology and its homonymy] Vesnik zoologii, 38 (3), 67-72. [In Ukrainian]. [2004.za].

Zaldívar-Riverón, A., Shaw, M.R., Sáez, A. G., Mori, M., Belokoblylskij, S.A., Shaw, S.R. \& Quicke, D.L.J. (2008) Evolution of the parasitic wasp subfamily Rogadinae (Braconidae): phylogeny and evolution of lepidopteran host ranges and mummy characteristics. BMC evolutionary Biology, 8 (329), 1-20 + 5 additional files. https://doi.org/10.1186/1471-2148-8-329

Zeng, X., Fu, J., Chen, L., Tian, Y. \& Chen, X. (2006) Cryptic species and systematics of the hynobiid salamanders of the Liua-Pseudohynobius complex: molecular and phylogenetic perspectives. Biochemical Systematics and Ecology, 34, 467477. https://doi.org/10.1016/j.bse.2006.01.006

Zhang, P., Chen, Y.-Q., Zhou, H., Liu, Y.-F., Wang, X.-L., Papenfuss, T.J., Wake, D.B. \& Qu, L.-H. (2006) Phylogeny, evolution, and biogeography of Asiatic salamanders (Hynobiidae). Proceedings of the national Academy of Sciences, 103, 7360 7365 .

https://doi.org/10.1073/pnas.0602325103

Zhang, P., Liang, D., Mao, R.-L., Hillis, D., Wake, D. \& Cannatella, D. (2013) Efficient sequencing of anuran mtDNAs and a mitogenomic exploration of the phylogeny and evolution of frogs. Molecular Biology and Evolution, 30, 1899-1915. [2013.za]. https://doi.org/10.1093/molbev/mst091

Zhang, P., Papenfuss, T.J., Wake, M.H., Qu, L. \& Wake, D.B. (2008) Phylogeny and biogeography of the family Salamandridae (Amphibia: Caudata) inferred from complete mitochondrial genomes. Molecular Phylogenetics and Evolution, 49, 586597.

https://doi.org/10.1016/j.ympev.2008.08.020

Zhang, P. \& Wake, D.B. (2009) Higher-level salamander relationships and divergence dates inferred from complete mitochondrial genomes. Molecular Phylogenetics and Evolution, 53, 492-508. \{Q\}. https://doi.org/10.1016/j.ympev.2008.08.020

Zhang, Z.-Q. (Ed.) (2011a) Animal biodiversity: an outline of higher-level classification and survey of taxonomic richness. Zootaxa, 3148 (1), 1-237.

https://doi.org/10.11646/zootaxa.3148.1.1

Zhang, Z.-Q. (2011b) Animal biodiversity: an introduction to higher-level classification and taxonomic richness. In: Zhang, Z.-Q. (Ed.), Animal biodiversity: an outline of higher-level classification and survey of taxonomic richness. Zootaxa, 3148 (1), pp. 7-12.

https://doi.org/10.11646/zootaxa.3148.1.3

Zhang, Z.-Q. (2011c) Phylum Arthropoda von Siebold, 1848, In: Zhang, Z.-Q. (Ed.), Animal biodiversity: an outline of higher-level classification and survey of taxonomic richness. Zootaxa, 3148 (1), 99-103.

https://doi.org/10.11646/zootaxa.3148.1.14

Zhang, Z.-Q., Fan, Q.-H., Pesic, V., Smit, H., Bochkov, A. V., Khaustov, A. A., Baker, A., Wohltmann, A., Wen, T., Amrine, J. W., Beron, P., Lin, J., Gabrys, G. \& Husband, R. (2011) Order Trombidiformes Reuter, 1909. In: Zhang, Z.-Q. (Ed.), Animal biodiversity: an outline of higher-level classification and survey of taxonomic richness. Zootaxa, 3148 (1), 129138.

https://doi.org/10.11646/zootaxa.3148.1.24

Zhang, Z.-Q. (Ed.) (2013a) Animal biodiversity: an outline of higher-level classification and survey of taxonomic richness (Addenda 2013). Zootaxa, 3703 (1), 1-82.

https://doi.org/10.11646/zootaxa.3703.1.6

Zhang, Z.-Q. (2013b) Animal biodiversity: an update of classification and diversity in 2013. In: Zhang, Z.-Q. (Ed.), Animal biodiversity: an outline of higher-level classification and survey of taxonomic richness (Addenda 2013). Zootaxa, 3703 (1), pp. 5-11.

https://doi.org/10.11646/zootaxa.3703.1.3

Zhang, Z.-Q. (2013c) Phylum Arthropoda. In: Zhang, Z.-Q. (Ed.), Animal biodiversity: an outline of higher-level classificationand survey of taxonomic richness (Addenda 2013). Zootaxa, 3703 (1), 17-26. https://doi.org/10.11646/zootaxa.3703.1.6

Zimkus, B.M., Lawson, L., Loader, S.P. \& Hanken, J. (2012) Terrestrialization, miniaturization and rates of diversification in African Puddle Frogs (Anura: Phrynobatrachidae). PloS One, 7 (e35118), 1-11.

https://doi.org/10.1371/journal.pone.0035118

Zimkus, B.M., Rödel, M.-O. \& Hillers, A. (2010) Complex patterns of continental speciation: molecular phylogenetics and biogeography of sub-Saharan puddle frogs (Phrynobatrachus). Molecular Phylogenetics and Evolution, 55, 883-900. https://doi.org/10.1016/j.ympev.2009.12.012

Zittel, K.A. (1888) Handbuch der Palaeontologie. I Abtheilung. Palaeozoologie. III Band. Vertebrata (Pisces, Amphibia, Reptilia, Aves). Second instalment. R. Oldenbourg, München \& Leipzig, pp. 257-436. [1888.za].

Zug, G.R. (1993) Herpetology. An introductory biology of Amphibians and Reptiles. Academic Press, San Diego, i-xv +527 pp. $\{\mathrm{Q}\}$. 
https://doi.org/10.2307/1447112

Zug, G.R., Vitt, L.J. \& Caldwell, J.P. (2001) Herpetology. An introductory biology of Amphibians and Reptiles. Second Edition. Academic Press, San Diego, i-xiv + 630 pp. [2001. $\mathrm{za}$.

Zweifel, R.G. (1956) Two pelobatid frogs from the Tertiary of North America and their relationships to fossil and recent forms. American Museum Novitates, 1762, 1-45. [1956.za].

Zweifel, R.G. (1957) A new frog of the genus Rana from Michoacán, Mexico. Copeia, 1957, 78-83.

https://doi.org/10.2307/1439391

Zweifel, R.G. (1972) Results of the Archbold Expeditions. No. 97. A revision of the frogs of the subfamily Asterophryinae, family Microhylidae. Bulletin of the American Museum of natural History, 148, 411-546.

Zweifel, R.G. (1986) A new genus and species of Microhylid frog from the Cerro de la Neblina region of Venezuela and a discussion of relationships among New World Microhylid genera. American Museum Novitates, 2863, 1-24.

\section{Notes concerning the publication dates of some works:}

$\lceil\mathrm{P} 00\rfloor$ Year of publication appearing on the title page of the volume.

[P01 J Brongniart 1800.ba > Latreille 1800.la, where the former is cited (p. ix).

[P02 Duméril 1805.da [before 15 november 1805] according to Gregory (2010).
[P03 J Oppel 1811.oa [January-March 1811] > Oppel 1811.ob [11 May 1811], according to Sherborn (1914).

[P04 Cuvier 1816.ca [7 December 1816] according to Cowan (1969).

〔P05 J Bonaparte 1838.ba and Bonaparte 1839.bd: dates according to Salvadori (1888).

(P06」 Agassiz 1843.aa and Agassiz 1847.aa: dates according to Bowley $^{+1}$ (1968).

〔P07 Schlegel 1850.sa, cited in Bonaparte 1850.bb > Bonaparte 1850.bb [March 1850, according to Duméril ${ }^{+2}$ 1854.da: 32 .] > Baird 1850.ba, cited in Gray 1850.ga > Gray 1850.ga [June 1850, according to Duméril ${ }^{+2} 1854$.da: 32 .]. Note: Given the precise publication dates given by Duméril ${ }^{+2}$, the citations of Gray in Bonaparte 1850.bb must be based on reading by Bonaparte of letters or of manuscript from Gray.

[P08 J Günther 1859.ga [12 February 1859] according to Sherborn (1934)

\P09」 Cope 1869.ca: date according to Vanzolini (1977).

[P10] Brocchi 1881.ba: date of livraison acccording to Crosnier ${ }^{+1}$ (1998)

〔P11 Stipanicic ${ }^{+1}$ 1956.sa: date according to Anonymous (1967: 322).

[P12 J Thorn 1966.ta: date according to Thorn (1968).

\{P13 J Špinar 1979.sa: date according to Sanchiz (1998).

[P14 Laurent 1980.la [18 April 1980]: date according to the last page of the fascicle 104 (4).

[P15] Roček 1981.ra: date according to the first page of the paper.

\{P16] Dubois 1987.da [13 May 1987] < Dubois 1987.db [1 October 1987] according to Dubois (1988b). 


\section{APPENDICES}

\section{Appendix A1.GLO. Glossary}

Technical taxonomic, nomenclatural and other terms used here, and their correspondence with terms used in the Code, if available.

\section{Structure of entries}

For each term used here, this Glossary provides: (1) the grammatical category of the term; (2) the domain of application of the term; (3) the etymology of the term (only for technical terms coined especially for nomenclature and taxonomy); (4) a definition, with comments and/or mention of related terms and antonyms (terms of opposite meaning) if relevant; (5) the reference to first publication of the term, or mention that it is introduced here (Hoc loco); (6) the equivalent term or expression used in the Code for the same concept, if available.

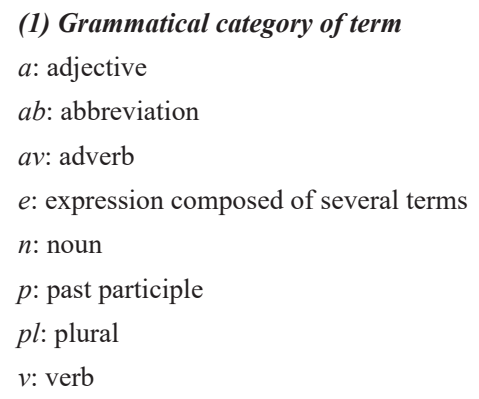

(4) Abbreviation and definition of term, with comments and/or mention of related terms and antonyms (terms of opposite meaning) if relevant ANG: Angionym: term designating a superordinate class

ANT: Antonym: term of opposite meaning

END: Endonym: term designating a subordinate class

ETY: Etymology of term

SYN: Synonym: term of same meaning

(5) Reference to first publication of the term, or mention that it is introduced here (hoc loco)

(6) Equivalent term or expression used in the Code for the same concept, if available

\section{Use of italics and bold and other conventions}

Bold characters are used only for the titles of entries.

In definitions, terms in bold italics are defined elsewhere in this Glossary, but terms between 'simple quotation marks' are not.

Terms in italics are involved in the etymology of a term used here.

The Latin expression Hoc loco means: in the present work. 
A-availability, e. • AV. • Availability of airesy (nomenclatural act). • Dubois 2015c: 24. • Code: no term.

Absolute rank, e. • NO. • Nomenclatural rank conceived and used as permanently attached to taxa, as if they expressed their 'nature' or 'essence', in biological or historical terms. • Dubois 2006c: 21, 2007a: 34. • Code: no term.

Acceptable tolerance, e. $\bullet$ AV. • Qualification of information, particularly regarding taxognosis, provided in a work introducing a new nomen that allows its nomenclatural availability in borderline situations. $\bullet$ Hoc loco. • Code: no term.

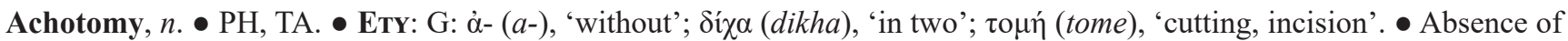
partition of a set into subsets. $\bullet$ Hoc loco. $\bullet$ Code: no term.

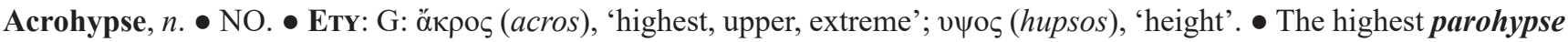
of a nomen in force in a given ergotaxonomy. $\bullet$ Hoc loco. $\bullet$ Code: no term.

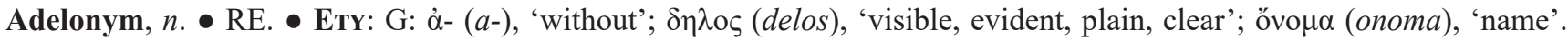
- Unregistered nomen, thus unprotected against potential invalidation of its availability. • ANT: delonym. • Dubois 2011a: 77. • Code: no term.

Adelphotaxa, $n$. Plural of adelphotaxon.

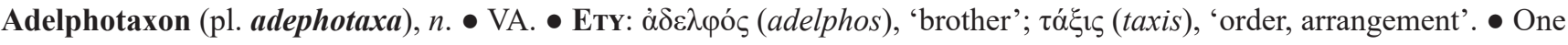
of two branches that are supposed, in a given hypothetical cladogeny, to be derived from a common ancestor. $\bullet$ Ax 1984. $\bullet$ Code: no term.

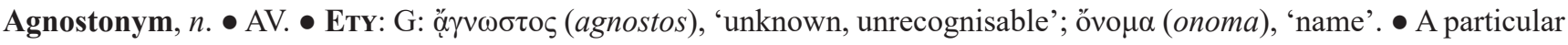
case of anoplonym: published but nomenclaturally unavailable nomen according to the Code, for having been published after 1999 without explicit statement that it is intentionally new (Article 16.1). $\bullet$ Hoc loco. • Code: unavailable name.

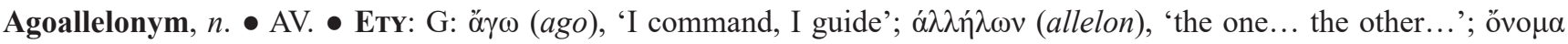
(onoma), 'name'. • One among two parallelonyms which was clearly given preference over the other one (its epomallelonym) in the original publication, and which for this reason has precedence over it. $\bullet$ Dubois $2015 c$ : $43,70$. - Code: no term.

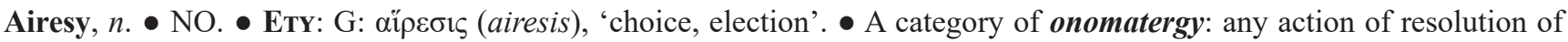
uncertainties and ambiguities which may have remained after a catastasy (original publication of a nomen). Airesies consist either in choices between several possibilities (e.g., designation of a single specimen or nominal taxon as onomatophore of a nomen introduced without this information, or fixation of precedence between synchronous doxisonyms or symprotographs) or in the brand new introduction of missing information (e.g., listing subsequently included specimens or nominal taxa in a nominal taxon which until then missed them). Choices made in airesies are left to the freedom of individual authors, but in some cases the Code provides Recommendations in this respect (e.g., the Recommendations of Article 74 concerning the designations of lectotypes). Once published, an airesy is irreversible and cannot be modified by individual authors but only through archoidy. • Dubois 2013: 3. • Code: first reviser action.

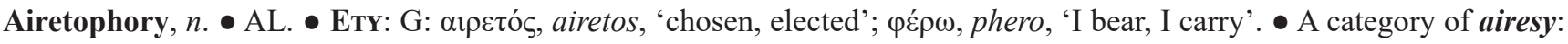
subsequent restriction or designation of onomatophore for a nomen. • Dubois 2013: 5. • Code: no term.

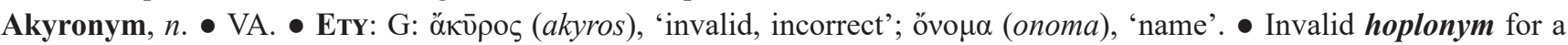
given taxon in a given ergotaxonomy. Its invalidity may be conditional (junior doxisonym, junior asthenomonym, lethakyronym) or permanent (junior isonym, junior hadromonym, archakyronym, archanecdidonym). • ANT: Kyronym. Dubois 2000b: 51. Code: no term.

Alienogenera, $n$. $\bullet$ Plural of Alienogenus.

Alienogenus (pl. alienogenera), n. • AL. • ETy: L: alienus, 'foreign, unrelated'; genus, 'birth, origin, class, kind'. • Genusseries taxomen expressly excluded from the protaxon for which a class-series nomen was promulgated, serving as onomatostasis of this class-series nomen. • END: coalienogenus, unialienogenus. $\bullet$ Dubois 2005c: 203. • Code: no term.

Alienordinate, a. • NO, TA. • ETy: L: alienus, 'foreign'; ordo, 'series, line, row, order'. Qualification of any of two or more taxa that have no direct hierarchical or sister-taxa relation in a given ergotaxonomy. $\bullet$ Dubois $2006 b$ : 827 (as xenordinate), 2008f: 60. Code: no term.

Alienordination, $n$. $\bullet$ NO, TA. $\bullet$ ETY: see Alienordinate. $\bullet$ The absence of relation of ordination between two alienordinate taxa in a given ergotaxonomy. • Dubois 2008f: 60. $\bullet$ Code: no term.

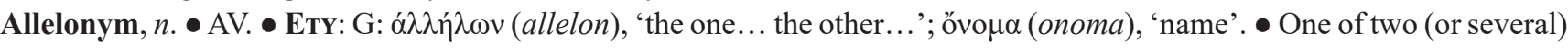
synonymous nomina used both (or all) as valid for the same taxon (having the same content) in the same publication. $\bullet$ END: archaeoallelonym, neoallelonym and parallelonym. • Dubois 2006a: 183, 2011a: 41. • Code: no term.

All-fossil, e. $\bullet$ XE. • For a taxon of AMPHiBia: that is not known to include a single extant species.

Allocate, $v$. AL. $\bullet$ See Allocation.

Allocated, $p$. $\bullet$ AL. $\bullet$ Qualification of a nomen (aptonym) that conforms to the conditions of taxonomic allocation as regulated by the nomenclatural system. • ANT: Unallocated. - Dubois 2005b: 396. • Code: no term. 
Allocation, $n$. • AL. • Onomatergy regulated by a nomenclatural system by which a nomen becomes attached to a taxon or several taxa in zoological nomenclature, under a given system of connexion between nomina and taxa (e.g., through onomatophores or through 'phylogenetic definitions'). $\bullet$ Dubois 2005b: 369. • Code: no term.

Allochronous, a. • AV, VA. • ETY: see Allochrony. - Qualification of distinct events that occurred at different dates. In the context of zoological nomenclature, the fact that two publications were distributed at different dates. $\bullet$ ANT: Synchronous. • Common language term; Dubois 2013: 5. • Code: no term.

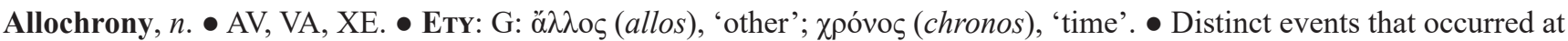
different dates. • ANT: Synchrony. • Common language term; Dubois \& Aescht 2019f: 50-51. • Code: no term.

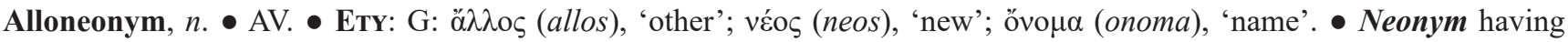
a partially or totally different etymology from its archaeonym, i.e., not directly derived from it through unjustified emendation. • ANT: Autoneonym. • Dubois 2000b: 52. • Code: new replacement name, nomen novum.

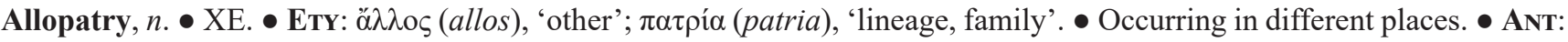
Sympatry. $\bullet$ Common term in evolutionary biology. $\bullet$ Code: no term.

Allot, $v$. $\bullet$ VA. - Process of choice between the family-series or the class-series for the nominal-series assignment of a nomen in borderline situations (see $\{\mathrm{k} 4\}$ and $[\mathrm{FPC}]$ in text). $\bullet$ Hoc loco. $\bullet$ Code: no term.

Allotment, $n$. $\bullet$ VA. $\bullet$ Result of a choice between the family-series or the class-series for the nominal-series assignment of a nomen in borderline situations (see $\{\mathrm{k} 4\}$ and $[\mathrm{FPC}]$ in text). $\bullet$ Hoc loco. $\bullet$ Code: no term.

Ambiostensional, n. • AL. • ETY: L: ambo, 'both, two together'; ostensio, 'action of showing'. • Qualification of a nomenclatural system, the Ambiostensional Nomenclatural System (AONS), which makes use of a double or alternative way of allocating nomina to taxa according to [1] the presence of or [2] the absence of intragenera in the metrotaxon: i.e., either [1] relying only on onomatophores (conucleogenera) (see Metrostensional) or [2] relying on both onomatophores (conucleogenera) and onomatostases (alienogenera) (see Orostensional) (see Dubois 2006a, $d$, 2007a, 2008f, 2015c; Dubois \& Ohler 2009). • Dubois 2011a: 39. • Code: no term.

Ambiostensional Nomenclatural System (AONS), e. • NO. • A composite class-series nomenclatural system in which nesonyms and ellitonyms are allocated to taxa through MONS whereas oronyms are so through OONS. • Dubois 2005c, 2006a, 2011a, 2015c. • Code: no term.

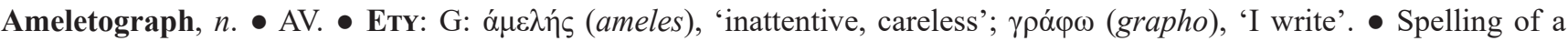
nomen used inadvertently in a publication by an author, editor or publisher. • ANT: meletograph. $\bullet$ Dubois $2000 b$ : 54 (as ameletonym), 2010a: 7. • Code: no term.

Ameletonym, $n$. • Obsolete for Ameletograph. • Dubois 2000b: 54.

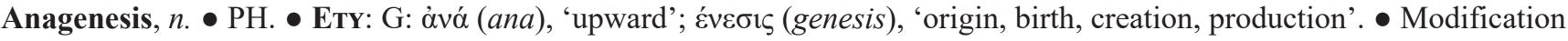
of characters within an evolutionary lineage, that may lead to speciation without cladogenesis (see Vaux et al. 2016, 2017 and Allmon 2017). • Rensch 1947: 95. • Code: no term.

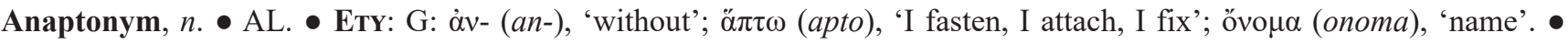
Nomenclaturally unallocated nomen [1] for not being clearly attached to an onomatophore in the three lower nominalseries covered by the Code, or under MONS in the case of CS distagmonyms, or [2] for being a gephyronym under OONS in the case of CS sozonymorphs. - ANT: aptonym. - Dubois 2011a: 25, 78. • Code: one among several meanings of the unclear term nomen dubium.

Anchor, $v$. $\bullet$ AL. $\bullet$ To perform an onomatergy consisting in the designation of an onomatophore for a taxon. $\bullet$ Hoc loco. - Code: no term.

Anchorage, $n$. $\bullet$ AL. $\bullet$ Result of an onomatergy consisting in the designation of an onomatophore for a taxon. $\bullet$ Hoc loco. - Code: no term.

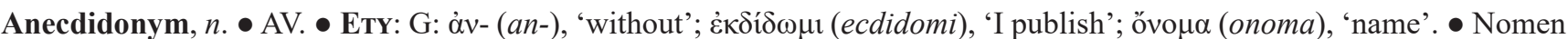
unavailable (anoplonym) for having been introduced in a publication unavailable under the Code or made unavailable by the Commission under the Plenary Power. • END: Archanecdidonym, Nomanecdidonym. AnT: Ecdidonym. • Dubois 2015c: 24, 71; redefined here. $\bullet$ Code: no term.

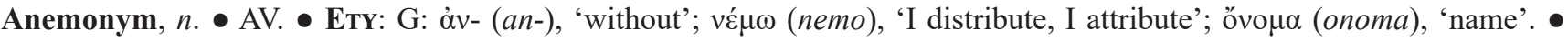
A nomen that is not unambiguously assigned or assignable to a nominal-series in the original publication where it is established. • AnT: nemonym. $\bullet$ Hoc loco. • Code: no term.

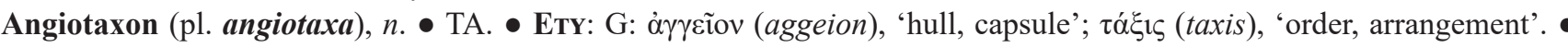
Any taxon which is superordinate to another taxon (its endotaxon) in a given ergotaxonomy. $\bullet$ Dubois 2005b: $406 . \bullet$ Code: no term.

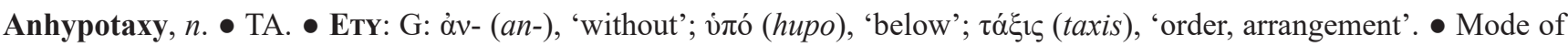
hypotaxy of a taxon that includes no subordinate taxon, being the 'terminal' lower taxon in a nomenclatural hierarchy. Given the current Rules of the Code, this can occur only in two cases, when the 'final' taxon is either a species or a subspecies. All nomina at ranks above the rank species designate taxa that include at least one species, even possibly 
still unnamed and undescribed, so they cannot fall in this category of hypotaxy. • Dubois \& Raffaëlli 2009: 12. • Code: no term.

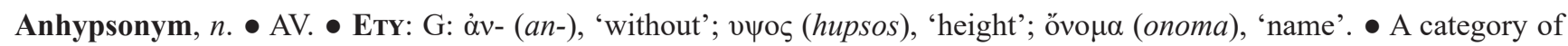
ectonym: nomen proposed under a nomenclatural system explicitly unranked or pseudoranked and therefore unavailable under the Code or under DONS. • AnT: hypsonym. • Dubois \& Frétey 2020a: 5, 38. • Code: no term.

Anoplonym, $n$. • AV. • ETY: G: ơvo $\pi \lambda$ os (anoplos), 'unarmed'; ővo $\mu \alpha($ onoma), 'name'. • Published but nomenclaturally unavailable nomen according to the Rules of the Code. • END: agnostonym, anecdidonym, atelonym, barbaronym, caconym, eulabonym, gymnonym. • AnT: hoplonym. • Dubois 2000b: 50. • Code: unavailable name.

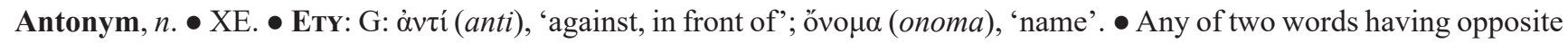
meanings. • Term in traditional use in general language, grammar and linguistics; Dubois \& Aescht 2019h: 75. • Code: no term.

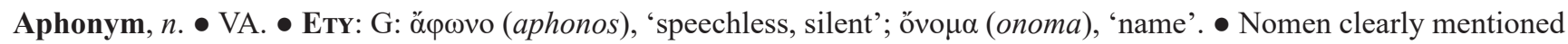
as nomenclaturally available (in some cases as an available senior homonym making a junior homonym invalid) but never used as valid by any author and in any publication after 31 December 1899. • Dubois 2005a: 85, 2005b: 411 . $\bullet$ Code: no term.

Aphoric, a. $\bullet$ See Aphory.

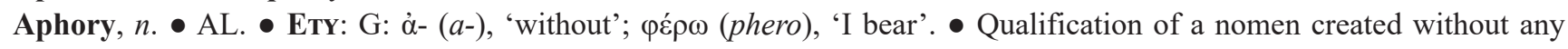
onomatophore. $\bullet$ Dubois 2005b: 404.

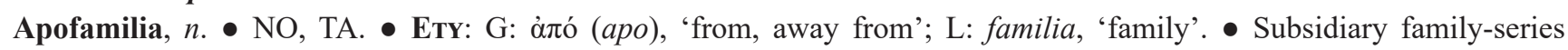
taxonominal rank, between family and epifamily. • SYN: apofamily. $\bullet$ Hoc loco. • Code: no term.

Apofamily, n. • NO, TA. • ETY: G: àjó (apo), 'from, away from'; L: familia, 'family'. • Subsidiary family-series taxonominal rank, between family and epifamily. • SYN: apofamilia. • Hoc loco. • Code: no term.

Apognosable, $a$. • TA. • ETY: see apognosis. • For a taxon, that can be distinguished from another taxon on the basis of character states that are considered to be shared by all members of the taxon and absent in all non-members, and that are considered, on the basis of a cladistic analysis and hypothesis, to be autapomorphic for the taxon. • Hoc loco. • Code: no term.

Apognoses, $n$. • Plural of Apognosis.

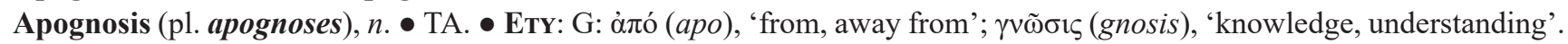
- A cladognosis of a taxon based on character states that are considered to be shared by all members of the taxon and absent in all non-members, and that are considered, on the basis of a cladistic analysis and hypothesis, to be autapomorphic for the taxon. • SYN: 'apomorphy-based definition' (de Queiroz \& Gauthier 1990). • Dubois 1997: 135, 2007a: 43; 2017d: 71. • Code: no term.

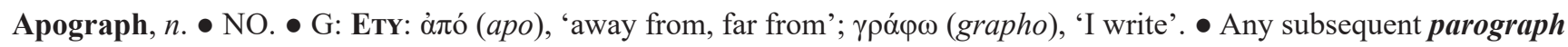
of an existing nomen. ANT: protograph. • Dubois 2010a: 6. Code: subsequent spelling.

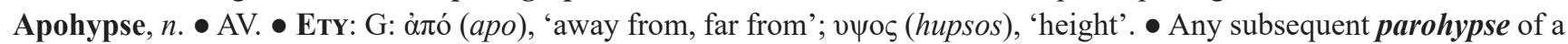
nomen. • ANT: protohypse. • Dubois 2010a: 6. Code: no term.

Apomorphic, $n$. • See Apomorphy.

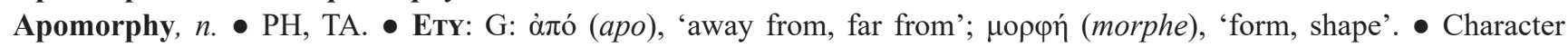
state observed in a taxon which is considered derived relative to the plesiomorphic state of this character in a taxon considered as ancestral. • Hennig 1950. • Code: no term.

Aponym, $n$. • AV. • ETY: G: àjó (apo), 'away from, far from'; óvo $\mu \alpha$ (onoma), 'name'. • Any subsequent paronym of an existing nomen, modified in spelling (apograph), rank (apohypse) and/or, if relevant, onymorph (aponymorph). An aponym is first-used by its scriptor. • AnT: protonym. • Dubois 2000b: 51. • Code: no term.

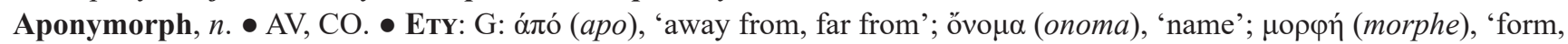
shape'. • Any subsequent paronymorph of a nomen. • AnT: protonymorph. • Dubois 2010a: 6. • Code: no term.

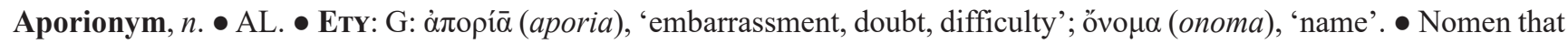
cannot be clearly referred to a taxon in an ergotaxonomy, either for nomenclatural (anaptonym, heterosynaptonym) or for taxonomic (nyctonym) reasons. • Dubois 2008d: 378. Code: one of the meanings of the ambiguous designation nomen dubium.

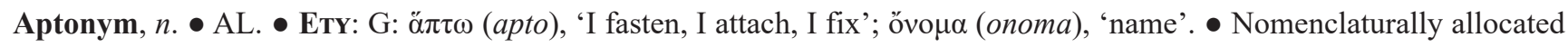
nomen according to the Rules of the Code, i.e., being clearly attached to an onomatophore. • AnT: anaptonym. Dubois 2011a: 25, 79. • Code: no term.

Arbiter, $n$. • NO. • ETY: L: arbiter, 'umpire, arbitrator'. • Author of an airesy, i.e. an onomatergy resolving a conflict of zygoidy. • Dubois 2013: 3. • Code: first reviser.

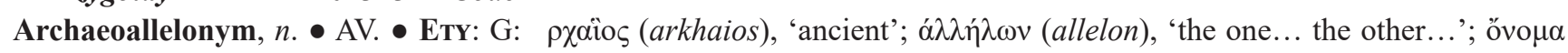
(onoma), 'name'. • One of two (or several) allelonyms which is an already available nomen. • Dubois $2015 c: 43,71$. 
- Code: no term.

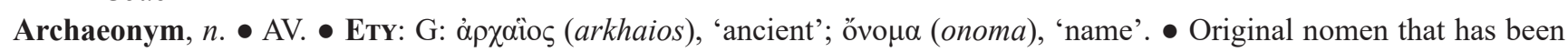
replaced by a neonym. - Dubois 2005a: 88, 2006a: 169, 182. • Code: no term.

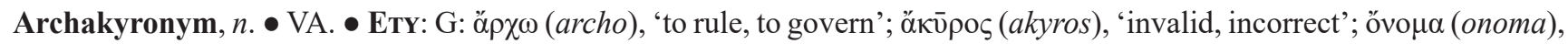
'name'. - Hoplonym permanently invalidated as a result of a specific action of the Commission under its Plenary Power as follows: availability of nomen maintained but removal of its validity (juniorisation) in order to validate another nomen. $\bullet$ New term. $\bullet$ Code: no term.

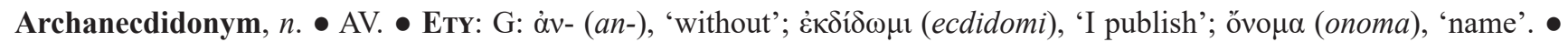
Nomen permanently made unavailable by the Commission under the Plenary Power, through removal of availability of the publication where this nomen had been established. • ANG: Anecdidonym. $\bullet$ Hoc loco. • Code: no term.

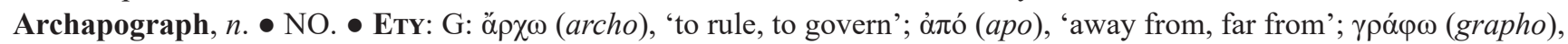
'I write'. - Autoneonym which has been given the status of apograph by the Code (Articles 33.2.3.1, 35.4.1) or by the Commission under the Plenary Power. • Hoc loco. • Code: no term.

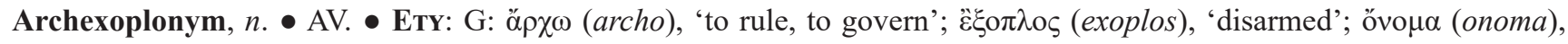
'name'. $\bullet$ Nomen permanently made unavailable by the Commission under the Plenary Power through removal of availability of the nomen itself. • ANG: Exoplonym. • Dubois 2011a: 28, 79; redefined hoc loco. • Code: no term.

Archograph, n. • AV, VA. • ETY: G: å $\rho \chi \omega($ archo), 'to rule, to govern'; $\gamma \rho \alpha ́ \varphi \omega ~(g r a p h o)$, 'I write'. • Eugraph that is imposed to a given nomen following a decision of the Commission under the Plenary Power. $\bullet$ Hoc loco. $\bullet$ Code: no term.

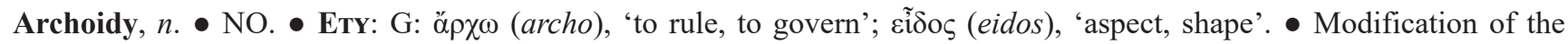
nomenclatural status of a nomen resulting from a specific action of the Commission under the Plenary Power. $\bullet$ Dubois \& Aescht 2019q: 146. • Code: no term.

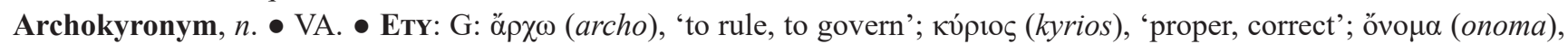
'name'. $\bullet$ Kyronym as a result of a specific action of the Commission under its Plenary Power through removal of validity to another nomen. $\bullet$ Dubois $2011 a: 28,79$. $\bullet$ Code: no term.

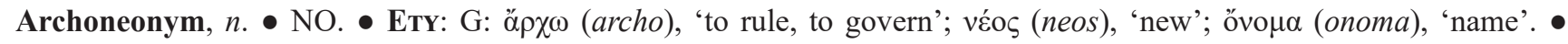
Ameletograph which has been afforded the status of available neonym by the Commission under the Plenary Power. $\bullet$ Hoc loco. $\bullet$ Code: no term.

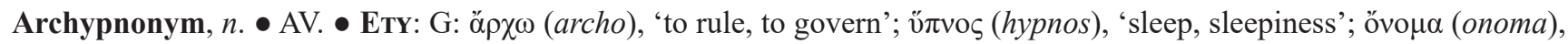
'name'. Hypnonym the availability or validity of which was conditionally removed by the Commission under the Plenary Power. • Dubois 2011a: 28, 79. • Code: no term.

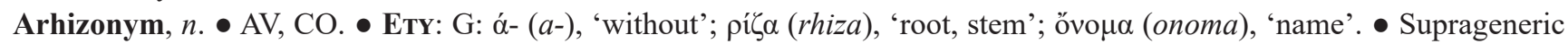
nomen $\mathbf{H N}$ not based on the stem of a genus-series nomen. If proposed as a family-series nomen, it is incorrectly formed according to Article 13.2 of the Code, and is therefore a family-series anoplonym (nomenclaturally unavailable). If proposed as a class-series nomen, it may be available under DONS Criteria (if the other conditions of nomenclatural availability are complied with), and if so it should be used under the spelling which has obtained general acceptance in the literature, if it exists. Apart for a few endings (e.g., -BRANCHIA, -GLOSSA, -PHORA), most endings are used only within limited zoological groups. In all cases where several nomina referred to the same taxonomic group share a common ending, the use of this ending should be homogenised in all of them in order to follow its most common spelling (e.g., -BATraCHia instead of -BATRACHI). • Dubois 2006a: 178, 2015c: 52. • Code: no term.

Assign, v. • AS, AV. • To implement an onomatergy of nominal-series assignment of a nomen. • Common language term, introduced in zoological nomenclature by Dubois (2015a: 6). $\bullet$ Code: no term.

Assigned, $p$. $\bullet \mathrm{AS}, \mathrm{AV}$. $\bullet$ Qualification of a nomen (nemonym) that conforms to the conditions of nominal-series assignment of nomina. - ANT: unassigned. • Common language term, introduced in zoological nomenclature by Dubois (2015a: 29). $\bullet$ Code: no term.

Assignment, $n$. • AS. • Onomatergy regulated by the Code by which a nomen is referred to a nominal-series (e.g., through original statement of the author of the nomen or through objective criteria). $\bullet$ Common language term, introduced in zoological nomenclature by Dubois (2015a: 71). • Code: no term.

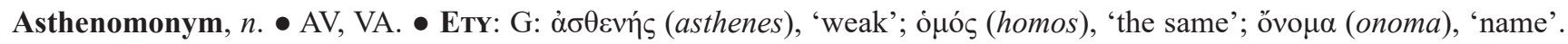
- Any of two (or more) available species-series epithets that are conditional homonyms for being homographs or paromographs (but not pseudomographs) and having been introduced for distinct taxomina and originally referred to different genera but subsequently referred to the same genus not being the first published among them, as long as both epithets remain referred to this genus. $\bullet$ Dubois 2000b: 57 . $\bullet$ Code: secondary homonym (in part).

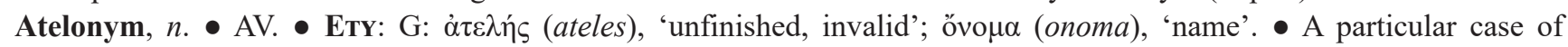
anoplonym: published but nomenclaturally unavailable nomen according to the Code, for not being conform to the provisions of Articles 10, 11 and 14 to 20. Dubois 2011a: 19, 79. - Code: unavailable name. 
Attribute, $v$. See Attribution.

Attribution, $n$. $\bullet$ NO. Rank attribution of a nomen: the referring of a nomen to a nomenclatural rank within its nominalseries. This attribution is labile, being liable to change whenever the ergotaxonomy changes. $\bullet$ Hoc loco. • Code: no term.

Auctor (pl. Auctores), n. • NO, TA. • ETY: L: auctor, 'author, founder'. • In the context of zoological nomenclature, name(s) of the person(s) to whom a published work, nomen or onomatergy is credited, i.e., whose name(s) appear(s) as signatory in the work itself-not through subsequent investigation (see Dubois 2008b). • Dubois 2013: 3. • Hoc loco. - Code: author.

Auctorship, $n$. $\bullet$ NO, TA. • ETY: see Scriptor. $\bullet$ In the context of zoological nomenclature, statement of the auctor of a published work, nomen or onomatergy. $\bullet$ Code: no term.

Author, $n$. $\bullet$ NO, TA. $\bullet$ Person(s) to whom a published work is credited. $\bullet$ Traditional term in science. $\bullet$ Code: author.

Authorship, $n$. $\bullet$ NO, TA. $\bullet$ Statement of the author of a published work is credited. $\bullet$ Traditional term in science. $\bullet$ Code: authorship.

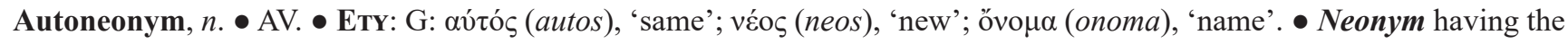
same etymology as its archaeonym, i.e., directly derived from it through unjustified emendation. • ANT: Alloneonym. - Dubois 2000b: 52. • Code: unjustified emendation.

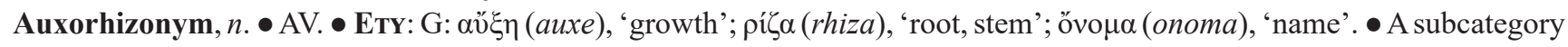
of pseudorhizonym: suprageneric nomen HN (designating a taxon HT) [1] based on the stem of a then available generic nomen GN referred as valid to HT in the ergotaxonomy adopted in the publication where HN was introduced, but [2] combined with an ending derived from another or several other terms (e.g., -formes, -morpha, -phora, etc.). If proposed as a family-series nomen, it is incorrectly formed according to the Code, and is therefore a FS anoplonym. If proposed as a class-series nomen, common particular cases are those of such nomina the original endings of which were derived from the roots forma (Latin) or $\mu \rho \rho \varphi$, morphe (Greek) meaning 'form, shape': under DONS as emended by Dubois \& Frétey $(2020 a)$, it should be fixed under the respective standard endings -IFORMIA or -OMORPHA, which are not in a relation of hierarchy but may be both used at whatever rank. • Dubois 2015c: 22; Dubois \& Frétey $2020 a$ - Code: no term.

Availability, $n$. $\bullet$ AV. $\bullet$ Result of an onomatergy regulated by the Code by which a nomen is promulgated in zoological nomenclature complying with the conditions of this code (hoplonym) or by which an airesy is made effective. • AnT: unavailability. • Term in traditional use in zoological nomenclature. • Code: availability.

Available, $a$. $\bullet$ AV. $\bullet$ Qualification of a nomen (hoplonym) or of an airesy that conforms to the conditions of nomenclatural availability as regulated by the Code. - AnT: unavailable. $\bullet$ Traditional term in nomenclature. • Code: available, potentially valid.

Avatar, $n$. • NO, TA. • ETY: Sanskrit: अवतार (ava-tara), 'successive incarnation of a divinity'. • One of several forms or manifestations that an entity (object, person, organism, concept, term, etc.) has taken or can take. In zoological nomenclature, one of the forms that a nomen can take, regarding its spelling, rank and/or onymorph. • Common language term, recently introduced in zoological nomenclature (Dubois 2005b: 396). • Code: no term.

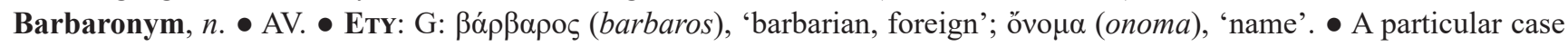
of anoplonym: published but nomenclaturally unavailable nomen according to the Code, for having been published in non-Latinised form and not having been Latinised and adopted as valid before 1900, or for having been published after 1899 (Articles 11.7.1.1, 11.7.2). • Hoc loco. • Code: unavailable name.

Bidirectional ostension, e. $\bullet$ AL. • Composite system of ostension by inclusion and exclusion, pointing both to one or several member(s) and non-member(s) of a class (such as a taxon) (see Dubois 2006c: 25). $\bullet$ Dubois 2007a: $46 . \bullet$ Code: no term.

Bijection, $n$. • PH, TA. • ETY: L: bis, 'twice'; iniectio, 'forcing a fluid into a body'. • One-to-one correspondence (every element of one domain is related exactly to one element of the other domain). $\bullet$ Mathematical term coined by the Bourbaki group (Bourbaki 1970). • SYN: Isomorphism. • Code: no term.

Bijective, $a$. • PH, TA. • ETY: L: bis, 'twice'; iniectio, 'forcing a fluid into a body'. $\bullet$ Qualification of a relation between two domains which follows a function of bijection. - Mathematical term coined by the Bourbaki group (Bourbaki 1970), introduced in zoological taxonomy by Dubois \& Aescht (2019e). • Code: no term.

Binomen (pl. binomina), n. • AV, CO. • ETY: L: bis, 'twice'; nomen, 'name'. • Nomen of rank species, composed of two terms, the generic substantive and the specific epithet. $\bullet$ Traditional term in zoological nomenclature. • Code: binomen.

Binomina, $n$. $\bullet$ Plural of binomen.

Binominal, a. • NO. • ETy: see Binomen. • Qualification of a nomenclatural system like that of the Code, in which taxa of the rank species, and only them, are designated by binomina. $\bullet$ Code: no term.

Biodiversity crisis, $e$. TA, XE. • The fact that the biosphere is facing one of the most severe and violent aggressions of its 
history, because of its exceptional speed. $\bullet$ Wilson 1985. C Code: no term.

Boleogenus, $n$. • TA. • ETY: English: BOL, abbreviation of 'Barcode of Life'; L: genus, 'race, lineage'. • Phenetic genus concept used in the framework of barcode studies, which relies on molecular 'distances' and 'thresholds' between entities to discriminate genus. $\bullet$ Hoc loco. $\bullet$ Code: no term.

Boleon (pl. boleons), $n$. • TA. • ETy: English: BOL, abbreviation of 'Barcode of Life'. • Phenetic taxonomic concept used in the framework of barcode studies, which relies on molecular 'distances' and 'thresholds' between entities to discriminate taxa. • Dubois 2017c: 17. $\bullet$ Code: no term.

Boleospecies, $n$. • TA. • ETY: English: BOL, abbreviation of 'Barcode of Life'; L: species, 'species'. • Phenetic species concept used in the framework of barcode studies, which relies on molecular 'distances' and 'thresholds' between entities to discriminate species. $\bullet$ Hoc loco. $\bullet$ Code: no term.

Branch, $n$. $\bullet$ NO, PH, TA, XE. • A portion of a phylogenetic tree situated between two nodes (dichotomies or polytomies). - Term in traditional use in evolutionary biology. $\bullet$ Code: no term.

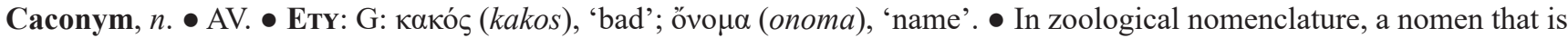
not acceptable for linguistic reasons and is therefore an anoplonym. $\bullet$ Term in use in biological nomenclature. $\bullet$ Code: no term.

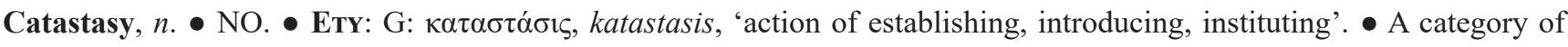
onomatergy: any published founder action of promulgation of a new nomen. $\bullet$ Dubois 2013: 3. • Code: no term.

Categories of usage, e. • AL, VA. • Under the Duplostensional Nomenclatural System, precisely defined categories of usage of class-series nomina, according to [1] the numbers of mentions of a nomen and of its alternative nomina and [2] the dates of these mentions. • END: sozonymorph and distagmonym. • Common language terms; Dubois 2005b, 2010 c. $\bullet$ Code: no term.

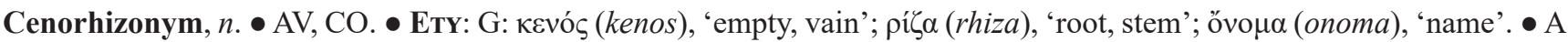
category of pseudorhizonym: suprageneric nomen HN (designating a taxon HT) [1] based on the stem of an available or unavailable genus-series nomen GN, followed by a simple ending denoting plural (e.g., -ae, -idae, -inae, -idi, oidea, -acea, etc), but [2] this nomen not being referred as valid to the taxon HT in the ergotaxonomy adopted in the publication where $\mathbf{H N}$ was introduced. If proposed as a family-series nomen, it is incorrectly formed according to the Code, and is therefore a family-series anoplonym. If proposed as a class-series nomen and available, it should be used with the standard ending -ACEI, which is not in a relation of hierarchy and may be used at whatever rank. • Dubois \& Bour 2011: 157; Dubois 2015c: 53; Dubois \& Frétey 2020a. • Code: no term.

Century of extinctions, e. $\bullet$ XE. $\bullet$ The $21^{\text {st }}$ century, which will witness much more extinctions of biological species than all other centuries in the history of mankind. • Dubois 2003: S9, S18, 2010c,f). $\bullet$ Code: no term.

Character, $n$. TA, AV. • Any intrinsic feature of organisms used for recognising, comparing, differentiating or classifying taxa. In a given taxon, the same character may occur under several distinct alternative character states. $\bullet$ Traditional term in zoological taxonomy. $\bullet$ Code: character.

Character state, e. $\bullet \mathrm{TA}, \mathrm{AV}$. $\bullet$ Any form that a particular character can take. $\bullet$ Traditional term in zoological taxonomy. - Code: no term.

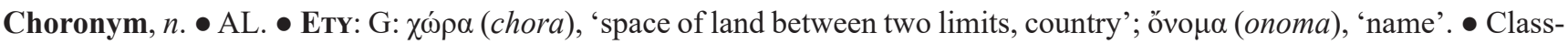
series nomen sozonymorph, taxonomically allocated within the frame of a given ergotaxonomy under DONS Criteria through its orotaxon if present, and being therefore its oronym. This is possible only if all the coalienogenera of this nomen are still its extragenera (excluded from its metrotaxon). If this is not the case, the nomen is a gephyronym and therefore an anaptonym. • Dubois 2006a: 187. • Code: no term.

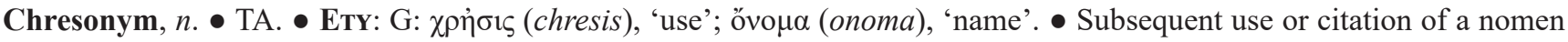
under any of its avatars or paronyms (parographs, parohypses or paronymorphs). • END: orthochresonym and heterochresonym. • Dubois 1982c: 267. • Code: no term.

Chronogenera, $n$. $\bullet$ Plural of chronogenus.

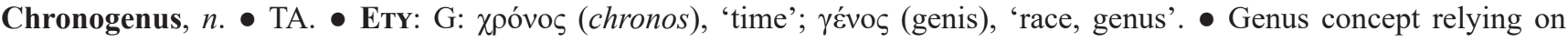
estimates of the absolute geological age of taxa. $\bullet$ Hoc loco. $\bullet$ Code: no term.

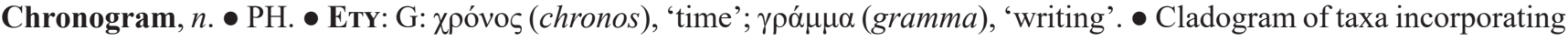
estimates of the absolute geological age of taxa. • Santamaría \& Therón 2009; Brower 2016: 573. • Code: no term.

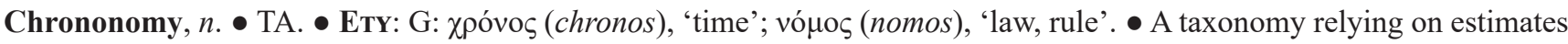
of the absolute geological age of taxa. $\bullet$ Hoc loco. $\bullet$ Code: no term.

Chronotaxa, $n$. $\bullet$ Plural of chronotaxon.

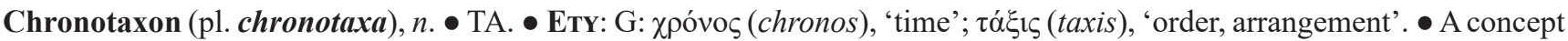
of taxon relying on estimates of the absolute geological age of taxa. $\bullet$ Hoc loco. $\bullet$ Code: no term.

Circumscription, $n$. • AL. • A synonym of extension. • Traditional term in philosophy, logics and didactics. • Code: no term. 
Circumspecific, a. • TA. • ETY: L: circum, 'around, near'; species, 'view, sight, shape, form, kind, species'. • That deals with taxa at ranks just above and below the ranks species and subspecies. $\bullet$ Kiriakoff 1953: 451; Dubois 2011a: 80. $\bullet$ Code: no term.

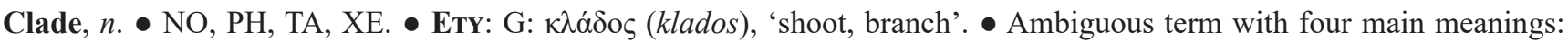
[1] in zoological taxonomy and nomenclature, a key rank (cladus) of the class-series, between phylum and class (Haeckel 1866b; Lankester 1911); [2] in zoological taxonomy and nomenclature, any key rank or 'pseudo-rank' of the family- or class-series, or not even clearly referred to a nominal-series (usual practice in many taxonomic publications nowadays; see e.g. Williams et al. 2016); [3] in evolutionary biology, a homophyletic group of organisms (derived from a common ancestor species), whether complete or not (Huxley 1957); [4] in evolutionary biology, a holophyletic group of organisms, including an ancestor species and all its descendants (Hennig 1950). See also Cladon. $\bullet$ Code: no term.

Cladification, $n$. • TA. • Biological classification based exclusively on the result of a cladistic analysis. • Mayr 1997. • Code: no term.

Cladistic, $a$. $\bullet$ PH, TA. • Referring to an analysis of genealogical relationships between organisms. • Cain \& Harrison 1960. - Code: no term.

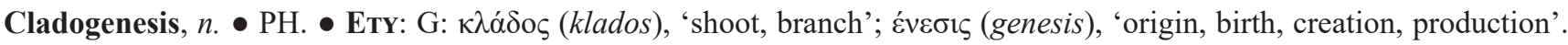
- Splitting of an evolutionary lineage, leading to speciation (see Vaux et al. 2016, 2017 and Allmon 2017). • Rensch 1947: 95. • Code: no term.

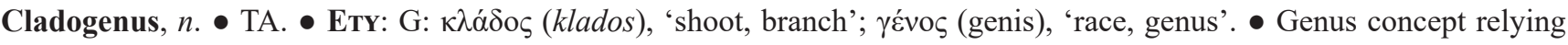
exclusively on the result of a cladistic analysis, applying to a group of species considered holophyletic but without any statement about the limits of this group. $\bullet$ Hoc loco. $\bullet$ Code: no term.

Cladognoses, $n$. Plural of Cladognosis.

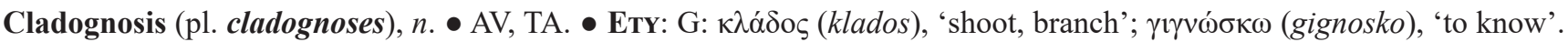
- An intensional definition of a taxon based on a cladistic hypothesis concerning its relationships with other taxa. $\bullet$ Dubois 1997: 135, 2007a: 43, 2017d: 70. • Code: no term.

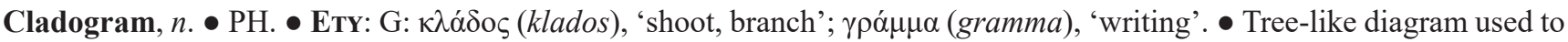
show the genealogical relations between organisms as resulting from a cladistic analysis. $\bullet$ Mayr 1965: 81 (see Dupuis 1984: 3 and Brower 2016). • Code: no term.

Cladon (pl. cladons), $n$. $\bullet$ TA. $\bullet$ Taxon based exclusively on the result of a cladistic analysis. • Mayr 1995. • Code: no term.

Cladonomy, $n$. $\bullet$ TA. $\bullet$ Taxonomy based exclusively on the result of a cladistic analysis. • Brummitt 1997 ; Dubois 1997. - Code: no term.

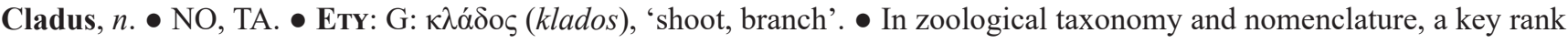
of the class-series, between phylum and class. $\bullet$ Haeckel 1866b. $\bullet$ SYN: clade [1]. $\bullet$ Code: no term.

Clan, n. • NO, TA. • ETY: Scottish Gaelic: clann, 'offspring, children of the family, clan'. $\bullet$ Secondary family-series key rank in zoological taxonomy and nomenclature, below tribe. • Bour \& Dubois 1985: 83. • SyN: clanus. • Code: no term.

Clanus, $n$. • NO, TA. • ETY: Scottish Gaelic: clann, 'offspring, children of the family, clan'. • Secondary family-series key rank in zoological taxonomy and nomenclature, below tribe. • Dubois 2006a: 208. • SyN: clanus. • Code: no term.

Class, $n$. • NO, TA. • ETY: L: classis, 'group, division, class'. • In zoological taxonomy and nomenclature, a key rank of the class-series, between phylum and order. $\bullet$ Term in traditional use in taxonomy. $\bullet$ Syn: classis. $\bullet$ Code: class.

Classification, $n$. • NO, TA. • ETY: L: classis, 'group, division, class'. • [1] Any process or system of ordering objects according to a priori criteria. [2] The result of this process (see ergotaxonomy). $\bullet$ Term in traditional use in biology. $\bullet$ Code: classification.

Classis, $n$. • NO, TA. • ETY: L: classis, 'group, division, class'. • In zoological taxonomy and nomenclature, a key rank of the class-series, between phylum and order. $\bullet$ Term in traditional use in taxonomy. $\bullet$ SyN: class. $\bullet$ Code: no term.

Class-series (CS), e. • NO. • In the nomenclatural hierarchy, the nominal-series ranked above the family-series, which is not fully regulated by the Code. It includes nomina of taxa at the ranks of phylum, class, order, and any additional ranks that may be required. $\bullet$ Dubois 2000b: 40. $\bullet$ Code: no term.

Class-series branch (CS-branch), e. $\bullet$ NO. $\bullet$ Any section of a cladistic tree below the rank order and above the rank family in the corresponding ergotaxonomy. $\bullet$ Hoc loco. $\bullet$ Code: no term.

Coalienogenus (pl. coalienogenera), n. • AL. • ETY: L: cum, 'with'; alienus, 'foreign, unrelated'; genus, 'birth, origin, class, kind'. A category of alienogenus: any member of the indissoluble set of several genus-series taxomina originally excluded from the protaxon for which a new class-series nomen was promulgated. $\bullet$ Hoc loco. • Code: no term.

Code, $n$. • NO. • The International Code on Zoological Nomenclature (see Anonymous 1999).

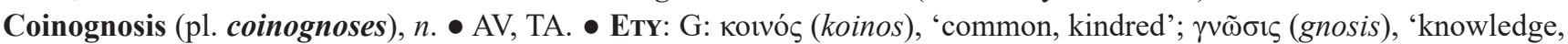
understanding'. Extensional cladognosis of a taxon based directly on the hypothesised cladistic relationships of this 
taxon derived from a cladistic analysis. • Dubois 2008f: 63. Code: no term.

Combination, $n$ • NO, TA. • ETY: L: combinatio, 'mating, assemblage of objects by two'. • A category of onymorph: any paronym of a nomen implying an association between a generic substantive and a specific or subspecific final epithet, irrespective of potential other words in the binomen or trinomen. $\bullet$ Term in traditional use in zoological nomenclature. - Code: combination.

Commission, $n$. $\bullet$ NO. $\bullet$ The International Commission on Zoological Nomenclature (see Anonymous 1999).

Comprehension, $n$. $\bullet$ See Intension.

Compulsory rank, e. $\bullet$ See Mandatory rank.

Connector, $n$. • NO. • Group of letters (e.g., -AID, -OID, -ID, -IN, -IT) connecting (if present) the stem of a family-series nomen (based on a genus-series nomen) to its suffix, and thus contributing to indicating the rank of the taxon to which it applies. • Alonso-Zarazaga 2005: 191; Dubois 2006a: 211; Dubois \& Aescht 2019m: 103. • Code: no term.

Conucleogenera, $n$. $\bullet$ Plural of conucleogenus.

Conucleogenus (pl. conucleogenera), n. • AL. • ETY: L: cum, 'with'; nucleus, 'kernel, nut'; genus, 'birth, origin, class, kind'. Any member of the indissoluble set of several genus-series taxomina originally referred to the protaxon for which a new class-series nomen was promulgated. • Dubois 2006a: 180. C Code: no term.

Coordinated, $p$. $\bullet$ AV. $\bullet$ In the context of zoological nomenclature, qualification of a nomen which exists under several paronyms that are in a relation of coordination. $\bullet$ Traditional term in zoological nomenclature. $\bullet$ Code: no term.

Coordination, $n$. $\bullet$ AV. $\bullet$ In the context of zoological nomenclature, the fact that any nomen created for a taxon at any rank within a nominal-series is deemed to have been simultaneously created for all taxa of other (higher or lower) ranks within that nominal-series including its onomatophore that might have to be recognised. • Traditional term in zoological nomenclature. $\bullet$ Code: coordination.

Correct, $a$. $\bullet$ CO. In the context of zoological nomenclature, qualification of a nomen (eunym) that conforms to the nomenclatural Rules regarding spelling, rank and, if relevant, onymorph. • ANT: incorrect. • Traditional term in nomenclature. $\bullet$ Code: correct.

Correctness, $n$. $\bullet$ CO. • Qualification of a valid nomen (kyronym) which bears a paronym-i.e. a spelling (parograph), rank (parohypse) and, if relevant, onymorph (paronymorph) - that is in agreement with the Rules of the Code. • ANT: incorrectness. - Traditional term in nomenclature. • Code: no term.

Criteria, $n$. Plural of criterion.

Criterion (pl. criteria), $n$. $\bullet$ NO, TA. • In zoological nomenclature, a rule proposed for implementation as a Rule in the Code, but which until this is done does not have the force of law. It may be followed by the zootaxonomists and zoologists who wish so, especially in domains where the Code is silent, such as the taxonomic allocation, validity and correctness of class-series nomina. $\bullet$ Term in traditional use in common language; Dubois 2015c. $\bullet$ Code: no term.

CS, ab. • See Class-series.

CS-branch, e. • See Class-series branch.

Date, $n$. $\bullet$ See Publication date.

Define, $v$. $\bullet$ See Definition.

Definition, $n$. $\bullet$ AV. • Common language term used with several meanings in zoological taxonomy and nomenclature. [1] Regarding the availability of a new nomen: a statement in words of character states, which, in combination, are considered to uniquely distinguish the taxon for which the new nomen is proposed from at least one other taxon of the same rank, the latter being explicitly mentioned. [2] Regarding the taxonomic allocation of a new nomen: see intensional definition and extensional definition. [3] Regarding taxonomic categories: a statement of the kind of information used to refer, if relevant, a taxon to a taxonomic category, and consequently to a nomenclatural rank. $\bullet$ Traditional term in zoological taxonomy. $\bullet$ Code: definition.

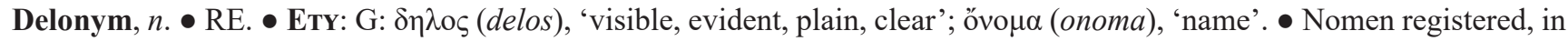
an international nomenclatural database recognised by the Code, thus permanently available in zoological nomenclature.

- ANT: adelonym. - Dubois 2011a: 81. • Code: no term.

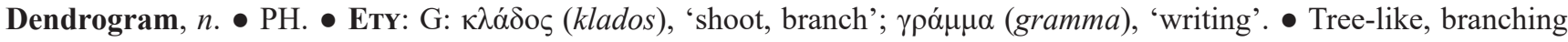
diagram used to indicate 'degrees of relationships' between organisms. • Mayr et al. 1953: 58 81 (see Brower 2016).

- Code: no term.

Description, $n$. (describe, v). • TA, AV. • A statement in words of some taxonomic character states of a specimen. • Traditional term in zoological taxonomy. $\bullet$ Code: description.

Designate, $v$. $\bullet$ AL. $\bullet$ In the context of zoological nomenclature, see Designation.

Designation, $n$. $\bullet$ AL. $\bullet$ In the context of zoological nomenclature, an onomatergy consisting in electing, by an explicit statement, the onomatophore of a newly (original designation) or previously (subsequent designation) established nomen. - Traditional term in zoology and philosophy; Dubois 2006a: 181, 251. Code: typification.

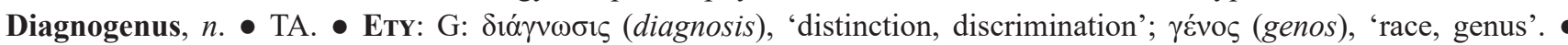


Genus concept relying on two Criteria: [1] genera should be groups of species considered to be strictly holophyletic; [2] they should be diagnosable through characters accessible to the external examination of specimens, i.e. mostly morphological and ecological, but excluding internal anatomical characters, cytogenetic or molecular data. • Hoc loco. - Code: no term.

Diagnosability, a. $\bullet$ TA. ETy: see diagnosis. • For a taxon, the fact that it can be distinguished from another taxon on the basis of characters accessible to the external examination of specimens or to the study of animals in their natural habitat, i.e. mostly morphological, behavioural and ecological, but excluding internal anatomical characters, cytogenetic or molecular data. • Vences et al. 2013: 217-218. • Code: no term.

Diagnosable, $a$. $\bullet$ TA. $\bullet$ ETY: see diagnosis. $\bullet$ For a taxon, that can be distinguished from another taxon on the basis of characters. $\bullet$ Common language term, here used with a precise technical meaning proper to taxonomy. $\bullet$ Code: no term.

Diagnoses, $n$. $\bullet$ Plural of diagnosis.

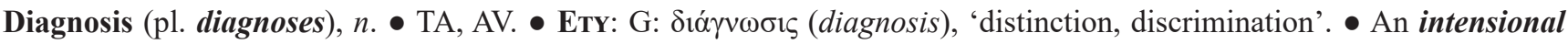
definition of a taxon based on character states, both apomorphic and plesiomorphic, that are considered to be differential for the taxon, i.e., shared by all members of the taxon and absent in all non-members. • Traditional term in taxonomy; Dubois 2017d: 71. Code: diagnosis.

Diagnostic, $a$. • TA. • ETY: see diagnosis. • For a character, that allows distinction between two taxa or more. $\bullet$ Common language term, here used with a precise technical meaning proper to taxonomy. $\bullet$ Code: no term.

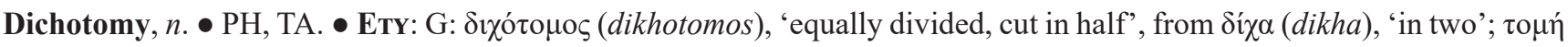
(tome), 'cutting, incision'. • Partition of a set into two subsets. • Common language term. $\bullet$ Code: no term.

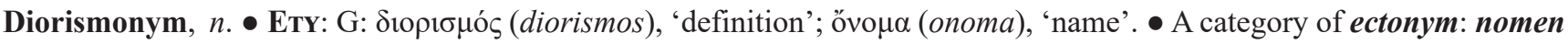
proposed under a nomenclatural system using explicitly intensional definitions instead of ostension for the allocation of nomina to taxa and therefore unavailable under DONS. • Dubois \& Frétey 2020a: 5, 42. • Code: no term.

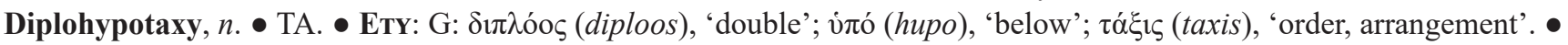
Mode of hypotaxy of a taxon that includes two parordinate taxa of just lower rank. In a phylogenetic taxonomic frame, the meaning of this situation is that a simple hypothesis of relationships between these two taxa is adopted, these two parordinate taxa being considered as sister-taxa. Although this interpretation can be challenged by subsequent works, as long as it is not such a taxonomy appears like a 'final' one. • Dubois \& Raffaëlli 2009: 12. • Code: no term.

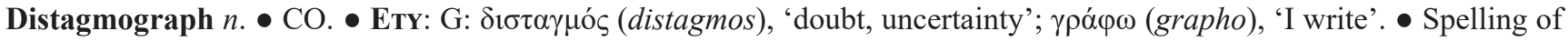
class-series nomen that has not had a universal or significant use in the literature after 31 December 1899 (i.e., that did not appear in at least 100 titles of publications since then). $\bullet$ ANT: sozograph. $\bullet$ Hoc loco. $\bullet$ Code: no term.

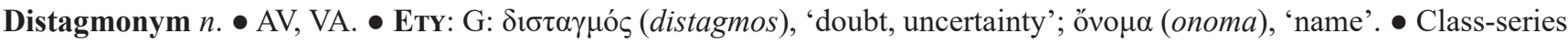
nomen that has not had a universal or significant use in the literature after 31 December 1899 (i.e., that did not appear in at least 100 titles of publications since then). • ANT: sozonymorph. • Dubois 2005a: 86, 2005b: 412. • Code: no term.

Distributed, $p$. $\bullet$ NO, TA. • For a work produced on paper, on an electronic disc or released by electronic means: publicly issued and dissiminated. - Common language term, here issued with a technical meaning relating to zoological taxonomy or nomenclature. $\bullet$ Code: distributed.

Distribution, $n$. $\bullet$ NO, TA. - The public issue and dissemination of a work produced on paper, on an electronic disc or released by electronic means. - Common language term, here issued with a technical meaning relating to zoological taxonomy or nomenclature. $\bullet$ Code: distribution.

DONS, $a b$. • See Duplostensional Nomenclatural System.

Double auctorship, e. • VA. • Qualification of the auctorship (and date) of a family-series junior synonym validated through Articles 35.4 .1 or 40.2 (see Dubois 2015a: 31-34). See primary auctorship and secondary auctorship. $\bullet$ Hoc loco. $\bullet$ Code: no term.

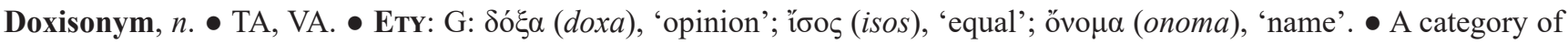
synonym: any of two or more nomina based on different onomatophores but considered, for subjective (taxonomic) reasons, to denote the same taxon, whose inclusive extension includes both their onomatophores. • Dubois $2000 b$ : 57. - Code: subjective synonym.

D-publication, $n$. • AV. • Publication released on optical disc (CD-Rom, DVD). • Dubois et al. 2013: 5. • Code: work on optical disc.

Duplostensional Nomenclatural System (DONS), e. • NO. • A composite class-series nomenclatural system in which distagmonyms are allocated to taxa through MONS whereas sozonymorphs are so through OONS if they are oronyms, through MONS if they are ellitonyms, or are unallocated if they are gephyronyms. • Dubois 2015a: 13. • Code: no term.

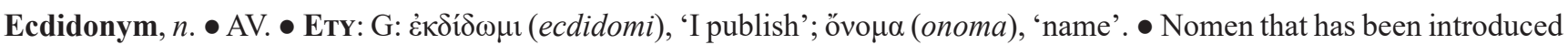


in a publication available under the Code. $\bullet$ ANT: Anecdidonym. $\bullet$ Hoc loco. $\bullet$ Code: no term.

Ecogenus, $n$. • TA. • ETY: G: oĩ which genera should be groups of species being morphological and ecological units, sharing closely related ecological niches and adaptive zones. $\bullet$ Hoc loco. $\bullet$ Code: no term.

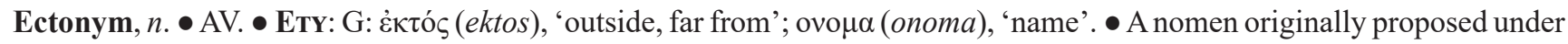
a nomenclatural system different from that of the Code and incompatible with it. This applies to nomina proposed within the framework of alternative nomenclatural systems, or simply which do not respect some of the basic requirements of the Code such as binominal nomenclature for species, the assignment of nomina to nominal-series and ranks, or the taxonomic allocation of nomina through ostension with onomatophores but not through verbal intensional definitions (see e.g. Dubois 2011a, 2015c; Dubois \& Frétey 2020a). • Dubois 2020a: 7, 38. • Code: no term.

Effective, $a$. • AL. • Qualification of an onomatergy that makes it actual under the Rules of the Code. $\bullet$ Traditional term in common language, introduced in zoological nomenclature by Dubois \& Aescht (2019s: 166). • Code: no term.

Elect, $v$. $\bullet$ AL. $\bullet$ In the context of zoological nomenclature, see Election.

Election, $n$. $\bullet$ AL. $\bullet$ A general term for the fixation of the onomatophore of a nomen, whether by original or by subsequent designation. • END: designation, monophory, tautonymy. • Traditional term in nomenclature; Dubois \& Aescht $2017 e$ : 33. Code: no term.

Electonucleogenera, $n$. • Plural of electonucleogenus.

Electonucleogenus (pl. electonucleogenera). • AL. • ETY: L: eligo, 'pick up, choose'; nucleus, 'nucleus, core, stone' (from nux, 'nut'); genus, 'birth, origin, class, kind'. • Nominal genus subsequently designated among the prenucleogenera of a family-series being an arhizonym. $\bullet$ Hoc loco. $\bullet$ Code: no term.

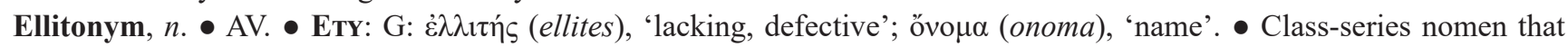
misses an onomatostasis (alienogenera) and that therefore can be validated only as a metronym under the Ostensional Nomenclatural Systems. - One of the two meanings of the term nesonym as defined by Dubois (2015c: 65), hereby distinguished from the latter. $\bullet$ Code: no term.

Empire, $n$. $\bullet$ NO, TA. • ETY: L: imperium, 'supreme power, empire'. • Highest class-series key rank in biological taxonomy and nomenclature. $\bullet$ Term in traditional use in taxonomy. $\bullet$ SYN: imperium. $\bullet$ Code: no term.

Ending, $n$. $\bullet$ NO. $\bullet$ For the purpose of zoological nomenclature, the letter or group of letters at the end of a nomen. In the species- and genus-series, the ending is composed of the suffix alone; in the family-series, the ending indicates the rank of the taxon and is composed of the connector (if present) and the suffix. • END: fixed ending and variable ending. - Term of grammar, in traditional use in biological nomenclature, redefined by Dubois \& Aescht 2019j,r). • Code: ending.

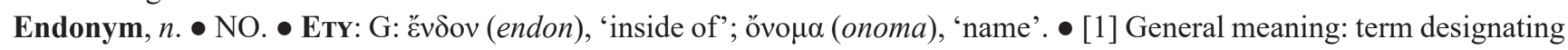
a subordinate class. [2] Specialised meaning in nomenclature: nomen which applies to an endotaxon in a given ergotaxonomy. • Dubois \& Aescht 2019h: 76. • Code: no term.

Endotaxa, $n$. $\bullet$ One of the two plurals of endotaxon.

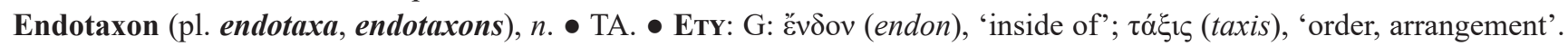
- Any taxon which is subordinate to another taxon (its angiotaxon) in a given ergotaxonomy. • Dubois $2005 b$ : 406.

- Code: no term.

Endotaxons, $n$. $\bullet$ One of the two plurals of endotaxon.

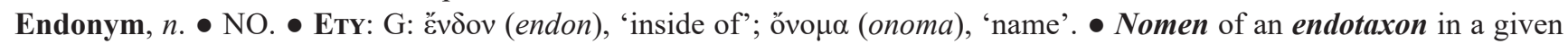
ergotaxonomy. • Dubois \& Aescht 2019i: 76. • Code: no term.

Endotaxa, $n$. $\bullet$ One of the two plurals of endotaxon.

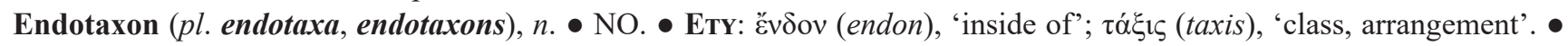
Any taxon which is subordinate to another taxon (its angiotaxon) in a given ergotaxonomy. • Dubois $2005 b$ : $406 . \bullet$ Code: no term.

Endotaxons, $n$. $\bullet$ One of the two plurals of endotaxon.

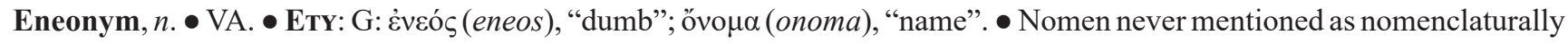
available by any author and in any publication after 31 December 1899. • Dubois 2005a: 85, 2005b: 411. • Code: no term.

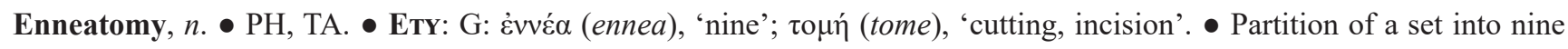
subsets. $\bullet$ Hoc loco. $\bullet$ Code: no term.

Epifamilia, $n$. • NO, TA. • EтY: G: غ̇ंí (epi), 'on, over'; L: familia, 'family’ • Subsidiary family-series taxonominal rank, between apofamily and superfamily. • SyN: epifamily. • Bour \& Dubois 1985. • Code: no term.

Epifamily, n. • NO, TA. • ETY: G: ė $\pi$ í (epi), 'on, over'; L: familia, 'family'. • Subsidiary family-series taxonominal rank, between apofamily and superfamily. • SYN: epifamilia. • Bour \& Dubois 1985. • Code: no term.

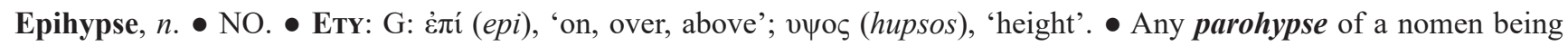


superordinate to another parohypse of the same nomen. • ANT: hypohypse. • Dubois 2006b: 828 (as 'epinym'), 2011a: 22, 82. - Code: no term.

EPITA, $a b$. See Explicit internal airesy.

Epithet, $n$. • NO. • Specific or subspecific nomen, never bearing a capital, being part of a binomen or trinomen. • Traditional term in zoological nomenclature. • Code: species-group name [English text]; nom du niveau espèce [French text].

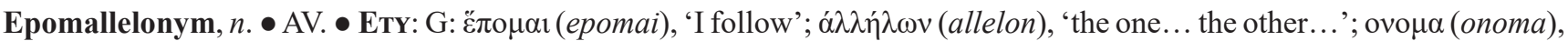
'name'. • One among two parallelonyms which was clearly not given preference over the other one (its agoallelonym) in the original publication, and which for this reason does not have precedence over it. $\bullet$ Dubois 2015c: 43, 73. • Code: no term.

E-publication, $n$. • AV. • Publication distributed electronically online. • Dubois et al. 2013: 5. • Code: work issued and distributed electronically.

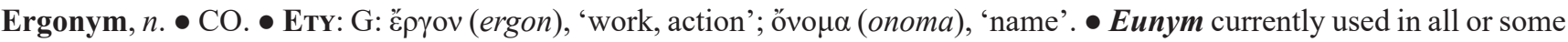
ergotaxonomies. • ANT: argionym. • Dubois 2000b: 54. $\bullet$ Code: no term.

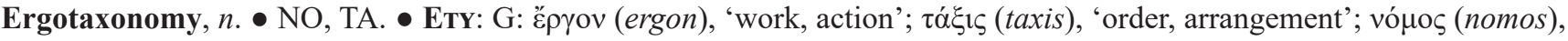
'law, rule'. • Any classification considered valid in a certain work by a given author. • Dubois 2005b: 406. $\bullet$ Code: no term.

ETA, $a b$. $\bullet$ See External airesy.

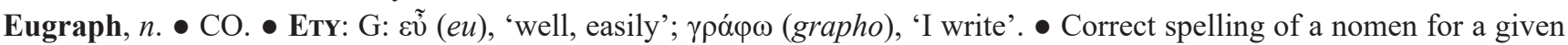
taxon in a given ergotaxonomy. This spelling may be imposed by the Code or by DONS to a given nomen, superseding its protograph if necessary: [1] either for being a nomograph (eunomograph or legonomograph); [2] or for being an archograph; [3] or for being a legethograph. • ANT: nothograph. • Dubois 2010a: 7, 40. • Code: correct original spelling, justified emendation, mandatory change.

Eugraphy, n. CO. • ETY: see Eugraph. • Rules and Criteria allowing to establish the eugraph of a nomen for a given taxon in a given ergotaxonomy, following the Code for SS, GS and FS nomina (nomographs and archographs) or DONS for CS nomina (legethographs). $\bullet$ Hoc loco. $\bullet$ Code: no term.

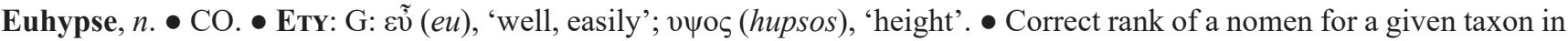
a given ergotaxonomy. $\bullet$ Dubois 2010a: 7. • Code: no term.

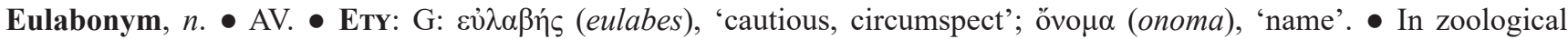
nomenclature, a nomen that is proposed conditionally after 1960 and is therefore an anoplonym. • Hoc loco. • Code: no term.

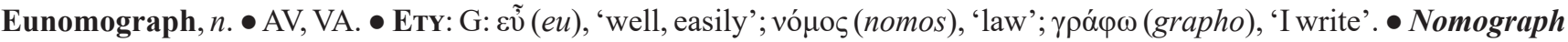
that is imposed by the Code to a given nomen in a given ergotaxonomy, superseding the protograph because the protograph is an original nothograph and must go through a mandatory spelling correction (Dubois 2013: 10). • Hoc loco. - Code: justified emendation.

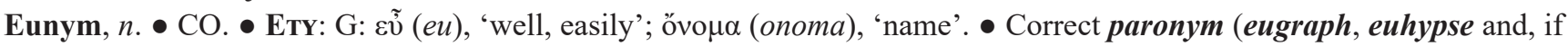
relevant, eunymorph) of a nomen for a given taxon in a given ergotaxonomy. • ANT: nothonym. • Dubois $2000 b$ : 54. - Code: no term.

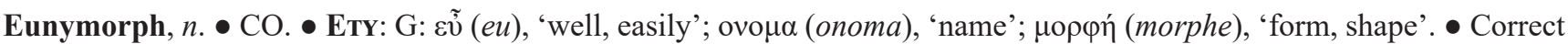
onymorph of a nomen for a given taxon in a given ergotaxonomy. • Dubois 2010a: 7. • Code: no term.

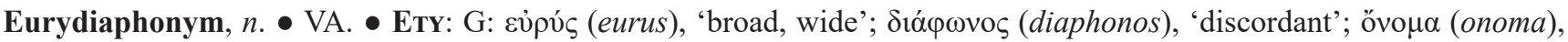
'name'. - Nomen that has been used as valid for a given taxon, or for taxa having totally or partially identical extensions, in the titles of 100 scientific works after 31 December 1899 . D Dubois 2005a: 85, 2005b: 412. • Code: no term.

Exclusive extension, $e$. $\bullet$ AL. $\bullet$ System of extension by exclusion, listing all non-member(s) of a class (such as a taxon). $\bullet$ Dubois 2005b: 379 . $\bullet$ Code: no term.

Exclusive ostension, e. $\bullet$ AL. $\bullet$ System of ostension by exclusion, pointing to one or several non-member(s) of a class (such as a taxon). $\bullet$ Dubois 2006c: 25. $\bullet$ Code: no term.

Exhaustive taxonomy, e. • TA, XE. • Development of a strategy for deploying enough manpower and funds for field work to approach an (almost) exhaustive taxonomic inventory of a taxonomic group worldwide. • Dubois 2008e. $\bullet$ Code: no term.

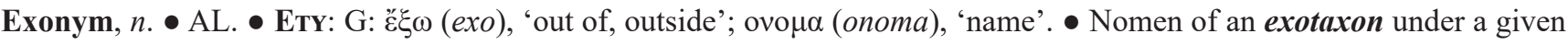
ergotaxonomy. $\bullet$ Hoc loco. $\bullet$ Code: no term.

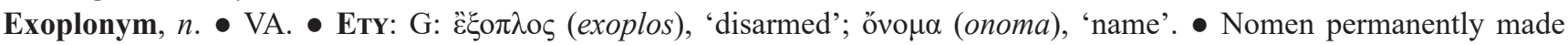
unavailable by the Commission under the Plenary Power, through one of the following actions: [1] removal of availability of the publication where this nomen had been established (archanecdidonym); [2] removal of availability of the nomen itself (archexoplonym). • Dubois 2000b: 51. • Code: no term. 
Exotaxa, $n$. One of the two plurals of exotaxon.

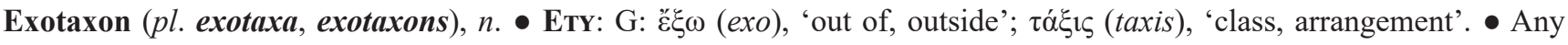
external (or 'sister') taxon of a given taxon of the same rank (parordinate) in a given ergotaxonomy. Under DONS, this concept applies to any CS taxon including one or several alienogenera of a CS nomen. • Dubois \& Frétey $2020 a$.

- Code: no term.

Exotaxons, $n$. One of the two plurals of endotaxon.

Explicit internal airesy (EPITA), e. • VA, CO. • An internal airesy which is explicit i.e., all competing spellings being mentioned and one of them being designated as correct. $\bullet$ Dubois 2013: 12. $\bullet$ Code: no term.

Extant, $n$. $\bullet$ XE. $\bullet$ For a taxon of AMPHibia: that includes at least one living (non-fossil) species.

Extension, $n$. $\bullet$ AL. $\bullet$ System of allocation of a nomen to a concept or class (such as a taxon) through providing a list of all objects that satisfy the intensional definition of a concept (inclusive extension), or that do not satisfy it (exclusive extension). • Traditional term in philosophy, logics and didactics (see Dubois 2005a: 74, 2005b: 379). Code: no term.

Extensional, $a$. • AL. • See Extension.

Extensional definition, $e$. $\bullet$ AL. $\bullet$ Definition of a concept or class (such as a taxon) based on extension. $\bullet$ Traditional term in philosophy, logics and didactics (see Dubois 2005b: 379). $\bullet$ Code: no term.

External airesy (ETA), e. • VA, CO. • An airesy taken in case of zygography under Article 24.2.3 of the Code by an author or authors not being the original auctor(s) of the nomen. To be valid, an external airesy must be explicit, i.e., both competing spellings must be mentioned and one of them must be unambiguously designated as correct. $\bullet$ Dubois 2013: 12. Code: no term.

Extinct, $n$. XE. • For a taxon of AMPHiBia: an extant taxon all members of which are considered to have become extinct during the Anthropocene.

Extragenera, $n$. • Plural of extragenus.

Extragenus (pl. extragenera), n. • AL. • ETY: L: extra-, 'out of, outside'; genus, 'birth, origin, class, kind'. • Any of the alienogenera allowing to ascertain the external limits of a class-series taxon and therefore to identify its sister-taxon or -taxa of same rank (parordinate) under the Orostensional Nomenclatural System. • Dubois 2006a: 230. • Code: no term.

Familia, $n$. $\bullet$ NO, TA. • ETY: L: familia, 'family'. • Highest family-series key rank in zoological taxonomy and nomenclature. - Term in traditional use in taxonomy. $\bullet$ SYN: family. $\bullet$ Code: no term.

Family, $n$. $\bullet$ NO, TA. • ETY: L: familia, 'family'. Highest family-series key rank in zoological taxonomy and nomenclature. - Term in traditional use in taxonomy. $\bullet$ SYN: familia. $\bullet$ Code: no term.

Family-series (FS), e. • NO. • In the nomenclatural hierarchy, the highest-ranking nominal-series fully regulated by the Code. It includes nomina of taxa at the ranks of family, subfamily, tribe, subtribe, superfamily, and any additional ranks that may be required. $\bullet$ Dubois 2000b: 40. $\bullet$ Code: family group [English text]; niveau famille [French text].

Family-series branch (FS-branch), e. $\bullet$ NO. • Any section of a cladistic tree below the lowest class-series rank and above the rank genus in the corresponding ergotaxonomy. $\bullet$ Hoc loco. $\bullet$ Code: no term.

Final epithet, $e$. $\bullet$ NO.• Epithet designating a taxon, either of specific or of subspecific rank, which is the lowest ranked one in a given classification. - Term in use in botanical nomenclature (Turland et al. 2018), introduced in zoological nomenclature by Dubois (2011a: 58,83). • Code: no term.

First reviser, e. $\bullet$ NO. $\bullet$ Code: first reviser. $\bullet$ See Arbiter.

First-use, $n$. $\bullet$ AV. $\bullet$ The result of the process by which a scriptor uses for the first time in the taxonomic literature a new aponym (apograph, apohypse or aponymorph) for a hoplonym. • Term in common use with various meanings in common language, here used with a precise technical meaning proper to nomenclature; Dubois 2000b: 44. • Code: no term.

First-use, $v$. $\bullet$ AV. $\bullet$ The process by which a scriptor uses for the first time in the taxonomic literature a new aponym (apograph, apohypse or aponymorph) for a hoplonym. - Term in common use with various meanings in common language, here used in a specialised technical sense proper to nomenclature; hoc loco. • Code: no term.

First-user, e. $\bullet \mathrm{AV}$. $\bullet$ Name(s) of the scriptor who appear(s) as author of the work where an aponym was first published. - Dubois 2000b: 42. - See Scriptor.

Fixed ending, e. $\bullet$ NO. Ending of a nomen that is not liable to change according to the ergotaxonomy adopted. This includes in particular the following two situations: [1] species-series epithet in the genitive case: suffix reflecting in some cases the genders and numbers of the persons or places referred to by the epithet; [2] genus-series substantive: suffix indicating its grammatical gender. • Dubois \& Aescht 2019j: 103. • Code: no term.

FRR, ab. • See Fully regulated family-series ranks.

FS, $a b$. $\bullet$ See Family-series.

FS-branch, e. • See Family-series branch. 
Fully regulated family-series ranks (FRR), e. • NO. • Ranks of the family-series for which mandatory endings are prescribed by the Code (Articles 29.2 and 34.1): superfamily (-OIDEA), family (-IDAE), subfamily (-INAE), tribe (-INI) and subtribe (-INA). • Dubois \& Aescht 2019o: 128. $\bullet$ Code: no term.

Gender, $n$. $\bullet$ NO. • In some languages (e.g. Latin languages or German), each of the classes (masculine, feminine, common, neuter) of nouns and pronouns distinguished by different inflections in words syntaxically associated with them. $\bullet$ Term of grammar, in traditional use in zoological nomenclature. • Code: gender.

Generic substantive, $e$. $\bullet$ NO. Generic or subgeneric nomen, always bearing a capital, being part of a binomen or trinomen. Dubois 2000b: 40. - Code: generic name, genus name, name of a genus.

Genion, $n$. $\bullet$ TA. $\bullet$ A taxonomic category of nomenclatural rank genus. $\bullet$ Dubois 2009c: 29, 45. $\bullet$ Code: no term.

Genus, $n$. $\bullet$ NO, TA. • ETY: L: genus, 'birth, origin, class, kind'. • Only genus-series key rank in zoological taxonomy and nomenclature. $\bullet$ Term in traditional use in taxonomy. $\bullet$ Code: genus.

Genus-series, e. $\bullet$ NO. $\bullet$ In the nomenclatural hierarchy, the nominal-series ranked between the species-series and the family-series. It includes taxa at the ranks of genus and subgenus. $\bullet$ Dubois 2000b: 40. $\bullet$ Code: genus group [English text]; niveau genre [French text].

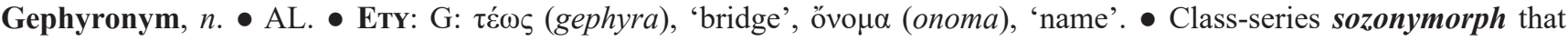
cannot be taxonomically allocated within the frame of a given ergotaxonomy because at least one of its coalienogenera is now one of its intragenera. This nomen is therefore an anaptonym. $\bullet$ Hoc loco. $\bullet$ Code: no term.

Gephyrotaxa, $n$. • One of the two plurals of gephyrotaxon. • Dubois 2005b: 407.

Gephyrotaxic, a. N, TA. • ETY: see Gephyrotaxy. Qualification of two distinct taxa being in a relation of partial overlap of their extensions, in a given ergotaxonomy, and whose nomina are assigned to the same or different nominalseries. • Dubois 2005b: 407. • Code: no term.

Gephyrotaxon (pl.gephyrotaxa, gephyrotaxons), $n$. • NO, TA. • ETY: see Gephyrotaxy. • One of two distinct taxa being in a relation of partial overlap of their extensions, in a given ergotaxonomy, and whose nomina are assigned to the same or different nominal-series. • Dubois 2005b: 407. • Code: no term.

Gephyrotaxons, $n$. $\bullet$ One of the two plurals of gephyrotaxon. $\bullet$ Hoc loco.

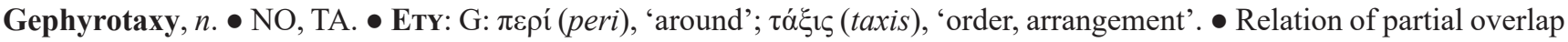
of their extensions, in a given ergotaxonomy, and whose nomina are assigned to the same or different nominal-series.

- Dubois 2005b: 407. • Code: no term.

Getalienogenera, $n$. $\bullet$ Plural of getalienogenus.

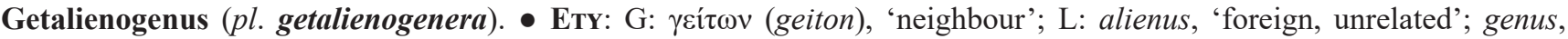
'birth, origin, class, kind'. - Closest alienogenus of a CS nomen allowing to ascertain the external limits of the CS taxon designated by this nomen and therefore to identify its parordinate sister-taxon or -taxa under the Orostensional Nomenclatural System. • Dubois 2006a: 189, 253 (as 'getextragenus'); renamed by Dubois \& Frétey (2020a). • Code: no term.

Getangiotaxa, $n$. • Plural of getangiotaxon.

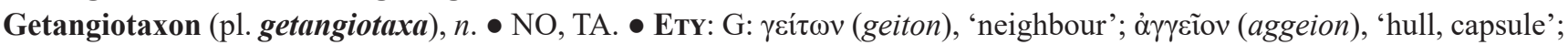

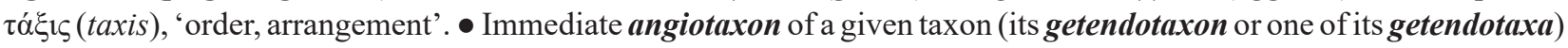
in a given ergotaxonomy. • Dubois \& Berkani 2013: 53. • Code: no term.

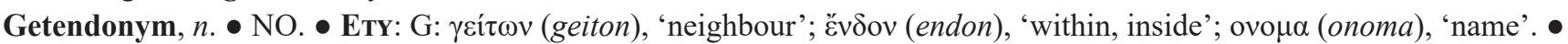
Nomen of a getendotaxon under a given ergotaxonomy. Under DONS Criteria, class-series nomen/nomina allowing the taxonomic allocation of another class-series taxon through inclusion only. $\bullet$ Hoc loco. $\bullet$ Code: no term.

Getendotaxa, $n$. P Plural of getendotaxon.

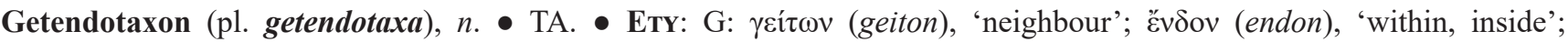
$\tau \alpha \dot{\xi} \xi \varsigma$ (taxis), 'order, arrangement'. - Immediate subordinate taxon of a given taxon (its getangiotaxon) in a given ergotaxonomy. • Dubois \& Berkani 2013: 53. • Code: no term.

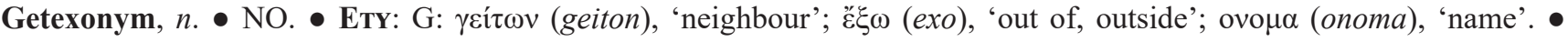
Nomen of a getexotaxon under a given ergotaxonomy. Under DONS Criteria, class-series nomen/nomina allowing the taxonomic allocation of another class-series taxon through both inclusion and exclusion. $\bullet$ Dubois \& Frétey $2020 a$. $\bullet$ Code: no term.

Getexotaxa, $n$. Plural of getexotaxon.

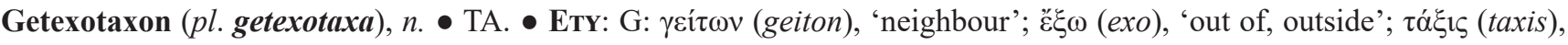
'order, arrangement'. - A category of exotaxon: closest external (or 'sister') taxon of a given taxon of the same rank (parordinate) in a given ergotaxonomy. Under DONS, this concept applies to the closest CS taxon including one or several alienogenera of a CS nomen and allowing to ascertain the external limits of the CS taxon designated by an oronym and therefore to identify, through its getalienogenus or getalienogenera, its parordinate CS taxon or taxa under the Orostensional Nomenclatural System. - Dubois 2015c: 74. • Code: no term. 
Getextragenus, $n$. $\bullet$ NO. • Obsolete for Getalienogenus.

Getonucleogenera, $n$. $\bullet$ Plural of getonucleogenus.

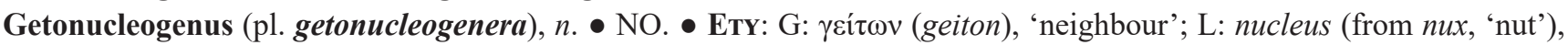
'nucleus, core, stone'; genus, 'birth, origin, class, kind'. • One of the closest conucleogenera of a class-series taxon, allowing to identify its class-series metronym under the Metrostensional Nomenclatural System. • Hoc loco. • Code: no term.

Grade, $n$. • PH. • A level of biological organisation and complexity of organisms, term devoid of cladistic meaning. • Lankester 1877: 399. $\bullet$ Code: no term.

GS, $a b$. • See Genus-series.

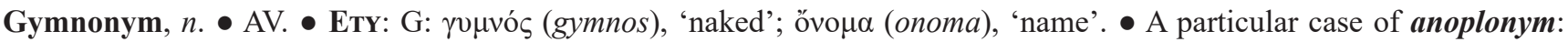
published but nomenclaturally unavailable nomen according to the Code, for failing to comply with the provisions of Articles 12 or 13 (i.e., missing a diagnosis or description, and in some cases an onomatophore). Dubois 2000b: 49-50.

- Code: nomen nudum.

Gymnonymy, $n$. • AV. • ETY: see Gymnonym. $\bullet$ The fact that a new nomen is nomenclaturally unavailable nomen according to the Code, for failing to comply with the provisions of Articles 12 or 13 (i.e., missing a diagnosis or description, and in some cases an onomatophore). $\bullet$ Hoc loco. $\bullet$ Code: no term.

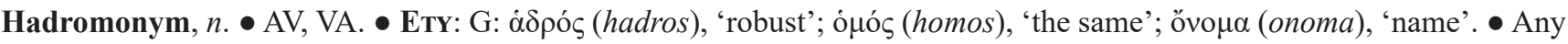
of two or more available nomina introduced for distinct taxomina and being permanently homonyms for being either: [1] in the family-series, rhizomographs; or [2] in the genus-series, homographs; or [3] in the species-series, epithets being homographs or paromographs (but not pseudomographs) originally referred to the same priscogenus. • Dubois 2000b: 57. Code: [1] and [2] homonym; [3] primary homonym and secondary homonym (in part).

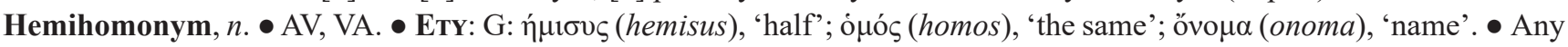
of two or more distinct nomina that are homographs but that belong in different nominal-series (in zoology) or which depend on different Codes (e.g., zoological, botanical and bateriological). • Starobogatov 1984, 1991: 8; Shipunov 2011: 65. - Code: no term.

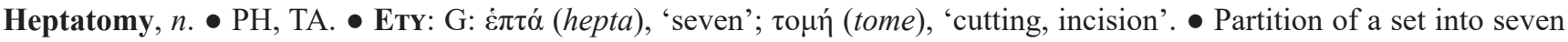
subsets. $\bullet$ Hoc loco. $\bullet$ Code: no term.

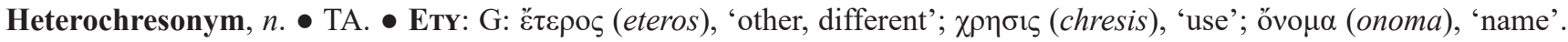
- Chresonym inappropriately used to designate a taxon (misidentification). • ANT: orthochresonym. • Dubois 2000b: 59. - Code: no term.

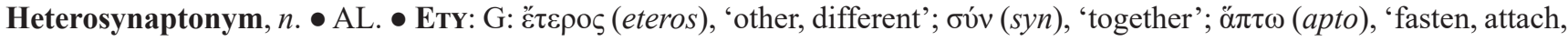
fix'; ővora (onoma), 'name'. - Synaptonym considered taxonomically heterogeneous (composed of specimens or taxomina currently referred to different taxa). • ANT: homosynaptonym. • Dubois 2011a: 25, 84. • Code: one of the meanings of the ambiguous designation nomen dubium.

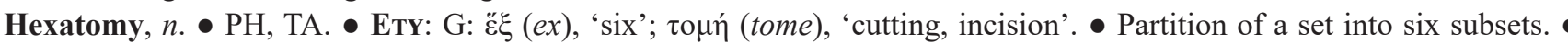
Common language term. $\bullet$ Code: no term.

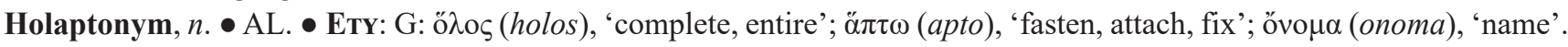
- Monaptonym whose monophoric onomatophore (holophoront, nucleospecies or nucleogenus) was designated in the original publication where the nomen was promulgated. • Dubois 2011a: 25, 84. • Code: no term.

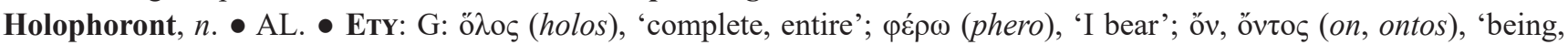
individual'. • Single specimen originally elected as onymophoront of a species-series nomen. • Dubois $2005 b$ : 403. - Code: holotype.

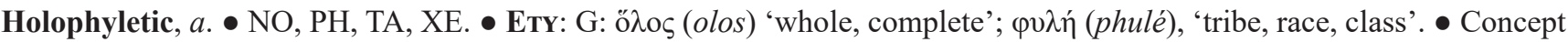
applying to taxa: qualification of a taxon considered to include all the descendants of its most recent common ancestor as well as the latter. • SyN: monophyletic sensu Hennig (1950). Ashlock 1971: 63. • Code: no term.

Holophyly, $n$. $\bullet$ See Holophyletic.

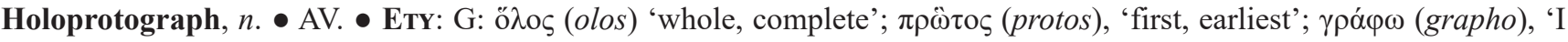
write'. $\bullet$ A category of protograph: unique original spelling of a nomen. • AnT: symprotograph. Dubois \& Aescht 2019l: 112. Code: original spelling.

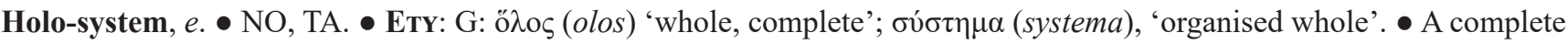
taxonomic or nomenclatural system for a given group of organisms, i.e., allowing unambiguous, objective, repeatable and universal decisions in all cases and situations. $\bullet$ Dubois 2015c: 8, 74. • Code: no term.

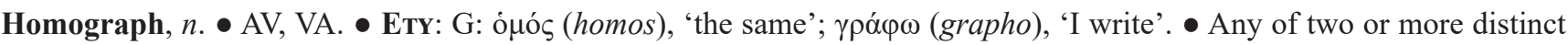
nomina (having different auctors, dates and onomatophores) of the same nominal-series having the exactly same spelling (even if having different grammatical genders). • Term in traditional use in common language, introduced in zoological nomenclature by Dubois (2012a: 64). • Code: no term. 
Homographic, $n$. $\bullet$ AV, VA. • ETY: see Homograph. • Term having the exactly same spelling as another one. • Dubois 2012a: 64. Code: no term.

Homography, $n$. • AV, VA. • ETY: see Homograph. • The fact that two distinct nomina are homographs. • Dubois 2012a: 64. Code: no term.

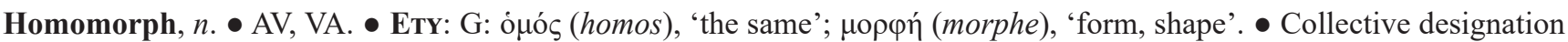
for the set of all the homonymorphs, i.e., nomina based on the same stem, irrespective of their nominal-series and of their ending. • Term in traditional use in common language; Dubois 2015c: 17, 74. • Code: no term.

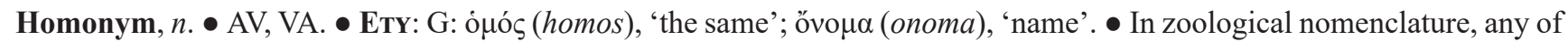
two or more distinct hoplonyms (having different authors, dates and onomatophores) of the same nominal-series having spellings deemed to be identical under the Code. • Endonyms: [1] homograph, rhizomograph and paromograph; [2] asthenomonym and hadromonym. - Term in traditional use in common language and in zootaxonomy. • Code: homonym.

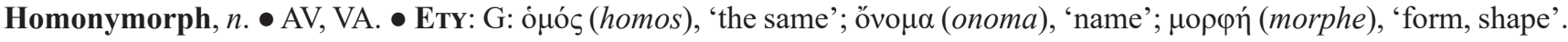
- Any member of a homomorph. • Dubois 2015c: 17, 74. • Code: no term.

Homonymous, a. • AV, VA. • ETY: see Homonym. • In zoological nomenclature, the qualification of two distinct nomina of the same nominal-series that are homonyms under the Code. $\bullet$ Term in traditional use in common language and in zootaxonomy. $\bullet$ Code: homonymous.

Homonymy, $n$. • AV, VA. • ETY: see Homonym. • In zoological nomenclature, the fact that two distinct nomina of the same nominal-series are homonyms under the Code. $\bullet$ Term in traditional use in common language and in zootaxonomy. $\bullet$ Code: homonymy.

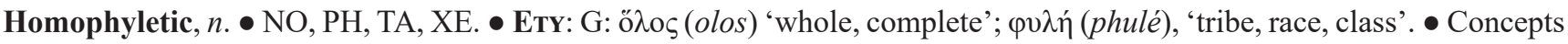
applying to taxa: qualification of a non-polyphyletic taxon, considered to be composed of descendants of a common ancestor (see monophyletic), but including either all of them (see holophyletic) or only some of them (see paraphyletic) - ETy: monophyletic sensu Haeckel (1866b). • Dubois 1986b. • Code: no term.

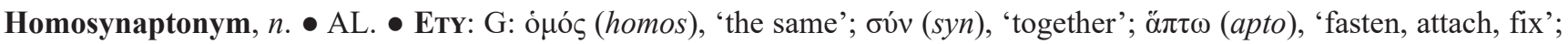
övo $\mu \alpha$ (onoma), 'name'. - Synaptonym which is either indissoluble (members of a hapantotype as defined in the Code; conucleogenera of a class-series nomen under DONS) or considered taxonomically homogeneous (composed of specimens or taxomina which are referred to the same taxon). • ANT: heterosynaptonym. Dubois 2011a: $25,84$. - Code: no term.

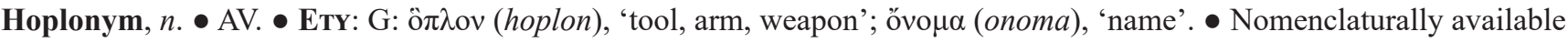
nomen according to the Rules of the Code. • ANT: anoplonym. Dubois 2000b: 50. $\bullet$ Code: available name.

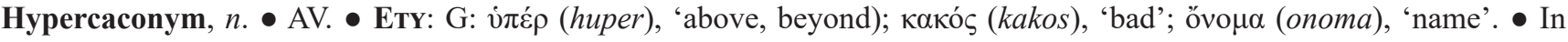
zoological nomenclature, a category of caconym: genus-, family- or class-series nomen which is not a uninomen and is therefore an anoplonym. $\bullet$ Hoc loco. $\bullet$ Code: no term.

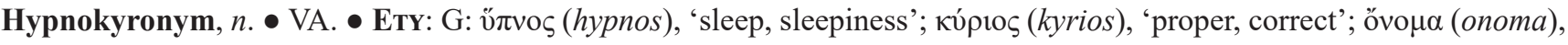
'name'. - Nomen which under DONS Criteria could potentially be used at valid at a rank lower than a teokyronym (e.g., following the resolution of a polytomy), as long as this does not occur, even in a single work, during a 25-year period subsequent to 31 December 2015. Through an exception to regular DONS Criteria, during this period, this nomen remains permanently allocated to this taxon: if this taxon is not recognised as valid in a given ergotaxonomy, this nomen is simply treated as invalid, and cannot be transferred to a more inclusive taxon, even if under regular DONS Criteria it would have to be so. • Dubois 2015c: 74, 2016: 15. $\bullet$ Code: no term.

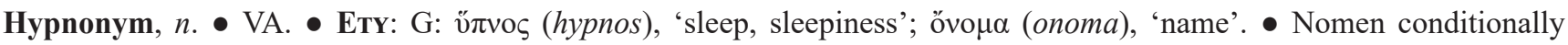
invalidated (i.e., liable to be reinstored as valid as a result of taxonomic changes), either as a result of the Rules of the Code or of an archoidy. • Dubois 2000b: 51. • Code: no term.

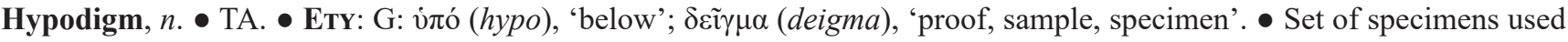
by a taxonomist to recognise and describe a new species-series taxon. $\bullet$ Simpson 1940: 418. $\bullet$ Code: no term.

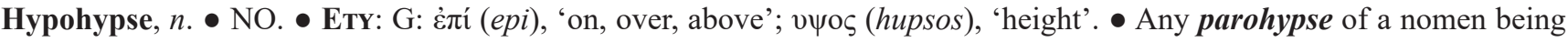
subordinate to another parohypse of the same nomen. • Ant: epihypse. • Dubois 2006b: 828 (as 'hyponym'), 2011a: 22, 85. • Code: nominotypical.

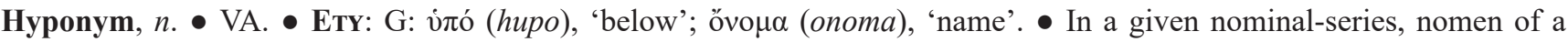
subordinate taxon bearing the same nomen (with the same author, date and onomatophore) as its superordinate taxon. - Dubois 2006b: 828 . Code: nominotypical name.

Hyponymous, $a$. $\bullet$ See Hyponym.

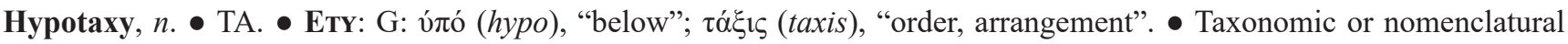
subordination. - END: anhypotaxy, diplohypotaxy, monohypotaxy and polyhypotaxy. If used in a phylogenetic taxonomic frame, they correspond to different topologies of trees, with or without polytomies, thus partly reflecting the 
resolution of the tree. $\bullet$ Dubois \& Raffaëlli 2009: 11. • Code: no term.

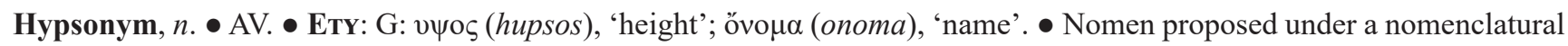
system explicitly or implicitly ranked. • ANT: Anhypsonym. $\bullet$ Hoc loco. • Code: no term.

Idiognoses, $n$. • Plural of idiognosis.

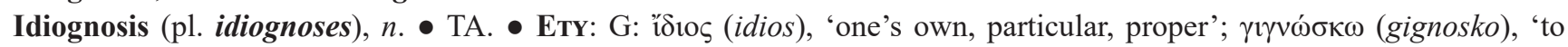
know'. An intensional definition of a taxon based on character states that are considered to provide a brief description or characterisation of a taxon, including both diagnostic (differential) characters and characters shared with other taxa. - Dubois \& Raffaëlli 2009: 15. • Code: no term.

Imperium, n. • NO, TA. • ETY: L: imperium, 'supreme power, empire'. • Highest class-series key rank in biological taxonomy and nomenclature. $\bullet$ Term in traditional use in taxonomy. $\bullet$ SyN: empire. $\bullet$ Code: no term.

Implicit etymological nucleogenus designation, $e$. AL. $\bullet$ In the family-series, implicit designation of the nucleogenus of a new family-series nomen, derived from the fact that a single nominal genus included in the new family-series taxon bears a nomen the stem of which is unambiguously the stem of the new family-series nomen. Such a mode of designation is invalid after 1999 (Art. 16.2). • Dubois 1984b: 24. • Code: no term.

Implicit internal airesy (IPITA), e. • VA. • An internal airesy which is implicit i.e., only one of the competing spellings being mentioned, which is considered by the Code to designating it as correct. $\bullet$ Dubois 2013: 12. $\bullet$ Code: no term.

Inclusive extension, e. $\bullet$ AL. • System of extension by inclusion, listing all member(s) of a class (such as a taxon). • Dubois 2005b: 379. $\bullet$ Code: no term.

Inclusive ostension, $e$. AL. • System of ostension by inclusion, pointing to one or several member(s) of a class (such as a taxon). $\bullet$ Dubois 2006c: 25. $\bullet$ Code: no term.

Incorrect, $a$. $\bullet \mathrm{CO}$. $\bullet$ In the context of zoological nomenclature, qualification of a nomen (nothonym) that fails to conform to the Rules of the Code regarding spelling, rank and, if relevant, onymorph. • ANT: correct. • Traditional term in nomenclature. $\bullet$ Code: incorrect.

Incorrectness, $n$. • CO. • Qualification of an available nomen (kyronym) which bears a paronym-i.e., a spelling (parograph), rank (parohypse) and, if relevant, onymorph (paronymorph) — that is not in agreement with the Rules of the Code. • ANT: correctness. $\bullet$ Traditional term in nomenclature. $\bullet$ Code: no term.

Indication, $n$. $\bullet \mathrm{AV}$. $\bullet$ A reference to a previously published information or to an onomatergy which, in the absence of a description, definition or diagnosis, provides availability to a new nomen, if it satisfies the relevant provisions of Articles 10 and 11 (if published before 1931) and 16.2 (if published before 2000) of the Code. • Code: indication.

Intension, $n$. $\bullet$ AL. $\bullet$ System of allocation of a nomen to a concept or class (such as a taxon) through providing a set of properties or attributes that characterise a concept or a class. - Traditional term in philosophy, logics and didactics (see Dubois 2005a: 74, 2005b: 379). • SyN: comprehension. • Code: no term.

Intensional, $a$. $\bullet$ See intension.

Intensional definition, e. $\bullet$ AL. • Definition of a concept or class (such as a taxon) based on intension. • Traditional term in philosophy, logics and didactics (see Dubois 2005b: 379). $\bullet$ Code: no term.

Intensionally, $a v$. $\bullet$ See intension.

Intention, $n$. $\bullet$ NO, TA, XE. • Purpose, aim. • Traditional term in coomon language. • Code: intention.

Internal airesy (ITA), e. • An airesy taken in case of zygography under Article 24.2.4 of the Code by the original auctor(s) of the nomen. • END: explicit internal airesy and implicit internal airesy. • Dubois 2013: 12. • Code: no term.

Intragenera, $n$. $\bullet$ Plural of intragenus.

Intragenus (pl. intragenera), n. • AL. • ETy: L: intra-, 'within, inside'; genus, 'birth, origin, class, kind'. • Alienogenus of a class-series nomen that in a given ergotaxonomy is included in the least inclusive class-series taxon (metrotaxon) including all the conucleogenera of this class-series taxon. $\bullet$ Dubois 2006a: 187. • Code: no term.

Invalid, $a$. • VA. - In the context of zoological nomenclature, qualification of a nomen (akyronym) that does not conform to the conditions of nomenclatural validity as regulated by the Code (nomakyronym, lethakyronym) or that has been invalidated by the Commission (archakyronym). • ANT: valid. • Traditional term in zoological nomenclature. $\bullet$ Code: invalid.

Invalidate, $v$. $\bullet$ VA. $\bullet$ Common language term, proposed by Dubois (2000b: 46) to designate the action of withdrawing the availability or validity to a hoplonym either by an author following the Rules of the Code or by the Commission under the Plenary Power. $\bullet$ Code: suppress, invalidate.

Invalidation, $n$. $\bullet$ VA. C Common language term, proposed by Dubois (2000b: 47) to designate the result of the action of withdrawing the availability or validity to a hoplonym either by an author following the Rules of the Code or by the Commission under the Plenary Power. $\bullet$ Code: suppression.

Invalidity, $n$. • VA. • Statement regulated by the Code according to which a nomen is determined not to be the one that must be used for to a taxon or several taxa in zoological nomenclature. • ANT: validity. $\bullet$ Traditional term in zoological nomenclature. • Code: invalidity. 
IPITA, $a b . \bullet$ See Implicit internal airesy.

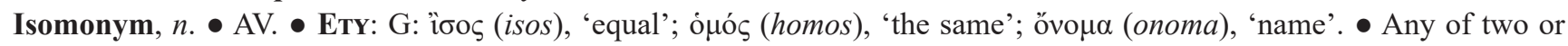
more distinct hoplonyms (having different authors, dates and onomatophores) of the same nominal-series having the exactly same onomatophore (or onomatophore and onomatostasis if relevant) and that are homonyms under the Code. - Dubois 2012a: 66, 77. • Code: no term.

Isomorphism, $n$. $\bullet \mathrm{PH}, \mathrm{TA}$. $\bullet$ See Bijection. $\bullet$ Code: no term.

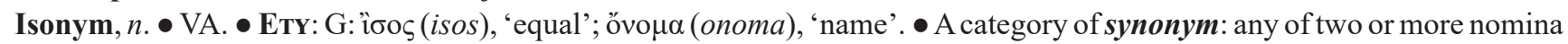
of the same nominal-series based on the same onomatophore. $\bullet$ Dubois 2000b: 57. • Code: objective synonym.

Isotaxa, $n$. $\bullet$ One of the two plurals of isotaxon. $\bullet$ Dubois 2005b: 406 .

Isotaxic, $a$. $\bullet$ NO, TA. $\bullet$ ETY: see Isotaxy. $\bullet$ Qualification of two distinct taxa of the same or different nominal-series having exactly the same extension in a given ergotaxonomy. $\bullet$ Dubois 2005b: 407. • Code: no term.

Isotaxon (pl. isotaxa, isotaxons), $n$. • NO, TA. • ETy: see Isotaxy. • One of two distinct taxa of the same or different nominal-series having exactly the same extension in a given ergotaxonomy. $\bullet$ Dubois 2005b: 407. $\bullet$ Code: no term.

Isotaxons, $n$. $\bullet$ One of the two plurals of isotaxon. • Hoc loco.

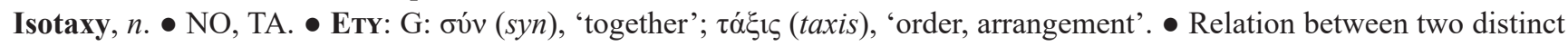
taxa of the same or different nominal-series having exactly the same extension in a given ergotaxonomy. $\bullet$ Dubois 2005b: 406. • Code: no term.

ITA, $a b$. $\bullet$ See Internal airesy.

Junior, $a . \bullet$ NO. $\bullet$ In the context of zoological nomenclature, and concerning a nomen, an airetophory or or a spelling: published at a date subsequent to that of publication of another nomen, onomatergy or spelling, qualified as senior. $\bullet$ Traditional term in nomenclature. $\bullet$ Code: junior.

Juniorisation, $n$. $\bullet$ NO. • In the context of zoological nomenclature, and concerning a conflict of zygoidy between synchronous nomina, spellings or airetophories, airesy by which a nomen, spelling or airetophory is denied precedence in favour of another one, which is then seniorised relative to it. $\bullet$ Dubois 2000b: 47. • Code: no term.

Juniorise, $v$. $\bullet$ See Juniorisation.

Key rank, e. $\bullet$ NO, TA. $\bullet$ Main nomenclatural rank of traditional use in zoological nomenclature: e.g., classis, ordo, familia, tribus, genus, species. • ANT: subsidiary rank. $\bullet$ END: primary key rank, secondary key rank. $\bullet$ Common language terms; Dubois 2006a: 208. • Code: no term.

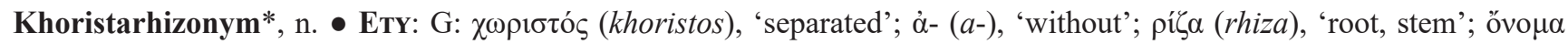
(onoma), 'name'. • Arhizonym ending with a complex original ending in -form- or -morph-. $\bullet$ Hoc loco. $\bullet$ Code: no term.

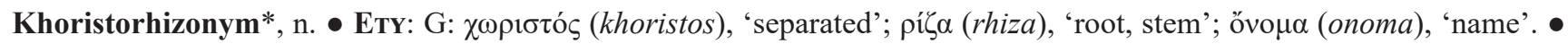
Pseudorhizonym based on the stem of an available genus-series nomen referred or not as valid to the class-series taxon for which it is proposed, or on the stem of a nomen of another nominal-series or of a non-scientific name of animal, with a complex original ending in -form- or -morph-. • Dubois \& Frétey 2020a: 18, 46. $\bullet$ Code: no term.

Kingdom, $n$. $\bullet$ NO, TA. $\bullet$ A class-series key rank in biological taxonomy and nomenclature, between imperium and phylum. - Term in traditional use in taxonomy. SYN: regnum. Code: no term.

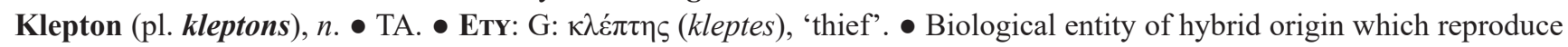
sexually or parasexually through sexual parasitism at each generation of one or several other entity/ies (mayron/s or klepton/s) closely related phylogenetically; heredity may be clonal, hemiclonal or meroclonal (for details see Dubois 2008c, 2009c, 2011b). • Dubois \& Günther 1982: 290. • Code: no term.

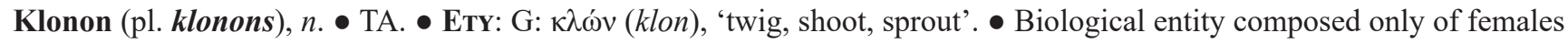
which reproduce parasexually or asexually through pathenogenesis, cutting, vegetative multiplication or any other reproductive system by which an organism transmits its genome unchanged (except for new mutations) to its offspring; heredity is clonal in mitoklonons and clonal or meroclonal in meioklonons; many klonons are of hybrid origin (for details see Dubois 2008c, 2009c, 2011b). • Dubois 1991: 68. • Code: no term.

Kyon (pl. kyons), $n$. $\bullet$ TA. $\bullet$ Artificial term based on the last two letters of the patronym of Theodosius Dobzhansky (19001975), in replacement of his term pseudospecies (Dobzhansky 1970). • Biological entity having either a gametogenesis implying ameiosis or metameiosis or a germonogenesis implying gynogenesis or parthenogenesis, or both; some of these entities (klonons) maintain themselves independently in nature, whereas others (kleptons) depend at each generation on the gametes produced by another entity (mayron or klepton), thus practicing 'sexual parasitism'; most kyons are of hybrid origin (for details see Dubois 2008c, 2009c, 2011b). • Dubois 2008c: 189. • Code: no term.

Kyronym, $n$. • VA. • ETY: G: kúplos (kyrios), 'proper, correct'; övo $\mu \alpha($ onoma), 'name'. • Valid nomen for a given taxon in a given ergotaxonomy. $\bullet$ ANT: akyronym. $\bullet$ Dubois 2000b: 51. Code: valid name.

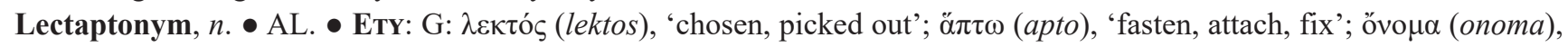
'name'. Monaptonym whose monophoric onomatophore (lectophoront, neophoront, nucleospecies or nucleogenus) 
was designated in a publication subsequent to that where the nomen was promulgated. • Dubois 2011a: $25,86 . \bullet$ Code: no term.

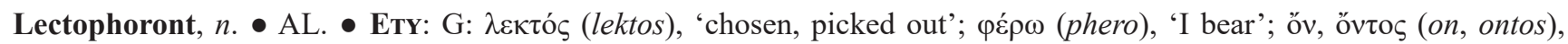
'being, individual'. - Single specimen subsequently designated among a series of symphoronts for designation as onymophoront of a species-series nomen. • Dubois 2005b: 403. $\bullet$ Code: lectotype.

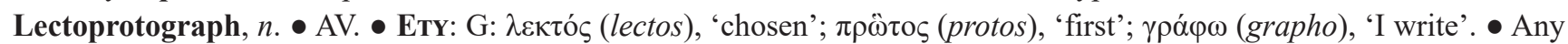
original spelling among symprotographs validated by an airesy under Article 24.2. • Dubois 2010a: 15. • Code: correct original spelling.

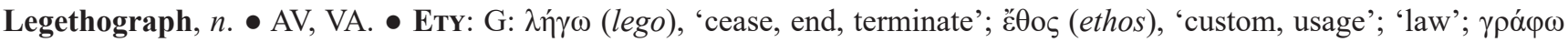
(grapho), 'I write'. $\bullet$ Eugraph that is imposed to a given class-series nomen according to the DONS Criteria. $\bullet$ Hoc loco. $\bullet$ Code: no term.

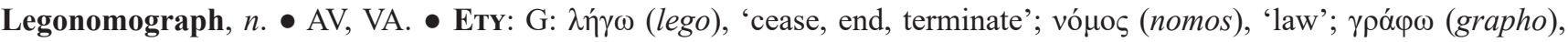
'I write'. - Nomograph that is imposed by the Code to a given nomen in a given ergotaxonomy, superseding the protograph because the ending of the latter must be corrected as a result of a mandatory ending correction (Dubois 2013: 10): either a change of combination in the species-series or of rank in the family-series. $\bullet$ SrN: $\bullet$ Hoc loco. $\bullet$ Code: mandatory change.

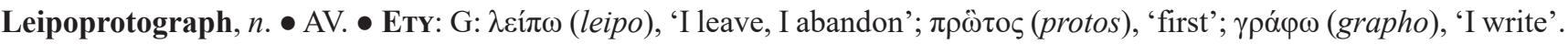
- Any original spelling among symprotographs rejected by an airesy under Article 24.2. • Dubois 2010a: 15. • Code: incorrect original spelling.

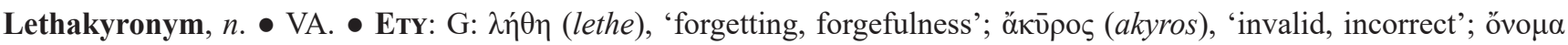
(onoma), 'name'. A Akyronym invalidated (juniorised) for complying with the conditions for being a nomen oblitum as defined in Article 23.9 of the 1999 Code. $\bullet$ Hoc loco. $\bullet$ Code: no term.

Lineage, $n$. • NO, PH, TA, XE. • A single line of direct ancestry and descent. Biological entities at different levels of organisation form lineages: for example, genes, cells and organisms all replicate or reproduce to form lineages. Lineages at one level of organisation often make up, or are contained within, lineages at higher levels of organisation; for example, numerous cell lineages often make up an organism lineage. • Term in traditional use in evolutionary biology; Queiroz 1998, Avise 2008. • Code: no term.

Mandatory, $a$. $\bullet$ NO. $\bullet$ Required by the nomenclatural Rules. • Common language term; Dubois \& Aescht 2019o: 129. • Code: mandatory.

Linz Zoocode Committee (LZC), e. • NO. • International Committee, founded in 2014, working on the Zoocode, a set of proposals of improvements to the Code. • See Dubois et al. 2016, 2019.

Linz Zoocode Proposals (LZP), e. • NO. • Proposals of improvements to the Code published by the Linz Zoocode Committee. • See Dubois et al. 2016, 2019.

LZC, $a b . \bullet$ NO. $\bullet$ See Linz Zoocode Committee.

LZP, ab. • NO. • See Linz Zoocode Proposals.

Mandatory ending correction, $e$. CO. A category of nomographic correction: correction of the ending of a nothograh required by the nomenclatural Rules. $\bullet$ Dubois 2013: 11. • Code: mandatory change.

Mandatory spelling correction, $e$. $\bullet \mathrm{CO}$. $\bullet$ A category of nomographic correction: correction of a nothograh or of its the stem required by the nomenclatural Rules. • Dubois 2013: 11. • Code: justified emendation.

Mandatory rank, e. - NO, TA. • Any of the seven taxonominal ranks (kingdom, phylum, class, order, family, genus, species) to which any animal organism should be referred in zoological taxonomy and nomenclature. Dubois $2007 a$ : 57 (as compulsory rank), 2020a: 6. • Code: no term.

Mayron (pl. mayrons), $n$. $\bullet$ TA. • Taxonomic species corresponding to the nondimensional 'mixiological species concept' or 'biological species concept' (BSC): independent bisexual panmictic entity, constituting a 'closed' or 'protected' gene pool, composed of organisms with eumeiosis, breeding freely among them but usually not with organisms belonging to other similar entities (see Dubois 2011b). • See Dubois 2007a: 48. • Code: no term.

Median, $n$. $\bullet$ NO, XE. $\bullet$ Second quartile of a data set, i.e. the value separating the higher half from the lower half of its data set: $50 \%$ of the data lie below this point, and $50 \%$ lie above. $\bullet$ Term in traditional use in statistics and probability. $\bullet$ Code: no term.

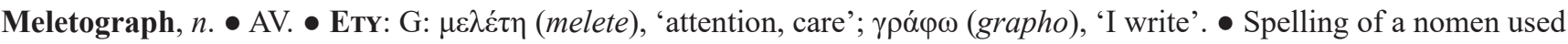
voluntarily/intentionally in a publication by an author, scriptor, editor, printer or publisher. • ANT: ameletograph. Dubois 2000b: 54 (as ameletonym), 2010b: 7. • Code: no term.

Meletonym, $n$. See Meletograph.

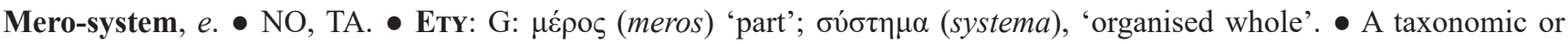
nomenclatural pro-system which covers only some taxa or ranks only. • Dubois $2015 c$ : 8, 75. • Code: system.

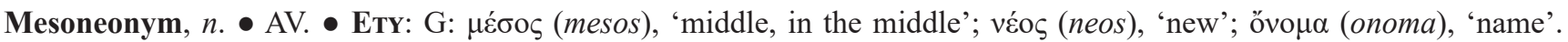


- Neonym whose etymology is not clearly different or the same as that of its archaeonym. • Hoc loco. • Code: no term.

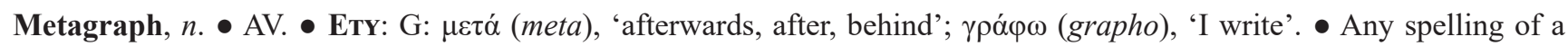
nomen different from the correct original spelling and which may be either an autoneonym or a symprotograph, a leipoprotograph or a nomographic correction. $\bullet$ Hoc loco. • Code: no term.

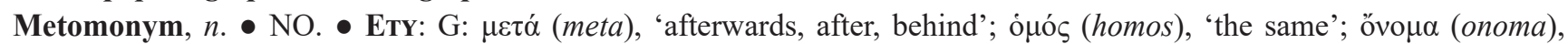
'name'. • Junior homonym resulting from a replacement or modification of the onomatophore (or onomatophore and onomatostasis if relevant) of a previously introduced hoplonym. • Dubois 2012a: 66, 77. • Code: no term.

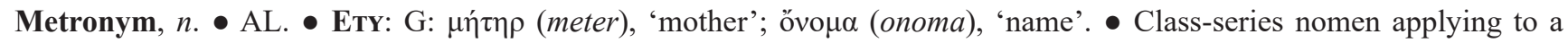
metrotaxon within the frame of a given ergotaxonomy, i.e. to the least inclusive (lowest ranked) class-series taxon including all its conucleogenera. • Dubois 2011a: 88; redefined in Dubois 2015c: 77. • Code: no term.

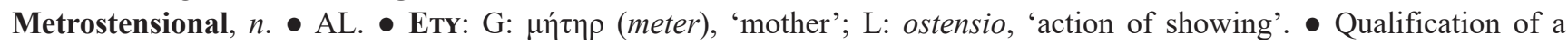
nomenclatural system, the Metrostensional Nomenclatural System (MONS), that relies only on onomatophores (conucleogenera) for the taxonomic allocation of class-series nomina (inclusive ostension): within a given taxonomic frame, a nomen applies to the least inclusive taxon that includes all its conucleogenera. • Dubois \& Raffaëlli $2012: 88$. - Code: no term.

Metrostensional Nomenclatural System (MONS), e. • NO. • A class-series nomenclatural system which relies only on onomatophores for the taxonomic allocation of nomina through inclusive ostension: within a given taxonomic frame, a nomen applies to the least inclusive taxon that includes all its onomatophore (see Dubois 2006c). • Dubois \& Raffaelli 2012: 88; Dubois 2015c: 13.

Metrotaxa, $n$. P Plural of metrotaxon.

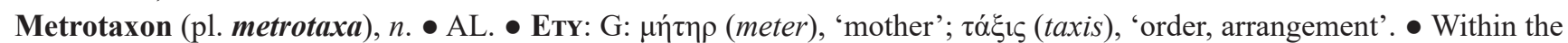
frame of a given ergotaxonomy, the least inclusive class-series taxon including all the conucleogenera of a class-series nomen. $\bullet$ Dubois 2006a: 188. $\bullet$ Code: no term.

Microtaxonomy, $n$. $\bullet$ TA. $\bullet$ Discipline of taxonomy dealing with the study of species and circumspecific taxa. $\bullet$ Mayr \& Ashlock 1991. Code: no term.

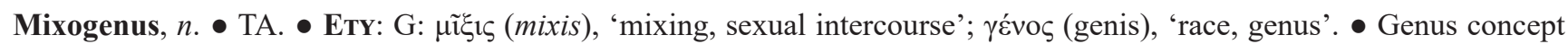
according to which whenever two species are documented to have produced, whether in natural or in artificial conditions, true viable adult diploid hybrids, they should be referred to the same genus, as well as all the other species which by other criteria are considered congeneric with them (Dubois 1981a,c, 1982a, 1983a, 1988a,c, 2004d). See also syngameon [2]. • Hoc loco. • Code: no term.

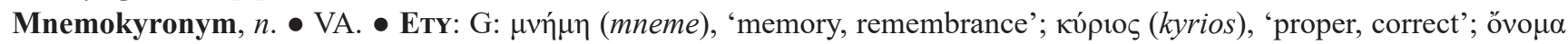
(onoma), 'name'. • Kyronym validated (seniorised) for complying with the conditions for being a nomen protectum as defined in Article 23.9 of the 1999 Code. $\bullet$ Hoc loco. $\bullet$ Code: nomen protectum.

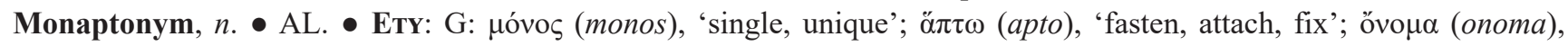
'name'. A Aptonym whose onomatophore is monophoric, being composed of a single specimen (in the species-series: holophoront, lectophoront or neophoront) or taxomen (in the genus-series: nucleospecies; in the family-series and class-series: nucleogenus). • ANT: synaptonym. • END: holaptonym and lectaptonym. • Dubois 2011a: 25, 86. • Code: no term.

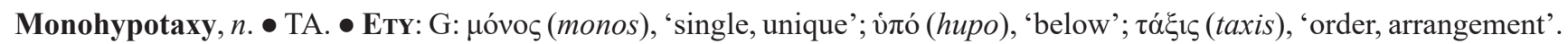
- Mode of hypotaxy of a taxon that includes only one immediately subordinate taxon. In a phylogenetic taxonomic frame, the two successive ranks are clearly redundant, as they do not provide distinct taxonomic information, but they may be useful for mere nomenclatural reasons (see Dubois, 2007a, 2008f). • Dubois \& Raffaëlli 2009: 12. • Code: no term.

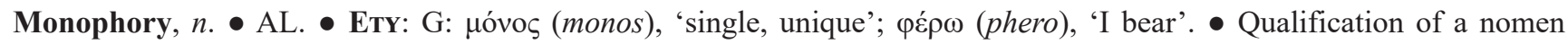
supported by an onomatophore composed of a single specimen (in the species-series) or taxomen (in the three other nominal-series). The designation of this onomatophore may have been original or subsequent. $\bullet$ Dubois 2005b: 404 . $\bullet$ Code: monotypy.

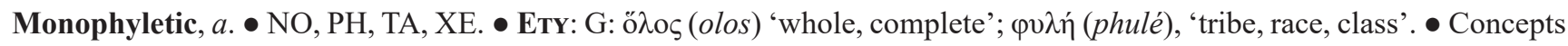
applying to taxa: [1] Haeckel's (1866b) concept: non-polyphyletic taxon, considered to be composed of descendants of a common ancestor (see homophyletic), but including either all of them (see holophyletic or only some of them (see paraphyletic); [2] Hennig's (1950) concept: non-polyphyletic and non-paraphyletic taxon, considered to include all the descendants of its most recent common ancestor as well as the latter (see holophyletic). • Ashlock 1971; Dubois 1986 . - Code: no term.

Monophyly, $n$. See Monophyletic.

Monosemic, a. • NO. • ETY: see Monosemy. • In the context of zoological nomenclature, the qualification of either [1] 
a nomenclatural system that does not allow the same nomen to designate distinct taxa, or [2] any nomen being in this situation (see Dubois 2007a: 41). • ANT: polysemic. • Term in traditional use in linguistics and grammar. $\bullet$ Code: no term.

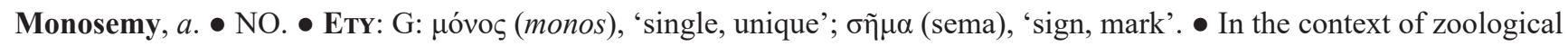
nomenclature, the fact that a nomenclatural system does not allow the same nomen to designate distinct taxa. $\bullet$ ANT: polysemy. • Term in traditional use in linguistics and grammar. • Code: no term.

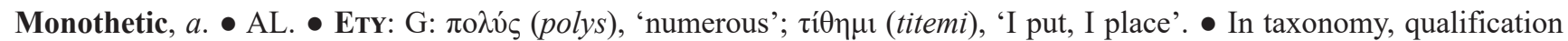
of a diagnosis of taxon involving a unique combination of character states that are both necessary and sufficient for membership in the taxon. • ANT: Polythetic. • Sneath 1962; Van Regenmortel 2016; Dubois 2017d. • Code: no term.

Monothetic diagnosis, e. • AL. • A diagnosis of taxon involving a unique combination of character states that are both necessary and sufficient for membership in the taxon. • ANT: Polythetic diagnosis. • Sneath 1962; Van Regenmortel 2016; Dubois 2017d. • Code: no term.

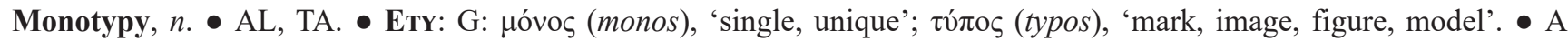
confusing term, used in systematics in two distinct senses: [1] a taxonomic one (see monohypotaxy and anhypotaxy); [2] a nomenclatural one (see monophory). The use of this term in nomenclature is here discouraged (see Dubois \& Raffaëlli 2009: 401-405). • Traditional term in nomenclature. • Code: monotypy.

MONS, ab. • See Metrostensional Nomenclatural System.

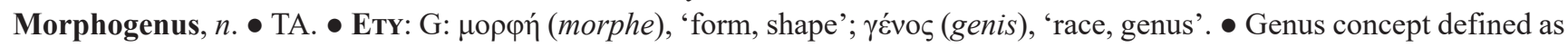
a group of species sharing morphological characters. $\bullet$ Term in use in taxonomy. $\bullet$ Code: no term.

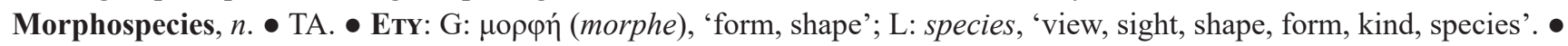
Species concept defined as a group of organisms sharing morphological characters. $\bullet$ Term in common use in taxonomy. - Code: no term.

Name, $n$. $\bullet$ NO, TA. Ambiguous and confusing term used in various senses in the Code: [1] scientific name (see Nomen); [2] spelling; [3] rank; [4] combination; [5] onymorph; [6] 'vernacular' name; [7] name of an author in the sense given to this term in the Code (see Auctor); [8] name of the first-user of a new spelling, rank or combination for an available scientific name (see Scriptor); [9] various other 'names' (or persons, localities, plants, etc.). $\bullet$ Because of this ambiguity, the use of this term in nomenclature to designate a scientific name is here discouraged (see Dubois 2000b: 39-40; Dubois \& Aescht 2016) and the term Nomen is used instead for this purpose. • Traditional term in various domains of biology, including nomenclature. • Code: name.

N-availability, e. • AV. • Availability of nomen. • Dubois 2015c: 24. • Code: no term.

Nemonym, $n$. A AV. • ETY: G: vé $\mu \omega$ (nemo), 'I distribute, I attribute'; óvo $\mu \alpha($ onoma), 'name'. • Anomen that is unambiguously assigned to a nominal-series in the original publication where it is established. $\bullet$ Hoc loco. $\bullet$ Code: no term.

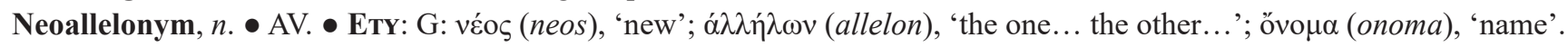
- One of two (or several) allelonyms which is a brand new nomen whereas its allelonym(s) is/are already available nomen/nomina. • Dubois 2015c: 43, 71. • Code: no term.

Neonym, $n$. AV. • ETy: G: véos (neos), 'new'; ővo $\mu \alpha$ (onoma), 'name'. • Nomen proposed expressly to replace an available nomen (its archaeonym), and having the same onomatophore (and onomatostasis in the case of CS sozonymorphs). $\bullet$ ANT: poieonym. • END: alloneonym and autoneonym. • Dubois 2000b: 52. • Code: new replacement name, nomen novum, unjustified emendation.

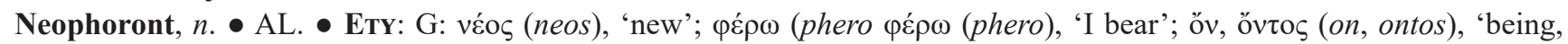
individual'. • Single specimen designated as onymophoront of a species-series nomen when the original or subsequent onymophoront(s) is/are considered to have been lost or destroyed. • Dubois 2005b: 403. • Code: neotype.

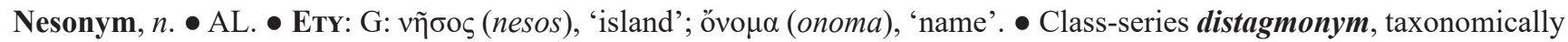
allocated within the frame of a given ergotaxonomy under DONS Criteria through its metrotaxon, without reference to its orotaxon if present, and being therefore its metronym. Dubois 2006a: 188. One of the two meanings of the term nesonym as defined by Dubois (2015c: 65), hereby distinguished from the term ellitonym and used in this restricted meaning. • Code: no term.

New replacement name, $e$. $\bullet$ See Neonym.

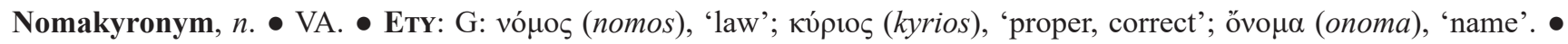
Akyronym as a result of the regular Rules of the Code concerning precedence between zygonyms. $\bullet$ Hoc loco. $\bullet$ Code: no term.

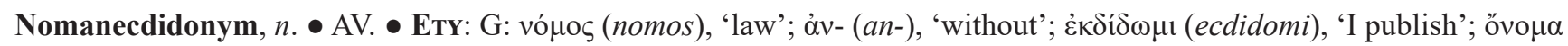
(onoma), 'name'. • Nomen not published, after 1757, in the meaning of Articles 3.2, 8-9, 11.1 and 21.8 of the Code, or published after 1950 with anonymous authorship (Article 14). • ANG: Anecdidonym. • Hoc loco. • Code: no term.

Nomen (pl. nomina), n. • NO, TA. • ETY: L: nomen, 'name'. • Scientific name as defined, and regulated if relevant, by the Code. • Dubois 2000b: 39. • Code: scientific name. 
Nomen dubium (pl. nomina dubia), e. $\bullet$ NO. • See anaptonym, aporionym, heterosynaptonym and nyctonym.

Nomen novum (pl. nomina nova), e. • NO. • See neonym.

Nomen nudum (pl. nomina nuda), e. • NO. • See anoplonym, atelonym and gymnonym.

Nomen oblitum (pl. nomina oblita), e. • NO. • See aphonym, distagmonyn, lethakyronym, eneonym, schizeurydiaphonym and stenodiaphonym.

Nomen protectum (pl. nomina protecta), e. $\bullet$ NO. • See sozodiaphonym and sozonym.

Nomenclatural act, e. • NO. • See Onomatergy.

Nomenclatural ambiguity, e. $\bullet \mathrm{VA}, \mathrm{CO}$. • Any situation in which the nomenclatural status of a nomen is ambiguous. $\bullet$ Dubois 2011a: 22. • Code: no term.

Nomenclatural foundation, e. • See Principle of Nomenclatural Foundation.

Nomenclatural hierarchy, e. • VA. • The sequence of nominal-series and nomenclatural ranks having increasing levels of inclusiveness, used to account for the phylogenetic relationships between taxa. $\bullet$ Term in traditional use in zoological nomenclature and taxonomy. $\bullet$ Code: taxonomic hierarchy.

Nomenclatural independence, e. • See Principle of Nomenclatural Independence.

Nomenclatural parsimony, e. $\bullet \mathrm{AV}, \mathrm{VA}$. $\bullet$ The need of fewer nomina than taxa to name the latter. $\bullet$ Dubois $2006 c: 838$, 2008f: 55, 61. See also Nomenclatural thrift. $\bullet$ Code: no term.

Nomenclatural Parsimony Index (NPI), e. • AV, VA. • The ratio, expressed in percent, of the number of nomina to the number of their parohypses used as valid in a given ergotaxonomy. $\bullet$ Hoc loco. $\bullet$ Code: no term.

Nomenclatural Process, $e$. $\bullet$ NO. - The process through which the valid nomen of a taxon is established. It consists of four main stages, steps or 'floors' (Dubois 2005a,b,d, 2015c; Dubois et al. 2019): availability (including nominal-series assignment), allocation, validity (including correctness) and registration. Dubois 2005b: 381, 2010a: 11, $2011 a$ : 11. - Code: no term.

Nomenclatural rank, e. $\bullet \mathrm{AV}, \mathrm{VA}$. $\bullet$ The place of a nomen in a nomenclatural hierarchy. In the Code, each rank is referred to a given nominal-series. $\bullet$ Term in traditional use in zoological nomenclature and taxonomy. • Code: rank.

Nomenclatural robustness, e. • NO. • Qualification of a nomenclatural system which displays both stability (i.e., the nomina of taxa do not change as long as the ergotaxonomies do not change) and flexibility (i.e., in some cases nomina do not change even if the ergotaxonomies change). • Dubois 2005b, 2011a. $\bullet$ Code: no term.

Nomenclatural stability, $e$ • NO. $\bullet$ Qualification of a nomenclatural system in which the nomina of taxa change as little as possible, or not at all, even if ergotaxonomies change. $\bullet$ Term in traditional use in zoological nomenclature. $\bullet$ Code: stability.

Nomenclatural status of nomen, $e$. $\bullet$ NO. $\bullet$ The dimensions of the status of a nomen which depend only on nomenclatural Rules, and not on the ergotaxonomy adopted: nominal-series assignment and nomenclatural availability. • Term in traditional use in zootaxonomy, precisely defined by Dubois $(2017 b: 36)$. $\bullet$ Code: no term.

Nomenclatural thrift, e. $\bullet$ VA. $\bullet$ The attention given, in order to reduce the synonymy load of taxonomy, to the need to prevent the creation of 'needless nomina' through appropriate nomenclatural acts - e.g., the adequate designation of onomatophores for nomina that still miss them. See also Nomenclatural parsimony. • Dubois 2019: 75. • Code: no term.

Nomenclature, $n$. • NO, TA, XE. • ETY: L: nomenclatura, 'nomenclature', from nomen, 'name' and calo, 'I call' • [1] A subdiscipline of taxonomy which is in charge of providing the valid nomina for the taxa. [2] Any system of nomina that applies to the taxa used in a given ergotaxonomy. • Traditional term in taxonomy. $\bullet$ Code: nomenclature.

Nomina, $n$. • Plural of nomen.

Nomina dubia, e. • Plural of nomen dubium.

Nomina nova, e. • Plural of nomen novum.

Nomina nuda, $e \bullet$ Plural of nomen nudum.

Nomina oblita, e. $\bullet$ Plural of nomen oblitum.

Nomina protecta, $e$. $\bullet$ Plural of nomen protectum.

Nominal taxon, e. $\bullet$ See Taxomen.

Nominal-series (NS), e. • NO. • Any of the sets of coordinated nomina interacting for priority and validity regarding synonymy, homonymy and onomatergies (species-series, genus-series, family-series or class-series). • Dubois 2000b: 40. $\bullet$ Code: group of names [English text]; niveau nomenclatural [French text].

Nominal-series branch (NS-branch), e. • NO. • Any section of a cladistic tree including only some ranks in the corresponding ergotaxonomy. $\bullet$ Hoc loco. $\bullet$ Code: no term.

Nominal-series saturation, e. $\bullet$ VA. $\bullet$ Situation in which all the ranks allowed by the Code in a given nominal-series have been used in a formal ergotaxonomy and nomenclature. $\bullet$ Hoc loco. • Code: no term.

Nominal-set, $e$. $\bullet$ NO. Any of the sets of nomina referred to the same nominal-series and the rank designation of which includes the same key term: e.g., the family-set and the tribe-set within the family-series, including respectively the 
ranks family, subfamily and superfamily, and tribe and subtribe. • Dubois \& Aescht 2017c: 27. • Code: no term.

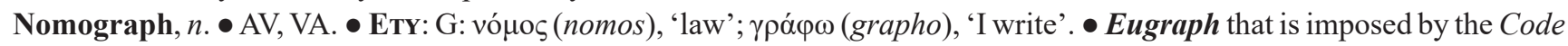
to a given nomen in a given ergotaxonomy, superseding the protograph if necessary. Two categories: [1] eunomograph because the protograph is an original nothograph; [2] legonomograph because the ending of the protograph must be corrected as a result of a change of combination in the species-series or of rank in the family-series. • Dubois $2013: 10$. - Code: [1] justified emendation; [2] mandatory change.

Nomographic correction, e. • AV, VA. • ETY: see Nomograph. • Any correction in the spelling, stem or ending of a nothograph required by the nomenclatural Rules. - Dubois 2013: 11. - Code: justified emendation, mandatory change.

Nomography, $n$. • AV, VA. • ETY: see Nomograph. • A Principle of the Code according to which a spelling (eugraph) is imposed to a given nomen, superseding the protograph if necessary. $\bullet$ Dubois 2013: 10. $\bullet$ Code: no term.

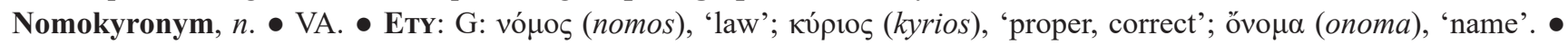
Kyronym as a result of the regular Rules of the Code concerning precedence between zygonyms. $\bullet$ Hoc loco. $\bullet$ Code: no term.

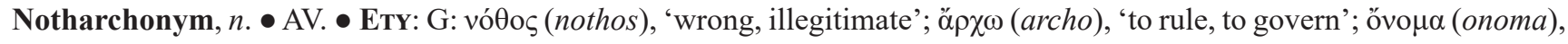
'name'. - Nomen proposed within the frame of a nomenclatural system alternative to the current Code and incompatible with it. $\bullet$ Hoc loco. $\bullet$ Code: no term.

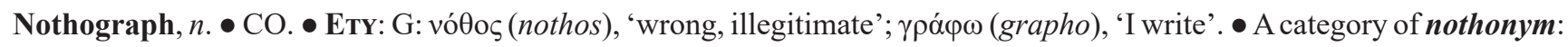
incorrect spelling of a nomen for a given taxon at a given rank in a given ergotaxonomy. • AnT: eugraph. • Dubois 2010a: 29. Code: incorrect spelling.

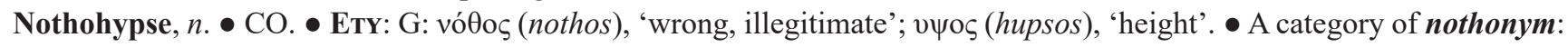
incorrect rank of a nomen for a given taxon in a given taxonomy. • ANT: euhypse. • Dubois 2010a: 7. • Code: no term.

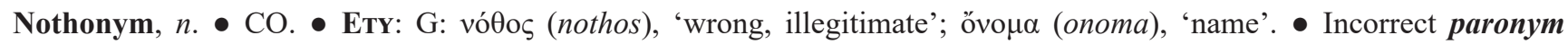
(nothograph, nothohypse and/or nothonymorph) of a nomen for a given taxon in an ergotaxonomy. • ANT: eunym. • Dubois 2000b: 54. • Code: no term.

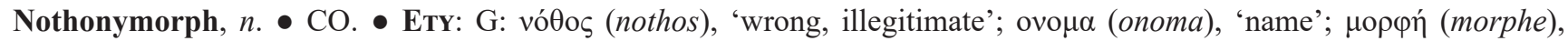
'form, shape'. • A category of nothonym: incorrect onymorph of a nomen for a given taxon in an taxonomy. • ANT: eunymorph. • Dubois 2010a: 7. • Code: no term.

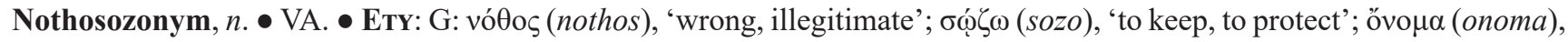
'name'. • Sozonymorph that has not been used in any title of scientific publication since 31 December 1899. • Dubois 2015c: 21. C Code: no term.

NPI, $a b$. • See Nomenclatural Parsimony Index.

NS, $a b$. • See Nominal-series.

NS-branch, e. • See Nominal-series branch.

Nucleogenera, $n$. $\bullet$ Plural of nucleogenus.

Nucleogenus (pl. nucleogenera), n. • AL. • ETY: L: nucleus (from nux, 'nut'), 'nucleus, core, stone'; genus, 'birth, origin, class, kind'. - Genus-series taxomen serving as onomatophore of a family-series or class-series nomen. - END: conucleogenus, uninucleogenus. • Dubois 2005a: 77, 2005b: 404. • Code: type genus.

Nucleomen (pl. nucleomina), n. • AL. • ETY: L: nucleus (from nux, 'nut'), 'nucleus, core, stone'; nomen, 'name'. • Taxomen serving as onomatophore of a nomen of a nominal-series above the species-series. • END: nucleospecies, nucleogenus. • Dubois 2005a: 77, 2005b: 403. • Code: no term.

Nucleomina, $n$. $\bullet$ Plural of nucleomen

Nucleospecies, $n$. • AL. • ETy: L: nucleus (from nux, 'nut'), 'nucleus, core, stone'; species, 'idea, kind, species'. • Speciesseries taxomen serving as onomatophore of a genus-series nomen. • Dubois 2005a: 77, 2005b: 404. • Code: type species.

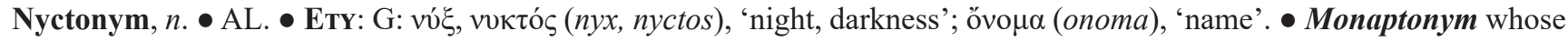
monophoric onomatophore (lectophoront, neophoront, nucleospecies or nucleogenus) cannot be referred to a known ergotaxon. • ANT: photonym. - Dubois 2011a: 54, 88. • Code: one of the meanings of the ambiguous designation nomen dubium.

Objective, $a$. $\bullet$ NO. $\bullet$ Actual, existing outside and independent of the mind. $\bullet$ Common language term. $\bullet$ Code: objective.

Obtainable, $a$. $\bullet$ AV. $\bullet$ [1] In Articles 8.1.3 and 8.4.2.1 of the Code: producible, that can be produced. [2] In Article 8.1.2 of the Code: acquirable, that can be acquired. $\bullet$ Common language term, introduced in zoological nomenclature with a formal definition by Dubois \& Aescht (2017f). $\bullet$ Code: no term.

Obtained, $p$. • AV. • In Article 9.12 of the Code: produced and acquired. • Common language term, introduced in zoological nomenclature with a formal definition by Dubois \& Aescht (2017f). • Code: no term. 


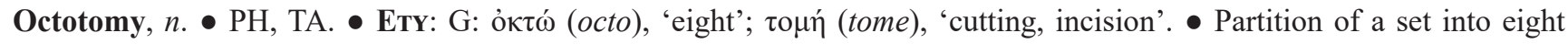
subsets. $\bullet$ Hoc loco. $\bullet$ Code: no term.

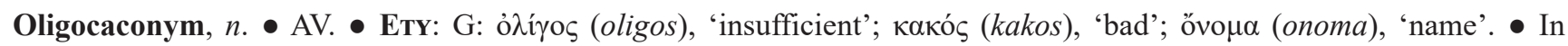
zoological nomenclature, a category of caconym: a nomen established in a work that is not consistently binominal for nomina of rank species and is therefore an anoplonym (Article 11.4). $\bullet$ Hoc loco. $\bullet$ Code: no term.

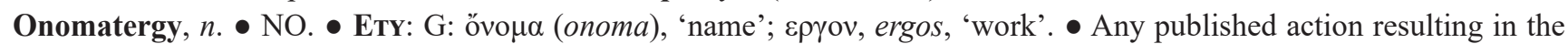
establishment of a new nomen (catastasy) or in affecting the nomenclatural status of an available nomen (airesy). • Dubois 2013: 3. • Code: nomenclatural act.

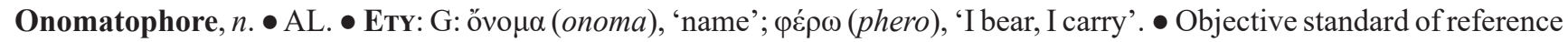
of inclusive ostension determining the taxonomic allocation of a nomen: within a given ergotaxonomic frame, the nomen can be potentially applied to any taxon that includes its onomatophore. In the species-series, onomatophores are specimens, whereas in the genus- and family-series they are taxomina. • END: onymophoront, nucleomen. • Simpson 1940: 421. • Code: type, name-bearing type.

Onomatostases, $n$. Plural of onomatostasis.

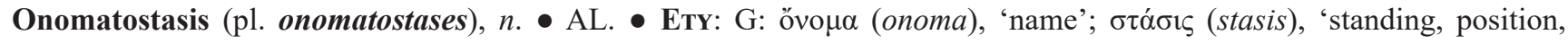
station'. - Objective standard of reference of exclusive ostension determining the taxonomic allocation of a class-series nomen: within a given taxonomic frame, the nomen applies to the taxon that includes its onomatophore and excludes its onomatostasis. Onomatostases are taxomina. • Dubois 2005a: 79, 2005b: 203, 2006a: 189, 2011a: 39. • Code: no term.

ONS, ab. • See Ostensional Nomenclatural System.

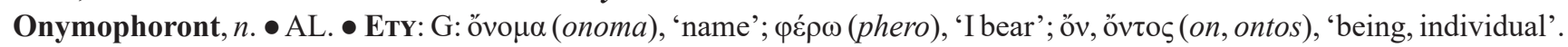
- Specimen(s) serving as onomatophore of a nomen of the species-series, which may be either single (holophoront, lectophoront or neophoront) or multiple (symphoronts). Dubois 2005a: 77, 2005b: 403. • Code: type specimen.

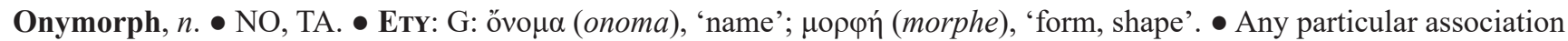
between genus-series substantive(s) and species-series epithet(s), used to designate a species-series taxon. Acombination is a particular case of onymorph. • Smith \& Pérez-Higareda 1986: 422. • Code: no term.

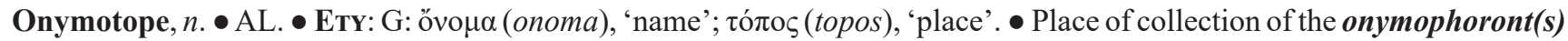
of a species-series taxomen. $\bullet$ Dubois 2005b: 404. • Code: type locality.

OONS, ab. • See Orostensional Nomenclatural System.

Order, $n$. • NO, TA. • ETY: L: ordo, 'series, line, row, order'. • In zoological taxonomy and nomenclature, a key rank of the class-series, between class and phalanx. $\bullet$ Term in traditional use in taxonomy. $\bullet$ SrN: ordo. $\bullet$ Code: order.

Ordination, $n$. • NO, TA. • ETy: L: ordo, 'series, line, row, order'. $\bullet$ The relation between two taxa in a given hierarchy and ergotaxonomy: alienordination, parordination, subordination or superordination. See also Topotaxy. • Dubois \& Berkani 2013: 53. • Code: no term.

Ordo, $n$. • NO, TA. • ETY: L: ordo, 'series, line, row, order'. • In zoological taxonomy and nomenclature, a key rank of the class-series, between class and phalanx. $\bullet$ Term in traditional use in taxonomy. $\bullet$ SyN: order. $\bullet$ Code: order.

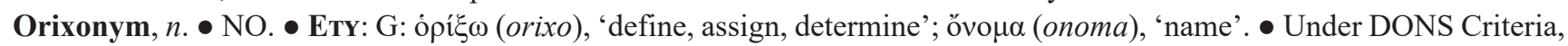
class-series nomen/nomina allowing the taxonomic allocation of another class-series taxon either through inclusion only (getendonym) or through both inclusion and exclusion (getexonym). $\bullet$ Hoc loco. • Code: no term.

Oronym, $n$. • AL. • ETY: G: ópos (oros), 'mountain'; ǒvo $\mu \alpha$ (onoma), 'name'. • Class-series nomen applying to an orotaxon within the frame of a given ergotaxonomy, i.e. to the most inclusive (highest ranked) class-series taxon including all its conucleogenera and excluding all its coalienogenera. • Dubois 2011a: 88; redefined in Dubois 2015c: 77. • Code: no term.

Orostensional, n. • AL • ETY: G: őpos (oros), 'limit, frontier'; L: ostensio, 'action of showing'. • Qualification of a nomenclatural system, the Orostensional Nomenclatural System (OONS), that relies on bidirectional ostension for the taxonomic allocation of nomina. $\bullet$ Dubois 2015c: 13. $\bullet$ Code: no term.

Orostensional Nomenclatural System (OONS), e. • NO. • A class-series nomenclatural system which relies both on onomatophores (nucleogenera) and onomatostases (alienogenera) for the taxonomic allocation of class-series nomina (bidirectional ostension): within a given taxonomic frame, a nomen [1] either applies to the most inclusive taxon that includes all its conucleogenera and excludes all its alienogenera (orotaxon) if it exists, or [2] is an anaptonym if such a taxon does not exist because of overlapping between the onomatophore and the onomatostasis (gephyronym) (see Dubois 2006a: 188). • Dubois 2015c: 13. C Code: no term.

Orotaxa, $n$. Plural of orotaxon.

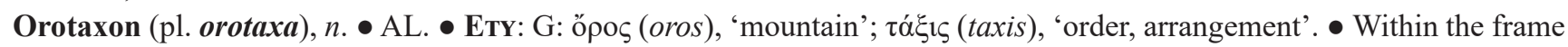
of a given ergotaxonomy, the most inclusive class-series taxon including all the conucleogenera of a class-series taxon and excluding all its coalienogenera. • Dubois 2006a: 188. • Code: no term. 


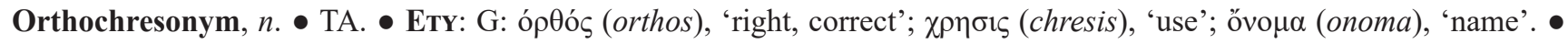
Chresonym appropriately used to designate a taxon. • AnT: heterochresonym. $\bullet$ Dubois 2000b: 59. • Code: no term.

Ostension, $n$. $\bullet$ AL. $\bullet$ System of allocation of a nomen to a concept or class (such as a taxon) through pointing to an object being an example or member of the class (inclusive ostension), or a non-example or non-member of the class (exclusive ostension), or both (bidirectional ostension), without providing an intensional or closed extensional definition, or information on the boundaries the class. - Traditional term in philosophy, logics and didactics (see Keller et al. 2003: 99; Dubois 2005b: 380, 2011a: 89). • Code: no term.

Ostensional, $a$. $\bullet$ AL. $\bullet$ See Ostension.

Ostensional Nomenclatural System (ONS), e. • NO. • A nomenclatural system that relies on ostension for the taxonomic allocation of nomina. • Dubois 2015a. Code: no term.

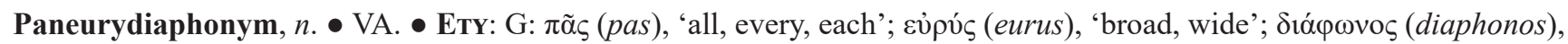

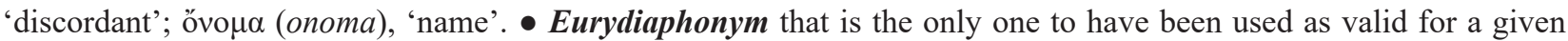
taxon, or for taxa having totally or partially identical extensions, in the titles of 100 scientific works. $\bullet$ ETY: G: $\pi \tilde{\alpha} \varsigma$

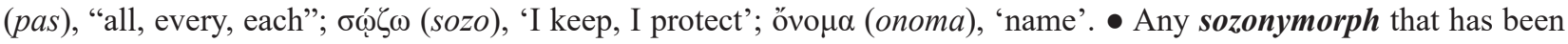
used as valid in the title of at least one scientific publication after 1899. • END: sozonym and sozodiaphonym. • Dubois 2020a: 41. • Code: no term.

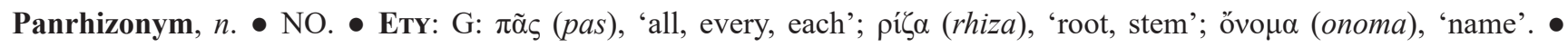
Suprageneric nomen the stem of which is a nomen of the genus-series or of another nominal-series, or a vernacular name of animal. - END: rhizonyms, pseudorhizonyms, auxorhizonyms, cenorhizonyms, xenorhizonyms and quasirhizonyms. • Code: no term.

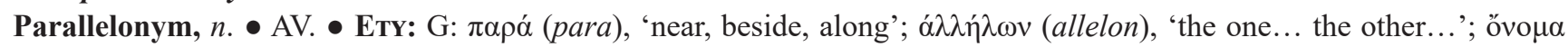
(onoma), 'name'. • One of two (or several) allelonyms which are all new nomina. • Dubois 2015c: 43, 78. • END: agoallelonym and epomallelonym. • Code: no term.

Paraphyletic, $a$. • PH, TA. • Concept applying to taxa: qualification of a homophyletic group that includes its most recent common ancestor but not all of the descendants of the latter. $\bullet$ Hennig 1950; Ashlock 1971; Dubois 1986b. $\bullet$ Code: no term.

Paraphyly, $n$. • See Paraphyletic.

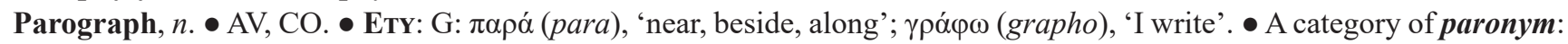
any spelling, either original (protograph) or subsequent (apograph), ever used in the literature for a nomen. $\bullet$ Dubois 2010a: 6. Code: no term.

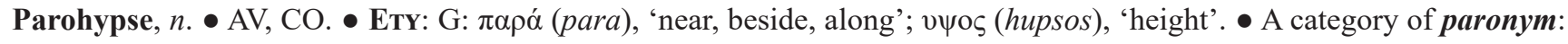
any of the avatars, either original (protohypse) or subsequent (apohypse), of the rank of a nomen. $\bullet$ Dubois $2010 a$ : 6. - Code: no term.

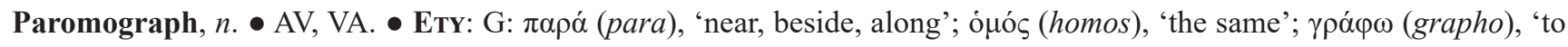
write'. - Any of two or more distinct hoplonyms (having different auctors, dates and onomatophores) of the same nominal-series having the same etymology and meaning, and spellings deemed to be identical under Article 58 of the Code. • Dubois 2012a: 64. • Code: variant spelling.

Paromography, $n$. See Paromograph.

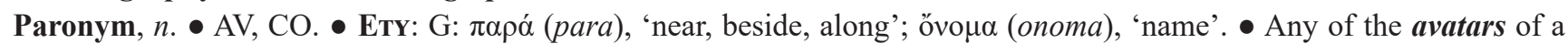
nomen, either original (protonym) or subsequent (aponym), and concerning its spelling (parograph), rank (parohypse) and/or, if relevant, onymorph (paronymorph). • Dubois 2000b: 53. • Code: no term.

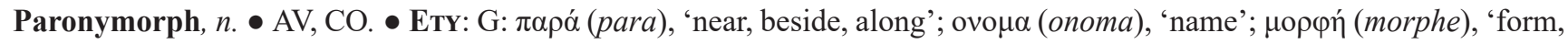
shape'. - A category of paronym: any of the avatars, either original (protonymorph) or subsequent (aponymorph), of the onymorph of a nomen. $\bullet$ Dubois 2010a: 6. Code: no term.

Parordinate, a. • NO, TA. • ETY: L: par, 'equal, same'; ordo, 'series, line, row, order'. $\bullet$ Qualification of any of two or more taxa that have the same hierarchical rank and are immediately subordinate to the same superordinate taxon in a given ergotaxonomy. • Dubois 2006a: 827, 2007a: 33, 2008a: $60 \bullet$ Code: no term.

Parordination, $n$. • NO, TA. • ETY: L: par, 'equal, same'; ordo, 'series, line, row, order'. • The relation of ordination between two parordinate taxa in a given ergotaxonomy. • Dubois 2007a, 2008a. • Code: no term.

Partially regulated family-series ranks (PRR), e. • NO. • Ranks of the family-series for which the Code does not prescribe mandatory endings but only that their ending nominative indicates plural. $\bullet$ Dubois \& Aescht 2019o: 128. $\bullet$ Code: no term.

P-availability, e. • AV. • Availability of publication. • Dubois 2015c: 24. • Code: no term.

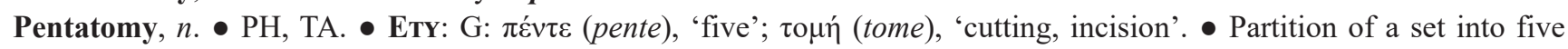
subsets. $\bullet$ Common language term. • Code: no term.

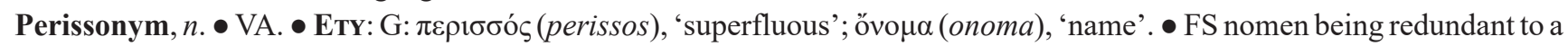


superordinate CS nomen in a given ergotaxonomy, that should therefore preferably not be used under the nomenclatural Criteria used in the present work as its purpose is only to comply with tradition but it carries no cladistic information.

- Hoc loco. - Code: no term.

Peritaxa, $n$. $\bullet$ One of the two plurals of peritaxon. $\bullet$ Hoc loco.

Peritaxic, a. • NO, TA. • ETY: see Peritaxy. • Qualification of two distinct taxa (an angiotaxon and an endotaxon) being in a relation of inclusion, and whose nomina are assigned to the same or different nominal-series. $\bullet$ Dubois $2005 b: 407$. - Code: no term.

Peritaxon (pl. peritaxa, peritaxons), $n$. • NO, TA. • ETy: see Peritaxy. • One of two distinct taxa (an angiotaxon and an endotaxon) being in a relation of inclusion, and whose nomina are assigned to the same or different nominal-series. $\bullet$ Dubois 2006a: 255. • Code: no term.

Peritaxons, $n$. $\bullet$ One of the two plurals of peritaxon. $\bullet$ Hoc loco.

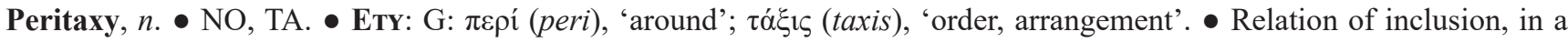
given ergotaxonomy, between two taxa (an angiotaxon and an endotaxon) whose nomina are assigned to the same or different nominal-series. • Dubois 2005b: 406. Code: no term.

Phalanx, $n$. $\bullet$ NO, TA. • Exy: L: phalanx, 'phalanx, body of soldiers'. • In zoological taxonomy and nomenclature, a key rank of the class-series, between order and family. $\bullet$ Term in traditional use in taxonomy; Dubois 2006a. $\bullet$ Code: no term.

Phenetic, $n$. $\bullet$ TA. $\bullet$ Concerning overall similarity and difference between organisms without regard to phylogeny. $\bullet$ Term in traditional use in evolutionary biology. $\bullet$ Code: no term.

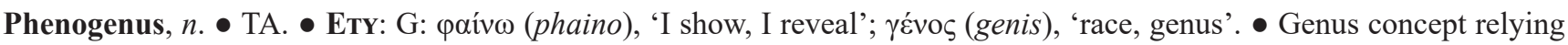
on the presence of phenetic characters shared by species. $\bullet$ Hoc loco. $\bullet$ Code: no term.

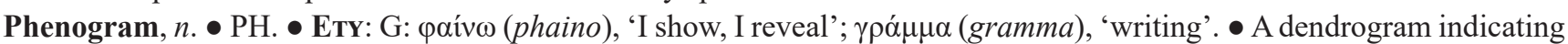
degree of overall similarity or distance. • Mayr 1965: 81 (see Brower 2016). $\bullet$ Code: no term.

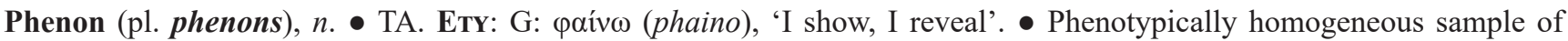
organisms. • Camp \& Gilly (1943: 335). $\bullet$ Code: no term.

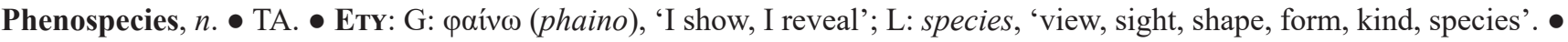
Species concept defined as a phenotypically homogeneous group of organisms. $\bullet$ Term in common use in taxonomy. - Code: no term.

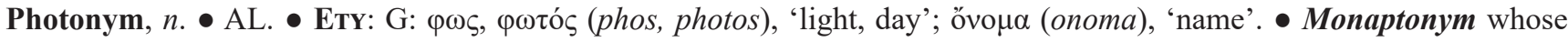
monophoric onomatophore (lectophoront, neophoront, nucleospecies or nucleogenus) is referred to a known ergotaxon. • ANT: nyctonym. Dubois 2011a: 54, 89. • Code: no term.

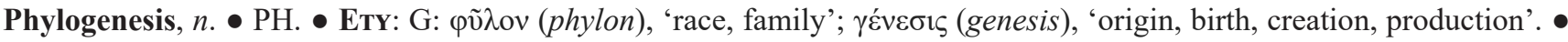
Biological process of differentiation and diversification of organisms during evolution, including speciation. • SrN: phylogeny. $\bullet$ Haeckel 1866a: 60. Code: no term.

Phylogenetic, $n$. $\bullet$ PH. • See Phylogenesis.

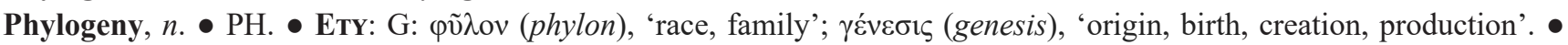
Biological process of differentiation and diversification of organisms during evolution, including speciation. • SYN: phylogenesis. • Haeckel 1866a: 60. • Code: no term.

Phylogram, $n$. • PH. • ETY: G: $\varphi \tilde{u} \lambda$ ov (phylon), 'race, family'; $\gamma \rho \alpha ́ \mu \mu \alpha$ (gramma), 'writing'. • A dendrogram indicating both cladistic branching and the relative amount of anagenetic change that has occurred between nodes. $\bullet$ Mayr 1969: 256 (see Brower 2016). • Code: no term.

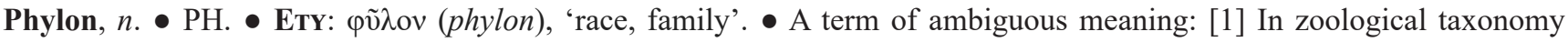
and nomenclature, a rank of the class-series, between kingdom and class (Haeckel 1866a: 61) (see phylum); [2] in evolutionary biology, a holophyletic evolutionary group (Dubois 1991: 65) (see lineage). $\bullet$ Code: no term.

Phylonomy, $n$. • TA. • Taxonomy based on a phylogram. • Hoc loco. • Code: no term.

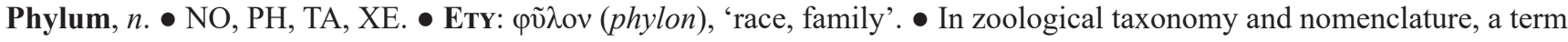
of ambiguous meaning: [1] a key rank of the class-series, between kingdom and class (Haeckel 1866b); [2] any rank of the family- or class-series (see e.g. Zhang 2011a-b). $\bullet$ Code: no term.

Plenary Power, e. • NO. • The power of the Commission to suspend or modify the application of Art. 1 to 76 of the Code in the way that it considers necessary to serve the interests of stability and universality of nomenclature in certains cases. - Code: plenary power.

Plesiomorphic, $n$. • See Plesiomorphy.

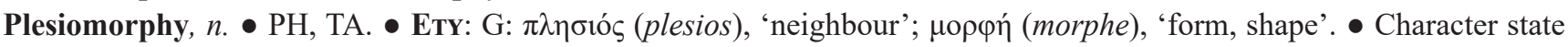
observed in a taxon which is considered derived primitive to the apomorphic state of this character in a taxon considered as descendant. • Hennig 1950. • Code: no term.

Plurinomen. (pl. plurinomina), n. • AV, CO. • L: plures, 'more numerous'; nomen, 'name'. • Nomen composed of two or 
more terms, including at least a generic substantive and a specific epithet. $\bullet$ Traditional term in zoological nomenclature. - Code: binomen.

Plurinomina, $n$. Plural of plurinomen.

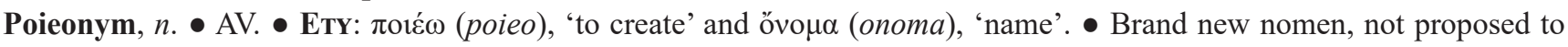
replace an existing one. ANT: neonym. • Dubois 2017a: 12. • Code: no term.

Polychotomy, $n$. $\bullet$ See Polytomy.

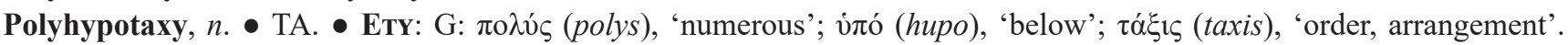
- Mode of hypotaxy of a taxon that includes more than two parordinate taxa of just lower rank. In a phylogenetic taxonomic frame, the meaning of this situation is unclear, as two different situations may account for it: [1] these parordinate taxa are the members of a still unresolved polytomy, which subsequent work can possibly resolve; [2] a hypothesis already exists regarding the relationships between the members of the polytomy, but it was not implemented into the ergotaxonomy in order to limit the number of ranks of this taxonomy. • Dubois \& Raffaëlli 2009: 12. • Code: no term.

Polyphyletic, $a$. $\bullet$ PH, TA. • Concept applying to taxa: qualification of a non-homophyletic group, i.e. that does not include its most recent common ancestor. $\bullet$ Haeckel 1874; Hennig 1950; Ashlock 1971; Dubois 1986b. $\bullet$ Code: no term.

Polyphyly, $a$. • See Polyphyletic.

Polysemic, a. • NO. • ETY: see Polysemy. • In the context of zoological nomenclature, the qualification of either [1] a nomenclatural system that allows the same nomen to designate distinct taxa, or [2] any nomen being in this situation (see Dubois 2007a: 41). • ANT: monosemic. • Term in traditional use in linguistics and grammar. • Code: no term.

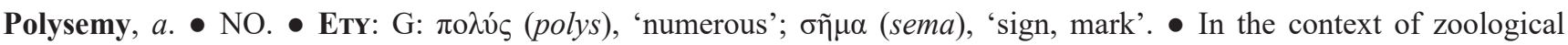
nomenclature, the fact that a nomenclatural system allows the same nomen to designate distinct taxa. $\bullet$ ANT: monosemy. - Term in traditional use in linguistics and grammar. • Code: no term.

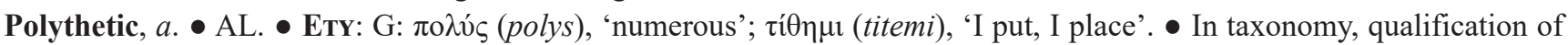
a diagnosis of taxon involving a variable, but unique to the taxon, combination of alternative character states, none of which is necessarily present in every member of the taxon. • ANT: Monothetic. • Sneath 1962; Van Regenmortel 2016; Dubois $2017 d$. $\bullet$ Code: no term.

Polythetic diagnosis, $e$. $\bullet$ AL. $\bullet$ In taxonomy, a diagnosis of taxon involving a variable, but unique to the taxon, combination of alternative character states, none of which is necessarily present in every member of the taxon. • ANT: Monothetic diagnosis. • Sneath 1962; Van Regenmortel 2016; Dubois 2017d. • Code: no term.

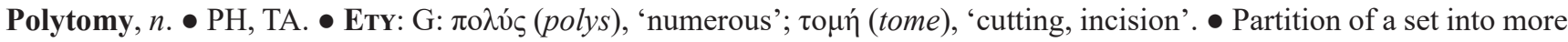
than two subsets. - SYN: polychotomy. • END: trichotomy, tetratomy, pentatomy, hexatomy, heptatomy, octotomy, enneatomy. $\bullet$ Common language term. $\bullet$ Code: no term.

Potentially valid, e. $\bullet \mathrm{AV}$, VA. $\bullet$ An available and allocated nomen which is not invalid but which may become so for reason of synonymy, homonymy, proedry, airesy or archoidy. • Traditional term in zoological and botanical nomenclature. • SYN: available. Code: potentially valid.

P-publication, $n$. $\bullet$ AV. $\bullet$ Publication printed on paper. $\bullet$ Dubois et al. 2013: 5. • Code: work printed on paper.

Precedence, $n$. $\bullet$ VA. $\bullet$ In zoological nomenclature, the fact that a nomen must be used as valid against its potential synonyms and homonyms, as a result of one of the Principles of Validity of the Code. • AnT: Subservience. $\bullet$ Traditional term in zoological nomenclature. • Code: precedence.

Prefix, $n$. $\bullet$ NO. A letter or group of letters preceding a word having its independent existence in order to modify its meaning. $\bullet$ Common language term. $\bullet$ Code: prefix.

Prenucleogenera, $n$. $\bullet$ Plural of prenucleogenus.

Prenucleogenus (pl. prenucleogenera). • AL. • ETY: L: prae, in the sense of 'before'; nucleus, 'nucleus, core, stone' (from nux, 'nut'); genus, 'birth, origin, class, kind'. • One of several nominal genera originally included in a new nominal family-series at its first publication (generic symphory), before subsequent designation among them of a single elitonucleogenus. • Hoc loco. • Code: no term.

Prenucleospecies, n. AL. • Eтr: L: prae, in the sense of 'before'; nucleus, 'nucleus, core, stone' (from nux, 'nut'); species, 'species'. - One of several nominal species originally included in a new nominal genus or subgenus at its first publication (specific symphory), before subsequent designation among them of a single nucleospecies. $\bullet$ Dubois 2005b: 404. Code: originally included nominal species.

Pre-registration, $n$. $\bullet$ AV, VA. • A category of registration of a nomen or an onomatergy that occurred before the publication of the latter, e.g. registration in Zoobank before an electronic publication. • Dubois \& Aescht 2019a: 12. • Code: no term.

Prevailing usage, e. • VA. • An ambiguous formula, used in different parts of the Code under different meanings: see details in Dubois (2010a: 13-14, 2017b: 24) and Löbl (2015). This formula is not used in the present work, which relies on well-defined categories of usage (Dubois 2006a, 2010a, 2015c). • Code: prevailing usage. 
Preventive archaeology, e. $\bullet \mathrm{XE}$. $\bullet$ The domain of archaeology devoted to the protection of threatened archaeological sites (see e.g. Bozóki-Ernycy 2007).

Preventive taxonomy, $e$. TA, XE. $\bullet$ The organisation of special field work parties for the collection of specimens of animal species threatened with extinction by predictable destruction or major alteration of habitats or ecosystems. Dubois 2008e. $\bullet$ Code: no term.

Primary auctorship, e. • VA. - In case of double auctorship of a family-series junior synonym validated before 1961 through Article 40.2 (see Dubois 2015a: 31-34), the auctorship (and date) of the junior nomen which are validated against those of its senior synonym. In the present work, this primary auctorship is presented between double vertical bars: e.g. DENDROBATIDAE \|Bonaparte, 1850\|-Cope, 1865. • Hoc loco. • Code: no term.

Primary key rank, e. • NO, TA. • Any of the seven mandatory taxonominal key ranks (kingdom, phylum, class, order, family, genus, species) of zoological taxonomy and nomenclature. • ANT: secondary key rank. $\bullet$ Common language terms; Dubois 2006a: 217. • Code: no term.

Primary homonym, e. • VA. • See Hadromonym.

Primogenera, $n$. Plural of primogenus.

Primogenus (pl. primogenera), n. • NO. • ETY: L: primus, 'original, primary'; genus, 'birth, origin, class, kind'. • Genusseries nomen expressly mentioned as valid and included in (conucleogenus or uninucleogenus) or excluded from (alienogenus) a new class-series nomen in the original publication of the latter. $\bullet$ Dubois 2015c: 78. $\bullet$ Code: no term.

Primoscriptor, $n$. • AV, CO. • ETY: L: primus, 'first'; and scriptor, 'writer, author'. $\bullet$ See Scriptor. • Dubois $2000 b$ (as first-user), 2013. $\bullet$ Code: no term.

Principle, $n$. $\bullet$ NO. - Within the frame of the Code, a general statement of general value which applies to all relevant nomenclatural acts and which is the basis for all particular and specific Rules of the Code. • Traditional term in zoological nomenclature; Dubois 2011a: 90. • Code: no definition.

Principle of Airesy, e. • VA. - In any situation of synchronous zygoidy between nomina of the same nominal-series, precedence among zygonyms (homonyms or synonyms), zygographs (competing parographs of a nomen) or zygophories (competing airetophories for a nomen) is fixed by the action of an arbiter publishing an explicit act of airesy, i.e. seniorisation of one item and juniorisation of the other(s), removing this ambiguity. This airesy is definitive and irreversible by subsequent actions of individual authors. It may however be superseded by other Principles of Validity. • Dubois 2011a (as 'Principle of First-Reviser'), 2013; Dubois \& Aescht 2019m. • Code: no term.

Principle of Archoidy, e. $\bullet$ NO. In case of nomenclatural ambiguity, uncertainty or conflict, liable to disturb the universality of zoological nomenclature and to cause confusion, the Commission may be conferred Plenary Power to take a specific action aiming at solving the problem. In order to do so, it is entitled to set aside, as needed, any existing Rule of the Code (except those concerning the powers and duties of the respective internationally accepted regulatory body). $\bullet$ Dubois \& Aescht 2019q. • Code: no term.

Principle of Binomina, e. $\bullet \mathrm{AV}, \mathrm{CO}$. $\bullet$ The nomen of a taxon of rank species is a binomen. The nomen of a taxon of rank subspecies is a trinomen. The nomina of all taxa above the species-series are uninomina. Nomina of subgenera, aggregates of species and aggregates of subspecies are uninomina that, when used in a binomen or trinomen, must be interpolated in parentheses between those of their superordinate and subordinate taxa; such nomina are not counted in the number of words of a binomen or trinomen. • Dubois 2011a, 2013; Dubois \& Aescht 2019b. • Code: Principle of Binominal Nomenclature (Articles 4-6, 11.4; pages 4-6, 10-11).

Principle of Coordination, $e \cdot \mathrm{AV}, \mathrm{VA}$. $\bullet$ In the family-, genus- and species-series, a nomen introduced for a taxon at any rank of the nominal-series is deemed to be simultaneously introduced for any other taxon at any other rank of the same nominal-series (e.g., genus Rana, subgenus Rana, or superfamily RANOIDEA, family RANIDAE, subfamily RANINAE, tribe RANINI, subtribe RANINA). These different paronyms of the same nomen may be used in parallel at different ranks in a given ergotaxonomy. Whenever indeed used for such other taxa, these are not different nomina (synonyms) but they are all avatars of the same nomen, having the same onomatophore, author and date. $\bullet$ Dubois 2011a, 2013; Dubois \& Aescht 2019d. • Code: Principle of Coordination (Article 36, p. 45; Article 43, p. 48; Article 46, p. 50).

Principle of Homonymy, e. • VA. • Whenever two nomina of the same nominal-series are strictly identical (homographs) or deemed to be identical under the Rules of the Code (rhizomographs or paromographs), only one can be potentially valid (if not invalid for another reason). In the genus- and family-series, homonymy is absolute and irreversible (hadromonymy), but in the species-series it can be either absolute and irreversible (hadromonymy) or relative and reversible (asthenomonymy). The potentially valid nomen among homonyms is determined, according to the situation, by one of the Principles regulating nomenclatural precedence among nomina involved in a relation of zygoidy. The Principle of Homonymy does not apply between homonymous epithets combined with homonymous but distinct generic substantives (pseudomographs). • Dubois 2011a, 2013; Dubois \& Aescht 2019i. • Code: Principle of Homonymy (Article 52, p. 56).

Principle of Neonymy, e. • AV. • The publication of the clearly intentional replacement of an available nomen by a 
different nomen results in the introduction in zoological nomenclature of a neonym, which has the same onomatophore as the replaced nomen (archaeonym) but a different author and a different date. A neonym having the same etymology as its archaeonym is an autoneonym, whereas a neonym having a partially or completely different etymology is an alloneonym. Allelonyms are alternative nomina published in the same work for the same taxon. They have the same onomatophore, author and date. • Dubois 2011a, 2013; Dubois \& Aescht 2019f. • Code: no term.

Principle of Nomenclatural Foundation, $e$. $\bullet$ NO. • The nomenclatural status of a nomen is fixed once and for all in the original publication where this nomen is introduced, or if relevant by the Principle of Airesy, and cannot be modified by subsequent actions of individual zoologists. • Dubois 2011a, 2013; Dubois \& Aescht 2017b. • Code: no term.

Principle of Nominal-Series, e. • AS, AV. • The Code's nomenclatural hierarchy covers all taxa recognised by taxonomists in the animal kingdom. This hierarchy is divided in four nominal-series: the species-, genus-, family- and class-series. Each nominal-series accommodates several ranks (four in the species-series, two in the genus-series, an unlimited number in the family- and class-series). To become available, a new nomen must be introduced as unambiguously referred, either implicitly or explicitly to one of these nominal-series, and it must follow the Principle of Binomina. $\bullet$ Dubois 2011a, 2013; Dubois \& Aescht 2017d. • Code: no term.

Principle of Nomography, e. • CO. • In a given ergotaxonomy, any kyronym at a given rank can have a single correct spelling (eugraph), which can be either its protograph or one of its apographs, particularly in cases of mandatory spelling or ending correction. • Dubois 2013; Dubois \& Aescht 2019o. • Code: not stated as a Principle, but implemented as Rules in Articles 19 (p. 21), 27 (p. 32), 28 (p. 32), 32.2 (p. 39), 32.5 (p. 39-42), 33.2 (p. 42 ), 34 (p. $43-44)$ and 58.

Principle of Onomatophores, e. • AL. • Each nomen has, actually or potentially, an onomatophore, i.e., an objective standard of reference of inclusive ostension whereby the taxonomic allocation of the nomen can be determined. In any given ergotaxonomy, the nomen can be potentially applied to any taxon that includes its onomatophore. In the speciesseries, onomatophores are specimens, whereas in the genus-, family- and class-series they are taxomina. • Dubois 2011a, 2013; Dubois \& Aescht 2019f. • Code: Principle of Typification (Article 61, p. 63-64).

Principle of Priority, e. • VA. • In a given nominal-series, in any situation of allochronous zygoidy, the first published zygonym (homonym or synonym), zygograph (competing parograph) or zygophory (competing airetophory) has precedence, except if the Principles of Nomography or Sozoidy apply. • Dubois 2011a, 2013; Dubois \& Aescht 2019 l. - Code: part of the Principle of Priority (Article 23, p. 24).

Principle of Proedry, e. • VA. • In a given nominal-series, whenever zygonyms (homonyms or synonyms) are introduced simultaneously, but proposed at different ranks within their nominal-series, the nomen proposed at higher rank has precedence. The same applies between synchronous zygophories (competing airetophories) if they concern taxa at different ranks: the designation made for the taxon at higher rank has precedence. • Dubois 2013; Dubois \& Aescht 2019n. Code: not stated as a Principle, but implemented as a Rule in Articles 24.1 (p. 30), 55.5 (p. 58), 56.3 (p. 58 ), 57.7 (p. 60) and 61.2 .1 (p. 64).

Principle of Registration, $e$. $\bullet$ RE. $\bullet$ The nomenclatural status of publications, nomina, spellings and onomatergies may be fixed and registered online, and therefore protected from oblivion and rejection, in an international open database recognised by the Commission or its successor body as appropriate for this purpose. Three kinds or categories of registrations exist: [1] post-registration of decisions of the Commission under the Plenary Power regarding nomenclatural availability (of works, nomina and/or onomatergies), taxonomic allocation (of nomina) and validity and correctness (validity of nomina and/or onomatergies; correctness of spellings of nomina); [2] post-registration of availability/ unavailability of nomina duly listed in Lists of Available Names; [3] pre-registration on Zoobank, respecting all the Code's requirements in this respect, of new works, nomina and onomatergies before online publication of the work. $\bullet$ Dubois 2011a, 2013; Dubois \& Aescht 2019r. • Code: no term.

Principle of Sozoidy, e. • NO. • In the class-series, among two or more synonyms or homonyms, whenever one qualifies as sozonym or sozodiaphonym, it must be given precedence for validity (if not invalid for another reason) over its synonym(s) or homonym(s) that would have precedence over it according to the usual criteria of Priority, Airesy or Proedry; however these usual criteria apply among sozodiaphonyms. The same Principle applies to two or more spellings, the sozograph being the correct spelling, or to two or more zygophories, if one of them qualifies as a sozairetophory. $\bullet$ Dubois 2011 (as 'Principle of Sozonymy'), 2013; Dubois \& Aescht 2019p. • Code: not stated as a Principle, but some of the conditions listed here appear in Article 23.9 on Reversal of precedence (p. 27-29).

Principle of Synonymy, e. • VA. • Whenever two nomina of the same nominal-series are based on the same onomatophore (isonyms, which include allelonyms) or considered as synonyms in a given ergotaxonomy despite being based on different onomatophores (doxisonyms), only one can be potentially valid (if not invalid for another reason). $\bullet$ Dubois 2011a, 2013; Dubois \& Aescht 2019k. • Code: part of the Principle of Priority (Article 23, p. 24).

Principle of Zoological Nomenclature Independence, e. • NO. • Zoological nomenclature as regulated by the Code and by DONS is independent from [1] taxonomy (i.e. it does not interfere with taxonomic thought and action), and [2] all other codes of nomenclature, whether in force for non-animal living beings or based on other basic premices 
incompatible with those of the Code. • Dubois 2011a, 2013 (both as 'Principle of Nomenclatural Independence'); Dubois \& Aescht 2017a. $\bullet$ Code: no term.

Principle of Zygoidy, e. • AL, VA, CO. • In the frame of a given ergotaxonomy, a taxon at a given rank must bear a single nomen with a single spelling. Different situations of conflict of zygoidy may be distinguished: [1] zygonymy: conflict between homonymous or synonymous nomina competing for validity; [2] zygography: conflict between spellings competing for correctness; and [3] zygophory: conflict between onomatophore restrictions or designations competing for validity. These conflicts must be resolved, according to the situation, through use of the appropriate one among the following five Principles: Priority, Airesy, Proedry, Nomography and Sozoidy. • Dubois 2013; Dubois \& Aescht $2019 g$. - Code: no term.

Priority, $n$. $\bullet$ VA. • In the context of zoological nomenclature, a qualification of a nomen, an onomatergy or a spelling published previously to another one and having therefore nomenclatural precedence on the latter. $\bullet$ Traditional term in zoological nomenclature. $\bullet$ Code: priority.

Priscogenus, $n$. $\bullet$ AV, VA. • ETY: L: prisco, 'primitive'; genus, 'race, kind, genus'. • The generic substantive with which a new species-series epithet was combined in the publication where it was made available. $\bullet$ Dubois \& Aescht $2019 h$ : 77. $\bullet$ Code: no term.

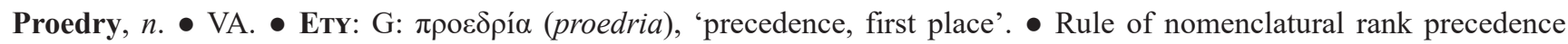
between synchronous synonyms or homonyms under the Code (Articles 24, 55.5, 56.3 and 57.7) which states that if one of these nomina was proposed at a higher rank than the other(s), it takes precedence over it/them whenever they are considered synonyms. $\bullet$ Dubois 2013: 7. • Code: no term.

Promulgate, $v$. AV. $\bullet$ Publish a new work, a new nomen or a new onomatergy complying with the Rules of the Code for nomenclatural availability. • Dubois 2020b: 51. • Code: one of the meanings of the verb 'establish'.

Promulgation, $n$. $\bullet \mathrm{AV}$. • Publication of a new work, a new nomen or a new onomatergy complying with the Rules of the Code for nomenclatural availability (Articles 8-9). • Dubois 2020b: 51. • Code: one of the meanings of the term establishment.

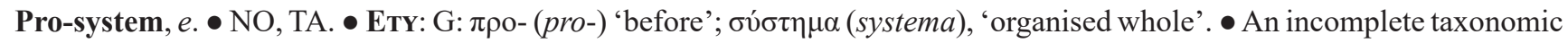
or nomenclatural system for a given group of organisms, i.e., allowing unambiguous, objective, repeatable and universal decisions only in some cases and situations. • END: mero-systems and pseudo-systems. • Dubois 2015c: 8, 79. • Code: system.

Protaxa, $n$. $\bullet$ One of the two plurals of protaxon.

Protaxon (pl. protaxa, protaxon), $n$. • AL. • ETY: G: $\pi \rho 0-($ pro-), in the sense of 'first, primitive, original'; $\tau \alpha \dot{\xi} \xi 1 \zeta$ (taxis), 'order, arrangement'. • Taxon with its complete original extension (i.e., members, circumscription) in the publication where it was first proposed. • Dubois 2005b: 405. • Code: no term.

Protaxons, $n$. $\bullet$ One of the two plurals of protaxon.

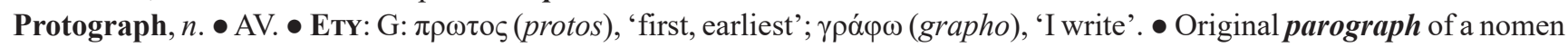
in the publication where it was originally introduced. - ANG: protonym. - END: holoprotograph, symprotograph, lectoprotograph, leipoprotograph. • ANT: apograph. • Dubois 2010a: 6. C Code: original spelling.

Protohypse, $n$. $\bullet \mathrm{AV}$. $\bullet$ ETY: G: $\pi \rho \omega \tau \circ \varsigma$ (protos), 'first, earliest'; $v \psi \mathrm{\psi}$ (hypsos), 'height' • A category of protonym: original rank of a nomen. • ANT: apohypse. • Dubois 2010a: 6. Code: no term.

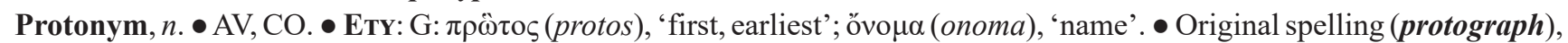
rank (protohypse) and/or, if relevant, onymorph (protonymorph) of a nomen. • ANT: aponym. • Dubois 2000b: 51. • Code: no term.

Protonymorph, $n$. • AV. • ETY: G: $\pi \rho \omega \tau$ shape'. • A category of protonym: original onymorph of a nomen. • ANT: aponymorph. • Dubois 2010a: 6. Code: no term.

PRR, ab. • See Partially regulated family-series ranks.

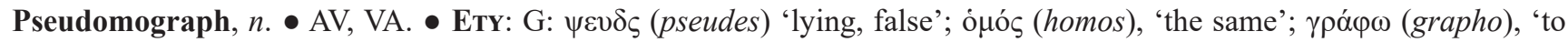
write'. - Any of two or more distinct identical or 'deemed to be identical' (under Article 58 of the Code) epithets originally referred to genera designated by homonymous but distinct generic substantives. • Dubois \& Aescht $2019 h$ : 69, 77. • Code: no term.

Pseudorank, $n$. NO. • ETY: see Pseudoranked. • So-called ranks used by some authors in pseudoranked nomenclatural system, in which the attribution of nomina to 'ranks' does not provide information on their place in the taxonominal hierarchy. $\bullet$ Hoc loco. $\bullet$ Code: no term.

Pseudoranked, $p$. • NO. • ETY: G: $\psi \varepsilon v \delta \varsigma$ (pseudes) 'lying, false'; Frankish: hring, 'circle, ring', from Proto-Germanic hringaz, 'circle, ring, something curved'. - Qualification of a nomenclatural system in which ranks of nomina are mentioned but used in an inconsistent manner, for example assigning different ranks to parordinate taxa, or having different hierarchies between the same ranks in different parts of the classification, or using ranks for some taxa but 
no rank for others, simply referred to as 'taxa' or 'clades'. Ranks used in such a system provide no information on the hierarchical relationships between nomina, and by way of consequence on the structure of the tree adopted as a basis for the taxonomy. $\bullet$ Dubois 2007a: 34 . $\bullet$ Code: no term.

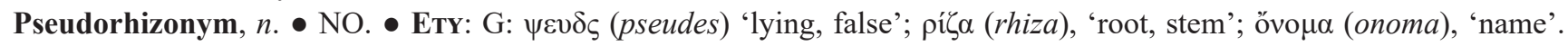
- Suprageneric nomen HN (designating a taxon HT) based on the stem of a genus-series nomen but the latter not complying with the conditions of the Code for the availability of FS nomina (available GS nomen included as valid in HT). If proposed as a family-series nomen, it is incorrectly formed according to Article 13.2 of the Code, and is therefore a family-series anoplonym (nomenclaturally unavailable). If proposed as a class-series nomen, it may be available under DONS Criteria (if the other conditions of nomenclatural availability are complied with). • END: auxorhizonym, cenorhizonym and xenorhizonym. Dubois 2015c: 22, 79. • Code: no term.

Pseudorhizonymy, $n$. • NO. • ETy: see Pseudorhizonym. • The fact that a nomen is a pseudorhizonym. • Hoc loco. • Code: no term.

Pseudospecies, $n$. $\bullet$ See Kyon.

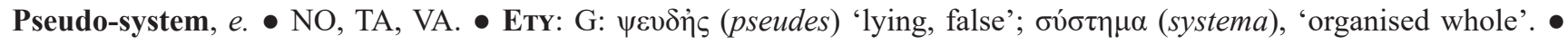
A taxonomic or nomenclatural pro-system which leaves some decisions unsettled and therefore requires recourse to subjectivity and personal opinions. $\bullet$ Dubois $2015 c$ : 8 , 79. $\bullet$ Code: no term._

Publication, $n$. $\bullet$ NO, TA. • [1] General meaning: [1a] the act of distribution of a work; [1b] the result of this act: a work distributed. [2] In the context of zoological nomenclature: [2a] the act of promulgation of a work conforming to the provisions of Articles 8-9 of the Code (i.e., mostly, printed with ink on paper and distributed as several identical copies, or released electronically after 2011) (see promulgation); [2b] the result of this act: a work promulgated. • Traditional term in zoological nomenclature. $\bullet$ Code: publication.

Publication date, e. • NO. - In the context of zoological nomenclature, the actual date of public distribution of a publication — not its date of writing, submission, acceptance, printing or any other date that may appear in the document itself. $\bullet$ Term in traditional use in nomenclature. $\bullet$ Code: date.

Published, $p$. $\bullet$ NO. $\bullet$ In the context of zoological nomenclature, work issued conforming to the provisions of Articles 8-9 of the Code. • See Publication.

Quantile, $n$. $\bullet$ NO, XE. • A cut point dividing the range of a probability distribution or of observations in a sample into continuous intervals with equal probabilities. There is one fewer quantile than the number of groups thus created. $\bullet$ Term in traditional use in statistics and probability. $\bullet$ Code: no term.

Quartile, n. $\bullet$ NO, XE. • A quantile dividing the number of data points into four more or less equal parts, or quarters. $\bullet$ Term in traditional use in statistics and probability. $\bullet$ Code: no term.

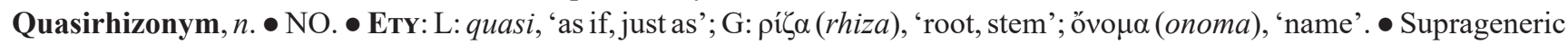
nomen HN based on the stem of either a nomen of the SS, FS or CS or of a non-scientific name of animal, this stem being combined with an ending derived from another or several other terms (e.g., -formes, -morpha, -phora, etc.). If proposed as a family-series nomen, it is incorrectly formed according to Article 13.2 of the Code, and is therefore a family-series anoplonym (nomenclaturally unavailable). If proposed as a class-series nomen and available, common particular cases are those of such nomina the original endings of which were derived from the roots forma (Latin) or

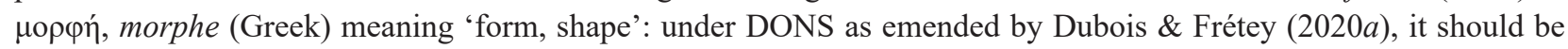
used under the respective standard endings -IFORMES or -OMORPHES, which are not in a relation of hierarchy but may be both used at whatever rank $\bullet$ Dubois \& Frétey $2020 a$. $\bullet$ Code: no term.

Quasirhizonymy, $n$. • NO. • ETY: see Quasirhizonym. • The fact that a nomen is a quasirhizonym. • Hoc loco. • Code: no term.

Radicogenera, $n$. • Plural of radicogenus.

Radicogenus (pl. radicogenera), n. • NO. • ETY: L: radix, 'root'; genus, 'birth, origin, class, kind'. • Genus-series nomen playing the function of radiconomen of a suprageneric nomen. $\bullet$ Hoc loco. $\bullet$ Code: no term.

Radiconomen (pl. radiconomina), $n$. • NO. • ETY: L: radix, 'root'; nomen, 'name'. • Nomen or non-scientific name on which a rhizonym, a pseudorhizonym or a quasirhizonym is based. • Dubois 2015c: 23, 79. • Code: no term.

Radiconomina, $n$. $\bullet$ Plural of radiconomen.

Rank, $n$. • AV, VA. • ETY: Frankish: hring, 'circle, ring', from Proto-Germanic hringaz, 'circle, ring, something curved'. • The place of a nomen in a nomenclatural hierarchy or of a taxon in a taxonominal hierarchy. See Nomenclatural rank, Absolute rank, Relative rank and Taxonominal level. - Traditional term in nomenclature and taxonomy, precisely defined by Dubois \& Malécot (2005: 101) and Dubois (2005b: 412). • Code: rank.

Ranked, $p$. • NO. • ETY: see Rank. $\bullet$ Qualification of a nomenclatural system in which ranks are assigned to all nomina of supraspecific and infraspecific taxa. In a consistent such system, parordinate taxa are always assigned to the same rank, the hierarchy of primary key ranks used in different parts of the classification is the same, and all taxa are referred to ranks, but some of these qualifications at least are missing in pseudoranked and unranked nomenclatural systems. 
- Traditional term in zoological nomenclature. $\bullet$ Code: no term.

Rapid Ecological Assessment, e. • XE. • A methodology devised to provide multiple scale information required to guide actions of ecological conservation (see e.g. Muchoney et al. 1991).

Recent, $n$. $\bullet$ XE. $\bullet$ For a taxon of AMPHibia: that is referred to the LisSAMPHibia.

Recommendation, $n$. $\bullet$ NO. • A suggestion of 'good practice' which zootaxonomists are encouraged to follow, but failure to do so has no bearing on the availability or validity of onomatergies. A Recommendation has no juridical function and is therefore not part of the effective regulations of the Code. $\bullet$ Code: recommendation.

Redundant taxon, e. • VA. • A taxon whose formal recognition in an ergotaxonomy does not bring any supplementary phylogenetic information additional to that alreadty provided by an immediately subordinate or superordinate taxon. $\bullet$ Term in traditional use in nomenclature. $\bullet$ Code: no term.

Referred to, e. • TA. • General language term, used sometimes in taxonomy with two precise technical meanings: [1] in the species-series, the statement that a species-series epithet is referred to a nominal genus may be made through actual combination with the generic substantive or through virtual combination, by simple mention that it belongs to this genus, whether considered as valid or as an invalid synonym; [2] the statement that a specimen or a taxon belongs to a taxon recognised in a given ergotaxonomy. • Dubois \& Aescht 2019h: 77. • Code: no term.

Registered, $p$. $\bullet$ RE. $\bullet$ Qualification of a nomen (delonym) that conforms to the conditions of nomenclatural registration of the Code. • ANT: unregistered. $\bullet$ Traditional term in many domains. $\bullet$ Code: no term.

Registration, $n$. $\bullet$ RE. • Onomatergy by which a nomen registered in an international nomenclatural database recognised by the Code becomes permanently available in zoological nomenclature (delonym). $\bullet$ Traditional term in many domains. - Code: registration.

Regnum, $n$. • NO, TA. • ETY: L: regnum, 'kingdom'. • A class-series key rank in biological taxonomy and nomenclature, between imperium and phylum. $\bullet$ Term in traditional use in taxonomy. • SYN: kingdom. $\bullet$ Code: no term.

Relacter, $n$. $\bullet$ TA. $\bullet$ A taxonomic criterion relying on the relations that may exist, in natural or artificial conditions, between two entities composed of organisms, such as crossability, sympatry-parapatry-allopatry, parasitic specificity, ecological competitive exclusion, or presence-absence of a hybrid zone and of a gene flow in their contact zone. $\bullet$ Dubois $1988 c$ : 57 (as 'relational taxinomic criterion'), 2004d: 45. • Code: no term.

Relational, $n$. $\bullet$ TA. $\bullet$ In taxonomy, qualification of a Criterion relying on the relations that may exist, in natural or artificial conditions, between two entities composed of organisms. $\bullet$ Dubois $1988 c$ : 57 . $\bullet$ Code: no term.

Relative rank, e. $\bullet \mathrm{AV}, \mathrm{VA}$. $\bullet$ Nomenclatural rank conceived and used as provisionally attached to taxa, the same taxon being liable to shift from one rank to another in order to express the hierarchical relationships between taxa, according to the phylogenetic hypothesis adopted. $\bullet$ Dubois 2007a: 34. $\bullet$ Code: no term.

Rescue archaeology, e. $\bullet$ XE. $\bullet$ The domain of archaeology devoted to the rescue or salvage by excavation of threatened archaeological sites (see e.g. Demoule 2002).

Resurrection, $n$. $\bullet$ See Revalidation.

Reversal of precedence, $e$. $\bullet$ VA. $\bullet$ In the context of the Code, the suspension of the Principle of Priority in cases covered by Article 23.9. - See Lethakyronym and Sozoidy.

Revalidation, $n$. $\bullet$ VA. - Process opposite to that of synonymisation, by which a nomen once considered an invalid doxisonym is reinstated as valid. • Common language term, here used with a precise technical meaning proper to nomenclature; equivalent to the term 'resurrection' often used in the taxonomic literature to designate this process. Code: no term.

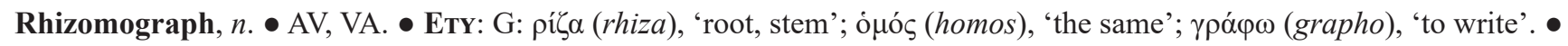
Any of two or more distinct protographs of the family or class-series having different spellings but derived from the same stem or from homographic terms. • Dubois 2012a: 64. • Code: no term.

Rhizomography, $n$. $\bullet$ AV, VA. • ETY: see Rhizomograph. $\bullet$ The fact that two distinct nomina are rhizomographs. $\bullet$ Dubois 2012a: 65. Code: no term.

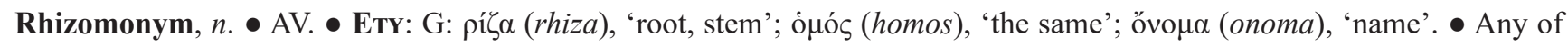
two or several family-series or class-series nomina derived from identical or homonymous stems, which according to the Rules of the Code or the Criteria of DONS must be considered homonyms, even if they have different endings. $\bullet$ Dubois 2012a: 65, 79. • Code: no term.

Rhizonym, $n$. • NO. • ETY: G: pí̧ $\alpha$ (rhiza), 'root, stem'; ővo $\mu \alpha$ (onoma), 'name'. • Suprageneric nomen HN (designating a taxon HT) based on the stem of a then available genus-series nomen GN referred as valid to HT, followed by a simple ending denoting plural (e.g., -AE, -IDAE, -INAE, -IDI, -OIDEA, -ACEA, etc). If proposed as a family-series nomen, it may be available under Article 13.2 of the Code (if all other criteria of nomenclatural availability are complied with), but then, according to the rank where it is used, it should be so with a correct ending according to the Code's Rules or to DONS' proposals (T.HIE). If proposed as a class-series nomen, it may be available under DONS Criteria (if all other criteria of nomenclatural availability are complied with), but then, it should be so with the standard ending -ACEA, which is not in 
a relation of hierarchy and may be used at whatever rank. $\bullet$ Dubois 2006c: 8, 2015c: 80. $\bullet$ Code: no term.

Rhizonymy, $n$. $\bullet$ NO. • ETY: see Rhizonym. $\bullet$ The fact that a nomen is a rhizonym. • Dubois \& Frétey $2020 a$. $\bullet$ Code: no term.

Robustness, $n$. • TA. • In taxonomy and nomenclature, a combination of stability and flexibility, according to which an ergotaxonomy and its associated nomenclature should be flexible enough to be able to change, in order to take new information or ideas into account, but that it cannot do so 'easily'. • Dubois 2005b: 373. • Code: no term.

Rule, $n$. $\bullet$ NO. $\bullet$ Within the frame of the Code, a mandatory prescription, compatible with its Principles, which applies in particular nomenclatural situations and cases, and regulates the availability, allocation and validity of nomina and onomatergies. • Term in traditional use in nomenclature and in common language. $\bullet$ Code: rule.

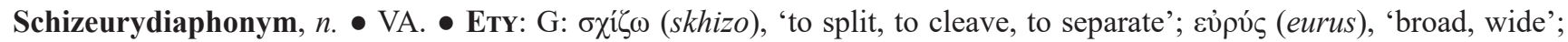

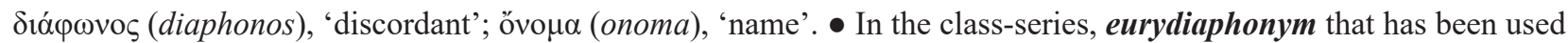
as valid for a given taxon, or for taxa having totally or partially identical extensions, in the titles of at least 100 works in the scientific literature after 31 December 1899, but alternatively to another eurydiaphonym that has also been used significantly for the same taxon or for taxa having totally or partially identical extensions. $\bullet$ Dubois 2005a: 85, 2005b: 412. Code: no term.

Scientific name, $e . \bullet$ NO, TA. • See Nomen.

Scriptor (pl. Scriptores), n. • AV, CO. • ETY: L: scriptor, 'writer, author'. • In the context of zoological nomenclature, name(s) of the person(s) to whom the first use of an aponym is credited, i.e., whose name(s) appear(s) as signatory of the work where this aponym first appeared itself—not established through subsequent investigation. $\bullet$ Dubois $2000 b$ : 42 (as first-user), 2013: 3 (as primoscriptor), 2015a: 15. • Code: no term.

Scriptores, $n . \bullet$ Plural of scriptor.

Scriptorship, $n$. $\bullet$ NO, TA. • ETY: see Scriptor. • In the context of zoological nomenclature, statement of the scriptor of an aponym. - Dubois et al. 2019: 15. • Code: no term.

Secondary auctorship, e. • VA. • In case of double auctorship of a family-series junior synonym validated before 1961 through Article 35.4.1 (see Dubois 2015a: 31,33-34), the auctorship (and date) of the senior nomen which are transferred to its junior synonym. In the present work, this secondary auctorship is presented between simple vertical bars: e.g. MEgOPHRYIDAE Bonaparte, 1850-|Noble, 1931|. • Hoc loco. • Code: no term.

Secondary homonym, e. • VA. • See Asthenomonym and Hadromonym.

Secondary key rank, e. • NO, TA. • Any taxonominal key rank that is not part of the seven mandatory ranks of zoological taxonomy and nomenclature:e.g., province, circle, legion, phalanx, stirps, tribe, clan, caste. • ANT: primary key rank. - Common language terms; Dubois 2006a: 217. • Code: no term.

Senior, $a$. $\bullet$ NO. $\bullet$ In the context of zoological nomenclature, and concerning a nomen, an onomatergy or a spelling: published at a date prior to that of publication of another nomen, onomatergy or spelling, qualified as junior. $\bullet$ Traditional term in nomenclature. $\bullet$ Code: senior.

Seniorisation, $n$. $\bullet$ NO. - In the context of zoological nomenclature, and concerning a conflict of zygoidy between synchronous nomina, spellings or airetophories, airesy by which a nomen, spelling or airetophory is granted precedence over another one, which is then juniorised relative to it. $\bullet$ Dubois 2000b: 47. $\bullet$ Code: junior.

Seniorise, $v$. $\bullet$ See Seniorisation.

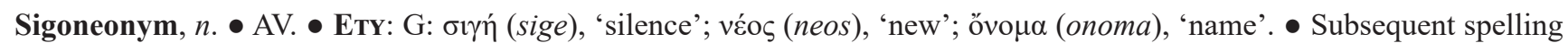
which, being clearly a meletograph, must be considered a neonym although it does not meet the restrictive criteria of Article 33.2.1 (see NS1-NS5 in column 3 of Table T8.NS-2). • Hoc loco. • Code: no term.

Signatory, $n$. $\bullet$ NO, TA. $\bullet$ Name(s) of the person(s) which appear(s) as the 'author' on the cover or at the beginning or end of a published work. • Dubois \& Aescht 2019o: 131. • Code: author.

Simpson, $n$. $\bullet$ TA. $\bullet$ Species concept relying on the result of a cladistic analysis: set of organisms that can be defined by an apognosis and are considered to represent a separate lineage. $\bullet$ Dubois 2007a: 48. $\bullet$ Code: no term.

Sister-taxon (pl. sister-taxa), e. $\bullet$ PH, TA. • One of two or several taxa that correspond to two or several branches resulting from the splitting in two (dichotomy) or more (polytomy) of a branch in a cladistic tree. $\bullet$ Term in traditional use in phylogeny and taxonomy. $\bullet$ Code: no term.

SLI, $a b$. • See Synonymy Load Index.

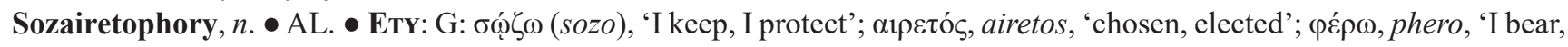
I carry'. Airetophory that is the only one that has been treated as valid in at least 100 titles of publications since then, and which for this reason must be treated as valid. • Dubois \& Aescht 2019p: 139-140. • Code: no term.

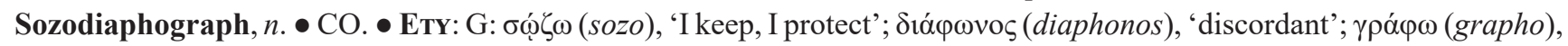
'I write'. • Spelling of a class-series nomen that has been used as correct in at least 100 titles of scientific publications after 31 December 1899, but alternatively to (an)other sozodiaphograph(s) for the same taxon or for taxa having totally or partially identical extensions. • Hoc loco. • Code: no term. 


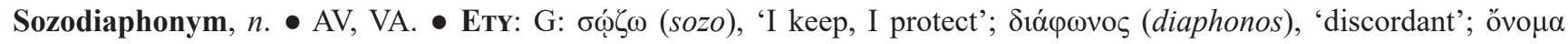
(onoma), 'name'. • Class-series nomen that has been used as valid in at least 100 titles of scientific publications after 31 December 1899, but alternatively to (an)other sozodiaphonym(s) for the same taxon or for taxa having totally or partially identical extensions. • Dubois \& Raffaëlli 2012: 90; Dubois 2016: 11. • Code: no term.

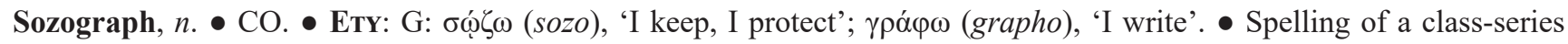
nomen that has been used as valid in at least 100 titles of scientific publications after 31 December 1899 , whereas no other spelling has been used so for the same nomen, and which for this reason must be treated as the correct spelling of this nomen. • ANT: distagmograph. - Dubois 2013: 12. Code: no term.

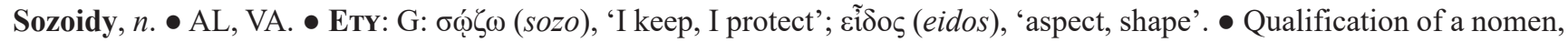
spelling or onomatergy that has had a really important usage in the literature, having been mentioned as valid or correct in at least 100 titles of scientific publications after 31 December 1899, and which therefore under DONS Criteria should be given precedence over a senior nomen, spelling or onomatergy. • Dubois 2013: 8. • Code: no general term, but 'reversal of precedence' applies to some cases of sozoidy.

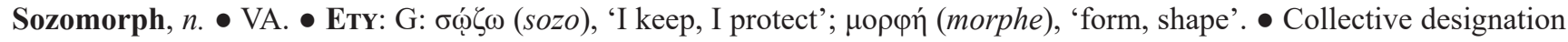
for all the sozonymorphs based on the same stem. Dubois 2015c: 19, 80. $\bullet$ Code: no term.

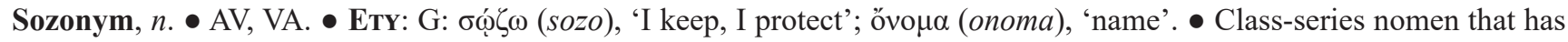
been used as valid in at least 100 titles of scientific publications after 31 December 1899, whereas none of its synonyms has been used so for the same taxon or for taxa having totally or partially identical extensions. Such a nomen must be validated even if this requires to make an exception to the DONS Criteria, e.g., against a senior synonym or homonym. - Dubois 2005a: 86, 2005b: 412, 2016: 11. • AnT: distagmonym. • Code: no term.

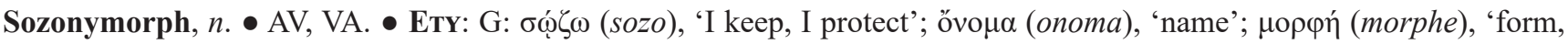
shape'. Any nomen being member of a set of CS homonymorphs, which collectively have been used as valid in at least 100 titles of scientific publications after 31 December 1899. • END: pansozonym, sozodiaphonym and nothosozonym. - Dubois 2015c: 19, 2016: 11, 16. - Code: no term for this precise concept, but the concept of nomen protectum corresponds partially to it.

Sozonymy, $n$. • VA. • ETY: see Sozonym. • Situation in zoological nomenclature where, among two or more synonyms or homonyms, one or several qualify as sozonymorph(s). In such cases, the sozonym or one of the sozodiaphonyms must be given precedence for validity (if not invalid for another reason) over its senior synonym(s) or homonym(s). • Dubois 2011a: 92. C Code: prevailing usage.

Speciation, $n$. $\bullet$ PH, TA. • ETy: L: species, 'species'. • Phenomenon of emergence of a new species (see Barigozzi 1982). - Cook 1906, 1908. •. Code: no term.

Species, $n$. • NO, TA. • ETY: L: species, 'species'. • Ambiguous term used with several meanings in biology, including: [1] a basic unit of evolution, resulting either from cladogenesis or from anagenesis; [2] a taxonomic category, defined e.g. as a panmictic bisexual entity or as a holophyletic group of organisms (see specion, mayron, simpson, kyon, etc.); [3] a primary key rank in the nomenclatural hierarchy, below genus. • Traditional term in biology (see Mayden 1997; Dubois 2008d, 2009c, 2011b). •. Code: species.

Species-series (SS), e. • NO. • In the nomenclatural hierarchy, the lowest nominal-series which is fully regulated by the Code, ranked below the genus-series. It includes nomina of taxa at the ranks of species, subspecies, species aggregate and subspecies aggregate. • Dubois 2000b: 40. • Code: species group [English text]; niveau espèce [French text].

Specific epithet, $e$. $\bullet$ NO. $\bullet$ Epithet designating a taxon of specific rank. $\bullet$ Traditional term in zoological nomenclature. $\bullet$ Code: no term.

Specion, $n$. $\bullet$ TA. $\bullet$ A taxonomic category of nomenclatural rank genus. $\bullet$ Dubois 2009c: 10, 16, 47. $\bullet$ Code: no term.

Spelling, $n$. AV, CO. • The arrangement of letters that form a word. In nomenclature, the same nomen can take different spellings, its parographs. $\bullet$ Term in traditional use in common language and in nomenclature. • Code: spelling.

SS, $a b$. $\bullet$ See Species-series.

Stage, $n$. $\bullet$ NO. $\bullet$ One of the three or four stages, steps or 'floors' of the Nomenclatural Process leading to the valid nomen of any given taxon (Dubois 2005a-b,d): assignment-availability, allocation, validity-correctness and in some cases registration. - Dubois 2005b: 381, 2010a: 11. • Code: no term.

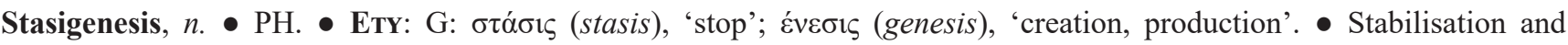
persistence of characters in an evolutionary lineage. • Huxley 1957. • Code: no term.

Status of nomen, e. • NO, TA. • The status of a nomen regarding nominal-series assignment, nomenclatural availability, taxonomic allocation, taxonomic validity and nomenclatural correctness. • END: nomenclatural status of nomen, taxonomic status of nomen. • SYN: taxonominal status of nomen. • Term in traditional use in zootaxonomy, precisely defined by Dubois (2017b: 35-37). • Code: no term.

Stem, $n$. $\bullet$ NO. $\bullet$ For the purpose of zoological nomenclature, the first part of a nomen, which is invariable and which is followed by a fixed or variable ending. In the family-series, the stem is usually the part of a genus-series nomen, derived 
from its Latin or Latinised genitive, to which is added a family-series ending; after 1999, it may also be the whole of this genus-series nomen, which is then treated as being an arbitrary combination of letters. In the species-series, epithets that are adjectives or past participles consist of an invariable stem, to which a variable ending indicating grammatical gender and number is added. For other species-series epithets, the whole nomen (stem and ending) is indeclinable. • Term of grammar, in traditional use in biological nomenclature; Dubois \& Aescht 2019j. • Code: stem.

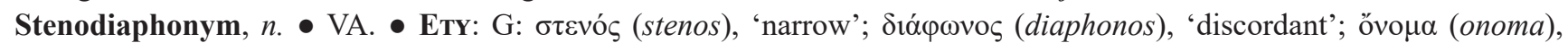
'name'. - Nomen that has not been used as valid in the titles of at least 100 works in the scientific literature after 31 December 1899. • Dubois 2005a: 85, 2005b: 411. • Code: no term.

Subfamilia, $n$. • NO, TA. • ETY: L: sub, 'below'; familia, 'family'. $\bullet$ Subsidiary family-series taxonominal rank, just below family. $\bullet$ Term in traditional use in taxonomy. $\bullet$ SYN: subfamily. $\bullet$ Code: subfamily.

Subfamily, $n$. • NO, TA. • ETY: L: sub, 'below'; familia, 'family'. • Subsidiary family-series taxonominal rank, just below family. $\bullet$ Term in traditional use in taxonomy. $\bullet$ SYN: subfamilia. $\bullet$ Code: subfamily.

Subjective, $a$. $\bullet$ NO. $\bullet$ Based on or influenced by personal feelings, tastes or opinions. $\bullet$ Common language term. $\bullet$ Code: subjective.

Subordinate, a. • NO, TA. • ETY: L: sub, 'below'; ordo, 'series, line, row, order'. • Qualification of a taxon that is at a lower hierarchical rank than another taxon, which is superordinate to it. • Traditional term in zoological taxonomy and nomenclature. $\bullet$ Code: subordinate.

Subordination, $n$. • NO, TA. • ETY: L: sub, 'below'; ordo, 'series, line, row, order'. • The relation of ordination of a subordinate taxon to its superordinate taxon in a given ergotaxonomy. • Dubois 2007a, 2008f. $\bullet$ Code: no term.

Subservience. • VA. • In zoological nomenclature, the fact that a nomen must be rejected as invalid for being a junior synonym or homonym, as a result either of one of the Principles of Validity of the Code. $\bullet$ Common language term, hereby introduced as a technical term in zoological nomenclature. • ANT: Precedence. $\bullet$ Hoc loco. • Code: no term.

Subsidiary rank, e. • NO, TA. • Nomenclatural rank related to a $\boldsymbol{k}$ ey rank (e.g., classis, ordo, familia, tribus, genus, species) by the adjunction of a prefix (e.g., super-, sub-, infra-). • ANT: key rank. • Common language terms; Dubois 2006a: 220. $\bullet$ Code: no term.

Subspecific epithet, e. $\bullet$ NO. $\bullet$ Epithet designating a taxon of subspecific rank. $\bullet$ Traditional term in zoological nomenclature. - Code: no term.

Substantive, $n$. • NO. • Generic or subgeneric nomen, always bearing a capital, being part of a binomen or trinomen. • Term of grammar, introduced in zoological nomenclature by Dubois (2000b: 40). $\bullet$ Code: genus-group name [English text]; nom du niveau genre [French text].

Subtribe, $n$. • NO, TA. • Eтr: L: sub, 'below'; tribus, 'tribe'. • Subsidiary family-series taxonominal rank, just below tribe. - Term in traditional use in taxonomy. $\bullet$ SYN: subtribus. $\bullet$ Code: subtribe.

Subtribus, $n$. $\bullet$ NO, TA. • ETY: L: sub, 'below'; tribus, 'tribe'. • Subsidiary family-series taxonominal rank, just below tribe. $\bullet$ Term in traditional use in taxonomy. $\bullet$ SYN: subtribe. $\bullet$ Code: subtribe.

Suffix, $n$. $\bullet$ NO. $\bullet$ For the purpose of zoological nomenclature, a letter or group of letters at the end of a nomen which may carry a standard, identified meaning or usage, such as indicating Latin cases (e.g. -ae or -i), or small size (e.g. -ella or -ita), or resemblance (e.g. -oides or -ops). In the species- and genus-series, the suffix when it exists is identical with the ending. In the family-series, the suffix is the letter or group of letters (e.g., $-A E,-I,-A,-E A,-I A$ ) indicating nominative plural in Latin and pointing to the rank of the taxon, following either directly the stem of a family-series nomen based on a genus-series nomen, or the connector which follows it, if present. • Common language term; Alonso-Zarazaga 2005: 191 (as 'ending proper'); Dubois \& Aescht 2019j: 103. • Code: suffix.

Superfamilia, $n$. $\bullet$ NO, TA. • ETY: L: sub, 'below'; familia, 'family'. • Subsidiary family-series taxonominal rank, above family. $\bullet$ Term in traditional use in taxonomy. $\bullet$ SYN: superfamily. $\bullet$ Code: superfamily.

Superfamily, $n$. • NO, TA. • ETY: L: sub, 'below'; familia, 'family'. • Subsidiary family-series taxonominal rank, just below family. $\bullet$ Term in traditional use in taxonomy. $\bullet$ SYN: superfamilia. $\bullet$ Code: superfamily.

Superordinate, a. • NO, TA. • ETY: L: super, 'above'; ordo, 'series, line, row, order'. • Qualification of a taxon that is at a higher hierarchical rank than another taxon, which is subordinate to it in a given ergotaxonomy. Immediately superordinate taxon: see getangiotaxon. - Traditional term in zoological taxonomy and nomenclature. • Code: no term.

Superordination, $n$. • NO, TA. • ETY: L: sub, 'below'; ordo, 'series, line, row, order'. • The relation of ordination of a superordinate taxon to its subordinate taxa in a given ergotaxonomy. • Dubois $2007 a$, 2008f. Code: no term.

Supraspecies, $n$. • NO, TA. • ETY: L: supra, 'above, beyond'; species, 'species'. • Subsidiary species-series taxonominal rank, above species. • Génermont \& Lamotte 1980; Dubois \& Raffaëlli 2009. • Code: aggregate of species.

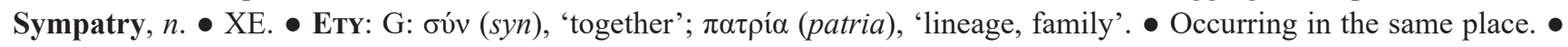
ANT: Allopatry. $\bullet$ Common term in evolutionary biology. $\bullet$ Code: no term.

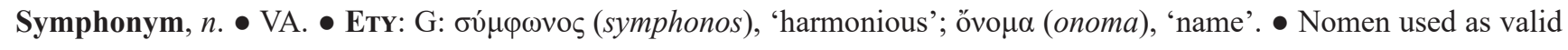


for the taxon it denotes, or for taxa having totally or partially identical extensions, by all authors and in all publications after 31 December 1899. • Dubois 2005a: 85, 2005b: 411. • Code: no term.

Symphoric, $a$. $\bullet$ See Symphory.

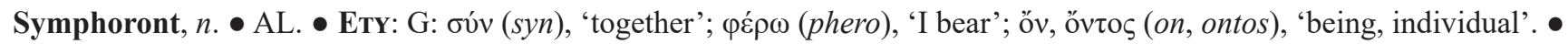
One of several specimens originally used collectively as onomatophore of a species-series nomen. • Dubois $2005 b$ : 403. - Code: syntype.

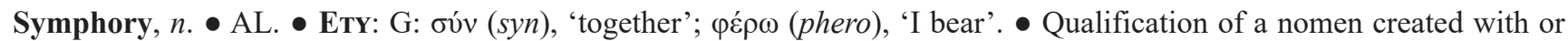
supported by an onomatophore composed of a series of specimens (in the species-series) or of taxomina (in the other three nominal-series). • Dubois 2005b: 404.

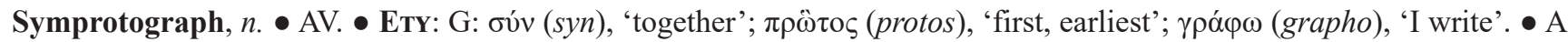
category of protograph: one of two or more alternative original spellings of a nomen. $\bullet$ ETY: holoprotograph. $\bullet$ Dubois 2010a: 8, 42. Code: one of multiple original spellings.

Synapomorphic, $n$. $\bullet$ See Synapomorphy.

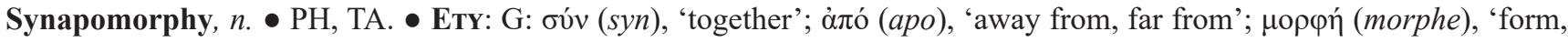
shape'. A Apomorphy shared by two or more taxa. $\bullet$ Hennig 1950. $\bullet$ Code: no term.

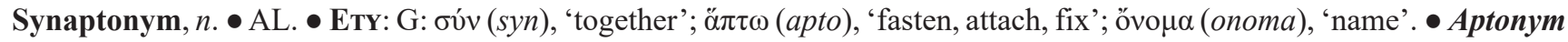
whose onomatophore is symphoric, being composed of more than one specimen (in the species-series: symphoronts) or taxomen (in the genus-series: prenucleospecies; in the class-series: conucleogenera). Synaptonyms may be original (symphory fixed in the original publication) or subsequent (symphory being subsequent to aphory in the original publication). They may also be indissoluble or considered taxonomically homogeneous (homosynaptonyms) or considered taxonomically heterogeneous (heterosynaptonyms). • ANT: monaptonym. • Dubois 2011a: 25, 94. • Code: one among several meanings of the unclear term nomen dubium.

Synchronous, $a$. $\bullet$ AV, VA. • ETY: see Synchrony. $\bullet$ Qualification of distinct events that occurred at the same date. In the context of zoological nomenclature, the fact that two publications were distributed at the same date. $\bullet$ Ant: allochronous. - Common language term; Dubois 2013: 5. • Code: no term.

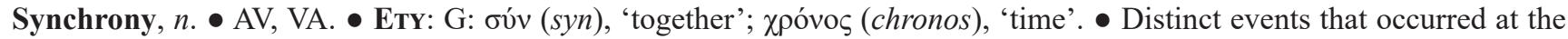
same date. • ANT: allochrony. • Common language term; Dubois \& Aescht 2019f: 50, 52. • Code: no term.

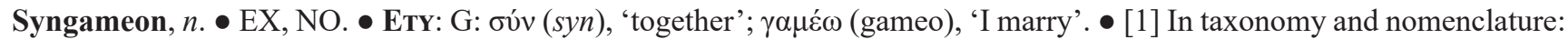
taxon of taxonominal rank supraspecies composed of two or more species liable to produce rare hybrids in their contact zone (Lotsy 1918); [2] in evolutionary biology, the set of organisms liable to produce viable hybrids, in natural or artificial conditions (Cuénot \& Tétry 1951). • Code: no term.

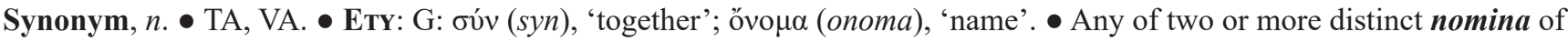
the same nominal-series considered, either for objective (isonyms) or for subjective (doxisonyms) reasons, to denote the same taxon in a given ergotaxonomic frame. $\bullet$ Traditional term in zootaxonomy. $\bullet$ ANT: xenonym. Code: synonym.

Synonymic list, e. • TA, VA. • ETY: see Synonym. • List of synonyms. • Traditional term in zootaxonomy. • Code: no term.

Synonymisation, $n$. $\bullet$ TA, VA. • ETY: see Synonym. • Process by which a nomen is invalidated for being considered an invalid synonym or homonym. • Traditional term in zootaxonomy. • Code: no term.

Synonymous, $a$. $\bullet$ TA, VA. • ETY: see Synonym. • In zoological nomenclature, the qualification of two distinct nomina of the same nominal-series that are synonyms under the Code. $\bullet$ Term in traditional use in common language and in zootaxonomy. • Code: synonymous.

Synonymy, $n$. $\bullet$ TA, VA. • ETY: see Synonym. $\bullet$ The fact that two distinct nomina of the same nominal-series are considered to denote the same taxon in a given ergotaxonomy, either for objective (isonymy) or for subjective (doxisonymy) reasons. • Traditional term in zootaxonomy. • Code: synonymy.

Synonymy load, e. $\bullet$ NO, TA. • ETY: see Synonym. • The quantitative importance of synonyms (mainly doxisonyms) in a given ergotaxonomy. $\bullet$ Dubois 2008a: 857. C Code: no term.

Synonymy Load Index (SLI), e. • AV, VA. • The ratio, expressed in percent, of the number of nomina treated as invalid (akyronyms) in a given ergotaxonomy to the number of available nomina in the taxonomic group covered by the study (hoplonyms). • Hoc loco. • Code: no term.

Synotaxa, $n$. $\bullet$ One of the two plurals of synotaxon. $\bullet$ Dubois 2005b: 406.

Synotaxic, a. • NO, TA. • ETy: see Synotaxy. • Qualification of two distinct taxa, being either isotaxic, peritaxic or gephyrotaxic, whose nomina, of the same or different nominal-series, are considered to denote (are allocated to) the same taxon in a given ergotaxonomy. $\bullet$ Dubois 2005b: 411. Code: no term.

Synotaxic list, e. • NO, TA. • ETY: see Synotaxy. • List of synotaxa. $\bullet$ Hoc loco. • Code: no term.

Synotaxon (pl. synotaxa, synotaxons), $n$. $\bullet$ NO, TA. • ETY: see Synotaxy. • One of two distinct taxa, being either isotaxic, peritaxic or gephyrotaxic, whose nomina, of the same or different nominal-series, are considered to denote (are allocated 
to) the same taxon in a given ergotaxonomy. $\bullet$ Dubois $2005 b$ : 406 . $\bullet$ Code: no term.

Synotaxons, $n$. $\bullet$ One of the two plurals of synotaxon. $\bullet$ Hoc loco.

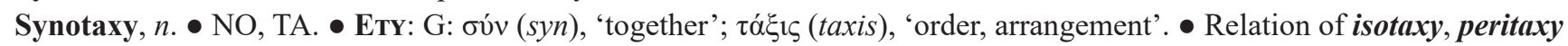
or gephyrotaxy between two distinct taxa, whose nomina, of the same or different nominal-series, are considered to denote (are allocated to) the same taxon in a given ergotaxonomy . $\bullet$ Dubois 2005b: 405. $\bullet$ Code: no term.

Synotaxic, $a$. $\bullet$ NO, TA. • ETY: see Synotaxy. • The fact that two distinct nomina of the same or different nominal-series are considered to denote the same taxon in a given ergotaxonomy. $\bullet$ Hoc loco. $\bullet$ Code: no term.

Synotaxic list, e. • NO, TA. • ETY: see Synotaxy. • List of synotaxa. • Hoc loco. • Code: no term.

Synotaxon (pl. synotaxa, synotaxons), n. • NO, TA. • ETy: see Synotaxy. $\bullet$ One of two distinct taxa of the same or different nominal-series that are considered to correspond to the same taxon (same extension) in a given ergotaxonomy. - Hoc loco. - Code: no term.

Synotaxons, $n$. $\bullet$ One of the two plurals of synotaxon.

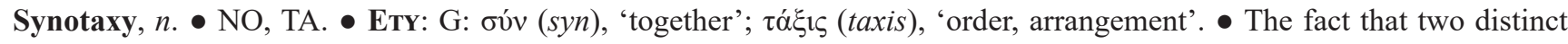
taxa of the same or different nominal-series are considered to correspond to the same taxon (same extension) in a given ergotaxonomy. • Dubois \& Ohler 2019: 19. • Code: no term.

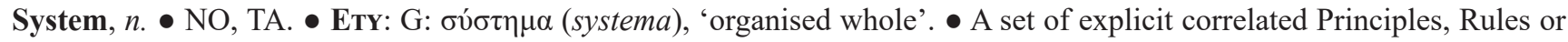
Criteria allowing to establish a classification of organisms (in taxonomy) or the nomina of the taxa recognised by this classification (in nomenclature). • END: holo-systems, pro-systems. • Term in traditional use in common language. • Code: system.

Systematics, $n$. $\bullet$ NO, TA. • EтY: G: $\sigma u ́ \sigma \tau \eta \mu \alpha(s y s t e m a)$, 'group, troup, system of doctrines, institutions, political constitution, philosophical system'. - The domain of biology devoted to the study of the diversity of living organisms (biodiversity), of their evolution (evolutionary biology), their relationships (phylogeny), their classification (taxonomy) and their nomination (nomenclature). $\bullet$ Term in traditional use in biology. $\bullet$ Code: no term.

Tautonymy, $n$. $\bullet$ AV, AL. $\bullet$ The use of the same word for the substantive of the nomen of a genus-series taxon and the final epithet of the nomen of one of its included species-series taxa. Tautonymy is qualified as absolute when the substantive and the epithet were both published within the frame of binominal nomenclature. Tautonymy is qualified as Linnaean when the substantive was introduced before 1931 and the epithet was a pre-1758 nomen cited as a synonym of only one of the species-series taxon originally included in that genus-series taxon. Both kinds of tautonymy may result in the election of the nucleospecies of a genus-series nomen, if it has not been effected previously by original designation or monophory. • Code: tautonymy.

Taxa, $n$. $\bullet$ One of the two plurals of taxon.

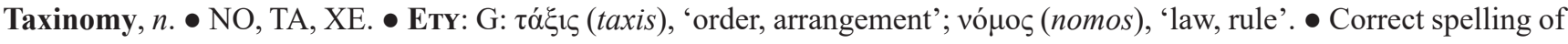
the term taxonomy (see Tardieu 2011), often used in French scientific publications (e.g. Dubois 1987b, 1987d) but not in publications in other languages. • Code: no term.

Taxognoses, $n$. • Plural of taxognosis.

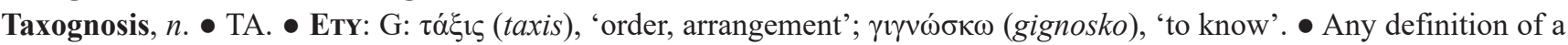
taxon, whether based on characters or on hypothesised cladistic relationships between taxa. $\bullet$ Dubois \& Raffaëlli 2009: 15. Code: no term.

Taxomen (pl. taxomina), n. • NO. • ETY: G: $\tau \alpha$ ákı (taxis), 'order, arrangement'; L: nomen, 'name'. • The permanent association between a nomen and an onomatophore, allowing objective, non-ambiguous and stable allocation of nomina to taxa. • Dubois 2000a: 21, 2000b: 40. • Code: nominal taxon.

Taxomina, $n$. $\bullet$ Plural of taxomen.

Taxon (pl. taxons, taxa), n. • NO, TA, XE. • G: $\tau$ ákı (taxis), 'order, arrangement'. • Ambiguous term used with two main meanings in zoological taxonomy and nomenclature: [1] any taxonomic unit recognised in an ergotaxonomy, whether named or not (Meyer 1926); [2], any rank of the family- or class-series (incorrect but usual practice in many recent taxonomic publications nowadays; see e.g. Frost et al. 2006) (see cladon). • Code: [1] taxon, 'taxonomic taxon' (!); nominal taxon; [2] no term.

Taxonomic category, e. $\bullet$ TA. $\bullet$ A group of taxa that share certain biological (e.g., crossability) or historical-chronological (e.g., geological age) characteristics (see Dubois \& Malécot 2005: 98; Dubois 2005b: 412-413, 2006a: 219-220, 2007a, 2008f). Taxonomic categories may be ranked (corresponding to nomenclatural ranks of the nomenclatural hierarchy: e.g., species, genus, tribe) or unranked (categories that do not correspond to nomenclatural ranks: e.g., kyon, klepton, klonon). • Traditional term in nomenclature and taxonomy. $\bullet$ Code: no term.

Taxonomic consistency, e. • NO, TA. • In class-series zoological nomenclature, the requirement that all suprageneric nomina introduced in the same publication for taxa that were originally assigned to the same taxonomic rank must be referred to the same nominal-series. This requires to give pre-eminence to the family-series for this assignment in case of incorrect formation (as arhizonyms) of some suprageneric nomina referred to parordinate taxa in a publication. 
Common language terms; Dubois 2006a: 178. Code: no term.

Taxonomic crisis, $e$. TA, XE. - The fact that the scientific discipline of taxonomy is facing a major crisis since the last decades of the $20^{\text {th }}$ century, showing at best an 'inertia' (see Tancoigne $\&$ Dubois 2013) but not the drastic development that would be required by the biodiversity crisis. $\bullet$ Dubois 2003, 2010c. $\bullet$ Code: no term.

Taxonomic gap, e. $\bullet$ TA, XE. $\bullet$ The fact that our taxonomic inventory of the living species of our planet is highly incomplete. - Dubois 2010c: 260. • Code: no term.

Taxonomic hierarchy, e. • NO, PH, TA. • The hierarchical structure of a biological classification, which reflects both the phylogenetic relationships between taxa and the nomenclatural hierarchy of nominal-series and nomenclatural ranks used to designate the taxa. $\bullet$ Traditional term in nomenclature and taxonomy. $\bullet$ Code: taxonomic hierarchy.

Taxonomic impediment, e. $\bullet$ TA, XE. • The fact that, both quantitatively andqualitatively, our knowledge of the species and other taxa of our planet is very unsatisfying, in fact much below the standard required today by our society for all other scientific disciplines. • Anonymous 1994. $\bullet$ Code: no term.

Taxonomic parsimony, e. $\bullet$ NO. $\bullet$ The fact that the Code, through the Principle of Coordination, requires to have fewer nomina than taxa to name the latter unambiguously. $\bullet$ Dubois 2006b-d,2007b, 2008f. $\bullet$ Code: no term.

Taxonomic status of nomen, $e$. $\bullet$ NO, TA. $\bullet$ The dimensions of the status of a nomen which depend both on nomenclatural Rules and on the ergotaxonomy adopted: taxonomic allocation, taxonomic validity and nomenclatural correctness. $\bullet$ Term in traditional use in zootaxonomy, precisely defined by Dubois (2017b: 36-37). • Code: no term.

Taxonomic urgency, e. $\bullet$ TA, XE. $\bullet$ The need, resulting from the combination of the biodiversity crisis and of the taxonomic gap, to accelerate considerably the inventory of the species of the Earth before many of them are extinct. $\bullet$ Dubois 2010c: 260. • Code: no term.

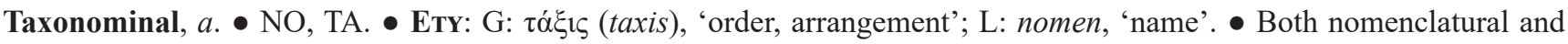
taxonomic. • Dubois 2011c: 51. $\bullet$ Code: no term.

Taxonominal level, $e$. $\bullet$ NO, TA. $\bullet$ The place of a nomen in a nomenclatural hierarchy of nominal-series and nomenclatural ranks and of the taxon it designates in the taxonomic hierarchy that the latter reflects. $\bullet$ Traditional term in nomenclature and taxonomy. • Hoc loco. • Code: rank.

Taxonominal status of nomen, e. • See Status of nomen.

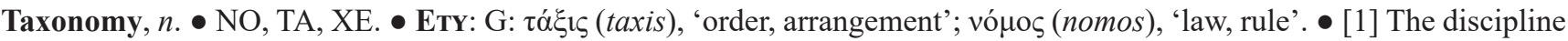
of systematics that deals with the theory and practice of the classification of living organisms. [2] Any system of taxa recognised as valid by an author (see ergotaxonomy). • Candolle 1813: 19 (as 'taxonomie'). Although this original spelling was erroneous (see Tardieu 2011), it has been adopted as valid in most scientific publications except in French language. - Syn: taxinomy. $\bullet$ Code: no term.

Taxons, $n$. $\bullet$ One of the two plurals of taxon.

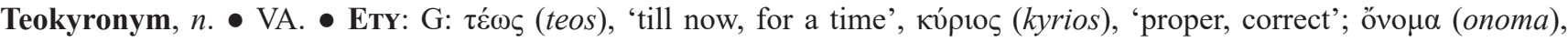
'name'. • Nomen used as valid under DONS Criteria during a 25-year period subsequent to 31 December 2015 for a single taxon at a given rank but not, even in a single work, for a taxon or taxa at lower ranks to which it could potentially apply (e.g., following the resolution of a polytomy). • Dubois 2016: 16. • Code: no term.

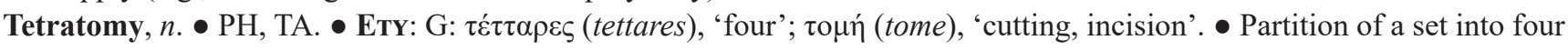
subsets. $\bullet$ Common language term. $\bullet$ Code: no term.

The Commission or its successor body, e. • NO. • The International Commission on Zoological Nomenclature (see Commission) or its successor internationally accepted regulatory body that will be in charge of implementing the Plenary Power whenever necessary under the next edition of the Code or under the Zoocode. • Dubois \& Aescht 2019q: 144. $\bullet$ Code: no term.

Theory-bound, e. • AL. • Concerning a nomenclatural system, the fact that it is linked to a taxonomic paradigm. In such a system, the allocation of nomina to taxa relies on intension, not on ostension or extension (see Dubois 2006a, $d, 2007 a$, 2008f). • Dubois 2010d: 5. • Code: no term.

Theory-free, $e$. AL. $\bullet$ Concerning a nomenclatural system, the fact that it is independent from all taxonomic paradigms. In such a system, the allocation of nomina to taxa relies exclusively on ostension or extension, never on intension (see Dubois 2006a, $d$, 2007a, 2008f). • Dubois 2007a: 37, 43, 2007b: 396. - Code: no term.

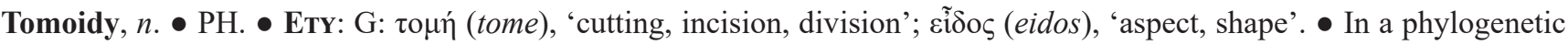
tree, the pattern of subdivision of a branch or of absence of subdivision. • END: dichotomy, polytomy, achotomy. $\bullet$ Hoc loco. $\bullet$ Code: no term.

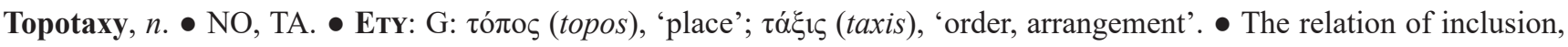
overlapping or exclusion between two taxa regarding their contents in a given ergotaxonomy. See also ordination. Dubois 2005b: 405; Dubois \& Berkani 2013: 53. • Code: no term.

Tree, $n$. $\bullet$ PH, XE. • Common language term used in evolutionary biology to designate a tree-like, branching diagram used to indicate 'degrees of relationships' between organisms. • Code: no term. 
Tribe, $n$. • NO, TA. • ETY: L: tribus, 'tribe'. • Secondary family-series key rank in zoological taxonomy and nomenclature, below family. $\bullet$ Term in traditional use in taxonomy. $\bullet$ SYN: tribus. $\bullet$ Code: no term.

Tribus, $n$. $\bullet$ NO, TA. • ETY: L: tribus, 'tribe'. • Secondary family-series key rank in zoological taxonomy and nomenclature, below family. $\bullet$ Term in traditional use in taxonomy. $\bullet$ SYN: tribe. $\bullet$ Code: no term.

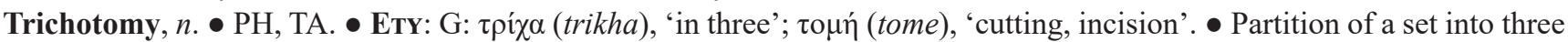
subsets. $\bullet$ Common language term. $\bullet$ Code: no term.

Trinomen (pl. trinomina), n. • AV, CO. • ETY: L: tres, 'three'; nomen, 'name'. • Nomen of rank subspecies, composed of three terms, the generic substantive and the specific and subspecific epithets. $\bullet$ Traditional term in zoological nomenclature. $\bullet$ Code: trinomen.

Trinomina, $n$. Plural of trinomen.

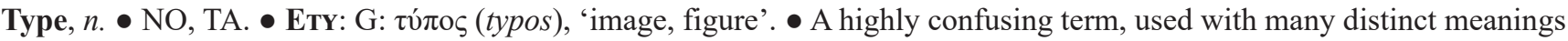
in common language as well as in biology, and in systematics with two distinct meanings, a taxonomic one (see hypodigm) and a nomenclatural one (see onomatophore). The use of this term in nomenclature is here discouraged (see Dubois 2005b: 401-405; Dubois \& Aescht 2019c, Dubois et al. 2019). • Traditional term in various domains of biology, including nomenclature. • Code: name-bearing type.

Unallocated, $p$. AL. Q Qualification of a nomen (anaptonym) that does not conform to the conditions of taxonomic allocation as regulated by the Code. • ANT: allocated. $\bullet$ Dubois 2005b: 396. • Code: no term.

Unassigned, $p$. • AS. • Qualification of a nomen (anemonym) that does not conform to the conditions of nomenclatural assignment as regulated by the Code, and is therefore unavailable. • ANT: assigned. • Common language term, introduced in zoological nomenclature by Dubois (2015a). $\bullet$ Code: no term.

Unavailability, $n$. $\bullet \mathrm{AV}$. Absence of a statement regulated by the Code according to which a nomen is promulgated in zoological nomenclature complying with the conditions of this code (hoplonym) or by which an airesy is made effective. • ANT: availability. • Term in traditional use in zoological nomenclature. • Code: no term.

Unavailable, $a$. $\bullet \mathrm{AV}$. $\bullet$ Qualification of a nomen (anoplonym) that does not conform to the conditions of nomenclatural availability as regulated by a code. • ANT: available. - Traditional term in zoological nomenclature. • Code: unavailable.

Unialienogenus (pl. unialienogenera), n. • AL. • ETy: L: unus, 'one'; alienus, 'foreign, unrelated'; genus, 'birth, origin, class, kind'. - The single genus-series taxomen originally excluded from the protaxon for which a new class-series nomen was promulgated. $\bullet$ Hoc loco. $\bullet$ Code: no term.

Uninomen (pl. uninomina), n. AL, CO. • ETY: L: unus, 'one'; nomen, 'name'. • Nomen of any rank composed of a single term. $\bullet$ Traditional term in zoological nomenclature. $\bullet$ Code: no term.

Uninomina, $n$. Plural of uninomen.

Uninucleogenera, $n$. • Plural of uninucleogenus.

Uninucleogenus (pl. uninucleogenera), n. • AL. • ETY: L: unus, 'one'; nucleus, 'kernel, nut'; genus, 'birth, origin, class, kind'. • [1] In the family-series: the genus-series taxomen originally explicitly or implicitly (before 2000) designated as onomatophore of a new family-series nomen; [2] in the class-series: the single genus-series taxomen originally referred to the protaxon for which a new class-series nomen was promulgated. $\bullet$ Dubois 2015c: 23, 81. • Code: [1] type genus; [2] no term.

Unjustified emendation, e. $\bullet$ See Autoneonym.

Unpublished, $p$. • AV. • In zoological nomenclature, work issued not conforming to the provisions of Articles 8-9 of the Code. See Publication. • Traditional term in many domains. • Code: no term.

Unranked, $p$. $\bullet$ NO. • ETY: see Rank. Qualification of a nomenclatural system in which no ranks are assigned to the nomina of supraspecific and infraspecific taxa. $\bullet$ Traditional term in zoological nomenclature. $\bullet$ Code: no term.

Unregistered, $p$. $\bullet$ RE. $\bullet$ Qualification of a nomen (adelonym) that does not conform to the conditions of nomenclatural registration of the Code. $\bullet$ ANT: registered. $\bullet$ Traditional term in many domains. $\bullet$ Code: no term.

Upper Quarter of nomina (UQN), e. NO, XE. • Upper quarter of usage of nomina of a data set, i.e. above the upper (third) quartile of this data set. $\bullet$ Hoc loco. $\bullet$ Code: no term.

Upper Quartile, e. $\bullet$ NO, XE. $\bullet$ Third quartile of a data set, i.e. the middle value between its median and its highest value: $75 \%$ of the data lie below this point, and $25 \%$ lie above. $\bullet$ Term in traditional use in statistics and probability. $\bullet$ Code: no term.

UQ-nomen, e. • NO, TA. • In family-series nomenclature, nomen designating a taxon considered valid and having a number of usages above the upper quartile of usages since 1758. $\bullet$ Hoc loco. $\bullet$ Code: no term.

UQN, e. • See Upper Quarter of nomina.

Usage, $n$. $\bullet$ VA. $\bullet$ In the context of zoological nomenclature, the fact that a nomen has been mentioned in some publications and during a given period. $\bullet$ Traditional term in nomenclature. $\bullet$ Code: usage.

Valid, $a$. VA. - In the context of zoological nomenclature, qualification of a nomen (kyronym) that conforms to the 
conditions of nomenclatural validity as regulated by the Code. • ANT: invalid. - Traditional term in zoological nomenclature. $\bullet$ Code: valid.

Validate, $v$. $\bullet$ VA. $\bullet$ Common language term, proposed by Dubois (2000b: 47) to designate the action of determining the validity of a hoplonym either by an author following the Rules of the Code or by the Commission under the Plenary Power. • Code: validate.

Validation, $n$. $\bullet$ VA. • Common language term, proposed by Dubois (2000b: 48) to designate the result of the action of determining the validity of a hoplonym either by an author following the Rules of the Code or by the Commission under the Plenary Power. • Code: no term.

Validity, $n$. $\bullet$ VA. • In zoological nomenclature: [1] statement regulated by the Code by which a nomen is determined to be the one that must be used for a taxon or several taxa in zoological nomenclature. [2] qualification of a valid nomen. $\bullet$ ANT: invalidity. $\bullet$ Traditional term in zoological nomenclature. $\bullet$ Code: validity.

Variable ending, e. $\bullet$ NO. • Ending of a nomen that is liable to change according to the ergotaxonomy adopted. Two situations: [1] species-series epithet being an adjective or a past participle: suffix indicating the grammatical gender of the epithet; [2] family-series nomen: ending indicating the rank, composed of two parts: the connector and the suffix proper. • Dubois \& Aescht 2019j: 103. • Code: no term.

Variety-series (VS), e. • NO. • In the nomenclatural hierarchy, the nominal-series ranked below the species-series, which is not fully regulated by the Code. It includes nomina of taxa at the ranks of variety, form and any additional ranks that may be required. • Dubois \& Malécot 2005: 102, Dubois 2005b: 408. • Code: no term.

Virtual combination, $e$. $\bullet$ NO, TA. • A combination that does not appear in a publication but that is implied by the explicit statement that a species-series epithet (whether considered as valid or as an invalid synonym) is referred to a nominal genus. • Dubois 1995b: 65; Dubois \& Aescht 2019h: 77. • Code: no term.

Voucher, $n$. $\bullet$ NO, TA. - Any reference specimen kept in a (preferably permanent and curated) collection, whether an onymophoront or not. • Traditional term used in biology. $\bullet$ Code: no term.

VS, $a b$. $\bullet$ See Variety-series.

Work, $n$. $\bullet$ NO, TA. - In the context of zoological taxonomy and nomenclature, a publication. $\bullet$ Traditional term in zoological nomenclature. $\bullet$ Code: work, published work.

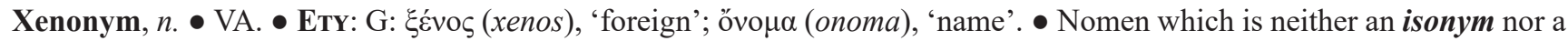
doxisonym of another nomen within a given ergotaxonomic frame. • ANT: synonym. • Hoc loco. • Code: no term.

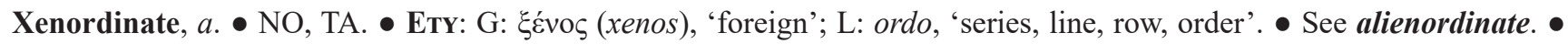
Dubois 2006b: 827. $\bullet$ Code: no term.

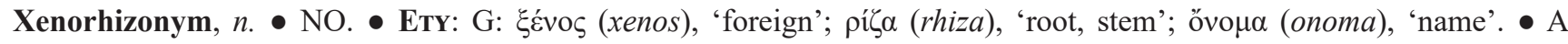
category of pseudorhizonym: suprageneric nomen HN (designating a taxon HT) [1] based on the stem of an available or unavailable genus-series nomen GN, but [2] this nomen not being referred as valid to the taxon HT in the ergotaxonomy adopted in the publication where $\mathbf{H N}$ was created and [3] its stem being combined with an ending derived from another or several other terms (e.g., -formes, -morpha, -phora, etc.). If proposed as a family-series nomen, it is incorrectly formed according to the Code, and is therefore a FS anoplonym. If proposed as a class-series nomen, common particular cases are those of such nomina the original endings of which were derived from the roots forma

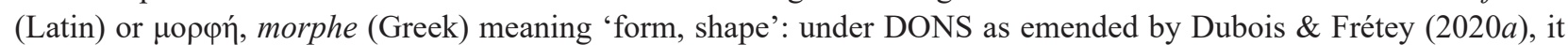
should be used under the respective standard endings -IFORMI or -OMORPHI, which are not in a relation of hierarchy but may be both used at whatever rank. - Dubois 2015c: 22, 82, 90. • Code: no term.

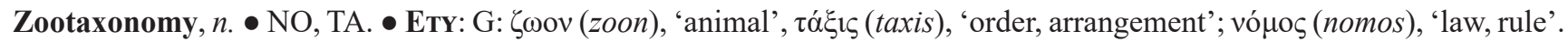
- Zoological taxonomy. • Term in use in recent publications dealing with zoological taxonomy. $\bullet$ Code: no term.

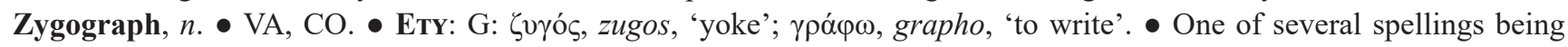
potentially the correct one for the same nomen. $\bullet$ Dubois 2013: 24. • Code: no term.

Zygography, n. • VA, CO. • ETY: see Zygograph. • Qualification of all situations of nomenclatural conflict between several spellings being potentially the correct one for the same nomen. $\bullet$ Dubois 2013: 5. • Code: no term.

Zygoid, n. $\bullet$ VA. • ETY: see Zygoidy. • Any item (zygonym, zygograph or zygonomatergy) involved in a situation of zygoidy. $\bullet$ Hoc loco. $\bullet$ Code: no term.

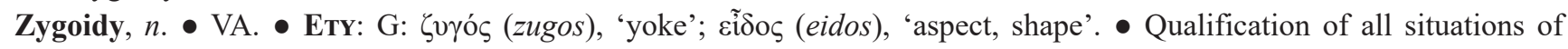
nomenclatural conflict between several nomina, spellings or onomatophore designations being potentially the valid one for the same taxon or nomen. $\bullet$ Dubois 2013: 5. • Code: no term.

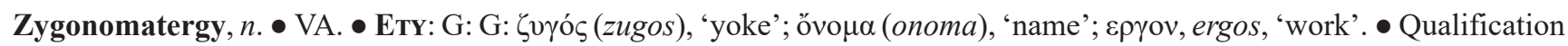
of all situations of nomenclatural conflict between several distinct onomatergies concerning an available nomen. $\bullet H o c$ loco. $\bullet$ Code: no term.

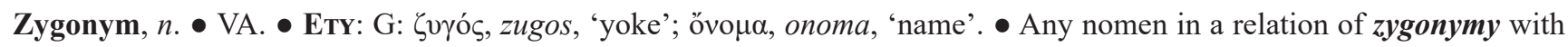
another nomen. - Dubois 2013: 24. • Code: no term. 
Zygonymy, n. • VA. • ETY: see Zygonym. Q Qualification of all situations of nomenclatural conflict between several nomina being potentially the valid one for the same taxon or set of related coordinated taxa. $\bullet$ Dubois 2013: 5. $\bullet$ Code: no term.

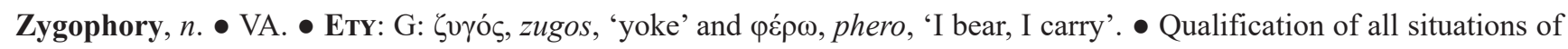
nomenclatural conflict between several distinct onomatophore restrictions or designations being potentially the valid one for the same nomen. • Dubois 2013: 5. • Code: no term. 
APPENDIX A2.TREE-1. Detailed phylogenetic tree of LISSAMPHIBIA, showing all species and higher supraspecific taxa recognised here as valid.

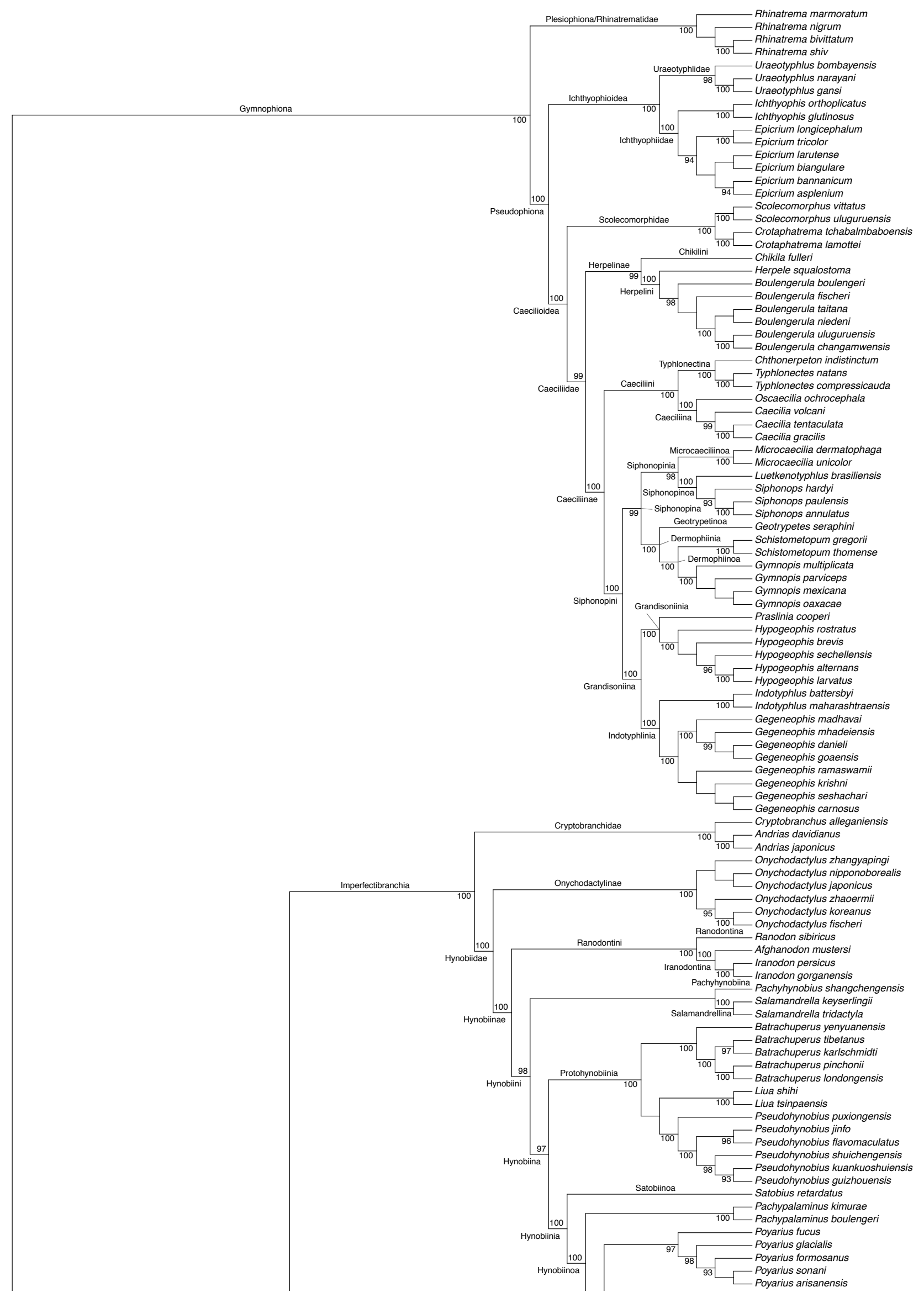


Pseudobranchus axanthus Pseudobranchus axanthus $100 \_$Pseudobranch ${ }_{100} \square$ S Siren intermedia Dicamptodon tenebrosus

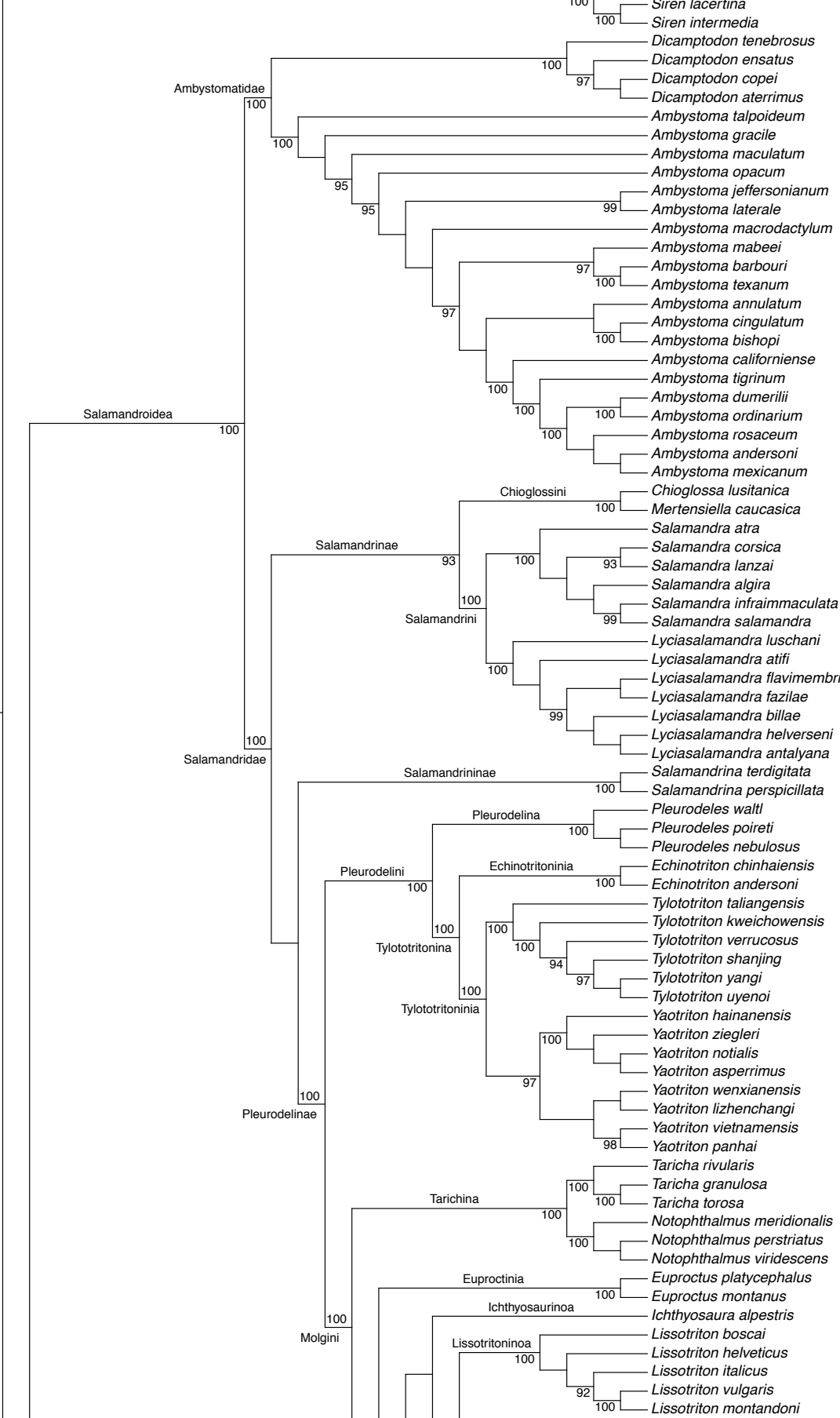




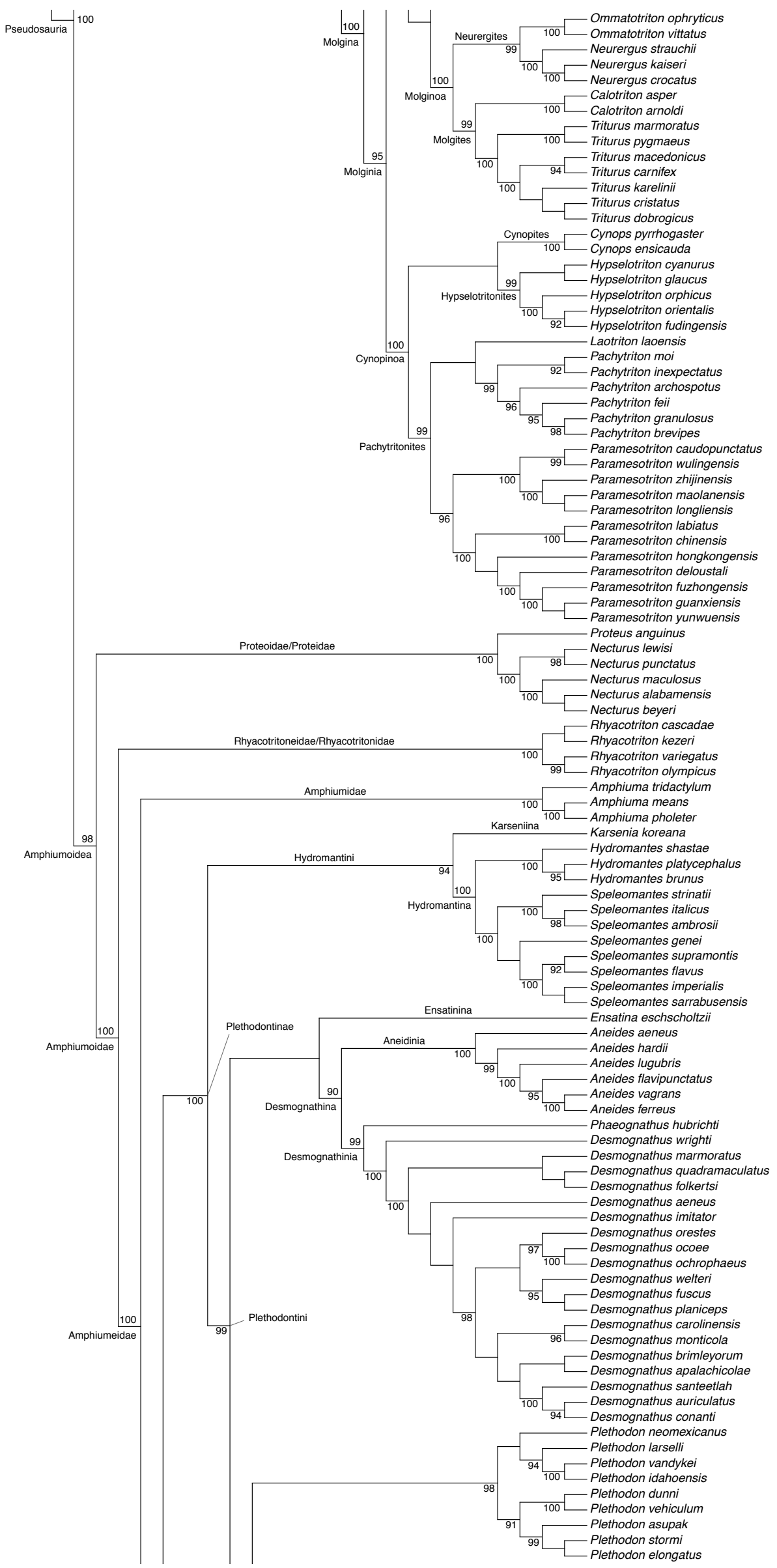




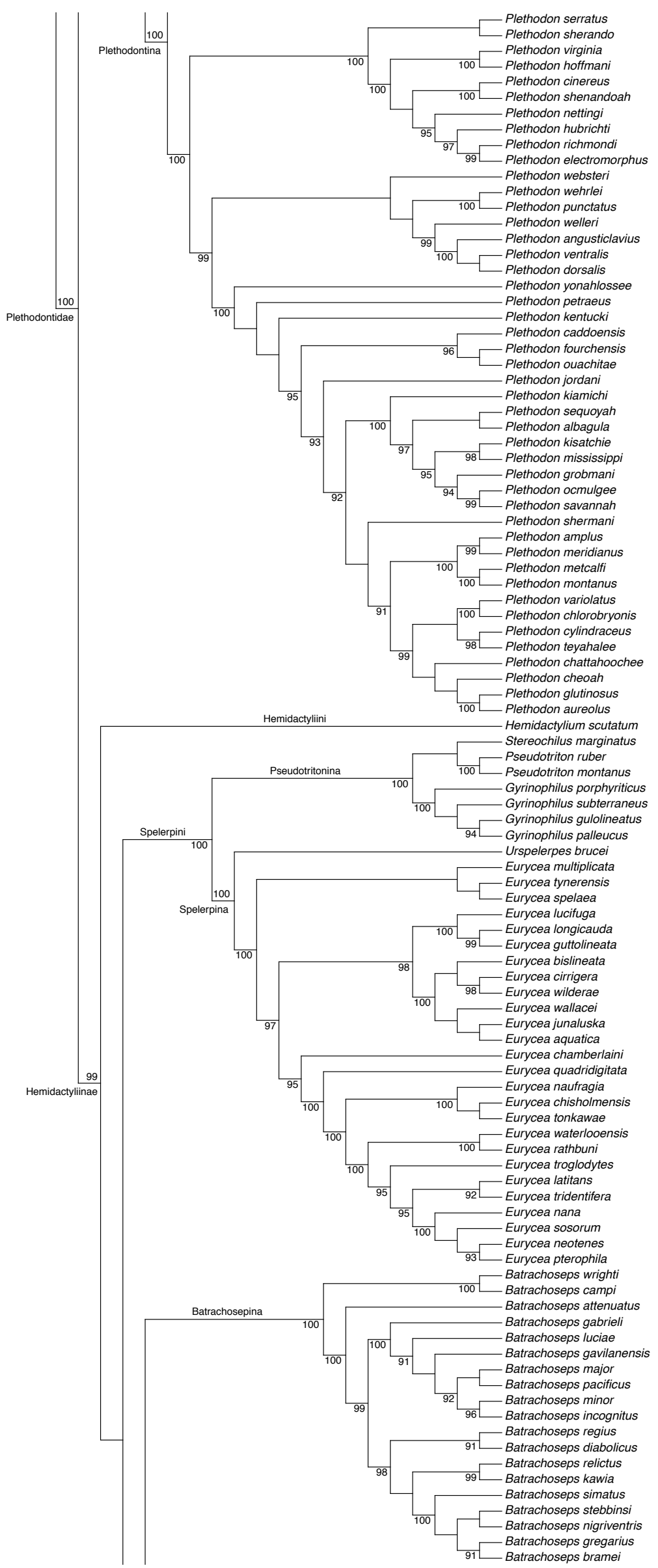




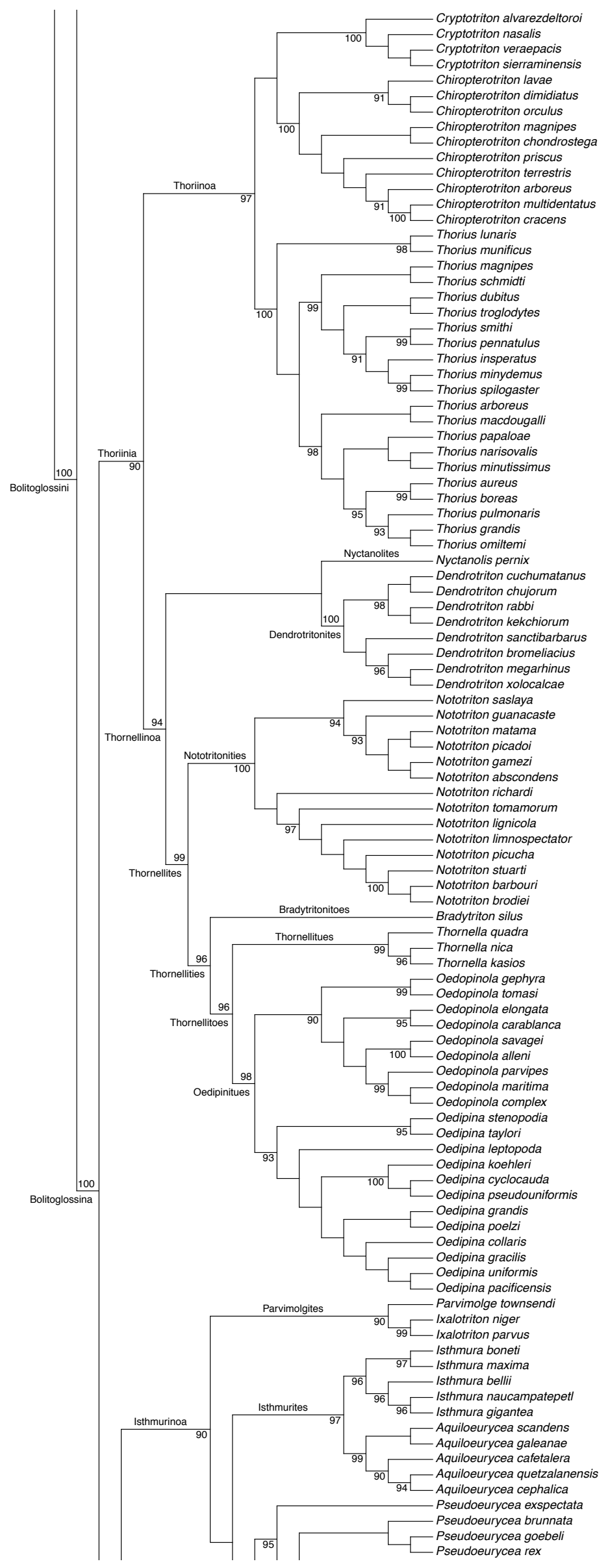




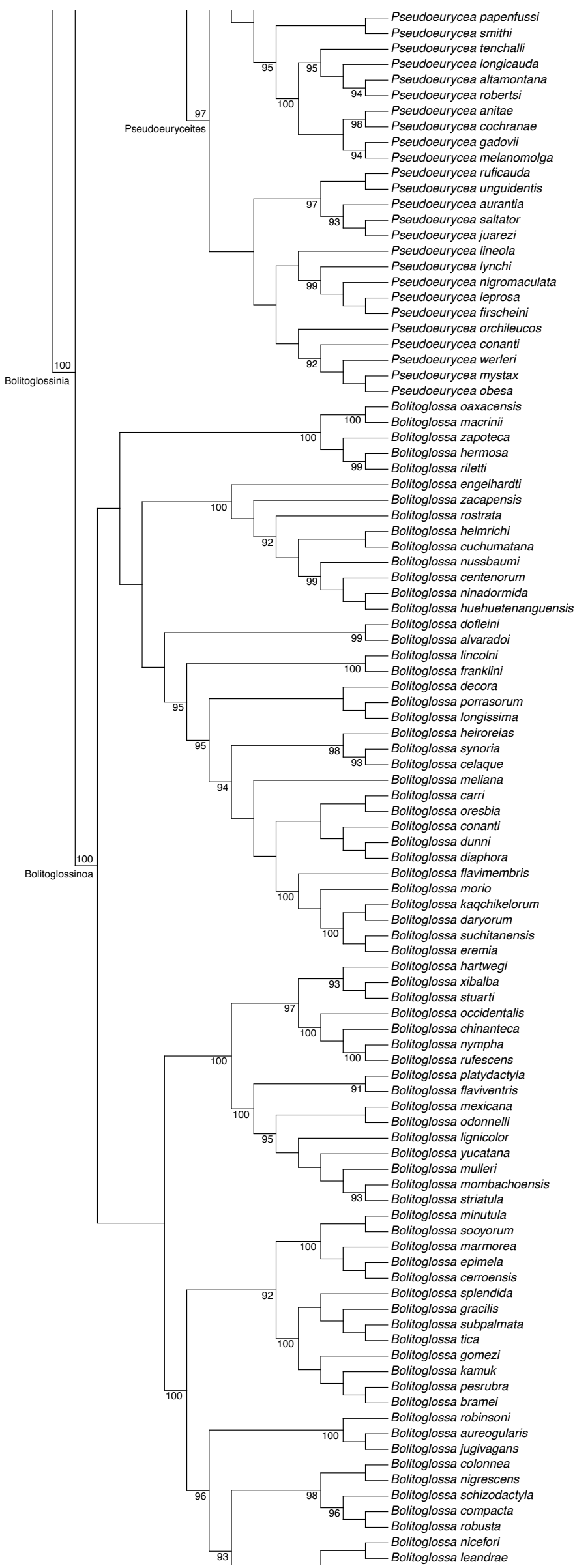




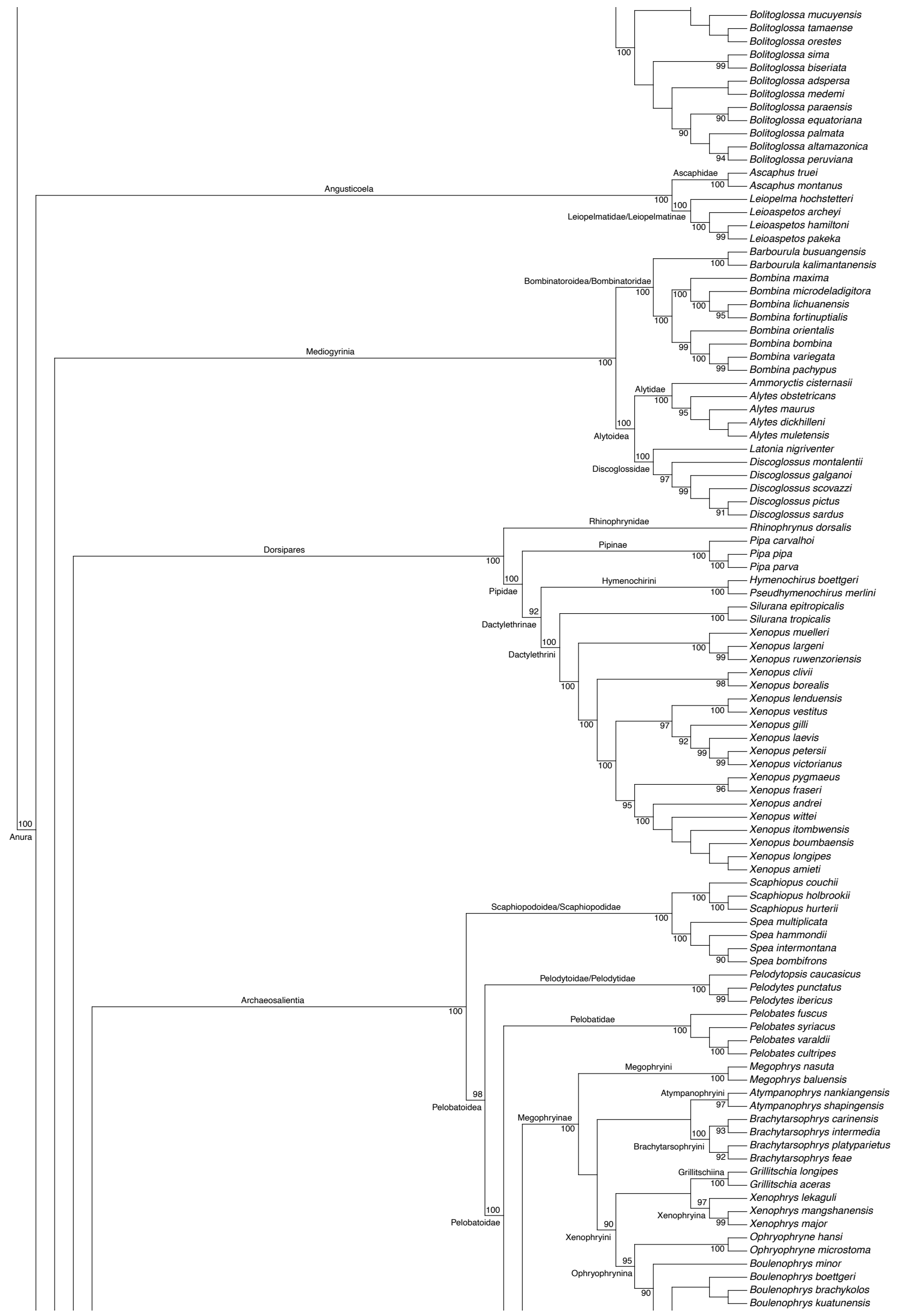




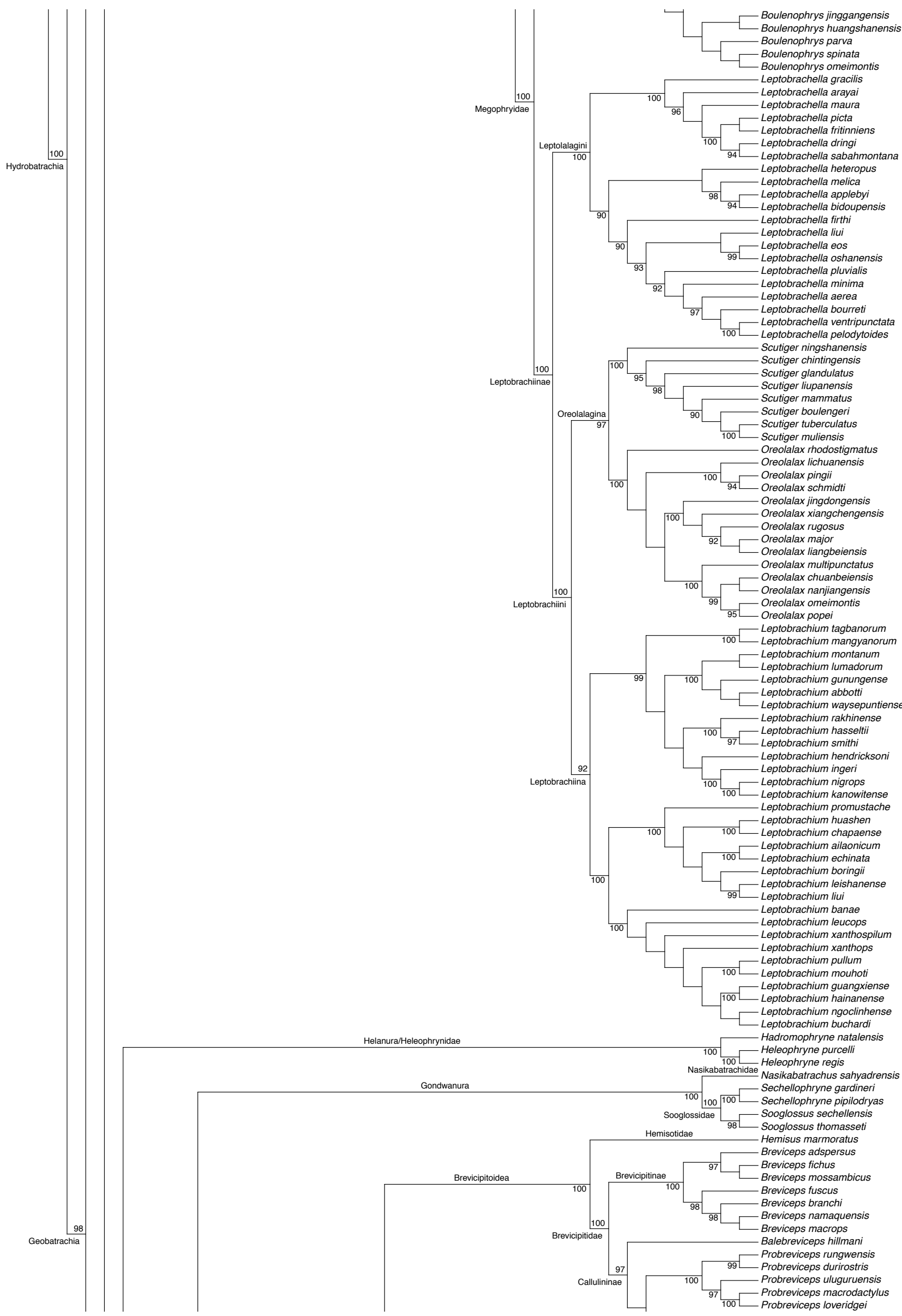




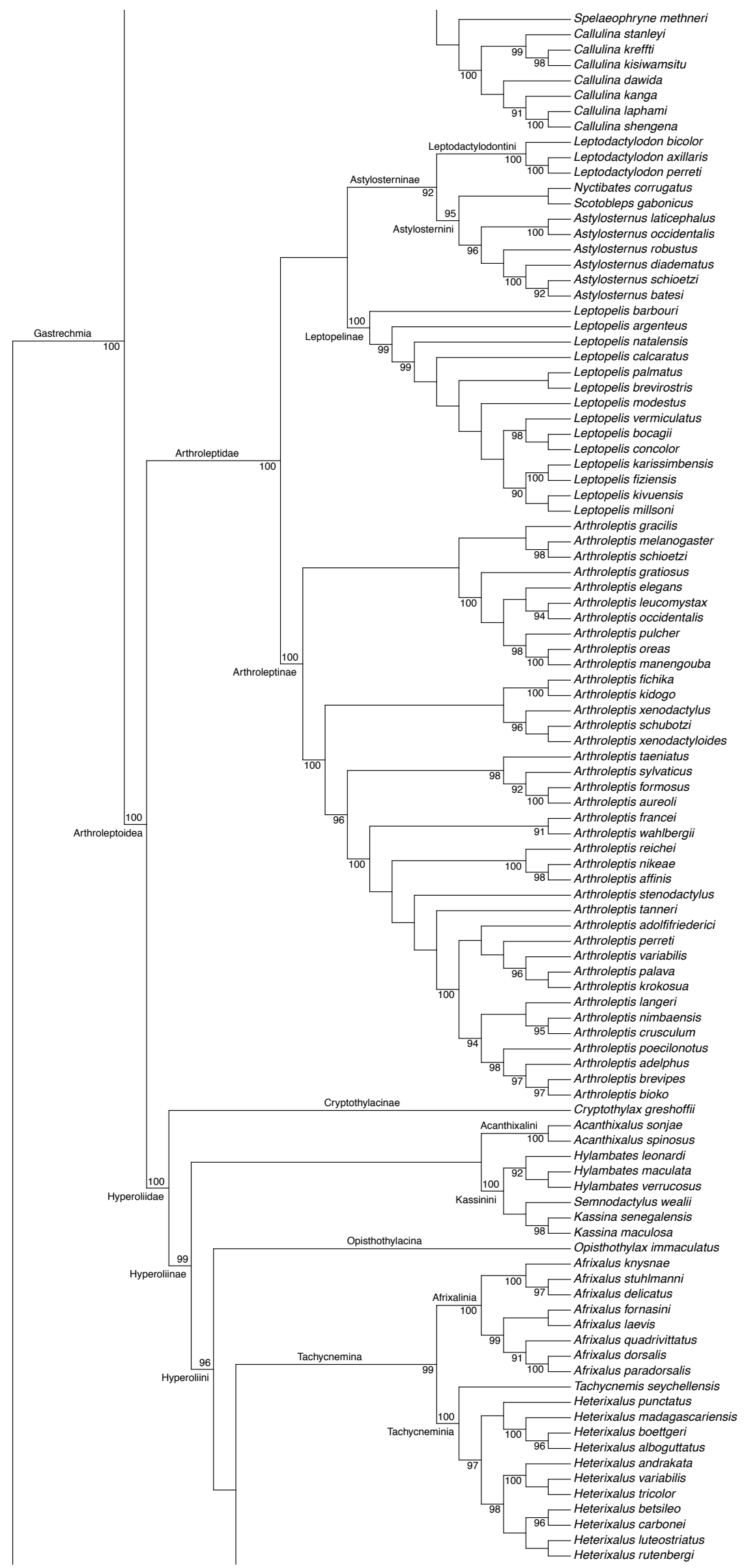




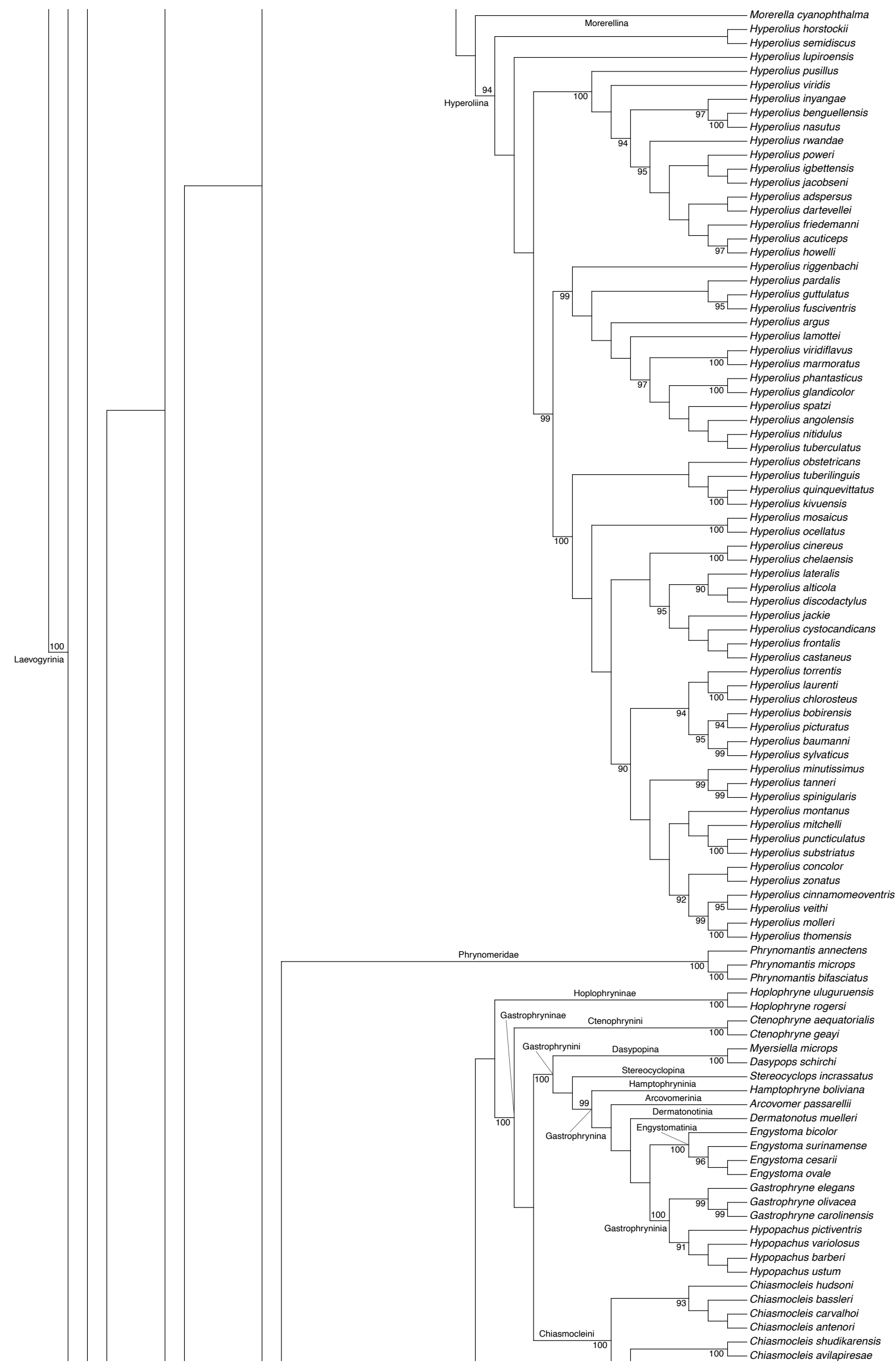




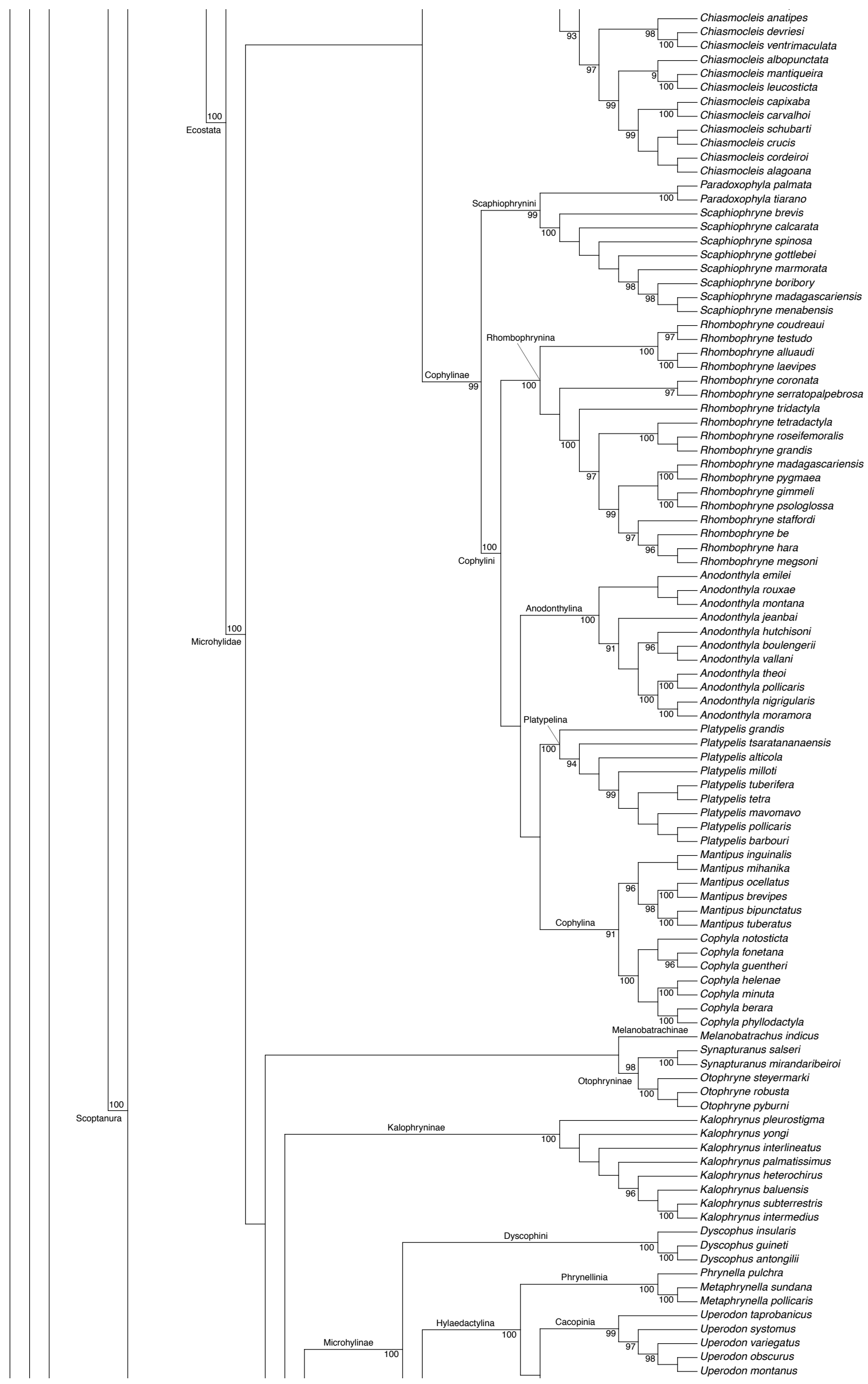



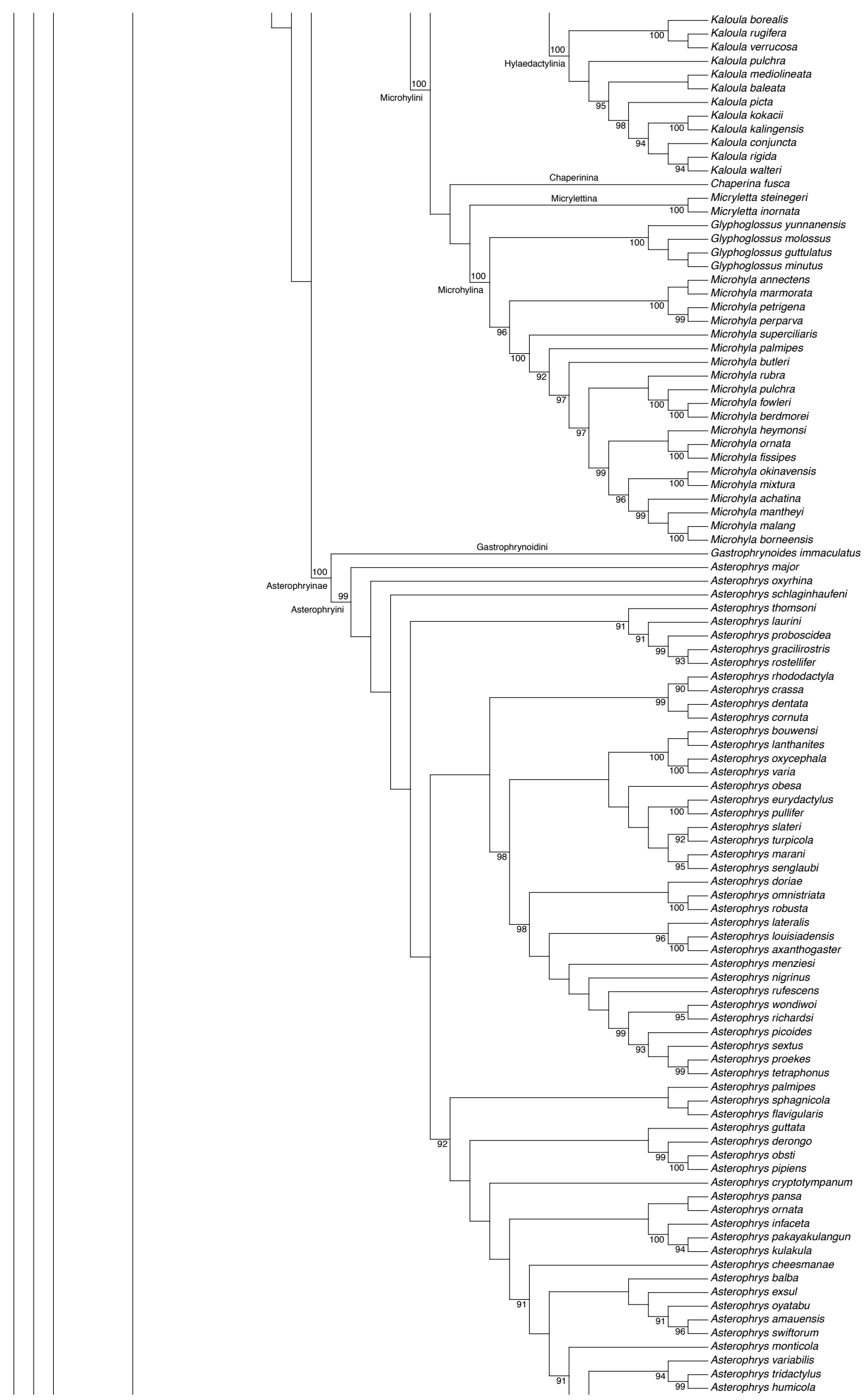


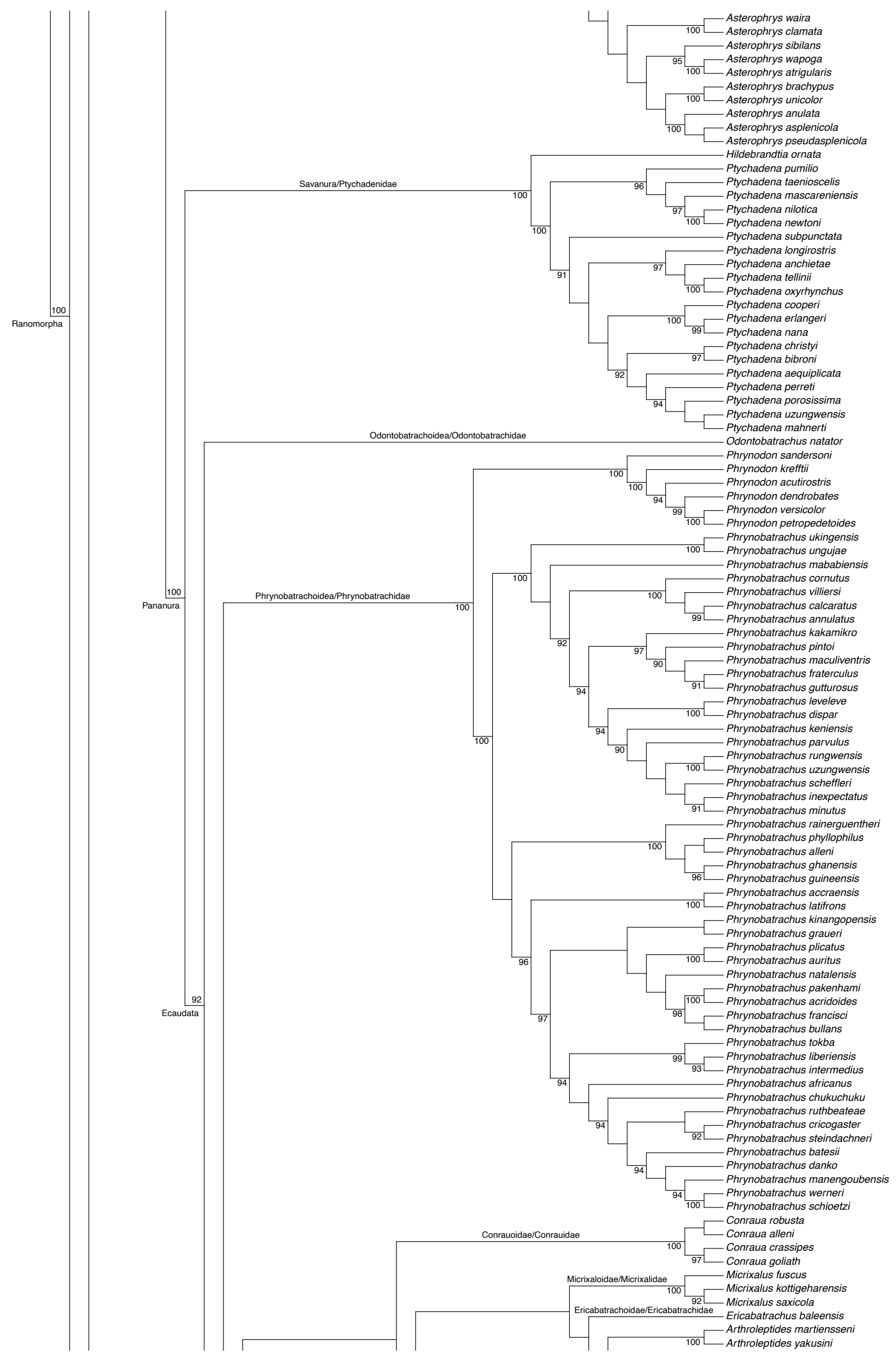



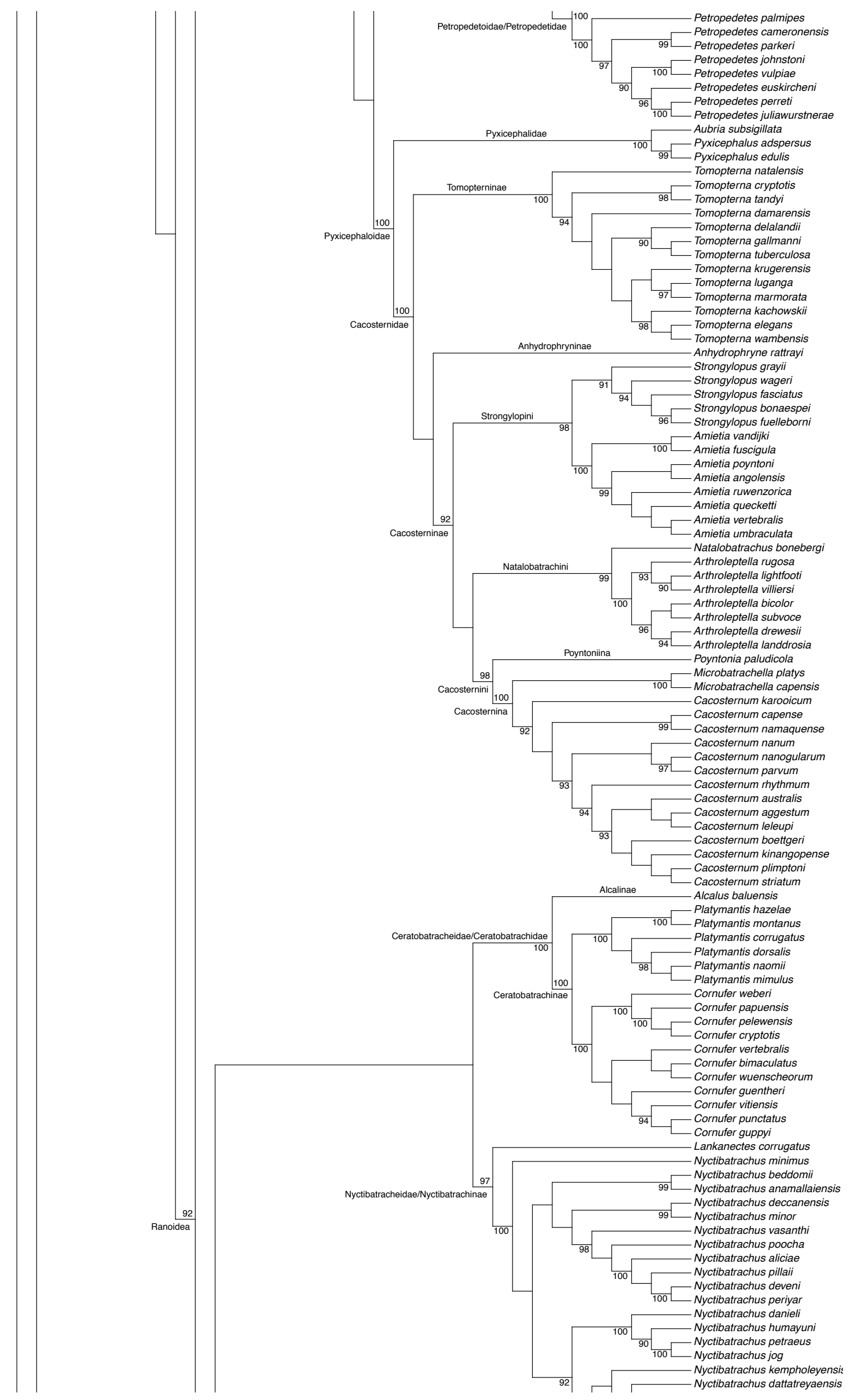


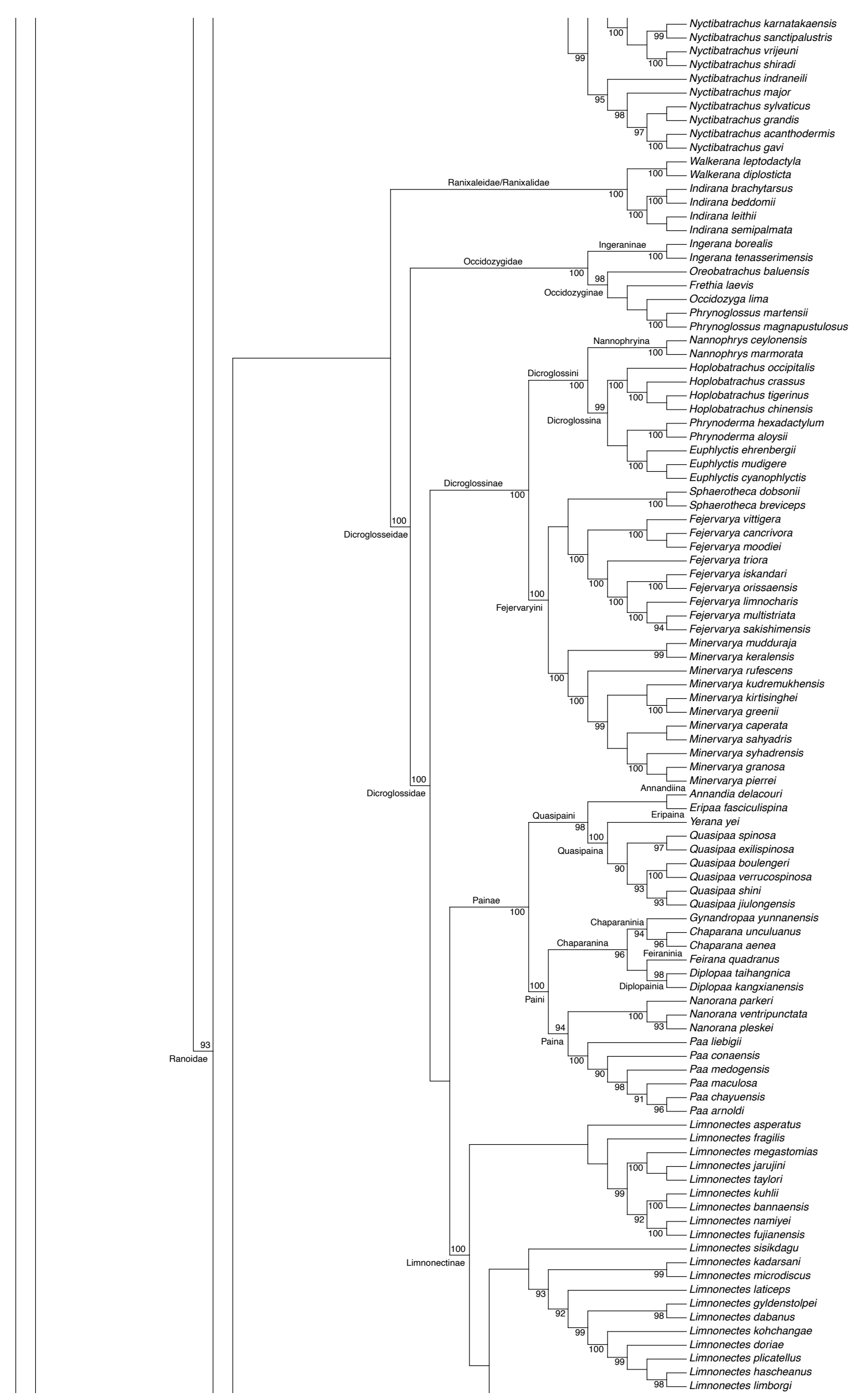




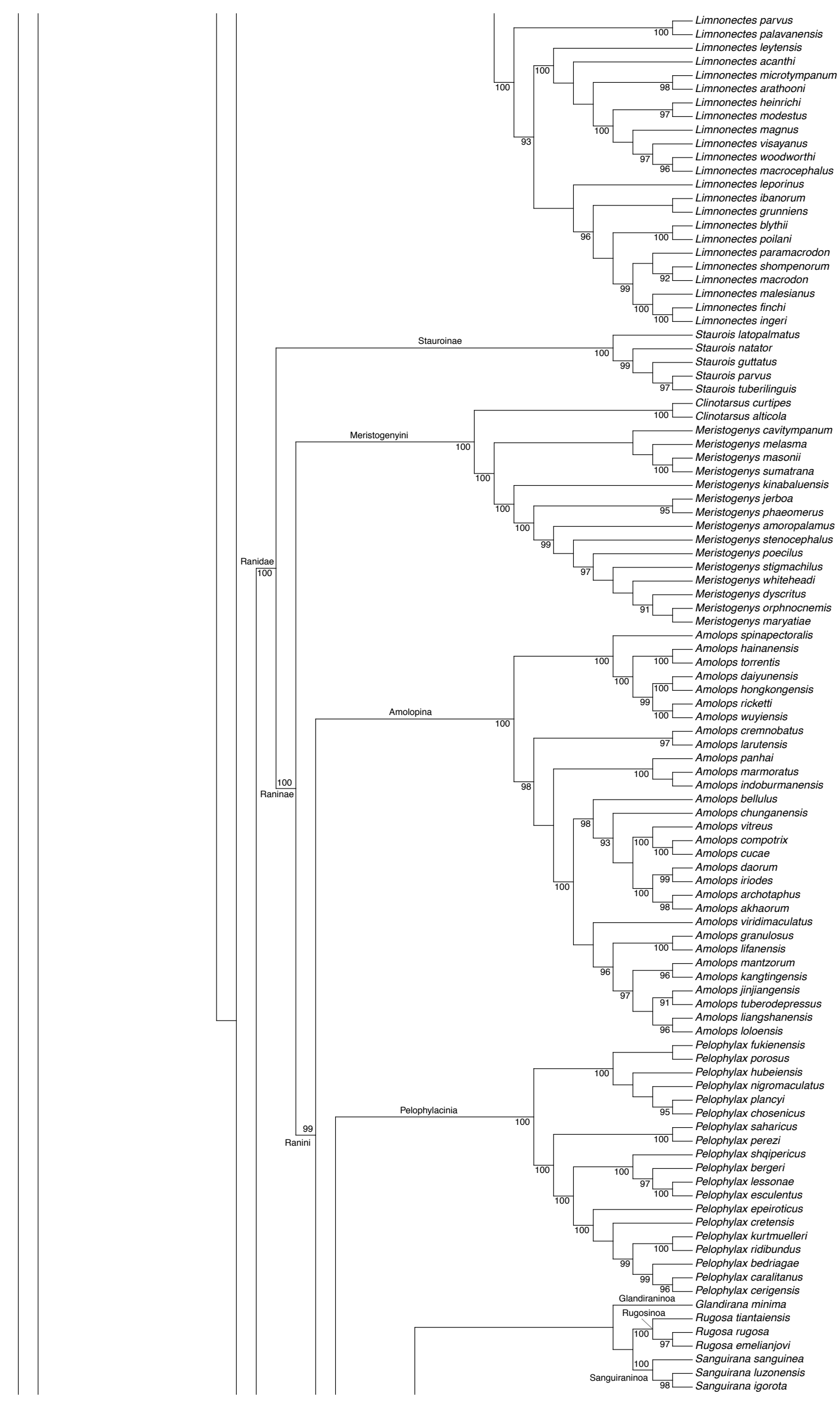



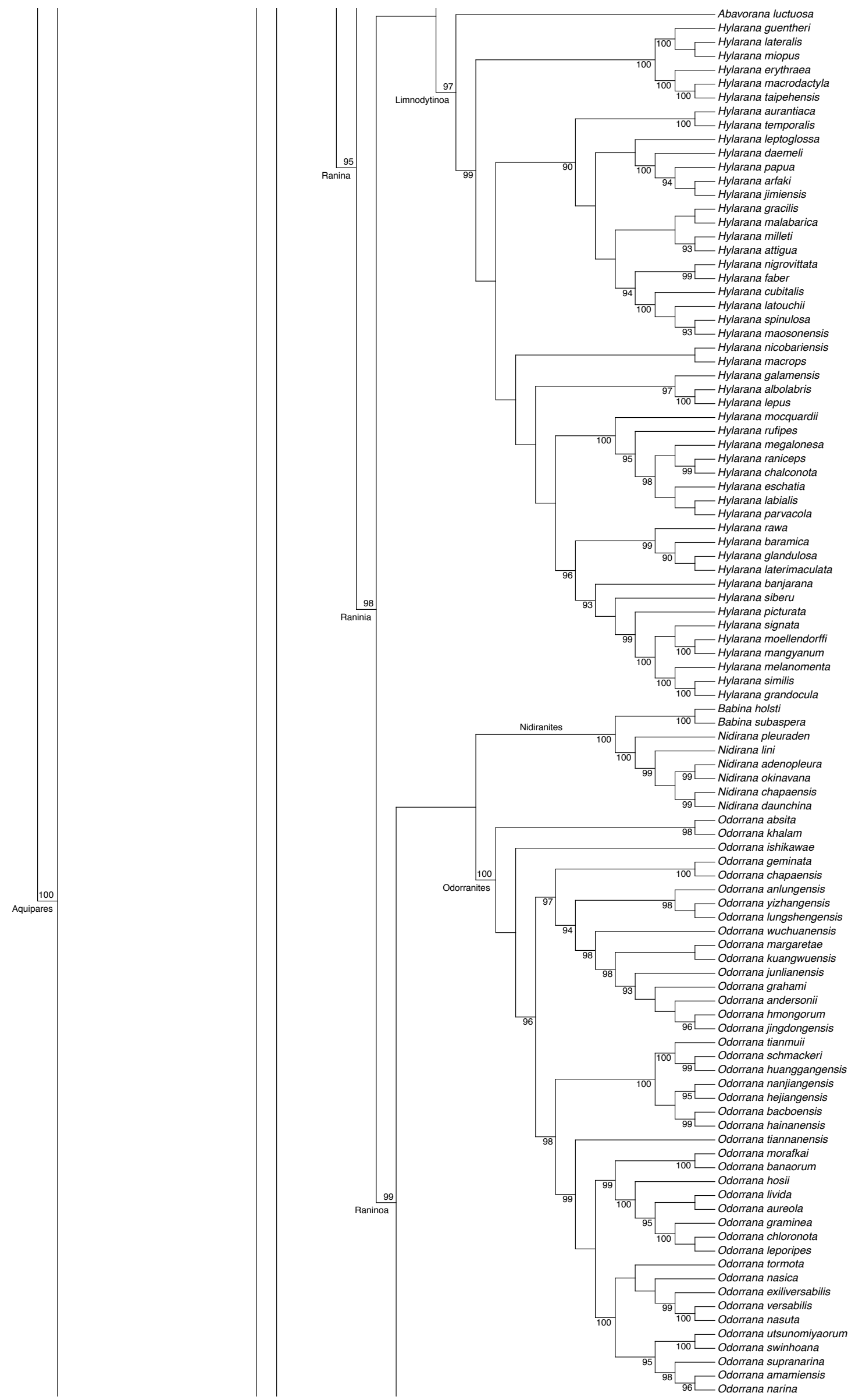


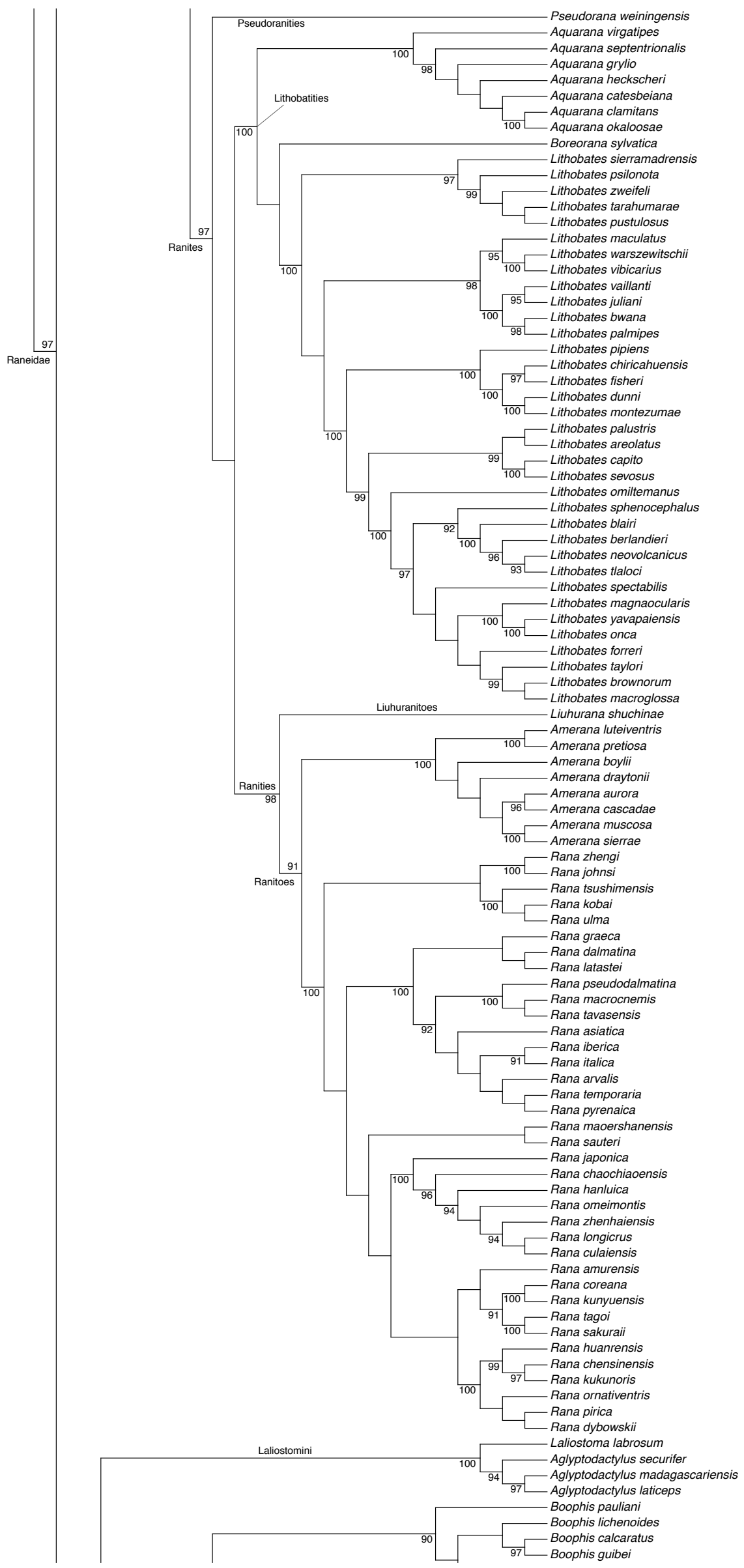




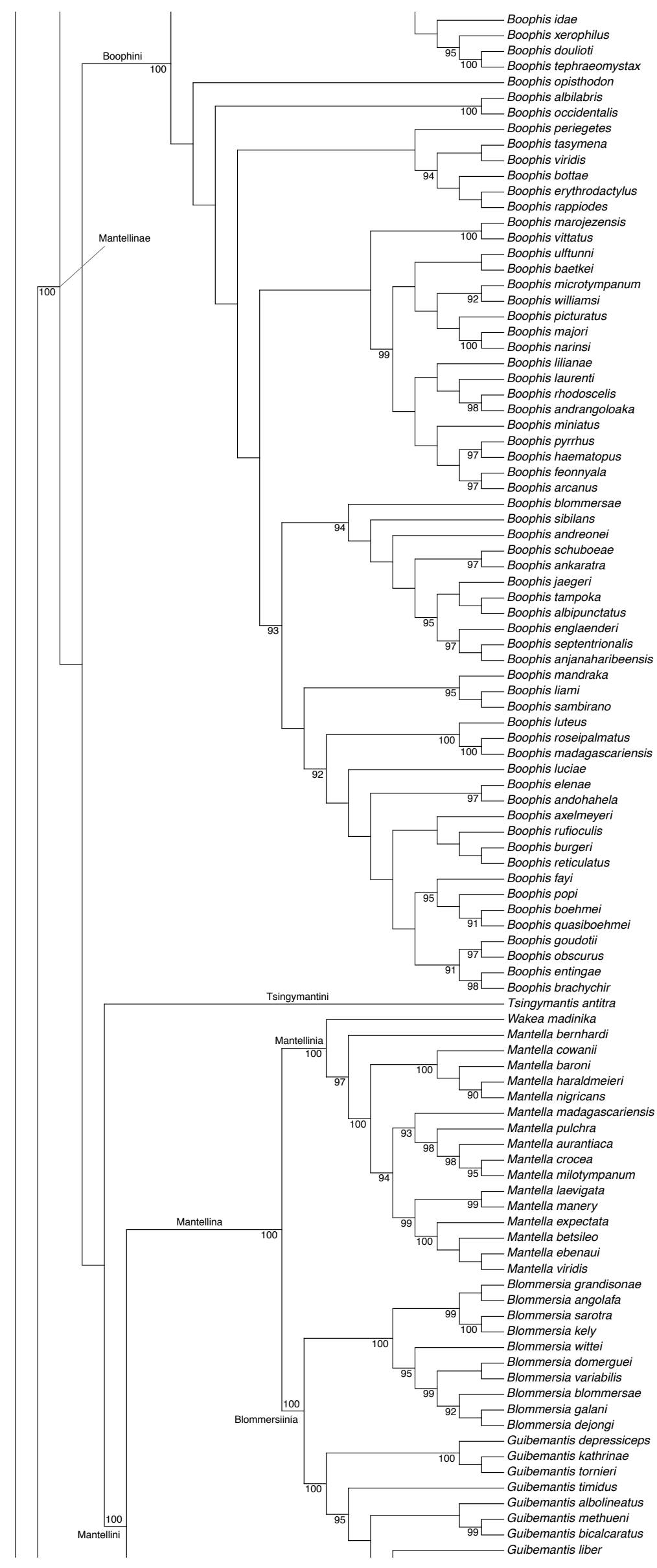




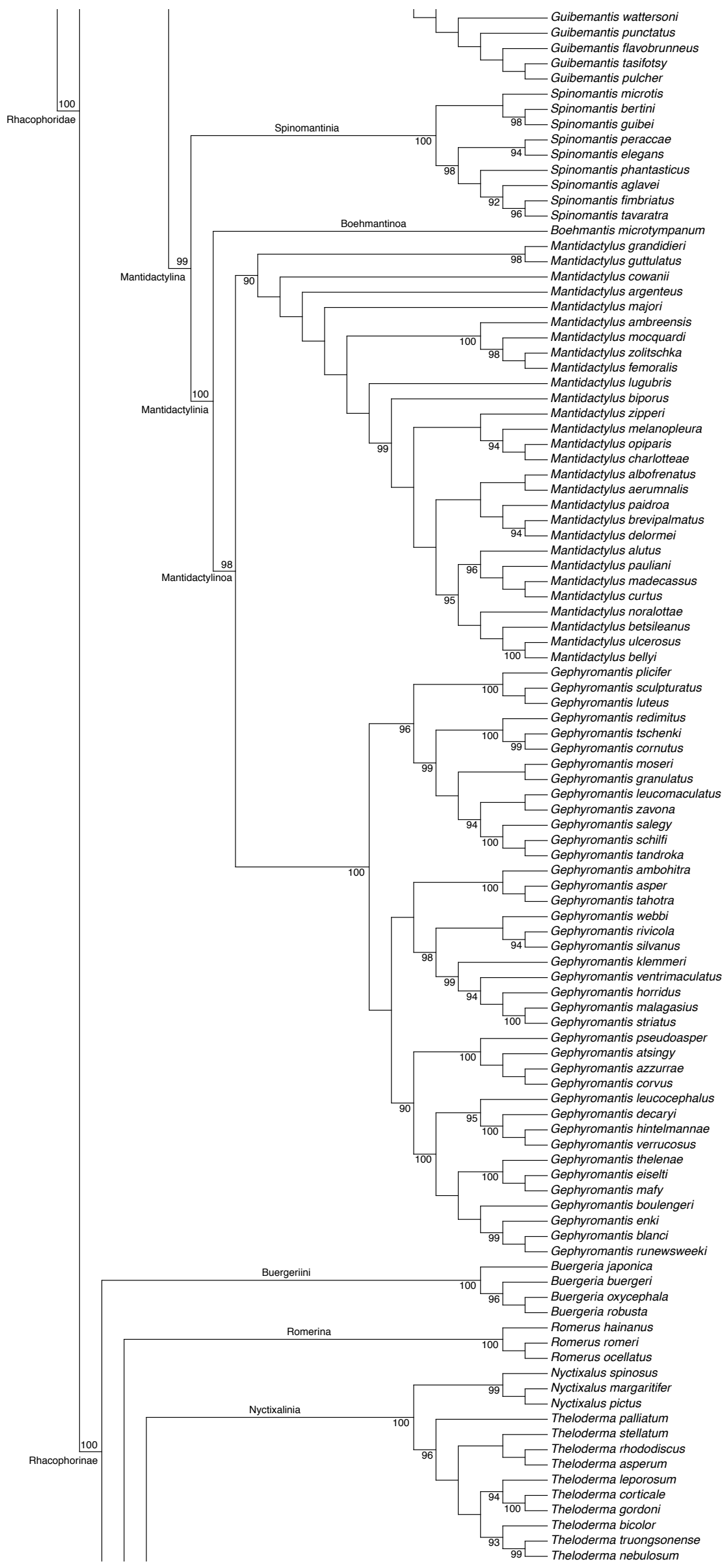




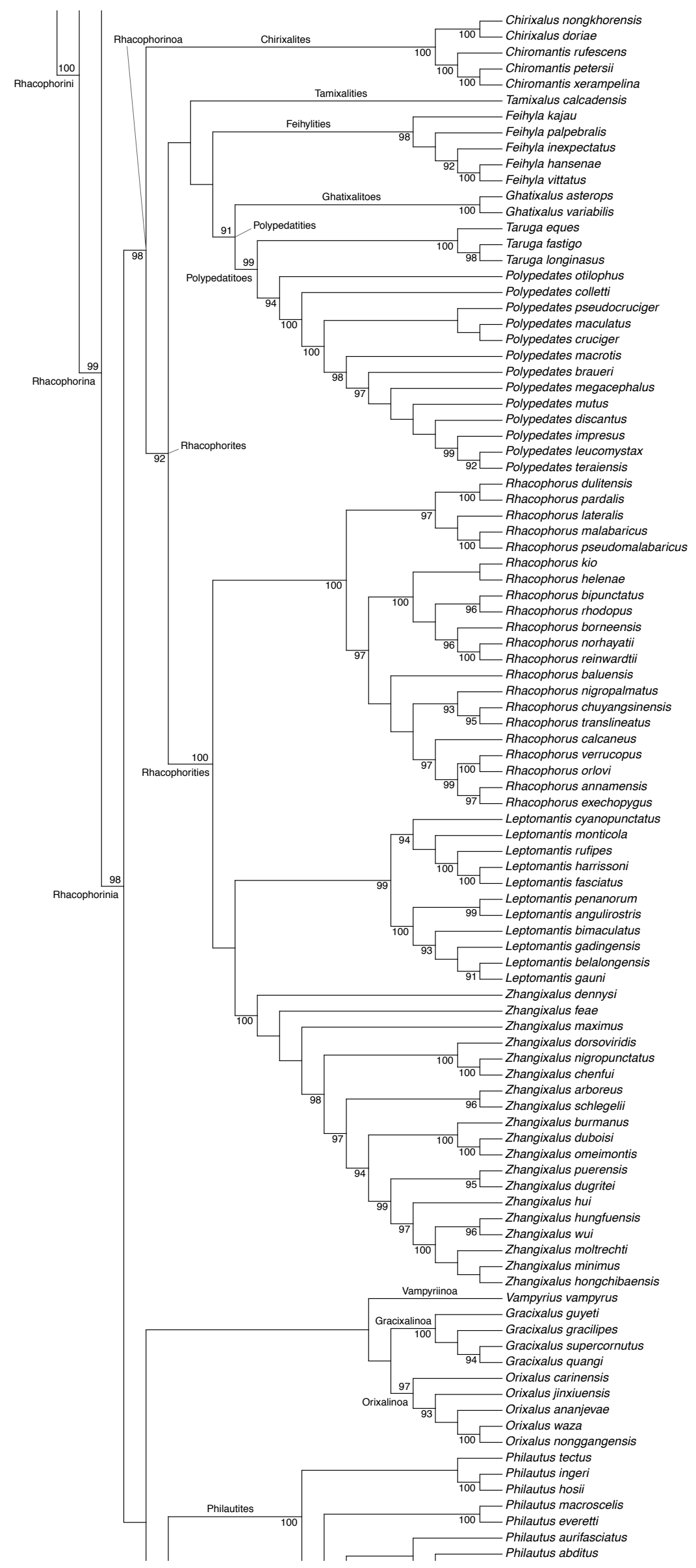




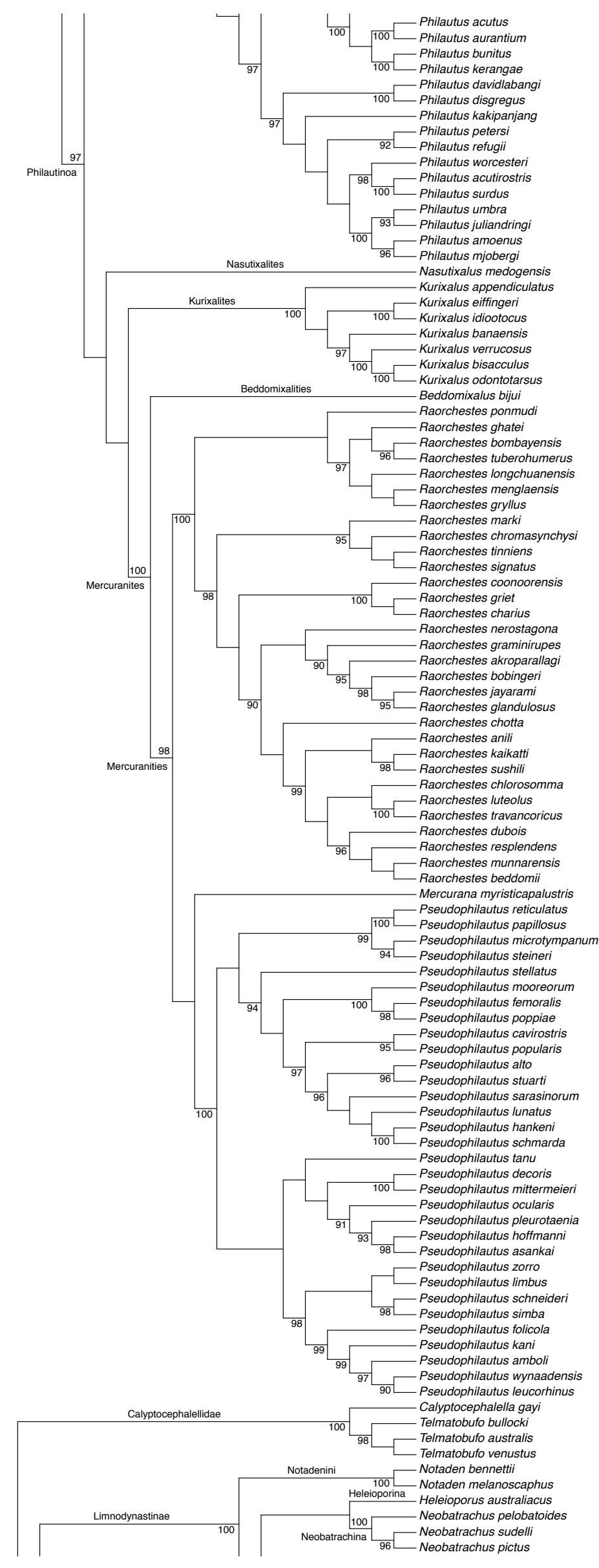




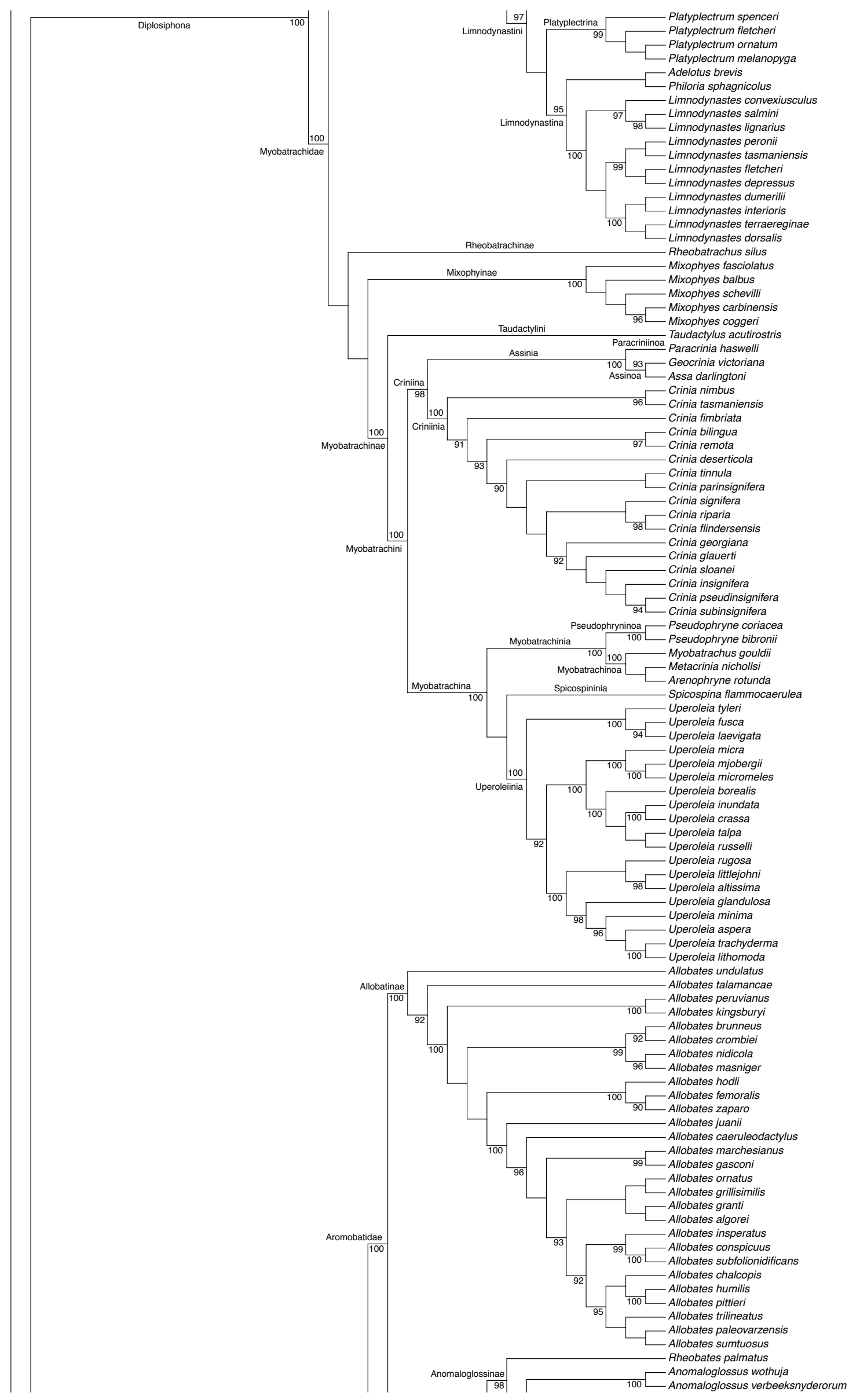




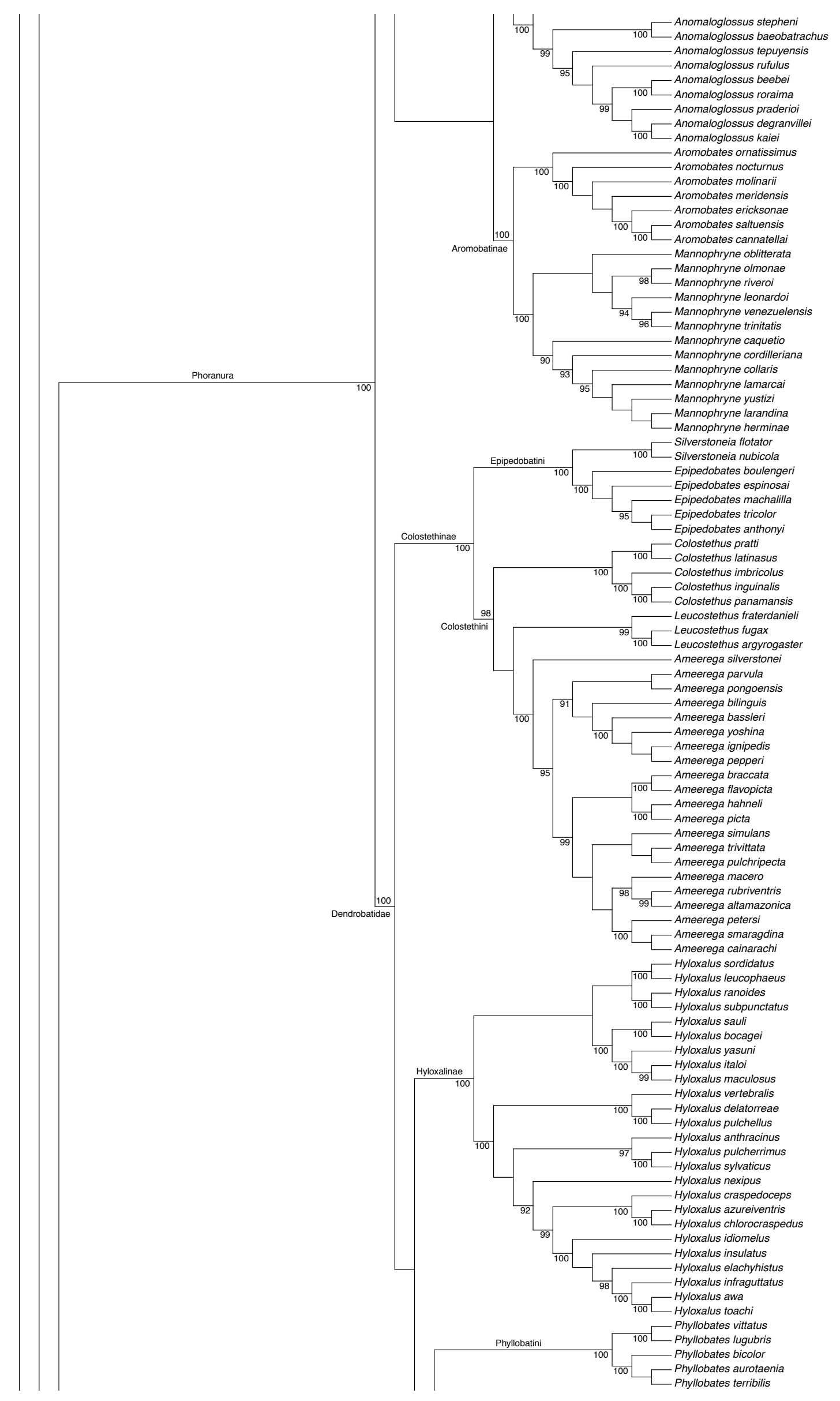



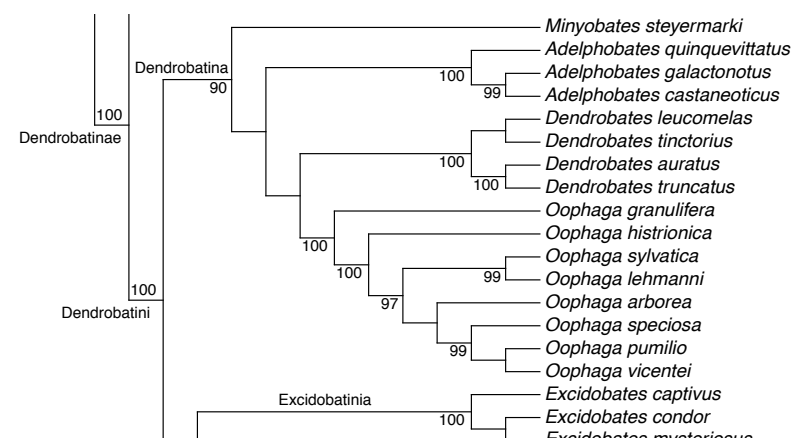

Excidobates mysterio

100

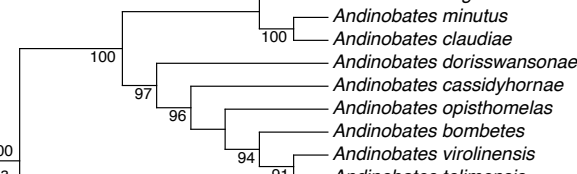
Andinobates tolimensis

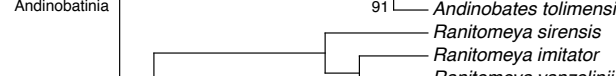

100

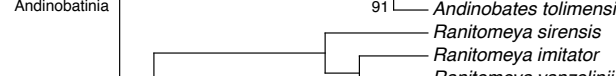

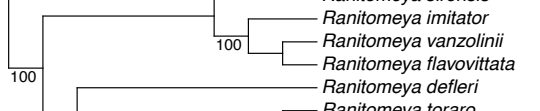
$99 \quad$ Ranitomeya toraro Ranitomeya variabilis
Ranitomeya amazonica Ranitomeya ventrimaculata Ranitomeya reticulata Ranitomeya uakarii Ranitomeya benedicta Ranitomeya fantastica - Cryptobatrachus boulenger

Cryptobatrachinae Cryptobatrachus boulenge
Hemiphractus helioi
96
Hemiphractus scutatus 96
Hemiphractinae

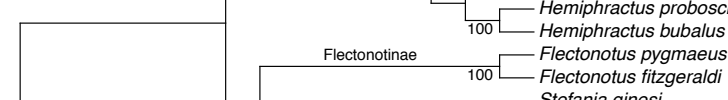

100 $1 0 0 \longdiv { 9 3 }$ Stefania satelles

Flectonotinae ectonotus pygmaeus Flectonotus fitzgeraldi Stefania ginesi Stetaninae $100 \quad$ Stefania woodleyi $100-$ Stefania roraima 100 - Stefania scala 45 Stefania riae - Stefania coxi ania ayangannae
iana goeldii

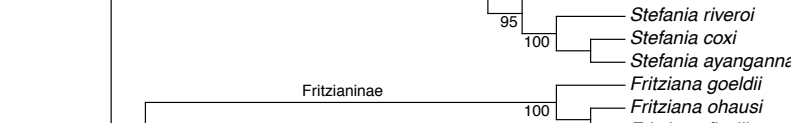

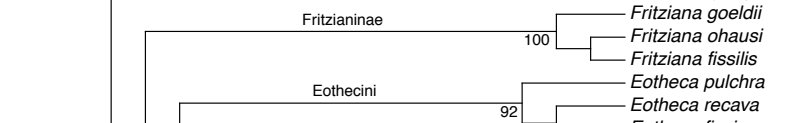

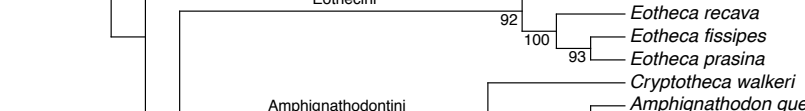

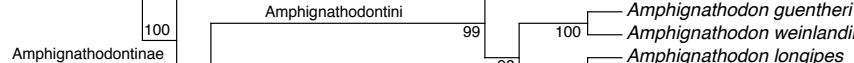
$98 \quad \sqrt{100} \square$ - Amphignathodon longipes _ Amphignathodon helenae

100 - Amphignathodon cornutus Alainia albolineata Alainia ernestoi

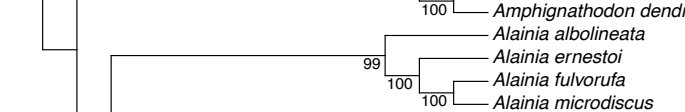

Alainia microdiscus

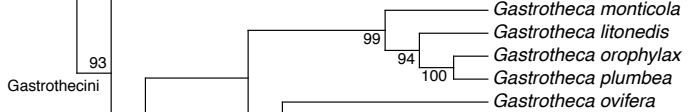
Gastrothecin

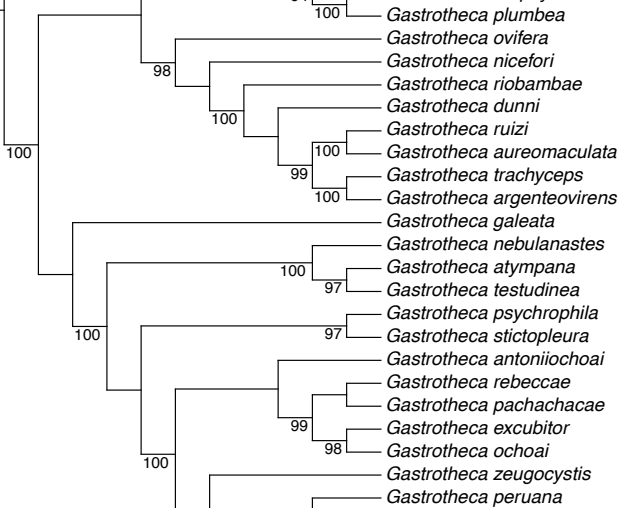




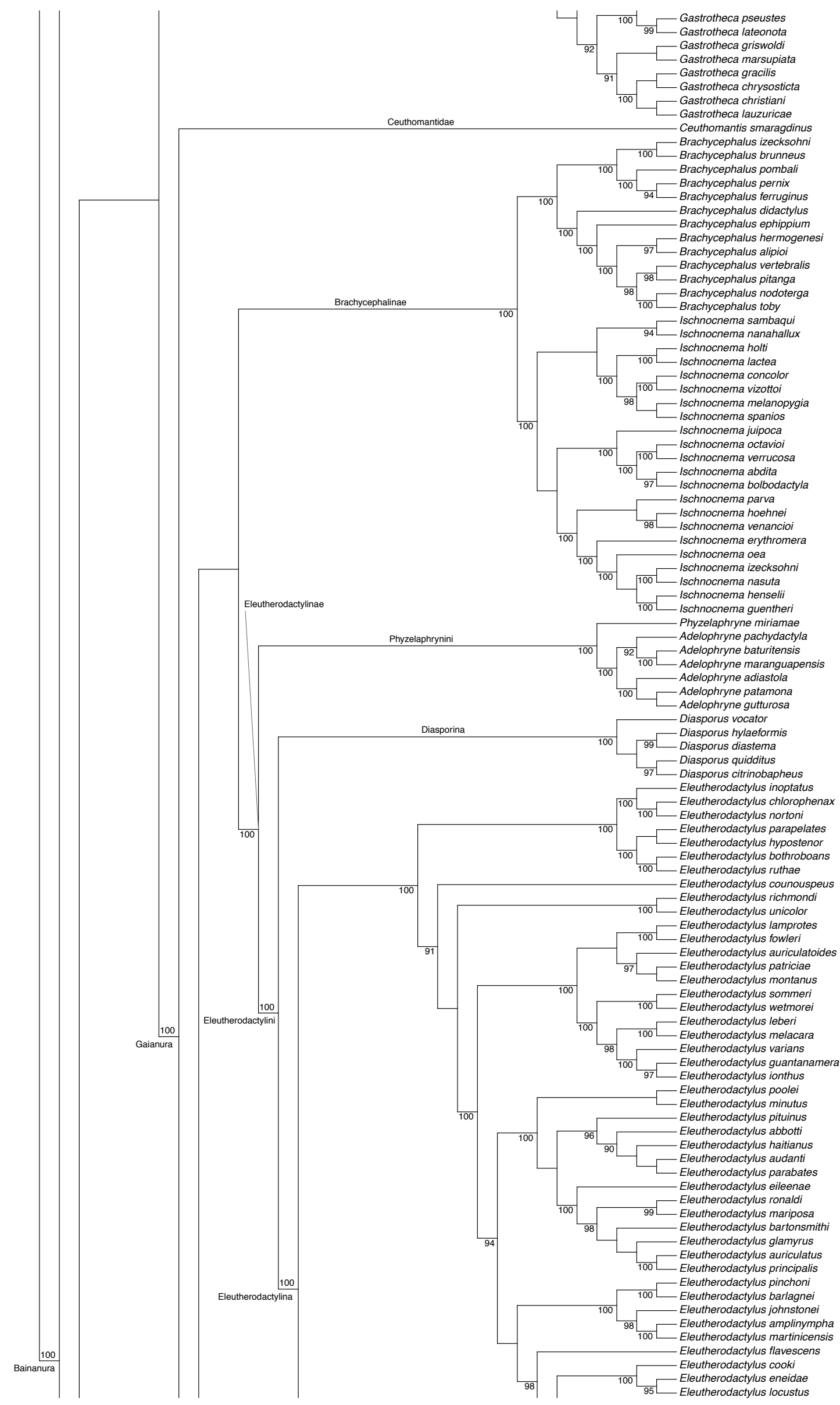



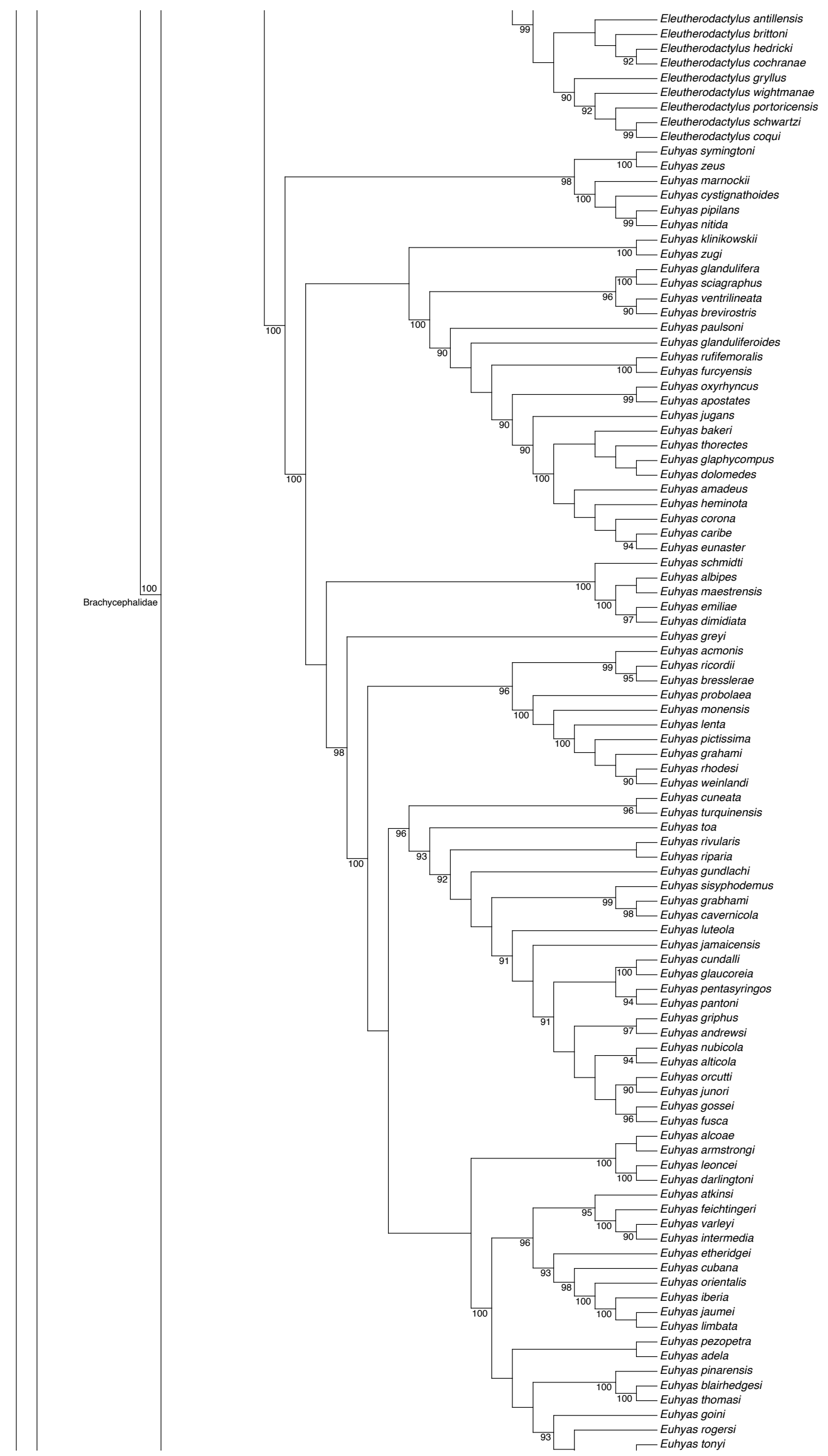


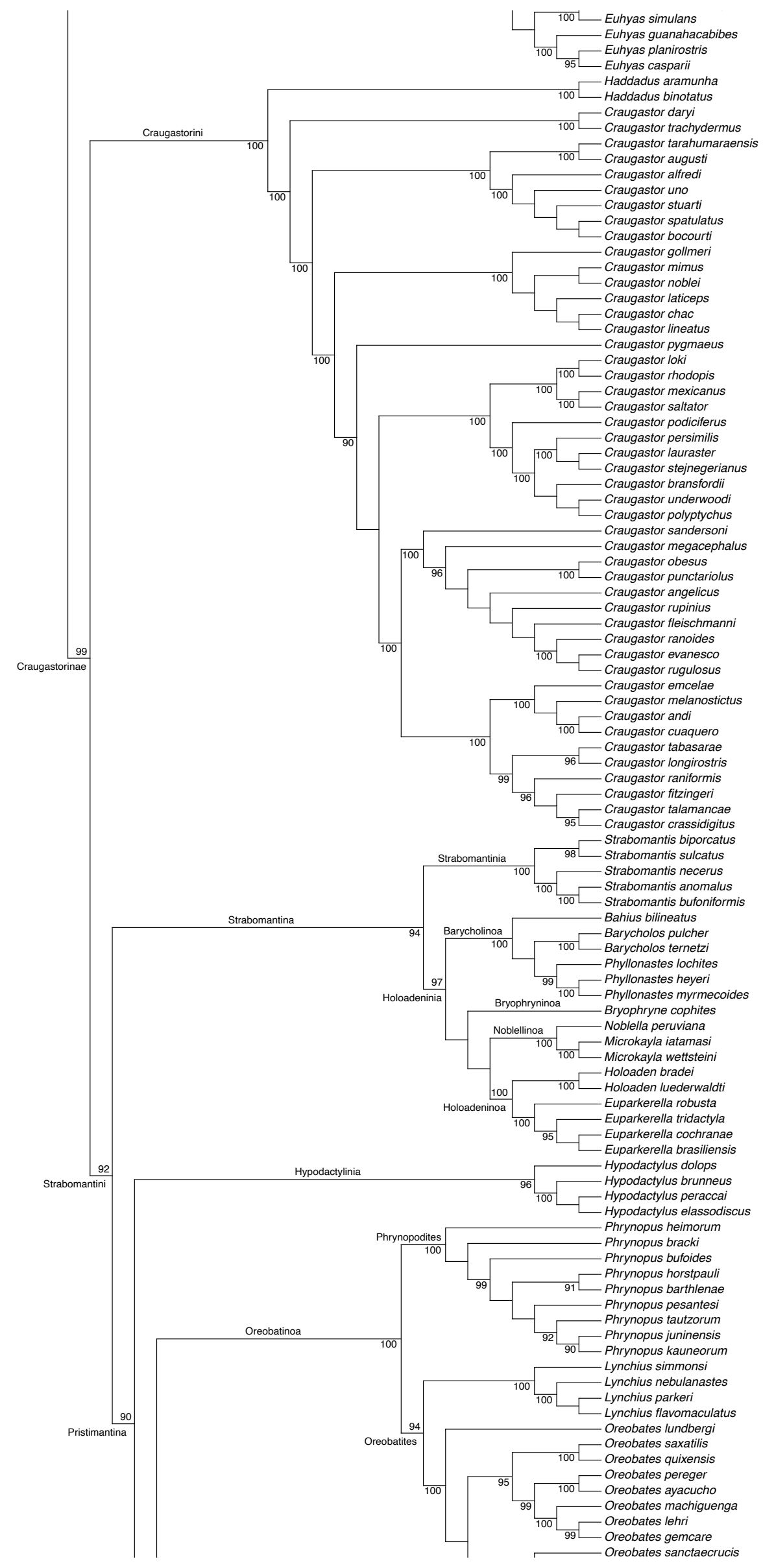




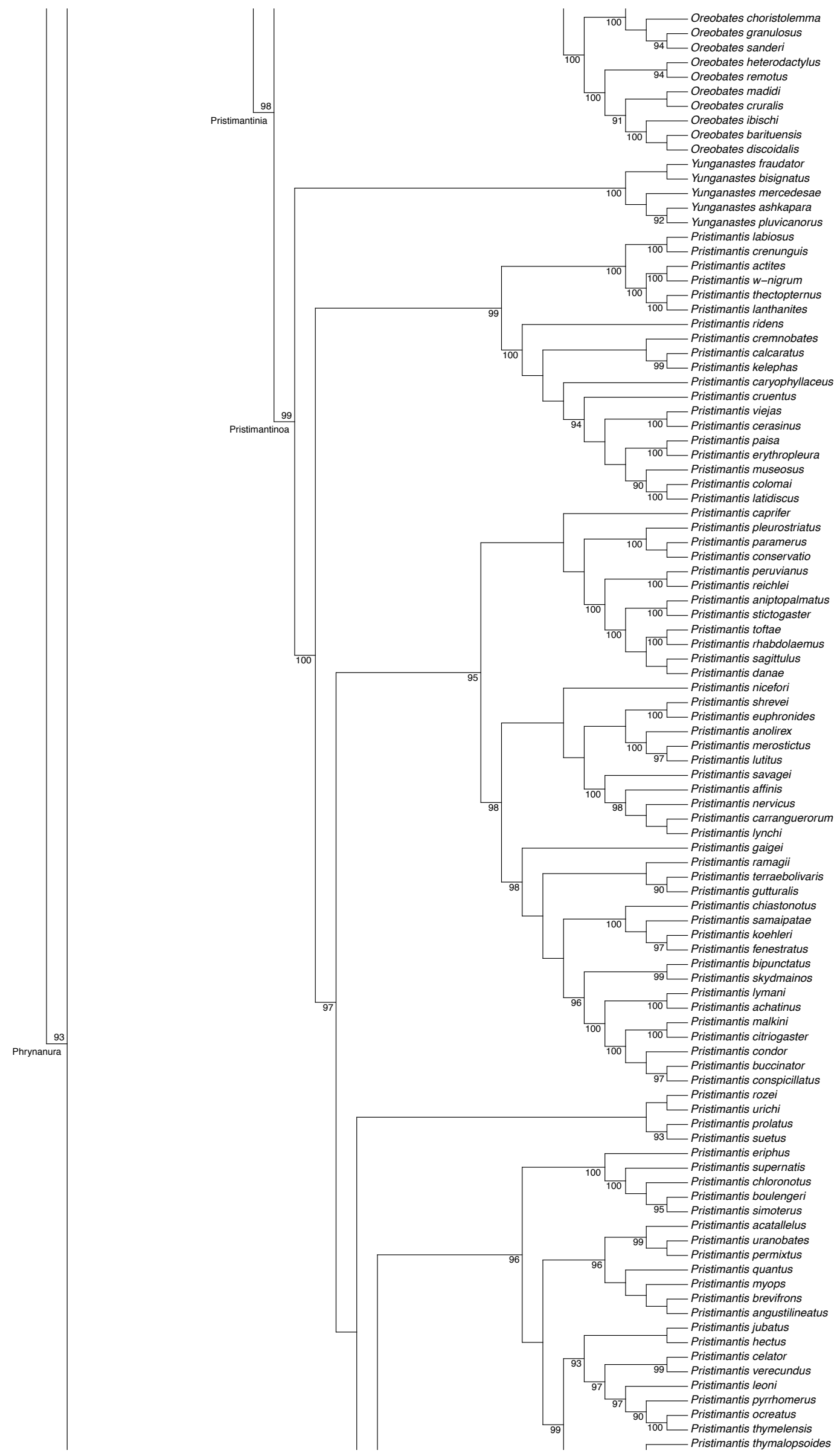




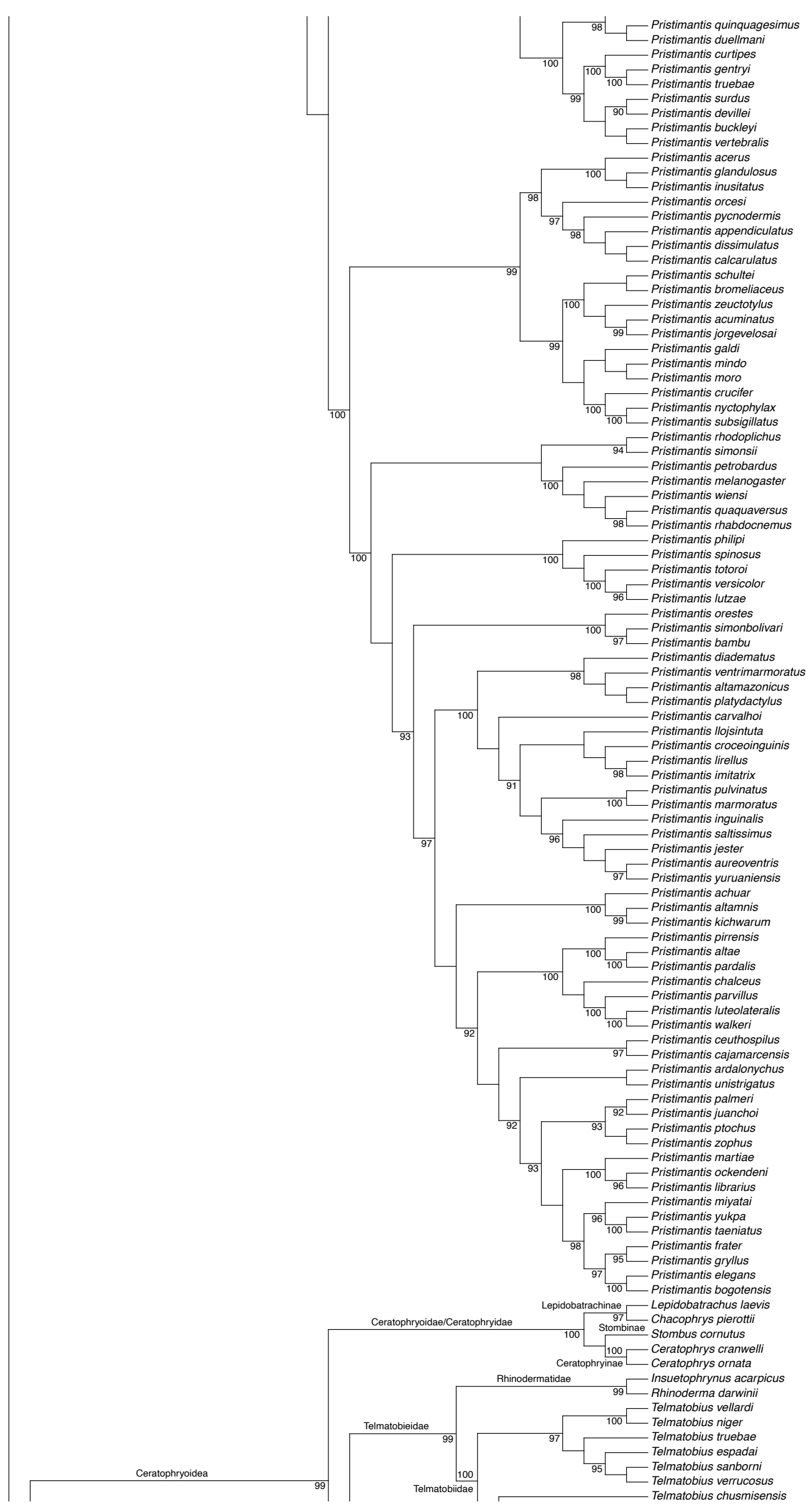




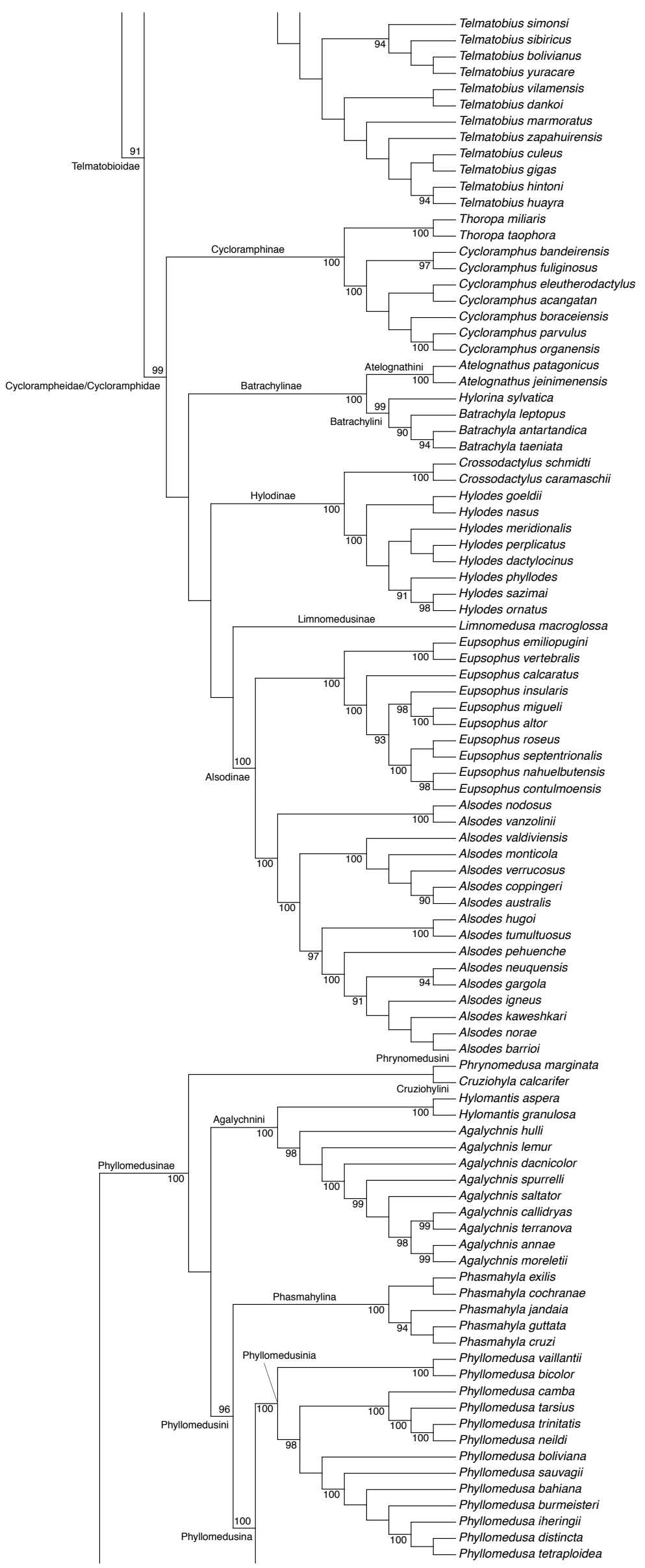




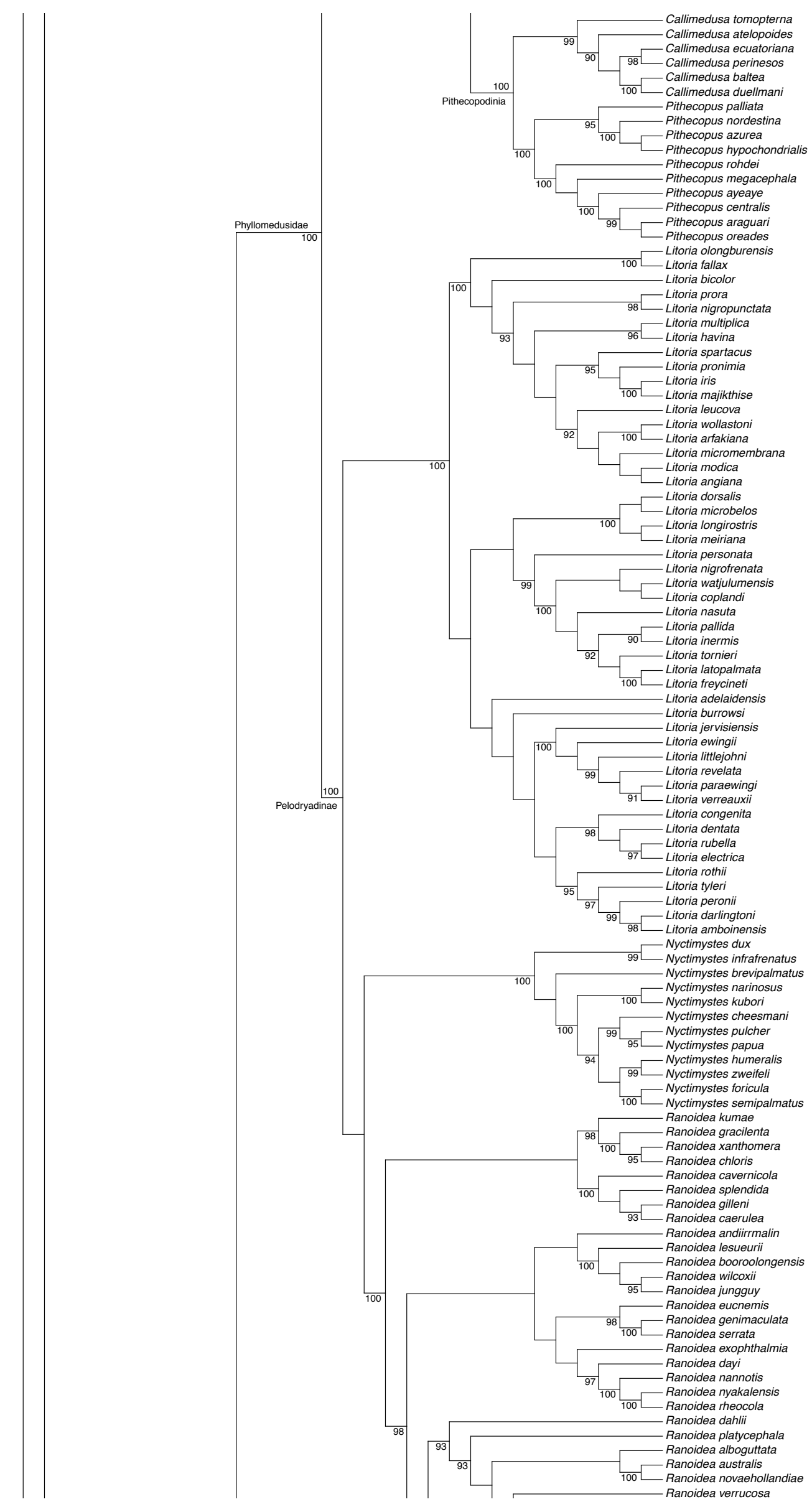




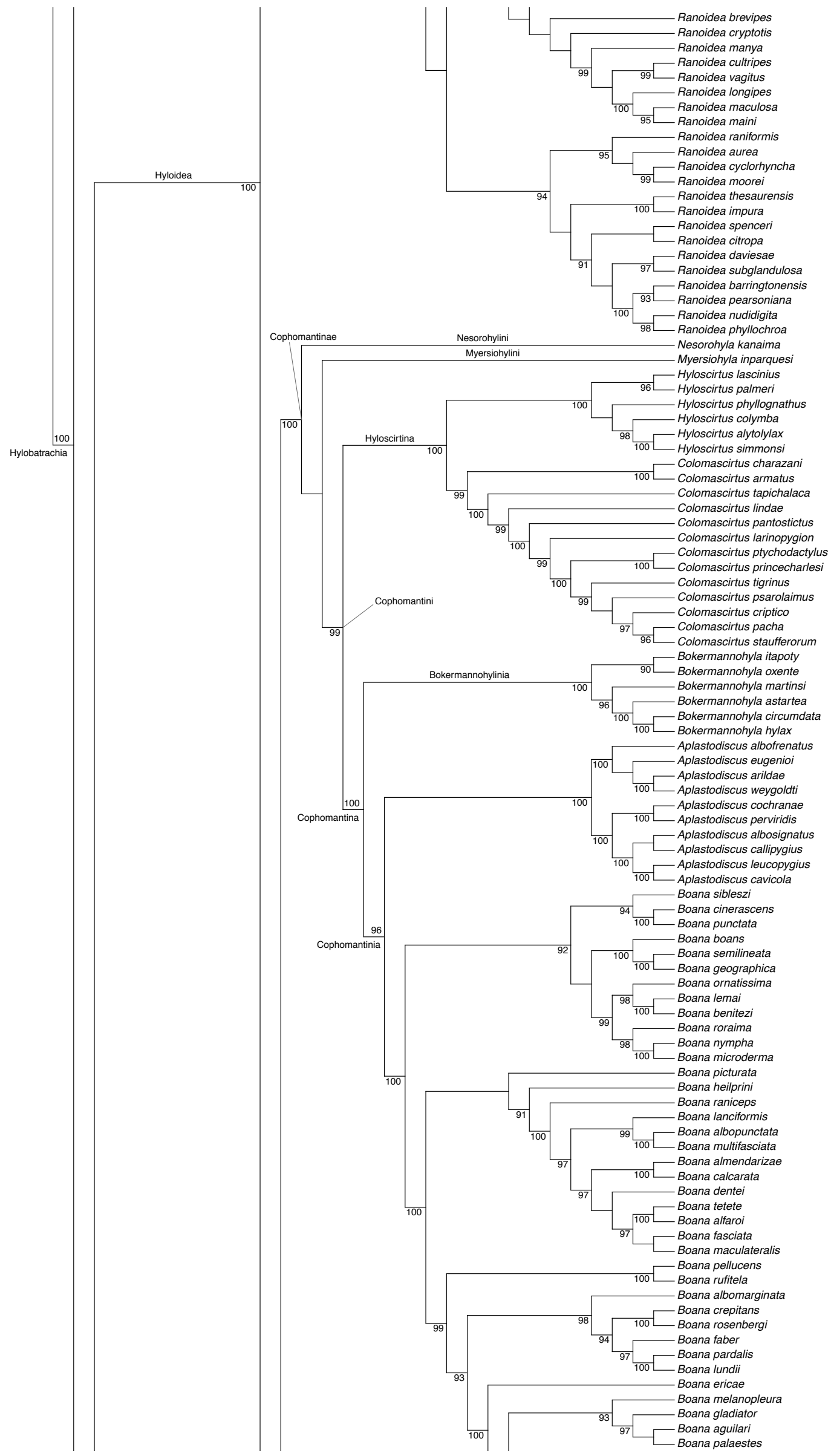




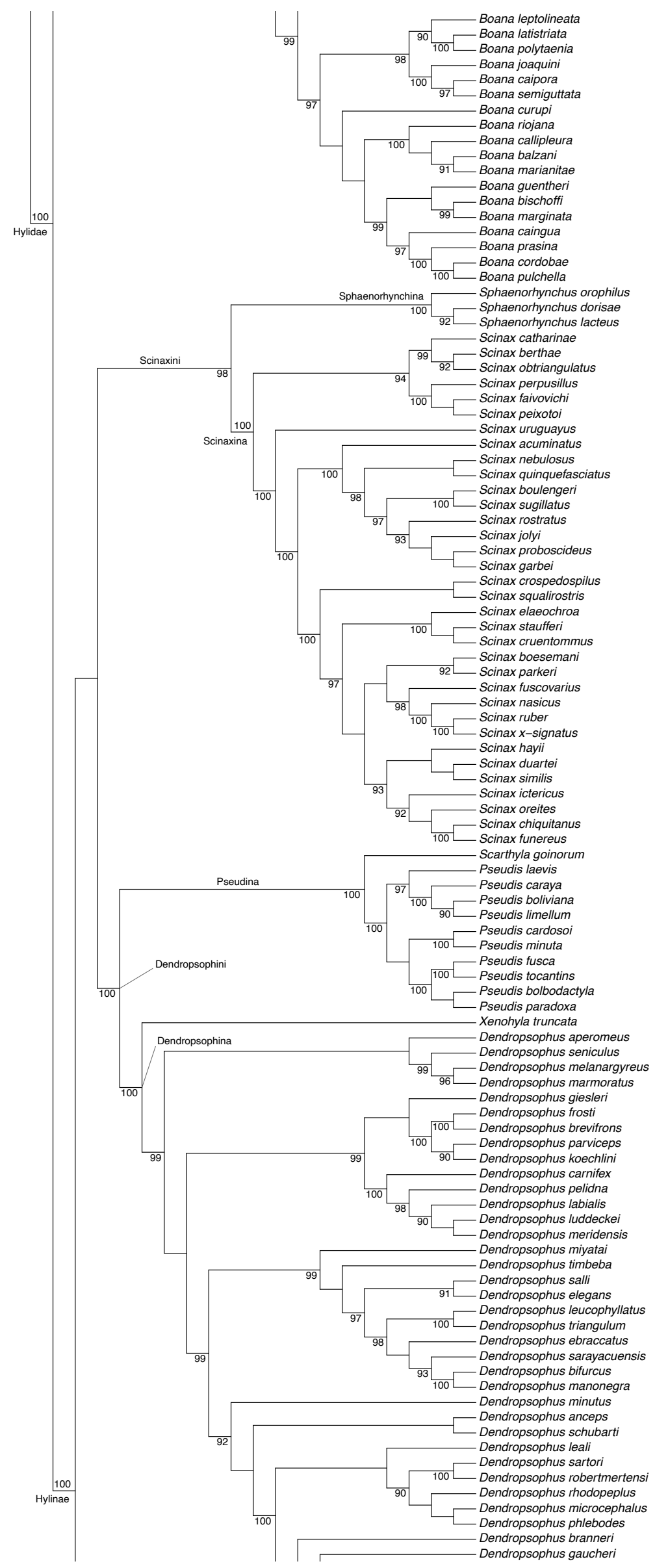




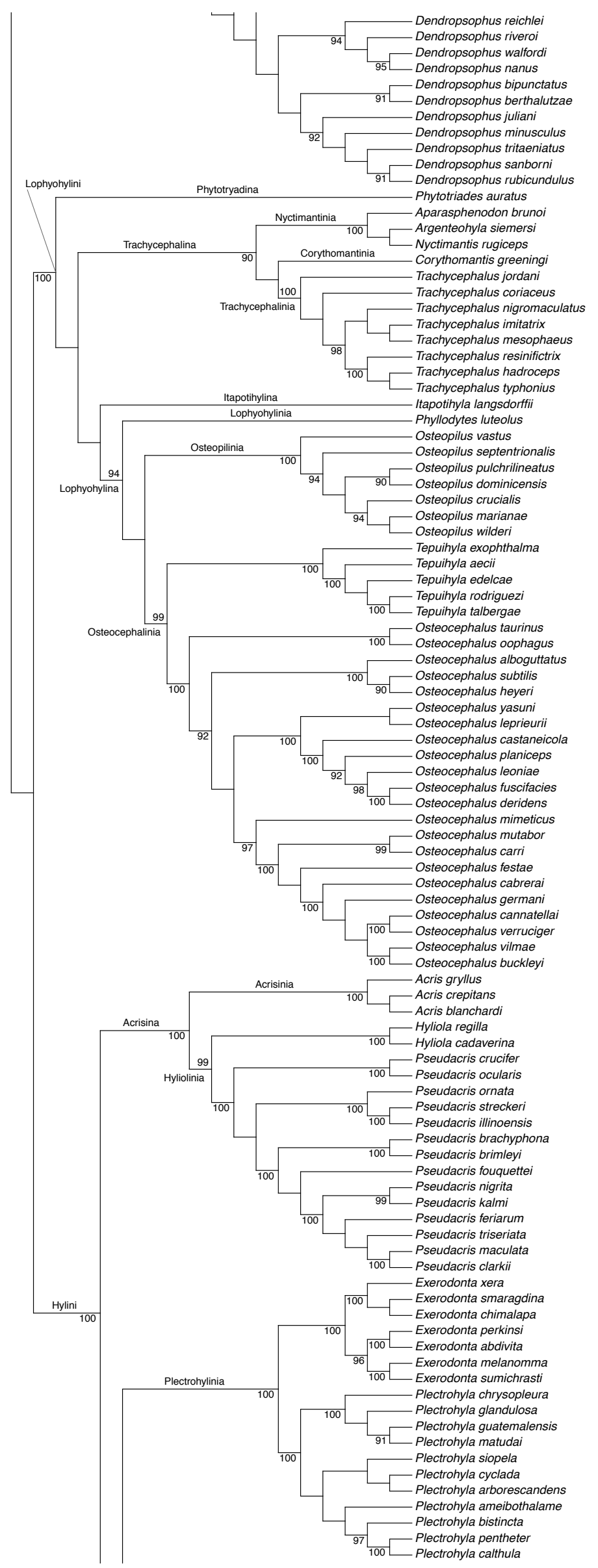




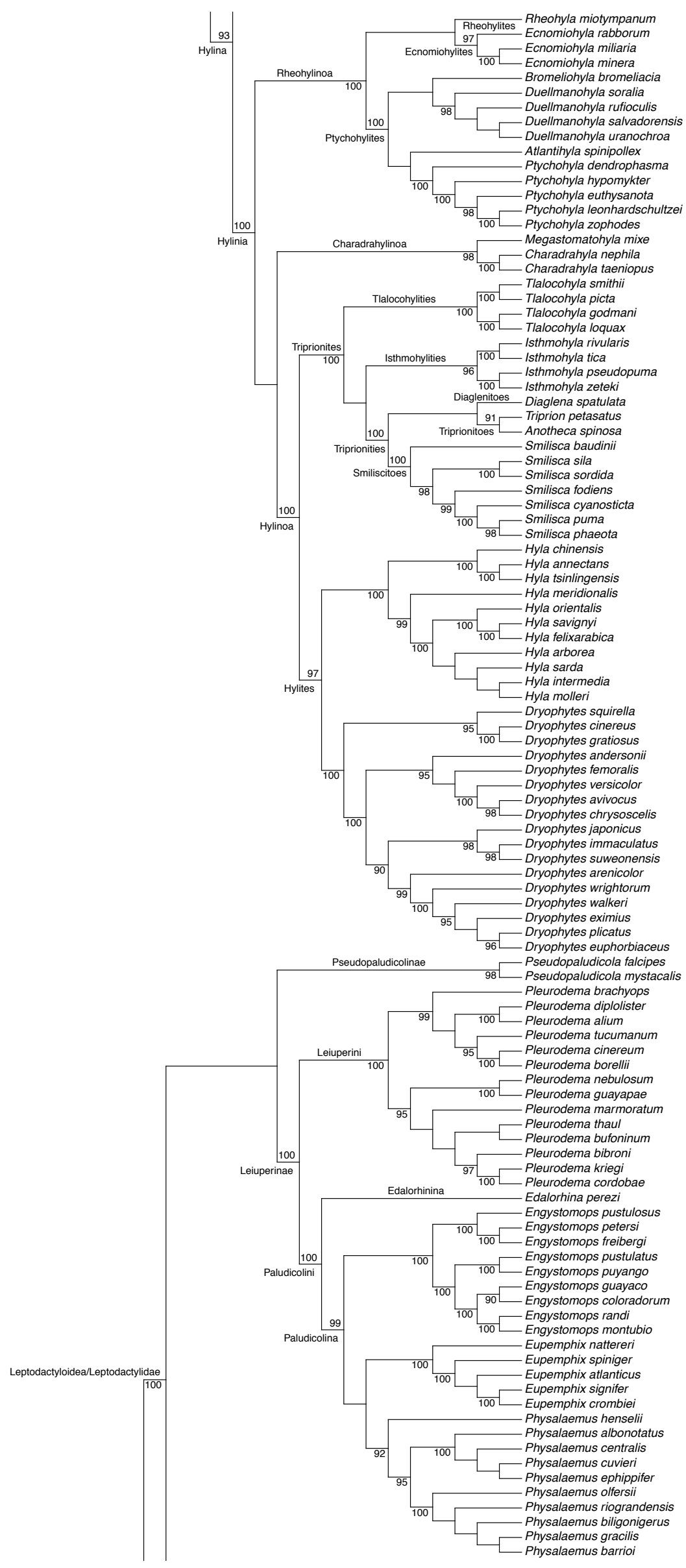




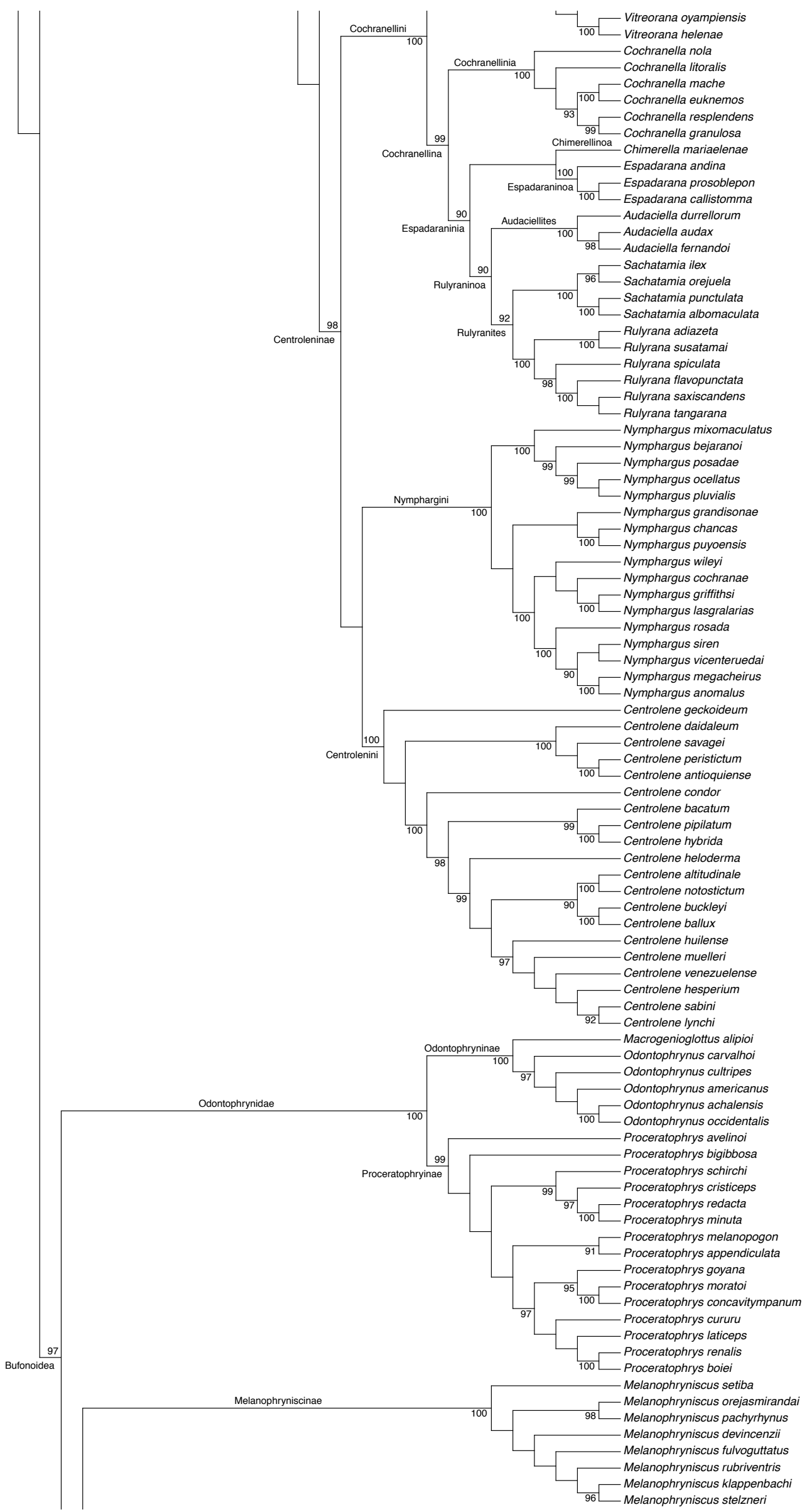




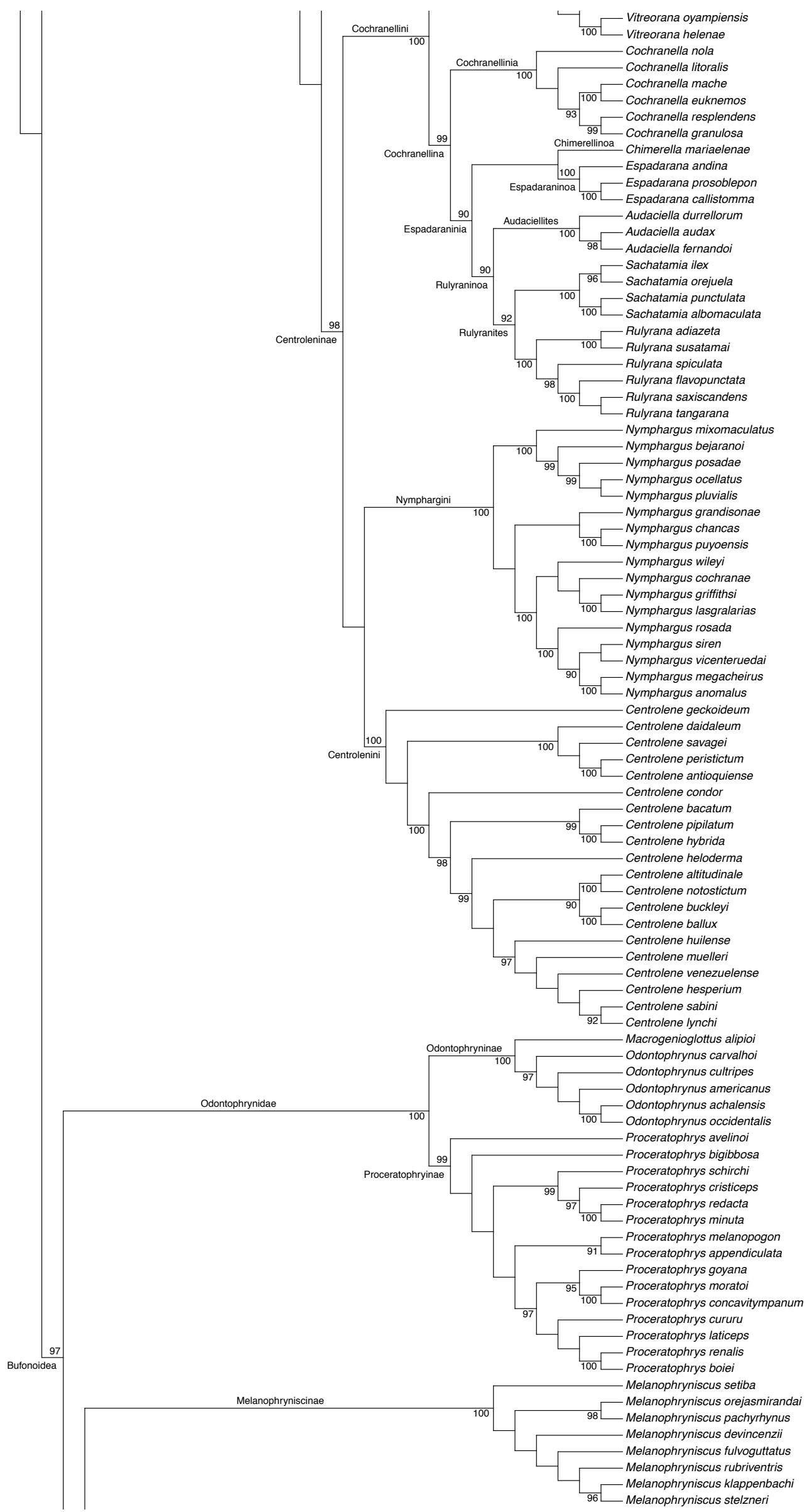




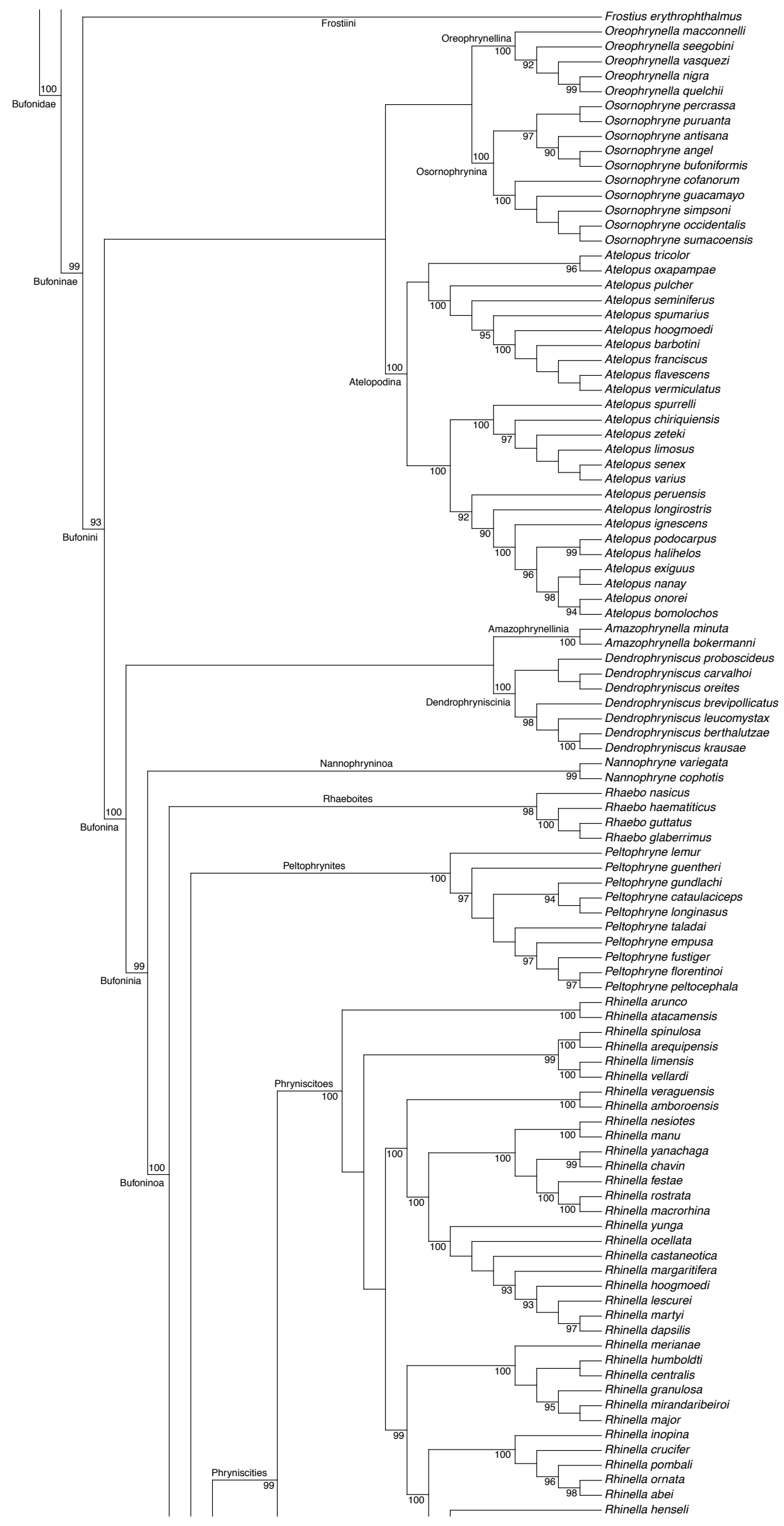




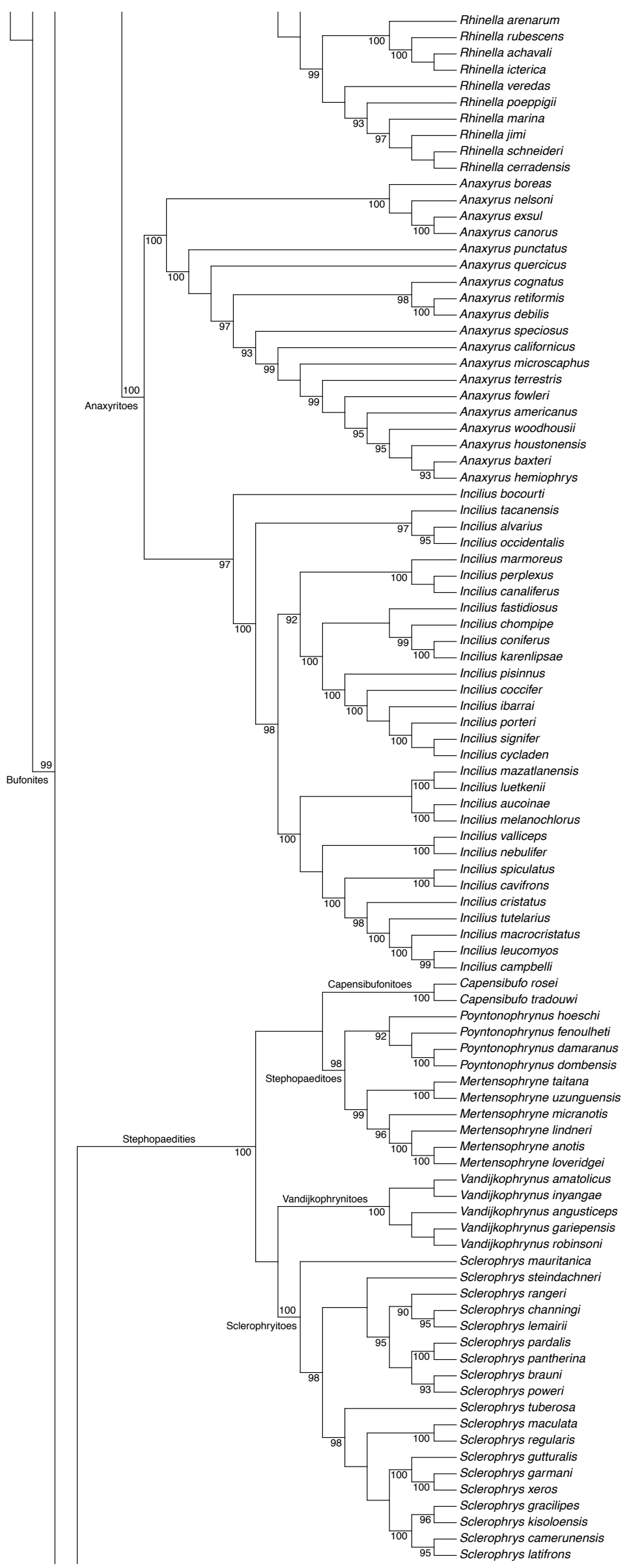




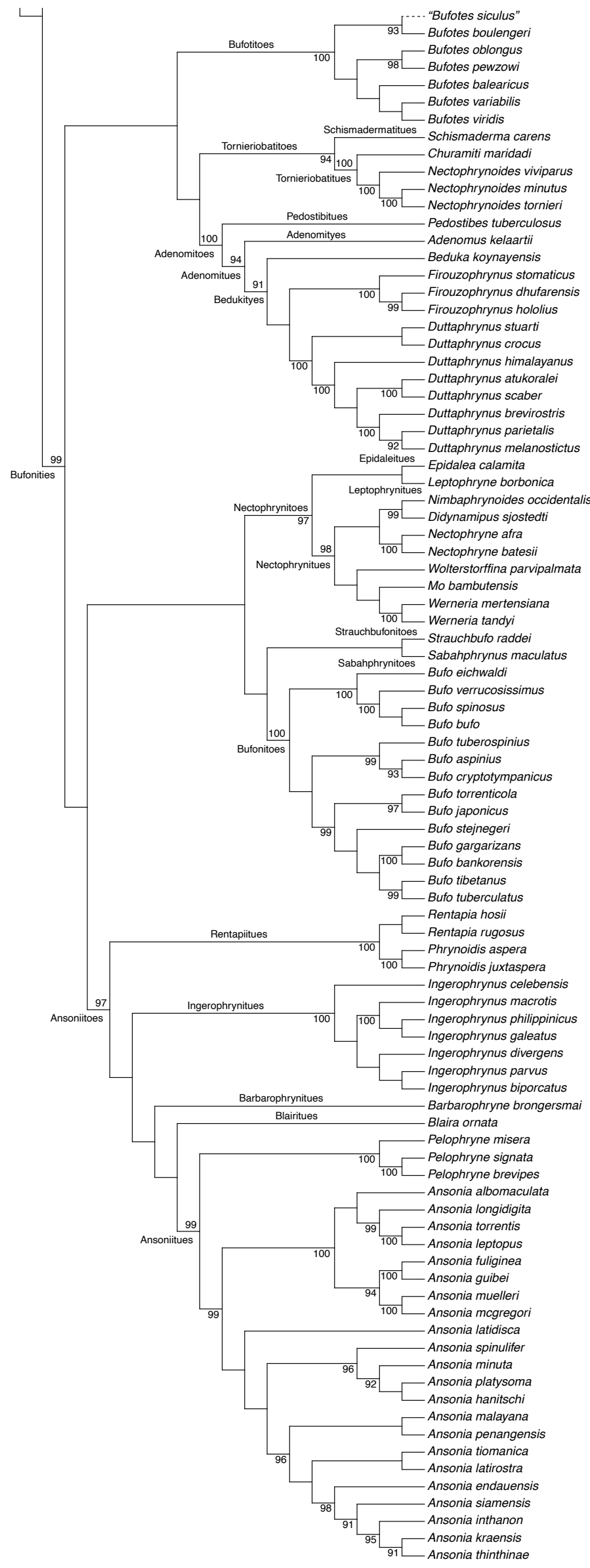


APPEndix A3.TREE-2. Simplified phylogenetic tree of Lissamphibia, showing all genera and higher supraspecific taxa recognised here as valid.

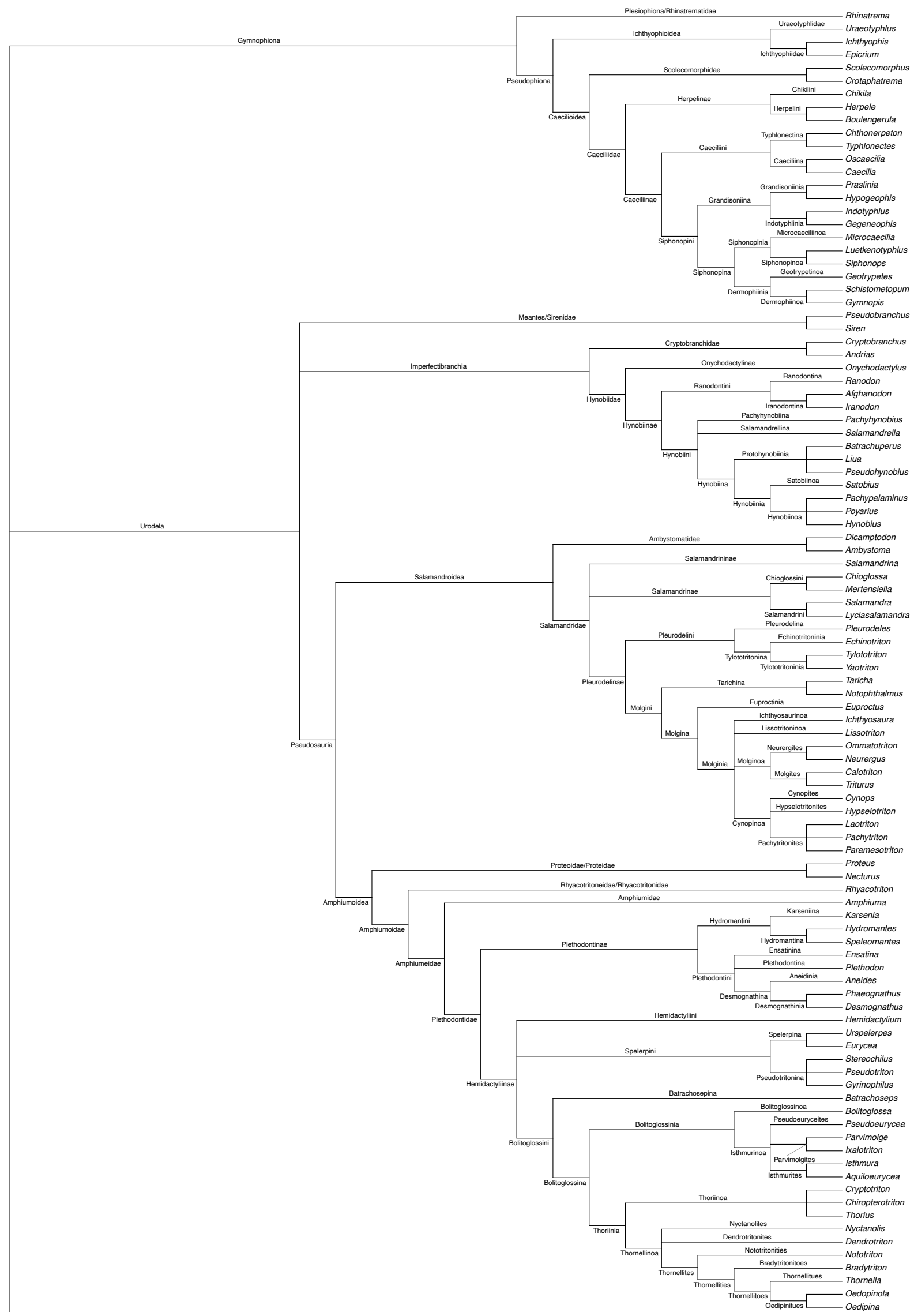




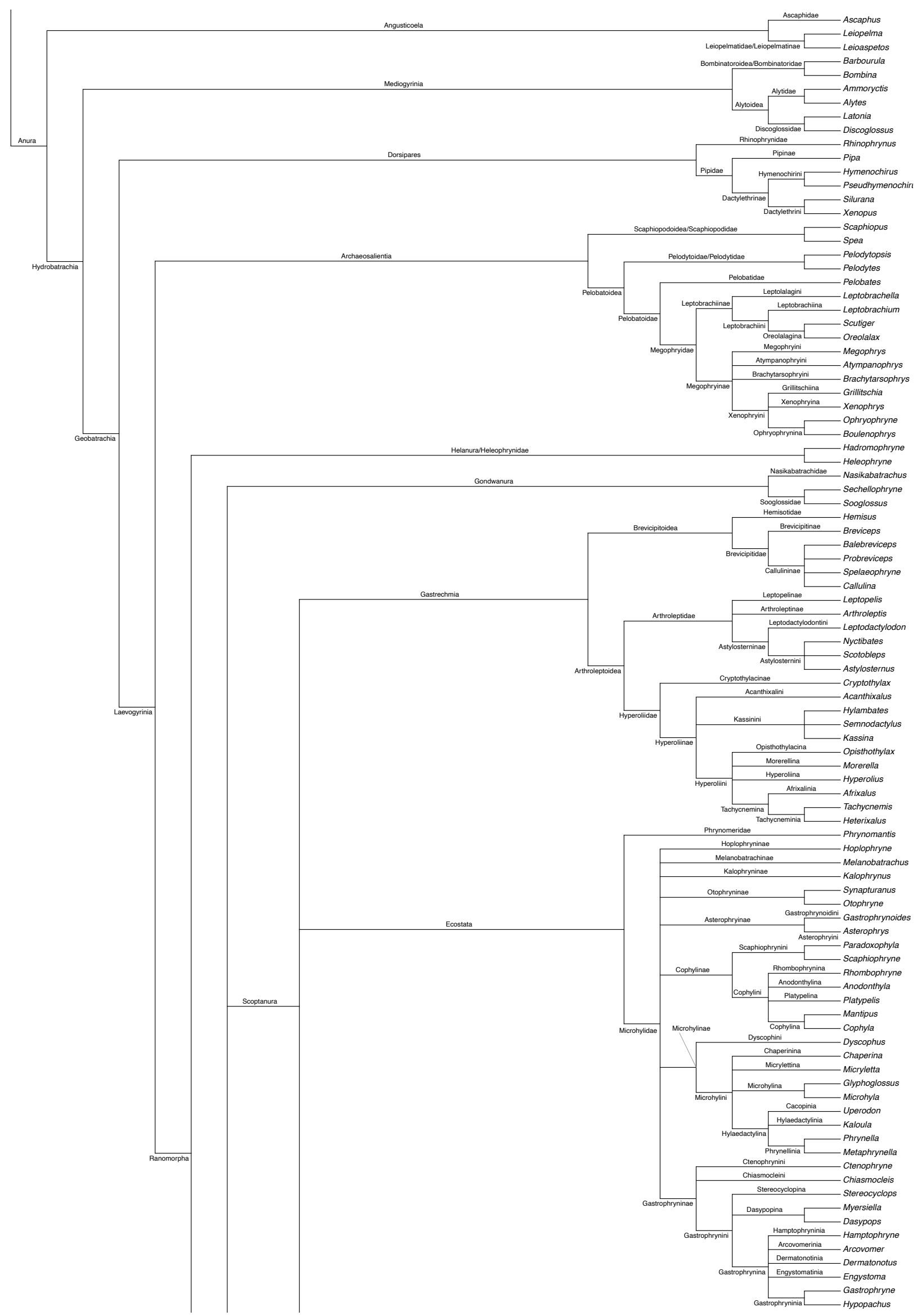




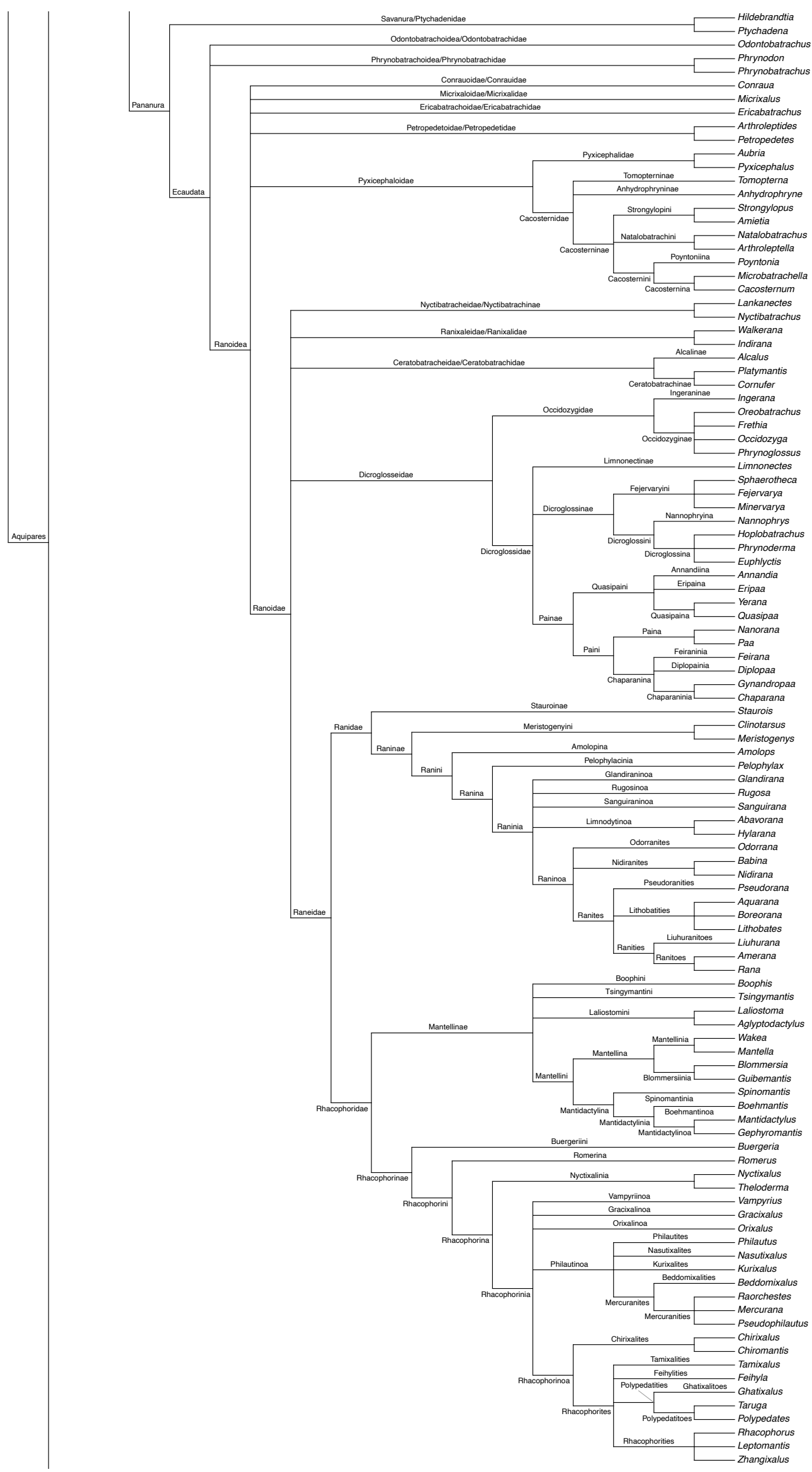




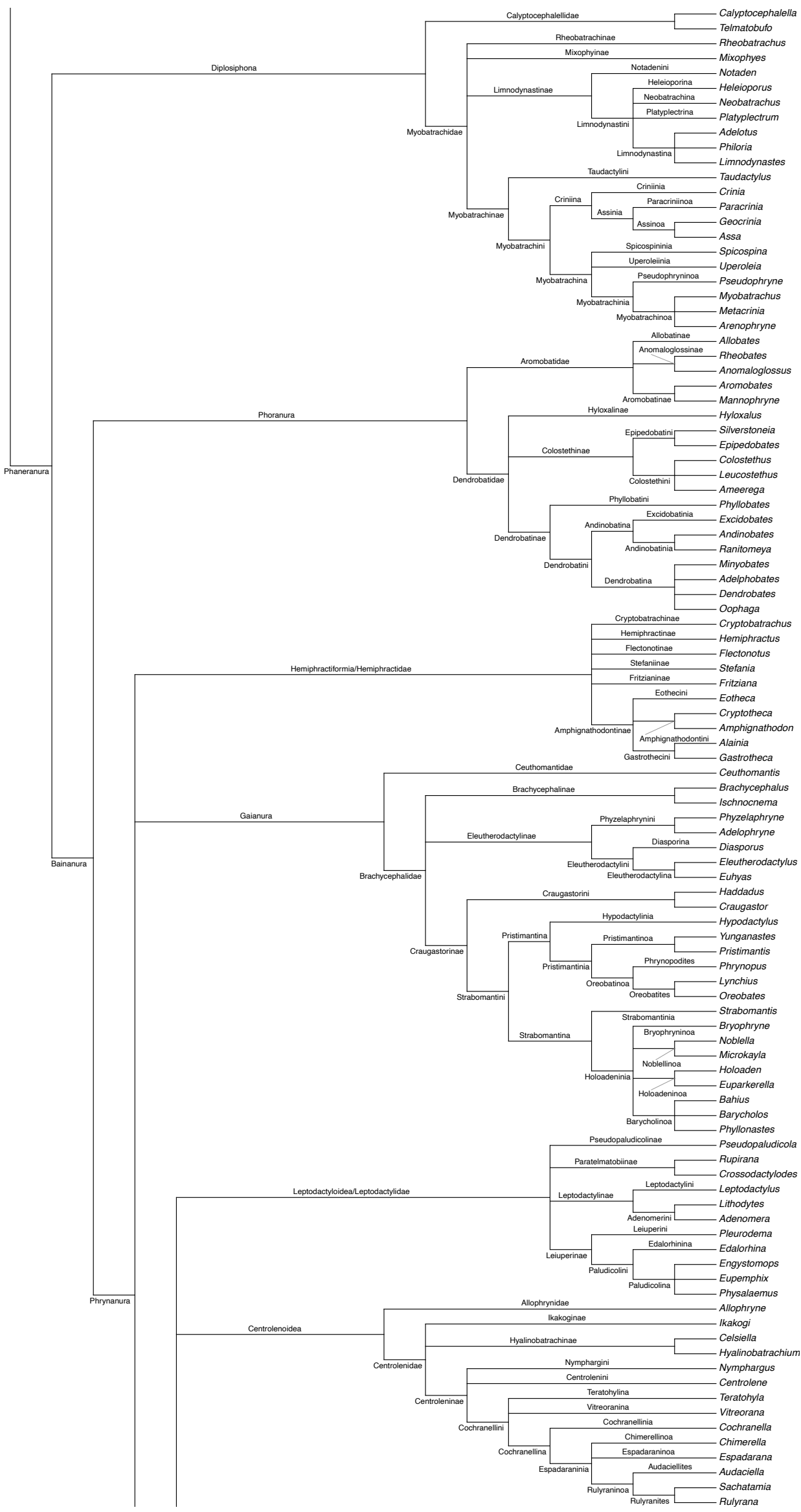




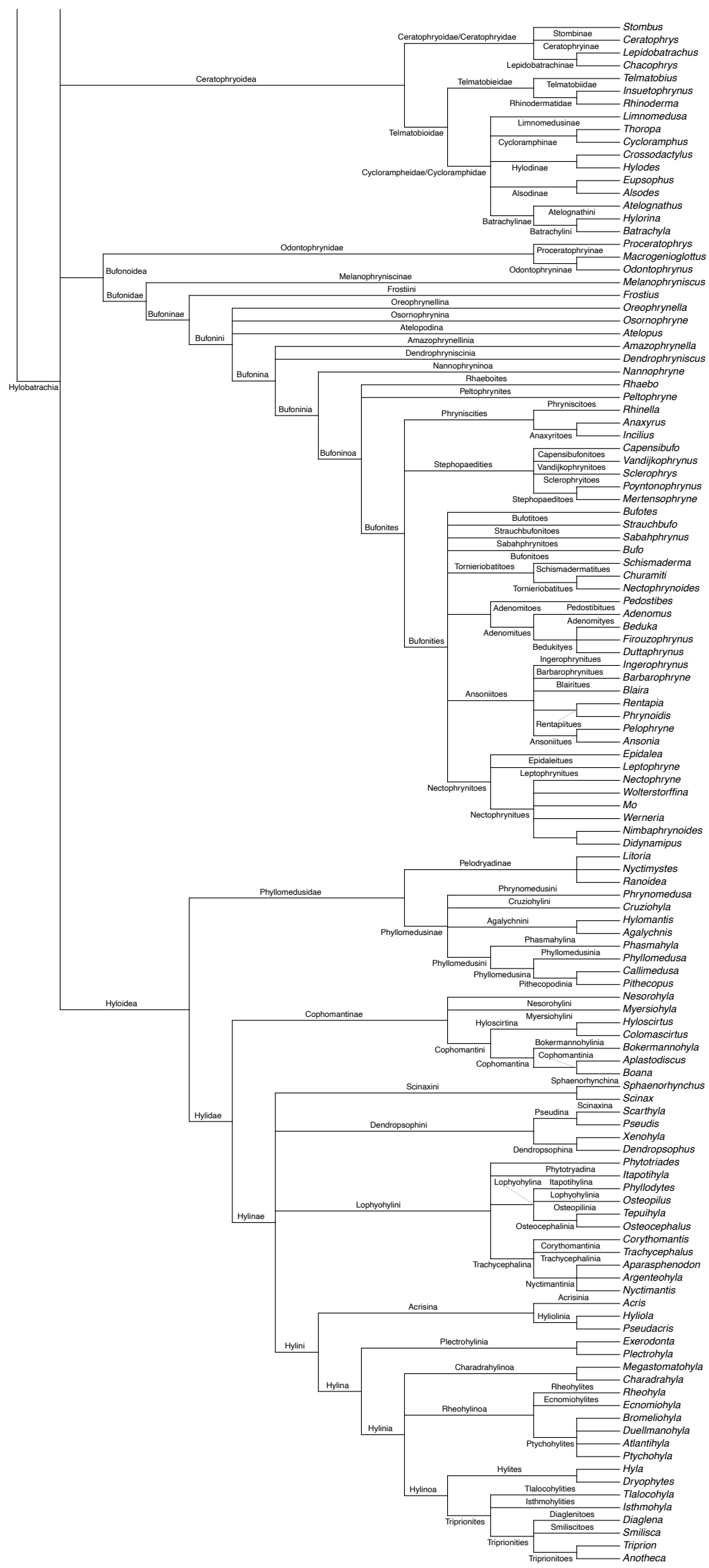


APPENDix A4.RNK. Abbreviations for ranks of taxa cited in Appendices A6.NFS, A7.NCS and A8.ECT

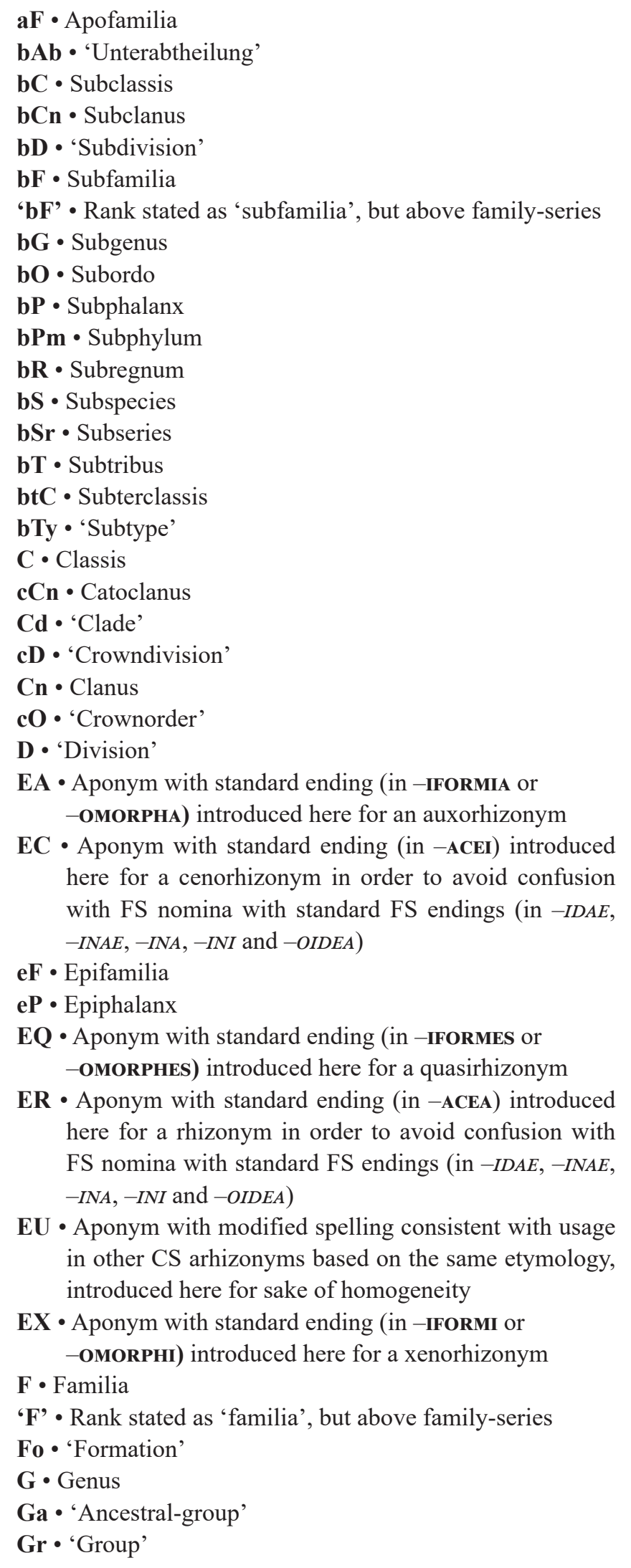

Gs • 'Gens'

hCn $\cdot$ Hypoclanus

He 'Heerde'

hO $\cdot$ Hypoordo

hP • Hypophalanx

hT $\cdot$ Hypotribus

iC ・ Infraclassis

iCn • Infraclanus

iO - Infraordo

iP - Infraphalanx

iT $\bullet$ Infratribus

Kl - 'Klan'

Kr • 'Kreis'

L • Legio

Li • 'Linie'

O - Ordo

P • Phalanx

pC - Superclassis

pF • Superfamilia

'pF' - Rank stated as 'superfamilia', but above familyseries

Pm • Phylum

'Pm' - Pseudo-rank stated as 'phylum', but this term is used at various hierarchical levels in the ergotaxonomy at stake, which is therefore pseudo-ranked, not really ranked

pO - Superordo

pP $\cdot$ Superphalanx

pvO • Parvordo

$\mathbf{R} \cdot$ Regnum

$\mathbf{R h} \cdot$ 'Reihe'

RNS $\cdot$ Rank not stated

$\mathbf{S} \cdot$ Species

Sc $\cdot$ Sectio

$\mathrm{Sr} \cdot$ Series

St $\cdot$ Stirps

T• Tribus

'T' $\mathbf{T}$ Rank stated as 'tribus', but above family-series

tAb • 'Hauptabtheilung'

tRh • 'Hauptreihe'

Tx • 'Taxon'

Ty • 'Type'

UC - Unspecified (or discussed) rank in class-series

UF • Unspecified (or discussed) rank in family-series

UU - Unspecified (or discussed) rank in unspecified (or discussed) nominal-series

$\mathbf{X} \cdot$ 'Taxon'

$\mathbf{Z g} \cdot$ 'Zug'

$\mathbf{Z t} \cdot$ 'Zunft' 


\section{APPENDIX A5.NGS. Genus-series nomina and taxa of LiSSAMPHIBIA.}

\section{Structure of accounts}

First line $\bullet$ Genus nomen or parograph.

Second line - ST (Taxonomic and nomenclatural status of nomen); and ID (Identifier of kyronym of genus-series taxon in

Appendix A9.CLAD-1) (only for lissamphibian genus-series nomina).

Third line $\cdot$ PN (Protonym of nucleospecies).

Fourth line $\bullet$ PK (Protonym of kyronym of nucleospecies).

Fifth line $\bullet$ KG (Kyronym of genus).

Sixth line • PF (Kyronym of family).

Genus-series nomen or apograph • Genus-series nomen (given as its protograph) or apograph mentioned in one of the Tables of this work, followed by its shortened authorship (auctorship or scriptorship) and date (year), by information whether its nucleospecies is based on extant or fossil (\$) onymophoront(s), and by an abbreviation giving the main characteristic of its taxonominal status.

Whenever the authorship consists in more than one author or scriptor, only the name of the first of them is given, followed by the number of other authors or scriptors, as follows: Duméril ${ }^{+1}$, Frost $^{+18}$. The complete authorship is given in our list of references of publications only if the work at stake is also cited in the text.

In this Table, we tried to include all hoplonyms (including neonyms and lectoprotographs) and anoplonyms (mostly gymnonyms) of lissamphibian GS nomina published after 1757, as well as non-lissamphibian senior homonyms of lissamphibian GS nomina. However, not all GS apographs (mostly ameletographs) appear in this Table (they play no role regarding zoological nomenclature, as an apograph is just a subsequent avatar of a nomen and does not have its own availability), but some are mentioned, when they have been cited in several publications and/or when they appear in another Table of this work (e.g., as a primogenus of a class-series nomen), and, if so, followed by their scriptorship and first known date of use.

In all cases where a lissamphibian GS nomen must be rejected as invalid for being a junior homonym, only the earliest senior homonym is given in this table, as its existence is sufficient to preoccupy the spelling of the generic nomen at stake over the whole zoology. No further information on these senior non-lissamphibian homonyms (such as their current allocation or validity) is provided here and these nomina do not appear in Appendices A9.CLAD-1 and A10.CLAD-2.

In the titles of accounts, lissamphibian GS nomina considered valid in this work are in bold italics and those considered invalid, unavailable or unallocated, as well as non-lissamphibian GS nomina, are in simple italics. Auctorship is indicated by the presence of a comma between the name of (first) auctor and date, and scriptorship by presence of a colon between the nomen and the name of its scriptor(s), which is not followed by a comma.

Meanings of abbreviations used for the main categories of taxonominal status of nomina and apographs in titles of accounts

AK - Lissamphibian akyronym: available lissamphibian GS nomen (hoplonym) considered invalid in the present work $(n=$ 871). Ex a mp le: Abrana Parker, 1931

AN $\bullet$ Lissamphibian anoplonym: unavailable lissamphibian GS nomen $(n=171) \cdot \bullet$ Exa mple: Adenomera: Fitzinger 1861.

EX - Lissamphibian exoplonym: lissamphibian GS nomen made unavailable by the Commission under its Plenary Power $(n=$ 14). Ex a m ple: Acrodytes Fitzinger, 1843.

KY - Lissamphibian kyronym: available lissamphibian GS nomen (hoplonym) considered valid in the present work $(n=771)$. - Exa mple: Acanthixalus Laurent, 1944.

ZA - Non-lissamphibian anoplonym: unavailable non-lissamphibian GS nomen being senior homograph of a lissamphibian available nomen (hoplonym) ( $n=11)$. Exa mple: Assa: Gray 1951.

ZH - Non-lissamphibian hoplonym: available non-lissamphibian GS nomen being homonym of a lissamphibian available nomen (hoplonym) $(n=102)$. E x a m ple: Abrana Strand, 1928.

ST - Detailed taxonominal status of genus-series nomen or apograph regarding: nomenclatural availability and taxonominal validity in the present work.

Meanings of abbreviations used for ST categories defined below

AL - Anoplonym: lissamphibian GS nomen unavailable for failing to comply with the Criteria of availability of publications or of nomina of the Code $(n=113)$. Ex a mple: Adenomera: Fitzinger 1861.

AM - Ameletograph (incorrect subsequent spelling): spelling of a lissamphibian GS nomen resulting from inadvertent change of original protograph, devoid of independent nomenclatural availability (anoplonym) $(n=41)$. Ex a mple: Aubrya: Schiøtz 1964.

CA - Archakyronym: lissamphibian GS nomen considered invalid in CLAD as a result of its rejection through the Plenary Power of the Commission $(n=9)$. - Ex a m ple: Autodax Boulenger, 1887.

CE - Archexoplonym: lissamphibian GS nomen made unavailable by the Commission under the Plenary Power, through removal of availability of nomen itself $(n=10)$. Exa mple: Acrodytes Fitzinger, 1843. 
CW Archanecdidonym: lissamphibian GS nomen considered invalid in CLAD as a result of the rejection through the Plenary Power of the Commission of the work where it had been published. $(n=4) \cdot \bullet$ Exa mple: Buffo La Cepède, 1788.

JD - Junior doxisonym: lissamphibian GS nomen considered invalid (nomakyronym) in CLAD as a result of our taxonomic analysis and for being considered a junior doxisonym (subjective synonym) of an available nomen considered as valid ( $n$ =604). Exa m ple: Abroscaphus Laurent, 1944.

JH - Junior homonym: invalid lissamphibian GS nomen (nomakyronym) for being a junior homonym of an available nomen, whether considered valid or not $(n=113)$. Ex a mple: Abrana Parker, 1931.

$\mathbf{J I} \cdot$ Junior isonym: lissamphibian GS nomen (nomakyronym) considered invalid in CLAD as a result of our taxonomic analysis and for being a junior isonym (objective synonym) of an available nomen considered as valid $(n=142)$. E x a mple: Alethotriton Fatio, 1872.

KC • Archokyronym: lissamphibian GS nomen considered valid in CLAD as a result of our taxonomic analysis and of its validation through the Plenary Power of the Commission $(n=2) \cdot \bullet$ Ex a mple: Epicrium Wagler, 1828.

KN - Nomokyronym: lissamphibian GS nomen considered valid in CLAD as a result of our taxonomic analysis and of the regular Rules of the Code concerning precedence between zygonyms (if relevant) ( $n=767) \cdot \bullet$ Exa mple: Acanthixalus Laurent, 1944.

LC $\cdot$ Lectoprotograph (correct original spelling): correct spelling of an available lissamphibian GS nomen, resulting from an airesy (first reviser action) among symprotographs (multiple original spellings). ( $n=16)$. Example: Aneides Baird, 1851.

LI - Leipoprotograph (incorrect original spelling): incorrect spelling of an available lissamphibian GS nomen, resulting from an airesy (first reviser action) among symprotographs (multiple original spellings), devoid of independent nomenclatural availability $(n=17) \cdot \bullet \mathbf{E x}$ a mp le: Anaides: Baird 1851 .

NC • Archoneonym: lissamphibian GS nomen given the status of available nomen novum by the Commission under the Plenary Power $(n=1)$. E x a mp le: Liopelma Günther, 1869.

$\mathbf{N L} \cdot$ Alloneonym (nomen novum, new replacement nomen): available lissamphibian GS neonym having a partially or totally different etymology from its archaeonym, i.e., not directly derived from it through unjustified emendation $(n=41)$. Exa mple: Adelotus Ogilby, 1907.

NS - Sigoneonym (nomen deemed to be a neonym): new meletograph of an available lissamphibian GS nomen considered here as available although it does not meet the restrictive criteria of Article 33.2.1 (see NS1-NS5 in column 3 of Table T8.NS2) $(n=48)$. Ex a mple: Anodontohyla Gadow, 1901.

NT - Autoneonym: available lissamphibian GS neonym having the same etymology as its archaeonym, i.e., directly derived from it through unjustified emendation $(n=83)$. Exa mple: Amblystoma Agassiz, 1844.

PO Poieonym: brand new available lissamphibian GS nomen, not proposed to replace an existing one, complying with the Rules of availability of the Code for both publications and nomina (hoplonym) $(n=1464) \cdot-$ Ex a mples: Abrana Parker, 1931; Acanthixalus Laurent, 1944.

RO - Lethakyronym: lissamphibian GS nomen considered invalid in CLAD as a result of our taxonomic analysis and of its rejection as nomen oblitum under Reversal of Precedence as defined in Article $23.9(n=2) \cdot \bullet$ Ex a mple: Atylodes Gistel, 1868.

RP - Mnemokyronym: lissamphibian GS nomen considered valid in CLAD as a result of our taxonomic analysis and of its validation as nomen protectum under Reversal of Precedence as defined in Article $23.9(n=2)$. Exa mple: Hyla Laurenti, 1768.

ZF - Non-lissamphibian GS radicogenus of a FS nomen: available non-lissamphibian GS nomen the stem of which has provided the stem of a family-series nomen and which is homographic with the stem of an available FS lissamphibian nomen, making both FS nomina homonyms $(n=3)$. - Ex a m ple: Acrida Linnaeus, 1758.

ZN $•$ Non-lissamphibian GS anoplonym: non-lissamphibian GS nomen unavailable for failing to comply with the Criteria of availability of publications or of nomina of the Code, homograph of a lissamphibian GS nomen $(n=11) \cdot \operatorname{Example}$ : Assa: Gray 1851.

zo Non-lissamphibian GS hoplonym: available non-lissamphibian GS nomen being homonym of a lissamphibian available GS nomen $(n=99)$. Ex a m ple: Abrana Strand, 1928.

AK $\bullet$ Categories of akyronyms of lissamphibians $(n=871)$

LC.JD • Lectoprotograph, junior doxisonym $(n=3) \cdot \bullet \mathbf{E} \mathbf{x}$ a m ple: Hyladactylus Tschudi, 1838.

LC.JH • Lectoprotograph, junior homonym $(n=2) \cdot \bullet \mathbf{E x}$ a mp le: Hyperoodon Philippi, 1902.

LC.JI • Lectoprotograph, junior isonym $(n=1)$. E x a m ple : Batrachychthis Pizarro, 1876.

LC.RO - Lectoprotograph, lethakyronym $(n=1)$. Ex a m ple: Ranetta Garsault, 1764.

NC.JI • Archoneonym, junior isonym $(n=1)$. E x a m p le: Liopelma Günther, 1869.

NL.CA • Alloneonym, archakyronym $(n=1)$. Ex a mple: Autodax Boulenger, 1887.

NL.JD • Alloneonym, junior doxisonym $(n=6)$. E x a mple: Bradybates Gistel, 1848. 
NL.JH • Alloneonym, junior homonym $(n=2)$. E xa mp le: Cordylus Wagler, 1828.

NL.JI • Alloneonym, junior isonym $(n=29)$. Ex a m ple: Apneumona Fleming, 1822.

NS.JD • Sigoneonym, junior doxisonym $(n=19)$. E x a m ple: Axoloth Gray, 1842.

NS.JH • Sigoneonym, junior homonym $(n=1)$. Ex a m ple: Trachycephalus Ferguson, 1875.

NS.JI • Sigoneonym, junior isonym $(n=27) \cdot \bullet \mathbf{E x}$ a m ple: Anodontohyla Gadow, 1901.

NT.JD • Autoneonym, junior doxisonym $(n=21)$. Exa mple: Amfignathodon Palacký, 1898.

NT.JH • Autoneonym, junior homonym $(n=3)$. $-\mathbf{E} \mathbf{x}$ a m p le: Hyperodon Agassiz, 1847.

NT.JI • Autoneonym, junior isonym $(n=55)$. E x a m p le : Amblystoma Agassiz, 1844.

PO.CA • Poieonym, archakyronym $(n=8) \cdot \bullet \mathbf{E x}$ a m p le: Axolot Bonaparte, 1831.

PO.JD • Poieonym, junior doxisonym $(n=555) \cdot \bullet$ Ex a m ple: Abroscaphus Laurent, 1941.

PO.JH • Poieonym, junior homonym $(n=106)$. E x a m ple: Abrana Parker, 1931.

PO.JI • Poieonym, junior isonym $(n=29)$. Ex a m ple: Abroscaphus Laurent, 1941.

PO.Ro • Poieonym, lethakyronym $(n=1)$. Ex a m ple: Atylodes Gistel, 1868.

AN $\bullet$ Categories of anoplonyms of lissamphibians $(n=171)$

AL • Anoplonym $(n=113)$. Ex a m ple: Adenomera: Fitzinger 1861.

AM $\cdot$ Ameletonym $(n=41)$. Ex a mple: Aubrya: Schiøtz 1964.

LI $\bullet$ Leipoprotograph $(n=17) \cdot \bullet \mathbf{E} \mathbf{x}$ a mple: Anaides: Baird 1851.

EX Categories of exoplonyms of lissamphibians $(n=14)$

NS.CE • Sigoneonym, archexoplonym $(n=1)$. Exa mple: Phyllhydrus Gray, 1831.

NT.CE • Autoneonym, archexoplonym $(n=1)$. Ex a m ple: Mycetoglossus Bonaparte, 1839.

NT.CW • Autoneonym, archanecdidonym $(n=1)$. $-\mathbf{E} \mathbf{x}$ a m p le: Buffo La Cepède, 1788.

PO.CE • Poieonym, archexoplonym $(n=8)$. Ex a m ple: Acrodytes Fitzinger, 1843.

PO.Cw • Poieonym, archanecdidonym $(n=3)$. $\bullet$ Ex a mple: Calamita Oken, 1816.

$\mathrm{KY} \cdot$ Categories of kyronyms of lissamphibians $(\boldsymbol{n}=\mathbf{7 7 1})$

LC.KN • Lectoprotograph, nomokyronym $(n=9)$. Exa m p le : Aneides Baird, 1851.

NL.KN • Alloneonym, nomokyronym $(n=3) \cdot \bullet \mathbf{E x a m p l e : ~ A d e l o t u s ~ O g i l b y , ~} 1907$.

NT.KN • Autoneonym, nomokyronym $(n=2)$. E x a mple: Estesiella Báez, 1995.

PO.KC Poieonym, archokyronym $(n=2) \cdot \bullet$ Exa mple: Epicrium Wagler, 1828.

PO.KN • Poieonym, nomokyronym $(n=753)$. E xa mple: Acanthixalus Laurent, 1944.

PO.RP • Poieonym, mnemokyronym $(n=2)$. Ex a m ple: Hyla Laurenti, 1768.

$\mathrm{ZA} \cdot$ Categories of non-lissamphibian anoplonyms $(\boldsymbol{n}=11)$

$\mathbf{Z N} \cdot$ Anoplonym $(n=11) \cdot \mathbf{E x}$ a mple: Assa: Gray 1851.

$\mathbf{Z H} \cdot$ Categories of non-lissamphibian hoplonyms $(\boldsymbol{n}=\mathbf{1 0 2})$

$\mathbf{Z F} \cdot$ Radicogenus $(n=3)$. Exa mp le: Acrida Linnaeus, 1758.

zo $\cdot$ Hoplonym $(n=99)$. $\cdot$ Ex a m ple: Abrana Strand, 1928.

CI • Category identifier of genus-series nomen $(n=1937)$.

e0001, e0002, etc. $・$ Numbers of genus-series exoplonyms designating lissamphibian taxa $(n=14)$.

h0001, h0002, etc. $\cdot$ Numbers of genus-series hoplonyms designating lissamphibian taxa $(n=1642)$.

n0001, n0002, etc. $・$ Numbers of genus-series anoplonyms designating lissamphibian taxa $(n=171)$.

zh001, zh002, etc. $\cdot$ Numbers of genus-series hoplonyms designating taxa non including lissamphibians $(n=102)$.

zn001, zn002, etc. $•$ Numbers of genus-series anoplonyms designating taxa non including lissamphibians $(n=11)$.

ID - Identifier of kyronym of genus-series taxon shown in KG (documented only for lissamphibian nomina) ( $n=779: 579$ extant, 200 all-fossil). This number appears preceded by G.28 in A.CLAD-1. It is preceded by $\uparrow$ for all-fossil genera, and followed by $\S$ for genera referred to only by anoplonyms or anecdidonyms but for which no hoplonyms were ever proposed ( $n=13: 4$ extant, 9 all-fossil).

PN - Protonym of nucleospecies - Protonym (original combination and spelling) of the nominal nucleospecies (typespecies) of nomen in PK.

AU $\cdot$ SS or GS anoplonym (unavailable nomen) of lissamphibian taxon for failing to comply with the Criteria of availability of publications or of nomina of the Code.

PK • Protonym of kyronym of nucleospecies - Original combination and spelling of the valid nomen in Appendix A9.CLAD-1 of the species-series taxon designated by the nomen in PK.

* The nucleospecies (type species) of the genus is represented in Appendix A2.TREE-1: Rana temporaria*.

${ }^{\circ}$ The nucleospecies (type species) of the genus is not represented in Appendix A2.TREE-1: Elosia duidensis ${ }^{\circ}$.

KG $•$ Kyronym of genus • Valid and correct nomen in Appendix A9.CLAD-1 of the genus taxon designated by the nomen of column 4 , followed by its author and identifier.

* The genus is represented in Appendix A2.TREE-1 by its nucleospecies or an isonym of the latter: Rana*. 
${ }^{1}$ The genus is represented in Appendix A2.TREE-1 by a doxisonym of its nucleospecies: Pipa ${ }^{1}$.

${ }^{2}$ The genus is represented in Appendix A2.TREE-1 by the nucleospecies of a generic nomen being its doxisonym: Latonia ${ }^{2}$.

${ }^{3}$ The genus is represented in Appendix A2.TREE-1 but only by species that include neither its nucleospecies, nor a doxisonym of the latter, nor the nucleospecies of a doxisonym of the generic nomen at stake: Uraeotyphlus ${ }^{3}$.

${ }^{\circ}$ The genus is not represented in Appendix A2.TREE-1: Dischidodactylus ${ }^{\circ}$.

KF - Kyronym of family $•$ Valid and correct nomen of family to which the kyronyms of KG are referred in Appendix A9.CLAD-1, followed by its identifier (see Appendix A6.NFS for its authorship).

\section{Other abbreviations and symbols}

$\$ \cdot$ Nomen based on a nucleospecies the onymophoront/s (type-specimen/s) of which is/are fossils.

$\# \cdot$ Nomen based on a nucleospecies the onymophoront/s (type-specimen/s) of which is/are fossil footprints.

$\dagger \cdot$ Nomen designating an all-fossil taxon.

- Nomen designating a taxon containing at least one non-recent lissamphibian species/taxon: detailed information on this nomen was not sought, not being necessary for the present work.

AG $\bullet$ Unavailable genus-series nomen having no available counterpart.

AS $\cdot$ Unavailable species-series nomen

INR - Information not relevant here.

\section{Note}

The following two words appear sometimes in lists of amphibian genera, but they are not nomina of taxa.

'Hybridus' as used by Peracca $(1886: 9,12)$, although presented in combination with a specific epithet, does not designate a genus or a taxon, but a taxonomic category like 'species', 'genus' or 'klepton'.

'Tartalina' as used by Duméril et al. (1854: 70) is not a nomen but an emendation of the vernacular name 'Tarantolina' mentioned by Savi (1823: 107). 
Abavorana Oliver $^{+3}, 2015 \cdot \mathbf{K Y}$

ST: PO.KN • CI: h0001 • ID: 408

PN: Limnodytes luctuosus Peters, 1871

PK: Limnodytes luctuosus* Peters, 1871

KG: Abavorana* Oliver $^{+3}, 2015$

KF: RANIDAE 1796.ba.f001

Abrana Strand, $1928 \cdot \mathbf{Z H}$

ST: zo • CI: zh001

Abrana Parker, $1931 \cdot$ AK

ST: PO.JH • CI: h0002 • ID: 464

PN: Abrana cotti Parker, 1931

PK: Rana schillukorum ${ }^{\circ}$ Werner, 1908

KG: Ptychadena* Boulenger, 1917

KF: PTYCHADENIDAE 1987.da.f002

Abranchus Boie, $1824 \cdot \mathbf{Z H}$

ST: zo • CI: zh002

Abranchus Harlan, 1825 • AK

ST: PO.JH • CI: h0003 • ID: 504

PN: Salamandra alleganiensis Sonnini ${ }^{+1}, 1801$

PK: Salamandra alleganiensis* Sonnini ${ }^{+1}, 1801$

KG: Cryptobranchus ${ }^{1}$ Leuckart, 1821

KF: CRYPTOBRANCHIDAE 1826.fb.f003

Abroscaphus Laurent, 1941 • AK

ST: PO.JD • CI: h0004 • ID: 320

PN: Arthroleptis adolfifriederici Nieden, 1911

PK: Arthroleptis adolfifriederici* Nieden, 1911

KG: Arthroleptis* Smith, 1849

KF: ARTHROLEPTIDAE 1869.mc.f011

Acanthixalus Laurent, $1944 \cdot \mathbf{K Y}$

ST: PO.KN • CI: h0005 • ID: 330

PN: Hyperolius spinosus Buchholz ${ }^{+1}$ in Peters, 1875

PK: Hyperolius spinosus* Buchholz ${ }^{+1}$ in Peters, 1875

KG: Acanthixalus* Laurent, 1944

KF: HYPEROLIIDAE 1943.lb.f001

Acrida Linnaeus, $1758 \cdot \mathbf{Z H}$

ST: $\mathbf{Z F} \bullet \mathbf{C I}$ : zh003

Acrides Macleay, $1821 \cdot \mathbf{Z H}$

ST: ZF • CI: zh004

Acris Duméril ${ }^{+1}, 1841 \cdot \mathbf{K Y}$

ST: PO.KN • CI: h0006・ ID: 198

PN: Rana gryllus Le Conte, 1825

PK: Rana gryllus* Le Conte, 1825

KG: Acris $^{*}$ Duméril $^{+1}, 1841$

KF: HYLIDAE 1815.ra.f002-|1825.gb.f001|

Acrodytes Fitzinger, $1843 \cdot \mathbf{E X}$

ST: PO.CE・CI: e0001・ID: 231

PN: Rana venulosa Laurenti, 1768

PK: Rana typhonia* Linnaeus, 1758

KG: Trachycephalus* Tschudi, 1838

KF: HYLIDAE 1815.ra.f002-|1825.gb.f001|

Adelastes Zweifel, 1986 • KY

ST: PO.KN • CI: h0008・ ID: 279

PN: Adelastes hylonomos Zweifel, 1986

PK: Adelastes hylonomos ${ }^{\circ}$ Zweifel, 1986

KG: Adelastes $^{\circ} \mathrm{Zweifel}, 1986$

KF: MICROHYLIDAE ||1843.fa.f012||-1931.na.f001
Adelophryne Hoogmoed ${ }^{+1}, 1984 \cdot \mathbf{K Y}$

ST: PO.KN • CI: h0009 • ID: 083

PN: Adelophryne adiastola Hoogmoed ${ }^{+1}, 1984$

PK: Adelophryne adiastola* Hoogmoed ${ }^{+1}, 1984$

KG: Adelophryne* Hoogmoed ${ }^{+1}, 1984$

KF: BRACHYCEPHALIDAE 1858.gc.f002

Adelotus Ogilby, 1907 • KY

ST: NL.KN • CI: h0010 • ID: 260

PN: Cryptotis brevis Günther, 1863

PK: Cryptotis brevis* Günther, 1863

KG: Adelotus* Ogilby, 1907

KF: MYOBATRACHIDAE 1850.sa.f001

Adelphesiren Goin $^{+1}, 1958+\cdot \mathbf{A K}$

ST: PO.JD • CI: h0011 • ID: $\uparrow 176$

PN: Adelphesiren olivae $\mathrm{Goin}^{+1}, 1958$ *

PK: Habrosaurus dilatus ${ }^{\circ}$ Gilmore, $1928 \uparrow$

KG: Habrosaurus ${ }^{\circ}$ Gilmore, $1928 \dagger$

KF: SIRENIDAE $1825 \mathrm{gb} . \mathrm{f} 005$

Adelphobates Grant ${ }^{+9}, 2006 \cdot \mathbf{K Y}$

ST: PO.KN • CI: h0012 • ID: 047

PN: Dendrobates castaneoticus Caldwel1 ${ }^{+1}, 1990$

PK: Dendrobates castaneoticus* Calwell ${ }^{+1}, 1990$

KG: Adelphobates* Grant ${ }^{+9}, 2006$

KF: DENDROBATIDAE ||1850.bb.f006||-1865.ca.f002

Adenomera: Fitzinger $1861 \cdot \mathbf{A N}$

ST: AL・CI: n0001・ID: 251

PN: Adenomera marmorata Steindachner, 1867

PK: Adenomera marmorata ${ }^{\circ}$ Steindachner, 1867

KG: Adenomera ${ }^{3}$ Steindachner, 1867

KF: LEPTODACTYLIDAE |1838.ta.f001\|-1896.wa.f001

Adenomera Steindachner, $1867 \bullet \mathbf{K Y}$

ST: PO.KN • CI: h0013・ID: 251

PN: Adenomera marmorata Steindachner, 1867

PK: Adenomera marmorata ${ }^{\circ}$ Steindachner, 1867

KG: Adenomera ${ }^{3}$ Steindachner, 1867

KF: LEPTODACTYLIDAE ||1838.ta.f001||-1896.wa.f001

Adenomus Cope, $1861 \cdot \mathbf{K Y}$

ST: PO.KN • CI: h0014 • ID: 104

PN: Adenomus badioflavus Cope, 1861

PK: Bufo kelaartii* Günther, 1858

KG: Adenomus $^{1}$ Cope, 1861

KF: BUFONIDAE 1825.gb.f004

Aelurolalax Dubois, 1987 • AK

ST: PO.JD • CI: h0015 • ID: 016

PN: Megalophrys weigoldi Vogt, 1924

PK: Megalophrys weigoldi ${ }^{\circ}$ Vogt, 1924

KG: Oreolalax* Myers $^{+1}, 1962$

KF: MEGOPHRYIDAE 1850.bb.f008-|1931.na.f003|

Aelurophryne Boulenger, 1919 • AK

ST: PO.JD・CI: h0016・ID: 017

PN: Bufo mammatus Günther, 1896

PK: Bufo mammatus* Günther, 1896

KG: Scutiger ${ }^{2}$ Theobald, 1868

KF: MEGOPHRYIDAE 1850.bb.f008-|1931.na.f003|

Aenigmanura Brown $^{+4}, 2015$ • AK

ST: PO.JD・CI: h0017 • ID: 369 
PN: Platymantis papuensis schmidti Brown $^{+1}, 1968$

PK: Platymantis papuensis schmidti ${ }^{\circ}$ Brown $^{+1}, 1968$

KG: Cornufer* Tschudi, 1838

KF: CERATOBATRACHIDAE 1884.ba.f001

Aerugoamnis Henrici ${ }^{+2}, 2013+\cdot \mathbf{K Y}$

ST: PO.KN • CI: h0018 •ID: $\uparrow 091$

PN: Aerugoamnis paulus Henrici ${ }^{+2}, 2013$ \$

PK: Aerugoamnis paulus ${ }^{\circ}$ Henrici $^{+2}, 2013 \dagger$

KG: Aerugoamnis ${ }^{\circ}$ Henrici $^{+2}, 2013 \dagger$

KF: PELODYTIDAE 1850.bb.f002

Afghanodon Dubois ${ }^{+1}, 2012 \cdot \mathbf{K Y}$

ST: PO.KN・CI: h0019・ID: 514

PN: Batrachuperus mustersi Smith, 1940

PK: Batrachuperus mustersi* Smith, 1940

KG: Afghanodon* Dubois ${ }^{+1}, 2012$

KF: HYNOBIIDAE ||1856.ha.f001||-1859.cb.f002

Afrana Dubois, 1992 • AK

ST: PO.JH • CI: h0020 • ID: 362

PN: Rana fuscigula Duméril ${ }^{+1}, 1841$

PK: Rana fuscigula* Duméril ${ }^{+1}, 1841$

KG: Amietia* Dubois, 1987

KF: CACOSTERNIDAE 1931.na.f008

Afrixalus Laurent, $1944 \cdot \mathbf{K Y}$

ST: PO.KN • CI: h0021 • ID: 334

PN: Euchnemis fornasinii Bianconi, 1849

PK: Euchnemis fornasinii* Bianconi, 1849

KG: Afrixalus* Laurent, 1944

KF: HYPEROLIIDAE 1943.lb.f001

Afrocaecilia Taylor, 1968 • AK

ST: PO.JD • CI: h0022 • ID: 496

PN: Boulengerula taitanus Loveridge, 1935

PK: Boulengerula taitanus* Loveridge, 1935

KG: Boulengerula* Tornier, 1896

KF: CAECILIIDAE 1814.ra.f003-|1825.gb.f008|

Agalychnis Cope, $1864 \cdot \mathbf{K Y}$

ST: PO.KN • CI: h0023・ID: 238

PN: Hyla callidryas Cope, 1862

PK: Hyla callidryas* Cope, 1862

KG: Agalychnis* Cope, 1864

KF: PHYLLOMEDUSIDAE 1858.gc.f009

Aglyptodactylus Boulenger, 1919• KY

ST: PO.KN • CI: h0024 • ID: 424

PN: Limnodytes madagascariensis Duméril, 1853

PK: Limnodytes madagascariensis* Duméril, 1853

KG: Aglyptodactylus* Boulenger, 1919

KF: RHACOPHORIDAE |1858.gc.f012||-1932.ha.f001

Alainia Duellman ${ }^{+1}, 2018 \cdot \mathbf{K Y}$

ST: PO.KN • CI: h0025 • ID: 090

PN: Nototrema microdiscus Andersson, 1910

PK: Nototrema microdiscus* Andersson, 1910

KG: Alainia* Duellman ${ }^{+1}, 2018$

KF: HEMIPHRACTIDAE 1862.pa.f001

Albanerpeton Estes $^{+1}, 1976+\bullet \mathbf{K Y}$

ST: PO.KN • CI: h0026 • ID: $\uparrow 002$

PN: Albanerpeton inexpectatum Estes $^{+1}, 1976$ \$

PK: Albanerpeton inexpectatum ${ }^{\circ}$ Estes $^{+1}, 1976 \dagger$
KG: Albanerpeton ${ }^{\circ}$ Estes $^{+1}, 1976 \dagger$

KF: ALBANERPETIDAE 1982.fa.f001 †

Albericus Burton ${ }^{+1}$, 1995 • AK

ST: PO.JD・CI: h0027 • ID: 280

PN: Cophixalus darlingtoni Loveridge, 1948

PK: Cophixalus darlingtoni ${ }^{\circ}$ Loveridge, 1948

KG: Asterophrys* Tschudi, 1838

KF: MICROHYLIDAE ||1843.fa.f012||-1931.na.f001

Albionbatrachus Meszoely ${ }^{+2}, 1984+\cdot \mathbf{K Y}$

ST: PO.KN • CI: h0028・ID: $\uparrow 068$

PN: Albionbatrachus wightensis Meszoely ${ }^{2}, 1984$ †

PK: Albionbatrachus wightensi ${ }^{\circ}$ Meszoely $^{+2}, 1984 \uparrow$

KG: Albionbatrachus ${ }^{\circ}$ Meszoely $^{+2}, 1984 \uparrow$

KF: PALAEOBATRACHIDAE 1865.ca.f001 †

Alcalus Brown ${ }^{+4}, 2015 \cdot \mathbf{K Y}$

ST: PO.KN • CI: h0029・ID: 368

PN: Micrixalus mariae Inger 1954

PK: Micrixalus mariae ${ }^{\circ}$ Inger 1954

KG: Alcalus $^{3}$ Brown $^{+4}, 2015$

KF: ALCALIDAE 2015.ba.f002

Alethotriton Fatio, 1872 • AK

ST: PO.JI • CI: h0030 • ID: 566

PN: Triton cristatus Laurenti, 1768

PK: Triton cristatus* Laurenti, 1768

KG: Triturus $*$ Rafinesque, 1815

KF: SALAMANDRIDAE 1820.ga.f002

Alexteroon Perret, 1988 - AK

ST: PO.JD • CI: h0031 • ID: 331

PN: Hyperolius obstetricans Ahl, 1931

PK: Hyperolius obstetricans* Ahl, 1931

KG: Hyperolius* Rapp, 1842

KF: HYPEROLIIDAE 1943.lb.f001

Algiandra Dubois $^{+1}, 2009 \cdot \mathbf{A K}$

ST: PO.JD • CI: h0032 • ID: 578

PN: Salamandra maculosa algira Bedriaga, 1883

PK: Salamandra maculosa algira* Bedriaga, 1883

KG: Salamandra ${ }^{1}$ Garsault, 1764

KF: SALAMANDRIDAE 1820.ga.f002

Allobates Zimmermann ${ }^{+1}$, 1988 • KY

ST: PO.KN • CI: h0033・ID: 034

PN: Prostherapis femoralis Boulenger, 1884

PK: Prostherapis femoralis* Boulenger, 1884

KG: Allobates $*$ Zimmermann $^{+1}, 1988$

KF: АROMOBATIDAE 2006.gc.f004

Allomesotriton Freytag, 1983 • AK

ST: PO.JD・CI: h0034・ID: 562

PN: Trituroides caudopunctatus $\mathrm{Liu}^{+1}$ in $\mathrm{Hu}^{+2}, 1973$

PK: Trituroides caudopunctatus* $\mathrm{Liu}^{+1}$ in $\mathrm{Hu}^{+2}, 1973$

KG: Paramesotriton* Chang, 1936

KF: SALAMANDRIDAE 1820.ga.f002

Allopaa Ohler $^{+1}, 2006 \bullet \mathbf{K Y}$

ST: PO.KN • CI: h0035 • ID: 381

PN: Rana (Paa) hazarensis Dubois ${ }^{+1}, 1979$

PK: Rana (Paa) hazarensis ${ }^{\circ}$ Dubois $^{+1}, 1979$

KG: Allopaa $^{\circ}$ Ohler $^{+1}, 2006$

KF: DiCROGLOSSIDAE 1987.da.f004 
Allophryne Gaige, $1926 \cdot \mathbf{K Y}$

ST: PO.KN • CI: h0036・ID: 155

PN: Allophryne ruthveni Gaige, 1926

PK: Allophryne ruthveni* Gaige, 1926

KG: Allophryne* Gaige, 1926

KF: ALLOPHRYNIDAE 1978.ga.f001

Alpandra Dubois $^{+1}, 2009$ • AK

ST: PO.JD • CI: h0037 • ID: 578

PN: Salamandra atra Laurenti, 1768

PK: Salamandra atra* Laurenti, 1768

KG: Salamandra ${ }^{1}$ Garsault, 1764

KF: SALAMANDRIDAE 1820.ga.f002

Alsodes Bell, $1843 \cdot \mathbf{K Y}$

ST: PO.KN • CI: h0038・ID: 173

PN: Alsodes monticola Bell, 1843

PK: Alsodes monticola* Bell, 1843

KG: Alsodes* Bell, 1843

KF: ALSODIDAE 1869.mc.f005

Altanulia Gubin, $1993+\bullet \mathbf{K Y}$

ST: PO.KN • CI: h0039 • ID: $\uparrow 008$

PN: Altanulia alifanovi Gubin, 1993 \$

PK: Altanulia alifanovi ${ }^{\circ}$ Gubin, $1993 \dagger$

KG: Altanulia $^{\circ}$ Gubin, $1993 \dagger$

KF: ANURA Familia INCERTAE SEDIS

Altigius Wild, 1995 • AK

ST: PO.JD • CI: h0040 • ID: 301

PN: Altigius alios Wild, 1995

PK: Altigius alios ${ }^{\circ}$ Wild, 1995

KG: Hamptophryne* Carvalho, 1954

KF: МiсRонYLIDAE ||1843.fa.f012||-1931.na.f001

Altiphrynoides Dubois, $1987 \bullet \mathbf{K Y}$

ST: PO.KN • CI: h0041 • ID: 102

PN: Nectophrynoides malcolmi Grandison, 1978

PK: Nectophrynoides malcolmi $i^{\circ}$ Grandison, 1978

KG: Altiphrynoides ${ }^{\circ}$ Dubois, 1987

KF: BUFONIDAE 1825.gb.f004

Altirana Stejneger, 1927 • AK

ST: PO.JD • CI: h0042 • ID: 387

PN: Altirana parkeri Stejneger, 1927

PK: Altirana parkeri* Stejneger, 1927

KG: Nanorana* Günther, 1896

KF: DICROGLOSSIDAE 1987.da.f004

Alytes Wagler, $1829 \bullet \mathbf{K Y}$

ST: PO.KN • CI: h0043 • ID: 467

PN: Bufo obstetricans Laurenti, 1768

PK: Bufo obstetricans* Laurenti, 1768

KG: Alytes* Wagler, 1829

KF: ALYTIDAE 1843.fa.f008

Amazonella Lundblad, $1931 \cdot \mathbf{z H}$

ST: zo • CI: zh005

Amazonella FoUQUET ${ }^{+9}, 2012$ • AK

ST: PO.JH • CI: h0044 • ID: 101

PN: Atelopus minutus Melin, 1941

PK: Atelopus minutus* Melin, 1941

KG: Amazophrynella* Fouquet $^{+9}, 2012$

KF: BUFONIDAE 1825.gb.f004
Amazophrynella Fouquet ${ }^{+9}, 2012 \cdot \mathbf{K Y}$

ST: PO.KN・CI: h0045 • ID: 101

PN: Atelopus minutus Melin, 1941

PK: Atelopus minutus* Melin, 1941

KG: Amazophrynella* Fouquet $^{+9}, 2012$

KF: BUFONIDAE 1825.gb.f004

Amblyphrynus Cochran ${ }^{+1}$, $1961 \bullet \mathbf{A K}$

ST: PO.JD・CI: h0046・ID: 073

PN: Amblyphrynus ingeri Cochran ${ }^{+1}, 1961$

PK: Amblyphrynus ingeri ${ }^{\circ}$ Cochran $^{+1}, 1961$

KG: Strabomantis* Peters, 1863

KF: BRACHYCEPHALIDAE 1858.gc.f002

Amblystoma Agassiz, 1844 • AK

ST: NT.JI • CI: h0047 • ID: 555

PN: Lacerta subviolacea Barton, 1804

PK: Lacerta maculata* Shaw, 1802

KG: Ambystoma ${ }^{1}$ Tschudi, 1838

KF: AмBYSTOMATIDAE 1850.ga.f004

Ambystoma Tschudi, $1838 \cdot \mathbf{K Y}$

ST: PO.KN • CI: h0048・ ID: 555

PN: Lacerta subviolacea Barton, 1804

PK: Lacerta maculata* Shaw, 1802

KG: Ambystoma ${ }^{1}$ Tschudi, 1838

KF: AмвYSTOMATIDAE 1850.ga.f004

Ambystomichnus Peabody, 1954 +i $\bullet \mathbf{K Y}$

ST: PO.KN • CI: h0049・ID: $\uparrow 185$

PN: Ammobatrachus montanensis Gilmore 1928 \$i

PK: Ammobatrachus montanensis ${ }^{\circ}$ Gilmore 1928 †

KG: Ambystomichnus ${ }^{\circ}$ Peabody, $1954 \dagger$

KF: AмBYSTOMATIDAE 1850.ga.f004

Ameerega Bauer, 1986 • KY

ST: PO.KN • CI: h0050 • ID: 039

PN: Hyla trivittata Spix, 1824

PK: Hyla trivittata* Spix, 1824

KG: Ameerega* Bauer, 1986

KF: DENDROBATIDAE $\|1850 . b b . f 006\|-1865 . c a . f 002$

Amerana Dubois, $1992 \cdot \mathbf{K Y}$

ST: PO.KN • CI: h0051 •ID: 418

PN: Rana boylii Baird, 1854

PK: Rana boylii* Baird, 1854

KG: Amerana* Dubois, 1992

KF: RANIDAE 1796.ba.f001

Amfignathodon Palacký, 1898 • AK

ST: NT.JD • CI: h0052・ ID: 087

PN: Amphignathodon guentheri Boulenger, 1882

PK: Amphignathodon guentheri* Boulenger, 1882

KG: Amphignathodon* Boulenger, 1882

KF: HEMIPHRACTIDAE 1862.pa.f001

Amietia Dubois, $1987 \bullet \mathbf{K Y}$

ST: PO.KN・CI: h0053・ID: 362

PN: Rana vertebralis Hewitt, 1927

PK: Rana vertebralis* Hewitt, 1927

KG: Amietia* Dubois, 1987

KF: CACOSTERNIDAE 1931.na.f008

Amietophrynus Frost ${ }^{+18}, 2006$ • AK

ST: PO.JD・CI: h0054 • ID: 140 
PN: Bufo regularis Reuss, 1833

PK: Bufo regularis* Reuss, 1833

KG: Sclerophrys* Tschudi, 1838

KF: BUFONIDAE 1825.gb.f004

Ammoryctis Lataste, $1879 \cdot \mathbf{K Y}$

ST: PO.KN • CI: h0055 • ID: 468

PN: Alytes cisternasii Boscá, 1879

PK: Alytes cisternasii* Boscá, 1879

KG: Ammoryctis* Wagler, 1829

KF: ALYTIDAE 1843.fa.f008

Amnirana Dubois, 1992 • AK

ST: PO.JD • CI: h0056・ ID: 409

PN: Rana amnicola Perret, 1977

PK: Rana amnicola ${ }^{\circ}$ Perret, 1977

KG: Hylarana* Tschudi, 1838

KF: RANIDAE 1796.ba.f001

Amo Dubois, $1992 \cdot \mathbf{A K}$

ST: PO.JD • CI: h0057 • ID: 405

PN: Rana larutensis Boulenger, 1899

PK: Rana larutensis* Boulenger, 1899

KG: Amolops $^{2}$ Cope, 1865

KF: RANIDAE 1796.ba.f001

Amolops Cope, 1865 • KY

ST: PO.KN • CI: h0058 • ID: 405

PN: Polypedates afghana Günther, 1859

PK: Polypedates afghana ${ }^{\circ}$ Günther, 1859

KG: Amolops $^{2}$ Cope, 1865

KF: RANIDAE 1796.ba.f001

Amphignathodon Boulenger, 1882 • KY

ST: PO.KN • CI: h0059 • ID: 087

PN: Amphignathodon guentheri Boulenger, 1882

PK: Amphignathodon guentheri* Boulenger, 1882

KG: Amphignathodon* Boulenger, 1882

KF: HEMIPHRACTIDAE 1862.pa.f001

Amphignathodontoides Kuhn, $1941+\bullet \mathbf{A K}$

ST: PO.JD・CI: h0060 • ID: $\uparrow 090$

PN: Amphignathodontoides eocenicus Kuhn, 1941 *

PK: Halleobatrachus hinschei ${ }^{\circ}$ Kuhn, 1941 †

KG: Eopelobates ${ }^{\circ}$ Parker, $1929 \dagger$

KF: PELOBATIDAE 1850.bb.f004

Amphirana: Aymard $1856+\cdot \mathbf{A N}$

ST: AL $・$ CI: n0002・ID: $\uparrow 009 \S$

PN: Amphirana palustris Aymard, $1856+\bullet$ AS

PK: Amphirana palustris ${ }^{\circ}$ Aymard, $1856+\bullet$ AS

KG: Amphirana ${ }^{\circ}$ Aymard, $1856 \uparrow \cdot \mathbf{A G}$

KF: ANURA Familia INCERTAE SEDIS

Amphitriton Rogers, $1976+\cdot \mathbf{K Y}$

ST: PO.KN •CI: h0061・ID: $\uparrow 186$

PN: Amphitriton brevis Rogers, 1976 \$

PK: Amphitriton brevis $^{\circ}$ Rogers, $1976 \dagger$

KG: Amphitriton ${ }^{\circ}$ Rogers, $1976 \dagger$

KF: AмвYSTOMATIDAE 1850.ga.f004

Amphiuma Garden in Smith, 1821 • KY

ST: PO.KN • CI: h0062 • ID: 520

PN: Amphiuma means Garden in Smith, 1821

PK: Amphiuma means* Garden in Smith, 1821
KG: Amphiuma* Garden in Smith, 1821

KF: AMPHIUMIDAE 1825.gb.f07

Amphiumophis Werner, 1900 • AK

ST: PO.JD・CI: h0063・ID: 474

PN: Amphiumophis andicola Werner, 1900

PK: Caecilia tentaculata* Linnaeus, 1758

KG: Caecilia* Linnaeus, 1758

KF: CAECILIIDAE 1814.ra.f003-|1825.gb.f008|

Amphodus Peters, 1873 • AK

ST: PO.JD • CI: h0064 • ID: 221

PN: Amphodus wuchereri Peters, 1873

PK: Amphodus wuchereri ${ }^{\circ}$ Peters, 1873

KG: Phyllodytes* Wagler, 1830

KF: HYLIDAE 1815.ra.f002-|1825.gb.f001|

Anaides Westwood, $1842 \cdot \mathbf{z H}$

ST: zo • CI: zh006

Anaides: Baird 1851 • AN

ST: $\mathbf{L I} ・$ CI: n0003・ID: 547

PN: Salamandra lugubris Hallowell, 1849

PK: Salamandra lugubris* Hallowell, 1849

KG: Aneides* Baird, 1851

KF: PLETHODONTIDAE 1850.ga.f002

Anaxyrus Tschudi, 1845 - KY

ST: PO.KN • CI: h0065 • ID: 136

PN: Anaxyrus melancholicus Tschudi, 1845

PK: Bufo compactilis ${ }^{\circ}$ Wiegmann, 1833

KG: Anaxyrus $^{3}$ Tschudi, 1845

KF: BUFONIDAE 1825.gb.f004

Anchylorana Taylor, $1942+\cdot \mathbf{A K}$

ST: PO.JD・CI: h0066・ID: 415

PN: Anchylorana moorei Taylor, 1942 +

PK: Anchylorana moorei ${ }^{\circ}$ Taylor, $1942 \dagger$

KG: Lithobates* Fitzinger, 1843

KF: RANIDAE 1796.ba.f001

Ancudia Philippi, 1902 • KY

ST: PO.KN • CI: h0067 • ID: 097

PN: Ancudia concolor Philippi, 1902

PK: Ancudia concolor ${ }^{\circ}$ Philippi, 1902

KG: Ancudia $^{\circ}$ Philippi, 1902

KF: Hylobatrachia Familia INCERTAE SEDIS

Andinobates Twomey ${ }^{+3}$ in Brown ${ }^{+13}, 2011 \cdot \mathbf{K Y}$

ST: PO.KN • CI: h0068・ ID: 044

PN: Dendrobates bombetes Myers ${ }^{+1}, 1980$

PK: Dendrobates bombetes* ${ }^{*}$ Mers $^{+1}, 1980$

KG: Andinobates * Twomey ${ }^{+3}$ in Brown ${ }^{+13}, 2011$

KF: DENDROBATIDAE $\|1850 . b b . f 006\|-1865 . c a . f 002$

Andinophryne Hoogmoed, 1985 • AK

ST: PO.JD・CI: h0069・ID: 145

PN: Andinophryne colomai Hoogmoed, 1985

PK: Andinophryne colomai ${ }^{\circ}$ Hoogmoed, 1985

KG: Rhaebo* Cope, 1862

KF: BUFONIDAE 1825.gb.f004

Andrias Tschudi, $1837+\bullet \mathbf{K Y}$

ST: PO.KN • CI: h0070 • ID: 503

PN: Salamandra scheuchzeri Holl, 1831 \$

PK: Salamandra scheuchzeri ${ }^{\circ}$ Holl, $1831 \dagger$ 
KG: Andrias ${ }^{2}$ Tschudi, 1837

KF: CRYPTOBRANCHIDAE 1826.fb.f003

Aneides Baird, 1851 • KY

ST: LC.KN • CI: h0071 • ID: 547

PN: Salamandra lugubris Hallowell, 1849

PK: Salamandra lugubris* Hallowell, 1849

KG: Aneides* Baird, 1851

KF: PLETHODONTIDAE 1850.ga.f002

Anhydrophryne Hewitt, 1919 • KY

ST: PO.KN • CI: h0072・ID: 356

PN: Anhydrophryne rattrayi Hewitt, 1919

PK: Anhydrophryne rattrayi* Hewitt, 1919

KG: Anhydrophryne* Hewitt, 1919

KF: CACOSTERNIDAE 1931.na.f008

Anilany Scherz ${ }^{+6}, 2016 \cdot \mathbf{A K}$

ST: PO.JD • CI: h0073・ID: 286

PN: Stumpffia helenae 2000

PK: Stumpffia helenae* 2000

KG: Cophyla* Boettger, 1880

KF: MICROHYLIDAE ||1843.fa.f012||-1931.na.f001

Annandia Dubois, $1992 \cdot \mathbf{K Y}$

ST: PO.KN • CI: h0074 • ID: 389

PN: Rana delacouri Angel, 1928

PK: Rana delacouri* Angel, 1928

KG: Annandia* Dubois, 1992

KF: DICROGLOSSIDAE 1987.da.f004

Anodonthyla Müller, $1892 \cdot \mathbf{K Y}$

ST: PO.KN • CI: h0075 • ID: 285

PN: Anodonthyla boulengerii Müller, 1892

PK: Anodonthyla boulengerii* Müller, 1892

KG: Anodonthyla* Müller, 1892

KF: MICROHYLIDAE ||1843.fa.f012||-1931.na.f001

Anodontohyla Gadow, 1901 • AK

ST: NS.JI • CI: h0076・ID: 285

PN: Anodonthyla boulengerii Müller, 1892

PK: Anodonthyla boulengerii* Müller, 1892

KG: Anodonthyla* Müller, 1892

KF: MICROHYLIDAE ||1843.fa.f012||-1931.na.f001

Anomaloglossus Grant ${ }^{+9}, 2006 \cdot \mathbf{K Y}$

ST: PO.KN • CI: h0077 • ID: 035

PN: Colostethus beebei Noble, 1923

PK: Colostethus beebei* Noble, 1923

KG: Anomaloglossus* Grant ${ }^{+9}, 2006$

KF: АROMOBATIDAE 2006.gc.f004

Anotheca Smith, 1939 • KY

ST: PO.KN • CI: h0078 • ID: 209

PN: Gastrotheca coronata Stejneger, 1911

PK: Hyla spinosa* Steindachner, 1864

KG: Anotheca ${ }^{1}$ Smith, 1939

KF: HYLIDAE 1815.ra.f002-|1825.gb.f001|

Anoualerpeton Gardner ${ }^{+2}, 2003+\bullet \mathbf{K Y}$

ST: PO.KN • CI: h0079 • ID: $\uparrow 003$

PN: Anoualerpeton unicus Gardner ${ }^{+2}, 2003$ \$

PK: Anoualerpeton unicus ${ }^{\circ}$ Gardner $^{+2}, 2003 \dagger$

KG: Anoualerpeton ${ }^{\circ}$ Gardner $^{+2}, 2003 \uparrow$

KF: ALBANERPETIDAE 1982.fa.f001 $\dagger$
Ansonia Stoliczka, 1870 • KY

ST: PO.KN • CI: h0080 • ID: 113

PN: Ansonia penangensis Stoliczka, 1870

PK: Ansonia penangensis* Stoliczka, 1870

KG: Ansonia* Stoliczka, 1870

KF: BUFONIDAE 1825.gb.f004

Aparasphenodon Miranda-Ribeiro, 1920 • KY

ST: PO.KN • CI: h0081 • ID: 228

PN: Aparasphenodon brunoi Miranda-Ribeiro, 1920

PK: Aparasphenodon brunoi* Miranda-Ribeiro, 1920

KG: Aparasphenodon* Miranda-Ribeiro, 1920

KF: HYLIDAE 1815.ra.f002-|1825.gb.f001|

Aphantophryne Fry, $1917 \cdot \mathbf{A K}$

ST: PO.JD・CI: h0082 • ID: 280

PN: Aphantophryne pansa Fry, 1917

PK: Aphantophryne pansa* Fry, 1917

KG: Asterophrys* Tschudi, 1838

KF: MICROHYLIDAE ||1843.fa.f012||-1931.na.f001

Aplastodiscus Lutz, $1950 \cdot \mathbf{K Y}$

ST: PO.KN • CI: h0083・ID: 188

PN: Aplastodiscus perviridis Lutz, 1950

PK: Aplastodiscus perviridis* Lutz, 1950

KG: Aplastodiscus* Lutz, 1950

KF: HYLIDAE 1815.ra.f002-|1825.gb.f001|

Apneumona Fleming, 1822 • AK

ST: NL.JI • CI: h0084 • ID: 554

PN: Proteus anguinus Laurenti, 1768

PK: Proteus anguinus * Laurenti, 1768

KG: Proteus* Laurenti, 1768

KF: PROTEIDAE 1831.ba.f002

Apodops Estes ${ }^{+1}, 1972$ † KY

ST: PO.KN • CI: h0085・ID: $\uparrow 121$

PN: Apodops pricei Estes $^{+1}, 1972$ \$

PK: Apodops pricei ${ }^{\circ}$ Estes $^{+1}, 1972 \dagger$

KG: Apodops $^{\circ}$ Estes $^{+1}, 1972 \dagger$

KF: GYMNOPHIONA Familia INCERTAE SEDIS

Apricosiren Evans ${ }^{+1}, 2002+\cdot \mathbf{K Y}$

ST: PO.KN • CI: h0086 •ID: $\uparrow 124$

PN: Apricosiren ensomi Evans $^{+1}, 2002$ *

PK: Apricosiren ensomi ${ }^{\circ}$ Evans $^{+1}, 2002 \dagger$

KG: Apricosiren ${ }^{\circ}$ Evans $^{+1}, 2002 \uparrow$

KF: Urodela Familia INCERTAE SEDIS

Aquarana Dubois, $1992 \cdot \mathbf{K Y}$

ST: PO.KN • CI: h0087 • ID: 413

PN: Rana catesbeiana Shaw, 1802

PK: Rana catesbeiana* Shaw, 1802

KG: Aquarana* Dubois, 1992

KF: RANIDAE 1796.ba.f001

Aquiloeurycea Rovito $^{+3}, 2015 \cdot \mathbf{K Y}$

ST: PO.KN • CI: h0088 • ID: 523

PN: Spelerpes cephalicus Cope, 1869

PK: Spelerpes cephalicus* Cope, 1869

KG: Aquiloeurycea ${ }^{*}$ Rovito $^{+3}, 2015$

KF: PLETHODONTIDAE 1850.ga.f002

Aquixalus Delorme ${ }^{+3}, 2005 \cdot \mathbf{A K}$

ST: PO.JD • CI: h0089 • ID: 441 
PN: Philautus odontotarsus $\mathrm{Ye}^{+1}, 1993$

PK: Philautus odontotarsus* $\mathrm{Ye}^{+1}, 1993$

KG: Kurixalus ${ }^{*} \mathrm{Fei}^{+2}$ in Fei, 1999

KF: RHACOPHORIDAE ||1858.gc.f012 |-1932.ha.f001

Aralobatrachus Nessov, 1981 • KY

ST: PO.KN • CI: h0090 • ID: $\uparrow 010$

PN: Aralobatrachus robustus Nessov, 1981 *

PK: Aralobatrachus robustus ${ }^{\circ}$ Nessov, $1981 \dagger$

KG: Aralobatrachus ${ }^{\circ}$ Nessov, 1981 †

KF: ANURA Familia INCERTAE SEDIS

Arariphrynus Leal $^{+1}, 2006+\cdot \mathbf{K Y}$

ST: PO.KN • CI: h0091 • ID: $\uparrow 011$

PN: Arariphrynus placidoi $\mathrm{Leal}^{+1}, 2006$ *

PK: Arariphrynus placido ${ }^{\circ}$ Leal $^{+1}, 2006 \dagger$

KG: Arariphrynus ${ }^{\circ}$ Leal $^{+1}, 2006 \dagger$

KF: ANURA Familia INCERTAE SEDIS

Archaeoovulus Capasso ${ }^{+3}, 2013+\bullet \mathbf{K Y}$

ST: PO.KN • CI: h0092・ID: $\dagger 001 \S$

PN: Archaeoovulus palenae Capasso ${ }^{+3}, 2013+$

PK: Archaeoovulus palenae ${ }^{\circ}$ Capasso $^{+3}, 2013 \dagger$

KG: Archaeoovulus ${ }^{\circ}$ Capasso $^{+3}, 2013 \dagger$

KF: LISSAMPHIBIA Familia INCERTAE SEDIS

Archaeopelobates Kuhn, $1941+\bullet$ AK

ST: PO.JD • CI: h0093 • ID: $\uparrow 090$

PN: Archaeopelobates efremovi Kuhn, 1941 \$

PK: Halleobatrachus hinschei ${ }^{\circ}$ Kuhn, $1941 \dagger$

KG: Eopelobates ${ }^{\circ}$ Parker, $1929 \dagger$

KF: PELOBATIDAE 1850.bb.f004

Archaeotriton Meyer, 1860 + KY

ST: PO.KN・CI: h0094・ID: $\uparrow 190$

PN: Triton basalticus Meyer, $1859+$

PK: Triton basalticus ${ }^{\circ}$ Meyer, $1859 \dagger$

KG: Archaeotriton ${ }^{\circ}$ Meyer, $1860 \dagger$

KF: SALAMANDRIDAE 1820.ga.f002

Archipelobates: Tatarinov 1970 - AN

ST: AL • CI: n0004 • ID: $\uparrow 012 \S$

PN: Archipelobates giganteum Tatarinov, 1970 • AS

PK: Archipelobates giganteum ${ }^{\circ}$ Tatarinov, $1970 \uparrow \cdot \mathbf{A S}$

KG: Archipelobates ${ }^{\circ}$ Tatarinov, $1970 \dagger \cdot \mathbf{A G}$

KF: ANURA Familia INCERTAE SEDIS

Arcovomer Carvalho, $1954 \cdot \mathbf{K Y}$

ST: PO.KN • CI: h0095 • ID: 296

PN: Arcovomer passarellii Carvalho, 1954

PK: Arcovomer passarellii* Carvalho, 1954

KG: Arcovomer* Carvalho, 1954

KF: MICROHYLIDAE ||1843.fa.f012||-1931.na.f001

Arenophryne Tyler, $1976 \bullet \mathbf{K Y}$

ST: PO.KN • CI: h0096・ID: 271

PN: Arenophryne rotunda Tyler, 1976

PK: Arenophryne rotunda* Tyler, 1976

KG: Arenophryne* Tyler, 1976

KF: MYOBATRACHIDAE 1850.sa.f001

Arethusa Montfort, $1808 \cdot \mathbf{Z H}$

ST: zo • CI: zh007

Arethusa: Bonaparte $1838 \cdot \mathbf{A N}$

ST: AL •CI: n0005 • ID: 026
PN: Bombina marmorata Koch in Sturm, 1828

PK: Bufo fuscus* Laurenti, 1768

KG: Pelobates* Wagler, 1830

KF: PELOBATIDAE 1850.bb.f004

Arethusa: Duméril ${ }^{+1}, 1841 \cdot \mathbf{A N}$

ST: $\mathbf{A L} ・ \mathbf{C I}:$ n0006・ID: 027

PN: Rana punctata Daudin, 1802

PK: Rana punctata* Daudin, 1802

KG: Pelodytes* Bonaparte, 1838

KF: PELODYTIDAE 1850.bb.f002

Argenteohyla Trueb, $1970 \cdot \mathbf{K Y}$

ST: PO.KN • CI: h0097・ID: 229

PN: Hyla siemersi Mertens, 1937

PK: Hyla siemersi* Mertens, 1937

KG: Argenteohyla* Trueb, 1970

KF: HYLIDAE 1815.ra.f002-|1825.gb.f001|

Arlequinus Perret, $1988 \cdot \mathbf{K Y}$

ST: PO.KN • CI: h0098 • ID: 326

PN: Hyperolius krebsi Mertens, 1938

PK: Hyperolius krebsi ${ }^{\circ}$ Mertens, 1938

KG: Arlequinus $^{\circ}$ Perret, 1988

KF: HYPEROLIIDAE 1943.lb.f001

Aromobates Myers ${ }^{+2}$, $1991 \bullet \mathbf{K Y}$

ST: PO.KN • CI: h0099 • ID: 037

PN: Aromobates nocturnus Myers ${ }^{+2}, 1991$

PK: Aromobates nocturnus* Myers $^{+2}, 1991$

KG: Aromobates* Myers ${ }^{+2}, 1991$

KF: АRомоватIDAE 2006.gc.f004

Arthroleptella Hewitt, 1926 • KY

ST: PO.KN・CI: h0100・ID: 360

PN: Arthroleptis lightfooti Boulenger, 1910

PK: Arthroleptis lightfooti* Boulenger, 1910

KG: Arthroleptella* Hewitt, 1926

KF: CACOSTERNIDAE 1931.na.f008

Arthroleptides Nieden, 1911 • KY

ST: PO.KN • CI: h0101・ID: 354

PN: Arthroleptides martiensseni Nieden, 1911

PK: Arthroleptides martiensseni* Nieden, 1911

KG: Arthroleptides* Nieden, 1911

KF: PETROPEDETIDAE 1931.na.f006

Arthroleptis Smith, $1849 \bullet \mathbf{K Y}$

ST: PO.KN • CI: h0102 • ID: 320

PN: Arthroleptis wahlbergii Smith, 1849

PK: Arthroleptis wahlbergii* Smith, 1849

KG: Arthroleptis* Smith, 1849

KF: ARTHROLEPTIDAE 1869.mc.f011

Arthroleptulus Laurent, 1941 • AK

ST: Po.JD・CI: h0103・ID: 320

PN: Arthroleptis xenodactylus Boulenger, 1909

PK: Arthroleptis xenodactylus* Boulenger, 1909

KG: Arthroleptis* Smith, 1849

KF: ARTHROLEPTIDAE 1869.mc.f011

Aruncus: Philippi $1899 \cdot$ AN

ST: $\mathbf{A L} ・ \mathbf{C I}: \mathrm{n} 0007 \bullet \mathbf{I D}: 138$

PN: Aruncus valdivianus Philippi, 1902

PK: Bufo spinulosus* Wiegmann, 1834 
KG: Rhinella ${ }^{2}$ Fitzinger, 1826

KF: BUFONIDAE 1825.gb.f004

Aruncus Philippi, $1902 \cdot \mathbf{A K}$

ST: PO.JD • CI: h0104 • ID: 138

PN: Aruncus valdivianus Philippi, 1902

PK: Bufo spinulosus* Wiegmann, 1834

KG: Rhinella ${ }^{2}$ Fitzinger, 1826

KF: BUFONIDAE 1825.gb.f004

Ascaphus Stejneger, $1899 \cdot \mathbf{K Y}$

ST: PO.KN • CI: h0105・ID: 004

PN: Ascaphus truei Stejneger, 1899

PK: Ascaphus truei* Stejneger, 1899

KG: Ascaphus* Stejneger, 1899

KF: ASCAPHIDAE 1923.fa.f001

Asperomantis Vences ${ }^{+10}, 2017 \cdot \mathbf{A K}$

ST: PO.JD・CI: h0106・ID: 431

PN: Rana aspera Boulenger, 1882

PK: Rana aspera $^{\circ}$ Boulenger, 1882

KG: Gephyromantis* Methuen, 1920

KF: RHACOPHORIDAE ||1858.gc.f012||-1932.ha.f001

Asphaerion Meyer, 1847 + $\mathbf{A K}$

ST: PO.JD • CI: h0107 • ID: 406

PN: Asphaerion reussi Meyer, 1847 \$

PK: Asphaerion reussi ${ }^{\circ}$ Meyer, $1847 \dagger$

KG: Pelophylax* Fitzinger, 1843

KF: RANIDAE 1796.ba.f001

Assa: Gray $1851 \cdot \mathbf{Z A}$

ST: $\mathbf{z N} \bullet \mathbf{C I}:$ zn001

Assa Tyler, $1972 \bullet \mathbf{K Y}$

ST: PO.KN • CI: h0108・ID: 267

PN: Crinia darlingtoni Loveridge, 1933

PK: Crinia darlingtoni* Loveridge, 1933

KG: Assa* Tyler, 1972

KF: MYOBATRACHIDAE 1850.sa.f001

Asterodactylus Wagler in Boie, 1827 • AK

ST: NL.JI • CI: h0109 • ID: 012

PN: Pipa americana Laurenti,1768

PK: Rana pipa* Linnaeus, 1758

KG: Pipa $^{1}$ Laurenti, 1768

KF: PIPIDAE 1825.gb.f003-|1826.fb.f002|

Asterofrys Palacký, 1898 • AK

ST: NT.JI • CI: h0110 • ID: 280

PN: Ceratophrys turpicola Schlegel, 1837

PK: Ceratophrys turpicola* Schlegel, 1837

KG: Asterophrys* Tschudi, 1838

KF: MICROHYLIDAE ||1843.fa.f012||-1931.na.f001

Asterophrys Tschudi, $1838 \cdot \mathbf{K Y}$

ST: PO.KN • CI: h0111 • ID: 280

PN: Ceratophrys turpicola Schlegel, 1837

PK: Ceratophrys turpicola* Schlegel, 1837

KG: Asterophrys* Tschudi, 1838

KF: MICROHYLIDAE ||1843.fa.f012||-1931.na.f001

Astrobatrachus Vijayakumar ${ }^{+8}, 2019 \cdot \mathbf{K Y}$

ST: PO.KN • CI: h0112 • ID: 398

PN: Astrobatrachus kurichiyana Vijayakumar ${ }^{+8}, 2019$

PK: Astrobatrachus kurichiyana ${ }^{\circ}$ Vijayakumar $^{+8}, 2019$
KG: Astrobatrachus ${ }^{\circ}$ Vijayakumar $^{+8}, 1838$

KF: NYCTIBATRACHIDAE 1993.ba.f001-01

Astrodactylus [Hogg, 1838] Hogg, 1839• AK

ST: NS.JI • CI: h0113 • ID: 012

PN: Pipa americana Laurenti,1768

PK: Rana pipa* Linnaeus, 1758

KG: Pipa $^{1}$ Laurenti, 1768

KF: PIPIDAE 1825.gb.f003-|1826.fb.f002|

Astylosternus Werner, 1898 • KY

ST: PO.KN・CI: h0114・ID: 321

PN: Astylosternus diadematus Werner, 1898

PK: Astylosternus diadematus* Werner, 1898

KG: Astylosternus* Werner, 1898

KF: ARTHROLEPTIDAE 1869.mc.f011

Ateleopus Agassiz, 1847 • AK

ST: NT.JI • CI: h0115 • ID: 100

PN: Atelopus flavescens Duméril ${ }^{+1}, 1841$

PK: Atelopus flavescens* Duméril ${ }^{+1}, 1841$

KG: Atelopus* Duméril ${ }^{+1}, 1841$

KF: BUFONIDAE 1825.gb.f004

Atelognathus Lynch, $1978 \cdot \mathbf{K Y}$

ST: PO.KN • CI: h0116 •ID: 175

PN: Batrachophrynus patagonicus Gallardo, 1962

PK: Batrachophrynus patagonicus* Gallardo, 1962

KG: Atelognathus* Lynch, 1978

KF: BATRACHYLIDAE 1965.ga.f002

Atelophryne Boulenger, 1906 • AK

ST: PO.JD • CI: h0117・ID: 124

PN: Atelophryne minuta Boulenger, 1906

PK: Didynamipus sjostedti* Andersson, 1903

KG: Didynamipus* Andersson, 1903

KF: BUFONIDAE 1825.gb.f004

Atelophryniscus $\mathrm{McCranie}^{+2}$, 1989 • AK

ST: PO.JD • CI: h0118・ID: 138

PN: Atelophryniscus chrysophorus $\mathrm{McCranie}^{+2}, 1989$

PK: Atelophryniscus chrysophorus ${ }^{\circ} \mathrm{McCranie}^{+2}, 1989$

KG: Rhinella ${ }^{2}$ Fitzinger, 1826

KF: BUFONIDAE 1825.gb.f004

Atelopus Duméril ${ }^{+1}, 1841 \cdot \mathbf{K Y}$

ST: PO.KN • CI: h0119・ID: 100

PN: Atelopus flavescens Duméril ${ }^{+1}, 1841$

PK: Atelopus flavescens* Duméril ${ }^{+1}, 1841$

KG: Atelopus $*$ Duméril $^{+1}, 1841$

KF: BUFONIDAE 1825.gb.f004

Atilophus Cuvier ${ }^{+1}, 1840$ • AK

ST: PO.JD・CI: h0120・ID: 138

PN: Rana margaritifera Laurenti, 1768

PK: Rana margaritifera* Laurenti, 1768

KG: Rhinella ${ }^{2}$ Fitzinger, 1826

KF: BUFONIDAE 1825.gb.f004

Atlantihyla Faivovich ${ }^{+15}, 2018 \cdot \mathbf{K Y}$

ST: PO.KN • CI: h0121 • ID: 212

PN: Atlantihyla spinipollex Faivovich ${ }^{+15}, 2018$

PK: Atlantihyla spinipollex* Faivovich ${ }^{+15}, 2018$

KG: Atlantihyla* Faivovich ${ }^{+15}, 2018$

KF: HYLIDAE 1815.ra.f002-|1825.gb.f001| 
Atopophrynus $\mathrm{Lynch}^{+1}, 1982 \cdot \mathbf{K Y}$

ST: PO.KN • CI: h0122 • ID: 055

PN: Atopophrynus syntomopus Lynch $^{+1}, 1982$

PK: Atopophrynus syntomopus ${ }^{\circ}$ Lynch $^{+1}, 1982$

KG: Atopophrynus ${ }^{\circ} \mathrm{Lynch}^{+1}, 1982$

KF: BRACHYCEPHALIDAE 1858.gc.f002

Atretochoana Nussbaum ${ }^{+1}$, 1995 • KY

ST: PO.KN • CI: h0123・ID: 476

PN: Typhlonectes eiselti Taylor, 1968

PK: Typhlonectes eiselti ${ }^{\circ}$ Taylor, 1968

KG: Atretochoana ${ }^{\circ}$ Nussbaum $^{+1}, 1995$

KF: CAECILIIDAE 1814.ra.f003-|1825.gb.f008|

Atylodes Gistel, 1868 • AK

ST: PO.Ro・CI: h0124 • ID: 545

PN: Salamandra genei Temminck $^{+1}, 1838$

PK: Salamandra genei* ${ }^{*}$ Temminck $^{+1}, 1838$

KG: Speleomantes* Dubois, 1984

KF: PLETHODONTIDAE 1850.ga.f002

Atympanolalax $\mathrm{Fei}^{+1}, 2016 \cdot \mathbf{A K}$

ST: PO.JD • CI: h0125 • ID: 016

PN: Scutiger rugosa Liu, 1943

PK: Scutiger rugosa* Liu, 1943

KG: Oreolalax* Myers ${ }^{+1}, 1962$

KF: MEGOPHRYIDAE 1850.bb.f008-|1931.na.f003|

Atympanophrys $\operatorname{Tian}^{+1}, 1983 \cdot \mathbf{K Y}$

ST: PO.KN • CI: h0126・ID: 019

PN: Megophrys shapingensis Liu, 1950

PK: Megophrys shapingensis* Liu, 1950

KG: Atympanophrys* Tian $^{+1}, 1983$

KF: MEGOPHRYIDAE 1850.bb.f008-|1931.na.f003|

Aubria Boulenger, 1917 • KY

ST: PO.KN • CI: h0127・ID: 366

PN: Rana subsigillata Duméril, 1856

PK: Rana subsigillata* Duméril, 1856

KG: Aubria* Boulenger, 1917

KF: PYXICEPHALIDAE 1850.bb.f005

Aubrya: Schiøtz 1964 - AN

ST: AM • CI: n0008 • ID: 366

PN: Rana subsigillata Duméril, 1856

PK: Rana subsigillata* Duméril, 1856

KG: Aubria* Boulenger, 1917

KF: PYXICEPHALIDAE 1850.bb.f005

Audaciella nov. $\bullet \mathrm{KY}$

ST: PO.KN • CI: h0128・ID: 160

PN: Centrolenella audax $\mathrm{Lynch}^{+1}, 1973$

PK: Centrolenella audax* $\mathrm{Lynch}^{+1}, 1973$

KG: Audaciella* nov.

KF: CENTROLENIDAE 1951.ta.f001

Auletris Wagler, $1830 \cdot \mathbf{A K}$

ST: PO.JI • CI: h0129 • ID: 189

PN: Rana boans Linnaeus, 1758

PK: Rana boans* Linnaeus, 1758

KG: Boana* Gray, 1825

KF: HYLIDAE 1815.ra.f002-|1825.gb.f001|

Aurana Walker, $1863 \cdot \mathbf{Z H}$

ST: zo • CI: zh008
Aurana Bauer, 1985 • AK

ST: PO.JH • CI: h0130・ID: 418

PN: Rana aurora Baird $^{+1}, 1852$

PK: Rana aurora* ${ }^{*}$ Baird $^{+1}, 1852$

KG: Amerana* Dubois, 1992

KF: RANIDAE 1796.ba.f001

Aurorana Dubois, $1992 \cdot \mathbf{A K}$

ST: PO.JD・CI: h0131・ID: 418

PN: Rana aurora Baird $^{+1}, 1852$

PK: Rana aurora* ${ }^{*}$ Baird $^{+1}, 1852$

KG: Amerana* Dubois, 1992

KF: RANIDAE 1796.ba.f001

Australobatrachus Tyler, $1976+\cdot$ KY

ST: PO.KN • CI: h0132 •ID: $\uparrow 102$

PN: Australobatrachus ilius Tyler, 1976 \$

PK: Australobatrachus ilius ${ }^{\circ}$ Tyler, 1976 †

KG: Australobatrachus ${ }^{\circ}$ Tyler, $1976 \dagger$

KF: PHYLLOMEDUSIDAE 1858.gc.f009

Australocrinia Heyer $^{+1}$, 1976 • AK

ST: PO.JD • CI: h0133・ID: 270

PN: Pterophrynus tasmaniensis Günther, 1864

PK: Pterophrynus tasmaniensis* Günther, 1864

KG: Crinia* Tschudi, 1838

KF: MYOBATRACHIDAE 1850.sa.f001

Australotheca Malinsky, $2009 \cdot \mathbf{Z H}$

ST: zo • CI: zh009

Australotheca Duellman, 2015 • AK

ST: PO.JH • CI: h0134 • ID: 090

PN: Nototrema microdiscus Andersson, 1910

PK: Nototrema microdiscus* Andersson, 1910

KG: Alainia* Duellman ${ }^{+1}, 2018$

KF: HEMIPHRACTIDAE 1862.pa.f001

Austrochaperina Fry, 1912 • AK

ST: PO.JD・CI: h0135・ID: 280

PN: Austrochaperina robusta Fry, 1912

PK: Austrochaperina robusta ${ }^{\circ}$ Fry, 1912

KG: Asterophrys* Tschudi, 1838

KF: MICROHYLIDAE ||1843.fa.f012||-1931.na.f001

Autodax Boulenger, 1887 - AK

ST: NL.CA • CI: h0136 • ID: 547

PN: Salamandra lugubris Hallowell, 1849

PK: Salamandra lugubris* Hallowell, 1849

KG: Aneides* Baird, 1851

KF: PLETHODONTIDAE 1850.ga.f002

Avitabatrachus Báez ${ }^{+2}, 2000$ • KY

ST: PO.KN • CI: h0137・ID: $\uparrow 061$

PN: Avitabatrachus uliana Báez $^{+2}, 2000$ †

PK: Avitabatrachus uliana ${ }^{\circ}$ Báez $^{+2}, 2000 \dagger$

KG: Avitabatrachus $^{\circ} \mathrm{Báez}^{+2}, 2000 \dagger$

KF: DorsIPARES Familia INCERTAE SEDIS

Aviturus Gubin, $1991+\bullet \mathbf{K Y}$

ST: PO.KN • CI: h0138・ID: $\uparrow 164$

PN: Aviturus exsecratus Gubin, 1991 \$

PK: Aviturus exsecratus ${ }^{\circ}$ Gubin, $1991+$

KG: Aviturus $^{\circ}$ Gubin, $1991 \uparrow$

KF: CRYPTOBRANCHIDAE 1826.fb.f003 
Axolot Bonaparte, 1831 • AK

ST: Po.CA • CI: h0139 • ID: 555

PN: Axolotus pisciformis Jarocki, 1822

PK: Gyrinus mexicanus* Shaw ${ }^{+1}, 1789$

KG: Ambystoma ${ }^{1}$ Tschudi, 1838

KF: AмBYSTOMATIDAE 1850.ga.f004

Axolotes Owen, 1844 • AK

ST: NT.JD • CI: h0140 • ID: 555

PN: Gyrinus mexicanus Shaw $^{+1}, 1789$

PK: Gyrinus mexicanus* Shaw ${ }^{+1}, 1789$

KG: Ambystoma ${ }^{1}$ Tschudi, 1838

KF: AмBYSTOMATIDAE 1850.ga.f004

Axoloth Gray, 1842 • AK

ST: NS.JD・CI: h0141 • ID: 555

PN: Siren pisciformis Shaw, 1802

PK: Gyrinus mexicanus* Shaw ${ }^{+1}, 1789$

KG: Ambystoma ${ }^{1}$ Tschudi, 1838

KF: AмвчSTомAтIDAE 1850.ga.f004

Axolotl: Oken 1821 • EX

ST: PO.CE • CI: e0002 • ID: 555

PN: Siren pisciformis Shaw, 1802

PK: Gyrinus mexicanus* Shaw $^{+1}, 1789$

KG: Ambystoma ${ }^{1}$ Tschudi, 1838

KF: AмвYSTOMATIDAE 1850.ga.f004

Axolotus Jarocki, 1822 • EX

ST: PO.CE • CI: e0003 • ID: 555

PN: Siren pisciformis Shaw 18022

PK: Gyrinus mexicanus* Shaw ${ }^{+1}, 1789$

KG: Ambystoma ${ }^{1}$ Tschudi, 1838

KF: AмвYSTOMATIDAE 1850.ga.f004

Aygroua Jones ${ }^{+2}, 2003+\bullet \mathbf{K Y}$

ST: PO.KN • CI: h0143・ID: $\dagger 013$

PN: Aygroua anoualensis Jones ${ }^{+2}, 2003$ *

PK: Aygroua anoualensis ${ }^{\circ}$ Jones $^{+2}, 2003 \dagger$

KG: Aygroua $^{\circ}$ Jones $^{+2}, 2003 \dagger$

KF: ANURA Familia INCERTAE SEDIS

Babina Thompson, $1912 \cdot \mathbf{K Y}$

ST: PO.KN • CI: h0144 • ID: 410

PN: Rana holsti Boulenger, 1892

PK: Rana holsti* Boulenger, 1892

KG: Babina* Thompson, 1912

KF: RANIDAE 1796.ba.f001

Babina Van Denburgh, 1912 • AK

ST: Po.JI • CI: h0145 • ID: 410

PN: Rana holsti Boulenger, 1892

PK: Rana holsti* Boulenger, 1892

KG: Babina* Thompson, 1912

KF: RANIDAE 1796.ba.f001

\section{Bahius nov. $\bullet$ KY}

ST: PO.KN • CI: h0146・ID: 063

PN: Eleutherodactylus bilineatus Bokermann, 1975

PK: Eleutherodactylus bilineatus* Bokermann, 1975

KG: Bahius* nov.

KF: BRACHYCEPHALIDAE 1858.gc.f002

Bakonybatrachus Szentesi ${ }^{+1}, 2012+\bullet \mathbf{K Y}$

ST: PO.KN • CI: h0147 • ID: $\uparrow 115$
PN: Bakonybatrachus fedori Szentesi ${ }^{+1}, 2012$ *

PK: Bakonybatrachus fedori ${ }^{\circ}$ Szentesi $^{+1}, 2012 \dagger$

KG: Bakonybatrachus ${ }^{\circ}$ Szentesi $^{+1}, 2012 \dagger$

KF: DISCOGLOSSIDAE 1858.gc.f004

Baleaphryne Sanchíz ${ }^{+1}, 1979$ † AK

ST: PO.JD • CI: h0148 • ID: 467

PN: Baleaphryne muletensis Sanchíz ${ }^{+1}, 1979$

PK: Baleaphryne muletensis* Sanchíz ${ }^{+1}, 1979$

KG: Alytes* Wagler, 1829

KF: ALYTIDAE 1843.fa.f008

Balebreviceps Largen ${ }^{+1}, 1989 \bullet \mathbf{K Y}$

ST: PO.KN • CI: h0149・ID: 343

PN: Balebreviceps hillmani Largen ${ }^{+1}, 1989$

PK: Balebreviceps hillmani* Largen $^{+1}, 1989$

KG: Balebreviceps* Largen $^{+1}, 1989$

KF: BREVICIPITIDAE 1850.bb.f012

Baliopygus Schulze, 1891 • AK

ST: PO.JD • CI: h0150 • ID: 406

PN: Rana ridibunda Pallas, 1771

PK: Rana ridibunda* Pallas, 1771

KG: Pelophylax* Fitzinger, 1843

KF: RANIDAE 1796.ba.f001

Balveherpeton: Skutschas ${ }^{+2} 2020 a+\cdot \mathbf{A N}$

ST: $\mathbf{A L} \bullet \mathbf{C I}:$ n0009・ID: $\uparrow 125$

PN: Balveherpeton hoennetalensis Skutschas ${ }^{+6}, 2020 a \ddagger$

PK: Balveherpeton hoennetalensi ${ }^{\circ}$ Skutschas $^{+6}, 2020 b \dagger$

KG: Balveherpeton ${ }^{\circ}$ Skutschas $^{+6}, 2020 b \dagger$

KF: Urodela Familia INCERTAE SEDIS

Balveherpeton Skutschas ${ }^{+2}, 2020 b$ \$ KY

ST: PO.KN • CI: h0151 • ID: $\uparrow 125$

PN: Balveherpeton hoennetalensis Skutschas ${ }^{+6}, 2020 b+$

PK: Balveherpeton hoennetalensi ${ }^{\circ}$ Skutschas $^{+6}, 2020 b \dagger$

KG: Balveherpeton ${ }^{\circ}$ Skutschas $^{+6}, 2020 b \dagger$

KF: Urodela Familia INCERTAE SEDIS

Bamburana $\mathrm{Fei}^{+2}$ in $\mathrm{Fei}^{+4}$, 2005 - AK

ST: PO.JD • CI: h0152・ID: 412

PN: Rana versabilis $\mathrm{Liu}^{+1}, 1962$

PK: Rana versabilis* $\mathrm{Liu}^{+1}, 1962$

KG: Odorrana $* \mathrm{Fei}^{+2}, 1990$

KF: RANIDAE 1796.ba.f001

Baranophrys: Kretzoi $1956+\cdot \mathbf{A N}$

ST: $\mathbf{A L} ・$ CI: $\mathrm{n} 0010 \bullet$ ID: $\uparrow 014 \S$

PN: Baranophrys discoglossoides Kretzoi, $1956+\cdot$ AS

PK: Baranophrys discoglossoides ${ }^{\circ} \mathrm{Kretzoi}, 1956 \uparrow \cdot \mathbf{A S}$

KG: Baranophrys ${ }^{\circ}$ Kretzoi, $1956 \uparrow \cdot \mathbf{A G}$

KF: ANURa Familia INCERTAE SEDIS

Barbarophryne Beukema ${ }^{+8}, 2013 \cdot \mathbf{K Y}$

ST: PO.KN・CI: h0153・ID: 115

PN: Bufo brongersmai Hoogmoed, 1972

PK: Bufo brongersmai* Hoogmoed, 1972

KG: Barbarophryne* Beukema ${ }^{+8}, 2013$

KF: BUFONIDAE 1825.gb.f004

Barbourula Taylor $^{+1}$, $1924 \cdot \mathbf{K Y}$

ST: PO.KN • CI: h0154 • ID: 471

PN: Barbourula busuangensis Taylor ${ }^{+1}, 1924$

PK: Barbourula busuangensis* Taylor ${ }^{+1}, 1924$ 
KG: Barbourula* Taylor $^{+1}, 1924$

KF: BOMBINATORIDAE 1825.gb.f002

Bargmannia Totton, $1954 \cdot \mathbf{Z H}$

ST: zo • CI: zh010

Bargmannia Herre, 1955 + $\mathbf{A K}$

ST: PO.JH • CI: h0155 • ID: $\uparrow 188$

PN: Bargmannia wettsteini Herre, 1955 \$

PK: Bargmannia wettsteini ${ }^{\circ}$ Herre, $1955 \dagger$

KG: Sanchizia ${ }^{\circ}$ Dubois $^{+1}, 2012 \dagger$

KF: AмвYSTомATIDAE 1850.ga.f004

Baryboas Gistel, 1848 + $\mathbf{A K}$

ST: NL.JI • CI: h0156・ID: $\uparrow 111$

PN: Pelophilus agassizii Tschudi, 1838 \$

PK: Pelophilus agassizii ${ }^{\circ}$ Tschudi, $1838 \dagger$

KG: Pelophilus ${ }^{\circ}$ Tschudi, $1838 \dagger$

KF: Mediogyrinia Familia InCERTAE SEDIS

Barycholos Heyer, $1969 \cdot \mathbf{K Y}$

ST: PO.KN・CI: h0157・ ID: 064

PN: Leptodactylus pulcher Boulenger, 1898

PK: Leptodactylus pulcher* Boulenger, 1898

KG: Barycholos* Heyer, 1969

KF: BRACHYCEPHALIDAE 1858.gc.f002

Barygenys Parker, 1936 • AK

ST: PO.JD • CI: h0158 • ID: 280

PN: Barygenys cheesmanae Parker, 1936

PK: Barygenys cheesmanae ${ }^{\circ}$ Parker, 1936

KG: Asterophrys* Tschudi, 1838

KF: MiCROHYLIDAE |1843.fa.f012||-1931.na.f001

Basanitia Miranda-Ribeiro, 1923 • AK

ST: PO.JD • CI: h0159・ID: 058

PN: Basanitia lactea Miranda-Ribeiro, 1923

PK: Basanitia lactea* Miranda-Ribeiro, 1923

KG: Ischnocnema* Reinhardt ${ }^{+1}, 1862$

KF: BRACHYCEPHALIDAE 1858.gc.f002

Bathysiredon Dunn, 1939 • AK

ST: PO.JD • CI: h0160 • ID: 555

PN: Siredon dumerilii Dugès, 1870

PK: Siredon dumerilii* Dugès, 1870

KG: Ambystoma ${ }^{1}$ Tschudi, 1838

KF: AмвYSTOMATIDAE 1850.ga.f004

Batrachohyperus Rye, 1881 • AK

ST: NT.JI • CI: h0161・ID: 509

PN: Desmodactylus pinchonii David, 1872

PK: Desmodactylus pinchonii* David, 1872

KG: Batrachuperus* Boulenger, 1878

KF: НYNOBIIDAE ||1856.ha.f001||-1859.cb.f002

Batrachophrynus Peters, 1873 • AK

ST: PO.JD・CI: h0162・ID: 186

PN: Batrachophrynus macrostomus Peters, 1873

PK: Batrachophrynus macrostomus ${ }^{\circ}$ Peters, 1873

KG: Telmatobius ${ }^{3}$ Wiegmann, 1834

KF: TELMATOBIIDAE 1843.fa.f006

Batrachopsis Fitzinger, 1843 • AK

ST: PO.JI • CI: h0163 • ID: 540

PN: Salamandra subfusca Green, 1818

PK: Salamandra rubra* Sonnini ${ }^{+1}, 1801$
KG: Pseudotriton ${ }^{1}$ Tschudi, 1838

KF: PLETHODONTIDAE 1850.ga.f002

Batrachopsis Boulenger, $1882 \cdot \mathbf{A K}$

ST: PO.JH • CI: h0164 • ID: 264

PN: Asterophrys melanopyga Doria, 1875

PK: Asterophrys melanopyga* Doria, 1875

KG: Platyplectrum ${ }^{1}$ Günther, 1863

KF: MYOBATRACHIDAE 1850.sa.f001

Batrachosauroides Taylor ${ }^{+1}, 1943+\mathbf{K Y}$

ST: PO.KN • CI: h0165・ID: $\uparrow 145$

PN: Batrachosauroides dissimulans Taylor ${ }^{+1}, 1943$ \$

PK: Batrachosauroides dissimulans ${ }^{\circ}$ Taylor $^{+1}, 1943 \dagger$

KG: Batrachosauroides ${ }^{\circ}$ Taylor $^{+1}, 1943 \dagger$

KF: HYLAEOBATRACHIDAE 1889.1a.f001 †

Batrachoseps Bonaparte, $1839 \cdot \mathbf{K Y}$

ST: PO.KN・CI: h0166・ID: 521

PN: Salamandrina attenuata Eschscholtz, 1833

PK: Salamandrina attenuata* Eschscholtz, 1833

KG: Batrachoseps* Bonaparte, 1839

KF: PLETHODONTIDAE 1850.ga.f002

Batrachulina Kuhn, $1962+\cdot \mathbf{K Y}$

ST: PO.KN • CI: h0167 • ID: $\uparrow 015$

PN: Batrachus lemanensis Pomel, 1853 \$

PK: Batrachus lemanensis ${ }^{\circ}$ Pomel, $1853 \dagger$

KG: Batrachulina ${ }^{\circ}$ Kuhn, $1962 \dagger$

KF: ANURA Familia INCERTAE SEDIS

Batrachuperus Boulenger, $1878 \cdot \mathbf{K Y}$

ST: PO.KN • CI: h0168・ID: 509

PN: Desmodactylus pinchonii David, 1872

PK: Desmodactylus pinchonii* David, 1872

KG: Batrachuperus* Boulenger, 1878

KF: HYNOBIIDAE ||1856.ha.f001||-1859.cb.f002

Batrachus Schaeffer, $1760 \cdot \mathbf{Z H}$

ST: zo • CI: zh011

Batrachus Rafinesque, 1814 • AK

ST: PO.JH • CI: h0169 • ID: 121

PN: Bufo viridis Laurenti, 1768

PK: Bufo viridis* Laurenti, 1768

KG: Bufotes* Rafinesque, 1815

KF: BUFONIDAE 1825.gb.f004

Batrachus Pomel, $1853+\bullet \mathbf{A K}$

ST: PO.JH • CI: h0170 • ID: $\uparrow 015$

PN: Batrachus lemanensis Pomel, 1853 *

PK: Batrachus lemanensis ${ }^{\circ}$ Pomel, $1853 \dagger$

KG: Batrachulina ${ }^{\circ}$ Kuhn, $1962 \dagger$

KF: ANURA Familia INCERTAE SEDIS

Batrachchythis: Garman 1877 • AN

ST: AM・CI: n0011・ID: 196

PN: Rana paradoxa Linnaeus, 1758

PK: Rana paradoxa* Linnaeus, 1758

KG: Pseudis* Wagler, 1830

KF: HYLIDAE 1815.ra.f002-|1825.gb.f001|

Batrachichthys: Garman 1877 - AN

ST: AM • CI: n0012 • ID: 196

PN: Rana paradoxa Linnaeus, 1758

PK: Rana paradoxa* Linnaeus, 1758 
KG: Pseudis* Wagler, 1830

KF: HYLIDAE 1815.ra.f002-|1825.gb.f001|

Batrachychthis Pizarro, 1876 • AK

ST: LC.JI • CI: h0171 • ID: 196

PN: Rana paradoxa Linnaeus, 1758

PK: Rana paradoxa* Linnaeus, 1758

KG: Pseudis* Wagler, 1830

KF: HYLIDAE 1815.ra.f002-|1825.gb.f001|

Batrachychthys: Pizarro 1876 • AN

ST: LI • CI: n0013・ID: 196

PN: Rana paradoxa Linnaeus, 1758

PK: Rana paradoxa* Linnaeus, 1758

KG: Pseudis* Wagler, 1830

KF: HYLIDAE 1815.ra.f002-|1825.gb.f001|

Batrachyla Bell, $1843 \cdot \mathbf{K Y}$

ST: PO.KN • CI: h0172・ID: 177

PN: Batrachyla leptopus Bell, 1843

PK: Batrachyla leptopus* Bell, 1843

KG: Batrachyla* Bell, 1843

KF: BATRACHYLIDAE 1965.ga.f002

Batrachylodes Boulenger, 1887 • AK

ST: PO.JD • CI: h0173 • ID: 369

PN: Batrachylodes vertebralis Boulenger, 1887

PK: Batrachylodes vertebralis* Boulenger, 1887

KG: Cornufer* Tschudi, 1838

KF: CERATOBATRACHIDAE 1884.ba.f001

Batrachyperus Boulenger, 1882 • AK

ST: NT.JI • CI: h0174 • ID: 509

PN: Desmodactylus pinchonii David, 1872

PK: Desmodactylus pinchonii* David, 1872

KG: Batrachuperus* Boulenger, 1878

KF: HYNOBIIDAE ||1856.ha.f001||-1859.cb.f002

Batracinus: Rafinesque 1815 - AN

ST: $\mathbf{A L} ・ \mathbf{C I}:$ n0014 •ID: 419

PN: Rana temporaria Linnaeus, 1758

PK: Rana temporaria* Linnaeus, 1758

KG: Rana* Linnaeus, 1758

KF: RANIDAE 1796.ba.f001

Baurubatrachus Báez ${ }^{+1}, 1990$ + KY

ST: PO.KN • CI: h0175 • ID: $\uparrow 097$

PN: Baurubatrachus pricei Báez $^{+1}, 1990$ \$

PK: Baurubatrachus pricei ${ }^{\circ} \mathrm{Báez}^{+1}, 1990 \dagger$

KG: Baurubatrachus ${ }^{\circ}$ Báez $^{+1}, 1990 \dagger$

KF: CERATOPHRYIDAE 1838.ta.f002

Bdellophis Boulenger, 1895 • AK

ST: PO.JD・CI: h0176・ID: 499

PN: Bdellophis vittatus Boulenger, 1895

PK: Bdellophis vittatus* Boulenger, 1895

KG: Scolecomorphus ${ }^{2}$ Boulenger, 1883

KF: SCOLECOMORPHIDAE 1969.ta.f001

Beddomixalus Abraham ${ }^{+4}, 2013$ • KY

ST: PO.KN • CI: h0177•ID: 442

PN: Polypedates bijui Zachariah ${ }^{+5}, 2011$

PK: Polypedates bijui* Zachariah ${ }^{+5}, 2011$

KG: Beddomixalus $*$ Abraham $^{+4}, 2013$

KF: RHACOPHORIDAE ||1858.gc.f012||-1932.ha.f001
Beduka nov. • KY

ST: PO.KN • CI: h0178 • ID: 105

PN: Bufo koynayensis Soman, 1963

PK: Bufo koynayensis* Soman, 1963

KG: Beduka* nov.

KF: BUFONIDAE 1825.gb.f004

Beelzebufo Evans $^{+1}, 2008+\cdot \mathbf{K Y}$

ST: PO.KN • CI: h0179・ID: $\uparrow 096$

PN: Beelzebufo ampinga Evans $^{+1}, 2008$ \$

PK: Beelzebufo amping ${ }^{\circ}$ Evans $^{+1}, 2008 \uparrow$

KG: Beelzebufo ${ }^{\circ}$ Evans $^{+1}, 2008 \dagger$

KF: CERATOPHRYIDAE 1838.ta.f002

Beiyanerpeton $\mathrm{Gao}^{+1}, 2012+\bullet \mathbf{K Y}$

ST: PO.KN • CI: h0180 •ID: $\uparrow 177$

PN: Beiyanerpeton jianpingensis $\mathrm{Gao}^{+1}, 2012$ *

PK: Beiyanerpeton jianpingensis ${ }^{\circ} \mathrm{Gao}^{+1}, 2012 \dagger$

KG: Beiyanerpeton ${ }^{\circ} \mathrm{Gao}^{+1}, 2012 \uparrow$

KF: Pseudosauria Familia InCERTae sedis

Berdmorea Stoliczka, 1872 • AK

ST: PO.JD • CI: h0181 • ID: 305

PN: Engystoma interlineatum Blyth, 1855

PK: Engystoma interlineatum* Blyth, 1855

KG: Kalophrynus* Tschudi, 1838

KF: MICROHYLIDAE ||1843.fa.f012||-1931.na.f001

Bijurana Chandramouli ${ }^{+3}, 2020 \bullet \mathbf{A K}$

ST: PO.JD・CI: h0182・ID: 409

PN: Hylorana nicobariensis Stoliczka, 1870

PK: Hylarana nicobariensis* (Stoliczka, 1870)

KG: Hylarana* Tschudi, 1838

KF: RANIDAE 1796.ba.f001

Bilaterana Bauer, 1985 • AK

ST: PO.JD・CI: h0183・ID: 406

PN: Rana ridibunda Pallas, 1771

PK: Rana ridibunda* Pallas, 1771

KG: Pelophylax* Fitzinger, 1843

KF: RANIDAE 1796.ba.f001

Bishara Nessov, 1997 • KY

ST: PO.KN • CI: h0184・ID: $\uparrow 126$

PN: Bishara backa Nessov, 1997 †

PK: Bishara backa ${ }^{\circ}$ Nessov, $1997 \dagger$

KG: Bishara ${ }^{\circ}$ Nessov, $1997 \dagger$

KF: Urodela Familia INCERTAE SEDIS

Bissektia Nessov, 1981 + KY

ST: PO.KN • CI: h0185・ID: $\uparrow 127$

PN: Bissektia nana Nessov, 1981 \$

PK: Bissektia nana ${ }^{\circ}$ Nessov, $1981 \dagger$

KG: Bissektia ${ }^{\circ}$ Nessov, $1981 \dagger$

KF: Urodela Familia INCERTAE SEDIS

Blaira nov. $\bullet \mathrm{KY}$

ST: PO.KN・CI: h0186・ID: 116

PN: Ansonia ornata Gunther, 1876

PK: Ansonia ornata* Gunther, 1876

KG: Blaira* nov.

KF: BUFONIDAE 1825.gb.f004

Blepsimolge Hillis ${ }^{+3}$, 2001 • AK

ST: PO.JD • CI: h0187・ID: 542 
PN: Eurycea nana Bishop, 1941

PK: Eurycea nana* Bishop, 1941

KG: Eurycea* Rafinesque, 1822

KF: PLETHODONTIDAE 1850.ga.f002

Blommersia Dubois, $1992 \cdot \mathbf{K Y}$

ST: PO.KN • CI: h0188・ID: 426

PN: Gephyromantis blommersae Guibé, 1975

PK: Gephyromantis blommersae* Guibé, 1975

KG: Blommersia* Dubois, 1992

KF: RHACOPHORIDAE ||1858.gc.f012||-1932.ha.f001

Blythophryne Chandramouli ${ }^{+7}, 2016 \cdot \mathbf{K Y}$

ST: PO.KN • CI: h0189 • ID: 106

PN: Blythophryne beryet Chandramouli ${ }^{+7}, 2016$

PK: Blythophryne beryet ${ }^{\circ}$ Chandramouli $^{+7}, 2016$

KG: Blythophryne ${ }^{\circ}$ Chandramouli $^{+7}, 2016$

KF: BUFONIDAE 1825.gb.f004

Boana Gray, $1825 \bullet \mathbf{K Y}$

ST: PO.KN • CI: h0190 • ID: 189

PN: Rana boans Linnaeus, 1758

PK: Rana boans* Linnaeus, 1758

KG: Boana* Gray, 1825

KF: HYLIDAE 1815.ra.f002-|1825.gb.f001|

Boehmantis Glaw ${ }^{+1}, 2006 \cdot \mathbf{K Y}$

ST: PO.KN • CI: h0191 • ID: 430

PN: Mantidactylus microtympanum Angel, 1935

PK: Mantidactylus microtympanum* Angel, 1935

KG: Boehmantis* Glaw $^{+1}, 2006$

KF: RHACOPHORIDAE ||1858.gc.f012||-1932.ha.f001

Bokermannohyla Faivovich ${ }^{+5}, 2005$ • KY

ST: PO.KN • CI: h0192 • ID: 187

PN: Hyla circumdata Cope, 1871

PK: Hyla circumdata* Cope, 1871

KG: Bokermannohyla* Faivovich ${ }^{+5}, 2005$

KF: HYLIDAE 1815.ra.f002-|1825.gb.f001|

Bolitoglossa Duméril ${ }^{+2}, 1854$ • KY

ST: PO.KN • CI: h0193・ID: 522

PN: Bolitoglossa mexicana Duméril ${ }^{+2}, 1854$

PK: Bolitoglossa mexicana* Duméril ${ }^{+2}, 1854$

KG: Bolitoglossa * Duméril ${ }^{+2}, 1854$

KF: PlethodontIDAE 1850.ga.f002

Bombina Oken, 1816 • CK • KY

ST: PO.KN • CI: h0194 • ID: 472

PN: Rana bombina Linnaeus, 1760

PK: Rana bombina* Linnaeus, 1760

KG: Bombina* Oken, 1816

KF: BOMBINATORIDAE 1825.gb.f002

Bombinator Merrem, 1820 • AK

ST: PO.JD • CI: h0195 • ID: 472

PN: Bufo igneus Laurenti, 1768

PK: Rana bombina* Linnaeus, 1760

KG: Bombina* Oken, 1816

KF: BOMBINATORIDAE 1825.gb.f002

Bombitator Wagler, 1830 • AK

ST: NT.JD • CI: h0196 • ID: 472

PN: Bufo igneus Laurenti, 1768

PK: Rana bombina* Linnaeus, 1760
KG: Bombina* Oken, 1816

KF: BOMBINATORIDAE 1825.gb.f002

Boophis Tschudi, $1838 \cdot \mathbf{K Y}$

ST: PO.KN • CI: h0197・ ID: 423

PN: Boophis goudotii Tschudi, 1838

PK: Boophis goudotii* Tschudi, 1838

KG: Boophis* Tschudi, 1838

KF: RHACOPHORIDAE ||1858.gc.f012||-1932.ha.f001

Borborocoetea Strand, 1928 • AK

ST: NT.JD • CI: h0198 • ID: 174

PN: Borborocoetes grayii Bell, 1843

PK: Cystignathus roseus* Duméril ${ }^{+1}, 1841$

KG: Eupsophus* Fitzinger, 1843

KF: ALSODIDAE 1869.mc.f005

Borborocoetes Schoenherr, $1842 \cdot \mathbf{Z H}$

ST: zo • CI: zh012

Borborocoetes Bell, 1843 • AK

ST: PO.JH • CI: h0199 • ID: 174

PN: Borborocoetes grayii Bell, 1843

PK: Cystignathus roseus* Duméril ${ }^{+1}, 1841$

KG: Eupsophus* Fitzinger, 1843

KF: ALSODIDAE 1869.mc.f005

Borborocoites Gistel, $1848+\cdot \mathbf{A K}$

ST: NL.JI • CI: h0200 • ID: $\uparrow 069$

PN: Palaeobatrachus goldfussii Tschudi, 1838 \$

PK: Rana diluviana ${ }^{\circ}$ Goldfuss, $1831 \dagger$

KG: Palaeobatrachus ${ }^{\circ}$ Tschudi, $1838 \uparrow$

KF: PALAEOBATRACHIDAE 1865.ca.f001 †

Borealophrys $\mathrm{Fei}^{+2}, 2016$ • AK

ST: PO.JD • CI: h0201 • ID: 019

PN: Megophrys nankiangensis $\mathrm{Liu}^{+1}, 1966$

PK: Megophrys nankiangensis* Liu $^{+1}, 1966$

KG: Atympanophrys* Tian $^{+1}, 1983$

KF: MEGOPHRYIDAE 1850.bb.f008-|1931.na.f003|

Boreorana nov. $\bullet \mathrm{KY}$

ST: PO.KN • CI: h0202 • ID: 414

PN: Rana sylvatica Le Conte, 1825

PK: Rana sylvatica* Le Conte, 1825

KG: Boreorana* nov.

KF: RANIDAE 1796.ba.f001

Borneophrys Delorme ${ }^{+3}, 2006$ • AK

ST: PO.JD • CI: h0203 • ID: 021

PN: Megophrys edwardinae Inger, 1989

PK: Megophrys edwardinae ${ }^{\circ}$ Inger, 1989

KG: Megophrys ${ }^{2} \mathrm{Kuhl}^{+1}, 1822$

KF: MEGOPHRYIDAE 1850.bb.f008-|1931.na.f003|

Boulengerana $\mathrm{Fei}^{+2}, 2010 \cdot \mathbf{A K}$

ST: PO.JD・CI: h0204・ID: 409

PN: Rana guentheri Boulenger, 1882

PK: Rana guentheri* Boulenger, 1882

KG: Hylarana* Tschudi, 1838

KF: RANIDAE 1796.ba.f001

Boulengerula Tornier, $1896 \cdot \mathbf{K Y}$

ST: PO.KN • CI: h0205・ID: 496

PN: Boulengerula boulengeri Tornier, 1896

PK: Boulengerula boulengeri* Tornier, 1896 
KG: Boulengerula* Tornier, 1896

KF: CAECILIIDAE 1814.ra.f003-|1825.gb.f008|

Boulenophrys $\mathrm{Fei}^{+2}, 2016 \cdot \mathbf{K Y}$

ST: PO.KN • CI: h0206・ID: 023

PN: Leptobrachium boettgeri Boulenger, 1899

PK: Leptobrachium boettgeri* Boulenger, 1899

KG: Boulenophrys* $\mathrm{Fei}^{+2}, 2016$

KF: MEGOPHRYIDAE 1850.bb.f008-|1931.na.f003

Bourretia Dubois, 1987 • AK

ST: PO.JD • CI: h0207 • ID: 380

PN: Rana toumanoffi Bourret, 1941

PK: Rana macrognathus dabana* Smith, 1922

KG: Limnonectes* Fitzinger, 1843

KF: DiCROGLOSSIDAE 1987.da.f004

Brachycephalus Fitzinger, 1826 • KY

ST: PO.KN • CI: h0208 • ID: 057

PN: Bufo ephippium Spix, 1824

PK: Bufo ephippium* Spix, 1824

KG: Brachycephalus* Fitzinger, 1826

KF: BRACHYCEPHALIDAE 1858.gc.f002

Brachycormus Meyer, $1860+\cdot \mathbf{K Y}$

ST: PO.KN • CI: h0209・ID: $\uparrow 191$

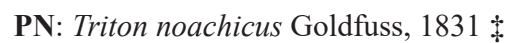

PK: Triton noachicus ${ }^{\circ}$ Goldfuss, $1831 \dagger$

KG: Brachycormus ${ }^{\circ}$ Meyer, $1860 \dagger$

KF: SALAMANDRIDAE 1820.ga.f002

Brachymerus: Dejean $1835 \cdot \mathbf{z A}$

ST: $\mathbf{z N} \bullet \mathbf{C I}: \mathbf{z n} 002$

Brachymerus Chevrolat in Hope, $1841 \cdot \mathbf{z H}$ ST: zo • CI: zh013

Brachymerus Smith, $1847 \cdot \mathbf{A K}$

ST: PO.JH • CI: h0210 • ID: 319

PN: Brachymerus bifasciatus Smith, 1847

PK: Brachymerus bifasciatus* Smith, 1847

KG: Phrynomantis* Peters, 1867

KF: PHRYNOMERIDAE 1931.na.f013

Brachytarsophrys $\operatorname{Tian}^{+1}, 1983 \cdot \mathbf{K Y}$

ST: PO.KN • CI: h0211 • ID: 020

PN: Leptobrachium carinensis Boulenger, 1889

PK: Leptobrachium carinensis* Boulenger, 1889

KG: Brachytarsophrys* Tian $^{+1}, 1983$

KF: MEGOPHRYIDAE 1850.bb.f008-|1931.na.f003|

Bradyarges Gistel, 1868 • AK

ST: NL.JI • CI: h0212 • ID: 557

PN: Euproctus rusconii Gené, 1839

PK: Molge platycephala* Gravenhorst, 1829

KG: Euproctus ${ }^{1}$ Gené, 1839

KF: SALAMANDRIDAE 1820.ga.f002

Bradybates Tschudi, $1838 \cdot \mathbf{A K}$

ST: PO.JD・CI: h0213・ID: 571

PN: Bradybates ventricosus Tschudi, 1838

PK: Pleurodeles walt ${ }^{*}$ Michahelles, 1830

KG: Pleurodeles* Michahelles, 1830

KF: SALAMANDRIDAE 1820.ga.f002

Bradymedusa Miranda-Ribeiro, 1926 • AK

ST: PO.JD • CI: h0214 • ID: 245
PN: Bradymedusa moschata Miranda-Ribeiro, 1926

PK: Phyllomedusa rohdei* Mertens, 1926

KG: Pithecopus* Cope, 1866

KF: PHYLLOMEDUSIDAE 1858.gc.f009

Bradytes: Dejean $1834 \cdot \mathbf{z A}$

ST: $\mathbf{z N} \cdot \mathbf{C I}: \mathbf{z n} 003$

Bradytes Gistel, $1848 \cdot \mathbf{A K}$

ST: NL.JD・CI: h0215・ID: 571

PN: Bradybates ventricosus Tschudi, 1838

PK: Pleurodeles walt * Michahelles, 1830

KG: Pleurodeles* Michahelles, 1830

KF: SALAMANDRIDAE 1820.ga.f002

Bradytriton Wake $^{+1}, 1983 \cdot \mathbf{K Y}$

ST: PO.KN • CI: h0216・ID: 533

PN: Bradytriton silus Wake $^{+1}, 1983$

PK: Bradytriton silus* Wake $^{+1}, 1983$

KG: Bradytriton* Wake $^{+1}, 1983$

KF: PLETHODONTIDAE 1850.ga.f002

Brasilotyphlus Taylor, $1968 \bullet \mathbf{K Y}$

ST: PO.KN • CI: h0217 • ID: 490

PN: Gymnopis braziliensis Dunn, 1945

PK: Gymnopis braziliensis ${ }^{\circ}$ Dunn, 1945

KG: Brasilotyphlus ${ }^{\circ}$ Taylor, 1968

KF: CAECILIIDAE 1814.ra.f003-|1825.gb.f008|

Brendanura Wells $^{+1}, 1985 \cdot \mathbf{A K}$

ST: PO.JD • CI: h0218・ID: 237

PN: Chiroleptes alboguttatus Günther, 1867

PK: Chiroleptes alboguttatus* Günther, 1867

KG: Ranoidea ${ }^{1}$ Tschudi, 1838

KF: PHYLLOMEDUSIDAE 1858.gc.f009

Breviceps Merrem, $1820 \bullet \mathbf{K Y}$

ST: PO.KN • CI: h0219・ID: 342

PN: Rana gibbosa Linnaeus, 1758

PK: Rana gibbosa ${ }^{\circ}$ Linnaeus, 1758

KG: Breviceps $^{3}$ Merrem, 1820

KF: BREVICIPITIDAE 1850.bb.f012

Bromeliohyla Faivovich $^{+5}, 2005 \cdot \mathbf{K Y}$

ST: PO.KN • CI: h0220 • ID: 213

PN: Hyla bromeliacia Schmidt, 1933

PK: Hyla bromeliacia* Schmidt, 1933

KG: Bromeliohyla* Faivovich ${ }^{+5}, 2005$

KF: HYLIDAE 1815.ra.f002-|1825.gb.f001|

Brygoomantis Dubois, 1992 • AK

ST: PO.JD • CI: h0221・ID: 432

PN: Limnodytes ulcerosus Boettger, 1880

PK: Limnodytes ulcerosus* Boettger, 1880

KG: Mantidactylus* Boulenger, 1895

KF: RHACOPHORIDAE ||1858.gc.f012||-1932.ha.f001

Bryobatrachus Rounsevell ${ }^{+4}, 1994 \cdot \mathbf{A K}$

ST: PO.JD • CI: h0222 • ID: 270

PN: Bryobatrachus nimbus Rounsevel1 ${ }^{+4}, 1994$

PK: Bryobatrachus nimbus* Rounsevell ${ }^{+4}, 1994$

KG: Crinia* Tschudi, 1838

KF: MYOBATRACHIDAE 1850.sa.f001

Bryophryne Hedges $^{+2}, 2008 \cdot \mathbf{K Y}$

ST: PO.KN • CI: h0223・ID: 066 
PN: Phrynopus cophites Lynch, 1975

PK: Phrynopus cophites* Lynch, 1975

KG: Bryophryne* Hedges $^{+2}, 2008$

KF: BRACHYCEPHALIDAE 1858.gc.f002

Bryotriton Dubois ${ }^{+1}, 2012$ • AK

ST: PO.JD • CI: h0224 • ID: 537

PN: Oedipus barbouri Schmidt, 1936

PK: Oedipus barbouri* Schmidt, 1936

KG: Nototriton* Wake ${ }^{+1}, 1983$

KF: PLETHODONTIDAE 1850.ga.f002

Bubonias Cope, 1874 • AK

ST: PO.JD • CI: h0225 • ID: 247

PN: Bubonias plicifrons Cope, 1874

PK: Edalorhina perezi* Jiménez de la Espada, 1870

KG: Edalorhina* Jiménez de la Espada, 1870

KF: LEIUPERIDAE 1850.bb.f010

Buccinator Gistel, 1848 • AK

ST: NL.JI • CI: h0226・ID: 423

PN: Boophis goudotii Tschudi, 1838

PK: Boophis goudotii* Tschudi, 1838

KG: Boophis* Tschudi, 1838

KF: RHACOPHORIDAE ||1858.gc.f012||-1932.ha.f001

Buergeria Tschudi, $1838 \cdot \mathbf{K Y}$

ST: PO.KN • CI: h0227・ID: 436

PN: Hyla buergeri Temminck $^{+1}, 1838$

PK: Hyla buergeri* ${ }^{*}$ Temminck $^{+1}, 1838$

KG: Buergeria* Tschudi, 1838

KF: RHACOPHORIDAE |1858.gc.f012||-1932.ha.f001

Bufavus Portis, $1885+\mathbf{A K}$

ST: PO.JD・CI: h0228 • ID: 120

PN: Bufavus meneghinii Portis, 1885 *

PK: Rana bufo* Linnaeus, 1758

KG: $B$ ufo* Garsault, 1764

KF: BUFONIDAE 1825.gb.f004

Buffo La Cepède, $1788 \cdot \mathbf{E X}$

ST: NT.CW • CI: e0004 • ID: 121

PN: Bufo viridis Laurenti, 1768

PK: Bufo viridis* Laurenti, 1768

KG: Bufotes* Rafinesque, 1815

KF: BUFONIDAE 1825.gb.f004

Buffo Montfort, $1810 \cdot \mathbf{Z H}$

ST: zo • CI: zh014

Bufo: Rösel von Rosenhof 1758 • AN

ST: AL $\cdot \mathbf{C I}:$ n0015 • ID: 120

PN: Rana bufo Linnaeus, 1758

PK: Rana bufo* Linnaeus, 1758

KG: Bufo* Garsault, 1764

KF: BUFONIDAE 1825.gb.f004

Bufo: Vogel $1758 \cdot \mathbf{A N}$

ST: AL $・$ CI: n0016・ID: 120

PN: Rana bufo Linnaeus, 1758

PK: Rana bufo* Linnaeus, 1758

KG: Bufo* Garsault, 1764

KF: BUFONIDAE 1825.gb.f004

Bufo Garsault, 1764 • KY

ST: PO.KN • CI: h0230 • ID: 120
PN: Rana bufo Linnaeus, 1758

PK: Rana bufo* Linnaeus, 1758

KG: Bufo* Garsault, 1764

KF: BUFONIDAE 1825.gb.f004

Bufo Laurenti, 1768 • AK

ST: PO.JH • CI: h0231・ID: 121

PN: Bufo viridis Laurenti, 1768

PK: Bufo viridis* Laurenti, 1768

KG: Bufotes* Rafinesque, 1815

KF: BUFONIDAE 1825.gb.f004

Bufoides Pillai ${ }^{+1}, 1974 \cdot \mathbf{K Y}$

ST: PO.KN • CI: h0232・ID: 107

PN: Ansonia meghalayana Yazdani ${ }^{+1}, 1971$

PK: Ansonia meghalayana ${ }^{\circ}$ Yazdani $^{+1}, 1971$

KG: Bufoides ${ }^{\circ}$ Pillai $^{+1}, 1974$

KF: BUFONIDAE 1825.gb.f004

Bufonella Girard, 1853 • AK

ST: PO.JD • CI: h0233 • ID: 274

PN: Bufonella crucifera Girard, 1853

PK: Bombinator australis ${ }^{\circ}$ Gray, 1835

KG: Pseudophryne ${ }^{3}$ Fitzinger, 1843

KF: MYOBATRACHIDAE 1850.sa.f001

Bufonopsis Kuhn, $1941+\bullet \mathbf{A K}$

ST: PO.JD • CI: h0234 • ID: $\uparrow 069$

PN: Bufonopsis dentatus Kuhn, 1941 \$

PK: Pelobatinopsis hinschei ${ }^{\circ}$ Kuhn, $1941 \dagger$

KG: Palaeobatrachus ${ }^{\circ}$ Tschudi, $1838 \dagger$

KF: PALAEOBATRACHIDAE 1865.ca.f001 †

Bufotes Rafinesque, $1815 \cdot \mathbf{K Y}$

ST: PO.KN • CI: h0235・ID: 121

PN: Bufo viridis Laurenti, 1768

PK: Bufo viridis* Laurenti, 1768

KG: Bufotes* Rafinesque, 1815

KF: BUFONIDAE 1825.gb.f004

Bulga Gistel, 1868 • AK

ST: NL.JI • CI: h0236・ID: 557

PN: Euproctus rusconii Gené, 1839

PK: Molge platycephala* Gravenhorst, 1829

KG: Euproctus ${ }^{1}$ Gené, 1839

KF: SALAMANDRIDAE 1820.ga.f002

Bulua Boulenger, 1904 • AK

ST: PO.JD • CI: h0237 • ID: 324

PN: Bulua ventrimarmorata Boulenger, 1904

PK: Bulua ventrimarmorata ${ }^{\circ}$ Boulenger, 1904

KG: Leptodactylodon ${ }^{3}$ Andersson, 1903

KF: ARTHROLEPTIDAE 1869.mc.f011

Cacophryne Davis, 1935 • AK

ST: PO.JD • CI: h0238 • ID: 123

PN: Hylaplesia borbonica Tschudi, 1838

PK: Hylaplesia borbonica* Tschudi, 1838

KG: Leptophryne ${ }^{2}$ Fitzinger, 1843

KF: BUFONIDAE 1825.gb.f004

Cacophrynus Cope, 1867 • AK

ST: PO.JD • CI: h0239 • ID: 347

PN: Kakophrynus sudanensis Steindachner, 1863

PK: Engystoma marmoratum* Peters, 1854 
KG: Hemisus ${ }^{2}$ Günther, 1859

KF: HEMISOTIDAE 1867.ca.f002

Cacopoides Barbour, 1908 • AK

ST: PO.JD • CI: h0240 • ID: 310

PN: Cacopoides borealis Barbour, 1908

PK: Cacopoides borealis* Barbour, 1908

KG: Kaloula* Gray, 1831

KF: MICROHYLIDAE ||1843.fa.f012||-1931.na.f001

Cacopus Günther, 1864 • AK

ST: NL.JI • CI: h0241 • ID: 309

PN: Engystoma marmoratum Guérin-Méneville, 1838

PK: Rana systoma* Schneider, 1799

KG: Uperodon ${ }^{1}$ Duméril $^{+1}, 1841$

KF: MICROHYLIDAE ||1843.fa.f012||-1931.na.f001

Cacosternum Boulenger, 1887 • KY

ST: PO.KN • CI: h0242 • ID: 357

PN: Cacosternum nanum Boulenger, 1887

PK: Cacosternum nanum* Boulenger, 1887

KG: Cacosternum* Boulenger, 1887

KF: CACOSTERNIDAE 1931.na.f008

Cacotus Günther, 1869 • AK

ST: PO.JD • CI: h0243 • ID: 173

PN: Cacotus maculatus Günther, 1869

PK: Cystignathus nodosus* Duméril ${ }^{+1}, 1841$

KG: Alsodes* Bell, 1843

KF: ALSODIDAE 1869.mc.f005

Caecilia Linnaeus, $1758 \bullet \mathbf{K Y}$

ST: LC.KN • CI: h0244・ID: 474

PN: Caecilia tentaculata Linnaeus, 1758

PK: Caecilia tentaculata* Linnaeus, 1758

KG: Caecilia* Linnaeus, 1758

KF: CAECILIIDAE 1814.ra.f003-|1825.gb.f008|

Caecilita Wake $^{+1}, 2009 \cdot \mathbf{A K}$

ST: PO.JD • CI: h0245 • ID: 492

PN: Caecilita iwokramae $\mathrm{Wake}^{+1}, 2009$

PK: Caecilita iwokramae ${ }^{\circ}$ Wake $^{+1}, 2009$

KG: Microcaecilia ${ }^{3}$ Taylor, 1968

KF: CAECILIIDAE 1814.ra.f003-|1825.gb.f008|

Caecilius Curtis, $1837 \cdot \mathbf{z H}$

ST: $\mathbf{z F} \bullet \mathbf{C I}$ : zh015

Calamita Schneider, 1799 • AK

ST: PO.JI • CI: h0246・ID: 204

PN: Rana arborea Linnaeus, 1758

PK: Rana arborea* Linnaeus, 1758

KG: Hyla* Laurenti, 1768

KF: HYLIDAE 1815.ra.f002-|1825.gb.f001|

Calamita Oken, 1816 • EX

ST: PO.CW • CI: e0005・ID: 122

PN: Bufo calamita Laurenti, 1768

PK: Bufo calamita* Laurenti, 1768

KG: Epidalea* Cope, 1864

KF: BUFONIDAE 1825.gb.f004

Calamita Fitzinger, 1826 • AK

ST: PO.JH • CI: h0248 • ID: 237

PN: Hyla cyanea Daudin, 1803

PK: Rana caerulea* White, 1890
KG: Ranoidea ${ }^{1}$ Tschudi, 1838

KF: PHYLLOMEDUSIDAE 1858.gc.f009

Calamites Guettard, $1770 \bullet \mathbf{z H}$

ST: zo • CI: zh016

Calamites Wagler, 1830 • AK

ST: PO.JH • CI: h0249 • ID: 237

PN: Rana caerulea White, 1890

PK: Rana caerulea* White, 1890

KG: Ranoidea ${ }^{1}$ Tschudi, 1838

KF: PHYLLOMEDUSIDAE 1858.gc.f009

Calamitus: Rafinesque 1815 • AN

ST: AL •CI: n0017・ID: 122

PN: Bufo calamita Laurenti, 1768

PK: Bufo calamita* Laurenti, 1768

KG: Epidalea* Cope, 1864

KF: BUFONIDAE 1825.gb.f004

Calamobates Witte, 1930 • AK

ST: PO.JD・CI: h0250・ID: 181

PN: Calamobates boulengeri Witte, 1930

PK: Calamobates boulengeri ${ }^{\circ}$ Witte, 1930

KG: Crossodactylus ${ }^{3}$ Duméril $^{+1}, 1841$

KF: HYLODIDAE 1858.gc.f010

Caledon Goldfuss, 1820 • AK

ST: NL.JI • CI: h0251 • ID: 554

PN: Proteus anguinus Laurenti, 1768

PK: Proteus anguinus* Laurenti, 1768

KG: Proteus* Laurenti, 1768

KF: PROTEIDAE 1831.ba.f002

Calliglutus Barbour ${ }^{+1}$, 1916 • AK

ST: PO.JD・CI: h0252・ID: 313

PN: Calliglutus smithi Barbour ${ }^{+1}, 1916$

PK: Calliglutus smithi ${ }^{\circ}$ Barbour $^{+1}, 1916$

KG: Glyphoglossus* Günther, 1869

KF: MICROHYLIDAE ||1843.fa.f012||-1931.na.f001

Callimedusa Duellman ${ }^{+2}, 2016 \bullet \mathbf{K Y}$

ST: PO.KN • CI: h0253・ID: 244

PN: Phyllomedusa perinesos Duellman, 1973

PK: Phyllomedusa perinesos* Duellman, 1973

KG: Callimedusa* Duellman ${ }^{+2}, 2016$

KF: PHYLLOMEDUSIDAE 1858.gc.f009

Calliopersa Safaei-Mahroo \& Ghaffari, 2020 • AK

ST: PO.JD • CI: h0254 • ID: 121

PN: Bufo surdus Boulenger, 1891

PK: Bufotes surdus ${ }^{\circ}$ (Boulenger, 1891)

KG: Bufotes* Rafinesque, 1815

KF: BUFONIDAE 1825.gb.f004

Calliphryne Agassiz, 1847 • AK

ST: NT.JI • CI: h0255 • ID: 305

PN: Kalophrynus pleurostigma Tschudi, 1838

PK: Kalophrynus pleurostigma* Tschudi, 1838

KG: Kalophrynus* Tschudi, 1838

KF: MICROHYLIDAE ||1843.fa.f012||-1931.na.f001

Callixalus Laurent, $1950 \bullet \mathbf{K Y}$

ST: PO.KN • CI: h0256・ID: 327

PN: Callixalus pictus Laurent, 1950

PK: Callixalus pictus ${ }^{\circ}$ Laurent, 1950 
KG: Callixalus ${ }^{\circ}$ Laurent, 1950

KF: HYPEROLIIDAE 1943.lb.f001

Callobatrachus Wang ${ }^{+1}, 1999+\cdot \mathbf{K Y}$

ST: PO.KN • CI: h0257• ID: $\uparrow 107$

PN: Callobatrachus sanyanensis $\mathrm{Wang}^{+1}, 1999$ *

PK: Callobatrachus sanyanensis ${ }^{\circ}$ Wang $^{+1}, 1999+$

KG: Callobatrachus ${ }^{\circ} \mathrm{Wang}^{+1}, 1999 \dagger$

KF: Mediogyrinia Familia INCERTAE SEDIS

Calluella Stoliczka, 1872 • AK

ST: PO.JD • CI: h0258・ID: 313

PN: Megalophrys guttulata Blyth, 1856

PK: Megalophrys guttulata* Blyth, 1856

KG: Glyphoglossus* Günther, 1869

KF: MICROHYLIDAE ||1843.fa.f012||-1931.na.f001

Callula Günther, 1864 • AK

ST: NT.JI • CI: h0259 • ID: 310

PN: Kaloula pulchra Gray, 1831

PK: Kaloula pulchra* Gray, 1831

KG: Kaloula* Gray, 1831

KF: MiCROHYLIDAE |1843.fa.f012 |-1931.na.f001

Callulina Nieden, $1911 \cdot \mathbf{K Y}$

ST: PO.KN • CI: h0260 • ID: 344

PN: Callulina kreffti Nieden, 1911

PK: Callulina kreffti* Nieden, 1911

KG: Callulina* Nieden, 1911

KF: BREVICIPITIDAE 1850.bb.f012

Callulops Boulenger, 1888 • AK

ST: PO.JD • CI: h0261 • ID: 280

PN: Callulops doriae Boulenger, 1888

PK: Callulops doriae* Boulenger, 1888

KG: Asterophrys* Tschudi, 1838

KF: MICROHYLIDAE ||1843.fa.f012||-1931.na.f001

Calofrynus Palacký, 1898 • AK

ST: NT.JI • CI: h0262 • ID: 305

PN: Kalophrynus pleurostigma Tschudi, 1838

PK: Kalophrynus pleurostigma* Tschudi, 1838

KG: Kalophrynus* Tschudi, 1838

KF: MICROHYLIDAE ||1843.fa.f012||-1931.na.f001

Calohyla Peters, 1863 - AK

ST: NT.JI • CI: h0263・ID: 310

PN: Kaloula pulchra Gray, 1831

PK: Kaloula pulchra* Gray, 1831

KG: Kaloula* Gray, 1831

KF: MICROHYLIDAE ||1843.fa.f012||-1931.na.f001

Calophryne Fitzinger, 1843 • AK

ST: NS.JI • CI: h0264・ID: 305

PN: Kalophrynus pleurostigma Tschudi, 1838

PK: Kalophrynus pleurostigma* Tschudi, 1838

KG: Kalophrynus* Tschudi, 1838

KF: MICROHYLIDAE |1843.fa.f012||-1931.na.f001

Calophrynus Cope, $1863 \cdot \mathbf{A K}$

ST: NS.JI • CI: h0265・ID: 305

PN: Kalophrynus pleurostigma Tschudi, 1838

PK: Kalophrynus pleurostigma* Tschudi, 1838

KG: Kalophrynus* Tschudi, 1838

KF: MICROHYLIDAE ||1843.fa.f012||-1931.na.f001
Calostethus Mivart, 1869 • AK

ST: NS.JI • CI: h0266・ ID: 040

PN: Phyllobates latinasus Cope, 1863

PK: Phyllobates latinasus* Cope, 1863

KG: Colostethus* Cope, 1866

KF: DENDROBATIDAE |1850.bb.f006||-1865.ca.f002

Calotriton Gray, $1858 \cdot \mathbf{K Y}$

ST: PO.KN • CI: h0267 • ID: 565

PN: Hemitriton punctulatus Dugès, 1852

PK: Hemitriton asper* Dugès, 1852

KG: Calotriton ${ }^{1}$ Gray, 1858

KF: SALAMANDRIDAE 1820.ga.f002

Calyptahyla Trueb $^{+1}, 1974 \cdot \mathbf{A K}$

ST: PO.JD・CI: h0268・ID: 225

PN: Trachycephalus lichenatus Gosse, 1851

PK: Hyla crucialis* Harlan, 1826

KG: Osteopilus ${ }^{1}$ Fitzinger, 1843

KF: HYLIDAE 1815.ra.f002-|1825.gb.f001|

Calyptocephala : Dejean $1834 \cdot \mathbf{z A}$

ST: $\mathbf{z N} \cdot \mathbf{C I}: \mathbf{z n} 004$

Calyptocephala Boheman, $1850 \cdot \mathbf{Z H}$

ST: zo • CI: zh017

Calyptocephala Nieden, 1923 • AK

ST: PO.JH • CI: h0269 • ID: 257

PN: Calyptocephalus gayi Duméril ${ }^{+1}, 1841$

PK: Calyptocephalus gayi* Duméril ${ }^{+1}, 1841$

KG: Calyptocephalella* Strand, 1928

KF: CALYPTOCEPHALELLIDAE 1960.ra.f001

Calyptocephalella Strand, 1928・ KY

ST: PO.KN • CI: h0270 • ID: 257

PN: Calyptocephalus gayi Duméril ${ }^{+1}, 1841$

PK: Calyptocephalus gayi* Duméril ${ }^{+1}, 1841$

KG: Calyptocephalella* Strand, 1928

KF: CALYPTOCEPHALELLIDAE 1960.ra.f001

Calyptocephalus Gray, $1832 \cdot \mathbf{z H}$

ST: zo • CI: zh018

Calyptocephalus Duméril ${ }^{+1}, 1841$ • AK

ST: PO.JH • CI: h0271 • ID: 257

PN: Calyptocephalus gayi Duméril ${ }^{+1}, 1841$

PK: Calyptocephalus gayi* Duméril ${ }^{+1}, 1841$

KG: Calyptocephalella* Strand, 1928

KF: CALYPTOCEPHALELLIDAE 1960.ra.f001

Camarataxis Cope, $1859 \cdot \mathbf{A K}$

ST: PO.JD・CI: h0272・ID: 555

PN: Ambystoma maculatum Hallowell, 1858

PK: Ambystoma mavortia ${ }^{\circ}$ Baird, 1850

KG: Ambystoma ${ }^{1}$ Tschudi, 1838

KF: AMBYSTOMATIDAE 1850.ga.f004

Camariolius Peters, 1863 • AK

ST: PO.JD・CI: h0273・ID: 270

PN: Camariolius varius Peters, 1863

PK: Crinia (Ranidella) signifera* Girard, 1853

KG: Crinia* Tschudi, 1838

KF: MYOBATRACHIDAE 1850.sa.f001

Campbellius Hedges ${ }^{+2}$, 2008 • AK

ST: PO.JD • CI: h0274 • ID: 059 
PN: Eleutherodactylus stadelmani Schmidt, 1936

PK: Eleutherodactylus stadelmani ${ }^{\circ}$ Schmidt, 1936

KG: Craugastor* Cope, 1862

KF: BRACHYCEPHALIDAE 1858.gc.f002

Capensibufo Grandison, $1980 \cdot \mathbf{K Y}$

ST: PO.KN • CI: h0275 • ID: 139

PN: Bufo tradouwi Hewitt, 1926

PK: Bufo tradouwi* Hewitt, 1926

KG: Capensibufo* Grandison, 1980

KF: BUFONIDAE 1825.gb.f004

Cardioglossa Boulenger, 1900 • AK

ST: PO.JD • CI: h0276・ ID: 320

PN: Cardioglossa gracilis Boulenger, 1900

PK: Cardioglossa gracilis* Boulenger, 1900

KG: Arthroleptis* Smith, 1849

KF: ARTHROLEPTIDAE 1869.mc.f011

Carpathotriton Venczel, $2008+\cdot \mathbf{K Y}$

ST: PO.KN • CI: h0277・ ID: $\uparrow 192$

PN: Carpathotriton matraensis Venczel, 2008 \$

PK: Carpathotriton matraensis ${ }^{\circ}$ Venczel, $2008 \uparrow$

KG: Carpathotriton $^{\circ}$ Venczel, $2008 \uparrow$

KF: SALAMANDRIDAE 1820.ga.f002

Carpophrys: Anonymous 1976 • AN

ST: $\mathbf{A L} \bullet \mathbf{C I}:$ n0018 •ID: 018

PN: Megophrys oshanensis Liu, 1950

PK: Megophrys oshanensis* Liu, 1950

KG: Leptobrachella ${ }^{\circ}$ Smith, 1925

KF: MEGOPHRYIDAE 1850.bb.f008-|1931.na.f003|

Cassina: Cope $1864 \cdot \mathbf{A N}$

ST: AL •CI: n0019・ID: 338

PN: Cystignathus senegalensis Duméril ${ }^{+1}, 1841$

PK: Cystignathus senegalensis* Duméril ${ }^{+1}, 1841$

KG: Kassina* Girard, 1853

KF: HYPEROLIIDAE 1943.lb.f001

Cassina Boulenger, 1882 • AK

ST: NT.JI • CI: h0278・ ID: 338

PN: Cystignathus senegalensis Duméril ${ }^{+1}, 1841$

PK: Cystignathus senegalensis* Duméril ${ }^{+1}, 1841$

KG: Kassina* Girard, 1853

KF: HYPEROLIIDAE 1943.lb.f001

Cassiniopsis Monard, 1937 • AK

ST: PO.JD • CI: h0279 • ID: 338

PN: Cassiniopsis kuvangensis Monard, 1937

PK: Cassiniopsis kuvangensis ${ }^{\circ}$ Monard, 1937

KG: Kassina* Girard, 1853

KF: HYPEROLIIDAE 1943.lb.f001

Castaneides Dubois ${ }^{+1}, 2012 \cdot \mathbf{A K}$

ST: PO.JD • CI: h0280 • ID: 547

PN: Plethodon aeneus Cope ${ }^{+1}, 1881$

PK: Plethodon aeneus* ${ }^{*}$ Cope $^{+1}, 1881$

KG: Aneides* Baird, 1851

KF: PLETHODONTIDAE 1850.ga.f002

Caudacaecilia Taylor, $1968 \cdot \mathbf{A K}$

ST: PO.JD • CI: h0281 • ID: 500

PN: Ichthyophis nigroflavus Taylor, 1960

PK: Ichthyophis nigroflavus ${ }^{\circ}$ Taylor, 1960
KG: Epicrium ${ }^{\circ}$ Wagler, 1828

KF: ICHTHYOPHIIDAE 1968.ta.f001

Cauphias Brocchi, 1877 • AK

ST: PO.JI • CI: h0282 • ID: 219

PN: Plectrohyla guatemalensis Brocchi, 1877

PK: Plectrohyla guatemalensis* Brocchi, 1877

KG: Plectrohyla* Brocchi, 1877

KF: HYLIDAE 1815.ra.f002-|1825.gb.f001|

Cavicola Ancey, $1887 \cdot \mathbf{Z H}$

ST: zo • CI: zh019

Cavicola Lutz, 1930 • AK

ST: PO.JH • CI: h0283・ID: 253

PN: Rana mystacea Spix, 1824

PK: Rana mystacea* Spix, 1824

KG: Leptodactylus ${ }^{1}$ Fitzinger, 1826

KF: LEPTODACTYLIDAE ||1838.ta.f001||-1896.wa.f001

Cecilia [Rafinesque, 1814] Rafinesque, 1815 • AK

ST: NS.JI • CI: h0284 • ID: 474

PN: Caecilia tentaculata Linnaeus, 1758

PK: Caecilia tentaculata* Linnaeus, 1758

KG: Caecilia* Linnaeus, 1758

KF: CAECILIIDAE 1814.ra.f003-|1825.gb.f008|

Celsiella Guayasamin ${ }^{+5}, 2009 \cdot \mathbf{K Y}$

ST: PO.KN • CI: h0285・ID: 166

PN: Centrolenella revocata Rivero, 1985

PK: Centrolenella revocata* Rivero, 1985

KG: Celsiella* Guayasamin ${ }^{+5}, 2009$

KF: CENTROLENIDAE 1951.ta.f001

Celtedens McGowan ${ }^{+1}, 1995+\bullet \mathbf{K Y}$

ST: PO.KN • CI: h0286・ID: $\uparrow 004$

PN: Triton megacephalus Costa, 1864 t

PK: Triton megacephalus ${ }^{\circ}$ Costa, $1864 \dagger$

KG: Celtedens ${ }^{\circ} \mathrm{McGowan}^{+1}, 1995 \dagger$

KF: ALBANERPETIDAE 1982.fa.f001 †

Centrolene Jiménez de la Espada, 1872 • KY

ST: PO.KN • CI: h0287・ID: 156

PN: Centrolene geckoideum Jiménez de la Espada, 1872

PK: Centrolene geckoideum* Jiménez de la Espada, 1872

KG: Centrolene* Jiménez de la Espada, 1872

KF: CENTROLENIDAE 1951.ta.f001

Centrolenella Noble, 1920 • AK

ST: PO.JD • CI: h0288・ID: 156

PN: Centrolenella antioquiensis Noble, 1920

PK: Centrolenella antioquiensis* Noble, 1920

KG: Centrolene* Jiménez de la Espada, 1872

KF: CENTROLENIDAE 1951.ta.f001

Centrotelma Burmeister, 1856 • AK

ST: PO.JD・CI: h0289・ID: 189

PN: Hyla infulata Neuwied, 1824

PK: Hyla albomarginata* Spix, 1824

KG: Boana* Gray, 1825

KF: HYLIDAE 1815.ra.f002-|1825.gb.f001|

Cephaloloxes: Gistel 1848 • AN

ST: AL • CI: n0020・ID: $001 \S$

PN: INR

PK: INR 
KG: INR

KF: LisSAMPHIBIA Familia INCERTAE SEDIS

Cephalopeltis Mueller, $1832 \cdot \mathbf{z H}$

ST: zo • CI: zh020

Cephalopeltis: Duméril ${ }^{+1} 1841 \cdot \mathbf{A N}$

ST: AL • CI: n0021 • ID: 257

PN: Calyptocephalus gayi Duméril ${ }^{+1}, 1841$

PK: Calyptocephalus gayi* Duméril ${ }^{+1}, 1841$

KG: Calyptocephalella* Strand, 1928

KF: CALYPTOCEPHALELLIDAE 1960.ra.f001

Cephalopeltis Jiménez de la Espada, 1875 • AK

ST: PO.JH • CI: h0290 • ID: 257

PN: Calyptocephalus gayi Duméril ${ }^{+1}, 1841$

PK: Calyptocephalus gayi* Duméril ${ }^{+1}, 1841$

KG: Calyptocephalella* Strand, 1928

KF: CALYPTOCEPHALELLIDAE 1960.ra.f001

Cephalophractus: Fitzinger $1843 \cdot \mathbf{A N}$

ST: $\mathbf{A L} \cdot \mathbf{C I}:$ n0022 ・ID: 231

PN: Cephalophractus galeatus Fitzinger, 1843 AN

PK: Trachycephalus nigromaculatus* Tschudi, 1838

KG: Trachycephalus* Tschudi, 1838

KF: HYLIDAE 1815.ra.f002-|1825.gb.f001|

Cerathyla Jiménez de la Espada, 1870 • AK

ST: PO.JD • CI: h0291 • ID: 095

PN: Cerathyla bubalus Jiménez de la Espada,1870

PK: Cerathyla bubalus* Jiménez de la Espada, 1870

KG: Hemiphractus ${ }^{1}$ Wagler, 1828

KF: HEMIPHRACTIDAE 1862.pa.f001

Ceratobatrachus Boulenger, 1884 • AK

ST: PO.JD • CI: h0292 • ID: 369

PN: Ceratobatrachus guentheri Boulenger, 1884

PK: Ceratobatrachus guentheri* Boulenger, 1884

KG: Cornufer* Tschudi, 1838

KF: CERATOBATRACHIDAE 1884.ba.f001

Ceratohyla Boulenger, 1882 • AK

ST: NT.JD • CI: h0293 • ID: 095

PN: Cerathyla bubalus Jiménez de la Espada, 1870

PK: Cerathyla bubalus* Jiménez de la Espada, 1870

KG: Hemiphractus ${ }^{1}$ Wagler, 1828

KF: HEMIPHRACTIDAE 1862.pa.f001

Ceratophris Cuvier, $1829 \cdot \mathbf{A K}$

ST: NS.JI • CI: h0294・ID: 169

PN: Ceratophrys varius Neuwied, 1824

PK: Bufo auritus ${ }^{\circ}$ Raddi, 1823

KG: Ceratophrys ${ }^{3}$ Neuwied, 1824

KF: CERATOPHRYIDAE 1838.ta.f002

Ceratophryne Schlegel, 1858 • AK

ST: NS.JI • CI: h0295・ID: 169

PN: Ceratophrys varius Neuwied, 1824

PK: Bufo auritus ${ }^{\circ}$ Raddi, 1823

KG: Ceratophrys ${ }^{3}$ Neuwied, 1824

KF: CERATOPHRYIDAE 1838.ta.f002

Ceratophryne Günther, 1859 • AK

ST: PO.JH • CI: h0296 • ID: 021

PN: Ceratophryne nasuta Schlegel, 1858

PK: Ceratophryne nasuta* Schlegel, 1858
KG: Megophrys ${ }^{2} \mathrm{Kuhl}^{+1}, 1822$

KF: MEGOPHRYIDAE 1850.bb.f008-|1931.na.f003|

Ceratophrys Neuwied, $1824 \cdot \mathbf{K Y}$

ST: PO.KN • CI: h0297・ID: 169

PN: Ceratophrys varius Neuwied, 1824

PK: Bufo auritus ${ }^{\circ}$ Raddi, 1823

KG: Ceratophrys ${ }^{3}$ Neuwied, 1824

KF: CERATOPHRYIDAE 1838.ta.f002

Ceuthomantis Heinicke ${ }^{+5}, 2009 \bullet \mathbf{K Y}$

ST: PO.KN • CI: h0298・ ID: 085

PN: Ceuthomantis smaragdinus Heinicke $^{+5}, 2009$

PK: Ceuthomantis smaragdinus* Heinicke ${ }^{+5}, 2009$

KG: Ceuthomantis* Heinicke ${ }^{+5}, 2009$

KF: CEUTHOMANTIDAE 2009.ha.f003

Chachaiphrynus Nicoli, $2017+\cdot \mathbf{K Y}$

ST: PO.KN • CI: h0299・ID: $\uparrow 095$

PN: Chachaiphrynus lynchi Nicoli, 2017 *

PK: Chachaiphrynus lynchi ${ }^{\circ}$ Nicoli, $2017 \dagger$

KG: Chachaiphrynus ${ }^{\circ}$ Nicoli, $2017 \dagger$

KF: ODONTOPHRYNIDAE 1971.la.f002

Chacophrys Reig ${ }^{+1}, 1963 \bullet \mathbf{K Y}$

ST: PO.KN • CI: h0300 • ID: 170

PN: Ceratophrys pierottii Vellard, 1948

PK: Ceratophrys pierottii* Vellard, 1948

KG: Chacophrys* Reig ${ }^{+1}, 1963$

KF: CERATOPHRYIDAE 1838.ta.f002

Chalcorana Dubois, 1992 • AK

ST: PO.JD・CI: h0301 • ID: 409

PN: Hyla chalconota Schlegel, 1837

PK: Hyla chalconota* Schlegel, 1837

KG: Hylarana* Tschudi, 1838

KF: RANIDAE 1796.ba.f001

Chaltenobatrachus Basso $^{+3}, 2011 ・ \mathbf{K Y}$

ST: PO.KN • CI: h0302 • ID: 176

PN: Telmatobius grandisonae Lynch, 1975

PK: Telmatobius grandisonae ${ }^{\circ}$ Lynch, 1975

KG: Chaltenobatrachus ${ }^{\circ}$ Basso $^{+3}, 2011$

KF: BATRACHYLIDAE 1965.ga.f002

Chaparana Bourret, $1939 \cdot \mathbf{K Y}$

ST: PO.KN • CI: h0303 • ID: 383

PN: Rana (Chaparana) fansipani Bourret, 1939

PK: Rana aenea* Smith, 1922

KG: Chaparana ${ }^{1}$ Bourret, 1939

KF: DiCROGLOSSIDAE 1987.da.f004

Chaperina Mocquard, $1892 \cdot \mathbf{K Y}$

ST: PO.KN • CI: h0304 • ID: 308

PN: Chaperina fusca Mocquard, 1892

PK: Chaperina fusca* Mocquard, 1892

KG: Chaperina* Mocquard, 1892

KF: MICROHYLIDAE ||1843.fa.f012||-1931.na.f001

Charadrahyla Faivovich ${ }^{+5}, 2005 \bullet \mathbf{K Y}$

ST: PO.KN • CI: h0305 • ID: 201

PN: Hyla taeniopus Günther, 1901

PK: Hyla taeniopus* Günther, 1901

KG: Charadrahyla* Faivovich ${ }^{+5}, 2005$

KF: HYLIDAE 1815.ra.f002-|1825.gb.f001| 
Chascax Ritgen, $1828 \cdot \mathbf{A K}$

ST: PO.JD・CI: h0306・ID: 138

PN: Bufo horridus Daudin, 1802

PK: Bufo spinulosus* Wiegmann, 1834

KG: Rhinella ${ }^{2}$ Fitzinger, 1826

KF: BUFONIDAE 1825.gb.f004

Chaunus Wagler, 1828 • AK

ST: PO.JD • CI: h0307・ ID: 138

PN: Chaunus marmoratus Wagler, 1828

PK: Bufo granulosus* Spix, 1824

KG: Rhinella ${ }^{2}$ Fitzinger, 1826

KF: BUFONIDAE 1825.gb.f004

Chelomophrynus Henrici, $1991+$ KY

ST: PO.KN • CI: h0308・ ID: $\uparrow 081$

PN: Chelomophrynus bayi Henrici, 1991 \$

PK: Chelomophrynus bayi ${ }^{\circ}$ Henrici, $1991 \dagger$

KG: Chelomophrynus ${ }^{\circ}$ Henrici, $1991 \dagger$

KF: RHINOPHRYNIDAE 1858.gc.f013

Chelotriton Pomel, $1853+\cdot \mathbf{K Y}$

ST: PO.KN • CI: h0309・ID: $\uparrow 193$

PN: Chelotriton paradoxus Pomel, 1853 \$

PK: Chelotriton paradoxus ${ }^{\circ}$ Pomel, $1853 \dagger$

KG: Chelotriton ${ }^{\circ}$ Pomel, $1853 \dagger$

KF: SALAMANDRIDAE 1820.ga.f002

Chelydobatrachus Günther, 1859 • AK

ST: PO.JD • CI: h0310 • ID: 273

PN: Breviceps gouldii Gray, 1841

PK: Breviceps gouldii* Gray, 1841

KG: Myobatrachus ${ }^{1}$ Schlegel, 1850

KF: MYOBATRACHIDAE 1850.sa.f001

Chianopelas: Tschudi $1845 a \cdot \mathbf{A N}$

ST: AL・CI: n0023・ID: 246

PN: Leiuperus viridis Tschudi, 1845

PK: Leiuperus marmoratus* Duméril ${ }^{+1}, 1841$

KG: Pleurodema* Tschudi, 1838

KF: LEIUPERIDAE 1850.bb.f010

Chiasmocleis Méhelÿ, 1904 • KY

ST: PO.KN • CI: h0311 • ID: 292

PN: Engystoma albopunctatum Boettger, 1885

PK: Engystoma albopunctatum* Boettger, 1885

KG: Chiasmocleis* Méhelÿ, 1904

KF: MICROHYLIDAE ||1843.fa.f012||-1931.na.f001

Chikila $\mathrm{Kamei}^{+9}$, 2012 • KY

ST: PO.KN・CI: h0312 • ID: 495

PN: Herpele fulleri Alcock, 1904

PK: Herpele fulleri* Alcock, 1904

KG: Chikila* $\mathrm{Kamei}^{+9}, 2012$

KF: CAECILIIDAE 1814.ra.f003-|1825.gb.f008|

Chilixalus Werner, 1899 • AK

ST: PO.JD・CI: h0313・ID: 415

PN: Ixalus warszewitschii Schmidt, 1857

PK: Ixalus warszewitschii* Schmidt, 1857

KG: Lithobates* Fitzinger, 1843

KF: RANIDAE 1796.ba.f001

Chilophryne Fitzinger, 1843 • AK

ST: PO.JD • CI: h0314 • ID: 138
PN: Bufo dorbignyi Duméril ${ }^{+1}, 1841$

PK: Bufo dorbignyi ${ }^{\circ}$ Duméril $^{+1}, 1841$

KG: Rhinella ${ }^{2}$ Fitzinger, 1826

KF: BUFONIDAE 1825.gb.f004

Chimerella Guayasamin ${ }^{+5}, 2009 \cdot \mathbf{K Y}$

ST: PO.KN • CI: h0315 • ID: 158

PN: Centrolene mariaelenae Cisneros-Heredia ${ }^{+1}, 2006$

PK: Centrolene mariaelenae* Cisneros-Heredia ${ }^{+1}, 2006$

KG: Chimerella* Guayasamin ${ }^{+5}, 2009$

KF: CENTROLENIDAE 1951.ta.f001

Chioglossa Bocage, $1864 \cdot \mathbf{K Y}$

ST: PO.KN・CI: h0316・ID: 575

PN: Chioglossa lusitanica Bocage, 1864

PK: Chioglossa lusitanica* Bocage, 1864

KG: Chioglossa* Bocage, 1864

KF: SALAMANDRIDAE 1820.ga.f002

Chionopelas: Tschudi $1845 b \cdot \mathbf{A N}$

ST: AL • CI: n0024・ID: 246

PN: Leiuperus viridis Tschudi, 1845

PK: Leiuperus marmoratus* Duméril ${ }^{+1}, 1841$

KG: Pleurodema* Tschudi, 1838

KF: LEIUPERIDAE 1850.bb.f010

Chirixalus Boulenger, $1893 \cdot \mathbf{K Y}$

ST: PO.KN • CI: h0317・ID: 448

PN: Chirixalus doriae Boulenger, 1893

PK: Chirixalus doriae* Boulenger, 1893

KG: Chirixalus* Boulenger, 1893

KF: RHACOPHORIDAE ||1858.gc.f012||-1932.ha.f001

Chirodryas Keferstein, 1867 • AK

ST: PO.JD・CI: h0318・ ID: 237

PN: Chirodryas raniformis Kefertsein, 1867

PK: Chirodryas raniformis* Kefertsein, 1867

KG: Ranoidea ${ }^{1}$ Tschudi, 1838

KF: PHYLLOMEDUSIDAE 1858.gc.f009

Chiroleptes Richardson, $1837 \cdot \mathbf{Z H}$

ST: zo・CI: zh021

Chiroleptes Günther, 1859 • AK

ST: PO.JH • CI: h0319 • ID: 237

PN: Alytes australis Gray, 1842

PK: Alytes australis* Gray, 1842

KG: Ranoidea ${ }^{1}$ Tschudi, 1838

KF: PHYLLOMEDUSIDAE 1858.gc.f009

Chiromantis Peters, $1854 \cdot \mathbf{K Y}$

ST: PO.KN • CI: h0320・ID: 449

PN: Chiromantis xerampelina Peters, 1854

PK: Chiromantis xerampelina* Peters, 1854

KG: Chiromantis* Peters, 1854

KF: RHACOPHORIDAE ||1858.gc.f012||-1932.ha.f001

Chiropterotriton Taylor, $1944 \cdot \mathbf{K Y}$

ST: PO.KN • CI: h0321・ID: 528

PN: Oedipus multidentatus Taylor, 1939

PK: Oedipus multidentatus* Taylor, 1939

KG: Chiropterotriton* Taylor, 1944

KF: PLETHODONTIDAE 1850.ga.f002

Chlorofilus Palacký, 1898 • AK

ST: NT.JI • CI: h0322・ID: 200 
PN: Rana nigrita Le Conte, 1825

PK: Rana nigrita* Le Conte, 1825

KG: Pseudacris* Fitzinger, 1843

KF: HYLIDAE 1815.ra.f002-|1825.gb.f001|

Chlorolius Perret, 1988 • AK

ST: PO.JD • CI: h0323・ID: 331

PN: Hyperolius koehleri Mertens, 1940

PK: Hyperolius koehleri ${ }^{\circ}$ Mertens, 1940

KG: Hyperolius* Rapp, 1842

KF: HYPEROLIIDAE 1943.lb.f001

Choanacantha Méhelÿ, 1898 • AK

ST: PO.JD • CI: h0324 • ID: 280

PN: Choanacantha rostrata Méhelÿ, 1898

PK: Choanacantha rostrata ${ }^{\circ}$ Méhelÿ, 1898

KG: Asterophrys* Tschudi, 1838

KF: MICROHYLIDAE ||1843.fa.f012||-1931.na.f001

Choerophryne Van Kampen, 1914 • AK

ST: PO.JD・CI: h0325・ID: 280

PN: Choerophryne proboscidea Van Kampen, 1914

PK: Choerophryne proboscidea ${ }^{\circ}$ Van Kampen, 1914

KG: Asterophrys* Tschudi, 1838

KF: MICROHYLIDAE ||1843.fa.f012||-1931.na.f001

Chondrodela: Rafinesque $1815 \cdot \mathbf{A N}$

ST: AL • CI: n0025 • ID: 419

PN: Rana temporaria Linnaeus, 1758

PK: Rana temporaria* Linnaeus, 1758

KG: Rana* Linnaeus, 1758

KF: RANIDAE 1796.ba.f001

Chondrotus Cope, 1887 • AK

ST: PO.JD・CI: h0326・ID: 556

PN: Amblystoma tenebrosum Baird $^{+1}, 1852$

PK: Amblystoma tenebrosum* Baird ${ }^{+1}, 1852$

KG: Dicamptodon* Strauch, 1870

KF: AмBYSTOMATIDAE 1850.ga.f004

Chonomantis Glaw ${ }^{+1}, 1994$ • AK

ST: PO.JD • CI: h0327 • ID: 432

PN: Rana albofrenata Müller, 1892

PK: Rana albofrenata* Müller, 1892

KG: Mantidactylus* Boulenger, 1895

KF: RHACOPHORIDAE ||1858.gc.f012||-1932.ha.f001

Chorophilus Baird, 1854 • AK

ST: PO.JI • CI: h0328・ ID: 200

PN: Rana nigrita Le Conte, 1825

PK: Rana nigrita* Le Conte, 1825

KG: Pseudacris* Fitzinger, 1843

KF: HYLIDAE 1815.ra.f002-|1825.gb.f001|

Chrysobatrachus Laurent, 1951・ KY

ST: PO.KN • CI: h0329・ ID: 328

PN: Chrysobatrachus cupreonitens Laurent, 1951

PK: Chrysobatrachus cupreonitens ${ }^{\circ}$ Laurent, 1951

KG: Chrysobatrachus ${ }^{\circ}$ Laurent, 1951

KF: HYPEROLIIDAE 1943.lb.f001

Chrysodonta Mitchill, $1822 \cdot \mathbf{A K}$

ST: PO.JD • CI: h0330 • ID: 520

PN: Chrysodonta larvaeformis Mitchill, 1822

PK: Amphiuma means* Garden in Smith, 1821
KG: Amphiuma* Garden in Smith, 1821

KF: AMPHIUMIDAE 1825.gb.f07

Chrysopaa Ohler $^{+1}, 2006 \cdot \mathbf{K Y}$

ST: PO.KN • CI: h0331 • ID: 372

PN: Rana sternosignata Murray, 1885

PK: Rana sternosignata ${ }^{\circ}$ Murray, 1885

KG: Chrysopaa ${ }^{\circ}$ Ohler $^{+1}, 2006$

KF: DiCROGLOSSIDAE 1987.da.f004

Chrysotriton Estes, $1981+\cdot \mathbf{K Y}$

ST: PO.KN • CI: h0332・ID: $\uparrow 187$

PN: Chrysotriton tiheni Estes, 1981 \$

PK: Chrysotriton tiheni $i^{\circ}$ Estes, $1981 \dagger$

KG: Chrysotriton ${ }^{\circ}$ Estes, $1981 \uparrow$

KF: AMBYSTOMATIDAE 1850.ga.f004

Chthonerpeton Peters, $1880 \bullet \mathbf{K Y}$

ST: PO.KN • CI: h0333・ID: 477

PN: Siphonops indistinctus Reinhardt ${ }^{+1}, 1862$

PK: Siphonops indistinctus* Reinhardt ${ }^{+1}, 1862$

KG: Chthonerpeton* Peters, 1880

KF: CAECILIIDAE 1814.ra.f003-|1825.gb.f008|

Chunerpeton $\mathrm{GaO}^{+1}, 2003+\cdot \mathbf{K Y}$

ST: PO.KN • CI: h0334 •ID: $\uparrow 165$

PN: Chunerpeton tianyiensis $\mathrm{Gao}^{+1}, 2003$ \$

PK: Chunerpeton tianyiensis ${ }^{\circ} \mathrm{Gao}^{+1}, 2003 \dagger$

KG: Chunerpeton $^{\circ} \mathrm{Gao}^{+1}, 2003 \uparrow$

KF: CRYPTOBRANCHIDAE 1826.fb.f003

Churamiti Channing ${ }^{+1}$, 2002 • KY

ST: PO.KN • CI: h0335・ID: 134

PN: Churamiti maridadi Channing $^{+1}, 2002$

PK: Churamiti maridadi $*$ Channing ${ }^{+1}, 2002$

KG: Churamiti* Channing ${ }^{+1}, 2002$

KF: BUFONIDAE 1825.gb.f004

Cinclidium Blyth, $1842 \cdot \mathbf{z H}$

ST: zo • CI: zh022

Cinclidium Cope, 1867 • AK

ST: PO.JH • CI: h0336・ID: 189

PN: Cinclidium granulatum Cope, 1867

PK: Rana boans* Linnaeus, 1758

KG: Boana* Gray, 1825

KF: HYLIDAE 1815.ra.f002-|1825.gb.f001|

Cincloscopus Cope, 1871 • AK

ST: PO.JD • CI: h0337・ID: 189

PN: Cinclidium granulatum Cope, 1867

PK: Rana boans* Linnaeus, 1758

KG: Boana* Gray, 1825

KF: HYLIDAE 1815.ra.f002-|1825.gb.f001|

Clinotarsus Mivart, $1869 \cdot \mathbf{K Y}$

ST: PO.KN • CI: h0338・ ID: 402

PN: Pachybatrachus robustus Mivart, 1869

PK: Rana curtipes* Jerdon, 1853

KG: Clinotarsus ${ }^{1}$ Mivart, 1869

KF: RANIDAE 1796.ba.f001

Cochranella Taylor, 1951 • KY

ST: PO.KN • CI: h0339・ID: 157

PN: Centrolenella granulosa Taylor, 1949

PK: Centrolenella granulosa* Taylor, 1949 
KG: Cochranella* Taylor, 1951

KF: CENTROLENIDAE 1951.ta.f001

Coecilia: Linnaeus 1758 • AN

ST: LI • CI: n0026 • ID: 474

PN: Caecilia tentaculata Linnaeus, 1758

PK: Caecilia tentaculata* Linnaeus, 1758

KG: Caecilia* Linnaeus, 1758

KF: CAECILIIDAE 1814.ra.f003-|1825.gb.f008|

Coecilia Sonnini ${ }^{+1}, 1801$ • AK

ST: NS.JI • CI: h0340 • ID: 474

PN: Caecilia tentaculata Linnaeus, 1758

PK: Caecilia tentaculata* Linnaeus, 1758

KG: Caecilia* Linnaeus, 1758

KF: CAECILIIDAE 1814.ra.f003-|1825.gb.f008|

Coelonotus Peters, $1855 \cdot \mathbf{Z H}$

ST: zo • CI: zh023

Coelonotus Miranda-Ribeiro, 1920 • AK

ST: PO.JH • CI: h0341 • ID: 094

PN: Coelonotus fissilis Miranda-Ribeiro, 1920

PK: Coelonotus fissilis* Miranda-Ribeiro, 1920

KG: Fritziana* Mello-Leitão, 1937

KF: HEMIPHRACTIDAE 1862.pa.f001

Cofofryne Palacký, 1898 • AK

ST: NT.JI • CI: h0342・ ID: 017

PN: Bombinator sikimmensis Blyth, 1854

PK: Bombinator sikimmensis ${ }^{\circ}$ Blyth, 1854

KG: Scutiger ${ }^{2}$ Theobald, 1868

KF: MEGOPHRYIDAE 1850.bb.f008-|1931.na.f003|

Coggerdonia Wells ${ }^{+1}, 1985$ • AK

ST: PO.JD • CI: h0343 • ID: 235

PN: Hyla adelaidensis Gray, 1841

PK: Hyla adelaidensis* Gray, 1841

KG: Litoria* Tschudi, 1838

KF: PHYLLOMEDUSIDAE 1858.gc.f009

Colleeneremia Wells $^{+1}$, $1985 \bullet \mathbf{A K}$

ST: PO.JD • CI: h0344 • ID: 235

PN: Hyla rubella Gray, 1842

PK: Hyla rubella* Gray, 1842

KG: Litoria* Tschudi, 1838

KF: PHYLLOMEDUSIDAE 1858.gc.f009

Colodactylus Tschudi, $1845 \bullet \mathbf{K Y}$

ST: PO.KN・CI: h0345・ID: 014

PN: Colodactylus coerulescens Tschudi, 1845

PK: Colodactylus coerulescens ${ }^{\circ}$ Tschudi, 1845

KG: Colodactylus ${ }^{\circ}$ Tschudi, 1845

KF: LAEVOGYrINIA Familia INCERTAE SEDIS

Colomascirtus Duellman ${ }^{+2}, 2016$ • KY

ST: PO.KN • CI: h0346・ID: 190

PN: Hyla larinopygion Duellman, 1973

PK: Hyla larinopygion* Duellman, 1973

KG: Colomascirtus* Duellman ${ }^{+2}, 2016$

KF: HYLIDAE 1815.ra.f002-|1825.gb.f001|

Colostethus Cope, 1866 • KY

ST: PO.KN • CI: h0347 • ID: 040

PN: Phyllobates latinasus Cope, 1863

PK: Phyllobates latinasus* Cope, 1863
KG: Colostethus* Cope, 1866

KF: DENDROBATIDAE \|1850.bb.f006"|-1865.ca.f002

Colosthetus Gadow, $1901 \cdot \mathbf{A K}$

ST: NS.JI • CI: h0348 • ID: 040

PN: Phyllobates latinasus Cope, 1863

PK: Phyllobates latinasus* Cope, 1863

KG: Colostethus* Cope, 1866

KF: DENDROBATIDAE \|1850.bb.f006"|-1865.ca.f002

Colpoglossus Boulenger, $1904 \cdot \mathbf{A K}$

ST: PO.JD • CI: h0349・ID: 313

PN: Colpoglossus brooksii Boulenger, 1904

PK: Colpoglossus brooksii ${ }^{\circ}$ Boulenger, 1904

KG: Glyphoglossus* Günther, 1869

KF: MICROHYLIDAE ||1843.fa.f012||-1931.na.f001

Comobatrachus Hecht ${ }^{+1}, 1960 \$ \cdot \mathbf{K Y}$

ST: PO.KN • CI: h0350 •ID: $\uparrow 016$

PN: Comobatrachus aenigmatis Hecht in $\mathrm{Hecht}^{+1}$, 1960 \%

PK: Comobatrachus aenigmatis ${ }^{\circ}$ Hecht in $\mathrm{Hecht}^{+1}, 1960 \dagger$

KG: Comobatrachus $^{\circ} \mathrm{Hecht}^{+1}, 1960 \dagger$

KF: ANURA Familia INCERTAE SEDIS

Comonecturoides Hecht ${ }^{+1}, 1960+\cdot \mathbf{K Y}$

ST: PO.KN • CI: h0351 • ID: $\uparrow 128$

PN: Comonecturoides marshi Estes in $\mathrm{Hecht}^{+1}, 1960$ \$

PK: Comonecturoides marsh $i^{\circ}$ Estes in $\mathrm{Hecht}^{+1}, 1960 \dagger$

KG: Comonecturoides ${ }^{\circ} \mathrm{Hecht}^{+1}, 1960 \dagger$

KF: Urodela Familia INCERTAE SEDIS

Conrana Boulenger, $1910 \bullet \mathbf{A K}$

ST: NS.JI • CI: h0352 • ID: 351

PN: Conraua robusta Nieden, 1908

PK: Conraua robusta* Nieden, 1908

KG: Conraua* Nieden, 1908

KF: CONRAUIDAE 1992.da.f001

Conrana Bauer, 1985 • AK

ST: PO.JH • CI: h0353・ID: 413

PN: Rana catesbeiana Shaw, 1802

PK: Rana catesbeiana* Shaw, 1802

KG: Aquarana* Dubois, 1992

KF: RANIDAE 1796.ba.f001

Conraua Nieden, 1908 • KY

ST: PO.KN • CI: h0354 • ID: 351

PN: Conraua robusta Nieden, 1908

PK: Conraua robusta* Nieden, 1908

KG: Conraua* Nieden, 1908

KF: CONRAUIDAE 1992.da.f001

Copea Steindachner, 1864 • AK

ST: PO.JD・CI: h0355・ID: 314

PN: Copea fulva Steindachner, 1864

PK: Engystoma rubrum* Jerdon, 1853

KG: Microhyla* Tschudi, 1838

KF: MICROHYLIDAE ||1843.fa.f012||-1931.na.f001

Copeicaecilia Taylor, $1968 \cdot \mathbf{A K}$

ST: PO.JD・CI: h0356・ID: 487

PN: Siphonops syntremus Cope, 1866

PK: Siphonops syntremus ${ }^{\circ}$ Cope, 1866

KG: Gymnopis* Peters, 1874

KF: CAECILIIDAE 1814.ra.f003-|1825.gb.f008| 
Copeotyphlinus Taylor, 1968 • AK

ST: PO.JD・CI: h0357・ID: 487

PN: Siphonops syntremus Cope, 1866

PK: Siphonops syntremus ${ }^{\circ}$ Cope, 1866

KG: Gymnopis* Peters, 1874

KF: CAECILIIDAE 1814.ra.f003-|1825.gb.f008|

Cophaeus Cope, 1889 • AK

ST: NL.JI • CI: h0358・ ID: 186

PN: Telmatobius peruvianus Wiegmann, 1834

PK: Telmatobius peruvianus ${ }^{\circ}$ Wiegmann, 1834

KG: Telmatobius ${ }^{3}$ Wiegmann, 1834

KF: TELMATOBIIDAE 1843.fa.f006

Cophixalus Boettger, 1892 • AK

ST: PO.JD • CI: h0359・ID: 280

PN: Sphenophryne verrucosa Boulenger, 1898

PK: Sphenophryne verrucosa ${ }^{\circ}$ Boulenger, 1898

KG: Asterophrys* Tschudi, 1838

KF: MICROHYLIDAE ||1843.fa.f012||-1931.na.f001

Cophomantis Peters, 1870 • AK

ST: PO.JD・CI: h0360 • ID: 189

PN: Cophomantis punctillata Peters, 1870

PK: Hyla geographica var. semilineata* Spix, 1824

KG: Boana* Gray, 1825

KF: HYLIDAE 1815.ra.f002-|1825.gb.f001|

Cophophryne Boulenger, 1887 • AK

ST: NT.JI • CI: h0361 • ID: 017

PN: Bombinator sikimmensis Blyth, 1854

PK: Bombinator sikimmensis ${ }^{\circ}$ Blyth, 1854

KG: Scutiger ${ }^{2}$ Theobald, 1868

KF: MEGOPHRYIDAE 1850.bb.f008-|1931.na.f003|

Cophyla Boettger, $1880 \bullet \mathbf{K Y}$

ST: PO.KN • CI: h0362・ID: 286

PN: Cophyla phyllodactyla Boettger, 1880

PK: Cophyla phyllodactyla* Boettger, 1880

KG: Cophyla* Boettger, 1880

KF: MICROHYLIDAE ||1843.fa.f012||-1931.na.f001

Copiula Méhelÿ, 1901 • AK

ST: PO.JD • CI: h0363 • ID: 280

PN: Phrynixalus oxyrhinus Boulenger, 1898

PK: Phrynixalus oxyrhinus* Boulenger, 1898

KG: Asterophrys* Tschudi, 1838

KF: MICROHYLIDAE ||1843.fa.f012||-1931.na.f001

Coplandia Wells $^{+1}, 1985$ • AK

ST: PO.JD・CI: h0364・ID: 262

PN: Kyarranus kundagungan Ingram $^{+1}, 1958$

PK: Kyarranus kundagungan ${ }^{\circ}$ Ingram $^{+1}, 1958$

KG: Philoria ${ }^{2}$ Spencer, 1901

KF: MYOBATRACHIDAE 1850.sa.f001

Coracodichus Laurent, 1941 • AK

ST: PO.JD・CI: h0365・ID: 320

PN: Arthroleptis whytii Boulenger, 1897

PK: Arthroleptis stenodactylus* Pfeffer, 1893

KG: Arthroleptis* Smith, 1849

KF: ARTHROLEPTIDAE 1869.mc.f011

Cordicephalus Wardle ${ }^{+2}$, $1947 \cdot \mathbf{z H}$

ST: zo • CI: zh024
Cordicephalus Nevo, $1968+\bullet \mathbf{A K}$

ST: PO.JH • CI: h0366・ID: $\uparrow 064$

PN: Cordicephalus gracilis Nevo, 1968 \$

PK: Cordicephalus gracilis ${ }^{\circ}$ Nevo, $1968 \uparrow$

KG: Nevobatrachus ${ }^{\circ}$ Mahony, $2019 \dagger$

KF: DorsIPARES Familia INCERTAE SEDIS

Cordylus Gronovius, $1763 \cdot \mathbf{Z A}$

ST: zN • CI: zn005

Cordylus Laurenti, $1768 \cdot \mathbf{Z H}$

ST: zo • CI: zh025

Cordylus Wagler, 1828 • AK

ST: NL.JH • CI: h0367・ ID: 554

PN: Proteus anguinus Laurenti, 1768

PK: Proteus anguinus* Laurenti, 1768

KG: Proteus* Laurenti, 1768

KF: PROTEIDAE 1831.ba.f002

Cornufer Tschudi, $1838 \bullet \mathbf{K Y}$

ST: PO.KN • CI: h0368・ ID: 369

PN: Halophila vitiensis Girard, 1853

PK: Halophila vitiensis* Girard, 1853

KG: Cornufer* Tschudi, 1838

KF: CERATOBATRACHIDAE 1884.ba.f001

Corsandra Dubois $^{+1}, 2009$ • AK

ST: PO.JD・CI: h0369・ID: 578

PN: Salamandra corsica Savi, 1838

PK: Salamandra corsica* Savi, 1838

KG: Salamandra ${ }^{1}$ Garsault, 1764

KF: SALAMANDRIDAE 1820.ga.f002

Corythomantis Boulenger, $1896 \cdot \mathbf{K Y}$

ST: PO.KN • CI: h0370 • ID: 227

PN: Corythomantis greeningi Boulenger, 1896

PK: Corythomantis greeningi* Boulenger, 1896

KG: Corythomantis* Boulenger, 1896

KF: HYLIDAE 1815.ra.f002-|1825.gb.f001|

Cosmus: Dejean $1821 \cdot \mathbf{Z A}$

ST: $\mathbf{z N} \cdot \mathbf{C I}:$ zn006

Cosmus: Gistel 1848 • AN

ST: AL $・$ CI: n0027・ID: $001 \S$

PN: INR

PK: INR

KG: INR

KF: LisSAMPHIBIA Familia INCERTAE SEDIS

Cotobotes Gistel, 1848 • AK

ST: NL.JI • CI: h0371・ID: 538

PN: Salamandra scutata Temminck $^{+1}, 1838$

PK: Salamandra scutata* Temminck $^{+1}, 1838$

KG: Hemidactylium* Tschudi, 1838

KF: PLETHODONTIDAE 1850.ga.f002

Cranophryne Cope, 1889 • AK

ST: PO.JD・CI: h0372・ID: 137

PN: Cranopsis fastidiosus Cope, 1875

PK: Cranopsis fastidiosus* Cope, 1875

KG: Incilius* Cope, 1863

KF: BUFONIDAE 1825.gb.f004

Cranopsis Adams, $1860 \bullet \mathbf{Z H}$

ST: zo • CI: zh026 
Cranopsis Cope, $1875 \cdot \mathbf{A K}$

ST: PO.JH • CI: h0373・ID: 137

PN: Cranopsis fastidiosus Cope, 1875

PK: Cranopsis fastidiosus* Cope, 1875

KG: Incilius* Cope, 1863

KF: BUFONIDAE 1825.gb.f004

Craspedoglossa Müller, 1922 • AK

ST: PO.JD • CI: h0374 • ID: 179

PN: Craspedoglossa santaecatharinae Müller, 1922

PK: Borborocoetes bolitoglossus ${ }^{\circ}$ Werner, 1897

KG: Cycloramphus* Tschudi, 1838

KF: CYCLORAMPHIDAE 1850.bb.f003-|1852.ba.f001|

Cratia Báez ${ }^{+2,} 2009$ + $\cdot$ KY

ST: PO.KN • CI: h0375 • ID: $\uparrow 017$

PN: Cratia gracilis $\mathrm{BÁEZ}^{+2}, 2009+$

PK: Cratia gracilis ${ }^{\circ}$ Báez $^{+2}, 2009 \dagger$

KG: Cratia $^{\circ}$ Báez $^{+2,} 2009 \dagger$

KF: ANURA Familia INCERTAE SEDIS

Cratopipa: Souza Carvalho ${ }^{+6} 2019 a+\cdot \mathbf{A N}$

ST: $\mathbf{A L} \bullet \mathbf{C I}:$ n0028 ・ ID: $\uparrow 071$

PN: Cratopipa novaolindensis Souza Carvalho ${ }^{+6}, 2019 a \ddagger$

PK: Cratopipa novaolindensis ${ }^{\circ}$ Souza Carvalho ${ }^{+6}, 2019 b \dagger$

KG: Cratopipa ${ }^{\circ}$ Souza Carvalho ${ }^{+6}, 2019 b \dagger$

KF: PIPIDAE 1825.gb.f003-|1826.fb.f002|

Cratopipa Souza Carvalho ${ }^{+6}, 2019 b+\bullet \mathbf{K Y}$

ST: PO.KN • CI: h0376・ID: $\uparrow 071$

PN: Cratopipa novaolindensis Souza Carvalho ${ }^{+6}, 2019 b$ \$

PK: Cratopipa novaolindensis ${ }^{\circ}$ Souza Carvalho ${ }^{+6}, 2019 b \dagger$

KG: Cratopipa $^{\circ}$ Souza Carvalho ${ }^{+6}, 2019 b \dagger$

KF: PIPIDAE 1825.gb.f003-|1826.fb.f002|

Craugastor Cope, $1862 \bullet \mathbf{K Y}$

ST: PO.KN・CI: h0377・ID: 059

PN: Hylodes fitzingeri Schmidt, 1857

PK: Hylodes fitzingeri* Schmidt, 1857

KG: Craugastor* Cope, 1862

KF: BRACHYCEPHALIDAE 1858.gc.f002

Crepidius Candèze, $1859 \cdot \mathbf{z H}$

ST: zo • CI: zh027

Crepidius Cope, 1875 • AK

ST: PO.JH • CI: h0378 • ID: 137

PN: Crepidius epioticus Cope, 1875

PK: Crepidius epioticus ${ }^{\circ}$ Cope, 1875

KG: Incilius* Cope, 1863

KF: BUFONIDAE 1825.gb.f004

Crepidophryne Cope, $1889 \bullet \mathbf{A K}$

ST: PO.JD • CI: h0379・ID: 137

PN: Crepidius epioticus Cope, 1875

PK: Crepidius epioticus ${ }^{\circ}$ Cope, 1875

KG: Incilius* Cope, 1863

KF: BUFONIDAE 1825.gb.f004

Cretasalia Gubin, $1999+\bullet \mathbf{K Y}$

ST: PO.KN • CI: h0380 • ID: $\uparrow 112$

PN: Cretasalia tsybini Gubin, 1999 \$

PK: Cretasalia tsybini* Gubin, 1999 †

KG: Cretasalia* Gubin, $1999+$

KF: GoBIATIDAE 1991.ra.f001 †
Crinia Tschudi, $1838 \cdot \mathbf{K Y}$

ST: PO.KN • CI: h0381 •ID: 270

PN: Crinia georgiana Tschudi, 1838

PK: Crinia georgiana* Tschudi, 1838

KG: Crinia* Tschudi, 1838

KF: MYOBATRACHIDAE 1850.sa.f001

Crossodactyle: Brocchi, $1879 \cdot \mathbf{A N}$

ST: AM • CI: n0029・ID: 181

PN: Crossodactylus gaudichaudii Duméril ${ }^{+1}, 1841$

PK: Crossodactylus gaudichaudii ${ }^{\circ}$ Duméril $^{+1}, 1841$

KG: Crossodactylus ${ }^{3}$ Duméril $^{+1}, 1841$

KF: HYLODIDAE 1858.gc.f010

Crossodactylodes Cochran, $1938 \cdot \mathbf{K Y}$

ST: PO.KN・CI: h0382・ID: 254

PN: Crossodactylodes pintoi Cochran, 1938

PK: Crossodactylodes pintoi ${ }^{\circ}$ Cochran, 1938

KG: Crossodactylodes ${ }^{2}$ Cochran, 1938

KF: PARATELMATOBIIDAE 2012.oa.f001

Crossodactylus Duméril ${ }^{+1}, 1841 \bullet \mathbf{K Y}$

ST: PO.KN・CI: h0383・ID: 181

PN: Crossodactylus gaudichaudii Duméril ${ }^{+1}, 1841$

PK: Crossodactylus gaudichaudii ${ }^{\circ}$ Duméril $^{+1}, 1841$

KG: Crossodactylus ${ }^{3}$ Duméril $^{+1}, 1841$

KF: HYLODIDAE 1858.gc.f010

Crotaphatrema Nussbaum, 1985 • KY

ST: PO.KN • CI: h0384 • ID: 498

PN: Herpele bornmuelleri Werner, 1899

PK: Herpele bornmuelleri ${ }^{\circ}$ Werner, 1899

KG: Crotaphatrema ${ }^{3}$ Nussbaum, 1985

KF: SCOLECOMORPHIDAE 1969.ta.f001

Crotaphitis Schulze, $1891 \bullet \mathbf{A K}$

ST: PO.JD・CI: h0385・ID: 419

PN: Rana arvalis Nilsson, 1842

PK: Rana arvalis* Nilsson, 1842

KG: Rana* Linnaeus, 1758

KF: RANIDAE 1796.ba.f001

Crumenifera Cope, 1862 • AK

ST: PO.JD • CI: h0386・ID: 331

PN: Crumenifera pusilla Cope, 1862

PK: Crumenifera pusilla* Cope, 1862

KG: Hyperolius* Rapp, 1842

KF: HYPEROLIIDAE 1943.lb.f001

Cruziohyla Faivovich $^{+5}, 2005 \cdot \mathbf{K Y}$

ST: PO.KN • CI: h0387・ID: 240

PN: Agalychnis calcarifer Boulenger, 1902

PK: Agalychnis calcarifer* Boulenger, 1902

KG: Cruziohyla* Faivovich ${ }^{+5}, 2005$

KF: PHYLLOMEDUSIDAE 1858.gc.f009

Cryptobatrachus Ruthven, $1916 \cdot \mathbf{K Y}$

ST: PO.KN・CI: h0388・ID: 092

PN: Cryptobatrachus boulengeri Ruthven, 1916

PK: Cryptobatrachus boulengeri* Ruthven, 1916

KG: Cryptobatrachus* Ruthven, 1916

KF: HEMIPHRACTIDAE 1862.pa.f001

Cryptobranchichnus Huene, 1941 +; • KY

ST: PO.KN • CI: h0389 • ID: $\uparrow 129$ 
PN: Cryptobranchichnus infericolor Huene, 1941 †i

PK: Cryptobranchichnus infericolor ${ }^{\circ}$ Huene, $1941 \dagger$

KG: Cryptobranchichnus ${ }^{\circ}$ Huene, $1941 \dagger$

KF: Urodela Familia INCERTAE SEDIS

Cryptobranchus Leuckart, 1821 • KY

ST: PO.KN • CI: h0390 • ID: 504

PN: Salamandra salamandroides Leuckart, 1821

PK: Salamandra alleganiensis* Sonnini ${ }^{+1}, 1801$

KG: Cryptobranchus ${ }^{1}$ Leuckart, 1821

KF: CRYPTOBRANCHIDAE 1826.fb.f003

Cryptophyllobates Lötters ${ }^{+1}, 2000$ • AK

ST: PO.JD • CI: h0391 • ID: 053

PN: Phyllobates azureiventris Kneller ${ }^{+1}, 1985$

PK: Phyllobates azureiventris* Kneller ${ }^{+1}, 1985$

KG: Hyloxalus ${ }^{2}$ Jiménez de la Espada, 1870

KF: DENDROBATIDAE $\|1850 . b b . f 006\|-1865 . c a . f 002$

Cryptopsophis Boulenger, 1883 • AK

ST: PO.JD • CI: h0392 • ID: 487

PN: Cryptopsophis multiplicatus Boulenger, 1883

PK: Gymnopis multiplicata* Peters, 1874

KG: Gymnopis* Peters, 1874

KF: CAECILIIDAE 1814.ra.f003-|1825.gb.f008|

Cryptotheca Duellman, 2015 • KY

ST: PO.KN • CI: h0393・ID: 088

PN: Gastrotheca walkeri Duellman, 1980

PK: Gastrotheca walkeri* Duellman, 1980

KG: Cryptotheca* Duellman, 2015

KF: HEMIPHRACTIDAE 1862.pa.f001

Cryptothylax Laurent $^{+1}$, $1950 \cdot \mathbf{K Y}$

ST: PO.KN・CI: h0394 • ID: 329

PN: Hylambates greshoffii Schilthuis, 1889

PK: Hylambates greshoffii* Schilthuis, 1889

KG: Cryptothylax* Laurent ${ }^{+1}, 1950$

KF: HYPEROLIIDAE 1943.lb.f001

Cryptotis Pomel, $1848 \cdot \mathbf{Z H}$

ST: zo • CI: zh028

Cryptotis Günther, 1863 • AK

ST: PO.JH • CI: h0395 • ID: 260

PN: Cryptotis brevis Günther, 1863

PK: Cryptotis brevis* Günther, 1863

KG: Adelotus* Ogilby, 1907

KF: MYOBATRACHIDAE 1850.sa.f001

Cryptotriton García-París ${ }^{+1}, 2000 \cdot \mathbf{K Y}$

ST: PO.KN • CI: h0396 • ID: 529

PN: Oedipus nasalis Dunn, 1924

PK: Oedipus nasalis* Dunn, 1924

KG: Cryptotriton* García-París ${ }^{+1}, 2000$

KF: PlethodontidAE 1850.ga.f002

Ctenocranius Melin, $1941 \cdot \mathbf{A K}$

ST: PO.JD • CI: h0397 • ID: 073

PN: Limnophys cornutus Jiménez de la Espada, 1870

PK: Limnophys cornutus ${ }^{\circ}$ Jiménez de la Espada, 1870

KG: Strabomantis* Peters, 1863

KF: BRACHYCEPHALIDAE 1858.gc.f002

Ctenophryne Mocquard, $1904 \cdot \mathbf{K Y}$

ST: PO.KN • CI: h0398・ID: 293
PN: Ctenophryne geayi Mocquard, 1904

PK: Ctenophryne geayi* Mocquard, 1904

KG: Ctenophryne* Mocquard, 1904

KF: MICROHYLIDAE ||1843.fa.f012||-1931.na.f001

Cultripes Müller, 1832 • AK

ST: PO.JD • CI: h0399 • ID: 026

PN: Rana cultripes Cuvier, 1829

PK: Rana cultripes* Cuvier, 1829

KG: Pelobates* Wagler, 1830

KF: PELOBATIDAE 1850.bb.f004

Cuttysarkus Estes, 1964 + $\mathbf{A K}$

ST: PO.JD • CI: h0400 • ID: $\uparrow 149$

PN: Cuttysarkus mcnallyi Estes, 1964 †

PK: Prodesmodon copei ${ }^{\circ}$ Estes, $1964 \uparrow$

KG: Prodesmodon ${ }^{\circ}$ Estes, $1964 \uparrow$

KF: HYLAEOBATRACHIDAE 1889.1a.f001 †

Cyclocephalus Berthold, $1827 \cdot \mathbf{Z H}$

ST: zo • CI: zh029

Cyclocephalus Jiménez de la Espada, 1875 • AK

ST: PO.JH • CI: h0401 • ID: 078

PN: Cyclocephalus lacrimosus Jiménez de la Espada, 1875

PK: Cyclocephalus lacrimosus ${ }^{\circ}$ Jiménez de la Espada, 1875

KG: Pristimantis* Jiménez de la Espada, 1870

KF: BRACHYCEPHALIDAE 1858.gc.f002

Cycloramphos: Tschudi $1838 \cdot \mathbf{A N}$

ST: LI • CI: n0030・ID: 179

PN: Cycloramphus fulginosus Tschudi, 1838

PK: Cycloramphus fuliginosus* Tschudi, 1838

KG: Cycloramphus* Tschudi, 1838

KF: CYCLORAMPHIDAE 1850.bb.f003-|1852.ba.f001|

Cycloramphos Agassiz, 1847 • AK

ST: NT.JI • CI: h0402 • ID: 179

PN: Cycloramphus fulginosus Tschudi, 1838

PK: Cycloramphus fuliginosus* Tschudi, 1838

KG: Cycloramphus* Tschudi, 1838

KF: CYCLORAMPHIDAE 1850.bb.f003-|1852.ba.f001|

Cycloramphus Tschudi, $1838 \cdot \mathbf{K Y}$

ST: LC.KN • CI: h0403 • ID: 179

PN: Cycloramphus fuliginosus Tschudi, 1838

PK: Cycloramphus fuliginosus* Tschudi, 1838

KG: Cycloramphus* Tschudi, 1838

KF: CYCLORAMPHIDAE 1850.bb.f003-|1852.ba.f001|

Cyclorana Steindachner, $1867 \cdot \mathbf{A K}$

ST: PO.JD • CI: h0404 • ID: 237

PN: Cyclorana novaehollandiae Steindachner, 1867

PK: Cyclorana novaehollandiae* Steindachner, 1867

KG: Ranoidea ${ }^{1}$ Tschudi, 1838

KF: PHYLLOMEDUSIDAE 1858.gc.f009

Cyclorhamphus Agassiz, $1847 \cdot \mathbf{A K}$

ST: NT.JI • CI: h0405・ID: 179

PN: Cycloramphus fulginosus Tschudi, 1838

PK: Cycloramphus fuliginosus* Tschudi, 1838

KG: Cycloramphus* Tschudi, 1838

KF: CYCLORAMPHIDAE 1850.bb.f003-|1852.ba.f001|

Cylindrosoma Tschudi, $1838 \cdot \mathbf{A K}$

ST: PO.JD・CI: h0406・ ID: 542 
PN: Salamandra longicauda Green, 1818

PK: Salamandra longicauda* Green, 1818

KG: Eurycea* Rafinesque, 1822

KF: PLETHODONTIDAE 1850.ga.f002

Cynops Tschudi, $1838 \cdot \mathbf{K Y}$

ST: PO.KN • CI: h0407 • ID: 558

PN: Salamandra subcristatus Temminck ${ }^{+1}, 1838$

PK: Molge pyrrhogaster* Boie, 1826

KG: Cynops ${ }^{1}$ Tschudi, 1838

KF: SALAMANDRIDAE 1820.ga.f002

Cynotriton Dubois ${ }^{+1}$, 2011 • AK

ST: PO.JD・CI: h0408・ID: 559

PN: Triton (Cynops) orientalis David, 1875

PK: Triton (Cynops) orientalis* David, 1875

KG: Hypselotriton ${ }^{2}$ Wolterstorff, 1934

KF: SALAMANDRIDAE 1820.ga.f002

Cystignathus Wagler, 1830 • AK

ST: PO.JD • CI: h0409・ ID: 253

PN: Rana pachypus Spix, 1824

PK: Rana latrans* Steffen, 1815

KG: Leptodactylus ${ }^{1}$ Fitzinger, 1826

KF: LEPTODACTYLIDAE ||1838.ta.f001||-1896.wa.f001

Czatkobatrachus Evans ${ }^{+1}, 1998+$ KY

ST: PO.KN • CI: h0410 • ID: $\uparrow 018$

PN: Czatkobatrachus polonicus Evans $^{+1}$, 1998 \$

PK: Czatkobatrachus polonicus ${ }^{\circ}$ Evans $^{+1}, 1998 \uparrow$

KG: Czatkobatrachus ${ }^{\circ}$ Evans $^{+1}, 1998 \dagger$

KF: ANURA Familia INCERTAE SEDIS

Dactylethra Cuvier, 1829 • AK

ST: PO.JD • CI: h0411 • ID: 009

PN: Bufo laevis Daudin, 1802

PK: Bufo laevis* Daudin, 1802

KG: Xenopus ${ }^{1}$ Wagler in Boie, 1827

KF: PIPIDAE 1825.gb.f003-|1826.fb.f002|

Dactyletra Hoffmann, 1878 • AK

ST: NS.JD • CI: h0412 • ID: 009

PN: Bufo laevis Daudin, 1802

PK: Bufo laevis* Daudin, 1802

KG: Xenopus ${ }^{1}$ Wagler in Boie, 1827

KF: PIPIDAE 1825.gb.f003-|1826.fb.f002|

Dactylonyx: Bonaparte $1839 \bullet \mathbf{A N}$

ST: $\mathbf{A L} \bullet \mathbf{C I}:$ n0031 • ID: 517

PN: Onychodactylus schlegeli Tschudi, 1838

PK: Salamandra japonica* Houttuyn, 1782

KG: Onychodactylus ${ }^{1}$ Tschudi, 1838

KF: HYNOBIIDAE ||1856.ha.f001\|-1859.cb.f002

Dalianbatrachus $\mathrm{Gao}^{+1}, 2004+\mathbf{A K}$

ST: PO.JD・CI: h0413・ID: $\uparrow 033$

PN: Dalianbatrachus mengi $\mathrm{Gao}^{+1}, 2004$ \$

PK: Mesophryne beipiaoensis ${ }^{\circ} \mathrm{Gao}^{+1}, 2001 \uparrow$

KG: Mesophryne ${ }^{\circ} \mathrm{Gao}^{+1}, 2001 \uparrow$

KF: ANURA Familia INCERTAE SEDIS

Dasypops Miranda Ribeiro, 1924 • KY

ST: PO.KN • CI: h0414 • ID: 294

PN: Dasypops schirchi Miranda Ribeiro, 1924

PK: Dasypops schirchi* Miranda Ribeiro, 1924
KG: Dasypops* Miranda Ribeiro, 1924

KF: MICROHYLIDAE ||1843.fa.f012||-1931.na.f001

Dehmiella Herre $^{+1}, 1950$ † AK

ST: PO.JD • CI: h0415・ID: 578

PN: Dehmiella schindewolfi $\mathrm{Herre}^{+1}, 1950$ †

PK: Salamandra sansaniensis ${ }^{\circ}$ Lartet, $1851 \uparrow^{\dagger}$

KG: Salamandra ${ }^{1}$ Garsault, 1764

KF: SALAMANDRIDAE 1820.ga.f002

Dendricus Gistel, 1848 • AK

ST: NL.JI • CI: h0416・ID: 436

PN: Hyla buergeri Temminck $^{+1}, 1838$

PK: Hyla buergeri* ${ }^{*}$ Temminck $^{+1}, 1838$

KG: Buergeria* Tschudi, 1838

KF: RHACOPHORIDAE ||1858.gc.f012||-1932.ha.f001

Dendrobates Wagler, $1830 \cdot \mathbf{K Y}$

ST: PO.KN • CI: h0417・ID: 048

PN: Rana tinctoria Cuvier, 1797

PK: Rana tinctoria* Cuvier, 1797

KG: Dendrobates* Wagler, 1830

KF: DENDROBATIDAE ||1850.bb.f006||-1865.ca.f002

Dendrobatorana Ahl, $1927 \cdot \mathbf{K Y}$

ST: PO.KN • CI: h0418・ID: 435

PN: Hylambates dorsalis Peters, 1875

PK: Hylambates dorsalis ${ }^{\circ}$ Peters, 1875

KG: Dendrobatorana ${ }^{\circ}$ Ahl, 1927

KF: RHACOPHORIDAE ||1858.gc.f012||-1932.ha.f001

Dendrohyas Wagler, 1830 • AK

ST: PO.JI • CI: h0419・ID: 204

PN: Rana arborea Linnaeus, 1758

PK: Rana arborea* Linnaeus, 1758

KG: Hyla* Laurenti, 1768

KF: HYLIDAE 1815.ra.f002-|1825.gb.f001|

Dendromanes Gistel, 1848 • AK

ST: NL.JI • CI: h0420 • ID: 314

PN: Microhyla achatina Tschudi, 1838

PK: Microhyla achatina* Tschudi, 1838

KG: Microhyla* Tschudi, 1838

KF: MICROHYLIDAE ||1843.fa.f012||-1931.na.f001

Dendromedusa Gistel, 1848 • AK

ST: NL.JD・CI: h0421・ID: 189

PN: Calamita punctatus Schneider, 1799

PK: Calamita punctatus* Schneider, 1799

KG: Boana* Gray, 1825

KF: HYLIDAE 1815.ra.f002-|1825.gb.f001|

Dendrophryniscus Jiménez de la Espada, 1870 • KY

ST: PO.KN • CI: h0422・ ID: 147

PN: Dendrophryniscus brevipollicatus Jiménez de la Espada, 1870

PK: Dendrophryniscus brevipollicatus* Jiménez de la Espada, 1870

KG: Dendrophryniscus * Jiménez de la Espada, 1870

KF: BUFONIDAE 1825.gb.f004

Dendropsophus Fitzinger, $1843 \cdot \mathbf{K Y}$

ST: PO.KN • CI: h0423・ID: 194

PN: Hyla frontalis Daudin, 1800

PK: Rana leucophyllata* Beireis, 1783

KG: Dendropsophus ${ }^{1}$ Fitzinger, 1843

KF: HYLIDAE 1815.ra.f002-|1825.gb.f001| 
Dendrotriton $\mathrm{Wake}^{+1}, 1983 \cdot \mathbf{K Y}$

ST: PO.KN • CI: h0424 • ID: 531

PN: Oedipus bromeliacia Schmidt, 1936

PK: Oedipus bromeliacia* Schmidt, 1936

KG: Dendrotriton $*$ Wake $^{+1}, 1983$

KF: PLETHODONTIDAE 1850.ga.f002

Dermatonotus Méhelÿ, $1904 \cdot \mathbf{K Y}$

ST: PO.KN • CI: h0425 • ID: 297

PN: Engystoma muelleri Boettger, 1885

PK: Engystoma muelleri* Boettger, 1885

KG: Dermatonotus* Méhelÿ, 1904

KF: MICROHYLIDAE ||1843.fa.f012||-1931.na.f001

Dermophis Peters, 1880 • AK

ST: PO.JD • CI: h0426・ ID: 487

PN: Siphonops mexicanus Duméril ${ }^{+1}, 1841$

PK: Siphonops mexicanus* Duméril ${ }^{+1}, 1841$

KG: Gymnopis* Peters, 1874

KF: CAECILIIDAE 1814.ra.f003-|1825.gb.f008|

Desmiostoma Sager, $1858 \cdot \mathbf{A K}$

ST: PO.JD • CI: h0427 • ID: 555

PN: Desmiostoma maculatus Sager, 1858

PK: Ambystoma mavortia ${ }^{\circ}$ Baird, 1850

KG: Ambystoma ${ }^{1}$ Tschudi, 1838

KF: АмвYSTомAтIDAE 1850.ga.f004

Desmodactylus Duméril ${ }^{+2}, 1854$ • AK

ST: NL.JI • CI: h0428 • ID: 538

PN: Salamandra scutata Temminck ${ }^{+1}, 1838$

PK: Salamandra scutata* Temminck ${ }^{+1}, 1838$

KG: Hemidactylium* Tschudi, 1838

KF: PLETHODONTIDAE 1850.ga.f002

Desmognathus Baird, $1850 \cdot \mathrm{KY}$

ST: PO.KN • CI: h0429 • ID: 548

PN: Triturus fuscus Rafinesque, 1820

PK: Triturus fuscus* Rafinesque, 1820

KG: Desmognathus* Baird, 1850

KF: Plethodontidae 1850.ga.f002

Diaglena Cope, $1887 \cdot \mathbf{K Y}$

ST: PO.KN • CI: h0430 • ID: 207

PN: Triprion spatulatus Günther, 1882

PK: Triprion spatulatus* Günther, 1882

KG: Diaglena* Cope, 1887

KF: HYLIDAE 1815.ra.f002-|1825.gb.f001|

Dianrana $\mathrm{Fei}^{+2}, 2010 \bullet \mathbf{A K}$

ST: PO.JD • CI: h0431 • ID: 411

PN: Rana pleuraden Boulenger, 1904

PK: Rana pleuraden* Boulenger, 1904

KG: Nidirana ${ }^{1}$ Dubois, 1992

KF: RANIDAE 1796.ba.f001

Diasporus Hedges ${ }^{+2}, 2008 \cdot \mathbf{K Y}$

ST: PO.KN • CI: h0432 • ID: 080

PN: Lithodytes diastema Cope, 1875

PK: Lithodytes diastema* Cope, 1875

KG: Diasporus ${ }^{*}$ Hedges $^{+2}, 2008$

KF: BRACHYCEPHALIDAE 1858.gc.f002

Dicamptodon Strauch, $1870 \cdot \mathbf{K Y}$

ST: PO.KN • CI: h0433・ ID: 556
PN: Triton ensatus Eschscholtz, 1833

PK: Triton ensatus* Eschscholtz, 1833

KG: Dicamptodon* Strauch, 1870

KF: AмвYSTOMATIDAE 1850.ga.f004

Dicroglossus Günther, 1860 • AK

ST: PO.JD • CI: h0434 • ID: 373

PN: Dicroglossus adolfi Günther, 1860

PK: Rana cyanophlyctis* Schneider, 1799

KG: Euphlyctis ${ }^{1}$ Fitzinger, 1843

KF: DiCROGLOSSIDAE 1987.da.f004

Didocus Cope, 1866 • AK

ST: PO.JD • CI: h0435・ID: 026

PN: Rana calcarata Michahelles, 1830

PK: Rana cultripes* Cuvier, 1829

KG: Pelobates* Wagler, 1830

KF: PELOBATIDAE 1850.bb.f004

Didynamipus Andersson, $1903 \cdot \mathbf{K Y}$

ST: PO.KN • CI: h0436・ID: 124

PN: Didynamipus sjostedti Andersson, 1903

PK: Didynamipus sjostedti* Andersson, 1903

KG: Didynamipus* Andersson, 1903

KF: BUFONIDAE 1825.gb.f004

Diemictylus Rafinesque, 1820 • AK

ST: PO.JD・CI: h0437・ ID: 569

PN: Triturus (Diemictylus) viridescens Rafinesque, 1820

PK: Triturus (Diemictylus) viridescens* Rafinesque, 1820

KG: Notophthalmus ${ }^{1}$ Rafinesque, 1820

KF: SALAMANDRIDAE 1820.ga.f002

Dilobates Boulenger, 1900 • AK

ST: PO.JD • CI: h0438 • ID: 321

PN: Dilobates platycephalus Boulenger, 1900

PK: Gampsosteonyx batesi* Boulenger, 1900

KG: Astylosternus* Werner, 1898

KF: ARTHROLEPTIDAE 1869.mc.f011

Dimorphognathus Boulenger, 1906 • AK

ST: PO.JD • CI: h0439 • ID: 350

PN: Heteroglossa africana Hallowell, 1858

PK: Heteroglossa africana* Hallowell, 1858

KG: Phrynobatrachus* Günther, 1862

KF: PHRYNOBATRACHIDAE 1941.lb.f001

Diplopaa nov. $\bullet \mathrm{KY}$

ST: PO.KN • CI: h0440 • ID: 385

PN: Paa (Feirana) taihangnicus $\mathrm{Chen}^{+1}, 2002$

PK: Paa (Feirana) taihangnicus ${ }^{*} \mathrm{Chen}^{+1}, 2002$

KG: Diplopaa* nov., 2006

KF: DICROGLOSSIDAE 1987.da.f004

Diplopelma Günther, 1859 • AK

ST: PO.JD • CI: h0441 • ID: 314

PN: Engystoma ornatum Duméril ${ }^{+1}, 1841$

PK: Engystoma ornatum* Duméril ${ }^{+1}, 1841$

KG: Microhyla* Tschudi, 1838

KF: MICROHYLIDAE ||1843.fa.f012||-1931.na.f001

Diplopelturus Depéret, 1897 • AK

ST: PO.JD • CI: h0442 • ID: 470

PN: Diplopelturus ruscinensis Depéret, 1897 *

PK: Rana gigantea ${ }^{\circ}$ Lartet, $1851 \dagger$ 
KG: Latonia ${ }^{3}$ Meyer, $1845 \dagger$

KF: DISCOGLOSSIDAE 1858.gc.f004

Dischidodactylus Lynch, $1979 \cdot \mathbf{K Y}$

ST: PO.KN • CI: h0443・ID: 086

PN: Elosia duidensis Rivero, 1968

PK: Elosia duidensis ${ }^{\circ}$ Rivero, 1968

KG: Dischidodactylus ${ }^{\circ}$ Lynch, 1979

KF: CEUTHOMANTIDAE 2009.ha.f003

Discodactylus Wagler in Michahelles, 1833 • AK

ST: NL.JI • CI: h0444 • ID: 204

PN: Rana arborea Linnaeus, 1758

PK: Rana arborea* Linnaeus, 1758

KG: Hyla* Laurenti, 1768

KF: HYLIDAE 1815.ra.f002-|1825.gb.f001|

Discodeles Boulenger, 1918 • AK

ST: PO.JD • CI: h0445 • ID: 369

PN: Rana guppyi Boulenger, 1884

PK: Rana guppyi* Boulenger, 1884

KG: Cornufer* Tschudi, 1838

KF: CERATOBATRACHIDAE 1884.ba.f001

Discoglossus Otth, 1837 • KY

ST: PO.KN • CI: h0446・ID: 469

PN: Discoglossus pictus Otth, 1837

PK: Discoglossus pictus* Otth, 1837

KG: Discoglossus* Otth, 1837

KF: DISCOGLOSSIDAE 1858.gc.f004

Docidophryne Fitzinger, 1843 • AK

ST: PO.JD • CI: h0447 • ID: 138

PN: Bufo agua Latreille in Sonnini ${ }^{+1}, 1801$

PK: Bufo ictericus* Spix, 1824

KG: Rhinella ${ }^{2}$ Fitzinger, 1826

KF: BUFONIDAE 1825.gb.f004

Doctylethra: Hoffmann 1878 - AN

ST: $\mathbf{A M} \cdot$ CI: n0032 • ID: 009

PN: Bufo laevis Daudin, 1802

PK: Bufo laevis* Daudin, 1802

KG: Xenopus ${ }^{1}$ Wagler in Boie, 1827

KF: PIPIDAE 1825.gb.f003-|1826.fb.f002|

Doctyletra: Hoffmann 1878 - AN

ST: $\mathbf{A M} \cdot \mathbf{C I}$ : n0033・ID: 009

PN: Bufo laevis Daudin, 1802

PK: Bufo laevis* Daudin, 1802

KG: Xenopus ${ }^{1}$ Wagler in Boie, 1827

KF: PIPIDAE 1825.gb.f003-|1826.fb.f002|

Doryphoros Mayer, 1835 • AK

ST: PO.JD • CI: h0448・ID: 253

PN: Rana pachypus Spix, 1824

PK: Rana latrans* Steffen, 1815

KG: Leptodactylus ${ }^{1}$ Fitzinger, 1826

KF: LEPTODACTYLIDAE ||1838.ta.f001||-1896.wa.f001

Dromoplectrus Camerano, 1879 • AK

ST: PO.JD • CI: h0449 • ID: 136

PN: Bufo anomalus Günther, 1859

PK: Bufo compactilis ${ }^{\circ}$ Wiegmann, 1833

KG: Anaxyrus $^{3}$ Tschudi, 1845

KF: BUFONIDAE 1825.gb.f004
Dryaderces Jungfer ${ }^{+24}, 2013 \cdot \mathbf{K Y}$

ST: PO.KN • CI: h0450 • ID: 222

PN: Hyla pearsoni Gaige, 1929

PK: Hyla pearsoni ${ }^{\circ}$ Gaige, 1929

KG: Dryaderces ${ }^{\circ}$ Jungfer $^{+24}, 2013$

KF: HYLIDAE 1815.ra.f002-|1825.gb.f001|

Drymomantis Peters, 1882 • AK

ST: PO.JD • CI: h0451 • ID: 235

PN: Hylomantis fallax Peters, 1880

PK: Hylomantis fallax* Peters, 1880

KG: Litoria* Tschudi, 1838

KF: PHYLLOMEDUSIDAE 1858.gc.f009

Dryomelictes Fitzinger, 1843 • AK

ST: PO.JI • CI: h0452 • ID: 234

PN: Hyla lactea Daudin, 1800

PK: Hyla lactea* Daudin, 1800

KG: Sphaenorhynchus* Tschudi, 1838

KF: HYLIDAE 1815.ra.f002-|1825.gb.f001|

Dryomelictes Cope, 1865 • AK

ST: PO.JH • CI: h0453 • ID: 234

PN: Hyla aurantiaca Daudin, 1802

PK: Hyla lactea* Daudin, 1800

KG: Sphaenorhynchus* Tschudi, 1838

KF: HYLIDAE 1815.ra.f002-|1825.gb.f001|

Dryophytes Fitzinger, $1843 \cdot \mathbf{K Y}$

ST: PO.KN • CI: h0454 • ID: 203

PN: Hyla versicolor Le Conte, 1825

PK: Hyla versicolor* Le Conte, 1825

KG: Dryophytes* Fitzinger, 1843

KF: HYLIDAE 1815.ra.f002-|1825.gb.f001|

Dryopsophus Fitzinger, 1843 • AK

ST: PO.JD • CI: h0455 • ID: 237

PN: Hyla citripoda Péron, 1807

PK: Hyla citropa* Péron, 1825

KG: Ranoidea ${ }^{1}$ Tschudi, 1838

KF: PHYLLOMEDUSIDAE 1858.gc.f009

Duboimantis $\mathrm{Glaw}^{+1}, 2006$ • AK

ST: PO.JD • CI: h0456 • ID: 431

PN: Limnodytes granulatus Boettger, 1881

PK: Limnodytes granulatus* Boettger, 1881

KG: Gephyromantis* Methuen, 1920

KF: RHACOPHORIDAE ||1858.gc.f012||-1932.ha.f001

Duellmania Dubois, 1987 • AK

ST: PO.JD • CI: h0457 • ID: 091

PN: Hyla argenteovirens Boettger, 1892

PK: Hyla argenteovirens* Boettger, 1892

KG: Gastrotheca* Fitzinger, 1843

KF: HEMIPHRACTIDAE 1862.pa.f001

Duellmanohyla Campbell ${ }^{+1}, 1992 \cdot \mathbf{K Y}$

ST: PO.KN • CI: h0458 • ID: 214

PN: Hyla uranochroa Cope, 1875

PK: Hyla uranochroa* Cope, 1875

KG: Duellmanohyla* Campbell ${ }^{+1}, 1992$

KF: HYLIDAE 1815.ra.f002-|1825.gb.f001|

Duttaphrynus Frost ${ }^{+18}, 2006 \cdot \mathbf{K Y}$

ST: PO.KN • CI: h0459 • ID: 108 
PN: Bufo melanostictus Schneider, 1799

PK: Bufo melanostictus* Schneider, 1799

KG: Duttaphrynus ${ }^{*}$ Frost $^{+18}, 2006$

KF: BUFONIDAE 1825.gb.f004

Dyscophina Van Kampen, 1905 • AK

ST: PO.JD • CI: h0460 • ID: 313

PN: Dyscophina volzi Van Kampen, 1905

PK: Dyscophina volzi ${ }^{\circ}$ Van Kampen, 1905

KG: Glyphoglossus* Günther, 1869

KF: MICROHYLIDAE ||1843.fa.f012||-1931.na.f001

Dyscophus Grandidier, 1872 • KY

ST: PO.KN • CI: h0461・ID: 307

PN: Dyscophus insularis Grandidier, 1872

PK: Dyscophus insularis* Grandidier, 1872

KG: Dyscophus* Grandidier, 1872

KF: MICROHYLIDAE ||1843.fa.f012||-1931.na.f001

Eburana Dubois, 1992 • AK

ST: PO.JD • CI: h0462 • ID: 412

PN: Rana narina Stejneger, 1901

PK: Rana narina* Stejneger, 1901

KG: Odorrana* $\mathrm{Fei}^{+2}, 1990$

KF: RANIDAE 1796.ba.f001

Echinotriton Nussbaum ${ }^{+1}, 1982$ • KY

ST: PO.KN • CI: h0463・ID: 572

PN: Tylototriton andersoni Boulenger, 1892

PK: Tylototriton andersoni* Boulenger, 1892

KG: Echinotriton ${ }^{*}$ Nussbaum $^{+1}, 1982$

KF: SALAMANDRIDAE 1820.ga.f002

Ecnomiohyla Faivovich ${ }^{+5}, 2005 \bullet \mathbf{K Y}$

ST: PO.KN • CI: h0464 • ID: 211

PN: Hypsiboas miliarius Cope, 1886

PK: Hypsiboas miliarius* Cope, 1886

KG: Ecnomiohyla* Faivovich ${ }^{+5}, 2005$

KF: HYLIDAE 1815.ra.f002-|1825.gb.f001|

Ectopoglossus Grant ${ }^{+7}, 2017 \cdot \mathbf{K Y}$

ST: PO.KN • CI: h0465 • ID: 052

PN: Ectopoglossus saxatilis Grant ${ }^{+7}, 2017$

PK: Ectopoglossus saxatilis ${ }^{\circ} \mathrm{Grant}^{+7}, 2017$

KG: Ectopoglossus ${ }^{\circ}$ Grant $^{+7}, 2017$

KF: DENDROBATIDAE $\|1850 . b b . f 006\|-1865 . c a . f 002$

Edalorhina Jiménez de la Espada, 1870 • KY

ST: PO.KN • CI: h0466・ID: 247

PN: Edalorhina perezi Jiménez de la Espada, 1870

PK: Edalorhina perezi* Jiménez de la Espada, 1870

KG: Edalorhina* Jiménez de la Espada, 1870

KF: LEIUPERIDAE 1850.bb.f010

Edaphotheca Duellman, 2015 • AK

ST: PO.JD • CI: h0467・ ID: 091

PN: Gastrotheca galeata Trueb $^{+1}, 1978$

PK: Gastrotheca galeata* Trueb $^{+1}, 1978$

KG: Gastrotheca* Fitzinger, 1843

KF: HEMIPHRACTIDAE 1862.pa.f001

Edwardtayloria Marx, 1975• AK

ST: PO.JD • CI: h0468 • ID: 437

PN: Hazelia spinosa Taylor, 1920

PK: Hazelia spinosa* Taylor, 1920
KG: Nyctixalus * Boulenger, 1882

KF: RHACOPHORIDAE ||1858.gc.f012||-1932.ha.f001

Egoria Skutschas $^{+6}, 2020$ + $\mathbf{K Y}$

ST: PO.KN • CI: h0469・ID: $\uparrow 130$

PN: Egoria malashichevi Skutschas ${ }^{+6}, 2020$ \$

PK: Egoria malashichevi ${ }^{\circ}$ Skutschas $^{+6}, 2020 \dagger$

KG: Egoria $^{\circ}$ Skutschas $^{+6}, 2020 \dagger$

KF: Urodela Familia INCERTAE SEDIS

Elachistocleis Parker, 1927 • AK

ST: PO.JI • CI: h0470 • ID: 298

PN: Rana ovalis Schneider, 1799

PK: Rana ovalis* Schneider, 1799

KG: Engystoma* Fitzinger, 1826

KF: MICROHYLIDAE ||1843.fa.f012||-1931.na.f001

Elachyglossa Andersson, 1916 • AK

ST: PO.JD • CI: h0471 • ID: 380

PN: Elachyglossa gyldenstolpei Andersson, 1916

PK: Elachyglossa gyldenstolpei* Andersson, 1916

KG: Limnonectes* Fitzinger, 1843

KF: DiCROGLOSSIDAE 1987.da.f004

Eladinea Miranda-Ribeiro, 1937 • AK

ST: PO.JD • CI: h0472 • ID: 522

PN: Eladinea estheri Miranda-Ribeiro, 1937

PK: Oedipus paraensis* Unterstein, 1930

KG: Bolitoglossa* Duméril ${ }^{+2}, 1854$

KF: PLETHODONTIDAE 1850.ga.f002

Elaphromantis Laurent, 1941 • AK

ST: PO.JD • CI: h0473・ID: 325

PN: Hylambates notatus Buchholz ${ }^{+1}$ in Peters, 1875

PK: Hylambates notatus ${ }^{\circ}$ Buchholz $^{+1}$ in Peters, 1875

KG: Leptopelis ${ }^{2}$ Günther, 1859

KF: ARTHROLEPTIDAE 1869.mc.f011

Electrorana Xing ${ }^{+3} ; 2018+\bullet \mathbf{K Y}$

ST: PO.KN • CI: h0474・ID: $\uparrow 108$

PN: Electrorana limoae $\mathrm{Xing}^{+3}, 2018$ \$

PK: Electrorana limoae ${ }^{\circ} \mathrm{Xing}^{+3}, 2018 \dagger$

KG: Electrorana ${ }^{\circ} \mathrm{Xing}^{+3}, 2018$ †

KF: Mediogyrinia Familia INCERTAE SEDIS

Eleutherodactylus Duméril ${ }^{+1}, 1841$ • KY

ST: PO.KN • CI: h0475 • ID: 081

PN: Hylodes martinicensis Tschudi, 1838

PK: Hylodes martinicensis* Tschudi, 1838

KG: Eleutherodactylus* Duméril ${ }^{+1}, 1841$

KF: BRACHYCEPHALIDAE 1858.gc.f002

Elkobatrachus Henrici ${ }^{+1}, 2006+\mathbf{K Y}$

ST: PO.KN • CI: h0476・ID: $\uparrow 085$

PN: Elkobatrachus brocki Henrici ${ }^{+1}, 2006+$

PK: Elkobatrachus brocki ${ }^{\circ}$ Henrici $^{+1}, 2006 \dagger$

KG: Elkobatrachus ${ }^{\circ}$ Henrici $^{+1}, 2006$ †

KF: Archaeosalientia Familia INCERTAE SEDIS

Ellipsoglossa Duméril ${ }^{+2}, 1854$ • AK

ST: PO.JD・CI: h0477• ID: 505

PN: Salamandra naevia Temminck $^{+1}, 1838$

PK: Salamandra naevia* Temminck $^{+1}, 1838$

KG: Hynobius* Tschudi, 1838

KF: HYNOBIIDAE ||1856.ha.f001||-1859.cb.f002 
Elophila Huebner, $1822 \cdot \mathbf{z H}$

ST: zo • CI: zh030

Elophila: Duméril ${ }^{+1} 1841 \cdot \mathbf{A N}$

ST: AL・CI: n0034・ID: 423

PN: Boophis goudotii Tschudi, 1838

PK: Boophis goudotii* Tschudi, 1838

KG: Boophis* Tschudi, 1838

KF: RHACOPHORIDAE ||1858.gc.f012||-1932.ha.f001

Elosia Tschudi, $1838 \cdot \mathbf{A K}$

ST: PO.JD • CI: h0478 • ID: 182

PN: Hyla nasus Lichtenstein, 1823

PK: Hyla nasus* Lichtenstein, 1823

KG: Hylodes ${ }^{1}$ Fitzinger, 1826

KF: HYLODIDAE 1858.gc.f010

Emydops Broom, 1912 • ZH

ST: zo • CI: zh031

Emydops Miranda-Ribeiro, 1920 • AK

ST: PO.JH • CI: h0479 • ID: 302

PN: Emydops hypomelas Miranda-Ribeiro, 1920

PK: Stereocyclops incrassatus* Cope, 1870

KG: Stereocyclops* Cope, 1870

KF: MICROHYLIDAE ||1843.fa.f012||-1931.na.f001

Engistoma Peracca, 1904 • AK

ST: NS.JI • CI: h0480 • ID: 298

PN: Rana ovalis Schneider, 1799

PK: Rana ovalis* Schneider, 1799

KG: Engystoma* Fitzinger, 1826

KF: MICROHYLIDAE ||1843.fa.f012||-1931.na.f001

Engystoma Fitzinger, $1826 \cdot \mathbf{K Y}$

ST: PO.KN・CI: h0481 • ID: 298

PN: Rana ovalis Schneider, 1799

PK: Rana ovalis* Schneider, 1799

KG: Engystoma* Fitzinger, 1826

KF: MICROHYLIDAE ||1843.fa.f012||-1931.na.f001

Engystomops Jiménez de la Espada, 1872 • KY

ST: PO.KN • CI: h0482 • ID: 248

PN: Engystomops petersi Jiménez de la Espada, 1872

PK: Engystomops petersi* Jiménez de la Espada, 1872

KG: Engystomops* Jiménez de la Espada, 1872

KF: LEIUPERIDAE 1850.bb.f010

Enigmatosaurus Nopcsa, $1908+\bullet$ AK

ST: NL.JI • CI: h0483 • ID: $\uparrow 045$

PN: Thaumastosaurus bottii Stefano, $1904 \$$

PK: Thaumastosaurus bottii ${ }^{\circ}$ Stefano, $1904 \dagger$

KG: Thaumastosaurus ${ }^{\circ}$ Stefano, $1904 \dagger$

KF: ANURa Familia INCERTAE SEDIS

Enneabatrachus Evans ${ }^{+1}, 1993$ + KY

ST: PO.KN • CI: h0484 • ID: $\uparrow 109$

PN: Enneabatrachus hechti Evans ${ }^{+1}, 1993$ \$

PK: Enneabatrachus hechti ${ }^{\circ}$ Evans $^{+1}, 1993 \dagger$

KG: Enneabatrachus ${ }^{\circ}$ Evans $^{+1}, 1993 \dagger$

KF: MEDIOGYrinia Familia INCERTAE SEDIS

Ensatina Gray, 1850 • KY

ST: PO.KN • CI: h0485 • ID: 550

PN: Ensatina eschscholtzii Gray, 1850

PK: Ensatina eschscholtzii* Gray, 1850
KG: Ensatina* Gray, 1850

KF: PLETHODONTIDAE 1850.ga.f002

Entomoglossus Peters, $1870 \bullet \mathbf{A K}$

ST: PO.JD • CI: h0486・ID: 253

PN: Entomoglossus pustulatus Peters, 1870

PK: Entomoglossus pustulatus ${ }^{\circ}$ Peters, 1870

KG: Leptodactylus ${ }^{1}$ Fitzinger, 1826

KF: LEPTODACTYLIDAE ||1838.ta.f001\|-1896.wa.f001

Enydrobius Wagler, 1830 • AK

ST: NL.JI • CI: h0487・ID: 182

PN: Hyla ranoides Spix, 1824

PK: Hyla nasus* Lichtenstein, 1823

KG: Hylodes ${ }^{1}$ Fitzinger, 1826

KF: HYLODIDAE 1858.gc.f010

Eobarbourula Folie ${ }^{+6}, 2013+\cdot \mathbf{K Y}$

ST: PO.KN • CI: h0488 • ID: $\uparrow 120$

PN: Eobarbourula delfinoi Folie $^{+6}, 2013$ *

PK: Eobarbourula delfino $i^{\circ}$ Folie $^{+6}, 2013 \dagger$

KG: Eobarbourula ${ }^{\circ}$ Folie $^{+6}, 2013 \dagger$

KF: BOMBINATORIDAE 1825.gb.f002

Eobatrachus Marsh, 1887 † KY

ST: PO.KN • CI: h0489 • ID: $† 019$

PN: Eobatrachus agilis Marsh, 1887 \$

PK: Eobatrachus agilis ${ }^{\circ}$ Marsh, $1887 \dagger$

KG: Eobatrachus ${ }^{\circ}$ Marsh, $1887 \dagger$

KF: AnURA Familia INCERTAE SEDIS

Eobufella Kuhn, 1941 + AK

ST: PO.JD • CI: h0490 • ID: $\uparrow 090$

PN: Eobufella parvula Kuhn, 1941 *

PK: Halleobatrachus hinsche $i^{\circ}$ Kuhn, $1941 \dagger$

KG: Eopelobates ${ }^{\circ}$ Parker, $1929 \dagger$

KF: PELOBATIDAE 1850.bb.f004

Eocaecilia Jenkins ${ }^{+1}, 1993+\cdot \mathbf{K Y}$

ST: PO.KN • CI: h0491・ID: $\uparrow 123$

PN: Eocaecilia micropodia Jenkins ${ }^{+1}, 1993$ \$

PK: Eocaecilia micropodia ${ }^{\circ}$ Jenkins $^{+1}, 1993 \dagger$

KG: Eocaecilia ${ }^{\circ}$ Jenkins $^{+1}, 1993 \dagger$

KF: EOCAECILIIDAE 1993.ja.f001 †

Eodiscoglossus Villalta, $1954+\bullet \mathbf{K Y}$

ST: PO.KN • CI: h0492 •ID: $\uparrow 116$

PN: Eodiscoglossus santonjae Villalta, 1954 \$

PK: Eodiscoglossus santonjae ${ }^{\circ}$ Villalta, $1954 \dagger$

KG: Eodiscoglossus ${ }^{\circ}$ Villalta, $1954 \dagger$

KF: DISCOGLOSSIDAE 1858.gc.f004

Eopelobates Parker, 1929 \$ $\mathbf{K Y}$

ST: PO.KN • CI: h0493・ID: $\uparrow 090$

PN: Eopelobates anthracinus Parker, 1929 \$

PK: Eopelobates anthracinus ${ }^{\circ}$ Parker, $1929 \dagger$

KG: Eopelobates ${ }^{\circ}$ Parker, $1929 \dagger$

KF: PELOBATIDAE 1850.bb.f004

Eophractus Schaeffer, $1949+\bullet \mathbf{A K}$

ST: PO.JD • CI: h0494 • ID: 257

PN: Eophractus casamayorensis Schaeffer, 1949 *

PK: Eophractus casamayorensis ${ }^{\circ}$ Schaeffer, $1949+$

KG: Calyptocephalella* Strand, 1928

KF: CALYPTOCEPHALELLIDAE 1960.ra.f001 
Eorhinophrynus Hecht, $1959+\cdot \mathbf{K Y}$

ST: PO.KN • CI: h0495 • ID: $\uparrow 082$

PN: Eorhinophrynus septentrionalis Hecht, 1959 \$

PK: Eorhinophrynus septentrionalis ${ }^{\circ}$ Hecht, $1959 \dagger$

KG: Eorhinophrynus ${ }^{\circ}$ Hecht, $1959 \dagger$

KF: RHINOPHRYNIDAE 1858.gc.f013

Eorubeta Hecht, $1960+\cdot \mathbf{K Y}$

ST: PO.KN • CI: h0496 • ID: $\uparrow 020$

PN: Eorubeta nevadensis Hecht, 1960 \$

PK: Eorubeta nevadensis ${ }^{\circ}$ Hecht, $1960 \dagger$

KG: Eorubeta ${ }^{\circ}$ Hecht, 1960 †

KF: ANURA Familia INCERTAE SEDIS

Eoscapherpeton Nessov, $1981+\cdot \mathbf{K Y}$

ST: PO.KN • CI: h0497・ID: $\uparrow 166$

PN: Eoscapherpeton asiaticum Nessov, 1981 *

PK: Eoscapherpeton asiaticum ${ }^{\circ}$ Nessov, $1981 \dagger$

KG: Eoscapherpeton ${ }^{\circ}$ Nessov, $1981 \dagger$

KF: CRYPTOBRANCHIDAE 1826.fb.f003

Eotheca Duellman, 2015 • KY

ST: PO.KN • CI: h0498 • ID: 089

PN: Nototrema fissipes Boulenger, 1888

PK: Nototrema fissipes* Boulenger, 1888

KG: Eotheca* Duellman, 2015

KF: HEMIPHRACTIDAE 1862.pa.f001

Eoxenopoides Haughton, 1931 \$ $\mathbf{K Y}$

ST: PO.KN • CI: h0499 • ID: $\uparrow 072$

PN: Eoxenopoides reuningi Haughton, 1931 *

PK: Eoxenopoides reuningi ${ }^{\circ}$ Haughton, $1931 \dagger$

KG: Eoxenopoides ${ }^{\circ}$ Haughton, $1931 \dagger$

KF: PIPIDAE 1825.gb.f003-|1826.fb.f002|

Epedaphus Cope, 1885 • AK

ST: PO.JD • CI: h0500 • ID: 203

PN: Hyla gratiosa Le Conte, 1856

PK: Hyla gratiosa* Le Conte, 1856

KG: Dryophytes* Fitzinger, 1843

KF: HYLIDAE 1815.ra.f002-|1825.gb.f001|

Ephippifer Agassiz, 1844 • AK

ST: NS.JI • CI: h0501 • ID: 057

PN: Bufo ephippium Spix, 1824

PK: Bufo ephippium* Spix, 1824

KG: Brachycephalus* Fitzinger, 1826

KF: BRACHYCEPHALIDAE 1858.gc.f002

Ephippiger: Gravenhorst $1845 \cdot \mathbf{A N}$

ST: AL • CI: n0035 •ID: 057

PN: Bufo ephippium Spix, 1824

PK: Bufo ephippium* Spix, 1824

KG: Brachycephalus* Fitzinger, 1826

KF: BRACHYCEPHALIDAE 1858.gc.f002

Ephippipher Cocteau, 1835 • AK

ST: NL.JI • CI: h0502・ID: 057

PN: Bufo ephippium Spix, 1824

PK: Bufo ephippium* Spix, 1824

KG: Brachycephalus* Fitzinger, 1826

KF: BRACHYCEPHALIDAE 1858.gc.f002

Epicrionops Boulenger, 1883 • AK

ST: PO.JD • CI: h0503 • ID: 473
PN: Epicrionops bicolor Boulenger, 1883

PK: Epicrionops bicolor ${ }^{\circ}$ Boulenger, 1883

KG: Rhinatrema* ${ }^{*}$ Duméril $^{+1}, 1841$

KF: RHINATREMATIDAE 1977.na.f001

Epicrium Wagler, $1828 \cdot \mathbf{K Y}$

ST: PO.KC • CI: h0504 • ID: 500

PN: Caecilia hypocyana Boie, 1827

PK: Caecilia hypocyana ${ }^{\circ}$ Boie, 1827

KG: Epicrium $^{\circ}$ Wagler, 1828

KF: ICHTHYOPHIIDAE 1968.ta.f001

Epidalea Cope, $1864 \cdot \mathbf{K Y}$

ST: PO.KN • CI: h0505 • ID: 122

PN: Bufo calamita Laurenti, 1768

PK: Bufo calamita* Laurenti, 1768

KG: Epidalea* Cope, 1864

KF: BUFONIDAE 1825.gb.f004

Epipedobates Myers, $1987 \cdot \mathbf{K Y}$

ST: PO.KN • CI: h0506 • ID: 042

PN: Prostherapis tricolor Boulenger, 1899

PK: Prostherapis tricolor* Boulenger, 1899

KG: Epipedobates* Myers, 1987

KF: DENDROBATIDAE ||1850.bb.f006||-1865.ca.f002

Epipole Gistel, $1848 \cdot \mathbf{A K}$

ST: NL.JI • CI: h0507 • ID: 331

PN: Hyla horstockii Schlegel, 1837

PK: Hyla horstockii* Schlegel, 1837

KG: Hyperolius* Rapp, 1842

KF: HYPEROLIIDAE 1943.lb.f001

Epipolysemia Brame, $1973 \$ \cdot \mathbf{A K}$

ST: PO.JD • CI: h0508・ID: $\uparrow 193$

PN: Salamandra ogygia Goldfuss, 1831 \%

PK: Salamandra ogygia ${ }^{\circ}$ Goldfuss, $1831 \uparrow$

KG: Chelotriton ${ }^{\circ}$ Pomel, $1853 \uparrow$

KF: SALAMANDRIDAE 1820.ga.f002

Epirhexis Cope, 1866 • EX

ST: PO.CE • CI: e0006 • ID: 082

PN: Batrachyla longipes Baird, 1859

PK: Batrachyla longipes ${ }^{\circ}$ Baird, 1859

KG: Euhyas* Fitzinger, 1843

KF: BRACHYCEPHALIDAE 1858.gc.f002

Eremiophilus Fitzinger, 1843 • EX

ST: PO.CE • CI: e0007 • ID: 338

PN: Cystignathus senegalensis Duméril ${ }^{+1}, 1841$

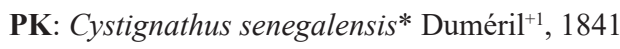

KG: Kassina* Girard, 1853

KF: HYPEROLIIDAE 1943.lb.f001

Ericabatrachus Largen, 1991 • KY

ST: PO.KN • CI: h0511 • ID: 352

PN: Ericabatrachus baleensis Largen, 1991

PK: Ericabatrachus baleensis* Largen, 1991

KG: Ericabatrachus* Largen, 1991

KF: ERICABATRACHIDAE nov. 2017.da.f96

Eripaa Dubois, $1992 \cdot \mathbf{K Y}$

ST: PO.KN • CI: h0512 • ID: 390

PN: Rana fasciculispina Inger, 1970

PK: Rana fasciculispina* Inger, 1970 
KG: Eripaa* Dubois, 1992

KF: DICROGLOSSIDAE 1987.da.f004

Esophus: Cope $1870 \bullet \mathbf{A N}$

ST: AM・CI: n0036・ID: 173

PN: Cystignathus nodosus Duméril ${ }^{+1}, 1841$

PK: Cystignathus nodosus* Duméril ${ }^{+1}, 1841$

KG: Alsodes* Bell, 1843

KF: ALSODIDAE 1869.mc.f005

Espadarana Guayasamin ${ }^{+5}, 2009 \cdot \mathbf{K Y}$

ST: PO.KN • CI: h0513・ID: 159

PN: Centrolenella andina Rivero, 1968

PK: Centrolenella andina* Rivero, 1968

KG: Espadarana* Guayasamin ${ }^{+5}, 2009$

KF: CENTROLENIDAE 1951.ta.f001

Estesiella Báez, 1995 † KY

ST: NT.KN • CI: h0514 • ID: $† 021$

PN: Estesius boliviensis Báez, 1991 \$

PK: Estesius boliviensis ${ }^{\circ}$ Báez, 1991 †

KG: Estesiella ${ }^{\circ}$ Báez, 1995 †

KF: ANURA Familia INCERTAE SEDIS

Estesina Roček $^{+1}, 1993$ + $\mathbf{K Y}$

ST: PO.KN • CI: h0515 • ID: $† 022$

PN: Estesina elegans Roček $^{+1}, 1993$ *

PK: Estesina elegans ${ }^{\circ}$ Roček $^{+1}, 1993 \dagger$

KG: Estesina ${ }^{\circ}$ Roček $^{+1}, 1993 \dagger$

KF: ANURA Familia INCERTAE SEDIS

Estesius Wallach, $1984 \cdot \mathbf{z H}$

ST: zo・CI: zh032

Estesius Báez, 1991 + AK

ST: PO.JH • CI: h0516・ID: $\uparrow 021$

PN: Estesius boliviensis Báez, 1991 \$

PK: Estesius boliviensis ${ }^{\circ}$ Báez, $1991 \dagger$

KG: Estesiella ${ }^{\circ}$ Báez, $1995 \dagger$

KF: ANURA Familia INCERTAE SEDIS

Etnabatrachus Hochnull, $2003+\mathbf{K Y}$

ST: PO.KN • CI: h0517 • ID: $\uparrow 099$

PN: Etnabatrachus maximus Hochnull, 2003 \$

PK: Etnabatrachus maximus ${ }^{\circ}$ Hochnull, $2003+$

KG: Etnabatrachus ${ }^{\circ}$ Hochnull, $2003 \dagger$

KF: HYLIDAE 1815.ra.f002-|1825.gb.f001|

Eubaphus Bonaparte, 1831 • AK

ST: PO.JI • CI: h0518・ID: 048

PN: Rana tinctoria Cuvier, 1797

PK: Rana tinctoria* Cuvier, 1797

KG: Dendrobates* Wagler, 1830

KF: DENDROBATIDAE $\| 1850$. bb.f006"|-1865.ca.f002

Eubates: Steindachner $1864 \cdot \mathbf{A N}$

ST: AL •CI: n0037・ID: 331

PN: Hyperoliuss heuglini Steindachner, 1864

PK: Crumenifera pusilla* Cope, 1862

KG: Hyperolius* Rapp, 1842

KF: HYPEROLIIDAE 1943.lb.f001

Eucnemis Ahrens, $1812 \cdot \mathbf{z H}$

ST: zo • CI: zh033

Eucnemis Tschudi, 1838 • AK

ST: PO.JH • CI: h0519 • ID: 331
PN: Hyla horstockii Schlegel, 1837

PK: Hyla horstockii* Schlegel, 1837

KG: Hyperolius* Rapp, 1842

KF: HYPEROLIIDAE 1943.lb.f001

Euhyas Fitzinger, $1843 \cdot \mathbf{K Y}$

ST: PO.KN • CI: h0520 • ID: 082

PN: Hylodes ricordii Duméril ${ }^{+1}, 1841$

PK: Hylodes ricordii ${ }^{*}$ Duméril $^{+1}, 1841$

KG: Euhyas* Fitzinger, 1843

KF: BRACHYCEPHALIDAE 1858.gc.f002

Euparkerella Griffiths, 1959 • KY

ST: PO.KN • CI: h0521 • ID: 067

PN: Sminthillus brasiliensis Parker, 1926

PK: Sminthillus brasiliensis* Parker, 1926

KG: Euparkerella* Griffiths, 1959

KF: BRACHYCEPHALIDAE 1858.gc.f002

Eupemfix Palacký, 1898 • AK

ST: NT.JI • CI: h0522 • ID: 249

PN: Eupemphix nattereri Steindachner, 1863

PK: Eupemphix nattereri* Steindachner, 1863

KG: Eupemphix* Steindachner, 1863

KF: LEIUPERIDAE 1850.bb.f010

Eupemphix Steindachner, 1863 • KY

ST: PO.KN • CI: h0523・ID: 249

PN: Eupemphix nattereri Steindachner, 1863

PK: Eupemphix nattereri* Steindachner, 1863

KG: Eupemphix* Steindachner, 1863

KF: LEIUPERIDAE 1850.bb.f010

Euphlyctis Fitzinger, $1843 \cdot \mathbf{K Y}$

ST: PO.KN・CI: h0524 • ID: 373

PN: Rana leschenaultii Duméril ${ }^{+1}, 1841$

PK: Rana cyanophlyctis* Schneider, 1799

KG: Euphlyctis ${ }^{1}$ Fitzinger, 1843

KF: DiCROGLOSSIDAE 1987.da.f004

Eupodion: Jan $1857 \cdot \mathbf{A N}$

ST: $\mathbf{A L} ・ \mathbf{C I}: \mathrm{n} 0038 \bullet \mathbf{I D}: 249$

PN: Eupemphix nattereri Steindachner, 1863

PK: Eupemphix nattereri* Steindachner, 1863

KG: Eupemphix* Steindachner, 1863

KF: LEIUPERIDAE 1850.bb.f010

Eupomplyx: Jan 1857 AN

ST: $\mathbf{A L} ・$ CI: n0039・ID: 249

PN: Eupemphix nattereri Steindachner, 1863

PK: Eupemphix nattereri* Steindachner, 1863

KG: Eupemphix* Steindachner, 1863

KF: LEIUPERIDAE 1850.bb.f010

Euproctus Gené, 1839 • KY

ST: PO.KN・CI: h0525・ID: 557

PN: Euproctus rusconii Gené, 1839

PK: Molge platycephala* Gravenhorst, 1829

KG: Euproctus ${ }^{1}$ Gené, 1839

KF: SALAMANDRIDAE 1820.ga.f002

Eupsophus Fitzinger, $1843 \cdot \mathbf{K Y}$

ST: PO.KN • CI: h0526・ID: 174

PN: Cystignathus roseus Duméril ${ }^{+1}, 1841$

PK: Cystignathus roseus* Duméril ${ }^{+1}, 1841$ 
KG: Eupsophus* Fitzinger, 1843

KF: ALSODIDAE 1869.mc.f005

Eurhina Fitzinger, 1843 • AK

ST: PO.JI • CI: h0527 • ID: 138

PN: Bufo proboscideus Spix, 1824

PK: Bufo proboscideus ${ }^{\circ}$ Spix, 1824

KG: Rhinella ${ }^{2}$ Fitzinger, 1826

KF: BUFONIDAE 1825.gb.f004

Eurycea Rafinesque, 1822 • KY

ST: PO.KN • CI: h0528・ID: 542

PN: Eurycea lucifuga Rafinesque, 1822

PK: Eurycea lucifuga* Rafinesque, 1822

KG: Eurycea* Rafinesque, 1822

KF: PLETHODONTIDAE 1850.ga.f002

Eurycea Rafinesque, 1832 • AK

ST: PO.JH • CI: h0529 • ID: 504

PN: Eurycea mucronata Rafinesque, 1832

PK: Salamandra alleganiensis* Sonnini ${ }^{+1}, 1801$

KG: Cryptobranchus ${ }^{1}$ Leuckart, 1821

KF: CRYPTOBRANCHIDAE 1826.fb.f003

Eurycephalella Báez ${ }^{+2}, 2009$ † KY

ST: PO.KN • CI: h0530 • ID: $† 023$

PN: Eurycephalella alcinae $\mathrm{Báez}^{+2}, 2009$ \$

PK: Eurycephalella alcinae ${ }^{\circ} \mathrm{Báez}^{+2}, 2009 \dagger$

KG: Eurycephalella ${ }^{\circ}$ Báez $^{+2}, 2009 \dagger$

KF: ANURA Familia INCERTAE SEDIS

Euscelis Fitzinger, 1843 • AK

ST: PO.JD • CI: h0531 • ID: 237

PN: Hyla lesueurii Duméril ${ }^{+1}, 1841$

PK: Hyla lesueurii* Duméril ${ }^{+1}, 1841$

KG: Ranoidea ${ }^{1}$ Tschudi, 1838

KF: PHYLLOMEDUSIDAE 1858.gc.f009

Eusophis Neave, 1940 • AK

ST: NT.JD • CI: h0532・ID: 174

PN: Cystignathus roseus Duméril ${ }^{+1}, 1841$

PK: Cystignathus roseus* Duméril ${ }^{+1}, 1841$

KG: Eupsophus* Fitzinger, 1843

KF: ALSODIDAE 1869.mc.f005

Eusophus Cope, 1865 • AK

ST: PO.JD • CI: h0533・ID: 173

PN: Cystignathus nodosus Duméril ${ }^{+1}, 1841$

PK: Cystignathus nodosus* Duméril ${ }^{+1}, 1841$

KG: Alsodes* Bell, 1843

KF: ALSODIDAE 1869.mc.f005

Exaeretus Fieber, $1864 \cdot \mathbf{Z H}$

ST: zo • CI: zh034

Exaeretus Waga, 1876 • AK

ST: PO.JH • CI: h0534 • ID: 576

PN: Exaeretus caucasicus Waga, 1876

PK: Exaeretus caucasicus* Waga, 1876

KG: Mertensiella* Wolterstorff, 1925

KF: SALAMANDRIDAE 1820.ga.f002

Excidobates Twomey ${ }^{+1}, 2008 \cdot \mathbf{K Y}$

ST: PO.KN • CI: h0535 • ID: 046

PN: Dendrobates mysteriosus Myers, 1982

PK: Dendrobates mysteriosus* Myers, 1982
KG: Excidobates* ${ }^{*}$ womey ${ }^{+1}, 2008$

KF: DENDROBATIDAE \|1850.bb.f006"|-1865.ca.f002

Exerodonta Brocchi, $1879 \cdot \mathbf{K Y}$

ST: PO.KN • CI: h0536・ID: 218

PN: Exerodonta sumichrasti Brocchi, 1879

PK: Exerodonta sumichrasti* Brocchi, 1879

KG: Exerodonta* Brocchi, 1879

KF: HYLIDAE 1815.ra.f002-|1825.gb.f001|

Exobranchia: Rafinesque 1815 - AN

ST: AL • CI: n0040 • ID: 554

PN: Proteus anguinus Laurenti, 1768

PK: Proteus anguinus* Laurenti, 1768

KG: Proteus* Laurenti, 1768

KF: PROTEIDAE 1831.ba.f002

Fanchonia Werner, 1893 • AK

ST: PO.JD • CI: h0537 • ID: 237

PN: Fanchonia elegans Werner, 1893

PK: Rana aurea* Lesson, 1829

KG: Ranoidea ${ }^{1}$ Tschudi, 1838

KF: PHYLLOMEDUSIDAE 1858.gc.f009

Feihyla Frost ${ }^{+18}, 2006 \cdot \mathbf{K Y}$

ST: PO.KN • CI: h0538 • ID: 450

PN: Philautus palpebralis Smith, 1924

PK: Philautus palpebralis* Smith, 1924

KG: Feihyla* Frost $^{+18}, 2006$

KF: RHACOPHORIDAE ||1858.gc.f012||-1932.ha.f001

Feirana Dubois, $1992 \cdot \mathbf{K Y}$

ST: PO.KN • CI: h0539・ID: 386

PN: Rana quadranus $\mathrm{Liu}^{+2}, 1960$

PK: Rana quadranus* $\mathrm{Liu}^{+2}, 1960$

KG: Feirana* Dubois, 1992

KF: DiCROGLOSSIDAE 1987.da.f004

Fejervarya Bolkay, $1915 \cdot \mathbf{K Y}$

ST: PO.KN • CI: h0540 • ID: 377

PN: Rana limnocharis Boie in Gravenhorst, 1829

PK: Rana limnocharis* Boie in Gravenhorst, 1829

KG: Fejervarya* Bolkay, 1915

KF: DICROGLOSSIDAE 1987.da.f004

Fergusonia Hoffmann, 1878 • AK

ST: NL.JD・CI: h0541 • ID: 376

PN: Trachucephalus ceylanicus Ferguson, 1874

PK: Nannophrys ceylonensis* Günther, 1869

KG: Nannophrys* Günther, 1869

KF: DICROGLOSSIDAE 1987.da.f004

Fichteria Scortecci, 1941 - AK

ST: PO.JD・CI: h0542・ID: 319

PN: Fichteria somalica Scortecci, 1941

PK: Fichteria somalica ${ }^{\circ}$ Scortecci, 1941

KG: Phrynomantis* Peters, 1867

KF: PhryNomeridae 1931.na.f013

Firouzophrynus Safaei-Mahroo \& Ghaffari, 2020 • KY

ST: PO.KN • CI: h0543・ID: 109

PN: Bufo olivaceus Blanford, 1874

PK: Firouzophrynus olivaceus ${ }^{\circ}$ (Blanford, 1874)

KG: Firouzophrynus ${ }^{3}$ Safaei-Mahroo \& Ghaffari, 2020

KF: BUFONIDAE 1825.gb.f004 
Flectonotus Miranda-Ribeiro, $1926 \cdot \mathbf{K Y}$

ST: PO.KN • CI: h0544 • ID: 093

PN: Nototrema pygmaeum Boettger, 1893

PK: Nototrema pygmaeum* Boettger, 1893

KG: Flectonotus* Miranda-Ribeiro, 1926

KF: HEMIPHRACTIDAE 1862.pa.f001

Frankixalus $\mathrm{Biju}^{+9}, 2016$ • AK

ST: PO.JD • CI: h0545 • ID: 446

PN: Polypedates jerdonii Günther, 1876

PK: Polypedates jerdonii ${ }^{\circ}$ Günther, 1876

KG: Nasutixalus ${ }^{*}$ Jiang $^{+3}$ in Jiang ${ }^{+5}, 2016$

KF: RHACOPHORIDAE |1858.gc.f012||-1932.ha.f001

Frethia nov. $\bullet \mathrm{KY}$

ST: PO.KN • CI: h0546・ID: 394

PN: Oxyglossus laevis Günther, 1859

PK: Oxyglossus laevis* Günther, 1859

KG: Frethia* nov.

KF: OCCIDOZYGIDAE 1990.fa.f002

Fritzia Cambridge, $1879 \cdot \mathbf{Z H}$

ST: zo • CI: zh035

Fritzia Miranda-Ribeiro, 1920 • AK

ST: PO.JH • CI: h0547 • ID: 094

PN: Hyla goeldii Boulenger, 1895

PK: Hyla goeldii* Boulenger, 1895

KG: Fritziana* Mello-Leitão, 1937

KF: HEMIPHRACTIDAE 1862.pa.f001

Fritziana Mello-Leitão, 1937 • KY

ST: NT.KN • CI: h0548・ ID: 094

PN: Hyla goeldii Boulenger, 1895

PK: Hyla goeldii* Boulenger, 1895

KG: Fritziana* Mello-Leitão, 1937

KF: HEMIPHRACTIDAE 1862.pa.f001

Frostius Cannatella, 1986 • KY

ST: PO.KN・CI: h0549・ID: 150

PN: Atelopus pernambucensis Bokermann, 1962

PK: Atelopus pernambucensis ${ }^{\circ}$ Bokermann, 1962

KG: Frostius ${ }^{3}$ Cannatella, 1986

KF: BUFONIDAE 1825.gb.f004

Gabohyla Araujo-Vieira ${ }^{+3}, 2020 \bullet \mathbf{K Y}$

ST: PO.KN • CI: h0550 • ID: 233

PN: Sphaenorhynchus pauloalvini Bokermann, 1973

PK: Sphaenorhynchus pauloalvini ${ }^{\circ}$ Bokermann, 1973

KG: Gabohyla ${ }^{\circ}$ Araujo-Vieira ${ }^{+3}, 2020$

KF: HYLIDAE 1815.ra.f002-|1825.gb.f001|

Galverpeton Estes $^{+1}, 1982+\bullet \mathbf{K Y}$

ST: PO.KN・CI: h0551・ID: $\uparrow 131$

PN: Galverpeton ibericum Estes $^{+1}, 1982$ *

PK: Galverpeton ibericum ${ }^{\circ}$ Estes $^{+1}, 1982 \dagger$

KG: Galverpeton ${ }^{\circ}$ Estes $^{+1}, 1982 \dagger$

KF: Urodela Familia INCERTAE SEDIS

Gampsosteonyx Boulenger, 1900 • AK

ST: PO.JD • CI: h0552 • ID: 321

PN: Gampsosteonyx batesi Boulenger, 1900

PK: Gampsosteonyx batesi* Boulenger, 1900

KG: Astylosternus* Werner, 1898

KF: ARTHROLEPTIDAE 1869.mc.f011
Garbeana Miranda-Ribeiro, 1926 • AK

ST: PO.JD・CI: h0553・ID: 232

PN: Garbeana garbei Miranda-Ribeiro, 1926

PK: Garbeana garbei* Miranda-Ribeiro, 1926

KG: Scinax ${ }^{2}$ Wagler, 1830

KF: HYLIDAE 1815.ra.f002-|1825.gb.f001|

Gastrophryne Fitzinger, $1843 \cdot \mathbf{K Y}$

ST: PO.KN • CI: h0554・ID: 299

PN: Engystoma rugosum Duméril ${ }^{+1}, 1841$

PK: Engystoma carolinense* Holbrook, 1836

KG: Gastrophryne ${ }^{1}$ Fitzinger, 1843

KF: MICROHYLIDAE ||1843.fa.f012||-1931.na.f001

Gastrophrynoides Noble, $1926 \cdot \mathbf{K Y}$

ST: PO.KN・CI: h0555・ID: 281

PN: Engystoma borneense Boulenger, 1897

PK: Engystoma borneense ${ }^{\circ}$ Boulenger, 1897

KG: Gastrophrynoides ${ }^{3}$ Noble, 1926

KF: MICROHYLIDAE ||1843.fa.f012||-1931.na.f001

Gastrotheca Fitzinger, $1843 \cdot \mathbf{K Y}$

ST: PO.KN • CI: h0556・ ID: 091

PN: Hyla marsupiata Duméril ${ }^{+1}, 1841$

PK: Hyla marsupiata* Duméril ${ }^{+1}, 1841$

KG: Gastrotheca* Fitzinger, 1843

KF: HEMIPHRACTIDAE 1862.pa.f001

Gegeneophis Peters, $1880 \bullet \mathbf{K Y}$

ST: NL.KN • CI: h0557 • ID: 485

PN: Epicrium carnosum Beddome, 1870

PK: Epicrium carnosum* Beddome, 1870

KG: Gegeneophis* Peters, 1880

KF: CAECILIIDAE 1814.ra.f003-|1825.gb.f008

Gegenes Hübner, 1819 • ZH

ST: zo • CI: zh036

Gegenes Günther, 1876 • AK

ST: PO.JH・CI: h0558・ID: 485

PN: Epicrium carnosum Beddome, 1870

PK: Epicrium carnosum* Beddome, 1870

KG: Gegeneophis* Peters, 1880

KF: CAECILIIDAE 1814.ra.f003-|1825.gb.f008|

Gegenophis Boulenger, 1882 • AK

ST: NT.JI • CI: h0559・ ID: 485

PN: Epicrium carnosum Beddome, 1870

PK: Epicrium carnosum* Beddome, 1870

KG: Gegeneophis* Peters, 1880

KF: CAECILIIDAE 1814.ra.f003-|1825.gb.f008|

Genibatrachus $\mathrm{Gao}^{+1}, 2017$ \$ $\mathbf{K Y}$

ST: PO.KN • CI: h0560・ID: $\uparrow 060$

PN: Genibatrachus baoshanensis $\mathrm{Gao}^{+1}, 2017$ \$

PK: Genibatrachus baoshanensis ${ }^{\circ} \mathrm{Gao}^{+1}, 2017 \dagger$

KG: Genibatrachus ${ }^{\circ} \mathrm{Gao}^{+1}, 2017$ †

KF: GeOBATRACHIA Familia INCERTAE SEDIS

Genyofryne Palacký, 1898 • AK

ST: NT.JD・CI: h0561・ID: 280

PN: Genyophryne thomsoni Boulenger, 1890

PK: Genyophryne thomsoni* Boulenger, 1890

KG: Asterophrys* Tschudi, 1838

KF: MICROHYLIDAE ||1843.fa.f012||-1931.na.f001 
Genyophryne Boulenger, 1890 • AK

ST: PO.JD・CI: h0562 • ID: 280

PN: Genyophryne thomsoni Boulenger, 1890

PK: Genyophryne thomsoni* Boulenger, 1890

KG: Asterophrys* Tschudi, 1838

KF: MICROHYLIDAE ||1843.fa.f012||-1931.na.f001

Geobatrachus Ruthven, 1915 • KY

ST: PO.KN • CI: h0563・ID: 056

PN: Geobatrachus walkeri Ruthven, 1915

PK: Geobatrachus walkeri ${ }^{\circ}$ Ruthven, 1915

KG: Geobatrachus ${ }^{\circ}$ Ruthven, 1915

KF: BRACHYCEPHALIDAE 1858.gc.f002

Geocrinia Blake, $1973 \cdot \mathbf{K Y}$

ST: PO.KN • CI: h0564 • ID: 268

PN: Pterophrynus laevis Günther, 1864

PK: Pterophrynus laevis ${ }^{\circ}$ Günther, 1864

KG: Geocrinia ${ }^{3}$ Blake, 1973

KF: MYOBATRACHIDAE 1850.sa.f001

Geognathus Dubois ${ }^{+1}, 2012 \cdot \mathbf{A K}$

ST: PO.JD • CI: h0565 • ID: 548

PN: Desmognathus wrighti King, 1936

PK: Desmognathus wrighti* King, 1936

KG: Desmognathus* Baird, 1850

KF: PLETHODONTIDAE 1850.ga.f002

Geomolge Boulenger, 1886 • AK

ST: PO.JD • CI: h0566・ID: 517

PN: Geomolge fischeri Boulenger, 1886

PK: Geomolge fischeri* Boulenger, 1886

KG: Onychodactylus ${ }^{1}$ Tschudi, 1838

KF: НYNOBIIDAE ||1856.ha.f001||-1859.cb.f002

Geophryne Brown $^{+1}, 2014+\cdot \mathbf{K Y}$

ST: PO.KN • CI: h0567・ID: $\uparrow 100$

PN: Pseudacris nordensis Chantell, $1964+$

PK: Pseudacris nordensis ${ }^{\circ}$ Chantell, $1964 \dagger$

KG: Geophryne ${ }^{\circ}$ Brown $^{+1}, 2014 \uparrow$

KF: HYLIDAE 1815.ra.f002-|1825.gb.f001|

Geotriton: Bonaparte 1831 • AN

ST: $\mathbf{A L} \bullet$ CI: n0041 • ID: 564

PN: Salamandra exigua Laurenti, 1768

PK: Lacerta vulgaris* Linnaeus, 1758

KG: Lissotriton ${ }^{1}$ Bell, 1839

KF: SALAMANDRIDAE 1820.ga.f002

Geotriton Bonaparte, 1832 • EX

ST: PO.CE • CI: e0008・ ID: 564

PN: Salamandra exigua Laurenti, 1768

PK: Lacerta vulgaris* Linnaeus, 1758

KG: Lissotriton ${ }^{1}$ Bell, 1839

KF: SALAMANDRIDAE 1820.ga.f002

Geotrypetes Peters, $1880 \cdot \mathbf{K Y}$

ST: PO.KN・CI: h0569・ID: 489

PN: Caecilia seraphini Duméril, 1859

PK: Caecilia seraphini* Duméril, 1859

KG: Geotrypetes* Peters, 1880

KF: CAECILIIDAE 1814.ra.f003-|1825.gb.f008|

Gephyromantis Methuen, $1920 \bullet \mathbf{K Y}$

ST: PO.KN・CI: h0570 • ID: 431
PN: Gephyromantis boulengeri Methuen, 1920

PK: Gephyromantis boulengeri* Methuen, 1920

KG: Gephyromantis* Methuen, 1920

KF: RHACOPHORIDAE |1858.gc.f012||-1932.ha.f001

Germanobatrachus Kuhn, $1941+\cdot \mathbf{A K}$

ST: PO.JD • CI: h0571 • ID: $\uparrow 110$

PN: Germanobatrachus beurleni Kuhn, 1941 \$

PK: Opisthocoelellus weigelti ${ }^{\circ}$ Kuhn, $1941 \dagger$

KG: Opisthocoelellus ${ }^{\circ}$ Kuhn, $1941 \dagger$

KF: Mediogyrinia Familia INCERTAE SEDIS

Geyeriella Herre, $1950+\cdot \mathbf{K Y}$

ST: PO.KN • CI: h0572 • ID: $\uparrow 171$

PN: Geyeriella mertensi Herre, 1950 †

PK: Geyeriella mertensi ${ }^{\circ}$ Herre, $1950 \dagger$

KG: Geyeriella ${ }^{\circ}$ Herre, $1950 \uparrow$

KF: HYNOBIIDAE $\|$ 1856.ha.f001\|-1859.cb.f002

Ghatixalus $\mathrm{Biju}^{+2}, 2008 \cdot \mathbf{K Y}$

ST: PO.KN • CI: h0573・ID: 451

PN: Polypedates variabilis Jerdon, 1853

PK: Polypedates variabilis* Jerdon, 1853

KG: Ghatixalus * $\mathrm{Biju}^{+2}, 2008$

KF: RHACOPHORIDAE ||1858.gc.f012 |-1932.ha.f001

Ghatophryne: $\mathrm{Biju}^{+4} 2009 \cdot \mathbf{A N}$

ST: $\mathbf{A L} \bullet \mathbf{C I}:$ n0042 • ID: 116

PN: Ansonia ornata Gunther, 1876

PK: Ansonia ornata* Gunther, 1876

KG: Blaira* nov.

KF: BUFONIDAE 1825.gb.f004

Gigantobatrachus Casamiquela, $1958+\mathbf{A K}$

ST: PO.JD・CI: h0574 • ID: 257

PN: Gigantobatrachus parodii Casamiquela, 1958 *

PK: Gigantobatrachus parodii ${ }^{\circ}$ Casamiquela, $1958 \dagger$

KG: Calyptocephalella* Strand, 1928

KF: CALYPTOCEPHALELLIDAE 1960.ra.f001

Gigantophrys $\mathrm{Fei}^{+2}, 2016$ • AK

ST: PO.JD • CI: h0575 • ID: 019

PN: Megophrys giganticus $\mathrm{Liu}^{+2}, 1960$

PK: Megophrys giganticus ${ }^{\circ} \mathrm{Liu}^{+2}, 1960$

KG: Atympanophrys* Tian $^{+1}, 1983$

KF: MEGOPHRYIDAE 1850.bb.f008-|1931.na.f003|

Gigantorana Noble, 1931 • AK

ST: PO.JD • CI: h0576・ID: 351

PN: Rana goliath Boulenger, 1906

PK: Rana goliath* Boulenger, 1906

KG: Conraua* Nieden, 1908

KF: CONRAUIDAE 1992.da.f001

Glandirana $\mathrm{Fei}^{+2}, 1990 \cdot \mathbf{K Y}$

ST: PO.KN • CI: h0577・ID: 407

PN: Rana minima Ting $^{+1}, 1979$

PK: Rana minima* Ting $^{+1}, 1979$

KG: Glandirana* $\mathrm{Fei}^{+1}, 1990$

KF: RANIDAE 1796.ba.f001

Glandula Stimpson, $1852 \cdot \mathbf{z H}$

ST: zo • CI: zh037

Glandula $\operatorname{Tian}^{+1}, 1985$ • AK

ST: PO.JH • CI: h0578 • ID: 472 
PN: Bombinator maximus Boulenger, 1905

PK: Bombinator maximus* Boulenger, 1905

KG: Bombina* Oken, 1816

KF: BOMBINATORIDAE 1825.gb.f002

Glauertia Loveridge, 1933 • AK

ST: PO.JD • CI: h0579 • ID: 276

PN: Glauertia russelli Loveridge, 1933

PK: Glauertia russelli* Loveridge, 1933

KG: Uperoleia ${ }^{2}$ Gray, 1841

KF: MYOBATRACHIDAE 1850.sa.f001

Glossiphus: Green in Rafinesque 1832 • AN

ST: $\mathbf{A L} ・$ CI: n0043・ID: 542

PN: Salamandra longicauda Green, 1818

PK: Salamandra longicauda* Green, 1818

KG: Eurycea* Rafinesque, 1822

KF: PLETHODONTIDAE 1850.ga.f002

Glossoliga Bonaparte, 1839 • AK

ST: PO.JD • CI: h0580 • ID: 571

PN: Triton poireti Gervais, 1835

PK: Triton poireti* Gervais, 1835

KG: Pleurodeles* Michahelles, 1830

KF: SALAMANDRIDAE 1820.ga.f002

Glossostoma Le Conte, $1851 \cdot \mathbf{z H}$

ST: zo • CI: zh038

Glossostoma Günther, 1901 • AK

ST: PO.JH • CI: h0581 • ID: 293

PN: Glossostoma aterrimum Günther, 1901

PK: Glossostoma aterrimum ${ }^{\circ}$ Günther, 1901

KG: Ctenophryne* Mocquard, 1904

KF: MICROHYLIDAE ||1843.fa.f012||-1931.na.f001

Glyfoglossus Palacký, 1898 • AK

ST: NT.JI • CI: h0582・ID: 313

PN: Glyphoglossus molossus Günther, 1869

PK: Glyphoglossus molossus* Günther, 1869

KG: Glyphoglossus* Günther, 1869

KF: MICROHYLIDAE ||1843.fa.f012||-1931.na.f001

Glyphoglossus Günther, 1869 • KY

ST: PO.KN • CI: h0583 • ID: 313

PN: Glyphoglossus molossus Günther, 1869

PK: Glyphoglossus molossus* Günther, 1869

KG: Glyphoglossus* Günther, 1869

KF: MICROHYLIDAE ||1843.fa.f012||-1931.na.f001

Gnathophryne Méhelÿ, 1901 • AK

ST: PO.JD • CI: h0584 • ID: 280

PN: Mantophryne robusta Boulenger, 1898

PK: Mantophryne robusta* Boulenger, 1898

KG: Asterophrys* Tschudi, 1838

KF: MICROHYLIDAE ||1843.fa.f012||-1931.na.f001

Gnathophysa Fitzinger, 1843 • AK

ST: PO.JD • CI: h0585 • ID: 253

PN: Rana labyrinthica Spix, 1824

PK: Rana labyrinthica* Spix, 1824

KG: Leptodactylus ${ }^{1}$ Fitzinger, 1826

KF: LEPTODACTYLIDAE ||1838.ta.f001||-1896.wa.f001

Gobiates: Špinar $1983+\bullet \mathbf{A N}$

ST: AL • CI: n0044 • ID: $\uparrow 113$
PN: Gobiates khermeentsavi Špinar ${ }^{+1}, 1986$ *

PK: Gobiates khermeentsavi ${ }^{\circ}$ Śpinar $^{+1}, 1986 \dagger$

KG: Gobiates ${ }^{\circ}$ S̆pinar $^{+1,} 1986 \dagger$

KF: GOBIATIDAE 1991.ra.f001 †

Gobiates Špinar ${ }^{+1,} 1986+\bullet \mathbf{K Y}$

ST: PO.KN • CI: h0586・ID: $\uparrow 113$

PN: Gobiates khermeentsavi Špinar ${ }^{+1}, 1986$ \$

PK: Gobiates khermeentsavi ${ }^{\circ}$ Śpinar $^{+1}, 1986 \dagger$

KG: Gobiates ${ }^{\circ}$ Špinar $^{+1,} 1986 \dagger$

KF: GOBIATIDAE 1991.ra.f001 †

Gobiatoides Roček ${ }^{+1}, 1993+\bullet \mathbf{K Y}$

ST: PO.KN • CI: h0587・ID: $\uparrow 024$

PN: Gobiatoides parvus Roček ${ }^{+1}, 1993$ *

PK: Gobiatoides parvus ${ }^{\circ}$ Roček $^{+1}, 1993$ †

KG: Gobiatoides ${ }^{\circ}$ Roček $^{+1}, 1993$ †

KF: ANURa Familia INCERTAE SEDIS

Gomphobates Reinhardt ${ }^{+1}, 1862 \cdot \mathbf{A K}$

ST: PO.JD • CI: h0588・ID: 250

PN: Gomphobates notatus Reinhardt ${ }^{+1}, 1862$

PK: Physalaemus cuvieri* Fitzinger, 1826

KG: Physalaemus* Fitzinger, 1826

KF: LEIUPERIDAE 1850.bb.f010

Gorhixalus Dubois, 1987 • AK

ST: PO.JD・CI: h0589・ID: 447

PN: Rhacophorus hosii Boulenger, 1895

PK: Rhacophorus hosii* Boulenger, 1895

KG: Philautus* Gistel, 1848

KF: RHACOPHORIDAE ||1858.gc.f012||-1932.ha.f001

Gracilibatrachus Báez, 2013 † KY

ST: PO.KN • CI: h0590 • ID: $\uparrow 062$

PN: Gracilibatrachus avallei Báez, 2013 \$

PK: Gracilibatrachus avallei ${ }^{\circ}$ Báez, $2013 \dagger$

KG: Gracilibatrachus ${ }^{\circ}$ Báez, 2013 †

KF: DorsIPARES Familia INCERTAE SEDIS

Gracixalus Delorme ${ }^{+3}, 2005 \cdot \mathbf{K Y}$

ST: PO.KN • CI: h0591 • ID: 439

PN: Philautus gracilipes Bourret, 1937

PK: Philautus gracilipes* Bourret, 1937

KG: Gracixalus* Delorme ${ }^{+3}, 2005$

KF: RHACOPHORIDAE ||1858.gc.f012||-1932.ha.f001

Gradwellia Wells ${ }^{+1}, 1985$ • AK

ST: PO.JD • CI: h0592 • ID: 274

PN: Pseudophryne major Parker, 1940

PK: Pseudophryne major ${ }^{\circ}$ Parker, 1940

KG: Pseudophryne ${ }^{3}$ Fitzinger, 1843

KF: MYOBATRACHIDAE 1850.sa.f001

Grandisonia Taylor, $1968 \bullet \mathbf{A K}$

ST: PO.JD・CI: h0593・ID: 482

PN: Hypogeophis alternans Stejneger, 1893

PK: Hypogeophis alternans* Stejneger, 1893

KG: Hypogeophis* Peters, 1880

KF: CAECILIIDAE 1814.ra.f003-|1825.gb.f008|

Grillitschia nov. $\bullet \mathrm{KY}$

ST: PO.KN • CI: h0594 • ID: 022

PN: Megalophrys longipes Boulenger, 1886

PK: Megalophrys longipes* Boulenger, 1886 
KG: Grillitschia* nov.

KF: MEGOPHRYIDAE 1850.bb.f008-|1931.na.f003|

Grippiella Herre, 1949 + AK

ST: PO.JD・CI: h0595 • ID: $\uparrow 193$

PN: Grippiella mohri Herre, 1949 \$

PK: Chelotriton paradoxus ${ }^{\circ}$ Pomel, $1853 \uparrow$

KG: Chelotriton ${ }^{\circ}$ Pomel, $1853 \dagger$

KF: SALAMANDRIDAE 1820.ga.f002

Grobina Dubois, 1987 • AK

ST: PO.JD • CI: h0596・ID: 472

PN: Bombinator maximus Boulenger, 1905

PK: Bombinator maximus* Boulenger, 1905

KG: Bombina* Oken, 1816

KF: BOMBINATORIDAE 1825.gb.f002

Gryphius: Gistel 1848 • AN

ST: AL・CI: n0045・ID: 002§

PN: INR

PK: INR

KG: INR

KF: LISSAMPHIBIA Familia INCERTAE SEDIS

Grypiscus Cope, 1867 • AK

ST: PO.JD • CI: h0597 • ID: 179

PN: Grypiscus umbrinus Cope, 1867

PK: Cycloramphus fuliginosus* Tschudi, 1838

KG: Cycloramphus* Tschudi, 1838

KF: CYCLORAMPHIDAE 1850.bb.f003-|1852.ba.f001|

Guentheria Bleeker, $1861 \cdot \mathbf{z H}$

ST: zo・CI: zh039

Guentheria Miranda-Ribeiro, 1926 • AK

ST: PO.JH • CI: h0598 • ID: 194

PN: Hyla dasynota Günther, 1869

PK: Hyla senicula* Cope, 1868

KG: Dendropsophus ${ }^{1}$ Fitzinger, 1843

KF: HYLIDAE 1815.ra.f002-|1825.gb.f001|

Guibemantis Dubois, $1992 \cdot \mathbf{K Y}$

ST: PO.KN • CI: h0599・ID: 427

PN: Rhacophorus depressiceps Boulenger, 1882

PK: Rhacophorus depressiceps* Boulenger, 1882

KG: Guibemantis* Dubois, 1992

KF: RHACOPHORIDAE ||1858.gc.f012 |-1932.ha.f001

Gymnophis Gadow, 1901 • AK

ST: NS.JI • CI: h0600 • ID: 487

PN: Gymnopis multiplicata Peters, 1874

PK: Gymnopis multiplicata* Peters, 1874

KG: Gymnopis* Peters, 1874

KF: CAECILIIDAE 1814.ra.f003-|1825.gb.f008|

Gymnopis Peters, $1874 \cdot \mathbf{K Y}$

ST: PO.KN • CI: h0601 • ID: 487

PN: Gymnopis multiplicata Peters, 1874

PK: Gymnopis multiplicata* Peters, 1874

KG: Gymnopis* Peters, 1874

KF: CAECILIIDAE 1814.ra.f003-|1825.gb.f008|

Gynandropaa Dubois, 1992 • KY

ST: PO.KN • CI: h0602 • ID: 384

PN: Rana yunnanensis Anderson, 1878

PK: Rana yunnanensis* Anderson, 1878
KG: Gynandropaa* Dubois, 1992

KF: DICROGLOSSIDAE 1987.da.f004

Gyrinophilus Cope, 1869 • KY

ST: PO.KN • CI: h0603・ID: 539

PN: Salamandra porphyritica Green, 1827

PK: Salamandra porphyritica* Green, 1827

KG: Gyrinophilus* Cope, 1869

KF: PLETHODONTIDAE 1850.ga.f002

Gyrinus Geoffroy, $1762 \cdot \mathbf{z H}$

ST: zo • CI: zh040

Gyrinus: Hermann 1783 - AN

ST: AL・CI: n0046・ID: 419

PN: Rana temporaria Linnaeus, 1758

PK: Rana temporaria* Linnaeus, 1758

KG: Rana* Linnaeus, 1758

KF: RANIDAE 1796.ba.f001

Gyrinus $\mathrm{Shaw}^{+1}, 1798$ • AK

ST: PO.JH • CI: h0604 • ID: 555

PN: Gyrinus mexicanus Shaw $^{+1}, 1798$

PK: Gyrinus mexicanus* Shaw $^{+1}, 1798$

KG: Ambystoma ${ }^{1}$ Tschudi, 1838

KF: AмвYSTOMATIDAE 1850.ga.f004

Habrahyla Goin, 1961 • AK

ST: PO.JD • CI: h0605 • ID: 325

PN: Habrahyla eiselti Goin, 1961

PK: Hylambates notatus ${ }^{\circ}$ Buchholz $^{+1}$ in Peters, 1875

KG: Leptopelis ${ }^{2}$ Günther, 1859

KF: ARTHROLEPTIDAE 1869.mc.f011

Habrosaurus Gilmore, $1928+\bullet \mathbf{K Y}$

ST: PO.KN • CI: h0606・ID: $\uparrow 176$

PN: Habrosaurus dilatus Gilmore, $1928+$

PK: Habrosaurus dilatus ${ }^{\circ}$ Gilmore, $1928+$

KG: Habrosaurus ${ }^{\circ}$ Gilmore, $1928 \dagger$

KF: SIRENIDAE 1825gb.f005

Haddadus Hedges ${ }^{+2}, 2008 \cdot \mathbf{K Y}$

ST: PO.KN • CI: h0607・ ID: 060

PN: Rana binotata Spix, 1824

PK: Rana binotata* Spix, 1824

KG: Haddadus* Hedges ${ }^{+2}, 2008$

KF: BRACHYCEPHALIDAE 1858.gc.f002

Hadromophryne Van Dijk, 2008 • KY

ST: PO.KN • CI: h0608・ID: 465

PN: Heleophryne natalensis Hewitt, 1913

PK: Heleophryne natalensis* Hewitt, 1913

KG: Hadromophryne* Van Dijk, 2008

KF: HELEOPHRYNIDAE 1931.na.f004

Haideotriton Carr, 1939 • AK

ST: PO.JD • CI: h0609・ ID: 542

PN: Haideotriton wallacei Carr, 1939

PK: Haideotriton wallacei* Carr, 1939

KG: Eurycea* Rafinesque, 1822

KF: PLETHODONTIDAE 1850.ga.f002

Halleobatrachus Kuhn, $1941+$ • AK

ST: PO.JD • CI: h0610 • ID: $\uparrow 090$

PN: Halleobatrachus hinschei Kuhn, 1941 \$

PK: Halleobatrachus hinschei ${ }^{\circ}$ Kuhn, $1941 \dagger$ 
KG: Eopelobates ${ }^{\circ}$ Parker, $1929+$

KF: PELOBATIDAE 1850.bb.f004

Halophila Gray, $1843 \cdot \mathbf{z H}$

ST: zo • CI: zh041

Halophila Girard, 1853 • AK

ST: PO.JH • CI: h0611 • ID: 369

PN: Halophila vitiensis Girard, 1853

PK: Halophila vitiensis* Girard, 1853

KG: Cornufer* Tschudi, 1838

KF: CERATOBATRACHIDAE 1884.ba.f001

Hammatodactylus Fitzinger, 1843 • AK

ST: PO.JD • CI: h0612 • ID: 173

PN: Cystignathus nodosus Duméril ${ }^{+1}, 1841$

PK: Cystignathus nodosus* Duméril ${ }^{+1}, 1841$

KG: Alsodes* Bell, 1843

KF: ALSODIDAE 1869.mc.f005

Hamptophryne Carvalho, $1954 \cdot \mathbf{K Y}$

ST: PO.KN • CI: h0613 • ID: 301

PN: Chiasmocleis boliviana Parker, 1927

PK: Chiasmocleis boliviana* Parker, 1927

KG: Hamptophryne* Carvalho, 1954

KF: MICROHYLIDAE ||1843.fa.f012||-1931.na.f001

Haptoglossa Cope, 1893 • AK

ST: PO.JD • CI: h0614 • ID: 534

PN: Haptoglossa pressicauda Cope, 1893

PK: Haptoglossa pressicauda* Cope, 1893

KG: Oedipina* Keferstein, 1868

KF: PLETHODONTIDAE 1850.ga.f002

Hatzegobatrachus Venczel ${ }^{+1}, 2003+\cdot \mathbf{K Y}$

ST: PO.KN • CI: h0615 • ID: $\uparrow 025$

PN: Hatzegobatrachus grigorescui Venczel $^{+1}, 2003$ \$

PK: Hatzegobatrachus grigorescui ${ }^{\circ}$ Venczel $^{+1}, 2003 \dagger$

KG: Hatzegobatrachus ${ }^{\circ}$ Venczel $^{+1}, 2003 \dagger$

KF: ANURA Familia INCERTAE SEDIS

Hazelia Walcott, $1920 \cdot \mathbf{Z H}$

ST: zo • CI: zh042

Hazelia Taylor, $1920 \bullet \mathbf{A K}$

ST: PO.JH • CI: h0616 • ID: 437

PN: Hazelia spinosa Taylor, 1920

PK: Hazelia spinosa* Taylor, 1920

KG: Nyctixalus* Boulenger, 1882

KF: RHACOPHORIDAE |1858.gc.f012||-1932.ha.f001

Hedronchus Cope, 1877 † KY

ST: PO.KN • CI: h0617 •ID: $\uparrow 155$

PN: Hedronchus sternbergii Cope 1877 t

PK: Hedronchus sternbergii ${ }^{\circ}$ Cope, $1877 \dagger$

KG: Hedronchus ${ }^{\circ}$ Cope, $1877 \dagger$

KF: SCAPHERPETIDAE 1959.aa.f001 †

Hekatobatrachus Špinar, $1972+\bullet \mathbf{A K}$

ST: PO.JD • CI: h0618 • ID: $\uparrow 069$

PN: Palaeophrynos grandipes Giebel, 1851 †

PK: Palaeophrynos grandipes $^{\circ}$ Giebel, $1851 \dagger$

KG: Palaeobatrachus ${ }^{\circ}$ Tschudi, $1838 \uparrow$

KF: PALAEOBATRACHIDAE 1865.ca.f001 †

Heleioforus Krefft, 1865 • AK

ST: NS.JI • CI: h0619 • ID: 259
PN: Heleioporus albopunctatus Gray, 1841

PK: Heleioporus albopunctatus ${ }^{\circ}$ Gray, 1841

KG: Heleioporus ${ }^{2}$ Gray, 1841

KF: MYOBATRACHIDAE 1850.sa.f001

Heleioporus Gray, 1841a • KY

ST: PO.KN • CI: h0620 • ID: 259

PN: Heleioporus albopunctatus Gray, 1841

PK: Heleioporus albopunctatus ${ }^{\circ}$ Gray, 1841

KG: Heleioporus ${ }^{2}$ Gray, 1841

KF: MYOBATRACHIDAE 1850.sa.f001

Heleophryne Sclater, 1898 • KY

ST: PO.KN・CI: h0621・ID: 466

PN: Heleophryne purcelli Sclater, 1898

PK: Heleophryne purcelli* Sclater, 1898

KG: Heleophryne* Sclater, 1898

KF: HELEOPHRYNIDAE 1931.na.f004

Heliarchon Meyer, 1860 + $\mathbf{A K}$

ST: PO.JD • CI: h0622・ID: $\uparrow 193$

PN: Heliarchon fuscillatus Meyer, 1860 †

PK: Chelotriton paradoxus ${ }^{\circ}$ Pomel, $1853+$

KG: Chelotriton $^{\circ}$ Pomel, $1853 \uparrow$

KF: SALAMANDRIDAE 1820.ga.f002

Heliophryne Heyer, 1975 • AK

ST: NS.JI • CI: h0623・ID: 466

PN: Heleophryne purcelli Sclater, 1898

PK: Heleophryne purcelli* Sclater, 1898

KG: Heleophryne* Sclater, 1898

KF: HELEOPHRYNIDAE 1931.na.f004

Helioporus: Gray $1841 b \cdot \mathbf{A N}$

ST: AM・CI: n0047・ID: 259

PN: Heleioporus albopunctatus Gray, 1841

PK: Heleioporus albopunctatus ${ }^{\circ}$ Gray, 1841

KG: Heleioporus ${ }^{2}$ Gray, 1841

KF: Myobatrachidae 1850.sa.f001

Heliorana Steindachner, $1867 \cdot \mathbf{A K}$

ST: PO.JD • CI: h0624・ID: 261

PN: Heliorana grayi Steindachner, 1867

PK: Limnodynastes (Platyplectron) dumerilii* Peters, 1863

KG: Limnodynastes* Fitzinger, 1843

KF: MYOBATRACHIDAE 1850.sa.f001

Helocaetes Baird, $1854 \cdot \mathbf{A K}$

ST: PO.JD・CI: h0625・ID: 200

PN: Hyla triseriata Wied-Neuwied, 1838

PK: Hyla triseriata* Wied-Neuwied, 1838

KG: Pseudacris* Fitzinger, 1843

KF: HYLIDAE 1815.ra.f002-|1825.gb.f001|

Heloecetes Baird, $1859 \cdot \mathbf{A K}$

ST: NT.JD • CI: h0626・ID: 200

PN: Hyla triseriata Wied-Neuwied, 1838

PK: Hyla triseriata* Wied-Neuwied, 1838

KG: Pseudacris* Fitzinger, 1843

KF: HYLIDAE 1815.ra.f002-|1825.gb.f001|

Hemidactylium Tschudi, 1838 • KY

ST: PO.KN • CI: h0627・ID: 538

PN: Salamandra scutata Temminck ${ }^{+1}, 1838$

PK: Salamandra scutata* Temminck $^{+1}, 1838$ 
KG: Hemidactylium* Tschudi, 1838

KF: PLETHODONTIDAE 1850.ga.f002

Hemimantis Peters, 1863 • AK

ST: PO.JD • CI: h0628 • ID: 350

PN: Hemimantis calcaratus Peters, 1863

PK: Hemimantis calcaratus* Peters, 1863

KG: Phrynobatrachus* Günther, 1862

KF: PHRYNOBATRACHIDAE 1941.lb.f001

Heminectes Philippi, 1902 • AK

ST: PO.JD • CI: h0629・ID: 185

PN: Heminectes rufus Philippi, 1902

PK: Heminectes rufus ${ }^{\circ}$ Philippi, 1902

KG: Rhinoderma* Duméril ${ }^{+1}, 1841$

KF: RHINODERMATIDAE 1850.bb.f011

Hemiphractus Wagler, 1828・ KY

ST: PO.KN • CI: h0630 • ID: 095

PN: Hemiphractus spixii Wagler, 1828

PK: Rana scutata* Spix, 1824

KG: Hemiphractus ${ }^{1}$ Wagler, 1828

KF: HEMIPHRACTIDAE 1862.pa.f001

Hemipipa Miranda-Ribeiro, 1937 • AK

ST: PO.JD • CI: h0631 • ID: 012

PN: Protopipa carvalhoi Miranda-Ribeiro, 1937

PK: Protopipa carvalhoi* Miranda-Ribeiro, 1937

KG: Pipa ${ }^{1}$ Laurenti, 1768

KF: PIPIDAE 1825.gb.f003-|1826.fb.f002|

Hemisalamandra Dugès, 1852 • AK

ST: PO.JI • CI: h0632・ID: 566

PN: Triton cristatus Laurenti, 1768

PK: Triton cristatus* Laurenti, 1768

KG: Triturus* Rafinesque, 1815

KF: SALAMANDRIDAE 1820.ga.f002

Hemisus Günther, 1859 • KY

ST: PO.KN • CI: h0633・ID: 347

PN: Engystoma guttatum Rapp, 1842

PK: Engystoma guttatum $^{\circ}$ Rapp, 1842

KG: Hemisus ${ }^{2}$ Günther, 1859

KF: HEMISOTIDAE 1867.ca.f002

Hemitriton Van der Hoeven, 1833 • AK

ST: PO.JI • CI: h0634 • ID: 554

PN: Proteus anguinus Laurenti, 1768

PK: Proteus anguinus* Laurenti, 1768

KG: Proteus* Laurenti, 1768

KF: PROTEIDAE 1831.ba.f002

Hemitriton Dugès, 1852 • AK

ST: PO.JH • CI: h0635 • ID: 563

PN: Triton alpestris Laurenti, 1768

PK: Triton alpestris* Laurenti, 1768

KG: Ichthyosaura ${ }^{1}$ Sonnini ${ }^{+1}, 1801$

KF: SALAMANDRIDAE 1820.ga.f002

Hemitrypus Cope, $1877+\bullet \mathbf{A K}$

ST: PO.JD • CI: h0636・ID: $\uparrow 155$

PN: Hemitrypus jordanianus Cope 1877 \$

PK: Hedronchus sternbergii ${ }^{\circ}$ Cope, $1877 \dagger$

KG: Hedronchus ${ }^{\circ}$ Cope, $1877 \uparrow$

KF: SCAPHERPETIDAE 1959.aa.f001 †
Hensonbatrachus Gardner ${ }^{+1}, 2015+\cdot \mathbf{K Y}$

ST: PO.KN • CI: h0637 • ID: $\uparrow 026$

PN: Hensonbatrachus kermiti Gardner ${ }^{+1}, 2015 \$$

PK: Hensonbatrachus kermiti ${ }^{\circ}$ Gardner $^{+1}, 2015 \dagger$

KG: Hensonbatrachus ${ }^{\circ}$ Gardner $^{+1}, 2015$ †

KF: ANURA Familia INCERTAE SEDIS

Heredia Girard, 1857 • AK

ST: PO.JD • CI: h0638 • ID: 550

PN: Heredia oregonensis Girard, 1857

PK: Ensatina eschscholtzii* Gray, 1850

KG: Ensatina* Gray, 1850

KF: PLETHODONTIDAE 1850.ga.f002

Herpele Peters, $1880 \cdot \mathrm{KY}$

ST: PO.KN・CI: h0639・ID: 497

PN: Caecilia squalostoma Stutchbury, 1834

PK: Caecilia squalostoma* Stutchbury, 1834

KG: Herpele* Peters, 1880

KF: CAECILIIDAE 1814.ra.f003-|1825.gb.f008|

Hesperocrinia Wells $^{+1}, 1985$ • AK

ST: PO.JD・CI: h0640・ID: 268

PN: Crinia leai Fletcher, 1898

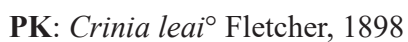

KG: Geocrinia ${ }^{3}$ Blake, 1973

KF: MYOBATRACHIDAE 1850.sa.f001

Heterixalus Laurent, $1944 \cdot \mathbf{K Y}$

ST: PO.KN • CI: h0641 • ID: 335

PN: Eucnemis madagascariensis Duméril ${ }^{+1}, 1841$

PK: Eucnemis madagascariensis* ${ }^{*}$ Duméril $^{+1}, 1841$

KG: Heterixalus* Laurent, 1944

KF: HYPEROLIIDAE 1943.lb.f001

Heteroclitotriton Stefano, $1903+\cdot \mathbf{A K}$

ST: PO.JD・CI: h0642・ID: 578

PN: Heteroclitotriton zitelli Stefano, 1903 *

PK: Salamandra sansaniensis ${ }^{\circ}$ Lartet, $1851 \dagger$

KG: Salamandra ${ }^{1}$ Garsault, 1764

KF: SALAMANDRIDAE 1820.ga.f002

Heteroglossa Nietner, $1856 \cdot \mathbf{Z H}$

ST: zo • CI: zh043

Heteroglossa: Hallowell 1857 • AN

ST: $\mathbf{A L} ・ \mathbf{C I}:$ n0048・ID: 550

PN: Heredia oregonensis Girard, 1856

PK: Ensatina eschscholtzii* Gray, 1850

KG: Ensatina* Gray, 1850

KF: PLETHODONTIDAE 1850.ga.f002

Heteroglossa Hallowell, 1858 • AK

ST: PO.JH • CI: h0643・ID: 350

PN: Heteroglossa africana Hallowell, 1858

PK: Heteroglossa africana* Hallowell, 1858

KG: Phrynobatrachus* Günther, 1862

KF: PHRYNobatrachidae 1941.lb.f001

Heteropelis Laurent, 1941 • AK

ST: PO.JD • CI: h0644 • ID: 325

PN: Leptopelis parkeri Barbour $^{+1}, 1928$

PK: Leptopelis parkeri ${ }^{\circ}$ Barbour $^{+1}, 1928$

KG: Leptopelis ${ }^{2}$ Günther, 1859

KF: ARTHROLEPTIDAE 1869.mc.f011 
Heterotriton Gray, 1850 • AK

ST: PO.JD • CI: h0645 • ID: 555

PN: Salamandra ingens Green, 1831

PK: Salamandra tigrina* Green, 1825

KG: Ambystoma ${ }^{1}$ Tschudi, 1838

KF: AMBYSTOMATIDAE 1850.ga.f004

Hightonia Vieites ${ }^{+3}, 2011 \cdot \mathbf{A K}$

ST: PO.JD • CI: h0646 • ID: 551

PN: Ambystoma vehiculum Cooper, 1869

PK: Ambystoma vehiculum* Cooper, 1869

KG: Plethodon* Tschudi, 1838

KF: PLETHODONTIDAE 1850.ga.f002

Hildebrandtia Nieden, $1907 \cdot \mathbf{K Y}$

ST: PO.KN • CI: h0647 • ID: 462

PN: Pyxicephalus ornatus Peters, 1878

PK: Pyxicephalus ornatus* Peters, 1878

KG: Hildebrandtia* Nieden, 1907

KF: PTYCHADENIDAE 1987.da.f002

Hiperoodon: Philippi $1902 \cdot \mathbf{A N}$

ST: LI • CI: n0049 • ID: 309

PN: Engystoma marmoratum Guérin-Méneville, 1838

PK: Rana systoma* Schneider, 1799

KG: Uperodon* Duméri ${ }^{+1}, 1841$

KF: MICROHYLIDAE ||1843.fa.f012|-1931.na.f001

Holoaden Miranda-Ribeiro, $1920 \bullet \mathbf{K Y}$

ST: PO.KN • CI: h0648 • ID: 068

PN: Holoaden luederwaldti Miranda-Ribeiro, 1920

PK: Holoaden luederwaldti* Miranda-Ribeiro, 1920

KG: Holoaden* Miranda-Ribeiro, 1920

KF: BRACHYCEPHALIDAE 1858.gc.f002

Holonectes Peters, 1863 • AK

ST: PO.JD • CI: h0649 • ID: 310

PN: Hylaedactylus (Holonectes) conjunctus Peters, 1863

PK: Hylaedactylus (Holonectes) conjunctus* Peters, 1863

KG: Kaloula* Gray, 1831

KF: MICROHYLIDAE ||1843.fa.f012||-1931.na.f001

Hoplobactrachus: Theobald 1868 • AN

ST: AM • CI: n0050 • ID: 374

PN: Hoplobatrachus ceylanicus Peters, 1863

PK: Rana crassa* Jerdon, 1853

KG: Hoplobatrachus ${ }^{1}$ Peters, 1863

KF: DICROGLOSSIDAE 1987.da.f004

Hoplobatrachus Peters, $1863 \cdot \mathbf{K Y}$

ST: PO.KN • CI: h0650 • ID: 374

PN: Hoplobatrachus ceylanicus Peters, 1863

PK: Rana crassa* Jerdon, 1853

KG: Hoplobatrachus ${ }^{1}$ Peters, 1863

KF: DICROGLOSSIDAE 1987.da.f004

Hoplophryne Barbour ${ }^{+1}, 1928 \cdot \mathbf{K Y}$

ST: PO.KN • CI: h0651 • ID: 303

PN: Hoplophryne uluguruensis Barbour ${ }^{+1}, 1928$

PK: Hoplophryne uluguruensis* Barbour ${ }^{+1}, 1928$

KG: Hoplophryne* Barbour ${ }^{+1}, 1928$

KF: MICROHYLIDAE ||1843.fa.f012||-1931.na.f001

Horezmia Nessov, $1981+\cdot \mathbf{K Y}$

ST: PO.KN • CI: h0652 • ID: $\uparrow 167$
PN: Horezmia gracile Nessov, 1981 :

PK: Horezmia gracile ${ }^{\circ}$ Nessov, $1981 \dagger$

KG: Horezmia ${ }^{\circ}$ Nessov, $1981 \dagger$

KF: CRYPTOBRANCHIDAE 1826.fb.f003

Hosmeria Wells ${ }^{+1}, 1985$ • AK

ST: PO.JD • CI: h0653 • ID: 276

PN: Uperoleia marmorata laevigata Keferstein, 1867

PK: Uperoleia marmorata laevigata* Keferstein, 1867

KG: Uperoleia ${ }^{2}$ Gray, 1841

KF: MYOBATRACHIDAE 1850.sa.f001

Houlema Gray, $1831 \cdot \mathbf{A K}$

ST: PO.JD • CI: h0654 •ID: 395

PN: Houlema obscura Gray, 1831

PK: Rana lima* Gravenhorst, 1829

KG: Occidozyga* Kuhl ${ }^{+1}, 1822$

KF: OCCIDOZYGIDAE 1990.fa.f002

Huangixalus $\mathrm{Fe}^{+2}$, 2012 • AK

ST: PO.JD • CI: h0655 •ID: 455

PN: Rhacophorus translineatus Wu, 1977

PK: Rhacophorus translineatus* Wu, 1977

KG: Rhacophorus* $\mathrm{Kuhl}^{+1}, 1822$

KF: RHACOPHORIDAE ||1858.gc.f012||-1932.ha.f001

Huia Yang, $1991 \cdot$ AK

ST: PO.JD • CI: h0656 • ID: 403

PN: Rana cavitympanum Boulenger, 1893

PK: Rana cavitympanum* Boulenger, 1893

KG: Meristogenys* Yang, 1991

KF: RANIDAE 1796.ba.f001

Huicundomantis Paéz \& Ron, 2019 • KY

ST: PO.KN • CI: h0657 • ID: 078

PN: Eleutherodactylus phoxocephalus Lynch, 1979

PK: Eleutherodactylus phoxocephalus* Lynch, 1979

KG: Pristimantis* Jiménez de la Espada, 1870

KF: BRACHYCEPHALIDAE 1858.gc.f002

Humerana Dubois, $1992 \cdot \mathbf{A K}$

ST: PO.JD • CI: h0658 • ID: 409

PN: Rana humeralis Boulenger, 1887

PK: Rana humeralis ${ }^{\circ}$ Boulenger, 1887

KG: Hylarana* ${ }^{*}$ Tschudi, 1838

KF: RANIDAE 1796.ba.f001

Hungarobatrachus Szentesi ${ }^{+1}, 2010 \ddagger \mathbf{K Y}$

ST: PO.KN・CI: h0659 • ID: $\uparrow 104$

PN: Hungarobatrachus szukacsi Szentesi ${ }^{+1}$, 2010

PK: Hungarobatrachus szukacsi ${ }^{\circ}$ Szentesi ${ }^{+1}, 2010 \dagger$

KG: Hungarobatrachus ${ }^{\circ}$ Szentesi ${ }^{+1}, 2010 \dagger$

KF: SCOPTANURA Familia INCERTAE SEDIS

Hyalinobatrachium Ruíz-Carranza ${ }^{+1}, 1991$ • KY

ST: PO.KN • CI: h0660 • ID: 167

PN: Hylella fleischmanni Boettger, 1893

PK: Hylella fleischmanni* Boettger, 1893

KG: Hyalinobatrachium* Ruíz-Carranza ${ }^{+1}, 1991$

KF: CENTROLENIDAE 1951.ta.f001

Hyas Leach, $1814 \cdot \mathbf{z H}$

ST: zo • CI: zh044

Hyas Wagler, $1830 \cdot \mathbf{A K}$

ST: PO.JH • CI: h0661 • ID: 204 
PN: Rana arborea Linnaeus, 1758

PK: Rana arborea* Linnaeus, 1758

KG: Hyla* Laurenti, 1768

KF: HYLIDAE 1815.ra.f002-|1825.gb.f001|

Hydochthon: Gray 1831 • AN

ST: AM •CI: n0051・ID: 554

PN: Proteus anguinus Laurenti, 1768

PK: Proteus anguinus* Laurenti, 1768

KG: Proteus* Laurenti, 1768

KF: PROTEIDAE 1831.ba.f002

Hydrobatrachus Stadie, 1962 • AK

ST: PO.JD・CI: h0662・ID: 351

PN: Rana beccarii Boulenger, 1911

PK: Rana beccarii ${ }^{\circ}$ Boulenger, 1911

KG: Conraua* Nieden, 1908

KF: CONRAUIDAE 1992.da.f001

Hydrognathus Dubois ${ }^{+1}, 2012 \cdot \mathbf{A K}$

ST: PO.JD・CI: h0663・ID: 548

PN: Desmognathus brimleyorum Stejneger, 1895

PK: Desmognathus brimleyorum* Stejneger, 1895

KG: Desmognathus* Baird, 1850

KF: PLETHODONTIDAE 1850.ga.f002

Hydrolaetare Gallardo, 1963 • AK

ST: PO.JD • CI: h0664 • ID: 253

PN: Limnomedusa schmidti Cochran ${ }^{+1}, 1959$

PK: Limnomedusa schmidti ${ }^{\circ}$ Cochran $^{+1}, 1959$

KG: Leptodactylus ${ }^{1}$ Fitzinger, 1826

KF: LEPTODACTYLIDAE ||1838.ta.f001||-1896.wa.f001

Hydromantes Gistel, $1848 \bullet \mathbf{K Y}$

ST: PO.KC・CI: h0665・ID: 544

PN: Spelerpes platycephalus Camp, 1916

PK: Spelerpes platycephalus* Camp, 1916

KG: Hydromantes* Gistel, 1848

KF: PLETHODONTIDAE 1850.ga.f002

Hydromantoides Lanza ${ }^{+1}$, 1981• AK

ST: PO.JI • CI: h0666・ID: 544

PN: Spelerpes platycephalus Camp, 1916

PK: Spelerpes platycephalus* Camp, 1916

KG: Hydromantes* Gistel, 1848

KF: PLETHODONTIDAE 1850.ga.f002

Hydrophylax Fitzinger, 1843 • AK

ST: PO.JD • CI: h0667・ID: 409

PN: Rana malabarica Tschudi, 1838

PK: Rana malabarica* Tschudi, 1838

KG: Hylarana* Tschudi, 1838

KF: RANIDAE 1796.ba.f001

Hydrosalamandra Leuckart, 1840 • AK

ST: PO.JD • CI: h0668・ID: 503

PN: Megalobatrachus sieboldi Tschudi, 1837 \$

PK: Triton japonicus* Temminck, 1836

KG: Andrias ${ }^{2}$ Tschudi, 1837

KF: CRYPTOBRANCHIDAE 1826.fb.f003

Hydroscopes Gistel, 1848 • AK

ST: NL.JD • CI: h0669 • ID: 505

PN: Salamandra naevia Temminck $^{+1}, 1838$

PK: Salamandra naevia* Temminck $^{+1}, 1838$
KG: Hynobius* Tschudi, 1838

KF: HYNOBIIDAE ||1856.ha.f001||-1859.cb.f002

Hydrospelaeus Leuckart, 1821 • AK

ST: NL.JI • CI: h0670 • ID: 554

PN: Proteus anguinus Laurenti, 1768

PK: Proteus anguinus* Laurenti, 1768

KG: Proteus* Laurenti, 1768

KF: PROTEIDAE 1831.ba.f002

Hydrostentor: Fitzinger 1861 AN

ST: AL・CI: n0052 • ID: 374

PN: Rana tigrina pantherina Steindachner, 1867

PK: Rana chinensis* Osbeck, 1765

KG: Hoplobatrachus ${ }^{1}$ Peters, 1863

KF: DiCROGLOSSIDAE 1987.da.f004

Hydryla: Rafinesque $1815 \bullet \mathbf{A N}$

ST: $\mathbf{A L} \bullet \mathbf{C I}:$ n0053・ID: 204

PN: Rana arborea Linnaeus, 1758

PK: Rana arborea* Linnaeus, 1758

KG: Hyla* Laurenti, 1768

KF: HYLIDAE 1815.ra.f002-|1825.gb.f001|

Hyla Laurenti, 1768 • KY

ST: PO.RP • CI: h0671 • ID: 204

PN: Hyla viridis Laurenti, 1768

PK: Rana arborea* Linnaeus, 1758

KG: Hyla* Laurenti, 1768

KF: HYLIDAE 1815.ra.f002-|1825.gb.f001

Hyla Ritgen, $1828 \cdot \mathbf{A K}$

ST: PO.JH • CI: h0672・ID: 243

PN: Rana bicolor Boddaert, 1772

PK: Rana bicolor* Boddaert, 1772

KG: Phyllomedusa* Wagler, 1830

KF: PHYLLOMEDUSIDAE 1858.gc.f009

Hyla Burmeister, 1856 • AK

ST: PO.JH・CI: h0673・ID: 189

PN: Rana boans Linnaeus, 1758

PK: Rana boans* Linnaeus, 1758

KG: Boana* Gray, 1825

KF: HYLIDAE 1815.ra.f002-|1825.gb.f001|

Hylactophryne Lynch, 1968 • AK

ST: PO.JD • CI: h0674 • ID: 059

PN: Hylodes augusti Dugés, 1879

PK: Hylodes augusti* Dugés, 1879

KG: Craugastor* Cope, 1862

KF: BRACHYCEPHALIDAE 1858.gc.f002

Hyladactyla: Tschudi, 1838 • AN

ST: LI •CI: n0054・ID: 310

PN: Bombinator baleatus Müller, 1836

PK: Bombinator baleatus* Müller, 1836

KG: Kaloula* Gray, 1831

KF: MICROHYLIDAE ||1843.fa.f012||-1931.na.f001

Hyladactylus Tschudi, 1838 • AK

ST: LC.JD・CI: h0675 • ID: 310

PN: Bombinator baleatus Müller, 1836

PK: Bombinator baleatus* Müller, 1836

KG: Kaloula* Gray, 1831

KF: MICROHYLIDAE ||1843.fa.f012||-1931.na.f001 
Hylaedactyla: Duméril ${ }^{+1} 1841$ • AN

ST: AL・CI: n0055・ID: 310

PN: Bombinator baleatus Müller, 1836

PK: Bombinator baleatus* Müller, 1836

KG: Kaloula* Gray, 1831

KF: MICROHYLIDAE ||1843.fa.f012||-1931.na.f001

Hylaedactylus Duméril ${ }^{+1}$, 1841 • AK

ST: NT.JD・CI: h0676・ID: 310

PN: Bombinator baleatus Müller, 1836

PK: Bombinator baleatus* Müller, 1836

KG: Kaloula* Gray, 1831

KF: MICROHYLIDAE ||1843.fa.f012||-1931.na.f001

Hylaemorphus: Jan 1857 • AN

ST: AL・CI: n0056・ID: 100

PN: Hylaemorphus pluto Schmidt, 1858

PK: Phrynidium varium* Lichtenstein $^{+2}, 1856$

KG: Atelopus $*$ Duméril $^{+1}, 1841$

KF: BUFONIDAE 1825.gb.f004

Hylaemorphus Schmidt, 1857 • AK

ST: PO.JD • CI: h0677•ID: 100

PN: Hylaemorphus dumerilii Schmidt, 1857

PK: Phrynidium varium* Lichtenstein ${ }^{+2}, 1856$

KG: Atelopus* Duméril ${ }^{+1}, 1841$

KF: BUFONIDAE 1825.gb.f004

Hylaeobatrachus Dollo, $1884+$ KY

ST: PO.KN • CI: h0678・ID: $\uparrow 146$

PN: Hylaeobatrachus croyii Dollo, 1884 †

PK: Hylaeobatrachus croyii ${ }^{\circ}$ Dollo, $1884 \uparrow$

KG: Hylaeobatrachus ${ }^{\circ}$ Dollo, $1884 \dagger$

KF: HYLAEOBATRACHIDAE 1889.la.f001 †

Hylambates Duméril, 1853 • KY

ST: PO.KN • CI: h0679・ID: 337

PN: Hylambates maculatus Duméril, 1853

PK: Hylambates maculatus* Duméril, 1853

KG: Hylambates* Duméril, 1853

KF: HYPEROLIIDAE 1943.lb.f001

Hylanus: Rafinesque $1815 \cdot \mathbf{A N}$

ST: AL •CI: n0057・ID: 204

PN: Rana arborea Linnaeus, 1758

PK: Rana arborea* Linnaeus, 1758

KG: Hyla* Laurenti, 1768

KF: HYLIDAE 1815.ra.f002-|1825.gb.f001|

Hylapesia: Savage ${ }^{+3} 2007 \cdot \mathbf{A N}$

ST: $\mathbf{A M} \bullet$ CI: n0058・ID: 189

PN: Calamita punctatus Schneider, 1799

PK: Calamita punctatus* Schneider, 1799

KG: Boana* Gray, 1825

KF: HYLIDAE 1815.ra.f002-|1825.gb.f001|

Hylaplesia Boie in Schlegel, $1826 b$ • AK

ST: PO.CA • CI: h0680 • ID: 189

PN: Calamita punctatus Schneider, 1799

PK: Calamita punctatus* Schneider, 1799

KG: Boana* Gray, 1825

KF: HYLIDAE 1815.ra.f002-|1825.gb.f001|

Hylaplesia Boie in Boie, 1828 • AK

ST: PO.JD • CI: h0681 • ID: 189
PN: Calamita punctatus Schneider, 1799

PK: Calamita punctatus* Schneider, 1799

KG: Boana* Gray, 1825

KF: HYLIDAE 1815.ra.f002-|1825.gb.f001|

Hylarana Tschudi, $1838 \cdot \mathbf{K Y}$

ST: PO.KN • CI: h0682・ID: 409

PN: Hyla erythraea Schlegel, 1827

PK: Hyla erythraea* Schlegel, 1827

KG: Hylarana* Tschudi, 1838

KF: RANIDAE 1796.ba.f001

Hylaria Rafinesque, 1814 • AK

ST: NT.JI • CI: h0683・ID: 204

PN: Hyla viridis Laurenti, 1768

PK: Rana arborea* Linnaeus, 1758

KG: Hyla* Laurenti, 1768

KF: HYLIDAE 1815.ra.f002-|1825.gb.f001|

Hylarthroleptis Ahl, 1925 • AK

ST: PO.JD・CI: h0684・ID: 350

PN: Hylarthroleptis accraensis Ahl, 1925

PK: Hylarthroleptis accraensis* Ahl, 1925

KG: Phrynobatrachus* Günther, 1862

KF: PHRYNOBATRACHIDAE 1941.lb.f001

Hyledactylus Casto de Elera, 1895 • AK

ST: NT.JD • CI: h0685 • ID: 310

PN: Bombinator baleatus Müller, 1836

PK: Bombinator baleatus* Müller, 1836

KG: Kaloula* Gray, 1831

KF: MICROHYLIDAE ||1843.fa.f012||-1931.na.f001

Hylella Reinhardt $^{+1}, 1862$ • AK

ST: PO.JD・CI: h0686・ID: 194

PN: Hylella tenera Reinhardt ${ }^{+1}, 1862$

PK: Hyla bipunctata* Spix, 1824

KG: Dendropsophus ${ }^{1}$ Fitzinger, 1843

KF: HYLIDAE 1815.ra.f002-|1825.gb.f001|

Hylesinus Fabricius, $1801 \cdot \mathbf{z H}$

ST: zo • CI: zh045

Hylesinus: Rafinesque 1815 - AN

ST: $\mathbf{A L} ・ \mathbf{C I}:$ n0059・ID: 204

PN: Rana arborea Linnaeus, 1758

PK: Rana arborea* Linnaeus, 1758

KG: Hyla* Laurenti, 1768

KF: HYLIDAE 1815.ra.f002-|1825.gb.f001|

Hyliola Mocquard, 1899 • KY

ST: PO.KN • CI: h0687•ID: 199

PN: Hyla regilla Baird $^{+1}, 1852$

PK: Hyla regilla* Baird $^{+1}, 1852$

KG: Hyliola* Mocquard, 1899

KF: HYLIDAE 1815.ra.f002-|1825.gb.f001|

Hylixalus Boulenger, 1882 • AK

ST: NT.JI • CI: h0688・ ID: 053

PN: Hyloxalus fuliginosus Jiménez de la Espada, 1870

PK: Hyloxalus fuliginosus ${ }^{\circ}$ Jiménez de la Espada, 1870

KG: Hyloxalus ${ }^{2}$ Jiménez de la Espada, 1870

KF: DENDROBATIDAE $\|1850 . b b . f 006\|-1865$.ca.f002

Hylobatrachus Laurent, 1943 • AK

ST: PO.JD・CI: h0689 • ID: 432 
PN: Rana cowanii Boulenger, 1882

PK: Rana cowanii* Boulenger, 1882

KG: Mantidactylus* Boulenger, 1895

KF: RHACOPHORIDAE ||1858.gc.f012||-1932.ha.f001

Hylodactylus Agassiz, $1847 \cdot \mathbf{A K}$

ST: NT.JD • CI: h0690 • ID: 310

PN: Bombinator baleatus Müller, 1836

PK: Bombinator baleatus* Müller, 1836

KG: Kaloula* Gray, 1831

KF: MICROHYLIDAE ||1843.fa.f012||-1931.na.f001

Hylodes Fitzinger, $1826 \bullet \mathbf{K Y}$

ST: PO.KN • CI: h0691・ID: 182

PN: Hyla ranoides Spix, 1824

PK: Hyla nasus* Lichtenstein, 1823

KG: Hylodes ${ }^{1}$ Fitzinger, 1826

KF: HyLODIDAE 1858.gc.f010

Hylomantis Peters, $1873 \cdot \mathbf{K Y}$

ST: PO.KN • CI: h0692 • ID: 239

PN: Hylomantis aspera Peters, 1873

PK: Hylomantis aspera* Peters, 1873

KG: Hylomantis* Peters, 1873

KF: PHYLLOMEDUSIDAE 1858.gc.f009

Hylomantis Peters, 1880 • AK

ST: PO.JH • CI: h0693 • ID: 235

PN: Hylomantis fallax Peters, 1880

PK: Hylomantis fallax* Peters, 1880

KG: Litoria* Tschudi, 1838

KF: PHYLLOMEDUSIDAE 1858.gc.f009

Hylomedusa Burmeister, 1856 • AK

ST: PO.JD・CI: h0694 • ID: 189

PN: Hyla crepitans Wied-Neuwied, 1824

PK: Hyla crepitans* Wied-Neuwied, 1824

KG: Boana* Gray, 1825

KF: HYLIDAE 1815.ra.f002-|1825.gb.f001|

Hylonomus Dawson, $1860 \cdot \mathbf{z H}$

ST: zo • CI: zh046

Hylonomus Peters, 1882 • AK

ST: PO.JH • CI: h0695 • ID: 191

PN: Hylonomus bogotensis Peters, 1882

PK: Hylonomus bogotensis ${ }^{\circ}$ Peters, 1882

KG: Hyloscirtus ${ }^{3}$ Peters, 1882

KF: HyLIDAE 1815.ra.f002-|1825.gb.f001|

Hylophorbus Macleay, 1878 • AK

ST: PO.JD • CI: h0696・ ID: 280

PN: Hylophorbus rufescens Macleay, 1878

PK: Hylophorbus rufescens* Macleay, 1878

KG: Asterophrys* Tschudi, 1838

KF: MICROHYLIDAE ||1843.fa.f012||-1931.na.f001

Hylophryne: Steindachner 1864 • AN

ST: AL •CI: n0060・ID: 310

PN: Hylaedactylus (Holonectes) conjunctus Peters, 1863

PK: Hylaedactylus (Holonectes) conjunctus* Peters, 1863

KG: Kaloula* Gray, 1831

KF: MICROHYLIDAE ||1843.fa.f012||-1931.na.f001

Hyloplesia Agassiz, 1847 • AK

ST: NT.JD・CI: h0697・ ID: 189
PN: Calamita punctatus Schneider, 1799

PK: Calamita punctatus* Schneider, 1799

KG: Boana* Gray, 1825

KF: HYLIDAE 1815.ra.f002-|1825.gb.f001|

Hylopsis: Rafinesque 1815 - AN

ST: AL • CI: n0061 • ID: 204

PN: Rana arborea Linnaeus, 1758

PK: Rana arborea* Linnaeus, 1758

KG: Hyla* Laurenti, 1768

KF: HYLIDAE 1815.ra.f002-|1825.gb.f001|

Hylopsis Werner, 1894 • AK

ST: PO.JD・CI: h0698 • ID: 234

PN: Hylopsis platycephalus Werner, 1894

PK: Hylopsis platycephalus ${ }^{\circ}$ Werner, 1894

KG: Sphaenorhynchus* Tschudi, 1838

KF: HYLIDAE 1815.ra.f002-|1825.gb.f001|

Hylorana Günther, 1864 • AK

ST: NT.JI • CI: h0699 • ID: 409

PN: Hyla erythraea Schlegel, 1827

PK: Hyla erythraea* Schlegel, 1827

KG: Hylarana* Tschudi, 1838

KF: RANIDAE 1796.ba.f001

Hylorhina Agassiz, 1847 • AK

ST: NT.JI • CI: h0700 • ID: 178

PN: Hylorina sylvatica Bell, 1843

PK: Hylorina sylvatica* Bell, 1843

KG: Hylorina* Bell, 1843

KF: BATRACHYLIDAE 1965.ga.f002

Hylorina Bell, 1843 • KY

ST: PO.KN・CI: h0701・ID: 178

PN: Hylorina sylvatica Bell, 1843

PK: Hylorina sylvatica* Bell, 1843

KG: Hylorina* Bell, 1843

KF: BATRACHYLIDAE 1965.ga.f002

Hyloscirtus Peters, 1882 - KY

ST: PO.KN • CI: h0702・ID: 191

PN: Hylonomus bogotensis Peters, 1882

PK: Hylonomus bogotensis ${ }^{\circ}$ Peters, 1882

KG: Hyloscirtus ${ }^{3}$ Peters, 1882

KF: HYLIDAE 1815.ra.f002-|1825.gb.f001|

Hyloxalus Jiménez de la Espada, 1870 • KY

ST: PO.KN • CI: h0703・ID: 053

PN: Hyloxalus fuliginosus Jiménez de la Espada, 1870

PK: Hyloxalus fuliginosus ${ }^{\circ}$ Jiménez de la Espada, 1870

KG: Hyloxalus ${ }^{2}$ Jiménez de la Espada, 1870

KF: DENDROBATIDAE $\|1850 . b b . f 006\|-1865 . c a . f 002$

Hymenochirus Boulenger, $1896 \bullet \mathbf{K Y}$

ST: PO.KN・CI: h0704 • ID: 010

PN: Xenopus boettgeri Tornier, 1896

PK: Xenopus boettgeri* Tornier, 1896

KG: Hymenochirus* Boulenger, 1896

KF: PIPIDAE 1825.gb.f003-|1826.fb.f002|

Hynobius Tschudi, $1838 \cdot \mathbf{K Y}$

ST: PO.KN • CI: h0705 • ID: 505

PN: Salamandra nebulosa Temminck $^{+1}, 1838$

PK: Salamandra nebulosa* Temminck ${ }^{+1}, 1838$ 
KG: Hynobius* Tschudi, 1838

KF: HYNOBIIDAE ||1856.ha.f001||-1859.cb.f002

Hyobates: Jan $1857 \cdot \mathbf{A N}$

ST: AL・CI: n0062・ID: 250

PN: Eupemphix fuscomaculatus Steindachner, 1864

PK: Liuperus biligonigerus* Cope, 1861

KG: Physalaemus* Fitzinger, 1826

KF: LEIUPERIDAE 1850.bb.f010

Hyogobatrachus Ikeda ${ }^{+2}, 2016+\bullet \mathbf{K Y}$

ST: PO.KN・CI: h0706・ID: $\uparrow 056$

PN: Hyogobatrachus wadai $\mathrm{Ikeda}^{+2}, 2016$ \$

PK: Hyogobatrachus wadai ${ }^{\circ} \mathrm{Ikeda}^{+2}, 2016 \uparrow$

KG: Hyogobatrachus ${ }^{\circ} \mathrm{Ikeda}^{+2}, 2016 \dagger$

KF: Hydrobatrachia Familia INCERTAE SEDIS

Hyophryne Carvalho, $1954 \cdot \mathbf{A K}$

ST: PO.JD • CI: h0707 • ID: 302

PN: Hyophryne histrio Carvalho, 1954

PK: Hyophryne histrio ${ }^{\circ}$ Carvalho, 1954

KG: Stereocyclops* Cope, 1870

KF: MICROHYLIDAE ||1843.fa.f012||-1931.na.f001

Hyperobatrachus Rye, 1881 • AK

ST: NT.JI • CI: h0708・ID: 509

PN: Desmodactylus pinchonii David, 1872

PK: Desmodactylus pinchonii* David, 1872

KG: Batrachuperus* Boulenger, 1878

KF: HYNOBIIDAE ||1856.ha.f001||-1859.cb.f002

Hyperodon Duméril, $1804 \cdot \mathbf{z H}$

ST: zo • CI: zh047

Hyperodon Agassiz, $1847 \bullet \mathbf{A K}$

ST: NT.JH • CI: h0709・ID: 309

PN: Engystoma marmoratum Guérin-Méneville, 1838

PK: Rana systoma* Schneider, 1799

KG: Uperodon* Duméril ${ }^{+1}, 1841$

KF: MICROHYLIDAE ||1843.fa.f012||-1931.na.f001

Hyperolia Agassiz, 1847 • AK

ST: NT.JI • CI: h0710 • ID: 276

PN: Uperoleia marmorata Gray, 1841

PK: Uperoleia marmorata ${ }^{\circ}$ Gray, 1841

KG: Uperoleia ${ }^{2}$ Gray, 1841

KF: MYOBATRACHIDAE 1850.sa.f001

Hyperolius Rapp, 1842 • KY

ST: PO.KN • CI: h0711 • ID: 331

PN: Hyla horstockii Schlegel, 1837

PK: Hyla horstockii* Schlegel, 1837

KG: Hyperolius* Rapp, 1842

KF: HYPEROLIIDAE 1943.lb.f001

Hyperolius: Boulenger $1882 \cdot$ AN

ST: $\mathbf{A L} ・ \mathbf{C I}: \mathrm{n} 0063 \cdot \mathbf{I D}: 276$

PN: Uperoleia marmorata Gray, 1841

PK: Uperoleia marmorata ${ }^{\circ}$ Gray, 1841

KG: Uperoleia ${ }^{2}$ Gray, 1841

KF: MYOBATRACHIDAE 1850.sa.f001

Hyperoodon La Cepède, $1804 \cdot \mathbf{z H}$

ST: zo • CI: zh048

Hyperoodon Philippi, 1902 • AK

ST: LC.JH • CI: h0712 • ID: 309
PN: Engystoma marmoratum Guérin-Méneville, 1838

PK: Rana systoma* Schneider, 1799

KG: Uperodon* Duméril ${ }^{+1}, 1841$

KF: MICROHYLIDAE ||1843.fa.f012||-1931.na.f001

Hypochthon Merrem, 1820 • AK

ST: NT.JI • CI: h0713・ID: 554

PN: Proteus anguinus Laurenti, 1768

PK: Proteus anguinus* Laurenti, 1768

KG: Proteus* Laurenti, 1768

KF: PROTEIDAE 1831.ba.f002

Hypodactylus Hedges ${ }^{+2}$, $2008 \cdot \mathbf{K Y}$

ST: PO.KN・CI: h0714・ID: 074

PN: Eleutherodactylus elassodiscus Lynch, 1973

PK: Eleutherodactylus elassodiscus* Lynch, 1973

KG: Hypodactylus * Hedges ${ }^{+2}, 2008$

KF: BRACHYCEPHALIDAE 1858.gc.f002

Hypodictyon Cope, 1885 • AK

ST: PO.JD・CI: h0715・ID: 078

PN: Phyllobates ridens Cope, 1866

PK: Phyllobates ridens* Cope, 1866

KG: Pristimantis* Jiménez de la Espada, 1870

KF: BRACHYCEPHALIDAE 1858.gc.f002

Hypogeophis Peters, $1880 \bullet \mathbf{K Y}$

ST: PO.KN • CI: h0716 • ID: 482

PN: Coecilia rostrata Cuvier, 1829

PK: Coecilia rostrata* Cuvier, 1829

KG: Hypogeophis* Peters, 1880

KF: CAECILIIDAE 1814.ra.f003-|1825.gb.f008|

Hypopachus Keferstein, $1867 \bullet \mathbf{K Y}$

ST: PO.KN・CI: h0717・ ID: 300

PN: Hypopachus seebachii Keferstein, 1867

PK: Engystoma variolosum* Cope, 1866

KG: Hypopachus* Keferstein, 1867

KF: MICROHYLIDAE ||1843.fa.f012||-1931.na.f001

Hypselotriton Wolterstorff, $1934 \cdot \mathbf{K Y}$

ST: PO.KN • CI: h0718 • ID: 559

PN: Molge wolterstorffi Boulenger, 1905

PK: Molge wolterstorffi $i^{\circ}$ Boulenger, 1905

KG: Hypselotriton ${ }^{2}$ Wolterstorff, 1934

KF: SALAMANDRIDAE 1820.ga.f002

Hypsiboas Wagler, 1830 • AK

ST: PO.JD・CI: h0719・ID: 189

PN: Hyla palmata Bonnaterre, 1789

PK: Rana boans* Linnaeus, 1758

KG: Boana* Gray, 1825

KF: HYLIDAE 1815.ra.f002-|1825.gb.f001|

Hypsipsophus Fitzinger, 1843 • AK

ST: PO.JD・CI: h0720・ID: 189

PN: Hyla xerophilla Duméril ${ }^{+1}, 1841$

PK: Hyla crepitans* Wied-Neuwied, 1824

KG: Boana* Gray, 1825

KF: HYLIDAE 1815.ra.f002-|1825.gb.f001|

Hypsirana Kinghorn, 1928 • AK

ST: PO.JD • CI: h0721 • ID: 369

PN: Hypsirana heffernani Kinghorn, 1928

PK: Hypsirana heffernani ${ }^{\circ}$ Kinghorn, 1928 
KG: Cornufer* Tschudi, 1838

KF: CERATOBATRACHIDAE 1884.ba.f001

Hysaplesia Boie in Schlegel, 1826a • AK

ST: PO.CA • CI: h0722 • ID: 189

PN: Calamita punctatus Schneider, 1799

PK: Calamita punctatus* Schneider, 1799

KG: Boana* Gray, 1825

KF: HYLIDAE 1815.ra.f002-|1825.gb.f001|

Iberobatrachus Báez, 2013 + KY

ST: PO.KN • CI: h0723 • ID: $\uparrow 027$

PN: Iberobatrachus angelae Báez, 2013 \$

PK: Iberobatrachus angelae ${ }^{\circ}$ Báez, $2013 \dagger$

KG: Iberobatrachus ${ }^{\circ}$ Báez, 2013 †

KF: ANURA Familia INCERTAE SEDIS

Ichthyophis Fitzinger, $1826 \bullet \mathbf{K Y}$

ST: PO.KN・CI: h0724 • ID: 501

PN: Caecilia glutinosa Linnaeus, 1758

PK: Caecilia glutinosa* Linnaeus, 1758

KG: Ichthyophis* Fitzinger, 1826

KF: ICHTHYOРHIIDAE 1968.ta.f001

Ichthyosaura Sonnini ${ }^{+1}, 1801 \cdot \mathbf{K Y}$

ST: PO.KN • CI: h0725 • ID: 563

PN: Proteus tritonius Laurenti, 1768

PK: Triton alpestris* Laurenti, 1768

KG: Ichthyosaura ${ }^{1}$ Sonnini ${ }^{+1}, 1801$

KF: SALAMANDRIDAE 1820.ga.f002

Idiocranium Parker, 1936 • KY

ST: PO.KN • CI: h0726・ID: 483

PN: Idiocranium russeli Parker, 1936

PK: Idiocranium russeli ${ }^{\circ}$ Parker, 1936

KG: Idiocranium ${ }^{\circ}$ Parker, 1936

KF: CAECILIIDAE 1814.ra.f003-|1825.gb.f008|

Ikakogi Guayasamin ${ }^{+5}, 2009 \cdot \mathbf{K Y}$

ST: PO.KN・CI: h0727・ID: 168

PN: Centrolene tayrona Ruiz-Carranza ${ }^{+1}, 1991$

PK: Centrolene tayrona* Ruiz-Carranza ${ }^{+1}, 1991$

KG: Ikakogi* Guayasamin ${ }^{+5}, 2009$

KF: CENTROLENIDAE 1951.ta.f001

Iliodiscus Miranda-Ribeiro, 1920 • AK

ST: PO.JD • CI: h0728 • ID: 179

PN: Iliodiscus dubius Miranda-Ribeiro, 1920

PK: Iliodiscus dubius ${ }^{\circ}$ Miranda-Ribeiro, 1920

KG: Cycloramphus* Tschudi, 1838

KF: CYCLORAMPHIDAE 1850.bb.f003-|1852.ba.f001|

Incilius Cope, $1863 \bullet \mathbf{K Y}$

ST: PO.KN • CI: h0729・ID: 137

PN: Bufo coniferus Cope, 1862

PK: Bufo coniferus* Cope, 1862

KG: Incilius* Cope, 1863

KF: BUFONIDAE 1825.gb.f004

Indirana: Bauer 1985 • AN

ST: AL・CI: n0064・ID: 461

PN: Rana leptodactyla Boulenger, 1882

PK: Rana leptodactyla* Boulenger, 1882

KG: Walkerana* Dahanukar ${ }^{+5}, 2016$

KF: RANIXALIDAE 1987.da.f005
Indirana Laurent, $1986 \bullet \mathbf{K Y}$

ST: PO.KN • CI: h0730 • ID: 460

PN: Polypedates beddomii Günther, 1875

PK: Polypedates beddomii* Günther, 1875

KG: Indirana* Laurent, 1986

KF: RANIXALIDAE 1987.da.f005

Indobatrachus Noble, $1930+\mathbf{K Y}$

ST: PO.KN • CI: h0731・ID: $\uparrow 103$

PN: Rana pusilla Owen, 1847 †

PK: Rana pusilla ${ }^{\circ}$ Owen, $1847 \dagger$

KG: Indobatrachus ${ }^{\circ}$ Noble, $1930 \dagger$

KF: MYOBATRACHIDAE 1850.sa.f001

Indorana $\mathrm{Folie}^{+6}, 2013+\bullet \mathbf{K Y}$

ST: PO.KN • CI: h0732 •ID: $\uparrow 106$

PN: Indorana prasadi Folie $^{+6}, 2013$ *

PK: Indorana prasadi ${ }^{\circ}$ Folie $^{+6}, 2013 \dagger$

KG: Indorana ${ }^{\circ}$ Folie $^{+6}, 2013 \dagger$

KF: RHACOPHORIDAE ||1858.gc.f012||-1932.ha.f001

Indosylvirana Oliver ${ }^{+3}, 2015 \cdot \mathbf{A K}$

ST: PO.JD • CI: h0733・ID: 409

PN: Rana flavescens Jerson, 1853

PK: Rana flavescens ${ }^{\circ}$ Jerson, 1853

KG: Hylarana* Tschudi, 1838

KF: RANIDAE 1796.ba.f001

Indotyphlus Taylor, $1960 \bullet \mathbf{K Y}$

ST: PO.KN • CI: h0734・ID: 486

PN: Indotyphlus battersbyi Taylor, 1960

PK: Indotyphlus battersbyi* Taylor, 1960

KG: Indotyphlus* Taylor, 1960

KF: CAECILIIDAE 1814.ra.f003-|1825.gb.f008|

Ingerana Dubois, 1987 • KY

ST: PO.KN • CI: h0735 • ID: 393

PN: Rana tenasserimensis Sclater, 1892

PK: Rana tenasserimensis* Sclater, 1892

KG: Ingerana* Dubois, 1987

KF: OCCIDOZYGIDAE 1990.fa.f002

Ingerophrynus Frost ${ }^{+18}, 2006 \cdot \mathbf{K Y}$

ST: PO.KN • CI: h0736 • ID: 117

PN: Bufo biporcatus Gravenhorst, 1829

PK: Bufo biporcatus* Gravenhorst, 1829

KG: Ingerophrynus* Frost $^{+18}, 2006$

KF: BUFONIDAE 1825.gb.f004

Insuetophrynus Barrio, 1970 • KY

ST: PO.KN • CI: h0737 • ID: 184

PN: Insuetophrynus acarpicus Barrio, 1970

PK: Insuetophrynus acarpicus* Barrio, 1970

KG: Insuetophrynus* Barrio, 1970

KF: RHINODERMATIDAE 1850.bb.f011

Iranodon Dubois ${ }^{+1}, 2012 \cdot \mathbf{K Y}$

ST: PO.KN • CI: h0738 • ID: 515

PN: Batrachuperus persicus Eiselt ${ }^{+1}, 1970$

PK: Batrachuperus persicus* Eiselt ${ }^{+1}, 1970$

KG: Iranodon* Dubois ${ }^{+1}, 2012$

KF: HYNOBIIDAE ||1856.ha.f001\|-1859.cb.f002

Iridotriton Evans $^{+4}, 2005+\cdot \mathbf{K Y}$

ST: PO.KN • CI: h0739 • ID: $\uparrow 132$ 
PN: Iridotriton hechti Evans $^{+4}, 2005$ \$

PK: Iridotriton hechti ${ }^{\circ}$ Evans $^{+4}, 2005 \dagger$

KG: Iridotriton ${ }^{\circ}$ Evans $^{+4}, 2005 \dagger$

KF: Urodela Familia INCERTAE SEDIS

Ischnocnema Reinhardt ${ }^{+1}, 1862$ • KY

ST: PO.KN • CI: h0740 • ID: 058

PN: Leiuperus verrucosus Reinhardt ${ }^{+1}, 1862$

PK: Leiuperus verrucosus* Reinhardt ${ }^{+1}, 1862$

KG: Ischnocnema* Reinhardt ${ }^{+1}, 1862$

KF: BRACHYCEPHALIDAE 1858.gc.f002

Isodactylium Strauch, 1870 • AK

ST: PO.JD • CI: h0741 • ID: 513

PN: Isodactylium schrenckii Strauch, 1870

PK: Salamandrella keyserlingii* Dybowski, 1870

KG: Salamandrella* Dybowski, 1870

KF: НYNOBIIDAE ||1856.ha.f001\|-1859.cb.f002

Isodactylus Gray, $1845 \cdot \mathbf{Z H}$

ST: zo • CI: zh049

Isodactylus Hedges ${ }^{+2}, 2008 \cdot \mathbf{A K}$

ST: PO.JH • CI: h0742 • ID: 073

PN: Eleutherodactylus elassodiscus Lynch, 1973

PK: Eleutherodactylus elassodiscus* Lynch, 1973

KG: Hypodactylus ${ }^{*}$ Hedges $^{+1}, 2008$

KF: BRACHYCEPHALIDAE 1858.gc.f002

Isthmohyla Faivovich ${ }^{+5}, 2005 \cdot \mathbf{K Y}$

ST: PO.KN • CI: h0743・ID: 205

PN: Hyla pseudopuma Günther, 1901

PK: Hyla pseudopuma* Günther, 1901

KG: Isthmohyla* Faivovich $^{+5}, 2005$

KF: HYLIDAE 1815.ra.f002-|1825.gb.f001|

Isthmura Dubois ${ }^{+1}, 2012 \cdot \mathbf{K Y}$

ST: PO.KN • CI: h0744 • ID: 524

PN: Spelerpes bellii Gray, 1850

PK: Spelerpes bellii* Gray, 1850

KG: Isthmura* ${ }^{*}$ Dubois $^{+1}, 2012$

KF: PLETHODONTIDAE 1850.ga.f002

Itapotihyla Faivovich ${ }^{+5}, 2005 \cdot \mathbf{K Y}$

ST: PO.KN • CI: h0745 • ID: 220

PN: Hyla langsdorffii Duméril ${ }^{+1}, 1841$

PK: Hyla langsdorffii* Duméril ${ }^{+1}, 1841$

KG: Itapotihyla* Faivovich ${ }^{+5}, 2005$

KF: HYLIDAE 1815.ra.f002-|1825.gb.f001|

Itemirella Nessov, $1981+\cdot \mathbf{K Y}$

ST: PO.KN • CI: h0746 • ID: $\uparrow 028$

PN: Itemirella cretacea Nessov, 1981 \$

PK: Itemirella cretacea ${ }^{\circ}$ Nessov, $1981 \dagger$

KG: Itemirella ${ }^{\circ}$ Nessov, $1981 \dagger$

KF: ANURA Familia INCERTAE SEDIS

Ixalotriton $\mathrm{Wake}^{+1}, 1989 \cdot \mathbf{K Y}$

ST: PO.KN • CI: h0747 • ID: 525

PN: Ixalotriton niger Wake $^{+1}, 1989$

PK: Ixalotriton niger* Wake $^{+1}, 1989$

KG: Ixalotriton* Wake $^{+1}, 1989$

KF: PLETHODONTIDAE 1850.ga.f002

Ixalus Ogilby, $1837 \bullet \mathbf{z H}$

ST: zo•CI: zh050
Ixalus Duméril ${ }^{+1}, 1841 \bullet \mathbf{A K}$

ST: PO.JH • CI: h0748 • ID: 447

PN: Hyla aurifasciata Schlegel, 1837

PK: Hyla aurifasciata* Schlegel, 1837

KG: Philautus* Gistel, 1848

KF: RHACOPHORIDAE ||1858.gc.f012||-1932.ha.f001

Jeholotriton Wang, $2000+\cdot \mathbf{K Y}$

ST: PO.KN • CI: h0749・ID: $\uparrow 133$

PN: Jeholotriton paradoxus Wang, 2000 \$

PK: Jeholotriton paradoxus ${ }^{\circ}$ Wang, $2000 \dagger$

KG: Jeholotriton ${ }^{\circ}$ Wang, $2000 \dagger$

KF: Urodela Familia INCERTAE SEDIS

Julianus Duellman ${ }^{+2}, 2016 \cdot \mathbf{A K}$

ST: PO.JD • CI: h0750 • ID: 232

PN: Hyla uruguaya Schmidt, 1944

PK: Hyla uruguaya* Schmidt, 1944

KG: Scinax ${ }^{2}$ Wagler, 1830

KF: HYLIDAE 1815.ra.f002-|1825.gb.f001|

Kababisha Evans $^{+2}, 1996+\bullet \mathbf{K Y}$

ST: PO.KN • CI: h0751・ID: $\uparrow 174$

PN: Kababisha humarensis Evans ${ }^{+2}, 1996$ *

PK: Kababisha humarensis ${ }^{\circ}$ Evans $^{+2}, 1996 \dagger$

KG: Kababisha ${ }^{\circ}$ Evans $^{+2}, 1996 \dagger$

KF: NOTERPETIDAE 1993.ra.f001

Kakophrynus Steindachner, 1863 • AK

ST: PO.JD • CI: h0752 • ID: 347

PN: Kakophrynus sudanensis Steindachner, 1863

PK: Engystoma marmoratum* Peters, 1854

KG: Hemisus ${ }^{2}$ Günther, 1859

KF: HEMISOTIDAE 1867.ca.f002

Kalophrynus Tschudi, $1838 \cdot \mathbf{K Y}$

ST: PO.KN • CI: h0753 • ID: 305

PN: Kalophrynus pleurostigma Tschudi, 1838

PK: Kalophrynus pleurostigma* Tschudi, 1838

KG: Kalophrynus* Tschudi, 1838

KF: MICROHYLIDAE ||1843.fa.f012||-1931.na.f001

Kalooula: Castro de Elera, 1895 • AN

ST: AM • CI: n0065 • ID: 310

PN: Kaloula pulchra Gray, 1831

PK: Kaloula pulchra* Gray, 1831

KG: Kaloula* Gray, 1831

KF: MICROHYLIDAE ||1843.fa.f012||-1931.na.f001

Kaloula Gray, 1831 • KY

ST: PO.KN • CI: h0754 • ID: 310

PN: Kaloula pulchra Gray, 1831

PK: Kaloula pulchra* Gray, 1831

KG: Kaloula* Gray, 1831

KF: MICROHYLIDAE ||1843.fa.f012||-1931.na.f001

Kankanophryne Heyer ${ }^{+1}, 1976$ • AK

ST: PO.JD • CI: h0755 • ID: 274

PN: Pseudophryne occidentalis Parker, 1940

PK: Pseudophryne occidentalis ${ }^{\circ}$ Parker, 1940

KG: Pseudophryne ${ }^{3}$ Fitzinger, 1843

KF: MYOBATRACHIDAE 1850.sa.f001

Karaurus Ivachnenko, $1978+\cdot \mathbf{K Y}$

ST: PO.KN • CI: h0756・ID: $\uparrow 152$ 
PN: Karaurus sharovi Ivachnenko 1978 \$

PK: Karaurus sharovi ${ }^{\circ}$ Ivachnenko $1978 \dagger$

KG: Karaurus $^{\circ}$ Ivachnenko, $1978 \dagger$

KF: KARAURIDAE 1978.ia.f001 †

Karsenia $\mathrm{Min}^{+5}, 2005 \cdot \mathbf{K Y}$

ST: PO.KN • CI: h0757 • ID: 546

PN: Karsenia koreana $\mathrm{Min}^{+5}, 2005$

PK: Karsenia koreana* Min $^{+5}, 2005$

KG: Karsenia* $\mathrm{Min}^{+5}, 2005$

KF: PLETHODONTIDAE 1850.ga.f002

Karstotriton $\mathrm{Fei}^{+1}, 2016 \bullet \mathbf{A K}$

ST: PO.JD • CI: h0758 • ID: 562

PN: Paramesotriton zhijinensis $\mathrm{Li}^{+2}, 2008$

PK: Paramesotriton zhijinensis* $\mathrm{Li}^{+2}, 2008$

KG: Paramesotriton* Chang, 1936

KF: SALAMANDRIDAE 1820.ga.f002

Kassina Girard, 1853 • KY

ST: PO.KN • CI: h0759 • ID: 338

PN: Cystignathus senegalensis Duméril ${ }^{+1}, 1841$

PK: Cystignathus senegalensis* Duméril ${ }^{+1}, 1841$

KG: Kassina* Girard, 1853

KF: HYPEROLIIDAE 1943.lb.f001

Kassinula Laurent, 1940 • KY

ST: PO.KN • CI: h0760 • ID: 339

PN: Kassinula wittei Laurent, 1940

PK: Kassinula wittei $i^{\circ}$ Laurent, 1940

KG: Kassinula ${ }^{\circ}$ Laurent, 1940

KF: HYPEROLIIDAE 1943.lb.f001

Kirtixalus Dubois, 1987 • AK

ST: PO.JD・CI: h0761・ID: 444

PN: Polypedates microtympanum Günther, 1859

PK: Polypedates microtympanum* Günther, 1859

KG: Pseudophilautus* Laurent, 1943

KF: RHACOPHORIDAE ||1858.gc.f012||-1932.ha.f001

Kiyatriton Averianov ${ }^{+1}, 2002+\bullet \mathbf{K Y}$

ST: PO.KN • CI: h0762 • ID: $\uparrow 134$

PN: Kiyatriton leshchinskiyi Averianov ${ }^{+1}, 2002 \ddagger$

PK: Kiyatriton leshchinskiyi Averianov ${ }^{+1}, 2002 \dagger$

KG: Kiyatriton Averianov ${ }^{+1}, 2002 \uparrow$

KF: Urodela Familia INCERTAE SEDIS

Kizylkuma Nessov, 1981 +・ KY

ST: PO.KN • CI: h0763・ID: $\uparrow 114$

PN: Kizylkuma antiqua Nessov, 1981 \$

PK: Kizylkuma antiqua ${ }^{\circ}$ Nessov, $1981 \dagger$

KG: Kizylkuma ${ }^{\circ}$ Nessov, $1981 \uparrow$

KF: ALYTIDAE 1843.fa.f008

Koalliella Herre, 1950 † KY

ST: PO.KN • CI: h0764 • ID: $\uparrow 194$

PN: Koalliella genzeli Herre, 1950 *

PK: Koalliella genzeli ${ }^{\circ}$ Herre, $1950 \dagger$

KG: Koalliella ${ }^{\circ}$ Herre, $1950 \uparrow$

KF: SALAMANDRIDAE 1820.ga.f002

Kokartus Nessov, $1988+\bullet \mathbf{K Y}$

ST: PO.KN • CI: h0765 • ID: $\uparrow 153$

PN: Kokartus honorarius Nessov, 1988 \$

PK: Kokartus honorarius ${ }^{\circ}$ Nessov, $1988 \dagger$
KG: Kokartus ${ }^{\circ}$ Nessov, $1988 \dagger$

KF: KARAURIDAE 1978.ia.f001 †

Kulgeriherpeton Skutschas $^{+6}, 2018+\mathbf{K Y}$

ST: PO.KN • CI: h0766・ID: $\uparrow 135$

PN: Kulgeriherpeton ultimum Skutschas ${ }^{+6}, 2018$ *

PK: Kulgeriherpeton ultimum Skutschas ${ }^{+6}, 2018$ †

KG: Kulgeriherpeton Skutschas ${ }^{+6}, 2018 \dagger$

KF: UrodeLA Familia INCERTAE SEDIS

Kurixalus $\mathrm{Fei}^{+2}$ in Fei, $1999 \cdot \mathbf{K Y}$

ST: PO.KN • CI: h0767•ID: 441

PN: Rana eiffingeri Boettger, 1895

PK: Rana eiffingeri* Boettger, 1895

KG: Kurixalus $* \mathrm{Fei}^{+2}$ in $\mathrm{Fei}, 1999$

KF: RHACOPHORIDAE ||1858.gc.f012||-1932.ha.f001

Kuruleufemia Gómez, 2016 † KY

ST: PO.KN • CI: h0768・ ID: $\uparrow 077$

PN: Kuruleufemia xenopoides Gómez, 2016 \$

PK: Kuruleufemia xenopoides ${ }^{\circ}$ Gómez, 2016 †

KG: Kuruleufemia ${ }^{\circ}$ Gómez, 2016

KF: PIPIDAE 1825.gb.f003-|1826.fb.f002|

Kururubatrachus: Agnolin ${ }^{+6} 2020 a+\bullet A N$

ST: AL • CI: n0066 - ID: $† 057$

PN: Kururubatrachus gondwanicus Agnolin ${ }^{+6}, 2020 a+$

PK: Kururubatrachus gondwanicus ${ }^{\circ}$ Agnolin $^{+6}, 2020 b \dagger$

KG: Kururubatrachus ${ }^{\circ}$ Agnolin $^{+6}, 2020 b \dagger$

KF: Hydrobatrachia Familia INCERTAE SEDIS

Kururubatrachus Agnolin ${ }^{+6}, 2020 b+\cdot \mathbf{K Y}$

ST: PO.KN • CI: h0769 • ID: $\uparrow 057$

PN: Kururubatrachus gondwanicus Agnolin ${ }^{+6}, 2020 b$ \$

PK: Kururubatrachus gondwanicus ${ }^{\circ}$ Agnolin $^{+6}, 2020 b \dagger$

KG: Kururubatrachus ${ }^{\circ}$ Agnolin $^{+6}, 2020 b \uparrow$

KF: Hydrobatrachia Familia INCERTAE SEDIS

Kyarranus Moore, $1959 \cdot \mathbf{A K}$

ST: PO.JD・CI: h0770 • ID: 262

PN: Kyarranus sphagnicolus Moore, 1958

PK: Kyarranus sphagnicolus* Moore, 1958

KG: Philoria ${ }^{2}$ Spencer, 1901

KF: MYOBATRACHIDAE 1850.sa.f001

Laccotriton $\mathrm{GaO}^{+2}, 1998+\cdot \mathbf{K Y}$

ST: PO.KN • CI: h0771 •ID: $\uparrow 136$

PN: Laccotriton subsolanus $\mathrm{Gao}^{+2}, 1998$ *

PK: Laccotriton subsolanus ${ }^{\circ} \mathrm{Gao}^{+2}, 1998 \dagger$

KG: Laccotriton $^{\circ} \mathrm{Gao}^{+2}, 1998 \uparrow$

KF: UrodeLA Familia INCERTAE SEDIS

Lacusirana Hillis ${ }^{+1}, 2005$ • AK

ST: PO.JD・CI: h0772・ID: 415

PN: Rana megapoda Taylor, 1942

PK: Rana megapoda ${ }^{\circ}$ Taylor, 1942

KG: Lithobates* Fitzinger, 1843

KF: RANIDAE 1796.ba.f001

Ladailadne Dubois, 1987 • AK

ST: PO.JD・CI: h0773・ID: 081

PN: Eleutherodactylus jasperi Drewry ${ }^{+1}, 1976$

PK: Eleutherodactylus jasperi ${ }^{\circ}$ Drewry $^{+1}, 1976$

KG: Eleutherodactylus* Duméril ${ }^{+1}, 1841$

KF: BRACHYCEPHALIDAE 1858.gc.f002 
Lahatnanguri $\mathrm{Brown}^{+4}, 2015 \cdot \mathbf{A K}$

ST: PO.JD • CI: h0774 • ID: 370

PN: Platymantis levigatus Brown ${ }^{+1}, 1974$

PK: Platymantis levigatus ${ }^{\circ}$ Brown $^{+1}, 1974$

KG: Platymantis ${ }^{1}$ Günther, 1859

KF: CERATOBATRACHIDAE 1884.ba.f001

Lalax Hamilton, $1990 \cdot \mathbf{Z H}$

ST: zo • CI: zh051

Lalax Delorme ${ }^{+3}, 2006 \cdot \mathbf{A K}$

ST: PO.JH • CI: h0775 • ID: 018

PN: Leptolalax bourreti Dubois, 1983

PK: Leptolalax bourreti* Dubois, 1983

KG: Leptobrachella ${ }^{\circ}$ Smith, 1925

KF: MEGOPHRYIDAE 1850.bb.f008-|1931.na.f003|

Laliostoma $\mathrm{Glaw}^{+2}$, $1998 \cdot \mathbf{K Y}$

ST: PO.KN • CI: h0776・ID: 425

PN: Tomopterna labrosa Cope, 1868

PK: Tomopterna labrosa* Cope, 1868

KG: Laliostoma* Glaw $^{+2}, 1998$

KF: RHACOPHORIDAE ||1858.gc.f012||-1932.ha.f001

Lalos Dubois ${ }^{+4}, 2010 \bullet \mathbf{A K}$

ST: PO.JD • CI: h0777 • ID: 018

PN: Leptolalax bourreti Dubois, 1983

PK: Leptolalax bourreti* Dubois, 1983

KG: Leptobrachella ${ }^{\circ}$ Smith, 1925

KF: MEGOPHRYIDAE 1850.bb.f008-|1931.na.f003|

Lanebatrachus Taylor, 1941 \$• AK

ST: PO.JD • CI: h0778 • ID: 555

PN: Lanebatrachus martini Taylor, 1941 \$

PK: Plioambystoma kansense ${ }^{\circ}$ Adams $^{+1}, 1929 \dagger$

KG: Ambystoma ${ }^{1}$ Tschudi, 1838

KF: AмBYSTOMATIDAE 1850.ga.f004

Lankanectes Dubois ${ }^{+1}, 2001 \cdot \mathrm{KY}$

ST: PO.KN • CI: h0779 • ID: 399

PN: Rana corrugata Peters, 1863

PK: Rana corrugata* Peters, 1863

KG: Lankanectes* Dubois ${ }^{+1}, 2001$

KF: NYCTIBATRACHIDAE 1993.ba.f001

Lanzarana Clarke, $1982 \cdot \mathbf{K Y}$

ST: PO.KN • CI: h0780 • ID: 463

PN: Hildebrandtia largeni Lanza, 1978

PK: Hildebrandtia largeni ${ }^{\circ}$ Lanza, 1978

KG: Lanzarana ${ }^{\circ}$ Clarke, 1982

KF: PTYCHADENIDAE 1987.da.f002

Laotriton Dubois ${ }^{+1}, 2009 \cdot \mathbf{K Y}$

ST: PO.KN • CI: h0781 •ID: 560

PN: Paramesotriton laoensis Stuart ${ }^{+1}, 2002$

PK: Paramesotriton laoensis* Stuart ${ }^{+1}, 2002$

KG: Laotriton* Dubois ${ }^{+1}, 2009$

KF: SALAMANDRIDAE 1820.ga.f002

Larvarius Rafinesque, 1815 • AK

ST: NT.JI • CI: h0782・ ID: 554

PN: Proteus anguinus Laurenti, 1768

PK: Proteus anguinus* Laurenti, 1768

KG: Proteus* Laurenti, 1768

KF: PROTEIDAE 1831.ba.f002
Latoglossus Hossini, 2000 \$ KY

ST: PO.KN • CI: h0783・ID: $\uparrow 117$

PN: Latoglossus zraus Hossini, 2000 \$

PK: Latoglossus zraus ${ }^{\circ}$ Hossini, $2000 \dagger$

KG: Latoglossus zraus ${ }^{\circ}$ Hossini, $2000 \dagger$

KF: DISCOGLOSSIDAE 1858.gc.f004

Latonia: Braun $1843 a+\cdot \mathbf{A N}$

ST: AL • CI: n0067 • ID: 470

PN: Latonia seyfriedii Braun, $1843 a \ddagger \cdot$ AS

PK: Latonia seyfriedii ${ }^{\circ}$ Meyer, $1845 \dagger$

KG: Latonia ${ }^{3}$ Meyer, 1845

KF: DISCOGLOSSIDAE 1858.gc.f004

Latonia: Meyer $1843 c \$ \cdot \mathbf{A N}$

ST: AL・CI: n0068・ID: 470

PN: Latonia (Ceratophrys) seyfriedii Meyer, $1843 c+\bullet$ AS

PK: Latonia seyfriedii ${ }^{\circ}$ Meyer, $1845 \dagger$

KG: Latonia ${ }^{3}$ Meyer, 1845

KF: DISCOGLOSSIDAE 1858.gc.f004

Latonia Meyer, $1845+\bullet^{\mathbf{K Y}}$

ST: PO.KN • CI: h0784 • ID: 470

PN: Latonia seyfriedii Meyer, 1845 *

PK: Latonia seyfriedii ${ }^{\circ}$ Meyer, $1845 \dagger$

KG: Latonia ${ }^{3}$ Meyer, 1845

KF: DISCOGLOSSIDAE 1858.gc.f004

Latonix: Meyer $1843 b+\cdot \mathbf{A N}$

ST: AL・CI: n0069・ID: 470

PN: Latonix (Ceratophrys) seyfriedii Meyer, 1843b $\ddagger \cdot$ AS

PK: Latonix seyfriedii ${ }^{\circ}$ Meyer, $1845 \uparrow$

KG: Latonia $^{3}$ Meyer, 1845

KF: DISCOGLOSSIDAE 1858.gc.f004

Laurasiarana: Hillis $^{+1} 2005$ - AN

ST: AL・CI: n0070・ID: 418

PN: Rana aurora Baird $^{+1}, 1852$

PK: Rana aurora* Baird ${ }^{+1}, 1852$

KG: Amerana* Dubois, 1992

KF: RANIDAE 1796.ba.f001

Laurentixalus Amiet, 2012 • AK

ST: PO.JD • CI: h0785 • ID: 334

PN: Megalixalus laevis Ahl, 1930

PK: Megalixalus laevis* Ahl, 1930

KG: Afrixalus* Laurent, 1944

KF: HYPEROLIIDAE 1943.lb.f001

Laurentomantis Dubois, 1980 • AK

ST: PO.JD・CI: h0786・ID: 431

PN: Microphryne malagasia Methuen ${ }^{+1}, 1913$

PK: Microphryne malagasia* Methuen ${ }^{+1}, 1913$

KG: Gephyromantis* Methuen, 1920

KF: RHACOPHORIDAE ||1858.gc.f012||-1932.ha.f001

Laurentophryne Tihen, $1960 \bullet \mathbf{K Y}$

ST: PO.KN・CI: h0787・ID: 125

PN: Wolterstorffina parkeri Laurent, 1950

PK: Wolterstorffina parkeri ${ }^{\circ}$ Laurent, 1950

KG: Laurentophryne ${ }^{\circ}$ Tihen, 1960

KF: BUFONIDAE 1825.gb.f004

Lechriodus Boulenger, 1882 • AK

ST: PO.JD・CI: h0788 • ID: 264 
PN: Asterophrys melanopyga Doria, 1875

PK: Asterophrys melanopyga* Doria, 1875

KG: Platyplectrum ${ }^{1}$ Günther, 1863

KF: МYOBATRACHIDAE 1850.sa.f001

Leioaspetos Wells ${ }^{+1}, 1985$ • KY

ST: PO.KN • CI: h0789 • ID: 005

PN: Liopelma hamiltoni McCulloch, 1919

PK: Liopelma hamiltoni* McCulloch, 1919

KG: Leioaspetos* Wells ${ }^{+1}, 1985$

KF: LEIOPELMATIDAE 1869.mc.f07-|1942.ta.f001|

Leiopelma Fitzinger, $1861 \bullet \mathbf{K Y}$

ST: PO.KN • CI: h0790 • ID: 006

PN: Leiopelma hochstetteri Fitzinger, 1861

PK: Leiopelma hochstetteri* Fitzinger, 1861

KG: Leiopelma* Fitzinger, 1861

KF: LEIOPELMATIDAE 1869.mc.f07-|1942.ta.f001|

Leiuperus Duméril $^{+1}, 1841$ • AK

ST: PO.JD • CI: h0791 • ID: 246

PN: Leiuperus marmoratus Duméril ${ }^{+1}, 1841$

PK: Leiuperus marmoratus* Duméril ${ }^{+1}, 1841$

KG: Pleurodema* Tschudi, 1838

KF: LEIUPERIDAE 1850.bb.f010

Leiyla Keferstein, 1868 • AK

ST: PO.JD • CI: h0792・ID: 059

PN: Leiyla guentherii Keferstein, 1868

PK: Hylodes fitzingeri* Schmidt, 1857

KG: Craugastor* Cope, 1862

KF: BRACHYCEPHALIDAE 1858.gc.f002

Lepidobatrachus Budgett, $1899 \cdot \mathbf{K Y}$

ST: PO.KN • CI: h0793・ID: 171

PN: Lepidobatrachus asper Budgett, 1899

PK: Lepidobatrachus asper ${ }^{\circ}$ Budgett, 1899

KG: Lepidobatrachus ${ }^{3}$ Budgett, 1899

KF: CERATOPHRYIDAE 1838.ta.f002

Lepthyla: Duméril ${ }^{+1} 1841$ • AN

ST: AL • CI: n0071・ID: 235

PN: Litoria freycineti Tschudi, 1838

PK: Litoria freycineti* Tschudi, 1838

KG: Litoria* Tschudi, 1838

KF: PHYLLOMEDUSIDAE 1858.gc.f009

Leptobrachella Smith, 1925 • KY

ST: PO.KN • CI: h0794・ID: 018

PN: Leptobrachella mjobergi Smith, 1925

PK: Leptobrachella mjobergi ${ }^{\circ}$ Smith, 1925

KG: Leptobrachella ${ }^{\circ}$ Smith, 1925

KF: MEGOPHRYIDAE 1850.bb.f008-|1931.na.f003|

Leptobrachium Tschudi, 1838 • KY

ST: PO.KN • CI: h0795・ID: 015

PN: Leptobrachium hasseltii Tschudi, 1838

PK: Leptobrachium hasseltii* Tschudi, 1838

KG: Leptobrachium* Tschudi, 1838

KF: MEGOPHRYIDAE 1850.bb.f008-|1931.na.f003|

Leptodactylodon Andersson, 1903 • KY

ST: PO.KN • CI: h0796 • ID: 324

PN: Leptodactylodon ovatus Andersson, 1903

PK: Leptodactylodon ovatus ${ }^{\circ}$ Andersson, 1903
KG: Leptodactylodon ${ }^{3}$ Andersson, 1903

KF: ARTHROLEPTIDAE 1869.mc.f011

Leptodactylus Fitzinger, $1826 \cdot \mathbf{K Y}$

ST: PO.KN • CI: h0797・ ID: 253

PN: Rana typhonia Latreille in Sonnini ${ }^{+1}, 1801$

PK: Rana fusca* Schneider, 1799

KG: Leptodactylus ${ }^{1}$ Fitzinger, 1826

KF: LEPTODACTYLIDAE ||1838.ta.f001||-1896.wa.f001

Leptolalax Dubois, $1980 \bullet \mathbf{A K}$

ST: PO.JD・CI: h0798 • ID: 018

PN: Leptobrachium gracile Günther, 1872

PK: Leptobrachium gracile* Günther, 1872

KG: Leptobrachella ${ }^{\circ}$ Smith, 1925

KF: MEGOPHRYIDAE 1850.bb.f008-|1931.na.f003|

Leptomantis Peters, $1867 \cdot \mathbf{K Y}$

ST: PO.KN • CI: h0799・ID: 454

PN: Leptomantis bimaculata Peters, 1867

PK: Leptomantis bimaculata* Peters, 1867

KG: Leptomantis* Peters, 1867

KF: RHACOPHORIDAE ||1858.gc.f012||-1932.ha.f001

Leptoparius Peters, 1863 • AK

ST: PO.JD • CI: h0800 • ID: 350

PN: Stenorhynchus natalensis Smith, 1849

PK: Stenorhynchus natalensis* Smith, 1849

KG: Phrynobatrachus* Günther, 1862

KF: PHRYNOBATRACHIDAE 1941.lb.f001

Leptopelis Günther, 1859 • KY

ST: PO.KN • CI: h0801・ID: 325

PN: Hyla aubryi Duméril, 1856

PK: Hyla aubryi ${ }^{\circ}$ Duméril, 1856

KG: Leptopelis ${ }^{2}$ Günther, 1859

KF: ARTHROLEPTIDAE 1869.mc.f011

Leptophryne Fitzinger, 1843 • KY

ST: PO.KN • CI: h0802 • ID: 123

PN: Bufo cruentatus Tschudi, 1838

PK: Bufo cruentatus $^{\circ}$ Tschudi, 1838

KG: Leptophryne ${ }^{2}$ Fitzinger, 1843

KF: BUFONIDAE 1825.gb.f004

Leptopus Latreille, $1809 \cdot \mathbf{Z H}$

ST: zo • CI: zh052

Leptopus Mayer, 1835 • AK

ST: PO.JH • CI: h0803・ID: 012

PN: Pipa americana Laurenti,1768

PK: Rana pipa* Linnaeus, 1758

KG: Pipa ${ }^{1}$ Laurenti, 1768

KF: PIPIDAE 1825.gb.f003-|1826.fb.f002|

Leptosooglossus Van der Meijden ${ }^{+5}, 2007 \bullet \mathbf{A K}$

ST: PO.JI • CI: h0804 • ID: 032

PN: Nectophryne gardineri Boulenger, 1911

PK: Nectophryne gardineri* Boulenger, 1911

KG: Sechellophryne* Nussbaum ${ }^{+1}, 2007$

KF: SOOGLOSSIDAE 1931.na.f002

Leucostethus Grant ${ }^{+7}, 2017 \cdot \mathbf{K Y}$

ST: PO.KN • CI: h0805・ID: 041

PN: Leucostethus argyrogaster Morales ${ }^{+1}, 1993$

PK: Leucostethus argyrogaster* Morales ${ }^{+1}, 1993$ 
KG: Leucostethus* Morales $^{+1}, 1993$

KF: DENDROBATIDAE \|1850.bb.f006"|-1865.ca.f002

Leurognathus Moore, $1899 \cdot \mathbf{A K}$

ST: PO.JD • CI: h0806 • ID: 548

PN: Leurognathus marmorata Moore, 1899

PK: Leurognathus marmorata* Moore, 1899

KG: Desmognathus* Baird, 1850

KF: PLETHODONTIDAE 1850.ga.f002

Levirana Cope, $1894 \cdot \mathbf{A K}$

ST: PO.JD・CI: h0807・ID: 415

PN: Levirana vibicaria Cope, 1894

PK: Levirana vibicaria* Cope, 1894

KG: Lithobates* Fitzinger, 1843

KF: RANIDAE 1796.ba.f001

Liangshantriton $\mathrm{Fei}^{+1}, 2012 \cdot \mathbf{A K}$

ST: PO.JD • CI: h0808 • ID: 573

PN: Tylototriton taliangensis Liu, 1950

PK: Tylototriton taliangensis* Liu, 1950

KG: Tylototriton* Anderson, 1871

KF: SALAMANDRIDAE 1820.ga.f002

Liaobatrachus $\mathrm{Ji}^{+1}, 1998+\cdot \mathbf{K Y}$

ST: PO.KN • CI: h0809 • ID: $† 029$

PN: Liaobatrachus grabaui $\mathrm{Ji}^{+1}, 1998$ \$

PK: Liaobatrachus grabaui ${ }^{\circ} \mathrm{Ji}^{+1}, 1998 \uparrow$

KG: Liaobatrachus ${ }^{\circ} \mathrm{Ji}^{+1}, 1998 \dagger$

KF: ANURA Familia INCERTAE SEDIS

Liaoxitriton Dong ${ }^{+1}, 1998+\bullet \mathbf{K Y}$

ST: PO.KN • CI: h0810 •ID: $\uparrow 159$

PN: Liaoxitriton zhongjiani Dong $^{+1}, 1998$ \$

PK: Liaoxitriton zhongjiani ${ }^{\circ}$ Dong $^{+1}, 1998 \dagger$

KG: Liaoxitriton ${ }^{\circ}$ Dong $^{+1}, 1998 \dagger$

KF: IMPERFECTIBRANCHIA Familia INCERTAE SEDIS

Libycus Špinar, 1980 + $\mathbf{A K}$

ST: PO.JD • CI: h0811 • ID: 009

PN: Xenopus (Libycus) hasaunus Špinar, 1980 \$

PK: Xenopus (Libycus) hasaunus ${ }^{\circ}$ pinar, $1980 \dagger$

KG: Xenopus ${ }^{1}$ Wagler in Boie, 1827

KF: PIPIDAE 1825.gb.f003-|1826.fb.f002|

Lihyla: Cope $1887 \cdot \mathbf{A N}$

ST: $\mathbf{A M} \bullet \mathbf{C I}$ : n0072・ID: 059

PN: Leiyla guentherii Keferstein, 1868

PK: Hylodes fitzingeri* Schmidt, 1857

KG: Craugastor* Cope, 1862

KF: BRACHYCEPHALIDAE 1858.gc.f002

Lihyperus O'Shaughnessy, 1875 • AK

ST: NT.JD • CI: h0812・ID: 244

PN: Leiuperus marmoratus Duméril ${ }^{+1}, 1841$

PK: Leiuperus marmoratus* Duméril ${ }^{+1}, 1841$

KG: Pleurodema* Tschudi, 1838

KF: LEIUPERIDAE 1850.bb.f010

Limnaoedus Mittleman ${ }^{+1}, 1953$ • AK

ST: PO.JD • CI: h0813 • ID: 200

PN: Hylodes ocularis Holbrook, 1838

PK: Hylodes ocularis* Holbrook, 1838

KG: Pseudacris* Fitzinger, 1843

KF: HYLIDAE 1815.ra.f002-|1825.gb.f001|
Limnarches Gistel, 1848 • AK

ST: NL.JI • CI: h0814 • ID: 555

PN: Lacerta subviolacea Barton, 1804

PK: Lacerta maculata* Shaw, 1802

KG: Ambystoma ${ }^{1}$ Tschudi, 1838

KF: AMBYSTOMATIDAE 1850.ga.f004

Limnocharis Berthold, $1827 \cdot \mathbf{Z H}$

ST: zo • CI: zh053

Limnocharis Bell, 1843 • AK

ST: PO.JH・CI: h0815・ID: 181

PN: Limnocharis fuscus Bell, 1843

PK: Crossodactylus gaudichaudii ${ }^{\circ}$ Duméril $^{+1}, 1841$

KG: Crossodactylus ${ }^{3}$ Duméril $^{+1}, 1841$

KF: HyLODIDAE 1858.gc.f010

Limnodynastes Fitzinger, $1843 \cdot \mathbf{K Y}$

ST: PO.KN • CI: h0816・ID: 261

PN: Cystignathus peronii Duméril ${ }^{+1}, 1841$

PK: Cystignathus peronii* Duméril ${ }^{+1}, 1841$

KG: Limnodynastes* Fitzinger, 1843

KF: MYOBATRACHIDAE 1850.sa.f001

Limnodytes Duméril ${ }^{+1}, 1841$ • AK

ST: NT.JI • CI: h0817 • ID: 409

PN: Hyla erythraea Schlegel, 1827

PK: Hyla erythraea* Schlegel, 1827

KG: Hylarana* Tschudi, 1838

KF: RANIDAE 1796.ba.f001

Limnomedusa Fitzinger, 1843・ KY

ST: PO.KN • CI: h0818・ID: 183

PN: Cystignathus macroglossus Duméril ${ }^{+1}, 1841$

PK: Cystignathus macroglossus* Duméril ${ }^{+1}, 1841$

KG: Limnomedusa* Fitzinger, 1843

KF: LIMNOMEDUSIDAE 2017.daf46

Limnonectes Fitzinger, $1843 \cdot \mathbf{K Y}$

ST: PO.KN・CI: h0819・ID: 380

PN: Rana kuhlii Tschudi, 1838

PK: Rana kuhlii* Tschudi, 1838

KG: Limnonectes* Fitzinger, 1843

KF: DiCROGLOSSIDAE 1987.da.f004

Limnophilus Burmeister, $1839 \cdot \mathbf{Z H}$ ST: zo • CI: zh054

Limnophilus Fitzinger, 1843 • AK

ST: PO.JH • CI: h0820 • ID: 464

PN: Rana mascareniensis Duméril ${ }^{+1}, 1841$

PK: Rana mascareniensis* Duméril ${ }^{+1}, 1841$

KG: Ptychadena* Boulenger, 1917

KF: PTYCHADENIDAE 1987.da.f002

Limnophys Jiménez de la Espada, 1870 • AK

ST: PO.JD・CI: h0821・ID: 073

PN: Limnophys cornutus Jiménez de la Espada, 1870

PK: Limnophys cornutus ${ }^{\circ}$ Jiménez de la Espada, 1870

KG: Strabomantis* Peters, 1863

KF: BRACHYCEPHALIDAE 1858.gc.f002

Lineatriton Tanner, $1950 \bullet \mathbf{A K}$

ST: PO.JD • CI: h0822 • ID: 527

PN: Spelerpes lineola Cope, 1865

PK: Spelerpes lineola* Cope, 1865 
KG: Pseudoeurycea* Taylor, 1944

KF: PLETHODONTIDAE 1850.ga.f002

Linglongtriton $\mathrm{Jia}^{+1}, 2019+\bullet \mathbf{K Y}$

ST: PO.KN • CI: h0823・ID: $\uparrow 160$

PN: Linglongtriton daxishanensis $\mathrm{Jia}^{+1}, 2019+$

PK: Linglongtriton daxishanensis ${ }^{\circ} \mathrm{Jia}^{+1}, 2019 \dagger$

KG: Linglongtriton ${ }^{\circ} \mathrm{Jia}^{+1}, 2019 \dagger$

KF: IMPERFECTIBRANCHIA Familia INCERTAE SEDIS

Linguaelapsus Cope, $1887 \cdot \mathbf{A K}$

ST: PO.JD • CI: h0824 • ID: 555

PN: Ambystoma annulatum Cope, 1886

PK: Ambystoma annulatum* Cope, 1886

KG: Ambystoma ${ }^{1}$ Tschudi, 1838

KF: АмвYSTOMATIDAE 1850.ga.f004

Liohyla: Günther 1900 • AN

ST: $\mathbf{A M} \bullet \mathbf{C I}:$ n0073・ID: 059

PN: Leiyla guentherii Keferstein, 1868

PK: Hylodes fitzingeri* Schmidt, 1857

KG: Craugastor* Cope, 1862

KF: BRACHYCEPHALIDAE 1858.gc.f002

Liopelma: Cope $1865 \cdot \mathbf{A N}$

ST: $\mathbf{A M} \bullet \mathbf{C I}:$ n0074 • ID: 006

PN: Leiopelma hochstetteri Fitzinger, 1861

PK: Leiopelma hochstetteri* Fitzinger, 1861

KG: Leiopelma* Fitzinger, 1861

KF: LEIOPELMATIDAE 1869.mc.f07-|1942.ta.f001|

Liopelma Günther, 1869 • AK

ST: NC.JI • CI: h0825・ID: 006

PN: Leiopelma hochstetteri Fitzinger, 1861

PK: Leiopelma hochstetteri* Fitzinger, 1861

KG: Leiopelma* Fitzinger, 1861

KF: LEIOPELMATIDAE 1869.mc.f07-|1942.ta.f001|

Liophryne Boulenger, 1897 • AK

ST: PO.JD • CI: h0826・ID: 280

PN: Liophryne rhododactyla Boulenger, 1897

PK: Liophryne rhododactyla* Boulenger, 1897

KG: Asterophrys* Tschudi, 1838

KF: MICROHYLIDAE ||1843.fa.f012||-1931.na.f001

Lisapsus Steindachner, 1867 • AK

ST: NS.JD・CI: h0827 • ID: 196

PN: Lysapsus limellum Cope, 1862

PK: Lysapsus limellum* Cope, 1862

KG: Pseudis* Wagler, 1830

KF: HYLIDAE 1815.ra.f002-|1825.gb.f001|

Lisserpeton Estes, $1965+\cdot \mathbf{K Y}$

ST: PO.KN • CI: h0828・ID: $\uparrow 156$

PN: Lisserpeton bairdi Estes, 1965 †

PK: Lisserpeton bairdi ${ }^{\circ}$ Estes, $1965 \dagger$

KG: Lisserpeton ${ }^{\circ}$ Estes, $1965 \dagger$

KF: SCAPHERPETIDAE 1959.aa.f001 †

Lissotriton Bell, 1839 • KY

ST: PO.KN • CI: h0829・ID: 564

PN: Salamandra punctata Latreille, 1800

PK: Lacerta vulgaris* Linnaeus, 1758

KG: Lissotriton ${ }^{1}$ Bell, 1839

KF: SALAMANDRIDAE 1820.ga.f002
Lithobates Fitzinger, $1843 \cdot \mathbf{K Y}$

ST: PO.KN • CI: h0830 • ID: 415

PN: Rana palmipes Spix, 1824

PK: Rana palmipes* Spix, 1824

KG: Lithobates* Fitzinger, 1843

KF: RANIDAE 1796.ba.f001

Lithobatrachus Parker, $1929+\mathbf{A K}$

ST: PO.JD • CI: h0831 • ID: $\uparrow 069$

PN: Hyla europaea Noble, 1929 \$

PK: Rana diluviana ${ }^{\circ}$ Goldfuss, $1831 \dagger$

KG: Palaeobatrachus ${ }^{\circ}$ Tschudi, $1838 \dagger$

KF: PALAEOBATRACHIDAE 1865.ca.f001 †

Lithodytes Fitzinger, $1843 \cdot \mathbf{K Y}$

ST: PO.KN • CI: h0832 • ID: 252

PN: Rana lineata Schneider, 1799

PK: Rana lineata* Schneider, 1799

KG: Lithodytes* Fitzinger, 1843

KF: LEPTODACTYLIDAE ||1838.ta.f001||-1896.wa.f001

Litopleura Jiménez de la Espada, 1875 • AK

ST: PO.JD・CI: h0833・ID: 183

PN: Litopleura maritimum Jiménez de la Espada, 1875

PK: Cystignathus macroglossus* Duméril ${ }^{+1}, 1841$

KG: Limnomedusa* Fitzinger, 1843

KF: LIMNOMEDUSIDAE 2017.daf46

Litoria Tschudi, $1838 \cdot \mathbf{K Y}$

ST: PO.KN • CI: h0834 • ID: 235

PN: Litoria freycineti Tschudi, 1838

PK: Litoria freycineti* Tschudi, 1838

KG: Litoria* Tschudi, 1838

KF: PHYLLOMEDUSIDAE 1858.gc.f009

Littlejohnophryne Wells ${ }^{+1}, 1985$ • AK

ST: PO.JD • CI: h0835・ID: 270

PN: Crinia riparia Littlejohn $^{+1}, 1965$

PK: Crinia riparia* Littlejohn $^{+1}, 1965$

KG: Crinia* Tschudi, 1838

KF: MYOBATRACHIDAE 1850.sa.f001

Liua $\mathrm{Zhao}^{+1}, 1983 \cdot \mathbf{K Y}$

ST: PO.KN • CI: h0836 • ID: 510

PN: Hynobius wushanensis $\mathrm{Liu}^{+2}, 1960$

PK: Hynobius shihi* Liu, 1950

KG: Liua ${ }^{1}$ Zhao $^{+1}, 1983$

KF: HYNOBIIDAE ||1856.ha.f001||-1859.cb.f002

Liuhurana $\mathrm{Fei}^{+4}$ in $\mathrm{Fei}^{+2}, 2010$ - KY

ST: PO.KN • CI: h0837・ ID: 417

PN: Rana shuchinae Liu, 1950

PK: Rana shuchinae* Liu, 1950

KG: Liuhurana* $\mathrm{Fei}^{+4}$ in $\mathrm{Fei}^{+2}, 2010$

KF: RANIDAE 1796.ba.f001

Liuia Frost, 1985 • AK

ST: NS.JI • CI: h0838・ ID: 510

PN: Hynobius wushanensis $\mathrm{Liu}^{+2}, 1960$

PK: Hynobius shihi* Liu, 1950

KG: Liua ${ }^{1} \mathrm{Zhao}^{+1}, 1983$

KF: HYNOBIIDAE \|1856.ha.f001\|-1859.cb.f002

Liuixalus: $\mathrm{Li}^{+4} 2008 \cdot \mathbf{A N}$

ST: AL • CI: n0075・ID: 459 
PN: Philautus romeri Smith, 1953

PK: Philautus romeri* Smith, 1953

KG: Romerus* nov.

KF: RHACOPHORIDAE ||1858.gc.f012 |-1932.ha.f001

Liuophrys $\mathrm{Fei}^{+2}, 2016 \cdot \mathbf{A K}$

ST: PO.JD • CI: h0839 • ID: 025

PN: Megophrys glandulosa $\mathrm{Fei}^{+2}, 1990$

PK: Megophrys glandulosa ${ }^{\circ} \mathrm{Fei}^{+2}, 1990$

KG: Xenophrys ${ }^{\circ}$ Günther, 1864

KF: MEGOPHRYIDAE 1850.bb.f008-|1931.na.f003|

Liuperus Cope, $1861 \cdot \mathbf{A K}$

ST: NS.JD・CI: h0840・ID: 244

PN: Leiuperus marmoratus Duméril ${ }^{+1}, 1841$

PK: Leiuperus marmoratus* Duméril ${ }^{+1}, 1841$

KG: Pleurodema* Tschudi, 1838

KF: LEIUPERIDAE 1850.bb.f010

Liurana Dubois, $1987 \cdot \mathbf{K Y}$

ST: PO.KN • CI: h0841 • ID: 371

PN: Cornufer xizangensis $\mathrm{Hu}, 1977$

PK: Cornufer xizangensis ${ }^{\circ} \mathrm{Hu}, 1977$

KG: Liurana ${ }^{\circ}$ Dubois, 1987

KF: LIURANIDAE 2010.ma.f0010

Liventsovkia Ratnikov, $1993+\cdot \mathbf{K Y}$

ST: PO.KN • CI: h0842 • ID: $\uparrow 030$

PN: Liventsovkia jucunda Ratnikov, $1993+$

PK: Liventsovkia jucunda ${ }^{\circ}$ Ratnikov, $1993 \dagger$

KG: Liventsovkia ${ }^{\circ}$ Ratnikov, $1993 \dagger$

KF: ANURA Familia INCERTAE SEDIS

Liyla: Cope $1870 \bullet \mathbf{A N}$

ST: AM・CI: n0076・ID: 059

PN: Leiyla guentherii Keferstein, 1868

PK: Hylodes fitzingeri* Schmidt, 1857

KG: Craugastor* Cope, 1862

KF: BRACHYCEPHALIDAE 1858.gc.f002

Liyperus Agassiz, 1847 • AK

ST: NT.JD • CI: h0843 • ID: 246

PN: Leiuperus marmoratus Duméril ${ }^{+1}, 1841$

PK: Leiuperus marmoratus* Duméril ${ }^{+1}, 1841$

KG: Pleurodema* Tschudi, 1838

KF: LEIUPERIDAE 1850.bb.f010

Llankibatrachus Báez ${ }^{+1}, 2003$ † KY

ST: PO.KN • CI: h0844 • ID: $\uparrow 073$

PN: Llankibatrachus truebae $\mathrm{Báez}^{+1}, 2003$ \$

PK: Llankibatrachus truebae ${ }^{\circ} \mathrm{Báez}^{+1}, 2003 \uparrow$

KG: Llankibatrachus ${ }^{\circ} \mathrm{Báez}^{+1}, 2003$ †

KF: PIPIDAE 1825.gb.f003-|1826.fb.f002|

Llewellynura Wells $^{+1}, 1985$ • AK

ST: PO.JD・CI: h0845・ID: 235

PN: Hyla dorsalis microbelos Cogger, 1966

PK: Hyla dorsalis microbelos* Cogger, 1966

KG: Litoria* Tschudi, 1838

KF: PHYLLOMEDUSIDAE 1858.gc.f009

Lobipes Cuvier, $1817 \bullet \mathbf{Z H}$

ST: zo • CI: zh055

Lobipes Fitzinger, 1843 • AK

ST: PO.JH・CI: h0846・ID: 189
PN: Hyla palmata Bonnaterre, 1789

PK: Rana boans* Linnaeus, 1758

KG: Boana* Gray, 1825

KF: HYLIDAE 1815.ra.f002-|1825.gb.f001|

Lophinus: Rafinesque 1815 • AN

ST: AL • CI: n0077 • ID: 564

PN: Salamandra punctata Latreille, 1800

PK: Lacerta vulgaris* Linnaeus, 1758

KG: Lissotriton ${ }^{1}$ Bell, 1839

KF: SALAMANDRIDAE 1820.ga.f002

Lophinus Gray, 1850 • AK

ST: PO.JI • CI: h0847・ ID: 564

PN: Salamandra punctata Latreille, 1800

PK: Lacerta vulgaris* Linnaeus, 1758

KG: Lissotriton ${ }^{1}$ Bell, 1839

KF: SALAMANDRIDAE 1820.ga.f002

Lophiohyla Miranda-Ribeiro, 1926 • AK

ST: NS.JD・CI: h0848・ID: 221

PN: Lophyohyla piperata Miranda-Ribeiro, 1923

PK: Hyla luteola* Wied-Neuwied, 1824

KG: Phyllodytes* Wagler, 1830

KF: HYLIDAE 1815.ra.f002-|1825.gb.f001|

Lophopus Dumortier, $1835 \cdot \mathbf{Z H}$

ST: zo • CI: zh056

Lophopus Tschudi, 1838 • AK

ST: PO.JH • CI: h0849 • ID: 194

PN: Bufo marmoratus Laurenti, 1768

PK: Bufo marmoratus* Laurenti, 1768

KG: Dendropsophus ${ }^{1}$ Fitzinger, 1843

KF: HYLIDAE 1815.ra.f002-|1825.gb.f001|

Lophyohila: Miranda-Ribeiro 1923 • AN

ST: LI • CI: n0078・ID: 221

PN: Lophyohyla piperata Miranda-Ribeiro, 1923

PK: Hyla luteola* Wied-Neuwied, 1824

KG: Phyllodytes* Wagler, 1830

KF: HYLIDAE 1815.ra.f002-|1825.gb.f001|

Lophyohyla Miranda-Ribeiro, 1923 • AK

ST: LC.JD • CI: h0850 • ID: 221

PN: Lophyohyla piperata Miranda-Ribeiro, 1923

PK: Hyla luteola* Wied-Neuwied, 1824

KG: Phyllodytes* Wagler, 1830

KF: HYLIDAE 1815.ra.f002-|1825.gb.f001|

Luetkenotyphlus Taylor, $1968 \bullet \mathbf{K Y}$

ST: PO.KN • CI: h0851・ID: 493

PN: Siphonops brasiliensis Lütken, 1852

PK: Siphonops brasiliensis* Lütken, 1852

KG: Luetkenotyphlus* Taylor, 1968

KF: CAECILIIDAE 1814.ra.f003-|1825.gb.f008|

Lupacolus Brown ${ }^{+4}, 2015$ • AK

ST: PO.JD・ CI: h0852 • ID: 370

PN: Cornufer dorsalis Duméril, 1853

PK: Cornufer dorsalis* Duméril, 1853

KG: Platymantis ${ }^{1}$ Günther, 1859

KF: CERATOBATRACHIDAE 1884.ba.f001

Lutetiobatrachus Wuttke, $1998+\bullet \mathbf{K Y}$

ST: PO.KN • CI: h0853・ID: $\uparrow 031$ 
PN: Lutetiobatrachus gracilis Wuttke, 1988 \$

PK: Lutetiobatrachus gracilis ${ }^{\circ}$ Wuttke, $1988 \dagger$

KG: Lutetiobatrachus ${ }^{\circ}$ Wuttke, $1998 \dagger$

KF: ANURA Familia INCERTAE SEDIS

Lutkenotyphlus Nussbaum, 1986 • AK

ST: NT.JI • CI: h0854 • ID: 493

PN: Siphonops brasiliensis Lütken, 1852

PK: Siphonops brasiliensis* Lütken, 1852

KG: Luetkenotyphlus* Taylor, 1968

KF: CAECILIIDAE 1814.ra.f003-|1825.gb.f008|

Lyciasalamandra Veith $^{+1}, 2004 \cdot \mathbf{K Y}$

ST: PO.KN • CI: h0855 • ID: 577

PN: Molge luschani Steindachner, 1891

PK: Molge luschani* Steindachner, 1891

KG: Lyciasalamandra* Veith $^{+1}, 2004$

KF: SALAMANDRIDAE 1820.ga.f002

Lynchius Hedges ${ }^{+2}, 2008 \cdot \mathbf{K Y}$

ST: PO.KN・CI: h0856・ID: 075

PN: Phrynopus parkeri Lynch, 1975

PK: Phrynopus parkeri* Lynch, 1975

KG: Lynchius* Hedges ${ }^{+2}, 2008$

KF: BRACHYCEPHALIDAE 1858.gc.f002

Lynchophrys Laurent, 1983 • AK

ST: PO.JD • CI: h0857 • ID: 186

PN: Batrachophrynus brachydactylus Peters, 1873

PK: Batrachophrynus brachydactylus ${ }^{\circ}$ Peters, 1873

KG: Telmatobius ${ }^{3}$ Wiegmann, 1834

KF: TELMATOBIIDAE 1843.fa.f006

Lysapsus Cope, 1862 • AK

ST: PO.JD・CI: h0858 • ID: 196

PN: Lysapsus limellum Cope, 1862

PK: Lysapsus limellum* Cope, 1862

KG: Pseudis* Wagler, 1830

KF: HYLIDAE 1815.ra.f002-|1825.gb.f001|

Lysapus Hoffmann, 1878 • AK

ST: NS.JD • CI: h0859 • ID: 196

PN: Lysapsus limellum Cope, 1862

PK: Lysapsus limellum* Cope, 1862

KG: Pseudis* Wagler, 1830

KF: HYLIDAE 1815.ra.f002-|1825.gb.f001|

Lystris Cope, $1869 \bullet \mathbf{A K}$

ST: PO.JD • CI: h0860 • ID: 246

PN: Lystris brachyops Cope, 1869

PK: Lystris brachyops* Cope, 1869

KG: Pleurodema* Tschudi, 1838

KF: LEIUPERIDAE 1850.bb.f010

Macrogenioglottus Carvalho, $1946 \bullet \mathbf{K Y}$

ST: PO.KN • CI: h0861・ID: 152

PN: Macrogenioglottus alipioi Carvalho, 1946

PK: Macrogenioglottus alipioi* Carvalho, 1946

KG: Macrogenioglottus* Carvalho, 1946

KF: ODONTOPHRYNIDAE 1971.la.f002

Macropelobates Noble, $1924+\cdot \mathbf{K Y}$

ST: PO.KN • CI: h0862 • ID: $\uparrow 086$

PN: Macropelobates osborni Noble, 1924 †

PK: Macropelobates osborni ${ }^{\circ}$ Noble, $1924 \dagger$
KG: Macropelobates $^{\circ}$ Noble, $1924 \dagger$

KF: ArChaeosalientia Familia INCERTAE SEDIS

Macrothaelacion Wagler in Michahelles, 1833 • AK

ST: PO.JD • CI: h0863・ID: 138

PN: Bufo nasutus Schneider, 1799

PK: Rana margaritifera* Laurenti, 1768

KG: Rhinella ${ }^{2}$ Fitzinger, 1826

KF: BUFONIDAE 1825.gb.f004

Maculopaa $\mathrm{Fei}^{+2}, 2010 \bullet \mathbf{A K}$

ST: PO.JD • CI: h0864・ID: 388

PN: Rana maculosa $\mathrm{Liu}^{+2}, 1960$

PK: Rana maculosa* $\mathrm{Liu}^{+2}, 1960$

KG: Paa* Dubois, 1975

KF: DiCROGLOSSIDAE 1987.da.f004

Madecassophryne Guibé, 1974 • KY

ST: PO.KN • CI: h0865・ID: 284

PN: Madecassophryne truebae Guibé, 1974

PK: Madecassophryne truebae ${ }^{\circ}$ Guibé, 1974

KG: Madecassophryne ${ }^{\circ}$ Guibé, 1974

KF: MICROHYLIDAE ||1843.fa.f012||-1931.na.f001

Magaelosia: Miranda-Ribeiro 1923 • AN

ST: $\mathbf{L I} \cdot \mathbf{C I}$ : n0079・ID: 182

PN: Helosia bufonium Girard, 1853

PK: Hyla nasus* Lichtenstein, 1823

KG: Hylodes ${ }^{1}$ Fitzinger, 1826

KF: HYLODIDAE 1858.gc.f010

Magnadigita Taylor, $1944 \cdot \mathbf{A K}$

ST: PO.JD • CI: h0866・ ID: 522

PN: Bolitoglossa nigroflavescens Taylor, 1941

PK: Oedipus franklini* Schmidt, 1936

KG: Bolitoglossa* Duméril ${ }^{+2}, 1854$

KF: PLETHODONTIDAE 1850.ga.f002

Mahonabatrachus Wells ${ }^{+1}, 1985$ • AK

ST: PO.JD • CI: h0867 • ID: 235

PN: Hyla meiriana Tyler, 1969

PK: Hyla meiriana* Tyler, 1969

KG: Litoria* Tschudi, 1838

KF: PHYLLOMEDUSIDAE 1858.gc.f009

Maitsomantis Glaw ${ }^{+1}, 2006 \cdot \mathbf{A K}$

ST: PO.JD • CI: h0868・ID: 432

PN: Mantidactylus argenteus Methuen, 1920

PK: Mantidactylus argenteus* Methuen, 1920

KG: Mantidactylus* Boulenger, 1895

KF: RHACOPHORIDAE ||1858.gc.f012||-1932.ha.f001

Makihynobius $\mathrm{Fei}^{+2}, 2012 \cdot \mathbf{A K}$

ST: PO.JD・CI: h0869・ID: 507

PN: Salamandrella sonani Maki, 1922

PK: Salamandrella sonani* Maki, 1922

KG: Poyarius* Dubois ${ }^{+1}, 2012$

KF: HYNOBIIDAE ||1856.ha.f001\|-1859.cb.f002

Malachylodes Cope, $1879 \bullet \mathbf{A K}$

ST: PO.JD • CI: h0870 • ID: 082

PN: Malachylodes guttilatus Cope, 1879

PK: Malachylodes guttilatus ${ }^{\circ}$ Cope, 1879

KG: Euhyas* Fitzinger, 1843

KF: BRACHYCEPHALIDAE 1858.gc.f002 
Maltzania Boettger, $1881 \cdot \mathbf{A K}$

ST: PO.JD • CI: h0871 • ID: 367

PN: Maltzania bufonia Boettger, 1881

PK: Pyxicephalus edulis* Peters, 1854

KG: Pyxicephalus* Tschudi, 1838

KF: PYXICEPHALIDAE 1850.bb.f005

Manculus Cope, $1869 \cdot \mathbf{A K}$

ST: PO.JD • CI: h0872 • ID: 542

PN: Salamandra quadridigitata Holbrook, 1842

PK: Salamandra quadridigitata* Holbrook, 1842

KG: Eurycea* Rafinesque, 1822

KF: PLETHODONTIDAE 1850.ga.f002

Mannophryne La Marca, $1992 \cdot \mathbf{K Y}$

ST: PO.KN • CI: h0873・ID: 038

PN: Colostethus yustizi La Marca, 1989

PK: Colostethus yustizi* La Marca, 1989

KG: Mannophryne* La Marca, 1992

KF: AROMOBATIDAE 2006.gc.f004

Mantella Boulenger, $1882 \cdot \mathbf{K Y}$

ST: PO.KN • CI: h0874 • ID: 428

PN: Dendrobates betsileo Grandidier, 1872

PK: Dendrobates betsileo* Grandidier, 1872

KG: Mantella* Boulenger, 1882

KF: RHACOPHORIDAE |1858.gc.f012||-1932.ha.f001

Mantidactylus Boulenger, $1895 \cdot \mathbf{K Y}$

ST: PO.KN • CI: h0875 • ID: 432

PN: Rana guttulata Boulenger, 1881

PK: Rana guttulata* Boulenger, 1881

KG: Mantidactylus* Boulenger, 1895

KF: RHACOPHORIDAE |1858.gc.f012||-1932.ha.f001

Mantiphrys Mocquard, 1895 • AK

ST: PO.JD・CI: h0876・ID: 289

PN: Mantiphrys laevipes Mocquard, 1895

PK: Mantiphrys laevipes* Mocquard, 1895

KG: Rhombophryne* Boettger, 1880

KF: MICROHYLIDAE ||1843.fa.f012||-1931.na.f001

Mantipus Peters, $1883 \cdot \mathbf{K Y}$

ST: PO.KN • CI: h0877•ID: 287

PN: Mantipus hildebrandti Peters, 1883

PK: Plethodontohyla inguinalis* Boulenger, 1882

KG: Mantipus $^{1}$ Peters, 1883

KF: MICROHYLIDAE ||1843.fa.f012||-1931.na.f001

Mantophryne Boulenger, 1897 • AK

ST: PO.JD • CI: h0878 • ID: 280

PN: Mantophryne lateralis Boulenger, 1897

PK: Mantophryne lateralis* Boulenger, 1897

KG: Asterophrys* Tschudi, 1838

KF: MICROHYLIDAE ||1843.fa.f012||-1931.na.f001

Mantophrys Mocquard, $1909 \cdot \mathbf{A K}$

ST: NT.JD・CI: h0879・ ID: 289

PN: Mantiphrys laevipes Mocquard, 1895

PK: Mantiphrys laevipes* Mocquard, 1895

KG: Rhombophryne* Boettger, 1880

KF: MICROHYLIDAE ||1843.fa.f012||-1931.na.f001

Marmorerpeton Evans ${ }^{+2}, 1988+\cdot \mathbf{K Y}$

ST: PO.KN • CI: h0880 •ID: $\uparrow 137$
PN: Marmorerpeton kermacki Evans $^{+2}, 1988$ \$

PK: Marmorerpeton kermacki $i^{\circ}$ Evans $^{+2}, 1988 \dagger$

KG: Marmorerpeton $^{\circ}$ Evans $^{+2}, 1988 \dagger$

KF: Urodela Familia INCERTAE SEDIS

Matsuirana $\mathrm{Fei}^{+2}, 2010 \bullet \mathbf{A K}$

ST: PO.JD • CI: h0881 • ID: 412

PN: Rana ishikawae Stejneger, 1901

PK: Rana ishikawae* Stejneger, 1901

KG: Odorrana $* \mathrm{Fei}^{+2}, 1990$

KF: RANIDAE 1796.ba.f001

Mayamandra Parra-Olea ${ }^{+2}, 2004 \cdot \mathbf{A K}$

ST: PO.JD • CI: h0882 • ID: 522

PN: Bolitoglossa hartwegi Wake ${ }^{+1}, 1969$

PK: Bolitoglossa hartwegi* Wake $^{+1}, 1969$

KG: Bolitoglossa* Duméril ${ }^{+2}, 1854$

KF: PLETHODONTIDAE 1850.ga.f002

Meantes: Rafinesque $1822 \cdot \mathbf{A N}$

ST: $\mathbf{A L} ・ \mathbf{C I}:$ n0080・ID: 519

PN: Siren lacertina Österdam, 1766

PK: Siren lacertina* Österdam, 1766

KG: Siren* Österdam, 1766

KF: SIRENIDAE 1825gb.f005

Megaelosia Miranda-Ribeiro, 1923 • AK

ST: LC.JD • CI: h0883 • ID: 182

PN: Helosia bufonium Girard, 1853

PK: Hyla nasus* Lichtenstein, 1823

KG: Hylodes ${ }^{1}$ Fitzinger, 1826

KF: HYLODIDAE 1858.gc.f010

Megalixalus Günther, 1869 • AK

ST: PO.JD・CI: h0884・ID: 336

PN: Megalixalus infrarufus Günther, 1869

PK: Eucnemis seychellensis* Tschudi, 1838

KG: Tachycnemis* Fitzinger, 1843

KF: HYPEROLIIDAE 1943.lb.f001

Megalobatrachus Tschudi, $1837+\cdot \mathbf{A K}$

ST: PO.JD • CI: h0885 • ID: 503

PN: Megalobatrachus sieboldi Tschudi, 1837 *

PK: Triton japonicus* Temminck, 1836

KG: Andrias ${ }^{2}$ Tschudi, 1837

KF: CRYPTOBRANCHIDAE 1826.fb.f003

Megalofrys Palacký, 1898 • AK

ST: NT.JI • CI: h0886・ ID: 021

PN: Megophrys montana $\mathrm{Kuhl}^{+1}, 1822$

PK: Megophrys montana ${ }^{\circ} \mathrm{Kuhl}^{+1}, 1822$

KG: Megophrys ${ }^{2} \mathrm{Kuhl}^{+1}, 1822$

KF: MEGOPHRYIDAE 1850.bb.f008-|1931.na.f003|

Megalophrys Wagler, 1830 • AK

ST: NS.JI • CI: h0887・ID: 021

PN: Megophrys montana $\mathrm{Kuhl}^{+1}, 1822$

PK: Megophrys montana ${ }^{\circ} \mathrm{Kuhl}^{+1}, 1822$

KG: Megophrys ${ }^{2} \mathrm{Kuhl}^{+1}, 1822$

KF: MEGOPHRYIDAE 1850.bb.f008-|1931.na.f003|

Megalophys: Gray 1842 • AN

ST: AM • CI: n0081 • ID: 021

PN: Megophrys montana $\mathrm{Kuhl}^{+1}, 1822$

PK: Megophrys montana ${ }^{\circ} \mathrm{Kuhl}^{+1}, 1822$ 
KG: Megophrys ${ }^{2} \mathrm{Kuhl}^{+1}, 1822$

KF: MEGOPHRYIDAE 1850.bb.f008-|1931.na.f003|

Megalotriton Zittel, $1890+\cdot \mathbf{K Y}$

ST: PO.KN • CI: h0888 •ID: $\uparrow 199$

PN: Megalotriton filholi Zittel, 1890 +

PK: Megalotriton filholi ${ }^{\circ}$ Zittel, $1890 \dagger$

KG: Megalotriton ${ }^{\circ}$ Zittel, $1890 \dagger$

KF: SALAMANDRIDAE 1820.ga.f002

Megapterna Savi, $1839 \cdot \mathbf{A K}$

ST: PO.JD • CI: h0889・ID: 557

PN: Megapterna montana Savi, 1839

PK: Megapterna montana* Savi, 1839

KG: Euproctus ${ }^{1}$ Gené, 1839

KF: SALAMANDRIDAE 1820.ga.f002

Megastomatohyla Faivovich ${ }^{+5}, 2005 \cdot \mathbf{K Y}$

ST: PO.KN • CI: h0890 • ID: 202

PN: Hyla mixe Duellman, 1965

PK: Hyla mixe* Duellman, 1965

KG: Megastomatohyla* Faivovich ${ }^{+5}, 2005$

KF: HYLIDAE 1815.ra.f002-|1825.gb.f001|

Megistolotis Tyler ${ }^{+2}, 1979 \cdot \mathbf{A K}$

ST: PO.JD • CI: h0891 • ID: 261

PN: Megistolotis lignarius Tyler ${ }^{+2}, 1979$

PK: Megistolotis lignarius* Tyler ${ }^{+2}, 1979$

KG: Limnodynastes* Fitzinger, 1843

KF: MYOBATRACHIDAE 1850.sa.f001

Megophrys Kuhl ${ }^{+1}, 1822 \bullet \mathbf{K Y}$

ST: LC.KN • CI: h0892 • ID: 021

PN: Megophrys montana $\mathrm{Kuhl}^{+1}, 1822$

PK: Megophrys montana ${ }^{\circ} \mathrm{Kuhl}^{+1}, 1822$

KG: Megophrys ${ }^{2} \mathrm{Kuhl}^{+1}, 1822$

KF: MEGOPHRYIDAE 1850.bb.f008-|1931.na.f003|

Mehelyia Wandolleck, 1911 • AK

ST: PO.JD • CI: h0893 • ID: 280

PN: Mehelyia lineata Wandolleck, 1911

PK: Sphenophryne biroi ${ }^{\circ}$ Méhelÿ, 1897

KG: Asterophrys* Tschudi, 1838

KF: MICROHYLIDAE ||1843.fa.f012||-1931.na.f001

Meinus: Rafinesque $1815 \cdot \mathbf{A N}$

ST: AL・CI: n0082・ID: 564

PN: Pelonectes boscai Lataste in Blanchard, 1879

PK: Pelonectes boscai* Lataste in Blanchard, 1879

KG: Lissotriton ${ }^{1}$ Bell, 1839

KF: SALAMANDRIDAE 1820.ga.f002

Meinus Dubois ${ }^{+1}, 2009 \cdot \mathbf{A K}$

ST: PO.JD・ CI: h0894 • ID: 564

PN: Pelonectes boscai Lataste in Blanchard, 1879

PK: Pelonectes boscai* Lataste in Blanchard, 1879

KG: Lissotriton ${ }^{1}$ Bell, 1839

KF: SALAMANDRIDAE 1820.ga.f002

Melanobatrachus Beddome, 1878 • KY

ST: PO.KN • CI: h0895 • ID: 306

PN: Melanobatrachus indicus Beddome, 1878

PK: Melanobatrachus indicus* Beddome, 1878

KG: Melanobatrachus* Beddome, 1878

KF: MICROHYLIDAE |1843.fa.f012||-1931.na.f001
Melanophryne $\mathrm{Lehr}^{+1}, 2007 \cdot \mathbf{A K}$

ST: PO.JD・CI: h0896・ID: 293

PN: Phrynopus carpish Lehr $^{+2}, 2002$

PK: Phrynopus carpish $^{\circ}$ Lehr $^{+2}, 2002$

KG: Ctenophryne* Mocquard, 1904

KF: MICROHYLIDAE ||1843.fa.f012||-1931.na.f001

Melanophryniscus Gallardo, 1961 • KY

ST: PO.KN • CI: h0897・ID: 151

PN: Phryniscus stelzneri Weyenbergh, 1875

PK: Phryniscus stelzneri* Weyenbergh, 1875

KG: Melanophryniscus* Gallardo, 1961

KF: BUFONIDAE 1825.gb.f004

Mengbatrachus $\operatorname{Tan}^{+3}, 2018+\cdot \mathbf{K Y}$

ST: PO.KN • CI: h0898 • ID: $\uparrow 032$

PN: Mengbatrachus moqi $\mathrm{Tan}^{+3}, 2018$ †

PK: Mengbatrachus moqi ${ }^{\circ} \operatorname{Tan}^{+3}, 2018 \dagger$

KG: Mengbatrachus ${ }^{\circ} \operatorname{Tan}^{+3}, 2018 \dagger$

KF: ANURA Familia INCERTAE SEDIS

Menobranchus Harlan, 1825 • AK

ST: PO.JD・CI: h0899・ID: 553

PN: Triton lateralis Say in James, 1822

PK: Sirena maculosa* Rafinesque, 1818

KG: Necturus* Rafinesque, 1819

KF: PRoteIDAE 1831.ba.f002

Menopoma Harlan, 1825 • AK

ST: PO.JD • CI: h0900 • ID: 504

PN: Salamandra alleganiensis Sonnini ${ }^{+1}, 1801$

PK: Salamandra alleganiensis* Sonnini ${ }^{+1}, 1801$

KG: Cryptobranchus ${ }^{1}$ Leuckart, 1821

KF: CRYPTOBRANCHIDAE 1826.fb.f003

Mercurana Abraham ${ }^{+4}, 2013 \cdot \mathbf{K Y}$

ST: PO.KN・CI: h0901 •ID: 443

PN: Mercurana myristicapalustris Abraham ${ }^{+4}, 2013$

PK: Mercurana myristicapalustris* ${ }^{*}$ braham $^{+4}, 2013$

KG: Mercurana* Abraham ${ }^{+4}, 2013$

KF: RHACOPHORIDAE |1858.gc.f012||-1932.ha.f001

Meristogenys Yang, 1991 • KY

ST: PO.KN • CI: h0902 • ID: 403

PN: Hylarana jerboa Günther, 1872

PK: Hylarana jerboa* Günther, 1872

KG: Meristogenys* Yang, 1991

KF: RANIDAE 1796.ba.f001

Merothaelacium Wagler in Michahelles, 1833 • AK

ST: PO.JD・CI: h0903・ID: 138

PN: Rana margaritifera Laurenti, 1768

PK: Rana margaritifera* Laurenti, 1768

KG: Rhinella ${ }^{2}$ Fitzinger, 1826

KF: BUFONIDAE 1825.gb.f004

Mertensiella Wolterstorff, $1925 \cdot \mathbf{K Y}$

ST: PO.KN • CI: h0904 • ID: 576

PN: Exaeretus caucasicus Waga, 1876

PK: Exaeretus caucasicus* Waga, 1876

KG: Mertensiella* Wolterstorff, 1925

KF: SALAMANDRIDAE 1820.ga.f002

Mertensophryne Tihen, 1960 • KY

ST: PO.KN • CI: h0905・ID: 141 
PN: Bufo micranotis rondoensis Loveridge, 1942

PK: Bufo micranotis* Loveridge, 1925

KG: Mertensophryne ${ }^{1}$ Tihen, 1960

KF: BUFONIDAE 1825.gb.f004

Mesophryne $\mathrm{GaO}^{+1}, 2001+\cdot \mathbf{K Y}$

ST: PO.KN • CI: h0906 • ID: $\uparrow 033$

PN: Mesophryne beipiaoensis $\mathrm{Gao}^{+1}, 2001$ \$

PK: Mesophryne beipiaoensis ${ }^{\circ} \mathrm{Gao}^{+1}, 2001 \dagger$

KG: Mesophryne ${ }^{\circ} \mathrm{Gao}^{+1}, 2001 \dagger$

KF: ANURA Familia INCERTAE SEDIS

Mesotriton Bolkay, $1927 \bullet \mathbf{A K}$

ST: PO.JD • CI: h0907 • ID: 563

PN: Triton alpestris Laurenti, 1768

PK: Triton alpestris* Laurenti, 1768

KG: Ichthyosaura ${ }^{1}$ Sonnini $^{+1}, 1801$

KF: SALAMANDRIDAE 1820.ga.f002

Mesotriton Bourret, 1934 • AK

ST: PO.JH • CI: h0908 • ID: 562

PN: Mesotriton deloustali Bourret, 1934

PK: Mesotriton deloustali* Bourret, 1934

KG: Paramesotriton* Chang, 1936

KF: SALAMANDRIDAE 1820.ga.f002

Messelobatrachus Wuttke, 1988 + AK

ST: PO.JD • CI: h0909・ ID: $\uparrow 069$

PN: Messelobatrachus tobieni Wuttke, 1988 *

PK: Messelobatrachus tobieni ${ }^{\circ}$ Wuttke, $1988 \uparrow$

KG: Palaeobatrachus ${ }^{\circ}$ Tschudi, $1838 \dagger$

KF: PALAEOBATRACHIDAE 1865.ca.f001 †

Metacrinia Parker, 1940 • KY

ST: PO.KN • CI: h0910 • ID: 272

PN: Pseudophryne nichollsi Harrison, 1927

PK: Pseudophryne nichollsi* Harrison, 1927

KG: Metacrinia* Parker, 1940

KF: MYOBATRACHIDAE 1850.sa.f001

Metaeus Girard, 1853 - AK

ST: PO.JD • CI: h0911 • ID: 246

PN: Metaeus timidus Girard, 1853

PK: Metaeus timidus ${ }^{\circ}$ Girard, 1853

KG: Pleurodema* Tschudi, 1838

KF: LEIUPERIDAE 1850.bb.f010

Metamagnusia Günther, 2009 • AK

ST: PO.JD・CI: h0912・ID: 280

PN: Metamagnusia marani Günther, 2009

PK: Metamagnusia marani* Günther, 2009

KG: Asterophrys* Tschudi, 1838

KF: MICROHYLIDAE ||1843.fa.f012||-1931.na.f001

Metaphrynella Parker, $1934 \cdot \mathbf{K Y}$

ST: PO.KN • CI: h0913・ID: 311

PN: Phrynella pollicaris Boulenger, 1890

PK: Phrynella pollicaris* Boulenger, 1890

KG: Metaphrynella* Parker, 1934

KF: MICROHYLIDAE ||1843.fa.f012||-1931.na.f001

Metaphryniscus Señaris ${ }^{+2}, 1994$ • KY

ST: PO.KN • CI: h0914 • ID: 098

PN: Metaphryniscus sosai Señaris ${ }^{+2}, 1994$

PK: Metaphryniscus sosai ${ }^{\circ}$ Señaris $^{+2}, 1994$
KG: Metaphryniscus ${ }^{\circ}$ Señaris $^{+2}, 1994$

KF: BUFONIDAE 1825.gb.f004

Metopostira Méhelÿ, 1901 • AK

ST: PO.JD • CI: h0915・ID: 280

PN: Metopostira ocellata Méhelÿ, 1901

PK: Hylophorbus rufescens* Macleay, 1878

KG: Asterophrys* Tschudi, 1838

KF: MICROHYLIDAE ||1843.fa.f012||-1931.na.f001

Micrarthroleptis Deckert, 1938 • AK

ST: PO.JD・CI: h0916・ID: 350

PN: Arthroleptis pygmaeus Ahl, 1925

PK: Arthroleptis pygmaeus ${ }^{\circ}$ Ahl, 1925

KG: Phrynobatrachus* Günther, 1862

KF: Phrynobatrachidae 1941.lb.f001

Micrhyla Duméril $^{+1}, 1841$ • AK

ST: NS.JI • CI: h0917 • ID: 314

PN: Microhyla achatina Tschudi, 1838

PK: Microhyla achatina* Tschudi, 1838

KG: Microhyla* Tschudi, 1838

KF: MICROHYLIDAE ||1843.fa.f012||-1931.na.f001

Micrixalus Boulenger, 1888 • KY

ST: PO.KN • CI: h0918 • ID: 353

PN: Ixalus fuscus Boulenger, 1882

PK: Ixalus fuscus* Boulenger, 1882

KG: Micrixalus* Boulenger, 1888

KF: MICRIXALIDAE 2001.db.f001

Microbatrachella Hewitt, 1926• KY

ST: PO.KN • CI: h0919 • ID: 358

PN: Phrynobatrachus capensis Boulenger, 1910

PK: Phrynobatrachus capensis* Boulenger, 1910

KG: Microbatrachella* Hewitt, 1926

KF: CACOSTERNIDAE 1931.na.f008

Microbatrachus Roux, 1910 • AK

ST: PO.JD • CI: h0920 • ID: 280

PN: Microbatrachus pusillus Roux, 1910

PK: Microbatrachus pusillus ${ }^{\circ}$ Roux, 1910

KG: Asterophrys* Tschudi, 1838

KF: MICROHYLIDAE ||1843.fa.f012||-1931.na.f001

Microbatrachus Hewitt, 1926 • AK

ST: PO.JH • CI: h0921 • ID: 358

PN: Phrynobatrachus capensis Boulenger, 1910

PK: Phrynobatrachus capensis* Boulenger, 1910

KG: Microbatrachella* Hewitt, 1926

KF: CACOSTERNIDAE 1931.na.f008

Microbatrachylus Taylor, $1939 \bullet \mathbf{A K}$

ST: PO.JD・CI: h0922・ID: 059

PN: Eleutherodactylus hobartsmithi Taylor, 1936

PK: Eleutherodactylus hobartsmithi ${ }^{\circ}$ Taylor, 1936

KG: Craugastor* Cope, 1862

KF: BRACHYCEPHALIDAE 1858.gc.f002

Microcaecilia Taylor, $1968 \bullet \mathbf{K Y}$

ST: PO.KN • CI: h0923・ID: 492

PN: Dermophis albiceps Boulenger, 1882

PK: Dermophis albiceps ${ }^{\circ}$ Boulenger, 1882

KG: Microcaecilia ${ }^{3}$ Taylor, 1968

KF: CAECILIIDAE 1814.ra.f003-|1825.gb.f008| 
Microdiscopus Peters, 1877 • AK

ST: PO.JD • CI: h0924 • ID: 397

PN: Microdiscopus sumatranus Peters, 1877

PK: Microdiscopus sumatranus ${ }^{\circ}$ Peters, 1877

KG: Phrynoglossus* Peters, 1867

KF: OCCIDOZYGIDAE 1990.fa.f002

Microhyla Tschudi, $1838 \cdot \mathbf{K Y}$

ST: PO.KN • CI: h0925 • ID: 314

PN: Microhyla achatina Tschudi, 1838

PK: Microhyla achatina* Tschudi, 1838

KG: Microhyla* Tschudi, 1838

KF: MICROHYLIDAE ||1843.fa.f012||-1931.na.f001

Microkayla Riva $^{+3}$, 2017 • KY

ST: PO.KN • CI: h0926・ID: 069

PN: Microkayla teqta Riva $^{+1}, 2014$

PK: Microkayla teqta ${ }^{\circ}$ Riva $^{+1}, 2014$

KG: Microkayla ${ }^{3}$ Riva $^{+3}, 2014$

KF: BRACHYCEPHALIDAE 1858.gc.f002

Microphryne Peters, $1873 \cdot \mathbf{A K}$

ST: PO.JD • CI: h0927 • ID: 248

PN: Paludicola pustulosa Cope, 1864

PK: Paludicola pustulosa* Cope, 1864

KG: Engystomops* Jiménez de la Espada, 1872

KF: LEIUPERIDAE 1850.bb.f010

Microphryne Methuen ${ }^{+1}, 1913$ • AK

ST: PO.JH • CI: h0928 • ID: 431

PN: Microphryne malagasia Methuen $^{+1}, 1913$

PK: Microphryne malagasia* Methuen $^{+1}, 1913$

KG: Gephyromantis* Methuen, 1920

KF: RHACOPHORIDAE |1858.gc.f012||-1932.ha.f001

Microps Dahl, $1823 \cdot \mathbf{Z H}$

ST: zo・CI: zh057

Microps Wagler, 1828 • AK

ST: PO.JH • CI: h0929 • ID: 298

PN: Microps unicolor Wagler, 1828

PK: Rana ovalis* Schneider, 1799

KG: Engystoma* Fitzinger, 1826

KF: MICROHYLIDAE ||1843.fa.f012 |-1931.na.f001

Micryletta Dubois, 1987 • KY

ST: PO.KN • CI: h0930 • ID: 315

PN: Microhyla inornata Boulenger, 1890

PK: Microhyla inornata* Boulenger, 1890

KG: Micryletta* Dubois, 1987

KF: MICROHYLIDAE ||1843.fa.f012||-1931.na.f001

Mimandra Dubois $^{+1}$, $2009 \cdot \mathbf{A K}$

ST: PO.JD • CI: h0931 • ID: 578

PN: Salamandra lanzai $\mathrm{Nascetti+3}^{+3}, 1988$

PK: Salamandra lanzai* Nascetti ${ }^{+3}, 1988$

KG: Salamandra ${ }^{1}$ Garsault, 1764

KF: SALAMANDRIDAE 1820.ga.f002

Mimosiphonops Taylor, $1968 \cdot \mathbf{K Y}$

ST: PO.KN • CI: h0932 • ID: 491

PN: Mimosiphonops vermiculatus Taylor, 1968

PK: Mimosiphonops vermiculatus ${ }^{\circ}$ Taylor, 1968

KG: Mimosiphonops ${ }^{\circ}$ Taylor, 1968

KF: CAECILIIDAE 1814.ra.f003-|1825.gb.f008|
Minascaecilia Wake $^{+1}, 1983 \cdot \mathbf{A K}$

ST: PO.JD • CI: h0933・ID: 487

PN: Minascaecilia sartoria Wake $^{+1}, 1983$

PK: Siphonops syntremus ${ }^{\circ}$ Cope, 1866

KG: Gymnopis ${ }^{\circ}$ Peters, 1874

KF: CAECILIIDAE 1814.ra.f003-|1825.gb.f008|

Minervarya Dubois $^{+2}, 2001 \cdot \mathbf{K Y}$

ST: PO.KN • CI: h0934 • ID: 378

PN: Minervarya sahyadris Dubois ${ }^{+2}, 2001$

PK: Minervarya sahyadris* Dubois $^{+2}, 2001$

KG: Minervarya* Dubois ${ }^{+2}, 2001$

KF: DICROGLOSSIDAE 1987.da.f004

Mini Scherz ${ }^{+10}$, 2019• AK

ST: PO.JD・CI: h0935・ID: 286

PN: Mini mum Scherz $^{+10}, 2019$

PK: Mini mum $^{\circ}$ Scherz $^{+10}, 2019$

KG: Cophyla* Boettger, 1880

KF: MICROHYLIDAE ||1843.fa.f012||-1931.na.f001

Minyobates Myers, $1987 \cdot \mathbf{K Y}$

ST: PO.KN • CI: h0936・ ID: 049

PN: Dendrobates steyermarki Rivero, 1971

PK: Dendrobates steyermarki* Rivero, 1971

KG: Minyobates* Myers, 1987

KF: DENDROBATIDAE ||1850.bb.f006"|-1865.ca.f002

Miopelobates Wettstein-Westersheimb, 1955 + $\mathbf{A K}$

ST: PO.JD • CI: h0937• ID: 470

PN: Miopelobates zapfei Wettstein-Westersheimb, 1955 :

PK: Rana gigantea ${ }^{\circ}$ Lartet, $1851 \dagger$

KG: Latonia ${ }^{3}$ Meyer, $1845 \dagger$

KF: DISCOGLOSSIDAE 1858.gc.f004

Miopelodytes Taylor, $1941+\cdot \mathbf{K Y}$

ST: PO.KN • CI: h0938 • ID: $\uparrow 092$

PN: Miopelodytes gilmorei Taylor, 1941 \$

PK: Miopelodytes gilmore $i^{\circ}$ Taylor, $1941 \uparrow$

KG: Miopelodytes ${ }^{\circ}$ Taylor, $1941 \dagger$

KF: PELODYTIDAE 1850.bb.f002

Mioproteus Estes $^{+1}, 1978+\cdot \mathbf{K Y}$

ST: Po.KN • CI: h0939 • ID: $\uparrow 182$

PN: Mioproteus cancasicus Estes $^{+1}, 1978$ :

PK: Mioproteus caucasicus ${ }^{\circ}$ Estes $^{+1}, 1978 \uparrow$

KG: Mioproteus ${ }^{\circ}$ Estes $^{+1}, 1978 \dagger$

KF: PROTEIDAE 1831.ba.f002

Mitrolysis Cope, 1889 • AK

ST: PO.JD • CI: h0940 • ID: 237

PN: Chiroleptes alboguttatus Günther, 1867

PK: Chiroleptes alboguttatus* Günther, 1867

KG: Ranoidea ${ }^{1}$ Tschudi, 1838

KF: PHYLLOMEDUSIDAE 1858.gc.f009

Mixophyes Günther, 1864 • KY

ST: PO.KN • CI: h0941 • ID: 266

PN: Mixophyes fasciolatus Günther, 1864

PK: Mixophyes fasciolatus* Günther, 1864

KG: Mixophyes* Günther, 1864

KF: MYOBATRACHIDAE 1850.sa.f001

Mixophys Ford ${ }^{+1}$, $1993 \cdot \mathbf{A K}$

ST: NS.JI • CI: h0942・ID: 266 
PN: Mixophyes fasciolatus Günther, 1864

PK: Mixophyes fasciolatus* Günther, 1864

KG: Mixophyes* Günther, 1864

KF: MYOBATRACHIDAE 1850.sa.f001

Mo nov. • KY

ST: PO.KN • CI: h0943 • ID: 126

PN: Bufo bambutensis Amiet, 1972

PK: Bufo bambutensis* Amiet, 1972

KG: $M o^{*}$ nov.

KF: BUFONIDAE 1825.gb.f004

Mocquardia Ahl, 1931 • AK

ST: NT.JD・CI: h0944 • ID: 340

PN: Rothschildia kounhiensis Mocquard, 1905

PK: Rothschildia kounhiensis ${ }^{\circ}$ Mocquard, 1905

KG: Paracassina ${ }^{\circ}$ Peracca, 1907

KF: HYPEROLIIDAE 1943.lb.f001

Mogophrys: $\mathrm{Kuhl}^{+1} 1822$ • AN

ST: $\mathbf{L I}$ • CI: n0083・ID: 021

PN: Megophrys montana $\mathrm{Kuhl}^{+1}, 1822$

PK: Megophrys montana ${ }^{\circ} \mathrm{Kuhl}^{+1}, 1822$

KG: Megophrys ${ }^{2} \mathrm{Kuhl}^{+1}, 1822$

KF: MEGOPHRYIDAE 1850.bb.f008-|1931.na.f003|

Molge Merrem, 1820 • AK

ST: NT.JI • CI: h0945・ID: 566

PN: Triton cristatus Laurenti, 1768

PK: Triton cristatus* Laurenti, 1768

KG: Triturus* Rafinesque, 1815

KF: SALAMANDRIDAE 1820.ga.f002

Monsechobatrachus Fejérváry, 1921 • KY

ST: PO.KN・CI: h0946・ID: $\uparrow 034$

PN: Palaeobatrachus gaudryi Vidal, 1902 \$

PK: Palaeobatrachus gaudryi $i^{\circ}$ Vidal, $1902 \dagger$

KG: Monsechobatrachus ${ }^{\circ}$ Fejérváry, $1921 \dagger$

KF: ANURA Familia INCERTAE SEDIS

Montorana Vogt, $1924 \cdot \mathbf{A K}$

ST: PO.JD・CI: h0947・ID: 387

PN: Montorana ahli Vogt, 1924

PK: Nanorana pleskei* Günther, 1896

KG: Nanorana* Günther, 1896

KF: DICROGLOSSIDAE 1987.da.f004

Montsechobatrachus: Simpson $1926+$ AN

ST: $\mathbf{A M} ・$ CI: n0084・ID: $\dagger 034$

PN: Palaeobatrachus gaudryi Vidal, 1902 \$

PK: Palaeobatrachus gaudryi $i^{\circ}$ Vidal, $1902 \dagger$

KG: Monsechobatrachus ${ }^{\circ}$ Fejérváry, $1921 \uparrow$

KF: ANURA Familia INCERTAE SEDIS

Morerella Rödel ${ }^{+4}$ in Rödel ${ }^{+12}, 2009$ • KY

ST: PO.KN・CI: h0948・ID: 332

PN: Morerella cyanophthalma Rödel ${ }^{+4}$ in $\mathrm{Rödel}^{+12}, 2009$

PK: Morerella cyanophthalma* Rödel ${ }^{+4}$ in Rödel $^{+12}, 2009$

KG: Morerella* Rödel ${ }^{+4}$ in Rödel ${ }^{+12} 2009$

KF: HYPEROLIIDAE 1943.lb.f001

Mosleyia Wells ${ }^{+1}, 1985 \cdot \mathbf{A K}$

ST: PO.JD • CI: h0949 • ID: 237

PN: Hyla nannotis Andersson, 1916

PK: Hyla nannotis* Andersson, 1916
KG: Ranoidea ${ }^{1}$ Tschudi, 1838

KF: PHYLLOMEDUSIDAE 1858.gc.f009

Mucubatrachus La Marca, 2007 • AK

ST: PO.JD • CI: h0950 • ID: 078

PN: Hylodes briceni Boulenger, 1903

PK: Hylodes briceni ${ }^{\circ}$ Boulenger, 1903

KG: Pristimantis* Jiménez de la Espada, 1870

KF: BRACHYCEPHALIDAE 1858.gc.f002

Muraenopsis Fitzinger, 1843 • AK

ST: PO.JD • CI: h0951 • ID: 520

PN: Amphiuma tridactylum Cuvier, 1827

PK: Amphiuma tridactylum* Cuvier, 1827

KG: Amphiuma* Garden in Smith, 1821

KF: AMPHIUMIDAE 1825.gb.f07

Musergus Dubois ${ }^{+1}, 2009 \cdot \mathbf{A K}$

ST: PO.JD・CI: h0952・ID: 567

PN: Molge strauchii Steindachner, 1888

PK: Molge strauchii* Steindachner, 1888

KG: Neurergus* Cope, 1862

KF: SALAMANDRIDAE 1820.ga.f002

Mycetoglossus Bonaparte, 1839 • EX

ST: NT.CE・CI: e0009・ID: 540

PN: Salamandra subfusca Green, 1818

PK: Salamandra rubra* Sonnini ${ }^{+1}, 1801$

KG: Pseudotriton ${ }^{1}$ Tschudi, 1838

KF: PLETHODONTIDAE 1850.ga.f002

Mycetoides: Duméril ${ }^{+2} 1854$ • AN

ST: AL • CI: n0085 • ID: 522

PN: Bolitoglossa mexicana Duméril ${ }^{+2}, 1854$

PK: Bolitoglossa mexicana* Duméril ${ }^{+2}, 1854$

KG: Bolitoglossa* Duméril ${ }^{+2}, 1854$

KF: PlethodontidaE 1850.ga.f002

Mycrohyla Casto de Elera, 1895 • AK

ST: NT.JI • CI: h0954 • ID: 314

PN: Microhyla achatina Tschudi, 1838

PK: Microhyla achatina* Tschudi, 1838

KG: Microhyla* Tschudi, 1838

KF: MICROHYLIDAE ||1843.fa.f012||-1931.na.f001

Myersiella Carvalho, $1954 \cdot \mathbf{K Y}$

ST: PO.KN • CI: h0955 • ID: 295

PN: Engystoma subnigrum Miranda-Ribeiro, 1924

PK: Engystoma microps* Duméril ${ }^{+1}, 1841$

KG: Myersiella ${ }^{1}$ Carvalho, 1954

KF: MICROHYLIDAE ||1843.fa.f012 |-1931.na.f001

Myersiohyla Faivovich ${ }^{+5}, 2005 \cdot \mathbf{K Y}$

ST: PO.KN・CI: h0956・ID: 192

PN: Hyla inparquesi Ayarzagüena ${ }^{+1}, 1994$

PK: Hyla inparquesi* Ayarzagüena ${ }^{+1}, 1994$

KG: Myersiohyla* Faivovich ${ }^{+5}, 2005$

KF: HYLIDAE 1815.ra.f002-|1825.gb.f001|

Myiobatrachus [Bonaparte, 1850] Schlegel, 1858 • AK

ST: NS.JI • CI: h0957 • ID: 273

PN: Myobatrachus paradoxus Schlegel, 1850

PK: Breviceps gouldii* Gray, 1841

KG: Myobatrachus ${ }^{1}$ Schlegel, 1850

KF: MYOBATRACHIDAE 1850.sa.f001 
Mynbulakia Nessov, 1981 \$ AK

ST: Po.JD • CI: h0958 • ID: $\uparrow 166$

PN: Mynbulakia surgayi Nessov, 1981 \$

PK: Eoscapherpeton asiaticum ${ }^{\circ}$ Nessov, $1981 \dagger$

KG: Eoscapherpeton ${ }^{\circ}$ Nessov, $1981 \dagger$

KF: CRYPTOBRANCHIDAE 1826.fb.f003

Myobatrachus Schlegel, $1850 \cdot \mathbf{K Y}$

ST: PO.KN • CI: h0959 • ID: 273

PN: Myobatrachus paradoxus Schlegel, 1850

PK: Breviceps gouldii* Gray, 1841

KG: Myobatrachus ${ }^{1}$ Schlegel, 1850

KF: MYOBATRACHIDAE 1850.sa.f001

Myraenopsis Agassiz, 1847 • AK

ST: PO.JD • CI: h0960 • ID: 520

PN: Amphiuma tridactylum Cuvier, 1827

PK: Amphiuma tridactylum* Cuvier, 1827

KG: Amphiuma* Garden in Smith, 1821

KF: AMPHIUMIDAE 1825.gb.f07

Mysticellus Garg ${ }^{+1}, 2019 \bullet \mathbf{K Y}$

ST: PO.KN • CI: h0961 • ID: 316

PN: Mysticellus franki $\mathrm{Garg}^{+1}, 2019$

PK: Mysticellus franki ${ }^{\circ} \mathrm{Garg}^{+1}, 2019$

KG: Mysticellus ${ }^{\circ} \mathrm{Garg}^{+1}, 2019$

KF: MICROHYLIDAE ||1843.fa.f012||-1931.na.f001

Myxophyes Krefft, 1865 • AK

ST: NS.JI • CI: h0962 • ID: 266

PN: Mixophyes fasciolatus Günther, 1864

PK: Mixophyes fasciolatus* Günther, 1864

KG: Mixophyes* Günther, 1864

KF: MYOBATRACHIDAE 1850.sa.f001

Nannobatrachus Boulenger, 1882 • AK

ST: PO.JD • CI: h0963・ID: 400

PN: Nannobatrachus beddomii Boulenger, 1882

PK: Nannobatrachus beddomii* Boulenger, 1882

KG: Nyctibatrachus* Boulenger, 1882

KF: NYCTIBATRACHIDAE 1993.ba.f001

Nannofrys Palacký, 1898 • AK

ST: NT.JI • CI: h0964 • ID: 376

PN: Nannophrys ceylonensis Günther, 1869

PK: Nannophrys ceylonensis* Günther, 1869

KG: Nannophrys* Günther, 1869

KF: DICROGLOSSIDAE 1987.da.f004

Nannophryne Günther, 1870 • KY

ST: PO.KN • CI: h0965・ID: 146

PN: Nannophryne variegata Günther, 1870

PK: Nannophryne variegata* Günther, 1870

KG: Nannophryne* Günther, 1870

KF: BUFONIDAE 1825.gb.f004

Nannophrys Günther, $1869 \cdot \mathbf{K Y}$

ST: PO.KN • CI: h0966・ ID: 376

PN: Nannophrys ceylonensis Günther, 1869

PK: Nannophrys ceylonensis* Günther, 1869

KG: Nannophrys* Günther, 1869

KF: DICROGLOSSIDAE 1987.da.f004

Nanorana Günther, 1896 • KY

ST: PO.KN • CI: h0967 • ID: 387
PN: Nanorana pleskei Günther, 1896

PK: Nanorana pleskei* Günther, 1896

KG: Nanorana* Günther, 1896

KF: DICROGLOSSIDAE 1987.da.f004

Nanotriton Parra-Olea ${ }^{+2}, 2004 \cdot \mathbf{A K}$

ST: PO.JD • CI: h0968 • ID: 522

PN: Oedipus rufescens Cope, 1869

PK: Oedipus rufescens* Cope, 1869

KG: Bolitoglossa* Duméril ${ }^{+2}, 1854$

KF: PLETHODONTIDAE 1850.ga.f002

Nasikabatrachus $\mathrm{Biju}^{+1}, 2003 \cdot \mathbf{K Y}$

ST: PO.KN • CI: h0969 • ID: 031

PN: Nasikabatrachus sahyadrensis $\mathrm{Biju}^{+1}, 2003$

PK: Nasikabatrachus sahyadrensis* $\mathrm{Biju}^{+1}, 2003$

KG: Nasikabatrachus* Biju $^{+1}, 2003$

KF: NASIKABATRACHIDAE 2003.bb.f001

Nasirana Dubois, 1992 • AK

ST: PO.JD • CI: h0970 • ID: 402

PN: Rana alticola Boulenger, 1882

PK: Rana alticola* Boulenger, 1882

KG: Clinotarsus* Mivart, 1869

KF: RANIDAE 1796.ba.f001

Nasutixalus $\mathrm{Jiang}^{+3}$ in $\mathrm{Jiang}^{+5}, 2016 \cdot \mathbf{K Y}$

ST: PO.KN • CI: h0971 • ID: 446

PN: Nasutixalus medogensis Jiang ${ }^{+3}$ in Jiang $^{+5}, 2016$

PK: Nasutixalus medogensis $*$ Jiang $^{+3}$ in Jiang ${ }^{+5}, 2016$

KG: Nasutixalus ${ }^{*}$ Jiang $^{+3}$ in Jiang ${ }^{+5}, 2016$

KF: RHACOPHORIDAE ||1858.gc.f012||-1932.ha.f001

Natalobatrachus Hewitt ${ }^{+1}, 1912 \cdot \mathbf{K Y}$

ST: PO.KN • CI: h0972 • ID: 361

PN: Natalobatrachus bonebergi Hewitt ${ }^{+1}, 1912$

PK: Natalobatrachus bonebergi* Hewitt ${ }^{+1}, 1912$

KG: Natalobatrachus* Hewitt ${ }^{+1}, 1912$

KF: CACOSTERNIDAE 1931.na.f008

Nattereria Steindachner, 1864 • AK

ST: PO.JD • CI: h0973・ID: 250

PN: Nattereria lateristriga Steindachner, 1864

PK: Nattereria lateristriga ${ }^{\circ}$ Steindachner, 1864

KG: Physalaemus* Fitzinger, 1826

KF: LEIUPERIDAE 1850.bb.f010

Nectes: Bleeker 1857 • AN

ST: AL • CI: n0086・ID: 111

PN: Nectes pleurotaenia Bleeker, 1857 uN

PK: Pseudobufo subasper ${ }^{\circ}$ Tschudi, 1838

KG: Pseudobufo ${ }^{\circ}$ Tschudi, 1838

KF: BUFONIDAE 1825.gb.f004

Nectes Cope, $1865 \cdot \mathbf{A K}$

ST: NT.JI • CI: h0974 • ID: 111

PN: Pseudobufo subasper Tschudi, 1838

PK: Pseudobufo subasper ${ }^{\circ}$ Tschudi, 1838

KG: Pseudobufo ${ }^{\circ}$ Tschudi, 1838

KF: BUFONIDAE 1825.gb.f004

Nectocaecilia Taylor, $1968 \bullet \mathbf{K Y}$

ST: PO.KN • CI: h0975 • ID: 478

PN: Chthonerpeton petersii Boulenger, 1882

PK: Chthonerpeton petersii ${ }^{\circ}$ Boulenger, 1882 
KG: Nectocaecilia ${ }^{\circ}$ Taylor, 1968

KF: CAECILIIDAE 1814.ra.f003-|1825.gb.f008|

Nectodactylus Miranda Ribeiro, 1924 • AK

ST: PO.JD • CI: h0976 • ID: 292

PN: Nectodactylus spinulosus Miranda Ribeiro, 1924

PK: Engystoma leucosticta* Boulenger, 1888

KG: Chiasmocleis* Méhelÿ, 1904

KF: MICROHYLIDAE |1843.fa.f012||-1931.na.f001

Nectofryne Palacký, 1898 • AK

ST: NT.JI • CI: h0977 • ID: 127

PN: Nectophryne afra Buchholz ${ }^{+1}$ in Peters, 1875

PK: Nectophryne afra* Buchholz ${ }^{+1}$ in Peters, 1875

KG: Nectophryne* Buchholz ${ }^{+1}$ in Peters, 1875

KF: BUFONIDAE 1825.gb.f004

Nectophryne Buchholz ${ }^{+1}$ in Peters, 1875 • KY

ST: PO.KN • CI: h0978 • ID: 127

PN: Nectophryne afra Buchholz ${ }^{+1}$ in Peters, 1875

PK: Nectophryne afra* Buchholz ${ }^{+1}$ in Peters, 1875

KG: Nectophryne* Buchholz ${ }^{+1}$ in Peters, 1875

KF: BUFONIDAE 1825.gb.f004

Nectophrynoides Noble, $1926 \cdot \mathbf{K Y}$

ST: PO.KN • CI: h0979 • ID: 135

PN: Nectophryne tornieri Roux, 1906

PK: Nectophryne tornieri* Roux, 1906

KG: Nectophrynoides* Noble, 1926

KF: BUFONIDAE 1825.gb.f004

Nectura: Neave 1940 AN

ST: $\mathbf{A M} ・$ CI: n0087・ ID: 553

PN: Sirena maculosa Rafinesque, 1818

PK: Sirena maculosa* Rafinesque, 1818

KG: Necturus* Rafinesque, 1819

KF: PROTEIDAE 1831.ba.f002

Necturus Rafinesque, $1819 \cdot \mathbf{K Y}$

ST: PO.KN • CI: h0980 • ID: 553

PN: Sirena maculosa Rafinesque, 1818

PK: Sirena maculosa* Rafinesque, 1818

KG: Necturus* Rafinesque, 1819

KF: PROTEIDAE 1831.ba.f002

Nectusus: Neave 1940 • AN

ST: $\mathbf{A M} \bullet$ CI: n0088・ ID: 553

PN: Sirena maculosa Rafinesque, 1818

PK: Sirena maculosa* Rafinesque, 1818

KG: Necturus* Rafinesque, 1819

KF: PROTEIDAE 1831.ba.f002

Negatchevkia Ratnikov, $1993+\bullet \mathbf{K Y}$

ST: PO.KN • CI: h0981 • ID: †035

PN: Negatchevkia donensis Ratnikov, 1993 †

PK: Negatchevkia donensis ${ }^{\circ}$ Ratnikov, $1993+$

KG: Negatchevkia ${ }^{\circ}$ Ratnikov, $1993 \dagger$

KF: ANURA Familia INCERTAE SEDIS

Nelsonophryne Frost, 1987 • AK

ST: PO.JD • CI: h0982 • ID: 293

PN: Glossostoma aterrimum Günther, 1901

PK: Glossostoma aterrimum ${ }^{\circ}$ Günther, 1901

KG: Ctenophryne* Mocquard, 1904

KF: MICROHYLIDAE ||1843.fa.f012||-1931.na.f001
Nenirana Hillis ${ }^{+1}, 2005 \cdot \mathbf{A K}$

ST: PO.JD・CI: h0983・ID: 415

PN: Rana areolata Baird $^{+1}, 1852$

PK: Rana areolata* Baird $^{+1}, 1852$

KG: Lithobates* Fitzinger, 1843

KF: RANIDAE 1796.ba.f001

Neobatrachus Peters, $1863 \cdot \mathbf{K Y}$

ST: PO.KN • CI: h0984・ID: 263

PN: Neobatrachus pictus Peters, 1863

PK: Neobatrachus pictus* Peters, 1863

KG: Neobatrachus* Peters, 1863

KF: MYOBATRACHIDAE 1850.sa.f001

Neobufo Bolkay, 1919 • AK

ST: PO.JD • CI: h0985 • ID: 120

PN: Bufo vulgaris Laurenti, 1768

PK: Rana bufo* Linnaeus, 1758

KG: $B u f o *$ Garsault, 1764

KF: BUFONIDAE 1825.gb.f004

Neophractops Wells ${ }^{+1}, 1985$ • AK

ST: PO.JD・CI: h0986・ID: 237

PN: Chiroleptes platycephalus Günther, 1873

PK: Chiroleptes platycephalus* Günther, 1873

KG: Ranoidea ${ }^{1}$ Tschudi, 1838

KF: PHYLLOMEDUSIDAE 1858.gc.f009

Neoprocoela Schaeffer, $1949+\cdot \mathbf{K Y}$

ST: PO.KN • CI: h0987 • ID: $\uparrow 098$

PN: Neoprocoela edentatus Schaeffer, 1949 \$

PK: Neoprocoela edentata ${ }^{\circ}$ Schaeffer, $1949 \dagger$

KG: Neoprocoela ${ }^{\circ}$ Schaeffer, $1949 \dagger$

KF: TELMATOBIIDAE 1843.fa.f006

Neoruinosus Wells ${ }^{+1}, 1985$ • AK

ST: PO.JD・CI: h0988・ID: 263

PN: Heleioporus sudelli Lamb, 1911

PK: Heleioporus sudelli* Lamb, 1911

KG: Neobatrachus* Peters, 1863

KF: MYOBATRACHIDAE 1850.sa.f001

Neoscaphiopus Taylor, $1942+\cdot \mathbf{A K}$

ST: PO.JD • CI: h0989 • ID: 030

PN: Neoscaphiopus noblei Taylor, 1941 *

PK: Neoscaphiopus noblei ${ }^{\circ}$ Taylor, $1941 \dagger$

KG: Spea* Cope, 1866

KF: SCAPHIOPODIDAE 1865.ca.f003

Neotriton Bolkay, 1927 • AK

ST: PO.JD・CI: h0990 • ID: 566

PN: Triton karelinii Strauch, 1870

PK: Triton karelinii* Strauch, 1870

KG: Triturus* Rafinesque, 1815

KF: SALAMANDRIDAE 1820.ga.f002

Nephelobates La Marca, 1994 • AK

ST: PO.JD • CI: h0991 • ID: 037

PN: Phyllobates alboguttatus Boulenger, 1903

PK: Phyllobates alboguttatus ${ }^{\circ}$ Boulenger, 1903

KG: Aromobates* Myers $^{+2}, 1991$

KF: AROMOBATIDAE 2006.gc.f004

Nesionixalus Perret, 1976 • AK

ST: PO.JD • CI: h0992 • ID: 331 
PN: Hyperolius thomensis Bocage, 1886

PK: Hyperolius thomensis* Bocage, 1886

KG: Hyperolius* Rapp, 1842

KF: HYPEROLIIDAE 1943.lb.f001

Nesobia Ancey, $1887 \cdot \mathbf{z H}$

ST: zo • CI: zh058

Nesobia Van Kampen, 1923 • AK

ST: PO.JH • CI: h0993 • ID: 018

PN: Leptobrachium natunae Günther, 1895

PK: Leptobrachium natunae ${ }^{\circ}$ Günther, 1895

KG: Leptobrachella ${ }^{\circ}$ Smith, 1925

KF: MEGOPHRYIDAE 1850.bb.f008-|1931.na.f003|

Nesomantis Boulenger, $1909 \cdot \mathbf{A K}$

ST: PO.JD • CI: h0994 • ID: 033

PN: Nesomantis thomasseti Boulenger, 1909

PK: Nesomantis thomasseti* Boulenger, 1909

KG: Sooglossus* Boulenger, 1906

KF: SOOGLOSSIDAE 1931.na.f002

Nesorohyla Pinheiro ${ }^{+4,} 2019$ • KY

ST: PO.KN • CI: h0995・ID: 193

PN: Hyla kanaima Goin ${ }^{+1}, 1969$

PK: Hyla kanaima* Goin ${ }^{+1}, 1969$

KG: Nesorohyla* Pinheiro ${ }^{+4,} 2019$

KF: HYLIDAE 1815.ra.f002-|1825.gb.f001|

Nesovtriton Skutschas, $2009+\cdot \mathbf{K Y}$

ST: PO.KN • CI: h0996・ID: $\uparrow 138$

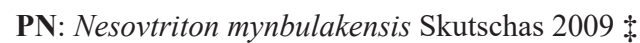

PK: Nesovtriton mynbulakensis ${ }^{\circ}$ Skutschas $2009 \dagger$

KG: Nesovtriton ${ }^{\circ}$ Skutschas $2009 \dagger$

KF: Urodela Familia INCERTAE SEDIS

Neurergus Cope, 1862 • KY

ST: PO.KN・CI: h0997・ID: 567

PN: Neurergus crocatus Cope, 1862

PK: Neurergus crocatus* Cope, 1862

KG: Neurergus* Cope, 1862

KF: SALAMANDRIDAE 1820.ga.f002

Neusibatrachus Seiffert, $1972+\cdot \mathbf{K Y}$

ST: PO.KN • CI: h0998 • ID: $\uparrow 063$

PN: Neusibatrachus wilferti Seiffert, 1972 *

PK: Neusibatrachus wilferti ${ }^{\circ}$ Seiffert, $1972 \dagger$

KG: Neusibatrachus ${ }^{\circ}$ Seiffert, $1972 \dagger$

KF: DORSIPARES Familia INCERTAE SEDIS

Nevobatrachus Mahony, $2019+\cdot \mathbf{K Y}$

ST: PO.KN • CI: h0999 • ID: $\uparrow 064$

PN: Cordicephalus gracilis Nevo, 1968 \$

PK: Cordicephalus gracilis $^{\circ}$ Nevo, $1968 \uparrow$

KG: Nevobatrachus ${ }^{\circ}$ Mahony, $2019 \dagger$

KF: DorsIPARES Familia INCERTAE SEDIS

Nezpercius Blob ${ }^{+4}, 2001+\cdot \mathbf{K Y}$

ST: PO.KN • CI: h1000・ID: $\uparrow 139$

PN: Nezpercius dodsoni $\mathrm{Blob}^{+4}, 2001$ \$

PK: Nezpercius dodsoni ${ }^{\circ} \mathrm{Blob}^{+4}, 2001 \dagger$

KG: Nezpercius $^{\circ} \mathrm{Blob}^{+4}, 2001 \dagger$

KF: Urodela Familia INCERTAE SEDIS

Niceforonia Goin ${ }^{+1}$, 1963 • KY

ST: PO.KN • CI: h1001 • ID: 061
PN: Niceforonia nana $\mathrm{Goin}^{+1}, 1963$

PK: Niceforonia nana ${ }^{\circ}$ Goin $^{+1}, 1963$

KG: Niceforonia ${ }^{\circ} \mathrm{Goin}^{+1}, 1963$

KF: BRACHYCEPHALIDAE 1858.gc.f002

Nidirana Dubois, $1992 \cdot \mathbf{K Y}$

ST: PO.KN • CI: h1002・ID: 411

PN: Rana psaltes Kuramoto, 1985

PK: Rana okinavana* Boettger, 1895

KG: Nidirana ${ }^{1}$ Dubois, 1992

KF: RANIDAE 1796.ba.f001

Niedenia Ahl, $1924 \cdot \mathbf{A K}$

ST: PO.JD・CI: h1003・ID: 179

PN: Niedenia spinulifer Ahl, 1923

PK: Cycloramphus asper ${ }^{\circ}$ Werner, 1899

KG: Cycloramphus* Tschudi, 1838

KF: CYCLORAMPHIDAE 1850.bb.f003-|1852.ba.f001|

Nimbaphrynoides Dubois, 1987 • KY

ST: PO.KN・CI: h1004・ID: 128

PN: Nectophrynoides occidentalis Angel, 1943

PK: Nectophrynoides occidentalis* Angel, 1943

KG: Nimbaphrynoides* Dubois, 1987

KF: BUFONIDAE 1825.gb.f004

Nireus Agassiz, $1847 \cdot \mathbf{Z H}$

ST: zo • CI: zh059

Nireus Theobald, $1880 \bullet \mathbf{A K}$

ST: PO.JH • CI: h1005 • ID: 015

PN: Nireus pulcherrimus Theobald, 1880

PK: Leptobrachium hasseltii* Tschudi, 1838

KG: Leptobrachium* Tschudi, 1838

KF: MEGOPHRYIDAE 1850.bb.f008-|1931.na.f003|

Noblella Barbour, $1930 \cdot \mathbf{K Y}$

ST: PO.KN • CI: h1006・ID: 070

PN: Sminthillus peruvianus Noble, 1921

PK: Sminthillus peruvianus* Noble, 1921

KG: Noblella* Barbour, 1930

KF: BRACHYCEPHALIDAE 1858.gc.f002

Notaden Günther, $1873 \cdot \mathbf{K Y}$

ST: PO.KN • CI: h1007 • ID: 265

PN: Notaden bennettii Günther, 1873

PK: Notaden bennettii* Günther, 1873

KG: Notaden* Günther, 1873

KF: MYOBATRACHIDAE 1850.sa.f001

Noterpeton Rage ${ }^{+2}, 1993+\mathbf{K Y}$

ST: PO.KN • CI: h1008 •ID: $\uparrow 175$

PN: Noterpeton bolivianum Rage $^{+2}, 1993$ †

PK: Noterpeton bolivianum ${ }^{\circ}$ Rage $^{+2}, 1993 \dagger$

KG: Noterpeton ${ }^{\circ}$ Rage $^{+2}, 1993 \dagger$

KF: NOTERPETIDAE 1993.ra.f001 †

Nothophryne Poynton, 1963 • KY

ST: PO.KN • CI: h1009・ID: 364

PN: Nothophryne broadleyi Poynton, 1963

PK: Nothophryne broadleyi ${ }^{\circ}$ Poynton, 1963

KG: Nothophryne ${ }^{\circ}$ Poynton, 1963

KF: CACOSTERNIDAE 1931.na.f008

Notiomolge Hillis ${ }^{+3}$, 2001 • AK

ST: PO.JD • CI: h1010 • ID: 542 
PN: Eurycea neotenes Bishop ${ }^{+1}, 1937$

PK: Eurycea neotenes* Bishop ${ }^{+1}, 1937$

KG: Eurycea* Rafinesque, 1822

KF: PLETHODONTIDAE 1850.ga.f002

Notobatrachus Reig in Stipanicic ${ }^{+1,} 1956+\bullet \mathbf{K Y}$

ST: PO.KN • CI: h1011 •ID: $\uparrow 055$

PN: Notobatrachus degiustoi Reig in Stipanicic ${ }^{+1}, 1956$ *

PK: Notobatrachus degiusto $i^{\circ}$ Reig in Stipanicic ${ }^{+1}, 1956 \dagger$

KG: Notobatrachus ${ }^{\circ}$ Reig in Stipanicic $^{+1}, 1956 \dagger$

KF: LEIOPELMATIDAE 1869.mc.f07-|1942.ta.f001|

Notodelphis Hoffmann, 1878 • AK

ST: NS.JD・CI: h1012・ID: 091

PN: Notodelphys ovifera Lichtenstein $^{+1}, 1854$

PK: Notodelphys ovifera* Lichtenstein ${ }^{+1}, 1854$

KG: Gastrotheca* Fitzinger, 1843

KF: HEMIPHRACTIDAE 1862.pa.f001

Notodelphys Alleman, $1847 \cdot \mathbf{Z H}$

ST: zo • CI: zh060

Notodelphys Lichtenstein ${ }^{+1}, 1854$ • AK

ST: PO.JH• CI: h1013・ID: 091

PN: Notodelphys ovifera Lichtenstein ${ }^{+1}, 1854$

PK: Notodelphys ovifera* Lichtenstein ${ }^{+1}, 1854$

KG: Gastrotheca* Fitzinger, 1843

KF: HEMIPHRACTIDAE 1862.pa.f001

Notokassina Drewes, 1985 • AK

ST: PO.JD・CI: h1014 • ID: 341

PN: Cassina wealii Boulenger, 1882

PK: Cassina wealii* Boulenger, 1882

KG: Semnodactylus ${ }^{1}$ Hoffman, 1939

KF: HYPEROLIIDAE 1943.1b.f001

Notophthalmus Rafinesque, $1820 \bullet \mathbf{K Y}$

ST: PO.KN • CI: h1015・ID: 569

PN: Triturus miniatus Rafinesque, 1820

PK: Triturus (Diemictylus) viridescens* Rafinesque, 1820

KG: Notophthalmus ${ }^{1}$ Rafinesque, 1820

KF: SALAMANDRIDAE 1820.ga.f002

Nototheca Bokermann, 1950 • AK

ST: PO.JD • CI: h1016 • ID: 094

PN: Coelonotus fissilis Miranda-Ribeiro, 1920

PK: Coelonotus fissilis* Miranda-Ribeiro, 1920

KG: Fritziana* Mello-Leitão, 1937

KF: HEMIPHRACTIDAE 1862.pa.f001

Nototrema Agassiz, $1847 \cdot \mathbf{z H}$

ST: zo • CI: zh061

Nototrema Günther, 1859 • AK

ST: PO.JH・CI: h1017 •ID: 091

PN: Hyla marsupiata Duméril ${ }^{+1}, 1841$

PK: Hyla marsupiata* Duméril ${ }^{+1}, 1841$

KG: Gastrotheca* Fitzinger, 1843

KF: HEMIPHRACTIDAE 1862.pa.f001

Nototriton Wake ${ }^{+1}, 1983$ • KY

ST: PO.KN • CI: h1018 • ID: 537

PN: Spelerpes picadoi Stejneger, 1911

PK: Spelerpes picadoi* Stejneger, 1911

KG: Nototriton* Wake $^{+1}, 1983$

KF: PLETHODONTIDAE 1850.ga.f002
Novirana: Hillis ${ }^{+1} 2005 \cdot \mathbf{A N}$

ST: AL・CI: n0089・ID: 415

PN: Rana pipiens Schreber, 1782

PK: Rana pipiens* Schreber, 1782

KG: Lithobates* Fitzinger, 1843

KF: RANIDAE 1796.ba.f001

Novooskolia Ratnikov, 1993 • KY

ST: PO.KN • CI: h1019・ID: $\uparrow 036$

PN: Novooskolia cristata Ratnikov, 1993 *

PK: Novooskolia cristata ${ }^{\circ}$ Ratnikov, $1993 \dagger$

KG: Novooskolia ${ }^{\circ}$ Ratnikov, $1993 \dagger$

KF: ANURA Familia INCERTAE SEDIS

Nuominerpeton $\mathrm{Jia}^{+1}, 2016+\mathbf{K Y}$

ST: PO.KN • CI: h1020・ID: $\uparrow 161$

PN: Nuominerpeton aquilonaris $\mathrm{Jia}^{+1}, 2016+$

PK: Nuominerpeton aquilonaris ${ }^{\circ} \mathrm{Jia}^{+1}, 2016 \dagger$

KG: Nuominerpeton ${ }^{\circ} \mathrm{Jia}^{+1}, 2016 \dagger$

KF: IMPERFECTIBRANCHIA Familia INCERTAE SEDIS

Nukusurus Nessov, $1981+\bullet \mathbf{K Y}$

ST: PO.KN • CI: h1021・ID: $† 005$

PN: Nukusurus insuetus Nessov, 1981 \$

PK: Nukusurus insuetus ${ }^{\circ}$ Nessov, $1981 \dagger$

KG: Nukusurus ${ }^{\circ}$ Nessov, $1981 \dagger$

KF: ALBANERPETIDAE 1982.fa.f001 †

Nyctanolis Elias $^{+1}, 1983 \cdot \mathbf{K Y}$

ST: PO.KN • CI: h1022・ID: 532

PN: Nyctanolis pernix Elias $^{+1}, 1983$

PK: Nyctanolis pernix* Elias $^{+1}, 1983$

KG: Nyctanolis* Elias $^{+1}, 1983$

KF: PLETHODONTIDAE 1850.ga.f002

Nyctibates Boulenger, $1904 \cdot \mathbf{K Y}$

ST: PO.KN・CI: h1023・ID: 322

PN: Nyctibates corrugatus Boulenger, 1904

PK: Nyctibates corrugatus* Boulenger, 1904

KG: Nyctibates* Boulenger, 1904

KF: ARTHROLEPTIDAE 1869.mc.f011

Nyctibatrachus Boulenger, 1882 • KY

ST: PO.KN • CI: h1024・ID: 400

PN: Nyctibatrachus major Boulenger, 1882

PK: Nyctibatrachus major* Boulenger, 1882

KG: Nyctibatrachus* Boulenger, 1882

KF: NYCTIBATRACHIDAE 1993.ba.f001

Nyctimantis Boulenger, $1882 \bullet \mathbf{K Y}$

ST: PO.KN • CI: h1025・ID: 230

PN: Nyctimantis rugiceps Boulenger, 1882

PK: Nyctimantis rugiceps* Boulenger, 1882

KG: Nyctimantis* Boulenger, 1882

KF: HYLIDAE 1815.ra.f002-|1825.gb.f001|

Nyctimystes Stejneger, $1916 \bullet \mathbf{K Y}$

ST: PO.KN・CI: h1026・ID: 236

PN: Nyctimantis papua Boulenger, 1897

PK: Nyctimantis papua* Boulenger, 1897

KG: Nyctimystes* Stejneger, 1916

KF: PHYLLOMEDUSIDAE 1858.gc.f009

Nyctixalus Boulenger, $1882 \cdot \mathbf{K Y}$

ST: PO.KN • CI: h1027•ID: 437 
PN: Nyctixalus margaritifer Boulenger, 1882

PK: Nyctixalus margaritifer* Boulenger, 1882

KG: Nyctixalus* Boulenger, 1882

KF: RHACOPHORIDAE |1858.gc.f012||-1932.ha.f001

Nymphargus Cisneros-Heredia ${ }^{+1}, 2007 \cdot \mathbf{K Y}$

ST: PO.KN • CI: h1028 • ID: 165

PN: Cochranella cochranae Goin, 1961

PK: Cochranella cochranae* Goin, 1961

KG: Nymphargus * Cisneros-Heredia ${ }^{+1}, 2007$

KF: CENTROLENIDAE 1951.ta.f001

Oaxakia Parra-Olea ${ }^{+2}, 2004 \cdot \mathbf{A K}$

ST: PO.JD • CI: h1029 • ID: 522

PN: Oedipus macrinii Lafrentz, 1930

PK: Oedipus macrinii* Lafrentz, 1930

KG: Bolitoglossa* Duméril ${ }^{+2}, 1854$

KF: PLETHODONTIDAE 1850.ga.f002

Obstetricans Dugès, 1834 • AK

ST: PO.JD • CI: h1030 • ID: 467

PN: Bufo obstetricans Laurenti, 1768

PK: Bufo obstetricans* Laurenti, 1768

KG: Alytes* Wagler, 1829

KF: ALYTIDAE 1843.fa.f008

Occidozyga $\mathrm{Kuhl}^{+1}, 1822 \cdot \mathbf{K Y}$

ST: PO.KN • CI: h1031 • ID: 395

PN: Rana lima Gravenhorst, 1829

PK: Rana lima* Gravenhorst, 1829

KG: Occidozyga* $\mathrm{Kuhl}^{+1}, 1822$

KF: OCCIDOZYGIDAE 1990.fa.f002

Ochthomantis $\mathrm{Glaw}^{+1}, 1994$ • AK

ST: PO.JD・CI: h1032 • ID: 432

PN: Rana femoralis Boulenger, 1882

PK: Rana femoralis* Boulenger, 1882

KG: Mantidactylus* Boulenger, 1895

KF: RHACOPHORIDAE ||1858.gc.f012 |-1932.ha.f001

Odontobatrachus Barej ${ }^{+3}$ in Barej $\mathrm{j}^{+10}, 2014$ • KY

ST: PO.KN • CI: h1033 • ID: 348

PN: Petropedetes natator Boulenger, 1905

PK: Petropedetes natator* Boulenger, 1905

KG: Odontobatrachus* Barej $^{+3}$ in Barej $^{+10}, 2014$

KF: ODONTOBATRACHIDAE 2014.ba.f001

Odontophrynus Reinhardt ${ }^{+1}, 1862 \cdot \mathbf{K Y}$

ST: PO.KN • CI: h1034 • ID: 153

PN: Odontophrynus cultripes Reinhardt ${ }^{+1}, 1862$

PK: Odontophrynus cultripes* Reinhardt ${ }^{+1}, 1862$

KG: Odontophrynus* Reinhardt ${ }^{+1}, 1862$

KF: ODONTOPHRYNIDAE 1971.la.f002

Odorrana $\mathrm{Fei}^{+2}, 1990 \cdot \mathbf{K Y}$

ST: PO.KN・CI: h1035・ID: 412

PN: Rana margaretae Liu, 1950

PK: Rana margaretae* Liu, 1950

KG: Odorrana* $\mathrm{Fei}^{+2}, 1990$

KF: RANIDAE 1796.ba.f001

Oedipina Keferstein, 1868 • KY

ST: PO.KN • CI: h1036・ID: 534

PN: Oedipina uniformis Keferstein, 1868

PK: Oedipina uniformis* Keferstein, 1868
KG: Oedipina* Keferstein, 1868

KF: PLETHODONTIDAE 1850.ga.f002

Oedipus Berthold, $1827 \cdot \mathbf{Z H}$

ST: zo • CI: zh062

Oedipus Tschudi, $1838 \cdot \mathbf{A K}$

ST: PO.JH • CI: h1037 • ID: 522

PN: Salamandra platydactylus Gray, 1831

PK: Salamandra platydactylus* Gray, 1831

KG: Bolitoglossa* Duméril ${ }^{+1}, 1854$

KF: PLETHODONTIDAE 1850.ga.f002

Oeditriton: McCranie ${ }^{+1} 2008$ - AN

ST: AL・CI: n0090 • ID: 536

PN: Oedipina quadra $\mathrm{McCranie}^{+1}, 2008$

PK: Oedipina quadra* $\mathrm{McCranie}^{+1}, 2008$

KG: Thornella nov.

KF: PLETHODONTIDAE 1850.ga.f002

Oedopinola Hilton, 1946 • KY

ST: PO.KN • CI: h1038 • ID: 535

PN: Oedipus complex Dunn, 1924

PK: Oedipus complex* Dunn, 1924

KG: Oedopinola* Hilton, 1946

KF: PLETHODONTIDAE 1850.ga.f002

Ogallalabatrachus Taylor, $1941+\bullet \mathbf{A K}$

ST: PO.JD・CI: h1039 • ID: 555

PN: Ogallalabatrachus horarium Taylor, 1941 \$

PK: Plioambystoma kansense ${ }^{\circ}$ Adams $^{+1}, 1929 \dagger$

KG: Ambystoma ${ }^{1}$ Tschudi, 1838

KF: АмвYSTOMATIDAE 1850.ga.f004

Oiacurus Leuckart, 1821 • AK

ST: NT.JI • CI: h1040 • ID: 566

PN: Triton cristatus Laurenti, 1768

PK: Triton cristatus* Laurenti, 1768

KG: Triturus $*$ Rafinesque, 1815

KF: SALAMANDRIDAE 1820.ga.f002

Oligosemia Navás, 1922 + KY

ST: PO.KN • CI: h1041 • ID: $\uparrow 195$

PN: Oligosemia spinosa Navás 1922 \$

PK: Oligosemia spinosa ${ }^{\circ}$ Navás $1922 \dagger$

KG: Oligosemia ${ }^{\circ}$ Navás $1922 \dagger$

KF: SALAMANDRIDAE 1820.ga.f002

Ollotis Cope, $1875 \bullet \mathbf{A K}$

ST: PO.JD・CI: h1042・ID: 137

PN: Ollotis coerulescens Cope, 1875

PK: Cranopsis fastidiosus* Cope, 1875

KG: Incilius* Cope, 1863

KF: BUFONIDAE 1825.gb.f004

Ologigon: Miranda-Ribeiro 1923 • AN

ST: AM • CI: n0091・ID: 232

PN: Hyla strigilata Spix, 1824

PK: Hyla strigilata ${ }^{\circ}$ Spix, 1824

KG: Scinax ${ }^{2}$ Wagler, 1830

KF: HYLIDAE 1815.ra.f002-|1825.gb.f001|

Ololigon: Miranda-Ribeiro $1923 \cdot \mathbf{A N}$

ST: AM • CI: n0092 - ID: 232

PN: Hyla strigilata Spix, 1824

PK: Hyla strigilata ${ }^{\circ}$ Spix, 1824 
KG: Scinax ${ }^{2}$ Wagler, 1830

KF: HYLIDAE 1815.ra.f002-|1825.gb.f001|

Ololygon Fitzinger, 1843 • AK

ST: PO.JD・CI: h1043・ID: 232

PN: Hyla strigilata Spix, 1824

PK: Hyla strigilata ${ }^{\circ}$ Spix, 1824

KG: Scinax ${ }^{2}$ Wagler, 1830

KF: HYLIDAE 1815.ra.f002-|1825.gb.f001|

Ombrana Dubois, 1992 • AK

ST: PO.JD • CI: h1044 • ID: 388

PN: Rana sikimensis Jerdon, 1870

PK: Rana sikimensis ${ }^{\circ}$ Jerdon, 1870

KG: Paa* Dubois, 1975

KF: DICROGLOSSIDAE 1987.da.f004

Ombropaa nov. $\bullet \mathrm{KY}$

ST: PO.KN・CI: h1045・ID: 382

PN: Rana gammii Anderson, 1871

PK: Rana gammii ${ }^{\circ}$ Anderson, 1871

KG: Ombropaa ${ }^{\circ}$ nov.

KF: DICROGLOSSIDAE 1987.da.f004

Ommatotriton Gray, 1850 • KY

ST: PO.KN • CI: h1046・ ID: 568

PN: Triton vittatus Gray, 1835

PK: Triton vittatus* Gray, 1835

KG: Ommatotriton* Gray, 1850

KF: SALAMANDRIDAE 1820.ga.f002

Oninia Günther ${ }^{+2}, 2010$ • AK

ST: PO.JD・CI: h1047・ID: 280

PN: Oninia senglaubi Günther ${ }^{+2}, 2010$

PK: Oninia senglaubi* Günther ${ }^{+2}, 2010$

KG: Asterophrys* Tschudi, 1838

KF: MICROHYLIDAE ||1843.fa.f012||-1931.na.f001

Onychodactylus Tschudi, 1838 - KY

ST: PO.KN・CI: h1048・ID: 517

PN: Onychodactylus schlegeli Tschudi, 1838

PK: Salamandra japonica* Houttuyn, 1782

KG: Onychodactylus ${ }^{1}$ Tschudi, 1838

KF: HYNOBIIDAE ||1856.ha.f001||-1859.cb.f002

Onychopus Fitzinger, $1843 \cdot \mathbf{z H}$

ST: zo • CI: zh063

Onychopus: Duméril ${ }^{+2} 1854$ • AN

ST: AM・CI: n0093・ID: 517

PN: Onychodactylus schlegeli Tschudi, 1838

PK: Salamandra japonica* Houttuyn, 1782

KG: Onychodactylus ${ }^{1}$ Tschudi, 1838

KF: НYNOBIIDAE ||1856.ha.f001||-1859.cb.f002

Onycopus: Duméril ${ }^{+1} 1841$ • AN

ST: AL •CI: n0094・ID: 503

PN: Megalobatrachus sieboldi Tschudi, 1837 \$

PK: Triton japonicus* Temminck, 1836

KG: Andrias $^{2}$ Tschudi, 1837

KF: CRYPTOBRANCHIDAE 1826.fb.f003

Oocormus Boulenger, 1905 • AK

ST: PO.JD • CI: h1049 • ID: 179

PN: Oocormus microps Boulenger, 1905

PK: Cystignathus parvulus* Girard, 1853
KG: Cycloramphus* Tschudi, 1838

KF: CYCLORAMPHIDAE 1850.bb.f003-|1852.ba.f001|

Ooeidozyga $\mathrm{Kuhl}^{+1}, 1822$ • AK

ST: PO.JI • CI: h1050 • ID: 395

PN: Rana lima Gravenhorst, 1829

PK: Rana lima* Gravenhorst, 1829

KG: Occidozyga* $\mathrm{Kuhl}^{+1}, 1822$

KF: OCCIDOZYGIDAE 1990.fa.f002

Oophaga Bauer, 1994 • KY

ST: PO.KN • CI: h1051・ID: 050

PN: Dendrobates pumilio Schmidt, 1857

PK: Dendrobates pumilio* Schmidt, 1857

KG: Oophaga* Bauer, 1994

KF: DENDROBATIDAE $\|1850 . b b . f 006\|-1865 . c a . f 002$

Ophiobatrachus Gray, 1868 • AK

ST: PO.JD・CI: h1052・ID: 534

PN: Ophiobatrachus vermicularis Gray, 1868

PK: Oedipina uniformis* Keferstein, 1868

KG: Oedipina* Keferstein, 1868

KF: PLETHODONTIDAE 1850.ga.f002

Ophryophryne Boulenger, $1903 \cdot \mathbf{K Y}$

ST: PO.KN • CI: h1053 • ID: 024

PN: Ophryophryne microstoma Boulenger, 1903

PK: Ophryophryne microstoma* Boulenger, 1903

KG: Ophryophryne* Boulenger, 1903

KF: MEGOPHRYIDAE 1850.bb.f008-|1931.na.f003

Opisthocoelellus Kuhn, 1941 \$ $\mathbf{K Y}$

ST: PO.KN・CI: h1054・ID: $\uparrow 110$

PN: Opisthocoelellus weigelti Kuhn, 1941 \$

PK: Opisthocoelellus weigelti ${ }^{\circ}$ Kuhn, $1941 \dagger$

KG: Opisthocoelellus ${ }^{\circ}$ Kuhn, $1941 \dagger$

KF: Mediogyrinia Familia InCERTAE SEDIS

Opisthodelphis Brocchi, 1881 • AK

ST: NS.JD・CI: h1055・ID: 091

PN: Notodelphys ovifera Lichtenstein $^{+1}, 1854$

PK: Notodelphys ovifera* Lichtenstein ${ }^{+1}, 1854$

KG: Gastrotheca* Fitzinger, 1843

KF: HEMIPHRACTIDAE 1862.pa.f001

Opisthodelphys Günther, 1859 • AK

ST: NT.JD・CI: h1056・ID: 091

PN: Notodelphys ovifera Lichtenstein $^{+1}, 1854$

PK: Notodelphys ovifera* Lichtenstein ${ }^{+1}, 1854$

KG: Gastrotheca* Fitzinger, 1843

KF: HEMIPHRACTIDAE 1862.pa.f001

Opisthodon Steindachner, 1867 • AK

ST: PO.JD・CI: h1057 • ID: 264

PN: Opisthodon frauenfeldi Steindachner, 1867

PK: Discoglossus ornatus* Gray, 1842

KG: Platyplectrum ${ }^{1}$ Günther, 1863

KF: MYOBATRACHIDAE 1850.sa.f001

Opisthothylax: Perret 1962 AN

ST: AL・CI: n0095・ID: 333

PN: Megalixalus immaculatus Boulenger, 1903

PK: Megalixalus immaculatus* Boulenger, 1903

KG: Opisthothylax* Perret, 1966

KF: HYPEROLIIDAE 1943.lb.f001 
Opisthothylax Perret, $1966 \bullet \mathbf{K Y}$

ST: PO.KN • CI: h1058 • ID: 333

PN: Megalixalus immaculatus Boulenger, 1903

PK: Megalixalus immaculatus* Boulenger, 1903

KG: Opisthothylax* Perret, 1966

KF: HYPEROLIIDAE 1943.lb.f001

Opisthotriton Auffenberg, 1961 \$ $\mathbf{K Y}$

ST: PO.KN • CI: h1059 • ID: $\uparrow 147$

PN: Opisthotriton kayi Auffenberg, 1961 \$

PK: Opisthotriton kayi ${ }^{\circ}$ Auffenberg, $1961 \uparrow$

KG: Opisthotriton ${ }^{\circ}$ Auffenberg, $1961 \dagger$

KF: HYLAEOBATRACHIDAE 1889.la.f001 †

Orchestes Illiger, $1798 \cdot \mathbf{z H}$

ST: zo • CI: zh064

Orchestes Tschudi, $1838 \bullet \mathbf{A K}$

ST: PO.JH・CI: h1060・ID: 447

PN: Hyla aurifasciata Schlegel, 1837

PK: Hyla aurifasciata* Schlegel, 1837

KG: Philautus* Gistel, 1848

KF: RHACOPHORIDAE ||1858.gc.f012 |-1932.ha.f001

Oreobates Jiménez de la Espada, $1872 \cdot \mathbf{K Y}$

ST: PO.KN • CI: h1061 • ID: 076

PN: Oreobates quixensis Jiménez de la Espada, 1872

PK: Oreobates quixensis* Jiménez de la Espada, 1872

KG: Oreobates* Jiménez de la Espada, 1872

KF: BRACHYCEPHALIDAE 1858.gc.f002

Oreobatrachus Boulenger, $1896 \bullet \mathbf{K Y}$

ST: PO.KN • CI: h1062 • ID: 396

PN: Oreobatrachus baluensis Boulenger, 1896

PK: Oreobatrachus baluensis* Boulenger, 1896

KG: Oreobatrachus* Boulenger, 1896

KF: OCCIDOZYGIDAE 1990.fa.f002

Oreolalax Myers $^{+1}, 1962 \cdot \mathbf{K Y}$

ST: PO.KN・CI: h1063・ID: 016

PN: Scutiger pingii Liu, 1943

PK: Scutiger pingii* Liu, 1943

KG: Oreolalax* Myers ${ }^{+1}, 1962$

KF: MEGOPHRYIDAE 1850.bb.f008-|1931.na.f003|

Oreophryne Boettger, $1895 \cdot \mathbf{A K}$

ST: PO.JD • CI: h1064 • ID: 280

PN: Oreophryne senckengergiana Boettger, 1895

PK: Microhyla achatina moluccensis ${ }^{\circ}$ Peters $^{+1}, 1878$

KG: Asterophrys* Tschudi, 1838

KF: MICROHYLIDAE ||1843.fa.f012||-1931.na.f001

Oreophryne Boulenger, 1895 • AK

ST: PO.JH • CI: h1065・ID: 148

PN: Oreophryne quelchii Boulenger, 1895

PK: Oreophryne quelchii* Boulenger, 1895

KG: Oreophrynella* Boulenger, 1895

KF: BUFONIDAE 1825.gb.f004

Oreophrynella Boulenger, $1895 \cdot \mathbf{K Y}$

ST: PO.KN • CI: h1066・ID: 148

PN: Oreophryne quelchii Boulenger, 1895

PK: Oreophryne quelchii* Boulenger, 1895

KG: Oreophrynella* Boulenger, 1895

KF: BUFONIDAE 1825.gb.f004
Oriandra Dubois $^{+1}, 2009 \cdot \mathbf{A K}$

ST: PO.JD・CI: h1067・ID: 578

PN: Salamandra maculosa infraimmaculata Martens, 1885

PK: Salamandra maculosa infraimmaculata* Martens, 1885

KG: Salamandra ${ }^{1}$ Garsault, 1764

KF: SALAMANDRIDAE 1820.ga.f002

Orixalus nov. $\bullet \mathrm{KY}$

ST: PO.KN • CI: h1068・ID: 440

PN: Gracixalus nonggangensis $\mathrm{Mo}^{+4}, 2013$

PK: Gracixalus nonggangensis* $\mathrm{Mo}^{+4}, 2013$

KG: Orixalus* nov.

KF: RHACOPHORIDAE ||1858.gc.f012||-1932.ha.f001

Orthophyia Meyer, $1845 \$ \cdot \mathbf{K Y}$

ST: PO.KN • CI: h1069・ID: $\uparrow 183$

PN: Orthophyia longa Meyer, 1845 \$

PK: Orthophyia longa ${ }^{\circ}$ Meyer, $1845 \dagger$

KG: Orthophyia ${ }^{\circ}$ Meyer, $1845 \dagger$

KF: PROTEIDAE 1831.ba.f002

Oscaecilia Taylor, $1968 \cdot \mathbf{K Y}$

ST: PO.KN・CI: h1070 • ID: 475

PN: Caecilia ochrocephala Cope, 1866

PK: Caecilia ochrocephala* Cope, 1866

KG: Oscaecilia* Taylor, 1968

KF: CAECILIIDAE 1814.ra.f003-|1825.gb.f008|

Osilophus Tschudi, $1838 \cdot \mathbf{A K}$

ST: PO.JD・CI: h1071・ID: 231

PN: Rana typhonia Linnaeus, 1758

PK: Rana typhonia* Linnaeus, 1758

KG: Trachycephalus* Tschudi, 1838

KF: HYLIDAE 1815.ra.f002-|1825.gb.f001|

Osornophryne Ruiz-Carranza ${ }^{+1}, 1976$ • KY

ST: PO.KN • CI: h1072・ID: 149

PN: Osornophryne percrassa Ruiz-Carranza ${ }^{+1}, 1976$

PK: Osornophryne percrassa* Ruiz-Carranza ${ }^{+1}, 1976$

KG: Osornophryne* Ruiz-Carranza ${ }^{+1}, 1976$

KF: BUFONIDAE 1825.gb.f004

Osteocephalus: Fitzinger 1843 • AN

ST: $\mathbf{A L} \cdot \mathbf{C I}: \mathrm{n} 0096 \cdot \mathbf{I D}: 223$

PN: Osteocephalus taurinus Steindachner, 1862

PK: Osteocephalus taurinus* Steindachner, 1862

KG: Osteocephalus* Steindachner, 1862

KF: HYLIDAE 1815.ra.f002-|1825.gb.f001|

Osteocephalus Steindachner, $1862 \cdot \mathbf{K Y}$

ST: PO.KN • CI: h1073・ID: 223

PN: Osteocephalus taurinus Steindachner, 1862

PK: Osteocephalus taurinus* Steindachner, 1862

KG: Osteocephalus* Steindachner, 1862

KF: HYLIDAE 1815.ra.f002-|1825.gb.f001|

Osteopilus Fitzinger, $1843 \cdot \mathbf{K Y}$

ST: PO.KN • CI: h1074・ ID: 225

PN: Trachycephalus marmoratus Duméril ${ }^{+1}, 1841$

PK: Hyla septentrionalis* Duméril ${ }^{+1}, 1841$

KG: Osteopilus ${ }^{1}$ Fitzinger, 1843

KF: HYLIDAE 1815.ra.f002-|1825.gb.f001|

Osteosternum $\mathrm{Wu}, 1929 \cdot \mathbf{A K}$

ST: PO.JD • CI: h1075 • ID: 395 
PN: Osteosternum amoyense Wu, 1929

PK: Rana lima* Gravenhorst, 1829

KG: Occidozyga* $\mathrm{Kuhl}^{+1}, 1822$

KF: OCCIDOZYGIDAE 1990.fa.f002

Otaspis Cope, 1869 • AK

ST: PO.JD • CI: h1076・ID: 144

PN: Peltaphryne empusa Cope, 1862

PK: Peltaphryne empusa* Cope, 1862

KG: Peltophryne* Fitzinger, 1843

KF: BUFONIDAE 1825.gb.f004

Otilopha Gray in Griffith, 1831 • AK

ST: PO.JD・CI: h1077•ID: 138

PN: Rana margaritifera Laurenti, 1768

PK: Rana margaritifera* Laurenti, 1768

KG: Rhinella ${ }^{2}$ Fitzinger, 1826

KF: BUFONIDAE 1825.gb.f004

Otilophes: Cuvier 1829 • AN

ST: AL • CI: n0097・ID: 138

PN: Rana margaritifera Laurenti, 1768

PK: Rana margaritifera* Laurenti, 1768

KG: Rhinella ${ }^{2}$ Fitzinger, 1826

KF: BUFONIDAE 1825.gb.f004

Otilophis Cuvier ${ }^{+1}$, 1831 • AK

ST: PO.JD • CI: h1078 • ID: 138

PN: Rana margaritifera Laurenti, 1768

PK: Rana margaritifera* Laurenti, 1768

KG: Rhinella ${ }^{2}$ Fitzinger, 1826

KF: BUFONIDAE 1825.gb.f004

Otilophus Cuvier ${ }^{+1}, 1832$ • AK

ST: PO.JD・CI: h1079・ID: 138

PN: Rana margaritifera Laurenti, 1768

PK: Rana margaritifera* Laurenti, 1768

KG: Rhinella ${ }^{2}$ Fitzinger, 1826

KF: BUFONIDAE 1825.gb.f004

Otilophus Günther, 1859 • AK

ST: PO.JH • CI: h1080 • ID: 231

PN: Rana typhonia Linnaeus, 1758

PK: Rana typhonia* Linnaeus, 1758

KG: Trachycephalus* Tschudi, 1838

KF: HYLIDAE 1815.ra.f002-|1825.gb.f001|

Otolophus Fitzinger, 1843 - AK

ST: Po.JD • CI: h1081 • ID: 138

PN: Rana margaritifera Laurenti, 1768

PK: Rana margaritifera* Laurenti, 1768

KG: Rhinella ${ }^{2}$ Fitzinger, 1826

KF: BUFONIDAE 1825.gb.f004

Otophryne Boulenger, $1900 \bullet \mathbf{K Y}$

ST: PO.KN • CI: h1082 • ID: 317

PN: Otophryne robusta Boulenger, 1900

PK: Otophryne robusta* Boulenger, 1900

KG: Otophryne* Boulenger, 1900

KF: MICROHYLIDAE ||1843.fa.f012||-1931.na.f001

Otylophus: Cei $1953 \cdot \mathbf{A N}$

ST: $\mathbf{A M} \bullet \mathbf{C I}:$ n0098・ID: 138

PN: Rana margaritifera Laurenti, 1768

PK: Rana margaritifera* Laurenti, 1768
KG: Rhinella ${ }^{2}$ Fitzinger, 1826

KF: BUFONIDAE 1825.gb.f004

Oumtkoutia Rage $^{+1}, 2008$ + $\mathbf{K Y}$

ST: PO.KN • CI: h1083 • ID: $\uparrow 074$

PN: Oumtkoutia anae Rage $^{+1}, 2008$ *

PK: Oumtkoutia anae ${ }^{\circ}$ Rage $^{+1}, 2008 \dagger$

KG: Oumtkoutia ${ }^{\circ}$ Rage $^{+1}, 2008$ †

KF: PIPIDAE 1825.gb.f003-|1826.fb.f002|

Oxydactyla Van Kampen, 1913 • AK

ST: PO.JD・CI: h1084・ID: 280

PN: Oxydactyla brevicrus Van Kampen, 1913

PK: Oxydactyla brevicrus ${ }^{\circ}$ Van Kampen, 1913

KG: Asterophrys* Tschudi, 1838

KF: MICROHYLIDAE ||1843.fa.f012||-1931.na.f001

Oxyglossus Swainson, $1827 \bullet \mathbf{Z H}$

ST: zo • CI: zh065

Oxyglossus Tschudi, 1838 • AK

ST: PO.JH • CI: h1085 • ID: 395

PN: Rana lima Gravenhorst, 1829

PK: Rana lima* Gravenhorst, 1829

KG: Occidozyga* $\mathrm{Kuhl}^{+1}, 1822$

KF: OCCIDOZYGIDAE 1990.fa.f002

Oxyrhachis Germar, $1833 \cdot \mathbf{Z H}$

ST: zo • CI: zh066

Oxyrhachis: Nicholls $1916 \cdot \mathbf{A N}$

ST: AL • CI: n0099・ID: 394

PN: Oxyglossus laevis Günther, 1859

PK: Oxyglossus laevis* Günther, 1859

KG: Frethia* nov.

KF: OCCIDOZYGIDAE 1990.fa.f002

Oxyrhinchus: Duméril ${ }^{+1} 1841 \bullet$ AN

ST: $\mathbf{A M} \bullet \mathbf{C I}:$ n0100・ID: 138

PN: Bufo granulosus Spix, 1824

PK: Bufo granulosus* Spix, 1824

KG: Rhinella ${ }^{2}$ Fitzinger, 1826

KF: BUFONIDAE 1825.gb.f004

Oxyrhynchus Leach, $1818 \cdot \mathbf{z H}$

ST: zo • CI: zh067

Oxyrhynchus Spix, 1824 • AK

ST: PO.JH • CI: h1086・ID: 138

PN: Bufo granulosus Spix, 1824

PK: Bufo granulosus* Spix, 1824

KG: Rhinella ${ }^{2}$ Fitzinger, 1826

KF: BUFONIDAE 1825.gb.f004

Paa Dubois, 1975 • KY

ST: PO.KN・CI: h1087・ID: 388

PN: Rana liebigii Günther, 1860

PK: Rana liebigii* Günther, 1860

KG: Paa* Dubois, 1975

KF: DiCROGLOSSIDAE 1987.da.f004

Pachybatrachus Keferstein, 1868 • AK

ST: PO.JD・CI: h1088・ID: 309

PN: Pachybatrachus petersii Keferstein, 1868

PK: Rana systoma* Schneider, 1799

KG: Uperodon* Duméril ${ }^{+1}, 1841$

KF: MICROHYLIDAE ||1843.fa.f012 |-1931.na.f001 
Pachybatrachus Mivart, 1869 • AK

ST: PO.JH • CI: h1089・ID: 402

PN: Pachybatrachus robustus Mivart, 1869

PK: Rana curtipes* Jerdon, 1853

KG: Clinotarsus* Mivart, 1869

KF: Ranidae 1796.ba.f001

Pachybatrachus $\mathrm{Bá}^{+1,1998}$ \$ $\mathbf{A K}$

ST: PO.JH • CI: h1090 • ID: $\uparrow 075$

PN: Pachybatrachus taqueti Báez ${ }^{+1}, 1998$ :

PK: Pachybatrachus taqueti ${ }^{\circ}$ Báez $^{+1}, 1998 \dagger$

KG: Pachycentrata ${ }^{\circ}$ Báez $^{+1}, 2004 \dagger$

KF: PIPIDAE 1825.gb.f003-|1826.fb.f002|

Pachycentrata $\mathrm{Báez}^{+1}, 2004$ + KY

ST: PO.KN • CI: h1091・ID: $\uparrow 075$

PN: Pachybatrachus taqueti Báez ${ }^{+1}, 1998$ :

PK: Pachybatrachus taqueti ${ }^{\circ}$ Báez $^{+1}, 1998 \uparrow$

KG: Pachycentrata ${ }^{\circ}$ Báez $^{+1}, 2004 \uparrow$

KF: PIPIDAE 1825.gb.f003-|1826.fb.f002|

Pachyhynobius $\mathrm{Fei}^{+2}, 1983 \cdot \mathbf{K Y}$

ST: PO.KN • CI: h1092 • ID: 512

PN: Pachyhynobius shangchengensis $\mathrm{Fe}^{+1}, 1983$

PK: Pachyhynobius shangchengensis* $\mathrm{Fe}^{+1}, 1983$

KG: Pachyhynobius ${ }^{*} \mathrm{Fe}^{+1}, 1983$

KF: НYNOBIIDAE ||1856.ha.f001||-1859.cb.f002

Pachymandra Parra-Olea ${ }^{+2}, 2004 \cdot \mathbf{A K}$

ST: PO.JD • CI: h1093 • ID: 522

PN: Spelerpes dofleini Werner, 1903

PK: Spelerpes dofleini* Werner, 1903

KG: Bolitoglossa* Duméril ${ }^{+2}, 1854$

KF: PLETHODONTIDAE 1850.ga.f002

Pachymedusa Duellman, 1968 • AK

ST: PO.JD • CI: h1094・ID: 238

PN: Phyllomedusa dacnicolor Cope, 1864

PK: Phyllomedusa dacnicolor* Cope, 1864

KG: Agalychnis* Cope, 1864

KF: PHYLLOMEDUSIDAE 1858.gc.f009

Pachypalaminus Thompson, $1912 \cdot \mathbf{K Y}$

ST: Po.KN • CI: h1095 • ID: 506

PN: Pachypalaminus boulengeri Thompson, 1912

PK: Pachypalaminus boulengeri* Thompson, 1912

KG: Pachypalaminus* Thompson, 1912

KF: HYNOBIIDAE ||1856.ha.f001|-1859.cb.f002

Pachypus Billberg, 1820 • ZH

ST: zo • CI: zh068

Pachypus Lutz, $1930 \cdot \mathbf{A K}$

ST: PO.JH • CI: h1096 •ID: 253

PN: Rana pentadactyla Laurenti, 1768

PK: Rana pentadactyla* Laurenti, 1768

KG: Leptodactylus ${ }^{1}$ Fitzinger, 1826

KF: LEPTODACTYLIDAE |1838.ta.f001||-1896.wa.f001

Pachytriton Boulenger, $1878 \cdot \mathbf{K Y}$

ST: PO.KN • CI: h1097 • ID: 561

PN: Triton brevipes Sauvage, 1877

PK: Triton brevipes* Sauvage, 1877

KG: Pachytriton* Boulenger, 1878

KF: SALAMANDRIDAE 1820.ga.f002
Paedomolge Hillis ${ }^{+3}$, 2001 • AK

ST: PO.JD • CI: h1098 • ID: 542

PN: Eurycea tonkawae Chippindale ${ }^{+3}, 2000$

PK: Eurycea tonkawae* Chippindale ${ }^{+3}, 2000$

KG: Eurycea* Rafinesque, 1822

KF: PLETHODONTIDAE 1850.ga.f002

Paedophryne Kraus, 2010 • AK

ST: PO.JD • CI: h1099・ID: 280

PN: Paedophryne kathismaphlox Kraus, 2010

PK: Paedophryne kathismaphlox ${ }^{\circ}$ Kraus, 2010

KG: Asterophrys* Tschudi, 1838

KF: MICROHYLIDAE ||1843.fa.f012||-1931.na.f001

Palaeobatrachus Tschudi, 1838 \$ KY

ST: PO.KN • CI: h1100・ID: $† 069$

PN: Palaeobatrachus goldfussii Tschudi, 1838 \$

PK: Rana diluvian $a^{\circ}$ Goldfuss, $1831 \uparrow$

KG: Palaeobatrachus ${ }^{\circ}$ Tschudi, $1838 \uparrow$

KF: PALAEOBATRACHIDAE 1865.ca.f001 †

Palaeobufo Bolkay, 1919 • AK

ST: PO.JD • CI: h1101 •ID: 138

PN: Rana marina Linnaeus, 1758

PK: Rana marina* Linnaeus, 1758

KG: Rhinella ${ }^{2}$ Fitzinger, 1826

KF: BUFONIDAE 1825.gb.f004

Palaeopelobates Kuhn, $1941+\cdot \mathbf{A K}$

ST: PO.JD • CI: h1102 • ID: $\uparrow 090$

PN: Palaeopelobates geiseltalensis Kuhn, 1941 *

PK: Halleobatrachus hinschei $i^{\circ}$ Kuhn, $1941 \uparrow$

KG: Eopelobates ${ }^{\circ}$ Parker, $1929 \dagger$

KF: PELOBATIDAE $1850 . b b . f 004$

Palaeophryne: Fitzinger $1843+\cdot \mathbf{A N}$

ST: AM • CI: n0101・ID: $\uparrow 094$

PN: Palaeophrynos gessneri Tschudi, $1838+$

PK: Palaeophrynos gessneri $i^{\circ}$ Tschudi, $1838 \uparrow$

KG: Palaeophrynos ${ }^{\circ}$ Tschudi, $1838 \dagger$

KF: BUFONIDAE 1825.gb.f004

Palaeophrynos Tschudi, $1838+\bullet \mathbf{K Y}$

ST: PO.KN • CI: h1103・ ID: †094

PN: Palaeophrynos gessneri Tschudi, 1838 *

PK: Palaeophrynos gessneri ${ }^{\circ}$ Tschudi, $1838 \dagger$

KG: Palaeophrynos ${ }^{\circ}$ Tschudi, $1838 \uparrow$

KF: BUFONIDAE 1825.gb.f004

Palaeophrynus Agassiz, 1844 \$・AK

ST: NT.JI • CI: h1104 • ID: $\dagger 094$

PN: Palaeophrynos gessneri Tschudi, 1838 \$

PK: Palaeophrynos gessneri ${ }^{\circ}$ Tschudi, $1838 \dagger$

KG: Palaeophrynos ${ }^{\circ}$ Tschudi, $1838 \uparrow$

KF: BUFONIDAE 1825.gb.f004

Palaeoplethodon Poinar $^{+1}, 2015+\cdot \mathbf{K Y}$

ST: PO.KN • CI: h1105・ID: $\uparrow 181$

PN: Palaeoplethodon hispaniolae Poinar $^{+1}, 2015$ *

PK: Palaeoplethodon hispaniolae ${ }^{\circ}$ Poinar $^{+1}, 2015 \dagger$

KG: Palaeoplethodon ${ }^{\circ}$ Poinar $^{+1}, 2015 \dagger$

KF: PLETHODONTIDAE 1850.ga.f002

Palaeopleurodeles Herre, $1941+\cdot \mathbf{K Y}$

ST: PO.KN • CI: h1106・ ID: $\uparrow 196$ 
PN: Palaeopleurodeles hauffi Herre, 1941 \$

PK: Palaeopleurodeles hauffi ${ }^{\circ}$ Herre, $1941 \dagger$

KG: Palaeopleurodeles ${ }^{\circ}$ Herre, $1941 \dagger$

KF: SALAMANDRIDAE 1820.ga.f002

Palaeoproteus Herre, $1935+\cdot \mathbf{K Y}$

ST: PO.KN • CI: h1107 • ID: $\uparrow 148$

PN: Palaeoproteus klatti Herre, 1935 \$

PK: Palaeoproteus klatti ${ }^{\circ}$ Herre, $1935 \uparrow$

KG: Palaeoproteus ${ }^{\circ}$ Herre, $1935 \dagger$

KF: HYLAEOBATRACHIDAE 1889.la.f001 †

Palaeosalamandra Herre, 1949 + AK

ST: PO.JD • CI: h1108 • ID: 578

PN: Palaeosalamandra kohlitzi Herre, 1949 †

PK: Salamandra sansaniensis ${ }^{\circ}$ Lartet, $1851 \dagger$

KG: Salamandra ${ }^{1}$ Garsault, 1764

KF: SALAMANDRIDAE 1820.ga.f002

Palaeosalamandrina Herre, $1949+\bullet A K$

ST: PO.JD • CI: h1109・ID: $\uparrow 193$

PN: Palaeosalamandrina dehmi Herre, 1949 \$

PK: Chelotriton paradoxus ${ }^{\circ}$ Pomel, $1853 \dagger$

KG: Chelotriton ${ }^{\circ}$ Pomel, $1853 \dagger$

KF: SALAMANDRIDAE 1820.ga.f002

Palaeotaricha Frank, 1955 +・AK

ST: PO.JD • CI: h1110 • ID: 570

PN: Palaeotaricha oligocenica Frank, 1955 \$

PK: Palaeotaricha oligocenica ${ }^{\circ}$ Frank, $1955 \dagger$

KG: Taricha* Gray, 1850

KF: SALAMANDRIDAE 1820.ga.f002

Palaeotriton Fitzinger, 1837 + $\mathbf{A K}$

ST: PO.CA • CI: h1111 •ID: 503

PN: Salamandra gigantea Meyer, 1832 *

PK: Salamandra scheuchzeri ${ }^{\circ}$ Holl, $1831 \dagger$

KG: Andrias $^{2}$ Tschudi, 1837

KF: CRYPTOBRANCHIDAE 1826.fb.f003

Palaeotriton Kittl, $1894 \cdot \mathbf{Z H}$

ST: zo • CI: zh069

Palaeotriton Bolkay, 1927 • AK

ST: PO.JH • CI: h1112 • ID: 564

PN: Lacerta vulgaris Linnaeus, 1758

PK: Lacerta vulgaris* Linnaeus, 1758

KG: Lissotriton ${ }^{1}$ Bell, 1839

KF: SALAMANDRIDAE 1820.ga.f002

Paleoamphiuma Rieppel ${ }^{+1}, 1998+\cdot \mathbf{K Y}$

ST: PO.KN • CI: h1113 • ID: $\uparrow 179$

PN: Paleoamphiuma tetradactylum Rieppel ${ }^{+1}, 1998$ \$

PK: Paleoamphiuma tetradactylum ${ }^{\circ}$ Rieppel $^{+1}, 1998 \dagger$

KG: Paleoamphiuma ${ }^{\circ}$ Rieppel $^{+1}, 1998 \dagger$

KF: AмPHIUMIDAE 1825.gb.f07

Paleorana: Scortecci $1931 \cdot \mathbf{A N}$

ST: AL • CI: n0102・ID: 351

PN: Rana beccarii Boulenger, 1911

PK: Rana beccarii ${ }^{\circ}$ Boulenger, 1911

KG: Conraua* Nieden, 1908

KF: CONRAUIDAE 1992.da.f001

Paleotriton: Bronn $1838+\cdot \mathbf{A N}$

ST: $\mathbf{A M} ・$ CI: n0103・ID: 503
PN: Salamandra gigantea Burton, 1808 \$

PK: Salamandra scheuchzer ${ }^{\circ}$ Holl, $1831 \dagger$

KG: Andrias $^{2}$ Tschudi, 1837

KF: CRYPTOBRANCHIDAE 1826.fb.f003

Palmatorappia Ahl, 1927 • AK

ST: PO.JD • CI: h1114 • ID: 369

PN: Hylella solomonis Sternfeld, 1918

PK: Hypsirana heffernani ${ }^{\circ}$ Kinghorn, 1928

KG: Cornufer* Tschudi, 1838

KF: CERATOBATRACHIDAE 1884.ba.f001

Palmatotriton Smith, 1945 - AK

ST: PO.CA • CI: h1115・ID: 522

PN: Oedipus rufescens Cope, 1869

PK: Oedipus rufescens* Cope, 1869

KG: Bolitoglossa* Duméril ${ }^{+2}, 1854$

KF: PLETHODONTIDAE 1850.ga.f002

Palmirana Ritgen, 1828 • AK

ST: Po.JI • CI: h1116・ID: 419

PN: Rana temporaria Linnaeus, 1758

PK: Rana temporaria* Linnaeus, 1758

KG: Rana* Linnaeus, 1758

KF: RANIDAE 1796.ba.f001

Palmitus: Rafinesque 1815 • AN

ST: AL • CI: n0104 • ID: 564

PN: Lacerta helvetica Razoumowsky, 1789

PK: Lacerta helvetica* Razoumowsky, 1789

KG: Lissotriton ${ }^{1}$ Bell, 1839

KF: SALAMANDRIDAE 1820.ga.f002

Paludicola Wagler, 1830 • AK

ST: PO.JD・CI: h1117・ID: 250

PN: Bufo albifrons Spix, 1824

PK: Bufo albifrons ${ }^{\circ}$ Spix, 1824

KG: Physalaemus* Fitzinger, 1826

KF: LEIUPERIDAE 1850.bb.f010

Pandanusicola Glaw ${ }^{+1}, 1994$ • AK

ST: PO.JD・CI: h1118・ID: 427

PN: Rhacophorus bicalcaratus Boettger, 1913

PK: Rhacophorus bicalcaratus* Boettger, 1913

KG: Guibemantis* Dubois, 1992

KF: RHACOPHORIDAE ||1858.gc.f012||-1932.ha.f001

Pangerpeton Wang $^{+1}, 2006+\cdot \mathbf{K Y}$

ST: PO.KN • CI: h1119・ID: $\uparrow 162$

PN: Pangerpeton sinensis $\mathrm{Wang}^{+1}, 2006$ \$

PK: Pangerpeton sinensis ${ }^{\circ}$ Wang $^{+1}, 2006 \dagger$

KG: Pangerpeton ${ }^{\circ} \mathrm{Wang}^{+1}, 2006 \dagger$

KF: IMPERFECTIBRANCHIA Familia INCERTAE SEDIS

Panophrys: Dujardin $1840 \cdot \mathbf{Z A}$

ST: zN • CI: zn007

Panophrys Dujardin, $1841 \cdot \mathbf{z H}$

ST: zo • CI: zh070

Panophrys $\mathrm{Rao}^{+1}, 1997 \cdot \mathbf{A K}$

ST: PO.JH • CI: h1 $120 \bullet$ ID: 023

PN: Megophrys omeimontis Liu, 1950

PK: Megophrys omeimontis* Liu, 1950

KG: Boulenophrys* $\mathrm{Fe}^{+1}, 2016$

KF: MEGOPHRYIDAE 1850.bb.f008-|1931.na.f003| 
Pantherana Dubois, 1992 • AK

ST: PO.JD • CI: h1121 • ID: 415

PN: Rana pipiens Schreber, 1782

PK: Rana pipiens* Schreber, 1782

KG: Lithobates* Fitzinger, 1843

KF: RANIDAE 1796.ba.f001

Papurana Dubois, 1992 • AK

ST: PO.JD • CI: h1122 • ID: 409

PN: Rana papua Lesson, 1830

PK: Rana papua* Lesson, 1830

KG: Hylarana* Tschudi, 1838

KF: RANIDAE 1796.ba.f001

Parabufella Kuhn, 1941 \$ AK

ST: PO.JD・CI: h1123・ID: $\uparrow 090$

PN: Parabufella longipes Kuhn, 1941 \$

PK: Halleobatrachus hinschei ${ }^{\circ}$ Kuhn, $1941 \dagger$

KG: Eopelobates ${ }^{\circ}$ Parker, $1929 \dagger$

KF: PELOBATIDAE 1850.bb.f004

Paracassina Peracca, 1907 • KY

ST: PO.KN • CI: h1124 • ID: 340

PN: Cassina obscura Boulenger, 1895

PK: Cassina obscura ${ }^{\circ}$ Boulenger, 1895

KG: Paracassina ${ }^{\circ}$ Peracca, 1907

KF: HYPEROLIIDAE 1943.lb.f001

Paracophyla Millot ${ }^{+1}$, $1951 \cdot \mathbf{A K}$

ST: PO.JD・CI: h1125・ID: 288

PN: Paracophyla tuberculata Millot $^{+1}, 1951$

PK: Platypelis barbouri* Noble, 1940

KG: Platypelis ${ }^{2}$ Boulenger, 1882

KF: MICROHYLIDAE ||1843.fa.f012||-1931.na.f001

Paracrinia Heyer ${ }^{+1}, 1976 \bullet \mathbf{K Y}$

ST: PO.KN・CI: h1126・ID: 269

PN: Crinia haswelli Fletcher, 1894

PK: Crinia haswelli* Fletcher, 1894

KG: Paracrinia ${ }^{*}$ Heyer $^{+1}, 1976$

KF: MYOBATRACHIDAE 1850.sa.f001

Paradactylodon: Risch $1984 \cdot \mathbf{A N}$

ST: AL • CI: n0105 • ID: 515

PN: Batrachuperus gorganensis Clergue-Gazeau ${ }^{+1}, 1979$

PK: Batrachuperus gorganensis* Clergue-Gazeau ${ }^{+1}, 1979$

KG: Iranodon* Dubois ${ }^{+1}, 2012$

KF: HYNOBIIDAE ||1856.ha.f001\|-1859.cb.f002

Paradiscoglossus Estes ${ }^{+1}, 1982+\bullet \mathbf{K Y}$

ST: PO.KN • CI: h1127・ID: $\uparrow 118$

PN: Paradiscoglossus americanus Estes $^{+1}, 1982$ *

PK: Paradiscoglossus americanus ${ }^{\circ}$ Estes $^{+1}, 1982 \dagger$

KG: Paradiscoglossus ${ }^{\circ}$ Estes $^{+1}, 1982 \uparrow$

KF: DISCOGLOSSIDAE 1858.gc.f004

Paradoxophyla Blommers-Schlösser ${ }^{+1}, 1991$ • KY

ST: PO.KN • CI: h1128 • ID: 290

PN: Microhyla palmata Guibé, 1974

PK: Microhyla palmata* Guibé, 1974

KG: Paradoxophyla* Blommers-Schlösser ${ }^{+1}, 1991$

KF: MICROHYLIDAE ||1843.fa.f012||-1931.na.f001

Paraheleioporus Hoser, $2019 \bullet \mathbf{K Y}$

ST: PO.KN • CI: h1129・ID: 259
PN: Heleioporus barycragus Lee, 1967

PK: Heleioporus barycragus ${ }^{\circ}$ Lee, 1967

KG: Heleioporus ${ }^{2}$ Gray, 1841

KF: MYOBATRACHIDAE 1850.sa.f001

Parahynobius Venczel, $1999+\cdot \mathbf{K Y}$

ST: PO.KN • CI: h1130 IID: $\uparrow 172$

PN: Parahynobius betfianus Venczel, 1999 *

PK: Parahynobius betfianus ${ }^{\circ}$ Venczel, $1999 \dagger$

KG: Parahynobius ${ }^{\circ}$ Venczel, $1999 \dagger$

KF: HYNOBIIDAE $\| 1856$.ha.f001\|-1859.cb.f002

Paralatonia Venczel $^{+1}, 2003+\bullet \mathbf{K Y}$

ST: PO.KN • CI: h1131・ID: $\uparrow 119$

PN: Paralatonia transylvanica Venczel $^{+1}, 2003$ \$

PK: Paralatonia transylvanica ${ }^{\circ}$ Venczel $^{+1}, 2003 \dagger$

KG: Paralatonia $^{\circ}$ Venczel $^{+1}, 2003 \uparrow$

KF: DISCOGLOSSIDAE 1858.gc.f004

Paramegophrys: Liu $1964 \cdot \mathbf{A N}$

ST: $\mathbf{A L} ・ \mathbf{C I}: \mathrm{n} 0106 \cdot \mathbf{I D}: 018$

PN: Leptobrachium pelodytoides Boulenger, 1893

PK: Leptobrachium pelodytoides* Boulenger, 1893

KG: Leptobrachella ${ }^{\circ}$ Smith, 1925

KF: MEGOPHRYIDAE 1850.bb.f008-|1931.na.f003|

Paramesotriton Chang, 1936• KY

ST: PO.KN • CI: h1132・ID: 562

PN: Mesotriton deloustali Bourret, 1934

PK: Mesotriton deloustali* Bourret, 1934

KG: Paramesotriton* Chang, 1936

KF: SALAMANDRIDAE 1820.ga.f002

Paramophrynella La Marca, 2007 • AK

ST: PO.JD・CI: h1133・ID: 078

PN: Eupsophus ginesi Rivero, 1964

PK: Eupsophus ginesi $i^{\circ}$ Rivero, 1964

KG: Pristimantis* Jiménez de la Espada, 1870

KF: BRACHYCEPHALIDAE 1858.gc.f002

Paranecturus Demar, $2013+\bullet \mathbf{K Y}$

ST: PO.KN • CI: h1134 - ID: $\uparrow 184$

PN: Paranecturus garbanii Demar, 2013 *

PK: Paranecturus garbanii ${ }^{\circ}$ Demar, $2013 \dagger$

KG: Paranecturus ${ }^{\circ}$ Demar, 2013 †

KF: PROTEIDAE 1831.ba.f002

Parapelophryne $\mathrm{Fei}^{+2}, 2003 \cdot \mathrm{KY}$

ST: PO.KN • CI: h1135・ID: 103

PN: Nectophryne scalptus $\mathrm{Liu}^{+1}, 1973$

PK: Nectophryne scalptus ${ }^{\circ} \mathrm{Liu}^{+1}, 1973$

KG: Parapelophryne ${ }^{\circ} \mathrm{Fei}^{+1}, 2003$

KF: BUFONIDAE 1825.gb.f004

Paraphyllobates: Bauer 1994 • AN

ST: AL・CI: n0107・ID: 039

PN: Hyla trivittata Spix, 1824

PK: Hyla trivittata* Spix, 1824

KG: Ameerega* Bauer, 1986

KF: DENDROBATIDAE $\|1850 . b b . f 006\|-1865 . c a . f 002$

Parapseudacris Hardy ${ }^{+1}$, 1986 • AK

ST: PO.JD • CI: h1136 • ID: 200

PN: Hyla crucifer Wied-Neuwied, 1838

PK: Hyla crucifer* Wied-Neuwied, 1838 
KG: Pseudacris* Fitzinger, 1843

KF: HYLIDAE 1815.ra.f002-|1825.gb.f001|

Pararthroleptis Ahl, 1925 • AK

ST: PO.JD • CI: h1137 • ID: 350

PN: Pararthroleptis nanus Ahl, 1925

PK: Pararthroleptis nanus ${ }^{\circ}$ Ahl, 1925

KG: Phrynobatrachus* Günther, 1862

KF: PHRYNOBATRACHIDAE 1941.lb.f001

Paratelmatobius Lutz ${ }^{+1}, 1958$ • AK

ST: PO.JD • CI: h1138 • ID: 254

PN: Paratelmatobius lutzii Lutz ${ }^{+1}, 1958$

PK: Paratelmatobius lutzii ${ }^{\circ}$ Lutz $^{+1}, 1958$

KG: Crossodactylodes ${ }^{2}$ Cochran, 1938

KF: PARATELMATOBIIDAE 2012.oa.f001

Parhoplophryne Barbour ${ }^{+1}, 1928$ • KY

ST: PO.KN • CI: h1139・ID: 304

PN: Parhoplophryne usambarica Barbour $^{+1}, 1928$

PK: Parhoplophryne usambarica ${ }^{\circ}$ Barbour $^{+1}, 1928$

KG: Parhoplophryne ${ }^{\circ}$ Barbour $^{+1}, 1928$

KF: MICROHYLIDAE ||1843.fa.f012||-1931.na.f001

Parkerana Dubois, 1984 • AK

ST: PO.JD • CI: h1140 • ID: 464

PN: Abrana cotti Parker, 1931

PK: Rana schillukorum ${ }^{\circ}$ Werner, 1908

KG: Ptychadena* Boulenger, 1917

KF: PTYCHADENIDAE 1987.da.f002

Parrisia Denton ${ }^{+1}, 1998+\bullet \mathbf{K Y}$

ST: PO.KN • CI: h1141・ID: $\uparrow 149$

PN: Parrisia neocesariensis Denton ${ }^{+1}, 1998$ \$

PK: Parrisia neocesariensis ${ }^{\circ}$ Denton $^{+1}, 1998 \dagger$

KG: Parrisia $^{\circ}$ Denton $^{+1}, 1998 \dagger$

KF: HYLAEOBATRACHIDAE 1889.1a.f001 †

Paruwrobates Bauer, $1994 \cdot \mathbf{K Y}$

ST: PO.KN・CI: h1142・ID: 054

PN: Dendrobates andinus Myers $^{+1}, 1987$

PK: Dendrobates andinus ${ }^{\circ}$ Myers $^{+1}, 1987$

KG: Paruwrobates $^{\circ}$ Bauer, 1994

KF: DENDROBATIDAE |1850.bb.f006"|-1865.ca.f002

Parvibranchus Hogg, 1839 • AK

ST: NL.JI • CI: h1143・ID: 518

PN: Siren striata Le Conte, 1824

PK: Siren striata* Le Conte, 1824

KG: Pseudobranchus* Gray, 1825

KF: SIRENIDAE 1825gb.f005

Parvicaecilia Taylor, 1968 • AK

ST: PO.JD・CI: h1144 • ID: 492

PN: Gymnophis nicefori Barbour, 1924

PK: Gymnophis nicefori ${ }^{\circ}$ Barbour, 1924

KG: Microcaecilia ${ }^{3}$ Taylor, 1968

KF: CAECILIIDAE 1814.ra.f003-|1825.gb.f008|

Parvimolge Taylor, $1944 \cdot \mathbf{K Y}$

ST: PO.KN • CI: h1145 • ID: 526

PN: Oedipus townsendi Dunn, 1922

PK: Oedipus townsendi* Dunn, 1922

KG: Parvimolge* Taylor, 1944

KF: PLETHODONTIDAE 1850.ga.f002
Parvulus Lutz, 1930 • AK

ST: PO.JD・CI: h1146・ID: 251

PN: Leptodactylus nanus Müller, 1922

PK: Leptodactylus nanus ${ }^{\circ}$ Müller, 1922

KG: Adenomera ${ }^{3}$ Steindachner, 1867

KF: LEPTODACTYLIDAE ||1838.ta.f001||-1896.wa.f001

Parvurus Dubois ${ }^{+1}, 2012$ • AK

ST: PO.JD・CI: h1 $147 \bullet$ ID: 553

PN: Menobranchus punctatus Gibbes, 1850

PK: Menobranchus punctatus* Gibbes, 1850

KG: Necturus* Rafinesque, 1819

KF: PROTEIDAE 1831.ba.f002

Patagopipa Aranciaga Rolando ${ }^{+2}, 2019+\bullet \mathbf{K Y}$

ST: PO.KN • CI: h1148・ID: $\dagger 078$

PN: Patagopipa corsolinii Aranciaga Rolando ${ }^{+2}, 2019$ *

PK: Patagopipa corsolinii ${ }^{\circ}$ Aranciaga Rolando ${ }^{+2}, 2019 \dagger$

KG: Patagopipa ${ }^{\circ}$ Aranciaga Rolando ${ }^{+2}, 2019 \dagger$

KF: PIPIDAE 1825.gb.f003-|1826.fb.f002|

Pectoglossa Mivart, $1868 \cdot \mathbf{A K}$

ST: PO.JD・CI: h1149・ID: 555

PN: Plethodon persimilis Gray, 1859

PK: Salamandra jeffersoniana* Green, 1827

KG: Ambystoma ${ }^{1}$ Tschudi, 1838

KF: АмвуSTомAтIDAE 1850.ga.f004

Pedostibes Günther, 1876 • KY

ST: PO.KN • CI: h1150 • ID: 110

PN: Pedostibes tuberculosus Günther, 1876

PK: Pedostibes tuberculosus* Günther, 1876

KG: Pedostibes* Günther, 1876

KF: BUFONIDAE 1825.gb.f004

Pegaeus Gistel, 1868 • AK

ST: PO.JI • CI: h1151 • ID: 120

PN: Rana bufo Linnaeus, 1758

PK: Rana bufo* Linnaeus, 1758

KG: $B u f o^{*}$ Garsault, 1764

KF: BUFONIDAE 1825.gb.f004

Pelida Gistel, 1848 • AK

ST: NL.JD • CI: h1152 • ID: 310

PN: Bombinator baleatus Müller, 1836

PK: Bombinator baleatus* Müller, 1836

KG: Kaloula* Gray, 1831

KF: MICROHYLIDAE ||1843.fa.f012||-1931.na.f001

Pelobates Wagler, $1830 \cdot \mathbf{K Y}$

ST: PO.KN • CI: h1153・ID: 026

PN: Bufo fuscus Laurenti, 1768

PK: Bufo fuscus* Laurenti, 1768

KG: Pelobates* Wagler, 1830

KF: PELOBATIDAE 1850.bb.f004

Pelobatinopsis Kuhn, $1941+\cdot \mathbf{A K}$

ST: PO.JD • CI: h1154 • ID: $\uparrow 069$

PN: Pelobatinopsis hinschei Kuhn, 1941 \%

PK: Pelobatinopsis hinschei ${ }^{\circ}$ Kuhn, $1941 \dagger$

KG: Palaeobatrachus ${ }^{\circ}$ Tschudi, $1838 \dagger$

KF: PALAEOBATRACHIDAE 1865.ca.f001 †

Pelobatrachus Beddard, 1908 • AK

ST: PO.JD・CI: h1155・ID: 021 
PN: Ceratophryne nasuta Schlegel, 1858

PK: Ceratophryne nasuta* Schlegel, 1858

KG: Megophrys ${ }^{2} \mathrm{Kuhl}^{+1}, 1822$

KF: MEGOPHRYIDAE 1850.bb.f008-|1931.na.f003|

Pelobius Erichson, $1832 \cdot \mathbf{z H}$

ST: zo • CI: zh071

Pelobius Fitzinger, 1843 • AK

ST: PO.JH • CI: h1156•ID: 235

PN: Litoria freycineti Tschudi, 1838

PK: Litoria freycineti* Tschudi, 1838

KG: Litoria* Tschudi, 1838

KF: PHYLLOMEDUSIDAE 1858.gc.f009

Pelodryas: Günther 1858 • AN

ST: AL • CI: n0108・ID: 237

PN: Rana caerulea White, 1890

PK: Rana caerulea* White, 1890

KG: Ranoidea ${ }^{1}$ Tschudi, 1838

KF: PHYLLOMEDUSIDAE 1858.gc.f009

Pelodryas Günther, 1859 • AK

ST: PO.JD • CI: h1157 • ID: 237

PN: Rana caerulea White, 1890

PK: Rana caerulea* White, 1890

KG: Ranoidea ${ }^{1}$ Tschudi, 1838

KF: PHYLLOMEDUSIDAE 1858.gc.f009

Pelodytes Bonaparte, $1838 \cdot \mathbf{K Y}$

ST: PO.KN • CI: h1158・ID: 027

PN: Rana punctata Daudin, 1802

PK: Rana punctata* Daudin, 1802

KG: Pelodytes* Bonaparte, 1838

KF: PELODYTIDAE 1850.bb.f002

Pelodytes Gistel, 1848 • AK

ST: NL.JH • CI: h1159 • ID: 540

PN: Salamandra subfusca Green, 1818

PK: Salamandra rubra* Sonnini ${ }^{+1}, 1801$

KG: Pseudotriton ${ }^{1}$ Tschudi, 1838

KF: PlethodontidaE 1850.ga.f002

Pelodytopsis Nikolskii, $1896 \bullet \mathbf{K Y}$

ST: PO.KN • CI: h1160 • ID: 028

PN: Pelodytes caucasicus Boulenger, 1896

PK: Pelodytes caucasicus* Boulenger, 1896

KG: Pelodytopsis Nikolskii, 1896

KF: PELODYTIDAE 1850.bb.f002

Pelonectes Fitzinger, 1843 • AK

ST: PO.JD • CI: h1161 • ID: 557

PN: Molge platycephala Gravenhorst, 1829

PK: Molge platycephala* Gravenhorst, 1829

KG: Euproctus ${ }^{1}$ Gené, 1839

KF: SALAMANDRIDAE 1820.ga.f002

Pelonectes Lataste in Blanchard, 1879 • AK

ST: PO.JH • CI: h1162 • ID: 564

PN: Pelonectes boscai Lataste in Blanchard, 1879

PK: Pelonectes boscai* Lataste in Blanchard, 1879

KG: Lissotriton ${ }^{1}$ Bell, 1839

KF: SALAMANDRIDAE 1820.ga.f002

Pelopeltis Bauer, 1986 • AK

ST: PO.JD • CI: h1163 • ID: 325
PN: Leptopelis bufonides Schiøtz, 1967

PK: Leptopelis bufonides ${ }^{\circ}$ Schiøtz, 1967

KG: Leptopelis* Günther, 1859

KF: ARTHROLEPTIDAE 1869.ma.f011

Pelophilus Tschudi, $1838+\bullet \mathbf{K Y}$

ST: PO.KN • CI: h1164・ID: $\uparrow 111$

PN: Pelophilus agassizii Tschudi, 1838 †

PK: Pelophilus agassizii ${ }^{\circ}$ Tschudi, $1838 \dagger$

KG: Pelophilus ${ }^{\circ}$ Tschudi, $1838 \dagger$

KF: Mediogyrinia Familia INCERTAE SEDIS

Pelophryne Barbour, 1938 • KY

ST: PO.KN • CI: h1165・ID: 114

PN: Pelophryne albotaeniata Barbour, 1938

PK: Pelophryne albotaeniata ${ }^{\circ}$ Barbour, 1938

KG: Pelophryne 3 Barbour, 1938

KF: BUFONIDAE 1825.gb.f004

Pelophylax Fitzinger, $1843 \cdot \mathbf{K Y}$

ST: PO.KN • CI: h1166・ID: 406

PN: Rana esculenta Linnaeus, 1758

PK: Rana esculenta* Linnaeus, 1758

KG: Pelophylax* Fitzinger, 1843

KF: RANIDAE 1796.ba.f001

Pelorius Hedges, 1989 • AK

ST: PO.JD・CI: h1167・ID: 081

PN: Leptodactylus inoptatus Barbour, 1914

PK: Leptodactylus inoptatus* Barbour, 1914

KG: Eleutherodactylus* Duméril ${ }^{+1}, 1841$

KF: BRACHYCEPHALIDAE 1858.gc.f002

Peltocephalus Duméril ${ }^{+1}, 1835 \cdot \mathbf{Z H}$

ST: zo • CI: zh072

Peltocephalus Tschudi, 1838 • AK

ST: PO.JH • CI: h1168・ID: 257

PN: Peltocephalus quoyi Tschudi, 1838

PK: Calyptocephalus gayi* Duméril ${ }^{+1}, 1841$

KG: Calyptocephalella* Strand, 1928

KF: CALYPTOCEPHALELLIDAE 1960.ra.f001

Peltophryne Fitzinger, 1843 • KY

ST: PO.KN • CI: h1169・ID: 144

PN: Bufo peltocephalus Tschudi, 1838

PK: Bufo peltocephalus* Tschudi, 1838

KG: Peltophryne* Fitzinger, 1843

KF: BUFONIDAE 1825.gb.f004

Pelusius: Wagler $1830 \cdot \mathbf{A N}$

ST: $\mathbf{A M} ・ \mathbf{C I}:$ n0109・ID: 504

PN: Salamandra gigantea Barton, 1808

PK: Salamandra alleganiensis* Sonnini ${ }^{+1}, 1801$

KG: Cryptobranchus ${ }^{1}$ Leuckart, 1821

KF: CRYPTOBRANCHIDAE 1826.fb.f003

Pengilleyia Wells ${ }^{+1}, 1985$ • AK

ST: PO.JD・CI: h1170・ID: 235

PN: Litoria tyleri Martin ${ }^{+4}, 1979$

PK: Litoria tyleri* ${ }^{*}$ Martin $^{+4}, 1979$

KG: Litoria* Tschudi, 1838

KF: PHYLLOMEDUSIDAE 1858.gc.f009

Peralaimos Jiménez de la Espada, 1875 • AK

ST: PO.JD・CI: h1171 • ID: 248 
PN: Bufo stentor Jiménez de la Espada, 1872

PK: Paludicola pustulosa* Cope, 1864

KG: Engystomops* Jiménez de la Espada, 1872

KF: LEIUPERIDAE 1850.bb.f010

Peratosauroides Naylor in Estes, $1981+\cdot \mathbf{K Y}$

ST: PO.KN • CI: h1172 • ID: $\uparrow 150$

PN: Peratosauroides problematica Naylor in Estes, 1981 †

PK: Peratosauroides problematica ${ }^{\circ}$ Naylor in Estes, $1981 \uparrow$

KG: Peratosauroides ${ }^{\circ}$ Naylor in Estes, $1981 \dagger$

KF: HYLAEOBATRACHIDAE 1889.1a.f001 †

Perialia Gray, 1845 • AK

ST: PO.JD・CI: h1173・ID: 259

PN: Perialia eyrei Gray, 1845

PK: Perialia eyrei ${ }^{\circ}$ Gray, 1845

KG: Heleioporus ${ }^{2}$ Gray, 1841

KF: MYOBATRACHIDAE 1850.sa.f001

Petraponia Massalongo, 1853 • AK

ST: PO.JD • CI: h1174・ID: 566

PN: Petroponia nigra Massalongo, 1854

PK: Triton carnifex* Laurenti, 1768

KG: Triturus* Rafinesque, 1815

KF: SALAMANDRIDAE 1820.ga.f002

Petropedetes Reichenow, $1874 \cdot \mathbf{K Y}$

ST: PO.KN • CI: h1175 • ID: 355

PN: Petropedetes cameronensis Reichenow, 1874

PK: Petropedetes cameronensis* Reichenow, 1874

KG: Petropedetes* Reichenow, 1874

KF: PETROPEDETIDAE 1931.na.f006

Phaenerobranchus Fitzinger, 1826 • AK

ST: NS.JD・CI: h1176・ID: 553

PN: Phanerobranchus tetradactylus Leuckart, 1821

PK: Sirena maculosa* Rafinesque, 1818

KG: Necturus* Rafinesque, 1819

KF: PROTEIDAE 1831.ba.f002

Phaeognathus Highton, 1961 • KY

ST: PO.KN • CI: h1177•ID: 549

PN: Phaeognathus hubrichti Highton, 1961

PK: Phaeognathus hubrichti* Highton, 1961

KG: Phaeognathus* Highton, 1961

KF: PlethodontidaE 1850.ga.f002

Phanerabronchus: Baird 1849 • AN

ST: $\mathbf{A M} ・$ CI: n0110 • ID: 553

PN: Phanerobranchus tetradactylus Leuckart, 1821

PK: Sirena maculosa* Rafinesque, 1818

KG: Necturus* Rafinesque, 1819

KF: PROTEIDAE 1831.ba.f002

Phanerobranchus Leuckart, 1821 • AK

ST: PO.JD・CI: h1178・ID: 553

PN: Phanerobranchus tetradactylus Leuckart, 1821

PK: Sirena maculosa* Rafinesque, 1818

KG: Necturus* Rafinesque, 1819

KF: PROTEIDAE 1831.ba.f002

Phanerobronchus: Baird 1849 • AN

ST: AM • CI: n0111 • ID: 553

PN: Phanerobranchus tetradactylus Leuckart, 1821

PK: Sirena maculosa* Rafinesque, 1818
KG: Necturus* Rafinesque, 1819

KF: PROTEIDAE 1831.ba.f002

Phanerotis Boulenger, 1890 • AK

ST: PO.JD • CI: h1179・ID: 264

PN: Phanerotis fletcheri Boulenger, 1890

PK: Phanerotis fletcheri* Boulenger, 1890

KG: Platyplectrum ${ }^{1}$ Günther, 1863

KF: MYOBATRACHIDAE 1850.sa.f001

Pharyngodon Diesing, $1861 \bullet \mathbf{Z H}$

ST: zo • CI: zh073

Pharyngodon Cope, 1865 • AK

ST: PO.JH • CI: h1180 • ID: 210

PN: Pharyngodon petasatus Cope, 1865

PK: Pharyngodon petasatus* Cope, 1865

KG: Triprion* Cope, 1866

KF: HYLIDAE 1815.ra.f002-|1825.gb.f001|

Phasmahyla Cruz, 1991 • KY

ST: PO.KN • CI: h1181・ID: 242

PN: Phyllomedusa guttata Lutz, 1924

PK: Phyllomedusa guttata* Lutz, 1924

KG: Phasmahyla* Cruz, 1991

KF: PHYLLOMEDUSIDAE 1858.gc.f009

Phatnomatorhina: Bonaparte 1839 • AN

ST: AL • CI: n0112 • ID: 551

PN: Salamandra glutinosa Green, 1818

PK: Salamandra glutinosa* Green, 1818

KG: Plethodon* Tschudi, 1838

KF: PLETHODONTIDAE 1850.ga.f002

Pherohapsis Zweifel, 1972 • AK

ST: PO.JD・CI: h1182・ID: 280

PN: Pherohapsis menziesi Zweifel, 1972

PK: Pherohapsis menziesi* Zweifel, 1972

KG: Asterophrys* Tschudi, 1838

KF: MICROHYLIDAE ||1843.fa.f012 |-1931.na.f001

Philautus Gistel, 1848 • KY

ST: NL.KN • CI: h1183・ID: 447

PN: Hyla aurifasciata Schlegel, 1837

PK: Hyla aurifasciata* Schlegel, 1837

KG: Philautus* Gistel, 1848

KF: RHACOPHORIDAE ||1858.gc.f012||-1932.ha.f001

Philhydrus Brookes, 1828 • EX

ST: PO.CE • CI: e0010 • ID: 555

PN: Siren pisciformis Shaw, 1802

PK: Gyrinus mexicanus* Shaw $^{+1}, 1789$

KG: Ambystoma ${ }^{1}$ Tschudi, 1838

KF: AмвYSTOMATIDAE 1850.ga.f004

Philocryphus Fletcher, 1894 • AK

ST: PO.JD・CI: h1185・ID: 259

PN: Philocryphus flavoguttatus Fletcher, 1894

PK: Rana australiaca* Shaw $^{+1}, 1795$

KG: Heleioporus ${ }^{2}$ Gray, 1841

KF: MYOBATRACHIDAE 1850.sa.f001

Philoria Spencer, 1901 • KY

ST: PO.KN • CI: h1186・ID: 262

PN: Philoria frosti Spencer, 1901

PK: Philoria frosti ${ }^{\circ}$ Spencer, 1901 
KG: Philoria ${ }^{2}$ Spencer, 1901

KF: MYOBATRACHIDAE 1850.sa.f001

Phirix Schmidt, 1857 • AK

ST: PO.JD • CI: h1187 • ID: 100

PN: Phirix pachydermus Schmidt, 1857

PK: Phirix pachydermus ${ }^{\circ}$ Schmidt, 1857

KG: Atelopus* Duméril ${ }^{+1}, 1841$

KF: BUFONIDAE 1825.gb.f004

Phlyctimantis Laurent ${ }^{+1}, 1950 \bullet \mathbf{A K}$

ST: PO.JD • CI: h1188 • ID: 337

PN: Hylambates leonardi Boulenger, 1906

PK: Hylambates leonardi* Boulenger, 1906

KG: Hylambates* Duméril, 1853

KF: HYPEROLIIDAE 1943.lb.f001

Phobobates Zimmermann ${ }^{+1}$, 1988 • AK

ST: PO.JD • CI: h1189 • ID: 039

PN: Dendrobates silverstonei Myers ${ }^{+1}, 1979$

PK: Dendrobates silverstonei* Myers $^{+1}, 1979$

KG: Ameerega* Bauer, 1986

KF: DENDROBATIDAE $\| 1850$. bb.f006"|-1865.ca.f002

Phosphotriton: Tissier $^{+6} 2015+\mathbf{A N}$

ST: AL • CI: n0113 • ID: $\uparrow 197$

PN: Phosphotriton sigei Tissier $^{+6}, 2016$ \$

PK: Phosphotriton sige ${ }^{\circ}$ Tissier $^{+6}, 2016 \dagger$

KG: Phosphotriton ${ }^{\circ}$ Tissier $^{+6}, 2016 \dagger$

KF: SALAMANDRIDAE 1820.ga.f002

Phosphotriton Tissier ${ }^{+6}, 2016 \$ \cdot \mathbf{K Y}$

ST: PO.KN • CI: h1190・ID: $\uparrow 197$

PN: Phosphotriton sigei Tissier $^{+6}, 2016$ *

PK: Phosphotriton sigei ${ }^{\circ}$ Tissier $^{+6}, 2016 \uparrow$

KG: Phosphotriton ${ }^{\circ}$ Tissier $^{+6}, 2016 \dagger$

KF: SALAMANDRIDAE 1820.ga.f002

Phractops Peters, 1867 • AK

ST: PO.JD • CI: h1191 • ID: 237

PN: Phractops alutaceus Peters, 1867

PK: Cyclorana novaehollandiae* Steindachner, 1867

KG: Ranoidea ${ }^{1}$ Tschudi, 1838

KF: PHYLLOMEDUSIDAE 1858.gc.f009

Phreniscus: Gray 1841 • AN

ST: $\mathbf{A M} ・$ CI: n0114・ID: 138

PN: Phryniscus nigricans Wiegmann, 1834

PK: Bufo spinulosus* Wiegmann, 1834

KG: Rhinella ${ }^{2}$ Fitzinger, 1826

KF: BUFONIDAE 1825.gb.f004

Phrynacius: Rafinesque 1815 • AN

ST: $\mathbf{A L} \bullet \mathbf{C I}:$ n0115 • ID: 120

PN: Rana bufo Linnaeus, 1758

PK: Rana bufo* Linnaeus, 1758

KG: Bufo* Garsault, 1764

KF: BUFONIDAE 1825.gb.f004

Phrynanodus Ahl, 1933 • AK

ST: PO.JD • CI: h1192 • ID: 058

PN: Phrynanodus nanus Ahl, 1933

PK: Hylodes parvus* Girard, 1853

KG: Ischnocnema* Reinhardt ${ }^{+1}, 1862$

KF: BRACHYCEPHALIDAE 1858.gc.f002
Phryne Meigen, $1800 \cdot \mathbf{z H}$

ST: zo • CI: zh074

Phryne Oken, 1816 • EX

ST: PO.Cw • CI: e0011 • ID: 120

PN: Bufo vulgaris Laurenti, 1768

PK: Rana bufo* Linnaeus, 1758

KG: Bufo* Garsault, 1764

KF: BUFONIDAE 1825.gb.f004

Phryne Fitzinger, 1843 - AK

ST: PO.JH • CI: h1194 • ID: 120

PN: Bufo vulgaris Laurenti, 1768

PK: Rana bufo* Linnaeus, 1758

KG: $B$ ufo* Garsault, 1764

KF: BUFONIDAE 1825.gb.f004

Phrynella Boulenger, 1887 • KY

ST: PO.KN • CI: h1195・ID: 312

PN: Phrynella pulchra Boulenger, 1887

PK: Phrynella pulchra* Boulenger, 1887

KG: Phrynella* Boulenger, 1887

KF: MICROHYLIDAE ||1843.fa.f012||-1931.na.f001

Phrynidium Lichtenstein ${ }^{+2}, 1856$ • AK

ST: PO.JD • CI: h1196・ID: 100

PN: Phrynidium varium Lichtenstein ${ }^{+2}, 1856$

PK: Phrynidium varium* ${ }^{*}$ Lichtenstein $^{+2}, 1856$

KG: Atelopus* Duméril ${ }^{+1}, 1841$

KF: BUFONIDAE 1825.gb.f004

Phryniscus Wiegmann, 1834 • AK

ST: PO.JD・CI: h1197• ID: 138

PN: Phryniscus nigricans Wiegmann, 1834

PK: Bufo spinulosus* Wiegmann, 1834

KG: Rhinella ${ }^{2}$ Fitzinger, 1826

KF: BUFONIDAE 1825.gb.f004

Phrynixalus Boettger, 1895 • AK

ST: PO.JD • CI: h1198 • ID: 280

PN: Phrynixalus montanus Boettger, 1895

PK: Phrynixalus montanus ${ }^{\circ}$ Boettger, 1895

KG: Asterophrys* Tschudi, 1838

KF: MICROHYLIDAE ||1843.fa.f012||-1931.na.f001

Phrynobatrachus Günther, 1862 • KY

ST: PO.KN • CI: h1199・ID: 350

PN: Phrynobatrachus natalensis Günther, 1862

PK: Stenorhynchus natalensis* Smith, 1849

KG: Phrynobatrachus* Günther, 1862

KF: PHRYNOBATRACHIDAE 1941.lb.f001

Phrynocara Peters, 1883 - AK

ST: PO.JD・CI: h1200 • ID: 287

PN: Phrynocara tuberatum Peters, 1883

PK: Phrynocara tuberatum* Peters, 1883

KG: Mantipus $^{1}$ Peters, 1883

KF: MICROHYLIDAE ||1843.fa.f012||-1931.na.f001

Phrynoceros Tschudi, 1838 • AK

ST: PO.JD • CI: h1201・ID: 169

PN: Phrynoceros vaillanti Tschudi, 1838

PK: Rana cornuta* Linnaeus, 1758

KG: Ceratophrys ${ }^{3}$ Neuwied, 1824

KF: CERATOPHRYIDAE 1838.ta.f002 
Phrynocerus: Rafinesque 1815 • AN

ST: AL • CI: n0116 • ID: 120

PN: Rana bufo Linnaeus, 1758

PK: Rana bufo* Linnaeus, 1758

KG: $B u f o^{*}$ Garsault, 1764

KF: BUFONIDAE 1825.gb.f004

Phrynocerus Cope, $1862 \cdot$ AK

ST: NS.JD・CI: h1202 • ID: 169

PN: Phrynoceros vaillanti Tschudi, 1838

PK: Rana cornuta* Linnaeus, 1758

KG: Ceratophrys ${ }^{3}$ Neuwied, 1824

KF: CERATOPHRYIDAE 1838.ta.f002

Phrynoderma: Sturm $1843 \cdot \mathbf{Z A}$

ST: $\mathbf{z N} \bullet \mathbf{C I}: \mathbf{z n} 008$

Phrynoderma Fitzinger, $1843 \cdot \mathbf{K Y}$

ST: PO.KN • CI: h1203・ID: 375

PN: Rana cutipora Duméril $^{+1}, 1841$

PK: Rana hexadactyla* Lesson, 1834

KG: Phrynoderma ${ }^{1}$ Fitzinger, 1843

KF: DiCROGLOSSIDAE 1987.da.f004

Phrynoderma Boulenger, 1893 • AK

ST: PO.JH • CI: h1204 • ID: 438

PN: Phrynoderma asperum Boulenger, 1893

PK: Phrynoderma asperum* Boulenger, 1893

KG: Theloderma* Tschudi, 1838

KF: RHACOPHORIDAE ||1858.gc.f012||-1932.ha.f001

Phrynodon Parker, 1935 • KY

ST: PO.KN • CI: h1205・ID: 349

PN: Phrynodon sandersoni Parker, 1935

PK: Phrynodon sandersoni* Parker, 1935

KG: Phrynobatrachus* Günther, 1862

KF: PHRYNOBATRACHIDAE 1941.lb.f001

Phrynoglossus Peters, $1867 \cdot \mathbf{K Y}$

ST: PO.KN • CI: h1206 • ID: 397

PN: Phrynoglossus martensii Peters, 1867

PK: Phrynoglossus martensii* Peters, 1867

KG: Phrynoglossus* Peters, 1867

KF: OCCIDOZYGIDAE 1990.fa.f002

Phrynohyas Fitzinger, $1843 \cdot \mathbf{A K}$

ST: PO.JD • CI: h1207 • ID: 231

PN: Hyla zonata Spix, 1824

PK: Rana typhonia* Linnaeus, 1758

KG: Trachycephalus* Tschudi, 1838

KF: HYLIDAE 1815.ra.f002-|1825.gb.f001|

Phrynoidis Fitzinger in Treitschke, $1842 \cdot \mathbf{K Y}$

ST: PO.KN • CI: h1208・ID: 118

PN: Bufo asper Gravenhorst, 1829

PK: Bufo asper* Gravenhorst, 1829

KG: Phrynoidis* Fitzinger in Treitschke, 1842

KF: BUFONIDAE 1825.gb.f004

Phrynomantis Peters, $1867 \cdot \mathbf{K Y}$

ST: PO.KN • CI: h1209 • ID: 319

PN: Brachymerus bifasciatus Smith, 1847

PK: Brachymerus bifasciatus* Smith, 1847

KG: Phrynomantis* Peters, 1867

KF: PHRYNOMERIDAE 1931.na.f013
Phrynomedusa Miranda-Ribeiro, 1923 • KY

ST: PO.KN • CI: h1210 • ID: 241

PN: Phrynomedusa fimbriata Miranda-Ribeiro, 1923

PK: Phrynomedusa fimbriata $^{\circ}$ Miranda-Ribeiro, 1923

KG: Phrynomedusa ${ }^{3}$ Miranda-Ribeiro, 1923

KF: PHYLLOMEDUSIDAE 1858.gc.f009

Phrynomerus Noble, $1926 \bullet \mathbf{A K}$

ST: NT.JI • CI: h1211 • ID: 319

PN: Brachymerus bifasciatus Smith, 1847

PK: Brachymerus bifasciatus* Smith, 1847

KG: Phrynomantis* Peters, 1867

KF: PHRYNOMERIDAE 1931.na.f013

Phrynomorphus: Curtis $1829 \cdot \mathbf{z A}$

ST: zN • CI: zn009

Phrynomorphus Curtis, $1833 \cdot \mathbf{z H}$

ST: zo • CI: zh075

Phrynomorphus Fitzinger, 1843 • AK

ST: PO.JH • CI: h1212 • ID: 145

PN: Bufo leschenaulti Duméril ${ }^{+1}, 1841$

PK: Bufo guttatus* Schneider, 1799

KG: Rhaebo* Cope, 1862

KF: BUFONIDAE 1825.gb.f004

Phrynophrys: Bonaparte 1839 • AN

ST: AL • CI: n0117 • ID: 021

PN: Megophrys montana $\mathrm{Kuhl}^{+1}, 1822$

PK: Megophrys montana ${ }^{\circ} \mathrm{Kuhl}^{+1}, 1822$

KG: Megophrys ${ }^{2} \mathrm{Kuhl}^{+1}, 1822$

KF: MEGOPHRYIDAE 1850.bb.f008-|1931.na.f003|

Phrynopsis Rafinesque, $1815 \cdot \mathbf{z H}$

ST: zo • CI: zh076

Phrynopsis Pfeffer, 1893 • AK

ST: PO.JH • CI: h1213・ID: 367

PN: Phrynopsis boulengerii Pfeffer, 1893

PK: Pyxicephalus edulis* Peters, 1854

KG: Pyxicephalus* Tschudi, 1838

KF: PYXICEPHALIDAE 1850.bb.f005

Phrynopus Peters, $1873 \cdot \mathbf{K Y}$

ST: PO.KN • CI: h1214 • ID: 077

PN: Phrynopus peruanus Peters, 1873

PK: Phrynopus peruanus ${ }^{\circ}$ Peters, 1873

KG: Phrynopus ${ }^{3}$ Peters, 1873

KF: BRACHYCEPHALIDAE 1858.gc.f002

Phrynotes: Rafinesque $1815 \cdot \mathbf{A N}$

ST: $\mathbf{A L} \bullet \mathbf{C I}: \mathrm{n} 0118 \bullet \mathbf{I D}: 120$

PN: Rana bufo Linnaeus, 1758

PK: Rana bufo* Linnaeus, 1758

KG: Bufo* Garsault, 1764

KF: BUFONIDAE 1825.gb.f004

Phylacomantis Glaw ${ }^{+1}, 1994$ • AK

ST: PO.JD・CI: h1215・ID: 431

PN: Mantidactylus corvus Glaw $^{+1}, 1994$

PK: Mantidactylus corvus* ${ }^{*} \mathrm{Glaw}^{+1}, 1994$

KG: Gephyromantis* Methuen, 1920

KF: RHACOPHORIDAE ||1858.gc.f012||-1932.ha.f001

Phylhydrus Swainson, 1839 • AK

ST: PO.JD • CI: h1216・ID: 555 
PN: Siren pisciformis Shaw, 1802

PK: Gyrinus mexicanus* Shaw $^{+1}, 1789$

KG: Ambystoma ${ }^{1}$ Tschudi, 1838

KF: АмвYSTOMATIDAE 1850.ga.f004

Phyllhydrus Gray, 1831 • EX

ST: NS.CE • CI: e0012 • ID: 555

PN: Siren pisciformis Shaw, 1802

PK: Gyrinus mexicanus* ${ }^{*}$ Shaw $^{+1}, 1789$

KG: Ambystoma ${ }^{1}$ Tschudi, 1838

KF: AмвYSTOMATIDAE 1850.ga.f004

Phyllidrus Agassiz, 1845 • AK

ST: NT.JD・CI: h1218・ID: 555

PN: Siren pisciformis Shaw, 1802

PK: Gyrinus mexicanus* Shaw $^{+1}, 1789$

KG: Ambystoma ${ }^{1}$ Tschudi, 1838

KF: AмBYSTOMATIDAE 1850.ga.f004

Phyllobates Duméril ${ }^{+1}, 1841$ • KY

ST: PO.KN • CI: h1219・ID: 051

PN: Phyllobates bicolor Duméril ${ }^{+1}, 1841$

PK: Phyllobates bicolor* Duméril ${ }^{+1}, 1841$

KG: Phyllobates* Duméril ${ }^{+1}, 1841$

KF: DENDROBATIDAE $\| 1850$. bb.f006"|-1865.ca.f002

Phyllobius Schoenherr, $1824 \cdot \mathbf{z H}$

ST: zo • CI: zh077

Phyllobius Fitzinger, $1843 \cdot \mathbf{A K}$

ST: PO.JH • CI: h1220 • ID: 189

PN: Hyla albomarginata Spix, 1824

PK: Hyla albomarginata* Spix, 1824

KG: Boana* Gray, 1825

KF: HYLIDAE 1815.ra.f002-|1825.gb.f001|

Phyllodromus Jiménez de la Espada, 1875 • AK

ST: PO.JD・CI: h1221・ID: 053

PN: Phyllodromus pulchellum Jiménez de la Espada, 1875

PK: Phyllodromus pulchellum* Jiménez de la Espada, 1875

KG: Hyloxalus ${ }^{2}$ Jiménez de la Espada, 1870

KF: DENDROBATIDAE $\|1850 . b b . f 006\|-1865 . c a . f 002$

Phyllodytes Wagler, $1830 \cdot \mathbf{K Y}$

ST: PO.KN • CI: h1222 • ID: 221

PN: Hyla luteola Wied-Neuwied, 1824

PK: Hyla luteola* Wied-Neuwied, 1824

KG: Phyllodytes* Wagler, 1830

KF: HYLIDAE 1815.ra.f002-|1825.gb.f001|

Phyllodytes Gistel, 1848 • AK

ST: PO.JH • CI: h1223・ID: 369

PN: Halophila vitiensis Girard, 1853

PK: Halophila vitiensis* Girard, 1853

KG: Cornufer* Tschudi, 1838

KF: CERATOBATRACHIDAE 1884.ba.f001

Phyllomedusa Wagler, $1830 \cdot \mathbf{K Y}$

ST: PO.KN • CI: h1224・ID: 243

PN: Rana bicolor Boddaert, 1772

PK: Rana bicolor* Boddaert, 1772

KG: Phyllomedusa* Wagler, 1830

KF: PHYLLOMEDUSIDAE 1858.gc.f009

Phyllonastes Heyer, 1977 • KY

ST: PO.KN • CI: h1225・ID: 065
PN: Euparkerella myrmecoides Lynch, 1976

PK: Euparkerella myrmecoides* Lynch, 1976

KG: Phyllonastes* Heyer, 1977

KF: BRACHYCEPHALIDAE 1858.gc.f002

Physalaemus Fitzinger, 1826 • KY

ST: PO.KN • CI: h1226・ID: 250

PN: Physalaemus cuvieri Fitzinger, 1826

PK: Physalaemus cuvieri* Fitzinger, 1826

KG: Physalaemus* Fitzinger, 1826

KF: LEIUPERIDAE 1850.bb.f010

Physalamis: Gray 1831 • AN

ST: AL •CI: n0119・ID: 250

PN: Physalaemus cuvieri Fitzinger, 1826

PK: Physalaemus cuvieri* Fitzinger, 1826

KG: Physalaemus* Fitzinger, 1826

KF: LEIUPERIDAE 1850.bb.f010

Physalus La Cepède, $1804 \cdot \mathbf{Z H}$

ST: zo • CI: zh078

Physalus Jan, $1857 \cdot \mathbf{A N}$

ST: $\mathbf{A L} \bullet \mathbf{C I}:$ n0120 • ID: 100

PN: Phryniscus ignescens Cornalia, 1849

PK: Phryniscus ignescens* Cornalia, 1849

KG: Atelopus* Duméril ${ }^{+1}, 1841$

KF: BUFONIDAE 1825.gb.f004

Physodes Desmarest, $1825 \cdot \mathbf{z H}$

ST: zo • CI: zh079

Physodes: Jan $1857 \cdot \mathbf{A N}$

ST: AL・CI: n0121・ID: 246

PN: Lystris brachyops Cope, 1869

PK: Lystris brachyops* Cope, 1869

KG: Pleurodema* Tschudi, 1838

KF: LEIUPERIDAE 1850.bb.f010

Physolaemus Agassiz, 1847 • AK

ST: NT.JI • CI: h1227 • ID: 250

PN: Physalaemus cuvieri Fitzinger, 1826

PK: Physalaemus cuvieri* Fitzinger, 1826

KG: Physalaemus* Fitzinger, 1826

KF: LEIUPERIDAE 1850.bb.f010

Phytotriades Jowers ${ }^{+2}, 2009 \cdot \mathbf{K Y}$

ST: PO.KN • CI: h1228・ID: 226

PN: Amphodus auratus Boulenger, 1917

PK: Amphodus auratus* Boulenger, 1917

KG: Phytotriades* Jowers $^{+2}, 2009$

KF: HYLIDAE 1815.ra.f002-|1825.gb.f001|

Phyzelaphryne Heyer, $1977 \bullet \mathbf{K Y}$

ST: PO.KN・CI: h1229・ID: 084

PN: Phyzelaphryne miriamae Heyer, 1977

PK: Phyzelaphryne miriamae* Heyer, 1977

KG: Phyzelaphryne* Heyer, 1977

KF: BRACHYCEPHALIDAE 1858.gc.f002

Piceoerpeton Meszoely, 1967 • KY

ST: PO.KN • CI: h1230 • ID: $\uparrow 157$

PN: Piceoerpeton willwoodense Meszoely, 1967 \$

PK: Piceoerpeton willwoodense $e^{\circ}$ Meszoely, $1967 \uparrow$

KG: Piceoerpeton ${ }^{\circ}$ Meszoely, $1967 \dagger$

KF: SCAPHERPETIDAE 1959.aa.f001 † 
Pingia Chang, 1936 • AK

ST: PO.JD • CI: h1231・ID: 561

PN: Pachytriton granulosus Chang, 1933

PK: Pachytriton granulosus* Chang, 1933

KG: Pachytriton* Boulenger, 1878

KF: SALAMANDRIDAE 1820.ga.f002

Pipa Laurenti, 1768 • KY

ST: PO.KN • CI: h1232 • ID: 012

PN: Pipa americana Laurenti,1768

PK: Rana pipa* Linnaeus, 1758

KG: Pipa ${ }^{1}$ Laurenti, 1768

KF: PIPIDAE 1825.gb.f003-|1826.fb.f002|

Piparius Rafinesque, $1815 \cdot \mathbf{A K}$

ST: NT.JI • CI: h1233・ID: 012

PN: Pipa americana Laurenti,1768

PK: Rana pipa* Linnaeus, 1758

KG: Pipa ${ }^{1}$ Laurenti, 1768

KF: PIPIDAE 1825.gb.f003-|1826.fb.f002|

Pipra Linnaeus, $1758 \cdot \mathbf{Z H}$

ST: zo • CI: zh080

Pipra Gray, $1825 \cdot \mathbf{A K}$

ST: NT.JH • CI: h1234 • ID: 012

PN: Pipa americana Laurenti,1768

PK: Rana pipa* Linnaeus, 1758

KG: Pipa ${ }^{1}$ Laurenti, 1768

KF: PIPIDAE 1825.gb.f003-|1826.fb.f002|

Pithecopsis Duméril ${ }^{+1}, 1841$ • AK

ST: PO.JI • CI: h1235・ID: 179

PN: Cycloramphus fuliginosus Tschudi, 1838

PK: Cycloramphus fuliginosus* Tschudi, 1838

KG: Cycloramphus* Tschudi, 1838

KF: CYCLORAMPHIDAE 1850.bb.f003-|1852.ba.f001|

Pithecopus Cope, 1866 • KY

ST: PO.KN • CI: h1236・ID: 245

PN: Phyllomedusa azurea Cope, 1862

PK: Phyllomedusa azurea* Cope, 1862

KG: Pithecopus* Cope, 1866

KF: PHYLLOMEDUSIDAE 1858.gc.f009

Plagiodon Duméril, $1853 \cdot \mathbf{z H}$

ST: zo • CI: zh081

Plagiodon: Duméril ${ }^{+2} 1854$ • AN

ST: AL $・$ CI: n0122・ID: 555

PN: Lacerta subviolacea Barton, 1804

PK: Lacerta maculata* Shaw, 1802

KG: Ambystoma ${ }^{1}$ Tschudi, 1838

KF: AмвYSTOMATIDAE 1850.ga.f004

Plagiodons: Duméril ${ }^{+2} 1854$ • AN

ST: AL •CI: n0123・ID: 555

PN: Lacerta subviolacea Barton, 1804

PK: Lacerta maculata* Shaw, 1802

KG: Ambystoma ${ }^{1}$ Tschudi, 1838

KF: AмвYSTомAтIDAE 1850.ga.f004

Platosphus L'Isle, 1877 + $\mathbf{A K}$

ST: PO.JD • CI: h1237 • ID: 120

PN: Platosphus gervaisii L'Isle, 1877 *

PK: Rana bufo* Linnaeus, 1758
KG: Bufo* Garsault, 1764

KF: BUFONIDAE 1825.gb.f004

Platyhyla Boulenger, 1889 • AK

ST: PO.JD・CI: h1238 • ID: 288

PN: Platyhyla grandis Boulenger, 1889

PK: Platyhyla grandis* Boulenger, 1889

KG: Platypelis ${ }^{2}$ Boulenger, 1882

KF: MICROHYLIDAE ||1843.fa.f012||-1931.na.f001

Platymantis: Günther, 1858 • AN

ST: AL • CI: n0124・ID: 370

PN: Platymantis plicifera Günther, 1859

PK: Hylodes corrugatus* Duméril, 1853

KG: Platymantis ${ }^{1}$ Günther, 1859

KF: CERATOBATRACHIDAE 1884.ba.f001

Platymantis Günther, $1859 \cdot \mathbf{K Y}$

ST: PO.KN • CI: h1239・ID: 370

PN: Platymantis plicifera Günther, 1859

PK: Hylodes corrugatus* Duméril, 1853

KG: Platymantis ${ }^{1}$ Günther, 1859

KF: CERATOBATRACHIDAE 1884.ba.f001

Platypelis Boulenger, 1882 • KY

ST: PO.KN • CI: h1240 • ID: 288

PN: Platypelis cowanii Boulenger, 1882

PK: Platypelis cowanii ${ }^{\circ}$ Boulenger, 1882

KG: Platypelis ${ }^{2}$ Boulenger, 1882

KF: MICROHYLIDAE ||1843.fa.f012||-1931.na.f001

Platyplectron: Peters 1863 - AN

ST: AM • CI: n0125・ID: 264

PN: Platyplectrum marmoratum Günther, 1863

PK: Discoglossus ornatus* Gray, 1842

KG: Platyplectrum ${ }^{1}$ Günther, 1863

KF: MYOBATRACHIDAE 1850.sa.f001

Platyplectrum Günther, 1863・ KY

ST: PO.KN • CI: h1241 • ID: 264

PN: Platyplectrum marmoratum Günther, 1863

PK: Discoglossus ornatus* Gray, 1842

KG: Platyplectrum ${ }^{1}$ Günther, 1863

KF: MYOBATRACHIDAE 1850.sa.f001

Platyrhynchus Leuckart, 1816 • AK

ST: NL.JI • CI: h1242・ ID: 554

PN: Proteus anguinus Laurenti, 1768

PK: Proteus anguinus* Laurenti, 1768

KG: Proteus* Laurenti, 1768

KF: PROTEIDAE 1831.ba.f002

Plectrohyla Brocchi, 1877 • KY

ST: PO.KN・CI: h1243・ID: 219

PN: Plectrohyla guatemalensis Brocchi, 1877

PK: Plectrohyla guatemalensis* Brocchi, 1877

KG: Plectrohyla* Brocchi, 1877

KF: HYLIDAE 1815.ra.f002-|1825.gb.f001|

Plectromantis Peters, 1862 • AK

ST: PO.JD • CI: h1244 • ID: 253

PN: Plectromantis wagneri Peters, 1862

PK: Plectromantis wagneri* Peters, 1862

KG: Leptodactylus ${ }^{1}$ Fitzinger, 1826

KF: LEPTODACTYLIDAE ||1838.ta.f001||-1896.wa.f001 
Plectropus Kirby, $1826 \cdot \mathbf{z H}$

ST: zo • CI: zh082

Plectropus Duméril ${ }^{+1}, 1841$ • AK

ST: PO.JH • CI: h1245 • ID: 310

PN: Plectropus pictus Duméril ${ }^{+1}, 1841$

PK: Plectropus pictus* Duméril ${ }^{+1}, 1841$

KG: Kaloula* Gray, 1831

KF: MICROHYLIDAE ||1843.fa.f012||-1931.na.f001

Plethodon Tschudi, 1838 • KY

ST: PO.KN・CI: h1246・ID: 551

PN: Salamandra glutinosa Green, 1818

PK: Salamandra glutinosa* Green, 1818

KG: Plethodon* Tschudi, 1838

KF: PLETHODONTIDAE 1850.ga.f002

Plethodontohyla Boulenger, 1882 • AK

ST: PO.JD • CI: h1247 • ID: 286

PN: Callula notosticta Günther, 1877

PK: Callula notosticta* Günther, 1877

KG: Cophyla* Boettger, 1880

KF: МiCROHYLIDAE |1843.fa.f012||-1931.na.f001

Plethopsis Bishop, 1937 • AK

ST: PO.JD • CI: h1248 • ID: 521

PN: Plethopsis wrighti Bishop, 1937

PK: Plethopsis wrighti* Bishop, 1937

KG: Batrachoseps* Bonaparte, 1839

KF: PLETHODONTIDAE 1850.ga.f002

Pleurodeles Michahelles, $1830 \bullet \mathbf{K Y}$

ST: PO.KN • CI: h1249・ID: 571

PN: Pleurodeles waltl Michahelles, 1830

PK: Pleurodeles walt ${ }^{*}$ Michahelles, 1830

KG: Pleurodeles* Michahelles, 1830

KF: SALAMANDRIDAE 1820.ga.f002

Pleurodema Tschudi, $1838 \cdot \mathbf{K Y}$

ST: LC.KN • CI: h1250 • ID: 246

PN: Pleurodema bibroni Tschudi, 1838

PK: Pleurodema bibroni* Tschudi, 1838

KG: Pleurodema* Tschudi, 1838

KF: LEIUPERIDAE 1850.bb.f010

Pleuroderes: Hoffmann 1878 • AN

ST: AM • CI: n0126・ID: 571

PN: Pleurodeles waltl Michahelles, 1830

PK: Pleurodeles walt $*$ Michahelles, 1830

KG: Pleurodeles* Michahelles, 1830

KF: SALAMANDRIDAE 1820.ga.f002

Pleuroderma: Tschudi 1838 AN

ST: $\mathbf{L I} \bullet$ CI: n0127・ ID: 246

PN: Pleurodema bibroni Tschudi, 1838

PK: Pleurodema bibroni* Tschudi, 1838

KG: Pleurodema* Tschudi, 1838

KF: LEIUPERIDAE 1850.bb.f010

Plicagnathus Cook, $1917+\cdot \mathbf{A K}$

ST: PO.JD • CI: h1251 • ID: 503

PN: Plicagnathus matthewi Cook, 1917 *

PK: Plicagnathus matthewi ${ }^{\circ}$ Cook, $1917 \dagger$

KG: Andrias $^{2}$ Tschudi, 1837

KF: CRYPTOBRANCHIDAE 1826.fb.f003
Plioambystoma Adams in Adams ${ }^{+1}, 1929+\mathbf{A K}$

ST: PO.JD • CI: h1252・ID: 555

PN: Plioambystoma kansense Adams ${ }^{+1}, 1929+$

PK: Plioambystoma kansense ${ }^{\circ}$ Adams $^{+1}, 1929 \dagger$

KG: Ambystoma ${ }^{1}$ Tschudi, 1838

KF: AMBYSTOMATIDAE 1850.ga.f004

Pliobatrachus Fejérváry, 1917 + AK

ST: PO.JD • CI: h1253・ID: $\uparrow 069$

PN: Pliobatrachus langhae Fejérváry, 1917 \$

PK: Pliobatrachus langhae ${ }^{\circ}$ Fejérváry, 1917 †

KG: Palaeobatrachus ${ }^{\circ}$ Tschudi, $1838 \dagger$

KF: PALAEOBATRACHIDAE 1865.ca.f001 †

Podonectes: Steindachner 1864 • AN

ST: AL・CI: n0128・ID: 196

PN: Lysapsus limellum Cope, 1862

PK: Lysapsus limellum* Cope, 1862

KG: Pseudis* Wagler, 1830

KF: HYLIDAE 1815.ra.f002-|1825.gb.f001|

Pohlia Steindachner, 1867 • AK

ST: PO.JI • CI: h1254 • ID: 415

PN: Rana palmipes Spix, 1824

PK: Rana palmipes* Spix, 1824

KG: Lithobates* Fitzinger, 1843

KF: RANIDAE 1796.ba.f001

Polypedates Tschudi, $1838 \bullet \mathbf{K Y}$

ST: LC.KN • CI: h1255・ID: 452

PN: Hyla leucomystax Gravenhorst, 1829

PK: Hyla leucomystax* Gravenhorst, 1829

KG: Polypedates* Tschudi, 1838

KF: RHACOPHORIDAE ||1858.gc.f012||-1932.ha.f001

Polypedetes Whitney, $1890 \bullet \mathbf{A K}$

ST: NT.JI • CI: h1256・ID: 452

PN: Hyla leucomystax Gravenhorst, 1829

PK: Hyla leucomystax* Gravenhorst, 1829

KG: Polypedates* Tschudi, 1838

KF: RHACOPHORIDAE ||1858.gc.f012||-1932.ha.f001

Polypedotes: Tschudi $1838 \cdot \mathbf{A N}$

ST: $\mathbf{L I} \cdot \mathbf{C I}$ : n0129 • ID: 452

PN: Hyla leucomystax Gravenhorst, 1829

PK: Hyla leucomystax* Gravenhorst, 1829

KG: Polypedates* Tschudi, 1838

KF: RHACOPHORIDAE |1858.gc.f012||-1932.ha.f001

Polyphone Gistel, 1848 • AK

ST: NL.JI • CI: h1257 • ID: 237

PN: Ranoidea jacksoniensis Tschudi, 1838

PK: Rana aurea* Lesson, 1829

KG: Ranoidea ${ }^{1}$ Tschudi, 1838

KF: PHYLLOMEDUSIDAE 1858.gc.f009

Polysemia Guenée in Boisduval ${ }^{+1}, 1857 \cdot \mathbf{z H}$

ST: zo • CI: zh083

Polysemia Meyer, $1860+\mathbf{A K}$

ST: PO.JH • CI: h1258 • ID: $\uparrow 193$

PN: Salamandra ogygia Goldfuss, 1831 t

PK: Salamandra ogygia ${ }^{\circ}$ Goldfuss, $1831 \uparrow$

KG: Chelotriton $^{\circ}$ Pomel, $1853 \uparrow$

KF: SALAMANDRIDAE 1820.ga.f002 
Pomatops Barbour, 1910 • AK

ST: PO.JD • CI: h1259 • ID: 280

PN: Pomatops valvifera Barbour, 1910

PK: Pomatops valvifera ${ }^{\circ}$ Barbour, 1910

KG: Asterophrys* Tschudi, 1838

KF: MICROHYLIDAE ||1843.fa.f012||-1931.na.f001

Potamorana Brown ${ }^{+4}, 2015 \cdot \mathbf{A K}$

ST: PO.JD • CI: h1260 • ID: 369

PN: Rana bufoniformis Boulenger, 1884

PK: Rana bufoniformis ${ }^{\circ}$ Boulenger, 1884

KG: Cornufer* Tschudi, 1838

KF: CERATOBATRACHIDAE 1884.ba.f001

Potamotyphlus Taylor, $1968 \cdot \mathbf{K Y}$

ST: LC.KN・CI: h1261・ID: 479

PN: Caecilia kaupii Berthold, 1859

PK: Caecilia kaupii ${ }^{\circ}$ Berthold, 1859

KG: Potamotyphlus ${ }^{\circ}$ Taylor, 1968

KF: CAECILIIDAE 1814.ra.f003-|1825.gb.f008|

Potomotyphlus: Taylor 1968 - AN

ST: LI・CI: n0130・ID: 479

PN: Caecilia kaupii Berthold, 1859

PK: Caecilia kaupii ${ }^{\circ}$ Berthold, 1859

KG: Potamotyphlus ${ }^{\circ}$ Taylor, 1968

KF: CAECILIIDAE 1814.ra.f003-|1825.gb.f008|

Poyarius Dubois ${ }^{+1}, 2012 \cdot \mathbf{K Y}$

ST: PO.KN • CI: h1262 • ID: 507

PN: Hynobius formosanus Maki, 1922

PK: Hynobius formosanus* Maki, 1922

KG: Poyarius* ${ }^{*}$ Dubois $^{+1}, 2012$

KF: НYNOBIIDAE ||1856.ha.f001||-1859.cb.f002

Poyntonia Channing ${ }^{+1}, 1989 \bullet \mathbf{K Y}$

ST: PO.KN • CI: h1263・ID: 359

PN: Poyntonia paludicola Channing ${ }^{+1}, 1989$

PK: Poyntonia paludicola* Channing ${ }^{+1}, 1989$

KG: Poyntonia $*$ Channing ${ }^{+1}, 1989$

KF: CACOSTERNIDAE 1931.na.f008

Poyntonophrynus Frost $^{+18}, 2006 \cdot \mathbf{K Y}$

ST: PO.KN • CI: h1264・ID: 142

PN: Bufo vertebralis Smith, 1848

PK: Bufo vertebralis ${ }^{\circ}$ Smith, 1848

KG: Poyntonophrynus ${ }^{3}$ Frost $^{+18}, 2006$

KF: BUFONIDAE 1825.gb.f004

Prana Bauer, 1985 • AK

ST: PO.JD・CI: h1265・ID: 415

PN: Rana pipiens Schreber, 1782

PK: Rana pipiens* Schreber, 1782

KG: Lithobates* Fitzinger, 1843

KF: RANIDAE 1796.ba.f001

Praslinia Boulenger, 1909 • KY

ST: PO.KN・CI: h1266・ID: 484

PN: Praslinia cooperi Boulenger, 1909

PK: Praslinia cooperi* Boulenger, 1909

KG: Praslinia* Boulenger, 1909

KF: CAECILIIDAE 1814.ra.f003-|1825.gb.f008|

Pristimantis Jiménez de la Espada, 1870 • KY

ST: PO.KN • CI: h1267・ID: 078
PN: Pristimantis galdi Jiménez de la Espada, 1870

PK: Pristimantis galdi* Jiménez de la Espada, 1870

KG: Pristimantis* Jiménez de la Espada, 1870

KF: BRACHYCEPHALIDAE 1858.gc.f002

Proacris Holman, $1961+\cdot \mathbf{K Y}$

ST: PO.KN • CI: h1268・ID: $\uparrow 101$

PN: Proacris mintoni Holman, 1961 *

PK: Proacris mintoni $i^{\circ}$ Holman, $1961 \uparrow$

KG: Proacris ${ }^{\circ}$ Holman, $1961 \dagger$

KF: HYLIDAE 1815.ra.f002-|1825.gb.f001|

Proamphiuma Estes, $1969+\cdot \mathbf{K Y}$

ST: PO.KN • CI: h1269・ID: $\uparrow 180$

PN: Proamphiuma cretacea Estes, 1969 \$

PK: Proamphiuma cretacea ${ }^{\circ}$ Estes, $1969 \dagger$

KG: Proamphiuma ${ }^{\circ}$ Estes, $1969 \dagger$

KF: AMPHIUMIDAE 1825.gb.f07

Probatrachus Peters, $1878+\bullet \mathbf{K Y}$

ST: PO.KN・CI: h1270・ID: $\uparrow 070$

PN: Probatrachus vicetinus Peters 1878 *

PK: Probatrachus vicetinus ${ }^{\circ}$ Peters $1878 \dagger$

KG: Probatrachus ${ }^{\circ}$ Peters, $1878 \dagger$

KF: PALAEOBATRACHIDAE 1865.ca.f001 †

Probreviceps Parker, $1931 \bullet \mathbf{K Y}$

ST: PO.KN • CI: h1271 • ID: 345

PN: Breviceps macrodactylus Nieden, 1926

PK: Breviceps macrodactylus* Nieden, 1926

KG: Probreviceps* Parker, 1931

KF: BREVICIPITIDAE 1850.bb.f012

Proceratophrys Miranda-Ribeiro, 1920 • KY

ST: PO.KN・CI: h1272・ID: 154

PN: Ceratophrys bigibbosa Peters, 1872

PK: Ceratophrys bigibbosa* Peters, 1872

KG: Proceratophrys* Miranda-Ribeiro, 1920

KF: ODONTOPHRYNIDAE 1971.la.f002

Procerobatrachus Roček ${ }^{+1}, 1993+\bullet \mathbf{K Y}$

ST: PO.KN • CI: h1273・ID: $\uparrow 037$

PN: Procerobatrachus paulus Roček $^{+1}, 1993$ †

PK: Procerobatrachus paulus ${ }^{\circ}$ Roček $^{+1}, 1993$ †

KG: Procerobatrachus ${ }^{\circ}$ Roček $^{+1}, 1993$ †

KF: ANURA Familia INCERTAE SEDIS

Procynops Young, $1965+\bullet \mathbf{K Y}$

ST: PO.KN • CI: h1274・ID: $\uparrow 198$

PN: Procynops miocenicus Young, 1965 †

PK: Procynops miocenicus ${ }^{\circ}$ Young, $1965 \dagger$

KG: Procynops ${ }^{\circ}$ Young, $1965 \dagger$

KF: SALAMANDRIDAE 1820.ga.f002

Prodesmodon Estes, 1964 \$・ KY

ST: PO.KN・CI: h1275・ID: $\uparrow 151$

PN: Prodesmodon copei Estes, 1964 *

PK: Prodesmodon copei ${ }^{\circ}$ Estes, $1964 \dagger$

KG: Prodesmodon ${ }^{\circ}$ Estes, $1964 \uparrow$

KF: HyLAEOBATRACHIDAE 1889.la.f001 †

Prodiscoglossus Friant, $1944+\cdot \mathbf{A K}$

ST: PO.JD • CI: h1276・ID: 470

PN: Prodiscoglossus vertaizoni Friant, 1944 \$.

PK: Prodiscoglossus vertaizoni ${ }^{\circ}$ Friant, $1944 \uparrow$ 
KG: Latonia ${ }^{3}$ Meyer, $1845 \dagger$

KF: DISCOGLOSSIDAE 1858.gc.f004

Prohartia Wells ${ }^{+1}, 1985$ • AK

ST: PO.JD • CI: h1277 • ID: 276

PN: Pseudophryne fimbrianus Parker, 1926

PK: Pseudophryne rugosa* Andersson, 1916

KG: Uperoleia ${ }^{2}$ Gray, 1841

KF: MYOBATRACHIDAE 1850.sa.f001

Prohynobius $\mathrm{Fei}^{+1}, 1985+\cdot \mathbf{A N}$

ST: $\mathbf{A L} ・ \mathbf{C I}: \mathrm{n} 0131 \cdot \mathbf{I D}: \uparrow 173 \S$

PN: INR

PK: INR

KG: INR

KF: HYNOBIIDAE ||1856.ha.f001\|-1859.cb.f002

Propelodytes Weitzel, $1938+\cdot \mathbf{A K}$

ST: PO.JD・CI: h1278 • ID: $\uparrow 090$

PN: Propelodytes wagneri Weitzel, 1938 *

PK: Propelodytes wagneri ${ }^{\circ}$ Weitzel, $1938 \dagger$

KG: Eopelobates ${ }^{\circ}$ Parker, $1929 \uparrow$

KF: PELOBATIDAE 1850.bb.f004

Prosalirus Shubin ${ }^{+1}, 1995+\cdot \mathbf{K Y}$

ST: PO.KN • CI: h1279 • ID: $† 052$

PN: Prosalirus bitis Shubin $^{+1}, 1995$ *

PK: Prosalirus bitis ${ }^{\circ}$ Shubin $^{+1}, 1995 \dagger$

KG: Prosalirus ${ }^{\circ}$ Shubin $^{+1}, 1995 \dagger$

KF: PROSALIRIDAE 1995.sa.f001 †

Prosiren Goin ${ }^{+1}, 1958+\bullet \mathbf{K Y}$

ST: PO.KN • CI: h1280 •ID: $\uparrow 154$

PN: Prosiren elinorae Goin $^{+1}, 1958$ \$

PK: Prosiren elinorae ${ }^{\circ}$ Goin $^{+1}, 1958 \dagger$

KG: Prosiren ${ }^{\circ}$ Goin $^{+1}, 1958$ †

KF: PROSIRENIDAE 1969.ea.f001 †

Prospea: $\mathrm{Chen}^{+3} 2016+\cdot \mathbf{A N}$

ST: AL • CI: n0132 - ID: $\uparrow 093 \S$

PN: Prospea holoserisca $\mathrm{Chen}^{+3}, 2016+\cdot$ AS

PK: Prospea holoserisca ${ }^{\circ} \mathrm{Chen}^{+3}, 2016 \uparrow \bullet \mathbf{A S}$

KG: Prospea $^{\circ} \mathrm{Chen}^{+3}, 2016+\bullet \mathbf{A G}$

KF: SCAPHIOPODIDAE 1865.ca.f003 †

Prostherapis Cope, 1868 - AK

ST: PO.JD • CI: h1281 • ID: 040

PN: Prostherapis inguinalis Cope, 1868

PK: Prostherapis inguinalis* Cope, 1868

KG: Colostethus* Cope, 1866

KF: DENDROBATIDAE $\|1850 . b b . f 006\|-1865 . c a . f 002$

Prostheraspis Hoffmann, 1877 • AK

ST: NT.JD・CI: h1282・ ID: 040

PN: Prostherapis inguinalis Cope, 1868

PK: Prostherapis inguinalis* Cope, 1868

KG: Colostethus* Cope, 1866

KF: DENDROBATIDAE $\| 1850$. bb.f006"|-1865.ca.f002

Proteocordylus Eichwald, $1831+\bullet \mathbf{A K}$

ST: PO.CA • CI: h1283 • ID: 503

PN: Proteocordylus diluvii Eichwald, 1831 *

PK: Salamandra scheuchzeri ${ }^{\circ}$ Holl, $1831+$

KG: Andrias $^{2}$ Tschudi, 1837

KF: CRYPTOBRANCHIDAE 1826.fb.f003
Proteus Laurenti, $1768 \cdot \mathbf{K Y}$

ST: PO.KN • CI: h1284 • ID: 554

PN: Proteus anguinus Laurenti, 1768

PK: Proteus anguinus* Laurenti, 1768

KG: Proteus* Laurenti, 1768

KF: PROTEIDAE 1831.ba.f002

Protobatrachus Gistel, 1848 • AK

ST: PO.JD・CI: h1285・ID: 419

PN: Protobatrachus nodicaudatus Gistel, 1848

PK: Rana temporaria* Linnaeus, 1758

KG: Rana* Linnaeus, 1758

KF: RANIDAE 1796.ba.f001

Protobatrachus Piveteau, $1936+\mathbf{A K}$

ST: PO.JH • CI: h1286・ID: $\uparrow 054$

PN: Protobatrachus massinoti Piveteau, 1936 \$

PK: Protobatrachus massinoti ${ }^{\circ}$ Piveteau, $1936+$

KG: Triadobatrachus ${ }^{\circ}$ Kuhn, $1962 \uparrow$

KF: TRIADOBATRACHIDAE 1962.ka.f001 †

Protohynobius $\mathrm{Fei}^{+1}, 2000 \cdot \mathbf{A K}$

ST: PO.JD • CI: h1287 • ID: 511

PN: Protohynobius puxiongensis $\mathrm{Fe}^{+1}, 2000$

PK: Protohynobius puxiongensis* $\mathrm{Fei}^{+1}, 2000$

KG: Pseudohynobius* $\mathrm{Fei}^{+1}, 1983$

KF: HYNOBIIDAE $\| 1856$.ha.f001\|-1859.cb.f002

Protonophis: Tschudi $1838 \cdot \mathbf{A N}$

ST: AM • CI: n0133・ID: 504

PN: Salamandra horrida Barton, 1808

PK: Salamandra alleganiensis* Sonnini ${ }^{+1}, 1801$

KG: Cryptobranchus ${ }^{1}$ Leuckart, 1821

KF: CRYPTOBRANCHIDAE 1826.fb.f003

Protonopsis Le Conte, 1824 • AK

ST: PO.JD・CI: h1288・ID: 504

PN: Salamandra horrida Barton, 1808

PK: Salamandra alleganiensis* Sonnini ${ }^{+1}, 1801$

KG: Cryptobranchus ${ }^{1}$ Leuckart, 1821

KF: CRYPTOBRANCHIDAE 1826.fb.f003

Protopelobates Bieber, 1881 + $\mathbf{A K}$

ST: PO.JD • CI: h1289 • ID: $† 069$

PN: Protopelobates gracilis Bieber, 1881 \$

PK: Palaeobatrachus laube ${ }^{\circ}$ Bieber, $1881 \dagger$

KG: Palaeobatrachus ${ }^{\circ}$ Tschudi, $1838 \dagger$

KF: PALAEOBATRACHIDAE 1865.ca.f001 †

Protopelobates: Bauer $1986+\cdot \mathbf{A N}$

ST: $\mathbf{A L} ・ \mathbf{C I}: \mathrm{n} 0134 \cdot \mathbf{I D}: \dagger 084 \S$

PN: INR

PK: INR

KG: INR

KF: LAEVOGYRINIA Familia INCERTAE SEDIS

Protophrynos: Zittel $1888+\cdot \mathbf{A N}$

ST: $\mathbf{A L} ・ \mathbf{C I}:$ n0135・ID: $\uparrow 038 \S$

PN: Protophrynus arethusae Pomel, 1853 †

PK: Protophrynus arethusae ${ }^{\circ}$ Pomel, $1853 \dagger$

KG: Protophrynus ${ }^{\circ}$ Pomel, $1853 \dagger$

KF: ANURA Familia INCERTAE SEDIS

Protophrynus: Pomel $1853+\cdot \mathbf{A N}$

ST: $\mathbf{A L} \cdot \mathbf{C I}:$ n0136・ID: $\uparrow 038 \S$ 
PN: Protophrynus arethusae Pomel, 1853 \$

PK: Protophrynus arethusae ${ }^{\circ}$ Pomel, $1853 \dagger$

KG: Protophrynus $^{\circ}$ Pomel, $1853 \dagger$

KF: ANURA Familia INCERTAE SEDIS

Protopipa Noble, $1925 \cdot \mathbf{A K}$

ST: PO.JD • CI: h1290 • ID: 012

PN: Pipa aspera Müller, 1924

PK: Pipa aspera $^{\circ}$ Müller, 1924

KG: Pipa $^{1}$ Laurenti, 1768

KF: PIPIDAE 1825.gb.f003-|1826.fb.f002|

Pseudacris Fitzinger, 1843 • KY

ST: PO.KN • CI: h1291・ID: 200

PN: Rana nigrita Le Conte, 1825

PK: Rana nigrita* Le Conte, 1825

KG: Pseudacris* Fitzinger, 1843

KF: HyLIDAE 1815.ra.f002-|1825.gb.f001|

Pseudarthroleptis Deckert, 1938 • AK

ST: PO.JD • CI: h1292 • ID: 350

PN: Hemimantis calcaratus Peters, 1863

PK: Hemimantis calcaratus* Peters, 1863

KG: Phrynobatrachus* Günther, 1862

KF: PHRYNOBATRACHIDAE 1941.lb.f001

Pseudendrobates Bauer, 1987 • AK

ST: PO.JD • CI: h1293・ ID: 039

PN: Dendrobates silverstonei Myers ${ }^{+1}, 1979$

PK: Dendrobates silverstonei* Myers $^{+1}, 1979$

KG: Ameerega* Bauer, 1986

KF: DENDROBATIDAE $\|1850 . b b . f 006\|-1865 . c a . f 002$

Pseudengystoma Witte, 1930 • AK

ST: PO.JD・CI: h1294 • ID: 280

PN: Pseudengystoma bouwensi Witte, 1930

PK: Pseudengystoma bouwensi* Witte, 1930

KG: Asterophrys* Tschudi, 1838

KF: MICROHYLIDAE ||1843.fa.f012||-1931.na.f001

Pseudepidalea Frost ${ }^{+18}, 2006$ • AK

ST: PO.JI • CI: h1295・ID: 121

PN: Bufo viridis Laurenti, 1768

PK: Bufo viridis* Laurenti, 1768

KG: Bufotes* Rafinesque, 1815

KF: BUFONIDAE 1825.gb.f004

Pseudes Leunis, $1844 \cdot \mathbf{A K}$

ST: NS.JI • CI: h1296・ID: 196

PN: Rana paradoxa Linnaeus, 1758

PK: Rana paradoxa* Linnaeus, 1758

KG: Pseudis* Wagler, 1830

KF: HYLIDAE 1815.ra.f002-|1825.gb.f001|

Pseudhymenochirus Chabanaud, 1920 • KY

ST: Po.KN・CI: h1297・ID: 011

PN: Pseudhymenochirus merlini Chabanaud, 1920

PK: Pseudhymenochirus merlini* Chabanaud, 1920

KG: Pseudhymenochirus* Chabanaud, 1920

KF: PIPIDAE 1825.gb.f003-|1826.fb.f002|

Pseudis Wagler, $1830 \bullet \mathbf{K Y}$

ST: PO.KN • CI: h1298 • ID: 196

PN: Rana paradoxa Linnaeus, 1758

PK: Rana paradoxa* Linnaeus, 1758
KG: Pseudis* Wagler, 1830

KF: HYLIDAE 1815.ra.f002-|1825.gb.f001|

Pseudoamolops: Jiang ${ }^{+6} 1997$ • AN

ST: AL • CI: n0137・ID: 419

PN: Rana sauteri Boulenger, 1909

PK: Rana sauteri* Boulenger, 1909

KG: Rana* Linnaeus, 1758

KF: RANIDAE 1796.ba.f001

Pseudoamolops $\mathrm{Fei}^{+2}, 2000 \cdot \mathbf{A K}$

ST: PO.JD・CI: h1299・ID: 419

PN: Rana sauteri Boulenger, 1909

PK: Rana sauteri* Boulenger, 1909

KG: Rana* Linnaeus, 1758

KF: RANIDAE 1796.ba.f001

Pseudobatrachus Peters, 1873 • AK

ST: PO.JD・CI: h1300・ID: 186

PN: Pseudobatrachus jelskii Peters, 1873

PK: Pseudobatrachus jelskii ${ }^{\circ}$ Peters, 1873

KG: Telmatobius ${ }^{3}$ Wiegmann, 1834

KF: TELMATOBIIDAE 1843.fa.f006

Pseudobranchus Gray, 1825 • KY

ST: PO.KN • CI: h1301 • ID: 518

PN: Siren striata Le Conte, 1824

PK: Siren striata* Le Conte, 1824

KG: Pseudobranchus* Gray, 1825

KF: SIRENIDAE 1825gb.f005

Pseudobufo Tschudi, 1838 - KY

ST: PO.KN • CI: h1302・ID: 111

PN: Pseudobufo subasper Tschudi, 1838

PK: Pseudobufo subasper ${ }^{\circ}$ Tschudi, 1838

KG: Pseudobufo ${ }^{\circ}$ Tschudi, 1838

KF: BUFONIDAE 1825.gb.f004

Pseudocallulops Günther, 2009 • AK

ST: PO.JD • CI: h1303・ID: 280

PN: Callulops pullifer Günther, 2006

PK: Callulops pullifer* Günther, 2006

KG: Asterophrys* Tschudi, 1838

KF: MICROHYLIDAE ||1843.fa.f012||-1931.na.f001

Pseudocassina Ahl, 1924 • AK

ST: PO.JD・CI: h1304 • ID: 325

PN: Pseudocassina ocellata Ahl, 1923

PK: Megalixalus gramineus ${ }^{\circ}$ Boulenger, 1898

KG: Leptopelis ${ }^{2}$ Günther, 1859

KF: ARTHROLEPTIDAE 1869.mc.f011

Pseudoeurycea Taylor, 1944 • KY

ST: PO.KN • CI: h1305・ID: 527

PN: Spelerpes leprosus Cope, 1869

PK: Spelerpes leprosus* Cope, 1869

KG: Pseudoeurycea* Taylor, 1944

KF: PLETHODONTIDAE 1850.ga.f002

Pseudofryne Palacký, 1898 • AK

ST: NT.JI • CI: h1306・ID: 274

PN: Bombinator australis Gray, 1835

PK: Bombinator australis ${ }^{\circ}$ Gray, 1835

KG: Pseudophryne ${ }^{3}$ Fitzinger, 1843

KF: MYOBATRACHIDAE 1850.sa.f001 
Pseudohemisus Mocquard, 1895 • AK

ST: PO.JD • CI: h1307 • ID: 291

PN: Hemisus obscurus Grandidier, 1872

PK: Hemisus obscurus ${ }^{\circ}$ Grandidier, 1872

KG: Scaphiophryne* Boulenger, 1882

KF: MICROHYLIDAE ||1843.fa.f012||-1931.na.f001

Pseudohyla Andersson, 1946 • AK

ST: PO.JD • CI: h1308 • ID: 078

PN: Pseudohyla nigrogrisea Andersson, 1946

PK: Pseudohyla nigrogrisea ${ }^{\circ}$ Andersson, 1946

KG: Pristimantis* Jiménez de la Espada, 1870

KF: BRACHYCEPHALIDAE 1858.gc.f002

Pseudohynobius $\mathrm{Fei}^{+1}$, $1983 \bullet \mathbf{K Y}$

ST: PO.KN・CI: h1309・ID: 511

PN: Hynobius flavomaculatus $\mathrm{Hu}^{+1}, 1978$

PK: Hynobius flavomaculatus* $\mathrm{Hu}^{+1}, 1978$

KG: Pseudohynobius* ${ }^{*} \mathrm{Fe}^{+1}, 1983$

KF: HYNOBIIDAE ||1856.ha.f001||-1859.cb.f002

Pseudopaludicola Miranda-Ribeiro, 1926• KY

ST: PO.KN • CI: h1310 • ID: 256

PN: Liuperus falcipes Hensel, 1867

PK: Liuperus falcipes* Hensel, 1867

KG: Pseudopaludicola* Miranda-Ribeiro, 1926

KF: PSEUDOPALUDICOLIDAE 1965.ga.f003

Pseudopelobates Pasteur, 1958 • AK

ST: PO.JD • CI: h1311 • ID: 026

PN: Pelobates transcaucasicus Delwig, 1928

PK: Pelobates syriacus* Boettger, 1889

KG: Pelobates* Wagler, 1830

KF: PELOBATIDAE 1850.bb.f004

Pseudophilautus Laurent, 1943・ KY

ST: PO.KN・CI: h1312・ID: 444

PN: Ixalus temporalis Günther, 1864

PK: Ixalus temporalis ${ }^{\circ}$ Günther, 1864

KG: Pseudophilautus ${ }^{2}$ Laurent, 1943

KF: RHACOPHORIDAE ||1858.gc.f012||-1932.ha.f001

Pseudophryne Fitzinger, $1843 \cdot \mathbf{K Y}$

ST: PO.KN • CI: h1313 • ID: 274

PN: Bombinator australis Gray, 1835

PK: Bombinator australis $^{\circ}$ Gray, 1835

KG: Pseudophryne ${ }^{3}$ Fitzinger, 1843

KF: MYOBATRACHIDAE 1850.sa.f001

Pseudopipa Ritgen, 1828 • AK

ST: PO.JD • CI: h1314 • ID: 009

PN: Bufo laevis Daudin, 1802

PK: Bufo laevis* Daudin, 1802

KG: Xenopus ${ }^{1}$ Wagler in Boie, 1827

KF: PIPIDAE 1825.gb.f003-|1826.fb.f002|

Pseudorana $\mathrm{Fei}^{+2}$, $1990 \cdot \mathbf{K Y}$

ST: PO.KN • CI: h1315・ID: 416

PN: Rana weiningensis $\mathrm{Liu}^{+2}, 1962$

PK: Rana weiningensis* Liu $^{+2}, 1962$

KG: Pseudorana* $\mathrm{Fei}^{+2}, 1990$

KF: RANIDAE 1796.ba.f001

Pseudosalamandra Tschudi, 1838 • AK

ST: PO.JD • CI: h1316 • ID: 505
PN: Salamandra naevia Temminck $^{+1}, 1838$

PK: Salamandra naevia* Temminck $^{+1}, 1838$

KG: Hynobius* Tschudi, 1838

KF: HYNOBIIDAE ||1856.ha.f001||-1859.cb.f002

Pseudosiphonops Taylor, 1968 • AK

ST: PO.JD • CI: h1317 • ID: 491

PN: Pseudosiphonops ptychodermis Taylor, 1968

PK: Mimosiphonops vermiculatus ${ }^{\circ}$ Taylor, 1968

KG: Mimosiphonops ${ }^{\circ}$ Taylor, 1968

KF: CAECILIIDAE 1814.ra.f003-|1825.gb.f008|

Pseudotriton Tschudi, $1838 \cdot \mathbf{K Y}$

ST: PO.KN・CI: h1318・ ID: 540

PN: Salamandra subfusca Green, 1818

PK: Salamandra rubra* Sonnini ${ }^{+1}, 1801$

KG: Pseudotriton ${ }^{1}$ Tschudi, 1838

KF: PLETHODONTIDAE 1850.ga.f002

Pseudotyphlonectes Lescure ${ }^{+1}, 1986$ • AK

ST: PO.JD • CI: h1319・ID: 480

PN: Caecilia natans Fischer in Peters, 1880

PK: Caecilia natans* Fischer in Peters, 1880

KG: Typhlonectes* Peters, 1880

KF: CAECILIIDAE 1814.ra.f003-|1825.gb.f008|

Pseudoxenopus Barbour ${ }^{+1}$, 1927 • AK

ST: PO.JD • CI: h1320 • ID: 351

PN: Pseudoxenopus alleni Barbour ${ }^{+1}, 1927$

PK: Pseudoxenopus alleni* ${ }^{*}$ Barbour $^{+1}, 1927$

KG: Conraua* Nieden, 1908

KF: CONRAUIDAE 1992.da.f001

Psychrophrynella Hedges ${ }^{+2}, 2008$ - KY

ST: PO.KN • CI: h1321 • ID: 071

PN: Phrynopus bagrecito Lynch, 1986

PK: Phrynopus bagrecito ${ }^{\circ}$ Lynch, 1986

KG: Psychrophrynella ${ }^{\circ}$ Hedges $^{+2}, 2008$

KF: BRACHYCEPHALIDAE 1858.gc.f002

Psyllophryne Izecksohn, 1971 • AK

ST: PO.JD • CI: h1322・ID: 057

PN: Psyllophryne didactyla Izecksohn, 1971

PK: Psyllophryne didactyla* Izecksohn, 1971

KG: Brachycephalus* Fitzinger, 1826

KF: BRACHYCEPHALIDAE 1858.gc.f002

Pternohyla Boulenger, 1882 • AK

ST: PO.JD • CI: h1323・ID: 208

PN: Pternohyla fodiens Boulenger, 1882

PK: Pternohyla fodiens* Boulenger, 1882

KG: Smilisca ${ }^{1}$ Cope, 1865

KF: HYLIDAE 1815.ra.f002-|1825.gb.f001|

Pterophrynus Lütken, 1864 • AK

ST: PO.JD・CI: h1324・ID: 270

PN: Pterophrynus verrucosus Lütken, 1864

PK: Crinia (Ranidella) signifera* Girard, 1853

KG: Crinia* Tschudi, 1838

KF: MYOBATRACHIDAE 1850.sa.f001

Pterorana Kiyasetuo ${ }^{+1}, 1986$ • KY

ST: PO.KN • CI: h1325・ID: 401

PN: Pterorana khare Kiyasetuo ${ }^{+1}, 1986$

PK: Pterorana khare ${ }^{\circ}$ Kiyasetuo $^{+1}, 1986$ 
KG: Pterorana ${ }^{\circ}$ Kiyasetuo $^{+1}, 1986$

KF: RANIDAE 1796.ba.f001

Ptychadaena Parker, 1930 - AK

ST: NS.JI • CI: h1326・ID: 464

PN: Rana mascareniensis Duméril ${ }^{+1}, 1841$

PK: Rana mascareniensis* Duméril ${ }^{+1}, 1841$

KG: Ptychadena* Boulenger, 1917

KF: PTYCHADENIDAE 1987.da.f002

Ptychadena Boulenger, 1917 • KY

ST: PO.KN・CI: h1327・ID: 464

PN: Rana mascareniensis Duméril ${ }^{+1}, 1841$

PK: Rana mascareniensis * Duméril ${ }^{+1}, 1841$

KG: Ptychadena* Boulenger, 1917

KF: PTYCHADENIDAE 1987.da.f002

Ptychohyla Taylor, $1944 \cdot \mathbf{K Y}$

ST: PO.KN・CI: h1328 • ID: 215

PN: Ptychohyla adipoventris Taylor, 1944

PK: Hyla leonhardschultzei* Ahl, 1934

KG: Ptychohyla ${ }^{1}$ Taylor, 1944

KF: HYLIDAE 1815.ra.f002-|1825.gb.f001|

Pulchrana Dubois, 1992 • AK

ST: PO.JD • CI: h1329・ID: 407

PN: Polypedates signatus Günther, 1872

PK: Polypedates signatus* Günther, 1872

KG: Hylarana* Tschudi, 1838

KF: RANIDAE 1796.ba.f001

Pycnacris Fouquette ${ }^{+1}, 2014 \cdot \mathbf{A K}$

ST: PO.JD • CI: h1330 • ID: 200

PN: Rana ornata Holbrook, 1836

PK: Rana ornata* Holbrook, 1836

KG: Pseudacris* Fitzinger, 1843

KF: HYLIDAE 1815.ra.f002-|1825.gb.f001|

Pyleus Gistel, 1848 • AK

ST: NL.JI • CI: h1331・ID: 111

PN: Pseudobufo subasper Tschudi, 1838

PK: Pseudobufo subasper ${ }^{\circ}$ Tschudi, 1838

KG: Pseudobufo ${ }^{\circ}$ Tschudi, 1838

KF: BUFONIDAE 1825.gb.f004

Pyronicia Gray, 1858 • AK

ST: PO.JD • CI: h1332 • ID: 566

PN: Salamandra marmorata Latreille, 1800

PK: Salamandra marmorata* Latreille, 1800

KG: Triturus* Rafinesque, 1815

KF: SALAMANDRIDAE 1820.ga.f002

Pyxicephalus Tschudi, 1838 - KY

ST: PO.KN・CI: h1333・ID: 367

PN: Pyxicephalus adspersus Tschudi, 1838

PK: Pyxicephalus adspersus* Tschudi, 1838

KG: Pyxicephalus* Tschudi, 1838

KF: PYXICEPHALIDAE 1850.bb.f005

Qiantriton $\mathrm{Fei}^{+2}, 2012 \cdot \mathbf{A K}$

ST: PO.JD • CI: h1334 • ID: 573

PN: Tylototriton kweichowensis Fang $^{+1}, 1932$

PK: Tylototriton kweichowensis* Fang ${ }^{+1}, 1932$

KG: Tylototriton* Anderson, 1871

KF: SALAMANDRIDAE 1820.ga.f002
Qianotriton $\mathrm{Fei}^{+1}, 2016 \cdot \mathbf{A K}$

ST: NT.JI • CI: h1335 • ID: 573

PN: Tylototriton kweichowensis Fang ${ }^{+1}, 1932$

PK: Tylototriton kweichowensis* Fang ${ }^{+1}, 1932$

KG: Tylototriton* Anderson, 1871

KF: SALAMANDRIDAE 1820.ga.f002

Qinglongtriton $\mathrm{Jia}^{+1}, 2016+\cdot \mathbf{K Y}$

ST: PO.KN • CI: h1336・ID: $\uparrow 178$

PN: Qinglongtriton gangouensis $\mathrm{Jia}^{+1}, 2016$ \$

PK: Qinglongtriton gangouensis ${ }^{\circ} \mathrm{Jia}^{+1}, 2016 \uparrow$

KG: Qinglongtriton ${ }^{\circ} \mathrm{Jia}^{+1}, 2016 \dagger$

KF: Pseudosauria Familia InCERTAE SEDIS

Qiongbufo $\mathrm{Fei}^{+2}, 2012 \cdot \mathbf{A K}$

ST: PO.JD • CI: h1337 • ID: 117

PN: Bufo ledongensis $\mathrm{Fei}^{+1}, 2009$

PK: Bufo ledongensis ${ }^{\circ} \mathrm{Fei}^{+1}, 2009$

KG: Ingerophrynus* Frost $^{+18}, 2006$

KF: BUFONIDAE 1825.gb.f004

Qiongobufo $\mathrm{Fei}^{+1}, 2016$ • AK

ST: NS.JD • CI: h1338・ID: 117

PN: Bufo ledongensis $\mathrm{Fei}^{+2}, 2009$

PK: Bufo ledongensis ${ }^{\circ} \mathrm{Fei}^{+2}, 2009$

KG: Ingerophrynus* Frost $^{+18}, 2006$

KF: BUFONIDAE 1825.gb.f004

Qosqophryne Catenazzi ${ }^{+3}, 2020 \bullet \mathbf{K Y}$

ST: PO.KN • CI: h1339 • ID: 072

PN: Bryophryne gymnotis Lehr $^{+1}, 2009$

PK: Bryophryne gymnotis ${ }^{\circ}$ Lehr $^{+1}, 2009$

KG: Qosqophryne ${ }^{\circ}$ Catenazzi $^{+3}, 2020$

KF: BRACHYCEPHALIDAE 1858.gc.f002

Quadrana Caldwell ${ }^{+1}, 1952 \cdot \mathbf{Z H}$

ST: zo • CI: zh084

Quadrana $\mathrm{Fei}^{+2}, 1990$ • AK

ST: PO.JH • CI: h1340 • ID: 386

PN: Rana quadranus $\mathrm{Liu}^{+2}, 1960$

PK: Rana quadranus* Liu $^{+2}, 1960$

KG: Feirana* Dubois, 1992

KF: DiCROGLOSSIDAE 1987.da.f004

Quasipaa Dubois, $1992 \cdot \mathbf{K Y}$

ST: PO.KN • CI: h1341・ID: 391

PN: Rana boulengeri Günther, 1889

PK: Rana boulengeri* Günther, 1889

KG: Quasipaa* Dubois, 1992

KF: DiCROGLOSSIDAE 1987.da.f004

Quilticohyla Faivovich $^{+15}, 2018 \cdot \mathbf{K Y}$

ST: PO.KN・CI: h1342・ID: 216

PN: Quilticohyla sanctaecrucis Faivovich ${ }^{+15}, 2018$

PK: Quilticohyla sanctaecrucis ${ }^{\circ}$ Faivovich $^{+15}, 2018$

KG: Quilticohyla Faivovich $^{+15}, 2018$

KF: HYLIDAE 1815.ra.f002-|1825.gb.f001|

Quinquevertebron Kuhn, 1941 + $\mathbf{A K}$

ST: PO.JD • CI: h1343・ ID: $\uparrow 069$

PN: Quinquevertebron germanicum Kuhn, 1941 \$

PK: Pelobatinopsis hinschei ${ }^{\circ}$ Kuhn, 1941 †

KG: Palaeobatrachus ${ }^{\circ}$ Tschudi, $1838+$

KF: PALAEOBATRACHIDAE 1865.ca.f001 † 
Quinzhyla Bauer, 2005 • AK

ST: PO.JD・CI: h1344・ID: 194

PN: Bufo marmoratus Laurenti, 1768

PK: Bufo marmoratus* Laurenti, 1768

KG: Dendropsophus ${ }^{1}$ Fitzinger, 1843

KF: HYLIDAE 1815.ra.f002-|1825.gb.f001|

Racophorus Schlegel, 1826・AK

ST: NS.JI • CI: h1345 • ID: 455

PN: Rhacophorus moschatus $\mathrm{Kuhl}^{+1}, 1822$

PK: Hyla reinwardtii* Schlegel, 1840

KG: Rhacophorus* $\mathrm{Kuhl}^{+1}, 1822$

KF: RHACOPHORIDAE \|1858.gc.f012||-1932.ha.f001

Rafinus Dubois ${ }^{+1}, 2009 \cdot \mathbf{A K}$

ST: PO.JD・CI: h1346・ID: 569

PN: Diemyctylus miniatus meridionalis Cope, 1880

PK: Diemyctylus miniatus meridionalis* Cope, 1880

KG: Notophthalmus ${ }^{1}$ Rafinesque, 1820

KF: SALAMANDRIDAE 1820.ga.f002

Ramanella $\mathrm{RaO}^{+1}, 1925 \cdot \mathbf{A K}$

ST: PO.JD • CI: h1347・ID: 309

PN: Ramanella symbioitica $\mathrm{Rao}^{+1}, 1925$

PK: Callula variegata* Stoliczka, 1872

KG: Uperodon* Duméril ${ }^{+1}, 1841$

KF: MICROHYLIDAE ||1843.fa.f012||-1931.na.f001

Ramonellus $\mathrm{Nevo}^{+1}, 1969+\bullet \mathbf{K Y}$

ST: PO.KN • CI: h1348・ID: $\uparrow 140$

PN: Ramonellus longispinus $\mathrm{Nevo}^{+1}, 1969 \ddagger$

PK: Ramonellus longispinus ${ }^{\circ} \mathrm{Nevo}^{+1}, 1969 \dagger$

KG: Ramonellus ${ }^{\circ} \mathrm{Nevo}^{+1}, 1969 \dagger$

KF: Urodela Familia INCERTAE SEDIS

Rana Linnaeus, $1758 \cdot \mathbf{K Y}$

ST: PO.KN • CI: h1349・ID: 419

PN: Rana temporaria Linnaeus, 1758

PK: Rana temporaria* Linnaeus, 1758

KG: Rana* Linnaeus, 1758

KF: RANIDAE 1796.ba.f001

Rana: Rösel von Rosenhof 1758 • AN

ST: AL • CI: n0138・ID: 419

PN: Rana temporaria Linnaeus, 1758

PK: Rana temporaria* Linnaeus, 1758

KG: Rana* Linnaeus, 1758

KF: RANIDAE 1796.ba.f001

Rana: Vogel 1758 • AN

ST: $\mathbf{A L} \bullet \mathbf{C I}:$ n0139・ID: 419

PN: Rana temporaria Linnaeus, 1758

PK: Rana temporaria* Linnaeus, 1758

KG: Rana* Linnaeus, 1758

KF: RANIDAE 1796.ba.f001

Rana Ritgen, 1828 • AK

ST: PO.JH • CI: h1350 • ID: 252

PN: Rana schneideri Merrem, 1820

PK: Rana lineata* Schneider, 1799

KG: Lithodytes* Fitzinger, 1843

KF: LEPTODACTYLIDAE ||1838.ta.f001||-1896.wa.f001

Ranapes: Lockley ${ }^{+1} 2014+; \cdot A N$

ST: LI • CI: n0140 • ID: $\uparrow 039$
PN: Ranipes laci Lockley $^{+1}, 2014$ \$i

PK: Ranipes laci ${ }^{\circ}$ Lockley $^{+1}, 2014 \dagger$

KG: Ranipes ${ }^{\circ}$ Lockley $^{+1}, 2014 \dagger$

KF: ANURA Familia INCERTAE SEDIS

Ranaria Rafinesque, 1814 • AK

ST: NT.JI • CI: h1351 • ID: 419

PN: Rana temporaria Linnaeus, 1758

PK: Rana temporaria* Linnaeus, 1758

KG: Rana* Linnaeus, 1758

KF: RANIDAE 1796.ba.f001

Ranaster Macleay, 1878 • AK

ST: PO.JD・CI: h1352・ID: 261

PN: Ranaster convexiusculus Macleay, 1878

PK: Ranaster convexiusculus* Macleay, 1878

KG: Limnodynastes* Fitzinger, 1843

KF: MYOBATRACHIDAE 1850.sa.f001

Ranavus Portis, $1885+\bullet \mathbf{K Y}$

ST: PO.KN・CI: h1353・ID: $\uparrow 105$

PN: Ranavus scarabellii Portis, 1885 末

PK: Ranavus scarabellii ${ }^{\circ}$ Portis, $1885 \dagger$

KG: Ranavus $^{\circ}$ Portis, 1885 †

KF: RANIDAE 1796.ba.f001

Ranella: Garsault 1764 • AN

ST: $\mathbf{L I}$ •CI: n0141 • ID: 204

PN: Rana arborea Linnaeus, 1758

PK: Rana arborea* Linnaeus, 1758

KG: Hyla* Laurenti, 1768

KF: HYLIDAE 1815.ra.f002-|1825.gb.f001|

Ranetta Garsault, 1764 • AK

ST: LC.RO・CI: h1354 • ID: 204

PN: Rana arborea Linnaeus, 1758

PK: Rana arborea* Linnaeus, 1758

KG: Hyla* Laurenti, 1768

KF: HYLIDAE 1815.ra.f002-|1825.gb.f001|

Ranhyla: Girard $1858 \cdot \mathbf{A N}$

ST: AL・CI: n0142 • ID: 409

PN: Hyla erythraea Schlegel, 1827

PK: Hyla erythraea* Schlegel, 1827

KG: Hylarana* Tschudi, 1838

KF: RANIDAE 1796.ba.f001

Ranidella Girard, 1853 • AK

ST: PO.JD • CI: h1355・ID: 270

PN: Crinia (Ranidella) signifera Girard, 1853

PK: Crinia (Ranidella) signifera* Girard, 1853

KG: Crinia* Tschudi, 1838

KF: MYOBATRACHIDAE 1850.sa.f001

Ranidens Boulenger, 1882 • AK

ST: NT.JI • CI: h1356・ID: 516

PN: Ranodon sibiricus Kessler, 1866

PK: Ranodon sibiricus* Kessler, 1866

KG: Ranodon* Kessler, 1866

KF: HYNOBIIDAE \|1856.ha.f001\|-1859.cb.f002

Ranina Lamarck, $1801 \cdot \mathbf{Z H}$

ST: zo • CI: zh085

Ranina: Bibron in Bonaparte 1839 • AN

ST: AL • CI: n0143・ID: $007 \S$ 
PN: INR

PK: INR

KG: INR

KF: Hydrobatrachia Familia INCERTAE SEDIS

Ranina David, 1872 • AK

ST: PO.JH • CI: h1357 • ID: 314

PN: Ranina symetrica David, 1872

PK: Engystoma pulchrum* Hallowell, 1861

KG: Microhyla* Tschudi, 1838

KF: MICROHYLIDAE ||1843.fa.f012||-1931.na.f001

Ranipes Lockley ${ }^{+1}, 2014$ \$i $\bullet \mathbf{K Y}$

ST: LC.KN • CI: h1358・ ID: $\uparrow 039$

PN: Ranipes laci Lockley ${ }^{+1}, 2014$ \$

PK: Ranipes laci ${ }^{\circ}$ Lockley $^{+1}, 2014 \dagger$

KG: Ranipes $^{\circ}$ Lockley $^{+1}, 2014 \dagger$

KF: ANURA Familia INCERTAE SEDIS

Ranitomeya Bauer, $1985 \bullet \mathbf{K Y}$

ST: PO.KN・CI: h1359・ID: 045

PN: Dendrobates reticulatus Boulenger, 1884

PK: Dendrobates reticulatus* Boulenger, 1884

KG: Ranitomeya* Bauer, 1985

KF: DENDROBATIDAE $\| 1850$. bb.f006 |-1865.ca.f002

Ranixalus Dubois, 1986 • AK

ST: PO.JD • CI: h1360 • ID: 460

PN: Ranixalus gundia Dubois, 1986

PK: Ranixalus gundia ${ }^{\circ}$ Dubois, 1986

KG: Indirana* Laurent, 1986

KF: RANIXALIDAE 1987.da.f005

Ranodon Kessler, $1866 \bullet \mathbf{K Y}$

ST: PO.KN・CI: h1361・ID: 516

PN: Ranodon sibiricus Kessler, 1866

PK: Ranodon sibiricus* Kessler, 1866

KG: Ranodon* Kessler, 1866

KF: HyNOBIIDAE ||1856.ha.f001||-1859.cb.f002

Ranoidea Tschudi, $1838 \bullet \mathbf{K Y}$

ST: LC.KN • CI: h1362・ID: 237

PN: Ranoidea jacksoniensis Tschudi, 1838

PK: Rana aurea* Lesson, 1829

KG: Ranoidea ${ }^{1}$ Tschudi, 1838

KF: PHYLLOMEDUSIDAE 1858.gc.f009

Ranoides: Tschudi 1838 • AN

ST: $\mathbf{L I} ・$ CI: n0144 • ID: 237

PN: Ranoidea jacksoniensis Tschudi, 1838

PK: Rana aurea* Lesson, 1829

KG: Ranoidea ${ }^{1}$ Tschudi, 1838

KF: PHYLLOMEDUSIDAE 1858.gc.f009

Ranomorphus Ratnikov, $1993+\cdot \mathbf{K Y}$

ST: PO.KN • CI: h1363・ID: $\dagger 040$

PN: Ranomorphus similis Ratnikov, 1993 \$

PK: Ranomorphus similis ${ }^{\circ}$ Ratnikov, $1993 \dagger$

KG: Ranomorphus ${ }^{\circ}$ Ratnikov, 1993 †

KF: ANURA Familia INCERTAE SEDIS

Ranosoma Ahl, 1924 • AK

ST: PO.JD・CI: h1364 • ID: 374

PN: Ranosoma schereri Ahl, 1924

PK: Rana occipitalis* Günther, 1859
KG: Hoplobatrachus ${ }^{1}$ Peters, 1863

KF: DiCROGLOSSIDAE 1987.da.f004

Ranula: Schumacher $1817 \cdot \mathbf{Z A}$

ST: $\mathbf{z N} \bullet \mathbf{C I}: \mathrm{zn} 010$

Ranula Peters, 1859 • AK

ST: PO.JH • CI: h1365・ID: 415

PN: Ranula gollmeri Peters, 1859

PK: Rana palmipes* Spix, 1824

KG: Lithobates* Fitzinger, 1843

KF: RANIDAE 1796.ba.f001

Raorchestes $\mathrm{Biju}^{+4}, 2010 \bullet \mathbf{K Y}$

ST: PO.KN・CI: h1366・ID: 445

PN: Ixalus glandulosus Jerdon, 1854

PK: Ixalus glandulosus* Jerdon, 1854

KG: Raorchestes* $\mathrm{Biju}^{+4}, 2010$

KF: RHACOPHORIDAE |1858.gc.f012||-1932.ha.f001

Rappia Günther, 1865 • AK

ST: NL.JI・CI: h1367・ID: 331

PN: Hyla horstockii Schlegel, 1837

PK: Hyla horstockii* Schlegel, 1837

KG: Hyperolius* Rapp, 1842

KF: HYPEROLIIDAE 1943.lb.f001

Rawlinsonia Wells ${ }^{+1}, 1985$ • AK

ST: PO.JD・CI: h1368・ID: 235

PN: Hyla ewingi Duméril ${ }^{+1}, 1841$

PK: Hyla ewingi* Duméril ${ }^{+1}, 1841$

KG: Litoria* Tschudi, 1838

KF: PHYLLOMEDUSIDAE 1858.gc.f009

Regalerpeton Zhang $^{+3,} 2009+\cdot \mathbf{K Y}$

ST: PO.KN・CI: h1369・ID: $\uparrow 163$

PN: Regalerpeton weichangensis $\mathrm{Zhang}^{+3}, 2009+$

PK: Regalerpeton weichangensis ${ }^{\circ} \mathrm{Zhang}^{+3}, 2009 \dagger$

KG: Regalerpeton ${ }^{\circ} \mathrm{Zhang}^{+3}, 2009 \dagger$

KF: IMPERFECTIBRANCHIA Familia INCERTAE SEDIS

Relictivomer Carvalho, 1954 • AK

ST: PO.JD・CI: h1370 • ID: 298

PN: Hypopachus pearsei Ruthven, 1914

PK: Hypopachus pearsei ${ }^{\circ}$ Ruthven, 1914

KG: Engystoma* Fitzinger, 1826

KF: MICROHYLIDAE ||1843.fa.f012||-1931.na.f001

Relictocleis nov. $\bullet \mathrm{KY}$

ST: PO.KN • CI: h1371 • ID: 292

PN: Chiasmocleis gnoma Canedo $^{+2}, 2004$

PK: Chiasmocleis gnoma ${ }^{\circ}$ Canedo $^{+2}, 2004$

KG: Chiasmocleis* Méhelÿ, 1904

KF: MICROHYLIDAE ||1843.fa.f012||-1931.na.f001

Relictus Hubbs ${ }^{+1}$, 1972• ZH

ST: zo • CI: zh086

Relictus: $\mathrm{Sá}^{+8} 2018 \cdot \mathbf{A N}$

ST: AL •CI: n0145・ID: 292

PN: Chiasmocleis gnoma Canedo ${ }^{+2}, 2004$

PK: Chiasmocleis gnoma ${ }^{\circ}$ Canedo $^{+2}, 2004$

KG: Chiasmocleis* Méhelÿ, 1904

KF: MICROHYLIDAE ||1843.fa.f012||-1931.na.f001

Relictus: $\mathrm{Sá}^{+8} 2019$ •AN

ST: AL・CI: n0146・ID: 292 
PN: Chiasmocleis gnoma Canedo $^{+2}, 2004$

PK: Chiasmocleis gnoma ${ }^{\circ}$ Canedo $^{+2}, 2004$

KG: Chiasmocleis* Méhelÿ, 1904

KF: MICROHYLIDAE ||1843.fa.f012||-1931.na.f001

Rentapia $\mathrm{Chan}^{+4}, 2016$ • KY

ST: PO.KN • CI: h1372 • ID: 119

PN: Nectophryne hosii Boulenger, 1892

PK: Nectophryne hosii* Boulenger, 1892

KG: Rentapia* Chan $^{+4}, 2016$

KF: BUFONIDAE 1825.gb.f004

Rhacoforus Palacký, 1898 • AK

ST: NT.JI • CI: h1373・ID: 455

PN: Rhacophorus moschatus $\mathrm{Kuhl}^{+1}, 1822$

PK: Hyla reinwardtii* Schlegel, 1840

KG: Rhacophorus* $\mathrm{Kuhl}^{+1}, 1822$

KF: RHACOPHORIDAE \|1858.gc.f012||-1932.ha.f001

Rhacophorus $\mathrm{Kuhl}^{+1}, 1822 \cdot \mathbf{K Y}$

ST: PO.KN • CI: h1374・ID: 455

PN: Rhacophorus moschatus Kuhl ${ }^{+1}, 1822$

PK: Hyla reinwardtii* Schlegel, 1840

KG: Rhacophorus* $\mathrm{Kuhl}^{+1}, 1822$

KF: RHACOPHORIDAE |1858.gc.f012||-1932.ha.f001

Rhadinosteus Henrici, $1998+\bullet \mathbf{K Y}$

ST: PO.KN • CI: h1375 • ID: $\uparrow 083$

PN: Rhadinosteus parvus Henrici, 1998 \$

PK: Rhadinosteus parvus ${ }^{\circ}$ Henrici, $1998 \dagger$

KG: Rhadinosteus ${ }^{\circ}$ Henrici, $1998 \dagger$

KF: RHINOPHRYNIDAE 1858.gc.f013

Rhaeba: Boulenger 1882 • AN

ST: $\mathbf{A M} ・ \mathbf{C I}:$ n0147・ID: 145

PN: Bufo leschenaulti Duméril ${ }^{+1}, 1841$

PK: Bufo guttatus* Schneider, 1799

KG: Rhaebo* Cope, 1862

KF: BUFONIDAE 1825.gb.f004

Rhaebo Cope, $1862 \cdot \mathbf{K Y}$

ST: PO.KN・CI: h1376・ID: 145

PN: Bufo leschenaulti Duméril ${ }^{+1}, 1841$

PK: Bufo guttatus* Schneider, 1799

KG: Rhaebo* Cope, 1862

KF: BUFONIDAE 1825.gb.f004

Rhamphophryne Trueb, 1971 • AK

ST: PO.JD • CI: h1377・ID: 138

PN: Rhamphophryne acrolopha Trueb, 1971

PK: Rhamphophryne acrolopha ${ }^{\circ}$ Trueb, 1971

KG: Rhinella ${ }^{2}$ Fitzinger, 1826

KF: BUFONIDAE 1825.gb.f004

Rhaphidochir Wagler in Michahelles, 1833 • AK

ST: PO.JD・CI: h1378・ID: 009

PN: Bufo laevis Daudin, 1802

PK: Bufo laevis* Daudin, 1802

KG: Xenopus ${ }^{1}$ Wagler in Boie, 1827

KF: PIPIDAE 1825.gb.f003-|1826.fb.f002|

Rheobates Grant ${ }^{+9}, 2006$ • KY

ST: PO.KN • CI: h1379・ID: 036

PN: Phyllobates palmatus Werner, 1899

PK: Phyllobates palmatus* Werner, 1899
KG: Rheobates $* \mathrm{Grant}^{+9}, 2006$

KF: AROMOBATIDAE 2006.gc.f004

Rheobatrachus Liem, 1973 • KY

ST: PO.KN • CI: h1380・ID: 278

PN: Rheobatrachus silus Liem, 1973

PK: Rheobatrachus silus* Liem, 1973

KG: Rheobatrachus* Liem, 1973

KF: MYOBATRACHIDAE 1850.sa.f001

Rheohyla Duellman ${ }^{+2}, 2016 \cdot \mathbf{K Y}$

ST: PO.KN・CI: h1381 •ID: 217

PN: Hyla miotympanum Cope, 1863

PK: Hyla miotympanum* Cope, 1863

KG: Rheohyla* Duellman ${ }^{+2}, 2016$

KF: HYLIDAE 1815.ra.f002-|1825.gb.f001|

Rhinatrema Duméril $^{+1}, 1841$ • KY

ST: PO.KN • CI: h1382・ID: 473

PN: Caecilia bivittata Guérin-Méneville, 1838

PK: Caecilia bivittata* Guérin-Méneville, 1838

KG: Rhinatrema* Duméril ${ }^{+1}, 1841$

KF: RHINATREMATIDAE 1977.na.f001

Rhinella Fitzinger, 1826 • KY

ST: PO.KN • CI: h1383・ID: 138

PN: Bufo proboscideus Spix, 1824

PK: Bufo proboscideus ${ }^{\circ}$ Spix, 1824

KG: Rhinella ${ }^{2}$ Fitzinger, 1826

KF: BUFONIDAE 1825.gb.f004

Rhinellus Cuvier ${ }^{+1}, 1831 \cdot \mathbf{A K}$

ST: NS.JD・CI: h1384・ID: 138

PN: Bufo proboscideus Spix, 1824

PK: Bufo proboscideus ${ }^{\circ}$ Spix, 1824

KG: Rhinella ${ }^{2}$ Fitzinger, 1826

KF: BUFONIDAE 1825.gb.f004

Rhinoderma Duméril ${ }^{+1}, 1841$ • KY

ST: PO.KN・CI: h1385・ID: 185

PN: Rhinoderma darwinii Duméril ${ }^{+1}, 1841$

PK: Rhinoderma darwinii* Duméril ${ }^{+1}, 1841$

KG: Rhinoderma* Duméril ${ }^{+1}, 1841$

KF: RHINODERMATIDAE 1850.bb.f011

Rhinophrynus Duméril ${ }^{+1}, 1841$ • KY

ST: PO.KN • CI: h1386・ID: 013

PN: Rhinophrynus dorsalis Duméril ${ }^{+1}, 1841$

PK: Rhinophrynus dorsalis* Duméril ${ }^{+1}, 1841$

KG: Rhinophrynus* Duméril ${ }^{+1}, 1841$

KF: RHINOPHRYNIDAE 1858.gc.f013

Rhithrotriton Nesterov, $1916 \cdot \mathbf{A K}$

ST: PO.JD・CI: h1387・ID: 567

PN: Rhithrotriton derjugini Nesterov, 1916

PK: Rhithrotriton derjugini ${ }^{\circ}$ Nesterov, 1916

KG: Neurergus* Cope, 1862

KF: SALAMANDRIDAE 1820.ga.f002

Rhombofryne Palacký, 1898 • AK

ST: NT.JI • CI: h1388 • ID: 289

PN: Rhombophryne testudo Boettger, 1880

PK: Rhombophryne testudo* Boettger, 1880

KG: Rhombophryne* Boettger, 1880

KF: MICROHYLIDAE ||1843.fa.f012 |-1931.na.f001 
Rhomboglossus: Duméril ${ }^{+1} 1841$ • AN

ST: AL • CI: n0148・ID: 395

PN: Rana lima Gravenhorst, 1829

PK: Rana lima* Gravenhorst, 1829

KG: Occidozyga* $\mathrm{Kuhl}^{+1}, 1822$

KF: OCCIDOZYGIDAE 1990.fa.f002

Rhombophryne Boettger, 1880 • KY

ST: PO.KN • CI: h1389・ID: 289

PN: Rhombophryne testudo Boettger, 1880

PK: Rhombophryne testudo* Boettger, 1880

KG: Rhombophryne* Boettger, 1880

KF: MICROHYLIDAE ||1843.fa.f012||-1931.na.f001

Rhyacosiredon Dunn, 1928 • AK

ST: PO.JD・CI: h1390・ID: 555

PN: Amblystoma altamirani Dugès, 1895

PK: Amblystoma altamirani* Dugès, 1895

KG: Ambystoma ${ }^{1}$ Tschudi, 1838

KF: AмBYSTOMATIDAE 1850.ga.f004

Rhyacotriton Dunn, 1920 • KY

ST: PO.KN • CI: h1391 • ID: 552

PN: Ranodon olympicus Gaige, 1917

PK: Ranodon olympicus* Gaige, 1917

KG: Rhyacotriton* Dunn, 1920

KF: RHYACOTRITONIDAE 1958.ta.f002

Ribeirina Parker, 1934 • AK

ST: PO.JD • CI: h1392 • ID: 302

PN: Emydops hypomelas Miranda-Ribeiro, 1920

PK: Stereocyclops incrassatus* Cope, 1870

KG: Stereocyclops* Cope, 1870

KF: MICROHYLIDAE ||1843.fa.f012||-1931.na.f001

Rohanixalus $\mathrm{Biju}^{+9}, 2020 \bullet \mathbf{A K}$

ST: PO.JD・CI: h1653・ID: 450

PN: Ixalus vittatus Boulenger, 1887

PK: Ixalus vittatus* Boulenger, 1887

KG: Feihyla* Frost ${ }^{+18}, 2006$

KF: RHACOPHORIDAE $\| 1858 . g c . f 012||-1932 . h a . f 001$

Romerus nov. • KY

ST: PO.KN • CI: h1393・ID: 459

PN: Philautus romeri Smith, 1953

PK: Philautus romeri* Smith, 1953

KG: Romerus* nov.

KF: RHACOPHORIDAE $\| 1858 . g c . f 012||-1932$.ha.f001

Rothschildia Grote, $1896 \bullet \mathbf{Z H}$

ST: zo • CI: zh087

Rothschildia Mocquard, 1905 • AK

ST: $\mathbf{L C . J H} \bullet$ CI: h1394・ ID: 340

PN: Rothschildia kounhiensis Mocquard, 1905

PK: Rothschildia kounhiensis ${ }^{\circ}$ Mocquard, 1905

KG: Paracassina ${ }^{\circ}$ Peracca, 1907

KF: HYPEROLIIDAE 1943.lb.f001

Rotschildia: Mocquard 1905 - AN

ST: LI • CI: n0149・ID: 340

PN: Rothschildia kounhiensis Mocquard, 1905

PK: Rothschildia kounhiensis ${ }^{\circ}$ Mocquard, 1905

KG: Paracassina ${ }^{\circ}$ Peracca, 1907

KF: HYPEROLIIDAE 1943.lb.f001
Rubeta Fatio, $1872 \cdot \mathbf{A K}$

ST: PO.JI • CI: h1395・ID: 121

PN: Bufo calamita Laurenti, 1768

PK: Bufo calamita* Laurenti, 1768

KG: Epidalea* Cope, 1864

KF: BUFONIDAE 1825.gb.f004

Rubricacaecilia Evans $^{+1}, 2001+\cdot \mathbf{K Y}$

ST: PO.KN • CI: h1396・ID: $\uparrow 122$

PN: Rubricacaecilia monbaroni Evans $^{+1}, 2001$ \$

PK: Rubricacaecilia monbaroni ${ }^{\circ}$ Evans $^{+1}, 2001 \dagger$

KG: Rubricacaecilia ${ }^{\circ}$ Evans $^{+1}, 2001 \dagger$

KF: GyMnOPHIONA Familia INCERTAE SEDIS

Rugosa $\mathrm{Fei}^{+2}, 1990 \bullet \mathbf{K Y}$

ST: PO.KN • CI: h1397・ID: 420

PN: Rana rugosa Temminck $^{+1}, 1838$

PK: Rana rugosa* Temminck $^{+1}, 1838$

KG: Rugosa* $\mathrm{Fei}^{+2}, 1990$

KF: RANIDAE 1796.ba.f001

Rulyrana Guayasamin ${ }^{+5}, 2009$ • KY

ST: PO.KN・CI: h1398・ID: 161

PN: Centrolenella flavopunctata Lynch $^{+1}, 1973$

PK: Centrolenella flavopunctata* Lynch $^{+1}, 1973$

KG: Rulyrana* Guayasamin ${ }^{+5}, 2009$

KF: CENTROLENIDAE 1951.ta.f001

Rupirana Heyer, 1999 • KY

ST: PO.KN • CI: h1399・ID: 255

PN: Rupirana cardosoi Heyer, 1999

PK: Rupirana cardosoi* Heyer, 1999

KG: Rupirana* Heyer, 1999

KF: PARATELMATOBIIDAE 2012.oa.f001

Sabahphrynus Matsui ${ }^{+2}, 2007 \bullet \mathbf{K Y}$

ST: PO.KN・CI: h1400・ID: 131

PN: Nectophryne maculata Mocquard, 1890

PK: Nectophryne maculata* Mocquard, 1890

KG: Sabahphrynus* Matsui ${ }^{+2}, 2007$

KF: BUFONIDAE 1825.gb.f004

Sachatamia Guayasamin ${ }^{+5}, 2009$ • KY

ST: PO.KN • CI: h1401 • ID: 162

PN: Centrolenella albomaculata Taylor, 1949

PK: Centrolenella albomaculata* Taylor, 1949

KG: Sachatamia* Guayasamin ${ }^{+5}, 2009$

KF: CENTROLENIDAE 1951.ta.f001

Saevesoederberghia Roček $^{+1}, 1993$ † KY

ST: PO.KN • CI: h1402・ID: $\uparrow 041$

PN: Saevesoederberghia egredia Roček $^{+1}, 1993$ \$

PK: Saevesoederberghia egredia ${ }^{\circ}$ Roček $^{+1}, 1993 \dagger$

KG: Saevesoederberghia ${ }^{\circ}$ Roček $^{+1}, 1993 \dagger$

KF: ANURA Familia INCERTAE SEDIS

Saganura Wells ${ }^{+1}, 1985$ • AK

ST: PO.JD・CI: h1403・ID: 235

PN: Hyla burrowsi Scott, 1942

PK: Hyla burrowsi* Scott, 1942

KG: Litoria* Tschudi, 1838

KF: PHYLLOMEDUSIDAE 1858.gc.f009

Sahona Glaw $^{+1}, 2006 \cdot \mathbf{A K}$

ST: PO.JD • CI: h1404 • ID: 423 
PN: Polypedates tephraeomystax Duméril, 1853

PK: Polypedates tephraeomystax* Duméril, 1853

KG: Boophis* Tschudi, 1838

KF: RHACOPHORIDAE |1858.gc.f012||-1932.ha.f001

Salamandra: Gronovius 1763 • AN

ST: AL • CI: n0150・ID: 578

PN: Salamandra maculosa Laurenti, 1768

PK: Lacerta salamandra* Linnaeus, 1758

KG: Salamandra ${ }^{1}$ Garsault, 1764

KF: SALAMANDRIDAE 1820.ga.f002

Salamandra Garsault, $1764 \cdot \mathbf{K Y}$

ST: PO.KN • CI: h1405・ID: 578

PN: Salamandra terrestris Bonnaterre, 1789

PK: Lacerta salamandra* Linnaeus, 1758

KG: Salamandra ${ }^{1}$ Garsault, 1764

KF: SALAMANDRIDAE 1820.ga.f002

Salamandra Laurenti, 1768 • AK

ST: PO.JD・CI: h1406・ID: 578

PN: Salamandra maculosa Laurenti, 1768

PK: Lacerta salamandra* Linnaeus, 1758

KG: Salamandra ${ }^{1}$ Garsault, 1764

KF: SALAMANDRIDAE 1820.ga.f002

Salamandraches Gistel, 1848 • AK

ST: PO.JD • CI: h1407 • ID: 578

PN: Salamandraches crassicaudis Gistel, 1848

PK: Lacerta salamandra* Linnaeus, 1758

KG: Salamandra ${ }^{1}$ Garsault, 1764

KF: SALAMANDRIDAE 1820.ga.f002

Salamandrella Dybowski, 1870 • KY

ST: PO.KN・CI: h1408・ID: 513

PN: Salamandrella keyserlingii Dybowski, 1870

PK: Salamandrella keyserlingii* Dybowski, 1870

KG: Salamandrella* Dybowski, 1870

KF: НYNOBIIDAE ||1856.ha.f001\|-1859.cb.f002

Salamandrina Fitzinger, $1826 \cdot \mathbf{K Y}$

ST: PO.KN • CI: h1409・ID: 579

PN: Salamandra perspicillata Savi, 1821

PK: Salamandra perspicillata* Savi, 1821

KG: Salamandrina* Fitzinger, 1826

KF: SALAMANDRIDAE 1820.ga.f002

Salamandroidis Fitzinger, 1843 • AK

ST: PO.JI • CI: h1410・ID: 555

PN: Lacerta subviolacea Barton, 1804

PK: Lacerta maculata* Shaw, 1802

KG: Ambystoma ${ }^{1}$ Tschudi, 1838

KF: AмвYSTOMATIDAE 1850.ga.f004

Salamandrops Wagler, 1830 • AK

ST: PO.JD・CI: h1411 • ID: 504

PN: Salamandra gigantea Barton, 1808

PK: Salamandra alleganiensis* Sonnini ${ }^{+1}, 1801$

KG: Cryptobranchus ${ }^{1}$ Leuckart, 1821

KF: CRYPTOBRANCHIDAE 1826.fb.f003

Sallywalkerana Dahanukar ${ }^{+5}, 2016$ • AK

ST: NT.JI • CI: h1412 • ID: 461

PN: Ixalus diplostictus Günther, 1875

PK: Ixalus diplostictus* Günther, 1875
KG: Walkerana* Dahanukar ${ }^{+5}, 2016$

KF: RANIXALIDAE 1987.da.f005

Saltenia Reig, $1959+\bullet \mathbf{K Y}$

ST: PO.KN • CI: h1413・ID: $† 079$

PN: Saltenia ibanezi Reig, 1959 \$

PK: Saltenia ibanezi ${ }^{\circ}$ Reig, $1959 \dagger$

KG: Saltenia ${ }^{\circ}$ Reig, $1959 \dagger$

KF: PIPIDAE 1825.gb.f003-|1826.fb.f002|

Sanchizia Dubois $^{+1}, 2012+\bullet \mathbf{K Y}$

ST: PO.KN • CI: h1414・ID: $\uparrow 188$

PN: Bargmannia wettsteini Herre, 1955 \$

PK: Bargmannia wettsteini $i^{\circ}$ Herre, $1955 \dagger$

KG: Sanchizia ${ }^{\circ}$ Dubois $^{+1}, 2012 \dagger$

KF: AмвYSTOMATIDAE 1850.ga.f004

Sandyrana Wells ${ }^{+1}, 1985$ • AK

ST: PO.JD・CI: h1415・ID: 236

PN: Hyla infrafrenata Günther, 1867

PK: Hyla infrafrenata* Günther, 1867

KG: Nyctimystes* Stejneger, 1916

KF: PHYLLOMEDUSIDAE 1858.gc.f009

Sanguirana Dubois, $1992 \cdot \mathbf{K Y}$

ST: PO.KN • CI: h1416・ID: 421

PN: Rana sanguinea Boettger, 1893

PK: Rana sanguinea* Boettger, 1893

KG: Sanguirana* Dubois, 1992

KF: RANIDAE 1796.ba.f001

Sanshuibatrachus Wang ${ }^{+2}, 2017$ † KY

ST: PO.KN • CI: h1417 • ID: $† 089$

PN: Sanshuibatrachus sinensis Wang ${ }^{+2}, 2017$ †

PK: Sanshuibatrachus sinensis ${ }^{\circ}$ Wang $^{+2}, 2017 \dagger$

KG: Sanshuibatrachus ${ }^{\circ}$ Wang $^{+2}, 2017 \dagger$

KF: Pelobatoidae Familia INCERTAE SEDIS

Sarcohyla Duellman ${ }^{+2}, 2016$ • AK

ST: PO.JD • CI: h1418・ID: 219

PN: Cauphias crassus Brocchi, 1877

PK: Cauphias crassus $^{\circ}$ Brocchi, 1877

KG: Plectrohyla* Brocchi, 1877

KF: HYLIDAE 1815.ra.f002-|1825.gb.f001|

Satobius Adler ${ }^{+1}, 1990 \bullet \mathbf{K Y}$

ST: PO.KN • CI: h1419・ID: 508

PN: Hynobius retardatus Dunn, 1923

PK: Hynobius retardatus* Dunn, 1923

KG: Satobius* Adler ${ }^{+1}, 1990$

KF: HYNOBIIDAE \|1856.ha.f001\|-1859.cb.f002

Saurocercus Fitzinger, 1843 • AK

ST: PO.JD・CI: h1420・ID: 542

PN: Salamandra longicauda Green, 1818

PK: Salamandra longicauda* Green, 1818

KG: Eurycea* Rafinesque, 1822

KF: PLETHODONTIDAE 1850.ga.f002

Saurophis Fitzinger, $1826 \cdot \mathbf{Z H}$

ST: zo • CI: zh088

Saurophis Gray, 1850 • AK

ST: NT.JH • CI: h1421 • ID: 551

PN: Salamandra erythronota Rafinesque, 1818

PK: Salamandra cinerea* Green, 1818 
KG: Plethodon* Tschudi, 1838

KF: PLETHODONTIDAE 1850.ga.f002

Sauropsis Agassiz, $1832 \cdot \mathbf{z H}$

ST: zo • CI: zh089

Sauropsis Fitzinger, 1843 • AK

ST: PO.JH • CI: h1422 • ID: 551

PN: Salamandra erythronota Rafinesque, 1818

PK: Salamandra cinerea* Green, 1818

KG: Plethodon* Tschudi, 1838

KF: PLETHODONTIDAE 1850.ga.f002

Scafiopus Palacký, 1898 • AK

ST: NT.JI • CI: h1423・ID: 029

PN: Scaphiopus solitarius Holbrook, 1836

PK: Rana holbrooki* Harlan, 1835

KG: Scaphiopus ${ }^{1}$ Holbrook, 1836

KF: SCAPHIOPODIDAE 1865.ca.f003

Scafiorhina Palacký, 1898 • AK

ST: NT.JI • CI: h1424・ID: 291

PN: Scaphiophryne marmorata Boulenger, 1882

PK: Scaphiophryne marmorata* Boulenger, 1882

KG: Scaphiophryne* Boulenger, 1882

KF: MICROHYLIDAE ||1843.fa.f012||-1931.na.f001

Scapherpeton Cope, $1877+\bullet \mathbf{A K}$

ST: PO.JD・CI: h1425・ID: $\uparrow 155$

PN: Scapherpeton tectum Cope, 1877 \$

PK: Hedronchus sternbergii ${ }^{\circ}$ Cope, $1877 \dagger$

KG: Hedronchus ${ }^{\circ}$ Cope, $1877 \dagger$

KF: SCAPHERPETIDAE 1959.aa.f001 †

Scaphiophryne Boulenger, $1882 \bullet \mathbf{K Y}$

ST: PO.KN • CI: h1426・ID: 291

PN: Scaphiophryne marmorata Boulenger, 1882

PK: Scaphiophryne marmorata* Boulenger, 1882

KG: Scaphiophryne* Boulenger, 1882

KF: MICROHYLIDAE ||1843.fa.f012||-1931.na.f001

Scaphiopus Holbrook, 1836 • KY

ST: PO.KN・CI: h1427・ ID: 029

PN: Scaphiopus solitarius Holbrook, 1836

PK: Rana holbrooki* Harlan, 1835

KG: Scaphiopus ${ }^{1}$ Holbrook, 1836

KF: SCAPHIOPODIDAE 1865.ca.f003

Scaptophryne: Fitzinger 1861 • AN

ST: AL $・$ CI: n0151・ID: 314

PN: Engystoma pulchrum Hallowell, 1861

PK: Engystoma pulchrum* Hallowell, 1861

KG: Microhyla* Tschudi, 1838

KF: MICROHYLIDAE ||1843.fa.f012||-1931.na.f001

Scarthyla Duellman ${ }^{+1}, 1988$ • KY

ST: PO.KN • CI: h1428・ID: 197

PN: Scarthyla ostinodactyla Duellman ${ }^{+1}, 1988$

PK: Hyla goinorum* Bokermann, 1962

KG: Scarthyla ${ }^{1}$ Duellman $^{+1}, 1988$

KF: HYLIDAE 1815.ra.f002-|1825.gb.f001|

Schismaderma Smith, 1849 • KY

ST: PO.KN • CI: h1429 • ID: 133

PN: Schismaderma lateralis Smith, 1849

PK: Bufo carens* Smith, 1848
KG: Schismaderma ${ }^{1}$ Smith, 1849

KF: BUFONIDAE 1825.gb.f004

Schistometopum Parker, 1941 • KY

ST: PO.KN • CI: h1430 • ID: 488

PN: Dermophis gregorii Boulenger, 1895

PK: Dermophis gregorii* Boulenger, 1895

KG: Schistometopum* Parker, 1941

KF: CAECILIIDAE 1814.ra.f003-|1825.gb.f008|

Schmibufo $\mathrm{Fei}^{+1}, 2016$ • AK

ST: PO.JD・CI: h1431 • ID: 120

PN: Bufo stejnegeri Schmidt, 1931

PK: Bufo stejnegeri* Schmidt, 1931

KG: $B$ ufo* Garsault, 1764

KF: BUFONIDAE 1825.gb.f004

Schoutedenella Witte, 1921 • AK

ST: PO.JD・CI: h1432・ID: 320

PN: Schoutedenella globosa Witte, 1921

PK: Arthroleptis xenochirus ${ }^{\circ}$ Boulenger, 1905

KG: Arthroleptis* Smith, 1849

KF: ARTHROLEPTIDAE 1869.mc.f011

Schwartzius Hedges ${ }^{+2}$, 2008 • AK

ST: PO.JD・CI: h1433・ID: 081

PN: Eleutherodactylus counouspeus Schwartz, 1964

PK: Eleutherodactylus counouspeus* Schwartz, 1964

KG: Eleutherodactylus* Duméril ${ }^{+1}, 1841$

KF: BRACHYCEPHALIDAE 1858.gc.f002

Sciaphos Gray, 1845 • AN

ST: AL $・$ CI: n0152・ID: $003 \S$

PN: INR

PK: INR

KG: INR

KF: ANURA Familia INCERTAE SEDIS

Scinacodes Fitzinger, 1843 - AK

ST: PO.JD • CI: h1434 • ID: 182

PN: Hyla nasus Lichtenstein, 1823

PK: Hyla nasus* Lichtenstein, 1823

KG: Hylodes ${ }^{1}$ Fitzinger, 1826

KF: HYLODIDAE 1858.gc.f010

Scinax Wagler, 1830 • KY

ST: PO.KN • CI: h1435 • ID: 232

PN: Hyla aurata Wied-Neuwied, 1821

PK: Hyla aurata ${ }^{\circ}$ Wied-Neuwied, 1821

KG: Scinax ${ }^{2}$ Wagler, 1830

KF: HYLIDAE 1815.ra.f002-|1825.gb.f001|

Sclerophrys Tschudi, $1838 \cdot \mathbf{K Y}$

ST: PO.KN • CI: h1436・ID: 140

PN: Sclerophrys capensis Tschudi, 1838

PK: Sclerophrys capensis* Tschudi, 1838

KG: Sclerophrys* Tschudi, 1838

KF: BUFONIDAE 1825.gb.f004

Scolecomorphus Boulenger, $1883 \bullet \mathbf{K Y}$

ST: PO.KN • CI: h1437 • ID: 499

PN: Scolecomorphus kirkii Boulenger, 1883

PK: Scolecomorphus kirkii ${ }^{\circ}$ Boulenger, 1883

KG: Scolecomorphus ${ }^{2}$ Boulenger, 1883

KF: SCOLECOMORPHIDAE 1969.ta.f001 
Scotiophryne Estes, $1969+\cdot \mathbf{K Y}$

ST: Po.KN • CI: h1438 • ID: $\uparrow 042$

PN: Scotiophryne pustulosa Estes, 1969 †

PK: Scotiophryne pustulosa $a^{\circ}$ Estes, $1969 \dagger$

KG: Scotiophryne ${ }^{\circ}$ Estes, $1969 \dagger$

KF: ANURA Familia INCERTAE SEDIS

Scotobius Germar, 1824 • ZH

ST: zo • CI: zh090

Scotobius Gistel, 1848 • AN

ST: AL • CI: n0153・ID: $002 \S$

PN: INR

PK: INR

KG: INR

KF: LISSAMPHIBIA Familia INCERTAE SEDIS

Scotobleps Boulenger, $1900 \bullet \mathbf{K Y}$

ST: PO.KN • CI: h1439・ID: 323

PN: Scotobleps gabonicus Boulenger, 1900

PK: Scotobleps gabonicus* Boulenger, 1900

KG: Scotobleps* Boulenger, 1900

KF: ARTHROLEPTIDAE 1869.mc.f011

Scurrilirana Hillis ${ }^{+1}$, 2005 • AK

ST: PO.JD • CI: h1440 • ID: 415

PN: Rana berlandieri Baird, 1854

PK: Rana berlandieri* Baird, 1854

KG: Lithobates* Fitzinger, 1843

KF: RANIDAE 1796.ba.f001

Scutiger Theobald, $1868 \cdot \mathbf{K Y}$

ST: PO.KN • CI: h1441 • ID: 017

PN: Bombinator sikimmensis Blyth, 1854

PK: Bombinator sikimmensis ${ }^{\circ}$ Blyth, 1854

KG: Scutiger ${ }^{2}$ Theobald, 1868

KF: MEGOPHRYIDAE 1850.bb.f008-|1931.na.f003|

Scythrophrys Lynch, 1971 • AK

ST: PO.JD • CI: h1442 • ID: 254

PN: Zachaenus sawayae Cochran, 1953

PK: Zachaenus sawayae* Cochran, 1953

KG: Crossodactylodes ${ }^{2}$ Cochran, 1938

KF: PARATELMATOBIIDAE 2012.oa.f001

Scytopis Cope, $1862 \cdot \mathbf{A K}$

ST: PO.JD • CI: h1443 • ID: 231

PN: Scytopis hebes Cope, 1862

PK: Rana typhonia* Linnaeus, 1758

KG: Trachycephalus* Tschudi, 1838

KF: HYLIDAE 1815.ra.f002-|1825.gb.f001|

Scytopsis Knauer, $1878 \bullet \mathbf{A K}$

ST: NS.JD • CI: h1444 • ID: 231

PN: Scytopis hebes Cope, 1862

PK: Rana typhonia* Linnaeus, 1758

KG: Trachycephalus* Tschudi, 1838

KF: HYLIDAE 1815.ra.f002-|1825.gb.f001|

Sechellophryne Nussbaum ${ }^{+1}, 2007 \cdot \mathbf{K Y}$

ST: PO.KN • CI: h1445 • ID: 032

PN: Nectophryne gardineri Boulenger, 1911

PK: Nectophryne gardineri* Boulenger, 1911

KG: Sechellophryne* Nussbaum ${ }^{+1}, 2007$

KF: SOOGLOSSIDAE 1931.na.f002
Seiranota Barnes, $1826 \cdot \mathbf{A K}$

ST: PO.JD・CI: h1446•ID: 579

PN: Seiranota condylura Barnes, 1826

PK: Salamandra perspicillata* Savi, 1821

KG: Salamandrina* Fitzinger, 1826

KF: SALAMANDRIDAE 1820.ga.f002

Seminobatrachus Skutschas ${ }^{+1}, 2012+\cdot \mathbf{K Y}$

ST: PO.KN • CI: h1447 • ID: $\uparrow 141$

PN: Seminobatrachus boltyschkensis Skutschas ${ }^{+1}, 2012$ 末

PK: Seminobatrachus boltyschkensis Skutschas ${ }^{+1}, 2012 \dagger$

KG: Seminobatrachus Skutschas ${ }^{+1}, 2012 \dagger$

KF: Urodela Familia INCERTAE SEDIS

Semnodactylus Hoffman, 1939 • KY

ST: PO.KN・CI: h1448・ID: 341

PN: Semnodactylus thabanchuensis Hoffman, 1939

PK: Cassina wealii* Boulenger, 1882

KG: Semnodactylus ${ }^{1}$ Hoffman, 1939

KF: HyPEROLIIDAE 1943.lb.f001

Septentriomolge Hillis ${ }^{+3}$, $2001 \cdot \mathbf{A K}$

ST: PO.JD・CI: h1449 • ID: 542

PN: Eurycea chisholmensis Chippindale ${ }^{+3}, 2000$

PK: Eurycea chisholmensis ${ }^{*}$ Chippindale $^{+3}, 2000$

KG: Eurycea* Rafinesque, 1822

KF: PLETHODONTIDAE 1850.ga.f002

Septobrachium: Tschudi, $1838 \cdot \mathbf{A N}$

ST: $\mathbf{L I} \bullet \mathbf{C I}:$ n0154・ID: 015

PN: Leptobrachium hasseltii Tschudi, 1838

PK: Leptobrachium hasseltii* Tschudi, 1838

KG: Leptobrachium* Tschudi, 1838

KF: MEGOPHRYIDAE 1850.bb.f008-|1931.na.f003|

Shelania Casamiquela, $1960+\cdot \mathbf{K Y}$

ST: PO.KN • CI: h1450・ID: $\uparrow 080$

PN: Shelania pascuali Casamiquela, 1960 \$

PK: Shelania pascuali ${ }^{\circ}$ Casamiquela, $1960 \dagger$

KG: Shelania ${ }^{\circ}$ Casamiquela, $1960 \dagger$

KF: PIPIDAE 1825.gb.f003-|1826.fb.f002|

Shirerpeton Matsumoto ${ }^{+1}, 2018+\cdot \mathbf{K Y}$

ST: PO.KN • CI: h1451 • ID: $\uparrow 006$

PN: Shirerpeton isajii Matsumoto $^{+1}, 2018$ \$

PK: Shirerpeton isajii ${ }^{\circ}$ Matsumoto $^{+1}, 2018 \dagger$

KG: Shirerpeton ${ }^{\circ}$ Matsumoto $^{+1}, 2018 \uparrow$

KF: ALBANERPETIDAE 1982.fa.f001 †

Shomronella Estes $^{+2}, 1978+\cdot \mathbf{K Y}$

ST: PO.KN • CI: h1452 • ID: $\uparrow 065$

PN: Shomronella jordanica Estes $^{+2}, 1979$ \$

PK: Shomronella jordanica ${ }^{\circ}$ Estes $^{+2}, 1979 \dagger$

KG: Shomronella ${ }^{\circ}$ Estes $^{+2}, 1978 \dagger$

KF: DorsIPARES Familia INCERTAE SEDIS

Siamophryne Suwannapom ${ }^{+6}, 2018 \cdot \mathbf{K Y}$

ST: PO.KN • CI: h1453・ID: 282

PN: Siamophryne troglodytes Suwannapom ${ }^{+6}, 2018$

PK: Siamophryne troglodytes ${ }^{\circ}$ Suwannapom $^{+6}, 2018$

KG: Siamophryne $e^{\circ}$ Suwannapom $^{+6}, 2018$

KF: MICROHYLIDAE ||1843.fa.f012||-1931.na.f001

Sibilatrix Kaup, $1829 \cdot \mathbf{z H}$

ST: zo • CI: zh091 
Sibilatrix Fitzinger, $1843 \cdot$ AK

ST: PO.JD・CI: h1454・ID: 253

PN: Cystignathus gracilis Duméril ${ }^{+1}, 1840$

PK: Cystignathus gracilis* Duméril ${ }^{+1}, 1840$

KG: Leptodactylus ${ }^{1}$ Fitzinger, 1826

KF: LEPTODACTYLIDAE ||1838.ta.f001||-1896.wa.f001

Sieboldia Gray, $1838 \cdot \mathbf{A K}$

ST: PO.JD • CI: h1455 • ID: 503

PN: Megalobatrachus sieboldi Tschudi, 1837 \$

PK: Triton japonicus* Temminck, 1836

KG: Andrias ${ }^{2}$ Tschudi, 1837

KF: CRYPTOBRANCHIDAE 1826.fb.f003

Sieboldiana Ishikawa, $1904 \cdot \mathbf{A K}$

ST: NS.JD・CI: h1456・ID: 503

PN: Megalobatrachus sieboldi Tschudi, 1837 \$

PK: Triton japonicus* Temminck, 1836

KG: Andrias ${ }^{2}$ Tschudi, 1837

KF: CRYPTOBRANCHIDAE 1826.fb.f003

Sieboldtia Agassiz, 1839 • AK

ST: NS.JD • CI: h1457 • ID: 503

PN: Megalobatrachus sieboldi Tschudi, 1837 \$

PK: Triton japonicus* Temminck, 1836

KG: Andrias $^{2}$ Tschudi, 1837

KF: CRYPTOBRANCHIDAE 1826.fb.f003

Sierrana Dubois, $1992 \cdot \mathbf{A K}$

ST: PO.JD・CI: h1458・ID: 415

PN: Rana sierramadrensis Taylor, 1939

PK: Rana sierramadrensis* Taylor, 1939

KG: Lithobates* Fitzinger, 1843

KF: RANIDAE 1796.ba.f001

Sigalegalephrynus $\mathrm{Smart}^{+7}, 2017 \cdot \mathbf{K Y}$

ST: PO.KN・CI: h1459・ID: 112

PN: Sigalegalephrynus mandailinguensis Smart ${ }^{+7}, 2017$

PK: Sigalegalephrynus mandailinguensis ${ }^{\circ}$ Smart $^{+7}, 2017$

KG: Sigalegalephrynus ${ }^{\circ}$ Smart $^{+7}, 2017$

KF: BUFONIDAE 1825.gb.f004

Silurana Gray, $1864 \cdot \mathbf{K Y}$

ST: PO.KN • CI: h1460・ ID: 008

PN: Silurana tropicalis Gray, 1864

PK: Silurana tropicalis* Gray, 1864

KG: Silurana* Gray, 1864

KF: PIPIDAE 1825.gb.f003-|1826.fb.f002|

Silverstoneia Grant $^{+9}, 2006 \bullet \mathbf{K Y}$

ST: PO.KN • CI: h1461・ID: 043

PN: Phyllobates nubicola Dunn, 1924

PK: Phyllobates nubicola* Dunn, 1924

KG: Silverstoneia* Grant $^{+9}, 2006$

KF: DENDROBATIDAE ||1850.bb.f006"|-1865.ca.f002

Simomantis Boulenger, $1918 \cdot \mathbf{A K}$

ST: PO.JD・CI: h1462・ID: 422

PN: Ixalus latopalmatus Boulenger, 1887

PK: Ixalus latopalmatus* Boulenger, 1887

KG: Staurois* Cope, 1865

KF: RANIDAE 1796.ba.f001

Sinerpeton $\mathrm{GaO}^{+1}, 2001+\cdot \mathbf{K Y}$

ST: PO.KN • CI: h1463・ID: $\uparrow 142$
PN: Sinerpeton fengshanensis $\mathrm{Gao}^{+1}, 2001$ *

PK: Sinerpeton fengshanensis ${ }^{\circ} \mathrm{Gao}^{+1}, 2001 \dagger$

KG: Sinerpeton ${ }^{\circ} \mathrm{Gao}^{+1}, 2001 \dagger$

KF: Urodela Familia INCERTAE SEDIS

Singidella Báez $^{+1}, 2005 \$ \cdot \mathbf{K Y}$

ST: PO.KN • CI: h1464・ID: $\uparrow 076$

PN: Singidella latecostata Báez $^{+1}, 2005$ \$

PK: Singidella latecostata ${ }^{\circ} \mathrm{Báez}^{+1}, 2005 \dagger$

KG: Singidella ${ }^{\circ}$ Báez $^{+1}, 2005 \dagger$

KF: PIPIDAE 1825.gb.f003-|1826.fb.f002|

Sinobius Dubois, 1987 • AK

ST: PO.JD・CI: h1465・ID: 512

PN: Xenobius melanonychus Zhang ${ }^{+1}, 1985$

PK: Pachyhynobius shangchengensis* $\mathrm{Fei}^{+2}, 1983$

KG: Pachyhynobius * $\mathrm{Fei}^{+2}, 1983$

KF: HYNOBIIDAE ||1856.ha.f001\|-1859.cb.f002

Siphneus Brants, 1827 • ZH

ST: zo • CI: zh092

Siphneus Fitzinger, 1843 - AK

ST: PO.JH • CI: h1466・ID: 314

PN: Engystoma ornatum Duméril ${ }^{+1}, 1841$

PK: Engystoma ornatum* Duméril ${ }^{+1}, 1841$

KG: Microhyla* Tschudi, 1838

KF: MICROHYLIDAE ||1843.fa.f012||-1931.na.f001

Siphonops Wagler, 1828 • KY

ST: PO.KN • CI: h1467•ID: 494

PN: Caecilia annulata Mikan, 1820

PK: Caecilia annulata* Mikan, 1820

KG: Siphonops * Wagler, 1828

KF: CAECILIIDAE 1814.ra.f003-|1825.gb.f008|

Siredon Wagler, 1829 • AK

ST: PO.CA • CI: h1468・ID: 555

PN: Siredon axolotl Wagler, 1830

PK: Gyrinus mexicanus* Shaw ${ }^{+1}, 1789$

KG: Ambystoma ${ }^{1}$ Tschudi, 1838

KF: AмвYSTOMATIDAE 1850.ga.f004

Siren Österdam, $1766 \cdot \mathbf{K Y}$

ST: PO.KN • CI: h1469 • ID: 519

PN: Siren lacertina Österdam, 1766

PK: Siren lacertina* Österdam, 1766

KG: Siren* Österdam, 1766

KF: SIRENIDAE 1825gb.f005

Sirena: Fischer 1808 • AN

ST: AM • CI: n0155・ID: 519

PN: Siren lacertina Österdam, 1766

PK: Siren lacertina* Österdam, 1766

KG: Siren* Österdam, 1766

KF: SIRENIDAE $1825 \mathrm{gb} . \mathrm{f} 005$

Sirene Link, $1794 \cdot \mathbf{z H}$

ST: zo • CI: zh093

Sirene: Fischer 1813 • AN

ST: AM • CI: n0156・ID: 519

PN: Siren lacertina Österdam, 1766

PK: Siren lacertina* Österdam, 1766

KG: Siren* Österdam, 1766

KF: SIRENIDAE $1825 \mathrm{gb} . \mathrm{f} 005$ 
Sirene Oken, 1816 • Ex

ST: PO.CW・CI: e0013・ID: 519

PN: Siren lacertina Österdam, 1766

PK: Siren lacertina* Österdam, 1766

KG: Siren* Österdam, 1766

KF: SIRENIDAE $1825 \mathrm{gb} . f 005$

Sirenodon Wiegmann, 1832 • AK

ST: PO.CA • CI: h1471 • ID: 555

PN: Siredon axolotl Wagler, 1830

PK: Gyrinus mexicanus* Shaw ${ }^{+1}, 1789$

KG: Ambystoma ${ }^{1}$ Tschudi, 1838

KF: АмвYSTOMATIDAE 1850.ga.f004

Sirenoides Gray, 1850 • AK

ST: NS.JD・CI: h1472・ID: 520

PN: Amphiuma didactylum Cuvier, 1827

PK: Amphiuma means* Garden in Smith, 1821

KG: Amphiuma* Garden in Smith, 1821

KF: AMPHIUMIDAE 1825.gb.f07

Sirenoidis Fitzinger, 1843 • AK

ST: PO.JD・CI: h1473・ID: 520

PN: Amphiuma didactylum Cuvier, 1827

PK: Amphiuma means* Garden in Smith, 1821

KG: Amphiuma* Garden in Smith, 1821

KF: AMPHIUMIDAE 1825.gb.f07

Smilisca Cope, $1865 \bullet \mathbf{K Y}$

ST: PO.KN • CI: h1474・ID: 208

PN: Smilisca daulinia Cope, 1865

PK: Hyla baudinii* Duméril ${ }^{+1}, 1841$

KG: Smilisca ${ }^{1}$ Cope, 1865

KF: HYLIDAE 1815.ra.f002-|1825.gb.f001|

Sminthillus Barbour ${ }^{+1}, 1920$ • AK

ST: PO.JD・CI: h1475・ID: 082

PN: Phyllobates limbatus Cope, 1862

PK: Phyllobates limbatus* Cope, 1862

KG: Euhyas* Fitzinger, 1843

KF: BRACHYCEPHALIDAE 1858.gc.f002

Somuncuria Lynch, 1978 • AK

ST: PO.JD・CI: h1476・ID: 246

PN: Telmatobius somuncurensis Cei, 1969

PK: Telmatobius somuncurensis* Cei, 1969

KG: Pleurodema* Tschudi, 1838

KF: LEIUPERIDAE 1850.bb.f010

Sooglossus Boulenger, $1906 \cdot \mathbf{K Y}$

ST: PO.KN • CI: h1477・ID: 033

PN: Arthroleptis sechellensis Boettger, 1896

PK: Arthroleptis sechellensis* Boettger, 1896

KG: Sooglossus* Boulenger, 1906

KF: SOOGLOSSIDAE 1931.na.f002

Spea Cope, $1866 \bullet \mathbf{K Y}$

ST: PO.KN・CI: h1478・ID: 030

PN: Scaphiopus bombifrons Cope, 1863

PK: Scaphiopus bombifrons* Cope, 1863

KG: Spea* Cope, 1866

KF: SCAPHIOPODIDAE 1865.ca.f003

Spelaeophryne Ahl, $1924 \cdot \mathbf{K Y}$

ST: PO.KN • CI: h1479・ID: 346
PN: Spelaeophryne methneri Ahl, 1924

PK: Spelaeophryne methneri* Ahl, 1924

KG: Spelaeophryne* Ahl, 1924

KF: BREVICIPITIDAE 1850.bb.f012

Speleomantes Dubois, $1984 \cdot \mathbf{K Y}$

ST: PO.RP • CI: h1480 • ID: 545

PN: Hydromantes italicus Dunn, 1923

PK: Hydromantes italicus* Dunn, 1923

KG: Speleomantes* Dubois, 1984

KF: PLETHODONTIDAE 1850.ga.f002

Spelerpes Rafinesque, 1832 • AK

ST: PO.JI • CI: h1481 • ID: 542

PN: Eurycea lucifuga Rafinesque, 1822

PK: Eurycea lucifuga* Rafinesque, 1822

KG: Eurycea* Rafinesque, 1822

KF: PlethodontidaE 1850.ga.f002

Sphaenorhynchus Tschudi, 1838 • KY

ST: PO.KN・CI: h1482 • ID: 234

PN: Hyla lactea Daudin, 1800

PK: Hyla lactea* Daudin, 1800

KG: Sphaenorhynchus* Tschudi, 1838

KF: HYLIDAE 1815.ra.f002-|1825.gb.f001|

Sphaenorynchus Nieden, 1923 • AK

ST: NT.JI • CI: h1483・ ID: 234

PN: Hyla lactea Daudin, 1800

PK: Hyla lactea* Daudin, 1800

KG: Sphaenorhynchus* Tschudi, 1838

KF: HYLIDAE 1815.ra.f002-|1825.gb.f001

Sphaeroteca Dubois 1987 • AK

ST: NS.JI • CI: h1484 • ID: 379

PN: Sphaerotheca strigata Günther, 1859

PK: Rana breviceps* Schneider, 1799

KG: Sphaerotheca ${ }^{1}$ Günther, 1859

KF: DICROGLOSSIDAE 1987.da.f004

Sphaerotheca Günther, 1859 • KY

ST: PO.KN・CI: h1485・ID: 379

PN: Sphaerotheca strigata Günther, 1859

PK: Rana breviceps* Schneider, 1799

KG: Sphaerotheca ${ }^{1}$ Günther, 1859

KF: DICROGLOSSIDAE 1987.da.f004

Sphagepodium: Steindachner 1864 • AN

ST: $\mathbf{A L} \bullet \mathbf{C I}:$ n0157・ID: 250

PN: Leiuperus albonotatus Steindachner, 1864

PK: Leiuperus albonotatus* Steindachner, 1864

KG: Physalaemus* Fitzinger, 1826

KF: LEIUPERIDAE 1850.bb.f010

Sphenophryne Peters ${ }^{+1}, 1878 \cdot \mathbf{A K}$

ST: Po.JD・CI: h1486・ID: 280

PN: Sphenophryne cornuta Peters $^{+1}, 1878$

PK: Sphenophryne cornuta* Peters $^{+1}, 1878$

KG: Asterophrys* Tschudi, 1838

KF: MICROHYLIDAE ||1843.fa.f012 |-1931.na.f001

Sphoenohyla $\mathrm{Lutz}^{+1}, 1938 \cdot \mathbf{A K}$

ST: PO.JD・ CI: h1487・ID: 234

PN: Hyla aurantiaca Daudin, 1802

PK: Hyla lactea* Daudin, 1800 
KG: Sphaenorhynchus* Tschudi, 1838

KF: HYLIDAE 1815.ra.f002-|1825.gb.f001|

Spicospina Roberts ${ }^{+4}, 1997 \cdot \mathbf{K Y}$

ST: PO.KN • CI: h1488・ID: 275

PN: Spicospina flammocaerulea Roberts $^{+4}, 1997$

PK: Spicospina flammocaerulea* Roberts ${ }^{+4}, 1997$

KG: Spicospina* Roberts ${ }^{+4}, 1997$

KF: MYOBATRACHIDAE 1850.sa.f001

Spinomantis Dubois, $1992 \cdot \mathbf{K Y}$

ST: PO.KN • CI: h1489・ID: 433

PN: Rhacophorus aglavei Methuen ${ }^{+1}, 1913$

PK: Rhacophorus aglavei* Methuen $^{+1}, 1913$

KG: Spinomantis* Dubois, 1992

KF: RHACOPHORIDAE ||1858.gc.f012 |-1932.ha.f001

Spinophrynoides Dubois, 1987 • AK

ST: PO.JD・CI: h1490・ID: 102

PN: Bufo osgoodi Loveridge, 1932

PK: Bufo osgoodi $i^{\circ}$ Loveridge, 1932

KG: Altiphrynoides ${ }^{\circ}$ Dubois, 1987

KF: BUFONIDAE 1825.gb.f004

Spondylophryne: Kretzoi $1956+\cdot \mathbf{A N}$

ST: AL • CI: n0158 ・ ID: $\uparrow 043 \S$

PN: Spondylophryne villanyensis Kretzoi, $1956+\bullet$ AS

PK: Spondylophryne villanyensis ${ }^{\circ}$ Kretzoi, $1956+\bullet$ AS

KG: Spondylophryne ${ }^{\circ}$ Kretzoi, $1956 \uparrow \cdot \mathbf{A G}$

KF: ANURA Familia INCERTAE SEDIS

Staurois Cope, $1865 \cdot \mathbf{K Y}$

ST: PO.KN・CI: h1491・ID: 422

PN: Ixalus natator Günther, 1859

PK: Ixalus natator* Günther, 1859

KG: Staurois* Cope, 1865

KF: RANIDAE 1796.ba.f001

Stefania Rivero, 1968 • KY

ST: PO.KN・CI: h1492・ID: 096

PN: Hyla evansi Boulenger, 1904

PK: Hyla evansi* Boulenger, 1904

KG: Stefania* Rivero, 1968

KF: HEMIPHRACTIDAE 1862.pa.f001

Stegoporus Wiegmann, 1832 • EX

ST: PO.CE・CI: e0014 • ID: 555

PN: Siredon axolotl Wagler, 1830

PK: Gyrinus mexicanus* Shaw $^{+1}, 1789$

KG: Ambystoma ${ }^{1}$ Tschudi, 1838

KF: AмвYSTомAтIDAE 1850.ga.f004

Stelladerma Poyarkov ${ }^{+8}, 2015$ • AK

ST: PO.JD・CI: h1655・ID: 438

PN: Theloderma stellatum Taylor, 1962

PK: Theloderma stellatum* Taylor, 1962

KG: Theloderma* Tschudi, 1838

KF: RHACOPHORIDAE |1858.gc.f012||-1932.ha.f001

Stemobates: Bauer 1994 • AN

ST: $\mathbf{A L} \bullet \mathbf{C I}:$ n0159 • ID: 050

PN: Dendrobates pumilio Schmidt, 1857

PK: Dendrobates pumilio* Schmidt, 1857

KG: Oophaga* Bauer, 1994

KF: DENDROBATIDAE ||1850.bb.f006||-1865.ca.f002
Stenocephalus Latreille, $1829 \cdot \mathbf{z H}$

ST: zo • CI: zh094

Stenocephalus Tschudi, 1838 • AK

ST: PO.JH • CI: h1494 • ID: 298

PN: Microps unicolor Wagler, 1828

PK: Rana ovalis* Schneider, 1799

KG: Engystoma* Fitzinger, 1826

KF: MiCROHYLIDAE ||1843.fa.f012||-1931.na.f001

Stenodactylus Fitzinger, $1826 \cdot \mathbf{z H}$

ST: zo • CI: zh095

Stenodactylus Philippi, $1902 \cdot \mathbf{A K}$

ST: PO.JH • CI: h1495・ID: 138

PN: Bufo ventralis Philippi, 1902

PK: Bufo spinulosus* Wiegmann, 1834

KG: Rhinella ${ }^{2}$ Fitzinger, 1826

KF: BUFONIDAE 1825.gb.f004

Stenofryne Palacký, 1898 • AK

ST: NT.JD・CI: h1496・ID: 280

PN: Sphenophryne cornuta Peters $^{+1}, 1878$

PK: Sphenophryne cornuta* Peters $^{+1}, 1878$

KG: Asterophrys* Tschudi, 1838

KF: MICROHYLIDAE ||1843.fa.f012||-1931.na.f001

Stenoglossa Chaudoir, $1848 \cdot \mathbf{Z H}$

ST: zo • CI: zh096

Stenoglossa Andersson, 1903 • AK

ST: PO.JH • CI: h1497 • ID: 129

PN: Stenoglossa fulva Andersson, 1903

PK: Bufo preussi ${ }^{\circ}$ Matschie, 1893

KG: Werneria ${ }^{3}$ Poche, 1903

KF: BUFONIDAE 1825.gb.f004

Stenorhynchus Hemprich, $1820 \bullet \mathbf{z H}$

ST: zo • CI: zh097

Stenorhynchus Smith, 1849 • AK

ST: PO.JH • CI: h1498・ ID: 350

PN: Stenorhynchus natalensis Smith, 1849

PK: Stenorhynchus natalensis* Smith, 1849

KG: Phrynobatrachus* Günther, 1862

KF: PHRYNOBATRACHIDAE 1941.lb.f001

Stephopaedes Channing, 1979 • AK

ST: PO.JD・CI: h1499・ID: 141

PN: Bufo anotis Boulenger, 1907

PK: Bufo anotis* Boulenger, 1907

KG: Mertensophryne ${ }^{1}$ Tihen, 1960

KF: BUFONIDAE 1825.gb.f004

Stereochilus Cope, $1869 \bullet \mathbf{K Y}$

ST: PO.KN • CI: h1500 • ID: 541

PN: Pseudotriton marginatus Hallowell, 1856

PK: Pseudotriton marginatus* Hallowell, 1856

KG: Stereochilus* Cope, 1869

KF: Plethodontidae 1850.ga.f002

Stereocyclops Cope, $1870 \bullet \mathbf{K Y}$

ST: PO.KN • CI: h1501・ID: 302

PN: Stereocyclops incrassatus Cope, 1870

PK: Stereocyclops incrassatus* Cope, 1870

KG: Stereocyclops* Cope, 1870

KF: MICROHYLIDAE ||1843.fa.f012||-1931.na.f001 
Stertirana: Hillis ${ }^{+1} 2005$ • AN

ST: AL •CI: n0160・ID: 415

PN: Rana montezumae Baird, 1854

PK: Rana montezumae* Baird, 1854

KG: Lithobates* Fitzinger, 1843

KF: RANIDAE 1796.ba.f001

Stombus Gravenhorst, $1825 \cdot \mathbf{K Y}$

ST: PO.KN • CI: h1502 • ID: 172

PN: Rana cornuta Linnaeus, 1758

PK: Rana cornuta* Linnaeus, 1758

KG: Stombus* Gravenhorst, 1825

KF: CERATOPHRYIDAE 1838.ta.f002

Strabomantis Peters, $1863 \cdot \mathbf{K Y}$

ST: PO.KN・CI: h1503・ID: 073

PN: Strabomantis biporcatus Peters, 1863

PK: Strabomantis biporcatus* Peters, 1863

KG: Strabomantis* Peters, 1863

KF: BRACHYCEPHALIDAE 1858.gc.f002

Strauchbufo $\mathrm{Fei}^{+2}, 2012 \cdot \mathbf{K Y}$

ST: PO.KN • CI: h1504・ID: 132

PN: Bufo raddei Strauch, 1876

PK: Bufo raddei* Strauch, 1876

KG: Strauchbufo $* \mathrm{Fei}^{+2}, 2012$

KF: BUFONIDAE 1825.gb.f004

Strauchibufo $\mathrm{Fei}^{+1}, 2016 \cdot \mathbf{A K}$

ST: NT.JI • CI: h1505・ID: 132

PN: Bufo raddei Strauch, 1876

PK: Bufo raddei* Strauch, 1876

KG: Strauchbufo* $\mathrm{Fe}^{+1}, 2012$

KF: BUFONIDAE 1825.gb.f004

Strauchophryne Borkin ${ }^{+1}$, 2013 • AK

ST: PO.JI • CI: h1506・ID: 132

PN: Bufo raddei Strauch, 1876

PK: Bufo raddei* Strauch, 1876

KG: Strauchbufo $* \mathrm{Fei}^{+2}, 2012$

KF: BUFONIDAE 1825.gb.f004

Strombus: Gray 1831 • AN

ST: AM • CI: n0161 • ID: 172

PN: Rana cornuta Linnaeus, 1758

PK: Rana cornuta* Linnaeus, 1758

KG: Stombus* Gravenhorst, 1825

KF: CERATOPHRYIDAE 1838.ta.f002

Strongylopus Tschudi, $1838 \cdot \mathbf{K Y}$

ST: PO.KN • CI: h1507 • ID: 363

PN: Rana fasciata Smith, 1849

PK: Rana fasciata* Smith, 1849

KG: Strongylopus* Tschudi, 1838

KF: CACOSTERNIDAE 1931.na.f008

Stumpffia Boettger, 1881 • AK

ST: PO.JD・CI: h1508・ID: 289

PN: Stumpffia psologlossa Boettger, 1881

PK: Stumpffia psologlossa* Boettger, 1881

KG: Rhombophryne* Boettger, 1880

KF: MICROHYLIDAE ||1843.fa.f012||-1931.na.f001

Suleobatrachus Špinar, $1972+\cdot \mathbf{A K}$

ST: PO.JD・CI: h1509・ID: $\uparrow 069$
PN: Palaeobatrachus laubei Bieber, 1881 †

PK: Palaeobatrachus laube ${ }^{\circ}$ Bieber, $1881 \dagger$

KG: Palaeobatrachus ${ }^{\circ}$ Tschudi, $1838+$

KF: PALAEOBATRACHIDAE 1865.ca.f001 †

Sumaterana Arikin ${ }^{+5}, 2018 \bullet \mathbf{K Y}$

ST: PO.KN • CI: h1510 • ID: 404

PN: Sumaterana crassiovis Boulenger, 1920

PK: Sumaterana crassiovis ${ }^{\circ}$ Boulenger, 1920

KG: Sumaterana ${ }^{\circ}$ Boulenger, 1920

KF: RANIDAE 1796.ba.f001

Sunnybatrachus Evans ${ }^{+1}, 2002+\cdot \mathbf{K Y}$

ST: PO.KN • CI: h1511・ID: $\uparrow 044$

PN: Sunnybatrachus purbeckensis Evans ${ }^{+1}, 2002$ \$

PK: Sunnybatrachus purbeckensis ${ }^{\circ}$ Evans $^{+1}, 2002 \dagger$

KG: Sunnybatrachus ${ }^{\circ}$ Evans $^{+1}, 2002 \dagger$

KF: ANURA Familia INCERTAE SEDIS

Sylvacaecilia Wake, 1987 • KY

ST: PO.KN・CI: h1512・ID: 481

PN: Geotrypetes grandisonae Taylor, 1970

PK: Geotrypetes grandisonae ${ }^{\circ}$ Taylor, 1970

KG: Sylvacaecilia ${ }^{\circ}$ Wake, 1987

KF: CAECILIIDAE 1814.ra.f003-|1825.gb.f008

Sylvirana Dubois, 1992 • AK

ST: PO.JD • CI: h1513・ID: 409

PN: Limnodytes nigrovittatus Blyth, 1855

PK: Limnodytes nigrovittatus* Blyth, 1855

KG: Hylarana* Tschudi, 1838

KF: RANIDAE 1796.ba.f001

Synapturanus Carvalho, $1954 \cdot \mathbf{K Y}$

ST: PO.KN・CI: h1514・ID: 318

PN: Synapturanus mirandaribeiroi Nelson ${ }^{+1}, 1975$

PK: Synapturanus mirandaribeiroi* ${ }^{*}$ Nelson $^{+1}, 1975$

KG: Synapturanus* Carvalho, 1954

KF: MICROHYLIDAE ||1843.fa.f012||-1931.na.f001

Syncope Walker, 1973 • KY

ST: PO.KN・CI: h1515・ID: 292

PN: Syncope antenori Walker, 1973

PK: Syncope antenori* Walker, 1973

KG: Chiasmocleis* Méhelÿ, 1904

KF: MICROHYLIDAE ||1843.fa.f012||-1931.na.f001

Syren Freeman ${ }^{+1}, 1807 \cdot \mathbf{A K}$

ST: NS.JI • CI: h1516・ID: 519

PN: Siren lacertina Österdam, 1766

PK: Siren lacertina* Österdam, 1766

KG: Siren* Österdam, 1766

KF: SIRENIDAE 1825gb.f005

Syrrhaphus Günther, 1900 • AK

ST: NT.JD・CI: h1517・ID: 082

PN: Syrrhophus marnocki Cope, 1878

PK: Syrrhophus marnocki* Cope, 1878

KG: Euhyas* Fitzinger, 1843

KF: BRACHYCEPHALIDAE 1858.gc.f002

Syrrhophus Cope, 1878 • AK

ST: PO.JD • CI: h1518 • ID: 082

PN: Syrrhophus marnocki Cope, 1878

PK: Syrrhophus marnocki* Cope, 1878 
KG: Euhyas* Fitzinger, 1843

KF: BRACHYCEPHALIDAE 1858.gc.f002

Syrrhopus Boulenger, 1888 • AK

ST: NS.JD・CI: h1519・ ID: 082

PN: Syrrhophus marnocki Cope, 1878

PK: Syrrhophus marnocki* Cope, 1878

KG: Euhyas* Fitzinger, 1843

KF: BRACHYCEPHALIDAE 1858.gc.f002

Syrrophus Dickerson, 1907 • AK

ST: NS.JD • CI: h1520 • ID: 082

PN: Syrrhophus marnocki Cope, 1878

PK: Syrrhophus marnocki* Cope, 1878

KG: Euhyas* Fitzinger, 1843

KF: BRACHYCEPHALIDAE 1858.gc.f002

Systoma Wagler, 1830 • AK

ST: NT.JI • CI: h1521 • ID: 298

PN: Rana ovalis Schneider, 1799

PK: Rana ovalis* Schneider, 1799

KG: Engystoma* Fitzinger, 1826

KF: MICROHYLIDAE ||1843.fa.f012||-1931.na.f001

Tachiramantis Heinicke ${ }^{+2}, 2015 \cdot \mathbf{K Y}$

ST: PO.KN • CI: h1522 • ID: 062

PN: Eleutherodactylus prolixodiscus Lynch, 1978

PK: Eleutherodactylus prolixodiscus ${ }^{\circ}$ Lynch, 1978

KG: Tachiramantis ${ }^{\circ}$ Heinicke $^{+2}, 2015$

KF: GaIANURA Familia INCERTAE SEDIS

Tachycnemis Fitzinger, $1843 \cdot \mathbf{K Y}$

ST: PO.KN • CI: h1523・ID: 336

PN: Eucnemis seychellensis Tschudi, 1838

PK: Eucnemis seychellensis* Tschudi, 1838

KG: Tachycnemis* Fitzinger, 1843

KF: HYPEROLIIDAE 1943.lb.f001

Tahananpuno Brown ${ }^{+4}, 2015$ • AK

ST: PO.JD • CI: h1524 • ID: 370

PN: Cornufer guentheri Boulenger, 1882

PK: Cornufer guentheri* Boulenger, 1882

KG: Platymantis ${ }^{1}$ Günther, 1859

KF: CERATOBATRACHIDAE 1884.ba.f001

Talmalsodes Diaz, 1992 • AK

ST: PO.JI • CI: h1525・ ID: 173

PN: Telmatobius montanus Philippi, 1902

PK: Telmatobius montanus ${ }^{\circ}$ Philippi, 1902

KG: Alsodes* Bell, 1843

KF: ALSODIDAE 1869.mc.f005

Tambabatrachus Ikeda $^{+2}, 2016+\cdot \mathbf{K Y}$

ST: PO.KN • CI: h1526・ID: $\uparrow 058$

PN: Tambabatrachus kawazu $\mathrm{Ikeda}^{+2}, 2016$ \$

PK: Tambabatrachus kawazu ${ }^{\circ} \mathrm{Ikeda}^{+2}, 2016 \dagger$

KG: Tambabatrachus ${ }^{\circ}$ Ikeda $^{+2}, 2016 \dagger$

KF: Hydrobatrachia Familia INCERTAE SEDIS

Tamixalus nov. $\bullet \mathrm{KY}$

ST: PO.KN・CI: h1527・ID: 457

PN: Rhacophorus calcadensis Ahl, 1927

PK: Rhacophorus calcadensis* Ahl, 1927

KG: Tamixalus* nov.

KF: RHACOPHORIDAE ||1858.gc.f012||-1932.ha.f001
Taphriomantis Laurent, $1941 \cdot \mathbf{A K}$

ST: PO.JD • CI: h1528 • ID: 325

PN: Cystignathus bocagii Günther, 1865

PK: Cystignathus bocagii* Günther, 1865

KG: Leptopelis $^{2}$ Günther, 1859

KF: ARTHROLEPTIDAE 1869.mc.f011

Taricha Gray, $1850 \bullet \mathbf{K Y}$

ST: PO.KN • CI: h1529・ID: 570

PN: Triton torosus Rathke, 1833

PK: Triton torosus* Rathke, 1833

KG: Taricha* Gray, 1850

KF: SALAMANDRIDAE 1820.ga.f002

Tarsopterus Reinhardt ${ }^{+1}, 1862 \bullet \mathbf{A K}$

ST: PO.JD・CI: h1530・ID: 181

PN: Tarsopterus trachystomus Reinhardt ${ }^{+1}, 1862$

PK: Tarsopterus trachystomus ${ }^{\circ}$ Reinhardt $^{+1}, 1862$

KG: Crossodactylus ${ }^{3}$ Duméril $^{+1}, 1841$

KF: HYLODIDAE 1858.gc.f010

Taruga Meegaskumbura ${ }^{+6}, 2010 \bullet \mathbf{K Y}$

ST: PO.KN • CI: h1531・ID: 453

PN: Polypedates fastigo Manamendra-Arachchi ${ }^{+1}, 2001$

PK: Polypedates fastigo* Manamendra-Arachchi ${ }^{+1}, 2001$

KG: Taruga* Meegaskumbura ${ }^{+6}, 2010$

KF: RHACOPHORIDAE ||1858.gc.f012||-1932.ha.f001

Taudactylus Straughan ${ }^{+1}$, $1966 \bullet \mathbf{K Y}$

ST: PO.KN • CI: h1532 • ID: 277

PN: Taudactylus diurnus Straughan ${ }^{+1}, 1966$

PK: Taudactylus diurnus ${ }^{\circ}$ Straughan $^{+1}, 1966$

KG: Taudactylus ${ }^{3}$ Straughan $^{+1}, 1966$

KF: MYOBATRACHIDAE 1850.sa.f001

Taylorana Dubois, 1987 • AK

ST: PO.JD・CI: h1533・ID: 380

PN: Polypedates hascheanus Stoliczka, 1870

PK: Polypedates hascheanus* Stoliczka, 1870

KG: Limnonectes* Fitzinger, 1843

KF: DiCROGLOSSIDAE 1987.da.f004

Teletrema Miranda-Ribeiro, 1937 • AK

ST: PO.JD • CI: h1534 • ID: 076

PN: Teletrema heterodactylum Miranda-Ribeiro, 1937

PK: Teletrema heterodactylum* Miranda-Ribeiro, 1937

KG: Oreobates* Jiménez de la Espada, 1872

KF: BRACHYCEPHALIDAE 1858.gc.f002

Telmalsodes Diaz, 1989 • AK

ST: PO.JD・CI: h1535・ID: 173

PN: Telmatobius montanus Philippi, 1902

PK: Telmatobius montanus ${ }^{\circ}$ Philippi, 1902

KG: Alsodes* Bell, 1843

KF: ALSODIDAE 1869.mc.f005

Telmatobius Wiegmann, $1834 \cdot \mathbf{K Y}$

ST: PO.KN・CI: h1536・ID: 186

PN: Telmatobius peruvianus Wiegmann, 1834

PK: Telmatobius peruvianus ${ }^{\circ}$ Wiegmann, 1834

KG: Telmatobius $^{3}$ Wiegmann, 1834

KF: TELMATOBIIDAE 1843.fa.f006

Telmatobufo Schmidt, $1952 \cdot \mathbf{K Y}$

ST: PO.KN • CI: h1537•ID: 258 
PN: Telmatobufo bullocki Schmidt, 1952

PK: Telmatobufo bullocki* Schmidt, 1952

KG: Telmatobufo* Schmidt, 1952

KF: CALYPTOCEPHALELLIDAE 1960.ra.f001

Tenuirana $\mathrm{Fei}^{+2}, 1990$ • AK

ST: PO.JD • CI: h1538 • ID: 409

PN: Rana taipehensis Van Denburgh, 1909

PK: Rana taipehensis* Van Denburgh, 1909

KG: Hylarana* Tschudi, 1838

KF: RANIDAE 1796.ba.f001

Tephrodytes Henrici, $1994+\bullet \mathbf{K Y}$

ST: PO.KN • CI: h1539 • ID: $\dagger 087$

PN: Tephrodytes brassicarvalis Henrici, 1994 \$

PK: Tephrodytes brassicarvalis ${ }^{\circ}$ Henrici, $1994 \uparrow$

KG: Tephrodytes ${ }^{\circ}$ Henrici, $1994 \uparrow$

KF: Archaeosalientia Familia INCERTAE SEDIS

Tepuihyla Ayarzagüena ${ }^{+2}, 1993 \cdot \mathbf{K Y}$

ST: PO.KN • CI: h1540 • ID: 224

PN: Hyla rodriguezi Rivero, 1968

PK: Hyla rodriguezi* Rivero, 1968

KG: Tepuihyla* Ayarzagüena ${ }^{+2}, 1993$

KF: HYLIDAE 1815.ra.f002-|1825.gb.f001|

Teracophrys: Ameghino $1901+$ AN

ST: AL・CI: n0162・ID: 257

PN: Teracophrys rugata Ameghino, $1901+\bullet$ AS

PK: Teracophrys rugata ${ }^{\circ}$ Ameghino, $1901 \dagger \cdot$ AS

KG: Calyptocephalella* Strand, 1928

KF: CALYPTOCEPHALELLIDAE 1960.ra.f001

Teratohyla Taylor, 1951・ KY

ST: PO.KN • CI: h1541 • ID: 163

PN: Centrolenella spinosa Taylor, 1949

PK: Centrolenella spinosa* Taylor, 1949

KG: Teratohyla* Taylor, 1951

KF: CENTROLENIDAE 1951.ta.f001

Tetraprion Stejneger ${ }^{+1}$, 1891 • AK

ST: PO.JD • CI: h1542 • ID: 231

PN: Tetraprion jordani Stejneger ${ }^{+1}, 1891$

PK: Tetraprion jordani* ${ }^{*}$ Stejneger ${ }^{+1}, 1891$

KG: Trachycephalus* Tschudi, 1838

KF: HYLIDAE 1815.ra.f002-|1825.gb.f001|

Thaumastosaurus Stefano, $1904+$ KY

ST: PO.KN • CI: h1543 • ID: $\dagger 045$

PN: Thaumastosaurus bottii Stefano, $1904+$

PK: Thaumastosaurus bottii ${ }^{\circ}$ Stefano, $1904 \dagger$

KG: Thaumastosaurus ${ }^{\circ}$ Stefano, $1904 \dagger$

KF: ANURA Familia INCERTAE SEDIS

Theatonius Fox, $1976+\cdot \mathbf{K Y}$

ST: PO.KN • CI: h1544 • ID: $\uparrow 046$

PN: Theatonius lancensis Fox, 1976 \$

PK: Theatonius lancensis ${ }^{\circ}$ Fox, $1976 \dagger$

KG: Theatonius $^{\circ}$ Fox, $1976 \dagger$

KF: ANURA Familia INCERTAE SEDIS

Theloderma Tschudi, $1838 \cdot \mathbf{K Y}$

ST: PO.KN • CI: h1545 • ID: 438

PN: Theloderma leporosa Tschudi, 1838

PK: Theloderma leporosa* Tschudi, 1838
KG: Theloderma* Tschudi, 1838

KF: RHACOPHORIDAE ||1858.gc.f012||-1932.ha.f001

Thoraciliacus Nevo, $1968+\bullet \mathbf{K Y}$

ST: PO.KN • CI: h1546・ ID: $\uparrow 066$

PN: Thoraciliacus rostriceps Nevo, 1968 \$

PK: Thoraciliacus rostriceps ${ }^{\circ} \mathrm{Nevo}, 1968 \uparrow$

KG: Thoraciliacus $^{\circ}$ Nevo, $1968 \dagger$

KF: DorSIPARES Familia INCERTAE SEDIS

Thorius Cope, $1869 \mathrm{a} \cdot \mathbf{K Y}$

ST: PO.KN • CI: h1547 • ID: 530

PN: Thorius pennatribus Cope, $1869 a$

PK: Thorius pennatribus* Cope, $1869 a$

KG: Thorius* Cope, $1869 a$

KF: PLETHODONTIDAE 1850.ga.f002

\section{Thornella nov. $\bullet \mathrm{KY}$}

ST: PO.KN • CI: h1548・ID: 536

PN: Oedipina quadra $\mathrm{McCranie}^{+2}, 2008$

PK: Oedipina quadra* $\mathrm{McCranie}^{+2}, 2008$

KG: Thornella* nov.

KF: PLETHODONTIDAE 1850.ga.f002

Thoropa Cope, $1865 \bullet \mathbf{K Y}$

ST: PO.KN • CI: h1549・ID: 180

PN: Cystignathus missiessii Eydoux ${ }^{+1}, 1842$

PK: Rana miliaris* Spix, 1824

KG: Thoropa ${ }^{1}$ Cope, 1865

KF: CYCLORAMPHIDAE 1850.bb.f003-|1852.ba.f001|

Tianophrys $\mathrm{Fei}^{+2}, 2016 \cdot \mathbf{A K}$

ST: PO.JD・CI: h1550 • ID: 023

PN: Megophrys shuichengensis $\operatorname{Tian}^{+2}, 2000$

PK: Megophrys shuichengensis ${ }^{\circ} \operatorname{Tian}^{+2}, 2000$

KG: Boulenophrys* $\mathrm{Fei}^{+2}, 2016$

KF: MEGOPHRYIDAE 1850.bb.f008-|1931.na.f003|

Tibetuperus Dubois ${ }^{+1}, 2012 \cdot \mathbf{A K}$

ST: PO.JD • CI: h1551 • ID: 507

PN: Batrachuperus yenyuanensis Liu, 1950

PK: Batrachuperus yenyuanensis* Liu, 1950

KG: Batrachuperus* Boulenger, 1878

KF: HYNOBIIDAE ||1856.ha.f001||-1859.cb.f002

Tigrina Grevé, 1894 • ZH

ST: zo • CI: zh098

Tigrina $\mathrm{Fei}^{+2}$, $1990 \bullet \mathbf{A K}$

ST: PO.JH • CI: h1552 • ID: 374

PN: Rana tigerina Daudin, 1802

PK: Rana tigerina* Daudin, 1802

KG: Hoplobatrachus ${ }^{1}$ Peters, 1863

KF: DiCROGLOSSIDAE 1987.da.f004

Tirahanulap Brown ${ }^{+4}, 2015$ • AK

ST: PO.JD・CI: h1553・ID: 370

PN: Philautus hazelae Taylor, 1920

PK: Philautus hazelae* Taylor, 1920

KG: Platymantis ${ }^{1}$ Günther, 1859

KF: CERATOBATRACHIDAE 1884.ba.f001

Tischleriella Herre, $1949+\cdot \mathbf{A K}$

ST: PO.JD • CI: h1554 • ID: $\uparrow 193$

PN: Tischleriella buddenbrocki Herre, 1949 \$

PK: Chelotriton paradoxus ${ }^{\circ}$ Pomel, $1853 \dagger$ 
KG: Chelotriton ${ }^{\circ}$ Pomel, $1853 \dagger$

KF: SALAMANDRIDAE 1820.ga.f002

Tlalocohyla Faivovich ${ }^{+5}, 2005 \cdot \mathbf{K Y}$

ST: PO.KN・CI: h1555・ID: 206

PN: Hyla smithii Boulenger, 1902

PK: Hyla smithii* Boulenger, 1902

KG: Tlalocohyla* Faivovich ${ }^{+5}, 2005$

KF: HYLIDAE 1815.ra.f002-|1825.gb.f001|

Tomodactylus Günther, 1900 • AK

ST: PO.JD • CI: h1556・ID: 082

PN: Tomodactylus amulae Günther, 1900

PK: Liuperus nitidus* Peters, 1870

KG: Euhyas* Fitzinger, 1843

KF: BRACHYCEPHALIDAE 1858.gc.f002

Tomopterna Duméril ${ }^{+1}, 1841 \cdot \mathbf{K Y}$

ST: PO.KN • CI: h1557•ID: 365

PN: Pyxicephalus delalandii Tschudi, 1838

PK: Pyxicephalus delalandii* Tschudi, 1838

KG: Tomopterna* ${ }^{*}$ Duméril $^{+1}, 1841$

KF: CACOSTERNIDAE 1931.na.f008

Tornierella Ahl, 1924 • AK

ST: PO.JD • CI: h1558 • ID: 340

PN: Tornierella pulchra Ahl, 1924

PK: Rothschildia kounhiensis ${ }^{\circ}$ Mocquard, 1905

KG: Paracassina ${ }^{\circ}$ Peracca, 1907

KF: HYPEROLIIDAE 1943.lb.f001

Tornierobates: Neave $1940 \cdot \mathbf{A N}$

ST: AM • CI: n0163・ID: 135

PN: Pseudophryne vivipara Tornier, 1905

PK: Pseudophryne vivipara* Tornier, 1905

KG: Nectophrynoides* Noble, 1926

KF: BUFONIDAE 1825.gb.f004

Tornierobates Frost ${ }^{+18}, 2006 \cdot \mathbf{A K}$

ST: NS.JD・CI: h1559 • ID: 135

PN: Pseudophryne vivipara Tornier, 1905

PK: Pseudophryne vivipara* Tornier, 1905

KG: Nectophrynoides* Noble, 1926

KF: BUFONIDAE 1825.gb.f004

Tornieriobates Miranda-Ribeiro, 1926 • AK

ST: PO.JD • CI: h1560 • ID: 135

PN: Pseudophryne vivipara Tornier, 1905

PK: Pseudophryne vivipara* Tornier, 1905

KG: Nectophrynoides* Noble, 1926

KF: BUFONIDAE 1825.gb.f004

Torrentirana Hillis $^{+1}$, 2005 • AK

ST: PO.JD・CI: h1561・ID: 415

PN: Rana tarahumarae Boulenger, 1917

PK: Rana tarahumarae* Boulenger, 1917

KG: Lithobates* Fitzinger, 1843

KF: RANIDAE 1796.ba.f001

Torrentophryne: $\mathrm{Rao}^{+1} 1994 \cdot \mathbf{A N}$

ST: AL・CI: n0164・ID: 120

PN: Torrentophryne aspinia $\mathrm{Rao}^{+1}, 1994$

PK: Torrentophryne aspinia* $\mathrm{Rao}^{+1}, 1994$

KG: $B u f o^{*}$ Garsault, 1764

KF: BUFONIDAE 1825.gb.f004
Torrentophryne Yang in Yang $^{+2}, 1996$ • AK

ST: PO.JD • CI: h1562 • ID: 120

PN: Torrentophryne aspinia $\mathrm{Rao}^{+1}, 1994$

PK: Torrentophryne aspinia* $\mathrm{Rao}^{+1}, 1994$

KG: $B u f o *$ Garsault, 1764

KF: BUFONIDAE 1825.gb.f004

Trachucephalus Ferguson, 1874 • AK

ST: PO.JD・CI: h1563・ID: 376

PN: Trachucephalus ceylanicus Ferguson, 1874

PK: Nannophrys ceylonensis* Günther, 1869

KG: Nannophrys* Günther, 1869

KF: DICROGLOSSIDAE 1987.da.f004

Trachycara Tschudi, $1845 \cdot \mathbf{A K}$

ST: PO.JD・CI: h1564・ID: 138

PN: Trachycara fusca Tschudi, 1845

PK: Rana margaritifera* Laurenti, 1768

KG: Rhinella ${ }^{2}$ Fitzinger, 1826

KF: BUFONIDAE 1825.gb.f004

Trachycephalus Tschudi, $1838 \cdot \mathbf{K Y}$

ST: PO.KN・CI: h1565・ID: 231

PN: Trachycephalus nigromaculatus Tschudi, 1838

PK: Trachycephalus nigromaculatus* Tschudi, 1838

KG: Trachycephalus * Tschudi, 1838

KF: HYLIDAE 1815.ra.f002-|1825.gb.f001|

Trachycephalus Ferguson, 1875 • AK

ST: NS.JH • CI: h1566・ID: 376

PN: Trachucephalus ceylanicus Ferguson, 1874

PK: Nannophrys ceylonensis* Günther, 1869

KG: Nannophrys* Günther, 1869

KF: DiCROGLOSSIDAE 1987.da.f004

Trachyhyas Fitzinger, $1843 \cdot \mathbf{A K}$

ST: PO.JD・CI: h1567・ID: 452

PN: Polypedates rugosus Duméril ${ }^{+1}, 1841$

PK: Hyla leucomystax* Gravenhorst, 1829

KG: Polypedates* Tschudi, 1838

KF: RHACOPHORIDAE ||1858.gc.f012||-1932.ha.f001

Trachymantis Giglio-Tos, $1917 \cdot \mathbf{z H}$

ST: zo • CI: zh099

Trachymantis Methuen, $1920 \bullet \mathbf{A K}$

ST: PO.JH • CI: h1568・ID: 431

PN: Microphryne malagasia Methuen ${ }^{+1}, 1913$

PK: Microphryne malagasia* Methuen ${ }^{+1}, 1913$

KG: Gephyromantis* Methuen, 1920

KF: RHACOPHORIDAE ||1858.gc.f012||-1932.ha.f001

Trachyphrynus Goin ${ }^{+1}, 1963$ • AK

ST: PO.JD・CI: h1569・ID: 078

PN: Trachyphrynus myersi Goin $^{+1}, 1963$

PK: Trachyphrynus myersi ${ }^{\circ}$ Goin $^{+1}, 1963$

KG: Pristimantis* Jiménez de la Espada, 1870

KF: BRACHYCEPHALIDAE 1858.gc.f002

Tregobatrachus Holman, $1975+\bullet \mathbf{K Y}$

ST: PO.KN • CI: h1570 • ID: $\uparrow 053$

PN: Tregobatrachus hibbardi Holman, 1975 \$

PK: Tregobatrachus hibbardi $i^{\circ}$ Holman, $1975 \dagger$

KG: Tregobatrachus ${ }^{\circ}$ Holman, $1975 \dagger$

KF: TREGOBATRACHIDAE 1975.hb.f001 † 
Tremeropugus Smith, $1831 \cdot \mathbf{A K}$

ST: PO.JD・CI: h1571 • ID: 009

PN: Tremeropugus typicus Smith, 1831

PK: Bufo laevis* Daudin, 1802

KG: Xenopus ${ }^{1}$ Wagler in Boie, 1827

KF: PIPIDAE 1825.gb.f003-|1826.fb.f002|

Triadobatrachus Kuhn, $1962+\cdot \mathbf{K Y}$

ST: PO.KN • CI: h1572・ID: $\uparrow 054$

PN: Protobatrachus massinoti Piveteau, 1936 \$

PK: Protobatrachus massinoti ${ }^{\circ}$ Piveteau, $1936 \uparrow$

KG: Triadobatrachus ${ }^{\circ}$ Kuhn, $1962 \uparrow$

KF: TRIADOBATRACHIDAE 1962.ka.f001 †

Triassurus Ivachnenko, $1978+\cdot \mathbf{K Y}$

ST: PO.KN • CI: h1573・ID: $\uparrow 158$

PN: Triassurus sixtelae Ivachnenko, 1978 \$

PK: Triassurus sixtelae ${ }^{\circ}$ Ivachnenko, $1978 \dagger$

KG: Triassurus ${ }^{\circ}$ Ivachnenko, $1978 \dagger$

KF: TRIASSURIDAE 1978.ia.f002 †

Trichobatrachus Boulenger, 1900 • AK

ST: PO.JD・CI: h1574 • ID: 321

PN: Trichobatrachus robustus Boulenger, 1900

PK: Trichobatrachus robustus* Boulenger, 1900

KG: Astylosternus* Werner, 1898

KF: ARTHROLEPTIDAE 1869.mc.f011

Trigonophrys Hallowell, 1857 • AK

ST: PO.JD・CI: h1575・ID: 169

PN: Trigonophrys rugiceps Hallowell, 1857

PK: Uperodon ornatum* Bell, 1843

KG: Ceratophrys ${ }^{3}$ Neuwied, 1824

KF: CERATOPHRYIDAE 1838.ta.f002

Triprion Cope, $1866 \bullet \mathbf{K Y}$

ST: PO.KN • CI: h1576・ID: 210

PN: Pharyngodon petasatus Cope, 1865

PK: Pharyngodon petasatus* Cope, 1865

KG: Triprion* Cope, 1866

KF: HYLIDAE 1815.ra.f002-|1825.gb.f001|

Tristella: Gray $1850 \cdot \mathbf{A N}$

ST: AL • CI: n0165・ID: 569

PN: Salamandra symmetrica Harlan, 1825

PK: Triturus (Diemictylus) viridescens* Rafinesque, 1820

KG: Notophthalmus ${ }^{1}$ Rafinesque, 1820

KF: SALAMANDRIDAE 1820.ga.f002

Tritogenius Gistel, $1848 \cdot \mathbf{A K}$

ST: NL.JI • CI: h1577・ID: 503

PN: Salamandra scheuchzeri Holl, $1831+$

PK: Salamandra scheuchzeri ${ }^{\circ}$ Holl, $1831 \dagger$

KG: Andrias $^{2}$ Tschudi, 1837

KF: CRYPTOBRANCHIDAE 1826.fb.f003

Tritomegas Amyot ${ }^{+1}, 1843 \cdot \mathbf{Z H}$

ST: zo・CI: zh100

Tritomegas Duméril ${ }^{+1}, 1854$ • AK

ST: PO.JH • CI: h1578 • ID: 503

PN: Megalobatrachus sieboldi Tschudi, 1837 \$

PK: Triton japonicus* Temminck, 1836

KG: Andrias $^{2}$ Tschudi, 1837

KF: CRYPTOBRANCHIDAE 1826.fb.f003
Triton Linnaeus, $1758 \cdot \mathbf{Z H}$

ST: zo • CI: zh101

Triton Laurenti, 1768 • AK

ST: PO.JH・CI: h1579・ID: 566

PN: Triton cristatus Laurenti, 1768

PK: Triton cristatus* Laurenti, 1768

KG: Triturus* Rafinesque, 1815

KF: SALAMANDRIDAE 1820.ga.f002

Tritonella Swainson, 1839 • AK

ST: NT.JI • CI: h1580 • ID: 566

PN: Triton cristatus Laurenti, 1768

PK: Triton cristatus* Laurenti, 1768

KG: Triturus* Rafinesque, 1815

KF: SALAMANDRIDAE 1820.ga.f002

Trituroides Chang, $1936 \bullet \mathbf{A K}$

ST: PO.JD・CI: h1581 • ID: 562

PN: Cynops chinensis Gray, 1859

PK: Cynops chinensis* Gray, 1859

KG: Paramesotriton* Chang, 1936

KF: SALAMANDRIDAE 1820.ga.f002

Triturus Rafinesque, $1815 \bullet \mathbf{K Y}$

ST: PO.KN • CI: h1582・ID: 566

PN: Triton cristatus Laurenti, 1768

PK: Triton cristatus* Laurenti, 1768

KG: Triturus* Rafinesque, 1815

KF: SALAMANDRIDAE 1820.ga.f002

Troglobates Gistel, 1848 + $\mathbf{A K}$

ST: NL.JI • CI: h1583・ID: $\uparrow 094$

PN: Palaeophrynos gessneri Tschudi, 1838 *

PK: Palaeophrynos gessneri ${ }^{\circ}$ Tschudi, $1838 \uparrow$

KG: Palaeophrynos ${ }^{\circ}$ Tschudi, $1838 \dagger$

KF: BUFONIDAE 1825.gb.f004

Truebella Graybeal ${ }^{+1}, 1995 \bullet \mathbf{K Y}$

ST: PO.KN • CI: h1584・ID: 099

PN: Truebella skoptes Graybeal $^{+1}, 1995$

PK: Truebella skoptes ${ }^{\circ}$ Graybeal $^{+1}, 1995$

KG: Truebella ${ }^{\circ}$ Graybeal $^{+1}, 1995$

KF: BUFONIDAE 1825.gb.f004

Trypheropsis Cope, 1868 • AK

ST: PO.JD・CI: h1585・ID: 415

PN: Ranula chrysoprasina Cope, 1866

PK: Ixalus warszewitschii* Schmidt, 1857

KG: Lithobates* Fitzinger, 1843

KF: RANIDAE 1796.ba.f001

Tsingymantis $\mathrm{Glaw}^{+2}, 2006 \cdot \mathbf{K Y}$

ST: PO.KN・CI: h1586・ID: 434

PN: Tsingymantis antitra $\mathrm{Glaw}^{+2}, 2006$

PK: Tsingymantis antitra* Glaw $^{+2}, 2006$

KG: Tsingymantis* ${ }^{*} \mathrm{Glaw}^{+2}, 2006$

KF: RHACOPHORIDAE $\| 1858 . g c . f 012||-1932$.ha.f001

Tsinpa Dubois $^{+1}, 2012 \cdot \mathbf{A K}$

ST: PO.JD・CI: h1587 • ID: 510

PN: Ranodon tsinpaensis $\mathrm{Liu}^{+1}, 1966$

PK: Ranodon tsinpaensis* Liu $^{+1}, 1966$

KG: Liua $^{1}$ Zhao $^{+1}, 1983$

KF: HYNOBIIDAE |1856.ha.f001\|-1859.cb.f002 
Turanomolge Nikolsky, 1918 • AK

ST: PO.JD・CI: h1588 • ID: 566

PN: Turanomolge mensbieri Nikolsky, 1918

PK: Triton karelinii* Strauch, 1870

KG: Triturus* Rafinesque, 1815

KF: SALAMANDRIDAE 1820.ga.f002

Twittya Dubois ${ }^{+1}, 2009$ • AK

ST: PO.JD • CI: h1589 • ID: 570

PN: Triturus rivularis Twitty, 1935

PK: Triturus rivularis* Twitty, 1935

KG: Taricha* Gray, 1850

KF: SALAMANDRIDAE 1820.ga.f002

Tylerana Dubois, 1992 • AK

ST: PO.JD・CI: h1590・ID: 409

PN: Rana jimiensis Tyler, 1963

PK: Rana jimiensis* Tyler, 1963

KG: Hylarana* Tschudi, 1838

KF: RANIDAE 1796.ba.f001

Tylerdella Wells $^{+1}, 1985$ • AK

ST: PO.JD・CI: h1591・ID: 270

PN: Ranidella remota Tyler $^{+1}, 1974$

PK: Ranidella remota* Tyler $^{+1}, 1974$

KG: Crinia* Tschudi, 1838

KF: MYOBATRACHIDAE 1850.sa.f001

Tylototriton Anderson, $1871 \cdot \mathbf{K Y}$

ST: PO.KN • CI: h1592 • ID: 573

PN: Tylototriton verrucosus Anderson, 1871

PK: Tylototriton verrucosus* Anderson, 1871

KG: Tylototriton*Anderson, 1871

KF: SALAMANDRIDAE 1820.ga.f002

Tylotriton Boettger, 1885 • AK

ST: NT.JI • CI: h1593・ID: 573

PN: Tylototriton verrucosus Anderson, 1871

PK: Tylototriton verrucosus* Anderson, 1871

KG: Tylototriton* Anderson, 1871

KF: SALAMANDRIDAE 1820.ga.f002

Tympanoceros Bocage, $1895 \cdot \mathbf{A K}$

ST: PO.JD • CI: h1594 • ID: 355

PN: Tympanoceros newtonii Bocage, 1895

PK: Cornufer johnstoni* Boulenger, 1888

KG: Petropedetes* Reichenow, 1874

KF: PETROPEDETIDAE 1931.na.f006

Typhlomolge Stejneger, 1896 • AK

ST: PO.JD・CI: h1595・ID: 542

PN: Typhlomolge rathbuni Stejneger, 1896

PK: Typhlomolge rathbuni* Stejneger, 1896

KG: Eurycea* Rafinesque, 1822

KF: Plethodontidae 1850.ga.f002

Typhlonectes Peters, $1880 \cdot \mathbf{K Y}$

ST: PO.KN • CI: h1596・ID: 480

PN: Caecilia compressicauda Duméril ${ }^{+1}, 1841$

PK: Caecilia compressicauda* Duméril ${ }^{+1}, 1841$

KG: Typhlonectes* Peters, 1880

KF: CAECILIIDAE 1814.ra.f003-|1825.gb.f008|

Typhlotriton Stejneger, 1892 • AK

ST: PO.JD • CI: h1597 • ID: 542
PN: Typhlotriton spelaeus Stejneger, 1892

PK: Typhlotriton spelaeus* Stejneger, 1892

KG: Eurycea* Rafinesque, 1822

KF: PLETHODONTIDAE 1850.ga.f002

Tyrrellbatrachus Gardner, $2015+\mathbf{K Y}$

ST: PO.KN • CI: h1598 • ID: $\uparrow 047$

PN: Tyrrellbatrachus brinkmani Gardner, 2015 \$

PK: Tyrrellbatrachus brinkmani ${ }^{\circ}$ Gardner, $2015 \dagger$

KG: Tyrrellbatrachus ${ }^{\circ}$ Gardner, 2015 †

KF: ANURA Familia INCERTAE SEDIS

Uberabatrachus Báez ${ }^{+5}, 2012+\bullet \mathbf{K Y}$

ST: PO.KN • CI: h1599・ID: $\uparrow 048$

PN: Uberabatrachus carvalhoi $\mathrm{Bá}^{+5}{ }^{+5}, 2012$ *

PK: Uberabatrachus carvalhoi ${ }^{\circ} \mathrm{Báez}^{+5}, 2012 \dagger$

KG: Uberabatrachus ${ }^{\circ}$ Báez $^{+5}, 2012 \uparrow$

KF: ANURa Familia INCERTAE SEDIS

Ukrainurus Vasilyan $^{+4,} 2013$ \$ KY

ST: PO.KN • CI: h1600 IID: $\uparrow 168$

PN: Ukrainurus hypsognathus Vasilyan ${ }^{+4}, 2013$ *

PK: Ukrainurus hypsognathus ${ }^{\circ}$ Vasilyan $^{+4}, 2013 \dagger$

KG: Ukrainurus ${ }^{\circ}$ Vasilyan $^{+4,} 2013 \dagger$

KF: CRYPTOBRANCHIDAE 1826.fb.f003

Ulanurus Gubin, $1991+\bullet \mathbf{K Y}$

ST: PO.KN • CI: h1601・ID: $\uparrow 169$

PN: Ulanurus fractus Gubin, 1991 \$

PK: Ulanurus fractus ${ }^{\circ}$ Gubin, $1991 \dagger$

KG: Ulanurus $^{\circ}$ Gubin, $1991 \dagger$

KF: CRYPTOBRANCHIDAE 1826.fb.f003

Uldzinia Gubin, 1996 • KY

ST: PO.KN • CI: h1602・ID: $\uparrow 088$

PN: Uldzinia kurochkini Gubin, 1996 \$

PK: Uldzinia kurochkini ${ }^{\circ}$ Gubin, $1996 \dagger$

KG: Uldzinia ${ }^{\circ}$ Gubin, $1996 \dagger$

KF: Archaeosalientia Familia InCERTAE SEDIS

Unculuana $\mathrm{Fei}^{+2}, 1990$ • AK

ST: PO.JD • CI: h1603・ID: 383

PN: Rana unculuana $\mathrm{Liu}^{+2}, 1960$

PK: Rana unculuana* Liu $^{+2}, 1960$

KG: Chaparana ${ }^{1}$ Bourret, 1939

KF: DICROGLOSSIDAE 1987.da.f004

Unicus: $\mathrm{Sá}^{+8} 2019 a \cdot \mathbf{A N}$

ST: AL •CI: n0166・ID: 292

PN: Chiasmocleis gnoma Canedo $^{+2}, 2004$

PK: Chiasmocleis gnoma ${ }^{\circ}$ Canedo $^{+2}, 2004$

KG: Chiasmocleis* Méhelÿ, 1904

KF: MICROHYLIDAE ||1843.fa.f012||-1931.na.f001

Unicus: $\mathrm{Sá}^{+8} 2019 b \cdot \mathbf{A N}$

ST: AL •CI: n0167・ID: 292

PN: Chiasmocleis gnoma Canedo $^{+2}, 2004$

PK: Chiasmocleis gnoma ${ }^{\circ}$ Canedo $^{+2}, 2004$

KG: Chiasmocleis* Méhelÿ, 1904

KF: MICROHYLIDAE ||1843.fa.f012||-1931.na.f001

Uperodon Duméril ${ }^{+1}, 1841 \cdot \mathbf{K Y}$

ST: PO.KN • CI: h1604 • ID: 309

PN: Engystoma marmoratum Guérin-Méneville, 1838

PK: Rana systoma* Schneider, 1799 
KG: Uperodon* Duméril ${ }^{+1}, 1841$

KF: MICROHYLIDAE ||1843.fa.f012||-1931.na.f001

Uperoleia Gray, $1841 \cdot \mathbf{K Y}$

ST: PO.KN • CI: h1605 • ID: 276

PN: Uperoleia marmorata Gray, 1841

PK: Uperoleia marmorata ${ }^{\circ}$ Gray, 1841

KG: Uperoleia ${ }^{2}$ Gray, 1841

KF: MYOBATRACHIDAE 1850.sa.f001

Uperoleja: Gray in Grey 1841 • AN

ST: AM・CI: n0168・ID: 276

PN: Uperoleia marmorata Gray, 1841

PK: Uperoleia marmorata ${ }^{\circ}$ Gray, 1841

KG: Uperoleia ${ }^{2}$ Gray, 1841

KF: MYOBATRACHIDAE 1850.sa.f001

Uraeotyphlus Peters, $1880 \bullet \mathbf{K Y}$

ST: PO.KN • CI: h1606・ID: 502

PN: Coecilia oxyura Duméril ${ }^{+1}, 1841$

PK: Coecilia oxyura ${ }^{\circ}$ Duméril $^{+1}, 1841$

KG: Uraeotyphlus ${ }^{3}$ Peters, 1880

KF: URAEOTYPHLIDAE 1979.na.f001

Urotropis Rafinesque, $1822 \cdot \mathbf{A K}$

ST: PO.JD • CI: h1607 • ID: 504

PN: Urotropis mucronata Rafinesque, 1822

PK: Salamandra alleganiensis* Sonnini ${ }^{+1}, 1801$

KG: Cryptobranchus ${ }^{1}$ Leuckart, 1821

KF: CRYPTOBRANCHIDAE 1826.fb.f003

Urotropis Jiménez de la Espada, 1875 • AK

ST: PO.JH • CI: h1608 • ID: 550

PN: Urotropis platensis Jimenez de la Espada, 1875

PK: Ensatina eschscholtzii* Gray, 1850

KG: Ensatina* Gray, 1850

KF: PLETHODONTIDAE 1850.ga.f002

Urspelerpes Camp ${ }^{+5}, 2009 \cdot \mathbf{K Y}$

ST: PO.KN • CI: h1609 • ID: 543

PN: Urspelerpes brucei $\mathrm{Camp}^{+5}, 2009$

PK: Urspelerpes brucei* Camp $^{+5}, 2009$

KG: Urspelerpes ${ }^{*}$ Camp $^{+5}, 2009$

KF: PLETHODONTIDAE 1850.ga.f002

Urupia Skutschas $^{+1}, 2011+\cdot \mathbf{K Y}$

ST: PO.KN • CI: h1610 • ID: $\uparrow 143$

PN: Urupia monstrosa Skutschas $^{+1}, 2011$ t

PK: Urupia monstrosa ${ }^{\circ}$ Skutschas $^{+1}, 2011 \dagger$

KG: Urupia $^{\circ}$ Skutschas $^{+1}, 2011 \dagger$

KF: Urodela Familia INCERTAE SEDIS

Valdotriton Evans $^{+1}, 1996$ + $\mathbf{K Y}$

ST: PO.KN・CI: h1611・ID: $\uparrow 144$

PN: Valdotriton gracilis Evans ${ }^{+1}, 1996$ \$

PK: Valdotriton gracilis $^{\circ}$ Evans $^{+1}, 1996 \dagger$

KG: Valdotriton ${ }^{\circ}$ Evans $^{+1}, 1996 \dagger$

KF: Urodela Familia INCERTAE SEDIS

Vampyrius nov. • KY

ST: PO.KN • CI: h1612 • ID: 458

PN: Rhacophorus vampyrus Rowley ${ }^{+4}, 2010$

PK: Rhacophorus vampyrus* Rowley ${ }^{+4}, 2010$

KG: Vampyrius* nov.

KF: RHACOPHORIDAE ||1858.gc.f012 |-1932.ha.f001
Vandijkophrynus Frost ${ }^{+18}, 2006 \bullet \mathbf{K Y}$

ST: PO.KN • CI: h1613・ID: 143

PN: Bufo angusticeps Smith, 1848

PK: Bufo angusticeps* Smith, 1848

KG: Vandijkophrynus $*$ Frost $^{+18}, 2006$

KF: BUFONIDAE 1825.gb.f004

Vanzolinius Heyer, 1974 • AK

ST: PO.JD • CI: h1614 • ID: 253

PN: Leptodactylus discodactylus Boulenger, 1883

PK: Leptodactylus discodactylus* Boulenger, 1883

KG: Leptodactylus ${ }^{1}$ Fitzinger, 1826

KF: LEPTODACTYLIDAE ||1838.ta.f001\|-1896.wa.f001

Varibatrachus Parmley ${ }^{+2}, 2015+\mathbf{K Y}$

ST: PO.KN・CI: h1615・ID: $† 049$

PN: Varibatrachus abraczinskasae Parmley ${ }^{+2}, 2015$

PK: Varibatrachus abraczinskasae ${ }^{\circ}$ Parmley $^{+2}, 2015$

KG: Varibatrachus ${ }^{\circ}$ Parmley $^{+2}, 2015$

KF: ANURA Familia INCERTAE SEDIS

Vatomantis $\mathrm{Glaw}^{+1}, 2006 \cdot \mathbf{A K}$

ST: PO.JD • CI: h1616・ID: 431

PN: Rhacophorus webbi Grandison, 1953

PK: Rhacophorus webbi* Grandison, 1953

KG: Gephyromantis* Methuen, 1920

KF: RHACOPHORIDAE ||1858.gc.f012||-1932.ha.f001

Vibrissaphora Liu, 1945 • AK

ST: PO.JD • CI: h1617 • ID: 015

PN: Vibrissaphora boringii Liu, 1945

PK: Vibrissaphora boringii* Liu, 1945

KG: Leptobrachium* Tschudi, 1838

KF: MEGOPHRYIDAE 1850.bb.f008-|1931.na.f003|

Vieraella Reig, 1961 + $\mathbf{K Y}$

ST: PO.KN・CI: h1618・ID: $\dagger 050$

PN: Vieraella herbstii Reig, 1961 \$

PK: Vieraella herbstii ${ }^{\circ}$ Reig, $1961 \dagger$

KG: Vieraella ${ }^{\circ}$ Reig, $1961 \dagger$

KF: ANURA Familia INCERTAE SEDIS

Vierella: Cei $1962+\bullet \mathbf{A N}$

ST: AM • CI: n0169 • ID: $\uparrow 050$

PN: Vieraella herbstii Reig, 1961 \$

PK: Vieraella herbstii ${ }^{\circ}$ Reig, $1961 \dagger$

KG: Vieraella ${ }^{\circ}$ Reig, $1961 \dagger$

KF: ANURA Familia INCERTAE SEDIS

Vierella Gardner $^{+1}, 2015+\mathbf{A K}$

ST: NT.JI • CI: h1619・ID: $\uparrow 050$

PN: Vieraella herbstii Reig, 1961 *

PK: Vieraella herbstii ${ }^{\circ}$ Reig, $1961 \dagger$

KG: Vieraella ${ }^{\circ}$ Reig, $1961 \dagger$

KF: ANURA Familia INCERTAE SEDIS

Vietnamophryne Poyarkov ${ }^{+6}, 2018$ • KY

ST: PO.KN • CI: h1620・ID: 283

PN: Vietnamophryne inexpectata Poyarkov ${ }^{+6}, 2018$

PK: Vietnamophryne inexpectata ${ }^{\circ}$ Poyarkov $^{+6}, 2018$

KG: Vietnamophryne ${ }^{\circ}$ Poyarkov $^{+6}, 2018$

KF: MICROHYLIDAE ||1843.fa.f012||-1931.na.f001

Vitreorana Guayasamin ${ }^{+5}, 2009 \cdot \mathbf{K Y}$

ST: PO.KN • CI: h1621 • ID: 164 
PN: Centrolenella antisthenesi Goin, 1963

PK: Centrolenella antisthenesi* Goin, 1963

KG: Vitreorana* Guayasamin ${ }^{+5}, 2009$

KF: CENTROLENIDAE 1951.ta.f001

Voigtiella Herre, $1949+\bullet \mathbf{A K}$

ST: PO.JD • CI: h1622 • ID: 578

PN: Voigtiella ludwigi Herre, 1949 \$

PK: Salamandra sansaniensis ${ }^{\circ}$ Lartet, $1851 \dagger$

KG: Salamandra ${ }^{1}$ Garsault, 1764

KF: SALAMANDRIDAE 1820.ga.f002

Vulcanobatrachus Trueb ${ }^{+2}, 2005+\mathbf{K Y}$

ST: PO.KN・CI: h1623・ID: $\uparrow 067$

PN: Vulcanobatrachus mandelai Trueb $^{+2}, 2005$ 末

PK: Vulcanobatrachus mandelai ${ }^{\circ}$ Trueb $^{+2}, 2005 \dagger$

KG: Vulcanobatrachus ${ }^{\circ}$ Trueb $^{+2}, 2005 \dagger$

KF: DORSIPARES Familia INCERTAE SEDIS

Wagleria Girard, 1853 • AK

ST: Po.JI • CI: h1624・ID: 261

PN: Cystignathus peronii Duméril ${ }^{+1}, 1841$

PK: Cystignathus peronii* Duméril ${ }^{+1}, 1841$

KG: Limnodynastes* Fitzinger, 1843

KF: MYOBATRACHIDAE 1850.sa.f001

Wakea Glaw ${ }^{+1}, 2006 \bullet \mathbf{K Y}$

ST: PO.KN • CI: h1625・ID: 429

PN: Mantidactylus madinika Vences $^{+3}, 2002$

PK: Mantidactylus madinika* Vences ${ }^{+3}, 2002$

KG: Wakea* Glaw ${ }^{+1}, 2006$

KF: RHACOPHORIDAE |1858.gc.f012||-1932.ha.f001

Walkerana: Otte $^{+1} 2009 \cdot \mathbf{Z A}$

ST: $\mathbf{z N} \bullet \mathbf{C I}: \mathbf{z n} 011$

Walkerana Dahanukar ${ }^{+5}, 2016 \cdot \mathbf{K Y}$

ST: PO.KN・CI: h1626・ID: 461

PN: Ixalus diplostictus Günther, 1875

PK: Ixalus diplostictus* Günther, 1875

KG: Walkerana* Dahanukar ${ }^{+5}, 2016$

KF: RANIXALIDAE 1987.da.f005

Wawelia Casamiquela, $1959+\bullet$ AK

ST: PO.JD • CI: h1627 • ID: 257

PN: Wawelia gerholdi Casamiquela, 1959 \$

PK: Wawelia gerhold $i^{\circ}$ Casamiquela, $1959 \dagger$

KG: Calyptocephalella* Strand, 1928

KF: CALYPTOCEPHALELLIDAE 1960.ra.f001

Wealdenbatrachus Fey, $1988+\bullet \mathbf{K Y}$

ST: PO.KN・CI: h1628・ID: $\uparrow 059$

PN: Wealdenbatrachus jucarensis Fey, 1988 \$

PK: Wealdenbatrachus jucarensis ${ }^{\circ}$ Fey, $1988 \dagger$

KG: Wealdenbatrachus ${ }^{\circ}$ Fey, $1988 \dagger$

KF: Hydrobatrachia Familia InCERTAE SEDIS

Werneria Poche, 1903 • KY

ST: PO.KN・CI: h1629・ID: 129

PN: Stenoglossa fulva Andersson, 1903

PK: Bufo preussi ${ }^{\circ}$ Matschie, 1893

KG: Werneria ${ }^{3}$ Poche, 1903

KF: BUFONIDAE 1825.gb.f004

Wesserpeton Sweetman ${ }^{+1}, 2013+\mathbf{K Y}_{\mathbf{H}}$

ST: PO.KN • CI: h1630 • ID: $\uparrow 007$
PN: Wesserpeton evansae Sweetman ${ }^{+1}, 2013$ \$

PK: Wesserpeton evansae ${ }^{\circ}$ Sweetman $^{+1}, 2013 \dagger$

KG: Wesserpeton ${ }^{\circ}$ Sweetman $^{+1}, 2013 \dagger$

KF: ALBANERPETIDAE 1982.fa.f001 †

Wolterstorffiella: Herre $1939+\bullet \mathbf{A N}$

ST: AL $・$ CI: n0170 • ID: $\uparrow 189$

PN: Wolterstorffiella wiggeri Herre, 1950 末

PK: Wolterstorffiella wigger $i^{\circ}$ Herre, $1950 \dagger$

KG: Wolterstorffiella ${ }^{\circ}$ Herre, $1950 \dagger$

KF: AмBYSTOMATIDAE 1850.ga.f004

Wolterstorffiella Herre, $1950+\cdot \mathbf{K Y}$

ST: PO.KN・CI: h1631・ID: $\uparrow 189$

PN: Wolterstorffiella wiggeri Herre, 1950 *

PK: Wolterstorffiella wiggeri ${ }^{\circ}$ Herre, $1950 \dagger$

KG: Wolterstorffiella ${ }^{\circ}$ Herre, $1950 \dagger$

KF: АмвYSTOMATIDAE 1850.ga.f004

Wolterstorffina Mertens, $1939 \bullet \mathbf{K Y}$

ST: PO.KN・CI: h1632・ID: 130

PN: Nectophryne parvipalmata Werner, 1898

PK: Nectophryne parvipalmata* Werner, 1898

KG: Wolterstorffina* Mertens, 1939

KF: BUFONIDAE 1825.gb.f004

Wurana $\mathrm{Li}^{+2}, 2006 \cdot \mathbf{A K}$

ST: PO.JD・CI: h1633・ID: 412

PN: Rana tormotus Wu, 1977

PK: Rana tormotus* Wu, 1977

KG: Odorrana $* \mathrm{Fei}^{+2}, 1990$

KF: RANIDAE 1796.ba.f001

Xanthophryne: $\mathrm{Biju}^{+4} 2009 \cdot \mathbf{A N}$

ST: AL・CI: n0171・ID: 105

PN: Bufo koynayensis Soman, 1963

PK: Bufo koynayensis* Soman, 1963

KG: Duttaphrynus* Frost ${ }^{+18}, 2006$

KF: BUFONIDAE 1825.gb.f004

Xenobatrachus Peters ${ }^{+1}$, 1878 • AK

ST: PO.JD • CI: h1634 • ID: 280

PN: Xenobatrachus ophiodon Peters ${ }^{+1}, 1878$

PK: Xenobatrachus ophiodon ${ }^{\circ}$ Peters $^{+1}, 1878$

KG: Asterophrys* Tschudi, 1838

KF: MICROHYLIDAE ||1843.fa.f012||-1931.na.f001

Xenobius Borgmeier, $1931 \cdot \mathbf{z H}$

ST: zo • CI: zh102

Xenobius Zhang ${ }^{+1}, 1985$ • AK

ST: PO.JH • CI: h1635 • ID: 512

PN: Xenobius melanonychus Zhang ${ }^{+1}, 1985$

PK: Pachyhynobius shangchengensis* $\mathrm{Fe}^{+1}, 1983$

KG: Pachyhynobius* $\mathrm{Fei}^{+1}, 1983$

KF: HYNOBIIDAE ||1856.ha.f001\|-1859.cb.f002

Xenohyla Izecksohn, $1998 \bullet \mathbf{K Y}$

ST: PO.KN・CI: h1636・ID: 195

PN: Hyla truncata Izecksohn, 1959

PK: Hyla truncata* Izecksohn, 1959

KG: Xenohyla* Izecksohn, 1998

KF: HYLIDAE 1815.ra.f002-|1825.gb.f001|

Xenophrys Günther, 1864 • KY

ST: PO.KN • CI: h1637•ID: 025 
PN: Xenophrys monticola Günther, 1864

PK: Xenophrys monticola ${ }^{\circ}$ Günther, 1864

KG: Xenophrys ${ }^{\circ}$ Günther, 1864

KF: MEGOPHRYIDAE 1850.bb.f008-|1931.na.f003|

Xenopus Wagler in Boie, 1827 • KY

ST: PO.KN • CI: h1638 • ID: 009

PN: Xenopus boiei Wagler, 1827

PK: Bufo laevis* Daudin, 1802

KG: Xenopus ${ }^{1}$ Wagler in Boie, 1827

KF: PIPIDAE 1825.gb.f003-|1826.fb.f002|

Xenorhina Peters, 1863 • AK

ST: PO.JD • CI: h1639 • ID: 280

PN: Bombinator oxycephalus Schlegel, 1858

PK: Bombinator oxycephalus* Schlegel, 1858

KG: Asterophrys* Tschudi, 1838

KF: MICROHYLIDAE ||1843.fa.f012||-1931.na.f001

Xiphoctonus Gistel, 1848 • $\mathbf{A K}$

ST: NL.JD • CI: h1640 • ID: 555

PN: Salamandra jeffersoniana Green, 1827

PK: Salamandra jeffersoniana* Green, 1827

KG: Ambystoma ${ }^{1}$ Tschudi, 1838

KF: AмвYSTOMATIDAE 1850.ga.f004

Xiphonura Tschudi, 1838 - AK

ST: PO.JD • CI: h1641 • ID: 555

PN: Salamandra jeffersoniana Green, 1827

PK: Salamandra jeffersoniana* Green, 1827

KG: Ambystoma ${ }^{1}$ Tschudi, 1838

KF: АмвYSTомAтIDAE 1850.ga.f004

Yaksha $\mathrm{Daza}^{+8}, 2020$ † KY

ST: PO.KN • CI: h1654 •ID: $† 200$

PN: Yaksha perettii Daza $^{+8}, 2020$ †

PK: Yaksha perettii ${ }^{\circ}$ Daza $^{+8}, 2020 \dagger$

KG: Yaksha ${ }^{\circ} \mathrm{Daza}^{+8}, 2020 \dagger$

KF: ALBANERPETIDAE 1982.fa.f001

Yaotriton Dubois ${ }^{+1}, 2009 \cdot \mathbf{K Y}$

ST: PO.KN • CI: h1642 • ID: 574

PN: Tylototriton asperrimus Unterstein, 1830

PK: Tylototriton asperrimus* Unterstein, 1830

KG: Yaotriton* ${ }^{*}$ Dubois $^{+1}, 2009$

KF: SALAMANDRIDAE 1820.ga.f002

Yerana Jiang ${ }^{+2}, 2006 \cdot \mathbf{K Y}$

ST: PO.KN • CI: h1643・ID: 392

PN: Paa (Feirana) yei $\mathrm{Chen}^{+2}, 2002$

PK: Paa (Feirana) yei* $\mathrm{Chen}^{+2}, 2002$

KG: Yerana* Jiang ${ }^{+2}, 2006$

KF: DiCROGLOSSIDAE 1987.da.f004

Yizhoubatrachus $\mathrm{GaO}^{+1}, 2004+\cdot \mathbf{K Y}$

ST: PO.KN • CI: h1644 • ID: $\uparrow 051$

PN: Yizhoubatrachus macilentus $\mathrm{Gao}^{+1}, 2004$ 末

PK: Yizhoubatrachus macilentus ${ }^{\circ} \mathrm{Gao}^{+1}, 2004 \uparrow$
KG: Yizhoubatrachus ${ }^{\circ} \mathrm{Gao}^{+1}, 2004 \dagger$

KF: AnURA Familia INCERTAE SEDIS

Yunganastes Padial $^{+4}, 2007 \cdot \mathbf{K Y}$

ST: PO.KN • CI: h1645 • ID: 079

PN: Eleutherodactylus pluvicanorus Riva ${ }^{+1}, 1997$

PK: Eleutherodactylus pluvicanorus $* \mathrm{Riva}^{+1}, 1997$

KG: Yunganastes* Padial $^{+4}, 2007$

KF: BRACHYCEPHALIDAE 1858.gc.f002

Zachaenus Cope, 1866 • AK

ST: PO.JD・CI: h1646・ID: 179

PN: Cystignathus parvulus Girard, 1853

PK: Cystignathus parvulus* Girard, 1853

KG: Cycloramphus* Tschudi, 1838

KF: CYCLORAMPHIDAE 1850.bb.f003-|1852.ba.f001|

Zaissanurus Chernov, $1959+\cdot \mathbf{K Y}$

ST: PO.KN • CI: h1647•ID: $\uparrow 170$

PN: Zaissanurus beliajevae Chernov, 1959 \$

PK: Zaissanurus beliajevae ${ }^{\circ}$ Chernov, $1959 \dagger$

KG: Zaissanurus $^{\circ}$ Chernov, $1959 \dagger$

KF: CRYPTOBRANCHIDAE 1826.fb.f003

Zakerana Howlader, 2011 • AK

ST: PO.JD • CI: h1648 • ID: 378

PN: Rana limnocharis syhadrensis Annandale, 1919

PK: Rana limnocharis syhadrensis* Annandale, 1919

KG: Minervarya* Dubois ${ }^{+2}, 2001$

KF: DiCROGLOSSIDAE 1987.da.f004

Zaphrissa Cope, $1866+\mathbf{A K}$

ST: PO.JD・CI: h1649・ID: 026

PN: Zaphrissa eurypelis Cope, 1866 *

PK: Pelobates decheni ${ }^{\circ}$ Troschel, $1861 \dagger$

KG: Pelobates* Wagler, 1830

KF: PELOBATIDAE 1850.bb.f004

Zhangixalus $\mathrm{Li}^{+3}$ in Jiang ${ }^{+4}, 2019 \cdot \mathbf{K Y}$

ST: PO.KN・CI: h1650・ID: 456

PN: Polypedates dugritei David, 1872

PK: Polypedates dugrite ${ }^{*}$ David, 1872

KG: Zhangixalus ${ }^{*} \mathrm{Li}^{+3}$ in $\mathrm{Jiang}^{+4}, 2009$

KF: RHACOPHORIDAE ||1858.gc.f012||-1932.ha.f001

Zoodioctes Gistel, 1848 • AK

ST: NL.JI • CI: h1651・ID: 409

PN: Hyla erythraea Schlegel, 1827

PK: Hyla erythraea* Schlegel, 1827

KG: Hylarana* Tschudi, 1838

KF: RANIDAE 1796.ba.f001

Zweifelia Dubois, 1992 • AK

ST: PO.JD・CI: h1652・ID: 415

PN: Rana tarahumarae Boulenger, 1917

PK: Rana tarahumarae* Boulenger, 1917

KG: Lithobates* Fitzinger, 1843

KF: RANIDAE 1796.ba.f001 


\section{APPENDix A6.NFS. Family-series nomina and taxa of LiSSAMPHIBIA.}

This table provides, in alphabetical order, all lissamphibian family-series (FS) nomina published from 1758 to 31 October 2020, and a few non-lissamphibian senior homonyms of these nomina (in cases where there exist several non-lissamphibian senior homonyms, only that first published is mentioned in this table, as it is enough to make all its junior homonyms invalid). The nomina are listed under the alphabetical order of their protonyms, followed by their status regarding availability, allocation and validity. Then their serial identifier and category identifier are given, as well as their status. In the following lines their relationships (such as neonymy or homonymy) with other nomina, their paronyms, onomatophores, as well as, if relevant, their eunyms and family allocation in the present ergotaxonomy, are indicated. In this table, some of these abbreviations (AP, AN, CI, JH) are used for nomina of both the genus- and family-series.

Protonym of family-series nomen.

Nomen appearing in one of the Tables of this work, followed by its shortened auctorship and publication date (year), with the following general structure: auctorship + publication year + publication identifier in year + nomen identifier in publication (+ paronym identifier). Whenever the auctorship consists in more than one auctor, only the name of the first one is given, followed by the number of other auctors, as follows: Duméril ${ }^{+1}$, Frost ${ }^{+18}$ (see examples at the end of this legend). The complete auctorship is given in References in the text above. All family-series aponyms and leipoprotographs appear here followed by their known scriptorship and first date of use.

\section{Available (hoplonyms) and valid (kyronyms) FS nomina of lissamphibian taxa}

$\mathbf{K Y} \cdot \mathrm{FS}$ nomokyronym (nomen considered valid in CLAD).

CK $•$ FS archokyronym (nomen validated through the Plenary Power of the Commission).

MK • Valid FS nomen through validation under Article 35.4.1.

PK - FS nomen valid at low ranks but invalid at high ranks because of partial invalidation through airesy, or under Articles 23.9 or 40.2 , or of action of the Commission.

RK • FS mnemokyronym (nomen protectum under Reversal of Precedence as defined in Article 23.9).

SK • Valid FS nomen through validation under Article 40.2.

Available and allocated but invalid FS nomina of lissamphibian taxa

CG $•$ FS archakyronym (invalidated FS nomen) through invalidation of its nucleogenus under the Plenary Power of the Commission.

CI • FS archakyronym (invalidated FS nomen) through the Plenary Power of the Commission.

JD - Invalid FS nomen (nomakyronym) for being junior doxisonym of an available and valid FS lissamphibian nomen.

JG - Invalid FS nomen (nomakyronym) for being based on a GS nomen being a junior homonym or isonym of an available GS nomen.

$\mathbf{J H} \cdot$ Invalid FS nomen (nomakyronym) for being junior homonym of an available FS nomen. In such cases only the earliest senior homonym is given in this table, as its existence is sufficient to preoccupy the spelling of the generic nomen at stake over the whole zoology.

JI Invalid FS nomen (nomakyronym) for being junior isonym of an available and valid FS lissamphibian nomen.

IM - Invalid FS nomen (nomakyronym) for being based on a GS being a metagraph - i.e., an autoneonym or an ameletograph (incorrect subsequent spelling) of the nomen of its nucleogenus (Article 35.4.1).

RI - FS lethakyronym (nomen oblitum under Reversal of Precedence as defined in Article 23.9).

SG - Invalid FS nomen (nomakyronym) for having been based on a GS nomen treated as a junior synonym before 1961, having then been replaced by a junior FS synonym and being then in 'prevailing usage' (Article 40.2), and having then taken the original date of the senior synonym although not its author.

Unavailable or unallocated FS nomina or graphs of lissamphibian taxa

AN - FS anoplonym (unavailable nomen) of lissamphibian taxon for failing to comply with the criteria of availability of publications or of nomina of the Code.

AP $\cdot$ FS anaptonym (nomenclaturally available but taxonomically unallocated lissamphibian nomen).

FS nomina of non-lissamphibian taxa being involved in relations of homonymy with available FS lissamphibian nomina. No further information on these nomina (such as their current validity) is provided here and they do not appear in Tables CLAD.

ZA - Available (hoplonym) non-amphibian FS nomen being homonym of a lissamphibian available FS nomen.

Cases under study

UI - Status of nomen posing problems, case submitted to the Commission for resolution, nomen considered here as invalid.

$\mathbf{U V} \cdot$ Status of nomen posing problems, case submitted to the Commission for resolution, nomen considered here as valid.

SI, Serial identifier of family-series nomen; CI, Category identifier of family-series patronym; ST, Status of FS nomen

(As.Av-Al.Va): assignment, availability \& allocation, validity \& correctness of nomen.

SI. Serial identifier of FS nomen $(n=596)$.

CI. Category identifier of FS nomen.

h001, h002, etc. • Numbers of family-series hoplonyms designating recent amphibians taxa (LiSSAMPHIBIA) $(n=488)$.

n001, n002, etc. • Numbers of family-series anoplonyms designating recent amphibian taxa (LISSAMPHIBIA) $(n=104)$.

zh01, zh02, etc. $\cdot$ Numbers of family-series hoplonyms designating taxa non including lissamphibians $(n=4)$. 
ST • Nomenclatural and taxonomic status of FS nomen (As.Av-Al.Va): As (assignment) + Av-Al (availability and allocation) + Va (validity) of nomen (indicated as a three number code: e.g. 0.10 .30 stands for a family-nomen, the nucleogenus of which has been explicitly designated, and it is available and valid).

\section{As • Criterion of assignment to the family-series (see Table CS-FS):}

0 Explicit family-series assignment and rhizonymy (FS1).

1 Implicit family-series assignment though unclear nominal-series assignment and rhizonymy (FS2).

2 Explicit or implicit family-series assignment through rank parordination or subordination to clear family-series nomen or nomina and arhizonymy or pseudarhizonymy (FS3).

Av-Al • Category of nomen regarding nomenclatural availability and taxonomic allocation:

$\mathbf{1 0}$ • Hoplonym (available nomen), aptonym (taxonomically allocated nomen) and photonym (taxonomically identified nomen).

22 - Anoplonym (agnostonym), for missing after 1999 the express mention that the nomen is introduced as a new scientific name (Article 16.1).

23 - Anoplonym (barbaronym) for having been published in non-Latinised form and not having been Latinised and adopted as valid before 1900.

$24 \cdot$ Anoplonym (arhizonym), for being based on the stem of an unavailable genus-series nomen.

25 - Anoplonym (arhizonym), for not being based on the stem of an available or unavailable genus-series nomen followed by a simple ending.

26 - Anoplonym (pseudorhizonym, cenorhizonym), for being based on the stem of an available or unavailable genus-series nomen, but the latter not being referred as valid to the family-series taxon in the publication where the nomen is introduced.

27 - Anoplonym (pseudorhizonym, auxorhizonym), for being based on the stem of an available or unavailable genus-series nomen, but combined with an ending derived from another or several other terms.

28 - Anoplonym (gymnonym), for missing after 1930 a description, definition or diagnosis of the taxon for which the new nomen is proposed, or missing reference to such a published statement, and for not being an explicit neonym (Article 13.1).

29 - Anoplonym (eulabonym), for having been proposed conditionally after 1960 (Article 15.1).

Va $・$ Category of nomen validity in $C L A D$ (see Dubois 2011a: figure 5):

30 Kyronym through publication priority over junior homonyms or synonyms.

31 Kyronym through airesy among synchronous nomina.

32 - Kyronym through proedry among synchronous nomina.

33 - Kyronym (nomen protectum, mnemokyronym) through reversal of precedence (Article 23.9).

34 Kyronym for being validated through Article 35.4.1 (rejection of senior isonym based on an autoneonym or an incorrect spelling of the nomen of its nucleogenus).

35 - Kyronym for being validated through Article 40.2 (rejection of nomen having been replaced before 1961 because of synonymy of the nucleogenus and not being in 'prevailing usage').

36 - Kyronym through precedence given to it among isonyms or doxisonyms, or by permanent invalidation of the latter, by the Commission under its Plenary Power.

37 - Kyronym for low-ranked taxa but exoplonym for higher-ranked taxa as a result of airesy or proedry, or of use of Articles 23.9 (reversal of precedence) or 40.2 (replacement of family-series nomen by a junior nomen because of doxisonymy of nucleogenus), or of conditional invalidation by the Commission under its Plenary Power.

38 - Case under study, nomen treated here as kyronym.

40 • Hypnonym for being a junior doxisonym.

$41 \cdot$ Hypnonym through airesy among doxisonyms.

$\mathbf{4 2} \cdot$ Hypnonym through proedry among doxisonyms.

43 - Hypnonym (nomen oblitum) through reversal of precedence among doxisonyms (Article 23.9).

44 - Hypnonym for having been replaced before 1961 because of doxisonymy of the nucleogenus and not being in 'prevailing usage' (Article 40.2).

45 Hypnonym (archypnonym) through subservience given to it among doxisonyms by the Commission under its Plenary Power.

46 $\cdot$ Hypnonym (anaptonym) for being so far taxonomically unallocated.

$\mathbf{5 0} \cdot$ Exoplonym for being an anoplonym.

$\mathbf{5 2} \cdot$ Exoplonym for being a junior isonym.

53 - Exoplonym for being based on a nucleogenus being a junior homonym.

$\mathbf{5 7}$ - Exoplonym (nomen oblitum) through reversal of precedence among isonyms.

58 - Exoplonym for being based on a metagraph, i.e., an autoneonym or an incorrect spelling of the nomen of its nucleogenus (Article 35.4.1).

61 - Exoplonym (archexoplonym) through having been invalidated by the Commission under its Plenary Power.

62 - Exoplonym (archexoplonym) through its nucleogenus having been invalidated by the Commission under its Plenary Power.

99 - Hoplonym, nomenclatural status regarding validity not explored here, being irrelevant for this study.

RL • Relationships of neonymy, allelonymy, homonymy and precedence (other than publication priority) of nomen $\mathrm{N}$ with other nomina [Whenever relevant]. 
$\leftrightarrow$ Allelonym of.

$\downarrow$ Junior homonym of (only earliest one is cited in case of multiple senior homonyms).

$\leftarrow$ Neonym of

$\rightarrow$ Spelling modified by the Commission under the Plenary Power.

$\geq$ Given precedence over synchronous synonym or homonym. $\bullet$ Reference.

$\leq$ Given subservience under synchronous synonym or homonym. • Reference.

$>$ Given precedence over senior synonym or homonym. $\bullet$ Reference.

$<$ Given subservience under junior synonym or homonym. $\bullet$ Reference.

AI • Precedence established through airesy (first-reviser action). $\bullet$ Reference.

PI - Precedence established through senior nucleogenus being invalid as a result of an action of the Commission under its Plenary Power (see A.NGS).

PM - Precedence established through senior synonym being based on a metagraph [i.e., an autoneonym or an incorrect spelling of the nomen of its nucleogenus (Article 35.4.1)], taking the original author and date of the latter.

PP • Precedence established through Plenary Power of the Commission • Reference.

PR • Precedence established through proedry (rank precedence).

PS - Precedence among family-series nomina established through junior synonym having replaced the senior synonym before 1961 because of synonymy of the nucleogenera and being in 'prevailing usage' (Article 40.2), and taking the original date of the senior synonym although not its author.

RI • Precedence established through 'Reversal of precedence' (Article 23.9). • Reference.

SP • Spelling emended through Plenary Power of the Commission $\bullet$ Reference.

US • Case under study.

PR • Paronyms of FS nomen, in the chronological order of their publication.

Each paronym is given with mention of its scriptor, reference, page and original rank. For abbreviations of ranks, see A.RNK.

1758.la., 1801.sa., etc. • Identifier of publication (see '6. References').

.f001, f002, etc. $・$ Identifier of FS nomen in publication.

$-00 \cdot$ Protonym of nomen.

$-01,-02$, etc. Aponyms of nomen (by order of publication).

$-\mathrm{c} 0$. Lectoprotograph of nomen.

-i1 - Leipoprotograph of nomen.

For each nomen, paronyms are given in chronological order of their publication, followed by their original rank.

Information is also given in this field, if relevant, for:

The resolution of conflicts of zygography among symprotographs (see Dubois 2013):

EEA • Explicit external airesy.

IIA- Implicit internal airesy.

The mention of placement of the nomen on an Official List or Index by the Commission:

IG • Nomen placed on the Official Index of Rejected and Invalid Familial Names in Zoology (Article 80).

LG • Nomen placed on the Official List of Familial Names in Zoology (Article 80).

OS • Onomatophore: nucleospecies (type species) of GS nomen and its mode of designation.

AM - Unavailable GS ameletograph (incorrect subsequent spelling) of lissamphibian taxon resulting from inadvertent change of spelling of original protograph.

AN - GS anoplonym (unavailable nomen) of lissamphibian taxon for failing to comply with the criteria of availability of publications or of nomina of the Code.

AP • GS anaptonym (nomenclaturally available but taxonomically unallocated lissamphibian nomen).

CI • GS archakyronym (invalidated nomen) through the Plenary Power of the Commission.

IN - Available GS nomen (hoplonym) but not mentioned as valid in the FS taxon for which a new FS nomen is proposed, thus making the latter unavailable (Article 11.7.1.1).

JH - Invalid GS nomen (nomakyronym) for being junior homonym of an available GS nomen.

OA - Original aphory (no included GS taxon mentioned in original work) (for arhizonyms).

OD • Original explicit designation (for rhizonyms and arhizonyms).

OE - Original implicit etymological designation (for rhizonyms and pseudorhizonyms).

$\mathbf{O M} \cdot$ Original monophory (for arhizonyms).

PD • Present designation of nucleogenus for new FS nomen or of electonucleogenus among prenucleogenera (for arhizonyms).

PN • Number of prenucleogenera, among which a electonucleogenus was subsequently designated (for arhizonyms).

The nucleogenus (type genus) of a family-series nomen is a nominal, not biological, genus. In this field, this nominal genus is mentioned first (N1), whether considered valid or invalid in CLAD. When the date of this nomen is followed by a second date between parentheses, this means that the first date is that of the protonym and the second date that of first publication of an aponym (which plays no role 
regarding zoological nomenclature, as an aponym is just a subsequent avatar of a nomen and does not have its own availability). If it is considered invalid in CLAD, it is followed by the valid nomen (N2) that applies to this genus in CLAD, with the following distinctions between two situations (see also A.NGS):

$\mathrm{N} 1 \equiv \mathrm{N} 2 \cdot \mathrm{N} 1$ is an invalid isonym (objective synonym) of N2.

$\mathrm{N} 1 \approx \mathrm{N} 2 \cdot \mathrm{In} C L A D, \mathrm{~N} 1$ is an invalid doxisonym (subjective synonym) of $\mathrm{N} 2$.

For arhizonyms, to save space, the complete list of prenucleogenera (which may be as numerous as 187) is not given here, but the number of prenucleogenera is indicated before the nomen of the electonucleogenus and preceded by the sign ».

EN - Eunyms of kyronym of FS taxa recognised as valid in $C L A D$.

To save space, nomina in this field are given followed only by their identifiers (see examples below), without their auctorship (given for each nomen of this table).

If more than one taxon bears this nomen in CLAD:

- In the line of the valid nomen, all paronyms used as valid in CLAD are given.

- In the lines of synonyms of the valid nomen, only the highest and the lowest ranked paronyms used as valid in $C L A D$ are mentioned here, separated by the sign "')"' and preceded by a number from (1) for the highest ranked to ( $n$ ) for the lowest ranked.

EF • Eunym of kyronym of family including the nucleogenus of FS nomen in CLAD.

\section{AbBreViations AND SYMBOLS PRESENT IN SEVERAL FIELDS:}

DOP. • Part of the identifier of a nomen established as new in the present work ('Dubois, Ohler \& Pyron').

INR • Information not relevant here (item does not exist).

- Nomen designating a taxon containing at least one non-recent amphibian (non-LiSSAMPHIBIA) species/taxon: detailed information on this nomen was not sought, not being necessary for the present work.

$\dagger \cdot$ Nomen designating an all-fossil taxon.

\section{Examples of citation of FS nomina}

Standard case

RANINA Batsch, 1796.ba.f001 • Original authorship and identifier of FS protonym.

RANIDAE 1796.ba.f001-05 - Shortened identifier of eunym for a FS taxon at a given rank.

Double authorship following Article 35.4.1 of the Code (format of writing modified from Dubois 2015a, see '2.3.7.2')

HYLARINIA Rafinesque, 1815.ra.f002 - Original authorship and identifier of FS protonym based on a GS metagraph (autoneonym or ameletograph).

HYLINA Gray, 1825.gb.f001 • Original authorship and identifier of FS protonym based on a GS archaeonym.

HYLIDAE 1815.ra.f002-|1825.gb.f001|-09 • Shortened identifier of eunym with double authorship for a FS taxon at a given rank.

Double authorship following Article 40.2 of the Code (format of writing modified from Dubois 2015a, see '2.3.7.2')

POLYPEDATIDAE Günther, 1858.gc.f012 - Original authorship and identifier of protonym based on a GS nomen considered before 1961 as an invalid junior synonym.

RHACOPHORIDAE Hoffman, 1932.ha.f001 - Original authorship and identifier of protonym based on a GS nomen considered before 1961 as a valid senior synonym of a GS nomen on which a senior FS nomen was based.

RHACOPHORIDAE ||1858.gc.f012||-1932.ha.f001-00 - Shortened identifier of eunym with double authorship for a FS taxon at a given rank. 
ACanthixalini nov., DOP.da.f094 • KY

SI: $535 \bullet$ CI: h428・ST: 0.10 .30

RL: INR

PA: $00 \cdot$ ACANTHIXALINI $\bullet$ Hoc loco $\bullet$ T

OS: Acanthixalus $1944 \cdot \mathbf{P D}$

EN: ACANTHIXALINI DOP.da.f094-00 • T

EF: HYPEROLIIDAE 1943.lb.f001

ACHоLотіDA Stannius, 1856.sa.f001 • AN

SI: $126 \bullet$ CI: n042 • ST: 2.25 .50

RL: INR

PA: $00 \cdot$ ACHOLOTIDA $\bullet$ Stannius 1856.sa: $4 \cdot \mathbf{F}$

OS: Siredon $1829 \approx$ Ambystoma $1838 \cdot \mathbf{O M}$

EN: AMBYSTOMATIDAE 1850.ga.f002-08 • F

EF: AMBYSTOMATIDAE 1850.ga.f002

ACoelonotae Miranda-Ribeiro, 1926.ma.f002 • AN

SI: $209 \bullet$ CI: $n 060 \bullet$ ST: 2.25 .50

RL: INR

PA: $00 \cdot$ ACOELONOTAE $・$ Miranda-Ribeiro 1926.ma: $64 \cdot \mathbf{U F}$

OS: » 11 PN, including: Hyla 1768 PD

EN: (1) HYLOIDEA 1815.ra.f002-|1825.ga.f001|-20 • pF "n'm"

(8) HYLITES 1815.ra.f002-|1825.ga.f001|-26 • Cn

EF: HYLIDAE 1815.ra.f002-|1825.ga.f001|

ACRIDINA Macleay, 1821.ma.f001 • ZA-UI

SI: $014 \cdot$ CI: zh01 • ST: 0.10 .99

RL: > ACRIDINA Mivart, 1869.ma.f008

$>$ ACRIDODEA Karsch, 1893.ka.f001

PA: $00 \cdot$ ACRIDINA $・$ Macleay 1821.ma: $436 \bullet \mathbf{T}$

OS: Acrides $1821 \cdot$ OD

EN: •

EF: $\bullet$

ACRIDINa Mivart, 1869.ma.f008 • JH-UV

SI: $168 \bullet$ CI: h116 • ST: 0.10 .38

RL: < ACRIDINA Macleay, 1821.ma.f001 • PR: Dubois ${ }^{+2}$ 2017.da: 54

> ACRIDODEA Karsch, 1893.ka.f001 • PR: Dubois ${ }^{+2}$ 2017.da: 54

PA: $00 \cdot$ ACRIDINA $\bullet$ Mivart 1869.ma: $292 \cdot \mathbf{b F}$

01 - ACRIDINAE $\bullet$ Kuhn 1965.ka: $96 \bullet \mathbf{b F}$

02 - ACRISINAE $\bullet$ Dubois $^{+2}$ 2017.da: $54 \cdot \mathbf{b F}$

03 - ACRISINI $\bullet$ Dubois $^{+2}$ 2017.da: $55 \cdot$ T

$04 \cdot$ ACRIDINI Dubois $^{+2}$ 2017.da: $55 \cdot \mathrm{T}$

05 - ACRISINA $\bullet$ Hoc loco $\bullet \mathbf{b T}$

$06 \cdot$ ACRISINIA $\bullet$ Hoc loco $\bullet$ iT

OS: Acris $1841 \cdot \mathbf{O E}$

EN: (1) ACRISINA 1869.ma.f008-05 • bT

(2) ACRISINIA 1869.ma.f008-06 • iT

EF: HYLIDAE 1815.ra.f002-|1825.ga.f001|

ACRIDODEA Karsch, 1893.ka.f001 • uv

SI: $193 \cdot$ CI: zh04 • ST: 0.10 .99

RL: < ACRIDINA Macleay, 1821.ma.f001

$<$ ACRIDINA Mivart, 1869.ma.f008

PA: 00 • ACRIDODEA • Karsch 1893.ka: 51 • UF

OS: Acrida $1758 \cdot \mathbf{O E}$

EN: •

EF: •

Adelastinae Peloso ${ }^{+10}$, 2016.pa.f001 • KY

SI: $435 \bullet$ CI: h328 • ST: 0.10 .30
RL: INR

PA: $00 \cdot$ ADELASTINAE $・$ Peloso $^{+10}$ 2016.pa: $131 \cdot$ bF

OS: Adelastes $1986 \bullet$ OD

EN: ADELASTINAE 2016.pa.f001-00 • bF

EF: MICROHYLIDAE |1843.fa.f012|-1931.na.f001

AdenOMERIDAE Hoffmann, 1878.ha.f003 • KY

SI: $181 \bullet$ CI: h125 • ST: 0.10 .30

RL: INR

PA: $00 \cdot$ ADENOMERIDAE $\bullet$ Hoffmann 1878.ha: $613 \cdot \mathbf{b F}$

$01 \cdot$ ADENOMERINI $\bullet$ Hoc loco $\bullet \mathbf{T}$

OS: Adenomera $1867 \cdot \mathbf{O E}$

EN: ADENOMERINI 1878.ha.f003-01 • bF

EF: LEPTODACTYLIDAE |1838.ta.f001|-1896.wa.f001

AdenOmidae Cope, 1861.ca.f001 • KY

SI: $147 \bullet$ CI: h098 • ST: 0.10 .30

RL: INR

PA: 00 • ADENOMINAE • Cope 1861.ca: $371 \cdot \mathbf{F}$

$01 \cdot$ ADENOMIDAE $\bullet$ Hoffmann 1878.ha: $614 \cdot \mathbf{b F}$

02 $•$ ADENOMINAE $\bullet$ Dubois 1983.da: $273 \cdot \mathbf{b F}$

$03 \cdot$ ADENOMITOES $\bullet$ Hoc loco $\bullet \mathbf{i C n}$

$04 \cdot$ ADENOMITUES $\bullet$ Hoc loco $\bullet \mathbf{h C n}$

OS: Adenomus $1861 \cdot \mathbf{O E}$

EN: (1) ADENOMITOES 1861.ca.f001-03 • iCn

(2) ADENOMITUES 1861.ca.f001-04 • hCn

EF: BUFONIDAE 1825.ga.f004

AFrIXalinia nov., DOP.da.f097 • KY

SI: $538 \bullet$ CI: h431 • ST: 0.10 .30

RL: INR

PA: $00 \cdot$ AFRIXALINIA $\bullet$ Hoc loco $\bullet$ iT

OS: Afrixalus $1944 \cdot \mathbf{P D}$

EN: AFRIXALINIA DOP.da.f097-00 • iT

EF: HYPEROLIIDAE 1943.1b.f001

AFROCAECILIITI Lescure ${ }^{+2}$, 1986.lb.f005 • JD

SI: $324 \bullet$ CI: h232 • ST: 0.10 .40

RL: INR

PA: $00 \cdot$ AFROCAECILIITI $・$ Lescure $^{+2}$ 1986.lb: $164 \cdot$ bT

OS: Afrocaecilia $1968 \approx$ Boulengerula $1896 \cdot$ OE

EN: (1) HERPELINAE 1984.la.f001-00 • bF

(2) HERPELINI 1984.la.f001-02 • T

EF: CAECILIIDAE 1814.ra.f003-|1825.ga.f008|

AgalyChNINI nov., DOP.da.f067 • KY

SI: $508 \bullet$ CI: h401 • ST: 0.10 .30

RL: INR

PA: $00 \cdot$ AGALYCHNINI $\bullet$ Hoc loco $\bullet \mathbf{T}$

OS: Agalychnis $1864 \cdot \mathbf{P D}$

EN: AGALYCHNINI DOP.da.f067-00 • T

EF: PhyLLOMEDUSIDAE 1858.gc.f009

AGLOSSA Wiegmann in Wiegmann ${ }^{+1}$, 1832.wa.f001 • AN

SI: $039 \bullet \mathbf{C I}: \mathrm{n} 017 \cdot \mathbf{S T}: 2.25 .50$

RL: INR

PA: $00 \cdot$ AGLOSSA - Wiegmann $^{+1}$ 1832.wa: $200 \cdot \mathbf{F}$

$01 \cdot$ AGLOSSA $\bullet$ Leunis 1844.la: $128 \cdot \mathbf{U F}$

02 $\bullet$ AGLOSSIDAE $\bullet$ Mayer 1849.ma: $37 \cdot \mathbf{F}$

03 - AGLOSSA $\bullet$ Leunis 1860.la: $335 \cdot \mathbf{T}$

04 $\bullet$ AGLOSSA $\bullet$ Huene 1931.ha: $311 \cdot \mathbf{p F}$

OS: » 2 PN, including: Pipa $1768 \bullet$ PD 
EN: (1) PIPIDAE 1825.gb.f003-|1826.fb.f002|-07 • F

(2) PIPINAE 1825.gb.f003-|1826.fb.f002|-13 • bF

EF: PIPIDAE 1825.gb.f003-|1826.fb.f002|

AlbanerpetontidaE Fox $^{+1}$, 1982.fa.f001 +

SI: $309 \cdot \mathbf{C I}:$ h220 • ST: 0.10 .30

RL: INR

PA: $00 \cdot$ ALBANERPETONTIDAE Fox $^{+1}$ 1982.fa: 118, 120 • F

01 - ALBANERPETONTINAE • Wiechmann 2003.wa: [2], 20 • bF

$02 \cdot$ ALBANERPETONTOIDEA • Dubois 2005.da: $6 \cdot \mathbf{p F}$

$03 \cdot$ ALBANERPETONTOIDIA $\bullet$ Dubois 2005.da: $6 \cdot \mathbf{e F}$

$04 \cdot$ ALBANERPETIDAE $^{-}$Averianov $^{+1}$ 2012.aa: 466 • F

OS: Albanerpeton $1976+\cdot \mathbf{O E}$

EN: ALBANERPETIDAE 1982.fa.f001-04 † $\mathbf{F}$

EF: ALBANERPETIDAE 1982.fa.f001 †

AlchlinaE Brown ${ }^{+4}$, 2015.ba.f002 • KY

SI: 434 • CI: h327 • ST: 0.10 .30

RL: INR

PA: $00 \cdot$ ALCALINAE $\bullet$ Brown $^{+4}$ 2015.ba: $142 \cdot \mathbf{b F}$

OS: Alcalus 2015 • OD

EN: ALCALINAE 2015.ba.f002-00 • bF

EF: CERATOBATRACHIDAE 1884.ba.f001

AllobatinaE Grant ${ }^{+9}$, 2006.gb.f003 • KY

SI: 372 • CI: h278 • ST: 0.10 .30

RL: INR

PA: $00 \cdot$ ALLOBATINAE $\bullet$ Grant $^{+9}$ 2006.gb: $4 \cdot \mathbf{b F}$

OS: Allobates $1988 \cdot$ OD

EN: ALLOBATINAE 2006.gb.f003-00 • bF

EF: АROMOBATIDAE 2006.gb.f001

ALLOPHRYNIDAE Savage, 1973.sa.f002 • AN

SI: $294 \bullet \mathbf{C I}:$ n083・ST: 0.28 .50

RL: INR

PA: $00 \cdot$ ALLOPHRYNIDAE $\bullet$ Savage 1973.sa: $354 \cdot \mathbf{F}$

OS: Allophryne $1926 \cdot \mathbf{O E}$

EN: ALLOPHRYNIDAE 1978.ga.f001-00 • F

EF: ALLOPHRYNIDAE 1978.ga.f001

AllophrynidaE Goin ${ }^{+2}$, 1978.ga.f001 • KY

SI: $301 \bullet \mathbf{C I}:$ h2 $14 \cdot \mathbf{S T}: 0.10 .30$

RL: INR

PA: 00 • ALLOPHRYNIDAE $\bullet$ Goin $^{+2}$ 1978.ga: $240 \cdot$ F

01 ALLOPHRYNINAE • Dubois 1983.da: $274 \cdot \mathbf{b F}$

OS: Allophryne $1926 \cdot \mathbf{O E}$

EN: ALLOPHRYNIDAE 1978.ga.f001-00 • F

EF: ALLOPHRYNIDAE 1978.ga.f001

Alsodina Mivart, 1869.ma.f005 • KY

SI: $165 \cdot \mathbf{C I}:$ h113 • ST: 0.10 .31

RL: > CACOTINA 1869.ma.f006 • AI: Lynch 1971.la: 9

PA: $00 \cdot$ ALSODINA • Mivart 1869.ma: $290 \cdot \mathbf{b F}$

$01 \cdot$ ALSODINI $\bullet$ Lynch 1969.1b: $3 \cdot \mathbf{T}$

02 ALSODIDAE $\cdot$ Pyron $^{+1}$ 2011.pa: $543 \cdot \mathbf{F}$

03 - ALSODINAE $^{\circ}$ Pyron $^{+1}$ 2011.pa: $546 \bullet \mathbf{b F}$

OS: Alsodes $1843 \cdot \mathbf{O E}$

EN: ALSODINAE 1869.ma.f005-03 • bF

EF: CYCLORAMPHIDAE 1850.bb.f003-|1852.ba.f001|

AlytaE Fitzinger, 1843.fa.f008 • KY

SI: 073 • CI: h041 • ST: 0.10 .30

RL: INR
PA: $00 \cdot A L Y T A E \bullet$ Fitzinger 1843.fa: $32 \cdot \mathbf{F}$

01 ALYTINA • Bonaparte 1850.bb: pl. • bF

$02 \cdot$ ALYTIDAE $\bullet$ Günther 1858.gc: $346 \cdot \mathbf{F}$

$03 \cdot$ ALYTIDAE $\cdot$ Hoffmann 1878.ha: $613 \cdot \mathbf{b F}$

$04 \cdot$ ALYTINI $\bullet$ Sanchíz 1984.sa: $61 \cdot$ T

05 - ALYTINAE • Dubois 1987.da: $12 \cdot \mathbf{b F}$

$06 \cdot$ ALITIDAE $\bullet$ Spadola $^{+1}$ 2010.sa: $271 \bullet \mathbf{F}$

$07 \cdot$ ALYTOIDEA $\bullet$ Hoc loco $\bullet \mathbf{p F}$

OS: Alytes $1829 \cdot \mathbf{O E}$

EN: (1) ALYTOIDEA 1843.fa.f008-07 • pF

(2) ALYTIDAE 1843.fa.f008-02 • F

EF: ALYTIDAE 1843.fa.f008

AMazophrynellinia nov., DOP.da.f015 • KY

SI: $456 \bullet \mathbf{C I}:$ h349 • ST: 0.10 .30

RL: INR

PA: $00 \cdot$ AMAZOPHRYNELLINIA $・ H O c$ loco $\bullet$ iT

OS: Amazophrynella 2012 • PD

EN: AMAZOPHRYNELLINIA DOP.da.f015-00 • iT

EF: BUFONIDAE 1825.gb.f004

AMBLYOPES Goldfuss, 1820.ga.f003 • AN

SI: $013 \cdot \mathbf{C I}:$ n008 • ST: 2.25 .50

RL: INR

PA: $00 \cdot$ AMBLYOPES $\bullet$ Goldfuss 1820.ga: xi • F

OS: » 3 PN, including: Coecilia $1801 \equiv$ Caecilia $1758 \cdot \mathbf{P D}$

EN: (1) CAECILIOIDEA 1814.ra.f003-|1825.gb.f008|-19 • pF "')"')

(5) CAECILIINA 1814.ra.f003-|1825.gb.f008|-26・bT

EF: CAECILIIDAE 1814.ra.f003-|1825.gb.f008|

Amblystomata Cope, 1861.ca.f002 • JD

SI: $148 \cdot \mathbf{C I}:$ h099 • ST: 0.10 .52

RL: INR

PA: $00 \cdot$ AMBLYSTOMATA • Cope 1861.ca: 373 • UF

01 AMBLYSTOMIDAE $\bullet$ Cope 1863.ca: $343 \cdot \mathbf{F}$

$02 \cdot$ AMBLYSTOMIDA $・$ Knauer 1878.ka: $98 \cdot \mathbf{F}$

$03 \cdot$ AMBLYSTOMATINAE $\bullet$ Boulenger 1882.bc: vii, $31 \cdot \mathbf{b F}$

$04 \cdot$ AMBLYSTOMATIDAE $\bullet$ Garman 1884.ga: $37 \cdot \mathbf{F}$

OS: Amblystoma $1846 \equiv$ Ambystoma $1838 \cdot \mathbf{O E}$

EN: AмвYSTOMATIDAE 1850.ga.f002-08 • F

EF: AмBYSTOMATIDAE 1850.ga.f002

AmbYStomina Gray, 1850.ga.f002 • KY

SI: $113 \cdot \mathbf{C I}:$ h075 • ST: 1.10 .36

RL: AмвYSTOMATIDAE 1850.ga.f002-08 • SP: Opinion 649 (Riley $^{+1}$ 1963.rb: 102)

PA: $00 \cdot$ AмвYSTOMINA $\bullet$ Gray 1850.ga: $32 \cdot \mathbf{U F}$

$01 \cdot$ AMBYSTOMINA $\bullet$ Hallowell 1856.ha: $6 \cdot \mathbf{b F}$

$02 \cdot$ AMBYSTOMATA $・$ Hallowell 1856.ha: 7, 9 • UF

$03 \cdot$ AмвYSTOMIDAE $\bullet$ Hallowell 1856.ha: $11 \cdot \mathbf{b F}$

$04 \cdot$ AMBYSTOMINAE $\bullet$ Cope 1859.cb: $122 \cdot \mathbf{b F}$

$05 \cdot$ AMBYSTIDAE $\bullet$ Hoffmann 1878.ha: 585 • F

$06 \cdot$ AMBYLSTOMIDAE $\bullet$ Hoffmann 1878.ha: 585 • F

07 - AMBYSTOMIDAE $\bullet$ Hoffmann 1878.ha: $726 \cdot \mathbf{F}$

08 • AMBYSTOMATIDAE • Hay 1892.ha: $415 \cdot \mathbf{F}$

09 A AMBSTOMOIDEA • Herre 1950.ha: $293 \cdot \mathbf{p F}$

10 AMBYSTOMATINAE • Tihen 1958.ta: 1 • bF • IG: Smith $^{+1}$ 1961.sa: 215

11 - AMBYSTOMATOIDEA • Dubois 2005.da: $19 \cdot \mathbf{p F}$ 
12 - AMBYSTOMATOIDAE • Dubois ${ }^{+1}$ 2012.da: $147 \cdot \mathbf{e F}$

OS: Ambystoma $1838 \bullet \mathbf{O E}$

EN: АмвYSTOMATIDAE 1850.ga.f002-08 • F

EF: AMBYSTOMATIDAE 1850.ga.f002

AMOLOPINAE Yang, 1989.ya.f001 • AN

SI: $341 \cdot \mathbf{C I}: \mathrm{n} 089 \bullet \mathbf{S T}$ : 0.28 .50

RL: INR

PA: 00 • AMOLOPINAE • Yang 1989.ya: 256 • bF

OS: Amolops $1865 \cdot \mathbf{O E}$

EN: AMOLOPINA 1990.fa.f001-03 • bT

EF: RANIDAE 1796.ba.f001

AmolopinaE $\mathrm{Fei}^{+2}$, 1990.fa.f001 • KY

SI: $344 \cdot \mathbf{C I}:$ h251 • ST: 0.10 .30

RL: INR

PA: $00 \cdot$ AMOLOPINAE $\bullet \mathrm{Fei}^{+2}$ 1990.fa: 4, $123 \cdot \mathbf{b F}$

01 $\cdot$ AMOLOPSINAE $\bullet$ Yang 1991.ya: $172 \cdot \mathbf{b F}$

$02 \cdot$ AMOLOPINI $\bullet$ Scott 2005.sa: 4, $527 \cdot \mathbf{T}$

$03 \cdot$ AMOLOPINA $\cdot$ Hoc loco $\bullet \mathbf{b T}$

OS: Amolops $1865 \cdot$ OE

EN: AMOLOPINA 1990.fa.f001-03 • bT

EF: RANIDAE 1796.ba.f001

AmphignathodontidaE Boulenger, 1882.bb.f002 • KY

SI: $186 \bullet \mathbf{C I}:$ h128 • ST: 0.10 .30

RL: INR

PA: 00 • AMPHIGNATHODONTIDAE • Boulenger 1882.bb: xvi, $449 \cdot \mathbf{F}$ 01 - AMPHIGNATHODONTINAE • Gadow 1901.ga: xi, $188 \cdot \mathbf{b F}$ $02 \cdot$ AMPHIGNATHODONTINI $\cdot$ Hoc loco $\bullet$ T

OS: Amphignathodon $1882 \cdot \mathbf{O E}$

EN: (1) AMPHIGNATHODONTINAE 1882.bb.f002-01 • bF (2) AMPHIGNATHODONTINI 1882.bb.f002-02 • T

EF: HEMIPHRACTIDAE 1862.pa.f001

AмрніUMida Gray, 1825.gb.f007 • KY

SI: $021 \bullet \mathbf{C I}:$ h011 • ST: 0.10 .30

RL: INR

PA: $00 \bullet$ AMPHIUMIDAE $\bullet$ Gray $1825 . \mathrm{gb}: 216 \bullet \mathbf{F}$

$01 \cdot$ AMPHIUMOIDEA $•$ Fitzinger 1828.fa: $24 \cdot \mathbf{F}$

$02 \cdot$ AMPHIUMIDEA $\bullet$ Jourdan 1834.ja: $61 \cdot \mathbf{F}$

$03 \cdot$ AMPHIUMOIDAE $\bullet$ Jourdan 1834.ja: $61 \cdot \mathbf{F}$

04 AMPHIUMINA • Bonaparte 1838.bb: $393 \cdot \mathbf{b F}$

05 - AMPHIUMOIDES • Duméril ${ }^{+1}$ 1841.da: $52 \cdot \mathbf{F}$

$06 \cdot$ AMPHIUMIDES Duméril ${ }^{+1}$ 1841.da: table after page $53 \cdot \mathbf{F}$

07 • AMPHIUMININA • Gray 1850.ga: 54, 70 • UF

08 - AMPHIUMIDA • Jan 1857.ja: $55 \cdot \mathbf{F}$

09 A AMPHIUMOIDEAE $\bullet$ Stejneger 1907.sa: $3 \cdot \mathbf{p F}$

10 - AMPHIUMOIDEA • Dunn 1922.da: $426 \cdot \mathbf{p F}$

11 - AMPHIUMOIDAE $\bullet$ Hay 1929.ha: $843 \cdot \mathbf{p F}$

12 - AMPHIUMOIDAE $・$ Dubois $^{+1}$ 2012.da: $138 \cdot \mathbf{e F}$

13 - AMPHIUMEIDAE $\bullet$ Hoc loco $\bullet \mathbf{a F}$

OS: Amphiuma $1821 \cdot \mathbf{O E}$

EN: (1) AMPHIUMOIDEA 1825.gb.f007-10 • pF

(2) AMPHIUMOIDAE 1825.gb.f007-12 • eF

(3) AMPHIUMEIDAE 1825.gb.f007-13 • aF

(4) AMPHIUMIDAE 1825.gb.f007-00 • F

EF: AMPHIUMIDAE 1825.gb.f007

ANAXYRItoes nov., DOP.da.f028 • KY

SI: $469 \bullet$ CI: h362 • ST: 0.10 .30
RL: INR

PA: $00 \cdot$ ANAXYRITOES $\bullet$ HOC loco $\bullet$ iCn

OS: Anaxyrus $1845 \cdot$ PD

EN: ANAXYRITOES DOP.da.f028-00 • iCn

EF: BUFONIDAE 1825.gb.f004

Andinobatina nov., DOP.da.f004 • KY

SI: 445 • CI: h338 • ST: 0.10 .30

RL: INR

PA: $00 \cdot$ AndINOBATINA $\bullet$ Hoc loco $\bullet$ bT

$01 \cdot$ AndINOBATINIA $\cdot$ Hoc loco $\bullet$ iT

OS: Andinobates $2011 \cdot \mathbf{P D}$

EN: (1) ANDINOBATINA DOP.da.f004-00 • bT

(2) ANDINOBATINIA DOP.da.f004-01 • iT

EF: DENDROBATIDAE |1850.bb.f006|-1865.ca.f002 ANDRIADINA Bonaparte, 1839.bd.f001 +

SI: $056 \bullet$ CI: h029・ST: 0.10 .40

RL: INR

PA: 00 - ANDRIADINA • Bonaparte 1839.bd: [260] • bF

01 - ANDRIADIDAE • Bonaparte 1845.ba: $378 \cdot \mathbf{F}$

$02 \cdot$ ANDRIANTIDAE $\bullet$ Bonaparte 1850.bb: pl. • F

03 - ANDRIANTINA $\bullet$ Bonaparte 1850.bb: pl. • bF

OS: Andrias $1837+\cdot \mathbf{O E}$

EN: CRYPTOBRANCHIDAE 1826.fb.f003-04 • F

EF: CRYPTOBRANCHIDAE 1826.fb.f003

ANEIDINI Dubois, 2008.da.f002 • AN

SI: $375 \bullet$ CI: n092 • ST: 0.22 .50

RL: INR

PA: $00 \cdot$ ANEIDINI $\bullet$ Dubois 2008.da: $72 \cdot \mathbf{T}$

01 ANEIDITOI • Dubois 2008.da: $74 \cdot \mathbf{C n}$

OS: Aneides $1851 \cdot \mathbf{O E}$

EN: ANEIDINIA 2012.wa.f002-01・iT

EF: PLETHODONTIDAE 1850.ga.f001

ANEIDINI Vieites ${ }^{+3}$, 2011.va.f001 • AN

SI: $411 \cdot \mathbf{C I}:$ n099 • ST: 0.28 .50

RL: INR

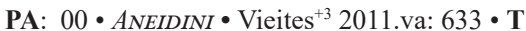

OS: Aneides $1851 \cdot$ OD

EN: ANEIDINIA 2012.wa.f002-01・iT

EF: PLETHODONTIDAE 1850.ga.f001

AneIdINI Wake, 2012.wa.f002 • KY

SI: $416 \bullet \mathbf{C I}:$ h310 • ST: 0.10 .30

RL: INR

PA: $00 \cdot$ ANEIDINI • Wake 2012.wa: 79 • T

$01 \cdot$ ANEIDINIA $\bullet$ Hoc loco $\bullet \mathbf{i T}$

OS: Aneides $1851 \cdot$ OD

EN: ANEIDINIA 2012.wa.f002-01・iT

EF: PLETHODONTIDAE 1850.ga.f001

ANEIDINI Dubois ${ }^{+1}$, 2012.da.f007 • JD

SI: $424 \bullet$ CI: h318・ST: 0.10 .40

RL: INR

PA: $00 \cdot$ ANEIDINI Dubois $^{+1}$ 2012.da: $117 \cdot \mathbf{T}$

OS: Aneides $1851 \cdot$ OD

EN: ANEIDINIA 2012.wa.f002-01 • iT

EF: PlethodontidaE 1850.ga.f001

ANGUINEA Leunis, 1844.la.f004 • AN

SI: $089 \bullet \mathbf{C I}:$ n035 • ST: 2.25 .50 
RL: INR

PA: $00 \cdot$ ANGUINEA $\bullet$ Leunis 1844.la: $129 \cdot \mathbf{F}$

OS: » 2 PN, including: Coecilia $1801 \equiv$ Caecilia $1758 \bullet \mathbf{P D}$

EN: (1) CAECILIOIDEA 1814.ra.f003-|1825.gb.f008|-19 • pF "'m!'

(5) CAECILIINA 1814.ra.f003-|1825.gb.f008|-26 • bT

EF: CAECILIIDAE 1814.ra.f003-|1825.gb.f008| ANGUINEA Van der Hoeven, 1855.va.f001 • AN

SI: $119 \bullet \mathbf{C I}: \mathrm{n} 038 \bullet \mathbf{S T}: 2.25 .50$

RL: INR

PA: 00 • ANGUINEA $•$ Van der Hoeven 1855.va: 462 • P

OS: » 3 PN, including: Siren $1766 \bullet \mathbf{P D}$

EN: SIRENIDAE 1825.gb.f005-00 • F

EF: SIRENIDAE 1825.gb.f005

ANGUSTICOELA Huene, 1948.ha.f001 • AN

SI: $245 \bullet$ CI: n066 • ST: 0.25 .50

RL: INR

PA: 00 • ANGUSTICOELA $\bullet$ Huene 1948.ha: $71 \cdot \mathbf{F}$

OS: OA: Leiopelma $1861 \cdot \mathbf{P D}$

EN: (1) LEIOPELMATIDAE 1869.ma.f007-|1942.ta.f001|-02 • F

(2) LEIOPELMATINAE 1869.ma.f007-|1942.ta.f001|-03 • bF

EF: LEIOPELMATIDAE 1869.ma.f007-|1942.ta.f001|

ANHYDROPHRYNINAE nov., DOP.da.f100 • KY

SI: $541 \cdot$ CI: h434 • ST: 0.10 .30

RL: INR

PA: 00 • ANHYDROPHRYNINAE $\bullet$ HOc loco $\bullet \mathbf{b F}$

OS: Anhydrophryne $1919 \cdot \mathbf{P D}$

EN: ANHYDROPHRYNINAE DOP.da.f100-00 • bF

EF: CACOSTERNIDAE 1931.na.f008

Annandini $\mathrm{Fei}^{+2}$, 2010.fa.f008 • PK

SI: $399 \bullet$ CI: h299 • ST: 0.10 .37

RL: $\leq$ QUASIPAINI 2010.fa.f007 • AI: hoc loco

PA: $00 \cdot$ ANNANDIINI $\bullet \mathrm{Fei}^{+2}$ 2010.fa: $17 \cdot \mathbf{T}$

$01 \cdot$ ANNANDIINA $\bullet$ Hoc loco $\bullet \mathbf{b T}$

OS: Annandia $1992 \cdot$ OD

EN: ANNANDIINA 2010.fa.f008-01 • bT

EF: DICROGLOSSIDAE 1987.da.f004

ANODONTHYLINA nov., DOP.da.f081 • KY

SI: $522 \cdot$ CI: h415 • ST: 0.10 .30

RL: INR

PA: $00 \cdot$ ANODONTHYLINA $\bullet$ Hoc loco $\bullet \mathbf{b T}$

OS: Anodonthyla $1892 \cdot \mathbf{P D}$

EN: ANODONTHYLINA DOP.da.f081-00 • bT

EF: MICROHYLIDAE |1843.fa.f012|-1931.na.f001

ANOMALOCOELA Huene, 1948.ha.f003 • AN

SI: $247 \cdot$ CI: n068 • ST: 0.25 .50

RL: INR

PA: 00 • ANOMALOCOELA $・$ Huene 1948.ha: $71 \cdot$ F

OS: OA: Pelobates $1830 \bullet \mathbf{P D}$

EN: (1) PELOBATOIDEA 1850.bb.f004-13 • pF

"'1")

(3) PeLOBatidae $1850 . \mathrm{bb} . \mathrm{f004-00} \cdot \mathbf{F}$

EF: PELOBATIDAE 1850.bb.f004

AnOMaloglossinae Grant ${ }^{+9}, 2006 . g b . f 002 \cdot \mathbf{K Y}$

SI: 371 • CI: h277 • ST: 0.10 .30

RL: INR
PA: $00 \cdot$ ANOMALOGLOSSINAE $\bullet \mathrm{Grant}^{+9}$ 2006.gb: $4 \cdot \mathbf{b F}$

OS: Anomaloglossus 2006 • OD

EN: ANOMALOGLOSSINAE 2006.gb.f002-00 • bF

EF: АRомоватIDAE 2006.gb.f001

ANOURA Latreille, 1825.la.f002 • AN

SI: $024 \cdot \mathbf{C I}: \mathrm{n} 011 \bullet \mathbf{S T}: 2.25 .50$

RL: INR

PA: $00 \cdot$ ANOURA $\bullet$ Latreille 1825.la: $104 \cdot \mathbf{F}$

01 ANURI $\bullet$ Eichwald 1831.eb: $165 \cdot \mathbf{F}$

02 • ANURA $\bullet$ Giebel 1846.ga: $306 \cdot \mathbf{F}$

OS: » 4 PN, including: Rana $1758 \cdot \mathbf{P D}$

EN: (1) RANOIDEA 1796.ba.f001-28 • pF

"'I!"

(12) RANITOES 1796.ba.f001-38 • iCn

EF: RANIDAE 1796.ba.f001

ANSONITTOES nov., DOP.da.f017 • KY

SI: $458 \bullet$ CI: h351 • ST: 0.10 .30

RL: INR

PA: $00 \cdot$ ANSONITOES $\bullet$ Hoc loco $\bullet$ iCn

$01 \cdot$ ANSONITUES $\bullet$ Hoc loco $\bullet \mathbf{h C n}$

OS: Ansonia $1870 \bullet \mathbf{P D}$

EN: (1) ANSONITTOES DOP.da.f017-00 • iCn

(2) ANSONIITUES DOP.da.f017-01 • hCn

EF: BUFONIDAE 1825.gb.f004

APNEUMIDAE Brookes, 1828.bc.f002 • AN

SI: $034 \cdot \mathbf{C I}: \mathrm{n} 015 \bullet \mathbf{S T}: 2.25 .50$

RL: INR

PA: $00 \cdot$ APNEUMIDAE $\bullet$ Brookes 1828.bc: $16 \cdot \mathbf{F}$

OS: Philhydrus 1828 CI $\approx$ Ambystoma $1838 \cdot \mathbf{O M}$

EN: AMBYSTOMATIDAE 1850.ga.f002-08 • F

EF: АмвYSTOMATIDAE 1850.ga.f002

APODA Oppel, 1811.oc.f001 • AN

SI: $145 \bullet$ CI: n046 • ST: 2.25 .50

RL: INR

PA: 00 • APODA • Oppel 1811.oc: $72 \cdot \mathbf{F}$

OS: Caecilia $1758 \cdot \mathbf{P D}$

EN: (1) CAECILIOIDEA 1814.ra.f003-|1825.gb.f008|-19 • pF "'m!'

(5) CAECILIINA 1814.ra.f003-|1825.gb.f008|-26 • bT

EF: CAECILIIDAE 1814.ra.f003-|1825.gb.f008|

AQUIPARES Blainville, 1835.ba.f002 • AN

SI: $046 \bullet \mathbf{C I}: \mathrm{n} 024 \bullet \mathbf{S T}: 2.25 .50$

RL: INR

PA: 00 • AQUIPARES $•$ Blainville 1835.ba: $277 \cdot \mathbf{F}$

OS: » 29 PN, including: Rana $1758 \cdot \mathbf{P D}$

EN: (1) RANOIDEA 1796.ba.f001-28 • pF

"')"।

(12) RANITOES 1796.ba.f001-38 • iCn

EF: RANIDAE 1796.ba.f001

Arcovomerinia nov., DOP.da.f086 • KY

SI: $527 \bullet$ CI: h420 • ST: 0.10 .30

RL: INR

PA: 00 • ARCOVOMERINIA $・ H O C$ loco $・$ iT

OS: Arcovomer $1954 \cdot \mathbf{P D}$

EN: ARCOVOMERINIA DOP.da.f086-00 • iT

EF: MICROHYLIDAE |1843.fa.f012|-1931.na.f001 
АвомоватIDAE Grant ${ }^{+9}$, 2006.gb.f001 • KY

SI: $370 \bullet$ CI: h276 • ST: 0.10 .30

RL: INR

PA: 00 • AROMOBATIDAE $\bullet \mathrm{Grant}^{+9}$ 2006.gb: 4 • F • PR $01 \cdot$ AROMOBATINAE $\bullet \mathrm{Grant}^{+9}$ 2006.gb: $4 \cdot \mathbf{b F}$

OS: Aromobates $1991 \cdot$ OD

EN: (1) АROMOBATIDAE 2006.gb.f001-00 • F

(2) AROMOBATINAE 2006.gb.f001-01 • bF

EF: АRомовАтIDAE 2006.gb.f001

Arthroleptina Mivart, 1869.ma.f011 • KY

SI: $171 \cdot \mathbf{C I}:$ h119 • ST: 0.10 .30

RL: INR

PA: 00 • ARTHROLEPTINA $・$ Mivart 1869.ma: $294 \cdot \mathbf{b F}$

01 ARTHROLEPTINAE • Noble 1931.na: $515 \cdot \mathbf{b F}$

02 - ARTHROLEPTIDAE • Laurent 1972.1a: $200 \cdot \mathbf{F}$

03 - ARTHROLEPTOIDAE $\bullet$ Dubois 1992.da: $309 \cdot \mathbf{e F}$

$04 \cdot$ ARTHROLEPTINI $\bullet$ Frost $^{+18}$ 2006.fa: $234 \cdot$ T

05 $\cdot$ ARTHROLEPTOIDEA $\bullet$ Hoc loco $\bullet \mathbf{p F}$

OS: Arthroleptis $1849 \cdot \mathbf{O E}$

EN: (1) ARTHROLEPTOIDEA 1869.ma.f011-05 • pF

(2) ARTHROLEPTIDAE 1869.ma.f011-02 • $\mathbf{F}$

(3) ARTHROLEPTINAE 1869.ma.f011-01 • bF

EF: ARTHROLEPTIDAE 1869.ma.f011

ASCAPHIDAE Fejérváry, 1923.fa.f001 • KY

SI: $206 \bullet$ CI: h1 $143 \cdot$ ST: 0.10 .30

RL: INR

PA: 00 • ASCAPHIDAE $\bullet$ Fejérváry 1923.fa: $178 \cdot \mathbf{F}$

$01 \cdot$ ASCAPHOIDEA $\bullet$ Lynch 1973.lb: $162 \cdot \mathbf{b F}$

OS: Ascaphus $1899 \cdot \mathbf{O E}$

EN: ASCAPHIDAE 1923.fa.f001-00 • $\mathbf{F}$

EF: ASCAPHIDAE 1923.fa.f001

ASSINIA nov., DOP.da.f076 • KY

SI: $517 \bullet$ CI: h4 $410 \bullet$ ST: 0.10 .30

RL: INR

PA: $00 \cdot$ ASSINIA $\bullet$ Hoc loco $\bullet$ iT

$01 \cdot$ ASSINOA $\bullet$ Hoc loco $\bullet \mathbf{h T}$

OS: Assa $1972 \cdot \mathbf{P D}$

EN: (1) ASSINIA DOP.da.f076-00 • iT

(2) ASSINOA DOP.da.f076-01 • hT

EF: MYOBATRACHIDAE 1850.sa.f001

ASTEROPHRYDIDAE Günther, 1858.gc.f006 • KY

SI: $134 \cdot \mathbf{C I}$ : h088 • ST: 0.10 .30

RL: INR

PA: 00 • ASTEROPHRYDIDAE $\bullet$ Günther 1858.gc: $346 \bullet \mathbf{F}$ $01 \cdot$ ASTEROPHRYDINA $\bullet$ Mivart 1869.ma: $294 \cdot \mathbf{b F}$ 02 $\bullet$ ASTOPHRYDIDAE $\bullet$ Hoffmann 1878.ha: $589 \cdot \mathbf{b F}$ 03 - ASTEROPHRYDIDAE $\bullet$ Hoffmann 1878.ha: $613 \cdot \mathbf{b F}$ 04 - ASTEROPHRYIDAE $\bullet$ Fejérváry 1923.fa: $181 \cdot \mathbf{F}$ 05 ASTEROPHRYINAE $\bullet$ Fejérváry 1923.fa: $181 \cdot \mathbf{b F}$ 06 ASTEROPHRYNAE • Fejérváry 1923.fa: $181 \cdot \mathbf{b F}$ $07 \cdot$ ASTEROPHRYNIDAE $\bullet$ Parker 1940.pa: $1 \cdot \mathbf{F}$ 08 • ASTEROPHRYNINAE $\bullet$ Tatarinov 1964.ta: $133 \cdot \mathbf{b F}$ 09 ASTEROPHRYNINI • Burton 1986.bb: $444 \cdot \mathbf{T}$

OS: Asterophrys $1838 \cdot \mathbf{O E}$

EN: (1) ASTEROPHRYINAE 1858.gc.f006-05 • bF (2) ASTEROPHRYINI 1858.gc.f006-09 • T
EF: MICROHYLIDAE |1843.fa.f012|-1931.na.f001

AStrobatraChINAE Vijayakumar ${ }^{+8}$, 2019.va.f001 • KY

SI: 592 • CI: h485 • ST: 0.10 .30

RL: INR

PA: $00 \cdot$ ASTROBATRACHINAE $・$ Vijayakumar $^{+8} 2019$.va: $1 \cdot$ bF

$01 \cdot$ ASTROBATRACHIDAE $\bullet$ Hoc loco $\bullet \mathbf{F}$

OS: Astrobatrachus 2019 • OD

EN: ASTROBATRACHIDAE 2019.va.f001-01 • F

EF: ASTROBATRACHIDAE 2019.va.f001

ASTRODACTYLIDAE Hogg 1838.ha.f002 • JI

SI: $049 \bullet$ CI: h023 • ST: 0.10 .52

RL: INR

PA: $00 \cdot$ ASTRODACTLIIDAE $\bullet$ Hogg 1838.ha: $152 \cdot \mathbf{F}$

01 ASTRODACTYLAE $\bullet$ Duméril 1863.da: $300 \bullet \mathbf{F}$

OS: Astrodactylus [1838] 1839 $\equiv$ Pipa $1768 \bullet \mathbf{O E}$

EN: (1) PIPIDAE 1825.gb.f003-|1826.fb.f002|-07 • F

(2) PIPINAE 1825.gb.f003-|1826.fb.f002|-13 • bF

EF: PIPIDAE 1825.gb.f003-|1826.fb.f002|

Astylosterninae Noble, 1927.na.f002 • KY

SI: $215 \bullet$ CI: h150 • ST: 0.10 .30

RL: INR

PA: $00 \bullet$ ASTYLOSTERNINAE $\bullet$ Noble 1927.na: $110 \bullet \mathbf{b F}$

01 ASTYLOSTERNIDAE $\bullet$ Bauer 1986.ba: ii $\bullet \mathbf{F}$

02 - ASTYLOSTERNOIDEA $\bullet$ Bauer 1986.ba: iv $\cdot \mathbf{p F}$

03 - ASTYLOSTERNINI $・$ Frost $^{+18}$ 2006.fa: $234 \cdot \mathbf{T}$

OS: Astylosternus $1898 \cdot \mathbf{O E}$

EN: (1) ASTYLOSTERNINAE 1927.na.f002-00 • bF

(2) ASTYLOSTERNINI 1927.na.f002-03 • T

EF: ARTHROLEPTIDAE 1869.ma.f011

Atelognathini nov., DOP.da.f048 • KY

SI: $489 \bullet$ CI: h382 • ST: 0.10 .30

RL: INR

PA: $00 \cdot$ ATELOGNATHINI $\bullet$ Hoc loco $\bullet \mathbf{T}$

OS: Atelognathus $1978 \cdot \mathbf{P D}$

EN: ATELOGNATHINI DOP.da.f048-00 • T

EF: CYCLORAMPHIDAE 1850.bb.f003-|1852.ba.f001|

ATELOPODA Fitzinger, 1843.fa.f005 • KY

SI: $070 \bullet$ CI: h038 • ST: 0.10 .30

RL: INR

PA: $00 \cdot A T E L O P O D A \cdot$ Fitzinger 1843.fa: $32 \cdot \mathbf{F}$

01 ATELOPODES $\bullet$ Fitzinger 1861.fa: $414 \cdot \mathbf{F}$

02 ATELOPODIDAE $\bullet$ Parker 1934.pa: $8 \cdot \mathbf{F}$

03 - ATELOPODINAE $\bullet$ Davis 1935.da: $91 \cdot \mathbf{b F}$

$04 \cdot$ ATELOPODIDADE $\bullet$ Lutz 1954.1a: $172 \cdot \mathbf{F}$

05 • ATELOPIDIDAE $\bullet$ Gallardo 1961.ga: $205 \cdot \mathbf{F}$

06 ATELOPIDAE $\bullet$ Hellmich 1963.ha: $659 \cdot \mathbf{F}$

$07 \cdot$ ATELOPODINA $\bullet$ Hoc loco $\bullet \mathbf{b T}$

OS: Atelopus $1841 \cdot \mathbf{O E}$

EN: ATELOPODINA 1843.fa.f005.07 • bT

EF: BUFONIDAE 1825.gb.f004

ATYMPANOPHRYNI nov., DOP.da.f001 • KY

SI: $442 \cdot$ CI: h335 • ST: 0.10 .30

RL: INR

PA: 00 • ATYMPANOPHRYNI $\bullet$ Hoc loco $\bullet \mathbf{T}$

OS: Atympanophrys $1983 \cdot \mathbf{P D}$

EN: ATYMPANOPHRYNI DOP.da.f001-00 • T 
EF: MEGOPHRYIDAE 1850.bb.f008-|1931.na.f003|

AUdACIElates nov., DOP.da.f043 • KY

SI: $484 \cdot$ CI: h377 • ST: 0.10 .30

RL: INR

PA: $00 \cdot$ AUdACIELLITES $\bullet$ Hoc loco $・$ Cn

OS: Audaciella nov. $・$ PD

EN: AUDACIELLITES DOP.da.f043-00 • Cn

EF: CENTROLENIDAE 1951.ta.f001

AVIturinaE Gubin, 1991.ga.F001 +

SI: $346 \bullet$ CI: h253・ST: 0.10 .40

RL: INR

PA: 00 • AVITURINAE • Gubin 1991.ga: $97 \cdot \mathbf{b F}$

OS: Aviturus $1991+\cdot \mathbf{O E}$

EN: CRYPTOBRANCHIDAE 1826.fb.f003-04 • F

EF: CRYPTOBRANCHIDAE 1826.fb.f003

BARBAROPHRYNITUES nov., DOP.da.f018 • KY

SI: $459 \bullet \mathbf{C I}:$ h352 • ST: 0.10 .30

RL: INR

PA: $00 \cdot$ BARBAROPHRYNITUES $\bullet$ HOc loco $\bullet \mathbf{h C n}$

OS: Barbarophryne 2013 • PD

EN: BARBAROPHRYNITUES DOP.da.f018-00 • hCn

EF: BUFONIDAE 1825.gb.f004

BARYCHOLINOA nov., DOP.da.f006 • KY

SI: $447 \cdot \mathbf{C I}:$ h3 $340 \cdot \mathbf{S T}: 0.10 .30$

RL: INR

PA: $00 \cdot$ BARYCHOLINOA $\cdot$ Hoc loco $\bullet \mathbf{h T}$

OS: Barycholos $1969 \cdot \mathbf{P D}$

EN: BARYCHOLINOA DOP.da.f006-00 • hT

EF: BRACHYCEPHALIDAE 1858.gc.f002

BARYGENYINI Burton, 1986.bb.f001 • JD

SI: $317 \cdot \mathbf{C I}:$ h225 • ST: 0.10 .40

RL: INR

PA: 00 • BARYGENYINI • Burton 1986.bb: $444 \cdot$ T

OS: Barygenys $1936 \cdot \mathbf{O E}$

EN: (1) ASTEROPHRYINAE 1858.gc.f006-05 • bF

(2) ASTEROPHRYINI 1858.gc.f006-09 • T

EF: MICROHYLIDAE|1843.fa.f012|-1931.na.f001

BATRACHI Batsch, 1788.ba.f001 • AN

SI: $001 \cdot \mathbf{C I}: \mathrm{n} 001 \bullet \mathbf{S T}: 2.25 .50$

RL: INR

PA: 00 - BATRACHI • Batsch 1788.ba: 437 • F

$01 \cdot$ BATRACHIA $\cdot$ Schinz 1833.sa: $213 \cdot \mathbf{F}$

$02 \cdot$ BATRACHOIDEA $~$ Van der Hoeven 1833.va: iii, $308 \cdot \mathbf{F}$

$03 \cdot$ BATRACHII $・$ Van der Hoeven 1855.va: 468 • F

OS: » 4 PN, including: Rana $1758 \cdot \mathbf{P D}$

EN: (1) RANOIDEA 1796.ba.f001-28 • pF

"')"')

(12) RANITOES 1796.ba.f001-38 • iCn

EF: RANIDAE 1796.ba.f001

BATRACHOPHRYNIDAE Cope, 1875.ca.f001 • JD

SI: $176 \bullet \mathbf{C I}:$ h122 • ST: 0.10 .40

RL: INR

PA: 00 • BATRACHOPHRYNIDAE • Cope 1875.ca: 9 • F

OS: Batrachophrynus $1873 \approx$ Telmatobius $1834 \cdot \mathbf{O E}$

EN: (1) TELMATOBIOIDAE 1843.fa.f006-04 • eF

(")।")
(3) TELMATOBIIDAE $1843 . \mathrm{fa} . \mathrm{f006}-01 \cdot \mathbf{F}$

EF: TELMATOBIIDAE 1843.fa.f006

BATRACHOSAUROIDIDAE Auffenberg, 1958.aa.f001 +

SI: $255 \bullet$ CI: h1 $180 \bullet$ ST: 0.10 .40

RL: INR

PA: 00 • BATRACHOSAUROIDIDAE • Auffenberg 1958.aa: $172 \cdot \mathbf{F}$

$01 \cdot$ BATRACHOSAURIDAE $\bullet$ Vorobyeva $^{+1}$ 1996.va: $69 \cdot \mathbf{F}$

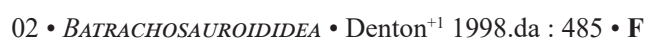

03 - BATRACHOSAURIDIDAE • Wilson 2006.wb: 61; Sullivan ${ }^{+1}$ 2015.sb: $110 \cdot \mathbf{F}$

$04 \cdot$ BATRACHOSAUROIDAE $・$ Böhme $^{+2}$ 2011.bb: online supplementary [5] $\cdot \mathbf{F}$

OS: Batrachosauroides $1943+\cdot \mathbf{O E}$

EN: HYLAEOBATRACHIDAE 1889.1a.f001-00 † $\mathbf{F}$

EF: HYLAEOBATRACHIDAE 1889.1a.f001 †

BATRACHOSEPSINI Dubois, 2008.da.f001 • AN

SI: $374 \cdot \mathbf{C I}:$ n091 - ST: 0.22 .50

RL: INR

PA: 00 - BATRACHOSEPSINI • Dubois 2008.da: 71 • T

01 BATRACHOSEPSITA • Dubois 2008.da: $73 \cdot \mathbf{i T}$

OS: Batrachoseps $1839 \cdot \mathbf{O E}$

EN: BATRACHOSEPINA 2012.wa.f001-01 • bT

EF: PLETHODONTIDAE 1850.ga.f001

BATRACHOSEPSINI Vieites ${ }^{+3}$, 2011.va.f003 • AN

SI: $413 \bullet \mathbf{C I}: \mathrm{n} 101 \bullet \mathbf{S T}: 0.28 .50$

RL: INR

PA: $00 \cdot$ BATRACHOSEPSINI • Vieites ${ }^{+3}$ 2011.va: 633 • T

OS: Batrachoseps $1839 \cdot$ OD

EN: BATRACHOSEPINA 2012.wa.f001-01・bT

EF: PLETHODONTIDAE 1850.ga.f001

BATRACHOSEPSINI Jockusch ${ }^{+3}$ 2012.ja.f001 • AN

SI: $414 \cdot \mathbf{C I}: \mathrm{n} 102 \bullet \mathbf{S T}: 0.28 .50$

RL: INR

PA: 00 - BATRACHOSEPINI $\bullet$ Jockusch $^{+3}$ 2012.ja: $1 \cdot$ T

OS: Batrachoseps $1839 \cdot$ OD

EN: BATRACHOSEPINA 2012.wa.f001-01・bT

EF: PlETHODONTIDAE 1850.ga.f001

BatraCHOSEPINI Wake, 2012.wa.f001 • KY

SI: 415 • CI: h309 • ST: 0.10 .30

RL: INR

PA: 00 • BATRACHOSEPINI • Wake 2012.wa: 76 • T

$01 \cdot$ BATRACHOSEPINA $\bullet H O c$ loco $\bullet \mathbf{b T}$

OS: Batrachoseps $1839 \cdot$ OD

EN: BATRACHOSEPINA 2012.wa.f001-01・bT

EF: PlETHODONTIDAE 1850.ga.f001

BATRACHOSEPINA Dubois ${ }^{+1}$, 2012.da.f005 • JD

SI: $422 \bullet$ CI: h316・ST: 0.10 .40

RL: INR

PA: 00 • BATRACHOSEPINA • Dubois ${ }^{+1}$ 2012.da: $115 \cdot$ bT

OS: Batrachoseps $1839 \cdot$ OD

EN: BATRACHOSEPINA 2012.wa.f001-01 • bT

EF: PlethodontidaE 1850.ga.f001

BATRACHYLINAE Gallardo, 1965.ga.f002 • KY

SI: $270 \bullet$ CI: h1 $188 \bullet$ ST: 0.10 .30

RL: INR

PA: 00 • BATRACHYLINAE • Gallardo 1965.ga: $83 \cdot \mathbf{b F}$ 
01 BATRACHYLINI $\bullet$ Lynch 1971.la: $123 \bullet \mathbf{T}$

02 BATRACHYLIDAE $\bullet$ Pyron $^{+1}$ 2011.pa: 546 • F

OS: Batrachyla $1843 \cdot \mathbf{O E}$

EN: (1) BATRACHYLINAE 1965.ga.f002-00 • bF

(2) BATRACHYLINI 1965.ga.f002-01 • T

EF: CYCLORAMPHIDAE 1850.bb.f003-|1852.ba.f001| BATRACINIA Rafinesque, 1815.ra.f003 • AN

SI: $008 \cdot \mathbf{C I}:$ n004 • ST: 2.25 .50

RL: INR

PA: $00 \cdot$ BATRACINIA $・$ Rafinesque 1815.ra: $78 \cdot \mathbf{b F}$

OS: Batracinus 1815 AN $\equiv$ Rana $1758 \cdot \mathbf{O E}$

EN: (1) RANOIDEA 1796.ba.f001-28・pF ")"!)

(12) RANITOES 1796.ba.f001-38 • iCn

EF: RANIDAE 1796.ba.f001

BATRACOPHIDES Bonaparte, 1831.ba.f001 • AN

SI: $035 \bullet \mathbf{C I}: \mathrm{n} 016 \bullet \mathbf{S T}: 2.25 .50$

RL: $\leftrightarrow$ CAECILIIDAE 1814.ra.f003-|1825.gb.f008|-10

PA: c0 • BATRACOPHIDES • Bonaparte 1831.ba: 66 • F • EEA: Hoc loco

i1 • BATROCHOPHIDES • Bonaparte 1831.ba: 66 • F

OS: Caecilia $1758 \cdot \mathbf{O M}$

EN: (1) CAECILIOIDEA 1814.ra.f003-|1825.gb.f008|-19 • pF (")"।

(5) CAECILIINA 1814.ra.f003-|1825.gb.f008|-26 • bT

EF: CAECILIIDAE 1814.ra.f003-|1825.gb.f008|

BEDDOMIXALITIES nov., DOP.da.f121 • KY

SI: $562 \cdot$ CI: h455 • ST: 0.10 .30

RL: INR

PA: $00 \bullet$ BEDDOMIXALITIES $\bullet$ Hoc loco $\bullet \mathbf{b C n}$

OS: Beddomixalus $2013 \cdot \mathbf{P D}$

EN: BEDDOMIXALITIES DOP.da.f121-00 • bCn

EF: RHACOPHORIDAE |1858.gc.f012|-1932.ha.f001

BLAIRITUES nov., DOP.da.f019 • KY

SI: 460 • CI: h353 • ST: 0.10 .30

RL: INR

PA: $00 \cdot$ BLAIRITUES $\bullet$ Hoc loco $\bullet \mathbf{h C n}$

OS: Blaira nov. $・$ PD

EN: BLAIRITUES DOP.da.f019-00 • hCn

EF: BUFONIDAE 1825.gb.f004

BLOMMERSIINIA nov., DOP.da.f1 12 • KY

SI: $553 \bullet$ CI: h446・ST: 0.10 .30

RL: INR

PA: 00 • BLOMMERSIINIA $・ H O$ Hoco $\bullet \mathbf{i T}$

OS: Blommersia $1992 \cdot \mathbf{P D}$

EN: BLOMMERSIINIA DOP.da.f112-00 • iT

EF: RHACOPHORIDAE |1858.gc.f012|-1932.ha.f001

BоEHMANTINOA nov., DOP.da.f1 14 • KY

SI: $555 \bullet$ CI: h448 • ST: 0.10 .30

RL: INR

PA: $00 \cdot$ BoEhMANTINOA $\cdot$ Hoc loco $\bullet \mathbf{h T}$

OS: Boehmantis $2006 \cdot \mathbf{P D}$

EN: BOEHMANTINOA DOP.da.f114-00 • hT

EF: RHACOPHORIDAE |1858.gc.f012|-1932.ha.f001

BOKERMANNOHYLINIA nOV., DOP.da.f050 • KY

SI: $491 \cdot \mathbf{C I}:$ h384 • ST: 0.10 .30
RL: INR

PA: $00 \cdot$ BOKERMANNOHYLINIA $・ H O c$ loco $\bullet$ iT

OS: Bokermannohyla 2005 • PD

EN: BOKERMANNOHYLINIA DOP.da.f050-00 • iT

EF: HYLIDAE 1815.ra.f002-|1825.gb.f001|

BolitoglossidaE Hallowell, 1856.ha.f002 • KY

SI: $122 \cdot \mathbf{C I}:$ h081 • ST: 0.10 .37

RL: $\leq$ HEMIDACTYLIDAE 1856.ha.f003 • AI: Dubois 2005.da: 5

PA: $00 \cdot$ BOLITOGLOSSIDAE $\bullet$ Hallowell 1856.ha: $11 \cdot \mathbf{b F}$

01 BOLITOGLOSSIDAE $\bullet$ Hoffmann 1878.ha: $585 \cdot \mathbf{F}$

02 BOLITOGLOSSINAE $\bullet$ Regal 1966.ra: $405 \cdot \mathbf{b F}$

03 - BoLITOGLOSSINI • Wake 1966.wa: $1 \cdot$ T

$04 \cdot$ BolitoGLOSSINA $\cdot$ Hoc loco $\bullet$ bT

$05 \cdot$ BOLITOGLOSSINIA $\bullet$ Hoc loco $\bullet$ iT

$06 \cdot$ BOLITOGLOSSINOA $\cdot$ Hoc loco $\bullet \mathbf{h T}$

OS: Bolitoglossa $1854 \cdot \mathbf{O E}$

EN: (1) BoLITOGLOSSINI 1850.ha.f002-03 • T

(2) BoLitoglossina 1850.ha.f002-04 • bT

(3) BoLitoglossinia 1850.ha.f002-05 • iT

(4) BolitogLOSSINOA 1850.ha.f002-06 • hT

EF: PlethodontidaE 1850.ga.f0014

BombINATORINA Gray, 1825.gb.f002 • KY

SI: $016 \bullet \mathbf{C I}:$ h007 • ST: 0.10 .30

RL: INR

PA: 00 • BOMBINATORINA • Gray 1825.gb: $214 \cdot \mathbf{U F}$

01 - BOMBINATOROIDEA • Fitzinger 1826.fb: $37 \cdot \mathbf{F}$

02 • BOMBINATORIDAE • Gray 1831.gb: $38 \cdot \mathbf{F}$

03 - BOMBINATORES • Goldfuss 1832.ga: $332 \cdot \mathbf{Z t}$

$04 \cdot$ BOMBINATORES $・$ Tschudi 1838.ta: $26 \cdot \mathbf{F}$

05 BOMBINATORES $・$ Leunis 1844.1a: $128 \cdot \mathbf{U F}$

$06 \cdot$ BOMBINATORINA • Bonaparte 1850.bb: pl. • bF

$07 \cdot$ BOMBINATORINA • Günther 1858.gc: $344 \cdot$ Sc

08 • BOMBINATORES • Leunis 1860.1a: $337 \cdot \mathbf{T}$

$09 \cdot$ BOMBINATOROIDES $\cdot$ Hoffmann 1878.ha: 581 • F

10 BOMBINATORINA • Hoffmann 1878.ha: 613. • F

11 BOMBINATORIDAE • Hoffmann 1878.ha: 613. • bF

12 • BOMBINATORIDES • Lataste 1878.1b: 3. • F

13 - BOMBINATORIDA • Bayer 1885.ba: $18 \cdot \mathbf{F}$

14 • BOMBINATORINAE • Dubois 1983.da: $271 \cdot \mathbf{b F}$

$15 \cdot$ BOMBINATOROIDIA Dubois 2005.da: $7 \cdot \mathbf{e F}$

16 BOMBINATOROIDEA • Dubois 2005.da: $7 \cdot \mathbf{p F}$

OS: Bombinator $1820 \approx$ Bombina $1816 \cdot \mathbf{O E}$

EN: (1) BOMBINATOROIDEA 1825.gb.f002-16 • pF

(2) BOMBINATORIDAE 1825.gb.f002-02 • F

EF: BOMBINATORIDAE 1825.gb.f002

BombininaE Fejérváry, 1921.fb.f002 • JD

SI: $205 \bullet \mathbf{C I}:$ h142 • ST: 0.10 .40

RL: INR

PA: $00 \cdot$ BOMBININAE • Fejérváry 1921.fb: $24 \cdot \mathbf{b F}$

01 BOMBINIDAE $\bullet$ Tatarinov 1964.ta: $8,128 \cdot \mathbf{F}$

02 BомвIDAE • Aubekerova-Tleuberdina 1977.ab: $76 \cdot \mathbf{F}$

03 - BOMBINOIDEA • Špinar 1983.sa: $53 \cdot \mathbf{p F}$

OS: Bombina $1816 \cdot \mathbf{O E}$

EN: (1) BOMBINATOROIDEA 1825.gb.f002-16 • pF

(2) BOMBINATORIDAE 1825.gb.f002-02 • F

EF: BOMBINATORIDAE 1825.gb.f002 
BомвітAтоRоIDEA Fitzinger, 1832.fa.f002 • JD

SI: 038 • CI: h021 • ST: 2.10 .40

RL: INR

PA: 00 • BоMBITATOROIDEA $\bullet$ Fitzinger 1832.fa: $329 \cdot \mathbf{F}$ $01 \cdot$ BOMBITATORES $\bullet$ Fitzinger 1843.fa: $32 \cdot \mathbf{F}$

OS: Bombitator $1830 \approx$ Bombina $1816 \cdot$ OE

EN: (1) BOMBINATOROIDEA 1825.gb.f002-16 • pF (2) BOMBINATORIDAE 1825.gb.f002-02 • F

EF: BOMBINATORIDAE 1825.gb.f002

BoophinaE Vences $^{+1}$, 2001.va.f001 • KY

SI: $357 \cdot$ CI: h264 • ST: 0.10 .30

RL: INR

PA: 00 • BOOPHINAE $・$ Vences $^{+1}$ 2001.va: $85 \cdot$ bF

$01 \cdot$ BOOPHINI $\bullet$ Dubois 2005.da: $16 \cdot \mathbf{T}$

02 $\bullet$ BOOPHINAE $\bullet \mathrm{Glaw}^{+1}$ 2006.ga: $238 \cdot \mathbf{b F}$

OS: Boophis $1838 \cdot$ OD

EN: BOOPHINI 2001.va.f001-01 • T

EF: RHACOPHORIDAE |1858.gc.f012|-1932.ha.f001

BRACHYCEPHALINA Günther, 1858.gc.f002 • KY

SI: $130 \bullet$ CI: h084 • ST: 0.10 .30

RL: INR

PA: 00 • BRACHYCEPHALINA • Günther 1858.gc: $344 \cdot \mathbf{S c}$ $01 \cdot B R A C H Y C E P H A L I D A E \cdot$ Günther 1858.gc: $346 \cdot \mathbf{F}$ 02 BRACHYCEPHALINA $\bullet$ Hoffmann 1878.ha: $613 \cdot \mathbf{F}$ 03 • BRACHYCEPHALIDAE $\bullet$ Hoffmann 1878.ha: 613 • bF 04 BRACHYCEPHALINAE $\bullet$ Noble 1931.na: $507 \cdot \mathbf{b F}$ 05 $\bullet$ BRACHICEPHALIDAE $\bullet$ Smith 1939.sb: $37 \cdot \mathbf{F}$ 06 BRACHYCEPHALOIDEA Padial $^{+2}$ 2014.pa: $49 \cdot \mathbf{p F}$

OS: Brachycephalus $1826 \cdot \mathbf{O E}$

EN: (1) BRACHYCEPHALIDAE 1858.gc.f002-01 • F

(2) BRACHYCEPHALINAE 1858.gc.f002-04 • bF

EF: BRACHYCEPHALIDAE $1858 . \mathrm{gc} . \mathrm{f002}$

BRACHYMERIDAE Günther, 1858.gc.f011 • JG

SI: $139 \cdot$ CI: h092 • ST: 0.10 .53

RL: INR

PA: 00 • BRACHYMERIDAE $\bullet$ Günther 1858.gc: $346 \cdot \mathbf{F}$ 01 BRACHYMERIDAE $\bullet$ Hoffmann 1878.ha: $614 \cdot \mathbf{b F}$

OS: Brachymerus $1847 \mathbf{J H} \equiv$ Phrynomantis $1867 \cdot \mathbf{O E}$

EN: PHRYNOMERIDAE 1931.na.f013-01 • F

EF: PHRYNOMERIDAE 1931.na.f013

BRACHYTARSOPHRYINI nov., DOP.da.f002 • KY

SI: $443 \cdot \mathbf{C I}:$ h336 • ST: 0.10 .30

RL: INR

PA: 00 • BRACHYTARSOPHRYINI $\bullet$ Hoc loco $\bullet \mathbf{T}$

OS: Brachytarsophrys $1983 \cdot \mathbf{P D}$

EN: BRACHYTARSOPHRYINI DOP.da.f002-00 • T

EF: MEGOPHRYIDAE 1850.bb.f008-|1931.na.f003|

BRADYBATINA Bonaparte, 1850.bb.f013 • JD

SI: $105 \bullet$ CI: h067 • ST: 0.10 .40

RL: INR

PA: 00 • BRADYBATINA $\bullet$ Bonaparte 1850.bb: pl. $\bullet \mathbf{b F}$

OS: Bradybates $1838 \approx$ Pleurodeles $1830 \bullet \mathbf{O E}$

EN: (1) PLEURODELINAE 1838.ta.f005-08 • bF "'m!"

(3) PLeURodelina 1838.ta.f005-10 • b T

EF: SALAMANDRIDAE 1820.ga.f002
BRADYTRITONITOES nOV., DOP.da.f137 • KY

SI: $578 \bullet$ CI: h471 • ST: 0.10 .30

RL: INR

PA: 00 • BRADYTRITONITOES $\bullet$ HOc loco $\bullet \mathbf{i C n}$

OS: Bradytriton $1983 \cdot$ PD

EN: BRADYTRITONITOES DOP.da.f137-00 • iCn

EF: PLETHODONTIDAE 1850.ga.f001

BRANCHIATA Gravenhorst, 1843.ga.f001 • AN

SI: $084 \bullet \mathbf{C I}: \mathrm{n} 031 \cdot \mathbf{S T}: 2.25 .50$

RL: INR

PA: 00 • BRANCHIATA Gravenhorst 1843.ga: $393 \cdot \mathbf{F}$

OS: » 4 PN, including: Siren $1766 \bullet$ PD

EN: SIRENIDAE 1825.gb.f005-00 • F

EF: SIRENIDAE 1825.gb.f005

BRASILOTYPHLILI Lescure ${ }^{+2}$, 1986.lb.f008 • JD

SI: $327 \cdot$ CI: h235 • ST: 0.10 .40

RL: INR

PA: 00 • BRASILOTYPHLILI $\bullet$ Lescure $^{+2}$ 1986.lb: $166 \cdot \mathbf{i T}$

OS: Brasilotyphlus $1968 \cdot \mathbf{O E}$

EN: SIPHONOPINI 1850.bb.f017-08 • T

EF: CAECILIIDAE 1814.ra.f003-|1825.gb.f008|

BREVICIPITINA Bonaparte, 1850.bb.f012 • KY

SI: $104 \cdot$ CI: h066 • ST: 0.10 .30

RL: INR

PA: $00 \cdot$ BREVICIPITINA $\bullet$ Bonaparte 1850.bb: pl. $\bullet \mathbf{b F}$

01 BREVICIPITIDAE $\bullet$ Cope 1867.ca: $191 \cdot \mathbf{F}$

02 BREVICIPITINAE $\bullet$ Van Kampen 1923.va: $\mathbf{x} \cdot \mathbf{b F}$

03 - BREVICIPETIDAE $\bullet$ Romer 1933.ra: $437 \cdot \mathbf{F}$

$04 \cdot$ BREVICEPITIDAE $\bullet$ Miranda Ribeiro 1937.ma: $56 \cdot \mathbf{F}$

05 BREVICIPINAE $\bullet$ Lynch 1971.la: $203 \cdot \mathbf{b F}$

06 BREVICIPEDIDAE $\bullet$ Ardila-Robayo 1979.aa: $456 \cdot \mathbf{F}$

$07 \cdot$ BREVICEPINAE $\bullet$ Bogart $^{+1}$ 1981.ba: 59 • bF

08 BREVICEPTIDAE $\bullet$ Du Preez ${ }^{+1}$ 2009.pa: 4 • F

09 • BREVICIPITOIDAE $・$ Zhang $^{+5}$ 2013.za: $1904 \cdot$ UF

$10 \cdot$ BREVICIPITOIDEA $\bullet$ Hoc loco $\bullet \mathbf{p F}$

OS: Breviceps $1820 \bullet \mathbf{O E}$

EN: (1) BREVICIPITOIDEA 1850.bb.f012-10 • pF

(2) BREVICIPITIDAE 1850.bb.f012-01 • F

(3) BREVICIPITINAE $1850 . \mathrm{bb} . \mathrm{f012}-02 \cdot \mathbf{b F}$

EF: BREVICIPITIDAE 1850.bb.f012

BRYOPHRYNINOA nov., DOP.da.f007 • KY

SI: $448 \bullet$ CI: h341 • ST: 0.10 .30

RL: INR

PA: 00 • BRYOPHRYNINOA $\bullet$ Hoc loco $\bullet \mathbf{h T}$

OS: Bryophryne $2008 \cdot \mathbf{P D}$

EN: BRYOPHRYNINOA DOP.da.f007-00 • hT

EF: BRACHYCEPHALIDAE 1858.gc.f002

BUERGERIINAE Channing, 1989.ca.f002 • KY

SI: $343 \cdot$ CI: h250 • ST: 0.10 .30

RL: INR

PA: 00 • BUERGERIINAE $\bullet$ Channing 1989.ca: $116 \bullet \mathbf{b F}$

$01 \cdot$ BUERGERIINI $\bullet$ Dubois 1992.da: $335 \bullet \mathbf{T}$

OS: Buergeria $1838 \cdot \mathbf{O E}$

EN: BUERGERIINI 1989.ca.f002-01・T

EF: RHACOPHORIDAE |1858.gc.f012|-1932.ha.f001 
BUFAVIDAE Fejérváry, 1921.fa.f002 + • JD

SI: 203 • CI: h140 • ST: 0.10 .40

RL: INR

PA: 00 • BUFAVIDAE $\bullet$ Fejérváry 1921.fa: $30 \cdot \mathbf{F}$

OS: Bufavus $1885 \div \approx$ Bufo $1764 \cdot$ OE

EN: (1) BUFONOIDEA 1825.gb.f004-20 • pF ")"»"

(10) BUFONITOES 1825.gb.f004-33 • iCn

EF: BUFONIDAE 1825.gb.f004

BUFONIFORMES Duméril ${ }^{+1}$, 1841.da.f003 • AN

SI: $062 \bullet$ CI: n029・ST: 2.27 .50

RL: INR

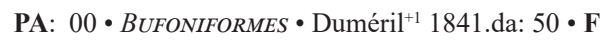
01 BUFONIFORMES $\bullet$ Desmarest 1856.da: $4 \cdot \mathbf{F}$

OS: Bufo $1764 \cdot$ OE

EN: (1) BUFONOIDEA 1825.gb.f004-20 • pF "'m!" (10) BUFONITOES 1825.gb.f004-33 • iCn

EF: BUFONIDAE 1825.gb.f004

BUFONINA Gray, 1825.gb.f004 • KY

SI: $018 \bullet \mathbf{C I}:$ h009 • ST: 0.10 .30

RL: INR

PA: 00 • BUFONINA • Gray 1825.gb: $214 \cdot \mathbf{U C}$ $01 \cdot$ BUFONOIDEA $\bullet$ Fitzinger 1826.fb: $37 \cdot \mathbf{F}$ 02 BUFONIDEA $\bullet$ Fitzinger 1827.fa: $264 \cdot \mathbf{F}$ 03 - BUFONES • Fitzinger 1832.fa: 328;

Wiegmann $^{+1} 1832$ wa: $202 \cdot \mathbf{F}$ $04 \cdot$ BUFONOIDEA $•$ Fitzinger 1832.fa: $328 \cdot \mathbf{G r}$ 05 BUFONES $\bullet$ Goldfuss 1832 .ga: $330 \cdot \mathbf{Z t}$ 06 BUFONINA $\bullet$ Bonaparte 1838.ba: [195] • bF 07 BUFONINI • Bonaparte 1838.ba: [196] • UF $08 \cdot$ BUFONIDAE $\bullet$ Bell 1839.ba: $105 \cdot \mathbf{F}$ 09 BUFOIDAE $\bullet$ Swainson 1839.sa: $88 \cdot \mathbf{F}$ $10 \cdot$ BUFONIA $\bullet$ Gravenhorst 1843.ga: $393 \cdot \mathbf{L}$ $11 \cdot$ BUFONES $\bullet$ Leunis 1844.la: $128 \cdot \mathbf{U F}$ $12 \cdot$ BUFONIA $\bullet$ Gravenhorst 1845.ga: $43 \cdot \mathbf{F}$ 13 - BUFONINA $\cdot$ Stannius 1856.sa: $5 \cdot \mathbf{F}$ $14 \cdot$ BUFONINA $\bullet$ Günther 1858.gc: $344 \cdot$ Sc $15 \cdot$ BUFONES $\bullet$ Leunis 1860.la: $337 \cdot \mathbf{T}$ $16 \cdot$ BUFONIDES $\bullet$ Bruch 1862.ba: $221 \cdot \mathbf{F}$ $17 \cdot$ BUFONIDA $\bullet$ Haeckel 1866.ha: cxxxii • F 18 • BUFONOIDES $\bullet$ Hoffmann 1878.ha: $581 \cdot \mathbf{F}$ 19 BUFONIDAE $\bullet$ Hoffmann 1878.ha: $581 \cdot \mathbf{b F}$ $20 \cdot$ BUFONOIDEA • Gill 1884.gb: 621 • pF $21 \cdot$ BUFONIDAE $\bullet$ Boulenger 1893.ba: $39 \cdot \mathbf{F}$ $22 \cdot$ BUFONIDI $\bullet$ Acloque 1900.aa: $489 \cdot \mathbf{F}$ 23 - BUFONINAE • Fejérváry 1917.fa: $152 \cdot$ bF $24 \cdot$ BUFONOIDEA • Bolkay 1919.ba: $356 \cdot \mathbf{G a}$ $25 \cdot$ BUFONINAE • Fejérváry 1921.fb: $26 \cdot \mathbf{b F}$ $26 \cdot$ BUFONIDEA $\bullet$ Lynch 1973.lb: $165 \cdot \mathbf{p F}$

$27 \cdot$ BUFONINI $\bullet$ Hoc loco $\bullet \mathbf{T}$

$28 \cdot$ BUFONINA $\bullet$ Hoc loco $\bullet$ bT

$29 \cdot$ BUFONINIA $\bullet$ Hoc loco $\bullet$ iT

$30 \cdot$ BUFONINOA $\bullet$ Hoc loco $\bullet \mathbf{h T}$

$31 \cdot$ BUFONITES $\cdot$ Hoc loco $\cdot \mathbf{C n}$

$32 \cdot$ BUFONITIES $\bullet$ Hoc loco $\bullet$ bCn
$33 \cdot$ BUFONITOES $\bullet$ Hoc loco $\bullet$ iCn

OS: Bufo $1764 \cdot$ OE

EN: (1) BUFONOIDEA 1825.gb.f004-20 • pF

(2) BUFONIDAE 1825.gb.f004-08 • F

(3) BUFONINAE 1825.gb.f004-23 • bF

(4) BUFONINI 1825.gb.f004-27 • T

(5) BUFONINA 1825.gb.f004-28 • bT

(6) BUFONINIA 1825.gb.f004-29 • iT

(7) BUFONINOA 1825.gb.f004-30 • hT

(8) BUFONITES 1825.gb.f004-31 • Cn

(9) BUFONITIES 1825.gb.f004-32 • bCn

(10) BUFONITOES 1825.gb.f004-33 • iCn

EF: BUFONIDAE 1825.gb.f004

BUfotitoes nov., DOP.da.f022 • KY

SI: $463 \cdot \mathbf{C I}:$ h356 • ST: 0.10 .30

RL: INR

PA: $00 \cdot$ BUFOTITOES $\bullet$ Hoc loco $\bullet$ iCn

OS: Bufotes $1815 \cdot \mathbf{P D}$

EN: BUFOTITOES DOP.da.f022-00 • iCn

EF: BUFONIDAE 1825.gb.f004

CACOPINAE Noble, 1931.na.f011 • KY

SI: $226 \bullet$ CI: h161 • ST: 0.10 .30

RL: INR

PA: $00 \cdot$ CACOPINAE $\bullet$ Noble 1931.na: $532 \cdot$ bF 01 • CACOPINIA $\bullet$ Hoc loco $\bullet$ iT

OS: Cacopus $1864 \equiv$ Uperodon $1841 \cdot \mathbf{O E}$

EN: CACOPINIA 1931.na.f011-01 • iT

EF: MICROHYLIDAE |1843.fa.f012|-1931.na.f001

CacosterninaE Noble, 1931.na.f008 • KY

SI: $223 \bullet$ CI: h158 • ST: 0.10 .30

RL: INR

PA: $00 \bullet$ CACOSTERNINAE $\bullet$ Noble 1931.na: $527 \bullet$ bF

01 • CACOSTERNIDAE $\bullet$ Hoc loco $\bullet \mathbf{F}$

$02 \cdot$ CACOSTERNINI $\bullet$ Hoc loco $\bullet \mathbf{T}$

$03 \cdot$ CACOSTERNINA $\bullet$ Hoc loco $\bullet \mathbf{b T}$

OS: Cacosternum $1887 \cdot \mathbf{O E}$

EN: (1) CACOSTERNIDAE 1931.na.f008-01 • F

(2) CACOSTERNINAE 1931.na.f008-00 • bF

(3) CACOSTERNINI 1931.na.f008-02 • $\mathbf{T}$

(4) CACOSTERNINA 1931.na.f008-03 • bT

EF: CACOSTERNIDAE 1931.na.f008

CACotina Mivart, 1869.ma.f006 • JD

SI: $166 \bullet$ CI: h114 • ST: 0.10 .41

RL: < ALSODINA 1869.ma.f005 - AI: Lynch 1971.la: 9

PA: $00 \cdot$ CACOTINA $\bullet$ Mivart 1869.ma: $290 \bullet \mathbf{b F}$

OS: Cacotus $1869 \approx$ Alsodes $1843 \cdot \mathbf{O E}$

EN: ALSODIDAE 1869.ma.f005-02 • F

EF: ALSODIDAE 1869.ma.f005

CAECILIADAE Gray, 1825.gb.f008 • CK

SI: $022 \cdot$ CI: h012 • ST: 0.10 .36

RL: > CECILINIA 1814.ra.f003 • PP: Opinion 1830 (Anonymous 1996.aa: 68)

> CAECILIINI Kolbe, 1880.ka.f001 • PP: Opinion 1830

(Anonymous 1996.aa: 68)

PA: $00 \cdot C_{A E C I L I A D A E} \cdot$ Gray 1825.gb: $217 \cdot \mathbf{F}$

01 CAECILIOIDES Fitzinger 1826.fc: $348 \cdot \mathbf{F}$ 
02 CAECILIARIA • Hemprich 1829.ha: xix, $374 \cdot \mathbf{F}$

$03 \cdot$ CAECILIDAE $\bullet$ Bonaparte 1831.ba: $66 \cdot \mathbf{F}$

$04 \cdot$ CAECILIOIDEI $\bullet$ Eichwald 1831.eb: $177 \cdot \mathbf{F}$

05 C CAECILIADEA $・$ Jourdan 1834.ja: $235 \cdot \mathbf{F}$

$06 \cdot$ CAECILINA $\bullet$ Bonaparte 1839.bf: $16 \cdot \mathbf{b F}$

07 CAECILOIDES $\cdot$ Duméril $^{+1}$ 1841.da: table after page $53 \cdot \mathbf{F}$

08 - CAECILINIA • Rafinesque 1845.ra: 226. - F

09 • CAECILIOIDEA • Gistel 1848.gb: $102 \cdot \mathbf{F}$

10 CAECILIOIDES $\bullet$ Gray 1850.ga: $56 \cdot \mathbf{U F}$

11 - CAECILIIDAE $\bullet$ Bonaparte 1850.bb: pl. • F

12 - CAECILIINA $\bullet$ Bonaparte 1850.bb: pl. • bF

13 - CAECILIADE • Bonaparte 1852.ba: 480 - F

14 - CAECILIAE • Van der Hoeven 1855.va: 460 • F

15 - CAECILOIDAE $・$ Keferstein 1867.ka: $361 \cdot \mathbf{F}$

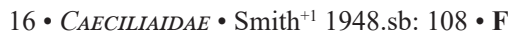

17 • CAECILIINAE • Taylor 1969.ta: $303 \cdot \mathbf{b F}$

18 - CAECILIOIDES $・$ Lescure $^{+2}$ 1986.1b: $167 \cdot \mathbf{h F}$

19 - CAECILIOIDEA $・$ Lescure $^{+2}$ 1986.1b: $167 \cdot \mathbf{p F}$

$20 \cdot$ CAECILIOIDAE Lescure $^{+2}$ 1986.1b: $168 \cdot \mathbf{e F}$

$21 \cdot$ CAECILIILAE Lescure $^{+2}$ 1986.1b: $168 \cdot \mathbf{i F}$

22 - CAECILIAOIDEA Lescure $^{+1}$ 1988.1a: $20 \cdot \mathbf{p F}$

23 - CAECILIAINAE $\bullet$ Hedges $^{+2}$ 1993.ha: $72 \cdot \mathbf{b F}$

24 - CAECILIOIDIA $・$ Dubois 2005.da: $21 \cdot \mathbf{e F}$

$25 \cdot$ CAECILIINI $\bullet$ Hoc loco $\bullet \mathbf{T}$

$26 \cdot$ CAECILIINA $\cdot$ Hoc loco $\bullet \mathbf{b T}$

OS: Caecilia $1758 \cdot \mathbf{O E}$

EN: (1) CAECILIOIDEA 1814.ra.f003-|1825.gb.f008|-19 • pF

(2) CAECILIIDAE 1814.ra.f003-|1825.gb.f008|-11 • F

(3) CAECILIINAE 1814.ra.f003-|1825.gb.f008|-17 • bF

(4) CAECILIINI 1814.ra.f003-|1825.gb.f008|-25 • T

(5) CAECILIINA 1814.ra.f003-|1825.gb.f008|-26・bT

EF: CAECILIIDAE 1814.ra.f003-|1825.gb.f008|

CAECILIINI Kolbe, 1880.ea.f001 • ZA-CI

SI: $184 \cdot \mathbf{C I}: \mathrm{zh} 03 \cdot \mathbf{S T}: 0.10 .99$

RL: < CAECILIADAE 1825.gb.f008 • PP: Opinion 1830

(Anonymous 1996.aa: 68)

PA: $00 \cdot$ CAECILIINI $・$ Kolbe 1880.ka: $183 \cdot$ T

OS: Caecilius $1837 \cdot \mathbf{O E}$

EN: $\bullet$

EF: •

CALAMITAE Wiegmann in Wiegmann ${ }^{+1}$, 1832.wa.f002 • AN

SI: $040 \bullet \mathbf{C I}: \mathrm{n} 018 \bullet \mathbf{S T}: 2.25 .50$

RL: INR

PA: $00 \cdot$ CALAMITAE $\bullet$ Wiegmann $^{+1}$ 1832.wa: $200 \cdot \mathbf{U F}$ 01 CALAMITINA $\cdot$ Gravenhorst 1843.ga: $393 \cdot \mathbf{L}$

$02 \cdot$ CALAMITAE $^{*} \mathrm{Wiegmann}^{+1}$ 1843.wa: $200 \cdot \mathbf{F}$

03 CALAMITINA $\bullet$ Gravenhorst 1845.ga: $43 \cdot \mathbf{F}$

OS: Hyla $1768 \bullet \mathbf{O M}$

EN: (1) HYLOIDEA 1815.ra.f002-|1825.gb.f001|-20 • pF ")"')

(8) HYLITES 1815.ra.f002-|1825.gb.f001|-26 • Cn

EF: HYLIDAE 1815.ra.f002-|1825.gb.f001|

CALAMITAE Leunis, 1844.1a.f002 • JG

SI: $087 \bullet$ CI: h052 • ST: 1.10 .53

RL: INR

PA: $00 \cdot$ CALAMITAE $\bullet$ Leunis 1844.1a: $128 \cdot \mathbf{U F}$
01 • CALAMITAE $\bullet$ Leunis 1860.1a: $336 \bullet \mathbf{T}$

OS: Calamites $1830 \mathbf{J H} \approx$ Ranoidea $1838 \bullet \mathbf{O E}$

EN: PELODRYADINAE 1859.ga.f001-01・bF

EF: PHYLLOMEDUSIDAE 1858.gc.f009

CalluellinaE $\mathrm{Fei}^{+2}$ in $\mathrm{Fei}^{+4}$, 2005.fb.f001 • JD

SI: 392 • CI: h292 • ST: 0.10 .40

RL: INR

PA: $00 \cdot$ CALLUELLIINAE $-\mathrm{Fei}^{+2}$ in $\mathrm{Fei}^{+4}$ 2005.fb: 4, 177, 271 • bF

OS: Calluella $1872 \approx$ Glyphoglossus $1869 \cdot \mathbf{O E}$

EN: (1) MICROHYLIDAE |1843.fa.f012|-1931.na.f001-01 • F "'m"')

(4) MICROHYLINA |1843.fa.f012|-1931.na.f001-08 • bT

EF: MICROHYLIDAE |1843.fa.f012|-1931.na.f001

CallulininaE nov., DOP.da.f098 • KY

SI: $539 \bullet \mathbf{C I}:$ h432 • ST: 0.10 .30

RL: INR

PA: $00 \cdot$ CALLULININAE $\bullet$ Hoc loco $\bullet \mathbf{b F}$

OS: Callulina $1911 \cdot \mathbf{P D}$

EN: CALLULININAE DOP.da.f098-00 • bF

EF: BREVICIPITIDAE 1850.bb.f012

CALLULOPINI Dubois, 1988.da.f001 • JD

SI: 339 • CI: h247 • ST: 0.10 .40

RL: INR

PA: $00 \cdot$ CALLULOPINI $・$ Dubois 1988.da: $3 \cdot$ T

OS: Callulops $1888 \approx$ Asterophrys $1838 \cdot$ OD

EN: (1) ASTEROPHRYINAE 1858.gc.f006-05 • bF

(2) ASTEROPHRYINI 1858.gc.f006-09・ T

EF: MICROHYLIDAE|1843.fa.f012|-1931.na.f001

Calostethina Mivart, 1869.ma.f009 • JI

SI: $169 \cdot \mathbf{C I}:$ h117 • ST: 0.10 .52

RL: INR

PA: $00 \cdot$ CALOSTETHINA • Mivart 1869.ma: $293 \cdot \mathbf{b F}$

01 CALOSTETHIDAE $\bullet$ Cope 1875.ca: $7 \cdot \mathbf{F}$

OS: Calostethus $1869 \equiv$ Colostethus $1866 \bullet \mathbf{O E}$

EN: (1) COLOSTETHINAE 1867.ca.f001-01 • bF

(2) Colostethini 1867.ca.f001-02 • T

EF: DENDROBATIDAE |1850.bb.f006|-1865.ca.f002

CALYPTOCEPHALELLINAE Reig, 1960.ra.f001 • KY

SI: $263 \bullet \mathbf{C I}:$ h1 $184 \cdot \mathbf{S T}: 0.10 .30$

RL: INR

PA: $00 \cdot$ CALYPTOCEPHALELLINAE • Reig 1960.ra: $113 \cdot \mathbf{b F}$

01 - CALYPTOCEPHALELLINI - Lynch 1978.1a: $42 \cdot \mathbf{T}$

02 CALYPTOCEPHALELLIDAE • Bossuyt ${ }^{+1}$ 2009.ba: $359 \cdot \mathbf{F}$

OS: Calyptocephalella $1928 \cdot \mathbf{O E}$

EN: CALYPTOCEPHALELLIDAE 1960.ra.f001-02 • F

EF: CALYPTOCEPHALELLIDAE 1960.ra.f001

CALYPTOCEPHALINAE Cei, 1962.ca.f001 • JG

SI: $266 \bullet$ CI: h1 $186 \bullet$ ST: 0.10 .53

RL: INR

PA: 00 • CALYPTOCEPHALINAE • Cei 1962.ca: $104 \cdot$ bF

OS: Calyptocephalus $1841 \mathbf{J H} \equiv$ Calyptocephalella $1928 \cdot \mathbf{O E}$

EN: CALYPTOCEPHALELLIDAE 1960.ra.f001-02 • F

EF: CALYPTOCEPHALELLIDAE 1960.ra.f001

CAPENSIBUfONITOES nov., DOP.da.f029 • KY

SI: 470 • CI: h363 • ST: 0.10 .30

RL: INR 
PA: $00 \cdot$ CAPENSIBUFONITOES $\bullet$ Hoc loco $\bullet$ iCn

OS: Capensibufo $1980 \cdot$ PD

EN: CAPENSIBUFONITOES DOP.da.f029-00 • iCn

EF: BUFONIDAE 1825.gb.f004

CAUDATA Oppel, 1811.oc.f003 • AN

SI: $088 \bullet \mathbf{C I}: \mathrm{n} 034 \cdot \mathbf{S T}: 2.25 .50$

RL: INR

PA: $00 \cdot$ CAUDATA $・$ Oppel 1811.oc: $22 \cdot \mathbf{F}$

OS: » 4 PN, including: Salamandra $1768 \approx$ Salamandra $1764 \cdot$ PD

EN: (1) SALAMANDROIDEA 1820.ga.f002-21・pF

"')"'

(4) SALAMANDRINI 1820.ga.f002-28 • T

EF: SALAMANDRIDAE 1820.ga.f002

CECILINIA Rafinesque, 1814.ra.f003 • CG

SI: $005 \bullet$ CI: h003 • ST: 0.10 .61

RL: < CAECILIADAE 1825.gb.f008 • PP: Opinion 1830

(Anonymous 1996.aa: 68)

PA: 00 • CECILINIA • Rafinesque 1814.ra: $104 \cdot \mathbf{F}$

01 CECILIDAE $\bullet$ Bonaparte 1839.be: $272 \cdot \mathbf{F}$

$02 \cdot$ CECILOIDES $・$ Duméril 1839.da: $581 \cdot \mathbf{F}$

$03 \cdot$ CECILIODES $\bullet$ Gray 1850.ga: $56 \cdot \mathbf{U F}$

$04 \cdot$ CECILIINA $\cdot$ Bonaparte 1852.ba: $480 \cdot \mathbf{b F}$

$05 \cdot$ CECILIES $\bullet$ Lataste $1878.1 \mathrm{~b}: 2 \cdot \mathbf{F}$

$06 \cdot$ CECILIIDAE $\bullet$ Dubois 1985.da: $71 \cdot \mathbf{F}$

OS: Cecilia $1814 \mathbf{C I} \equiv$ Caecilia $1758 \cdot \mathbf{O E}$

EN: (1) CAECILIOIDEA 1814.ra.f003-|1825.gb.f008|-18 • pF "')"'

(5) CAECILIINA 1814.ra.f003-|1825.gb.f008|-25 • bT

EF: CAECILIIDAE 1814.ra.f003-|1825.gb.f008|

CENTROLENIDAE Taylor, 1951.ta.f001 • KY

SI: $250 \bullet$ CI: h176 • ST: 0.10 .30

RL: INR

PA: $00 \bullet$ CENTROLENIDAE • Taylor 1951.ta: $36 \bullet \mathbf{F}$

01 - CENTROLENINAE • Barrio 1968.ba: 165; Lutz 1968.la: $22 \cdot \mathbf{b F}$

$02 \cdot$ CENTROLENOIDEA $・ H O c$ loco $\bullet \mathbf{p F}$

$03 \cdot$ CENTROLENINI $\cdot$ Hoc loco $\bullet \mathbf{T}$

OS: Centrolene $1872 \cdot \mathbf{O E}$

EN: (1) CENTROLENOIDEA 1951.ta.f001-02・pF

(2) CENTROLENIDAE 1951.ta.f001-00 • F

(3) CENTROLENINAE 1951.ta.f001-01 • bF

(4) CENTROLENINI 1951.ta.f001-03 • T

EF: CENTROLENIDAE 1951.ta.f001

CEPHALOPHRYNAE Tschudi, 1845.ta.f002 • AN

SI: $091 \bullet \mathbf{C I}: \mathrm{n} 036 \bullet \mathbf{S T}: 2.25 .50$

RL: INR

PA: $00 \cdot$ CEPHALOPHRYNAE • Tschudi 1845.ta: $169 \cdot \mathbf{F}$

OS: Trachycara $1845 \approx$ Rhinella $1826 \cdot \mathbf{O M}$

EN: (1) PHRYNISCITIES 1858.gc.f005-04 • bCn: F.11.01.04

(2) PHRYNISCITOES 1858.gc.f005-05 • iCn: F.12.02.05

EF: BUFONIDAE 1825.gb.f004

CERATOBATRACHIDAE Boulenger, 1884.ba.f001 • KY

SI: $187 \cdot \mathbf{C I}:$ h129 • ST: 0.10 .30

RL: INR

PA: 00 - CERATOBATRACHIDAE • Boulenger 1884.ba: $212 \cdot \mathbf{F}$

01 CERATOBATRACHINAE • Gadow 1901.ga: xi, $237 \cdot \mathbf{b F}$

02 • CERATOBRACHIDAE • Kuhn 1961.ka: 22 • F
03 - CERATOBRACHINI • Dubois 1981.da: 231 • T

$04 \cdot$ CERATOBATRACHEIDAE $\bullet$ Hoc loco $\bullet \mathbf{a F}$

OS: Ceratobatrachus $1884 \approx$ Cornufer $1838 \cdot$ OD

EN: (1) CERATOBATRACHEIDAE 1884.ba.f001-04 • aF

(2) CERATOBATRACHIDAE 1884.ba.f001-00 • F

(3) CERATOBATRACHINAE 1884.ba.f001-01 • bF

EF: CERATOBATRACHIDAE 1884.ba.f001

CERATOPHREIDAE Bonaparte, 1850.bb.f007 • JI

SI: $099 \bullet \mathbf{C I}:$ h061 - ST: 0.10 .52

RL: $\leftarrow$ CERATOPHRYDES 1838.ta.f002

PA: 00 - CERATOPHREIDAE • Bonaparte 1850.bb: pl. • F

01 - CERATOPHREIDINA • Bonaparte 1850.bb: pl. • bF

$02 \cdot$ CERATOPHRIIDAE • Waite 1927.wa: $328 \cdot \mathbf{F}$

OS: Ceratophris $1829 \equiv$ Ceratophrys $1824 \cdot \mathbf{O E}$

EN: (1) CERATOPHRYOIDEA 1838.ta.f002-14 • pF "')"'।

(4) CERATOPHRYINAE 1838.ta.f002-06 • bF

EF: CERATOPHRYIDAE 1838.ta.f002

CERATOPHRYDES Tschudi 1838.ta.f002 • KY

SI: $052 \bullet \mathbf{C I}:$ h026 • ST: 0.10 .30

RL: $\leq$ CYSTIGNATHI 1838.ta.f001 - AI: Cope 1866.ca: 88

PA: $00 \cdot$ CERATOPHRYDES • Tschudi 1838.ta: $26 \cdot \mathbf{F}$

$01 \cdot$ CERATOPHRYDES • Bronn 1849.ba: $684 \cdot \mathbf{U F}$

$02 \cdot$ CERATOPHRYDIDAE $\bullet$ Cope 1863.cb: $50 \cdot \mathbf{F}$

03 - CERATOPHRYDES • Cope 1866.ca: $89 \cdot \mathbf{G r}$

$04 \cdot$ CERATOPHRYDIDEAS • Miranda-Ribeiro 1926.ma: 153 • F

05 • CERATOPHRYIDAE • Parker 1933.pa: $12 \cdot \mathbf{F}$

06 • CERATOPHRYINAE • Parker 1935.pa: $511 \bullet \mathbf{b F}$

07 - CERATOPHYDES • Parker 1940.pa: 1 - UC

08 • CERATOPHYINAE • Parker 1940.pa: $2 \cdot \mathbf{b F}$

09 • CERATOPHYDAE $\bullet$ Lutz 1954.la: $156 \cdot \mathbf{F}$

10 CERATOPHRYNINAE • Reig 1960.ra: $117 \cdot \mathbf{b F}$

11 - CERATOPHRYNIDAE $\bullet \mathrm{Reig}^{+1}$ 1963.ra: $125 \cdot \mathbf{F}$

12 - CERATOPHRYNAE - Cei 1970.ca: $183 \cdot \mathbf{b F}$

13 - CERATOPHRYNINI $・$ Laurent $^{+1}$ 1981.la: 7 • T

$14 \cdot$ CERATOPHRYOIDEa $\cdot$ Hoc loco $\cdot \mathbf{p F}$

$15 \cdot$ CERATOPHRYOIDAE $\cdot$ Hoc loco $\bullet \mathbf{e F}$

OS: Ceratophrys $1824 \cdot \mathbf{O E}$

EN: (1) CERATOPHRYOIDEA 1838.ta.f002-14 • pF

(2) CERATOPHRYOIDAE 1838.ta.f002.15 • eF

(3) CERATOPHRYIDAE 1838.ta.f002-05 • F

(4) CERATOPHRYINAE 1838.ta.f002-06 • bF

EF: CERATOPHRYIDAE 1838.ta.f002

CEUTHOMANTIDaE Heinicke ${ }^{+5}$, 2009.ha.f001 • KY

SI: $391 \bullet \mathbf{C I}:$ h291・ST: 0.10 .30

RL: INR

PA: 00 • CEUTHOMANTIDAE $\bullet$ Heinicke $^{+5}$ 2009.ha: $1 \cdot \mathbf{F}$

01 C CEUTHOMANTINAE $\bullet$ Padial $^{+2}$ 2014.pa: $599 \bullet \mathbf{b F}$

OS: Ceuthomantis 2009 • OD

EN: CEUTHOMANTIDAE 2009.ha.f001-00 • F

EF: CEUTHOMANTIDAE 2009.ha.f001

Chaparanina nov., DOP.da.f103・ Ky

SI: $544 \cdot$ CI: h437 • ST: 0.10 .30

RL: INR

PA: $00 \cdot$ CHAPARANINA $-H o c$ loco $\bullet \mathbf{b T}$

01 • CHAPARANINIA $\cdot$ Hoc loco $・ \mathbf{i T}$ 
OS: Chaparana $1939 \cdot \mathbf{P D}$

EN: (1) CHAPARANINA DOP.da.f103-00 • bT

(2) CHAPARANINIA DOP.da.f103-01 • iT

EF: DicRoglossidae 1987.da.f004

Chaperininae Peloso $^{+10}$, 2016.pa.f002 • KY

SI: 436 • CI: h329 • ST: 0.10 .30

RL: INR

PA: $00 \cdot$ CHAPERININAE Peloso $^{+10}$ 2016.pa: $135 \cdot \mathbf{b F}$ $01 \cdot$ CHAPERININA $\bullet$ Hoc loco $\bullet \mathbf{b T}$

OS: Chaperina $1892 \cdot$ OD

EN: CHAPERININA 2016.pa.f002-01 • bT

EF: MICROHYLIDAE |1843.fa.f012|-1931.na.f001

Charadrahylinoa nov., DOP.da.f054 - KY

SI: $495 \bullet$ CI: h388 • ST: 0.10 .30

RL: INR

PA: 00 • CHARADRAHYLINOA $\bullet$ Hoc loco $\bullet \mathbf{h}$ T

OS: Charadrahyla $2005 \cdot$ PD

EN: CHARADRAHYLINOA DOP.da.f054-00 • hT

EF: HYLIDAE 1815.ra.f002-|1825.gb.f001|

ChIASMOCLEINI nov., DOP.da.f083 • KY

SI: $524 \bullet$ CI: h417 • ST: 0.10 .30

RL: INR

PA: $00 \cdot$ CHIASMOCLEINI $・ H o c$ loco $・ \mathbf{T}$

OS: Chiasmocleis $1904 \cdot \mathbf{P D}$

EN: CHIASMOCLEINI DOP.da.f083-00 • T

EF: MICROHYLIDAE |1843.fa.f012|-1931.na.f001

CHIKILIDAE Kamei ${ }^{+9}$, 2012.ka.f001 • KY

SI: 427 • CI: h321 • ST: 0.10 .30

RL: INR

PA: $00 \bullet$ CHIKILIDAE $\bullet \mathrm{Kamei}^{+9}$ 2012.ka: 1 • F

$01 \cdot$ CHIKILINI $\bullet$ Hoc loco $\bullet \mathbf{F}$

OS: Chikila 2012 - OD

EN: CHIKILINI 2012.ka.f001-01 • T

EF: CAECILIIDAE 1814.ra.f003-|1825.gb.f008|

ChIMERELLINOA nov., DOP.da.f041 • KY

SI: 482 •CI: h375 • ST: 0.10 .30

RL: INR

PA: $00 \cdot$ CHIMERELLINOA $・ H o c$ loco $\bullet \mathrm{hT}$

OS: Chimerella $2009 \cdot \mathbf{P D}$

EN: CHIMERELLINOA DOP.da.f041-00 • hT

EF: CENTROLENIDAE 1951.ta.f001

Chioglossini Dubois ${ }^{+1}$, 2009.db.f004 • KY

SI: $388 \bullet$ CI: h2 $288 \bullet$ ST: 0.10 .30

RL: INR

PA: 00 • CHIOGLOSSINI $\bullet$ Dubois $^{+1}$ 2009.db: $60 \cdot$ T

OS: Chioglossa $1864 \cdot$ OD

EN: CHIOGLOSSINI 2009.db.f004-00 • T

EF: SALAMANDRIDAE 1820.ga.f002

ChIRIXALITES nov., DOP.da.f123 • KY

SI: $564 \bullet$ CI: h457 • ST: 0.10 .30

RL: INR

PA: $00 \cdot$ ChIRIXALITES $\bullet$ Hoc loco $\bullet \mathbf{C n}$

OS: Chirixalus $1893 \cdot \mathbf{P D}$

EN: CHIRIXALITES DOP.da.f123-00 • Cn

EF: RHACOPHORIDAE |1858.gc.f012|-1932.ha.f001
Chiroleptina Mivart, 1869.ma.f010 • JG-JD

SI: $170 \bullet$ CI: h118 • ST: 0.10 .53

RL: INR

PA: 00 • CHIRoleptina • Mivart 1869.ma: $294 \cdot \mathbf{b F}$

OS: Chiroleptes $1859 \mathbf{J H} \approx$ Ranoidea $1838 \cdot \mathbf{O E}$

EN: PELODRYADINAE 1859.ga.f001-01 • bF

EF: PHYLLOMEDUSIDAE 1858.gc.f009

Clinotarsini $\mathrm{Fei}^{+2}$, 2010.fa.f011 • JD

SI: 402 • CI: h302 • ST: 0.10 .42

RL: $\leq$ MERISTOGENYINAE 2010.fa.f003 • PR: hoc loco

PA: 00 $\bullet$ CLINOTARSINI $\bullet \mathrm{Fei}^{+2}$ 2010.fa: $18 \cdot \mathbf{T}$

OS: Clinotarsus $1869 \cdot$ OD

EN: MERISTOGENYINI 2010.fa.f003-02 • T

EF: RANIDAE 1796.ba.f001

CoChranellini Guayasamin ${ }^{+5}$, 2009.ga.f001 • KY

SI: $389 \bullet$ CI: h289 • ST: 0.10 .30

RL: INR

PA: $00 \cdot$ COCHRANELLINI $^{*}$ Guayasamin $^{+5} 2009$.ga: $3 \cdot \mathbf{T}$

$01 \cdot$ CochRANELLINA $\bullet$ Hoc loco $\bullet \mathbf{b T}$

$02 \cdot$ COCHRANELLINIA $\bullet$ Hoc loco $\bullet$ iT

OS: Cochranella $1951 \cdot$ OD

EN: (1) COCHRANELLINI 2009.ga.f001-00・ T

(2) Cochranellina 2009.ga.f001-01 • bT

(3) CoChraNELLINIA 2009.ga.f001-02 • iT

EF: CENTROLENIDAE 1951.ta.f001

CoECILIOIDEA Fitzinger, 1826.fb.f001 • JI

SI: $027 \bullet$ CI: h013 • ST: 0.10 .52

RL: INR

PA: $00 \cdot$ COECILIOIDEA $\bullet$ Fitzinger 1826.fb: $35 \cdot \mathbf{F}$

$01 \cdot$ COECILIADAE $\bullet$ Brookes 1828.bc: $16 \cdot \mathbf{F}$

$02 \cdot$ COECILIAE $\bullet$ Goldfuss 1832.ga: $326 \cdot \mathbf{F}$

03 COECILINA • Bonaparte 1838.bb: $392 \cdot \mathbf{b F}$

$04 \cdot$ COECILIEA $\bullet$ Tschudi 1845.tb: $80 \cdot \mathbf{F}$

05 $\bullet$ COECILOIDEI $\bullet$ Troschel 1848.ta: $661 \cdot \mathbf{F}$

$06 \cdot$ COECILIIDAE $\bullet$ Gray 1850.ga: 6, 56, $57 \cdot \mathbf{F}$

$07 \cdot$ COECILIOIDEI $\bullet$ Gray 1850 ga: $56 \cdot \mathbf{U F}$

$08 \cdot$ COECILIINA $\bullet$ Gray 1850.ga: $56 \cdot \mathbf{U F}$

09 COECILOIDES $\bullet$ Bruch 1862.ba: $221 \cdot \mathbf{F}$

10 $\operatorname{COECILODES} \bullet$ Hoffmann 1878.ha: $590 \cdot \mathbf{F}$

$11 \cdot$ COECILIIDA $\bullet$ Knauer 1878.ka: $92 \cdot \mathbf{F}$

OS: Coecilia $1801 \equiv$ Caecilia $1758 \cdot \mathbf{O E}$

EN: (1) CAECILIOIDEA 1814.ra.f003-|1825.gb.f008|-19 • pF "1"1!"

(5) CAECILIINA 1814.ra.f003-|1825.gb.f008|-26 • bT

EF: CAECILIIDAE 1814.ra.f003-|1825.gb.f008|

Coelonotae Miranda-Ribeiro, 1926.ma.f003 • JG

SI: $210 \bullet \mathbf{C I}:$ h1 $146 \bullet \mathbf{S T}: 0.10 .53$

RL: INR

PA: $00 \cdot$ COELONOTAE $・$ Miranda-Ribeiro 1926.ma: $64 \cdot \mathbf{U F}$

OS: Coelonotus $1920 \mathbf{J H} \approx$ Fritziana $1937 \cdot \mathbf{O E}$

EN: FRITZIANINAE DOP.da.f013-00 • bF

EF: HEMIPHRACTIDAE 1862.pa.f001

ColodaCtYlI Tschudi, 1845.ta.f001 • AP

SI: $090 \bullet$ CI: h053 • ST: 0.10 .46

RL: INR

PA: $00 \cdot$ COLODACTYLI $\bullet$ Tschudi 1845.ta: $167 \cdot \mathbf{F}$ 
01 • COLODACTYLIDAE • Dubois 1987.da: 11 • F

OS: Colodactylus 1845 AP・ OE

EN: LAEVOGYRINIA INCERTAE SEDIS

EF: LAEVOGYRINIA INCERTAE SEDIS

ColostethidaE Cope, 1867.ca.f001 • KY

SI: $158 \bullet \mathbf{C I}:$ h106 - ST: 0.10 .30

RL: INR

PA: 00 • COLOSTETHIDAE • Cope 1867.ca: 191 • F 01 $\operatorname{COLOSTETHiNAE} \bullet$ Bauer 1987.bb: $5 \cdot \mathbf{b F}$ $02 \cdot \operatorname{ColOSTETHINI} \cdot$ Hoc loco $\bullet \mathbf{T}$

OS: Colostethus $1866 \cdot \mathbf{O E}$

EN: (1) CoLOSTEthinaE 1867.ca.f001-01 • bF (2) Colostethini 1867.ca.f001-02 • T

EF: DENDROBATIDAE |1850.bb.f006|-1865.ca.f002

ConRaUINI Dubois, 1992.da.f001・ KY

SI: $348 \bullet$ CI: h255 • ST: 0.10 .30

RL: INR

PA: 00 • CONRAUINI • Dubois 1992.da: $314 \cdot \mathbf{T}$

$01 \cdot$ CONRAUINAE $・$ Dubois 2005.da: $16 \cdot \mathbf{b F}$

02 - CONRAUIDAE • Pyron $^{+1}$ 2011.pa: 547 • F

$03 \cdot$ CONRAUOIDAE $\bullet$ Hoc loco $\bullet \mathbf{e F}$

OS: Conraua $1908 \cdot$ OD

EN: (1) CONRAUOIDAE 1992.da.f001-03 • eF

(2) ConRAUidaE 1992.da.f001-02 • F

EF: CONRAUIDAE 1992.da.f001

Cophomantina Hoffmann, 1878.ha.f004 • KY

SI: $182 \bullet \mathbf{C I}:$ h126・ST: 0.10 .30

RL: INR

PA: 00 - COPHOMANTINA • Hoffmann 1878.ha: $614 \cdot \mathbf{F}$

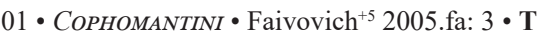

02 - COPHOMANTINAE • Duellman ${ }^{+2}$ 2016.fa: $3 \cdot \mathbf{b F}$

$03 \cdot$ COPHOMANTINA $\bullet$ Hoc loco $\bullet \mathbf{b T}$

$04 \cdot$ COPHOMANTINIA $・ H o c$ loco $\bullet$ iT

OS: Cophomantis $1870 \approx$ Boana $1825 \cdot \mathbf{O E}$

EN: (1) COPHOMANTINAE 1878.ha.f004-02 • bF

(2) Cophomantini 1878.ha.f004-01 • T

(3) CoPHOMAnTINa 1878.ha.f004-03 • b T

(4) COPHOMantinia 1878.ha.f004-04 • iT

EF: HYLIDAE 1815.ra.f002-|1825.gb.f001|

COPHYLIDAE Cope, 1889.ca.f001 • KY

SI: $189 \bullet \mathbf{C I}:$ h130 - ST: 0.10 .30

RL: INR

PA: $00 \cdot$ COPHYLIDAE $・$ Cope 1889.ca: 248 - F

$01 \cdot$ COPHYLINAE $\bullet$ Parker 1934.pa: v • bF

$02 \cdot \mathrm{COPHYLINI} \cdot \mathrm{Hoc}$ loco $\bullet \mathbf{T}$

$03 \cdot$ COPHYLINA $\cdot$ Hoc loco $\bullet \mathbf{b T}$

OS: Cophyla $1880 \cdot \mathbf{O E}$

EN: (1) COPHYLINAE 1889.ca.f001-01・bF

(2) CopHYLINI 1889.ca.f001-02 • T

(3) CopHYLINA 1889.ca.f001-03 • b T

EF: MICROHYLIDAE |1843.fa.f012|-1931.na.f001

Cordulina Van der Hoeven, 1855.va.f002 • AN

SI: $120 \bullet \mathbf{C I}: \mathrm{n} 039 \bullet \mathbf{S T}: 2.25 .50$

RL: INR

PA: $00 \cdot$ CoRdulina $・$ Van der Hoeven 1855.va: $464 \cdot \mathbf{P}$

OS: » 3 PN, including: Cryptobranchus 1821 • PD
EN: CRYPTOBRANCHIDAE 1826.fb.f003-04 • F

EF: CRYPTOBRANCHIDAE 1826.fb.f003

CORNUFERINAE Noble, 1931.na.F007 • JD

SI: $222 \bullet \mathbf{C I}:$ h157・ST: 0.10 .40

RL: INR

PA: 00 • CORNUFERINAE • Noble 1931.na: $521 \cdot \mathbf{b F}$

OS: Cornufer $1838 \cdot \mathbf{O E}$

EN: (1) CERATOBATRACHEIDAE 1884.ba.f001-04 • aF

(2) CERATOBATRACHIDAE 1884.ba.f001-00 • F

EF: CERATOBATRACHIDAE 1884.ba.f001

Corythomantinia nov., DOP.da.f065 • KY

SI: $506 \bullet \mathbf{C I}:$ h399・ST: 0.10 .30

RL: INR

PA: $00 \cdot$ CORYTHOMANTINIA $\bullet$ Hoc loco $\bullet$ iT

OS: Corythomantis 1896 P PD

EN: CORYTHOMANTINIA DOP.da.f065-00 • iT

EF: HYLIDAE 1815.ra.f002-|1825.gb.f001|

CRAUGASTORIDAE Hedges ${ }^{+2}$, 2008.ha.f001 • KY

SI: $381 \bullet \mathbf{C I}:$ h281・ST: 0.10 .31

RL: $\geq$ STRABOMANTIDAE 2008.ha.f003 - AI: Padial ${ }^{+2}$ 2014.pa: 52

PA: 00 • CRAUGASTORIDAE $\bullet$ Hedges $^{+2}$ 2008.ha: 3 • F

01 • CRAUGASTORINAE $\bullet$ Pyron $^{+1}$ 2011.pa: $547 \cdot \mathbf{b F}$

$02 \cdot$ CRAUGASTORINI $\bullet$ Hoc loco $\bullet \mathbf{T}$

OS: Craugastor $1862 \cdot$ OD

EN: (1) CRAUGASTORINAE 2008.ha.f001-01 • bF

(2) CRAUGASTORINI 2008.ha.f001-02 • T

EF: BRACHYCEPHALIDAE 1858.gc.f002

CRINiaE Cope, 1866.ca.f001・ KY

SI: $154 \cdot \mathbf{C I}:$ h104 $\bullet$ ST: 0.10 .30

RL: INR

PA: $00 \bullet$ CRINIAE $\bullet$ Cope 1866.ca: $89 \bullet \mathbf{G r}$

01 • CRINIINAE • Noble 1931.na: 496 •bF

$02 \cdot$ CRINIINA $\bullet$ Hoc loco $\bullet \mathbf{b T}$

$03 \cdot$ CRINIINIA $\bullet$ Hoc loco $\bullet$ iT

OS: Crinia $1838 \cdot \mathbf{O E}$

EN: (1) CRINIINA 1866.ca.f001-02 • bT

(2) CRINIINIA 1866.ca.f001-03 • iT

EF: MYOBATRACHIDAE 1850.sa.f001

CROSSODACTYLODINAE Fouquet ${ }^{+6}$, 2013.fa.f001 • JD

SI: $430 \bullet \mathbf{C I}:$ h324 • ST: 0.10 .40

RL: INR

PA: 00 • CROSSODACTYLODINAE $\bullet$ Fouquet $^{+6} 2013$ fa: $445 \cdot \mathbf{b F}$

OS: Crossodactylodes $1938 \cdot$ OD

EN: PARATELMATOBIIDAE 2012.oa.f001-01・F

EF: PARATELMATOBIIDAE 2012.oa.f001

CRUZIoHylini nov., DOP.da.f068・ KY

SI: $509 \bullet \mathbf{C I}: \mathrm{h} 402 \bullet \mathbf{S T}: 0.10 .30$

RL: INR

PA: $00 \bullet$ CRUZIOHYLINI $\bullet$ Hoc loco $\bullet \mathbf{T}$

OS: Cruziohyla 2005 • PD

EN: CRUZIOHYLINI DOP.da.f068-00 • T

EF: Phyllomedusidae 1858.gc.f009

CRYPTOBATRACHIDAE Frost ${ }^{+18}$, 2006.fa.f001 • KY

SI: $367 \bullet \mathbf{C I}:$ h273 • ST: 0.10 .30

RL: INR

PA: c0 • CRYPTOBATRACHIDAE - Frost $^{+18} 2006$ fa: 6 • F • EEA: PD 
i1 - CRYPTOBRANCHIDAE • Frost ${ }^{+18}$ 2006.fa: 155 • F

02 CRYPTOBATRACHINAE • Castroviejo-Fischer ${ }^{+7}$ 2015.ca: 20 - bF

OS: Cryptobatrachus $1916 \cdot$ OD

EN: CRYPTOBATRACHINAE 2006.fa.f001-02 • bF

EF: HEMIPHRACTIDAE 1862.pa.f001

CRYPTOBRANCHOIDEA Fitzinger, 1826.fb.f003 • KY

SI: $029 \bullet \mathbf{C I}:$ h015 • ST: 0.10 .30

RL: INR

PA: 00 - CRYPTOBRANCHOIDEA • Fitzinger 1826.fb: $41 \cdot \mathbf{F}$ 01 C CRYPTOBRANCHOIDEI • Eichwald 1831.eb: $164 \cdot \mathbf{F}$ 02 • CRYPTOBRANCHOIDEAE • Gray 1850.ga: $51 \cdot \mathbf{F}$ 03 - CRYPTOBRANCHOIDES • Duméril ${ }^{+2}$ 1854.da: 22 • F $04 \cdot$ CRYPTOBRANCHIDAE $\bullet$ Claus 1868.cb: 586 • F $05 \cdot$ CRYPTOBRANCHIATA • Wiedersheim 1877.wa: 356 • T 06 - CRYPTOBRANCHIOIDES • Hoffmann 1878.ha: 581 • F $07 \cdot$ CRYPTOBRANCHIATA • Leunis 1883.la: $624 \cdot \mathbf{F}$ $08 \cdot$ CRYPTOBRANCHIA $・$ Zittel 1888.za: $418 \cdot \mathbf{F}$ 09 • CRYPTOBRANCHOIDA • Cope 1889.ca: $18 \cdot \mathbf{F}$ 10 - CRYPTOBRANCHIIDAE • Cope 1889.ca: 30 • F 11 - CRYPTOCHIDAE • Cope 1889.cb: $861 \cdot \mathbf{F}$ 12 - CRYPTOBRANCHOIDEA • Dunn 1922.da: $427 \cdot \mathbf{p F}$ 13 - CRYPTOBRANCHIAE • Chang 1936.ca: $118 \cdot \mathbf{F}$

14 - CRYPTOBRANCHINAE • Regal 1966.ra: $405 \cdot \mathbf{b F}$ 15 - CRPTOBRANCHIDAE $\cdot \mathrm{Ye}^{+2}$ 1993.ya: $64 \cdot \mathbf{F}$ 16 - CRYPTODONTIDAE • Crespo 2001.ca: $112 \cdot \mathbf{F}$ 17 - CRYPTOBRANCHOIDIA • Dubois 2005.da: $48 \cdot \mathbf{e F}$ OS: Cryptobranchus $1821 \cdot \mathbf{O E}$

EN: CRYPTOBRANCHIDAE 1826.fb.f003-04 • F

EF: CRYPTOBRANCHIDAE 1826.fb.f003

CRYPTOThylaCinaE nov., DOP.da.f093 • KY

SI: $534 \cdot$ CI: h427 • ST: 0.10 .30

RL: INR

PA: $00 \cdot$ CRYPTOTHYLACINAE $\bullet$ Hoc loco $\bullet \mathbf{b F}$

OS: Cryptothylax $1950 \cdot$ PD

EN: CRYPTOTHYLACINAE DOP.da.f093-00 • bF

EF: HYPEROLIIDAE 1943.lb.f001

CTENOPHRYNINI nov., DOP.da.f084 • KY

SI: 525 • CI: h418 • ST: 0.10 .30

RL: INR

PA: $00 \cdot$ CTENOPHRYNINI $\cdot$ Hoc loco $\bullet$ T

OS: Ctenophryne $1904 \cdot \mathbf{P D}$

EN: CTENOPHRYNINI DOP.da.f084-00 • T

EF: MICROHYLIDAE |1843.fa.f012|-1931.na.f001

CyCloramphina Bonaparte, 1852.ba.f001 • MK

SI: $117 \bullet \mathbf{C I}:$ h079 • ST: 0.10 .34

RL: > CYCLORHAMPHINA 1850.bb.f003 - MK: Dubois 1985.da: 66

PA: $00 \cdot$ CYCLORAMPHINA • Bonaparte 1852.ba: $477 \cdot \mathbf{b F}$

01 • CYCLORAMPHIINAE • Gallardo 1965.ga: $84 \cdot \mathbf{b F}$

$02 \cdot$ CYCLORAMPHINAE $・$ Ardila-Robayo 1979.aa: $455 \cdot \mathbf{b F}$

03 $・$ CYCLORAMPHINI • Dubois 1985.da: $66 \cdot \mathbf{T}$

$04 \cdot$ CYCLORAMPHIDAE $・$ Frost $^{+18}$ 2006.fa: $6 \cdot \mathbf{F}$

05 $\cdot$ CYCLORAMPHEIDAE $\bullet$ Hoc loco $\bullet \mathbf{a F}$

OS: Cycloramphus $1838 \cdot \mathbf{O E}$

EN: (1) CYCLORAMPHEIDAE 1850.bb.f003-|1852.ba.f001|-05 • aF (2) CYCLORAMPHIDAE 1850.bb.f003-|1852.ba.f001|-04 • F
(3) CYCLORAMPHINAE 1850.bb.f003-|1852.ba.f001|-02 • bF EF: CYCLORAMPHIDAE 1850.bb.f003-|1852.ba.f001| CYCLORANINAE Parker, 1940.pa.f001・JD

SI: $234 \cdot \mathbf{C I}:$ h167 • ST: 0.10 .40

RL: INR

PA: 00 • CYCLORANINAE $・$ Parker 1940.pa: $2 \cdot \mathbf{b F}$ 01 C CYCLORANINI $・$ Lynch 1969.1b: $3 \cdot \mathbf{T}$

02 $\bullet$ CYCLORANIINAE $・$ Reig 1972.ra: $34 \cdot \mathbf{b F}$

OS: Cyclorana $1867 \approx$ Litoria $1838 \cdot \mathbf{O E}$

EN: PELODRYADINAE 1859.ga.f001-01・bF

EF: PHYLLOMEDUSIDAE 1858.gc.f009

CYCLORHAMPHINA Bonaparte, 1850.bb.f003 • MK

SI: $095 \bullet \mathbf{C I}:$ h057 • ST: 0.10 .58

RL: < CYCLORAMPHINA 1852.ba.f001 - MK: Dubois 1985.da: 66

PA: $00 \cdot$ CYCLORHAMPHINA • Bonaparte 1850.bb: pl. • bF

01 • CYCLORHAMPHIINAE • Lutz 1954.la: $157 \cdot \mathbf{b F}$

02 • CYCLORHAMPHINAE • Lutz 1954.la: $175 \bullet \mathbf{b F}$

03 $・$ CYCLORHAMPHINI $・$ Dubois 1983.da: 273 • T

OS: Cyclorhamphus $1847 \equiv$ Cycloramphus $1838 \cdot \mathbf{O E}$

EN: (1) CYCLORAMPHEIDAE 1850.bb.f003-|1852.ba.f001|-05 • aF

(2) CYCLORAMPHIDAE 1850.bb.f003-|1852.ba.f001|-04 • F

EF: CYCLORAMPHIDAE 1850.bb.f003-|1852.ba.f001|

CYNOPITA Dubois ${ }^{+1}$, 2009.db.f001 • KY

SI: $385 \bullet$ CI: h285 • ST: 0.10 .30

RL: INR

PA: $00 \cdot$ CYNOPITA Dubois $^{+1}$ 2009.db: $44 \cdot$ iT

$01 \cdot$ CYNOPINOA $\cdot$ Hoc loco $\bullet \mathbf{h T}$

$02 \cdot$ CYNOPITES $\bullet$ Hoc loco $\bullet \mathbf{C n}$

OS: Cynops $1838 \cdot \mathbf{O D}$

EN: (1) CYNOPINOA 2009.db.f001-01・hT

(2) CYNOPITES 2009.db.f001-02 • Cn

EF: SALAMANDRIDAE 1820.ga.f002

CYSTIGNATHI Tschudi 1838.ta.f001 • SG

SI: $051 \bullet \mathbf{C I}:$ h025 • ST: 0.10 .44

RL: $\geq$ CERATOPHRYDES 1838.ta.f002 - AI: Cope 1866.ca: 88 $<$ LEPTODACTYLIDAE |1838.ta.f001|-1896.wa.f001 • PS: Dubois 1983.da: 273

PA: $00 \cdot$ CYSTIGNATHI $\bullet$ Tschudi 1838.ta: $25 \cdot \mathbf{F}$

01 • CYSTIGNATHIDAE • Günther 1858.gc: $346 \cdot \mathbf{F}$

02 - CYSTIGNATHI • Cope 1866.ca: $90 \cdot \mathbf{G r}$

03 - CYSTIGNATHINA • Mivart 1869.ma: $293 \cdot \mathbf{b F}$

$04 \cdot$ CYSTIGNATHIDAE $\bullet$ Hoffmann 1878.ha: $613 \cdot \mathbf{b F}$

05 $\operatorname{CYSTIGNATHINAE} \bullet$ Gadow 1901.ga: xi, $211 \cdot \mathbf{b F}$

06 CYSTYGNATHINAE • Fejérváry 1918.fa: $119 \cdot \mathbf{b F}$

OS: Cystignathus $1830 \approx$ Leptodactylus $1826 \cdot \mathbf{O E}$

EN: (1) LEPTODACTYLOIDEA |1838.ta.f001|-1896.wa.f001-03 • pF (")"')

(3) LEPTODACTYLINAE |1838.ta.f001|-1896.wa.f001-01 • bF

EF: LEPTODACTYLIDAE |1838.ta.f001|-1896.wa.f001

DaCtYlethridaE Hogg 1838.ha.f017 • KY

SI: $048 \bullet$ CI: h022 • ST: 0.10 .30

RL: INR

PA: $00 \cdot$ DACTYLETHRIDAE $\bullet$ Hogg 1838.ha: $152 \cdot \mathbf{F}$

01 - DACTYLETHRINA $・$ Bonaparte 1850.bb: pl. • bF

$02 \cdot$ DACTYLETHRIDA $・$ Knauer 1878.ka: $103 \cdot \mathbf{F}$

03 - DACTYLETHRAE • Peters 1882.pa: xv, 179 • F 
04 DACTYLETHRINAE • Metcalf 1923.ma: 391 • bF

05 - DACTYLETHRINI $\bullet$ Hoc loco $・$ T

OS: Dactylethra $1829 \approx$ Xenopus $1827 \cdot$ OE

EN: (1) DACTYLETHRINAE 1838.ha.f001-04 • bF

(2) DACTYLETHRINI 1838.ha.f001-05 • T

EF: PIPIDAE 1825.gb.f003-|1826.fb.f002|

DACTYLETRIDAE Hoffmann, 1878.ha.f001 • JI

SI: $179 \bullet \mathbf{C I}: \mathrm{h} 123 \cdot \mathbf{S T}: 0.10 .52$

RL: INR

PA: 00 - DACTYLETRIDAE • Hoffmann 1878.ha: 584 • F

OS: Dactyletra $1878 \approx$ Xenopus $1827 \cdot \mathbf{O E}$

EN: (1) DACTYLETHRINAE 1838.ha.f001-04 • bF

(2) DACTYLETHRINI 1838.ha.f001-05 • T

EF: PIPIDAE 1825.gb.f003-|1826.fb.f002|

DasyPoPINA nov., DOP.da.f085 • KY

SI: $526 \bullet \mathbf{C I}: \mathrm{h} 419 \cdot \mathbf{S T}: 0.10 .30$

RL: INR

PA: $00 \bullet$ DASYPOPINA $\bullet$ Hoc loco $\bullet \mathbf{b T}$

OS: Dasypops $1924 \cdot \mathbf{P D}$

EN: DASYPOPINA DOP.da.f085-00・bT

EF: MICROHYLIDAE |1843.fa.f012|-1931.na.f001

DendrobatidaE Cope, 1865.ca.f002 • CK

SI: 152 • CI: h102 • ST: 0.10 .36

RL: > PHYLLOBATAE 1843.fa.f007 • PP: Opinion 2223 (Anonymous 2009.aa)

PA: $00 \cdot$ DENDROBATIDAE $\bullet$ Cope 1865.ca: $100 \cdot \mathbf{F}$

01 - DENDROBATINAE • Gadow 1901.ga: xi, $272 \cdot \mathbf{b F}$

02 DENDRONATINAE • Bauer 1988.ba: $6 \cdot \mathbf{b F}$

$03 \cdot$ DENDROBATOIDAE $・$ Dubois 1992.da: $309 \cdot \mathbf{e F}$

$04 \cdot$ DENDROBATINI $^{\bullet}$ Grant $^{+7}$ 2017.ga: $27 \cdot \mathbf{T}$

05 - DENDROBATINA $\bullet$ Hoc loco $\bullet \mathbf{b T}$

OS: Dendrobates $1830 \cdot \mathbf{O E}$

EN: (1) DENDROBATIDAE $\mid 1850$. bb.f006|-1865.ca.f002-00 • F

(2) DENDROBATINAE |1850.bb.f006|-1865.ca.f002-01 • bF

(3) DENDROBATINI |1850.bb.f006|-1865.ca.f002-04• T

(4) DENDROBATINA |1850.bb.f006|-1865.ca.f002-05 • b T

EF: DENDROBATIDAE |1850.bb.f006|-1865.ca.f002

DENDROHYADOIDEA Fitzinger, 1832.fa.f001 • JI

SI: $037 \bullet \mathbf{C I}:$ h020 • ST: 2.10 .52

RL: INR

PA: 00 - DENDRIOHYADOIDEA • Fitzinger 1832.fa: $327 \cdot \mathbf{G r}$

OS: Dendrohyas $1830 \equiv$ Hyla $1768 \cdot \mathbf{O E}$

EN: (1) HYLOIDEA 1815.ra.f002-|1825.gb.f001|-20・pF (1)"

(8) HYLITES 1815.ra.f002-|1825.gb.f001|-26 • Cn

EF: HYLIDAE 1815.ra.f002-|1825.gb.f001|

DENDROPHRYNISCINA Jiménez de la Espada, 1870.ja.f001 - KY

SI: $173 \bullet \mathbf{C I}: \mathrm{h} 121 \bullet \mathbf{S T}: 0.10 .30$

RL: INR

PA: 00 • DENDROPHRYNISCINA • Jiménez de la Espada 1870.ja: 65 - Sc

01 • DENDROPHRYNISCIDAE • Jiménez de la Espada 1870.ja: 65 • F

02 - DENDROPHRYNISCINAE • Gadow 1901.ga: xi, $224 \cdot \mathbf{b F}$

$03 \cdot$ DENDROPHRYNISCINIA $\cdot$ HOC lOCO $\bullet \mathbf{i F}$

OS: Dendrophryniscus $1870 \cdot$ OD
EN: DENDROPHRYNISCINIA 1870.ja.f001-03 • iT

EF: BUFONIDAE 1825.gb.f004

DENDROPSOPHI Fitzinger, 1843.fa.f003 • KY

SI: $068 \bullet \mathbf{C I}:$ h036 • ST: 0.10 .30

RL: INR

PA: $00 \cdot$ DENDROPSOPHI $\bullet$ Fitzinger 1843.fa: $31 \cdot \mathbf{F}$ $01 \cdot$ DENDROPSOPHINI Faivovich $^{+5}$ 2005.fa: $3 \cdot$ T

02 - DENDROPSOPHINAE • Duellman+2 2016.fa: $3 \cdot \mathbf{b F}$

$03 \cdot$ DENDROPSOPHINA $\cdot$ Hoc loco $\bullet \mathbf{b T}$

OS: Dendropsophus $1843 \cdot \mathbf{O E}$

EN: (1) DENDROPSOPHINI 1843.fa.f003.01・ T

(3) DENDROPSOPHINA 1843.fa.f003.03 • bT

EF: HYLIDAE 1815.ra.f002-|1825.gb.f001|

DENDROTRITONITES nOV., DOP.da.f136 • KY

SI: $577 \bullet$ CI: h470 • ST: 0.10 .30

RL: INR

PA: $00 \cdot$ DENDROTRITONITES $\bullet$ Hoc loco $・ \mathbf{C n}$

OS: Dendrotriton 1983 • PD

EN: DENDROTRITONITES DOP.da.f136-00 • Cn

EF: PlethodontidaE 1850.ga.f001

DERMatonotinia nov., DOP.da.f087 • KY

SI: $528 \cdot \mathbf{C I}:$ h421 • ST: 0.10 .30

RL: INR

PA: $00 \cdot$ DERMATONOTINIA $\cdot$ Hoc loco $\bullet$ iT

OS: Dermatonotus $1904 \cdot \mathbf{P D}$

EN: DERMATONOTINIA DOP.da.f087-00 • iT

EF: MICROHYLIDAE |1843.fa.f012|-1931.na.f001

DERMOPHINAE Taylor, 1969.ta.f002 • KY

SI: $286 \bullet \mathbf{C I}:$ h201・ST: 0.10 .30

RL: INR

PA: 00 • DERMOPHINAE • Taylor 1969.ta: $303 \cdot \mathbf{b F}$

01 • DERMOPHIIDAE • Laurent 1984.la: $199 \cdot \mathbf{F}$

02 • DERMOPHIINAE • Laurent 1984.1a: 199 • bF

03 - DERMOPHINI $・$ Lescure $^{+2}$ 1986.1b: 166 T

$04 \cdot$ DERMOPHIINIA $・ H O c$ loco $・$ iT

05 - DERMOPHIINOA $・$ Hoc loco $\bullet \mathbf{h T}$

OS: Dermophis $1880 \cdot \mathbf{O E}$

EN: (1) DERMOPHIINIA 1969.ta.f002-04 • iT

(2) DERMOPHIINOA 1969.ta.f002-05 • hT

EF: CAECILIIDAE 1814.ra.f003-|1825.gb.f008|

Derotremata Schinz, 1833.sa.f001 • AN

SI: $041 \bullet \mathbf{C I}: \mathrm{n} 019 \bullet \mathbf{S T}: 2.25 .50$

RL: INR

PA: $00 \cdot$ DEROTREMATA $・$ Schinz 1833.sa: $196 \cdot \mathbf{F}$

OS: » 6 PN, including: Siren 1766 PD

EN: SIRENIDAE 1825.gb.f005-00 • F

EF: SIRENIDAE 1825.gb.f005

DEROTREMEN Haeckel, 1866.ha.f001 • AN

SI: $156 \bullet \mathbf{C I}: \mathrm{n} 049 \bullet \mathbf{S T}: 2.25 .50$

RL: INR

PA: 00 • DEROTREMEN • Haeckel 1866.ha: cxxxi • F

01 - DEROTREMATA • Zittel 1888.za: $418 \cdot \mathbf{F}$

OS: » 2 PN, including: Cryptobranchus 1821 • PD

EN: CRYPTOBRANCHIDAE 1826.fb.f003-04 - F

EF: CRYPTOBRANCHIDAE 1826.fb.f003 
DERotreta Van der Hoeven, 1833.va.f001 • AN

SI: $043 \bullet$ CI: n021 • ST: 2.25 .50

RL: INR

PA: $00 \cdot$ DEROTRETA $・$ Van der Hoeven 1833.va: iii, $302 \cdot \mathbf{F}$

OS: » 5 PN, including: Caecilia $1758 \cdot$ PD

EN: (1) CAECILIOIDEA 1814.ra.f003-|1825.gb.f008|-19 • pF "'m!'

(5) CAECILIINA 1814.ra.f003-|1825.gb.f008|-26 • bT

EF: CAECILIIDAE 1814.ra.f003-|1825.gb.f008|

Desmognathina Gray, 1850.ga.f003 • KY

SI: $114 \cdot$ CI: h076 • ST: 1.10 .30

RL: INR

PA: 00 • DESMOGNATHINA • Gray 1850.ga: $40 \cdot \mathbf{U F}$

$01 \cdot$ DESMOGNATHIDAE $\bullet$ Cope 1866.ca: $103 \cdot \mathbf{F}$

$02 \cdot$ DESMOGNATHINAE $\bullet$ Boulenger 1882.bc: viii, $76 \cdot \mathbf{b F}$

03 DISMOGNATHINAE • Dunn 1917.da: $399 \cdot \mathbf{b F}$

$04 \cdot$ DESMOGNATHINI $・$ Dubois 2005.da: $20 \bullet \mathbf{T}$

$05 \cdot$ DESMOGNATHINA $\bullet$ Hoc loco $\bullet \mathbf{b T}$

$06 \cdot$ DESMOGNATHINIA $\bullet$ Hoc loco $\bullet$ iT

OS: Desmognathus $1850 \bullet \mathbf{O E}$

EN: (1) DESMOGNATHINA 1850.ga.f003-05 • bT

(2) DESMOGNATHINIA 1850.ga.f003-06 • iT

EF: PLETHODONTIDAE 1850.ga.f001

Diaglenitoes nov., DOP.da.f149 • KY

SI: $590 \bullet$ CI: h483 • ST: 0.10 .30

RL: INR

PA: 00 • DIAGLENITOES $\bullet$ Hoc loco $\bullet$ iCn

OS: Diaglena $1887 \cdot \mathbf{P D}$

EN: DIAGLENITOES DOP.da.f149-00 • iCn

EF: HYLIDAE 1815.ra.f002-|1825.gb.f001|

DIASPORINa nov., DOP.da.f148 • KY

SI: $589 \bullet$ CI: h482 • ST: 0.10 .30

RL: INR

PA: $00 \cdot$ DIASPORINA $\bullet$ Hoc loco $\bullet$ bT

OS: Diasporus $2008 \cdot \mathbf{P D}$

EN: DIASPORINA DOP.da.f148-05 • bT

EF: BRACHYCEPHALIDAE 1858.gc.f002

DiCAMPTODONTINAE Tihen, 1958.ta.f001 • JD

SI: 256 • CI: h181 • ST: 0.10 .40

RL: > RHYACOTRITONINAE 1958.ta.f002 • AI: Regal 1966.ra: 405

PA: 00 • DICAMPTODONTINAE $\bullet$ Tihen 1958.ta: $1 \cdot \mathbf{b F}$

01 • DICAMPTODONTIDAE $\bullet$ Edwards 1976.ea: $325 \cdot \mathbf{F}$

OS: Dicamptodon $1870 \cdot \mathbf{O E}$

EN: AMBYSTOMATIDAE 1850.ga.f002-08 • F

EF: AMBYSTOMATIDAE 1850.ga.f002

DiCroglossini Dubois, 1987.da.f004 • US

SI: $336 \bullet$ CI: h244 • ST: 0.10 .30

RL: INR

PA: 00 • DICROGLOSSINI • Dubois 1987.da: $57 \cdot \mathbf{T}$

$01 \cdot$ DICROGLISSINI $\bullet$ Laurent 1991.la: $4 \cdot \mathbf{T}$

02 - DiCROGLOSSINAE $\bullet$ Dubois 1992.da: $313 \cdot \mathbf{b F}$

03 DICROGLOSSIDAE $\cdot$ Frost $^{+18}$ 2006.fa: $7 \cdot \mathbf{F}$

$04 \cdot$ DICRGLOSSINAE $\bullet \mathrm{Fei}^{+2} 2010$.fa: $12 \cdot \mathbf{b F}$

$05 \cdot$ DicroglosSEIDAE $\bullet$ Hoc loco $\cdot \mathbf{a F}$

$06 \cdot$ DicroglosSINA $\bullet$ Hoc loco $\bullet \mathbf{b T}$

OS: Dicroglossus $1860 \approx$ Euphlyctis $1843 \cdot$ OD
EN: (1) DICROGLOSSEIDAE 1987.da.f004-05 • aF

(2) DiCROGLOSSIDAE 1987.da.f004-03 • F

(3) DiCRogLOSSINAE 1987.da.f004-02 • bF

(4) DICROGLOSSINI 1987.da.f004-00 • T

(5) DicRoglossina 1987.da.f004-06 • bT

EF: DiCROGLOSSIDAE 1987.da.f004

DiPLASIOCOELA Huene, 1948.ha.f005 • AN

SI: $249 \bullet$ CI: n070 • ST: 0.25 .50

RL: INR

PA: $00 \cdot$ DIPLASIOCOELA $\bullet$ Huene 1948.ha: $71 \cdot \mathbf{F}$

OS: » OA: Rana $1758 \cdot \mathbf{P D}$

EN: (1) RANOIDEA 1796.ba.f001-28 • pF

"'I!"

(12) RANITOES 1796.ba.f001-38 • iCn

EF: RANIDAE 1796.ba.f001

Diplopainia nov., DOP.da.f104 • KY

SI: $545 \bullet$ CI: h438 • ST: 0.10 .30

RL: INR

PA: $00 \cdot$ DIPLOPAINIA $\bullet$ Hoc loco $\bullet$ iT

OS: Diplopaa nov. $2016 \cdot \mathbf{P D}$

EN: DIPLOPAINIA DOP.da.f104-00 • iT

EF: DICROGLOSSIDAE 1987.da.f004

DISCOGLOSSIDAE Günther, 1858.gc.f004 • UV

SI: $132 \cdot \mathbf{C I}:$ h086 • ST: 0.10 .30

RL: INR

PA: $00 \cdot$ DISCOGLOSSIDAE $\bullet$ Günther 1858.gc: $346 \bullet \mathbf{F}$

01 - DISCOGLOSSINA $\bullet$ Mivart 1869.ma: $294 \cdot \mathbf{b F}$

02 • DicroglOSSIDAE $\bullet$ Anderson 1871.aa: $38 \cdot \mathbf{F}$

03 - DISCOGLOSSIDAE $\bullet$ Hoffmann 1878.ha: $613 \cdot \mathbf{b F}$

$04 \cdot$ DISCOGLOSSOIDEA $\bullet$ Gill 1884.gb: $621 \cdot \mathbf{p F}$

$05 \cdot$ DISCOGLOSSINAE $\bullet$ Fejérváry 1921.fb: $25 \bullet \mathbf{b F}$

06 DISCOGLOSSIDYAE $\bullet$ Morescalchi 1995.ma: $868 \cdot \mathbf{F}$

$07 \cdot$ DICRGLOSSINAE $\bullet \mathrm{Fei}^{+2} 2010$ fa: $12 \cdot \mathbf{b F}$

08 $\bullet$ DCRGLOSSINAE $\bullet \mathrm{Fei}^{+2}$ 2010.fa: $17 \cdot \mathbf{b F}$

OS: Discoglossus $1837 \cdot \mathbf{O E}$

EN: DISCOGLOSSIDAE 1858.gc.f004-00 • F

EF: DISCOGLOSSIDAE 1858.gc.f004

DorSIPARES Blainville, 1835.ba.f001 • AN

SI: 045 • CI: n023 • ST: 2.25 .50

RL: INR

PA: $00 \cdot$ DORSIPARES $\bullet$ Blainville 1835.ba: $276 \cdot \mathbf{F}$

OS: Pipa $1768 \cdot \mathbf{O M}$

EN: (1) PIPIDAE 1825.gb.f003-|1826.fb.f002|-07 • F

(2) PIPINAE 1825.gb.f003-|1826.fb.f002|-13 • bF

EF: PIPIDAE 1825.gb.f003-|1826.fb.f002|

DRYOPHYTAE Fitzinger, 1843.fa.f002 • JD

SI: $067 \bullet$ CI: h035 • ST: 0.10 .40

RL: INR

PA: $00 \cdot$ DRYOPHYTAE $\bullet$ Fitzinger 1843.fa: $31 \cdot \mathbf{F}$

OS: Dryophytes $1843 \approx$ Hyla $1768 \cdot \mathrm{OE}$

EN: (1) HYLOIDEA 1815.ra.f002-|1825.gb.f001|-20 • pF "'1"')

(8) HYLITES 1815.ra.f002-|1825.gb.f001|-26 • Cn

EF: HYLIDAE 1815.ra.f002-|1825.gb.f001|

DYSCOPHIDAE Boulenger, 1882.bb.f001 • KY

SI: 185 • CI: h127 • ST: 0.10 .30 
RL: INR

PA: $00 \cdot$ DYSCOPHIDAE $\bullet$ Boulenger 1882.bb: x, $179 \cdot \mathbf{F}$

$01 \cdot$ DYSCOPHINAE $\bullet$ Gadow 1901.ga: xi, $235 \cdot \mathbf{b F}$

$02 \cdot$ DISCOPHIDAE $\bullet$ Miranda-Ribeiro 1924.ma: $143 \cdot \mathbf{F}$

03 - DISCOPHYNAE • Tatarinov 1964.ta: $133 \cdot \mathbf{F}$

$04 \cdot$ DYSCOPHIIDINAE $\bullet$ Kuhn 1965.ka: $843 \cdot \mathbf{F}$

05 $\bullet$ DYSCOPHINI $\bullet$ Hoc loco $\bullet$ T

OS: Dyscophus $1872 \cdot \mathbf{O E}$

EN: DYSCOPHINI $1882 . \mathrm{bb} . \mathrm{f001-05} \bullet \mathbf{T}$

EF: MICROHYLIDAE |1843.fa.f012|-1931.na.f001

ECAUDATA Oppel, 1811.oc.f002 • AN

SI: $086 \bullet$ CI: n033 • ST: 2.25 .50

RL: INR

PA: 00 $\bullet$ ECAUDATA $\bullet$ Oppel 1811.oc: $72 \cdot \mathbf{F}$

OS: » 4 PN, including: Rana $1758 \cdot \mathbf{P D}$

EN: (1) RANOIDEA 1796.ba.f001-28 • pF

"'"!"

(12) RANITOES 1796.ba.f001-38 • iCn

EF: RANIDAE 1796.ba.f001

ECHINOTRITONINIA nov., DOP.da.f147 • KY

SI: $588 \bullet$ CI: h481 • ST: 0.10 .30

RL: INR

PA: $00 \cdot$ ECHINOTRITONINIA $\bullet$ Hoc loco $\bullet$ iT

OS: Echinotriton $1982 \cdot \mathbf{P D}$

EN: ECHINOTRITONINIA DOP.da.f147-00 • iT

EF: SALAMANDRIDAE 1820.ga.f002

ECNOMIOHYLITES nov., DOP.da.f058 • KY

SI: $499 \cdot$ CI: h392 • ST: 0.10 .30

RL: INR

PA: $00 \cdot$ ECNOMIOHYLITES $\bullet$ Hoc loco $・ \mathbf{C n}$

OS: Ecnomiohyla 2005 • PD

EN: ECNOMIOHYLITES DOP.da.058-00 • Cn

EF: HYLIDAE 1815.ra.f002-|1825.gb.f001|

EDALORHININA nov., DOP.da.f071 • KY

SI: $512 \bullet$ CI: h405 • ST: 0.10 .30

RL: INR

PA: $00 \cdot$ EDALORHININA $\bullet$ Hoc loco $\bullet \mathbf{b T}$

OS: Edalorhina $1870 \cdot \mathbf{P D}$

EN: EDALORHININI DOP.da.f071-00 • bT

EF: LEPTODACTYLIDAE |1838.ta.f001|-1896.wa.f001

ELEUTHERODACTYLINAE Lutz, 1954.1a.f001・ KY

SI: $251 \cdot \mathbf{C I}:$ h177 • ST: 0.10 .30

RL: INR

PA: $00 \cdot$ ELEUTHERODACTYLINAE $・$ Lutz 1954.la: $157 \cdot \mathbf{b F}$

$01 \cdot$ ELEUTHERODACTYLYNAE $\bullet$ Lutz 1954.1b: $229 \bullet \mathbf{b F}$

02 ELEUTHERODACTYLINI $\bullet$ Lynch 1969.1b: $3 \cdot$ T

03 • ELEUTHERODACTYLIDAE $\bullet$ Hedges $^{+2}$ 2008.ha: $47 \cdot \mathbf{F}$

$04 \cdot$ ELEUTHERODACTYLOIDIA $•$ Fouquette $^{+1}$ 2014.fa: $6 \cdot \mathbf{e F}$

05 ELEUTHERODACTYLINA Hoc loco • bT

OS: Eleutherodactylus $1841 \cdot \mathbf{O E}$

EN: (1) ELEUTHERODACTYLINAE 1954.la.f001-00 • bF

(2) ELEUTHERODACTYLINI 1954.la.f001-02 • T

(3) ELEUTHERODACTYLINA 1954.1a.f001-05 • b T

EF: BRACHYCEPHALIDAE 1858.gc.f002

ELEUTHEROGNATHINAE Méhely, 1901.ma.f002 • AN

SI: $196 \bullet$ CI: n058 • ST: 2.25 .50
RL: INR

PA: 00 • ELEUTHEROGNATHINAE • Méhely 1901.ma: $171 \bullet \mathbf{b F}$

01 ELEUTHEROGNATHIDAE $\bullet$ Kuhn 1967.kb: $22 \cdot \mathbf{F}$

OS: » 6 PN, including: Sphenophryne $1878 \approx$ Asterophrys $1838 \bullet$ PD

EN: (1) ASTEROPHRYINAE 1858.gc.f006-05 • bF

(2) ASTEROPHRYINI 1858.gc.f006-09・ T

EF: MICROHYLIDAE |1843.fa.f012|-1931.na.f001

ELLIPSOGLOSSIDAE Hallowell, 1856.ha.f001 • sG

SI: $121 \bullet$ CI: h080 • ST: 0.10 .44

RL: < HYNOBIINAE 1859.cb.f002 • PS: Dubois 1984.da: 114

PA: $00 \cdot$ ELLIPSOGLOSSIDAE $\bullet$ Hallowell 1856.ha: $11 \cdot \mathbf{b F}$

$01 \cdot$ ELLIPSOGLOSSIDAE $\bullet$ Hoffmann 1878.ha: $585 \cdot \mathbf{F}$

OS: Ellipsoglossa $1854 \approx$ Hynobius $1838 \cdot \mathbf{O E}$

EN: (1) HYNOBIIDAE |1856.ha.f001|-1859.cb.f002-01 • F

"1"1)

(6) HYNOBIINOA |1856.ha.f001|-1859.cb.f002-07 • hT

EF: HYNOBIIDAE |1856.ha.f001|-1859.cb.f002

ELOSIIDAE Miranda-Ribeiro, 1923.mb.f001 • JD

SI: $207 \bullet$ CI: h144 • ST: 0.10 .40

RL: INR

PA: $00 \bullet$ ELOSIIDAE $\bullet$ Miranda-Ribeiro 1923.mb: $827 \cdot \mathbf{F}$

$01 \cdot$ ELOSIINAE $\bullet$ Lutz 1930.la: $195 \cdot \mathbf{b F}$

$02 \cdot$ ELOSIINI Ardila-Robayo 1979.aa: $385 \cdot \mathbf{T}$

OS: Elosia $1838 \approx$ Hylodes $1826 \cdot \mathbf{O E}$

EN: HYLODIDAE 1858.gc.f010-00 • F

EF: HYLODIDAE 1858.gc.f010

ENGISTOMATIDAE Methuen ${ }^{+1}$, 1913.ma.f001 • JD

SI: $198 \bullet$ CI: h135 $\bullet$ ST: 0.10 .52

RL: INR

PA: 00 ENGISTOMATIDAE $\bullet$ Methuen $^{+1}$ 1913.ma: $58 \cdot \mathbf{F}$

01 ENGISTOMATINAE $\bullet$ Methuen $^{+1}$ 1913.ma: $58 \cdot \mathbf{b F}$

OS: Engistoma 1904 Elachistocleis $1927 \cdot$ OE

EN: ENGYSTOMATINIA 1850.bb.f009-08・ iT

EF: MICROHYLIDAE |1843.fa.f012|-1931.na.f001

ENGYSTOMIDAE Bonaparte, 1850.bb.f009 • KY

SI: $101 \cdot$ CI: h063 • ST: 0.10 .30

RL: INR

PA: $00 \cdot$ ENGYSTOMIDAE $\bullet$ Bonaparte 1850.bb: pl. $\bullet \mathbf{F}$

$01 \cdot$ ENGYSTOMINA $\bullet$ Bonaparte 1850.bb: pl. $\bullet \mathbf{b F}$

02 ENGYSTOMATIDAE • Günther 1858.gc: $346 \bullet \mathbf{F}$

03 ENGYSTOMIDAE $\bullet$ Hoffmann 1878.ha: $613 \cdot \mathbf{b F}$

$04 \cdot$ ENGYSTOMITIDAE $\bullet$ Hoffmann 1878.ha: $617 \cdot \mathbf{b F}$

05 ENGYSTOMIDA $\bullet$ Knauer 1878.ka: $108 \cdot \mathbf{F}$

06 ENGYSTOMATA $\bullet$ Peters 1882.pa: xv, $172 \cdot \mathbf{F}$

$07 \cdot$ ENGYSTOMATINAE $\bullet$ Gadow 1901.ga: xi, $225 \cdot \mathbf{b F}$

$08 \cdot$ ENGYSTOMATINIA $\bullet$ Hoc loco $\bullet$ iT

OS: Engystoma $1826 \cdot \mathbf{O E}$

EN: ENGYSTOMATINIA 1850.bb.f009-08• iT

EF: MICROHYLIDAE |1843.fa.f012|-1931.na.f001

ENSATININA Gray, 1850.ga.f005 • KY

SI: $116 \bullet$ CI: h078 • ST: 1.10 .37

RL: $\leq$ PLETHODONTIDAE 1850.ga.f001 • PR: Dubois ${ }^{+1}$ 2012.da: 98

PA: $00 \bullet$ ENSATININA $\bullet$ Gray 1850.ga: $48 \cdot \mathbf{U F}$

$01 \cdot$ ENSATININI Vieites $^{+3}$ 2011.va: $633 \cdot \mathbf{T}$

$02 \cdot$ ENSATININA $\bullet$ Hoc loco $\bullet \mathbf{b T}$

OS: Ensatina $1850 \bullet \mathbf{O E}$ 
EN: ENSATININA 1850.ga.f005-02 • bT

EF: PlethodontidaE 1850.ga.f001

EOCAECILIAIDAE Jenkins ${ }^{+1}$, 1993.ja.f001 +• KY

SI: $351 \bullet \mathbf{C I}:$ h258・ST: 0.10 .30

RL: INR

PA: $00 \cdot$ EOCAECILIAIDAE $\bullet$ Jenkins $^{+1}$ 1993.ja: $246 \bullet$ F

$01 \cdot$ EOCAECILIDAE Heatwole $^{+1} 2000$ ha: $1468 \cdot \mathbf{F}$

$02 \cdot$ EOCAECILIOIDIA $・$ Dubois 2005.da: $22 \cdot \mathbf{e F}$

03 EOCAECILIOIDEA • Dubois 2005.da: $22 \cdot \mathbf{p F}$

$04 \cdot$ EOCAECILIIDAE $\bullet$ Dubois 2005.da: $22 \cdot \mathbf{F}$

OS: Eocaecilia $1993+\cdot \mathbf{O E}$

EN: EOCAECILIIDAE 1993.ja.f001-04 † • F

EF: EOCAECILIIDAE 1993.ja.f001 †

EOPELOBATINAE $\breve{S}_{\text {pinar }}^{+2}$, 1971.sa.f001 + JD

SI: $289 \bullet$ CI: h204 • ST: 0.10 .40

RL: INR

PA: $00 \cdot$ EOPELOBATINAE $\bullet \mathrm{SLpinar}^{+2}$ 1971.sa: $279 \cdot \mathbf{b F}$

$01 \cdot$ EOPELOBATIDA • Eiselt 1988.ea: $54 \cdot \mathbf{F}$

$02 \cdot$ EOPELOBATIDAE $\bullet$ Gaudant 1997.ga: 435, $443 \cdot \mathbf{F}$

OS: Eopelobates $1929+\cdot \mathbf{O E}$

EN: (1) PELOBATOIDEA 1850.bb.f004-13 • pF "')!"

(3) Pelobatidae 1850. bb.f004-00 • F

EF: PELOBATIDAE 1850.bb.f004

EOSCAPHERPETONTINAE Nessov, 1981.na.f001 + JD

SI: $308 \bullet \mathbf{C I}:$ h2 $19 \bullet$ ST: 0.10 .40

RL: INR

PA: $00 \cdot$ EOSCAPHERPETONTINAE • NesSOV 1981.na: $60 \cdot \mathbf{b F}$ 01 • EOSCAPHERPETINAE • Marjanović ${ }^{+1}$ 2014.ma: $543 \cdot \mathbf{b F}$

OS: Eoscapherpeton $1981+\cdot \mathbf{O E}$

EN: CRYPTOBRANCHIDAE 1826.fb.f003-04 • F

EF: CRYPTOBRANCHIDAE 1826.fb.f003

EоTHECINI nov., DOP.da.f011 • KY

SI: 452 • CI: h345 • ST: 0.10 .30

RL: INR

PA: $00 \cdot$ EOTHECINI $\bullet$ Hoc loco $・$ T

OS: Eotheca 2015 • PD

EN: EOTHECINI DOP.da.f011-00 • T

EF: HEMIPHRACTIDAE 1862.pa.f001

EOXENOPOIDIDAE Laurent, 1948.la.f001 +• JD

SI: $244 \cdot \mathbf{C I}:$ h175 • ST: 0.10 .40

RL: INR

PA: $00 \cdot$ EOXENOPOIDIDAE $\bullet$ Laurent 1948.1a: $1 \cdot \mathrm{F}$ 01 EOXENOPIDIDAE • Casamiquela 1959.ca: $7 \cdot \mathrm{F}$ $02 \cdot$ EOXENOPODIDAE $\bullet$ Casamiquela 1960.ca: $20 \cdot \mathrm{F}$ $03 \cdot$ EOXENOPOIDIDADE $\bullet$ Casamiquela 1961.ca: $108 \cdot \mathrm{F}$

OS: Eoxenopoides $1931+\cdot \mathbf{O E}$

EN: PIPIDAE 1825.gb.f003-|1826.fb.f002|-07 • F

EF: PIPIDAE 1825.gb.f003-|1826.fb.f002|

EPICRIA Fitzinger, 1843.fa.f017 • CI

SI: $082 \bullet$ CI: h050 • ST: 1.10 .45

RL: < ICHTHYOPHIIDAE 1968.ta.f001 • PP: Opinion 1749

(Anonymous 1993.aa: 261)

PA: $00 \cdot$ EPICRIA $\bullet$ Fitzinger 1843.fa: $34 \cdot \mathbf{F}$

$01 \cdot$ EPICRINA $\bullet$ Bonaparte 1845.ba: $378 \cdot \mathbf{b F}$

$02 \cdot$ EPICRIINA $・$ Bonaparte 1850.bb: pl. • bF
03 • EPICRIIDAE $\bullet$ Dubois 1984.da: $113 \cdot \mathbf{F}$

$04 \cdot$ EPICRIOIDES $\bullet$ Lescure $^{+2}$ 1986.lb: 154 . $\cdot \mathbf{h F}$

05 E EPICRIOIDEA $・$ Lescure $^{+2}$ 1986.lb: 154. • pF

$06 \cdot$ EPICRIOIDAE $・$ Lescure $^{+2}$ 1986.lb: 154. e eF

$07 \cdot$ EPICRIINAE $\bullet$ Lescure $^{+2}$ 1986.lb: 155 . bF

$08 \cdot$ EPICRIILAE $\bullet$ Lescure $^{+2}$ 1986.lb: 155 . iF

09 EPICRIUMIDAE • Anonymous 1993.aa: $261 \cdot \mathbf{F}$

OS: Epicrium $1828 \approx$ Ichthyophis $1826 \cdot \mathbf{O E}$

EN: (1) IСНTHYОРНIOIDEA 1968.ta.f001-04 • F

(2) ICHTHYOРHIDAE 1968.ta.f001-00 • F

EF: ICHTHYOРHIIDAE 1968.ta.f001

EPIDALEITUES nov., DOP.da.f023 • KY

SI: $464 \cdot \mathbf{C I}:$ h357 • ST: 0.10 .30

RL: INR

PA: $00 \cdot$ EPIDALEITUES $\bullet$ Hoc loco $\bullet \mathbf{h C n}$

OS: Epidalea $1864 \cdot \mathbf{P D}$

EN: EPIDALEITUES DOP.da.f023-00 • hCn

EF: BUFONIDAE 1825.gb.f004

EPIPEDOBATINI nov., DOP.da.f003 • KY

SI: $444 \cdot \mathbf{C I}:$ h337 • ST: 0.10 .30

RL: INR

PA: $00 \cdot$ EPIPEDOBATINI $\bullet$ Hoc loco $\bullet \mathbf{T}$

OS: Epipedobates $1987 \cdot \mathbf{P D}$

EN: EPIPEDOBATINI DOP.da.f003-00 • T

EF: DENDROBATIDAE |1850.bb.f006|-1865.ca.f002

ERICABATRACHIDAE nOV., DOP.da.f099 • KY

SI: $540 \bullet$ CI: h433 - ST: 0.10 .30

RL: INR

PA: $00 \cdot$ ERICABATRACHOIDAE $\bullet$ Hoc loco $\bullet \mathbf{e F}$

$01 \cdot$ ERICABATRACHIDAE $\bullet$ Hoc loco $\bullet \mathbf{F}$

OS: Ericabatrachus $1991 \cdot$ PD

EN: (1) ERICABATRACHOIDAE DOP.da.f099-00 • eF

(2) ERICABATRACHIDAE DOP.da.f099-01 • F

EF: ERICABATRACHIDAE 2017.da.997

ERIPAINA nov., DOP.da.f106・ KY

SI: 547 • CI: h440 • ST: 0.10 .30

RL: INR

PA: $00 \cdot$ ERIPAINA $\bullet$ Hoc loco $\bullet$ bT

OS: Eripaa $1992 \cdot \mathbf{P D}$

EN: ERIPAINA DOP.da.f106-00 • bT

EF: DICROGLOSSIDAE 1987.da.f004

ESPADARANINIA nov., DOP.da.f040 • KY

SI: $481 \bullet \mathbf{C I}:$ h374 • ST: 0.10 .30

RL: INR

PA: $00 \cdot$ ESPADARANINIA $\bullet$ Hoc loco $\bullet$ iT

$01 \cdot$ ESPADARANINOA $\cdot H o c$ loco $\bullet \mathbf{h T}$

OS: Espadarana $2009 \cdot \mathbf{P D}$

EN: (1) ESPADARANINIA DOP.da.f040-00・iT

(2) ESPADARANINOA DOP.da.040-01・hT

EF: CENTROLENIDAE 1951.ta.f001

EUBAPHIDAE Bonaparte, 1850.bb.f006• SG

SI: 098 • CI: h060 • ST: 0.10 .57

RL: < DENDROBATIDAE 1865.ca.f002 • RI: Dubois 1982.dc: 273

PA: $00 \cdot$ EUBAPHIDAE $\bullet$ Bonaparte 1850.bb: pl. • F

$01 \cdot$ EUBAPHINA $\bullet$ Bonaparte 1850.bb: pl. $\bullet \mathbf{b F}$

OS: Eubaphus $1831 \equiv$ Dendrobates $1830 \bullet \mathbf{O E}$ 
EN: (1) DENDROBATIDAE |1850.bb.f006|-1865.ca.f002-00 • F ")!')"

(4) DENDROBATINA |1850.bb.f006|-1865.ca.f002-05• b T

EF: DENDROBATIDAE |1850.bb.f006|-1865.ca.f002

EUPROCTITA Dubois ${ }^{+1}$, 2009.db.f002 • KY

SI: $386 \bullet \mathbf{C I}:$ h2 $286 \bullet \mathbf{S T}: 0.10 .30$

RL: INR

PA: $00 \cdot$ EUPROCTITA $・$ Dubois $^{+1}$ 2009.db: 50 • iT $01 \cdot$ EUPROCTINIA $\bullet$ Hoc loco $\bullet \mathbf{i T}$

OS: Euproctus $1839 \cdot$ OD

EN: EUPROCTINIA 2009.db.f002-01・iT

EF: SALAMANDRIDAE 1820.ga.f002

EUPSOPHIINAE Lutz, 1969.la.f003・JD

SI: $282 \bullet$ CI: h199・ST: 0.10 .40

RL: INR

PA: 00 • EUPSOPHIINAE • Lutz 1969.1a: 281 • bF

OS: Eupsophus $1843 \cdot \mathbf{O E}$

EN: ALSODIDAE 1869.ma.f005-02 • F

EF: ALSODIDAE 1869.ma.f005

EхCIDOBATINIA nov., DOP.da.f005 • KY

SI: $446 \cdot \mathbf{C I}:$ h339 • ST: 0.10 .30

RL: INR

PA: $00 \cdot$ EXCIDOBatINIA $\bullet$ Hoc loco $\bullet$ iT

OS: Excidobates $2008 \cdot \mathbf{P D}$

EN: EXCIDOBATINIA DOP.da.f005-00 • iT

EF: DENDROBATIDAE |1850.bb.f006|-1865.ca.f002

EXOBRANCHES Lataste, 1878.1b.f001 • AN

SI: $183 \bullet \mathbf{C I}:$ n055 • ST: 2.25 .50

RL: $\leftarrow$ PROTEINA 1831.ba.f002

PA: $00 \cdot$ EXOBRANCHES $\bullet$ Lataste 1878.1b: $3 \cdot \mathbf{F}$

OS: Proteus $1768 \cdot \mathbf{A N}$

EN: (1) PROTEOIDAE 1831.ba.f002-10 • eF

(2) PRoteidae 1831.ba.f002-02 • F

EF: PROTEIDAE 1831.ba.f002

FEIHYLITIES nov., DOP.da.f124・ KY

SI: $565 \bullet$ CI: h458 • ST: 0.10 .30

RL: INR

PA: $00 \cdot$ FEIHYLITIES $\bullet$ Hoc loco $\bullet$ Cn

OS: Feihyla $2006 \cdot \mathbf{P D}$

EN: FEIHYLITIES DOP.da.f124-00 • Cn

EF: RHACOPHORIDAE |1858.gc.f012|-1932.ha.f001

FEIRANINIA nov., DOP.da.f105 • KY

SI: $546 \bullet$ CI: h439・ST: 0.10 .30

RL: INR

PA: $00 \bullet$ FEIRANINIA $\cdot$ Hoc loco $\bullet$ iT

OS: Feirana $1992 \cdot$ PD

EN: FEIRANINIA DOP.da.f105-00・iT

EF: DiCROGLOSSIDAE 1987.da.f004

FEJERVARYINI $\mathrm{Fei}^{+2}$, 2010.fa.f005 • KY

SI: $396 \bullet \mathbf{C I}:$ h296・ST: 0.10 .30

RL: INR

PA: c0 • FEJERVARYINI $\bullet \mathrm{Fei}^{+2} 2010 . \mathrm{fa}: 17 \cdot$ T $~$ EEA: PD i1 - FEJERVAYINI $\bullet \mathrm{Fei}^{+2}$ 2010.fa: $28 \cdot \mathbf{T}$

OS: Fejervarya $1915 \cdot$ OD

EN: FEJERVARYINI 2010.fa.f005-c0 • T

EF: DICROGLOSSIDAE 1987.da.f004
FlectonotinaE nov., DOP.da.f012 • KY

SI: $453 \cdot \mathbf{C I}:$ h3 $46 \cdot \mathbf{S T}: 0.10 .30$

RL: INR

PA: $00 \cdot$ FLECTONOTINAE $\bullet$ Hoc loco $\bullet \mathbf{b F}$

OS: Flectonotus $1926 \cdot \mathbf{P D}$

EN: FLECTONOTINAE DOP.da.f012-00 • bF

EF: HEMIPHRACTIDAE 1862.pa.f001

FritzianinaE nov., DOP.da.f013 • KY

SI: 454 • CI: h347 • ST: 0.10 .30

RL: INR

PA: $00 \cdot$ FRITZIANINAE $\bullet$ Hoc loco $\bullet \mathbf{b F}$

OS: Fritziana $1937 \cdot$ PD

EN: FRITZIANINAE DOP.da.f013-00 • bF

EF: HEMIPHRACTIDAE 1862.pa.f001

Frostini nov., DOP.da.f037 • KY

SI: 478 • CI: h371 • ST: 0.10 .30

RL: INR

PA: $00 \cdot$ FROSTIINI $\bullet$ Hoc loco $\bullet$ T

OS: Frostius 1986 • PD

EN: FROSTIINI DOP.da.f037-00 • T

EF: BUFONIDAE 1825.gb.f004

GASTROPHRYNAE Fitzinger, 1843.fa.f011 • PK

SI: $076 \bullet \mathbf{C I}:$ h044 • ST: 0.10 .37

RL: $\geq$ HYLAEDACTYLI 1843.fa.f009 • AI: Parker 1934.pa: 16

< MICROHYLIDAE 1931.na.f001 • PS: Dubois 1983.da: 274

PA: 00 • GASTROPHRYNAE • Fitzinger 1843.fa: 33 • F

01 - GASTROPHRYNIDAE • Metcalf 1923.ma: $25 \cdot \mathbf{F}$

02 - GASTROPHRYNINAE • Metcalf 1923.ma: $294 \cdot \mathbf{b F}$

03 - GASTROPHRYNINI • Dubois 2005.da: $15 \cdot \mathbf{T}$

$04 \cdot$ GASTROPHRYNINA $\cdot$ Hoc loco $\bullet$ bT

$05 \cdot$ GASTROPHRYNINIA $\cdot$ Hoc loco $\bullet$ iT

OS: Gastrophryne $1843 \cdot \mathbf{O E}$

EN: (1) GASTROPHRYNINAE 1843.fa.f011-02 • bF

(2) GASTROPHRYNINI 1843.fa.f011-03 • T

(3) GASTROPHRYNINA 1843.fa.f011-04 • bT

(4) GASTROPHRYNINIA 1843.fa.f011-05 • iT

EF: MICROHYLIDAE |1843.fa.f012|-1931.na.f001

GASTROPHRYNOIDINI nov., DOP.da.f080 • KY

SI: $521 \cdot \mathbf{C I}: \mathrm{h} 414 \cdot \mathbf{S T}: 0.10 .30$

RL: INR

PA: $00 \cdot$ GASTROPHRYNOIDINI $\bullet$ Hoc loco $・$ T

OS: Gastrophrynoides $1926 \cdot \mathbf{P D}$

EN: GASTROPHRYNOIDINI DOP.da.f080-00 • T

EF: MICROHYLIDAE |1843.fa.f012|-1931.na.f001

GASTROTHECINAE Noble, 1927.na.f001 • KY

SI: $214 \cdot \mathbf{C I}:$ h1 $149 \bullet \mathbf{S T}: 0.10 .30$

RL: INR

PA: $00 \bullet$ GASTROTHECINAE • Noble 1927.na: 93・bF

$01 \cdot$ GASTROTHECINI $\bullet$ Hoc loco $\bullet$ T

OS: Gastrotheca $1843 \cdot \mathbf{O E}$

EN: GASTROTHECINI 1927.na.f001-01・ T

EF: HEMIPHRACTIDAE 1862.pa.f001

GENYOPHRYNIDAE Boulenger, 1890.ba.f001 • JD

SI: $191 \bullet \mathbf{C I}:$ h132 • ST: 0.10 .40

RL: INR

PA: 00 - GENYOPHRYNIDAE • Boulenger 1890.ba: $327 \cdot \mathbf{F}$ 
01 - GENYOPHRYNINAE • Gadow 1901.ga: xi, 236 • bF

OS: Genyophryne $1890 \approx$ Asterophrys $1838 \cdot$ OD

EN: (1) ASTEROPHRYINAE 1858.gc.f006-05 • bF

(2) ASTEROPHRYINI 1858.gc.f006-09 • T

EF: MICROHYLIDAE |1843.fa.f012|-1931.na.f001

GEOTRITONIDAE Bonaparte, 1850.bb.f016 • CG

SI: $108 \cdot$ CI: h070 • ST: 0.10 .62

RL: INR

PA: $00 \cdot$ GEOTRITONIDAE • Bonaparte 1850.bb: pl. • F $01 \cdot$ GEOTRITONINA $\bullet$ Bonaparte 1850.bb: pl. $\bullet \mathbf{b F}$

OS: Geotriton $1832 \mathbf{C I} \approx$ Lissotriton $1839 \cdot \mathbf{O E}$

EN: LISSOTRITONITA 2017.da.fe2-00 • hT

EF: SALAMANDRIDAE 1820.ga.f002

GEOTRYPETIDAE Lescure ${ }^{+2}$, 1986.1b.f001・ KY

SI: $320 \bullet \mathbf{C I}:$ h228 • ST: 0.10 .30

RL: INR

PA: 00 • GEOTRYPETIDAE • Lescure ${ }^{+2}$ 1986.lb: 145 • F

$01 \cdot$ GEOTRYPETOIDAE $\bullet$ Lescure $^{+2}$ 1986.lb: $162 \cdot \mathbf{e F}$

$02 \cdot$ GEOTRYPETINOA $•$ HOC loco $\bullet \mathbf{h T}$

OS: Geotrypetes $1880 \cdot \mathbf{O E}$

EN: GEOTRYPETINOA 1986.1b.f001-02 • hT

EF: CAECILIIDAE 1814.ra.f003-|1825.gb.f008|

GEYERIELLINAE Brame, 1958.ba.f004 + AN

SI: $260 \bullet \mathbf{C I}: \mathrm{n} 075 \bullet \mathbf{S T}: 0.28 .50$

RL: INR

PA: $00 \cdot$ GEYERIELLINAE • Brame 1958.ba: 5 • bF

OS: Geyeriella $1950+\cdot \mathbf{O E}$

EN: HYNOBIIDAE |1856.ha.f001|-1859.cb.f002-01 • F

EF: HYNOBIIDAE |1856.ha.f001|-1859.cb.f002

GHATIXALITOES nov., DOP.da.f125・ KY

SI: $566 \bullet$ CI: h459・ST: 0.10 .30

RL: INR

PA: $00 \cdot$ GHATIXALITOES $\bullet$ Hoc loco $\bullet$ iCn

OS: Ghatixalus $2008 \cdot \mathbf{P D}$

EN: GHATIXALITOES DOP.da.f125-00 • iCn

EF: RHACOPHORIDAE |1858.gc.f012|-1932.ha.f001

GLANDIRANINI $\mathrm{Fei}^{+2}$, 2010.fa.f016 • KY

SI: $407 \cdot \mathbf{C I}:$ h307 • ST: 0.10 .30

RL: INR

PA: $00 \bullet$ GLANDIRANINI $\bullet \mathrm{Fei}^{+2}$ 2010.fa: $18 \cdot \mathbf{T}$

$01 \cdot$ GLANDIRANINOA $\bullet$ Hoc loco $\bullet \mathbf{h T}$

OS: Glandirana 1990• OD

EN: GLANDIRANINOA 2010.fa.f016-01 • hT

EF: RANIDAE 1796.ba.f001

GOBIATIDAE Roček ${ }^{+1}$, 1991.ra.f001 +• KY

SI: $347 \cdot \mathbf{C I}:$ h254 - ST: 0.10 .30

RL: INR

PA: 00 • GobiatidaE $\bullet$ Roček $^{+1}$ 1991.ra: 78 • F

01 $\cdot$ GOBIATINAE $\bullet$ Barbadillo $^{+2}$ 1997.ba: $55 \cdot \mathbf{b F}$

OS: Gobiates 1986 \% OE

EN: GOBIATIDAE 1991.ra.f001-00†・bT

EF: GOBIATIDAE 1991.ra.f001 †

GRACIXALINOA nov., DOP.da.f117 • KY

SI: $558 \cdot \mathbf{C I}: \mathrm{h} 451 \cdot \mathbf{S T}: 0.10 .30$

RL: INR

PA: $00 \cdot$ GRACIXALINOA $\bullet$ Hoc loco $\bullet \mathbf{h T}$
OS: Gracixalus 2005 • PD

EN: GRACIXALINOA DOP.da.f117-00 • hT

EF: RHACOPHORIDAE |1858.gc.f012|-1932.ha.f001

GRANDISONIILAE Lescure ${ }^{+2}$, 1986.1b.f004 • KY

SI: $323 \cdot \mathbf{C I}:$ h231 - ST: 0.10 .30

RL: INR

PA: $00 \cdot$ GRANDISONIILAE $\bullet$ Lescure $^{+2}$ 1986.1b: $163 \cdot \mathbf{i F}$

01 - GRANDISONIINA $\cdot$ Hoc loco $\bullet \mathbf{b T}$

$02 \cdot$ GRANDISONIINIA $\cdot$ Hoc loco $\bullet$ iT

OS: Grandisonia $1968 \cdot \mathbf{O E}$

EN: (1) GRANDISONIINA 1986.lb.f004-01 • bT

(2) GRANDISONIINIA 1986.lb.f004-02 • iT

EF: CAECILIIDAE 1814.ra.f003-|1825.gb.f008|

GRILLITSCHIINA nov., DOP.da.f148 • KY

SI: $594 \cdot \mathbf{C I}:$ h486 • ST: 0.10 .30

RL: INR

PA: $00 \cdot$ GRILLITSCHIINA $\bullet$ Hoc loco $\bullet \mathbf{b T}$

OS: Grillitschia DOP • PD

EN: GRILLITSCHIINA DOP.da.f148-00 • bT

EF: MEGOPHRYIDAE 1850.bb.f008-|1931.na.f003|

GRYPISCINA Mivart, 1869.ma.f012 • JD

SI: $172 \cdot \mathbf{C I}:$ h120 • ST: 0.10 .40

RL: INR

PA: $00 \cdot$ GRYPISCINA • Mivart 1869.ma: $295 \cdot \mathbf{b F}$

01 - GRYPISCINI • Lynch 1969.1b: $3 \cdot$ T

$02 \cdot$ GRYPISCINAE $\bullet$ Ardila-Robayo 1979.aa: $455 \cdot \mathbf{b F}$

OS: Grypiscus $1867 \approx$ Cycloramphus $1838 \cdot \mathbf{O E}$

EN: (1) CYCLORAMPHEIDAE 1850.bb.f003-|1852.ba.f001|-05 • aF

(2) CYCLORAMPHIDAE 1850.bb.f003-|1852.ba.f001|-04 • F

EF: CYCLORAMPHIDAE 1850.bb.f003-|1852.ba.f001|

GYMNODERMIA Rafinesque, 1815.ra.f001 • AN

SI: $006 \bullet \mathbf{C I}: \mathrm{n} 003 \cdot \mathbf{S T}: 2.25 .50$

RL: INR

PA: $00 \bullet$ GYMNODERMIA $\bullet$ Rafinesque 1815.ra: $78 \cdot \mathbf{F}$

OS: „ 2 PN, including: Cecilia 1814 E Caecilia 1758 - PD

EN: (1) CAECILIOIDEA 1814.ra.f003-|1825.gb.f008|-19・pF "'!")"

(5) CAECILIINA 1814.ra.f003-|1825.gb.f008|-26・bT

EF: CAECILIIDAE 1814.ra.f003-|1825.gb.f008|

GYMNOPHIDES Latreille, 1825.la.f001 • AN

SI: $023 \cdot \mathbf{C I}: \mathrm{n} 010 \bullet \mathbf{S T}: 2.25 .50$

RL: INR

PA: $00 \cdot$ GYMNOPHIDES $\bullet$ Latreille 1825.la: $103 \cdot \mathbf{F}$

OS: Caecilia $1758 \cdot \mathbf{O M}$

EN: (1) CAECILIOIDEA 1814.ra.f003-|1825.gb.f008|-19 • pF ")!"!

(5) CAECILIINA 1814.ra.f003-|1825.gb.f008|-26・bT

EF: CAECILIIDAE 1814.ra.f003-|1825.gb.f008|

GYMNOPIILAE Lescure Le $^{+2}$ 1986.lb.f009 • JD

SI: $328 \bullet \mathbf{C I}:$ h236 • ST: 0.10 .40

RL: INR

PA: $00 \cdot$ GYMNOPILAE $\bullet$ Lescure $^{+2}$ 1986.lb: $168 \cdot \mathbf{i F}$

OS: Gymnopis $1874 \cdot \mathbf{O E}$

EN: (1) DERMOPHIINIA 1969.ta.f002-04 • iT

(2) DERMOPHIINOA 1969.ta.f002-05 • hT

EF: CAECILIIDAE 1814.ra.f003-|1825.gb.f008| 
GYRINOPHILITA Dubois, 2008.da.f006• AN

SI: $379 \bullet$ CI: n096 • ST: 0.22 .50

RL: INR

PA: 00 • GYRINOPHILITA • Dubois 2008.da: $74 \cdot$ iT

OS: Gyrinophilus $1869 \cdot \mathbf{O E}$

EN: PSEUDOTRITONINA 2012.da.f005-00 • bT

EF: PLETHODONTIDAE 1850.ga.f001

HAMPTOPHRYNINIA nov., DOP.da.f088 • KY

SI: $529 \bullet$ CI: h422 • ST: 0.10 .30

RL: INR

PA: 00 • HAMPTOPHRYNINIA $\bullet$ Hoc loco $\bullet \mathbf{i T}$

OS: Hamptophryne $1954 \cdot \mathbf{P D}$

EN: HAMPTOPHRYNINIA DOP.da.f088-00 • iT

EF: MICROHYLIDAE |1843.fa.f012|-1931.na.f001

HELEIOPORIDAE Bauer, 1986.ba.f001 • AN

SI: $316 \bullet$ CI: n088 • ST: 0.28 .50

RL: INR

PA: 00 • HELEIOPORIDAE • Bauer 1986.ba: $7 \bullet \mathrm{F}$

OS: Heleioporus $1841 \cdot \mathrm{PD}$

EN: HELEIOPORINA 2017.da.f71-00 • bT

EF: MYOBATRACHIDAE 1850.sa.f001

HeLEIOPORIDAE Bauer, 1987.bc.f002 • KY

SI: 332 • CI: h240 • ST: 0.10 .30

RL: INR

PA: 00 • HELEIOPORIDAE $\bullet$ Bauer 1987.bc: $52 \cdot \mathbf{F}$ $01 \cdot$ HELEIOPORINA $\bullet$ Hoc loco $\bullet \mathbf{b T}$

OS: Heleioporus $1841 \cdot \mathbf{P D}$

EN: HELEIOPORINA 1987.bc.f002-01 • bT

EF: MYOBATRACHIDAE 1850.sa.f001

HeleOphryninaE Noble, 1931.na.f004 • KY

SI: $219 \bullet$ CI: h154 • ST: 0.10 .30

RL: INR

PA: 00 • HELEOPHRYNINAE • Noble 1931.na: $498 \cdot \mathbf{b F}$ $01 \cdot$ HELEOPHRYNIDAE $\bullet$ Hoffman 1935.ha: $2 \cdot \mathbf{F}$ 02 - HELEOPHRYNOIDEA • Dubois 2005.da: $9 \cdot \mathbf{p F}$

OS: Heleophryne $1898 \cdot \mathbf{O E}$

EN: HELEOPHRYNIDAE 1931.na.f004-01 • F

EF: HELEOPHRYNIDAE 1931.na.f004

HELIOPHRYNIDAE Heyer, 1975.ha.f001 • JD

SI: $296 \bullet$ CI: h209 • ST: 0.10 .52

RL: INR

PA: 00 • HELIOPHRYNIDAE $\bullet$ Heyer 1975.ha: $48 \cdot \mathbf{F}$ $01 \cdot$ HELIOPHRYNINAE $\bullet$ Laurent 1980.1a: $417 \cdot \mathbf{b F}$

OS: Heliophryne $1975 \equiv$ Heleophryne $1898 \cdot \mathbf{O E}$

EN: HELEOPHRYNIDAE 1931.na.f004-01 • F

EF: HELEOPHRYNIDAE 1931.na.f004

Hemidactylidae Hallowell, 1856.ha.f003 • KY

SI: $123 \cdot$ CI: h082 • ST: 0.10 .31

RL: $\geq$ BoLITOGLOSSIDAE 1856.ha.f002 - AI: Dubois 2005.da: 5

PA: $00 \bullet$ HEMIDACTYLIDAE $\bullet$ Hallowell 1856.ha: $11 \cdot \mathbf{b F}$

01 HEMIDACTYLIDAE $\bullet$ Hoffmann 1878.ha: $585 \cdot \mathbf{F}$

02 $\cdot$ HEMIDACTYLIIDAE $\bullet$ Cope 1889.ca: $119 \cdot \mathbf{b F}$

03 HEMIDACTYLIINI $\bullet$ Wake 1966.wa: $1 \cdot$ T

04 HEMIDACTYLINI • Brame 1967.ba: $13 \cdot \mathbf{T}$

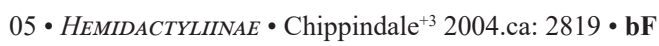

OS: Hemidactylium $1838 \cdot \mathbf{O E}$
EN: (1) HEMIDACTYLIINAE 1856.ha.f003-05 • bF

(2) HEMIDACTYLIINI 1856.ha.f003-03 • T

EF: PLETHODONTIDAE 1850.ga.f001

HEMIGNATHODONTINAE Miranda-Ribeiro, 1926.ma.f006 • AN

SI: $213 \bullet$ CI: $\mathrm{n} 061 \bullet \mathbf{S T}: 2.25 .50$

RL: INR

PA: $00 \bullet$ HEMIGNATHODONTINAE • Miranda-Ribeiro 1926.ma: 65 $\cdot \mathbf{b F}$

OS: » 5 PN, including: Gastrotheca $1843 \cdot \mathbf{P D}$

EN: GASTROTHECINI 1927.na.f001-01 • T

EF: HEMIPHRACTIDAE 1862.pa.f001

HEMIMANTIDAE Hoffmann, 1878.ha.f002 • CI

SI: $180 \bullet$ CI: h124 $\bullet$ ST: 0.10 .45

RL: < PHRYNOBATRACHINAE 1941.lb.f001 • PP: Opinion 1921

(Anonymous 1999.aa)

PA: 00 • HEMIMANTIDAE $\bullet$ Hoffmann 1878.ha: $613 \cdot \mathbf{b F}$

$01 \cdot$ HEMIMANTINAE $\bullet$ Dubois 1982.db: $136 \cdot \mathbf{b F}$

OS: Hemimantis $1863 \approx$ Phrynobatrachus $1862 \cdot$ OE

EN: (1) PHRYNOBATRACHOIDEA 1941.lb.f001-02 • pF

(2) PHRYNOBATRACHIDAE 1941.lb.f001-01 • F

EF: PHRYNOBATRACHIDAE 1941.lb.f001

HemiphractidaE Peters, 1862.pa.f001 • KY

SI: $149 \bullet$ CI: h100 • ST: 0.10 .30

RL: INR

PA: $00 \cdot$ HEMIPHRACTIDAE $\bullet$ Peters 1862.pa: $146 \cdot \mathbf{F}$

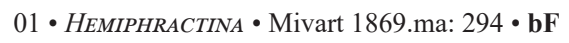

02 • HEMIPHRACTINA • Jiménez de la Espada 1870.ja: $62 \cdot$ Sc

03 • HEMIPHRACTINAE $\bullet$ Gadow 1901.ga: xi, $210 \bullet \mathbf{b F}$

$04 \cdot$ HEMIPHRACTYDAE $\bullet$ Miranda-Ribeiro 1926.ma: $119 \cdot \mathbf{F}$

OS: Hemiphractus $1828 \cdot \mathbf{O E}$

EN: (1) HEMIPHRACTIDAE 1862.pa.f001-00 • F

(2) HEMIPHRACTINAE 1862.pa.f001-03 • bF

EF: HEMIPHRACTIDAE 1862.pa.f001

HEMISALAMANDRAE Goldfuss, 1820.ga.f001 • AN

SI: $011 \cdot \mathbf{C I}: \mathrm{n} 007$ • ST: 2.25 .50

RL: INR

PA: $00 \cdot$ HEMISALAMANDRAE $\bullet$ Goldfuss 1820.ga: $\mathbf{x} \cdot \mathbf{F}$

$01 \cdot$ HEMISALAMANDRAE $\bullet$ Jourdan 1834.ja: $585 \bullet \mathbf{T}$

OS: » 2 PN, including: Siren $1766 \cdot \mathbf{P D}$

EN: SIRENIDAE 1825.gb.f005-00 • F

EF: SIRENIDAE 1825.gb.f005

HeMisIDAE Cope, 1867.ca.f002・ KY

SI: $159 \bullet \mathbf{C I}:$ h107 • ST: 0.10 .30

RL: INR

PA: $00 \bullet$ HEMISIDAE $\bullet$ Cope 1867.ca: $198 \cdot \mathbf{F}$

01 $\operatorname{HEMISINA} \bullet$ Mivart 1869.ma: $288 \cdot \mathbf{b F}$

$02 \cdot$ HEMISOTINA $\bullet$ Günther 1870.ga: $119 \cdot \mathbf{b F}$

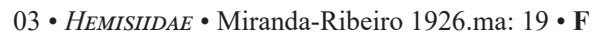

$04 \cdot$ HEMISINAE $\bullet$ Noble 1931.na: $540 \bullet \mathbf{b F}$

05 HEMISOTIDAE Frost $^{+1}$ 1987.fa: $24 \cdot \mathbf{F}$

06 • HEMISOTOIDAE $\bullet$ Dubois 1992.da: $209 \cdot \mathbf{e F}$

OS: Hemisus $1859 \cdot \mathbf{O E}$

EN: HEMISOTIDAE 1867.ca.f002-05 • F

EF: HEMISOTIDAE 1867.ca.f002

HERPELINAE Laurent, 1984.1a.f001 • KY

SI: $313 \bullet$ CI: h223 • ST: 0.10 .30 
RL: INR

PA: 00 • HERPELINAE • Laurent 1984.la: $199 \cdot \mathbf{b F}$

$01 \cdot$ HERPELOIDI $・$ Lescure $^{+2}$ 1986.lb: $163 \cdot \mathbf{b T}$

$02 \cdot$ HERPELINI $\bullet$ Lescure $^{+2}$ 1986.1b: $163 \cdot \mathbf{T}$

03 $\cdot$ HERPELITI $・$ Lescure $^{+2}$ 1986.lb: $164 \cdot$ iT

OS: Herpele $1880 \cdot \mathbf{O E}$

EN: (1) HERPELINAE 1984.la.f001-00 • bF

(2) HERPELINI 1984.la.f001-02 • T

EF: CAECILIIDAE 1814.ra.f003-|1825.gb.f008|

HoloadeninaE Hedges $^{+2}$, 2008.ha.f004 • KY

SI: $384 \cdot \mathbf{C I}:$ h2 $284 \cdot$ ST: 0.10 .37

RL: < STRABOMANTIDAE 2008.ha.f004 - PR: Hedges ${ }^{+2}$ 2008: 5

PA: $00 \bullet$ HOLOADENINAE $\bullet$ Hedges $^{+2}$ 2008.ha: $5 \cdot$ bF

$01 \cdot$ HOLOADENINIA $\bullet$ Hoc loco $\bullet$ iT

$02 \cdot$ HOLOADENINOA $\bullet$ Hoc loco $\bullet \mathbf{h T}$

OS: Holoaden $1920 \cdot$ OD

EN: (1) HOLOADENINIA 2008.ha.f004-01・iT

(2) HOLOADENINOA 2008.ha.f004-02 • hT

EF: BRACHYCEPHALIDAE 1858.gc.f002

HoplobatrachinI $\mathrm{Fei}^{+2}, 2010 . \mathrm{fa} . \mathrm{f004}$ • JD

SI: 395 • CI: h295 • ST: 0.10 .40

RL: INR

PA: $00 \cdot$ HOPLOBATRACHINI $\bullet \mathrm{Fei}^{+2}$ 2010.fa: $17 \cdot \mathbf{T}$

OS: Hoplobatrachus $1863 \cdot$ OD

EN: (1) DICROGLOSSEIDAE 1987.da.f004-03 • aF ")"')

(5) DICROGLOSSINA 1987.da.f004-04 • bT

EF: DiCROGLOSSIDAE 1987.da.f004

HoPlopHRYNINAE Noble, 1931.na.f016・ KY

SI: $231 \bullet \mathbf{C I}:$ h165 • ST: 0.10 .30

RL: INR

PA: 00 • HOPLOPHRYNINAE • Noble 1931.na: 539 • bF

$01 \cdot$ HOPLOPHRYNIDAE Bossuyt $^{+1}$ 2009.ba: $358 \cdot \mathbf{F}$

OS: Hoplophryne $1928 \cdot \mathbf{O E}$

EN: HOPLOPHRYNINAE 1931.na.f016-00 • bF

EF: MICROHYLIDAE |1843.fa.f012|-1931.na.f001

HYALINOBATRACHINAE Guayasamin ${ }^{+5}$, 2009.ga.f002 • KY

SI: $390 \bullet \mathbf{C I}:$ h2 $290 \cdot \mathbf{S T}: 0.10 .30$

RL: INR

PA: $00 \cdot$ HYALINOBATRACHINAE • Guayasamin ${ }^{+5}$ 2009.ga: $3 \cdot \mathbf{b F}$

OS: Hyalinobatrachium 1991 • OD

EN: HYALINOBATRACHINAE 2009.ga.f002-00 • F

EF: CENTROLENIDAE 1951.ta.f001

HYDROMANTINI Dubois, 2008.da.f003 • AN

SI: $376 \bullet \mathbf{C I}: \mathrm{n} 093 \cdot \mathbf{S T}: 0.22 .50$

RL: INR

PA: $00 \cdot$ HYDROMANTINI • Dubois 2008.da: $72 \cdot \mathbf{T}$

01 HYDROMANTINA • Dubois 2008.da: $74 \cdot \mathbf{T}$

OS: Hydromantes $1848 \cdot \mathbf{O E}$

EN: (1) HYDROMANTINI 2012.wa.f003-00 • T

(2) HYDROMANTINA 2012.wa.f003-01 • bT

EF: PLETHODONTIDAE 1850.ga.f001

HYDROMANTINI Vieites ${ }^{+3}$, 2011.va.f002 • AN

SI: $412 \bullet \mathbf{C I}: \mathrm{n} 100 \bullet \mathbf{S T}: 0.28 .50$

RL: INR

PA: 00 • HYDROMANTINI • Vieites ${ }^{+3}$ 2011.va: $633 \cdot \mathbf{T}$
OS: Hydromantes $1848 \cdot$ OD

EN: (1) HYDROMANTINI 2012.wa.f003-00 • T

(2) HYDROMANTINA 2012.wa.f003-01 • b T

EF: PlethodontidaE 1850.ga.f001

HydRomantini Wake, 2012.f003 • KY

SI: 417 • CI: h311 • ST: 0.10 .30

RL: INR

PA: $00 \bullet$ HYDROMANTINI • Wake 2012.wa: $80 \bullet$ T

01 • HYDROMANTINA • Hoc loco • b T

OS: Hydromantes 1848 - OD

EN: (1) HYDROMANTINI 2012.wa.f003-00 • T

(2) HYdRomantina 2012.wa.f003-01 • b T

EF: PLETHODONTIDAE 1850.ga.f001

HYDROMANTINA Dubois ${ }^{+1}$, 2012.da.f009 • JI

SI: $426 \bullet \mathbf{C I}:$ h320 • ST: 0.10 .40

RL: INR

PA: $00 \cdot$ HYDROMANTINA • Dubois ${ }^{+1}$ 2012.da: $118 \cdot$ bT

OS: Hydromantes 1848 - OD

EN: (1) HYDROMANTINI 2012.wa.f003-00・ T

(2) HYDROMANTINA 2012.wa.f003-01 • bT

EF: PleTHODONTIDAE 1850.ga.f001

HYLAEDACTYLI Fitzinger, 1843.fa.f009 • KY

SI: 074 • CI: h042 • ST: 0.10 .37

RL: $\leq$ GASTROPHRYNAE 1843.fa.f012 • AI: Parker 1934.pa: 16

PA: $00 \cdot$ HYLAEDACTYLI $\bullet$ Fitzinger 1843.fa: $33 \cdot \mathbf{F}$

01 - HYLAEDACTYLIDAE $\bullet$ Bonaparte 1850.bb: pl. • F

$02 \cdot H Y L A E D A C T Y L I N A \cdot$ Bonaparte 1850.bb: pl. • bF

03 - HYLAEDACTYLIDAE • Hoffmann 1878.ha: 614 • bF

$04 \cdot$ HYLAEDACTYLIDA $・$ Knauer 1878.ka: $112 \cdot \mathbf{F}$

$05 \cdot H Y L A E D A C T Y L I N A \cdot H o c$ loco $\bullet \mathbf{b T}$

$06 \cdot$ HYLAEDACTYLINIA $\cdot$ Hoc loco $\bullet \mathbf{i T}$

OS: Hylaedactylus $1841 \approx$ Kaloula $1831 \cdot$ OE

EN: (1) HYLAEDACTYLINA 1843.fa.f009-05 • bT

(2) HYLAEDACTYLINIA 1843.fa.f009-06・ iT

EF: MICROHYLIDAE |1843.fa.f012|-1931.na.f001

HYLAEFORMES Duméril ${ }^{+1}$, 1841.da.f002 • AN

SI: $061 \bullet \mathbf{C I}: \mathrm{n} 028 \cdot \mathbf{S T}: 2.27 .50$

RL: INR

PA: $00 \cdot$ HYLAEFORMES • Duméril ${ }^{+1}$ 1841.da: $50 \cdot \mathbf{F}$

01 HYLAEFORMES • Desmarest 1857.da: $13 \cdot \mathbf{F}$

OS: Hyla $1768 \cdot \mathbf{O E}$

EN: (1) HYLOIDEA 1815.ra.f002-|1825.gb.f001|-20・pF "')"।

(8) HYLITES 1815.ra.f002-|1825.gb.f001|-26 • Cn

EF: HYLIDAE 1815.ra.f002-|1825.gb.f001|

HYLAEOBATRACHIDAE Lydekker, 1889.1a.f001 + KY

SI: $190 \bullet \mathbf{C I}:$ h131 • ST: 0.10 .30

RL: INR

PA: $00 \bullet$ HYLAEOBATRACHIDAE $\bullet$ Lydekker 1889.1a: 1040 • F

01 - HYAELOBATRACHOIDEA • Huene 1931.ha: $310 \cdot \mathbf{p F}$

02 HYALLOBATRACHIDAE • Huene 1931.ha: 310 • F

03 - HYLAEOBATRACHOIDEA • Kuhn 1965.ka: 39 • F

OS: Hylaeobatrachus $1884+\cdot \mathbf{O E}$

EN: HYLAEOBATRACHIDAE 1889.la.f001-00 † • F

EF: HyLAEOBATRACHIDAE 1889.1a.f001 † 
HYLAPLESINA Günther, 1858.gc.f001 • CG

SI: $129 \bullet$ CI: h083 • ST: 0.10 .62

RL: INR

PA: 00 • HYLAPLESINA • Günther 1858.gc: $345 \cdot$ Sc $01 \cdot$ HYLAPLESIDAE $\bullet$ Günther 1858.gc: $341 \cdot \mathbf{F}$ 02 • HYLAPLESURA • Wood 1863.wa: $174 \cdot$ Sc 03 • HYLAPLESIINA • Günther 1868.gb: $148 \cdot \mathbf{U F}$ $04 \cdot H_{Y L A P L E S I I D A E} \bullet$ Cope 1875.ca: $8 \cdot \mathbf{F}$ 05 HYLAPLESINA $\bullet$ Hoffmann 1878.ha: $614 \cdot \mathbf{F}$ 06 HYLAPLESIDAE $\bullet$ Hoffmann 1878.ha: $614 \cdot \mathbf{b F}$ $07 \cdot$ HYLAPLESIIDA $^{\circ}$ Knauer 1878.ka: $112 \cdot \mathbf{F}$

OS: Hylaplesia $1826 \mathbf{C I} \approx$ Boana $1825 \cdot \mathbf{O E}$

EN: (1) COPHOMANTINAE 1878.ha.f004-02 • bF "1")"

(4) Cophomantinia 1878.ha.f004-04 • iT

EF: HYLIDAE 1815.ra.f002-|1825.gb.f001|

HYLARANINI Fei ${ }^{+2}$, 2010.fa.f012 • JD

SI: $403 \cdot \mathbf{C I}:$ h303 • ST: 0.10 .40

RL: INR

PA: $00 \cdot$ HYLARANINI $\bullet \mathrm{Fei}^{+2} 2010 . \mathrm{fa}: 18 \cdot \mathbf{T}$

OS: Hylarana $1838 \cdot$ OD

EN: LIMNODYTINOA 1843.fa.f001-02 • hT

EF: RANIDAE 1796.ba.f001

HYLARANINI Fei ${ }^{+2}$, 2010.fa.f012 • JD

SI: $403 \cdot \mathbf{C I}:$ h303 • ST: 0.10 .40

RL: INR

PA: $00 \cdot$ HYLARANINI $\bullet \mathrm{Fei}^{+2} 2010$.fa: $18 \cdot \mathbf{T}$

OS: Hylarana $1838 \cdot$ OD

EN: LIMNODYTINOA 1843.fa.f001-02 • hT

EF: RANIDAE 1796.ba.f001

HyLARINIA Rafinesque, 1815.ra.f002 • MK

SI: $007 \bullet$ CI: h004 • ST: 0.10 .58

RL: < HYLINA 1825.gb.f001 • MK: Dubois 1983.da: 274

PA: 00 $\operatorname{HYLARINIA} \bullet$ Rafinesque 1815.ra: $78 \cdot \mathbf{F}$

OS: Hylaria $1814 \equiv$ Hyla $1768 \cdot \mathbf{O E}$

EN: (1) HYLOIDEA 1815.ra.f002-|1825.gb.f001|-20 • pF "m)"

(8) HYLITES 1815.ra.f002-|1825.gb.f001|-26 • Cn

EF: HYLIDAE 1815.ra.f002-|1825.gb.f001|

HyLINA Gray, 1825.gb.f001 • MK

SI: $015 \bullet$ CI: h006 • ST: 0.10 .34

RL: > HYLARINIA 1815.ra.f002 • MK: Dubois 1983.da: 274

PA: $00 \cdot$ HYLINA $\bullet$ Gray 1825.gb: $213 \cdot \mathbf{U F}$

01 $H_{Y L A D A E} \cdot$ Boie 1828.ba: $363 \cdot \mathbf{F}$

$02 \cdot H_{Y L E N A E} \cdot$ Gray 1829 .ga: $203 \cdot \mathbf{U F}$

$03 \cdot H_{Y L A E} \bullet$ Fitzinger 1832.fa: $327 \cdot \mathbf{F}$

$04 \cdot$ HYLINA $\bullet$ Jourdan 1834.ja: $621 \cdot \mathbf{F}$

05 HYLADINA $\bullet$ Bonaparte 1838.ba: [195] $\bullet \mathbf{b F}$

06 ILADINI • Bonaparte 1838.ba: [196] • UF

$07 \cdot$ HYLOIDEA $\bullet$ Holbrook 1842.ha: $113 \cdot \mathbf{F}$

08 HYLAINA Bonaparte 1845.ba: $378 \cdot \mathbf{b F}$

09 HYLIDAE Bonaparte 1850.bb: pl. $\bullet \mathbf{F}$

$10 \cdot$ HYLINA Bonaparte 1850.bb: pl. $\bullet \mathbf{b F}$

$11 \cdot H_{Y T I D A E} \bullet$ Bonaparte 1852.ba: $477 \cdot \mathbf{F}$

12 • HYLINA • Günther 1858.gc: $344 \cdot$ Sc

$13 \cdot$ HYLOIDES $\bullet$ Bruch 1862.ba: $221 \cdot \mathbf{F}$
14 • HYLAEIDES • Gouriet 1868.ga: $206 \cdot \mathbf{F}$

15 - HYLIDAE $\bullet$ Hoffmann 1878.ha: $614 \cdot \mathbf{b F}$

16 HYLIDA $\bullet$ Knauer 1878.ka: $109 \cdot \mathbf{F}$

17 • HYLIDA • Bayer 1885.ba: $18 \cdot \mathbf{F}$

18 HYLIDI $^{*}$ Acloque 1900.aa: $489 \cdot \mathbf{F}$

19 • HYLINAE • Gadow 1901.ga: xii, $189 \cdot \mathbf{b F}$

20 HYLOIDEA • Dubois 1983.da: $272 \cdot \mathbf{p F}$

$21 \cdot$ HYLINI Faivovich $^{+5}$ 2005.fa: $3 \cdot$ T

$22 \cdot$ HYLOIDIA $^{\circ}$ Fouquette $^{+1}$ 2014.fa: $7 \cdot \mathbf{e F}$

$23 \cdot$ HYLINA $\cdot H o c l o c o \cdot \mathbf{b T}$

$24 \cdot$ HYLINIA $\cdot H o c$ loco $\bullet \mathbf{i T}$

$25 \cdot$ HYLINOA $\cdot$ Hoc loco $\bullet \mathbf{h T}$

$26 \cdot$ HYLITES $\bullet H O c$ loco $\bullet \mathbf{C n}$

OS: Hyla $1768 \cdot \mathbf{O E}$

EN: (1) HYLOIDEA 1815.ra.f002-|1825.gb.f001|-20 • pF

(2) HYLIDAE 1815.ra.f002-|1825.gb.f001|-09 • F

(3) HYLINAE 1815.ra.f002-|1825.gb.f001|-19 • bF

(4) HYLINI 1815.ra.f002-|1825.gb.f001|-21 • T

(5) HYLINA 1815.ra.f002-|1825.gb.f001|-23 • bT

(6) HYLINIA 1815.ra.f002-|1825.gb.f001|-24 • iT

(7) HYLINOA 1815.ra.f002-|1825.gb.f001|-25 • hT

(8) HYLITES 1815.ra.f002-|1825.gb.f001|-26 • Cn

EF: HYLIDAE 1815.ra.f002-|1825.gb.f001|

HyLiOlinae Dubois ${ }^{+2}$, 2017.da.f001 • KY

SI: $439 \bullet$ CI: h332 • ST: 0.10 .30

RL: INR

PA: $00 \cdot$ HYLIOLINAE $・$ Dubois $^{+2} 2017$.da: $55 \cdot$ bF

$01 \cdot$ HYLIOLINI $\bullet$ Dubois $^{+2} 2017$.da: $51 \cdot$ T

$02 \cdot$ HYLIOLINIA $\bullet$ Hoc loco $\bullet$ iT

OS: Hyliola $1899 \cdot$ OD

EN: HYLIOLINIA 2017.da.f001-02 • iT

EF: HYLIDAE 1815.ra.f002-|1825.gb.f001|

HyLODIDAE Günther, 1858.gc.f010 • KY

SI: $138 \bullet \mathbf{C I}:$ h091 • ST: 0.10 .30

RL: INR

PA: 00 • HYLODIDAE • Günther 1858.gc: $346 \bullet \mathbf{F}$

$01 \cdot H_{Y L O D E S} \bullet$ Cope 1866.ca: $90 \cdot \mathbf{G r}$

02 • HYLODINA • Mivart 1869.ma: $293 \cdot \mathbf{b T}$

03 $\cdot$ HYLODIDAE $\bullet$ Hoffmann 1878.ha: $614 \cdot \mathbf{b F}$

$04 \cdot H_{Y L O D I N A} \bullet$ Knauer 1878.ka: $112 \cdot \mathbf{b F}$

05 • HEYLODIDAE • Miranda-Ribeiro 1923.mb: $827 \cdot \mathbf{F}$

06 $\bullet$ HYLODINAE $\bullet$ Savage 1973.sa: $354 \cdot \mathbf{b F}$

OS: Hylodes $1826 \cdot \mathbf{O E}$

EN: HYLODINAE 1858.gc.f010-03 • bF

EF: CYCLORAMPHIDAE 1850.bb.f003-|1852.ba.f001|

HyLOSCIRTINa nov., DOP.da.f051 • KY

SI: 492 • CI: h385 • ST: 0.10 .30

RL: INR

PA: $00 \cdot$ HyLOSCIRTINA $\bullet$ Hoc loco $\bullet$ bT

OS: Hyloscirtus $1882 \cdot \mathbf{P D}$

EN: HYLOSCIRTINA DOP.da.f051-00 • bT

EF: HYLIDAE 1815.ra.f002-|1825.gb.f001|

HyLOXALINAE Grant ${ }^{+9}$, 2006.gb.f004 • KY

SI: $373 \cdot$ CI: h279 • ST: 0.10 .30

RL: INR

PA: $00 \cdot$ HYLOXALINAE Grant $^{+9}$ 2006.gb: $4 \cdot \mathbf{F}$ 
OS: Hyloxalus $1870 \cdot$ OD

EN: HYLOXALINAE 2006.gb.f004-00 • bF

EF: DENDROBATIDAE |1850.bb.f006|-1865.ca.f002

HYMENOCHIRIDAE Bolkay, 1919.ba.f001 • KY

SI: $201 \cdot \mathbf{C I}:$ h138 • ST: 0.10 .30

RL: INR

PA: $00 \cdot$ HYMENOCHIRIDAE $\bullet$ Bolkay 1919.ba: $343 \cdot \mathbf{F}$ $01 \cdot$ HYMENOCHIRINI $\bullet$ Bewick $^{+3}$ 2012.ba: $914 \cdot \mathbf{T}$

OS: Hymenochirus $1896 \cdot \mathbf{O E}$

EN: HYMENOCHIRINI 1919.ba.f001-01 ・ T

EF: PIPIDAE 1825.gb.f003-|1826.fb.f002|

HynobinnaE Cope, 1859.cb.f002 • SK

SI: $143 \cdot$ CI: h096 $\bullet$ ST: 0.10 .35

RL: > ELLIPSOGLOSSIDAE Hallowell, 1856.ha.f001 • PS: Dubois 1984.da: 114

PA: $00 \bullet$ HYNOBIINAE $\bullet$ Cope 1859.cb: $125 \cdot \mathbf{b F}$

$01 \cdot$ HYNOBIIDAE $\bullet$ Cope 1866.ca: $107 \cdot \mathbf{F}$

02 HYNOBIINAE $\cdot$ Hoffmann 1878.ha: $585 \cdot \mathbf{F}$

03 HYNOBIDAE $\cdot$ Highton 1940.ha: $40 \cdot \mathbf{F}$

$04 \cdot$ HYNOBIINI $^{\bullet}$ Dubois $^{+1}$ 2012.da: $113 \cdot \mathbf{T}$

05 HYNOBIINA Dubois $^{+1}$ 2012.da: $113 \cdot$ bT

$06 \cdot$ HYNOBIINIA $\bullet$ Hoc loco $\bullet$ iT

$07 \cdot \mathrm{HYNOBINOA} \cdot \mathrm{HOc}$ loco $\bullet \mathbf{h T}$

OS: Hynobius $1838 \cdot \mathbf{O E}$

EN: (1) HYNOBIIDAE |1856.ha.f001|-1859.cb.f002-01 • F

(2) HYNOBIINAE |1856.ha.f001|-1859.cb.f002-00 • bF

(3) HYNOBIINI |1856.ha.f001|-1859.cb.f002-04 • T

(4) HYNOBIINA |1856.ha.f001|-1859.cb.f002-05 • bT

(5) HYNOBIINIA |1856.ha.f001|-1859.cb.f002-06 • iT

(6) HYNOBIINOA |1856.ha.f001|-1859.cb.f002-07 • hT

EF: HYNOBIIDAE |1856.ha.f001|-1859.cb.f002

HYPEROLIINAE Laurent, 1943.lb.f001・ KY

SI: $240 \bullet$ CI: h172 • ST: 0.10 .30

RL: INR

PA: 00 • HYPEROLIINAE • Laurent 1943.lb: $16 \bullet \mathbf{b F}$

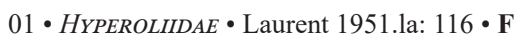

02 $\cdot$ HYPEROLIDAE $\bullet$ Casamiquela 1961.ca: $81 \cdot \mathbf{F}$

$03 \cdot$ HYPEROLIINI $\bullet$ Laurent 1972.la: $201 \cdot \mathbf{T}$

$04 \cdot$ HYPEROLIINA $\bullet$ Hoc loco $\bullet \mathbf{b T}$

OS: Hyperolius $1842 \cdot \mathbf{O E}$

EN: (1) HYPEROLIIDAE 1943.1b.f001-01 • F

(2) HYPEROLIINAE 1943.1b.f001-00 • bF

(3) HYPEROLIINI 1943.lb.f001-03 • T

(4) HYPEROLIINA 1943.lb.f001-04 • b T

EF: HYPEROLIIDAE 1943.1b.f001

HYPOCHTONINA Bonaparte, 1840.ba.f002 • JI

SI: $059 \bullet \mathbf{C I}:$ h031 • ST: 0.10 .52

RL: INR

PA: $00 \cdot$ HYPOCHTONINA $^{\circ}$ Bonaparte 1840.ba: $287 \cdot \mathbf{b F}$

$01 \cdot$ HYPOCHTHONINA $\bullet$ Bonaparte 1840.bb: $395 \cdot \mathbf{b F}$

02 $\bullet$ HYPOCHTHONIDAE $\bullet$ Bonaparte 1850.bb: pl. $・ \mathbf{F}$

OS: Hypochthon $1820 \equiv$ Proteus $1768 \cdot \mathbf{O E}$

EN: (1) PROTEOIDAE 1831.ba.f002-10 • eF

(2) PROTEIDAE 1831.ba.f002-02 • F

EF: PROTEIDAE 1831.ba.f002
HyPODACTYLINAE Heinicke ${ }^{+4}$, 2018.ha.f001 • KY

SI: $440 \bullet$ CI: h333 • ST: 0.10 .30

RL: INR

PA: 00 HYPODACTYLINAE Heinicke $^{+4}$ 2018.ha: $152 \cdot$ bF

$01 \cdot$ HYPODACTYLINIA $\bullet$ Hoc loco $\bullet$ iT

OS: Hypodactylus $2008 \cdot \mathbf{P D}$

EN: HYPODACTYLINIA 2018.ha.f001-01 • iT

EF: BRACHYCEPHALIDAE 1858.gc.f002

HyPSELOTRITONITES nov., DOP.da.f141 • KY

SI: $582 \bullet$ CI: h475 • ST: 0.10 .30

RL: INR

PA: 00 • HYPSELOTRITONITES $\bullet$ Hoc loco $\bullet \mathbf{C n}$

OS: Hypselotriton $1934 \cdot \mathbf{P D}$

EN: HYPSELOTRITONITES DOP.da.f141-00 • Cn

EF: SALAMANDRIDAE 1820.ga.f002

ICHTHYОРНHDAE Taylor, 1968.ta.f001・ CK

SI: $277 \bullet \mathbf{C I}:$ h194 • ST: 0.10 .36

RL: > EPICRIA 1843.fa.f018 • PP: Opinion 1749 (Anonymous 1993. aa: 261)

PA: $00 \bullet$ ICHTHYOPHIIDAE $\bullet$ Taylor 1968.ta: x, $46 \bullet \mathbf{F}$

01 ICHTHYOPHIDAE $\bullet$ Taylor 1969.ta: $303 \cdot \mathbf{F}$

02 $・$ ICHTHYOPHIINAE $\bullet$ Nussbaum 1979.na: $13 \cdot \mathbf{b F}$

$03 \cdot$ ICHTHYOPHIDINAE Wollenberg $^{+1}$ 2009.wb: $1050 \bullet \mathbf{b F}$

$04 \cdot$ ICHTHYOPHIOIDEA $\bullet$ Hoc loco $\bullet \mathbf{p F}$

OS: Ichthyophis $1826 \cdot$ OD

EN: (1) ICHTHYOРHIOIDEA 1968.ta.f001-04 • F

(2) ICHTHYOРHIIDAE 1968.ta.f001-00 • F

EF: IСНTHYOРНIIDAE 1968.ta.f001

ICHTHYOSAURINOA nOv., DOP.da.f143・KY

SI: $584 \bullet$ CI: h477 • ST: 0.10 .30

RL: INR

PA: $00 \cdot$ ICHTHYOSAURINOA $\bullet$ Hoc loco $\bullet \mathbf{h T}$

OS: Ichthyosaura $1801 \cdot$ PD

EN: ICHTHYOSAURINOA DOP.da.f143-00 • hT

EF: SALAMANDRIDAE 1820.ga.f002

ICHTYOIDA Latreille, 1825.la.f004 • AN

SI: $026 \bullet \mathbf{C I}: \mathrm{n} 013 \bullet \mathbf{S T}: 2.25 .50$

RL: INR

PA: $00 \cdot$ ICHTYOIDA $\bullet$ Latreille 1825.la: $105 \cdot \mathbf{F}$

01 ICHTHYOIDA Berthold 1827.ba: $103 \cdot \mathbf{F}$

$02 \cdot$ ICHTHYOIDEI $\bullet$ Eichwald 1831.eb: $163 \cdot \mathbf{F}$

03 - ICHTHYODEA $\bullet$ Goldfuss 1832.ga: 325 ; Wiegmann ${ }^{+1}$ 1832.wa: $203 \cdot \mathbf{F}$

$04 \cdot$ ICHTHYODEA $・$ Leunis 1844.1a: $129 \cdot \mathbf{U F}$

$05 \cdot$ ICHTHYODEA $\bullet$ Leunis 1860.1a: $341 \cdot \mathbf{T}$

$06 \cdot$ ICHTHYOIDEA $\bullet$ Wiedersheim 1877.wa: $356 \cdot \mathbf{F}$

07 - ICHTHYOIDEA $・$ Huene 1931.ha: $310 \bullet \mathbf{p F}$

OS: » 2 PN, including: Siren 1766• PD

EN: SIRENIDAE 1825.gb.f005-00 • F

EF: SIRENIDAE 1825.gb.f005

ICHTHYODEA Goldfuss, 1834.ga.f001 • AN

SI: $044 \bullet$ CI: n022 • ST: 2.25 .50

RL: INR

PA: $00 \cdot$ ICHTHYODEA $\bullet$ Goldfuss 1834.ga: $453 \cdot \mathbf{F}$

OS: » 2 PN, including: Amphiuma $1821 \cdot \mathbf{P D}$

EN: (1) AMPHIUMOIDEA 1825.gb.f007-10 • pF 
(4) AMPHIUMIDAE 1825.gb.f007-00 • F

EF: AMPHIUMIDAE 1825.gb.f007

ICHTHYODEA Schreiber, 1875.sa.f001 • AN

SI: $177 \cdot \mathbf{C I}: \mathrm{n} 053 \bullet \mathbf{S T}: 2.25 .50$

RL: INR

PA: $00 \cdot$ ICHTHYODEA $・$ Schreiber 1875.sa: 8 • F

OS: Proteus $1768 \cdot \mathbf{O M}$

EN: (1) PROTEOIDAE 1831.ba.f002-10 • eF

(2) PROTEIDAE 1831.ba.f002-02 • F

EF: PROTEIDAE 1831.ba.f002

IKAKOGINAE nov., DOP.da.f047 • KY

SI: $488 \bullet \mathbf{C I}:$ h381 • ST: 0.10 .30

RL: INR

PA: $00 \cdot$ IKAKOGINAE $\bullet$ Hoc loco $\bullet \mathbf{b F}$

OS: Ikakogi 2009・PD

EN: IKAKOGINAE DOP.da.f047-00 • bF

EF: CENTROLENIDAE 1951.ta.f001

INDIRANINAE Blommers-Schlösser, 1993.ba.f002 • JD

SI: $352 \cdot \mathbf{C I}:$ h259・ST: 0.10 .40

RL: INR

PA: 00 • INDIRANINAE • Blommers-Schlösser 1993.ba: 199 • bF

OS: Indirana $1986 \cdot \mathbf{O E}$

EN: (1) RANIXALEIDAE 1987.da.f005-03 • aF

(2) RANIXALIDAE 1987.da.f005-02 • F

EF: RANIXALIDAE 1987.da.f005

INDOTYPHLINI Lescure ${ }^{+2}$, 1986.lb.f006 • KY

SI: $325 \cdot$ CI: h233・ST: 0.10 .30

RL: INR

PA: 00 - INDOTYPHLINI $\bullet$ Lescure $^{+2}$ 1986.1b: $164 \cdot$ T

01 INDOTYPHLIDAE $\bullet$ Wilkinson $^{+3}$ 2011.wa: $43 \cdot \mathbf{F}$

$02 \cdot$ INDOTYPHLINIA $・ H O c$ loco $\bullet \mathbf{i T}$

OS: Indotyphlus $1960 \cdot \mathbf{O E}$

EN: INDOTYPHLINIA 1986.1b.f006-02 • iT

EF: CAECILIIDAE 1814.ra.f003-|1825.gb.f008|

INGERANINI $\mathrm{Fei}^{+2}$, 2010.fa.f009 • KY

SI: $400 \bullet \mathbf{C I}:$ h300 • ST: 0.10 .30

RL: INR

PA: $00 \cdot \operatorname{INGERANINI} \cdot \mathrm{Fei}^{+2} 2010 . \mathrm{fa}: 17 \cdot \mathbf{T}$

$01 \cdot$ INGERANINAE $\bullet$ Hoc loco $\bullet \mathbf{b F}$

OS: Ingerana $1987 \cdot$ OD

EN: INGERANINAE 2010.fa.f009-01 • bF

EF: OCCIDOZYGIDAE 1990.fa.f002

INGEROPHRYNITUES nOV., DOP.da.f020 • KY

SI: $461 \bullet \mathbf{C I}:$ h354 • ST: 0.10 .30

RL: INR

PA: $00 \cdot$ INGEROPHRYNITUES $\bullet$ HOc loco $\bullet \mathbf{h C n}$

OS: Ingerophrynus $2006 \cdot \mathbf{P D}$

EN: INGEROPHRYNITUES DOP.da.f020-00 • hCn

EF: BUFONIDAE 1825.gb.f004

IRANODONTINA nov., DOP.da.f131 • KY

SI: $572 \bullet$ CI: h465 • ST: 0.10 .30

RL: INR

PA: $00 \cdot$ IRANODONTINA $\cdot$ Hoc loco $\bullet \mathbf{h T}$

OS: Iranodon $2012 \cdot \mathbf{P D}$

EN: IRANODONTINA DOP.da.f131-00 • hT
EF: HYNOBIIDAE |1856.ha.f001|-1859.cb.f002

ISTHMOHYLITIES nov., DOP.da.f055 • KY

SI: 496 • CI: h389 • ST: 0.10 .30

RL: INR

PA: $00 \cdot$ ISTHMOHYLITIES $\bullet$ Hoc loco $\bullet \mathbf{b C n}$

OS: Isthmohyla $2005 \cdot \mathbf{P D}$

EN: ISTHMOHYLITIES DOP.da.f055-00 • bCn

EF: HYLIDAE 1815.ra.f002-|1825.gb.f001|

ISTHMURINOA nov., DOP.da.f132 • KY

SI: $573 \cdot \mathbf{C I}: \mathrm{h} 466 \cdot \mathbf{S T}: 0.10 .30$

RL: INR

PA: $00 \cdot$ ISTHMURINOA $・ H O C$ loco $\bullet \mathbf{h T}$

$01 \cdot$ ISTHMURITES $\bullet$ Hoc loco $\bullet \mathbf{C n}$

OS: Isthmura $2012 \cdot$ PD

EN: (1) ISTHMURINOA DOP.da.f132-00 • hT

(2) ISTHMURITES DOP.da.f132-01 • Cn

EF: PLETHODONTIDAE 1850.ga.f001

ITAPOTIHYLINA nov., DOP.da.f061 • KY

SI: 502 • CI: h395 • ST: 0.10 .30

RL: INR

PA: $00 \cdot$ ITAPOTIHYLINA $・$ Hoc loco $\bullet \mathbf{b T}$

OS: Itapotihyla $2005 \cdot$ PD

EN: ITAPOTIHYLINA DOP.da.f061-00 • bT

EF: HYLIDAE 1815.ra.f002-|1825.gb.f001|

KaLOPHRYNINa Mivart, 1869.ma.f003 • KY

SI: $163 \cdot \mathbf{C I}:$ h111 • ST: 1.10 .30

RL: INR

PA: $00 \cdot$ KALOPHRYNINA • Mivart 1869.ma: 289 • bF

01 $\operatorname{KALOPHRYNINAE} \bullet$ Noble 1931.na: $536 \cdot \mathbf{b F}$

$02 \cdot$ KALOPHRYNIDAE $\bullet$ Bossuyt $^{+1}$ 2009.ba: $358 \cdot \mathbf{F}$

OS: Kalophrynus $1838 \cdot \mathbf{O E}$

EN: KALOPHRYNINAE 1869.ma.f003-01 • bF

EF: MICROHYLIDAE |1843.fa.f012|-1931.na.f001

KaLOULINAE Noble, 1931.na.F014 • JD

SI: $229 \bullet$ CI: h163 • ST: 0.10 .40

RL: INR

PA: $00 \cdot$ KALOULINAE $\bullet$ Noble 1931.na: $538 \cdot \mathbf{b F}$

01 $\operatorname{KALOULIDAE} \bullet$ Parker 1934.pa: $16 \cdot \mathbf{F}$

OS: Kaloula $1831 \cdot$ OE

EN: (1) HYLAEDACTYLINA 1843.fa.f009-05 • b T

(2) HYLAEDACTYLINIA 1843.fa.f009-06 • iT

EF: MICROHYLIDAE |1843.fa.f012|-1931.na.f001

KARAURIDAE Ivachnenko, 1978.ia.f001 +

SI: $302 \cdot \mathbf{C I}:$ h2 $15 \bullet \mathbf{S T}: 0.10 .30$

RL: INR

PA: 00 - KARAURIDAE $・$ Ivachnenko 1978.ia: $85 \cdot \mathbf{F}$

01 - KARAURURIDAE • Nessov 1993.na: $30 \cdot \mathbf{F}$

02 KARAUROIDIA $・$ Dubois 2005.da: $19 \cdot \mathbf{e F}$

$03 \cdot$ KARAUROIDEA $・$ Dubois 2005.da: $19 \cdot \mathbf{p F}$

OS: Karaurus $1978+\cdot$ OE

EN: KARAURIDAE 1978.ia.f001-00 † - F

EF: KARAURIDAE 1978.ia.f001 †

KARSENIINI Dubois, 2008.da.f004 • AN

SI: 377 • CI: n094 • ST: 0.22 .50

RL: INR

PA: $00 \cdot$ KARSENIINI • Dubois 2008.da: $72 \cdot \mathbf{T}$ 
01 - KARSENIINA • Dubois 2008.da: 74 • bT

OS: Karsenia $2005 \cdot \mathbf{O E}$

EN: KARSENIINA 2012.da.f008-01 • bT

EF: Plethodontidae 1850.ga.f001

KARSENIINI Dubois ${ }^{+1}$, 2012.da.f008 • KY

SI: $425 \bullet \mathbf{C I}:$ h319・ST: 0.10 .30

RL: INR

PA: 00 • KARSENIINI $\bullet$ Dubois $^{+1}$ 2012.da: $117 \cdot \mathbf{T}$ $01 \cdot$ KARSENIINA $^{\circ}$ Dubois $^{+1}$ 2012.da: $118 \cdot \mathbf{b T}$

OS: Karsenia $2005 \cdot$ OD

EN: KARSENIINA 2012.da.f008-01 • bT

EF: PLETHODONTIDAE 1850.ga.f001

KASSININI Laurent, 1972.1a.f001・ KY

SI: 290 • CI: h205・ST: 0.10 .30

RL: INR

PA: $00 \bullet \operatorname{KASSININI} \bullet$ Laurent 1972.1a: 201 • T

01 - KASSININAE • Dubois 1981.da: $227 \bullet \mathbf{b F}$

OS: Kassina $1853 \cdot \mathbf{O E}$

EN: KASSININI 1972.la.f001-00・T

EF: HyPEROLIIDAE 1943.lb.f001

KURIXALITES nov., DOP.da.f119・ KY

SI: $560 \bullet \mathbf{C I}: \mathrm{h} 453 \bullet \mathbf{S T}: 0.10 .30$

RL: INR

PA: $00 \cdot$ KURIXALITES $\bullet$ Hoc loco $\bullet \mathbf{C n}$

OS: Kurixalus $1999 \cdot \mathbf{P D}$

EN: KURIXALITES DOP.da.f119-00 • Cn

EF: RHACOPHORIDAE |1858.gc.f012|-1932.ha.f001

LALIOSTOMINaE Vences $^{+1}$, 2001.va.f002 • KY

SI: $358 \bullet \mathbf{C I}:$ h265 - ST: 0.10 .30

RL: INR

PA: 00 • LALIOSTOMINAE $・$ Vences $^{+1}$ 2001.va: $85 \cdot$ bF

01 • LALIOSTOMINI • Dubois 2005.da: $16 \cdot \mathbf{T}$

02 • LALIOSTOMATINAE • Glaw ${ }^{+1}$ 2006.ga: $238 \cdot \mathbf{b F}$

OS: Laliostoma $1998 \cdot$ OD

EN: LALIOSTOMINI 2001.va.f002-01・T

EF: RHACOPHORIDAE |1858.gc.f012|-1932.ha.f001

LANKANECTINAE Dubois ${ }^{+1}$, 2001.da.f001・JD

SI: $359 \bullet$ CI: h266・ST: 0.10 .40

RL: INR

PA: 00 • LANKANECTINAE • Dubois ${ }^{+1}$ 2001.da: $84 \cdot$ bF

OS: Lankanectes $2001 \cdot$ OD

EN: (1) NYCTIBATRACHEIDAE 1993.ba.f001-02 • aF (1)"

(3) NYCTIBATRACHINAE 1993.ba.f001-00 • bF

EF: NYCTIBATRACHIDAE 1993.ba.f001

LATONIIDAE Špinar, 1979.sa.f001 + AN

SI: $305 \bullet \mathbf{C I}: \mathrm{n} 084 \bullet \mathbf{S T}: 0.28 .50$

RL: INR

PA: $00 \bullet$ LATONIIDAE • Špinar 1979.sa: 289, $290 \bullet \mathbf{F}$

OS: Latonia $1843+\cdot \mathbf{O E}$

EN: DISCOGLOSSIDAE 1858.gc.f004-00 • F

EF: DISCOGLOSSIDAE 1858.gc.f004

LECHRIODONTA Strauch, 1870.sa.f002 • AN

SI: $175 \bullet \mathbf{C I}: \mathrm{n} 052 \bullet \mathbf{S T}: 2.25 .50$

RL: INR

PA: $00 \cdot$ LECHRIODONTA • Strauch 1870.sa: $53 \cdot$ T
$01 \cdot$ LECHRIODONTA • Hoffmann 1878 ha: 665 • bF

02 • LECHRIODONTA • Leunis 1883.la: 624 • F

03 - LECHRIODONTA • Gadow 1901.ga: $95 \cdot \mathbf{U F}$

OS: » 11 PN, including: Plethodon $1838 \cdot \mathbf{P D}$

EN: (1) Plethodontidae 1850.ga.f001-00 • F (")")"

(4) PLETHodontina 1850.ga.f001-09 • bT

EF: Plethodontidae 1850.ga.f001

LEIODERMES Bory de Saint-Vincent, 1828.bb.f001 • AN

SI: $032 \bullet \mathbf{C I}: \mathrm{n} 014 \cdot \mathbf{S T}: 2.25 .50$

RL: INR

PA: 00 - LEIODERMES • Bory de Saint-Vincent 1828.bb: $215 \cdot \mathbf{F}$

OS: Coecilia $1801 \equiv$ Caecilia $1758 \cdot \mathbf{O M}$

EN: (1) CAECILIOIDEA 1814.ra.f003-|1825.gb.f008|-19・pF "')"!

(5) CAECILIINA 1814.ra.f003-|1825.gb.f008|-26 • bT

EF: CAECILIIDAE 1814.ra.f003-|1825.gb.f008|

LEIOPELMIDAE Turbott, 1942.ta.f001 • CK

SI: $239 \bullet \mathbf{C I}:$ h171・ST: 0.10 .36

RL: > LIOPELMATINA 1869.ma.f007 • PP: Opinion 1071 (Melville 1977.ma)

PA: 00 • LEIOPELMIDAE • Turbott 1942.ta: 247 • F • IG: Melville 1977.ma

01 - LEIOPELMOIDEA • Laurent 1948.1a: 1 • F

$02 \cdot$ LEIOPELMATIDAE $・$ Stephenson 1951.sa: 18 • F • LG Melville 1977.ma

$03 \cdot$ LEIOPELMATINAE $・$ Kuhn 1965.ka: 86 • bF

$04 \cdot$ LEIOPELMATOIDIA $・$ Dubois 2005.da: $8 \cdot \mathbf{e F}$

05 - LEIOPELMATOIDEA • Dubois 2005.da : $8 \cdot \mathbf{p F}$

$06 \cdot$ LEIOPELMATOIDIA $・$ Fouquette $^{+1} 2014$ fa: $6 \bullet \mathbf{p F}$

OS: Leiopelma $1861 \cdot \mathbf{O E}$

EN: (1) LEIOPELMATIDAE 1869.ma.f007-|1942.ta.f001|-02 • F

(2) LEIOPELMATINAE 1869.ma.f007-|1942.ta.f001|-03 • bF

EF: LEIOPELMATIDAE 1869.ma.f007-|1942.ta.f001|

LEIUPERINA Bonaparte, 1850.bb.f010 • KY

SI: $102 \bullet \mathbf{C I}:$ h064・ST: 0.10 .30

RL: INR

PA: $00 \cdot$ LEIUPERINA $・$ Bonaparte 1850.bb: pl. • bF

01 - LEINPERINA • Bonaparte 1852.ba: $478 \cdot \mathbf{b F}$

$02 \cdot$ LEIUPERIDAE $^{\circ}$ Grant $^{+9}$ 2006.gb: $4 \cdot \mathbf{F}$

03 $\bullet$ LEIUPERINAE $\bullet$ Pyron $^{+1}$ 2011.pa: $574 \cdot \mathbf{b F}$

$04 \cdot$ LEIUPERINI $\bullet$ Hoc loco $・$ T

OS: Leiuperus $1841 \approx$ Pleurodema $1838 \cdot \mathbf{O E}$

EN: (1) LEIUPERINAE 1850.bb.f010-03 • bF

(2) LEIUPERINI 1850.bb.f010-04 • T

EF: LEPTODACTYLIDAE |1838.ta.f001|-1896.wa.f001

LEPIDOBATRACHIDAE Bauer, 1987.ba.f001 • KY

SI: $330 \bullet \mathbf{C I}: \mathrm{h} 238 \cdot \mathbf{S T}: 0.10 .40$

RL: INR

PA: $00 \bullet$ LEPIDOBATRACHIDAE • Bauer 1987.ba: $5 \bullet \mathbf{F}$

01 - LEPIDOBATRACHINAE Hoc loco $\bullet \mathbf{b F}$

OS: Lepidobatrachus $1899 \cdot \mathbf{O E}$

EN: LEPIDOBATRACHINAE 1987.ba.f001-01・bF

EF: CERATOPHRYIDAE 1838.ta.f002

LEPTOBRACHIINI Dubois, 1980.da.f001 • AN

SI: 306 • CI: n085 • ST: 0.28 .50 
RL: INR

PA: 00 - LEPTOBRACHIINI • Dubois 1980.da: 471 • T

$$
\text { 01 - LEPTOBRACHIINAE • Dubois 1983.da: } 272 \cdot \mathbf{b F}
$$

OS: Leptobrachium $1838 \cdot \mathbf{O E}$

EN: (1) LEPTOBRACHIINAE 1983.db.f001-00 • bF

$$
\text { "')"' }
$$

(3) LEPTOBRACHIINA 1983.db.f001-02 • b T

EF: MEGOPHRYIDAE 1850.bb.f008-|1931.na.f003|

LEPTOBRACHINAE Dubois, 1983.db.f001 • KY

SI: $311 \cdot \mathbf{C I}:$ h221 • ST: 0.10 .30

RL: INR

PA: 00 • LEPTOBRACHIINAE • Dubois 1983.db: $147 \cdot \mathbf{b F}$

$01 \cdot \operatorname{LEPTOBRACHIINI} \cdot H o c$ loco $・ \mathbf{T}$

$02 \cdot$ LEPTOBRACHIINA $・ H O c$ loco $\bullet \mathbf{b T}$

OS: Leptobrachium $1838 \cdot \mathbf{O E}$

EN: (1) LEPTOBRACHIINAE 1983.db.f001-00 • bF

(2) LEPTOBRACHIINI 1983.db.f001-01 • T

(3) LEPTOBRACHIINA 1983.db.f001-02 • bT

EF: MEGOPHRYIDAE 1850.bb.f008-|1931.na.f003|

LEPTODACTYLIDAE Werner, 1896.wa.f001 • SK

SI: $194 \cdot \mathbf{C I}:$ h134 • ST: 0.10 .35

RL: > CYSTIGNATHI 1838.ta.f001 • PS: Dubois 1983.da: 273 > PLECTROMANTIDAE 1869.ma.f002 • PS: Dubois 1983.da: 273

PA: 00 - LEPTODACTYLIDAE • Werner 1896.wa: $357 \cdot \mathbf{F}$

01 - LEPTODACTYLINAE • Metcalf 1923.ma: $272 \cdot \mathbf{b F}$

02 - LEPTODACTYLYDAE • Lutz 1954.1a: $172 \cdot \mathbf{F}$

$03 \cdot$ LEPTODACTILYDAE • Cei 1958.ca: $274 \cdot \mathbf{F}$

$04 \cdot$ LEPTODACTYLOIDEA $・$ Reig 1972.ra: $29 \cdot \mathbf{p F}$

05 - LEPTODACYLIDAE • Melville 1978.ma: $224 \cdot \mathbf{F}$

$06 \cdot$ LEPTIDACTYLIDAE $・$ Crespo 2001.ca: $109 \cdot \mathbf{F}$

$07 \cdot$ LEPTODACTILIDAE • Crespo 2001.ca: $109 \cdot \mathbf{F}$

$08 \cdot \operatorname{LEPTODACTYLINI} \bullet H O C l o c o \cdot \mathbf{T}$

OS: Leptodactylus $1826 \cdot \mathbf{O E}$

EN: (1) LEPTODACTYLOIDEA |1838.ta.f001|-1896.wa.f001-04 • pF

(2) LEPTODACTYLIDAE |1838.ta.f001|-1896.wa.f001-00 • F

(3) LEPTODACTYLINAE |1838.ta.f001|-1896.wa.f001-01 • bF

(4) LEPTODACTYLINI |1838.ta.f001|-1896.wa.f001-08 • T

EF: LEPTODACTYLIDAE |1838.ta.f001|-1896.wa.f001

LEPTODACTYLODONTINI nov., DOP.da.f092 • KY

SI: $533 \cdot$ CI: h426 • ST: 0.10 .30

RL: INR

PA: $00 \cdot$ LEPTODACTYLODONTINI $・ H O C$ loco • bT

OS: Leptodactylodon $1903 \cdot \mathbf{P D}$

EN: LEPTODACTYLODONTINI DOP.da.f092-00 • bT

EF: ARTHROLEPTIDAE 1869.ma.f011

LEPTOLALAGINAE Delorme ${ }^{+3}$, 2006.da.f001 • KY

SI: $365 \bullet$ CI: h271 • ST: 0.10 .30

RL: INR

PA: $00 \cdot$ LEPTOLALAGINAE $・$ Delorme $^{+3}$ 2006.da: $7 \cdot$ bF

$01 \cdot$ LEPTOLALAGINI $\bullet$ Hoc loco $・$ T

OS: Leptolalax $1980 \cdot$ OD

EN: LEPTOLALAGINI 2006.da.f001-01・T

EF: MEGOPHRYIDAE 1850.bb.f008-|1931.na.f003|

LEPTOPELINI Laurent, 1972.la.f002 • KY

SI: 291 • CI: h206 • ST: 0.10 .30

RL: INR
PA: $00 \bullet$ LEPTOPELINI • Laurent 1972.1a: 201 • T

01 - LEPTOPELINAE $・$ Dubois 1981.da: $227 \cdot \mathbf{b F}$

02 - LEPTOPELIDAE $\bullet$ Bauer 1986.ba: iii • F

OS: Leptopelis $1859 \cdot \mathbf{O E}$

EN: LEPTOPELINAE 1972.la.f002-01 • bF

EF: ARTHROLEPTIDAE 1869.ma.f011

LEPTOPHRYNITUES nov., DOP.da.f024 • KY

SI: $465 \cdot \mathbf{C I}:$ h358 • ST: 0.10 .30

RL: INR

PA: $00 \cdot$ LEPTOPHRYNITUES $・ H O c$ loco $・ \mathbf{h C n}$

OS: Leptophryne $1843 \cdot \mathbf{P D}$

EN: LEPTOPHRYNITUES DOP.da.f024-00 • hCn

EF: BUFONIDAE 1825.gb.f004

LIMNODYNASTINI Lynch, 1969.1b.f001 • AN

SI: $283 \bullet \mathbf{C I}: \mathrm{n} 080 \bullet \mathbf{S T}: 0.28 .50$

RL: INR

PA: $00 \bullet$ LIMNODYNASTINI $\bullet$ Lynch 1969.1b: $3 \cdot$ T

OS: Limnodynastes $1843 \cdot \mathbf{O E}$

EN: (1) LIMNODYNASTINAE 1971.la.f001-01 • bF

(2) LIMNODYNASTINI 1971.la.f001-00 • T

(3) LIMNODYNASTINA 1971.la.f001-03・bT

EF: MYOBATRACHIDAE 1850.sa.f001

LIMNODYNASTINI Lynch, 1971.la.f001 • KY

SI: $287 \bullet$ CI: h202 • ST: 0.10 .30

RL: INR

PA: 00 - LIMNODYNASTINI - Lynch 1971.la: 83 • T

01 - LIMNODYNASTINAE • Heyer ${ }^{+1}$ 1976.ha: 5 • bF

02 - LIMNODYNASTIDAE • Zug ${ }^{+2} 2001 . z a: ~ 411 \cdot \mathbf{F}$

$03 \cdot$ LIMNODYNASTINA $\cdot$ Hoc loco $\bullet \mathbf{b T}$

OS: Limnodynastes $1843 \cdot \mathbf{O E}$

EN: (1) LIMNODYNASTINAE 1971.la.f001-01 • bF

(2) LIMNODYNASTINI 1971.la.f001-00 • T

(3) LIMNODYNASTINA 1971.la.f001-03・bT

EF: MYOBATRACHIDAE 1850.sa.f001

LIMNODYTAE Fitzinger, 1843.fa.f001 • KY

SI: $066 \bullet \mathbf{C I}:$ h034 • ST: 0.10 .30

RL: INR

PA: $00 \cdot$ LIMNODYTAE • Fitzinger 1843.fa: $31 \cdot \mathbf{F}$

01 - LIMNODYTINI • Dubois 1981.da: $231 \cdot \mathbf{F}$

$02 \cdot$ LIMNODYTINOA $\cdot$ Hoc loco $\bullet \mathbf{h T}$

OS: Limnodytes $1841 \equiv$ Hylarana $1838 \cdot \mathbf{O E}$

EN: LIMNODYTINOA 1843.fa.f001-02・hT

EF: RANIDAE 1796.ba.f001

LIMNOMEDUSINAE nov., DOP.da.f049 • KY

SI: $490 \bullet \mathbf{C I}:$ h383 - ST: 0.10 .30

RL: INR

PA: $00 \cdot$ LIMNOMEDUSINAE $\bullet$ Hoc loco $\bullet \mathbf{b F}$

OS: Limnomedusa $1843 \cdot \mathbf{P D}$

EN: LIMNOMEDUSINAE DOP.da.f049-00 • bF

EF: CYCLORAMPHIDAE 1850.bb.f003-|1852.ba.f001|

LIMNONECTINI Dubois, 1992.da.f002 • KY

SI: $349 \bullet$ CI: h256 • ST: 0.10 .30

RL: INR

PA: 00 - LIMNONECTINI • Dubois 1992.da: $315 \cdot$ T

01 $\operatorname{LIMNONETINAE} \cdot \mathrm{Fei}^{+2}$ 2010.fa: $12 \cdot \mathbf{b F}$

$02 \cdot \operatorname{LIMNONECTINAE} \bullet \mathrm{Fei}^{+2} 2010 . \mathrm{fa}: 27 \cdot \mathbf{b F}$ 
OS: Limnonectes 1843 • OD

EN: LIMNONECTINAE 1992.da.f002-02 • bF

EF: DICROGLOSSIDAE 1987.da.f004

LINGUATA Gravenhorst, 1843.ga.f002 • AN

SI: $085 \bullet \mathbf{C I}: \mathrm{n} 032 \bullet \mathbf{S T}: 2.25 .50$

RL: INR

PA: $00 \cdot$ LINGUATA $\bullet$ Gravenhorst 1843.ga: $393 \cdot$ F

OS: » 9 PN, including: Rana 1758 • PD

EN: (1) RANOIDEA 1796.ba.f001-28 • pF

")"!"

(12) RANITOES 1796.ba.f001-38 • iCn

EF: RANIDAE 1796.ba.f001

LIOPELMATINA Mivart, 1869.ma.f007 • CG

SI: $167 \cdot \mathbf{C I}:$ h115 • ST: 0.10 .61

RL: < LEIOPELMIDAE 1942.ta.f001 • PP: Opinion 1071 (Melville 1977.ma)

PA: 00 • LIOPELMATINA • Mivart 1869.ma: 291 • bF • IG: Melville 1977.ma

01 • LIOPELMIDAE • Noble 1924.na: 9 • F • IG: Melville 1977.ma

02 - LIPELMIDAE • Kuhn 1939.ka: 92 • F・IG: Melville 1977.ma

03 - LIOPELMOIDEA • Laurent 1948.1a: $1 \cdot \mathbf{p F}$

OS: Liopelma $1865 \mathbf{C I} \equiv$ Leiopelma $1861 \cdot \mathbf{O E}$

EN: (1) LEIOPELMATIDAE 1869.ma.f007-|1942.ta.f001|-02 • F

(2) LEIOPELMATINAE 1869.ma.f007-|1942.ta.f001|-03 • bF

EF: LEIOPELMATIDAE 1869.ma.f007-|1942.ta.f001|

LIPELUCIDAE Huene, 1956.ha.f001 \$• AN

SI: $252 \bullet \mathbf{C I}: \mathrm{n} 071 \bullet \mathbf{S T}: 0.25 .50$

RL: INR

PA: $00 \cdot$ LIPELUCIDAE $・$ Huene 1956.ha: $113 \cdot \mathbf{b F}$

OS: » 5 PN, including: Eobatrachus 1887 \$・PD

EN: ANURA Familia INCERTAE SEDIS

EF: ANURA Familia INCERTAE SEDIS

LIPOTREMEN Haeckel, 1866.ha.f002 • AN

SI: $157 \bullet \mathbf{C I}: \mathrm{n} 050 \bullet \mathbf{S T}: 2.25 .50$

RL: INR

PA: 00 • LIPOTREMEN • Haeckel 1866.ha: cxxxii • F

OS: » [2 PN, including: ] Salamandra $1768 \approx$ Salamandra 1764 - PD

EN: (1) SALAMANDROIDEA 1820.ga.f002-21・pF ")"')

(4) SALAMANDRINI 1820.ga.f002-28 • T

EF: SALAMANDRIDAE 1820.ga.f002

LISSOTRITONINOA nOv., DOP.da.f144 • KY

SI: $585 \bullet$ CI: h478 • ST: 0.10 .30

RL: INR

PA: $00 \cdot$ LiSSOTRITONINOA $\bullet$ Hoc loco $\bullet \mathbf{h T}$

OS: Lissotriton $1839 \cdot \mathbf{P D}$

EN: LISSOTRITONINOA DOP.da.f144-00 • hT

EF: SALAMANDRIDAE 1820.ga.f002 MaNTELLINAE Laurent, 1946. la.f001

LITHOBatITIES nov., DOP.da.f108 • KY

SI: $549 \bullet$ CI: h442 • ST: 0.10 .30

RL: INR

PA: $00 \cdot$ LITHOBATITIES $\bullet$ Hoc loco $\bullet$ bCn

OS: Lithobates $1843 \cdot \mathbf{P D}$

EN: LITHOBATITIES DOP.da.f108-00 • bCn
EF: RANIDAE 1796.ba.f001

LITORIINAE Dubois ${ }^{+1}$, 2016.da.f001 • JD

SI: 438 • CI: h331 • ST: 0.10 .40

RL: INR

PA: $00 \cdot$ LITORIINAE $・$ Dubois $^{+1}$ 2016.da: $19 \cdot \mathbf{b F}$

OS: Litoria $1838 \cdot$ OD

EN: PELODRYADINAE 1859.ga.f001-01 • bF

EF: PHYLLOMEDUSIDAE 1858.gc.f009

LIUHURANITOES nov., DOP.da.f110 • KY

SI: $551 \cdot \mathbf{C I}: \mathrm{h} 444 \cdot \mathbf{S T}: 0.10 .30$

RL: INR

PA: $00 \cdot$ LIUHURANITOES $\bullet$ Hoc loco $\bullet$ bCn

OS: Liuhurana 2010 • PD

EN: LIUHURANITOES DOP.da.f110-00 • bCn

EF: RANIDAE 1796.ba.f001

LIUIXALINI Hertwig ${ }^{+3}$ 2013.ha.f001 • AN

SI: $431 \bullet \mathbf{C I}: \mathrm{n} 103 \bullet \mathbf{S T}: 0.22 .50,0.24 .50,0.28 .50$

RL: INR

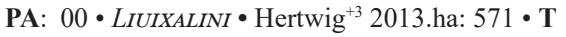

OS: Liuixalus 2008 AN $\equiv$ Romeus nov. $\bullet$ OE

EN: ROMERINA DOP.da.f128-00 • bT

EF: RHACOPHORIDAE |1858.gc.f012|-1932.ha.f001

LIURANINAE $\mathrm{Fei}^{+2}$, 2010.fa.f010 • KY

SI: $401 \bullet \mathbf{C I}:$ h301 • ST: 0.10 .30

RL: INR

PA: $00 \cdot$ LIURANINAE $\bullet \mathrm{Fei}^{+2}$ 2010.fa: $12 \cdot \mathbf{b F}$

01 $\operatorname{LIURANINI} \bullet \mathrm{Fei}^{+2}$ 2010.fa: $17 \cdot \mathbf{T}$

OS: Liurana $1987 \cdot$ OD

EN: LIURANINAE 2010.fa.010-00 • F

EF: CERATOBATRACHIDAE 1884.ba.f001

LoPHIOHYLINAE Miranda-Ribeiro, 1926.ma.f004 • мK

SI: $211 \cdot \mathbf{C I}:$ h1 $147 \cdot \mathbf{S T}: 0.10 .58$

RL: $\leq$ LOРHYOHYLINI 2014.fa.f001 • MK: Fouquette ${ }^{+1}$ 2014.fa: 368

PA: $00 \cdot$ LOPHIOHYLINAE • Miranda-Ribeiro 1926.ma: $64 \cdot \mathbf{F}$

$01 \cdot$ LOPHIOHYLINI $^{*}$ Faivovich $^{+5}$ 2005.fa: $4 \cdot$ T

$02 \cdot$ LOPHIOHYLINA $^{*}$ Faivovich $^{+5} 2005$ fa: 4 • bT

$03 \cdot$ LOPHIOHYLINIA $^{*}$ Faivovich $^{+5}$ 2005.fa: 4 • iT

OS: Lophiohyla $1926 \approx$ Phyllodytes $1830 \cdot \mathbf{O E}$

EN: (1) LOPHYOHYLINI 1926.ma.f004-|2014.fa.f001|-00 • T "')"')

(3) LOPHYOHYLINIA 1926.ma.f004-|2014.fa.f001|-03 • iT

EF: HYLIDAE 1815.ra.f002-|1825.gb.f001|

LорнуонуцIN Fouquette ${ }^{+1}$, 2014.fa.f001 • мк

SI: 432 • CI: h325 • ST: 0.10 .34

RL: $\geq$ LOPHIOHYLINAE 1926.ma.f004 - MK: Fouquette ${ }^{+1}$ 2014.fa: 368

PA: $00 \cdot$ LOPHYOHYLINI $・$ Fouquette $^{+1}$ 2014.fa: 7 • T・NO

$01 \cdot$ LOPHYOHYLINAE $・$ Duellman $^{+2}$ 2016.db: $3 \cdot \mathbf{T} ・$ NO

$02 \cdot$ LOPHYOHYLINA $・ H o c$ loco $・ \mathbf{b T}$

$03 \cdot$ LOPHYOHYLINIA $\cdot$ Hoc loco $・$ iT

OS: Lophyohyla $1923 \approx$ Phyllodytes $1830 \cdot \mathbf{O E}$

EN: (1) LоРнYОНYLINI 1926.ma.f004-|2014.fa.f001|-00 • T

(2) LOPHYOHYLINA 1926.ma.f004-|2014.fa.f001|-02 • bT

(3) LOPHYOHYLINIA 1926.ma.f004-|2014.fa.f001|-03 • iT

EF: HYLIDAE 1815.ra.f002-|1825.gb.f001|

MACROGENIOGLOTTIDAE Reig, 1972.ra.f001 • JD

SI: 292 • CI: h207 • ST: 0.10 .40 
RL: INR

PA: 00 • MACROGENIOGLOTTIDAE • Reig 1972.ra: 30 • F

OS: Macrogenioglottus $1946 \cdot \mathbf{O E}$

EN: (1) ODONTOPHRYNIDAE 1971.la.f002-03 • F

(2) ODONTOPHRYNINAE 1971.la.f002-04 • bF

EF: ODONTOPHRYNIDAE 1971.la.f002

MantellinaE Laurent, 1946.la.f001・ KY

SI: 242 •CI: h173 • ST: 0.10 .30

RL: INR

PA: 00 • MANTELLINAE $\bullet$ Laurent 1946.la: $336 \cdot \mathbf{b F}$ 01 MANTELLIDAE $\bullet$ Bauer 1985.ba: $3 \cdot \mathbf{F}$

02 $\cdot$ MANTELLINI $\bullet$ Dubois 2005.da: $16 \cdot \mathbf{T}$

$03 \cdot$ MANTELLINA $\bullet$ Hoc loco $\bullet$ bT

$04 \cdot$ MANTELLINIA $\bullet$ Hoc loco $\bullet$ iT

OS: Mantella $1882 \cdot \mathbf{O E}$

EN: (1) MANTELLINAE 1946.la.f001-00 • bF

(2) MANTELLINI 1946.la.f001-02 • T

(3) MANTELLINA 1946.la.f001-03 • bT

(4) MANTELLINIA 1946.la.f001-04 • iT

EF: RHACOPHORIDAE |1858.gc.f012|-1932.ha.f001

MANTIDACTYLINa nov., DOP.da.f113 • KY

SI: $554 \cdot \mathbf{C I}: \mathrm{h} 447 \cdot \mathbf{S T}: 0.10 .30$

RL: INR

PA: $00 \cdot$ MANTIDACTYLINA $\bullet$ Hoc loco $\bullet$ bT

$01 \cdot$ MaNTIDACTYLINIA $\bullet$ Hoc loco $\bullet$ iT

02 $\cdot$ MANTIDACTYLINOA $\bullet$ Hoc loco $\bullet \mathbf{h T}$

OS: Mantidactylus $1895 \cdot$ PD

EN: (1) MANTIDACTYLINA DOP.da.f113-00 • bT

(2) MANTIDACTYLINIA DOP.da.f113-01 • iT

(3) MANTIDACTYLINOA DOP.da.f113-02 • hT

EF: RHACOPHORIDAE |1858.gc.f012|-1932.ha.f001

MEANTIA Rafinesque, 1814.ra.f002 • AN

SI: $004 \cdot$ CI: n002 • ST: 2.25 .50

RL: INR

PA: 00 • MEANTIA $・$ Rafinesque 1814.ra: $103 \cdot \mathbf{F}$

OS: » Subsequent mention in Rafinesque 1815.ra: 78: » 3 PN, including: Sirena 1808 AM $\equiv$ Siren 1766 PD

EN: SIRENIDAE 1825.gb.f005-00 • F

EF: SIRENIDAE 1825.gb.f005

MECODONTA Strauch, 1870.sa.f001 • AN

SI: $174 \cdot$ CI: n051 • ST: 2.25 .50

RL: INR

PA: $00 \cdot$ MECODONTA $\bullet$ Strauch 1870.sa: $28 \cdot \mathbf{T}$

01 MECODONTA $\bullet$ Hoffmann 1878.ha: $662 \cdot \mathbf{b F}$

02 $\cdot$ MECODONTA $\bullet$ Leunis 1883.1a: $624 \cdot \mathbf{F}$

03 $\cdot$ MECODONTA $\bullet$ Gadow 1901.ga: $95 \cdot \mathbf{U F}$

OS: » 6 PN, including: Salamandra $1768 \approx$ Salamandra $1764 \cdot$ PD

EN: (1) SALAMANDROIDEA 1820.ga.f002-21 • pF "m)"

(4) SALAMANDRINI 1820.ga.f002-28 • T

EF: SALAMANDRIDAE 1820.ga.f002

MEGALOBATRACHI Fitzinger, 1843.fa.f014 • JD

SI: $079 \bullet$ CI: h047 • ST: 0.10 .40

RL: INR

PA: 00 • MEGALOBATRACHI $\bullet$ Fitzinger 1843.fa: $34 \cdot \mathbf{F}$

01 MEGALOBATRACHIDAE • Jánossy 1979.ja: 22 • F
OS: Megalobatrachus $1837 \approx$ Andrias $1837+\cdot$ OE

EN: CRYPTOBRANCHIDAE 1826.fb.f003-04 • F

EF: CRYPTOBRANCHIDAE 1826.fb.f003

MEGALOPHREIDINA Bonaparte, 1850.bb.f008 • MK

SI: $100 \bullet$ CI: h062 • ST: 0.10 .58

RL: $\leq$ PELOBATIDAE 1850.bb.f004 • PR: Dubois 1983.da: 271 $\leq$ MEGOPHRYIDAE 1850.bb.f008-|1931.na.F003| • MK: Dubois 1983.da: 272

PA: 00 • MEGALOPHREIDINA $\bullet$ Bonaparte 1850.bb: pl. $\bullet \mathbf{b F}$ $01 \cdot$ MEGALOPHRYINAE $\bullet$ Fejérváry 1921.fb: $25 \cdot \mathbf{b F}$ 02 $\cdot$ MEGALOPHRYNINAE $\bullet$ Tatarinov 1964.ta: $129 \cdot \mathbf{b F}$

OS: Megalophrys $1830 \equiv$ Megophrys $1822 \cdot \mathbf{O E}$

EN: (1) MEGOPHRYIDAE 1850.bb.f008-|1931.na.f003|-04 • F "111"

(3) MEGOPHRYINI 1850.bb.f008-|1931.na.f003|-02 • T

EF: MEGOPHRYIDAE 1850.bb.f008-|1931.na.f003|

MEGOPHRYINAE Noble, 1931.na.f003・ MK

SI: $218 \bullet$ CI: h153 • ST: 0.10 .34

RL: > MEGALOPHREIDINA 1850.bb.f008 • MK: Dubois 1983.da: 272

PA: $00 \bullet$ MEGOPHRYINAE $\bullet$ Noble 1931.na: $492 \cdot \mathbf{b F}$

01 $\operatorname{MEGOPHRYNAE} \bullet$ Casamiquela 1961.ca: $79 \cdot \mathbf{b F}$

02 $\bullet$ MEGOPHRYINI $\bullet$ Dubois 1980.da: $471 \bullet$ T

03 $\cdot$ MEGOPHRYNIDAE $\bullet$ Špinar 1983.sa: $55 \cdot \mathbf{F}$

$04 \cdot$ MEGOPHRYIDAE $\bullet$ Špinar 1983.sa: $55 \cdot \mathbf{F}$

05 MEGAPHRYINAE Chaimanee $^{+2}$ 1993.cb: $46 \cdot \mathbf{b F}$

OS: Megophrys $1822 \cdot \mathbf{O E}$

EN: (1) MEGOPHRYIDAE 1850.bb.f008-|1931.na.f003|-04 • F

(2) MEGOPHRYINAE 1850.bb.f008-|1931.na.f003|-00 • bF

(3) MEGOPHRYINI 1850.bb.f008-|1931.na.f003|-02 • T

EF: MEGOPHRYIDAE 1850.bb.f008-|1931.na.f003|

MELANOBATRACHINAE Noble, 1931.na.f015 • KY

SI: $230 \bullet$ CI: h164 • ST: 0.10 .30

RL: INR

PA: 00 • MELANOBATRACHINAE $\bullet$ Noble 1931.na: $538 \cdot \mathbf{b F}$

01 MEGALOBATRACHINAE $\bullet$ Kuhn 1962.ka: $348 \cdot \mathbf{b F}$

02 $\operatorname{MEGALOBATRACHIDAE} \bullet$ Bossuyt $^{+1}$ 2009.ba: $358 \cdot \mathbf{F}$

OS: Melanobatrachus $1878 \cdot \mathbf{O E}$

EN: MELANOBATRACHINAE 1931.na.f015-00 • bF

EF: MICROHYLIDAE |1843.fa.f012|-1931.na.f001

MELANOPHRYNISCINAE nov., DOP.da.f038 • KY

SI: $479 \bullet$ CI: h372 • ST: 0.10 .30

RL: INR

PA: $00 \cdot$ MELANOPHRYNISCINAE $\bullet$ Hoc loco $\bullet \mathbf{b F}$

OS: Melanophryniscus $1961 \cdot \mathbf{P D}$

EN: MELANOPHRYNISCINAE DOP.da.f038-00 • bF

EF: BUFONIDAE 1825.gb.f004

MENOBRANCHIDAE Gray, 1842.ga.f002 • JD

SI: $065 \bullet$ CI: h033 • ST: 0.10 .40

RL: INR

PA: 00 • MENOBRANCHIDAE $\bullet$ Gray 1842.ga: $114 \cdot \mathbf{F}$

$01 \cdot$ MENOBRANCHIA $\cdot$ Lichtenstein $^{+2}$ 1856.la: $45 \cdot \mathbf{F}$

02 MENOBRANCHIDA $\bullet$ Knauer 1878.ka: $96 \bullet \mathbf{F}$

OS: Menobranchus $1825 \approx$ Necturus $1819 \cdot \mathbf{O E}$

EN: (1) PROTEOIDAE 1831.ba.f002-10 • eF

(2) PROTEIDAE 1831.ba.f002-02 • F

EF: PRoteIdaE 1831.ba.f002 
MENOPOMATIDAE Hogg 1838.ha.f003 • JD

SI: $050 \bullet$ CI: h024 • ST: 0.10 .40

RL: INR

PA: $00 \cdot$ MENOPOMATIDAE $\bullet$ Hogg 1838.ha: $152 \cdot \mathbf{F}$

01 $\cdot$ MENOPOMINA $\bullet$ Bonaparte 1839.bf: $16 \cdot \mathbf{U F}$

02 - MENOPOMIDAE • Baird 1851.ba: $252 \cdot \mathbf{U F}$

03 $\cdot$ MENOPOMAE $\bullet$ Duméril 1863.da: $303 \cdot \mathbf{F}$

04 $\bullet$ MENOPOMIDAE $\bullet$ Claus 1868.cb: $586 \bullet \mathbf{F}$

05 MENOPOMIDA $\bullet$ Smith 1877.sa: tab. [10-11], 19, $21 \cdot \mathbf{U F}$

$06 \cdot$ MENOPOMIDA $\bullet$ Knauer 1878.ka: $96 \cdot \mathbf{F}$

OS: Menopoma $1825 \approx$ Cryptobranchus $1821 \cdot \mathbf{O E}$

EN: CRYPTOBRANCHIDAE 1826.fb.f003-04 • F

EF: CRYPTOBRANCHIDAE 1826.fb.f003

MERCURANITES nov., DOP.da.f120 • KY

SI: $561 \bullet$ CI: h454 • ST: 0.10 .30

RL: INR

PA: $00 \cdot$ MERCURANITES $\bullet$ Hoc loco $\bullet \mathbf{C n}$

$01 \cdot$ MERCURANITIES $\bullet$ Hoc loco $\bullet \mathbf{b C n}$

OS: Mercurana $2013 \cdot \mathbf{P D}$

EN: (1) MERCURANITES DOP.da.f120-00 • Cn

(2) MERCURANITIES DOP.da.f120-01 • bCn

EF: RHACOPHORIDAE |1858.gc.f012|-1932.ha.f001

MeristogenYINAE $\mathrm{Fei}^{+2}$, 2010.fa.f003 • KY

SI: $394 \cdot$ CI: h294 • ST: 0.10 .32

RL: $\geq$ CLINOTARSINI 2010.fa.f011 • PR: hoc loco

PA: c0 • MERISTOGENYINAE $\bullet \mathrm{Fei}^{+2}$ 2010.fa: $18 \cdot \mathbf{b F} \bullet$ EEA: PD i1 $\bullet$ MERISTOGENINAE $\bullet \mathrm{Fei}^{+2}$ 2010.fa: $17 \cdot \mathbf{b F}$

02 $\bullet$ MERISTOGENYINI $\bullet \mathrm{Fei}^{+2}$ 2010.fa: $18 \cdot \mathbf{T}$

OS: Meristogenys $1991 \cdot$ OD

EN: MERISTOGENYINI 2010.fa.f003-02 • T

EF: RANIDAE 1796.ba.f001

MICRHYLINA Günther, 1858.gc.f003 • MK

SI: $131 \cdot$ CI: h085 • ST: 0.10 .58

RL: < MICROHYLIDAE |1843.fa.f012|-1931.na.f001 • MK: Dubois 1983.da: 275

PA: $00 \bullet$ MICRHYLINA • Günther 1858.gc: $344 \cdot \mathbf{S c}$

01 MICRHYLIDAE $\bullet$ Günther 1858.gc: $346 \cdot \mathbf{F}$

02 - MICHRYLINA • Mivart 1869.ma: $288 \cdot \mathbf{b F}$

03 - MICHRYLINA • Jiménez de la Espada 1870.ja: $65 \cdot$ Sc

$04 \cdot$ MICHRYLIDAE $\bullet$ Fatio 1872.fa: $230 \cdot \mathbf{F}$

05 MICRHYLINA $\bullet$ Hoffmann 1878.ha: $614 \cdot \mathbf{F}$

06 MICRIHYLINA $\bullet$ Brocchi 1881.ba: $28 \cdot$ Sc

OS: Micrhyla $1841 \equiv$ Microhyla $1838 \cdot \mathbf{O E}$

EN: (1) MICROHYLIDAE |1843.fa.f012|-1931.na.f001-01 • F ")"')"

(4) MICROHYLINA |1843.fa.f012|-1931.na.f001-08 • bT

EF: MICROHYLIDAE |1843.fa.f012|-1931.na.f001

MICRIXALINAE Bossuyt ${ }^{+1}$, 2001.ba.f001 • AN

SI: $361 \cdot$ CI: n090 • ST: 0.28 .50

RL: INR

PA: $00 \cdot$ MICRIXALINAE $\bullet$ Bossuyt $^{+1}$ 2001.ba: $94 \cdot \mathbf{b F}$

OS: Micrixalus $1888 \cdot \mathbf{O E}$

EN: (1) MICRIXALOIDAE 2001.db.f001-02・eF

(2) MICRIXALIDAE 2001.db.f001-01 • F

EF: MICRIXALIDAE 2001.db.f001
MiCRIXALINAE Dubois ${ }^{+2}$, 2001.db.f001 • KY

SI: $362 \bullet$ CI: h268 • ST: 0.10 .30

RL: INR

PA: $00 \cdot$ MICRIXALINAE $\bullet$ Dubois $^{+2} 2001 . \mathrm{db}: 56 \cdot \mathbf{b F}$

$01 \cdot \operatorname{MICRIXALIDAE} \cdot$ Frost $^{+18}$ 2006.fa: $7 \cdot \mathbf{F}$

02 $\cdot$ MICRIXALOIDAE $\bullet$ Hoc loco $\bullet \mathbf{e F}$

OS: Micrixalus $1888 \cdot$ OD

EN: (1) MICRIXALOIDAE 2001.db.f001-02 • eF

(2) MICRIXALIDAE 2001.db.f001-01 • F

EF: MICRIXALIDAE 2001.db.f001

MICROCAECILIINOA nov., DOP.da.f129 • KY

SI: $570 \bullet$ CI: h463 • ST: 0.10 .30

RL: INR

PA: 00 • MICROCAECILIINOA $\bullet$ Hoc loco $\bullet \mathbf{h T}$

OS: Microcaecilia $1968 \cdot \mathbf{P D}$

EN: MICROCAECILIINOA DOP.da.f129-00 • hT

EF: CAECILIIDAE 1814.ra.f003-|1825.gb.f008|

MiCROHYLINAE Noble, 1931.na.f001 • SK

SI: $216 \bullet$ CI: h151 • ST: 0.10 .35

RL: > GASTROPHRYNAE 1843.fa.f012 • PS: Dubois 1983.da: 274

> MICRHYLINA 1858.gc.f003 • MK: Dubois 1983.da: 275

PA: $00 \bullet$ MICROHYLINAE $\bullet$ Noble 1931.na: 451 • bF

01 $\cdot$ MICROHYLIDAE $\bullet$ Parker 1934.pa: i $\bullet$ F

02 $\cdot$ MICROHYLOIDEA $・$ Laurent 1948.1a: $3 \cdot \mathbf{b F}$

03 - MICROCHYLIDAE $\bullet$ Casamiquela 1961.ca: $81 \cdot \mathbf{F}$

$04 \cdot$ MICROPHYLIDAE $\bullet$ Richards $^{+4}$ 1977.ra: $387 \cdot \mathbf{e F}$

05 - MICROHYLOIDAE $\bullet$ Dubois 1992.da: $309 \cdot$ eF

06 - MICRIHYLIDAE $\bullet$ Ota 1995.oa: $72 \cdot$ F

07 - MICROHYLINI $\bullet$ Dubois 2005.da: $15 \cdot \mathbf{T}$

$08 \cdot$ MICROHYLINA $\bullet$ Hoc loco $\bullet$ bT

OS: Microhyla $1838 \cdot \mathbf{O E}$

EN: (1) MICROHYLIDAE |1843.fa.f012|-1931.na.f001-01 • F

(2) MICROHYLINAE |1843.fa.f012|-1931.na.f001-00 • bF

(3) MiCROHYLINI |1843.fa.f012|-1931.na.f001-07• T

(4) MiCROHYLINA |1843.fa.f012|-1931.na.f001-08 • bT

EF: MICROHYLIDAE |1843.fa.f012|-1931.na.f001

MICRYLETTINA nov., DOP.da.f091 • KY

SI: $532 \bullet$ CI: h425 • ST: 0.10 .30

RL: INR

PA: $00 \cdot$ MiCRYLETTINA $\bullet$ Hoc loco $\bullet$ bT

OS: Micryletta $1987 \cdot \mathbf{P D}$

EN: MICRYLETTINA DOP.da.f091-00 • bT

EF: MICROHYLIDAE |1843.fa.f012|-1931.na.f001

MIXOPHYINAE nov., DOP.da.f075 • KY

SI: $516 \bullet$ CI: h409 • ST: 0.10 .30

RL: INR

PA: $00 \cdot$ MIXOPHYINAE $\bullet$ Hoc loco $\bullet \mathbf{b T}$

OS: Mixophyes $1864 \cdot \mathbf{P D}$

EN: MIXOPHYINAE DOP.da.f075-00 • bT

EF: MYOBATRACHIDAE 1850.sa.f001

MolgINA Bonaparte, 1850.bb.f015 • KY

SI: $107 \cdot$ CI: h069 • ST: 0.10 .30

RL: INR

PA: $00 \cdot$ MoLGINA $\bullet$ Bonaparte 1850.bb: pl. $\bullet \mathbf{b F}$

$01 \cdot \operatorname{MoLGIDAE} \bullet$ Gray 1850 ga: 5, $30 \cdot \mathbf{F}$

02 • MOLGIDA • Knauer 1878.ka: 97. • F 
03 $\cdot$ MOLGINAE $\bullet$ Dubois 1985 da: $68 \cdot \mathbf{b F}$

$04 \cdot$ MOLGINI Dubois $^{+1}$ 2009.db: 30 • T

05 MOLGINA Dubois $^{+1}$ 2009.db: $30 \cdot \mathbf{b T}$

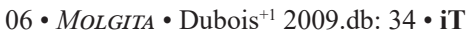

$07 \cdot$ MolGINIA $\cdot H o c$ loco $\bullet$ iT

$08 \cdot \mathrm{MoLGINOA} \cdot \mathrm{Hoc}$ loco $\bullet \mathbf{h T}$

$09 \cdot \mathrm{MolGITES} \cdot \mathrm{Hoc}$ loco $\bullet \mathbf{C n}$

OS: Molge $1820 \equiv$ Triturus $1815 \cdot \mathbf{O E}$

EN: (1) MoLGINI 1850.bb.f015-04 • T

(2) Molgina 1850.bb.f015-05 • bT

(3) MolGiNIA 1850.bb.f015-07 • iT

(4) Molginoa 1850.bb.f015-08 • hT

(5) Molgites 1850.bb.f015-09 • Cn

EF: SALAMANDRIDAE 1820.ga.f002

MONOMORPHA Van der Hoeven, 1833.va.f002 • AN

SI: 042 • CI: n020 • ST: 2.25 .50

RL: INR

PA: 00 - MONOMORPHA • Van der Hoeven 1833.va: iii, $304 \cdot \mathbf{F}$

OS: » 5 PN, including: Siren 1766 • PD

EN: SIRENIDAE 1825.gb.f005-00 • F

EF: SIRENIDAE 1825.gb.f005

MONTSECHOBATRACHIDAE Romer, 1945.ra.f001 • AN

SI: $241 \cdot$ CI: n065 $\bullet$ ST: 0.28 .50

RL: INR

PA: 00 • MONTSECHOBATRACHIDAE • Romer 1945.ra: 591 • F

OS: Montsechobatrachus 1921 (1926) $+\cdot \mathbf{A M} \equiv$ Monsechobatrachus $1921+\cdot \mathbf{O E}$

EN: ANURA Familia INCERTAE SEDIS

EF: ANURA Familia INCERTAE SEDIS

MoNTSECHOBATRACHIDAE Casamiquela, 1961.ca.f001 †・AP

SI: $264 \cdot$ CI: h1 $185 \bullet$ ST: 0.10 .46

RL: INR

PA: $00 \bullet$ MONTSECHOBATRACHIDAE • Casamiquela 1961.ca: 81, 97 • F

OS: Montsechobatrachus $1926+\cdot \mathbf{A M} \equiv$ Monsechobatrachus $1921+\cdot \mathbf{O E}$

EN: ANURA Familia INCERTAE SEDIS

EF: ANURA Familia INCERTAE SEDIS

Morerellina nov., DOP.da.f095 • KY

SI: 536 • CI: h429 • ST: 0.10 .30

RL: INR

PA: $00 \cdot$ MORERELLINA $\cdot$ Hoc loco $\bullet \mathbf{b T}$

OS: Morerella 2009 PD

EN: MORERELLINA DOP.da.f095-00 • bT

EF: HYPEROLIIDAE 1943.lb.f001

MURAENOPSES Fitzinger, 1843.fa.f016 • JD

SI: $081 \bullet$ CI: h049 • ST: 0.10 .40

RL: INR

PA: $00 \cdot$ MURAENOPSES • Fitzinger 1843.fa: $34 \cdot \mathbf{F}$

OS: Muraenopsis $1843 \approx$ Amphiuma $1821 \cdot \mathbf{O E}$

EN: (1) AMPHIUMOIDEA 1825.gb.f007-10 • pF

"1")"

(4) AMPHIUMIDAE 1825.gb.f007-00 • F

EF: AMPHIUMIDAE 1825.gb.f007

MYCETOGLOSSINA Bonaparte, 1850.bb.f017 • CG

SI: $109 \bullet$ CI: h071 • ST: 0.10 .61

RL: INR
PA: 00 • MYCETOGLOSSINA • Bonaparte 1850.bb: pl. • bF • IG: Opinion 1873 (Anonymous 1997.aa)

01 $\cdot$ MYCETOGLOSSINI $・$ Dubois 1984.da: $113 \cdot \mathbf{b F}$

OS: Mycetoglossus 1839 CI $\equiv$ Pseudotriton $1838 \cdot \mathbf{O E}$

EN: PSEUDOTRITONINA 2012.da.f006-00 • bT

EF: PLETHODONTIDAE 1850.ga.f001

MYCTODERA Lichtenstein ${ }^{+2}$, 1856.1a.f001 • AN

SI: $125 \cdot \mathbf{C I}:$ n041 - ST: 2.25 .50

RL: INR

PA: $00 \cdot$ MYCTODERA $・$ Lichtenstein $^{+2}$ 1856.la: $43 \cdot \mathbf{F}$

OS: „ 11 PN, including: Salamandra $1768 \approx$ Salamandra 1764 - PD

EN: (1) SALAMANDROIDEA 1820.ga.f002-21 • pF "')"')

(4) SALAMANDRINI 1820.ga.f002-28・ T

EF: SALAMANDRIDAE 1820.ga.f002

MYERSIOHYLINI nov., DOP.da.f052 • KY

SI: $494 \bullet \mathbf{C I}: \mathrm{h} 387 \bullet \mathbf{S T}: 0.10 .30$

RL: INR

PA: $00 \cdot$ MYERSIOHYLINI $\bullet$ Hoc loco $\bullet$ T

OS: Myersiohyla $2005 \cdot \mathbf{P D}$

EN: MYERSIOHYLINI DOP.da.f052-00 • T

EF: HYLIDAE 1815.ra.f002-|1825.gb.f001|

MYIOBATRACHIDAE Bonaparte, 1850.bb.f001 • JI

SI: $093 \cdot \mathbf{C I}:$ h055 - ST: 0.10 .52

RL: INR

PA: $00 \cdot$ MYIOBATRACHIDAE $\bullet$ Bonaparte 1850.bb: pl. • F

01 - MYIOBATRACHINA • Bonaparte 1850.bb: pl. • bF

OS: Myiobatrachus [1850] $1858 \equiv$ Myobatrachus $1850 \bullet \mathbf{O E}$

EN: (1) MYOBATRACHIDAE 1850.sa.f001-00・ F

"')"')

(6) MYOBATRACHINOA 1850.sa.f001-07 • hT

EF: MYOBATRACHIDAE 1850.sa.f001

MYOBATRACHIDAE Schlegel, 1850.sa.f001 • KY

SI: $092 \bullet \mathbf{C I}:$ h054 • ST: 0.10 .30

RL: INR

PA: $00 \cdot$ MYOBATRACHIDAE $\bullet$ Schlegel 1850.sa: $10 \cdot \mathbf{F}$

01 - MYOBATRACHIDA • Knauer 1878.ka: $104 \cdot \mathbf{F}$

02 $\operatorname{MYOBATRACHINAE} \bullet$ Parker 1940.pa: $2 \cdot \mathbf{b F}$

03 - MYOBATRACHIDAAE • Laurent 1991.1a: 6 • F

$04 \cdot$ MYOBATRACHOIDEA $\bullet$ Bossuyt $^{+1}$ 2009.ba: $359 \cdot \mathbf{p F}$

$05 \cdot$ MYOBATRACHINI $\cdot$ Hoc loco $・$ T

06 - MYOBATRACHINA $\cdot$ Hoc loco $\bullet \mathbf{b T}$

$07 \cdot$ MYOBATRACHINIA $\cdot$ Hoc loco $\bullet$ iT

$08 \cdot$ MYOBATRACHINOA $\cdot$ Hoc loco $\bullet \mathbf{h T}$

OS: Myobatrachus $1850 \bullet \mathbf{O E}$

EN: (1) MYOBATRACHIDAE 1850.sa.f001-00 • F

(2) MYOBATRACHINAE 1850.sa.f001-02 • bF

(3) MYOBATRACHINI 1850.sa.f001-05 • T

(4) MYOBATRACHINA 1850.sa.f001-06 • bT

(5) MYOBATRACHINIA 1850.sa.f001-07 • iT

(6) MYOBATRACHINOA 1850.sa.f001-08 • hT

EF: MYOBATRACHIDAE 1850.sa.f001

NANNOPHRYINI $\mathrm{Fei}^{+2}$, 2010.fa.f006 • KY

SI: $397 \bullet$ CI: h297 • ST: 0.10 .30

RL: INR 
PA: 00 • NANNOPHRYINI $\bullet \mathrm{Fei}^{+2}$ 2010.fa: $17 \cdot \mathbf{T}$

$01 \cdot$ NANNOPHRYINA $\bullet$ Hoc loco $\bullet \mathbf{b T}$

OS: Nannophrys $1869 \cdot$ OD

EN: NANNOPHRYINA 2010.fa.f006-01 • bT

EF: DicRogLOSSIDAE 1987.da.f004

NANNOPHRYNINOA nov., DOP.da.f034 • KY

SI: $475 \bullet$ CI: h368 - ST: 0.10 .30

RL: INR

PA: $00 \cdot$ NANNOPHRYNINOA $\bullet$ Hoc loco $\bullet \mathbf{h T}$

OS: Nannophryne $1870 \cdot \mathbf{P D}$

EN: NANNOPHRYNINOA DOP.da.f034-00 • hT

EF: BUFONIDAE 1825.gb.f004

NASIKABATRACHIDAE $\mathrm{Biju}^{+1}$, 2003.bb.f001 • KY

SI: $360 \bullet \mathbf{C I}:$ h267 • ST: 0.10 .30

RL: INR

PA: $00 \cdot$ NASIKABATRACHIDAE $・ \mathrm{Biju}^{+1}$ 2003.bb: $711 \cdot \mathbf{F}$

OS: Nasikabatrachus 2003・OD

EN: NASIKABATRACHIDAE 2003.bb.f001-00・F

EF: NASIKABATRACHIDAE 2003.bb.f001

NASUTIXALITES nov., DOP.da.f122・ KY

SI: $563 \bullet \mathbf{C I}: \mathrm{h} 456 \bullet \mathbf{S T}: 0.10 .30$

RL: INR

PA: $00 \cdot$ NASUTIXALITES $\bullet$ Hoc loco $\bullet \mathbf{C n}$

OS: Nasutixalus $2016 \cdot \mathbf{P D}$

EN: NASUTIXALITES DOP.da.f122-00 • Cn

EF: RHACOPHORIDAE |1858.gc.f012|-1932.ha.f001

NatalobatraChini nov., DOP.da.f102 • KY

SI: $543 \bullet$ CI: h436 • ST: 0.10 .30

RL: INR

PA: $00 \bullet$ NataLOBATRACHINI $\bullet$ Hoc loco $\bullet$ T

OS: Natalobatrachus $1912 \cdot \mathbf{P D}$

EN: NATALOBATRACHINI DOP.da.f102-00・ T

EF: CACOSTERNIDAE 1931.na.f008

NECTOPHRYNIDAE Laurent, 1942.la.f001・ KY

SI: $238 \bullet \mathbf{C I}:$ h170 • ST: 0.10 .30

RL: INR

PA: $00 \bullet$ NECTOPHRYNIDAE • Laurent 1942.la: 6 • F

$01 \cdot N E C T O P H R Y N I N I \cdot$ Dubois 1987.da: 27 • T

$02 \cdot$ NECTOPHRYNITOES $\bullet$ HOC lOCO $\bullet \mathbf{i C n}$

$03 \cdot$ NECTOPHRYNITUES $・ H O c$ loco $\bullet \mathbf{h C n}$

OS: Nectophryne $1875 \cdot \mathbf{O E}$

EN: (1) NECTOPHRYNITOES 1942.1a.f001-02 • iCn

(2) NECTOPHRYNITUES 1942.la.f001-03 • hCn

EF: BUFONIDAE 1825.gb.f004

NECTOPHRYNOIDINI Dubois, 1982.f001 • AN

SI: $310 \bullet \mathbf{C I}: \mathrm{n} 086 \bullet \mathbf{S T}: 0.28 .50$

RL: INR

PA: 00 • NECTOPHRYNOIDINI • Dubois 1982.da: 50 • T

OS: Nectophrynoides $1926 \cdot \mathbf{O E}$

EN: (1) TORNIERIOBATITOES 1926.ma.f001-03・iCn

(2) ToRNIERIOBATITUES 1926.ma.f001-04 • hCn

EF: BUFONIDAE 1825.gb.f004

NECTURI Fitzinger, 1843.fa.f018 • JD

SI: $083 \bullet \mathbf{C I}:$ h051・ST: 0.10 .40

RL: INR

PA: $00 \bullet N E C T U R I \cdot$ Fitzinger 1843.fa: $35 \cdot \mathbf{F}$
01 - NECTURINA • Bonaparte 1845.ba: $378 \cdot \mathbf{b F}$

$02 \cdot$ NECTURIDAE $\bullet$ Bonaparte 1850.bb: pl. • F

03 - NECTURINAE $\bullet$ Blackburn $^{+1}$ 2011.ba: $47 \cdot \mathbf{b F}$

OS: Necturus $1819 \cdot \mathbf{O E}$

EN: (1) PROTEOIDAE 1831.ba.f002-10 • eF

(2) Proteidae 1831.ba.f002-02 • F

EF: PROTEIDAE 1831.ba.f002

NeOBATRACHINA nov., DOP.da.f072 • KY

SI: $513 \bullet \mathbf{C I}: \mathrm{h} 406 \bullet \mathbf{S T}$ : 0.10 .30

RL: INR

PA: $00 \bullet$ NEOBATRACHINA $・ H O c$ loco $\bullet \mathbf{b T}$

OS: Neobatrachus $1863 \cdot \mathbf{P D}$

EN: NEOBATRACHINA DOP.da.f072-00 • bT

EF: MYOBATRACHIDAE 1850.sa.f001

NeSOROHYLINI nov., DOP.da.f053 • KY

SI: 493 • CI: h386・ST: 0.10 .30

RL: INR

PA: $00 \bullet$ NESOROHYLINI $・ H o c$ loco $・$ T

OS: Nesorohyla 2019・PD

EN: NESOROHYLINI DOP.da.f053-00 • T

EF: HYLIDAE 1815.ra.f002-|1825.gb.f001|

NEURERGITES nov., DOP.da.f145 • KY

SI: $586 \bullet$ CI: h479 • ST: 0.10 .30

RL: INR

PA: $00 \cdot$ NEURERGITES $\bullet$ Hoc loco $\bullet \mathbf{h T}$

OS: Neurergus $1862 \cdot \mathbf{P D}$

EN: NEURERGITES DOP.f145-00 • Cn

EF: SALAMANDRIDAE 1820.ga.f002

NidiRANINI $\mathrm{Fei}^{+2}$, 2010.fa.f013 • KY

SI: $404 \cdot \mathbf{C I}:$ h304 $\bullet$ ST: 0.10 .30

RL: INR

PA: $00 \bullet$ NIDIRANINI $\bullet \mathrm{Fei}^{+2}$ 2010.fa: $18 \cdot \mathbf{T}$

$01 \cdot$ NIDIRANITES $\bullet$ Hoc loco $\bullet \mathbf{C n}$

OS: Nidirana $1992 \cdot$ OD

EN: NIDIRANITES 2010.fa.f013-01 • Cn

EF: RANIDAE 1796.ba.f001

Noblellinoa nov., DOP.da.f008 • KY

SI: $449 \bullet$ CI: h342 • ST: 0.10 .30

RL: INR

PA: $00 \cdot$ NOBLELLINOA $\cdot$ Hoc loco $\bullet \mathbf{h T}$

OS: Noblella $1930 \cdot \mathbf{P D}$

EN: NOBLELLINOA DOP.da.f008-00 • hT

EF: BRACHYCEPHALIDAE 1858.gc.f002

NotadenINI nov., DOP.da.f074 • KY

SI: $515 \bullet \mathbf{C I}:$ h408 • ST: 0.10 .30

RL: INR

PA: $00 \cdot$ NotadENINI $\bullet$ Hoc loco $\bullet \mathbf{b T}$

OS: Notaden $1873 \cdot \mathbf{P D}$

EN: NOTADENINI DOP.da.f074-00 • bT

EF: MYOBATRACHIDAE 1850.sa.f001

NoterPETONTIDAE Rage ${ }^{+2}$, 1993.ra.f001 † KY

SI: $353 \bullet \mathbf{C I}: \mathrm{h} 260 \bullet \mathbf{S T}$ : 0.10 .30

RL: INR

PA: 00 • NOTERPETONTIDAE $\bullet$ Rage $^{+2}$ 1993.ra: $516 \bullet \mathbf{F}$

01 - NOTERPETIDAE $\bullet$ Dubois $^{+1}$ 2012.da: $102 \cdot \mathbf{F}$

OS: Noterpeton $1993+\cdot$ OD 
EN: NOTERPETIDAE 1993.ra.f001-01 † • F

EF: NOTERPETIDAE 1993.ra.f001-01 †

NotobatrachidaE Reig in Stepanicic ${ }^{+1}$, 1956.sa.f001 - KY

SI: $253 \bullet \mathbf{C I}:$ h178 $\bullet$ ST: 0.10 .30

RL: INR

PA: 00 • NотовATRACHIDAE • Reig in Stipanicic ${ }^{+1}$ 1956.sa: $219 \bullet \mathbf{F}$ $01 \cdot$ NOTOBATRACIDAE $・$ Reig 1958.ra: $114 \cdot \mathbf{F}$ 02 - NOTOBATRACHINAE • Barbadillo ${ }^{+2}$ 1997.ba: $55 \cdot \mathbf{b F}$

OS: Notobatrachus $1956+\cdot \mathbf{O E}$

EN: NOTOBATRACHINAE 1956.sa.f001-02 • bF

EF: LEIOPELMATIDAE 1869.ma.f007-|1942.ta.f001|

Nototritonities nov., DOP.da.f139 • KY

SI: 580 - CI: h473 • ST: 0.10 .30

RL: INR

PA: 00 • NototRitonities $\bullet$ Hoc loco $\bullet$ bCn

OS: Nototriton $1983 \cdot \mathbf{P D}$

EN: NototRITONITIES DOP.da.f139-00 • bCn

EF: PLETHODONTIDAE 1850.ga.f001

NyCtanolites nov., DOP.da.f140 • KY

SI: $581 \bullet \mathbf{C I}: \mathrm{h} 474 \cdot \mathbf{S T}: 0.10 .30$

RL: INR

PA: $00 \cdot$ NYCTANOLITES $\bullet$ Hoc loco $\bullet \mathbf{C n}$

OS: Nyctanolis $1983 \cdot \mathbf{P D}$

EN: NYCTANOLITES DOP.da.f140-00 • Cn

EF: PLETHODONTIDAE 1850.ga.f001

NYCTIBATRACHINAE Blommers-Schlösser, 1993.ba.f001・ KY

SI: $354 \cdot \mathbf{C I}:$ h261 - ST: 0.10 .30

RL: INR

PA: $00 \bullet$ NYCTIBATRACHINAE • Blommers-Schlösser 1993.ba: 199 $\cdot \mathbf{b F}$

01 - NYCTIBATRACHIDAE $\bullet$ Frost $^{+18}$ 2006.fa: 7 • F

$02 \cdot$ NYCTIBATRACHEIDAE $\bullet H O c$ loco $\bullet \mathbf{a F}$

OS: Nyctibatrachus $1882 \cdot \mathbf{O E}$

EN: (1) NYCTIBATRACHEIDAE 1993.ba.f001-02 • aF

(2) NYCTIBATRACHIDAE 1993.ba.f001-01 • F

EF: NYCTIBATRACHIDAE 1993.ba.f001

NyCtimantiNia nov., DOP.da.f066 • KY

SI: $507 \bullet$ CI: h400 • ST: 0.10 .30

RL: INR

PA: $00 \cdot$ NYCTIMANTINIA $\bullet$ Hoc loco $\bullet$ iT

OS: Nyctimantis $1882 \cdot \mathbf{P D}$

EN: NYCTIMANTINIA DOP.da.f066-00 • iT

EF: HYLIDAE 1815.ra.f002-|1825.gb.f001|

NYCTIMYSTINAE Laurent, 1975.la.f001 • JD

SI: $297 \bullet \mathbf{C I}:$ h2 $10 \bullet \mathbf{S T}: 0.10 .40$

RL: INR

PA: $00 \cdot$ NYCTIMYSTINAE $\bullet$ Laurent 1975.la: $183 \cdot \mathbf{b F}$

OS: Nyctimystes $1916 \cdot \mathbf{O E}$

EN: PELODRYADINAE 1859.ga.f001-01・bF

EF: PHYLLOMEDUSIDAE 1858.gc.f009

NyCTIXALINI Grosjean ${ }^{+3}$, 2008.ga.f001 • KY

SI: $380 \bullet \mathbf{C I}:$ h2 $280 \bullet \mathbf{S T}: 0.10 .30$

RL: INR

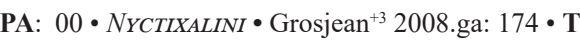

$01 \cdot$ NYCTIXALINIA $\bullet$ Hoc loco $\bullet$ iT
OS: Nyctixalus $1882 \cdot$ OD

EN: NYCTIXALINIA 2008.ga.f001-01・iT

EF: RHACOPHORIDAE |1858.gc.f012|-1932.ha.f001

NyMPHARGINI nov., DOP.da.f046 • KY

SI: $487 \bullet \mathbf{C I}:$ h3 380 - ST: 0.10 .30

RL: INR

PA: $00 \cdot$ NYMPHARGINI $\bullet$ Hoc loco $\bullet$ T

OS: Nymphargus 2007 • PD

EN: NYMPHARGINI DOP.da.f046-00 • T

EF: CENTROLENIDAE 1951.ta.f001

OCCIDOZYGINAE $\mathrm{Fei}^{+2}$, 1990.fa.f002 • KY

SI: $345 \bullet$ CI: h252 • ST: 0.10 .30

RL: INR

PA: 00 - OCCIDOZYGINAE $\bullet \mathrm{Fei}^{+2}$ 1990.fb: 4, 123 • bF

01 OCCIDOZYINAE $\cdot \mathrm{Ye}^{+2}$ 1993.ya: $309 \cdot \mathbf{b F}$

$02 \cdot$ OCCIDOZYGINI • Dubois 2005.da: $16 \cdot \mathbf{T}$

03 OCCIDOZYGIDAE $\bullet$ Borah $^{+5}$ 2013.ba: $39 \cdot \mathbf{F}$

OS: Occidozyga $1822 \cdot \mathbf{O E}$

EN: (1) OCCIDOZYGIDAE 1990.fa.f002-03 • F

(2) OCCIDOZYGINAE 1990.fa.f002-00 • bF

EF: OCCIDOZYGIDAE 1990.fa.f002

ODONTOBATRACHIDAE Barej $^{+5}$, 2014.ba.f001 • KY

SI: $433 \cdot \mathbf{C I}: \mathrm{h} 326 \cdot \mathbf{S T}: 0.10 .30$

RL: INR

PA: $00 \cdot$ ODONTOBATRACHIDAE • Barej ${ }^{+5}$ 2014.ba: $1 \cdot \mathbf{F}$

01 - ODONTOBATRACHOIDEA $\bullet$ HOc loco $\bullet \mathbf{p F}$

OS: Odontobatrachus $2014 \cdot$ OD

EN: (1) ODONTOBATRACHOIDEA 2014.ba.f001-01 • pF

(2) ODONTOBATRACHIDAE 2014.ba.f001-00 • F

EF: ODONTOBATRACHIDAE 2014.ba.f001

ODONTOPHRYNINI Lynch, 1969.lb.f002 • AN

SI: $284 \cdot \mathbf{C I}: \mathrm{n} 081 \cdot \mathbf{S T}: 0.28 .50$

RL: INR

PA: 00 • ODONTOPHRYNINI - Lynch 1969.1b: 3 • T

OS: Odontophrynus $1862 \cdot \mathbf{O E}$

EN: (1) ODONTOPHRYNIDAE 1971.la.f002-03 • F

(2) ODONTOPHRYNINAE 1971.la.f002-04 • bF

EF: ODONTOPHRYNIDAE 1971.1a.f002

ODONTOPHRYNINI Lynch, 1971.la.f002 • KY

SI: $288 \bullet$ CI: h203・ST: 0.10 .30

RL: INR

PA: $00 \cdot$ ODONTOPHRYNINI $\bullet$ Lynch 1971.1a: $130 \cdot$ T

01 - ODONTOPHRYINI - Lynch 1973.1a: $497 \cdot \mathbf{T}$

$02 \cdot$ ODONTOPHRYNAE $\bullet$ Ardila-Robayo 1979.aa: pl. p. 474-475

- bF

03 - ODONTOPHRYNIDAE $・$ Pyron $^{+1}$ 2011.pa: $543 \cdot \mathbf{F}$

$04 \cdot$ ODONTOPHRYNINAE $\bullet$ Hoc loco $\bullet \mathbf{F}$

OS: Odontophrynus $1862 \cdot \mathbf{O E}$

EN: (1) ODONTOPHRYNIDAE 1971.1a.f002-03 • F

(2) ODONTOPHRYNINAE 1971.1a.f002-04 • bF

EF: ODONTOPHRYNIDAE 1971.1a.f002

ODORRANINI $\mathrm{Fei}^{+2}$, 2010.fa.f015 • KY

SI: $406 \bullet \mathbf{C I}:$ h306・ST: 0.10 .30

RL: INR

PA: $00 \cdot$ ODORRANINI $\bullet \mathrm{Fei}^{+2}$ 2010.fa: $18 \cdot \mathbf{T}$

$01 \cdot$ ODORRANITES $\bullet \mathrm{HOC}$ loco $\bullet \mathbf{C n}$ 
OS: Odorrana 1990 • OD

EN: ODORRANITES 2010.fa.f015-01 • Cn

EF: RANIDAE 1796.ba.f001

OEDIPINA Gray, 1850.ga.f004 • JG

SI: $115 \bullet \mathbf{C I}:$ h077 • ST: 1.10 .53

RL: INR

PA: $00 \cdot$ OEDIPINA $・$ Gray 1850.ga: $42 \cdot \mathbf{U F}$

OS: Oedipus $1838 \mathbf{J H} \approx$ Bolitoglossa $1854 \cdot \mathbf{O E}$

EN: (1) BoLITOGLOSSINI 1850.ha.f002-03 • T ")"»"

(4) BolitoglossinOA 1850.ha.f002-06 • hT

EF: Plethodontidae 1850.ga.f001

OEDIPINITUES nov., DOP.da.f138・ KY

SI: $579 \bullet$ CI: h472 • ST: 0.10 .30

RL: INR

PA: $00 \bullet$ OEDIPINITUES $\bullet$ Hoc loco $\bullet \mathbf{h C n}$

OS: Oedipina $1868 \cdot \mathbf{P D}$

EN: OEDIPINITUES DOP.da.f138-00 • hCn

EF: PLETHODONTIDAE 1850.ga.f001

ONYCHODACTYLINAE Dubois ${ }^{+1}$, 2012.da.f001 • KY

SI: $418 \bullet \mathbf{C I}:$ h3 $12 \cdot \mathbf{S T}: 0.10 .30$

RL: INR

PA: $00 \cdot$ ONYCHODACTYLINAE $・$ Dubois $^{+1}$ 2012.da: $113 \cdot$ bF

OS: Onychodactylus $1838 \cdot$ OD

EN: ONYCHODACTYLINAE 2012.da.f001-00 • bF

EF: HYNOBIIDAE |1856.ha.f001|-1859.cb.f002

OPHIOSOMES Duméril, 1839.da.f001 • AN

SI: $057 \bullet \mathbf{C I}: \mathrm{n} 026 \bullet \mathbf{S T}: 2.25 .50$

RL: INR

PA: 00 • OPHIOSOMES • Duméril 1839.da: $581 \cdot \mathbf{F}$

$01 \cdot$ ОРніоОомА $\bullet$ Gray 1850.ga: $56 \cdot \mathbf{F}$

$02 \cdot$ OPHIOSOMES • Desmarest 1857.da: $17 \cdot \mathbf{F}$

OS: » 4 PN, including: Caecilia $1758 \cdot \mathbf{P D}$

EN: (1) CAECILIOIDEA 1814.ra.f003-|1825.gb.f008|-19 • pF ")"')

(5) CAECILIINA 1814.ra.f003-|1825.gb.f008|-26 • bT

EF: CAECILIIDAE 1814.ra.f003-|1825.gb.f008|

OPHRYOPHRYNINA nov., DOP.da.f149 • KY

SI: 595 • CI: h487 • ST: 0.10 .30

RL: INR

PA: $00 \cdot$ OPHRYOPHRYNINA $\cdot$ HOC loco $\bullet$ bT

OS: Ophryophryne 1903 • PD

EN: OPHRYOPHRYNINA DOP.da.f149-00 • bT

EF: MEGOPHRYIDAE 1850.bb.f008-|1931.na.f003|

OPISTHOCOELA Huene, 1948.ha.f002 • AN

SI: $246 \bullet \mathbf{C I}: \mathrm{n} 067 \bullet \mathbf{S T}: 0.25 .50$

RL: INR

PA: 00 • OPISTHOCOELA • Huene 1948.ha: $71 \cdot$ F

OS: » OA, PD: Bombina $1816 \cdot \mathbf{O E}$

EN: (1) BOMBINATOROIDEA 1825.gb.f002-16 • pF

(2) BOMBINATORIDAE 1825.gb.f002-02 • F

EF: BOMBINATORIDAE 1825.gb.f002

OPISTHOCOELELLIDAE Tatarinov, 1964.ta.f001 + AN

SI: $268 \cdot \mathbf{C I}: \mathrm{n} 077 \cdot \mathbf{S T}: 0.28 .50$

RL: INR

PA: 00 • OPISTHOCOELELLIDAE • Tatarinov 1964.ta: 8, 129 • F
OS: Opisthocoelellus $1941+\cdot \mathbf{O E}$

EN: DISCOGLOSSIDAE 1858.gc.f004-00 • F

EF: DISCOGLOSSIDAE 1858.gc.f004

OPISTHODELPHYNAE Lutz, 1968.1a.f001 • JD

SI: $276 \bullet \mathbf{C I}:$ h193 • ST: 0.10 .40

RL: INR

PA: 00 - OPISTHODELPHYNAE • Lutz 1968.1a: 3, 8, 13 • bF

01 - OPISTHODELPHYINAE • Lutz 1969.1a: $275 \cdot \mathbf{b F}$

OS: Opisthodelphys $1859 \approx$ Gastrotheca $1843 \cdot \mathbf{O E}$

EN: GASTROTHECINI 1927.na.f001-01・T

EF: HEMIPHRACTIDAE 1862.pa.f001

OPISTHOTHYLACINA nov., DOP.da.f096 • KY

SI: $537 \bullet \mathbf{C I}:$ h430 • ST: 0.10 .30

RL: INR

PA: $00 \cdot$ OPISTHOTHYLACINA $\bullet$ Hoc loco $\bullet \mathbf{b T}$

OS: Opisthothylax 1966 • PD

EN: OPISTHOTHYLACINA DOP.da.f096-00 • bT

EF: HYPEROLIIDAE 1943.lb.f001

OrEobatinoa nov., DOP.da.f009 • KY

SI: $450 \bullet \mathbf{C I}: \mathrm{h} 343 \cdot \mathbf{S T}: 0.10 .30$

RL: INR

PA: $00 \cdot$ OREOBATINOA $\bullet$ Hoc loco $\bullet \mathbf{h T}$

01 - OREOBATITES $\bullet$ Hoc loco $\bullet \mathbf{C n}$

OS: Oreobates $1872 \cdot$ PD

EN: (1) OREOBATINOA DOP.da.f009-00 • hT

(2) OREOBATITES DOP.da.f009-01 • Cn

EF: BRACHYCEPHALIDAE 1858.gc.f002

OrEOLALAXINAE Tian't, 1985.ta.f001 • KY

SI: $315 \bullet \mathbf{C I}:$ h224 • ST: 0.10 .30

RL: INR

PA: $00 \cdot$ OREOLALAXINAE $・ \operatorname{Tian}^{+1}$ 1985.ta: $221 \bullet \mathbf{b F}$

01 - OREOLALAGINAE • Dubois 1987.db: $173 \cdot \mathbf{b F}$

$02 \cdot$ OREOLALAGINA $\cdot$ Hoc loco $\bullet$ bT

OS: Oreolalax $1962 \cdot \mathbf{O E}$

EN: OREOLALAGINA 1985.ta.f001-02・bT

EF: MEGOPHRYIDAE 1850.bb.f008-|1931.na.f003|

OREOPHRYNELLINA nOV., DOP.da.f035 • KY

SI: 476 • CI: h369 • ST: 0.10 .30

RL: INR

PA: 00 - OREOPHRYNELLINA $\cdot$ Hoc loco • bT

OS: Oreophrynella $1895 \cdot \mathbf{P D}$

EN: OREOPHRYNELLINA DOP.da.f035-00 • bT

EF: BUFONIDAE 1825.gb.f004

OrIXALINOA nov., DOP.da.f118 • KY

SI: $559 \bullet \mathbf{C I}: \mathrm{h} 452 \bullet \mathbf{S T}: 0.10 .30$

RL: INR

PA: $00 \cdot$ ORIXALINOA $\bullet$ Hoc loco $\bullet \mathbf{h T}$

OS: Orixalus nov. • PD

EN: ORIXALINOA DOP.da.f118-00 • hT

EF: RHACOPHORIDAE |1858.gc.f012|-1932.ha.f001

OSCAECILIIDAE Lescure ${ }^{+2}$, 1986.lb.f002 • JD

SI: $321 \cdot \mathbf{C I}:$ h229 • ST: 0.10 .40

RL: INR

PA: $00 \cdot$ OSCAECILIIDAE $・$ Lescure $^{+2}$ 1986.1b: $145 \cdot \mathbf{F}$

$01 \cdot$ OSCAECILIOIDAE $\bullet$ Lescure $^{+2}$ 1986.1b: $167 \cdot \mathbf{e F}$

OS: Oscaecilia $1968 \cdot \mathbf{O E}$ 
EN: (1) CAECILIOIDEA 1814.ra.f003-|1825.gb.f008|-19 • pF "')"'

(5) CAECILIINA 1814.ra.f003-|1825.gb.f008|-26 • bT

EF: CAECILIIDAE 1814.ra.f003-|1825.gb.f008|

OSORNOPHRYNINA nov., DOP.da.f036 • KY

SI: 477 • CI: h370 • ST: 0.10 .30

RL: INR

PA: 00 • OSORNOPHRYNINA $\cdot H O C$ loco $・$ bT

OS: Osornophryne $1976 \cdot \mathbf{P D}$

EN: OSORNOPHRYNINA DOP.da.f036-00 • bT

EF: BUFONIDAE 1825.gb.f004

OSTEOCEPHALINIA nov., DOP.da.f062 • KY

SI: $503 \cdot \mathbf{C I}:$ h396 • ST: 0.10 .30

RL: INR

PA: $00 \cdot$ OSTEOCEPHALINIA $\bullet$ Hoc loco $\bullet$ iT

OS: Osteocephalus $1862 \cdot \mathbf{P D}$

EN: OSTEOCEPHALINIA DOP.da.f062-00 • iT

EF: HYLIDAE 1815.ra.f002-|1825.gb.f001|

OSTEOPILINIA nov., DOP.da.f063 • KY

SI: $504 \cdot \mathbf{C I}:$ h397 • ST: 0.10 .30

RL: INR

PA: $00 \cdot$ OSTEOPILINIA $\cdot$ Hoc loco $\bullet$ iT

OS: Osteopilus $1843 \cdot \mathbf{P D}$

EN: OSTEOPILINIA DOP.da.f063-00 • iT

EF: HYLIDAE 1815.ra.f002-|1825.gb.f001|

OTOPHRYNINAE Wassersug ${ }^{+1}$, 1987.wa.f001・ KY

SI: $338 \cdot \mathbf{C I}:$ h2 $246 \cdot \mathbf{S T}: 0.10 .30$

RL: INR

PA: 00 • OTOPHRYNINAE • Wassersug ${ }^{+1}$ 1987.wa: $137 \cdot \mathbf{b F}$

OS: Otophryne $1900 \cdot \mathbf{O E}$

EN: OTOPHRYNINAE 1987.wa.f001-00 • bF

EF: MICROHYLIDAE |1843.fa.f012|-1931.na.f001

РАСНYHYNOBiIn Dubois ${ }^{+1}$, 2012.da.f002 • KY

SI: $419 \bullet$ CI: h313 • ST: 0.10 .30

RL: INR

PA: 00 - PACHYHYNOBIINI $\bullet$ Dubois $^{+1} 2012$ da: $113 \cdot$ T

$01 \cdot$ PACHYHYNOBIINA $\bullet$ Hoc loco $\bullet \mathbf{b T}$

OS: Pachyhynobius $1983 \cdot$ OD

EN: PACHYHYNOBIINA 2012.da.f002-01 • bT

EF: HYNOBIIDAE |1856.ha.f001|-1859.cb.f002

PAChytritonites nov., DOP.da.f142 • KY

SI: $583 \bullet$ CI: h476・ST: 0.10 .30

RL: INR

PA: $00 \cdot$ PACHYTRITONITES $\bullet$ Hoc loco $\bullet$ Cn

OS: Pachytriton $1878 \cdot \mathbf{P D}$

EN: PACHYTRITONITES DOP.da.f142-00 • Cn

EF: SALAMANDRIDAE 1820.ga.f002

PAINI Dubois, 1992.da.f003 • KY

SI: $350 \bullet \mathbf{C I}:$ h257• ST: 0.10 .30

RL: INR

PA: $00 \cdot P_{A I N I} \cdot$ Dubois 1992.da: $317 \cdot \mathbf{T}$

$01 \cdot P_{A I N I N A E} \cdot \mathrm{Fei}^{+2} 2010 \mathrm{fa}: 12 \cdot \mathbf{b F}$

$02 \cdot P_{A I N A E} \cdot \mathrm{Fei}^{+2} 2010$.fa: $17 \cdot \mathbf{b F}$

$03 \cdot P_{A I N A} \cdot$ Hoc loco $\bullet \mathbf{b T}$

OS: Paa $1975 \cdot$ OD

EN: (1) PAINAE 1992.da.f003-02 • bF
(2) PAINI 1992.da.f003-00 • T

(3) PAINA 1992.da.f003-03 • b T

EF: DICROGLOSSIDAE 1987.da.f004

PalaeobatrachidaE Cope, 1865.ca.f001 + KY

SI: $151 \bullet \mathbf{C I}:$ h101・ST: 0.10 .30

RL: INR

PA: 00 - PALAEOBATRACHIDAE • Cope 1865.ca: $99 \cdot \mathbf{F}$

01 PALAEOBATRACHOIDEA • Bolkay 1919.ba: $348 \cdot \mathbf{G a}$

$02 \cdot$ PALAEOBATRACHYDAE Stipanicic $^{+1}$ 1956.sa: $216 \cdot \mathbf{F}$

03 - PALEOBATRACHIDAE - Zweifel 1956.za: 10 • F

$04 \cdot$ PALEOBATRACIDAE • Casamiquela 1959.ca: 6 • F

05 • PALAEOBATRACIDAE • Casamiquela 1961.ca: $111 \cdot \mathbf{F}$

$06 \cdot$ PALAEOBATRACHOIDEA • Špinar 1972.sa: $33 \cdot \mathbf{U F}$

07 • PALAEOBATRACHINAE • Špinar 1976.sa: 286, $287 \cdot \mathbf{b F}$

08 • PALAEOBATRACHOIDEA • Śpinar 1983.sa: $53 \cdot \mathbf{p F}$

09 PALAEOBATRACHIA • Haas 2003.ha: 43 • UF

OS: Palaeobatrachus $1838+\bullet$ OE

EN: PALAEOBATRACHIDAE 1865.ca.f001-00 † - F

EF: PALAEOBATRACHIDAE 1865.ca.f001 †

PALAEURODELIDEA Brame, 1958.ba.f001 † AN

SI: $257 \bullet \mathbf{C I}: \mathrm{n} 072 \bullet \mathbf{S T}: 0.25 .50,0.28 .50$

RL: INR

PA: 00 - PALAEURODELIDEA • Brame 1958.ba: 2 • F

$01 \cdot$ PALAEURODELIDAE $・$ Martín $^{+2}$ 2012.ma: 160 • F

OS: Hylaeobatrachus $1884+\cdot \mathbf{O M}$

EN: HYLAEOBATRACHIDAE 1889.1a.f001-00 † ・ F

EF: HYLAEOBATRACHIDAE 1889.1a.f001 †

Paludicolina Mivart, 1869.ma.f004 • KY

SI: $164 \cdot \mathbf{C I}:$ h112 • ST: 0.10 .30

RL: INR

PA: 00 - PALUDICOLINA • Mivart 1869.ma: 290 • bF

$01 \cdot$ PALUDICOLIDAE • Miranda-Ribeiro 1924.ma: $143 \cdot \mathbf{F}$

$02 \cdot$ PALUDICOLINAE $・$ Lutz 1929.1a: $5 \cdot \mathbf{b F}$

$03 \cdot$ PALUDICOLINI $\bullet$ Hoc loco $\bullet \mathbf{T}$

$04 \cdot$ PALUdICOLINA $\cdot$ Hoc loco $\bullet \mathbf{b T}$

OS: Paludicola $1830 \approx$ Physalaemus $1826 \cdot \mathbf{O E}$

EN: (1) PALUdicolinaE 1869.ma.f004-02 • bF

(2) PALUDICOLINI 1869.ma.f004-03 • T

(3) PALUdicolina 1869.ma.f004-04 • b T

EF: LEPTODACTYLIDAE |1838.ta.f001|-1896.wa.f001

PARACRINIINOA nov., DOP.da.f077 • KY

SI: $518 \cdot \mathbf{C I}: \mathrm{h} 411 \cdot \mathbf{S T}: 0.10 .30$

RL: INR

PA: $00 \cdot$ PARACRINIINOA $\bullet$ Hoc loco $\bullet \mathbf{b T}$

OS: Paracrinia $1976 \cdot$ PD

EN: PARACRINIINOA DOP.da.f077-00 • hT

EF: MYOBATRACHIDAE 1850.sa.f001

Paratelmatobinae Pyron ${ }^{+1}$, 2011.pa.f001 • AN

SI: $409 \cdot \mathbf{C I}:$ n097 • ST: 0.28 .50

RL: INR

PA: 00 • PARATELMATOBINAE $\bullet$ Pyron $^{+1}$ 2011.pa: 547, 579, $580 \bullet$ bF

OS: Paratelmatobius $1958 \cdot$ OD

EN: PARATELMATOBIINAE 2012.oa.f001-00 • bF

EF: LEPTODACTYLIDAE |1889.ta.f001|-1896.wa.f001

ParatelmatobinnaE Ohler $^{+1}$, 2012.0a.f001 • KY

SI: 428 • CI: h322 • ST: 0.10 .30 
RL: INR

PA: 00 • PARATELMATOBIINAE $\bullet$ Ohler $^{+1}$ 2012.oa: $165 \cdot \mathbf{b F}$

OS: Paratelmatobius $1958 \cdot$ OD

EN: PARATELMATOBIIDAE 2012.oa.f001-00 • bF

EF: LEPTODACTYLIDAE |1838.ta.f001|-1896.wa.f001

ParVIMolgITES nov., DOP.da.f133 • KY

SI: $574 \cdot$ CI: h467 • ST: 0.10 .30

RL: INR

PA: $00 \cdot$ PARVIMOLGITES $\bullet$ Hoc loco $\bullet$ Cn

OS: Parvimolge $1944 \cdot \mathbf{P D}$

EN: PARVIMOLGITES DOP.da.f133-00 • Cn

EF: PLETHODONTIDAE 1850.ga.f001

Pedostibitues nov., DOP.da.f016 • KY

SI: $457 \cdot$ CI: h350 • ST: 0.10 .30

RL: INR

PA: $00 \cdot$ PEDOSTIBITUES $\bullet$ Hoc loco $\bullet \mathbf{h C n}$

OS: Pedostibes $1876 \cdot \mathbf{P D}$

EN: PeDostibitues DOP.da.f016-00 • hCn

EF: BUFONIDAE 1825.gb.f004

PelObatidaE Bonaparte, 1850.bb.f004 • KY

SI: $096 \bullet$ CI: h058 • ST: 0.10 .32

RL: $\geq$ MEGALOPHREIDINA 1850.bb.f008 • PR: Dubois 1983.da: 271 $\geq$ PELODYTINA 1850.bb.f002 - PR: Dubois 1983.da: 271

PA: 00 P PLLOBATIDAE $\bullet$ Bonaparte 1850.bb: pl. • F

$01 \cdot$ PELOBATINA $\bullet$ Bonaparte 1850.bb: pl. $\bullet \mathbf{b F}$

$02 \cdot$ PELOBATOIDEI $\bullet$ Lichtenstein $^{+2}$ 1856.1a: $40 \cdot \mathbf{F}$

03 P PLOBATOIDEA $\bullet$ Stannius 1856.sa: $4 \cdot \mathbf{F}$

$04 \cdot$ PELOBATIDES $\bullet$ Bruch 1862.ba: $221 \cdot \mathbf{F}$

05 PELOBATIDEA $\bullet$ Huxley 1871.ha: $189 \cdot \mathbf{U F}$

$06 \cdot$ PELOBATIDAS $\bullet$ Knauer 1878.ka: $107 \cdot \mathbf{F}$

$07 \cdot$ PELOBATIDA $\bullet$ Bayer 1885.ba: $3 \cdot \mathbf{F}$

08 PELOBATINA $\bullet$ Schulze 1891.sa: $168 \cdot \mathbf{T}$

09 PELOBATOIDEA • Bolkay 1919.ba: $348 \cdot \mathbf{G a}$

10 PELOBATINAE $\bullet$ Fejérváry 1921.fb: $25 \cdot \mathbf{b F}$

$11 \cdot$ PELOBATOIDEA $・$ Bolkay 1929.ba: $58 \cdot \mathbf{p F}$

12 PALOBATIDAE $\cdot \mathrm{Fei}^{+1}$ 1990.fa: $420 \cdot \mathbf{F}$

13 P PELABATIDAE $\cdot \mathrm{Fei}^{+1}$ 1990.fa: $428 \cdot \mathbf{F}$

$14 \cdot P_{\text {EELOBATIDAE }} \cdot \mathrm{Fei}^{+3}$ 1995.fa: $237 \cdot \mathbf{F}$

$15 \cdot$ PELOBATOIDIA $・$ Dubois 2005.da: $8 \cdot \mathbf{e F}$

16 P PLOBATOIDAE $\bullet$ Hoc loco $\bullet \mathbf{e F}$

OS: Pelobates $1830 \cdot \mathbf{O E}$

EN: (1) PELOBATOIDEA 1850.bb.f004-11 • pF

(2) PELOBATOIDAE $1850 . b b . f 004-16 \cdot \mathbf{e F}$

(3) PELOBATIDAE 1850.bb.f004-00 • F

EF: PELOBATIDAE 1850.bb.f004

PeLOBATINOPSIDINAE Špinar, 1976.sa.f001 † • JD

SI: $299 \bullet$ CI: h212 • ST: 0.10 .40

RL: INR

PA: $00 \cdot$ PELOBATINOPSIDINAE $\bullet$ Špinar 1976.sa: $287 \cdot \mathbf{b F}$

$01 \cdot$ PELOBATINOPSINAE Haubold in Krumbiegel ${ }^{+2}$ 1983.ka: $122 \cdot \mathbf{b F}$

OS: Pelobatinopsis $1941 \$ \approx$ Palaeobatrachus $1838 \$ \cdot$ OE

EN: PALAEOBATRACHIDAE 1865.ca.f001-00 $\bullet \cdot \mathbf{F}$

EF: PALAEOBATRACHIDAE 1865.ca.f001 †

PELOBIINI Erichson, 1837.ea.f001 • ZA

SI: $047 \cdot$ CI: zh02 • ST: 0.10 .99
RL: INR

PA: $00 \bullet$ PELOBIINI $\bullet$ Erichson 1837.ea: $182 \cdot \mathbf{G r}$

OS: Pelobius $1832 \cdot \bullet \cdot$ OE

EN: •

EF: •

PELOBII Fitzinger, 1843.fa.f004 • JG-JI

SI: $069 \cdot$ CI: h037 • ST: 0.10 .53

RL: $\downarrow$ PELOBIINI 1837.ea.f001

PA: $00 \cdot$ PELOBII $\bullet$ Fitzinger 1843.fa: $31 \cdot \mathbf{F}$

01 P PLOBIINAE $\bullet$ Duellman $^{+2} 2016 . \mathrm{db}: 3 \cdot \mathbf{b F}$

OS: Pelobius $1843 \mathbf{J H} \equiv$ Litoria $1838 \cdot \mathbf{O E}$

EN: PELODRYADINAE 1859.ga.f001-01 • bF

EF: PhylLOMEDUSIDAE 1858.gc.f009

PELODRYADIDAE Günther, 1858.gc.f008 • AN

SI: $136 \bullet \mathbf{C I}: \mathrm{n} 045 \cdot \mathbf{S T}: 0.24 .50$

RL: INR

PA: $00 \bullet$ PELODRYADIDAE $\bullet$ Günther 1858.gc: $346 \bullet \mathbf{F}$

OS: Pelodryas $1858 \mathbf{A N} \approx$ Ranoidea $1838 \cdot \mathbf{O E}$

EN: PELODRYADINAE 1859.ga.f001-01 • bF

EF: PHYLLOMEDUSIDAE 1858.gc.f009

PELODRYADIDAE Günther, 1859.ga.f001 • KY

SI: 144 • CI: h097 • ST: 0.10 .30

RL: INR

PA: $00 \cdot$ PELODRYADIDAE $\bullet$ Günther 1859.ga: ix, $119 \cdot \mathbf{F}$

01 PELODRYADIDAE $\bullet$ Hoffmann 1878.ha: $614 \cdot \mathbf{b F}$

02 PELODRYADINAE $\bullet$ Dowling $^{+1}$ 1978.da: $37.1 \cdot \mathbf{b F}$

OS: Pelodryas $1859 \approx$ Ranoidea $1838 \cdot \mathbf{O E}$

EN: PELODRYADINAE 1859.ga.f001-01 • bF

EF: PHYLlOMEdusidae 1858.gc.f009

Pelodytina Bonaparte, 1850.bb.f002 • KY

SI: $094 \cdot$ CI: h056 • ST: 0.10 .37

RL: $\leq$ PELOBATIDAE 1850.bb.f004 • PR: Dubois 1983.da: 271

PA: $00 \cdot$ PELODYTINA $\bullet$ Bonaparte 1850.bb: pl. $\bullet \mathbf{b F}$

01 PELODYTIDES $\bullet$ Bruch 1862.ba: $221 \cdot \mathbf{F}$

02 PELODYTIDAE $\bullet$ Cope 1866.ca: $68 \cdot \mathbf{F}$

$03 \cdot$ PELODYTINAE $\bullet$ Fejérváry 1923.fa: $181 \cdot \mathbf{b F}$

$04 \cdot$ PELODYTOIDAE $\bullet$ Hoc loco $\bullet \mathbf{e F}$

OS: Pelodytes $1838 \cdot \mathbf{O E}$

EN: (1) PELODYTOIDAE 1850.bb.f002-04 • eF

(2) PELODYTIDAE $1850 . \mathrm{bb} . \mathrm{f002}-02 \cdot \mathbf{F}$

EF: PELODYTIDAE 1850.bb.f002

Pelophylacinia nov., DOP.da.f107 • KY

SI: $548 \cdot$ CI: h441 • ST: 0.10 .30

RL: INR

PA: $00 \cdot$ PELOPHYLACINIA $\bullet$ Hoc loco $\bullet \mathbf{b T}$

OS: Pelophylax $1843 \cdot \mathbf{P D}$

EN: PELOPHYLACINIA DOP.da.f107-00 • iT

EF: RANIDAE 1796.ba.f001

Peltophrynites nov., DOP.da.f032 • Ky

SI: $473 \bullet$ CI: h366 • ST: 0.10 .30

RL: INR

PA: $00 \cdot$ PELTOPHRYNITES $・$ Hoc loco $・ \mathbf{C n}$

OS: Peltophryne $1843 \cdot \mathbf{P D}$

EN: Peltophrynites DOP.da.f032-00 • Cn

EF: BUFONIDAE 1825.gb.f004 
PERENNIBRANCHIA Betta, 1864.ba.f001 • AN

SI: $150 \bullet$ CI: n048・ST: 2.25 .50

RL: INR

PA: $00 \cdot$ PERENNIBRANCHIA • Betta 1864.ba: $505 \cdot \mathbf{F}$

OS: » 4 PN, including: Proteus $1768 \cdot \mathbf{P D}$

EN: (1) PROTEOIDAE 1831.ba.f002-10 • eF

(2) PROTEIDAE 1831.ba.f002-02 • F

EF: PROTEIDAE 1831.ba.f002

PERENNIBRANCHIATA Zittel, 1888.za.f001 • AN

SI: $188 \bullet \mathbf{C I}: \mathrm{n} 056 \bullet \mathbf{S T}: 2.25 .50$

RL: $\leftrightarrow$ PHANEROBRANCHIA 1827.fa.f001-05

PA: $00 \cdot$ PERENNIBRANCHIATA $・$ Zittel 1888.za: $418 \cdot \mathbf{F}$

OS: Phanerobranchus $1821 \approx$ Necturus $1819 \cdot$ AN

EN: (1) PROTEOIDAE 1831.ba.f002-10 • eF

(2) PROTEIDAE 1831.ba.f002-02 • F

EF: PROTEIDAE 1831.ba.f002

PEROBRANCHES Duméril ${ }^{+2}$, 1854.da.f001 • AN

SI: $118 \cdot \mathbf{C I}: \mathrm{n} 037 \cdot \mathbf{S T}: 2.25 .50$

RL: INR

PA: $00 \cdot$ PEROBRANCHES Duméril $^{+2}$ 1854.da: xii, xix, 35, $199 \cdot \mathbf{F}$

01 PEROBRANCHIA $\bullet$ Betta 1864.ba: $505 \cdot \mathbf{F}$

OS: » 2 PN, including: Amphiuma $1821 \cdot \mathbf{P D}$

EN: (1) AMPHIUMOIDEA 1825.gb.f007-10 • pF

"m!n

(4) AMPHIUMIDAE 1825.gb.f007-00 • F

EF: AMPHIUMIDAE 1825.gb.f007

Petropedetinae Noble, 1931.na.f006 • KY

SI: $221 \cdot$ CI: h156 • ST: 0.10 .30

RL: INR

PA: $00 \bullet$ PETROPEDETINAE $\bullet$ Noble 1931.na: $520 \bullet \mathbf{b F}$

01 P PETROPEDATINAE • Tatarinov 1964.ta: $132 \cdot \mathbf{b F}$

02 PETROPEDETIDAE $\bullet$ Bauer 1985.ba: $3 \cdot \mathbf{F}$

$03 \cdot$ PETROPEDETOIDAE $\bullet$ Hoc loco $\bullet \mathbf{e F}$

OS: Petropedetes $1874 \cdot \mathbf{O E}$

EN: (1) PetropedetoIDAE 1931.na.f006-03 • eF

(2) PETROPEDETIDAE 1931.na.f006-02 • F

EF: PETROPEDETIDAE 1931.na.f006

PHAENEROBRANCHOIDEA Fitzinger, 1826.fb.f004 • RI

SI: $030 \bullet$ CI: h016 • ST: 0.10 .43

RL: < PROTEINA 1831.ba.f002 • RI: Dubois ${ }^{+1} 2015$. da: 44

PA: 00 • PHAENEROBRANCHOIDEA $\bullet$ Fitzinger 1826.fb: $43 \cdot \mathbf{F}$

OS: Phaenerobranchus $1826 \approx$ Necturus $1819 \cdot \mathbf{O E}$

EN: (1) PROTEOIDAE 1831.ba.f002-10 • eF

(2) PROTEIDAE 1831.ba.f002-02 • F

EF: PROTEIDAE 1831.ba.f002

PhANEROBRANCHOIDEA Fitzinger, 1827.fa.f001 • RI

SI: $031 \cdot$ CI: h017 • ST: 0.10 .43

RL: $\leftarrow$ PHAENEROBRANCHOIDEA 1826.fb.f004

< PROTEINA 1831.ba.f002 • RI: Dubois ${ }^{+1}$ 2015.da: 44

PA: $00 \cdot$ PHANEROBRANCHOIDEA • Fitzinger 1827.fa: $264 \cdot \mathbf{F}$

$01 \cdot$ PhANEROBRANCHIDEAE $\bullet$ Gray 1850 ga: $64 \cdot \mathbf{F}$

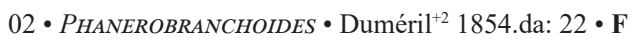

03 PHANEROBRANCHIATA $\bullet$ Wied 1865.wa: viii, $138 \cdot \mathbf{F}$

04 PHANEROBRANCHIOIDES $\bullet$ Hoffmann 1878.ha: $582 \cdot \mathbf{F}$

05 P PANEROBRANCHIA $\bullet$ Zittel 1888.za: $418 \cdot \mathbf{F}$

06 PHANEROBRANCHOIDA $\bullet$ Cope 1889.ca: $18 \cdot \mathbf{F}$
07 • PHANEROBRANCHIDAE $・$ Huene 1931.ha: 310 • F

$08 \cdot$ PHANEROBRANCHINAE Dubois $^{+1}$ 2012.da: $118,146 \cdot \mathbf{b F}$

OS: Phanerobranchus $1821 \approx$ Necturus $1819 \cdot \mathbf{O E}$

EN: (1) PROTEOIDAE 1831.ba.f002-10 • eF

(2) PROTEIDAE 1831.ba.f002-02 • F

EF: PROTEIDAE 1831.ba.f002

Phanerobranchiata Wiedersheim, 1877.wa.f001 • AN

SI: $178 \cdot \mathbf{C I}:$ n054 • ST: 2.25 .50

RL: INR

PA: 00 - PHANEROBRANCHIATA • Wiedersheim 1877.wa: 356 - UF

OS: » 3 PN, including: Proteus $1768 \cdot$ PD

EN: (1) PROTEOIDAE 1831.ba.f002-10 • eF

(2) PROTEIDAE 1831.ba.f002-02 • F

EF: PRoteIdaE 1831.ba.f002

Phaneroglossa Huene, 1931.ha.f001 • AN

SI: $232 \bullet$ CI: n063 • ST: 2.25 .50

RL: INR

PA: 00 P PAANEROGLOSSA $\bullet$ Huene 1931.ha: $311 \cdot \mathbf{p F}$

OS: » 17 PN, including: Rana $1758 \cdot \mathbf{P D}$

EN: (1) RANOIDEA 1796.ba.f001-28 • pF

"II!)

(12) RANITOES 1796.ba.f001-38 • iCn

EF: RANIDAE 1796.ba.f001

Phasmahylina nov., DOP.da.f070 • KY

SI: $511 \bullet$ CI: h404 • ST: 0.10 .30

RL: INR

PA: $00 \cdot$ PHASMAHYLINA $・$ Hoc loco $\bullet \mathbf{b T}$

OS: Phasmahyla $1991 \cdot \mathbf{P D}$

EN: PHASMAHYLINA DOP.da.f070-00 • bT

EF: PhYLLOMEDUSIDAE 1858.gc.f009

Philautinae Dubois, 1981.da.f001 • KY

SI: $307 \cdot$ CI: h218 • ST: 0.10 .30

RL: INR

PA: $00 \cdot$ PHILAUTINAE $\bullet$ Dubois 1981.da: $227 \cdot \mathbf{b F}$

01 PhILAUTINI $\bullet$ Dubois 1987.da: $69 \cdot \mathbf{T}$

02 PhilautinOA $\bullet$ Hoc loco $\bullet \mathbf{h T}$

$03 \cdot$ PhilaUtites $\bullet$ Hoc loco $\bullet \mathbf{C n}$

OS: Philautus $1848 \cdot \mathbf{O E}$

EN: (1) Philautinoa 1981.da.f001-02 • hT

(2) Philautites 1981.da.f001-03 • Cn

EF: RHACOPHORIDAE |1858.gc.f012|-1932.ha.f001

PhrYNaCINIA Rafinesque, 1815.ra.f004 • AN

SI: $009 \bullet$ CI: n005 $\bullet$ ST: 2.25 .50

RL: INR

PA: $00 \cdot$ PHRYNACINIA $・$ Rafinesque 1815.ra: $78 \cdot \mathbf{b F}$

OS: Phrynacius $1815 \mathrm{AN} \equiv$ Bufo $1764 \cdot \mathbf{O E}$

EN: (1) BUFONOIDEA 1825.gb.f004-20 • pF

(')!')

(10) BUFONITOES 1825.gb.f004-33 • iCn

EF: BUFONIDAE 1825.gb.f004

Phrynellinia nov., DOP.da.f090 • KY

SI: $531 \cdot$ CI: h424 • ST: 0.10 .30

RL: INR

PA: $00 \cdot$ PHRYNELLINIA $\bullet$ Hoc loco $\bullet$ iT

OS: Phrynella $1887 \cdot \mathbf{P D}$

EN: PHRYNELLINIA DOP.da.f090-00 • iT 
EF: MICROHYLIDAE |1843.fa.f012|-1931.na.f001

PhrYNiscidaE Günther, 1858.gc.f005 • KY

SI: 133 • CI: h087 • ST: 0.10 .30

RL: INR

PA: $00 \cdot$ PHRYNISCIDAE • Günther 1858.gc: $346 \bullet$ F

$01 \cdot$ PHRYNISCINA • Mivart 1869.ma: $288 \cdot \mathbf{b F}$

$02 \cdot$ PHRYNISEIDAE $\bullet$ Hoffmann 1878.ha: $591 \cdot \mathbf{F}$

03 - PHRYNISCIDAE $\bullet$ Hoffmann 1878.ha: $613 \cdot \mathbf{b F}$

$04 \cdot$ PHRYNISCITIES $\cdot$ Hoc loco $\bullet \mathbf{b C n}$

05 $\cdot$ PHRYNISCITOES $\bullet$ HOC loco $\bullet \mathbf{b C n}$

OS: Phryniscus $1834 \approx$ Rhinella $1826 \cdot \mathbf{O E}$

EN: (1) PHRYNISCITIES 1858.gc.f005-04 • bCn: F.11.01.04

(2) PhrYNisCitoes 1858.gc.f005-05 • iCn: F.12.02.05

EF: BUFONIDAE 1825.gb.f004

PhrynobatrachinaE Laurent, 1941.la.f001 • AN

SI: $236 \bullet \mathbf{C I}:$ n064 • ST: 0.28 .50

RL: INR

PA: 00 • PHRYNOBATRACHINAE • Laurent 1941.1a: 79 • bF

OS: Phrynobatrachus $1862 \cdot \mathbf{O E}$

EN: (1) PHRYNOBATRACHOIDEA 1941.lb.f001-02 • pF

(2) PHRYNOBATRACHIDAE 1941.lb.f001-01 • F

EF: PHRYNOBATRACHIDAE 1941.lb.f001

PhrynobatrachinaE Laurent, 1941.lb.f001 • CK

SI: $237 \bullet \mathbf{C I}:$ h169 • ST: 0.10 .36

RL: > HEMIMANTIDAE 1878.ha.f002 • PP: Opinion 1921 (Anonymous 1999.aa)

PA: 00 • PhRYNOBATRACHINAE • Laurent 1941.lb: 192 • bF 01 - PHRYNOBATRACHIDAE • Dubois 1992.da: $309 \cdot \mathbf{F}$

$02 \cdot$ PHRYNOBATRACHOIDEA $\cdot$ Hoc loco $\bullet \mathbf{p F}$

OS: Phrynobatrachus $1862 \cdot \mathbf{O E}$

EN: (1) PHRYNOBATRACHOIDEA 1941.lb.f001-02 • pF

(2) PhrynobatrachidaE 1941.lb.f001-01 • F

EF: PhrYNobatraCHIDAE 1941.lb.f001

PhrYNomantini Burton, 1986.bb.f002 • JD

SI: 318 • CI: h226 • ST: 0.10 .40

RL: INR

PA: 00 • PHRYNOMANTINI • Burton 1986.bb: $444 \cdot$ T

OS: Phrynomantis $1867 \cdot$ OE

EN: PHRYNOMERIDAE 1931.na.f013-01 • F

EF: PHRYNOMERIDAE 1931.na.f013

Phrynomedusini nov., DOP.da.f069 • KY

SI: $510 \bullet$ CI: h403・ST: 0.10 .30

RL: INR

PA: $00 \cdot$ PhRYNOMEdUSINI $\bullet$ Hoc loco $\bullet$ T

OS: Phrynomedusa 1923 • PD

EN: PHRYNOMEDUSINI DOP.da.f069-00 • T

EF: PHYLLOMEDUSIDAE 1858.gc.f009

PhrynomerinaE Noble, 1931.na.f013 • KY

SI: $228 \bullet \mathbf{C I}:$ h162 • ST: 0.10 .30

RL: INR

PA: 00 • PHRYNOMERINAE • Noble 1931.na: 538 • bF

01 P PHRYNOMERIDAE $\bullet$ Parker 1934.pa: $9 \cdot \mathbf{F}$

OS: Phrynomerus $1926 \equiv$ Phrynomantis $1867 \cdot \mathbf{O E}$

EN: PHRYNOMERIDAE 1931.na.f013-01 • F

EF: PHRYNOMERIDAE 1931.na.f013
Phrynopodites nov., DOP.da.f010 • KY

SI: $451 \cdot \mathbf{C I}:$ h3 $44 \cdot \mathbf{S T}: 0.10 .30$

RL: INR

PA: $00 \cdot$ PHRYNOPODITES $\cdot$ HOc loco $・ \mathbf{C n}$

OS: Phrynopus $1873 \cdot$ PD

EN: PHRYNOPODITES DOP.da.f010-00 • Cn

EF: BRACHYCEPHALIDAE 1858.gc.f002

PhrYNopsinaE Noble, 1931.na.f005 • JG

SI: $220 \bullet \mathbf{C I}:$ h155 • ST: 0.10 .53

RL: INR

PA: $00 \cdot$ PHRYNOPSINAE $・$ Noble 1931.na: $518 \cdot \mathbf{b F}$

01 P PRYNOSPINAE $\bullet$ Tatarinov 1964.ta: $132 \cdot \mathbf{b F}$

OS: Phrynopsis $1893 \mathbf{J H} \approx$ Pyxicephalus $1838 \cdot \mathbf{O E}$

EN: (1) PYXICEPHALOIDAE 1850.bb.f005-04 • eF

(2) PYXICEPHALIDAE 1850.bb.f005-03 • F

EF: PYXICEPHALIDAE 1850.bb.f005

Phyllobatae Fitzinger, 1843.fa.f007 • PK

SI: $072 \cdot$ CI: h040 • ST: 0.10 .37

RL: < DENDROBATIDAE 1865.ca.f002 • PP: Opinion 2223

(Anonymous 2009.aa: 104)

PA: $00 \cdot$ PHYLLOBATAE $\bullet$ Fitzinger 1843.fa: $32 \cdot$ F

01 • PHYLLOBATIDAE • Parker 1933.pa: $12 \cdot \mathbf{F}$

02 • PHYLLOBATINAE • Ardila-Robayo 1979.aa: $385 \cdot \mathbf{b F}$

$03 \cdot$ PHYLLOBATINI $\bullet$ Hoc loco $\bullet$ T

OS: Phyllobates $1841 \cdot \mathbf{O E}$

EN: PHYLLOBATINI 1843.fa.f007.03 • T

EF: DENDROBATIDAE |1850.bb.f006|-1865.ca.f002

Phyllomedusidae Günther, 1858.gc.f009 • KY

SI: $137 \bullet \mathbf{C I}:$ h090 • ST: 0.10 .30

RL: INR

PA: 00 • PHYLLOMEDUSIDAE $\bullet$ Günther 1858.gc: $346 \bullet$ F

01 • PHYLLOMEDUSIDAE $・$ Hoffmann 1878.ha: $614 \cdot \mathbf{b F}$

$02 \cdot$ PHYLLOMEDUSIDA $・$ Knauer 1878.ka: $113 \cdot \mathbf{F}$

03 PHYLLOMEDUSINAE • Miranda-Ribeiro 1926.ma: $64 \cdot \mathbf{b F}$

$04 \cdot$ PHYLLOMEDUSINI $\bullet H o c$ loco $・$ T

$05 \cdot$ PHYLLOMEDUSINA $\cdot$ Hoc loco $\bullet \mathbf{b T}$

$06 \cdot$ PHYLLOMEDUSINIA $・ H O c$ loco $\bullet$ iT

OS: Phyllomedusa $1830 \cdot \mathbf{O E}$

EN: (1) PHYLLOMEDUSIDAE 1858.gc.f009-00 • F

(2) PhyllOMEdusinaE 1858.gc.f009-03 • bF

(3) PHYLLOMEDUSINI 1858.gc.f009-04 • T

(4) Phyllomedusina 1858.gc.f009-05 • bT

(3) Phyllomedusinia 1858.gc.f009-06 • iT

EF: PHYLLOMEDUSIDAE 1858.gc.f009

Phytotriadina nov., DOP.da.f064 • Ky

SI: $505 \bullet \mathbf{C I}:$ h398 • ST: 0.10 .30

RL: INR

PA: $00 \cdot$ PHYTOTRIADINA $\bullet$ Hoc loco $\bullet \mathbf{b T}$

OS: Phytodriades $2009 \cdot \mathbf{P D}$

EN: PHYTOTRIADINA DOP.da.f064-00 • bT

EF: HYLIDAE 1815.ra.f002-|1825.gb.f001|

Phyzelaphryninae Hedges ${ }^{+2}$, 2008.ha.f002 • KY

SI: $382 \bullet \mathbf{C I}:$ h2 $282 \cdot \mathbf{S T}: 0.10 .30$

RL: INR

PA: $00 \cdot$ PHYZELAPHRYNINAE Hedges $^{+2}$ 2008.ha: $5 \cdot$ bF

$01 \cdot$ PHYZELAPHRYNINI $\bullet$ Hoc loco $\bullet$ T 
OS: Phyzelaphryne $1977 \cdot$ OD

EN: PHYZELAPHRYNINI 2008.ha.f002-01 • T

EF: BRACHYCEPHALIDAE 1858.gc.f002

PIPAEFORMES Duméril ${ }^{+1}$, 1841.da.f004 • AN

SI: $063 \bullet$ CI: n030 • ST: 2.27 .50

RL: INR

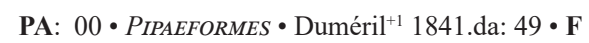
01 PIPAEFORMES $\bullet$ Desmarest 1856.da: $19 \cdot \mathbf{F}$

OS: Pipa $1768 \cdot \mathbf{O E}$

EN: (1) PIPIDAE 1825.gb.f003-|1826.fb.f002|-07 • F (2) PIPINAE 1825.gb.f003-|1826.fb.f002|-13 • bF EF: PIPIDAE 1825.gb.f003-|1826.fb.f002|

PIPOIDEA Fitzinger, 1826.fb.f002 • MK SI: $028 \bullet$ CI: h014 $\bullet$ ST: 0.10 .34

RL: > PIPRINA 1825.gb.f003 • MK

PA: $00 \cdot$ PIPOIDEA $\bullet$ Fitzinger 1826.fb: $37 \cdot \mathbf{F}$ $01 \cdot$ PIPARIA $\bullet$ Hemprich 1829.ha: xix, $373 \cdot \mathbf{F}$ $02 \cdot$ PIPINA $\bullet$ Gray 1829.ga: $203 \cdot \mathbf{U F}$ 03 $P$ PIPAE $\bullet$ Goldfuss 1832 .ga: $330 \cdot \mathbf{Z t}$ 04 PIPINA $\bullet$ Bonaparte 1838.ba: [195] • bF $05 \cdot$ PIPAE $\bullet$ Tschudi 1838 ta: $26 \bullet \mathbf{F}$ $06 \cdot$ PIPINI Bonaparte $1839 . \mathrm{bc}:[225] \cdot \mathbf{b F}$ $07 \cdot$ PIPIDAE $\bullet$ Swainson 1839.sa: $88 \cdot \mathbf{F}$ 08 PIPAE $\bullet$ Bronn 1849.ba: 684• UF 09 PIPADAE Hallowell 1858.ha: $65 \cdot \mathbf{F}$ $10 \bullet$ PIPOIDES $\bullet$ Bruch 1862.ba: $221 \cdot \mathbf{F}$ $11 \cdot$ PIPAEIDES • Gouriet 1868.ga: $206 \cdot \mathbf{F}$ $12 \cdot P_{I P I D A} \cdot$ Knauer 1878.ka: $103 \cdot \mathbf{F}$ 13 PIPINAE $\bullet$ Metcalf 1923.ma: $3 \cdot \mathbf{b F}$ 14 $\cdot$ PIPOIDEA $\bullet$ Laurent 1948.la: $1 \cdot \mathbf{p F}$ $15 \cdot$ PIPOIDIA $\bullet$ Dubois 2005.da: $8 \cdot \mathbf{e F}$

OS: Pipa $1768 \cdot$ OE

EN: (1) PIPIDAE 1825.gb.f003-|1826.fb.f002|-07 • F

(2) PIPINAE 1825.gb.f003-|1826.fb.f002|-13 • bF

EF: PIPIDAE 1825.gb.f003-|1826.fb.f002|

PIPRINA Gray, 1825.gb.f003 • MK

SI: $017 \bullet$ CI: h008 • ST: 0.10 .53

RL: < PIPOIDEA 1826.fb.f002 • MK

PA: $00 \cdot$ PIPRINA Gray $1825 . \mathrm{gb}: 214 \cdot \mathbf{U C}$

$01 \cdot$ PIPRIDAE $\bullet$ Gray 1842 .ga: $112 \cdot \mathbf{F}$

OS: Pipra $1825 \mathbf{J H} \equiv$ Pipa $1768 \cdot \mathbf{O E}$

EN: (1) PIPIDAE 1825.gb.f003-|1826.fb.f002|-07 • F (2) PIPINAE 1825.gb.f003-|1826.fb.f002|-13 • bF EF: PIPIDAE 1825.gb.f003-|1826.fb.f002|

Pithecopinae Lutz, 1969.1a.f001 • KY

SI: $280 \bullet$ CI: h197 • ST: 0.10 .30

RL: INR

PA: 00 P PITHECOPINAE $\bullet$ Lutz 1969.la: $274 \cdot \mathbf{b F}$ $01 \cdot$ PITHECOPODINIA $\bullet$ Hoc loco $\bullet$ iT

OS: Pithecopus $1866 \cdot \mathbf{O E}$

EN: PITHECOPODINIA 1969.la.f001-01 • iT

EF: Phyllomedusidae 1858.gc.f009

Platosphinae Fejérváry, 1917.fa.f001 † • JD

SI: $199 \bullet$ CI: h136 • ST: 0.10 .40

RL: INR

PA: 00 • PLATOSPHINAE $・$ Fejérváry 1917.fa: $151 \cdot \mathbf{b F}$
OS: Platosphus $1877 \dagger \approx$ Bufo $1764 \cdot \mathbf{O E}$

EN: (1) BUFONOIDEA 1825.gb.f004-20 • pF

"'1")

(10) BUFONITOES 1825.gb.f004-33 • iCn

EF: BUFONIDAE 1825.gb.f004

PlatymantinaE Savage, 1973.sa.f001 • AN

SI: 293 • CI: n082 • ST: 0.28 .50

RL: INR

PA: $00 \bullet$ PLATYMANTINAE $・$ Savage 1973.sa: $354 \cdot \mathbf{b F}$

OS: Platymantis $1859 \cdot \mathbf{O E}$

EN: (1) CERATOBATRACHEIDAE 1884.ba.f001-04 • aF

(2) CERATOBATRACHIDAE 1884.ba.f001-00 • F

EF: CERATOBATRACHIDAE 1884.ba.f001

Platymantinae Bauer, 1985.ba.f001 • AN

SI: $314 \cdot$ CI: n087 • ST: 0.28 .50

RL: INR

PA: 00 • PLATYMANTINAE $・$ Bauer 1985.ba: $3 \cdot \mathbf{b F}$

OS: Platymantis $1859 \cdot \mathbf{O E}$

EN: (1) CERATOBATRACHEIDAE 1884.ba.f001-04 • aF

(2) CERATOBatraCHidaE 1884.ba.f001-00 • F

EF: CERATOBATRACHIDAE 1884.ba.f001

PLATYMANTINI Laurent, 1986.la.f001・JD

SI: $319 \bullet \mathbf{C I}:$ h227 • ST: 0.10 .40

RL: INR

PA: $00 \cdot$ PLATYMANTINI $_{\text {L Laurent 1986.la: } 760 \bullet \text { T }}$

OS: Platymantis $1859 \cdot \mathbf{O E}$

EN: (1) CERATOBATRACHEIDAE 1884.ba.f001-04 • aF

(2) CERATOBATRACHIDAE 1884.ba.f001-00 • F

EF: CERATOBATRACHIDAE 1884.ba.f001

Platypelina nov., DOP.da.f082 • KY

SI: $523 \cdot$ CI: h416 • ST: 0.10 .30

RL: INR

PA: $00 \cdot$ PLATYPELINA $\bullet$ Hoc loco $\bullet \mathbf{b T}$

OS: Platypelis $1882 \cdot \mathbf{P D}$

EN: PLATYPELINA DOP.da.f082-00 • bT

EF: MICROHYLIDAE |1843.fa.f012|-1931.na.f001

PlatyPlectrina nov., DOP.da.f073 • KY

SI: $514 \cdot$ CI: h407 • ST: 0.10 .30

RL: INR

PA: $00 \cdot$ PLATYPLECTRINA $\bullet$ Hoc loco $\bullet \mathbf{b T}$

OS: Platyplectrum $1863 \cdot \mathbf{P D}$

EN: PLATYPLECTRINA DOP.da.f073-00 • bT

EF: MYOBATRACHIDAE 1850.sa.f001

Plectrohylinia nov., DOP.da.f060 • Ky

SI: $501 \bullet \mathbf{C I}:$ h394 • ST: 0.10 .30

RL: INR

PA: $00 \bullet$ PLECTROHYLINIA $・$ Hoc loco $\bullet$ iT

OS: Plectrohyla $1877 \cdot \mathbf{P D}$

EN: PLECTROHYLINIA DOP.da.f060-00 • iT

EF: HYLIDAE 1815.ra.f002-|1825.gb.f001|

Plectromantidae Mivart, 1869.ma.f002 • SG

SI: $162 \bullet$ CI: h110 • ST: 0.10 .44

RL: < LEPTODACTYLIDAE |1838.ta.f001|-1896.wa.f001 • PS: Dubois 1983.da: 273

PA: 00 - PLECTROMANTIDAE • Mivart 1869.ma: 286 • F

$01 \cdot$ PLECTROMANTIDAE $\bullet$ Hoffmann 1878.ha: $614 \cdot \mathbf{b F}$ 
OS: Plectromantis $1862 \approx$ Leptodactylus $1826 \cdot \mathbf{O E}$

EN: (1) LEPTODACTYLOIDEA |1838.ta.f001|-1896.wa.f001-03 • pF ")"»"

(3) LEPTODACTYLINAE |1838.ta.f001|-1896.wa.f001-01 • bF

EF: LEPTODACTYLIDAE |1838.ta.f001|-1896.wa.f001

Plethodontidae Gray, 1850.ga.f001 • KY

SI: $112 \cdot \mathbf{C I}:$ h074 • ST: 0.10 .32

RL: $\geq$ ENSATININA 1850.ga.f007 • PR: Dubois ${ }^{+1}$ 2012: 98

PA: $00 \bullet$ PLETHODONTIDAE • Gray 1850.ga: 5, 31 • F

$01 \cdot$ PLETHODONTINA • Gray 1850.ga: 38 • UF

$02 \cdot$ PLETHODONTIDAE $・$ Hallowell 1856.ha: $10 \cdot \mathbf{b F}$

$03 \cdot P_{\text {LETHODONTAE }} \cdot$ Cope 1859.cb: $124 \cdot \mathbf{U F}$

$04 \cdot$ PLETHODONTIDA $\cdot$ Knauer 1878.ka: $97 \cdot \mathbf{F}$

05 PLETHODONTINAE $\bullet$ Boulenger 1882.bc: vii, $51 \cdot \mathbf{b F}$

$06 \cdot$ PLETHODONTINA $・$ Schulze 1891.sa: $5 \cdot$ T

07 P PETHODONTINI • Wake 1966.wa: 1 • T

08 • PLETHODONTOIDEA • Milner 2000.ma: $1429 \bullet \mathbf{p F}$

$09 \cdot$ PLETHODONTINA $・ H o c$ loco $\bullet \mathbf{b T}$

OS: Plethodon $1838 \cdot \mathbf{O E}$

EN: (1) PLETHODONTIDAE 1850.ga.f001-00 • F

(2) Plethodontinae 1850.ga.f001-05 • bF

(3) Plethodontini 1850.ga.f001-07 • T

(4) Plethodontina 1850.ga.f001-09 • bT

EF: PlethodontidAE 1850.ga.f001

PleURodeles Tschudi 1838.ta.f005 • KY

SI: $055 \bullet$ CI: h028 • ST: 0.10 .30

RL: INR

PA: $00 \cdot$ PLEURODELES $・$ Tschudi 1838.ta: $56 \cdot$ F

$01 \cdot$ PLEURODELINA $\cdot$ Bonaparte 1838.bd: $125 \cdot \mathbf{b F}$

02 PLEURODELIDINA • Bonaparte 1840.ba: $287 \cdot \mathbf{b F}$

$03 \cdot$ PLEURODELAE $・$ Fitzinger 1843.fa: $33 \cdot \mathbf{F}$

$04 \cdot$ PLEURODELIDAE $\bullet$ Bonaparte 1850.bb: pl. • F

05 PLEURODELIDAE $\cdot$ Hallowell 1856.ha: $10 \cdot \mathbf{b F}$

06 PLEURODELAE • Cope 1859.cb: $125 \cdot \mathbf{U F}$

$07 \cdot$ PLEURODELIDAE $・$ Cope 1859.cb: $125 \cdot \mathbf{U F}$

08 PLEURODELINAE • Brame 1957.ba: $2 \cdot \mathbf{b F}$

$09 \cdot$ PLEURODELINI Dubois $^{+1}$ 2009.db: $30 \cdot \mathbf{T}$

$10 \cdot$ PleURodelina $\cdot$ Hoc loco $\bullet \mathbf{b T}$

OS: Pleurodeles $1830 \cdot \mathbf{O E}$

EN: (1) PLEURODELINAE 1838.ta.f005-08 • bF

(2) PLEURODELINI 1838.ta.f005-09 • T

(3) PlEurodelina 1838.ta.f005-10 • bT

EF: SALAMANDRIDAE 1820.ga.f002

PleurodemaE Cope, 1866.ca.f002 • JD

SI: $155 \bullet$ CI: h105 • ST: 0.10 .52

RL: INR

PA: $00 \cdot$ PLEURODEMAE • COPE 1866.ca: $90 \cdot \mathbf{G r}$

$01 \cdot$ PLEURODEMAE $\bullet$ Cope 1869.ca: $312 \cdot \mathbf{T}$

OS: Pleurodema $1838 \cdot \mathbf{O E}$

EN: (1) LEIUPERIDAE 1850.bb.F010-02 • F

(2) LEIUPERINAE 1850. bb.F010-03 • bF

EF: LEIUPERIDAE 1850.bb.F010

PolyPEDAtida Günther, 1858.gc.f012 • PK

SI: 140 • CI: h093 - ST: 0.10 .37

RL: < RHACOPHORIDAE 1932.ha.f001 • PS: Dubois 1983.da: 276

PA: $00 \bullet$ POLYPEDATIDAE $\bullet$ Günther 1858.gc: $346 \bullet$ F
01 POLYPEDATYDAE $\bullet$ Krefft 1865.ka: $18 \cdot \mathbf{F}$

$02 \cdot$ POLYPEDATINA $・$ Mivart 1869.ma: $292 \cdot \mathbf{b F}$

$03 \cdot$ POLYPEDATIDAE $\bullet$ Hoffmann 1878.ha: $614 \cdot \mathbf{b F}$

$04 \cdot$ POLYPEDATINAE $\bullet$ Boulenger 1888.ba: $205 \cdot \mathbf{b F}$

$05 \cdot$ POLYPEDATITIES $\bullet$ Hoc loco $\bullet \mathbf{b C n}$

$06 \cdot$ POLYPEDATITOES $\bullet$ Hoc loco $\bullet \mathbf{i C n}$

OS: Polypedates 1838

EN: (1) POLYPEDATITIES 1858.gc.f012-05 • bCn

(2) POLYPEDATITOES 1858.gc.f012-06 • iCn

EF: RHACOPHORIDAE |1858.gc.f012|-1932.ha.f001

PolyPEDETIDAE Whitney, 1890.wa.f001 • JI

SI: $192 \bullet \mathbf{C I}:$ h133 • ST: 0.10 .52

RL: INR

PA: $00 \cdot$ POLYPEDETIDAE • Whitney 1890.wa: 4606 • F

OS: Polypedetes $1890 \equiv$ Polypedates 1890

EN: (1) POLYPEDATITIES 1858.gc.F012-04 • bCn

(2) POLYPEDATITOES 1858.gc.F012-05 • iCn

EF: RHACOPHORIDAE |1858.gc.f012|-1932.ha.f001

Polysemiaden Meyer, $1860 . \mathrm{mb} . \mathrm{f00}+\bullet \mathbf{A N}$

SI: $146 \cdot \mathbf{C I}:$ n047 • ST: 0.23 .50

RL: INR

PA: $00 \cdot$ POLYSEMIADEN • Meyer 1860.mb: $559 \cdot \mathbf{F}$

01 POLYSEMIIDAE $\cdot$ Martín $^{+2}$ 2012.ma: $174 \cdot \mathbf{F}$

OS: Polysemia $1860 \uparrow \approx$ Chelotriton $1853 \uparrow \cdot \mathbf{O E}$

EN: (1) PLEURODELINAE 1838.ta.f005-08 • bF "')"')

(3) PLEURODELINA 1838.ta.f005-10 • bT

EF: SALAMANDRIDAE 1820.ga.f002

POTAMOTYPHLIDAE Lescure ${ }^{+2}$, 1986.lb.f003 • JD

SI: $322 \bullet \mathbf{C I}:$ h230 • ST: 0.10 .40

RL: INR

PA: c0 • РОтАмОтYРНLIDAE - Lescure ${ }^{+2}$ 1986.1b: 145 • F • EEA: Hoc loco

i1 • РотAмотYPHILIDAE • Lescure ${ }^{+2}$ 1986.lb: 160 - F

02 - РОтАМОТYРHLOIDAE $\bullet$ Lescure $^{+2}$ 1986.1b: $169 \cdot \mathbf{e F}$

03 POTAMOTYPHLINAE - Lescure ${ }^{+2}$ 1986.lb: $169 \cdot \mathbf{b F}$

$04 \cdot$ POTAMOTYPHLILAE $・$ Lescure $^{+2}$ 1986.1b: $169 \cdot \mathbf{i F}$

05 • POTAMOTYPHLINI • Lescure ${ }^{+2}$ 1986.1b: $170 \bullet \mathbf{T}$

OS: Potamotyphlus $1968 \cdot$ OE

EN: TYPHLONECTINA 1968.ta.f002-09 • bT

EF: CAECILIIDAE 1814.ra.f003-|1825.gb.f008|

Poyntonitna nov., DOP.da.f101 • KY

SI: $542 \cdot \mathbf{C I}: \mathrm{h} 435 \cdot \mathbf{S T}: 0.10 .30$

RL: INR

PA: $00 \cdot$ POYNTONINA $\bullet$ Hoc loco $\bullet \mathbf{b T}$

OS: Poyntonia $1989 \cdot \mathbf{P D}$

EN: POYNTONIINA DOP.da.f101-00 • bT

EF: CACOSTERNIDAE 1931.na.f008

PRistimantinaE Pyron ${ }^{+1}$, 2011.pa.f002 • AN

SI: $410 \bullet \mathbf{C I}:$ n098 • ST: 0.28 .50

RL: INR

PA: $00 \bullet$ PRISTIMANTINAE $\bullet$ Pyron $^{+1}$ 2011.pa: 547, 579, $580 \bullet \mathbf{b F}$

OS: Pristimantis $1870 \cdot$ OD

EN: (1) PRISTIMANTINA 2012.oa.f002-01 • bT

(2) PRISTIMAntinia 2012.oa.f002-02 • iT

(3) PRistimantinoa 2012.oa.f002-03 • hT 
EF: BRACHYCEPHALIDAE 1858.gc.f002

Pristimantinae Ohler $^{+1}$, 2012.oa.f002 • KY

SI: $429 \bullet \mathbf{C I}:$ h323 • ST: 0.10 .30

RL: INR

PA: 00 • PRISTIMANTINAE $\bullet$ Ohler $^{+1}$ 2012.oa: $165 \cdot$ bF

$01 \cdot$ PRISTIMANTINA $\bullet$ Hoc loco $\bullet \mathbf{b T}$

$02 \cdot$ Pristimantinia $\bullet$ Hoc loco $\bullet \mathbf{i T}$

$03 \cdot$ PRISTIMANTINOA $\bullet$ Hoc loco $\bullet \mathbf{h T}$

OS: Pristimantis $1870 \cdot$ OD

EN: (1) PRISTIMANTINA 2012.oa.f002-01 • bT

(2) Pristimantinia 2012.oa.f002-02 • iT

(3) Pristimantinoa 2012.oa.f002-03 • h T

EF: BRACHYCEPHALIDAE 1858.gc.f002

Proceratophryinae nov., DOP.da.f039 • Ky

SI: $480 \bullet$ CI: h373 • ST: 0.10 .30

RL: INR

PA: 00 • PROCERATOPHRYINAE $\bullet$ Hoc loco $\bullet \mathbf{b F}$

OS: Proceratophrys $1920 \bullet \mathbf{P D}$

EN: PROCERATOPHRYINAE DOP.da.f039-00 • bF

EF: ODONTOPHRYNIDAE 1971.la.f002

PROCOELA Huene, 1948.ha.f004 • AN

SI: $248 \bullet$ CI: n069 • ST: 0.25 .50

RL: INR

PA: $00 \cdot$ PROCOELA Huene 1948.ha: $71 \cdot \mathbf{F}$

OS: » OA, PD: Bufo $1764 \cdot$ OE

EN: (1) BUFONOIDEA 1825.gb.f004-20 • pF "'m!'

(10) BUFONITOES 1825.gb.f004-33 • iCn

EF: BUFONIDAE 1825.gb.f004

PRosalamandrIDEA Stefano, 1903.sa.f001 • AN

SI: $197 \bullet \mathbf{C I}: \mathrm{n} 059 \bullet \mathbf{S T}: 2.25 .50$

RL: INR

PA: 00 • PROSALAMANDRIDEA • Stefano 1903.sa: $49 \cdot \mathbf{F}$

01 PROSALAMANDRIDAE $\bullet$ Martín $^{+2}$ 2012.ma: $174 \cdot \mathbf{F}$

OS: » 2 PN, including: Heteroclitotriton $1903 \uparrow \approx$ Salamandra 1764 - PD

EN: (1) SALAMANDROIDEA 1820.ga.f002-21 • pF "nm!n

(4) SALAMANDRINI 1820.ga.f002-28 • T

EF: SALAMANDRIDAE 1820.ga.f002

ProsaliridaE Shubin ${ }^{+1}$, 1995.sa.f001 † $\bullet$ KY

SI: $355 \bullet$ CI: h262 • ST: 0.10 .30

RL: INR

PA: 00 • PROSALIRIDAE $\bullet$ Shubin $^{+1}$ 1995.sa: $49 \cdot \mathbf{F}$

OS: Prosalirus $1995 \dagger \cdot$ OE

EN: PRoSALIRIDAE 1995.sa.f001-00 $\bullet \cdot \mathbf{F}$

EF: PROSALIRIDAE 1995.sa.f001 †

Prosirenidae Estes, 1969.ea.f001 † • KY

SI: $279 \bullet$ CI: h196 • ST: 0.10 .30

RL: INR

PA: $00 \cdot$ PROSIRENIDAE $\bullet$ Estes 1969.ea: $87 \cdot \mathbf{F}$

01 P PROSIRINIDAE $\bullet$ Rowe $^{+3}$ 1992.ra: $492 \cdot \mathbf{F}$

02 PROTOSIRENIDAE $\bullet$ Vorobyeva $^{+1}$ 1996.va: $69 \cdot \mathbf{F}$

OS: Prosiren $1958+\bullet$ OE

EN: PROSIRENIDAE 1969.ea.f001-00 $\uparrow \cdot \mathbf{F}$

EF: PROSIRENIDAE 1969.ea.f001 †
Proteina Gray, 1825.gb.f006 • AN

SI: $020 \bullet \mathbf{C I}: \mathrm{n} 009 \bullet \mathbf{S T}: 2.26 .50$

RL: INR

PA: $00 \cdot$ PROTEINA $\bullet$ Gray 1825.gb: $215 \cdot \mathbf{U F}$

OS: » 2 PN, including: Hypochthon $1820 \equiv$ Proteus $1768 \cdot \mathbf{P D}$

EN: (1) PROTEOIDAE 1831.ba.f002-10 • eF

(2) PROTEIDAE 1831.ba.f002-02 • F

EF: PROTEIDAE 1831.ba.f002

Proteina Bonaparte, 1831.ba.f002 • RK

SI: $036 \bullet$ CI: h019 • ST: 0.10 .33

RL: > PHAENEROBRANCHOIDEA 1826.fb.f004• RI: Dubois ${ }^{+1}$ 2015.da: 44

> PHANEROBRANCHOIDEA 1827.fa.f001・ RI: Dubois ${ }^{+1}$ 2015.da: 44

PA: $00 \cdot$ PROTEINA $\bullet$ Bonaparte 1831.ba: $78 \cdot$ UF

$01 \cdot$ PROTEIDEA $\bullet$ Goldfuss 1832 ga: $323 \cdot \mathbf{F}$

02 $\bullet$ PROTEIDAE $\bullet$ Hogg 1838.ha: $152 \cdot \mathbf{F}$

03 PROTEIDES $\bullet$ Duméril $^{+1}$ 1841.da: $52 \cdot \mathbf{F}$

04 P PROTEIDA $・$ Jan 1857.ja: $55 \cdot \mathbf{F}$

05 PROTEIDEA • Huxley 1871.ha: $173 \cdot \mathbf{U F}$

06 PROTOIDEA $\bullet$ Stefano 1903.sa: $47 \cdot \mathbf{F}$

$07 \cdot$ PROTAEIDAE $\bullet$ Laurent 1948.1b: $3 \cdot \mathbf{F}$

$08 \cdot P_{R O T E O I D E A} \cdot$ Dubois 2005.da: $20 \cdot \mathbf{p F}$

09 PROTEINAE Blackburn ${ }^{+1}$ 2011.ba: $46 \cdot \mathbf{b F}$

10 P PROTEOIDAE $\bullet$ Dubois $^{+1}$ 2012.da: $98 \cdot \mathbf{e F}$

OS: Proteus $1768 \cdot \mathbf{O E}$

EN: (1) PROTEOIDAE 1831.ba.f002-10 • eF

(2) PROTEIDAE 1831.ba.f002-02 • F

EF: PROTEIDAE 1831.ba.f002

PROTOBATRACHIDAE Kuhn, 1941.ka.f001 † • JI

SI: $235 \bullet$ CI: h168 $\bullet$ ST: 0.10 .53

RL: INR

PA: 00 • PROTOBATRACHIDAE $・$ Kuhn 1941.ka: $346 \bullet$ F

01 PROTOBATRACHIDAE $\bullet$ Tatarinov 1964.ta: $127 \cdot \mathbf{F}$

OS: Protobatrachus $1936 \uparrow \cdot \mathbf{J H} \equiv$ Triadobatrachus $1962 \uparrow \cdot \mathbf{O E}$

EN: TRIADOBATRACHIDAE 1962.ka.f001-00 $\uparrow \cdot \mathbf{F}$

EF: TRIADOBATRACHIDAE 1962.ka.f001 †

PRotohynobinnaE $\mathrm{Fei}^{+1}$, 2000.fa.f001 • KY

SI: $356 \cdot$ CI: h263 • ST: 0.10 .30

RL: INR

PA: $00 \cdot$ PROTOHYNOBIINAE $\bullet \mathrm{Fei}^{+1} 2000$ fa: $64 \cdot \mathbf{F}$

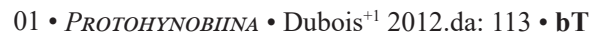

$02 \cdot$ PROTOHYNOBIINIA $\bullet$ Hoc loco $\bullet$ iT

OS: Protohynobius $2000 \approx$ Pseudohynobius $1983 \cdot$ OD

EN: РROTOHYNOBIINIA 2000.fa.f001-02 • iT

EF: HYNOBIIDAE |1856.ha.f001|-1859.cb.f002

PRotonopsidina Bonaparte, 1840.ba.f001 • JD

SI: $058 \bullet$ CI: h030 • ST: 0.10 .40

RL: INR

PA: 00 • PROTONOPSIDINA • Bonaparte 1840.ba: $287 \cdot \mathbf{b F}$

$01 \cdot$ PROTONOPSINA $\bullet$ Bonaparte 1845.ba: $378 \cdot \mathbf{b F}$

$02 \cdot$ PROTONOPSIDAE $\bullet$ Gray 1850.ga: 6, $52 \cdot \mathbf{F}$

03 PROTONOPSEIDAE $\bullet$ Bonaparte 1850.bb: pl. • F

04 PROTONOPSEINA $\bullet$ Bonaparte 1850.bb: pl. $\bullet \mathbf{b F}$

OS: Protonopsis $1824 \approx$ Cryptobranchus $1821 \cdot$ OE

EN: CRYPTOBRANCHIDAE 1826.fb.f003-04 • F 
EF: CRYPTOBRANCHIDAE 1826.fb.f003

PRotopelobatidae Fejérváry, 1921.fb.f001 † • JD

SI: $204 \cdot$ CI: h141 • ST: 0.10 .40

RL: INR

PA: 00 • PROTOPELOBATIDAE • Fejérváry 1921.fb: 24 • F

OS: Protopelobates $1881 \uparrow \approx$ Palaeobatrachus $1838 \dagger \cdot \mathbf{O E}$

EN: PALAEOBATRACHIDAE 1865.ca.f001-00 $\uparrow$ F

EF: PALAEOBATRACHIDAE 1865.ca.f001 $\dagger$

PSEUDAE Fitzinger, 1843.fa.f010 • KY

SI: $075 \bullet$ CI: h043 • ST: 0.10 .30

RL: INR

PA: $00 \cdot P_{S E U D A E} \cdot$ Fitzinger 1843.fa: $33 \cdot \mathbf{F}$

$01 \cdot$ PSEUDES $\bullet$ Cope $1866 . \mathrm{ca}: 89 \cdot \mathbf{G r}$

02 P PEUDINAE $\bullet$ Noble 1931.na: $496 \cdot \mathbf{b F}$

03 $\bullet$ PSEUDIDAE $\bullet$ Savage $^{+1}$ 1953.sa: $198 \cdot \mathbf{F}$

$04 \cdot$ PSEUDINA $\bullet$ Hoc loco $\bullet \mathbf{b T}$

OS: Pseudis $1830 \bullet \mathbf{O E}$

EN: PSEUDINA 1843.fa.f010-04 • bT

EF: HYLIDAE 1815.ra.f002-|1825.gb.f001|

PSEUdoeURYCEITES nov., DOP.da.f134 • KY

SI: $575 \bullet$ CI: h468・ST: 0.10 .30

RL: INR

PA: $00 \cdot$ PSEUDOEURYCEITES $・$ Hoc loco $・ \mathbf{C n}$

OS: Pseudoeurycea $1944 \cdot \mathbf{P D}$

EN: PSEUdoevryceItes DOP.da.f134-00 • Cn

EF: PLETHODONTIDAE 1850.ga.f001

PSEUDOHEMISIINAE Tatarinov, 1964.ta.f002 • AN

SI: $269 \bullet$ CI: n078 • ST: 0.28 .50

RL: INR

PA: 00 P PSEUDOHEMISIINAE $・$ Tatarinov 1964.ta: $132 \cdot \mathbf{F}$

OS: Pseudohemisus $1895 \approx$ Scaphiophryne $1882 \cdot$ OE

EN: SCAPHIOPHRYNINI Laurent, 1946.1a.f002-03 • T

EF: MICROHYLIDAE |1843.fa.f012|-1931.na.f001

PSEUdopaludicolinae Gallardo, 1965.ga.f003 • KY

SI: $271 \bullet$ CI: h1 $189 \bullet$ ST: 0.10 .30

RL: INR

PA: 00 • PSEUDOPALUDICOLINAE • Gallardo 1965.ga: $84 \cdot$ bF

OS: Pseudopaludicola $1926 \cdot \mathbf{O E}$

EN: PSEUDOPALUDICOLINAE 1965.ga.f003-00 • bF

EF: LEPTODACTYLIDAE |1838.ta.f001|-1896.wa.f001

PSEUdophrynoidea Bauer, 1987.bc.f001 • KY

SI: $331 \cdot \mathbf{C I}:$ h239 • ST: 0.10 .30

RL: INR

PA: $00 \cdot$ PSEUDOPHRYNOIDEA $・$ Bauer 1987.bc: $51 \cdot \mathbf{p F}$

$01 \cdot$ PSEUDOPHRYNINOA $\bullet$ Hoc loco $\bullet \mathbf{h T}$

OS: Pseudophryne $1843 \cdot \mathbf{P D}$

EN: PSEUDOPHRYNINOA 1987.bc.f001-01 • pF

EF: MYOBATRACHIDAE 1850.sa.f001

PseUdoranities nov., DOP.da.f109 • KY

SI: $550 \bullet$ CI: h443 • ST: 0.10 .30

RL: INR

PA: 00 • PSEUDORANITIES $\bullet$ Hoc loco $\bullet$ bCn

OS: Pseudorana $1990 \cdot \mathbf{P D}$

EN: PSEUDORANITIES DOP.da.f109-00 • bCn

EF: RANIDAE 1796.ba.f001
PSEUDOSIPHONOPITI Lescure ${ }^{+2}$, 1986.lb.f007 • JD

SI: $326 \bullet$ CI: h234 • ST: 0.10 .40

RL: INR

PA: 00 • PSEUDOSIPHONOPITI $・$ Lescure $^{+2}$ 1986.1b: $166 \cdot$ bT

$01 \cdot$ PSEUDOSIPHONOPILI $\bullet$ Lescure $^{+2}$ 1986.1b: $166 \bullet$ iT

OS: Pseudosiphonops $1968 \approx$ Mimosiphonops $1968 \cdot \mathbf{O E}$

EN: (1) SIPHONOPINI 1850.bb.f017-08 • T

"1"1)

(4) SIPHONOPINOA 1850.bb.f017-12 • hT

EF: CAECILIIDAE 1814.ra.f003-|1825.gb.f008|

PSEUdotritonina Dubois, 2008.da.f005 • AN

SI: $378 \bullet$ CI: n095 • ST: 0.22 .50

RL: INR

PA: 00 • PSEUDOTRITIONINA • Dubois 2008.da: $73 \cdot$ bT

$01 \cdot$ PSEUDOTRITONITA $\bullet$ Dubois 2008.da: $74 \bullet$ iT

OS: Pseudotriton $1838 \cdot \mathbf{O E}$

EN: PSEUDOTRITONINA 2012.da.f006-00 • bT

EF: PLETHODONTIDAE 1850.ga.f001

PSEUdotritonina Dubois ${ }^{+1}$, 2012.da.f006 • KY

SI: $423 \cdot$ CI: h317 • ST: 0.10 .30

RL: INR

PA: $00 \bullet$ PSEUDOTRITONINA $・$ Dubois $^{+1}$ 2012.da: $115 \bullet$ bT

OS: Pseudotriton $1838 \cdot$ OD

EN: PSEUDOTRITONINA 2012.da.f006-00 • bT

EF: PLETHODONTIDAE 1850.ga.f001

PSEUDOTYPHLONECTINI Lescure ${ }^{+2}$, 1986.lb.f010 • JD

SI: $329 \bullet$ CI: h237 • ST: 0.10 .40

RL: INR

PA: 00 PSEUDOTYPHLONECTINI $・$ Lescure $^{+2}$ 1986.1b: $170 \bullet$ T

OS: Pseudotyphlonectes $1986 \approx$ Typhlonectes $1880 \bullet$ OE

EN: TYPHLONECTINA 1968.ta.f002-09 • bT

EF: CAECILIIDAE 1814.ra.f003-|1825.gb.f008|

Pteroranini $\mathrm{Fei}^{+2}$, 2010.fa.f014 • AP

SI: $405 \bullet$ CI: h305 • ST: 0.10 .46

RL: INR

PA: $00 \bullet$ PTERORANINI $\bullet \mathrm{Fei}^{+2}$ 2010.fa: $18 \cdot \mathbf{T}$

OS: Pterorana $1986 \cdot$ OD

EN: (1) RANOIDEA 1796.ba.f001-28・pF

"'m!'

(5) RANINAE 1796.ba.f001-23 • bF

EF: RANIDAE 1796.ba.f001

Ptychadenini Dubois, 1987.da.f002 • KY

SI: $334 \cdot$ CI: h242 • ST: 0.10 .30

RL: INR

PA: 00 • PTYCHADENINI $\bullet$ Dubois 1987.da: $55 \cdot \mathbf{T}$

01 P PTYCHADENINAE $\bullet$ Dubois 1992.da: $316 \bullet \mathbf{b F}$

$02 \cdot$ PTYCHADENIDAE Frost $^{+18}$ 2006.fa: $7 \cdot \mathbf{F}$

OS: Ptychadena $1917 \cdot$ OD

EN: PTYCHADENIDAE 1987.da.f002-02 • F

EF: PTYCHADENIDAE 1987.da.f002

PTYCHOHYLITES nov., DOP.da.f059 • KY

SI: $500 \bullet$ CI: h393 • ST: 0.10 .30

RL: INR

PA: $00 \cdot$ PTYCHOHYLITES $\bullet$ HOC loco $\bullet \mathbf{C n}$

OS: Ptychohyla $1944 \cdot \mathbf{P D}$

EN: PTYCHOHYLITES DOP.da.f059-00 • Cn 
EF: HYLIDAE 1815.ra.f002-|1825.gb.f001|

PYXICEPHALINA Bonaparte, 1850.bb.f005 • KY

SI: $097 \cdot$ CI: h059 • ST: 0.10 .30

RL: INR

PA: $00 \cdot$ PYXICEPHALINA • Bonaparte 1850.bb: pl. • bF

01 PYXICEPHALINI • Dubois 1987.da: 66 • T

02 PYXICEPHALINAE • Dubois 1992.da: $317 \cdot \mathbf{b F}$

03 PYXICEPHALIDAE Roelants $^{+7}$ 2007.ra: $889 \cdot \mathbf{F}$

$04 \cdot$ PYXICEPHALOIDAE $\bullet$ Hoc loco $\bullet \mathbf{e F}$

OS: Pyxicephalus $1838 \cdot \mathbf{O E}$

EN: (1) PYXICEPHALOIDAE 1850.bb.f005-04 • eF

(2) PYXICEPHALIDAE 1850.bb.f005-03 • F

EF: PYXICEPHALIDAE 1850.bb.f005

QUASIPAINI $\mathrm{Fei}^{+2}$, 2010.fa.f007 • KY

SI: 398 • CI: h298 • ST: 0.10 .31

RL: $\geq$ ANNANDIINI 2010.fa.f008 • AI: hoc loco

PA: $00 \cdot$ QUASIPAINI $\bullet \mathrm{Fei}^{+2} 2010 \mathrm{fa}: 17 \cdot \mathbf{T}$

$01 \cdot$ QuasipaINa $\bullet$ Hoc loco $\bullet \mathbf{b T}$

OS: Quasipaa 1992 • OD

EN: (1) QUASIPAINI 2010.fa.f007-00 • T

(2) QuASIPAINA 2010.fa.f007-01 • b T

EF: DICROGLOSSIDAE 1987.da.f004

RACOPHORIDAE Hellmich, 1957.ha.f001 • JD

SI: 254 • CI: h179 • ST: 0.10 .52

RL: INR

PA: 00 • RACOPHORIDAE $\cdot$ Hellmich 1957.ha: $28 \cdot \mathbf{F}$

OS: Racophorus $1826 \equiv$ Rhacophorus $1822 \cdot \mathrm{OE}$

EN: (1) RACOPHORIDAE |1858.gc.f012|-1932.ha.f001-00 • F ")!)!

(8) RHACOPHORITIES |1858.gc.f012|-1932.ha.f001-09 • bCn

EF: RHACOPHORIDAE |1858.gc.f012|-1932.ha.f001

RANARIDIA Rafinesque, 1814.ra.f001 • JI

SI: $003 \cdot \mathbf{C I}:$ h002 • ST: 0.10 .52

RL: INR

PA: $00 \cdot$ RANARIDIA $・$ Rafinesque 1814.ra: $102 \cdot \mathbf{F}$

01 $\operatorname{RANARINIA} \cdot$ Rafinesque 1815.ra: $78 \cdot \mathbf{F}$

OS: Ranaria $1814 \equiv$ Rana $1758 \cdot \mathbf{O E}$

EN: (1) RANOIDEA 1796.ba.f001-28・pF

"')"!

(12) RANITOES 1796.ba.f001-38 • iCn

EF: RANIDAE 1796.ba.f001

RANAVINAE Fejérváry, 1921.fa.f001 † • JD

SI: $202 \cdot \mathbf{C I}:$ h139・ST: 0.10 .40

RL: INR

PA: $00 \cdot \operatorname{RANAVIDAE} \cdot$ Fejérváry 1921.fa: $29 \cdot \mathbf{F}$

$01 \cdot$ RANAVINAE $\bullet$ Fejérváry 1921.fa: $29 \cdot \mathbf{b F}$

OS: Ranavus $1885 \dagger \cdot \mathbf{O E}$

EN: (1) RANOIDEA 1796.ba.f001-28 • pF

"'"!"

(6) RANINI 1796.ba.f001-30 • T

EF: RANIDAE 1796.ba.f001

RANIFORMES Duméril ${ }^{+1}$, 1841.da.f001 • AN

SI: $060 \bullet \mathbf{C I}: \mathrm{n} 027 \bullet \mathbf{S T}: 2.27 .50$

RL: INR

PA: $00 \cdot$ RANIFORMES $・$ Duméril $^{+1}$ 1841.da: 50 • F 01 - RANIFORMES $・$ Desmarest 1857.da: $21 \cdot \mathbf{F}$
02 • RANIFORMIA $・$ Cope 1864.ca: $51 \cdot \mathbf{F}$

OS: Rana $1758 \cdot \mathbf{O E}$

EN: (1) RANOIDEA 1796.ba.f001-28・ pF

"'"1)"

(12) RANITOES 1796.ba.f001-38 • iCn

EF: RANIDAE 1796.ba.f001

RANINA Batsch, 1796.ba.f001 • KY

SI: $002 \cdot \mathbf{C I}:$ h001 • ST: 0.10 .30

RL: INR

PA: $00 \cdot R_{A N I N A} \cdot$ Batsch 1796.ba: $179 \cdot \mathbf{F}$

$01 \cdot R_{A N A E} \cdot$ Goldfuss 1820.ga: xi $\bullet \mathbf{F}$

$02 \cdot \operatorname{RANADAE} \bullet$ Gray $1825 . \mathrm{gb}: 213 \cdot \mathbf{F}$

$03 \cdot R_{A N I N A} \cdot$ Gray 1825.gb: $214 \cdot \mathbf{U F}$

$04 \cdot$ RANOIDEA $\cdot$ Fitzinger 1826.fb: $37 \cdot \mathbf{F}$

$05 \cdot \operatorname{RANIDAE} \cdot$ Boie 1828.ba: $363 \cdot \mathbf{F}$

$06 \cdot R_{A N A} \cdot$ Wilbrand 1829.wa: $273 \cdot \mathbf{F}$

$07 \cdot$ RANARIA $\cdot$ Hemprich 1829.ha: xix, 373 • F

$08 \cdot R A N I A D A E \bullet$ Smith 1831.sa: $18 \cdot \mathbf{F}$

$09 \cdot$ RANOIDEA $\cdot$ Fitzinger 1832.fa: $328 \cdot \mathbf{G r}$

$10 \cdot R_{A N A E} \cdot$ Goldfuss 1832 ga: $336 \cdot \mathbf{Z t}$

11 - RANADEA $\bullet$ Jourdan 1834.jb: $356 \cdot \mathbf{F}$

12 $\cdot$ RANINA $\bullet$ Bonaparte 1838.ba: [195] • bF

13 - RAMIDAE $\bullet$ Hogg 1838.ha: $152 \cdot \mathbf{F}$

$14 \cdot$ RANINA $\bullet$ Gravenhorst 1843.ga: $393 \cdot \mathbf{L}$

$15 \cdot R_{A N A E} \cdot$ Leunis $1844.1 \mathrm{la}: 128 \cdot \mathbf{U F}$

$16 \cdot R_{A N I N I} \cdot$ Bronn 1849.ba: $684 \cdot \mathbf{U F}$

$17 \cdot$ RANINA • Günther 1858.gc: $344 \cdot$ Sc

$18 \cdot R_{A N A E} \cdot$ Leunis 1860.la: $336 \bullet \mathbf{T}$

19 - RANOIDES • Bruch 1862.ba: $221 \cdot \mathbf{F}$

$20 \cdot$ RANIDA $\bullet$ Haeckel 1866.ha: cxxxii • F

$21 \cdot$ RANIDES $\bullet$ Gouriet 1868.ga: $206 \cdot \mathbf{F}$

$22 \cdot$ RANIDAE $\bullet$ Hoffmann 1878.ha: $613 \cdot \mathbf{b F}$

$23 \cdot$ RANINAE $\bullet$ Boulenger 1888.ba: $205 \cdot \mathbf{b F}$

$24 \cdot R_{A N I D I} \cdot$ Acloque 1900.aa: $489 \cdot \mathbf{F}$

25 • RANOIIDEA • Gill 1903.ga: $71 \cdot \mathbf{F}$

$26 \cdot$ RANOIDEA $\cdot$ Bolkay 1919.ba: $345 \cdot \mathbf{G a}$

$27 \cdot$ RANOIDA • Bolkay 1919.ba: $345 \cdot$ Ga

$28 \cdot R_{A N O I D E A} \cdot$ Bolkay 1929.ba: $58 \cdot \mathbf{p F}$

$29 \cdot$ RANOIDAE $・$ Dubois 1992.da: $309 \cdot \mathbf{e F}$

$30 \cdot \operatorname{RANINI} \cdot$ Dubois 1992.da: $320 \cdot \mathbf{T}$

31 - RANOIDIA Dubois 2005.da: $3 \cdot \mathbf{e F}$

$32 \cdot$ RANEIDAE $\bullet H o c$ loco $\cdot \mathbf{a F}$

$33 \cdot$ RANINA $\cdot H o c l o c o \cdot \mathbf{b T}$

$34 \cdot$ RANINIA $\cdot$ Hoc loco $\bullet \mathbf{i T}$

$35 \cdot$ RANINOA $\cdot$ Hoc loco $\bullet \mathbf{h T}$

$36 \cdot$ RANITES $\bullet$ Hoc loco $\bullet$ Cn

$37 \cdot$ RANITIES $\cdot$ Hoc loco $\bullet \mathbf{b C n}$

$38 \cdot$ RANITOES $\bullet$ Hoc loco $\bullet$ iCn

OS: Rana $1758 \cdot \mathbf{O E}$

EN: (1) RANOIDEA 1796.ba.f001-28 • pF

(2) RANOIDAE 1796.ba.f001-29 • eF

(3) RANEIDAE 1796.ba.f001-32 • aF

(4) RANIDAE 1796.ba.f001-05 • F

(5) RANINAE 1796.ba.f001-23 • bF

(6) RANINI 1796.ba.f001-30 • T

(7) RANINA 1796.ba.f001-33 • bT 

(8) RANINIA 1796.ba.f001-34 • iT
(9) RANINOA 1796.ba.f001-35 • hT
(10) RANITES $1796 . \mathrm{ba} . \mathrm{f001-36} \cdot \mathbf{C n}$
(11) RANITIES 1796.ba.f001-37 • bCn
(12) RANITOES 1796.ba.f001-38 • iCn

EF: RANIDAE 1796.ba.f001

RANIXALINI Dubois, 1987.da.f005 • KY

SI: 337 • CI: h245 • ST: 0.10 .30

RL: INR

PA: $00 \cdot R_{A N I X A L I N I} \cdot$ Dubois 1987.da: $66 \cdot \mathbf{T}$ 01 - RANIXALINAE $\cdot$ Dubois 1992.da: $334 \cdot \mathbf{b F}$ $02 \cdot$ RANIXALIDAE $・$ Van Bocxlaer ${ }^{+4}$ 2006.va: $2 \cdot \mathbf{F}$ $03 \cdot$ RANIXALEIDAE $\bullet$ Hoc loco $\bullet \mathbf{a F}$

OS: Ranixalus $1986 \approx$ Indirana $1986 \cdot$ OD

EN: (1) RANIXALEIDAE 1987.da.f005-03 • aF

(2) RANIXALIDAE 1987.da.f005-02 • F

EF: RANIXALIDAE 1987.da.f005

RANODONTIDAE Thorn, 1966.ta.f001 • KY

SI: $275 \bullet \mathbf{C I}:$ h192 • ST: 0.10 .30

RL: INR

PA: $00 \cdot$ RANODONTIDAE $・$ Thorn 1966.ta: $108 \cdot \mathbf{F}$

$01 \cdot$ RANODONTINI $\bullet$ Hoc loco $\bullet \mathbf{b T}$

$02 \cdot$ RANODONTINA $\bullet$ Hoc loco $\bullet \mathbf{b T}$

OS: Ranodon $1866 \cdot \mathbf{O E}$

EN: (1) RANODONTINI 1966.ta.f001-01 • T

(2) RANODONTINA 1966.ta.f001-02 • b T

EF: HYNOBIIDAE |1856.ha.f001|-1859.cb.f002

$\boldsymbol{R A N O D O N T I N I}$ Dubois $^{+1}$, 2012.da.f003 • JI

SI: $420 \bullet \mathbf{C I}:$ h314 • ST: 0.10 .52

RL: INR

PA: $00 \cdot$ RANODONTINI $・$ Dubois $^{+1}$ 2012.da: $113 \cdot$ T

OS: Ranodon $1866 \cdot \mathbf{O E}$

EN: (1) RANODONTINI 1966.ta.f001-01 • T

(2) RANODONTINA 1966.ta.f001-02 • bT

EF: HYNOBIIDAE |1856.ha.f001|-1859.cb.f002

RENTAPIITUES nov., DOP.da.f021・ KY

SI: $462 \bullet \mathbf{C I}:$ h355 • ST: 0.10 .30

RL: INR

PA: $00 \cdot$ RENTAPIITUES $\bullet$ Hoc loco $\bullet \mathbf{h C n}$

OS: Rentapia $2016 \cdot \mathbf{P D}$

EN: RENTAPIITUES DOP.da.f021-00 • hCn

EF: BUFONIDAE 1825.gb.f004

RHACOPHORIDAE Hoffman, 1932.ha.f001 • SK

SI: $233 \bullet \mathbf{C I}:$ h166 • ST: 0.10 .35

RL: > POLYPEDATIDAE 1858.gc.f012 • PS: Dubois 1983.da: 276

PA: 00 - RHACOPHORIDAE • Hoffman 1932.ha: $562 \cdot$ F

01 - RHACOPHORINAE $\bullet$ Laurent 1943.la: $16 \cdot \mathbf{b F}$

$02 \cdot \operatorname{RHACOPHRIDAE} \cdot \mathrm{Fei}^{+2}$ 1990.fa: $170 \cdot \mathbf{F}$

03 - RHACOPHORINI $・$ Dubois 1992.da: $336 \cdot \mathbf{T}$

$04 \cdot$ RHCOPHORIDAE $\bullet \mathrm{Fei}^{+4} 2005 . \mathrm{fb}: 256 \cdot \mathbf{F}$

$05 \cdot$ RHACOPHORINA $\cdot$ HOC lOCO $\bullet \mathbf{b T}$

$06 \cdot$ RHACOPHORINIA $\cdot$ HOC lOCO $\bullet$ iT

$07 \cdot$ RHACOPHORINOA $\cdot$ HOC loco $\bullet \mathbf{h T}$

$08 \cdot$ RHACOPHORITES $・ H O C$ lOCO $・ \mathbf{C n}$

$09 \cdot$ RHACOPHORITIES $\bullet$ HOc lOCO $\bullet \mathbf{b C n}$

OS: Rhacophorus $1822 \cdot \mathbf{O E}$
EN: (1) RHACOPHORIDAE |1858.gc.f012|-1932.ha.f001-00 • F

(2) RHACOPHORINAE |1858.gc.f012|-1932.ha.f001-01 • bF

(3) RHACOPHORINI |1858.gc.f012|-1932.ha.f001-03 • T

(4) RHACOPHORINA |1858.gc.f012|-1932.ha.f001-05 • bT

(5) RHACOPHORINIA |1858.gc.f012|-1932.ha.f001-06 • iT

(6) RHACOPHORINOA |1858.gc.f012|-1932.ha.f001-07 • hT

(7) RHACOPHORITES |1858.gc.f012|-1932.ha.f001-08 • Cn

(8) RHACOPHORITIES |1858.gc.f012|-1932.ha.f001-09 • bCn

EF: RHACOPHORIDAE |1858.gc.f012|-1932.ha.f001

RHAEBOITES nov., DOP.da.f033 • KY

SI: $474 \cdot \mathbf{C I}:$ h367 • ST: 0.10 .30

RL: INR

PA: $00 \cdot$ RHAEBOITES $\bullet$ Hoc loco $\bullet$ Cn

OS: Rhaebo $1862 \cdot \mathbf{P D}$

EN: RHAEBOITES DOP.da.f033-00 • Cn

EF: BUFONIDAE 1825.gb.f004

RHINODERMiNa Bonaparte, 1850.bb.f011 • KY

SI: $103 \cdot \mathbf{C I}:$ h065 • ST: 0.10 .30

RL: INR

PA: 00 - RHINODERMinA • Bonaparte 1850.bb: pl. • bF

01 • RHINODERMATIDAE $\bullet$ Günther 1858.gc: $346 \bullet \mathbf{b F}$

02 • RHINODERMATINAE $・$ Noble 1931.na: $506 \cdot \mathbf{b F}$

OS: Rhinoderma $1841 \cdot \mathbf{O E}$

EN: RHINODERMATIDAE 1850.bb.f011-01 • F

EF: RHINODERMATIDAE 1850.bb.f011

RHEOBATRACHINAE Heyer ${ }^{+1}$, 1976.ha.f001 • KY

SI: $298 \bullet \mathbf{C I}:$ h2 $11 \bullet \mathbf{S T}: 0.10 .30$

RL: INR

PA: $00 \cdot$ RHEOBATRACHINAE $\cdot$ Heyer $^{+1}$ 1976.ha: $11 \cdot \mathbf{b F}$

01 • RHEOBATRACHIDAE • Laurent 1980.1a: 401 • F

OS: Rheobatrachus 1973 • OE

EN: RHEOBATRACHINAE 1976.ha.f001-00 • F

EF: MYOBATRACHIDAE 1850.sa.f001

RHEOHYLINOA nov., DOP.da.f057 • KY

SI: $498 \bullet \mathbf{C I}:$ h391・ST: 0.10 .30

RL: INR

PA: $00 \cdot$ RHEOHYLINOA $\bullet$ Hoc loco $\bullet \mathbf{h T}$

$01 \cdot$ RHEOHYLITES $\bullet$ Hoc loco $\bullet \mathbf{C n}$

OS: Rheohyla 2016 • PD

EN: (1) RHEOHYLINOA DOP.da.f057-00 • hT

(2) RHEOHYLITES DOP.da.057-01 • Cn

EF: HYLIDAE 1815.ra.f002-|1825.gb.f001|

RHINATREMATIDAE Nussbaum, 1977.na.f001 • KY

SI: $300 \cdot \mathbf{C I}:$ h2 $13 \cdot \mathbf{S T}: 0.10 .30$

RL: INR

PA: 00 • RHINATREMATIDAE • Nussbaum 1977.na: 1 • F

01 • RHINATREMIDAE $\bullet$ Laurent 1984.1a: $199 \cdot \mathbf{F}$

$02 \cdot$ RHINATREMATOIDES $\bullet$ Lescure $^{+2}$ 1986.lb: $158 \cdot \mathbf{h F}$

03 - RHINATREMATOIDEA $\cdot$ Lescure $^{+2}$ 1986.lb: $158 \cdot \mathbf{p F}$

$04 \cdot$ RHINATREMATOIDAE $\bullet$ Lescure $^{+2}$ 1986.1b: $158 \cdot \mathbf{e F}$

OS: Rhinatrema $1841 \cdot \mathbf{O E}$

EN: RHINATREMATIDAE 1977.na.f001-00 • F

EF: RHINATREMATIDAE 1977.na.f001

RHINOPHRYNIDAE Günther, 1858.gc.f013 • KY

SI: $141 \cdot \mathbf{C I}:$ h094 • ST: 0.10 .30

RL: INR 
PA: $00 \bullet$ RHINOPHRYNIDAE • Günther 1858.gc: $348 \cdot \mathbf{F}$

01 $\bullet$ RHINOPHRYNINA • Günther 1859.ga: xiv • Sc

$02 \cdot$ RHINOPHRYNIDA $・$ Knauer 1878.ka: $108 \cdot \mathbf{F}$

03 $\bullet$ RHINOPHRYNINAE $・$ Noble 1931.na: $500 \cdot \mathbf{b F}$

$04 \cdot$ RHYNOPHRYNIDAE Casamiquela 1961.ca: $79 \cdot \mathbf{F}$

OS: Rhinophrynus $1841 \cdot$ OD

EN: RHINOPHRYNIDAE 1858.gc.f013-00 • F

EF: RHINOPHRYNIDAE 1858.gc.f013

RHOMbOPHRYNINAE Noble, 1931.na.f009 • KY

SI: $224 \cdot$ CI: h159 • ST: 0.10 .30

RL: INR

PA: 00 • RHOMBOPHRYNINAE $・$ Noble 1931.na: 529 • bF

$01 \cdot$ RHOMBOPHRYNINA $\bullet$ HOc loco $\bullet \mathbf{b T}$

OS: Rhombophryne $1880 \cdot \mathbf{O E}$

EN: RHOMBOPHRYNINA 1931.na.f009-01 • bT

EF: MICROHYLIDAE |1843.fa.f012|-1931.na.f001

RHYACOTRITONINAE Tihen, 1958.ta.f002 • KY

SI: $261 \bullet \mathbf{C I}:$ h1 $82 \cdot$ ST: 0.10 .30

RL: < DICAMPTODONTINAE 1958.ta.f001 - AI: Regal 1966.ra: 405

PA: $00 \bullet$ RHYACOTRITONINAE $・$ Tihen 1958.ta: $1 \cdot \mathbf{b F}$

01 RHYACOTRITONIDAE $\cdot \mathrm{Good}^{+1}$ 1992.ga: v, xi, 1, 13 • F

$02 \cdot$ RHYACOTRITONOIDEA • Dubois 2005.da: $20 \bullet \mathbf{p F}$

$03 \cdot$ RHYACOTRITONEIDAE $\bullet$ Hoc loco $\bullet \mathbf{a F}$

OS: Rhyacotriton $1920 \cdot \mathbf{O E}$

EN: (1) RHYACOTRITONEIDAE 1958.ta.f002-03 • aF

(2) RHYACOTRITONIDAE 1958.ta.f002-01 • F

EF: RHYACOTRITONIDAE 1958.ta.f002

RoMERINA nov., DOP.da.f128 • KY

SI: $569 \bullet$ CI: h462 • ST: 0.10 .30

RL: INR

PA: $00 \cdot$ ROMERINA $\cdot$ Hoc loco $\bullet \mathbf{b T}$

OS: Romerus nov. • PD

EN: ROMERINA DOP.da.f128-00 • bT

EF: RHACOPHORIDAE |1858.gc.f012|-1932.ha.f001

RUGosinoa nov., DOP.da.f111 • KY

SI: 552 • CI: h445 • ST: 0.10 .30

RL: INR

PA: $00 \cdot$ RUGOSINOA $\cdot$ Hoc loco $\bullet \mathbf{b C n}$

OS: Rugosa $1990 \cdot \mathbf{P D}$

EN: RUGOSINOA DOP.da.f111-00 • bCn

EF: RANIDAE 1796.ba.f001

RULYRaniNoa nov., DOP.da.f042 • KY

SI: $483 \bullet$ CI: h376 • ST: 0.10 .30

RL: INR

PA: $00 \cdot$ RULYRANINOA $\cdot$ Hoc loco $\bullet \mathbf{h T}$

$01 \cdot$ RULYRANITES $\bullet$ Hoc loco $\bullet \mathbf{C n}$

OS: Rulyrana $2009 \cdot$ PD

EN: (1) RULYRANINOA DOP.da.f042-00 • hT

(2) RULYRANITES DOP.da.f042-01 • Cn

EF: CENTROLENIDAE 1951.ta.f001

SABAHPHRYNitoes nov., DOP.da.f025 • KY

SI: $466 \bullet \mathbf{C I}:$ h359・ST: 0.10 .30

RL: INR

PA: $00 \cdot$ SABAHPHRYNITOES $\bullet$ Hoc loco $\bullet \mathbf{i C n}$

OS: Sabahphrynus 2007 PD

EN: SABAHPHRYNITOES DOP.da.f025-00 • iCn
EF: BUFONIDAE 1825.gb.f004

SalamandRaE Goldfuss, 1820.ga.f002 • KY

SI: $012 \bullet \mathbf{C I}:$ h005 • ST: 0.10 .30

RL: INR

PA: $00 \cdot \operatorname{SALAMANDRAE} \bullet$ Goldfuss 1820.ga: xi • F

$01 \cdot S_{A L A M A N D R I D A E} \bullet$ Gray $1825 . \mathrm{gb}: 215 \cdot \mathbf{F}$

$02 \cdot$ SALAMANDROIDEA $\cdot$ Fitzinger 1826.fb: $37 \cdot \mathbf{F}$

03 - SALAMANDRINA • Hemprich 1829.ha: xix, $373 \cdot \mathbf{F}$

$04 \cdot S_{A L A M A N D R O I D E A} \cdot$ Fitzinger 1832.fa: $329 \cdot \mathbf{G r}$

05 - SALAMANDRINA $・$ Bonaparte 1839.bd: [259] • bF

06 - SALMANDRIDAE • Bonaparte 1839.be: $272 \cdot \mathbf{F}$

$07 \cdot$ SALAMANDROIDES • Duméril ${ }^{+1}$ 1841.da: $52 \cdot \mathbf{F}$

08 - SALAMANDRIDES $\bullet$ Duméril $^{+1}$ 1841.da: table after page $53 \cdot \mathbf{F}$

09 - SALAMANDRINA • Leunis 1844.1a: $129 \cdot$ UC

10 - SALAMANDRINAE • Bronn 1849.ba: $683 \cdot \mathbf{U F}$

11 - SALAMANDRIDAE • Bronn 1849.ba: 683 • UF

12 - SALAMANDRINAE • Baird 1851.ba: $253 \cdot \mathbf{F}$

13 - SALAMANDRINES • Desmarest 1856.da: $152 \cdot \mathbf{F}$

$14 \cdot$ SALAMANDRIDA $・$ Jan 1857.ja: $54 \cdot \mathbf{F}$

15 - SALAMANDRINAE $\bullet$ Cope 1859.cb: $125 \cdot \mathbf{b F}$

16 - SALAMANDRAE $\bullet$ Cope 1859.cb: $125 \cdot \mathbf{U C}$

17 - SALAMANDRINA • Leunis 1860.1a: $339 \cdot \mathbf{T}$

18 - SALAMANDRAE • Betta 1864.ba: $512 \cdot \mathbf{b F}$

19 - SALAMANDRIDEA • Huxley 1871.ha: $173 \cdot \mathbf{U F}$

$20 \cdot$ SALAMANDRIAE $・$ Hoffmann 1878.ha: $583 \cdot \mathbf{F}$

21 - SALAMANDROIDEA • Garman 1884.ga: $37 \cdot \mathbf{p F}$

$22 \cdot$ SALAMANDRIDI • Acloque 1900.aa: $494 \cdot \mathbf{F}$

$23 \cdot$ SALAMANDRIDEA - Stefano 1903.sa: $42 \cdot \mathbf{F}$

$24 \cdot$ SALAMANDROIDEAE $・$ Stejneger 1907.sa: $3 \cdot \mathbf{p F}$

25 - SALAMANDROIDAE • Hay 1929.ha: $848 \cdot \mathbf{p F}$

26 - SALAMANDRINA • Huene 1931.ha: $311 \cdot \mathbf{p F}$

$27 \cdot$ SALAMANDROIDIA • Dubois 2005.da: $19 \cdot \mathbf{e F}$

28 - SALAMANDRINI Dubois $^{+1}$ 2009.db: 60 - T

29 • SALAMANDROIDAE • Dubois ${ }^{+1}$ 2012.da: $148 \cdot \mathbf{e F}$

OS: Salamandra $1768 \approx$ Salamandra $1764 \cdot$ OE

EN: (1) SALAMANDROIDEA 1820.ga.f002-21 • pF

(2) SALAMANDRIDAE 1820.ga.f002-01・F

(3) SALAMANDRINAE 1820.ga.f002-15 • bF

(4) SALAMANDRINI 1820.ga.f002-28・ T

EF: SALAMANDRIDAE 1820.ga.f002

SALAMANDRELLINA Dubois ${ }^{+1}$, 2012.da.f004 • KY

SI: $421 \cdot \mathbf{C I}:$ h315 • ST: 0.10 .30

RL: INR

PA: $00 \cdot$ SALAMANDRELLINA • Dubois ${ }^{+1}$ 2012.da: $113 \cdot$ bT

OS: Salamandrella 1870 • OD

EN: SALAMANDRELLINA 2012.da.f004-00 • bT

EF: HYNOBIIDAE |1856.ha.f001|-1859.cb.f002

SALAMANDRINAE Fitzinger, 1843.fa.f013 • KY

SI: $078 \cdot \mathbf{C I}:$ h046 • ST: 0.10 .30

RL: INR

PA: $00 \cdot$ SALAMANDRINAE $\bullet$ Fitzinger 1843.fa: $33 \cdot \mathbf{F}$

01 - SALAMANDRININAE $・$ Dubois $^{+1}$ 2009.db: $29 \cdot \mathbf{b F}$

OS: Salamandrina $1826 \cdot \mathbf{O E}$

EN: SALAMANDRININAE 1843.fa.f013-01 • bF

EF: SALAMANDRIDAE 1820.ga.f002 
SALAMANDROPES Fitzinger, 1843.fa.f015 • JD

SI: $080 \bullet$ CI: h048 • ST: 0.10 .40

RL: INR

PA: $00 \cdot$ SALAMANDROPES $\bullet$ Fitzinger 1843.fa: $34 \cdot \mathbf{F}$

OS: Salamandrops $1830 \approx$ Cryptobranchus $1821 \cdot$ OE

EN: CRYPTOBRANCHIDAE 1826.fb.f003-04 • F

EF: CRYPTOBRANCHIDAE 1826.fb.f003

SALTENIIDAE Kuhn, 1965.ka.f002 † • AN

SI: $274 \bullet$ CI: n079 • ST: 0.29 .50

RL: INR

PA: 00 • SALTENIIDAE $\bullet$ Kuhn 1965.ka: $88 \cdot \mathbf{F}$

OS: Saltenia $1959+\bullet$ OE

EN: SALTENIINAE DOP.da.f148-00 †

EF: PIPIDAE 1825.gb.f003-|1826.fb.f002|

SALTENIINAE nov., DOP.da.f148 † $\mathbf{K Y}$

SI: $593 \cdot \mathbf{C I}: \mathrm{n} 104 \cdot \mathbf{S T}: 0.10 .30$

RL: INR

PA: $00 \cdot$ SALTENIINAE $\bullet$ Hoc loco $\bullet$ bF

OS: Saltenia $1959+\cdot$ PD

EN: SALTENIINAE DOP.da.f148-00 †

EF: PIPIDAE 1825.gb.f003-|1826.fb.f002|

SANGUIRANINI $\mathrm{Fei}^{+2}$, 2010.fa.f017 • KY

SI: $408 \bullet$ CI: h308 • ST: 0.10 .30

RL: INR

PA: $00 \cdot$ SANGUIRANINI $\bullet \mathrm{Fei}^{+2} 2010$ fa: $18 \cdot \mathbf{T}$ $01 \cdot$ SANGUIRANINOA $\bullet$ Hoc loco $\bullet \mathbf{h T}$

OS: Sanguirana $1992 \cdot$ OD

EN: SANGUIRANINOA 2010.fa.f017-01 • hT

EF: RANIDAE 1796.ba.f001

SATOBIINOA nov., DOP.da.f130 • KY

SI: $571 \bullet$ CI: h464 • ST: 0.10 .30

RL: INR

PA: $00 \cdot$ SATOBIINOA $\bullet$ Hoc loco $\bullet$ hT

OS: Satobius $1990 \cdot \mathbf{P D}$

EN: SATOBINOA DOP.da.f130-00 $\bullet \mathbf{h T}$

EF: HYNOBIIDAE |1856.ha.f001|-1859.cb.f002

SCAPHERPETONIDaE Auffenberg ${ }^{+1}$, 1959.aa.f001 † ・ KY

SI: $262 \bullet$ CI: h1 $183 \bullet$ ST: 0.10 .30

RL: INR

PA: $00 \cdot$ SCAPHERPETONIDAE $\bullet$ Auffenberg $^{+1}$ 1959.aa: 5 • F 01 • SCAPHERPETONTIDAE • Estes 1965.ea: $321 \cdot \mathbf{F}$ 02 - SCAPHERPETONTINAE • Edwards 1976.ea: $325 \cdot \mathbf{b F}$ 03 - SCAPHERPETONINAE $・$ Brame $^{+3}$ 1978.ba: $45 \cdot \mathbf{b F}$ 04 - SCAPHERPETODONTIDAE $\cdot$ Vorobyeva $^{+1}$ 1996.va: $69 \cdot$ F 05 - SCAPHERPETIDAE • Skutschas 2009.sa: $663 \cdot \mathbf{F}$

OS: Scapherpeton $1877 \dagger \approx$ Hedronchus $1877 \uparrow \cdot \mathbf{O E}$

EN: SCAPHERPETIDAE 1959.aa.f001-05 $\bullet$ F

EF: SCAPHERPETIDAE 1959.aa.f001 $\dagger$

SCAPHIOPHRYNINAE Laurent, 1946.la.f002 • KY

SI: $243 \bullet \mathbf{C I}:$ h174 • ST: 0.10 .30

RL: INR

PA: $00 \bullet$ SCAPHIOPHRYNINAE $\bullet$ Laurent 1946.la: $337 \bullet \mathbf{b F}$

01 $\bullet$ SCAPHIOPHRYNIDAE $\bullet$ Kuhn 1967.kb: $37 \cdot \mathbf{F}$

02 • SCAPHIOPHRYNIINAE $\bullet$ Guibé 1978.gb: $8 \cdot \mathbf{b F}$

$03 \cdot$ SCAPHIOPHRYNINI $\bullet$ Hoc loco $\bullet \mathbf{T}$

OS: Scaphiophryne Boulenger, $1882 \cdot \mathbf{O E}$
EN: SCAPHIOPHRYNINI 1946.la.f002-03 • T

EF: MICROHYLIDAE |1843.fa.f012|-1931.na.f001

SCAPHIOPODIDAE Cope, 1865.ca.f003 • KY

SI: $153 \cdot \mathbf{C I}:$ h103 • ST: 0.10 .30

RL: INR

PA: c0 • SCAPHIOPODIDAE $・$ Cope 1865.ca: $104 \cdot$ F • IIA: Cope 1866.ca: 68

i1 $\bullet$ SCAPHIOPIDAE $\bullet$ Cope 1865.ca: $107 \cdot \mathbf{F}$

02 • SCAPHIOPODINA • Mivart 1869.ma: $291 \cdot \mathbf{b F}$

03 - SCAPHIOPINAE $\bullet$ Śpinar $^{+2}$ 1971.sa: $284 \cdot \mathbf{b F}$

$04 \cdot$ SCAPHIOPODINAE $\bullet$ Dubois 1983.da: $271 \cdot \mathbf{b F}$

$05 \cdot$ SCAPHIOPODOIDEA $\bullet$ Hoc loco $\cdot \mathbf{p F}$

OS: Scaphiopus $1836 \cdot \mathbf{O E}$

EN: (1) SCAPHIOPODOIDEA 1865.ca.f003-05 • pF

(2) SCAPHIOPODIDAE 1865.ca.f003-c0 • F

EF: SCAPHIOPODIDAE 1865.ca.f003

SCHISMADERMATITUES nov., DOP.da.f027 • KY

SI: 468 • CI: h361 • ST: 0.10 .30

RL: INR

PA: $00 \cdot$ SCHISMADERMATITUES $\bullet$ Hoc loco $\bullet \mathbf{h C n}$

OS: Schismaderma $1849 \cdot \mathbf{P D}$

EN: SCHISMADERMATITUES DOP.da.f027-00 • hCn

EF: BUFONIDAE 1825.gb.f004

SCINAXINAE Duellman ${ }^{+2}$, 2016.db.f002 • KY

SI: $437 \bullet$ CI: h330 • ST: 0.10 .30

RL: INR

PA: 00 - SCINAXINAE $\cdot$ Duellman $^{+2}$ 2016.db: 3, $25 \cdot \mathbf{b F}$

01 $\cdot$ SCINAGINAE $\bullet$ Dubois $^{+1}$ 2019.db: $125 \cdot \mathbf{b F}$

$02 \cdot \operatorname{SCINAXINI} \bullet \mathrm{Hoc}$ loco $\bullet$ T

$03 \cdot$ SCINAXINA $\bullet$ Hoc loco $\bullet$ bT

$04 \cdot$ SCINACINAE $\bullet$ Hoc loco $\bullet \mathbf{b F}$

OS: Scinax $1830 \bullet$ PD

EN: (1) SCINAXINI 2016.db.f002-02 • T

(2) SCINAXINA 2016.db.f002-03 • bT

EF: HYLIDAE 1815.ra.f002-|1825.gb.f001|

SCLEROPHRYITOES nov., DOP.da.f030 • KY

SI: $471 \bullet$ CI: h364 • ST: 0.10 .30

RL: INR

PA: 00 • SCLEROPHRYITOES $\bullet$ HOc loco $・$ iCn

OS: Sclerophrys $1838 \cdot \mathbf{P D}$

EN: SCLEROPHRYITOES DOP.da.f030-00 • iCn

EF: BUFONIDAE 1825.gb.f004

SCOLECOMORPHIDAE Taylor, 1969.ta.f001 • KY

SI: $285 \bullet$ CI: h200 • ST: 0.10 .30

RL: INR

PA: 00 • SCOLECOMORPHIDAE • Taylor 1969.ta: $297 \bullet \mathbf{F}$

$01 \cdot$ SCOLECOMORPHOIDES $\bullet$ Lescure $^{+2}$ 1986.lb: $159 \cdot \mathbf{h F}$

$02 \cdot$ SCOLECOMORPHOIDEA $\cdot$ Lescure $^{+2}$ 1986.lb: $159 \cdot \mathbf{p F}$

03 - SCOLECOMORPHOIDAE $\bullet$ Lescure $^{+2}$ 1986.lb: $159 \cdot \mathbf{e F}$

$04 \cdot$ SCOLECOMORPHINAE $・$ Lescure $^{+2}$ 1986.lb: $159 \cdot \mathbf{b F}$

OS: Scolecomorphus $1883 \cdot$ OD

EN: SCOLECOMORPHIDAE 1969.ta.f001-00 • F

EF: SCOLECOMORPHIDAE 1969.ta.f001

SEIRANotina Bonaparte, 1850.bb.f014 • JD

SI: $106 \bullet$ CI: h068 • ST: 1.10 .40

RL: INR 
PA: 00 - SEIRANOTINA • Bonaparte 1850.bb: pl. • F 01 - SEIRANOTINA • Gray 1850.ga: $29 \cdot \mathbf{U F}$ 02 - SEIRANOTIDAE • Hallowell 1856.ha: 10. • bF $03 \cdot$ SEIRANOTIDAE $\bullet$ Gray 1858.gb: $137 \cdot \mathbf{F}$ $04 \cdot$ SIRANOTIDAE $\bullet$ Cope 1866.ca: $108 \cdot \mathbf{F}$ $05 \cdot$ SEIRANODONTIDAE $\bullet$ Kuhn 1967.kb: $38 \cdot \mathbf{F}$

OS: Seiranota $1826 \approx$ Salamandrina $1826 \cdot \mathbf{O E}$

EN: SALAMANDRININAE 1843.fa.f013-01 • bF

EF: SALAMANDRIDAE 1820.ga.f002

SIEBOLDIIDAE Bonaparte, 1850.bb.f017 • JD

SI: $110 \bullet \mathbf{C I}:$ h072 • ST: 0.10 .40

RL: INR

PA: $00 \cdot$ SIEBOLDIIDAE $\bullet$ Bonaparte 1850.bb: pl. • F $01 \cdot$ SIEBOLDIINA $\bullet$ Bonaparte 1850.bb: pl. • bF

OS: Sieboldia $1838 \approx$ Andrias $1837 \uparrow \cdot \mathbf{O E}$

EN: CRYPTOBRANCHIDAE 1826.fb.f003-04 • F

EF: CRYPTOBRANCHIDAE 1826.fb.f003

SiluraninaE Cannatella ${ }^{+1}$, 1988.ca.f001・JD

SI: $340 \bullet$ CI: h248 • ST: 0.10 .40

RL: INR

PA: 00 • SILURANINAE • Cannatella ${ }^{+1}$ 1988.ca: 1 • bF

OS: Silurana $1864 \cdot \mathbf{O E}$

EN: (1) DACTYLETHRINAE 1838.ha.f001-04 • bF (2) DACTYLETHRINI 1838.ha.f001-05 • T

EF: PIPIDAE 1825.gb.f003-|1826.fb.f002

SiPHONOPINA Bonaparte, 1850.bb.f019 • KY

SI: $111 \bullet \mathbf{C I}:$ h073 • ST: 0.10 .30

RL: INR

PA: $00 \cdot$ SIPHONOPINA • Bonaparte 1850.bb: pl. • bF 01 - SIPHONOPIDAE $\bullet$ Dubois 1984.da: $113 \cdot \mathbf{F}$ 02 - SIPHONOPINAE • Dubois 1984.da: $113 \cdot \mathbf{b F}$ $03 \cdot$ SIPHONOPOIDES - Lescure $^{+2}$ 1986.lb: $162 \cdot \mathbf{h F}$ $04 \cdot$ SIPHONOPOIDEA $\cdot$ Lescure $^{+2}$ 1986.1b: $162 \cdot \mathbf{p F}$ $05 \cdot$ SIPHONOPOIDAE - Lescure $^{+2}$ 1986.lb: $163 \cdot \mathbf{p F}$ $06 \cdot$ SIPHONOPILAE $・$ Lescure $^{+2}$ 1986.lb: $162 \cdot \mathbf{i F}$ $07 \cdot$ SIPHONOPOIDI $\bullet$ Lescure $^{+2} 1986.1 \mathrm{~b}: 166 \cdot \mathbf{p T}$ $08 \cdot$ SIPHONOPINI $\cdot$ Lescure $^{+2}$ 1986.1b: $166 \cdot \mathbf{T}$ $09 \cdot$ SIPHONOPITI $・$ Lescure $^{+2}$ 1986.lb: $167 \cdot \mathbf{b T}$ $10 \cdot$ SIPHONOPINA $\cdot$ Hoc loco $\bullet \mathbf{b T}$ 11 - SIPHONOPINIA $\cdot$ Hoc loco $\bullet$ iT

$12 \cdot$ SIPHONOPINOA $\bullet$ Hoc loco $\bullet \mathbf{h T}$

OS: Siphonops $1828 \cdot \mathbf{O E}$

EN: (1) SIPHONOPINI 1850.bb.f019-08 • T

(2) SIPHONOPINA $1850 . \mathrm{bb} . \mathrm{f019}-10 \cdot \mathbf{b T}$

(3) SIPHONOPINIA 1850.bb.f019-11・iT

(4) SIPHONOPINOA 1850.bb.f019-12 • hT

EF: CAECILIIDAE 1814.ra.f003-|1825.gb.f008|

SIREDONIDAE Gray, 1842.ga.f001 • CG

SI: $064 \cdot$ CI: h032 • ST: 0.10 .62

RL: INR

PA: $00 \cdot$ SIREDONIDAE • Gray 1842.ga: $114 \cdot \mathbf{F}$ $01 \cdot$ SIREDONTIDAE $\bullet$ Bonaparte 1850.bb: pl. • F $02 \cdot$ SIREDONTINA • Bonaparte 1850.bb: pl. • bF

OS: Siredon 1829 CI $\approx$ Ambystoma $1838 \cdot \mathbf{O E}$

EN: АмвYSTOMATIDAE 1850.ga.f002-08 • F

EF: AмBYSTOMATIDAE 1850.ga.f002
SirenidaE Gray, 1825.gb.f005 • KY

SI: $019 \bullet \mathbf{C I}:$ h010 • ST: 0.10 .30

RL: INR

PA: $00 \cdot$ SIRENIDAE $\bullet$ Gray 1825.gb: $215 \cdot \mathbf{F}$

$01 \cdot$ SERENINA • Gray 1825.gb: $216 \cdot \mathbf{U F}$

$02 \cdot$ SIRENEA • Hemprich 1829.ha: xix, $373 \cdot \mathbf{F}$

$03 \cdot$ SIRENINA • Gray 1829.ga: $205 \cdot$ UF

$04 \cdot$ SIRENIDEA $\cdot$ Jourdan 1834.jb: $438 \cdot \mathbf{F}$

05 - SIRENA • Blainville 1835.ba: $282 \cdot \mathbf{F}$

$06 \cdot$ SIRENINA $\bullet$ Bonaparte 1838.bc: $393 \cdot \mathbf{b F}$

$07 \cdot$ SIRENES $\bullet$ Fitzinger 1843.fa: $35 \cdot \mathbf{F}$

08 - SIRENOIDEI • Bronn 1849.ba: 682 • UF

$09 \cdot$ SIRENIDES • Gouriet 1868.ga: $206 \cdot \mathbf{F}$

$10 \cdot$ SIRENIDA $・$ Knauer 1878.ka: 95 • F

11 - SIRENOIDAE • Hay 1929.ha: $842 \cdot \mathbf{p F}$

12 • SIRENOIDEA • Milner 2000.ma: $1412 \cdot \mathbf{p F}$

13・SIRENOIDIA • Dubois 2005.da: $21 \cdot \mathbf{e F}$

OS: Siren $1766 \cdot \mathbf{O E}$

EN: SIRENIDAE 1825.gb.f005-00 • F

EF: SIRENIDAE 1825.gb.f005

SMiLISCITOES nov., DOP.da.f150 • KY

SI: $591 \cdot \mathbf{C I}: \mathrm{h} 484 \cdot \mathbf{S T}: 0.10 .30$

RL: INR

PA: $00 \cdot$ SMILISCITOES $\bullet$ Hoc loco $\bullet$ iCn

OS: Smilisca $1865 \cdot \mathbf{P D}$

EN: SMILISCITOES DOP.da.f150-00 • iCn

EF: HYLIDAE 1815.ra.f002-|1825.gb.f001|

SooglossinaE Noble, 1931.na.f002 • KY

SI: $217 \bullet \mathbf{C I}:$ h152 • ST: 0.10 .30

RL: INR

PA: $00 \cdot \operatorname{SoOGLOSSINAE} \bullet$ Noble 1931.na: 492 • bF

$01 \cdot$ SOOGLOSSIDAE $\bullet$ Griffiths 1963.ga: $273 \cdot \mathbf{F}$

$02 \cdot$ SOOGLOSSOIDEA • Dubois 2005.da: $17 \cdot \mathbf{p F}$

OS: Sooglossus $1906 \cdot$ OE

EN: SOOGLOSSIDAE 1931.na.f002-01 • F

EF: SoOGLOSSIDAE 1931.na.f002

SPEIDAE Špinar, 1983.sa.f001 • JD

SI: 312 • CI: h222 • ST: 0.10 .40

RL: INR

PA: $00 \cdot$ SPEIDAE $\bullet$ Špinar 1983.sa: $55 \cdot \mathbf{F}$

OS: Spea $1866 \cdot \mathbf{O E}$

EN: (1) SCAPHIOPODOIDEA 1865.ca.f003-05 • pF (2) SCAPHIOPODIDAE 1865.ca.f003-c0 • F

EF: SCAPHIOPODIDAE 1865.ca.f003

SPElerpINaE Cope, 1859.cb.f001 • KY

SI: $142 \bullet$ CI: h095 - ST: 0.10 .30

RL: INR

PA: $00 \cdot$ SPELERPINAE $\bullet$ Cope 1859.cb: $123 \cdot \mathbf{b F}$

$01 \cdot$ SPELERPEAE $・$ Cope 1859.cb: $124 \cdot \mathbf{U F}$

$02 \cdot$ SPELERPINE $・$ Cope 1863.ca: $343 \cdot \mathbf{U F}$

$03 \cdot$ SPELERPINAE $\cdot$ Hoffmann 1878.ha: $585 \cdot \mathbf{F}$

$04 \cdot$ SPELERPES • Cope 1889.ca: $121 \cdot \mathbf{U F}$

05 - SPELERPESIDI • Acloque 1900.aa: $493 \cdot \mathbf{F}$

06 - SPELERPINI • Dubois 2005.da: $20 \bullet \mathbf{T}$

$07 \cdot$ SPELERPINA $\cdot$ Hoc loco $\bullet \mathbf{b T}$

OS: Spelerpes $1832 \equiv$ Eurycea $1822 \cdot \mathbf{O E}$ 
EN: (1) SPELERPINI 1859.cb.f001-06・T

(2) SPELERPINA 1859.cb.f001-07 • bT

EF: PlethodontidaE 1850.ga.f001

SPHAENORHYNChina Faivovich ${ }^{+15}$, 2018.fa.f001 • JD

SI: $441 \bullet \mathbf{C I}:$ h334 • ST: 0.10 .40

RL: INR

PA: 00 - SPHAENORHYNCHINA • Faivovich ${ }^{+15}$ 2018.fa: $25 \cdot \mathbf{b T}$ 01 SPHAENORHYNCHINI • Araujo-Vieira ${ }^{+3}$ 2020.aa: $81 \cdot \mathrm{T}$

OS: Sphaenorhynchus $1838 \cdot \mathbf{P D}$

EN: SPHAENORHYNCHINA • Faivovich ${ }^{+15} 2018$.fa 00 • bT

EF: HYLIDAE 1815.ra.f002-|1825.gb.f001|

SPHENOPHRYNINAE Noble, 1931.na.f010 • JD

SI: $225 \bullet$ CI: h160 • ST: 0.10 .40

RL: INR

PA: $00 \cdot$ SPHENOPHRYNINAE • Noble 1931.na: 531 • bF

01 - SPHAENOPHRYNINAE • Tatarinov 1964.ta: $133 \cdot \mathbf{b F}$

OS: Sphenophryne $1878 \approx$ Asterophrys $1838 \cdot \mathbf{O E}$

EN: (1) ASTEROPHRYINAE 1858.gc.f006-05 • bF

(2) ASTEROPHRYINI 1858.gc.f006-09 • T

EF: MICROHYLIDAE |1843.fa.f012|-1931.na.f001

SPICOSPININIA nov., DOP.da.f078 • KY

SI: $519 \bullet \mathbf{C I}:$ h412 • ST: 0.10 .30

RL: INR

PA: $00 \cdot$ SPICOSPININIA $\bullet$ Hoc loco $\bullet$ iT

OS: Spicospina $1997 \cdot \mathbf{P D}$

EN: SPICOSPININIA DOP.da.f078-00・iT

EF: MYOBATRACHIDAE 1850.sa.f001

SPINOMANTINIA nov., DOP.da.f115 • KY

SI: $556 \bullet$ CI: h449・ST: 0.10 .30

RL: INR

PA: $00 \cdot$ SPINOMANTINIA $\cdot$ Hoc loco $\bullet$ iT

OS: Spinomantis $1992 \cdot \mathbf{P D}$

EN: SPINOMANTINIA DOP.da.f115-00 • iT

EF: RHACOPHORIDAE |1858.gc.f012|-1932.ha.f001

StaURoINI Dubois, 2005.da.f001 • KY

SI: $363 \bullet$ CI: h269 • ST: 0.10 .30

RL: INR

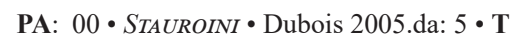
$01 \cdot$ STAUROINAE $\cdot$ Hoc loco $\cdot \mathbf{b F}$

OS: Staurois $1865 \cdot$ OD

EN: STAUROINAE 2005.da.f001-01 • F

EF: RANIDAE 1796.ba.f001

STAUROINAE • $\mathrm{Fei}^{+2}$, 2010.fa.f002 • JD

SI: $393 \cdot \mathbf{C I}:$ h293・ST: 0.10 .52

RL: INR

PA: $00 \cdot$ STAUROINAE $\bullet \mathrm{Fei}^{+2}$ 2010.fa: $17 \cdot \mathbf{b F}$

OS: Staurois $1865 \cdot$ OD

EN: STAUROINAE 2005.da.f001-01 • F

EF: RANIDAE 1796.ba.f001

StefanitNaE nov., DOP.da.f014 • KY

SI: 455 • CI: h348 • ST: 0.10 .30

RL: INR

PA: $00 \cdot$ STEFANIINAE $\bullet$ Hoc loco $\bullet \mathbf{b F}$

OS: Stefania $1968 \cdot \mathbf{P D}$

EN: STEFANIINAE DOP.da.f014-00 • bF

EF: HEMIPHRACTIDAE 1862.pa.f001
STEPHOPAEDINI Dubois, 1987.da.f001 • KY

SI: 333 • CI: h241 • ST: 0.10 .30

RL: INR

PA: 00 - STEPHOPAEDINI • Dubois 1987.da: $27 \cdot \mathbf{T}$

$01 \cdot$ STEPHOPAEDITIES $\bullet$ Hoc loco $\bullet$ bCn

$02 \cdot$ STEPHOPAEDITOES $\bullet$ HOc loco $\bullet$ iCn

OS: Stephopaedes $1979 \approx$ Mertensophryne $1960 \cdot$ OD

EN: (1) STEPHOPAEDITIES 1987.da.f001-01 • bCn

(2) STEPHOPAEDITOES 1987.da.f001-02 • iCn

EF: BUFONIDAE 1825.gb.f004

STEREOCYCLOPINA nov., DOP.da.f089 • KY

SI: 530 • CI: h423 - ST: 0.10 .30

RL: INR

PA: $00 \cdot$ STEREOCYCLOPINA $\cdot$ HOC loco $\bullet \mathbf{b T}$

OS: Stereocyclops $1870 \cdot \mathbf{P D}$

EN: STEREOCYCLOPINA DOP.da.f089-00 • bT

EF: MICROHYLIDAE |1843.fa.f012|-1931.na.f001

StombinaE Gallardo, 1965.ga.f001 • JD

SI: 272 • CI: h190 • ST: 0.10 .40

RL: INR

PA: $00 \cdot$ STOMBINAE $\bullet$ Gallardo 1965.ga: $82 \cdot \mathbf{b F}$

OS: Stombus $1825 \approx$ Ceratophrys $1824 \cdot \mathbf{O E}$

EN: STOMBINAE 1965.ga.f001-00

EF: CERATOPHRYIDAE 1838.ta.f002

StrabomantidaE Hedges ${ }^{+2}$, 2008.ha.f003 • PK

SI: $383 \cdot \mathbf{C I}:$ h283 • ST: 0.10 .37

RL: $\leq$ CRAUGASTORIDAE 2008.ha.f001 • AI: Padial ${ }^{+2}$ 2014.pa: 52 $\geq$ HOLOADENINAE 2008.ha.f004 • PR: Hedges ${ }^{+2}$ 2008: 5

PA: $00 \cdot$ STRABOMANTIDAE $\bullet$ Hedges $^{+2}$ 2008.ha: 5 • F

01 - STRABOMANTINAE $\bullet$ Hedges $^{+2}$ 2008.ha: $5 \cdot$ bF

$02 \cdot$ STRABOMANTINI $\bullet$ Hoc loco $\bullet$ T

$03 \cdot$ StRaBOMANTINA $\bullet$ Hoc loco $\bullet \mathbf{b T}$

$04 \cdot$ STRABOMANTINIA $\bullet$ Hoc loco $\bullet$ iT

OS: Strabomantis $1863 \cdot$ OD

EN: (1) STRABOMANTINI 2008.ha.f003-02 • T

(2) STRABOMANTINA 2008.ha.f003-03 • bT

(3) STRABOMANTINIA 2008.ha.f003-04 • iT

EF: BRACHYCEPHALIDAE 1858.gc.f002

STRAUCHBUFONITOES nov., DOP.da.f026 • KY

SI: 467 • CI: h360 • ST: 0.10 .30

RL: INR

PA: $00 \cdot$ STRAUCHBUFONITOES $\bullet$ Hoc loco $\bullet \mathbf{i C n}$

OS: Strauchbufo 2012 • PD

EN: STRAUCHBUFONITOES DOP.da.f026-00 • iCn

EF: BUFONIDAE 1825.gb.f004

StrongylopinaE Scott, 2005.sa.f001 • KY

SI: $364 \cdot \mathbf{C I}:$ h270 • ST: 0.10 .30

RL: INR

PA: $00 \cdot$ STRONGYLOPINAE $・$ Scott 2005.sa: $507 \cdot$ bF

$01 \cdot$ STRONGYLOPINI $\bullet$ Hoc loco $\bullet$ T

OS: Strongylopus $1838 \cdot$ OD

EN: STRONGYLOPINI 2005.sa.f001-01・F

EF: CACOSTERNIDAE 1931.na.f008

SYMPHYGNATHINAE Méhely, 1901.ma.f001 • AN

SI: 195 • CI: n057 • ST: 2.25 .50

RL: INR 
PA: 00 • SYMPHYGNATHINAE • Méhely 1901.ma: 171 • bF OS: » 3 PN, including: Xenorhina 1863 • PD

EN: (1) ASTEROPHRYINAE 1858.gc.f006-05 • bF

(2) ASTEROPHRYINI 1858.gc.f006-09 • T

EF: MICROHYLIDAE|1843.fa.f012|-1931.na.f001

SYMPHYGNATHINAE Noble, 1931.na.f012 • AN

SI: $227 \bullet \mathbf{C I}:$ n062 • ST: 0.25 .50

RL: INR

PA: $00 \cdot$ SYMPHYGNATHINAE • Noble 1931.na: $534 \cdot \mathbf{b F}$

OS: » 5 PN, including: Glyphoglossus 1869 • PD

EN: (1) MICROHYLIDAE |1843.fa.f012|-1931.na.f001-01 • F ")"»"

(4) MICROHYLINA |1843.fa.f012|-1931.na.f001-08 • bT

EF: MICROHYLIDAE |1843.fa.f012|-1931.na.f001

SYRENIADAE Brookes, 1828.bc.f001 • JI

SI: $033 \bullet$ CI: h018 • ST: 0.10 .52

RL: $\leftarrow$ SIRENIDAE $1825 . \mathrm{gb} . \mathrm{f005}$

PA: $00 \cdot$ SYRENIADAE $\bullet$ Brookes 1828.bc: $15 \cdot \mathbf{F}$

OS: Syren $1807 \equiv$ Siren $1766 \cdot \mathbf{O E}$

EN: SIRENIDAE 1825.gb.f005-00 • F

EF: SIRENIDAE 1825.gb.f005

SYSTOMata Stannius, 1856.sa.f003 • AN

SI: 128 • CI: n044 • ST: 2.25 .50

RL: INR

PA: $00 \cdot$ SYSTOMATA $・$ Stannius 1856.sa: $5 \cdot \mathbf{F}$

OS: Systoma $1830 \equiv$ Engystoma $1826 \cdot \mathbf{O M}$

EN: ENGYSTOMATINIA 1850.bb.f009-08・iT

EF: MICROHYLIDAE |1843.fa.f012|-1931.na.f001

TaChYCNEMinaE Channing, 1989.ca.f001 • KY

SI: 342 • CI: h249 • ST: 0.10 .30

RL: INR

PA: $00 \cdot$ TACHYCNEMINAE $\bullet$ Channing 1989.ca: $116 \bullet \mathbf{b F}$

$01 \cdot$ TACHYCNEMINA $\cdot H o c$ loco $\bullet \mathbf{b T}$

$02 \cdot$ TACHYCNEMINIA $\bullet$ Hoc loco $\bullet \mathbf{i T}$

OS: Tachycnemis $1843 \cdot \mathbf{O E}$

EN: (1) TACHYCNEMINA 1989.ca.f001-01・bT

(2) TACHYCNEMINIA 1989.ca.f001-02 • iT

EF: HYPEROLIIDAE 1943.lb.f001

TAMIXALITIES nov., DOP.da.f126・ KY

SI: $567 \bullet$ CI: h460 • ST: 0.10 .30

RL: INR

PA: $00 \cdot$ TAMIXALITIES $\bullet$ Hoc loco $\bullet \mathbf{b C n}$

OS: Tamixalus nov. • PD

EN: TAMIXALITIES DOP.da.f126-00 • bCn

EF: RHACOPHORIDAE |1858.gc.f012|-1932.ha.f001

TARICHINA Dubois ${ }^{+1}$, 2009.db.f003 • KY

SI: $387 \cdot \mathbf{C I}:$ h2 $87 \cdot$ ST: 0.10 .30

RL: INR

PA: $00 \cdot$ TARICHINA • Dubois ${ }^{+1} 2009 . d b: 57 \cdot$ bT

01 - TARICHINI • Litvinchuk ${ }^{+1}$ 2009.1a: $464 \cdot \mathbf{T}$

OS: Taricha $1850 \cdot$ OD

EN: TARICHINA 2009.db.f003-00 • bT

EF: SALAMANDRIDAE 1820.ga.f002

TAUDACTYLINI nov., DOP.da.f079 • KY

SI: 520 • CI: h413 • ST: 0.10 .30

RL: INR
PA: $00 \cdot$ TAUDACTYLINI $\cdot$ Hoc loco $\bullet \mathbf{T}$

OS: Taudactylus $1966 \cdot \mathbf{P D}$

EN: TAUDACTYLINI DOP.da.f079-00 • T

EF: MYOBATRACHIDAE 1850.sa.f001

TеLматові Fitzinger, 1843.fa.f006 • KY

SI: 071 • CI: h039 • ST: 0.10 .30

RL: INR

PA: $00 \cdot$ TELMATOBII $\bullet$ Fitzinger 1843.fa: $32 \cdot \mathbf{F}$

01 - TELMATOBIIDAE • Miranda-Ribeiro 1920.ma: 320 • F

02 - TELMATOBIINAE • Vellard 1951.va: $3 \cdot \mathbf{b F}$

03 - TELMATOBIINI $\bullet$ Lynch 1969.1b: $3 \cdot \mathbf{T}$

$04 \cdot$ TELMATOBIOIDAE $\bullet$ Hoc loco $\bullet \mathbf{e F}$

05 - TELMATOBIEIDAE $\bullet$ Hoc loco $\bullet \mathbf{a F}$

OS: Telmatobius $1834 \cdot \mathbf{O E}$

EN: (1) TELMATOBIOIDAE 1843.fa.f006-04 • eF

(2) TELMATOBIEIDAE 1843.fa.f006-05 • aF

(3) TELMATOBIIDAE 1843.fa.f006-01 • F

EF: TELMATOBIIDAE 1843.fa.f006

TERATOHYLINA nov., DOP.da.f044 • KY

SI: $485 \bullet$ CI: h378 • ST: 0.10 .30

RL: INR

PA: $00 \cdot$ TERATOHYLINA $・$ Hoc loco $\bullet \mathbf{b T}$

OS: Teratohyla $1951 \cdot \mathbf{P D}$

EN: TERATOHYLINA DOP.da.f044-00 • bT

EF: CENTROLENIDAE 1951.ta.f001

THORIIDaE Cope, 1869.cb.f001 • KY

SI: $160 \bullet$ CI: h108 • ST: 0.10 .30

RL: INR

PA: $00 \cdot$ THORIIDAE $\bullet$ Cope 1869.cb: $110 \cdot \mathbf{F}$

01 $\cdot$ THORINAE $\bullet$ Hay 1892.ha: $489 \cdot \mathbf{b F}$

$02 \cdot$ THORIINIA $\cdot$ Hoc loco $\bullet$ iT

03 - THORIINOA $\cdot$ Hoc loco $\bullet \mathbf{h T}$

OS: Thorius $1869 \cdot \mathbf{O E}$

EN: (1) THORIINIA 1869.cb.f001-02 • iT

(2) THORIINOA 1869.cb.f001-03 • hT

EF: PlethodontidaE 1850.ga.f001

THORNELLINOA nov., DOP.da.f135 • KY

SI: $576 \bullet$ CI: h469 • ST: 0.10 .30

RL: INR

PA: $00 \cdot$ THORNELLINOA $・$ Hoc loco $\bullet \mathbf{h T}$

$01 \cdot$ THORNELLITES $\bullet$ Hoc loco $\bullet \mathbf{C n}$

02 • THORNELLITIES $・$ Hoc loco $\bullet \mathbf{b C n}$

03 - THORNELLITOES $・ H O C$ loco • iCn

$04 \cdot$ THORNELLITUES $・ H O c$ loco $\bullet \mathbf{h C n}$

OS: Thornella nov. • PD

EN: (1) THORNELLINOA DOP.da.f135-00 • hT

(2) THORNELLITES DOP.da.f135-01 • Cn

(3) THORNELLTIES DOP.da.f135-02 • bCn

(4) THORNELLITOES DOP.da.f135-03 • iCn

(5) THORNELLITUES DOP.da.f135-04 • hCn

EF: PLETHODONTIDAE 1850.ga.f001

THOROPIDAE Frost ${ }^{+18}$, 2006.fa.f002 • JD

SI: $368 \cdot \mathbf{C I}:$ h2 $274 \cdot$ ST: 0.10 .40

RL: INR

PA: $00 \cdot$ THOROPIDAE $\bullet$ Frost $^{+18}$ 2006.fa: 7 • F

OS: Thoropa $1865 \cdot$ OD 
EN: (1) CYCLORAMPHEIDAE 1850.bb.f003-|1852.ba.f001|-05 • aF (2) CYCLORAMPHIDAE 1850.bb.f003-|1852.ba.f001|-04 • F

EF: CYCLORAMPHIDAE 1850.bb.f003-|1852.ba.f001|

ThalOCOHYLITIES nov., DOP.da.f056 • KY

SI: $497 \cdot \mathbf{C I}:$ h390 • ST: 0.10 .30

RL: INR

PA: $00 \cdot$ TLALOCOHYLITIES $\bullet$ Hoc loco $\bullet \mathbf{b C n}$

OS: Tlalocohyla $2005 \cdot \mathbf{P D}$

EN: TLALOCOHYLITIES DOP.da.f056-00 • bCn

EF: HYLIDAE 1815.ra.f002-|1825.gb.f001|

Tomopternini Dubois, 1987.da.f003 • KY

SI: $335 \bullet$ CI: h243 • ST: 0.10 .30

RL: INR

PA: 00 • TOMOPTERNINI • Dubois 1987.da: $56 \cdot \mathbf{T}$

01 TOMOPTERNINAE • Dubois 1992.da: $336 \cdot \mathbf{b F}$

OS: Tomopterna $1841 \cdot$ OD

EN: TOMOPTERNINAE 1987.da.f003-01 • bF

EF: CACOSTERNIDAE 1931.na.f008

TornIERIOBATIDAE Miranda-Ribeiro, 1926.ma.f001 • KY

SI: $208 \cdot \mathbf{C I}:$ h1 $145 \cdot \mathbf{S T}: 0.10 .30$

RL: INR

PA: 00 • TORNIERIOBATIDAE • Miranda-Ribeiro 1926.ma: $19 \cdot \mathbf{F}$

01 - TORNIERIOBATINAE • Dubois 1983.da: $273 \cdot \mathbf{b F}$

02 • TORNIERIOBATINI • Dubois 1987.da: $25 \cdot \mathbf{T}$

$03 \cdot$ TORNIERIOBATITOES $\bullet$ Hoc loco $\bullet \mathbf{i C n}$

$04 \cdot$ TORNIERIOBATITUES $\bullet$ HOC lOCO $\bullet \mathbf{h C n}$

OS: Tornieriobates $1926 \approx$ Nectophrynoides $1926 \cdot \mathbf{O E}$

EN: (1) TORNIERIOBATITOES 1926.ma.f001-03 • iCn

(2) ToRNIERIOBATITUES 1926.ma.f001-04 • hCn

EF: BUFONIDAE 1825.gb.f004

TORNIEROBATIDAE Frost $^{+18}$, 2006.fa.f003 • JD

SI: $369 \bullet$ CI: h275 • ST: 0.10 .52

RL: INR

PA: 00 • TORNIEROBATIDAE • Frost ${ }^{+18}$ 2006.fa: 213・ F

OS: Tornierobates $1940 \approx$ Nectophrynoides $1926 \cdot \mathbf{O E}$

EN: (1) TORNIERIOBATITOES 1926.ma.f001-03 • iCn

(2) ToRNIERIOBATITUES 1926.ma.f001-04 • hCn

EF: BUFONIDAE 1825.gb.f004

TRACHYCEPHALINAE Lutz, 1969.la.f002 • KY

SI: $281 \bullet$ CI: h198 • ST: 0.10 .30

RL: INR

PA: $00 \cdot$ TRACHYCEPHALINAE • Lutz 1969.1a: $275 \bullet \mathbf{b F}$

$01 \cdot$ TRACHYCEPHALINA $-H o c$ loco $\bullet \mathbf{b T}$

$02 \cdot$ TRACHYCEPHALINIA $\cdot$ Hoc loco $\bullet \mathbf{i T}$

OS: Trachycephalus $1838 \cdot \mathbf{O E}$

EN: (1) TRACHYCEPHALINA 1969.la.f002-01 • bT

(2) TRACHYCEPHALINIA 1969.1a.f002-02 • iT

EF: HYLIDAE 1815.ra.f002-|1825.gb.f001|

TRACHYSTOMATA Stannius, 1856.sa.f002 • AN

SI: $127 \cdot \mathbf{C I}: \mathrm{n} 043 \cdot \mathbf{S T}: 2.25 .50$

RL: INR

PA: $00 \cdot$ TRACHYSTOMATA $・$ Stannius 1856.sa: 4 • F

OS: Siren $1766 \cdot \mathbf{O M}$

EN: SIRENIDAE 1825.gb.f005-00 • F

EF: SIRENIDAE 1825.gb.f005
TREGOBATRACHIDAE Holman, 1975.hb.f001 † KY

SI: $295 \bullet \mathbf{C I}:$ h208・ST: 0.10 .30

RL: INR

PA: 00 - TREGOBATRACHIDAE • Holman 1975.hb: 50, 54 • F

OS: Tregobatrachus $1975+\bullet \mathbf{O E}$

EN: TREGOBATRACHIDAE 1975.hb.f001-00†・ F

EF: TREGOBATRACHIDAE 1975.hb.f001 †

TREMATODERA Lichtenstein ${ }^{+2}$, 1856.la.f002 • AN

SI: $124 \cdot \mathbf{C I}: \mathrm{n} 040 \bullet \mathbf{S T}: 2.25 .50$

RL: INR

PA: $00 \cdot$ TREMATODERA $^{-}$Lichtenstein $^{+2}$ 1856.la: $45 \cdot \mathbf{F}$

OS: » 2 PN, including: Amphiuma $1821 \cdot \mathbf{P D}$

EN: (1) AMPHIUMOIDEA 1825.gb.f007-10 • pF

"')"।

(4) AMPHIUMIDAE 1825.gb.f007-00 • F

EF: AMPHIUMIDAE 1825.gb.f007

TRIADOBATRACHIDAE Kuhn, 1962.ka.f001 † KY

SI: $267 \bullet \mathbf{C I}: \mathrm{h} 187 \bullet \mathbf{S T}: 0.10 .30$

RL: INR

PA: 00 • TRIADOBATRACHIDAE • Kuhn 1962.ka: $328 \cdot \mathbf{F}$

01 - TRIADOBATRADIDAE $\bullet \mathrm{Rage}^{+1}$ 1989.ra: $4 \cdot \mathbf{F}$

02 - TRIADOBATRACHOIDIA • Dubois 2005.da: $18 \cdot \mathbf{e F}$

03 - TRIADOBATRACHOIDEA • Dubois 2005.da: $18 \cdot \mathbf{p F}$

OS: Triadobatrachus $1962 \uparrow \bullet \mathbf{O E}$

EN: TRIADOBATRACHIDAE 1962.ka.f001-00† • F

EF: TRIADOBATRACHIDAE 1962.ka.f001 †

TRIASSURIDAE Ivachnenko, 1978.ia.f002 † KY

SI: $303 \cdot \mathbf{C I}: \mathrm{h} 216 \bullet \mathbf{S T}: 0.10 .30$

RL: INR

PA: $00 \bullet$ TRIASSURIDAE $・$ Ivachnenko 1978.ia: $87 \cdot \mathbf{F}$

OS: Triassurus $1978+\bullet \mathbf{O E}$

EN: TRIASSURIDAE 1978.ia.f002-00 $\bullet \cdot \mathbf{F}$

EF: TRIASSURIDAE 1978.ia.f002 †

TrIPRIONINAE Miranda-Ribeiro, 1926.ma.f005 • KY

SI: $212 \bullet \mathbf{C I}:$ h1 $148 \cdot \mathbf{S T}: 0.10 .30$

RL: INR

PA: 00 • TRIPRIONINAE • Miranda-Ribeiro 1926.ma: 64 • F

$01 \cdot$ TRIPRIONITES $\bullet$ Hoc loco $\bullet \mathbf{C n}$

$02 \cdot$ TRIPRIONITIES $\bullet$ HOC loco $\bullet \mathbf{b C n}$

$03 \cdot$ TRIPRIONITOIES $\bullet$ HOC lOCO $\bullet \mathbf{i C n}$

OS: Triprion $1866 \cdot \mathbf{O E}$

EN: (1) TRIPRIONITES 1926.ma.f005-01 • Cn

(2) TRIPRIONITIES 1926.ma.f005-02 • bCn

(3) TRIPRIONITOES 1926.ma.f005-03・oCn

EF: HYLIDAE 1815.ra.f002-|1825.gb.f001|

TRITONIA Rafinesque, 1815.ra.f005 • AN

SI: $010 \bullet \mathbf{C I}:$ n006 • ST: 2.26 .50

RL: INR

PA: $00 \cdot$ TRITONIA - Rafinesque 1815.ra: $78 \cdot \mathbf{F}$

01 - TRITONIDAE • Boie 1828.ba: $363 \cdot \mathbf{F}$

OS: » 5 PN, including: Triturus $1815 \equiv$ Triton 1768 JH • PD

EN: (1) MoLGINI 1850.ga.f001-04 • T

"'I")"

(5) MolGITES 1850.ga.f001-09 • Cn

EF: SALAMANDRIDAE 1820.ga.f002 
TRITONES Tschudi, 1838.ta.f003 • JG

SI: $053 \bullet$ CI: h027 • ST: 0.10 .53

RL: INR

PA: $00 \cdot$ TRITONES - Tschudi 1838.ta: $26 \cdot \mathbf{F}$

01 - TRITONES • Bronn 1849.ba: $683 \cdot \mathbf{U F}$

02 - TRITONINA $\bullet$ Bonaparte 1850.bb: pl. • bF

03 - TRITONIDAE $\bullet$ Hallowell 1856.ha: $10 \cdot \mathbf{b F}$

04 TRITONINAE $\bullet$ Cope 1863.ca: $343 \cdot \mathbf{b F}$

05 TRITONES • Betta 1864.ba: $513 \cdot \mathbf{b F}$

06 ${ }^{\text {TRITONIDAE }}$ Claus 1868.cb: $587 \cdot \mathbf{F}$

$07 \cdot$ TRITONINA $\bullet$ Fatio 1872 .fa: $486 \bullet \mathbf{T}$

08 $\bullet$ TRITONIDI $\bullet$ Acloque 1900.aa: $494 \cdot \mathbf{F}$

OS: Triton $1768 \mathbf{J H} \equiv$ Triturus $1815 \cdot \mathbf{O E}$

EN: (1) MoLGINI 1850.ga.f001-04 • T

"')"'

(5) MoLGites 1850.ga.f001-09 • Cn

EF: SALAMANDRIDAE 1820.ga.f002

TRITONIDES Tschudi, 1838.ta.f004 • AN

SI: $054 \cdot \mathbf{C I}: \mathrm{n} 025 \cdot \mathbf{S T}: 2.25 .50$

RL: INR

PA: 00 • TRITONIDES $\bullet$ Tschudi 1838.ta: $26 \bullet \mathbf{F}$

$01 \cdot$ TRITONIDES $\bullet$ Bronn 1849.ba: $683 \cdot \mathbf{U F}$

OS: » 3 PN, including: Menopoma 1825 $\approx$ Cryptobranchus 1821 - PD

EN: CRYPTOBRANCHIDAE 1826.fb.f003-04 • F

EF: CRYPTOBRANCHIDAE 1826.fb.f003

TRITURINAE Brame, 1958.ba.f003 • AN

SI: $259 \bullet$ CI: n074 • ST: 0.28 .50

RL: INR

PA: 00 • TRITURINAE $\bullet$ Brame 1958.ba: $4 \cdot \mathbf{b F}$

OS: Triturus $1815 \cdot \mathbf{O E}$

EN: (1) MoLGINI 1850.ga.f001-04 • T

"n!n!

(5) MoLGITES 1850.ga.f001-09 • Cn

EF: SALAMANDRIDAE 1820.ga.f002

TRITURINAE Kuhn, 1965.ka.f001 • JD

SI: 273 • CI: h191 • ST: 0.10 .40

RL: INR

PA: 00 • TRITURINAE • Kuhn 1965.ka: 37 • F

OS: Triturus $1815 \cdot \mathbf{O E}$

EN: (1) MOLGINI 1850.ga.f001-04 • T "'m!'

(5) MoLGITES 1850.ga.f001-09 • Cn

EF: SALAMANDRIDAE 1820.ga.f002

TSINGYMANTINI nov., DOP.da.f116 • KY

SI: $557 \cdot$ CI: h450 • ST: 0.10 .30

RL: INR

PA: $00 \cdot$ TSINGYMANTINI $\bullet$ Hoc loco $\bullet \mathbf{T}$

OS: Tsingymantis $2006 \cdot \mathbf{P D}$

EN: TSINGYMANTINI DOP.da.f116-00 • T

EF: RHACOPHORIDAE |1858.gc.f012|-1932.ha.f001

TyLOtotritonina nov., DOP.da.f146 • KY

SI: $587 \bullet$ CI: h480 • ST: 0.10 .30

RL: INR

PA: $00 \cdot$ TYLOTOTRITONINA $\bullet$ Hoc loco $\bullet \mathbf{h T}$

$01 \cdot$ TYLOTOTRITONINIA $\bullet$ Hoc loco $\bullet$ iT
OS: Tylototriton $1871 \cdot \mathbf{P D}$

EN: (1) TYLOTOTRITONINA DOP.da.f146-00 • hT

(2) TYLototritoniNIA DOP.da.f146-01 • iT

EF: SALAMANDRIDAE 1820.ga.f002

TYPHLOMOLGIDAE Stejneger ${ }^{+1}$, 1917.sa.f001 • JD

SI: 200 • CI: h137 • ST: 0.10 .40

RL: INR

PA: $00 \cdot$ TYPHLOMOLGIDAE $\bullet$ Stejneger ${ }^{+1}$ 1917.sa: $6 \cdot \mathbf{F}$

OS: Typhlomolge $1896 \approx$ Eurycea $1822 \cdot \mathbf{O E}$

EN: (1) SPELERPINI 1859.cb.f001-06・T

(2) SPELERPINA 1859.cb.f001-07 • bT

EF: PLETHODONTIDAE 1850.ga.f001

TYPHLONECTIDAE Taylor, 1968.ta.f002 • KY

SI: $278 \bullet$ CI: h195 $\mathbf{S T}: 0.10 .30$

RL: INR

PA: 00 • TYPHLONECTIDAE • Taylor 1968.ta: xi, 231 • F

$01 \cdot$ TYPHLONECTOIDES $\cdot$ Lescure $^{+2}$ 1986.lb: $169 \cdot \mathbf{h F}$

02 TYPHLONECTOIDEA $・$ Lescure $^{+2}$ 1986.1b: $169 \cdot \mathbf{p F}$

03 - TYPHLONECTOIDAE $・$ Lescure $^{+2}$ 1986.1b: $170 \cdot \mathbf{e F}$

$04 \cdot$ TYPHLONECTINAE $\cdot$ Lescure $^{+2}$ 1986.lb: $170 \cdot \mathbf{b F}$

05 TYPHLONECTILAE $\cdot$ Lescure $^{+2}$ 1986.lb: $170 \bullet \mathbf{i F}$

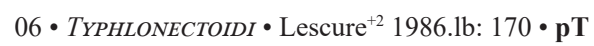

$07 \cdot$ TYPHLONECTINI $\bullet$ Lescure $^{+2}$ 1986.lb: $171 \cdot$ T

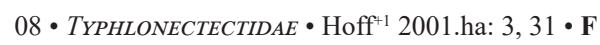

09 $\cdot$ TYPHLONECTINA $\bullet$ Hoc loco $\bullet \mathbf{b T}$

OS: Typhlonectes $1880 \cdot$ OD

EN: TYPHLONECTINA 1968.ta.f002-09 • bT

EF: CAECILIIDAE 1814.ra.f003-|1825.gb.f008|

UPEROLIIDAE Günther, 1858.gc.f007 • KY

SI: $135 \bullet$ CI: h089 - ST: 0.10 .30

RL: INR

PA: 00 • UPEROLIIDAE • Günther 1858.gc: $346 \bullet$ F

01 - UPEROLEIIDAE $\bullet$ Krefft 1865.ka: $17 \cdot \mathbf{F}$

02 - UPEROLEIIDAE $\bullet$ Keferstein 1867.ka: $349 \cdot \mathbf{F}$

03 - UPEROLIINA • Mivart 1869.ma: $291 \cdot \mathbf{b F}$

$04 \cdot$ UPEROLIIDAE $\bullet$ Hoffmann 1878.ha: $613 \cdot \mathbf{b F}$

$05 \cdot$ UPEROLEIINIA $\bullet$ Hoc loco $\bullet$ iT

OS: Uperoleia $1841 \cdot \mathbf{O E}$

EN: UPEROLEIINIA 1858.gc.f007-04 • iT

EF: MYOBATRACHIDAE 1850.sa.f001

URAEOTYPHLINAE Nussbaum, 1979.na.f001 • KY

SI: $304 \cdot$ CI: h217 • ST: 0.10 .30

RL: INR

PA: $00 \cdot$ URAEOTYPHLINAE $\bullet$ Nussbaum 1979.na: $14 \cdot \mathbf{b F}$

01 URAEOTYPHLIDAE $\cdot$ Lescure $^{+2}$ 1986.lb: $145 \cdot \mathbf{F}$

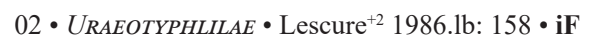

03 $-U_{\text {RAEOTYPHLIDINAE }} \cdot$ Wollenberg $^{+1}$ 2009.wb: $1050 \bullet \mathbf{b F}$

OS: Uraeotyphlus $1880 \cdot \mathbf{O E}$

EN: URAEOTYPHLIDAE 1979.na.f001-01 • F

EF: URAEOTYPHLIDAE 1979.na.f001

URODELA Latreille, 1825.la.f003 • AN

SI: $025 \bullet$ CI: n012 • ST: 2.25 .50

RL: INR

PA: $00 \cdot U_{R O D E L A} \cdot$ Latreille 1825.la: $105 \cdot \mathbf{F}$

$01 \cdot$ URODELI $\bullet$ Eichwald 1831.eb: $164 \cdot \mathbf{F}$

OS: » 3 PN, including: Salamandra $1768 \approx$ Salamandra $1764 \cdot$ PD 
EN: (1) SALAMANDROIDEA 1820.ga.f002-21・pF

"')"!"

(4) SALAMANDRINI 1820.ga.f002-28 • T

EF: SALAMANDRIDAE 1820.ga.f002

VAMPYRINOA nOV., DOP.da.f127 • KY

SI: $568 \bullet \mathbf{C I}:$ h461 • ST: 0.10 .30

RL: INR

PA: $00 \cdot$ VAMPYRIINOA $\bullet$ Hoc loco $\bullet \mathbf{h T}$

OS: Vampyrius nov. • PD

EN: VAMPYRIINOA DOP.da.f127-00 • hT

EF: RHACOPHORIDAE |1858.gc.f012|-1932.ha.f001

VANDIJKOPHRYNITOES nOv., DOP.da.f031 • KY

SI: $472 \bullet$ CI: h365 • ST: 0.10 .30

RL: INR

PA: 00 • VANDIJKOPHRYNITOES • HOC loco • iCn

OS: Vandijkophrynus 2006 • PD

EN: VANDIJKOPHRYNITOES DOP.da.f031-00 • iCn

EF: BUFONIDAE 1825.gb.f004

VIERAELLIDAE Reig, 1961.ra.f001 † • AN

SI: $265 \cdot \mathbf{C I}: \mathrm{n} 076 \bullet \mathbf{S T}: 0.29 .50$

RL: INR

PA: 00 • VIERAELLIDAE • Reig 1961.ra: 77• F

OS: Vieraella $1961+\bullet \mathbf{O E}$

EN: ANURA Familia INCERTAE SEDIS

EF: ANURA Familia INCERTAE SEDIS

VITREORANINA nov., DOP.da.f045 • KY

SI: $486 \bullet$ CI: h379・ST: 0.10 .30

RL: INR

PA: 00 - VITREORANINA $\cdot$ Hoc loco $\bullet \mathbf{b T}$

OS: Vitreorana $2009 \cdot \mathbf{P D}$

EN: VITREORANINA DOP.da.f045-00 • bT

EF: CENTROLENIDAE 1951.ta.f001

VOIGTIELLINAE Brame, 1958.ba.f002 † A AN

SI: $258 \bullet \mathbf{C I}: \mathrm{n} 073 \bullet \mathbf{S T}: 0.28 .50$

RL: INR

PA: 00 • VOIGTIELLINAE • Brame 1958.ba: $4 \cdot \mathbf{b F}$

OS: Voigtiella $1949+\approx$ Salamandra $1764 \cdot$ OE
EN: (1) SALAMANDROIDEA 1820.ga.f002-21 • pF

("I)"

(4) SALAMANDRINI 1820.ga.f002-28 • T

EF: SALAMANDRIDAE 1820.ga.f002

XENOPHRYINI Delorme ${ }^{+3}$, 2006.da.f002 • KY

SI: $366 \bullet \mathbf{C I}:$ h272 • ST: 0.10 .30

RL: INR

PA: $00 \cdot$ XENOPHRYINI $・$ Delorme $^{+3}$ 2006.da: $7 \cdot$ T

$01 \cdot$ XENOPHRYINA $\bullet$ Hoc loco $\bullet \mathbf{b T}$

OS: Xenophrys $1864 \cdot$ OD

EN: (1) XENOPHRYINI 2006.da.f002-00 • T

(2) XENOPHRYINA 2006.da.f002-01 • b T

EF: MEGOPHRYIDAE 1850.bb.f008-|1931.na.f003|

XENOPODA Fitzinger, 1843.fa.f012 • JD

SI: $077 \bullet \mathbf{C I}:$ h045 • ST: 0.10 .40

RL: INR

PA: $00 \cdot X_{E N O P O D A} \cdot$ Fitzinger 1843.fa: $33 \cdot \mathbf{F}$

$01 \cdot X_{E N O P O D E S} \cdot$ Fitzinger 1861.fa: $416 \cdot \mathrm{UF}$

02 XENOPIDAE $^{*}$ Cope 1889.ca: $253 \cdot \mathbf{F}$

$03 \cdot$ XENOPODIDAE $\bullet$ Abel 1919.aa: xii, 322; Bolkay 1919.ba: $277 \cdot \mathbf{F}$

$04 \cdot X_{E N O P O D I N A E} \cdot$ Metcalf 1923.ma: $3 \cdot \mathbf{b F}$

$05 \cdot$ XENOPINAE $\bullet$ Noble 1931.na: $489 \cdot \mathbf{b F}$

OS: Xenopus $1827 \cdot \mathbf{O E}$

EN: (1) DACTYLETHRINAE 1838.ha.f001-04 • bF

(2) DACTYLETHRINI 1838.ha.f001-05 • T

EF: PIPIDAE 1825.gb.f003-|1826.fb.f002|

XENORHINIDAE Mivart, 1869.ma.f001 • JD

SI: $161 \bullet \mathbf{C I}:$ h109・ST: 0.10 .40

RL: INR

PA: $00 \bullet$ XENORHINIDAE $\bullet$ Mivart 1869.ma: $286 \bullet \mathbf{F}$

01 - XENORHININI • Burton 1986.bb: $444 \cdot \mathbf{T}$

OS: Xenorhina $1863 \approx$ Asterophrys $1838 \cdot \mathbf{O E}$

EN: (1) ASTEROPHRYINAE 1858.gc.f006-05 • bF

(2) ASTEROPHRYINI 1858.gc.f006-09 • T

EF: MICROHYLIDAE |1843.fa.f012|-1931.na.f001 


\section{APPENDIX A7.NCS. Class-series nomina and taxa of LisSAMPHIBIA.}

The table provides all CS nomina of LissamphiBIa published from 1758 to 31 October 2020, and some of their nonlissamphibian senior homonyms (in all cases where there exist several such homonyms, only that which was first published is mentioned in this table, as it is enough to make all its junior homonyms invalid under DONS Criteria). All nomina are listed by alphabetical order of their eugraph as defined by DONS Criteria. Then in the second line their serial and category identifier and the status of the nomen are indicated. For each of the nomina the protograph and the paronyms are given. In the following lines, if relevant, its relationships (such as neonymy or homonymy) with other nomina, its getendonyms, getexonyms and its eunym with status and rank are listed. Technical terms employed here are defined in Appendix A1.GLO.

\section{EUG • Eugraph of protonym of CS nomen.}

SI, Serial identifier of CS nomen $(n=443)$; CI, Category identifier of CS nomen; ST, Status of CS nomen (A.U.T.V.C.): allocation, usage, availability validity \& correctness of nomen.

c001, c002, etc. - Numbers of class-series hoplonyms designating recent amphibians taxa (LissaMPHIBIA) and two of their angiotaxa

(Amphibia, Vertebrata) $(n=404)$, including valid ones $(n=37)$ and invalid ones $(n=367)$.

cn01, cn02, etc. - Numbers of class-series anoplonyms designating recent amphibian taxa (LisSAMPHIBIA) and their getangiotaxon

(AMPhiBIA) $(n=7)$

mc01, mc02, etc. $\bullet$ Numbers of class-series hoplonyms designating taxa including both recent amphibian taxa (LISSAMPHIBIA) and taxa not

belonging in them $(n=9)$.

zh01, zh02, etc. $\cdot$ Numbers of class-series hoplonyms designating taxa not including lissamphibians $(n=22)$.

zn01 $\bullet$ Number of class-series anoplonym designating taxon not including lissamphibians $(n=1)$.

ST • Status of CS nomen (A.U.T.V.C.): A, allocation; U, usage; T, availability; V, validity; C, correctness of nomen.

A $\bullet$ Criterion of assignment to the class-series (see T.ASN):

$\mathbf{1} \cdot$ Explicit class-series allocation [CS1].

2 Implicit class-series allocation through consistent arhizonymy, pseudorhizonymy or quasirhizonymy [CS2].

3 Implicit class-series allocation through rank superordination or parordination to a rank of the class-series [CS3].

4 Implicit class-series allocation through rank superordination to the rank family before 1858 [CS4].

5 Implicit class-series allocation through neonymy or allelonymy for a class-series nomen [CS5].

$\mathbf{U} \cdot$ Category of nomen regarding usage:

D • Distagmonym.

$\mathbf{N} \cdot$ Nothosozonym.

S • Sozodiaphonym.

U Unknown and irrelevant here (non-lissamphibian nomen, mentioned here only for purposes of homonymy).

$\mathbf{T} \cdot$ Category of nomen regarding system of taxonomic allocation in the ergotaxonomy adopted:

Sozonymorphs:

E $\cdot$ Nesonym being a sozonymorph epomallelonym of a distagmonym, taxonomically allocated through its metronym only.

G • Gephyronym being a sozonymorph, taxonomically unallocated because of presence of intragenera in the metrotaxon.

$\mathbf{O} \cdot$ Choronym being a sozonymorph, taxonomically allocated through both its metronym and its oronym.

$\mathbf{R} \cdot$ Nesonym being a sozonymorph ellitonym (missing an oronym), taxonomically allocated through its metronym only.

Distagmonyms:

A $\cdot$ Choronym being a distagmonym, allelonym or neonym of a sozonymorph, taxonomically allocated through both its metronym and its oronym

M Nesonym being a distagmonym, taxonomically allocated through its metronym only.

Others:

U Unknown and irrelevant here (non-lissamphibian nomen, mentioned here only for purposes of homonymy).

$\mathbf{V} \cdot$ Category of nomen regarding availability, taxonomic allocation and validity in CLAD:

Anoplonym, anaptonym or hypnokyronym:

00 - Gymnonym: anoplonym (unavailable nomen) under the Code, for missing an indication, description, definition or diagnosis in words of the taxon for which the new nomen is proposed.

02 - Hoplonym (available nomen) and sozonymorph but anaptonym (taxonomically unallocated nomen) under DONS Rules because of presence of intragenera in taxon $\mathbf{T}$ designated by $\mathbf{N}$ in the frame of CLAD.

03 - Hoplonym (available nomen) and aptonym (taxonomically allocated nomen) under DONS Rules but hypnokyronym (invalid nomen in $C L A D$ ) because of absence of taxon $\mathbf{T}$ designated by $\mathbf{N}$ in the frame of CLAD.

04 - Anoplonym (agnostonym), for missing after 1999 the express mention that the nomen is introduced as a new scientific name (Article 16.1). 
Kyronym (nomen available and valid):

10 - Valid nomen through sozodiaphonymy.

11 V Valid nomen among distagmonyms through publication priority over junior homonyms and/or synonyms.

12 - Valid nomen among distagmonyms through airesy (first-reviser action) over synchronous homonyms and/or synonyms, and if relevant through publication priority over other junior homonyms and/or synonyms.

Akyronym (nomen available but non valid) for being an invalid homonym:

20 - Invalid nomen for being a (senior or junior) homonym of a sozodiaphonym.

21 - Invalid junior homonym through publication priority among distagmonyms.

Akyronym (nomen available but non valid) for being an invalid synonym:

30 - Invalid nomen for being a (senior or junior) synonym of a sozodiaphonym.

31 - Invalid junior synonym through publication priority among distagmonyms.

32 - Invalid junior synonym through airesy (first-reviser action) among distagmonyms.

Akyronym (nomen available but non valid) for being both an invalid homonym and an invalid synonym:

40 - Invalid nomen for being both a (senior or junior) homonym or synonym of a sozodiaphonym and a (senior or junior) homonym or synonym of another nomen.

41 Invalid junior homonym and synonym through publication priority among distagmonyms.

42 - Invalid junior homonym and synonym through publication priority and/or airesy (first-reviser action) and/or proedry (rank precedence) among distagmonyms.

99 Hoplonym, nomenclatural status regarding validity not explored here, being irrelevant for this study.

C • Category of nomen regarding correctness of spelling (see T.RHI and T. LEG):

A - Auxorhizonym: correct under DONS Criteria with one of the standard endings -IFORMIA or - OMORPHA.

C • Cenorhizonym: correct under DONS Criteria with the standard ending-ACEI.

E - Arhizonym with incorrect original ending or spelling under DONS Criteria, corrected (apograph) with an ending or spelling following the usage of other nomina having the same ending (legethograph) or spelling (eunomograph).

K • Khoristarhizonym: correct under DONS Criteria with one of the standard endings -IFORMIES or - OMORPHIES.

$\mathbf{O} \cdot$ Arhizonym with correct original spelling (protograph) under DONS Criteria.

$\mathbf{R} \cdot$ Rhizonym: nomen correct under DONS Criteria, if valid, with the standard ending in -ACEA.

$\mathbf{X} \cdot$ Xenorhizonym: correct under DONS Criteria with one of the standard endings -IFORMI or -OMORPHI.

\section{PN • Protonym of CS nomen $\mathrm{N}$ of taxon $\mathrm{T}$ with its auctor and date}

Note: The auctorship 'DOP.da' designates nomina established as new in the present work.

AK - Hoplonym but akyronym (invalid nomen) in CLAD.

AN - CS anoplonym (unavailable nomen) of lissamphibian taxon for failing to comply with the criteria of availability of publications of the Code or of the DONS criteria of availability of CS nomina.

AP - Anaptonym (nomenclaturally available but taxonomically unallocated lissamphibian nomen).

HK - Hypnokyronym: akyronym in this work, but potentially valid class-series nomen in CLAD following the potential resolution of a polytomy.

$\mathbf{K Y} \cdot$ Kyronym: valid class-series nomen of an ergotaxon in CLAD.

ZA - Available (hoplonym) CS nomen established for a taxon including both lissamphibian and non-lissamphibian species/taxa and being homonym of a lissamphibian CS nomen.

zz - Available (hoplonym) CS nomen established for a taxon including only non-lissamphibian species/taxa and being homonym of a lissamphibian CS nomen.

\section{PA • Paronyms of CS nomen $\mathbf{N} \bullet$ Scriptor, reference \& page $\bullet$ Rank}

For each nomen, paronyms are given in chronological order of their publication, followed by their original rank.

They are given followed by their original rank (for the meaning of abbreviations of ranks, see Table A.RNK) or of one of the following abbreviations for emended spellings proposed here to comply with DONS Criteria:

EA - Aponym with standard ending (in -IFORMIA or-OMORPHA) introduced for an auxorhizonym.

EC • Aponym with standard ending (in - ACEI) introduced for a cenorhizonym in order to avoid confusion with FS nomina with standard FS endings (in $-I D A E,-I N A E,-I N A,-I N I$ and $-O I D E A$ ) .

$\mathbf{E E} \cdot$ Aponym with modified ending or spelling under DONS Criteria, corrected here in order to be consistent with usage in other CS arhizonyms based on the same etymology and using the same ending (see Table T.ENZ) or spelling, introduced for sake of homogeneity.

EK • Aponym with standard ending (in -IFORMIES or -OMORPHIES) introduced here for a khoristarhizonym.

EQ • Aponym with standard ending (in -IFORMES or -OMORPHES) introduced for a quasirhizonym.

ER - Aponym with standard ending (in - ACEA) introduced for a rhizonym in order to avoid confusion with FS nomina with standard FS endings (in $-I D A E,-I N A E,-I N A,-I N I$ and $-O I D E A$ ).

EX • Aponym with standard ending (in-IFORMI or-OMORPHI) introduced for a xenorhizonym.

Information is also given in this column, when appropriate, for the resolution of conflicts of zygoidy among symprotographs 
EEA • Explicit external airesy.

IIA - Implicit internal airesy.

Identifiers of nomina and paronyms

1758.la., 1801.sa., etc. • Identifier of publication (see 6. References).

.c01, .c02, etc. $\cdot$ Identifier of CS nomen in publication.

$-00 \cdot$ Protonym of nomen.

$-01,-02$, etc. $\cdot$ Aponyms of nomen (by order of publication).

-c0. - Lectoprotograph of nomen.

-i1, -i2, etc. - Leipoprotographs of nomen (by order of appearance in publication).

RL • Relationships of neonymy, allelonymy, homonymy and precedence (other than publication priority) of nomen $\mathbf{N}$ with other nomina, indicated if relevant.

$\leftrightarrow$ Archeoallelonym of

$\leftrightarrow \mid$ Neoallelonym of

$\leftrightarrow$ Parallelonym of

i Agoallelonym of

$\leftrightarrow$ ! Epomallelonym of

$\downarrow$ Junior homonym of (only earliest one is cited in case of multiple senior homonyms)

$\leftarrow$ Neonym of

$>$ Given precedence over synchronous synonym or homonym $\bullet$ Reference

$<$ Given subservience under synchronous synonym or homonym $\bullet$ Reference

AI - Precedence established through airesy (first-reviser action).

PR • Precedence established through proedry (rank precedence).

GN • Nomen/nomina of CS getendonyms of taxon T (including all its conucleogenera).

$\mathbf{G Z} \cdot$ If the CS nomen $\mathbf{N}$ is a sozomorph in CLAD, one of the two following possibilities:

GX - The nomen/nomina of the CS getexonyms (based on their getextragenera).

GI - Intranyms (based on their intragenera) of taxon $\mathbf{T}$ (including all its conucleogenera).

EN • Eunym of CS taxon used in $\boldsymbol{C L A D}$ if it exists under DONS Rules, or mention that the nomen $\mathbf{N}$ is an anaptonym.

ANAPTONYM • Anaptonym in CLAD.

HYP. • Hypnokyronym of taxon in CLAD.

KYR. $\cdot$ Kyronym of taxon in CLAD.

TEO. $・$ Teokyronym of taxon in CLAD.

Various abbreviations and conventions found in several columns:

DOP. • Part of the identifier of a nomen established as new in the present work ('Dubois, Ohler \& Pyron').

HL $\cdot$ Hoc loco (present designation or airesy).

INR - Information not relevant here (item does not exist).

OA - Original aphory (no included taxon mentioned in original work).

SD - Subsequent designation, followed by its reference.

NL - Nomen designating a taxon containing at least one non-recent lissamphibian species/taxon: detailed information on this nomen was not sought, not being necessary for the present work.

$\uparrow \cdot$ Nomen designating an all-fossil taxon. 
AblePhara Miranda Ribeiro, 1937

SI: 359 • CI: c329 • ST: 2.D.M.31.O

PN: Ablephara Miranda Ribeiro, 1937.ma.c02 • aK

PA: $00 \cdot$ ABLEPHARA $・$ Miranda Ribeiro 1937.ma: $56 \cdot$ UC

RL: INR

GN: DoRSIPARES 1816.ba.c06

GZ: INR

EN: KYR. C.07.01. Hypoordo DorsIPARES Blainville, 1816. ba.c06-02

ABRANCHIA Schaeffer, 1760

SI: $003 \bullet$ CI: zn01 • ST: 1.U.U.00.E

PN: Abranchiales Schaeffer, 1760.sa.c01 • AN-ZZ

PA: $00 \cdot$ AbranChiales $\bullet$ Schaeffer 1760.sa: $14 \cdot \mathbf{C}$

01 • ABRANCHIA $\bullet$ Hoc loco $\bullet$ EE

RL, GN, GZ, EN: •

ABRANCHIA Cuvier, 1816

SI: $047 \bullet$ CI: zh12 • ST: 1.U.U.99.E

PN: Abranches Cuvier, 1816.ca.c02 • zz

PA: 00 • ABRANCHES • Cuvier 1816.ca: $527 \bullet$ O

$01 \cdot$ AbranChia $\bullet$ Jourdan 1834.ja: $4 \cdot$ O

01 • AbranChiaE • Agassiz 1843.aa: $1 \cdot \mathbf{U C}$

RL, GN, GZ, EN: •

ABRANCHIA Wagler, 1830

SI: $107 \bullet$ CI: c088 • ST: 2.D.M.42.E

PN: AbRanchiales Wagler, 1830.wa.c06 • AK

PA: c0 • ABRANChiales • Wagler 1828.wa: 131 • 'T' • EEA: HL i1 • Ebranchiales • Wagler 1830.wa: 131 • 'T'

02 • ABRANCHIA • Bell 1836.ba: $91 \cdot$ O

RL: $\downarrow$ Abranches $1816 . c a . c 02$

$<$ Hedraeoglossi 1830.wa.c05 • PR

$<$ Branchiales 1830.wa.c07 • AI: HL

GN: IMPERFECTIBRANCHIA 1838.ha.c03

Pseudosauria 1816.ba.c08

GZ: INR

EN: KYR. C.04.03. Ordo Urodela Duméril, 1805.da.c02-12 [HYP. Subordo NulLibranchia Bonaparte, 1831.ba.c01-01] ABRANCHIA Hogg, 1838

SI: $129 \bullet$ CI: c109・ST: 1.D.M.41.O

PN: Abranchia Hogg, 1838.ha.c02 • AK

PA: 00 • ABRANCHIA • Hogg 1838.ha: $152 \cdot$ O

RL: $\downarrow$ AbRanChes 1816. ca.c02

GN: PSEUDOPHIONA 1816.ba.c11

GZ: INR

EN: KYR. C.05.04. Subordo Pseudophiona Blainville, 1816. ba.c11-06

ACERCI Wagler, 1828

SI: $100 \bullet$ CI: c081 • ST: 3.D.M.31.O

PN: ACERCI Wagler, 1828.wb.c07 • AK

PA: 00 • ACERCI Wagler 1828.wb: 859 • 'F'

RL: INR

GN: Phaneranura DOP.da.c02

SCOPTANURa 1973. sb.c02

GZ: INR

EN: TEO. C.09.01. Epiphalanx AQUIPARES Blainville, 1816.ba.c07-02

[HYP. Phalanx AQUIPARES Blainville, 1816.ba.c07-03]
ACHELATA Fischer, 1808

SI: $025 \bullet$ CI: c014 • ST: 2.D.A.30.O

PN: ACHElata Fischer, 1808.fa.c02 • AK

PA: 00 • ACHELATA • Fischer 1808.fa: [25] • UC

RL: $\leftarrow$ BatraCiENS 1800.ba.c01

GN: ANURA 1805.da.c01

Urodela 1805.da.c02

GZ: » GI:

GYMNOPHIONA 1814.ra.c01

EN: ANAPTONYM

[HYP. Superordo BaTRACHIA Brongniart, 1800.ba.c01-14]

ACOSMANURA Starrett, 1973

SI: $399 \bullet$ CI: c359 • ST: 1.D.M.31.O

PN: ACOSManURa Starrett, 1973.sb.c04 • AK

PA: 00 • ACosmanURA • Starrett 1973.sb: $251 \cdot$ UC

01 ACOSManURA • Savage 1973.sa: $354 \bullet$ bO

RL: INR

GN: ARCHAEOSALIENTIA 1981.ra.c01

RANOMORPHA 1921.fb.c08

GZ: INR

EN: KYR. C.07.02. Hypoordo LaEvogyrinia Lataste, 1878. la.c01-04

AEIBRANCHIA Leuckart, 1840

SI: $148 \bullet$ CI: c126・ST: 2.D.M.31.E

PN: AeIBRANChiata Leuckart, 1840.la.c01 • AK

PA: 00 • AEIBRANChiata • Leuckart 1840.1a: 19 • 'F'

01 - Aeibranchiata $・$ Leuckart 1841.la: $29 \cdot$ UC

$02 \cdot$ AeIBRANCHIA $\bullet$ Hoc loco $\bullet$ EE

RL: INR

GN: Meantes 1767.la.c01

Pseudosauria 1816.ba.c08

GZ: INR

EN: KYR. C.04.03. Ordo Urodela Duméril, 1805.da.c02-12

[HYP. Subordo PNeumobranchia Sonnini ${ }^{+1}$, 1801.sa.c01-02] Aglossa Wagler, 1830

SI: $103 \bullet$ CI: c084 • ST: 2.D.M.31.E

PN: Aglossae Wagler, 1830.wa.c02 • AK

PA: 00 • AGLOSSAE • Wagler 1830.wa: 131 • 'F'

01 • AGLOSSAE • Holbrook 1842.ha: $74 \cdot \mathbf{S c}$

02 - AGLOSSA • Gravenhorst 1845.ga: $43 \cdot \mathbf{U C}$

03 - AgLOSSA • Stannius 1856.sa: 4 • bO

$04 \cdot$ AGLOSSAE • Günther 1858.gc: $339 \cdot \mathbf{G r}$

$05 \cdot$ AGLossA • Günther 1858.gc: $339 \cdot \mathbf{G r}$

06 - AgLossa • Hoffmann 1878.ha: 582 • 'F'

07 • Aglossa • Lataste 1879.1b: 339 • 'T'

08 • AGLossa • Haeckel 1902.ha: $640 \cdot 0$

09 • Aglossa • Casamiquela 1961.ca: $81 \cdot$ bO

RL: INR

GN: DORSIPARES 1816.ba.c06

GZ: INR

EN: KYR. C.07.01. Hypoordo DorsIPARES Blainville, 1816. ba.c06-02

Aglossa Knauer, 1878

SI: 267 • CI: c241 • ST: 1.D.M.41.O

PN: Aglossa Knauer, 1878.ka.c03 • AK

PA: $00 \cdot$ AgLOSSA • Knauer 1878.ka: $103 \cdot$ bO 
RL: $\downarrow$ Aglossae 1830.wa.c02

GN: DORSIPARES 1816.ba.c06

LAEVOGYRINIA 1878.1a.c01

GZ: INR

EN: KYR. C.06.01. Infraordo GEOBATRACHIA Ritgen, 1828.ra.c18-02

Allocaudata $\mathrm{Fox}^{+1}, 1982$

SI: 408 • CI: c368 • ST: 1.D.M.11.O

PN: Allocaudata Fox ${ }^{+1}$, 1982.fa.c01

PA: 00 - Allocaudata - Fox ${ }^{+1}$ 1982.fa: 120 • O

$01 \cdot$ Allocaudata $・$ Dubois 2005.da: $6 \cdot$ pO

RL: INR

GN: Allocaudata 1982.fa.c01

GZ: INR

EN: KYR. C.04.†01. Ordo Allocaudata Fox ${ }^{+1}$, 1982.fa.c01-00 AmblyStOMATACEA Romer, 1933

SI: $356 \bullet$ CI: c403・ST: 1.D.M.00.R

PN: AMbLySTOMOIdeA Romer, 1933.ra.c02 • AN

PA: 00 • AMBLYSTOMOIDEA • Romer 1933.ra: $437 \bullet$ bO

$01 \cdot$ AMbLYSTOMATACEA $\cdot$ Hoc loco $\bullet$ ER

RL: INR

GN: PSeudosauria 1816.ba.c08

GZ: INR

EN: KYR. C.05.07. Subordo Pseudosauria Blainville, 1816.ba.c08-07

Amblystomatacea Romer, 1945

SI: 362 • CI: c404 • ST: 1.D.M.00.R

PN: AMblystomoidea Romer, 1945.ra.c01 • AN

PA: 00 • AMbLystomoideA • Romer 1945.ra: 592 • bO

01 AMblySTOMOIDAES • Pearse 1948.pa: $20 \bullet$ bO

02 - AMblySTOMATACEA $\cdot$ Hoc loco $・$ ER

RL: < SALAMANDROIDEA 1945.ra.c02 • AI: HL

$>$ ProteIda 1945.ra.c03 • AI: HL

GN: PSEUdosauria 1816.ba.c08

|URODELA INCERTAE SEDIS|

GZ: INR

EN: KYR. C.04.03. Ordo Urodela Duméril, 1805.da.c02-12 AmbYstomataceA Noble, 1931

SI: 352 • CI: c325 • ST: 1.D.M.31.R

PN: АмвYSTOMOIDEA Noble, 1931.na.c02 • AK

PA: 00 • AMвYSTOMOIDEA • Noble 1931.na: 471 • bO

01 - АмвуSTOMina • Pearse 1936.pa: $20 \bullet \mathbf{b O}$

02 - АмвYstomatoideA • Tihen 1958.ta: $1 \cdot$ bO

03 - AмbystomatoideI - Dubois 1983.da: $113 \cdot$ bO

$04 \cdot$ AMbYSTOMATACEA $\cdot$ Hoc loco $・$ ER

RL: INR

GN: PSEUdOSAURIa 1816.ba.c08

GZ: INR

EN: KYR. C.05.07. Subordo Pseudosauria Blainville, 1816.ba.c08-07

AmbystomataceA Tatarinov, 1964

SI: 388 • CI: c348 • ST: 1.D.M.40.R

PN: AMвySTOMATOIDEI Tatarinov, 1964.tb.c01 • AK

PA: 00 - AмbystomatoideI • Tatarinov 1964.tb: 9, $161 \cdot$ bO

01 - АмвYstomatoIDEA • Dowling ${ }^{+1}$ 1978.da: 4.1, 14.1 • bO

$02 \cdot$ AMbYSTOMATACEA $\cdot$ Hoc loco $\bullet$ ER
RL: $\downarrow$ AмвystomoideA 1931.na.c02

< SAlaMandroideI 1964.tb.c02 • AI: HL

GN: Pseudosauria 1816.ba.c08

|URODELA INCERTAE SEDIS|

GZ: INR

EN: KYR. C.04.03. Ordo URODELA Duméril, 1805.da.c02-12 AmbystomataceA Kuhn, 1965

SI: 391 • CI: c351 • ST: 1.D.M.41.R

PN: Aмвystomatoidea Kuhn, 1965.ka.c02 • AK

PA: 00 • AMbYSTOMATOIDEA • Kuhn 1965.ka: $35 \cdot$ bO

$01 \cdot$ AMbYSTOMATACEA $\cdot H o c$ loco $\bullet$ ER

RL: $\downarrow$ AMBystomoIdeA 1931.na.c02

> Plethodontoidea 1965.ka.c04 • AI: HL

GN: IMPERFECTIBRANCHIA 1838.ha.c03

PSEUdosauria 1816.ba.c08

GZ: INR

EN: KYR. C.04.03. Ordo URODELA Duméril, 1805.da.c02-12

[HYP. Subordo Nullibranchia Bonaparte, 1831.ba.c01-01]

AmbystomataceA Estes, 1981

SI: 404 • CI: c364 • ST: 1.D.M.40.R

PN: Амвystomatoidea Estes, 1981.ea.c03 • AK

PA: 00 • AмвYSTOMATOIDEA • Estes 1981.ea: xiv, 45 • bO

01 - AmbYSTOMATACEA • Hoc loco • ER

RL: $\downarrow$ AMBYSTOMOIDEA 1931.na.c02

$>$ KARAUROIDEA 1981.ea.c01 • AI: HL

< SAlamandroidea 1981.ea.c04 • AI: HL

GN: IMPERFECTIBRANCHIA 1838.ha.c03

Pseudosauria 1816.ba.c08

|URODELA INCERTAE SEDIS|

GZ: INR

EN: KYR. C.04.03. Ordo Urodela Duméril, 1805.da.c02-12

AмpHIBIA Linnaeus, 1758

SI: $001 \bullet \mathbf{C I}: \mathrm{mc01} \bullet \mathbf{S T}: 1 . \mathrm{N} . \mathrm{G} .02 . \mathrm{O}$

PN: АMPHiBIA Linnaeus, 1758.1a.c01 • AP-ZA

PA: 00 • AMPHibia • Linnaeus 1758.1a: $12 \cdot \mathbf{C}$

RL: INR

GN: AмPHiBIA 1816.ba.c02

|Aмniota|

|PISCES|

GZ: » GI:

|Amniota|

|Pisces|

EN: ANAPTONYM

AмpнiBIA Garsault, 1764

SI: 004 • CI: $\mathrm{mc02} \bullet \mathbf{S T}$ : 1.N.G.02.E

PN: AMPHIBIES Garsault, 1764.ga.c01 • AP-ZA

PA: $00 \cdot$ AMPHIBIES • Garsault 1764.ga: $18 \cdot$ UC

01 AMPHiBia • Batsch 1788.ba: $88 \cdot \mathbf{C}$

RL: $\downarrow$ AMPHiBia 1758.la.c01

GN: Амphibia 1816.ba.c02

|Aмniota|

GZ: » GI:

|Amniota|

EN: ANAPTONYM

AMPHIBIA Latreille, 1806

SI: $022 \bullet$ CI: $\mathrm{c} 012 \cdot$ ST: $1 . N . O .40 . O$ 
PN: АмpHibia Latreille, 1806.la.c01・AK

PA: 00 • AмPHiBIA • Latreille 1806.la: 2; Latreille 1825.1a: 103 • C

01 - AMPHibiES • Latreille 1824.la: $9 \cdot \mathbf{C}$

02 АMPHiBia • Bonaparte 1831.ba: $66 \cdot$ bC

RL: $\downarrow$ AMPHiBia 1758.la.c01

GN: » OA, SD: Latreille 1825.1a: 103:

ANURA 1805.da.c01

Urodela 1805.da.c02

GZ: » GI:

» OA, SD: Latreille 1825.la: 103:

GYMNOPHIONA 1814.ra.c01

EN: ANAPTONYM

[HYP. Superordo BATRACHIA Brongniart, 1800.ba.c01-14]

Aмphibia Blainville, 1816

SI: $034 \cdot \mathbf{C I}$ : $\mathrm{c021} \bullet \mathbf{S T}$ : 1. S.O.10.E

PN: АмтнувiEns Blainville, 1816.ba.c02

PA: 00 • AMPHYBIENS • Blainville 1816.ba: '107' [115] • C

01 - AMPhibiens • Blainville 1816.bb: $246 \cdot \mathbf{C}$

02 - AMPHibien • Blainville 1818.ba: $1368 \cdot \mathbf{C}$

03 - АмрнівіA • Macleay 1821.ma: $275 \cdot \mathbf{C}$

04 - АмPHiвia • Bonaparte 1831.ba: $66 \cdot$ bC

05 - АмРнівII • Jourdan 1834.ja: $59 \cdot \mathbf{C}$

06 • АмРніві • Desmarest 1856.da: 150 • O

07 - АмрнівеA • Pearse 1936.pa: $20 \cdot \mathbf{C}$

08 - АмрнувіA $\cdot$ Moreno $^{+1}$ 1978.mb: $93 \cdot \mathbf{C}$

09 • АмрнiвiA • Gardiner 1982.ga: 228 • D

10 • Амрніва • Borkin ${ }^{+1}$ 2013.bb: 501 • C

RL: $\downarrow$ AMPhibia 1758.1a.c01

$\leftrightarrow>$ NUDIPELLIFERES 1816.ba.c01 • AI: HL

$\leftrightarrow>$ ICTYOIDES 1816.ba.c03 • AI: HL

$\leftrightarrow>$ NUDS 1816.ba.c04 • AI: HL

GN: ANURA 1805.da.c01

GYMNOPHIONA 1814.ra.c01

Urodela 1805.da.c02

GZ: » GX:

$\mid$ AMniota $\mid$

|Pisces|

EN: KYR. C.02.01. Classis AMPHibia Blainville, 1816.ba.c02-03 AmpHiBIA Blainville, 1816

SI: 042 • CI: c029 • ST: 1.N.G.02.E

PN: AMPHIBIENS Blainville, 1816.ba.c10 • AP

PA: 00 • AMPHiBIENS • Blainville 1816.ba: '111' [119]• O

$01 \cdot$ AMPHibians $\bullet$ Kirby $1835 . \mathrm{ka}: 415 \cdot \mathbf{O}$

$02 \cdot$ AMPHibia $\cdot$ Hoc loco $\bullet \mathbf{E E}$

RL: $\downarrow$ AMPhibia 1758.1a.c01

GN: MeANTES 1767.la.c01

Pseudosauria 1816.ba.c08

GZ: » GI:

Pseudosauria 1816.ba.c08

EN: ANAPTONYM

AMPHicoela Meyer, 1860

SI: $216 \bullet \mathbf{C I}: \mathrm{c} 193 \cdot \mathbf{S T}:$ 2.D.M.31.E

PN: AMPHICOELI Meyer, 1860.mb.c04 • AK

PA: 00 - AMPHiCOELI • Meyer 1860.mb: $559 \cdot$ UC

$01 \cdot$ AMPHicOeLA $\cdot H o c l o c o \cdot \mathbf{E E}$

RL: INR
GN: IMPERFECTIBRANCHIA 1838.ha.c03

GZ: INR

EN: KYR. C.05.05. Subordo IMPERFECTIBRANCHIA Hogg, 1838.ha.c03-02

Amphicoela Owen, 1860

SI: $217 \bullet$ CI: zh19 • ST: 1.U.U.99.E

PN: AMPHicoelia Owen, 1860.oa.c01 • zz

PA: 00 - AMPHICOELIA • Owen 1860.oa: x, $271 \cdot$ bO

$01 \cdot$ AMPHicOeLA $\cdot$ Hoc loco $\bullet$ EE

RL: $\downarrow$ AMPHICOELI 1860.mb.c04

GN, GZ, EN: •

Amphicoela Noble, 1931

SI: $353 \bullet$ CI: c326・ST: 1.D.M.21.O

PN: AMPHicoela Noble, 1931.na.c03 • AK

PA: 00 - AMPHICOELA • Noble 1931.na: 485 • bO

01 - AMPHicoelina • Pearse 1936.pa: $20 \bullet$ bO

02 - АMPHicoelia • Kuhn 1939.ka: $92 \cdot$ bO

RL: $\downarrow$ AMPHICOELI $1860 . \mathrm{mb} . \mathrm{c0} 4$

GN: ANGUSTICOELA 1958.ra.c01

GZ: INR

EN: KYR. C.05.01. Subordo ANGUSTICOELA Reig, 1958.ra.c01-00 AMPHicoELA Romer, 1933

SI: $357 \bullet \mathbf{C I}: \mathrm{mc06} \bullet \mathbf{S T}: 1 . \mathrm{D} . \mathrm{M} .00 . \mathrm{O}$

PN: AMPHicoela Romer, 1933.ra.c03 • AN

PA: 00 • AMPHicoela • Romer 1933.ra: $437 \cdot$ bO

RL: $\downarrow$ AMPHICOELI 1860.mb.c04

GN: ANGUSTICOELA 1958.ra.c01

|ANURA INCERTAE SEDIS|

GZ: INR

EN: KYR. C.04.01. Ordo ANURa Duméril, 1805.da.c01-07 AmpHicoela Kuhn, 1961

SI: $379 \bullet \mathbf{C I}: \mathrm{mc09} \bullet \mathbf{S T}: 1 . D . M .99 . O$

PN: AMPHicoela Kuhn, 1961.ka.c05 • zA

PA: 00 • AMPHicoelA • Kuhn 1961.ka: 23・ bO

RL: $\downarrow$ AMPHicOELI $1860 . \mathrm{mb} . \mathrm{c0} 4$

GN: AмPHiBia 1816.ba.c02

|AMniota|

GZ: INR

EN: •

AmpHiCOELA Kuhn, 1962

SI: 380 • CI: c340 • ST: 1.D.M.40.O

PN: AMPHicoela Kuhn, 1962.ka.c01 • AK

PA: 00 • AMPHICOELA • Kuhn 1962.ka: $329 \bullet$ bO

RL: $\downarrow$ AMPHICOELI 1860.mb.c04

$<$ ArChaeobatrachia 1962.ka.c02 • AI: HL

< NeObatrachia 1962.ka.c03 • AI: HL

GN: ANGUSTICOELA 1958.ra.c01

|ANURA INCERTAE SEDIS|

GZ: INR

EN: KYR. C.04.01. Ordo ANURa Duméril, 1805.da.c01-07 AMPHIGYRINIA Blanchard, 1885

SI: 285 • CI: c259 • ST: 1.D.M.30.E

PN: AMPHIGYRINIDES Blanchard, 1885.bb.c01 • AK

PA: 00 • AMPHIGYRINIDES • Blanchard 1885.bb: 587 • UC

01 • AMPHIGYRINIDAE • Lataste 1888.1a: 240 • UC

$02 \cdot$ AMPHIGYRINIA $\bullet$ Hoc loco $\bullet$ EE 
RL: INR

GN: DORSIPARES 1816.ba.c06

GZ: INR

EN: KYR. C.07.01. Hypoordo DorsIPARES Blainville, 1816.ba.c06-02

AmphiPNEUSTA Merrem, 1820

SI: 053 • CI: c035 • ST: 2.D.M.31.O

PN: AMPHIPNEUSTA Merrem, 1820.ma.c04• AK

PA: c0 • AMPHIPNEUSTA • Merrem 1820.ma: 163 • 'T' • IIA: Merrem 1822.ma: 695

i1 • AMPHYPNEUSTA • Merrem 1820.ma: 166 • 'T'

02 • AMPHIPNEUSTA $・$ Bonaparte 1831.ba: $67 \cdot$ O

03 • AMPHIPNEUSTA • Bonaparte 1831.bb: 135; Gray 1831.ga: $107 \cdot$ Sc

$04 \cdot$ AMPHIPNEURTA $\cdot$ Swainson 1839.sa: 86, $95 \cdot \mathbf{O}$

05 • AMPHIPNEURA • Swainson 1839.sa: $339 \bullet$ O

RL:INR

GN: Meantes 1767.la.c01

PSEUdosauria 1816.ba.c08

GZ:INR

EN:KYR. C.04.03. Ordo Urodela Duméril, 1805.da.c02-12 [HYP. Subordo PNeumobranchia Sonnini ${ }^{+1}$, 1801.sa.c01-02] AMPHISACRALIA Bolkay, 1919

SI: 310 • CI: c283 • ST: 1.D.M.31.O

PN: AMPHISACRaLIa Bolkay, 1919.ba.c01 • AK

PA: 00 • AMPHISACRALIA • Bolkay 1919.ba: 348 •bO

RL: INR

GN: GEOBATRACHIA 1828.ra.c18 Mediogyrinia 1878.1a.c02

GZ: INR

EN: KYR. C.05.02. Subordo Hydrobatrachia Ritgen, 1828.ra.c14-01

Amphiumacea Duméril ${ }^{+1}, 1841$

SI: $158 \bullet$ CI: c135 • ST: 4.D.M.31.R

PN: AMPhiumoides $\bullet$ Duméril $^{+1}$, 1841.da.c05 • AK

PA: 00 • AMPHIUMOIDES • Duméril ${ }^{+1} 1841$ da: $52 \cdot \mathbf{G r} / \mathbf{S c} /{ }^{\circ} \mathbf{T}$ '

01 • Amphiumoidea $・$ Cope 1888.ca: $464 \cdot \mathbf{U C}$

02 • AMPHiUMOIDEA $・$ Regal 1966.ra: $405 \cdot$ bO

03 - AMPHIUMACEA $\cdot$ Hoc loco $\bullet$ ER

RL: < ATRETODERES 1841.da.c03 • AI: HL

$\leftrightarrow>$ Perobranches 1841.da.c04 • AI: HL

$>$ EXOBRANCHES 1841.da.c06 • AI: HL

$>$ Trematoderes 1841.da.c08 • AI: HL

GN: Pseudosauria 1816.ba.c08

GZ: INR

EN: KYR. C.05.07. Subordo Pseudosauria Blainville, 1816.ba.c08-07

ANGUiformi Hogg, 1839

SI: $139 \bullet$ CI: c117 • ST: 1.D.M.41.X

PN: ANGUIFORMIA Hogg, 1839.ha.c04 • AK

PA: 00 • ANGUiformia • Hogg 1839.ha: $271 \cdot$ O

01 $\bullet$ ANGUIFORMES $\bullet$ Dubois 2015.da: $54 \cdot \mathbf{E X}$

02 • ANGUIFORMI $\bullet$ Hoc loco $\bullet$ EX

RL: $\downarrow$ ANGUIFORMIA 1811.oa.c03

< UROPHORA 1839.ha.c01 • AI: HL

$>$ TETRAPODA 1839.ha.c03 • AI: HL
GN: GYMNOPHIONA 1814.ra.c01

Urodela 1805.da.c02

GZ: INR

EN: KYR. C.03.01. Subclassis Lissamphibia Gadow, 1898.ga.c01-00

[HYP. Superordo Derotreta Van der Hoeven, 1833.va.c01-01]

ANGUIFORMI Gouriet, 1868

SI: $252 \bullet$ CI: c226・ST: 1.D.M.41.X

PN: ANGUIFORMES Gouriet, 1868.ga.c07 • AK

PA: 00 • ANGUIFORMES • Gouriet 1868.ga: $210 \bullet \mathbf{b S r}$

$01 \cdot$ AngUIfORMi $\cdot H o c l o c o \cdot \mathbf{E X}$

RL: $\downarrow$ ANGUiformia 1811.oa.c03

GN: Meantes 1767.la.c01

Pseudosauria 1816.ba.c08

GZ: INR

EN: KYR. C.04.03. Ordo Urodela Duméril, 1805.da.c02-12

[HYP. Subordo Pneumobranchia Sonnini ${ }^{+1}$, 1801.sa.c01-02]

ANGUIFORMIA Oppel, 1811

SI: 028 • CI: zh08 • ST: 1.U.U.99.A

PN: ANGUIFORMes Oppel, 1811.oa.c03 • zZ

PA: 00 • ANGUIFORMES • Oppel 1811.oa: $264 \cdot$ C

01 - ANGUIFORMia - Dubois 2015.da: $54 \cdot \mathbf{C}$

RL, GN, GZ, EN: •

ANGUINACEI Wiegmann ${ }^{+1}, 1832$

SI: 117 • CI: c097 • ST: 1.D.M.31.C

PN: ANguinea Wiegmann ${ }^{+1}$, 1832.wa.c01 • AK

PA: 00 - ANGUINEA • Wiegmann ${ }^{+1}$ 1832.wa: $199 \cdot$ O

01 - ANGUINEA • Leunis 1844.1a: 149 • 'F'

02 ANGUINACEI - Dubois 2015.da: 90 • EC

RL: INR

GN: Pseudophiona 1816.ba.c11

GZ: INR

EN: KYR. C.05.04. Subordo Pseudophiona Blainville, 1816.ba.c11-06

ANGUSTICOELA Reig, 1958

SI: $366 \bullet$ CI: c333・ST: 1.D.M.11.O

PN: AnguSTICOELA Reig, 1958.ra.c01

PA: 00 • ANGUSTICOELA • Reig 1958.ra: $111 \cdot$ bO

RL: $\leftrightarrow \mid$ AMPHICOELA 1931.na.c03

GN: ANGUSTICOELA 1958.ra.c01

GZ: INR

EN: KYR. C.05.01. Subordo ANGUSTICOELA Reig, 1958.ra.c01-00 ANISOBATRACHIA Fejérváry, 1921

SI: 316 • CI: c289 • ST: 1.D.M.30.E

PN: ANisobatrachoidea Fejérváry, 1921.fb.c04 • AK

PA: 00 - ANISOBATRACHOIDEA • Fejérváry 1921.fb: 24 • bO

$01 \cdot$ Anisobatrachia • Dubois 2015.da: $106 \cdot \mathbf{E E}$

RL: > PelobatomorPha 1921.fb.c05 • PR

> CYSTIGNATHOMORPHA 1921.fb.c07 • PR

GN: ANGUSTICOELA 1958.ra.c01

Hydrobatrachia 1828.ra.c14

GZ: INR

EN: KYR. C.04.01. Ordo ANURa Duméril, 1805.da.c01-07

ANOMOCOELA Nicholls, 1916

SI: $303 \bullet \mathbf{C I}: \mathrm{c} 276 \bullet \mathbf{S T}: 1 . D . M .31 .0$ 
PN: ANomocoela Nicholls, 1916.na.c02 • AK

PA: 00 • ANOMOCOELA • Nicholls 1916.na: 86 • 'T'

01 - Anomocoelina • Pearse 1936.pa: 20 • bO

02 • ANOMOCOELA • Tatarinov 1964.ta: $129 \cdot$ bO

RL: INR

GN: DORSIPARES 1816.ba.c06

LAEVOGYRINIA 1878.1a.c01

GZ: INR

EN: KYR. C.06.01. Infraordo GEOBATRACHIA Ritgen, 1828.ra.c18-02

AnOmocoela Noble, 1922

SI: $322 \cdot \mathbf{C I}$ : c295 • ST: 1.D.M.21.O

PN: ANOMOcoela Noble, 1922.na.c01 • AK

PA: 00 • ANOMOCOELA • Noble 1922.na: $22 \cdot$ bO

RL: $\downarrow$ ANOMOCOELA 1916.na.c02

GN: Archaeosalientia 1981.ra.c01

GZ: INR

EN: KYR. C.08.01. Superphalanx ArChaEosalientia Roček, 1981.ra.c01-01

AnOmocoela Noble, 1931

SI: $354 \cdot$ CI: c327 • ST: 1.D.M.41.O

PN: ANomocoela Noble, 1931.na.c04 • AK

PA: 00 • ANOMOCOELA • Noble 1931.na: 491 • bO

RL: $\downarrow$ ANOMOCOELA 1916.na.c02

GN: Archaeosalientia 1981.ra.c01 RANOMORPHA 1921.fb.c08

GZ: INR

EN: KYR. C.07.02. Hypoordo LAEvogYRINIA Lataste, 1878.la.c01-04

Anomocoela Tatarinov, 1964

SI: $386 \bullet$ CI: c346•ST: 1.D.M.41.O

PN: ANomocoela Tatarinov, 1964.ta.c01・aK

PA: 00 • ANOMOcoelA • Tatarinov 1964.ta: 8, 126 • bO

RL: $\downarrow$ ANOMOCOELA 1916.na.c02

< Procoela 1964.ta.c02 • AI: HL

GN: GEOBATRACHIA 1828.ra.c18

Mediogyrinia 1878.1a.c02

GZ: INR

EN: KYR. C.05.02. Subordo HYDROBATRACHIA Ritgen, 1828.ra.c14-01

ANONYXIA Miranda-Ribeiro, 1924

SI: $329 \bullet$ CI: c302 • ST: 2.D.M.30.O

PN: ANONYXIa Miranda-Ribeiro, 1924.ma.c04 • AK

PA: 00 • ANONYXIA • Miranda-Ribeiro 1924.ma: 141 • UC

RL: < GYMNOBATRACHIA 1924.ma.c02 • PR

$>$ THORACECHMIA 1924.ma.c05 • PR

< Protosternia 1924.ma.c08 • PR

$\leftrightarrow>$ THEROSTERnIA 1924.ma.c09 • AI: HL

GN: AnguSTiCOELA 1958.ra.c01

HYdrobatrachia 1828.ra.c14

GZ: INR

EN: KYR. C.04.01. Ordo ANURA Duméril, 1805.da.c01-07

ANura Duméril, 1805

SI: $017 \bullet \mathbf{C I}: \mathrm{c008} \bullet$ ST: 2. S.O.10.E

PN: ANOURes Duméril, 1805.da.c01

PA: 00 • ANOURES • Duméril 1805.da: 91 • 'F'
01 - ANUREN • Meckel in Cuvier 1810.ca: pl. 3 • UC

$02 \cdot$ ANURI $\cdot$ Fischer 1813.fa: $58 \cdot$ UC

03 - ANURIA $・$ Rafinesque 1815.ra: $78 \cdot$ bO

$04 \cdot$ ANOURA $・$ Gray 1825.ga: $213 \cdot$ O

$05 \cdot$ ANURA $^{- \text {Ficinus }^{+1}}$ 1826.fa: pl. • UC

$06 \cdot$ ANOURA • Bell 1836.ba: $91 \cdot$ O

$07 \cdot$ ANURA $・$ Hogg 1839.ha: $270 \bullet \mathbf{O}$

08 • ANOURES • Gray 1842.ga: $111 \cdot$ O

$09 \cdot$ ANOURI • Mayer 1849.ma: $198 \cdot \mathbf{b O}$

10 ANURI • Massalongo 1854.ma: 421 • UC

11 - ANOURES • Desmarest 1857.da: 2 - bO

12 • ANURA • Girard 1858.ga: vii • 'T'

13 - ANOURA • Cooper 1859.ca: 303 • 'T'

14 - ANURA • Haeckel 1889.ha: 625 • L

15 - AnURA - Abel 1919.aa: xii, $311 \cdot$ bC

16 ANURA • Milner 1988.ma: 82 • cO

$17 \cdot$ ANURAN $^{-S_{\text {Sarania }}}{ }^{+4}$ 2015.sa: $413 \cdot \mathbf{O}$

RL: $\leftrightarrow>$ ECAUDATI 1805.da.c03 • AI

GN: ANURA 1805.da.c01

GZ: » GX:

GYMNOPHIONA 1814.ra.c01

URODELA 1805.da.c02

EN: KYR. C.04.01. Ordo ANURA Duméril, 1805.da.c01-07 APHANOBRANCHIA Leuckart, 1840

SI: 149 • CI: c127 • ST: 2.D.M.30.E

PN: APHANOBRANChiata Leuckart, 1840.1a.c02 • AK

PA: 00 - APHANOBRANCHIATA • Leuckart 1840.1a: 20 • UC

01 - APHANEROBRANCHIATA - Kuhn 1967.kb: 13 • UC

02 - APHANOBRANCHIA - Dubois 2015.da: $107 \cdot \mathbf{E E}$

RL: INR

GN: ANURA 1805.da.c01

GYMNOPHIONA 1814.ra.c01

Urodela 1805.da.c02

GZ: INR

EN: KYR. C.03.01. Subclassis LisSAMPhibia Gadow, 1898.ga.c01-00

Apneuma Brookes, 1828

SI: 073 • CI: c055 • ST: 1.D.M.31.O

PN: APNEUMA Brookes, 1828.bc.c01 • AK

PA: 00 - APNEUMA • Brookes 1828.bc: $16 \cdot$ O

RL: INR

GN: Pseudosauria 1816.ba.c08

GZ: INR

EN: KYR. C.05.07. Subordo Pseudosauria Blainville, 1816.ba.c08-07

APODA Linnaeus, 1758

SI: $002 \bullet$ CI: zh01・ST: 1.U.U.99.E

PN: APODES Linnaeus, 1758.1a.c02 $\bullet \mathbf{z Z}$

PA: 00 - APODES • Linnaeus 1758.1a: 241 • O

$01 \cdot$ APODA $\cdot H o c l o c o \cdot \mathbf{E E}$

RL, GN, GZ, EN: •

Apoda Oppel, 1811

SI: $029 \bullet$ CI: c017 • ST: 2.S.O.40.O

PN: APODA Oppel, 1811.ob.c01 • AK

PA: 00 • APODA • Oppel 1811.ob: $409 \cdot$ 'F'

01 APODA • Merrem 1820.ma: 163 • O 
02 - APODA • Gravenhorst 1843.ga: $393 \cdot \mathbf{Z t}$

$03 \cdot$ APODA • Gravenhorst 1845.ga: $433 \cdot \mathbf{U C}$

$04 \cdot$ APODIDA $・$ Pearse 1936.pa: $20 \bullet \mathbf{O}$

$05 \cdot \mathbf{A P O A D} \cdot \mathrm{Fei}^{+2}$ 1990.fb: $1,5 \cdot \mathbf{O}$

RL: $\downarrow$ APODES 1758.1a.c02

GN: GYMNOPHIONA 1814.ra.c01

GZ: » GX:

ANURA 1805.da.c01

URODELA 1805.da.c02

EN: KYR. C.04.02. Ordo GyMnOPHIONA Rafinesque, 1814.ra.c01-02

Aquipares Blainville, 1816

SI: $039 \bullet$ CI: c026・ST: 1.D.M.11.O

PN: AQUIPARES Blainville, 1816.ba.c07

PA: 00 • AQUIPARES • Blainville 1816.ba: “111” [119] • bO

01 AQUIPARIA - Jourdan 1834.ja: $102 \cdot \mathbf{D}$

$02 \cdot$ AQUiPARES $\cdot H o c$ loco $\bullet \mathbf{e P}$

$03 \cdot$ AQUiPares $\cdot H o c l o c o \cdot \mathbf{P}$

RL: INR

GN: » OA, SD: Ducrotay Blainville 1822.da: 5:

Phaneranura DOP.da.c02

SCOPTANURA 1973.sb.c02

GZ: INR

EN: TEO. C.09.01. Epiphalanx AQUIPARES Blainville, 1816.ba.c07-02

[HYP. Phalanx AQUiPARES Blainville, 1816.ba.c07-03]

Aralobatrachia Bauer, 1987

SI: $420 \bullet$ CI: c380 • ST: 1.D.M.30.O

PN: Aralobatrachia Bauer, 1987.bc.c05 • AK

PA: 00 • Aralobatrachia • Bauer 1987.bc: 52 • UC

RL: INR

GN: ANURA 1805.da.c01

GZ: INR

EN: KYR. C.04.01. Ordo ANURa Duméril, 1805.da.c01-07 ARCHAEOBATRACHIA Reig, 1958

SI: 367 • CI: c334 • ST: 1.D.M.31.O

PN: Archaeobatrachia Reig, 1958.ra.c02 • AK

PA: 00 • ARCHAEObatrachia • Reig 1958.ra: 113 • bO

01 - ArCHEOBATRACHIA • Casamiquela 1961.ca: 95 • bO

RL: INR

GN: GEOBATRACHIA 1828.ra.c18 Mediogyrinia 1878.la.c02

GZ: INR

EN: KYR. C.05.02. Subordo Hydrobatrachia Ritgen, 1828.ra.c14-01

ArCHAEOBATRACHia Kuhn, 1962

SI: $381 \bullet$ CI: c341 • ST: 1.D.M.40.O

PN: Archaeobatrachia Kuhn, 1962.ka.c02 • AK

PA: 00 • ARCHAEOBATRACHIA • Kuhn 1962.ka: $334 \cdot$ bO

RL: $\downarrow$ Archaeobatrachia 1958.ra.c02

$>$ AMPHICOELA 1962.ka.c01 • AI: HL

< NeObatraChIa 1962.ka.c03 • AI: HL

GN: GEOBATRACHIA 1828.ra.c18

Mediogyrinia 1878.1a.c02

|ANURA INCERTAE SEDIS|

GZ: INR
EN: KYR. C.04.01. Ordo ANURa Duméril, 1805.da.c01-07 ARCHAEOBATRACHIA Laurent, 1967

SI: 395 • CI: c355 • ST: 1.D.M.40.O

PN: ArChaeobatrachia Laurent, 1967.la.c01 • AK

PA: 00 • ARCHAEOBATRACHIA • Laurent 1967.la: 209 • bO

RL: $\downarrow$ ARChaEobatrachia 1958.ra.c02

GN: ANGUSTICOELA 1958.ra.c01

HYdRobatraCHIA 1828.ra.c14

GZ: INR

EN: KYR. C.04.01. Ordo ANURA Duméril, 1805.da.c01-07 ArCHAEOCOELA Kuhn, 1967

SI: 394 • CI: c354 • ST: 1.D.M.31.O

PN: ArChaeocoela Kuhn, 1967.ka.c01 • AK

PA: 00 • ArCHAEOCOELA • Kuhn 1967.ka: 186 • bO

RL: $\leftrightarrow \mid$ AMPHICOELA 1931.na.c03

GN: ANGUSTICOELA 1958.ra.c01

GZ: INR

EN: KYR. C.05.01. Subordo ANguSTICOELA Reig, 1958.ra.c01-00 ArchaeOSAlientia Roček, 1981

SI: $406 \bullet$ CI: c366 • ST: 1.D.M.11.O

PN: Archaeosalientia Roček, 1981.ra.c01

PA: 00 • ARChaEOSALIENTIA • Roček 1981.ra: 1 • O

$01 \cdot$ ArchaeOSAlientia $\cdot H o c$ loco $\bullet$ pP

RL: INR

GN: ArChaeosalientia 1981.ra.c01

GZ: INR

EN: KYR. C.08.01. Superphalanx ARCHAEOSALIENTIA Roček, 1981.ra.c01-01

ARCIFERa Cope, 1864

SI: 230 • CI: c204 • ST: 1.D.M.31.E

PN: ARCIFERI Cope, 1864.cb.c02 • AK

PA: $00 \cdot$ ARCIFERI • Cope 1864.cb: $182 \cdot$ bO

01 - ARCIFERA • Cope 1865.ca: $97 \cdot$ bO

02 - ARCIFORMia • Mivart 1869.ma: $281 \cdot \mathbf{S r}$

03 ARCIFERI - Hoffmann 1878.ha: $598 \cdot$ UC

$04 \cdot$ ARCIFERA $\cdot$ Boulenger 1882.bb: vii, $183 \cdot \mathbf{S r}$

05 - ARCIFERA - Zittel 1888.za: $429 \cdot$ UC

06 ARCIFERA $\cdot$ Goodrich 1930.ga: xxi • Sc

RL: INR

GN: GEOBATRACHIA 1828.ra.c18

Mediogyrinia 1878.1a.c02

GZ: INR

EN: KYR. C.05.02. Subordo Hydrobatrachia Ritgen, 1828.ra.c14-01

ARCIFERa Cope, 1889

SI: $292 \bullet$ CI: c265・ST: 2.D.M.40.O

PN: ArCIFERA Cope, 1889.ca.c02 • AK

PA: 00 - ARCIFERA • Cope 1889.ca: 246 • 'T'/bO

01 - ARCIFERA - Abel 1919.aa: xii, $246 \cdot \mathbf{R}$

02 - ARCIFERA • Miranda-Ribeiro 1924.ma: 139 • UC

RL: $\downarrow$ ARCIFERI 1864.cb.c02

GN: ANGUSTICOELA 1958.ra.c01

Hydrobatrachia 1828.ra.c14

GZ: INR

EN: KYR. C.04.01. Ordo ANURA Duméril, 1805.da.c01-07 
ArCuCADENTIA Hogg, 1839

SI: $146 \bullet$ CI: c124 • ST: 1.D.M.30.O

PN: ArCuCAdentia Hogg, 1839.hb.c03 • AK

PA: 00 • ARCUCADENTIA • Hogg 1839.hb: 376 • 'T'

RL: INR

GN: ANURA 1805.da.c01

URODELA 1805.da.c02

GZ: INR

EN: KYR. C.03.01. Subclassis LissamPHIBIA Gadow, 1898.ga.c01-00

[HYP. Superordo BATRACHIA Brongniart, 1800.ba.c01-14] Arcumanentia Hogg, 1839

SI: 147 • CI: c125 • ST: 1.D.M.32.O

PN: Arcumanentia Hogg, 1839.hb.c04 • AK

PA: 00 • ARCuMANENTIA • Hogg 1839.hb: 376 • 'T'

RL: < INTERNIBRANCHIA 1839.hb.c01 • AI: HL

GN: IMPERFECTIBRANCHIA 1838.ha.c03

GZ: INR

EN: KYR. C.05.05. Subordo IMPERFECTIBRANCHIA Hogg, 1838.ha.c03-02

\section{ASCAPHACEA}

SI: $369 \bullet$ CI: cn01・ST: 1.D.M.00.R

PN: ASCAPHOIDEA Laurent in Fuhn, 1960.fa.c01 • AN

PA: 00 • ASCAPHOIDEA • Laurent in Fuhn 1960.fa: $163 \cdot$ bO

$01 \cdot$ ASCAPHACEA $\bullet H o c l o c o \cdot$ ER

RL: INR

GN: ANGUSTICOELA 1958.ra.c01

GZ: INR

EN: KYR. C.05.01. Subordo ANGusticoela Reig, 1958.ra.c01-00 AstatodipNOA Laurent in Fuhn, 1960

SI: $098 \bullet$ CI: c079 • ST: 3.D.M.30.O

PN: AstatodipnOa Wagler, 1828.wb.c05 • AK

PA: 00 • AstaTOdiPNOA • Wagler 1828.wb: 859 • 'T'

RL: INR

GN: ANURA 1805.da.c01

URODELA 1805.da.c02

GZ: INR

EN: KYR. C.03.01. Subclassis LISSAMPHIBIA Gadow, 1898.ga.c01-00

[HYP. Superordo BaTRACHIA Brongniart, 1800.ba.c01-14] Asterophryomorpha Fejérváry, 1923

SI: $325 \bullet$ CI: c298 • ST: 2.D.M.30.A

PN: ASTEROPHRYOMORPHA Fejérváry, 1923.fa.c01 • AK

PA: 00 • ASTEROPHRYOMORPHA • Fejérváry 1923 fa: $180 \cdot$ Gs 01 • ASTEROPHRYNOMORPHA • Kuhn 1967.ka: $14 \cdot$ UC

RL: $\leftarrow$ Pelobatomorpha 1921.fb.c05

GN: ANGUSTICOELA 1958.ra.c01

HYDROBATRACHIA 1828.ra.c14

GZ: INR

EN: KYR. C.04.01. Ordo ANURA Duméril, 1805.da.c01-07 AtARsata Meyer, 1860

SI: $215 \bullet$ CI: c192 • ST: 2.D.M.31.E

PN: ATARSIDEN Meyer, 1860.mb.c03 • AK

PA: $00 \cdot$ ATARSIDEN $・$ Meyer 1860.mb: $559 \cdot$ UC

$01 \cdot$ ATARSATa $\cdot H o c l o c o \cdot \mathbf{E E}$

RL: INR
GN: Pseudosauria 1816.ba.c08

GZ: INR

EN: KYR. C.05.07. Subordo Pseudosauria Blainville, 1816.ba.c08-07

AtARsata Meyer, 1863

SI: $227 \bullet \mathbf{C I}: \mathrm{c} 201 \bullet \mathbf{S T}$ : 2.D.M.41.E

PN: AtARSIDEN Meyer, 1863.mb.c01 • AK

PA: 00 • ATARSIDEN • Meyer 1863.mb: 296 • UC

$01 \cdot$ Atarsata $\cdot$ Hoc loco $\bullet$ EE

RL: INR

GN: IMPERFECTIBRANCHIA 1838.ha.c03 Pseudosauria 1816.ba.c08

GZ: INR

EN: KYR. C.04.03. Ordo Urodela Duméril, 1805.da.c02-12

[HYP. Subordo Nullibranchia Bonaparte, 1831.ba.c01-01] ATretodera Duméril+1, 1841

SI: $156 \bullet \mathbf{C I}: \mathrm{c} 133 \bullet \mathbf{S T}: 2 . \mathrm{D} . \mathrm{M} .31 . \mathrm{E}$

PN: ATretoderes Duméril+1, 1841.da.c03 • AK

PA: 00 • ATretoderes • Duméril ${ }^{+1}$ 1841.da: 52 • Gr/Sc/'T'

01 - ATRETODERA • Baird 1850.ba: $281 \cdot \mathbf{G r}$

02 • ATrETODERA • Baird 1851.ba: $250 \bullet$ bO

$03 \cdot$ AleTroderes • Desmarest 1856.da: $152 \cdot \mathbf{G r}$

04 - ATretodeira • Girard 1858.ga: vii • ' $T$ '

05 - ARETODERES • Cope 1859.cb: $122 \cdot \mathbf{U C}$

RL: $\leftrightarrow \mid$ SALamandres 1816.ba.c09

$>$ Perobranches 1841.da.c04 - AI: HL

$>$ AMPhiUmoIdes 1841.da.c05 - AI: HL

$>$ EXoBRANCHES 1841.da.c06 • AI: HL

$>$ Trematoderes 1841.da.c08 • AI: HL

GN: PSEUdosauria 1816.ba.c08

GZ: INR

EN: KYR. C.05.07. Subordo Pseudosauria Blainville, 1816.ba.c08-07

ATretodera Gouriet, 1868

SI: $250 \bullet \mathbf{C I}: \mathrm{c} 224 \bullet \mathbf{S T}: 2 . \mathrm{D} . M .40 . \mathrm{E}$

PN: Atretoderes Gouriet, 1868.ga.c05 • AK

PA: 00 • ATRETODERES • Gouriet 1868.ga: 206 • UC

01 - ATRETOderA • Dubois 2015.da: 107 • EE

RL: $\downarrow$ ATretoderes 1841.da.c03

< Pulmones 1868.ga.c01 • PR

$<$ Eubatraciens 1868.ga.c02 • AI: HL

GN: ANURA 1805.da.c01

GYMNOPHIONA 1814.ra.c01

URODELA 1805.da.c02

GZ: INR

EN: KYR. C.03.01. Subclassis LisSAMPhiBIa Gadow, 1898.ga.c01-00

Atretodera Brocchi, 1881

SI: $283 \bullet$ CI: c257・ST: 2.D.M.41.E

PN: ATretoderes Brocchi, 1881.ba.c05 • AK

PA: 00 • ATRETOdERES • Brocchi 1881.ba: 102 • UC

$01 \cdot$ ATretodera $\cdot H o c$ loco $\bullet$ EE

RL: $\downarrow$ ATretoderes 1841 .da.c03

GN: IMPERFECTIBRANCHIA 1838.ha.c03

Pseudosauria 1816.ba.c08

GZ: INR 
EN: KYR. C.04.03. Ordo Urodela Duméril, 1805.da.c02-12

[HYP. Subordo Nullibranchia Bonaparte, 1831.ba.c01-01] Australobatrachia Bauer, 1987

SI: $419 \bullet \mathbf{C I}:$ c379 • ST: 1.D.M.30.O

PN: Australobatrachia Bauer, 1987.bc.c04 • AK

PA: 00 • AUSTRalobatraChia • Bauer 1987.bc: 52 • UC

RL: INR

GN: Phaneranura DOP.da.c02 SCOPTANURA 1973.sb.c02

GZ: INR

EN: TEO. C.09.01. Epiphalanx AQUIPARES Blainville, 1816.ba.c07-02

[HYP. Phalanx AQUIPARES Blainville, 1816.ba.c07-03]

\section{BAINANURA nov.}

SI: $436 \bullet$ CI: c395 • ST: 1.D.M.11.O

PN: BaINANURA nov., DOP.da.c03

PA: $00 \cdot$ BAINANURA $・ H o c l o c o \cdot \mathbf{b P}$

RL: INR

GN: BAINANURA DOP.da.c03

GZ: INR

EN: KYR. C.11.01. Subphalanx BaINANURA nov., DOP.da.c03-00 BATRACHIA Brongniart, 1800

SI: $014 \cdot$ CI: $0005 \cdot$ ST: 1.S.O.03.E

PN: BATRACIENS Brongniart, 1800.ba.c01 • HK

PA: 00 - BATRACIENS • Brongniart 1800.ba: 82 • O

$01 \cdot$ BATRACHIENS $・$ Latreille 1800.1a: xxxvii • O

02 - BATRACHII • Latreille 1800.1a: xxxvii • O

03 - BatraChia - Macartney in Cuvier 1802.ca: pl. 3 - UC

$04 \cdot$ BATRACII • Duméril 1805.da: 90 - O

05 - BATRACHIA - Rafinesque 1814.ra: $102 \cdot$ O

06 • BATRACHIA • Leuckart 1821.1a: 258 • 'F'

07 - BATRACHI • Wagler 1828.wb: 859 • O

08 - BATRACHIA • Bonaparte 1831.bb: $135 \cdot$ bC

09 • BATRACHIA • Carus 1834.ca: $25 \cdot$ bO

10 • BATRACHII • Bronn 1849.ba: 683 • O

11 • Batrachia • Giebel 1852.ga: 239, 301 • Kr

12 - BatraCianI • Massalongo 1854.ma: $421 \cdot$ O

13 - BATRACI • Betta 1857.ba: 22 - O

$14 \cdot$ BATRACHIA $・$ Dubois 2005.da: $6 \cdot \mathbf{p O}$

RL: INR

GN: ANURA 1805.da.c01

URODELA 1805.da.c02

GZ: » GI:

GYMNOPHIONA 1814.ra.c01

EN: ANAPTONYM

[HYP. Superordo BATRACHIA Brongniart, 1800.ba.c01-14]

Batrachia Oppel, 1811

SI: $027 \bullet \mathbf{C I}: \mathrm{c016} \bullet \mathbf{S T}: 1 . \mathrm{S} . \mathrm{E} .20 . \mathrm{E}$

PN: BATRACIENS Oppel, 1811.oa.c02 • AK

PA: 00 • BATRACIENS • Oppel 1811.oa: 260 • O

01 • BATRACII • Oppel 1811.ob: $394 \cdot$ O

02 • BatraChIA • Merrem 1820.ma: 4 • C

$03 \cdot$ BATRACHA $・$ Brookes 1828.bc: $15 \cdot \mathbf{O}$

$04 \cdot$ BATRACHII • Bonaparte 1838.ba: [193] • bC

$05 \cdot$ BATRACHIA $・$ Bonaparte 1838. bd: $124 \cdot$ Sc

06 • BATRACHIANS • Gray 1842.ga: $111 \cdot$ UC
07 • BATRACHOIDEA • Van der Hoeven 1864.va: 288 • O

08 • BATRACHIA - Milner 1988.ma: $82 \cdot$ pO

RL: $\downarrow$ BATRACIENS 1800.ba.c01 • SD $\leftrightarrow$ ! < NUDA 1811.oa.c01 • AI: HL

GN: ANURA 1805.da.c01

GYMNOPHIONA 1814.ra.c01

Urodela 1805.da.c02

GZ: INR

EN: KYR. C.03.01. Subclassis LiSSAMPHiBIa Gadow, 1898.ga.c01-00

BATRACHIA Blainville, 1816

SI: $037 \bullet$ CI: $\mathrm{c024} \bullet$ ST: 1. N.O.40.E

PN: Batraciens Blainville, 1816.ba.c05 • AK

PA: 00 • BATRACIENS • Blainville 1816.ba: "111" [119]• O

01 • BATRACHII - Ritgen 1828.ra: $278 \cdot \mathbf{H e}$

02 • BATRACHIA • Müller 1831.ma: 711 • O

03 - BATRACIA - Swainson 1839.sa: 86 • O

04 • BATRACHII • Mayer 1849.ma: 198 • bO

05 • BATRACHII • Van der Hoeven 1855.va: x, 468 • O

06 - BatraCHIA - Stannius 1856.sa: 4 - O

07 • BATRACHIA • Huxley 1871.ha: $173 \cdot$ UC

08 • BATRACHIA • Haeckel 1889.ha: 625 • L

RL: $\downarrow$ BATRACIENS 1800 .ba.c01

GN: ANURA 1805.da.c01

GZ: » GX:

GYMNOPHIONA 1814.ra.c01

Urodela 1805.da.c02

EN: KYR. C.04.01. Ordo ANURA Duméril, 1805.da.c01-07 BATRACHIA Meyer, 1832

SI: $116 \bullet$ CI: $\mathrm{mc0} 4$ - ST: 2. N.G.02.E

PN: BATRACHIER von Meyer, 1832.ma.c01 • AP-ZA

PA: 00 • BATRACHIER • von Meyer 1832.ma: 101 • UC

01 - BatRaCHIA $\cdot H o c$ loco $\bullet$ EE

RL: $\downarrow$ BATRACIENS 1800 .ba.c01

GN: LiSSAMPHIBIA 1898.ga.c01 |Non-LiSSAMPHIBIAN AMPHIBIA|

GZ: » GI:

|Non-LisSamphibian AMPHibia|

|Aмniota|

EN: ANAPTONYM

BATRACHOIDEI Leuckart, 1840

SI: $150 \bullet \mathbf{C I}$ : c128・ST: 1.N.G.02.E

PN: BATRACHOIDEA Leuckart, 1840.la.c03 • AP

PA: 00 - BATRACHOIDEA - Leuckart 1840.la: 20 • 'F'

01 • BATRACHI • Leuckart 1841.la: 2 • UC

02 - BATRACHIA - Leuckart 1841.1a: 30 • UC

$03 \cdot$ BATRACHOIDEI $\cdot H o c$ loco $\bullet$ EE

RL: $\downarrow$ BATRACIENS 1800 .ba.c01

GN: ANURA 1805.da.c01

Urodela 1805.da.c02

GZ: » GI:

GYMNOPHIONA 1814.ra.c01

URODELA 1805.da.c02

EN: ANAPTONYM

BATRACHIA Owen, 1841

SI: $166 \bullet$ CI: c143・ST: $1 . S . O .40 .0$ 
PN: Batrachia Owen, 1841.oa.c01 • AK

PA: 00 • BATRACHIA • Owen 1841.oa: 179 • O

01 - Batrachia • Goodrich 1930.ga: xxi • C

RL: $\downarrow$ BATRACIENS 1800 .ba.c01

GN: АмpHiBia 1816.ba.c02

GZ: » GX:

|Amniota $\mid$

EN: KYR. C.02.01. Classis AMPHibia Blainville, 1816.ba.c02-03 BATRACHIA Mayer, 1849

SI: $183 \bullet$ CI: c160 • ST: 1.N.O.40.E

PN: BATRACHOIDEI Mayer, 1849.ma.c02 • AK

PA: 00 • BATRACHOIDEI • Mayer 1849.ma: 198 • bO

01 - BATRACHIA • Dubois 2015.da: $107 \cdot \mathbf{E E}$

RL: $\downarrow$ BATRACIENS 1800 .ba.c01

$\leftrightarrow \mid$ UrodeLES 1805.da.c02

$>$ HolodactYLI 1849.ma.c03 • PR

$>$ COLOBODACTYLI 1849.ma.c04 $・$ PR

GN: URODELA 1805.da.c02

GZ: » GX:

ANURA 1805.da.c01

GYMNOPHIONA 1814.ra.c01

EN: KYR. C.04.03. Ordo URoDELA Duméril, 1805.da.c02-12

BATRACHOPHIONA Latreille, 1825

SI: 069 • CI: c051 • ST: 3.D.M.31.E

PN: BATRACHOPHIDES Latreille, 1825.la.c01 • AK

PA: 00 • BATRACHOPHIDES • Latreille 1825.1a: $102 \cdot$ Sc 01 - BATRACHOPHIDII • Bonaparte 1831.bb: $134 \cdot$ O

02 - BATRACHOPIDII • Bonaparte 1839.bf: $16 \cdot$ O

03 - BATRACOPHIDII • Bonaparte 1852.ba: $480 \cdot$ O

04 - BATRACHOPHIDIENS • Gouriet 1868.ga: $204 \cdot$ UC

05 - BATRACHOPHIDIA • Hoffmann 1878.ha: $583 \cdot$ O

06 - BatraCHOPHIDia - Miranda-Ribeiro 1924.ma: 137 - UC

07 - BatrachOPHIONA $\cdot$ Hoc loco $\bullet$ EE

RL: INR

GN: PSEUDOPHIONA 1816.ba.c11

GZ: INR

EN: KYR. C.05.04. Subordo Pseudophiona Blainville, 1816.ba.c11-06

BATRACHOPHIONA Gray, 1842

SI: 168 • CI: c145 • ST: 3.D.M.40.E

PN: BATRACHOPHILIA Gray, 1842.ga.c02 • AK

PA: 00 - BATRACHOPHILIA: Gray 1842.ga: 113 • O

01 - BATRACHOPHIONA - Dubois 2015.da: 107 • EE

RL: $\downarrow$ BATRACHOPHIDES 1825.la.c01

GN: Plesiophiona DOP.da.c10

PSEUDOPHIONA 1816.ba.c11

GZ: INR

EN: KYR. C.04.02. Ordo GYMnOPHIONA Rafinesque, 1814.ra.c01-02

Batrachosauria Miranda-Ribeiro, 1924

SI: 328 • CI: c301 • ST: 2.D.M.31.O

PN: Batrachosauria Miranda-Ribeiro, 1924.ma.c03 • AK

PA: 00 - BATRACHOSAURIA • Miranda-Ribeiro 1924.ma: 138 - UC

RL: > BRANCHIPULMONADOS 1924.ma.c01 • AI: HL

GN: Meantes 1767.la.c01

Pseudosauria 1816.ba.c08
GZ: INR

EN: KYR. C.04.03. Ordo Urodela Duméril, 1805.da.c02-12 [HYP. Subordo Pneumobranchia Sonnini ${ }^{+1}$, 1801.sa.c01-02] BATRACHOSAUROIDACEA Kuhn, 1961

SI: $376 \bullet$ CI: c337 • ST: 1.D.M.30.R

PN: Batrachosauroidoidea Kuhn, 1961.ka.c02 † • AK

PA: 00 - BATRACHOSAUROIDOIDEA • Kuhn 1961.ka: 13 • bO $01 \cdot$ BatrachOSAUROIDACEA $\cdot$ Hoc loco $・$ ER

RL: < CRYPTOBRANCHOIDEA 1961.ka.c01 • AI: HL

< Proteida 1961.ka.c03 - AI: HL

$<$ Meantes 1961.ka.c04 • AI: HL

GN: |URODELA INCERTAE SEDIS|

GZ: INR

EN: KYR. C.04.03. Ordo Urodela Duméril, 1805.da.c02-12 BDALSIPODOBATRACHIA Ritgen, 1828

SI: $089 \bullet$ CI: c070 • ST: 2.D.M.32.E

PN: BDALSIPODOBATRACHI Ritgen, 1828.ra.c15 • AK

PA: 00 • BDALSIPODOBATRACHI • Ritgen 1828.ra: 278 • 'F'

01 - BDALLIPODOBATRACHI • Jourdan 1834.ja: 149 • 'F'

02 - BDALSIPODOBATRACHIA $\cdot H o c$ loco $\bullet$ EE

RL: $\leftrightarrow<$ HYLOBATRACHI 1828.ra.c16 • AI: HL

GN: HYLOBATRACHIA 1828.ra.c16

GZ: INR

EN: KYR. C.13.03. Hypophalanx Hylobatrachia Ritgen, 1828.ra.c16-01

Blepharosa Miranda Ribeiro, 1937

SI: 358 • CI: c328 • ST: 2.D.M.31.O

PN: BLEPHAROSA Miranda Ribeiro, 1937.ma.c01 • AK

PA: 00 - BLEPHAROSA • Miranda Ribeiro 1937.ma: 55 • UC

RL: INR

GN: Phaneranura DOP.da.c02

SCOPTANURA 1973.sb.c02

GZ: INR

EN: TEO. C.09.01. Epiphalanx AQUIPARES Blainville, 1816.ba.c07-02

[HYP. Phalanx AQuiPares Blainville, 1816.ba.c07-03]

BRACHYCEPHALOMORPHA Fejérváry, 1921

SI: 321 • CI: c294 • ST: 2.D.M.31.A

PN: BRACHYCEPHALOMORPHA Fejérváry, 1921.fb.c09 • AK

PA: 00 - BRACHYCEPHALOMORPHA • Fejérváry 1921.fb: 28 - Gs

RL: INR

GN: Phaneranura DOP.da.c02

SCOPTANURA 1973.sb.c02

GZ: INR

EN: TEO. C.09.01. Epiphalanx AQUIPAREs Blainville, 1816.ba.c07-02

[HYP. Phalanx AQuiPares Blainville, 1816.ba.c07-03]

Branchiata Pallas, 1814

SI: $030 \bullet$ CI: zh09・ST: 1.U.U.99.O

PN: BRANChiata Pallas, 1814.pa.c01 • zz

PA: 00 - BRANCHIATA • Pallas 1814.pa: 70 • O

RL, GN, GZ, EN:

BRANCHIATA Jarocki, 1822

SI: $061 ・$ CI: c043・ST: 2.D.M.41.E

PN: BRANCHIATA Jarocki, 1822.ja.c04 • AK 
PA: 00 • BRANCHIATA - Jarocki 1822.ja: 137 • O

$01 \cdot$ BRanchiales • Wagler 1830.wa: 131 • ' $T$ '

02 - BRANCHiata - Carus 1834.ca: 25 • bO

$03 \cdot$ BRANCHIATA $・$ Fitzinger 1843.fa: $35 \cdot \mathbf{S c}$

RL: $\downarrow$ BRANCHIATA 1814.pa.c01

$<$ Hedraeoglossi 1830.wa.c05 • PR

$>$ Abranchiales 1830.wa.c06 • AI: HL

GN: Meantes 1767.la.c01

Pseudosauria 1816.ba.c08

GZ: INR

EN: KYR. C.04.03. Ordo URODELA Duméril, 1805.da.c02-12

[HYP. Subordo Pneumobranchia Sonnini ${ }^{+1}$, 1801.sa.c01-02]

BRANCHIATA Ficinus ${ }^{+1}, 1826$

SI: $070 \bullet$ CI: c052 • ST: 2.D.M.41.O

PN: BRANChiata Ficinus ${ }^{+1}$, 1826.fa.c01 • AK

PA: 00 • Branchiata - Ficinus $^{+1}$ 1826.fa: pl. • UC

RL: $\downarrow$ BRANCHIATA 1814 .pa.c01

GN: ANURA 1805.da.c01

GYMNOPHIONA 1814.ra.c01

URODELA 1805.da.c02

GZ: INR

EN: KYR. C.03.01. Subclassis LisSAMPHibia Gadow, 1898.ga.c01-00

BRANCHIPULMONATA Miranda-Ribeiro, 1924

SI: $326 \bullet$ CI: c299 • ST: 2.D.M.31.E

PN: BRANChipUlmonados Miranda-Ribeiro, 1924.ma.c01 • AK

PA: 00 - BRANCHIPUlMonados - Miranda-Ribeiro 1924.ma: $137 \cdot \mathbf{U C}$

$01 \cdot$ Branchipulmonata $\cdot H o c$ loco $\bullet$ EE

RL: < BATRACHOSAURIA 1924.ma.c03 • AI: HL

GN: Meantes 1767.la.c01

Pseudosauria 1816.ba.c08

GZ: INR

EN: KYR. C.04.03. Ordo Urodela Duméril, 1805.da.c02-12

[HYP. Subordo Pneumobranchia Sonnini ${ }^{+1}$, 1801.sa.c01-02]

BranCHIUROMOLGAE Ritgen, 1828

SI: $078 \bullet$ CI: c059・ST: 2.D.M.31.E

PN: BranchiUromolgaei Ritgen, 1828.ra.c04 • AK

PA: c0 • BRANCHIUROMOLGAEI • Ritgen 1828.ra: 274 - Zg • EEA: HL

i1 - BRANCHiUROMALgaEI • Ritgen 1828.ra: $277 \cdot \mathbf{Z g}$

$02 \cdot$ BranchiUromolgaE $\bullet$ Hoc loco $\bullet$ EE

RL: $\leftrightarrow<$ Dysmolgae 1828.ra.c05 • AI: HL

GN: MeanTes 1767.la.c01

Pseudosauria 1816.ba.c08

GZ: INR

EN: KYR. C.04.03. Ordo Urodela Duméril, 1805.da.c02-12

[HYP. Subordo Pneumobranchia Sonnini ${ }^{+1}$, 1801.sa.c01-02] BuFONACEA Haeckel, 1889

SI: 294 • CI: c267 • ST: 1.D.M.31.R

PN: BUfONACEA Haeckel, 1889.ha.c01 • AK

PA: 00 • BUFONACEA • Haeckel 1889.ha: 640 • O

RL: INR

GN: Hylobatrachia 1828.ra.c16

GZ: INR

EN: KYR. C.13.03. Hypophalanx Hylobatrachia Ritgen, 1828.ra.c16-01

BufonaCEA Laurent in Fuhn, 1960

SI: 373 • CI: cn05 • ST: 1.D.M.00.R

PN: BUFONOIDEA Laurent in Fuhn, 1960.fa.c05 • AN

PA: 00 • BUFONOIDEA • Laurent in Fuhn 1960.fa: 163 • bO

$01 \cdot$ BUfONACEA $\bullet$ Hoc loco $・$ ER

RL: $\downarrow$ BUFONACEA 1889.ha.c01

GN: Phoranura DOP.da.c04

Phrynanura DOP.da.c05

GZ: INR

EN: KYR. C.11.01. Subphalanx BaINANURA nov., DOP.da.c03-00 Bufoniformia Cope, 1864

SI: $231 \bullet \mathbf{C I}: \mathrm{c} 205 \bullet \mathbf{S T}: 1 . \mathrm{D} . \mathrm{M} .31 . \mathrm{A}$

PN: BUfONIFORMES Cope, 1864.cb.c03 • AK

PA: 00 • BUFONIFORMES • Cope 1864.cb: 182 • bO

$01 \cdot$ BUfONIFORMia - Cope 1865.ca: $97 \cdot$ bO

02 - BUFONIFORMES • Brocchi 1881.ba: $9 \cdot$ UC

03 • BUFONIFORMES • Boulenger 1882.ba: $12 \cdot$ UC

RL: INR

GN: DORSIPARES 1816.ba.c06

LAEVOGYRINIA 1878.1a.c01

GZ: INR

EN: KYR. C.06.01. Infraordo GEOBATRACHIA Ritgen, 1828.ra.c18-02

BUFONIFORMIA Steindachner, 1867

SI: 244 • CI: c218 • ST: 1.D.M.41.A

PN: BUFONIFORMia Steindachner, 1867.sa.c02 • AK

PA: 00 - BUFONIFORMiA - Steindachner 1867.sa: 34 • Sc

01 • BUfONIFORMES • Philippi 1902.pa: ix • UC

RL: $\downarrow$ BUFONIFORMIA $1864 . c b . c 03$

< HylaPlesiformia 1867.sa.c03 • AI: HL

GN: Phaneranura DOP.da.c02

SCOPTANURA 1973.sb.c02

GZ: INR

EN: TEO. C.09.01. Epiphalanx AQUIPARES Blainville, 1816.ba.c07-02

[HYP. Phalanx AQUiPAREs Blainville, 1816.ba.c07-03]

Bufoniformia Hay, 1929

SI: $349 \bullet$ CI: c322 • ST: 1.D.M.40.A

PN: BUFONIFORMES Hay, 1929.ha.c06 • AK

PA: 00 • BUFONIFORMES • Hay 1929.ha: 521, $852 \cdot$ bO

01 • BUFONIFORMia - Dubois 2015.da: $105 \cdot$ EA

RL: $\downarrow$ BUFONIFORMIA $1864 . c b . c 03$

GN: LiSSAMPHiBIA 1898.ga.c01

|TEMNOSPONDYLI|

GZ: INR

EN: KYR. C.02.01. Classis AMPHIBIA Blainville, 1816.ba.c02-03

BufONOMORPHA Fejérváry, 1921

SI: 318 • CI: c291・ST: 2.D.M.31.A

PN: BufonimorPha Fejérváry, 1921.fb.c06 • AK

PA: 00 • BUFONIMORPHA • Fejérváry 1921.fb: 24 • Gs

01 - BufONOMORPHA • Dubois 2015.da: 90 - EA

RL: INR

GN: DoRSIPARES 1816.ba.c06

LAEVOGYRINIA 1878.la.c01

GZ: INR 
EN: KYR. C.06.01. Infraordo GEOBATRACHIA Ritgen, 1828.ra.c18-02

CADUCIBRANChIA Latreille 1824

SI: $062 \bullet$ CI: c044 • ST: 1.D.M.30.E

PN: CADUCIBRanches Latreille 1824.la.c01 • AK

PA: 00 - CAdUCIBRANCHES - Latreille 1824.1a: 9 • O

01 - Caducibranchia - Latreille 1825.1a: $104 \cdot$ O

02 CAduCibranchiata - Owen 1835.oa: 214 • UC

03 CAdNABRANChia - Hogg 1838.ha: $152 \cdot$ O

04 - Caducibranchiata Cope 1859 .cb: $122 \cdot$ O

RL: INR

GN: » OA, SD: Latreille 1825.1a: 104-105:

ANURA 1805.da.c01

URODELA 1805.da.c02

GZ: INR

EN: KYR. C.03.01. Subclassis LisSAMPHibia Gadow, 1898.ga.c01-00

[HYP. Superordo BATRACHIA Brongniart, 1800.ba.c01-14]

CAducibranchia Betta, 1864

SI: $228 \bullet$ CI: c202 • ST: 1.D.M.41.O

PN: CAduCibranchia Betta, 1864.ba.c01 • AK

PA: 00 - CAdUCIBRANCHIA - Betta 1864.ba: 512 • bO

01 - Caducibranchia • Haeckel 1889.ha: 625 • O

RL: $\downarrow$ CAduCibranches 1824.1a.c01

GN: Pseudosauria 1816.ba.c0 8

GZ: INR

EN: KYR. C.05.07. Subordo Pseudosauria Blainville, 1816.ba.c08-07

Caducibranchia Cope, 1866

SI: 234 • CI: c208 • ST: 1.D.M.41.E

PN: Caducibranchiata Cope, 1866.ca.c03 • AK

PA: 00 - CADUCIBRANCHIATA • Cope 1866.ca: 97 • bO

01 CAducibranchiates - Cope 1866.ca: $98 \cdot$ bO

$02 \cdot$ CAdUCIBRANCHES • Lataste 1878.1b: $3 \cdot$ Sc

03 C Caducibranchia - Goodrich 1930.ga: xxi • bO

RL: $\downarrow$ CADUCibranCHes 1824 la.c01

GN: IMPERFECTIBRANCHIA 1838.ha.c03 Pseudosauria 1816.ba.c08

GZ: INR

EN: KYR. C.04.03. Ordo URODELA Duméril, 1805.da.c02-12 [HYP. Subordo Nullibranchia Bonaparte, 1831.ba.c01-01]

Caeciliacea Wagler, 1830

SI: $102 \cdot$ CI: c083 • ST: 1.D.M.31.R

PN: Caeciliae Wagler, 1830.wa.c01 • AK

PA: 00 - CAECILIAE • Wagler 1830.wa: $131 \cdot$ O

01 - CAECILIOIDEI $・$ Lescure $^{+2}$ 1986.1b: $145 \cdot$ bO

$02 \cdot$ CAeciliIdeI $\bullet$ Lescure $^{+2}$ 1986.1b: $145 \cdot$ iO

03 CAECILIAOIdeI $\cdot$ Lescure $^{+1}$ 1988.1a: $20 \bullet$ bO

$04 \cdot$ CAECILIAIDEA $\cdot$ Lescure $^{+1}$ 1988.1a: $20 \cdot$ iO

$05 \cdot$ CAECILIACEA $\cdot H o c$ loco $\bullet$ ER

RL: INR

GN: PSEUdophiona 1816.ba.c11

GZ: INR

EN: KYR. C.05.04. Subordo Pseudophiona Blainville, 1816.ba.c11-06
Caeciliacea Sarasin ${ }^{+1}, 1890$

SI: $299 \bullet$ CI: c272 • ST: 1.D.M.41.R

PN: CAeCIloidea Sarasin ${ }^{+1}$, 1890.sa.c03 • AK

PA: 00 - CAECILOIDEA • Sarasin ${ }^{+1} 1890$. sa: $245 \cdot$ bO

$01 \cdot$ CAeciliacea • Dubois 2015.da: $106 \cdot$ ER

RL: $\downarrow$ Caeciliae 1830 .wa.c01

< NeObatrachi 1890.sa.c01 • PR

GN: GYMNOPHIONA 1814.ra.c01

URODELA 1805.da.c02

GZ: INR

EN: KYR. C.03.01. Subclassis Lissamphibia Gadow, 1898.ga.c01-00

[HYP. Superordo Derotreta Van der Hoeven, 1833.va.c01-01]

Calamitacei Link, 1807

SI: 023 • CI: c013 • ST: 1.D.M.30.C

PN: Calamitae Link, 1807.la.c01 • AK

PA: 00 - Calamitae - Link 1807.la: $53 \cdot$ O

01 - CAlamitaCeI - Dubois 2015.da: 90 • EC

RL: INR

GN: ANURA 1805.da.c01

URODELA 1805.da.c02

GZ: INR

EN: KYR. C.03.01. Subclassis Lissamphibia Gadow, 1898.ga.c01-00

[HYP. Superordo BATraCHIA Brongniart, 1800.ba.c01-14]

Callulacea Haeckel, 1889

SI: 295 • CI: c268 • ST: 1.D.M.31.R

PN: Callulacea Haeckel, 1889.ha.c02 • AK

PA: 00 - CallulaceA • Haeckel 1866.ha: 640 • O

RL: INR

GN: Phaneranura DOP.da.c02

SCOPTANURA 1973.sb.c02

GZ: INR

EN: TEO. C.09.01. Epiphalanx AQUIPAREs Blainville, 1816.ba.c07-02

[HYP. Phalanx AQUIPAREs Blainville, 1816.ba.c07-03] Cathetura Duméril ${ }^{+1}, 1839$

SI: $134 \cdot$ CI: zh16 • ST: 2.U.U.99.E

PN: Cathetures Duméril ${ }^{+1}, 1839 . d b . c 01 \cdot \mathbf{z Z}$

PA: 00 - Cathetures • Duméril ${ }^{+1} 1839 . \mathrm{db}: 18 \cdot \mathbf{G r} / \mathbf{T}$

$01 \cdot$ CATHETURA $\cdot H o c$ loco $\bullet$ EE

RL: $\leftrightarrow$ COMPRESSICAUDES 1839.db.c02

GN, GZ, EN: •

Cathetura Duméril ${ }^{+2}, 1854$

SI: $196 \bullet$ CI: c173・ST: 2.D.M.41.E

PN: Cathetures Duméril ${ }^{+2}$, 1854.da.c02 • AK

PA: 00 - Cathetures • Duméril ${ }^{+2}$ 1854.da: 38 - UC

$01 \cdot$ Cathetura $\cdot H o c l o c o \cdot \mathbf{E E}$

RL: $\downarrow$ Cathetures 1839 .db.c01

$\leftrightarrow$ > CoMPressiCAUdes 1854.da.c01 - AI: HL

GN: Pseudosauria 1816.ba.c0 0

GZ: INR

EN: KYR. C.05.07. Subordo Pseudosauria Blainville, 1816.ba.c08-07 
Caudata Scopoli, 1777

SI: $009 \bullet$ CI: zh03 • ST: 2.N.O.99.O

PN: Caudata Scopoli, 1777.sa.c02 • zZ

PA: 00 • CAUdata • Scopoli 1777.sa: $411 \cdot$ Gs

RL: $\downarrow>$ CAUdaTa 1777. sa.c02 $・$ PR

GN, GZ, EN: •

Caudata Scopoli, 1777

SI: $012 \bullet$ CI: $\mathrm{c} 003 \bullet$ ST: 1. N.G.02.O

PN: Caudata Scopoli, 1777.sa.c05 • AP-ZA

PA: 00 • CAUdATA $・$ Scopoli 1777.sa: $463 \cdot$ O

RL: $\downarrow<$ CaUdata 1777 .sa.c02 • PR

GN: AMPHIBIA 1816.ba.c02

|АмNiota|

GZ: » GI:

AMPHIBIA 1816.ba.c02

|АмNiota|

EN: ANAPTONYM

CaUdata Duméril 1805

SI: $020 \bullet$ CI: $\mathrm{c} 011$ • ST: 2.S.O.40.E

PN: Caudati Duméril, 1805.da.c04 • AK

PA: 00 • Caudati • Duméril 1805.da: 94 • 'F'

01 C Caudata $\bullet$ Oppel 1811.ob: 409 • 'F'

$02 \cdot$ CAUdata • Leuckart 1821.la: 260 • UC

03 C CAUdata • Hemprich 1829.ha: xix, $373 \cdot$ Fo

$04 \cdot$ CaUdata - Wiegmann $^{+1}$ 1832.wa: $198 \cdot$ bO

05 C CAUdata • Gravenhorst 1843.ga: $393 \cdot \mathbf{Z t}$

06 CAudata $\cdot$ Hoffmann 1878.ha: $615 \cdot \mathbf{U C}$

$07 \cdot$ Caudata $・$ Boulenger 1882.bc: vii, $1 \cdot$ O

$08 \cdot$ Caudata $\cdot$ Haeckel 1889.ha: $625 \cdot \mathbf{L}$

09 • Caudata • Gill 1903.ga: 72 • 'F'

10 C Caudatida • Pearse 1936.pa: $20 \cdot \mathbf{O}$

RL: $\downarrow$ Caudata 1777.sa.c02

$\leftrightarrow<$ UrodeLES 1805.da.c02 • AI: Zittel, 1888.za: 412

GN: URODELA 1805.da.c02

GZ: » GX:

ANURA 1805.da.c01

GYMNOPHIONA 1814.ra.c01

EN: KYR. C.04.03. Ordo Urodela Duméril, 1805.da.c02-12

CAUDATA Leuckart, 1821

SI: $056 \bullet$ CI: $038 \bullet$ ST: 2.N.G.02.O

PN: CAUDATA Leuckart, 1821.la.c03 • AP

PA: $00 \cdot$ CAUdata $・$ Leuckart 1821.la: $260 \bullet \mathbf{U C}$

$01 \cdot$ CaUdatae • Wagler 1830.wa: $131 \cdot \mathbf{D}$

RL: $\downarrow$ CAudata 1777. sa.c02

GN: Pseudosauria 1816.ba.c08

GZ: » GI:

PSeudosauria 1816.ba.c08

EN: ANAPTONYM

Caudata Leuckart, 1840

SI: $151 \bullet$ CI: c129・ST: 2.N.G.02.O

PN: Caudata Leuckart, 1840.la.c04 • AP

PA: 00 • CAUDATA • Leuckart 1840.1a: 20 • 'bF'

RL: $\downarrow$ Caudata 1777. sa.c02

GN: IMPERFECTIBRANCHIA 1838.ha.c03

Pseudosauria 1816.ba.c08

GZ: » GI:
IMPERFECTIBRANCHIA 1838.ha.c03

MeANTES 1767.la.c01

Pseudosauria 1816.ba.c08

EN: ANAPTONYM

CaUdata Haeckel, 1866

SI: $238 \bullet$ CI: c212 • ST: 1 .N.E.40.O

PN: CaUdata Haeckel, 1866.ha.c04 • AK

PA: $00 \cdot$ CAUdATA $・$ Haeckel 1866.ha: cxxxi • O

RL: $\downarrow$ CAUdata 1777. sa.c02

< Sozobranchia 1866.ha.c02 • AI: HL

$\leftrightarrow$ ! < SozURA 1866.ha.c03 • AI: HL

GN: IMPERFECTIBRANCHIA 1838.ha.c03

Pseudosauria 1816.ba.c08

GZ: INR

EN: KYR. C.04.03. Ordo Urodela Duméril, 1805.da.c02-12

[HYP. Subordo Nullibranchia Bonaparte, 1831.ba.c01-01]

Caudata Goodrich, 1930

SI: $340 \bullet$ CI: c313・ST: 1. N.G.02.O

PN: CAUdaTa Goodrich, 1930.ga.c02 • AP

PA: 00 • CAudata • Goodrich 1930.ga: xxi • bO

RL: $\downarrow$ Caudata 1777. sa.c02

GN: URODELA 1805.da.c02

|Non-LisSAMPHIBIAN AMPHIBIA|

GZ: » GI:

|Non-LisSAMPHIBIAN AMPHIBIA|

EN: ANAPTONYM

Caudata Hay, 1929

SI: 345 • CI: c318 • ST: 1.N.G.02.E

PN: Caudati Hay, 1929.ha.c02 • AP

PA: $00 \cdot$ CAUdATI $\bullet$ Hay 1929.ha: 521, $839 \cdot$ bO

$01 \cdot$ Caudata $\cdot$ Hoc loco $\bullet$ EE

RL: INR

GN: URODELA 1805.da.c02

|Non-LisSAMPHIBIAN AMPHIBIA|

GZ: » GI:

|Non-LISSAMPHIBIAN AMPHIBIA|

EN: ANAPTONYM

Caudata Trueb ${ }^{+1}, 1991$

SI: 425 • CI: c385 • ST: 1.N.G.02.O

PN: Caudata Trueb ${ }^{+1}$, 1991.ta.c01 • AP

PA: 00 - CAUdATA - Trueb ${ }^{+1}$ 1991.ta: 233 - O

RL: $\downarrow$ Caudata 1777. sa.c02

GN: URODELA 1805.da.c02

GZ: » GI:

Urodela 1805.da.c02

EN: ANAPTONYM

Ceciliacea Fatio, 1872

SI: $254 \bullet$ CI: c228 • ST: 1.D.M.30.R

PN: CeCILIDES Fatio, 1872.fa.c01 • AK

PA: 00 - CeCILIDES $\cdot$ Fatio 1872.fa: $7 \cdot 0$

01 - Ceciliacea • Dubois 2015.da: 107 • ER

RL: $\leftrightarrow \mid$ OPHIOMORPHI 1855.va.c02

GN: PLesiophiona DOP.da.c10

PSEUdOPhIONA 1816.ba.c11

GZ: INR

EN: KYR. C.04.02. Ordo GyMnOPHIONA Rafinesque, 
1814.ra.c01-02

Celatibranchia Hogg, 1841

SI: 162 • CI: c139 • ST: 1.D.M.31.O

PN: Celatibranchia Hogg, 1841.ha.c01 • AK

PA: 00 • Celatibranchia • Hogg 1841.ha: 357 • 'T'

RL: INR

GN: PSEUDOPHIONA 1816.ba.c11

GZ: INR

EN: KYR. C.05.04. Subordo Pseudophiona Blainville, 1816.ba.c11-06

Cercopi Wagler, 1828

SI: 099 • CI: c080 • ST: 3.D.M.31.O

PN: CERCOPI Wagler, 1828.wb.c06 • AK

PA: $00 \cdot$ CerCoPI • Wagler 1828.wb: $859 \cdot$ 'F'

RL: INR

GN: PSEUdosauria 1816.ba.c08

GZ: INR

EN: KYR. C.05.07. Subordo Pseudosauria Blainville, 1816.ba.c08-07

Chersobatae Fitzinger, 1843

SI: 172 • CI: c149 • ST: 2.D.M.31.O

PN: Chersobatae Fitzinger, 1843.fa.c04 • AK

PA: 00 • CHERSOBATAE • Fitzinger 1843.fa: $32 \cdot \mathbf{S c}$ $01 \cdot$ Chersobates $\cdot$ Tschudi 1845.tb: $69 \cdot$ Sc

RL: < Hydronectae 1843.fa.c03 • AI: HL

GN: GEOBATRACHIA 1828.ra.c18 Mediogyrinia 1878.1a.c02

GZ: INR

EN: KYR. C.05.02. Subordo Hydrobatrachia Ritgen, 1828.ra.c14-01

Chirodysmolgae Ritgen, 1828

SI: $080 \bullet$ CI: c061 • ST: 2.D.M.31.O

PN: Chirodysmolgae Ritgen, 1828.ra.c06 • AK

PA: 00 • ChirodysmolgaE • Ritgen 1828.ra: 277 • 'F'

01 Chirodysmolgae $\bullet$ Dubois 2020.dra: $32 \cdot$ bO

RL: INR

GN: Meantes 1767.la.c01

GZ: INR

EN: KYR. C.05.06. Subordo Meantes Linné, 1767.la.c01-01

Coeciliacea Blainville, 1816

SI: $044 \bullet$ CI: c031 • ST: 1.D.M.32.R

PN: CoeciLIes Blainville, 1816.ba.c12 • AK

PA: 00 • Coecilies • Blainville 1816.ba: "111" [119] • O

$01 \cdot$ Coeciliae $\cdot$ Wagler 1828.wa: $736 \cdot \mathbf{U C}$

02 $\bullet$ CoeciliaE $\bullet$ Müller 1831.ma: $711 \cdot$ O

03 - Coecilia $・$ Kuhn 1939.ka: $18 \cdot$ O

$04 \cdot$ COECILIACEA $\bullet H o c$ loco $\bullet$ ER

RL: $\leftrightarrow<$ PSEUDOPHYDIENS 1816.ba.c11 • AI: HL

GN: PSEUDOPHIONA 1816.ba.c11

GZ: INR

EN: KYR. C.05.04. Subordo Pseudophiona Blainville, 1816.ba.c11-06

Coeciliacea Knauer, 1878

SI: $265 \bullet$ CI: c239 • ST: 1.D.M.40.R

PN: COECILIOIDEA Knauer, 1878.ka.c01 • AK

PA: $00 \cdot$ COECILIOIDEA $・$ Knauer 1878.ka: $91 \cdot$ O
01 $\cdot$ CoECILIAE $\bullet$ Goodrich 1930.ga: xxi $\bullet$ bC

02 COECILIAE $\bullet$ Goodrich 1930.ga: xxi • O

03 - Coeciliacea $・$ Dubois 2015.da: $107 \cdot$ ER

RL: $\downarrow$ COECILIES 1816.ba.c12

GN: Plesiophiona DOP.da.c10

PSEUDOPHIONA 1816.ba.c11

GZ: INR

EN: KYR. C.04.02. Ordo GyMnophiona Rafinesque, 1814. ra.c01-02

Coeciliformia Zagorodniuk, 2004

SI: $431 \bullet$ CI: c391・ST: 5.D.N.30.A

PN: CoeCiLIformes Zagorodniuk, 2004.za.c01 • AK

PA: 00 • COECILIFORMES $・$ Zagorodniuk 2004.za: 70 • O

$01 \cdot$ CoeCiliformia $・$ Dubois 2015.da: $107 \cdot$ EA

RL: $\downarrow$ APODEs 1758.la.c02

$\leftarrow$ APODa 1811.ob.c01

GN: GYMNOPHIONA 1814.ra.c01

GZ: ANura 1805.da.c01

URODELA 1805.da.c02

EN: KYR. C.04.02. Ordo GyMnophiona Rafinesque, 1814.ra.c01-02

Colobodactyla Mayer, 1849

SI: 185 • CI: c162 • ST: 2.N.O.31.E

PN: ColObodactYli Mayer, 1849.ma.c04 • AK

PA: 00 • Colobodactyl $・$ Mayer 1849.ma: $198 \cdot$ UC

$01 \cdot$ Colobodactyla $\cdot$ Hoc loco $\bullet$ EU

RL: < Malacopoda 1849.ma.c01 • PR

$<$ Holodactyli 1849.ma.c03 • PR

GN: Meantes 1767.la.c01

Pseudosauria 1816.ba.c08

GZ: INR

EN: KYR. C.04.03. Ordo Urodela Duméril, 1805.da.c02-12

[HYP. Subordo Pneumobranchia Sonnini ${ }^{+1}$, 1801.sa.c01-02]

CompressiCAUdata Duméril ${ }^{+1}, 1839$

SI: $135 \bullet$ CI: zh17 • ST: 2.U.U.99.E

PN: CoMPresSICAUdes Duméril ${ }^{+1}$, 1839.db.c02 • zz

PA: 00 • CoMPRESSICAUdeS $・$ Duméril $^{+1} 1839 . \mathrm{db}: 40 \cdot \mathbf{G r} / \mathbf{T}$

$01 \cdot$ COMPRESSICAUDATA $\cdot H o c$ loco $\bullet$ EE

RL: $\leftrightarrow$ Cathetures 1839.db.c01

GN, GZ, EN: •

COMPRESSICAUDATA Duméril ${ }^{+2}, 1854$

SI: 195 • CI: c172 • ST: 2.D.M.41.E

PN: CoMPresSicaudes Duméril ${ }^{+2}, 1854$.da.c01 • AK

PA: $00 \cdot$ COMPRESSICAUDES $・$ Duméril $^{+2} 1854$ da: $38 \cdot$ UC

$01 \cdot$ COMPRESSICAUDATA $\cdot$ Hoc loco $\cdot$ EE

RL: $\downarrow$ COMPRESSICAUdes 1839 .db.c02

$\leftrightarrow<$ Cathetures 1854.da.c02 • AI: HL

GN: Pseudosauria 1816.ba.c08

GZ: INR

EN: KYR. C.05.07. Subordo Pseudosauria Blainville, 1816.ba.c08-07

Costata Müller, 1840

SI: $153 \bullet$ CI: zh18・ST: 1.U.U.99.O

PN: Costata Müller, 1840.ma.c01 • zZ

PA: 00 • Costata • Müller 1840.ma: 25 • 'F'

RL, GN, GZ, EN: • 
Costata Lataste, 1878

SI: 272 • CI: c246 • ST: 1.D.M.41.E

PN: CostaTI Lataste, 1878.lb.c02 • AK

PA: 00 • CostaTI • Lataste 1879.1b: 339 • 'bT'

$01 \cdot$ Costata $\bullet$ Stejneger 1907.sa: v, $50 \bullet$ bO

RL: $\downarrow$ COSTATA 1840.ma.c01

GN: MEDIOGYRINIA 1878.la.c02

GZ: INR

EN: KYR. C.06.02. Infraordo Mediogyrinia Lataste, 1878.1a.c02-02

Costata Stejneger ${ }^{+1}, 1917$

SI: $306 \bullet$ CI: c279 • ST: 1.D.M.41.O

PN: CosTaTA Stejneger ${ }^{+1}$, 1917.sa.c01 • AK

PA: $00 \cdot$ CostaTA $\bullet$ Stejneger ${ }^{+1}$ 1917.sa: $25 \cdot$ bO

RL: $\downarrow$ CostaTa 1840.ma.c01

GN: ANGUSTICOELA 1958.ra.c01

HydRobatrachia 1828.ra.c14

GZ: INR

EN: KYR. C.04.01. Ordo ANURa Duméril, 1805.da.c01-07

Cryptobranchacea Noble, 1931

SI: 351 • CI: c324 • ST: 1.D.M.41.R

PN: CRyptobranchoidea Noble, 1931.na.c01 • AK

PA: 00 • CRYPTOBRANChOIDEA • Noble 1931.na: $465 \cdot$ bO

01 Cryptobranchina • Pearse 1936.pa: $20 \cdot$ bO

02 - CRYPtobranchoidei • Tatarinov 1964.ta: 9, 159 • bO

$03 \cdot$ Cryptobranchacea $\cdot$ Hoc loco $\bullet$ ER

RL: $\downarrow$ CRYPtobranches 1805 .da.c05

GN: IMPERFECTIBRANCHIA 1838.ha.c03

GZ: INR

EN: KYR. C.05.05. Subordo IMPERFECTIBRANCHIA Hogg, 1838.ha.c03-02

Cryptobranchacea Romer, 1933

SI: $355 \bullet$ CI: $\mathrm{mc} 05 \bullet$ ST: 1.D.M.00.R

PN: Cryptobranchoidea Romer, 1933.ra.c01 • AN

PA: 00 • CRYPTOBRANCHOIDEA • Romer 1933.ra: $437 \cdot$ bO

$$
01 \cdot \text { Cryptobranchacea } \cdot \text { Hoc loco } \bullet \text { ER }
$$

RL: $\downarrow$ Cryptobranches 1805 .da.c05

GN: IMPERFECTIBRANCHIA 1838.ha.c03 |URODELA INCERTAE SEDIS|

GZ: INR

EN: KYR. C.04.03. Ordo Urodela Duméril, 1805.da.c02-12 Cryptobranchacea Kuhn, 1961

SI: $375 \bullet$ CI: c336 • ST: 1.D.M.40.R

PN: Cryptobranchoidea Kuhn, 1961.ka.c01 • AK

PA: 00 • CryPtobranchoidea • Kuhn 1961.ka: 12 • bO $01 \cdot$ CRYPtobranchacea $・$ Dubois 2015 da: $107 \cdot$ ER

RL: $\downarrow$ CRyptobranches 1805 .da.c05 > Batrachosauroidoidea 1961.ka.c02 • AI: HL

> ProteIDa 1961.ka.c03 • AI: HL

< Meantes 1961.ka.c04 • AI: HL

GN: IMPERFECTIBRANCHIA 1838.ha.c03

|URODELA INCERTAE SEDIS|

GZ: INR

EN: KYR. C.04.03. Ordo Urodela Duméril, 1805.da.c02-12

CRYPtobranchacea Kuhn, 1965

SI: 390 • CI: c350 • ST: 1.D.M.40.R
PN: Cryptobranchoidea Kuhn, 1965.ka.c01 • AK

PA: 00 • CRYPTOBRANChoidea • Kuhn 1965.ka: $33 \cdot$ bO

01 - Cryptobranchacea - Dubois 2015.da: $105 \cdot$ ER

RL: $\downarrow$ Cryptobranches 1805 .da.c05

GN: URODELA 1805.da.c02

|AMPHIBIA INCERTAE SEDIS|

GZ: INR

EN: KYR. C.02.01. Classis AMPHIBIA Blainville, 1816.ba.c02-03 Cryptobranchia Duméril, 1805

SI: $021 \bullet$ CI: zh06 • ST: 1.U.U.99.E

PN: Cryptobranches Duméril, 1805.da.c05 • zZ

PA: 00 • Cryptobranches • Duméril 1805.da: $97 \cdot$ O

01 - Cryptobranchia • Jourdan 1834.ja: $340 \bullet$ O

02 CRyptobranchiata $・$ Jourdan 1834 .ja: $340 \bullet$ O

RL, GN, GZ, EN: •

Cryptobranchia Wagler, 1828

SI: $097 \bullet$ CI: $\mathrm{c} 078 \bullet$ ST: 3.D.M.41.E

PN: CRYPtobranchi Wagler, 1828.wb.c04 • AK

PA: $00 \cdot$ CRYPTOBRANCHI • Wagler 1828.wb: 859 • 'F'

$01 \cdot$ Cryptobranchia $\cdot$ Hoc loco $\bullet$ EE

RL: $\downarrow$ Cryptobranches 1805 .da.c05

GN: Pseudosauria 1816.ba.c08

GZ: INR

EN: KYR. C.05.07. Subordo Pseudosauria Blainville, 1816.ba.c08-07

Cryptobranchia Bonaparte, 1831

SI: $110 \bullet$ CI: $\mathrm{c091} \bullet$ ST: 1.D.M.40.O

PN: CRYPtobranchia Bonaparte, 1831.bb.c01 • AK

PA: $00 \cdot$ CRYPTOBRANCHIA $・$ Bonaparte 1831.bb: $136 \cdot \mathbf{O}$

$01 \cdot$ Cryptobranchiae $\bullet$ Gray 1842.ga: $113 \cdot$ O

RL: $\downarrow$ Cryptobranches 1805 .da.c05

GN: IMPERFECTIBRANCHIA 1838.ha.c03

Pseudosauria 1816.ba.c08

GZ: INR

EN: KYR. C.04.03. Ordo Urodela Duméril, 1805.da.c02-12

[HYP. Subordo Nullibranchia Bonaparte, 1831.ba.c01-01]

Cryptobranchiformia Milner, 2000

SI: $429 \bullet$ CI: c389 • ST: 1.D.M.30.A

PN: Cryptobranchiformes Milner, 2000.ma.c02 • AK

PA: 00 • CRYPTOBRANChiformes • Milner 2000.ma: $1412 \cdot$ iO

$01 \cdot$ CRYptobranchiformia $\cdot H o c l o c o \cdot$ EA

RL: INR

GN: IMPERFECTIBRANCHIA 1838.ha.c03

GZ: INR

EN: KYR. C.05.05. Subordo IMPERFECTIBRANCHIA Hogg, 1838.ha.c03-02

Cryptopleurae Fitzinger, 1843

SI: $175 \bullet$ CI: $\mathrm{c} 152 \bullet$ ST: 2.D.M.31.O

PN: Cryptopleurae Fitzinger, 1843.fa.c07 • aK

PA: 00 • CRYPTOPLeURAe $\bullet$ Fitzinger 1843.fa: $33 \cdot \mathbf{S c}$

$01 \cdot$ Cryptopleura $\bullet$ Gray 1850.ga: $14,15,70 \cdot$ UC

RL: > Phaeneropleurae 1843.fa.c06 • AI: HL

GN: PSEUdosauRIa 1816.ba.c08

GZ: INR

EN: KYR. C.05.07. Subordo Pseudosauria Blainville, 1816.ba.c08-07 
Cycloglena Bruch, 1862

\section{SI: 221 • CI: c195 • ST: 2.D.M.31.E}

PN: CyCloglenides Bruch, 1862.ba.c01 • AK

PA: 00 - CyCloglenides • Bruch 1862.ba: 221 • 'F'

$$
01 \cdot \text { CyClOGLena } \cdot \text { Hoc loco } \cdot \text { EE }
$$

RL: > Plagioglenides 1862.ba.c02 • AI: HL $>$ Plagioglena 1862.ba.c03 • AI: HL

GN: Phaneranura DOP.da.c02 SCOPTANURA 1973.sb.c02

GZ: INR

EN: TEO. C.09.01. Epiphalanx AQUIPARES Blainville, 1816.ba.c07-02

[HYP. Phalanx AQUIPARES Blainville, 1816.ba.c07-03]

Cystignathomorpha Fejérváry, 1921

SI: $319 \bullet$ CI: c292 • ST: 2.D.M.30.A

PN: CystignathomorPha Fejérváry, 1921.fb.c07 • AK

PA: 00 • CystignathomorPHa • Fejérváry 1921.fb: 26 • Gs

RL: < ANISOBATRACHOIDEA 1921.fb.c04 • PR

$$
<\text { Pelobatomorpha 1921.fb.c05 • AI: HL }
$$

GN: ANGUSTICOELA 1958.ra.c01

$$
\text { HYDROBATRACHIA 1828.ra.c14 }
$$

GZ: INR

EN: KYR. C.04.01. Ordo ANURa Duméril, 1805.da.c01-07

DACTYLETHRIFORMIA Brocchi, 1881

SI: $281 \bullet$ CI: c255 • ST: 2.D.M.31.A

PN: DACTYLERIFORMES Brocchi, 1881.ba.c03 • AK

PA: 00 • DACTYLERIFORMES • Brocchi 1881.ba: 9 • UC $01 \cdot$ DACTYLETHRIFORMES • Boulenger 1882.ba: $12 \cdot$ UC

$02 \cdot$ Dactylethriformia $\cdot$ Hoc loco $\bullet$ EA

RL: INR

GN: DoRSIPARES 1816.ba.c06

GZ: INR

EN: KYR. C.07.01. Hypoordo DorsIPARES Blainville, 1816.ba.c06-02

Delesura Jan, 1857

SI: $204 \bullet$ CI: c181 • ST: 1.D.A.30.O

PN: Delesura Jan, 1857.ja.c01 • AK

PA: 00 • DELESURA • Jan 1857.ja: $54 \cdot$ O

RL: $\leftrightarrow \mid$ URODELES 1805.da.c02

GN: URODELA 1805. da.c02

GZ: » GX:

ANURA 1805.da.c01

GYMNOPHIONA 1814.ra.c01

EN: KYR. C.04.03. Ordo Urodela Duméril, 1805.da.c02-12

Dentata Scopoli, 1777

SI: $011 \bullet$ CI: zh05・ST: 2.D.M.99.E

PN: DeNTati Scopoli, 1777.sa.c04 • zZ

PA: $00 \cdot$ DENTATI $\cdot$ Scopoli 1777.sa: $452 \cdot$ D

$01 \cdot$ Dentata $\cdot$ Hoc loco $\bullet$ EE

RL, GN, GZ, EN: •

DENTATA Fatio, 1872

SI: $255 \bullet$ CI: c229 • ST: 1.D.M.31.O

PN: Dentata Fatio, 1872.fa.c02 • AK

PA: $00 \cdot$ DENTATA $・$ Fatio 1872.fa: 230, $293 \cdot$ D

RL: $\downarrow<$ DENTATA 1777. sa.c04 $\bullet$ AI: HL

$>$ DENTATA 1872.fa.c04 • AI: HL
GN: GEOBATRACHIA 1828.ra.c18

MediogYRInIA 1878.1a.c02

GZ: INR

EN: KYR. C.05.02. Subordo Hydrobatrachia Ritgen, 1828.ra.c14-01

Dentata Fatio, 1872
SI: 257 • CI: c231 • ST: 1.D.M.41.O
PN: Dentata Fatio, 1872.fa.c04 • AK
PA: $00 \cdot$ DENTATA $・$ Fatio 1872.fa: $230 \cdot$ D
RL: $\downarrow<$ DENTATA 1777. sa.c04 - AI: HL
$>$ EDENTATA 1872 .fa.c03 • AI: HL
GN: Phaneranura DOP.da.c02
SCOPTANURA 1973.sb.c02
GZ: INR
EN: TEO. C.09.01. Epiphalanx AQUIPARES Blainville, 1816.ba.c07-02

[HYP. Phalanx AquiPares Blainville, 1816.ba.c07-03]

DERMATOPHIONA Ritgen, 1828

SI: $075 \bullet$ CI: c056・ST: 2.D.M.31.E

PN: DERMATOPHIDES Ritgen, 1828.ra.c01 • AK

PA: 00 • DERMATOPHIDES • Ritgen 1828.ra: $258 \cdot$ He

01 - [DERMATO]PHES • Agassiz 1847.aa: 346 • UC

02 - DeRMATOPHIONA $\cdot$ Hoc loco $\bullet$ EE

RL: $\leftrightarrow<$ SCOLECODES 1828.ra.c02 • AI: HL

$\leftrightarrow>$ STOLIDOPHIDES 1828.ra.c03 • PR

GN: PseUdophiona 1816.ba.c11

GZ: INR

EN: KYR. C.05.04. Subordo Pseudophiona Blainville, 1816.ba.c11-06

Derotremata Müller, 1831

SI: 112 • CI: c093 • ST: 1.D.M.32.O

PN: Derotremata Müller, 1831.ma.c01 • AK

PA: 00 - Derotremata • Müller 1831.ma: 711 • O

01 - DeIRETREMATA - Leuckart 1840.1a: $19 \cdot$ 'F'

02 - DeIRETREMATA • Leuckart 1841.1a: 30 • UC

03 - DEROTREMATA • Fitzinger 1843.fa: $34 \cdot$ Sc

$04 \cdot$ Derotremata $\cdot$ Stannius $1856 . \mathrm{sa}: 4 \cdot \mathbf{b O}$

05 - Derotermata - Meyer 1860.ma: 50 • O

$06 \cdot$ Derotrema $\cdot$ Claus 1868.cb: $585 \cdot$ bO

07 - DeRotrema - Knauer 1878.ka: $96 \cdot \mathbf{U C}$

RL: < NUlLIBRANCHIA 1831.ba.c01 - AI: Dubois 2015: 49

GN: IMPERFECTIBRANCHIA 1838.ha.c03

Pseudosauria 1816.ba.c08

GZ: INR

EN: KYR. C.04.03. Ordo Urodela Duméril, 1805.da.c02-12

[HYP. Subordo Nullibranchia Bonaparte, 1831.ba.c01-01]

Derotreta Van der Hoeven, 1833

SI: $118 \bullet$ CI: c098 • ST: 1.D.M.03.O

PN: Derotreta Van der Hoeven, 1833.va.c01 • HK

PA: 00 - Derotreta • Van der Hoeven 1833.va: iii, 302 • O

01 - Derotreta - Dubois 2015.da: 51 • pO

RL: INR

GN: GYMNOPHIONA 1814.ra.c01

URODELA 1805.da.c02

GZ: INR

EN: KYR. C.03.01. Subclassis Lissamphibia Gadow, 
1898.ga.c01-00

[HYP. Superordo Derotreta Van der Hoeven, 1833.va.c01-01]

DiADACTYlobatrachia Ritgen, 1828

SI: $091 \bullet$ CI: c072 • ST: 2.D.M.32.E

PN: DiaDaCtYlobatrachi Ritgen, 1828.ra.c17 • AK

PA: 00 - DiaDACTYLOBATRACHI • Ritgen 1828.ra: 278 • 'F' $01 \cdot$ DiadaCtYlobatrachia $\cdot$ Hoc loco $\bullet$ EE

RL: $\leftrightarrow<$ GEOBATRACHI 1828.ra.c18 • AI: HL

GN: DORSIPARES 1816.ba.c06

LAEVOGYRINIA 1878.la.c01

GZ: INR

EN: KYR. C.06.01. Infraordo GEOBATRACHIA Ritgen, 1828.ra.c18-02

Dimela Gouriet, 1868

SI: 248 • CI: c222 • ST: 1.D.M.31.E

PN: DiMeles Gouriet, 1868.ga.c03 • AK

PA: 00 • DimeLES • Gouriet 1868.ga: $206 \cdot$ UC

$01 \cdot$ DimeLA $\cdot$ Hoc loco $\bullet$ EE

RL: INR

GN: Meantes 1767.la.c01

GZ: INR

EN: KYR. C.05.06. Subordo MeanTeS Linné, 1767.la.c01-01

DiPLASIOCOELA Nicholls, 1916

SI: $305 \bullet$ CI: c278 • ST: 1.D.M.31.O

PN: DiPlasiocoela Nicholls, 1916.na.c04 • AK

PA: 00 - DiPLASIOCOELA • Nicholls 1916.na: 87 • 'T' $01 \cdot$ DiPLASIOCOELINA $\cdot$ Pearse 1936.pa: $20 \cdot$ bO

RL: INR

GN: Phaneranura DOP.da.c02 SCOPTANURA 1973.sb.c02

GZ: INR

EN: TEO. C.09.01. Epiphalanx AQUIPARES Blainville, 1816.ba.c07-02

[HYP. Phalanx AQuiPares Blainville, 1816.ba.c07-03]

DiPlasiocOELA Noble 1922

SI: $324 \cdot$ CI: c297 • ST: 1.D.M.21.O

PN: DiPlasiocoela Noble 1922.na.c03 • AK

PA: 00 - DiPLASIOCOELA • Noble 1922.na: 22 • bO

RL: $\downarrow$ DiPlasiocoela 1916.na.c04

GN: GASTRECHMIA 1867. ca.c02

Pananura DOP.da.c07

GZ: INR

EN: TEO. C.10.03. Phalanx Scoptanura Starrett, 1973.sb.c02-02 [HYP. Subphalanx unnamed]

DiPlasiocoEla Ahl, 1930

SI: 338 • CI: c311 • ST: 1.D.M.21.O

PN: DiPlasiocoela Ahl, 1930.aa.c03 • AK

PA: 00 • DiPLASIOCOELA • Ahl 1930.aa: $85 \cdot$ bO

01 - DisPlaciocoela • Casamiquela 1961.ca: 77, 80 • bO

RL: $\downarrow$ DiPlasiocoela 1916.na.c04

GN: ECOSTATA 1879.1b.c04

GASTRECHMIA 1867.ca.c02

PANANURA DOP.da.c07

GZ: INR

EN: TEO. C.10.03. Phalanx Scoptanura Starrett, 1973.sb.c02-02
DiplopNeuma Hogg, 1838

SI: $131 \bullet$ CI: c111 • ST: 1.D.M.31.E

PN: Diplopneumena Hogg, 1838.ha.c04 • AK

PA: 00 • DiPLOPNEUMENA • Hogg 1838.ha: $152 \cdot$ bC

01 - DiplopNeUmona - Agassiz 1847.aa: $363 \cdot$ UC

$02 \cdot$ DiplopNeUMA $\cdot H o c$ loco $\bullet$ EE

RL: > MANentibranchia 1838.ha.c06 • PR

GN: MEANTES 1767.la.c01

Pseudosauria 1816.ba.c08

GZ: INR

EN: KYR. C.04.03. Ordo Urodela Duméril, 1805.da.c02-12

[HYP. Subordo Pneumobranchia Sonnini ${ }^{+1}$, 1801.sa.c01-02]

DiPlopNeUMA Hogg, 1838

SI: $143 \bullet$ CI: c121・ST: 1.D.M.40.E

PN: DiPlopNeumena Hogg, 1839.ha.c08 • AK

PA: 00 - DiPlopNeumena • Hogg 1839.ha: $274 \bullet$ bC

01 - Diplopneumona - Agassiz 1847.aa: $363 \cdot$ bC

02 - Diplopneuma • Duméril 1863.da: $301 \cdot$ bC

RL: $\downarrow$ DiPlopNeumena 1838 .ha.c05

$>$ LACERTIFORMI 1839.ha.c06 • PR

GN: IMPERFECTIBRANCHIA 1838.ha.c03

Meantes 1767.la.c01

Pseudosauria 1816.ba.c08

GZ: INR

EN: KYR. C.04.03. Ordo Urodela Duméril, 1805.da.c02-12

DIPLOPNOA Bonaparte, 1838

SI: $127 \bullet$ CI: c107 • ST: 1.D.M.30.O

PN: DiPLOPNOA Bonaparte, 1838.bd.c02 • AK

PA: $00 \cdot$ DiPLOPNOA • Bonaparte 1838.bd: $124 \cdot$ bC

$01 \cdot$ DiPlopNOA • Van der Hoeven 1864.va: 288 • Sc

RL: $\leftrightarrow$ ! $<$ DIPNOA 1838.bd.c01

GN: ANURA 1805.da.c01

GYMNOPHIONA 1814.ra.c01

Urodela 1805.da.c02

GZ: INR

EN: KYR. C.03.01. Subclassis Lissamphibia Gadow, 1898.ga.c01-00

Diplosiphona Günther, 1859

SI: 212 • CI: c189 • ST: 2.D.M.11.O

PN: Diplosiphona Günther, 1859.ga.c02

PA: 00 - DiPlosiPHONA • Günther 1859.ga: vii, $3 \cdot \mathbf{S r}$

$01 \cdot$ Diplosiphona $\cdot$ Hoc loco $\bullet$ bP

RL: INR

GN: DiPLOSIPHONA 1859.ga.c02

GZ: INR

EN: KYR. C.11.02. Subphalanx DiplosiphoNa Günther, 1859.ga.c02-01

DiPNOA Leuckart, 1821

SI: $055 \bullet$ CI: c037 • ST: 1.D.M.30.O

PN: DIPNOA Leuckart, 1821.la.c02 • AK

PA: 00 - DiPNOA • Leuckart 1821.1a: 258 • O

$01 \cdot$ DiPNOA $・$ Van der Hoeven 1833.va: iii, $302 \cdot$ Sc

02 - DIPNOA - Leuckart 1840.1a: $19 \cdot \mathbf{D}$

03 - DiPNOA $\cdot$ Stannius 1856.sa: $3 \cdot \mathbf{b C}$

RL: INR

GN: ANURA 1805.da.c01 
URODELA 1805.da.c02

GZ: INR

EN: KYR. C.03.01. Subclassis LisSAMPHibia Gadow, 1898.ga.c01-00

[HYP. Superordo BATRACHIA Brongniart, 1800.ba.c01-14]

DiPNOA Wagler, 1828

SI: 094 • CI: c075 • ST: 3.D.M.41.O

PN: DiPNOA Wagler, 1828.wb.c01 • AK

PA: 00 • DIPNOA • Wagler 1828.wb: 859 • 'T'

RL: $\downarrow$ DIPNOA 1821.la.c02

GN: Pseudosauria 1816.ba.c08

GZ: INR

EN: KYR. C.05.07. Subordo Pseudosauria Blainville, 1816.ba.c08-07

DIPNOA Bonaparte, 1838

SI: $126 \bullet$ CI: c106・ST: 1.D.M.40.O

PN: DiPNOA Bonaparte, 1838.bd.c01 • AK

PA: 00 • DiPNOA • Bonaparte 1838.bd: $124 \cdot$ bC

01 - DiPNOA • Leuckart 1840.1a: $20 \cdot \mathbf{A b}$

$02 \cdot$ DiPNOA • Fitzinger 1843.fa: $12 \cdot$ O

03 - DiPNOA • Tschudi 1845.ta: $167 \cdot \mathbf{S r}$

$04 \cdot$ DiPnOA • Van der Hoeven 1855.va: x, $459 \cdot$ Sc

RL: $\downarrow$ DIPNOA 1821.la.c02 i > DiPlOPNOA 1837.ba.c02

GN: ANURA 1805.da.c01

GYMNOPHIONA 1814.ra.c01

URODELA 1805.da.c02

GZ: INR

EN: KYR. C.03.01. Subclassis LisSAMPhibia Gadow, 1898.ga.c01-00

DipoDA Blainville, 1816

SI: 045 • CI: zh10 • ST: 1.U.U.99.E

PN: DiPODEs Blainville, 1816.da.c13 • zz

PA: 00 • DiPODES • Blainville 1816.ba: "112” [120] • O

01 - DIPODA • Jourdan 1834.ja: $397 \cdot$ UC

RL, GN, GZ, EN: •

DipodA Hogg, 1839

SI: $137 \bullet$ CI: c115 • ST: 1.D.M.41.O

PN: DiPODA Hogg, 1839.ha.c02 • AK

PA: $00 \cdot$ DIPODA • Hogg 1839.ha: $271 \cdot$ O

RL: $\downarrow$ DIPODES 1816.da.c13

GN: Meantes 1767.la.c01

GZ: INR

EN: KYR. C.05.06. Subordo Meantes Linné, 1767.la.c01-01

DiscoDACTYla Knauer, 1878

SI: $268 \bullet$ CI: c242 • ST: 1.D.M.31.E

PN: Discodactylia Knauer, 1878.ka.c04 • AK

PA: 00 - DiscodACTYLIA $・$ Knauer 1878.ka: $109 \cdot$ Gr $01 \cdot$ DiscodaCTYLA $\cdot H o c$ loco $\bullet$ EE

RL: INR

GN: Phaneranura DOP.da.c02 SCOPTANURA 1973.sb.c02

GZ: INR

EN: TEO. C.09.01. Epiphalanx AQUIPARES Blainville, 1816.ba.c07-02

[HYP. Phalanx AQUIPARES Blainville, 1816.ba.c07-03]
DiscODACTYLA Blanchard, 1885

SI: $286 \bullet$ CI: c260 • ST: 1.D.M.40.E

PN: DisCODACTYLES Blanchard, 1885.bb.c02 • AK

PA: 00 - DisCODACTYLES • Blanchard 1885.bb: 588 • UC

$01 \cdot$ DisCODACTYLA $\cdot$ Hoc loco $\bullet$ EE

RL: $\downarrow$ DisCODACTYLA $1878 . \mathrm{ka} . \mathrm{c} 04$

GN: Phoranura DOP.da.c04

Phrynanura DOP.da.c05

GZ: INR

EN: KYR. C.11.01. Subphalanx BAINANURA nov., DOP.da.c03-00 DiscoglossaceA Laurent in Fuhn, 1960

SI: 370 • CI: cn02 • ST: 1.D.M.00.R

PN: Discoglossoidea Laurent in Fuhn, 1960.fa.c02 • AN

PA: 00 - DiscoglossoideA • Laurent in Fuhn 1960.fa: 163 • bO

$01 \cdot$ DiscogLOSSACEA $\bullet$ Hoc loco $\bullet$ ER

RL: INR

GN: GEOBATRACHIA 1828.ra.c18

Mediogyrinia 1878.1a.c02

GZ: INR

EN: KYR. C.05.02. Subordo Hydrobatrachia Ritgen, 1828.ra.c14-01

DiscoglossacEA Sokol, 1977

SI: 400 • CI: c360 • ST: 1.D.M.30.R

PN: DiscoglossoIDEI Sokol, 1977.sa.c01 • AK

PA: 00 • DiscoglossoIDEI • Sokol 1977.sa: 505 • bO

01 - DisCOGLOSSACEA • Dubois 2015.da: 106 • ER

RL: INR

GN: ANGUSTICOELA 1958.ra.c01 Hydrobatrachia 1828.ra.c14

GZ: INR

EN: KYR. C.04.01. Ordo AnURa Duméril, 1805.da.c01-07

Dorsipares Blainville, 1816

SI: $038 \bullet$ CI: $\mathrm{c} 025 \bullet$ ST: 1.D.M.11.O

PN: DorsIPARES Blainville, 1816.ba.c06

PA: 00 • DorSIPARES • Blainville 1816.ba: '111' [119] • bO

$01 \cdot$ DoRSIPARI $・$ Jourdan 1834.ja: $409 \cdot$ bO

$02 \cdot$ DoRSIPARES $\cdot$ Hoc loco $\bullet$ hO

RL: INR

GN: » OA, SD: Ducrotay Blainville 1822.da: 5: DORSIPARES 1816.ba.c06

GZ: INR

EN: KYR. C.07.01. Hypoordo DorsIPARES Blainville, 1816.ba.c06-02

DuPlogYrinia Lataste, 1888

SI: $288 \bullet$ CI: c262 • ST: 1.D.M.30.E

PN: DUPLOGYRINIDAE Lataste, 1888.la.c01・AK

PA: c0 • DUPLOGYRINIDAE • Lataste 1888.1a: 240 - UC

i1・DUPlOgYRINIDES • Lataste 1888.1a: 240 • UC

$02 \cdot$ DuplOGYRINIA $\cdot$ Hoc loco $\bullet$ EE

RL: $\leftarrow$ AMPHIGYRINIA 1885.bb.c01

GN: DoRSIPARES 1816.ba.c06

GZ: INR

EN: KYR. C.07.01. Hypoordo DorsIPARES Blainville, 1816.ba.c06-02

Dysmolgae Ritgen, 1828

SI: $079 \bullet \mathbf{C I}: \mathrm{c060} \bullet$ ST: 2.D.M.31.O 
PN: Dysmolgae Ritgen, 1828.ra.c05 • AK

PA: 00 • DySMOLGAE • Ritgen 1828.ra: $277 \cdot \mathbf{Z g}$

RL: $\leftrightarrow>$ BRANCHIUROMOLGAEI 1828.ra.c04 • AI: HL

GN: Meantes 1767.la.c01

Pseudosauria 1816.ba.c08

GZ: INR

EN: KYR. C.04.03. Ordo URODELA Duméril, 1805.da.c02-12 [HYP. Subordo Pneumobranchia Sonnini ${ }^{+1}$, 1801.sa.c01-02]

Ecaudata Scopoli, 1777

SI: $013 \cdot$ CI: c004 • ST: 1.D.M.11.O

PN: ECAUdata Scopoli, 1777.sa.c06

PA: 00 - ECAUdATA - Scopoli 1777.sa: $464 \cdot$ O

$01 \cdot$ ECAUdATA $\cdot H o c$ loco $\bullet$ iP

RL: INR

GN: ECAUDATA 1777 .sa.c06

GZ: INR

EN: KYR. C.12.03. Infraphalanx ECAUdATA Scopoli, 1777.sa.c06-01

ECAUDATA Duméril, 1805

SI: $019 \bullet \mathbf{C I}$ : $\mathrm{c010} \bullet$ ST: 2.D.A.40.E

PN: ECAUdATI Duméril, 1805.da.c03 • AK

PA: 00 - ECAUdATI • Duméril 1805.da: 929 • 'F'

01 - ECAUDATA • Oppel 1811.ob: 409 • 'F'

$02 \cdot$ ECAUDATA $・$ Leuckart 1821.1a: $259 \cdot$ UC

$03 \cdot$ ECAUDATA $\cdot$ Hemprich 1829.ha: xix, $373 \cdot$ Fo

$04 \cdot$ ECAUDATA $^{*}$ Wiegmann $^{+1}$ 1832.wa: $198 \cdot$ bO

$05 \cdot$ ECAUDATA $\cdot$ Gravenhorst 1843.ga: $393 \cdot \mathbf{Z t}$

$06 \cdot$ ECAUdATA $・ H a e c k e l$ 1866.ha: cxxxii • O

$07 \cdot$ ExAUdATA - Zittel 1888.za: $421 \cdot$ O

$08 \cdot$ ECAUDATA $・ H a e c k e l ~ 1889$.ha: $625 \cdot \mathbf{L}$

09 • EuCAUdATA • Tilak ${ }^{+1}$ 1977.ta: $196 \cdot$ bC

RL: $\downarrow$ ECAUDATA 1777 .sa.c06

$\leftrightarrow<$ ANOURES 1805.da.c01・SD

GN: ANURA 1805.da.c01

GZ: » GX:

GYMNOPHIONA 1814.ra.c01

URODELA 1805.da.c02

EN: KYR. C.04.01. Ordo ANURA Duméril, 1805.da.c01-07

ECAUDATA Jarocki, 1822

SI: 059 • CI: c041 • ST: 1.D.M.41.E

PN: ECAUDATA Jarocki, 1822.ja.c02 • AK

PA: 00 • ECAUDATA • Jarocki 1822.ja: $137 \cdot$ O

RL: $\downarrow$ ECAUDATA 1777 .sa.c06

GN: DoRSIPARES 1816.ba.c06

LAEVOGYRINIA 1878.1a.c01

GZ: INR

EN: KYR. C.06.01. Infraordo GEOBATRACHIA Ritgen, 1828.ra.c18-02

EcAudata Van der Hoeven, 1828

SI: 093 • CI: c074 • ST: 3.D.M.41.E

PN: ECAUdATI Van der Hoeven, 1828.va.c01 • AK

PA: 00 - ECAUdATI • Van der Hoeven 1828.va: pl. • 'F'

$$
01 \cdot \text { ECAUdATA } \cdot H o c \text { loco } \cdot \mathbf{E E}
$$

RL: $\downarrow$ ECAUDATA 1777 .sa.c06

GN: Phaneranura DOP.da.c02

ScoptanURA 1973.sb.c02
GZ: INR

EN: TEO. C.09.01. Epiphalanx AQUIPARES Blainville, 1816.ba.c07-02

[HYP. Phalanx AQUIPAREs Blainville, 1816.ba.c07-03]

ECAUDATA Wagler, 1830

SI: 105 • CI: c086 • ST: 2.D.M.41.E

PN: ECAUdatae Wagler, 1830.wa.c04 • AK

PA: 00 - ECAUDATAE • Wagler 1830.wa: $131 \cdot \mathbf{D}$

$01 \cdot$ ECAUdATA • Leunis 1844.1a: $144 \cdot$ 'F'

$02 \cdot$ ACAUdata - Knauer 1878.ka: $100 \cdot \mathbf{O}$

$03 \cdot$ ECAUDATA $・$ Lataste 1879.1a: $339 \cdot \mathbf{O}$

RL: $\downarrow$ ECAUDATA 1777 .sa.c06

GN: GEOBATRACHIA 1828.ra.c18

Mediogyrinia 1878.1a.c02

GZ: INR

EN: KYR. C.05.02. Subordo Hydrobatrachia Ritgen, 1828.ra.c14-01

ECAUdATA Hoffmann, 1878

SI: $261 \bullet$ CI: c235 • ST: 1.D.M.41.O

PN: ECAUDATa Hoffmann, 1878.ha.c01 • AK

PA: 00 - ECAUDATA - Hoffmann 1878.ha: $615 \cdot$ O

RL: $\downarrow$ ECAUDATA 1777 .sa.c06

< THERIOMORPHI 1878.ha.c02 • AI: HL

GN: ANGUSTICOELA 1958.ra.c01

Hydrobatrachia 1828.ra.c14

GZ: INR

EN: KYR. C.04.01. Ordo ANura Duméril, 1805.da.c01-07

ECostata Lataste, 1879

SI: 278 • CI: c252 • ST: 1.D.M.11.E

PN: ECOSTATi Lataste, 1879.1b.c04

PA: $00 \cdot$ ECosTaTI • Lataste 1879.1b: $339 \cdot$ 'bT'

$01 \cdot$ ECosTaTa $\cdot$ Dubois $^{+2} 2016$. da: $49 \cdot \mathbf{E E}$

$02 \cdot \mathbf{E C O S T A T A} \cdot H$ Hoc loco $\bullet \mathbf{b P}$

RL: INR

GN: ECOSTATA 1879.1b.c04

GZ: INR

EN: KYR. C.11.03. Subphalanx Ecostata Lataste, 1879.1b.c04-02

EDEnTATa Scopoli, 1777
SI: $010 \bullet$ CI: zh04 • ST: 2.D.M.99.E
PN: EdENTATI Scopoli, 1777.sa.c03 • zz
PA: $00 \cdot$ EdENTATI $・$ Scopoli 1777.sa: $452 \cdot$ D
$01 \cdot$ Edentata $\cdot$ Hoc loco $\bullet$ EE
RL, GN, GZ, EN: •

EDENTATA Fatio, 1872

SI: $256 \bullet \mathbf{C I}: \mathrm{c} 230 \bullet \mathbf{S T}: 1 . D . M .41 . \mathrm{O}$

PN: Edentata Fatio, 1872.fa.c03 • AK

PA: 00 - EDENTATA • Fatio 1872.fa: 230 - D

RL: $\downarrow$ Edentati 1777 .sa.c03

$<$ Dentata 1872.fa.c04 • AI: HL

$<$ Edentata 1872.fa.c05 • AI: HL

GN: Phaneranura DOP.da.c02

SCOPTANURA 1973.sb.c02

GZ: INR

EN: TEO. C.09.01. Epiphalanx AQUIPARES Blainville, 1816.ba.c07-02

[HYP. Phalanx AquiPares Blainville, 1816.ba.c07-03] 
EDENTATA Fatio, 1872

SI: $258 \bullet$ CI: c232 • ST: 1.D.M.41.O

PN: EDENTATA Fatio, 1872.fa.c05 • AK

PA: 00 • EdENTATA $・$ Fatio 1872 fa: $230,417 \cdot \mathbf{D}$

RL: $\downarrow$ EDENTATI 1777.sa.c03

$>$ EDENTATA 1872.fa.c03 • AI: HL

GN: HyLOBATRACHIA 1828.ra.c16

GZ: INR

EN: KYR. C.13.03. Hypophalanx Hylobatrachia Ritgen, 1828.ra.c16-01

EOAPODA Duellman ${ }^{+1}, 2007$

SI: 432 • CI: c392 • ST: 1.D.M.31.O

PN: EOAPODA Duellman ${ }^{+1}$, 2007.da.c01 • AK

PA: $00 \cdot$ EOAPODA $・$ Duellman $^{+1}$ 2007.da: $2129 \bullet$ bO

RL: INR

GN: |GYMNOPHIONA INCERTAE SEDIS|

GZ: INR

EN: KYR. C.04.02. Ordo GyMnophiona Rafinesque, 1814.ra.c01-02

EPICRIACEA Lescure ${ }^{+2}, 1986$

SI: $414 \bullet$ CI: c374 • ST: 1.D.M.31.R

PN: EPICRIIDEI Lescure ${ }^{+2}, 1986.1 \mathrm{~b} . c 02 \cdot \mathbf{A K}$

PA: $00 \cdot$ EPICRIIDEI $\bullet$ Lescure $^{+2}$ 1986.lb: $152 \cdot$ iO $01 \cdot$ EPICRIACEA $\bullet$ Hoc loco $\bullet$ ER

RL: INR

GN: PSEUDOPHIONA 1816.ba.c11

GZ: INR

EN: KYR. C.05.04. Subordo Pseudophiona Blainville, 1816.ba.c11-06

EUAMPHIBIA Goodrich, 1930

SI: $343 \cdot$ CI: c316 • ST: 3.D.M.30.O

PN: EUAMPHIBIA Goodrich, 1930.ga.c05 • AK

PA: 00 • EUAMPHIBIA • Goodrich 1930.ga: $319 \cdot$ UC

RL: INR

GN: ANURA 1805.da.c01

GyMNOPHIONA 1814.ra.c01

URODELA 1805.da.c02

GZ: INR

EN: KYR. C.03.01. Subclassis LisSAMPHIBIA Gadow, 1898.ga.c01-00

EuANura Piveteau, 1937

SI: $360 \bullet$ CI: c330 • ST: 1.D.A.31.E

PN: EuANOURA Piveteau, 1937.pa.c01 • AK

PA: $00 \cdot$ EUANOURA • Piveteau 1937.pa: $169 \cdot$ bO/O

$01 \cdot$ EU-ANURA $・$ Kuhn 1939.ka: $18 \cdot$ bO

02 • EUANURA $・$ Kuhn 1939.ka: $91 \cdot$ bO

03 - EOANURA • Pearse 1948.pa: $20 \cdot \mathbf{O}$

RL: $\leftarrow$ ANOures 1805.da.c01

> Proanoura 1937.pa.c02 • AI: HL

GN: ANURA 1805.da.c01

GZ: » GX:

GYMNOPHIONA 1814.ra.c01

URODELA 1805.da.c02

EN: KYR. C.04.01. Ordo ANURa Duméril, 1805.da.c01-07

EubATRACHIA Gouriet, 1868

SI: 247 • CI: c221 • ST: 1.D.M.30.E
PN: EUbatraciens Gouriet, 1868.ga.c02 • AK

PA: 00 - EUBATRACIENS • Gouriet 1868.ga: $204 \cdot \mathbf{S r}$

01 - Eubatrachia • Miranda-Ribeiro 1924.ma: 137 • UC

RL: > PulMones 1868.ga.c01 • AI: HL

$>$ ATRETODERES 1868.ga.c05 - AI: HL

GN: ANURA 1805.da.c01

GYMNOPHIONA 1814.ra.c01

URODELA 1805.da.c02

GZ: INR

EN: KYR. C.03.01. Subclassis Lissamphibia Gadow, 1898.ga.c01-00

Euglossa Bauer, 1987

SI: 418 • CI: c378 • ST: 1.D.M.30.O

PN: Euglossa Bauer, 1987.bc.c03 • AK

PA: 00 • EUGLOSSA • Bauer 1987.bc: 52 • UC

RL: INR

GN: DORSIPARES 1816.ba.c06

LAEVOGYRINIA 1878.1a.c01

GZ: INR

EN: KYR. C.06.01. Infraordo GEOBATRACHIA Ritgen, 1828.ra.c18-02

EXOBRANCHIA Duméril ${ }^{+1}, 1841$

SI: 159 • CI: c136 • ST: 2.D.M.31.E

PN: ExobranCheS Duméril ${ }^{+1}, 1841 . d a . c 06 \cdot$ AK

PA: 00 • ExobrancheS • Duméril ${ }^{+1} 1841$ da: 52 • Gr/Sc/'T'

$01 \cdot$ Exobranchia $\cdot$ Hoc loco $\bullet$ EE

RL: < ATRETODERES 1841.da.c03 • AI: HL

$>$ Perobranches 1841.da.c04 • AI: HL

$<$ AMPHIUMOIDES 1841.da.c05 • AI: HL

< Trematoderes 1841.da.c08 • AI: HL

GN: Pseudosauria 1816.ba.c0 8

GZ: INR

EN: KYR. C.05.07. Subordo Pseudosauria Blainville, 1816.ba.c08-07

EXTERNIBRANCHIA Hogg, 1839

SI: 145 • CI: c123 • ST: 1.D.M.31.O

PN: EXTERNibRanChia Hogg, 1839.hb.c02 • AK

PA: 00 • EXTERNIBRANCHIA • Hogg 1839.hb: 375 • 'T'

RL: INR

GN: MEANTES 1767.la.c01

Pseudosauria 1816.ba.c08

GZ: INR

EN: KYR. C.04.03. Ordo Urodela Duméril, 1805.da.c02-12

[HYP. Subordo Pneumobranchia Sonnini ${ }^{+1}$, 1801.sa.c01-02]

Firmisternia Cope, 1875

SI: $259 \bullet \mathbf{C I}: \mathrm{c} 233 \bullet \mathbf{S T}: 1 . \mathrm{D} . \mathrm{M} .31 . \mathrm{O}$

PN: Firmisternia Cope, 1875.ca.c01 • AK

PA: 00 - FirmisTernia - Cope 1875.ca: 8 • bO

$01 \cdot$ Firmisternia $\cdot$ Boulenger 1882.bb: vii, $2 \cdot \mathbf{S r}$

02 - Firmisternia - Zittel 1888.za: $428 \cdot$ UC

03 • Firmisternia • Cope 1889.ca: $246 \bullet$ 'pF'

RL: INR

GN: Phaneranura DOP.da.c02

SCOPTANURA 1973.sb.c02

GZ: INR

EN: TEO. C.09.01. Epiphalanx AQUIPARES Blainville, 


\section{6.ba.c07-02}

[HYP. Phalanx AQUIPARES Blainville, 1816.ba.c07-03]

FIRMISTERNIA Zittel, 1888

SI: $290 \bullet$ CI: c263・ST: 2.D.M.41.O

PN: Firmisternia Zittel, 1888.za.c02 • AK

PA: 00 • FIRMISTERNIA - Zittel 1888.za: viii, 428 • UC

RL: $\downarrow$ FIRMISTERNIA 1875.ca.c01

GN: ECAUDATA 1777.sa.c06

|HYDROBATRACHIA INCERTAE SEDIS|

GZ: INR

EN: KYR. C.05.02. Subordo Hydrobatrachia Ritgen, 1828.ra.c14-01

FIRMiSTERnia Abel, 1919

SI: 308 • CI: c281・ST: 2.D.M.21.O

PN: Firmisternia Abel, 1919.aa.c01 • AK

PA: 00 • Firmisternia • Abel 1919.aa: xii, $324 \cdot \mathbf{R}$

RL: $\downarrow$ FIRMISTERNIA 1875. ca.c0 1

GN: ECOSTATA 1879.1b.c04

Pananura DOP.da.c07

GZ: INR

EN: TEO. C.10.03. Phalanx ScOPTANura Starrett, 1973.sb.c02-02 [HYP. Subphalanx unnamed]

Firmisternia Goodrich, 1930

SI: 339 • CI: c312 • ST: 2.D.M.41.O

PN: Firmisternia Goodrich, 1930.ga.c01 • AK

PA: 00 • Firmisternia • Goodrich 1930.ga: xxi • Sc

RL: $\downarrow$ FIRMISTERNIA 1875 .ca.c01

GN: DoRSIPARES 1816.ba.c06 LAEVOGYRINIA 1878.1a.c01

GZ: INR

EN: KYR. C.06.01. Infraordo GEObaTRACHIA Ritgen, 1828.ra.c18-02

Gaianura nov.

SI: $439 \bullet \mathbf{C I}: \mathrm{c} 398 \bullet \mathbf{S T}: 1 . \mathrm{D} . \mathrm{M} .11 . \mathrm{O}$

PN: GaIANURA nov., DOP.da.c06

PA: 00 • GaIANURA $\cdot H o c$ loco $\bullet \mathbf{h P}$

RL: INR

GN: GaIANURA DOP.da.c06

GZ: INR

EN: KYR. C.13.01. Hypophalanx GAIANURA nov., DOP.da.c06-00 Gastrechmia Cope, 1867

SI: $242 \bullet$ CI: c216 • ST: 1.D.M.11.O

PN: Gastrechmia Cope, 1867.ca.c02

PA: 00 - GASTREChMia - Cope 1867.ca: 190 • bO

01 - GASTRECHMIA • Hoffmann 1878.ha: $598 \cdot$ UC

02 - GASTRECHMia • Cope 1889.ca: 246 • 'pF'

$03 \cdot \mathbf{G A S T R E C H M I A} \cdot H o c$ loco $\bullet \mathbf{b P}$

RL: INR

GN: GASTRECHMIA 1867.ca.c02

GZ: INR

EN: KYR. C.11.04. Subphalanx GaStrechmia Cope, 1867.ca.c02-03

Gastrechmia Miranda-Ribeiro, 1924

SI: $331 \bullet \mathbf{C I}$ : c304 • ST: 2.D.M.21.O

PN: Gastrechmia Miranda-Ribeiro, 1924.ma.c06 • AK

PA: 00 • GASTREChMia • Miranda-Ribeiro 1924.ma: 143 • UC
RL: $\downarrow$ GASTRECHMIA $1867 . c a . c 02$

GN: ECOSTATA 1879.1b.c04

GASTRECHMIA 1867.ca.c02

GZ: INR

EN: TEO. C.10.03. Phalanx Scoptanura Starrett, 1973.sb.c02-02

[HYP. Subphalanx ScoptanURa Starrett, 1973.sb.c02-02]

Geobatrachia Ritgen, 1828

SI: 092 • CI: c073 • ST: 2.D.M.12.E

PN: GeObatraChI Ritgen, 1828.ra.c18

PA: 00 • GEOBATRACHI • Ritgen 1828.ra: 278 • 'F'

01 - GEObatrachia - Dubois ${ }^{+2} 2016 . d b: 49 \cdot \mathbf{E E}$

$02 \cdot$ GeOBATRACHIA $\cdot$ Hoc loco $\bullet$ iO

RL: $\leftrightarrow>$ DIADACTYLOBATRACHI 1828.ra.c17 • AI: HL

GN: DoRSIPARES 1816.ba.c06

LAEVOGYRINIA 1878.1a.c01

GZ: INR

EN: KYR. C.06.01. Infraordo GEOBATRACHIA Ritgen, 1828.ra.c18-02

Geodytae Fitzinger, 1843

SI: $173 \bullet$ CI: c150 • ST: 2.D.M.31.O

PN: GeOdyTaE Fitzinger, 1843.fa.c05 • AK

PA: 00 - GEODYTAE • Fitzinger 1843.fa: $33 \cdot$ Sc

$01 \cdot$ GEODITAE $・$ Tschudi 1845. tb: $78 \cdot \mathbf{S c}$

RL: INR

GN: DORSIPARES 1816.ba.c06

LAEVOGYRINIA 1878.1a.c01

GZ: INR

EN: KYR. C.06.01. Infraordo GEOBATRACHIA Ritgen, 1828.ra.c18-02

Geomolgae Ritgen, 1828

SI: $085 \bullet$ CI: c066 • ST: 2.D.M.31.0

PN: Geomolgae Ritgen, 1828.ra.c11 • AK

PA: 00 • GeOMolgaE • Ritgen 1828.ra: 279 • 'F'

RL: > Pododysmolgae 1828.ra.c07 • AI: HL

$<$ Morphiuromolgaei 1828.ra.c08 • PR

$<$ Molgae 1828.ra.c09 • AI: HL

$>$ Hydromolgae 1828.ra.c10 • AI: HL

GN: Pseudosauria 1816.ba.c0 8

GZ: INR

EN: KYR. C.05.07. Subordo Pseudosauria Blainville, 1816.ba.c08-07

GeOPHILI Menke, 1828

SI: $074 \bullet$ CI: zh15 • ST: 1.U.U.99.E

PN: GeOPHILAE Menke, 1828.ma.c01 • zz

PA: 00 • GEOPHILAE • Menke 1828.ma: 7 • bO

01 - GEOPHILA • Jourdan 1834.ja: 542 • UC

$02 \cdot \mathbf{G E O P H I L I} \cdot \mathrm{Hoc} l o c o \cdot \mathbf{E E}$

RL, GN, GZ, EN:

GeOPHILI Fitzinger, 1843

SI: $176 \bullet$ CI: c153・ST: 2.D.M.40.E

PN: GeOPHILI Fitzinger, 1843.fa.c08 • AK

PA: 00 • GEOPHILI • Fitzinger 1843.fa: 33 • Sc

$01 \cdot$ ELOPHILE • Gray 1850.ga: 14, 70 •UC

RL: $\downarrow$ GEOPHILA 1828.ma.c01

> HYDROPHILI 1843.fa.c09 • AI: HL

GN: IMPERFECTIBRANCHIA 1838.ha.c03 
Pseudosauria 1816.ba.c08

GZ: INR

EN: KYR. C.04.03. Ordo Urodela Duméril, 1805.da.c02-12

[HYP. Subordo Nullibranchia Bonaparte, 1831.ba.c01-01]

\section{GONDWANURA nov.}

SI: 434 • CI: c393 • ST: 1.D.M.11.O

PN: GoNDWANura nov., DOP.da.c01

PA: $00 \cdot$ GONDWANURA $\cdot H o c$ loco $\bullet$ P

RL: INR

GN: GONDWANURA DOP.da.c01

GZ: INR

EN: KYR. C.10.01. Phalanx GoNdwanura nov., DOP.da.c01-00 GongYlura Duméril ${ }^{+2}, 1854$

SI: 198 • CI: c175 • ST: 2.D.M.31.E

PN: GoNGYLURES Duméril ${ }^{+2}$, 1854.da.c04 • AK

PA: 00 • GONGYLURES • Duméril ${ }^{+2}$ 1854.da: 38 • UC

$01 \cdot$ GONGYLURA $\cdot H o c$ loco $\bullet$ EE

RL: $\leftrightarrow>$ RoTONDICAUDES 1854.da.c03 • AI: HL

GN: PSEUdosauria 1816.ba.c0 8

GZ: INR

EN: KYR. C.05.07. Subordo Pseudosauria Blainville, 1816.ba.c08-07

GradiENTIA Laurenti, 1768

SI: $007 \bullet$ CI: $\mathrm{mc03} \bullet$ ST: 1.D.M.99.O

PN: Gradientia Laurenti, 1768.1a.c02 • ZA

PA: 00 - GRADIENTIA - Laurenti 1768.la: 36 • O

RL: INR

GN: AMPHIBIA 1816.ba.c02 |Amniota|

GZ: INR

EN: •

Gradientia Merrem, 1820

SI: $051 \bullet \mathbf{C I}: \mathrm{c033} \bullet \mathbf{S T}:$ 1.D.M.41.O

PN: Gradientia Merrem, 1820.ma.c02 • AK

PA: 00 - GRADIENTIA • Merrem 1820.ma: 163 • O

RL: $\downarrow$ GRADIENTIA 1768.la.c02

GN: MeanTes 1767.la.c01

Pseudosauria 1816.ba.c08

GZ: INR

EN: KYR. C.04.03. Ordo Urodela Duméril, 1805.da.c02-12 [HYP. Subordo Pneumobranchia Sonnini ${ }^{+1}$, 1801.sa.c01-02] GradiEnTia Gray, 1850

SI: $189 \bullet$ CI: c166・ST: 1.D.M.40.O

PN: Gradientia Gray, 1850.ga.c01・AK

PA: 00 • Gradientia • Gray 1850.ga: $5,13 \cdot$ bO

RL: $\downarrow$ GRADIENTIA 1768 .la.c02

$<$ Pseudosauria 1850.ga.c02 • PR

$<$ Meantia 1850.ga.c04 • PR

GN: IMPERFECTIBRANCHIA 1838.ha.c03 Pseudosauria 1816.ba.c08

GZ: INR

EN: KYR. C.04.03. Ordo URODELA Duméril, 1805.da.c02-12 [HYP. Subordo Nullibranchia Bonaparte, 1831.ba.c01-01] GymnOBATRACHIA Miranda-Ribeiro, 1924

SI: $327 \bullet$ CI: C300 • ST: 2.D.O.31.O

PN: GYMnObatraChia Miranda-Ribeiro, 1924.ma.c02 • AK
PA: 00 • Gymnobatrachia • Miranda-Ribeiro 1924.ma: 138 - UC

RL: $\leftrightarrow \mid$ ANOURES 1805.da.c01

$>$ ANONYXIA 1924.ma.c04 • PR

$>$ THORACECHMIA 1924.ma.c05 • PR

$\leftrightarrow>$ Protosternia 1924.ma.c08 • AI: HL

$>$ THEROSTERNIA 1924.ma.c09 • PR

GN: ANURA 1805.da.c01

GZ: » GX:

GYMNOPHIONA 1814.ra.c01

Urodela 1805.da.c02

EN: KYR. C.04.01. Ordo ANURA Duméril, 1805.da.c01-07 GYMNODERMA Rüppell, 1845

SI: $180 \bullet$ CI: c157 • ST: 1.D.M.30.O

PN: GYMNODERMa Rüppell, 1845.ra.c01 • AK

PA: 00 • GYMNODERMA • Rüppell 1845.ra: 313 • O

RL: INR

GN: ANURA 1805.da.c01

GYMNOPHIONA 1814.ra.c01

URODELA 1805.da.c02

GZ: INR

EN: KYR. C.03.01. Subclassis LisSAMPhibia Gadow, 1898.ga.c01-00

Gymnophiona Rafinesque, 1814

SI: 031 • CI: $\mathrm{c} 018 \bullet \mathbf{S T}$ : 1.S.O.10.E

PN: GYMNOPHIA Rafinesque, 1814.ra.c01

PA: 00 • GYMNOPHia • Rafinesque 1814.ra: 104 • O

01 • GYMNOPHIDIA • Müller 1831.ma: 711 • O

$02 \cdot$ GyMnOPHiONa • Müller 1832.mb: 198 • O

$03 \cdot$ GYMNOPHiONA • Huxley 1871.ha: $173 \cdot \mathbf{U C}$

$04 \cdot$ GyMnOPHiOna • Abel 1919.aa: xii, $332 \cdot$ bC

05 • GYMNOPHIONES • Goodrich 1930.ga: xxi • bC

06 - Gymnophiones • Goodrich 1930.ga: xxi • O

07 • GYMnOPHiONA • Von Huene 1948.ha: 66 • bO

08 • GyMNOPHiONA • Milner 1988.ma: $82 \cdot$ pO

09 • GYMONOPHIONA • Dubois ${ }^{+1}$ 2005.db: 356 • O

$10 \cdot$ GYMNOPHONA $・$ Wilkinson $^{+2}$ 2009.wa: $413 \cdot \mathbf{O}$

RL: INR

GN: GYMNOPHIONA 1814.ra.c01

GZ: » GX:

ANURA 1805.da.c01

Urodela 1805.da.c02

EN: KYR. C.04.02. Ordo GYMNOPHIONA Rafinesque, 1814.ra.c01-02

HAPLOSIPHONA Günther, 1859

SI: $211 \bullet$ CI: c188 • ST: 2.D.M.31.O

PN: HAPlosiphona Günther, 1859.ga.c01 • AK

PA: $00 \cdot$ HAPlosiphona • Günther 1859.ga: vii, $1 \cdot \mathbf{S r}$

RL: INR

GN: DORSIPARES 1816.ba.c06

GZ: INR

EN: KYR. C.07.01. Hypoordo DorsIPARES Blainville, 1816.ba.c06-02

Hedraeoglossa Wagler, 1828

SI: $101 \bullet$ CI: c082 • ST: 3.D.M.31.E

PN: HedraeOglossi Wagler, 1828.wb.c08 • AK

PA: 00 • HedRAEOGLOSSI • Wagler 1828.wb: 859 • 'F' 
01 - Hedraeoglossae - Wagler 1830.wa: 131 • 'F'

$02 \cdot$ Hedraeoglossa $\cdot$ Hoc loco $\bullet$ EE

RL: INR

GN: PSEUdophiona 1816.ba.c11

GZ: INR

EN: KYR. C.05.04. Subordo Pseudophiona Blainville, 1816.ba.c11-06

Hedraeoglossa Wagler, 1830

SI: $106 \bullet$ CI: $\mathbf{c 0 8 7} \bullet$ ST: 2.D.M.40.E

PN: Hedraeoglossi Wagler, 1830.wa.c05 • AK

PA: 00 - HedraeOglossi • Wagler 1830.wa: 131 • 'F' 01 - Hedraeoglossa • Dubois 2015.da: 107 • EE

RL: $\downarrow$ Hedraeoglossi 1828.wb.c08

$>$ ABranchiales 1830.wa.c06 • PR

$>$ BRANCHIALES 1830.wa.c07 • PR

GN: IMPERFECTIBRANCHIA 1838.ha.c03

MeANTeS 1767.la.c01

Pseudosauria 1816.ba.c08

GZ: INR

EN: KYR. C.04.03. Ordo Urodela Duméril, 1805.da.c02-12

Helanura nov.

SI: $442 \bullet \mathbf{C I}: \mathrm{c} 401 \bullet \mathbf{S T}$ : 1.D.M.11.O

PN: Helanura nov., DOP.da.c09

PA: 00 • HelanURa $\cdot$ Hoc loco $\bullet$ eP

RL: INR

GN: HELANURA DOP.da.c09

GZ: INR

EN: KYR. C.09.02. Epiphalanx HelanURA nov., DOP.da.c09-00 HemiBATRACHIA Fitzinger, 1843

SI: $169 \bullet$ CI: c146・ST: 1.D.M.30.O

PN: HeMibatrachia Fitzinger, 1843.fa.c01 • AK

PA: 00 • HemibatraChia • Fitzinger 1843.fa: 12 • O

RL: INR

GN: IMPERFECTIBRANCHIA 1838.ha.c03

Pseudosauria 1816.ba.c08

GZ: INR

EN: KYR. C.04.03. Ordo Urodela Duméril, 1805.da.c02-12 [HYP. Subordo Nullibranchia Bonaparte, 1831.ba.c01-01]

Hemiphractiformia Brocchi, 1881

SI: $279 \bullet$ CI: c253 • ST: 2.D.M.11.A

PN: HeMIPHRACTIFORMES Brocchi, 1881.ba.c01

PA: 00 • HEMIPHRACTIFORMES • Brocchi 1881.ba: 9 • UC $01 \cdot$ HeMiPHRACTIFORMia $\cdot$ Hoc loco $\bullet \mathbf{h P}$

RL: INR

GN: HEMIPHRACTIFORMIA 1881.ba.c01

GZ: INR

EN: KYR. C.13.02. Hypophalanx HeMIPHRACTIFORMIA Brocchi, 1881.ba.c01-01

HemisalamandRaE Fitzinger, 1843

SI: $178 \bullet$ CI: c155 • ST: 2.D.M.31.O

PN: Hemisalamandrae Fitzinger, 1843.fa.c10 • AK

PA: 00 • HemisalamandraE • Fitzinger 1843.fa: $34 \cdot$ Sc

RL: INR

GN: IMPERFECTIBRANCHIA 1838.ha.c03

GZ: INR

EN: KYR. C.05.05. Subordo IMPERFECTIBRANCHIA Hogg, 1838.ha.c03-02

HeTEROMORPHIES Hübner, 1816

SI: $048 \bullet$ CI: zh13・ST: 1.U.U.99.K

PN: HeteromorPhae Hübner, 1816.ha.c01 • zZ

PA: 00 • HETEROMORPHAE • Hübner 1816.ha: 193 • St

01 • HETEROMORPHIES $\bullet$ Hoc loco $\bullet$ EK

RL, GN, GZ, EN: •

HETEROMORPHIES Fitzinger, 1832

SI: $114 \bullet$ CI: $0095 \bullet$ ST: 2.D.M.41.K

PN: HeteromorPHA Fitzinger, 1832.fa.c01 • AK

PA: 00 - HETEROMORPHA • Fitzinger 1832.fa: 327 • Ab

01 - HETEROMORPHA • Fitzinger 1835.fa: $107 \cdot$ bO

02 - HeteromorPHiES • Hoc loco • EK

RL: $\downarrow$ HeTEROMORPHIES 1816.ha.c01

GN: GEOBATRACHIA 1828.ra.c18

Mediograinia 1878.la.c02

GZ: INR

EN: KYR. C.05.02. Subordo Hydrobatrachia Ritgen, 1828.ra.c14-01

HolodactYla Mayer, 1849

SI: $184 \bullet$ CI: c161・ST: 2.N.O.30.E

PN: HolodaCtyli Mayer, 1849.ma.c03 • AK

PA: 00 • HolOdACTYLI - Mayer 1849.ma: 198 • UC

$01 \cdot$ HolOdaCTYLA $\cdot$ Hoc loco $\bullet$ EU

RL: < MALACOPODA 1849.ma.c01・PR

$>$ ColobodactYli 1849.ma.c04 • PR

GN: IMPERFECTIBRANCHIA 1838.ha.c03 Pseudosauria 1816.ba.c08

GZ: INR

EN: KYR. C.04.03. Ordo Urodela Duméril, 1805.da.c02-12

[HYP. Subordo Nullibranchia Bonaparte, 1831.ba.c01-01]

HOMOMORPHIES Fitzinger, 1832

SI: $115 \bullet$ CI: c096・ST: 2.D.M.41.K

PN: НомомоRPHA Fitzinger, 1832.fa.c02 • AK

PA: 00 • НоMOMORPHA • Fitzinger 1832.fa: 329 • Ab

01 • НоMOMORPHAE • Dubois 2015.da: 106 • EK

$02 \cdot$ HoMOMORPHIES $・ H O c$ loco $・$ EK

RL: INR

GN: Pseudosauria 1816.ba.c08

GZ: INR

EN: KYR. C.05.07. Subordo Pseudosauria Blainville, 1816.ba.c08-07

HOMOMORPHIES Fitzinger, 1835

SI: 124 • CI: c104 • ST: 1.D.M.31.K

PN: HoмомоRPHA Fitzinger, 1835.fa.c02 • AK

PA: 00 • НомомоRPHA • Fitzinger 1835.fa: $107 \cdot$ bO

$01 \cdot$ HomomorPHIES • Hoc loco • EK

RL: $\downarrow$ HoMOMORPHIES 1832 .fa.c02

GN: » [OA, SD: HL]

GYMNOPHIONA 1814.ra.c01

URODELA 1805.da.c02

GZ: INR

EN: KYR. C.03.01. Subclassis Lissamphibia Gadow, 1898.ga.c01-00

[HYP. Superordo Derotreta Van der Hoeven, 1833.va.c01-01] 
Horizontalia Bauer, 1987

SI: $416 \bullet$ CI: c376 • ST: 1.D.M.30.O

PN: Horizontalia Bauer, 1987.bc.c01 • AK

PA: 00 • Horizontalia • Bauer 1987.bc: $49 \cdot$ UC

RL: INR

GN: GONDWANURA DOP.da.c01

Phaneranura DOP.da.c02

SCOPTANURA 1973.sb.c02

GZ: INR

EN: TEO. C.09.01. Epiphalanx AQUIPAREs Blainville, 1816.ba.c07-02

[HYP. Epiphalanx HoRizontalia Bauer, 1987.bc.c01]

Hydrobatrachia Ritgen, 1828

SI: $088 \bullet$ CI: c069 • ST: 2.D.M.12.E

PN: Hydrobatrachi Ritgen, 1828.ra.c14

PA: 00 • Hydrobatrachi • Ritgen 1828.ra: 278 • 'F'

$01 \cdot$ Hydrobatrachia $\bullet$ Hoc loco $\bullet$ bO

RL: $\leftrightarrow>$ Phyllopodobatrachi 1828.ra.c13 • AI: HL

GN: GEOBATRACHIA 1828.ra.c18

MediogYRINIA 1878.la.c02

GZ: INR

EN: KYR. C.05.02. Subordo Hydrobatrachia Ritgen, 1828.ra.c14-01

Hydromolgae Ritgen, 1828

SI: $084 \bullet$ CI: c065 • ST: 2.D.M.31.O

PN: HydRomolgae Ritgen, 1828.ra.c10 • AK

PA: 00 • HydroMOLGaE • Ritgen 1828.ra: 279 • 'F'

RL: > PododysMolgae 1828.ra.c07 • AI: HL

$<$ Morphiuromolgaei 1828.ra.c08 • PR

$<$ Molgae 1828.ra.c09 • AI: HL

$<$ GeOMOLGAE 1828.ra.c11 • AI: HL

GN: Pseudosauria 1816.ba.c08

GZ: INR

EN: KYR. C.05.07. Subordo Pseudosauria Blainville, 1816.ba.c08-07

HYdRONECTAE Fitzinger, 1843

SI: $171 \bullet$ CI: c148 • ST: 2.D.M.31.O

PN: HydroneCtaE Fitzinger, 1843.fa.c03 • AK

PA: 00 • HydroneCTAE • Fitzinger 1843.fa: $30 \cdot \mathbf{S c}$

RL: > ChersobataE 1843.fa.c04 • AI: HL

GN: GEOBATRACHIA 1828.ra.c18

MEDIOGYRINIA 1878.la.c02

GZ: INR

EN: KYR. C.05.02. Subordo Hydrobatrachia Ritgen, 1828.ra.c14-01

HydroneCtae Fitzinger, 1861

SI: $220 \bullet$ CI: c194 • ST: 2.D.M.41.O

PN: HydroneCtaE Fitzinger, 1861.fb.c01 • AK

PA: 00 • HYdRONECTAE • Fitzinger 1861.fb: $217 \bullet$ bO

RL: $\downarrow$ Hydronectae 1843 .fa.c03

GN: ANGUSTICOELA 1958.ra.c01

Hydrobatrachia 1828.ra.c14

GZ: INR

EN: KYR. C.04.01. Ordo ANURa Duméril, 1805.da.c01-07

Hydrophili Fitzinger, 1861

SI: 177 • CI: c154 • ST: 2.D.M.30.O
PN: HydROPHILI Fitzinger, 1843.fa.c09 • AK

PA: $00 \cdot$ HYdROPHILI $\cdot$ Fitzinger 1843.fa: $33 \cdot$ Sc

RL: < GeOPHIL 1843.fa.c08 • AI: HL

GN: IMPERFECTIBRANCHIA 1838.ha.c03 Pseudosauria 1816.ba.c08

GZ: INR

EN: KYR. C.04.03. Ordo URodela Duméril, 1805.da.c02-12 [HYP. Subordo NulLibranchia Bonaparte, 1831.ba.c01-01] HyLaCea Haeckel, 1889

SI: 296 • CI: c269 • ST: 1.D.M.41.R

PN: HylaCEA Haeckel, 1889.ha.c03 • AK

PA: 00 • HylaCEA • Haeckel 1866.ha: $640 \cdot$ O

RL: $\downarrow$ HyLAEAE 1816 .ha.c02

GN: HyLOBATRACHIA 1828.ra.c16

GZ: INR

EN: KYR. C.13.03. Hypophalanx Hylobatrachia Ritgen, 1828.ra.c16-01

HYLACEI Hübner, 1816

SI: $049 \bullet$ CI: zh14 • ST: 1.U.U.99.C

PN: Hylaeae Hübner, 1816.ha.c02 • zz

PA: 00 • HYLAEAE • Hübner 1816.ha: $283 \bullet$ St

$01 \cdot$ HyLACEI $\bullet$ Hoc loco $\bullet$ EC

RL, GN, GZ, EN: •

HylaeObatrachiacea Goodrich, 1930

SI: $341 \bullet$ CI: c314 • ST: 1.D.M.30.R

PN: Hylaeobatrachia Goodrich, 1930.ga.c03 • AK

PA: 00 • Hylaeobatrachia • Goodrich 1930.ga: xxi • bO

01 • HylaeObatrachiacea • Dubois 2015.da: 107 • ER

RL: INR

GN: |URODELA INCERTAE SEDIS|

GZ: INR

EN: KYR. C.04.03. Ordo Urodela Duméril, 1805.da.c02-12

HYLAPLESIFORMIA Steindachner, 1867

SI: $245 \bullet$ CI: c219 • ST: 1.D.M.31.A

PN: HyLAPLESIFORMIA Steindachner, 1867.sa.c03 • AK

PA: 00 • HyLAPLESIFORMIA $•$ Steindachner 1867 .sa: $68 \cdot$ Sc

RL: > BUFONIFORMIA 1867.sa.c02 • AI: HL

GN: Phaneranura DOP.da.c02

SCOPTANURA 1973.sb.c02

GZ: INR

EN: TEO. C.09.01. Epiphalanx AQUIPARES Blainville, 1816.ba.c07-02

[HYP. Phalanx AQUIPARES Blainville, 1816.ba.c07-03]

HYLAPLESIFORMIA Brocchi, 1881

SI: $280 \bullet$ CI: c254 • ST: 2.D.M.41.A

PN: HylaPLeSIFORMES Brocchi, 1881.ba.c02 • aK

PA: 00 • HylaPLesiformes $\bullet$ Brocchi 1881.ba: $9 \cdot$ UC

$01 \cdot$ HyLAPLESIFORMia $\cdot$ Hoc loco $\bullet$ EA

RL: $\downarrow$ HyLAPLeSIFORMia 1867 .sa.c03

GN: HyLOBATRACHIA 1828.ra.c16

GZ: INR

EN: KYR. C.13.03. Hypophalanx Hylobatrachia Ritgen,

$$
\text { 1828.ra.c16-01 }
$$

Hyliformia Cope, 1863

SI: $226 \bullet$ CI: c200 • ST: 2.D.M.31.A

PN: HylaEFormia Cope, 1863.cb.c01 • AK 
PA: 00 • HylaEFORMia • Cope 1863.cb: 352 • Sr

$01 \cdot$ Hylaeformia $・$ Steindachner 1867.sa: $47 \cdot$ Sc

02 • Hylaeformes • Brocchi 1881.ba: 9 • UC

03 - HyLiformes • Boulenger 1882.ba: 12 • UC

04 • Hyliformia • Dubois 2015.da: 90 • EA

RL: $\leftarrow$ Platydactyla 1858.gc.c04

GN: Phaneranura DOP.da.c02

SCOPTANURA 1973.sb.c02

GZ: INR

EN: TEO. C.09.01. Epiphalanx AQUIPARES Blainville, 1816.ba.c07-02

[HYP. Phalanx AQUIPARES Blainville, 1816.ba.c07-03]

Hylobatrachia Ritgen, 1828

SI: $090 \bullet$ CI: c071 • ST: 2.D.M.12.E

PN: HyLObatrachi Ritgen, 1828.ra.c16

PA: 00 • HyLOBATRACHI • Ritgen 1828.ra: 278 • 'F'

$01 \cdot$ Hylobatrachia $\cdot H o c l o c o \cdot \mathbf{h P}$

RL: $\leftrightarrow>$ BDALSIPODOBATRACHI 1828.ra.c15 • AI: HL

GN: HyLOBATRACHIA 1828.ra.c16

GZ: INR

EN: KYR. C.13.03. Hypophalanx HyLOBatrachia Ritgen, 1828.ra.c16-01

HyPSIBATAE Fitzinger, 1843

SI: 170 • CI: c147 • ST: 2.D.M.31.O

PN: HyPsibatae Fitzinger, 1843.fa.c02 • AK

PA: $00 \cdot$ HYPSIBATAE • Fitzinger 1843.fa: $30 \bullet$ Sc

RL: INR

GN: Phaneranura DOP.da.c02

SCOPTANURA 1973.sb.c02

GZ: INR

EN: TEO. C.09.01. Epiphalanx AQuiPares Blainville, 1816.ba.c07-02

[HYP. Phalanx AQUIPARES Blainville, 1816.ba.c07-03]

ICHTHYODI Blainville, 1816

SI: $035 \bullet$ CI: $\mathrm{c} 022 \bullet$ ST: 1.D.A.32.E

PN: ICTYOIDES Blainville, 1816.ba.c03 • AK

PA: 00 • ICTYOIDES • Blainville 1816.ba: "111" [119] • bC

01 - ICTHYOIDES • Blainville 1816.bb: 254 • bC

02 - ICHTHYOIDES • Ducrotay Blainville 1821.da: 10 • C

03 - ICTHYOIDES • Macleay 1821.ma: $262 \cdot \mathbf{C}$

$04 \cdot$ ICHTHYODES • Hallowell 1856.ha: $6 \cdot$ bC

$05 \cdot$ ICHTHYODI • Dubois 2015.da: $105 \cdot \mathbf{E E}$

RL: $\leftrightarrow<$ NUDIPELLIFERES 1816.ba.c01 • AI: HL

$\leftrightarrow<$ AMphybiens 1816.ba.c02 • AI: HL

$\leftrightarrow>$ NUDS 1816.ba.c04 • AI: HL

GN: ANURA 1805.da.c01

GYMNOPHIONA 1814.ra.c01

URODELA 1805.da.c02

GZ: » GX:

|Амniota|

|PISCES|

EN: KYR. C.02.01. Classis AmphiBIA Blainville, 1816.ba.c02-03

ICHTHYODI Leuckart, 1821

SI: $054 \bullet$ CI: $\mathrm{c} 036 \bullet \mathbf{S T}: 2 . \mathrm{D} . \mathrm{M} .40 . \mathrm{E}$

PN: ICHTHYOIDEA Leuckart, 1821.la.c01 • AK

PA: 00 • ICHTHYOIDEA • Leuckart 1821.1a: 258 • 'F'
$01 \cdot$ ICHTHYODI • Wagler 1830.wa: $131 \bullet \mathbf{O}$

02 - ICHTHYOIDEA • Leuckart 1840.1a: $19 \cdot \mathbf{b A b}$

03・ ICHTHYODES • Duméril ${ }^{+2}$ 1854.da: 199 • O

$04 \cdot$ ICHTHYODI • Wied 1865.wa: viii, $132 \cdot \mathbf{U C}$

05 - ICHTHYOIDEA • Dubois ${ }^{+1}$ 2012.da: $78 \cdot$ bO

RL: $\downarrow$ ICTYOIDES 1816.ba.c03

GN: IMPERFECTIBRANCHIA 1838.ha.c03

Pseudosauria 1816.ba.c08

GZ: INR

EN: KYR. C.04.03. Ordo Urodela Duméril, 1805.da.c02-12

[HYP. Subordo Nullibranchia Bonaparte, 1831.ba.c01-01]

ICHTHYODI Wagler, 1828

SI: $095 \bullet \mathbf{C I}: \mathrm{c076} \bullet \mathbf{S T}$ : 3.D.M.41.O

PN: ICHTHYODI Wagler, 1828.wb.c02 • AK

PA: 00 ・ ICHTHYODI • Wagler 1828.wb: $859 \bullet$ O

RL: $\downarrow$ ICTYOIDES 1816.ba.c03

GN: Pseudosauria 1816.ba.c08

GZ: INR

EN: KYR. C.05.07. Subordo Pseudosauria Blainville, 1816.ba.c08-07

ICHTHYODI Bonaparte, 1831

SI: $109 \bullet \mathbf{C I}: \mathrm{c090} \bullet \mathbf{S T}$ : 1.D.M.41.E

PN: ICHTYOIDA Bonaparte, 1831.ba.c02 • AK

PA: 00 - ICHTYOIDA • Bonaparte 1831.ba: 78 • O

$01 \cdot$ ICHTHYOIDI • Bonaparte 1838.bc: $393 \cdot$ O

02 - ICHTHYODI • Bonaparte 1838.bd: $657 \cdot \mathbf{O}$

03 - ICHTHYOIDEA • Bonaparte 1838.bd: 125 • O

04 - ICHTHYODEA • Bonaparte 1839.bf: $16 \cdot \mathbf{O}$

05 - ICHTHYODEI • Bonaparte 1840.ba: $287 \cdot \mathbf{O}$

06 - ICHTHYODEA • Claus 1868.cb: 584 • bO

07 - ICHTHYOdEA • Gadow 1901.ga: 95 • UC

RL: $\downarrow$ ICTYOIDES 1816.ba.c03

GN: MeanTes 1767.la.c01

PseUdosauria 1816.ba.c08

GZ: INR

EN: KYR. C.04.03. Ordo Urodela Duméril, 1805.da.c02-12

[HYP. Subordo PNeumobranchia Sonnini ${ }^{+1}$, 1801.sa.c01-02]

ICHTHYODI Hoffmann, 1878

SI: $264 \bullet$ CI: c238 • ST: 2.D.M.40.E

PN: ICHTHYOIDEA Hoffmann, 1878.ha.c04 • AK

PA: 00 - ICHTHYOIDEA • Hoffmann 1878.ha: 674 • UC

$01 \cdot$ ICHTHYODEA • Knauer 1878.ka: $95 \cdot \mathbf{b O}$

02 - ICHTHYOIDEA • Zittel 1888.za: viii, $418 \cdot \mathbf{b O}$

03 - ICHTHYODI • Dubois 2015.da: $107 \cdot \mathbf{E E}$

RL: $\downarrow$ ICTYOIDES 1816.ba.c03

$<$ ICHTHYOMORPHI 1866.oa.c01 • PR

GN: IMPERFECTIBRANCHIA 1838.ha.c03

MeanTes 1767.la.c01

Pseudosauria 1816.ba.c08

GZ: INR

EN: KYR. C.04.03. Ordo Urodela Duméril, 1805.da.c02-12 ICHTHYOMORPHI Owen, 1866

SI: 239 • CI: c213・ST: 1.D.M.31.X

PN: ICHTHYOMORPHA Owen, 1866.oa.c01 • AK

PA: 00 • ICHTHYOMORPHA • Owen 1866.oa: 15 • bO

01 • ICHTHYOMORPHI • Dubois 2015.da: 90 • EX 
RL: INR

GN: Meantes 1767.la.c01

Pseudosauria 1816.ba.c08

GZ: INR

EN: KYR. C.04.03. Ordo Urodela Duméril, 1805.da.c02-12

[HYP. Subordo Pneumobranchia Sonnini ${ }^{+1}$, 1801.sa.c01-02]

ICHTHYOMORPHI Hoffmann, 1878

SI: 263 • CI: c237 • ST: 1.D.M.40.X

PN: IсHTHYOMORPHA Hoffmann, 1878.ha.c03 • AK

PA: 00 • ICHTHYOMORPHA • Hoffmann 1878.ha: $661 \cdot$ O

$01 \cdot$ ICHTHYOMORPHI $\bullet$ Hoc loco $\bullet$ EK

RL: $\downarrow$ ICHTHYOMORPHI 1866.oa.c01 $>$ ICHTHYOIDEA 1878.ha.c04 • PR

GN: IMPERFECTIBRANCHIA 1838.ha.c03

Meantes 1767.la.c01

Pseudosauria 1816.ba.c08

GZ: INR

EN: KYR. C.04.03. Ordo Urodela Duméril, 1805.da.c02-12

ICHTHYOSTERNIA Miranda-Ribeiro, 1924

SI: 335 • CI: c308 • ST: 2.D.M.31.O

PN: ICHTHYOSTERNIA Miranda-Ribeiro, 1924.ma.c10 • AK

PA: 00 • ICHTHYOSTERNIA • Miranda-Ribeiro 1924.ma: 12 • UC

RL: $\leftrightarrow<$ ProtonYXIA 1924.ma.c07 • AI: HL

GN: DORSIPARES 1816.ba.c06

GZ: INR

EN: KYR. C.07.01. Hypoordo DorsIPAREs Blainville, 1816.ba.c06-02

IMMUTABILIA Haworth, 1825

SI: 068 • CI: c050 • ST: 3.D.M.31.O

PN: ImMUtABiLia Haworth, 1825.ha.c02 • AK

PA: 00 - ImmUtabilia • Haworth 1825.ha: $372 \cdot$ UC

$01 \cdot$ ImmutaBilia $・$ Fitzinger 1826.fb: $36 \cdot$ 'T'

02 - ImMUtABILIA • Bonaparte 1831.bb: $135 \cdot$ Sc

RL: INR

GN: Meantes 1767.la.c01

Pseudosauria 1816.ba.c08

GZ: INR

EN: KYR. C.04.03. Ordo Urodela Duméril, 1805.da.c02-12

[HYP. Subordo Pneumobranchia Sonnini ${ }^{+1}$, 1801.sa.c01-02]

IMMUTABILIA Gray, 1842

SI: $167 \bullet$ CI: c144 • ST: 1.D.M.40.O

PN: IMmUtabiLia Gray, 1842.ga.c01 • AK

PA: 00 • ImMUTABILIA • Gray 1842.ga: $113 \cdot$ Se

$01 \cdot$ ImMUtabiLia $\bullet$ Gill 1903.ga: $73 \cdot$ T

RL: $\downarrow$ IMMUTABILIA 1825 .ha.c02

GN: IMPERFECTIBRANCHIA 1838.ha.c03

Meantes 1767.la.c01

Pseudosauria 1816.ba.c08

GZ: INR

EN: KYR. C.04.03. Ordo Urodela Duméril, 1805.da.c02-12

IMPERFECTIBRANCHIA Hogg, 1838

SI: $130 \bullet$ CI: c110 • ST: 1.D.M.11.O

PN: IMPERFECTIBRANCHIA Hogg, 1838.ha.c03

PA: 00 • IMPERFECTIBRANCHIA $\cdot$ Hogg 1838.ha: $152 \cdot$ O

01 - IMPERFECTIBRANCHIA $・$ Dubois $^{+1} 2012$ da: $78 \cdot$ iO

02 - IMPERFECTIBRANCHIA • Dubois 2015.da: $49 \cdot$ bO
RL: INR

GN: IMPERFECTIBRANCHIA 1838.ha.c03

GZ: INR

EN: KYR. C.05.05. Subordo IMPERFECTIBRANCHIA Hogg, 1838.ha.c03-02

INTERNIBRANCHIA Hogg, 1839

SI: 144 • CI: c122 • ST: 1.D.M.31.O

PN: INTERNIBRANCHIA Hogg, 1839.hb.c01 • AK

PA: 00 - INTERNIBRANCHIA • Hogg 1839.hb: 375 • 'T'

RL: > ARCUMANENTIA 1839.hb.c04 • AI: HL

GN: IMPERFECTIBRANCHIA 1838 .ha.c03

GZ: INR

EN: KYR. C.05.05. Subordo IMPERFECTIBRANCHIA Hogg, 1838.ha.c03-02

Karauracea Estes, 1981

SI: 402 • CI: c362 • ST: 1.D.M.30.R

PN: Karauroidea Estes, 1981.ea.c01 $\uparrow \cdot$ AK

PA: 00 - Karauroidea • Estes 1981.ea: xiii, 10 •bO

01 KaraURACEA $・$ Dubois 2015.da: $107 \cdot$ ER

RL: < AMBYstomatoideA 1981.ea.c03 • AI: HL

$<$ SALAMANDROIDEA 1981.ea.c04 • AI: HL

GN: |URODELA INCERTAE SEDIS|

GZ: INR

EN: KYR. C.04.03. Ordo URodela Duméril, 1805.da.c02-12 LACERTACEI Gray, 1850

SI: $194 \bullet$ CI: c171 • ST: 1.D.M.30.C

PN: LACERTINI Gray, 1850.ga.c06 • AK

PA: $00 \cdot$ LACERTINI $\bullet$ Gray 1850.ga: $10 \bullet$ bC

01 - LACERTACEI • Dubois 2015.da: 90 • EC

RL: INR

GN: ANURA 1805.da.c01

Urodela 1805.da.c02

GZ: INR

EN: KYR. C.03.01. Subclassis LISSAMPHIBIA Gadow, 1898.ga.c01-00

[HYP. Superordo BATRACHIA Brongniart, 1800.ba.c01-14]

LACERTIFORMI Jarocki, 1822

SI: $060 \bullet$ CI: c042 • ST: 1.D.M.30.X

PN: LACERTIFORMIA Jarocki, 1822.ja.c03 • AK

PA: $00 \cdot$ LACERTIFORMIA • Jarocki 1822.ja: $137 \cdot$ O

$01 \cdot \mathbf{L A C E R T I F O R M I} \cdot H o c$ loco $\bullet$ EX

RL: INR

GN: PSEUdosauria 1816.ba.c08

GZ: INR

EN: KYR. C.05.07. Subordo Pseudosauria Blainville, 1816.ba.c08-07

LACERTIFORMI Hogg, 1839

SI: $141 \bullet$ CI: c119 • ST: 1.D.M.30.X

PN: LACERTIFORMIA Hogg, 1839.ha.c06 • AK

PA: 00 • LACERTIFORMIA $・$ Hogg 1839.ha: $271 \bullet$ O

$01 \cdot$ LACERTINIFORMia $・$ Gray 1850.ga: $51,71 \cdot$ O

02 - LACERTIFORMES $\bullet$ Dubois 2015.da: $90 \cdot$ EX

03 - LACERTIFORMI $\cdot$ Hoc loco $\bullet$ EX

RL: $\downarrow$ LACERTIFORMI 1822 .ja.c03

$<$ DiplopNeUmena 1839.ha.c08 • PR

GN: IMPERFECTIBRANCHIA 1838.ha.c03 
Pseudosauria 1816.ba.c08

GZ: INR

EN: KYR. C.04.03. Ordo Urodela Duméril, 1805.da.c02-12

[HYP. Subordo Nullibranchia Bonaparte, 1831.ba.c01-01]

LAEVOGYRINIA Lataste, 1878

SI: $269 \bullet$ CI: c243・ST: 2.D.M.11.E

PN: LAEVOGYRINIDAE Lataste, 1878.1a.c01

PA: 00 • LAEVOGYRINIDAE • Lataste 1878.1a: $491 \cdot$ UC

01 • LAEVOGYRINIDES • Lataste 1879.1a: $984 \cdot$ bO

$02 \cdot$ LAEVOGYRINIDAE $・$ Lataste 1879.1b: $339 \bullet$ bO

03 - LAEvogYRINIA - Dubois ${ }^{+2} 2016 . d b: 49 \cdot$ EE

$04 \cdot$ LAEVOGYRINIA $\cdot$ Hoc loco $\bullet$ hO

RL: INR

GN: ArchaeOSAlientia 1981.ra.c01

RANOMORPHA 1921.fb.c08

GZ: INR

EN: KYR. C.07.02. Hypoordo LAEvoGYRINIA Lataste, 1878.la.c01-04

LEMMANURA Starrett, 1973

SI: 398 • CI: c358 • ST: 1.D.M.31.O

PN: LeMmanura Starrett, 1973.sb.c03 • AK

PA: 00 - LeMmANURA • Starrett 1973.sb: $251 \cdot$ UC

$01 \cdot$ LemmanuRA • Savage 1973.sa: $354 \cdot$ bO

RL: INR

GN: ANGUSTICOELA 1958.ra.c01 HYDROBATRACHIA 1828.ra.c14

GZ: INR

EN: KYR. C.04.01. Ordo ANURA Duméril, 1805.da.c01-07 LINGUATA Gravenhorst, 1845

SI: $179 \bullet$ CI: c156 • ST: 4.D.M.31.O

PN: LiNGUATA Gravenhorst, 1845.ga.c01 • AK

PA: 00 • LingUATA • Gravenhorst 1845.ga: 43 • UC

$01 \cdot$ LINGUATA $\cdot$ Stejneger 1907.sa: v, $54 \cdot \mathbf{b O}$

RL: INR

GN: Phaneranura DOP.da.c02

SCOPTANURA 1973.sb.c02

GZ: INR

EN: TEO. C.09.01. Epiphalanx AQUIPARES Blainville, 1816.ba.c07-02

[HYP. Phalanx AQUIPARES Blainville, 1816.ba.c07-03]

LINGUATA Stejneger ${ }^{+1}, 1917$

SI: $307 \bullet$ CI: c280 • ST: 1.D.M.41.O

PN: LINGUATA Stejneger ${ }^{+1}$, 1917.sa.c02 • AK

PA: 00 • LINGUATA • Stejneger ${ }^{+1}$ 1917.sa: $25 \cdot$ bO

RL: INR

GN: Archaeosalientia 1981.ra.c01

RANOMORPHA 1921.fb.c08

GZ: INR

EN: KYR. C.07.02. Hypoordo LAEvogYRINIA Lataste,

$$
\text { 1878.la.c01-04 }
$$

LiPOBRANCHIA Haeckel, 1879

SI: 273 • CI: c247 • ST: 1.D.M.31.O

PN: LiPOBRANCHIA Haeckel, 1879.ha.c01 • AK

PA: 00 • LIPOBRANCHIA • Haeckel 1879.ha: 539 • UC

RL: INR

GN: PSeudosauria 1816.ba.c08
GZ: INR

EN: KYR. C.05.07. Subordo Pseudosauria Blainville, 1816.ba.c08-07

LiSSAMPHIBIA Haeckel, 1866

SI: 235 • CI: c209・ST: 1.N.O.30.O

PN: LiSSAMPHIBIA Haeckel, 1866.ha.c01 • AK

PA: 00 - LISSAMPHiBIA • Haeckel 1866.ha: x, cxxxi • bC

RL: INR

GN: ANURA 1805.da.c01

Urodela 1805.da.c02

GZ: » GI:

GYMNOPHIONA 1814.ra.c01

|Non-LisSAmphibian Amphibia|

EN: ANAPTONYM

[HYP. Superordo BATRACHIA Brongniart, 1800.ba.c01-14]

LiSSAMPHIBIA Gadow, 1898

SI: $300 \bullet \mathbf{C I}: \mathrm{c} 273 \bullet \mathbf{S T}$ : $1 . \mathrm{S} . \mathrm{O} .10 . \mathrm{O}$

PN: LisSAMPHibia Gadow, 1898.ga.c01

PA: 00 - LiSSAMPHibia • Gadow 1898.ga: xii, $13 \cdot$ bC

$01 \cdot$ LISSAMPHIBIA $\cdot$ Gardiner 1982.ga: $228 \cdot$ bD

$02 \cdot \operatorname{LiSSAMPHiBIA} \cdot$ Milner 1988.ma: 82 • cD

RL: $\downarrow$ LISSAMPHIBIA 1866 .ha.c01

GN: ANURA 1805.da.c01

GYMNOPHIONA 1814.ra.c01

Urodela 1805.da.c02

GZ: » GX:

|Non-Lissamphibian AMphibia|

EN: KYR. C.03.01. Subclassis LisSAMPHibia Gadow, 1898.ga.c01-00

LiSSAMPHIBIA Hay, 1929

SI: 344 • CI: c317 • ST: 1.N.G.02.O

PN: LisSAMPHibia Hay, 1929.ha.c01 • AP

PA: 00 • LiSSAMPHibIA • Hay 1929.ha: 521, 839 • bC

RL: $\downarrow$ LISSAMPHIBIA 1866. ha.c01

GN: LiSSAMPHIBIA 1901.ga.c01

|Non-LisSamphibian AMPHibia|

GZ: » GI:

|Non-Lissamphibian AMphibia|

EN: ANAPTONYM

Malacoderma Kirby, 1835

SI: $125 \bullet$ CI: c105 • ST: 1.D.M.30.O

PN: Malacoderma Kirby, 1835.ka.c01 • AK

PA: 00 • MALACODERMA • Kirby $1835 . \mathrm{ka}: 414 \cdot$ bC

RL: INR

GN: ANURA 1805.da.c01

Urodela 1805.da.c02

GZ: INR

EN: KYR. C.03.01. Subclassis LisSAMPHibia Gadow, 1898.ga.c01-00

[HYP. Superordo BATRACHIA Brongniart, 1800.ba.c01-14]

Malacopoda Mayer, 1849

SI: $182 \bullet$ CI: c159 • ST: 1.D.A.30.O

PN: MalaCOPODA Mayer, 1849.ma.c01 • AK

PA: 00 - MALACOPODA • Mayer 1849.ma: 198 • O

RL: $\leftrightarrow \mid$ BATRACIENS 1800.ba.c01

GN: ANURA 1805.da.c01 
URODELA 1805.da.c02

GZ: » GI:

GYMNOPHIONA 1814.ra.c01

EN: ANAPTONYM

[HYP. Superordo BATRACHIA Brongniart, 1800.ba.c01-14]

ManentibranCHIA Hogg, 1838

SI: 132 • CI: c112 • ST: 1.D.M.31.O

PN: Manentibranchia Hogg, 1838.ha.c05 • AK

PA: 00 - MANENTIBRANCHIA - Hogg 1838.ha: 152 • O

$01 \cdot$ Mancabranchia - Gray 1850 .ga: $51,71 \cdot$ UC

RL: < DiPLOPNEUMENA 1838.ha.c05 • PR

GN: MEANTES 1767.la.c01

PSEUdosauria 1816.ba.c08

GZ: INR

EN: KYR. C.04.03. Ordo Urodela Duméril, 1805.da.c02-12

[HYP. Subordo Pneumobranchia Sonnini ${ }^{+1}$, 1801.sa.c01-02]

MeANTES Linnaeus, 1767

SI: $005 \bullet \mathbf{C I}: \mathrm{c001} \bullet \mathbf{S T}: 1 . \mathrm{D} . \mathrm{M} .11 . \mathrm{O}$

PN: Meantes Linnaeus, 1767.1a.c01

PA: 00 - MEANTES • Linné 1767.la: unnumbered additional page • O

01 MeanTES • Stejneger ${ }^{+1}$ 1917.sa: $24 \cdot \mathbf{b O}$

02 Meantina - Pearse 1936.pa: $20 \cdot \mathbf{b O}$

03 - Meantes • Dubois 2015.da: $49 \cdot$ iO

RL: INR

GN: Meantes 1767.la.c01

GZ: INR

EN: KYR. C.05.06. Subordo Meantes Linné, 1767.la.c01-01

Meantes Gray, 1850

SI: $192 \bullet$ CI: c169・ST: 1.D.M.41.E

PN: Meantia Gray, 1850.ga.c04 • AK

PA: $00 \cdot$ Meantia • Gray 1850.ga: 6, 63 • O

01 - MeAnTeS $\cdot H o c$ loco $・$ EE

RL: $\downarrow$ Meantes 1767.la.c01

$>$ Gradientia 1850.ga.c01 • PR

< Pseudosauria 1850.ga.c02 • AI: HL

GN: MEANTES 1767.la.c01

Pseudosauria 1816.ba.c08

GZ: INR

EN: KYR. C.04.03. Ordo Urodela Duméril, 1805.da.c02-12 [HYP. Subordo Pneumobranchia Sonnini ${ }^{+1}$, 1801.sa.c01-02]

MeAntes Kuhn, 1961

SI: $378 \bullet$ CI: c339 • ST: 1.D.M.40.O

PN: Meantes Kuhn, 1961.ka.c04 • AK

PA: 00 • MEANTES • Kuhn 1961.ka: $14 \cdot$ bO

RL: $\downarrow$ Meantes 1767.la.c01

> CRYPTOBRANCHOIDEA 1961.ka.c01 • AI: HL

> BatrachosaUroIdoIDEA 1961.ka.c02 • AI: HL

> ProteIda 1961.ka.c03 • AI: HL

GN: MEANTES 1767.la.c01

|URODELA INCERTAE SEDIS|

GZ: INR

EN: KYR. C.04.03. Ordo Urodela Duméril, 1805.da.c02-12

Mediogyrinia Lataste, 1878

SI: $270 \bullet \mathbf{C I}: \mathrm{c} 244 \bullet \mathbf{S T}: 2 . \mathrm{D} . \mathrm{M} .11 . \mathrm{E}$

PN: Mediogyrinidae Lataste, 1878.la.c02

PA: 00 • Mediogyrinidae • Lataste 1878.1a: 491 • UC
01 • Mediogyrinia • Dubois 2015.da: 12 - EE

$02 \cdot$ Mediogyrinia $\bullet H o c l o c o \cdot \mathbf{i O}$

RL: INR

GN: Mediogyrinia 1878.la.c02

GZ: INR

EN: KYR. C.06.02. Infraordo Mediogyrinia Lataste, 1878.1a.c02-02

Mediogyrinia Lataste, 1879

SI: $274 \bullet$ CI: c248 • ST: 1.D.M.41.E

PN: Mediogyrinides Lataste, 1879.la.c01 • AK

PA: 00 • MEDIOGYRINIDES • Lataste 1879.1a: $984 \cdot$ bO

01 MediogYRINIDAE • Lataste 1879.1b: $339 \cdot$ bO

02 - Mediogyrinia $\cdot H o c l o c o \cdot$ EE

RL: $\downarrow$ Mediogyrinidae 1878.1a.c02

GN: GEOBATRACHIA 1828.ra.c18

Mediogrrinia 1878.la.c02

GZ: INR

EN: KYR. C.05.02. Subordo Hydrobatrachia Ritgen, 1828.ra.c14-01

Mesobatrachia Laurent, 1980

SI: $401 ・$ CI: c361・ST: 1.D.M.31.O

PN: Mesobatrachia Laurent, 1980.la.c01・aK

PA: 00 • MesobatraCHIA • Laurent 1980.1a: 398 • bO

RL: INR

GN: DORSIPARES 1816.ba.c06

LAEVOGYRINIA 1878.1a.c01

GZ: INR

EN: KYR. C.06.01. Infraordo GEOBATRACHIA Ritgen, 1828.ra.c18-02

Miura Van der Hoeven, 1833

SI: $120 \bullet \mathbf{C I}$ : c100 • ST: 1.D.M.31.O

PN: MiUra Van der Hoeven, 1833.va.c03 • AK

PA: 00 • MiUra • Van der Hoeven 1833.va: iii, 307 • O

RL: INR

GN: GEOBATRACHIA 1828.ra.c18

Mediogyrinia 1878.la.c02

GZ: INR

EN: KYR. C.05.02. Subordo Hydrobatrachia Ritgen, 1828.ra.c14-01

MolgaceA Ritgen, 1828

SI: $083 \bullet$ CI: c064 • ST: 4.D.M.31.R

PN: MolgaE Ritgen, 1828.ra.c09 • AK

PA: $00 \cdot$ MolGAE • Ritgen 1828.ra: $277 \cdot \mathbf{Z g}$

01 MolgaEI - Jourdan 1834.jb: $100 \cdot \mathbf{O}$

02 - MolgaCEA $\cdot H o c l o c o \cdot$ ER

RL: > PododysMolgae 1828.ra.c07 • PR

$\leftrightarrow<$ MorphiUromolgaeI 1828.ra.c08 • AI: HL

$>$ HYDROMOLGAE 1828.ra.c10 • PR

$>$ GeOMOLGAE 1828.ra.c11 • PR

GN: Pseudosauria 1816.ba.c0 8

GZ: INR

EN: KYR. C.05.07. Subordo Pseudosauria Blainville, 1816.ba.c08-07

Monopneuma Hogg, 1838

SI: $128 \bullet$ CI: c108・ST: 1.D.M.31.E

PN: Monopneumena Hogg, 1838.ha.c01 • AK 
PA: $00 \cdot$ MonOPNEUMena $・$ Hogg 1838.ha: $152 \cdot$ bC 01 MonOPNEUMA • Duméril 1863.da: $300 \cdot$ bC

RL: INR

GN: ANURA 1805.da.c01

GYMNOPHIONA 1814.ra.c01

URODELA 1805.da.c02

GZ: INR

EN: KYR. C.03.01. Subclassis LisSAMPHibia Gadow, 1898.ga.c01-00

MonOSACRALIA Bolkay, 1919

SI: 311 • CI: c284 • ST: 1.D.M.31.O

PN: MonosaCralia Bolkay, 1919.ba.c02 • AK

PA: $00 \bullet$ MonosACRALIA • Bolkay 1919.ba: $348 \cdot$ bO

RL: INR

GN: ANGUSTICOELA 1958.ra.c01

HydrobatraChia 1828.ra.c14

GZ: INR

EN: KYR. C.04.01. Ordo ANURA Duméril, 1805.da.c01-07 MorPhiUROMOLGAE Ritgen, 1828

SI: 082 • CI: c063・ST: 2.D.M.31.E

PN: MorPhiUromolgaei Ritgen, 1828.ra.c08 • AK

PA: c0 • MorPhiUromolgaeI • Ritgen 1828.ra: 274 • Zg • EEA:

HL

i1 • MorphuromolgaeI • Ritgen 1828.ra: $274 \cdot \mathbf{Z g}$

02 - MorPhUROMOLGAEI • Jourdan 1834.jb: 112 • Sc

$03 \cdot$ MorphiuromolgaE $\cdot$ Hoc loco $\bullet$ EE

RL: > PoDODYSMOLGAE 1828.ra.c07 • PR

$\leftrightarrow>$ MolgaE 1828.ra.c09 • AI: HL

$>$ HydromolgaE 1828.ra.c10 • PR

$>$ GEOMOLGAE 1828.ra.c11 • PR

GN: PSEUdosauria 1816.ba.c0 8

GZ: INR

EN: KYR. C.05.07. Subordo Pseudosauria Blainville, 1816.ba.c08-07

Mutabilia Merrem, 1820

SI: $052 \bullet \mathbf{C I}: \mathrm{c034} \bullet \mathbf{S T}$ : 2.D.M.31.O

PN: Mutabilia Merrem, 1820.ma.c03 • AK

PA: 00 • MutabiLIA • Merrem 1820.ma: 163 • 'T'

RL: INR

GN: Pseudosauria 1816.ba.c08

GZ: INR

EN: KYR. C.05.07. Subordo Pseudosauria Blainville, 1816.ba.c08-07

Mutabilia Fitzinger, 1826

SI: $072 \bullet$ CI: c054 • ST: 2.D.M.30.O

PN: MutabiLia Fitzinger, 1826.fb.c02 • AK

PA: 00 • Mutabilia • Fitzinger 1826.fb: 36 • 'T'

$01 \cdot$ Mutabilia • Gray 1831.ga: $99 \cdot$ O

$02 \cdot$ MutaBiLia • Fitzinger 1832.fa: $327 \cdot \mathbf{Z t}$

03 • Mutabilia • Gray 1842.ga: $111 \cdot$ Sc

RL: $\downarrow$ Mutabilia 1820.ma.c03

GN: ANURA 1805.da.c01

URODELA 1805.da.c02

GZ: INR

EN: KYR. C.03.01. Subclassis LisSAMPHIBIA Gadow, 1898.ga.c01-00
[HYP. Superordo BATRACHIA Brongniart, 1800.ba.c01-14] Mutabilia Hay, 1929

SI: $348 \bullet$ CI: c321・ST: 1.D.M.40.O

PN: Mutabilia Hay, 1929.ha.c05 • AK

PA: 00 • Mutabilia • Hay 1929.ha: 521, 839 • $\boldsymbol{O}$

RL: $\downarrow$ Mutabilia 1820.ma.c03

GN: LISSAMPHIBIA 1901.ga.c01

|LEPOSPONDYLI|

GZ: INR

EN: KYR. C.02.01. Classis AмpHiBia Blainville, 1816.ba.c02-03 Myctodera Stannius, 1856

SI: $203 \bullet$ CI: c180 • ST: 1.D.M.31.O

PN: MyCTODERA Stannius, 1856.sa.c01 • AK

PA: $00 \cdot$ MyctoderA • Stannius 1856.sa: 4 • bO

01 - MYCTODERA • Cope 1888.ca: $464 \cdot \mathbf{U C}$

RL: INR

GN: PSEUdosauria 1816.ba.c08

GZ: INR

EN: KYR. C.05.07. Subordo Pseudosauria Blainville, 1816.ba.c08-07

NeOBatrachia Sarasin ${ }^{+1}, 1890$

SI: $297 \bullet$ CI: c270 • ST: 1.D.M.30.E

PN: NeOBatrachI Sarasin ${ }^{+1}$, 1890.sa.c01 • AK

PA: 00 - NEOBATRACHI $\cdot \operatorname{Sarasin}^{+1} 1890 . \mathrm{sa}: 245 \cdot$ bC

01 - NeObatrachia • Dubois 2015.da: $107 \cdot \mathbf{E E}$

RL: > CAECILOIDEA 1890. sa.c03 • PR

GN: ANURA 1805.da.c01

GYMNOPHIONA 1814.ra.c01

URODELA 1805.da.c02

GZ: INR

EN: KYR. C.03.01. Subclassis Lissamphibia Gadow, 1898.ga.c01-00

NeOBATRACHIA Reig, 1958

SI: $368 \bullet$ CI: c335・ST: 1.D.M.41.O

PN: NeObatrachia Reig, 1958.ra.c03 • AK

PA: 00 • NeObATRACHIA • Reig 1958.ra: $114 \bullet$ bO

RL: $\downarrow$ NeOBatrachi 1890 .sa.c01

GN: AQUIPARES 1816.ba.c07

Helanura DOP.da.c09

GZ: INR

EN: KYR. C.08.02. Superphalanx RANOMORPHA Fejérváry, 1921.fb.c08-01

NeObatrachia Kuhn, 1962

SI: 382 • CI: c342 • ST: 1.D.M.41.O

PN: NeObatrachia Kuhn, 1962.ka.c03 • AK

PA: 00 • NeObatraChia • Kuhn 1962.ka: 341 • bO

RL: $\downarrow$ NeOBATRACHI 1890. sa.c01

$>$ AMPHICOELA 1962.ka.c01 • AI: HI

$>$ ARCHAEOBATRACHIA 1962.ka.c02 • AI: HL

GN: GEOBATRACHIA 1828.ra.c18

Mediogyrinia 1878.1a.c02

|ANURA INCERTAE SEDIS|

GZ: INR

EN: KYR. C.04.01. Ordo ANURA Duméril, 1805.da.c01-07

NeObatrachia Kuhn, 1965

SI: $392 \bullet \mathbf{C I}$ : c352・ST: 1.D.M.41.O 
PN: NeOBatrachia Kuhn, 1965.ka.c03 • AK

PA: 00 • NeObatraCHIA • Kuhn 1965.ka: 92 • bO

RL: $\downarrow$ NeOBatrachi 1890 .sa.c01

GN: DORSIPARES 1816.ba.c06

LAEVOGYRINIA 1878.1a.c01

GZ: INR

EN: KYR. C.06.01. Infraordo GEOBATRACHIA Ritgen, 1828.ra.c18-02

NeOcaudata Milner, 2000

SI: 428 • CI: c388 • ST: 1.D.M.30.O

PN: NeOCAUdATA Milner, 2000.ma.c01 • AK

PA: 00 • NEOCAUDATA • Milner 2000.ma: 1412 • bO

RL: INR

GN: IMPERFECTIBRANCHIA 1838.ha.c03

Pseudosauria 1816.ba.c08

GZ: INR

EN: KYR. C.04.03. Ordo Urodela Duméril, 1805.da.c02-12

[HYP. Subordo Nullibranchia Bonaparte, 1831.ba.c01-01]

NeONAMPHIBIA Haeckel, 1902

SI: $301 \cdot \mathbf{C I}$ : c274 • ST: 1.D.A.30.O

PN: NeONAMPhibia Haeckel, 1902.ha.c01 • AK

PA: 00 - NeONAMPHiBia • Haeckel 1866.ha: 640 • bC

RL: $\leftrightarrow \mid$ LISSAMPHIBIA 1866.ha.c01

GN: ANURA 1805.da.c01

URODELA 1805.da.c02

GZ: » GI:

GYMNOPHIONA 1814.ra.c01

|Non-LiSSAMPHibian AMPHIBIA|

EN: ANAPTONYM

[HYP. Superordo BATRACHIA Brongniart, 1800.ba.c01-14]

Neosalientia Roček, 1981

SI: $407 \bullet$ CI: c367•ST: 1.D.M.31.O

PN: NeOSAlientia Roček, 1981.ra.c02 • AK

PA: 00 • NeOSALIENTIA • Roček 1981.ra: 1 • O

RL: INR

GN: ANGUSTICOELA 1958.ra.c01

HYdrobatrachia 1828.ra.c14

|ANURA INCERTAE SEDIS|

GZ: INR

EN: KYR. C.04.01. Ordo ANURA Duméril, 1805.da.c01-07

Notocentrophora von Huene, 1920

SI: $312 \bullet$ CI: c285 • ST: 2.D.A.31.E

PN: NotOCENTROPHORI von Huene, 1920.ha.c01 • AK

PA: 00 • NOTOCENTROPHORI • Von Huene 1920.ha: 211 • Ga 01 - NotoCENTROPHORI • Von Huene 1931.ha: 302 • UC

02 - NotOCENTROPHORI • Von Huene 1956.ha: 110 • O

03 - NOTOCENTROPHORA • Dubois 2015.da: $106 \cdot \mathbf{E E}$

RL: $\leftrightarrow$ ! ANOURES 1805.da.c01

GN: ANURA 1805.da.c01

GZ: » GX:

GYMNOPHIONA 1814.ra.c01

URODELA 1805.da.c02

EN: KYR. C.04.01. Ordo ANURA Duméril, 1805.da.c01-07

NuDA Scopoli, 1777

SI: 008 • CI: zh02 • ST: 2.D.M.99.O

PN: NUDA Scopoli, 1777.sa.c01・zz
PA: $00 \cdot$ NUdA • Scopoli 1777.sa: $381 \cdot$ Gs

RL, GN, GZ, EN: •

NudA Oppel, 1811

SI: $026 \bullet \mathbf{C I}: \mathrm{c015} \bullet \mathbf{S T}: 1 . \mathrm{D} . \mathrm{M} .20 . \mathrm{O}$

PN: NUDA Oppel, 1811.oa.c01 • AK

PA: $00 \cdot$ NUDA $・$ Oppel 1811.oa: $260 \cdot \mathbf{O}$

$01 \cdot$ NUDA $\cdot$ Bonaparte $1838 . b d: 124 \cdot$ Sc

$02 \cdot$ NUdA $・$ Leunis $1851.1 \mathrm{a}: 101 \cdot \mathbf{b C}$

RL: $\downarrow$ NUDA 1777. sa.c01

i↔> BatraCIENS 1811.oa.c02 • AI: HL

GN: » [The conucleogenera of BATRACIENS 1800.ba.c01 + Caecilia 1758.1a]:

ANURA 1805.da.c01

GYMNOPHIONA 1814.ra.c01

URODELA 1805.da.c02

GZ: INR

EN: KYR. C.03.01. Subclassis Lissamphibia Gadow, 1898.ga.c01-00

NudA Blainville, 1816

SI: $036 \bullet \mathbf{C I}$ : $\mathrm{c} 023 \cdot \mathbf{S T}$ : 1.D.A.40.S

PN: Nuds Blainville, 1816.ba.c04 • AK

PA: 00 • Nuds • Blainville 1816.ba: '111' [119] • bC

$01 \cdot$ NUDA $・$ Dubois 2015.da: $105 \cdot \mathbf{E E}$

RL: $\downarrow$ NUDA 1777. sa.c01

$\leftrightarrow$ NUDIPELLIFERES 1816.ba.c01 • AI: HL

$\leftrightarrow<$ AMPHYBIENS 1816.ba.c02 $\bullet$ SD

$\leftrightarrow<$ ICTYOIDES 1816.ba.c03 • AI: HL

GN: ANURA 1805.da.c01

GYMNOPHIONA 1814.ra.c01

Urodela 1805.da.c02

GZ: » GX:

|Aмniota|

|Pisces|

EN: KYR. C.02.01. Classis AмpHIBIA Blainville, 1816.ba.c02-03 NuDA Blainville, 1816

SI: $071 \bullet \mathbf{C I}: \mathrm{c053} \bullet \mathbf{S T}: 2 . \mathrm{D} . \mathrm{M} .41 . \mathrm{O}$

PN: NuDA Fitzinger, 1826.fb.c01 • AK

PA: $00 \cdot$ NUdA • Fitzinger 1826.fb: $4 \cdot$ 'T'

$01 \cdot$ NUDA $\cdot$ Bonaparte 1831.bb: $134 \cdot \mathbf{O}$

RL: $\downarrow$ NudA 1777 .sa.c01

GN: PSEUdophiona 1816.ba.c11

GZ: INR

EN: KYR. C.05.04. Subordo Pseudophiona Blainville, 1816.ba.c11-06

NuDA Leuckart, 1841

SI: $165 \bullet$ CI: c142 • ST: 2.D.M.40.O

PN: NUDA Leuckart, 1841.la.c01 • AK

PA: $00 \cdot$ NUDA • Leuckart 1841.1a: $30 \cdot$ UC

RL: $\downarrow$ NUDA 1777. sa.c01

GN: IMPERFECTIBRANCHIA 1838.ha.c03

Pseudosauria 1816.ba.c08

GZ: INR

EN: KYR. C.04.03. Ordo Urodela Duméril, 1805.da.c02-12

[HYP. Subordo Nullibranchia Bonaparte, 1831.ba.c01-01]

NUDIPELLIFERA Blainville, 1816

SI: $033 \bullet$ CI: c020 • ST: 1.D.A.32.E 
PN: NUdIPELLIFERES Blainville, 1816.ba.c01 • AK

PA: 00 • NUDIPELLIFERES • Blainville 1816.ba: “107” [115] • C

$01 \cdot$ NUDIPELLIFERA $\cdot$ Jourdan 1834.jb: $151 \cdot \mathbf{C}$

$02 \cdot$ NUDIPELLIFERES • Hallowell 1856.ha: 6 • bC

RL: $\leftrightarrow<$ AMPHYBIENS 1816.ba.c02 • SD

$\leftrightarrow>$ ICTYOIDES 1816 ba.c03 - AI: HL

$\leftrightarrow>$ NuDs 1816.ba.c04 • AI: HL

GN: ANURA 1805.da.c01

GYMNOPHIONA 1814.ra.c01

URODELA 1805.da.c02

GZ: » GX:

|АмNiota

|Pisces|

EN: KYR. C.02.01. Classis AмpHibia Blainville, 1816.ba.c02-03

NullibRANCHIA Bonaparte, 1831

SI: $108 \bullet$ CI: c089 • ST: 1.D.M.03.O

PN: Nullibranchia Bonaparte, 1831.ba.c01 • HK

PA: 00 - Nullibranchia • Bonaparte 1831.ba: 67 • O

01 • Nullibranchia • Dubois 2015.da: 49 • bO

RL: > Derotremata 1831.ma.c01 • AI: Dubois 2015: 49

GN: IMPERFECTIBRANCHIA 1838.ha.c03

PSEUdOSAURIa 1816.ba.c08

GZ: INR

EN: KYR. C.04.03. Ordo Urodela Duméril, 1805.da.c02-12

[HYP. Subordo Nullibranchia Bonaparte, 1831.ba.c01-02]

Odontoglossa Cope, 1875

SI: $260 \bullet \mathbf{C I}:$ c234 • ST: 1.D.M.31.O

PN: Odontaglossa Cope, 1875.ca.c02 • AK

PA: $00 \cdot$ Odontaglossa • Cope 1875.ca: $8 \cdot$ bO

RL: INR

GN: DORSIPARES 1816.ba.c06

GZ: INR

EN: KYR. C.07.01. Hypoordo DorsiPares Blainville, 1816.ba.c06-02

OpHIDIOBATRACHIA Duvernoy, 1849

SI: $181 \bullet$ CI: c158 • ST: 1.D.M.31.E

PN: OPHIDIO-BATRACIENS Duvernoy, 1849.da.c01 • AK

PA: c0 • OPHIDIO-BATRACIENS • Duvernoy 1849.da: 186, 189 • O - EEA: HL

i1 - OPHOdio-Batraciens • Duvernoy 1849.da: 185 • O

02 - OPHIDIOBATRACHIA $\cdot$ Hoc loco $\bullet$ EE

RL: INR

GN: PSEUdophiona 1816.ba.c11

GZ: INR

EN: KYR. C.05.04. Subordo Pseudophiona Blainville, 1816.ba.c11-06

OPHIOMORPHI Van der Hoeven, 1855

SI: $200 \bullet$ CI: c177• ST: 1.D.M.30.X

PN: OPHiomorpha Van der Hoeven, 1855.va.c02 • AK

PA: 00 • ОрніомоRPHA • Van der Hoeven 1855.va: x, 460 • O

$01 \cdot$ OPHIOMORPHA $・$ Huxley 1863.ha: $68 \cdot \mathbf{U C}$

02 • OPHIOMORPHA • Owen 1866.oa: $15 \cdot$ bO

03 - OPHIOMORPHES • Fatio 1872.fa: 7 • O

04 - OPHIOMORPHI • Dubois 2015.da: 90 • EX

RL: $\leftrightarrow \mid$ Peromeles 1839.da.c01

GN: Plesiophiona DOP.da.c10
Pseudophiona 1816.ba.c11

GZ: INR

EN: KYR. C.04.02. Ordo GymnOPHIONA Rafinesque, 1814.ra.c01-02

Ophiosoma Duméril ${ }^{+1}, 1841$

SI: $160 \bullet$ CI: c137 • ST: 2.D.M.30.E

PN: OPHIOSOMES Duméril ${ }^{+1}, 1841 . d a . c 07 \cdot \mathbf{A K}$

PA: 00 - OPHIOSOMES • Duméril ${ }^{+1}$ 1841.da: plate after page 53 - $\mathrm{Gr} / \mathrm{Sc} /{ }^{\prime T}$ '

$01 \cdot$ OPHIOSOMA $\cdot$ Lichtenstein $^{+2}$ 1856.la: $35 \cdot \mathbf{O}$

$02 \cdot$ OPHIOSOMA $・$ Jan 1857.ja: $52 \cdot \mathbf{U C}$

RL: INR

GN: Plesiophiona DOP.da.c10

PSEUDOPHIONA 1816.ba.c11

GZ: INR

EN: KYR. C.04.02. Ordo Gymnophiona Rafinesque, 1814.ra.c01-02

OpISTHOCOELA Meyer, 1860

SI: $213 \bullet \mathbf{C I}: \mathrm{c} 190 \bullet \mathbf{S T}$ : 2.D.M.31.E

PN: OpISTHOcoeli Meyer, 1860.mb.c01 • AK

PA: 00 • OPISTHOCOELI - Meyer 1860.mb: $559 \bullet$ UC

RL: INR

GN: Pseudosauria 1816.ba.c08

GZ: INR

EN: KYR. C.05.07. Subordo Pseudosauria Blainville, 1816.ba.c08-07

OpISTHOCOELA Owen, 1860

SI: $218 \bullet$ CI: zh20 • ST: 1.U.U.99.E

PN: OpISTHOCOELIA Owen, 1860.oa.c02 • zz

PA: 00 - OPISTHOCOELIA - Owen 1860.oa: x, 272 • bO

$01 \cdot$ OPITHOCOELA $\cdot H o c$ loco $\bullet$ EE

RL: $\downarrow$ OPISTHOCOELI $1860 . \mathrm{mb} . c 01$

GN, GZ, EN: •

OpISTHOCOELA Lataste, 1879

SI: $277 \bullet \mathbf{C I}: \mathrm{c} 251 \bullet \mathbf{S T}: 1 . \mathrm{D} . \mathrm{M} .41 . \mathrm{E}$

PN: OPISTHOCOELIDAE Lataste, 1879.1b.c03 • AK

PA: 00 • OPISTHOCOELIDAE • Lataste 1879.1b: $339 \bullet$ bO

01 - OPISTHOCOELA • Noble 1922.na: $21 \cdot$ bO

02 • OPISTHOCOELIA $・$ Kuhn 1939.ka: 92 • bO

$04 \cdot$ OPISTOCOELA • Casamiquela 1961.ca: $77 \cdot$ bO

03 - OPISTHOCALIDAE $・$ Kuhn 1967.kb: $31 \cdot$ UC

RL: $\downarrow$ OPISTHOCOELI $1860 . \mathrm{mb} . \mathrm{c} 01$

$\leftrightarrow \mid$ Mediogyrinides 1879.1a.c01

GN: GEOBATRACHIA 1828.ra.c18

Mediogyrinia 1878.1a.c02

GZ: INR

EN: KYR. C.05.02. Subordo Hydrobatrachia Ritgen, 1828.ra.c14-01

Opisthocoela Nicholls, 1916

SI: $302 \bullet \mathbf{C I}:$ c275・ST: 1.D.M.41.O

PN: OpISTHOCOela Nicholls, 1916.na.c01 • AK

PA: 00 • OPISTHOCOELA • Nicholls 1916.na: 86 • 'T'

01 - OPISTHOCOELINA - Pearse 1936.pa: 20 • bO

$02 \cdot$ OPISTHOCOELA $・ \mathrm{Fei}^{+1}$ 2016.fa: ix • bO

RL: $\downarrow$ OPISTHOCOELI $1860 . \mathrm{mb} . \mathrm{c} 01$

$\leftrightarrow \mid$ Mediogyrinidae 1878.la.c02 
GN: Mediogyrinia 1878.la.c02

GZ: INR

EN: KYR. C.06.02. Infraordo Mediogyrinia Lataste, 1878.1a.c02-02

OpISTHOCOELA Ahl, 1930

SI: $336 \bullet$ CI: c309・ST: 1.D.M.41.O

PN: OPISTHOCOELA Ahl, 1930.aa.c01 • AK

PA: 00 • OPISTHOCOELA • Ahl 1930.aa: $83 \cdot$ bO

RL: $\downarrow$ OPISTHOCOeli $1860 . \mathrm{mb} . \mathrm{c} 01$

GN: ANGUSTICOELA 1958.ra.c01 Hydrobatrachia 1828.ra.c14

GZ: INR

EN: KYR. C.04.01. Ordo ANURA Duméril, 1805.da.c01-07 OpISTHOGLOSSA Günther, 1858

SI: $205 \bullet$ CI: c182 • ST: 2.D.M.31.O

PN: OpISThoglossa Günther, 1858.gc.c01 • AK

PA: 00 • OPISTHOGLOSSA • Günther 1858.gc: 339 • Gr

01 - Opistoglossa • Fatio 1872.fa: 232 -UC

02 • OPISTHOGLOSSA • Hoffmann 1878.ha: 616 • UC

$03 \cdot$ OPISTHOGLOSSES • Lataste 1879.lb: 276 • UC

04 • OPISTHOGLOSSA • Lataste 1879.1b: 339 • 'T'

$05 \cdot$ OPHISTOGLOSSES • Brocchi 1881.ba: $5 \cdot$ Sc

RL: > OXYDACTYLA 1858.gc.c03 • PR

GN: GEOBATRACHIA 1828.ra.c18

MEDIOGYRINIA 1878.la.c02

GZ: INR

EN: KYR. C.05.02. Subordo HYdrobatrachia Ritgen, 1828.ra.c14-01

Orthoglena Bruch, 1862

SI: $224 \bullet$ CI: c198・ST: 2.D.M.41.O

PN: ORTHOGLENIDES Bruch, 1862.ba.c04 • AK

PA: 00 • ORTHOGLENIDES • Bruch 1862.ba: 221 • 'F'

$01 \cdot$ OrthogLena $\cdot H o c l o c o \cdot \mathbf{E U}$

RL: $\leftrightarrow<$ ORTHOGLENA 1862 .ba.c05 • AI: HL

GN: GEOBATRACHIA 1828.ra.c18

Mediogyrinia 1878.la.c02

GZ: INR

EN: KYR. C.05.02. Subordo Hydrobatrachia Ritgen, 1828.ra.c14-01

Orthoglena Bruch, 1862

SI: $225 \bullet$ CI: c199 • ST: 2.D.M.31.O

PN: ORTHOGLENA Bruch, 1862.ba.c05 • AK

PA: 00 - ORTHOGLENA • Bruch 1862.ba: 221 • 'F'

RL: $\leftrightarrow>$ ORTHOGLENIDES 1862.ba.c04 • AI: HL

GN: GEOBATRACHIA 1828.ra.c18

Mediogyrinia 1878.1a.c02

GZ: INR

EN: KYR. C.05.02. Subordo Hydrobatrachia Ritgen, 1828.ra.c14-01

OXYDACTYLA Günther, 1858

SI: $207 \bullet$ CI: c184・ST: 2.D.M.31.O

PN: OXYDACTYLa Günther, 1858.gc.c03 • AK

PA: 00 • OXYDACTYLA • Günther 1858.gc: $341 \cdot \mathbf{S r}$

$01 \cdot$ OXYDACTILA $\cdot$ Hoffmann 1878.ha: $616 \cdot \mathbf{U C}$

$02 \cdot$ OXYDACTYLIA $・$ Knauer 1878.ka: $104 \cdot$ Gr

$03 \cdot$ OXYDACTYLES • Lataste 1879.1b: $276 \cdot \mathbf{U C}$
04 • OXYDACTYLES • Brocchi 1881.ba: 5 • Gr

RL: < OPISTHOgLOSSA 1858.gc.c01・PR

GN: GeOBATRACHIA 1828.ra.c18

Mediogyrinia 1878.1a.c02

GZ: INR

EN: KYR. C.05.02. Subordo HydrobatraChIA Ritgen, 1828.ra.c14-01

PAlaEOBATRACHACEA Fejérváry, 1921

SI: $313 \bullet$ CI: c286 • ST: 2.D.M.31.R

PN: Palaeobatrachoidea Fejérváry, 1921.fb.c01 • AK

PA: 00 - PalaeObatrachoidea • Fejérváry 1921.fb: 16 • bO

$01 \cdot$ Palaeobatrachacea $\cdot$ Hoc loco $\bullet$ ER

RL: > PIPOMORPHA 1921.fb.c02 • PR

$>$ Palaeobatrachomorpha 1921.fb.c03 • PR

GN: DoRSIPARES 1816.ba.c06

GZ: INR

EN: KYR. C.07.01. Hypoordo DorsIPAREs Blainville, 1816.ba.c06-02

Palaeobatrachacea Bauer, 1987

SI: $417 \bullet$ CI: c377• ST: 1.D.M.41.R

PN: Palaeobatrachia Bauer, 1987.bc.c02 • AK

PA: 00 • Palaeobatrachia • Bauer 1987.bc: 52 • UC

01 - Palaeobatrachacea $\cdot$ Hoc loco $\bullet$ ER

RL: $\downarrow$ Palaeobatrachoidea $1987 . b c . c 06$

GN: DORSIPARES 1816.ba.c06

LAEVOGYRINIA 1878.1a.c01

GZ: INR

EN: KYR. C.06.01. Infraordo GEOBATRACHIA Ritgen, 1828.ra.c18-02

PalaeobatrachomorPha Fejérváry, 1921

SI: 315 • CI: c288 • ST: 2.D.M.31.A

PN: Palaeobatrachomorpha Fejérváry, 1921.fb.c03 • aK

PA: 00 - PalaeobatrachomorPha $\uparrow$ Fejérváry 1921.fb: 24 - Gs

RL: < Palaeobatrachoidea 1921.fb.c01 • PR

< Pipomorpha 1921.fb.c02 • AI: HL

GN: DoRSIPARES 1816.ba.c06

GZ: INR

EN: KYR. C.07.01. Hypoordo DorsiPares Blainville, 1816.ba.c06-02

Pananura nov.

SI: $440 \bullet$ CI: c399・ST: 1.D.M.11.O

PN: Pananura nov., DOP.da.c07

PA: $00 \cdot$ PananURa $\cdot$ Hoc loco $\bullet$ bP

RL: INR

GN: Pananura DOP.da.c07

GZ: INR

EN: KYR. C.11.05. Subphalanx Pananura nov., DOP.da.c07-00 Parotoidia Gardiner, 1982

SI: 409 • CI: c369 • ST: 1.D.M.30.O

PN: Parotoidia Gardiner, 1982.ga.c01 • AK

PA: 00 • Parotoidia • Gardiner 1982.ga: $228 \bullet$ pO

01 • Paratoidea • Milner 1988.ma: $74 \cdot$ pO

RL: INR

GN: ANURA 1805.da.c01

URODELA 1805.da.c02

GZ: INR 
EN: KYR. C.03.01. Subclassis LisSAMPhiBia Gadow, 1898.ga.c01-00

[HYP. Superordo BATRACHIA Brongniart, 1800.ba.c01-14]

PeDATA Haworth, 1825

SI: $067 \bullet$ CI: c049 • ST: 2.D.M.30.O

PN: Pedata Haworth, 1825.ha.c01 • AK

PA: 00 • PEDATA • Haworth 1825.ha: 372 • UC

RL: INR

GN: ANURA 1805.da.c01

URODELA 1805.da.c02

GZ: INR

EN: KYR. C.03.01. Subclassis LisSAMPhibia Gadow, 1898.ga.c01-00

[HYP. Superordo BATRACHIA Brongniart, 1800.ba.c01-14]

Pelobatacea Laurent in Fuhn, 1960

SI: $372 \bullet$ CI: cn04 • ST: 1.D.M.00.R

PN: Pelobatoidea Laurent in Fuhn, 1960.fa.c04 • AN

PA: 00 - Pelobatoidea • Laurent in Fuhn 1960.fa: 163 • bO

$01 \cdot$ Pelobatacea $\cdot H o c l o c o \cdot$ ER

RL: INR

GN: ArChaeOSAlientia 1981.ra.c01

GZ: INR

EN: KYR. C.08.01. Superphalanx ARCHAEOSALIENTIA Roček, 1981.ra.c01-01

Pelobatomorpha Fejérváry, 1921

SI: 317 • CI: c290 • ST: 1.D.M.31.A

PN: Pelobatomorpha Fejérváry, 1921.fb.c05 • AK

PA: 00 • PelobatomorPha • Fejérváry 1921.fb: 24 • Gs

RL: < ANiSOBATRACHOIDEA 1921.fb.c04 • PR

> CYSTIGNATHOMORPHA 1921.fb.c07 • AI: HL

GN: ANGUSTICOELA 1958.ra.c01

Hydrobatrachia 1828.ra.c14

GZ: INR

EN: KYR. C.04.01. Ordo ANURA Duméril, 1805.da.c01-07

PERENNIBRANCHIA Latreille 1824

SI: 063 • CI: c045 • ST: 1.D.M.31.E

PN: Perennibranches Latreille 1824.la.c02 • AK

PA: 00 - PerenNibranches • Latreille 1824.la: 9 • O

01 - Perennibranchia • Latreille 1825.la: 105 • O

02 - Perennibranchiata - Jourdan 1834.jb: 234 • Gr

03 - Perennibranchiata - Jones 1841.ja: 589 • UC

04 - Perennibrachiae • Gray 1842 .ga: $113 \cdot$ O

05 - Perennobrachia • Gray 1842.ga: $114 \cdot$ O

06 Perennibranchiata - Stannius $1856 . \mathrm{sa}: 4 \cdot$ bO

07 - Perennibranchiata • Haeckel 1866.ha: cxxxi • O

RL: INR

GN: » OA, SD: Latreille 1825.1a: 105:

Meantes 1767.la.c01

Pseudosauria 1816.ba.c08

GZ: INR

EN: KYR. C.04.03. Ordo Urodela Duméril, 1805.da.c02-12

[HYP. Subordo Pneumobranchia Sonnini ${ }^{+1}$, 1801.sa.c01-02]

PERENNIBRANCHIA Hunter, 1834

SI: $121 \cdot \mathbf{C I}$ : c101 • ST: 1.D.M.21.E

PN: Perennibranchiata Hunter, 1834.ha.c01 • AK

PA: 00 - Perennibranchiata • Hunter 1834.ha: 145 • UC
$01 \cdot$ Perennibranchia $\cdot$ Hoc loco $\bullet$ EE

RL: $\downarrow$ Perennibranches 1824.1a.c02

GN: IMPERFECTIBRANCHIA 1838.ha.c03

Meantes 1767.la.c01

GZ: INR

EN: KYR. C.04.03. Ordo Urodela Duméril, 1805.da.c02-12

[HYP. Subordo Pseudosalamandrae Bonaparte, 1850.bb.c02-02]

Perennibranchia Lataste, 1878

SI: $271 \bullet$ CI: c245 • ST: 2.D.M.41.E

PN: Perennibranches Lataste, 1878.1b.c01 • AK

PA: 00 - Perennibranches - Lataste 1878.1b: 3 • Sc

01 - Perennibranchia • Goodrich 1930.ga: xxi • bO

RL: $\downarrow$ Perennibranches 1824.1a.c02

GN: PSEUdosauria 1816.ba.c08

GZ: INR

EN: KYR. C.05.07. Subordo Pseudosauria Blainville, 1816.ba.c08-07

Perobranchia Duméril ${ }^{+1}, 1841$

SI: 157 • CI: c134 • ST: 2.D.M.31.E

PN: Perobranches Duméril ${ }^{+1}$, 1841.da.c04 • AK

PA: 00 - Perobranches • Duméril ${ }^{+1} 1841$ da: 52 - Gr/Sc/'T'

01 - Perobranchia - Dubois 2016.da: 9 • iO

02 - Perobranchia - Dubois 2016.da: $9 \cdot$ hO

RL: < ATRETODERES 1841.da.c03 • AI: HL

$\leftrightarrow<$ AMPHIUMOIDES 1841.da.c05 • AI: HL

$<$ ExobranCHES 1841.da.c06 • AI: HL

$<$ Trematoderes 1841.da.c08 • AI: HL

GN: Pseudosauria 1816.ba.c0 8

GZ: INR

EN: KYR. C.05.07. Subordo Pseudosauria Blainville, 1816.ba.c08-07

Peromela Duméril, 1839

SI: 133 • CI: c113 • ST: 1.D.M.30.E

PN: Peromeles Duméril, 1839.da.c01 • AK

PA: 00 - Peromeles • Duméril 1839.da: 583 • bO

01 Peromeles • Baird 1851.ba: 249, $261 \cdot$ O

02 - Peromela - Van der Hoeven 1855.va: 460 - O

RL: INR

GN: Plesiophiona DOP.da.c10

PSEUDOPHIONA 1816.ba.c11

GZ: INR

EN: KYR. C.04.02. Ordo GYMNOPHIONA Rafinesque, 1814.ra.c01-02

Peromela Gouriet, 1868

SI: $251 \bullet$ CI: c225・ST: 1.D.M.41.E

PN: Peromeles Gouriet, 1868.ga.c06 • AK

PA: 00 • Peromeles • Gouriet 1868.ga: 206 • UC

$01 \cdot$ Peromela $\cdot H o c$ loco $\cdot$ EE

RL: $\downarrow$ Peromeles 1839.da.c01

GN: PSEUdOPHIONA 1816.ba.c11

GZ: INR

EN: KYR. C.05.04. Subordo Pseudophiona Blainville, 1816.ba.c11-06

Phaeneropleurae Fitzinger, 1843

SI: 174 • CI: c151 • ST: 2.D.M.31.O 
PN: Phaeneropleurae Fitzinger, 1843.fa.c06 • AK

PA: 00 - PhaENEROPleUrae • Fitzinger 1843.fa: 33 - Sc

$01 \cdot$ Phaeneropleura • Gray 1850 .ga: $15,71 \cdot$ UC

$02 \cdot$ Phaneropleurae $\cdot$ Kuhn 1967.kb: $33 \cdot$ UC

RL: < CRYPTOPLEURAE 1843.fa.c07 • AI: HL

GN: PSEUdosauria 1816.ba.c0 8

GZ: INR

EN: KYR. C.05.07. Subordo Pseudosauria Blainville, 1816.ba.c08-07

Phaneranura nov.

SI: 435 • CI: c394 • ST: 1.D.M.11.O

PN: Phaneranura nov., DOP.da.c02

PA: $00 \cdot$ PhanERANURA $\cdot$ Hoc loco $\bullet \mathbf{P}$

RL: INR

GN: Phaneranura DOP.da.c02

GZ: INR

EN: KYR. C.10.02. Phalanx Phaneranura nov., DOP.da.c02-00

Phanerobranchia Wagler, 1828

SI: 096 • CI: c077 • ST: 3.D.M.31.E

PN: Phanerobranchi Wagler, 1828.wb.c03 • AK

PA: 00 • PhanerobranChi • Wagler 1828.wb: 859 • 'F'

$01 \cdot$ Phanerobranchia $\cdot H o c$ loco $\bullet$ EE

RL: INR

GN: Pseudosauria 1816.ba.c08

GZ: INR

EN: KYR. C.05.07. Subordo Pseudosauria Blainville, 1816.ba.c08-07

Phanerobranchia Bonaparte, 1831

SI: $111 \bullet$ CI: $092 \bullet$ ST: 1.D.M.41.O

PN: Phanerobranchia Bonaparte, 1831.bb.c02 • AK

PA: 00 - Phanerobranchia • Bonaparte 1831.bb: 136 • O 01 - Phanaerobranchia • Bonaparte 1838.ba: [194] • O

$02 \cdot$ Phanerobanchia - Gray 1850 .ga: $64 \cdot$ O

RL: $\downarrow$ Phanerobranchi 1828.wb.c03

GN: Meantes 1767.la.c01 PSEUdosauria 1816.ba.c08

GZ: INR

EN: KYR. C.04.03. Ordo Urodela Duméril, 1805.da.c02-12 [HYP. Subordo Pneumobranchia Sonnini ${ }^{+1}$, 1801.sa.c01-02]

Phaneroglossa Wagler, 1830

SI: $104 \cdot \mathbf{C I}$ : c085 • ST: 2.D.M.30.E

PN: Phaneroglossae Wagler, 1830.wa.c03 • AK

PA: 00 • Phaneroglossae • Wagler 1830.wa: 131 • 'F' 01 • Phaneroglossa • Hoffmann 1878.ha: 582 • 'F'

RL: INR

GN: ANURA 1805.da.c01 Urodela 1805.da.c02

GZ: INR

EN: KYR. C.03.01. Subclassis LisSAMPHibia Gadow, 1898.ga.c01-00

[HYP. Superordo BATRACHIA Brongniart, 1800.ba.c01-14]

Phaneroglossa Duméril ${ }^{+1}, 1841$

SI: $154 \cdot$ CI: c131 • ST: 2.D.M.41.E

PN: Phaneroglosses Duméril ${ }^{+1}, 1841$.da.c01 • AK

PA: 00 • Phaneroglosses • Duméril ${ }^{+1} 1841$ da: 49 • Gr/Sc/'T' $01 \cdot$ Phaneroglossae • Gray 1842 .ga: $112 \cdot$ Sc
02 • Phaneroylossae • Holbrook 1842.ha: $74 \cdot$ Sc

03 - Phaneroglossa • Gervais 1847.ga: $721 \cdot$ Gr

$04 \cdot$ Phaneroglossa - Baird 1851.ba: $257 \cdot$ bO

05 P Phaneroglosses • Desmarest 1857.da: 19 • 'T'

06 Phaneroglossae • Günther 1858.gc: $339 \cdot \mathbf{G r}$

07 - Phaneroglosses • Blanchard 1885.bb: 588 - UC

08 • Phaneroglossa • Nicholls 1916.na: $81 \cdot$ UC

RL: $\downarrow$ Phaneroglossae 1830 .wa.c03

GN: GEOBATRACHIA 1828.ra.c18

Mediogyrinia 1878.la.c02

GZ: INR

EN: KYR. C.05.02. Subordo Hydrobatrachia Ritgen, 1828.ra.c14-01

Phaneroglossa Boulenger, 1882

SI: $284 \bullet$ CI: c258・ST: 1.D.M.41.O

PN: Phaneroglossa Boulenger, 1882.bb.c01 c aK

PA: 00 - Phaneroglossa • Boulenger 1882.bb: vii, $1 \cdot$ bO

$01 \cdot$ Phaneroglossa • Abel 1919.aa: xii, $322 \cdot$ O

RL: $\downarrow$ Phaneroglossae 1830.wa.c03

GN: ANGUSTICOELA 1958.ra.c01

Hydrobatrachia 1828.ra.c14

GZ: INR

EN: KYR. C.04.01. Ordo ANURA Duméril, 1805.da.c01-07

Phoranura nov.

SI: 437 • CI: c396 • ST: 1.D.M.11.O

PN: Phoranura nov., DOP.da.c04

PA: $00 \cdot$ PhORANURA $\cdot$ Hoc loco $\bullet$ iP

RL: INR

GN: Phoranura DOP.da.c04

GZ: INR

EN: KYR. C.12.01. Infraphalanx PhOranura nov., DOP.da.c04-00

Phrynanura nov.

SI: 438 • CI: c397 • ST: 1.D.M.11.O

PN: Phrynanura nov., DOP.da.c05

PA: 00 - PhryNANURA $\cdot$ Hoc loco $\bullet$ iP

RL: INR

GN: Phrynanura DOP.da.c05

GZ: INR

EN: KYR. C.12.02. Infraphalanx Phrynanura nov., DOP.da.c05-00

PhrYNiA Bauer, 1986

SI: 412 • CI: c372 • ST: 2.D.M.31.O

PN: Phrynia Bauer, 1986.ba.c02 • AK

PA: 00 • PhrYNia • Bauer 1986.ba: 6 • UC

RL: INR

GN: Phaneranura DOP.da.c02

SCOPTANURA 1973.sb.c02

GZ: INR

EN: TEO. C.09.01. Epiphalanx AQUIPARES Blainville, 1816.ba.c07-02

[HYP. Phalanx AQuiPares Blainville, 1816.ba.c07-03]

PhrynOBATRACHIA Bauer, 1987

SI: 421 • CI: c381 • ST: 1.D.M.30.O

PN: Phrynobatrachia Bauer, 1987.bc.c06 • AK

PA: 00 - PhrynObatraChia • Bauer 1987.bc: 52 • UC

RL: INR 
GN: AQUIPARES 1816.ba.c07

Helanura DOP.da.c09

GZ: INR

EN: KYR. C.08.02. Superphalanx RANOMORPHA Fejérváry, 1921.fb.c08-01

PHRYNOBATRACHIA Bauer, 1988

SI: 424 • CI: c384 • ST: 1.D.M.30.O

PN: Phrynobatrachia Bauer, 1988.ba.c01 • AK

PA: 00 • Phrynobatrachia • Bauer 1988.ba: E 2 - UC

RL: $\downarrow$ Phrynobatrachia 1987. bc.c06

GN: AQUIPARES 1816.ba.c07 HelanURa DOP.da.c09

GZ: INR

EN: KYR. C.08.02. Superphalanx RANOMORPHA Fejérváry, 1921.fb.c08-01

PhrYnoglossa Duméril ${ }^{+1}, 1841$

SI: $155 \bullet$ CI: c132 • ST: 2.D.M.31.E

PN: Phrynaglosses Duméril ${ }^{+1}$, 1841.da.c02 • AK

PA: 00 • Phrynaglosses • Duméril ${ }^{+1}$ 1841.da: 49 • Gr/Sc/'T'

$01 \cdot$ Phrynaglossae • Gray 1842.ga: $112 \cdot$ Sc

02 • Phrynoglossae • Agassiz 1847.aa: 830 • UC

03 • Phrynaglossa • Baird 1851.ba: $257 \cdot$ bO

04 • Phrynaglosses • Desmarest 1856.da: 156 • 'T'

05 • PhrYNOglOSSES • Desmarest 1857.da: 19 • 'T'

$06 \cdot$ Phrynoglossa $\cdot$ Hoc loco $・$ ER

RL: $\leftarrow$ AgLOSSAE 1830.wa.c02

GN: DORSIPARES 1816.ba.c06

GZ: INR

EN: KYR. C.07.01. Hypoordo DorsIPARES Blainville, 1816.ba.c06-02

Phyllopodobatrachia Ritgen, 1828

SI: $087 \bullet$ CI: $\mathrm{c068} \bullet$ ST: 2.D.M.32.E

PN: Phyllopodobatrachi Ritgen, 1828.ra.c13 • AK

PA: 00 • Phyllopodobatrachi • Ritgen 1828.ra: 278 • 'F'

$01 \cdot$ Phyllopodobatrachia $\cdot H o c l o c o \cdot$ EE

RL: $\leftrightarrow<$ HYDROBATRACHI 1828.ra.c14 • AI: HL

GN: GEOBATRACHIA 1828.ra.c18

MEDIOGYRINIA 1878.1a.c02

GZ: INR

EN: KYR. C.05.02. Subordo Hydrobatrachia Ritgen, 1828.ra.c14-01

PIPACEA Laurent in Fuhn, 1960

SI: $371 \cdot \mathbf{C I}$ : cn03 • ST: 1.D.M.00.R

PN: PiPOIdea Laurent in Fuhn, 1960.fa.c03 • AN

PA: 00 • PIPOIDEA • Laurent in Fuhn 1960.fa: 163 • bO

$01 \cdot \operatorname{PIPACEA} \bullet H o c$ loco $\bullet$ ER

RL: INR

GN: DoRSIPARES 1816.ba.c06

GZ: INR

EN: KYR. C.07.01. Hypoordo DorsiPares Blainville, 1816.ba.c06-02

PIPACEA Dubois, 1983

SI: $410 \bullet \mathbf{C I}$ : c370 • ST: 1.D.M.31.R

PN: PIPOIDEI Dubois, 1983.da.c01 • AK

PA: 00 • PIPOIDEI • Dubois 1983.da: 271 • bO

01 • PIPACEA • Dubois 2015.da: 90 • ER
RL: $\leftarrow$ Mesobatrachia 1980.la.c01

GN: DORSIPARES 1816.ba.c06

LAEVOGYRINIA 1878.1a.c01

GZ: INR

EN: KYR. C.06.01. Infraordo GEOBATRACHIA Ritgen, 1828.ra.c18-02

PIPIFORMIA Brocchi, 1881

SI: 282 • CI: c256 • ST: 2.D.M.31.A

PN: PiPAeformes Brocchi, 1881.ba.c04 • AK

PA: 00 • PIPAEFORMES • Brocchi 1881.ba: 9 • UC

$01 \cdot$ PiPIfORMes • Boulenger 1882.ba: $12 \cdot \mathbf{U C}$

02 PIPIFORMIA • Dubois 2015.da: 90 • UC

RL: INR

GN: DoRSIPARES 1816.ba.c06

GZ: INR

EN: KYR. C.07.01. Hypoordo DorsIPARES Blainville, 1816.ba.c06-02

PipomorPHa Fejérváry, 1921

SI: 314 • CI: c287・ST: 2.D.M.31.A

PN: Pipaemorpha Fejérváry, 1921.fb.c02 • AK

PA: 00 • PiPAEMORPHa • Fejérváry 1921.fb: 16 • Gs

01 • PIPOMORPHA • Dubois 2015.da: 90 • EA

RL: < Palaeobatrachoidea 1921.fb.c01 • PR

$>$ PalaeobatrachomorPha 1921.fb.c03 - AI: HL

GN: DORSIPARES 1816.ba.c06

GZ: INR

EN: KYR. C.07.01. Hypoordo DorsiPares Blainville, 1816.ba.c06-02

PiscIFORMi Hogg, 1839

SI: $142 \bullet$ CI: c120 • ST: 1.D.M.31.X

PN: Pisciformia Hogg, 1839.ha.c07 • AK

PA: 00 • Pisciformia • Hogg 1839.ha: 271 • O

01 • PISCIFORMES • Dubois 2015.da: 90 • EX

$02 \cdot \operatorname{PisCIFORMI} \cdot H o c l o c o \cdot \mathbf{E X}$

RL: INR

GN: Pseudosauria 1816.ba.c08

GZ: INR

EN: KYR. C.05.07. Subordo Pseudosauria Blainville, 1816.ba.c08-07

Plagioglena Bruch, 1862

SI: $222 \bullet$ CI: c196 • ST: 2.D.M.31.O

PN: Plagioglenides Bruch, 1862.ba.c02 • AK

PA: 00 • Plagioglenides • Bruch 1862.ba: 221 • 'F',

$01 \cdot$ Plagioglena $\cdot$ Hoc loco $\bullet$ EE

RL: < CyCLOGLENIDES 1862.ba.c01 • AI: HL

$>$ Plagioglena 1862.ba.c03 • AI: HL

GN: HyLobatrachia 1828.ra.c16

GZ: INR

EN: KYR. C.13.03. Hypophalanx HyLobatrachia Ritgen, 1828.ra.c16-01

Plagioglena Bruch, 1862

SI: $223 \bullet$ CI: c197・ST: 2.D.M.31.O

PN: Plagioglena Bruch, 1862.ba.c03 • AK

PA: 00 - Plagioglena • Bruch 1862.ba: 221 • 'F'

RL: < CyClOGLENIDES 1862.ba.c01 • AI: HL

$>$ Plagioglenides 1862.ba.c02 • AI: HI 
GN: Phaneranura DOP.da.c02

SCOPTANURA 1973.sb.c02

GZ: INR

EN: TEO. C.09.01. Epiphalanx AQUIPAREs Blainville, 1816.ba.c07-02

[HYP. Phalanx AQUIPARES Blainville, 1816.ba.c07-03]

Platydactyla Günther, 1858

SI: $208 \bullet$ CI: c185 • ST: 2.D.M.31.O

PN: Platydactyla Günther, 1858.gc.c04 • AK

PA: 00 • Platydactyla • Günther 1858.gc: $341 \cdot \mathbf{S r}$ 01 - Platydactyla • Hoffmann 1878.ha: 645 • UC 02 - Platydactyles • Brocchi 1881.ba: 5 • Gr

RL: INR

GN: Phaneranura DOP.da.c02 SCOPTANURA 1973.sb.c02

GZ: INR

EN: TEO. C.09.01. Epiphalanx AQUIPARES Blainville, 1816.ba.c07-02

[HYP. Phalanx AQuiPaRes Blainville, 1816.ba.c07-03]

Platydactyla Lataste, 1879

SI: 275 • CI: c249・ST: 2.D.M.41.E

PN: Platydactyles Lataste, 1879.1b.c01・aK

PA: $00 \bullet$ PlatydaCtYles • Lataste 1879.1b: $276 \bullet$ UC

$01 \cdot \operatorname{PlatydaCtyla} \cdot H o c l o c o \cdot \mathbf{E E}$

RL: $\downarrow$ Platydactyla $1858 . g c . c 04$

GN: HYLOBATRACHIA 1828.ra.c16

GZ: INR

EN: KYR. C.13.03. Hypophalanx HyLOBatrachia Ritgen, 1828.ra.c16-01

\section{Plesiophiona nov.}

SI: $443 \bullet$ CI: $c 402 \bullet$ ST: 1.D.M.11.O

PN: Plesiophiona nov., DOP.da.c10

PA: $00 \cdot$ Plesiophiona $\cdot H o c$ loco $\bullet$ bO

RL: INR

GN: Plesiophiona DOP.da.c10

GZ: INR

EN: KYR. C.05.03. Subordo Plesiophiona nov., DOP.da.c10-00 Plethodontacea Smith ${ }^{+1}, 1948$

SI: $365 \bullet$ CI: c332 • ST: 1.D.M.31.R

PN: Plethodontoidea Smith ${ }^{+1}$, 1948.sa.c01 • AK

PA: 00 • PlethodontoIdeA • Smith $^{+1}$ 1948.sa: iii, 16 • bO $01 \cdot$ Plethodontacea $\cdot$ Hoc loco $\bullet$ ER

RL: INR

GN: Pseudosauria 1816.ba.c08

GZ: INR

EN: KYR. C.05.07. Subordo Pseudosauria Blainville, 1816.ba.c08-07

Plethodontacea Kuhn, 1962

SI: 384 • CI: c344・ST: 1.D.M.40.R

PN: Plethodontoidea Kuhn, 1962.ka.c05 • AK

PA: 1962.ka.c05.00 • PlethodontoideA • Kuhn 1962.ka: 363 • bO $01 \cdot$ Plethodontacea $・ H o c l o c o \bullet$ ER

RL: $\downarrow$ Plethodontoidea 1948.sa.c01

< Salamandroidea 1962.ka.c04 • AI: HI

< Proteida 1962.ka.c06 • AI: HL

GN: IMPERFECTIBRANCHIA 1838.ha.c03
Pseudosauria 1816.ba.c08

GZ: INR

EN: KYR. C.04.03. Ordo Urodela Duméril, 1805.da.c02-12

[HYP. Subordo NULLIBRANCHIA Bonaparte, 1831.ba.c01-01]

Plethodontacea Kuhn, 1965

SI: 393 • CI: c353・ST: 1.D.M.40.R

PN: Plethodontoidea Kuhn, 1965.ka.c04 • AK

PA: 00 • Plethodontoidea • Kuhn 1965.ka: 38 • bO

01 - Plethodontacea • Dubois 2015.da: 107 • ER

RL: $\downarrow$ Plethodontoidea 1948.sa.c01

< AMbystomatoidea 1965.ka.c02 • AI: HL

GN: Pseudosauria 1816.ba.c0 8

|URODELA INCERTAE SEDIS|

GZ: INR

EN: KYR. C.04.03. Ordo Urodela Duméril, 1805.da.c02-12 Pneumobranchia Sonnini ${ }^{+1}, 1801$

SI: $016 \bullet \mathbf{C I}: \mathrm{c007} \bullet \mathrm{ST}$ : 1.D.M.03.E

PN: Pneumobranchiens Sonnini ${ }^{+1}$, 1801.sa.c01 • HK

PA: 00 • Pneumobranchiens • Sonnini ${ }^{+1}$ 1801.sa: 309 • O

01 - Pneumobranches • Bory de Saint-Vincent 1828.bb: $218 \cdot \mathbf{O}$

02 • Pneumobranchia $・$ Dubois 2015.da: $49 \cdot$ bO

03 P Peumobranchia • Dubois 2016.da: $9 \cdot$ iO

RL:INR

GN: Meantes 1767.la.c01

PSEUdOSAURIA 1816.ba.c08

GZ:INR

EN:KYR. C.04.03. Ordo Urodela Duméril, 1805.da.c02-12

[HYP. Subordo Pneumobranchia Sonnini ${ }^{+1}$, 1801.sa.c01-02]

PNEUMOBRANCHIA Hunter, 1834

SI: $122 \bullet$ CI: c102・ST: 1.D.M.41.E

PN: Pneumobranchiata Hunter, 1834.ha.c02 • AK

PA: 00 • Pneumobranchiata • Hunter 1834.ha: 145 • UC

01 • Pneumobranchia • Owen 1835.oa: 214 • UC

RL: $\leftarrow$ Perennibranchiata 1834.ha.c01

GN: IMPERFECTIBRANCHIA 1838.ha.c03

Meantes 1767.la.c01

GZ: INR

EN: KYR. C.04.03. Ordo Urodela Duméril, 1805.da.c02-12

[HYP. Subordo Pseudosalamandrae Bonaparte, 1850.bb.c02-02]

Pododysmolgae Ritgen, 1828

SI: $081 \bullet$ CI: c062 • ST: 2.D.M.31.O

PN: Pododysmolgae Ritgen, 1828.ra.c07 • AK

PA: 00 • PododysMolgaE • Ritgen 1828.ra: 277 • 'F'

RL: < MorPHIUROMOLGAEI 1828.ra.c08 • PR

$<$ Molgae 1828.ra.c09・PR

$<$ Hydromolgae 1828.ra.c10 • AI: HL

< GeOMolgae 1828.ra.c11 • AI: HL

GN: Pseudosauria 1816.ba.c08

GZ: INR

EN: KYR. C.05.07. Subordo Pseudosauria Blainville, 1816.ba.c08-07

Proanura Piveteau, 1937

SI: $361 \bullet \mathbf{C I}$ : c331 • ST: 1.D.M.31.E

PN: Proanoura Piveteau, 1937.pa.c02 + • AK 
PA: 00 • Proanoura • Piveteau 1937.pa: $169 \cdot$ bO/O

01 Proanura - Kuhn 1939.ka: $18 \cdot$ bO

02 - Proanura • Romer 1945.ra: $591 \cdot$ O

$03 \cdot$ Proanoura $\cdot$ Kuhn 1961.ka: $23 \cdot$ O

$04 \cdot$ ProanuPa • Romer 1966.rb: $364 \cdot$ O

RL: < EUANOURA 1937.pa.c01 • AI: HL

GN: |ANURA INCERTAE SEDIS|

GZ: INR

EN: KYR. C.04.01. Ordo ANURA Duméril, 1805.da.c01-07 Procera Feller ${ }^{+1}, 1998$

SI: $427 \bullet$ CI: c387 • ST: 1.D.M.30.O

PN: Procera Feller+1, 1998.fa.c01・aK

PA: 00 • Procera • Feller ${ }^{+1}$ 1998.fa: $511 \cdot$ pO

RL: INR

GN: GYMNOPHIONA 1814.ra.c01

URODELA 1805.da.c02

|LISSAMPHIBIA INCERTAE SEDIS|

GZ: INR

EN: KYR. C.03.01. Subclassis LisSAMPHibia Gadow, 1898.ga.c01-00

Procoela Owen, 1860

SI: 219 • CI: zh21 • ST: 1.U.U.99.E

PN: Procoelia Owen, 1860.oa.c03 • zz

PA: 00 - Procoelia • Owen 1860.oa: x, 273 • bO $01 \cdot \operatorname{ProcoeLA} \cdot H o c$ loco $\cdot$ EE

RL, GN, GZ, EN: •

Procoela Lataste, 1879

SI: $276 \bullet$ CI: c250 • ST: 1.D.M.41.E

PN: Procoelidae Lataste, 1879.1b.c02 • AK

PA: $00 \cdot$ Procoelidae • Lataste 1879.1b: $339 \cdot$ bO $01 \cdot \operatorname{Procoela} \cdot H o c l o c o \cdot \mathbf{E E}$

RL: $\downarrow$ Procoelia 1860.oa.c03 $\leftrightarrow \mid$ LAEVOGYRINIDAE 1878.1a.c01

GN: Archaeosalientia 1981.ra.c01 RANOMORPHA 1921.fb.c08

GZ: INR

EN: KYR. C.07.02. Hypoordo LAEvogYrinia Lataste, 1878.la.c01-04

Procoela Nicholls, 1916

SI: 304 • CI: c277 • ST: 1.D.M.21.O

PN: Procoela Nicholls, 1916.na.c03 • AK

PA: 00 • ProcoeLA • Nicholls 1916.na: 87 • 'T' 01 Procoelina • Pearse 1936.pa: 20 • bO 02 - Procela • Pearse 1949.pa: 20 • bO

RL: $\downarrow$ Procoelia 1860.oa.c03

GN: BAINANURA DOP.da.c03 DiPLOSIPHONA 1859.ga.c02

GZ: INR

EN: KYR. C.12.02. Infraphalanx Phrynanura nov., DOP.da.c05-00

[HYP. Unnamed]

Procoela Noble, 1922

SI: $323 \bullet$ CI: c296 • ST: 1.D.M.21.O

PN: Procoela Noble, 1922.na.c02 • AK

PA: 00 • ProcoeLA • Noble 1922.na: 22 • bO

RL: $\downarrow$ Procoelia 1860.oa.c03
GN: GaIANURA DOP.da.c06

HylobatraChia 1828.ra.c16

GZ: INR

EN: KYR. C.12.02. Infraphalanx Phrynanura nov., DOP.da.c05-00

Procoela Ahl, 1930
SI: 337 • CI: c310 • ST: 1.D.M.41.O
PN: Procoela Ahl, 1930.aa.c02 • AK
PA: 00 - Procoela • Ahl 1930.aa: $84 \cdot$ bO
RL: $\downarrow$ Procoelia 1860.oa.c03
GN: DoRSIPARES 1816.ba.c06
LAEVOGYRINIA 1878.1a.c01
GZ: INR
EN: KYR. C.06.01. Infraordo GEOBATRACHIA Ritgen, 1828.ra.c18-02

Procoela Tatarinov, 1964

SI: 387 • CI: c347 • ST: 1.D.M.41.O

PN: Procoela Tatarinov, 1964.ta.c02 • AK

PA: 00 - Procoela • Tatarinov 1964.ta: 8, $126 \cdot$ bO

RL: $\downarrow$ Procoelia 1860.oa.c03

$>$ ANOMOCOELA 1964.ta.c01 • AI: HL

GN: GEOBATRACHIA 1828.ra.c18

Mediogyrinia 1878.1a.c02

GZ: INR

EN: KYR. C.05.02. Subordo Hydrobatrachia Ritgen, 1828.ra.c14-01

Procoela Fei ${ }^{+1} 2016$

SI: 433 • CI: cn07 • ST: 1.D.M.00-04.O

PN: Procoela Fei ${ }^{+1}$ 2016.fa.c01• AN

PA: $00 \cdot$ ProcoeLA $\bullet \mathrm{Fei}^{+1}$ 2016.fa: xii • bO

RL: INR

GN: HYLOBATRACHIA 1828.ra.c16

GZ: INR

EN: KYR. C.13.03. Hypophalanx Hylobatrachia Ritgen, 1828.ra.c16-01

Prolatibranchia Hogg, 1841

SI: $163 \bullet$ CI: c140 • ST: 1.D.M.30.O

PN: Prolatibranchia Hogg, 1841.ha.c02 • AK

PA: 00 • Prolatibranchia • Hogg 1841.ha: 357 • 'T'

RL: INR

GN: ANURA 1805.da.c01

URODELA 1805.da.c02

GZ: INR

EN: KYR. C.03.01. Subclassis LisSAMPhibia Gadow, 1898.ga.c01-00

[HYP. Superordo BATRACHIA Brongniart, 1800.ba.c01-14]

Prosirenacea Estes, 1981

SI: 403 • CI: c363 • ST: 1.D.M.30.R

PN: Prosirenoidea Estes, 1981.ea.c02 † • AK

PA: 00 - ProsirenoIdea • Estes 1981.ea: xiii, 18 •bO

$01 \cdot$ ProsirenaCEA • Dubois 2015.da: 107 - ER

RL: INR

GN: |URODELA INCERTAE SEDIS|

|LISSAMPHIBIA INCERTAE SEDIS|

GZ: INR

EN: KYR. C.03.01. Subclassis Lissamphibia Gadow, 


\section{8.ga.c01-00}

Proteacea Müller, 1831

SI: $113 \bullet$ CI: c094 • ST: 1.D.M.31.R

PN: Proteidea Müller, 1831.ma.c02 • AK

PA: 00 • ProteideA • Müller 1831.ma: $711 \cdot$ O

$01 \cdot$ Proteideae $・$ Tschudi 1838.ta: $26 \cdot \mathbf{O}$

02 - РroteI • Bonaparte 1850.bb: pl. • O

03 - Proteacea $・$ Dubois 2015.da: 90 • ER

RL: INR

GN: Meantes 1767.la.c01

Pseudosauria 1816.ba.c08

GZ: INR

EN: KYR. C.04.03. Ordo Urodela Duméril, 1805.da.c02-12

[HYP. Subordo Pneumobranchia Sonnini ${ }^{+1}$, 1801.sa.c01-02]

Proteacea Cope 1866

SI: 233 • CI: c207 • ST: 1.D.M.41.R

PN: Proteida Cope, 1866.ca.c02 • aK

PA: 00 • ProteIDA • Cope 1866.ca: $102 \cdot$ bO

$01 \cdot$ Proteida $\bullet$ Cope 1868.ca: $208 \cdot \mathbf{O}$

02 - Proteina • Pearse 1936.pa: $20 \bullet$ bO

03 - Proteidea • Boettger 1952.ba: $279 \cdot$ bO

$04 \cdot$ Proteoidea $・$ Edwards 1976.ea: $325 \cdot$ bO

05 • ProteOIDEI $\bullet$ Dubois 1983.da: $113 \cdot$ bO

$06 \cdot$ Proteacea $\cdot$ Hoc loco $\bullet$ ER

RL: $\downarrow$ Proteidea 1831.ma.c02

GN: Pseudosauria 1816.ba.c08

GZ: INR

EN: KYR. C.05.07. Subordo Pseudosauria Blainville, 1816.ba.c08-07

Proteacea Huxley, 1871

SI: 253 • CI: c227 • ST: 1.D.M.40.R

PN: Proteidea Huxley, 1871.ha.c01 • AK

PA: $00 \cdot$ Proteidea $\bullet$ Huxley 1871.ha: $173 \cdot$ bO

$01 \cdot$ Proteida $\bullet$ Knauer 1878.ka: $95 \cdot$ bO

02 • Proteacea • Dubois 2015.da: $107 \cdot$ ER

RL: $\downarrow$ Proteidea 1831.ma.c02

GN: IMPERFECTIBRANCHIA 1838.ha.c03

Meantes 1767.la.c01

Pseudosauria 1816.ba.c08

GZ: INR

EN: KYR. C.04.03. Ordo URodela Duméril, 1805.da.c02-12

Proteacea Hay, 1929

SI: 347 • CI: c320 • ST: 1.D.M.40.R

PN: Proteida Hay, 1929.ha.c04 • AK

PA: $00 \cdot$ ProteIdA $\cdot$ Hay 1929.ha: 521, $841 \cdot$ O

$01 \cdot$ Proteacea $\cdot$ Hoc loco $\bullet$ ER

RL: $\downarrow$ Proteidea 1831.ma.c02

GN: LiSSAMPHIBIA 1898.ga.c01

|LEPOSPONDYLI|

|AMPHIBIA INCERTAE SEDIS|

GZ: INR

EN: KYR. C.02.01. Classis AMPHIBIA Blainville, 1816.ba.c02-03

Proteacea Romer, 1945

SI: $364 \bullet$ CI: $\mathrm{mc} 08 \bullet$ ST: 1.D.M.00.R

PN: ProteIDa Romer, 1945.ra.c03 • AN

PA: 00 • ProteIDA • Romer 1945.ra: $592 \cdot$ bO
$01 \cdot$ Proteacea $\cdot$ Hoc loco $\bullet$ ER

RL: $\downarrow$ Proteidea 1831.ma.c02

< Amblystomatoidea 1945.ra.c01 • AI: HL

< SAlamandroidea 1945.ra.c02 • AI: HL

GN: Pseudosauria 1816.ba.c08

|URODELA INCERTAE SEDIS|

GZ: INR

EN: KYR. C.04.03. Ordo URoDela Duméril, 1805.da.c02-12 Proteacea Kuhn, 1961

SI: 377 • CI: c338 • ST: 1.D.M.40.R

PN: Proteida Kuhn, 1961.ka.c03 • AK

PA: 00 • Proteida • Kuhn 1961.ka: $13 \cdot$ bO

01 - Proteoidea • Estes 1981.ea: xiii, 26 • bO

02 - Proteacea • Dubois 2015.da: 107 • ER

RL: $\downarrow$ Proteidea 1831.ma.c02

< Cryptobranchoidea 1961.ka.c01 • AI: HL

> BATRACHOSAURoIdoIdea 1961.ka.c02 • AI: HL

< Meantes 1961.ka.c04 • AI: HL

GN: PSEUdosauria 1816.ba.c08

|URODELA INCERTAE SEDIS|

GZ: INR

EN: KYR. C.04.03. Ordo URODELA Duméril, 1805.da.c02-12 ProteaceA Kuhn, 1962

SI: $385 \bullet$ CI: c345 • ST: 1.D.M.40.R

PN: Proteida Kuhn, 1962.ka.c06• AK

PA: 00 • ProteIdA • Kuhn 1962.ka: 366 • bO

01 Proteacea - Dubois 2015.da: $107 \cdot$ ER

RL: $\downarrow$ Proteidea 1831.ma.c02

< Salamandroidea 1962.ka.c04 • AI: HL

$>$ Plethodontoidea 1962.ka.c05 • AI: HL

GN: Meantes 1767.la.c01

Pseudosauria 1816.ba.c08

|URODELA INCERTAE SEDIS|

GZ: INR

EN: KYR. C.04.03. Ordo Urodela Duméril, 1805.da.c02-12 Proteroglossa Günther, 1858

SI: $206 \bullet$ CI: c183・ST: 2.D.M.31.O

PN: Proteroglossa Günther, 1858.gc.c02 • AK

PA: 00 • Proteroglossa • Günther 1858.gc: 339 • Gr

01 • Proteroglossa • Fatio 1872.fa: $232 \cdot$ UC

02 - Proteroglosses • Brocchi 1881.ba: 5 • Sc

RL: INR

GN: DoRSIPARES 1816.ba.c06

GZ: INR

EN: KYR. C.07.01. Hypoordo DorsIPARES Blainville, 1816.ba.c06-02

Protonyxia Miranda-Ribeiro, 1924

SI: $332 \bullet$ CI: c305 • ST: 2.D.M.31.O

PN: Рrotonyxia Miranda-Ribeiro, 1924.ma.c07 • AK

PA: 00 • ProtonyXia • Miranda-Ribeiro 1924.ma: 12 • UC

RL: $\leftrightarrow>$ ICHTHYOSTERNIA 1924.ma.c10 • AI: HL

GN: DORSIPARES 1816.ba.c06

GZ: INR

EN: KYR. C.07.01. Hypoordo DorsIPARES Blainville, 1816.ba.c06-02 
Protosternia Miranda-Ribeiro, 1924

SI: 333 • CI: c306 • ST: 2.D.O.31.O

PN: Рrotosternia Miranda-Ribeiro, 1924.ma.c08 • AK

PA: 00 • Protosternia • Miranda-Ribeiro 1924.ma: 143 • UC

RL: $\leftrightarrow \mid$ ANOURES 1805.da.c01

$\leftrightarrow<$ GYMNOBATRACHIA 1924.ma.c02 • AI: HL

$>$ ANONYXIA 1924.ma.c04 • PR

$>$ THORACECHMIA 1924.ma.c05 • PR

$>$ Therosternia 1924.ma.c09 • PR

GN: ANURA 1805.da.c01

GZ: » GX:

GyMNOPHIONA 1814.ra.c01

URODELA 1805.da.c02

EN: KYR. C.04.01. Ordo ANura Duméril, 1805.da.c01-07

Pseudophiona Blainville, 1816

SI: $043 \bullet$ CI: c030 • ST: 1.D.M.12.E

PN: PseudophydiEns Blainville, 1816.ba.c11

PA: 00 • PseUdophydiens • Blainville 1816.ba: "111" [119] • O

$01 \cdot$ PSEUdophidiens • Blainville 1816.bb: $254 \cdot$ O

02 • PseUdOPHIDI • Gray 1825 .ga: $217 \cdot$ O

03 - Pseudo-Phidia • Blainville 1835.ba: $282 \cdot$ O

$04 \cdot$ Pseudophidia • Blainville 1839.bb: $673 \cdot$ O

05 P PseUdophidia $\cdot$ Gervais 1848.ga: $61 \cdot$ UC

06 P PSEUdOPHIONA $\cdot$ Hoc loco $\bullet$ bO

RL: $\leftrightarrow$ C CoECILIES 1816.ba.c12 • AI: HL

GN: PSEUdophiona 1816.ba.c11

GZ: INR

EN: KYR. C.05.04. Subordo Pseudophiona Blainville, 1816.ba.c11-06

Pseudophiona Gray, 1850

SI: $191 \bullet$ CI: c168・ST: 1.D.M.40.E

PN: Pseudophidia Gray, 1850.ga.c03 • AK

PA: $00 \cdot$ Pseudophidia • Gray 1850.ga: $6,56 \bullet$ O

01 PSEUdophiona • Dubois 2015.da: $107 \cdot$ EE

RL: $\downarrow$ Pseudophydiens 1816.ba.c11

GN: Plesiophiona DOP.da.c10

PseUdophiona 1816.ba.c11

GZ: INR

EN: KYR. C.04.02. Ordo GyMnophiona Rafinesque, 1814.ra.c01-02

Pseudophrynia Bauer, 1987

SI: 422 • CI: c382 • ST: 1.D.M.30.O

PN: Pseudophrynia Bauer, 1987.bc.c07 • AK

PA: 00 • PseUdoPhrynia • Bauer 1987.bc: 52 • UC

RL: INR

GN: AQUIPARES 1816.ba.c07

Helanura DOP.da.c09

GZ: INR

EN: KYR. C.08.02. Superphalanx RANOMORPHA Fejérváry, 1921.fb.c08-01

Pseudosalamandrae Bonaparte, 1850

SI: $188 \bullet$ CI: c165 • ST: 1.D.M.03.E

PN: Pseudo-SAlamandrae Bonaparte, 1850.bb.c02 • HK

PA: 00 - PSEUdo-SAlaMandrae • Bonaparte 1850.bb: pl. • O

$01 \cdot$ Pseudosalamandrae • Duméril 1863.da: $302 \cdot$ O

02 PSeudosalamandrae • Dubois 2015.da: $49 \cdot$ bO
RL: INR

GN: IMPERFECTIBRANCHIA 1838.ha.c03

Meantes 1767.la.c01

GZ: INR

EN: KYR. C.04.03. Ordo Urodela Duméril, 1805.da.c02-12

[HYP. Subordo Pseudosalamandrae Bonaparte, 1850.bb.c02-02]

Pseudosauria Blainville, 1816

SI: $040 \bullet$ CI: c027・ST: 1.D.M.12.E

PN: Pseudo Sauriens Blainville, 1816.ba.c08

PA: 00 • PSEUdo SAURIENS • Blainville 1816.ba: "111" [119] • O

$01 \cdot$ Pseudo-Sauriens • Blainville 1816.bb: $254 \cdot$ O

02 Pseudosauriens • Ducrotay Blainville 1822.da: tab. $5 \cdot \mathbf{O}$

03 P Pseudosauri • Gray 1825.ga: $215 \cdot \mathbf{O}$

$04 \cdot$ Pseudo-Sauria • Blainville 1835.ba: $280 \cdot$ O

$05 \cdot$ Pseudosauria $\cdot$ Gervais 1848.ga: $61 \cdot$ UC

06 PSeudosauria $・$ Dubois $^{+1}$ 2012.da: $78 \cdot$ iO

$07 \cdot$ PseUdosauria • Dubois 2016.da: $9 \cdot$ bO

08 PSeudosauria • Dubois 2016.da: $9 \cdot$ hO

RL: $\leftrightarrow$ > SALAMANDRES 1816.ba.c09 • AI: HL

GN: Pseudosauria 1816.ba.c08

GZ: INR

EN: KYR. C.05.07. Subordo Pseudosauria Blainville, 1816.ba.c08-07

PSEUdOSAURIA Gray, 1850

SI: $190 \bullet$ CI: c167 • ST: 1.D.M.40.O

PN: Pseudosauria Gray, 1850.ga.c02 • AK

PA: $00 \cdot$ PseUdosaURIA $・$ Gray 1850 .ga: $6,51 \cdot 0$

RL: $\downarrow$ Pseudo SAuriens 1816.ba.c08

$>$ GRADIENTIA 1850.ga.c01 • PR

$>$ Meantia 1850.ga.c04 • AI: HL

GN: IMPERFECTIBRANCHIA 1838.ha.c03

Pseudosauria 1816.ba.c08

GZ: INR

EN: KYR. C.04.03. Ordo Urodela Duméril, 1805.da.c02-12

[HYP. Subordo NulLibranchia Bonaparte, 1831.ba.c01-01]

PSEUdosauria Cope, 1889

SI: $293 \bullet$ CI: c266・ST: 1.D.M.41.O

PN: Pseudosauria Cope, 1889.cb.c01 • AK

PA: 00 • Pseudosauria • Cope 1889.cb: $861 \cdot$ O

RL: $\downarrow$ Pseudo SAURIENS 1816.ba.c08

GN: GyMnOPHIONA 1814.ra.c01

Urodela 1805.da.c02

GZ: INR

EN: KYR. C.03.01. Subclassis LisSAMPHIBIA Gadow, 1898.ga.c01-00

[HYP. Superordo Derotreta Van der Hoeven, 1833.va.c01-01]

Psiloderma Van der Hoeven, 1855

SI: $199 \bullet$ CI: c176・ST: 3.D.M.30.O

PN: Psiloderma Van der Hoeven, 1855.va.c01 • AK

PA: 00 • PsiLOderma • Van der Hoeven 1855.va: 459 • Sc

RL: $\leftrightarrow \mid$ DiPNOA 1838.bd.c01

GN: ANURA 1805.da.c01

GYMNOPHIONA 1814.ra.c01

URODELA 1805.da.c02 
GZ: INR

EN: KYR. C.03.01. Subclassis LisSAMPHIBIA Gadow, 1898.ga.c01-00

Pulmonata Cuvier, 1816

SI: $046 \bullet$ CI: zh11・ST: 1.U.U.99.E

PN: Pulmones Cuvier, 1816.ca.c01・ zz

PA: 00 - Pulmones • Cuvier 1816.ca: 387 • O 01 - Pulmonea • Bonaparte 1831.ba: $63 \cdot$ bC $02 \cdot$ Pulmonata $\bullet$ Ehrenberg 1831.ea: [85] • O 03 Pulmonea - Jourdan 1834.jb: $332 \cdot$ O

RL, GN, GZ, EN: •

Pulmonata Gouriet, 1868

SI: $246 \bullet$ CI: c220 • ST: 1.D.M.40.E

PN: Pulmones Gouriet, 1868.ga.c01 • AK

PA: 00 • Pulmones • Gouriet 1868.ga: 203 • UC 01 - Pulmonados • Miranda-Ribeiro 1924.ma: 137 • UC 02 - Pulmonata - Dubois 2015.da: $107 \cdot$ EE

RL: $\downarrow$ Pulmonata 1816.ca.c01

$>$ ATretoderes 1868.ga.c05 • PR

$<$ EUbatraciENS 1868.ga.c02 • AI: HL

GN: ANURA 1805.da.c01

GYMNOPHIONA 1814.ra.c01

URODELA 1805.da.c02

GZ: INR

EN: KYR. C.03.01. Subclassis Lissamphibia Gadow, 1898.ga.c01-00

Pygomolgae Ritgen, 1828

SI: $086 \bullet$ CI: c067 • ST: 2.D.A.31.E

PN: Pygomolgaei Ritgen, 1828.ra.c12 • AK

PA: 00 • PygomolgaeI - Ritgen 1828.ra: 278 • He 01 • Pygomolgaei • Jourdan 1834.jb: $335 \cdot$ bO

$02 \cdot$ Pygomolgae $・$ Dubois 2015.da: $106 \cdot \mathbf{E E}$

RL: $\leftrightarrow \mid$ BATRACIENS 1816.ba.c05

GN: ANURA 1805.da.c01

GZ: » GX:

GYMNOPHIONA 1814.ra.c01

Urodela 1805.da.c02

EN: KYR. C.04.01. Ordo ANURA Duméril, 1805.da.c01-07

RAMIBRANCHIA Hogg, 1841

SI: 164 • CI: c141 • ST: 1.D.M.31.O

PN: RAMibranChIa Hogg, 1841.ha.c03 • AK

PA: 00 • RAMIBRANCHIA • Hogg 1841.ha: 361 • 'T'

RL: INR

GN: Meantes 1767.la.c01 Pseudosauria 1816.ba.c08

GZ: INR

EN: KYR. C.04.03. Ordo Urodela Duméril, 1805.da.c02-12 [HYP. Subordo Pneumobranchia Sonnini ${ }^{+1}$, 1801.sa.c01-02]

RANACEA Wilbrand, 1814

SI: $032 \bullet \mathbf{C I}$ : c019・ST: 1.D.M.30.R

PN: RANACEA Wilbrand, 1814.wa.c01 • AK

PA: 00 - RANACEA - Wilbrand 1814.wa: 117 • O

$01 \cdot$ RANINA $\cdot$ Gravenhorst 1817.ga: pl. $9 \cdot \mathbf{O}$

$02 \cdot \operatorname{RANAE} \cdot$ Bonaparte 1838. bc: $392 \cdot \mathbf{O}$

RL: INR

GN: ANURA 1805.da.c01
URODELA 1805.da.c02

GZ: INR

EN: KYR. C.03.01. Subclassis LisSAMPhibia Gadow, 1898.ga.c01-00

[HYP. Superordo BATRaChIA Brongniart, 1800.ba.c01-14]

RANACEA Spix, 1824

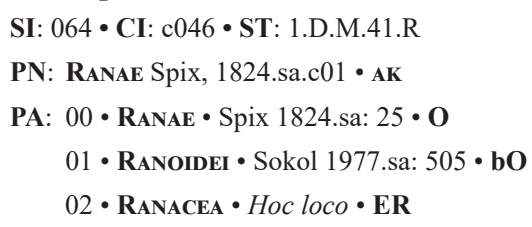

RL: $\downarrow$ RANACEA 1814 .wa.c01

GN: DoRSIPARES 1816.ba.c06

LAEVOGYRINIA 1878.1a.c01

GZ: INR

EN: KYR. C.06.01. Infraordo GEOBATRACHIA Ritgen, 1828.ra.c18-02

RANACEA Bonaparte, 1850

SI: 187 • CI: c164 • ST: 1.D.M.41.R

PN: RANAE Bonaparte, 1850.bb.c01 • AK

PA: $00 \cdot \operatorname{RANAE} \cdot$ Bonaparte 1850. bb: pl. • O

01 - RANACEA • Haeckel 1889.ha: 625. • O

RL: $\downarrow$ RANACEA 1814 .wa.c0 1

GN: GEOBATRACHIA 1828.ra.c18

Mediogyrinia 1878.1a.c02

GZ: INR

EN: KYR. C.05.02. Subordo Hydrobatrachia Ritgen, 1828.ra.c14-01

RANACEA Laurent in Fuhn, 1960

SI: 374 • CI: cn06 • ST: 1.D.M.00.R

PN: RANOIDEA Laurent in Fuhn, 1960.fa.c06 • AN

PA: 00 • RANOIDEA • Laurent in Fuhn 1960.fa: 163 • bO

$01 \cdot \mathbf{R A N A C E A} \cdot \mathrm{Hoc}$ loco $\bullet$ ER

RL: $\downarrow$ RANACEA 1814 .wa.c01

GN: ECOSTATA 1879.1b.c04

Pananura DOP.da.c07

GZ: INR

EN: TEO. C.10.03. Phalanx Scoptanura Starrett, 1973.sb.c02-02 [HYP. Subphalanx unnamed]

RANIFORMIA Hogg, 1839

SI: $140 \bullet$ CI: c118 • ST: 1.D.M.31.A

PN: RANIFORMIA Hogg, 1839.ha.c05 • AK

PA: 00 • RANIFORMIA • Hogg 1839.ha: $271 \cdot$ O

01 - RANIFORMES • Brocchi 1881.ba: $9 \cdot \mathbf{U C}$

$02 \cdot$ RANIFORMES $\bullet$ Boulenger 1882.ba: $12 \cdot \mathbf{U C}$

RL: INR

GN: GEOBATRACHIA 1828.ra.c18

Mediogyrinia 1878.1a.c02

GZ: INR

EN: KYR. C.05.02. Subordo Hydrobatrachia Ritgen,

$$
\text { 1828.ra.c14-01 }
$$

RANIFORMIA Cope, 1864

SI: $229 \bullet$ CI: c203 • ST: 1.D.M.41.A

PN: RANIFORMIA Cope, 1864.cb.c01 • AK

PA: c0 • RANIFORMia - Cope 1864.cb: 183 - bO • IIA: Cope 1865. ca: 114 
i1 • RANifORMeS • Cope 1864.cb: $181 \cdot$ bO

$02 \cdot$ RANIFORMIA $\cdot$ Mivart 1869.ma: $281 \cdot \mathbf{S r}$

RL: $\downarrow$ RANIFORMIA 1839 .ha.c05

GN: GASTRECHMIA 1867.ca.c02

PANANURA DOP.da.c07

GZ: INR

EN: TEO. C.10.03. Phalanx Scoptanura Starrett, 1973.sb.c02-02 [HYP. Subphalanx unnamed]

RANIFORMIA Cope, 1867

SI: $241 \bullet$ CI: c215 • ST: 1.D.M.41.A

PN: RANIFORMIA Cope, 1867.ca.c01 • AK

PA: 00 • RANIFORMIA - Cope 1867.ca: $189 \cdot$ bO $01 \cdot$ RANIFORMIA • Hoffmann 1878.ha: $608 \cdot$ UC

02 - RANIFORMES • Philippi 1902.pa: $x \cdot$ UC

RL: $\downarrow$ RANIFORMIA 1839.ha.c05

GN: Phaneranura DOP.da.c02 SCOPTANURA 1973.sb.c02

GZ: INR

EN: TEO. C.09.01. Epiphalanx AQUIPARES Blainville, 1816.ba.c07-02

[HYP. Phalanx AQUIPAREs Blainville, 1816.ba.c07-03]

RANIFORMIA Steindachner, 1867

SI: 243 • CI: c217 • ST: 1.D.M.41.A

PN: RANIFORMIA Steindachner, 1867.sa.c01 • AK

PA: 00 - RANIFORMIA - Steindachner 1867.sa: 6 • Sc

RL: $\downarrow$ RANIFORMIA 1839.ha.c05

GN: ANGUSTICOELA 1958.ra.c01 Hydrobatrachia 1828.ra.c14

GZ: INR

EN: KYR. C.04.01. Ordo ANURA Duméril, 1805.da.c01-07 RANIFORMIA Hay, 1929

SI: $350 \bullet$ CI: c323 • ST: 1.D.M.41.A

PN: RANIFORMES Hay, 1929.ha.c07 • AK

PA: 00 • RANIFORMES • Hay 1929.ha: 521, $854 \cdot$ O

$01 \cdot$ RANIFORMIA $\cdot$ Hoc loco $\bullet$ EA

RL: $\downarrow$ RANIFORMIA 1839.ha.c05

GN: ECAUDATA 1777.sa.c06

GZ: INR

EN: KYR. C.12.03. Infraphalanx ECAUdATA Scopoli, 1777.sa.c06-01

RANOMORPHA Fejérváry, 1921

SI: 320 • CI: c293 • ST: 1.D.M.11.A

PN: RANOMORPHA Fejérváry, 1921.fb.c08

PA: 00 • RANOMORPHA • Fejérváry 1921.fb: 16 • Gs

RL: INR

GN: AQUIPARES 1816.ba.c07

Helanura DOP.da.c09

GZ: INR

EN: KYR. C.08.02. Superphalanx RANOMORPHA Fejérváry, 1921.fb.c08-01

RHINATREMATACEA Lescure ${ }^{+2}, 1986$

SI: $413 \bullet$ CI: c373 • ST: 1.D.M.30.R

PN: RHINATREMATOIDEI Lescure ${ }^{+2}$, 1986.lb.c01 • AK

PA: 00 - RHINATREMATOIDEI • Lescure ${ }^{+2}$ 1986.1b: $145 \cdot$ bO

$01 \cdot$ RHINATREMATIDEI $\bullet$ Lescure $^{+2}$ 1986.lb: $152 \cdot$ iO

02 • RHINATREMATACEA • Dubois 2015.da: 107 • ER
RL: INR

GN: Plesiophiona DOP.da.c10

PSEUDOPHIONA 1816.ba.c11

GZ: INR

EN: KYR. C.04.02. Ordo GyMnophiona Rafinesque, 1814.ra.c01-02

RotONDICAUDATA Duméril ${ }^{+2}, 1854$

SI: 197 • CI: c174 • ST: 2.D.M.31.E

PN: RotondiCAUdES Duméril ${ }^{+2}, 1854$.da.c03 • AK

PA: 00 - RotondiCAUDES • Duméril ${ }^{+2}$ 1854.da: 38 - UC

$01 \cdot \operatorname{RotONDICAUDATA} \cdot \mathrm{Hoc}$ loco $\bullet \mathbf{E E}$

RL: $\leftrightarrow<$ GONGYLURES 1854.da.c04 • AI: HL

GN: Pseudosauria 1816.ba.c0 0

GZ: INR

EN: KYR. C.05.07. Subordo Pseudosauria Blainville, 1816.ba.c08-07

SAlAmandracea Blainville, 1816

SI: $041 \bullet$ CI: c028・ST: 1.D.M.32.R

PN: SALAMANDRES Blainville, 1816.ba.c09 • AK

PA: 00 • SALAMANDRES • Blainville 1816.ba: "111" [119] • O

01 - SAlaMandRina • Müller 1831.ma: $711 \cdot$ O

$02 \cdot$ SAlamandRINAE • Tschudi 1838.ta: $26 \cdot \mathbf{O}$

03 - SAlamandROIDES • Duméril ${ }^{+1}$ 1841.da: 52 • Gr/Sc/'T'

$04 \cdot$ SALAMANDRAE • Bonaparte 1850.bb: pl. • O

$05 \cdot$ SALAMANDRINA - Claus 1868.cb: 586 • bO

06 - Salamandridea $\cdot$ Huxley 1871.ha: $173 \cdot$ bO.

07 - SALAMANDROIDEA • Noble 1931.na: 473 • bO

08 - SAlamandriodae • Pearse 1948.pa: 20 • bO

09 - SALAMANDRIOdEA • Pearse 1949.pa: 20 • bO

10 - SAlamandroideI • Dubois 1983.da: $113 \cdot$ bO

11 - Salamandracea $\cdot$ Hoc loco $\bullet$ ER

RL: $\leftrightarrow<$ PSEUdo SaUriens 1816.ba.c08 • AI: HL

GN: PSEudosauria 1816.ba.c08

GZ: INR

EN: KYR. C.05.07. Subordo Pseudosauria Blainville, 1816.ba.c08-07

SALAMANDRACEA Knauer, 1878

SI: 266 • CI: c240 • ST: 1.D.M.40.R

PN: SALAMANDrina Knauer, 1878.ka.c02 • AK

PA: 00 - SALAMANDRINA - Knauer 1878.ka: $96 \cdot$ bO

01 - SALAMANDRINES • Brocchi 1881.ba: $102 \cdot$ UC

$02 \cdot$ SALAMANDRACEA $\cdot H o c$ loco $\bullet$ ER

RL: $\downarrow$ SALAMANDres 1816 .ba.c09

GN: IMPERFECTIBRANCHIA 1838.ha.c03

Pseudosauria 1816.ba.c08

GZ: INR

EN: KYR. C.04.03. Ordo Urodela Duméril, 1805.da.c02-12

[HYP. Subordo Nullibranchia Bonaparte, 1831.ba.c01-01]

SALAMANDRACEA Sarasin ${ }^{+1}, 1887$

SI: $287 \bullet$ CI: c261・ST: 2.D.M.41.R

PN: SAlamandrina $\operatorname{Sarasin}^{+1}$, 1887.sa.c01 • AK

PA: 00 - SALAMANDRINA • $\operatorname{Sarasin}^{+1} 1887 . \mathrm{sa}: 29 \cdot$ UC

01 - SAlamandRaCEA • Dubois 2015.da: 106 • ER

RL: $\downarrow$ SALAMandres 1816 .ba.c09

GN: GYMNOPHIONA 1814.ra.c01

URODELA 1805.da.c02 
GZ: INR

EN: KYR. C.03.01. Subclassis LisSAMPHIBIA Gadow, 1898.ga.c01-00

[HYP. Superordo Derotreta Van der Hoeven, 1833.va.c01-01]

SALAMANDRACEA Sarasin ${ }^{+1}, 1890$

SI: 298 • CI: c271 • ST: 1.D.M.40.R

PN: SALAMANDroidea $\operatorname{Sarasin}^{+1}$, 1890.sa.c02 • AK

PA: 00 - SALAMANDROIDEA • $\operatorname{Sarasin}^{+1} 1890$.sa: 245 • bO 01 - SALAMANDRACEA • Dubois 2015.da: $107 \cdot$ ER

RL: $\downarrow$ SALAMANDRES 1816 .ba.c09

GN: IMPERFECTIBRANCHIA 1838.ha.c03

MeANTES 1767.la.c01

Pseudosauria 1816.ba.c08

GZ: INR

EN: KYR. C.04.03. Ordo URODELA Duméril, 1805.da.c02-12

SALAMANDRACEA Romer, 1945

SI: $363 \bullet$ CI: $\mathrm{mc07} \bullet$ ST: 1.D.M.00.R

PN: SAlaMandroidea Romer, 1945.ra.c02 • AN

PA: 00 • SALAMANDROIDEA • Romer 1945.ra: 592 • bO

$$
01 \cdot \text { SAlaMANDRACEA } \cdot \text { Hoc loco } \bullet \text { ER }
$$

RL: $\downarrow$ SALAMANDRES 1816 .ba.c09

$>$ AMblystomatoidea 1945.ra.c01 • AI: HL

> ProteIda 1945.ra.c03 • AI: HL

GN: PSEUdosauria 1816. ba.c0 8 |URODELA INCERTAE SEDIS|

GZ: INR

EN: KYR. C.04.03. Ordo URODELA Duméril, 1805.da.c02-12 SALAMANDRACEA Kuhn, 1962

SI: 383 • CI: c343 • ST: 1.D.M.40.R

PN: Salamandroidea Kuhn, 1962.ka.c04 • AK

PA: 00 • SALAMANDROIDEA • Kuhn 1962.ka: 356 • bO $01 \cdot$ SAlamandridea $\cdot$ Kuhn 1965.ka: 37 • bO 02 - SAlamandRaCEA • Dubois 2015.da: 107 • ER

RL: $\downarrow$ SALAMANDRES 1816 .ba.c09

> Plethodontoidea 1962.ka.c05 - AI: HL

$>$ ProteIda 1962.ka.c06 • AI: HL

GN: Pseudosauria 1816.ba.c0 8 |URODELA INCERTAE SEDIS|

GZ: INR

EN: KYR. C.04.03. Ordo Urodela Duméril, 1805.da.c02-12 SALAMANDRACEA Tatarinov, 1964

SI: $389 \bullet$ CI: c349・ST: 1.D.M.40.R

PN: SAlamandroidei Tatarinov, 1964.tb.c02 • AK

PA: 00 • SALAMANDROIDEI - Tatarinov 1964.tb: 9, $161 \cdot$ bO 01 - SALAMANDRACEA • Dubois 2015.da: 107 - ER

RL: $\downarrow$ SALAMANDRES 1816 .ba.c09 $>$ AMbYSTOMATOIDEI 1964.tb.c01 • AI: HL

GN: IMPERFECTIBRANCHIA 1838.ha.c03 Pseudosauria 1816.ba.c08 |URODELA INCERTAE SEDIS|

GZ: INR

EN: KYR. C.04.03. Ordo Urodela Duméril, 1805.da.c02-12 SALAMANDRACEA Estes, 1981

SI: $405 \bullet$ CI: c365 - ST: 1.D.M.41.R

PN: Salamandroidea Estes, 1981.ea.c04 • AK
PA: 00 • SAlamandroidea • [Naylor 1978.na: 607]; Estes 1981.ea: xiv, $63 \cdot \mathbf{b O}$

$01 \cdot$ SAlamandracea $\cdot H o c l o c o \cdot$ ER

RL: $\downarrow$ SALAMandres 1816 .ba.c09

$>$ KARAUROIDEA 1981.ea.c01 • AI: HL

$>$ AMBYstomatoidea 1981.ea.c03 • AI: HL

GN: MeANTES 1767.la.c01

Pseudosauria 1816.ba.c08

GZ: INR

EN: KYR. C.04.03. Ordo Urodela Duméril, 1805.da.c02-12

[HYP. Subordo Pneumobranchia Sonnini ${ }^{+1}$, 1801.sa.c01-02]

SALAMANDRACEA Trueb ${ }^{+1}, 1991$

SI: $426 \bullet$ CI: c386 • ST: $1 . D . M .40 . R$

PN: SALAMANDroidea Trueb ${ }^{+1}$, 1991.ta.c02 • AK

PA: 00 - SAlamandROIDEA - Trueb ${ }^{+1}$ 1991.ta: 233 •bO

01 - SALAMANDRACEA • Dubois 2015.da: 107 - ER

RL: INR

GN: Pseudosauria 1816.ba.c08

|LISSAMPHIBIA INCERTAE SEDIS|

GZ: INR

EN: KYR. C.03.01. Subclassis Lissamphibia Gadow, 1898.ga.c01-00

SALAMANDRIFORMIA Milner, 2000

SI: 430 • CI: c390 • ST: 1.D.M.30.A

PN: SALAMANDriformes Milner, 2000.ma.c03 • AK

PA: 00 - SALAMANDRIFORMES • Milner 2000.ma: $1412 \cdot$ iO

01 - SALAMANDRIFORMIA - Dubois 2016.da: 9 • iO

RL: INR

GN: Pseudosauria 1816.ba.c08

GZ: INR

EN: KYR. C.05.07. Subordo Pseudosauria Blainville, 1816.ba.c08-07

SALIENTIA Laurenti, 1768

SI: $006 \bullet$ CI: $\mathrm{c002} \bullet$ ST: 1.N.G.02.O

PN: SAlientia Laurenti, 1768.1a.c01・AP

PA: $00 \cdot$ SALIENTIA $・$ Laurenti 1768.1a: $24 \cdot$ O

RL: INR

GN: ANURA 1805.da.c01

URODELA 1805.da.c02

GZ: » GI:

Urodela 1805.da.c02

EN: ANAPTONYM

SALIENTIA Merrem, 1820

SI: $050 \bullet \mathbf{C I}$ : $\mathrm{c032} \bullet \mathbf{S T}$ : $1 . \mathrm{S} .0 .41 . \mathrm{O}$

PN: SAlientia Merrem, 1820.ma.c01・AK

PA: 00 - SAlientia - Merrem 1820.ma: $163 \cdot$ O

$01 \cdot$ SALIENTIA $\bullet$ Gray 1850.ga: $5 \cdot$ bO

02 - SAlientia • Goodrich 1930.ga: xxi • bC

03 - SAliENTIDA - Pearse 1936.pa: 20 • O

$04 \cdot$ SALIENTIA $・$ Romer 1945.ra: $591 \cdot$ pO

05 - SALENTIA • Anonymous 1976.aa: 128 • UC

RL: $\downarrow$ SALIENTIA 1768.la.c01

GN: ANURA 1805.da.c01

GZ: » GX:

GYMNOPHIONA 1814.ra.c01

URODELA 1805.da.c02 
EN: KYR. C.04.01. Ordo ANURA Duméril, 1805.da.c01-07 Salientia Hay, 1929

SI: 346 • CI: c319 • ST: 1.N.G.02.O

PN: Salientia Hay, 1929.ha.c03 • AP

PA: 00 - SAlientia • Hay 1929.ha: 521, 850 • O

RL: $\downarrow$ SALIENTIA 1768.la.c01

GN: LISSAMPHIBIA 1898.ga.c01-00 |Non-LiSSAMPHibIAN AMPHIBIA|

GZ: » GI: |Non-LisSAMPHibIAN AMPHIBIA|

EN: ANAPTONYM

SAURICHTHYODI Jourdan, 1834

SI: $123 \bullet$ CI: c103・ST: 1.D.M.31.O

PN: SAURIChthyi Jourdan, 1834.jb.c01 • AK

PA: c0 • SAURICHTHYI • Jourdan 1834.jb: 398 • O • EEA: HL i1 - SAURichtyenS • Jourdan 1834.jb: $398 \cdot$ O

$01 \cdot$ SAURICHTYODI $\cdot$ Hoc loco $\bullet$ EE

RL: INR

GN: Meantes 1767.la.c01 Pseudosauria 1816.ba.c08

GZ: INR

EN: KYR. C.04.03. Ordo Urodela Duméril, 1805.da.c02-12 [HYP. Subordo Pneumobranchia Sonnini ${ }^{+1}$, 1801.sa.c01-02] SaUrobatrachia Van der Hoeven, 1855

SI: $201 \bullet$ CI: c178 • ST: 1.D.M.30.E

PN: Saurobatrachi Van der Hoeven, 1855.va.c03 • AK

PA: 00 - SAUrobatrachi • Van der Hoeven 1855.va: x, 461 • O $01 \cdot$ SAURobatraChia - Huxley 1863.ha: 66 • UC 02 - SaurobatraChia • Van der Hoeven 1864.va: 288 • O 03 - SaurobatraChII - Fatio 1872.fa: 7 • O 04 - Saurabatrachia • Noble 1931.na: $465 \cdot$ O

RL: $\leftrightarrow>$ SozURA 1855.va.c04 - AI: HL

GN: IMPERFECTIBRANCHIA 1838.ha.c03

MEANTES 1767.la.c01

Pseudosauria 1816.ba.c08

GZ: INR

EN: KYR. C.04.03. Ordo Urodela Duméril, 1805.da.c02-12

\section{SaVanura nov.}

SI: $441 \bullet \mathbf{C I}: \mathrm{c} 400 \bullet \mathbf{S T}: 1 . \mathrm{D} . \mathrm{M} .11 . \mathrm{O}$

PN: SAVANURA nov., DOP.da.c08

PA: 00 - SAVANURA $\cdot$ Hoc loco $\cdot$ iP

RL: INR

GN: SAVANURA .DOP.da.c08

GZ: INR

EN: KYR. C.12.04. Infraphalanx SAVANURA nov., DOP.da.c08-00 SCOLECODES Ritgen, 1828

SI: $076 \bullet$ CI: $\mathrm{c057} \bullet$ ST: 2.D.M.31.O

PN: SCOLECODES Ritgen, 1828.ra.c02 • AK

PA: $00 \cdot$ SCOLECODES • Ritgen 1828.ra: $263 \cdot$ He $01 \cdot$ SCOLECODES $•$ Jourdan 1834.jb: $405 \cdot$ bO

RL: $\leftrightarrow>$ DERMATOPHIDES 1828.ra.c01 • AI: HL $\leftrightarrow>$ STOLIDOPHIDES 1828.ra.c03 • PR

GN: PSEUdOPHIONA 1816.ba.c11

GZ: INR

EN: KYR. C.05.04. Subordo Pseudophiona Blainville, 1816.ba.c11-06
SCOPTANURA Starrett, 1973

SI: 397 • CI: c357 • ST: 1.D.M.11.O

PN: SCOPTANURA Starrett, 1973.sb.c02

PA: 00 • SCOPTANURA • Starrett 1973.sb: 251 • UC

$01 \cdot$ SCOPTANURA • Savage 1973.sa: $353 \cdot$ bO

$02 \cdot$ SCOPTANURA $\cdot$ Hoc loco $\cdot \mathbf{P}$

$03 \cdot \mathrm{SCOPTANURA} \cdot H O c$ loco $\bullet \mathbf{b P}$

RL: INR

GN: ECOSTATA 1879.1b.c04 GastrechMia 1867.ca.c02

GZ: INR

EN: TEO. C.10.03. Phalanx ScOPtanura Starrett, 1973.sb.c02-02 [HYP. Subphalanx ScOPTANURA Starrett, 1973.sb.c02-02]

SERPENTIFORMI Leuckart, 1840

SI: $152 \bullet$ CI: c130 • ST: 2.D.M.31.X

PN: SERPENTIFORMia Leuckart, 1840.1a.c05 • AK

PA: 00 - SERPENTIFORMIA - Leuckart 1840.1a: 20 • 'F'

01 - SERPENTIFORMIA - Leuckart 1841.1a: 30 • UC

02 - SERPENTIFORMES • Dubois 2015.da: 90 • EX

$03 \cdot$ SERPENTIFORMI $\cdot$ Hoc loco $\bullet$ EX

RL: INR

GN: PSEUdoPHIONA 1816.ba.c11

GZ: INR

EN: KYR. C.05.04. Subordo Pseudophiona Blainville, 1816.ba.c11-06

SiPHONOPACEA Lescure ${ }^{+2}, 1986$

SI: 415 • CI: c375 • ST: 1.D.M.31.R

PN: SIPHONOPIDEI Lescure ${ }^{+2}$, 1986.lb.c03 • AK

PA: $00 \cdot$ SIPHONOPIDEI $・$ Lescure $^{+2} 1986.1 b: 152 \cdot$ iO

$01 \cdot$ SIPHONOPACEA $・ H o c$ loco $・$ ER

RL: < EPICRIIDEI 1986.1b.c02 • PR

GN: PSEUDOPHIONA 1816.ba.c11

GZ: INR

EN: KYR. C.05.04. Subordo Pseudophiona Blainville, 1816.ba.c11-06

SIRENACEA Jarocki, 1822

SI: 058 • CI: c040 • ST: 1.D.M.30.R

PN: SIRENIA Jarocki, 1822.ja.c01 • AK

PA: 00 - SIRENIA • Jarocki 1822.ja: $135 \cdot$ C

$01 \cdot \operatorname{SIRENACEA} \cdot$ Hoc loco $\bullet$ ER

RL: INR

GN: ANURA 1805.da.c01

Urodela 1805.da.c02

GZ: INR

EN: KYR. C.03.01. Subclassis Lissamphibia Gadow, 1898.ga.c01-00

[HYP. Superordo BATRACHIA Brongniart, 1800.ba.c01-14]

SIRENACEA Gray, 1825

SI: $066 \bullet$ CI: c048・ST: 1.D.M.31.R

PN: SiRENES Gray, 1825.ga.c02 • AK

PA: 00 • SIRENES • Gray 1825.ga: $215 \bullet$ O

$01 \cdot \operatorname{SIRENACEA} \cdot H o c$ loco $\bullet$ ER

RL: $\downarrow$ SIRENIA 1822 .ja.c01

GN: Meantes 1767.la.c01

Pseudosauria 1816.ba.c08

GZ: INR 
EN: KYR. C.04.03. Ordo Urodela Duméril, 1805.da.c02-12 [HYP. Subordo Pneumobranchia Sonnini ${ }^{+1}$, 1801.sa.c01-02] SIRENACEA Goodrich, 1930

SI: $342 \bullet$ CI: c315 • ST: 1.D.M.41.R

PN: SIRENOIDEA Goodrich, 1930.ga.c04 • AK

PA: $00 \cdot$ SIRENOIDEA • Goodrich 1930.ga: xxi • bO 01 - SIRENOIDEI • Dubois 1983.da: $113 \cdot$ bO $02 \cdot$ SIRENACEA $\cdot H o c$ loco $\bullet$ ER

RL: $\downarrow$ SIRENIA 1822.ja.c01 $\leftrightarrow \mid$ Meantes 1767.la.c01

GN: MeANTES 1767.la.c01

GZ: INR

EN: KYR. C.05.06. Subordo MeanTES Linné, 1767.la.c01-01 SozoBRANCHIA Haeckel, 1866

SI: $236 \bullet$ CI: c210 • ST: 1.D.M.41.O

PN: SozobranCHIA Haeckel, 1866.ha.c02 • AK

PA: 00 - SozobranCHIA - Haeckel 1866.ha: cxxxi • O $01 \cdot$ Socobranchia $・$ Kuhn 1967.kb: $38 \cdot$ UC

RL: $\leftrightarrow \mid$ Perennibranches 1824.la.c02

$>$ SozUra 1866.ba.c03 • AI: HL

$>$ CAUdata 1866.ha.c04 - AI: HL

GN: Meantes 1767.la.c01

PSEUdosauria 1816.ba.c08

GZ: INR

EN: KYR. C.04.03. Ordo Urodela Duméril, 1805.da.c02-12 [HYP. Subordo Pneumobranchia Sonnini ${ }^{+1}$, 1801.sa.c01-02] SozUra Van der Hoeven, 1833

SI: $119 \bullet \mathbf{C I}$ : $099 \cdot$ ST: 1.D.M.31.O

PN: Sozura Van der Hoeven, 1833.va.c02 • AK

PA: 00 • SozURA • Van der Hoeven 1833.va: iii, $304 \cdot$ O

RL: INR

GN: Meantes 1767.la.c01 Pseudosauria 1816.ba.c08

GZ: INR

EN: KYR. C.04.03. Ordo Urodela Duméril, 1805.da.c02-12 [HYP. Subordo Pneumobranchia Sonnini ${ }^{+1}$, 1801.sa.c01-02] Sozura Van der Hoeven, 1855

SI: $202 \bullet$ CI: c179 • ST: 1.D.M.30.O

PN: Sozura Van der Hoeven, 1855.va.c04 • AK

PA: 00 • SozURA • Van der Hoeven 1855.va: 461 • O

RL: $\downarrow$ SozURA 1833.va.c02 $\leftrightarrow<$ SAUrobatrachi 1855.va.c03 • AI: HL

GN: IMPERFECTIBRANCHIA 1838.ha.c03

Meantes 1767.la.c01

Pseudosauria 1816.ba.c08

GZ: INR

EN: KYR. C.04.03. Ordo Urodela Duméril, 1805.da.c02-12

SozUra Haeckel, 1866

SI: 237 • CI: c211 • ST: 1.D.M.40.O

PN: SozUra Haeckel, 1866.ha.c03 • AK

PA: $00 \cdot$ SozURA • Haeckel 1866.ha: cxxxi • O

RL: $\downarrow$ SozURA 1833 .va.c02

$<$ Sozobranchia 1866.ha.c02 • AI: HL

i↔> CAUdATA 1866.ha.c04 • AI: HL

GN: IMPERFECTIBRANCHIA 1838.ha.c03

Pseudosauria 1816.ba.c08
GZ: INR

EN: KYR. C.04.03. Ordo Urodela Duméril, 1805.da.c02-12 [HYP. Subordo Nullibranchia Bonaparte, 1831.ba.c01-01] StOLIDOPHIONA Ritgen, 1828

SI: $077 \bullet \mathbf{C I}: \mathrm{c058} \bullet \mathbf{S T}$ : 2.D.M.31.E

PN: STOLIDOPHIDES Ritgen, 1828.ra.c03 • AK

PA: 00 - STOLIDOPHIDES • Ritgen 1828.ra: 258 • 'F' $01 \cdot$ STOLIDOPHIONA $\cdot H o c$ loco $\bullet$ EE

RL: $\leftrightarrow<$ DERMATOPHIDES 1828.ra.c01 • PR $\leftrightarrow<$ SCOLECODES 1828.ra.c02 • PR

GN: Pseudophiona 1816.ba.c11

GZ: INR

EN: KYR. C.05.04. Subordo Pseudophiona Blainville, 1816.ba.c11-06

Subichthyodi Ducrotay Blainville, 1822

SI: 057 • CI: c039 • ST: 1.D.M.31.E

PN: SubiChTHYens Ducrotay Blainville, 1822.da.c01 • AK

PA: 00 - Subichthyens - Ducrotay Blainville 1822.da: t.ab. 5 • O

$01 \cdot$ SUbiCHTHYI $\cdot$ Jourdan 1834.jb: $486 \cdot \mathbf{O}$

02 - Subichthiens • Gray 1850.ga: $64 \cdot$ O

$03 \cdot \operatorname{SUBICHTHYODI} \cdot$ Hoc loco $\bullet \mathbf{E E}$

RL: INR

GN: MeANTES 1767.la.c01 Pseudosauria 1816.ba.c08

GZ: INR

EN: KYR. C.04.03. Ordo URoDELA Duméril, 1805.da.c02-12 [HYP. Subordo Pneumobranchia Sonnini ${ }^{+1}$, 1801.sa.c01-02] TARSATA Meyer, 1860

SI: $214 \cdot$ CI: c191・ST: 2.D.M.31.E

PN: TARSIDEN Meyer, 1860.mb.c02 • AK

PA: 00 • TARSIDEN • Meyer 1860.mb: $559 \cdot$ UC

$01 \cdot$ TARSATA $\cdot H o c l o c o \cdot \mathbf{E E}$

RL: INR

GN: Pseudosauria 1816.ba.c0 8

GZ: INR

EN: KYR. C.05.07. Subordo Pseudosauria Blainville, 1816.ba.c08-07

TEMnOSPONDYLI Zittel, 1888

SI: 289 • CI: zh22 • ST: 1.U.U.99.O

PN: Temnospondyu Zittel, 1888.za.c01 • zz

PA: 00 • TEMNOSPONDYLI - Zittel 1888.za: viii, 384 • bO

RL, GN, GZ, EN: •

Tetramela Gouriet, 1868

SI: $249 \bullet$ CI: c223・ST: 2.D.M.31.E

PN: Tetrameles Gouriet, 1868.ga.c04 • AK

PA: 00 - Tetrameles - Gouriet 1868.ga: 206 • UC

$01 \cdot$ Tetramela $\cdot H o c$ loco $\bullet$ EE

RL: INR

GN: Pseudosauria 1816.ba.c08

GZ: INR

EN: KYR. C.05.07. Subordo Pseudosauria Blainville, 1816.ba.c08-07

Tetrapoda Fischer, 1808

SI: $024 \cdot$ CI: zh07 • ST: 1.U.U.99.E

PN: Tetrapodes Fischer, 1808.fa.c01 • zz

PA: 00 • TETRAPODES • Fischer 1808.fa: [13] • UC 
$01 \cdot$ Tetrapoda $\cdot H o c l o c o \cdot \mathbf{E E}$

RL, GN, GZ, EN: •

Tetrapoda Hogg, 1839

SI: $138 \cdot$ CI: c116 • ST: 1.D.M.40.O

PN: TeTraPOda Hogg, 1839.ha.c03 • AK

PA: 00 • TETRAPODA • Hogg 1839.ha: 271 • O

RL: $\downarrow$ TeTraPODES 1808 .fa.c01

< UROPHORA 1839.ha.c01 • AI: HL

$>$ ANGUIFORMI 1839.ha.c04 • AI: HL

GN: ANURA 1805.da.c01

Urodela 1805.da.c02

GZ: INR

EN: KYR. C.03.01. Subclassis LisSAMPHibia Gadow, 1898.ga.c01-00

[HYP. Superordo BATRACHIA Brongniart, 1800.ba.c01-14]

THERIOMORPHI Owen, 1866

SI: 240 • CI: c214 • ST: 1.D.M.31.X

PN: THeriomorPha Owen, 1866.oa.c02 • AK

PA: 00 - THERIOMORPHA • Owen 1866.oa: $15 \cdot$ bO

01 - THERIOMORPHI • Dubois 2015.da: $90 \cdot \mathbf{E X}$

RL: INR

GN: DORSIPARES 1816.ba.c06

LAEVOGYRINIA 1878.1a.c01

GZ: INR

EN: KYR. C.06.01. Infraordo GEOBATRACHIA Ritgen, 1828.ra.c18-02

THERIOMORPHI Hoffmann, 1878

SI: $262 \cdot$ CI: c236 • ST: 1.D.M.41.X

PN: TheriomorPha Hoffmann, 1878.ha.c02 • AK

PA: 00 - THERIOMORPHA - Hoffmann 1878.ha: 615 • O

01 • THERIOMORPHI • Dubois 2015.da: $90 \cdot \mathbf{E X}$

RL: $\downarrow$ THERIOMORPHI 1866.oa.c02 • bO

$>$ ECAUDATA 1878.ha.c01 - AI: HL

GN: ANGUSTICOELA 1958.ra.c01

HYDROBATRACHIA 1828.ra.c14

GZ: INR

EN: KYR. C.04.01. Ordo ANURa Duméril, 1805.da.c01-07

Therosternia Miranda-Ribeiro, 1924

SI: 334 • CI: c307 • ST: 2.D.M.31.O

PN: Therosternia Miranda-Ribeiro, 1924.ma.c09 • AK

PA: 00 • Therosternia • Miranda-Ribeiro 1924.ma: 143 • UC

RL: < GYMNOBATRACHIA 1924.ma.c02 • PR

$\leftrightarrow<$ ANONYXIA 1924.ma.c04 • AI: HL

$>$ THORACECHMIA 1924.ma.c05 • PR

< Protosternia 1924.ma.c08 • PR

GN: AnguSTiCOELA 1958.ra.c01

HYDROBATRACHIA 1828.ra.c14

GZ: INR

EN: KYR. C.04.01. Ordo ANURA Duméril, 1805.da.c01-07

ThoraceCHMia Miranda-Ribeiro, 1924

SI: 330 • CI: c303 • ST: 2.D.M.31.O

PN: ThORACEChMia Miranda-Ribeiro, 1924.ma.c05 • AK

PA: 00 • ThORACECHMIA • Miranda-Ribeiro 1924.ma: 141 • UC

RL: INR

GN: ANGUSTICOELA 1958.ra.c01

HYDROBATRACHIA 1828.ra.c14
GZ: INR

EN: KYR. C.04.01. Ordo ANURa Duméril, 1805.da.c01-07

TraCHYSTOMATA Cope, 1866

SI: 232 • CI: c206・ST: 1.D.M.31.O

PN: Trachystomata Cope, 1866.ca.c01 • AK

PA: 00 - Trachystomata - Cope 1866.ca: $102 \cdot$ bO

$01 \cdot$ TraChySTOMATA $・$ Cope 1868.ca: $208 \cdot \mathbf{O}$

RL: INR

GN: MEANTES 1767.la.c01

GZ: INR

EN: KYR. C.05.06. Subordo Meantes Linné, 1767.la.c01-01

Trematodera Duméril ${ }^{+1}, 1841$

SI: $161 \bullet$ CI: c138 • ST: 2.D.M.31.E

PN: Trematoderes Duméril ${ }^{+1}$, 1841.da.c08 • AK

PA: 00 - Trematoderes - Duméril $^{+1}$ 1841.da: plate after page $53 \cdot \mathbf{G r} / \mathbf{S c} /{ }^{\prime T}$ '

01 - TrematOdereS • Gouriet 1868.ga: $206 \cdot$ UC

02 - Trematodera • Dubois 2016.da: $9 \cdot$ bO

03 - Trematodera - Dubois 2016.da: 9 • iO

RL: < ATRETODERES 1841.da.c03 • AI: HL

$>$ Perobranches 1841.da.c04 - AI: HL

$<$ AMphiumoides 1841.da.c05 • AI: HL

$>$ EXoBRANCHES 1841.da.c06 • AI: HL

GN: Pseudosauria 1816.ba.c0 0

GZ: INR

EN: KYR. C.05.07. Subordo Pseudosauria Blainville, 1816.ba.c08-07

Trematodera Baird, 1850

SI: $186 \bullet$ CI: c163・ST: 2.D.M.40.O

PN: Trematodera Baird, 1850.ba.c01 • AK

PA: 00 - Trematodera • Baird 1850.ba: $289 \cdot \mathbf{G r}$

01 - Trematodera - Baird 1851.ba: $250 \cdot \mathbf{b O}$

02 - ThrEMATODERES $・$ Desmarest 1856.da: $25 \cdot \mathbf{G r}$

03 - Trematodeira • Girard 1858.ga: vii • UC

RL: $\downarrow$ Trematoderes 1841 .da.c08

GN: IMPERFECTIBRANCHIA 1838.ha.c03

MeANTes 1767.la.c01

PSEUdosauria 1816.ba.c08

GZ: INR

EN: KYR. C.04.03. Ordo URODELA Duméril, 1805.da.c02-12 Trematodera Cope, 1859

SI: $210 \bullet$ CI: c187・ST: 2.D.M.41.E

PN: Trematoderes Cope, 1859.cb.c02 • AK

PA: 00 - TrEMATODERES - Cope 1859.cb: $122 \cdot$ UC

01 - Trematodera $\cdot$ Cope 1888.ca: $464 \cdot \mathbf{U C}$

RL: $\downarrow$ Trematoderes 1841 .da.c08

GN: IMPERFECTIBRANCHIA 1838.ha.c03

GZ: INR

EN: KYR. C.05.05. Subordo IMPERFECTIBRANCHIA Hogg, 1838.ha.c03-02

TritonaCEA Gray, 1850

SI: 193 • CI: c170 • ST: 3.D.M.30.R

PN: Tritones Gray, 1850.ga.c05 • AK

PA: $00 \bullet$ TRITONES • Gray 1850 .ga: $10 \bullet \mathbf{U C}$

01 - TritonaCEA • Dubois 2015.da: 107 - ER

RL: INR 
GN: ANURA 1805.da.c01

GYMNOPHIONA 1814.ra.c01

Urodela 1805.da.c02

GZ: INR

EN: KYR. C.03.01. Subclassis LissamphiBIA Gadow, 1898.ga.c01-00

Urodela Duméril, 1805

SI: $018 \bullet \mathbf{C I}: \mathrm{c009} \bullet$ ST: 2.S.O.10.E

PN: URODELES Duméril, 1805.da.c02

PA: 00 • URODELES • Duméril 1805.da: 91 • 'F'

01 - Urodelen - Meckel in Cuvier 1810.ca: pl. 3 • UC

$02 \cdot$ URODELI • Fischer 1813.fa: $58 \cdot$ UC

03 - URODELIA • Rafinesque 1815.ra: $78 \cdot$ bO

04 • UrodeLeS • Duméril ${ }^{+1}$ 1841.da: 4 • bO

05 - UrODELES • Gray 1842.ga: $111 \cdot$ O

06 • UrodelI • Mayer 1849.ma: 198 • bO

07 • UrodeLI • Massalongo 1854.ma: 430 • UC

08 • Urodela • Girard 1858.ga: vii • 'T'

09 • UrODELAE • Günther 1858.gc: 344 • 'T'

10 • Urodela • Huxley 1871.ha: 172 • UC

11 - UrOdELIA • Fatio 1872.fa: 7 • O

12 - UrodelA • Knauer 1878.ka: 93 • O

13 - URODELA • Haeckel 1889.ha: 625 - L

14 • URODELA • Säve-Söderbergh 1935.sa: 202 • C

15 - Urodela • Von Huene 1948.ha: 66 • bO

16 - URODELA • Milner 1988.ma: 82 • cO

17 - Urodela - Trueb $^{+1}$ 1991.ta: $233 \cdot$ pO

RL: $\leftrightarrow$ > CAUDATI 1805.da.c04 • AI: Zittel, 1888.za: 412

GN: URODELA 1805.da.c02

GZ: » GX:

ANURA 1805.da.c01

GYMNOPHIONA 1814.ra.c01

EN: KYR. C.04.03. Ordo Urodela Duméril, 1805.da.c02-12 URODELA Gray, 1825

SI: $065 \bullet$ CI: c047•ST: 1.N.G.02.O

PN: Urodela Gray, 1825.ga.c01 • AP

PA: 00 • URODELA • Gray 1825.ga: 215 • O

$01 \cdot$ UrodeLA $・$ Ficinus $^{+1}$ 1826.fa: pl. • UC

$02 \cdot$ Urodela • Bell 1836.ba: $91 \cdot$ O

$03 \cdot$ UrodeLES • Gray 1842.ga: $113 \cdot$ O

04 • URADELA • Cooper 1859.ca: 305 • 'T'

RL: $\downarrow$ URODELES 1805 .da.c02

GN: PSEUdosauria 1816.ba.c08

GZ: » GI:

PSEUdOSAURIa 1816.ba.c0 8

EN: ANAPTONYM

URODELA Cope, 1859

SI: $209 \bullet$ CI: c186・ST: 1.N.R.40.E

PN: Urodela Cope, 1859.cb.c01 • AK

PA: 00 • URODELA • Cope 1859.cb: 122 • bO

01 - Urodela • Cope 1875.ca: $11 \cdot$ O

RL: $\downarrow$ URODELES 1805.da.c02

GN: IMPERFECTIBRANCHIA 1838.ha.c03

PSEUdOSAURIA 1816.ba.c08

GZ: INR

EN: KYR. C.04.03. Ordo Urodela Duméril, 1805.da.c02-12
[HYP. Subordo Nullibranchia Bonaparte, 1831.ba.c01-01] Urodela Cope, 1889

SI: $291 \bullet$ CI: c264・ST: 1.N.G.02.O

PN: Urodela Cope, 1889.ca.c01 • AP

PA: 00 • Urodela • Cope 1889.ca: 5 • O

RL: $\downarrow$ URODELES 1805 .da.c02

GN: GYMNOPHIONA 1814.ra.c01 Urodela 1805.da.c02

GZ: » GI:

Urodela 1805.da.c02

EN: ANAPTONYM

UrodELA Abel, 1919

SI: $309 \bullet$ CI: c282 • ST: 1.N.G.02.O

PN: Urodela Abel, 1919.aa.c02 • AP

PA: 00 • Urodela • Abel 1919.aa: xii, 324 • bC

01 - Urodela • Goodrich 1930.ga: xxi, • O

02 • Urodela • Von Huene 1952.ha: 7 • bO

RL: $\downarrow$ URODELES 1805.da.c02

GN: URODELA 1805.da.c02 |Non-LiSSAMPHibian AMPHIBIa||

GZ: » GI:

ANURA 1805.da.c01

GYMNOPHIONA 1814.ra.c01

|Non-LissamphibIan AMPHIBIA|

EN: ANAPTONYM

UROPHORA Hogg, 1839

SI: $136 \bullet \mathbf{C I}$ : c114・ST: 1.D.M.31.O

PN: UrOPHORA Hogg, 1839.ha.c01 • AK

PA: 00 - UROPHORA • Hogg 1839.ha: 270 • O

RL: > TETRAPODA 1839.ha.c03 • AI: HL

$>$ ANGUIFORMI 1839.ha.c04 • AI: HL

GN: GYMNOPHIONA 1814.ra.c01

URODELA 1805.da.c02

GZ: INR

EN: KYR. C.03.01. Subclassis LissamPhibIA Gadow, 1898.ga.c01-00

[HYP. Superordo Derotreta Van der Hoeven, 1833.va.c01-01]

Vertebrata Cuvier, 1800

SI: $015 \bullet$ CI: $\mathrm{c006} \bullet$ ST: 1.S.O.10.E

PN: VERTÉBRÉS Cuvier, 1800.ca.c01

PA: 00 - VERTEBRES - Cuvier 1800.ca: first unnumbered table • UC

$01 \cdot$ VERTEBRATA $・$ Cuvier 1816.ca: $58 \cdot$ UC

$02 \cdot$ VERTEBRATA $・$ Ruggiero ${ }^{+8} 2015$.ra: $50 \cdot$ bPm

RL: INR

GN: VERTEBRATA 1800.ca.c01

GZ: » GX:

|AsCidiaCEa|

EN: KYR. C.01.01. Subphylum VeRTEBRATa Cuvier, 1800.ca.c01-02

VERTICALIA Bauer, 1986

SI: $411 \bullet \mathbf{C I}$ : c371・ST: 1.D.M.31.O

PN: VERTICALIA Bauer, 1986.ba.c01 • AK

PA: 00 • VerTiCalia • Bauer 1986.ba: 2 • UC

RL: INR

GN: ARChaEOSALIENTIA 1981.ra.c01 
RANOMORPHA 1921.fb.c08

GZ: INR

EN: KYR. C.07.02. Hypoordo LAEvogYrinia Lataste, 1878.la.c01-04

Xenoanura Starrett, 1973

SI: $396 \bullet$ CI: c356・ST: 1.D.M.31.O

PN: XeNOanura Starrett, 1973.sb.c01 • AK

PA: 00 - XenOanURa - Starrett 1973.sb: $251 \cdot$ UC

$01 \cdot$ XeNOANURA • Savage 1973.sa: $353 \cdot$ bO

RL: INR

GN: DoRSIPARES 1816.ba.c06
GZ: INR

EN: KYR. C.07.01. Hypoordo DorsiPares Blainville, 1816.ba.c06-02

Xenobatrachia Bauer, 1987

SI: $423 \bullet \mathbf{C I}: \mathrm{c} 383 \bullet \mathbf{S T}$ : 1.D.M.31.O

PN: XeNObatraChia Bauer, 1987.bc.c08 • AK

PA: 00 - XeNObATRACHIA • Bauer 1987.bc: 53 • UC

RL: INR

GN: ECOSTATA 1879.1b.c04

GZ: INR

EN: KYR. C.11.03. Subphalanx Ecostata Lataste, 1879.1b.c04-02 


\section{APPENDix A8.ECT. Ectonyms of LisSAMPHibia.}

This Table provides all ectonyms (names proposed under an unranked or pseudoranked nomenclatural system such as the Phylocode) for lissamphibian taxa published from 1992 to 31 October 2020.

The name is presented under its protonym and with the reason for considering it an ectonym, its author and date, and the kind of diagnoses used to validate the taxon. For each of the ectonyms, a serial identifier is attributed by date of publication, and its hemihomonyms among available zoological nomina, its getendotaxa and synotaxa are provided. Reference to justification and category of ectonym are given if relevant. Abbreviations used are given below. See Glossary for onymological terms and definition of unusual technical terms.

\section{Protonym of ectonym}

«XX»・ Nomen expressly proposed as unranked (anhypsonym).

$<\mathrm{YYY}>$ • Nomen expressly proposed as following the International Code for Phylogenetic Nomenclature (Cantino \& Queiroz 2020) (notharchonym).

DI - Nomen for which a diagnosis based on characters was provided.

ND - Nomen for which a 'phylogenetic definition' (cladognosis) but no diagnosis based on characters was provided.

SI - Serial identifier of ectonym

PA - Ectonym, its author and the taxonomic category to which it was originally referred Cd $\cdot$ Clade.

Tx $\cdot$ Taxon.

UU • Unspecified (or discussed) rank in unspecified (or discussed) nominal-series

$\mathrm{HH} \cdot$ If relevant, older senior hemihomonym of ectonym in the zoological class-series or/and family-series

GT • Getendotaxa of ectonym in $C L A D$

$\boldsymbol{n} \mathbf{G} \dot{\dagger} \cdot \bullet$ Number of all-fossil genus or genera, not listed here.

$\mathrm{SY} \cdot$ Synotaxa of ectonym in $C L A D$

HYP $\cdot$ Hypnokyronym.

KYR $\cdot$ Kyronym.

TEO $\cdot$ Teokyronym.

$\mathrm{JU} \cdot$ Statement in the original work justifying the treatment of this nomen as an ectonym

$\mathrm{C} \cdot$ Category of ectonym

$\mathbf{A H} \cdot$ Anhypsonym.

NH $\cdot$ Notharchonym.

Various abbreviations and conventions found in several columns

INR • Information not relevant here (item does not exist). 
«ACOSMAnURA» Frost $^{+18}$, 2006.fa.e16 • DI

SI: 040

PA: 00 • AcosmanuRA $・$ Frost $^{+18}$ 2006.fa: $6,185 \cdot$ Tx

HH: INR

GT:ARCHAEOSALIENTIA 1981.ra.c01 RANOMORPHA 1921.fb.c08

SY: KYR. C.07.02. Hypoordo LAEVOGYRINIA Lataste, 1878.1a.c01-03

JU: Nomen presented expressly (p. 141 sq., 185) as unranked

C: $\mathbf{A H}$

«AFRICANURA» Frost ${ }^{+18}$, 2006.fa.e42・DI

SI: 066

PA: 00 - AFricanURA $・$ Frost $^{+18}$ 2006.fa: 7, $237 \cdot$ Tx

HH: INR

GT:ECAUDATA 1777.sa.c06

SY: KYR. C.12.03. Infraphalanx ECAUDATA Scopoli, 1777. sa.c06-01

JU: Nomen presented expressly (p. 141 sq., 237) as unranked

C: $\mathbf{A H}$

«AFrobatraChIA» Frost $^{+18}$, 2006.fa.e35 • DI

SI: 059

PA: 00 • AfrobATRACHIA $・$ Frost $^{+18}$ 2006.fa: 7, 231 • Tx

HH: INR

GT:GASTRECHMIA 1867.ca.c02

SY: KYR. C.11.04. Subphalanx GaStrechmia Cope, 1867. ca.c02-03

JU: Nomen presented expressly (p. 141 sq., 231) as unranked

C: $\mathbf{A H}$

«AgASTOROPHRYNIA» Frost ${ }^{+18}$, 2006.fa.e32 • DI

SI: 056

PA: 00 • AGASTOROPHRYNIA • Frost ${ }^{+18}$ 2006.fa: $6,210 \bullet$ Tx

HH: INR

GT:PhORANURa DOP.da.c04

Phrynanura DOP.da.c05

SY: KYR. C.11.01. Subphalanx BAINANURA nov., DOP. da.c03-00

JU: Nomen presented expressly (p. 141 sq., 210) as unranked

C: $\mathbf{A H}$

«Aglaioanura» Frost ${ }^{+18}$, 2006.fa.e44 • DI

SI: 068

PA: $00 \cdot$ AglaiOanura $・$ Frost $^{+18}$ 2006.fa: $7,243 \cdot$ Tx

HH: INR

GT: RANOIDAE 1796.ba.f001

SY:KYR. F.15.10. Epifamilia RANOIDAE Batsch, 1796. ba.f001-29

JU: Nomen presented expressly (p. 141 sq., 243) as unranked

C: $\mathbf{A H}$

«Allocentroleniae» Guayasamin ${ }^{+5}$, 2009.ga.e01

$$
\text { - DI }
$$

SI: 079

PA: 00 - Allocentroleniae $\bullet$ Guayasamin $^{+5}$ 2009.ga: 3
- $\mathbf{T x}$

HH: INR

GT: CENTROLENOIDEA 1951.ta.f001

SY: KYR. F.14.04. Superfamilia CENTROLENOIDEA Taylor, 1951.ta.f001-02

JU: Nomen presented expressly (p. 19-20) as unranked

C: $\mathbf{A H}$

«AllodaPanura» Frost ${ }^{+18}, 2006$. fa.e34 • DI

SI: 057

PA: 00 • AllodapanuRa $・$ Frost $^{+18}$ 2006.fa: 7, $224 \bullet$ Tx

HH: INR

GT:ECosTaTA 1879.1b.c04

GASTRECHMIA 1867.ca.c02

SY: TEO. C.10.03. Phalanx SCOPTANURA Starrett, 1973. sb.c02-02

[HYP. Infraphalanx unnamed]

JU: Nomen presented expressly (p. 141 sq., 224) as unranked

C: $\mathbf{A H}$

«AMAZORANA» Streicher ${ }^{+7}$, 2018.sa.e03 • DI

SI: 089

PA: 00 • AMAZORANA $\bullet$ Streicher $^{+7}$ 2018.sa: $139,142 \cdot \mathbf{C d}$

HH: INR

GT:PhORANURA DOP.da.c04

Phrynanura DOP.da.c05

SY: KYR. C.11.01. Subphalanx BaINANURA nov., DOP. da.c03-00

JU: Nomen presented expressly (p. 139, 142) as unranked

C: $\mathbf{A H}$

«AMETROBATRACHIA» Frost ${ }^{+18}$, 2006.fa.e41 • DI

SI: 065

PA: 00 • AMETROBATRACHIA • Frost ${ }^{+18}$ 2006.fa: 7, 237 • Tx

HH: INR

GT:ECAUDATA 1777.sa.c06

SY: KYR. C.12.03. Infraphalanx ECaUdata Scopoli, 1777. sa.c06-01

JU: Nomen presented expressly (p. 141 sq., 237) as unranked

C: $\mathbf{A H}$

«AмPнIBIA» Queiroz ${ }^{+1}$, 1992.qa.e02・ND

SI: 002

PA: 00 • AmPHiBia $・$ Queiroz ${ }^{+1}$ 1992.qa: $474 \cdot \mathbf{C d}$

HH: АMPHiBIA Blainville, 1816.ba.c02

GT:ANURA 1805.da.c01

GYMNOPHIONA 1814.ra.c01

URODELA 1805.da.c02

SY: KYR. C.03.01. Subclassis LissamphiBIa Gadow, 1898. ga.c01-00

JU: Unranked nomen presented expressly (p. 475) as a "node-based name"

C: $\mathbf{A H}$

$<$ AMPHIBIA $>$ Laurin ${ }^{+12}$ in Queiroz $^{+2}$, 2020.qa.e01 • DI

SI: 093

PA: $00 \cdot$ AMPHIBIA $・$ Laurin ${ }^{+12} 2020$ ga: $765 \cdot$ Cd

HH: АмPHiBIA Blainville, 1816.ba.c02

GT:LISSAMPHIBIA 1898.ga.c01 
Non-lissamphibian AMPHIBIA 1816.ba.c02

SY: KYR. C.02.01. Classis AMPHiBIA Blainville, 1816. ba.c02-03

JU: Unranked nomen adopted (p. 764) as a "converted clade name" for a "total clade" under the Phylocode

C: NH

«ANOMOCOELA» Frost ${ }^{+18}$, 2006.fa.e17 • DI

SI: 041

PA: 00 • ANOMOCOela $・$ Frost $^{+18}$ 2006.fa: 6, 186 • Tx

HH: ANOMOcoela Nicholls, 1916.na.c02

GT:ARCHAEOSALIENTIA 1981.ra.c01

SY: KYR. C.08.01. Superphalanx ArChaEOSALIENTIA Roček, 1981.ra.c01-01

JU: Nomen presented expressly (p. 141 sq., 186) as unranked

C: $\mathbf{A H}$

«ANURA» Queiroz ${ }^{+1}$, 1992.qa.e01・ ND

SI: 010

PA: 00 • ANURA • De Queiroz ${ }^{+1}$ 1992.qa: $474 \cdot$ Cd

HH: ANOURES Duméril, 1805.da.c01

GT:ANURA 1805.da.c01

SY: KYR. C.04.01. Ordo ANURA Duméril, 1805.da.c01-07

JU: Unranked nomen presented expressly (p. 475) as a "node-based name"

C: $\mathbf{A H}$

«ANURA» Ford ${ }^{+1}$, 1993.fa.e01・DI

SI: 013

PA: 00 • ANURA • Ford ${ }^{+1}$ 1993.fa: $94 \cdot$ Cd

HH: ANOURES Duméril, 1805.da.c01

GT:ANURA 1805.da.c01

SY: KYR. C.04.01. Ordo ANURA Duméril, 1805.da.c01-07

JU: Unranked nomen presented expressly (p. 99) as a "nodebased name"

C: $\mathbf{A H}$

«APODA» Queiroz ${ }^{+1}$, 1992.qa.e03 • ND

SI: 003

PA: $00 \cdot$ ApodA $・$ Queiroz ${ }^{+1}$ 1992.qa: $474 \cdot$ Cd

HH: APODES Linnaeus, 1758.la.c02

GT:GYMNOPHIONA 1814.ra.c01

SY: KYR. C.04.02. Ordo Gymnophiona Rafinesque, 1814. ra.c01-02

JU: Unranked nomen presented expressly (p. 475) as a "stem-based name"

C: $\mathbf{A H}$

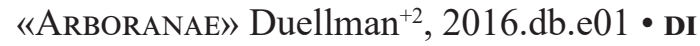

SI: 086

PA: 00 • ArboranaE $\bullet$ Duellman $^{+2}$ 2016.db: 1, 7 • Tx

HH: INR

GT:HYLOIDEA 1815.ra.f002-|1825.gb.f001|

SY: KYR. F.14.06. Superfamilia HYLOIDEA Rafinesque, 1815.ra.f002-|Gray, 1825.gb.f001|-20

JU: Nomen presented expressly (p. 1, 7) as unranked

C: $\mathbf{A H}$

«ATHESPHATANURA» Frost ${ }^{+18}$, 2006.fa.e27 • DI

SI: 051

PA: 00 • ATHESPHATANURA • Frost ${ }^{+18}$ 2006.fa: 6, 202 • Tx

\section{HH: INR}

GT:Phoranura DOP.da.c04

Phrynanura DOP.da.c05

SY: KYR. C.11.01. Subphalanx BaINANURA nov., DOP. da.c03-00

JU: Nomen presented expressly (p. 141 sq., 202) as unranked

C: $\mathbf{A H}$

«ATLANTICANURA» Frazão ${ }^{+2}$, 2015.fa.e01 • DI

SI: 084

PA: 00 • AtLanTiCANURA • Frazão ${ }^{+2}$ 2015.fa: 1 , 10 • Cd

HH: INR

GT:AQUIPARES 1816.ba.c07

Helanura DOP.da.c09

SY: KYR. C.08.02. Superphalanx RaNomorPHa Fejérváry, 1921.fb.c08-01

JU: Nomen presented expressly (p. 1, 6) as unranked

C: $\mathbf{A H}$

«Australobatrachia» Frost ${ }^{+18}$, 2006.fa.e22 • DI

SI: 046

PA: 00 • AustralOBATRACHIA • Frost ${ }^{+18} 2006 . f a: ~ 6,193 \cdot$ Tx

HH: DiPLosiPhona Günther, 1859.ga.c02

GT:DIPLOSIPHONA 1859.ga.c02

SY: KYR. C.11.02. Subphalanx Diplosiphona Günther, 1859.ga.c02-01

JU: Nomen presented expressly (p. 141 sq., 193) as unranked

C: $\mathbf{A H}$

«BATRACHIA» de Queiroz ${ }^{+1}$, 1992.qa.e01 • ND

SI: 006

PA: 00 • BATRACHIA • De Queiroz ${ }^{+1} 1992 . q a: 474 \cdot$ Cd

HH: BATRACIENS Brongniart, 1800.ba.c01

GT:ANURA 1805.da.c01

URODELA 1805.da.c02

SY: KYR. C.03.01. Subclassis LisSAMPHIBIA Gadow, 1898. ga.c01-00

[HYP. Superordo BATRACHIA Brongniart, 1800.ba.c01$14]$

JU: Unranked nomen presented expressly (p. 475) as a "node-based name"

C: $\mathbf{A H}$

«BATRACHIA» Frost $^{+18}$, 2006.fa.e03・DI

SI: 027

PA: 00 • BATRACHIA • Frost ${ }^{+18}$ 2006.fa: 5, $168 \cdot$ Tx

HH: Batraciens Brongniart, 1800.ba.c01

GT:ANURA 1805.da.c01

URODELA 1805.da.c02

SY: KYR. C.03.01. Subclassis LisSAMPHIBIA Gadow, 1898. ga.c01-00

[HYP. Superordo BATRACHIA Brongniart, 1800.ba.c0114]

JU: Nomen presented expressly (p. 141 sq., 168) as unranked

C: $\mathbf{A H}$

«BombInANURA» Ford $^{+1}$, 1993.fa.e03 • DI

SI: 015 
PA: 00 • BombinANURA • Ford ${ }^{+1}$ 1993.fa: $94 \cdot$ Cd

\section{HH: INR}

GT:GEOBATRACHIA 1828.ra.c18

MEDIOGYRINIA 1878.1a.c02

SY: KYR. C.05.02. Subordo Hydrobatrachia Ritgen, 1828.ra.c14-01

JU: Unranked nomen presented expressly (p. 101) as a "node-based name"

C: $\mathbf{A H}$

«CAlamitophrynia» Grant ${ }^{+9}$, 2006.gb.e02 • DI

SI: 071

PA: 00 • CALAMitophrynia • Grant ${ }^{+9}$ 2006.gb: 4, $154 \cdot$ Tx

HH: INR

GT:Phoranura DOP.da.c04

Phrynanura DOP.da.c05

SY: KYR. C.11.01. Subphalanx BaINANURA nov., DOP. da.c03-00

JU: Nomen presented expressly (p. 146 sq., 154) as unranked

C: $\mathbf{A H}$

«CAUDATA» de Queiroz ${ }^{+1}$, 1992.qa.e01 • ND

SI: 008

PA: 00 • CAUDATA • De Queiroz ${ }^{+1}$ 1992.qa: $474 \cdot$ Cd

HH: CAUdaTa Scopoli, 1777.sa.c02

GT:URODELA 1805.da.c02

SY: KYR. C.04.03. Ordo Urodela Duméril, 1805.da.c0212

JU: Unranked nomen presented expressly (p. 475) as a "node-based name"

C: $\mathbf{A H}$

«CAUdATA» Frost ${ }^{+18}$, 2006.fa.e04・DI

SI: 028

PA: $00 \cdot$ CAUDATA $・$ Frost $^{+18}$ 2006.fa: 5, 169 • Tx

HH: CAUdata Scopoli, 1777.sa.c02

GT:URODELA 1805.da.c02

SY: KYR. C.04.03. Ordo URodela Duméril, 1805.da.c0212

JU: Nomen presented expressly (p. 141 sq., 169) as unranked

C: $\mathbf{A H}$

$<$ CAUdATA $>$ D. Wake in Queiroz $^{+2}$, 2020.qa.e04 • ND SI: 094

PA: 00 • CAUDATA • D. Wake in Queiroz $^{+2}$, 2020.qa.e04: 785 - Cd

HH: CaUdaTA Scopoli, 1777.sa.c02

GT:URODELA 1805.da.c02

SY: KYR. C.04.03. Ordo Urodela Duméril, 1805.da.c0212

JU: Unranked nomen adopted as a "converted clade name" for a "crown clade" under the Phylocode

C: $\mathbf{N H}$

«CERATOBatrachia» Brown ${ }^{+4}, 2015$.ba.e01 • DI SI: 083

PA: 00 • PANCRYPTOBRANCHA $\bullet$ Brown $^{+4}$ 2015.ba: $138 \cdot$ Cd HH: INR

GT:CERATOBATRACHEIDAE 1884.ba.f001
SY: KYR. F.16.03. Apofamilia CERATOBATRACHEIDAE Boulenger, 1884.ba.f001-04

JU: Unranked nomen presented expressly (p. 138) as a "node-based name"

C: $\mathbf{A H}$

«Chthonobatrachia» Frost $^{+18}$, 2006.fa.e30 • DI

SI: 054

PA: $00 \bullet$ ChTHONOBATRACHIA $\bullet$ Frost $^{+18}$ 2006.fa: 6, 208 • Tx

HH: INR

GT:Phoranura DOP.da.c04 Phrynanura DOP.da.c05

SY: KYR. C.11.01. Subphalanx BaINANURA nov., DOP. da.c03-00

JU: Nomen presented expressly (p. 141 sq., 208) as unranked

C: $\mathbf{A H}$

«Cladophrynia» Frost ${ }^{+18}$, 2006.fa.e25 • DI

SI: 049

PA: 00 • CladoPhrynia $・$ Frost $^{+18}$ 2006.fa: $6,201 \cdot$ Tx

HH: INR

GT:Phoranura DOP.da.c04

Phrynanura DOP.da.c05

SY: KYR. C.11.01. Subphalanx BaINANURA nov., DOP. da.c03-00

JU: Nomen presented expressly (p. 141 sq., 201) as unranked

C: $\mathbf{A H}$

«CommutabiRana» Streicher $^{+7}$, 2018.sa.e04 • DI

SI: 090

PA: 00 • Commutabirana • Streicher ${ }^{+7}$ 2018.sa: 139, 142 - Cd

HH: INR

GT:Phoranura DOP.da.c04

Phrynanura DOP.da.c05

SY: KYR. C.11.01. Subphalanx BaINANURA nov., DOP. da.c03-00

JU: Nomen presented expressly (p. 139, 142) as unranked

C: $\mathbf{A H}$

«CORnUCOPIRANA» Streicher $^{+7}$, 2018.sa.e02 • DI

SI: 088

PA: $00 \bullet$ CORNUCOPIRANA $\bullet$ Streicher ${ }^{+7}$ 2018.sa: 139, 142 - Cd

HH: INR

GT: Phoranura DOP.da.c04

Phrynanura DOP.da.c05

SY: KYR. C.11.01. Subphalanx BaINANURA nov., DOP. da.c03-00

JU: Nomen presented expressly (p. 139, 142) as unranked

C: $\mathbf{A H}$

«Costata» Frost ${ }^{+18}$, 2006.fa.e15 • DI

SI: 039

PA: $00 \cdot$ CostaTA $・$ Frost $^{+18}$ 2006.fa: 6, 184 • Tx

HH: Costata Müller, 1840.ma.c01

GT:Mediogyrinia 1878.la.c02

SY: KYR. C.06.02. Infraordo Mediogyrinia Lataste, 1878. la.c02-01 
JU: Nomen presented expressly (p. 141 sq., 184) as unranked

C: $\mathbf{A H}$

«Cruciabatrachia» Grant ${ }^{+9}, 2006 . g b . e 01 ・ D I$

SI: 070

PA: 00 • CRUCIABATRACHIA • Grant ${ }^{+9}$ 2006.gb: 4, 151 • Tx

HH: INR

GT:PhORANURA DOP.da.c04

Phrynanura DOP.da.c05

SY: KYR. C.11.01. Subphalanx BaINANURA nov., DOP. da.c03-00

JU: Nomen presented expressly (p. 146 sq., 151) as unranked

C: $\mathbf{A H}$

«CRYPTOBRANCHOIDEI» Frost ${ }^{+18}, 2006$.fa.e05 • DI

SI: 029

PA: 00 • CRYPTOBRANCHOIDEI • Frost ${ }^{+18}$ 2006.fa: 5, 170 • Tx

HH: CRYPTOBRANCheS Duméril, 1805.da.c05

GT:IMPERFECTIBRANCHIA 1838.ha.c03

SY: KYR. C.05.05. Subordo IMPERFECTIBRANCHIA Hogg, 1838.ha.c03-02

JU: Nomen presented expressly (p. 141 sq., 170) as unranked

C: $\mathbf{A H}$

«DiadeCtOSALAMANDROIDEI» Frost ${ }^{+18}$, 2006.fa.e06 - DI

SI: 030

PA: 00 • DiAdECTOSALAMANDROIDEI • Frost ${ }^{+18}$ 2006.fa: 5, $171 \cdot \mathbf{T x}$

HH: INR

GT:MEANTES 1767.la.c01

PSEUdosauria 1816.ba.c08

SY: KYR. C.04.03. Ordo URODELA Duméril, 1805.da.c0212

[HYP. Phalanx Pneumobranchia Sonnini ${ }^{+1}, 1801$. sa.c01-02]

JU: Nomen presented expressly (p. 141 sq., 171) as unranked

C: $\mathbf{A H}$

«Diatriata» Wilkinson ${ }^{+1}$, 2006.wa.e02 • DI

SI: 074

PA: 00 • DiATRIATA • Wilkinson ${ }^{+1}$ 2006.wa: 46 • Tx

HH: INR

GT:ICHTHYOPHIOIDEA 1968.ta.f001

SY: KYR. F.14.16. Superfamilia ІСнтнуоРнIоIDEA Taylor, 1968.ta.f001-04

JU: Nomen presented expressly (p. 46) as unranked

C: $\mathbf{A H}$

«DIPHYABATRACHIA» Frost $^{+18}$, 2006.fa.e29 • DI

SI: 053

PA: 00 • DiPHYABATRACHIA • Frost ${ }^{+18}$ 2006.fa: 6, 205 • Tx

HH: INR

GT:HYLOBATRACHIA 1828.ra.c16

SY: KYR. C.13.03. Hypophalanx Hylobatrachia Ritgen, 1828.ra.c16-01

JU: Nomen presented expressly (p. 141 sq., 205) as unranked

C: $\mathbf{A H}$

«DiscoGlosSANURA» Ford $^{+1}$, 1993.fa.e04 • DI

SI: 016

PA: 00 • Discoglossanura • Ford ${ }^{+1}$ 1993.fa: $94 \cdot$ Cd

HH: INR

GT: GEOBATRACHIA 1828.ra.c18

MEDIOGYRINIA 1878.la.c02

SY: KYR. C.05.02. Subordo Hydrobatrachia Ritgen, 1828.ra.c14-01

JU: Unranked nomen presented expressly (p. 101) as a "node-based name"

C: $\mathbf{A H}$

«GYMnOPHIONA» Queiroz ${ }^{+1}$, 1992.qa.e04 • ND

SI: 004

PA: 00 • Gymnophiona • Queiroz ${ }^{+1}$ 1992.qa: 474 • Cd

HH: GYMnOPHIA Rafinesque, 1814.ra.c01

GT:GYMNOPHIONA 1814.ra.c01

SY: KYR. C.04.02. Ordo GYMnOPHIONA Rafinesque, 1814. ra.c01-02

JU: Unranked nomen presented expressly (p. 475) as a "node-based name"

C: $\mathbf{A H}$

«GYMnOPHIONA» Frost ${ }^{+18}$, 2006.fa.e01 • DI

SI: 025

PA: 00 • GymnOphiona • Frost ${ }^{+18}$ 2006.fa: 5, 165 • Tx

HH: Gyмnophia Rafinesque, 1814.ra.c01

GT:GYMNOPHIONA 1814.ra.c01

SY: KYR. C.04.02. Ordo GYMNOPHIONA Rafinesque, 1814. ra.c01-02

JU: Nomen presented expressly (p. 141 sq., 165) as unranked

C: $\mathbf{A H}$

$<$ Gymnophiona > M. H. Wake in Queiroz $^{+2}$, 2020.qa.e03 • ND

SI: 095

PA: 00 • Gymnophiona • M. H. Wake in Queiroz $^{+2}, 2020$. qa.e02: $779 \cdot \mathbf{C d}$

HH: GyMnOPHia Rafinesque, 1814.ra.c01

GT:GYMNOPHIONA 1814.ra.c01

SY: KYR. C.04.02. Ordo GYMNOPHIONA Rafinesque, 1814. ra.c01-02

JU: Unranked nomen adopted as a "converted clade name" for a "crown clade" under the Phylocode

C: $\mathbf{N H}$

$<$ GYMNOPHIONIFORMES $>$ Marjanović ${ }^{+1}$, 2008.ma.e01 - DI

SI: 077

PA: 00 • GYMNOPHIONIFORMES • Marjanović ${ }^{+1}$ 2008.ma: 152 - $\mathbf{T x}$

HH: INR

GT:GYMNOPHIONA 1814.ra.c01

SY: KYR. C.04.02. Ordo GYMnOphiona Rafinesque, 1814. ra.c01-02

JU: Nomen presented expressly (p. 152) as following the International Code for Phylogenetic 


\section{Nomenclature \\ C: $\mathbf{N H}$}

$<$ GYMNOPHIONOMORPHA $>$ Marjanović ${ }^{+1}$, 2008.ma.e02

$$
\text { - DI }
$$

SI: 078

PA: 00 • GYMNOPHIONOMORPHA • Marjanović ${ }^{+1}$ 2008.ma: $152 \cdot \mathbf{T x}$

\section{HH: INR}

GT:GYMNOPHIONA 1814.ra.c01

SY: KYR. C.04.02. Ordo Gymnophiona Rafinesque, 1814. ra.c01-02

JU: Nomen presented expressly (p. 152) as following the International Code for Phylogenetic Nomenclature

C: NH

«HeStiCOBATRACHIA» Frost $^{+18}$, 2006.fa.e31 • DI

SI: 055

PA: $00 \bullet$ HeSTiCOBATRACHIA $•$ Frost $^{+18}$ 2006.fa: $6,209 \bullet$ Tx

HH: INR

GT:PHORANURA DOP.da.c04

Phrynanura DOP.da.c05

SY: KYR. C.11.01. Subphalanx BaINANURA nov., DOP. da.c03-00

JU: Nomen presented expressly (p. 141 sq., 209) as unranked

C: $\mathbf{A H}$

«HydatinOSALAMANDROIDEI» Frost ${ }^{+18}$, 2006.fa.e07

$$
\text { - DI }
$$

SI: 031

PA: $00 \bullet$ HydatinOSALAMANDROIDeI • Frost ${ }^{+18}$ 2006.fa: 5, $171 \cdot \mathbf{T x}$

HH: INR

GT: Meantes 1767.la.c01

PseUdosauria 1816.ba.c08

SY: KYR. C.04.03. Ordo Urodela Duméril, 1805.da.c0212

[HYP. Phalanx Pneumobranchia Sonnini ${ }^{+1}, 1801$. sa.c01-02]

JU: Nomen presented expressly (p. 141 sq., 171) as unranked

C: $\mathbf{A H}$

«HyLOIDES» Frost ${ }^{+18}$, 2006.fa.e20 • DI

SI: 044

PA: $00 \cdot$ Hyloides $\bullet$ Frost $^{+18}$ 2006.fa: 6, 191 • Tx

HH: Hylaeae Hübner, 1816.ha.c02

HYLINA Gray, 1825.gb.f001

GT:GONDWANURA DOP.da.c01 Phaneranura DOP.da.c02

SY: TEO. C.09.01. Epiphalanx AQUIPARES de Blainville, 1816.ba.c07-02

[HYP. Phalanx unnamed]

JU: Nomen presented expressly (p. 141 sq., 191) as unranked

C: $\mathbf{A H}$

«INDIANURA» Frazão ${ }^{+2}$, 2015.fa.e02 • DI

SI: 085
PA: $00 \bullet$ IndianURA $\bullet$ Frazão $^{+2}$ 2015.fa: $1,6 \bullet$ Cd

HH: INR

GT:ECOSTATA 1879.lb.c04

GaSTRECHMIA 1867.ca.c02

Pananura DOP.da.c07

SY: TEO. C.10.03. Phalanx ScoptanURA Starrett, 1973. sb.c02-02

JU: Nomen presented expressly (p. 1, 6) as unranked

C: $\mathbf{A H}$

«LALAGOBATRACHIA» Frost ${ }^{+18}$, 2006.fa.e12 • DI

SI: 036

PA: 00 - LalaGOBATRACHia • Frost ${ }^{+18}$ 2006.fa:6, 180 • Tx

HH: INR

GT: GEOBATRACHIA 1828.ra.c18

Mediogyrinia 1878.la.c02

SY: KYR. C.05.02. Subordo Hydrobatrachia Ritgen, 1828.ra.c14-01

JU: Nomen presented expressly (p. 141 sq., 180) as unranked

C: $\mathbf{A H}$

«LAURENTOBATRACHIA» Frost ${ }^{+18}, 2006 . f a . e 37$ • DI

SI: 061

PA: 00 • LAURENTOBATRACHIA • Frost ${ }^{+18}$ 2006.fa: 7, 232 • Tx

HH: INR

GT:ARTHROLEPTOIDEA 1869.ma.f011

SY: KYR. F.14.08. Superfamilia ARTHROLEPTOIDEA Mivart, 1869.ma.f011-05

JU: Nomen presented expressly (p. 141 sq., 232) as unranked

C: $\mathbf{A H}$

«LEIOPELMATANURA» Ford ${ }^{+1}$, 1993.fa.e02 • DI

SI: 014

PA: 00 • Leiopelmatanura $\bullet$ Ford $^{+1}$ 1993.fa: $94 \cdot$ Cd

HH: INR

GT:ANGUSTICOELA 1958.ra.c01

HYdROBATRACHIA 1828.ra.c14

SY: KYR. C.04.01. Ordo ANURa Duméril, 1805.da.c01-07

JU: Unranked nomen presented expressly (p. 100) as a "node-based name"

C: $\mathbf{A H}$

«LEPTODACTYLIFORMES» Frost ${ }^{+18}$, 2006.fa.e28 • DI

SI: 052

PA: 00 • LEPTODACTYLIFORMES • Frost ${ }^{+18}$ 2006.fa: 6, 205 - $\mathbf{T x}$

HH: INR

GT:Phoranura DOP.da.c04

PhrYnanura DOP.da.c05

SY: KYR. C.11.01. Subphalanx BaINANURA nov., DOP. da.c03-00

JU: Nomen presented expressly (p. 141 sq., 205) as unranked

C: $\mathbf{A H}$

$<$ LISSAMPHIBIA $>$ Laurin $^{+12}$ in Queiroz $^{+2}$, 2020.qa.e02

$$
\text { - ND }
$$

SI: 096

PA: 00 - LISSAMPHIBIA • Laurin ${ }^{+12}$ in Queiroz $^{+2}$, 2020.qa.e02: 
$773 \cdot \mathbf{C d}$

HH: LiSSAMPHibia Gadow, 1898.ga.c01

GT:ANURA 1805.da.c01

GYMNOPHIONA 1814.ra.c01

URODELA 1805.da.c02

SY: KYR. C.03.01. Subclassis LisSAMPHibIa Gadow, 1898. ga.c01-00

JU: Unranked nomen adopted (p. 773) as a "converted clade name" for a "crown clade" under the Phylocode

C: NH

«Meridianura» Frost ${ }^{+18}$, 2006.fa.e24・DI

SI: 048

PA: $00 \bullet$ MERIdianura $・$ Frost $^{+18}$ 2006.fa: $6,196 \bullet$ Tx

HH: INR

GT:PhORANURA DOP.da.c04

Phrynanura DOP.da.c05

SY: KYR. C.11.01. Subphalanx BaINANURa nov., DOP. da.c03-00

JU: Nomen presented expressly (p. 141 sq., 196) as unranked

C: $\mathbf{A H}$

«Mesobatrachia» Ford $^{+1}$, 1993.fa.e06 • DI

SI: 018

PA: 00 • Mesobatrachia • Ford ${ }^{+1}$ 1993.fa: $94 \cdot$ Cd

HH: Mesobatrachia Laurent, 1980.la.c01

GT:DORSIPARES 1816.ba.c06

LAEVOGYRINIA 1878.1a.c01

SY: KYR. C.06.01. Infraordo GEObatraChia Ritgen, 1828. ra.c18-01

JU: Unranked nomen presented expressly (p. 102) as a "node-based name"

C: $\mathbf{A H}$

«NATATANURA» Frost ${ }^{+18}$, 2006.fa.e38 • DI

SI: 062

PA: $00 \bullet$ NATATANURA $・$ Frost $^{+18}$ 2006.fa: 7, $234 \cdot$ Tx

HH: INR

GT:ECAUDATA 1777.sa.c06 SAVANURA DOP.db.c08

SY: KYR. C.11.05. Subphalanx Pananura nov., DOP. da.c07-00

JU: Nomen presented expressly (p. 141 sq., 234) as unranked

C: $\mathbf{A H}$

«NeOAuSTRARANA» Streicher ${ }^{+7}$, 2018.sa.e01 • DI

SI: 087

PA: 00 • NeOAuSTRARANa $\bullet$ Streicher $^{+7}$ 2018.sa: 139, $142 \backslash$

$$
\text { - Cd }
$$

HH: INR

GT:CYCLORAMPHEIDAE 1850.bb.f002-|1852.ba.f001|

SY: KYR. F.16.01. Apofamilia CYCLORAMPHEIDAE Bonaparte, 1850.bb.f002-|Bonaparte, 1852. ba.f001|-05

JU: Nomen presented expressly (p. 139, 142) as unranked

C: $\mathbf{A H}$

«NeObaTraChia» Ford ${ }^{+1}$, 1993.fa.e08 • DI

SI: 020
PA: 00 • NeOBATRACHIA $\bullet$ Ford $^{+1}$ 1993.fa: $94 \cdot$ Cd

HH: NEOBATRACHI Sarasin ${ }^{+1}$, 1890.sa.c01

GT:AQUIPARES 1816.ba.c07

Helanura DOP.da.c09

SY: KYR. C.08.02. Superphalanx RANOMORPHA Fejérváry, 1921.fb.c08-01

JU: Unranked nomen presented expressly (p. 102) as a "node-based name"

C: $\mathbf{A H}$

«NeObatraChIA» Frost $^{+18}$, 2006.fa.e18 • DI

SI: 042

PA: 00 • NeOBATRACHia $・$ Frost $^{+18}$ 2006.fa: $6,189 \cdot$ Tx

HH: NEOBATRACHI Sarasin ${ }^{+1}$, 1890.sa.c01

GT:AQUIPARES 1816.ba.c07

HelanURa DOP.da.c09

SY: KYR. C.08.02. Superphalanx RANOMORPHA Fejérváry, 1921.fb.c08-01

JU: Nomen presented expressly (p. 141 sq., 189) as unranked

C: $\mathbf{A H}$

«NEOCAECILIA» Wilkinson ${ }^{+1}$, 2006.wa.e01・DI

SI: 073

PA: $00 \cdot$ NeOCAECILIA $・$ Wilkinson ${ }^{+1}$ 2006.wa: $44 \cdot$ Tx

HH: INR

GT:PSEUDOPHIONA 1816.ba.c11

SY: KYR. C.05.04. Subordo Pseudophiona de Blainville, 1816.ba.c11-06

JU: Nomen presented expressly (p. 44) as unranked

C: $\mathbf{A H}$

«NeOCAudata» Cannatella ${ }^{+1}$, 1993.ca.e01 • ND

SI: 011

PA: 00 • NeOCAUdATA $\bullet$ Cannatella $^{+1}$ 1993.ca: 2 - Cd

HH: NeOcaudata Milner, 2000.ma.c01

GT:IMPERFECTIBRANCHIA 1838.ha.c03

PseUdOSAURIA 1816.ba.c08

SY: KYR. C.04.03. Ordo Urodela Duméril, 1805.da.c0212

[HYP. Infraordo NULLIBRANCHIA Bonaparte, 1831. ba.c01-01]

JU: Unranked nomen presented expressly (p. 2) as a "nodebased name"

C: $\mathbf{A H}$

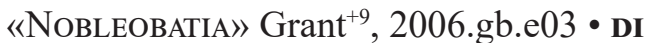

SI: 072

PA: $00 \cdot$ Nobleobatia $\bullet$ Grant ${ }^{+9}$ 2006.gb: 4, 155 • Tx

HH: INR

GT:Phoranura DOP.da.c04

Phrynanura DOP.da.c05

SY: KYR. C.11.01. Subphalanx BaINANURA nov., DOP. da.c03-00

JU: Nomen presented expressly (p. 146 sq., 155) as unranked

C: $\mathbf{A H}$

«Nobleobatrachia» Frost ${ }^{+18}, 2006 . f a . e 23$ • DI

SI: 047

PA: $00 \bullet$ NOBLEOBATRACHIA $•$ Frost $^{+18}$ 2006.fa: 6 , $196 \bullet$ Tx 


\section{HH: INR}

\section{GT:PhORANURA DOP.da.c04}

Phrynanura DOP.da.c05

SY: KYR. C.11.01. Subphalanx BaINANURA nov., DOP. da.c03-00

JU: Nomen presented expressly (p. 141 sq., 196) as unranked

C: $\mathbf{A H}$

«Notogaeanura» Frost ${ }^{+18}, 2006$. fa.e21 • DI

SI: 045

PA: $00 \bullet$ NotogaeAnURA $•$ Frost $^{+18}$ 2006.fa: $6,192 \cdot$ Tx

HH: INR

GT:BAINANURA DOP.da.c03

DiPLOSIPHONA 1859.ga.c02

SY: KYR. C.10.02. Phalanx Phaneranura nov., DOP. da.c02-00

JU: Nomen presented expressly (p. 141 sq., 192) as unranked

C: $\mathbf{A H}$

«Orthobatrachia» Heinicke ${ }^{+5}$, 2009.ha.e02 • DI

SI: 081

PA: 00 • ORTHOBATRACHIA • Heinicke ${ }^{+5}$ 2009.ha: 1, $24 \cdot$ Tx

HH: INR

GT: GAIANURA DOP.da.c06

HeMiPHRACTIFORMIA 1881.bd.c01

SY: TEO. C.12.02. Infraphalanx PhrYnanura nov., DOP. da.c05-00

[HYP. Catophalanx unnamed]

JU: Nomen presented expressly (p. 24) as unranked

C: $\mathbf{A H}$

«PANCRYPTOBRANCHA» Vasilyan ${ }^{+4}$, 2013.va.e01 • DI

SI: 082

PA: 00 • PANCRYPTOBRANCHA • Vasilyan ${ }^{+4}$ 2013.va: $301 \cdot$ Cd

HH: INR

GT:CRYPTOBRANCHIDAE 1826.fb.f003

SY: KYR. F.17.69. Familia CRYPTOBRANCHIDAE Fitzinger, 1826.fb.f003-04

JU: Unranked nomen presented expressly (p. 301) as a "stem-based name"

C: $\mathbf{A H}$

«PANPIPIDAE» Aranciaga Rolando ${ }^{+2}$, 2019.aa.e01・DI

SI: 091

PA: 00 PANPIPIDAE $\bullet$ Aranciaga Rolando ${ }^{+2}$ 2019.aa: 725 - Cd

HH: INR

GT:DACTYLETHRINAE 1838.ha.f001 PIPINAE 1825.ga.f003-|1826.fb.f002| SALTENIINAE DOP.da.f148

SY: KYR. F.17.69. Familia PIPIDAE 1825.ga.f003-|1826. fb.f002

JU: Unranked nomen presented expressly (p. 727) as the nomen of a "stem-based clade"

C: $\mathbf{A H}$

«PARABATRACHIA» Frost ${ }^{+18}$, 2006.fa.e45 • DI

SI: 069

PA: 00 • PARABATRACHIA • Frost ${ }^{+18}$ 2006.fa: $356 \bullet$ Tx

\section{HH: INR}

GT:GYMNOPHIONA 1814.ra.c01

SY: KYR. C.04.02. Ordo GYMnOPHIONA Rafinesque, 1814. ra.c01-02

JU: Nomen presented expressly (p. 141 sq., 356) as unranked

C: $\mathbf{A H}$

«PARATOIDEA» Queiroz ${ }^{+1}$, 1992.qa.e01・ ND

SI: 005

PA: 00 • PARATOIDEA • Queiroz ${ }^{+1}$ 1992.qa: $474 \cdot$ Cd

HH: Рarotoidia Gardiner, 1982.ga.c01

GT:ANURA 1805.da.c01

URODELA 1805.da.c02

SY: KYR. C.03.01. Subclassis LisSAMPhibia Gadow, 1898. ga.c01-00

[HYP. Superordo BATRACHIA Brongniart, 1800.ba.c0114]

JU: Unranked nomen presented expressly (p. 475) as a "stem-based name"

C: $\mathbf{A H}$

«Perennibranchia» Frost ${ }^{+18}, 2006 . f a . e 08$ - DI

SI: 032

PA: 00 • Perennibranchia • Frost ${ }^{+18}$ 2006.fa: 5, 172 • Tx

HH: PÉRenNibranches Latreille 1824.la.c02

GT:MeANTES 1767.la.c01

Pseudosauria 1816.ba.c08

SY: KYR. C.04.03. Ordo Urodela Duméril, 1805.da.c0212

[HYP. Phalanx Pneumobranchia Sonnini ${ }^{+1}, 1801$. sa.c01-02]

JU: Nomen presented expressly (p. 141 sq., 172) as unranked

C: $\mathbf{A H}$

«Phthanobatrachia» Frost ${ }^{+18}, 2006$. fa.e19 • DI

SI: 043

PA: $00 \bullet$ Phthanobatrachia $・$ Frost $^{+18}$ 2006.fa: $6,190 \bullet$ Tx

HH: INR

GT:GONDWANURA DOP.da.c01

Phaneranura DOP.da.c02

SCOPTANURA 1973.sb.c02

SY: TEO. C.09.01. Epiphalanx AQUIPARES de Blainville, 1816.ba.c07-02

JU: Nomen presented expressly (p. 141 sq., 190) as unranked

C: $\mathbf{A H}$

«PIPANURA» Ford ${ }^{+1}$, 1993.fa.e05・DI

SI: 017

PA: $00 \bullet$ PIPANURA $・$ Ford $^{+1}$ 1993.fa: $94 \cdot$ Cd

HH: INR

GT:DORSIPARES 1816.ba.c06

LAEVOGYRINIA 1878.1a.c01

SY: KYR. C.06.01. Infraordo GEOBATRACHIA Ritgen, 1828. ra.c18-01

JU: Unranked nomen presented expressly (p. 102) as a "node-based name"

C: $\mathbf{A H}$ 
«PIPIMORPHA» Ford ${ }^{+1}$, 1993.fa.e07・ ND

SI: 019

PA: 00 • PIPIMORPHA • Ford ${ }^{+1}$ 1993.fa: $94 \cdot$ Cd

HH: INR

GT:DORSIPARES 1816.ba.c06

SY: KYR. C.07.01. Hypoordo DorSIPARES de Blainville, 1816.ba.c06-02

JU: Unranked nomen presented expressly (p. 99) as a "stembased name"

C: $\mathbf{A H}$

«PIPINOMORPHA» Báez ${ }^{+1}$, 2003.ba.e01 • ND

SI: 023

PA: 00 • PIPINOMORPHA • Báez ${ }^{+1}$ 2003.ba: $454 \cdot$ Cd

HH: INR

GT:DACTYLETHRINAE 1838.ha.f001

PIPINAE 1825.gb.f003-|1826.fb.f002

SY: KYR. F.17.03. PIPIDAE Gray, 1825.gb.f003-|Fitzinger, 1826.fb.f002|-07

JU: Unranked nomen presented expressly (p. 454) as a "stem-based name"

C: $\mathbf{A H}$

«PlethosalamandroideI» Frost ${ }^{+18}$, 2006.fa.e10 • DI

SI: 034

PA: $00 \bullet$ PlethosalamandRoIdeI $\bullet$ Frost $^{+18}$ 2006.fa: 5, 175 - $\mathbf{T x}$

HH: INR

GT:AMPHIUMEIDAE 1825.gb.f007 RHYACOTRITONEIDAE 1958.ta.f002

SY: KYR. F.15.11. Epifamilia AMPHIUMOIDAE Gray, 1825. gb.f007-13

JU: Nomen presented expressly (p. 141 sq., 175) as unranked

C: $\mathbf{A H}$

«RANOIDES» Frost ${ }^{+18}$, 2006.fa.e33 • DI

SI: 058

PA: 00 - RANOIDES $・$ Frost $^{+18}$ 2006.fa: 7, $223 \cdot$ Tx

HH: RANACEA Wilbrand, 1814.wa.c01 RANINA Batsch, 1796.ba.f001

GT:ECosTATA 1879.1b.c04

GASTRECHMIA 1867.ca.c02

Pananura DOP.da.c07

SY: TEO. C.10.03. Phalanx Scoptanura Starrett, 1973. sb.c02-02

JU: Nomen presented expressly (p. 141 sq., 223) as unranked

C: $\mathbf{A H}$

«SAliEnTIA» de Queiroz ${ }^{+1}$, 1992.qa.e01・ND

SI: 009

PA: 00 - SAlientia • De Queiroz ${ }^{+1}$ 1992.qa: $474 \cdot$ Cd

HH: SALIENTIA Laurenti, 1768.la.c01

GT:ANURA 1805.da.c01

SY: KYR. C.04.01. Ordo ANURA Duméril, 1805.da.c01-07

JU: Unranked nomen presented expressly (p. 475) as a "stem-based name"

C: $\mathbf{A H}$
«SAlientia» Ford ${ }^{+1}$, 1993.fa.e10・DI

SI: 022

PA: 00 • SALIENTIA $\bullet$ Ford $^{+1}$ 1993.fa: $94 \cdot \mathbf{C d}$

HH: SALIENTIA Laurenti, 1768.la.c01

GT:ANURA 1805.da.c01

SY: KYR. C.04.01. Ordo AnURa Duméril, 1805.da.c01-07

JU: Unranked nomen presented expressly (p. 99) as a "stembased name"

C: $\mathbf{A H}$

«SAukrobatrachia» Frost ${ }^{+18}$, 2006.fa.e43 • DI

SI: 067

PA: 00 • SAUKROBATRACHIA $・$ Frost $^{+18}$ 2006.fa: 7, $241 \bullet$ Tx

HH: INR

GT:RANOIDAE 1796.ba.f001

SY: KYR. F.15.10. Epifamilia RANOIDAE Batsch, 1796. ba.f001-29

JU: Nomen presented expressly (p. 141 sq., 241) as unranked

C: $\mathbf{A H}$

«SCOPTANURA» Ford ${ }^{+1}$, 1993.fa.e09 • DI

SI: 021

PA: $00 \bullet$ SCOPTANURA $\bullet$ Ford $^{+1}$ 1993.fa: $94 \cdot \mathbf{C d}$

HH: SCOPTANURA Starrett, 1973.sb.c02

GT:ECOSTATA 1879.1b.c04

SY: KYR. C.11.03. Subphalanx Ecostata Lataste, 1879. lb.c04-01

JU: Unranked nomen presented expressly (p. 114) as a "stem-based name"

C: $\mathbf{A H}$

«SHELAnIINAE» Aranciaga Rolando ${ }^{+2}$, 2019.aa.e01・DI SI: 092

PA: $00 \bullet$ ShelaniInae $\bullet$ Aranciaga Rolando ${ }^{+2}$ 2019.aa: 727 - Cd

HH: INR

GT: $4 \mathbf{G}^{\dagger}$

SY: SALTENIINAE DOP.da.f148 †

JU: Unranked nomen presented expressly (p. 727) as the nomen of a "stem-based clade"

C: $\mathbf{A H}$

«SOKOLANURA» Frost ${ }^{+18}$, 2006.fa.e14・DI

SI: 038

PA: $00 \cdot$ SOKOLANURA $・$ Frost $^{+18}$ 2006.fa: $6,183 \cdot$ Tx

HH: INR

GT:GEOBATRACHIA 1828.ra.c18

Mediogyrinia 1878.1a.c02

SY: KYR. C.05.02. Subordo Hydrobatrachia Ritgen, 1828.ra.c14-01

JU: Nomen presented expressly (p. 141 sq., 183) as unranked

C: $\mathbf{A H}$

«STEgokrotaphia» Cannatella ${ }^{+1}$, 1993.ca.e02 • ND

SI: 012

PA: 00 • StEGOKROTAPHiA • Cannatella ${ }^{+1}$ 1993.ca: 2 • Cd

HH: INR

GT:PSEUDOPHIONA 1816.ba.c11

SY: KYR. C.05.04. Subordo Pseudophiona de Blainville, 
1816.ba.c11-06

JU: Unranked nomen presented expressly (p. 2) as a "nodebased name"

C: $\mathbf{A H}$

«STEGOKROTAPHIA» Frost $^{+18}$, 2006.fa.e02 • DI

SI: 026

PA: 00 • STEGOKROTAPHIA • Frost ${ }^{+18}$ 2006.fa: 5, $166 \bullet$ Tx

HH: INR

GT:PSEUDOPHIONA 1816.ba.c11

SY: KYR. C.05.04. Subordo Pseudophiona de Blainville, 1816.ba.c11-06

JU: Nomen presented expressly (p. 141 sq., 166) as unranked

C: $\mathbf{A H}$

«TelmatobatraChIA» Frost $^{+18}$, 2006.fa.e40 • DI

SI: 064

PA: 00 • Telmatobatrachia $・$ Frost $^{+18}$ 2006.fa: 7, $236 \bullet$ Tx

HH: INR

GT:ECAUDATA 1777.sa.c06

SY: KYR. C.12.03. Infraphalanx ECAUDATA Scopoli, 1777. sa.c06-01

JU: Nomen presented expressly (p. 141 sq., 236) as unranked

C: $\mathbf{A H}$

«TEMnOSPONDYLI» Queiroz ${ }^{+1}$, 1992.qa.e01 • ND

SI: 001

PA: 00 • TemnOSPONDYLI • Queiroz ${ }^{+1}$ 1992.qa: $474 \cdot$ Cd

HH: TeMnOSPONDYLI Zittel, 1888.za.c01

GT:AMPHIBIA 1816.ba.c02

SY: KYR. C.02.01. Classis AmpHibia Blainville, 1816. ba.c02-03

JU: Unranked nomen presented expressly (p. 475) as a "stem-based name"

C: $\mathbf{A H}$

«TERESOMATA» Wilkinson ${ }^{+1}$, 2006.wa.e03 • DI

SI: 075

PA: $00 \bullet$ Teresomata $・$ Wilkinson ${ }^{+1}$ 2006.wa: $46 \cdot \mathbf{T x}$

HH: INR

GT: CAECILIOIDEA Rafinesque, 1814.ra.f003-|Gray, 1825. gb.f008|

SY: KYR. F.14.15. Superfamilia CAECILIOIDEA Rafinesque, 1814.ra.f003-|Gray, 1825.gb.f008|-18

JU: Nomen presented expressly (p. 47) as unranked

C: $\mathbf{A H}$

«TERraranA» Hedges ${ }^{+2}$, 2008.ha.e01 • DI

SI: 076

PA: $00 \cdot$ Terrarana - Hedges $^{+2}$ 2008.ha: $1 \cdot$ Tx

$01 \cdot$ Terraranae $\bullet$ Dubois 2009.da: $171 \cdot \mathbf{U U}$

02 • Terranae • Dubois 2009.da: $171 \cdot \mathbf{U U}$

\section{HH: INR}

GT:GAIANURA DOP.da.c06

SY: KYR. C.13.01. Hypophalanx GaIANURA nov., DOP. da.c06-00

JU: Nomen presented expressly (p. 11, 21) as unranked

C: $\mathbf{A H}$
«TERrarana» Heinicke ${ }^{+5}$, 2009.ha.e01 • DI

SI: 080

PA: 00 - Terrarana $\bullet$ Heinicke ${ }^{+5}$ 2009.ha: 1, 5 • Tx

01 Terraranae $\bullet$ Duellman $^{+2}$ 2016.db: $8 \cdot \mathbf{T x}$

HH: «TERRARANA» Hedges ${ }^{+2}$, 2008.ha.e01

GT:GAIANURA DOP.da.c06

SY: KYR. C.13.01. Hypophalanx GaIANURA nov., DOP. da.c06-00

JU: Nomen presented expressly (p. 5) as unranked, with an etymology different from that of «TERRARANA» Hedges $^{+2}$, 2008.ha.e01, therefore resulting in the introduction of a new nomen, junior homonym of the latter

C: $\mathbf{A H}$

«TinctanURA» Frost ${ }^{+18}$, 2006.fa.e26・DI

SI: 050

PA: $00 \bullet$ TinCtanURA $・$ Frost $^{+18}$ 2006.fa: 6 , 201 • Tx

HH: INR

GT:Phoranura DOP.da.c04

PhrYNanura DOP.da.c05

SY: KYR. C.11.01. Subphalanx BAINANURA nov., DOP. da.c03-00

JU: Nomen presented expressly (p. 141 sq., 201) as unranked

C: $\mathbf{A H}$

«TREPTOBRANCHIA» Frost ${ }^{+18}$, 2006.fa.e09 • DI

SI: 033

PA: 00 • TREPTOBRANChia • Frost ${ }^{+18}$ 2006.fa: 5, 173 • Tx

HH: INR

GT:SALAMANDROIDEA 1820.ga.f002

SY: KYR. F.14.18. Superfamilia SALAMANDROIDEA Goldfuss, 1820.ga.f002-21

JU: Nomen presented expressly (p. 141 sq., 173) as unranked

C: $\mathbf{A H}$

«URODELA» de Queiroz ${ }^{+1}$, 1992.qa.e01 • ND

SI: 007

PA: 00 • URODELA • De Queiroz ${ }^{+1}$ 1992.qa: $474 \cdot$ Cd

HH: UrodÈLES Duméril, 1805.da.c02

GT:URODELA 1805.da.c02

SY: KYR. C.04.03. Ordo Urodela Duméril, 1805.da.c0212

JU: Unranked nomen presented expressly (p. 475) as a "stem-based name"

C: $\mathbf{A H}$

«VictoranURA» Frost ${ }^{+18}$, 2006.fa.e39・DI

SI: 063

PA: $00 \bullet$ ViCTORANURA $・$ Frost $^{+18}$ 2006.fa: 7, 235 • Tx

HH: INR

GT:ECAUDATA 1777.sa.c06

SY: KYR. C.12.03. Infraphalanx ECAUdATA Scopoli, 1777. sa.c06-01

JU: Nomen presented expressly (p. 141 sq., 235) as unranked

C: $\mathbf{A H}$ 
«Xenoanura» Frost $^{+18}$, 2006.fa.e13・DI

SI: 037

PA: 00 - XenOANURA $・$ Frost $^{+18}$ 2006.fa: 6, 181 • Tx

HH: XeNOANURA Starrett, 1973.sb.c01

GT:DORSIPARES 1816.ba.c06

SY: KYR. C.07.01. Hypoordo DorsIPARES de Blainville, 1816.ba.c06-02

JU: Nomen presented expressly (p. 141 sq., 181) as unranked

C: $\mathbf{A H}$

«Xenopodinomorpha» Báez ${ }^{+1}$, 2003.ba.e02 • ND

SI: 024

PA: 00 • XENOPODINOMORPHA • Báez ${ }^{+1}$ 2003.ba: 454 • Cd

HH: INR

GT:DACTYLETHRINI 1838.ha.f001

SY: KYR. F.19.01. Tribus DACTYLEThrini Hogg, 1838. ha.f001-05

JU: Unranked nomen presented expressly (p. 464) as a "stem-based name"

C: $\mathbf{A H}$

«XenosalamandroIDEI» Frost ${ }^{+18}$, 2006.fa.e11 • DI

SI: 035
PA: 00 - XenosalamandroideI • Frost $^{+18}$ 2006.fa:5, 176

$$
\text { - } \mathbf{T x}
$$

HH: INR

GT:AMPHIUMIDAE 1825.gb.f007 PLETHODONTIDAE 1850.ga.f001

SY: KYR. F.16.08. Apofamilia AMPHIUMEIDAE Gray, 1825. gb.f007-13

JU: Nomen presented expressly (p. 141 sq., 176) as unranked

C: $\mathbf{A H}$

«XenOSYneUnitanURA» Frost ${ }^{+18}$, 2006.fa.e36 • DI

SI: 060

PA: $00 \bullet$ XenOSYNEUnitANURA $\bullet$ Frost $^{+18}$ 2006.fa: 7, $231 \cdot$ Tx

HH: INR

GT:BREVICIPITOIDEA 1850.bb.f012

SY: KYR. F.14.09. Superfamilia BREVICIPITOIDEA Bonaparte, 1850.bb.f012-09

JU: Nomen presented expressly (p. 141 sq., 231) as unranked 
Appendix A9.CLAD-1. Complete cladonomy and nomenclature of LISSAMPHIBIA proposed here

Hierarchy adopted in this taxonomy:

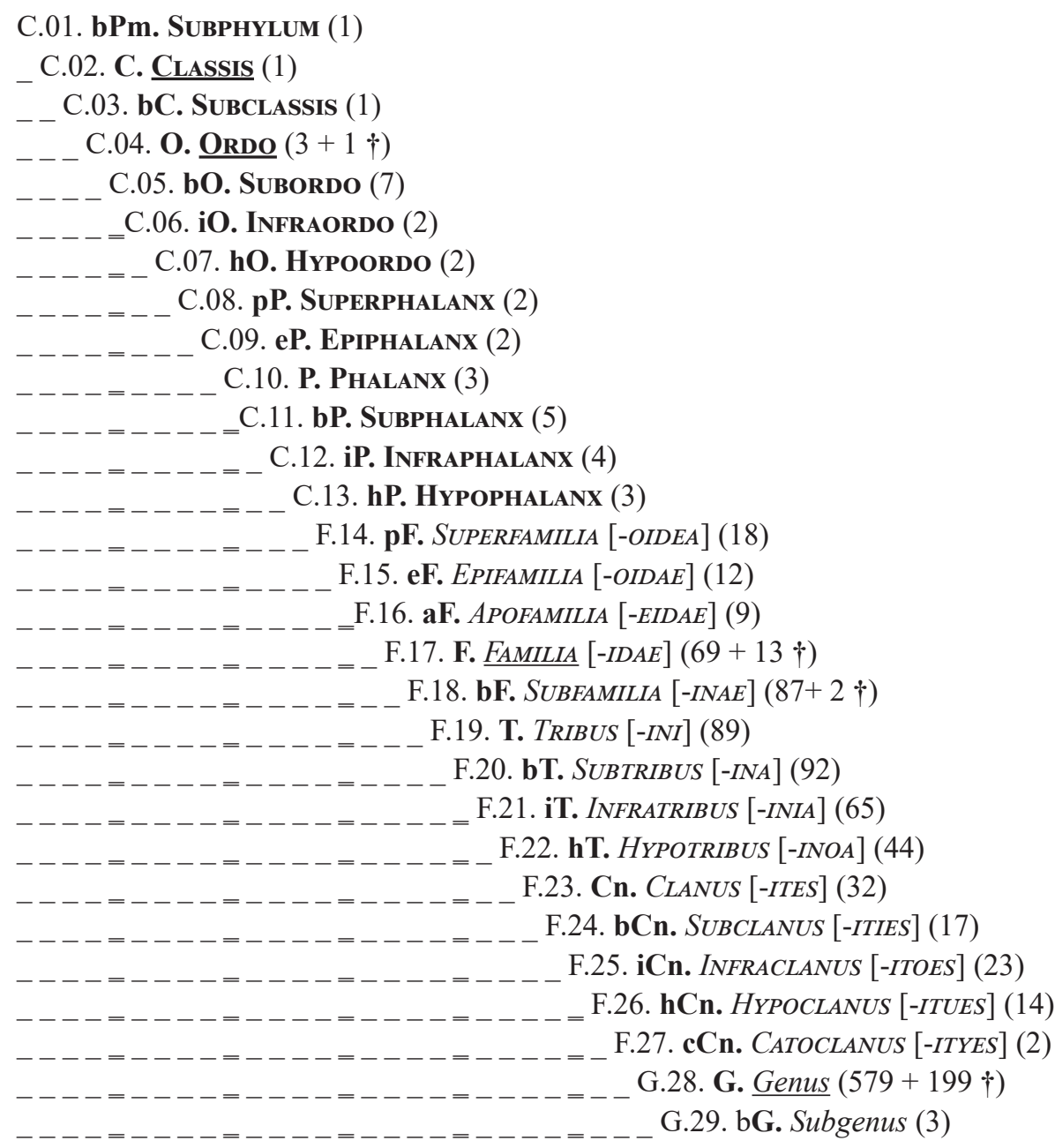

Nomina are numbered sequentially in each rank, in the order of their appearance in this table. Each rank is designated by a letter and a number, as above. The number of each all-fossil taxon is preceded by the sign $\uparrow$. The numbers of taxa referred to only by anoplonyms or anecdidonyms but for which no hoplonyms were ever proposed are immediately followed by the sign $\S$.

Two kinds of identifiers are used below, for taxa and for nomina:

[1] Identifiers of taxa recognised as valid in this work (e.g. C.01.01) precede the nomina of the taxa. They start with capital letters referring to the nominal-series at stake (C, class-series; F, family-series; $\mathrm{G}$, genus-series), followed by two numbers: the first one designates the rank (see hierarchy above) and the second one the sequential number (by order of appearance in the document). Two distinct sequences of numbers are used, one for all-fossil taxa (preceded by $\uparrow$ ) and one for taxa that include at least one recent species (without $\uparrow$ ).

[2] Identifiers of nomina (e.g. 1816.ba.c02-03) follow the authors of the nomina of the taxa. They start with an identifier of the publication where they were established (see our section 'References'), followed by the sequential number of the paronym at stake (see respectively Apendices A7.NCS, A6.NFS and A5.NGS).

Taxa are presented below strictly by alphabetical order within ranks.

Nomina underlined are nomina used at mandatory suprageneric ranks (classis, ordo, familia). 
Criteria for assignment of a taxon to the rank familia, indicated between brackets after the valid nominal-complex of the family:

[M] 'Mandatory Rank Criterion', which imposes the use of this rank for this taxon even if this makes it redundant with its superordinate taxon.

[N] 'Non-Redundancy Criterion'.

$[\mathrm{P}]$ 'Conflict of Precedence Criterion'.

[Q] 'Upper Quartile Criterion'. [Q+] 'Upper Quartile Criterion’ with > 90 \% usage after 1999. [Q-] 'Upper Quartile Criterion' with $0 \%$ usage after 1999.

[S] 'Sister-Taxa Criterion'.

[T] 'Nomenclatural Thrift Criterion'.

All taxa recognised here on the basis of our molecular TREE have a SHL-aLRT support value of $90 \%$ or more. Taxa including a single getendotaxon (taxon of just lower rank) have no support, but are recognised if they are parordinate (sister-taxa) to taxa having a support of $\{\mathbf{9 0}\}$ or more. All-fossil taxa have no support but are recognised on the basis of the literature of the groups at stake, just like some recent taxa that have no representative in TREE.

Generic nomina listed are those considered valid in this work. They are followed by their nucleospecies (type species) between parentheses, then by their synonyms, including unavailable ones, followed by their nucleospecies between parentheses, and finally by their support in TREE, in bold between braces, e.g. \{97\}. However the list does not mention most generic apographs (subsequent spellings) that do not clearly qualify as autoneonyms (see Tables T7.NS-1 and T8.NS-2, and Appendix A5.NGS), i.e. ameletographs (incorrect subsequent spellings), except when the latter have been used as nucleogenera of FS nomina or conucleogenera of CS nomina.

Indications regarding species-series nomina:

* The nucleospecies (nominal type species) of the genus is represented in TREE: Rana temporaria*.

- The nucleospecies of the genus is not represented in TREE: Leptobrachella mjobergi ${ }^{\circ}$.

Indications regarding genus-series nomina:

* The genus is represented in TREE by its nucleospecies or an isonym (objective synonym) of the latter: Rana*.

${ }^{1}$ The genus is represented in TREE by a doxisonym (subjective synonym) of its nucleospecies: Pipa ${ }^{1}$.

${ }^{2}$ The genus is represented in TREE by the nucleospecies of a generic nomen being its doxisonym: Leptobrachella ${ }^{2}$.

${ }^{3}$ The genus is represented in TREE but only by species that include neither its nucleospecies, nor a doxisonym of the latter, nor the nucleospecies of a doxisonym of the generic nomen at stake: Latonia ${ }^{3}$.

- The genus is not represented in TREE: Adelastes ${ }^{\circ}$.

Generic nomina which are invalid synonyms are presented after the valid nomen of the genus:

[1] preceded by the sign $\equiv$ if they are isonyms (objective synonyms): Rana $1758 \equiv$ Ranaria 1814 ;

[2] preceded by the sign $\approx$ if they are doxisonyms (subjective synonyms): Alytes $1829 \approx$ Baleaphryne 1979.

Nomina of nucleospecies which are invalid synonyms are followed by their valid nomen:

[1] preceded by the sign $\equiv$ if they are isonyms (objective synonyms): Hemiphractus 1828 (spixii 1828 $\equiv$ scutatus* 1824);

[2] preceded by the sign $\approx$ if they are doxisonyms (subjective synonyms): Ichthyosaura 1801 (tritonius $1768 \approx$ alpestris* 1768).

Two genus-series isonyms have by definition the same nucleospecies, so in this table the latter is not mentioned again after the nomen of the junior isonym.

The abbreviation NINS means 'no included nominal species'.

Generic and specific nomina followed by AN are anoplonyms (unavailable nomina).

Generic and specific nomina followed by AM are anecdidonyms (taxonomically unassigned available nomina).

Generic and specific nomina followed by CI are archakyronyms (resulting from action of the Commission) under CLAD.

Generic and specific nomina followed by RI are lethakyronyms.

Generic nomina followed by $\mathbf{J H}$ are preoccupied by senior homonyms.

$\mathbf{A M} \cdot$ Unavailable GS ameletograph (incorrect subsequent spelling) of lissamphibian taxon resulting from unvolontary change of spelling of original protograph.

AN - Unavailable GS nomen (anoplonym) of lissamphibian taxon for failing to comply with the criteria of availability of publications or of nomina of the Code. 
LT - GS lectoprotograph (correct original spelling) of an available lissamphibian GS nomen, resulting from an airesy (first reviser action) among symprotographs (multiple original spellings).

LP • Unvailable GS leipoprotograph (incorrect original spelling) of an available lissamphibian GS nomen resulting from an airesy (first reviser action) among symprotographs (multiple original spellings).

C.01.01. Subphylum VERTEBRATA Cuvier, 1800.ca.c01-02

C.02.01. Classis AMPHIBIA Blainville, 1816.ba.c02-03

_ C.03.01. Subclassis Lissamphibia Gadow, 1898.ga.c01-00

C.04.†00. Ordo INCERTAE SEDIS

G.28.†001§. Archaeoovulus ${ }^{\circ} 2013 \dagger$ (palenae $\left.^{\circ} 2013 \dagger\right)$ AM

G.28.001§. Cephaloloxes $^{\circ} 1848$ NT-AN-AP (NINS) $\equiv$ Cosmus $^{\circ} 1848$ AN-AP-JH

G.28.002§. Gryphius ${ }^{\circ} 1848$ NT-AN-AP (NINS) $\equiv$ Scotobius $^{\circ} 1848$ AN-AP-JH

C.04. $\dagger 01$. Ordo Allocaudata Fox ${ }^{+1}$, 1982.fa.c01-00 $\dagger$

F.17.†01. Familia ALBANERPETIDAE Fox ${ }^{+1}$, 1982.fa.f001-04 †

G.28.†002. Albanerpeton $^{\circ} 1976 \dagger$ (inexpectatum $^{\circ} 1976 \dagger$ )

G.28.†003. Anoualerpeton ${ }^{\circ} 2003 \dagger$ unicus $\left.^{\circ} 2003 \dagger\right)$

G.28.†004. Celtedens $s^{\circ} 1995 \dagger\left(\right.$ megacephalus $\left.{ }^{\circ} 1864 \dagger\right)$

G. $28 . \dagger 005$. Nukusurus $^{\circ} 1981 \dagger$ (insuetus $^{\circ} 1981 \dagger$ )

G.28.†006. Shirerpeton ${ }^{\circ} 2018 \dagger\left(\right.$ isajii $\left.^{\circ} 2018 \dagger\right)$

G.28.†007. Wesserpeton ${ }^{\circ} 2013 \dagger\left(\right.$ evansae $\left.^{\circ} 2013 \dagger\right)$

C.04.01. Ordo ANURA Duméril, 1805.da.c01-07 \{100\}

C.05.†0a. Subordo INCERTAE SEDIS †

F.17.†0a. Familia INCERTAE SEDIS $\dagger$

G.28. $\dagger$ 008. Altanulia $^{\circ} 1993 \dagger$ (alifanovi $\left.^{\circ} 1993 \dagger\right)$

G.28.†009§. Amphirana ${ }^{\circ} 1856 \uparrow$ AN (palustris ${ }^{\circ} 1856 \uparrow$ AN)

G.28.†010. Aralobatrachus $^{\circ} 1981 \dagger$ (robustus $\left.^{\circ} 1981 \dagger\right)$

G.28.†011. Arariphrynus $^{\circ} 2006 \dagger$ (placido $\left.^{\circ} 2006 \dagger\right)$

G.28. $\uparrow 012 \S$. Archipelobates $^{\circ} 1970 \dagger \mathbf{A N}$ (giganteum $\left.{ }^{\circ} 1970 \dagger \mathbf{A N}\right)$

G.28.†013. Aygrou $^{\circ} 2003 \dagger\left(\right.$ anoualensis $\left.^{\circ} 2003 \dagger\right)$

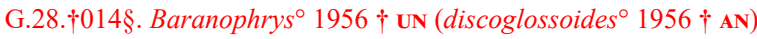

G.28.†015. Batrachulina ${ }^{\circ} 1962 \dagger$ (lemanensis $\left.^{\circ} 1853 \dagger\right) \equiv$ Batrachus $1853 \mathbf{J H}$

G.28.†016. Comobatrachus ${ }^{\circ} 1960 \dagger\left(\right.$ aenigmatis $\left.^{\circ} 1960 \dagger\right)$

G.28.†017. Cratia $^{\circ} 2009 \dagger\left(\right.$ gracilis $\left.^{\circ} 2009 \dagger\right)$

G.28.†018. Czatkobatrachus ${ }^{\circ} 1998 \dagger$ (polonicus $\left.^{\circ} 1998 \dagger\right)$

G.28.†019. Eobatrachus ${ }^{\circ} 1887 \dagger\left(\right.$ agilis $\left.^{\circ} 1887 \dagger\right)$

G.28.†020. Eorubeta $^{\circ} 1960 \dagger\left(\right.$ nevadensis $\left.^{\circ} 1960 \dagger\right)$

G.28. $\dagger 021$. Estesiella ${ }^{\circ} 1995 \dagger$ (boliviensis $\left.^{\circ} 1991 \dagger\right) \equiv$ Estesius $1991 \mathbf{J H}$

G.28. $\uparrow 022$. Estesina $^{\circ} 1993 \dagger\left(\right.$ elegans $\left.^{\circ} 1993 \dagger\right)$

G.28. $\dagger 023$. Eurycephalella ${ }^{\circ} 2009 \dagger\left(\right.$ alcinae $\left.^{\circ} 2009 \dagger\right)$

G.28.†024. Gobiatoides ${ }^{\circ} 1993 \dagger$ (parvus $\left.^{\circ} 1993 \dagger\right)$

G.28.†025. Hatzegobatrachus ${ }^{\circ} 2003 \dagger\left(\right.$ grigorescui $\left.^{\circ} 2003 \dagger\right)$

G.28.†026. Hensonbatrachus ${ }^{\circ} 2015 \dagger\left(\right.$ kermiti $\left.^{\circ} 2015 \dagger\right)$

G.28. $\dagger 027$. Iberobatrachus ${ }^{\circ} 2013 \dagger\left(\right.$ angelae $\left.^{\circ} 2013 \dagger\right)$

G.28. $\dagger 028$. Itemirella $1981 \dagger\left(\right.$ cretacea $\left.^{\circ} 1981 \dagger\right)$

G.28.†029. Liaobatrachus ${ }^{\circ} 1998 \dagger\left(\right.$ grabaui $\left.^{\circ} 1998 \dagger\right)$

G.28.†030. Liventsovkia $1993 \dagger\left(\right.$ jucunda $\left.{ }^{\circ} 1993 \dagger\right)$

G.28.†031. Lutetiobatrachus ${ }^{\circ} 1998 \dagger$ (gracilis $\left.^{\circ} 1998 \dagger\right)$

G.28.†032. Mengbatrachus $2018 \dagger\left(\right.$ moqi $\left.^{\circ} 2018 \dagger\right)$

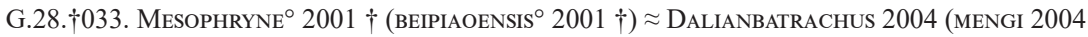

$\approx$ BEIPIAOENSIS $\left.^{\circ} 2001 \dagger\right)$

G.28.†034. Monsechobatrachus ${ }^{\circ} 1921 \dagger\left(\right.$ gaudryi $\left.^{\circ} 1902 \dagger\right) \equiv$ Montsechobatrachus 1926 AM

G.28.†035. Negatchevkia ${ }^{\circ} 1993 \dagger\left(\right.$ donensis $\left.^{\circ} 1993 \dagger\right)$

G.28.†036. Novooskolia $1993 \dagger\left(\right.$ cristata $\left.^{\circ} 1993 \dagger\right)$

G.28.†037. Procerobatrachus $^{\circ} 1993 \dagger$ paulus $\left.^{\circ} 1993 \dagger\right)$

G.28.†038§. Protophrynus $^{\circ} 1853 \uparrow \mathbf{A N - A P}\left(\right.$ arethusae $\left.^{\circ} 1853 \uparrow \mathbf{A N}\right) \equiv$ Protophrynos 1888 NT-AP 
G.28.†039. Ranipes $^{\circ} 2014 \dagger\left(\right.$ laci $\left.^{\circ} 2014 \dagger\right) \equiv$ Ranapes 2014 AN

G.28.†040. Ranomorphus ${ }^{\circ} 1993 \dagger\left(\right.$ similis $\left.^{\circ} 1993 \dagger\right)$

G.28.†041. Saevesoederberghia ${ }^{\circ} 1993 \uparrow\left(\right.$ egredia $\left.^{\circ} 1993 \uparrow\right)$

G.28.†042. Scotiophryne $1969 \dagger\left(\right.$ pustulosa $\left.^{\circ} 1969 \dagger\right)$

G.28.†043§. Spondylophryne ${ }^{\circ} 1956+$ AN (vilanyensis ${ }^{\circ} 1856 \dagger \mathbf{A N}$ )

G.28.†044. Sunnybatrachus ${ }^{\circ} 2002 \dagger$ (purbeckensis $\left.{ }^{\circ} 2002 \dagger\right)$

G.28.†045. Thaumastosaurus $^{\circ} 1904 \uparrow\left(\right.$ bottii $\left.^{\circ} 1904 \uparrow\right) \equiv$ Enigmatosaurus 1908

G.28.†046. Theatonius $^{\circ} 1976 \uparrow$ (lancensis $\left.^{\circ} 1976 \dagger\right)$

G.28.†047. Tyrrellbatrachus ${ }^{\circ} 2015 \dagger\left(\right.$ brinkmani $\left.^{\circ} 2015 \dagger\right)$

G.28.†048. Uberabatrachus $^{\circ} 2012 \dagger\left(\right.$ carvalhoi $\left.^{\circ} 2012 \dagger\right)$

G.28.†049. Varibatrachus ${ }^{\circ} 2015 \dagger\left(\right.$ abraczinskasae $\left.^{\circ} 2015 \dagger\right)$

G.28.†050. Vieraella $1961 \dagger\left(\right.$ herbstii $\left.^{\circ} 1961 \dagger\right) \equiv$ Vierella 1962 AM $\equiv$ Vierella 2015

G.28.†051. Yizhoubatrachus ${ }^{\circ} 2004 \uparrow$ (macilentus $\left.^{\circ} 2004 \uparrow\right)$

G.28.003§. Sciaphos ${ }^{\circ} 1845$ AN-AP (NINS)

F.17.†02. Familia PROSALIRIDAE Shubin $^{+1}$, 1995.sa.f001-00 $\dagger$

G.28.†052. Prosalirus ${ }^{\circ} 1995 \dagger\left(\right.$ bitis $\left.^{\circ} 1995 \dagger\right)$

F.17.†03. Familia TREGOBATRACHIDAE Holman, 1975.hb.f001-00 $\dagger$

G.28.†053. Tregobatrachus ${ }^{\circ} 1975 \dagger\left(\right.$ hibbardi $\left.^{\circ} 1974 \dagger\right)$

F.17.†04. Familia TRIADOBATRACHIDAE Kuhn, 1962.ka.f001-00 †

G.28.†054. Triadobatrachus ${ }^{\circ} 1962 \dagger\left(\right.$ massinoti $\left.^{\circ} 1936 \dagger\right) \equiv$ Protobatrachus $1936 \mathbf{J H}$

C.05.01. Subordo ANGuSTicoela Reig, 1958.ra.c01-00 \{100\}

F.17.01. Familia ASCAPHIDAE Fejérváry, 1923.fa.f001-00 \{100\} [S] [N]

G.28.004. Ascaphus* 1899 (truei* 1899)

F.17.02. Familia LEIOPELMATIDAE Mivart, 1869.ma.f007-|Turbott, 1942.ta.f001|-02 \{100\} [Q]

F.18.†01. Subfamilia NotobatraChinaE Reig in Stipanicic ${ }^{+1}$, 1956.sa.f001-02 †

G.28.†055. Notobatrachus ${ }^{\circ} 1956 \dagger$ (degiustoi $\left.^{\circ} 1956 \dagger\right)$

F.18.01. Subfamilia LEIOPELMATINAE Mivart, 1869.ma.f007-|Turbott, 1942.ta.f001|-03 \{100\}

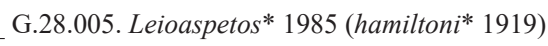

G.28.006. Leiopelma* 1861 (hochstetteri* 1861) $\equiv$ Liopelma 1865 AM $\equiv$ Liopelma 1869 NC-CI

C.05.02. Subordo Hydrobatrachia Ritgen, 1828.ra.c14-01 $\{100\}$

F.17.†0b. Familia INCERTAE SEDIS †

G.28.†056. Hyogobatrachus ${ }^{\circ} 2016 \dagger\left(\right.$ wadai $\left.^{\circ} 2016 \dagger\right)$

G.28.†057. Kururubatrachus ${ }^{\circ} 2020 b \dagger\left(\right.$ gondwanicus $\left.^{\circ} 2020 b \dagger\right) \equiv$ Kururubatrachus $2020 a$ AN $^{\circ}$

G.28.†058. Tambabatrachus ${ }^{\circ} 2016 \dagger\left(\right.$ kawazu $\left.^{\circ} 2016 \dagger\right)$

G.28.†059. Wealdenbatrachus ${ }^{\circ} 1988 \dagger$ (jucarensis $\left.^{\circ} 1988 \dagger\right)$

G.28.007§. Ranina ${ }^{\circ} 1839$ (NINS) AM

C.06.01. Infraordo GeObatraChIA Ritgen, 1828.ra.c18-02 \{98\}

F.17.†0c. Familia INCERTAE SEDIS †

G.28.†060. Genibatrachus ${ }^{\circ} 2017 \dagger$ (baoshanensis $\left.^{\circ} 2017 \dagger\right)$

C.07.01. Hypoordo Dorsipares Blainville, 1816.ba.c06-02 \{100\}

F.17.†0d. Familia INCERTAE SEDIS †

G.28.†061. Avitabatrachus ${ }^{\circ} 2000 \dagger\left(\right.$ uliana $\left.^{\circ} 2000 \dagger\right)$

G.28.†062. Gracilibatrachus ${ }^{\circ} 2013 \dagger$ (avallei $\left.^{\circ} 2013 \dagger\right)$

G.28.†063. Neusibatrachus ${ }^{\circ} 1972 \dagger\left(\right.$ wilferti $\left.^{\circ} 1972 \dagger\right)$

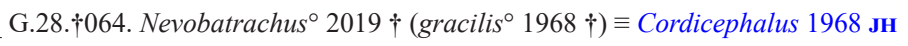

G.28.†065. Shomronella ${ }^{\circ} 1978 \dagger\left(\right.$ jordanica $\left.^{\circ} 1978 \dagger\right)$

G.28.†066. Thoraciliacus $^{\circ} 1968 †$ (rostriceps $\left.^{\circ} 1968 \uparrow\right)$

G.28.†067. Vulcanobatrachus ${ }^{\circ} 2005 \dagger\left(\right.$ mandelai $\left.^{\circ} 2005 \dagger\right)$

F.17.†05. Familia PALAEOBATRACHIDAE Cope, 1865.ca.f001-00 †

G.28.†068. Albionbatrachus ${ }^{\circ} 1984 \dagger$ (wightensis $\left.^{\circ} 1984 \dagger\right)$

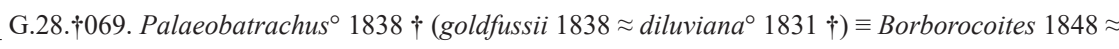

Protopelobates 1881 (gracilis $1881 \approx$ laubei $^{\circ} 1881 \dagger$ ) $\approx$ Pliobatrachus 1917 (langhae $\left.1917 \dagger\right) \approx$

Lithobatrachus 1929 (europaea $1929 \approx$ diluviana $^{\circ} 1831 \dagger$ ) $\approx$ Bufonopsis 1941 (dentatus $1941 \approx$

hinschei $^{\circ} 1941 \dagger$ ) $\approx$ Pelobatinopsis 1941 (hinschei ${ }^{\circ} 1941 \dagger$ ) $\approx$ Quinquevertebron 1941 (germanicum

$1941 \approx$ hinschei $\left.^{\circ} 1941 \dagger\right) \approx$ Hekatobatrachus 1972 (grandipes $\left.^{\circ} 1851 \dagger\right) \approx$ Suleobatrachus 1972

$\left(\right.$ laubei $\left.^{\circ} 1881 \dagger\right) \approx$ Messelobatrachus 1988 (tobieni $\left.i^{\circ} 1988 \dagger\right)$ 
G.28.†070. Probatrachus ${ }^{\circ} 1878 \dagger$ (vicetinus $^{\circ} 1877 \dagger$ )

F.17.03. Familia PIPIDAE Gray, 1825.ga.f003-|Fitzinger, 1826.fb.f002|-07 \{100\} [Q]

F.18.†0a. Subfamilia INCERTAE SEDIS $\dagger$

G.28.†071. Cratopipa ${ }^{\circ} 2019 b \dagger\left(\right.$ novaolindensis $\left.^{\circ} 2019 b \dagger\right) \equiv$ Cratopipa $2019 a$ AN

G.28. $\uparrow 072$. Eoxenopoides $^{\circ} 1931 \uparrow\left(\right.$ reuningi $\left.^{\circ} 1931 \dagger\right)$

G.28.†073. Llankibatrachus ${ }^{\circ} 2003 \uparrow\left(\right.$ truebae $\left.^{\circ} 2003 \dagger\right)$

G.28.†074. Oumtkoutia ${ }^{\circ} 2008+\left(\right.$ anae $\left.^{\circ} 2008 \dagger\right)$

G.28.†075. Pachycentrata ${ }^{\circ} 2004 \dagger\left(\right.$ taquet $\left.^{\circ} 1998 \dagger\right) \equiv$ Pachybatrachus $1998 \mathbf{J H}$

G.28.†076. Singidella $2005 \dagger\left(\right.$ latecostata $\left.^{\circ} 2005 \dagger\right)$

F.18.†02. Subfamilia SALTENIINAE nov., DOP.da.f148-00 †

G.28.†077. Kuruleufemia $2016 \dagger$ (xenopoides $^{\circ} 2016 \dagger$ )

G.28.†078. Patagopipa ${ }^{\circ} 2019 \dagger\left(\right.$ corsolinii $\left.^{\circ} 2019 \dagger\right)$

G.28.†079. Saltenia ${ }^{\circ} 1959 \dagger\left(\right.$ ibanezi $\left.^{\circ} 1959 \dagger\right)$

G.28.†080. Shelania $1960 \dagger\left(\right.$ pascuali $\left.^{\circ} 1960 \dagger\right)$

F.18.02. Subfamilia DACTYLETHRINAE Hogg, 1838.ha.f001-04 \{92\}

F.19.01. Tribus DACTYLETHRINI Hogg, 1838.ha.f001-05 \{100\} G.28.008. Silurana* 1864 (tropicalis* 1864) $\{\mathbf{1 0 0}\}$

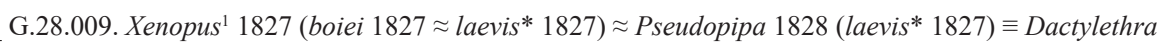

$1829 \equiv$ Rhaphidochir 1833 三 Dactyletra $1878 \equiv$ Doctylethra 1878 AM $\equiv$ Doctyletra 1878 AM $\approx$

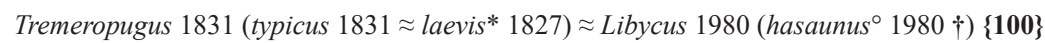

F.19.02. Tribus HYMENOCHIRINI Bolkay, 1919.ba.f001-01 \{100\}

G.28.010. Hymenochirus* 1896 (boettgeri* 1896)

G.28.011. Pseudhymenochirus* 1920 (merlini* 1920)

F.18.03. Subfamilia PIPINAE Gray, 1825.ga.f003-|Fitzinger, 1826.fb.f002|-13 $\{\mathbf{1 0 0}\}$

G.28.012. Pipa 1768 (americana $1768 \approx$ pipa $^{*} 1758$ ) $\equiv$ Piparius $1815 \equiv$ Pipra 1825 JH

$\equiv$ Asterodactylus $1827 \equiv$ Astrodactylus [1838] $1839 \equiv$ Leptopus $1835 \mathbf{J H} \approx$ Protopipa 1925 (aspera ${ }^{\circ}$

1924) ₹ Hemipipa 1937 (carvalhoi* 1937)

F.17.04. Familia RHINOPHRYNIDAE Günther, 1858.gc.f013-00 [Q]

G.28.†081. Chelomophrynus ${ }^{\circ} 1991 \dagger\left(\right.$ bayi $\left.^{\circ} 1991 \dagger\right)$

G.28.†082. Eorhinophrynus ${ }^{\circ} 1959 \dagger\left(\right.$ septentrionalis $\left.^{\circ} 1959 \dagger\right)$

G.28.†083. Rhadinosteus ${ }^{\circ} 1998 \dagger$ (parvus $\left.^{\circ} 1998 \dagger\right)$

G.28.013. Rhinophrynus* 1841 (dorsalis* 1841)

C.07.02. Hypoordo LAEVogYrinia Lataste, 1878.1a.c01-04 \{100\}

C.08.0a. Superphalanx INCERTAE SEDIS

F.17.†0e. Familia INCERTAE SEDIS †

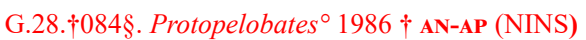

G.28.014. Colodactylus ${ }^{\circ} 1845$ (coerulescens $^{\circ} 1845$ )

C.08.01. Superphalanx ArCHaeOsalientia Roček, 1981.ra.c01-01 \{100\}

F.14.†0a. Superfamilia INCERTAE SEDIS †

F.17.†0f. Familia INCERTAE SEDIS †

G.28.†085. Elkobatrachus ${ }^{\circ} 2006 \dagger\left(\right.$ brocki $\left.^{\circ} 2006 \dagger\right)$

G.28.†086. Macropelobates $^{\circ} 1924 \uparrow\left(\right.$ osborni $\left.^{\circ} 1924 \dagger\right)$

G.28.†087. Tephrodytes ${ }^{\circ} 1994 \dagger$ (brassicarvalis $\left.^{\circ} 1994 \dagger\right)$

G.28.†088. Uldzinia ${ }^{\circ} 1996 \uparrow\left(\right.$ kurochkini $\left.^{\circ} 1996 \dagger\right)$

F.14.01. Superfamilia PELOBATOIDEA Bonaparte, 1850.bb.f004-11 \{98\}

F.15.01. Epifamilia PELOBATOIDAE Bonaparte, 1850.bb.f004-16 \{100\}

F.17.†0g. Familia INCERTAE SEDIS †

G.28.†089. Sanshuibatrachus ${ }^{\circ} 2017 \dagger\left(\right.$ sinensis $\left.^{\circ} 2017 \dagger\right)$

F.17.05. Familia MEGOPHRYIDAE Bonaparte, 1850.bb.f008-|Noble, 1931.na.f003|-04 \{100\} [Q+] [S]

F.18.04. Subfamilia LEPTOBRACHIINAE Dubois, 1983.db.f001-00 \{100\}

F.19.03. Tribus LEPTOBRACHIINI Dubois, 1983.db.f001-01 \{100\}

F.20.01. Subtribus LEPTOBRACHIINA Dubois, 1983.db.f001-02 \{92\}

G.28.015. Leptobrachium* 1838 (hasseltii* 1838) $\equiv$ Septobrachium 1838 AN $\approx$ Nireus $1880 \mathrm{JH}$

(pulcherrimus $1880 \approx$ hasseltii* 1838) $\approx$ Vibrissaphora 1945 (boringii* 1945)

F.20.02. Subtribus OREOLALAGINA Tian ${ }^{+1}$, 1985.ta.f001-02 \{97\}

G.28.016. Oreolalax* 1962 (pingii* 1943) $\approx$ Aelurolalax 1987 (weigoldi $\left.{ }^{\circ} 1924\right) \approx$ Atympanolalax $^{*} 016$ 
(rugosa* 1943) $\{\mathbf{1 0 0}\}$

G.28.017. Scutiger ${ }^{2} 1868$ (sikimmensis $\left.^{\circ} 1854\right) \equiv$ Cophophryne $1887 \equiv$ Cofofryne $1898 \approx$ Aelurophryne

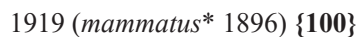

F.19.04. Tribus LEPTOLALAGINI Delorme ${ }^{+3}, 2006$. da.f001-01 $\{\mathbf{1 0 0}\}$

G.28.018. Leptobrachella ${ }^{2} 1925$ (mjobergi $\left.^{\circ} 1925\right) \approx$ Nesobia 1923 JH (natunae ${ }^{\circ} 1895$ )

$\approx$ Paramegophrys 1964 AN (pelodytoides* 1893) $\approx$ Carpophrys 1976 AN (oshanensis* 1950) $\approx$

Leptolalax 1980 (gracile* 1872) $\approx$ Lalax 2006 JH $\left(\right.$ bourreti $\left.^{*} 1983\right) \equiv$ Lalos 2010

F.18.05. Subfamilia MEGOPHRYINAE Bonaparte, 1850.bb.f008-|Noble, 1931.na.f003|-00 \{100\}

F.19.05. Tribus ATYMPANOPHRYINI nov., DOP.da.f001-00 \{97\}

G.28.019. Atympanophrys* 1983 (shapingensis* 1950) $\approx$ Borealophrys 2016 (nankiangensis* 1966)

$\approx$ Gigantophrys 2016 (giganticus $^{\circ} 1960$ )

F.19.06. Tribus BRACHYTARSOPHRYINI nov., DOP.da.f002-00 $\{\mathbf{1 0 0}\}$

G.28.020. Brachytarsophrys* 1983 (carinensis* 1899)

F.19.07. Tribus MEGOPHRYINI Bonaparte, 1850.bb.f008-|Noble, 1931.na.f003|-02 \{100\}

G.28.021. Megophrys ${ }^{2} 1822$ LT (montana $^{\circ}$ 1822) $\equiv$ Mogophrys 1822 LP $\equiv$ Megalophrys 1830

$\equiv$ Phrynophrys 1839 AN $\equiv$ Megalophys 1842 AM $\equiv$ Megalofrys $1898 \approx$ Ceratophryne $1859 \mathbf{J H}$

(nasuta* $^{1858)} \equiv$ Pelobatrachus $1908 \approx$ Borneophrys 2006 (edwardinae $\left.{ }^{\circ} 1989\right)$

F.19.08. Tribus XENOPHRYINI Delorme ${ }^{+3}$, 2006.da.f002-00 \{90\}

F.20.03. Subtribus GRILLITSCHIINA nov. DOP.da.f148-00 \{100\}

G.28.022. Grillitschia* nov. (longipes* 1886)

F.20.04. Subtribus OPHRYOPHRYNINA nov. DOP.da.f149-00 \{95\}

G.28.023. Boulenophrys* 2016 (boettgeri* 1899) $\approx$ Panophrys* 1997 JH (omeimontis* 1950)

$\approx$ Tianophrys 2016 (shuichengensis $\left.{ }^{\circ} 2000\right)\{\mathbf{9 0}\}$

G.28.024. Ophryophryne* 1903 (microstoma* 1903) $\{\mathbf{1 0 0}\}$

F.20.05. Subtribus XENOPHRYINA Delorme ${ }^{+3}, 2006$. da.f002-01 \{97\}

G.28.025. Xenophrys ${ }^{3} 1864$ (monticola $\left.^{\circ} 1864\right) \approx$ Liuophrys $_{2016}\left(\right.$ glandulosa $\left.^{\circ} 1990\right)$

F.17.06. Familia PELOBATIDAE Bonaparte, 1850.bb.f004-00 \{100\} [Q]

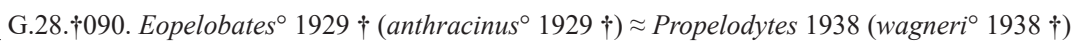

$\approx$ Amphignathodontoides 1941 (eocenicus $1941 \approx$ hinschei $^{\circ} 1941 \dagger$ ) $\approx$ Archaeopelobates 1941 (efremovi $1941 \approx$ hinsche $^{\circ} 1941 \dagger$ ) $\approx$ Eobufella 1941 (parvula $1941 \approx$ hinsche $^{\circ} 1941 \dagger$ ) $\approx$ Halleobatrachus 1941

(hinschei $1941 \dagger$ ) $\approx$ Palaeopelobates 1941 (geiseltalensis $1941 \approx$ hinschei $^{\circ} 1941 \dagger$ ) $\approx$ Parabufella 1941 (longipes $1941 \approx$ hinschei $^{\circ} 1941 \dagger$ )

G.28.026. Pelobates* 1830 (fuscus* 1768) $\approx$ Cultripes 1832 (cultripes* 1829) $\approx$ Arethusa 1838 AN-JH

(marmorata $1828 \approx$ fuscus $* 1768) \approx$ Didocus 1866 (calcarata $1830 \approx$ cultripes $\left.^{*} 1829\right) \approx$ Zaphrissa

1866 (eurypelis $1866 \approx$ decheni $^{\circ} 1861 \dagger$ ) $\approx$ Pseudopelobates 1958 (transcaucasicus $1928 \approx$ syriacus $*$

1889) $\{\mathbf{1 0 0}\}$

F.15.02. Epifamilia PELODYTOIDAE Bonaparte, 1850.bb.f002-04 \{100\}

F.17.07. Familia PELODYTIDAE Bonaparte, 1850.bb.f002-02 \{100\}[Q]

G.28.†091. Aerugoamnis ${ }^{\circ} 2013 \dagger\left(\right.$ paulus $\left.^{\circ} 2013 \dagger\right)$

G.28.†092. Miopelodytes ${ }^{\circ} 1941 \dagger\left(\right.$ gilmorei $\left.^{\circ} 1941 \dagger\right)$

G.28.027. Pelodytes* 1838 (punctata* 1802)-三Arethusa 1841 AN-JH \{99\}

G.28.028. Pelodytopsis* 1896 (caucasicus* 1896)

F.14.02. Superfamilia SCAPHIOPODOIDEA Cope, 1865.ca.f003-05 \{100\}

F.17.08. Familia SCAPHIOPODIDAE Cope, 1865.ca.f003-c0 \{100\} [M]

G.28.†093. Prospea $2016 \uparrow$ AN (holoserisca ${ }^{\circ} \uparrow 1863$ AN)

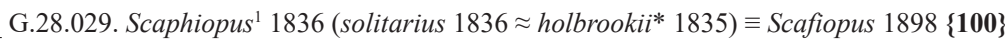

G.28.030. Spea* 1866 (bombifrons* 1863) $\approx$ Neoscaphiopus 1942 (noblei $\left.{ }^{\circ} 1941 \dagger\right)\{\mathbf{1 0 0}\}$

C.08.02. Superphalanx RANOMORPHA Fejérváry, 1921.fb.c08-01 \{100\}

C.09.01. Epiphalanx AQUIPAREs Blainville, 1816.ba.c07-02 \{100\}

C.10.01. Phalanx Gondwanura nov., DOP.da.c01-00 $\{\mathbf{1 0 0}\}$

F.17.09. Familia NASIKABATRACHIDAE Biju ${ }^{+1}$, 2003.bb.f001-00 [S] [N] G.28.031. Nasikabatrachus* 2003 (sahyadrensis* 2003)

F.17.10. Familia SOOGLOSSIDAE Noble, 1931.na.f002-01 \{100\} [Q]

G.28.032. Sechellophryne* 2007 (gardineri* 1911) 三Leptosooglossus $2007\{\mathbf{1 0 0}\}$

G.28.033. Sooglossus* 1906 (sechellensis* 1896) $\approx$ Nesomantis 1909 (thomasseti* 1909) \{98\}

C.10.02. Phalanx Phaneranura nov., DOP.da.c02-00 $\{\mathbf{1 0 0}\}$ 
C.11.01. Subphalanx BaInanura nov., DOP.da.c03-00 $\{\mathbf{1 0 0}\}$

C.12.01. Infraphalanx Phoranura nov., DOP.da.c04-00 $\{\mathbf{1 0 0}\}$

_ F.17.11. Familia AROMOBATIDAE Grant $^{+9}, 2006 . g b . f 001-00\{\mathbf{1 0 0}\}[\mathrm{S}][\mathrm{N}]$

F.18.06. Subfamilia ALLOBATINAE Grant ${ }^{+9}, 2006 . g b . f 006-00\{\mathbf{1 0 0}\}$

G.28.034. Allobates* 1988 (femoralis* 1884)

F.18.07. Subfamilia ANOMALOGLOSSINAE Grant ${ }^{+9}, 2006 . \mathrm{gb} . \mathrm{f002}-00\{\mathbf{9 8}\}$

G.28.035. Anomaloglossus* 2006 (beebei* 1923) $\{\mathbf{1 0 0}\}$

G.28.036. Rheobates* 2006 (palmatus* 1899)

F.18.08. Subfamilia AROмоватINAE Grant ${ }^{+9}, 2006$. gb.f001-01 $\{\mathbf{1 0 0}\}$

\left. _ G.28.037. Aromobates* $1991{\text { (nocturnus } * 1991) \approx \text { Nephelobates }^{*} 1994 \text { (alboguttatus }}^{\circ} 1903\right)\{\mathbf{1 0 0}\}$

G.28.038. Mannophryne* 1992 (yustizi* 1989) $\{\mathbf{1 0 0}\}$

F.17.12. Familia DENDROBATIDAE $\|$ Bonaparte, 1850.bb.f006\|-Cope, 1865.ca.f002-00 \{100\} [Q]

F.18.09. Subfamilia ColostethinaE Cope, 1867.ca.f001-01 $\{\mathbf{1 0 0}\}$

F.19.09. Tribus Colostethini Cope, 1867.ca.f001-02 \{98\}

G.28.039. Ameerega* 1986 (trivittata* 1824) $\equiv$ Paraphyllobates 1994 AN $\approx$ Pseudendrobates 1987

(silverstonei* 1979) $\equiv$ Phobobates $1988\{\mathbf{1 0 0}\}$

G.28.040. Colostethus* 1866 (latinasus* 1863) 三 Calostethus $1869 \equiv$ Colosthetus $1901 \approx$ Prostherapis

1868 (inguinalis* 1868) $\equiv$ Prostheraspis $1877\{\mathbf{1 0 0}\}$

G.28.041. Leucostethus* 2017 (argyrogaster* 1993) \{99\}

F.19.10. Tribus EPIPEDOBATINI nov., DOP.da.f003-00 $\{\mathbf{1 0 0}\}$

G.28.042. Epipedobates* 1987 (tricolor* 1899) $\{\mathbf{1 0 0}\}$

G.28.043. Silverstoneia* 2006 (nubicola* 1924) $\{\mathbf{1 0 0}\}$

F.18.10. Subfamilia DENDROBATINAE ||Bonaparte, 1850.bb.f006||-Cope, 1865.ca.f002-01 \{100\}

F.19.11. Tribus DENDROBATINI ||Bonaparte, 1850.bb.f006||-Cope, 1865.ca.f002-04 \{100\}

F.20.06. Subtribus ANDINOBATINA nov., DOP.da.f004-00 \{100\}

F.21.01. Infratribus ANDINOBATINIA nov., DOP.da.f004-01 $\{\mathbf{1 0 0}\}$ G.28.044. Andinobates* 2011 (bombetes* 1980) $\{\mathbf{1 0 0}\}$

G.28.045. Ranitomeya* 1985 (reticulatus* 1884) $\{\mathbf{1 0 0}\}$

F.21.02. Infratribus EXCIDOBATINIA nov., DOP.da.f005-00 \{100\}

G.28.046. Excidobates* 2008 (mysteriosus* 1982)

F.20.07. Subtribus DENDROBATINA ||Bonaparte, 1850.bb.f006||-Cope, 1865.ca.f002-05 \{91\} G.28.047. Adelphobates* 2006 (castaneoticus* 1990) $\{\mathbf{1 0 0}\}$

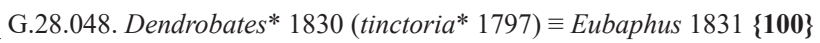

G.28.049. Minyobates* 1987 (steyermarki* 1971)

G.28.050. Oophaga* 1994 (pumilio* 1857) $\equiv$ Stemobates 1994 AN $\{\mathbf{1 0 0}\}$

_F.19.12. Tribus PHYLLOBATINI Fitzinger, 1843.fa.f007-03 \{100\}

G.28.051. Phyllobates* 1841 (bicolor* 1841)

F.18.11. Subfamilia HYLOXALINAE Grant ${ }^{+9}$, 2006.gb.f004-00 \{100\}

G.28.052. Ectopoglossus ${ }^{\circ} 2017$ (saxatilis $^{\circ}$ 2017)

G.28.053. Hyloxalus ${ }^{2} 1870$ (fuliginosus $^{\circ} 1870$ ) $\equiv$ Hylixalus $1882 \approx$ Phyllodromus 1875 (pulchellum $^{*}$

1875 ) $\approx$ Cryptophyllobates 2000 (azureiventris* 1985)

G.28.054. Paruwrobates ${ }^{\circ} 1994$ (andinus $^{\circ}$ 1987)

C.12.02. Infraphalanx PhrYnanura nov., DOP.da.c05-00 $\{\mathbf{1 0 0}\}$

C.13.01. Hypophalanx GaIANURA nov., DOP.da.c06-00 $\{\mathbf{1 0 0}\}$

F.17.13. Familia BRACHYCEPHALIDAE Günther, 1858.gc.f002-01 \{100\} [Q]

F.18.0a. Subfamilia INCERTAE SEDIS

G.28.055. Atopophrynus ${ }^{\circ} 1982$ (syntomopus $^{\circ} 1982$ )

G.28.056. Geobatrachus ${ }^{\circ} 1915$ (walkeri $^{\circ}$ 1915)

F.18.12. Subfamilia BRACHYCEPHALINAE Günther, 1858.gc.f002-04 \{100\}

G.28.057. Brachycephalus* 1826 (ephippium* 1825) EEphippipher 1835 E Ephippifer 1844

$\equiv$ Ephippiger 1845 AN $\approx$ Psyllophryne 1971 (didactyla* 1971) $\{\mathbf{1 0 0}\}$

G.28.058. Ischnocnema* 1862 (verrusosus* 1862) $\approx$ Basanitia 1923 (lactea* 1923) $\approx$ Phrynanodus

1933 (nanus $1933 \approx$ parvus $\left.^{*} 1853\right)\{\mathbf{1 0 0}\}$

F.18.13. Subfamilia CRAUGASTORINAE Hedges $^{+2}$, 2008.ha.f001-01 \{99\}

F.19.13. Tribus CRAUGASTORINI Hedges ${ }^{+2}, 2008$.ha.f001-02 \{100\} 


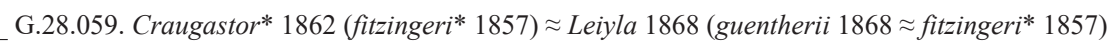

$\equiv$ Lihyla $1887 \mathbf{A M} \equiv$ Liohyla $1900 \mathbf{A M} \equiv$ Liyla $1870 \mathbf{A M} \approx$ Microbatrachylus 1939 (hobartsmithi ${ }^{\circ} 1936$ )

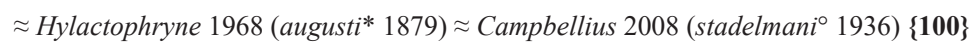

G.28.060. Haddadus* 2008 (binotata* 1824) $\{\mathbf{1 0 0}\}$

F.19.14. Tribus STRABOMANTINI Hedges ${ }^{+2}$, 2008.ha.f003-02 \{92\}

F.20.08. Subtribus STRABOMANTINA Hedges ${ }^{+2}$, 2008.ha.f003-03 \{94\}

$=$ F.21.03. Infratribus HoLOADENINIA Hedges ${ }^{+2}$, 2008.ha.f005-01 $\{\mathbf{1 0 0}\}$

F.22.0a. Hypotribus INCERTAE SEDIS

G.28.061. Niceforonia ${ }^{\circ} 1963$ (nana $^{\circ} 1963$ )

G.28.062. Tachiramantis $^{\circ} 2015$ (prolixodiscus $^{\circ}$ 1978)

F.22.01. Hypotribus BARYCHOLINOA nov., DOP.da.f006-00 \{100\}

G.28.063. Bahius* nov. (bilineatus* 1975)

G.28.064. Barycholos* 1969 (pulcher* 1898) $\{\mathbf{1 0 0}\}$

G.28.065. Phyllonastes* 1977 (myrmecoides* 1976) \{99\}

F.22.02. Hypotribus BRYOPHRYNINOA nov., DOP.da.f007-00-

G.28.066. Bryophryne* 2008 (cophites* 1975)

F.22.03. Hypotribus HOLOADENINOA Hedges ${ }^{+2}$, 2008.ha.f005-02 \{100\}

G.28.067. Euparkerella* 1959 (brasiliensis* 1925) $\{\mathbf{1 0 0}\}$

G.28.068. Holoaden* 1920 (luederwaldti* 1920) $\{\mathbf{1 0 0}\}$

F.22.04. Hypotribus NOBLELLINOA nov., DOP.da.f008-00 $\{\mathbf{1 0 0}\}$ G.28.069. Microkayla 2017 (teqta ${ }^{\circ}$ 2014) $\{\mathbf{1 0 0}\}$

G.28.070. Noblella* 1930 (peruvianus* 1921)

G.28.071. Psychrophrynella 2008 (bagrecito $\left.^{\circ} 1986\right)$

G.28.072. Qosqophryne 2020 (gymnotis $^{\circ} 2020$ )

F.21.04. Infratribus STRABOMANTINIA Hedges ${ }^{+2}$, 2008.ha.f003-04 \{100\}

\left. G.28.073. Strabomantis* $1863{\text { (biporcatus } * 1863) \approx \text { Limnophys }^{*} 1870 \text { (cornutus }}^{\circ} 1870\right) \equiv$ Ctenocranius $^{2}$ $1941 \approx$ Amblyphrynus 1961 (ingeri $^{\circ} 1961$ )

F.20.09. Subtribus Pristimantina Ohler ${ }^{+1}$, 2012.oa.f002-01 \{90\}

F.21.05. Infratribus HYPODACTYLINIA Heinicke ${ }^{+4}$, 2018.f001-01 \{96\}

G.28.074. Hypodactylus* 2008 (elassodiscus* 1973) $\equiv$ Isodactylus $2008 \mathbf{J H}$

F.21.06. Infratribus PRISTIMANTINIA Ohler ${ }^{+1}$, 2012.oa.f002-02 \{98\}

F.22.05. Hypotribus OREOBATINOA nov., DOP.da.f009-00 $\{\mathbf{1 0 0}\}$

F.23.01. Clanus OREOBATITES nov., DOP.da.f009-01 \{94\}

G.28.075. Lynchius* 2008 (parkeri* 1975) $\{\mathbf{1 0 0}\}$

G.28.076. Oreobates* 1872 (quixensis* 1872) $\approx$ Teletrema 1937 (heterodactylum* 1937) \{100\}

F.23.02. Clanus PHRYNOPODITES nov., DOP.da.f010-00 $\{\mathbf{1 0 0}\}$

G.28.077. Phrynopus ${ }^{3} 1873$ (peruanus $^{\circ}$ 1873)

F.22.06. Hypotribus PRISTIMANTINOA Ohler ${ }^{+1}$, 2012.oa.f002-03 \{99\}

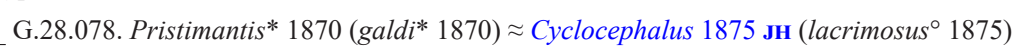

$\approx$ Hypodictyon 1885 (ridens* 1866) $\approx$ Pseudohyla 1946 (nigrogrisea $\left.{ }^{\circ} 1946\right) \approx$ Trachyphrynus 1963

(myersi $\left.{ }^{\circ} 1963\right) \approx$ Mucubatrachus 2007 (briceni $\left.^{\circ} 1903\right) \approx$ Paramophrynella $2007 \approx$ Huicundomantis

2019 (phoxocephalus* 1979) $\{\mathbf{1 0 0}\}$

G.28.079. Yunganastes* 2007 (pluvicanorus* 1997) $\{\mathbf{1 0 0}\}$

F.18.14. Subfamilia ELEUTHERODACTYLINAE Lutz, 1954.la.f001-00 \{100\}

F.19.15. Tribus ELEUTHERODACTYLINI Lutz, 1954.1a.f001-02 \{100\}

F.20.10. Subtribus DIASPORINA nov., DOP.da.f148-00 \{100\}

G.28.080. Diasporus* 2008 (diastema* 1875)

F.20.11. Subtribus ELEUTHERODACTYLINA Lutz, 1954.1a.f001-05 \{100

G.28.081. Eleutherodactylus* 1841 (martinicensis* 1838) $\approx$ Ladailadne 1987 (jasperi ${ }^{\circ} 1976$ )

$\approx$ Pelorius 1989 (inoptatus* 1914) $\approx$ Schwartzius 2008 (counouspeus* 1964) $\{\mathbf{1 0 0}\}$

G.28.082. Euhyas* 1843 (ricordii* 1841) $\approx$ Epirhexis 1866 (longipes ${ }^{\circ} 1859$ ) CI $\approx$ Syrrhophus 1878

(marnockii* 1878) $\equiv$ Syrrhopus $1888 \equiv$ Syrrhaphus $1900 \equiv$ Syrrophus $1907 \approx$ Malachylodes 1879

(guttilatus $\left.^{\circ} 1879\right) \approx$ Tomodactylus 1900 (amulae $1900 \approx$ nitidus $\left.^{*} 1870\right) \approx$ Sminthillus 1920 (limbatus*

1862) $\{\mathbf{1 0 0}\}$

F.19.16. Tribus PHYZELAPHRYNINI Hedges ${ }^{+2}$, 2008.ha.f002-01 $\{\mathbf{1 0 0}\}$

G.28.083. Adelophryne* 1984 (adiastola* 1984) $\{\mathbf{1 0 0}\}$ 
G.28.084. Phyzelaphryne* 1977 (miriamae* 1977)

F.17.14. Familia CEUTHOMANTIDAE Heinicke ${ }^{+5}$, 2009.ha.f001-00 [S] [N]

G.28.085. Ceuthomantis* 2009 (smaragdinus* 2009)

G.28.086. Dischidodactylus ${ }^{\circ} 1979$ (duidensis $^{\circ}$ 1968)

C.13.02. Hypophalanx Hemiphractiformia Brocchi, 1881.ba.c01-01 \{100\}

F.17.15. Familia HEMIPHRACTIDAE Peters, 1862.pa.f001-00 \{100\} [Q] [T]

F.18.15. Subfamilia AMPHIGNATHODONTINAE Boulenger, 1882.bb.f002-01 \{100\}

F.19.17. Tribus AMPHIGNATHODONTINI Boulenger, 1882.bb.f002-02 \{99\}

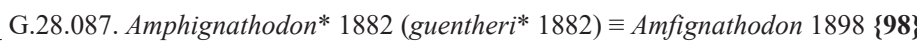

G.28.088. Cryptotheca* 2015 (walkeri* 1980)

F.19.18. Tribus EOTHECINI nov., DOP.da.f011-00 \{92\}

G.28.089. Eotheca* 2015 (fissipes* 1888)

F.19.19. Tribus GASTROTHECINI Noble, 1927.na.f001-01 \{93\}

G.28.090. Alainia* 2018 (microdiscus* 1910) $\equiv$ Australotheca 2015 JH \{99\}

G.28.091. Gastrotheca* 1843 (marsupiata* 1841) $\equiv$ Nototrema $1859 \mathbf{J H} \approx$ Notodelphys $1854 \mathbf{J H}$

(ovifera* 1854$) \equiv$ Opisthodelphys $1859 \equiv$ Notodelphis $1878 \equiv$ Opisthodelphis $1881 \approx$ Duellmania

1987 (argenteovirens* 1892) $\approx$ Edaphotheca 2015 (galeata* 1978) \{100\}

F.18.16. Subfamilia CRYPTOBATRACHINAE Frost $^{+18}$, 2006.fa.f001-02

G.28.092. Cryptobatrachus* 1916 (boulengeri* 1916)

F.18.17. Subfamilia FLECTONOTINAE nov., DOP.da.f012-00 \{100\}

G.28.093. Flectonotus* 1926 (pygmaeum* 1893)

F.18.18. Subfamilia FRITZIANINAE nov., DOP.da.f013-00 \{100\}

G.28.094. Fritziana* 1937 (goeldii* 1895) EFritzia 1920 JH $\approx$ Coelonotus 1920 JH (fissilis* 1920)

Nototheca 1950

F.18.19. Subfamilia HeMIPHRACTINAE Peters, 1862.pa.f001-03 \{96\}

G.28.095. Hemiphractus ${ }^{1} 1828$ (spixii $1828 \equiv$ scutata $\left.^{*} 1824\right) \approx$ Cerathyla 1870 (bubalus $\left.^{*} 1870\right)$

$\equiv$ Ceratohyla 1882

F.18.20. Subfamilia STEFANIINAE nov., DOP.da.f014-00 $\{\mathbf{1 0 0}\}$

G.28.096. Stefania* 1968 (evansi* 1904) $^{*}$

C.13.03. Hypophalanx Hylobatrachia Ritgen, 1828.ra.c16-01 \{100\}

F.14.0a. Superfamilia INCERTAE SEDIS

G.28.097. Ancudia 1902 (concolor ${ }^{\circ}$ 1902)

F.14.03. Superfamilia BUFONOIDEA Gray, 1825.ga.f004-20 \{97\}

F.17.16. Familia BUFONIDAE Gray, 1825.ga.f004-08 \{100\} [Q]

F.18.21. Subfamilia BUFONINAE Gray, 1825.ga.f004-23 \{99\}

F.19.20. Tribus BUFONINI Gray, 1825.ga.f004-27 \{93\}

F.20.0a. Subtribus INCERTAE SEDIS

G.28.098. Metaphryniscus ${ }^{\circ} 1994$ (sosai ${ }^{\circ}$ 1994)

G.28.099. Truebella 1995 (skoptes $\left.{ }^{\circ} 1995\right)$

F.20.12. Subtribus ATELOPODINA Fitzinger, 1843.fa.f005-07 \{100\}

G.28.100. Atelopus* 1841 (flavescens* 1841$) \equiv$ Ateleopus $1847 \approx$ Phrynidium 1856 (varium* 1856 )

$\approx$ Hylaemorphus 1857 a (dumerilii $1857 \approx$ varium $\left.^{*} 1856\right) \approx$ Hylaemorphus 1857 b AN (pluto $1858 \approx$

varium $\left.^{*} 1856\right) \approx$ Phirix 1857 (pachydermus $\left.{ }^{\circ} 1857\right) \approx$ Physalus 1857 AN-JH (ignescens* 1849)

F.20.13. Subtribus BUFONINA Gray, 1825.ga.f004-28 \{100\}

F.21.07. Infratribus AMAZOPHRYNELLINIA nov., DOP.da.f015-00 $\{\mathbf{1 0 0}\}$

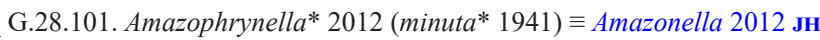

F.21.08. Infratribus BUFONINIA Gray, 1825.ga.f004-29 \{99\}

F.22.07. Hypotribus BUFONINOA Gray, 1825.ga.f004-30 \{100\}

F.23.03. Clanus BUFONITES Gray, 1825.ga.f004-31 \{99\}

F.24.01. Subclanus BUFONITIES Gray, 1825.ga.f004-32 \{99\}

F.25.0a. Infraclanus INCERTAE SEDIS

G.28.†094. Palaeophrynos ${ }^{\circ} 1838 \uparrow\left(\right.$ gessneri $\left.^{\circ} 1838 \dagger\right) \equiv$ Palaeophryne 1843 AM $\equiv$ Palaeophrynus

$1844 \equiv$ Troglobates 1848

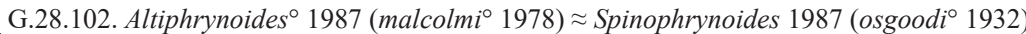

G.28.103. Parapelophryne 2003 (scalptus ${ }^{\circ} 1973$ )

F.25.01. Infraclanus ADENOMITOES Cope, 1861.ca.f001-03 \{100\} 
F.26.01. Hypoclanus ADENOMITUES Cope, 1861.ca.f001-04 \{94\}

F.27.01. Catoclanus ADENOMITYES Cope, 1861.ca.f001-04

G.28.104. Adenomus ${ }^{1} 1861$ (badioflavus $1860 \approx$ kelaartii* $^{*} 1858$ )

F.27.02. Catoclanus BEDUKITYES nov. \{91\}

G.28.105. Beduka* nov. (koynayensis* 1963) $\equiv$ Xanthophryne 2009 AN

G.28.106. Blythophryne 2016 (beryet $^{\circ}$ 2016)

G.28.107. Bufoides ${ }^{\circ} 1973$ (meghalayana ${ }^{\circ}$ 1971)

G.28.108. Duttaphrynus* 2006 (melanostictus* 1799) $\{\mathbf{1 0 0}\}$

G.28.109. Firouzophrynus ${ }^{3} 2020$ (olivaceus $^{\circ}$ 1874) $\{\mathbf{1 0 0}\}$

F.26.02. Hypoclanus PEDOSTIBITUES nov., DOP.da.f016-00

G.28.110. Pedostibes* 1876 (tuberculosus* 1876 )

F.25.02. Infraclanus ANSONIITOES nov., DOP.da.f017-00 \{97\}

F.26.0a. Hypoclanus INCERTAE SEDIS

G.28.111. Pseudobufo ${ }^{\circ} 1838$ (subasper $\left.{ }^{\circ} 1838\right) \equiv$ Pyleus $1848 \equiv$ Nectes $1865 \approx$ Nectes 1857 AN

(pleurotaenia $1857 \approx$ subasper $^{\circ} 1838$ )

G.28.112. Sigalegalephrynus ${ }^{\circ} 2017$ (mandailinguensis $^{\circ}$ 2017)

F.26.03. Hypoclanus ANSONIITUES nov., DOP.da.f017-01 \{99\}

G.28.113. Ansonia* 1870 (penangensis* 1870) $\{\mathbf{1 0 0}\}$

G.28.114. Pelophryne 1938 (albotaeniata $^{\circ} 1938$ )

F.26.04. Hypoclanus BARBAROPHRYNITUES nov., DOP.da.f018-00

G.28.115. Barbarophryne* 2013 (brongersmai* 1972)

F.26.05. Hypoclanus BLAIRITUES nov., DOP.da.f019-00

G.28.116. Blaira* nov. (ornata* 1876) $\equiv$ Ghatophryne 2009 AN

F.26.06. Hypoclanus INGEROPHRYNITUES nov., DOP.da.f020-00 $\{\mathbf{1 0 0}\}$

G.28.117. Ingerophrynus* 2006 (biporcatus* $^{*}$ 1829) $\approx$ Qiongbufo 2012 (ledongensis ${ }^{\circ} 2009$ )

E Qiongobufo 2016

F.26.07. Hypoclanus RENTAPIITUES nov., DOP.da.f021-00 $\{\mathbf{1 0 0}\}$

G.28.118. Phrynoidis* 1842 (asper* 1829) $\{\mathbf{1 0 0}\}$

G.28.119. Rentapia* 2016 (hosii* 1892)

F.25.03. Infraclanus BUFONITOES Gray, 1825.ga.f004-33 \{100\}

G.28.120. Bufo* 1764 (bufo* 1758) $\equiv$ Bufo $1758 a$ AN $\equiv$ Bufo $1758 b$ aN $\equiv$ Phrynacius 1815 AN

$\equiv$ Phrynocerus 1815 AN $\equiv$ Phrynotes $1815 \mathrm{AN} \equiv$ Phryne $1816 \mathrm{CI} \equiv$ Pegaeus $1868 \approx$ Phryne $1843 \mathbf{~ J H}$

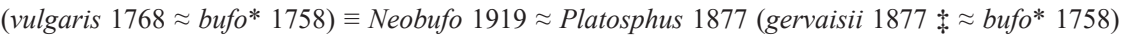

$\approx$ Bufavus 1885 (meneghinii $1885 \$ \approx$ bufo* 1758) $\approx$ Torrentophryne 1994 AN (aspinia* 1994) $\equiv$

Torrentophryne $1996 \approx$ Schmibufo 2016 (stejnegeri* 1931)

F.25.04. Infraclanus BUFOTITOES nov., DOP.da.f022-00 $\{\mathbf{1 0 0}\}$

G.28.121. Bufotes* 1815 (viridis* 1768) $\equiv$ Bufo $1768 \mathbf{J H} \equiv$ Buffo $1788 \mathbf{C I} \equiv$ Batrachus $1814 \mathbf{J H}$

$\equiv$ Pseudepidalea $2006 \approx$ Calliopersa 2020 (surdus $\left.{ }^{\circ} 1931\right)$

F.25.05. Infraclanus NECTOPHRYNITOES Laurent, 1942.la.f001-02 \{100\}

F.26.08. Hypoclanus EPIDALEITUES nov., DOP.da.f023-00

G.28.122. Epidalea* 1864 (calamita* 1768) $\equiv$ Calamitus 1815 AN $\equiv$ Calamita 1816 CI $\equiv$ Rubeta 1872

F.26.09. Hypoclanus LEPTOPHRYNITUES nov., DOP.da.f024-00

G.28.123. Leptophryne ${ }^{2} 1843$ (cruentatus $\left.^{\circ} 1838\right) \approx$ Cacophryne 1935 (borbonica* 1838)

F.26.10. Hypoclanus NECTOPHRYNITUES Laurent, 1942.1a.f001-03 $\{\mathbf{1 0 0}\}$

G.28.124. Didynamipus* 1903 (sjostedt $*$ 1903) ₹ Atelophryne 1906 (minuta $1906 \approx$ sjostedti* 1903)

G.28.125. Laurentophryne ${ }^{\circ} 1960$ (parkeri $\left.^{\circ} 1950\right)$

G.28.126. Mo* nov. (bambutensis* 1972)

G.28.127. Nectophryne* 1875 (afra* 1875) $\equiv$ Nectofryne $1898\{\mathbf{1 0 0}\}$

G.28.128. Nimbaphrynoides* 1987 (occidentalis* 1943)

G.28.129. Werneria 1903 (fulva $1903 \approx$ preussi $^{\circ}$ 1893) $\equiv$ Stenoglossa JH $1903\{\mathbf{1 0 0}\}$

G.28.130. Wolterstorffina* 1939 (parvipalmata* 1898 )

F.25.06. Infraclanus SABAHPHRYNITOES-nov., DOP.da.f025-00

G.28.131. Sabahphrynus* 2007 (maculata* 1890)

F.25.07. Infraclanus STRAUCHBUFONITOES nov., DOP.da.f026-00

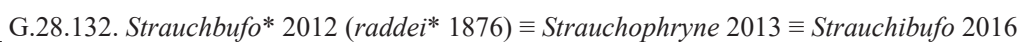

F.25.08. Infraclanus TORNIERIOBATITOES Miranda-Ribeiro, 1926.ma.f001-03 \{94\} 
F.26.11. Hypoclanus SCHISMADERMATITUES nov., DOP.da.f027-00

G.28.133. Schismaderma 1849 (lateralis $1849 \approx$ carens $^{*} 1848$ )

F.26.12. Hypoclanus ToRNIERIOBATITUES Miranda-Ribeiro, 1926.ma.f001-04 \{100\}

G.28.134. Churamiti* 2002 (maridadi* 2002)

G.28.135. Nectophrynoides* 1926 (tornieri* 1906) $\approx$ Tornieriobates 1926 (vivipara* 1905)

$\equiv$ Tornierobates 1940 AM $\equiv$ Tornierobates $2006\{\mathbf{1 0 0}\}$

F.24.02. Subclanus PHRYNISCITIES Günther, 1858.gc.f005-04 \{99\}

F.25.09. Infraclanus ANAXYRITOES nov., DOP.da.f028-00 $\{\mathbf{1 0 0}\}$

G.28.136. Anaxyrus ${ }^{3} 1845$ (melancholicus $1845 \approx$ compactilis $^{\circ} 1833$ ) $\approx$ Dromoplectrus 1879

(anomalus $1858 \approx$ compactilis $\left.^{\circ} 1833\right)\{\mathbf{1 0 0}\}$

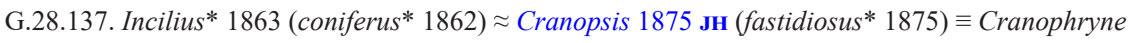

$1889 \approx$ Crepidius $1875^{\mathbf{J H}}$ (epioticus $\left.^{\circ} 1875\right) \equiv$ Crepidophryne $1889 \approx$ Ollotis 1875 (coerulescens 1875

$\approx$ fastidiosus $\left.^{*} 1875\right)\{97\}$

F.25.10. Infraclanus PHRYNISCITOES Günther, 1858.gc.f005-05 \{100\}

G.28.138. Rhinella ${ }^{2} 1826$ (proboscideus $\left.^{\circ} 1824\right) \equiv$ Rhinellus $1831 \equiv$ Eurhina $1843 \approx$ Oxyrhynchus 1824 JH (granulosus* 1824) $\equiv$ Oxyrhinchus 1841 AM $\approx$ Chascax 1828 (horridus $1802 \approx$ spinulosus* 1768)

$\approx$ Chaunus 1828 (marmoratus $1828 \approx$ granulosus $* 1824) \approx$ Otilophes 1829 AN (margaritifera* 1768 )

$\equiv$ Otilophis $1831 \equiv$ Otilopha $1831 \equiv$ Otilophus $1832 \equiv$ Merothaelacium $1833 \equiv$ Atilophus $1840 \equiv$ Otolophus $1843 \equiv$ Otylophus 1953 AM $\approx$ Macrothaelacion 1833 (nasutus $1799 \approx$ margaritifera* 1768) $\approx$ Phryniscus 1834 (nigricans $1834 \approx$ spinulosus $* 1834$ ) $\equiv$ Phreniscus 1841 AM $\approx$ Chilophryne 1843

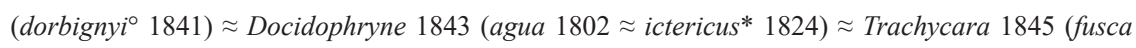
$1845 \approx$ margaritifera $* 1768) \approx$ Aruncus 1899 AN (valdivianus $1902 \approx$ spinulosus $* 1834) \equiv$ Aruncus $1902 \approx$ Stenodactylus 1902 JH (ventralis $1902 \approx{\text { spinulosus } * 1834) \approx \text { Palaeobufo* }^{*} 1919 \text { (marina* }}^{*}$ $1758) \approx$ Rhamphophryne 1971 (acrolopha $\left.{ }^{\circ} 1971\right) \approx$ Atelophryniscus 1989 (chrysophorus $\left.{ }^{\circ} 1989\right)$

F.24.03. Subclanus STEPHOPAEDITIES Dubois, 1987.da.f001-01 \{100\}

F.25.11. Infraclanus CAPENSIBUFONITOES nov., DOP.da.f029-00 $\{\mathbf{1 0 0}\}$

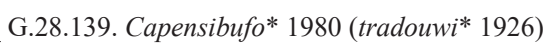

F.25.12. Infraclanus SCLEROPHRYITOES nov., DOP.da.f030-00 \{100\}

G.28.140. Sclerophrys* 1838 (capensis* 1838) ₹ Amietophrynus 2006 (regularis* 1833)

F.25.13. Infraclanus STEPHOPAEDITOES Dubois, 1987.da.f001-02 \{98\}

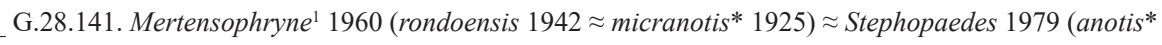
1907) $\{99\}$

G.28.142. Poyntonophrynus ${ }^{3} 2006$ (vertebralis $^{\circ}$ 1848) $\{92\}$

F.25.14. Infraclanus VANDIJKOPHRYNITOES nov., DOP.da.f031-00 \{100\}

G.28.143. Vandijkophrynus* 2006 (angusticeps* 1848)

F.23.04. Clanus PELTOPHRYNITES nov., DOP.da.f032-00 \{100\}

G.28.144. Peltophryne* 1843 (peltocephala* 1838) $\approx$ Otaspis 1869 (empusa* 1862)

F.23.05. Clanus RHAEBoITES nov., DOP.da.f033-00 \{98\}

G.28.145. Rhaebo* 1862 (haematiticus* 1862) ERhaeba $1882 \mathbf{A M} \approx$ Phrynomorphus 1843 JH

(leschenaulti $1841 \approx$ guttatus $\left.^{*} 1799\right) \approx$ Andinophryne 1985 (colomai ${ }^{\circ} 1985$ )

F.22.08. Hypotribus NANNOPHRYNINOA nov., DOP.da.f034-00 \{99\}

G.28.146. Nannophryne* 1870 (variegata* 1870)

F.21.09. Infratribus DENDROPHRYNISCINIA Jiménez de la Espada, 1870.ja.f001-03 \{100\}

G.28.147. Dendrophryniscus* 1870 (brevipollicatus* 1870)

F.20.14. Subtribus OREOPHRYNELLINA nov., DOP.da.f035-00 $\{\mathbf{1 0 0}\}$

G.28.148. Oreophrynella* 1895 (quelchii* 1895) 三 Oreophryne $1895 \mathbf{J H}$

F.20.15. Subtribus OSORNOPHRYNINA nov., DOP.da.f036-00 $\{\mathbf{1 0 0}\}$

G.28.149. Osornophryne* 1976 (percrassa* 1976)

F.19.21. Tribus FROSTIINI nov., DOP.da.f037-00

G.28.150. Frostius ${ }^{3} 1986$ (pernambucensis $^{\circ}$ 1962)

F.18.22. Subfamilia MELANOPHRYNISCINAE nov., DOP.da.f038-00 \{100\}

G.28.151. Melanophryniscus* 1961 (stelzneri* 1875)

F.17.17. Familia ODONTOPHRYNIDAE Lynch, 1971.la.f002-03 \{100\} [S] [N]

F.18.†0b. Subfamilia INCERTAE SEDIS $\dagger$

G.28.†095. Chachaiphrynus ${ }^{\circ} 2017 \dagger\left(\right.$ lynchi $\left.^{\circ} 2017 \dagger\right)$

F.18.23. Subfamilia ODONTOPHRYNINAE Lynch, 1971.la.f002-04 \{100\} 
G.28.152. Macrogenioglottus* 1946 (alipioi* 1946)

G.28.153. Odontophrynus* 1862 (cultripes* 1862) $\{\mathbf{1 0 0}\}$

F.18.24. Subfamilia PROCERATOPHRYINAE nov., DOP.da.f039-00 \{99\}

G.28.154. Proceratophrys* 1920 (bigibbosa* 1872)

F.14.04. Superfamilia CENTROLENOIDEA Taylor, 1951.ta.f001-02 \{100\}

F.17.18. Familia ALLOPHRYNIDAE Goin ${ }^{+2}$, 1978.ga.f001-00 \{100\} [S] [N]

G.28.155. Allophryne* 1926 (ruthveni* 1926)

F.17.19. Familia CENTROLENIDAE Taylor, 1951.ta.f001-00 \{100\} [Q]

F.18.25. Subfamilia CENTROLENINAE Taylor, 1951.ta.f001-01 \{100\}

F.19.22. Tribus CENTROLENINI Taylor, 1951.ta.f001-03 $\{\mathbf{1 0 0}\}$

G.28.156. Centrolene* 1872 (geckoideum* 1872) $\approx$ Centrolenella 1920 (antioquiensis* 1920)

F.19.23. Tribus CoCHRANELLINI Guayasamin ${ }^{+5}$, 2009.ga.f001-00 $\{\mathbf{1 0 0}\}$

F.20.16. Subtribus COCHRANELLINA Guayasamin ${ }^{+5}$, 2009.ga.f001-01 \{99\}

F.21.10. Infratribus COCHRANELLINIA Guayasamin ${ }^{+5}$, 2009.ga.f001-02 $\{\mathbf{1 0 0}\}$

G.28.157. Cochranella* 1951 (granulosa* 1949)

F.21.11. Infratribus ESPADARANINIA nov., DOP.da.f040-00 \{90\}

F.22.09. Hypotribus CHIMERELLINOA nov., DOP.da.f041-00

G.28.158. Chimerella* 2009 (mariaelenae* 2006)

F.22.10. Hypotribus ESPADARANINOA nov., DOP.da.f040-01 \{100\}

G.28.159. Espadarana* 2009 (andina* 1968)

F.22.11. Hypotribus RULYRANINOA nov., DOP.da.f042-00 \{90\}

F.23.06. Clanus AUDACIELLITES nov., DOP.da.f043-00 \{100\}

G.28.160. Audaciella* nov. (audax* 1973)

F.23.07. Clanus RULYRANITES nov., DOP.da.f042-01 \{92\}

G.28.161. Rulyrana* 2009 (flavopunctata* 1973) $\{\mathbf{1 0 0}\}$

G.28.162. Sachatamia* 2009 (albomaculata* 1949) $\{\mathbf{1 0 0}\}$

F.20.17. Subtribus TERATOHYLINA nov., DOP.da.f044-00 \{91\}

G.28.163. Teratohyla* 1951 (spinosa* 1949)

F.20.18. Subtribus VITREORANINA nov., DOP.da.f045-00 $\{\mathbf{1 0 0}\}$

G.28.164. Vitreorana* 2009 (antisthenesi* 1963)

F.19.24. Tribus NYMPHARGINI nov., DOP.da.f046-00 \{100\}

G.28.165. Nymphargus* 2007 (cochranae* 1961)

F.18.26. Subfamilia HYALINOBATRACHINAE Guayasamin ${ }^{+5}$, 2009.ga.f002-00 \{100\} G.28.166. Celsiella* 2009 (revocata* 1985) $\{\mathbf{1 0 0}\}$

G.28.167. Hyalinobatrachium* 1991 (fleischmanni* 1893) \{98\}

F.18.27. Subfamilia IKAKOGINAE nov., DOP.da.f047-00

G.28.168. Ikakogi* 2009 (tayrona* 1991)

F.14.05. Superfamilia CERATOPHRYOIDEA Tschudi, 1838.ta.f002-14 \{99\}

F.15.03. Epifamilia CERATOPHRYOIDAE Tschudi, 1838.ta.f002-15 \{100\}

F.17.20. Familia CERATOPHRYIDAE Tschudi, 1838.ta.f002-05 \{100\} [M]

F.18.28. Subfamilia CERATOPHRYINAE Tschudi, 1838.ta.f002-06 \{100\}

G.28.†096. Beelzebufo ${ }^{\circ} 2008 \dagger\left(\right.$ ampinga $\left.^{\circ} 2008 \dagger\right)$

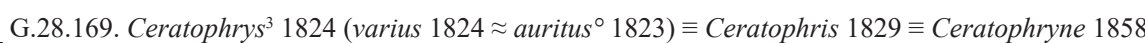

$\approx$ Phrynoceros 1838 (vaillanti $1838 \approx$ cornuta $^{*} 1758$ ) $\equiv$ Phrynocerus $1862 \approx$ Trigonophrys 1857

(rugiceps $1857 \approx$ ornatum* 1843 )

F.18.29. Subfamilia LEPIDOBATRACHINAE Bauer, 1987.ba.f001-01 \{97\}

G.28.†097. Baurubatrachus ${ }^{\circ} 1990 \dagger\left(\right.$ pricei $\left.^{\circ} 1990 \dagger\right)$

G.28.170. Chacophrys* 1963 (pierottii* 1948)

G.28.171. Lepidobatrachus ${ }^{3} 1899$ (asper ${ }^{\circ}$ 1899)

F.18.30. Subfamilia STOMBINAE Gallardo 1965.ga.f001-00

G.28.172. Stombus* 1825 (cornuta* 1758) $\equiv$ Strombus 1831 AM

F.15.04. Epifamilia TELMATOBIOIDAE Fitzinger, 1843.fa.f006-04 \{91\}

F.16.01. Apofamilia CYCLORAMPHEIDAE Bonaparte, 1850.bb.f003-|Bonaparte, 1852.ba.f001|-05 \{99\} [T]

F.17.21. Familia CYCLORAMPHIDAE Bonaparte, 1850.bb.f003-|Bonaparte, 1852.ba.f001|-04 \{100\} [N] [M] [T]

F.18.31. Subfamilia ALSODINAE Mivart, 1869.ma.f005-02 \{100\} [N] [M]

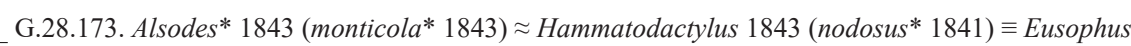


$1865 \equiv$ Esophus 1870 AM $\approx$ Cacotus 1869 (maculatus $1869 \approx$ nodosus* 1841) $\approx$ Telmalsodes 1989 (montanus $^{\circ}$ 1902) $\equiv$ Talmalsodes $1992\{\mathbf{1 0 0}\}$

G.28.174. Eupsophus* 1843 (roseus* 1841) EEusophis $1940 \approx$ Borborocoetes 1843 JH (grayii 1843

$\approx$ roseus $* 1841) \equiv$ Borborocoetea $1928\{\mathbf{1 0 0}\}$

F.18.32. Subfamilia BATRACHYLINAE Gallardo, 1965.ga.f002-02 \{100\} [N] [M] [T]

F.19.25. Tribus ATELOGNATHINI nov., DOP.da.f048-00 \{100\}

G.28.175. Atelognathus* 1978 (patagonicus* 1962) $\{\mathbf{1 0 0}\}$

G.28.176. Chaltenobatrachus ${ }^{\circ} 2011$ (grandisonae $^{\circ}$ 1975)

F.19.26. Tribus BATRACHYLINI Gallardo, 1965.ga.f002-00 \{99\}

G.28.177. Batrachyla* 1843 (leptopus* 1843) \{90\}

G.28.178. Hylorina* 1843 (sylvatica* 1843 ) $\equiv$ Hylorhina 1847

F.18.33. Subfamilia CYCLORAMPHINAE Bonaparte, 1850.bb.f003-|Bonaparte, 1852.ba.f001|-04 \{100\} [N] [M] [T] G.28.179. Cycloramphus* 1838 LT (fulginosus $1838 \equiv$ fuliginosus* 1838) $\equiv$ Cycloramphos 1838 LP

$\equiv$ Pithecopsis $1841 \equiv$ Cycloramphos $1847 \equiv$ Cyclorhamphus $1847 \approx$ Zachaenus 1866 (parvulus*

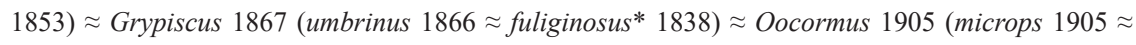
parvulus* 1853$) \approx$ Iliodiscus 1920 (dubius $\left.{ }^{\circ} 1920\right) \approx$ Craspedoglossa 1922 (santaecatharinae $1922 \approx$ bolitoglossus $\left.^{\circ} 1897\right) \approx$ Niedenia 1924 (spinulifer $1923 \approx$ asper $\left.^{\circ} 1899\right)\{\mathbf{1 0 0}\}$

G.28.180. Thoropa ${ }^{1} 1865$ (missiessii $1842 \approx$ miliaris* 1824$)\{\mathbf{1 0 0}\}$

F.18.34. Subfamilia HYLODINAE Günther, 1858.gc.f010-00 \{100\} [N] [M] [T]

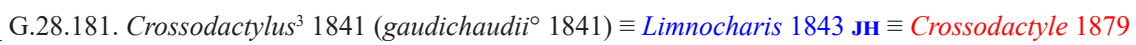

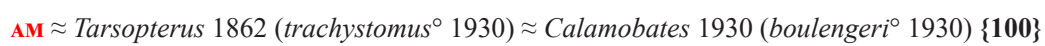

G.28.182. Hylodes ${ }^{1} 1826$ (ranoides $1824 \approx$ nasus $\left.^{*} 1823\right) \equiv$ Enydrobius $1830 \approx$ Elosia 1838 (nasus $*$

1823 ) $\equiv$ Scinacodes $1843 \approx$ Megaelosia $^{1} 1923$ (bufonium $1923 \approx$ nasus* 1823) $\equiv$ Magaelosia 1923 $\{100\}$

F.18.35. Subfamilia LIMNOMEDUSINAE nov., DOP.da.f049-00 [N] [M] [T]

G.28.183. Limnomedusa* 1843 (macroglossus* 1841) ₹ Litopleura 1875 (maritimum 1875

$\approx$ macroglossus* 1841 )

F.16.02. Apofamilia TELMATOBIEIDAE Fitzinger, 1843.fa.f006-05 \{99\}

F.17.22. Familia RHINODERMATIDAE Bonaparte, 1850.bb.f011-01 \{99\}[Q]

G.28.184. Insuetophrynus* 1970 (acarpicus* 1970)

G.28.185. Rhinoderma* 1841 (darwinii* 1841) $\approx$ Heminectes 1902 (rufus ${ }^{\circ} 1902$ )

F.17.23. Familia TELMATOBIIDAE Fitzinger, 1843.fa.f006-01 \{100\} [S] [P]

G.28.†098. Neoprocoela ${ }^{\circ} 1949 \dagger\left(\right.$ EDENTATA $\left.^{\circ} 1949 \dagger\right)$

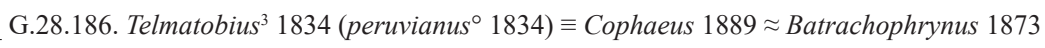

(macrostomus $^{\circ} 1873$ ) $\approx$ Pseudobatrachus 1873 (jelskii ${ }^{\circ} 1873$ ) $\approx$ Lynchophrys 1983 (brachydactylus ${ }^{\circ}$ 1873)

F.14.06. Superfamilia HYLOIDEA Rafinesque, 1815.ra.f002-|Gray, 1825.ga.f001|-20 \{100\}

F.17.24. Familia HYLIDAE Rafinesque, 1815.ra.f002-|Gray, 1825.ga.f001|-09 \{100\}[Q]

F.18.†0c. Subfamilia INCERTAE SEDIS $\dagger$

G.28.†099. Etnabatrachus ${ }^{\circ} 2003 \dagger\left(\right.$ MAXIMUS $\left.^{\circ} 2003 \dagger\right)$

G.28.†100. Geophryne ${ }^{\circ} 2014 \dagger\left(\right.$ nordensis $\left.^{\circ} 1964 \dagger\right)$

G.28.†101. Proacris ${ }^{\circ} 1961 \dagger($ MINTONI $1961 \dagger)$

F.18.36. Subfamilia COPHOMANTINAE Hoffmann, 1878.ha.f004-02 \{100\}

F.19.27. Tribus COPHOMANTINI Hoffmann, 1878.ha.f004-01 \{99\}

F.20.19. Subtribus COPHOMANTINA Hoffmann, 1878.ha.f004-03 \{100\}

F.21.12. Infratribus BOKERMANNOHYLINIA nov., DOP.da.f050-00 $\{\mathbf{1 0 0}\}$

G.28.187. Bokermannohyla* 2005 (circumdata* 1871)

F.21.13. Infratribus COPHOMANTINIA Hoffmann, 1878.ha.f004-04 \{96\}

G.28.188. Aplastodiscus* 1950 (perviridis* 1950) $\{\mathbf{1 0 0}\}$

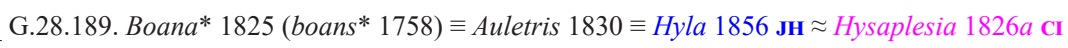

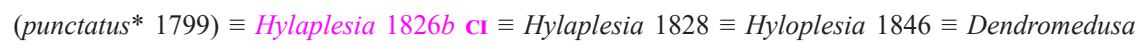
$1848 \equiv$ Hylapesia 2007 AM $\approx$ Hypsiboas 1830 (palmata $1789 \approx$ boans* 1758) $\equiv$ Lobipes $1843 \mathbf{J H}$ $\approx$ Hypsipsophus 1843 (xerophilla $1841 \approx$ crepitans $* 1824) \approx$ Phyllobius 1843 JH (albomarginata* $^{*}$ 1824 ) $\approx$ Centrotelma 1856 (infulata $1824 \approx$ albomarginata* 1824 ) $\approx$ Hylomedusa 1856 (crepitans* $1824) \approx$ Cinclidium 1867 JH (granulatum $1867 \approx$ boans* 1758) $\equiv$ Cincloscopus $1871 \approx$ Cophomantis 1870 (punctillata $1870 \approx$ semilineata $* 1824)_{100} \mathbf{1 0 0}$ 
F.20.20. Subtribus HyLOSCIRTINA nov., DOP.da.f051-00 \{100\}

G.28.190. Colomascirtus* 2016 (larynopigion* 1973) $\{99\}$

G.28.191. Hyloscirtus ${ }^{3} 1882$ (bogotensis $^{\circ}$ 1882) EHylonomus $1882 \mathbf{J H}\{\mathbf{1 0 0}\}$

F.19.28. Tribus MYERSIOHYLINI nov., DOP.da.f052-00

G.28.192. Myersiohyla* 2005 (inparquesi* 1994)

F.19.29. Tribus NESOROHYLINI nov., DOP.da.f053-00

G.28.193. Nesorohyla* 2019 (kanaima* 1969)

F.18.37. Subfamilia HYLINAE Rafinesque, 1815.ra.f002-|Gray, 1825.ga.f001|-19 \{100\}

F.19.30. Tribus DENDROPSOPHINI Fitzinger, 1843.fa.f003-01 \{100\}

F.20.21. Subtribus DENDROPSOPHINA Fitzinger, 1843.fa.f003-02 \{100\}

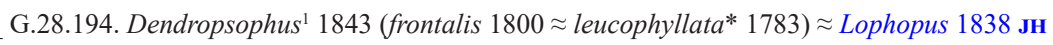

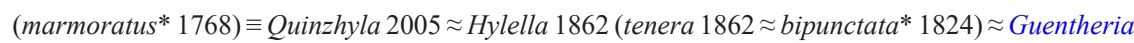

1926 JH (dasynota $1869 \approx$ senicula* 1868) $\{99\}$

G.28.195. Xenohyla* 1998 (truncata* 1959)

F.20.22. Subtribus PSEUDINA Fitzinger, 1843.fa.f010-04 \{100\}

G.28.196. Pseudis* 1830 (paradoxa* 1758) 三 Pseudes $1844 \equiv$ Batrachychthis 1876 LT

E Batrachychthys $1876 \mathbf{L P} \equiv$ Batrachchythis 1877 AM $\equiv$ Batrachichthys 1877 AM $\approx$ Lysapsus 1862

(limellum* 1862) $\equiv$ Lisapsus $1867 \equiv$ Lysapus $1878 \equiv$ Podonectes 1864 AN $\{\mathbf{1 0 0}\}$

G.28.197. Scarthyla1 1988 (ostinodactyla $1988 \approx$ goinorum $* 1962)^{*}$

F.19.31. Tribus HYLINI Rafinesque, 1815.ra.f002-|Gray, 1825.ga.f001|-21 \{100\}

F.20.23. Subtribus ACRISINA Mivart, 1869.ma.f008-05 \{100\}

F.21.14. Infratribus ACRISINIA Mivart, 1869.ma.f008-06 \{100\}

G.28.198. Acris* 1841 (gryllus* 1825)

F.21.15. Infratribus HYLIOLINIA Dubois ${ }^{+2}$, 2017.da.f001-02 \{99\}

G.28.199. Hyliola* 1899 (regilla* 1852) $\{\mathbf{1 0 0}\}$

G.28.200. Pseudacris* 1843 (nigrita* $^{*} 1825$ ) $\equiv$ Chorophilus $1854 \equiv$ Chlorofilus $1898 \approx$ Helocaetes

1854 (triseriata* 1838) $\equiv$ Heloecetes $1859 \approx$ Limnaoedus 1953 (ocularis* 1801 ) $\approx$ Parapseudacris

1986 (crucifer* 1838) $\approx$ Pycnacris 2014 (ornata* 1836) $\{\mathbf{1 0 0}\}$

F.20.24. Subtribus HYLINA Rafinesque, 1815.ra.f002-|Gray, 1825.ga.f001|-23 \{93\}

F.21.16. Infratribus HYLINIA Rafinesque, 1815.ra.f002-|Gray, 1825.ga.f001|-24 \{100\}

F.22.12. Hypotribus CHARADRAHYLINOA nov., DOP.da.f054-00 \{98\}

G.28.201. Charadrahyla* 2005 (taeniopus* 1901) $\{\mathbf{1 0 0}\}$

G.28.202. Megastomatohyla* 2005 (mixe* 1965)

F.22.13. Hypotribus HYLINOA Rafinesque, 1815.ra.f002-|Gray, 1825.ga.f001|-25 \{100\}

F.23.08. Clanus HYLITES Rafinesque, 1815.ra.f002-|Gray, 1825.ga.f001|-26 \{97\}

G.28.203. Dryophytes* 1843 (versicolor* 1825) $\approx$ Epedaphus 1885 (gratiosa* 1825$)\{\mathbf{1 0 0}\}$

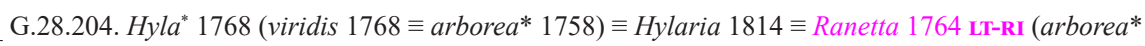

1758) $\equiv$ Ranella 1764 LP $\equiv$ Calamita $1799 \equiv$ Hydryla 1815 AN $\equiv$ Hylanus 1815 AN $\equiv$ Hylesinus 1815

AN $\equiv$ Hylopsis 1815 AN $\equiv$ Hyas $1830 \equiv$ Dendrohyas $1830 \equiv$ Discodactylus $1833\{\mathbf{1 0 0}\}$

F.23.09. Clanus TRIPRIONITES Miranda-Ribeiro, 1926.ma.f005-01 \{100\}

F.24.04. Subclanus ISTHMOHYLITIES nov., DOP.da.f055-00 \{96\}

G.28.205. Isthmohyla* 2005 (pseudopuma* 1901)

F.24.05. Subclanus TLALOCOHYLITIES nov., DOP.da.f056-00 \{100\}

G.28.206. Tlalocohyla* 2005 (smithii* 1902)

F.24.06. Subclanus TRIPRIONITIES Miranda-Ribeiro, 1926.ma.f005-02 \{100\}

F.25.15. Infraclanus DIAGLENITOES nov., DOP.da.f149-000 \{96\}

G.28.207. Diaglena* 1887 (spatulatus* 1882)

F.25.16. Infraclanus SMILISCITOES nov., DOP.da.f150-000 \{96\}

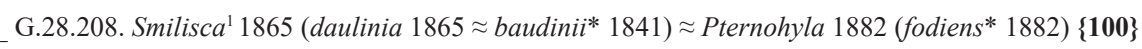

F.25.17. Infraclanus TRIPRIONITOES Miranda-Ribeiro, 1926.ma.f005-03 \{96\}

G.28.209. Anotheca 1939 (coronata $1911 \approx$ spinosa* $^{*} 1864$ )

G.28.210. Triprion* 1866 (petasatus* 1865) $\equiv$ Pharyngodon $1865 \mathbf{J H}$

F.22.14. Hypotribus RHEOHYLINOA nov., DOP.da.f057-00 \{100\}

F.23.10. Clanus ECNOMIOHYLITES nov., DOP.da.f058-00 \{97\}

G.28.211. Ecnomiohyla* 2005 (miliarius $* 1886$ )

F.23.11. Clanus PTYCHOHYLITES nov., DOP.da.f059-00\{100\} 
G.28.212. Atlantihyla* 2018 (spinipollex* 1936)

G.28.213. Bromeliohyla* 2005 (bromeliacea* 1933)

G.28.214. Duellmanohyla* 1992 (uranochroa* 1875) $\{\mathbf{9 8}\}$

G.28.215. Ptychohyla 1944 (adipoventris $1944 \approx$ leonardschultzei* 1934) $\{\mathbf{1 0 0}\}$

G.28.216. Quilticohyla 2018 (sanctaecrucis ${ }^{\circ}$ 1922)

F.23.12. Clanus RHEOHYLITES nov., DOP.da.f057-01

G.28.217. Rheohyla* 2016 (miotympanum* 1863)

F.21.17. Infratribus PLECTROHYLINIA nov., DOP.da.f060-00 $\{\mathbf{1 0 0}\}$

G.28.218. Exerodonta* 1879 (sumichrasti* 1879) $\{\mathbf{1 0 0}\}$

G.28.219. Plectrohyla* 1877 (guatemalensis* 1877) $\equiv$ Cauphias $1877 \approx$ Sarcohyla 2016

(crassus $^{\circ}$ 1877) $\{\mathbf{1 0 0}\}$

F.19.32. Tribus LорнYонYLINI Miranda-Ribeiro, 1926.ma.f004-|Fouquette ${ }^{+1}$, 2014.fa.f001|-00 \{100\}

F.20.25. Subtribus ITAPOTIHYLINA nov., DOP.da.f061-00

G.28.220. Itapotihyla* 2005 (langsdorffii* 1841)

F.20.26. Subtribus LOPHYOHYLINA Miranda-Ribeiro, 1926.ma.f004-|Fouquette ${ }^{+1}$, 2014.fa.f001|-02 \{94\}

= F.21.18. Infratribus LOPHYOHYLINIA Miranda-Ribeiro, 1926.ma.f004-|Fouquette ${ }^{+1}$, 2014.fa.f001|-03

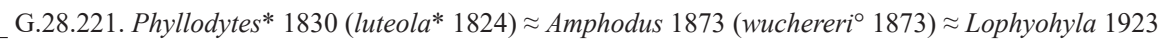

LT (piperata $1923 \approx$ luteola* 1824) $\equiv$ Lophyohila 1926 LP $\equiv$ Lophiohyla 1926

F.21.19. Infratribus OSTEOCEPHALINIA nov., DOP.da.f062-00 \{99\}

G.28.222. Dryaderces $^{\circ} 2013$ (pearsoni $^{\circ} 1929$ )

G.28.223. Osteocephalus* 1862 (taurinus* 1862) $\equiv$ Osteocephalus 1843 AN $\{\mathbf{1 0 0}\}$

G.28.224. Tepuihyla* 1993 (rodriguezi* 1968) $\{\mathbf{1 0 0}\}$

F.21.20. Infratribus OSTEOPILINIA nov., DOP.da.f063-00 $\{\mathbf{1 0 0}\}$

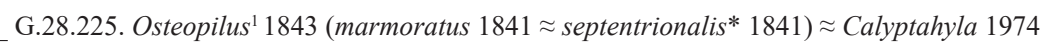

(lichenatus $1851 \approx$ crucialis* 1826 ) $^{*}$

F.20.27. Subtribus PHYTOTRYADINA nov., DOP.da.f064-00

G.28.226. Phytotriades* 2009 (auratus* 1917)

F.20.28. Subtribus TRACHYCEPHALINA Lutz, 1969.1a.f002-01 \{90\}

F.21.21. Infratribus CORYTHOMANTINIA nov., DOP.da.f065-00

G.28.227. Corythomantis* 1896 (greeningi* 1896)

F.21.22. Infratribus NYCTIMANTINIA nov., DOP.da.f066-00 \{100\}

G.28.228. Aparasphenodon* 1920 (brunoi* 1920)

G.28.229. Argenteohyla* 1970 (siemersi* 1937)

G.28.230. Nyctimantis* 1882 (rugiceps* $^{*}$ 1882)

F.21.23. Infratribus TRACHYCEPHALINIA Lutz, 1969.1a.f002-02 \{100\}

G.28.231. Trachycephalus* 1838 (nigromaculatus* 1838) $\approx$ Osilophus 1838 (typhonia* 1758)

$\equiv$ Otilophus $1859 \mathbf{J H} \approx$ Acrodytes $1843 \mathbf{C I}($ venulosa $1768 \approx$ typhonia* 1758) $\approx$ Cephalophractus 1843

AN $\left(\right.$ galeatus $^{\circ} 1843$ AN $\approx$ nigromaculatus $\left.* 1758\right) \approx$ Phrynohyas 1843 (zonata $1824 \approx$ typhonia* 1758 )

$\approx$ Scytopis 1862 (hebes $1862 \approx$ typhonia* 1758) Scytopsis $1878 \approx$ Tetraprion 1891 (jordani* 1891)

F.19.33. Tribus SCINAXINI Duellman ${ }^{+2}, 2016 . d b . f 002-01\{98\}$

F.20.29. Subtribus SCINAXINA Duellman ${ }^{+2}, 2016 . \mathrm{db} . \mathrm{f002}-03\{\mathbf{1 0 0}\}$

G.28.232. Scinax ${ }^{2} 1830\left(\right.$ aurata $\left.^{\circ} 1821\right) \approx$ Ololygon 1843 (strigilata $\left.{ }^{\circ} 1824\right) \equiv$ Ologigon $1923 \mathrm{AM}$

$\equiv$ Ololigon $1923 \mathrm{AM} \approx$ Garbeana $1926\left(\right.$ garbei* $^{*}$ 1926) $\approx$ Julianus $^{*} 2016$ (uruguaya* 1877) $\{\mathbf{1 0 0}\}$

F.20.30. Subtribus SPHAENORHYNCHINA Faivovich ${ }^{+15}$, 2018.fa.f001-00

G.28.233. Gabohyla 2020 (pauloalvini ${ }^{\circ} 2020$ )

G.28.234. Sphaenorhynchus* 1838 (lactea* 1800) $\equiv$ Dryomelictes $1843 \approx$ Dryomelictes 1865 JH

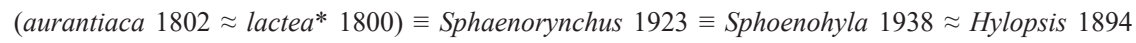

(platycephalus $\left.{ }^{\circ} 1894\right)\{\mathbf{1 0 0}\}$

F.17.25. Familia PHYLLOMEDUSIDAE Günther, 1858.gc.f009-00 \{100\} [S] [N]

F.18.38. Subfamilia PELODRYADINAE Günther, 1859.ga.f001-01 \{100\}

G.28.†102. Australobatrachus ${ }^{\circ} 1976 \dagger\left(\right.$ ilius $\left.^{\circ} 1976 \dagger\right)$

G.28.235. Litoria* 1838 (freycinet $*$ 1838$) \equiv$ Lepthyla 1841 AN $\equiv$ Pelobius $1843 \mathbf{J H} \approx$ Hylomantis 1880

JH $($ fallax* 1880$) \equiv$ Drymomantis $1882 \approx$ Coggerdonia 1985 (adelaidensis* 1841$) \approx$ Colleeneremia

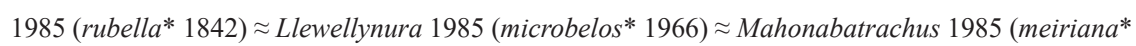

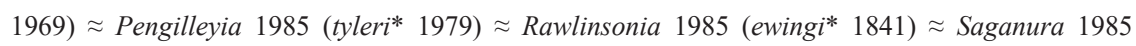

(burrowsi* 1942) $\{\mathbf{1 0 0}\}$ 
G.28.236. Nyctimystes* 1916 (papua* 1897) $\approx$ Sandyrana 1985 (infrafrenata* 1867) \{100\}

G.28.237. Ranoidea 1838 LT (jacksoniensis $1838 \approx$ aurea $\left.^{*} 1829\right) \equiv$ Ranoides 1838 LP $\equiv$ Polyphone

$1848 \approx$ Calamita $1826 \mathbf{J H}$ (caerulea* 1790) $\equiv$ Calamites $1830 \mathbf{J H} \equiv$ Pelodryas 1858 AN $\equiv$ Pelodryas

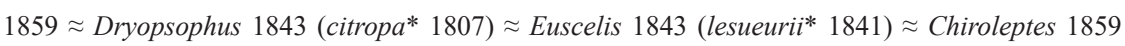

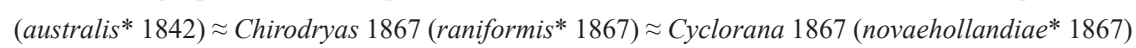

$\approx$ Phractops 1867 (alutaceus $1867 \approx$ novaehollandiae* 1867$) \approx$ Mitrolysis 1889 (alboguttatus* 1867)

$\equiv$ Brendanura $1985 \approx$ Fanchonia 1893 (elegans $1893 \approx$ aurea* 1829) $\approx$ Mosleyia 1985 (nannotis*

$1916) \approx$ Neophractops 1985 (platycephalus* 1873) $\{\mathbf{1 0 0}\}$

F.18.39. Subfamilia PHYLLOMEDUSINAE Günther, 1858.gc.f009-03 \{100\}

F.19.34. Tribus AGALYCHNINI nov., DOP.da.f067-00 \{100\}

G.28.238. Agalychnis* 1864 (callidryas* 1862) ₹ Pachymedusa 1968 (dacnicolor* 1864) \{98\}

G.28.239. Hylomantis* 1873 (aspera* 1873) $\{\mathbf{1 0 0}\}$

F.19.35. Tribus CRUZIOHYLINI nov., DOP.da.f068-00

G.28.240. Cruziohyla* 2005 (calcarifer* 1902)

F.19.36. Tribus PHRYNOMEDUSINI nov., DOP.da.f069-00

G.28.241. Phrynomedusa ${ }^{3} 1923$ (fimbriata ${ }^{\circ}$ 1923)

F.19.37. Tribus PHYLLOMEDUSINI Günther, 1858.gc.f009-04 \{96\}

F.20.31. Subtribus PHASMAHYLINA nov., DOP.da.f070-00 \{100\}

G.28.242. Phasmahyla* 1991 (guttata* 1924)

F.20.32. Subtribus PHYLLOMEDUSINA Günther, 1858.gc.f009-05 \{100\}

F.21.24. Infratribus PHYLLOMEDUSINIA Günther, 1858.gc.f009-06 \{100\}

G.28.243. Phyllomedusa* 1830 (bicolor* 1772) $\equiv$ Hyla $1828 \mathbf{J H}$

F.21.25. Infratribus PITHECOPODINIA Lutz, 1969.la.f001-01 \{100\}

G.28.244. Callimedusa* 2016 (perinesos* 1973) $\{99\}$

G.28.245. Pithecopus* 1866 (azurea* 1862) $\approx$ Bradymedusa 1926 (moschata $1926 \approx$ rohdei* 1926)

$\{100\}$

F.14.07. Superfamilia LEPTODACTYLOIDEA ||Tschudi, 1838.ta.f001||-Werner, 1896.wa.f001-03 \{100\}

F.17.26. Familia LEPTODACTYLIDAE ||Tschudi, 1838.ta.f001\|-Werner, 1896.wa.f001-00 \{100\} [Q] [T]

F.18.40. Subfamilia LEIUPERINAE Bonaparte, 1850.bb.f010-02 \{100\} [T]

F.19.38. Tribus LEIUPERINI Bonaparte, 1850.bb.f010-03 \{100\}

G.28.246. Pleurodema* 1838 LT (bibroni* 1838) $\equiv$ Pleuroderma 1838 LP $\approx$ Leiuperus 1841

(marmoratus $* 1841) \approx$ Chianopelas 1845 a AN (viridis $1845 \approx$ marmoratus $* 1841) \equiv$ Chionopelas

1845 AN $\equiv$ Liyperus $1847 \equiv$ Liuperus $1861 \equiv$ Lihyperus $1875 \approx$ Metaeus 1853 (timidus* 1853)

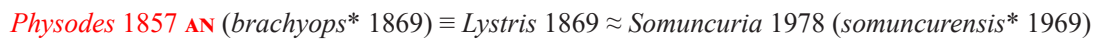

F.19.39. Tribus PALUDICOLINI Mivart, 1869.ma.f004-02 \{100\}

F.20.33. Subtribus EDALORHININA nov., DOP.da.f071-00

G.28.247. Edalorhina* 1870 (perezi* 1870) $\approx$ Bubonias 1874 (plicifrons $1874 \approx$ perezi* 1870 )

F.20.34. Subtribus PALUDICOLINA Mivart, 1869.ma.f004-03 \{99\}

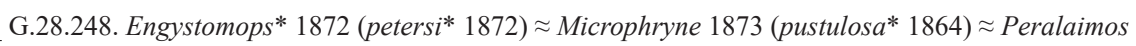

1875 (stentor $1872 \approx$ pustulosa* 1864) $\{\mathbf{1 0 0}\}$

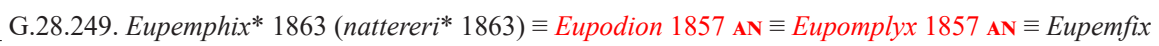
$1898\{\mathbf{1 0 0}\}$

G.28.250. Physalaemus* 1826 (cuvieri* 1826) $\equiv$ Physalamis 1831 AM $\equiv$ Physolaemus 1846

$\approx$ Paludicola 1830 (albifrons ${ }^{\circ} 1824$ ) $\approx$ Hyobates 1857 AN (fuscomaculatus $1864 \approx$ biligonigerus $*$

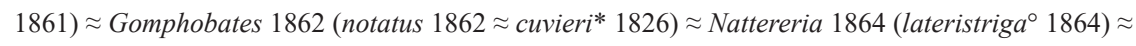
Sphagepodium 1864 AN (albonotatus* 1864) \{92\}

F.18.41. Subfamilia LEPTODACTYLINAE ||Tschudi, 1838.ta.f001\|-Werner, 1896.wa.f001-00 \{100\} [Q] [T]

F.19.40. Tribus ADENOMERINI Hoffmann, 1878.ha.f003-01 \{100\}

G.28.251. Adenomera 1867 (marmorata $\left.{ }^{3} 1867\right) \equiv$ Adenomera 1861 AN $\approx$ Parvulus 1930

(nanus ${ }^{\circ}$ 1922) $\{\mathbf{1 0 0}\}$

G.28.252. Lithodytes* 1843 (lineata* 1799) $\approx$ Rana 1828 JH (schneideri $1820 \approx$ lineata $\left.^{*} 1799\right)$

F.19.41. Tribus LEPTODACTYLINI ||Tschudi, 1838.ta.f001||-Werner, 1896.wa.f001-01 \{92\}

G.28.253. Leptodactylus 1826 (typhonius $1801 \approx$ fuscus $\left.^{*} 1799\right) \approx$ Cystignathus 1830 (pachypus 1824

$\approx$ latrans $\left.^{*} 1815\right) \equiv$ Doryphoros $1835 \approx$ Gnathophysa 1843 (labyrinthica* 1824$) \approx$ Sibilatrix 1843

$\left(\right.$ gracilis $\left.^{*} 1841\right) \approx$ Plectromantis 1862 (wagneri $\left.* 1862\right) \approx$ Entomoglossus 1870 (pustulatus $\left.{ }^{\circ} 1870\right) \approx$

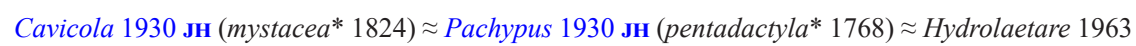


(schmidti $\left.{ }^{\circ} 1959\right) \approx$ Vanzolinius 1974 (discodactylus $\left.{ }^{*} 1883\right)\{99\}$

F.18.42. Subfamilia PARATELMatobiINAE Ohler $^{+1}$, 2012.oa.f001-01 \{99\} [T]

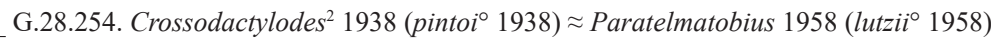

$\approx$ Scythrophrys 1971 (sawayae* 1953) $\{\mathbf{1 0 0}\}$

G.28.255. Rupirana* 1999 (cardosoi* 1999)

F.18.43. Subfamilia PSEUDOPALUDICOLINAE Gallardo, 1965.ga.f003-01 \{98\} [T]

G.28.256. Pseudopaludicola* 1926 (falcipes* 1867)

C.11.02. Subphalanx DiplosiPHona Günther, 1859.ga.c02-01 $\{\mathbf{1 0 0}\}$

F.17.27. Familia CALYPTOCEPHALELLIDAE Reig, 1960.ra.f001-02 \{100\} [S] [N]

G.28.257. Calyptocephalella* 1928 (gayi* 1841) $\equiv$ Calyptocephalus $1841 \mathbf{J H} \equiv$ Cephalopeltis 1841

AN $\equiv$ Cephalopeltis $1875 \equiv$ Calyptocephala $1923 \mathbf{J H} \equiv$ Peltocephalus $1838 \mathbf{J H}$ (quoyi $1838 \equiv$ gayi*

$1841) \approx$ Teracophrys 1901 AN (rugata 1901 AN $\$) \approx$ Eophractus 1949 (casamayorensis $\left.{ }^{\circ} \$ 1949\right) \approx$

Gigantobatrachus 1958 (parodii $^{\circ} 1958 \ddagger$ ) $\approx$ Wawelia $^{\circ} 1959$ (gerholdi $1959 \ddagger$ )

G.28.258. Telmatobufo* 1952 (bullocki* 1952) \{98\}

F.17.28. Familia MYOBATRACHIDAE Schlegel, 1850.sa.f001-00 \{100\} [Q]

F.18.†0d. Subfamilia INCERTAE SEDIS †

G.28.†103. Indobatrachus ${ }^{\circ} 1930 \dagger\left(\right.$ pusilla $\left.^{\circ} 1847 \dagger\right)$

F.18.44. Subfamilia LIMNODYNASTINAE Lynch, 1971.1a.f001-01 \{100\}

F.19.42. Tribus LIMNODYNASTINI Lynch, 1971.1a.f001-00 \{97\}

F.20.35. Subtribus HELEIOPORINA Bauer, 1987.bc.f002-01

G.28.259. Heleioporus ${ }^{2} 1841$ a (albopunctatus $^{\circ}$ 1841) $\equiv$ Helioporus 1841 b AM $\equiv$ Heleioforus 1865

$\approx$ Perialia 1845 (eyrei $\left.^{\circ} 1845\right) \approx$ Philocryphus 1894 (flavoguttatus $1894 \approx$ australiaca* $\left.^{*} 1795\right) \approx$

Paraheleioporus 2019 (barycragus $^{\circ}$ 1967)

F.20.36. Subtribus LIMNODYNASTINA Lynch, 1971.la.f001-03 \{95\}

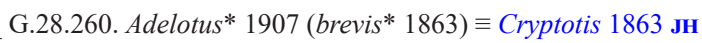

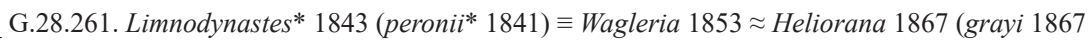

$\approx$ dumerilii* 1863$) \approx$ Ranaster 1878 (canvexiusculus* 1878) $\approx$ Megistolotis 1979 (lignarius* 1979)

$\{\mathbf{1 0 0}\}$

G.28.262. Philoria ${ }^{2} 1901$ (frosti $\left.^{\circ} 1901\right) \approx$ Kyarranus 1959 (sphagnicolus* 1958) $\approx$ Coplandia 1985

(kundagungan ${ }^{\circ}$ 1958)

F.20.37. Subtribus NEOBATRACHINA nov., DOP.da.f072-00 $\{\mathbf{1 0 0}\}$

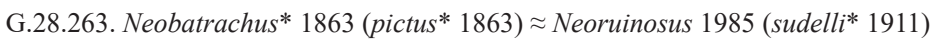

F.20.38. Subtribus PLATYPLECTRINA nov., DOP.da.f073-00 \{99\}

G.28.264. Platyplectrum ${ }^{1} 1863$ (marmoratum $1863 \approx$ ornatus $^{*} 1842$ ) $\equiv$ Platyplectron 1863 AM

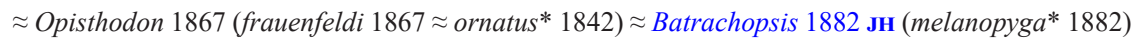

$\equiv$ Lechriodus $1882 \approx$ Phanerotis 1890 (fletcheri* 1890 )

F.19.43. Tribus NOTADENINI nov., DOP.da.f074-00 $\{\mathbf{1 0 0}\}$

G.28.265. Notaden* 1873 (bennettii* 1873)

F.18.45. Subfamilia MIXOPHYINAE nov., DOP.da.f075-00 \{100\}

G.28.266. Mixophyes* 1864 (fasciolatus* 1864) $\equiv$ Myxophyes $1865 \equiv$ Mixophys 1993

F.18.46. Subfamilia MYoBATRACHINAE Schlegel, 1850.sa.f001-02 \{100\}

F.19.44. Tribus MYOBATRACHINI Schlegel, 1850.sa.f001-05 \{100\}

F.20.39. Subtribus CRINIINA Cope, 1866.ca.f001-02 \{98\}

F.21.26. Infratribus ASSINIA nov., DOP.da.f076-00 \{100\}

F.22.15. Hypotribus ASSINOA nov., DOP.da.f076-01 \{93\}

G.28.267. Assa* 1972 (darlingtoni* 1933)

G.28.268. Geocrinia ${ }^{3} 1973$ (laevis $\left.^{\circ} 1864\right) \approx$ Hesperocrinia 1985 (leai $^{\circ} 1898$ )

F.22.16. Hypotribus PARACRINIINOA nov., DOP.da.f077-00

G.28.269. Paracrinia* 1976 (haswelli* 1894)

F.21.27. Infratribus CRINIINIA Cope, 1866.ca.f001-03 $\{\mathbf{1 0 0}\}$

G.28.270. Crinia* 1838 (georgiana* 1838) $\approx$ Ranidella 1853 (signifera* 1853) $\approx$ Camariolius 1863

(varius $1863 \approx$ signifera $\left.^{*} 1853\right) \approx$ Pterophrynus 1864 (verrucosus $1864 \approx$ signifera $^{*} 1853$ ) $\approx$

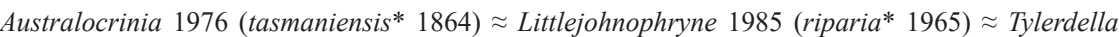

1985 (remota* 1974) $\approx$ Bryobatrachus 1994 (nimbus* 1994)

F.20.40. Subtribus MYOBATRACHINA Schlegel, 1850.sa.f001-06 \{100\}

F.21.28. Infratribus MYOBATRACHINIA Schlegel, 1850.sa.f001-07 \{100\} 
F.22.17. Hypotribus MYOBATRACHINOA Schlegel, 1850.sa.f001-08 \{100\}

G.28.271. Arenophryne* 1976 (rotunda* 1976)

G.28.272. Metacrinia* 1940 (nichollsi* 1927)

G.28.273. Myobatrachus ${ }^{1} 1850$ (paradoxus $1850 \approx$ gouldii* $^{*} 1841$ ) $\equiv$ Myiobatrachus [1850] 1858

$\approx$ Chelydobatrachus 1859 (gouldii* 1841)

F.22.18. Hypotribus PSEUDOPHRYNINOA Bauer, 1987.bc.f001-01 \{100\}

G.28.274. Pseudophryne 1843 (australis $^{\circ} 1835$ ) $\equiv$ Bufonella $1853 \equiv$ Pseudofryne $1898 \approx$ Kankanophryne

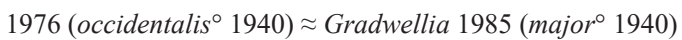

F.21.29. Infratribus SPICOSPININIA nov., DOP.da.f078-00

G.28.275. Spicospina* 1997 (flammocaerulea* 1997)

F.21.30. Infratribus UPEROLEIINIA Günther 1858.gc.f007-04 \{100\}

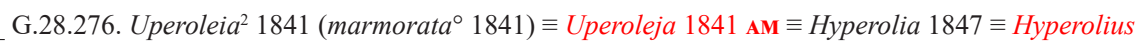

1882 Aм $\approx$ Glauertia 1933 (russell $*$ 1933) $\approx$ Hosmeria 1985 (laevigata* 1867 ) $\approx$ Prohartia 1985

(fimbrianus $1926 \approx$ rugosa* 1916)

F.19.45. Tribus TAUDACTYLINI nov., DOP.da.f079-00

G.28.277. Taudactylus ${ }^{3} 1966$ (diurnus $^{\circ}$ 1966)

F.18.47. Subfamilia RHEOBATRACHINAE Heyer ${ }^{+1}$, 1976.ha.f001-00

G.28.278. Rheobatrachus* 1973 (silus* 1973)

C.10.03. Phalanx Scoptanura Starrett, 1973.sb.c02-02 $\{\mathbf{1 0 0}\}$

C.11.†0a. Subphalanx INCERTAE SEDIS

G.28.†104. Hungarobatrachus ${ }^{\circ} 2010 \dagger\left(\right.$ szukacsi $\left.^{\circ} 2010 \dagger\right)$

C.11.03. Subphalanx Ecostata Lataste, 1879.1b.c04-02 \{100\}

F.17.29. Familia MICROHYLIDAE $\|$ Fitzinger, 1843.fa.f012\|-Noble, 1931.na.f001-01 \{100\} [Q]

F.18.48. Subfamilia ADELASTINAE Peloso ${ }^{+10}$, 2016.pa.f001-00

G.28.279. Adelastes $^{\circ} 1986$ (hylonomos $\left.^{\circ} 1986\right)$

F.18.49. Subfamilia ASTEROPHRYINAE Günther, 1858.gc.f006-05 \{100\}

F.19.46. Tribus ASTEROPHRYINI Günther, 1858.gc.f006-09 \{99\}

G.28.280. Asterophrys* 1838 (turpicola* 1837 ) $\equiv$ Asterofrys $1898 \approx$ Xenorhina 1863 (oxycephalus*

$1858) \approx$ Hylophorbus 1878 (rufescens $* 1878) \approx$ Sphenophryne 1878 (cornuta* 1878) $\approx$ Xenobatrachus 1878 (ophiodon $\left.{ }^{\circ} 1878\right) \equiv$ Stenofryne $1898 \approx$ Callulops 1888 (doriae* 1888) $\approx$ Genyophryne 1890 (thomsoni* 1890) $\equiv$ Genyofryne $1898 \approx$ Cophixalus 1892 (verrucosa $\left.{ }^{\circ} 1898\right) \approx$ Oreophryne 1895 (senckengergiana $1895 \approx$ moluccensis $\left.{ }^{\circ} 1878\right) \approx$ Phrynixalus 1895 (montanus $\left.^{\circ} 1895\right) \approx$ Liophryne $^{2}$ 1897 (rhododactyla* 1897) $\approx$ Mantophryne 1897 (lateralis* 1897) $\approx$ Choanacantha 1898 (rostrata ${ }^{\circ}$ $1898) \approx$ Copiula 1901 (oxyrhinus* 1898) $\approx$ Gnathophryne 1901 (robusta* 1889) $\approx$ Metopostira 1901 (ocellata $1901 \approx$ rufescens $\left.^{*} 1878\right) \approx$ Microbatrachus 1910 (pusillus ${ }^{\circ} 1910$ ) $\approx$ Pomatops $1910\left(\right.$ valvifera $\left.^{\circ} 1910\right) \approx$ Mehelyia 1911 (lineata $1911 \approx$ biroi $\left.^{\circ} 1897\right) \approx$ Austrochaperina 1912 $\left(\right.$ robusta $\left.{ }^{\circ} 1912\right) \approx$ Oxydactyla 1913 (brevicrus $\left.^{\circ} 1913\right) \approx$ Choerophryne $1914_{\text {(proboscidea }}$ 1914) $^{2}$ Aphantophryne 1917 (pansa* 1917) $\approx$ Pseudengystoma 1930 (bouwensi* $\left.{ }^{*} 1930\right) \approx$ Barygenys 1936 $\left(\right.$ cheesmanae $\left.^{\circ} 1936\right) \approx$ Pherohapsis 1972 (menziesi* 1972$) \approx$ Albericus 1995 (darlingtoni $\left.{ }^{\circ} 1948\right)$ $\approx$ Metamagnusia 2009 (marani* 2009) $\approx$ Pseudocallulops 2009 (pullifer* 2009) $\approx$ Oninia 2010 (senglaubi* 2010) $\approx$ Paedophryne 2010 (kathismaphlox $\left.{ }^{\circ} 2010\right)\{99\}$

F.19.47. Tribus GASTROPHRYNOIDINI nov., DOP.da.080-00

G.28.281. Gastrophrynoides ${ }^{3} 1926$ (borneense $^{\circ} 1897$ )

G.28.282. Siamophryne S $^{\circ} 018$ (troglodytes ${ }^{\circ} 2018$ )

G.28.283. Vietnamophryne 2018 (inexpectata ${ }^{\circ} 2018$ )

F.18.50. Subfamilia CoPHYLINAE Cope, 1889.ca.f001-01 \{99\}

F.19.48. Tribus COPHYLINI Cope, 1889.ca.f001-02 \{100\}

F.20.0b. Subtribus INCERTAE SEDIS

G.28.284. Madecassophryne 1974 (truebae ${ }^{\circ} 1974$ )

F.20.41. Subtribus ANODONTHYLINA nov., DOP.da.f081-00 \{100\}

G.28.285. Anodonthyla* 1892 (boulengerii* 1892) $\equiv$ Anodontohyla 1901

F.20.42. Subtribus COPHYLINA Cope, 1889.ca.f001-03 \{91\}

G.28.286. Cophyla* 1880 (phyllodactyla* 1880) $\approx$ Plethodontohyla 1882 (notosticta* 1877)

$\approx$ Anilany* $^{*} 2016$ (helenae* 2000) $\approx$ Mini $^{\circ} 2019\left(\right.$ mum $\left.^{\circ} 2019\right)\{\mathbf{1 0 0}\}$

G.28.287. Mantipus ${ }^{1} 1883$ (hildebrandti $1833 \approx$ inguinalis $^{*} 1882$ ) $\approx$ Phrynocara 1883 (tuberatum* 1883) $\{96\}$ 
F.20.43. Subtribus PLATYPELINA nov., DOP.da.f082-00 $\{\mathbf{1 0 0}\}$

G.28.288. Platypelis ${ }^{2} 1882\left(\right.$ cowanii $\left.^{\circ} 1882\right) \approx$ Platyhyla 1889 (grandis* 1889$) \approx$ Paracophyla 1951

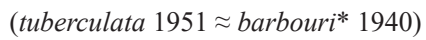

F.20.44. Subtribus RHOMBOPHRYNINA Noble, 1931.na.f009-01 \{100\}

G.28.289. Rhombophryne* 1880 (testudo* 1880) $\equiv$ Rhombofryne $1898 \approx$ Stumpffia 1881 (psologlossa*

$1881) \approx$ Mantiphrys 1895 (laevipes* 1895) $\equiv$ Mantophrys 1909

F.19.49. Tribus SCAPHIOPHRYNINI Laurent, 1946.la.f002-03 \{99\}

G.28.290. Paradoxophyla* 1991 (palmata* 1974) $\{\mathbf{1 0 0}\}$

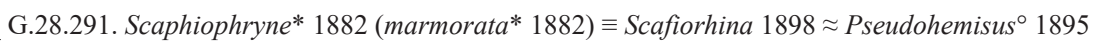

(obscurus $\left.{ }^{\circ} 1895\right)\{\mathbf{1 0 0}\}$

F.18.51. Subfamilia GASTROPHRYNINAE Fitzinger, 1843.fa.f011-02 \{100\}

F.19.50. Tribus CHIASMOCLEINI nov., DOP.da.f083-00 \{100\}

G.28.292. Chiasmocleis* 1904 (albopunctatum* 1885) ₹ Nectodactylus 1924 (spinulosus 1924

$\approx$ leucosticta* 1888 ) $^{*}$

G.29.001. Chiasmocleis* 1904 (albopunctatum* 1885) $\approx$ Nectodactylus 1924 (spinulosus 1924

$\approx$ leucosticta*1888) $\{\mathbf{9 3}\}$

G.29.002. Relictocleis ${ }^{\circ}$ nov. $\left(\right.$ gnoma $^{\circ}$ 2004) $\equiv$ Relictus 2018 AN $\equiv$ Relictus 2019 AN $\equiv$ Unicus $2019 a$

$\mathbf{A N} \equiv$ Unicus 2019 b AN

G.29.003. Syncope* 1973 (antenori* 1973) \{93\}

F.19.51. Tribus CTENOPHRYNINI nov., DOP.da.f084-00 $\{\mathbf{1 0 0}\}$

G.28.293. Ctenophryne* 1904 (geayi* 1904) $\approx$ Glossostoma $1901 \mathbf{J H}\left(\right.$ aterrimum $\left.^{\circ} 1900\right)$

$\equiv$ Nelsonophryne $1987 \approx$ Melanophryne 2007 (carpish $^{\circ}$ 2002)

F.19.52. Tribus GASTROPHRYNINI Fitzinger, 1843.fa.f011-03 \{100\}

F.20.45. Subtribus DASYPOPINA nov., DOP.da.f085-00 $\{\mathbf{1 0 0}\}$

G.28.294. Dasypops* 1924 (schirchi* 1924)

G.28.295. Myersiella 1954 (subnigrum $1920 \approx$ microps $\left.^{*} 1841\right)$

F.20.46. Subtribus GASTROPHRYNINA Fitzinger, 1843.fa.f011-04 \{99\}

F.21.31. Infratribus ARCOVOMERINIA nov., DOP.da.f086-00

G.28.296. Arcovomer* 1954 (passarellii* 1954)

F.21.32. Infratribus DERMATONOTINIA nov., DOP.da.f087-00

G.28.297. Dermatonotus* 1904 (muelleri* 1885)

F.21.33. Infratribus ENGYSTOMATINIA Bonaparte, 1850.bb.f009-08 \{100\}

G.28.298. Engystoma* 1826 (ovalis* 1799) $\equiv$ Systoma $1830 \equiv$ Engistoma 1904 AM $\equiv$ Elachistocleis

$1927 \approx$ Microps 1828 JH (unicolor $1828 \approx$ ovalis* 1799) $\equiv$ Stenocephalus 1838 JH $\approx$ Relictivomer

1954 (pearsei $^{\circ}$ 1914)

F.21.34. Infratribus GASTROPHRYNINIA Fitzinger, 1843.fa.f011-05 \{100\}

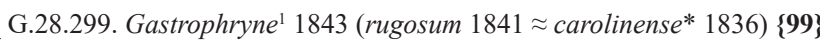

G.28.300. Hypopachus* 1867 (seebachii $1867 \approx$ variolosum $\left.^{*} 1866\right)\{91\}$

F.21.35. Infratribus HAMPTOPHRYNINIA nov., DOP.da.f088-00

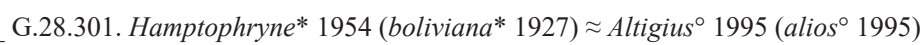

F.20.47. Subtribus STEREOCYCLOPINA nov., DOP.da.f089-00

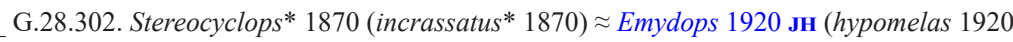

$\approx$ incrassatus $\left.^{*} 1870\right) \equiv$ Ribeirina $1934 \approx$ Hyophryne 1954 (histrio ${ }^{\circ} 1954$ )

F.18.52. Subfamilia HoplopHRYNINAE Noble, 1931.na.f016-00 \{100\}

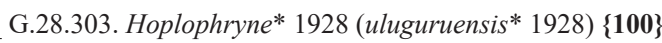

G.28.304. Parhoplophryne 1928 (usambarica ${ }^{\circ}$ 1928)

F.18.53. Subfamilia KALOPHRYNINAE Mivart, 1869.ma.f003-01 \{100\}

G.28.305. Kalophrynus* 1838 (pleurostigma* 1838) 三 Calophryne $1843 \equiv$ Calliphryne 1847

$\equiv$ Calophrynus $1863 \equiv$ Calofrynus $1898 \approx$ Berdmorea 1872 (interlineatum $* 1855$ )

F.18.54. Subfamilia MELANOBATRACHINAE Noble, 1931.na.f015-00

G.28.306. Melanobatrachus* 1878 (indicus* 1878)

F.18.55. Subfamilia MICROHYLINAE ||Fitzinger, 1843.fa.f012||-Noble, 1931.na.f001-00 \{100\}

F.19.53. Tribus DYSCOPHINI Boulenger, 1882.bb.f001-05 \{100\}

G.28.307. Dyscophus* 1872 (insularis* 1872)

F.19.54. Tribus MICROHYLINI ||Fitzinger, 1843.fa.f012||-Noble, 1931.na.f001-07 \{100\}

F.20.48. Subtribus CHAPERININA Peloso ${ }^{+10}$, 2016.pa.f002-01 
G.28.308. Chaperina* 1892 (fusca* 1892)

F.20.49. Subtribus HYLAEDACTYLINA Fitzinger, 1843.fa.f009-05 \{100\}

F.21.36. Infratribus CACOPINIA Noble, 1931.na.f011-01 \{99\}

G.28.309. Uperodon 1841 (marmoratum $1837 \approx$ systoma $\left.^{*} 1799\right) \equiv$ Hyperodon $1847 \equiv$ Cacopus 1864

$\equiv$ Hyperoodon $1902 \equiv$ Hiperoodon 1902 AN $\approx$ Pachybatrachus 1868 (petersii $1868 \approx$ systoma* 1799 )

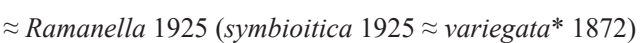

F.21.37. Infratribus HYLAEDACTYLINIA Fitzinger, 1843.fa.f009-06 \{100\}

G.28.310. Kaloula* 1831 (pulchra* 1831) 三 Calohyla $1863 \equiv$ Callula $1864 \equiv$ Kalooula 1895 AM

$\approx$ Hyladactylus 1838 LT (baleatus* 1836) $\equiv$ Hyladactyla 1838 LP $\equiv$ Hylaedactylus $1841 \equiv$ Hylaedactyla

1841 AN $\equiv$ Hylodactylus $1847 \equiv$ Pelida $1848 \approx$ Plectropus 1841 (pictus* 1841) $\approx$ Holonectes 1863

(conjunctus* 1863$) \equiv$ Hylophryne 1864 AN $\equiv$ Hyledactylus $1895 \approx$ Cacopoides 1908 (borealis* 1908)

F.21.38. Infratribus PHRYNELLINIA nov., DOP.da.f090-00 \{100\}

G.28.311. Metaphrynella* 1934 (pollicaris* 1890) $\{\mathbf{1 0 0}\}$

G.28.312. Phrynella* 1887 (pulchra* 1887)

F.20.50. Subtribus MICROHYLINA ||Fitzinger, 1843.fa.f012||-Noble, 1931.na.f001-08 \{100\}

G.28.313. Glyphoglossus* 1869 (molossus* 1869) $\equiv$ Glyfoglossus $1898 \approx$ Calluella 1872 (guttulata*

$1856) \approx$ Colpoglossus 1904 (brooksii $\left.{ }^{\circ} 1904\right) \approx$ Dyscophina 1905 (volzi $\left.{ }^{\circ} 1905\right) \approx$ Calliglutus 1916 (smithi ${ }^{\circ}$ 1916) $\{\mathbf{1 0 0}\}$

G.28.314. Microhyla* 1838 (achatina* 1838$) \equiv$ Micrhyla $1841 \equiv$ Dendromanes $1848 \equiv$ Mycrohyla

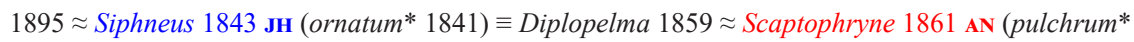

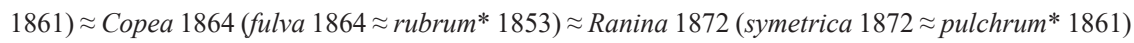
$\{96\}$

F.20.51. Subtribus MICRYLETTINA nov., DOP.da.f091-00 \{100\}

G.28.315. Micryletta* 1987 (inornata* 1890)

G.28.316. Mysticellus $^{\circ} 2019$ (franki $^{\circ}$ 2019)

F.18.56. Subfamilia OTOPHRYNINAE Wassersug ${ }^{+1}$, 1987.wa.f001-00 \{98 $\}$

G.28.317. Otophryne* 1900 (robusta* 1900) $\{\mathbf{1 0 0}\}$

G.28.318. Synapturanus* 1954 (mirandaribeiroi* 1975) $\{\mathbf{1 0 0}\}$

F.17.30. Familia PHRYNOMERIDAE Noble, 1931.na.f013-01 \{100\} [S] [N]

G.28.319. Phrynomantis* 1867 (bifasciatus* 1847) 三 Brachymerus $1847 \mathbf{J H} \equiv$ Phrynomerus 1926

$\approx$ Fichteria 1941 (somalica ${ }^{\circ}$ 1941)

C.11.04. Subphalanx Gastrechmia Cope, 1867.ca.c02-03 \{100\}

F.14.08. Superfamilia ARTHROLEPTOIDEA Mivart, 1869.ma.f011-05 \{100\}

F.17.31. Familia ARTHROLEPTIDAE Mivart, 1869.ma.f011-02 \{100\} [S] [P]

F.18.57. Subfamilia ARTHROLEPTINAE Mivart, 1869.ma.f011-01 \{100\}

G.28.320. Arthroleptis* 1849 (wahlbergii* 1849) $\approx$ Cardioglossa 1900 (gracilis* 1900)

$\approx$ Schoutedenella 1921 (globosa $1921 \approx$ xenochirus $\left.^{\circ} 1905\right) \approx$ Abroscaphus 1941 (adolfifriederici* $^{*}$

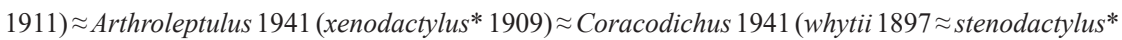
1893)

F.18.58. Subfamilia ASTYLOSTERNINAE Noble, 1927.f002-00 \{92\}

F.19.55. Tribus ASTYLOSTERNINI Noble, 1927.f002-03 \{95\}

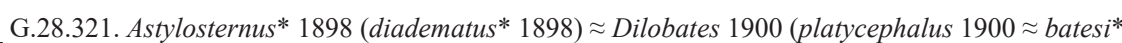

$1900) \approx$ Gampsosteonyx 1900 (batesi* 1900) ₹ Trichobatrachus 1900 (robustus* 1900) \{96\}

G.28.322. Nyctibates* 1904 (corrugatus* 1904)

G.28.323. Scotobleps* 1900 (gabonicus* 1900)

F.19.56. Tribus LEPTODACTYLODONTINI nov., DOP.da.f092-00 \{100\}

G.28.324. Leptodactylodon ${ }^{3} 1903$ (ovatus $^{\circ} 1903$ ) $\approx$ Bulua 1904 (ventrimarmorata $^{\circ} 1904$ )

F.18.59. Subfamilia LEPTOPELINAE Laurent, 1972.1a.f002-01 \{100\}

G.28.325. Leptopelis $^{2} 1859$ (aubryi $\left.^{\circ} 1856\right) \approx$ Pseudocassina 1924 (ocellata $1923 \approx$ gramineus $^{\circ} 1898$ )

$\approx$ Elaphromantis 1941 (notatus $\left.^{\circ} 1875\right) \approx$ Heteropelis 1941 (parkeri $\left.{ }^{\circ} 1928\right) \approx$ Taphriomantis 1941

(bocagii* $\left.^{*} 1865\right) \approx$ Habrahyla 1961 (eiselti $1961 \approx$ notatus $\left.^{\circ} 1875\right) \approx$ Pelopeltis 1986 (bufonides $^{\circ}$

1967)

F.17.32. Familia HYPEROLIIDAE Laurent, 1943.lb.f001-01 \{100\} [Q]

F.18.0b. Subfamilia INCERTAE SEDIS

G.28.326. Arlequinus $^{\circ} 1988$ (krebsi $\left.^{\circ} 1938\right)$ 
G.28.327. Callixalus $^{\circ} 1950$ (pictus $^{\circ}$ 1950)

G.28.328. Chrysobatrachus ${ }^{\circ} 1951$ (cupreonitens ${ }^{\circ}$ 1951)

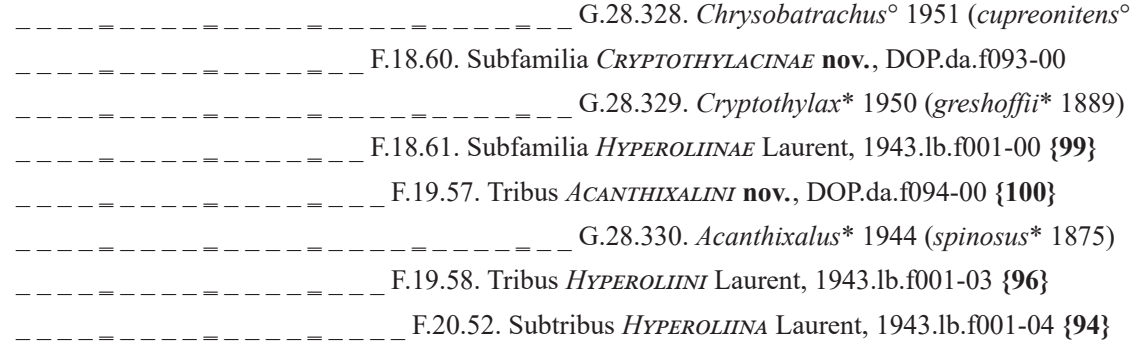

G.28.331. Hyperolius* 1842 (horstockii* 1837) $\equiv$ Eucnemis $1838 \mathbf{J H} \equiv$ Epipole $1848 \equiv$ Rappia 1865

$\approx$ Crumenifera 1862 (pusilla* 1862) $\approx$ Eubates 1864 AN (heuglini 1864 pusilla* 1862) Nesionixalus

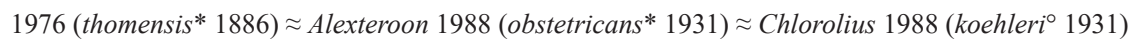

F.20.53. Subtribus MORERELLINA nov., DOP.da.f095-00

G.28.332. Morerella* 2009 (cyanophthalma* 2009)

F.20.54. Subtribus OPISTHотнYLACINA nov., DOP.da.f096-00

G.28.333. Opisthothylax*1966 (immaculatus*1903) 三Opisthothylax 1962 AN

F.20.55. Subtribus TACHYCNEMINA Channing, 1989.ca.f001-01 \{99\}

F.21.39. Infratribus AFRIXALINIA nov., DOP.da.f097-00 \{100\}

G.28.334. Afrixalus* 1944 (fornasinii* 1849) $\approx$ Laurentixalus 2012 (laevis* 1930)

F.21.40. Infratribus TACHYCNEMINIA Channing, 1989.ca.f001-02 \{100\}

G.28.335. Heterixalus* 1944 (madagascariensis* 1841) \{97\}

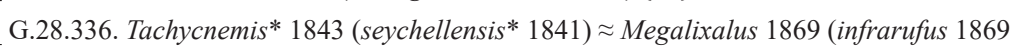

$\approx$ seychellensis* 1841 )

F.19.59. Tribus KASSININI Laurent, 1972.la.f001-00 \{100\}

G.28.337. Hylambates* 1853 (maculatus* 1853) $\approx$ Phlyctimantis 1950 (leonardi* 1906) \{92\}

G.28.338. Kassina* 1853 (senegalensis* 1841$) \equiv$ Eremiophilus 1843 CI $\equiv$ Cassina 1864 AN $\equiv$ Cassina

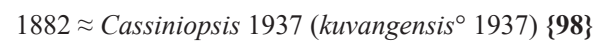

G.28.339. Kassinula 1940 (witte $i^{\circ}$ 1940)

G.28.340. Paracassina ${ }^{\circ} 1907$ (obscura $\left.^{\circ} 1895\right) \approx$ Rothschildia 1905 LT-JH $\left(\right.$ kounhiensis $\left.^{\circ} 1905\right)$

$\equiv$ Rotschildia 1905 LP $\equiv$ Tornierella $1924 \equiv$ Mocquardia 1931

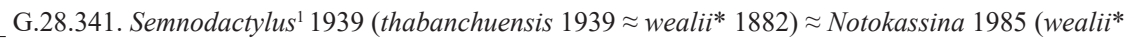

1882)

F.14.09. Superfamilia BREVICIPITOIDEA Bonaparte, 1850.bb.f012-10 \{100\}

F.17.33. Familia BREVICIPITIDAE Bonaparte, 1850.bb.f012-01 \{100\} [S] [P]

F.18.62. Subfamilia BREVICIPITINAE Bonaparte, 1850.bb.f012-02 \{100\}

G.28.342. Breviceps ${ }^{3} 1820$ (gibbosa $\left.{ }^{\circ} 1758\right)$

F.18.63. Subfamilia CALLULININAE nov., DOP.da.f098-00 \{97\}

G.28.343. Balebreviceps* 1989 (hillmani* 1989)

G.28.344. Callulina* 1911 (kreffti* 1911) \{100\}

G.28.345. Probreviceps* 1931 (macrodactylus* 1926) $\{\mathbf{1 0 0}\}$

G.28.346. Spelaeophryne* 1924 (methneri* 1924)

F.17.34. Familia HEMISOTIDAE Cope, 1867.ca.f002-05 [Q]

G.28.347. Hemisus $^{2} 1859$ (guttatum $^{\circ} 1842$ ) $\approx$ Kakophrynus 1863 (sudanensis $1863 \approx$ marmoratum $^{*}$

1854) $\equiv$ Cacophrynus 1867

C.11.05. Subphalanx Pananura nov., DOP.da.c07-00 $\{\mathbf{1 0 0}\}$

C.12.03. Infraphalanx ECAUDATA Scopoli, 1777.sa.c06-01 \{92\}

F.14.10. Superfamilia ODONTOBATRACHOIDEA Barej ${ }^{+5}$, 2014.ba.f001-01

F.17.35. Familia ODONTOBATRACHIDAE Barej $^{+5}$, 2014.ba.f001-00 [M]

G.28.348. Odontobatrachus* 2014 (natator* $^{*}$ 1905)

F.14.11. Superfamilia PHRYNOBATRACHOIDEA Laurent, 1941.lb.f001-02 \{100\}

F.17.36. Familia PHRYNOBATRACHIDAE Laurent, 1941.lb.f001-01 [M]

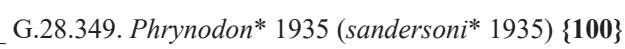

G.28.350. Phrynobatrachus ${ }^{1} 1862$ (natalensis $1862 \approx$ natalensis $\left.^{*} 1849\right) \approx$ Stenorhynchus $1849 \mathbf{J H}$

(natalensis* 1849$) \equiv$ Leptoparius $1863 \approx$ Heteroglossa 1858 JH (africana* 1858$) \equiv$ Dimorphognathus $1906 \approx$ Hemimantis 1863 (calcaratus* 1863) $\equiv$ Pseudarthroleptis $1938 \approx$ Hylarthroleptis 1925 (accraensis* 1925$) \approx$ Pararthroleptis 1925 (nanus $\left.^{\circ} 1925\right) \approx$ Micrarthroleptis 1938 (pygmaeus $^{\circ}$ 
1925) $\{\mathbf{1 0 0}\}$

F.14.12. Superfamilia RANOIDEA Batsch, 1796.ba.f001-28 \{92\}

F.15.05. Epifamilia CONRAUOIDAE Dubois, 1992.da.f001-03 \{100\}

F.17.37. Familia CONRAUIDAE Dubois, 1992.da.f001-02 \{100\} [M]

G.28.351. Conraua* 1908 (robusta* 1908) EConrana $1910 \approx$ Pseudoxenopus 1927 (alleni* 1927)

$\approx$ Gigantorana 1931 (goliath* 1906) $\approx$ Paleorana 1931 AN (beccarii $\left.^{\circ} 1911\right) \equiv$ Hydrobatrachus 1962

F.15.06. Epifamilia ERICABATRACHOIDAE nov., DOP.da.f099-00

F.17.38. Familia ERICABATRACHIDAE nov., DOP.da.f099-01 [M]

G.28.352. Ericabatrachus* 1991 (baleensis* 1991)

F.15.07. Epifamilia MICRIXALOIDAE Dubois $^{+2}, 2001 . d b . f 001-02\{100\}$

F.17.39. Familia MICRIXALIDAE Dubois $^{+2}$, 2001.db.f001-01 [M]

G.28.353. Micrixalus* 1888 (fuscus* 1882)

F.15.08. Epifamilia PETROPEDETOIDAE Noble, 1931.na.f006-03 $\{\mathbf{1 0 0}\}$

F.17.40. Familia PETROPEDETIDAE Noble, 1931.na.f006-02 [M]

G.28.354. Arthroleptides* 1911 (martiensseni* 1911) $\{\mathbf{1 0 0}\}$

G.28.355. Petropedetes* 1874 (cameronensis* 1874) ₹ Tympanoceros 1895 (newtoni 1895

$\approx$ johnstoni* 1888$)\{\mathbf{1 0 0}\}$

F.15.09. Epifamilia PYXICEPHALOIDAE Bonaparte, 1850.bb.f005-04 \{100\}

F.17.41. Familia CACOSTERNIDAE Noble, 1931.na.f008-01 \{100\} [N] [M]

F.18.64. Subfamilia ANHYDROPHRYNINAE nov., DOP.da.f100-00 G.28.356. Anhydrophryne* 1919 (rattrayi* 1919)

F.18.65. Subfamilia CACOSTERNINAE Noble, 1931.na.f008-00 \{92\}

F.19.60. Tribus CACOSTERNINI Noble, 1931.na.f008-02 \{93\}

F.20.56. Subtribus CACOSTERNINA Noble, 1931.na.f008-03 $\{\mathbf{1 0 0}\}$ G.28.357. Cacosternum* 1887 (nanum* 1887) \{92\}

G.28.358. Microbatrachella* 1926 (capensis* 1910) $\equiv$ Microbatrachus 1926 JH $\{\mathbf{1 0 0}\}$

F.20.57. Subtribus POYNTONIINA nov., DOP.da.f101-00 G.28.359. Poyntonia* 1989 (paludicola* 1989)

F.19.61. Tribus NATALOBATRACHINI nov., DOP.da.f102-00 \{99\} G.28.360. Arthroleptella* 1926 (lightfooti* 1910) $\{\mathbf{1 0 0}\}$

G.28.361. Natalobatrachus* 1912 (bonebergi* 1912)

F.19.62. Tribus STRONGYLOPINI Scott, 2005.sa.f001-01 \{98\}

G.28.362. Amietia* 1987 (vertebralis*1927) $\approx$ Afrana 1992 (fuscigula* 1841) $\{\mathbf{1 0 0}\}$

G.28.363. Strongylopus* 1838 (fasciata* 1849) \{91\}

F.18.66. Subfamilia TOMOPTERNINAE Dubois, 1987.da.f003-01 \{100\} G.28.364. Nothophryne 1963 (broadleyi $^{\circ}$ 1963)

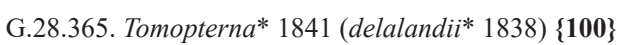

F.17.42. Familia PYXICEPHALIDAE Bonaparte, 1850.bb.f005-03 $\{\mathbf{1 0 0}\}[\mathrm{M}]$

G.28.366. Aubria* 1917 (subsigillata* 1856) $\equiv$ Aubrya 1964 AM

G.28.367. Pyxicephalus* 1838 (adspersus* 1838) ${ }^{*}$ Maltzania 1881 (bufonia $1881 \approx$ edulis* 1854 )

$\approx$ Phrynopsis $1893 \mathbf{J H}$ (boulengerii $1893 \approx$ edulis* 1854) \{99\}

F.15.10. Epifamilia RANOIDAE Batsch, 1796.ba.f001-29 \{93\}

F.16.03. Apofamilia CERATOBATRACHEIDAE Boulenger, 1884.ba.f001-04 \{100\}

F.17.43. Familia CERATOBATRACHIDAE Boulenger, 1884.ba.f001-00 \{100\} [N] [M] [T]

F.18.67. Subfamilia ALCALINAE Brown $^{+4}$, 2015.ba.f002-01 [N] [M] [T]

G.28.368. Alcalus $^{3} 2015$ (mariae $^{\circ}$ 1954)

F.18.68. Subfamilia CERATOBATRACHINAE Boulenger, 1884.ba.f001-00 \{100\} [N] [M] [T]

G.28.369. Cornufer* 1838 (vitiensis* 1853) $\equiv$ Phyllodytes $1848 \mathbf{~ J H} \equiv$ Halophila $1853 \mathbf{~ J H}$

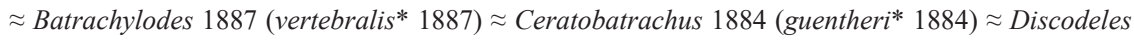

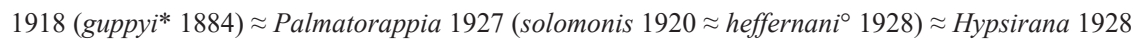

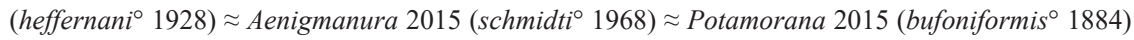
$\{100\}$

G.28.370. Platymantis ${ }^{1} 1859$ (plicifera $1858 \approx$ corrugatus* $^{*} 1853$ ) $\approx$ Platymantis 1858 AN (corrugatus* $1853) \approx$ Lahatnanguri 2015 (levigatus $\left.^{\circ} 1974\right) \approx$ Lupacolus 2015 (dorsalis* 1853) $\approx$ Tahananpuno 2015 (guentheri* 1882) $\approx$ Tirahanulap 2015 (hazelae* 1920) $\{\mathbf{1 0 0}\}$

F.18.69. Subfamilia LIURANINAE $\mathrm{Fei}^{+2}$, 2010.ma.f010-02 [N] [M] [T] 
F.16.04. Apofamilia DiCRoGlosseIDAE Dubois, 1987.da.f004-05 \{100\}

F.17.44. Familia DICROGLOSSIDAE Dubois, 1987.da.f004-03 \{100\} [N] [M]

F.18.0c. Subfamilia INCERTAE SEDIS

G.28.372. Chrysopaa ${ }^{\circ} 2006$ (sternosignata ${ }^{\circ}$ 1885)

F.18.70. Subfamilia DICROGLOSSINAE Dubois, 1987.da.f004-02 \{100\}

F.19.63. Tribus DiCRoglossinI Dubois, 1987.da.f004-00 \{100\}

F.20.58. Subtribus DICROGLOSSINA Dubois, 1987.da.f004-06 \{99\}

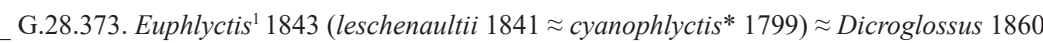

(adolfi $1860 \approx$ cyanophlyctis* 1799) $\{\mathbf{1 0 0}\}$

G.28.374. Hoplobatrachus ${ }^{1} 1863$ (ceylanicus $1863 \equiv$ crassus $\left.^{*} 1853\right) \equiv$ Hoplobactrachus 1868 AM

$\approx$ Hydrostentor 1861 AN (pantherina $1867 \approx$ chinensis* 1765$) \approx$ Ranosoma 1924 (schereri $1924 \approx$

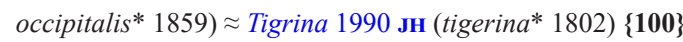

G.28.375. Phrynoderma 1843 (cutipora $1841 \approx$ hexadactyla* 1834 ) $\{\mathbf{1 0 0}\}$

F.20.59. Subtribus NANNOPHRYINA $\mathrm{Fei}^{+2}, 2010 . \mathrm{fa} . \mathrm{f006}-01\{\mathbf{1 0 0}\}$

G.28.376. Nannophrys* 1869 (ceylonensis* 1869) 三Nannofrys $1898 \approx$ Trachucephalus 1874

(ceylanicus $1874 \approx$ ceylonensis* 1869) $\equiv$ Trachycephalus $1875 \mathbf{J H} \equiv$ Fergusonia 1878

F.19.64. Tribus FEJERVARYINI Fei ${ }^{+2}, 2010 . \mathrm{fa} . \mathrm{f005}-\mathrm{c} 0\{\mathbf{1 0 0}\}$

G.28.377. Fejervarya* 1915 (limnocharis* 1829) $\{\mathbf{1 0 0}\}$

G.28.378. Minervarya* 2001 (sahyadris* 2001) ₹ Zakerana 2011 (syhadrensis* 1919) \{100\}

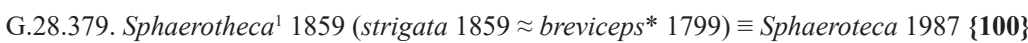

F.18.71. Subfamilia LIMNONECTINAE Dubois, 1992.da.f002-02 \{100\}

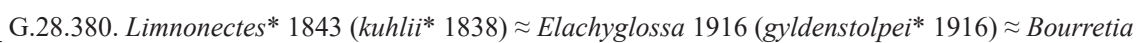

1987 (toumanoffi $1941 \approx$ dabana* 1922) $\approx$ Taylorana 1987 (hascheanus* 1870)

F.18.72. Subfamilia PAINAE Dubois, 1992.da.f003-02 \{100\}

F.19.0a. Tribus INCERTAE SEDIS

G.28.381. Allopaa $^{\circ} 2006$ (hazarensis ${ }^{\circ}$ 1979)

F.19.65. Tribus PAINI Dubois, 1992.da.f003-00 \{100\}

F.20.60. Subtribus CHAPARANINA nov., DOP.da.f103-00 \{96\}

F.21.0a. Infratribus INCERTAE SEDIS

G.28.382. Ombropaa $^{\circ}$ nov. ( gammii $^{\circ}$ 1871)

F.21.41. Infratribus CHAPARANINIA nov., DOP.da.f103-01 \{93\}

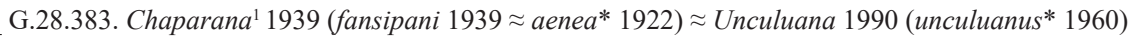

$\{96\}$

G.28.384. Gynandropaa* 1992 (yunnanensis* 1870)

F.21.42. Infratribus DIPLOPAINIA nov., DOP.da.f104-00 \{98\}

G.28.385. Diplopaa* nov. (taihangnicus* 2002)

F.21.43. Infratribus FEIRANINIA nov., DOP.da.f105-00

G.28.386. Feirana* 1992 (quadranus* 1960) $\equiv$ Quadrana 1990 JH

F.20.61. Subtribus PAINA Dubois, 1992.da.f003-03 \{94\}

G.28.387. Nanorana* 1896 (pleskei* 1896) $\approx$ Montorana 1924 (ahli $1924 \approx$ pleskei $\left.^{*} 1896\right) \approx$ Altirana

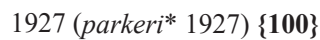

G.28.388. Paa* 1975 (liebigii* 1860) $\approx$ Ombrana 1992 (sikimensis $\left.{ }^{\circ} 1870\right) \approx$ Maculopaa 2010

(maculosa* 1960) $\{\mathbf{1 0 0}\}$

F.19.66. Tribus QUASIPAINI $\left.\mathrm{Fei}^{+2}, 2010 . \mathrm{fa} . \mathrm{f007-00 \{ 98}\right\}$

F.20.62. Subtribus ANNANDIINA $\mathrm{Fei}^{+2}$, 2010.fa.f008-01

G.28.389. Annandia* 1992 (delacouri* 1928)

F.20.63. Subtribus ERIPAINA nov., DOP.da.f106-00

G.28.390. Eripaa* 1992 (fasciculispina* 1970)

F.20.64. Subtribus QUASIPAINA Fei ${ }^{+2}, 2010 . f a . f 007-01\{\mathbf{1 0 0}\}$

G.28.391. Quasipaa* 1992 (boulengeri* 1889) $\{\mathbf{9 0}\}$

G.28.392. Yerana* 2006 (yei* 2002)

F.17.45. Familia OCCIDOZYGIDAE $\mathrm{Fei}^{+2}$, 1990.fa.f002-03 \{100\} [N] [M]

F.18.73. Subfamilia INGERANINAE $\mathrm{Fei}^{+2}, 2010$.fa.f009-01 \{100\}

G.28.393. Ingerana* 1987 (tenasserimensis* 1892)

F.18.74. Subfamilia OCCIDOZYGINAE $\mathrm{Fei}^{+2}$, 1990.fa.f002-00 \{98\} 
G.28.394. Frethia* nov. (laevis* 1859) $\equiv$ Oxyrhachis* 1916 AN

G.28.395. Occidozyga* 1822 a (lima* 1829) $\equiv$ Ooeidozyga $1822 b \equiv$ Oxyglossus 1838 JH

$\equiv$ Rhomboglossus 1841 AN $\approx$ Houlema 1831 (obscura $1831 \approx$ lima* 1829) $\approx$ Osteosternum 1929

(amoyense 1929 $\approx$ lima* 1829)

G.28.396. Oreobatrachus* 1896 (baluensis* 1896)

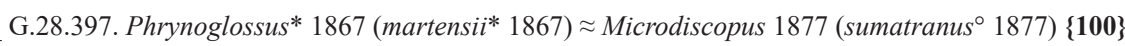

F.16.05. Apofamilia NYCTIBATRACHEIDAE Blommers-Schlösser, 1993.ba.f001-02 \{97\}

F.17.46. Familia ASTROBATRACHIDAE Vijayakumar ${ }^{+8}$, 2019.va.f001-00 [N] [M]

G.28.398. Astrobatrachus $^{\circ} 2019$ (kurichiyana ${ }^{\circ}$ 2019)

F.17.47. Familia NYCTIBATRACHIDAE Blommers-Schlösser, 1993.ba.f001-01 [N] [M]

G.28.399. Lankanectes* 2001 (corrugata* 1863)

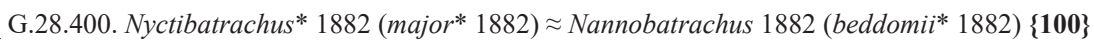

F.16.06. Apofamilia RANEIDAE Batsch, 1796.ba.f001-32 \{97\}

F.17.48. Familia RANIDAE $_{\text {Batsch, 1796.ba.f001-05 \{100\} [Q] }}$

F.18.75. Subfamilia RANINAE Batsch, 1796.ba.f001-23 \{100\}

F.19.0b. Tribus INCERTAE SEDIS

G.28.401. Pterorana ${ }^{\circ} 1986$ (khare ${ }^{\circ}$ 1986)

F.19.67. Tribus MERISTOGENYINI Fei ${ }^{+2}$, 2010.fa.f003-02 \{100\}

G.28.402. Clinotarsus ${ }^{1} 1869$ (robustus $1869 \approx$ curtipes $^{*} 1853$ ) $\equiv$ Pachybatrachus 1869 JH $\approx$ Nasirana

1992 (alticola* 1882) $\{\mathbf{1 0 0}\}$

G.28.403. Meristogenys* 1991 (jerboa* $^{*}$ 1872) $\approx$ Huia 1991 (cavitympanum* ${ }^{*}$ 1893) $\{\mathbf{1 0 0}\}$

G.28.404. Sumaterana ${ }^{\circ} 2018$ (crassiovis $^{\circ} 1920$ )

F.19.68. Tribus RANINI Batsch, 1796.ba.f001-30 \{99\}

F.20.†0a. Subtribus INCERTAE SEDIS †

G.28.†105. Ranavus ${ }^{\circ} 1885 \dagger\left(\right.$ scarabellii $\left.^{\circ} 1885 \dagger\right)$

F.20.65. Subtribus AMOLOPINA $\mathrm{Fei}^{+2}$, 1990.fa.f001-03 $\{\mathbf{1 0 0}\}$

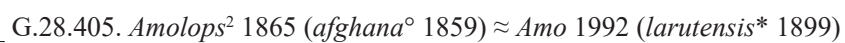

F.20.66. Subtribus RANINA Batsch, 1796.ba.f001-33 \{95\}

F.21.44. Infratribus PELOPHYLACINIA nov., DOP.da.f107-00 $\{\mathbf{1 0 0}\}$

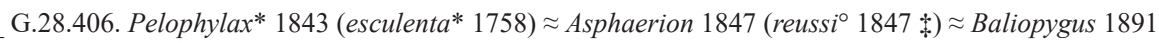

(ridibunda* 1771) $\equiv$ Bilaterana 1985

F.21.45. Infratribus RANINIA Batsch, 1796.ba.f001-34 \{98\}

F.22.19. Hypotribus GLANDIRANINOA $\mathrm{Fei}^{+2}$, 2010.fa.f016-01

G.28.407. Glandirana* 1990 (minima* 1979)

F.22.20. Hypotribus LIMNODYTINOA Fitzinger, 1843.fa.f001-02 \{97\}

G.28.408. Abavorana* 2015 (luctuosus* 1871)

G.28.409. Hylarana* 1838 (erythraea* 1827 ) $\equiv$ Limnodytes $1841 \equiv$ Zoodioctes $1848 \equiv$ Ranhyla

1858 AN $\equiv$ Hylorana $1864 \approx$ Hydrophylax 1843 (malabarica* 1838) $\approx$ Tenuirana 1990 (taipehensis* $1909) \approx$ Amnirana 1992 (amnicola $\left.{ }^{\circ} 1977\right) \approx$ Chalcorana 1992 (chaconota* 1837) $\approx$ Humerana 1992

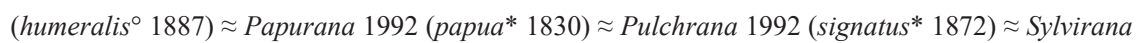
1992 (nigrovittatus* $^{*} 1856$ ) $\approx$ Tylerana 1992 (jimiensis* 1856) $\approx$ Boulengerana 2010 (guentheri* 1882 ) $\approx$ Indosylvirana 2015 (flavescens $^{\circ} 1853$ ) $\approx$ Bijurana 1992 (nicobariensis* 1870) \{99\}

F.22.21. Hypotribus RANINOA Batsch, 1796.ba.f001-35 \{99\}

F.23.13. Clanus NIDIRANITES Fei ${ }^{+2}, 2010 . f a . f 013-01\{100\}$

G.28.410. Babina* $1912 a$ (holsti* 1892) $\equiv$ Babina $1912 b\{\mathbf{1 0 0}\}$

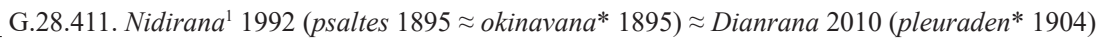

$\{100\}$

F.23.14. Clanus ODORRANITES Fei ${ }^{+2}$, 2010.fa.f015-01 $\{\mathbf{1 0 0}\}$

G.28.412. Odorrana* 1990 (margaretae* 1950) $\approx$ Eburana 1992 (narina* 1901) $\approx$ Bamburana 2005

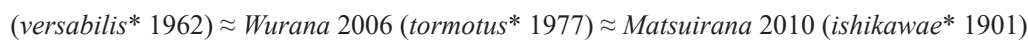

F.23.15. Clanus RANITES Batsch, 1796.ba.f001-36 \{97\}

F.24.07. Subclanus LITHOBATITIES nov., DOP.da.f108-00 $\{\mathbf{1 0 0}\}$

G.28.413. Aquarana* 1992 (catesbeiana* 1802) $\equiv$ Conrana 1985 JH $\{\mathbf{1 0 0}\}$

G.28.414. Boreorana* nov. (sylvatica* 1825)

G.28.415. Lithobates* 1843 (palmipes* 1824) $\equiv$ Pohlia $1867 \approx$ Ranula 1859 JH (gollmeri 1859

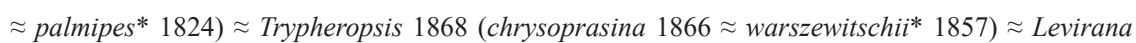




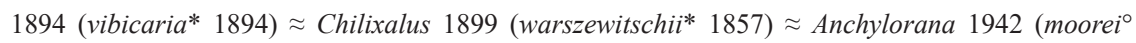

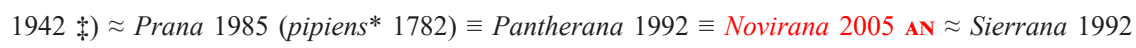

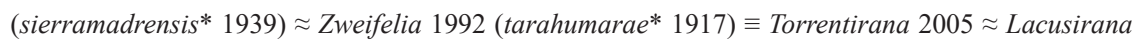
2005 (megapoda $\left.{ }^{\circ} 1942\right) \approx$ Nenirana 2005 (areolata* 1852) $\approx$ Scurrilirana 2005 (berlandieri* ${ }^{*}$ 1854)

$\approx$ Stertirana 2005 AN (montezumae* 1854) $\{\mathbf{1 0 0}\}$

F.24.08. Subclanus PSEUDORANITIES nov., DOP.da.f109-00

G.28.416. Pseudorana* 1990 (weiningensis* 1962)

F.24.09. Subclanus RANITIES Batsch, 1796.ba.f001-37 \{98\}

F.25.18. Infraclanus LIUHURANITOES nov., DOP.da.f110-00 G.28.417. Liuhurana* 2010 (shuchinae* 1950)

F.25.19. Infraclanus RANITOES Batsch, 1796.ba.f001-38 \{96\}

G.28.418. Amerana* 1992 (boylii* 1854) $\approx$ Aurana 1985 JH (aurora* 1852) $\equiv$ Aurorana 1992 $\equiv$ Laurasiarana 2005 AN $\{\mathbf{1 0 0}\}$

G.28.419. Rana* 1758 a (temporaria* 1758) $\equiv$ Rana $1758 b$ AN $\equiv$ Rana $1758 c$ AN $\equiv$ Gyrinus 1783 AN

$\equiv$ Ranaria $1814 \equiv$ Batracinus $1815 \mathrm{AN} \equiv$ Chondrodela $1815 \mathrm{AN} \equiv$ Palmirana $1828 \approx$ Protobatrachus 1848 (nodicaudatus $1848 \approx$ temporaria* 1758 ) $\approx$ Crotaphitis 1891 (arvalis* 1907) $\approx$ Pseudoamolops 1997 AN (sauteri* 1909$) \equiv$ Pseudoamolops $2000\{\mathbf{1 0 0}\}$

F.22.22. Hypotribus RUGOSINOA nov., DOP.da.f111-00 \{100\}

G.28.420. Rugosa* 1990 (rugosa* 1838)

F.22.23. Hypotribus SANGUIRANINOA $\mathrm{Fei}^{+2}$, 2010.fa.f017-01 $\{\mathbf{1 0 0}\}$

G.28.421. Sanguirana* 1992 (sanguinea* 1893)

F.18.76. Subfamilia STAUROINAE Dubois, 2005.da.f001-01 \{100\}

G.28.422. Staurois* 1865 (natator* 1858$) \approx$ Simomantis 1918 (latopalmatus* 1887)

F.17.49. Familia $\underline{\text { RHACOPHORIDAE }} \|$ Günther, 1858.gc.f012\|-Hoffman, 1932.ha.f001-00 \{100\} [Q]

F.18.77. Subfamilia MANTELLINAE Laurent, 1946.1a.f001-00 \{100\}

F.19.69. Tribus BOOPHINI Vences ${ }^{+1}$, 2001.va.f001-01 $\{\mathbf{1 0 0}\}$

G.28.423. Boophis* 1838 (goudotii* 1838) EElophila 1841 AN $\equiv$ Buccinator $1848 \approx$ Sahona 2006

(tephraeomystax*1853)

F.19.70. Tribus LALIOSTOMINI Vences ${ }^{+1}$, 2001.va.f002-01 \{100\}

G.28.424. Aglyptodactylus* 1919 (mascareniensis* 1853) \{94\}

G.28.425. Laliostoma* 1998 (labrosa* 1868)

F.19.71. Tribus MANTELLINI Laurent, 1946.la.f001-02 \{100\}

F.20.67. Subtribus MANTELLINA Laurent, 1946.1a.f001-03 \{100\}

F.21.46. Infratribus BLOMMERSIINIA nov., DOP.da.f112-00 \{100\} G.28.426. Blommersia* 1992 (blommersae* 1975) $\{\mathbf{1 0 0}\}$

G.28.427. Guibemantis* 1992 (depressiceps* 1882) $\approx$ Pandanusicola 1994 (bicalcaratus* 1913) $\{100\}$

F.21.47. Infratribus MANTELLINIA Laurent, 1946.1a.f001-04 \{100\}

G.28.428. Mantella* 1882 (betsileo* 1872) \{97\}

G.28.429. Wakea* 2006 (madinika* 2002)

F.20.68. Subtribus MANTIDACTYLINA nov., DOP.da.f113-00 \{99\}

F.21.48. Infratribus MANTIDACTYLINIA nov., DOP.da.f113-01 $\{\mathbf{1 0 0}\}$

F.22.24. Hypotribus BOEHMANTINOA nov., DOP.da.f114-00

G.28.430. Boehmantis* 2006 (microtympanum* 1935)

F.22.25. Hypotribus MANTIDACTYLINOA nov., DOP.da.f113-02 \{98\}

G.28.431. Gephyromantis*1920 (boulengeri* 1920) Microphryne 1913 JH (malagasia*1913)

$\equiv$ Trachymantis $1920 \mathbf{J H} \equiv$ Laurentomantis $1980 \approx$ Phylacomantis 1994 (corvus* 1994) $\approx$ Duboimantis 2006 (granulatus* 1881) $\approx$ Vatomantis 2006 (webbi* 1953) $\approx$ Asperomantis 2017 (aspera* 1882) $\{\mathbf{1 0 0}\}$

G.28.432. Mantidactylus* 1895 (guttulata* 1881) $\approx$ Brygoomantis 1992 (ulcerosus* 1880)

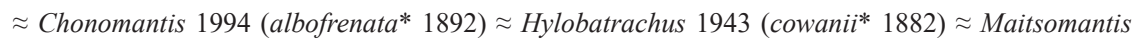
2006 (argenteus* 1920) $\approx$ Ochthomantis 1994 (femoralis* 1882) \{90\}

F.21.49. Infratribus SPINOMANTINIA nov., DOP.da.f115-00 $\{\mathbf{1 0 0}\}$

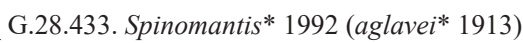

F.19.72. Tribus TSINGYMANTINI nov., DOP.da.f116-00

G.28.434. Tsingymantis* 2006 (antitra* 2006)

F.18.78. Subfamilia RHACOPHORINAE ||Günther, 1858.gc.f012||-Hoffman, 1932.ha.f001-01 \{100\} 
F.19.†0a. Tribus INCERTAE SEDIS

G.28.†106. Indorana $2013 \dagger\left(\right.$ prasadi $\left.^{\circ} 2013 \dagger\right)$

F.19.0c. Tribus INCERTAE SEDIS

G.28.435. Dendrobatorana ${ }^{\circ} 1927$ (dorsalis $^{\circ} 1875$ )

F.19.73. Tribus BUERGERIINI Channing, 1989.ca.f002-01 \{100\}

G.28.436. Buergeria* 1838 (buergeri* 1838) $\equiv$ Dendricus 1848

F.19.74. Tribus RHACOPHORINI |Günther, 1858.gc.f012||-Hoffman, 1932.ha.f001-03 \{100\}

F.20.69. Subtribus RHACOPHORINA \|Günther, 1858.gc.f012||-Hoffman, 1932.ha.f001-05 \{99\}

F.21.50. Infratribus NYCTIXALINIA Grosjean ${ }^{+3}$, 2008.ga.f001-01 $\{\mathbf{1 0 0}\}$

G.28.437. Nyctixalus* 1882 (margaritifer* 1882) $\approx$ Hazelia 1920 JH (spinosa* 1920)

$\equiv$ Edwardtayloria 1975 \{99\}

G.28.438. Theloderma* 1838 (leporosa* 1838) $\approx$ Phrynoderma $1893 \mathbf{~ J H ~ ( a s p e r u m * ~ 1 8 9 3 ) ~ \{ 9 6 \} ~}$

F.21.51. Infratribus RHACOPHORINIA ||Günther, 1858.gc.f012||-Hoffman, 1932.ha.f001-06 \{98\}

F.22.26. Hypotribus GRACIXALINOA nov., DOP.da.f117-00 $\{\mathbf{1 0 0}\}$

G.28.439. Gracixalus* 2005 (gracilipes* 1937)

F.22.27. Hypotribus ORIXALINOA nov., DOP.da.f118-00 \{97\}

G.28.440. Orixalus* nov. (nonggangensis* 2013)

F.22.28. Hypotribus PHILAUTINOA Dubois, 1981.da.f001-02 \{97\}

F.23.16. Clanus KURIXALITES nov., DOP.da.f119-00 \{100\}

G.28.441. Kurixalus* 1999 (eiffingeri* $\left.{ }^{*} 1895\right) \approx$ Aquixalus 2005 (odontotarsus* 1993)

F.23.17. Clanus MERCURANITES nov., DOP.da.f120-00 $\{\mathbf{1 0 0}\}$

F.24.10. Subclanus BEDDOMIXALITIES nov., DOP.da.f121-00

G.28.442. Beddomixalus* 2013 (bijui* 2011)

F.24.11. Subclanus MERCURANITIES nov., DOP.da.f120-01 \{98

G.28.443. Mercurana* 2013 (myristicapalustris* 2013)

G.28.444. Pseudophilautus ${ }^{2} 1943$ (temporalis $\left.{ }^{\circ} 1864\right) \approx$ Kirtixalus 1987 (microtympanum* 1858) \{100\}

G.28.445. Raorchestes* 2010 (glandulosus* 1854) $\{\mathbf{1 0 0}\}$

F.23.18. Clanus NASUTIXALITES nov., DOP.da.f122-00

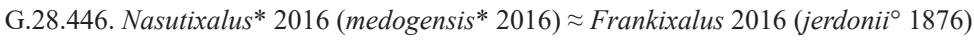

F.23.19. Clanus PhilaUtites Dubois, 1981.da.f001-03 \{100\}

G.28.447. Philautus* 1848 (aurifasciata* 1837) $\equiv$ Orchestes $1838 \mathbf{J H} \equiv$ Ixalus $1841 \mathbf{J H} \approx$ Gorhixalus 1987 (hosii* 1895)

F.22.29. Hypotribus RHACOPHORINOA ||Günther, 1858.gc.f012||-Hoffman, 1932.ha.f001-07 \{98\}

F.23.20. Clanus CHIRIXALITES nov., DOP.da.f123-00 $\{\mathbf{1 0 0}\}$ G.28.448. Chirixalus* 1893 (doriae* 1893) $\{\mathbf{1 0 0}\}$ G.28.449. Chiromantis* 1854 (xerampelina* 1854) $\{100\}$

F.23.21. Clanus RHACOPHORITES ||Günther, 1858.gc.f012||-Hoffman, 1932.ha.f001-08 \{92\}

F.24.12. Subclanus FEIHYLITIES nov., DOP.da.f124-00 \{98\}

G.28.450. Feihyla* 2006 (palpebralis* 1924)

F.24.13. Subclanus POLYPEDATITIES Günther, 1858.gc.f012-05 \{91\}

F.25.20. Infraclanus GHATIXALITOES nov., DOP.da.f125-00 $\{\mathbf{1 0 0}\}$

G.28.451. Ghatixalus* 2008 (variabilis* 1853)

F.25.21. Infraclanus POLYPEDATITOES Günther, 1858.gc.f012-06 \{99\}

G.28.452. Polypedates* 1838 LT (leucomystax* 1829) $\equiv$ Polypedotes 1838 LP $\equiv$ Polypedetes 1890

$\approx$ Trachyhyas 1843 (rugosus $1841 \approx$ leucomystax* 1829) \{94\}

G.28.453. Taruga* 2010 (fastigo* 2001) $\{\mathbf{1 0 0}\}$

F.24.14. Subclanus RHACOPHORITIES ||Günther, 1858.gc.f012||-Hoffman, 1932.ha.f001-09 \{100\} G.28.454. Leptomantis* 1867 (bimaculata* 1867) $\{99\}$

G.28.455. Rhacophorus ${ }^{1} 1822$ (moschatus $1822 \approx$ reinwardtii* $\left.^{*} 1840\right) \equiv$ Racophorus 1826

$\equiv$ Rhacoforus $1898 \approx$ Huangixalus 2012 (translineatus* 1977) $\{\mathbf{1 0 0}\}$

G.28.456. Zhangixalus* 2019 (dugritei* 1872) $\{\mathbf{1 0 0}\}$

F.24.15. Subclanus TAMIXALITIES nov., DOP.da.f126-00

G.28.457. Tamixalus* nov. (calcadensis* 1927)

F.22.30. Hypotribus VAMPYRIINOA nov., DOP.da.f127-00 \{97\}

G.28.458. Vampyrius* nov. (vampyrus* 2010)

F.20.70. Subtribus ROMERINA nov., DOP.da.fc128-00 $\{\mathbf{1 0 0}\}$ 
G.28.459. Romerus* nov. (romeri* 1953) ${ }^{*}$ Liuixalus 2008 AN

F.16.07. Apofamilia RANIXALEIDAE Dubois, 1987.da.f005-03 \{100\}

F.17.50. Familia RANIXALIDAE Dubois, 1987.da.f005-02 [M]

G.28.460. Indirana* 1986 (beddomii* 1875) $\approx$ Ranixalus 1986 (gundia ${ }^{\circ} 1986$ )

G.28.461. Walkerana* 2016 (diplostictus* 1876) $\equiv$ Sallywalkerana* $2016 \approx$ Indirana 1985 AN

(leptodactyla* 1882)

C.12.04. Infraphalanx SAVANURA nov., DOP.da.c08-00 $\{\mathbf{1 0 0}\}$

F.17.51. Familia PTYCHADENIDAE Dubois, 1987.da.f002-02 \{100\} [M]

G.28.462. Hildebrandtia* 1907 (ornatus* 1878)

G.28.463. Lanzarana $^{\circ} 1982$ (largeni $\left.^{\circ} 1978\right)$

G.28.464. Ptychadena* 1917 (mascareniensis* 1841) ELimnophilus $1843 \mathbf{J H} \equiv$ Ptychadaena 1930

$\approx$ Abrana 1931 JH $\left(\right.$ schillukorum ${ }^{\circ}$ 1908) $\equiv$ Parkerana $1984\{\mathbf{1 0 0}\}$

C.09.02. Epiphalanx Helanura nov., DOP.da.c09-00 $\{\mathbf{1 0 0}\}$

F.17.52. Familia HELEOPHRYNIDAE Noble, 1931.na.f004-01 \{100\} [Q]

G.28.465. Hadromophryne* 2008 (natalensis* 1913)

G.28.466. Heleophryne* 1898 (purcelli* 1898) 三 Heliophryne 1975 \{100\}

C.06.02. Infraordo Mediogyrinia Lataste, 1878.1a.c02-02 \{100\}

F.14.†0b. Superfamilia INCERTAE SEDIS †

F.17.†0h. Familia INCERTAE SEDIS †

G.28.†107. Callobatrachus ${ }^{\circ} 1999 \div\left(\right.$ sanyanensis $\left.^{\circ} 1999 \dagger\right)$

G.28.†108. Electrorana ${ }^{\circ} 2018 \dagger\left(\right.$ limoae $\left.^{\circ} 2018 \dagger\right)$

G.28.†109. Enneabatrachus ${ }^{\circ} 1993 \dagger\left(\right.$ hechti $\left.^{\circ} 1993 \dagger\right)$

G.28.†110. Opisthocoelellus $^{\circ} 1941 \dagger\left(\right.$ weigelt $\left.^{\circ} 1941 \dagger\right) \approx$ Germanobatrachus $1941 \dagger($ beurelni 1941

$\approx$ weigelti $\left.^{\circ} 1941 \dagger\right)$

G.28.†111. Pelophilus ${ }^{\circ} 1838 \dagger$ (agassizii $\left.^{\circ} 1838 \dagger\right) \equiv$ Baryboas $1848 \dagger$

F.17.†06. Familia GOBIATIDAE Roček $^{+1}$, 1991.ra.f001-00 †

G.28.†112. Cretasalia ${ }^{\circ} 1999 \dagger\left(\right.$ tsybini $\left.^{\circ} 1999 \dagger\right)$

G.28.†113. Gobiates $^{\circ} 1986 \dagger\left(\right.$ khermeentsavi $\left.^{\circ} 1986 \dagger\right) \equiv$ Gobiates 1983 AN

F.14.13. Superfamilia ALYTOIDEA Fitzinger, 1843.fa.f008-07 \{100\}

F.17.53. Familia $\underline{\text { ALYTIDAE }}$ Fitzinger, 1843.fa.f008-02 \{100\} [S] [P]

G.28.†114. Kizylkuma ${ }^{\circ} 1981 \dagger\left(\right.$ antiqua $\left.^{\circ} 1981 \dagger\right)$

G.28.467. Alytes* 1829 (obstetricans* 1768) $\equiv$ Obstetricans $1834 \approx$ Baleaphryne $1979 \$$ (muletensis* 1979)

G.28.468. Ammoryctis* 1879 (cisternasii* 1879)

F.17.54. Familia DISCOGLOSSIDAE Günther, 1858.gc.f004-00 \{100\} [Q]

G.28.†115. Bakonybatrachus $^{\circ} 2012 \dagger\left(\right.$ fedori $\left.^{\circ} 2012 \dagger\right)$

G.28.†116. Eodiscoglossus ${ }^{\circ} 1954 \dagger$ (santonjae $1954 \dagger$ )

G.28.†117. Latoglossus ${ }^{\circ} 2000 \dagger\left(\right.$ zraus $\left.^{\circ} 2000 \dagger\right) 99$

G.28.†118. Paradiscoglossus ${ }^{\circ} 1982 \dagger$ (americanus $^{\circ} 1982 \dagger$ )

G.28.†119. Paralatonia $^{\circ} 2003 \uparrow\left(\right.$ transylvatica $\left.^{\circ} 2003 \dagger\right)$

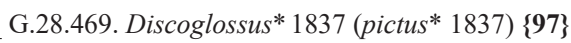

G.28.470. Latonia $^{3} 1845 \dagger\left(\right.$ seyfried $\left.^{\circ} 1845 \dagger\right) \equiv$ Latonia $1843 a$ AN $\equiv$ Latonix $1843 b$ AN $\equiv$ Latonia

1843 c AN $\approx$ Diplopelturus 1897 (gigantea ${ }^{\circ} 1851 \dagger$ ) $\equiv$ Miopelobates $1955 \dagger \approx$ Prodiscoglossus 1944

(vetaizoni $1944 \dagger$ )

F.14.14. Superfamilia BOMBINATOROIDEA Gray, 1825.ga.f002-16 $\{\mathbf{1 0 0}\}$

F.17.55. Familia BOMBINATORIDAE Gray, 1825.ga.f002-02 \{100\} [Q]

G.28.†120. Eobarbourula $2013 \dagger\left(\right.$ delfinoi $\left.^{\circ} 2013 \dagger\right)$

G.28.471. Barbourula* 1924 (busuangensis* 1924) $\{\mathbf{1 0 0}\}$

G.28.472. Bombina* 1816 (bombina* 1760) $\approx$ Bombinator 1820 (igneus $1768 \approx$ bombina* 1760)

$\equiv$ Bombitator $1830 \approx$ Glandula 1985 JH (maximus* 1905) $\equiv$ Grobina $1987\{\mathbf{1 0 0}\}$

C.04.02. Ordo GYMnOPHIONA Rafinesque, 1814.ra.c01-02 \{100\}

C.05.†0b. Subordo INCERTAE SEDIS †

F.17.†0i. Familia INCERTAE SEDIS †

G.28.†121. Apodops ${ }^{\circ} 1972 \dagger\left(\right.$ pricei $\left.^{\circ} 1972 \dagger\right)$

G.28. $\uparrow 122$ Rubricacaecilia $^{\circ} 2001 \dagger\left(\right.$ monbaroni $\left.^{\circ} 2001 \dagger\right)$

F.17.†07. Familia EOCAECILIIDAE Jenkins ${ }^{+1}$, 1993.ja.f001-04 † 
G.28.†123. Eocaecilia $1993 \dagger\left(\right.$ micropodia $\left.^{\circ} 1993 \dagger\right)$

C.05.03. Subordo Plesiophiona nov., DOP.da.c10-00 $\{\mathbf{1 0 0}\}$

F.17.56. Familia RHINATREMATIDAE Nussbaum, 1977.na.f001-00 \{100\} [Q]

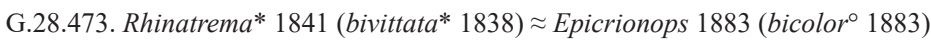

C.05.04. Subordo PSEudophiona Blainville, 1816.ba.c11-06 \{100\}

F.14.15. Superfamilia CAECILIOIDEA Rafinesque, 1814.ra.f003-|Gray, 1825.ga.f008|-18 \{100\}

F.17.57. Familia CAECILIIDAE Rafinesque, 1814.ra.f003-|Gray, 1825.ga.f008|-10 \{99\} [Q]

F.18.79. Subfamilia CAECILIINAE Rafinesque, 1814.ra.f003-|Gray, 1825.ga.f008|-16 \{100\}

F.19.75. Tribus CAECILIINI Rafinesque, 1814.ra.f003-|Gray, 1825.ga.f008|-24 \{100\}

F.20.71. Subtribus CAECILIINA Rafinesque, 1814.ra.f003-|Gray, 1825.ga.f008|-25 $\{\mathbf{1 0 0}\}$

G.28.474. Caecilia* 1758 LT (tentaculata* 1758) $\equiv$ Coecilia 1758 LP $\equiv$ Coecilia $1801 \equiv$ Cecilia 1814

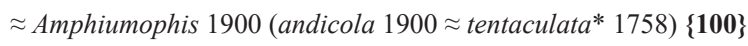

G.28.475. Oscaecilia* 1968 (ochrocephala* 1866)

F.20.72. Subtribus TYPHLONECTINA Taylor, 1968.ta.f002-09 \{100\}

G.28.476. Atretochoana ${ }^{\circ} 1995$ (eiselti $^{\circ}$ 1968)

G.28.477. Chthonerpeton* 1880 (indistinctus* 1862)

G.28.478. Nectocaecilia ${ }^{\circ} 1968$ (petersii ${ }^{\circ}$ 1882)

G.28.479. Potamotyphlus ${ }^{\circ} 1968$ LT (kaupii ${ }^{\circ}$ 1859) $\equiv$ Potomotyphlus 1968 LP

G.28.480. Typhlonectes* 1880 (compressicauda* 1841) $\approx$ Pseudotyphlonectes 1986 (natans* 1880)\}

$\{100\}$

F.19.76. Tribus SIPHONOPINI Bonaparte, 1850.bb.f019-08 \{100\}

F.20.73. Subtribus GRANDISONIINA Lescure ${ }^{+2}$, 1986.1b.f004-01 $\{\mathbf{1 0 0}\}$

F.21.0b. Infratribus INCERTAE SEDIS

G.28.481. Sylvacaecilia ${ }^{\circ} 1987$ (grandisonae ${ }^{\circ}$ 1970)

F.21.52. Infratribus GRANDISONIINIA Lescure ${ }^{+2}, 1986.1 \mathrm{~b}$. f004-02 $\{\mathbf{1 0 0}\}$

G.28.482. Hypogeophis* 1880 (rostrata* 1829) $\approx$ Grandisonia 1968 (alternans* 1893)\{100\}

G.28.483. Idiocranium $^{\circ} 1936$ (russeli $^{\circ}$ 1936)

G.28.484. Praslinia* 1909 (cooperi* 1909)

F.21.53. Infratribus INDOTYPHLINIA Lescure ${ }^{+2}, 1986.1 b . f 006-02\{\mathbf{1 0 0}\}$

G.28.485. Gegeneophis* 1880 (carnosum*1870) $\equiv$ Gegenes $1876 \mathbf{J H} \equiv$ Gegenophis $1882\{\mathbf{1 0 0}\}$

G.28.486. Indotyphlus* 1960 (battersbyi* 1960) $\{\mathbf{1 0 0}\}$

F.20.74. Subtribus SIPHONOPINA Bonaparte, 1850.bb.f019-10 \{99\}

F.21.54. Infratribus DERMOPHIINIA Taylor, 1969.ta.f002-04 \{100\}

F.22.31. Hypotribus DERMOPHIINOA Taylor, 1969.ta.f002-05 \{100\}

G.28.487. Gymnopis* 1874 (multiplicata* 1874) $\equiv$ Gymnophis $1901 \approx$ Cryptopsophis 1883

(multiplicatus $1883 \approx$ multiplicata* 1874$) \approx$ Dermophis 1880 (mexicanus* 1841) $\approx$ Copeicaecilia

1968 (syntremus $\left.^{\circ} 1866\right) \equiv$ Copeotyphlinus $1968 \approx$ Minascaecilia 1983 (sartoria $1983 \approx$ syntremus $^{\circ}$ 1866)

G.28.488. Schistometopum* 1941 (gregorii* 1895) $\{\mathbf{1 0 0}\}$

F.22.32. Hypotribus GEOTRYPETINOA Lescure ${ }^{+2}$, 1986.1b.f001-02

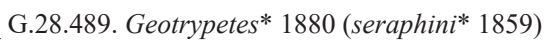

F.21.55. Infratribus SIPHONOPINIA Bonaparte, 1850.bb.f019-11\{98\}

F.22.0b. Hypotribus INCERTAE SEDIS

G.28.490. Brasilotyphlus ${ }^{\circ} 1968$ (braziliensis $\left.^{\circ} 1945\right)$

G.28.491. Mimosiphonops ${ }^{\circ} 1968$ (vermiculatus $\left.^{\circ} 1968\right) \approx$ Pseudosiphonops $^{\circ} 1968$ (ptychodermis 1968

$\approx$ vermiculatus $\left.^{\circ} 1968\right)$

F.22.33. Hypotribus MICROCAECILIINOA nov., DOP.da.f129-00 \{100\}

G.28.492. Microcaecilia ${ }^{3} 1968$ (albiceps $\left.^{\circ} 1882\right) \approx$ Parvicaecilia 1968 (nicefori $\left.^{\circ} 1924\right) \approx$ Caecilita $^{2}$

2009 (iwokramae 2009 )

F.22.34. Hypotribus SIPHONOPINOA Bonaparte, 1850.bb.f019-12 \{100\}

G.28.493. Luetkenotyphlus* 1968 (brasiliensis* 1852) $\equiv$ Lutkenotyphlus 1986

G.28.494. Siphonops* 1828 (annulata* 1820) \{93\}

F.18.80. Subfamilia HERPELINAE Laurent, 1984.1a.f001-00 \{99\}

F.19.77. Tribus CHIKILINI $\mathrm{Kamei}^{+9}$, 2012.ka.f001-01

G.28.495. Chikila* 2012 (fulleri* 1904)

F.19.78. Tribus HERPELINI Laurent, 1984.la.f001-02 \{100\} 


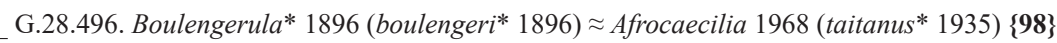

G.28.497. Herpele* 1880 (squalostoma* 1834)

F.17.58. Familia SCOLECOMORPHIDAE Taylor, 1969.ta.f001-00 \{100\} [N]

G.28.498. Crotaphatrema ${ }^{3} 1985$ (bornmuelleri $^{\circ}$ 1899) $\{\mathbf{1 0 0}\}$

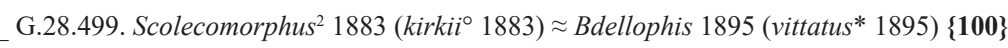

F.14.16. Superfamilia ICHTHYOРHIOIDEA Taylor, 1968.ta.f001-04 \{100\}

F.17.59. Familia ICHTHYOPHIIDAE Taylor, 1968.ta.f001-00 \{100\} [Q]

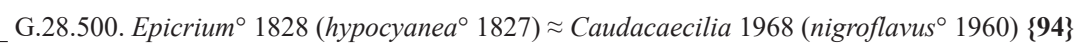

G.28.501. Ichthyophis* 1826 (glutinosa* 1758) $\{\mathbf{1 0 0}\}$

F.17.60. Familia URAEOTYPHLIDAE Nussbaum, 1979.na.f001-01 \{98\} [S] [N]

G.28.502. Uraeotyphlus ${ }^{3} 1880$ (oxyura $^{\circ} 1841$ )

C.04.03. Ordo Urodela Duméril, 1805.da.c02-12 \{100\}

C.05.†0c. Subordo INCERTAE SEDIS †

F.17.†0j. Familia INCERTAE SEDIS ${ }^{-} \dagger$

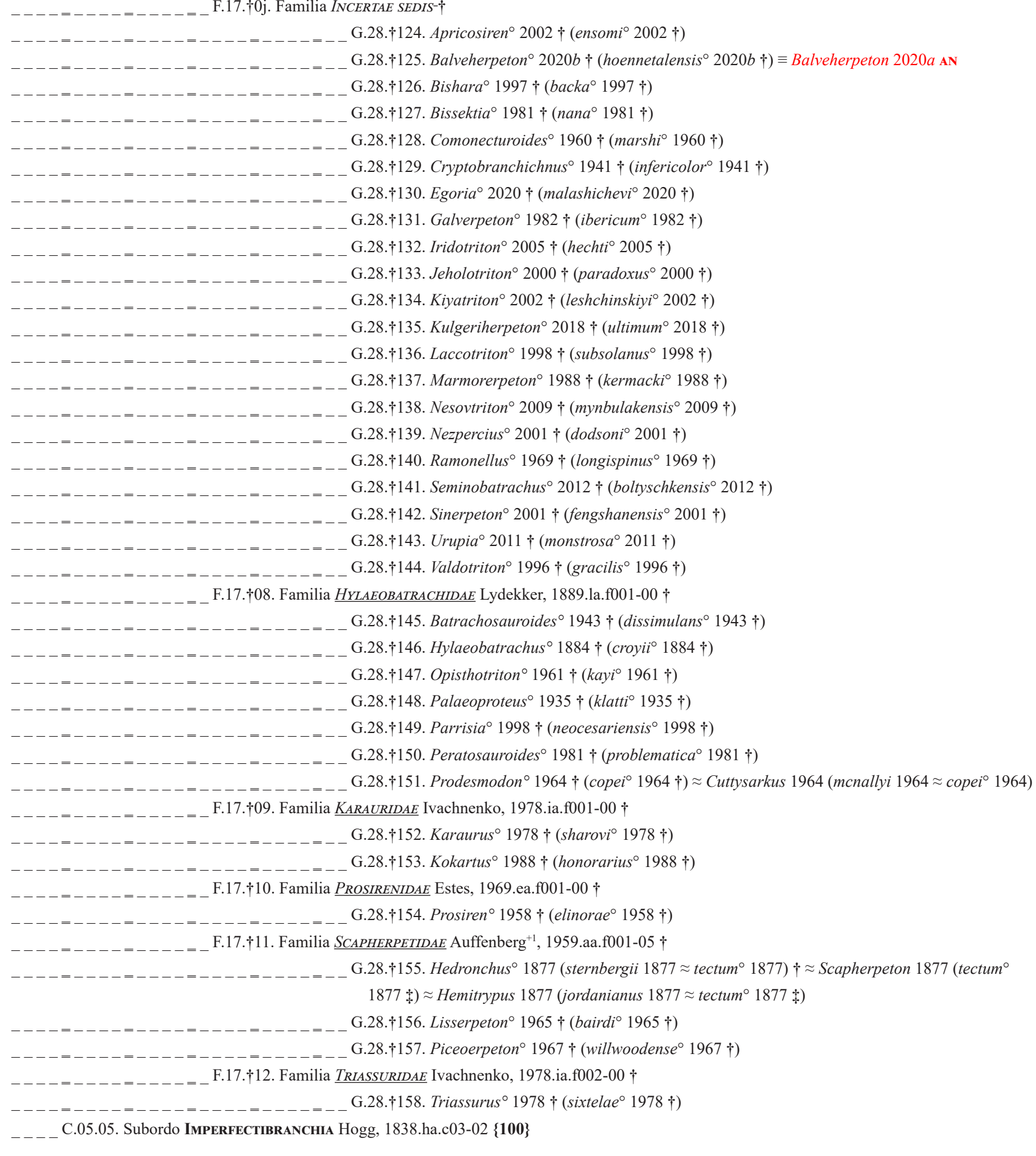


F.17.†0k. Familia INCERTAE SEDIS $\dagger$

G.28.†159. Liaoxitriton $^{\circ} 1998 \dagger\left(\right.$ zhongjiani $\left.^{\circ} 1998 \dagger\right)$

G.28. $\uparrow 160$. Linglongtriton $^{\circ} 2019 \uparrow\left(\right.$ daxishanensis $\left.^{\circ} 2019 \dagger\right)$

G.28.†161. Nuominerpeton ${ }^{\circ} 2016 \dagger$ (aquilonaris $\left.^{\circ} 2016 \dagger\right)$

G.28.†162. Pangerpeton ${ }^{\circ} 2006 \uparrow\left(\right.$ sinensis $\left.^{\circ} 2006 \dagger\right)$

G.28.†163. Regalerpeton ${ }^{\circ} 2009 \dagger\left(\right.$ weichangensis $\left.^{\circ} 2009 \dagger\right)$

F.17.61. Familia CRYPTOBRANCHIDAE Fitzinger, 1826.fb.f003-04 \{100\} [Q+] [S] [P]

G.28.†164. Aviturus $^{\circ} 1991 \dagger$ exsecratus $\left.^{\circ} 1991 \dagger\right)$

G.28.†165. Chunerpeton ${ }^{\circ} 2003 \dagger\left(\right.$ tianyiensis $\left.^{\circ} 2003 \dagger\right)$

G.28.†166. Escapherpeton ${ }^{\circ} 1981 \dagger$ (asiaticum $\left.^{\circ} 1981 \dagger\right) \equiv$ Mynbulakia 1981 (surgayi 1981

$\approx$ asiaticum $^{\circ} 1981$ :)

G. $28 . \dagger 167$. Horezmia ${ }^{\circ} 1981 \dagger\left(\right.$ gracile $\left.^{\circ} 1981 \dagger\right)$

G.28.†168. Ukrainurus ${ }^{\circ} 2013 \dagger\left(\right.$ hypsognathus $\left.^{\circ} 2013 \dagger\right)$

G.28.†169. Ulanurus $^{\circ} 1991 \dagger\left(\right.$ fractus $\left.^{\circ} 1991 \dagger\right)$

G.28.†170. Zaissanurus ${ }^{\circ} 1959 \dagger\left(\right.$ beliajevae $\left.^{\circ} 1959 \dagger\right)$

G.28.503. Andrias ${ }^{2} 1837 \dagger\left(\right.$ scheuchzeri $\left.^{\circ} 1831 \dagger\right) \equiv$ Tritogenius $1848 \equiv$ Proteocordylus $1831 \mathrm{CI} \dagger$

(diluvii $1831 \equiv$ scheuchzeri $\left.^{\circ} 1831\right) \approx$ Megalobatrachus 1837 (sielboldi 1837 इjaponicus* 1836) $\equiv$

Sieboldia $1838 \equiv$ Sieboldtia $1839 \equiv$ Hydrosalamandra $1840 \equiv$ Tritomegas 1854 JH $\equiv$ Sieboldiana

$1904 \equiv$ Onycopus 1841 AN $\equiv$ Palaeotriton 1837 (gigantea $1832 \equiv$ scheuchzeri $^{\circ} 1831$ ) CI $\equiv$ Paleotriton

1838 AN $\approx$ Plicagnathus $1917+\left(\right.$ matthewi $\left.^{\circ} 1917 \dagger\right)\{\mathbf{1 0 0}\}$

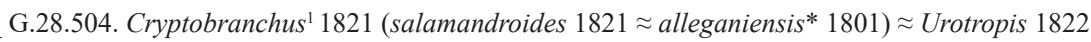

(mucronata $1822 \approx$ alleganiensis* 1801) EEurycea $1832 \mathbf{J H} \approx$ Protonopsis 1824 (horrida 1808

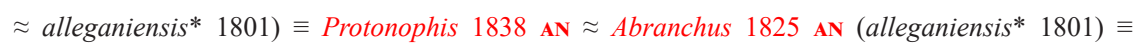

Menopoma $1825 \approx$ Salamandrops 1830 (gigantea $1808 \approx$ alleganiensis* 1801) $\equiv$ Pelusius 1830 AN

F.17.62. Familia $\underline{\text { HYNOBIIDAE }} \|$ Hallowell, 1856.ha.f001\|-Cope, 1859.cb.f002-01 \{100\} [Q]

F.18.†0e. Subfamilia INCERTAE SEDIS †

G.28.†171. Geyeriella ${ }^{\circ} 1950 \dagger\left(\right.$ mertensi $\left.i^{\circ} 1950 \dagger\right)$

G.28.†172. Parahynobius $^{\circ} 1999 \dagger$ (betfianus $\left.^{\circ} 1999 \dagger\right)$

G.28.†173. Prohynobius $^{\circ} 1985 \uparrow$ AN-AP (NINS)

F.18.81. Subfamilia HyNOBIINAE ||Hallowell, 1856.ha.f001||-Cope, 1859.cb.f002-00 \{100\}

F.19.79. Tribus HYNOBIINI ||Hallowell, 1856.ha.f001||-Cope, 1859.cb.f002-04 \{98\}

F.20.75. Subtribus HYNOBIINA \|Hallowell, 1856.ha.f001\|-Cope, 1859.cb.f002-05 \{97\}

F.21.56. Infratribus HYNOBIINIA \|Hallowell, 1856.ha.f001||-Cope, 1859.cb.f002-06 \{100\}

F.22.35. Hypotribus HYNOBIINOA ||Hallowell, 1856.ha.f001\|-Cope, 1859.cb.f002-07 \{100\}

G.28.505. Hynobius* 1838 (nebulosa* 1838) $\approx$ Pseudosalamandra 1838 (naevia* 1838)

$\equiv$ Hydroscopes $1848 \equiv$ Ellipsoglossa $1854\{\mathbf{1 0 0}\}$

G.28.506. Pachypalaminus* 1912 (boulengeri* 1912) $\{\mathbf{1 0 0}\}$

G.28.507. Poyarius* 2012 (formosanus* 1922) $\approx$ Makihynobius 2012 (sonani* 1922) \{97\}

F.22.36. Hypotribus SATOBIINOA nov., DOP.da.f130-00

G.28.508. Satobius* 1990 (retardatus* 1923)

F.21.57. Infratribus PROTOHYNOBIINIA $\mathrm{Fei}^{+1}$, 2000.fa.f001-02 $\{\mathbf{1 0 0}\}$

G.28.509. Batrachuperus* 1878 (pinchonii* 1872) 三Batrachohyperus $1881 \equiv$ Hyperobatrachus 1881

$\equiv$ Batrachyperus $1882 \approx$ Tibetuperus 2012 (yenyuanensis* 1950) $\{\mathbf{1 0 0}\}$

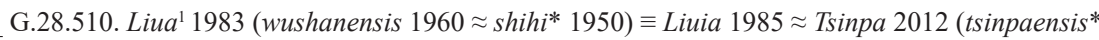
1966) $\{\mathbf{1 0 0}\}$

G.28.511. Pseudohynobius* 1983 (flavomaculatus* 1978) ₹ Protohynobius 2000 (puxiongensis*

2000)\} $\{\mathbf{1 0 0}\}$

F.20.76. Subtribus PACHYнYNobina Dubois ${ }^{+1}$, 2012.da.f002-01

G.28.512. Pachyhynobius* 1983 (shangchengensis* 1983) $\approx$ Xenobius 1985 JH (melanonychus 1985

$\approx$ shangchengensis* 1983) $\equiv$ Sinobius 1987

F.20.77. Subtribus SALAMANDRELLINA Dubois ${ }^{+1}$, 2012.da.f004-00 $\{\mathbf{1 0 0}\}$

G.28.513. Salamandrella* 1870 (keyserlingii* 1870) $\approx$ Isodactylium 1870 (schrenckii 1870

$\approx$ keyserlingii* 1870 )

F.19.80. Tribus RANODONTINI Thorn, 1966.ta.f001-01 \{100\}

F.20.78. Subtribus IRANODONTINA nov., DOP.da.f131-00 $\{\mathbf{1 0 0}\}$

G.28.514. Afghanodon* 2012 (mustersi* 1940) 
G.28.515. Iranodon* 2012 (persicus* 1970) $\approx$ Paradactylodon 1984 AN (gorganensis* 1979) \{100\}

F.20.79. Subtribus RANODONTINA Thorn, 1966.ta.f001-02

G.28.516. Ranodon* 1866 (sibiricus* 1866) $\equiv$ Ranidens 1882

F.18.82. Subfamilia ONYCHODACTYLINAE Dubois ${ }^{+1}, 2012$. da.f001-00 $\{\mathbf{1 0 0}\}$

G.28.517. Onychodactylus ${ }^{1} 1838$ (schlegeli $1838 \approx$ japonica* 1782) $\equiv$ Dactylonyx 1839 AN

$\equiv$ Onychopus 1854 AM $\approx$ Geomolge 1886 (fischeri* 1886)

C.05.06. Subordo MeanTes Linné, 1767.1a.c01-01 \{100\}

F.17.†13. Familia NOTERPETIDAE Rage ${ }^{+2}, 1993$. ra.f001-00 $†$

G.28.†174. Kababisha $1996 \uparrow$ (humarensis $\left.^{\circ} 1996 \uparrow\right)$

G.28.†175. Noterpeton ${ }^{\circ} 1993 \dagger$ (bolivianum $\left.^{\circ} 1993 \dagger\right)$

F.17.63. Familia SIRENIDAE Gray, 1825.ga.f005-00 \{100\} [Q]

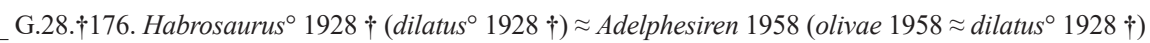

G.28.518. Pseudobranchus* 1825 (striata* 1824) $\equiv$ Parvibranchus $1839\{\mathbf{1 0 0}\}$

G.28.519. Siren* 1766 (lacertina* 1766) $\equiv$ Sirena 1808 AN $\equiv$ Sirene 1813 AN $\equiv$ Sirene 1816 CI

$\equiv$ Meantes $1822 \mathrm{AN} \equiv$ Syren $1828\{\mathbf{1 0 0}\}$

C.05.07. Subordo Pseudosauria Blainville, 1816.ba.c08-07 $\{\mathbf{1 0 0}\}$

F.14.†0c. Superfamilia INCERTAE SEDIS †

F.17.†01. Familia INCERTAE SEDIS †

_ G.28.†177. Beiyanerpeton ${ }^{\circ} 2012 \dagger$ (jianpingensis $^{\circ} 2012 \dagger$ )

G.28.†178. Qinglongtriton ${ }^{\circ} 2016 \dagger\left(\right.$ gangouensis $\left.^{\circ} 2016 \dagger\right)$

F.14.17. Superfamilia AMPHIUMOIDEA Gray, 1825.ga.f007-10 \{98\}

F.15.11. Epifamilia AMPHIUMOIDAE Gray, 1825.ga.f007-12 \{100\}

F.16.08. Apofamilia AMPHIUMEIDAE Gray, 1825.ga.f007-13 \{100\}

F.17.64. Familia AMPHIUMIDAE Gray, 1825.ga.f007-00 \{100\} [Q]

G.28.†179. Paleoamphiuma ${ }^{\circ} 1998 \dagger\left(\right.$ (tetradactylum $\left.{ }^{\circ} 1998 \dagger\right)$

G.28.†180. Proamphiuma $1969 \dagger\left(\right.$ cretacea $\left.^{\circ} 1969 \dagger\right)$

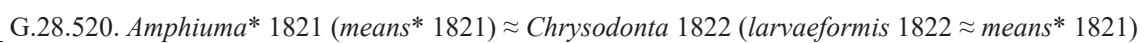

$\approx$ Muraenopsis 1843 (tridactylum* 1827) $\equiv$ Myraenopsis $1847 \approx$ Sirenoidis 1843 (didactylum $1827 \approx$

means* 1821) $\equiv$ Sirenoides 1850

F.17.65. Familia PLETHODONTIDAE Gray, 1850.ga.f001-00 \{100\} [Q]

F.18.†0f. Subfamilia INCERTAE SEDIS †

G.28.†181. Palaeoplethodon ${ }^{\circ} 2015 \dagger$ (hispaniolae ${ }^{\circ} 2015 \dagger$ )

F.18.83. Subfamilia HEMIDACTYLIINAE Hallowell, 1856.ha.f003-05 \{99\}

F.19.81. Tribus BoLItogLOSSINI Hallowell, 1856.ha.f002-03 \{100\}

F.20.80. Subtribus BATRACHOSEPINA Wake, 2012.wa.f001-01 \{100\}

G.28.521. Batrachoseps* 1839 (attenuata* 1833) $\approx$ Plethopsis 1937 (wrighti* 1937)

F.20.81. Subtribus BoLITOGLOSSINA Hallowell, 1856.ha.f002-04 \{100\}

F.21.58. Infratribus BolitoglossinIA Hallowell, 1856.ha.f002-05 $\{\mathbf{1 0 0}\}$

F.22.37. Hypotribus Bolitoglossinoa Hallowell, 1856.ha.f002-06 \{100\}

G.28.522. Bolitoglossa* 1854 (mexicana* 1854) $\equiv$ Mycetoides 1854 AN $\approx$ Oedipus 1838 JH

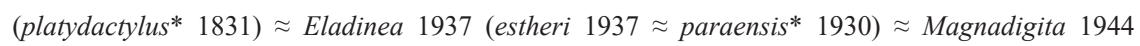

(nigloflavescens $1941 \approx$ franklini $\left.^{*} 1936\right) \approx$ Palmatotriton 1945 CI $($ rufescens $* 1869) \equiv$ Nanotriton

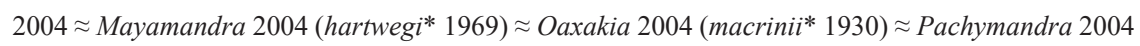
(dofleini* 1903)

F.22.38. Hypotribus ISTHMURINOA nov., DOP.da.f132-00 \{90\}

F.23.22. Clanus ISTHMURITES-nov., DOP.da.f132-01 \{97\}

G.28.523. Aquiloeurycea* 2015 (cephalicus* 1869) $\{99\}$

G.28.524. Isthmura* 2012 (bellii* 1850) \{96\}

F.23.23. Clanus PARVIMOLGITES nov., DOP.da.f133-00 \{90\}

G.28.525. Ixalotriton* 1989 (niger* 1989) \{99\}

G.28.526. Parvimolge* 1944 (townsendi* 1922)

F.23.24. Clanus PSEUDOEURYCEITES nov., DOP.da.f134-00 \{97\}

G.28.527. Pseudoeurycea* 1944 (leprosus* 1869) $\approx$ Lineatriton 1950 (lineola* 1865)

F.21.59. Infratribus THORIINIA Cope, 1869.cb.f001-02 \{90\}

F.22.39. Hypotribus THORIINOA Cope, 1869.cb.f001-03 \{95\}

G.28.528. Chiropterotriton* 1944 (multidentatus* 1939) $\{\mathbf{1 0 0}\}$ 
G.28.529. Cryptotriton* 2000 (nasalis* 1924) $\{\mathbf{1 0 0}\}$

G.28.530. Thorius* 1869 a (pennatribus* $\left.1869 a^{*}\right)\{\mathbf{1 0 0}\}$

F.22.40. Hypotribus THORNELLINOA nov., DOP.da.f135-00 \{94\}

F.23.25. Clanus DENDROTRITONITES nov., DOP.da.f136-00 \{100\}

G.28.531. Dendrotriton* 1983 (bromeliacia* 1936)

F.23.26. Clanus NYCTANOLITES nov., DOP.da.f140-00

G.28.532. Nyctanolis* 1983 (pernix* 1983)

F.23.27. Clanus THORNELLITES nov., DOP.da.f135-01 \{99\}L

F.24.16. Subclanus THORNELLITIES nov., DOP.da.f135-02 \{96\}

F.25.22. Infraclanus BRADYTRITONITOES nov., DOP.da.f137-00

G.28.533. Bradytriton* 1983 (silus* 1983)

F.25.23. Infraclanus THORNELLITOES nov., DOP.da.f135-03 \{96\}

F.26.13. Hypoclanus OEDIPINITUES nov., DOP.da.f138-00 \{98\}

G.28.534. Oedipina* 1868 (uniformis* 1868) $\approx$ Ophiobatrachus 1868 (vermicularis $1868 \approx$ uniformis*

1868 ) $\approx$ Haptoglossa 1893 (pressicauda* 1893) \{93\}

G.28.535. Oedopinola* 1946 (complex* 1924) \{90\}

F.26.14. Hypoclanus THORNELLITUES nov., DOP.da.f135-04 \{99\}

G.28.536. Thornella* nov. (quadra* 2008) $\equiv$ Oeditriton 2008 AN

F.24.17. Subclanus Nototritonities nov., DOP.da.f139-00 \{100\}

G.28.537. Nototriton* 1983 (picadoi* 1911) $\approx$ Bryotriton 2012 (barbouri* 1936)

F.19.82. Tribus HEMIDACTYLIINI Hallowell, 1856.ha.f003-03

G.28.538. Hemidactylium* 1838 (scutata* 1838) $\equiv$ Cotobotes $1848 \equiv$ Desmodactylus 1854

F.19.83. Tribus SPELERPINI Cope, 1859.cb.f001-06 \{100\}

F.20.82. Subtribus PSEUDOTRITONINA Dubois ${ }^{+1}, 2012$. da.f006-00 $\{\mathbf{1 0 0}\}$

G.28.539. Gyrinophilus* 1869 (porphyritica* 1827) $\{\mathbf{1 0 0}\}$

G.28.540. Pseudotriton 1838 (subfusca $1818 \approx$ rubra $\left.^{*} 1801\right) \equiv$ Mycetoglossus $1839 \equiv$ Batrachopsis

$1843 \equiv$ Pelodytes $1848 \mathbf{J H}\{\mathbf{1 0 0}\}$

G.28.541. Stereochilus* 1869 (marginatus* 1856)

F.20.83. Subtribus SPELERPINA Cope, 1859.cb.f001-07 $\{\mathbf{1 0 0}\}$

G.28.542. Eurycea* 1822 (lucifuga* 1822) ESpelerpes $1832 \approx$ Glossiphus 1832 AN (longicauda*

1818 ) $\equiv$ Cylindrosoma 1838 三Saurocercus $1843 \approx$ Manculus 1869 (quadridigitata* 1842) $\approx$

Typhlotriton 1892 (spelaeus* 1842) $\approx$ Typhlomolge 1896 (rathbuni* 1896) $\approx$ Haideotriton 1939

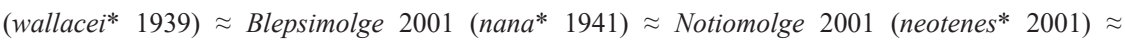

Paedomolge 2001 (tonkawae* 2000) $\approx$ Septentriomolge 2001 (chisholmensis* 2000) \{100\}

G.28.543. Urspelerpes* 2009 (brucei* 2009)

F.18.84. Subfamilia PLETHODONTINAE Gray, 1850.ga.f001-05 \{100\}

F.19.84. Tribus HYDROMANTINI Wake, 2012.wa.f003-00 \{94\}

F.20.84. Subtribus HYDROMANTINA Wake, 2012.wa.f003-01 \{100\}

_ G.28.544. Hydromantes* 1848 (platycephalus* 1916) $\equiv$ Hydromantoides $1981\{\mathbf{1 0 0}\}$

G.28.545. Speleomantes* 1984 (italicus* 1923) $\approx$ Atylodes 1868 RI (genei* 1838) \{100\}

F.20.85. Subtribus KARSENIINA Dubois ${ }^{+1}$, 2012.da.f008-01

G.28.546. Karsenia* 2005 (koreana* 2005)

F.19.85. Tribus PLETHODONTINI Gray, 1850.ga.f001-07 \{99\}

F.20.86. Subtribus DESMOGNATHINA Gray, 1850.ga.f003-05 \{90\}

F.21.60. Infratribus ANEIDINIA Wake, 2012.wa.f002-01 $\{\mathbf{1 0 0}\}$

G.28.547. Aneides* 1851 LT (lugubris* 1849) $\equiv$ Anaides 1851 LP-CI $\equiv$ Autodax 1887 CI $\approx$ Castaneides 2012 (aeneus* 1881)

F.21.61. Infratribus DESMOGNATHINIA Gray, 1850.ga.f003-06 \{99\}

G.28.548. Desmognathus* 1850 (fuscus* 1820) ₹ Leurognathus 1899 (marmorata* 1899)

$\approx$ Geognathus 2012 (wrighti* 1936) $\approx$ Hydrognathus 2012 (brimleyorum* 1895) \{100\}

G.28.549. Phaeognathus* 1961 (hubrichti* 1961)

F.20.87. Subtribus ENSATININA Gray, 1850.ga.f005-02

G.28.550. Ensatina* 1850 (eschscholtzii* 1850) $\approx$ Heredia 1857 (oregonensis $1857 \approx$ eschscholtzii*

$1850) \equiv$ Heteroglossa 1857 AN $\approx$ Urotropis $1875 \mathbf{J H}$ (platensis $1875 \approx$ eschscholtzii* 1850 )

F.20.88. Subtribus Plethodontina Gray, 1850.ga.f001-09 \{100\}

G.28.551. Plethodon* 1838 (glutinosa* 1818) $\equiv$ Phatnomatorhina 1839 AN $\approx$ Sauropsis $1843 \mathbf{~ J H}$ 
(erythronota $1818 \approx$ cinerea* $\left.^{*} 1818\right) \equiv$ Saurophis $1850 \approx$ Hightonia 2011 (vehiculum* 1859)

F.16.09. Apofamilia RHYACOTRITONEIDAE Tihen, 1958.ta.f002-03 $\{\mathbf{1 0 0}\}$

F.17.66. Familia RHYACOTRITONIDAE Tihen, 1958.ta.f002-01 [Q+] [C]

G.28.552. Rhyacotriton* 1920 (olympicus* 1917)

F.15.12. Epifamilia PROTEOIDAE Bonaparte, 1831.ba.f002-10 \{100\}

F.17.67. Familia PROTEIDAE Bonaparte, 1831.ba.f002-02 [Q]

G.28.†182. Mioproteus $^{\circ} 1978 \dagger$ (caucasicus $\left.^{\circ} 1978 \dagger\right)$

G. $28 . \dagger 183$. Orthophyia ${ }^{\circ} 1845 \dagger\left(\right.$ longa $\left.a^{\circ} 1845 \dagger\right)$

G.28.†184. Paranecturus $^{\circ} 2013 \dagger\left(\right.$ garbanii $\left.^{\circ} 2013 \dagger\right)$

G.28.553. Necturus* 1819 (maculosa* 1818) $\equiv$ Nectura 1940 AM $\equiv$ Nectusus 1940 am

$\approx$ Phanerobranchus 1821 (tetradactylus $1821 \approx$ maculosa* 1818) $\equiv$ Phaenerobranchus $1826 \equiv$ Phanerabronchus 1849 AM $\equiv$ Phanerobronchus 1849 AM $\approx$ Menobranchus 1825 (lateralis $1822 \approx$ maculosa* 1818) $\approx$ Parvurus 2012 (punctatus* 1850) $\{\mathbf{1 0 0}\}$

G.28.554. Proteus* 1768 (anguinus* 1768 ) $\equiv$ Exobranchia $1815 \mathrm{AN} \equiv$ Larvarius $1815 \equiv$ Platyrhynchus $1816 \equiv$ Hypochthon $1820 \equiv$ Caledon $1820 \equiv$ Hydrospelaeus $1821 \equiv$ Apneumona $1822 \equiv$ Cordylus $1828 \mathbf{J H} \equiv$ Hydochthon 1831 AM $\equiv$ Hemitriton 1833

F.14.18. Superfamilia SALAMANDROIDEA Goldfuss, 1820.ga.f002-21 \{100\} F.17.68. Familia AMBYSTOMATIDAE Gray, 1850.ga.f002-08 \{100\} [Q]

G.28.†185. Ambystomichnus ${ }^{\circ} 1954 \dagger$ (montanensis $\left.^{\circ} 1928 \dagger\right)$

G.28.†186. Amphitriton $^{\circ} 1976 \dagger\left(\right.$ brevis $\left.^{\circ} 1976 \dagger\right)$

G.28.†187. Chrysotriton ${ }^{\circ} 1981 \dagger\left(\right.$ tiheni $\left.^{\circ} 1981 \dagger\right)$

G.28.†188. Sanchizia $^{\circ} 2012 \dagger\left(\right.$ wettstein $\left.^{\circ} 1955 \dagger\right) \equiv$ Bargmannia $1955 \mathbf{J H}$

G.28. $\uparrow 189$. Wolterstorffiella ${ }^{\circ} 1950 \dagger\left(\right.$ wiggeri $\left.^{\circ} 1950 \dagger\right) \equiv$ Wolterstorffiella 1939 AN

G.28.555. Ambystoma 1838 (subviolacea $1804 \approx$ maculata* 1802) $\equiv$ Salamandroidis 1843

$\equiv$ Amblystoma $1844 \mathrm{CI} \equiv$ Limnarches $1848 \equiv$ Plagiodon $1854 \mathrm{AN} \equiv$ Plagiodons $1854 \mathrm{AN} \approx$ Gyrinus

1798 JH (mexicanus* 1798$) \equiv$ Axolotes $1844 \approx$ Axolotl 1821 AN (pisciformis $1802 \approx$ mexicanus $* 1798$ )

$\equiv$ Axolotus $1822 \mathrm{CI} \equiv$ Philhydrus $1828 \mathrm{CI} \equiv$ Axolot $1831 \mathrm{NC}-\mathrm{CI} \equiv$ Phyllhydrus $1831 \mathrm{CI} \equiv$ Phylhydrus $1839 \equiv$ Axoloth $1842 \equiv$ Phyllidrus $1844 \approx$ Siredon 1829 CI (axolotl $1829 \approx$ mexicanus* 1798$) \equiv$ Sirenodon $1832 \mathrm{CI} \equiv$ Stegoporus $1832 \mathrm{CI} \approx$ Xiphonura 1838 (jeffersoniana* 1827) $\equiv$ Xiphoctonus $1848 \approx$ Heterotriton 1850 (ingens $1831 \approx$ tigrina* 1825 ) $\approx$ Desmiostoma 1858 (maculatum $1858 a$ $\approx$ mavortia $^{\circ} 1850$ ) $\approx$ Camarataxis 1859 (maculatum $1858 b \approx$ mavortia $^{\circ} 1850$ ) $\approx$ Pectoglossa 1868 (persimilis $1859 \approx$ jeffersoniana* 1827$) \approx$ Linguaelapsus 1887 (annulatum* 1886) $\approx$ Rhyacosiredon

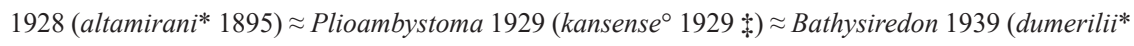

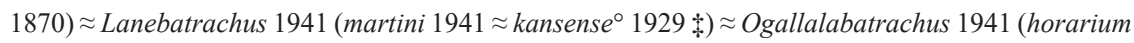
$1941 \approx$ kansense $\left.^{\circ} 1929 \$\right)\{\mathbf{1 0 0}\}$

G.28.556. Dicamptodon* 1870 (ensatus* 1833) Chondrotus 1887 (tenebrosum* 1852) \{100\}

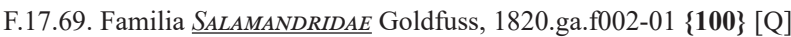

F.18.85. Subfamilia PLEURODELINAE Tschudi, 1838.ta.f005-08 $\{\mathbf{1 0 0}\}$

F.19.†0b. Tribus INCERTAE SEDIS $\dagger$

G. $28 . \dagger 190$. Archaeotriton $^{\circ} 1860 \dagger\left(\right.$ basalticus $\left.^{\circ} 1859 \dagger\right)$

G.28.†191. Brachycormus ${ }^{\circ} 1860 \dagger$ (noachicus $\left.^{\circ} 1831 \dagger\right)$

G.28. $\uparrow$ 192. Carpathotriton $^{\circ} 2008 \dagger$ (matraensis $\left.^{\circ} 2008 \uparrow\right)$

G.28.†193. Chelotriton $^{\circ} 1853 \dagger$ (paradoxus $\left.^{\circ} 1853 \dagger\right) \approx$ Heliarchon 1860 (fuscillatus 1860

$\approx$ paradoxus $\left.^{\circ} 1853 \dagger\right) \approx$ Polysemia $1860 \mathbf{J H}\left(\right.$ ogygia $\left.^{\circ} 1831 \dagger\right) \equiv$ Epipolysemia $1973 \approx$ Grippiella 1949 $\left(\right.$ mohri $1949 \approx$ paradoxus $\left.^{\circ} 1853 \dagger\right) \approx$ Palaeosalamandrina $1949\left(\right.$ dehmi $1949 \approx$ paradoxus $\left.^{\circ} 1853 \dagger\right) \approx$ Tischleriella 1949 (buddenbrocki $1949 \approx$ paradoxus $^{\circ} 1853 \dagger$ )

G.28.†194. Koalliella ${ }^{\circ} 1950 \dagger\left(\right.$ genzeli $\left.^{\circ} 1950 \dagger\right)$

G.28.†195. Oligosemia ${ }^{\circ} 1923 \dagger\left(\right.$ spinosa $\left.^{\circ} 1922 \dagger\right)$

G.28.†196. Palaeopleurodeles ${ }^{\circ} 1941 \dagger\left(\right.$ hauffi $\left.^{\circ} 1941 \dagger\right)$

G.28.†197. Phosphotriton ${ }^{\circ} 2016 \dagger\left(\right.$ sigei $\left.^{\circ} 2016 \dagger\right) \equiv$ Phosphotriton $2015 \dagger$ AN

G.28.†198. Procynops ${ }^{\circ} 1965 \dagger$ (miocenicus $\left.^{\circ} 1965 \dagger\right)$

F.19.86. Tribus MolgINI Bonaparte, 1850.bb.f015-04 \{100\}

F.20.89. Subtribus MoLGINA Bonaparte, 1850.bb.f015-05 \{100\}

F.21.62. Infratribus EUPROCTINIA Dubois ${ }^{+1}$, 2009.db.f002-01 $\{\mathbf{1 0 0}\}$

G.28.557. Euproctus ${ }^{1} 1839$ (rusconii 1839 $\approx$ platycephala* $^{*} 1829$ ) $\equiv$ Bradyarges $1868 \equiv$ Bulga 1868

$\approx$ Megapterna 1839 (montana* 1839$) \approx$ Pelonectes 1843 (platycephala* 1829$)$ 
F.21.63. Infratribus MoLGINIA Bonaparte, 1850.bb.f015-07 \{95\}

F.22.41. Hypotribus CYNOPINOA Dubois ${ }^{+1}, 2009 . d b . f 001-01\{\mathbf{1 0 0}\}$

F.23.28. Clanus CYNOPITES Dubois ${ }^{+1}$, 2009.db.f001-02 \{100\}

G.28.558. Cynops ${ }^{1} 1838$ (subcristatus $1838 \approx$ pyrrhogaster* $^{*} 1826$ )

F.23.29. Clanus HYPSELOTRITONITES nov., DOP.da.f141-00 \{99\}

G.28.559. Hypselotriton ${ }^{2} 1934$ (wolterstorffi $\left.^{\circ} 1905\right) \approx$ Cynotriton $^{2011}$ (orientalis $^{*}$ 1875)

F.23.30. Clanus PACHYTRITONITES nov., DOP.da.f142-00 \{99\}

G.28.560. Laotriton* 2009 (laoensis* 2002)

G.28.561. Pachytriton* 1878 (brevipes* 1877) $\approx$ Pingia 1936 (granulosus* 1933) \{99\}

G.28.562. Paramesotriton* 1935 (deloustali* 1934) $\equiv$ Mesotriton $1934 \mathbf{J H} \approx$ Trituroides 1936

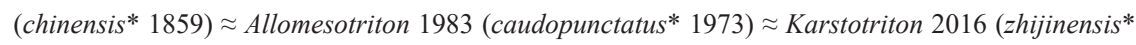
2008) $\{96\}$

F.22.42. Hypotribus ICHTHYOSAURINOA nov., DOP.da.f143-00

G.28.563. Ichthyosaura 1801 (tritonius $1768 \approx$ alpestris $^{*} 1768$ ) $\approx$ Hemitriton 1852 JH (alpestris*

1768) $\equiv$ Mesotriton 1927

F.22.43. Hypotribus LISSOTRITONINOA nov., DOP.da.f144-00 $\{\mathbf{1 0 0}\}$

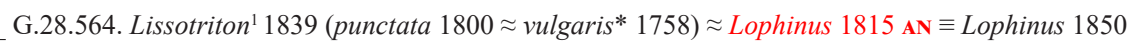

$\approx$ Meinus 1815 AN $($ boscai* 1879) $\equiv$ Pelonectes 1879 JH $\equiv$ Meinus $2009 \approx$ Palmitus 1815 AN (helvetica*

1879 ) $\approx$ Geotriton 1831 AN (exigua $1768 \approx$ vulgaris* 1758) E Geotriton 1832 CI $\approx$ Palaeotriton 1927 (vulgaris* 1758)

F.22.44. Hypotribus MoLGINOA Bonaparte, 1850.bb.f015-08 \{100\}

F.23.31. Clanus MoLGITES Bonaparte, 1850.bb.f015-09 \{99\}

G.28.565. Calotriton ${ }^{1} 1858$ (punctulatus $1852 \approx$ asper* $\left.^{*} 1852\right)\{\mathbf{1 0 0}\}$

G.28.566. Triturus* 1815 (cristatus* 1868) $\equiv$ Triton $1768 \mathbf{J H} \equiv$ Molge $1820 \equiv$ Oiacurus 1821

$\equiv$ Tritonella $1839 \equiv$ Hemisalamandra $1852 \equiv$ Alethotriton $1872 \approx$ Petraponia 1853 (nigra 1854

$\approx$ carnifex* 1768 ) $\approx$ Pyronicia 1858 (marmorata* 1800$) \approx$ Turanomolge 1918 (mensbieri $1918 \approx$

karelinii* $^{*}$ 1870) $\approx$ Neotriton 1927 (karelinii* 1870) $\{\mathbf{1 0 0}\}$

F.23.32. Clanus NEURERGITES nov., DOP.da.f145-00 \{99\}

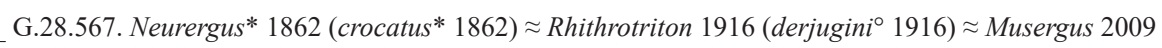

(strauchii* 1888$)\{\mathbf{1 0 0}\}$

G.28.568. Ommatotriton* 1850 (vittatus* 1835) $\{\mathbf{1 0 0}\}$

F.20.90. Subtribus TARICHINA Dubois ${ }^{+1}, 2009 . d b . f 003-00\{100\}$

G.28.569. Notophthalmus ${ }^{1} 1820$ (miniatus $1820 \approx$ viridescens $^{*} 1820$ ) $\approx$ Diemictylus 1820 (viridescens*

$1820) \approx$ Tristella $^{1} 1850$ AN (symmetrica $1825 \approx$ viridescens $\left.* 1820\right) \approx$ Rafinus 2009 (meridionalis* 1880 )

$\{100\}$

G.28.570. Taricha* 1850 (torosus* 1833) $\approx$ Palaeotaricha 1955 (oligocenica $\left.{ }^{\circ} 1955 \dagger\right) \approx$ Twittya 2009

(rivularis* 1935) $\{\mathbf{1 0 0}\}$

F.19.87. Tribus PLEURODELINI Tschudi, 1838.ta.f005-09 $\{\mathbf{1 0 0}\}$

F.20.91. Subtribus PLEURODELINA Tschudi, 1838.ta.f005-10 \{100\}

G.28.571. Pleurodeles* 1830 (walt ${ }^{*} 1830$ ) $\equiv$ Pleuroderes 1878 AM $\approx$ Bradybates 1838 (ventricosus $1838 \approx$ waltl $^{*} 1830$ ) $\equiv$ Bradytes $1848 \approx$ Glossoliga 1839 (poireti* 1835 )

F.20.92. Subtribus TYLOTOTRITONINA nov., DOP.da.f146-00 $\{\mathbf{1 0 0}\}$

F.21.64. Infratribus ECHINOTRITONINIA nov., DOP.da.f147-00 $\{\mathbf{1 0 0}\}$

G.28.572. Echinotriton* 1982 (andersoni* 1892)

F.21.65. Infratribus TYLOTOTRITONINIA nov., DOP.da.f146-01 $\{\mathbf{1 0 0}\}$

G.28.573. Tylototriton* 1871 (verrucosus* 1871) $\equiv$ Tylotriton $1885 \approx$ Qiantriton 2012

(kweichowensis* 1932) $\equiv$ Qianotriton $2016 \approx$ Liangshantriton 2012 (taliangensis* 1950) \{100\}

G.28.574. Yaotriton* 2009 (asperrimus* 1830) $\{97\}$

F.18.86. Subfamilia SALAMANDRINAE Goldfuss, 1820.ga.f002-15 \{93\}

F.19.†0c. Tribus INCERTAE SEDIS †

G.28.†199. Megalotriton $^{\circ} 1890 \dagger\left(\right.$ filholi $\left.^{\circ} 1890 \dagger\right)$

F.19.88. Tribus CHIOGLOSSINI Dubois ${ }^{+1}$, 2009.db.f004-00 $\{\mathbf{1 0 0}\}$

G.28.575. Chioglossa* 1864 (lusitanica* 1864)

G.28.576. Mertensiella* 1925 (caucasicus* 1876) E Exaeretus $1876 \mathbf{J H}$

F.19.89. Tribus SALAMANDRINI Goldfuss, 1820.ga.f002-28 $\{\mathbf{1 0 0}\}$

G.28.577. Lyciasalamandra* 2004 (luschani* 1891) $\{\mathbf{1 0 0}\}$ 


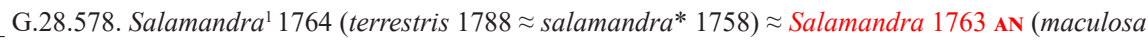
$1768 \approx$ salamandra* 1758$) \equiv$ Salamandra $1768 \approx$ Salamandraches 1848 (crassicaudis $1848 \approx$ salamandra* 1758$) \approx$ Heteroclitotriton 1903 (zitteli $1903 \approx$ sansaniensis $^{\circ} 1851 \ddagger$ ) $\approx$ Palaeosalamandra 1949 (kohlitzi $1949 \approx$ sansaniensis $^{\circ} 1851$ \$) $\approx$ Voigtiella 1949 (ludwigi $1949 \approx$ sansaniensis $^{\circ} 1851$ $\$) \approx$ Dehmiella 1950 (schindewolfi $1950 \approx$ sansaniensis $^{\circ} 1851$ \$) Algiandra 2009 (algira* 1883)

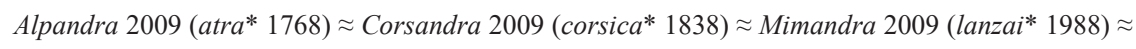
Oriandra 2009 (infraimmaculata* 1885) $\{\mathbf{1 0 0}\}$

F.18.87. Subfamilia SALAMANDRININAE Fitzinger, 1843.fa.f013-01 $\{\mathbf{1 0 0}\}$

G.28.579. Salamandrina* 1826 (perspicillata* 1821$) \approx$ Seiranota 1826 (condylura $1826 \approx$ perspicillata* 1821$)^{*}$ 
Appendix A10.CLAD-2. Simplified cladonomy and nomenclature of LiSSAMPHIBIA proposed here, showing all taxa from classis to subfamily and all genera.

Unavailable and invalid genera nomina are not listed here (see Appendix A9.CLAD-1).

$\mathrm{G} \cdot$ genus including at least one recent species.

$\mathrm{G} \uparrow \cdot$ all-fossil genus.

For the meaning of all other identifiers, see legend of Appendix A9.CLAD-1.

C.01.01. Subphylum VERTEBRATA 1800

_C.02.01. Classis AMPHIBIA 1816

_ _ C.03.01. Subclassis LissamphiBia 1898

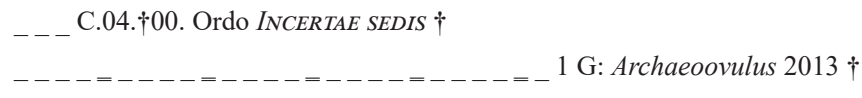

C.04. $\dagger 01$. Ordo Allocaudata $1982 \dagger$

F.17.†01. Familia $\underline{\text { ALBANERPETIDAE } 1982 \dagger}$

$6 \mathrm{G} \uparrow$ : Albanerpeton $1976 \dagger$; Anoualerpeton $2003 \dagger$; Celtedens $1995 \dagger$; Nukusurus 1981 †; Shirepeton $2018 \uparrow$; Wesserpeton $2013 \dagger$

C.04.01. Ordo ANURA 1805

C.05.†0a. Subordo INCERTAE SEDIS †

F.17.†0a. Familia INCERTAE SEDIS $\dagger$

$39 \mathrm{G} \uparrow$ : Altanulia 1993 †; Aralobatrachus $1981 \uparrow$; Arariphrynus $2006 \uparrow ;$ Aygroua 2003 †; Batrachulina $1962 \uparrow$; Comobatrachus 1960 †; Cratia $2009 \dagger$; Czatkobatrachus 1998 †; Eobatrachus 1887; Eorubeta $1960 \uparrow ;$ Estesiella $1995 \dagger ;$ Estesina $1993 \uparrow ;$ Eurycephalella $2009 \dagger ;$ Gobiatoides $1993 \uparrow ;$; Hatzegobatrachus 2003 †; Hensonbatrachus 2015 †; Iberobatrachus 2013 †; Itemirella 1981 †; Liaobatrachus 1998 †; Liventsovkia 1993 †; Lutetiobatrachus 1998 †; Mengbatrachus 2018 †; Mesophryne $2001 \dagger$; Monsechobatrachus 1921 †; Negatchevkia 1993 †; Novooskolia 1993 †; Procerobatrachus 1993 †; Ranipes 2014 †; Ranomorphus 1993 †; Saevesoederberghia 1993 †; Scotiophryne 1969 †; Sunnybatrachus 2002 †; Thaumastosaurus 1904 †; Theatonius 1976 †; Tyrrellbatrachus 2015 †; Uberabatrachus 2012 †; Varibatrachus 2015 †; Vieraella 1961 †; Yizhoubatrachus $2004 \uparrow$

F.17.†02. Familia PROSALIRIDAE $1995 \dagger$

$1 \mathrm{G} \dagger$ : Prosalirus $1995 \dagger$

F.17.†03. Familia $\underline{\text { TREGOBATRACHIDAE }} 1975 \dagger$

$1 \mathrm{G} \uparrow$ : Tregobatrachus $1975 \dagger$

F.17.†04. Familia TRIADOBATRACHIDAE $1962 \dagger$

$1 \mathrm{G} \uparrow$ : Triadobatrachus $1962 \dagger$

C.05.01. Subordo ANGUSTICOELA 1958

F.17.01. Familia ASCAPHIDAE 1923

$1 \mathrm{G}:$ Ascaphus 1899

F.17.02. Familia LEIOPELMATIDAE 1869-|1942| [Q]

F.18.†01. Subfamilia NOTOBATRACHINAE $1956 \dagger$ $1 \mathrm{G} \uparrow$ : Notobatrachus $1956 \uparrow$

F.18.01. Subfamilia LEIOPELMATINAE 1869-|1942|

2 G: Leioaspetos 1985; Leiopelma 1861

C.05.02. Subordo Hydrobatrachia 1828

F.17.†0b. Familia INCERTAE SEDIS $\dagger$

$4 \mathrm{G} \uparrow$ : Hyogobatrachus 2016 †; Kururubatrachus 2020 †; Tambabatrachus 2016 †; Wealdenbatrachus $1988 \dagger$

C.06.01. Infraordo Geobatrachia 1828

F.17.†0c. Familia INCERTAE SEDIS $\dagger$

$1 \mathrm{G} \uparrow$ : Genibatrachus $2017 \dagger$

C.07.01. Hypoordo DoRsIPARES 1816

F.17.†0d. Familia INCERTAE SEDIS † 
7 G †: Avitabatrachus $2000 \dagger$; Gracilibatrachus $2013 \uparrow ;$ Neusibatrachus $1972+;$ Nevobatrachus $2019+$;

Shomronella 1978 †; Thoraciliacus 1968 †; Vulcanobatrachus 2005 †

F.17.†05. Familia PALAEOBATRACHIDAE $1865 \dagger$

$3 \mathrm{G} \uparrow$ : Albionbatrachus $1984 \dagger$; Palaeobatrachus $1838 \dagger$; Probatrachus $1878 \dagger$

F.17.03. Familia PIPIDAE 1825-|1826| [Q]

F.18.†0a. Subfamilia INCERTAE SEDIS †

$6 \mathrm{G} \uparrow$ : Cratopipa 2019 †; Eoxenopoides 1931 †; Llankibatrachus 2003 †; Oumtkoutia 2008 †;

Pachycentrata $2004 \uparrow ;$ Singidella $2005 \dagger$

F.18.†02. Subfamilia SALTENIINAE nov. $\dagger$

$4 \mathrm{G} \uparrow:$ Kuruleufemia $2016 \uparrow$; Saltenia $1959 \dagger$; Shelania 1960 †; Patagopipa $2019 \dagger$

F.18.02. Subfamilia DACTYLETHRINAE 1838

4 G: Hymenochirus 1896; Pseudhymenochirus 1920; Silurana 1864; Xenopus 1827

F.18.03. Subfamilia PIPINAE 1825-|1826

1 G: Pipa 1768

F.17.04. Familia RHINOPHRYNIDAE 1858 [Q]

$3 \mathrm{G} \uparrow$ : Chelomophrynus $1991 \uparrow$; Eorhinophrynus $1959+;$ Rhadinosteus $1998 \dagger$

$1 \mathrm{G}$ : Rhinophrynus 1841

C.07.02. Hypoordo LaevogYrinia 1878

C.08.0a. Superphalanx INCERTAE SEDIS

F.17.0a. Familia INCERTAE SEDIS

$1 \mathrm{G}:$ Colodactylus 1845

C.08.01. Superphalanx Archaeosalientia 1981

F.14.†0a. Superfamilia INCERTAE SEDIS †

F.17.†Of. Familia INCERTAE SEDIS †

$4 \mathrm{G} \dagger$ : Elkobatrachus $2006+$; Macropelobates $1924 \uparrow$; Tephrodytes 1994 †; Uldzinia $1996 \dagger$

F.14.01. Superfamilia PELOBATOIDEA 1850

F.15.01. Epifamilia PELOBATOIDAE 1850

F.17.†0g. Familia INCERTAE SEDIS †

$1 \mathrm{G} \uparrow$ : Sanshuibatrachus $2017 \dagger$

F.17.05. Familia MEGOPHRYIDAE 1850-|1931| [Q+]

F.18.04. Subfamilia LEPTOBRACHIINAE 1983

4 G: Leptobrachella 1925; Leptobrachium 1838; Oreolalax 1962; Scutiger 1868

F.18.05. Subfamilia MEGOPHRYINAE 1850-|931|

7 G: Atympanophrys 1983; Boulenophrys 2016; Brachytarsophrys 1983; Grillitschia nov.; Megophrys

1822; Ophryophryne 1903; Xenophrys 1864

F.17.06. Familia PELOBATIDAE 1850 [Q]

$1 \mathrm{G} \uparrow$ : Eopelobates $1929 \dagger$

$1 \mathrm{G}$ : Pelobates 1830

F.15.02. Epifamilia PELODYTOIDAE 1850

F.17.07. Familia PELODYTIDAE 1850 [Q]

$2 \mathrm{G} \dagger$ : Aerugoamnis $2013 \uparrow$; Miopelodytes $1941 \uparrow$.

2 G: Pelodytes 1838; Pelodytopsis 1896

F.14.02. Superfamilia SCAPHIOPODOIDEA 1865

F.17.08. Familia SCAPHIOPODIDAE 1865

2 G: Scaphiopus 1836; Spea 1866

C.08.02. Superphalanx RANOMORPHA 1921

C.09.01. Epiphalanx AQUIPARES 1816

C.10.01. Phalanx Gondwanura nov.

F.17.09. Familia NASIKABATRACHIDAE 2003

1 G: Nasikabatrachus 2003

F.17.10. Familia SooglossidaE 1931 [Q]

2 G: Sechellophryne 2007; Sooglossus 1906

C.10.02. Phalanx Phaneranura nov.

C.11.01. Subphalanx BaINANURA nov.

C.12.01. Infraphalanx Phoranura nov.

F.17.11. Familia AROMOBATIDAE 2006 
F.18.06. Subfamilia ALLOBATINAE 2006 $1 \mathrm{G}$ : Allobates 1988

F.18.07. Subfamilia ANOMALOGLOSSINAE 2006 2 G: Anomaloglossus 2006; Rheobates 2006

F.18.08. Subfamilia AROMOBATINAE 2006 2 G: Aromobates 1991; Mannophryne 1992

F.17.12. Familia DENDROBATIDAE $\|1850\|-1865$ [Q]

F.18.09. Subfamilia ColostethinaE 1867 5 G: Ameerega 1986; Colostethus 1866; Epipedobates 1987; Leucostethus 2017; Silverstoneia 2006 F.18.10. Subfamilia DENDROBATINAE $\|1850\|-1865$ 8 G: Adelphobates 2006; Andinobates 2011; Dendrobates 1830; Excidobates 2008; Minyobates 1987; Oophaga 1994; Phyllobates 1841; Ranitomeya 1985

F.18.11. Subfamilia HYLOXALINAE 2006 3 G: Ectopoglossus 2017; Hyloxalus 1870; Paruwrobates 1994

C.12.02. Infraphalanx Phrynanura nov.

C.13.01. Hypophalanx Gaianura nov.

F.17.13. Familia BRACHYCEPHALIDAE 1858 [Q]

F.18.0a. Subfamilia INCERTAE SEDIS 2 G: Atopophrynus 1982; Geobatrachus 1915

F.18.12. Subfamilia BRACHYCEPHALINAE 1858 2 G: Brachycephalus 1826; Ischnocnema 1862

F.18.13. Subfamilia CRAUGASTORINAE 2008

21 G: Bahius nov.; Barycholos 1969; Bryophryne 2008; Craugastor 1862; Euparkerella 1959; Haddadus 2008; Holoaden 1920; Hypodactylus 2008; Lynchius 2008; Microkayla 2017; Niceforonia 1963; Noblella 1930; Oreobates 1872; Phrynopus 1873; Phyllonastes 1977; Pristimantis 1870; Psychrophrynella 2008; Qosqophryne 2020; Strabomantis 1863; Tachiramantis 2015; Yunganastes 2007

F.18.14. Subfamilia ELEUTHERODACTYLINAE 1954 5 G: Adelophryne 1984; Diasporus 2008; Euhyas 1843; Eleutherodactylus 1841; Phyzelaphryne 1977

F.17.14. Familia CEUTHOMANTIDAE 2009 2 G: Ceuthomantis 2009; Dischidodactylus 1979

C.13.02. Hypophalanx HeMiphractiformia 188

F.17.15. Familia HEMIPHRACTIDAE 1862 [Q]

F.18.15. Subfamilia AMPHIGNATHODONTINAE 1882 5 G: Alainia 2018; Amphignathodon 1882; Cryptotheca 2015; Eotheca 2015; Gastrotheca 1843

F.18.16. Subfamilia CRYPTOBATRACHINAE 2006 1 G: Cryptobatrachus 1916

F.18.17. Subfamilia FLECTONOTINAE nov. $1 \mathrm{G}:$ Flectonotus 1926

F.18.18. Subfamilia FRITZIANINAE nov. 1 G: Fritziana 1937

F.18.19. Subfamilia HEMIPHRACTINAE 1862 $1 \mathrm{G}$ : Hemiphractus 1828

F.18.20. Subfamilia STEFANIINAE nov. 1 G: Stefania 1968

C.13.03. Hypophalanx HyLOBATRaCHIA 1828

F.14.0a. Superfamilia INCERTAE SEDIS 1 G: Ancudia 1902

F.14.03. Superfamilia BUFONOIDEA 1825 F.17.16. Familia BUFONIDAE 1825 [Q] F.18.21. Subfamilia BUFONINAE 1825 $1 \mathrm{G} \uparrow$ : Palaeophrynos $1838 \dagger$ 53 G: Adenomus 1861; Altiphrynoides 1987; Amazophrynella 2012; Anaxyrus 1845; Ansonia 1870; Atelopus 1841; Barbarophryne 2013; Beduka nov.; Blaira nov.; Blythophryne 2016; Bufo 1764; Bufoides 1973; Bufotes 1815; Capensibufo 1980; Churamiti 2002; Dendrophryniscus 1870; Didynamipus 1903; Duttaphrynus 2006; Epidalea 1864; Firouzophrynus 2020; Frostius 1986; Incilius 1863; Ingerophrynus 2006; Laurentophryne 1960; Leptophryne 1843; Mertensophryne 1960; Metaphryniscus 1994; Mo nov.; 
Nannophryne 1870; Nectophryne 1875; Nectophrynoides 1926; Nimbaphrynoides 1987; Oreophrynella 1895; Osornophryne 1976; Parapelophryne 2003; Pedostibes 1876; Pelophryne 1938; Peltophryne 1843; Phrynoidis 1842; Poyntonophrynus 2006; Pseudobufo 1838; Rentapia 2016; Rhaebo 1862; Rhinella 1826; Sabahphrynus 2007; Schismaderma 1849; Sclerophrys 1838; Sigalegalephrynus 2017; Strauchbufo 2012; Truebella 1995; Vandijkophrynus 2006; Werneria 1903; Wolterstorffina 1939

F.18.22. Subfamilia MELANOPHRYNISCINAE nov.

1 G: Melanophryniscus 1961

F.17.17. Familia ODONTOPHRYNIDAE 1971

F.18.†0b. Subfamilia INCERTAE SEDIS † $1 \mathrm{G} \dagger$ : Chachaiphrynus $2017 \dagger$

F.18.23. Subfamilia ODONTOPHRYNINAE 1971

2 G: Macrogenioglottus 1946; Odontophrynus 1862

F.18.24. Subfamilia PROCERATOPHRYINAE nov.

1 G: Proceratophrys 1920

F.14.04. Superfamilia CENTROLENOIDEA 1951

F.17.18. Familia ALLOPHRYNIDAE 1978

$1 \mathrm{G}$ : Allophryne 1926

F.17.19. Familia CENTROLENIDAE 1951 [Q]

F.18.25. Subfamilia CENTROLENINAE 1951 10 G: Audaciella nov.; Centrolene 1872; Chimerella 2009; Cochranella 1951; Espadarana 2009; Nymphargus 2007; Rulyrana 2009; Sachatamia 2009; Teratohyla 1951; Vitreorana 2009

F.18.26. Subfamilia HYALINOBATRACHINAE 2009

2 G: Celsiella 2009; Hyalinobatrachium 1991

F.18.27. Subfamilia IKAKOGINAE nov.

1 G: Ikakogi 2009

F.14.05. Superfamilia CERATOPHRYOIDEA 1838

F.15.03. Epifamilia CERATOPHRYOIDAE 1838

F.17.20. Familia CERATOPHRYIDAE 1838

F.18.28. Subfamilia CERATOPHRYINAE Tschudi, 1838.ta.f002-06 $\{\mathbf{1 0 0}\}$ $1 \mathrm{G} \uparrow$ : Beelzebufo $2008 \uparrow$ 1 G: Ceratophrys 1824

F.18.29. Subfamilia LEPIDOBATRACHINAE Bauer, 1987.ba.f001-01 \{97\}

1 G. $\dagger$ : Baurubatrachus 1990 2 G: Chacophrys 1963; Lepidobatrachus 1899

F.18.30. Subfamilia SтомвINAE Gallardo 1965.ga.f001-00 1 G: Stombus 1825

F.15.04. Epifamilia TELMATOBIOIDAE 1843

F.16.01. Apofamilia CYCLORAMPHEIDAE 1850-|1852|

F.17.21. Familia CYCLORAMPHIDAE 1850-|1852|

F.18.31. Subfamilia ALSODINAE 1869 2 G: Alsodes 1843; Eupsophus 1843

F.18.32. Subfamilia BATRACHYLINAE 1965 4 G: Atelognathus 1978; Batrachyla 1843; Chaltenobatrachus 2011; Hylorina 1843

F.18.33. Subfamilia CYCLORAMPHINAE 1850-|1852| 2 G: Cycloramphus 1838; Thoropa 1865

F.18.34. Subfamilia HYLODINAE 1858 2 G: Crossodactylus 1841; Hylodes 1826

F.18.35. Subfamilia LIMNOMEDUSINAE nov. 1 G: Limnomedusa 1843

F.16.02. Apofamilia TELMATOBIEIDAE 1843

F.17.22. Familia RHINODERMATIDAE 1850 [Q] 2 G: Insuetophrynus 1970; Rhinoderma 1841

F.17.23. Familia TELMATOBIIDAE 1843 $1 \mathrm{G} \dagger$ : Neoprocoela $1949 \dagger$ $1 \mathrm{G}$ : Telmatobius 1834

F.14.06. Superfamilia HYLOIDEA 1815-|1825| 
F.17.24. Familia HYLIDAE $1815-|1825|$ [Q]

F.18.†0c. Subfamilia INCERTAE SEDIS $\dagger$ $3 \mathrm{G} \dagger$ : Etnabatrachus $2003 \uparrow$; Geophryne $2014 \uparrow$; Proacris $1961 \dagger$

F.18.36. Subfamilia CopHOMANTINAE 1878

7 G: Aplastodiscus 1950; Bokermannohyla 2005; Boana 1825; Colomascirtus 2016; Hyloscirtus 1882; Myersiohyla 2005; Nesorohyla 2019

F.18.37. Subfamilia HYLINAE 1815-|1825|

41 G: Acris 1841; Anotheca 1939; Aparasphenodon 1920; Argenteohyla 1970; Atlantihyla 2018;

Bromeliohyla 2005; Charadrahyla 2005; Corythomantis 1896; Dendropsophus 1843; Diaglena 1887; Dryaderces 2013; Dryophytes 1843; Duellmanohyla 1992; Ecnomiohyla 2005; Exerodonta 1879; Gabohyla 2020; Hyla 1768; Hyliola 1899; Isthmohyla 2005; Itapotihyla 2005; Megastomatohyla 2005; Nyctimantis 1882; Osteocephalus 1862; Osteopilus 1843; Phyllodytes 1830; Phytotriades 2009; Plectrohyla 1877; Pseudacris 1843; Pseudis 1830; Ptychohyla 1944; Quilticohyla 2018; Rheohyla 2016; Scarthyla 1988; Scinax 1830; Smilisca 1865; Sphaenorhynchus 1838; Tepuihyla 1993; Tlalocohyla 2005; Trachycephalus 1838; Triprion 1866; Xenohyla 1998

F.17.25. Familia PHYLLOMEDUSIDAE 1858

F.18.38. Subfamilia PELODRYADINAE 1859

$1 \mathrm{G} \uparrow$ : Australobatrachus 1976

3 G: Litoria 1838; Nyctimystes 1916; Ranoidea 1838

F.18.39. Subfamilia PHYLLOMEDUSINAE 1858 8 G: Agalychnis 1864; Callimedusa 2016; Cruziohyla 2005; Hylomantis 1873; Phasmahyla 1991;

Phrynomedusa 1923; Phyllomedusa 1830; Pithecopus 1866

F.14.07. Superfamilia LEPTODACTYLOIDEA $\|1838\|-1896$

F.17.26. Familia LEPTODACTYLIDAE $\|1838\|-1896$ [Q]

F.18.40. Subfamilia LEIUPERINAE 1850 5 G: Edalorhina 1870; Engystomops 1872; Eupemphix 1863; Physalaemus 1826; Pleurodema 1838 F.18.41. Subfamilia LEPTODACTYLINAE $\|1838\|-1896$ 3 G: Adenomera 1867; Leptodactylus 1826; Lithodytes 1843

F.18.42. Subfamilia PaRATELmatobiInAE 2012 2 G: Crossodactylodes 1938; Rupirana 1999

F.18.43. Subfamilia PSEUDOPALUDICOLINAE_ 1965 1 G: Pseudopaludicola 1926

C.11.02. Subphalanx Diplosiphona 1859

F.17.27. Familia CALYPTOCEPHALELLIDAE 1960

2 G: Calyptocephalella 1928; Telmatobufo 1952

F.17.28. Familia MYOBATRACHIDAE 1850 [Q]

F.18.†0d. Subfamilia INCERTAE SEDIS † $1 \mathrm{G} \uparrow$ : Indobatrachus $1930 \dagger$

F.18.44. Subfamilia LIMNODYNASTINAE 1971 7 G: Adelotus 1907; Heleioporus 1841; Limnodynastes 1843; Neobatrachus 1863; Notaden 1873; Philoria 1901; Platyplectrum 1863

F.18.45. Subfamilia MIXOPHYINAE nov. $1 \mathrm{G}$ : Mixophyes 1864

F.18.46. Subfamilia MYoBatrachinaE 1850

11 G: Arenophryne 1976; Assa 1972; Crinia 1838; Geocrinia 1973; Metacrinia 1940; Myobatrachus 1850; Paracrinia 1976; Pseudophryne 1843; Spicospina 1997; Taudactylus 1966; Uperoleia 1841 F.18.47. Subfamilia RHEOBATRACHINAE 1976

$1 \mathrm{G}$ : Rheobatrachus 1973

C.10.03. Phalanx Scoptanura 1973

C.11.†0a. Subphalanx INCERTAE SEDIS

$1 \mathrm{G} \dagger$ : Hungarobatrachus $2010 \dagger$

C.11.03. Subphalanx Ecostata 1879

F.17.29. Familia MICROHYLIDAE $\|1843\|-1931$ [Q]

F.18.48. Subfamilia ADELASTINAE 2016

$1 \mathrm{G}$ : Adelastes 1986

F.18.49. Subfamilia ASTEROPHRYINAE 1858 
4 G: Asterophrys 1838; Gastrophrynoides 1926; Siamophryne 2018; Vietnamophryne 2018

F.18.50. Subfamilia COPHYLINAE 1889

8 G: Anodonthyla 1892; Cophyla 1880; Madecassophryne 1974; Mantipus 1883; Paradoxophyla 1991;

Platypelis 1882; Rhombophryne 1880; Scaphiophryne 1882

F.18.51. Subfamilia GASTROPHRYNINAE 1843

11 G: Arcovomer 1954; Chiasmocleis 1904; Ctenophryne 1904; Dasypops 1924; Dermatonotus 1904;

Engystoma 1826; Gastrophryne 1843; Hamptophryne 1954; Hypopachus 1867; Myersiella 1954;

Stereocyclops 1870

F.18.52. Subfamilia HoPLOPHRYNINAE 1931

2 G: Hoplophryne 1928; Parhoplophryne 1928

F.18.53. Subfamilia KALOPHRYNINAE 1869

$1 \mathrm{G}:$ Kalophrynus 1838

F.18.54. Subfamilia MELANOBATRACHINAE 1931

$1 \mathrm{G}$ : Melanobatrachus 1878

F.18.55. Subfamilia MICROHYLINAE $\|1843\|-1931$

10 G: Chaperina 1892; Dyscophus 1872; Glyphoglossus 1869; Kaloula 1831; Metaphrynella 1934;

Micryletta 1987; Microhyla 1838; Mysticellus 2019; Phrynella 1887; Uperodon 1841

F.18.56. Subfamilia OTOPHRYNINAE 1987

2 G: Otophryne 1900; Synapturanus 1954

F.17.30. Familia PHRYNOMERIDAE 1931

$1 \mathrm{G}$ : Phrynomantis 1867

C.11.04. Subphalanx GaSTREChMia 1867

F.14.08. Superfamilia ARTHROLEPTOIDEA 1869

F.17.31. Familia ARTHROLEPTIDAE 1869

F.18.57. Subfamilia ARTHROLEPTINAE 1869

$1 \mathrm{G}$ : Arthroleptis 1849

F.18.58. Subfamilia ASTYLOSTERNINAE 1927

4 G: Astylosternus 1898; Leptodactylodon 1903; Nyctibates 1904; Scotobleps 1900

F.18.59. Subfamilia LEPTOPELINAE 1972

$1 \mathrm{G}$ : Leptopelis 1859

F.17.32. Familia HYPEROLIIDAE 1943 [Q]

F.18.0b. Subfamilia INCERTAE SEDIS

3 G: Arlequinus 1988; Callixalus 1950; Chrysobatrachus 1951

F.18.60. Subfamilia CRYPTOTHYLACINAE nov.

$1 \mathrm{G}:$ Cryptothylax 1950

F.18.61. Subfamilia HYPEROLIINAE 1943

12 G: Acanthixalus 1944; Afrixalus 1944; Heterixalus 1944; Hylambates 1853; Hyperolius 1842;

Kassina 1853; Kassinula 1940; Morerella 2009; Opisthothylax 1966; Paracassina 1907; Semnodactylus 1939; Tachycnemis 1843

F.14.09. Superfamilia BREVICIPITOIDEA 1850

F.17.33. Familia BREVICIPITIDAE 1850

F.18.62. Subfamilia BREVICIPITINAE 1850

$1 \mathrm{G}$ : Breviceps 1820

F.18.63. Subfamilia CALLULININAE nov.

4 G: Balebreviceps 1989; Callulina 1911; Probreviceps 1931; Spelaeophryne 1924

F.17.34. Familia HEMISOTIDAE 1867 [Q]

$1 \mathrm{G}$ : Hemisus 1859

C.11.05. Subphalanx Pananura nov.

C.12.03. Infraphalanx ECAUdATA 1777

F.14.10. Superfamilia ODONTOBATRACHOIDEA 2014

F.17.35. Familia ODONTOBATRACHIDAE 2014

$1 \mathrm{G}$ : Odontobatrachus 2014

F.14.11. Superfamilia PHRYNOBATRACHOIDEA 1941

F.17.36. Familia PHRYNOBATRACHIDAE 1941

2 G: Phrynobatrachus 1862; Phrynodon 1935

F.14.12. Superfamilia RANOIDEA 1796 
F.15.05. Epifamilia CONRAUOIDAE 1992

F.17.37. Familia CONRAUIDAE 1992

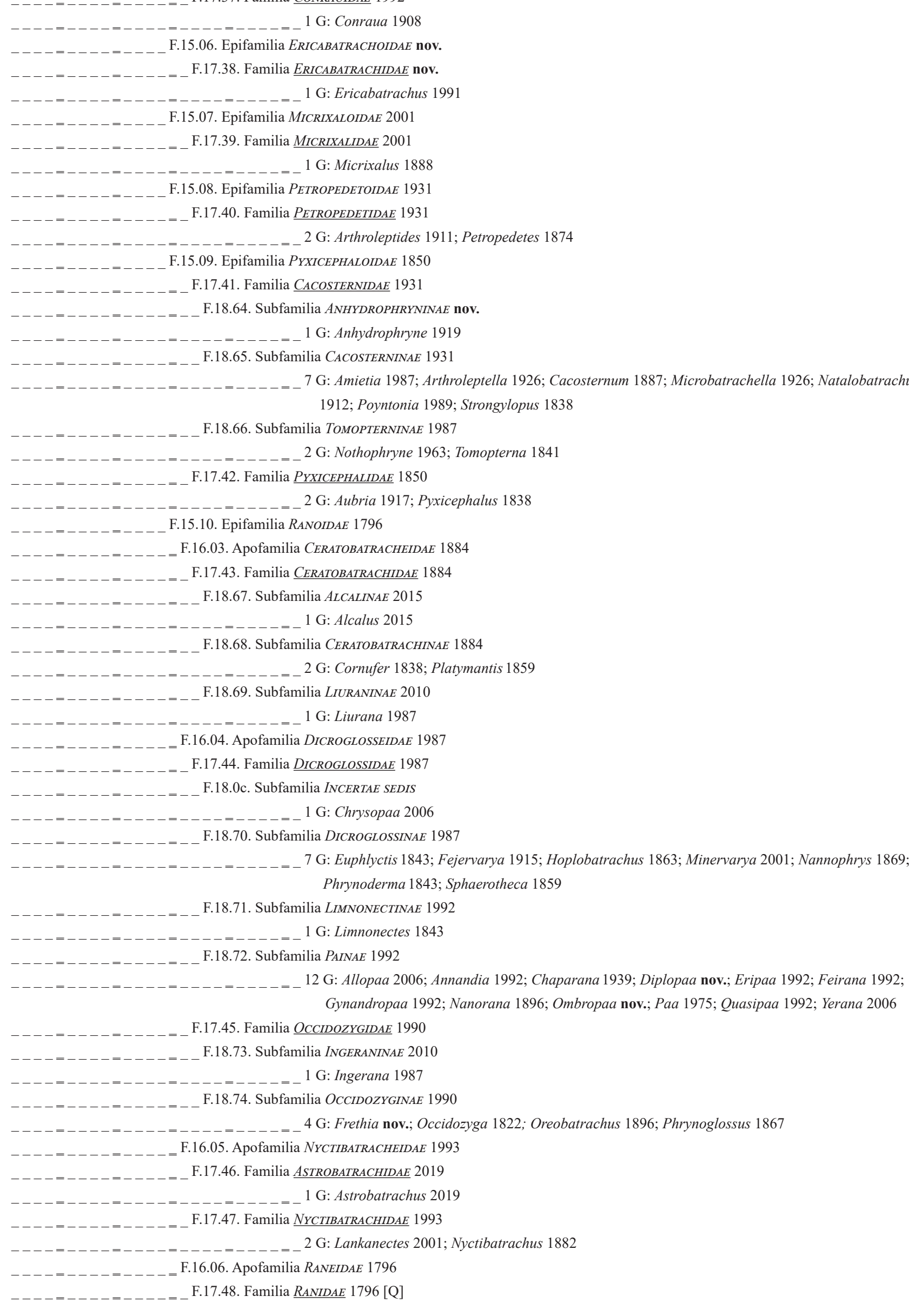


F.18.75. Subfamilia RANINAE 1796

$1 \mathrm{G} \uparrow$ : Ranavus $1885+$

21 G: Abavorana 2015; Amerana 1992; Amolops 1865; Aquarana 1992; Babina 1912; Boreorana nov.; Clinotarsus 1869; Glandirana 1990; Hylarana 1838; Lithobates 1843; Liuhurana 2010; Meristogenys 1991; Nidirana 1992; Odorrana 1990; Pelophylax 1843; Pseudorana 1990; Pterorana 1986; Rana 1758; Rugosa 1990; Sanguirana 1992; Sumaterana 2018

F.18.76. Subfamilia STAUROINAE 2005

1 G: Staurois 1865

F.17.49. Familia RHACOPHORIDAE $\|1858\|-1932$ [Q]

F.18.77. Subfamilia MANTELLINAE 1946

12 G: Aglyptodactylus 1919; Blommersia 1992; Boehmantis 2006; Boophis 1838; Gephyromantis 1920; Guibemantis 1992; Laliostoma 1998; Mantella 1882; Mantidactylus 1895; Spinomantis 1992; Tsingymantis 2006; Wakea 2006

F.18.78. Subfamilia RHACOPHORINAE $\|1858\|-1932$

$1 \mathrm{G} \dagger$ : Indorana $2013 \dagger$

25 G: Beddomixalus 2013; Buergeria 1838; Chirixalus 1893; Chiromantis 1854; Dendrobatorana 1927; Feihyla 2006; Ghatixalus 2008; Gracixalus 2005; Kurixalus 1999; Leptomantis 1867; Mercurana 2013; Nasutixalus 2016; Nyctixalus 1882; Orixalus nov.; Philautus 1848; Polypedates 1838; Pseudophilautus 1943; Raorchestes 2010; Rhacophorus 1822; Romerus nov.; Tamixalus nov.; Taruga 2010; Theloderma 1838; Vampyrius nov.; Zhangixalus 2019

F.16.07. Apofamilia RANIXALEIDAE 1987

F.17.50. Familia RANIXALIDAE 1987

2 G: Indirana 1986; Walkerana 2016

C.12.04. Infraphalanx SAVANura nov.

F.17.51. Familia PTYCHADENIDAE 1987

3 G: Hildebrandtia 1907; Lanzarana 1982; Ptychadena 1917

C.09.02. Epiphalanx Helanura nov.

F.17.52. Familia HELEOPHRYNIDAE 1931 [Q]

2 G: Hadromophryne 2008; Heleophryne 1898

C.06.02. Infraordo Mediogyrinia 1878

F.14.†0b. Superfamilia INCERTAE SEDIS †

F.17.†0h. Familia INCERTAE SEDIS †

$5 \mathrm{G} \dagger$ : Callobatrachus $1999 \dagger$; Electrorana 2018 †; Enneabatrachus 1993 †; Opisthocoelellus $1941 \uparrow$; Pelophilus $1838+$

F.17.†06. Familia GobIatIDAE $1991 \dagger$ $2 \mathrm{G} \uparrow$ : Cretasalia $1999+$; Gobiates $1986 \uparrow$

F.14.13. Superfamilia ALYTOIDEA 1843

F.17.53. Familia ALYTIDAE 1843

$1 \mathrm{G} \uparrow:$ Kizylkuma $1981 \dagger$

2 G: Alytes 1829; Ammoryctis 1879

F.17.54. Familia DISCOGLOSSIDAE 1858 [Q]

$5 \mathrm{G} \uparrow:$ Bakonybatrachus $2012 \uparrow ;$ Eodiscoglossus $1954 \uparrow ;$ Latoglossus $2000 \uparrow ;$ Paradiscoglossus $1982 \uparrow ;$

Paralatonia 2003 †

2 G: Discoglossus 1837; Latonia 1843

F.14.14. Superfamilia BOMBINATOROIDEA 1825

F.17.55. Familia BOMBINATORIDAE 1825 [Q]

$1 \mathrm{G} \uparrow$ : Eobarbourula 2013 †

2 G: Barbourula 1924; Bombina 1816

C.04.02. Ordo GYMNOPHIONA 1814

C.05.†0b. Subordo INCERTAE SEDIS $\dagger$

F.17.†0i. Familia INCERTAE SEDIS † $1 \mathrm{G} \uparrow:$ Rubricacaecilia $2001 \uparrow$

F.17.†07. Familia EOCAECILIIDAE $1993 \dagger$

$1 \mathrm{G} \uparrow$ : Eocaecilia $1993+$

C.05.03. Subordo Plesiophiona nov.

F.17.56. Familia RHINATREMATIDAE 1977 


\section{$1 \mathrm{G}:$ Rhinatrema 1841}

C.05.04. Subordo Pseudophiona 1816

F.14.15. Superfamilia CAECILIOIDEA 1814-|1825|

F.17.57. Familia CAECILIIDAE 1814-|1825| [Q]

F.18.79. Subfamilia CAECILIINAE 1814-|1825|

$1 \mathrm{G} \uparrow$ : Apodops $1972 \dagger$

21 G: Atretochoana 1995; Brasilotyphlus 1968; Caecilia 1758; Chthonerpeton 1880; Gegeneophis 1880; Geotrypetes 1880; Gymnopis 1874; Hypogeophis 1880; Idiocranium 1936; Indotyphlus 1960; Luetkenotyphlus 1968; Microcaecilia 1968; Mimosiphonops 1968; Nectocaecilia 1968; Oscaecilia 1968; Potamotyphlus 1968; Praslinia 1909; Schistometopum 1941; Siphonops 1828; Sylvacaecilia 1987; Typhlonectes 1880

F.18.80. Subfamilia HERPELINAE 1984

3 G: Boulengerula 1896; Chikila 2012; Herpele 1880

F.17.58. Familia SCOLECOMORPHIDAE 1969

2 G: Crotaphatrema 1985; Scolecomorphus 1883

F.14.16. Superfamilia ICHTHYOPHIOIDEA 1968

F.17.59. Familia ICHTHYOPHIIDAE 1968 [Q]

2 G: Epicrium 1828; Ichthyophis 1826

F.17.60. Familia URAEOTYPHLIDAE 1979

$1 \mathrm{G}$ : Uraeotyphlus 1880

C.04.03. Ordo URODELA 1805

C.05.†0c. Subordo INCERTAE SEDIS †

F.17.†0j. Familia INCERTAE SEDIS-†

$21 \mathrm{G} \uparrow$ : Apricosiren 2002 †; Balveherpeton $2020 \dagger$; Bishara 1997 †; Bissektia 1981 †; Comonecturoides 1960 †; Cryptobranchichnus $1941 \dagger ;$ Egoria $2020 \dagger$; Galverpeton 1982 †; Iridotriton 2005 †; Jeholotriton $2000 \uparrow$; Kiyatriton $2002 \uparrow$; Kulgeriherpeton 2018 †; Laccotriton $1998 \uparrow$; Marmorerpeton $1988 \uparrow$; Nesovtriton 2009 †; Nezpercius 2001 †; Ramonellus $1969 \dagger$; Seminobatrachus 2012 †; Sinerpeton $2001 \uparrow$; Urupia $2011 \uparrow$; Valdotriton $1996 \uparrow$

F.17.†08. Familia HYLAEOBATRACHIDAE $1889 \dagger$

7 G †: Batrachosauroides $1943 \uparrow$; Hylaeobatrachus $1884 \uparrow$; Opisthotriton $1961 \uparrow ;$ Palaeoproteus 1935 †; Parrisia 1998 †; Peratosauroides 1981 †; Prodesmodon 1964 †

F.17.†09. Familia KARAURIDAE $1978 \dagger$

$2 \mathrm{G} \uparrow$ : Karaurus $1978 \dagger$; Kokartus $1988 \dagger$

F.17.†10. Familia PROSIRENIDAE $1969 \dagger$

$1 \mathrm{G} \uparrow$ : Prosiren $1958 \dagger$

F.17.†11. Familia SCAPHERPETIDAE $1959 \dagger$

$3 \mathrm{G} \uparrow$ : Hedronchus $1877 \dagger$; Lisserpeton $1965 \uparrow ;$ Piceoerpeton $1967 \dagger$

F.17.†12. Familia TRIASSURIDAE $1978 \dagger$

$1 \mathrm{G} \dagger$ : Triassurus $1978 \dagger$

C.05.05. Subordo IMPERFECTIBRANCHIA 1838

F.17.†0k. Familia INCERTAE SEDIS †

$5 \mathrm{G} \uparrow$ : Liaoxitriton $1998 \uparrow$; Linglongtriton $2019 \uparrow ;$ Nuominerpeton $2016 \uparrow ;$ Pangerpeton $2006 \uparrow$;

Regalerpeton $2009+$;

F.17.61. Familia CRYPTOBRANCHIDAE $1826[\mathrm{Q}+]$

$7 \mathrm{G} \uparrow$ : Aviturus $1991 \uparrow$; Chunerpeton $2003 \uparrow$; Eoscapherpeton $1981 \uparrow$; Horezmia $1981 \uparrow$; Ukrainurus

$2013 \uparrow$; Ulanurus $1991 \uparrow ;$ Zaissanurus $1959 \dagger$

2 G: Andrias 1837; Cryptobranchus 1821

F.17.62. Familia HYNOBIIDAE $\|1856\|-1859$ [Q]

F.18.†0d. Subfamilia INCERTAE SEDIS †

$2 \mathrm{G}+$ : Geyeriella $1950 \uparrow ;$ Parahynobius $1999+$

F.18.81. Subfamilia HYNOBIINAE \|1856\|-1859

12 G: Afghanodon 2012; Batrachuperus 1878; Hynobius 1838; Iranodon 2012; Liua 1983;

Pachyhynobius 1983; Pachypalaminus 1912; Poyarius 2012; Pseudohynobius 1983; Ranodon 1866;

Salamandrella 1870; Satobius 1990

F.18.82. Subfamilia ONYCHODACTYLINAE 2012

$1 \mathrm{G}$ :. Onychodactylus 1838 
C.05.06. Subordo Meantes 1767

F.17.†13. Familia NOTERPETIDAE $1983 \dagger$

$2 \mathrm{G} \uparrow$ : Kababisha 1996 †; Noterpeton $1993 \dagger$

F.17.63. Familia SIRENIDAE 1825 [Q]

$1 \mathrm{G} \dagger$ : Habrosaurus $1928 \dagger$

2 G: Pseudobranchus 1825; Siren 1766

C.05.07. Subordo Pseudosauria 1816

F.14.†0c. Superfamilia INCERTAE SEDIS †

F.17.†01. Familia INCERTAE SEDIS †

$2 \mathrm{G} \dagger$ : Beiyanerpeton $2012 \uparrow$; Qinglongtriton ${ }^{\circ} 2016 \dagger$

F.14.17. Superfamilia AMPHIUMOIDEA 1825

F.15.11. Epifamilia AMPHIUMOIDAE 1825

F.16.08. Apofamilia AMPHIUMEIDAE 1825

F.17.64. Familia AMPHIUMIDAE 1825 [Q]

2 G †: Paleoamphiuma 1998 †; Proamphiuma 1969 †

$1 \mathrm{G}$ : Amphiuma 1821

F.17.65. Familia PLETHODONTIDAE 1850 [Q]

F.18.†0f. Subfamilia INCERTAE SEDIS $\dagger$

$1 \mathrm{G} \uparrow$ : Palaeoplethodon $2015 \dagger$

F.18.83. Subfamilia HEMIDACTYLIINAE 1856

23 G: Aquiloeurycea 2015; Batrachoseps 1839; Bolitoglossa 1854; Bradytriton 1983; Chiropterotriton

1944; Cryptotriton 2000; Dendrotriton 1983; Eurycea 1822; Gyrinophilus 1869; Hemidactylium 1838;

Isthmura 2012; Ixalotriton 1989; Nototriton 1983; Nyctanolis 1983; Oedipina 1868; Oedopinola 1946;

Parvimolge 1944; Pseudoeurycea 1944; Pseudotriton 1838; Stereochilus 1869; Thorius 1869; Thornea

nov.; Urspelerpes 2009

F.18.84. Subfamilia PLETHODONTINAE 1850

8 G: Aneides 1851; Desmognathus 1850; Ensatina 1850; Hydromantes 1848; Karsenia 2005;

Phaeognathus 1961; Plethodon 1838; Speleomantes 1984

F.16.09. Apofamilia RHYACOTRITONEIDAE 1958

F.17.66. Familia RHYACOTRITONIDAE $1958[\mathrm{Q}+]$

1 G: Rhyacotriton 1920

F.15.12. Epifamilia PROTEOIDAE Bonaparte, 1831.ba.f002-11

F.17.67. Familia PROTEIDAE Bonaparte, 1831.ba.f002-02 [Q]

$3 \mathrm{G} \dagger$ : Mioproteus $1978+$; Orthophyia $1845+$; Paranecturus $2013 \dagger$

2 G: Necturus 1819; Proteus 1768

F.14.18. Superfamilia SALAMANDROIDEA 1820

F.17.68. Familia AMBYSTOMATIDAE 1850 [Q]

$5 \mathrm{G} \uparrow$ : Ambystomichnus $1954 \uparrow ;$ Amphitriton $1976 \uparrow ;$ Chrysotriton $1981 \uparrow ;$ Sanchizia $2012 \uparrow$;

Wolterstorffiella $1950 \dagger$

2 G: Ambystoma 1838; Dicamptodon 1870

F.17.69. Familia SALAMANDRIDAE 1820 [Q]

F.18.85. Subfamilia PLEURODELINAE 1838

$9 \mathrm{G} \uparrow$ : Archaeotriton $1860 \uparrow ;$ Brachycormus $1860 \uparrow ;$ Carpathotriton $2008 \uparrow ;$ Chelotriton $1853 \uparrow ;$

Koalliella 1950 †; Oligosemia 1923 †; Palaeopleurodeles 1941 †; Phosphotriton 2016 †; Procynops $1965 \dagger$

18 G: Calotriton 1858; Cynops 1838; Echinotriton 1982; Euproctus 1839; Hypselotriton 1934;

Ichthyosaura 1801; Laotriton 2009; Lissotriton 1839; Neurergus 1862; Notophthalmus 1820;

Ommatotriton 1850; Pachytriton 1878; Paramesotriton 1935; Pleurodeles 1830; Taricha 1850;

Triturus 1815; Tylototriton 1871; Yaotriton 2009

F.18.86. Subfamilia SALAMANDRINAE 1820

$1 \mathrm{G} \dagger$ : Megalotriton $1890 \dagger$

4 G: Chioglossa 1864; Lyciasalamandra 2004; Mertensiella 1925; Salamandra 1764

F.18.87. Subfamilia SALAMANDRININAE 1843

1 G: Salamandrina 1826 
Appendix A11.CLAD-3. Families and subfamilies of Lissamphibia here considered valid.

$\dagger \cdot$ all-fossil taxon.

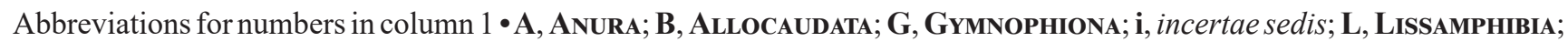
U, Urodela.

For the meaning of all other identifiers, see legend of Appendix A9.CLAD-1.

\begin{tabular}{|c|c|c|c|}
\hline NR. & FAMILY [NUMBER OF GENERA] & SUBFAMILIES [NUMBER OF GENERA] & $\begin{array}{l}\text { LOWEST CLASS-SERIES ANGIONYM OF THIS } \\
\text { FAMILY [RANK] }\end{array}$ \\
\hline$B \nmid 1$ & ALBANERPETIDAE $1982 \dagger[6 \dagger]$ & - & ALLOCAUDATA $1982 \uparrow[$ Ordo] \\
\hline BT & TOTAL Allocaudata: $1 \uparrow[6+]$ & {$[0+1 \dagger]$} & ALLOCAUDATA $1982 \dagger[$ Ordo] \\
\hline A01 & ALLOPHRYNIDAE 1978 [1] & - & HYLOBATRACHIA 1828 [Hypophalanx] \\
\hline A02 & ALYTIDAE $1843[2+1 \dagger]$ & - & MEDIOGYRINIA 1878 [Infraordo] \\
\hline \multirow[t]{3}{*}{$\mathbf{A 0 3}$} & AROMOBATIDAE 2006 [5] & ALLOBATINAE 2006 [1] & PhORANURa nov. [Infraphalanx] \\
\hline & & ANOMALOGLOSSINAE 2006 [2] & \\
\hline & & AROMOBATINAE 2006 [2] & \\
\hline \multirow[t]{3}{*}{ A04 } & ARTHROLEPTIDAE 1869 [6] & ARTHROLEPTINAE 1869 [1] & GASTRECHMIA 1867 [Subphalanx] \\
\hline & & ASTYLOSTERNINAE 1927 [4] & \\
\hline & & LEPTOPELINAE 1972 [1] & \\
\hline A05 & ASCAPHIDAE $1923[1]$ & - & ANGUSTICOELA 1958 [Subordo] \\
\hline A06 & ASTROBATRACHIDAE 2019 [1] & - & ECAUDATA 1777 [Infraphalanx] \\
\hline A07 & BOMBINATORIDAE $1825[2+1 \dagger]$ & - & MEDIOGYRINIA 1878 [Infraordo] \\
\hline \multirow[t]{4}{*}{ A08 } & BRACHYCEPHALIDAE 1858 [30] & BRACHYCEPHALINAE 1858 [2] & GAIANURA nov. [Hypophalanx] \\
\hline & & CRAUGASTORINAE 2008 [21] & \\
\hline & & ELEUTHERODACTYLINAE 1954 [5] & \\
\hline & & Incertae sedis BRACHYCEPHALIDAE [2] & \\
\hline \multirow[t]{2}{*}{ A09 } & BREVICIPITIDAE 1850 [5] & BREVICIPITINAE $1850[1]$ & GASTRECHMIA 1867 [Subphalanx] \\
\hline & & CALLULININAE nov. [4] & \\
\hline \multirow[t]{2}{*}{ A10 } & BUFONIDAE $1825[54+1 \dagger]$ & BUFONINAE $1825[53+1 \dagger]$ & HYLOBATRACHIA 1828 [Hypophalanx] \\
\hline & & MELANOPHRYNISCINAE nov. [1] & \\
\hline \multirow[t]{3}{*}{ A11 } & CACOSTERNIDAE 1931 [10] & ANHYDROPHRYNINAE nov. [1] & ECAUDATA 1777 [Infraphalanx] \\
\hline & & CACOSTERNINAE 1931 [7] & \\
\hline & & TOMOPTERNINAE 1987 [2] & \\
\hline A12 & CALYPTOCEPHALELLIDAE 1960 [2] & - & DIPLOSIPHONA 1859 [Subphalanx] \\
\hline \multirow[t]{3}{*}{ A13 } & CENTROLENIDAE 1951 [13] & CENTROLENINAE 1951 [10] & HYLOBATRACHIA 1828 [Hypophalanx] \\
\hline & & HYALINOBATRACHINAE 2009 [2] & \\
\hline & & IKAKOGINAE nov. [1] & \\
\hline \multirow[t]{3}{*}{ A14 } & CERATOBATRACHIDAE 1884 [4] & ALCALINAE 2015 [1] & ECAUDATA 1777 [Infraphalanx] \\
\hline & & CERATOBATRACHINAE 1884 [2] & \\
\hline & & LIURANINAE $2010[1]$ & \\
\hline
\end{tabular}

...Continued on the next page 
Appendix A11. (Continued)

\begin{tabular}{|c|c|c|c|}
\hline NR. & FAMILY [NUMBER OF GENERA] & SUBFAMILIES [NUMBER OF GENERA] & $\begin{array}{l}\text { LOWEST CLASS-SERIES ANGIONYM OF THIS } \\
\text { FAMILY [RANK] }\end{array}$ \\
\hline A15 & CERATOPHRYIDAE $1838[4+2 \dagger]$ & $\begin{array}{l}\text { CERATOPHRYINAE } 1838[1+1 \dagger] \\
\text { LEPIDOBATRACHINAE } 1987[2+1 \dagger] \\
\text { STOMBINAE } 1965[1]\end{array}$ & HYLOBATRACHIA 1828 [Hypophalanx] \\
\hline A16 & CEUTHOMANTIDAE 2009 [2] & - & GAIANURA nov. [Hypophalanx] \\
\hline A17 & CONRAUIDAE $1992[1]$ & - & ECAUDATA 1777 [Infraphalanx] \\
\hline A18 & $\begin{array}{l}\text { CYCLORAMPHIDAE } 1850-|1852| \\
{[11]}\end{array}$ & $\begin{array}{l}\text { ALSODINAE } 1869 \text { [2] } \\
\text { BATRACHYLINAE } 1965 \text { [4] } \\
\text { CYCLORAMPHINAE 1850-|1852| [2] } \\
\text { HYLODINAE } 1858 \text { [2] } \\
\text { LIMNOMEDUSINAE nov. [1] }\end{array}$ & HYLOBATRACHIA 1828 [Hypophalanx] \\
\hline
\end{tabular}

\begin{tabular}{|c|c|c|c|}
\hline A19 & $\begin{array}{l}\text { DENDROBATIDAE }\|1850\|-1865 \\
{[16]}\end{array}$ & $\begin{array}{l}\text { COLOSTETHINAE } 1867 \text { [5] } \\
\text { DENDROBATINAE }\|1850\|-1865 \text { [8] } \\
\text { HYLOXALINAE } 2006 \text { [3] }\end{array}$ & Phoranura nov. [Infraphalanx] \\
\hline A20 & DICROGLOSSIDAE 1987 [21] & $\begin{array}{l}\text { DICROGLOSSINAE } 1987 \text { [7] } \\
\text { LIMNONECTINAE } 1992[1] \\
\text { PAINAE } 1992 \text { [12] } \\
\text { Incertae sedis DICROGLOSSIDAE [1] }\end{array}$ & ECAUDATA 1777 [Infraphalanx] \\
\hline
\end{tabular}

\begin{tabular}{|c|c|c|c|}
\hline A21 & DISCOGLOSSIDAE $1858[2+5 \dagger]$ & - & MEDIOGYRINIA 1878 [Infraordo] \\
\hline A22 & ERICABATRACHIDAE nov. [1] & - & ECAUDATA 1777 [Infraphalanx] \\
\hline A23 & HELEOPHRYNIDAE 1931 [2] & - & HelanuRa nov. [Epiphalanx] \\
\hline \multirow[t]{6}{*}{ A24 } & \multirow[t]{6}{*}{ HEMIPHRACTIDAE 1862 [10] } & AMPHIGNATHODONTINAE 1882 [5] & \multirow{6}{*}{$\begin{array}{l}\text { HEMIPHRACTIFORMIA } 1881 \\
\text { [Hypophalanx] }\end{array}$} \\
\hline & & CRYPTOBATRACHINAE 2006 [1] & \\
\hline & & FLECTONOTINAE nov. [1] & \\
\hline & & FRITZIANINAE nov. [1] & \\
\hline & & HEMIPHRACTINAE 1862 [1] & \\
\hline & & STEFANIINAE nov. [1] & \\
\hline A25 & HEMISOTIDAE 1867 [1] & - & GASTRECHMIA 1867 [Subphalanx] \\
\hline \multirow[t]{3}{*}{ A26 } & \multirow[t]{3}{*}{ HYLIDAE 1815-|1825| [48+3†] } & COPHOMANTINAE 1878 [7] & \multirow[t]{3}{*}{ HYLOBATRACHIA 1828 [Hypophalanx] } \\
\hline & & HYLINAE 1815-|1825| [41] & \\
\hline & & Incertae sedis HYLIDAE $\dagger[3 \dagger]$ & \\
\hline \multirow[t]{3}{*}{ A27 } & \multirow[t]{3}{*}{ HYPEROLIIDAE 1943 [16] } & CRYPTOTHYLACINAE nov. [1] & \multirow[t]{3}{*}{ GASTRECHMIA 1867 [Subphalanx] } \\
\hline & & HYPEROLIINAE 1943 [12] & \\
\hline & & Incertae sedis HYPEROLIIDAE [3] & \\
\hline \multirow[t]{2}{*}{ A28 } & \multirow{2}{*}{$\begin{array}{l}\text { LEIOPELMATIDAE 1869-|1942| [2 } \\
+1 \dagger]\end{array}$} & LEIOPELMATINAE 1869-|1942| [2] & \multirow[t]{2}{*}{ ANGUSTICOELA 1958 [Subordo] } \\
\hline & & NOTOBATRACHINAE $1956 \dagger[1 \dagger]$ & \\
\hline \multirow[t]{4}{*}{ A29 } & \multirow{4}{*}{$\begin{array}{l}\text { LEPTODACTYLIDAE }\|1838\|-1896 \\
\text { [11] }\end{array}$} & LEIUPERINAE 1850 [5] & \multirow[t]{4}{*}{ HYLOBATRACHia 1828 [Hypophalanx] } \\
\hline & & LEPTODACTYLINAE \|1838\|-1896 [3] & \\
\hline & & PARATELMATOBIINAE 2012 [2] & \\
\hline & & PSEUDOPALUDICOLINAE 1965 [1] & \\
\hline
\end{tabular}


Appendix A11. (Continued)

\begin{tabular}{|c|c|c|c|}
\hline NR. & FAMILY [NUMBER OF GENERA] & SUBFAMILIES [NUMBER OF GENERA] & $\begin{array}{l}\text { LOWEST CLASS-SERIES ANGIONYM OF THIS } \\
\text { FAMILY [RANK] }\end{array}$ \\
\hline \multirow[t]{2}{*}{ A30 } & MEGOPHRYIDAE 1850-|1931| [11] & LEPTOBRACHIINAE 1983 [4] & \multirow{2}{*}{$\begin{array}{l}\text { ArChaEOSALIENTIA } 1981 \\
\text { [Superphalanx] }\end{array}$} \\
\hline & & MEGOPHRYINAE 1850-|1931| [7] & \\
\hline A31 & MICRIXALIDAE 2001 [1] & - & ECAUDATA 1777 [Infraphalanx] \\
\hline \multirow[t]{9}{*}{ A32 } & МICROHYLIDAE ||1843\|-1931 [40] & ADELASTINAE 2016 [1] & ECOSTATA 1879 [Subphalanx] \\
\hline & & ASTEROPHRYINAE 1858 [4] & \\
\hline & & COPHYLINAE 1889 [8] & \\
\hline & & GASTROPHRYNINAE 1843 [11] & \\
\hline & & HOPLOPHRYNINAE 1931 [2] & \\
\hline & & KALOPHRYNINAE 1869 [1] & \\
\hline & & MELANOBATRACHINAE 1931 [1] & \\
\hline & & MICROHYLINAE ||1843\|-1931 [10] & \\
\hline & & OTOPHRYNINAE 1987 [2] & \\
\hline
\end{tabular}

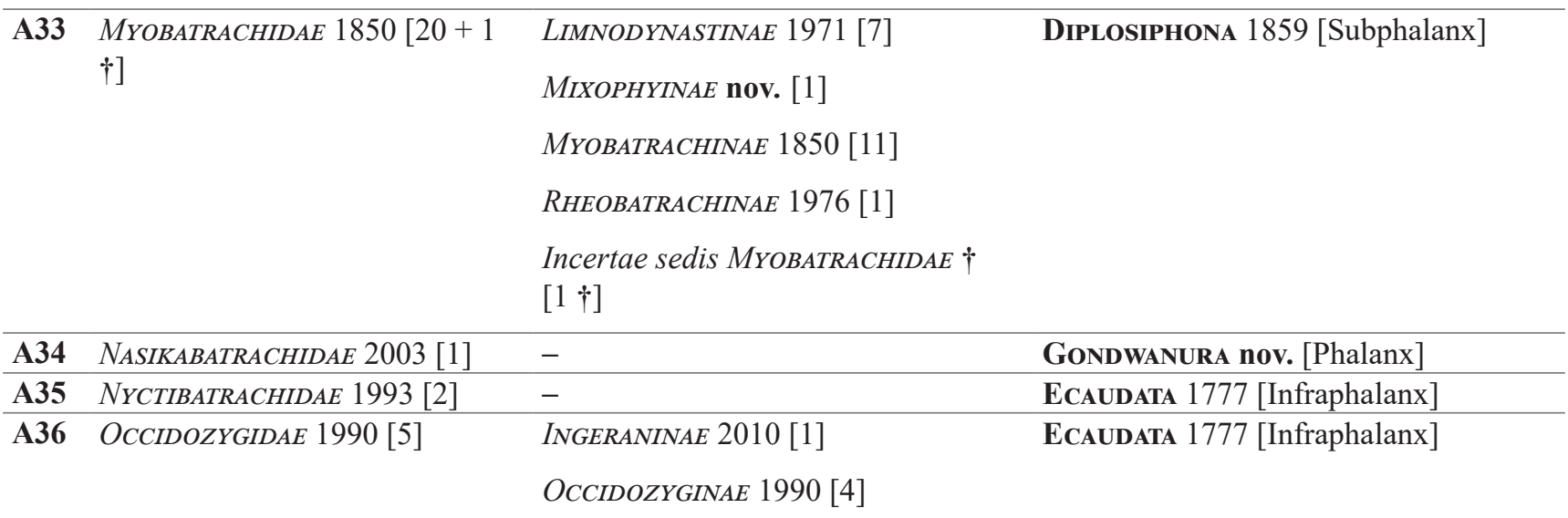

\begin{tabular}{llll}
\hline A37 & ODONTOBATRACHIDAE 2014 [1] & - & ECAUdATA 1777 [Infraphalanx] \\
\hline A38 & ODONTOPHRYNIDAE 1971 [3+ & ODONTOPHRYNINAE 1971 [2] & HyLOBATRACHIA 1828 [Hypophalanx] \\
& $1 \dagger]$ & PROCERATOPHRYINAE nov. [1] & \\
& & Incertae sedis ODONTOPHRYNIDAE $\dagger$ & \\
& & {$[1 \dagger]$}
\end{tabular}

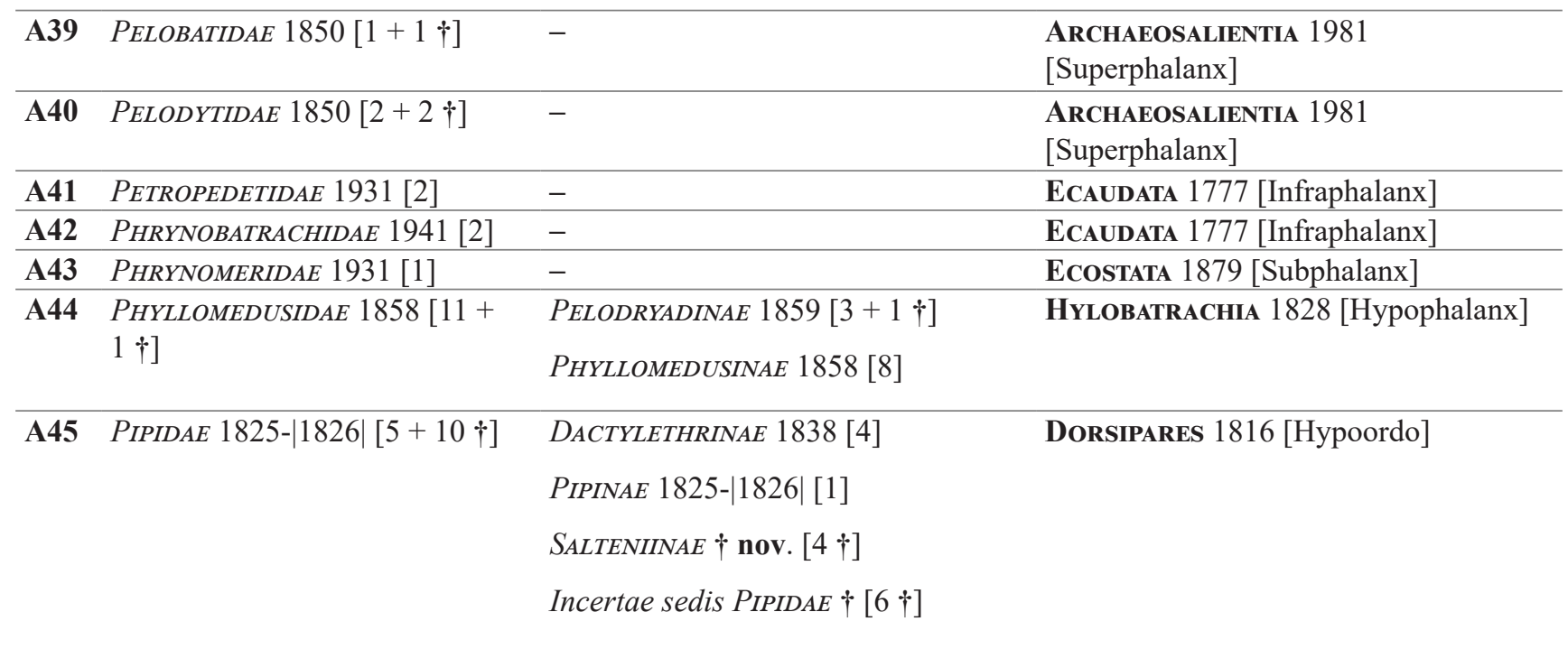

... Continued on the next page 
Appendix A11. (Continued)

\begin{tabular}{|c|c|c|c|}
\hline NR. & FAMILY [NUMBER OF GENERA] & SUBFAMILIES [NUMBER OF GENERA] & $\begin{array}{l}\text { LOWEST CLASS-SERIES ANGIONYM OF THIS } \\
\text { FAMILY [RANK] }\end{array}$ \\
\hline A46 & PTYCHADENIDAE 1987 [3] & - & SAVANURA nov. [Infraphalanx] \\
\hline A47 & PYXICEPHALIDAE 1850 [2] & - & ECAUDATA 1777 [Infraphalanx] \\
\hline \multirow[t]{2}{*}{ A48 } & RANIDAE $1796[22+1 \dagger]$ & RANINAE $1796[21+1 \dagger]$ & ECAUDATA 1777 [Infraphalanx] \\
\hline & & STAUROINAE 2005 [1] & \\
\hline
\end{tabular}

\begin{tabular}{lll}
\hline A49 & RANIXALIDAE $1987[2]$ & - \\
\hline A50 & RHACOPHORIDAE $\|1858\|-1932$ & MANTELLINAE 1946 [12] \\
& {$[37+1 \dagger]$} & RHACOPHORINAE $\|1858\|-1932[25+$ \\
& & $1 \dagger]$
\end{tabular}

\begin{tabular}{lll}
\hline A51 & RHINODERMATIDAE $1850[2]$ & - \\
\hline A52 & RHINOPHRYNIDAE $1858[1+3 \dagger]$ & - \\
\hline $\mathbf{A 5 3}$ & SCAPHIOPODIDAE $1865[2]$ & -
\end{tabular}

ECAUDATA 1777 [Infraphalanx]

ECAUDATA 1777 [Infraphalanx]

A53 SCAPHIOPODIDAE 1865 [2]

Hylobatrachia 1828 [Hypophalanx]

DorsiPares 1816 [Hypoordo]

ArChaeosalientia 1981

[Superphalanx]

\begin{tabular}{lll}
\hline A54 & SOOGLOSSIDAE $1931[2]$ & - \\
\hline A55 & TelmatobitdaE $1843[1+1 \dagger]$ & - \\
\hline Ai1 & Incertae sedis HylOBATRACHIA & -
\end{tabular}

GondWANURA nov. [Phalanx]

Hylobatrachia 1828 [Hypophalanx]

[1]

Ai2 Incertae sedis LAEVOGYRINIA [1] -

$\mathbf{A} \dagger \mathbf{1}$ GoBIATIDAE $1991 \dagger[2 \dagger]$ -

$\mathbf{A} \dagger 2$ PALAEOBATRACHIDAE $1865 \dagger[3 \dagger]-$

$\mathbf{A} \dagger \mathbf{3}$ PROSALIRIDAE $1995+[1 \dagger]$ -

A†4 TREGOBATRACHIDAE $1975 \dagger[1 \dagger]-$

A †5 TRIADOBATRACHIDAE $1962 \dagger[1 \dagger]-$

A†i1 Incertae sedis ANURA $\uparrow[39 \dagger]-$

A†i2 Incertae sedis -

Archaeosalientia $\uparrow[5 \dagger]$

Hylobatrachia nov. [Hypophalanx]

A $†$ i3 Incertae sedis DorsIPARES $\uparrow[7 \quad-$

$\dagger]$

A†i4 Incertae sedis Geobatrachia $\uparrow-$

$[1 \dagger]$

A†i5 Incertae sedis Hydrobatrachia $\dagger[4 \dagger]$

A $\dagger$ i6 Incertae sedis Mediogyrinia $\uparrow \quad-$

$[5 \dagger]$

$\mathbf{A} \dagger \mathbf{7}$ Incertae sedis Scoptanura $\dagger-$

LAEVOGYRINIA 1878 [Hypoordo]

Mediogyrinia 1878 [Infraordo]

DORSIPARES 1816 [Hypoordo]

ANURA 1805 [Ordo]

ANURA 1805 [Ordo]

ANURA 1805 [Ordo]

ANURA 1805 [Ordo]

ArChaeosalientia 1981

[Superphalanx]

DorsiPaRES 1816 [Hypoordo]

GEOBATRACHIA 1828 [Infraordo]

HYDrobatrachia 1828 [Subordo]

Mediogyrinia 1878 [Infraordo]

$[1 \dagger]$

\begin{tabular}{|c|c|c|}
\hline AT & $\begin{array}{l}\text { TOTAL ANURA: } 55+5 \dagger[470+[78+2 \dagger] \\
105 \dagger]\end{array}$ & ANURA 1805 [Ordo] \\
\hline
\end{tabular}

G01 CaECILIIDAE 1814-|1825| [24+ CAECILIINAE 1814-|1825| [21 + $1 \dagger$ Pseudophiona 1816 [Subordo] $1 \dagger]$

HERPELINAE 1984 [3]

\begin{tabular}{|c|c|c|c|}
\hline G02 & ICHTHYОРНIIDAE 1968 [2] & - & PSEUDOPHIONA 1816 [Subordo] \\
\hline G03 & RHINATREMATIDAE 1977 [1] & - & PLESIOPHIONA nov. [Subordo] \\
\hline G04 & SCOLECOMORPHIDAE 1969 [2] & - & PSEUDOPHIONA 1816 [Subordo] \\
\hline G05 & URAEOTYPHLIDAE 1979 [1] & - & PSEUDOPHIONA 1816 [Subordo] \\
\hline $\mathrm{G} \dagger 1$ & EOCAECILIIDAE $1993 \dagger[1 \dagger]$ & - & GYMNOPHIONA 1814 [Ordo] \\
\hline G†i1 & $\begin{array}{l}\text { Incertae sedis GYMNOPHIONA } \dagger \\
{[2 \dagger]}\end{array}$ & - & GYMNOPHIONA 1814 [Ordo] \\
\hline GT & $\begin{array}{l}\text { TOTAL GYMNOPHIONA: } \mathbf{5}+\mathbf{1} \dagger \\
{[30+4 \dagger]}\end{array}$ & {$[2]$} & GYMNOPHIONA 1814 [Ordo] \\
\hline U01 & AMBYSTOMATIDAE $1850[2+5 \dagger]$ & - & PSEUdosauria 1816 [Subordo] \\
\hline
\end{tabular}

... Continued on the next page 
Appendix A11. (Continued)

\begin{tabular}{|c|c|c|c|}
\hline NR. & FAMILY [NUMBER OF GENERA] & SUBFAMILIES [NUMBER OF GENERA] & $\begin{array}{l}\text { LOWEST CLASS-SERIES ANGIONYM OF THIS } \\
\text { FAMILY [RANK] }\end{array}$ \\
\hline U02 & AMPHIUMIDAE $1825[1+2 \dagger]$ & - & PSEUdOSAURIa 1816 [Subordo] \\
\hline U03 & $\begin{array}{l}\text { CRYPTOBRANCHIDAE } 1826[2+ \\
7 \dagger]\end{array}$ & - & IMPERFECTIBRANCHIA 1838 [Subordo] \\
\hline U04 & $\begin{array}{l}\text { HYNOBIIDAE }\|1856\|-1859[13+ \\
2 \dagger]\end{array}$ & $\begin{array}{l}\text { HYNOBIINAE }\|1856\|-1859[12] \\
\text { ONYCHODACTYLINAE } 2012[1] \\
\text { Incertae sedis HYNOBIIDAE } \dagger[2 \dagger]\end{array}$ & IMPERFECTIBRANCHIA 1838 [Subordo] \\
\hline U05 & $\begin{array}{l}\text { PLETHODONTIDAE } 1850[31+1 \\
\dagger]\end{array}$ & $\begin{array}{l}\text { HEMIDACTYLIINAE } 1856[23] \\
\text { PLETHODONTINAE } 1850[8] \\
\text { Incertae sedis PLETHODONTIDAE } \dagger \\
{[1 \dagger]}\end{array}$ & PSEUdosauria 1816 [Subordo] \\
\hline U06 & PROTEIDAE $1831[2+3 \dagger]$ & - & Pseudosauria 1816 [Subordo] \\
\hline U07 & RHYACOTRITONIDAE 1958 [1] & - & PSEudosauria 1816 [Subordo] \\
\hline U08 & $\begin{array}{l}\text { SALAMANDRIDAE } 1820[23+10 \\
\dagger]\end{array}$ & $\begin{array}{l}\text { PLEURODELINAE } 1838[18+9 \dagger] \\
\text { SALAMANDRINAE } 1820[4+1 \dagger] \\
\text { SALAMANDRININAE } 1843[1]\end{array}$ & Pseudosauria 1816 [Subordo] \\
\hline U09 & SIRENIDAE $1825[2+1 \dagger]$ & - & MEANTES 1767 [Subordo] \\
\hline $\mathrm{U} \dagger 1$ & $\begin{array}{l}\text { HYLAEOBATRACHIDAE } 1889+[7 \\
\dagger]\end{array}$ & - & URODELA 1805 [Ordo] \\
\hline $\mathrm{U} \dagger \mathbf{2}$ & KARAURIDAE $1978 \dagger[2 \dagger]$ & - & URODELA 1805 [Ordo] \\
\hline $\mathrm{U} \dagger 3$ & NOTERPETIDAE $1983 \dagger[2 \dagger]$ & - & MEANTES 1767 [Subordo] \\
\hline $\mathrm{U} \uparrow 4$ & PROSIRENIDAE $1969 \dagger[1 \dagger]$ & - & URODELA 1805 [Ordo] \\
\hline $\mathrm{U}+5$ & SCAPHERPETIDAE $1959 \dagger[3 \dagger]$ & - & URODELA 1805 [Ordo] \\
\hline $\mathrm{U} \uparrow 6$ & TRIASSURIDAE $1978 \dagger[1 \dagger]$ & - & URODELA 1805 [Ordo] \\
\hline $\mathrm{U} \dagger \mathrm{i1}$ & $\begin{array}{l}\text { Incertae sedis } \\
\text { IMPERFECTIBRANCHIA } \dagger[5 \dagger]\end{array}$ & - & IMPERFECTIBRANCHIA 1838 [Subordo] \\
\hline $\mathrm{U} \dagger \mathrm{i} 2$ & $\begin{array}{l}\text { Incertae sedis PSEUdosauria } \uparrow \\
{[2 \dagger]}\end{array}$ & - & Pseudosauria 1816 [Subordo] \\
\hline $\mathrm{U} \dagger \mathrm{i} 3$ & Incertae sedis URODELA $\uparrow[21 \dagger]$ & - & URODELA 1805 [Ordo] \\
\hline UT & $\begin{array}{l}\text { TOTAL URODELA: } 9+6+[77+ \\
75 \dagger]\end{array}$ & [7] & URODELA 1805 [Ordo] \\
\hline LT & $\begin{array}{l}\text { TOTAL LISSAMPHIBIA: } \mathbf{6 9}+\mathbf{1 3} \dagger \\
{[575+190 \dagger]}\end{array}$ & {$[87+2 \dagger]$} & АмРнIвIA 1816 [Classis] \\
\hline
\end{tabular}


Appendix A12.CLAD-4. Class-series cladonomy and nomenclature of LiSSAMPHIBIA proposed here.

Class-series partial hierarchy used here (see A.CLAD-1):

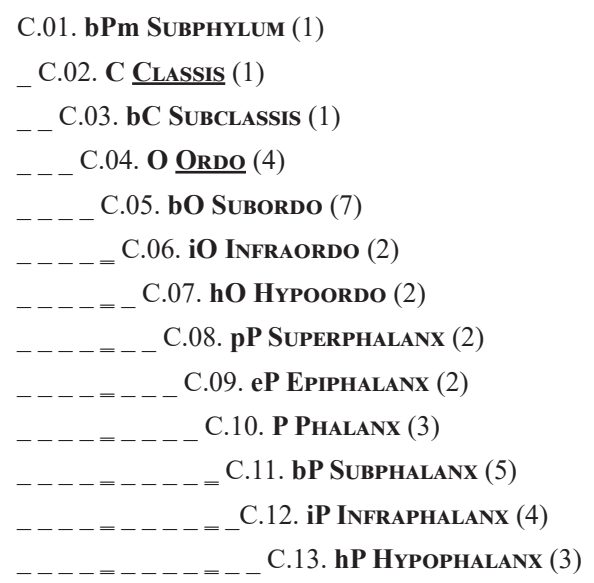

TOTAL: 35 lissamphibian taxa of the class-series +2 of their angiotaxa including also non-lissamphibians. 


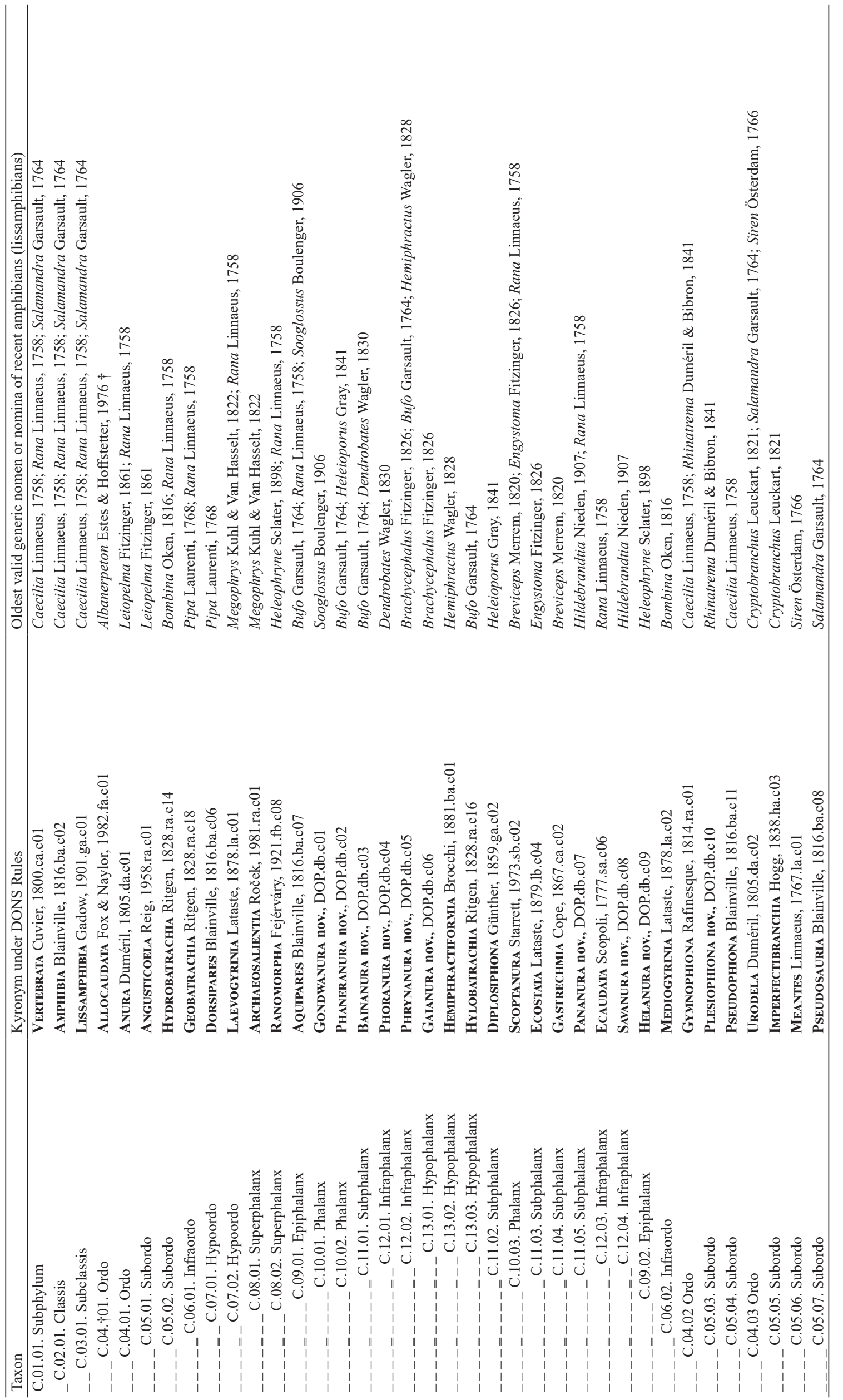


Appendix A13.QUA. Usage of nomina of families of extant LisSaMPHibia from 1796 to 2014.

For each nomen and each period, the Table gives the number of uses, followed between parentheses by the percentage of these uses among the publications of the period. In order to standardise for sample size, the number for the total period is the mean of the percentages of the five periods. The quarters are numbered from Q1 (lower quarter) to Q4 (upper quarter). According to the Upper Quartile Criterion [UQC] described in M\&M 2.4.5.2.1, nomina in the upper quarter Q4 are validated, except those marked [Q4-] that do not appear in any of the publications of the 2000-2014 period (exception [E1]), whereas those of the quarter Q3 having $90 \%$ or more usages in the same period [Q3+] are validated (exception [E2]).

Chronological list of references used for the establishment of the number of usages of family-series nomina in LISSAMPHIBIA (see References for details): Batsch 1796; Rafinesque 1815; Goldfuss 1820; Gray 1825, 1850; Fitzinger 1826, 1843; Bonaparte 1838, 1850; Hogg 1838, $1839 a-b, 1841$; Tschudi 1838; Duméril \& Bibron 1841; Agassiz 1847; Gistel 1850; Desmarest 1856; Lichtenstein et al. 1956; Stannius 1856; Günther 1859; Bruch 1862; Cope 1865, 1866 1867, 1875 , 1889a-b; Gouriet 1868; Mivart 1869; Fatio 1872; Hoffmann 1878; Boulenger 1882b-c, 1910, 1914; Sauvage 1885; Lydekker 1889, 1896; Gadow 1901; Lydekker et al. 1912; Werner 1912; Bolkay 1919; Fejérváry 1921b; Metcalf 1923; Nieden 1923; Miranda-Ribeiro 1926; Ahl 1931; Noble 1931; Lameere 1941; Romer 1945, 1966; Reig 1958; Fuhn 1960; Kuhn 1960, 1965, 1967b; Cochran 1962; Goin \& Goin 1962; Hellmich 1962; Griffiths 1963; Tatarinov 1964a; Gorham 1966, 1974; Laurent 1967, 1979, 1986; Taylor 1968; Burton \& Burton 1970; Porter 1972; Savage 1973; Breen 1974; Freytag 1974; Duellman 1975, 1977, 1979, 1988, 2003; Dowling \& Duellman 1978; Goin et al. 1978; Estes 1981; Dubois 1984b, 1985, 2005e; Duellman \& Trueb 1985; Frost 1985; Mattison 1987; Ananjeva et al. 1988; Benton 1993; Zug 1993; Glaw et al. 1998; Pough et al. 1998; Sanchiz 1998; McDiarmid \& Altig 1999; Fhutchins et al. 2003; Larson et al. 2003; Frost et al. 2006; Duellman \& Adler 2007; Raffaelli 2007, 2011; Roelants et al. 2007; Stuart et al. 2008; Vitt \& Caldwell 2009, 2014; Zhang \& Wake 2009; Blackburn \& Wake 2011; Pyron \& Wiens 2011.

Some of these references are used together to represent a complete classification: Hogg 1838, 1839a,b; Cope 1866, 1867; Boulenger (1882b-c); Nieden (1923) and Ahl (1931); Gorham (1966); Duellman (1977).

This list includes 107 references which represent 101 analysed classifications, 94 for the ANURA, 76 for the UrodELA and 64 for the GYMNOPHIONA.

\begin{tabular}{|c|c|c|c|c|c|c|c|}
\hline Familial nomen & $1796-1849$ & $1850-1899$ & 1900-1949 & 1950-1999 & $2000-2014$ & $1796-2014$ & Quartile \\
\hline \multicolumn{8}{|c|}{ ANURA } \\
\hline Number of works & 12 & 20 & 13 & 38 & 11 & 94 & - \\
\hline RANIDAE & $12(100 \%)$ & $20(100 \%)$ & $12(92.3 \%)$ & $38(100 \%)$ & $11(100 \%)$ & 98.5 & Q4 \\
\hline BUFONIDAE & $6(50 \%)$ & $18(90 \%)$ & $13(100 \%)$ & $37(97.4 \%)$ & $11(100 \%)$ & 87.5 & Q4 \\
\hline HYLIDAE & $5(41.7 \%)$ & $17(85 \%)$ & $13(100 \%)$ & $38(100 \%)$ & $11(100 \%)$ & 85.3 & Q4 \\
\hline PIPIDAE & $3(25 \%)$ & $15(75 \%)$ & $11(84.6 \%)$ & $37(97.4 \%)$ & $11(100 \%)$ & 76.4 & Q4 \\
\hline PELOBATIDAE & $0(0 \%)$ & $8(40 \%)$ & $11(84.6 \%)$ & $38(100 \%)$ & $11(100 \%)$ & 64.9 & Q4 \\
\hline DISCOGLOSSIDAE & $0(0 \%)$ & $12(60 \%)$ & $13(100 \%)$ & $37(97.4 \%)$ & $5(45.5 \%)$ & 60.6 & Q4 \\
\hline DENDROBATIDAE & $0(0 \%)$ & $8(40 \%)$ & $3(23.1 \%)$ & $25(65.8 \%)$ & $11(100 \%)$ & 45.8 & Q4 \\
\hline LEIOPELMATIDAE & $0(0 \%)$ & $0(0 \%)$ & $3(23.1 \%)$ & $33(86.8 \%)$ & $12(109.1 \%)$ & 43.8 & Q4 \\
\hline MICROHYLIDAE & $0(0 \%)$ & $3(15 \%)$ & $1(7.7 \%)$ & $35(92.1 \%)$ & $11(100 \%)$ & 43.0 & Q4 \\
\hline LEPTODACTYLIDAE & $0(0 \%)$ & $1(5 \%)$ & $2(15.4 \%)$ & $35(92.1 \%)$ & $11(100 \%)$ & 42.5 & Q4 \\
\hline RHINOPHRYNIDAE & $0(0 \%)$ & $4(20 \%)$ & $0(0 \%)$ & $35(92.1 \%)$ & $11(100 \%)$ & 42.4 & Q4 \\
\hline BRACHYCEPHALIDAE & $0(0 \%)$ & $3(15 \%)$ & $4(30.8 \%)$ & $24(63.2 \%)$ & $10(90.9 \%)$ & 40.0 & Q4 \\
\hline PELODYTIDAE & $0(0 \%)$ & $4(20 \%)$ & $2(15.4 \%)$ & $23(60.5 \%)$ & $10(90.9 \%)$ & 37.4 & Q4 \\
\hline CENTROLENIDAE & $0(0 \%)$ & $0(0 \%)$ & $0(0 \%)$ & $30(78.9 \%)$ & $11(100 \%)$ & 35.8 & Q4 \\
\hline MYOBATRACHIDAE & $0(0 \%)$ & $2(10 \%)$ & $0(0 \%)$ & $19(50 \%)$ & $11(100 \%)$ & 32.0 & Q4 \\
\hline RHACOPHORIDAE & $0(0 \%)$ & $0(0 \%)$ & $0(0 \%)$ & $29(76.3 \%)$ & $9(81.8 \%)$ & 31.6 & Q4 \\
\hline BOMBINATORIDAE & $3(25 \%)$ & $5(25 \%)$ & $0(0 \%)$ & $3(7.9 \%)$ & $11(100 \%)$ & 31.6 & Q4 \\
\hline SOOGLOSSIDAE & $0(0 \%)$ & $0(0 \%)$ & $0(0 \%)$ & $22(57.9 \%)$ & $11(100 \%)$ & 31.6 & Q4 \\
\hline HELEOPHRYNIDAE & $0(0 \%)$ & $0(0 \%)$ & $0(0 \%)$ & $19(50 \%)$ & $11(100 \%)$ & 30.0 & Q4 \\
\hline HYPEROLIIDAE & $0(0 \%)$ & $0(0 \%)$ & $0(0 \%)$ & $22(57.9 \%)$ & $10(90.9 \%)$ & 29.8 & Q4 \\
\hline HEMISOTIDAE & $0(0 \%)$ & $3(15 \%)$ & $1(7.7 \%)$ & $9(23.7 \%)$ & $10(90.9 \%)$ & 27.5 & Q4 \\
\hline RHINODERMATIDAE & $0(0 \%)$ & $2(10 \%)$ & $0(0 \%)$ & $23(60.5 \%)$ & $6(54.5 \%)$ & 25.0 & Q4 \\
\hline CYSTIGNATHIDAE & $1(8.3 \%)$ & $10(50 \%)$ & $8(61.5 \%)$ & $1(2.6 \%)$ & $0(0 \%)$ & 24.5 & Q4- \\
\hline
\end{tabular}


Appendix A13. (Continued)

\begin{tabular}{|c|c|c|c|c|c|c|c|}
\hline Familial nomen & $1796-1849$ & $1850-1899$ & 1900-1949 & 1950-1999 & 2000-2014 & $1796-2014$ & Quartile \\
\hline ENGYSTOMATIDAE & $0(0 \%)$ & $12(60 \%)$ & $8(61.5 \%)$ & $0(0 \%)$ & $0(0 \%)$ & 24.3 & Q4- \\
\hline HEMIPHRACTIDAE & $0(0 \%)$ & $6(30 \%)$ & $4(30.8 \%)$ & $0(0 \%)$ & $6(54.5 \%)$ & 23.1 & Q4 \\
\hline BREVICIPITIDAE & $0(0 \%)$ & $3(15 \%)$ & $2(15.4 \%)$ & $1(2.6 \%)$ & $8(72.7 \%)$ & 21.1 & Q3 \\
\hline ASCAPHIDAE & $0(0 \%)$ & $0(0 \%)$ & $1(7.7 \%)$ & $14(36.8 \%)$ & $6(54.5 \%)$ & 19.8 & Q3 \\
\hline ARTHROLEPTIDAE & $0(0 \%)$ & $0(0 \%)$ & $0(0 \%)$ & $6(15.8 \%)$ & $9(81.8 \%)$ & 19.5 & Q3 \\
\hline MEGOPHRYIDAE & $0(0 \%)$ & $0(0 \%)$ & $0(0 \%)$ & $2(5.3 \%)$ & $10(90.9 \%)$ & 19.2 & Q3+ \\
\hline PSEUDIDAE & $1(8.3 \%)$ & $0(0 \%)$ & $0(0 \%)$ & $32(84.2 \%)$ & $0(0 \%)$ & 18.5 & Q3 \\
\hline CERATOPHRYIDAE & $1(8.3 \%)$ & $1(5 \%)$ & $1(7.7 \%)$ & $2(5.3 \%)$ & $7(63.6 \%)$ & 18.0 & Q3 \\
\hline SCAPHIOPODIDAE & $0(0 \%)$ & $5(25 \%)$ & $0(0 \%)$ & $0(0 \%)$ & $7(63.6 \%)$ & 17.7 & Q3 \\
\hline ALYTIDAE & $1(8.3 \%)$ & $3(15 \%)$ & $0(0 \%)$ & $0(0 \%)$ & $7(63.6 \%)$ & 17.4 & Q3 \\
\hline CERATOBATRACHIDAE & $0(0 \%)$ & $3(15 \%)$ & $1(7.7 \%)$ & $0(0 \%)$ & $7(63.6 \%)$ & 17.3 & Q3 \\
\hline ALLOPHRYNIDAE & $0(0 \%)$ & $0(0 \%)$ & $0(0 \%)$ & $5(13.2 \%)$ & $7(63.6 \%)$ & 15.4 & Q3 \\
\hline MANTELLIDAE & $0(0 \%)$ & $0(0 \%)$ & $0(0 \%)$ & $1(2.6 \%)$ & $8(72.7 \%)$ & 15.1 & Q3 \\
\hline LIMNODYNASTIDAE & $0(0 \%)$ & $0(0 \%)$ & $0(0 \%)$ & $0(0 \%)$ & $8(72.7 \%)$ & 14.5 & Q3 \\
\hline PETROPEDETIDAE & $0(0 \%)$ & $0(0 \%)$ & $0(0 \%)$ & $2(5.3 \%)$ & $7(63.6 \%)$ & 13.8 & Q3 \\
\hline AMPHIGNATHODONTIDAE & $0(0 \%)$ & $3(15 \%)$ & $3(23.1 \%)$ & $0(0 \%)$ & $3(27.3 \%)$ & 13.1 & Q3 \\
\hline CYCLORHAMPHIDAE & $0(0 \%)$ & $0(0 \%)$ & $0(0 \%)$ & $0(0 \%)$ & $7(63.6 \%)$ & 12.7 & Q3 \\
\hline DICROGLOSSIDAE & $0(0 \%)$ & $0(0 \%)$ & $0(0 \%)$ & $0(0 \%)$ & $7(63.6 \%)$ & 12.7 & Q3 \\
\hline MICRIXALIDAE & $0(0 \%)$ & $0(0 \%)$ & $0(0 \%)$ & $0(0 \%)$ & $7(63.6 \%)$ & 12.7 & Q3 \\
\hline NYCTIBATRACHIDAE & $0(0 \%)$ & $0(0 \%)$ & $0(0 \%)$ & $0(0 \%)$ & $7(63.6 \%)$ & 12.7 & Q3 \\
\hline PYXICEPHALIDAE & $0(0 \%)$ & $0(0 \%)$ & $0(0 \%)$ & $0(0 \%)$ & $7(63.6 \%)$ & 12.7 & Q3 \\
\hline PTYCHADENIDAE & $0(0 \%)$ & $0(0 \%)$ & $0(0 \%)$ & $0(0 \%)$ & $7(63.6 \%)$ & 12.7 & Q3 \\
\hline HYLODIDAE & $0(0 \%)$ & $2(10 \%)$ & $1(7.7 \%)$ & $0(0 \%)$ & $5(45.5 \%)$ & 12.6 & Q3 \\
\hline DACTYLETHRIDAE & $2(16.7 \%)$ & $8(40 \%)$ & $0(0 \%)$ & $0(0 \%)$ & $0(0 \%)$ & 11.3 & Q3 \\
\hline NASIKABATRACHIDAE & $0(0 \%)$ & $0(0 \%)$ & $0(0 \%)$ & $0(0 \%)$ & $6(54.5 \%)$ & 10.9 & Q3 \\
\hline PHRYNOBATRACHIDAE & $0(0 \%)$ & $0(0 \%)$ & $0(0 \%)$ & $0(0 \%)$ & $6(54.5 \%)$ & 10.9 & Q3 \\
\hline XENOPODIDAE & $1(8.3 \%)$ & $3(15 \%)$ & $4(30.8 \%)$ & $0(0 \%)$ & $0(0 \%)$ & 10.8 & Q3 \\
\hline DENDROPHRYNISCIDAE & $0(0 \%)$ & $3(15 \%)$ & $4(30.8 \%)$ & $0(0 \%)$ & $0(0 \%)$ & 9.2 & Q2 \\
\hline RANIXALIDAE & $0(0 \%)$ & $0(0 \%)$ & $0(0 \%)$ & $0(0 \%)$ & $5(45.5 \%)$ & 9.1 & Q2 \\
\hline PELODRYADIDAE & $0(0 \%)$ & $3(15 \%)$ & $0(0 \%)$ & $8(21.1 \%)$ & $1(9.1 \%)$ & 9.0 & Q2 \\
\hline POLYPEDATIDAE & $0(0 \%)$ & $3(15 \%)$ & $3(23.1 \%)$ & $1(2.6 \%)$ & $0(0 \%)$ & 8.1 & Q2 \\
\hline DYSCOPHIDAE & $0(0 \%)$ & $3(15 \%)$ & $3(23.1 \%)$ & $0(0 \%)$ & $0(0 \%)$ & 7.6 & Q2 \\
\hline ATELOPODIDAE & $1(8.3 \%)$ & $0(0 \%)$ & $0(0 \%)$ & $11(28.9 \%)$ & $0(0 \%)$ & 7.4 & Q2 \\
\hline AROMOBATIDAE & $0(0 \%)$ & $0(0 \%)$ & $0(0 \%)$ & $0(0 \%)$ & $4(36.4 \%)$ & 7.3 & Q2 \\
\hline CALYPTOCEPHALELLIDAE & $0(0 \%)$ & $0(0 \%)$ & $0(0 \%)$ & $0(0 \%)$ & $4(36.4 \%)$ & 7.3 & Q2 \\
\hline LEIUPERIDAE & $0(0 \%)$ & $0(0 \%)$ & $0(0 \%)$ & $0(0 \%)$ & $4(36.4 \%)$ & 7.3 & Q2 \\
\hline ASTEROPHRYIDAE & $0(0 \%)$ & $7(35 \%)$ & $0(0 \%)$ & $0(0 \%)$ & $0(0 \%)$ & 7.0 & Q2 \\
\hline PHRYNISCIDAE & $0(0 \%)$ & $7(35 \%)$ & $0(0 \%)$ & $0(0 \%)$ & $0(0 \%)$ & 7.0 & Q2 \\
\hline TELMATOBIIDAE & $1(8.3 \%)$ & $0(0 \%)$ & $1(7.7 \%)$ & $0(0 \%)$ & $2(18.2 \%)$ & 6.8 & Q2 \\
\hline PHRYNOMERIDAE & $0(0 \%)$ & $0(0 \%)$ & $0(0 \%)$ & $9(23.7 \%)$ & $1(9.1 \%)$ & 6.6 & Q2 \\
\hline BATRACHOPHRYNIDAE & $0(0 \%)$ & $1(5 \%)$ & $0(0 \%)$ & $0(0 \%)$ & $3(27.3 \%)$ & 6.5 & Q2 \\
\hline ALSODIDAE & $0(0 \%)$ & $0(0 \%)$ & $0(0 \%)$ & $0(0 \%)$ & $3(27.3 \%)$ & 5.5 & Q2 \\
\hline CEUTHOMANTIDAE & $0(0 \%)$ & $0(0 \%)$ & $0(0 \%)$ & $0(0 \%)$ & $3(27.3 \%)$ & 5.5 & Q2 \\
\hline CRAUGASTORIDAE & $0(0 \%)$ & $0(0 \%)$ & $0(0 \%)$ & $0(0 \%)$ & $3(27.3 \%)$ & 5.5 & Q2 \\
\hline CRYPTOBATRACHIDAE & $0(0 \%)$ & $0(0 \%)$ & $0(0 \%)$ & $0(0 \%)$ & $3(27.3 \%)$ & 5.5 & Q2 \\
\hline ELEUTHERODACTYLIDAE & $0(0 \%)$ & $0(0 \%)$ & $0(0 \%)$ & $0(0 \%)$ & $3(27.3 \%)$ & 5.5 & Q2 \\
\hline HYMENOCHIRIDAE & $0(0 \%)$ & $0(0 \%)$ & $3(23.1 \%)$ & $0(0 \%)$ & $0(0 \%)$ & 4.6 & Q2 \\
\hline PHYLLOMEDUSIDAE & $0(0 \%)$ & $2(10 \%)$ & $0(0 \%)$ & $1(2.6 \%)$ & $1(9.1 \%)$ & 4.3 & Q2 \\
\hline SCAPHIOPHRYNIDAE & $0(0 \%)$ & $0(0 \%)$ & $0(0 \%)$ & $1(2.6 \%)$ & $2(18.2 \%)$ & 4.2 & Q2 \\
\hline COLOSTETHIDAE & $0(0 \%)$ & $4(20 \%)$ & $0(0 \%)$ & $0(0 \%)$ & $0(0 \%)$ & 4.0 & Q2 \\
\hline HYLAEDACTYLIDAE & $1(8.3 \%)$ & $2(10 \%)$ & $0(0 \%)$ & $0(0 \%)$ & $0(0 \%)$ & 3.7 & Q2 \\
\hline BATRACHYLIDAE & $0(0 \%)$ & $0(0 \%)$ & $0(0 \%)$ & $0(0 \%)$ & $2(18.2 \%)$ & 3.6 & Q2 \\
\hline ODONTOPHRYNIDAE & $0(0 \%)$ & $0(0 \%)$ & $0(0 \%)$ & $0(0 \%)$ & $2(18.2 \%)$ & 3.6 & Q1 \\
\hline
\end{tabular}


Appendix A13. (Continued)

\begin{tabular}{|c|c|c|c|c|c|c|c|}
\hline Familial nomen & $1796-1849$ & $1850-1899$ & 1900-1949 & 1950-1999 & $2000-2014$ & 1796-2014 & Quartile \\
\hline STRABOMANTIDAE & $0(0 \%)$ & $0(0 \%)$ & $0(0 \%)$ & $0(0 \%)$ & $2(18.2 \%)$ & 3.6 & Q1 \\
\hline ASTRODACTYLIDAE & $2(16.7 \%)$ & $0(0 \%)$ & $0(0 \%)$ & $0(0 \%)$ & $0(0 \%)$ & 3.3 & Q1 \\
\hline GASTROPHRYNIDAE & $1(8.3 \%)$ & $0(0 \%)$ & $1(7.7 \%)$ & $0(0 \%)$ & $0(0 \%)$ & 3.2 & Q1 \\
\hline PHYLLOBATIDAE & $1(8.3 \%)$ & $0(0 \%)$ & $0(0 \%)$ & $2(5.3 \%)$ & $0(0 \%)$ & 2.7 & Q1 \\
\hline BRACHYMERIDAE & $0(0 \%)$ & $2(10 \%)$ & $0(0 \%)$ & $0(0 \%)$ & $0(0 \%)$ & 2.0 & Q1 \\
\hline COPHYLIDAE & $0(0 \%)$ & $2(10 \%)$ & $0(0 \%)$ & $0(0 \%)$ & $0(0 \%)$ & 2.0 & Q1 \\
\hline HYLAPLESIIDAE & $0(0 \%)$ & $2(10 \%)$ & $0(0 \%)$ & $0(0 \%)$ & $0(0 \%)$ & 2.0 & Q1 \\
\hline SYSTOMATIDAE & $0(0 \%)$ & $2(10 \%)$ & $0(0 \%)$ & $0(0 \%)$ & $0(0 \%)$ & 2.0 & Q1 \\
\hline UPEROLEIIDAE & $0(0 \%)$ & $2(10 \%)$ & $0(0 \%)$ & $0(0 \%)$ & $0(0 \%)$ & 2.0 & Q1 \\
\hline CONRAUIDAE & $0(0 \%)$ & $0(0 \%)$ & $0(0 \%)$ & $0(0 \%)$ & $1(9.1 \%)$ & 1.8 & Q1 \\
\hline THOROPIDAE & $0(0 \%)$ & $0(0 \%)$ & $0(0 \%)$ & $0(0 \%)$ & $1(9.1 \%)$ & 1.8 & Q1 \\
\hline DENDROPSOPHIDAE & $1(8.3 \%)$ & $0(0 \%)$ & $0(0 \%)$ & $0(0 \%)$ & $0(0 \%)$ & 1.7 & Q1 \\
\hline DRYOPHYTIDAE & $1(8.3 \%)$ & $0(0 \%)$ & $0(0 \%)$ & $0(0 \%)$ & $0(0 \%)$ & 1.7 & Q1 \\
\hline LYMNODYTIDAE & $1(8.3 \%)$ & $0(0 \%)$ & $0(0 \%)$ & $0(0 \%)$ & $0(0 \%)$ & 1.7 & Q1 \\
\hline PELOBIIDAE & $1(8.3 \%)$ & $0(0 \%)$ & $0(0 \%)$ & $0(0 \%)$ & $0(0 \%)$ & 1.7 & Q1 \\
\hline RHEOBATRACHIDAE & $0(0 \%)$ & $0(0 \%)$ & $0(0 \%)$ & $3(7.9 \%)$ & $0(0 \%)$ & 1.6 & Q1 \\
\hline ELOSIIDAE & $0(0 \%)$ & $0(0 \%)$ & $1(7.7 \%)$ & $0(0 \%)$ & $0(0 \%)$ & 1.5 & Q1 \\
\hline GENYOPHRYNIDAE & $0(0 \%)$ & $0(0 \%)$ & $1(7.7 \%)$ & $0(0 \%)$ & $0(0 \%)$ & 1.5 & Q1 \\
\hline PALUDICOLIDAE & $0(0 \%)$ & $0(0 \%)$ & $1(7.7 \%)$ & $0(0 \%)$ & $0(0 \%)$ & 1.5 & Q1 \\
\hline COPHOMANTIDAE & $0(0 \%)$ & $1(5 \%)$ & $0(0 \%)$ & $0(0 \%)$ & $0(0 \%)$ & 1.0 & Q1 \\
\hline EUBAPHIDAE & $0(0 \%)$ & $1(5 \%)$ & $0(0 \%)$ & $0(0 \%)$ & $0(0 \%)$ & 1.0 & Q1 \\
\hline PLECTROMANTIDAE & $0(0 \%)$ & $1(5 \%)$ & $0(0 \%)$ & $0(0 \%)$ & $0(0 \%)$ & 1.0 & Q1 \\
\hline XENORHINIDAE & $0(0 \%)$ & $1(5 \%)$ & $0(0 \%)$ & $0(0 \%)$ & $0(0 \%)$ & 1.0 & Q1 \\
\hline
\end{tabular}

\begin{tabular}{|c|c|c|c|c|c|c|c|}
\hline & & & YMNOPHIO & & & & \\
\hline Number of works & 9 & 12 & 7 & 26 & 10 & 64 & - \\
\hline CAECILIIDAE & $9(100 \%)$ & $12(100 \%)$ & $7(100 \%)$ & $26(100 \%)$ & $10(100 \%)$ & 100.0 & Q4 \\
\hline ICHТНYОРНIIDAЕ & $0(0 \%)$ & $0(0 \%)$ & $0(0 \%)$ & $15(57.7 \%)$ & $10(100 \%)$ & 31.5 & Q4 \\
\hline RHINATREMATIDAE & $0(0 \%)$ & $0(0 \%)$ & $0(0 \%)$ & $11(42.3 \%)$ & $10(100 \%)$ & 28.5 & Q4 \\
\hline SCOLECOMORPHIDAE & $0(0 \%)$ & $0(0 \%)$ & $0(0 \%)$ & $15(57.7 \%)$ & $6(60 \%)$ & 23.5 & Q3 \\
\hline TYPHLONECTIDAE & $0(0 \%)$ & $0(0 \%)$ & $0(0 \%)$ & $17(65.4 \%)$ & $5(50 \%)$ & 23.1 & Q3 \\
\hline URAEOTYPHLIDAE & $0(0 \%)$ & $0(0 \%)$ & $0(0 \%)$ & $6(23.1 \%)$ & $4(40 \%)$ & 12.6 & Q3 \\
\hline DERMOPHIIDAE & $0(0 \%)$ & $0(0 \%)$ & $0(0 \%)$ & $1(3.8 \%)$ & $2(20 \%)$ & 4.8 & Q2 \\
\hline SIPHONOPIDAE & $0(0 \%)$ & $0(0 \%)$ & $0(0 \%)$ & $1(3.8 \%)$ & $2(20 \%)$ & 4.8 & Q2 \\
\hline HERPELIDAE & $0(0 \%)$ & $0(0 \%)$ & $0(0 \%)$ & $0(0 \%)$ & $2(20 \%)$ & 4.0 & Q2 \\
\hline INDOTYPHLIDAE & $0(0 \%)$ & $0(0 \%)$ & $0(0 \%)$ & $0(0 \%)$ & $2(20 \%)$ & 4.0 & Q1 \\
\hline EPICRIIDAE & $1(11.1 \%)$ & $0(0 \%)$ & $0(0 \%)$ & $1(3.8 \%)$ & $0(0 \%)$ & 3.0 & Q1 \\
\hline CHIKILIDAE & $0(0 \%)$ & $0(0 \%)$ & $0(0 \%)$ & $0(0 \%)$ & $1(10 \%)$ & 2.0 & Q1 \\
\hline
\end{tabular}

\section{URODELA}

Number of works

\begin{tabular}{lccccccc} 
Number of Works & 11 & 14 & 9 & 28 & 14 & - \\
\hline SALAMANDRIDAE & $10(90.9 \%)$ & $14(100 \%)$ & $9(100 \%)$ & $28(100 \%)$ & $14(100 \%)$ & 98.2 & Q4 \\
PROTEIDAE & $6(54.5 \%)$ & $11(78.6 \%)$ & $7(77.8 \%)$ & $28(100 \%)$ & $14(100 \%)$ & 82.2 & Q4 \\
SIRENIDAE & $5(45.5 \%)$ & $12(85.7 \%)$ & $7(77.8 \%)$ & $28(100 \%)$ & $14(100 \%)$ & 81.8 & Q4 \\
AMPHIUMIDAE & $3(27.3 \%)$ & $13(92.9 \%)$ & $7(77.8 \%)$ & $27(96.4 \%)$ & $14(100 \%)$ & 78.9 & Q4 \\
AMBYSTOMATIDAE & $0(0 \%)$ & $4(28.6 \%)$ & $3(33.3 \%)$ & $28(100 \%)$ & $14(100 \%)$ & 52.4 & Q4 \\
HYNOBIIDAE & $0(0 \%)$ & $4(28.6 \%)$ & $3(33.3 \%)$ & $26(92.9 \%)$ & $14(100 \%)$ & 51.0 & Q4 \\
PLETHODONTIDAE & $0(0 \%)$ & $5(35.7 \%)$ & $2(22.2 \%)$ & $27(96.4 \%)$ & $14(100 \%)$ & 50.9 & Q4 \\
CRYPTOBRANCHIDAE & $1(9.1 \%)$ & $2(14.3 \%)$ & $3(33.3 \%)$ & $27(96.4 \%)$ & $14(100 \%)$ & 50.6 & Q3+ \\
DICAMPTODONTIDAE & $0(0 \%)$ & $0(0 \%)$ & $0(0 \%)$ & $9(32.1 \%)$ & $10(71.4 \%)$ & 20.7 & Q3 \\
RHYACOTRITONIDAE & $0(0 \%)$ & $0(0 \%)$ & $0(0 \%)$ & $1(3.6 \%)$ & $14(100 \%)$ & 20.7 & Q3+ \\
PLEURODELIDAE & $2(18.2 \%)$ & $5(35.7 \%)$ & $0(0 \%)$ & $0(0 \%)$ & $0(0 \%)$ & 10.8 & Q3 \\
MENOPOMIDAE & $2(18.2 \%)$ & $2(14.3 \%)$ & $0(0 \%)$ & $0(0 \%)$ & $0(0 \%)$ & 6.5 & Q3 \\
DESMOGNATHIDAE & $0(0 \%)$ & $4(28.6 \%)$ & $0(0 \%)$ & $1(3.6 \%)$ & $0(0 \%)$ & 6.4 & Q3 \\
PROTONOPSIDAE & $0(0 \%)$ & $4(28.6 \%)$ & $0(0 \%)$ & $0(0 \%)$ & $0(0 \%)$ & 5.7 & Q3 \\
\hline
\end{tabular}


Appendix A13. (Continued)

\begin{tabular}{lccccccc}
\hline Familial nomen & $1796-1849$ & $1850-1899$ & $1900-1949$ & $1950-1999$ & $2000-2014$ & $1796-2014$ & Quartile \\
\hline TRITONIDAE & $3(27.3 \%)$ & $0(0 \%)$ & $0(0 \%)$ & $0(0 \%)$ & $0(0 \%)$ & 5.5 & Q2 \\
THORIIDAE & $0(0 \%)$ & $3(21.4 \%)$ & $0(0 \%)$ & $0(0 \%)$ & $0(0 \%)$ & 4.3 & Q2 \\
NECTURIDAE & $1(9.1 \%)$ & $1(7.1 \%)$ & $0(0 \%)$ & $1(3.6 \%)$ & $0(0 \%)$ & 4.0 & Q2 \\
MEGALOBATRACHIDAE & $1(9.1 \%)$ & $0(0 \%)$ & $0(0 \%)$ & $0(0 \%)$ & $0(0 \%)$ & 1.8 & Q2 \\
MURAENOPSIDAE & $1(9.1 \%)$ & $0(0 \%)$ & $0(0 \%)$ & $0(0 \%)$ & $0(0 \%)$ & 1.8 & Q2 \\
PHAENEROBRANCHIDAE & $1(9.1 \%)$ & $0(0 \%)$ & $0(0 \%)$ & $0(0 \%)$ & $0(0 \%)$ & 1.8 & Q2 \\
SALAMANDRINIDAE & $1(9.1 \%)$ & $0(0 \%)$ & $0(0 \%)$ & $0(0 \%)$ & $0(0 \%)$ & 1.8 & Q2 \\
SALAMANDROPSIDAE & $1(9.1 \%)$ & $0(0 \%)$ & $0(0 \%)$ & $0(0 \%)$ & $0(0 \%)$ & 1.8 & Q1 \\
GEOTRITONIDAE & $0(0 \%)$ & $1(7.1 \%)$ & $0(0 \%)$ & $0(0 \%)$ & $0(0 \%)$ & 1.4 & Q1 \\
HYPOCHTONIDAE & $0(0 \%)$ & $1(7.1 \%)$ & $0(0 \%)$ & $0(0 \%)$ & $0(0 \%)$ & 1.4 & Q1 \\
MENOBRANCHIDAE & $0(0 \%)$ & $1(7.1 \%)$ & $0(0 \%)$ & $0(0 \%)$ & $0(0 \%)$ & 1.4 & Q1 \\
MOLGIDAE & $0(0 \%)$ & $1(7.1 \%)$ & $0(0 \%)$ & $0(0 \%)$ & $0(0 \%)$ & 1.4 & Q1 \\
SIEBOLDIIDAE & $0(0 \%)$ & $1(7.1 \%)$ & $0(0 \%)$ & $0(0 \%)$ & $0(0 \%)$ & 1.4 & Q1 \\
SIREDONTIDAE & $0(0 \%)$ & $1(7.1 \%)$ & $0(0 \%)$ & $0(0 \%)$ & $0(0 \%)$ & 1.4 & Q1 \\
\hline
\end{tabular}




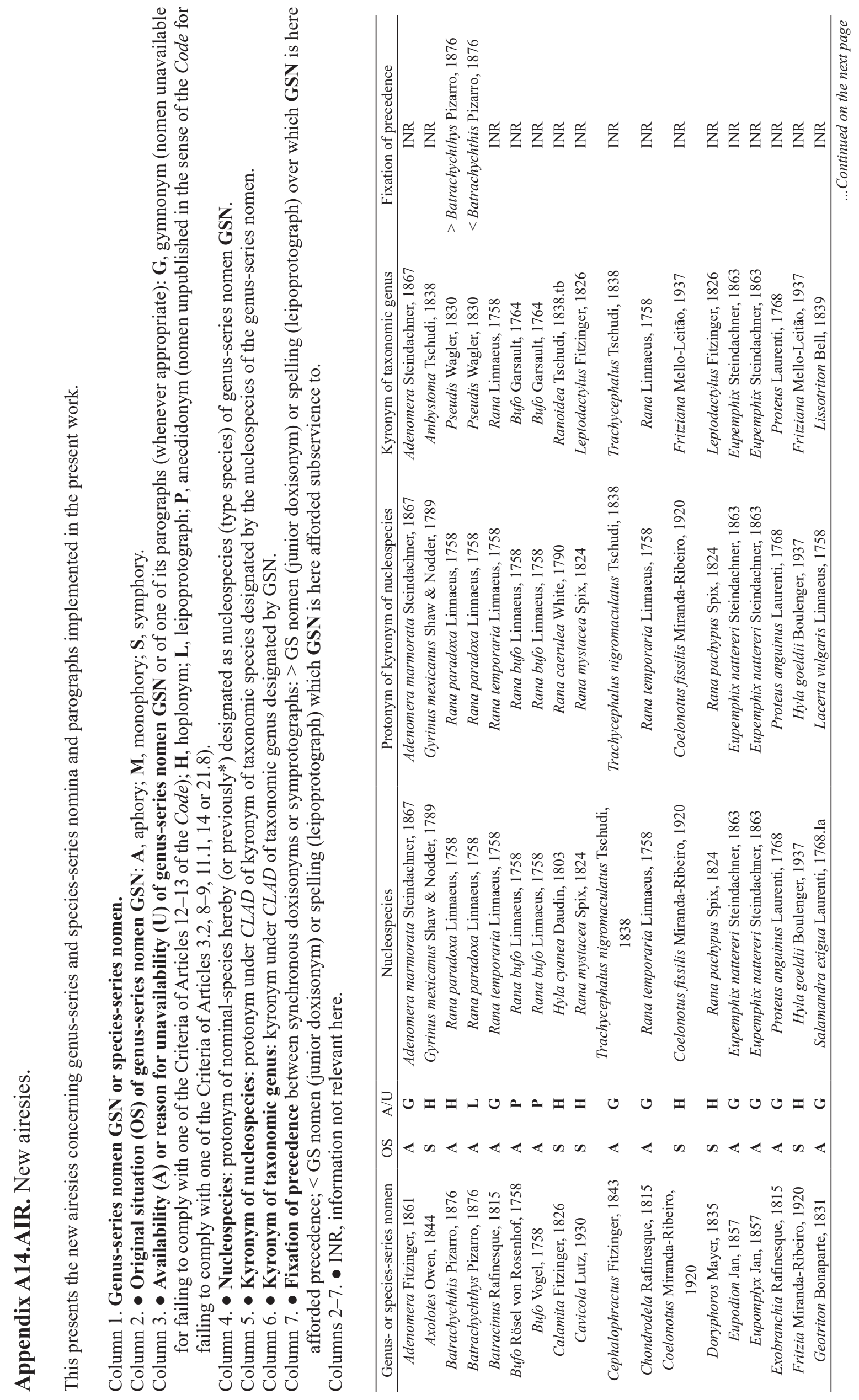




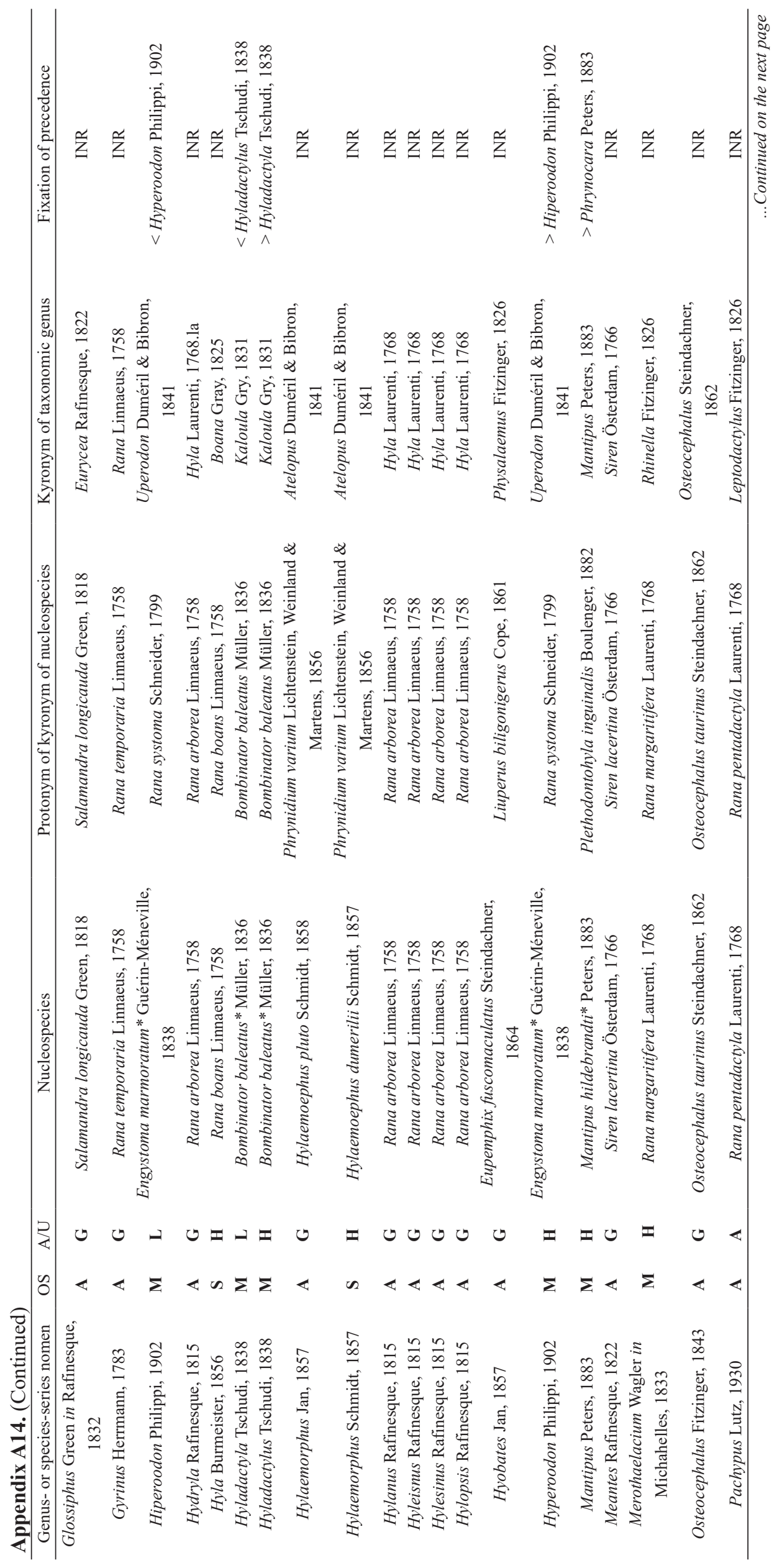




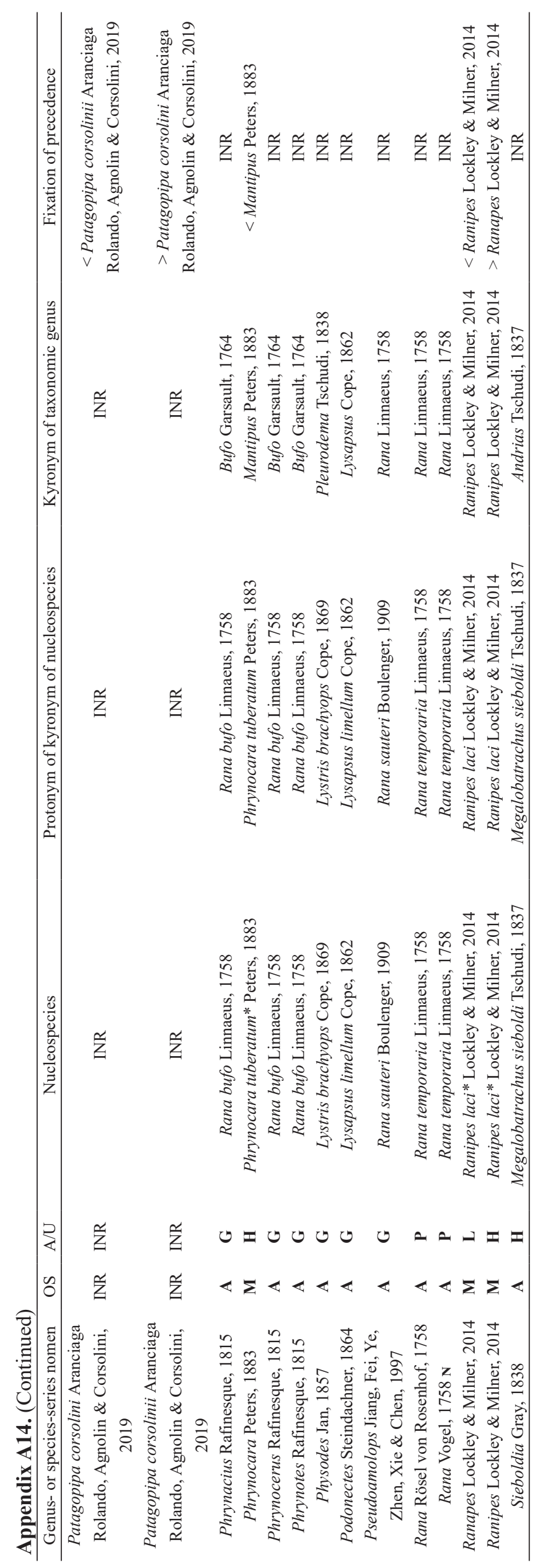


Appendix A15.MIS. Missing molecular data.

This Table lists the genera for which no molecular data from the onymophoront(s) of the valid nomen was available for the building of TREE.

Columns 1, 2, 3 and ${ }^{\circ}$ :

${ }^{1}$ The nominal genus is represented in TREE by specimens referred to a doxisonym of its nucleospecies: Pipa ${ }^{1}$.

${ }^{2}$ The nominal genus is represented in TREE by specimens referred to the nucleospecies of a generic nomen being its doxisonym: Andrias ${ }^{2}$.

${ }^{3}$ The nominal genus is represented in TREE but only by specimens referred to species that include neither its nucleospecies, nor a doxisonym of the latter, nor the nucleospecies of a doxisonym of the generic nomen at stake: Latonia ${ }^{3}$.

${ }^{\circ}$ The nominal genus is not represented in TREE: Dischidodactylus ${ }^{\circ}$.

\begin{tabular}{|c|c|c|c|c|}
\hline Family or higher taxon & 1 & 2 & 3 & $\circ$ \\
\hline \multicolumn{5}{|l|}{ ANURA } \\
\hline ARTHROLEPTIDAE & & Leptopelis & & \\
\hline ASTROBATRACHIDAE & & & & Astrobatrachus \\
\hline \multirow[t]{5}{*}{ BRACHYCEPHALIDAE } & & & Microkayla & Atopophrynus \\
\hline & & & Phrynopus & Geobatrachus \\
\hline & & & & Niceforonia \\
\hline & & & & Qosqophryne \\
\hline & & & & Tachiramantis \\
\hline BREVICIPITIDAE & & & Breviceps & \\
\hline \multirow[t]{10}{*}{ BUFONIDAE } & Adenomus & Leptophryne & Anaxyrus & Altiphrynoides \\
\hline & Mertensophryne & Rhinella & Firouzophrynus & Blythophryne \\
\hline & Schismaderma & & Frostius & Bufoides \\
\hline & & & Pelophryne & Calliopersa \\
\hline & & & Poyntonophrynus & Laurentophryne \\
\hline & & & Werneria & Metaphryniscus \\
\hline & & & & Parapelophryne \\
\hline & & & & Pseudobufo \\
\hline & & & & Sigalegalephrynus \\
\hline & & & & Truebella \\
\hline CACOSTERNIDAE & & & & Nothophryne \\
\hline CERATOBATRACHIDAE & Platymantis & & Alcalus & Liurana \\
\hline \multirow{2}{*}{ CERATOPHRYIDAE } & & & Ceratophrys & \\
\hline & & & Lepidobatrachus & \\
\hline CEUTHOMANTIDAE & & & & Dischidodactylus \\
\hline \multirow[t]{2}{*}{ CYCLORAMPHIDAE } & Hylodes & & Crossodactylus & Chaltenobatrachus \\
\hline & Thoropa & & & \\
\hline \multirow[t]{2}{*}{ DENDROBATIDAE } & & Hyloxalus & & Ectopoglossus \\
\hline & & & & Paruwrobates \\
\hline \multirow[t]{5}{*}{ DICROGLOSSIDAE } & Chaparana & & & Allopaa \\
\hline & Euphlyctis & & & Chrysopaa \\
\hline & Hoplobatrachus & & & Ombropaa \\
\hline & Phrynoderma & & & \\
\hline & Sphaerotheca & & & \\
\hline DisCOGLOSSIDAE & & & Latonia & \\
\hline HEMIPHRACTIDAE & Hemiphractus & & & \\
\hline HEMISOTIDAE & & Hemisus & & \\
\hline
\end{tabular}


Appendix A15. (Continued)

\begin{tabular}{|c|c|c|c|c|}
\hline Family or higher taxon & 1 & 2 & 3 & $\circ$ \\
\hline HYLIDAE & $\begin{array}{l}\text { Anotheca } \\
\text { Dendropsophus } \\
\text { Osteopilus } \\
\text { Ptychohyla } \\
\text { Scarthyla } \\
\text { Smilisca }\end{array}$ & Scinax & & $\begin{array}{l}\text { Dryaderces } \\
\text { Gabohyla } \\
\text { Quilticohyla }\end{array}$ \\
\hline HYPEROLIIDAE & Semnodactylus & & & $\begin{array}{l}\text { Arlequinus } \\
\text { Callixalus } \\
\text { Chrysobatrachus } \\
\text { Kassinula } \\
\text { Paracassina }\end{array}$ \\
\hline LEPTODACTYLIDAE & Leptodactylus & & Adenomera & \\
\hline MEGOPHRYIDAE & & $\begin{array}{l}\text { Leptobrachella } \\
\text { Scutiger }\end{array}$ & Xenophrys & \\
\hline MICROHYLIDAE & $\begin{array}{l}\text { Gastrophryne } \\
\text { Mantipus } \\
\text { Myersiella } \\
\text { Uperodon }\end{array}$ & Platypelis & Gastrophrynoides & $\begin{array}{l}\text { Adelastes } \\
\text { Madecassophryne } \\
\text { Mysticellus } \\
\text { Siamophryne } \\
\text { Vietnamophryne }\end{array}$ \\
\hline MYOBATRACHIDAE & $\begin{array}{l}\text { Myobatrachus } \\
\text { Platyplectrum }\end{array}$ & $\begin{array}{l}\text { Heleioporus } \\
\text { Philoria } \\
\text { Uperoleia }\end{array}$ & $\begin{array}{l}\text { Geocrinia } \\
\text { Pseudophryne } \\
\text { Taudactylus }\end{array}$ & \\
\hline $\begin{array}{l}\text { PHRYNOBATRACHIDAE } \\
\text { PHYLLOMEDUSIDAE } \\
\text { PIPIDAE }\end{array}$ & $\begin{array}{l}\text { Phrynobatrachus } \\
\text { Ranoidea } \\
\text { Pipa } \\
\text { Xenopus }\end{array}$ & & & \\
\hline PTYCHADENIDAE & & & & Lanzarana \\
\hline RANIDAE & $\begin{array}{l}\text { Clinotarsus } \\
\text { Nidirana }\end{array}$ & Amolops & & $\begin{array}{l}\text { Pterorana } \\
\text { Sumaterana }\end{array}$ \\
\hline RHACOPHORIDAE & & Pseudophilautus & & \\
\hline RHACOPHORIDAE & & & & Dendrobatorana \\
\hline SCAPHIOPODIDAE & Scaphiopus & & & \\
\hline TELMATOBIIDAE & & & Telmatobius & \\
\hline $\begin{array}{l}\text { HYLOBATRACHIA } \\
\text { GYMNOPHIONA }\end{array}$ & & & & Ancudia \\
\hline CAECILIIDAE & & & Microcaecilia & $\begin{array}{l}\text { Athretochoana } \\
\text { Brasilotyphlus } \\
\text { Idiocranium } \\
\text { Mimosiphonops } \\
\text { Nectocaecilia } \\
\text { Potamotyphlus } \\
\text { Sylvacaecilia }\end{array}$ \\
\hline ICHTHYОРНIIDAE & & & & Epicrium \\
\hline $\begin{array}{l}\text { SCOLECOMORPHIDAE } \\
\text { URAEOTYPHLIDAE }\end{array}$ & & Scolecomorphus & $\begin{array}{l}\text { Crotaphatrema } \\
\text { Uraeotyphlus }\end{array}$ & \\
\hline URODELA & & & & \\
\hline AMBYSTOMATIDAE & Ambystoma & & & \\
\hline $\begin{array}{l}\text { CRYPTOBRANCHIDAE } \\
\text { HYNOBIIDAE }\end{array}$ & $\begin{array}{l}\text { Cryptobranchus } \\
\text { Liua } \\
\text { Onychodactylus }\end{array}$ & Andrias & & \\
\hline PLETHODONTIDAE & Pseudotriton & & & \\
\hline
\end{tabular}


Appendix A15. (Continued)

\begin{tabular}{lllll}
\hline Family or higher taxon & 1 & 2 & 3 & $\circ$ \\
\hline SALAMANDRIDAE & Calotriton & Hypselotriton & & \\
& Cynops & & \\
& Euproctus & & \\
& Ichthyosaura & & \\
& Lissotriton \\
& Notophthalmus & & & \\
& Salamandra & & $\mathbf{2 1}$ & $\mathbf{4 4}$ \\
\hline Total ANURA & $\mathbf{3 3}$ & $\mathbf{1 4}$ & $\mathbf{3}$ & $\mathbf{0}$ \\
\hline Total GYMNOPHIONA & $\mathbf{0}$ & $\mathbf{1}$ & $\mathbf{0}$ & $\mathbf{5 2}$ \\
\hline Total URODELA & $\mathbf{1 2}$ & $\mathbf{2}$ & $\mathbf{2 4}$ & \\
\hline Total LiSSAMPHIBIA & $\mathbf{4 5}$ & $\mathbf{1 7}$ & & \\
\hline
\end{tabular}


Appendix A16.BUF. The Buffon Declaration.

The Buffon International Symposium was held at the Paris Muséum National d'Histoire Naturelle on 18-19 October 2007, on the occasion of the tercentenary of the birth of Buffon, one of the great founding fathers of the scientific study of the diversity of life. Four major institutions were co-organisers of this symposium: the Muséum National d'Histoire Naturelle (Paris, France), the Natural History Museum (London, UK), the Royal Botanic Gardens (Kew, UK) and the National Museum of Natural History of the Smithsonian Institution (Washington, USA). More than 200 participants, including representatives of 93 natural history institutions (natural history museums and research institutes, botanic gardens, zoos, etc.) from 36 countries and four continents discussed the following theme: "Natural History Museums and Institutions in the $21^{\text {st }}$ century: impact on our common future". The symposium adopted the following concluding message (Anonymous 2008):

\section{The Buffon Declaration \\ "Natural history institutions and the environmental crisis" \\ Concluding Message from the Buffon Symposium \\ (October 18th and 19th, 2007; Muséum National d'Histoire Naturelle, Paris, France)}

Given that science is critical for sustainable management of biodiversity and ecosystems and, through it, survival of human populations on this planet, the vital contributions of these institutions are fourfold.

- They are the primary repositories of the scientific samples on which understanding of the variety of life is ultimately based.

- Through leading-edge research, they extend knowledge of the structure and dynamics of biodiversity in the present and in the past.

- Through partnerships, and through programs of training and capacity-building, they strengthen the global capability to address current and future environmental challenges.

- They are a forum for direct engagement with civil society, which is indispensable for helping bring about the changes of behaviour on which our common future and the future of nature depend.

Today natural history institutions have particular responsibilities because global biodiversity is collapsing. Current approaches are inadequate in the face of this challenge. We therefore reaffirm our commitment to work together, and to develop new integrated approaches to understand and address the environmental crisis, and to communicate the issues to the public, policy makers and a broad range of stakeholders.

We make three recommendations:

(1) Collections of specimens and other databases on nature are a model of nature's variability and are a part of the world's scientific infrastructure (as exemplified by the OECD Global Science Forum). They are crucial tools for understanding the impact of climate change, of biodiversity loss, and other environmental challenges, but natural history collections are nowadays disappearing in many countries due to lack of funding.

We therefore call on governments and organisations to give the conservation of these vital collections increased levels of support.

(2) Naturalist research in the field is essential for the continued gathering and dissemination of information, as well as training and capacity-building initiatives. As a group, natural history institutions have developed, and will continue to develop and implement, best practice in this area. However, current policy changes derived from the U.N. Convention on Biological Diversity have made research, and the management of collections for scientific research on biodiversity, increasingly difficult and expensive. 
We therefore call on governments and the Convention on Biological Diversity:

(a) to recognize the difference between profit-oriented bioprospecting and science-oriented research for the public good, and

(b) to facilitate non-commercial biodiversity collecting and the movement of specimens, in their approaches to Access and Benefit-Sharing (ABS), including through their development of policy and regulations.

(3) Evolution is without doubt the most acceptable explanation for the diversity of life. It is crucial that only such empirical and testable approaches are accepted as "scientific" when discussing evolution. We strongly urge that support be given for the dissemination of scientific perspectives, which is our duty as outreach organisations, and for the teaching of evolution in schools.

In conclusion, the participants in the Buffon Symposium express the desire that scientists, policy makers and civil society unite in their efforts to achieve sustainable management of nature and the maintenance and restoration of ecosystems and their services upon which civilization depends. We reaffirm our conviction that a flourishing development model that is compatible with a sustainable natural world is possible. We are enthusiastic regarding the contributions we can make through our missions in this context, which consist of extending human knowledge of nature, training specialists of all kinds, and sharing knowledge with the public, particularly young people. We strongly affirm our capacity to provide an unbiased forum for the development of new ideas and new approaches among all the stakeholders concerned. 
Appendix A.17.ADD. Notes added in proofs.

Here above we presented the results of our survey of all the supraspecific nomina of LISSAMPHIBIA published from 1 January 1758 to 15 November 2020. At this latter date, these nomina amounted to 2935 (1827 of the genus-series, 592 of the family-series, 420 of the class-series and 96 ectonyms).

Four new nomina of the genus-series were published during our long work of correction of the proofs of this paper, and could therefore not be included in the text and tables above.

Wilkinson et al. (2021) described the new rhinatrematid genus and species Amazops amazops, which they regarded as the sister-taxon to the genus Rhinatrema Duméril \& Bibron, 1841.

Gorin et al. (2021) described the new microlylid genus Nanohyla (type species Microhyla annectens Boulenger, 1900), which they regarded as the sister-genus to Microhyla Tschudi, 1838. The separation of these two genera is supported by our data (see Appendix A2.TREE-1). They constitute together the sister-taxon to the genus Glyphoglossus Günther, 1869. According to our methodology, the recognition of Nanohyla requires to recognise two infratribes in the subtribe MICROHYLINA: the CALLUELLINIA Fei, Ye \& Jiang, 2005 for Glyphoglossus, and the MICROHYLINIA ||Fitzinger, 1843.fa.f012||-Noble, 1931 for Microhyla and Nanohyla.

Motta et al. (2021) provided the new generic nomen Heyerus for the single species Eleutherodactylus bilineatus Bokermann, 1975, which is also the type species of the genus Bahius described above. Since 2 February 2021, their paper is available on the website of the journal as an "early view" version, its pages being numbered from 1 to 17, and it is not included in the issue 59 (2) of February 2021 of the journal or in any other issue. According to Articles 9.9 and 21.8.3 of the 2012 Amendment of the Code (Anonymous 2012), such "preliminary versions of works accessible electronically in advance of publication" do not constitute published work and the nomen Heyerus, although preregistered on Zoobank, was not made available through this version. It will be made available by the publication online of the "final version" of this paper, in a subsequent issue of the journal, presumably with a different numbering of pages and perhaps other differences. The fact that the website of the journal states that the early view is the "Version of Record online" of this paper is of no relevance here, as the concept of "version of record" is absent from the version of the Code currently in force. The respective priority between Bahius and Heyerus will be settled by the dates of publication of the final versions of their respective papers.

Rage et al. (2021) described a series of fossil bone fragments as the new genus and species of incertae sedis anurans $\uparrow$ Rocekophryne ornata. The status of these two new nomina is similar to the previous one, having been distributed first, on 9 February 2021, as a document stated to be 'in press', i.e. as a preregistered but still unpublished preliminary version. They will become available when the final version of this paper is published first, either on paper or online.

17 February 2021

\section{References}

Fei, L., Ye, C. \& Jiang, J. (2005) A taxonomic study of the genus Calluella. In: Fei et al. (2005): 273-280. [In Chinese].

Gorin, V.A., Scherz, M.D., Korost, D.V. \& Poyarkov, N.A. Jr. (2021) Consequences of parallel miniaturisation in Microhylinae (Anura, Microhylidae), with the description of a new genus of diminutive South East Asian frogs. Zoosystematics and Evolution, 97, 2154.

Motta, A.P., Goulart Taucce, P.P., Haddad, C.F.B. \& Canedo, C. (2021) A new terraranan genus from the Brazilian Atlantic Forest with comments on the systematics of Brachycephaloidea (Amphibia: Anura). Journal of zoological Systematics and evolutionary Research, 2021, 1-17. [Early view, preregistered but still unpublished preliminary version, online since 2 February 2021].

Rage, J.-C., Adaci, M., Bensalah, M., Mahdoubi, M., Marivaux, L., Mebrouk, F. \& Tabuce, R. (2021) Latest early-early middle Eocene deposits of Algeria (Glib Zegdou, HGL50), yield the richest and most diverse fauna of amphibians and squamate reptiles from the Palaeogene of Africa. Palaeovertebrata, 43 (2)-e2, 1-32. [Early view, preregistered but still unpublished preliminary version, online since 9 February 2021].

Wilkinson, M., Reynolds, R.P. \& Jacobs, J.F. (2021) A new genus and species of rhinatrematid caecilian (Amphibia: Gymnophiona: Rhinatrematidae) from Ecuador. Herpetological Journal, 31, 27-34. 